\title{
Insights on Absolute and Relative Stereocontrol in Stereodivergent Cooperative
}

\section{Catalysis}

Avtar Changotra, ${ }^{\mathrm{a}}$ Bangaru Bhaskararao, ${ }^{\mathrm{a}}$ Christopher M. Hadad, ${ }^{\mathrm{b}}$ and Raghavan B. Sunoj ${ }^{\mathrm{a}, *}$

${ }^{a}$ Department of Chemistry, Indian Institute of Technology Bombay,

Powai, Mumbai 400076, India

${ }^{\mathrm{b}}$ Department of Chemistry and Biochemistry, The Ohio State University, Columbus, OH 43210, USA

E-mail: sunoj@chem.iitb.ac.in 
Part-A

\begin{tabular}{|c|c|c|}
\hline Section & Table of Contents & Page No. \\
\hline \multirow[t]{4}{*}{1.1} & Generation of Ir- $\pi$-allyl intermediate & S3 \\
\hline & (i) Activation of cinnamyl methyl carbonate & S3 \\
\hline & (ii) Optimized geometries of transition states (TSs) & S5 \\
\hline & (iii) Formation of $\mathrm{CO}_{2}$ and $\mathrm{CH}_{3} \mathrm{OH}$ by the action of $[\mathrm{DBU}-\mathrm{H}]^{+}$ & S7 \\
\hline 1.2 & Activation strain analysis of TSs $[12-13]_{\text {endo-re }}$ and $[12-13]_{\text {exo-si }}$ & S7 \\
\hline \multirow[t]{3}{*}{1.3} & Generation of $\mathrm{Cu}$-enolate & S8 \\
\hline & (i) Activation of azaaryl acetamide & S8 \\
\hline & (ii) Product release and the catalyst regeneration & S9 \\
\hline 1.4 & Energetic Span Model & $\mathrm{S} 12$ \\
\hline 1.5 & Kinetic Analysis & $\mathrm{S} 12$ \\
\hline \multirow[t]{4}{*}{1.6} & Reaction between $\mathrm{Cu}$-enolate and $\mathrm{Ir}-\pi$-allyl & S17 \\
\hline & $\begin{array}{l}\text { (i) Configurational and conformational study for the } \mathrm{C}-\mathrm{C} \text { bond } \\
\text { formation transition states }\end{array}$ & S17 \\
\hline & (ii) Energetic details of stereodivergence & S18 \\
\hline & $\begin{array}{l}\text { (iii) Conformational analysis of the } \mathrm{C}-\mathrm{C} \text { bond formation transition } \\
\text { states }\end{array}$ & S19 \\
\hline 1.7 & Space filling model for C2-P1 catalytic dyad & $\mathrm{S} 24$ \\
\hline 1.8 & $\begin{array}{l}\text { Global contact surface area, catalyst cavity volume and solvent } \\
\text { accessible surface area }\end{array}$ & $\mathrm{S} 24$ \\
\hline 1.9 & $\begin{array}{l}\text { Activation strain analysis for various modes of additions between } \\
\text { the nucleophile ( } \mathrm{Cu} \text {-enolate) and the electrophile (Ir- } \pi \text {-allyl) }\end{array}$ & $\mathrm{S} 27$ \\
\hline \multirow[t]{3}{*}{1.10} & Optimized geometries of stereocontrolling transition states & $\mathrm{S} 29$ \\
\hline & (i) C1-P1 catalyst dyad & $\mathrm{S} 29$ \\
\hline & (ii) C2-P1 catalyst dyad & $\mathrm{S} 32$ \\
\hline 1.11 & Space filling model for the lowest energy TS for all catalyst dyads & $\mathrm{S} 35$ \\
\hline 1.12 & Interaction wheel diagram & $\mathrm{S} 36$ \\
\hline 1.13 & Quantification of Noncovalent Interactions & S38 \\
\hline
\end{tabular}

\section{Part-B}

Optimized Cartesian Coordinates of All Stationary Points (pp S42-S386) 


\subsection{Generation of Ir- $\pi$-allyl intermediate}

\section{(i) Activation of cinnamyl methyl carbonate}

Cinnamyl methyl carbonate (10) gets activated in the form of Ir- $\pi$-allyl intermediate with the assistance of prononated base, $[\mathrm{DBU}-\mathrm{H}]^{+}$, generated during the $\mathrm{Cu}-$ Walphos enolate formation (Figure S1(a)). Depending on the mode of binding of the allyl moiety to the Ir center, an endo or an exo diastereomer of the Ir- $\pi$-allyl intermediate can be envisaged. A syn disposition of the allyl $\mathrm{C} 4-\mathrm{H}$ and the $\mathrm{Ir}-\mathrm{C}$ bond of the phosphoramidite ligand is denoted as endo whereas an anti orientation is designated as exo Figure S1(b). These notations are followed for both the $\mathbf{P 1}(R, R, R)$ and $\mathbf{P 2}(S, S, S)$ Ir-phosphoramidite catalysts. The elementary step barrier for the formation of the exo Ir- $\pi$-allyl intermediate is only about a kcal/mol higher than that for the endo intermediate in the case of P1 catalyst, suggesting that both endo and exo intermediates could be produced under the reaction conditions (Figure S1(a)). The relative Gibbs free energy of the transition states for the formation of an endo and an exo Ir$\pi$-allyl intermediate is provided in Tables S1.

(a)

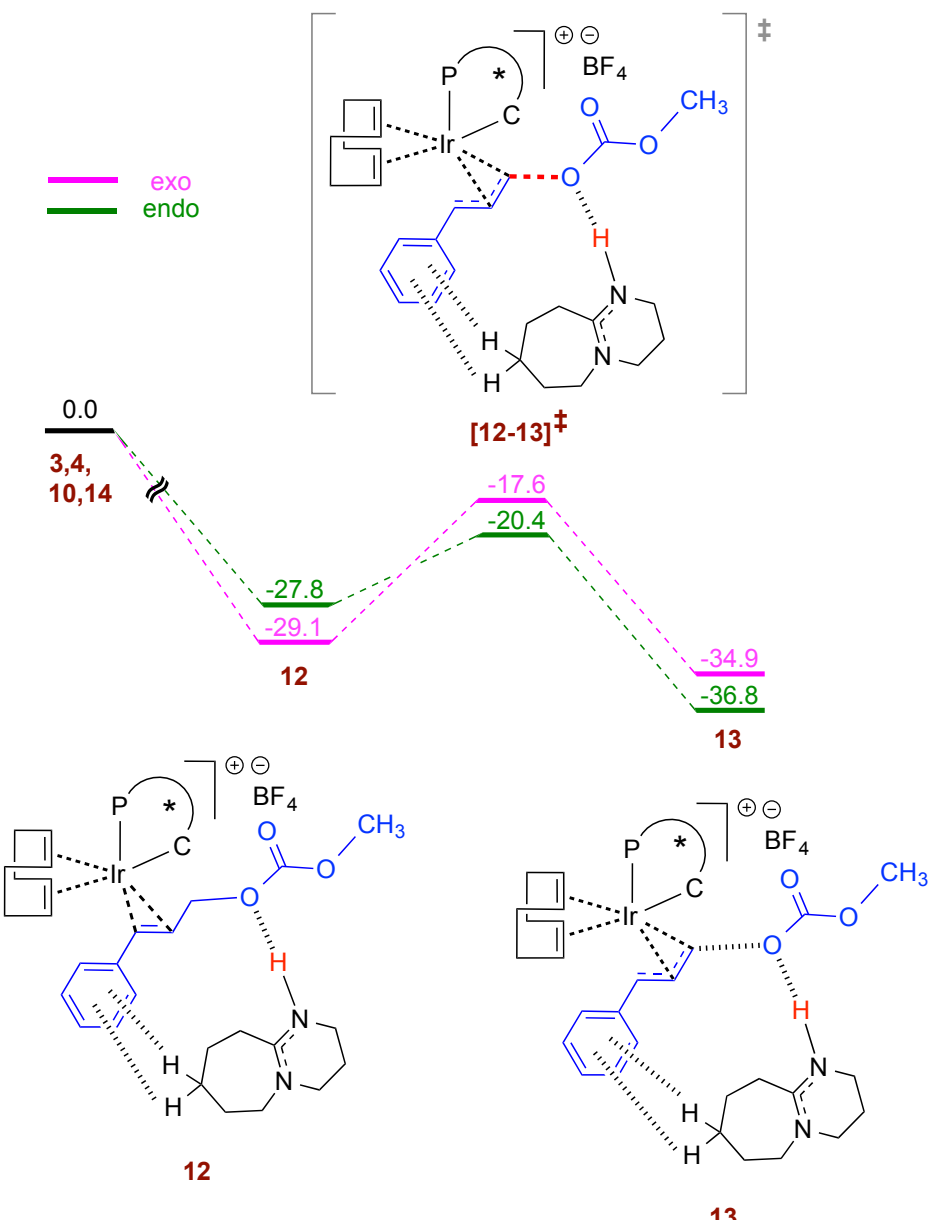


(b)
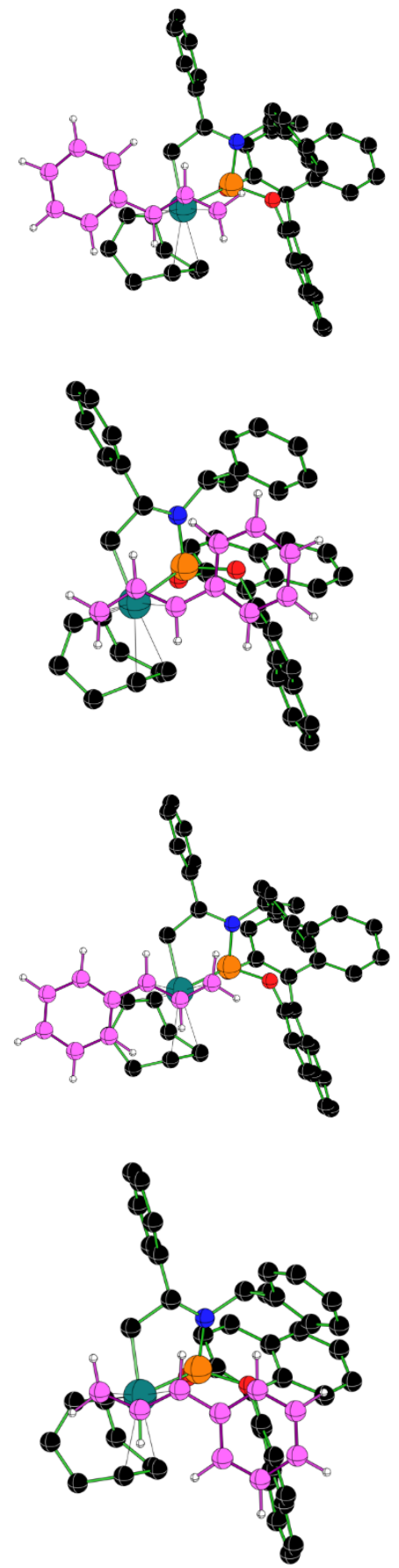

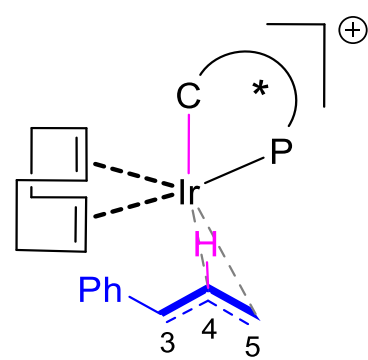

endo-re

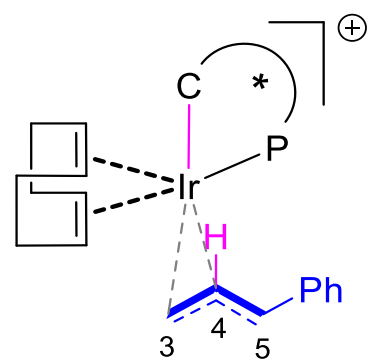

endo-si

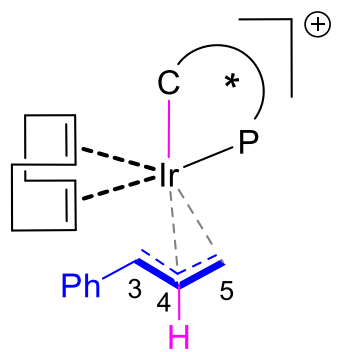

exo-si

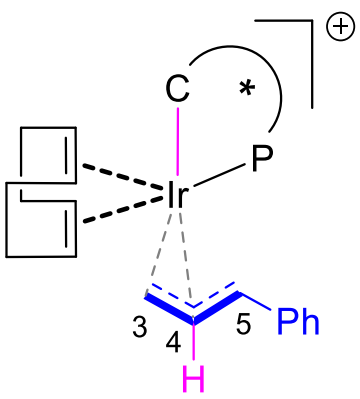

exo-re

$\ominus \mathrm{C} \oplus \mathrm{H} \odot \mathrm{Ir} \ominus \mathrm{P} \ominus \mathrm{O} \odot \mathrm{N}$ Allyl moiety is shown in pink color

Figure S1. (a) Gibbs free energy profile for the formation of Ir- $\pi$-allyl intermediate with P1 catalyst. (b) Optimized geometries and qualitative representation of an endo and an exo diastereomer of the Ir- $\pi$-allyl intermediates with the P1 catalyst. The re and si notations represents the prochiral face of the substrate which is readily available for bond formation with the incoming nucleophile. Although the transition states consist of the counter ion $\left(\mathrm{BF}^{-}\right)$in the actual computations, it is removed from this figure for improved clarity. 
Table S1. Relative Gibbs Free Energies (kcal/mol) of endo and exo Transition States [12-13] for the Formation of Ir- $\pi$-allyl Intermediate with Respect to the Lowest Energy Transition State for $\mathbf{P} 1$ and $\mathbf{P} 2$ catalysts

(a) without $\mathrm{BF}_{4}$ Counter Ion

\begin{tabular}{ccccc}
\hline & P1 & & P2 & \\
\hline endo-re & & 0.0 & endo-si & 0.0 \\
endo-si & 9.5 & endo-re & 9.5 \\
exo-re & & exo-si & 2.8 \\
exo-si & & 1.8 & exo-re & 1.8 \\
\hline
\end{tabular}

(b) with $\mathrm{BF}_{4}^{-}$Counter Ion

\begin{tabular}{ccccc}
\hline & P1 & & P2 & \\
\hline endo-re & & 0.0 & endo-si & 0.0 \\
endo-si & 10.8 & endo-re & 10.8 \\
exo-re & 6.2 & exo-si & 6.2 \\
exo-si & 3.6 & exo-re & 3.6 \\
\hline
\end{tabular}

\footnotetext{
${ }^{a-}$ The $r e$ and si notations represent the prochiral faces of the substrate, which is available for the bond formation
} with the incoming nucleophile

(ii) Optimized geometries of transition states for the formation of endo and exo Ir- $\pi$ allyl intermediate

(a)
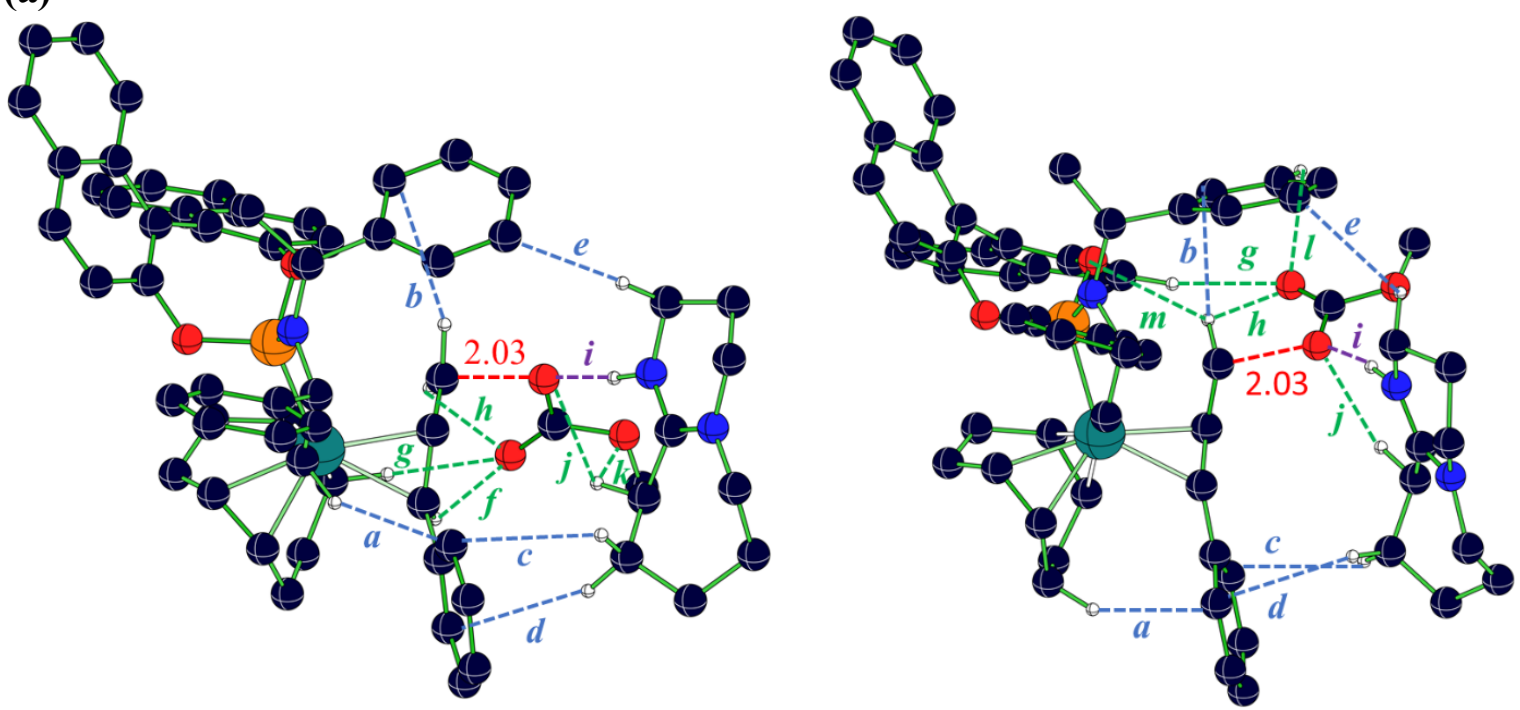

$\odot \mathrm{C} \oplus \mathrm{H} \odot \mathrm{Ir} \oplus \mathrm{Cu} \odot \mathrm{Fe} \odot \mathrm{Br} \oplus \mathrm{F} \odot \mathrm{P} \odot \mathrm{O} \odot \mathrm{N}$ 
(b)
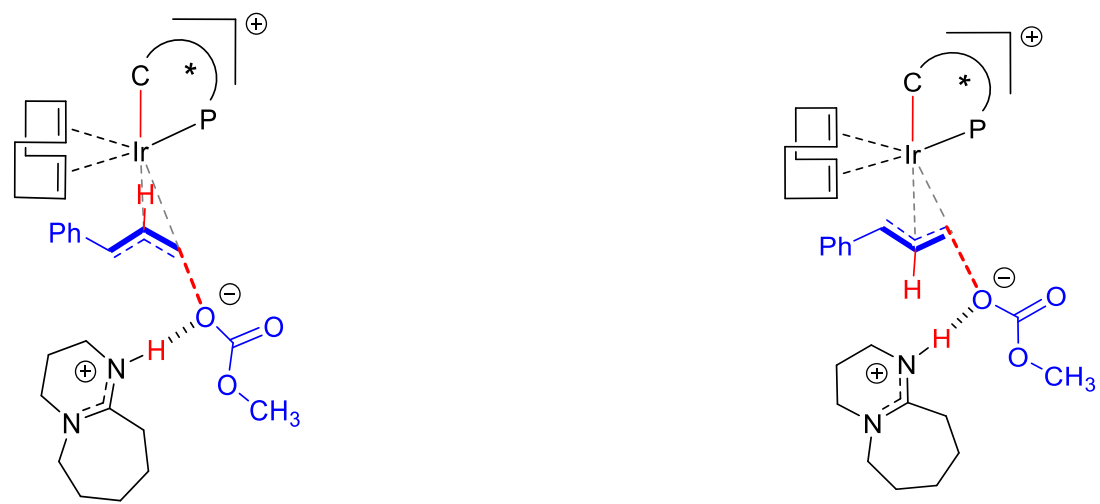

$[12-13]_{\text {endo-re }}{ }^{\ddagger}(0.0)$

$[12-13]_{\text {exo-si }}{ }^{\ddagger}(1.8)$

\begin{tabular}{|c|c|c|c|c|c|c|c|}
\hline \multirow[b]{2}{*}{$\begin{array}{l}\text { Bond } \\
\text { path }\end{array}$} & \multirow[b]{2}{*}{$\begin{array}{c}\text { Type of } \\
\text { interaction }\end{array}$} & \multicolumn{3}{|c|}{$[12-13]_{\text {endo-re }}{ }^{\dagger}$} & \multicolumn{3}{|c|}{$[12-13]_{\text {exo-si }}$} \\
\hline & & $(\AA)$ & $\rho \times 10^{-2}$ & $(\theta)$ & $(\AA)$ & $\rho \times 10^{-2}$ & $(\theta)$ \\
\hline$a$ & $\mathrm{C}-\mathrm{H} \cdots \pi$ & 2.37 & 1.4 & 23.8 & 2.64 & 0.9 & 25.4 \\
\hline$b$ & $\mathrm{C}-\mathrm{H} \cdots \pi$ & 3.57 & 0.1 & 25.5 & 2.88 & 0.5 & 66.8 \\
\hline$c$ & $\mathrm{C}-\mathrm{H} \cdots \pi$ & 2.89 & 0.6 & 68.6 & 2.89 & 0.6 & 60.1 \\
\hline$d$ & $\mathrm{C}-\mathrm{H} \cdots \pi$ & 2.83 & 0.7 & 37.3 & 2.85 & 0.6 & 47.3 \\
\hline$e$ & $\mathrm{C}-\mathrm{H} \cdots \pi$ & 2.74 & 0.7 & 17.8 & 2.86 & 0.6 & 47.6 \\
\hline$f$ & $\mathrm{C}-\mathrm{H} \cdots \mathrm{O}$ & 2.65 & 0.7 & 130.2 & $-^{a}$ & $-{ }^{a}$ & $-^{a}$ \\
\hline$g$ & $\mathrm{C}-\mathrm{H} \cdots \mathrm{O}$ & 2.39 & 1.1 & 175.8 & 2.42 & 1.0 & 170.1 \\
\hline$h$ & $\mathrm{C}-\mathrm{H} \cdots \mathrm{O}$ & 2.31 & 1.5 & 115.1 & 2.33 & 1.5 & 117.4 \\
\hline$i$ & $\mathrm{C}-\mathrm{H} \cdots \mathrm{O}$ & 1.75 & 4.1 & 172.5 & 1.72 & 4.3 & 169.2 \\
\hline$j$ & $\mathrm{C}-\mathrm{H} \cdots \mathrm{O}$ & 2.61 & 0.8 & 140.7 & 2.40 & 1.2 & 146.5 \\
\hline$k$ & $\mathrm{C}-\mathrm{H} \cdots \mathrm{O}$ & 2.70 & 0.7 & 12.4 & $-^{a}$ & $-^{a}$ & $-{ }^{a}$ \\
\hline$l$ & $\mathrm{C}-\mathrm{H} \cdots \mathrm{O}$ & $-^{a}$ & $-^{a}$ & $-^{a}$ & 2.59 & 0.8 & 122.9 \\
\hline$m$ & $\mathrm{C}-\mathrm{H} \cdots \mathrm{O}$ & $-{ }^{a}$ & $-{ }^{a}$ & $-^{a}$ & 2.89 & 0.5 & 112.5 \\
\hline
\end{tabular}

Figure S2. Optimized geometries of the transition states in the case of P1 catalyst, highlighting important noncovalent interactions in the $[12-13]_{\text {endo-re }}$ and $[12-13]_{\text {exo-si }}{ }^{*}$. Interatomic contacts that correspond to the NCIs are in $\AA$, and the corresponding electron densities $\left(\rho \times 10^{-2}\right)$ at the bond critical points as well as the angle of interaction $\left(\theta\right.$, in $\left.{ }^{\circ}\right)$ for various NCIs are provided in the table. Only selected hydrogen atoms are shown. Although the transition states consist of the counter ion $\left(\mathrm{BF}^{-}\right)$in the actual computations, it is removed from this figure for improved clarity. ${ }^{a-}$ No $b c p$ found 


\section{(iii) Formation of $\mathrm{CO}_{2}$ and $\mathrm{CH}_{3} \mathrm{OH}$ by the action of [DBU-H $]^{+}$}

Intermediate 13 , produced via the $\mathrm{C}-\mathrm{O}$ bond breaking TS $[\mathbf{1 2 - 1 3}]^{\ddagger}$, can provide the vital Ir- $\pi$ allyl intermediate and a carbonate-DBU adduct. The protonation of the carbonate oxygen of the $-\mathrm{OCH}_{3}$ group of the Ir-bound carbonate ester by [DBU-H] $]^{+}$via $\mathbf{T S}\left[\mathbf{1 3}^{\prime}-\mathbf{1 4} \mathbf{1}^{\prime}\right]$ is key to substrate activation. The action of $[\mathrm{DBU}-\mathrm{H}]^{+}$on methyl carbonate ester can then lead to $\mathrm{CO}_{2}$ and $\mathrm{CH}_{3} \mathrm{OH}\left(\mathbf{1 4}^{\prime}\right)$ as shown in Figure $\mathrm{S} 3$.

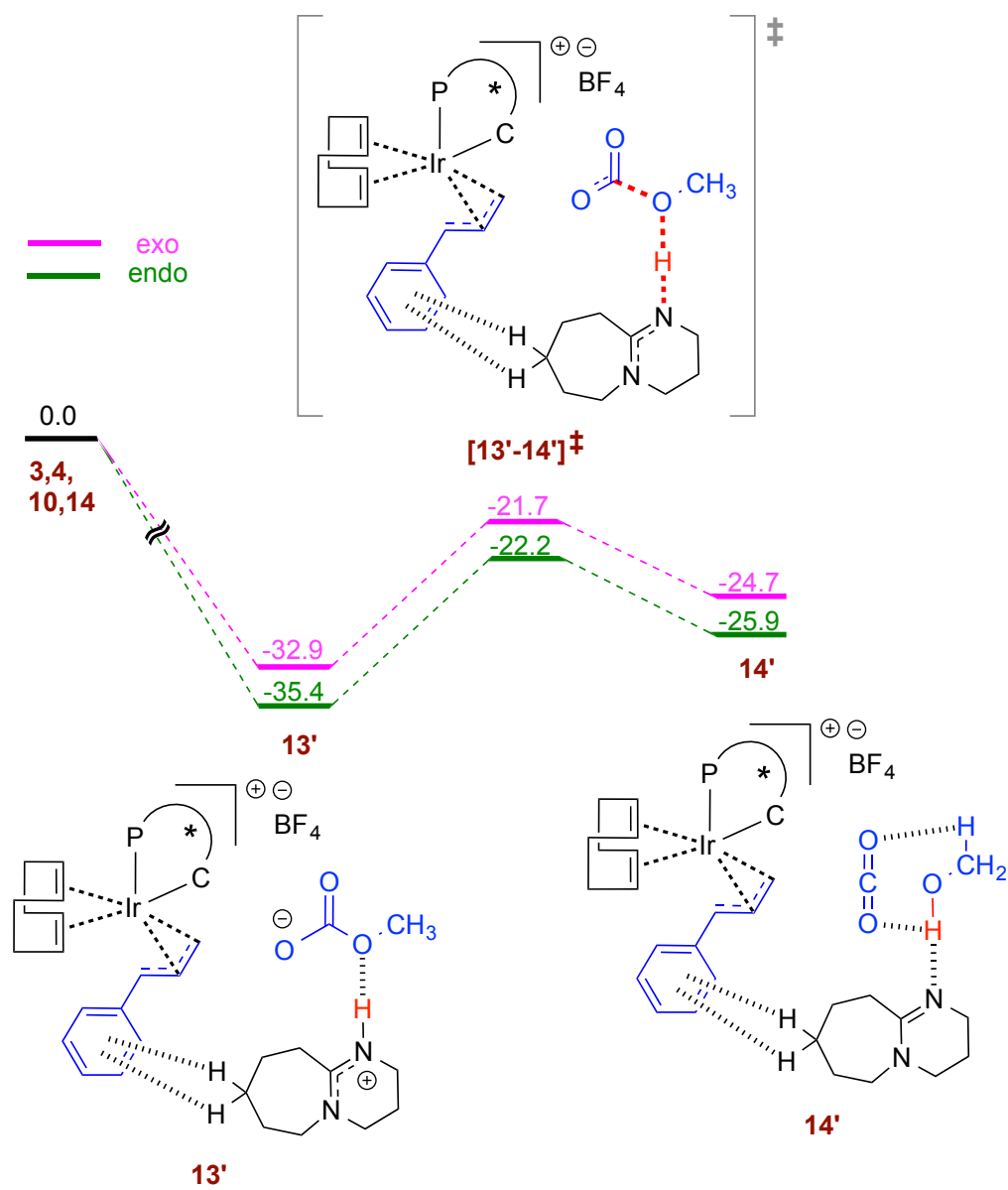

Figure S3. Gibbs free energy profile for the $[\mathrm{DBU}-\mathrm{H}]^{+}$assisted removal of methyl carbonate as $\mathrm{CO}_{2}$ and $\mathrm{MeOH}$.

\subsection{Activation strain analysis}

The activation strain analysis is applied to rationalize the origin of the energy difference between the TSs $[12-13]_{\text {endo-re }}$ and $\left._{[12-13}\right]_{\text {exo-si }}{ }^{*}$ for the formation of the endo and exo Ir- $\pi$ allyl intermediates. The activation strain energy $\left(\Delta \Delta \mathrm{E}^{\ddagger}\right)$ is partitioned to the (a) destabilizing distortion energy in the reacting partners $\left(\Delta \Delta \mathrm{E}_{\mathrm{d}}^{*}\right)$ while going from their respective ground state geometries to that in the transition states, and (b) stabilizing interaction energy between the deformed reactants $\left(\Delta \Delta \mathrm{E}_{\mathrm{i}}^{\mathrm{t}}\right)$ in the TS geometry. Here, the TS is divided into two 
fragments, $\mathrm{fl}=[\mathrm{Ir}-\pi \text {-allyl }]^{+} \mathrm{BF}_{4}^{-}$and $\mathrm{f} 2=[\mathrm{DBU}-\mathrm{H}]^{+} \mathrm{OCO}_{2} \mathrm{CH}_{3}^{-}$. The distortion energy of each fragment and the total interaction energy between the two fragments with respect to the lower energy [12-13 $]_{\text {endo-re }}{ }^{*}$ are given in Table S2.

Table S2. Computed Relative Distortion Energies in each Fragment, Total Relative Distortion Energies $\left(\Delta \Delta \mathrm{E}_{\mathrm{d}}^{\ddagger}\right)$ and Relative Interaction Energies $\left(\Delta \Delta \mathrm{E}_{\mathrm{i}}^{\dagger}\right)$ (in kcal/mol) in the TSs [12-13 $]_{\text {endo-re }}$ and $\left.^{112-13}\right]_{\text {exo-si }}{ }^{*}$ with P1 Catalyst Obtained at the $\mathrm{SMD}_{(\mathrm{THF})} / \mathrm{B} 3 \mathrm{LYP}-\mathrm{D} 3 / 6-$ $31 \mathrm{G}^{* *}, \mathrm{SDD}(\mathrm{Ir}, \mathrm{Cu}, \mathrm{Fe}, \mathrm{Br})$ Level of Theory. ${ }^{a}$ All Energies are in $\mathrm{kcal} / \mathrm{mol}$

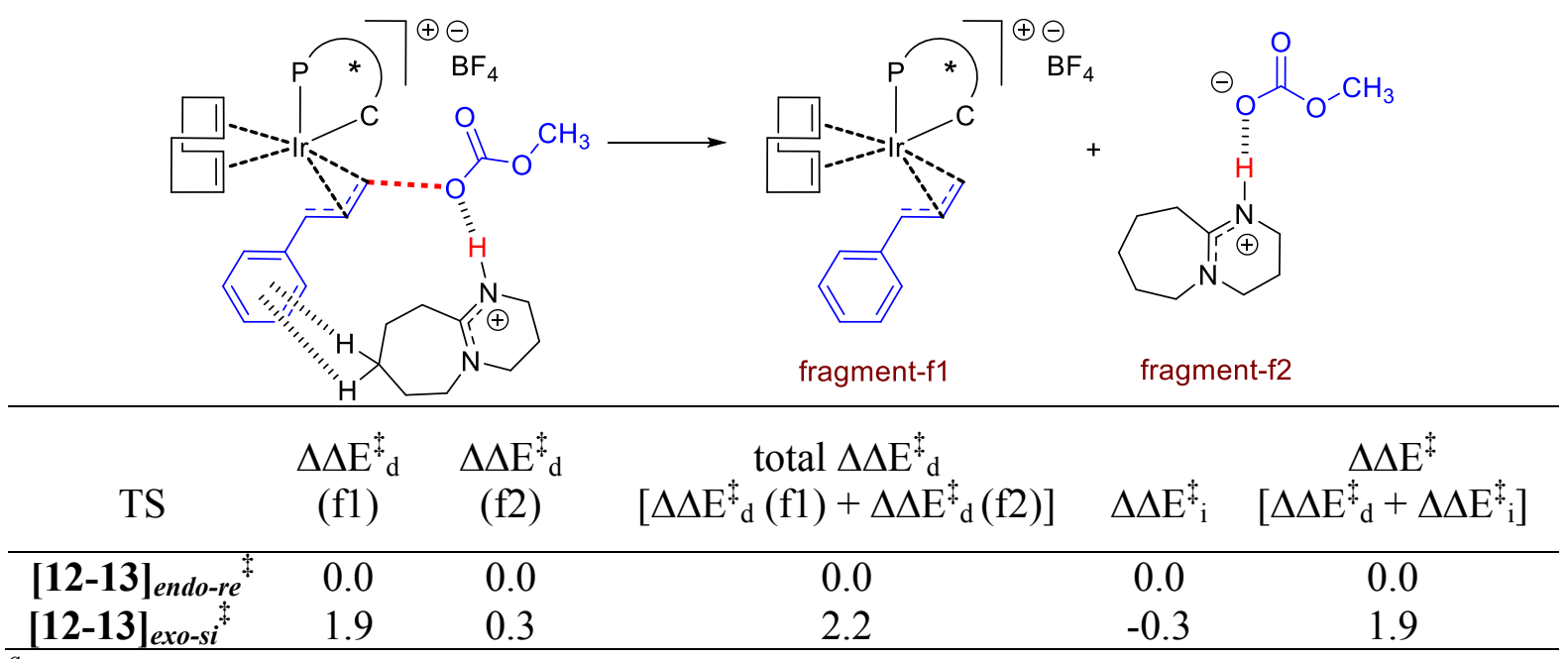

${ }^{a-}$ Relative energies with respect to lower energy TS

The distortion energies of fragments, $\mathrm{fl}$ and $\mathrm{f} 2$ are $1.9 \mathrm{kcal} / \mathrm{mol}$ and $0.3 \mathrm{kcal} / \mathrm{mol}$ higher in $[12-13]_{\text {exo-si }}^{*}$ compared to $[12-13]_{\text {endo-re }}^{*}$. On the other hand, the stabilizing interaction between the deformed fragments at the transition state geometry is $0.3 \mathrm{kcal} / \mathrm{mol}$ more in the higher energy $[\mathbf{1 2 - 1 3}]_{\text {exo-si }}{ }^{*}$ than that in the lower energy $[12-13]_{\text {endo-re }}{ }^{*}$. The overall balance between the destabilizing distortion and stabilization interaction energies is found to be in favor of [12-13 $]_{\text {endo-re }}{ }^{*}$ by $1.9 \mathrm{kcal} / \mathrm{mol}$. The higher distortion energy $(1.9 \mathrm{kcal} / \mathrm{mol})$ in the Ir$\pi$-allyl moiety is a major factor contributing to the energy difference between the stereodetermining transition states.

\subsection{Generation of Cu-enolate}

\section{(i) Activation of azaaryl acetamide}

Azaaryl acetamide 2 gets activated in the form of $\mathrm{Cu}$-Walphos enolate by the action of base DBU that then serves as the nucleophilic component in the reaction. Subsequent action of DBU generates a chiral $\mathrm{Cu}$-enolate intermediate 6 via $[\mathbf{5 - 6}]^{\ddagger}$ (as described in the main text). Ir- $\pi$-allyl intermediate enters the primary catalytic cycle, centered around the $\mathrm{Cu}$-Walphos enolate, by displacing $[\mathrm{DBU}-\mathrm{H}]^{+} \mathrm{PF}_{6}^{-}(7)$ and thereby leading to a lower energy intermediate 8 
(Figure S4). Alternatively, the release of the $[\mathrm{DBU}-\mathrm{H}]^{+} \mathrm{PF}_{6}^{-}$from intermediate 6 leads to $\mathbf{6}^{\prime}$, which is $9.9 \mathrm{kcal} / \mathrm{mol}$ higher in energy than $\mathbf{6}$. In the next step, the addition of the $\mathrm{Cu}-$ Walphos enolate to the Ir- $\pi$-allyl intermediate leads to a lower energy intermediate 8.

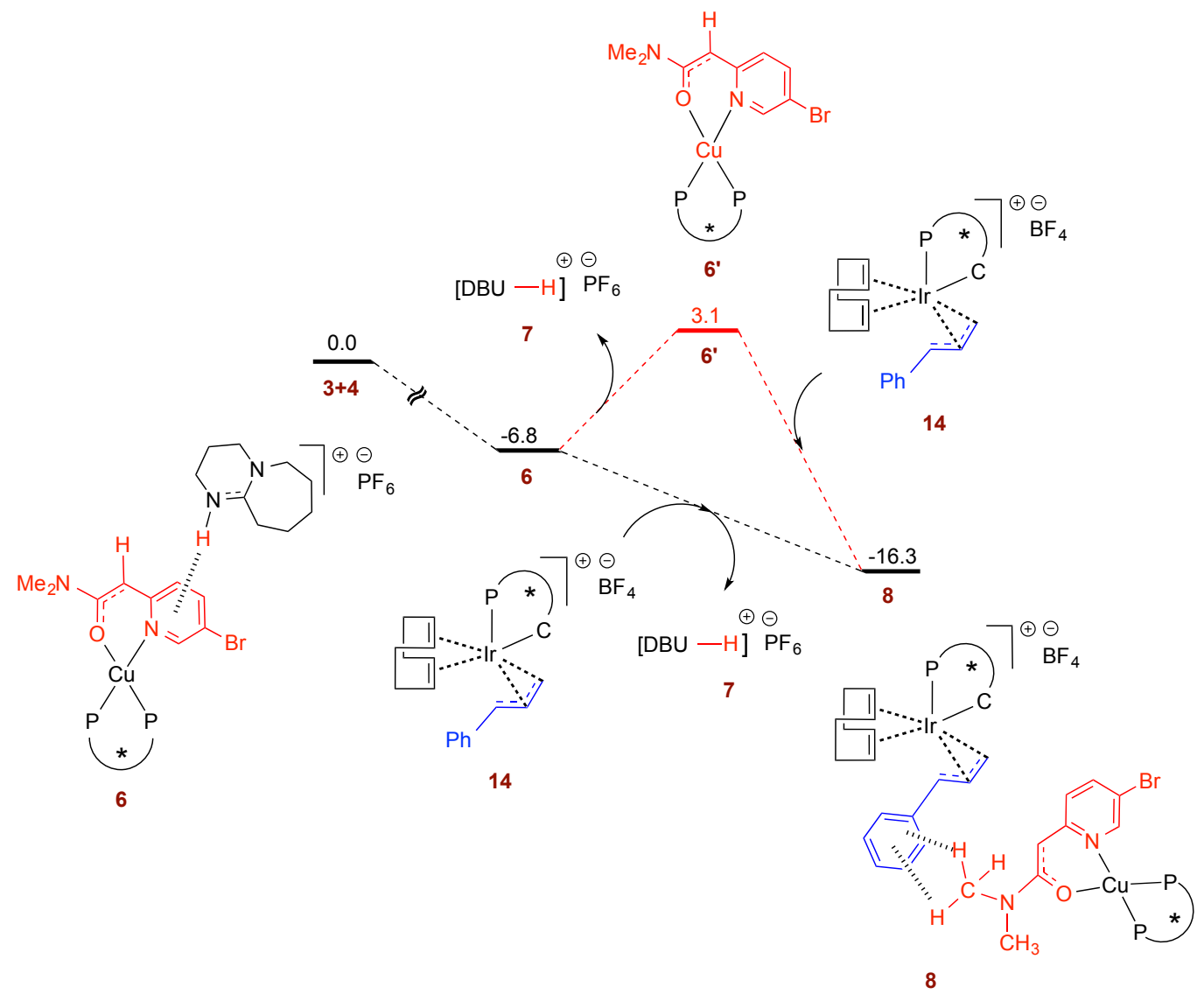

Figure S4. Gibbs Free energy profile for the formation of an intermediate $\mathbf{6}$ ' from $\mathbf{6}$.

\section{(ii) Different Possibilities for Product Release and the Catalyst Regeneration}

One can envisage a step-wise process for the release of the final product $\mathbf{1 1}$ from the catalystproduct complex 9. For instance, incorporation of a molecule of acetonitrile (native ligand in the catalyst precursor) can weaken the binding of $\mathbf{1 1}$ to the copper center and thereby open up a coordination site to generate more room for a new molecule of aryl amide (2). Similarly, allyl carbonate $\mathbf{1 0}$ uptake can help displace the $\operatorname{Ir}(\operatorname{cod})$ phosphoramidite from the product complex 9. Details of different possibilities considered for product release and catalyst regeneration are provided in the following section.

(I) Cinnamyl methyl carbonate $\mathbf{1 0}$ uptake along with 7 (protonated DBU) can help displace the $\operatorname{Ir}(\mathrm{cod})$ phophoramidite from the product complex 9 thereby resulting in the formation of complex 15 and 12 as shown in Scheme S1(a)-(c). Further uptake by the azaaryl acetamide 2 as shown in Scheme S1(a) or by two molecules of acetonitrile in consecutive steps as in Scheme S1(b) results in the release of the product 11 along with regeneration of the Walphos- 
ligated catalyst-substrate adduct $\mathbf{3}$ and catalyst precursor $\mathbf{1}$ respectively. The formation of a new catalyst-substrate complex 12 (Scheme 1(c), wherein allyl carbonate $\mathbf{1 0}$ is bound to the iridium center is relevant to the ensuing steps furnishing the Ir- $\pi$-allyl required for sustaining the catalytic cycle. The intermediate 13 produced via the $\mathrm{C}-\mathrm{O}$ bond breaking TS $[\mathbf{1 2 - 1 3}]^{\ddagger}$ can provide the vital Ir- $\pi$-allyl intermediate and a carbonate-DBU adduct.

(a)

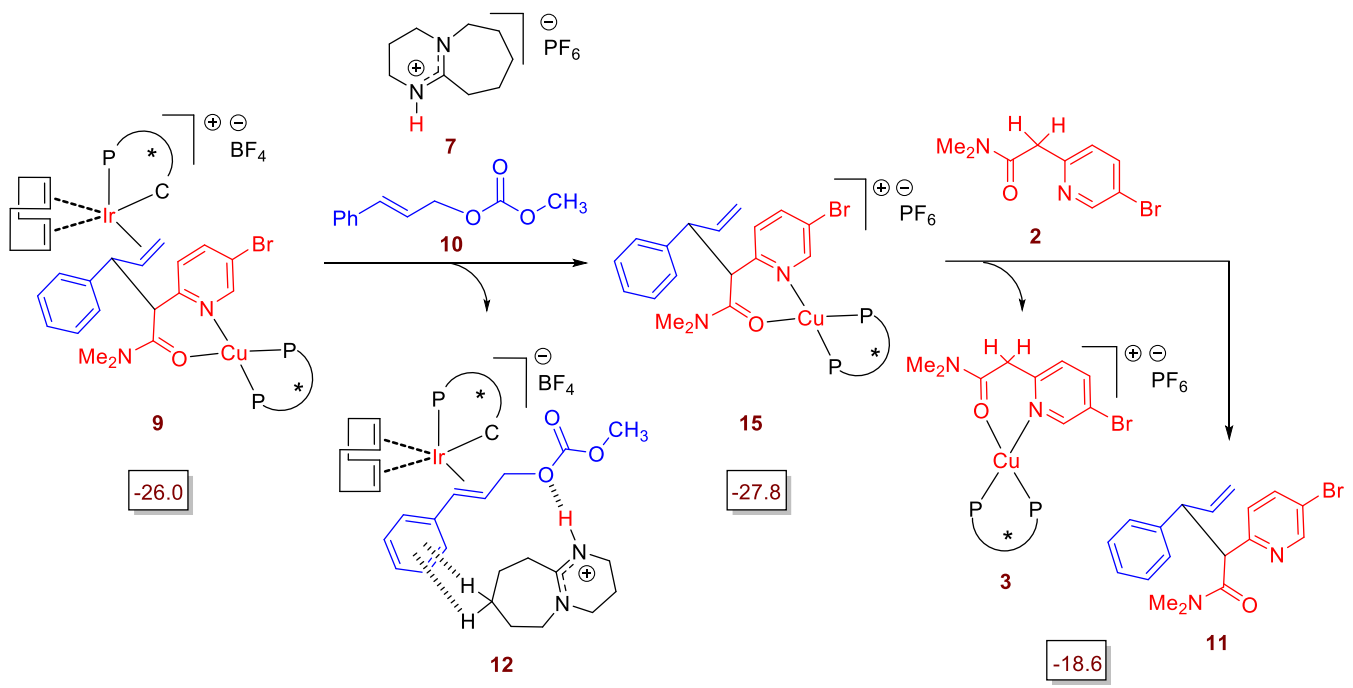

(b)

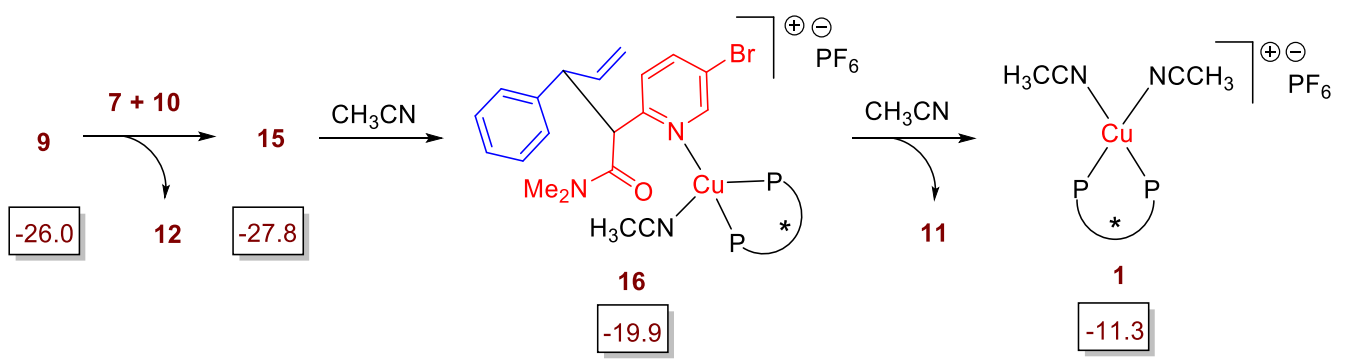

(c)

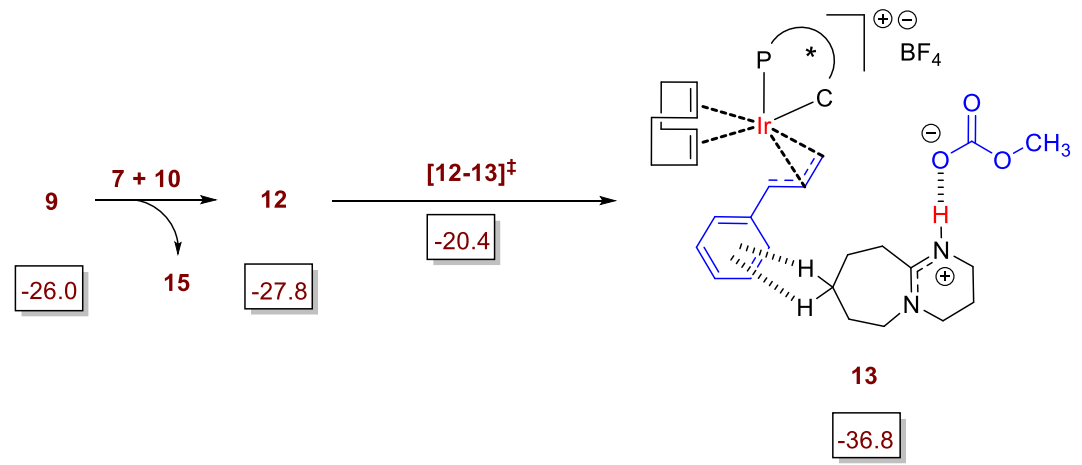

Scheme S1. Step-wise process for the release of the final product $\mathbf{1 1}$ from the catalystproduct complex $\mathbf{9}$ by the uptake of cinnamyl methyl carbonate $\mathbf{1 0}$ along with $\mathbf{7}$ (protonated $\mathrm{DBU}$ ). The relative Gibbs free energies (in $\mathrm{kcal} / \mathrm{mol}$ ) of the intermediates are provided in the square boxes. 
(II) Alternatively, an uptake of azaaryl acetamide 2 along with 7 (protonated DBU) by the catalyst-product complex 9, as shown in Scheme S2, results in the formation of an intermediate $\mathbf{1 7}$ which upon the incorporation of cinnamyl methyl carbonate $\mathbf{1 0}$ results in the final release of the product $\mathbf{1 1}$ and catalyst-substrate complex $\mathbf{1 2}$.

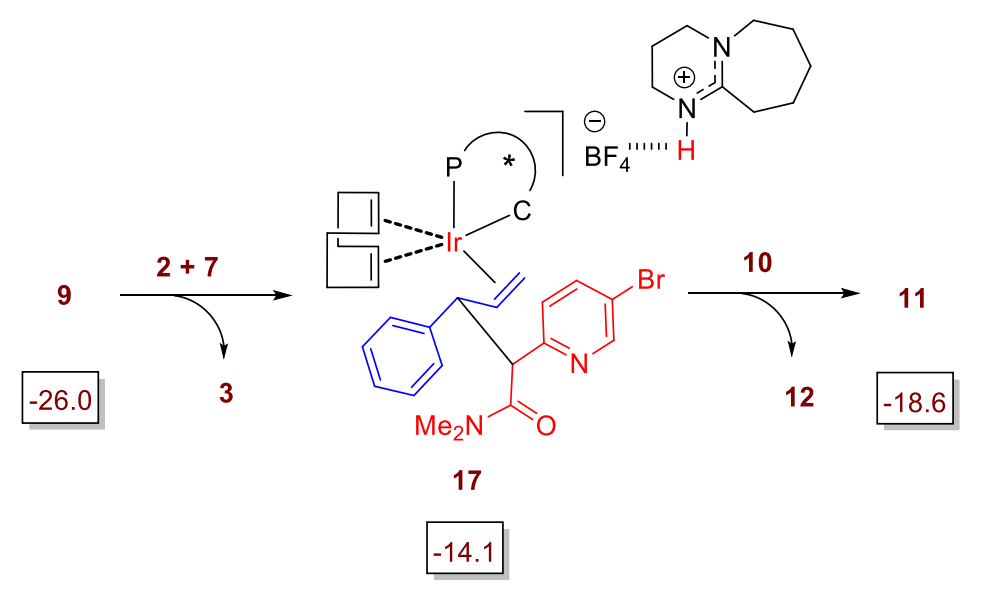

Scheme S2. Step-wise process for the release of the final product 11 from the catalystproduct complex 9 by the uptake of azaaryl acetamide 2 along with 7 (protonated DBU). The relative Gibbs free energies (in $\mathrm{kcal} / \mathrm{mol}$ ) of the intermediates are provided in the square boxes. Refer to Scheme S1 for additional details of numbering of various species shown here.

(III) Incorporation of a molecule of acetonitrile can weaken the binding of $\mathbf{1 1}$ to the copper center and thereby open up a coordination site to generate an intermediate $\mathbf{1 8}$ which subsequently takes up another molecule of acetonitrile along with 7 to generate intermediate 17 as shown in Scheme S3. Intermediate $\mathbf{1 7}$ provides the key Ir- $\pi$-allyl intermediate and a carbonate-DBU adduct 13, produced via the $\mathrm{C}-\mathrm{O}$ bond breaking TS $[\mathbf{1 2 - 1 3}]^{+}$after the incorporation of cinnamyl methyl carbonate $\mathbf{1 0}$.

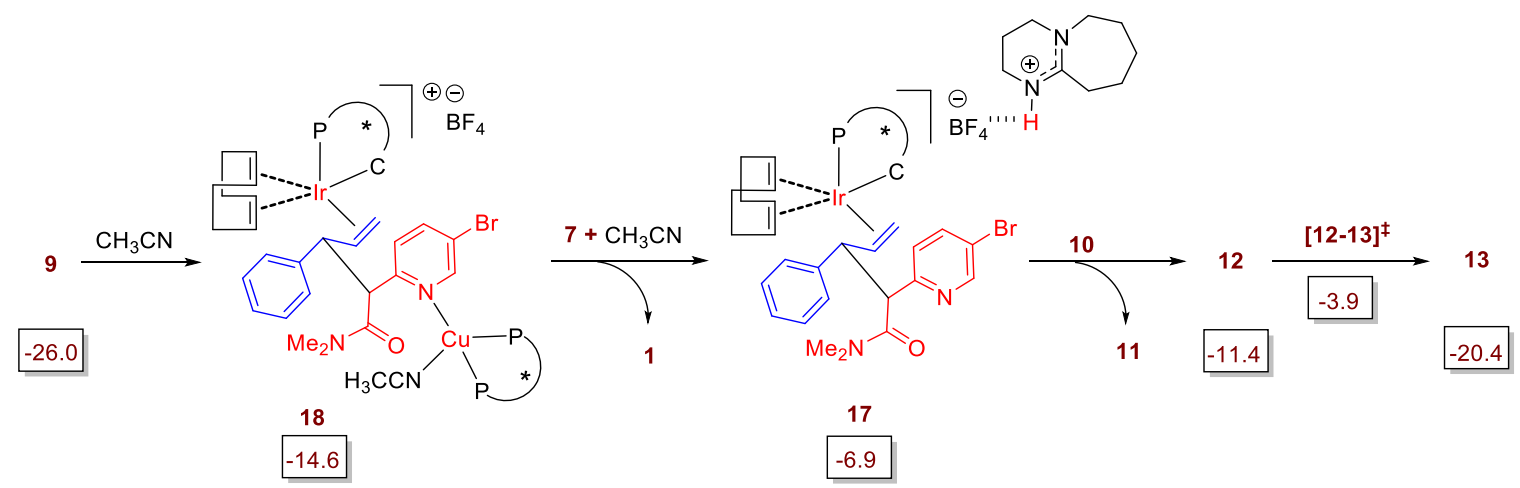

Scheme S3. Step-wise process for the release of the final product 11 from the catalystproduct complex 9 by the consecutive uptake of two molecules of acetonitrile followed by cinnamyl methyl carbonate 10. The relative Gibbs free energies (in $\mathrm{kcal} / \mathrm{mol}$ ) of the intermediates are provided in the square boxes. Refer to Scheme S1 for additional details of 
numbering of various species shown here.

Comparison of the different possibilities for the final release of the product 11, reveals that the uptake of substrate, cinnamyl methyl carbonate $\mathbf{1 0}$ along with $\mathbf{7}$ (protonated DBU) resulting in the formation of complexes $\mathbf{1 5}$ and $\mathbf{1 2}$ as shown as Scheme S1(c) is energetically more feasible. Complex 15 results in the regeneration of the Walphos-ligated catalystsubstrate adduct 3 (Scheme S1(a)) and catalyst precursor 1 (Scheme S1(b)) respectively. To sustain the catalytic process, the regeneration of the Ir-catalyst, should therefore involve the displacement of the product $\mathbf{1 1}$ from the product-catalyst complex $\mathbf{9}$ by a molecule of unreacted substrate $\mathbf{1 0}$ (cinnamyl carbonate).

\subsection{Energetic Span Model}

The energetic span $(\delta \mathrm{E})$ can be calculated from TOF-determining transition state (TDTS) and TOF-determining intermediate (TDI) as follows,

$\delta \mathrm{E}=\mathrm{TDTS}-\mathrm{TDI}, \quad$ if TDTS appears after the TDI

$\delta \mathrm{E}=\mathrm{TDTS}-\mathrm{TDI}+\Delta \mathrm{G}_{\mathrm{r}}$, if TDTS appears before the TDI

$\Delta \mathrm{G}_{\mathrm{r}}$ is the Gibbs free energy of the reaction.

Table S3. Calculation of Energetic Span ( $\delta \mathrm{E}$, in $\mathrm{kcal} / \mathrm{mol}$ ) using Different Likely Combinations of TDI and TDTS for Asymmetric Allylation of Azaaryl Acetamide using Cinnamyl Methyl Carbonate under Dual Catalytic Condition ${ }^{a}$

\begin{tabular}{|c|c|c|c|c|c|c|c|c|}
\hline TDI & TDTS & $\delta \mathrm{E}$ & TDI & TDTS & $\delta \mathrm{E}$ & TDI & TDTS & $\delta \mathrm{E}$ \\
\hline \multirow[t]{3}{*}{5} & {$[5-6]^{+}$} & 7.3 & 6 & {$[5-6]^{t}$} & -13.0 & 8 & {$[5-6]^{\dagger}$} & -3.5 \\
\hline & {$[8-9]^{\ddagger}$} & -5.5 & & {$[8-9]^{*}$} & -5.6 & & {$[8-9]^{*}$} & 3.9 \\
\hline & {$[12-13]^{\ddagger}$} & -13.5 & & {$[12-13]^{\ddagger}$} & -13.6 & & {$[12-13]^{\ddagger}$} & -4.1 \\
\hline \multirow[t]{3}{*}{9} & {$[5-6]^{+}$} & 6.2 & 12 & {$[5-6]^{*}$} & 8.0 & 13 & {$[5-6]^{\dagger}$} & 17.0 \\
\hline & {$[8-9]^{\ddagger}$} & -6.6 & & {$[8-9]^{*}$} & -4.8 & & {$[8-9]^{*}$} & 4.2 \\
\hline & {$[12-13]^{*}$} & 5.6 & & {$[12-13]^{\ddagger}$} & 7.4 & & {$[12-13]^{\ddagger}$} & -3.8 \\
\hline
\end{tabular}

${ }^{a-}$ The energy span calculations were done using the Gibbs free energy profile diagram (Figure 1) shown in main text.

\subsection{Details of Kinetic Analysis}

Kinetic analysis was applied to this catalytic reaction consisting of the $\mathrm{Cu}$-enolate formation, $\mathrm{C}-\mathrm{C}$ bond formation, generation of Ir- $\pi$-allyl intermediate, and $\mathrm{Cu}$-walphos-substrate complex (3). Table S4 provides a list of the reactions steps of significance to the kinetic model along with the corresponding free energies of activation for the forward and backward 
reactions, and the respective rate constants. The rate constants were calculated using the Eyring equation, at $24.85{ }^{\circ} \mathrm{C}(298.15 \mathrm{~K})$ as follows,

$$
k=\frac{k_{B} T}{h} e^{\frac{-\Delta G^{\ddagger}}{R T}}
$$

where $k$ is the rate constant, $k_{\mathrm{B}}$ is the Boltzmann constant $\left(\mathrm{kcal} \mathrm{K}^{-1}\right)$, T is the temperature $(\mathrm{K})$, $h$ is the Plank's constant ( $\mathrm{kcal} \mathrm{s}$ ), $\Delta \mathrm{G}^{\ddagger}$ is the free energy of activation ( $\mathrm{kcal} \mathrm{mol}^{-1}$ ), R is the gas constant $\left(\mathrm{kcal} \mathrm{mol}^{-1} \mathrm{~K}^{-1}\right)$. For barrierless steps, the rate constants were assumed to be equal to the pre-exponential factor in the Eyring equation, $k_{B} T / h=6.212 \times 10^{12} \mathrm{~s}^{-1} \mathrm{~mol}^{-2}\left(\right.$ at $\left.24.85^{\circ} \mathrm{C}\right){ }^{1}$

Table S4. Rate Constants ${ }^{2}$ and Gibbs Free Energies of Activation (kcal mol ${ }^{-1}$ )

\begin{tabular}{|c|c|c|c|c|c|c|}
\hline \multirow{2}{*}{$\begin{array}{l}\text { reaction } \\
\text { step }\end{array}$} & \multicolumn{2}{|r|}{ kinetic model } & \multirow{2}{*}{$\begin{array}{c}\Delta \mathrm{G}_{\mathrm{f}}^{\ddagger} \\
\text { barrier- } \\
\text { less }\end{array}$} & \multirow{2}{*}{$\begin{array}{c}\Delta \mathrm{G}_{\mathrm{b}}^{*} \\
6.9\end{array}$} & \multirow{2}{*}{$\begin{array}{c}k_{\mathrm{f}} \\
\text { forward }\end{array}$} & \multirow{2}{*}{$\begin{array}{c}k_{\mathrm{b}} \\
\text { backward }\end{array}$} \\
\hline & 1 & $3+4 \underset{k_{-1}}{\stackrel{k_{1}}{\rightleftharpoons}} 5$ & & & & \\
\hline $\begin{array}{l}\text { formation of } \\
\text { Cu-enolate }\end{array}$ & 2 & $5+14 \underset{k_{-2}}{\stackrel{k_{2}}{\rightleftharpoons}} 7+8$ & 7.3 & 16.7 & $\begin{array}{l}2.754 \times 10^{7} \\
\left(\mathrm{~s}^{-1} \mathrm{~mol}^{-1}\right)\end{array}$ & $\begin{array}{c}3.522 \\
\left(\mathrm{~s}^{-1} \mathrm{~mol}^{-1}\right)\end{array}$ \\
\hline $\begin{array}{l}\mathrm{C}-\mathrm{C} \text { bond } \\
\text { formation }\end{array}$ & 3 & $7+8+10 \frac{k_{3}}{k_{-3}} 12+15$ & 3.9 & 15.4 & $\begin{array}{l}8.576 \times 10^{9} \\
\left(\mathrm{~s}^{-1} \mathrm{~mol}^{-2}\right)\end{array}$ & $\begin{array}{c}3.163 \times 10^{1} \\
\left(\mathrm{~s}^{-1} \mathrm{~mol}^{-1}\right)\end{array}$ \\
\hline $\begin{array}{l}\text { formation of } \\
\text { Ir- } \pi \text {-allyl }\end{array}$ & 4 & $12 \underset{k_{-4}}{\stackrel{k_{4}}{\rightleftharpoons}} 13$ & 7.4 & 16.4 & $\begin{array}{c}2.326 \times 10^{7} \\
\left(\mathrm{~s}^{-1}\right)\end{array}$ & $\begin{array}{c}5.845 \\
\left(\mathrm{~s}^{-1}\right)\end{array}$ \\
\hline $\begin{array}{l}\text { regeneration } \\
\text { of } 14\end{array}$ & 5 & $13 \stackrel{k_{5}}{\frac{k_{-5}}{[D B U-H]}\left[\mathrm{OCO}_{2} \mathrm{CH}_{3}\right]}$ & 16.6 & $\begin{array}{l}\text { barrier } \\
\text {-less }\end{array}$ & $\begin{array}{c}4.170 \\
\left(\mathrm{~s}^{-1}\right)\end{array}$ & $\begin{array}{l}6.212 \times 10^{12} \\
\left(\mathrm{~s}^{-1} \mathrm{~mol}^{-1}\right)\end{array}$ \\
\hline $\begin{array}{l}\text { regeneration } \\
\quad \text { of } 3\end{array}$ & 6 & $15+2 \underset{k_{-6}}{\frac{k_{6}}{k_{1}}} 3+11$ & 9.2 & $\begin{array}{l}\text { barrier } \\
\text {-less }\end{array}$ & $\begin{array}{l}1.113 \times 10^{6} \\
\left(\mathrm{~s}^{-1} \mathrm{~mol}^{-1}\right)\end{array}$ & $\begin{array}{l}6.212 \times 10^{12} \\
\left(\mathrm{~s}^{-1} \mathrm{~mol}^{-1}\right)\end{array}$ \\
\hline
\end{tabular}

1) (a) Kalek, M.; Himo, F. Mechanism and selectivity of cooperatively catalyzed Meyer-Schuster rearrangement/Tsuji-Trost allylic substitution. Evaluation of synergistic catalysis by means of combined DFT and kinetics simulations. J. Am. Chem. Soc. 2017, 139, 10250-10266. (b) Kefalidis, C. E.; Davi, M.; Holstein, P. M.; Clot, E.; Baudoin, O. Mechanistic study of the selectivity of olefin versus cyclobutene formation by palladium(0)-catalyzed intramolecular $\mathrm{C}\left(s p^{3}\right)-\mathrm{H}$ activation. J. Org. Chem. 2014, 79, 11903-11910.

2) (a) The calculated rate constants should be regarded only as a decent estimate of the actual rate constants, as the errors in the computed entropies (i) that relies on harmonic oscillator and rigid rotor approximations besides (ii) those in translation entropy introduced through DFT calculations could be large. (b) Harvey, J. N.; Himo, F.; Maseras, F.; Perrin, L. ACS Catal. 2019, 9, 6803-6813. (c) Besora, M.; Vidossich, P.; Lledos, A.; Ujaque, G.; Maseras, F. Calculation of reaction free energies in solution: A comparison of current approaches. J. Phys. Chem. A 2018, 122, 1392-1399. (d) Grimme, S. Supramolecular binding thermodynamics by dispersioncorrected density functional theory. Chem.- Eur. J. 2012, 18, 9955-9964. (e) Marenich, A. V.; Olson, R. M.; Kelly, C. P.; Cramer, C. J.; Truhlar, D. G. Self-consistent reaction field model for aqueous and nonaqueous solutions based on accurate polarized partial charges. J. Chem. Theory Comput. 2007, 3, 2011-2033. (f) Piccini, G. M.; Sauer, J. Effect of anharmonicity on adsorption thermodynamics. J. Chem. Theory Comput. 2014, 10, 2479-2487. 


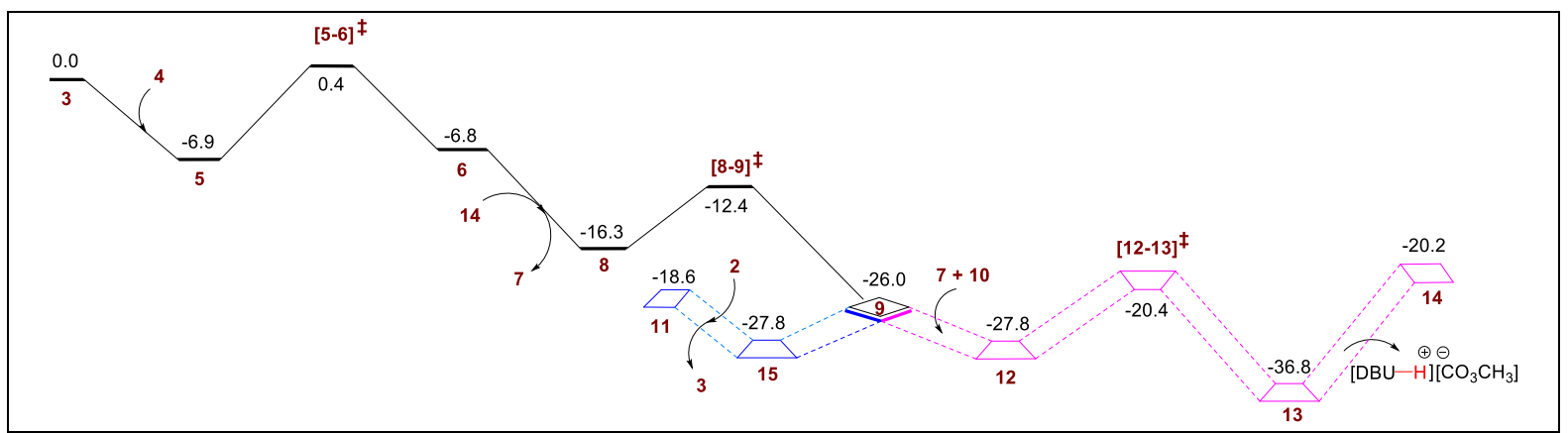

It can be noted from Table S4 that the rate constants of most of the forward reactions (entries 1-4, Table S4) are higher than the corresponding backward reactions, except that for 13 and 15. According to this analysis, complex 13 is the rate-limiting intermediate, which is in line with the TDI identified through the energetic span model. ${ }^{3}$ We have carried out microkinetic modeling ${ }^{4}$ to examine the concentration evolution of various species with time by using the rate constants of elementary reactions shown in entries 1-6 in Table S4. Shown below are the differential equations (1) to (13) associated with the kinetic model, which were solved for the initial concentrations as given in the experimental procedure. ${ }^{5}$ These concentrations are $3(0.100 \mathrm{mmol}), \mathbf{1 0}(0.110 \mathrm{mmol}), 4(0.0020 \mathrm{mmol}), 14(0.0010 \mathrm{mmol})$, and $2(0.100 \mathrm{mmol})$. The time evolution obtained using the Euler's numerical integration ${ }^{6}$ as shown in Figure S5 indicates the change in the concentration of various species with respect to time. The change in concentration can be expressed as,

$$
\left\{\mathrm{C}\left(t_{n+1}\right)=\mathrm{C}\left(t_{n}\right)+\Delta t \times \frac{d}{d t} \mathrm{C}\left(t_{n}\right)\right\}
$$

where, $\mathrm{C}\left(t_{\mathrm{n}}\right)$ is the concentration of any species at time $t_{\mathrm{n}}, \Delta t$ is the time interval between $t_{\mathrm{n}}$ and $t_{\mathrm{n}+1}, \mathrm{~d} / \mathrm{dt}$ is the rate of change of concentration of respective species given by the differential equations below,

$$
\begin{gathered}
\frac{d[3]}{d t}=-k_{1}[\mathbf{3}][\mathbf{4}]+k_{-1}[\mathbf{5}]+k_{6}[\mathbf{1 5}][2]-k_{-6}[\mathbf{3}][\mathbf{1 1}] \\
\frac{d[\mathbf{4}]}{d t}=-k_{1}[3][\mathbf{4}]+k_{-1}[\mathbf{5}]
\end{gathered}
$$

3) For energetic span model, see Table S3.

4) (a) Fernandez-Alvarez, V. M.; Nappi, M.; Melchiorre, P.; Maseras, F. Computational study with DFT and kinetic models on the mechanism of photoinitiated aromatic perfluoroalkylations. Org. Lett. 2015, 17, 2676-2679. (g) Guo, W.; Kuniyil, R.; Gomez, J. E.; Maseras, F.; Kleij, A. W. A domino process toward functionally dense quaternary carbons through $\mathrm{Pd}$-catalyzed decarboxylative $\mathrm{C}\left(s p^{3}\right)-\mathrm{C}\left(s p^{3}\right)$ bond formation. $J$. Am. Chem. Soc. 2018, 140, 3981-3987. (c) Rush, L. E.; Pringle, P. G.; Harvey, J. N. Computational kinetics of cobalt-catalyzed alkene hydroformylation. Angew. Chem., Int. Ed. 2014, 53, 8672-8676. (d) Besora, M.; Maseras, F. Microkinetic modeling in homogeneous catalysis. WIREs Comput Mol Sci. 2018, e1372.

5) Jiang, X.; Boehm, P.; Hartwig, J. F. Stereodivergent allylation of azaaryl acetamides and acetates by synergistic iridium and copper catalysis. J. Am. Chem. Soc. 2018, 140, 1239-1242.

6) Chapra, Steven C.; Raymond P. Canale. Numerical methods for engineers. Vol. 2. New York: Mcgraw-hill, 2011. 


$$
\begin{gathered}
\frac{d[\mathbf{5}]}{d t}=k_{1}[\mathbf{3}][\mathbf{4}]-k_{-1}[\mathbf{5}]-k_{2}[\mathbf{5}][\mathbf{1 4}]+k_{-2}[\mathbf{7}][\mathbf{8}] \\
\frac{d[\mathbf{1 4}]}{d t}=-k_{2}[\mathbf{5}][\mathbf{1 4}]+k_{-2}[\mathbf{7}][\mathbf{8}]+k_{5}[\mathbf{1 3}]-k_{-5}[\mathbf{1 4}]\left[(\mathrm{DBUH})\left(\mathrm{CO}_{3} \mathrm{CH}_{3}\right)\right] \\
\frac{d[\mathbf{7}]}{d t}=k_{2}[\mathbf{5}][\mathbf{1 4}]-k_{-2}[\mathbf{7}][\mathbf{8}]-k_{3}[\mathbf{7}][\mathbf{8}][\mathbf{1 0}]+k_{-3}[\mathbf{1 2}][\mathbf{1 5}] \\
\frac{d[\mathbf{8}]}{d t}=k_{2}[\mathbf{5}][\mathbf{1 4}]-k_{-2}[\mathbf{7}][\mathbf{8}]-k_{3}[\mathbf{7}][\mathbf{8}][\mathbf{1 0}]+k_{-3}[\mathbf{1 2}][\mathbf{1 5}] \\
\frac{d[\mathbf{1 0}]}{d t}=-k_{3}[\mathbf{7}][\mathbf{8}][\mathbf{1 0}]+k_{-3}[\mathbf{1 2}][\mathbf{1 5}] \\
\frac{d[\mathbf{1 5}]}{d t}=k_{3}[\mathbf{7}][\mathbf{8}][\mathbf{1 0}]-k_{-3}[\mathbf{1 2}][\mathbf{1 5}]-k_{6}[\mathbf{1 5}][\mathbf{2}]+k_{-6}[\mathbf{3}][\mathbf{1 1}] \\
\frac{d[\mathbf{1 2}]}{d t}=k_{3}[\mathbf{7}][\mathbf{8}][\mathbf{1 0}]-k_{-3}[\mathbf{1 2}][\mathbf{1 5}]-k_{4}[\mathbf{1 2}]+k_{-4}[\mathbf{1 3}] \\
\frac{d \mathbf{1 3}]}{d t}=k_{4}[\mathbf{1 2}]-k_{-4}[\mathbf{1 3}]-k_{5}[\mathbf{1 3}]+k_{-5}[\mathbf{1 4}]\left[(\mathrm{DBUH})\left(\mathrm{CO}_{3} \mathrm{CH}_{3}\right)\right] \\
\frac{d\left[(\mathrm{DBUH})\left(\mathrm{CO}_{3} \mathrm{CH}\right)\right]}{d t}=k_{5}[\mathbf{1 3}]-k_{-5}[\mathbf{1 4}]\left[(\mathrm{DBUH})\left(\mathrm{CO}_{3} \mathrm{CH}_{3}\right)\right] \\
\frac{d[\mathbf{2}]}{d t}=-k_{6}[\mathbf{1 5}][\mathbf{2}]+k_{-6}[\mathbf{3}][\mathbf{1 1}]
\end{gathered}
$$

For example, the concentration profile shown in Figure S5(c) for specie $\mathbf{3}$ is calculated as follows,

$$
[3]_{t_{n+1}}=[3]_{t_{n}}+\Delta t \times\left(-k_{1}[\mathbf{3}][4]+k_{-1}[\mathbf{5}]+k_{6}[\mathbf{1 5}][2]-k_{-6}[\mathbf{3}][\mathbf{1 1}]\right)
$$

$t_{\mathrm{n}}$ is the current time, $t_{\mathrm{n}+1}$ is the next time, $\Delta t$ is time interval. The equation (14) is solved from time $\mathrm{t}=0$ to $\mathrm{t}=5 \times 10^{-5}$ as shown in Figure S5(c).

Figure S5(a) depicts the variation in concentration of various intermediates $(\mathbf{7}, \mathbf{8}, \mathbf{1 2}$, 13 and 15) generated during the course of the reaction. The nearly overlapping curves for species 7 (green) and $\mathbf{8}$ (blue), indicate a rapid increase in concentration and then a gradual decrease, consistent with their consumption as the reaction proceeds. The concentration of species 15 and 13, shown using overlapping yellow and indigo colored curves, indicates a gradual increase as both of these species are generated in the forward reactions. To inspect the evolution of species 12 (orange) and buildup of the final product 11 (black), an expanded scale of the Y-axis as shown in Figure S5(b) was employed. The concentration of product (11) shows a sharp increase. It is noted that $\mathbf{1 2}$ is readily consumed as soon as it is formed, thereby making it appear in near-zero concentrations in Figure S5(a). A zoomed out representation of the concentration variation for the primary reactants such as $\mathbf{3 , 4 , 1 0}$ and $\mathbf{2}$ is provided in Figure S5(c-f). A drop in concentration of these species suggests their consumption as the reaction proceeds in the forward direction. Zoomed out picture shown in 
Figure S5(g-h) shows a gradual increase in the concentration of species such as $\mathbf{1 4}$ and $\mathbf{3}$ as both of these catalytic active species are regenerated in the later part of the reaction.

(a)

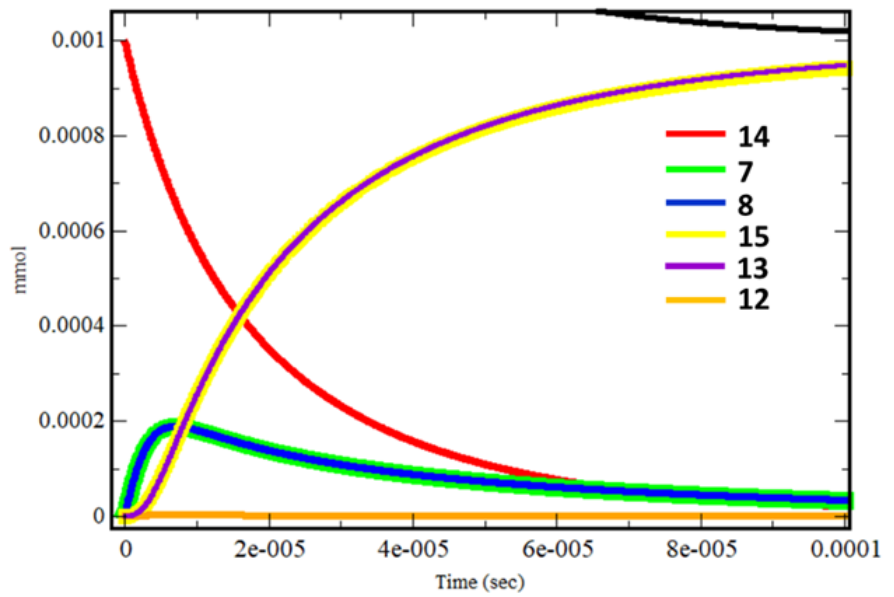

(b)

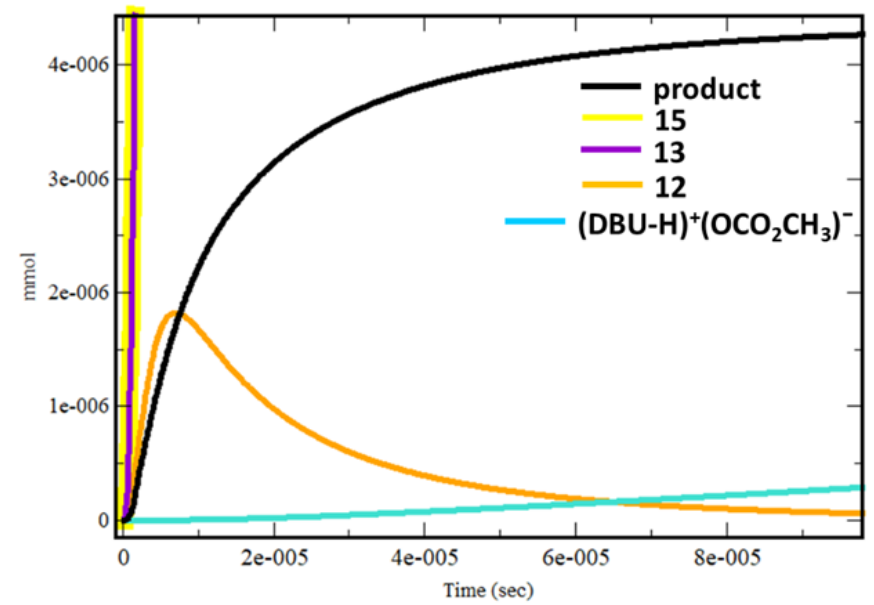

(c)

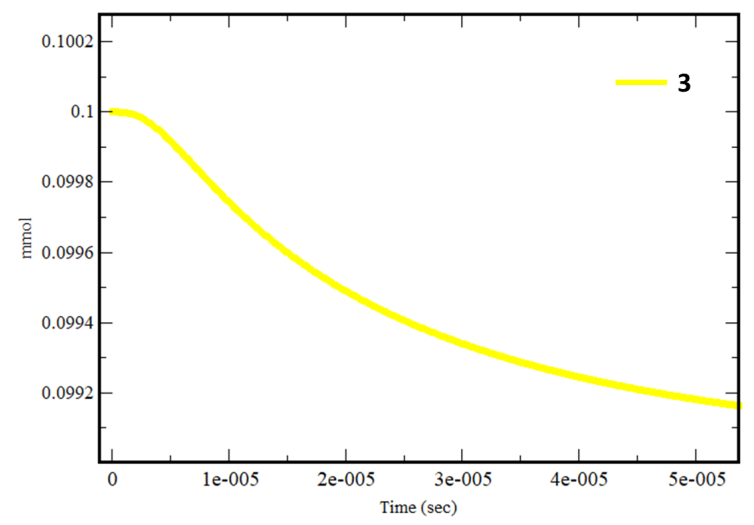

(d)

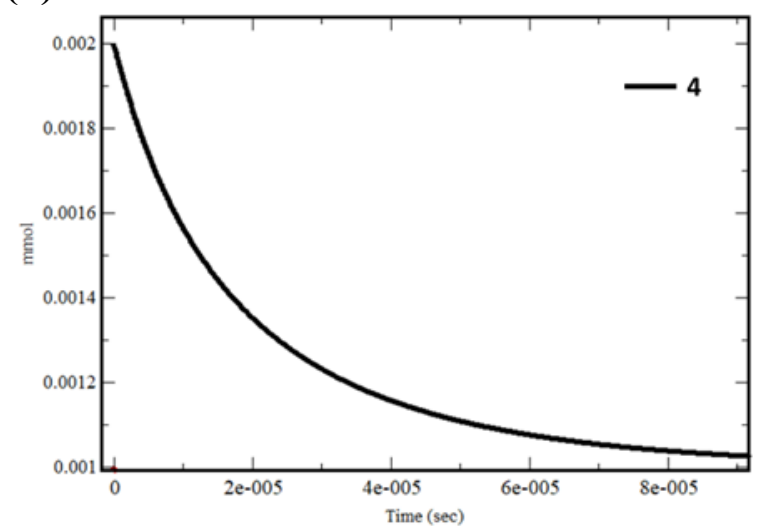


Figure S5 continued...

(e)

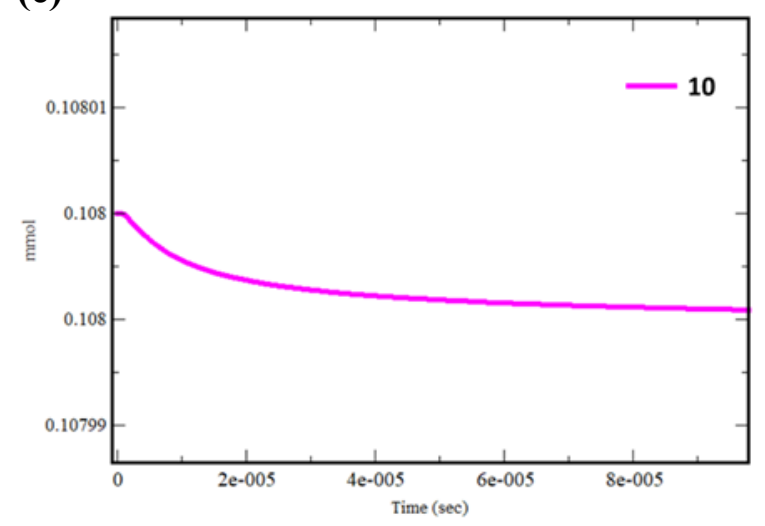

(g)

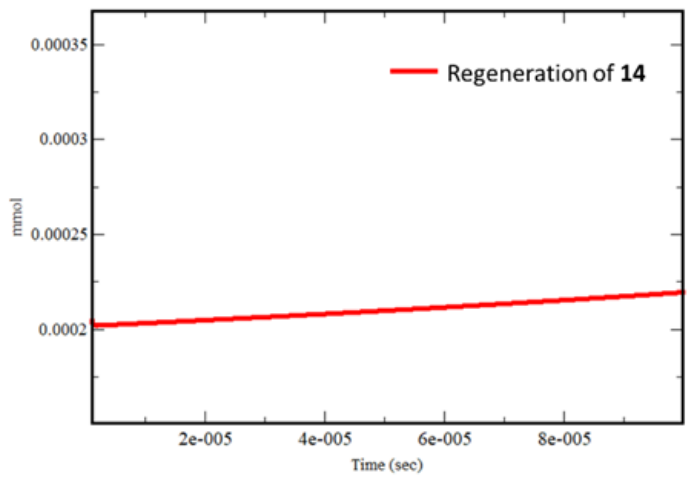

(f)

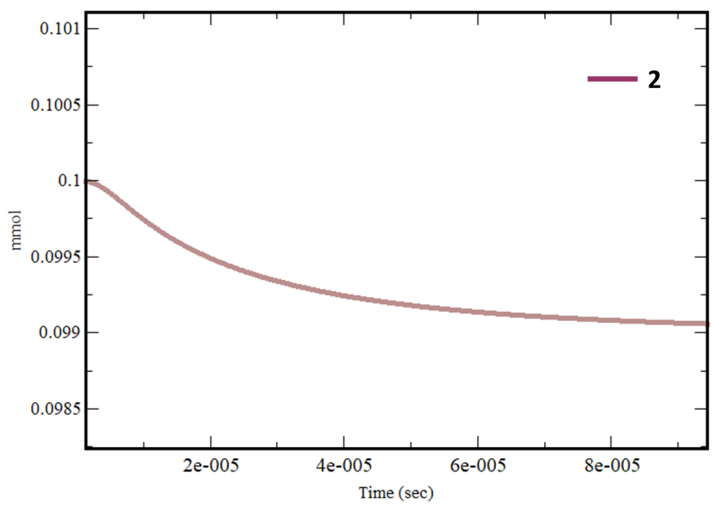

(h)

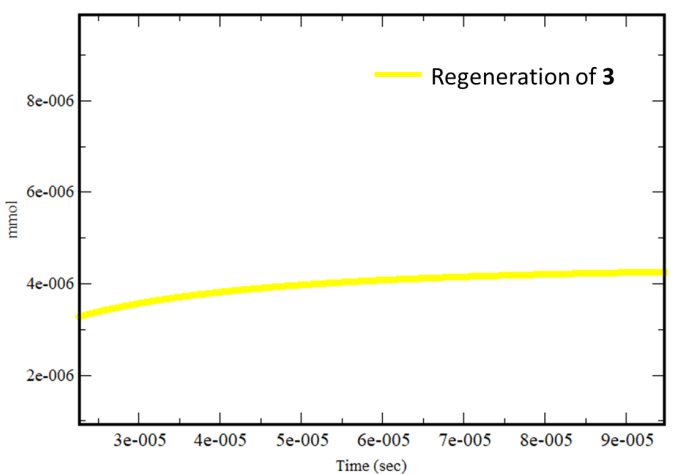

Figure S5. (a) Computed concentration (mmol) of various species and product over time (sec) obtained with DFT method. (b) Zoomed out region of the concentration variation for some selected species.

\subsection{Reaction between Cu-enolate and Ir- $\pi$-allyl}

(i) Different stereochemical modes of addition for the $\mathbf{C}-\mathbf{C}$ bond formation transition states between $\mathrm{Cu}$-enolate and $\mathrm{Ir}$ - $\pi$-allyl: Configurational and conformational study

The two reacting partners or substrates are activated by different transition-metal catalysts in separate catalytic events, which resulted in the formation of $\mathrm{Cu}$-enolate and Ir- $\pi$-allyl intermediates. These transition-metal catalytic cycles converge in the stereocontrolling $\mathrm{C}-\mathrm{C}$ bond formation step wherein the nucleophilic and electrophilic reacting partners react through their prochiral faces. This $\mathrm{C}-\mathrm{C}$ bond formation furnishes two new stereogenic carbon centers in the product. We have considered different stereochemical modes of addition for the $\mathrm{C}-\mathrm{C}$ bond formation that differ primarily in terms of the open prochiral face provided by the nucleophilic and the electrophilic partners (Figure S6). 

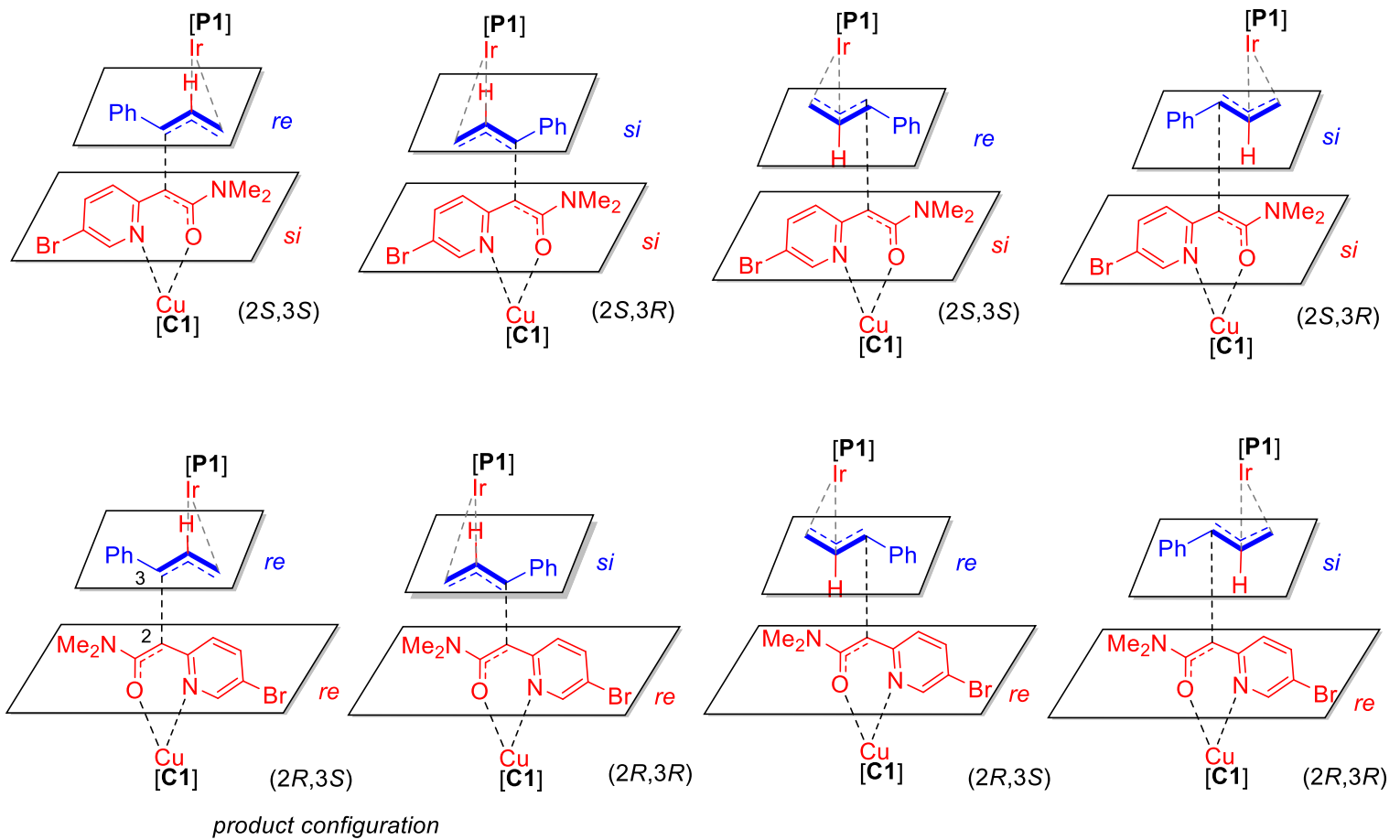

C1 $=\left(R, R_{\mathrm{p}}\right)$-Walphos ligand P1 $=(R, R, R)$-Phosphoramidite

Figure S6. Qualitative representation of different modes of $\mathrm{C}-\mathrm{C}$ bond formation transition states between $\mathrm{Cu}$-enolate and $\mathrm{Ir}-\pi$-allyl for the C1-P1 catalyst dyad. Product configuration at the ensuing stereogenic carbon centers is provided in parentheses. Although the transition states consist of the counter ion $\left(\mathrm{BF}^{-}\right)$in the actual computations, it is removed from this figure for improved clarity.

\section{(ii) Energetic details of stereodivergence}

Table S5. Relative Gibbs Free Energies (kcal/mol) for the $\mathrm{C}-\mathrm{C}$ Bond Formation Transition States Involving Different Prochiral Faces of the $\mathrm{Cu}$-enolate (nucleophile) and the Ir- $\pi$-allyl Intermediate (electrophile) for Different Catalyst Dyads (Cu-Walphos (C) and IrPhosphoramidite (P)) without $\mathrm{BF}_{4}{ }^{-}$Counter Ion Obtained at Different Level of Theories ${ }^{a}$

(a) C1-P1

\begin{tabular}{cccccccc}
\hline $\begin{array}{c}\text { prochiral } \\
\text { faces of C-P }\end{array}$ & $\begin{array}{c}\text { product } \\
\text { configuration }\end{array}$ & $\begin{array}{c}\text { orientation } \\
\text { of Ir- } \pi \text {-allyl }\end{array}$ & L1 & L2 & L3 & L4 & L5 \\
\hline re-re & $(2 R, 3 S)$ & endo & $\mathbf{0 . 0}$ & $\mathbf{0 . 0}$ & $\mathbf{0 . 0}$ & $\mathbf{0 . 0}$ & $\mathbf{0 . 0}$ \\
si-re & $(2 S, 3 S)$ & endo & 6.4 & 5.7 & 3.7 & 6.3 & 3.0 \\
si-si & $(2 S, 3 R)$ & endo & 6.7 & 6.4 & 4.3 & 5.9 & 5.3 \\
re-si & $(2 R, 3 R)$ & endo & 6.1 & 8.1 & 7.6 & 7.0 & 7.5 \\
si-si & $(2 S, 3 R)$ & exo & 7.7 & 4.8 & 3.0 & 4.5 & 4.1 \\
re-si & $(2 R, 3 R)$ & exo & 1.2 & 1.6 & 1.5 & 1.8 & 2.2 \\
si-re & $(2 S, 3 S)$ & exo & 16.3 & 17.1 & 12.9 & 17.0 & 14.8 \\
re-re & $(2 R, 3 S)$ & exo & 9.4 & 10.7 & 8.9 & 10.6 & 10.5
\end{tabular}


Table S5 continued...

\begin{tabular}{|c|c|c|c|c|c|c|c|}
\hline \multicolumn{8}{|c|}{ (b) C2-P1 } \\
\hline $\begin{array}{c}\text { prochiral } \\
\text { faces of } \mathbf{C}-\mathbf{P}\end{array}$ & $\begin{array}{c}\text { product } \\
\text { configuration }\end{array}$ & $\begin{array}{l}\text { orientation } \\
\text { of Ir- } \pi \text {-allyl }\end{array}$ & L1 & $\mathbf{L} 2$ & $\mathbf{L 3}$ & L4 & L5 \\
\hline re-re & $(2 R, 3 S)$ & endo & 6.8 & 7.4 & 5.6 & 5.7 & 6.1 \\
\hline si-re & $(2 S, 3 S)$ & endo & 0.0 & 0.0 & 0.0 & 0.0 & 0.0 \\
\hline$s i-s i$ & $(2 S, 3 R)$ & endo & 5.8 & 7.4 & 6.9 & 4.4 & 6.2 \\
\hline$r e-s i$ & $(2 R, 3 R)$ & endo & 13.1 & 14.0 & 11.4 & 11.8 & 10.8 \\
\hline$s i-s i$ & $(2 S, 3 R)$ & exo & 2.7 & 4.1 & 2.2 & 2.9 & 3.0 \\
\hline$r e-s i$ & $(2 R, 3 R)$ & exo & 11.6 & 12.5 & 8.7 & 12.6 & 8.7 \\
\hline si-re & $(2 S, 3 S)$ & exo & 11.8 & 13.0 & 13.1 & 13.0 & 12.0 \\
\hline re-re & $(2 R, 3 S)$ & exo & 9.9 & 10.0 & 8.8 & 9.5 & 10.1 \\
\hline \multicolumn{8}{|c|}{ (c) C2-P2 } \\
\hline $\begin{array}{c}\text { prochiral } \\
\text { faces of } \mathbf{C}-\mathbf{P}\end{array}$ & $\begin{array}{c}\text { product } \\
\text { configuration }\end{array}$ & $\begin{array}{l}\text { orientation } \\
\text { of Ir- } \pi \text {-allyl }\end{array}$ & L1 & L2 & $\mathbf{L 3}$ & L4 & L5 \\
\hline re-re & $(2 R, 3 S)$ & endo & 6.7 & 6.4 & 4.3 & 5.9 & 5.3 \\
\hline si-re & $(2 S, 3 S)$ & endo & 6.1 & 8.1 & 7.6 & 7.0 & 7.5 \\
\hline$s i-s i$ & $(2 S, 3 R)$ & endo & 0.0 & 0.0 & 0.0 & 0.0 & 0.0 \\
\hline re-si & $(2 R, 3 R)$ & endo & 6.4 & 5.7 & 3.7 & 6.3 & 3.0 \\
\hline$s i-s i$ & $(2 S, 3 R)$ & exo & 9.4 & 10.7 & 8.9 & 10.6 & 10.5 \\
\hline re-si & $(2 R, 3 R)$ & exo & 16.3 & 17.1 & 12.9 & 17.0 & 14.8 \\
\hline si-re & $(2 S, 3 S)$ & exo & 1.2 & 1.6 & 1.5 & 1.8 & 2.2 \\
\hline re-re & $(2 R, 3 S)$ & exo & 7.7 & 4.8 & 3.0 & 4.5 & 4.1 \\
\hline \multicolumn{8}{|c|}{ (d) C1-P2 } \\
\hline $\begin{array}{c}\text { prochiral } \\
\text { faces of } \mathbf{C}-\mathbf{P}\end{array}$ & $\begin{array}{c}\text { product } \\
\text { configuration }\end{array}$ & $\begin{array}{l}\text { orientation } \\
\text { of Ir- } \pi \text {-allyl }\end{array}$ & L1 & $\mathbf{L} 2$ & $\mathbf{L 3}$ & L4 & L5 \\
\hline re-re & $(2 R, 3 S)$ & endo & 5.8 & 7.4 & 6.9 & 4.4 & 6.2 \\
\hline si-re & $(2 S, 3 S)$ & endo & 13.1 & 14.0 & 11.4 & 11.8 & 10.8 \\
\hline$s i-s i$ & $(2 S, 3 R)$ & endo & 6.8 & 7.4 & 5.6 & 5.7 & 6.1 \\
\hline$r e-s i$ & $(2 R, 3 R)$ & endo & 0.0 & 0.0 & 0.0 & 0.0 & 0.0 \\
\hline$s i-s i$ & $(2 S, 3 R)$ & exo & 9.9 & 10.0 & 8.8 & 9.5 & 10.1 \\
\hline$r e-s i$ & $(2 R, 3 R)$ & exo & 11.8 & 13.0 & 13.1 & 13.0 & 12.0 \\
\hline si-re & $(2 S, 3 S)$ & exo & 11.6 & 12.5 & 8.7 & 12.6 & 8.7 \\
\hline re-re & $(2 R, 3 S)$ & exo & 2.7 & 4.1 & 2.2 & 2.9 & 3.0 \\
\hline \multicolumn{8}{|c|}{$\mathrm{SMD}_{(\mathrm{THF})} / \mathrm{B} 3 \mathrm{LYP}-\mathrm{D} 3 / 6-31 \mathrm{G}^{* *}, \mathrm{SDD}(\mathrm{Ir}, \mathrm{Cu}, \mathrm{Fe}, \mathrm{Br})$} \\
\hline
\end{tabular}

(iii) Conformational analysis of transition states for the $\mathrm{C}-\mathrm{C}$ bond formation between Cu-enolate and Ir- $\pi$-allyl intermediate

Key dihedral angles $\Phi 1, \Phi 2, \Phi 3, \Phi 4, \Phi 5$ and $\Phi 6$ are employed in the conformational sampling for the stereoselective $\mathrm{C}-\mathrm{C}$ bond formation transition states. 
Table S6. Relative Gibbs Free Energies (kcal/mol) for the $\mathrm{C}-\mathrm{C}$ Bond Formation Transition States Involving Different Prochiral Faces of the $\mathrm{Cu}$-enolate (nucleophile) and the Ir- $\pi$-allyl Intermediate (electrophile) Obtained at the $\mathrm{SMD}_{(\mathrm{THF})} / \mathrm{B} 3 \mathrm{LYP}-\mathrm{D} 3 / 6-31 \mathrm{G}^{* *}, \mathrm{SDD}(\mathrm{Ir}, \mathrm{Cu}, \mathrm{Fe}, \mathrm{Br})$ Level of Theory for Different Catalyst Dyad (Cu-Walphos $(\mathbf{C})$ and Ir-Phosphoramidite $(\mathbf{P})$ ) without $\mathrm{BF}_{4}^{-}$Counter Ion

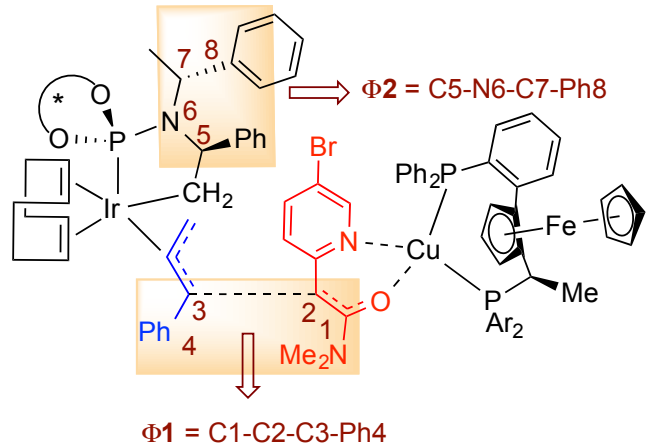

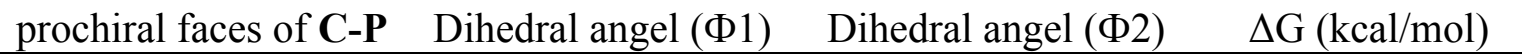

\begin{tabular}{|c|c|}
\hline re-re_1 & 47.9 \\
\hline re-re_2 & 92.1 \\
\hline re-re_3 & 48.5 \\
\hline re-re_4 & 91.4 \\
\hline re-re_5 & -97.3 \\
\hline re-re_6 & -94.8 \\
\hline si-re_1 & 164.8 \\
\hline si-re_3 & 165.3 \\
\hline si-re_6 & 166.2 \\
\hline si-re_8 & 164.9 \\
\hline$s i-s i \_1$ & 46.1 \\
\hline$s i-s i \_2$ & 179.2 \\
\hline$s i-s i \_3$ & 151.3 \\
\hline$s i-s i \_4$ & -169.4 \\
\hline$s i-s i \_5$ & 150.8 \\
\hline$s i-s i \_6$ & 151.1 \\
\hline$s i-s i \_7$ & -169.4 \\
\hline$s i-s i \_8$ & 179.1 \\
\hline si-si_9 & 105.6 \\
\hline re-si_1 & -103.1 \\
\hline re-si_2 & 45.9 \\
\hline re-si_3 & -169.4 \\
\hline re-si_4 & 68.1 \\
\hline re-si_5 & 27.9 \\
\hline re-re_1 & -99.8 \\
\hline re-re_2 & -178.0 \\
\hline re-re_3 & -118.2 \\
\hline re-re_4 & 56.9 \\
\hline
\end{tabular}

\section{C1-P1}

74.4

$-85.9$

$-73.0$

$-86.2$

70.6

65.9

70.1

$-72.9$

$-135.9$

70.4

67.6

72.3

69.1

$-88.7$

$-73.1$

$-70.7$

$-88.7$

72.2

70.1

$-67.1$

54.8

$-88.6$

56.4

75.4

C2-P1

64.5

$-135.8$

69.7

74.6
0.0

0.8

3.8

0.6

5.9

1.8

7.3

8.6

11.5

6.4

12.0

7.8

8.1

7.7

9.3

8.4

7.6

7.8

11.9

5.4

3.2

7.7

1.1

2.4

14.4

16.9

15.5

6.7 
Table S6 continued...

\begin{tabular}{lccc}
\hline re-re_5 & 56.4 & -76.9 & 11.2 \\
si-re_1 & 172.5 & 76.2 & 0 \\
si-re_2 & 172.8 & -87.6 & 4.5 \\
si-re_3 & 173.2 & -71.6 & 3.9 \\
si-re_4 & -32.5 & 128.2 & 7.6 \\
si-re_5 & 75.4 & 67.4 & 6.7 \\
si-re_6 & 107.7 & 74.9 & 6.5 \\
si-re_7 & 171.6 & -141.4 & 6.2 \\
si-re_8 & 173.2 & -71.6 & 3.8 \\
si-si_1 & 158.1 & -88.9 & 7.4 \\
si-si_2 & 150.9 & 72.6 & 2.7 \\
si-si_3 & 158.6 & -68.6 & 6.9 \\
si-si_4 & 79.0 & 71.4 & 5.7 \\
si-si_5 & 150.9 & 72.6 & 2.7 \\
si-si_6 & 152.1 & -72.8 & 5.1 \\
re-si_1 & 143.4 & 44.3 & 19.9 \\
re-si_2 & -56.2 & -142.3 & 17.4 \\
re-si_3 & -55.7 & 65.9 & 11.6 \\
re-si_4 & 152.5 & -83.7 & 13.9 \\
re-si_5 & -58.9 & -68.7 & 14.6 \\
\hline
\end{tabular}

Table S7. Relative Gibbs Free Energies (kcal/mol) for the $\mathrm{C}-\mathrm{C}$ Bond Formation Transition States Involving Different Prochiral Faces of the Cu-enolate (nucleophile) and the Ir- $\pi$-allyl Intermediate (electrophile) and Different Conformers of Walphos-ligated Cu-enolate Obtained at the $\mathrm{SMD}_{(\mathrm{THF})} / \mathrm{B} 3 \mathrm{LYP}-\mathrm{D} 3 / 6-31 \mathrm{G}^{* *}, \mathrm{SDD}(\mathrm{Ir}, \mathrm{Cu}, \mathrm{Fe}, \mathrm{Br})$ Level of Theory for C1-P1 Catalyst Dyad (Cu-Walphos $(\mathbf{C})$ and Ir-Phosphoramidite $(\mathbf{P}))$ without $\mathrm{BF}_{4}^{-}$Counter Ion

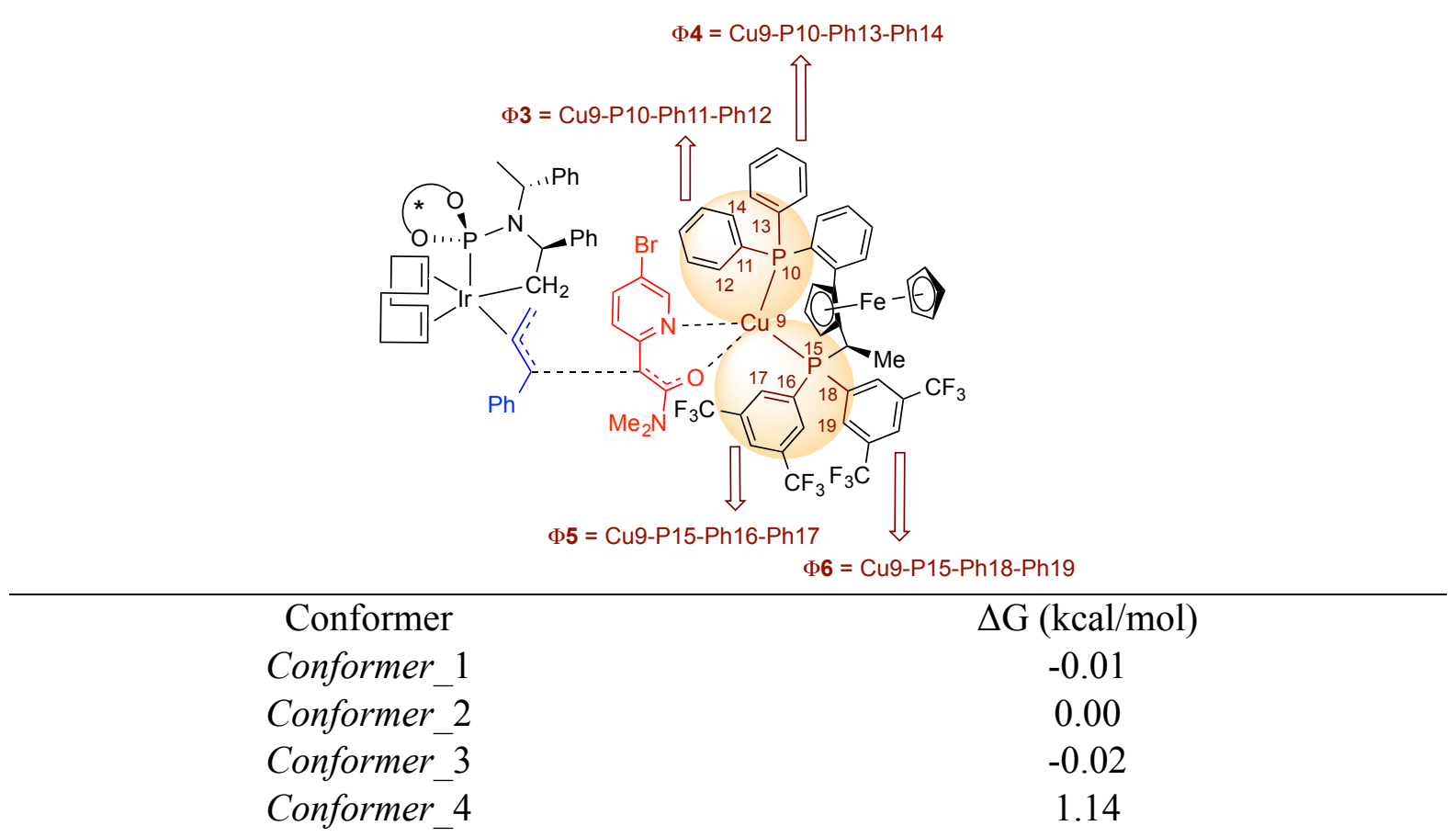


Table S7 continued...

\begin{tabular}{|c|c|}
\hline Conformer_5 & -0.01 \\
\hline Conformer_6 & -0.01 \\
\hline Conformer 7 & -0.01 \\
\hline Conformer_8 & -0.01 \\
\hline Conformer_9 & 0.00 \\
\hline Conformer_10 & 0.00 \\
\hline Conformer_11 & -0.01 \\
\hline Conformer-12 & 0.01 \\
\hline Conformer_13 & 0.01 \\
\hline Conformer_14 & 0.00 \\
\hline Conformer_15 & 0.00 \\
\hline Conformer_16 & -0.01 \\
\hline Conformer_17 & -0.01 \\
\hline Conformer_18 & -0.01 \\
\hline \multicolumn{2}{|c|}{ 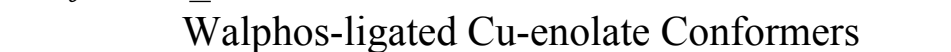 } \\
\hline Conformer_1 & 1.47 \\
\hline Conformer_2 & 0.73 \\
\hline Conformer_3 & 0.02 \\
\hline Conformer_4 & 0.01 \\
\hline Conformer_5 & 0.00 \\
\hline Conformer_6 & 0.87 \\
\hline Conformer 7 & 0.60 \\
\hline Conformer_8 & 0.59 \\
\hline Conformer_9 & 0.61 \\
\hline Conformer_10 & 0.22 \\
\hline Conformer_11 & 0.22 \\
\hline Conformer_12 & 0.60 \\
\hline Conformer_13 & 0.61 \\
\hline Conformer_14 & 0.59 \\
\hline Conformer_15 & 0.74 \\
\hline Conformer_16 & 0.63 \\
\hline Conformer_17 & 0.60 \\
\hline Conformer_18 & 0.60 \\
\hline Conformer_19 & 0.60 \\
\hline Conformer_20 & 0.76 \\
\hline Conformer_21 & 0.60 \\
\hline Conformer_22 & 0.60 \\
\hline Conformer_23 & 0.61 \\
\hline Conformer_24 & 0.62 \\
\hline Conformer_25 & 0.60 \\
\hline Conformer_26 & 0.60 \\
\hline Conformer_27 & 0.60 \\
\hline Conformer_28 & 0.60 \\
\hline Conformer_29 & 0.87 \\
\hline Conformer_30 & 0.59 \\
\hline
\end{tabular}


Table S8. Relative Gibbs Free Energies $(\mathrm{kcal} / \mathrm{mol})$ for the $\mathrm{C}-\mathrm{C}$ Bond Formation Transition States Involving Different Prochiral Faces of the $\mathrm{Cu}$-enolate (nucleophile) and the Ir- $\pi$-allyl Intermediate (electrophile) for Different Catalyst Dyads (Cu-Walphos (C) and IrPhosphoramidite $(\mathbf{P})$ ) with $\mathrm{BF}_{4}^{-}$Counter Ion Obtained at Different Level of Theories ${ }^{a}$

(a) C1-P1

\begin{tabular}{|c|c|c|c|c|c|c|c|}
\hline $\begin{array}{c}\text { prochiral } \\
\text { faces of } \mathbf{C}-\mathbf{P}\end{array}$ & $\begin{array}{c}\text { product } \\
\text { configuration }\end{array}$ & $\begin{array}{l}\text { orientation } \\
\text { of Ir- } \pi \text {-allyl }\end{array}$ & L1 & $\mathbf{L 2}$ & $\mathbf{L 3}$ & L4 & L5 \\
\hline re-re & $(2 R, 3 S)$ & endo & 0.0 & 0.0 & 0.0 & 0.0 & 0.0 \\
\hline si-re & $(2 S, 3 S)$ & endo & 7.6 & 6.0 & 6.5 & 6.4 & 5.3 \\
\hline$s i-s i$ & $(2 S, 3 R)$ & exo & 12.8 & 8.2 & 6.6 & 6.9 & 8.2 \\
\hline$r e-s i$ & $(2 R, 3 R)$ & exo & 2.7 & 5.2 & 2.2 & 2.0 & 5.4 \\
\hline \multicolumn{8}{|c|}{ (b) C2-P1 } \\
\hline $\begin{array}{c}\text { prochiral } \\
\text { faces of } \mathbf{C}-\mathbf{P}\end{array}$ & $\begin{array}{c}\text { product } \\
\text { configuration }\end{array}$ & $\begin{array}{l}\text { orientation } \\
\text { of Ir- } \pi \text {-allyl }\end{array}$ & L1 & $\mathbf{L 2}$ & $\mathbf{L 3}$ & L4 & L5 \\
\hline re-re & $(2 R, 3 S)$ & endo & 4.8 & 5.5 & 4.9 & 5.6 & 3.7 \\
\hline si-re & $(2 S, 3 S)$ & endo & 0.0 & 0.0 & 0.0 & 0.0 & 0.0 \\
\hline$s i-s i$ & $(2 S, 3 R)$ & exo & 1.5 & 3.1 & 3.2 & 4.6 & 3.1 \\
\hline$r e-s i$ & $(2 R, 3 R)$ & exo & 9.3 & 11.2 & 7.3 & 6.1 & 10.3 \\
\hline \multicolumn{8}{|c|}{ (c) C2-P2 } \\
\hline $\begin{array}{c}\text { prochiral } \\
\text { faces of } \mathbf{C}-\mathbf{P}\end{array}$ & $\begin{array}{c}\text { product } \\
\text { configuration }\end{array}$ & $\begin{array}{l}\text { orientation } \\
\text { of Ir- } \pi \text {-allyl }\end{array}$ & L1 & $\mathbf{L 2}$ & $\mathbf{L 3}$ & L4 & $\mathbf{L 5}$ \\
\hline re-re & $(2 R, 3 S)$ & exo & 12.8 & 8.2 & 6.5 & 6.8 & 8.1 \\
\hline si-re & $(2 S, 3 S)$ & exo & 2.7 & 5.2 & 2.2 & 2.0 & 5.3 \\
\hline$s i-s i$ & $(2 S, 3 R)$ & endo & 0.0 & 0.0 & 0.0 & 0.0 & 0.0 \\
\hline$r e-s i$ & $(2 R, 3 R)$ & endo & 7.6 & 6.0 & 6.5 & 6.4 & 5.3 \\
\hline \multicolumn{8}{|c|}{ (d) C1-P2 } \\
\hline $\begin{array}{c}\text { prochiral } \\
\text { faces of } \mathbf{C}-\mathbf{P}\end{array}$ & $\begin{array}{c}\text { product } \\
\text { configuration }\end{array}$ & $\begin{array}{l}\text { orientation } \\
\text { of Ir- } \pi \text {-allyl }\end{array}$ & L1 & $\mathbf{L 2}$ & $\mathbf{L 3}$ & L4 & $\mathbf{L 5}$ \\
\hline re-re & $(2 R, 3 S)$ & exo & 1.5 & 3.1 & 3.2 & 4.6 & 3.1 \\
\hline si-re & $(2 S, 3 S)$ & exo & 9.3 & 11.2 & 7.3 & 6.1 & 10.3 \\
\hline$s i-s i$ & $(2 S, 3 R)$ & endo & 4.8 & 5.5 & 4.9 & 5.6 & 3.7 \\
\hline$r e-s i$ & $(2 R, 3 R)$ & endo & 0.0 & 0.0 & 0.0 & 0.0 & 0.0 \\
\hline
\end{tabular}




\subsection{Space filling model for C2-P1 catalytic dyad}

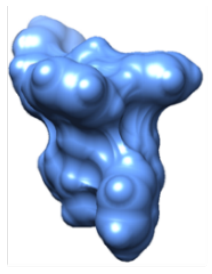

re

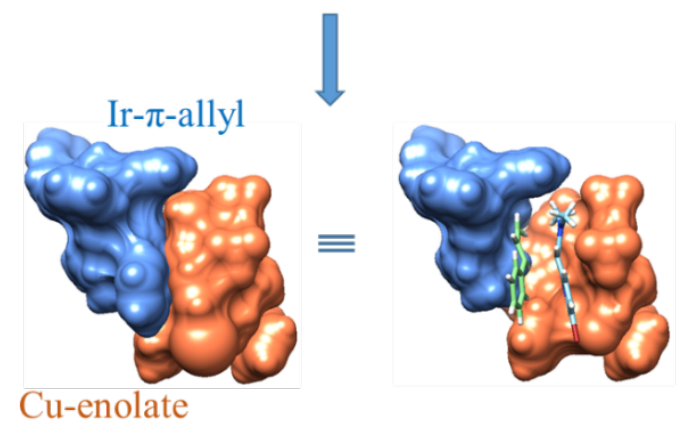

si-re

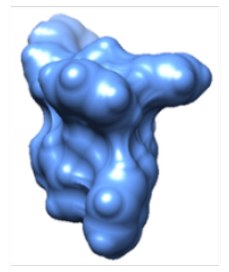

$s i$

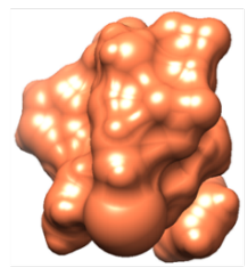

$s i$

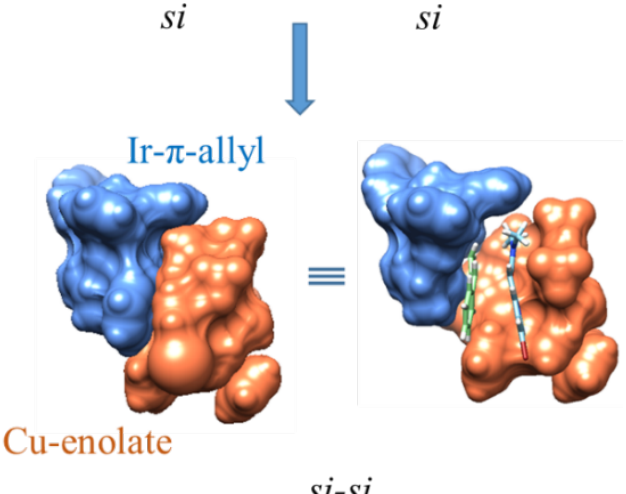

Figure S7. The space-filling representations for the C2-P1 catalytic dyad for the reacting partners ( $\mathrm{Cu}$-enolate and $\mathrm{Ir}-\pi$-allyl) are shown in the top row and the corresponding $\mathrm{C}-\mathrm{C}$ bond formation TSs conveying the contact between the chiral catalysts and the chiral cavity provided by the catalysts in the bottom row. In the second row, chiral catalysts are shown using space-filling model and a stick model is used for the substrates. Although the transition states consist of the counter ion $\left(\mathrm{BF}^{-}\right)$in the actual computations, it is removed from this figure for improved clarity.

\subsection{Global Contact Surface Area, Catalyst Cavity Volume, and Solvent Accessible}

\section{Surface Area}

Global contact surface area $^{7}$ between the two interacting catalysts, namely, chiral $\mathrm{Cu}$ Walphos and Ir-phosphoramidite catalysts is calculated as follows,

Contact_area $=[(\mathrm{Cu}-$ Walphos_area + Ir-phosphoramidite_area $)-($ Complex_area $)] / 2$

If only a single global contact surface is calculated, a selection named "contact" is created that includes all receptor atoms within $3.9 \AA$ of any atom of the catalyst. The area has to be normalized since it is counted twice (once on $\mathrm{Cu}$-Walphos and once on Ir-phosphoramidite).

7) (a) Ribeiro, J.; Rios-Vera, C.; Melo, F.; Schuller, A. Calculation of accurate interatomic contact surface areas for the quantitative analysis of non-bonded molecular interactions. Bioinformatics 2019, 35, 3499-3501. (b) Klenin, K. V.; Tristram, F.; Strunk, T.; Wenzel, W. Derivatives of molecular surface area and volume: Simple and exact analytical formulas. J. Comput. Chem. 2011, 32, 2647-2653. (c) Bauer, T. L.; Buchholz, P. C. F.; Pleiss, J. FEBS J. 2019. https://doi.org/10.1111/febs.15071 
The complex area takes into account the non-normal portions of the area that lie buried inside the other catalyst surface and hence need not be counted (Table S9).

Table S9. Calculation of Global Contact Surface Area (in $\AA^{2}$ ) between the two chiral CuWalphos and Ir-phosphoramidite catalysts for all the catalyst dyads ${ }^{a}$

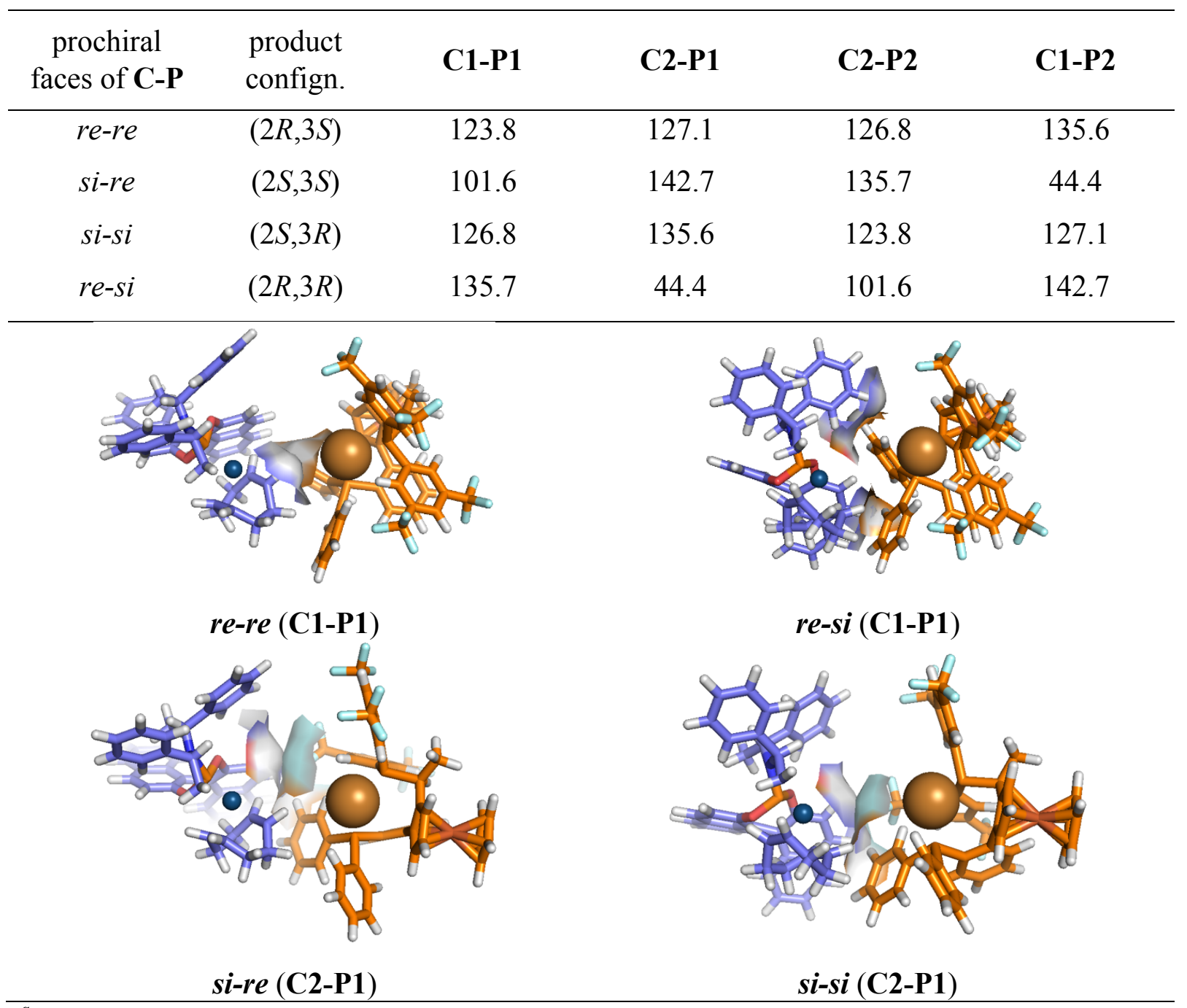

${ }^{a-}$ counter ion $\left(\mathrm{BF}^{-}\right)$is removed from figures for improved clarity.

Analysis of contact surface area reveals that the contact region between the chiral catalysts in the lower energy re-re mode of addition is lesser than that in the re-si mode for the C1-P1 catalyst dyad whereas an opposite trend is noticed for the C2-P1 catalyst dyad where the lower energy si-re mode of addition have larger contact area than that in the corresponding higher energy si-si mode of addition. The same is evident from the noncovalent interactions mapped through AIM analysis wherein examples with lower contact area exhibited lesser number of favourable interactions as compared to the ones with higher contact area results in more number of favourable interactions between the two chiral catalysts as shown in Figures S9 and S10. 
Table S10. Calculation of Catalyst Cavity Volume $\left(\AA^{3}\right)$ for re-re (low) and re-si (high) TSs with C1-P1 Catalyst

Both catalyst of re-re at probe 6

Shell volume

(probe radius of $6.0 \AA$ )

$$
2226
$$

Solvent

Solvent Volume

Both catalyst of re-si at probe 6

Shell volume

(probe radius of $6.0 \AA$ )
Both catalyst of re-re at probe 1.5

Solvent-excluded volume

(probe radius of $1.5 \AA$ )

\section{2}

volume

314

Both catalyst of $r e-s i$ at probe 1.5

Solvent-excluded volume

(probe radius of $1.5 \AA$ ) 1895

Solvent Volume $=$ shell volume-solvent-excluded volume

Solvent Volume 304

Table S11. Calculation of Catalyst Cavity Volume $\left(\AA^{3}\right)$ for si-re (low) and si-si (high) with C2-P1 Catalyst

\begin{tabular}{|c|c|}
\hline $\begin{array}{c}\text { Both catalyst of si-re at probe } 6 \\
\text { shell volume } \\
\text { (probe radius of } 6.0 \AA \text { ) }\end{array}$ & $\begin{array}{c}\text { Both catalyst of si-re at probe } 1.5 \\
\text { solvent-excluded volume } \\
\text { (probe radius of } 1.5 \AA \text { ) }\end{array}$ \\
\hline 2217 & 1911 \\
\hline \multicolumn{2}{|c|}{ Solvent Volume $=$ shell volume-solvent-excluded volume } \\
\hline Solvent Volume & 306 \\
\hline $\begin{array}{c}\text { Both catalyst of } s i-s i \text { at probe } 6 \\
\text { shell volume } \\
\text { (probe radius of } 6.0 \AA \text { ) }\end{array}$ & $\begin{array}{c}\text { Both catalyst of } s i-s i \text { at probe } 1.5 \\
\text { solvent-excluded volume } \\
\text { (probe radius of } 1.5 \AA \text { ) }\end{array}$ \\
\hline 2245 & 1936 \\
\hline \multicolumn{2}{|c|}{ solvent solume $=$ shell volume-solvent-excluded volume } \\
\hline Solvent Volume & 309 \\
\hline
\end{tabular}

Table S12. Calculation of Solvent Accessible Surface Area $\left(\AA^{2}\right)$ for C1-P1 and C2-P1

\begin{tabular}{cccccc}
\hline & C1-P1 & \multicolumn{3}{c}{ C2-P1 } \\
\hline & $r e-r e$ & $r e-s i$ & & $s i-r e$ & $s i-s i$ \\
\hline TS & 1046 & 1029 & TS & 1039 & 1035 \\
catalyst & 1038 & 1003 & catalyst & 1009 & 1013 \\
substrates & 295 & 302 & substrates & 293 & 297
\end{tabular}

\begin{tabular}{|c|c|c|c|}
\hline \multicolumn{4}{|c|}{ Solvent Accessible Surface Area $=($ Catalyst + Substrates $)-T S$ at probe $3 \AA^{2}$} \\
\hline re-re & $r e-s i$ & si-re & $s i-s i$ \\
\hline 287 & 276 & 263 & 275 \\
\hline
\end{tabular}




\subsection{Activation strain analysis}

For rationalizing the energetic preference for various modes of additions between the nucleophile (Cu-enolate) and electrophile (Ir- $\pi$-allyl), the activation strain analysis is applied to the stereo-determining transition states. Following fragments, $\mathrm{f} 1=\mathrm{Ir}-\pi$-allyl and $\mathrm{f} 2=\mathrm{Cu}-$ enolate are used in our computations (Table S13).

Table S13. Computed Relative Distortion Energies in each Fragment, Total Relative Distortion Energies $\left(\Delta \Delta \mathrm{E}_{\mathrm{d}}^{*}\right)$ and Relative Interaction Energies $\left(\Delta \Delta \mathrm{E}_{\mathrm{i}}^{*}\right)$ (in kcal/mol) in the Stereocontrolling TSs at the $\mathrm{SMD}_{(\mathrm{THF})} / \mathrm{B} 3 \mathrm{LYP}-\mathrm{D} 3 / 6-31 \mathrm{G}^{* *}, \mathrm{SDD}(\mathrm{Ir}, \mathrm{Cu}, \mathrm{Fe}, \mathrm{Br})$ Level of Theory $^{a}$ All Energies are in $\mathrm{kcal} / \mathrm{mol}$

\begin{tabular}{|c|c|c|c|c|c|}
\hline & * & $\mathrm{P}^{\mathrm{u}}$ * & $\begin{array}{c}\mathrm{Ph} \\
\text { fragment-f1 }\end{array}$ & $\mathrm{Me}$ & agment-f2 \\
\hline $\begin{array}{l}\text { mode of } \\
\text { addition }\end{array}$ & $\begin{array}{l}\Delta \Delta \mathrm{E}_{\mathrm{f}}^{\dagger} \\
(\mathrm{f} 1)\end{array}$ & $\begin{array}{l}\Delta \Delta \mathrm{E}_{\mathrm{d}}^{+} \\
(\mathrm{f} 2)\end{array}$ & $\begin{array}{c}\text { total } \Delta \Delta \mathrm{E}_{\mathrm{d}}^{\ddagger} \\
{\left[\Delta \Delta \mathrm{E}_{\mathrm{d}}^{\ddagger}(\mathrm{f} 1)+\Delta \Delta \mathrm{E}_{\mathrm{d}}^{\ddagger}(\mathrm{f} 2)\right]}\end{array}$ & $\Delta \Delta \mathrm{E}_{\mathrm{i}}^{\ddagger}$ & $\begin{array}{c}\Delta \Delta \mathrm{E}^{\dagger} \\
{\left[\Delta \Delta \mathrm{E}_{\mathrm{d}}^{\dagger}+\Delta \Delta \mathrm{E}_{\mathrm{i}}^{\dagger}\right]}\end{array}$ \\
\hline & & & C1-P1 & & \\
\hline re-re & 0.0 & 0.0 & 0.0 & 0.0 & 0.0 \\
\hline$r e-s i$ & 5.5 & 0.6 & 6.1 & -3.5 & 2.6 \\
\hline$s i-s i$ & 8.8 & 7.6 & $\begin{array}{r}16.4 \\
\text { C2-P1 }\end{array}$ & -3.0 & 13.4 \\
\hline si-re & 0.0 & 0.0 & 0.0 & 0.0 & 0.0 \\
\hline$s i-s i$ & 6.7 & -0.1 & 6.6 & -4.5 & 2.1 \\
\hline$r e-s i$ & 0.5 & 5.4 & $\begin{array}{c}5.9 \\
\text { C2-P2 }\end{array}$ & 5.7 & 11.6 \\
\hline$s i-s i$ & 0.0 & 0.0 & 0.0 & 0.0 & 0.0 \\
\hline si-re & 5.5 & 0.6 & 6.1 & -3.5 & 2.6 \\
\hline re-re & 8.8 & 7.6 & $\begin{array}{c}16.4 \\
\text { C1-P2 }\end{array}$ & -3.0 & 13.4 \\
\hline$r e-s i$ & 0.0 & 0.0 & 0.0 & 0.0 & 0.0 \\
\hline re-re & 6.7 & -0.1 & 6.6 & -4.5 & 2.1 \\
\hline si-re & 0.5 & 5.4 & 5.9 & 5.7 & 11.6 \\
\hline
\end{tabular}

${ }^{a-}$ Relative energies with respect to lower energy TS in each catalyst dyad.

The distortion energy of both the fragments $\mathrm{f} 1$ and $\mathrm{f} 2$ of the higher energy transition state is presented with respect to the fragments $\mathrm{f} 1$ and $\mathrm{f} 2$ of the lowest energy transition state 
(Table S13). For the C1-P1 catalyst combination, the distortion energies of $\mathrm{f1}$ and $\mathrm{f} 2$ are 5.5 $\mathrm{kcal} / \mathrm{mol}$ and $0.6 \mathrm{kcal} / \mathrm{mol}$ higher in re-si TS compared to re-re TS. However, the interaction energy between the two fragments in the re-si TS is $3.5 \mathrm{kcal} / \mathrm{mol}$ lower than in the re-re TS. Thus, the net balance between the destabilizing distortion and stabilization interaction results in an overall stabilization of re-re TS by $2.6 \mathrm{kcal} / \mathrm{mol}$. Similarly in the case of C2-P1 catalyst combination, the fragment $\mathrm{fl}$ of $s i$-si TS is distorted by $6.7 \mathrm{kcal} / \mathrm{mol}$ as compared to the $\mathrm{fl}$ of si-re TS whereas $\mathrm{f} 2$ of si-re TS is distorted by only $0.1 \mathrm{kcal} / \mathrm{mol}$ as compared to $\mathrm{f} 2$ of $s i$-si TS. In si-si TS, an improved interaction energy by $4.5 \mathrm{kcal} / \mathrm{mol}$ is noted than that in si-re TS. The net balance between the destabilizing distortion and stabilizing interaction energies results in an overall stabilization of si-re TS by $2.1 \mathrm{kcal} / \mathrm{mol}$. It can be noted from Figure 8 that Ir- $\pi$-allyl fragment experiences a higher distortion and thus can be considered as a major factor contributing to the energy difference between the stereo-determining TSs in both the catalyst combination.

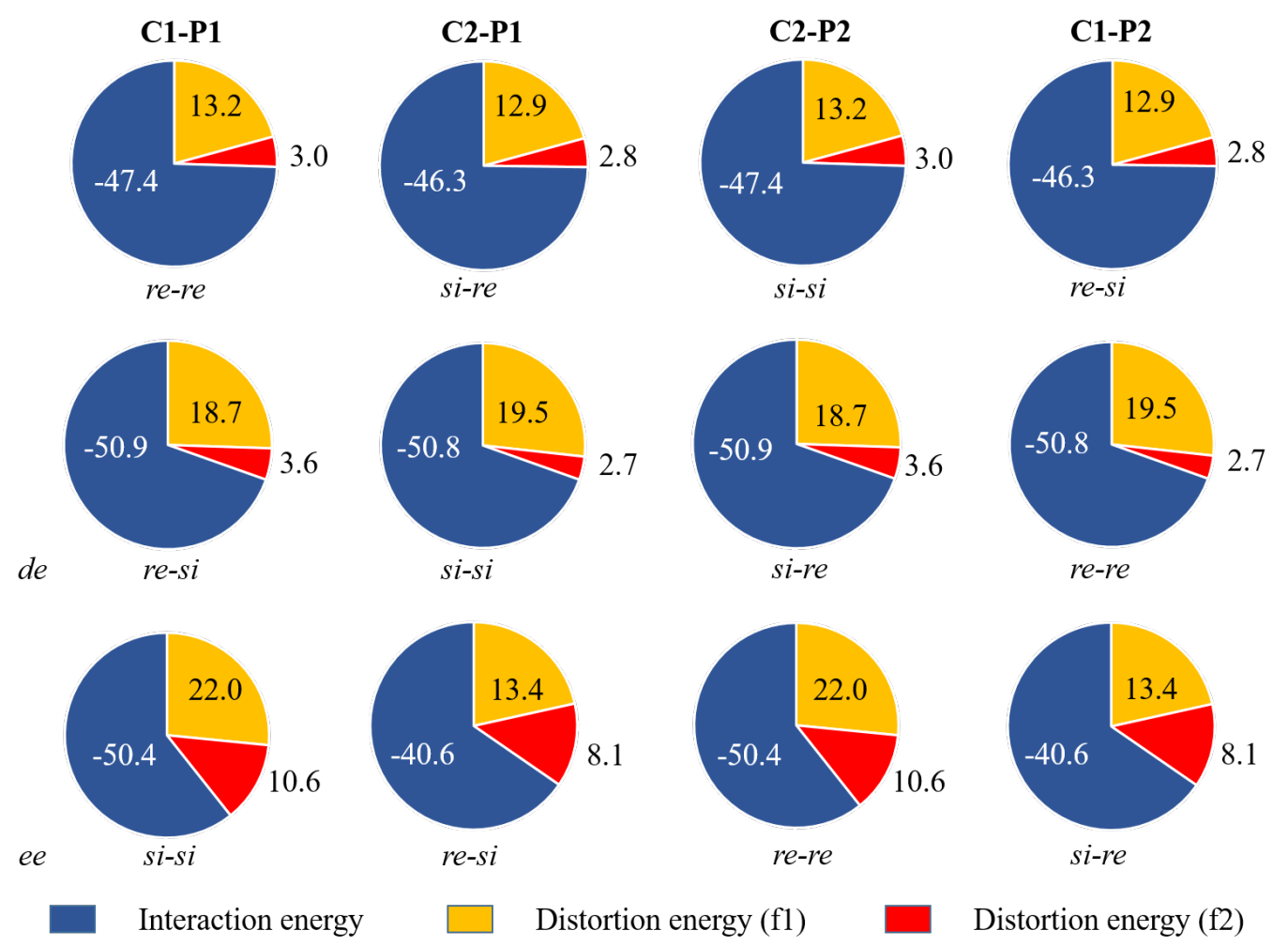

Figure 8. Computed distortion $\left(\Delta \Delta \mathrm{E}_{\mathrm{d}}^{\ddagger}\right)$ and interaction $\left(\Delta \Delta \mathrm{E}_{\mathrm{i}}^{\ddagger}\right)$ energies $(\mathrm{kcal} / \mathrm{mol})$ for the stereocontrolling TSs with respect to the separated reactants for each of the catalyst dyad. 


\subsection{Optimized Geometries of Stereocontrolling Transition States}

(i) C1-P1 Catalyst Dyad

(a) Space filling models of TSs [8-9] $]_{\text {re-re }}$ and $[8-9]_{r e-s i}^{+}$for the re-re and re-si modes of addition between $\mathrm{Cu}$-enolate (nucleophile) and $\mathrm{Ir}-\pi$-allyl intermediate (electrophile)

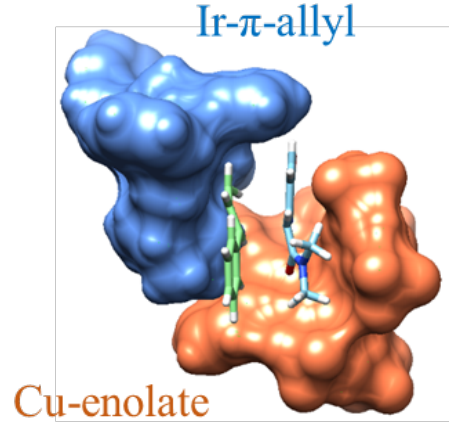

re-re

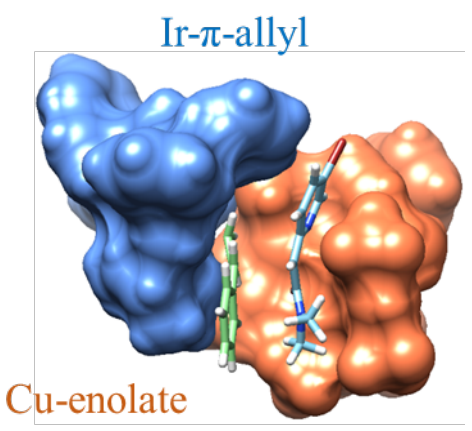

$r e-s i$

(b) NCIs interactions in the TSs
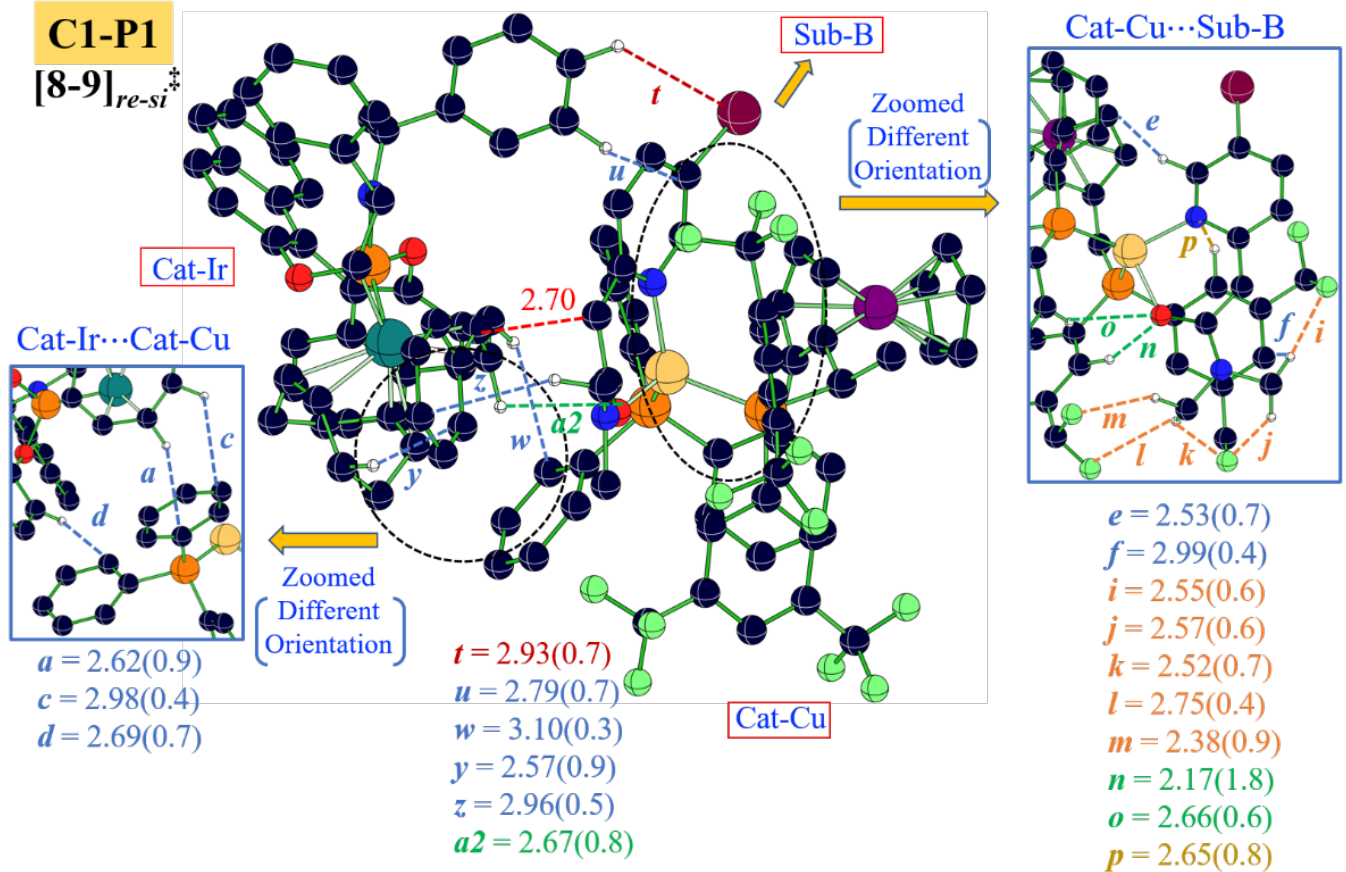
Figure S9 continued...

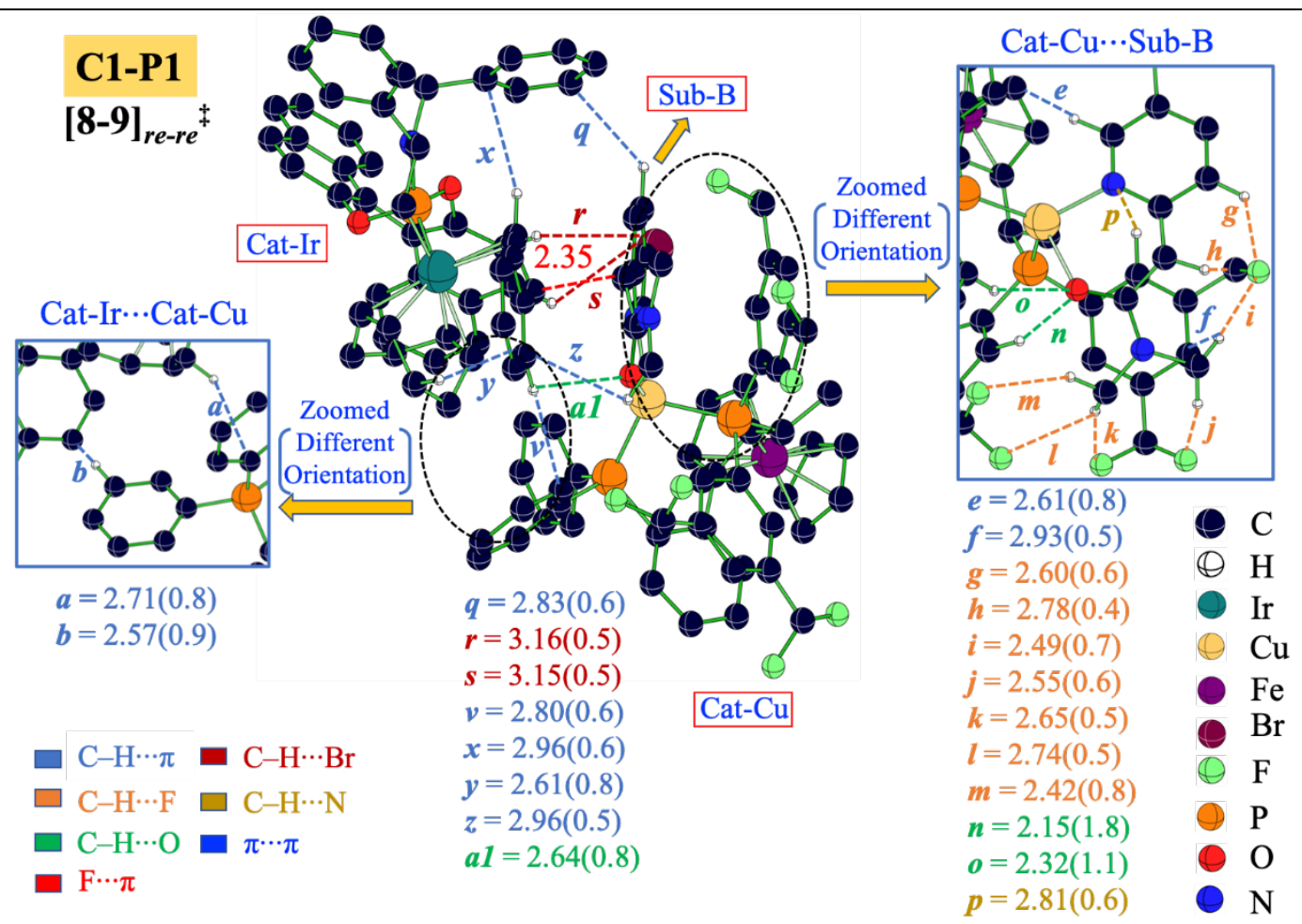

\begin{tabular}{|c|c|c|c|c|c|c|c|}
\hline \multirow[b]{2}{*}{ Bond Path } & \multirow{2}{*}{$\begin{array}{c}\text { Type of } \\
\text { Interaction }\end{array}$} & \multicolumn{3}{|c|}{ re-re } & \multicolumn{3}{|c|}{ re-si } \\
\hline & & $(\AA)$ & $\rho \times 10^{-2}$ & $(\theta)$ & $(\AA)$ & $\rho \times 10^{-2}$ & $(\theta)$ \\
\hline$a$ & $\mathrm{C}-\mathrm{H} \cdots \pi$ & 2.71 & 0.8 & 20.7 & 2.62 & 0.94 & 35.8 \\
\hline$b$ & $\mathrm{C}-\mathrm{H} \cdots \pi$ & 2.57 & 0.9 & 5.4 & $-^{a}$ & $-^{a}$ & $-^{a}$ \\
\hline$c$ & $\mathrm{C}-\mathrm{H} \cdots \pi$ & $-^{a}$ & $-^{a}$ & $-{ }^{a}$ & 2.98 & 0.47 & 63.5 \\
\hline$d$ & $\mathrm{C}-\mathrm{H} \cdots \pi$ & $-^{a}$ & $-^{a}$ & $-{ }^{a}$ & 2.69 & 0.79 & 30.9 \\
\hline$e$ & $\mathrm{C}-\mathrm{H} \cdots \pi$ & 2.61 & 0.8 & 31.2 & 2.53 & 0.7 & 20.0 \\
\hline$f$ & $\mathrm{C}-\mathrm{H} \cdots \pi$ & 2.93 & 0.5 & 62.2 & 2.99 & 0.4 & 50.9 \\
\hline$g$ & $\mathrm{C}-\mathrm{H} \cdots \mathrm{F}$ & 2.60 & 0.6 & 107.1 & $-{ }^{a}$ & $-^{a}$ & $-{ }^{a}$ \\
\hline$h$ & $\mathrm{C}-\mathrm{H} \cdots \mathrm{F}$ & 2.78 & 0.4 & 111.6 & $-{ }^{a}$ & $-{ }^{a}$ & $-{ }^{a}$ \\
\hline$i$ & $\mathrm{C}-\mathrm{H} \cdots \mathrm{F}$ & 2.49 & 0.7 & 172.8 & 2.55 & 0.6 & 173.5 \\
\hline$j$ & $\mathrm{C}-\mathrm{H} \cdots \mathrm{F}$ & 2.55 & 0.6 & 150.5 & 2.55 & 0.6 & 142.7 \\
\hline$k$ & $\mathrm{C}-\mathrm{H} \cdots \mathrm{F}$ & 2.65 & 0.5 & 162.2 & 2.52 & 0.7 & 128.0 \\
\hline$l$ & $\mathrm{C}-\mathrm{H} \cdots \mathrm{F}$ & 2.74 & 0.5 & 104.6 & 2.75 & 0.4 & 105.9 \\
\hline$m$ & $\mathrm{C}-\mathrm{H} \cdots \mathrm{F}$ & 2.42 & 0.8 & 144.1 & 2.38 & 0.9 & 142.2 \\
\hline$n$ & $\mathrm{C}-\mathrm{H} \cdots \mathrm{O}$ & 2.15 & 1.8 & 153.3 & 2.17 & 1.8 & 152.9 \\
\hline$o$ & $\mathrm{C}-\mathrm{H} \cdots \mathrm{O}$ & 2.32 & 1.1 & 150.0 & 2.66 & 0.6 & 139.6 \\
\hline$p$ & $\mathrm{C}-\mathrm{H} \cdots \mathrm{N}$ & 2.81 & 0.6 & 34.1 & 2.65 & 0.8 & 33.14 \\
\hline$q$ & $\mathrm{C}-\mathrm{H} \cdots \pi$ & 2.83 & 0.6 & 46.2 & $-{ }^{a}$ & $-^{a}$ & $-^{a}$ \\
\hline$r$ & $\mathrm{C}-\mathrm{H} \cdots \mathrm{Br}$ & 3.16 & 0.5 & 121.4 & $-^{a}$ & $-^{a}$ & $-{ }^{a}$ \\
\hline$s$ & $\mathrm{C}-\mathrm{H} \cdots \mathrm{Br}$ & 3.15 & 0.5 & 122.7 & $-^{a}$ & $-^{a}$ & $-^{a}$ \\
\hline$t$ & $\mathrm{C}-\mathrm{H} \cdots \mathrm{Br}$ & $-{ }^{a}$ & $-{ }^{a}$ & $-^{a}$ & 2.93 & 0.7 & 129.7 \\
\hline$u$ & $\mathrm{C}-\mathrm{H} \cdots \pi$ & $-^{a}$ & $-{ }^{a}$ & $-{ }^{a}$ & 2.79 & 0.7 & 57.5 \\
\hline$v$ & $\mathrm{C}-\mathrm{H} \cdots \pi$ & 2.80 & 0.6 & 16.8 & $-{ }^{a}$ & $-^{a}$ & $-{ }^{a}$ \\
\hline$w$ & $\mathrm{C}-\mathrm{H} \cdots \pi$ & $-^{a}$ & $-{ }^{a}$ & $-^{a}$ & 3.10 & 0.3 & 39.7 \\
\hline
\end{tabular}


Figure S9 continued...

\begin{tabular}{|c|c|c|c|c|c|c|c|}
\hline$x$ & $\mathrm{C}-\mathrm{H} \cdots \pi$ & 2.96 & 0.6 & 8.3 & $-{ }^{a}$ & $-^{a}$ & $-{ }^{a}$ \\
\hline$y$ & $\mathrm{C}-\mathrm{H} \cdots \pi$ & 2.61 & 0.8 & 18.3 & 2.57 & 0.9 & 21.6 \\
\hline$z$ & $\mathrm{C}-\mathrm{H} \cdots \pi$ & 2.96 & 0.5 & 73.5 & 2.96 & 0.5 & 33.2 \\
\hline$a 1$ & $\mathrm{C}-\mathrm{H} \cdots \mathrm{O}$ & 2.64 & 0.8 & 107.5 & $-^{a}$ & $-^{a}$ & $-{ }^{a}$ \\
\hline$a 2$ & $\mathrm{C}-\mathrm{H} \cdots \mathrm{O}$ & $-{ }^{a}$ & $-^{a}$ & $-{ }^{a}$ & 2.67 & 0.8 & 105.0 \\
\hline
\end{tabular}

${ }^{a-}$ No $b c p$ found

(c) Topological analysis of the electron density
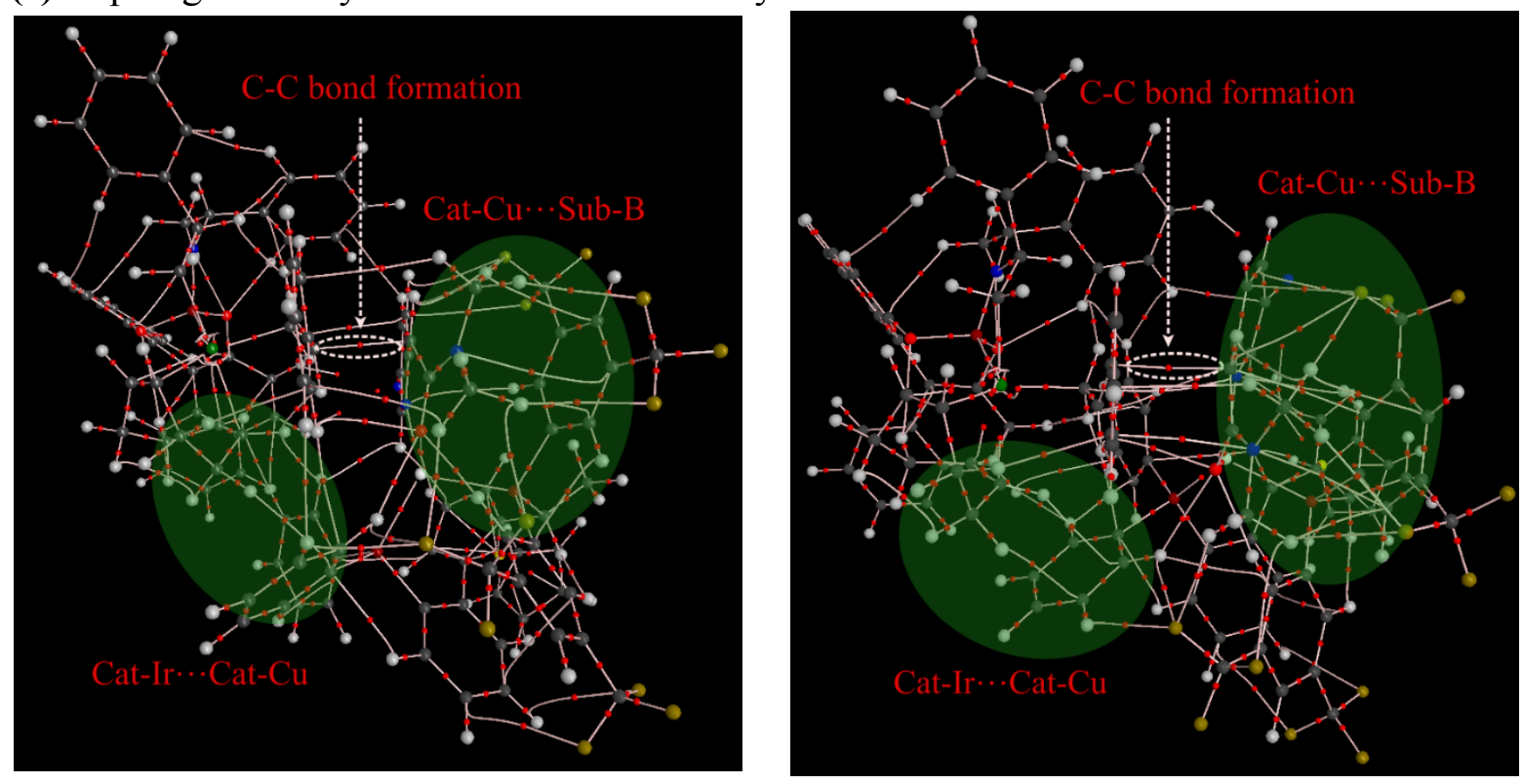

(d) NCI plots
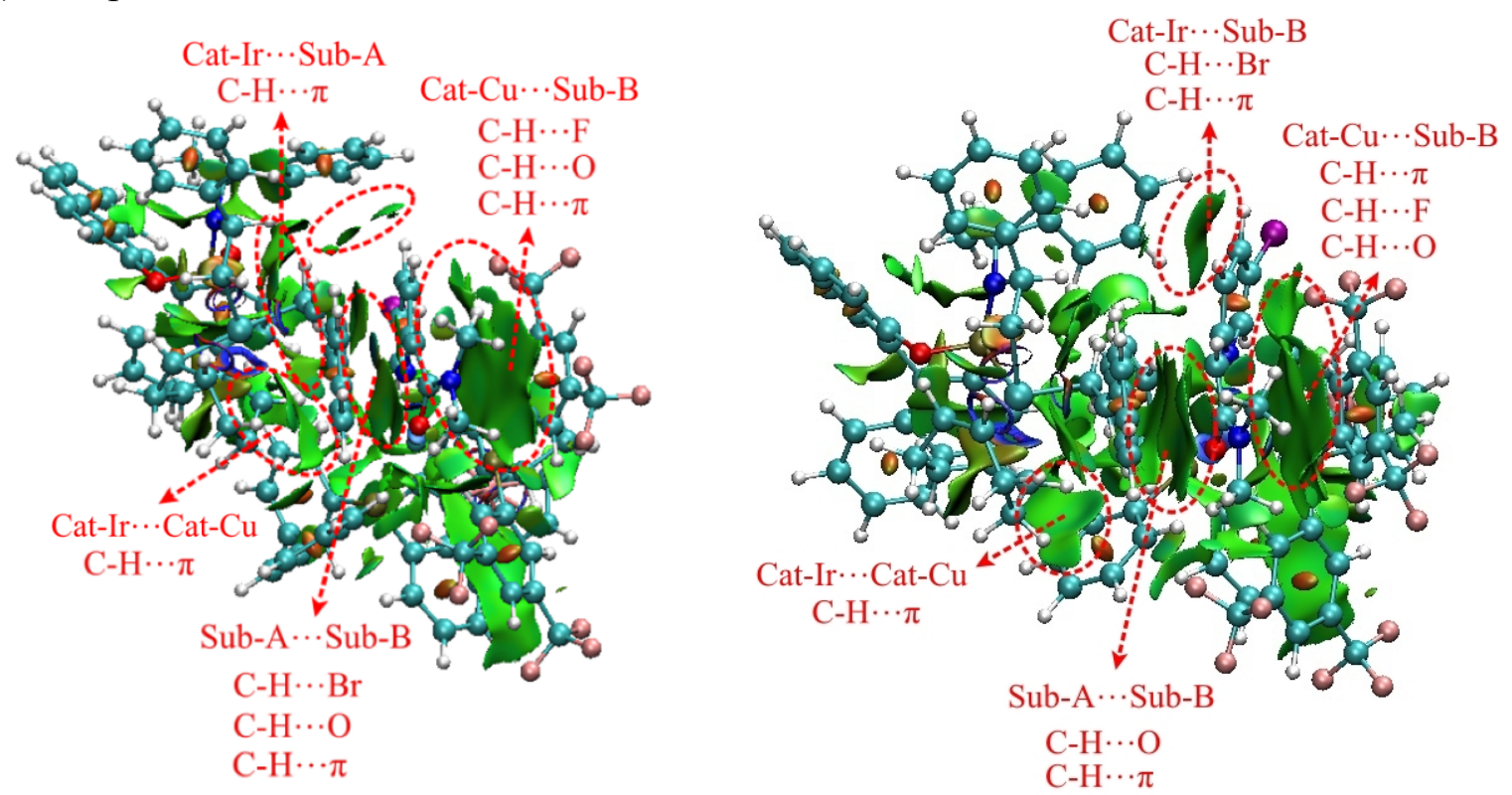

re-re

$r e-s i$ 
Figure S9. (a) Space-filling models for re-re and re-si diastereomeric TSs, (b) Important NCIs in the stereocontrolling TSs, (c) The bond critical points and the bond paths obtained using the atoms-in-molecules formalism, and (d) NCI plots highlighting the different interactions. Distances $(\AA)$, electron densities $\left(\rho \times 10^{-2}\right)$ at the bond critical points as well as the angle of interaction $\left(\theta\right.$, in $\left.{ }^{\circ}\right)$ for various NCIs for C1-P1 catalyst dyad are provided. Although the transition states consist of the counter ion $\left(\mathrm{BF}^{-}\right)$in the actual computations, it is removed from this figure for improved clarity.

(ii) C2-P1 Catalyst Dyad

(a) Space filling models of TSs $[8-9]_{s i-r e}$ and $[8-9]_{s i-s i}$ for the si-re and si-si modes of addition between $\mathrm{Cu}$-enolate (nucleophile) and $\mathrm{Ir}-\pi$-allyl intermediate (electrophile)

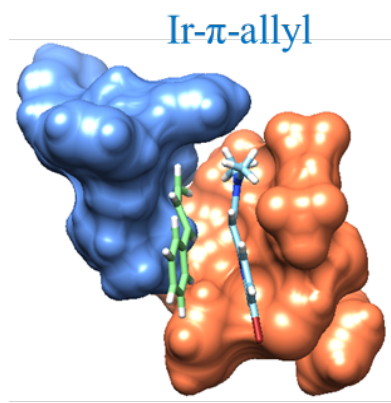

$\mathrm{Cu}$-enolate

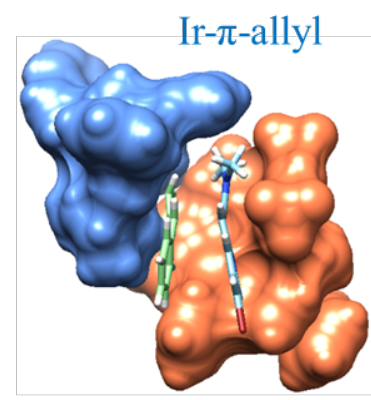

Cu-enolate

si-re

$s i-s i$

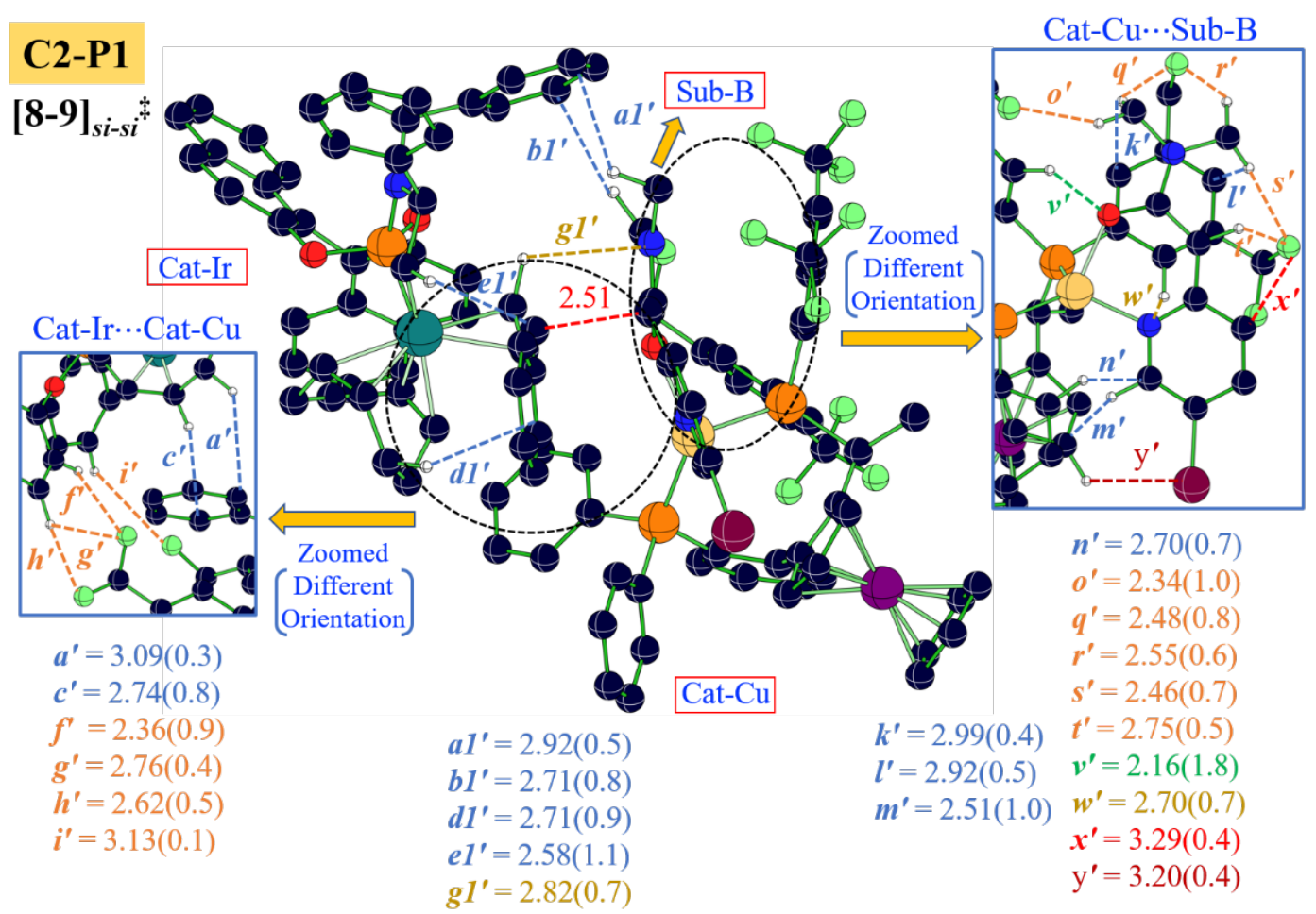


Figure S10 continued...

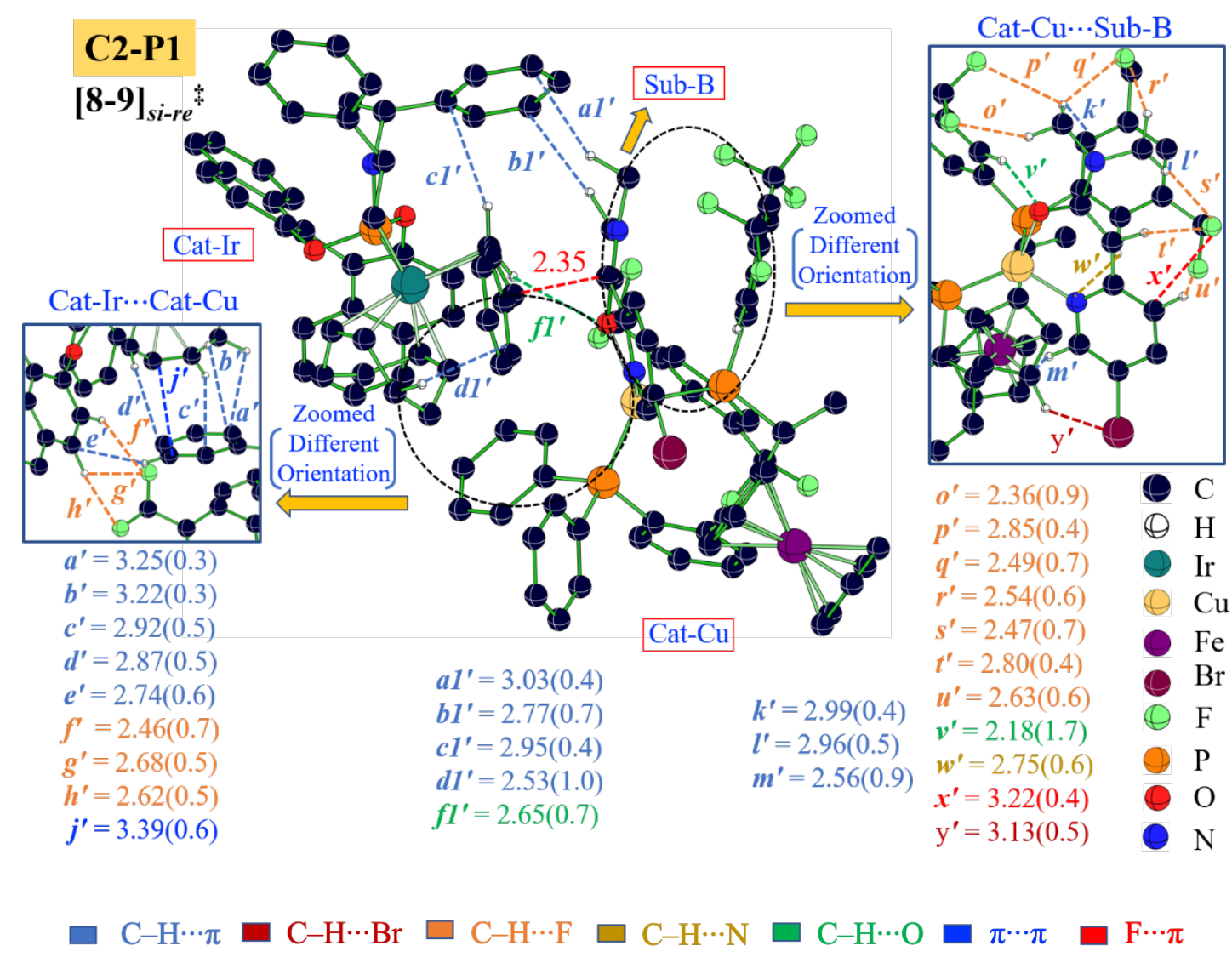

\begin{tabular}{|c|c|c|c|c|c|c|c|}
\hline \multirow[b]{2}{*}{$\begin{array}{l}\text { Bond } \\
\text { Path }\end{array}$} & \multirow[b]{2}{*}{$\begin{array}{c}\text { Type of } \\
\text { Interaction }\end{array}$} & \multicolumn{3}{|c|}{ si-re } & \multicolumn{3}{|c|}{$s i-s i$} \\
\hline & & $(\AA)$ & $\rho \times 10^{-2}$ & $(\theta)$ & $(\AA)$ & $\rho \times 10^{-2}$ & $(\theta)$ \\
\hline$a^{\prime}$ & $\mathrm{C}-\mathrm{H} \cdots \pi$ & 3.25 & 0.3 & 74.7 & 3.09 & 0.3 & 55.5 \\
\hline$b^{\prime}$ & $\mathrm{C}-\mathrm{H} \cdots \pi$ & 3.22 & 0.3 & 65.0 & $-{ }^{a}$ & $-^{a}$ & $-^{a}$ \\
\hline$c^{\prime}$ & $\mathrm{C}-\mathrm{H} \cdots \pi$ & 2.92 & 0.5 & 50.2 & 2.74 & 0.8 & 36.8 \\
\hline$d^{\prime}$ & $\mathrm{C}-\mathrm{H} \cdots \pi$ & 2.87 & 0.5 & 50.4 & $-a$ & $-^{a}$ & $-a$ \\
\hline$e^{\prime}$ & $\mathrm{C}-\mathrm{H} \cdots \pi$ & 2.74 & 0.6 & 39.9 & $-a$ & $-^{a}$ & $-a$ \\
\hline$f^{\prime}$ & $\mathrm{C}-\mathrm{H} \cdots \mathrm{F}$ & 2.46 & 0.7 & 120.9 & 2.36 & 0.9 & 125.2 \\
\hline$g^{\prime}$ & $\mathrm{C}-\mathrm{H} \cdots \mathrm{F}$ & 2.68 & 0.5 & 112.9 & 2.76 & 0.4 & 110.2 \\
\hline$h^{\prime}$ & $\mathrm{C}-\mathrm{H} \cdots \mathrm{F}$ & 2.62 & 0.5 & 154.8 & 2.62 & 0.5 & 148.6 \\
\hline$i^{\prime}$ & $\mathrm{C}-\mathrm{H} \cdots \mathrm{F}$ & $-{ }^{a}$ & $-^{a}$ & $-^{a}$ & 3.13 & 0.1 & 128.2 \\
\hline$j^{\prime}$ & $\pi \cdots \pi$ & 3.39 & 0.6 & $-^{a}$ & $-^{a}$ & $-^{a}$ & $-^{a}$ \\
\hline$k^{\prime}$ & $\mathrm{C}-\mathrm{H} \cdots \pi$ & 2.99 & 0.4 & 55.5 & 2.99 & 0.4 & 84.5 \\
\hline$l^{\prime}$ & $\mathrm{C}-\mathrm{H} \cdots \pi$ & 2.96 & 0.5 & 60.1 & 2.92 & 0.5 & 59.0 \\
\hline$m^{\prime}$ & $\mathrm{C}-\mathrm{H} \cdots \pi$ & 2.56 & 0.9 & 34.9 & 2.51 & 1.0 & 31.0 \\
\hline$n^{\prime}$ & $\mathrm{C}-\mathrm{H} \cdots \pi$ & $-^{a}$ & $-^{a}$ & $-^{a}$ & 2.70 & 0.7 & 28.0 \\
\hline$o^{\prime}$ & $\mathrm{C}-\mathrm{H} \cdots \mathrm{F}$ & 2.36 & 0.9 & 145.8 & 2.34 & 1.0 & 145.9 \\
\hline$p^{\prime}$ & $\mathrm{C}-\mathrm{H} \cdots \mathrm{F}$ & 2.85 & 0.4 & 103.1 & $-a$ & $-{ }^{a}$ & $-{ }^{a}$ \\
\hline$q^{\prime}$ & $\mathrm{C}-\mathrm{H} \cdots \mathrm{F}$ & 2.49 & 0.7 & 129.8 & 2.48 & 0.8 & 129.5 \\
\hline$r^{\prime}$ & $\mathrm{C}-\mathrm{H} \cdots \mathrm{F}$ & 2.54 & 0.6 & 143.3 & 2.55 & 0.6 & 139.7 \\
\hline
\end{tabular}


Figure S10 continued...

\begin{tabular}{|c|c|c|c|c|c|c|c|}
\hline$s^{\prime}$ & $\mathrm{C}-\mathrm{H} \cdots \mathrm{F}$ & 2.47 & 0.7 & 174.4 & 2.46 & 0.7 & 171.3 \\
\hline$t^{\prime}$ & $\mathrm{C}-\mathrm{H} \cdots \mathrm{F}$ & 2.80 & 0.4 & 111.4 & 2.75 & 0.5 & 110.0 \\
\hline$u^{\prime}$ & $\mathrm{C}-\mathrm{H} \cdots \mathrm{F}$ & 2.63 & 0.6 & 107.8 & $-{ }^{a}$ & $-^{a}$ & $-{ }^{a}$ \\
\hline$v^{\prime}$ & $\mathrm{C}-\mathrm{H} \cdots \mathrm{O}$ & 2.18 & 1.7 & 150.5 & 2.16 & 1.8 & 148.5 \\
\hline$w^{\prime}$ & $\mathrm{C}-\mathrm{H} \cdots \mathrm{N}$ & 2.75 & 0.6 & 31.9 & 2.70 & 0.7 & 125.3 \\
\hline$x^{\prime}$ & $\mathrm{C}-\mathrm{F} \cdots \pi$ & 3.22 & 0.4 & 51.8 & 3.29 & 0.4 & 49.7 \\
\hline$y^{\prime}$ & $\mathrm{C}-\mathrm{H} \cdots \mathrm{Br}$ & 3.13 & 0.5 & 121.9 & 3.20 & 0.4 & 123.3 \\
\hline$a 1^{\prime}$ & $\mathrm{C}-\mathrm{H} \cdots \pi$ & 3.03 & 0.4 & 45.8 & 2.92 & 0.5 & 54.5 \\
\hline$b 1^{\prime}$ & $\mathrm{C}-\mathrm{H} \cdots \pi$ & 2.77 & 0.7 & 14.2 & 2.71 & 0.8 & 11.1 \\
\hline$c 1^{\prime}$ & $\mathrm{C}-\mathrm{H} \cdots \pi$ & 2.95 & 0.4 & 5.9 & $-{ }^{a}$ & $-^{a}$ & $-{ }^{a}$ \\
\hline$d 1^{\prime}$ & $\mathrm{C}-\mathrm{H} \cdots \pi$ & 2.53 & 1.0 & 17.9 & 2.71 & 0.9 & 20.9 \\
\hline$e 1^{\prime}$ & $\mathrm{C}-\mathrm{H} \cdots \pi$ & $-^{a}$ & $-^{a}$ & $-{ }^{a}$ & 2.58 & 1.1 & 109.6 \\
\hline$f 1^{\prime}$ & $\mathrm{C}-\mathrm{H} \cdots \mathrm{O}$ & 2.65 & 0.7 & 128.5 & $-{ }^{a}$ & $-^{a}$ & $-{ }^{a}$ \\
\hline$g 1^{\prime}$ & $\mathrm{C}-\mathrm{H} \cdots \mathrm{N}$ & $-{ }^{a}$ & $-^{a}$ & $-{ }^{a}$ & 2.82 & 0.7 & 110.9 \\
\hline
\end{tabular}

(c) Topological analysis of the electron density
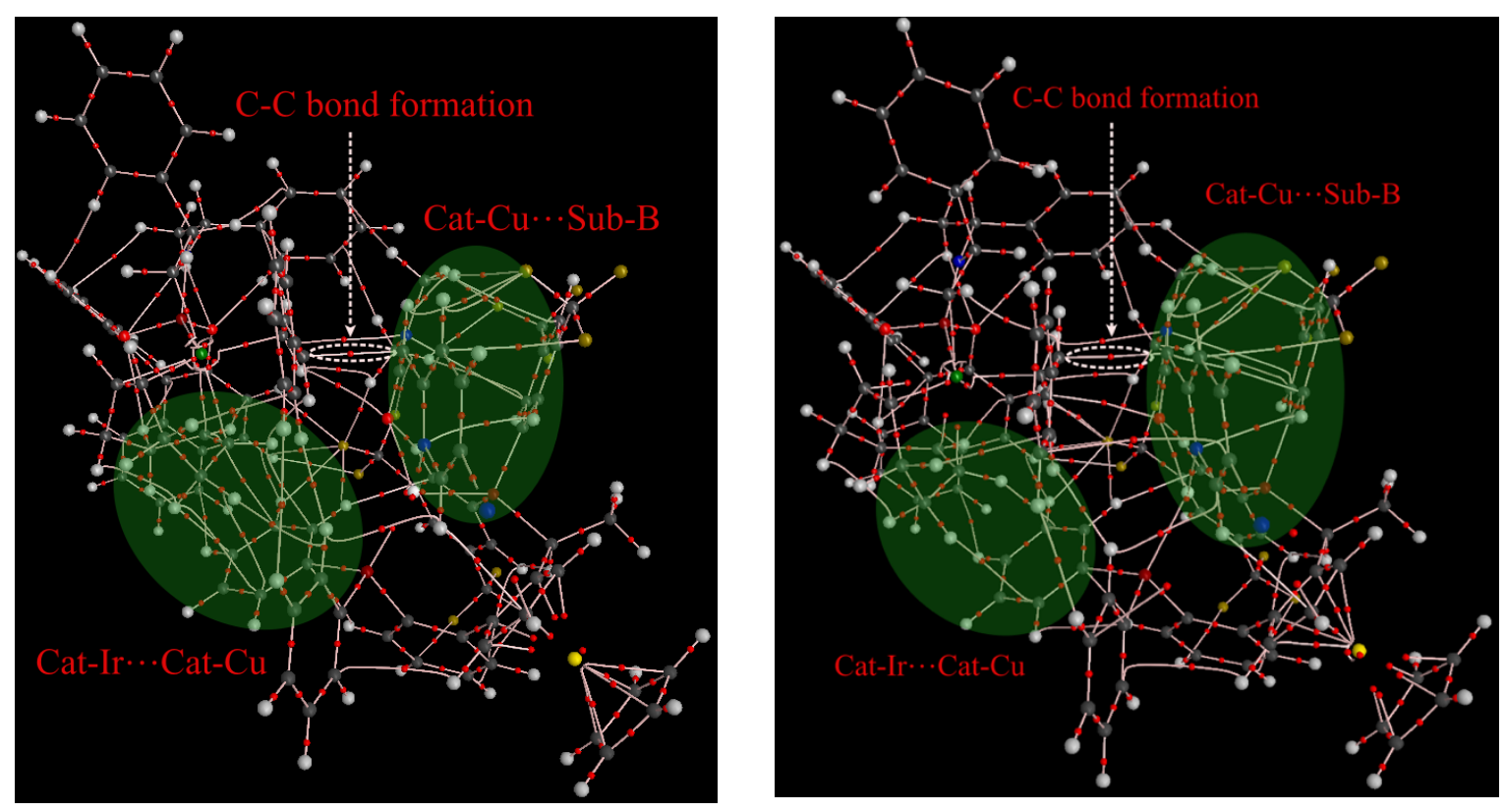
Figure S10 continued...

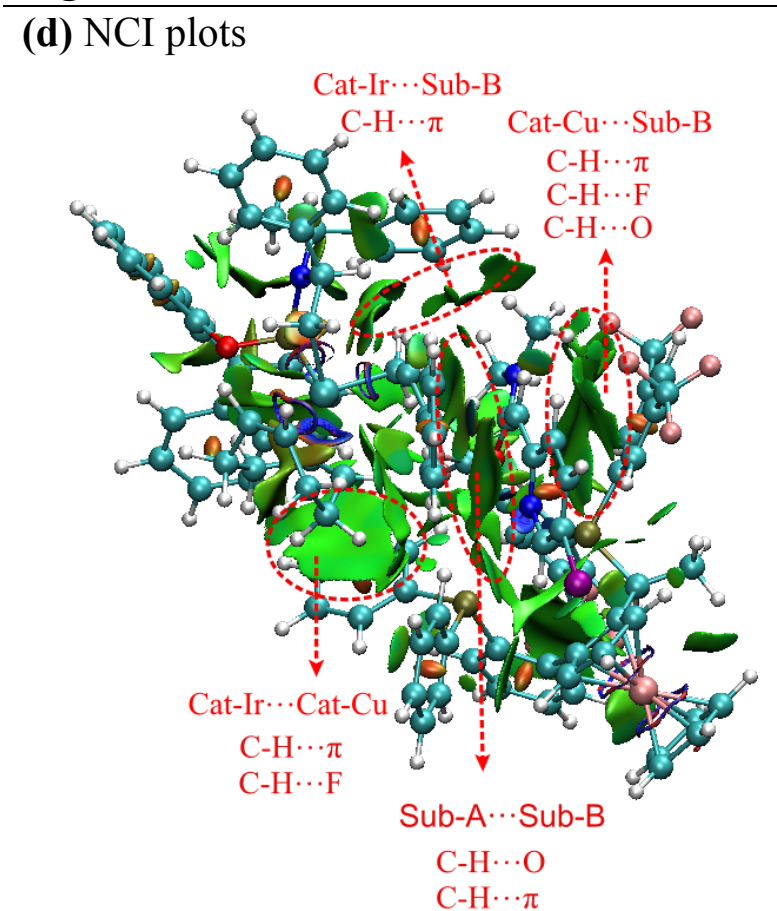

si-re

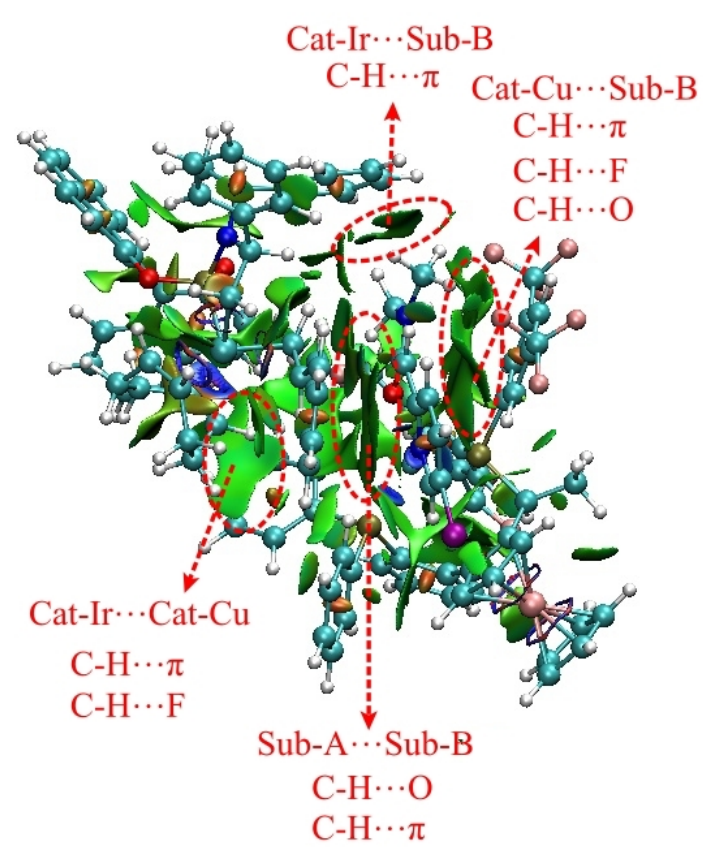

$s i-s i$

Figure S10. (a) Space-filling models for si-re and si-si diastereomeric TSs, (b) Important NCIs in the stereocontrolling TSs, (c) The bond critical points and the bond paths obtained using the atoms-in-molecules formalism, and (d) NCI plots highlighting the different interactions. Distances $(\AA)$, electron densities $\left(\rho \times 10^{-2}\right)$ at the bond critical points as well as the angle of interaction $\left(\theta\right.$, in $\left.{ }^{\circ}\right)$ for various NCIs for C2-P1 catalyst combination are provided. Although the transition states consist of the counter ion $\left(\mathrm{BF}^{-}\right)$in the actual computations, it is removed from this figure for improved clarity.

\subsection{Space filling model for the lowest energy TS for all catalyst dyads}
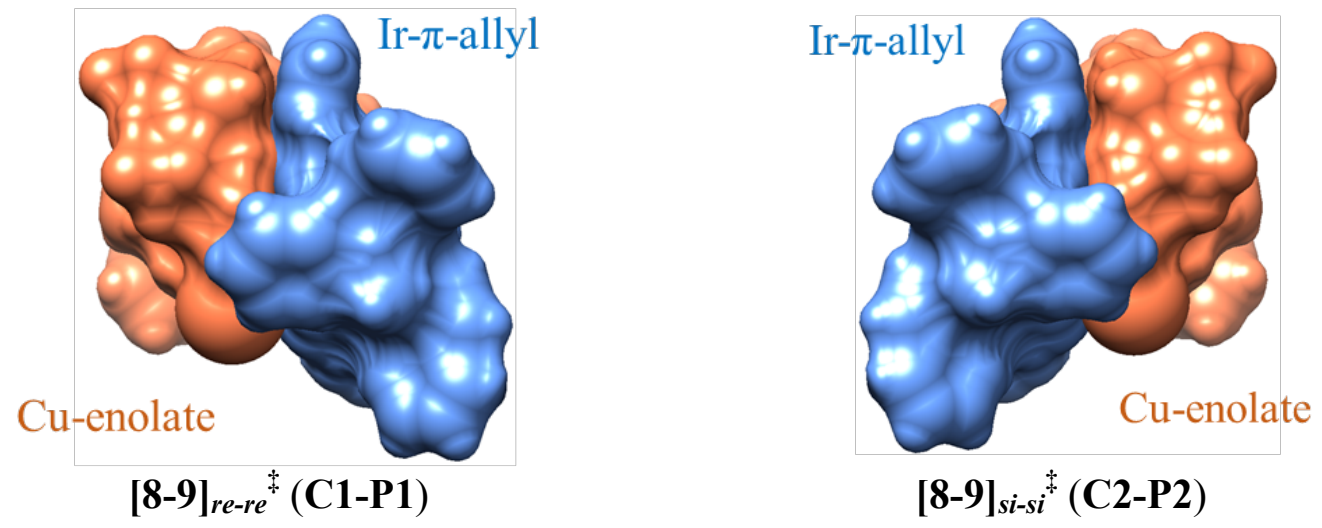


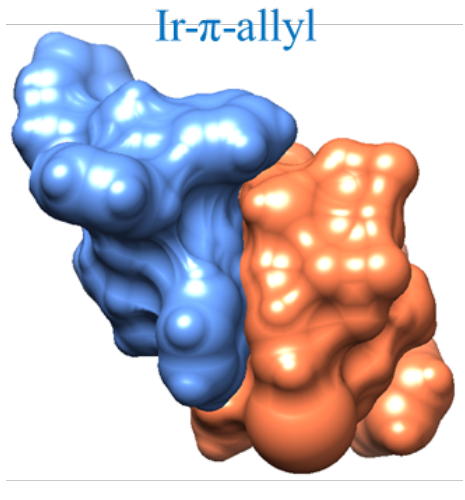

Cu-enolate

[8-9 $]_{\text {si-re }}{ }^{\ddagger}($ C2-P1)

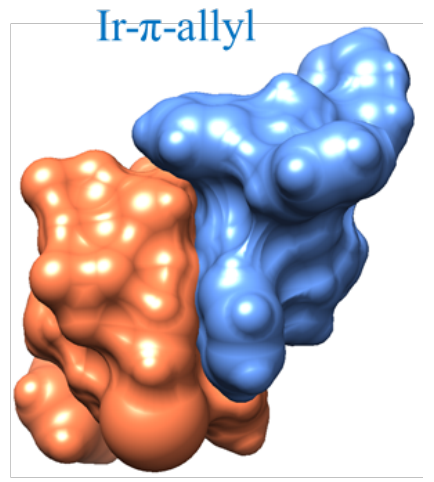

Cu-enolate

$[8-9]_{\text {resi }}^{*}(\mathbf{C 1 - P 2})$

Figure S11. The space-filling representations for all the catalyst dyads for the lowest energy stereocontrolling $\mathrm{C}-\mathrm{C}$ bond formation TSs conveying the contact between the chiral catalysts. Although the transition states consist of the counter ion $\left(\mathrm{BF}^{-}\right)$in the actual computations, it is removed from this figure for improved clarity.

1.12. Interaction wheel diagram: Interaction wheel diagram capturing all NCIs in the stereocontrolling TSs in each catalyst dyad is provided in Figures S12 and S13. As shown in the Figure 4 of the main text, the key NCIs are categorized into six types. Each such type of NCI could differ in nature (such as $\mathrm{C}-\mathrm{H} \cdots \pi, \mathrm{C}-\mathrm{H} \cdots \mathrm{F}, \mathrm{C}-\mathrm{H} \cdots \mathrm{O}, \mathrm{C}-\mathrm{H} \cdots \mathrm{N}, \mathrm{C}-\mathrm{H} \cdots \mathrm{Br}, \pi \cdots \pi$ and lone pair $\cdots \pi)$ and number of times it appears in a given TS geometry. Inspection of these NCIs in the stereocontrolling TSs conveys important molecular insights. This diagram helps us directly compare the key differences in the pattern of NCIs upon changing the catalyst configuration without having to inspect complex geometries of the TSs. It can be noticed from the total number of NCIs that the lowest energy TSs for each catalyst dyads exhibit more number of NCIs as compared to the corresponding higher energy TSs. Among the different categories of major NCIs in the stereocontrolling TSs, an important interaction is Cat-Ir ${ }^{\cdots}$ Cat-Cu operating between two chiral catalysts. In C2-P1 and C1-P2 catalyst dyads, the catalyst interact through $\mathrm{C}-\mathrm{H} \cdots \pi, \mathrm{C}-\mathrm{H} \cdots \mathrm{F}$ and $\pi \cdots \pi$ NCIs whereas $\mathrm{C}-\mathrm{H}^{\cdots} \cdots \pi$ interactions are noticed in the case of C1-P1and C2-P2 catalyst dyads. 


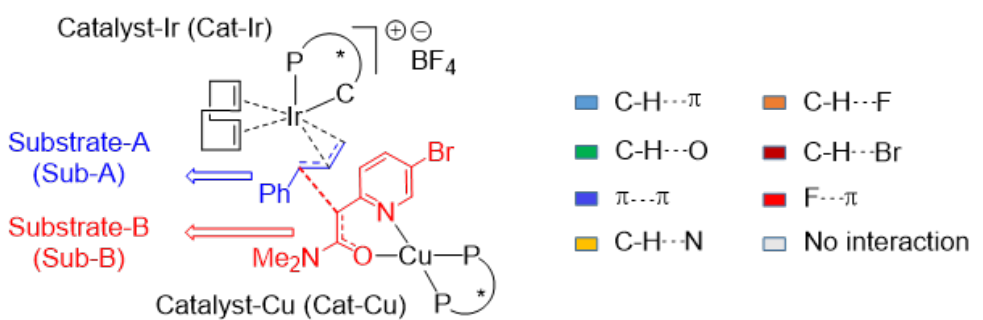

$\begin{array}{cccccccc}\text { C1-P1 } & \text { C1-P1 } & \text { C2-P1 } & \text { C2-P1 } & \text { C2-P2 } & \text { C2-P2 } & \text { C1-P2 } & \text { C1-P2 } \\ r e-r e & r e-s i & s i-r e & s i-s i & s i-s i & s i-r e & r e-s i & r e-r e\end{array}$

(1) Cat-Ir $\cdots$ Cat-Cu
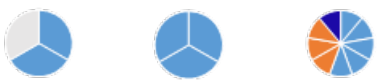

(2) Cat-Ir $\cdots$ Sub-A
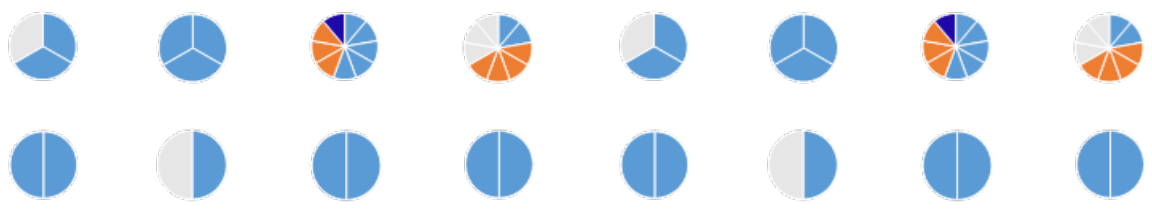

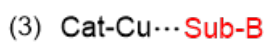
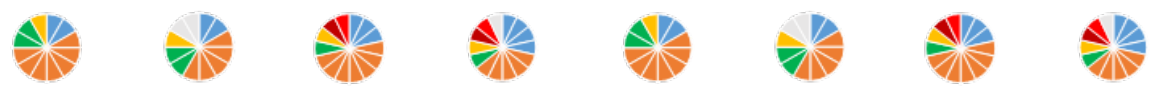

(4) Cat-Ir $\cdots$ Sub-B
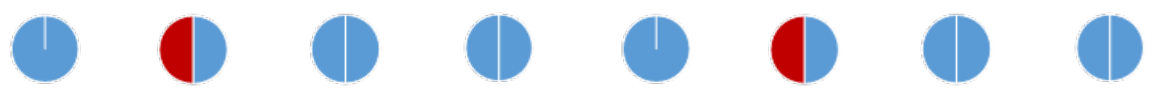

(5) Cat-Cu $\cdots$ Sub-A
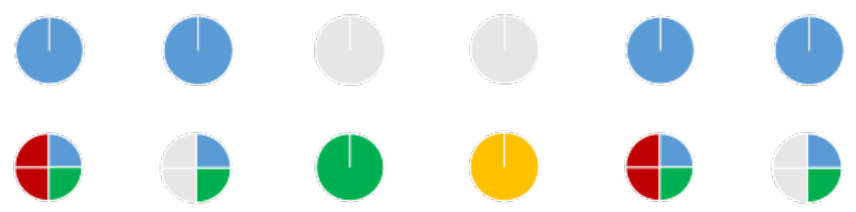

(6) Sub-A $\cdots$ Sub-B
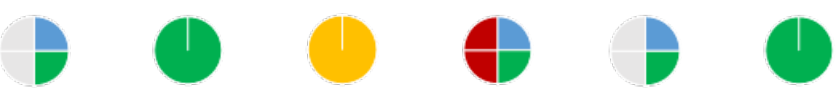

(7) Total NCls
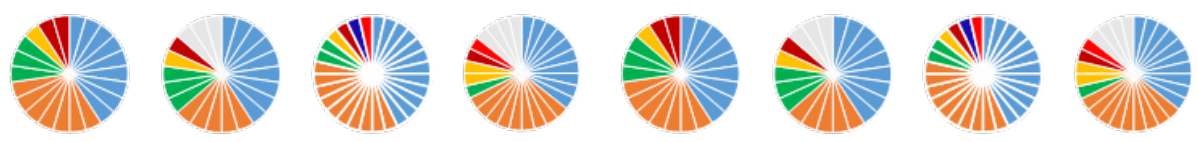

(8) Total Number

22

19

28

24

22

19

28

24

Product
Configuration

(2R,3S)

$(2 R, 3 R)$

$(2 S, 3 S) \quad(2 S, 3 R)$

$(2 S, 3 R)$

$(2 S, 3 S)$

$(2 R, 3 R)$

$(2 R, 3 S)$

Figure S12. Interaction wheel diagram capturing all NCIs in the stereocontrolling TSs in each catalyst dyad. 


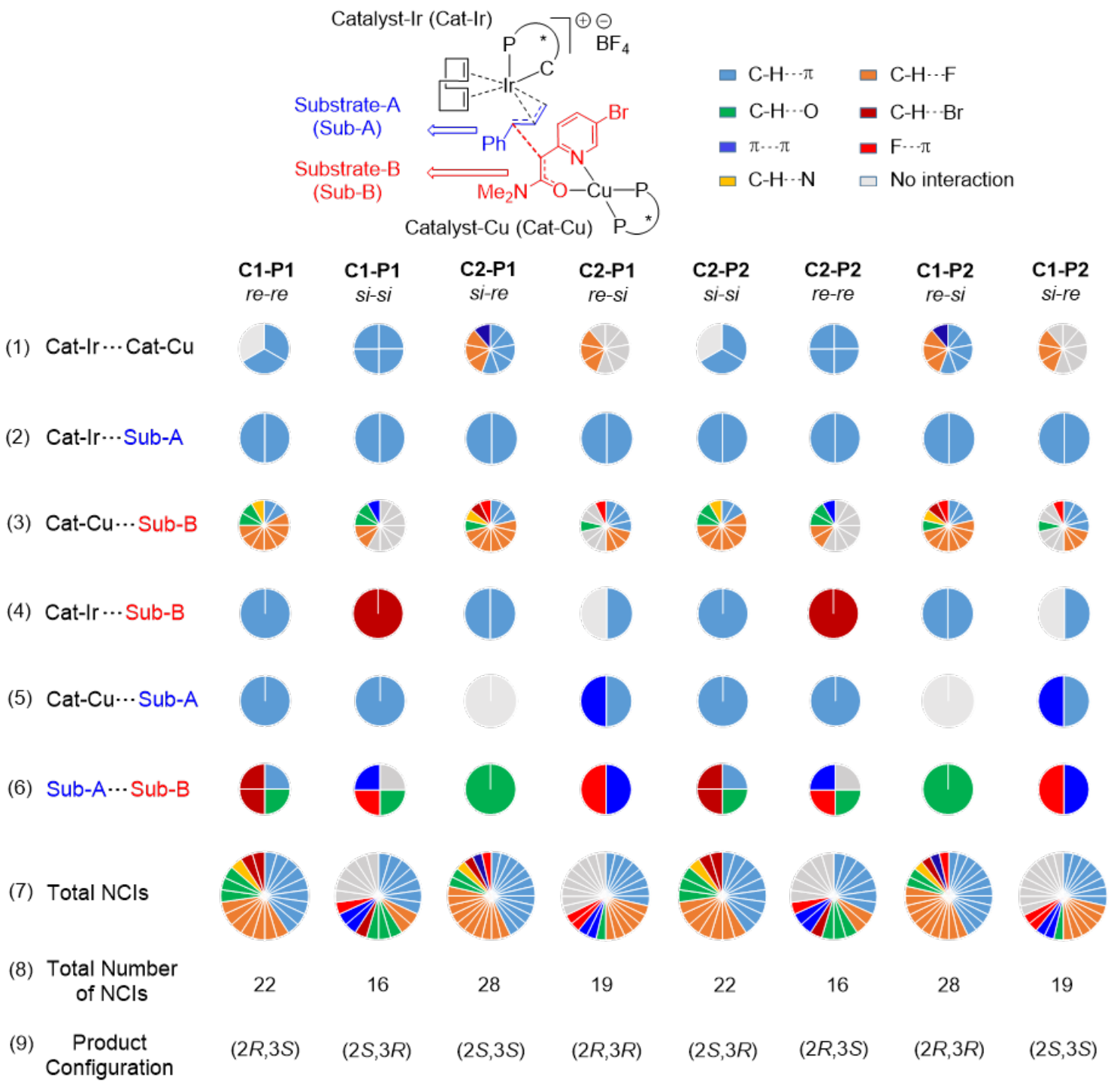

Figure S13. Interaction wheel diagram capturing all NCIs in the stereocontrolling TSs in each catalyst dyad.

\subsection{Quantification of Noncovalent Interactions using the Espinosa's Formulation}

We have employed Espinosa's formulation, that relies the topological parameters such as the electron density at the bond critical point $\left(\rho_{\mathrm{bcp}}\right)$, the corresponding Laplacian of the electron density $\left(\mathrm{V}^{2} \rho\right)$, and kinetic energy $(G)$ toward quantification of the overall strength of the NCIs. Sum of the strength of key NCIs (in $\mathrm{kcal} / \mathrm{mol}$ ) calculated using Espinosa's method for the stereocontrolling transition states is provided in Table S14.

Table S14. Estimated Strengths of the Important Noncovalent Interactions in the Stereocontrolling TSs for the C1-P1 and C2-P1 catalyst dyad. The Topological Quantities are Derived from the Atoms-in-Molecules (AIM) Analysis ${ }^{a}$ 
C1-P1

\begin{tabular}{|c|c|c|c|c|c|c|}
\hline re-re & & $\rho$ & $\nabla^{2} \rho$ & $\mathrm{G}$ & $\mathrm{V}=1 / 4 \nabla^{2} \rho-2 \mathrm{G}$ & $\mathrm{E}=1 / 2(\mathrm{~V})(\mathrm{kcal} / \mathrm{mol})$ \\
\hline \multirow{9}{*}{$\mathrm{C}-\mathrm{H} \cdots \pi$} & $a$ & 0.008 & -0.006 & 0.005 & -0.012 & -3.8 \\
\hline & $b$ & 0.009 & -0.007 & 0.006 & -0.014 & -4.3 \\
\hline & $e$ & 0.009 & -0.007 & 0.006 & -0.013 & -4.1 \\
\hline & $f$ & 0.005 & -0.004 & 0.003 & -0.008 & -2.5 \\
\hline & $q$ & 0.006 & -0.005 & 0.004 & -0.009 & -2.8 \\
\hline & $v$ & 0.006 & -0.005 & 0.004 & -0.009 & -2.8 \\
\hline & $x$ & 0.005 & -0.004 & 0.003 & -0.007 & -2.1 \\
\hline & $y$ & 0.009 & -0.007 & 0.005 & -0.013 & -4.0 \\
\hline & $z$ & 0.006 & -0.005 & 0.004 & -0.008 & -2.6 \\
\hline \multirow{7}{*}{$\mathrm{C}-\mathrm{H} \cdots \mathrm{F}$} & $g$ & 0.007 & -0.008 & 0.007 & -0.015 & -4.8 \\
\hline & $\boldsymbol{h}$ & 0.005 & -0.006 & 0.004 & -0.010 & -3.2 \\
\hline & $i$ & 0.007 & -0.008 & 0.006 & -0.015 & -4.7 \\
\hline & $j$ & 0.006 & -0.007 & 0.006 & -0.013 & -4.2 \\
\hline & $\boldsymbol{k}$ & 0.005 & -0.006 & 0.005 & -0.011 & -3.3 \\
\hline & $l$ & 0.005 & -0.006 & 0.005 & -0.011 & -3.6 \\
\hline & $m$ & 0.009 & -0.009 & 0.008 & -0.018 & -5.7 \\
\hline \multirow{3}{*}{$\mathrm{C}-\mathrm{H} \cdots \mathrm{O}$} & $a 1$ & 0.009 & -0.008 & 0.007 & -0.015 & -4.7 \\
\hline & $n$ & 0.019 & -0.014 & 0.014 & -0.031 & -9.8 \\
\hline & $\boldsymbol{o}$ & 0.012 & -0.010 & 0.010 & -0.022 & -6.9 \\
\hline \multirow{2}{*}{$\mathrm{C}-\mathrm{H} \cdots \mathrm{Br}$} & $r$ & 0.005 & -0.005 & 0.003 & -0.008 & -2.5 \\
\hline & $s$ & 0.005 & -0.004 & 0.003 & -0.008 & -2.4 \\
\hline $\mathrm{C}-\mathrm{H} \cdots \mathrm{N}$ & $p$ & 0.006 & -0.005 & 0.004 & -0.010 & -3.2 \\
\hline \multicolumn{6}{|c|}{ Total Strength } & -88.2 \\
\hline \multicolumn{7}{|c|}{ C1-P1 } \\
\hline re-si & & $\rho$ & $\nabla^{2} \rho$ & $\mathrm{G}$ & $\mathrm{V}=1 / 4 \nabla^{2} \rho-2 \mathrm{G}$ & $\mathrm{E}=1 / 2(\mathrm{~V})(\mathrm{kcal} / \mathrm{mol})$ \\
\hline \multirow{9}{*}{$\mathrm{C}-\mathrm{H} \cdots \pi$} & $a$ & 0.009 & -0.007 & 0.006 & -0.014 & -4.5 \\
\hline & $c$ & 0.005 & -0.004 & 0.003 & -0.007 & -2.2 \\
\hline & $d$ & 0.008 & -0.006 & 0.005 & -0.012 & -3.6 \\
\hline & $e$ & 0.007 & -0.006 & 0.005 & -0.011 & -3.5 \\
\hline & $f$ & 0.005 & -0.004 & 0.003 & -0.007 & -2.2 \\
\hline & $u$ & 0.007 & -0.006 & 0.005 & -0.011 & -3.5 \\
\hline & $w$ & 0.004 & -0.003 & 0.002 & -0.005 & -1.6 \\
\hline & $y$ & 0.009 & -0.007 & 0.006 & -0.014 & -4.3 \\
\hline & $z$ & 0.005 & -0.004 & 0.003 & -0.008 & -2.4 \\
\hline \multirow{5}{*}{$\mathrm{C}-\mathrm{H} \cdots \mathrm{F}$} & $i$ & 0.006 & -0.007 & 0.006 & -0.013 & -4.1 \\
\hline & $j$ & 0.006 & -0.007 & 0.006 & -0.013 & -4.2 \\
\hline & $\boldsymbol{k}$ & 0.007 & -0.008 & 0.007 & -0.015 & -4.8 \\
\hline & $l$ & 0.005 & -0.006 & 0.005 & -0.011 & -3.5 \\
\hline & $m$ & 0.010 & -0.010 & 0.009 & -0.020 & -6.3 \\
\hline \multirow{3}{*}{$\mathrm{C}-\mathrm{H} \cdots \mathrm{O}$} & $n$ & 0.018 & -0.013 & 0.013 & -0.030 & -9.4 \\
\hline & $o$ & 0.006 & -0.006 & 0.005 & -0.011 & -3.6 \\
\hline & $a 2$ & 0.008 & -0.008 & 0.006 & -0.015 & -4.6 \\
\hline $\mathrm{C}-\mathrm{H} \cdots \mathrm{Br}$ & $t$ & 0.008 & -0.007 & 0.005 & -0.012 & -3.8 \\
\hline $\mathrm{C}-\mathrm{H} \cdots \mathrm{N}$ & $p$ & 0.008 & -0.007 & 0.006 & -0.013 & -4.2 \\
\hline
\end{tabular}




\begin{tabular}{|c|c|c|c|c|c|c|}
\hline \multicolumn{6}{|c|}{ Total Strength } & \multirow[t]{2}{*}{-76.3} \\
\hline \multicolumn{6}{|c|}{ C2-P1 } & \\
\hline si-re & & $\rho$ & $\nabla^{2} \rho$ & $\mathrm{G}$ & $\mathrm{V}=1 / 4 \nabla^{2} \rho-2 \mathrm{G}$ & $\mathrm{E}=1 / 2(\mathrm{~V})(\mathrm{kcal} / \mathrm{mol})$ \\
\hline \multirow{12}{*}{$\mathrm{C}-\mathrm{H} \cdots \pi$} & $a^{\prime}$ & 0.004 & -0.003 & 0.002 & -0.005 & -1.7 \\
\hline & $b^{\prime}$ & 0.004 & -0.003 & 0.002 & -0.005 & -1.7 \\
\hline & $c^{\prime}$ & 0.006 & -0.004 & 0.003 & -0.008 & -2.5 \\
\hline & $d^{\prime}$ & 0.005 & -0.004 & 0.003 & -0.008 & -2.5 \\
\hline & $e^{\prime}$ & 0.007 & -0.006 & 0.005 & -0.011 & -3.4 \\
\hline & $a 1^{\prime}$ & 0.004 & -0.003 & 0.002 & -0.006 & -1.8 \\
\hline & $b 1^{\prime}$ & 0.007 & -0.005 & 0.004 & -0.010 & -3.1 \\
\hline & $c 1^{\prime}$ & 0.005 & -0.004 & 0.003 & -0.007 & -2.1 \\
\hline & $d 1^{\prime}$ & 0.010 & -0.008 & 0.006 & -0.015 & -4.6 \\
\hline & $\boldsymbol{k}^{\prime}$ & 0.004 & -0.004 & 0.003 & -0.006 & -2.0 \\
\hline & $l^{\prime}$ & 0.005 & -0.004 & 0.003 & -0.008 & -2.4 \\
\hline & $m^{\prime}$ & 0.009 & -0.008 & 0.006 & -0.015 & -4.7 \\
\hline \multirow{10}{*}{$\mathrm{C}-\mathrm{H} \cdots \mathrm{F}$} & $f^{\prime}$ & 0.008 & -0.009 & 0.008 & -0.017 & -5.5 \\
\hline & $g^{\prime}$ & 0.005 & -0.007 & 0.005 & -0.012 & -3.8 \\
\hline & $h^{\prime}$ & 0.005 & -0.006 & 0.005 & -0.011 & -3.4 \\
\hline & $o^{\prime}$ & 0.010 & -0.010 & 0.009 & -0.021 & -6.5 \\
\hline & $p^{\prime}$ & 0.004 & -0.005 & 0.004 & -0.009 & -2.9 \\
\hline & $q^{\prime}$ & 0.008 & -0.008 & 0.007 & -0.016 & -5.1 \\
\hline & $r^{\prime}$ & 0.006 & -0.007 & 0.006 & -0.014 & -4.3 \\
\hline & $s^{\prime}$ & 0.007 & -0.008 & 0.007 & -0.016 & -4.9 \\
\hline & $t^{\prime}$ & 0.005 & -0.006 & 0.004 & -0.010 & -3.1 \\
\hline & $u^{\prime}$ & 0.006 & -0.008 & 0.006 & -0.014 & -4.4 \\
\hline \multirow[b]{2}{*}{$\mathrm{C}-\mathrm{H} \cdots \mathrm{O}$} & $v^{\prime}$ & 0.018 & -0.013 & 0.013 & -0.030 & -9.3 \\
\hline & $f 1^{\prime}$ & 0.008 & -0.007 & 0.006 & -0.014 & -4.4 \\
\hline $\mathrm{C}-\mathrm{H} \cdots \mathrm{Br}$ & $y^{\prime}$ & 0.005 & -0.005 & 0.003 & -0.008 & -2.5 \\
\hline $\mathrm{C}-\mathrm{H} \cdots \mathrm{N}$ & $w^{\prime}$ & 0.007 & -0.006 & 0.005 & -0.011 & -3.5 \\
\hline$\pi \cdots \pi$ & $j^{\prime}$ & 0.006 & -0.005 & 0.004 & -0.008 & -2.6 \\
\hline $\mathrm{C}-\mathrm{F} \cdots \pi$ & $x^{\prime}$ & 0.005 & -0.005 & 0.004 & -0.010 & -3.1 \\
\hline \multicolumn{6}{|c|}{ Total Strength } & -101.7 \\
\hline \multicolumn{7}{|c|}{ C2-P1 } \\
\hline$s i-s i$ & & $\rho$ & $\nabla^{2} \rho$ & $\mathrm{G}$ & $\mathrm{V}=1 / 4 \nabla^{2} \rho-2 \mathrm{G}$ & $\mathrm{E}=1 / 2(\mathrm{~V})(\mathrm{kcal} / \mathrm{mol})$ \\
\hline \multirow{10}{*}{$\mathrm{C}-\mathrm{H} \cdots \pi$} & $a^{\prime}$ & 0.004 & -0.003 & 0.002 & -0.006 & -1.7 \\
\hline & $c^{\prime}$ & 0.008 & -0.006 & 0.005 & -0.012 & -3.8 \\
\hline & $a 1^{\prime}$ & 0.006 & -0.004 & 0.003 & -0.008 & -2.5 \\
\hline & $b 1^{\prime}$ & 0.008 & -0.006 & 0.005 & -0.011 & -3.5 \\
\hline & $d 1^{\prime}$ & 0.007 & -0.005 & 0.004 & -0.010 & -3.1 \\
\hline & $e 1^{\prime}$ & 0.011 & -0.011 & 0.009 & -0.020 & -6.4 \\
\hline & $\boldsymbol{k}^{\prime}$ & 0.004 & -0.004 & 0.003 & -0.006 & -2.0 \\
\hline & $l^{\prime}$ & 0.005 & -0.004 & 0.003 & -0.008 & -2.5 \\
\hline & $m^{\prime}$ & 0.010 & -0.008 & 0.007 & -0.016 & -5.0 \\
\hline & $n^{\prime}$ & 0.007 & -0.007 & 0.005 & -0.012 & -3.8 \\
\hline \multirow{2}{*}{$\mathrm{C}-\mathrm{H} \cdots \mathrm{F}$} & $f^{\prime}$ & 0.010 & -0.011 & 0.009 & -0.021 & -6.6 \\
\hline & $g^{\prime}$ & 0.005 & -0.006 & 0.005 & -0.011 & -3.3 \\
\hline
\end{tabular}




\begin{tabular}{ccccccc} 
& $\boldsymbol{h}^{\prime}$ & 0.005 & -0.006 & 0.005 & -0.011 & -3.4 \\
& $\boldsymbol{I}^{\prime}$ & 0.001 & -0.002 & 0.001 & -0.003 & -1.0 \\
& $\boldsymbol{o}^{\prime}$ & 0.010 & -0.010 & 0.009 & -0.021 & -6.7 \\
& $\boldsymbol{q}^{\prime}$ & 0.008 & -0.009 & 0.007 & -0.017 & -5.3 \\
& $\boldsymbol{r}^{\prime}$ & 0.006 & -0.007 & 0.006 & -0.013 & -4.2 \\
& $\boldsymbol{s}^{\prime}$ & 0.008 & -0.008 & 0.007 & -0.016 & -5.0 \\
& $\boldsymbol{t}^{\prime}$ & 0.005 & -0.006 & 0.005 & -0.011 & -3.5 \\
\hline $\mathrm{C}-\mathrm{H} \cdots \mathrm{O}$ & $\boldsymbol{v}^{\prime}$ & 0.019 & -0.014 & 0.014 & -0.031 & -9.8 \\
\hline $\mathrm{C}-\mathrm{H} \cdots \mathrm{Br}$ & $\boldsymbol{y}^{\prime}$ & 0.005 & -0.004 & 0.003 & -0.007 & -2.2 \\
\hline \multirow{2}{*}{$\mathrm{C}-\mathrm{H} \cdots \mathrm{N}$} & $\boldsymbol{g} \boldsymbol{1}^{\prime}$ & 0.007 & -0.006 & 0.005 & -0.011 & -3.6 \\
& $\boldsymbol{w}^{\prime}$ & 0.007 & -0.006 & 0.005 & -0.012 & -3.7 \\
\hline $\mathrm{C}-\mathrm{F} \cdots \pi$ & $\boldsymbol{x}^{\prime}$ & 0.005 & -0.006 & 0.004 & -0.010 & -3.1 \\
\hline \multicolumn{7}{c}{ Total Strength } \\
\hline
\end{tabular}

${ }^{a-} \rho=$ electron density, $\nabla^{2} \rho=$ Laplacian of the electron density; G, V, and H are respectively the kinetic, potential, and total electron energy densities at the bond critical points. 
Cartesian Coordinates of the optimized geometries of various stationary points obtained at the $\mathrm{SMD}_{(\mathrm{THF})} / \mathrm{B} 3 \mathrm{LYP}-\mathrm{D} 3 / 6-31 \mathrm{G} * *, \mathrm{SDD}(\mathrm{Ir}, \mathrm{Cu}, \mathrm{Fe}, \mathrm{Br})$ Level of Theory.

\begin{tabular}{|c|c|c|c|c|}
\hline \multicolumn{5}{|c|}{ Cinnamyl methyl carbonate (10) } \\
\hline \multicolumn{5}{|c|}{ Number of imaginary frequencies $=0$} \\
\hline \multicolumn{3}{|c|}{$\begin{array}{l}\text { Zero-point correction }= \\
\text { (Hartree/Particle) }\end{array}$} & \multicolumn{2}{|c|}{0.210817} \\
\hline \multicolumn{3}{|c|}{ Thermal correction to Energy= } & \multicolumn{2}{|c|}{0.224114} \\
\hline \multicolumn{3}{|c|}{ Thermal correction to Enthalpy } & \multicolumn{2}{|c|}{0.225058} \\
\hline \multicolumn{5}{|c|}{ Thermal correction to Gibbs Free Energy $=0.168302$} \\
\hline \multicolumn{4}{|c|}{ Sum of electronic and zero-point Energies $=$} & -651.890185 \\
\hline \multicolumn{4}{|c|}{ Sum of electronic and thermal Energies $=$} & -651.876888 \\
\hline \multicolumn{4}{|c|}{ Sum of electronic and thermal Enthalpies= } & -651.875943 \\
\hline \multicolumn{4}{|c|}{ Sum of electronic and thermal Free Energies $=$} & -651.932700 \\
\hline \multicolumn{5}{|c|}{ Cartesian Coordinates } \\
\hline 6 & 1.516024 & 1.780614 & -0.550798 & \\
\hline 6 & 0.193960 & 1.358750 & 0.007132 & \\
\hline 1 & -0.006454 & 1.671078 & 1.029984 & \\
\hline 6 & -0.696249 & 0.640132 & -0.693749 & \\
\hline 1 & -0.423230 & 0.343237 & -1.706136 & \\
\hline 6 & -2.025882 & 0.191147 & -0.254591 & \\
\hline 6 & -2.798699 & -0.589059 & -1.134469 & \\
\hline 6 & -2.568863 & 0.501693 & 1.008087 & \\
\hline 6 & -4.065313 & -1.046754 & -0.770139 & \\
\hline 1 & -2.396187 & -0.838589 & -2.113245 & \\
\hline 6 & -3.832777 & 0.044346 & 1.371891 & \\
\hline 1 & -2.001430 & 1.105533 & 1.709843 & \\
\hline 6 & -4.588228 & -0.731937 & 0.485539 & \\
\hline 1 & -4.642523 & -1.648175 & -1.467041 & \\
\hline 1 & -4.233150 & 0.295194 & 2.350421 & \\
\hline 1 & -5.574417 & -1.085416 & 0.772962 & \\
\hline 8 & 2.614595 & 1.211153 & 0.224168 & \\
\hline 6 & 2.943114 & -0.051114 & -0.085806 & \\
\hline 8 & 2.492483 & -0.714081 & -0.997779 & \\
\hline 8 & 3.876975 & -0.453476 & 0.786918 & \\
\hline 6 & 4.362124 & -1.794071 & 0.575967 & \\
\hline 1 & 4.830872 & -1.886997 & -0.407626 & \\
\hline 1 & 5.098930 & -1.964469 & 1.361511 & \\
\hline 1 & 3.547244 & -2.518447 & 0.658483 & \\
\hline 1 & 1.625263 & 1.496823 & -1.600119 & \\
\hline 1 & 1.667398 & 2.858977 & -0.449882 & \\
\hline
\end{tabular}

Azaaryl acetamide (2)

Number of imaginary frequencies $=0$

The smallest frequencies are $=35.402638 .164358 .7669 \mathrm{~cm}(-1)$ Electronic energy $=-547.7347365$

Zero-point correction $=$

0.190565

(Hartree/Particle)

Thermal correction to Energy= $\quad 0.203811$

Thermal correction to Enthalpy $=\quad 0.204755$

Thermal correction to Gibbs Free Energy $=\quad 0.147968$

Sum of electronic and zero-point Energies $=\quad-547.544171$

Sum of electronic and thermal Energies $=\quad-547.530926$

Sum of electronic and thermal Enthalpies $=\quad-547.529982$

Sum of electronic and thermal Free Energies $=\quad-547.586768$

Cartesian Coordinates

$\begin{array}{llll}6 & 3.063768 & -0.616644 & 0.135642 \\ 6 & 2.129034 & -0.552623 & 1.352389 \\ 6 & 0.686016 & -0.417611 & 0.914638\end{array}$

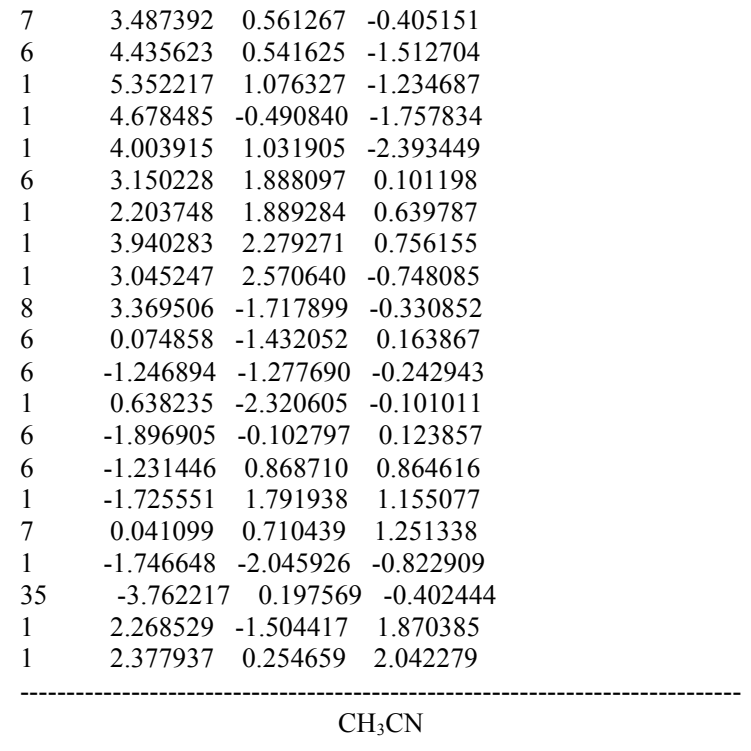

Number of imaginary frequencies $=0$

The smallest frequencies are $=398.1343399 .5145933 .8101$ $\mathrm{cm}(-1)$

Electronic energy $=-132.7645774$

Zero-point correction $=$

(Hartree/Particle)

Thermal correction to Energy= $\quad 0.048895$

Thermal correction to Enthalpy $=\quad 0.049839$

Thermal correction to Gibbs Free Energy $=\quad 0.022356$

Sum of electronic and zero-point Energies $=\quad-132.719261$

Sum of electronic and thermal Energies $=\quad-132.715682$

Sum of electronic and thermal Enthalpies $=\quad-132.714738$

Sum of electronic and thermal Free Energies $=\quad-132.742221$

Cartesian Coordinates

$\begin{array}{rrrrr}7 & 0.000000 & -0.000000 & 1.438627 \\ 6 & 0.000000 & -0.000000 & 0.277792 \\ 6 & -0.000000 & 0.000000 & -1.179680 \\ 1 & 0.000000 & 1.028348 & -1.553019 \\ 1 & 0.890576 & -0.514174 & -1.553019 \\ 1 & -0.890576 & -0.514174 & -1.553019\end{array}$

$\left[\mathrm{CH}_{3} \mathrm{OCO}^{-}\right]^{-}$

Number of imaginary frequencies $=0$

The smallest frequencies are $=126.8441185 .6375331 .5602$ $\mathrm{cm}(-1)$

Electronic energy $=-303.8128844$

Zero-point correction $=$

0.053897

(Hartree/Particle)

Thermal correction to Energy= $\quad 0.058925$

Thermal correction to Enthalpy= $\quad 0.059869$

Thermal correction to Gibbs Free Energy $=\quad 0.025702$

Sum of electronic and zero-point Energies $=\quad-303.758988$

Sum of electronic and thermal Energies $=\quad-303.753959$

Sum of electronic and thermal Enthalpies $=\quad-303.753015$

Sum of electronic and thermal Free Energies $=\quad-303.787183$

Cartesian Coordinates 


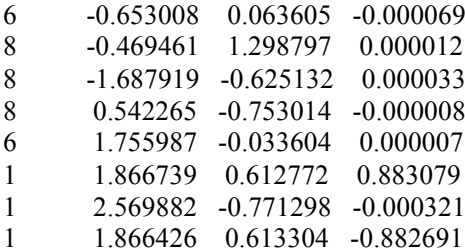

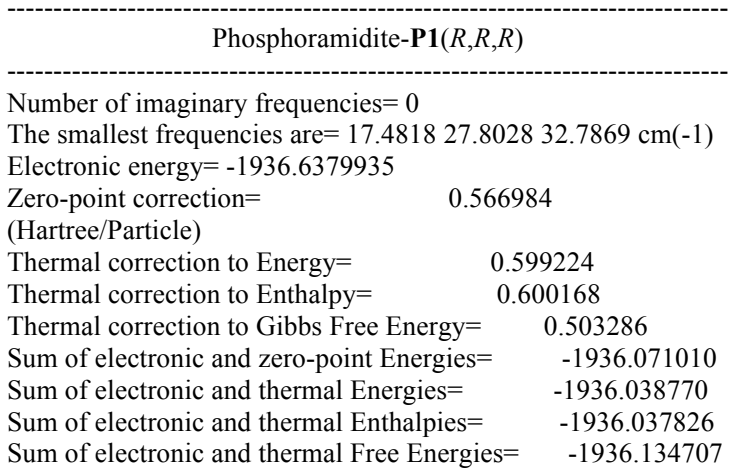
$\begin{array}{llll}2.640711 & -1.900983 & -2.500892\end{array}$
$2.743924-2.681893-1.741107$
$\begin{array}{lll}3.374569 & -2.095537 & -3.289521\end{array}$
$\begin{array}{llll}2.059358 & -0.851482 & 0.453855\end{array}$
$\begin{array}{llll}2.707732 & -1.703007 & 0.226591\end{array}$
$\begin{array}{lll}2.851751 & 0.047152 & 1.405529\end{array}$
$\begin{array}{lll}3.999748 & -0.462661 & 2.025185\end{array}$
$\begin{array}{lll}4.327706 & -1.472089 & 1.791414\end{array}$
$\begin{array}{lll}4.744991 & 0.315766 & 2.913219\end{array}$
$\begin{array}{lll}5.641207 & -0.095975 & 3.369643\end{array}$
$\begin{array}{lll}4.347384 & 1.623166 & 3.199536\end{array}$
$\begin{array}{lll}4.927932 & 2.235502 & 3.884177\end{array}$
$\begin{array}{lll}3.197543 & 2.138086 & 2.594550\end{array}$
$\begin{array}{lll}2.877945 & 3.154072 & 2.812167\end{array}$
$\begin{array}{lll}2.455358 & 1.357020 & 1.706919\end{array}$
$\begin{array}{lll}1.568032 & 1.768500 & 1.241489\end{array}$
$\begin{array}{lll}0.829988 & -1.441126 & 1.158117\end{array}$
$\begin{array}{lll}0.315681 & -2.155629 & 0.513194\end{array}$
$\begin{array}{lll}0.123820 & -0.673344 & 1.470486\end{array}$
$\begin{array}{lll}1.173641 & -1.973550 & 2.050993\end{array}$
$\begin{array}{llll}1.640175 & -1.984324 & -2.933694\end{array}$

Walphos-C1 $\left(R, R_{\mathrm{p}}\right)$

Number of imaginary frequencies $=0$

The smallest frequencies are $=10.458314 .051316 .9985 \mathrm{~cm}(-1)$ Electronic energy $=-3777.10338$

Zero-point correction $=$

0.673765

(Hartree/Particle)

Thermal correction to Energy $=\quad 0.727139$

Thermal correction to Enthalpy= $\quad 0.728083$

Thermal correction to Gibbs Free Energy $=\quad 0.580332$

Sum of electronic and zero-point Energies $=\quad-3776.429615$

Sum of electronic and thermal Energies= $\quad-3776.376242$

Sum of electronic and thermal Enthalpies $=\quad-3776.375297$

Sum of electronic and thermal Free Energies $=\quad-3776.523048$

Cartesian Coordinates

$\begin{array}{llll}-5.423441 & 0.360777 & -0.265305 \\ -4.32548 & -0.114547\end{array}$

$\begin{array}{llll}-6.423661 & 2.586332 & -0.548818\end{array}$

$\begin{array}{lll}-7.408867 & 3.038274 & -0.618270\end{array}$

$\begin{array}{lll}-6.267459 & 1.196438 & -0.768040\end{array}$

$\begin{array}{llll}-7.134225 & 0.590848 & -1.017461\end{array}$

$\begin{array}{lll}-5.026635 & 0.604317 & -0.675683\end{array}$

$\begin{array}{llll}-4.926576 & -0.458999 & -0.858119\end{array}$

$\begin{array}{lll}-3.867699 & 1.368099 & -0.357728\end{array}$

$\begin{array}{lll}-2.557103 & 0.783173 & -0.248853\end{array}$

$\begin{array}{lll}-2.323610 & -0.684383 & -0.363717\end{array}$

$\begin{array}{lll}-2.966482 & -1.637094 & 0.500830\end{array}$

$\begin{array}{lll}-3.760269 & -1.246754 & 1.616555\end{array}$

$\begin{array}{lll}-3.883408 & -0.192491 & 1.834875\end{array}$

$\begin{array}{lll}-4.358799 & -2.185046 & 2.429159\end{array}$

$\begin{array}{llll}-4.953316 & -1.859505 & 3.278176\end{array}$

$\begin{array}{lll}-4.201292 & -3.568543 & 2.173646\end{array}$

$\begin{array}{lll}-4.684099 & -4.296955 & 2.818734\end{array}$

$\begin{array}{llll}-3.422625 & -3.983028 & 1.116783\end{array}$

$\begin{array}{lll}-3.276997 & -5.042065 & 0.919008\end{array}$

$\begin{array}{llll}-2.778749 & -3.041600 & 0.268240\end{array}$

$-1.934604-3.465784-0.793484$

$\begin{array}{llll}-1.809654 & -4.529910 & -0.975029\end{array}$

$\begin{array}{llll}-1.258808 & -2.546425 & -1.556903\end{array}$

$\begin{array}{lll}-0.579981 & -2.849822 & -2.346880\end{array}$

$\begin{array}{llll}-1.433161 & -1.162316 & -1.317913\end{array}$

$\begin{array}{llll}2.825421 & -0.493564 & -1.922818\end{array}$

$\begin{array}{llll}2.612122 & 0.212976 & -2.736666\end{array}$

$\begin{array}{llll}4.233335 & -0.171579 & -1.432953\end{array}$

$\begin{array}{llll}4.556800 & 1.165401 & -1.158220\end{array}$

$\begin{array}{llll}3.804441 & 1.934181 & -1.313593\end{array}$

$\begin{array}{lll}5.810725 & 1.510719 & -0.660654\end{array}$

$\begin{array}{llll}6.038932 & 2.550281 & -0.442627\end{array}$

$\begin{array}{llll}6.770621 & 0.518351 & -0.432466\end{array}$

$\begin{array}{llll}7.747782 & 0.784302 & -0.039030\end{array}$

$\begin{array}{llll}6.465055 & -0.812585 & -0.715334\end{array}$

$\begin{array}{llll}7.204701 & -1.590292 & -0.545083\end{array}$

$\begin{array}{llll}5.203175 & -1.154774 & -1.213892\end{array}$

$4.981232 \quad-2.196768-1.419853$

\begin{tabular}{|c|c|c|}
\hline-1.460011 & -3.999265 & 0.460496 \\
\hline-3.295172 & 0.449487 & -1.140468 \\
\hline 1.462048 & -0.471072 & -1.034055 \\
\hline-1.695627 & -1.961604 & 0.080355 \\
\hline-0.363545 & -2.394101 & -0.278461 \\
\hline-0.497618 & -3.412716 & -1.275251 \\
\hline 0.319974 & -3.949892 & -1.736711 \\
\hline-1.883416 & -3.626626 & -1.526460 \\
\hline-2.302605 & -4.364708 & -2.197484 \\
\hline-2.621729 & -2.742207 & -0.689198 \\
\hline-3.697017 & -2.691975 & -0.611306 \\
\hline-0.678462 & -4.641360 & 2.271135 \\
\hline 0.090908 & -4.120295 & 2.825220 \\
\hline-0.462643 & -5.629507 & 1.261211 \\
\hline 0.497561 & -5.984799 & 0.911577 \\
\hline-1.737768 & -6.030607 & 0.757589 \\
\hline-1.911045 & -6.741714 & -0.039432 \\
\hline-2.741368 & -5.291400 & 1.455476 \\
\hline-3.807632 & -5.341527 & 1.277938 \\
\hline-2.087587 & -4.433423 & 2.389913 \\
\hline-2.572798 & -3.720926 & 3.043196 \\
\hline-2.025809 & -0.870247 & 1.033539 \\
\hline-1.576944 & -0.957089 & 2.361845 \\
\hline-1.031205 & -1.843441 & 2.664133 \\
\hline-1.824814 & 0.056440 & 3.284740 \\
\hline-1.458371 & -0.034926 & 4.302509 \\
\hline-2.535722 & 1.188470 & 2.888162 \\
\hline-2.726705 & 1.991873 & 3.591787 \\
\hline-2.988896 & 1.293965 & 1.573628 \\
\hline-3.533680 & 2.184091 & 1.278907 \\
\hline-2.748532 & 0.282145 & 0.629457 \\
\hline 0.944190 & -1.826119 & 0.213582 \\
\hline 0.783299 & -1.358561 & 1.186573 \\
\hline 2.032302 & -2.899380 & 0.371014 \\
\hline 2.924988 & -2.490573 & 0.849920 \\
\hline 2.335163 & -3.327961 & -0.589690 \\
\hline
\end{tabular}




\begin{tabular}{|c|c|c|c|}
\hline & 433 & 257 & 37 \\
\hline & -3.843691 & 2.216358 & -1.234871 \\
\hline & -2.932039 & 3.139034 & -1.771542 \\
\hline & -1.958113 & 2.798685 & -2.107078 \\
\hline & -3.263845 & 4.490664 & -1.885143 \\
\hline & -2.541887 & 5.188440 & -2.295445 \\
\hline & -4.522451 & 4.936370 & -1.475986 \\
\hline & -4.786079 & 5.986306 & -1.569735 \\
\hline & -5.444971 & 4.025926 & -0.952890 \\
\hline & -6.427651 & 4.366402 & -0.637426 \\
\hline & -5.109128 & 2.676087 & -0.832036 \\
\hline & -5.834423 & 1.980379 & -0.421750 \\
\hline & -4.931098 & -0.422175 & -1.120340 \\
\hline & -5.629854 & -0.752934 & 0.051707 \\
\hline & -5.220735 & -0.483377 & 1.020544 \\
\hline & -6.849217 & -1.431987 & -0.016260 \\
\hline 1 & -7.377053 & -1.683249 & 0.899913 \\
\hline & -7.390124 & -1.783581 & -1.255584 \\
\hline 1 & -8.337320 & -2.313189 & -1.307252 \\
\hline & -6.705132 & -1.454417 & -2.428569 \\
\hline 1 & -7.118063 & -1.727168 & -3.395945 \\
\hline & -5.482209 & -0.784963 & -2.360387 \\
\hline 1 & -4.947858 & -0.548986 & -3.277671 \\
\hline & 0.961950 & 1.123769 & -0.229528 \\
\hline & 0.775531 & 2.211057 & -1.093114 \\
\hline 1 & 0.846473 & 2.069342 & -2.167037 \\
\hline & 0.504532 & 3.485316 & -0.587208 \\
\hline & 0.401296 & 3.698063 & 0.783802 \\
\hline 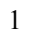 & 0.187593 & 4.686647 & 1.174899 \\
\hline & 0.581802 & 2.616461 & 1.647830 \\
\hline & 0.857613 & 1.342390 & 1.153397 \\
\hline 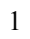 & 1.005135 & 0.532762 & 1.856534 \\
\hline & 3.304601 & -0.387603 & -0.786489 \\
\hline & 4.086099 & -1.171553 & -1.646468 \\
\hline 1 & 3.611178 & -1.769379 & -2.418706 \\
\hline & 5.476939 & -1.203091 & -1.517637 \\
\hline 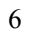 & 6.119557 & -0.438036 & -0.547847 \\
\hline 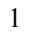 & 7.199144 & -0.455405 & -0.454892 \\
\hline & 5.345307 & 0.357832 & 0.298005 \\
\hline 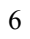 & 3.955398 & 0.383553 & 0.187259 \\
\hline & 3.390291 & 1.015348 & 0.861509 \\
\hline & 6.028413 & 1.145864 & 1.382050 \\
\hline 6 & 6.279026 & -2.030825 & -2.483922 \\
\hline & 0.399263 & 4.651126 & -1.531019 \\
\hline & 0.540464 & 2.882641 & 3.127922 \\
\hline & -0.602285 & 3.509912 & 3.496061 \\
\hline & 0.641325 & 1.756871 & 3.866281 \\
\hline & 1.558033 & 3.695825 & 3.505102 \\
\hline & 1.612620 & 5.193997 & -1.790977 \\
\hline & -0.374224 & 5.642262 & -1.033835 \\
\hline & -0.125688 & 4.292117 & -2.728535 \\
\hline & 6.321109 & 0.367692 & 2.453464 \\
\hline & 5.263935 & 2.163762 & 1.834780 \\
\hline & 7.198777 & 1.675169 & 0.957951 \\
\hline & 7.517788 & -2.303530 & -2.018788 \\
\hline & 5.676047 & -3.213438 & -2.748370 \\
\hline & 6.433557 & -1.401437 & -3.67395 \\
\hline
\end{tabular}

DBU $(4)$

Number of imaginary frequencies $=0$

The smallest frequencies are $=67.260888 .1080194 .8032 \mathrm{~cm}(-1)$ Electronic energy $=-462.138808$

Zero-point correction=

0.246331

(Hartree/Particle)

Thermal correction to Energy $=\quad 0.256112$

Thermal correction to Enthalpy= $\quad 0.257056$

Thermal correction to Gibbs Free Energy $=0.211362$

Sum of electronic and zero-point Energies $=\quad-461.892477$

Sum of electronic and thermal Energies $=\quad-461.882696$

Sum of electronic and thermal Enthalpies $=\quad-461.881752$

Sum of electronic and thermal Free Energies $=\quad-461.927446$

Cartesian Coordinates

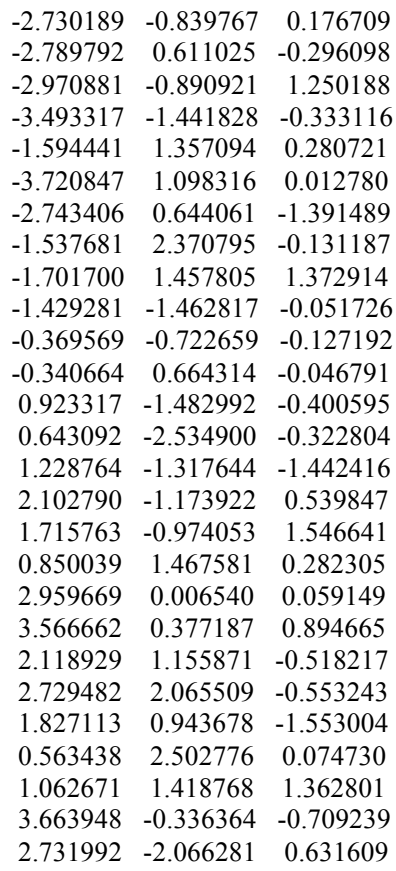

\section{[DBU-H]}

Number of imaginary frequencies $=0$

The smallest frequencies are $=62.338490 .0186176 .0142 \mathrm{~cm}(-1)$ Electronic energy $=-462.6281218$

Zero-point correction=

0.261261

(Hartree/Particle)

Thermal correction to Energy $=\quad 0.271195$

Thermal correction to Enthalpy= $\quad 0.272139$

Thermal correction to Gibbs Free Energy $=\quad 0.226194$

Sum of electronic and zero-point Energies $=\quad-462.366861$

Sum of electronic and thermal Energies $=\quad-462.356927$

Sum of electronic and thermal Enthalpies $=\quad-462.355983$

Sum of electronic and thermal Free Energies $=\quad-462.401928$

Cartesian Coordinates

$\begin{array}{lrrr}6 & -2.801083 & 0.775280 & -0.051813 \\ 6 & -2.742659 & -0.696100 & 0.327110 \\ 1 & -3.148163 & 0.905295 & -1.082967 \\ 1 & -3.467795 & 1.336491 & 0.606957 \\ 6 & -1.575810 & -1.357535 & -0.394627 \\ 1 & -3.673743 & -1.195420 & 0.049098 \\ 1 & -2.610317 & -0.800336 & 1.409031 \\ 1 & -1.457759 & -2.389065 & -0.060865 \\ 1 & -1.736384 & -1.369064 & -1.479135 \\ 7 & -1.456816 & 1.345398 & 0.075834 \\ 6 & -0.310051 & 0.666630 & 0.063758 \\ 7 & -0.301077 & -0.651490 & -0.115485 \\ 6 & 0.933187 & 1.505036 & 0.278244 \\ 1 & 0.646536 & 2.539711 & 0.075768 \\ 1 & 1.189872 & 1.461783 & 1.343523 \\ 6 & 2.148086 & 1.126988 & -0.584706 \\ 1 & 1.804455 & 0.847761 & -1.587296 \\ 6 & 0.911430 & -1.504030 & -0.245576 \\ 6 & 2.980500 & -0.005030 & 0.030787 \\ 1 & 3.639574 & -0.423854 & -0.738220 \\ 6 & 2.105455 & -1.116002 & 0.627247 \\ 1 & 2.707896 & -2.018790 & 0.771541 \\ 1 & 1.735777 & -0.836014 & 1.620270 \\ 1 & 0.580131 & -2.504010 & 0.038075 \\ 1 & 1.192014 & -1.545898 & -1.304951 \\ 1 & 3.628031 & 0.397242 & 0.818530 \\ 1 & 2.768117 & 2.019329 & -0.712627 \\ 1 & -1.387327 & 2.346064 & 0.208277\end{array}$

$[\mathrm{DBU}-\mathrm{H}]^{+} \mathrm{PF}_{6}^{-}(7)$ 
Number of imaginary frequencies $=0$

The smallest frequencies are $=24.244846 .959056 .9877 \mathrm{~cm}(-1)$ Electronic energy $=-1403.3760898$

$\begin{array}{lc}\begin{array}{lc}\text { Zero-point correction= } \\ \text { (Hartree/Particle) }\end{array} & 0.281777 \\ \text { Thermal correction to Energy= } & 0.299642 \\ \text { Thermal correction to Enthalpy= } & 0.300586 \\ \text { Thermal correction to Gibbs Free Energy= } & 0.235422 \\ \text { Sum of electronic and zero-point Energies= } & -1403.094313 \\ \text { Sum of electronic and thermal Energies= } & -1403.076448 \\ \text { Sum of electronic and thermal Enthalpies }= & -1403.075504 \\ \text { Sum of electronic and thermal Free Energies }= & -1403.140668\end{array}$

Sum of electronic and thermal Free Energies $=\quad-1403.140668$

Cartesian Coordinates

\begin{tabular}{llcc}
6 & -0.633493 & 2.841783 & 0.867752 \\
6 & -1.352312 & 3.047155 & -0.456565 \\
1 & 0.450706 & 2.825219 & 0.726586 \\
1 & -0.884619 & 3.623705 & 1.589052 \\
6 & -1.233388 & 1.780102 & -1.293870 \\
1 & -0.907254 & 3.885997 & -0.997415 \\
1 & -2.409090 & 3.276812 & -0.282835 \\
1 & -1.817043 & 1.871137 & -2.210910 \\
1 & -0.192190 & 1.577092 & -1.558605 \\
7 & -1.046939 & 1.556092 & 1.432108 \\
6 & -1.529073 & 0.516059 & 0.753462 \\
7 & -1.747165 & 0.602647 & -0.554599 \\
6 & -1.820598 & -0.714317 & 1.588780 \\
1 & -1.229852 & -0.609218 & 2.500964 \\
1 & -2.873116 & -0.678579 & 1.895257 \\
6 & -1.490205 & -2.060397 & 0.924686 \\
1 & -0.560971 & -1.954111 & 0.362128 \\
6 & -2.236855 & -0.489456 & -1.432130 \\
6 & -2.621419 & -2.570519 & 0.023664 \\
1 & -2.226512 & -3.348113 & -0.640660 \\
6 & -3.252628 & -1.449189 & -0.812473 \\
1 & -3.830403 & -1.885504 & -1.634341 \\
1 & -3.965562 & -0.868673 & -0.215478 \\
1 & -2.706893 & 0.020998 & -2.275046 \\
1 & -1.364908 & -1.027366 & -1.818316 \\
1 & -3.403679 & -3.040916 & 0.631761 \\
1 & -1.289761 & -2.788918 & 1.716763 \\
1 & -0.796427 & 1.381750 & 2.395562 \\
15 & 2.135173 & -0.334644 & -0.105164 \\
9 & 2.387260 & -1.932406 & 0.102813 \\
9 & 0.830128 & -0.670356 & -1.051599 \\
9 & 3.417250 & 0.016308 & 0.838463 \\
9 & 1.844537 & 1.272193 & -0.316216 \\
9 & 3.088243 & -0.336788 & -1.428504 \\
9 & 1.161308 & -0.322300 & 1.218442 \\
& & & \\
\hline & &
\end{tabular}

\section{$\left[\mathrm{PF}_{6}^{-}\right]$}

Number of imaginary frequencies $=0$

The smallest frequencies are $=288.2990292 .0495292 .6025$ $\mathrm{cm}(-1)$

Electronic energy $=-940.7146345$

Zero-point correction=

0.019013

(Hartree/Particle)

Thermal correction to Energy $=\quad 0.025167$

Thermal correction to Enthalpy $=\quad 0.026111$

Thermal correction to Gibbs Free Energy $=\quad-0.011213$

Sum of electronic and zero-point Energies $=\quad-940.695634$

Sum of electronic and thermal Energies=

Sum of electronic and thermal Enthalpies=

$-940.689479$

Sum of electronic and thermal Free Energies=

$-940.688535$

$-940.725860$ $\begin{array}{llll}9 & -1.378879 & 0.212263 & 0.856835\end{array}$

$0.189284 \quad-1.481211 \quad 0.671320$

$12 \mathrm{P} 1$ endo-re $\left[\mathrm{BF}_{4}^{-}\right]$

Number of imaginary frequencies $=0$

The smallest frequencies are $=17.803918 .427923 .4608 \mathrm{~cm}(-1)$ Electronic energy $=-3892.024528$

Zero-point correction=

1.235566

(Hartree/Particle)

Thermal correction to Energy= $\quad 1.307751$

Thermal correction to Enthalpy= $\quad 1.308695$

Thermal correction to Gibbs Free Energy $=\quad 1.126716$

Sum of electronic and zero-point Energies $=\quad-3890.788962$

Sum of electronic and thermal Energies $=\quad-3890.716778$

Sum of electronic and thermal Enthalpies $=\quad-3890.715833$

Sum of electronic and thermal Free Energies $=\quad-3890.897812$

Cartesian Coordinates

\begin{tabular}{|c|c|c|c|}
\hline 7 & 0.415599 & -0.950357 & -1.679610 \\
\hline 5 & 1.847695 & 0.324431 & -0.412166 \\
\hline 8 & 2.336083 & 0.082439 & 1.164795 \\
\hline 8 & 3.368986 & 0.385324 & -1.142132 \\
\hline 7 & 1.204835 & 1.856749 & -0.243591 \\
\hline 6 & 3.230557 & -0.932877 & 1.466050 \\
\hline 6 & 2.716606 & -2.054362 & 2.155567 \\
\hline 1 & 1.666850 & -2.056252 & 2.425911 \\
\hline 6 & 3.546375 & -3.104477 & 2.460948 \\
\hline 1 & 3.164149 & -3.969690 & 2.995435 \\
\hline 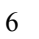 & 4.908030 & -3.089197 & 2.054282 \\
\hline 6 & 5.757251 & -4.203927 & 2.292286 \\
\hline 1 & 5.347782 & -5.063728 & 2.816319 \\
\hline . & 7.063396 & -4.204595 & 1.857635 \\
\hline 1 & 7.701724 & -5.064200 & 2.040080 \\
\hline 6 & 7.570656 & -3.085592 & 1.154843 \\
\hline 1 & 8.593819 & -3.098191 & 0.790188 \\
\hline 6 & 6.778196 & -1.982369 & 0.922234 \\
\hline 1 & 7.181289 & -1.139319 & 0.373829 \\
\hline 6 & 5.430188 & -1.936034 & 1.376618 \\
\hline 6 & 4.573141 & -0.802173 & 1.143263 \\
\hline 6 & 5.079614 & 0.465041 & 0.547225 \\
\hline 6 & 6.164433 & 1.205799 & 1.138028 \\
\hline 6 & 6.737596 & 0.859741 & 2.394418 \\
\hline 1 & 6.345129 & 0.010075 & 2.940301 \\
\hline 6 & 7.769997 & 1.596042 & 2.934272 \\
\hline 1 & 8.183021 & 1.314114 & 3.898727 \\
\hline 6 & 8.292850 & 2.719015 & 2.249695 \\
\hline 1 & 9.111204 & 3.285700 & 2.684238 \\
\hline 6 & 7.748301 & 3.0965 & 1.043042 \\
\hline 1 & 8.124950 & 3.968 & 0.514125 \\
\hline 6 & 6.671935 & 2.369170 & 0.466482 \\
\hline 6 & 6.075483 & & -0.753613 \\
\hline 1 & 6.476658 & 3.658364 & -1.265499 \\
\hline 6 & 4.985210 & 2.126068 & -1.259206 \\
\hline 1 & 4.490750 & 2.449364 & -2.169012 \\
\hline 6 & 4.476278 & 0.991509 & -0.586267 \\
\hline 6 & 0.063619 & 2.124954 & -1.162461 \\
\hline 1 & -0.857211 & 2.112406 & -0.570727 \\
\hline 6 & 0.198019 & 3.495410 & -1.811726 \\
\hline 6 & 1.434295 & 3.930380 & -2.313488 \\
\hline 1 & 2.307863 & 3.299366 & -2.180049 \\
\hline 6 & 1.546794 & 5.161045 & -2.959809 \\
\hline 1 & 2.512605 & 5.486852 & -3.337643 \\
\hline 6 & 0.420402 & 5.975439 & -3.119000 \\
\hline 1 & 0.506538 & 6.935180 & -3.621288 \\
\hline 6 & -0.813055 & 5.549085 & -2.622282 \\
\hline 1 & -1.692109 & 6.178442 & -2.734330 \\
\hline 6 & -0.924239 & 4.317988 & -1.968554 \\
\hline 1 & -1.880128 & 3.998626 & -1.566790 \\
\hline 6 & -0.029422 & 1.048594 & -2.261908 \\
\hline 1 & 0.675794 & 1.320092 & -3.056903 \\
\hline 1 & -1.027725 & 1.107511 & -2.698554 \\
\hline 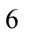 & 1.340775 & 2.822481 & 0.892652 \\
\hline & 0.876986 & 3.731527 & 0.500590 \\
\hline
\end{tabular}


$\begin{array}{lll}0.514971 & 2.424099 & 2.115494\end{array}$

$\begin{array}{lll}-0.841676 & 2.775714 & 2.160816\end{array}$

$\begin{array}{lll}-1.295477 & 3.307921 & 1.331351\end{array}$

$\begin{array}{lll}-1.632080 & 2.461573 & 3.269106\end{array}$

$\begin{array}{lll}-2.675927 & 2.755023 & 3.267321\end{array}$

$\begin{array}{lll}-1.072913 & 1.787818 & 4.356384\end{array}$

$\begin{array}{llll}-1.680885 & 1.548197 & 5.224906\end{array}$

$\begin{array}{lll}0.279635 & 1.434608 & 4.326527\end{array}$

$\begin{array}{lll}0.727524 & 0.918832 & 5.172212\end{array}$

$\begin{array}{lll}1.066187 & 1.753999 & 3.218308\end{array}$

$\begin{array}{lll}2.114188 & 1.479425 & 3.215598\end{array}$

$\begin{array}{llll}2.786943 & 3.198534 & 1.212303\end{array}$

$\begin{array}{llll}3.275905 & 3.592268 & 0.319114\end{array}$

$\begin{array}{lll}3.374569 & 2.357629 & 1.579286\end{array}$

$\begin{array}{lll}2.791010 & 3.978355 & 1.980506\end{array}$

$\begin{array}{lll}-0.915498 & -1.786142 & 1.036857\end{array}$

$\begin{array}{llll}-0.284866 & -2.645809 & 0.828984\end{array}$

$\begin{array}{lll}-0.482212 & -1.199110 & 1.846847\end{array}$

$\begin{array}{lll}-1.183656 & -0.918335 & -0.160136\end{array}$

$\begin{array}{lll}-1.474076 & 0.089622 & 0.127134\end{array}$

$\begin{array}{llll}-1.763869 & -1.445409 & -1.365414\end{array}$

$\begin{array}{llll}-1.865634 & -2.524627 & -1.418835\end{array}$

$\begin{array}{lll}1.820420 & -1.070098 & -3.374078\end{array}$

$\begin{array}{llll}2.160061 & -0.063998 & -3.609343\end{array}$

$\begin{array}{llll}0.509480 & -1.411283 & -3.822833\end{array}$

$\begin{array}{llll}-0.036468 & -0.643061 & -4.371103\end{array}$

$\begin{array}{llll}0.064782 & -2.820786 & -4.175433\end{array}$

$\begin{array}{lll}0.455494 & -3.103462 & -5.165778\end{array}$

$\begin{array}{lll}-1.024429 & -2.807489 & -4.272756\end{array}$

$\begin{array}{llll}0.468669 & -3.886573 & -3.133052\end{array}$

$\begin{array}{llll}1.425485 & -4.341094 & -3.409102\end{array}$

$\begin{array}{llll}-0.265821 & -4.699032 & -3.148716\end{array}$

$\begin{array}{llll}0.567011 & -3.361586 & -1.705449\end{array}$

$\begin{array}{llll}-0.219427 & -3.684374 & -1.032111\end{array}$

$\begin{array}{llll}1.732970 & -2.894686 & -1.129948\end{array}$

$\begin{array}{lll}1.792430 & -2.903497 & -0.046066\end{array}$

$\begin{array}{llll}3.060879 & -2.746118 & -1.845223\end{array}$

$\begin{array}{llll}3.712586 & -2.141031 & -1.212841\end{array}$

$\begin{array}{llll}3.534935 & -3.736052 & -1.926269\end{array}$

$\begin{array}{llll}2.960630 & -2.084980 & -3.238424\end{array}$

$\begin{array}{llll}2.857084 & -2.850532 & -4.015424\end{array}$

$\begin{array}{llll}3.906946 & -1.575089 & -3.444249\end{array}$

$\begin{array}{llll}-2.790816 & -0.759606 & -2.182567\end{array}$

$\begin{array}{llll}-3.214648 & 0.561719 & -1.951824\end{array}$

$\begin{array}{llll}-3.396124 & -1.461522 & -3.244796\end{array}$

$\begin{array}{llll}-4.172080 & 1.165895 & -2.766648\end{array}$

$\begin{array}{llll}-2.824193 & 1.118103 & -1.112457\end{array}$

$\begin{array}{llll}-4.353360 & -0.859147 & -4.061415\end{array}$

$\begin{array}{llll}-3.109739 & -2.495071 & -3.424716\end{array}$

$\begin{array}{llll}-4.742986 & 0.465117 & -3.831752\end{array}$

$\begin{array}{llll}-4.491988 & 2.177263 & -2.534665\end{array}$

$\begin{array}{lll}-4.800682 & -1.426320 & -4.873956\end{array}$

$\begin{array}{llll}-5.492441 & 0.935039 & -4.462601\end{array}$

$\begin{array}{llll}-1.974633 & -4.283907 & 0.467015\end{array}$

$\begin{array}{llll}-2.553368 & -3.542462 & 1.235678\end{array}$

$\begin{array}{lll}-2.167530 & -2.324811 & 1.634992\end{array}$

$\begin{array}{llll}-3.715057 & -3.813847 & 1.849191\end{array}$

$\begin{array}{lll}-4.338297 & -5.059257 & 1.471590\end{array}$

$\begin{array}{llll}-3.688980 & -5.903998 & 1.714037\end{array}$

$\begin{array}{llll}-5.258611 & -5.109879 & 2.053378\end{array}$

$\begin{array}{lll}-4.564181 & -5.064728 & 0.402074\end{array}$

$\begin{array}{lll}-4.258411 & -0.208393 & 4.007465\end{array}$

$\begin{array}{lll}-5.552041 & 0.331450 & 4.594393\end{array}$

$\begin{array}{lll}-3.553810 & 0.600239 & 3.794171\end{array}$

$\begin{array}{lll}-3.772338 & -0.920635 & 4.679674\end{array}$

$\begin{array}{lll}-6.313442 & 1.088312 & 3.513422\end{array}$

$\begin{array}{lll}-5.337235 & 1.005446 & 5.427745\end{array}$

$\begin{array}{lll}-6.166123 & -0.492678 & 4.973581\end{array}$

$\begin{array}{lll}-7.299863 & 1.380182 & 3.878271\end{array}$

$\begin{array}{llll}-5.774741 & 1.990362 & 3.207945\end{array}$

$\begin{array}{lll}-4.558717 & -0.898895 & 2.757694\end{array}$

$\begin{array}{llll}-5.579213 & -0.625244 & 1.950694\end{array}$

$\begin{array}{lll}-6.517529 & 0.248907 & 2.307518\end{array}$

$\begin{array}{llll}-5.581463 & -1.408452 & 0.651309\end{array}$

$\begin{array}{lll}-4.537389 & -1.659717 & 0.458898\end{array}$ $\begin{array}{lll}-6.101659 & -2.358215 & 0.828814\end{array}$

$\begin{array}{llll}-6.146878 & -0.693146 & -0.580699\end{array}$

$\begin{array}{lll}-5.800914 & 0.341163 & -0.571778\end{array}$

$\begin{array}{lll}-7.668144 & 0.667204 & 1.467725\end{array}$

$\begin{array}{lll}-7.673940 & -0.751398 & -0.669852\end{array}$

$\begin{array}{lll}-8.011017 & -0.023741 & -1.417705\end{array}$

$\begin{array}{lll}-8.343941 & -0.457179 & 0.679486\end{array}$

$\begin{array}{lll}-9.389294 & -0.171379 & 0.520014\end{array}$

$\begin{array}{lll}-8.366871 & -1.355227 & 1.308822\end{array}$

$\begin{array}{lll}-8.389127 & 1.086529 & 2.171471\end{array}$

$\begin{array}{llll}-7.334512 & 1.480060 & 0.814647\end{array}$

$\begin{array}{llll}-7.996208 & -1.739188 & -1.022717\end{array}$

$\begin{array}{llll}-5.703029 & -1.151636 & -1.468709\end{array}$

$\begin{array}{lll}-3.813964 & -1.467622 & 2.370723\end{array}$

$\begin{array}{lll}-4.241391 & 2.595743 & 0.758064\end{array}$

$\begin{array}{llll}-5.428019 & 2.546514 & -0.000666\end{array}$

$\begin{array}{llll}-4.474309 & 3.322277 & 1.945486\end{array}$

$\begin{array}{lll}-3.229787 & 3.229096 & 0.004838\end{array}$

$\begin{array}{llll}-3.822177 & 1.292051 & 1.084775\end{array}$

[12-13 $]^{\ddagger} \mathrm{P} 1$ endo-re $\left[\mathrm{BF}_{4}{ }^{-}\right]$

Number of imaginary frequencies $=1$

The smallest frequencies are $=-255.16722 .747217 .2882 \mathrm{~cm}(-1)$ Electronic energy $=-3892.0082688$

Zero-point correction $=$

1.232693

(Hartree/Particle)

Thermal correction to Energy= $\quad 1.305191$

Thermal correction to Enthalpy= $\quad 1.306135$

Thermal correction to Gibbs Free Energy $=\quad 1.120481$

Sum of electronic and zero-point Energies $=\quad-3890.775575$

Sum of electronic and thermal Energies $=\quad-3890.703078$

Sum of electronic and thermal Enthalpies $=\quad-3890.702134$

Sum of electronic and thermal Free Energies $=\quad-3890.887788$

Cartesian Coordinates

$\begin{array}{llcc}77 & 0.345172 & -0.749393 & -1.663200 \\ 15 & 1.865085 & 0.399471 & -0.366913 \\ 8 & 2.367387 & 0.028979 & 1.173071 \\ 8 & 3.361442 & 0.460262 & -1.128141 \\ 7 & 1.250107 & 1.921557 & -0.080379 \\ 6 & 3.216797 & -1.049475 & 1.377807 \\ 6 & 2.659084 & -2.194612 & 1.988527 \\ 1 & 1.615950 & -2.167761 & 2.283603 \\ 6 & 3.442969 & -3.304019 & 2.188646 \\ 1 & 3.029502 & -4.190434 & 2.661333 \\ 6 & 4.794290 & -3.321466 & 1.747992 \\ 6 & 5.589789 & -4.493100 & 1.870408 \\ 1 & 5.148598 & -5.371741 & 2.333779 \\ 6 & 6.883067 & -4.523203 & 1.399942 \\ 1 & 7.479317 & -5.426260 & 1.492462 \\ 6 & 7.431944 & -3.376397 & 0.777798 \\ 1 & 8.444332 & -3.410112 & 0.385495 \\ 6 & 6.693227 & -2.219243 & 0.658219 \\ 1 & 7.126886 & -1.354466 & 0.170151 \\ 6 & 5.360210 & -2.144250 & 1.151163 \\ 6 & 4.556033 & -0.955658 & 1.031123 \\ 6 & 5.107313 & 0.332235 & 0.524846 \\ 6 & 6.237819 & 0.973944 & 1.145270 \\ 6 & 6.828208 & 0.494858 & 2.348843 \\ 1 & 6.413907 & -0.382138 & 2.831668 \\ 6 & 7.907706 & 1.135485 & 2.917543 \\ 1 & 8.334311 & 0.751798 & 3.839980 \\ 6 & 8.461626 & 2.291139 & 2.317016 \\ 1 & 9.316497 & 2.781798 & 2.773166 \\ 6 & 7.901898 & 2.796756 & 1.165553 \\ 1 & 8.303722 & 3.694650 & 0.702988 \\ 6 & 6.778591 & 2.169147 & 0.561463 \\ 6 & 6.170055 & 2.717418 & -0.599653 \\ 1 & 6.594225 & 3.612529 & -1.045837 \\ 6 & 5.042032 & 2.145240 & -1.131916 \\ 1 & 4.541396 & 2.565470 & -1.997589 \\ 6 & 4.504896 & 0.976953 & -0.546153 \\ 6 & 0.103443 & 2.286204 & -0.957887\end{array}$


$\begin{array}{lll}-0.807465 & 2.267591 & -0.352441\end{array}$

$\begin{array}{llll}0.279870 & 3.687233 & -1.528283\end{array}$

$\begin{array}{llll}1.526876 & 4.106158 & -2.016698\end{array}$

$\begin{array}{llll}2.378056 & 3.437738 & -1.930226\end{array}$

$\begin{array}{llll}1.678559 & 5.367828 & -2.591139\end{array}$

$\begin{array}{llll}2.652283 & 5.680619 & -2.959304\end{array}$

$\begin{array}{llll}0.581152 & 6.229788 & -2.689884\end{array}$

$\begin{array}{llll}0.697826 & 7.214070 & -3.135181\end{array}$

$\begin{array}{llll}-0.662862 & 5.819223 & -2.206548\end{array}$

$\begin{array}{llll}-1.519333 & 6.485058 & -2.272460\end{array}$

$\begin{array}{llll}-0.813595 & 4.556574 & -1.625068\end{array}$

$-1.7773594 .247306-1.235075$

$\begin{array}{lll}-0.046841 & 1.284463 & -2.117512\end{array}$

$\begin{array}{llll}0.650242 & 1.575718 & -2.911331\end{array}$

$\begin{array}{llll}-1.050477 & 1.388480 & -2.529976\end{array}$

$\begin{array}{lll}1.428969 & 2.798384 & 1.123797\end{array}$

$\begin{array}{llll}0.968895 & 3.740363 & 0.813496\end{array}$

$\begin{array}{lll}0.623891 & 2.307402 & 2.326377\end{array}$

$\begin{array}{lll}-0.738227 & 2.630915 & 2.409315\end{array}$

$\begin{array}{lll}-1.206136 & 3.226824 & 1.632868\end{array}$

$\begin{array}{lll}-1.517272 & 2.199320 & 3.485622\end{array}$

$\begin{array}{lll}-2.567881 & 2.466814 & 3.509668\end{array}$

$\begin{array}{lll}-0.940194 & 1.441041 & 4.505731\end{array}$

$\begin{array}{lll}-1.540073 & 1.107665 & 5.348376\end{array}$

$\begin{array}{lll}0.420218 & 1.124798 & 4.443317\end{array}$

$\begin{array}{lll}0.882480 & 0.545029 & 5.238140\end{array}$

$\begin{array}{lll}1.194648 & 1.557190 & 3.365281\end{array}$

$\begin{array}{lll}2.247528 & 1.303700 & 3.334259\end{array}$

$\begin{array}{llll}2.890210 & 3.123840 & 1.427729\end{array}$

$\begin{array}{llll}3.358559 & 3.581610 & 0.553939\end{array}$

$\begin{array}{lll}3.473021 & 2.245857 & 1.705269\end{array}$

$\begin{array}{lll}2.933011 & 3.837335 & 2.256630\end{array}$

$\begin{array}{llll}-0.851757 & -1.559771 & 0.737787\end{array}$

$\begin{array}{lll}-0.468930 & -2.540368 & 0.514719\end{array}$

$\begin{array}{lll}-0.480045 & -1.078942 & 1.634681\end{array}$

$\begin{array}{lll}-1.429154 & -0.742545 & -0.260370\end{array}$

$\begin{array}{llll}-1.749904 & 0.241068 & 0.063647\end{array}$

$\begin{array}{llll}-1.863349 & -1.245447 & -1.537543\end{array}$

$\begin{array}{llll}-1.926323 & -2.324934 & -1.623625\end{array}$

$\begin{array}{llll}1.712052 & -0.792680 & -3.384965\end{array}$

$2.065244 \quad 0.221436 \quad-3.554053$

$\begin{array}{llll}0.385367 & -1.070559 & -3.828446\end{array}$

$\begin{array}{llll}0.152138 & -0.249052 & -4.302797\end{array}$

$\begin{array}{llll}-0.119228 & -2.432369 & -4.267230\end{array}$

$\begin{array}{llll}0.185656 & -2.616676 & -5.308908\end{array}$

$\begin{array}{lll}-1.210766 & -2.388695 & -4.273312\end{array}$

$\begin{array}{llll}0.332592 & -3.612794 & -3.379527\end{array}$

$1.279277 \quad-4.020643 \quad-3.745861$

$\begin{array}{llll}-0.401044 & -4.420695 & -3.470065\end{array}$

$\begin{array}{llll}0.481916 & -3.269912 & -1.906858\end{array}$

$\begin{array}{llll}-0.315692 & -3.607419 & -1.251121\end{array}$

$\begin{array}{llll}1.655748 & -2.864922 & -1.321173\end{array}$

$\begin{array}{llll}1.726754 & -2.936059 & -0.240332\end{array}$

$\begin{array}{llll}2.964936 & -2.625006 & -2.041913\end{array}$

$\begin{array}{llll}3.619984 & -2.077699 & -1.363668\end{array}$

$\begin{array}{llll}3.450394 & -3.593659 & -2.231094\end{array}$

$\begin{array}{llll}2.839717 & -1.829324 & -3.359426\end{array}$

$\begin{array}{llll}2.708730 & -2.511937 & -4.205758\end{array}$

$\begin{array}{llll}3.786785 & -1.309977 & -3.532985\end{array}$

$-2.888593 \quad-0.554450-2.351258$

$\begin{array}{lll}-3.306159 & 0.769412 & -2.115451\end{array}$

$\begin{array}{llll}-3.526448 & -1.266058 & -3.386778\end{array}$

$\begin{array}{llll}-4.299895 & 1.360077 & -2.894878\end{array}$

$\begin{array}{llll}-2.889915 & 1.337783 & -1.296167\end{array}$

$-4.515689-0.673616-4.172116$

$\begin{array}{lll}-3.255788 & -2.304545 & -3.559585\end{array}$

$\begin{array}{llll}-4.906168 & 0.647664 & -3.932791\end{array}$

$\begin{array}{llll}-4.618050 & 2.371627 & -2.660406\end{array}$

$\begin{array}{llll}-4.991128 & -1.248844 & -4.962286\end{array}$

$\begin{array}{llll}-5.683787 & 1.108392 & -4.535533\end{array}$

$\begin{array}{llll}-2.075980 & -4.191779 & 0.261948\end{array}$

$\begin{array}{llll}-2.674533 & -3.592988 & 1.159182\end{array}$

$\begin{array}{lll}-2.351052 & -2.487712 & 1.745757\end{array}$

$\begin{array}{lll}-3.854337 & -4.078724 & 1.667474\end{array}$

$\begin{array}{lll}-4.363488 & -5.253991 & 1.026634\end{array}$ $\begin{array}{llll}-3.659111 & -6.087914 & 1.103893\end{array}$

$\begin{array}{llll}-5.290258 & -5.501843 & 1.548827\end{array}$

$-4.573006-5.067658-0.031865$

$\begin{array}{lll}-4.227563 & -0.458300 & 4.064739\end{array}$

$\begin{array}{lll}-5.517976 & 0.076815 & 4.663464\end{array}$

$\begin{array}{lll}-3.499875 & 0.345379 & 3.917012\end{array}$

$\begin{array}{lll}-3.768168 & -1.220006 & 4.700977\end{array}$

$\begin{array}{lll}-6.244236 & 0.910651 & 3.615346\end{array}$

$\begin{array}{lll}-5.303713 & 0.696271 & 5.538515\end{array}$

$\begin{array}{lll}-6.156305 & -0.753932 & 4.984170\end{array}$

$\begin{array}{lll}-7.229094 & 1.206297 & 3.982257\end{array}$

$\begin{array}{lll}-5.680110 & 1.815775 & 3.369987\end{array}$

$\begin{array}{lll}-4.512901 & -1.065720 & 2.770222\end{array}$

$\begin{array}{lll}-5.520907 & -0.735840 & 1.975976\end{array}$

$\begin{array}{lll}-6.448270 & 0.143985 & 2.363939\end{array}$

$\begin{array}{llll}-5.534675 & -1.458412 & 0.642029\end{array}$

$\begin{array}{llll}-4.499931 & -1.749034 & 0.460486\end{array}$

$\begin{array}{lll}-6.097023 & -2.391913 & 0.766489\end{array}$

$\begin{array}{lll}-6.047361 & -0.660679 & -0.562315\end{array}$

$\begin{array}{llll}-5.666121 & 0.358947 & -0.494406\end{array}$

$\begin{array}{lll}-7.564035 & 0.645309 & 1.523997\end{array}$

$\begin{array}{llll}-7.573198 & -0.657041 & -0.685823\end{array}$

$\begin{array}{llll}-7.866744 & 0.121105 & -1.400784\end{array}$

$\begin{array}{lll}-8.262595 & -0.410310 & 0.663219\end{array}$

$\begin{array}{lll}-9.293246 & -0.077785 & 0.497653\end{array}$

$\begin{array}{lll}-8.331673 & -1.338863 & 1.242714\end{array}$

$\begin{array}{llll}-8.286476 & 1.053424 & 2.233363\end{array}$

$\begin{array}{lll}-7.191628 & 1.479278 & 0.919450\end{array}$

$\begin{array}{llll}-7.922678 & -1.612156 & -1.09788\end{array}$

$\begin{array}{llll}-5.604138 & -1.088595 & -1.465537\end{array}$

$\begin{array}{lll}-3.765447 & -1.656066 & 2.374982\end{array}$

$\begin{array}{llll}-4.092145 & 2.513634 & 0.882186\end{array}$

$\begin{array}{lll}-5.273454 & 2.566180 & 0.118187\end{array}$

$\begin{array}{lll}-4.309797 & 3.123722 & 2.134319\end{array}$

$\begin{array}{lll}-3.060909 & 3.196992 & 0.195695\end{array}$

$\begin{array}{lll}-3.698369 & 1.176428 & 1.072420\end{array}$

$13 \mathrm{P} 1$ endo-re $\left[\mathrm{BF}_{4}^{-}\right]$

Number of imaginary frequencies $=0$

The smallest frequencies are=14.9609 $21.214924 .3543 \mathrm{~cm}(-1)$ Electronic energy $=-3892.037264$

Zero-point correction=

(Hartree/Particle)

Thermal correction to Energy $=\quad 1.306566$

Thermal correction to Enthalpy=

1.307511

Thermal correction to Gibbs Free Energy=

Sum of electronic and zero-point Energies=

Sum of electronic and thermal Energies=

Sum of electronic and thermal Enthalpies=

Sum of electronic and thermal Free Energies=

$-3890.803185$

$-3890.730697$

$-3890.729753$

$-3890.911592$

Cartesian Coordinates

$\begin{array}{lccc}77 & 0.081108 & -0.765564 & -1.177739 \\ 15 & 1.898081 & 0.304239 & -0.336870 \\ 8 & 2.545166 & 0.017399 & 1.146451 \\ 8 & 3.230861 & 0.054672 & -1.303845 \\ 7 & 1.541035 & 1.924671 & -0.175895 \\ 6 & 3.401344 & -1.050882 & 1.391500 \\ 6 & 2.906897 & -2.065528 & 2.238591 \\ 1 & 1.911145 & -1.952804 & 2.654311 \\ 6 & 3.691326 & -3.158912 & 2.509198 \\ 1 & 3.327342 & -3.947302 & 3.161667 \\ 6 & 4.975891 & -3.289533 & 1.915375 \\ 6 & 5.764655 & -4.452578 & 2.128440 \\ 1 & 5.372498 & -5.232033 & 2.776402 \\ 6 & 6.990360 & -4.597758 & 1.519174 \\ 1 & 7.582416 & -5.492966 & 1.685142 \\ 6 & 7.472864 & -3.582288 & 0.659474 \\ 1 & 8.428767 & -3.710318 & 0.159702 \\ 6 & 6.740087 & -2.435300 & 0.443850 \\ 1 & 7.122110 & -1.673997 & -0.225681 \\ 6 & 5.479555 & -2.239778 & 1.074611 \\ 6 & 4.684667 & -1.056073 & 0.867773\end{array}$


$\begin{array}{llll}5.188786 & 0.115340 & 0.096664\end{array}$

$\begin{array}{llll}6.401469 & 0.804764 & 0.456086\end{array}$

$\begin{array}{lll}7.132289 & 0.505513 & 1.640197\end{array}$

$\begin{array}{llll}6.768835 & -0.266047 & 2.308380\end{array}$

$\begin{array}{lll}8.284163 & 1.191934 & 1.958210\end{array}$

$\begin{array}{lll}8.818351 & 0.949056 & 2.872338\end{array}$

$\begin{array}{llll}8.774305 & 2.214318 & 1.111187\end{array}$

$\begin{array}{lll}9.687176 & 2.741942 & 1.371604\end{array}$

$\begin{array}{llll}8.080984 & 2.546278 & -0.030509\end{array}$

$\begin{array}{llll}8.433696 & 3.342583 & -0.680654\end{array}$

$\begin{array}{llll}6.880608 & 1.869855 & -0.379488\end{array}$

$\begin{array}{llll}6.138554 & 2.245323 & -1.531680\end{array}$

$\begin{array}{llll}6.519110 & 3.036203 & -2.171671\end{array}$

$\begin{array}{llll}4.940324 & 1.639022 & -1.816275\end{array}$

$\begin{array}{llll}4.344273 & 1.924314 & -2.675878\end{array}$

$\begin{array}{llll}4.466246 & 0.606415 & -0.979111\end{array}$

$\begin{array}{llll}0.353408 & 2.335476 & -0.970103\end{array}$

$\begin{array}{llll}-0.483813 & 2.470332 & -0.282354\end{array}$

$\begin{array}{llll}0.586092 & 3.650289 & -1.698737\end{array}$

$\begin{array}{llll}1.823891 & 3.946264 & -2.285807\end{array}$

$\begin{array}{llll}2.646803 & 3.250928 & -2.162385\end{array}$

$\begin{array}{llll}2.006571 & 5.131219 & -2.999331\end{array}$

$\begin{array}{llll}2.974267 & 5.349972 & -3.443324\end{array}$

$\begin{array}{llll}0.950216 & 6.036664 & -3.138522\end{array}$

$\begin{array}{llll}1.091788 & 6.960559 & -3.692513\end{array}$

$\begin{array}{llll}-0.284903 & 5.750015 & -2.552332\end{array}$

$\begin{array}{llll}-1.108628 & 6.452617 & -2.646146\end{array}$

$\begin{array}{llll}-0.466554 & 4.565850 & -1.832910\end{array}$

$\begin{array}{llll}-1.422219 & 4.353690 & -1.363955\end{array}$

$\begin{array}{llll}-0.016703 & 1.231628 & -1.970625\end{array}$

$\begin{array}{llll}0.652264 & 1.289833 & -2.836860\end{array}$

$\begin{array}{llll}-1.029778 & 1.408615 & -2.337288\end{array}$

$\begin{array}{lll}1.873130 & 2.837923 & 0.973830\end{array}$

$\begin{array}{lll}1.535547 & 3.812520 & 0.610240\end{array}$

$\begin{array}{lll}1.030984 & 2.515419 & 2.209114\end{array}$

$\begin{array}{lll}-0.281996 & 3.005693 & 2.286816\end{array}$

$\begin{array}{lll}-0.673288 & 3.643761 & 1.501281\end{array}$

$\begin{array}{lll}-1.113626 & 2.682270 & 3.361658\end{array}$

$\begin{array}{lll}-2.125515 & 3.070797 & 3.376824\end{array}$

$\begin{array}{lll}-0.636077 & 1.870715 & 4.392062\end{array}$

$\begin{array}{lll}-1.276851 & 1.619021 & 5.232941\end{array}$

$\begin{array}{llll}0.679409 & 1.399456 & 4.343707\end{array}$

$\begin{array}{lll}1.067127 & 0.781523 & 5.149425\end{array}$

$\begin{array}{llll}1.504915 & 1.721872 & 3.264768\end{array}$

$\begin{array}{llll}2.520071 & 1.344821 & 3.246445\end{array}$

$\begin{array}{lll}3.373320 & 2.973530 & 1.226001\end{array}$

$\begin{array}{llll}3.880133 & 3.273139 & 0.305634\end{array}$

$\begin{array}{lll}3.832386 & 2.051579 & 1.580122\end{array}$

$\begin{array}{lll}3.538275 & 3.748729 & 1.980782\end{array}$

$\begin{array}{lll}-0.797901 & -0.556323 & 0.832504\end{array}$

$\begin{array}{lll}-0.909992 & -1.557046 & 1.241357\end{array}$

$\begin{array}{lll}-0.359438 & 0.167964 & 1.513359\end{array}$

$\begin{array}{lll}-1.792780 & -0.060783 & -0.062755\end{array}$

$\begin{array}{llll}-1.937868 & 1.007780 & -0.113934\end{array}$

$\begin{array}{llll}-2.494227 & -0.890914 & -0.942858\end{array}$

$\begin{array}{llll}-2.550465 & -1.943718 & -0.707468\end{array}$

$\begin{array}{llll}1.215118 & -1.334645 & -3.054527\end{array}$

$\begin{array}{llll}1.718759 & -0.425451 & -3.366964\end{array}$

$\begin{array}{llll}-0.160638 & -1.424467 & -3.331261\end{array}$

$\begin{array}{lll}-0.626276 & -0.586558 & -3.847826\end{array}$

$\begin{array}{llll}-0.935344 & -2.725354 & -3.428599\end{array}$

$-0.742413 \quad-3.177583-4.412996$

$\begin{array}{lll}-1.997908 & -2.476435 & -3.413006\end{array}$

$\begin{array}{llll}-0.638725 & -3.754565 & -2.312720\end{array}$

$\begin{array}{llll}0.147978 & -4.445391 & -2.633138\end{array}$

$\begin{array}{llll}-1.528492 & -4.365218 & -2.136830\end{array}$

$-0.239322-3.153104-0.972415$

$\begin{array}{lll}-0.963134 & -3.249632 & -0.171644\end{array}$

$\begin{array}{llll}1.060643 & -2.871623 & -0.598456\end{array}$

$\begin{array}{llll}1.254491 & -2.787459 & 0.466280\end{array}$

$\begin{array}{llll}2.288174 & -3.064335 & -1.463835\end{array}$

$\begin{array}{llll}3.122846 & -2.546139 & -0.989103\end{array}$

$\begin{array}{llll}2.548761 & -4.132301 & -1.470979\end{array}$

$\begin{array}{llll}2.131862 & -2.548233 & -2.909982\end{array}$

$\begin{array}{llll}1.760699 & -3.346449 & -3.559908\end{array}$
$3.120577 \quad-2.276680 \quad-3.290732$

$\begin{array}{llll}-3.446594 & -0.415410 & -1.949106\end{array}$

$\begin{array}{llll}-3.678550 & 0.949348 & -2.219405\end{array}$

$-4.203636-1.374250 \quad-2.653023$

$\begin{array}{lll}-4.617960 & 1.330121 & -3.175924\end{array}$

$\begin{array}{llll}-3.158207 & 1.718427 & -1.661701\end{array}$

$-5.134834-0.989355-3.615961$

$\begin{array}{lll}-4.070459 & -2.423941 & -2.412918\end{array}$

$\begin{array}{llll}-5.342773 & 0.366720 & -3.885055\end{array}$

$\begin{array}{llll}-4.793032 & 2.386599 & -3.358053\end{array}$

$\begin{array}{lll}-5.706164 & -1.746243 & -4.146285\end{array}$

$\begin{array}{llll}-6.072830 & 0.671070 & -4.629641\end{array}$

$\begin{array}{lll}-2.592960 & -5.278050 & 0.062653\end{array}$

$\begin{array}{lll}-3.149945 & -4.217242 & 0.371099\end{array}$

$\begin{array}{lll}-2.792918 & -3.327258 & 1.200019\end{array}$

$\begin{array}{lll}-4.346546 & -3.871142 & -0.285703\end{array}$

$\begin{array}{llll}-4.836628 & -4.824285 & -1.225002\end{array}$

$\begin{array}{llll}-5.028652 & -5.793919 & -0.752585\end{array}$

$\begin{array}{llll}-5.773168 & -4.415682 & -1.615719\end{array}$

$\begin{array}{llll}-4.134005 & -4.982715 & -2.051243\end{array}$

$\begin{array}{llll}-3.219964 & -0.805930 & 3.383365\end{array}$

$\begin{array}{lll}-4.056176 & -0.219466 & 4.510655\end{array}$

$\begin{array}{lll}-2.525191 & -0.057396 & 2.990493\end{array}$

$\begin{array}{lll}-2.645054 & -1.675655 & 3.709805\end{array}$

$\begin{array}{lll}-5.006721 & 0.825854 & 3.941938\end{array}$

$\begin{array}{llll}-3.413741 & 0.247347 & 5.262282\end{array}$

$\begin{array}{lll}-4.627402 & -1.015278 & 5.001437\end{array}$

$\begin{array}{lll}-5.731052 & 1.127713 & 4.702915\end{array}$

$\begin{array}{lll}-4.465115 & 1.715692 & 3.608039\end{array}$

$\begin{array}{lll}-4.105137 & -1.239039 & 2.306228\end{array}$

$\begin{array}{lll}-5.262891 & -0.666938 & 2.021548\end{array}$

$\begin{array}{lll}-5.774864 & 0.303675 & 2.790745\end{array}$

$\begin{array}{llll}-5.971478 & -1.206999 & 0.797595\end{array}$

$\begin{array}{llll}-5.259251 & -1.849139 & 0.278656\end{array}$

$\begin{array}{lll}-6.785431 & -1.863395 & 1.129117\end{array}$

$\begin{array}{lll}-6.507039 & -0.127540 & -0.159919\end{array}$

$\begin{array}{llll}-5.816658 & 0.719841 & -0.172521\end{array}$

$\begin{array}{lll}-6.888151 & 1.203517 & 2.397949\end{array}$

$\begin{array}{lll}-7.917491 & 0.348166 & 0.210460\end{array}$

$\begin{array}{lll}-8.120158 & 1.295256 & -0.304045\end{array}$

$\begin{array}{lll}-8.090239 & 0.537106 & 1.724749\end{array}$

$\begin{array}{lll}-8.967173 & 1.164746 & 1.918448\end{array}$

$\begin{array}{lll}-8.285642 & -0.420225 & 2.221563\end{array}$

$\begin{array}{lll}-7.218882 & 1.662471 & 3.332043\end{array}$

$\begin{array}{lll}-6.480024 & 2.001578 & 1.767826\end{array}$

$\begin{array}{lll}-8.664130 & -0.372466 & -0.146409\end{array}$

$-6.505459-0.535221-1.174321$

$\begin{array}{lll}-3.751751 & -2.034506 & 1.726647\end{array}$

$\begin{array}{lll}-3.794071 & 2.881229 & 0.938245\end{array}$

$\begin{array}{lll}-4.987295 & 2.926385 & 0.197786\end{array}$

$\begin{array}{lll}-3.858625 & 3.774108 & 2.018488\end{array}$

$\begin{array}{lll}-2.703408 & 3.226892 & 0.091625\end{array}$

$\begin{array}{lll}-3.585717 & 1.577272 & 1.422688\end{array}$

$12 \mathrm{P} 1$ exo-si $\left[\mathrm{BF}_{4}^{-}\right]$

Number of imaginary frequencies $=0$

The smallest frequencies are $=8.059014 .000020 .0176 \mathrm{~cm}(-1)$

Electronic energy $=-3892.0254436$

Zero-point correction=

(Hartree/Particle)

Thermal correction to Energy= $\quad 1.307835$

Thermal correction to Enthalpy= $\quad 1.308780$

Thermal correction to Gibbs Free Energy $=\quad 1.123718$

Sum of electronic and zero-point Energies $=\quad-3890.790007$

Sum of electronic and thermal Energies $=$

$-3890.717608$

Sum of electronic and thermal Enthalpies=

Sum of electronic and thermal Free Energies=

Cartesian Coordinates

$\begin{array}{llll}77 & 0.280289 & -0.887088 & -1.457943 \\ 15 & 1.823501 & 0.525010 & -0.502219 \\ 8 & 2.409054 & 0.389417 & 1.059551\end{array}$

$8 \quad 3.287942 \quad 0.500013 \quad-1.327303$ 
$\begin{array}{lll}1.222260 & 2.089228 & -0.419812\end{array}$

$\begin{array}{lll}3.162010 & -0.732651 & 1.372839\end{array}$

$\begin{array}{llll}2.542044 & -1.720402 & 2.170526\end{array}$

$\begin{array}{llll}1.533244 & -1.544301 & 2.528410\end{array}$

$\begin{array}{llll}3.229125 & -2.872370 & 2.466531\end{array}$

$\begin{array}{llll}2.771449 & -3.639934 & 3.084578\end{array}$

$\begin{array}{lll}4.529178 & -3.096518 & 1.935699\end{array}$

$\begin{array}{llll}5.211000 & -4.324244 & 2.156739\end{array}$

$\begin{array}{llll}4.725455 & -5.083756 & 2.764153\end{array}$

$\begin{array}{llll}6.449583 & -4.556794 & 1.602502\end{array}$

$\begin{array}{llll}6.957788 & -5.501596 & 1.772390\end{array}$

$\begin{array}{lll}7.055511 & -3.565672 & 0.793661\end{array}$

$\begin{array}{llll}8.021433 & -3.761095 & 0.336551\end{array}$

$\begin{array}{llll}6.429749 & -2.357506 & 0.574803\end{array}$

$\begin{array}{llll}6.904126 & -1.613694 & -0.054571\end{array}$

$\begin{array}{lll}5.158222 & -2.074055 & 1.147539\end{array}$

$\begin{array}{llll}4.471338 & -0.827801 & 0.928032\end{array}$

$\begin{array}{llll}5.113062 & 0.329719 & 0.243388\end{array}$

$\begin{array}{llll}6.348849 & 0.897768 & 0.722964\end{array}$

$\begin{array}{lll}6.964766 & 0.483636 & 1.937967\end{array}$

$\begin{array}{lll}6.488786 & -0.280074 & 2.541120\end{array}$

$\begin{array}{llll}8.146206 & 1.048102 & 2.368682\end{array}$

$\begin{array}{lll}8.589026 & 0.716952 & 3.303819\end{array}$

$\begin{array}{llll}8.783774 & 2.058771 & 1.610798\end{array}$

$\begin{array}{lll}9.718036 & 2.489745 & 1.958841\end{array}$

$\begin{array}{lll}8.204461 & 2.502358 & 0.443500\end{array}$

$\begin{array}{llll}8.670063 & 3.292571 & -0.140083\end{array}$

$\begin{array}{llll}6.978593 & 1.952445 & -0.020288\end{array}$

$\begin{array}{llll}6.354075 & 2.446828 & -1.197223\end{array}$

$\begin{array}{llll}6.843652 & 3.233658 & -1.764330\end{array}$

$\begin{array}{llll}5.133790 & 1.962004 & -1.593031\end{array}$

$\begin{array}{llll}4.620897 & 2.348275 & -2.467641\end{array}$

$\begin{array}{llll}4.509106 & 0.931039 & -0.853055\end{array}$

$\begin{array}{llll}-0.096578 & 2.247768 & -1.089002\end{array}$

$\begin{array}{lll}-0.867912 & 2.286071 & -0.316724\end{array}$

$\begin{array}{llll}-0.196137 & 3.541765 & -1.880318\end{array}$

$\begin{array}{llll}0.894082 & 4.057442 & -2.594448\end{array}$

$\begin{array}{llll}1.854240 & 3.552659 & -2.529977\end{array}$

$\begin{array}{llll}0.754068 & 5.211183 & -3.367756\end{array}$

$\begin{array}{lll}1.609577 & 5.603994 & -3.911281\end{array}$

$\begin{array}{lll}1.6 .481726 & 5.861064 & -3.443100\end{array}$

$\begin{array}{llll}-0.591106 & 6.758869 & -4.045329\end{array}$

$\begin{array}{llll}-1.574600 & 5.348135 & -2.739217\end{array}$

$\begin{array}{llll}-2.540306 & 5.843577 & -2.795584\end{array}$

$\begin{array}{llll}-1.432801 & 4.197847 & -1.959703\end{array}$

$\begin{array}{llll}-2.285540 & 3.787990 & -1.425734\end{array}$

$\begin{array}{llll}-0.401965 & 1.067247 & -2.027742\end{array}$

$\begin{array}{llll}0.069062 & 1.287118 & -2.993278\end{array}$

$\begin{array}{llll}-1.476905 & 1.059331 & -2.195684\end{array}$

$\begin{array}{llll}1.552378 & 3.173464 & 0.563456\end{array}$

$\begin{array}{lll}1.199505 & 4.081610 & 0.067117\end{array}$

$\begin{array}{llll}0.732530 & 3.042683 & 1.846937\end{array}$

$\begin{array}{lll}-0.518730 & 3.672914 & 1.914289\end{array}$

$\begin{array}{lll}-0.856312 & 4.277230 & 1.076123\end{array}$

$\begin{array}{lll}-1.335074 & 3.537141 & 3.039381\end{array}$

$\begin{array}{lll}-2.292569 & 4.047273 & 3.070770\end{array}$

$\begin{array}{llll}-0.915439 & 2.756908 & 4.118545\end{array}$

$\begin{array}{lll}-1.553706 & 2.635561 & 4.989619\end{array}$

$\begin{array}{lll}0.342528 & 2.148973 & 4.076587\end{array}$

$\begin{array}{lll}0.687108 & 1.551825 & 4.916268\end{array}$

$\begin{array}{lll}1.163042 & 2.301321 & 2.957122\end{array}$

$\begin{array}{llll}2.132120 & 1.819237 & 2.941243\end{array}$

$3.047649 \quad 3.382500 \quad 0.795249$

$\begin{array}{llll}3.551889 & 3.586202 & -0.151746\end{array}$

$\begin{array}{lll}3.534633 & 2.528556 & 1.264544\end{array}$

$3.181278 \quad 4.252034 \quad 1.446486$

$\begin{array}{lll}-0.961835 & 0.017795 & 1.320345\end{array}$

$\begin{array}{lll}-0.887734 & -1.154229 & 0.391660\end{array}$

$\begin{array}{lll}-0.578646 & -2.065297 & 0.903770\end{array}$

$\begin{array}{llll}-1.822406 & -1.251346 & -0.688526\end{array}$

$\begin{array}{llll}-2.352666 & -0.334528 & -0.914939\end{array}$

$\begin{array}{llll}1.306264 & -0.972740 & -3.419171\end{array}$

$\begin{array}{llll}1.545197 & 0.041971 & -3.729282\end{array}$

$\begin{array}{llll}-0.050527 & -1.379777 & -3.584972\end{array}$

$\begin{array}{llll}-0.734935 & -0.649375 & -4.016010\end{array}$ $\begin{array}{llll}-0.489957 & -2.816892 & -3.807362\end{array}$

$\begin{array}{llll}-0.290737 & -3.116922 & -4.848306\end{array}$

$\begin{array}{lll}-1.573860 & -2.850019 & -3.677060\end{array}$

$\begin{array}{llll}0.155301 & -3.822045 & -2.831345\end{array}$

$\begin{array}{llll}1.053839 & -4.265174 & -3.273324\end{array}$

$\begin{array}{llll}-0.541450 & -4.649136 & -2.661630\end{array}$

$\begin{array}{llll}0.516843 & -3.220289 & -1.476527\end{array}$

$\begin{array}{llll}-0.078368 & -3.572446 & -0.643202\end{array}$

$\begin{array}{llll}1.764550 & -2.694567 & -1.165032\end{array}$

$\begin{array}{llll}2.056869 & -2.696261 & -0.120841\end{array}$

$\begin{array}{llll}2.908351 & -2.542082 & -2.149089\end{array}$

$\begin{array}{llll}3.655563 & -1.894012 & -1.689333\end{array}$

$\begin{array}{llll}3.389410 & -3.521550 & -2.294204\end{array}$

$2.489028-1.941601 \quad-3.508108$

$\begin{array}{llll}2.247174 & -2.740792 & -4.217831\end{array}$

$\begin{array}{llll}3.346816 & -1.410861 & -3.933784\end{array}$

$\begin{array}{llll}-2.616872 & -2.439120 & -1.065867\end{array}$

$\begin{array}{llll}-2.561920 & -3.673620 & -0.392732\end{array}$

$\begin{array}{llll}-3.544054 & -2.307767 & -2.120588\end{array}$

$\begin{array}{llll}-3.359333 & -4.748872 & -0.792333\end{array}$

$\begin{array}{llll}-1.908931 & -3.790534 & 0.466704\end{array}$

$\begin{array}{lll}-4.347774 & -3.376092 & -2.511694\end{array}$

$\begin{array}{lll}-3.635255 & -1.343888 & -2.612787\end{array}$

$-4.252488-4.610268 \quad-1.857969$

$\begin{array}{llll}-3.293112 & -5.692734 & -0.256837\end{array}$

$\begin{array}{llll}-5.055358 & -3.245141 & -3.326420\end{array}$

$\begin{array}{llll}-4.876945 & -5.445007 & -2.163981\end{array}$

$\begin{array}{lll}-0.499159 & -1.357947 & 3.673214\end{array}$

$\begin{array}{lll}-1.583371 & -0.846119 & 3.478640\end{array}$

$\begin{array}{lll}-1.977030 & -0.196112 & 2.380778\end{array}$

$\begin{array}{llll}-2.612111 & -0.843606 & 4.338932\end{array}$

$\begin{array}{lll}-2.365656 & -1.522679 & 5.587867\end{array}$

$\begin{array}{llll}-1.540996 & -1.048246 & 6.126033\end{array}$

$\begin{array}{lll}-3.291043 & -1.430454 & 6.156594\end{array}$

$\begin{array}{lll}-2.130908 & -2.576043 & 5.413907\end{array}$

$\begin{array}{lll}-4.689821 & 2.458676 & 2.142115\end{array}$

$\begin{array}{lll}-6.143169 & 2.904872 & 2.127427\end{array}$

$\begin{array}{llll}-4.152301 & 2.813976 & 1.258921\end{array}$

$\begin{array}{lll}-4.171316 & 2.814845 & 3.034690\end{array}$

$\begin{array}{lll}-6.900305 & 2.110597 & 1.071811\end{array}$

$\begin{array}{lll}-6.205484 & 3.971508 & 1.896961\end{array}$

$\begin{array}{lll}-6.600096 & 2.742989 & 3.109927\end{array}$

$\begin{array}{lll}-7.965064 & 2.348151 & 1.107850\end{array}$

$\begin{array}{lll}-6.524349 & 2.335985 & 0.068599\end{array}$

$\begin{array}{lll}-4.636140 & 0.997326 & 2.152839\end{array}$

$\begin{array}{lll}-5.596641 & 0.178019 & 1.741127\end{array}$

$\begin{array}{lll}-6.762293 & 0.654840 & 1.303344\end{array}$

$\begin{array}{lll}-5.269631 & -1.297499 & 1.861118\end{array}$

$\begin{array}{lll}-4.183608 & -1.362493 & 1.950035\end{array}$

$\begin{array}{lll}-5.678458 & -1.656476 & 2.814146\end{array}$

$\begin{array}{llll}-5.734206 & -2.183124 & 0.696248\end{array}$

$\begin{array}{llll}-5.576038 & -1.637460 & -0.235301\end{array}$

$\begin{array}{lll}-7.821276 & -0.152172 & 0.652284\end{array}$

$\begin{array}{lll}-7.191958 & -2.636191 & 0.822780\end{array}$

$\begin{array}{llll}-7.531010 & -3.009935 & -0.150766\end{array}$

$\begin{array}{lll}-8.117385 & -1.507503 & 1.297508\end{array}$

$\begin{array}{lll}-9.157851 & -1.763369 & 1.069834\end{array}$

$\begin{array}{lll}-8.064734 & -1.390314 & 2.386374\end{array}$

$\begin{array}{lll}-8.719120 & 0.466302 & 0.706123\end{array}$

$\begin{array}{lll}-7.561539 & -0.257312 & -0.407728\end{array}$

$\begin{array}{lll}-7.266249 & -3.475729 & 1.525247\end{array}$

$\begin{array}{lll}-5.077346 & -3.054881 & 0.647891\end{array}$

$\begin{array}{lll}-3.738099 & 0.574635 & 2.374174\end{array}$

$\begin{array}{llll}-4.260230 & 1.507815 & -1.503559\end{array}$

$\begin{array}{llll}-5.114480 & 0.494028 & -1.024193\end{array}$

$\begin{array}{llll}4.985446 & 2.695822 & -1.678470\end{array}$

$\begin{array}{lll}-3.689714 & 1.091122 & -2.719877\end{array}$

$\begin{array}{lll}-3.231313 & 1.742844 & -0.550796\end{array}$

$\begin{array}{lll}-0.023692 & 0.229364 & 1.825980\end{array}$

$\begin{array}{lll}-1.333832 & 0.905816 & 0.821690\end{array}$

$[12-13]^{\dagger} \mathbf{P 1}$ exo-si $\left[\mathrm{BF}_{4}^{-}\right]$

Number of imaginary frequencies $=1$

The smallest frequencies are $=-270.067213 .944520 .2724 \mathrm{~cm}(-1)$ 
Electronic energy $=-3892.005384$

Zero-point correction=

(Hartree/Particle)

$\begin{array}{lc}\text { Thermal correction to Energy= } & 1.305490 \\ \text { Thermal correction to Enthalpy= }= & 1.306434 \\ \text { Thermal correction to Gibbs Free Energy= } & 1.123402 \\ \text { Sum of electronic and zero-point Energies= } & -3890.772142 \\ \text { Sum of electronic and thermal Energies }= & -3890.699894 \\ \text { Sum of electronic and thermal Enthalpies }= & -3890.698950 \\ \text { Sum of electronic and thermal Free Energies }= & -3890.881982\end{array}$

\subsection{2}

Cartesian Coordinates

\begin{tabular}{|c|c|c|c|}
\hline 7 & 0.226104 & -1.203028 & -1.187019 \\
\hline 15 & 1.784042 & 0.389046 & -0.548924 \\
\hline 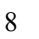 & 2.411021 & 0.541438 & 0.989640 \\
\hline 8 & 3.206367 & 0.199570 & -1.405077 \\
\hline 7 & 1.154773 & 1.922685 & -0.756350 \\
\hline 6 & 3.223634 & -0.469974 & 1.486275 \\
\hline 6 & 2.675914 & -1.294212 & 2.495926 \\
\hline 1 & 1.680900 & -1.077567 & 2.874760 \\
\hline 6 & 3.422680 & -2.339850 & 2.981476 \\
\hline 1 & 3.023887 & -2.979916 & 3.763500 \\
\hline 6 & 4.709236 & -2.623094 & 2.446122 \\
\hline 6 & 5.448411 & -3.759268 & 2.874352 \\
\hline 1 & 5.017737 & -4.398081 & 3.641128 \\
\hline 6 & 6.674328 & -4.055903 & 2.322605 \\
\hline 1 & 7.226681 & -4.930986 & 2.652462 \\
\hline 6 & 7.209581 & -3.224817 & 1.309592 \\
\hline 1 & 8.165715 & -3.474427 & 0.858443 \\
\hline 6 & 6.527362 & -2.105184 & 0.885014 \\
\hline 1 & 6.948192 & -1.485048 & 0.102216 \\
\hline 6 & 5.266733 & -1.756938 & 1.445445 \\
\hline 6 & 4.519788 & -0.603755 & 1.014034 \\
\hline 6 & 5.088277 & 0.417516 & 0.088399 \\
\hline 6 & 6.312577 & 1.114690 & 0.394044 \\
\hline 6 & 6.989144 & 0.969362 & 1.638128 \\
\hline 1 & 6.570137 & 0.321431 & 2.398368 \\
\hline 6 & 8.158354 & 1.650992 & 1.898626 \\
\hline 1 & 8.648957 & 1.526037 & 2.859786 \\
\hline 6 & 8.721824 & 2.516982 & 0.931657 \\
\hline 1 & 9.647297 & 3.042524 & 1.148441 \\
\hline 6 & 8.082192 & 2.701601 & -0.273114 \\
\hline 1 & 8.490665 & 3.37 & -1.018576 \\
\hline 6 & 6.866765 & 2.025902 & -0.567328 \\
\hline 6 & 6.179912 & 2.257105 & -1.789773 \\
\hline 1 & 6.612640 & 2.936828 & -2.518447 \\
\hline 6 & 4.970262 & 1.656803 & -2.032142 \\
\hline 1 & & & -2.941418 \\
\hline 6 & 4.424522 & 0.766948 & -1.078959 \\
\hline 6 & -0.180027 & 1.931476 & -1.416734 \\
\hline 1 & -0.934716 & 2.109685 & -0.646888 \\
\hline 6 & -0.310641 & 3.045179 & -2.443232 \\
\hline 6 & 0.758367 & 3.406513 & -3.274699 \\
\hline 1 & 1.722493 & 2.924013 & -3.136205 \\
\hline 6 & 0.594210 & 4.385799 & -4.255716 \\
\hline 1 & 1.433809 & 4.663121 & -4.887917 \\
\hline 6 & -0.645728 & 5.009908 & -4.424056 \\
\hline 1 & -0.774287 & 5.772302 & -5.187552 \\
\hline 6 & -1.717761 & 4.646712 & -3.604600 \\
\hline 1 & -2.686906 & 5.121816 & -3.731495 \\
\hline 6 & -1.551893 & 3.672003 & -2.617735 \\
\hline 1 & -2.389626 & 3.382057 & -1.991083 \\
\hline 6 & -0.482399 & 0.594145 & -2.113088 \\
\hline 1 & -0.022378 & 0.630842 & -3.106912 \\
\hline 1 & -1.556903 & 0.544887 & -2.264413 \\
\hline 6 & 1.464753 & 3.192150 & -0.019329 \\
\hline 1 & 1.203396 & 3.974081 & -0.737171 \\
\hline 6 & 0.542411 & 3.389548 & 1.183223 \\
\hline 6 & -0.538466 & 4.275632 & 1.072326 \\
\hline 1 & -0.704790 & 4.797377 & 0.133176 \\
\hline 6 & -1.405229 & 4.490889 & 2.146920 \\
\hline 1 & -2.235791 & 5.183043 & 2.038892 \\
\hline 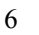 & -1.207454 & 3.811649 & 3.350735 \\
\hline & -1.883639 & 3.968060 & 4.186793 \\
\hline
\end{tabular}

$\begin{array}{lrrr}1 & -5.729896 & -1.234112 & 3.129456 \\ 6 & -5.575899 & -2.020339 & 1.098048 \\ 1 & -5.341072 & -1.581035 & 0.126047 \\ 6 & -7.666640 & 0.004262 & 0.714091 \\ 6 & -7.046753 & -2.451315 & 1.153918 \\ 1 & -7.321450 & -2.899226 & 0.191350 \\ 6 & -7.992155 & -1.284159 & 1.471518 \\ 1 & -9.018807 & -1.565574 & 1.212235 \\ 1 & -7.999928 & -1.066541 & 2.545760\end{array}$




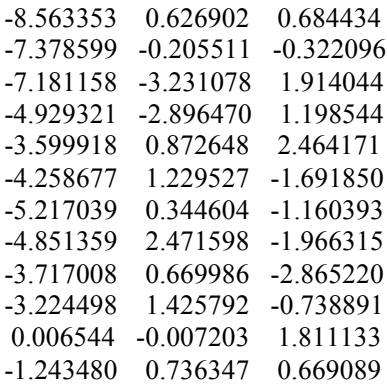

$13 \mathrm{P} 1$ exo-si $\left[\mathrm{BF}_{4}^{-}\right]$

Number of imaginary frequencies $=0$

The smallest frequencies are $=17.205621 .598024 .4186 \mathrm{~cm}(-1)$ Electronic energy $=-3892.0341591$

Zero-point correction=

1.233950

(Hartree/Particle)

Thermal correction to Energy= $\quad 1.30647$

Thermal correction to Enthalpy=

1.307420

Thermal correction to Gibbs Free Energy=

Sum of electronic and zero-point Energies=

Sum of electronic and thermal Energies=

1.125030

Sum of electronic and thermal Enthalpies=

$-3890.800210$

$-3890.727683$

$-3890.726739$

Sum of electronic and thermal Free Energies=

$-3890.909129$

Cartesian Coordinates

\begin{tabular}{|c|c|c|c|}
\hline 77 & -0.092677 & -0.550660 & -1.41 \\
\hline 15 & 1.724292 & 0.408089 & -0.45271 \\
\hline 8 & 2.330121 & -0.049145 & 1.005987 \\
\hline 8 & 3.084228 & 0.248169 & -1.402666 \\
\hline 7 & 1.377697 & 2.006248 & -0.134633 \\
\hline 6 & 3.155526 & -1.164009 & 1.143497 \\
\hline 6 & 2.600856 & -2.273846 & 1.814801 \\
\hline 1 & 1.567305 & -2.236259 & 2.142528 \\
\hline 6 & 3.363096 & -3.403252 & 1.981787 \\
\hline 1 & 2.949119 & -4.268078 & 2.492757 \\
\hline 6 & 4.678360 & -3.477326 & 1.449698 \\
\hline 6 & 5.443463 & -4.670901 & 1.554054 \\
\hline 1 & 5.012015 & -5.516138 & 2.083933 \\
\hline 6 & 6.693057 & -4.763426 & 0.984661 \\
\hline 1 & 7.266377 & -5.682300 & 1.065923 \\
\hline 6 & 7.224033 & -3.660991 & 0.273328 \\
\hline 1 & 8.198449 & -3.744950 & -0.199395 \\
\hline 6 & 6.514996 & -2.484055 & 0.167153 \\
\hline 1 & 6.935213 & -1.656365 & -0.391433 \\
\hline 6 & 5.231541 & -2.341789 & 0.766158 \\
\hline 6 & 4.459324 & -1.128349 & 0.672922 \\
\hline 6 & 5.004743 & 0.108351 & 0.04 \\
\hline 6 & 6.221111 & 0.727926 & 0.505867 \\
\hline 6 & 6.903870 & 0.297984 & 1.678277 \\
\hline 1 & 6.497283 & -0.522716 & 2.257067 \\
\hline 6 & 8.061192 & 0.918635 & 2.095638 \\
\hline 1 & 8.558181 & 0.576303 & 2.998839 \\
\hline 6 & 8.605083 & 2.001421 & 1.364158 \\
\hline 1 & 9.521990 & 2.475975 & 1.701267 \\
\hline 6 & 7.958660 & 2.459243 & 0.238486 \\
\hline 1 & 8.352754 & 3.303162 & -0.321715 \\
\hline 6 & 6.753053 & 1.853727 & -0.209317 \\
\hline 6 & 6.055584 & 2.359221 & -1.339767 \\
\hline 1 & 6.475660 & 3.197623 & -1.888080 \\
\hline 6 & 4.849493 & 1.819492 & -1.712200 \\
\hline 1 & 4.280345 & 2.208519 & -2.549468 \\
\hline 6 & 4.324222 & 0.724003 & -0.992862 \\
\hline 6 & 0.183475 & 2.511130 & -0.860039 \\
\hline 1 & -0.626135 & 2.637508 & -0.136762 \\
\hline 6 & 0.438112 & 3.859571 & -1.517160 \\
\hline 6 & 1.709974 & 4.235651 & -1.968976 \\
\hline 1 & 2.551972 & 3.574173 & -1.793116 \\
\hline 6 & 1.899954 & 5.457829 & -2.617251 \\
\hline 1 & 2.894080 & 5.739758 & -2.954595 \\
\hline 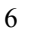 & 0.818589 & 6.317798 & -2.826715 \\
\hline
\end{tabular}

6

6

6

1

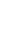

6

6
1

6

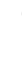

$$
6
$$

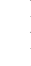$$
\begin{aligned}
& 6 \\
& 1 \\
& 1
\end{aligned}
$$

6

6

6
1
6

6

1
1
6

1
1
6

6

6
6
6

6
6
1
6

6
1
1

6
1
6

8

8

1
6
8

6
8
8

\begin{tabular}{l}
8 \\
6 \\
\hline
\end{tabular}

6
1
1

1
1
6

6

6

1
1
6
1
1 $\begin{array}{llll}0.966227 & 7.269683 & -3.329427\end{array}$

$\begin{array}{llll}-0.453375 & 5.946734 & -2.381224\end{array}$

$\begin{array}{llll}-1.300163 & 6.609624 & -2.538037\end{array}$

$\begin{array}{llll}-0.643062 & 4.727641 & -1.727273\end{array}$

$\begin{array}{llll}-1.633960 & 4.432060 & -1.394672\end{array}$

$\begin{array}{llll}-0.286322 & 1.506985 & -1.928331\end{array}$

$\begin{array}{llll}0.298015 & 1.666256 & -2.841844\end{array}$

$\begin{array}{llll}-1.324133 & 1.744874 & -2.161872\end{array}$

$\begin{array}{lll}1.770627 & 2.832584 & 1.060260\end{array}$

$\begin{array}{llll}1.483279 & 3.842454 & 0.755928\end{array}$

$\begin{array}{llll}0.935771 & 2.503068 & 2.297333\end{array}$

$\begin{array}{lll}-0.292388 & 3.154415 & 2.480142\end{array}$

$\begin{array}{llll}-0.628207 & 3.883739 & 1.750165\end{array}$

$\begin{array}{lll}-1.098923 & 2.877561 & 3.586018\end{array}$

$\begin{array}{lll}-2.053008 & 3.384937 & 3.691425\end{array}$

$\begin{array}{lll}-0.679817 & 1.953193 & 4.543113\end{array}$

$\begin{array}{lll}-1.301727 & 1.736673 & 5.407228\end{array}$

$\begin{array}{lll}0.551919 & 1.310936 & 4.383936\end{array}$

$\begin{array}{lll}0.894302 & 0.598255 & 5.129079\end{array}$

$\begin{array}{lll}1.350842 & 1.582585 & 3.272114\end{array}$

$\begin{array}{lll}2.300509 & 1.072828 & 3.167570\end{array}$

$\begin{array}{lll}3.275095 & 2.885700 & 1.318082\end{array}$

$\begin{array}{lll}3.801516 & 3.209986 & 0.418329\end{array}$

$\begin{array}{lll}3.691666 & 1.929692 & 1.631494\end{array}$

$\begin{array}{lll}3.466981 & 3.614840 & 2.111423\end{array}$

$\begin{array}{lll}-0.990672 & -0.136245 & 0.566230\end{array}$

$\begin{array}{llll}-1.942050 & -0.949769 & -0.112319\end{array}$

$\begin{array}{llll}-2.036683 & -1.976022 & 0.212869\end{array}$

$\begin{array}{lll}-2.630170 & -0.529879 & -1.251041\end{array}$

$\begin{array}{llll}-2.715538 & 0.527815 & -1.455395\end{array}$

$\begin{array}{llll}1.076643 & -0.864021 & -3.314059\end{array}$

$\begin{array}{llll}1.506410 & 0.105795 & -3.547407\end{array}$

$\begin{array}{llll}-0.289807 & -1.027791 & -3.615240\end{array}$

$\begin{array}{llll}-0.801673 & -0.167622 & -4.045661\end{array}$

$\begin{array}{lll}-0.970171 & -2.347763 & -3.916634\end{array}$

$\begin{array}{llll}-0.772709 & -2.607717 & -4.967404\end{array}$

$\begin{array}{lll}-2.046689 & -2.194412 & -3.839574\end{array}$

$\begin{array}{lll}-0.569473 & -3.535468 & -3.011342\end{array}$

$\begin{array}{llll}0.293398 & -4.056696 & -3.437061\end{array}$

$\begin{array}{lll}-1.395242 & -4.253305 & -3.005575\end{array}$

$\begin{array}{llll}-0.251748 & -3.161609 & -1.578401\end{array}$

$\begin{array}{llll}-1.001811 & -3.365906 & -0.823554\end{array}$

$\begin{array}{llll}0.994161 & -2.835432 & -1.120988\end{array}$

$\begin{array}{llll}1.125733 & -2.847391 & -0.045010\end{array}$

$\begin{array}{lll}2.243401 & -2.773419 & -1.970206\end{array}$

$\begin{array}{llll}3.032576 & -2.304720 & -1.382184\end{array}$

$\begin{array}{llll}2.580236 & -3.800189 & -2.172191\end{array}$

$\begin{array}{llll}2.091052 & -2.009298 & -3.307849\end{array}$

$\begin{array}{llll}1.820331 & -2.705805 & -4.108415\end{array}$

$\begin{array}{lll}3.067789 & -1.597513 & -3.575520\end{array}$

$\begin{array}{llll}-3.559767 & -1.380589 & -1.999433\end{array}$

$\begin{array}{llll}-3.792340 & -2.735487 & -1.685485\end{array}$

$\begin{array}{lll}-4.261160 & -0.811880 & -3.079381\end{array}$

$\begin{array}{llll}-4.673489 & -3.495576 & -2.451854\end{array}$

$\begin{array}{llll}-3.307034 & -3.188329 & -0.827075\end{array}$

$\begin{array}{lll}-5.144006 & -1.574758 & -3.842477\end{array}$

$\begin{array}{llll}-4.107477 & 0.239056 & -3.310547\end{array}$

$\begin{array}{lll}-5.346849 & -2.923579 & -3.537551\end{array}$

$\begin{array}{llll}-4.842216 & -4.538139 & -2.196480\end{array}$

$\begin{array}{lll}-5.674506 & -1.116949 & -4.672667\end{array}$

$-6.031340 \quad-3.521953-4.132084$

$\begin{array}{lll}-0.542092 & -3.202564 & 1.797532\end{array}$

$\begin{array}{lll}-1.644973 & -3.021731 & 2.347505\end{array}$

$\begin{array}{lll}-2.807431 & -3.147623 & 1.865013\end{array}$

$\begin{array}{lll}-1.664668 & -2.595023 & 3.680181\end{array}$

$\begin{array}{lll}-0.394670 & -2.315317 & 4.265284\end{array}$

$\begin{array}{llll}0.100112 & -1.472144 & 3.771478\end{array}$

$\begin{array}{llll}-0.589446 & -2.052908 & 5.309393\end{array}$

$\begin{array}{lll}0.270442 & -3.184559 & 4.225305\end{array}$

$\begin{array}{lll}-3.349668 & 0.153670 & 3.214971\end{array}$

$\begin{array}{lll}-4.291371 & 1.017153 & 4.038671\end{array}$

$\begin{array}{lll}-2.781112 & 0.773134 & 2.516840\end{array}$

$\begin{array}{lll}-2.650435 & -0.411742 & 3.829956\end{array}$

$\begin{array}{llll}-5.353359 & 1.604428 & 3.120084\end{array}$

$\begin{array}{llll}-3.743158 & 1.830321 & 4.520284\end{array}$ 


$\begin{array}{lrrr}1 & -4.762794 & 0.413756 & 4.822575 \\ 1 & -6.138774 & 2.087216 & 3.707815 \\ 1 & -4.922779 & 2.354933 & 2.449897 \\ 7 & -4.143383 & -0.811389 & 2.454596 \\ 6 & -5.358471 & -0.568152 & 1.993058 \\ 7 & -6.007851 & 0.563232 & 2.296102 \\ 6 & -5.959921 & -1.668922 & 1.142396 \\ 1 & -5.144839 & -2.359002 & 0.916010 \\ 1 & -6.681918 & -2.224869 & 1.752885 \\ 6 & -6.613456 & -1.192974 & -0.167735 \\ 1 & -6.023588 & -0.368529 & -0.574023 \\ 6 & -7.202962 & 1.072908 & 1.582551 \\ 6 & -8.075859 & -0.767744 & 0.012307 \\ 1 & -8.383263 & -0.177202 & -0.859206 \\ 6 & -8.301668 & 0.047688 & 1.294175 \\ 1 & -9.249777 & 0.591447 & 1.218044 \\ 1 & -8.399055 & -0.611302 & 2.164767 \\ 1 & -7.610990 & 1.846039 & 2.236787 \\ 1 & -6.860989 & 1.570391 & 0.668489 \\ 1 & -8.723380 & -1.653181 & 0.036998 \\ 1 & -6.548337 & -2.004051 & -0.898137 \\ 1 & -3.704882 & -1.735803 & 2.252817 \\ 5 & -3.863585 & 2.584311 & -0.063201 \\ 9 & -4.531308 & 1.342272 & -0.066773 \\ 9 & -4.766417 & 3.596043 & 0.280623 \\ 9 & -3.319403 & 2.816863 & -1.347713 \\ 9 & -2.813068 & 2.551496 & 0.883014 \\ 1 & -0.500613 & -0.593007 & 1.420278 \\ 1 & -1.178418 & 0.926374 & 0.654774 \\ ----------------------------------------------------------------------\end{array}$

\section{$12 \mathrm{P} 1$ endo-si $\left[\mathrm{BF}_{4}^{-}\right]$}

Number of imaginary frequencies $=0$

The smallest frequencies are $=16.456419 .704225 .7201 \mathrm{~cm}(-1)$ Electronic energy $=-3892.0137547$

Zero-point correction=

1.236467

(Hartree/Particle)

Thermal correction to Energy $=\quad 1.308353$

Thermal correction to Enthalpy= $\quad 1.309298$

Thermal correction to Gibbs Free Energy $=\quad 1.128982$

Sum of electronic and zero-point Energies $=\quad-3890.777288$

Sum of electronic and thermal Energies= $\quad-3890.705401$

Sum of electronic and thermal Enthalpies $=\quad-3890.704457$

Sum of electronic and thermal Free Energies $=\quad-3890.884773$

Cartesian Coordinates

$\begin{array}{lccc}77 & 0.437709 & -1.012148 & -1.534254 \\ 15 & 1.602326 & 0.546412 & -0.279050 \\ 8 & 2.125032 & 0.389506 & 1.301939 \\ 8 & 3.097934 & 0.877761 & -0.999321 \\ 7 & 0.707371 & 1.933192 & -0.135631 \\ 6 & 2.972578 & -0.683353 & 1.552121 \\ 6 & 2.409924 & -1.801576 & 2.203641 \\ 1 & 1.377073 & -1.737439 & 2.521659 \\ 6 & 3.172762 & -2.924530 & 2.404120 \\ 1 & 2.754028 & -3.791210 & 2.908227 \\ 6 & 4.506652 & -2.984509 & 1.914718 \\ 6 & 5.274208 & -4.175928 & 2.024075 \\ 1 & 4.827447 & -5.035004 & 2.518007 \\ 6 & 6.546100 & -4.249883 & 1.502777 \\ 1 & 7.120379 & -5.168063 & 1.585605 \\ 6 & 7.100170 & -3.128335 & 0.840647 \\ 1 & 8.093958 & -3.196470 & 0.407275 \\ 6 & 6.389711 & -1.952156 & 0.734422 \\ 1 & 6.827069 & -1.107485 & 0.215544 \\ 6 & 5.080274 & -1.831420 & 1.279297 \\ 6 & 4.302029 & -0.622877 & 1.166642 \\ 6 & 4.873956 & 0.642789 & 0.632130 \\ 6 & 6.054760 & 1.233430 & 1.214375 \\ 6 & 6.647471 & 0.741560 & 2.411702 \\ 1 & 6.194447 & -0.099648 & 2.922454 \\ 6 & 7.777475 & 1.325988 & 2.942731 \\ 1 & 8.202393 & 0.933197 & 3.862215 \\ 6 & 8.384320 & 2.435358 & 2.308270\end{array}$

$9.278250 \quad 2.881918 \quad 2.733955$

$\begin{array}{lll}7.825144 & 2.953165 & 1.161521\end{array}$

$\begin{array}{lll}8.267009 & 3.817487 & 0.672081\end{array}$

$\begin{array}{lll}6.651876 & 2.383249 & 0.597869\end{array}$

$\begin{array}{llll}6.045901 & 2.945010 & -0.558412\end{array}$

$6.510827 \quad 3.803216-1.035696$

$\begin{array}{lll}4.871299 & 2.433977 & -1.045590\end{array}$

$\begin{array}{lll}4.371808 & 2.873327 & -1.902449\end{array}$

$\begin{array}{llll}4.270395 & 1.310157 & -0.426681\end{array}$

$-0.397629 \quad 2.079083-1.110835$

$\begin{array}{lll}-1.263440 & 2.429663 & -0.541896\end{array}$

$-0.071255 \quad 3.141846-2.156301$

$1.208152 \quad 3.249866-2.721776$

$\begin{array}{llll}2.006003 & 2.606744 & -2.367969\end{array}$

$\begin{array}{lll}1.466305 & 4.182722 & -3.728284\end{array}$

$2.464189 \quad 4.252682-4.154282$

$\begin{array}{lll}0.449774 & 5.027859 & -4.182646\end{array}$

$\begin{array}{llll}0.650825 & 5.755867 & -4.963912\end{array}$

$\begin{array}{llll}-0.823517 & 4.936270 & -3.614697\end{array}$

$\begin{array}{llll}-1.617441 & 5.599237 & -3.949034\end{array}$

$\begin{array}{llll}-1.081271 & 4.003093 & -2.606770\end{array}$

$\begin{array}{llll}-2.064347 & 3.950034 & -2.149236\end{array}$

$\begin{array}{llll}-0.797251 & 0.734166 & -1.793452\end{array}$

$\begin{array}{llll}-0.841688 & 0.920722 & -2.870256\end{array}$

$\begin{array}{llll}-1.819334 & 0.517797 & -1.486283\end{array}$

$\begin{array}{lll}0.898748 & 3.131718 & 0.736590\end{array}$

$\begin{array}{llll}0.473726 & 3.950811 & 0.149270\end{array}$

$\begin{array}{lll}0.083074 & 3.060248 & 2.024215\end{array}$

$\begin{array}{llll}-1.150140 & 3.722150 & 2.087766\end{array}$

$\begin{array}{lll}-1.551897 & 4.200513 & 1.200729\end{array}$

$\begin{array}{lll}-1.885161 & 3.756437 & 3.275753\end{array}$

$\begin{array}{llll}-2.846717 & 4.261400 & 3.295011\end{array}$

$\begin{array}{lll}-1.391333 & 3.131315 & 4.421230\end{array}$

$\begin{array}{lll}-1.956229 & 3.161199 & 5.349205\end{array}$

$\begin{array}{lll}-0.172603 & 2.448108 & 4.361533\end{array}$

$\begin{array}{lll}0.211078 & 1.942604 & 5.243830\end{array}$

$\begin{array}{lll}0.555958 & 2.408409 & 3.172413\end{array}$

$\begin{array}{lll}1.497404 & 1.872270 & 3.136282\end{array}$

$\begin{array}{llll}2.359661 & 3.512077 & 0.977842\end{array}$

$\begin{array}{lll}2.859681 & 3.706443 & 0.027417\end{array}$

$\begin{array}{lll}2.918034 & 2.744272 & 1.514128\end{array}$

$\begin{array}{lll}2.390858 & 4.430173 & 1.572408\end{array}$

$\begin{array}{llll}1.528747 & -0.458461 & -3.376994\end{array}$

$\begin{array}{llll}1.451932 & 0.615264 & -3.528807\end{array}$

$\begin{array}{llll}0.371060 & -1.214668 & -3.726655\end{array}$

$\begin{array}{llll}-0.474755 & -0.651257 & -4.118798\end{array}$

$\begin{array}{llll}0.403646 & -2.647938 & -4.232393\end{array}$

$\begin{array}{llll}0.779167 & -2.667838 & -5.267366\end{array}$

$\begin{array}{llll}-0.628449 & -3.007313 & -4.280009\end{array}$

$\begin{array}{llll}1.234814 & -3.605212 & -3.353791\end{array}$

$2.247401-3.702956-3.760229$

$\begin{array}{llll}0.794686 & -4.607190 & -3.394346\end{array}$

$\begin{array}{llll}1.335742 & -3.179693 & -1.893160\end{array}$

$\begin{array}{llll}0.832860 & -3.817752 & -1.173470\end{array}$

$\begin{array}{llll}2.370684 & -2.395795 & -1.408236\end{array}$

$\begin{array}{llll}2.609816 & -2.480813 & -0.354045\end{array}$

$\begin{array}{llll}3.463280 & -1.818320 & -2.286999\end{array}$

$\begin{array}{llll}4.092001 & -1.180555 & -1.667503\end{array}$

$\begin{array}{llll}4.103547 & -2.646614 & -2.625546\end{array}$

$\begin{array}{llll}2.952991 & -1.010687 & -3.506222\end{array}$

$\begin{array}{llll}3.007792 & -1.627057 & -4.412336\end{array}$

$\begin{array}{llll}3.634403 & -0.170756 & -3.669383\end{array}$

$\begin{array}{llll}-1.890596 & -3.318538 & -1.721643\end{array}$

$\begin{array}{lll}-1.157496 & -4.119335 & -1.653084\end{array}$

$\begin{array}{llll}-2.242639 & -3.223162 & -2.748749\end{array}$

$\begin{array}{lll}-1.452934 & -2.010493 & -1.131343\end{array}$

$\begin{array}{llll}-2.256499 & -1.284698 & -1.222429\end{array}$

$\begin{array}{lll}-0.719836 & -1.966008 & 0.111046\end{array}$

$\begin{array}{lll}-0.201497 & -2.888948 & 0.360870\end{array}$

$\begin{array}{lll}-1.246648 & -1.325034 & 1.344309\end{array}$

$\begin{array}{lll}-1.960421 & -0.114586 & 1.372576\end{array}$

$\begin{array}{lll}-1.106026 & -2.028837 & 2.557585\end{array}$

$\begin{array}{lll}-2.485067 & 0.378380 & 2.565188\end{array}$

$\begin{array}{llll}-2.133691 & 0.446243 & 0.465402\end{array}$

$\begin{array}{lll}-1.631584 & -1.535336 & 3.754142\end{array}$ 


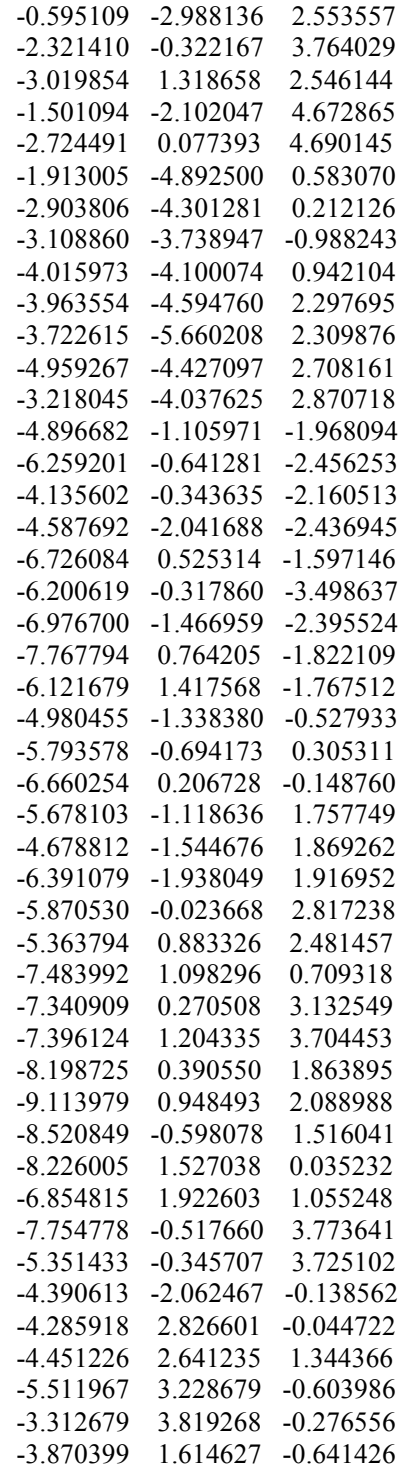

\section{$[12-13]^{\ddagger} \mathbf{P} 1$ endo-si $\left[\mathrm{BF}_{4}^{-}\right]$}

Number of imaginary frequencies $=1$

The smallest frequencies are $=-296.05967 .617712 .5028 \mathrm{~cm}(-1)$ Electronic energy $=-3891.9933958$

Zero-point correction=

(Hartree/Particle)

Thermal correction to Energy= $\quad 1.305136$

Thermal correction to Enthalpy= $\quad 1.306081$

Thermal correction to Gibbs Free Energy $=\quad 1.122892$

Sum of electronic and zero-point Energies $=\quad-3890.760459$

Sum of electronic and thermal Energies $=\quad-3890.688260$

Sum of electronic and thermal Enthalpies $=\quad-3890.687315$

Sum of electronic and thermal Free Energies $=\quad-3890.870504$

Cartesian Coordinates

$\begin{array}{llll}77 & 0.269459 & -0.838541 & -1.564084 \\ 15 & 1.698583 & 0.513268 & -0.374822 \\ 8 & 2.242742 & 0.305578 & 1.186499 \\ 8 & 3.183851 & 0.528667 & -1.162115 \\ 7 & 1.092388 & 2.055708 & -0.264265 \\ 6 & 2.979719 & -0.839862 & 1.467945 \\ 6 & 2.325853 & -1.848747 & 2.207038 \\ 1 & 1.316197 & -1.656432 & 2.546851 \\ 6 & 2.975306 & -3.029414 & 2.467275 \\ 1 & 2.485111 & -3.811520 & 3.040346 \\ 6 & 4.281287 & -3.259787 & 1.955078\end{array}$

$\begin{array}{lll}4.926766 & -4.514117 & 2.130024\end{array}$

$\begin{array}{lll}4.409826 & -5.288082 & 2.691466\end{array}$

$6.169143-4.752982 \quad 1.587420$

$\begin{array}{lll}6.649982 & -5.717657 & 1.721147\end{array}$

$\begin{array}{lll}6.814138 & -3.741071 & 0.837187\end{array}$

$7.782574 \quad-3.939900 \quad 0.386974$

$\begin{array}{lll}6.223981 & -2.507368 & 0.666128\end{array}$

$6.729564-1.749612 \quad 0.079645$

$\begin{array}{lll}4.950287 & -2.216365 & 1.230504\end{array}$

$\begin{array}{lll}4.297669 & -0.942841 & 1.053293\end{array}$

$\begin{array}{lll}4.985491 & 0.214555 & 0.419257\end{array}$

$\begin{array}{llll}6.240142 & 0.714578 & 0.925925\end{array}$

$\begin{array}{lll}6.813794 & 0.252768 & 2.144117\end{array}$

$\begin{array}{lll}6.290570 & -0.494932 & 2.728160\end{array}$

$\begin{array}{lll}8.013300 & 0.753399 & 2.603302\end{array}$

$\begin{array}{lll}8.422432 & 0.388968 & 3.541387\end{array}$

$\begin{array}{lll}8.712296 & 1.742068 & 1.871323\end{array}$

$\begin{array}{lll}9.659763 & 2.122470 & 2.241840\end{array}$

$\begin{array}{lll}8.176322 & 2.228997 & 0.700359\end{array}$

$\begin{array}{lll}8.690067 & 3.003518 & 0.136537\end{array}$

$\begin{array}{lll}6.933619 & 1.746017 & 0.208521\end{array}$

$\begin{array}{llll}6.354044 & 2.283971 & -0.972690\end{array}$

$6.892143 \quad 3.049868-1.524263$

$\begin{array}{llll}5.114931 & 1.870890 & -1.388867\end{array}$

$\begin{array}{llll}4.632191 & 2.298234 & -2.261548\end{array}$

$\begin{array}{llll}4.422343 & 0.869084 & -0.667807\end{array}$

$\begin{array}{llll}-0.153727 & 2.297525 & -1.037682\end{array}$

$\begin{array}{llll}-0.971132 & 2.413785 & -0.323843\end{array}$

$\begin{array}{lll}-0.051609 & 3.583446 & -1.848548\end{array}$

$\begin{array}{llll}1.086761 & 3.849038 & -2.626417\end{array}$

$1.917601 \quad 3.148468 \quad-2.603554$

$\begin{array}{llll}1.162513 & 5.003073 & -3.404867\end{array}$

$\begin{array}{llll}2.052860 & 5.199398 & -3.996701\end{array}$

$\begin{array}{llll}0.094977 & 5.908095 & -3.422944\end{array}$

$\begin{array}{llll}0.152189 & 6.807669 & -4.029729\end{array}$

$\begin{array}{lll}-1.039782 & 5.650637 & -2.651757\end{array}$

$\begin{array}{llll}-1.870441 & 6.351540 & -2.654216\end{array}$

$\begin{array}{llll}-1.112157 & 4.496896 & -1.864032\end{array}$

$-1.985654 \quad 4.307376-1.248736$

$\begin{array}{llll}-0.461968 & 1.132555 & -2.006279\end{array}$

$\begin{array}{lll}-0.022090 & 1.389570 & -2.974612\end{array}$

$\begin{array}{lll}-1.541866 & 1.110382 & -2.146042\end{array}$

$\begin{array}{lll}1.493232 & 3.174045 & 0.644083\end{array}$

$\begin{array}{lll}1.149496 & 4.063851 & 0.111592\end{array}$

$\begin{array}{lll}0.765262 & 3.191210 & 1.986318\end{array}$

$\begin{array}{lll}-0.364783 & 4.007625 & 2.129180\end{array}$

$\begin{array}{llll}-0.760263 & 4.528227 & 1.262445\end{array}$

$\begin{array}{lll}-0.987422 & 4.164659 & 3.369675\end{array}$

$\begin{array}{lll}-1.861884 & 4.803952 & 3.455041\end{array}$

$\begin{array}{llll}-0.486697 & 3.500954 & 4.490599\end{array}$

$\begin{array}{lll}-0.963262 & 3.624745 & 5.459360\end{array}$

$\begin{array}{lll}0.625569 & 2.664854 & 4.355176\end{array}$

$\begin{array}{lll}1.014992 & 2.133637 & 5.219564\end{array}$

$\begin{array}{lll}1.245613 & 2.511073 & 3.114907\end{array}$

$\begin{array}{lll}2.113030 & 1.867900 & 3.028457\end{array}$

$\begin{array}{lll}3.004287 & 3.346539 & 0.793098\end{array}$

$\begin{array}{llll}3.465926 & 3.493600 & -0.184692\end{array}$

$\begin{array}{lll}3.486882 & 2.499124 & 1.279905\end{array}$

$\begin{array}{lll}3.196184 & 4.237322 & 1.398623\end{array}$

$\begin{array}{llll}1.571138 & -0.629194 & -3.375351\end{array}$

$\begin{array}{llll}1.760843 & 0.432514 & -3.506815\end{array}$

$\begin{array}{llll}0.304620 & -1.100795 & -3.801680\end{array}$

$\begin{array}{llll}0.380009 & -0.369936 & -4.229347\end{array}$

$\begin{array}{llll}0.054570 & -2.522972 & -4.274651\end{array}$

$\begin{array}{llll}0.483786 & -2.647656 & -5.280692\end{array}$

$\begin{array}{llll}-1.023811 & -2.657693 & -4.387847\end{array}$

$\begin{array}{llll}0.611680 & -3.617246 & -3.334896\end{array}$

$\begin{array}{llll}1.599687 & -3.939163 & -3.679170\end{array}$

$\begin{array}{llll}-0.032071 & -4.500725 & -3.397270\end{array}$

$\begin{array}{llll}0.719383 & -3.209838 & -1.869072\end{array}$

$0.024912-3.697004-1.191402$

$\begin{array}{llll}1.857085 & -2.649123 & -1.320346\end{array}$

$\begin{array}{llll}1.993378 & -2.735147 & -0.246941\end{array}$

$\begin{array}{llll}3.099010 & -2.321069 & -2.122981\end{array}$

$3.791149 \quad-1.781654 \quad-1.479368$ 


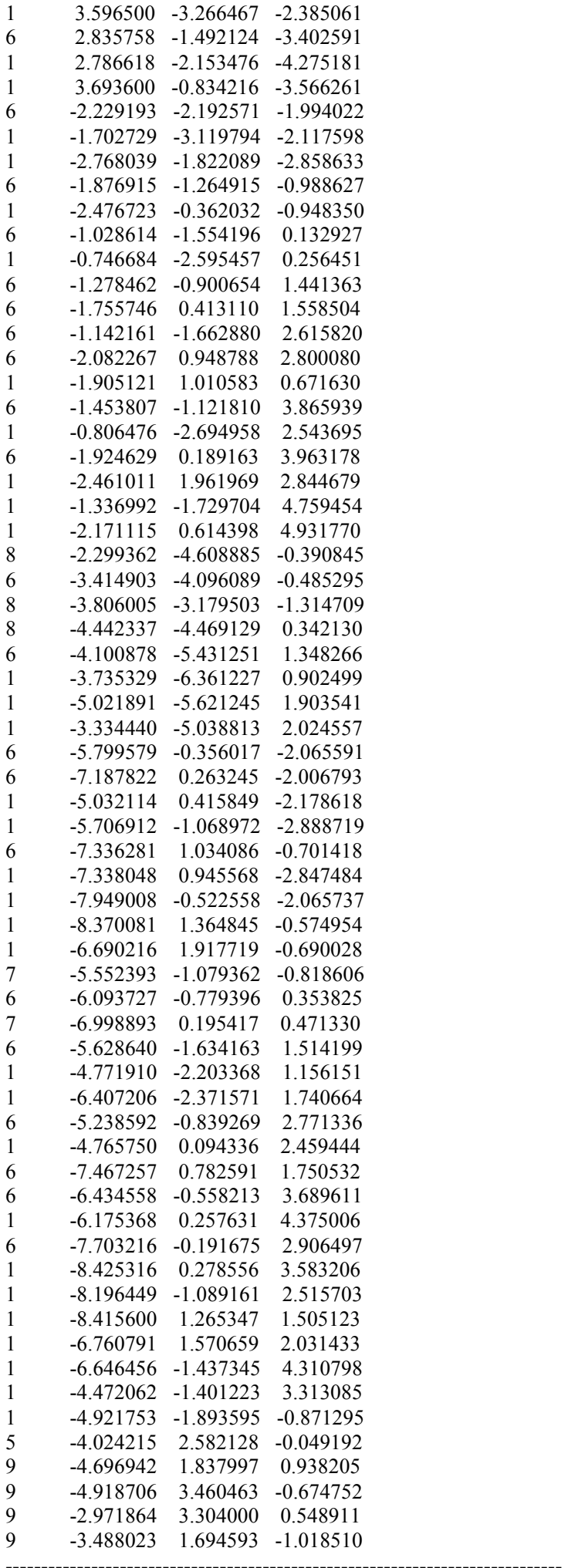

13 P1 endo-si $\left[\mathrm{BF}_{4}^{-}\right]$

Number of imaginary frequencies $=0$

The smallest frequencies are $=11.355816 .253419 .6476 \mathrm{~cm}(-1)$ Electronic energy $=-3892.021813$

Zero-point correction $=$

(Hartree/Particle)

Thermal correction to Energy= $\quad 1.306443$

Thermal correction to Enthalpy= $\quad 1.307387$

Thermal correction to Gibbs Free Energy $=\quad 1.122445$

Sum of electronic and zero-point Energies $=\quad$\begin{tabular}{l}
-3890.788168 \\
\hline
\end{tabular}
Sum of electronic and thermal Energies=

$-3890.715370$

Sum of electronic and thermal Enthalpies=

$-3890.714426$

Sum of electronic and thermal Free Energies=

$-3890.899368$

\begin{tabular}{|c|c|c|c|}
\hline \multicolumn{4}{|c|}{ Cartesian Coordinates } \\
\hline 77 & -0.006548 & -0.626941 & $1-1.664708$ \\
\hline 15 & 1.656638 & 0.530226 & -0.463480 \\
\hline 8 & 2.192144 & 0.258133 & 1.084798 \\
\hline 8 & 3.110657 & 0.388282 & -1.279410 \\
\hline 7 & 1.177141 & 2.114969 & -0.318685 \\
\hline 6 & 2.888299 & -0.918199 & 1.353740 \\
\hline 6 & 2.198726 & -1.906076 & 2.088034 \\
\hline 1 & 1.194774 & -1.685190 & 2.428715 \\
\hline 6 & 2.811121 & -3.105569 & 2.352560 \\
\hline 1 & 2.293740 & -3.871697 & 2.923382 \\
\hline 6 & 4.113951 & -3.375336 & 1.852016 \\
\hline 6 & 4.721141 & -4.647308 & 2.036060 \\
\hline 1 & 4.178412 & -5.403781 & 2.596922 \\
\hline 6 & 5.959298 & -4.924586 & 1.502252 \\
\hline 1 & 6.410607 & -5.902474 & 1.642423 \\
\hline 6 & 6.638381 & -3.935294 & 0.752175 \\
\hline 1 & 7.603166 & -4.164354 & 0.308662 \\
\hline 6 & 6.085769 & -2.685649 & 0.572057 \\
\hline 1 & 6.616970 & -1.946311 & -0.015069 \\
\hline 6 & 4.818088 & -2.354980 & 1.127724 \\
\hline 6 & 4.202834 & -1.064061 & 0.940506 \\
\hline 6 & 4.922111 & 0.061032 & 0.283030 \\
\hline 6 & 6.192803 & 0.537307 & 0.769657 \\
\hline 6 & 6.761749 & 0.086925 & 1.994098 \\
\hline 1 & 6.222821 & -0.634602 & 2.596630 \\
\hline 6 & 7.976933 & 0.566088 & 2.434006 \\
\hline 1 & 8.383576 & 0.211986 & 3.377032 \\
\hline 6 & 8.696150 & 1.519703 & 1.675189 \\
\hline 1 & 9.655748 & 1.883094 & 2.031166 \\
\hline 6 & 8.165839 & 1.994593 & 0.496937 \\
\hline 1 & 8.696056 & 2.742426 & -0.087187 \\
\hline 6 & 6.907401 & 1.533783 & 0.023755 \\
\hline 6 & 6.334526 & 2.060333 & -1.165595 \\
\hline 1 & 6.889015 & 2.799154 & -1.737342 \\
\hline 6 & 5.081513 & 1.671649 & -1.565057 \\
\hline 1 & 4.604102 & 2.092572 & -2.443612 \\
\hline 6 & 4.372043 & 0.706027 & -0.815036 \\
\hline 6 & -0.092803 & 2.451080 & -1.028884 \\
\hline 1 & -0.902216 & 2.471395 & -0.294359 \\
\hline 6 & -0.008847 & 3.821943 & -1.688416 \\
\hline 6 & 1.092398 & 4.166071 & -2.488047 \\
\hline 1 & 1.912991 & 3.461002 & -2.593431 \\
\hline 6 & 1.147714 & 5.405191 & -3.124569 \\
\hline 1 & 2.009613 & 5.662008 & -3.734808 \\
\hline 6 & 0.096438 & 6.317064 & -2.976508 \\
\hline 1 & 0.138427 & 7.283029 & -3.472161 \\
\hline 6 & -1.002695 & 5.980212 & -2.184618 \\
\hline 1 & -1.821036 & 6.684343 & -2.060183 \\
\hline 6 & -1.054989 & 4.740006 & -1.540597 \\
\hline 1 & -1.902100 & 4.479761 & -0.915259 \\
\hline 6 & -0.411199 & 1.415403 & -2.119011 \\
\hline 1 & 0.180269 & 1.660717 & -3.006594 \\
\hline 1 & -1.461640 & 1.541382 & -2.387116 \\
\hline 6 & 1.664238 & 3.158587 & 0.640860 \\
\hline 1 & 1.323446 & 4.089303 & 0.184176 \\
\hline 6 & 1.008448 & 3.121743 & 2.020389 \\
\hline 6 & -0.156914 & 3.872933 & 2.230113 \\
\hline 1 & -0.627716 & 4.375383 & 1.391193 \\
\hline 6 & -0.710043 & 4.001218 & 3.505944 \\
\hline 1 & -1.610734 & 4.592962 & 3.644047 \\
\hline 6 & -0.100716 & 3.377757 & 4.596677 \\
\hline 1 & -0.519814 & 3.484925 & 5.593550 \\
\hline 6 & 1.042535 & 2.599708 & 4.394506 \\
\hline 1 & 1.514660 & 2.097869 & 5.234917 \\
\hline 6 & 1.590599 & 2.470757 & 3.117533 \\
\hline 1 & 2.485741 & 1.875313 & 2.980314 \\
\hline 6 & 3.185777 & 3.272306 & 0.706351 \\
\hline 1 & 3.593327 & 3.450734 & -0.290288 \\
\hline 1 & 3.665523 & 2.387117 & 1.123160 \\
\hline
\end{tabular}


$\begin{array}{llll}3.445850 & 4.125243 & 1.340241\end{array}$

$1.428138 \quad-0.655055 \quad-3.375624$

$\begin{array}{llll}1.794779 & 0.359979 & -3.495472\end{array}$

$\begin{array}{llll}0.126832 & -0.905390 & -3.871088\end{array}$

$\begin{array}{llll}-0.396054 & -0.064449 & -4.322682\end{array}$

$\begin{array}{llll}-0.368589 & -2.253792 & -4.353556\end{array}$

$\begin{array}{llll}0.034642 & -2.423926 & -5.362554\end{array}$

$\begin{array}{llll}-1.454127 & -2.193725 & -4.466891\end{array}$

$\begin{array}{llll}-0.013165 & -3.446967 & -3.437076\end{array}$

$\begin{array}{llll}0.921061 & -3.910624 & -3.768712\end{array}$

$\begin{array}{lll}-0.797124 & -4.203717 & -3.522531\end{array}$

$\begin{array}{llll}0.104858 & -3.105716 & -1.961320\end{array}$

$-0.719335-3.462948-1.355984$

$1.272538-2.746522 \quad-1.333312$

$\begin{array}{llll}1.313257 & -2.832258 & -0.250466\end{array}$

$\begin{array}{llll}2.599252 & -2.556468 & -2.035114\end{array}$

$3.286712-2.079744-1.339205$

$\begin{array}{llll}3.020371 & -3.546969 & -2.259075\end{array}$

$\begin{array}{llll}2.530881 & -1.715168 & -3.330090\end{array}$

$\begin{array}{llll}2.408605 & -2.369279 & -4.199798\end{array}$

$\begin{array}{llll}3.490615 & -1.208155 & -3.457018\end{array}$

$-2.127190 \quad-1.018911-2.111282$

$\begin{array}{llll}-2.319165 & -2.069909 & -2.282321\end{array}$

$\begin{array}{llll}-2.563734 & -0.366625 & -2.863488\end{array}$

$\begin{array}{llll}-2.124127 & -0.529901 & -0.764722\end{array}$

$\begin{array}{llll}-2.470465 & 0.473926 & -0.559011\end{array}$

$\begin{array}{lll}-1.564992 & -1.297345 & 0.258118\end{array}$

$\begin{array}{lll}-1.432081 & -2.355623 & 0.070034\end{array}$

$\begin{array}{lll}-1.491824 & -0.937084 & 1.675494\end{array}$

$\begin{array}{lll}-1.726063 & 0.360685 & 2.163625\end{array}$

$\begin{array}{lll}-1.265357 & -1.977215 & 2.598610\end{array}$

$\begin{array}{lll}-1.774589 & 0.590706 & 3.535503\end{array}$

$\begin{array}{lll}-1.908391 & 1.183084 & 1.484892\end{array}$

$\begin{array}{lll}-1.292690 & -1.737280 & 3.971612\end{array}$

$\begin{array}{lll}-1.077594 & -2.980837 & 2.229444\end{array}$

$\begin{array}{lll}-1.556633 & -0.449652 & 4.444337\end{array}$

$\begin{array}{llll}-1.979931 & 1.591645 & 3.893805\end{array}$

$\begin{array}{lll}-1.118006 & -2.553195 & 4.66727\end{array}$

$\begin{array}{lll}-1.590658 & -0.254888 & 5.512533\end{array}$

$\begin{array}{llll}-2.523274 & -4.899215 & -1.673397\end{array}$

$\begin{array}{lll}-3.361327 & -4.162840 & -1.127568\end{array}$

$\begin{array}{llll}-4.214449 & -3.392014 & -1.642950\end{array}$

$\begin{array}{llll}-3.397418 & -4.139376 & 0.283223\end{array}$

$\begin{array}{llll}-2.509448 & -5.036134 & 0.941737\end{array}$

$\begin{array}{lll}-2.701960 & -6.076776 & 0.657377\end{array}$

$\begin{array}{lll}-2.686235 & -4.912756 & 2.013896\end{array}$

$\begin{array}{llll}-1.458791 & -4.816493 & 0.716918\end{array}$

$\begin{array}{llll}-5.640663 & -0.316534 & -1.863089\end{array}$

$\begin{array}{llll}-7.031126 & 0.295391 & -1.763084\end{array}$

$\begin{array}{llll}-4.874810 & 0.466228 & -1.848460\end{array}$

$\begin{array}{lll}-5.515280 & -0.911502 & -2.770114\end{array}$

$\begin{array}{llll}-7.184343 & 0.951412 & -0.397200\end{array}$

$\begin{array}{llll}-7.181945 & 1.048540 & -2.541357\end{array}$

$\begin{array}{lll}-7.787022 & -0.486643 & -1.896252\end{array}$

$\begin{array}{lll}-8.231582 & 1.216151 & -0.220614\end{array}$

$\begin{array}{llll}-6.587506 & 1.865678 & -0.333318\end{array}$

$\begin{array}{llll}-5.437840 & -1.204994 & -0.720857\end{array}$

$\begin{array}{lll}-5.941964 & -0.973259 & 0.478171\end{array}$

$\begin{array}{lll}-6.775962 & 0.051644 & 0.704582\end{array}$

$\begin{array}{llll}-5.531731 & -1.943564 & 1.562550\end{array}$

$\begin{array}{lll}-4.802516 & -2.625198 & 1.122404\end{array}$

$\begin{array}{lll}-6.400838 & -2.549210 & 1.843808\end{array}$

$\begin{array}{lll}-4.926675 & -1.252605 & 2.800354\end{array}$

$\begin{array}{lll}-4.373490 & -0.368629 & 2.472818\end{array}$

$\begin{array}{llll}-7.029499 & 0.652105 & 2.036513\end{array}$

$\begin{array}{lll}-5.985898 & -0.860169 & 3.839202\end{array}$

$\begin{array}{lll}-5.562190 & -0.104601 & 4.512046\end{array}$

$\begin{array}{lll}-7.271222 & -0.318056 & 3.195979\end{array}$

$\begin{array}{lll}-7.856935 & 0.217064 & 3.951821\end{array}$

$\begin{array}{lll}-7.908838 & -1.136197 & 2.842326\end{array}$

$\begin{array}{lll}-7.923218 & 1.265354 & 1.903219\end{array}$

$\begin{array}{lll}-6.197781 & 1.331561 & 2.256000\end{array}$

$\begin{array}{llll}-6.241939 & -1.727737 & 4.460225\end{array}$

$\begin{array}{lll}-4.187416 & -1.920817 & 3.251132\end{array}$

$\begin{array}{lll}-4.914199 & -2.097306 & -0.908272\end{array}$

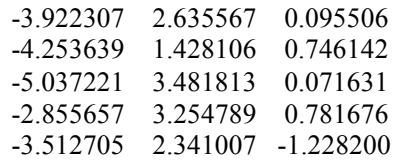

$12 \mathrm{P} 1$ exo-re $\left[\mathrm{BF}_{4}^{-}\right]$

Number of imaginary frequencies $=0$

The smallest frequencies are=12.4762 $18.640921 .2242 \mathrm{~cm}(-1)$ Electronic energy $=-3892.02497$

Zero-point correction=

(Hartree/Particle)

Thermal correction to Energy= $\quad 1.306842$

Thermal correction to Enthalpy=

1.307787

Thermal correction to Gibbs Free Energy=

Sum of electronic and zero-point Energies=

Sum of electronic and thermal Energies=

Sum of electronic and thermal Enthalpies=

Sum of electronic and thermal Free Energies=

$-3890.790429$

$-3890.718128$

$-3890.717183$

$-3890.900391$

Cartesian Coordinates

\begin{tabular}{|c|c|c|c|}
\hline 7 & 0.283642 & -1.076457 & 082 \\
\hline & 1.811814 & 0.251717 & -0.447251 \\
\hline & 2.428580 & 0.106688 & 1.093736 \\
\hline & 3.272360 & 0.267477 & -1.298908 \\
\hline & 1.187064 & 1.800674 & -0.320672 \\
\hline & 3.388416 & -0.853439 & 1.380846 \\
\hline & 2.975322 & -1.954511 & 2.163446 \\
\hline & 1.945898 & -1.989289 & 2.499775 \\
\hline 6 & 3.873678 & -2.945121 & 2.472150 \\
\hline & 3.567202 & -3.793394 & 3.077950 \\
\hline & 5.204955 & -2.892557 & 1.978046 \\
\hline 6 & 6.122044 & -3.949918 & 2.226143 \\
\hline & 5.789898 & -4.791923 & 2.828099 \\
\hline & 7.396180 & -3.919219 & 1.706052 \\
\hline 1 & 8.087020 & -4.735165 & 1.897700 \\
\hline 6 & 7.800566 & -2.826029 & 0.903083 \\
\hline 1 & 8.797424 & -2.815558 & 0.471486 \\
\hline 6 & 6.940867 & -1.777353 & 0.656988 \\
\hline 1 & 7.266373 & -0.955385 & 0.030569 \\
\hline 6 & 5.624302 & -1.761942 & 1.198513 \\
\hline 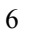 & 4.698714 & -0.684845 & 0.956638 \\
\hline 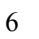 & 5.099276 & 0.555297 & 0.236793 \\
\hline 6 & 6.185781 & 1.386732 & 0.685200 \\
\hline 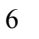 & 6.865301 & 1.163845 & 1.916047 \\
\hline 1 & 6.556052 & 0.341522 & 2.550420 \\
\hline 6 & 7.896630 & 049 & 2.319727 \\
\hline 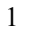 & 8.393091 & 1.795605 & 3.267505 \\
\hline 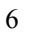 & 8.310603 & 3.073419 & 1.516413 \\
\hline 1 & 9.129297 & 3.70 & 1.843906 \\
\hline 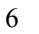 & 7.661208 & 3.332725 & 0.330567 \\
\hline 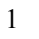 & 7.955233 & 4.177046 & -0.287744 \\
\hline 6 & 6.583555 & 2.515941 & -0.107509 \\
\hline 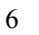 & 5.881795 & 2.808140 & -1.308350 \\
\hline 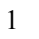 & 6.202078 & 3.650024 & -1.915840 \\
\hline 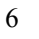 & 4.794168 & 2.058162 & -1.679866 \\
\hline 1 & 4.227207 & 2.279626 & -2.577529 \\
\hline 6 & 4.389 & 0.962109 & -0.884003 \\
\hline 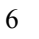 & 0.023753 & 2.030938 & -1.219447 \\
\hline 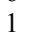 & -0.889431 & 2.019137 & -0.614401 \\
\hline 6 & 0.109218 & 3.375422 & -1.926864 \\
\hline 6 & 1.334729 & 3.882107 & -2.381731 \\
\hline 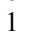 & 2.244492 & 3.334510 & -2.160794 \\
\hline 6 & 1.389547 & 5.080419 & -3.094734 \\
\hline 1 & 2.348831 & 5.463321 & -3.433776 \\
\hline 6 & 0.214747 & 5.786581 & -3.370839 \\
\hline 1 & 0.255069 & 6.720611 & -3.924769 \\
\hline 6 & -1.010606 & 5.284511 & -2.924879 \\
\hline 1 & -1.928815 & 5.828990 & -3.129648 \\
\hline 6 & -1.064543 & 4.088584 & -2.204271 \\
\hline & -2.016490 & 3.708910 & -1.849186 \\
\hline & -0.063315 & 0.913045 & -2.276691 \\
\hline & 0.689150 & 1.119825 & -3.04970 \\
\hline
\end{tabular}




$\begin{array}{rrrrrrrr}-1.028903 & 1.008757 & -2.774766 & 1 & -4.198564 & -0.843420 & 0.827439 \\ 1.363874 & 2.797551 & 0.785485 & 1 & -5.319478 & -1.837596 & 1.739586 \\ 0.820706 & 3.671594 & 0.416267 & 6 & -4.197414 & -0.397092 & 2.934691 \\ 0.665446 & 2.384419 & 2.082274 & 1 & -4.104724 & 0.691365 & 2.933073 \\ -0.718461 & 2.577257 & 2.193734 & 6 & -6.855945 & 0.628028 & 3.513842 \\ -1.271419 & 3.031674 & 1.380537 & 6 & -4.903521 & -0.889794 & 4.200050 \\ -1.405016 & 2.204220 & 3.351261 & 1 & -4.510202 & -0.344509 & 5.066603 \\ -2.478365 & 2.356677 & 3.403968 & 6 & -6.425352 & -0.709755 & 4.119425 \\ -0.714039 & 1.650067 & 4.429679 & 1 & -6.860674 & -0.770620 & 5.122871 \\ -1.245451 & 1.359455 & 5.331985 & 1 & -6.885607 & -1.517730 & 3.538772 \\ 0.670095 & 1.474233 & 4.339434 & 1 & -7.878867 & 0.842421 & 3.829967 \\ 1.221603 & 1.051176 & 5.175176 & 1 & -6.226722 & 1.447537 & 3.883735 \\ 1.351969 & 1.835955 & 3.175813 & 1 & -4.674782 & -1.949892 & 4.364245 \\ 2.422826 & 1.679336 & 3.120660 & 1 & -3.177455 & -0.782899 & 2.902666 \\ 2.812682 & 3.245438 & 0.973973 & 1 & -5.381207 & -0.128063 & -0.676228 \\ 3.209417 & 3.640301 & 0.036813 & 5 & -3.894855 & 2.637886 & 0.121432 \\ 3.463234 & 2.435968 & 1.303030 & 9 & -5.119102 & 2.932846 & 0.752909 \\ 2.848517 & 4.039688 & 1.726556 & 9 & -4.117661 & 2.337286 & -1.241577 \\ 1.636331 & -1.427519 & -3.281295 & 9 & -3.039196 & 3.749333 & 0.222674 \\ 2.035212 & -0.468409 & -3.602342 & 9 & -3.301018 & 1.521755 & 0.745506 \\ 0.292782 & -1.705418 & -3.666846 & 1 & -2.676457 & -0.718767 & -3.226457 \\ -0.205743 & -0.945226 & -4.270399 & 1 & -2.836277 & 0.475393 & -1.899457\end{array}$

$\begin{array}{lll}0.95465 & -3.091367 & -3.842844\end{array}$

$\begin{array}{llll}-0.036427 & -3.504464 & -4.827907\end{array}$

$\begin{array}{llll}-1.394164 & -2.979157 & -3.836675\end{array}$

$\begin{array}{llll}0.096749 & -4.082073 & -2.729990\end{array}$

$\begin{array}{llll}1.016781 & -4.607901 & -3.001813\end{array}$

$\begin{array}{llll}-0.676337 & -4.853125 & -2.641589\end{array}$

$\begin{array}{llll}0.279852 & -3.427971 & -1.363722\end{array}$

$\begin{array}{llll}-0.494136 & -3.640502 & -0.634315\end{array}$

$\begin{array}{llll}1.490767 & -2.984497 & -0.854907\end{array}$

$\begin{array}{lll}1.570232 & -2.908899 & 0.225635\end{array}$

$2.810736-2.994563-1.603789$

$3.505448-2.349828-1.061335$

$\begin{array}{llll}3.235304 & -4.009410 & -1.571107\end{array}$

$2.708664-2.499903-3.063486$

$2.524281 \quad-3.342602-3.738492$

$\begin{array}{llll}3.679270 & -2.086921 & -3.356205\end{array}$

$\begin{array}{llll}-2.822550 & -0.581163 & -2.152972\end{array}$

$\begin{array}{llll}-1.866037 & -1.389723 & -1.326321\end{array}$

$\begin{array}{llll}-2.085980 & -2.448164 & -1.418639\end{array}$

$\begin{array}{llll}-1.356021 & -0.908812 & -0.072611\end{array}$

$\begin{array}{llll}-1.539250 & 0.138959 & 0.147126\end{array}$

$\begin{array}{llll}-1.308765 & -1.726076 & 1.169380\end{array}$

$\begin{array}{lll}-2.024954 & -2.927110 & 1.319496\end{array}$

$\begin{array}{lll}-0.603886 & -1.240708 & 2.286748\end{array}$

$\begin{array}{lll}-2.019841 & -3.626152 & 2.530502\end{array}$

$\begin{array}{llll}-2.621174 & -3.301707 & 0.492497\end{array}$

$\begin{array}{lll}-0.605176 & -1.930877 & 3.497771\end{array}$

$\begin{array}{lll}-0.056868 & -0.308739 & 2.201084\end{array}$

$\begin{array}{lll}-1.309603 & -3.133591 & 3.627480\end{array}$

$\begin{array}{lll}-2.587148 & -4.549383 & 2.618657\end{array}$

$\begin{array}{llll}-0.059358 & -1.521511 & 4.343327\end{array}$

$\begin{array}{llll}-1.312545 & -3.671123 & 4.571609\end{array}$

$\begin{array}{llll}-3.984331 & -2.901416 & -3.083684\end{array}$

$\begin{array}{llll}-4.607373 & -2.180320 & -2.332196\end{array}$

$\begin{array}{llll}-4.209298 & -0.995919 & -1.848801\end{array}$

$\begin{array}{llll}-5.829322 & -2.426096 & -1.838898\end{array}$

$\begin{array}{lll}-6.426914 & -3.663970 & -2.277527\end{array}$

$\begin{array}{lll}-6.548881 & -3.669725 & -3.363742\end{array}$

$\begin{array}{llll}-7.399995 & -3.705600 & -1.788065\end{array}$

$\begin{array}{llll}-5.813391 & -4.516344 & -1.974165\end{array}$

$\begin{array}{llll}-7.068959 & 1.144614 & -0.799140\end{array}$

$\begin{array}{lll}-8.286631 & 1.349258 & 0.088299\end{array}$

$\begin{array}{llll}-6.565523 & 2.089187 & -1.017211\end{array}$

$\begin{array}{llll}-7.334490 & 0.662673 & -1.744087\end{array}$

$\begin{array}{lll}-7.831838 & 1.706901 & 1.498696\end{array}$

$\begin{array}{lll}-8.913313 & 2.152640 & -0.307449\end{array}$

$\begin{array}{lll}-8.888600 & 0.434316 & 0.119033\end{array}$

$\begin{array}{llll}-8.688306 & 1.749447 & 2.174077\end{array}$

$\begin{array}{lll}-7.327389 & 2.677441 & 1.512885\end{array}$

$\begin{array}{llll}-6.122083 & 0.274750 & -0.108556\end{array}$

$\begin{array}{lll}-6.033107 & 0.099924 & 1.201686\end{array}$

$\begin{array}{lll}-6.893713 & 0.692767 & 2.032702\end{array}$

$\begin{array}{lll}-4.916232 & -0.821834 & 1.646950\end{array}$

Cartesian Coordinates

$\begin{array}{lccc}\ldots \ldots \ldots \ldots \ldots \ldots \ldots \ldots \ldots \ldots \ldots \ldots \\ 17 & 0.257279 & -0.999197 & -1.604095 \\ 8 & 1.706408 & 0.380305 & -0.478071 \\ 8 & 2.257166 & 0.230394 & 1.079584 \\ 7 & 3.184543 & 0.406234 & -1.276050 \\ 6 & 1.047438 & 1.899782 & -0.367617 \\ 6 & 3.143873 & -0.785352 & 1.413337 \\ 1 & 2.627496 & -1.835889 & 2.201127 \\ 6 & 1.595096 & -1.778069 & 2.516523 \\ 1 & 3.437442 & -2.889551 & 2.541928 \\ 6 & 3.050764 & -3.700856 & 3.152339 \\ 6 & 4.776540 & -2.951218 & 2.069251 \\ 1 & 5.597138 & -4.080519 & 2.336767 \\ 6 & 5.185934 & -4.887026 & 2.938475 \\ 1 & 6.875965 & -4.162479 & 1.833884 \\ 6 & 7.491553 & -5.033381 & 2.039548 \\ 1 & 7.383034 & -3.113389 & 1.030701 \\ 6 & 8.382563 & -3.191210 & 0.612439 \\ 1 & 6.619854 & -1.996071 & 0.768319 \\ 6 & 7.022744 & -1.208436 & 0.142897 \\ 6 & 5.302317 & -1.865211 & 1.290374 \\ 6 & 4.472228 & -0.716786 & 1.022455 \\ 6 & 4.992149 & 0.487807 & 0.318548 \\ 6 & 6.144025 & 1.209471 & 0.799045 \\ 1 & 6.765579 & 0.919927 & 2.046409 \\ 6 & 6.357620 & 0.135506 & 2.672558 \\ 1 & 7.864373 & 1.631282 & 2.477992 \\ 6 & 8.313368 & 1.394218 & 3.438405 \\ 1 & 8.408888 & 2.671307 & 1.687765 \\ 6 & 9.279717 & 3.218281 & 2.037188 \\ 1 & 7.818948 & 2.995414 & 0.486916 \\ 6 & 8.211740 & 3.805608 & -0.121960 \\ 6 & 6.674255 & 2.293553 & 0.021263 \\ 1 & 6.031952 & 2.663205 & -1.191551 \\ 6 & 6.448319 & 3.473075 & -1.784104 \\ & 4.879688 & 2.033710 & -1.587767\end{array}$

$[12-13]^{\ddagger} \mathrm{P} 1$ exo-re $\left[\mathrm{BF}_{4}^{-}\right]$

Number of imaginary frequencies $=1$

The smallest frequencies are $=-282.729012 .863215 .5595 \mathrm{~cm}(-1)$ Electronic energy $=-3891.9994043$

Zero-point correction=

1.232196

(Hartree/Particle)

Thermal correction to Energy= $\quad 1.304676$

Thermal correction to Enthalpy=

1.305620

Thermal correction to Gibbs Free Energy=

Sum of electronic and zero-point Energies=

Sum of electronic and thermal Energies=

.121500

Sum of electronic and thermal Enthalpies=

$-3890.767209$

$-3890.694729$

$-3890.693785$

Sum of electronic and thermal Free Energies=

$-3890.877904$ 


\begin{tabular}{|c|c|c|c|c|c|c|}
\hline 4.345769 & 2.327494 & -2.485471 & 6 & -6.136733 & -4.570760 & -0.474794 \\
\hline 4.351506 & 0.976968 & -0.811120 & 1 & -6.168978 & -5.235008 & -1.344293 \\
\hline-0.147833 & 2.115463 & -1.222177 & 1 & -7.120032 & -4.535338 & -0.000063 \\
\hline-1.014765 & 2.203870 & -0.562708 & 1 & -5.397431 & -4.958313 & 0.234048 \\
\hline 0.000989 & 3.395888 & -2.033904 & 6 & -6.899990 & 0.806217 & -1.435552 \\
\hline 1.207798 & 3.676756 & -2.694535 & 6 & -8.211204 & 1.191948 & -0.769071 \\
\hline 2.045685 & 2.995169 & -2.577401 & 1 & -6.289061 & 1.684823 & -1.664599 \\
\hline 1.339230 & 4.822159 & -3.478194 & 1 & -7.064047 & 0.249232 & -2.361979 \\
\hline 2.281607 & 5.030016 & -3.978585 & 6 & -7.925469 & 1.700360 & 0.638068 \\
\hline 0.261313 & 5.703894 & -3.617447 & 1 & -8.718768 & 1.973198 & -1.341185 \\
\hline 0.362414 & 6.598062 & -4.226537 & 1 & -8.874877 & 0.321522 & -0.721288 \\
\hline-0.941225 & 5.430090 & -2.963336 & 1 & -8.860450 & 1.862555 & 1.179915 \\
\hline-1.780980 & 6.113405 & -3.059554 & 1 & -7.373493 & 2.645719 & 0.606094 \\
\hline-1.071556 & 4.283659 & -2.172283 & 7 & -6.141414 & -0.050369 & -0.530748 \\
\hline-1.999978 & 4.083309 & -1.648175 & 6 & -6.224548 & -0.034627 & 0.786370 \\
\hline-0.385008 & 0.940726 & -2.198088 & 7 & -7.123798 & 0.733038 & 1.417738 \\
\hline 0.152676 & 1.152275 & -3.128405 & 6 & -5.258395 & -0.961424 & 1.499741 \\
\hline-1.447512 & 0.965488 & -2.440742 & 1 & -4.517365 & -1.252380 & 0.754280 \\
\hline 1.313570 & 2.992204 & 0.627118 & 1 & -5.795254 & -1.877007 & 1.774084 \\
\hline 0.822553 & 3.857259 & 0.174420 & 6 & -4.547539 & -0.352502 & 2.719413 \\
\hline 0.619157 & 2.772120 & 1.969245 & 1 & -4.303306 & 0.688124 & 2.496400 \\
\hline-0.711729 & 3.185215 & 2.113605 & 6 & -7.098329 & 1.040843 & 2.865172 \\
\hline-1.237391 & 3.628085 & 1.276210 & 6 & -5.364668 & -0.465252 & 4.011222 \\
\hline-1.389189 & 3.017507 & 3.323585 & 1 & -4.946540 & 0.220741 & 4.758065 \\
\hline-2.428105 & 3.325570 & 3.400622 & 6 & -6.851381 & -0.150019 & 3.794698 \\
\hline-0.730604 & 2.463888 & 4.422419 & 1 & -7.324687 & 0.073444 & 4.757124 \\
\hline-1.250062 & 2.340600 & 5.369047 & 1 & -7.380740 & -1.020239 & 3.389454 \\
\hline 0.604765 & 2.067484 & 4.295831 & 1 & -8.083619 & 1.458360 & 3.082423 \\
\hline 1.127141 & 1.635516 & 5.145301 & 1 & -6.363679 & 1.838165 & 3.033054 \\
\hline 1.270477 & 2.212904 & 3.078438 & 1 & -5.272207 & -1.476488 & 4.426631 \\
\hline 2.300042 & 1.885201 & 2.994586 & 1 & -3.588459 & -0.861808 & 2.843356 \\
\hline 2.788317 & 3.368798 & 0.749513 & 1 & -5.463741 & -0.702564 & -0.962936 \\
\hline 3.186806 & 3.645782 & -0.228180 & 5 & -3.780778 & 2.501141 & 0.171625 \\
\hline 3.401379 & 2.564296 & 1.155229 & 9 & -4.999817 & 2.539161 & 0.876944 \\
\hline 2.879180 & 4.232386 & 1.415748 & 9 & -3.990363 & 2.073134 & -1.152833 \\
\hline 1.659588 & -1.023922 & -3.363291 & 9 & -3.215811 & 3.794163 & 0.167400 \\
\hline 1.865756 & 0.017073 & -3.597556 & 9 & -2.901631 & 1.603566 & 0.815475 \\
\hline 0.402761 & -1.514425 & -3.787223 & 1 & -2.549606 & -2.110958 & -2.997726 \\
\hline-0.242848 & -0.812387 & -4.313887 & 1 & -2.693733 & -0.402947 & -2.317522 \\
\hline
\end{tabular}

$13 \mathrm{P1}$ exo-re $\left[\mathrm{BF}_{4}^{-}\right]$

$\begin{array}{llll}0.423876 & -3.188316 & -5.131176\end{array}$
$\begin{array}{lll}-6.136733 & -4.570760 & -0.474794 \\ -6.168978 & -5.235008 & -1.344293\end{array}$
$\begin{array}{lll}20032 & -4.535338 & -0.000063\end{array}$
$\begin{array}{llll}-6.899990 & 0.806217 & -1.435552\end{array}$
$\begin{array}{lll}-8.211204 & 1.191948 & -0.769071\end{array}$
$\begin{array}{llll}-7.925469 & 1.700360 & 0.638068\end{array}$
$\begin{array}{lll}-8.718768 & 1.973198 & -1.341185\end{array}$
$\begin{array}{rrr}-8.874877 & 0.321522 & -0.721288 \\ -8.860450 & 1.862555 & 1.179915\end{array}$
$\begin{array}{lll}-7.373493 & 2.645719 & 0.606094\end{array}$
$\begin{array}{lll}-6.141414 & -0.050369 & -0.530748\end{array}$
$\begin{array}{lll}488 & -0.034627 & 0.786370\end{array}$
$\begin{array}{lll}-5.258395 & -0.961424 & 1.499741\end{array}$
$\begin{array}{lll}5.57365 & -1.252380 & 0.754280\end{array}$
$\begin{array}{lll}-4.547539 & -0.352502 & 2.719413\end{array}$
$\begin{array}{lll}4.303306 & 0.688124 & 2.496400\end{array}$
$\begin{array}{lll}-4.946540 & 0.220741 & 4.758065\end{array}$
$\begin{array}{lll}-0.150019 & 3.794698\end{array}$
$\begin{array}{lll}-7.380740 & -1.020239 & 3.389454\end{array}$
$\begin{array}{lll}8.083619 & 1.458360 & 3.082423\end{array}$
$\begin{array}{lll}-6.363679 & 1.838165 & 3.033054 \\ -5.272207 & -1.476488 & 4.426631\end{array}$
$\begin{array}{lll}-3.588459 & -0.861808 \quad 2.843356\end{array}$
$\begin{array}{llll}-5.463741 & -0.702564 & -0.962936\end{array}$
$\begin{array}{lll}.780778 & 2.501141 & 0.171625\end{array}$
$\begin{array}{rrr}-4.999817 & 2.539161 & 0.876944 \\ -3.990363 & 2.073134 & -1.152833\end{array}$
$\begin{array}{lll}-3.215811 & 3.794163 & 0.167400\end{array}$

$\begin{array}{llll}-0.999913 & -3.079319 & -4.115065\end{array}$

$\begin{array}{llll}0.694935 & -4.001957 & -3.136845\end{array}$

$1.697314 \quad-4.284524 \quad-3.471255$

$\begin{array}{llll}0.090654 & -4.913933 & -3.178329\end{array}$

$\begin{array}{llll}0.772441 & -3.546110 & -1.692510\end{array}$

$\begin{array}{llll}0.013527 & -3.934988 & -1.025441\end{array}$

$\begin{array}{llll}1.864132 & -2.944324 & -1.130629\end{array}$

$\begin{array}{llll}1.920454 & -2.904811 & -0.046758\end{array}$

$\begin{array}{llll}3.130084 & -2.599718 & -1.884759\end{array}$

$\begin{array}{llll}3.743765 & -1.968740 & -1.244257\end{array}$

$3.700829-3.526496 \quad-2.042323$

$\begin{array}{llll}2.918610 & -1.886324 & -3.242541\end{array}$

$\begin{array}{llll}2.911790 & -2.621018 & -4.055175\end{array}$

$3.785564-1.244152 \quad-3.419668$

$\begin{array}{llll}-2.479398 & -1.447392 & -2.148939\end{array}$

$\begin{array}{llll}-1.744504 & -1.867286 & -1.025413\end{array}$

$\begin{array}{llll}-1.708355 & -2.940396 & -0.893634\end{array}$

$\begin{array}{lll}-1.269332 & -1.012120 & 0.027780\end{array}$

$\begin{array}{lll}-1.681751 & -0.008864 & 0.061182\end{array}$

$-0.971518-1.554689 \quad 1.379139$

$\begin{array}{lll}-0.766289 & -2.923170 & 1.639557\end{array}$

$\begin{array}{lll}-0.937996 & -0.668205 & 2.471268\end{array}$

$\begin{array}{lll}-0.551907 & -3.388496 & 2.936796\end{array}$

$\begin{array}{lll}-0.782518 & -3.640800 & 0.827818\end{array}$

$-0.725103 \quad-1.133859 \quad 3.770177$

$\begin{array}{llll}-1.106307 & 0.389226 & 2.298060\end{array}$

$\begin{array}{lll}-0.533150 & -2.496478 & 4.013240\end{array}$

$\begin{array}{lll}-0.400728 & -4.451469 & 3.106030\end{array}$

$\begin{array}{lll}-0.714019 & -0.424759 & 4.591266\end{array}$

$\begin{array}{lll}-0.369409 & -2.857822 & 5.024581\end{array}$

$\begin{array}{lll}-3.888501 & -4.004140 & -1.733525\end{array}$

$-4.626700-3.050896-1.490700$

$-4.430958-1.799393-1.754330$

$\begin{array}{lll}-5.835210 & -3.224620 & -0.855592\end{array}$

Number of imaginary frequencies $=0$

The smallest frequencies are $=17.505723 .421824 .2100 \mathrm{~cm}(-1)$ Electronic energy $=-3892.0269636$

Zero-point correction $=$

1.234003

(Hartree/Particle)

Thermal correction to Energy= $\quad 1.306562$

Thermal correction to Enthalpy= $\quad 1.307506$

Thermal correction to Gibbs Free Energy $=\quad 1.126267$

Sum of electronic and zero-point Energies $=\quad-3890.792960$

Sum of electronic and thermal Energies $=\quad-3890.720402$

Sum of electronic and thermal Enthalpies $=\quad-3890.719457$

Sum of electronic and thermal Free Energies $=\quad-3890.900697$

Cartesian Coordinates

$\begin{array}{lccc}77 & -0.322562 & -0.488929 & -1.507096 \\ 15 & 1.518730 & 0.483351 & -0.447623 \\ 8 & 2.152032 & 0.134077 & 1.036977 \\ 8 & 2.873891 & 0.241120 & -1.394470 \\ 7 & 1.198051 & 2.091871 & -0.204835 \\ 6 & 2.876920 & -1.033819 & 1.253740 \\ 6 & 2.263798 & -2.001343 & 2.075820 \\ 1 & 1.300199 & -1.768399 & 2.507892 \\ 6 & 2.900349 & -3.194431 & 2.307960 \\ 1 & 2.440034 & -3.945403 & 2.943895 \\ 6 & 4.152060 & -3.473825 & 1.695402 \\ 6 & 4.780342 & -4.738522 & 1.856388 \\ 1 & 4.292330 & -5.482586 & 2.480547 \\ 6 & 5.970656 & -5.023140 & 1.226328 \\ 1 & 6.439048 & -5.994994 & 1.351722 \\ 6 & 6.577273 & -4.049272 & 0.397883 \\ 1 & 7.503093 & -4.284503 & -0.119347 \\ 6 & 6.003198 & -2.806863 & 0.235226\end{array}$


$\begin{array}{lll}6.479510 & -2.078902 & -0.410654\end{array}$

$\begin{array}{llll}4.784925 & -2.468427 & 0.888547\end{array}$

$\begin{array}{lll}4.151340 & -1.182925 & 0.729323\end{array}$

$\begin{array}{lll}4.811961 & -0.061442 & 0.006683\end{array}$

$\begin{array}{lll}6.112040 & 0.428854 & 0.390717\end{array}$

$\begin{array}{lll}6.784758 & -0.016815 & 1.563196\end{array}$

$\begin{array}{lll}6.307218 & -0.748794 & 2.203289\end{array}$

$\begin{array}{lll}8.023929 & 0.479472 & 1.905949\end{array}$

$\begin{array}{lll}8.510563 & 0.128431 & 2.811471\end{array}$

$\begin{array}{lll}8.665095 & 1.446543 & 1.095792\end{array}$

$\begin{array}{lll}9.644740 & 1.823317 & 1.374897\end{array}$

$\begin{array}{llll}8.034215 & 1.917660 & -0.033220\end{array}$

$\begin{array}{llll}8.504298 & 2.675700 & -0.654341\end{array}$

$\begin{array}{llll}6.748327 & 1.440051 & -0.405503\end{array}$

$\begin{array}{llll}6.072830 & 1.966335 & -1.539719\end{array}$

$\begin{array}{llll}6.568379 & 2.717799 & -2.147856\end{array}$

$\begin{array}{llll}4.796495 & 1.561741 & -1.838891\end{array}$

$\begin{array}{llll}4.243166 & 1.980103 & -2.672985\end{array}$

$\begin{array}{llll}4.168306 & 0.580006 & -1.040730\end{array}$

$\begin{array}{llll}0.010881 & 2.603770 & -0.932674\end{array}$

$\begin{array}{llll}-0.785443 & 2.783634 & -0.206621\end{array}$

$\begin{array}{llll}0.325796 & 3.914189 & -1.642499\end{array}$

$\begin{array}{llll}1.508600 & 4.053691 & -2.385634\end{array}$

$\begin{array}{llll}2.219567 & 3.232317 & -2.409353\end{array}$

$\begin{array}{llll}1.779766 & 5.236634 & -3.071655\end{array}$

$\begin{array}{llll}2.702920 & 5.334722 & -3.636872\end{array}$

$\begin{array}{llll}0.866666 & 6.296156 & -3.029472\end{array}$

$\begin{array}{llll}1.077251 & 7.219196 & -3.562649\end{array}$

$\begin{array}{llll}-0.312372 & 6.162549 & -2.293719\end{array}$

$\begin{array}{llll}-1.022789 & 6.983822 & -2.250385\end{array}$

$\begin{array}{llll}-0.581597 & 4.978948 & -1.598900\end{array}$

$\begin{array}{llll}-1.487506 & 4.880986 & -1.009125\end{array}$

$\begin{array}{llll}-0.482739 & 1.591639 & -1.977518\end{array}$

$\begin{array}{lll}0.096453 & 1.734534 & -2.895417\end{array}$

$\begin{array}{llll}-1.511905 & 1.865048 & -2.204685\end{array}$

$\begin{array}{lll}1.764278 & 3.033094 & 0.820584\end{array}$

$\begin{array}{lll}1.430770 & 4.007507 & 0.456548\end{array}$

$\begin{array}{lll}1.147211 & 2.876743 & 2.208433\end{array}$

$\begin{array}{lll}-0.061739 & 3.529934 & 2.486204\end{array}$

$\begin{array}{lll}-0.571831 & 4.084046 & 1.706767\end{array}$

$\begin{array}{lll}-0.633512 & 3.473064 & 3.759488\end{array}$

$\begin{array}{lll}-1.579256 & 3.974866 & 3.942869\end{array}$

$\begin{array}{llll}0.010977 & 2.778264 & 4.784442\end{array}$

$\begin{array}{lll}-0.422890 & 2.742323 & 5.780049\end{array}$

$\begin{array}{lll}1.217433 & 2.123883 & 4.518583\end{array}$

$\begin{array}{lll}1.723351 & 1.574245 & 5.307784\end{array}$

$\begin{array}{llll}1.776787 & 2.166982 & 3.240820\end{array}$

$\begin{array}{lll}2.709915 & 1.648591 & 3.052512\end{array}$

$\begin{array}{llll}3.289679 & 3.096310 & 0.828531\end{array}$

$\begin{array}{llll}3.659588 & 3.336759 & -0.169479\end{array}$

$\begin{array}{lll}3.755597 & 2.166446 & 1.153091\end{array}$

$\begin{array}{llll}3.605021 & 3.889067 & 1.513754\end{array}$

$\begin{array}{llll}0.984375 & -0.727007 & -3.325820\end{array}$

$\begin{array}{llll}1.424658 & 0.248889 & -3.505731\end{array}$

$\begin{array}{lll}-0.356846 & -0.882895 & -3.727492\end{array}$

$\begin{array}{lll}-0.839579 & -0.013042 & -4.169430\end{array}$

$\begin{array}{llll}-0.997676 & -2.200249 & -4.118389\end{array}$

$-0.696373 \quad-2.431128 \quad-5.150833$

$-2.080298-2.060792-4.144412$

$\begin{array}{llll}-0.661279 & -3.397992 & -3.202587\end{array}$

$\begin{array}{llll}0.235925 & -3.907148 & -3.567633\end{array}$

$\begin{array}{llll}-1.480033 & -4.120387 & -3.245878\end{array}$

$\begin{array}{llll}-0.453783 & -3.039225 & -1.743356\end{array}$

$\begin{array}{lll}-1.265658 & -3.299113 & -1.076831\end{array}$

$\begin{array}{llll}0.760293 & -2.728925 & -1.186246\end{array}$

$\begin{array}{llll}0.852461 & -2.773876 & -0.104046\end{array}$

$2.056547 \quad-2.682964-1.968438$

$2.830417-2.267182-1.325380$

$\begin{array}{llll}2.360028 & -3.716114 & -2.188658\end{array}$

$\begin{array}{llll}2.000373 & -1.869617 & -3.284814\end{array}$

$\begin{array}{llll}1.795686 & -2.534223 & -4.130474\end{array}$

$2.990592 \quad-1.442787 \quad-3.460142$

$\begin{array}{llll}-2.584029 & -0.471786 & -1.830342\end{array}$

$\begin{array}{llll}-2.328039 & -0.933019 & -0.526498\end{array}$

$\begin{array}{lll}-2.547562 & -1.973046 & -0.323480\end{array}$ $\begin{array}{lll}-1.602941 & -0.199338 & 0.441134\end{array}$

$\begin{array}{lll}-1.719120 & 0.877091 & 0.468075\end{array}$

$\begin{array}{lll}-1.279457 & -0.838111 & 1.739700\end{array}$

$\begin{array}{lll}-1.216953 & -2.235780 & 1.899483\end{array}$

$\begin{array}{lll}-1.121311 & -0.031686 & 2.876734\end{array}$

$\begin{array}{lll}-1.032573 & -2.806412 & 3.157054\end{array}$

$\begin{array}{lll}-1.318064 & -2.888989 & 1.043380\end{array}$

$\begin{array}{lll}-0.928903 & -0.603706 & 4.135741\end{array}$

$\begin{array}{lll}-1.194247 & 1.044634 & 2.777275\end{array}$

$\begin{array}{lll}-0.888474 & -1.991549 & 4.285201\end{array}$

$\begin{array}{lll}-0.991647 & -3.887881 & 3.253343\end{array}$

$\begin{array}{llll}-0.818956 & 0.042719 & 5.000056\end{array}$

$\begin{array}{lll}-0.743371 & -2.434240 & 5.266582\end{array}$

$-3.160837-5.197594 \quad-1.940024$

$\begin{array}{llll}-3.649834 & -4.197325 & -1.401633\end{array}$

$-4.275014 \quad-3.221286-1.899766$

$\begin{array}{lll}-3.491933 & -4.058957 & 0.003595\end{array}$

$\begin{array}{lll}-2.854141 & -5.146283 & 0.669024\end{array}$

$\begin{array}{llll}-3.391245 & -6.086699 & 0.505695\end{array}$

$\begin{array}{lll}-2.861074 & -4.900093 & 1.734216\end{array}$

$\begin{array}{lll}-1.820570 & -5.282734 & 0.329715\end{array}$

$-6.086163-0.477983-2.084700$

$\begin{array}{llll}-7.117146 & 0.579789 & -1.722981\end{array}$

$\begin{array}{lll}-5.233198 & -0.023631 & -2.601700\end{array}$

$\begin{array}{llll}-6.501124 & -1.247561 & -2.741701\end{array}$

$\begin{array}{llll}-6.578153 & 1.398716 & -0.557433\end{array}$

$\begin{array}{llll}-7.307386 & 1.231813 & -2.579631\end{array}$

$\begin{array}{llll}-8.064361 & 0.108731 & -1.437791\end{array}$

$\begin{array}{llll}-7.305073 & 2.143215 & -0.231729\end{array}$

$\begin{array}{llll}-5.659855 & 1.924416 & -0.830682\end{array}$

$\begin{array}{llll}-5.607749 & -1.141614 & -0.872181\end{array}$

$\begin{array}{lll}-5.743630 & -0.679434 & 0.363630\end{array}$

$\begin{array}{lll}-6.287493 & 0.519082 & 0.599272\end{array}$

$\begin{array}{lll}-5.250303 & -1.606776 & 1.454762\end{array}$

$\begin{array}{lll}-4.559414 & -2.307033 & 0.980945\end{array}$

$\begin{array}{lll}-6.101263 & -2.214338 & 1.788926\end{array}$

$\begin{array}{lll}-4.575889 & -0.917554 & 2.650605\end{array}$

$\begin{array}{lll}-3.997814 & -0.060194 & 2.295553\end{array}$

$\begin{array}{lll}-6.479167 & 1.150020 & 1.931494\end{array}$

$\begin{array}{lll}-5.583581 & -0.461351 & 3.711850\end{array}$

$\begin{array}{lll}-5.090982 & 0.243775 & 4.392052\end{array}$

$\begin{array}{lll}-6.816929 & 0.200414 & 3.081690\end{array}$

$\begin{array}{lll}-7.350210 & 0.783727 & 3.840487\end{array}$

$\begin{array}{lll}-7.527581 & -0.553365 & 2.723225\end{array}$

$\begin{array}{lll}-7.311234 & 1.843457 & 1.793173\end{array}$

$\begin{array}{lll}-5.595600 & 1.751402 & 2.158592\end{array}$

$\begin{array}{lll}-5.905305 & -1.316852 & 4.318972\end{array}$

$\begin{array}{lll}-3.852401 & -1.610161 & 3.090432\end{array}$

$-5.078031-2.028075-1.049828$

$\begin{array}{lll}-3.508248 & 3.096797 & 0.706736\end{array}$

$\begin{array}{lll}-4.840984 & 3.527291 & 0.758643\end{array}$

$\begin{array}{llll}-3.275042 & 2.420481 & -0.516414\end{array}$

$\begin{array}{lll}-2.647918 & 4.212380 & 0.788403\end{array}$

$\begin{array}{lll}-3.245634 & 2.217853 & 1.778632\end{array}$

$\begin{array}{lll}-3.042864 & -1.191488 & -2.497118\end{array}$

$\begin{array}{lll}-2.806596 & 0.569487 & -2.017161\end{array}$

\section{$[12-13]^{\ddagger} \mathrm{P} 2$ endo-re $\left[\mathrm{BF}_{4}^{-}\right]$}

Number of imaginary frequencies $=1$

The smallest frequencies are $=-274.259212 .518915 .7429 \mathrm{~cm}(-1)$ Electronic energy $=-3891.9936916$

Zero-point correction=

(Hartree/Particle)

Thermal correction to Energy= $\quad 1.305602$

Thermal correction to Enthalpy= $\quad 1.306546$

Thermal correction to Gibbs Free Energy= $\quad 1.123316$

Sum of electronic and zero-point Energies $=\quad-3890.760316$

Sum of electronic and thermal Energies $=\quad-3890.688090$

Sum of electronic and thermal Enthalpies $=\quad-3890.687145$

Sum of electronic and thermal Free Energies $=\quad-3890.870376$

Cartesian Coordinates

$77 \quad-0.218836-0.826181 \quad-1.527954$ 
$[12-13]^{\ddagger} \mathbf{P 2}$ exo-re $\left[\mathrm{BF}_{4}^{-}\right]$ 
Number of imaginary frequencies $=1$

The smallest frequencies are $=-269.982413 .941720 .1960 \mathrm{~cm}(-1)$ Electronic energy $=-3892.0053835$

$\begin{array}{lc}\text { Zero-point correction }= & 1.233234 \\ \text { (Hartree/Particle) } & 1.305488 \\ \text { Thermal correction to Energy= } & 1.306432 \\ \text { Thermal correction to Enthalpy= } & 1.123336 \\ \text { Thermal correction to Gibbs Free Energy= } & -3890.772149 \\ \text { Sum of electronic and zero-point Energies }= & -3890.699895 \\ \text { Sum of electronic and thermal Energies }= & -3890.698951 \\ \text { Sum of electronic and thermal Enthalpies }= & -3890.882048 \\ \text { Sum of electronic and thermal Free Energies }= & -390\end{array}$

felectronic and thermal Free Energies $=\quad-3890.882048$ $\begin{array}{lll}2.235742 & 5.182311 & 2.039846\end{array}$

$\begin{array}{llll}1.207043 & 3.811071 & 3.351565\end{array}$

$1.883174 \quad 3.967356 \quad 4.187690$

$\begin{array}{llll}0.136158 & 2.921960 & 3.471489\end{array}$

$\begin{array}{lll}-0.019338 & 2.373761 & 4.395619\end{array}$

$\begin{array}{lll}-0.733816 & 2.717312 & 2.400136\end{array}$

$\begin{array}{lll}-1.555841 & 2.021507 & 2.506959\end{array}$

$\begin{array}{lll}-2.940988 & 3.417718 & 0.313606\end{array}$

$\begin{array}{llll}-3.553768 & 3.354788 & -0.588037\end{array}$

$\begin{array}{lll}-3.331096 & 2.715887 & 1.049447\end{array}$

$\begin{array}{lll}-3.043346 & 4.428541 & 0.720564\end{array}$

$\begin{array}{lll}0.826263 & -0.141132 & 1.140591\end{array}$

$1.115001 \quad-1.416749 \quad 0.610166$

$\begin{array}{llll}0.814914 & -2.272378 & 1.207314\end{array}$

$\begin{array}{lll}1.949597 & -1.567744 & -0.540960\end{array}$

$2.426239-0.656242-0.877761$

$\begin{array}{llll}-1.313638 & -1.590701 & -3.060713\end{array}$

$-1.510950-0.632866-3.534911$

$\begin{array}{llll}0.013634 & -2.091106 & -3.197436\end{array}$

$\begin{array}{llll}0.712201 & -1.478168 & -3.766196\end{array}$

$\begin{array}{llll}0.375620 & -3.564617 & -3.190859\end{array}$

$\begin{array}{llll}0.129990 & -4.003840 & -4.169888\end{array}$

$\begin{array}{llll}1.458962 & -3.640429 & -3.082757\end{array}$

$\begin{array}{llll}-0.300450 & -4.371819 & -2.064209\end{array}$

$\begin{array}{llll}-1.238090 & -4.812069 & -2.418037\end{array}$

$\begin{array}{llll}0.348389 & -5.209418 & -1.790914\end{array}$

$\begin{array}{llll}-0.581512 & -3.560220 & -0.807010\end{array}$

$\begin{array}{lll}0.046013 & -3.786724 & 0.045323\end{array}$

$\begin{array}{llll}-1.781755 & -2.925184 & -0.546988\end{array}$

$\begin{array}{llll}-2.020289 & -2.707760 & 0.488691\end{array}$

$\begin{array}{llll}-2.944524 & -2.886599 & -1.516630\end{array}$

$-3.680198-2.179824-1.134653$

$\begin{array}{llll}-3.430866 & -3.873094 & -1.518528\end{array}$

$-2.552835-2.484886-2.957339$

$\begin{array}{llll}-2.391757 & -3.378074 & -3.57083\end{array}$

$\begin{array}{llll}-3.396396 & -1.954150 & -3.407744\end{array}$

$\begin{array}{llll}2.736242 & -2.764038 & -0.889123\end{array}$

$2.686558 \quad-3.981230 \quad-0.186481$

$3.644718-2.653912-1.961555$

$\begin{array}{llll}3.489334 & -5.059069 & -0.563923\end{array}$

$\begin{array}{llll}2.041131 & -4.083603 & 0.679738\end{array}$

$\begin{array}{llll}4.448659 & -3.729036 & -2.334682\end{array}$

$3.724520 \quad-1.704265 \quad-2.482701$

$\begin{array}{llll}4.369902 & -4.942680 & -1.643019\end{array}$

$\begin{array}{llll}3.435016 & -5.989002 & -0.003932\end{array}$

$\begin{array}{llll}5.143954 & -3.617692 & -3.162474\end{array}$

$\begin{array}{llll}4.996692 & -5.781592 & -1.932051\end{array}$

$\begin{array}{llll}0.343626 & -0.480232 & 4.069378\end{array}$

$\begin{array}{lll}1.479828 & -0.048110 & 3.859399\end{array}$

$\begin{array}{llll}2.069962 & 0.119413 & 2.724496\end{array}$

$\begin{array}{llll}2.300750 & 0.322753 & 4.893977\end{array}$

$\begin{array}{llll}1.745665 & 0.160642 & 6.203860\end{array}$

$\begin{array}{lll}0.849309 & 0.774867 & 6.338019\end{array}$

$\begin{array}{lll}2.521969 & 0.485072 & 6.900647\end{array}$

$\begin{array}{lll}1.484603 & -0.884295 & 6.400191\end{array}$

$\begin{array}{lll}4.492098 & 2.713187 & 1.844292\end{array}$

$\begin{array}{llll}5.929911 & 3.204149 & 1.790315\end{array}$

$\begin{array}{lll}3.977373 & 2.882015 & 0.894110\end{array}$

$\begin{array}{lll}3.922622 & 3.205687 & 2.634763\end{array}$

$\begin{array}{llll}6.737015 & 2.285141 & 0.882658\end{array}$

$\begin{array}{lll}5.968348 & 4.224991 & 1.401015\end{array}$

$\begin{array}{lll}6.364336 & 3.208481 & 2.796315\end{array}$

$\begin{array}{lll}7.796534 & 2.549117 & 0.919182\end{array}$

$\begin{array}{llll}6.394139 & 2.364320 & -0.153970\end{array}$

$\begin{array}{lll}4.486633 & 1.278995 & 2.122420\end{array}$

$\begin{array}{llll}5.470711 & 0.440877 & 1.837435\end{array}$

$\begin{array}{lll}6.620380 & 0.871895 & 1.306254\end{array}$

$\begin{array}{lll}5.203446 & -1.008001 & 2.194371\end{array}$

$\begin{array}{llll}4.137087 & -1.075146 & 2.417005\end{array}$

$\begin{array}{llll}5.729648 & -1.234462 & 3.129787\end{array}$

$\begin{array}{llll}5.575897 & -2.020594 & 1.098321\end{array}$

$\begin{array}{llll}5.341110 & -1.581279 & 0.126305\end{array}$

$\begin{array}{lll}7.666210 & 0.004198 & 0.714095\end{array}$

$\begin{array}{lll}7.046784 & -2.451408 & 1.154333\end{array}$

$\begin{array}{llll}7.321556 & -2.899499 & 0.191870\end{array}$ 


$\begin{array}{lrrr}6 & 7.992007 & -1.284041 & 1.471705 \\ 1 & 9.018701 & -1.565311 & 1.212433 \\ 1 & 7.999772 & -1.066243 & 2.545912 \\ 1 & 8.562808 & 0.626994 & 0.684274 \\ 1 & 7.378121 & -0.205794 & -0.322011 \\ 1 & 7.181254 & -3.230981 & 1.914642 \\ 1 & 4.929392 & -2.896789 & 1.198709 \\ 1 & 3.599787 & 0.872262 & 2.465218 \\ 5 & 4.259057 & 1.229798 & -1.692465 \\ 9 & 5.216392 & 0.343814 & -1.160922 \\ 9 & 4.853201 & 2.471141 & -1.967010 \\ 9 & 3.716788 & 0.670848 & -2.865847 \\ 9 & 3.225028 & 1.427290 & -0.739575 \\ 1 & -0.006743 & -0.007798 & 1.811175 \\ 1 & 1.243045 & 0.736398 & 0.669312\end{array}$

$[12-13]^{\ddagger} \mathrm{P2}$ endo-si $\left[\mathrm{BF}_{4}^{-}\right]$

Number of imaginary frequencies $=1$

The smallest frequencies are $=-255.16722 .747217 .2882 \mathrm{~cm}(-1)$ Electronic energy $=-3892.0082688$

Zero-point correction=

(Hartree/Particle)

Thermal correction to Energy=

Thermal correction to Enthalpy=

Thermal correction to Gibbs Free Energy=
Sum of electronic and zero-point Energies=

Sum of electronic and thermal Energies=

Sum of electronic and thermal Enthalpies=

Sum of electronic and thermal Free Energies=

1.305191

1.306135

1.120481

$-3890.775575$

$-3890.703078$

$-3890.702134$

$-3890.887788$

Cartesian Coordinates

\begin{tabular}{llll}
77 & -0.419600 & -0.945471 & -1.678424 \\
15 & -1.853109 & 0.327691 & -0.412251 \\
8 & -2.343180 & 0.082196 & 1.163651 \\
8 & -3.373646 & 0.390573 & -1.143701 \\
7 & -1.209938 & 1.859478 & -0.239721 \\
6 & -3.238937 & -0.932406 & 1.462986 \\
6 & -2.726887 & -2.054525 & 2.152940 \\
1 & -1.677572 & -2.057194 & 2.424907 \\
6 & -3.557951 & -3.104041 & 2.456808 \\
1 & -3.177222 & -3.969693 & 2.991653 \\
6 & -4.918991 & -3.087527 & 2.048126 \\
6 & -5.769496 & -4.201598 & 2.284594 \\
1 & -5.361459 & -5.061969 & 2.808810 \\
6 & -7.075108 & -4.200908 & 1.848335 \\
1 & -7.714444 & -5.060003 & 2.029655 \\
6 & -7.580493 & -3.081157 & 1.145383 \\
1 & -8.603226 & -3.092678 & 0.779487 \\
6 & -6.786751 & -1.978550 & 0.914208 \\
1 & -7.188385 & -1.134869 & 0.365687 \\
6 & -5.439269 & -1.933620 & 1.370279 \\
6 & -4.581028 & -0.800293 & 1.138735 \\
6 & -5.085940 & 0.468253 & 0.544173 \\
6 & -6.170675 & 1.208842 & 1.135437 \\
6 & -6.745177 & 0.860985 & 2.390728 \\
1 & -6.353868 & 0.010034 & 2.935425 \\
6 & -7.777404 & 1.597160 & 2.931091 \\
1 & -8.191427 & 1.313816 & 3.894704 \\
6 & -8.298770 & 2.721811 & 2.248149 \\
1 & -9.117026 & 3.288369 & 2.683046 \\
6 & -7.752863 & 3.101123 & 1.042670 \\
1 & -8.128327 & 3.974223 & 0.515045 \\
6 & -6.676597 & 2.373931 & 0.465691 \\
6 & -6.078672 & 2.794677 & -0.753037 \\
1 & -6.478694 & 3.666242 & -1.263680 \\
6 & -4.988458 & 2.132765 & -1.258856 \\
1 & -4.493056 & 2.457284 & -2.167705 \\
6 & -4.481039 & 0.996477 & -0.587665 \\
6 & -0.066882 & 2.128842 & -1.156284 \\
1 & 0.852631 & 2.116360 & -0.562683 \\
6 & -0.200911 & 3.499706 & -1.804869 \\
1 & -1.437344 & 3.935823 & -2.305215 \\
\hline & -2.311245 & 3.305521 & -2.170835
\end{tabular}

$\begin{array}{llll}-1.549587 & 5.166615 & -2.951361\end{array}$

$\begin{array}{llll}-2.515579 & 5.493192 & -3.328077\end{array}$

$\begin{array}{lll}-0.422745 & 5.980085 & -3.111845\end{array}$

$\begin{array}{llll}-0.508652 & 6.939905 & -3.614022\end{array}$

$\begin{array}{llll}0.810932 & 5.552634 & -2.616588\end{array}$

$\begin{array}{lll}1.690398 & 6.181222 & -2.729740\end{array}$

$\begin{array}{llll}0.921880 & 4.321424 & -1.963056\end{array}$

$\begin{array}{llll}1.878055 & 4.001018 & -1.562787\end{array}$

$\begin{array}{llll}0.030007 & 1.053350 & -2.256778\end{array}$

$\begin{array}{lll}-0.671368 & 1.326155 & -3.054743\end{array}$

$\begin{array}{llll}1.030385 & 1.111935 & -2.688963\end{array}$

$\begin{array}{lll}-1.345457 & 2.821894 & 0.899632\end{array}$

$\begin{array}{lll}-0.884186 & 3.732953 & 0.509214\end{array}$

$\begin{array}{lll}-0.515870 & 2.420537 & 2.119032\end{array}$

$\begin{array}{lll}0.839222 & 2.777879 & 2.163170\end{array}$

$\begin{array}{lll}1.287537 & 3.318898 & 1.336664\end{array}$

$\begin{array}{lll}1.635298 & 2.456605 & 3.265301\end{array}$

$\begin{array}{lll}2.678175 & 2.753749 & 3.261929\end{array}$

$\begin{array}{lll}1.083234 & 1.770195 & 4.348223\end{array}$

$\begin{array}{lll}1.695757 & 1.523969 & 5.211691\end{array}$

$\begin{array}{lll}-0.268427 & 1.413273 & 4.320733\end{array}$

$\begin{array}{lll}-0.711235 & 0.888572 & 5.163597\end{array}$

$\begin{array}{lll}-1.060594 & 1.739555 & 3.218428\end{array}$

$\begin{array}{lll}-2.107632 & 1.461461 & 3.217639\end{array}$

$\begin{array}{lll}-2.791570 & 3.194359 & 1.223718\end{array}$

$\begin{array}{llll}-3.283516 & 3.589477 & 0.332756\end{array}$

$\begin{array}{lll}-3.376745 & 2.351489 & 1.590030\end{array}$

$\begin{array}{lll}-2.795088 & 3.972289 & 1.993853\end{array}$

$\begin{array}{lll}0.901324 & -1.770982 & 1.046882\end{array}$

$\begin{array}{lll}0.263962 & -2.626614 & 0.842369\end{array}$

$\begin{array}{lll}0.471926 & -1.176896 & 1.853784\end{array}$

$\begin{array}{lll}1.175531 & -0.910780 & -0.154508\end{array}$

$\begin{array}{llll}1.468306 & 0.098074 & 0.126839\end{array}$

$1.757192-1.447408-1.354617$

$\begin{array}{llll}1.854273 & -2.527429 & -1.400286\end{array}$

$\begin{array}{lll}-1.817916 & -1.063099 & -3.377810\end{array}$

$\begin{array}{llll}-2.155010 & -0.056419 & -3.614424\end{array}$

$-0.505496 \quad-1.406227 \quad-3.821306$

$\begin{array}{llll}0.043683 & -0.638756 & -4.367446\end{array}$

$\begin{array}{llll}-0.061367 & -2.816340 & -4.172380\end{array}$

$\begin{array}{lll}-0.448202 & -3.098344 & -5.164451\end{array}$

$\begin{array}{llll}1.028307 & -2.804570 & -4.265025\end{array}$

$\begin{array}{llll}-0.471298 & -3.882110 & -3.132144\end{array}$

$\begin{array}{llll}-1.427293 & -4.335473 & -3.412983\end{array}$

$\begin{array}{llll}0.262377 & -4.695373 & -3.144821\end{array}$

$\begin{array}{lll}-0.575865 & -3.358051 & -1.704695\end{array}$

$\begin{array}{llll}0.206573 & -3.681777 & -1.027164\end{array}$

$\begin{array}{llll}-1.743359 & -2.889066 & -1.134495\end{array}$

$\begin{array}{llll}-1.807124 & -2.897470 & -0.050850\end{array}$

$-3.067907 \quad-2.737717 \quad-1.855280$

$\begin{array}{lll}-3.721306 & -2.131776 & -1.225467\end{array}$

$\begin{array}{llll}-3.543302 & -3.726810 & -1.938775\end{array}$

$\begin{array}{llll}-2.960289 & -2.076316 & -3.247734\end{array}$

$\begin{array}{llll}-2.854260 & -2.841885 & -4.024393\end{array}$

$\begin{array}{llll}-3.904927 & -1.565060 & -3.457911\end{array}$

$\begin{array}{llll}2.791959 & -0.774246 & -2.172438\end{array}$

$\begin{array}{lll}3.220179 & 0.547831 & -1.954518\end{array}$

$3.402854-1.492107 \quad-3.220878$

$\begin{array}{llll}4.188944 & 1.136456 & -2.767501\end{array}$

$2.823961 \quad 1.117521 \quad-1.126812$

$\begin{array}{llll}4.370521 & -0.904990 & -4.036269\end{array}$

$\begin{array}{lll}3.112776 & -2.526441 & -3.390265\end{array}$

$\begin{array}{lll}4.765809 & 0.419642 & -3.818678\end{array}$

$\begin{array}{llll}4.512746 & 2.148612 & -2.544782\end{array}$

$\begin{array}{lll}4.821820 & -1.484419 & -4.837900\end{array}$

$\begin{array}{llll}5.523787 & 0.877763 & -4.448037\end{array}$

$\begin{array}{llll}1.935613 & -4.282494 & 0.492926\end{array}$

$\begin{array}{lll}2.523032 & -3.537765 & 1.251741\end{array}$

$\begin{array}{llll}2.149404 & -2.315854 & 1.645889\end{array}$

$\begin{array}{lll}3.689014 & -3.811840 & 1.858439\end{array}$

$\begin{array}{lll}4.307731 & -5.059181 & 1.479959\end{array}$

$\begin{array}{lll}3.658706 & -5.902070 & 1.729278\end{array}$

$\begin{array}{lll}5.231989 & -5.109533 & 2.055439\end{array}$

$\begin{array}{lll}4.526197 & -5.067554 & 0.408945\end{array}$

$\begin{array}{lll}4.270413 & -0.208789 & 3.977487\end{array}$ 
$\begin{array}{llll}5.544958 & 0.359104 & 4.579675\end{array}$

$\begin{array}{lll}3.564846 & 0.586440 & 3.721669\end{array}$

$3.774009 \quad-0.906197 \quad 4.657404$

$\begin{array}{lll}6.321204 & 1.102576 & 3.500039\end{array}$

$\begin{array}{lll}5.305322 & 1.048671 & 5.393266\end{array}$

$\begin{array}{llll}6.158855 & -0.450041 & 4.990086\end{array}$

$\begin{array}{lll}7.300747 & 1.402844 & 3.877159\end{array}$

$\begin{array}{lll}5.784533 & 1.998653 & 3.174522\end{array}$

$\begin{array}{llll}4.610587 & -0.932117 & 2.756897\end{array}$

$\begin{array}{lll}5.636546 & -0.653514 & 1.958677\end{array}$

$\begin{array}{lll}6.549763 & 0.249960 & 2.307534\end{array}$

$\begin{array}{llll}5.681224 & -1.462798 & 0.677179\end{array}$

$\begin{array}{lll}4.660267 & -1.804241 & 0.504227\end{array}$

$\begin{array}{llll}6.280585 & -2.363215 & 0.861511\end{array}$

$\begin{array}{llll}6.172683 & -0.715988 & -0.569216\end{array}$

$\begin{array}{llll}5.774887 & 0.299328 & -0.546491\end{array}$

$\begin{array}{lll}7.679515 & 0.707188 & 1.458063\end{array}$

$\begin{array}{llll}7.698570 & -0.691765 & -0.693383\end{array}$

$\begin{array}{llll}7.980019 & 0.063523 & -1.436781\end{array}$

$\begin{array}{lll}8.382519 & -0.382373 & 0.645755\end{array}$

$\begin{array}{llll}9.410834 & -0.049597 & 0.467530\end{array}$

$\begin{array}{lll}8.458798 & -1.284528 & 1.264649\end{array}$

$\begin{array}{lll}8.395975 & 1.140675 & 2.158089\end{array}$

$\begin{array}{llll}7.312879 & 1.516104 & 0.817624\end{array}$

$\begin{array}{llll}8.064356 & -1.656051 & -1.067997\end{array}$

$\begin{array}{llll}5.731128 & -1.193292 & -1.448376\end{array}$

$\begin{array}{lll}3.887864 & -1.530760 & 2.375749\end{array}$

$\begin{array}{lll}4.237095 & 2.576399 & 0.748688\end{array}$

$\begin{array}{llll}5.412397 & 2.540281 & -0.028078\end{array}$

$\begin{array}{lll}4.475113 & 3.316651 & 1.926521\end{array}$

$\begin{array}{llll}3.204162 & 3.188177 & 0.006140\end{array}$

$\begin{array}{lll}3.845180 & 1.268503 & 1.091348\end{array}$

$[12-13]^{\ddagger} \mathbf{P 1}$ endo-re $\left[\mathrm{BF}_{4}^{-}\right]$conf2

Number of imaginary frequencies $=1$

The smallest frequencies are $=-186.729021 .288823 .2630 \mathrm{~cm}(-1)$ Electronic energy $=-3891.974268$

Zero-point correction=

1.233459

(Hartree/Particle)

Thermal correction to Energy=

1.305441

Thermal correction to Enthalpy=

1.306385

Thermal correction to Gibbs Free Energy=

Sum of electronic and zero-point Energies=

Sum of electronic and thermal Energies=

1.125899

$-3890.740809$

$-3890.668827$

$-3890.667883$

Sum of electronic and thermal Enthalpies=

$-3890.848369$

Cartesian Coordinates

$\begin{array}{lccc}77 & -0.988681 & -0.952320 & -1.148636 \\ 15 & -1.415841 & 1.014355 & 0.057077 \\ 8 & -0.558353 & 1.613307 & 1.356140 \\ 8 & -1.334703 & 2.360433 & -0.947464 \\ 7 & -2.955665 & 0.927735 & 0.684750 \\ 6 & 0.790181 & 1.846682 & 1.114195 \\ 6 & 1.709934 & 0.941265 & 1.686349 \\ 1 & 1.322906 & 0.183277 & 2.354767 \\ 6 & 3.044088 & 1.054212 & 1.385745 \\ 1 & 3.759652 & 0.361517 & 1.814968 \\ 6 & 3.497024 & 2.048338 & 0.475619 \\ 6 & 4.857818 & 2.111236 & 0.070550 \\ 1 & 5.555272 & 1.392733 & 0.488804 \\ 6 & 5.283985 & 3.045578 & -0.847187 \\ 1 & 6.325324 & 3.076355 & -1.154550 \\ 6 & 4.356200 & 3.958193 & -1.403460 \\ 1 & 4.688507 & 4.678980 & -2.144936 \\ 6 & 3.033761 & 3.938774 & -1.014811 \\ 1 & 2.334467 & 4.640218 & -1.454778 \\ 6 & 2.561596 & 2.998307 & -0.056642 \\ 6 & 1.180963 & 2.935271 & 0.348436 \\ 6 & 0.195375 & 3.986917 & -0.020382 \\ 6 & 0.424943 & 5.373061 & 0.308061 \\ 6 & 1.526992 & 5.804277 & 1.099561 \\ 1 & 2.218219 & 5.069784 & 1.494518\end{array}$

$\begin{array}{lll}1.720742 \quad 7.138102 & 1.388738\end{array}$

$\begin{array}{lll}2.566733 & 7.435824 & 2.001849\end{array}$

$\begin{array}{llll}0.825968 & 8.121385 & 0.904898\end{array}$

$\begin{array}{lll}0.995000 & 9.169310 & 1.134933\end{array}$

$\begin{array}{lll}-0.266633 & 7.739000 & 0.159841\end{array}$

$\begin{array}{llll}-0.976851 & 8.478339 & -0.201609\end{array}$

$\begin{array}{llll}-0.502898 & 6.370851 & -0.143012\end{array}$

$\begin{array}{llll}-1.658380 & 5.971584 & -0.867449\end{array}$

$\begin{array}{llll}-2.350766 & 6.731515 & -1.218948\end{array}$

$\begin{array}{llll}-1.913257 & 4.643935 & -1.094783\end{array}$

$\begin{array}{lll}-2.804423 & 4.317966 & -1.619705\end{array}$

$\begin{array}{llll}-1.000123 & 3.658795 & -0.644801\end{array}$

$\begin{array}{lll}-3.777983 & -0.141818 & 0.075417\end{array}$

$\begin{array}{lll}-4.439020 & -0.498498 & 0.875069\end{array}$

$\begin{array}{llll}-4.701007 & 0.343305 & -1.041724\end{array}$

$\begin{array}{llll}-4.624425 & 1.623258 & -1.604531\end{array}$

$\begin{array}{llll}-3.870638 & 2.316767 & -1.251598\end{array}$

$\begin{array}{llll}5.488752 & 2.008330 & -2.633748\end{array}$

$\begin{array}{lll}-5.407499 & 3.006026 & -3.057406\end{array}$

$\begin{array}{llll}-6.448025 & 1.117288 & -3.117011\end{array}$

$\begin{array}{lll}-7.118871 & 1.413641 & -3.918529\end{array}$

$\begin{array}{llll}-6.544235 & -0.158816 & -2.552540\end{array}$

$\begin{array}{llll}-7.293379 & -0.858925 & -2.912553\end{array}$

$\begin{array}{llll}-5.684655 & -0.535977 & -1.520628\end{array}$

$\begin{array}{llll}-5.775168 & -1.525828 & -1.079792\end{array}$

$\begin{array}{llll}-2.892001 & -1.348419 & -0.294858\end{array}$

$\begin{array}{lll}-3.448587 & -2.009814 & -0.961475\end{array}$

$\begin{array}{llll}-2.709765 & -1.878522 & 0.638717\end{array}$

$\begin{array}{lll}-3.721545 & 1.843098 & 1.580179\end{array}$

$\begin{array}{lll}-4.563495 & 2.219153 & 0.986838\end{array}$

$\begin{array}{lll}-4.297858 & 1.055502 & 2.750770\end{array}$

$\begin{array}{lll}-5.658307 & 1.153304 & 3.064192\end{array}$

$\begin{array}{lll}-6.308688 & 1.767443 & 2.444981\end{array}$

$\begin{array}{lll}-6.184735 & 0.462839 & 4.159531\end{array}$

$\begin{array}{llll}-7.243375 & 0.544266 & 4.392057\end{array}$

$\begin{array}{lll}-5.352423 & -0.339795 & 4.943408\end{array}$

$\begin{array}{lll}-5.761226 & -0.885865 & 5.789534\end{array}$

$\begin{array}{llll}-3.993959 & -0.447046 & 4.629594\end{array}$

$\begin{array}{lll}-3.334491 & -1.079292 & 5.214062\end{array}$

$\begin{array}{lll}-3.468556 & 0.251315 & 3.541653\end{array}$

$\begin{array}{lll}-2.417662 & 0.154051 & 3.292738\end{array}$

$\begin{array}{lll}-2.959741 & 3.064002 & 2.105221\end{array}$

$\begin{array}{lll}-2.573237 & 3.690092 & 1.300374\end{array}$

$\begin{array}{lll}-2.135098 & 2.777230 & 2.758227\end{array}$

$\begin{array}{lll}-3.664901 & 3.666013 & 2.686278\end{array}$

$\begin{array}{llll}0.991199 & -2.098481 & 0.558391\end{array}$

$\begin{array}{llll}1.722319 & -1.827313 & -0.180345\end{array}$

$\begin{array}{lll}1.086356 & -1.656730 & 1.533418\end{array}$

$\begin{array}{lll}-0.251985 & -2.642823 & 0.213463\end{array}$

$\begin{array}{lll}-0.919291 & -2.815201 & 1.045686\end{array}$

$\begin{array}{llll}-0.593858 & -3.182743 & -1.074462\end{array}$

$\begin{array}{llll}-1.785700 & 0.143742 & -2.916547\end{array}$

$\begin{array}{llll}-2.756187 & 0.550380 & -2.663711\end{array}$

$\begin{array}{llll}-1.762325 & -1.248020 & -3.189629\end{array}$

$-2.710210-1.778425-3.100351$

$\begin{array}{lll}-0.804282 & -1.908220 & -4.164174\end{array}$

$-1.162413-1.724081-5.188371$

$\begin{array}{llll}-0.865621 & -2.985415 & -4.014139\end{array}$

$\begin{array}{lll}0.672965 & -1.465971 & -4.049607\end{array}$

$\begin{array}{llll}0.865524 & -0.622047 & -4.719176\end{array}$

$1311688-2.283760-4.399315$

$\begin{array}{llll}1.103180 & -1.088665 & -2.646109\end{array}$

$\begin{array}{lll}1.674800 & -1.832036 & -2.104117\end{array}$

$\begin{array}{llll}1.063156 & 0.188688 & -2.157081\end{array}$

$\begin{array}{llll}1.617095 & 0.427425 & -1.252241\end{array}$

$\begin{array}{llll}0.555515 & 1.354427 & -2.977814\end{array}$

$\begin{array}{llll}0.562058 & 2.244528 & -2.352221\end{array}$

$\begin{array}{llll}1.278301 & 1.541011 & -3.785219\end{array}$

$\begin{array}{llll}-0.858807 & 1.161820 & -3.59195\end{array}$

$\begin{array}{llll}-0.769185 & 0.880771 & -4.648148\end{array}$

$\begin{array}{lll}-1.363126 & 2.130993 & -3.575000\end{array}$

$\begin{array}{llll}-1.673051 & -4.195422 & -1.221156\end{array}$

$\begin{array}{llll}-2.592529 & -4.510066 & -0.198958\end{array}$

$\begin{array}{lll}-1.767959 & -4.924270 & -2.422390\end{array}$

$-3.589134-5.465008-0.398860$ 


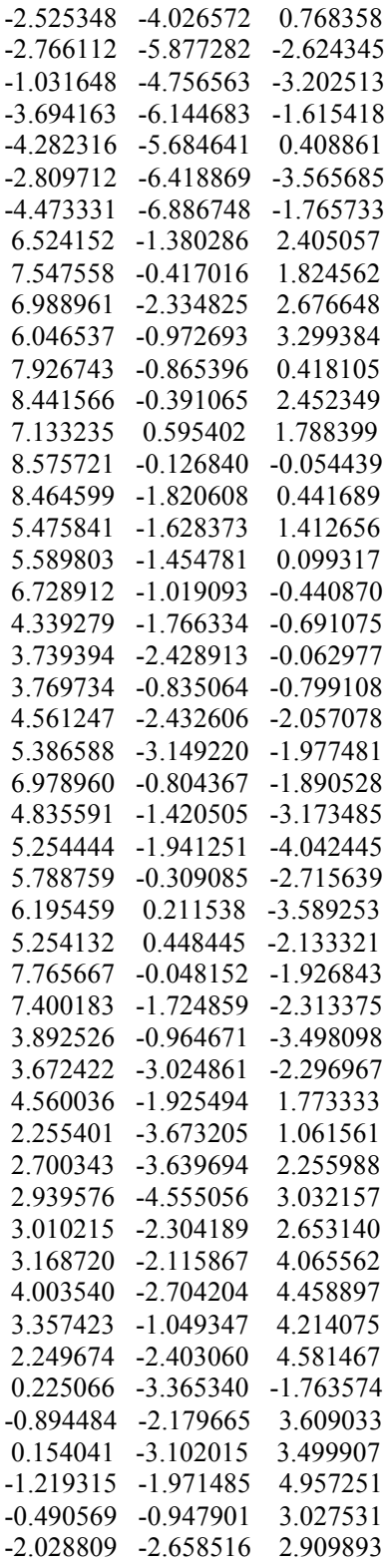

\section{$[12-13]^{\ddagger} \mathbf{P} 1$ exo-si $\left[\mathrm{BF}_{4}^{-}\right]$conf2}

Number of imaginary frequencies $=$

The smallest frequencies are $=-276.438417 .135820 .9824 \mathrm{~cm}(-1)$ Electronic energy $=-3891.9790924$

Zero-point correction=

1.234326

(Hartree/Particle)

Thermal correction to Energy= $\quad 1306301$

Thermal correction to Entha

1.307245

Thermal correction to Gibbs Free Energy=

Sum of electronic and zero-point Energies=

Sum of electronic and thermal Energies=

Sum of electronic and thermal Enthalpies=

Sum of electronic and thermal Free Energies=

1.125898

$-3890.744766$

$-3890.672792$

$-3890.671848$

Cartesian Coordinates

$\begin{array}{lrrr}77 & 1.663330 & -1.477615 & 0.248704 \\ 15 & -0.377807 & -0.520652 & 0.745886 \\ 8 & -1.545644 & -0.357906 & -0.455652 \\ 8 & -1.340781 & -1.300088 & 1.889970 \\ 7 & -0.139436 & 1.071939 & 1.211104 \\ 6 & -2.168594 & -1.521306 & -0.900078 \\ 6 & -1.767950 & -2.062409 & -2.143938 \\ 1 & -1.002209 & -1.562557 & -2.721774\end{array}$

$-2.357106-3.214513-2.605156$

$\begin{array}{lll}-2.064236 & -3.630994 & -3.564728\end{array}$

$\begin{array}{lll}-3.330898 & -3.893309 & -1.824539\end{array}$

$\begin{array}{lll}-3.896059 & -5.122678 & -2.260272\end{array}$

$-3.584176 \quad-5.525038 \quad-3.220534$

$-4.806680-5.797438-1.479322$

$-5.228534-6.738849-1.818808$

$\begin{array}{llll}-5.183222 & -5.269767 & -0.220679\end{array}$

$\begin{array}{lll}-5.884558 & -5.816595 & 0.403000\end{array}$

$\begin{array}{lll}-4.665564 & -4.072705 & 0.224177\end{array}$

$\begin{array}{llll}-4.959687 & -3.687097 & 1.193361\end{array}$

$\begin{array}{lll}-3.737742 & -3.337318 & -0.565333\end{array}$

$\begin{array}{llll}-3.175041 & -2.085924 & -0.133736\end{array}$

$\begin{array}{lll}-3.619563 & -1.391496 & 1.103132\end{array}$

$\begin{array}{lll}-4.987881 & -0.985819 & 1.293749\end{array}$

$\begin{array}{llll}-5.978034 & -1.128401 & 0.280608\end{array}$

$-5.705187-1.563520-0.673196$

$\begin{array}{lll}-7.274726 & -0.709545 & 0.488954\end{array}$

$\begin{array}{lll}-8.009301 & -0.824359 & -0.303134\end{array}$

$\begin{array}{lll}-7.658049 & -0.129277 & 1.721704\end{array}$

$\begin{array}{lll}-8.685321 & 0.188332 & 1.874443\end{array}$

$\begin{array}{lll}-6.721580 & 0.042639 & 2.716175\end{array}$

$\begin{array}{lll}-6.996444 & 0.503140 & 3.661582\end{array}$

$\begin{array}{lll}-5.372029 & -0.361197 & 2.526899\end{array}$

$\begin{array}{lll}-4.388724 & -0.133030 & 3.527243\end{array}$

$\begin{array}{lll}-4.687798 & 0.321893 & 4.467355\end{array}$

$\begin{array}{lll}-3.072708 & -0.449832 & 3.301041\end{array}$

$\begin{array}{lll}-2.309621 & -0.245806 & 4.043173\end{array}$

$\begin{array}{lll}-2.692690 & -1.042597 & 2.074644\end{array}$

$\begin{array}{lll}1.254678 & 1.314269 & 1.685894\end{array}$

$\begin{array}{lll}1.499232 & 2.336688 & 1.401901\end{array}$

$\begin{array}{lll}1.429568 & 1.252078 & 3.197105\end{array}$

$\begin{array}{lll}0.629349 & 0.481691 & 4.047038\end{array}$

$\begin{array}{lll}-0.171948 & -0.116321 & 3.632707\end{array}$

$\begin{array}{llll}0.865380 & 0.457044 & 5.425648\end{array}$

$\begin{array}{lll}0.231945 & -0.149114 & 6.068419\end{array}$

$\begin{array}{lll}1.909742 & 1.204142 & 5.972217\end{array}$

$\begin{array}{lll}2.093670 & 1.188197 & 7.042970\end{array}$

$\begin{array}{lll}2.718549 & 1.974493 & 5.129106\end{array}$

$\begin{array}{lll}3.535006 & 2.559853 & 5.543629\end{array}$

$\begin{array}{lll}2.477457 & 2.001394 & 3.756118\end{array}$

$\begin{array}{lll}3.104742 & 2.597120 & 3.097753\end{array}$

$\begin{array}{lll}2.272353 & 0.446893 & 0.914783\end{array}$

$\begin{array}{llll}3.157652 & 0.337657 & 1.539686\end{array}$

$\begin{array}{llll}2.589185 & 1.034969 & 0.059404\end{array}$

$\begin{array}{lll}-1.185600 & 2.026340 & 1.710812\end{array}$

$\begin{array}{lll}-1.510005 & 1.691920 & 2.701866\end{array}$

$\begin{array}{lll}-0.605965 & 3.425207 & 1.874304\end{array}$

$\begin{array}{lll}-0.656837 & 4.057413 & 3.119593\end{array}$

$\begin{array}{lll}-1.073765 & 3.526213 & 3.971138\end{array}$

$\begin{array}{lll}-0.162372 & 5.354982 & 3.281564\end{array}$

$\begin{array}{lll}-0.206766 & 5.831066 & 4.257104\end{array}$

$\begin{array}{lll}0.403653 & 6.027254 & 2.198037\end{array}$

$\begin{array}{lll}0.803280 & 7.029499 & 2.323641\end{array}$

$\begin{array}{llll}0.467608 & 5.397744 & 0.950438\end{array}$

$\begin{array}{lll}0.928492 & 5.908020 & 0.109670\end{array}$

$\begin{array}{llll}-0.042509 & 4.110137 & 0.786898\end{array}$

$\begin{array}{llll}0.032183 & 3.613248 & -0.175997\end{array}$

$\begin{array}{lll}-2.421492 & 2.118459 & 0.804499\end{array}$

$\begin{array}{llll}-2.971364 & 1.182409 & 0.742744\end{array}$

$\begin{array}{llll}-2.132524 & 2.417564 & -0.203759\end{array}$

$\begin{array}{lll}-3.092289 & 2.880115 & 1.212988\end{array}$

$\begin{array}{llll}1.149446 & 0.065674 & -1.983420\end{array}$

$\begin{array}{llll}1.407647 & 0.960458 & -1.442988\end{array}$

$\begin{array}{llll}0.116919 & -0.048537 & -2.253545\end{array}$

$\begin{array}{llll}1.983991 & -1.072679 & -1.974848\end{array}$

$\begin{array}{llll}1.668417 & -1.918209 & -2.580733\end{array}$

$3.273427-1.065875-1.361001$

$\begin{array}{lll}1.975357 & -2.265943 & 2.286751\end{array}$

$\begin{array}{llll}1.896041 & -1.438253 & 2.984025\end{array}$

$\begin{array}{lll}3.205695 & -2.358081 & 1.580237\end{array}$

$\begin{array}{llll}3.943272 & -1.583251 & 1.780653\end{array}$

$3.816162 \quad-3.660132 \quad 1.097886$

$\begin{array}{llll}4.226327 & -4.200184 & 1.964902\end{array}$

$\begin{array}{llll}4.666725 & -3.419223 & 0.460833\end{array}$ 


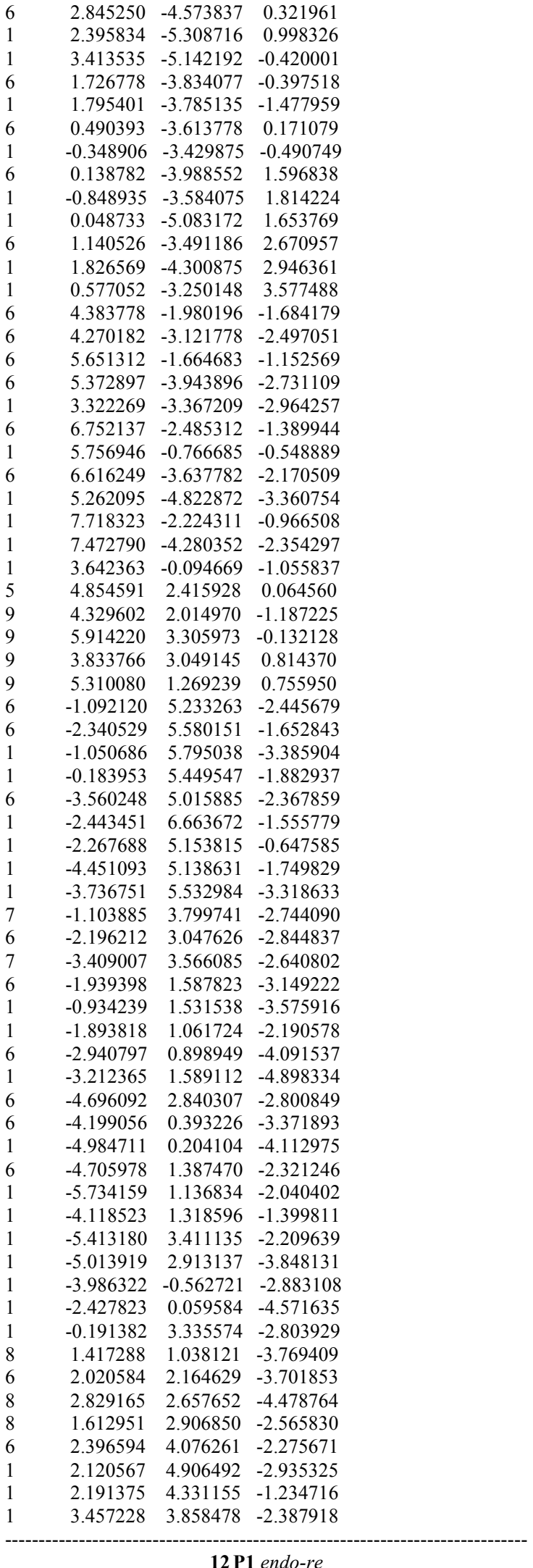

\section{P1 endo-re}

Number of imaginary frequencies $=0$

The smallest frequencies are $=16.221118 .565021 .2891 \mathrm{~cm}(-1)$ Electronic energy $=-3467.4000971$

Zero-point correction $=$

1.219937

(Hartree/Particle)

Thermal correction to Energy=
Thermal correction to Enthalpy=

1.286673

Thermal correction to Gibbs Free Energy $=\quad 1.118570$

Sum of electronic and zero-point Energies $=\quad-3466.180160$

Sum of electronic and thermal Energies=

Sum of electronic and thermal Enthalpies=

Sum of electronic and thermal Free Energies=

$-3466.114368$

$-3466.113424$

$-3466.281527$ 
$\begin{array}{llll}2.834650 & 2.803076 & 1.594531\end{array}$

$\begin{array}{llll}3.527669 & 2.998651 & 0.774954\end{array}$

$\begin{array}{lll}3.135023 & 1.885762 & 2.097736\end{array}$

$\begin{array}{llll}2.916147 & 3.626870 & 2.310815\end{array}$

$\begin{array}{lll}-0.964924 & -1.702983 & 1.473936\end{array}$

$\begin{array}{lll}-0.491988 & -2.628466 & 1.157113\end{array}$

$\begin{array}{lll}-0.329423 & -1.198695 & 2.201444\end{array}$

$\begin{array}{lll}-1.321360 & -0.775027 & 0.345929\end{array}$

$\begin{array}{llll}-1.458010 & 0.242351 & 0.705796\end{array}$

$\begin{array}{llll}-2.155884 & -1.193185 & -0.750791\end{array}$

$\begin{array}{llll}-2.369275 & -2.254952 & -0.828870\end{array}$

$\begin{array}{llll}1.097376 & -1.073366 & -3.309855\end{array}$

$1.464798-0.087289-3.584095$

$\begin{array}{llll}-0.285382 & -1.318574 & -3.553869\end{array}$

$\begin{array}{llll}-0.852367 & -0.507245 & -4.011984\end{array}$

$\begin{array}{lll}-0.877461 & -2.691854 & -3.823696\end{array}$

$\begin{array}{llll}-0.673055 & -2.994335 & -4.862605\end{array}$

$\begin{array}{lll}-1.963864 & -2.602325 & -3.741067\end{array}$

$\begin{array}{llll}-0.387055 & -3.787288 & -2.853461\end{array}$

$\begin{array}{llll}0.487071 & -4.297772 & -3.268572\end{array}$

$\begin{array}{lll}-1.163953 & -4.552976 & -2.754973\end{array}$

$-0.042543 \quad-3.276206-1.458651$

$\begin{array}{llll}-0.737187 & -3.559827 & -0.674149\end{array}$

$\begin{array}{llll}1.227248 & -2.891524 & -1.063898\end{array}$

$\begin{array}{lll}1.450862 & -2.926163 & -0.002020\end{array}$

$2.442859-2.815836-1.966410$

$\begin{array}{llll}3.204063 & -2.231202 & -1.446549\end{array}$

$\begin{array}{lll}2.856805 & -3.827063 & -2.096040\end{array}$

$\begin{array}{llll}2.171413 & -2.165533 & -3.340577\end{array}$

$\begin{array}{llll}1.890306 & -2.928767 & -4.074192\end{array}$

$\begin{array}{llll}3.105814 & -1.727662 & -3.705247\end{array}$

$\begin{array}{llll}-3.229854 & -0.368885 & -1.350897\end{array}$

$\begin{array}{llll}-3.563889 & 0.925729 & -0.908633\end{array}$

$\begin{array}{llll}-3.965366 & -0.890990 & -2.434324\end{array}$

$\begin{array}{llll}-4.549281 & 1.678187 & -1.551009\end{array}$

$\begin{array}{lll}-3.041774 & 1.355414 & -0.061567\end{array}$

$\begin{array}{llll}-4.947415 & -0.141495 & -3.079138\end{array}$

$\begin{array}{llll}-3.748676 & -1.899192 & -2.776346\end{array}$

$\begin{array}{llll}-5.242129 & 1.156658 & -2.646308\end{array}$

$\begin{array}{llll}-4.775559 & 2.679160 & -1.193439\end{array}$

$\begin{array}{lll}-5.488607 & -0.572239 & -3.917286\end{array}$

$\begin{array}{llll}-6.003739 & 1.745996 & -3.148501\end{array}$

$\begin{array}{lll}-2.368462 & -4.056260 & 1.126560\end{array}$

$\begin{array}{llll}-2.773917 & -3.202118 & 1.887472\end{array}$

$\begin{array}{lll}-2.171193 & -2.070524 & 2.247806\end{array}$

$\begin{array}{lll}-3.959825 & -3.248402 & 2.533899\end{array}$

$\begin{array}{lll}-4.753909 & -4.433837 & 2.290910\end{array}$

$\begin{array}{llll}-4.221869 & -5.320298 & 2.642813\end{array}$

$\begin{array}{llll}-5.670581 & -4.289694 & 2.862278\end{array}$

$\begin{array}{lll}-4.976629 & -4.537056 & 1.226453\end{array}$

$\begin{array}{llll}-3.992805 & 0.643847 & 2.887108\end{array}$

$\begin{array}{llll}-4.886883 & 1.833230 & 3.196447\end{array}$

$\begin{array}{lll}-3.158232 & 0.931918 & 2.240253\end{array}$

$\begin{array}{llll}-3.572910 & 0.197337 & 3.789737\end{array}$

$\begin{array}{llll}-5.649722 & 2.228769 & 1.939337\end{array}$

$\begin{array}{lll}-4.287234 & 2.679023 & 3.536697\end{array}$

$\begin{array}{llll}-5.593889 & 1.572761 & 3.991023\end{array}$

$\begin{array}{lll}-6.380719 & 3.005920 & 2.168147\end{array}$

$\begin{array}{lll}-4.969397 & 2.617598 & 1.173929\end{array}$

$\begin{array}{lll}-4.798694 & -0.375357 & 2.214644\end{array}$

$\begin{array}{lll}-5.916535 & -0.154899 & 1.528223\end{array}$

$\begin{array}{lll}-6.390840 & 1.078810 & 1.369617\end{array}$

$\begin{array}{lll}-6.581626 & -1.405387 & 0.986869\end{array}$

$\begin{array}{lll}-5.800938 & -2.167416 & 0.949071\end{array}$

$\begin{array}{lll}-7.311842 & -1.744607 & 1.732212\end{array}$

$\begin{array}{lll}-7.234229 & -1.288529 & -0.397714\end{array}$

$\begin{array}{llll}-6.590132 & -0.685739 & -1.043410\end{array}$

$\begin{array}{lll}-7.555372 & 1.447240 & 0.523548\end{array}$

$\begin{array}{lll}-8.649212 & -0.701026 & -0.350174\end{array}$

$\begin{array}{lll}-8.938665 & -0.393206 & -1.361703\end{array}$

$\begin{array}{lll}-8.751667 & 0.496195 & 0.604900\end{array}$

$\begin{array}{lll}-9.652586 & 1.074317 & 0.374470\end{array}$

$\begin{array}{lll}-8.860858 & 0.161669 & 1.643026\end{array}$

$\begin{array}{lll}-7.858324 & 2.433604 & 0.876277\end{array}$

$\begin{array}{lll}-7.210046 & 1.564345 & -0.509804\end{array}$ $\begin{array}{lll}-9.364518 & -1.470204 & -0.035932\end{array}$

$\begin{array}{lll}-7.261152 & -2.287609 & -0.843990\end{array}$

$\begin{array}{lll}-4.514281 & -1.341990 & 2.330775\end{array}$

$[12-13]^{\ddagger} \mathbf{P 1}$ endo-re

Number of imaginary frequencies $=1$

The smallest frequencies are $=-284.68919 .571318 .2824 \mathrm{~cm}(-1)$ Electronic energy $=-3467.3771617$

Zero-point correction $=$

1.217131

(Hartree/Particle)

Thermal correction to Energy= $\quad 1.283460$

Thermal correction to Enthalpy= $\quad 1.284404$

Thermal correction to Gibbs Free Energy $=\quad 1.113738$

Sum of electronic and zero-point Energies $=\quad-3466.160031$

Sum of electronic and thermal Energies $=\quad-3466.093702$

Sum of electronic and thermal Enthalpies $=\quad-3466.092758$

Sum of electronic and thermal Free Energies $=\quad-3466.263424$

Cartesian Coordinates

$15 \quad 1.654786 \quad 0.299371-0.313796$

$\begin{array}{lll}2.353620 & -0.055739 & 1.148765\end{array}$

$\begin{array}{lll}3.010407 & 0.274415 & -1.288046\end{array}$

$\begin{array}{llll}1.155017 & 1.868291 & -0.016817\end{array}$

$\begin{array}{lll}3.277293 & -1.090399 & 1.264369\end{array}$

$2.856176-2.234179 \quad 1.976345$

$1.857401-2.242556 \quad 2.400262$

$\begin{array}{lll}3.711059 & -3.300140 & 2.109676\end{array}$

$\begin{array}{lll}3.403409 & -4.185472 & 2.658737\end{array}$

$\begin{array}{lll}4.996947 & -3.273047 & 1.505386\end{array}$

$\begin{array}{lll}5.859725 & -4.401039 & 1.569945\end{array}$

$\begin{array}{lll}5.524248 & -5.279006 & 2.115762\end{array}$

$\begin{array}{lll}7.085744 & -4.390432 & 0.944369\end{array}$

$\begin{array}{lll}7.734730 & -5.259800 & 0.994687\end{array}$

$\begin{array}{llll}7.494954 & -3.246549 & 0.218063\end{array}$

$\begin{array}{llll}8.452031 & -3.249961 & -0.295577\end{array}$

$\begin{array}{llll}6.689656 & -2.130437 & 0.149865\end{array}$

$\begin{array}{llll}7.016709 & -1.268254 & -0.418971\end{array}$

$\begin{array}{llll}5.425029 & -2.096545 & 0.801638\end{array}$

$\begin{array}{llll}4.553805 & -0.951396 & 0.741445\end{array}$

$\begin{array}{llll}4.968587 & 0.331221 & 0.106187\end{array}$

$\begin{array}{llll}6.130434 & 1.064201 & 0.536200\end{array}$

$\begin{array}{lll}6.900099 & 0.685879 & 1.672074\end{array}$

$6.607224-0.185686 \quad 2.245325$

$\begin{array}{lll}8.001209 & 1.417386 & 2.061241\end{array}$

$\begin{array}{lll}8.567112 & 1.111106 & 2.936407\end{array}$

$\begin{array}{llll}8.398573 & 2.566987 & 1.337379\end{array}$

$\begin{array}{llll}9.272668 & 3.129437 & 1.652180\end{array}$

$\begin{array}{lll}7.665434 & 2.974531 & 0.246185\end{array}$

$\begin{array}{llll}7.947502 & 3.865588 & -0.308502\end{array}$

$\begin{array}{llll}6.515285 & 2.252158 & -0.173293\end{array}$

$\begin{array}{llll}5.730662 & 2.700301 & -1.270451\end{array}$

$\begin{array}{llll}6.039398 & 3.589565 & -1.812620\end{array}$

$\begin{array}{llll}4.579963 & 2.038037 & -1.620340\end{array}$

$\begin{array}{llll}3.947206 & 2.376459 & -2.433575\end{array}$

$\begin{array}{llll}4.199761 & 0.880249 & -0.907595\end{array}$

$\begin{array}{lll}-0.120481 & 2.214871 & -0.703926\end{array}$

$\begin{array}{lll}-0.916896 & 2.200184 & 0.049953\end{array}$

$\begin{array}{llll}-0.080660 & 3.611310 & -1.302987\end{array}$

$\begin{array}{llll}1.026224 & 4.048950 & -2.043942\end{array}$

$\begin{array}{llll}1.889814 & 3.397271 & -2.138511\end{array}$

$\begin{array}{llll}1.031112 & 5.314659 & -2.629678\end{array}$

$\begin{array}{lll}1.898528 & 5.644419 & -3.195069\end{array}$

$\begin{array}{llll}-0.074841 & 6.159503 & -2.487893\end{array}$

$\begin{array}{llll}-0.070079 & 7.145933 & -2.942813\end{array}$

$\begin{array}{llll}-1.181531 & 5.731071 & -1.751909\end{array}$

$-2.041757 \quad 6.383838-1.631045$

$\begin{array}{llll}-1.180527 & 4.465027 & -1.159729\end{array}$

$\begin{array}{llll}-2.038630 & 4.138746 & -0.576375\end{array}$

$\begin{array}{llll}-0.450284 & 1.191667 & -1.802743\end{array}$

$\begin{array}{llll}0.158460 & 1.425697 & -2.683874\end{array}$

$\begin{array}{lll}-1.491402 & 1.326993 & -2.097518\end{array}$

$\begin{array}{lll}1.499748 & 2.809569 & 1.097966\end{array}$ 
$\begin{array}{lll}1.335130 & 3.797202 & 0.658936\end{array}$ $\begin{array}{lll}0.512124 & 2.677738 & 2.255443\end{array}$ $\begin{array}{lll}-0.450956 & 3.676102 & 2.452802\end{array}$ $\begin{array}{lll}-0.483038 & 4.525034 & 1.774321\end{array}$ $\begin{array}{lll}-1.360461 & 3.597731 & 3.511727\end{array}$ $\begin{array}{lll}-2.090405 & 4.389775 & 3.655783\end{array}$ $\begin{array}{lll}-1.326726 & 2.506707 & 4.382652\end{array}$ $\begin{array}{lll}-2.032625 & 2.440849 & 5.205618\end{array}$ $\begin{array}{llll}-0.375563 & 1.499918 & 4.189547\end{array}$ $\begin{array}{llll}-0.342374 & 0.647264 & 4.862217\end{array}$ $\begin{array}{lll}0.539954 & 1.588031 & 3.139535\end{array}$ $\begin{array}{llll}1.282028 & 0.809597 & 3.008279\end{array}$ $\begin{array}{lll}2.957990 & 2.808329 & 1.561836\end{array}$ $\begin{array}{llll}3.631698 & 2.961945 & 0.717363\end{array}$ $\begin{array}{llll}3.245555 & 1.896062 & 2.081424\end{array}$ $\begin{array}{lll}3.085722 & 3.647355 & 2.253047\end{array}$ $\begin{array}{ccc}-0.941432 & -1.308808 & 1.263265\end{array}$ $\begin{array}{lll}-0.692001 & -2.346221 & 1.120099\end{array}$ $\begin{array}{llll}-0.382235 & -0.754047 & 2.004741\end{array}$ $\begin{array}{lll}-1.673278 & -0.588937 & 0.298082\end{array}$ $\begin{array}{lll}-1.874721 & 0.450926 & 0.529651\end{array}$ $\begin{array}{llll}-2.315178 & -1.197052 & -0.833010\end{array}$ $\begin{array}{llll}-2.435135 & -2.274055 & -0.791494\end{array}$ $1.040843 \quad-1.057630 \quad-3.172155$ $\begin{array}{llll}1.422360 & -0.073879 & -3.431139\end{array}$ $\begin{array}{llll}-0.338914 & -1.284003 & -3.429373\end{array}$ $\begin{array}{llll}-0.896792 & -0.460331 & -3.874609\end{array}$ $\begin{array}{llll}-0.946020 & -2.642993 & -3.720237\end{array}$ $\begin{array}{llll}-0.734326 & -2.914647 & -4.765471\end{array}$ $\begin{array}{llll}-2.029502 & -2.538389 & -3.648936\end{array}$ $\begin{array}{lll}-0.480909 & -3.782319 & -2.785086\end{array}$ $\begin{array}{llll}0.380079 & -4.298232 & -3.220284\end{array}$ $\begin{array}{lll}-1.278749 & -4.528772 & -2.716947\end{array}$ $\begin{array}{llll}-0.120513 & -3.342727 & -1.374554\end{array}$ $\begin{array}{llll}-0.830245 & -3.598416 & -0.591740\end{array}$ $\begin{array}{llll}1.145827 & -2.984705 & -0.976445\end{array}$ $\begin{array}{lll}1.353099 & -2.996266 & 0.089269\end{array}$ $\begin{array}{llll}2.360553 & -2.865506 & -1.869636\end{array}$ $\begin{array}{llll}3.119769 & -2.304986 & -1.322099\end{array}$ $\begin{array}{llll}2.776189 & -3.869033 & -2.041021\end{array}$ $\begin{array}{llll}2.100344 & -2.161970 & -3.218300\end{array}$ $\begin{array}{llll}1.809957 & -2.894310 & -3.978733\end{array}$ $\begin{array}{llll}3.041353 & -1.722551 & -3.561315\end{array}$ $\begin{array}{llll}-3.387306 & -0.529516 & -1.601868\end{array}$ $\begin{array}{llll}-3.745879 & 0.823016 & -1.433387\end{array}$ $\begin{array}{llll}-4.110649 & -1.278397 & -2.551627\end{array}$ $\begin{array}{llll}-4.741272 & 1.409003 & -2.216839\end{array}$ $\begin{array}{llll}-3.246944 & 1.427811 & -0.684663\end{array}$

$\begin{array}{lll}-5.104114 & -0.694653 & -3.336457\end{array}$ $\begin{array}{llll}-3.893691 & -2.336872 & -2.662478\end{array}$ $\begin{array}{llll}-5.420145 & 0.658594 & -3.181170\end{array}$ $\begin{array}{llll}-4.984888 & 2.458087 & -2.072343\end{array}$ $\begin{array}{llll}-5.639596 & -1.299786 & -4.062693\end{array}$ $\begin{array}{lll}-6.192014 & 1.117645 & -3.791745\end{array}$ $\begin{array}{lll}-2.390362 & -3.858158 & 1.440567\end{array}$ $\begin{array}{lll}-2.838274 & -3.016869 & 2.225827\end{array}$ $\begin{array}{llll}-2.323333 & -1.932149 & 2.659650\end{array}$ $\begin{array}{lll}-4.119881 & -3.190738 & 2.759438\end{array}$ $\begin{array}{lll}-4.796519 & -4.395898 & 2.375261\end{array}$ $\begin{array}{llll}-4.237273 & -5.276894 & 2.703277\end{array}$ $\begin{array}{lll}-5.768545 & -4.368508 & 2.872651\end{array}$

$\begin{array}{llll}-4.931713 & -4.450974 & 1.290601\end{array}$

$\begin{array}{lll}-3.987614 & 0.684722 & 3.103566\end{array}$

$\begin{array}{lll}-4.733033 & 2.008584 & 3.134350\end{array}$

$\begin{array}{lll}-3.052611 & 0.760935 & 2.541887\end{array}$

$\begin{array}{llll}-3.735606 & 0.330696 & 4.104997\end{array}$

$\begin{array}{lll}-5.336572 & 2.274942 & 1.761479\end{array}$

$\begin{array}{lll}-4.048924 & 2.817428 & 3.395609\end{array}$

$\begin{array}{lll}-5.530417 & 1.979438 & 3.884524\end{array}$

$\begin{array}{llll}-5.941146 & 3.182593 & 1.774807\end{array}$

$\begin{array}{lll}-4.551139 & 2.403795 & 1.006895\end{array}$

$\begin{array}{lll}-4.839104 & -0.324805 & 2.473327\end{array}$

$\begin{array}{lll}-5.875490 & -0.088118 & 1.678025\end{array}$

$\begin{array}{lll}-6.213573 & 1.158295 & 1.341390\end{array}$

$\begin{array}{lll}-6.601958 & -1.323058 & 1.187781\end{array}$

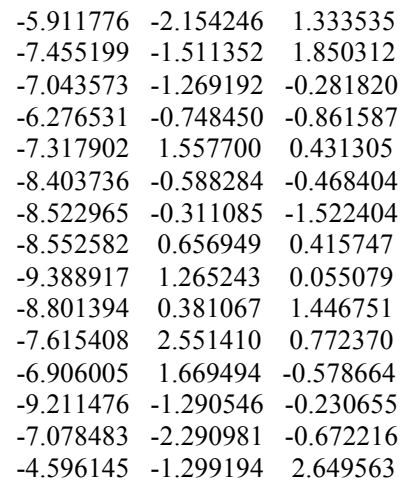

13 P1 endo-re

Number of imaginary frequencies $=0$

The smallest frequencies are $=14.425420 .383221 .2828 \mathrm{~cm}(-1)$ Electronic energy $=-3467.4048875$

Zero-point correction=

(Hartree/Particle)

Thermal correction to Energy $=\quad 1.284576$

Thermal correction to Enthalpy= $\quad 1.285520$

Thermal correction to Gibbs Free Energy= 1.115855

Sum of electronic and zero-point Energies $=\quad-3466.186865$

Sum of electronic and thermal Energies $=\quad-3466.120312$

Sum of electronic and thermal Enthalpies $=\quad-3466.119368$

Sum of electronic and thermal Free Energies $=\quad-3466.289032$

Cartesian Coordinates

$\begin{array}{lccc}77 & -0.164312 & -0.885604 & -1.024073 \\ 15 & 1.634402 & 0.307852 & -0.323868 \\ 8 & 2.359290 & 0.080127 & 1.135904 \\ 8 & 2.923217 & 0.092674 & -1.345679 \\ 7 & 1.195202 & 1.910523 & -0.189881 \\ 6 & 3.256569 & -0.965552 & 1.354197 \\ 6 & 2.825848 & -1.980199 & 2.234656 \\ 1 & 1.846658 & -1.889004 & 2.693209 \\ 6 & 3.648363 & -3.051634 & 2.479197 \\ 1 & 3.334745 & -3.840368 & 3.156792 \\ 6 & 4.904469 & -3.160555 & 1.823259 \\ 6 & 5.728806 & -4.303313 & 2.009822 \\ 1 & 5.387791 & -5.082011 & 2.686857 \\ 6 & 6.924548 & -4.430013 & 1.340120 \\ 1 & 7.544287 & -5.309677 & 1.486657 \\ 6 & 7.339895 & -3.415928 & 0.444573 \\ 1 & 8.272042 & -3.529702 & -0.101246 \\ 6 & 6.571745 & -2.288061 & 0.252455 \\ 1 & 6.902887 & -1.527533 & -0.444433 \\ 6 & 5.341082 & -2.111565 & 0.944568 \\ 6 & 4.509877 & -0.948496 & 0.763528 \\ 6 & 4.946777 & 0.221865 & -0.049167 \\ 6 & 6.156962 & 0.946501 & 0.239571 \\ 6 & 6.954808 & 0.679365 & 1.387382 \\ 1 & 6.646551 & -0.093405 & 2.081280 \\ 6 & 8.103502 & 1.398045 & 1.637507 \\ 1 & 8.690545 & 1.179305 & 2.524840 \\ 6 & 8.522921 & 2.421922 & 0.754905 \\ 1 & 9.434789 & 2.974335 & 0.961802 \\ 6 & 7.763571 & 2.723458 & -0.352669 \\ 1 & 8.062453 & 3.520485 & -1.028367 \\ 6 & 6.563829 & 2.013383 & -0.631250 \\ 6 & 5.750580 & 2.361070 & -1.743740 \\ 1 & 6.074008 & 3.159842 & -2.404885 \\ 6 & 4.554436 & 1.722105 & -1.959844 \\ 1 & 3.896502 & 1.994729 & -2.778196 \\ 6 & 4.160169 & 0.680833 & -1.092735 \\ 6 & -0.113367 & 2.214763 & -0.830470 \\ 1 & -0.858207 & 2.310359 & -0.030282 \\ 6 & -0.077763 & 3.524944 & -1.598644 \\ 6 & 0.984368 & 3.820557 & -2.465421 \\ 1 & 1.818389 & 3.127325 & -2.537543\end{array}$


$\begin{array}{llll}0.983511 & 5.001829 & -3.206405\end{array}$

$\begin{array}{llll}1.815084 & 5.224365 & -3.869386\end{array}$

$\begin{array}{llll}-0.082450 & 5.901118 & -3.094214\end{array}$

$\begin{array}{llll}-0.082042 & 6.821662 & -3.670829\end{array}$

$\begin{array}{llll}-1.142850 & 5.613666 & -2.232452\end{array}$

$\begin{array}{llll}-1.970033 & 6.311274 & -2.134310\end{array}$

$\begin{array}{llll}-1.136222 & 4.432733 & -1.484708\end{array}$

$\begin{array}{llll}-1.955124 & 4.215591 & -0.802590\end{array}$

$\begin{array}{llll}-0.521199 & 1.075569 & -1.773994\end{array}$

$\begin{array}{llll}0.029346 & 1.187674 & -2.714381\end{array}$

$\begin{array}{llll}-1.581253 & 1.170374 & -2.017045\end{array}$

$\begin{array}{llll}1.654802 & 2.974700 & 0.764747\end{array}$

$\begin{array}{lll}1.517298 & 3.899790 & 0.19865\end{array}$

$\begin{array}{lll}0.737296 & 3.062366 & 1.981591\end{array}$

$\begin{array}{lll}-0.120924 & 4.161468 & 2.117395\end{array}$

$\begin{array}{lll}-0.135710 & 4.924381 & 1.343359\end{array}$

$\begin{array}{llll}-0.949387 & 4.290214 & 3.236122\end{array}$

$\begin{array}{lll}-1.600335 & 5.155276 & 3.329236\end{array}$

$\begin{array}{lll}-0.939127 & 3.309583 & 4.230342\end{array}$

$\begin{array}{lll}-1.583663 & 3.404679 & 5.099446\end{array}$

$\begin{array}{lll}-0.094072 & 2.203220 & 4.099111\end{array}$

$\begin{array}{llll}-0.079563 & 1.435364 & 4.867690\end{array}$

$\begin{array}{lll}0.740779 & 2.083120 & 2.986754\end{array}$

$\begin{array}{lll}1.401784 & 1.229124 & 2.901333\end{array}$

$\begin{array}{lll}3.134440 & 2.944206 & 1.152543\end{array}$

$\begin{array}{lll}3.768365 & 2.942519 & 0.265145\end{array}$

$\begin{array}{llll}3.398393 & 2.093427 & 1.778556\end{array}$

$3.345648 \quad 3.857816 \quad 1.716610$

$\begin{array}{lll}-0.853551 & -0.757506 & 1.058369\end{array}$

$\begin{array}{lll}-0.808569 & -1.758284 & 1.478672\end{array}$

$\begin{array}{lll}-0.429221 & 0.016430 & 1.691402\end{array}$

$\begin{array}{llll}-1.988338 & -0.378143 & 0.279741\end{array}$

$\begin{array}{llll}-2.240757 & 0.675370 & 0.235724\end{array}$

$\begin{array}{llll}-2.689783 & -1.264513 & -0.538596\end{array}$

$\begin{array}{lll}-2.606744 & -2.322702 & -0.337471\end{array}$

$\begin{array}{llll}0.900754 & -1.363822 & -2.989199\end{array}$

$\begin{array}{llll}1.309146 & -0.414701 & -3.322305\end{array}$

$\begin{array}{llll}-0.474094 & -1.558389 & -3.183592\end{array}$

$\begin{array}{llll}-1.029311 & -0.752987 & -3.662165\end{array}$

$\begin{array}{lll}-1.171339 & -2.903280 & -3.237100\end{array}$

$-1.047834 \quad-3.325234 \quad-4.245491$

$\begin{array}{llll}-2.241144 & -2.721768 & -3.112138\end{array}$

$-0.704548 \quad-3.932695 \quad-2.182696$

$\begin{array}{llll}0.121321 & -4.530816 & -2.579320\end{array}$

$\begin{array}{llll}-1.524885 & -4.633845 & -1.997717\end{array}$

$\begin{array}{llll}-0.279922 & -3.339795 & -0.846768\end{array}$

$\begin{array}{llll}-0.946899 & -3.501179 & -0.003104\end{array}$

$1.010283 \quad-2.960448 \quad-0.545574$

$\begin{array}{llll}1.260527 & -2.871688 & 0.506750\end{array}$

$\begin{array}{llll}2.193550 & -3.032816 & -1.485846\end{array}$

$3.012327-2.464989-1.043386$

$\begin{array}{llll}2.533540 & -4.076699 & -1.538750\end{array}$

$1.921749-2.497116 \quad-2.908501$

$\begin{array}{llll}1.589312 & -3.308187 & -3.563277\end{array}$

$2.864981 \quad-2.133078-3.324573$

$\begin{array}{llll}-3.775111 & -0.882968 & -1.445839\end{array}$

$\begin{array}{llll}-4.154045 & 0.453654 & -1.692630\end{array}$

$\begin{array}{llll}-4.496362 & -1.905758 & -2.094835\end{array}$

$\begin{array}{llll}-5.196850 & 0.748641 & -2.569282\end{array}$

$\begin{array}{llll}-3.641654 & 1.270201 & -1.195355\end{array}$

$\begin{array}{llll}-5.535910 & -1.608709 & -2.974609\end{array}$

$\begin{array}{llll}-4.240958 & -2.941553 & -1.890119\end{array}$

$\begin{array}{lll}-5.889117 & -0.279150 & -3.219515\end{array}$

$\begin{array}{llll}-5.470463 & 1.784914 & -2.746128\end{array}$

$\begin{array}{llll}-6.076301 & -2.415300 & -3.461299\end{array}$

$\begin{array}{lll}-6.700062 & -0.044472 & -3.902466\end{array}$

$\begin{array}{llll}-1.922487 & -3.728516 & 1.939530\end{array}$

$\begin{array}{lll}-2.942306 & -3.068636 & 2.215081\end{array}$

$\begin{array}{lll}-3.083599 & -2.110497 & 3.019027\end{array}$

$\begin{array}{lll}-4.134750 & -3.396187 & 1.545262\end{array}$

$\begin{array}{lll}-4.082189 & -4.520528 & 0.671966\end{array}$

$\begin{array}{llll}-3.806822 & -5.434678 & 1.209231\end{array}$

$\begin{array}{lll}-5.086423 & -4.630399 & 0.253454\end{array}$

$\begin{array}{llll}-3.363848 & -4.380424 & -0.144164\end{array}$

$\begin{array}{llll}-3.526379 & 1.244963 & 2.964453\end{array}$

\begin{abstract}
$\begin{array}{lll}-4.254369 & 2.574082 & 2.831852\end{array}$
$\begin{array}{lll}-2.599400 & 1.246273 & 2.383377\end{array}$

$\begin{array}{llll}-3.252311 & 1.038487 & 4.002704\end{array}$

$\begin{array}{lll}-4.963925 & 2.611655 & 1.484069\end{array}$

$\begin{array}{llll}-3.544050 & 3.399349 & 2.906208\end{array}$

$\begin{array}{lll}-4.995142 & 2.688106 & 3.630334\end{array}$

$\begin{array}{lll}-5.525060 & 3.538819 & 1.362881\end{array}$

$\begin{array}{lll}-4.238519 & 2.549086 & 0.661859\end{array}$

$\begin{array}{lll}-4.385029 & 0.152332 & 2.502552\end{array}$

$\begin{array}{lll}-5.525613 & 0.289774 & 1.841772\end{array}$

$\begin{array}{lll}-5.912715 & 1.484951 & 1.375342\end{array}$

$-6.324555-0.983569 \quad 1.666833$

$\begin{array}{lll}-5.615579 & -1.806719 & 1.777087\end{array}$

$\begin{array}{lll}-7.019046 & -1.057722 & 2.513453\end{array}$

$\begin{array}{lll}-7.073548 & -1.124564 & 0.334831\end{array}$

$\begin{array}{lll}-6.447742 & -0.724365 & -0.466582\end{array}$

$\begin{array}{lll}-7.157902 & 1.776903 & 0.625080\end{array}$

$\begin{array}{lll}-8.439220 & -0.429047 & 0.333091\end{array}$

$\begin{array}{lll}-8.786049 & -0.321282 & -0.70176\end{array}$

$\begin{array}{lll}-8.384330 & 0.947275 & 1.008287\end{array}$

$\begin{array}{llll}-9.270794 & 1.528516 & 0.732177\end{array}$

$\begin{array}{lll}-8.412275 & 0.849710 & 2.099388\end{array}$

$\begin{array}{lll}-7.371163 & 2.828118 & 0.829130\end{array}$

$\begin{array}{llll}-6.948328 & 1.690869 & -0.448796\end{array}$

$\begin{array}{lll}-9.179027 & -1.050151 & 0.852216\end{array}$

$\begin{array}{llll}-7.195837 & -2.190623 & 0.118450\end{array}$

$\begin{array}{lll}-4.027920 & -0.816795 & 2.710242\end{array}$
\end{abstract}

12 P1 exo-si

Number of imaginary frequencies $=0$

The smallest frequencies are $=12.139818 .542125 .5783 \mathrm{~cm}(-1)$ Electronic energy $=-3467.3991261$

Zero-point correction=

1.220003

(Hartree/Particle)

Thermal correction to Energy $=\quad 1.285930$

Thermal correction to Enthalpy= $\quad 1.286874$

Thermal correction to Gibbs Free Energy $=\quad 1.118885$

Sum of electronic and zero-point Energies $=\quad-3466.179123$

Sum of electronic and thermal Energies $=\quad-3466.113196$

Sum of electronic and thermal Enthalpies $=\quad-3466.112252$

Sum of electronic and thermal Free Energies $=\quad-3466.280241$

Cartesian Coordinates

$\begin{array}{lccc}77 & -0.252516 & -0.569104 & -1.454371 \\ 15 & 1.484306 & 0.554870 & -0.439404 \\ 8 & 2.132369 & 0.175996 & 1.050909 \\ 8 & 2.877306 & 0.502622 & -1.363128 \\ 7 & 1.038704 & 2.141823 & -0.125351 \\ 6 & 2.807576 & -1.032202 & 1.178033 \\ 6 & 2.153401 & -2.060320 & 1.892794 \\ 1 & 1.185844 & -1.855001 & 2.339002 \\ 6 & 2.757521 & -3.289090 & 2.001731 \\ 1 & 2.273965 & -4.090543 & 2.553357 \\ 6 & 4.002062 & -3.543079 & 1.362429 \\ 6 & 4.589643 & -4.837160 & 1.389712 \\ 1 & 4.076913 & -5.626847 & 1.932813 \\ 6 & 5.772072 & -5.092139 & 0.732498 \\ 1 & 6.208158 & -6.086579 & 0.754682 \\ 6 & 6.413649 & -4.056851 & 0.011335 \\ 1 & 7.333850 & -4.266716 & -0.526437 \\ 6 & 5.879599 & -2.786876 & -0.021262 \\ 1 & 6.379396 & -2.008455 & -0.585793 \\ 6 & 4.669417 & -2.481515 & 0.662295 \\ 6 & 4.076517 & -1.169314 & 0.638047 \\ 6 & 4.771028 & 0.012409 & 0.051926 \\ 6 & 6.074468 & 0.425596 & 0.509862 \\ 6 & 6.726019 & -0.179195 & 1.621855 \\ 1 & 6.227954 & -0.974911 & 2.162640 \\ 6 & 7.973055 & 0.239671 & 2.033125 \\ 1 & 8.443020 & -0.236337 & 2.889087 \\ 6 & 8.644173 & 1.286387 & 1.357725 \\ 1 & 9.629787 & 1.600813 & 1.688350 \\ 6 & 8.034206 & 1.911887 & 0.294071\end{array}$


$\begin{array}{llll}8.527309 & 2.731027 & -0.222993\end{array}$

$\begin{array}{llll}6.742250 & 1.514820 & -0.145543\end{array}$

$\begin{array}{llll}6.091537 & 2.195199 & -1.210011\end{array}$

$\begin{array}{llll}6.608325 & 3.008778 & -1.711421\end{array}$

$\begin{array}{llll}4.815198 & 1.852440 & -1.577331\end{array}$

$\begin{array}{llll}4.283504 & 2.380278 & -2.362164\end{array}$

$\begin{array}{llll}4.157756 & 0.785144 & -0.924251\end{array}$

$\begin{array}{llll}-0.265178 & 2.528490 & -0.716414\end{array}$

$\begin{array}{lll}-0.990892 & 2.585425 & 0.100583\end{array}$

$\begin{array}{lll}-0.202774 & 3.906214 & -1.356628\end{array}$

$\begin{array}{llll}0.865047 & 4.266942 & -2.191343\end{array}$

$\begin{array}{llll}1.681484 & 3.565345 & -2.338989\end{array}$

$\begin{array}{llll}0.888413 & 5.515235 & -2.812892\end{array}$

$\begin{array}{llll}1.724718 & 5.784070 & -3.452596\end{array}$

$\begin{array}{llll}-0.158593 & 6.421569 & -2.612410\end{array}$

$\begin{array}{llll}-0.138460 & 7.394359 & -3.095391\end{array}$

$\begin{array}{llll}-1.225574 & 6.070939 & -1.782913\end{array}$

$\begin{array}{llll}-2.039200 & 6.771564 & -1.615411\end{array}$

$\begin{array}{llll}-1.243130 & 4.821564 & -1.156025\end{array}$

$\begin{array}{llll}-2.069895 & 4.556262 & -0.501021\end{array}$

$\begin{array}{llll}-0.771384 & 1.493613 & -1.743523\end{array}$

$\begin{array}{llll}-0.377620 & 1.782100 & -2.724187\end{array}$

$\begin{array}{lll}-1.858424 & 1.609239 & -1.808300\end{array}$

$\begin{array}{llll}1.506917 & 3.062998 & 0.968730\end{array}$

$\begin{array}{llll}1.219136 & 4.053168 & 0.604392\end{array}$

$\begin{array}{lll}0.720812 & 2.831649 & 2.259381\end{array}$

$\begin{array}{lll}-0.492011 & 3.514245 & 2.447057\end{array}$

$\begin{array}{llll}-0.801083 & 4.255659 & 1.714629\end{array}$

$\begin{array}{llll}-1.302741 & 3.255629 & 3.555358\end{array}$

$\begin{array}{lll}-2.240957 & 3.790924 & 3.676156\end{array}$

$\begin{array}{lll}-0.900384 & 2.319689 & 4.510658\end{array}$

$\begin{array}{lll}-1.531507 & 2.104910 & 5.368369\end{array}$

$\begin{array}{lll}0.328547 & 1.671333 & 4.361572\end{array}$

$\begin{array}{llll}0.658947 & 0.953506 & 5.106834\end{array}$

$\begin{array}{lll}1.132634 & 1.928073 & 3.248999\end{array}$

$\begin{array}{lll}2.071855 & 1.400150 & 3.141082\end{array}$

$3.022029 \quad 3.108509 \quad 1.150422$

$\begin{array}{lll}3.505919 & 3.381760 & 0.210553\end{array}$

$\begin{array}{llll}3.444369 & 2.164064 & 1.490845\end{array}$

$\begin{array}{lll}3.259881 & 3.876344 & 1.893130\end{array}$

$\begin{array}{lll}-1.178848 & 0.070464 & 1.509438\end{array}$

$\begin{array}{llll}-1.322481 & -0.950251 & 0.424554\end{array}$

$\begin{array}{llll}-1.106096 & -1.954207 & 0.787393\end{array}$

$\begin{array}{lll}-2.327407 & -0.799920 & -0.586539\end{array}$

$\begin{array}{llll}-2.761485 & 0.194123 & -0.661762\end{array}$

$\begin{array}{llll}0.677258 & -0.521288 & -3.462554\end{array}$

$\begin{array}{llll}1.002019 & 0.494883 & -3.676368\end{array}$

$\begin{array}{lll}-0.720143 & -0.772508 & -3.600637\end{array}$

$\begin{array}{llll}-1.337557 & 0.067795 & -3.919608\end{array}$

$\begin{array}{llll}-1.320441 & -2.121606 & -3.958879\end{array}$

$\begin{array}{llll}-1.205306 & -2.311373 & -5.037365\end{array}$

$\begin{array}{llll}-2.394812 & -2.064593 & -3.767323\end{array}$

$\begin{array}{lll}-0.737177 & -3.294589 & -3.146325\end{array}$

$\begin{array}{llll}0.093608 & -3.761231 & -3.685277\end{array}$

$\begin{array}{llll}-1.503918 & -4.068210 & -3.037962\end{array}$

$\begin{array}{llll}-0.262295 & -2.898448 & -1.751959\end{array}$

$\begin{array}{llll}-0.860888 & -3.284190 & -0.935331\end{array}$

$\begin{array}{llll}1.044176 & -2.539052 & -1.445555\end{array}$

$\begin{array}{llll}1.371017 & -2.692581 & -0.422892\end{array}$

$\begin{array}{llll}2.157208 & -2.386506 & -2.463458\end{array}$

$\begin{array}{llll}2.993538 & -1.888212 & -1.970699\end{array}$

$\begin{array}{llll}2.514858 & -3.386863 & -2.750279\end{array}$

$\begin{array}{llll}1.754527 & -1.580327 & -3.717743\end{array}$

$\begin{array}{llll}1.410802 & -2.255835 & -4.509006\end{array}$

$\begin{array}{llll}1.646239 & -1.083015 & -4.111668\end{array}$

$\begin{array}{lll}-3.285597 & -1.833917 & -1.034368\end{array}$

$\begin{array}{llll}-3.342225 & -3.136854 & -0.505138\end{array}$

$\begin{array}{llll}-4.248320 & -1.482960 & -2.003057\end{array}$

$\begin{array}{lll}-4.290438 & -4.057862 & -0.955492\end{array}$

$\begin{array}{llll}-2.657830 & -3.427678 & 0.284917\end{array}$

$\begin{array}{lll}-5.202622 & -2.397195 & -2.446570\end{array}$

$\begin{array}{llll}-4.232450 & -0.478019 & -2.417422\end{array}$

$\begin{array}{llll}-5.224683 & -3.697656 & -1.929687\end{array}$

$\begin{array}{llll}-4.308970 & -5.057063 & -0.528574\end{array}$

$\begin{array}{llll}-5.927361 & -2.096789 & -3.198603\end{array}$

$\begin{array}{lll}-5.964867 & -4.413814 & -2.274174\end{array}$

$\begin{array}{lll}-0.830500 & -1.637017 & 3.631865\end{array}$

$\begin{array}{lll}-1.847047 & -0.976308 & 3.559998\end{array}$

$\begin{array}{lll}-2.176832 & -0.128620 & 2.578888\end{array}$

$\begin{array}{lll}-2.847747 & -0.977712 & 4.449327\end{array}$

$\begin{array}{lll}-2.659477 & -1.856603 & 5.580069\end{array}$

$\begin{array}{llll}-1.767847 & -1.570004 & 6.143209\end{array}$

$\begin{array}{lll}-3.551412 & -1.733491 & 6.194231\end{array}$

$\begin{array}{llll}-2.566103 & -2.894149 & 5.249386\end{array}$

$\begin{array}{llll}-3.940880 & 2.599569 & 0.759807\end{array}$

$\begin{array}{llll}-5.109188 & 3.145660 & -0.043671\end{array}$

$\begin{array}{llll}-3.090765 & 2.378114 & 0.106272\end{array}$

$\begin{array}{lll}-3.601923 & 3.303879 & 1.523553\end{array}$

$\begin{array}{lll}-5.740501 & 2.006594 & -0.834850\end{array}$

$\begin{array}{llll}-4.762363 & 3.922018 & -0.730071\end{array}$

$\begin{array}{lll}-5.856900 & 3.588322 & 0.622331\end{array}$

$\begin{array}{llll}-6.626855 & 2.352561 & -1.367476\end{array}$

$\begin{array}{llll}-5.036575 & 1.605427 & -1.573689\end{array}$

$\begin{array}{lll}-4.353636 & 1.368407 & 1.435624\end{array}$

$\begin{array}{lll}-5.400404 & 0.608764 & 1.116913\end{array}$

$\begin{array}{lll}-6.158968 & 0.904433 & 0.063584\end{array}$

$\begin{array}{llll}-5.632065 & -0.570664 & 2.039722\end{array}$

$\begin{array}{lll}-4.672917 & -0.775294 & 2.521384\end{array}$

$\begin{array}{lll}-6.307642 & -0.239535 & 2.838390\end{array}$

$\begin{array}{lll}-6.158776 & -1.849910 & 1.375241\end{array}$

$\begin{array}{lll}-5.662037 & -1.979821 & 0.411263\end{array}$

$\begin{array}{lll}-7.343084 & 0.135483 & -0.401347\end{array}$

$\begin{array}{lll}-7.679804 & -1.849385 & 1.191855\end{array}$

$\begin{array}{lll}-7.949911 & -2.635135 & 0.476796\end{array}$

$\begin{array}{lll}-8.201906 & -0.494748 & 0.696410\end{array}$

$\begin{array}{lll}-9.212078 & -0.615033 & 0.291219\end{array}$

$\begin{array}{lll}-8.289226 & 0.219098 & 1.523791\end{array}$

$\begin{array}{lll}-7.947530 & 0.859522 & -0.949831\end{array}$

$\begin{array}{llll}-7.002241 & -0.617281 & -1.121717\end{array}$

$\begin{array}{lll}-8.174781 & -2.096590 & 2.138489\end{array}$

$\begin{array}{lll}-5.855749 & -2.703667 & 1.989129\end{array}$

$\begin{array}{lll}-3.745079 & 1.013236 & 2.167599\end{array}$

$\begin{array}{lll}-0.199384 & 0.053978 & 1.976957\end{array}$

$\begin{array}{lll}-1.394710 & 1.076663 & 1.165231\end{array}$

\section{$[12-13]^{\ddagger}$ P1 exo-si}

Number of imaginary frequencies $=1$

The smallest frequencies are $=-255.499614 .185819 .1075 \mathrm{~cm}(-1)$ Electronic energy $=-3467.3760757$

Zero-point correction $=$

1.217512

(Hartree/Particle)

Thermal correction to Energy= $\quad 1.283614$

Thermal correction to Enthalpy $=\quad 1.284558$

Thermal correction to Gibbs Free Energy $=1.115566$

Sum of electronic and zero-point Energies $=\quad-3466.158563$

Sum of electronic and thermal Energies $=\quad-3466.092462$

Sum of electronic and thermal Enthalpies $=\quad-3466.091518$

Sum of electronic and thermal Free Energies $=\quad-3466.260510$

Cartesian Coordinates

$\begin{array}{lccc}77 & -0.266860 & -0.779174 & -1.283017 \\ 15 & 1.500190 & 0.503067 & -0.509601 \\ 8 & 2.167085 & 0.346184 & 1.005538 \\ 8 & 2.857760 & 0.288763 & -1.447764 \\ 7 & 1.048093 & 2.112566 & -0.462613 \\ 6 & 2.843672 & -0.828821 & 1.319525 \\ 6 & 2.214087 & -1.706809 & 2.230658 \\ 1 & 1.276071 & -1.411859 & 2.692822 \\ 6 & 2.815925 & -2.906923 & 2.522114 \\ 1 & 2.355329 & -3.593109 & 3.227190 \\ 6 & 4.028483 & -3.284698 & 1.882496 \\ 6 & 4.605898 & -4.564841 & 2.102888 \\ 1 & 4.110826 & -5.245408 & 2.790615 \\ 6 & 5.757657 & -4.943432 & 1.450828 \\ 1 & 6.186282 & -5.926629 & 1.621449 \\ 6 & 6.377946 & -4.052352 & 0.542716 \\ 1 & 7.273657 & -4.362435 & 0.012218 \\ 6 & 5.855035 & -2.796752 & 0.320888\end{array}$


$\begin{array}{llll}6.339184 & -2.130178 & -0.383124\end{array}$

$\begin{array}{lll}4.676053 & -2.364812 & 0.989803\end{array}$

$\begin{array}{llll}4.092315 & -1.066914 & 0.768680\end{array}$

$\begin{array}{lll}4.781361 & -0.002212 & -0.015497\end{array}$

$\begin{array}{lll}6.100108 & 0.459369 & 0.343445\end{array}$

$\begin{array}{llll}6.772294 & 0.024735 & 1.520402\end{array}$

$\begin{array}{llll}6.279515 & -0.671345 & 2.188036\end{array}$

$\begin{array}{llll}8.032091 & 0.487274 & 1.834221\end{array}$

$\begin{array}{lll}8.518085 & 0.143916 & 2.743003\end{array}$

$\begin{array}{lll}8.695558 & 1.409473 & 0.990643\end{array}$

$\begin{array}{llll}9.691168 & 1.759309 & 1.247274\end{array}$

$\begin{array}{llll}8.066103 & 1.871235 & -0.142904\end{array}$

$\begin{array}{llll}8.552981 & 2.595555 & -0.790774\end{array}$

$\begin{array}{llll}6.760649 & 1.426712 & -0.486925\end{array}$

$\begin{array}{llll}6.090857 & 1.944581 & -1.628086\end{array}$

$\begin{array}{llll}6.602858 & 2.665434 & -2.259238\end{array}$

$\begin{array}{llll}4.802902 & 1.565693 & -1.910226\end{array}$

$\begin{array}{llll}4.257093 & 1.973413 & -2.754488\end{array}$

$\begin{array}{llll}4.154438 & 0.619904 & -1.084925\end{array}$

$\begin{array}{llll}-0.297411 & 2.380870 & -1.029777\end{array}$

$\begin{array}{llll}-0.979037 & 2.543491 & -0.188416\end{array}$

$\begin{array}{llll}-0.318228 & 3.644723 & -1.874393\end{array}$

$\begin{array}{llll}0.691753 & 3.906509 & -2.810801\end{array}$

$\begin{array}{llll}1.532523 & 3.222199 & -2.888549\end{array}$

$\begin{array}{llll}0.629183 & 5.040585 & -3.620553\end{array}$

$\begin{array}{llll}1.421865 & 5.236493 & -4.337471\end{array}$

$\begin{array}{llll}-0.448146 & 5.926078 & -3.508943\end{array}$

$\begin{array}{llll}-0.494812 & 6.810242 & -4.138282\end{array}$

$\begin{array}{llll}-1.458397 & 5.671968 & -2.578858\end{array}$

$\begin{array}{llll}-2.294286 & 6.359236 & -2.480299\end{array}$

$\begin{array}{llll}-1.390022 & 4.538664 & -1.763731\end{array}$

$\begin{array}{llll}-2.169111 & 4.349678 & -1.028482\end{array}$

$\begin{array}{llll}-0.819546 & 1.202517 & -1.874223\end{array}$

$\begin{array}{llll}-0.445595 & 1.332970 & -2.894710\end{array}$

$\begin{array}{llll}-1.907625 & 1.303128 & -1.931238\end{array}$

$\begin{array}{lll}1.536907 & 3.211081 & 0.443367\end{array}$

$\begin{array}{llll}1.338437 & 4.119924 & -0.131411\end{array}$

$\begin{array}{lll}0.680812 & 3.298772 & 1.706450\end{array}$

$\begin{array}{lll}-0.354879 & 4.243571 & 1.761375\end{array}$

$\begin{array}{lll}-0.497805 & 4.922315 & 0.924412\end{array}$

$\begin{array}{lll}-1.207628 & 4.315237 & 2.866956\end{array}$

$\begin{array}{lll}-2.005199 & 5.053324 & 2.887025\end{array}$

$\begin{array}{lll}-1.031717 & 3.440172 & 3.941007\end{array}$

$\begin{array}{lll}-1.700934 & 3.477317 & 4.795702\end{array}$

$\begin{array}{lll}0.012886 & 2.513207 & 3.908013\end{array}$

$\begin{array}{lll}0.153486 & 1.818537 & 4.729693\end{array}$

$\begin{array}{lll}0.862037 & 2.444270 & 2.803214\end{array}$

$\begin{array}{lll}1.655329 & 1.708504 & 2.788902\end{array}$

$\begin{array}{lll}3.041442 & 3.209375 & 0.709648\end{array}$

$\begin{array}{llll}3.595213 & 3.216721 & -0.231430\end{array}$

$\begin{array}{lll}3.376608 & 2.359677 & 1.302719\end{array}$

$\begin{array}{lll}3.289409 & 4.124592 & 1.255815\end{array}$

$\begin{array}{lll}-1.069935 & -0.008775 & 1.210132\end{array}$

$\begin{array}{lll}-1.550106 & -1.137504 & 0.523459\end{array}$

$\begin{array}{lll}-1.337740 & -2.103505 & 0.970715\end{array}$

$\begin{array}{llll}-2.433708 & -1.026949 & -0.596673\end{array}$

$\begin{array}{llll}-2.829134 & -0.030175 & -0.767876\end{array}$

$\begin{array}{llll}0.697626 & -0.982567 & -3.264646\end{array}$

$\begin{array}{llll}0.982552 & 0.014533 & -3.590419\end{array}$

$\begin{array}{llll}-0.679623 & -1.312227 & -3.396549\end{array}$

$\begin{array}{lll}-1.327176 & -0.546289 & -3.822270\end{array}$

$-1.210177-2.720616-3.584466$

$\begin{array}{llll}-1.054987 & -3.026429 & -4.630082\end{array}$

$\begin{array}{llll}-2.290286 & -2.694595 & -3.429874\end{array}$

$\begin{array}{llll}-0.591297 & -3.764018 & -2.632545\end{array}$

$\begin{array}{llll}0.268867 & -4.248925 & -3.104480\end{array}$

$\begin{array}{llll}-1.327174 & -4.552071 & -2.448595\end{array}$

$\begin{array}{llll}-0.156668 & -3.199684 & -1.287444\end{array}$

$\begin{array}{lll}-0.762092 & -3.486822 & -0.437197\end{array}$

$\begin{array}{llll}1.120995 & -2.753368 & -1.011546\end{array}$

$\begin{array}{lll}1.430385 & -2.730703 & 0.028014\end{array}$

$2.234682-2.673858 \quad-2.034107$

$\begin{array}{llll}3.059320 & -2.116043 & -1.592264\end{array}$

$\begin{array}{llll}2.609281 & -3.691160 & -2.217683\end{array}$

$\begin{array}{lll}1.826970 & -2.011038 & -3.369617\end{array}$

$\begin{array}{llll}1.531492 & -2.774818 & -4.096911\end{array}$

$2.705684-1.512889-3.788104$

$\begin{array}{lll}-3.380270 & -2.066862 & -1.044151\end{array}$

$\begin{array}{llll}-3.437827 & -3.369275 & -0.515624\end{array}$

$-4.330046-1.711106-2.022517$

$\begin{array}{llll}-4.390785 & -4.283239 & -0.967230\end{array}$

$\begin{array}{lll}-2.758837 & -3.666632 & 0.276023\end{array}$

$\begin{array}{lll}-5.285194 & -2.621832 & -2.471154\end{array}$

$\begin{array}{llll}-4.309350 & -0.704216 & -2.431984\end{array}$

$\begin{array}{llll}-5.317349 & -3.918424 & -1.947525\end{array}$

$\begin{array}{llll}-4.417669 & -5.281601 & -0.539558\end{array}$

$\begin{array}{llll}-6.004238 & -2.320243 & -3.227747\end{array}$

$-6.060077-4.631410 \quad-2.292919$

$\begin{array}{lll}-0.694161 & -0.749025 & 4.093802\end{array}$

$\begin{array}{lll}-1.774912 & -0.170128 & 3.948454\end{array}$

$\begin{array}{lll}-2.315542 & 0.222844 & 2.846994\end{array}$

$\begin{array}{llll}-2.570205 & 0.127534 & 5.023995\end{array}$

$\begin{array}{llll}-2.058949 & -0.286013 & 6.296554\end{array}$

$\begin{array}{lll}-1.106296 & 0.203281 & 6.523512\end{array}$

$\begin{array}{lll}-2.809945 & 0.011606 & 7.031895\end{array}$

$\begin{array}{lll}-1.911750 & -1.370143 & 6.334923\end{array}$

$\begin{array}{lll}-3.909388 & 2.478587 & 0.582448\end{array}$

$\begin{array}{llll}-5.117911 & 3.006917 & -0.172022\end{array}$

$\begin{array}{llll}-3.118116 & 2.187448 & -0.116936\end{array}$

$\begin{array}{lll}-3.491190 & 3.220384 & 1.267665\end{array}$

$\begin{array}{llll}-5.818860 & 1.841830 & -0.860759\end{array}$

$\begin{array}{llll}-4.807436 & 3.741820 & -0.919072\end{array}$

$\begin{array}{lll}-5.812518 & 3.496158 & 0.518559\end{array}$

$\begin{array}{llll}-6.740585 & 2.177682 & -1.337958\end{array}$

$\begin{array}{llll}-5.179743 & 1.403951 & -1.637788\end{array}$

$\begin{array}{lll}-4.299034 & 1.309694 & 1.368404\end{array}$

$\begin{array}{lll}-5.359118 & 0.543999 & 1.141554\end{array}$

$\begin{array}{lll}-6.176009 & 0.782652 & 0.111569\end{array}$

$\begin{array}{lll}-5.545092 & -0.588152 & 2.132841\end{array}$

$\begin{array}{lll}-4.569003 & -0.739171 & 2.600569\end{array}$

$-6.212250-0.232609 \quad 2.927841$

$\begin{array}{lll}-6.060498 & -1.909859 & 1.544211\end{array}$

$\begin{array}{lll}-5.597077 & -2.071912 & 0.567487\end{array}$

$\begin{array}{llll}-7.356992 & -0.032071 & -0.272137\end{array}$

$\begin{array}{lll}-7.586926 & -1.953975 & 1.415563\end{array}$

$\begin{array}{lll}-7.863413 & -2.776148 & 0.745012\end{array}$

$\begin{array}{lll}-8.159890 & -0.634428 & 0.882607\end{array}$

$\begin{array}{lll}-9.180691 & -0.796289 & 0.520408\end{array}$

$\begin{array}{lll}-8.235537 & 0.111675 & 1.681991\end{array}$

$\begin{array}{llll}-8.001159 & 0.652181 & -0.827061\end{array}$

$-7.029583-0.809761 \quad-0.972936$

$\begin{array}{lll}-8.041496 & -2.171065 & 2.389495\end{array}$

$\begin{array}{lll}-5.714463 & -2.729204 & 2.182043\end{array}$

$\begin{array}{lll}-3.644068 & 0.997527 & 2.104145\end{array}$

$-0.239924-0.097071 \quad 1.891541$

$\begin{array}{lll}-1.346925 & 0.983948 & 0.893575\end{array}$

13 P1 exo-si

Number of imaginary frequencies $=0$

The smallest frequencies are $=13.373520 .014423 .8465 \mathrm{~cm}(-1)$ Electronic energy $=-3467.4009901$

Zero-point correction=

(Hartree/Particle)

Thermal correction to Energy= $\quad 1.284253$

Thermal correction to Enthalpy= $\quad 1.285197$

Thermal correction to Gibbs Free Energy $=\quad 1.116099$

Sum of electronic and zero-point Energies $=\quad-3466.183051$

Sum of electronic and thermal Energies $=\quad-3466.116737$

Sum of electronic and thermal Enthalpies $=\quad-3466.115793$

Sum of electronic and thermal Free Energies $=\quad-3466.284891$

Cartesian Coordinates

$\begin{array}{lccc}77 & -0.548661 & -0.080075 & -1.507703 \\ 15 & 1.398268 & 0.445635 & -0.505678 \\ 8 & 1.904927 & -0.303295 & 0.866087 \\ 8 & 2.679468 & 0.150807 & -1.517018 \\ 7 & 1.340096 & 2.031803 & -0.012790 \\ 6 & 2.513374 & -1.562714 & 0.834156\end{array}$


$\begin{array}{llll}1.778769 & -2.620004 & 1.411667\end{array}$

$\begin{array}{llll}0.817952 & -2.402815 & 1.871797\end{array}$

$\begin{array}{llll}2.314357 & -3.884841 & 1.405057\end{array}$

$\begin{array}{llll}1.770130 & -4.708898 & 1.858257\end{array}$

$\begin{array}{lll}3.566326 & -4.142072 & 0.784375\end{array}$

$\begin{array}{llll}4.088080 & -5.461822 & 0.704089\end{array}$

$\begin{array}{llll}3.518871 & -6.270880 & 1.154335\end{array}$

$\begin{array}{llll}5.278566 & -5.714527 & 0.061074\end{array}$

$\begin{array}{llll}5.665533 & -6.727563 & 0.001548\end{array}$

$\begin{array}{llll}5.992684 & -4.650353 & -0.539503\end{array}$

$\begin{array}{llll}6.918799 & -4.855506 & -1.068793\end{array}$

$\begin{array}{llll}5.523034 & -3.356848 & -0.464833\end{array}$

$\begin{array}{llll}6.080698 & -2.557871 & -0.938587\end{array}$

$\begin{array}{llll}4.309092 & -3.054126 & 0.213355\end{array}$

$\begin{array}{llll}3.786662 & -1.714135 & 0.307793\end{array}$

$\begin{array}{llll}4.555719 & -0.533624 & -0.173954\end{array}$

$\begin{array}{llll}5.871876 & -0.221015 & 0.319386\end{array}$

$\begin{array}{llll}6.478759 & -0.935044 & 1.390321\end{array}$

$\begin{array}{llll}5.935912 & -1.744443 & 1.863529\end{array}$

$\begin{array}{lll}7.736522 & -0.602061 & 1.843690\end{array}$

$\begin{array}{lll}8.174324 & -1.157405 & 2.668133\end{array}$

$\begin{array}{lll}8.461329 & 0.459842 & 1.251514\end{array}$

$\begin{array}{lll}9.454873 & 0.705232 & 1.614939\end{array}$

$\begin{array}{llll}7.896064 & 1.187251 & 0.229151\end{array}$

$\begin{array}{lll}8.432213 & 2.018124 & -0.221793\end{array}$

$\begin{array}{llll}6.593335 & 0.881970 & -0.251073\end{array}$

$\begin{array}{llll}5.986795 & 1.667740 & -1.268313\end{array}$

$\begin{array}{llll}6.546850 & 2.489623 & -1.704910\end{array}$

$\begin{array}{llll}4.698083 & 1.414620 & -1.669630\end{array}$

$\begin{array}{llll}4.201016 & 2.019002 & -2.420471\end{array}$

$\begin{array}{llll}3.994377 & 0.335267 & -1.094813\end{array}$

$\begin{array}{llll}0.197277 & 2.797738 & -0.582253\end{array}$

$\begin{array}{lll}-0.528544 & 2.946334 & 0.224880\end{array}$

$\begin{array}{llll}0.627742 & 4.168291 & -1.079218\end{array}$

$\begin{array}{llll}1.768783 & 4.317609 & -1.880849\end{array}$

$\begin{array}{llll}2.378543 & 3.446089 & -2.101466\end{array}$

$2.134609 \quad 5.573204 \quad-2.364693$

$3.025843 \quad 5.677400 \quad-2.977485$

$\begin{array}{llll}1.359591 & 6.696902 & -2.057996\end{array}$

$\begin{array}{llll}1.645578 & 7.675325 & -2.433403\end{array}$

$\begin{array}{llll}0.222204 & 6.556060 & -1.260370\end{array}$

$\begin{array}{llll}-0.379505 & 7.425560 & -1.010556\end{array}$

$\begin{array}{llll}-0.137868 & 5.297605 & -0.769807\end{array}$

$\begin{array}{llll}-1.011553 & 5.192140 & -0.131323\end{array}$

$\begin{array}{llll}-0.476708 & 2.022693 & -1.725595\end{array}$

$\begin{array}{llll}0.065909 & 2.226950 & -2.654891\end{array}$

$\begin{array}{lll}-1.485399 & 2.421811 & -1.857468\end{array}$

$\begin{array}{lll}1.996377 & 2.741256 & 1.137352\end{array}$

$\begin{array}{llll}2.256214 & 3.721290 & 0.726849\end{array}$

$\begin{array}{lll}0.990784 & 2.976072 & 2.259711\end{array}$

$\begin{array}{lll}0.648516 & 4.288324 & 2.610181\end{array}$

$\begin{array}{lll}1.095244 & 5.117743 & 2.068071\end{array}$

$\begin{array}{lll}-0.265682 & 4.538956 & 3.636704\end{array}$

$\begin{array}{llll}-0.520033 & 5.563338 & 3.895607\end{array}$

$\begin{array}{lll}-0.851544 & 3.472296 & 4.320982\end{array}$

$\begin{array}{lll}-1.563475 & 3.661511 & 5.120118\end{array}$

$\begin{array}{lll}-0.518755 & 2.156939 & 3.979390\end{array}$

$\begin{array}{lll}-0.977781 & 1.323140 & 4.499882\end{array}$

$\begin{array}{lll}0.396609 & 1.911246 & 2.952542\end{array}$

$\begin{array}{llll}0.645197 & 0.888463 & 2.686847\end{array}$

$\begin{array}{llll}3.304556 & 2.150150 & 1.666048\end{array}$

$\begin{array}{llll}4.042639 & 2.047867 & 0.870667\end{array}$

$\begin{array}{lll}3.169814 & 1.189257 & 2.160575\end{array}$

$\begin{array}{lll}3.701985 & 2.856281 & 2.401498\end{array}$

$\begin{array}{lll}-1.358909 & 0.075460 & 0.521297\end{array}$

$\begin{array}{llll}-2.525671 & -0.389285 & -0.178097\end{array}$

$-2.791467-1.433618-0.053285$

$\begin{array}{llll}-3.256292 & 0.376451 & -1.069195\end{array}$

$\begin{array}{llll}-3.086686 & 1.448930 & -1.077639\end{array}$

$\begin{array}{llll}0.537825 & -0.299630 & -3.514464\end{array}$

$\begin{array}{llll}1.139000 & 0.601818 & -3.588861\end{array}$

$\begin{array}{llll}-0.831212 & -0.153011 & -3.773810\end{array}$

$\begin{array}{lll}-1.177240 & 0.845240 & -4.039364\end{array}$

$\begin{array}{llll}-1.792335 & -1.252653 & -4.173613\end{array}$

$\begin{array}{lll}-1.757748 & -1.372636 & -5.266459\end{array}$ $\begin{array}{llll}-2.803920 & -0.912669 & -3.937997\end{array}$

$\begin{array}{llll}-1.560169 & -2.623780 & -3.501585\end{array}$

$\begin{array}{llll}-0.817280 & -3.196979 & -4.062497\end{array}$

$\begin{array}{lll}-2.494131 & -3.191452 & -3.556591\end{array}$

$\begin{array}{llll}-1.131746 & -2.555276 & -2.047144\end{array}$

$\begin{array}{llll}-1.897863 & -2.756565 & -1.307360\end{array}$

$\begin{array}{llll}0.163512 & -2.560369 & -1.610318\end{array}$

$\begin{array}{lll}0.336678 & -2.774180 & -0.560355\end{array}$

$\begin{array}{llll}1.387443 & -2.552623 & -2.494749\end{array}$

$\begin{array}{llll}2.246127 & -2.289083 & -1.877946\end{array}$

$\begin{array}{llll}1.566664 & -3.577467 & -2.849309\end{array}$

$\begin{array}{llll}1.326632 & -1.594774 & -3.708194\end{array}$

$\begin{array}{llll}0.912657 & -2.115824 & -4.576466\end{array}$

$\begin{array}{llll}2.352558 & -1.325282 & -3.972211\end{array}$

$\begin{array}{llll}-4.379205 & -0.085737 & -1.881645\end{array}$

$\begin{array}{llll}-4.678560 & -1.449784 & -2.084764\end{array}$

$\begin{array}{llll}-5.212837 & 0.874905 & -2.488704\end{array}$

$\begin{array}{llll}-5.783219 & -1.830947 & -2.841960\end{array}$

$\begin{array}{llll}-4.038467 & -2.215973 & -1.663694\end{array}$

$\begin{array}{llll}-6.320251 & 0.490991 & -3.244205\end{array}$

$\begin{array}{llll}-4.989363 & 1.930301 & -2.353929\end{array}$

$\begin{array}{llll}-6.612855 & -0.863542 & -3.420786\end{array}$

$\begin{array}{llll}-5.995533 & -2.886434 & -2.986045\end{array}$

$\begin{array}{llll}-6.953072 & 1.249262 & -3.696064\end{array}$

$\begin{array}{llll}-7.473911 & -1.164776 & -4.009908\end{array}$

$\begin{array}{lll}-0.941787 & -1.969263 & 3.002218\end{array}$

$\begin{array}{llll}-1.143092 & -1.261248 & 4.012636\end{array}$

$\begin{array}{lll}-2.199411 & -0.657624 & 4.358035\end{array}$

$\begin{array}{lll}-0.120143 & -1.068497 & 4.927315\end{array}$

$\begin{array}{lll}1.146204 & -1.640379 & 4.612086\end{array}$

$\begin{array}{lll}1.621865 & -1.124978 & 3.770352\end{array}$

$\begin{array}{lll}1.768343 & -1.513259 & 5.502479\end{array}$

$\begin{array}{lll}1.065962 & -2.704402 & 4.369241\end{array}$

$\begin{array}{lll}-3.972172 & 1.704619 & 2.460589\end{array}$

$\begin{array}{lll}-5.403726 & 2.183846 & 2.277558\end{array}$

$\begin{array}{lll}-3.364001 & 1.955104 & 1.582766\end{array}$

$\begin{array}{lll}-3.493437 & 2.155956 & 3.332552\end{array}$

$\begin{array}{lll}-6.082090 & 1.332268 & 1.211634\end{array}$

$\begin{array}{llll}-5.424935 & 3.233454 & 1.972892\end{array}$

$\begin{array}{lll}-5.949234 & 2.093701 & 3.222887\end{array}$

$\begin{array}{lll}-7.146720 & 1.568855 & 1.159431\end{array}$

$\begin{array}{lll}-5.651028 & 1.521688 & 0.221981\end{array}$

$\begin{array}{lll}-3.966597 & 0.257455 & 2.644716\end{array}$

$\begin{array}{lll}-4.903047 & -0.562308 & 2.198334\end{array}$

$\begin{array}{lll}-5.962978 & -0.112818 & 1.510507\end{array}$

$\begin{array}{lll}-4.665854 & -2.024711 & 2.529868\end{array}$

$\begin{array}{lll}-3.610188 & -2.105077 & 2.799132\end{array}$

$\begin{array}{lll}-5.233781 & -2.267078 & 3.436405\end{array}$

$\begin{array}{llll}-5.000165 & -3.020888 & 1.408383\end{array}$

$\begin{array}{lll}-4.710567 & -2.588317 & 0.445386\end{array}$

$\begin{array}{lll}-6.994371 & -0.954118 & 0.853665\end{array}$

$\begin{array}{lll}-6.481396 & -3.416951 & 1.374294\end{array}$

$\begin{array}{lll}-6.708316 & -3.875322 & 0.404097\end{array}$

$\begin{array}{lll}-7.409733 & -2.217884 & 1.609418\end{array}$

$\begin{array}{lll}-8.424938 & -2.473875 & 1.287505\end{array}$

$\begin{array}{lll}-7.479651 & -1.980138 & 2.676671\end{array}$

$\begin{array}{llll}-7.865825 & -0.304634 & 0.754066\end{array}$

$-6.659717-1.196842-0.161887$

$\begin{array}{lll}-6.681588 & -4.177298 & 2.138393\end{array}$

$\begin{array}{lll}-4.378517 & -3.912065 & 1.542797\end{array}$

$-3.208297-0.158346 \quad 3.261401$

$\begin{array}{lll}-0.964643 & -0.607712 & 1.268363\end{array}$

$\begin{array}{llll}-1.338901 & 1.115111 & 0.837835\end{array}$

\section{$[12-13]^{\ddagger} \mathbf{P} 1$ exo-re}

Number of imaginary frequencies $=1$

The smallest frequencies are $=-258.376510 .348817 .7271 \mathrm{~cm}(-1)$ Electronic energy $=-3467.373106$

Zero-point correction $=$

(Hartree/Particle)

Thermal correction to Energy= $\quad 1.282771$

Thermal correction to Enthalpy $=\quad 1.283715$

Thermal correction to Gibbs Free Energy $=\quad 1.114210$

Sum of electronic and zero-point Energies $=\quad-3466.156679$ 
Sum of electronic and thermal Energies=
Sum of electronic and thermal Enthalpies

Sum of electronic and thermal Free Energies=

Cartesian Coordinates

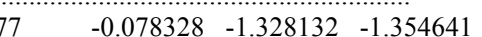

$\begin{array}{lllll}15 & 1.268110 & 0.351534 & -0.552403\end{array}$

$\begin{array}{lll}1.958149 & 0.442552 & 0.949140\end{array}$

$\begin{array}{llll}2.639965 & 0.468799 & -1.493453\end{array}$

$\begin{array}{llll}0.417265 & 1.795738 & -0.546154\end{array}$

$\begin{array}{llll}3.000282 & -0.416925 & 1.291618\end{array}$

$\begin{array}{lll}2.695582 & -1.438672 & 2.215488\end{array}$

$\begin{array}{lll}1.691587 & -1.488794 & 2.614238\end{array}$

$\begin{array}{lll}3.668099 & -2.337980 & 2.573593\end{array}$

$\begin{array}{lll}3.446645 & -3.125581 & 3.288217\end{array}$

$\begin{array}{lll}4.959728 & -2.276637 & 1.983685\end{array}$

$\begin{array}{llll}5.945043 & -3.258772 & 2.275131\end{array}$

$\begin{array}{llll}5.699467 & -4.042063 & 2.987492\end{array}$

$\begin{array}{lll}7.175208 & -3.232308 & 1.658067\end{array}$

$\begin{array}{lll}7.918115 & -3.992012 & 1.882553\end{array}$

$\begin{array}{llll}7.464916 & -2.220216 & 0.712347\end{array}$

$8.425582-2.216839 \quad 0.205430$

$\begin{array}{llll}6.538792 & -1.241769 & 0.422055\end{array}$

$6.777199-0.482557 \quad-0.312913$

$\begin{array}{lll}5.265662 & -1.222350 & 1.057602\end{array}$

$\begin{array}{llll}4.267452 & -0.223217 & 0.765049\end{array}$

$\begin{array}{llll}4.557436 & 0.949115 & -0.106380\end{array}$

$\begin{array}{llll}5.640907 & 1.856932 & 0.177834\end{array}$

$\begin{array}{lll}6.399412 & 1.792250 & 1.380400\end{array}$

$\begin{array}{lll}6.156542 & 1.040079 & 2.121332\end{array}$

$\begin{array}{lll}7.425242 & 2.679719 & 1.624480\end{array}$

$\begin{array}{lll}7.982344 & 2.612983 & 2.554609\end{array}$

$\begin{array}{llll}7.756035 & 3.680604 & 0.680343\end{array}$

$\begin{array}{llll}8.571863 & 4.368149 & 0.883287\end{array}$

$\begin{array}{llll}7.029478 & 3.787213 & -0.483911\end{array}$

$\begin{array}{llll}7.257599 & 4.563214 & -1.209953\end{array}$

$\begin{array}{llll}5.954173 & 2.899300 & -0.758433\end{array}$

$\begin{array}{llll}5.169911 & 3.042014 & -1.934964\end{array}$

$\begin{array}{llll}5.425981 & 3.819395 & -2.649234\end{array}$

$\begin{array}{llll}4.080512 & 2.235529 & -2.145968\end{array}$

$\begin{array}{llll}3.440636 & 2.351263 & -3.014494\end{array}$

$\begin{array}{llll}3.765183 & 1.224204 & -1.211088\end{array}$

$\begin{array}{llll}-0.950503 & 1.659123 & -1.101798\end{array}$

$\begin{array}{llll}-1.648994 & 1.512005 & -0.267707\end{array}$

$\begin{array}{llll}-1.403438 & 2.893434 & -1.861341\end{array}$

$\begin{array}{llll}-0.571097 & 3.523756 & -2.796803\end{array}$

$\begin{array}{llll}0.445370 & 3.162689 & -2.928745\end{array}$

$\begin{array}{llll}-1.036481 & 4.610913 & -3.536805\end{array}$

$\begin{array}{llll}-0.380052 & 5.096340 & -4.253700\end{array}$

$\begin{array}{llll}-2.344255 & 5.076008 & -3.359623\end{array}$

$\begin{array}{llll}-2.705269 & 5.921398 & -3.938203\end{array}$

$\begin{array}{llll}-3.179975 & 4.452198 & -2.430936\end{array}$

$\begin{array}{llll}-4.195484 & 4.810154 & -2.283760\end{array}$

$\begin{array}{llll}-2.706821 & 3.371154 & -1.682359\end{array}$

$\begin{array}{lll}-3.350649 & 2.891682 & -0.949228\end{array}$

$\begin{array}{llll}-1.025705 & 0.447859 & -2.043234\end{array}$

$\begin{array}{llll}-0.552448 & 0.723083 & -2.993253\end{array}$

$\begin{array}{llll}-2.082773 & 0.294357 & -2.262536\end{array}$

$\begin{array}{lll}0.636596 & 3.010566 & 0.308911\end{array}$

$\begin{array}{llll}0.049356 & 3.778300 & -0.199698\end{array}$

$\begin{array}{lll}0.036984 & 2.889527 & 1.708546\end{array}$

$\begin{array}{lll}-1.299665 & 3.268653 & 1.906814\end{array}$

$\begin{array}{lll}-1.880659 & 3.624759 & 1.059871\end{array}$

$\begin{array}{lll}-1.878083 & 3.229494 & 3.178633\end{array}$

$\begin{array}{lll}-2.908119 & 3.544246 & 3.316527\end{array}$

$\begin{array}{lll}-1.123279 & 2.814179 & 4.277219\end{array}$

$\begin{array}{lll}-1.566382 & 2.789925 & 5.268753\end{array}$

$\begin{array}{lll}0.212839 & 2.448318 & 4.093381\end{array}$

$\begin{array}{lll}0.813181 & 2.137500 & 4.943746\end{array}$

$\begin{array}{lll}0.786085 & 2.482104 & 2.821569\end{array}$

$\begin{array}{lll}1.822640 & 2.192944 & 2.700060\end{array}$

$\begin{array}{lll}2.073922 & 3.524850 & 0.304978\end{array}$

$\begin{array}{llll}2.402180 & 3.718000 & -0.717597\end{array}$

$\begin{array}{lll}2.779749 & 2.836581 & 0.768069\end{array}$
$-3466.090335$

$-3466.089391$

$-3466.258896$ $\begin{array}{lll}2.107182 & 4.467295 & 0.859869\end{array}$

$\begin{array}{llll}1.181073 & -1.375223 & -3.257803\end{array}$

$\begin{array}{llll}1.237280 & -0.344131 & -3.596685\end{array}$

$\begin{array}{llll}-0.030664 & -2.046959 & -3.503573\end{array}$

$-0.809564-1.482218-4.014672$

$\begin{array}{lll}-0.191038 & -3.551774 & -3.624829\end{array}$

$\begin{array}{llll}0.114665 & -3.863412 & -4.634868\end{array}$

$\begin{array}{lll}-1.256217 & -3.785653 & -3.543749\end{array}$

$\begin{array}{llll}0.586232 & -4.383768 & -2.579065\end{array}$

$\begin{array}{llll}1.588585 & -4.611369 & -2.953474\end{array}$

$\begin{array}{llll}0.081229 & -5.346844 & -2.455538\end{array}$

$\begin{array}{llll}0.715883 & -3.733164 & -1.213027\end{array}$

$\begin{array}{llll}0.089623 & -4.133246 & -0.426070\end{array}$

$\begin{array}{llll}1.769253 & -2.941606 & -0.834132\end{array}$

$\begin{array}{lll}1.917616 & -2.781247 & 0.229029\end{array}$

$\begin{array}{llll}2.928773 & -2.579287 & -1.738073\end{array}$

$\begin{array}{llll}3.524886 & -1.818521 & -1.238463\end{array}$

$\begin{array}{llll}3.575744 & -3.463203 & -1.832238\end{array}$

$\begin{array}{llll}2.537248 & -2.072734 & -3.148087\end{array}$

$\begin{array}{llll}2.555713 & -2.902312 & -3.862934\end{array}$

$\begin{array}{llll}3.305871 & -1.368842 & -3.477563\end{array}$

$\begin{array}{llll}-2.780642 & -2.150541 & -1.659657\end{array}$

$\begin{array}{llll}-1.945506 & -2.351877 & -0.549354\end{array}$

$\begin{array}{llll}-1.798543 & -3.391113 & -0.291384\end{array}$

$\begin{array}{lll}-1.472632 & -1.342246 & 0.365801\end{array}$

$\begin{array}{lll}-1.999334 & -0.391703 & 0.344652\end{array}$

$\begin{array}{llll}-1.017935 & -1.696157 & 1.738524\end{array}$

$\begin{array}{lll}-0.718613 & -3.010091 & 2.144410\end{array}$

$\begin{array}{lll}-0.898304 & -0.673503 & 2.696995\end{array}$

$\begin{array}{lll}-0.324359 & -3.288888 & 3.453490\end{array}$

$\begin{array}{lll}-0.806702 & -3.832586 & 1.444401\end{array}$

$\begin{array}{lll}-0.516218 & -0.952590 & 4.009565\end{array}$

$\begin{array}{lll}-1.103313 & 0.352555 & 2.410117\end{array}$

$\begin{array}{lll}-0.225919 & -2.263027 & 4.397458\end{array}$

$-0.102050-4.314136 \quad 3.736860$

$\begin{array}{lll}-0.441330 & -0.139922 & 4.724384\end{array}$

$\begin{array}{llll}0.074205 & -2.481419 & 5.418210\end{array}$

$\begin{array}{llll}-4.255760 & -4.350076 & -0.202320\end{array}$

$\begin{array}{llll}-4.928335 & -3.327710 & -0.306461\end{array}$

$-4.732794-2.327638-1.105936$

$\begin{array}{lll}-6.038477 & -3.112294 & 0.475688\end{array}$

$-6.330353-4.151980 \quad 1.417022$

$\begin{array}{lll}-6.500940 & -5.109243 & 0.914729\end{array}$

$\begin{array}{lll}-7.238570 & -3.839482 & 1.937326\end{array}$

$\begin{array}{lll}-5.514526 & -4.274795 & 2.136961\end{array}$

$\begin{array}{lll}-5.767933 & 1.000998 & -1.977352\end{array}$

$\begin{array}{llll}-6.816900 & 2.048404 & -1.633625\end{array}$

$\begin{array}{llll}-4.870840 & 1.472699 & -2.396651\end{array}$

$\begin{array}{llll}-6.139330 & 0.272339 & -2.701337\end{array}$

$\begin{array}{lll}-6.333798 & 2.884582 & -0.455954\end{array}$

$\begin{array}{llll}-7.003122 & 2.703783 & -2.488108\end{array}$

$\begin{array}{llll}-7.758028 & 1.552466 & -1.373934\end{array}$

$\begin{array}{llll}-7.151157 & 3.503675 & -0.079066\end{array}$

$\begin{array}{llll}-5.520042 & 3.555489 & -0.751752\end{array}$

$\begin{array}{llll}-5.407906 & 0.275025 & -0.759952\end{array}$

$\begin{array}{lll}-5.465504 & 0.785585 & 0.462966\end{array}$

$\begin{array}{lll}-5.869695 & 2.042743 & 0.673066\end{array}$

$\begin{array}{lll}-5.059991 & -0.170208 & 1.568725\end{array}$

$\begin{array}{lll}-4.505360 & -0.972884 & 1.082884\end{array}$

$\begin{array}{lll}-5.971280 & -0.634908 & 1.962279\end{array}$

$\begin{array}{lll}-4.217460 & 0.424335 & 2.706196\end{array}$

$\begin{array}{lll}-3.490025 & 1.128701 & 2.289147\end{array}$

$\begin{array}{lll}-5.775345 & 2.765777 & 1.966378\end{array}$

$\begin{array}{lll}-5.054976 & 1.110219 & 3.790019\end{array}$

$\begin{array}{lll}-4.395031 & 1.734137 & 4.404065\end{array}$

$\begin{array}{lll}-6.187358 & 1.965668 & 3.204474\end{array}$

$\begin{array}{lll}-6.539596 & 2.677160 & 3.958766\end{array}$

$\begin{array}{lll}-7.051748 & 1.344991 & 2.942216\end{array}$

$\begin{array}{lll}-6.439963 & 3.623945 & 1.860757\end{array}$

$\begin{array}{lll}-4.759144 & 3.161966 & 2.072815\end{array}$

$\begin{array}{lll}-5.487092 & 0.356056 & 4.458124\end{array}$

$\begin{array}{lll}-3.625792 & -0.380505 & 3.149650\end{array}$

$\begin{array}{llll}-5.202375 & -0.743013 & -0.843679\end{array}$

$-2.894130 \quad-2.961154 \quad-2.364822$

$\begin{array}{llll}-3.038540 & -1.167160 & -2.014478\end{array}$ 


\begin{tabular}{|c|c|c|c|}
\hline \multicolumn{4}{|c|}{$[12-13]^{\ddagger} \mathbf{P} 1$ endo-si } \\
\hline \multicolumn{4}{|c|}{ Number of imaginary frequencies $=1$} \\
\hline \multicolumn{4}{|c|}{ The smallest frequencies are $=-318.60$} \\
\hline \multicolumn{4}{|c|}{$=-3467.3649174$} \\
\hline \multicolumn{4}{|c|}{ Zero-point correction $=$} \\
\hline \multicolumn{4}{|c|}{ (Hartree/Particle) } \\
\hline \multicolumn{4}{|c|}{ Thermal correction to Energy= } \\
\hline \multicolumn{4}{|c|}{ Thermal correction to Enthalpy= } \\
\hline \multicolumn{4}{|c|}{ Thermal correction to Gibbs Free Energy= } \\
\hline \multicolumn{4}{|c|}{ Sum of electronic and zero-point Energies $=$} \\
\hline \multicolumn{4}{|c|}{ Sum of electronic and thermal Energies $=$} \\
\hline \multicolumn{4}{|c|}{ Sum of electronic and thermal Enthalpies $=$} \\
\hline & & & \\
\hline & & & \\
\hline 7 & 3003 & -1.185844 & -1.423240 \\
\hline 15 & 1.466482 & 0.237600 & -0.513100 \\
\hline 8 & 1.989831 & 0.376022 & 1.060634 \\
\hline 8 & 2.925015 & -0.131409 & -1.248109 \\
\hline 7 & 1.032365 & 1.829503 & -0.808606 \\
\hline & 2.735092 & -0.654320 & 1.629798 \\
\hline 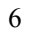 & & -1.427537 & \\
\hline 1 & 1. & -1.1 & 937 \\
\hline 6 & 2.746478 & -2.478526 & 3.201939 \\
\hline 1 & 2.260977 & -3.073092 & \\
\hline 6 & 4.0 & -2.8 & \\
\hline 6 & 4.722985 & -3.962178 & 915 \\
\hline 1 & 4.2 & -4.55 & \\
\hline 6 & 5. & -4.3 & \\
\hline 1 & 6.46 & -5.200 & \\
\hline 6 & & -3.5 & 68 \\
\hline 1 & 7.5 & $-3.85 \mathrm{C}$ & 25 \\
\hline 6 & 6.009 & -2.435845 & \\
\hline 1 & & -1.8 & \\
\hline & 4.724882 & -2.016 & 940 \\
\hline 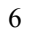 & 4.058382 & -0.8549 & \\
\hline 6 & & $0.0^{\top}$ & \\
\hline & 5.9893 & 0.711 & 0.6 \\
\hline 6 & 6.571387 & 0.646651 & \\
\hline 1 & 6.05 & 0.102 & \\
\hline 6 & 7.765516 & 1.276538 & 2.21 \\
\hline 1 & 8.1 & 1.217903 & 3.2 \\
\hline & 8.44 & 2.003812 & 1.216749 \\
\hline 1 & 9.393646 & 2.488879 & 1.4 \\
\hline 6 & 7.90 & 2.106 & -0.0 \\
\hline 1 & 8.40 & 2.676926 & 99 \\
\hline 6 & & & \\
\hline 6 & 6.08 & 1.629450 & 48 \\
\hline 1 & 6.609350 & 2.195298 & 46 \\
\hline 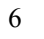 & & & \\
\hline 1 & 4.354731 & 1.230863 & 91 \\
\hline & 4.170963 & 0.357026 & -0.9 \\
\hline 6 & -0.218240 & 1.93 & 12 \\
\hline 1 & -1.061523 & 2.037065 & -0.921132 \\
\hline 6 & -0.222740 & 3.148038 & -2.532435 \\
\hline 6 & 0.85 & 3.414661 & -3.3 \\
\hline 1 & 1.735171 & 2.777643 & -3.348299 \\
\hline 6 & 0.811639 & 4.497222 & -4.267481 \\
\hline 1 & 1.656868 & 4.698337 & -4.919918 \\
\hline 6 & -0.315900 & 5.324844 & -4.308051 \\
\hline 1 & -0.349030 & 6.1682 & -4.9 \\
\hline 6 & -1.395093 & 5.064435 & -3.461548 \\
\hline 1 & -2.272448 & 5.705160 & -3.482974 \\
\hline 6 & -1.344258 & 3.983884 & -2.576062 \\
\hline 1 & -2.178941 & 3.790703 & -1.905706 \\
\hline 6 & -0.392744 & 0.672680 & -2.462339 \\
\hline 1 & 0.334452 & 0.713107 & -3.280899 \\
\hline 1 & -1.382378 & 0.699992 & -2.925770 \\
\hline 6 & 1.487972 & 3.089658 & -0.138730 \\
\hline 1 & 1.272473 & 3.859418 & -0.881559 \\
\hline 6 & 0.694854 & 3.491984 & 1.104881 \\
\hline & -0.300268 & 4.472819 & 0.981133 \\
\hline
\end{tabular}

\begin{tabular}{|c|c|c|}
\hline & & \\
\hline 0.941641 & 4.995604 & \\
\hline 1.698939 & 5.766368 & \\
\hline 1788 & 3745 & \\
\hline 1.076250 & 4.956473 & 4.261539 \\
\hline .382757 & 811 & \\
\hline 0.654467 & 3.186023 & \\
\hline 1.017653 & 3.026912 & 292 \\
\hline 134 & 2.26 & 039 \\
\hline 2.996101 & & 914 \\
\hline 3.537387 & 3.0 & 172 \\
\hline 272 & 2.4 & \\
\hline 3.226463 & 4.1 & \\
\hline 1.3 & & \\
\hline 1.7 & -0.8 & 000 \\
\hline 0.0 & -2.0 & \\
\hline 8880 & & \\
\hline 609 & -3 & 630 \\
\hline 908 & -4.0 & -4 \\
\hline 305 & -3.4 & \\
\hline 961 & -4.3 & 1727 \\
\hline 749 & -4.9 & \\
\hline 25 & -5 . & \\
\hline-0.2 & -3.5 & \\
\hline & & \\
\hline 0.9 & -3. & \\
\hline 0.9 & -2 & \\
\hline & & \\
\hline 3.0 & -2. & \\
\hline 521 & -4.2 & \\
\hline 2.3 & -2 & \\
\hline 2.2 & -3 & \\
\hline 775 & -2.4 & \\
\hline 6 & -1 . & \\
\hline-2.6 & -2 & \\
\hline 872 & & \\
\hline 63 & -0 & \\
\hline-2.6 & & \\
\hline & & \\
\hline 60 & -2 & \\
\hline 093 & -0.2 & \\
\hline-2.3 & & \\
\hline-1.4 & -0.7 & \\
\hline-2.4 & 1.8 & \\
\hline-2.6 & & \\
\hline-1.5 & & \\
\hline-1.0 & -1.7 & \\
\hline-2.1 & & \\
\hline-2.8 & 2.8 & \\
\hline-1.2 & -0.3 & \\
\hline-2.2 & & \\
\hline-4.3 & -4.1 & \\
\hline$-5.0^{\prime}$ & & \\
\hline-4.7 & -2.0 & \\
\hline-6.4 & -3.0 & \\
\hline-6.9 & -4.2 & \\
\hline & -5.1 & \\
\hline-7.9 & -4.0 & \\
\hline-6.3 & -4.3 & \\
\hline-5.2 & 1.5 & \\
\hline-6.0 & 2.6 & \\
\hline-4.2 & & \\
\hline-5.6 & 1.2 & -2. \\
\hline-5.6 & 2.8 & \\
\hline & & \\
\hline-7.1 & 2.4 & -0 . \\
\hline-6.2 & & \\
\hline-4.6 & 3.1 & \\
\hline$-5.2^{\prime}$ & 0.4 & -0 \\
\hline & & \\
\hline-5.7 & 1.5 & 1. \\
\hline-5.4 & -0.9 & \\
\hline & -1.4 & 0 \\
\hline & -1.442580 & 1.352044 \\
\hline-5.0 & -0.98 & 3.0 \\
\hline
\end{tabular}




$\begin{array}{rrrr}1 & -4.235081 & -0.306502 & 3.183309 \\ 6 & -6.130463 & 1.703232 & 2.943628 \\ 6 & -6.231649 & -0.650263 & 3.960403 \\ 1 & -5.827828 & -0.479867 & 4.965387 \\ 6 & -7.016643 & 0.584900 & 3.498819 \\ 1 & -7.588653 & 0.995671 & 4.337277 \\ 1 & -7.750160 & 0.314804 & 2.730308 \\ 1 & -6.665431 & 2.650538 & 3.022885 \\ 1 & -5.203623 & 1.806235 & 3.519778 \\ 1 & -6.916151 & -1.503610 & 4.034117 \\ 1 & -4.700524 & -1.989629 & 3.223639 \\ 1 & -5.111221 & -0.508628 & -0.868912 \\ -\end{array}$

$[12-13]^{\ddagger} \mathbf{P 2}$ endo-re

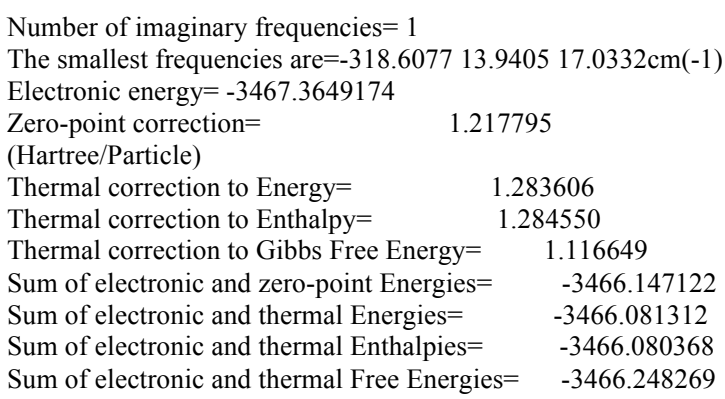

Cartesian Coordinates

\begin{tabular}{|c|c|c|c|}
\hline & 0.123998 & -1.192235 & -1.41 \\
\hline & -1.467843 & 0.239623 & -0.514578 \\
\hline & -1.995500 & 0.388896 & 1.056733 \\
\hline & -2.925134 & -0.129891 & -1.251601 \\
\hline & -1.029235 & 1.829155 & -0.817833 \\
\hline & -2.742115 & -0.637339 & 1.631039 \\
\hline & -2.096088 & -1.402701 & 2.625567 \\
\hline 1 & -1.086630 & -1.127724 & 2.903704 \\
\hline & -2.757875 & -2.449674 & 3.216774 \\
\hline 1 & -2.274346 & -3.037647 & 3.991951 \\
\hline & -4.071421 & -2.797455 & 2.800890 \\
\hline 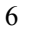 & -4.734362 & -3.933272 & 3.340078 \\
\hline 1 & -4.224940 & -4.516637 & 4.102703 \\
\hline 6 & -5.985518 & -4.298602 & 2.897802 \\
\hline 1 & -6.480297 & -5.172157 & 3.312060 \\
\hline 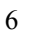 & -6.621179 & -3.541319 & 1.885354 \\
\hline 1 & -7.596465 & -3.847244 & 1.517676 \\
\hline 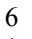 & -6.013826 & -2.423164 & 1.356048 \\
\hline 1 & -6.513464 & -1.863601 & 0.574607 \\
\hline 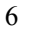 & -4.731598 & -1.999961 & 1.806065 \\
\hline 6 & -4.064125 & -0.841108 & 1.265388 \\
\hline 6 & -4.744534 & 0.077490 & 144 \\
\hline & -5.995726 & 0.718170 & 0.629390 \\
\hline , & -6.580340 & 0.659772 & 1.925701 \\
\hline 1 & -6.068223 & 0.123100 & 2.715619 \\
\hline 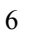 & -7.776856 & 1.288169 & 2.196398 \\
\hline 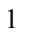 & -8.196077 & 1.235542 & 3.197115 \\
\hline & -8.461371 & 2.006397 & 1.187315 \\
\hline 1 & -9.406884 & 2.490532 & 1.413501 \\
\hline 6 & -7.914945 & 2.101861 & -0.072603 \\
\hline 1 & -8.418772 & 2.665834 & -0.853337 \\
\hline 6 & -6.673705 & 1.481333 & -0.379956 \\
\hline 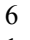 & -6.083381 & 1.621370 & -1.665476 \\
\hline t & -6.612129 & 2.181039 & -2.431860 \\
\hline 6 & -4.844854 & 1.090741 & -1.923566 \\
\hline 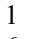 & -4.353830 & 1.221053 & -2.882281 \\
\hline 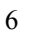 & -4.172323 & 0.358735 & -0.918630 \\
\hline 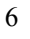 & 0.226456 & 1.929007 & -1.615918 \\
\hline 1 & 1.066409 & 2.022795 & -0.918893 \\
\hline 6 & 0.246227 & 3.137021 & -2.537124 \\
\hline 6 & -0.822466 & 3.409776 & -3.404258 \\
\hline 1 & -1.707400 & 2.779848 & -3.369770 \\
\hline & -0.762710 & 4.489736 & -4.284354 \\
\hline 1 & -1.600854 & 4.695632 & -4.944369 \\
\hline & 0.371486 & 5.308475 & -4.317624 \\
\hline
\end{tabular}

$\begin{array}{lll}0.417214 & 6.149228 & -5.003901\end{array}$

$\begin{array}{llll}1.441554 & 5.041750 & -3.461523\end{array}$

$2.324413 \quad 5.674997 \quad-3.477705$

$\begin{array}{llll}1.374747 & 3.963989 & -2.573787\end{array}$

$\begin{array}{llll}2.202821 & 3.765343 & -1.896804\end{array}$

$\begin{array}{llll}0.396243 & 0.660849 & -2.461386\end{array}$

$\begin{array}{llll}-0.330579 & 0.701703 & -3.280123\end{array}$

$\begin{array}{llll}1.386024 & 0.681293 & -2.924731\end{array}$

$\begin{array}{llll}-1.483422 & 3.092736 & -0.153533\end{array}$

$\begin{array}{llll}-1.263796 & 3.859980 & -0.897865\end{array}$

$\begin{array}{lll}-0.692759 & 3.496296 & 1.091421\end{array}$

$\begin{array}{llll}0.308987 & 4.470391 & 0.966807\end{array}$

$\begin{array}{llll}0.563234 & 4.845910 & -0.021046\end{array}$

$\begin{array}{llll}0.951452 & 4.992324 & 2.092926\end{array}$

$\begin{array}{lll}1.713882 & 5.757893 & 1.974142\end{array}$

$\begin{array}{llll}0.596566 & 4.546119 & 3.366798\end{array}$

$\begin{array}{llll}1.082033 & 4.958253 & 4.247257\end{array}$

$\begin{array}{lll}-0.384334 & 3.559634 & 3.501496\end{array}$

$\begin{array}{lll}-0.660065 & 3.199303 & 4.488649\end{array}$

$\begin{array}{lll}-1.020564 & 3.036673 & 2.375556\end{array}$

$\begin{array}{lll}-1.786617 & 2.280817 & 2.497011\end{array}$

$\begin{array}{lll}-2.992554 & 3.179151 & 0.076923\end{array}$

$\begin{array}{llll}-3.529474 & 3.047925 & -0.863499\end{array}$

$\begin{array}{llll}-3.362357 & 2.447805 & 0.794583\end{array}$

$\begin{array}{lll}-3.223967 & 4.176244 & 0.463231\end{array}$

$\begin{array}{llll}-1.305803 & -1.773006 & -3.015450\end{array}$

$\begin{array}{llll}-1.731846 & -0.857049 & -3.413670\end{array}$

$\begin{array}{lll}-0.002803 & -2.105762 & -3.467952\end{array}$

$\begin{array}{llll}0.461227 & -1.421906 & -4.177521\end{array}$

$\begin{array}{llll}0.512899 & -3.531673 & -3.556625\end{array}$

$\begin{array}{llll}0.047347 & -4.027539 & -4.421840\end{array}$

$\begin{array}{llll}1.584608 & -3.500187 & -3.768597\end{array}$

$\begin{array}{llll}0.265098 & -4.364061 & -2.279891\end{array}$

$\begin{array}{llll}-0.661551 & -4.937664 & -2.376821\end{array}$

$\begin{array}{llll}1.067862 & -5.099929 & -2.171657\end{array}$

$\begin{array}{llll}0.198058 & -3.543652 & -0.994635\end{array}$

$\begin{array}{llll}1.031296 & -3.689041 & -0.315313\end{array}$

$\begin{array}{llll}-0.980688 & -3.051170 & -0.449656\end{array}$

$\begin{array}{llll}-0.998903 & -2.853342 & 0.619198\end{array}$

$-2.337878-3.190033 \quad-1.109851$

$\begin{array}{llll}-3.039033 & -2.549038 & -0.576952\end{array}$

$\begin{array}{llll}-2.692592 & -4.222048 & -0.973561\end{array}$

$\begin{array}{llll}-2.352305 & -2.815364 & -2.610045\end{array}$

$-2.216805 \quad-3.710208 \quad-3.226859$

$\begin{array}{llll}-3.341741 & -2.420950 & -2.856658\end{array}$

$\begin{array}{llll}2.791428 & -1.893315 & -1.921336\end{array}$

$\begin{array}{llll}2.615357 & -2.934701 & -1.707791\end{array}$

$\begin{array}{llll}2.938886 & -1.625522 & -2.959653\end{array}$

$\begin{array}{llll}2.322445 & -0.886308 & -1.035044\end{array}$

$\begin{array}{llll}2.601424 & 0.133327 & -1.276933\end{array}$

$\begin{array}{llll}1.728904 & -1.137707 & 0.233955\end{array}$

$\begin{array}{llll}1.730649 & -2.171084 & 0.566555\end{array}$

$\begin{array}{lll}1.806811 & -0.217989 & 1.390004\end{array}$

$\begin{array}{llll}2.330617 & 1.084365 & 1.324308\end{array}$

$\begin{array}{lll}1.447580 & -0.719440 & 2.654774\end{array}$

$\begin{array}{lll}2.476637 & 1.853575 & 2.476586\end{array}$

$\begin{array}{llll}2.660733 & 1.486906 & 0.373732\end{array}$

$\begin{array}{lll}1.581570 & 0.052718 & 3.807590\end{array}$

$\begin{array}{lll}1.081850 & -1.740278 & 2.728809\end{array}$

$\begin{array}{lll}2.099605 & 1.346998 & 3.723406\end{array}$

$\begin{array}{lll}2.873221 & 2.859880 & 2.401394\end{array}$

$\begin{array}{llll}1.295778 & -0.361186 & 4.770521\end{array}$

$\begin{array}{lll}2.208635 & 1.955102 & 4.615775\end{array}$

$\begin{array}{llll}4.383369 & -4.106832 & -0.801221\end{array}$

$\begin{array}{llll}5.075835 & -3.128065 & -1.067046\end{array}$

$\begin{array}{llll}4.709926 & -2.022340 & -1.649260\end{array}$

$\begin{array}{llll}6.405784 & -3.076332 & -0.751672\end{array}$

$\begin{array}{llll}6.917109 & -4.235379 & -0.077466\end{array}$

$\begin{array}{llll}6.796823 & -5.134003 & -0.689495\end{array}$

$\begin{array}{llll}7.977710 & -4.038730 & 0.091239\end{array}$

$\begin{array}{llll}6.410912 & -4.392127 & 0.880362\end{array}$

$\begin{array}{llll}5.234318 & 1.586816 & -1.310025\end{array}$

$\begin{array}{llll}6.035585 & 2.716948 & -0.682848\end{array}$

$\begin{array}{llll}4.189468 & 1.886592 & -1.464635\end{array}$

$\begin{array}{llll}5.642308 & 1.287973 & -2.278894\end{array}$ 
$\begin{array}{lll}5.639725 & 2.858005 & 0.782262\end{array}$

$\begin{array}{llll}5.842385 & 3.656577 & -1.206628\end{array}$

$\begin{array}{lll}7.106858 & 2.503649 & -0.754502\end{array}$

$\begin{array}{llll}6.268143 & 3.598563 & 1.278072\end{array}$

$\begin{array}{lll}4.597628 & 3.185379 & 0.875529\end{array}$

$\begin{array}{llll}5.265896 & 0.420587 & -0.429674\end{array}$

$\begin{array}{llll}5.531519 & 0.434680 & 0.871984\end{array}$

$\begin{array}{llll}5.794952 & 1.577254 & 1.508280\end{array}$

$\begin{array}{llll}5.482668 & -0.933298 & 1.529008\end{array}$

$\begin{array}{llll}4.743386 & -1.492573 & 0.952832\end{array}$

$6.443979-1.429797 \quad 1.353133$

$\begin{array}{llll}5.087559 & -0.989744 & 3.008123\end{array}$

$\begin{array}{lll}4.241108 & -0.319197 & 3.176080\end{array}$

$\begin{array}{llll}6.133101 & 1.702786 & 2.947773\end{array}$

$\begin{array}{lll}6.236711 & -0.651509 & 3.962130\end{array}$

$\begin{array}{lll}5.827091 & -0.480382 & 4.964628\end{array}$

$\begin{array}{llll}7.020430 & 0.584775 & 3.501836\end{array}$

$\begin{array}{llll}7.592290 & 0.995220 & 4.340472\end{array}$

$\begin{array}{llll}7.754142 & 0.315882 & 2.733085\end{array}$

$\begin{array}{llll}6.666390 & 2.650967 & 3.028994\end{array}$

$\begin{array}{lll}5.205441 & 1.803318 & 3.523189\end{array}$

$\begin{array}{llll}6.923409 & -1.502507 & 4.041644\end{array}$

$4.718028-1.999336 \quad 3.214722$

$\begin{array}{llll}5.105147 & -0.500670 & -0.866590\end{array}$

\section{[12-13] $]^{\ddagger} \mathbf{P 2}$ endo-re conf2}

Number of imaginary frequencies : 1

The smallest frequency is : $-322.0638 \mathrm{~cm}(-1)$

Electronic energy $=-3467.3655764$

Zero-point correction $=$

1.217927

(Hartree/Particle)

Thermal correction to Energy= $\quad 1.283721$

Thermal correction to Enthalpy= $\quad 1.284666$

Thermal correction to Gibbs Free Energy= $\quad 1.116036$

Sum of electronic and zero-point Energies=

Sum of electronic and thermal Energies=

Sum of electronic and thermal Enthalpies=

Sum of electronic and thermal Free Energies=

$-3466.147649$

$-3466.081855$

$-3466.080911$

$-3466.249541$

Cartesian Coordinates

$0.123998-1.192235-1.414125$

$\begin{array}{lll}-1.467843 & 0.239623 & -0.514578\end{array}$

$\begin{array}{lll}-1.995500 & 0.388896 & 1.056733\end{array}$

$-2.925134-0.129891-1.251601$

$\begin{array}{lll}-1.029235 & 1.829155 & -0.817833\end{array}$

$\begin{array}{lll}-2.742115 & -0.637339 & 1.631039\end{array}$

$\begin{array}{lll}-2.096088 & -1.402701 & 2.625567\end{array}$

$\begin{array}{lll}-1.086630 & -1.127724 & 2.903704\end{array}$

$-2.757875 \quad-2.449674 \quad 3.216774$

$\begin{array}{lll}-2.274346 & -3.037647 & 3.991951\end{array}$

$-4.071421-2.797455 \quad 2.800890$

$\begin{array}{lll}-4.734362 & -3.933272 & 3.340078\end{array}$

$-4.224940-4.516637 \quad 4.102703$

$\begin{array}{llll}-5.985518 & -4.298602 & 2.897802\end{array}$

$\begin{array}{lll}-6.480297 & -5.172157 & 3.312060\end{array}$

$\begin{array}{lll}-6.621179 & -3.541319 & 1.885354\end{array}$

$\begin{array}{lll}-7.596465 & -3.847244 & 1.517676\end{array}$

$\begin{array}{lll}-6.013826 & -2.423164 & 1.356048\end{array}$

$\begin{array}{lll}-6.513464 & -1.863601 & 0.574607\end{array}$

$\begin{array}{lll}-4.731598 & -1.999961 & 1.806065\end{array}$

$\begin{array}{lll}-4.064125 & -0.841108 & 1.265388\end{array}$

$\begin{array}{llll}-4.744534 & 0.077490 & 0.312144\end{array}$

$\begin{array}{lll}-5.995726 & 0.718170 & 0.629390\end{array}$

$\begin{array}{lll}-6.580340 & 0.659772 & 1.925701\end{array}$

$\begin{array}{lll}-6.068223 & 0.123100 & 2.715619\end{array}$

$\begin{array}{llll}-7.776856 & 1.288169 & 2.196398\end{array}$

$\begin{array}{lll}-8.196077 & 1.235542 & 3.197115\end{array}$

$\begin{array}{lll}-8.461371 & 2.006397 & 1.187315\end{array}$

$\begin{array}{lll}-9.406884 & 2.490532 & 1.413501\end{array}$

$\begin{array}{lll}-7.914945 & 2.101861 & -0.072603\end{array}$ $\begin{array}{llll}-8.418772 & 2.665834 & -0.853337\end{array}$

$\begin{array}{llll}-6.673705 & 1.481333 & -0.379956\end{array}$

$\begin{array}{llll}-6.083381 & 1.621370 & -1.665476\end{array}$

$\begin{array}{lll}-6.612129 & 2.181039 & -2.431860\end{array}$

$\begin{array}{lll}-4.844854 & 1.090741 & -1.923566\end{array}$

$\begin{array}{llll}-4.353830 & 1.221053 & -2.882281\end{array}$

$\begin{array}{llll}-4.172323 & 0.358735 & -0.918630\end{array}$

$\begin{array}{llll}0.226456 & 1.929007 & -1.615918\end{array}$

$\begin{array}{llll}1.066409 & 2.022795 & -0.918893\end{array}$

$\begin{array}{llll}0.246227 & 3.137021 & -2.537124\end{array}$

$\begin{array}{llll}-0.822466 & 3.409776 & -3.404258\end{array}$

$\begin{array}{lll}-1.707400 & 2.779848 & -3.369770\end{array}$

$\begin{array}{llll}-0.762710 & 4.489736 & -4.284354\end{array}$

$\begin{array}{llll}-1.600854 & 4.695632 & -4.944369\end{array}$

$\begin{array}{llll}0.371486 & 5.308475 & -4.317624\end{array}$

$\begin{array}{llll}0.417214 & 6.149228 & -5.003901\end{array}$

$\begin{array}{llll}1.441554 & 5.041750 & -3.461523\end{array}$

$\begin{array}{llll}2.324413 & 5.674997 & -3.477705\end{array}$

$\begin{array}{llll}1.374747 & 3.963989 & -2.573787\end{array}$

$2.202821 \quad 3.765343 \quad-1.896804$

$\begin{array}{llll}0.396243 & 0.660849 & -2.461386\end{array}$

$\begin{array}{llll}-0.330579 & 0.701703 & -3.280123\end{array}$

$\begin{array}{llll}1.386024 & 0.681293 & -2.924731\end{array}$

$\begin{array}{llll}-1.483422 & 3.092736 & -0.153533\end{array}$

$\begin{array}{llll}-1.263796 & 3.859980 & -0.897865\end{array}$

$\begin{array}{lll}-0.692759 & 3.496296 & 1.091421\end{array}$

$\begin{array}{llll}0.308987 & 4.470391 & 0.966807\end{array}$

$\begin{array}{llll}0.563234 & 4.845910 & -0.021046\end{array}$

$\begin{array}{llll}0.951452 & 4.992324 & 2.092926\end{array}$

$\begin{array}{lll}1.713882 & 5.757893 & 1.974142\end{array}$

$\begin{array}{llll}0.596566 & 4.546119 & 3.366798\end{array}$

$\begin{array}{lll}1.082033 & 4.958253 & 4.247257\end{array}$

$\begin{array}{lll}-0.384334 & 3.559634 & 3.501496\end{array}$

$\begin{array}{lll}-0.660065 & 3.199303 & 4.488649\end{array}$

$\begin{array}{lll}-1.020564 & 3.036673 & 2.375556\end{array}$

$\begin{array}{lll}-1.786617 & 2.280817 & 2.497011\end{array}$

$\begin{array}{llll}-2.992554 & 3.179151 & 0.076923\end{array}$

$\begin{array}{llll}-3.529474 & 3.047925 & -0.863499\end{array}$

$\begin{array}{llll}-3.362357 & 2.447805 & 0.794583\end{array}$

$\begin{array}{lll}-3.223967 & 4.176244 & 0.463231\end{array}$

$\begin{array}{llll}-1.305803 & -1.773006 & -3.015450\end{array}$

$-1.731846 \quad-0.857049 \quad-3.413670$

$\begin{array}{llll}-0.002803 & -2.105762 & -3.467952\end{array}$

$\begin{array}{llll}0.461227 & -1.421906 & -4.177521\end{array}$

$\begin{array}{llll}0.512899 & -3.531673 & -3.556625\end{array}$

$\begin{array}{llll}0.047347 & -4.027539 & -4.421840\end{array}$

$\begin{array}{llll}1.584608 & -3.500187 & -3.768597\end{array}$

$\begin{array}{llll}0.265098 & -4.364061 & -2.279891\end{array}$

$\begin{array}{llll}-0.661551 & -4.937664 & -2.376821\end{array}$

$\begin{array}{llll}1.067862 & -5.099929 & -2.171657\end{array}$

$\begin{array}{llll}0.198058 & -3.543652 & -0.994635\end{array}$

$\begin{array}{llll}1.031296 & -3.689041 & -0.315313\end{array}$

$-0.980688-3.051170 \quad-0.449656$

$\begin{array}{lll}-0.998903 & -2.853342 & 0.619198\end{array}$

$\begin{array}{llll}-2.337878 & -3.190033 & -1.109851\end{array}$

$-3.039033-2.549038-0.576952$

$\begin{array}{llll}-2.692592 & -4.222048 & -0.973561\end{array}$

$\begin{array}{llll}-2.352305 & -2.815364 & -2.610045\end{array}$

$\begin{array}{llll}-2.216805 & -3.710208 & -3.226859\end{array}$

$\begin{array}{llll}-3.341741 & -2.420950 & -2.856658\end{array}$

$2.791428-1.893315-1.921336$

$\begin{array}{llll}2.615357 & -2.934701 & -1.707791\end{array}$

$\begin{array}{llll}2.938886 & -1.625522 & -2.959653\end{array}$

$\begin{array}{llll}2.322445 & -0.886308 & -1.035044\end{array}$

$\begin{array}{llll}2.601424 & 0.133327 & -1.276933\end{array}$

$\begin{array}{llll}1.728904 & -1.137707 & 0.233955\end{array}$

$\begin{array}{lll}1.730649 & -2.171084 & 0.566555\end{array}$

$\begin{array}{lll}1.806811 & -0.217989 & 1.390004\end{array}$

$\begin{array}{llll}2.330617 & 1.084365 & 1.324308\end{array}$

$\begin{array}{lll}1.447580 & -0.719440 & 2.654774\end{array}$ 


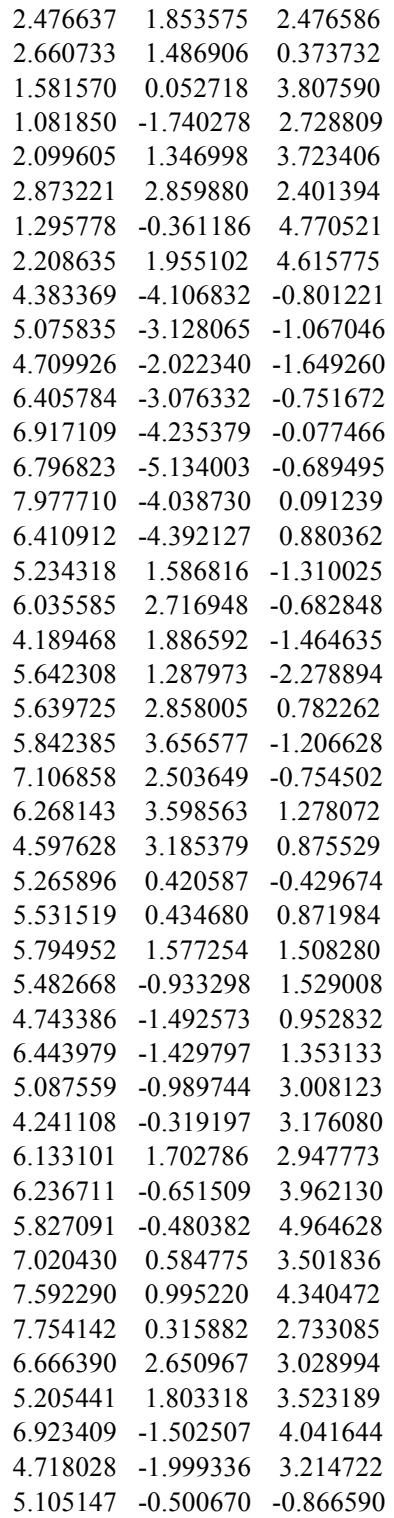

\section{$[12-13]^{\ddagger} \mathbf{P 2}$ endo-re conf2}

Number of imaginary frequencies : 1

The smallest frequency is : $-322.0722 \mathrm{~cm}(-1)$

Electronic energy $=-3467.3655765$

Zero-point correction $=\quad 1.217923$

(Hartree/Particle)

Thermal correction to Energy= $\quad 1.283719$

Thermal correction to Enthalpy= $\quad 1.284663$

Thermal correction to Gibbs Free Energy $=\quad 1.116010$

Sum of electronic and zero-point Energies $=\quad-3466.147654$

Sum of electronic and thermal Energies $=\quad-3466.081857$

Sum of electronic and thermal Enthalpies $=\quad-3466.080913$

Sum of electronic and thermal Free Energies $=\quad-3466.249567$

Cartesian Coordinates

$\begin{array}{llll}0.123979 & -1.192426 & -1.414026\end{array}$

$\begin{array}{lll}-1.467863 & 0.239514 & -0.514582\end{array}$

$\begin{array}{lll}-1.995528 & 0.388905 & 1.056716\end{array}$

$-2.925159-0.130038-1.251579$

$\begin{array}{lll}-1.029211 & 1.829015 & -0.817923\end{array}$

$\begin{array}{lll}-2.742193 & -0.637262 & 1.631077\end{array}$

$\begin{array}{lll}-2.096197 & -1.402615 & 2.625631\end{array}$

$\begin{array}{llll}-1.086717 & -1.127685 & 2.903741\end{array}$ $\begin{array}{lll}-2.758039 & -2.449516 & 3.216902\end{array}$

$\begin{array}{lll}-2.274534 & -3.037480 & 3.992100\end{array}$

$\begin{array}{lll}-4.071615 & -2.797235 & 2.801060\end{array}$

$\begin{array}{lll}-4.734619 & -3.932978 & 3.340326\end{array}$

$\begin{array}{llll}-4.225222 & -4.516329 & 4.102979\end{array}$

$\begin{array}{llll}-5.985804 & -4.298257 & 2.898089\end{array}$

$\begin{array}{lll}-6.480632 & -5.171755 & 3.312407\end{array}$

$\begin{array}{lll}-6.621431 & -3.540995 & 1.885605\end{array}$

$\begin{array}{lll}-7.596741 & -3.846881 & 1.517959\end{array}$

$\begin{array}{llll}-6.014015 & -2.422909 & 1.356223\end{array}$

$\begin{array}{llll}-6.513630 & -1.863364 & 0.574756\end{array}$

$\begin{array}{lll}-4.731756 & -1.999758 & 1.806197\end{array}$

$\begin{array}{lll}-4.064217 & -0.840978 & 1.265444\end{array}$

$\begin{array}{llll}-4.744567 & 0.077580 & 0.312119\end{array}$

$\begin{array}{llll}-5.995727 & 0.718357 & 0.629293\end{array}$

$\begin{array}{lll}-6.580358 & 0.660107 & 1.925603\end{array}$

$\begin{array}{lll}-6.068278 & 0.123476 & 2.715574\end{array}$

$\begin{array}{lll}-7.776843 & 1.288594 & 2.196231\end{array}$

$\begin{array}{lll}-8.196078 & 1.236080 & 3.196947\end{array}$

$\begin{array}{lll}-8.461307 & 2.006768 & 1.187075\end{array}$

$\begin{array}{lll}-9.406796 & 2.490975 & 1.413206\end{array}$

$\begin{array}{llll}-7.914864 & 2.102087 & -0.072847\end{array}$

$\begin{array}{llll}-8.418653 & 2.666016 & -0.853637\end{array}$

$\begin{array}{llll}-6.673655 & 1.481463 & -0.380131\end{array}$

$\begin{array}{llll}-6.083314 & 1.621344 & -1.665659\end{array}$

$\begin{array}{llll}-6.612026 & 2.180967 & -2.432102\end{array}$

$\begin{array}{llll}-4.844813 & 1.090624 & -1.923689\end{array}$

$\begin{array}{llll}-4.353776 & 1.220813 & -2.882414\end{array}$

$\begin{array}{llll}-4.172330 & 0.358680 & -0.918675\end{array}$

$\begin{array}{llll}0.226446 & 1.928795 & -1.616068\end{array}$

$\begin{array}{llll}1.066427 & 2.022631 & -0.919084\end{array}$

$\begin{array}{llll}0.246207 & 3.136734 & -2.537372\end{array}$

$\begin{array}{llll}-0.822534 & 3.409483 & -3.404447\end{array}$

$\begin{array}{llll}-1.707499 & 2.779604 & -3.369850\end{array}$

$\begin{array}{llll}-0.762785 & 4.489381 & -4.284621\end{array}$

$\begin{array}{llll}-1.600966 & 4.695276 & -4.944588\end{array}$

$\begin{array}{llll}0.371451 & 5.308058 & -4.318027\end{array}$

$\begin{array}{llll}0.417173 & 6.148763 & -5.004364\end{array}$

$\begin{array}{llll}1.441568 & 5.041335 & -3.461987\end{array}$

$\begin{array}{llll}2.324459 & 5.674534 & -3.478276\end{array}$

$\begin{array}{llll}1.374768 & 3.963640 & -2.574171\end{array}$

$\begin{array}{llll}2.202879 & 3.764993 & -1.897233\end{array}$

$\begin{array}{llll}0.396193 & 0.660571 & -2.461443\end{array}$

$\begin{array}{llll}-0.330654 & 0.701373 & -3.280162\end{array}$

$\begin{array}{llll}1.385957 & 0.680967 & -2.924825\end{array}$

$\begin{array}{llll}-1.483383 & 3.092657 & -0.153734\end{array}$

$\begin{array}{llll}-1.263757 & 3.859835 & -0.898134\end{array}$

$\begin{array}{lll}-0.692700 & 3.496316 & 1.091173\end{array}$

$\begin{array}{llll}0.309041 & 4.470404 & 0.966463\end{array}$

$\begin{array}{llll}0.563279 & 4.845833 & -0.021427\end{array}$

$\begin{array}{llll}0.951514 & 4.992439 & 2.092530\end{array}$

$\begin{array}{llll}1.713940 & 5.758000 & 1.973673\end{array}$

$\begin{array}{llll}0.596639 & 4.546345 & 3.366444\end{array}$

$\begin{array}{llll}1.082110 & 4.958560 & 4.246863\end{array}$

$\begin{array}{llll}-0.384253 & 3.559865 & 3.501238\end{array}$

$\begin{array}{llll}-0.659973 & 3.199620 & 4.488426\end{array}$

$\begin{array}{lll}-1.020490 & 3.036801 & 2.375350\end{array}$

$\begin{array}{llll}-1.786537 & 2.280950 & 2.496875\end{array}$

$\begin{array}{llll}-2.992510 & 3.179117 & 0.076737\end{array}$

$\begin{array}{llll}-3.529448 & 3.047810 & -0.863663\end{array}$

$\begin{array}{lll}-3.362314 & 2.447845 & 0.794473\end{array}$

$\begin{array}{llll}-3.223900 & 4.176250 & 0.462952\end{array}$

$\begin{array}{llll}-1.305909 & -1.773383 & -3.015211\end{array}$

$\begin{array}{llll}-1.732020 & -0.857472 & -3.413462\end{array}$

$\begin{array}{llll}-0.002932 & -2.106112 & -3.467792\end{array}$

$\begin{array}{llll}0.460999 & -1.422279 & -4.177447\end{array}$

$\begin{array}{llll}0.512895 & -3.531982 & -3.556370\end{array}$

$\begin{array}{llll}0.047444 & -4.027945 & -4.421584\end{array}$

$\begin{array}{llll}1.584612 & -3.500408 & -3.768281\end{array}$

$\begin{array}{llll}0.265105 & -4.364304 & -2.279596\end{array}$ 
$\begin{array}{llll}-0.661589 & -4.937843 & -2.376455\end{array}$

$\begin{array}{llll}1.067823 & -5.100226 & -2.171373\end{array}$

$\begin{array}{llll}0.198185 & -3.543816 & -0.994385\end{array}$

$\begin{array}{llll}1.031495 & -3.689138 & -0.315142\end{array}$

$\begin{array}{llll}-0.980525 & -3.051327 & -0.449323\end{array}$

$\begin{array}{lll}-0.998642 & -2.853432 & 0.619520\end{array}$

$\begin{array}{llll}-2.337775 & -3.190259 & -1.109383\end{array}$

$\begin{array}{llll}-3.038881 & -2.549208 & -0.576486\end{array}$

$\begin{array}{llll}-2.692486 & -4.222258 & -0.972962\end{array}$

$\begin{array}{llll}-2.352345 & -2.815733 & -2.609613\end{array}$

$\begin{array}{llll}-2.216880 & -3.710630 & -3.226356\end{array}$

$\begin{array}{llll}-3.341813 & -2.421367 & -2.856173\end{array}$

$2.791432 \quad-1.893481 \quad-1.921190$

$2.615410 \quad-2.934855 \quad-1.707541$

$2.938836 \quad-1.625784 \quad-2.959540$

$\begin{array}{llll}2.322411 & -0.886412 & -1.034989\end{array}$

$\begin{array}{llll}2.601360 & 0.133210 & -1.276968\end{array}$

$\begin{array}{llll}1.728878 & -1.137721 & 0.234034\end{array}$

$\begin{array}{llll}1.730651 & -2.171070 & 0.566724\end{array}$

$\begin{array}{lll}1.806791 & -0.217915 & 1.390015\end{array}$

$\begin{array}{llll}2.330630 & 1.084421 & 1.324227\end{array}$

$\begin{array}{lll}1.447542 & -0.719263 & 2.654822\end{array}$

$\begin{array}{llll}2.476670 & 1.853710 & 2.476450\end{array}$

$\begin{array}{llll}2.660757 & 1.486885 & 0.373623\end{array}$

$\begin{array}{llll}1.581552 & 0.052974 & 3.807582\end{array}$

$\begin{array}{llll}1.081795 & -1.740089 & 2.728932\end{array}$

$\begin{array}{lll}2.099623 & 1.347233 & 3.723305\end{array}$

$\begin{array}{lll}2.873280 & 2.860000 & 2.401184\end{array}$

$\begin{array}{llll}1.295749 & -0.360854 & 4.770542\end{array}$

$\begin{array}{llll}2.208672 & 1.955397 & 4.615631\end{array}$

$\begin{array}{llll}4.383741 & -4.106935 & -0.801323\end{array}$

$\begin{array}{llll}5.076031 & -3.128021 & -1.067058\end{array}$

$\begin{array}{llll}4.709925 & -2.022308 & -1.649172\end{array}$

$\begin{array}{llll}6.405967 & -3.076066 & -0.751671\end{array}$

$\begin{array}{llll}6.917476 & -4.235025 & -0.077455\end{array}$

$\begin{array}{llll}6.797385 & -5.133666 & -0.689497\end{array}$

$\begin{array}{llll}7.978033 & -4.038185 & 0.091301\end{array}$

$\begin{array}{llll}6.411265 & -4.391875 & 0.880348\end{array}$

$\begin{array}{llll}5.234177 & 1.586779 & -1.310020\end{array}$

$\begin{array}{llll}6.035510 & 2.716954 & -0.683001\end{array}$

$\begin{array}{llll}4.189315 & 1.886551 & -1.464565\end{array}$

$\begin{array}{llll}5.642082 & 1.287859 & -2.278901\end{array}$

$\begin{array}{llll}5.639807 & 2.858125 & 0.782139\end{array}$

$\begin{array}{llll}5.842264 & 3.656546 & -1.206829\end{array}$

$\begin{array}{lll}7.106774 & 2.503642 & -0.754751\end{array}$

$\begin{array}{llll}6.268308 & 3.598695 & 1.277829\end{array}$

$\begin{array}{llll}4.597733 & 3.185546 & 0.875501\end{array}$

$\begin{array}{llll}5.265836 & 0.420614 & -0.429586\end{array}$

$\begin{array}{llll}5.531603 & 0.434801 & 0.872038\end{array}$

$\begin{array}{llll}5.795073 & 1.577424 & 1.508234\end{array}$

$\begin{array}{llll}5.482953 & -0.933131 & 1.529169\end{array}$

$\begin{array}{llll}4.743946 & -1.492671 & 0.952904\end{array}$

$\begin{array}{llll}6.444436 & -1.429392 & 1.353561\end{array}$

$\begin{array}{llll}5.087578 & -0.989483 & 3.008224\end{array}$

$\begin{array}{lll}4.241063 & -0.318969 & 3.175983\end{array}$

$\begin{array}{llll}6.133121 & 1.703088 & 2.947738\end{array}$

$\begin{array}{lll}6.236558 & -0.651080 & 3.962386\end{array}$

$\begin{array}{llll}5.826755 & -0.479752 & 4.964776\end{array}$

$\begin{array}{lll}7.020367 & 0.585108 & 3.501998\end{array}$

$\begin{array}{llll}7.592187 & 0.995629 & 4.340623\end{array}$

$\begin{array}{llll}7.754118 & 0.316086 & 2.733330\end{array}$

$\begin{array}{llll}6.666430 & 2.651263 & 3.028906\end{array}$

$\begin{array}{llll}5.205413 & 1.803700 & 3.523065\end{array}$

$\begin{array}{llll}6.923236 & -1.502066 & 4.042191\end{array}$

$\begin{array}{lll}4.718075 & -1.999079 & 3.214853\end{array}$

$\begin{array}{llll}5.105109 & -0.500675 & -0.866445\end{array}$

\section{$[12-13]^{\ddagger} \mathbf{P} 2$ exo-si conf2}

Number of imaginary frequencies : 1

The smallest frequency is : $-259.0647 \mathrm{~cm}(-1)$ Electronic energy $=-3467.3737764$

Zero-point correction=

1.217210

(Hartree/Particle)

Thermal correction to Energy=

Thermal correction to Enthalpy=

1.283171

Thermal correction to Gibbs Free Energy $=\quad 1.117493$

Sum of electronic and zero-point Energies $=\quad-3466.156566$

Sum of electronic and thermal Energies $=\quad-3466.090605$

Sum of electronic and thermal Enthalpies $=\quad-3466.089661$

Sum of electronic and thermal Free Energies $=\quad-3466.256283$

\begin{tabular}{|c|c|c|c|}
\hline \multicolumn{4}{|c|}{ Cartesian Coordinates } \\
\hline 77 & 0.071396 & -1.323578 & -1.357272 \\
\hline 15 & -1.262961 & 0.363673 & -0.545280 \\
\hline 8 & -1.955104 & 0.448766 & 0.956521 \\
\hline 8 & -2.631730 & 0.500377 & -1.488270 \\
\hline 7 & -0.398777 & 1.799304 & -0.526791 \\
\hline 6 & -2.998884 & -0.412128 & 1.291052 \\
\hline 6 & -2.698848 & -1.438646 & 2.211167 \\
\hline 1 & -1.697475 & -1.489821 & 2.615978 \\
\hline 6 & -3.672202 & -2.341509 & 2.558038 \\
\hline 1 & -3.453490 & -3.132846 & 3.269359 \\
\hline 6 & -4.959474 & -2.280308 & 1.958421 \\
\hline 6 & -5.943206 & -3.269078 & 2.232267 \\
\hline 1 & -5.701121 & -4.057630 & 2.940041 \\
\hline 6 & -7.167660 & -3.242505 & 1.603892 \\
\hline 1 & -7.909245 & -4.007331 & 1.814830 \\
\hline 6 & -7.452923 & -2.223914 & 0.663781 \\
\hline 1 & -8.408827 & -2.220433 & 0.148018 \\
\hline 6 & -6.528209 & -1.239374 & 0.390038 \\
\hline 1 & -6.762510 & -0.475039 & -0.341082 \\
\hline 6 & -5.261019 & -1.220582 & 1.037312 \\
\hline 6 & -4.263668 & -0.216460 & 0.759848 \\
\hline 6 & -4.553034 & 0.963443 & -0.100789 \\
\hline 6 & -5.640339 & 1.864733 & 0.189854 \\
\hline 6 & -6.403455 & 1.783581 & 1.388504 \\
\hline 1 & -6.160842 & 1.023924 & 2.121769 \\
\hline 6 & -7.432890 & 2.665043 & 1.638901 \\
\hline 1 & -7.993254 & 2.585604 & 2.566077 \\
\hline 6 & -7.762685 & 3.676206 & 0.705426 \\
\hline 1 & -8.581383 & 4.358838 & 0.913407 \\
\hline 6 & -7.031722 & 3.798832 & -0.454531 \\
\hline 1 & -7.259198 & 4.582861 & -1.172132 \\
\hline 6 & -5.953026 & 2.916956 & -0.735451 \\
\hline 6 & -5.164960 & 3.074834 & -1.907441 \\
\hline 1 & -5.420457 & 3.859606 & -2.613766 \\
\hline 6 & -4.073202 & 2.273057 & -2.124469 \\
\hline 1 & -3.431934 & 2.400564 & -2.990270 \\
\hline 6 & -3.758066 & 1.251875 & -1.200068 \\
\hline 6 & 0.968566 & 1.656763 & -1.082182 \\
\hline 1 & 1.665988 & 1.503284 & -0.248152 \\
\hline 6 & 1.427679 & 2.890907 & -1.838497 \\
\hline 6 & 0.593357 & 3.532298 & -2.764683 \\
\hline 1 & -0.426865 & 3.179674 & -2.889892 \\
\hline 6 & 1.061420 & 4.618690 & -3.503974 \\
\hline 1 & 0.402963 & 5.112827 & -4.213017 \\
\hline 6 & 2.374611 & 5.071585 & -3.336031 \\
\hline 1 & 2.738180 & 5.916125 & -3.914207 \\
\hline 6 & 3.212647 & 4.436209 & -2.417378 \\
\hline 1 & 4.232640 & 4.784480 & -2.278561 \\
\hline 6 & 2.736854 & 3.356364 & -1.668493 \\
\hline 1 & 3.384261 & 2.868610 & -0.943978 \\
\hline 6 & 1.038847 & 0.448398 & -2.029324 \\
\hline 1 & 0.576518 & 0.733790 & -2.981536 \\
\hline 1 & 2.096615 & 0.287259 & -2.240963 \\
\hline 6 & -0.614604 & 3.013491 & 0.329804 \\
\hline 1 & -0.025510 & 3.780988 & -0.177156 \\
\hline 6 & -0.015701 & 2.888741 & 1.729370 \\
\hline
\end{tabular}


$\begin{array}{lll}1.320721 & 3.267756 & 1.928325\end{array}$

$\begin{array}{lll}1.901550 & 3.624567 & 1.081507\end{array}$

$\begin{array}{llll}1.898924 & 3.226988 & 3.200167\end{array}$

$\begin{array}{llll}2.928933 & 3.541825 & 3.338639\end{array}$

$\begin{array}{llll}1.144053 & 2.809058 & 4.297730\end{array}$

$\begin{array}{lll}1.587115 & 2.782901 & 5.289190\end{array}$

$\begin{array}{llll}-0.191914 & 2.442909 & 4.112998\end{array}$

$\begin{array}{lll}-0.792312 & 2.130638 & 4.962750\end{array}$

$\begin{array}{llll}-0.765214 & 2.479069 & 2.841213\end{array}$

$\begin{array}{lll}-1.801855 & 2.190414 & 2.718647\end{array}$

$\begin{array}{llll}-2.050776 & 3.531048 & 0.326714\end{array}$

$\begin{array}{llll}-2.377580 & 3.729559 & -0.695291\end{array}$

$\begin{array}{llll}-2.758674 & 2.842414 & 0.786277\end{array}$

$\begin{array}{llll}-2.083403 & 4.471252 & 0.885332\end{array}$

$\begin{array}{llll}-1.178316 & -1.344574 & -3.262683\end{array}$

$\begin{array}{llll}-1.222193 & -0.311422 & -3.597073\end{array}$

$\begin{array}{llll}0.027825 & -2.028960 & -3.505814\end{array}$

$\begin{array}{llll}0.815047 & -1.471234 & -4.012043\end{array}$

$\begin{array}{llll}0.166643 & -3.535105 & -3.641290\end{array}$

$\begin{array}{llll}-0.148255 & -3.832832 & -4.652622\end{array}$

$\begin{array}{llll}1.228805 & -3.784975 & -3.568728\end{array}$

$\begin{array}{llll}-0.617677 & -4.366286 & -2.599534\end{array}$

$\begin{array}{llll}-1.621350 & -4.585269 & -2.975899\end{array}$

$\begin{array}{llll}-0.120181 & -5.333668 & -2.479872\end{array}$

$-0.745510 \quad-3.721343 \quad-1.230595$

$\begin{array}{llll}-0.127223 & -4.131871 & -0.442513\end{array}$

$\begin{array}{llll}-1.792304 & -2.920623 & -0.852600\end{array}$

$\begin{array}{llll}-1.944792 & -2.765227 & 0.210565\end{array}$

$-2.944323 \quad-2.543756 \quad-1.760519$

$\begin{array}{llll}-3.537319 & -1.781950 & -1.259091\end{array}$

$\begin{array}{llll}-3.597513 & -3.422028 & -1.863778\end{array}$

$-2.541663 \quad-2.030034 \quad-3.164952$

$\begin{array}{llll}-2.564533 & -2.853537 & -3.886853\end{array}$

$\begin{array}{llll}-3.302475 & -1.316852 & -3.492530\end{array}$

$\begin{array}{llll}2.762095 & -2.178552 & -1.666111\end{array}$

$\begin{array}{lll}1.923740 & -2.369968 & -0.555147\end{array}$

$\begin{array}{llll}1.765553 & -3.407444 & -0.298067\end{array}$

$\begin{array}{llll}1.462151 & -1.358882 & 0.363868\end{array}$

$\begin{array}{llll}1.996984 & -0.413285 & 0.345906\end{array}$

$\begin{array}{lll}1.002871 & -1.712548 & 1.734944\end{array}$

$\begin{array}{llll}0.698985 & -3.025855 & 2.139093\end{array}$

$\begin{array}{llll}0.884455 & -0.690011 & 2.693744\end{array}$

$\begin{array}{llll}0.303287 & -3.304568 & 3.447755\end{array}$

$\begin{array}{lll}0.784825 & -3.848124 & 1.438538\end{array}$

$\begin{array}{llll}0.501287 & -0.969168 & 4.006019\end{array}$

$\begin{array}{lll}1.091748 & 0.335735 & 2.407156\end{array}$

$\begin{array}{llll}0.207794 & -2.279332 & 4.392680\end{array}$

$\begin{array}{lll}0.077621 & -4.329351 & 3.730162\end{array}$

$\begin{array}{llll}0.428188 & -0.157244 & 4.721988\end{array}$

$\begin{array}{lll}-0.092712 & -2.498118 & 5.413233\end{array}$

$\begin{array}{lll}4.223860 & -4.302933 & -0.054038\end{array}$

$\begin{array}{llll}4.912914 & -3.308837 & -0.264478\end{array}$

$\begin{array}{lll}4.710884 & -2.368590 & -1.132940\end{array}$

$\begin{array}{llll}6.058490 & -3.059600 & 0.454534\end{array}$

$\begin{array}{llll}6.350959 & -4.011921 & 1.483603\end{array}$

$\begin{array}{lll}6.476558 & -5.018405 & 1.072722\end{array}$

$\begin{array}{lll}7.285059 & -3.680703 & 1.942751\end{array}$

$\begin{array}{llll}5.557193 & -4.036476 & 2.237484\end{array}$

$\begin{array}{llll}5.768512 & 0.959196 & -2.008943\end{array}$

$\begin{array}{llll}6.842786 & 1.975943 & -1.651546\end{array}$

$\begin{array}{llll}4.890664 & 1.455238 & -2.440441\end{array}$

$\begin{array}{llll}6.128511 & 0.219501 & -2.727484\end{array}$

$\begin{array}{llll}6.366252 & 2.828187 & -0.482909\end{array}$

$\begin{array}{llll}7.061900 & 2.623387 & -2.504289\end{array}$

$\begin{array}{llll}7.764294 & 1.452455 & -1.375998\end{array}$

$\begin{array}{llll}7.194069 & 3.426530 & -0.095989\end{array}$

$\begin{array}{llll}5.573775 & 3.518789 & -0.791016\end{array}$

$\begin{array}{llll}5.371580 & 0.245016 & -0.795815\end{array}$

$\begin{array}{llll}5.430437 & 0.755004 & 0.428004\end{array}$

$\begin{array}{lll}5.864741 & 2.001096 & 0.641086\end{array}$

$\begin{array}{lll}4.985523 & -0.191452 & 1.528878\end{array}$

$\begin{array}{llll}4.374980 & -0.950408 & 1.038974\end{array}$

$\begin{array}{llll}5.873131 & -0.717030 & 1.898709\end{array}$

$\begin{array}{lll}4.192736 & 0.418812 & 2.692342\end{array}$

$\begin{array}{llll}3.473401 & 1.145326 & 2.301375\end{array}$

$\begin{array}{llll}5.792213 & 2.719203 & 1.938509\end{array}$

$\begin{array}{llll}5.073316 & 1.068419 & 3.764193\end{array}$

$\begin{array}{lll}4.443886 & 1.700774 & 4.401314\end{array}$

$\begin{array}{llll}6.213154 & 1.902558 & 3.163061\end{array}$

$\begin{array}{llll}6.598341 & 2.598698 & 3.915553\end{array}$

$\begin{array}{llll}7.056348 & 1.263439 & 2.876655\end{array}$

$\begin{array}{lll}6.463799 & 3.571189 & 1.829165\end{array}$

$\begin{array}{llll}4.782003 & 3.125738 & 2.060751\end{array}$

$\begin{array}{llll}5.500407 & 0.294958 & 4.413100\end{array}$

$\begin{array}{llll}3.590708 & -0.374708 & 3.142090\end{array}$

$\begin{array}{llll}5.159298 & -0.771408 & -0.879950\end{array}$

$\begin{array}{llll}2.860248 & -2.989804 & -2.373016\end{array}$

$\begin{array}{lll}3.024469 & -1.198240 & -2.025899\end{array}$

\section{$[12-13]^{\ddagger} \mathbf{P 2}$ endo-si conf2}

Number of imaginary frequencies : 1

The smallest frequency is : $-282.1375 \mathrm{~cm}(-1)$

Electronic energy=-3467.3779014

Zero-point correction $=$

1.217317

(Hartree/Particle)

Thermal correction to Energy=

Thermal correction to Enthalpy=

1.283551

correction to Enthalpy= 1.284495

Thermal correction to Gibbs Free Energy $=\quad 1.114537$

Sum of electronic and zero-point Energies $=\quad-3466.160585$

Sum of electronic and thermal Energies $=\quad-3466.094351$

Sum of electronic and thermal Enthalpies $=\quad-3466.093406$

Sum of electronic and thermal Free Energies $=\quad-3466.263365$

\begin{tabular}{|c|c|c|c|}
\hline & & \multicolumn{2}{|c|}{ Cartesian Coordinates } \\
\hline 77 & 0.096730 & -0.842829 & -1.277449 \\
\hline 15 & -1.656629 & 0.293603 & -0.305434 \\
\hline 8 & -2.361265 & -0.059379 & 1.155221 \\
\hline 8 & -3.008541 & 0.266324 & -1.284579 \\
\hline 7 & -1.160973 & 1.864901 & -0.012206 \\
\hline 6 & -3.290416 & -1.089531 & 1.269521 \\
\hline 6 & -2.879248 & -2.232135 & 1.989339 \\
\hline 1 & -1.883265 & -2.243878 & 2.419507 \\
\hline 6 & -3.740229 & -3.293158 & 2.123652 \\
\hline 1 & -3.440206 & -4.176581 & 2.679930 \\
\hline 6 & -5.021901 & -3.263988 & 1.510455 \\
\hline 6 & -5.888890 & -4.388895 & 1.572041 \\
\hline 1 & -5.561841 & -5.265654 & 2.124897 \\
\hline 6 & -7.109037 & -4.376732 & 0.935070 \\
\hline 1 & -7.761610 & -5.243473 & 0.983404 \\
\hline 6 & -7.507349 & -3.235031 & 0.199263 \\
\hline 1 & -8.459576 & -3.237821 & -0.323258 \\
\hline 6 & -6.697652 & -2.121956 & 0.133296 \\
\hline 1 & -7.016207 & -1.261324 & -0.442744 \\
\hline 6 & -5.439088 & -2.089652 & 0.796777 \\
\hline 6 & -4.563815 & -0.947591 & 0.739490 \\
\hline 6 & -4.972412 & 0.334316 & 0.099421 \\
\hline 6 & -6.134827 & 1.071021 & 0.521165 \\
\hline 6 & -6.912730 & 0.697090 & 1.653018 \\
\hline 1 & -6.625526 & -0.173547 & 2.230773 \\
\hline 6 & -8.014307 & 1.432576 & 2.033469 \\
\hline 1 & -8.586771 & 1.130070 & 2.905654 \\
\hline 6 & -8.403905 & 2.581725 & 1.304631 \\
\hline 1 & -9.278310 & 3.147426 & 1.612600 \\
\hline 6 & -7.662806 & 2.984985 & 0.217269 \\
\hline 1 & -7.938914 & 3.875695 & -0.340911 \\
\hline 6 & -6.512034 & 2.258560 & -0.193321 \\
\hline 6 & -5.719835 & 2.702045 & -1.286986 \\
\hline 1 & -6.022788 & 3.590868 & -1.833053 \\
\hline
\end{tabular}




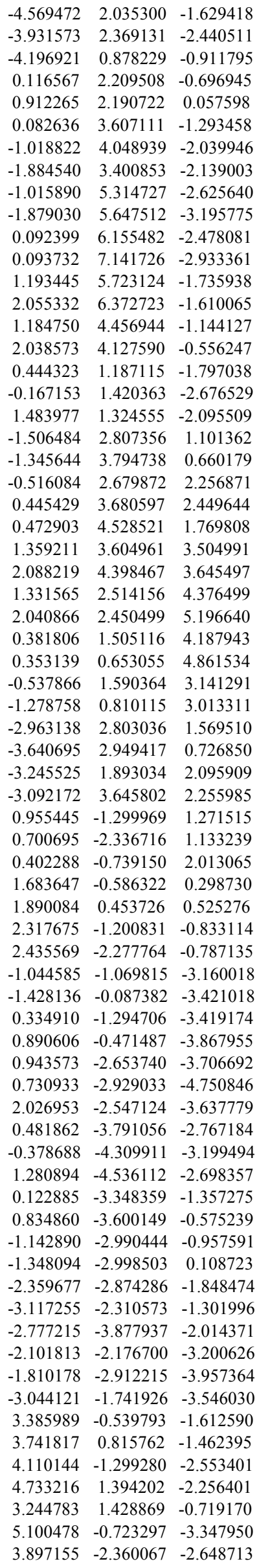

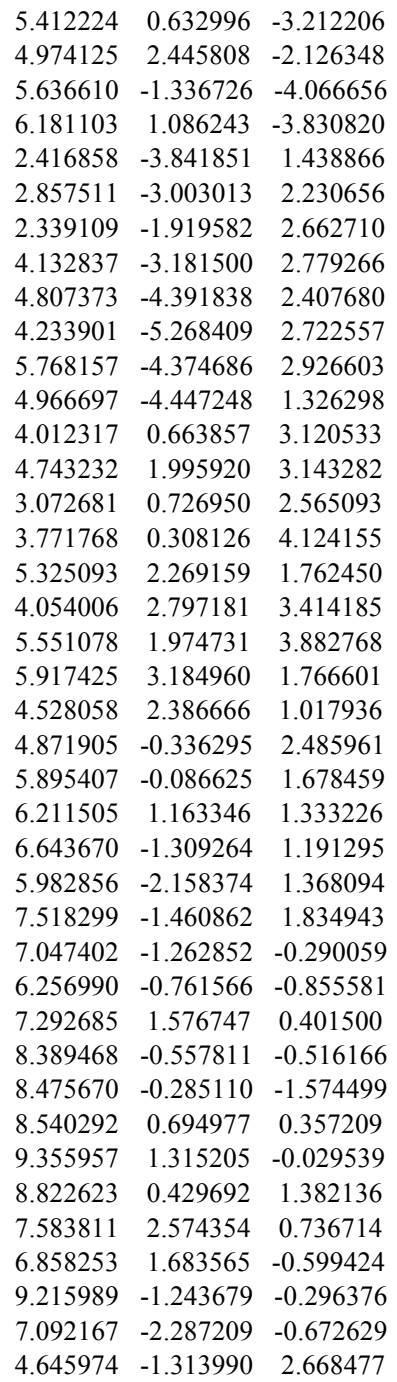

\section{$[12-13]^{\ddagger}$ P2 exo-re conf2}

Number of imaginary frequencies : 1

The smallest frequency is : $-254.3128 \mathrm{~cm}(-1)$

Electronic energy $=-3467.3768167$

Zero-point correction $=\quad 1.217893$

(Hartree/Particle)

Thermal correction to Energy = $\quad 1.283844$

Thermal correction to Enthalpy= $\quad 1.284789$

Thermal correction to Gibbs Free Energy $=\quad 1.117015$

Sum of electronic and zero-point Energies $=\quad-3466.158924$

Sum of electronic and thermal Energies $=\quad-3466.092972$

Sum of electronic and thermal Enthalpies $=\quad-3466.092028$

Sum of electronic and thermal Free Energies $=\quad-3466.259802$

Cartesian Coordinates

$\begin{array}{lll}0.266500 & -0.791632 & -1.277111\end{array}$

$\begin{array}{llll}-1.495907 & 0.505635 & -0.514856\end{array}$

$\begin{array}{lll}-2.159044 & 0.367545 & 1.004782\end{array}$

$\begin{array}{llll}-2.856635 & 0.285316 & -1.447120\end{array}$

$\begin{array}{llll}-1.038381 & 2.113320 & -0.489027\end{array}$

$\begin{array}{lll}-2.828568 & -0.807739 & 1.333993\end{array}$

$\begin{array}{lll}-2.194745 & -1.670688 & 2.256593\end{array}$

$\begin{array}{llll}-1.260767 & -1.363557 & 2.718808\end{array}$

$\begin{array}{lll}-2.788729 & -2.871514 & 2.560949\end{array}$

$\begin{array}{lll}-2.324831 & -3.545550 & 3.275480\end{array}$

$\begin{array}{llll}-3.996977 & -3.265818 & 1.923191\end{array}$

$\begin{array}{llll}-4.565746 & -4.547459 & 2.156878\end{array}$ 


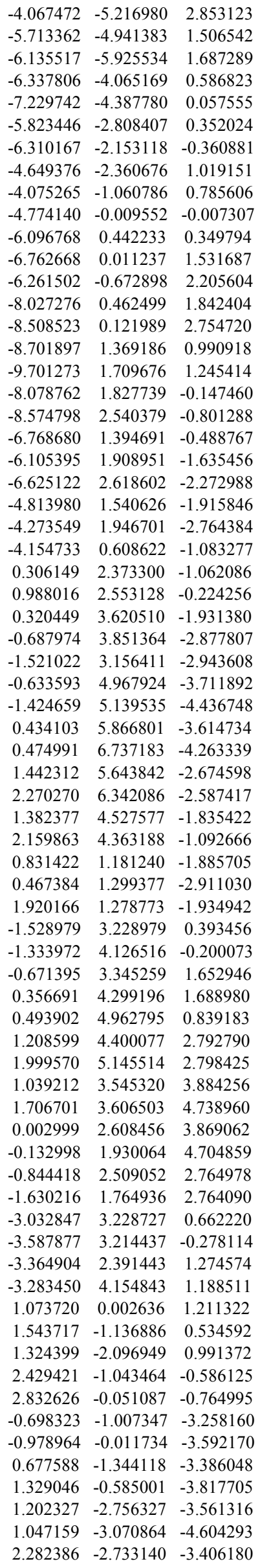

$\begin{array}{llll}0.578789 & -3.788604 & -2.600386\end{array}$

$\begin{array}{llll}-0.283604 & -4.273855 & -3.067801\end{array}$

$\begin{array}{llll}1.310737 & -4.578650 & -2.409438\end{array}$

$\begin{array}{llll}0.146519 & -3.209694 & -1.260745\end{array}$

$\begin{array}{llll}0.750067 & -3.491947 & -0.407506\end{array}$

$\begin{array}{llll}-1.129443 & -2.754997 & -0.989865\end{array}$

$\begin{array}{llll}-1.439842 & -2.723020 & 0.049096\end{array}$

$\begin{array}{llll}-2.242346 & -2.681483 & -2.013793\end{array}$

$-3.065578-2.117206-1.577653$

$\begin{array}{llll}-2.620537 & -3.699046 & -2.188025\end{array}$

$\begin{array}{llll}-1.831439 & -2.032404 & -3.355003\end{array}$

$\begin{array}{llll}-1.538321 & -2.803865 & -4.075077\end{array}$

$\begin{array}{llll}-2.707862 & -1.535211 & -3.779236\end{array}$

$\begin{array}{llll}3.370975 & -2.092926 & -1.021953\end{array}$

$\begin{array}{llll}3.423421 & -3.389309 & -0.478387\end{array}$

$\begin{array}{llll}4.321939 & -1.752524 & -2.004701\end{array}$

$\begin{array}{llll}4.372904 & -4.312211 & -0.919148\end{array}$

$\begin{array}{llll}2.743421 & -3.674858 & 0.316711\end{array}$

$\begin{array}{llll}5.273232 & -2.672288 & -2.442949\end{array}$

$\begin{array}{llll}4.305611 & -0.750197 & -2.425874\end{array}$

$\begin{array}{llll}5.300606 & -3.962686 & -1.903878\end{array}$

$\begin{array}{llll}4.396070 & -5.305361 & -0.479295\end{array}$

$\begin{array}{llll}5.992906 & -2.382457 & -3.203509\end{array}$

$\begin{array}{llll}6.040518 & -4.682541 & -2.240947\end{array}$

$\begin{array}{llll}0.649156 & -0.639936 & 4.111391\end{array}$

$\begin{array}{lll}1.744223 & -0.091197 & 3.957950\end{array}$

$\begin{array}{llll}2.310985 & 0.236781 & 2.847663\end{array}$

$\begin{array}{llll}2.527594 & 0.241074 & 5.031295\end{array}$

$\begin{array}{llll}1.986804 & -0.099046 & 6.313469\end{array}$

$\begin{array}{llll}1.040218 & 0.418548 & 6.499349\end{array}$

$\begin{array}{llll}2.730643 & 0.221300 & 7.046368\end{array}$

$\begin{array}{llll}1.818277 & -1.176898 & 6.403297\end{array}$

$\begin{array}{llll}3.917848 & 2.469188 & 0.573913\end{array}$

$\begin{array}{llll}5.133359 & 2.993195 & -0.172280\end{array}$

$\begin{array}{llll}3.133905 & 2.171031 & -0.130790\end{array}$

$\begin{array}{llll}3.491516 & 3.214771 & 1.249912\end{array}$

$\begin{array}{llll}5.836768 & 1.825114 & -0.853270\end{array}$

$\begin{array}{llll}4.831323 & 3.727385 & -0.923408\end{array}$

$\begin{array}{llll}5.823526 & 3.481660 & 0.523357\end{array}$

$\begin{array}{llll}6.763477 & 2.158443 & -1.322681\end{array}$

$\begin{array}{llll}5.203394 & 1.387571 & -1.635262\end{array}$

$\begin{array}{llll}4.303811 & 1.307487 & 1.372091\end{array}$

$\begin{array}{lll}5.363080 & 0.538373 & 1.153739\end{array}$

$\begin{array}{llll}6.182875 & 0.766132 & 0.123403\end{array}$

$\begin{array}{lll}5.547719 & -0.584476 & 2.155711\end{array}$

$\begin{array}{llll}4.573500 & -0.724839 & 2.630117\end{array}$

$\begin{array}{llll}6.221377 & -0.225097 & 2.943258\end{array}$

$\begin{array}{llll}6.051224 & -1.916017 & 1.578168\end{array}$

$\begin{array}{llll}5.582572 & -2.084653 & 0.605057\end{array}$

$\begin{array}{llll}7.350860 & -0.066829 & -0.262037\end{array}$

$\begin{array}{lll}7.576737 & -1.973650 & 1.443671\end{array}$

$\begin{array}{llll}7.843369 & -2.807033 & 0.783101\end{array}$

$\begin{array}{llll}8.158822 & -0.665948 & 0.891080\end{array}$

$\begin{array}{llll}9.173873 & -0.843196 & 0.520004\end{array}$

$\begin{array}{llll}8.252330 & 0.087179 & 1.681836\end{array}$

$\begin{array}{llll}7.997172 & 0.603051 & -0.831611\end{array}$

$\begin{array}{lll}7.007443 & -0.848569 & -0.950515\end{array}$

$\begin{array}{llll}8.033640 & -2.182072 & 2.418295\end{array}$

$\begin{array}{llll}5.701732 & -2.726397 & 2.225477\end{array}$

$\begin{array}{llll}3.646766 & 1.004222 & 2.109662\end{array}$

$\begin{array}{lll}0.239437 & -0.070804 & 1.889193\end{array}$

$\begin{array}{lll}1.358288 & 0.989268 & 0.882203\end{array}$

$[\mathbf{1 2 - 1 3}]^{\ddagger} \mathbf{P} 2$ exo-re conf2 $\left[\mathrm{BF} 4^{-}\right]$

Number of imaginary frequencies : 1

The smallest frequency is : $-271.3676 \mathrm{~cm}(-1)$

Electronic energy $=-3892.0061101$

Zero-point correction $=$

1.233671

(Hartree/Particle) 
Thermal correction to Energy=

Thermal correction to Enthalpy=

Thermal correction to Gibbs Free Energy=

Sum of electronic and zero-point Energies=

Sum of electronic and thermal Energies $=$

Sum of electronic and thermal Enthalpies=

Sum of electronic and thermal Free Energies=

Cartesian Coordinates

$\begin{array}{lll}-0.213677 & -1.186275 & -1.202814\end{array}$

$\begin{array}{lll}-1.784741 & 0.384507 & -0.543188\end{array}$

$\begin{array}{lll}-2.413816 & 0.510582 & 0.996290\end{array}$

$\begin{array}{lll}-3.204994 & 0.195754 & -1.403410\end{array}$

$\begin{array}{llll}-1.167057 & 1.925662 & -0.728283\end{array}$

$\begin{array}{lll}-3.230464 & -0.504940 & 1.477669\end{array}$

$\begin{array}{lll}-2.686488 & -1.344398 & 2.476825\end{array}$

$\begin{array}{lll}-1.689461 & -1.138683 & 2.856488\end{array}$

$\begin{array}{lll}-3.439175 & -2.390735 & 2.951621\end{array}$

$\begin{array}{lll}-3.042935 & -3.042284 & 3.725442\end{array}$

$\begin{array}{lll}-4.728689 & -2.659324 & 2.415709\end{array}$

$\begin{array}{lll}-5.475628 & -3.794152 & 2.834106\end{array}$

$\begin{array}{lll}-5.049273 & -4.443294 & 3.594510\end{array}$

$\begin{array}{lll}-6.704190 & -4.076862 & 2.280935\end{array}$

$\begin{array}{lll}-7.262637 & -4.950841 & 2.603292\end{array}$

$\begin{array}{lll}-7.234201 & -3.232938 & 1.275822\end{array}$

$\begin{array}{lll}-8.192160 & -3.471768 & 0.822847\end{array}$

$\begin{array}{lll}-6.544438 & -2.114267 & 0.861098\end{array}$

$\begin{array}{lll}-6.961548 & -1.484304 & 0.084202\end{array}$

$\begin{array}{lll}-5.281326 & -1.779545 & 1.424249\end{array}$

$\begin{array}{lll}-4.527334 & -0.626634 & 1.003944\end{array}$

$\begin{array}{lll}-5.089844 & 0.406095 & 0.087368\end{array}$

$\begin{array}{lll}-6.311894 & 1.105294 & 0.396919\end{array}$

$\begin{array}{lll}-6.990492 & 0.952541 & 1.639039\end{array}$

$\begin{array}{lll}-6.574767 & 0.296788 & 2.394441\end{array}$

$\begin{array}{lll}-8.157572 & 1.636392 & 1.903298\end{array}$

$\begin{array}{lll}-8.650280 & 1.505738 & 2.862639\end{array}$

$\begin{array}{lll}-8.716513 & 2.512030 & 0.942364\end{array}$

$\begin{array}{llll}-9.640167 & 3.039586 & 1.162046\end{array}$

$\begin{array}{lll}-8.074752 & 2.703830 & -0.260135\end{array}$

$\begin{array}{llll}-8.479881 & 3.388768 & -1.000572\end{array}$

$\begin{array}{llll}-6.861510 & 2.025958 & -0.558189\end{array}$

$\begin{array}{lll}-6.172208 & 2.264378 & -1.777845\end{array}$

$\begin{array}{llll}-6.601761 & 2.950854 & -2.502022\end{array}$

$\begin{array}{llll}-4.964198 & 1.661894 & -2.023103\end{array}$

$\begin{array}{llll}-4.400863 & 1.854352 & -2.930275\end{array}$

$\begin{array}{llll}-4.422859 & 0.763110 & -1.075928\end{array}$

$\begin{array}{llll}0.166521 & 1.953638 & -1.391089\end{array}$

$\begin{array}{llll}0.921190 & 2.125808 & -0.619908\end{array}$

$\begin{array}{llll}0.286680 & 3.083879 & -2.400908\end{array}$

$\begin{array}{llll}-0.787780 & 3.450839 & -3.222824\end{array}$

$\begin{array}{lll}-1.747729 & 2.959092 & -3.088871\end{array}$

$\begin{array}{llll}-0.634199 & 4.446965 & -4.188453\end{array}$

$\begin{array}{llll}-1.477913 & 4.727988 & -4.813464\end{array}$

$\begin{array}{llll}0.600341 & 5.083260 & -4.350462\end{array}$

$\begin{array}{llll}0.720380 & 5.858871 & -5.101870\end{array}$

$\begin{array}{lll}1.677632 & 4.715432 & -3.539997\end{array}$

$\begin{array}{llll}2.642236 & 5.201056 & -3.661524\end{array}$

$\begin{array}{llll}1.522399 & 3.723572 & -2.568580\end{array}$

$\begin{array}{llll}2.363709 & 3.430510 & -1.948096\end{array}$

$\begin{array}{llll}0.477857 & 0.628525 & -2.107219\end{array}$

$\begin{array}{llll}0.015266 & 0.675270 & -3.099499\end{array}$

$\begin{array}{lll}1.552451 & 0.590764 & -2.262163\end{array}$

$\begin{array}{lll}-1.482936 & 3.180762 & 0.031070\end{array}$

$\begin{array}{llll}-1.225218 & 3.976207 & -0.673085\end{array}$

$\begin{array}{lll}-0.561792 & 3.360897 & 1.237067\end{array}$

$\begin{array}{lll}0.519826 & 4.247685 & 1.140187\end{array}$

$\begin{array}{lll}0.687335 & 4.783302 & 0.209051\end{array}$

$\begin{array}{llll}1.385811 & 4.446311 & 2.218657\end{array}$

$\begin{array}{lll}2.216685 & 5.139749 & 2.121640\end{array}$
305756

1.306700

1.124792

$-3890.772439$

$-3890.700355$

$-3890.699410$

$-3890.881318$ $\begin{array}{lll}1.186359 & 3.749574 & 3.412172\end{array}$

$\begin{array}{llll}1.861820 & 3.893038 & 4.251114\end{array}$

$\begin{array}{llll}0.114852 & 2.859428 & 3.517903\end{array}$

$\begin{array}{llll}-0.041774 & 2.297098 & 4.433311\end{array}$

$\begin{array}{lll}-0.754769 & 2.671694 & 2.443315\end{array}$

$\begin{array}{llll}-1.577702 & 1.975541 & 2.539895\end{array}$

$\begin{array}{llll}-2.959837 & 3.394624 & 0.366955\end{array}$

$\begin{array}{llll}-3.572487 & 3.343571 & -0.535403\end{array}$

$\begin{array}{lll}-3.347370 & 2.680115 & 1.091802\end{array}$

$\begin{array}{llll}-3.067374 & 4.398562 & 0.789429\end{array}$

$\begin{array}{llll}0.825690 & -0.150043 & 1.140208\end{array}$

$\begin{array}{llll}1.129105 & -1.414473 & 0.591604\end{array}$

$\begin{array}{llll}0.837719 & -2.281844 & 1.175866\end{array}$

$\begin{array}{llll}1.965055 & -1.539749 & -0.561434\end{array}$

$2.433920 \quad-0.618972 \quad-0.883614$

$\begin{array}{llll}-1.299169 & -1.562156 & -3.079814\end{array}$

$\begin{array}{llll}-1.502635 & -0.600897 & -3.544427\end{array}$

$\begin{array}{llll}0.031355 & -2.052680 & -3.221801\end{array}$

$\begin{array}{llll}0.725145 & -1.430091 & -3.785785\end{array}$

$\begin{array}{llll}0.403067 & -3.523812 & -3.229784\end{array}$

$\begin{array}{llll}0.163311 & -3.954620 & -4.213879\end{array}$

$\begin{array}{llll}1.486530 & -3.593533 & -3.119801\end{array}$

$\begin{array}{llll}-0.269788 & -4.347201 & -2.112947\end{array}$

$\begin{array}{llll}-1.205841 & -4.786722 & -2.471742\end{array}$

$\begin{array}{llll}0.382184 & -5.185647 & -1.849831\end{array}$

$\begin{array}{llll}-0.553470 & -3.551098 & -0.846532\end{array}$

$\begin{array}{llll}0.076745 & -3.783278 & 0.002256\end{array}$

$\begin{array}{llll}-1.756127 & -2.923901 & -0.578918\end{array}$

$\begin{array}{llll}-1.995021 & -2.718020 & 0.459128\end{array}$

$\begin{array}{llll}-2.920195 & -2.883041 & -1.547191\end{array}$

$\begin{array}{llll}-3.659125 & -2.183661 & -1.158144\end{array}$

$\begin{array}{llll}-3.401062 & -3.872074 & -1.557468\end{array}$

$\begin{array}{llll}-2.532576 & -2.465294 & -2.984438\end{array}$

$\begin{array}{llll}-2.366332 & -3.351447 & -3.606684\end{array}$

$\begin{array}{llll}-3.379923 & -1.935987 & -3.429254\end{array}$

$\begin{array}{llll}2.759164 & -2.724549 & -0.931040\end{array}$

$\begin{array}{llll}2.720593 & -3.952572 & -0.246891\end{array}$

$\begin{array}{llll}3.660495 & -2.592358 & -2.006975\end{array}$

$\begin{array}{llll}3.526325 & -5.020137 & -0.646555\end{array}$

$\begin{array}{llll}2.081455 & -4.071767 & 0.621861\end{array}$

$\begin{array}{llll}4.467508 & -3.657272 & -2.402054\end{array}$

$\begin{array}{llll}3.731091 & -1.634693 & -2.514114\end{array}$

$\begin{array}{llll}4.399262 & -4.882166 & -1.729287\end{array}$

$\begin{array}{llll}3.480123 & -5.959174 & -0.101251\end{array}$

$\begin{array}{llll}5.156889 & -3.529376 & -3.232338\end{array}$

$\begin{array}{llll}5.028307 & -5.713185 & -2.035599\end{array}$

$\begin{array}{llll}0.344562 & -0.564620 & 4.055200\end{array}$

$\begin{array}{lll}1.481276 & -0.127089 & 3.859332\end{array}$

$\begin{array}{lll}2.065857 & 0.091566 & 2.730360\end{array}$

$\begin{array}{llll}2.309002 & 0.191864 & 4.905754\end{array}$

$\begin{array}{lll}1.760135 & -0.029732 & 6.209278\end{array}$

$\begin{array}{lll}0.852178 & 0.560628 & 6.368047\end{array}$

$\begin{array}{llll}2.531159 & 0.282796 & 6.917178\end{array}$

$\begin{array}{llll}1.521379 & -1.086678 & 6.367076\end{array}$

$\begin{array}{lll}4.478701 & 2.691374 & 1.903595\end{array}$

$\begin{array}{llll}5.914057 & 3.190027 & 1.852564\end{array}$

$\begin{array}{lll}3.956698 & 2.880196 & 0.961012\end{array}$

$\begin{array}{lll}3.912091 & 3.162543 & 2.709099\end{array}$

$\begin{array}{lll}6.719795 & 2.295766 & 0.919222\end{array}$

$\begin{array}{llll}5.945748 & 4.219903 & 1.487264\end{array}$

$\begin{array}{llll}6.354585 & 3.172268 & 2.855749\end{array}$

$\begin{array}{llll}7.777983 & 2.565022 & 0.954330\end{array}$

$\begin{array}{llll}6.369926 & 2.395459 & -0.113305\end{array}$

$\begin{array}{llll}4.481851 & 1.251141 & 2.148123\end{array}$

$\begin{array}{llll}5.469804 & 0.424953 & 1.842724\end{array}$

$\begin{array}{llll}6.613806 & 0.873470 & 1.314033\end{array}$

$\begin{array}{llll}5.212291 & -1.031310 & 2.176216\end{array}$

$\begin{array}{llll}4.144814 & -1.109267 & 2.390226\end{array}$

$\begin{array}{llll}5.733426 & -1.266380 & 3.112368\end{array}$

$\begin{array}{llll}5.598848 & -2.027001 & 1.070130\end{array}$ 
$\begin{array}{rrr}5.360857 & -1.579552 & 0.102688 \\ 7.662879 & 0.022176 & 0.704502 \\ 7.073802 & -2.443235 & 1.125428 \\ 7.354524 & -2.882035 & 0.160518 \\ 8.005945 & -1.267774 & 1.451750 \\ 9.036109 & -1.535147 & 1.191686 \\ 8.009586 & -1.057097 & 2.527468 \\ 8.552779 & 0.654176 & 0.671366 \\ 7.367835 & -0.182709 & -0.330671 \\ 7.215178 & -3.226639 & 1.880519 \\ 4.961517 & -2.910874 & 1.159473 \\ 3.598883 & 0.833184 & 2.487621 \\ 4.255145 & 1.272504 & -1.681636 \\ 5.189511 & 0.361984 & -1.150322 \\ 4.877662 & 2.502301 & -1.942749 \\ 3.709305 & 0.734881 & -2.863378 \\ 3.217816 & 1.483957 & -0.734641 \\ -0.009563 & -0.036380 & 1.811541 \\ 1.233702 & 0.739161 & 0.683293\end{array}$

$[\mathbf{1 2 - 1 3}]^{\ddagger} \mathbf{P 2}$ endo-re conf2 $\left[\mathrm{BF} 4^{-}\right]$

Number of imaginary frequencies : 1

The smallest frequency is : $-292.7258 \mathrm{~cm}(-1)$

Electronic energy $=-3891.9940773$

Zero-point correction $=$

1.233050

(Hartree/Particle)

Thermal correction to Energy= $\quad 1305225$

Thermal correction to Enthalpy=

1.306169

Thermal correction to Gibbs Free Energy=

Sum of electronic and zero-point Energies=

Sum of electronic and thermal Energies=

1.123094

$-3890.761027$

$-3890.688853$

Sum of electronic and thermal Enthalpies=

$-3890.687909$

Sum of electronic and thermal Free Energies=

$-3890.870983$

Cartesian Coordinates

$-0.267105-0.840835-1.558150$

$\begin{array}{llll}-1.695286 & 0.514853 & -0.369206\end{array}$

$\begin{array}{lll}-2.238515 & 0.314352 & 1.193875\end{array}$

$\begin{array}{llll}-3.181583 & 0.526864 & -1.155245\end{array}$

$\begin{array}{llll}-1.089988 & 2.058047 & -0.266244\end{array}$

$\begin{array}{lll}-2.968017 & -0.835005 & 1.479623\end{array}$

$\begin{array}{lll}-2.307248 & -1.836711 & 2.222266\end{array}$

$\begin{array}{lll}-1.300197 & -1.634757 & 2.563895\end{array}$

$\begin{array}{lll}-2.946382 & -3.022997 & 2.482362\end{array}$

$\begin{array}{lll}-2.450326 & -3.799947 & 3.057406\end{array}$

$\begin{array}{lll}-4.248795 & -3.266119 & 1.966848\end{array}$

$\begin{array}{llll}-4.882011 & -4.527041 & 2.139497\end{array}$

$\begin{array}{lll}-4.358574 & -5.296366 & 2.701266\end{array}$

$\begin{array}{llll}-6.120664 & -4.777931 & 1.593822\end{array}$

$\begin{array}{lll}-6.592246 & -5.747465 & 1.725329\end{array}$

$\begin{array}{lll}-6.774313 & -3.771712 & 0.843444\end{array}$

$\begin{array}{lll}-7.739655 & -3.979867 & 0.390855\end{array}$

$\begin{array}{lll}-6.196230 & -2.531999 & 0.674617\end{array}$

$\begin{array}{llll}-6.708471 & -1.779047 & 0.087809\end{array}$

$\begin{array}{lll}-4.926346 & -2.228911 & 1.241298\end{array}$

$\begin{array}{lll}-4.284563 & -0.949736 & 1.063608\end{array}$

$\begin{array}{llll}-4.981445 & 0.200615 & 0.426466\end{array}$

$\begin{array}{lll}-6.241746 & 0.689874 & 0.930082\end{array}$

$\begin{array}{lll}-6.814458 & 0.224029 & 2.147172\end{array}$

$\begin{array}{lll}-6.286556 & -0.518597 & 2.733357\end{array}$

$\begin{array}{lll}-8.019850 & 0.713794 & 2.602669\end{array}$

$\begin{array}{lll}-8.427971 & 0.345982 & 3.539905\end{array}$

$\begin{array}{llll}-8.726017 & 1.695327 & 1.868084\end{array}$

$\begin{array}{lll}-9.678103 & 2.067000 & 2.235618\end{array}$

$\begin{array}{lll}-8.191245 & 2.186351 & 0.698277\end{array}$

$\begin{array}{lll}-8.710311 & 2.955770 & 0.132383\end{array}$

$\begin{array}{lll}-6.942851 & 1.714428 & 0.210251\end{array}$

$\begin{array}{llll}-6.365422 & 2.256990 & -0.969872\end{array}$ $\begin{array}{lll}-6.909228 & 3.017942 & -1.522730\end{array}$

$\begin{array}{llll}-5.122176 & 1.854110 & -1.383531\end{array}$

$\begin{array}{llll}-4.641624 & 2.284901 & -2.255788\end{array}$

$\begin{array}{llll}-4.422310 & 0.858368 & -0.660853\end{array}$

$\begin{array}{llll}0.149001 & 2.300911 & -1.049905\end{array}$

$\begin{array}{llll}0.970054 & 2.430769 & -0.342586\end{array}$

$\begin{array}{llll}0.031939 & 3.576699 & -1.874122\end{array}$

$\begin{array}{llll}-1.118536 & 3.830411 & -2.638166\end{array}$

$\begin{array}{llll}-1.948099 & 3.129117 & -2.592983\end{array}$

$\begin{array}{llll}-1.206548 & 4.973267 & -3.431616\end{array}$

$\begin{array}{llll}-2.105927 & 5.161167 & -4.012447\end{array}$

$\begin{array}{llll}-0.139802 & 5.878223 & -3.478838\end{array}$

$\begin{array}{llll}-0.207192 & 6.769349 & -4.096956\end{array}$

$\begin{array}{llll}1.007059 & 5.631996 & -2.721873\end{array}$

$\begin{array}{llll}1.836783 & 6.333579 & -2.746794\end{array}$

$\begin{array}{llll}1.091956 & 4.489626 & -1.918926\end{array}$

$\begin{array}{llll}1.974808 & 4.308129 & -1.314323\end{array}$

$\begin{array}{llll}0.460061 & 1.129411 & -2.010474\end{array}$

$\begin{array}{llll}0.018620 & 1.378775 & -2.980013\end{array}$

$\begin{array}{llll}1.539926 & 1.110050 & -2.152124\end{array}$

$\begin{array}{llll}-1.495138 & 3.183507 & 0.631424\end{array}$

$\begin{array}{lll}-1.153573 & 4.070044 & 0.092056\end{array}$

$\begin{array}{lll}-0.769638 & 3.214670 & 1.974457\end{array}$

$\begin{array}{lll}0.357983 & 4.035528 & 2.111971\end{array}$

$\begin{array}{llll}0.753947 & 4.549439 & 1.241500\end{array}$

$\begin{array}{lll}0.978019 & 4.204921 & 3.352213\end{array}$

$\begin{array}{llll}1.850686 & 4.847091 & 3.433840\end{array}$

$\begin{array}{llll}0.477023 & 3.549588 & 4.478014\end{array}$

$\begin{array}{llll}0.951973 & 3.682797 & 5.446311\end{array}$

$\begin{array}{lll}-0.632714 & 2.709304 & 4.347938\end{array}$

$\begin{array}{lll}-1.022245 & 2.184511 & 5.216169\end{array}$

$\begin{array}{lll}-1.250096 & 2.542869 & 3.107966\end{array}$

$\begin{array}{lll}-2.115073 & 1.895917 & 3.025226\end{array}$

$\begin{array}{lll}-3.006864 & 3.352963 & 0.776609\end{array}$

$\begin{array}{llll}-3.467039 & 3.493947 & -0.202803\end{array}$

$\begin{array}{lll}-3.488239 & 2.507436 & 1.267766\end{array}$

$\begin{array}{llll}-3.202949 & 4.246582 & 1.376588\end{array}$

$\begin{array}{lll}-1.569802 & -0.638444 & -3.366711\end{array}$

$\begin{array}{llll}-1.758708 & 0.422769 & -3.502832\end{array}$

$-0.303777 \quad-1.112505 \quad-3.793821$

$\begin{array}{llll}0.380426 & -0.383572 & -4.225656\end{array}$

$\begin{array}{lll}-0.056964 & -2.536002 & -4.264486\end{array}$

$\begin{array}{llll}-0.490544 & -2.662925 & -5.268286\end{array}$

$\begin{array}{llll}1.020595 & -2.672415 & -4.382859\end{array}$

$\begin{array}{llll}-0.611427 & -3.626279 & -3.318610\end{array}$

$\begin{array}{llll}-1.600781 & -3.948777 & -3.658326\end{array}$

$\begin{array}{llll}0.031000 & -4.510848 & -3.379411\end{array}$

$-0.714672-3.212195-1.854160$

$\begin{array}{llll}-0.018229 & -3.696360 & -1.176447\end{array}$

$\begin{array}{llll}-1.851674 & -2.649808 & -1.305199\end{array}$

$\begin{array}{llll}-1.985875 & -2.731605 & -0.231258\end{array}$

$\begin{array}{llll}-3.095358 & -2.327039 & -2.107523\end{array}$

$\begin{array}{llll}-3.788283 & -1.788258 & -1.464357\end{array}$

$\begin{array}{llll}-3.591225 & -3.274235 & -2.366318\end{array}$

$\begin{array}{llll}-2.835415 & -1.500198 & -3.389283\end{array}$

$\begin{array}{llll}-2.789835 & -2.162739 & -4.261173\end{array}$

$\begin{array}{llll}-3.693057 & -0.841573 & -3.551212\end{array}$

$\begin{array}{llll}2.226152 & -2.176863 & -2.001601\end{array}$

$\begin{array}{llll}1.703167 & -3.106178 & -2.122938\end{array}$

$\begin{array}{llll}2.748144 & -1.796739 & -2.872089\end{array}$

$\begin{array}{llll}1.881470 & -1.256807 & -0.985457\end{array}$

$\begin{array}{llll}2.478317 & -0.352043 & -0.945374\end{array}$

$\begin{array}{llll}1.040601 & -1.552212 & 0.138563\end{array}$

$\begin{array}{llll}0.763691 & -2.594583 & 0.263463\end{array}$

$\begin{array}{lll}1.282661 & -0.891151 & 1.444368\end{array}$

$\begin{array}{llll}1.743474 & 0.428939 & 1.556166\end{array}$

$\begin{array}{llll}1.150268 & -1.648570 & 2.622365\end{array}$

$\begin{array}{llll}2.057128 & 0.975353 & 2.796294\end{array}$

$\begin{array}{llll}1.890150 & 1.023024 & 0.666433\end{array}$

$\begin{array}{llll}1.447910 & -1.096296 & 3.871033\end{array}$ 


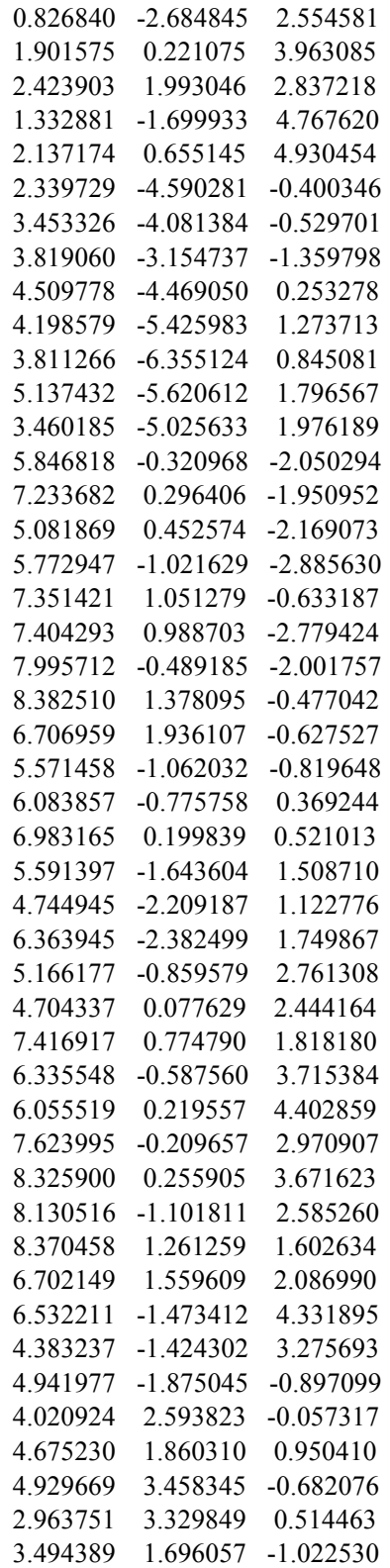

$[12-13]^{\ddagger} \mathbf{P 2}$ exo-si conf2 [BF4]

Number of imaginary frequencies : 1

The smallest frequency is : $-280.8071 \mathrm{~cm}(-1)$

Electronic energy $=-3892.0000815$

$\begin{array}{lc}\begin{array}{l}\text { Zero-point correction= } \\ \text { (Hartree/Particle) }\end{array} & 1.232644 \\ \text { Thermal correction to Energy= } & 1.305925 \\ \text { Thermal correction to Enthalpy= } & 1.122976 \\ \text { Thermal correction to Gibbs Free Energy= } & -3890.767438 \\ \text { Sum of electronic and zero-point Energies }= & -3890.695101 \\ \text { Sum of electronic and thermal Energies }= & -3890.694156 \\ \text { Sum of electronic and thermal Enthalpies }= & -3890.877105\end{array}$

Cartesian Coordinates

$\begin{array}{lll}-0.258071 & -1.003377 & -1.600391\end{array}$

$\begin{array}{lll}-1.707836 & 0.377278 & -0.476987\end{array}$

$\begin{array}{lll}-2.262255 & 0.229991 & 1.079327\end{array}$

$\begin{array}{lll}-3.183476 & 0.401724 & -1.278653\end{array}$

$\begin{array}{lll}-1.049992 & 1.897111 & -0.366015\end{array}$

$\begin{array}{lll}-3.151057 & -0.782980 & 1.414697\end{array}$ $\begin{array}{lll}-2.638345 & -1.828819 & 2.211178\end{array}$

$\begin{array}{lll}-1.606871 & -1.769631 & 2.530039\end{array}$

$\begin{array}{lll}-3.450798 & -2.878935 & 2.556829\end{array}$

$\begin{array}{lll}-3.067716 & -3.686104 & 3.174913\end{array}$

$\begin{array}{lll}-4.787966 & -2.942696 & 2.078977\end{array}$

$\begin{array}{lll}-5.609913 & -4.070127 & 2.350037\end{array}$

$\begin{array}{lll}-5.201760 & -4.872131 & 2.959742\end{array}$

$\begin{array}{lll}-6.885928 & -4.155912 & 1.840727\end{array}$

$\begin{array}{lll}-7.502363 & -5.025575 & 2.049082\end{array}$

$\begin{array}{lll}-7.388376 & -3.112835 & 1.026929\end{array}$

$\begin{array}{lll}-8.385320 & -3.193813 & 0.603101\end{array}$

$\begin{array}{lll}-6.623890 & -1.997242 & 0.760940\end{array}$

$\begin{array}{llll}-7.023257 & -1.214624 & 0.127085\end{array}$

$\begin{array}{lll}-5.309198 & -1.862433 & 1.289128\end{array}$

$\begin{array}{lll}-4.478153 & -0.715166 & 1.019073\end{array}$

$\begin{array}{llll}-4.996353 & 0.486911 & 0.309243\end{array}$

$\begin{array}{llll}-6.151497 & 1.208602 & 0.782310\end{array}$

$\begin{array}{lll}-6.779081 & 0.921417 & 2.027266\end{array}$

$\begin{array}{llll}-6.373715 & 0.138862 & 2.657454\end{array}$

$\begin{array}{lll}-7.880675 & 1.632790 & 2.451685\end{array}$

$\begin{array}{llll}-8.334273 & 1.397705 & 3.410366\end{array}$

$\begin{array}{lll}-8.422244 & 2.670416 & 1.656340\end{array}$

$\begin{array}{llll}-9.295025 & 3.217727 & 2.000208\end{array}$

$\begin{array}{lll}-7.826806 & 2.992028 & 0.457570\end{array}$

$\begin{array}{llll}-8.217457 & 3.800376 & -0.155060\end{array}$

$\begin{array}{llll}-6.679211 & 2.290133 & -0.000845\end{array}$

$\begin{array}{llll}-6.031598 & 2.657627 & -1.211565\end{array}$

$\begin{array}{llll}-6.445618 & 3.465771 & -1.808108\end{array}$

$\begin{array}{llll}-4.876920 & 2.028261 & -1.600672\end{array}$

$\begin{array}{llll}-4.338589 & 2.320506 & -2.496269\end{array}$

$\begin{array}{llll}-4.351757 & 0.973608 & -0.819142\end{array}$

$\begin{array}{llll}0.148715 & 2.111636 & -1.216443\end{array}$

$\begin{array}{llll}1.013752 & 2.197582 & -0.554054\end{array}$

$\begin{array}{llll}0.003358 & 3.394741 & -2.024843\end{array}$

$\begin{array}{llll}-1.196505 & 3.669834 & -2.700619\end{array}$

$\begin{array}{llll}-2.029870 & 2.979858 & -2.600902\end{array}$

$\begin{array}{llll}-1.327482 & 4.820402 & -3.476607\end{array}$

$\begin{array}{lll}-2.264254 & 5.023488 & -3.989327\end{array}$

$\begin{array}{llll}-0.256115 & 5.713473 & -3.592663\end{array}$

$\begin{array}{llll}-0.357448 & 6.612150 & -4.195031\end{array}$

$\begin{array}{llll}0.939453 & 5.445438 & -2.923715\end{array}$

$\begin{array}{llll}1.773705 & 6.137752 & -3.001833\end{array}$

$\begin{array}{llll}1.069379 & 4.293448 & -2.140547\end{array}$

$\begin{array}{llll}1.992248 & 4.097566 & -1.605067\end{array}$

$\begin{array}{llll}0.385362 & 0.937047 & -2.192850\end{array}$

$\begin{array}{llll}-0.151538 & 1.149210 & -3.123466\end{array}$

$\begin{array}{llll}1.447847 & 0.960839 & -2.435547\end{array}$

$\begin{array}{lll}-1.319415 & 2.989134 & 0.628295\end{array}$

$\begin{array}{lll}-0.824707 & 3.853583 & 0.178570\end{array}$

$\begin{array}{lll}-0.631801 & 2.767644 & 1.973628\end{array}$

$\begin{array}{lll}0.697288 & 3.183746 & 2.124832\end{array}$

$\begin{array}{llll}1.225599 & 3.628895 & 1.290361\end{array}$

$\begin{array}{lll}1.369749 & 3.016134 & 3.337605\end{array}$

$\begin{array}{llll}2.407487 & 3.326857 & 3.419967\end{array}$

$\begin{array}{llll}0.707788 & 2.458640 & 4.432371\end{array}$

$\begin{array}{llll}1.223445 & 2.334770 & 5.381016\end{array}$

$\begin{array}{llll}-0.625817 & 2.058541 & 4.298887\end{array}$

$\begin{array}{lll}-1.150618 & 1.623112 & 5.145093\end{array}$

$\begin{array}{llll}-1.286642 & 2.204711 & 3.078905\end{array}$

$\begin{array}{lll}-2.315056 & 1.874635 & 2.990234\end{array}$

$\begin{array}{lll}-2.794144 & 3.368336 & 0.742819\end{array}$

$\begin{array}{llll}-3.187034 & 3.645031 & -0.237275\end{array}$

$\begin{array}{lll}-3.410783 & 2.565625 & 1.146723\end{array}$

$\begin{array}{llll}-2.887815 & 4.233159 & 1.406957\end{array}$

$\begin{array}{llll}-1.659274 & -1.027587 & -3.360454\end{array}$

$\begin{array}{llll}-1.864472 & 0.013251 & -3.595992\end{array}$

$\begin{array}{llll}-0.402726 & -1.519124 & -3.783974\end{array}$

$\begin{array}{llll}0.243541 & -0.817878 & -4.310766\end{array}$

$\begin{array}{llll}-0.089499 & -2.969539 & -4.104269\end{array}$

$-0.426090 \quad-3.192836 \quad-5.128224$ 


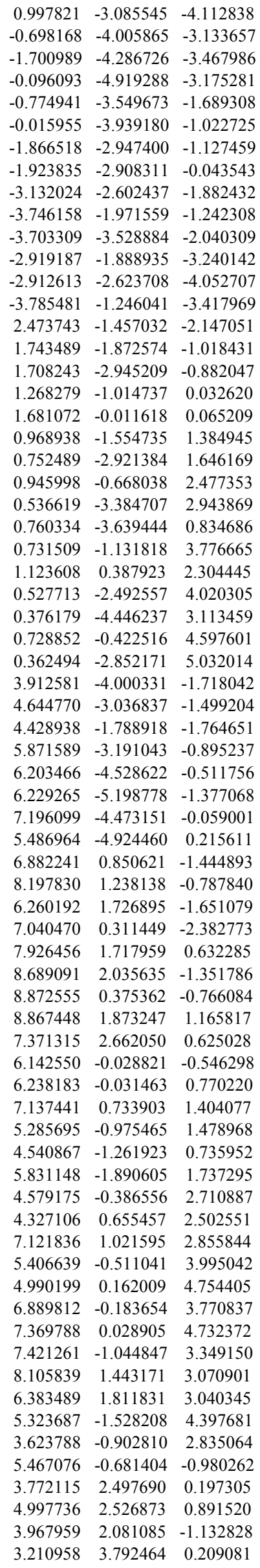

$\begin{array}{llll}2.896910 & 1.596639 & 0.841500\end{array}$

$\begin{array}{llll}2.542142 & -2.126307 & -2.991405\end{array}$

$2.681757 \quad-0.413068 \quad-2.325166$

$[12-13]^{\ddagger} \mathbf{P 2}$ endo-si conf2 [BF4ㄱ

Number of imaginary frequencies : 1

The smallest frequency is : $-253.4738 \mathrm{~cm}(-1)$

Electronic energy $=-3892.0090717$

Zero-point correction $=\quad 1.233359$

(Hartree/Particle)

Thermal correction to Energy= $\quad 1.305517$

Thermal correction to Enthalpy= $\quad 1.306461$

Thermal correction to Gibbs Free Energy $=\quad 1.124599$

Sum of electronic and zero-point Energies $=\quad-3890.775712$

Sum of electronic and thermal Energies $=\quad-3890.703555$

Sum of electronic and thermal Enthalpies $=\quad-3890.702611$

Sum of electronic and thermal Free Energies $=\quad-3890.884473$

Cartesian Coordinates

\begin{tabular}{|c|c|c|c|}
\hline 77 & -0.340824 & -0.762008 & -1.656276 \\
\hline 15 & -1.861696 & 0.404244 & -0.375316 \\
\hline 8 & -2.361907 & 0.054162 & 1.170997 \\
\hline 8 & -3.360287 & 0.456400 & -1.133758 \\
\hline 7 & -1.245725 & 1.929722 & -0.111420 \\
\hline 6 & -3.205184 & -1.026925 & 1.389104 \\
\hline 6 & -2.640669 & -2.162813 & 2.010790 \\
\hline 1 & -1.597903 & -2.127917 & 2.305638 \\
\hline 6 & -3.417469 & -3.275337 & 2.220572 \\
\hline 1 & -2.998281 & -4.155360 & 2.700069 \\
\hline 6 & -4.768727 & -3.304866 & 1.780818 \\
\hline 6 & -5.556170 & -4.480700 & 1.913588 \\
\hline 1 & -5.107923 & -5.352821 & 2.382475 \\
\hline 6 & -6.850318 & -4.522555 & 1.446556 \\
\hline 1 & -7.440418 & -5.428753 & 1.547565 \\
\hline 6 & -7.408133 & -3.383681 & 0.817791 \\
\hline 1 & -8.421442 & -3.426450 & 0.428782 \\
\hline 6 & -6.676959 & -2.222869 & 0.687249 \\
\hline 1 & -7.117589 & -1.364624 & 0.193827 \\
\hline 6 & -5.343080 & -2.135809 & 1.175962 \\
\hline 6 & -4.545300 & -0.944132 & 1.042917 \\
\hline 6 & -5.103159 & 0.335787 & 0.523894 \\
\hline 6 & -6.235832 & 0.978053 & 1.139653 \\
\hline 6 & -6.823323 & 0.506655 & 2.347675 \\
\hline 1 & -6.404670 & -0.364081 & 2.837874 \\
\hline 6 & -7.905684 & 1.146836 & 2.911320 \\
\hline 1 & -8.329804 & 0.768865 & 3.837286 \\
\hline 6 & -8.465653 & 2.294432 & 2.301040 \\
\hline 1 & -9.322951 & 2.784475 & 2.753305 \\
\hline 6 & -7.909025 & 2.792736 & 1.144868 \\
\hline 1 & -8.315550 & 3.684447 & 0.674396 \\
\hline 6 & -6.782944 & 2.165304 & 0.545721 \\
\hline 6 & -6.178586 & 2.705329 & -0.621348 \\
\hline 1 & -6.607729 & 3.594203 & -1.075160 \\
\hline 6 & -5.048722 & 2.133330 & -1.149973 \\
\hline 1 & -4.551282 & 2.548244 & -2.020099 \\
\hline 6 & -4.504947 & 0.973097 & -0.554115 \\
\hline 6 & -0.096392 & 2.281063 & -0.991611 \\
\hline 1 & 0.812638 & 2.273828 & -0.383416 \\
\hline 6 & -0.275429 & 3.672148 & -1.585838 \\
\hline 6 & -1.523049 & 4.078486 & -2.083501 \\
\hline 1 & -2.371925 & 3.408199 & -1.988392 \\
\hline 6 & -1.678632 & 5.330607 & -2.677373 \\
\hline 1 & -2.652857 & 5.633903 & -3.052048 \\
\hline 6 & -0.584737 & 6.195797 & -2.786361 \\
\hline 1 & -0.704378 & 7.172482 & -3.247299 \\
\hline 6 & 0.659839 & 5.797764 & -2.294169 \\
\hline 1 & 1.513652 & 6.466114 & -2.368421 \\
\hline 6 & 0.814544 & 4.544705 & -1.693148 \\
\hline
\end{tabular}


$\mathbf{P 1}$ endo-re $\left[\mathrm{BF}_{4}^{-}\right]$

Number of imaginary frequencies : 0

The smallest frequency is : $12.8582 \mathrm{~cm}(-1)$

Electronic energy $=-3125.4962645$

Zero-point correction=

(Hartree/Particle)

Thermal correction to Energy $=\quad 0.969328$

Thermal correction to Enthalpy= $\quad 0.970272$

Thermal correction to Gibbs Free Energy $=\quad 0.825821$

Sum of electronic and zero-point Energies $=\quad-3124.581715$

Sum of electronic and thermal Energies $=\quad-3124.526937$

Sum of electronic and thermal Enthalpies $=\quad-3124.525992$

Sum of electronic and thermal Free Energies $=\quad-3124.670443$

\begin{tabular}{crrr} 
& \multicolumn{3}{c}{ Cartesian Coordinates } \\
\hline 77 & -1.143406 & -1.367048 & -0.238216 \\
15 & 0.592702 & 0.095956 & -0.137958 \\
8 & 1.640024 & 0.205066 & 1.122888 \\
8 & 1.656429 & -0.181158 & -1.389421 \\
7 & -0.010229 & 1.640208 & -0.135852 \\
6 & 2.710553 & -0.662991 & 1.298692 \\
6 & 2.649576 & -1.504939 & 2.430322 \\
1 & 1.803104 & -1.404964 & 3.101529 \\
6 & 3.654405 & -2.414019 & 2.650767 \\
1 & 3.624957 & -3.065077 & 3.519806 \\
6 & 4.729562 & -2.537922 & 1.729272 \\
6 & 5.727925 & -3.535636 & 1.897440 \\
1 & 5.665549 & -4.190241 & 2.762757 \\
6 & 6.743294 & -3.682207 & 0.979527 \\
1 & 7.497265 & -4.452239 & 1.114117 \\
6 & 6.795451 & -2.835000 & -0.152940 \\
1 & 7.583765 & -2.968191 & -0.888294 \\
6 & 5.853002 & -1.846438 & -0.336447 \\
1 & 5.904764 & -1.214106 & -1.214821 \\
6 & 4.800355 & -1.652645 & 0.601224 \\
6 & 3.791706 & -0.638219 & 0.431518 \\
6 & 3.874576 & 0.388027 & -0.644230 \\
6 & 5.010146 & 1.265829 & -0.771956 \\
6 & 6.063035 & 1.303712 & 0.184742 \\
1 & 6.016499 & 0.655882 & 1.051763
\end{tabular}


$\begin{array}{llll}7.129281 & 2.163814 & 0.035320\end{array}$

$\begin{array}{lll}7.915619 & 2.178648 & 0.784603\end{array}$

$\begin{array}{llll}7.207109 & 3.032894 & -1.078979\end{array}$

$\begin{array}{llll}8.057010 & 3.700418 & -1.186349\end{array}$

$\begin{array}{llll}6.194854 & 3.040466 & -2.011463\end{array}$

$\begin{array}{llll}6.229551 & 3.716952 & -2.861430\end{array}$

$\begin{array}{llll}5.072453 & 2.178609 & -1.878527\end{array}$

$\begin{array}{llll}4.001733 & 2.223850 & -2.811408\end{array}$

$\begin{array}{lll}4.064760 & 2.904807 & -3.655308\end{array}$

$\begin{array}{llll}2.885841 & 1.445619 & -2.630138\end{array}$

$\begin{array}{llll}2.044281 & 1.486475 & -3.312160\end{array}$

$\begin{array}{llll}2.824829 & 0.561694 & -1.531820\end{array}$

$\begin{array}{llll}-1.422806 & 1.723442 & -0.608625\end{array}$

$\begin{array}{lll}-2.058174 & 1.905488 & 0.258410\end{array}$

$\begin{array}{lll}-1.584402 & 2.884713 & -1.580040\end{array}$

$\begin{array}{llll}-2.547015 & 3.876372 & -1.354980\end{array}$

$\begin{array}{lll}-3.175415 & 3.820868 & -0.473412\end{array}$

$\begin{array}{llll}-2.684972 & 4.939893 & -2.253010\end{array}$

$\begin{array}{llll}-3.433181 & 5.705369 & -2.065242\end{array}$

$\begin{array}{lll}-1.863306 & 5.025988 & -3.378003\end{array}$

$\begin{array}{llll}-1.969961 & 5.855755 & -4.071438\end{array}$

$\begin{array}{llll}-0.894966 & 4.041449 & -3.603861\end{array}$

$\begin{array}{llll}-0.245000 & 4.104260 & -4.472714\end{array}$

$\begin{array}{llll}-0.758639 & 2.980494 & -2.710074\end{array}$

$\begin{array}{llll}0.001388 & 2.224137 & -2.876975\end{array}$

$\begin{array}{llll}-1.845481 & 0.394193 & -1.251427\end{array}$

$\begin{array}{llll}-1.479506 & 0.352166 & -2.282386\end{array}$

$\begin{array}{llll}-2.935751 & 0.358775 & -1.294089\end{array}$

$\begin{array}{lll}0.479346 & 2.828685 & 0.650017\end{array}$

$\begin{array}{lll}-0.193978 & 3.625722 & 0.323355\end{array}$

$\begin{array}{lll}0.250778 & 2.649123 & 2.150128\end{array}$

$\begin{array}{lll}-1.046325 & 2.804976 & 2.659307\end{array}$

$\begin{array}{lll}-1.854307 & 3.128278 & 2.014144\end{array}$

$\begin{array}{lll}-1.325805 & 2.558796 & 4.005405\end{array}$

$\begin{array}{lll}-2.349326 & 2.652126 & 4.353498\end{array}$

$\begin{array}{lll}-0.299999 & 2.185772 & 4.875660\end{array}$

$\begin{array}{lll}-0.511476 & 1.995687 & 5.924555\end{array}$

$\begin{array}{lll}1.006040 & 2.071336 & 4.388800\end{array}$

$\begin{array}{lll}1.816194 & 1.798307 & 5.060119\end{array}$

$\begin{array}{lll}1.277231 & 2.298542 & 3.038378\end{array}$

$\begin{array}{lll}2.292887 & 2.184446 & 2.677120\end{array}$

$\begin{array}{lll}1.887350 & 3.262425 & 0.253282\end{array}$

$\begin{array}{llll}1.928429 & 3.446655 & -0.822401\end{array}$

$\begin{array}{lll}2.644945 & 2.518700 & 0.499929\end{array}$

$\begin{array}{llll}2.139193 & 4.190316 & 0.776166\end{array}$

$\begin{array}{lll}-1.568277 & -0.844508 & 1.838847\end{array}$

$\begin{array}{lll}-1.288653 & -1.697142 & 2.456207\end{array}$

$\begin{array}{lll}-1.203245 & 0.106353 & 2.216910\end{array}$

$\begin{array}{lll}-2.862544 & -0.820405 & 1.219203\end{array}$

$\begin{array}{lll}-3.300571 & 0.144262 & 0.995674\end{array}$

$\begin{array}{lll}-3.513452 & -1.977838 & 0.789035\end{array}$

$\begin{array}{lll}-3.190436 & -2.927945 & 1.198949\end{array}$

$\begin{array}{llll}-0.395876 & -2.135319 & -2.251574\end{array}$

$\begin{array}{llll}-0.199188 & -1.241114 & -2.833938\end{array}$

$\begin{array}{lll}-1.737120 & -2.518689 & -2.114060\end{array}$

$\begin{array}{llll}-2.490592 & -1.900936 & -2.600754\end{array}$

$\begin{array}{llll}-2.202931 & -3.921825 & -1.777671\end{array}$

$\begin{array}{lll}-2.151491 & -4.536090 & -2.688692\end{array}$

$\begin{array}{llll}-3.259595 & -3.863037 & -1.511942\end{array}$

$\begin{array}{lll}-1.429968 & -4.626582 & -0.636242\end{array}$

$\begin{array}{llll}-0.609666 & -5.223318 & -1.047303\end{array}$

$\begin{array}{lll}-2.105788 & -5.336642 & -0.149830\end{array}$

$\begin{array}{lll}-0.862587 & -3.701035 & 0.431413\end{array}$

$\begin{array}{lll}-1.338868 & -3.734999 & 1.407015\end{array}$

$\begin{array}{lll}0.393948 & -3.137661 & 0.377410\end{array}$

$\begin{array}{llll}0.818466 & -2.789486 & 1.313577\end{array}$

$\begin{array}{llll}1.376645 & -3.304376 & -0.761925\end{array}$

$\begin{array}{llll}1.3782499 & -2.584479 & -0.620799\end{array}$

$\begin{array}{llll}1.834151 & -4.300714 & -0.682620\end{array}$

$\begin{array}{llll}0.773521 & -3.114737 & -2.172080\end{array}$

$\begin{array}{llll}0.445781 & -4.077626 & -2.576059\end{array}$

$\begin{array}{lll}1.563804 & -2.755747 & -2.836747\end{array}$

$\begin{array}{llll}-4.761883 & -2.038592 & 0.026956\end{array}$

$\begin{array}{lll}-5.399234 & -0.904694 & -0.517642\end{array}$

$\begin{array}{llll}-5.358231 & -3.301254 & -0.171231\end{array}$ $\begin{array}{lll}-6.577667 & -1.042709 & -1.247879\end{array}$

$\begin{array}{llll}-4.996882 & 0.085964 & -0.347097\end{array}$

$\begin{array}{lll}-6.537502 & -3.433866 & -0.901620\end{array}$

$\begin{array}{lll}-4.888481 & -4.182400 & 0.258963\end{array}$

$\begin{array}{llll}-7.149365 & -2.303485 & -1.449600\end{array}$

$\begin{array}{lll}-7.057283 & -0.157180 & -1.655470\end{array}$

$\begin{array}{lll}-6.979074 & -4.416643 & -1.040634\end{array}$

$\begin{array}{lll}-8.068452 & -2.401647 & -2.020256\end{array}$

$\begin{array}{lll}-4.625540 & 2.553491 & 1.657912\end{array}$

$\begin{array}{lll}-4.394765 & 1.975914 & 0.374436\end{array}$

$\begin{array}{lll}-5.997009 & 2.685133 & 1.868376\end{array}$

$\begin{array}{lll}-4.051506 & 1.722915 & 2.638004\end{array}$

$\begin{array}{llll}-3.997421 & 3.815990 & 1.686558\end{array}$

P1 exo-si $\left[\mathrm{BF}_{4}^{-}\right]$

Number of imaginary frequencies : 0

The smallest frequency is : $13.3872 \mathrm{~cm}(-1)$

Electronic energy $=-3125.4910399$

Zero-point correction $=$

0.914402

(Hartree/Particle)

Thermal correction to Energy $=\quad 0.969248$

Thermal correction to Enthalpy= $\quad 0.970192$

Thermal correction to Gibbs Free Energy $=\quad 0.825039$

Sum of electronic and zero-point Energies $=\quad-3124.576637$

Sum of electronic and thermal Energies $=$

Sum of electronic and thermal Enthalpies=

$-3124.521792$

$-3124.520848$

Sum of electronic and thermal Free Energies=

$-3124.666001$

Cartesian Coordinates

$-1.255554-1.046700 \quad-0.340122$

$\begin{array}{llll}0.607243 & 0.237829 & -0.139153\end{array}$

$\begin{array}{lll}1.624092 & 0.193146 & 1.154207\end{array}$

$\begin{array}{lll}1.683399 & -0.076388 & -1.371409\end{array}$

$\begin{array}{llll}0.151698 & 1.834617 & -0.082783\end{array}$

$\begin{array}{lll}2.552362 & -0.829340 & 1.316434\end{array}$

$\begin{array}{lll}2.317362 & -1.727555 & 2.379469\end{array}$

$\begin{array}{lll}1.453394 & -1.561149 & 3.014056\end{array}$

$\begin{array}{lll}3.183937 & -2.772873 & 2.583121\end{array}$

$\begin{array}{lll}3.022778 & -3.468400 & 3.401733\end{array}$

$\begin{array}{lll}4.284330 & -2.978158 & 1.707122\end{array}$

$\begin{array}{lll}5.138539 & -4.104473 & 1.856834\end{array}$

$\begin{array}{lll}4.947237 & -4.793871 & 2.675055\end{array}$

$\begin{array}{lll}6.175119 & -4.327779 & 0.979186\end{array}$

$\begin{array}{llll}6.818104 & -5.194733 & 1.099212\end{array}$

$\begin{array}{lll}6.393561 & -3.431317 & -0.094060\end{array}$

$\begin{array}{llll}7.196681 & -3.623194 & -0.799755\end{array}$

$\begin{array}{llll}5.594782 & -2.320394 & -0.257274\end{array}$

$\begin{array}{llll}5.772423 & -1.651062 & -1.090417\end{array}$

$\begin{array}{lll}4.527677 & -2.044920 & 0.642825\end{array}$

$\begin{array}{llll}3.667077 & -0.898883 & 0.496393\end{array}$

$\begin{array}{lll}3.923750 & 0.161771 & -0.516833\end{array}$

$\begin{array}{llll}5.166955 & 0.888141 & -0.561609\end{array}$

$\begin{array}{lll}6.174098 & 0.746978 & 0.434057\end{array}$

$\begin{array}{lll}6.007258 & 0.075790 & 1.268124\end{array}$

$\begin{array}{lll}7.349502 & 1.462881 & 0.363539\end{array}$

$\begin{array}{llll}8.098320 & 1.342646 & 1.141216\end{array}$

$\begin{array}{llll}7.588154 & 2.358606 & -0.705902\end{array}$

$\begin{array}{llll}8.522176 & 2.910863 & -0.751279\end{array}$

$\begin{array}{llll}6.626524 & 2.537993 & -1.674195\end{array}$

$\begin{array}{lll}6.786332 & 3.237472 & -2.490597\end{array}$

$\begin{array}{llll}5.396087 & 1.828288 & -1.622341\end{array}$

$\begin{array}{llll}4.382180 & 2.049173 & -2.593018\end{array}$

$\begin{array}{llll}4.570904 & 2.744701 & -3.405775\end{array}$

$\begin{array}{llll}3.167626 & 1.418400 & -2.488425\end{array}$

$\begin{array}{llll}2.370795 & 1.591749 & -3.202895\end{array}$

$\begin{array}{llll}2.942413 & 0.509854 & -1.431864\end{array}$

$\begin{array}{llll}-1.242003 & 2.080790 & -0.535795\end{array}$

$\begin{array}{lll}-1.847879 & 2.326429 & 0.338245\end{array}$

$\begin{array}{llll}-1.308310 & 3.248551 & -1.510985\end{array}$

$\begin{array}{llll}-2.386001 & 4.141925 & -1.464008\end{array}$

$\begin{array}{llll}-3.141550 & 4.023570 & -0.695604\end{array}$

$\begin{array}{llll}-2.479317 & 5.181189 & -2.393962\end{array}$

$\begin{array}{llll}-3.319008 & 5.869455 & -2.345394\end{array}$ 
$\begin{array}{llll}-1.498216 & 5.342903 & -3.374844\end{array}$

$\begin{array}{lll}-1.570803 & 6.155022 & -4.093138\end{array}$

$\begin{array}{lll}-0.417162 & 4.457019 & -3.421848\end{array}$

$\begin{array}{llll}0.355958 & 4.578808 & -4.176160\end{array}$

$\begin{array}{llll}-0.325674 & 3.416777 & -2.496903\end{array}$

$\begin{array}{llll}0.518712 & 2.736472 & -2.522320\end{array}$

$\begin{array}{llll}-1.833144 & 0.826938 & -1.192831\end{array}$

$\begin{array}{llll}-1.514383 & 0.799464 & -2.240298\end{array}$

$\begin{array}{llll}-2.916970 & 0.924984 & -1.183406\end{array}$

$\begin{array}{lll}0.740812 & 2.942985 & 0.750535\end{array}$

$\begin{array}{llll}0.217015 & 3.826887 & 0.376310\end{array}$

$\begin{array}{lll}0.361647 & 2.797294 & 2.223519\end{array}$

$\begin{array}{lll}-0.914515 & 3.205241 & 2.640475\end{array}$

$\begin{array}{lll}-1.600227 & 3.667932 & 1.939504\end{array}$

$\begin{array}{lll}-1.337257 & 3.012719 & 3.957412\end{array}$

$\begin{array}{lll}-2.341992 & 3.311897 & 4.241111\end{array}$

$\begin{array}{lll}-0.475084 & 2.437264 & 4.892145\end{array}$

$\begin{array}{lll}-0.798229 & 2.289505 & 5.919079\end{array}$

$\begin{array}{lll}0.814275 & 2.065479 & 4.499037\end{array}$

$\begin{array}{llll}1.501437 & 1.634579 & 5.222705\end{array}$

$\begin{array}{lll}1.226978 & 2.242002 & 3.177082\end{array}$

$\begin{array}{lll}2.225979 & 1.935505 & 2.890271\end{array}$

$\begin{array}{llll}2.224657 & 3.179805 & 0.482264\end{array}$

$\begin{array}{llll}2.387493 & 3.353668 & -0.583647\end{array}$

$\begin{array}{llll}2.852933 & 2.344447 & 0.790049\end{array}$

$\begin{array}{lll}2.547785 & 4.069188 & 1.031999\end{array}$

$\begin{array}{lll}-1.616578 & -0.321375 & 1.728000\end{array}$

$\begin{array}{lll}-0.858593 & -0.560792 & 2.469023\end{array}$

$\begin{array}{lll}-1.889101 & 0.726198 & 1.688920\end{array}$

$\begin{array}{lll}-2.640522 & -1.290627 & 1.511440\end{array}$

$\begin{array}{lll}-2.540478 & -2.258725 & 1.991221\end{array}$

$\begin{array}{lll}-3.661638 & -1.071967 & 0.586403\end{array}$

$\begin{array}{lll}-3.866706 & -0.044663 & 0.311373\end{array}$

$\begin{array}{llll}-0.582521 & -1.659501 & -2.405807\end{array}$

$\begin{array}{llll}-0.329805 & -0.731267 & -2.908663\end{array}$

$\begin{array}{llll}-1.953607 & -1.956260 & -2.297842\end{array}$

$\begin{array}{llll}-2.649207 & -1.230715 & -2.717672\end{array}$

$\begin{array}{llll}-2.538998 & -3.346165 & -2.160992\end{array}$

$\begin{array}{llll}-2.591592 & -3.802096 & -3.160827\end{array}$

$\begin{array}{llll}-3.570673 & -3.247278 & -1.820413\end{array}$

$\begin{array}{lll}-1.781198 & -4.293303 & -1.204829\end{array}$

$\begin{array}{llll}-1.002185 & -4.836677 & -1.747278\end{array}$

$\begin{array}{llll}-2.485647 & -5.046225 & -0.838552\end{array}$

$\begin{array}{llll}-1.150294 & -3.614607 & -0.003450\end{array}$

$\begin{array}{lll}-1.674676 & -3.728033 & 0.938191\end{array}$

$\begin{array}{lll}0.136525 & -3.147481 & 0.042484\end{array}$

$\begin{array}{llll}0.568033 & -2.944504 & 1.018273\end{array}$

$\begin{array}{llll}1.106515 & -3.172336 & -1.117790\end{array}$

$\begin{array}{llll}1.950053 & -2.531262 & -0.864812\end{array}$

$\begin{array}{lll}1.509788 & -4.190358 & -1.214866\end{array}$

$\begin{array}{llll}0.521224 & -2.715463 & -2.474835\end{array}$

$\begin{array}{llll}0.134892 & -3.576328 & -3.029917\end{array}$

$\begin{array}{lll}1.340190 & -2.306999 & -3.072477\end{array}$

$\begin{array}{lll}-4.687049 & -2.035151 & 0.184115\end{array}$

$\begin{array}{lll}-4.716235 & -3.377980 & 0.610643\end{array}$

$\begin{array}{llll}-5.683235 & -1.593118 & -0.708339\end{array}$

$\begin{array}{llll}-5.698747 & -4.247789 & 0.143983\end{array}$

$\begin{array}{lll}-3.969436 & -3.747341 & 1.305587\end{array}$

$-6.663384-2.466638-1.176480$

$\begin{array}{llll}-5.680098 & -0.552430 & -1.021937\end{array}$

$-6.671714-3.799504 \quad-0.756547$

$\begin{array}{lll}-5.706825 & -5.280129 & 0.482517\end{array}$

$\begin{array}{llll}-7.422028 & -2.106538 & -1.865526\end{array}$

$\begin{array}{llll}-7.433594 & -4.482781 & -1.120592\end{array}$

$\begin{array}{lll}-4.582637 & 2.544256 & 1.343808\end{array}$

$\begin{array}{lll}-3.851638 & 3.754182 & 1.390241\end{array}$

$\begin{array}{lll}-3.973220 & 1.609828 & 2.202485\end{array}$

$\begin{array}{lll}-5.905690 & 2.768378 & 1.726946\end{array}$

$\begin{array}{lll}-4.550366 & 2.042617 & 0.015410\end{array}$

\section{P1 exo-re $\left[\mathrm{BF}_{4}^{-}\right]$}

Number of imaginary frequencies : 0

The smallest frequency is : $17.8007 \mathrm{~cm}(-1)$

Electronic energy=-3125.4892174
Zero-point correction=

(Hartree/Particle)

Thermal correction to Energy $=\quad 0.969867$

Thermal correction to Enthalpy=

0.915265

Thermal correction to Gibbs Free Energy=

Sum of electronic and zero-point Energies=

Sum of electronic and thermal Energies=

Sum of electronic and thermal Enthalpies=

Sum of electronic and thermal Free Energies=

0.970811

0.828279

$-3124.573952$

$-3124.519351$

$-3124.518407$

Cartesian Coordinates

$-3124.660938$

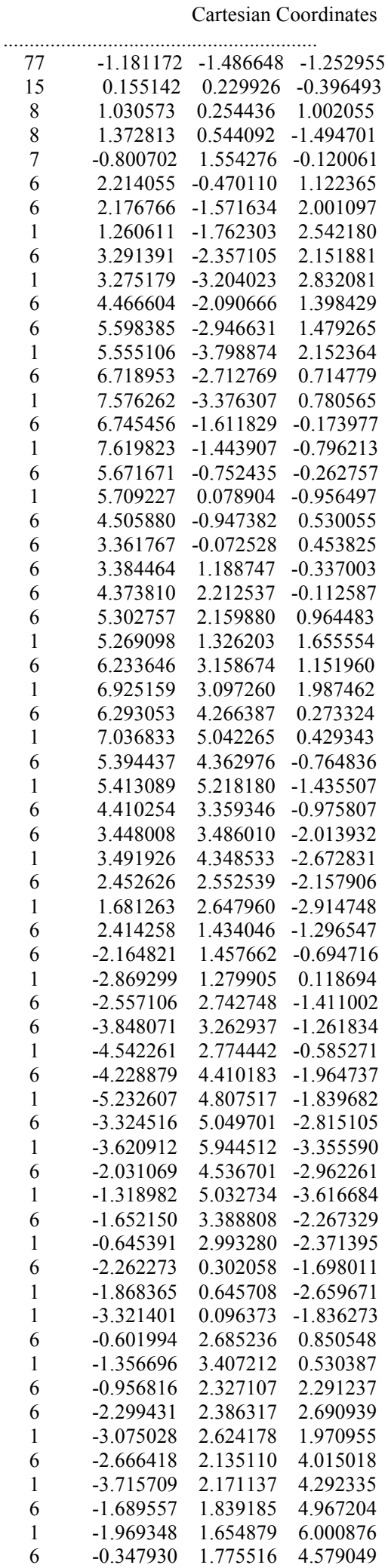


$\begin{array}{lll}0.419612 & 1.538914 & 5.311023\end{array}$

$\begin{array}{lll}0.014107 & 2.010621 & 3.252054\end{array}$

$\begin{array}{lll}1.058834 & 1.951876 & 2.969028\end{array}$

$\begin{array}{llll}0.740373 & 3.396387 & 0.700736\end{array}$

$\begin{array}{llll}0.867491 & 3.743327 & -0.326054\end{array}$

$\begin{array}{lll}1.590260 & 2.765121 & 0.957752\end{array}$

$\begin{array}{llll}0.753717 & 4.267734 & 1.362541\end{array}$

$\begin{array}{lll}-3.267377 & -2.452801 & -1.308640\end{array}$

$\begin{array}{llll}-3.935615 & -1.608142 & -1.411310\end{array}$

$\begin{array}{lll}-3.456239 & -3.286841 & -1.976321\end{array}$

$\begin{array}{lll}-2.675075 & -2.742627 & -0.066638\end{array}$

$\begin{array}{llll}-2.380813 & -3.768839 & 0.123561\end{array}$

$\begin{array}{lll}-2.235737 & -1.743516 & 0.835658\end{array}$

$\begin{array}{lll}-2.809285 & -0.826978 & 0.900658\end{array}$

$\begin{array}{llll}-0.098613 & -1.200136 & -3.219460\end{array}$

$\begin{array}{llll}-0.151983 & -0.135847 & -3.426586\end{array}$

$\begin{array}{llll}-1.272986 & -1.936210 & -3.466207\end{array}$

$\begin{array}{llll}-2.131717 & -1.380388 & -3.838581\end{array}$

$\begin{array}{llll}-1.316397 & -3.415318 & -3.796005\end{array}$

$\begin{array}{llll}-1.074435 & -3.535447 & -4.861986\end{array}$

$\begin{array}{llll}-2.346884 & -3.759969 & -3.680512\end{array}$

$\begin{array}{llll}-0.379578 & -4.307313 & -2.951297\end{array}$

$\begin{array}{llll}0.600601 & -4.382560 & -3.430475\end{array}$

$\begin{array}{llll}-0.788637 & -5.321987 & -2.933227\end{array}$

$\begin{array}{llll}-0.186413 & -3.846296 & -1.517255\end{array}$

$-0.724258-4.406791 \quad-0.762105$

$\begin{array}{llll}0.829315 & -3.026059 & -1.099398\end{array}$

$\begin{array}{llll}1.046458 & -2.983784 & -0.035230\end{array}$

$\begin{array}{llll}1.868494 & -2.427445 & -2.021943\end{array}$

$\begin{array}{llll}2.426066 & -1.677890 & -1.464264\end{array}$

$\begin{array}{llll}2.587401 & -3.215960 & -2.285707\end{array}$

$\begin{array}{llll}1.311095 & -1.784087 & -3.313625\end{array}$

$\begin{array}{llll}1.321679 & -2.509883 & -4.133092\end{array}$

$\begin{array}{llll}1.987828 & -0.978237 & -3.606767\end{array}$

$\begin{array}{lll}-1.521939 & -2.133423 & 2.076089\end{array}$

$\begin{array}{lll}-0.821903 & -3.349297 & 2.205882\end{array}$

$\begin{array}{lll}-1.596146 & -1.286443 & 3.193255\end{array}$

$\begin{array}{lll}-0.243915 & -3.717050 & 3.419383\end{array}$

$\begin{array}{lll}-0.712448 & -4.015070 & 1.357104\end{array}$

$\begin{array}{lll}-1.007060 & -1.651307 & 4.405184\end{array}$

$\begin{array}{lll}-2.145241 & -0.355789 & 3.114677\end{array}$

$\begin{array}{lll}-0.333552 & -2.868237 & 4.527519\end{array}$

$\begin{array}{lll}0.288061 & -4.661392 & 3.496337\end{array}$

$\begin{array}{lll}-1.083656 & -0.980178 & 5.254315\end{array}$

$\begin{array}{llll}0.120349 & -3.151880 & 5.472807\end{array}$

$\begin{array}{lll}-5.313314 & 0.484511 & 1.298851\end{array}$

$\begin{array}{lll}-4.872086 & 0.058579 & 0.017412\end{array}$

$\begin{array}{lll}-6.637685 & 0.088805 & 1.492278\end{array}$

$\begin{array}{lll}-4.482097 & -0.085402 & 2.281156\end{array}$

$\begin{array}{lll}-5.212647 & 1.893892 & 1.363578\end{array}$

$\mathbf{P 1}$ endo-si $\left[\mathrm{BF}_{4}^{-}\right]$

Number of imaginary frequencies : 0

The smallest frequency is : $14.5966 \mathrm{~cm}(-1)$

Electronic energy $=-3125.4843546$

Zero-point correction=

(Hartree/Particle)

Thermal correction to Energy=

Thermal correction to Enthalpy=

0.914562

Thermal correction to Gibbs Free Energy=

Sum of electronic and zero-point Energies=

Sum of electronic and thermal Energies=

Sum of electronic and thermal Enthalpies=

0.969392

0.970337

0.825586

$-3124.569793$

$-3124.514962$

$-3124.514018$

Sum of electronic and thermal Free Energies=

$-3124.658769$

Cartesian Coordinates

$\begin{array}{lll}1.000990 & -1.587439 & -1.276688\end{array}$

$\begin{array}{llll}-0.178764 & 0.300072 & -0.437323\end{array}$

$\begin{array}{lll}-1.012170 & 0.450911 & 0.990685\end{array}$

$\begin{array}{llll}-1.399511 & 0.690108 & -1.512200\end{array}$

$\begin{array}{llll}0.912374 & 1.530843 & -0.240434\end{array}$

$\begin{array}{lll}-2.165137 & -0.310488 & 1.161110\end{array}$

$\begin{array}{lll}-2.072186 & -1.398999 & 2.053246\end{array}$

$\begin{array}{lll}-1.140347 & -1.549109 & 2.582314\end{array}$

$\begin{array}{lll}-3.153330 & -2.225635 & 2.229081\end{array}$

$\begin{array}{lll}-3.095929 & -3.062903 & 2.918954\end{array}$

$\begin{array}{lll}-4.347102 & -2.016078 & 1.485839\end{array}$

$\begin{array}{lll}-5.440594 & -2.918380 & 1.586624\end{array}$

$\begin{array}{lll}-5.355807 & -3.759898 & 2.269296\end{array}$

$\begin{array}{lll}-6.575785 & -2.742907 & 0.827909\end{array}$

$\begin{array}{lll}-7.402756 & -3.442390 & 0.908142\end{array}$

$\begin{array}{llll}-6.656822 & -1.655397 & -0.073981\end{array}$

$\begin{array}{llll}-7.542095 & -1.533845 & -0.691581\end{array}$

$\begin{array}{lll}-5.621877 & -0.751626 & -0.181799\end{array}$

$\begin{array}{llll}-5.698905 & 0.068938 & -0.885140\end{array}$

$\begin{array}{lll}-4.442770 & -0.887090 & 0.603292\end{array}$

$\begin{array}{llll}-3.334686 & 0.029869 & 0.500333\end{array}$

$\begin{array}{llll}-3.409369 & 1.277704 & -0.308973\end{array}$

$\begin{array}{lll}-4.433373 & 2.267422 & -0.082823\end{array}$

$\begin{array}{lll}-5.345297 & 2.192867 & 1.007468\end{array}$

$\begin{array}{lll}-5.268833 & 1.371050 & 1.709281\end{array}$

$\begin{array}{lll}-6.313125 & 3.156175 & 1.194826\end{array}$

$\begin{array}{lll}-6.990965 & 3.077955 & 2.040096\end{array}$

$\begin{array}{lll}-6.428054 & 4.249032 & 0.303387\end{array}$

$\begin{array}{llll}-7.200111 & 4.996787 & 0.459420\end{array}$

$\begin{array}{llll}-5.547473 & 4.366874 & -0.748074\end{array}$

$\begin{array}{llll}-5.609032 & 5.211454 & -1.429586\end{array}$

$\begin{array}{llll}-4.528176 & 3.399441 & -0.960412\end{array}$

$\begin{array}{llll}-3.587897 & 3.547103 & -2.015902\end{array}$

$\begin{array}{llll}-3.674746 & 4.397706 & -2.686023\end{array}$

$\begin{array}{llll}-2.562529 & 2.648582 & -2.164860\end{array}$

$\begin{array}{llll}-1.808855 & 2.761903 & -2.937031\end{array}$

$\begin{array}{llll}-2.464899 & 1.542847 & -1.290326\end{array}$

$\begin{array}{llll}2.275715 & 1.269990 & -0.778472\end{array}$

$\begin{array}{lll}2.942202 & 1.088723 & 0.065762\end{array}$

$\begin{array}{llll}2.779918 & 2.473703 & -1.566401\end{array}$

$\begin{array}{llll}4.090762 & 2.934632 & -1.401112\end{array}$

$\begin{array}{llll}4.735153 & 2.455116 & -0.673120\end{array}$

$\begin{array}{llll}4.558133 & 4.012557 & -2.160094\end{array}$

$\begin{array}{llll}5.576867 & 4.364173 & -2.019727\end{array}$

$\begin{array}{llll}3.723128 & 4.640789 & -3.085796\end{array}$

$\begin{array}{llll}4.087443 & 5.481855 & -3.669377\end{array}$

$2.410818 \quad 4.184720 \quad-3.253548$

$1.751518 \quad 4.670925 \quad-3.967904$

$\begin{array}{llll}1.945318 & 3.107265 & -2.501306\end{array}$

$\begin{array}{llll}0.923213 & 2.758075 & -2.622459\end{array}$

$\begin{array}{llll}2.309592 & 0.042272 & -1.703586\end{array}$

$\begin{array}{llll}2.058624 & 0.373987 & -2.715634\end{array}$

$3.343048 \quad-0.304120 \quad-1.723455$

$\begin{array}{llll}0.793980 & 2.761057 & 0.610335\end{array}$

$\begin{array}{llll}1.601711 & 3.394846 & 0.237684\end{array}$

$\begin{array}{llll}1.108281 & 2.522537 & 2.085413\end{array}$

$\begin{array}{lll}2.450189 & 2.467371 & 2.490964\end{array}$

$\begin{array}{lll}3.248726 & 2.526455 & 1.759018\end{array}$

$\begin{array}{llll}2.787597 & 2.352002 & 3.841023\end{array}$

$\begin{array}{lll}3.834948 & 2.304012 & 4.124931\end{array}$

$\begin{array}{llll}1.784474 & 2.315769 & 4.811888\end{array}$

$\begin{array}{llll}2.043062 & 2.248980 & 5.865335\end{array}$

$\begin{array}{lll}0.443631 & 2.351948 & 4.417814\end{array}$

$\begin{array}{llll}-0.344563 & 2.309509 & 5.164907\end{array}$

$\begin{array}{lll}0.109181 & 2.446622 & 3.065935\end{array}$

$\begin{array}{lll}-0.935950 & 2.474160 & 2.778171\end{array}$

$\begin{array}{lll}-0.493043 & 3.544429 & 0.363807\end{array}$

$\begin{array}{llll}-0.574883 & 3.801195 & -0.694112\end{array}$

$\begin{array}{lll}-1.390455 & 2.999270 & 0.656422\end{array}$

$\begin{array}{llll}-0.460287 & 4.473391 & 0.940919\end{array}$

$\begin{array}{llll}2.810653 & -2.793300 & -1.123627\end{array}$

$\begin{array}{llll}3.645330 & -2.429675 & -1.717573\end{array}$

$\begin{array}{llll}2.609594 & -3.851752 & -1.268798\end{array}$

$\begin{array}{llll}2.678345 & -2.263186 & 0.215775\end{array}$

$\begin{array}{llll}3.284905 & -1.423765 & 0.539919\end{array}$

$\begin{array}{lll}1.735719 & -2.831414 & 1.067744\end{array}$

$\begin{array}{llll}1.289497 & -3.760636 & 0.729718\end{array}$

$\begin{array}{llll}0.042225 & -1.199155 & -3.220914\end{array}$

$\begin{array}{llll}0.182805 & -0.147713 & -3.454677\end{array}$

$\begin{array}{llll}1.179502 & -2.030177 & -3.423461\end{array}$

$\begin{array}{llll}2.079077 & -1.539531 & -3.791253\end{array}$

$\begin{array}{lll}1.131447 & -3.505261 & -3.766075\end{array}$ 


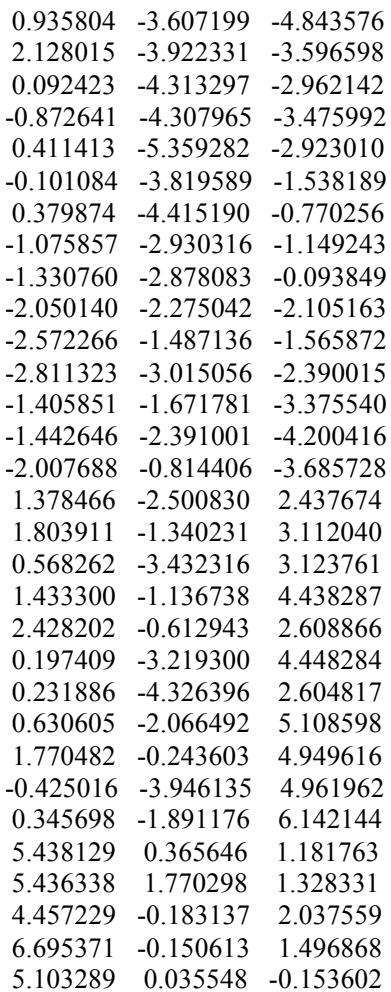

$\mathbf{P 2}$ endo-si $\left[\mathrm{BF}_{4}^{-}\right]$

Number of imaginary frequencies : 0

The smallest frequency is : $12.9605 \mathrm{~cm}(-1)$

Electronic energy $=-3125.4962636$

Zero-point correction $=$

0.914550

(Hartree/Particle)

Thermal correction to Energy=

Thermal correction to Enthalpy=

0.969328

0.970273

Thermal correction to Gibbs Free Energy=

Sum of electronic and zero-point Energies=

Sum of electronic and thermal Energies=

Sum of electronic and thermal Enthalpies=

Sum of electronic and thermal Free Energies=

0.825831

$-3124.581713$

$-3124.526935$

$-3124.525991$

Cartesian Coordinates

$\begin{array}{lll}1.143753 & -1.367025 & -0.238186\end{array}$

$\begin{array}{lll}-0.592515 & 0.095860 & -0.13798\end{array}$

$\begin{array}{lll}-1.639828 & 0.204811 & 1.122880\end{array}$

$-1.656212-0.181415-1.389414$

$\begin{array}{llll}0.010195 & 1.640202 & -0.135972\end{array}$

$\begin{array}{lll}-2.710391 & -0.663213 & 1.298616\end{array}$

$\begin{array}{lll}-2.649453 & -1.505158 & 2.430252\end{array}$

$\begin{array}{lll}-1.802996 & -1.405183 & 3.101477\end{array}$

$\begin{array}{lll}-3.654307 & -2.414207 & 2.650702\end{array}$

$\begin{array}{lll}-3.624891 & -3.065246 & 3.519755\end{array}$

$\begin{array}{lll}-4.729454 & -2.538093 & 1.729193\end{array}$

$\begin{array}{lll}-5.727848 & -3.535774 & 1.897372\end{array}$

$\begin{array}{lll}-5.665505 & -4.190354 & 2.762710\end{array}$

$\begin{array}{lll}-6.743205 & -3.682342 & 0.979446\end{array}$

$\begin{array}{lll}-7.497202 & -4.452347 & 1.114045\end{array}$

$\begin{array}{llll}-6.795310 & -2.835170 & -0.153049\end{array}$

$\begin{array}{llll}-7.583607 & -2.968363 & -0.888419\end{array}$

$\begin{array}{llll}-5.852828 & -1.846639 & -0.336564\end{array}$

$\begin{array}{lll}-5.904556 & -1.214338 & -1.214962\end{array}$

$\begin{array}{lll}-4.800200 & -1.652837 & 0.601126\end{array}$

$\begin{array}{lll}-3.791533 & -0.638424 & 0.431422\end{array}$

$\begin{array}{llll}-3.874376 & 0.387808 & -0.644346\end{array}$

$\begin{array}{llll}-5.009955 & 1.265581 & -0.772180\end{array}$

$\begin{array}{lll}-6.062914 & 1.303469 & 0.184440\end{array}$

$\begin{array}{lll}-6.016415 & 0.655682 & 1.051496\end{array}$

$\begin{array}{llll}-7.129194 & 2.163507 & 0.034889\end{array}$ $\begin{array}{lll}-7.915591 & 2.178340 & 0.784110\end{array}$

$\begin{array}{llll}-7.206980 & 3.032520 & -1.079464\end{array}$

$\begin{array}{lll}-8.056910 & 3.699989 & -1.186941\end{array}$

$\begin{array}{llll}-6.194648 & 3.040102 & -2.011865\end{array}$

$\begin{array}{lll}-6.229313 & 3.716542 & -2.861870\end{array}$

$\begin{array}{llll}-5.072212 & 2.178310 & -1.878797\end{array}$

$\begin{array}{llll}-4.001415 & 2.223575 & -2.811590\end{array}$

$\begin{array}{lll}-4.064396 & 2.904518 & -3.655505\end{array}$

$\begin{array}{llll}-2.885510 & 1.445386 & -2.630210\end{array}$

$\begin{array}{llll}-2.043865 & 1.486277 & -3.312129\end{array}$

$\begin{array}{llll}-2.824587 & 0.561463 & -1.531888\end{array}$

$\begin{array}{llll}1.422830 & 1.723561 & -0.608565\end{array}$

$\begin{array}{lll}2.058060 & 1.905755 & 0.258528\end{array}$

$\begin{array}{llll}1.584374 & 2.884763 & -1.580074\end{array}$

$\begin{array}{llll}2.546691 & 3.876680 & -1.354934\end{array}$

$\begin{array}{llll}3.174844 & 3.821485 & -0.473174\end{array}$

$\begin{array}{llll}2.684659 & 4.940064 & -2.253126\end{array}$

$\begin{array}{llll}3.432646 & 5.705743 & -2.065297\end{array}$

$\begin{array}{llll}1.863289 & 5.025758 & -3.378366\end{array}$

$\begin{array}{llll}1.969956 & 5.855413 & -4.071933\end{array}$

$\begin{array}{llll}0.895230 & 4.040957 & -3.604306\end{array}$

$\begin{array}{llll}0.245495 & 4.103450 & -4.473355\end{array}$

$\begin{array}{llll}0.758899 & 2.980143 & -2.710353\end{array}$

$\begin{array}{lll}-0.000912 & 2.223575 & -2.877325\end{array}$

$\begin{array}{llll}1.845808 & 0.394335 & -1.251204\end{array}$

$\begin{array}{llll}1.480138 & 0.352198 & -2.282263\end{array}$

$\begin{array}{llll}2.936094 & 0.359107 & -1.293574\end{array}$

$\begin{array}{llll}-0.479517 & 2.828576 & 0.649977\end{array}$

$\begin{array}{llll}0.193962 & 3.625625 & 0.323654\end{array}$

$\begin{array}{lll}-0.251476 & 2.648792 & 2.150147\end{array}$

$\begin{array}{lll}1.045508 & 2.804088 & 2.659778\end{array}$

$\begin{array}{llll}1.853921 & 3.126983 & 2.014958\end{array}$

$\begin{array}{lll}1.324442 & 2.557754 & 4.005974\end{array}$

$\begin{array}{lll}2.347929 & 2.650604 & 4.354352\end{array}$

$\begin{array}{lll}0.298189 & 2.185163 & 4.875884\end{array}$

$\begin{array}{lll}0.509227 & 1.994969 & 5.924847\end{array}$

$\begin{array}{lll}-1.007724 & 2.071237 & 4.388555\end{array}$

$\begin{array}{lll}-1.818224 & 1.798509 & 5.059579\end{array}$

$\begin{array}{lll}-1.278356 & 2.298566 & 3.038042\end{array}$

$\begin{array}{lll}-2.293932 & 2.184845 & 2.676440\end{array}$

$\begin{array}{lll}-1.887394 & 3.262417 & 0.252885\end{array}$

$\begin{array}{llll}-1.928154 & 3.446835 & -0.822773\end{array}$

$\begin{array}{lll}-2.645054 & 2.518633 & 0.499160\end{array}$

$\begin{array}{llll}-2.139414 & 4.190211 & 0.775855\end{array}$

$\begin{array}{lll}1.568350 & -0.844465 & 1.839002\end{array}$

$\begin{array}{llll}1.288474 & -1.697124 & 2.456209\end{array}$

$\begin{array}{llll}1.203290 & 0.106416 & 2.216984\end{array}$

$\begin{array}{llll}2.862666 & -0.820336 & 1.219535\end{array}$

$\begin{array}{llll}3.300652 & 0.144375 & 0.996049\end{array}$

$\begin{array}{llll}3.513615 & -1.977727 & 0.789261\end{array}$

$\begin{array}{llll}3.190687 & -2.927867 & 1.199154\end{array}$

$\begin{array}{llll}0.396198 & -2.135021 & -2.251670\end{array}$

$\begin{array}{llll}0.199360 & -1.240742 & -2.833863\end{array}$

$\begin{array}{llll}1.737492 & -2.518222 & -2.114263\end{array}$

$\begin{array}{llll}2.490883 & -1.900320 & -2.600889\end{array}$

$\begin{array}{llll}2.203391 & -3.921397 & -1.778132\end{array}$

$\begin{array}{llll}2.151666 & -4.535568 & -2.689198\end{array}$

$\begin{array}{llll}3.260136 & -3.862630 & -1.512736\end{array}$

$\begin{array}{llll}1.430737 & -4.626258 & -0.636545\end{array}$

$\begin{array}{llll}0.610588 & -5.223295 & -1.047481\end{array}$

$\begin{array}{llll}2.106810 & -5.336065 & -0.150118\end{array}$

$\begin{array}{llll}0.863152 & -3.700821 & 0.431121\end{array}$

$\begin{array}{llll}1.339276 & -3.734982 & 1.406797\end{array}$

$\begin{array}{lll}-0.393481 & -3.137621 & 0.377170\end{array}$

$\begin{array}{lll}-0.818106 & -2.789695 & 1.313388\end{array}$

$-1.376159-3.304438-0.762162$

$-2.182148-2.584699-0.621003$

$\begin{array}{llll}-1.833471 & -4.300870 & -0.682905\end{array}$

$\begin{array}{llll}-0.773046 & -3.114622 & -2.172290\end{array}$

$\begin{array}{llll}-0.445157 & -4.077439 & -2.576314\end{array}$

$\begin{array}{llll}-1.563359 & -2.755703 & -2.836958\end{array}$

$\begin{array}{llll}4.762073 & -2.038334 & 0.027214\end{array}$

$\begin{array}{llll}5.399473 & -0.904309 & -0.517047\end{array}$

$\begin{array}{llll}5.358415 & -3.300954 & -0.171258\end{array}$

$\begin{array}{llll}6.577940 & -1.042152 & -1.247263\end{array}$ 
$\begin{array}{llll}4.997108 & 0.086313 & -0.346306\end{array}$

$\begin{array}{llll}6.537723 & -3.433397 & -0.901615\end{array}$

$\begin{array}{lll}4.888638 & -4.182199 & 0.258705\end{array}$

$\begin{array}{llll}7.149630 & -2.302884 & -1.449279\end{array}$

$\begin{array}{llll}7.057587 & -0.156527 & -1.654608\end{array}$

$\begin{array}{llll}6.979294 & -4.416144 & -1.040847\end{array}$

$\begin{array}{llll}8.068746 & -2.400914 & -2.019910\end{array}$

$\begin{array}{lll}4.624460 & 2.554117 & 1.658511\end{array}$

$\begin{array}{llll}4.394401 & 1.976084 & 0.375118\end{array}$

$\begin{array}{lll}5.995781 & 2.686632 & 1.869339\end{array}$

$\begin{array}{lll}4.050647 & 1.723439 & 2.638662\end{array}$

$\begin{array}{lll}3.995538 & 3.816234 & 1.686703\end{array}$

P2 exo-re $\left[\mathrm{BF}_{4}^{-}\right]$

Number of imaginary frequencies : 0

The smallest frequency is : $8.5256 \mathrm{~cm}(-1)$

Electronic energy $=-3125.4910832$

Zero-point correction=

0.914283

(Hartree/Particle)

Thermal correction to Energy=

Thermal correction to Enthalpy=

0.969111

0.970055

Thermal correction to Gibbs Free Energy=

Sum of electronic and zero-point Energies=

Sum of electronic and thermal Energies=

Sum of electronic and thermal Enthalpies=

0.824645

$-3124.576800$

$-3124.521973$

$-3124.521029$

Sum of electronic and thermal Free Energies $=\quad-3124.666439$

\begin{tabular}{|c|c|c|c|}
\hline \multicolumn{4}{|c|}{ Cartesian Coordinates } \\
\hline 77 & 1.254169 & -1.042307 & -0.327675 \\
\hline 15 & -0.621314 & 0.214944 & -0.100960 \\
\hline 8 & -1.637232 & 0.129418 & 1.190687 \\
\hline 8 & -1.691112 & -0.080325 & -1.342798 \\
\hline 7 & -0.187471 & 1.816123 & -0.012046 \\
\hline 6 & -2.583829 & -0.881489 & 1.316792 \\
\hline 6 & -2.368811 & -1.813412 & 2.354893 \\
\hline 1 & -1.504240 & -1.680946 & 2.996778 \\
\hline 6 & -3.252910 & -2.849975 & 2.524765 \\
\hline 1 & -3.106323 & -3.571191 & 3.323684 \\
\hline 6 & -4.352792 & -3.011955 & 1.639418 \\
\hline 6 & -5.226017 & -4.127849 & 1.753937 \\
\hline 1 & -5.050224 & -4.842961 & 2.553317 \\
\hline 6 & -6.261641 & -4.309749 & 0.865693 \\
\hline 1 & -6.919237 & -5.169065 & 0.958477 \\
\hline 6 & -6.459783 & -3.380464 & -0.183262 \\
\hline 1 & -7.261926 & -3.539578 & -0.898172 \\
\hline 6 & -5.642334 & -2.278592 & -0.311571 \\
\hline 1 & -5.805131 & -1.584535 & -1.127275 \\
\hline 6 & -4.575604 & -2.045269 & 0.600918 \\
\hline 6 & -3.695981 & -0.909639 & 0.490125 \\
\hline 6 & -3.930392 & 0.179860 & -0.498011 \\
\hline 6 & -5.160760 & 0.927726 & -0.534487 \\
\hline 6 & -6.176056 & 0.782181 & 0.452040 \\
\hline 1 & -6.025563 & 0.089957 & 1.271981 \\
\hline 6 & -7.338551 & 1.519674 & 0.390385 \\
\hline 1 & -8.094122 & 1.395723 & 1.160907 \\
\hline 6 & -7.555332 & 2.442001 & -0.660984 \\
\hline 1 & -8.479458 & 3.011156 & -0.699660 \\
\hline 6 & -6.585385 & 2.624983 & -1.620293 \\
\hline 1 & -6.728736 & 3.343914 & -2.422758 \\
\hline 6 & -5.367587 & 1.893254 & -1.576947 \\
\hline 6 & -4.344952 & 2.116202 & -2.538232 \\
\hline 1 & -4.517698 & 2.830624 & -3.338064 \\
\hline 6 & -3.142073 & 1.461832 & -2.441104 \\
\hline 1 & -2.338006 & 1.634640 & -3.147785 \\
\hline 6 & -2.939095 & 0.529235 & -1.400951 \\
\hline 6 & 1.186464 & 2.091843 & -0.500280 \\
\hline 1 & 1.806560 & 2.358973 & 0.358193 \\
\hline 6 & 1.214328 & 3.249677 & -1.490285 \\
\hline 6 & 2.388290 & 4.002258 & -1.636481 \\
\hline 1 & 3.237475 & 3.788444 & -0.997911 \\
\hline 6 & 2.457210 & 5.020438 & -2.589731 \\
\hline 1 & 3.372547 & 5.597448 & -2.691748 \\
\hline 6 & 1.355956 & 5.306114 & -3.4017 \\
\hline
\end{tabular}

$\begin{array}{lll}1.410869 & 6.103469 & -4.137962\end{array}$

$\begin{array}{llll}0.180923 & 4.564740 & -3.253392\end{array}$

$-0.685529 \quad 4.784745 \quad-3.871845$

$\begin{array}{llll}0.113065 & 3.541717 & -2.305496\end{array}$

$\begin{array}{lll}-0.802576 & 2.975380 & -2.178609\end{array}$

$\begin{array}{llll}1.799971 & 0.847723 & -1.164398\end{array}$

$\begin{array}{llll}1.484724 & 0.822400 & -2.213059\end{array}$

$2.881988 \quad 0.965028-1.151249$

$\begin{array}{lll}-0.762896 & 2.895365 & 0.868626\end{array}$

$\begin{array}{lll}-0.292710 & 3.800325 & 0.474526\end{array}$

$\begin{array}{lll}-0.284506 & 2.742079 & 2.311196\end{array}$

$\begin{array}{llll}0.978700 & 3.241176 & 2.662972\end{array}$

$\begin{array}{lll}1.579317 & 3.773680 & 1.933864\end{array}$

$\begin{array}{lll}1.499014 & 3.045723 & 3.943907\end{array}$

$\begin{array}{llll}2.492853 & 3.416514 & 4.176537\end{array}$

$\begin{array}{llll}0.747807 & 2.373186 & 4.908878\end{array}$

$\begin{array}{lll}1.147243 & 2.221458 & 5.907965\end{array}$

$\begin{array}{llll}-0.529723 & 1.907456 & 4.582886\end{array}$

$\begin{array}{lll}-1.131923 & 1.400353 & 5.332425\end{array}$

$\begin{array}{lll}-1.039767 & 2.088224 & 3.295933\end{array}$

$\begin{array}{lll}-2.027120 & 1.709662 & 3.061066\end{array}$

$\begin{array}{lll}-2.268236 & 3.092354 & 0.703496\end{array}$

$\begin{array}{llll}-2.513836 & 3.254295 & -0.348319\end{array}$

$\begin{array}{lll}-2.853920 & 2.247509 & 1.063297\end{array}$

$\begin{array}{lll}-2.569788 & 3.979923 & 1.268368\end{array}$

$\begin{array}{lll}1.635117 & -0.323395 & 1.737355\end{array}$

$\begin{array}{llll}0.886795 & -0.567747 & 2.486396\end{array}$

$\begin{array}{lll}1.901579 & 0.725960 & 1.697189\end{array}$

$\begin{array}{lll}2.660878 & -1.287481 & 1.505398\end{array}$

$\begin{array}{lll}2.570689 & -2.258119 & 1.982155\end{array}$

$\begin{array}{lll}3.671357 & -1.060735 & 0.570725\end{array}$

$\begin{array}{llll}3.868273 & -0.032255 & 0.296431\end{array}$

$\begin{array}{llll}0.569498 & -1.646297 & -2.394667\end{array}$

$\begin{array}{llll}0.306132 & -0.715441 & -2.886927\end{array}$

$\begin{array}{llll}1.942869 & -1.934217 & -2.300984\end{array}$

$\begin{array}{llll}2.629896 & -1.199568 & -2.719272\end{array}$

$\begin{array}{lll}2.539930 & -3.320271 & -2.178056\end{array}$

$\begin{array}{llll}2.589729 & -3.768233 & -3.181630\end{array}$

$3.572891 \quad-3.215633 \quad-1.843251$

$\begin{array}{llll}1.795991 & -4.281145 & -1.224474\end{array}$

$\begin{array}{llll}1.015956 & -4.824210 & -1.765793\end{array}$

$\begin{array}{llll}2.508417 & -5.032858 & -0.871414\end{array}$

$\begin{array}{lll}1.170900 & -3.617954 & -0.011717\end{array}$

$\begin{array}{lll}1.704950 & -3.733650 & 0.924270\end{array}$

$\begin{array}{lll}-0.117823 & -3.158963 & 0.048856\end{array}$

$\begin{array}{lll}-0.542278 & -2.966991 & 1.029916\end{array}$

$\begin{array}{llll}-1.097493 & -3.181517 & -1.103309\end{array}$

$\begin{array}{lll}-1.943950 & -2.549211 & -0.837991\end{array}$

$\begin{array}{llll}-1.493993 & -4.201680 & -1.205424\end{array}$

$\begin{array}{llll}-0.527770 & -2.709064 & -2.461836\end{array}$

$\begin{array}{lll}-0.141059 & -3.562702 & -3.027679\end{array}$

$\begin{array}{lll}-1.355002 & -2.301052 & -3.048242\end{array}$

$\begin{array}{llll}4.699705 & -2.016048 & 0.157814\end{array}$

$\begin{array}{llll}4.737920 & -3.361502 & 0.575258\end{array}$

$\begin{array}{llll}5.690793 & -1.561591 & -0.734131\end{array}$

$\begin{array}{llll}5.723845 & -4.222406 & 0.099350\end{array}$

$\begin{array}{llll}3.995713 & -3.739466 & 1.270576\end{array}$

$\begin{array}{llll}6.674490 & -2.426246 & -1.211160\end{array}$

$\begin{array}{llll}5.681716 & -0.518013 & -1.037551\end{array}$

$\begin{array}{llll}6.691345 & -3.762110 & -0.801053\end{array}$

$\begin{array}{llll}5.739033 & -5.257033 & 0.430603\end{array}$

$\begin{array}{llll}7.429462 & -2.056864 & -1.899343\end{array}$

$\begin{array}{llll}7.455950 & -4.438445 & -1.172308\end{array}$

$\begin{array}{lll}4.604286 & 2.562936 & 1.225220\end{array}$

$\begin{array}{lll}3.802510 & 3.725451 & 1.204889\end{array}$

$\begin{array}{llll}4.023283 & 1.620200 & 2.094850\end{array}$

$\begin{array}{llll}5.898611 & 2.877546 & 1.641989\end{array}$

$\begin{array}{llll}4.647851 & 2.017130 & -0.087553\end{array}$

P2 exo-si $\left[\mathrm{BF}_{4}^{-}\right]$

Number of imaginary frequencies : 0

The smallest frequency is : $17.8206 \mathrm{~cm}(-1)$

Electronic energy $=-3125.4892183$ 
$\begin{array}{lll}-0.420984 & 1.536503 & 5.310683\end{array}$

$\begin{array}{lll}-0.015110 & 2.009143 & 3.252022\end{array}$

$\begin{array}{lll}-1.059747 & 1.950247 & 2.968676\end{array}$

$\begin{array}{lll}-0.740726 & 3.396445 & 0.701213\end{array}$

$\begin{array}{lll}-0.867666 & 3.743694 & -0.325491\end{array}$

$\begin{array}{lll}-1.590702 & 2.765176 & 0.957929\end{array}$

$\begin{array}{llll}-0.754094 & 4.267597 & 1.363277\end{array}$

$\begin{array}{llll}3.267612 & -2.452089 & -1.309120\end{array}$

$\begin{array}{llll}3.935896 & -1.607453 & -1.411650\end{array}$

$\begin{array}{llll}3.456521 & -3.286052 & -1.976885\end{array}$

$\begin{array}{llll}2.675342 & -2.742083 & -0.067161\end{array}$

$\begin{array}{lll}2.381226 & -3.768351 & 0.122971\end{array}$

$\begin{array}{llll}2.235762 & -1.743217 & 0.835285\end{array}$

$\begin{array}{llll}2.808977 & -0.826509 & 0.900633\end{array}$

$\begin{array}{llll}0.098832 & -1.199620 & -3.219845\end{array}$

$\begin{array}{llll}0.152101 & -0.135317 & -3.426908\end{array}$

$\begin{array}{llll}1.273303 & -1.935556 & -3.466611\end{array}$

$\begin{array}{llll}2.132019 & -1.379609 & -3.838829\end{array}$

$\begin{array}{llll}1.316902 & -3.414625 & -3.796540\end{array}$

$\begin{array}{lll}1.075166 & -3.534692 & -4.862580\end{array}$

$\begin{array}{llll}2.347390 & -3.759214 & -3.680870\end{array}$

$\begin{array}{llll}0.379988 & -4.306731 & -2.952083\end{array}$

$\begin{array}{llll}-0.600153 & -4.381847 & -3.431351\end{array}$

$\begin{array}{llll}0.788998 & -5.321428 & -2.934166\end{array}$

$\begin{array}{lll}0.186757 & -3.845954 & -1.517974\end{array}$

$\begin{array}{llll}0.724665 & -4.406548 & -0.762943\end{array}$

$\begin{array}{llll}-0.829092 & -3.025918 & -1.100025\end{array}$

$\begin{array}{llll}-1.046306 & -2.983781 & -0.035866\end{array}$

$\begin{array}{llll}-1.868258 & -2.427266 & -2.022564\end{array}$

$\begin{array}{llll}-2.425933 & -1.677841 & -1.464807\end{array}$

$\begin{array}{llll}-2.587080 & -3.215804 & -2.286488\end{array}$

$\begin{array}{lll}-1.310844 & -1.783663 & -3.314125\end{array}$

$\begin{array}{llll}-1.321373 & -2.509316 & -4.133719\end{array}$

$\begin{array}{lll}-1.987624 & -0.977810 & -3.607146\end{array}$

$\begin{array}{lll}1.522118 & -2.133807 & 2.075606\end{array}$

$\begin{array}{llll}0.821992 & -3.349689 & 2.204684\end{array}$

$\begin{array}{llll}1.596769 & -1.287736 & 3.193422\end{array}$

$\begin{array}{llll}0.244284 & -3.718344 & 3.418037\end{array}$

$\begin{array}{llll}0.712277 & -4.014734 & 1.355392\end{array}$

$\begin{array}{llll}1.008122 & -1.653572 & 4.405276\end{array}$

$\begin{array}{llll}2.145938 & -0.357067 & 3.115473\end{array}$

$\begin{array}{llll}0.334435 & -2.870475 & 4.526849\end{array}$

$\begin{array}{lll}-0.287824 & -4.662663 & 3.494360\end{array}$

$\begin{array}{llll}1.085194 & -0.983185 & 5.254947\end{array}$

$\begin{array}{lll}-0.119158 & -3.154842 & 5.472067\end{array}$

$\begin{array}{lll}5.313307 & 0.484656 & 1.298841\end{array}$

$\begin{array}{llll}4.873084 & 0.060778 & 0.016378\end{array}$

$\begin{array}{llll}6.637301 & 0.088013 & 1.492989\end{array}$

$\begin{array}{llll}4.480902 & -0.086281 & 2.279560\end{array}$

$\begin{array}{lll}5.213201 & 1.893969 & 1.365504\end{array}$

$\mathbf{P 2}$ endo-re $\left[\mathrm{BF}_{4}^{-}\right]$

Number of imaginary frequencies : 0
The smallest frequency is : $14.5966 \mathrm{~cm}(-1)$
Electronic energy $=-3125.4843546$

Zero-point correction $=$

(Hartree/Particle)

Thermal correction to Energy=

Thermal correction to Enthalpy=

Thermal correction to Gibbs Free Energy=

Sum of electronic and zero-point Energies=

Sum of electronic and thermal Energies=

Sum of electronic and thermal Enthalpies $=\quad-3124.514018$ 3124.658769

Cartesian Coordinates

$1.000990-1.587439-1.276688$

$\begin{array}{llll}-0.178764 & 0.300072 & -0.437323\end{array}$

$\begin{array}{lll}-1.012170 & 0.450911 & 0.990685\end{array}$

$\begin{array}{lll}-1.399511 & 0.690108 & -1.512200\end{array}$

$\begin{array}{llll}0.912374 & 1.530843 & -0.240434\end{array}$

$\begin{array}{llll}-2.165137 & -0.310488 & 1.161110\end{array}$

$\begin{array}{lll}-2.072186 & -1.398999 & 2.053246\end{array}$
0.969392

0.970337

0.825586

$-3124.569793$

$-3124.514962$

$-3124.514018$

$\begin{array}{llll}0.346687 & 1.773522 & 4.578990\end{array}$ 
$\begin{array}{lll}-1.140347 & -1.549109 & 2.582314\end{array}$

$\begin{array}{lll}-3.153330 & -2.225635 & 2.229081\end{array}$

$\begin{array}{lll}-3.095929 & -3.062903 & 2.918954\end{array}$

$\begin{array}{lll}-4.347102 & -2.016078 & 1.485839\end{array}$

$\begin{array}{lll}-5.440594 & -2.918380 & 1.586624\end{array}$

$\begin{array}{lll}-5.355807 & -3.759898 & 2.269296\end{array}$

$\begin{array}{llll}-6.575785 & -2.742907 & 0.827909\end{array}$

$\begin{array}{lll}-7.402756 & -3.442390 & 0.908142\end{array}$

$\begin{array}{llll}-6.656822 & -1.655397 & -0.073981\end{array}$

$\begin{array}{llll}-7.542095 & -1.533845 & -0.691581\end{array}$

$\begin{array}{lll}-5.621877 & -0.751626 & -0.181799\end{array}$

$\begin{array}{llll}-5.698905 & 0.068938 & -0.885140\end{array}$

$\begin{array}{lll}-4.442770 & -0.887090 & 0.603292\end{array}$

$\begin{array}{lll}-3.334686 & 0.029869 & 0.500333\end{array}$

$\begin{array}{llll}-3.409369 & 1.277704 & -0.308973\end{array}$

$\begin{array}{lll}-4.433373 & 2.267422 & -0.082823\end{array}$

$\begin{array}{lll}-5.345297 & 2.192867 & 1.007468\end{array}$

$\begin{array}{lll}-5.268833 & 1.371050 & 1.709281\end{array}$

$\begin{array}{lll}-6.313125 & 3.156175 & 1.194826\end{array}$

$\begin{array}{lll}-6.990965 & 3.077955 & 2.040096\end{array}$

$\begin{array}{lll}-6.428054 & 4.249032 & 0.303387\end{array}$

$\begin{array}{llll}-7.200111 & 4.996787 & 0.459420\end{array}$

$\begin{array}{llll}-5.547473 & 4.366874 & -0.748074\end{array}$

$\begin{array}{llll}-5.609032 & 5.211454 & -1.429586\end{array}$

$\begin{array}{llll}-4.528176 & 3.399441 & -0.960412\end{array}$

$\begin{array}{llll}-3.587897 & 3.547103 & -2.015902\end{array}$

$\begin{array}{llll}-3.674746 & 4.397706 & -2.686023\end{array}$

$\begin{array}{lll}-2.562529 & 2.648582 & -2.164860\end{array}$

$\begin{array}{llll}-1.808855 & 2.761903 & -2.937031\end{array}$

$\begin{array}{llll}-2.464899 & 1.542847 & -1.290326\end{array}$

$\begin{array}{llll}2.275715 & 1.269990 & -0.778472\end{array}$

$\begin{array}{llll}2.942202 & 1.088723 & 0.065762\end{array}$

$\begin{array}{llll}2.779918 & 2.473703 & -1.566401\end{array}$

$\begin{array}{llll}4.090762 & 2.934632 & -1.401112\end{array}$

$\begin{array}{llll}4.735153 & 2.455116 & -0.673120\end{array}$

$\begin{array}{llll}4.558133 & 4.012557 & -2.160094\end{array}$

$\begin{array}{llll}5.576867 & 4.364173 & -2.019727\end{array}$

$\begin{array}{llll}3.723128 & 4.640789 & -3.085796\end{array}$

$\begin{array}{llll}4.087443 & 5.481855 & -3.669377\end{array}$

$2.410818 \quad 4.184720 \quad-3.253548$

$1.751518 \quad 4.670925 \quad-3.967904$

$\begin{array}{llll}1.945318 & 3.107265 & -2.501306\end{array}$

$\begin{array}{llll}0.923213 & 2.758075 & -2.622459\end{array}$

$\begin{array}{llll}2.309592 & 0.042272 & -1.703586\end{array}$

$\begin{array}{llll}2.058624 & 0.373987 & -2.715634\end{array}$

$3.343048-0.304120-1.723455$

$\begin{array}{llll}0.793980 & 2.761057 & 0.610335\end{array}$

$\begin{array}{llll}1.601711 & 3.394846 & 0.237684\end{array}$

$\begin{array}{lll}1.108281 & 2.522537 & 2.085413\end{array}$

$\begin{array}{llll}2.450189 & 2.467371 & 2.490964\end{array}$

$\begin{array}{lll}3.248726 & 2.526455 & 1.759018\end{array}$

$\begin{array}{llll}2.787597 & 2.352002 & 3.841023\end{array}$

$\begin{array}{lll}3.834948 & 2.304012 & 4.124931\end{array}$

$\begin{array}{llll}1.784474 & 2.315769 & 4.811888\end{array}$

$\begin{array}{llll}2.043062 & 2.248980 & 5.865335\end{array}$

$\begin{array}{lll}0.443631 & 2.351948 & 4.417814\end{array}$

$\begin{array}{lll}-0.344563 & 2.309509 & 5.164907\end{array}$

$\begin{array}{lll}0.109181 & 2.446622 & 3.065935\end{array}$

$\begin{array}{lll}-0.935950 & 2.474160 & 2.778171\end{array}$

$\begin{array}{lll}-0.493043 & 3.544429 & 0.363807\end{array}$

$\begin{array}{lll}-0.574883 & 3.801195 & -0.694112\end{array}$

$\begin{array}{lll}-1.390455 & 2.999270 & 0.656422\end{array}$

$\begin{array}{llll}-0.460287 & 4.473391 & 0.940919\end{array}$

$\begin{array}{llll}2.810653 & -2.793300 & -1.123627\end{array}$

$\begin{array}{llll}3.645330 & -2.429675 & -1.717573\end{array}$

$\begin{array}{llll}2.609594 & -3.851752 & -1.268798\end{array}$

$\begin{array}{llll}2.678345 & -2.263186 & 0.215775\end{array}$

$\begin{array}{llll}3.284905 & -1.423765 & 0.539919\end{array}$

$\begin{array}{lll}1.735719 & -2.831414 & 1.067744\end{array}$

$\begin{array}{llll}1.289497 & -3.760636 & 0.729718\end{array}$

$\begin{array}{llll}0.042225 & -1.199155 & -3.220914\end{array}$

$\begin{array}{llll}0.182805 & -0.147713 & -3.454677\end{array}$

$\begin{array}{llll}1.179502 & -2.030177 & -3.423461\end{array}$

$\begin{array}{llll}2.079077 & -1.539531 & -3.791253\end{array}$

$\begin{array}{lll}1.131447 & -3.505261 & -3.766075\end{array}$ $\begin{array}{llll}0.935804 & -3.607199 & -4.843576\end{array}$

$\begin{array}{llll}2.128015 & -3.922331 & -3.596598\end{array}$

$\begin{array}{llll}0.092423 & -4.313297 & -2.962142\end{array}$

$\begin{array}{llll}-0.872641 & -4.307965 & -3.475992\end{array}$

$\begin{array}{llll}0.411413 & -5.359282 & -2.923010\end{array}$

$\begin{array}{llll}-0.101084 & -3.819589 & -1.538189\end{array}$

$\begin{array}{llll}0.379874 & -4.415190 & -0.770256\end{array}$

$-1.075857-2.930316-1.149243$

$\begin{array}{llll}-1.330760 & -2.878083 & -0.093849\end{array}$

$\begin{array}{lll}-2.050140 & -2.275042 & -2.105163\end{array}$

$\begin{array}{llll}-2.572266 & -1.487136 & -1.565872\end{array}$

$-2.811323-3.015056-2.390015$

$-1.405851-1.671781-3.375540$

$-1.442646 \quad-2.391001 \quad-4.200416$

$-2.007688-0.814406 \quad-3.685728$

$\begin{array}{lll}1.378466 & -2.500830 & 2.437674\end{array}$

$\begin{array}{llll}1.803911 & -1.340231 & 3.112040\end{array}$

$\begin{array}{lll}0.568262 & -3.432316 & 3.123761\end{array}$

$\begin{array}{llll}1.433300 & -1.136738 & 4.438287\end{array}$

$\begin{array}{lll}2.428202 & -0.612943 & 2.608866\end{array}$

$\begin{array}{llll}0.197409 & -3.219300 & 4.448284\end{array}$

$\begin{array}{llll}0.231886 & -4.326396 & 2.604817\end{array}$

$\begin{array}{lll}0.630605 & -2.066492 & 5.108598\end{array}$

$\begin{array}{lll}1.770482 & -0.243603 & 4.949616\end{array}$

$\begin{array}{lll}-0.425016 & -3.946135 & 4.961962\end{array}$

$\begin{array}{lll}0.345698 & -1.891176 & 6.142144\end{array}$

$\begin{array}{lll}5.438129 & 0.365646 & 1.181763\end{array}$

$\begin{array}{llll}5.436338 & 1.770298 & 1.328331\end{array}$

$\begin{array}{lll}4.457229 & -0.183137 & 2.037559\end{array}$

$\begin{array}{lll}6.695371 & -0.150613 & 1.496868\end{array}$

$\begin{array}{llll}5.103289 & 0.035548 & -0.153602\end{array}$

P1 endo-re

Number of imaginary frequencies : 0

The smallest frequency is : $19.0304 \mathrm{~cm}(-1)$

Electronic energy $=-2700.877626$

Zero-point correction $=$

(Hartree/Particle)

Thermal correction to Energy=

Thermal correction to Enthalpy=

0.946899

0.947843

Sum of electronic and zero-point Energies=

Sum of electronic and thermal Energies=

Sum of electronic and thermal Enthalpies=

Sum of electronic and thermal Free Energies=

$-2699.979282$

$-2699.930727$

$-2699.929783$

$-2700.059984$

Cartesian Coordinates

$\begin{array}{lll}-1.558666 & -1.024203 & -0.170337\end{array}$

$\begin{array}{llll}0.323955 & 0.213928 & -0.022867\end{array}$

$\begin{array}{lll}1.416958 & 0.044447 & 1.188513\end{array}$

$\begin{array}{lll}1.284709 & -0.021635 & -1.351950\end{array}$

$\begin{array}{lll}-0.093947 & 1.816913 & 0.177910\end{array}$

$\begin{array}{llll}2.396796 & -0.947401 & 1.180553\end{array}$

$\begin{array}{llll}2.289353 & -1.933277 & 2.184055\end{array}$

$\begin{array}{llll}1.479535 & -1.855752 & 2.901752\end{array}$

$\begin{array}{lll}3.208922 & -2.951572 & 2.229635\end{array}$

$\begin{array}{lll}3.145948 & -3.713957 & 3.000701\end{array}$

$\begin{array}{lll}4.237910 & -3.037578 & 1.253339\end{array}$

$\begin{array}{llll}5.148664 & -4.128718 & 1.244941\end{array}$

$\begin{array}{llll}5.056025 & -4.886030 & 2.018970\end{array}$

$\begin{array}{llll}6.117360 & -4.233604 & 0.272871\end{array}$

$\begin{array}{lll}6.805198 & -5.073984 & 0.272806\end{array}$

$6.207218-3.248958-0.739873$

$\begin{array}{llll}6.956830 & -3.347032 & -1.519769\end{array}$

$\begin{array}{llll}5.350773 & -2.169398 & -0.750585\end{array}$

$\begin{array}{llll}5.429827 & -1.430678 & -1.539298\end{array}$

$\begin{array}{lll}4.351570 & -2.015968 & 0.250564\end{array}$

$\begin{array}{llll}3.437845 & -0.902175 & 0.267210\end{array}$

$\begin{array}{llll}3.575572 & 0.250901 & -0.665966\end{array}$

$\begin{array}{llll}4.778720 & 1.039418 & -0.735859\end{array}$

$\begin{array}{llll}5.868364 & 0.858284 & 0.161344\end{array}$

$\begin{array}{llll}5.801386 & 0.102776 & 0.935084\end{array}$

$\begin{array}{llll}6.998394 & 1.642026 & 0.073922\end{array}$ 
$\begin{array}{llll}7.812173 & 1.490204 & 0.777127\end{array}$

$\begin{array}{llll}7.106968 & 2.647293 & -0.916364\end{array}$

$\begin{array}{llll}8.006993 & 3.251999 & -0.976460\end{array}$

$\begin{array}{llll}6.063380 & 2.865232 & -1.786809\end{array}$

$\begin{array}{llll}6.123749 & 3.647815 & -2.538517\end{array}$

$\begin{array}{llll}4.875620 & 2.087840 & -1.712420\end{array}$

$\begin{array}{llll}3.774987 & 2.351390 & -2.572107\end{array}$

$\begin{array}{llll}3.864605 & 3.134747 & -3.319308\end{array}$

$\begin{array}{llll}2.601358 & 1.651260 & -2.438176\end{array}$

$\begin{array}{llll}1.736690 & 1.856401 & -3.059890\end{array}$

$\begin{array}{llll}2.512171 & 0.632401 & -1.466560\end{array}$

$\begin{array}{llll}-1.526352 & 2.072561 & -0.127668\end{array}$

$\begin{array}{lll}-2.062940 & 2.150309 & 0.824255\end{array}$

$\begin{array}{llll}-1.725573 & 3.370642 & -0.889350\end{array}$

$\begin{array}{llll}-2.823100 & 4.186222 & -0.589602\end{array}$

$\begin{array}{lll}-3.491547 & 3.906575 & 0.221565\end{array}$

$\begin{array}{llll}-3.062314 & 5.352486 & -1.320887\end{array}$

$\begin{array}{lll}-3.917116 & 5.977162 & -1.077029\end{array}$

$\begin{array}{llll}-2.197994 & 5.718703 & -2.354964\end{array}$

$\begin{array}{llll}-2.377960 & 6.628859 & -2.919952\end{array}$

$\begin{array}{llll}-1.095050 & 4.912373 & -2.654460\end{array}$

$\begin{array}{llll}-0.415002 & 5.196042 & -3.453047\end{array}$

$\begin{array}{llll}-0.861756 & 3.744479 & -1.927931\end{array}$

$\begin{array}{llll}0.001504 & 3.124006 & -2.148673\end{array}$

$\begin{array}{llll}-2.109254 & 0.897961 & -0.920284\end{array}$

$\begin{array}{llll}-1.786461 & 0.975944 & -1.964587\end{array}$

$\begin{array}{llll}-3.200188 & 0.960431 & -0.913594\end{array}$

$\begin{array}{lll}0.540972 & 2.850550 & 1.073423\end{array}$

$\begin{array}{lll}0.106626 & 3.784086 & 0.705892\end{array}$

$\begin{array}{lll}0.069948 & 2.686570 & 2.516659\end{array}$

$\begin{array}{lll}-1.106593 & 3.336132 & 2.922183\end{array}$

$\begin{array}{lll}-1.619196 & 3.992534 & 2.223396\end{array}$

$\begin{array}{lll}-1.621278 & 3.160898 & 4.209184\end{array}$

$\begin{array}{lll}-2.535070 & 3.672046 & 4.499576\end{array}$

$\begin{array}{lll}-0.954020 & 2.340912 & 5.121244\end{array}$

$\begin{array}{lll}-1.348494 & 2.202672 & 6.123980\end{array}$

$\begin{array}{llll}0.236532 & 1.714980 & 4.740501\end{array}$

$\begin{array}{lll}0.774224 & 1.092517 & 5.450650\end{array}$

$\begin{array}{llll}0.744966 & 1.887267 & 3.451597\end{array}$

$\begin{array}{lll}1.666971 & 1.391238 & 3.175681\end{array}$

$\begin{array}{llll}2.051405 & 2.996177 & 0.903549\end{array}$

$\begin{array}{llll}2.298482 & 3.164127 & -0.146648\end{array}$

$\begin{array}{llll}2.613311 & 2.132606 & 1.255398\end{array}$

$\begin{array}{lll}2.377873 & 3.869133 & 1.477061\end{array}$

$\begin{array}{lll}-1.734601 & -0.732360 & 1.985759\end{array}$

$\begin{array}{lll}-1.538821 & -1.685764 & 2.473742\end{array}$

$\begin{array}{lll}-1.193436 & 0.096466 & 2.435225\end{array}$

$\begin{array}{lll}-3.069422 & -0.448659 & 1.535364\end{array}$

$\begin{array}{llll}-3.353861 & 0.595409 & 1.450113\end{array}$

$\begin{array}{lll}-3.960318 & -1.417008 & 1.088059\end{array}$

$\begin{array}{lll}-3.769634 & -2.455152 & 1.335979\end{array}$

$\begin{array}{llll}-1.035861 & -1.647580 & -2.319354\end{array}$

$\begin{array}{llll}-0.719090 & -0.727251 & -2.800262\end{array}$

$-2.413558-1.823776-2.151109$

$\begin{array}{lll}-3.068178 & -1.036128 & -2.521380\end{array}$

$-3.112933-3.144482-1.895557$

$\begin{array}{llll}-3.279385 & -3.651714 & -2.857244\end{array}$

$-4.103230 \quad-2.914660-1.494674$

$\begin{array}{llll}-2.385944 & -4.103231 & -0.923879\end{array}$

$\begin{array}{llll}-1.693797 & -4.747171 & -1.474031\end{array}$

$\begin{array}{llll}-3.130226 & -4.770077 & -0.478013\end{array}$

$\begin{array}{llll}-1.621993 & -3.421051 & 0.204592\end{array}$

$\begin{array}{lll}-2.045235 & -3.517666 & 1.199490\end{array}$

$\begin{array}{lll}-0.293689 & -3.047982 & 0.136767\end{array}$

$\begin{array}{lll}0.221289 & -2.892988 & 1.079860\end{array}$

$\begin{array}{llll}0.607947 & -3.208172 & -1.068081\end{array}$

$\begin{array}{llll}1.505206 & -2.611169 & -0.899378\end{array}$

$\begin{array}{llll}0.938347 & -4.255151 & -1.119612\end{array}$

$-0.029924 \quad-2.792529 \quad-2.411204$

$\begin{array}{llll}-0.520980 & -3.650358 & -2.879431\end{array}$

$\begin{array}{llll}0.769464 & -2.487474 & -3.091859\end{array}$

$\begin{array}{lll}-5.248730 & -1.154990 & 0.449524\end{array}$

$\begin{array}{llll}-5.693793 & 0.137181 & 0.097902\end{array}$

$\begin{array}{lll}-6.082448 & -2.251219 & 0.148529\end{array}$

$\begin{array}{lll}-6.918481 & 0.316301 & -0.539297\end{array}$ $\begin{array}{lll}-5.084592 & 1.005482 & 0.325595\end{array}$

$\begin{array}{llll}-7.308563 & -2.068947 & -0.488434\end{array}$

$\begin{array}{lll}-5.757260 & -3.252285 & 0.419581\end{array}$

$\begin{array}{llll}-7.729819 & -0.783971 & -0.839187\end{array}$

$\begin{array}{llll}-7.243712 & 1.318565 & -0.803327\end{array}$

$-7.933503 \quad-2.928724 \quad-0.711854$

$\begin{array}{lll}-8.683228 & -0.638160 & -1.338408\end{array}$

P1 exo-si

Number of imaginary frequencies : 0

The smallest frequency is : $10.9775 \mathrm{~cm}(-1)$

Electronic energy $=-2700.871334$

Zero-point correction=

0.897960

(Hartree/Particle)

Thermal correction to Energy=

Thermal correction to Enthalpy=

0.946703

0.947647

Thermal correction to Gibbs Free Energy=

Sum of electronic and zero-point Energies=

Sum of electronic and thermal Energies=

Sum of electronic and thermal Enthalpies=

0.816297

$-2699.973374$

$-2699.924631$

Sum of electronic and thermal Free Energies $=\quad-2700.055037$

Cartesian Coordinates

$\begin{array}{lll}-1.721212 & -0.612148 & -0.215835\end{array}$

$\begin{array}{lll}0.338494 & 0.299110 & -0.039200\end{array}$

$\begin{array}{lll}1.357153 & -0.053155 & 1.199999\end{array}$

$\begin{array}{lll}1.287281 & -0.070975 & -1.344971\end{array}$

$\begin{array}{llll}0.183532 & 1.949763 & 0.154884\end{array}$

$\begin{array}{lll}2.166914 & -1.187639 & 1.202910\end{array}$

$\begin{array}{llll}1.872038 & -2.159149 & 2.182916\end{array}$

$\begin{array}{llll}1.063147 & -1.963741 & 2.879010\end{array}$

$\begin{array}{llll}2.613645 & -3.313458 & 2.232641\end{array}$

$\begin{array}{llll}2.404711 & -4.068232 & 2.985228\end{array}$

$\begin{array}{lll}3.643539 & -3.549950 & 1.282549\end{array}$

$\begin{array}{llll}4.363416 & -4.775650 & 1.273881\end{array}$

$\begin{array}{lll}4.125352 & -5.521415 & 2.027689\end{array}$

$\begin{array}{llll}5.330788 & -5.020426 & 0.325873\end{array}$

$\begin{array}{lll}5.872041 & -5.961888 & 0.325171\end{array}$

$\begin{array}{llll}5.610418 & -4.046309 & -0.662002\end{array}$

$\begin{array}{llll}6.356994 & -4.252008 & -1.423559\end{array}$

$\begin{array}{llll}4.942287 & -2.841135 & -0.671760\end{array}$

$\begin{array}{llll}5.164449 & -2.112206 & -1.441777\end{array}$

$\begin{array}{lll}3.951589 & -2.543894 & 0.305339\end{array}$

$\begin{array}{llll}3.228980 & -1.297520 & 0.319679\end{array}$

$\begin{array}{llll}3.572405 & -0.172276 & -0.593966\end{array}$

$\begin{array}{llll}4.886730 & 0.415609 & -0.623314\end{array}$

$\begin{array}{llll}5.908098 & 0.055790 & 0.299831\end{array}$

$\begin{array}{lll}5.699976 & -0.687864 & 1.059590\end{array}$

$\begin{array}{lll}7.150513 & 0.649846 & 0.251832\end{array}$

$\begin{array}{llll}7.910510 & 0.363484 & 0.973022\end{array}$

$\begin{array}{llll}7.444752 & 1.633670 & -0.722111\end{array}$

$\begin{array}{llll}8.430782 & 2.087813 & -0.751029\end{array}$

$\begin{array}{llll}6.474312 & 2.021486 & -1.617718\end{array}$

$\begin{array}{llll}6.679695 & 2.789626 & -2.358575\end{array}$

$\begin{array}{llll}5.177078 & 1.440840 & -1.585825\end{array}$

$\begin{array}{llll}4.159020 & 1.875971 & -2.477055\end{array}$

$\begin{array}{llll}4.393806 & 2.636753 & -3.215868\end{array}$

$\begin{array}{llll}2.886566 & 1.368504 & -2.383424\end{array}$

$\begin{array}{lll}2.085320 & 1.704916 & -3.031715\end{array}$

$\begin{array}{llll}2.606643 & 0.376464 & -1.420544\end{array}$

$\begin{array}{llll}-1.177275 & 2.446631 & -0.176403\end{array}$

$\begin{array}{lll}-1.704285 & 2.647311 & 0.762206\end{array}$

$\begin{array}{llll}-1.134375 & 3.744866 & -0.964085\end{array}$

$\begin{array}{llll}-2.040240 & 4.768696 & -0.661434\end{array}$

$\begin{array}{llll}-2.736970 & 4.639555 & 0.163729\end{array}$

$\begin{array}{llll}-2.050670 & 5.951181 & -1.405910\end{array}$

$\begin{array}{llll}-2.757138 & 6.738734 & -1.158983\end{array}$

$\begin{array}{llll}-1.146215 & 6.124316 & -2.455846\end{array}$

$\begin{array}{lll}-1.147071 & 7.046104 & -3.030564\end{array}$

$\begin{array}{llll}-0.233945 & 5.108395 & -2.759022\end{array}$

$\begin{array}{llll}0.477146 & 5.240047 & -3.570091\end{array}$

$\begin{array}{llll}-0.230071 & 3.925603 & -2.019562\end{array}$

$\begin{array}{llll}0.485986 & 3.141510 & -2.244013\end{array}$ 
$\begin{array}{lll}-1.959458 & 1.378435 & -0.948157\end{array}$

$\begin{array}{llll}-1.632226 & 1.388701 & -1.994091\end{array}$

$\begin{array}{lll}-3.016533 & 1.651202 & -0.948540\end{array}$

$\begin{array}{llll}0.962901 & 2.869337 & 1.062344\end{array}$

$\begin{array}{lll}0.717363 & 3.858552 & 0.666438\end{array}$

$\begin{array}{lll}0.417474 & 2.816329 & 2.487746\end{array}$

$\begin{array}{lll}-0.619519 & 3.689892 & 2.850035\end{array}$

$\begin{array}{lll}-0.967531 & 4.428645 & 2.132320\end{array}$

$\begin{array}{lll}-1.205609 & 3.628452 & 4.117100\end{array}$

$\begin{array}{lll}-2.010569 & 4.311632 & 4.373615\end{array}$

$\begin{array}{lll}-0.749464 & 2.696960 & 5.051707\end{array}$

$\begin{array}{lll}-1.200183 & 2.645161 & 6.038839\end{array}$

$\begin{array}{lll}0.305249 & 1.843737 & 4.713939\end{array}$

$\begin{array}{lll}0.682103 & 1.130918 & 5.442604\end{array}$

$\begin{array}{lll}0.884482 & 1.903958 & 3.445439\end{array}$

$\begin{array}{lll}1.698788 & 1.233112 & 3.203153\end{array}$

$\begin{array}{lll}2.481550 & 2.743809 & 0.958535\end{array}$

$\begin{array}{llll}2.801444 & 2.844223 & -0.080253\end{array}$

$\begin{array}{lll}2.867902 & 1.803837 & 1.348435\end{array}$

$\begin{array}{lll}2.929827 & 3.558673 & 1.535253\end{array}$

$\begin{array}{lll}-1.863714 & -0.001862 & 1.901892\end{array}$

$\begin{array}{lll}-1.173019 & -0.490943 & 2.583444\end{array}$

$\begin{array}{lll}-1.847653 & 1.080739 & 1.983746\end{array}$

$\begin{array}{lll}-3.123664 & -0.656588 & 1.697806\end{array}$

$\begin{array}{lll}-3.243070 & -1.659546 & 2.094521\end{array}$

$\begin{array}{lll}-4.120821 & -0.129821 & 0.889266\end{array}$

$\begin{array}{llll}-4.058832 & 0.920211 & 0.622637\end{array}$

$\begin{array}{llll}-1.256574 & -1.261469 & -2.359852\end{array}$

$\begin{array}{llll}-0.850405 & -0.371777 & -2.831319\end{array}$

$\begin{array}{llll}-2.648469 & -1.291740 & -2.198089\end{array}$

$\begin{array}{llll}-3.201597 & -0.421446 & -2.549352\end{array}$

$\begin{array}{llll}-3.509202 & -2.523746 & -2.017158\end{array}$

$\begin{array}{llll}-3.773481 & -2.918277 & -3.009344\end{array}$

$\begin{array}{llll}-4.448857 & -2.208237 & -1.557297\end{array}$

$-2.887288-3.649370-1.164552$

$\begin{array}{llll}-2.253008 & -4.288517 & -1.784578\end{array}$

$\begin{array}{llll}-3.697354 & -4.284668 & -0.793784\end{array}$

$\begin{array}{llll}-2.081292 & -3.163760 & 0.025926\end{array}$

$\begin{array}{lll}-2.568707 & -3.226541 & 0.992951\end{array}$

$\begin{array}{lll}-0.729994 & -2.936306 & 0.019727\end{array}$

$\begin{array}{llll}-0.231500 & -2.858466 & 0.981400\end{array}$

$\begin{array}{llll}0.177274 & -3.090494 & -1.179590\end{array}$

$\begin{array}{llll}1.132602 & -2.619577 & -0.945576\end{array}$

$\begin{array}{llll}0.388969 & -4.159786 & -1.319523\end{array}$

$\begin{array}{llll}-0.366818 & -2.495992 & -2.498840\end{array}$

$\begin{array}{lll}-0.922157 & -3.256840 & -3.055403\end{array}$

$\begin{array}{llll}0.488481 & -2.223248 & -3.122318\end{array}$

$\begin{array}{lll}-5.344303 & -0.812175 & 0.471363\end{array}$

$\begin{array}{llll}-5.681489 & -2.116304 & 0.887175\end{array}$

$\begin{array}{lll}-6.199189 & -0.156672 & -0.436296\end{array}$

$\begin{array}{lll}-6.825304 & -2.741945 & 0.399170\end{array}$

$\begin{array}{llll}-5.047889 & -2.642410 & 1.593848\end{array}$

$\begin{array}{llll}-7.342047 & -0.786476 & -0.926177\end{array}$

$\begin{array}{llll}-5.952679 & 0.850928 & -0.761863\end{array}$

$\begin{array}{lll}-7.657698 & -2.083455 & -0.513265\end{array}$

$\begin{array}{lll}-7.070426 & -3.747221 & 0.729659\end{array}$

$\begin{array}{llll}-7.985495 & -0.266145 & -1.629737\end{array}$

$\begin{array}{llll}-8.546739 & -2.577116 & -0.894454\end{array}$

P1 exo-re

Number of imaginary frequencies : 0

The smallest frequency is : $18.4795 \mathrm{~cm}(-1)$

Electronic energy $=-2700.8703735$

Zero-point correction=

0.899973

(Hartree/Particle)

Thermal correction to Energy=

Thermal correction to Enthalpy=

0.948165

0.949110

Thermal correction to Gibbs Free Energy=

Sum of electronic and zero-point Energies=

Sum of electronic and thermal Energies=

0.821341

$-2699.970401$

$-2699.922208$

Sum of electronic and thermal Enthalpies=

$-2699.921264$

Sum of electronic and thermal Free Energies=

Cartesian Coordinates

$\begin{array}{lll}-1.857936 & -1.344757 & -0.888315\end{array}$

$\begin{array}{lll}-0.274482 & 0.273993 & -0.274676\end{array}$

$\begin{array}{lll}0.778709 & 0.227528 & 0.992651\end{array}$

$\begin{array}{llll}0.789283 & 0.484918 & -1.531431\end{array}$

$\begin{array}{lll}-1.081536 & 1.677005 & 0.107191\end{array}$

$\begin{array}{lll}1.916646 & -0.579676 & 0.955284\end{array}$

$\begin{array}{lll}1.925722 & -1.673985 & 1.843835\end{array}$

$\begin{array}{llll}1.083551 & -1.800085 & 2.510533\end{array}$

$\begin{array}{lll}2.993479 & -2.535888 & 1.844997\end{array}$

$\begin{array}{lll}3.015677 & -3.378289 & 2.530647\end{array}$

$\begin{array}{llll}4.065632 & -2.355838 & 0.929686\end{array}$

$\begin{array}{llll}5.133181 & -3.291581 & 0.858418\end{array}$

$\begin{array}{llll}5.126725 & -4.136898 & 1.541476\end{array}$

$\begin{array}{llll}6.144462 & -3.142405 & -0.063312\end{array}$

$\begin{array}{llll}6.952577 & -3.866278 & -0.113103\end{array}$

$\begin{array}{llll}6.121374 & -2.048641 & -0.960887\end{array}$

$6.906177-1.946948-1.704935$

$\begin{array}{llll}5.110895 & -1.113143 & -0.905181\end{array}$

$\begin{array}{llll}5.108161 & -0.289118 & -1.608474\end{array}$

$\begin{array}{lll}4.061150 & -1.221055 & 0.049544\end{array}$

$\begin{array}{llll}2.982424 & -0.266638 & 0.125913\end{array}$

$\begin{array}{llll}2.985178 & 0.985715 & -0.679244\end{array}$

$\begin{array}{llll}4.066824 & 1.935667 & -0.610325\end{array}$

$\begin{array}{llll}5.124543 & 1.826733 & 0.335302\end{array}$

$\begin{array}{lll}5.124685 & 1.005032 & 1.041423\end{array}$

$\begin{array}{lll}6.138789 & 2.758776 & 0.378876\end{array}$

$\begin{array}{lll}6.929461 & 2.657020 & 1.116701\end{array}$

$\begin{array}{llll}6.157717 & 3.850157 & -0.521732\end{array}$

$\begin{array}{llll}6.967887 & 4.572212 & -0.480179\end{array}$

$\begin{array}{llll}5.139047 & 3.999815 & -1.435155\end{array}$

$\begin{array}{llll}5.128638 & 4.844039 & -2.119484\end{array}$

$4.068081 \quad 3.067119-1.494504$

$\begin{array}{llll}2.987160 & 3.252263 & -2.398531\end{array}$

$\begin{array}{lll}3.005488 & 4.100408 & -3.076930\end{array}$

$\begin{array}{lll}1.914972 & 2.395812 & -2.386164\end{array}$

$\begin{array}{lll}1.056881 & 2.540622 & -3.034037\end{array}$

$\begin{array}{llll}1.914721 & 1.295403 & -1.501852\end{array}$

$\begin{array}{llll}-2.532426 & 1.650828 & -0.194638\end{array}$

$\begin{array}{lll}-3.071553 & 1.496437 & 0.748041\end{array}$

$\begin{array}{lll}-3.005088 & 2.962800 & -0.801819\end{array}$

$\begin{array}{lll}-4.195446 & 3.551818 & -0.362995\end{array}$

$\begin{array}{llll}-4.755447 & 3.088061 & 0.445702\end{array}$

$\begin{array}{llll}-4.665141 & 4.728420 & -0.954293\end{array}$

$\begin{array}{llll}-5.590425 & 5.176785 & -0.603464\end{array}$

$\begin{array}{llll}-3.941539 & 5.329960 & -1.985494\end{array}$

$\begin{array}{llll}-4.301665 & 6.247633 & -2.441670\end{array}$

$\begin{array}{llll}-2.746800 & 4.749219 & -2.425463\end{array}$

$\begin{array}{llll}-2.176719 & 5.216680 & -3.223610\end{array}$

$\begin{array}{lll}-2.283826 & 3.571673 & -1.840154\end{array}$

$\begin{array}{lll}-1.350551 & 3.123656 & -2.171260\end{array}$

$\begin{array}{llll}-2.870717 & 0.511519 & -1.165233\end{array}$

$\begin{array}{llll}-2.628209 & 0.841896 & -2.180906\end{array}$

$\begin{array}{llll}-3.953468 & 0.379782 & -1.133041\end{array}$

$\begin{array}{lll}-0.648315 & 2.809310 & 0.999146\end{array}$

$\begin{array}{lll}-1.344378 & 3.605611 & 0.727325\end{array}$

$\begin{array}{llll}-0.887475 & 2.535054 & 2.480481\end{array}$

$\begin{array}{lll}-2.148614 & 2.821839 & 3.025084\end{array}$

$\begin{array}{lll}-2.930528 & 3.212549 & 2.379145\end{array}$

$\begin{array}{lll}-2.400477 & 2.649523 & 4.388585\end{array}$

$\begin{array}{lll}-3.383073 & 2.880491 & 4.790677\end{array}$

$\begin{array}{lll}-1.381013 & 2.206977 & 5.234397\end{array}$

$\begin{array}{lll}-1.566252 & 2.085914 & 6.298003\end{array}$

$\begin{array}{lll}-0.118832 & 1.927255 & 4.703638\end{array}$

$\begin{array}{lll}0.680377 & 1.584473 & 5.354716\end{array}$

$\begin{array}{lll}0.123372 & 2.080235 & 3.338145\end{array}$

$\begin{array}{llll}1.106614 & 1.852031 & 2.944058\end{array}$

$\begin{array}{lll}0.741260 & 3.361247 & 0.693626\end{array}$

$\begin{array}{llll}0.804916 & 3.665791 & -0.352041\end{array}$

$\begin{array}{lll}1.543161 & 2.652829 & 0.896574\end{array}$

$\begin{array}{lll}0.905696 & 4.244401 & 1.318060\end{array}$

$\begin{array}{llll}-3.980664 & -2.177938 & -0.673163\end{array}$

$\begin{array}{llll}-4.610969 & -1.303335 & -0.775932\end{array}$

$\begin{array}{lll}-4.289302 & -3.034782 & -1.262547\end{array}$

$\begin{array}{lll}-3.284529 & -2.447490 & 0.521222\end{array}$ 


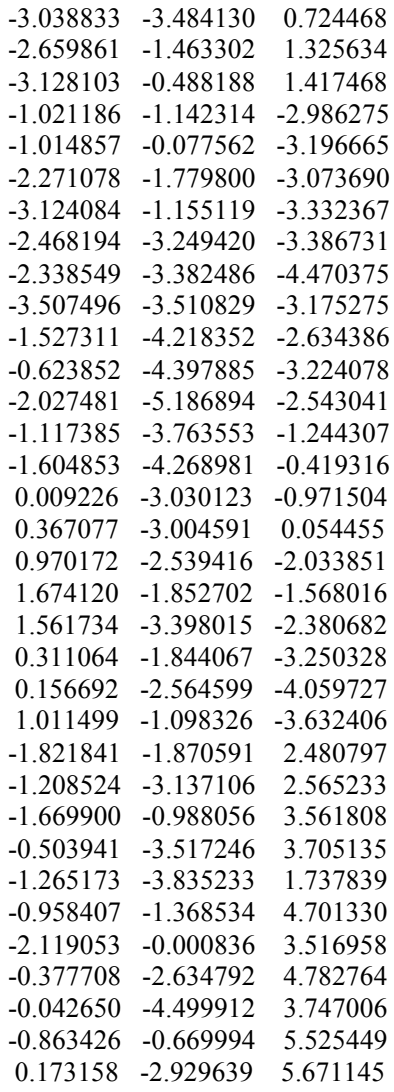

\section{P1 endo-si}

Number of imaginary frequencies :

The smallest frequency is : $18.7378 \mathrm{~cm}(-1)$ Electronic energy $=-2700.8687245$

Zero-point correction=

0.898446 (Hartree/Particle)

Thermal correction to Energy= $\quad 0.947129$

Thermal correction to Enthalpy=

0.948073

Thermal correction to Gibbs Free Energy=

Sum of electronic and zero-point Energies=

Sum of electronic and thermal Energies=

Sum of electronic and thermal Enthalpies=

Sum of electronic and thermal Free Energies=

0.817839

$-2699.970278$

$-2699.921595$

$-2699920651$

$-2700.050886$

Cartesian Coordinates

$\begin{array}{ccc}-1.401000 & -1.871831 & -0.598444 \\ -0.187747 & 0.144016 & -0.442284 \\ 0.710631 & 0.665208 & 0.847613 \\ 0.967343 & 0.195534 & -1.634461 \\ -1.279151 & 1.398079 & -0.573958 \\ 1.881597 & -0.017103 & 1.176994 \\ 1.842437 & -0.794383 & 2.353258 \\ 0.934409 & -0.783409 & 2.943801 \\ 2.944906 & -1.526023 & 2.718111 \\ 2.933856 & -2.120112 & 3.627700 \\ 4.102270 & -1.543079 & 1.892920 \\ 5.214435 & -2.369670 & 2.209289 \\ 5.173969 & -2.967175 & 3.116228 \\ 6.312041 & -2.426653 & 1.380658 \\ 7.154030 & -3.066078 & 1.628837 \\ 6.334669 & -1.660492 & 0.191247 \\ 7.189548 & -1.727408 & -0.475464 \\ 5.281140 & -0.833778 & -0.134240 \\ 5.315249 & -0.262882 & -1.054253 \\ 4.140599 & -0.729383 & 0.710245 \\ 3.015460 & 0.116089 & 0.392562 \\ 3.044247 & 1.068642 & -0.751590 \\ 4.078938 & 2.066394 & -0.877171\end{array}$

$\begin{array}{lll}5.031785 & 2.316648 & 0.149995\end{array}$

$\begin{array}{lll}4.982186 & 1.750071 & 1.072053\end{array}$

$\begin{array}{llll}6.007083 & 3.278755 & -0.001651\end{array}$

$\begin{array}{llll}6.716526 & 3.454036 & 0.802001\end{array}$

$\begin{array}{llll}6.089500 & 4.045150 & -1.188132\end{array}$

$6.867942 \quad 4.794756-1.295912$

$\begin{array}{llll}5.169548 & 3.847672 & -2.192786\end{array}$

$5.205288 \quad 4.442259-3.101860$

$\begin{array}{llll}4.141952 & 2.874788 & -2.061571\end{array}$

$\begin{array}{lll}3.162363 & 2.705333 & -3.077059\end{array}$

$3.227229 \quad 3.305760 \quad-3.979955$

$\begin{array}{llll}2.126132 & 1.824079 & -2.903106\end{array}$

$\begin{array}{llll}1.341066 & 1.709285 & -3.643096\end{array}$

$2.058351 \quad 1.045176 \quad-1.726399$

$\begin{array}{llll}-2.692738 & 0.952394 & -0.749354\end{array}$

$\begin{array}{llll}-3.164134 & 0.914919 & 0.240029\end{array}$

$\begin{array}{llll}-3.493068 & 1.904604 & -1.624183\end{array}$

$\begin{array}{llll}-4.776468 & 2.300931 & -1.232561\end{array}$

$\begin{array}{llll}-5.169399 & 1.970194 & -0.274066\end{array}$

$\begin{array}{llll}-5.550814 & 3.121376 & -2.058073\end{array}$

$\begin{array}{llll}-6.544952 & 3.422585 & -1.739777\end{array}$

$\begin{array}{llll}-5.043768 & 3.559022 & -3.282824\end{array}$

$\begin{array}{llll}-5.641048 & 4.202325 & -3.922600\end{array}$

$\begin{array}{llll}-3.760390 & 3.168886 & -3.680629\end{array}$

$\begin{array}{llll}-3.358516 & 3.509583 & -4.630868\end{array}$

$\begin{array}{llll}-2.992992 & 2.343494 & -2.859627\end{array}$

$\begin{array}{llll}-1.991751 & 2.048028 & -3.162904\end{array}$

$\begin{array}{llll}-2.743977 & -0.439694 & -1.396403\end{array}$

$\begin{array}{lll}-2.518238 & -0.325557 & -2.462567\end{array}$

$\begin{array}{llll}-3.771857 & -0.808037 & -1.331449\end{array}$

$\begin{array}{lll}-1.100226 & 2.832864 & -0.170926\end{array}$

$\begin{array}{llll}-1.862558 & 3.352547 & -0.753173\end{array}$

$\begin{array}{lll}-1.424693 & 3.131478 & 1.292532\end{array}$

$\begin{array}{lll}-2.705450 & 3.606594 & 1.611548\end{array}$

$\begin{array}{lll}-3.455389 & 3.677178 & 0.828165\end{array}$

$\begin{array}{lll}-3.016003 & 4.029098 & 2.906681\end{array}$

$\begin{array}{lll}-4.010467 & 4.407435 & 3.127518\end{array}$

$\begin{array}{lll}-2.043157 & 3.982984 & 3.906748\end{array}$

$\begin{array}{llll}-2.275587 & 4.319489 & 4.913320\end{array}$

$\begin{array}{llll}-0.769720 & 3.491981 & 3.606169\end{array}$

$\begin{array}{llll}-0.008811 & 3.444686 & 4.380314\end{array}$

$\begin{array}{lll}-0.462860 & 3.070293 & 2.312036\end{array}$

$\begin{array}{lll}0.534638 & 2.709490 & 2.093572\end{array}$

$\begin{array}{llll}0.231621 & 3.434597 & -0.614650\end{array}$

$\begin{array}{llll}0.356723 & 3.324730 & -1.693224\end{array}$

$1.093588 \quad 2.990228 \quad-0.117482$

$\begin{array}{llll}0.223342 & 4.502828 & -0.379673\end{array}$

$\begin{array}{llll}-3.145845 & -3.117454 & -0.100093\end{array}$

$\begin{array}{llll}-3.974293 & -3.056677 & -0.801911\end{array}$

$\begin{array}{lll}-2.849322 & -4.134435 & 0.137997\end{array}$

$\begin{array}{lll}-3.111242 & -2.134166 & 0.945024\end{array}$

$\begin{array}{lll}-3.824031 & -1.315945 & 0.926400\end{array}$

$\begin{array}{llll}-2.107257 & -2.139698 & 1.910997\end{array}$

$\begin{array}{lll}-1.506063 & -3.038753 & 2.005474\end{array}$

$-0.495987-2.092016-2.624186$

$-0.656192-1.152334-3.143501$

$\begin{array}{llll}-1.622612 & -2.944322 & -2.530532\end{array}$

$\begin{array}{llll}-2.544411 & -2.586691 & -2.985706\end{array}$

$\begin{array}{llll}-1.559705 & -4.453708 & -2.411988\end{array}$

$\begin{array}{llll}-1.367572 & -4.865609 & -3.413064\end{array}$

$\begin{array}{llll}-2.550606 & -4.811618 & -2.121327\end{array}$

$-0.504461 \quad-4.991082-1.418135$

$\begin{array}{llll}0.442660 & -5.165263 & -1.936488\end{array}$

$\begin{array}{llll}-0.835497 & -5.967057 & -1.051445\end{array}$

$-0.240632 \quad-4.094008 \quad-0.219743$

$\begin{array}{lll}-0.679521 & -4.408465 & 0.722539\end{array}$

$\begin{array}{llll}0.745930 & -3.142643 & -0.177890\end{array}$

$\begin{array}{lll}1.049197 & -2.761512 & 0.793347\end{array}$

$\begin{array}{llll}1.658274 & -2.809756 & -1.338441\end{array}$

$\begin{array}{llll}2.213919 & -1.909018 & -1.085087\end{array}$

$2.401491-3.613674 \quad-1.434224$

$\begin{array}{llll}0.945539 & -2.598128 & -2.694866\end{array}$

$\begin{array}{llll}0.946450 & -3.527834 & -3.273268\end{array}$

$\begin{array}{llll}1.526227 & -1.875537 & -3.272569\end{array}$

$\begin{array}{lll}1.993568 & -1.217634 & 3.041734\end{array}$ 


$\begin{array}{rrrr}6 & -2.770787 & -0.051024 & 3.177313 \\ 6 & -1.129609 & -1.576369 & 4.094946 \\ 6 & -2.697403 & 0.711149 & 4.337930 \\ 1 & -3.439618 & 0.261684 & 2.382621 \\ 6 & -1.043635 & -0.798982 & 5.248899 \\ 1 & -0.535080 & -2.482175 & 4.007948 \\ 6 & -1.831552 & 0.346470 & 5.374644 \\ 1 & -3.306522 & 1.602362 & 4.429086 \\ 1 & -0.371403 & -1.092753 & 6.049643 \\ 1 & -1.774420 & 0.953679 & 6.273211\end{array}$

\section{P2 endo-si}

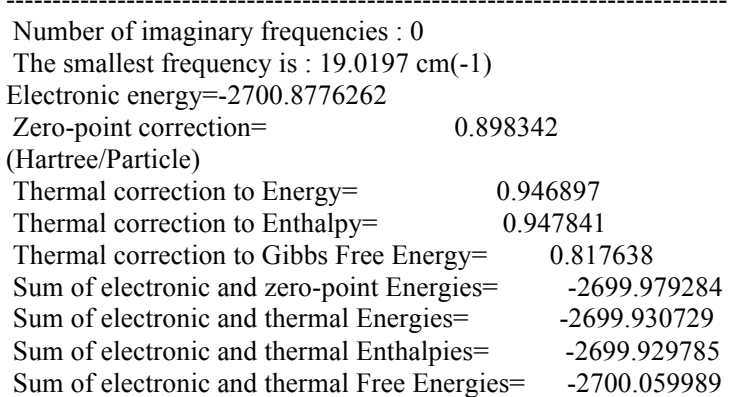

Cartesian Coordinates

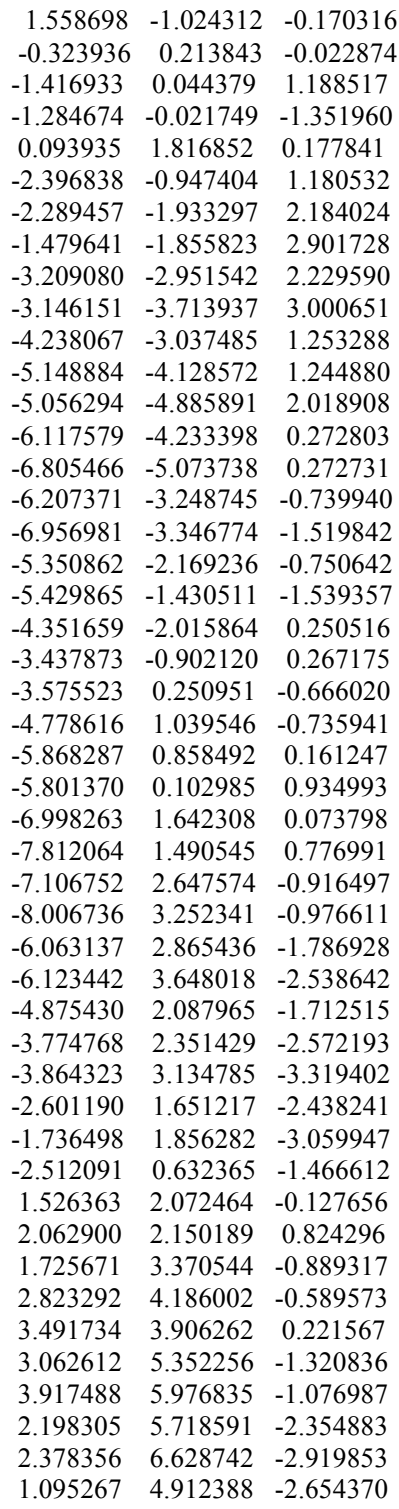

$\begin{array}{lll}0.415228 & 5.196152 & -3.452930\end{array}$

$\begin{array}{llll}0.861866 & 3.744499 & -1.927866\end{array}$

$\begin{array}{llll}-0.001469 & 3.124127 & -2.148608\end{array}$

$\begin{array}{llll}2.109277 & 0.897863 & -0.920262\end{array}$

$\begin{array}{llll}1.786496 & 0.975847 & -1.964569\end{array}$

$\begin{array}{llll}3.200211 & 0.960339 & -0.913562\end{array}$

$\begin{array}{lll}-0.540998 & 2.850485 & 1.073363\end{array}$

$\begin{array}{lll}-0.106582 & 3.784009 & 0.705885\end{array}$

$\begin{array}{lll}-0.070093 & 2.686473 & 2.516640\end{array}$

$\begin{array}{lll}1.106529 & 3.335850 & 2.922231\end{array}$

$\begin{array}{lll}1.619328 & 3.992105 & 2.223451\end{array}$

$\begin{array}{lll}1.621058 & 3.160616 & 4.209295\end{array}$

$\begin{array}{lll}2.534924 & 3.671604 & 4.499733\end{array}$

$\begin{array}{llll}0.953545 & 2.340842 & 5.121358\end{array}$

$\begin{array}{llll}1.347891 & 2.202610 & 6.124147\end{array}$

$\begin{array}{lll}-0.237101 & 1.715126 & 4.740556\end{array}$

$\begin{array}{lll}-0.774997 & 1.092844 & 5.450708\end{array}$

$\begin{array}{lll}-0.745367 & 1.887397 & 3.451584\end{array}$

$\begin{array}{llll}-1.667475 & 1.391570 & 3.175637\end{array}$

$\begin{array}{lll}-2.051409 & 2.996181 & 0.903351\end{array}$

$\begin{array}{llll}-2.298374 & 3.164183 & -0.146862\end{array}$

$\begin{array}{lll}-2.613389 & 2.132619 & 1.255099\end{array}$

$\begin{array}{lll}-2.377904 & 3.869123 & 1.476869\end{array}$

$\begin{array}{llll}1.734540 & -0.732549 & 1.985808\end{array}$

$\begin{array}{lll}1.538830 & -1.685997 & 2.473731\end{array}$

$\begin{array}{lll}1.193300 & 0.096211 & 2.435297\end{array}$

$\begin{array}{lll}3.069321 & -0.448730 & 1.535413\end{array}$

$\begin{array}{lll}3.353694 & 0.595358 & 1.450229\end{array}$

$\begin{array}{llll}3.960286 & -1.416994 & 1.088052\end{array}$

$\begin{array}{lll}3.769680 & -2.455170 & 1.335901\end{array}$

$\begin{array}{llll}1.035903 & -1.647660 & -2.319368\end{array}$

$\begin{array}{llll}0.719143 & -0.727324 & -2.800266\end{array}$

$\begin{array}{llll}2.413595 & -1.823863 & -2.151112\end{array}$

$3.068226 \quad-1.036216 \quad-2.521367$

$\begin{array}{lll}3.112954 & -3.144575 & -1.895560\end{array}$

$3.279404 \quad-3.651814 \quad-2.857244$

$\begin{array}{llll}4.103252 & -2.914756 & -1.494676\end{array}$

$\begin{array}{llll}2.385947 & -4.103305 & -0.923875\end{array}$

$\begin{array}{llll}1.693790 & -4.747236 & -1.474024\end{array}$

$\begin{array}{lll}3.130215 & -4.770160 & -0.478000\end{array}$

$\begin{array}{llll}1.622006 & -3.421093 & 0.204587\end{array}$

$\begin{array}{llll}2.045233 & -3.517728 & 1.199488\end{array}$

$\begin{array}{llll}0.293702 & -3.048000 & 0.136751\end{array}$

$\begin{array}{lll}-0.221283 & -2.893008 & 1.079842\end{array}$

$\begin{array}{lll}-0.607927 & -3.208224 & -1.068099\end{array}$

$\begin{array}{lll}-1.505194 & -2.611226 & -0.899417\end{array}$

$\begin{array}{lll}-0.938315 & -4.255208 & -1.119610\end{array}$

$\begin{array}{llll}0.029956 & -2.792600 & -2.411221\end{array}$

$\begin{array}{llll}0.521011 & -3.650440 & -2.879431\end{array}$

$\begin{array}{rrr}-0.769421 & -2.487545 & -3.091889\end{array}$

$\begin{array}{llll}5.248709 & -1.154847 & 0.449583\end{array}$

$\begin{array}{lll}5.693741 & 0.137388 & 0.098161\end{array}$

$\begin{array}{lll}6.082482 & -2.251003 & 0.148480\end{array}$

$\begin{array}{llll}6.918445 & 0.316638 & -0.538975\end{array}$

$\begin{array}{lll}5.084517 & 1.005641 & 0.325974\end{array}$

$\begin{array}{lll}7.308610 & -2.068602 & -0.488421\end{array}$

$\begin{array}{llll}5.757326 & -3.252116 & 0.419400\end{array}$

$\begin{array}{lll}7.729828 & -0.783564 & -0.838993\end{array}$

$\begin{array}{llll}7.243652 & 1.318950 & -0.802852\end{array}$

$\begin{array}{lll}7.933590 & -2.928326 & -0.711931\end{array}$

$8.683248-0.637651 \quad-1.338164$

P2 exo-re

Number of imaginary frequencies : 0

The smallest frequency is : $17.5090 \mathrm{~cm}(-1)$

Electronic energy $=-2700.8715565$

Zero-point correction $=$

(Hartree/Particle)

Thermal correction to Energy=

Thermal correction to Enthalpy=

0.897923

0.946654

0.947599

Thermal correction to Gibbs Free Energy=

Sum of electronic and zero-point Energies=

Sum of electronic and thermal Energies=

Sum of electronic and thermal Enthalpies= 
Cartesian Coordinates

$\begin{array}{lll}1.762335 & -0.535043 & -0.194108\end{array}$

$\begin{array}{lll}-0.333375 & 0.326232 & -0.026426\end{array}$

$\begin{array}{lll}-1.346982 & -0.042721 & 1.213206\end{array}$

$-1.269453-0.078068 \quad-1.329979$

$\begin{array}{llll}-0.218333 & 1.979073 & 0.155548\end{array}$

$\begin{array}{lll}-2.122520 & -1.201226 & 1.226005\end{array}$

$\begin{array}{lll}-1.801437 & -2.150256 & 2.219973\end{array}$

$\begin{array}{lll}-1.003592 & -1.919771 & 2.917951\end{array}$

$\begin{array}{lll}-2.501980 & -3.329268 & 2.279189\end{array}$

$\begin{array}{lll}-2.272059 & -4.066893 & 3.042520\end{array}$

$\begin{array}{lll}-3.514496 & -3.614027 & 1.323941\end{array}$

$\begin{array}{lll}-4.186905 & -4.866395 & 1.322936\end{array}$

$\begin{array}{lll}-3.927890 & -5.593620 & 2.087870\end{array}$

$\begin{array}{lll}-5.134113 & -5.159137 & 0.368261\end{array}$

$\begin{array}{lll}-5.638444 & -6.120862 & 0.372932\end{array}$

$\begin{array}{llll}-5.440248 & -4.207806 & -0.633789\end{array}$

$\begin{array}{llll}-6.169424 & -4.451078 & -1.401086\end{array}$

$\begin{array}{llll}-4.819147 & -2.977771 & -0.650677\end{array}$

$-5.060870-2.267238-1.431783$

$\begin{array}{llll}-3.851007 & -2.631024 & 0.332794\end{array}$

$\begin{array}{llll}-3.175051 & -1.358038 & 0.337911\end{array}$

$\begin{array}{lll}-3.553947 & -0.258155 & -0.592948\end{array}$

$\begin{array}{llll}-4.891167 & 0.274707 & -0.645399\end{array}$

$\begin{array}{lll}-5.908534 & -0.114534 & 0.270185\end{array}$

$\begin{array}{lll}-5.678876 & -0.836384 & 1.044533\end{array}$

$\begin{array}{llll}-7.175097 & 0.423194 & 0.196041\end{array}$

$\begin{array}{lll}-7.931724 & 0.114470 & 0.911527\end{array}$

$\begin{array}{llll}-7.498981 & 1.377255 & -0.797843\end{array}$

$\begin{array}{llll}-8.503875 & 1.785890 & -0.848259\end{array}$

$\begin{array}{llll}-6.533763 & 1.793863 & -1.686088\end{array}$

$\begin{array}{llll}-6.762249 & 2.539818 & -2.442664\end{array}$

$\begin{array}{llll}-5.212879 & 1.271730 & -1.627438\end{array}$

$\begin{array}{lll}-4.203355 & 1.737468 & -2.512783\end{array}$

$\begin{array}{llll}-4.461266 & 2.476570 & -3.265752\end{array}$

$\begin{array}{llll}-2.912147 & 1.285425 & -2.397178\end{array}$

$\begin{array}{llll}-2.117453 & 1.645806 & -3.040784\end{array}$

$\begin{array}{llll}-2.603146 & 0.318217 & -1.418073\end{array}$

$\begin{array}{llll}1.129309 & 2.514960 & -0.168656\end{array}$

$\begin{array}{lll}1.630637 & 2.753924 & 0.774898\end{array}$

$\begin{array}{llll}1.048925 & 3.796755 & -0.980615\end{array}$

$\begin{array}{llll}1.899236 & 4.865987 & -0.674470\end{array}$

$\begin{array}{lll}2.580232 & 4.782573 & 0.169434\end{array}$

$\begin{array}{llll}1.874378 & 6.035415 & -1.439112\end{array}$

$\begin{array}{llll}2.537960 & 6.858450 & -1.188933\end{array}$

$\begin{array}{llll}0.989774 & 6.149312 & -2.513632\end{array}$

$\begin{array}{llll}0.963389 & 7.060366 & -3.104630\end{array}$

$\begin{array}{llll}0.132432 & 5.087629 & -2.820710\end{array}$

$\begin{array}{llll}-0.563415 & 5.173115 & -3.650953\end{array}$

$\begin{array}{llll}0.163304 & 3.918627 & -2.060402\end{array}$

$\begin{array}{llll}-0.512403 & 3.100265 & -2.288356\end{array}$

$\begin{array}{lll}1.967987 & 1.465565 & -0.910636\end{array}$

$1.674738 \quad 1.464049 \quad-1.966263$

$\begin{array}{llll}3.013802 & 1.778545 & -0.877508\end{array}$

$\begin{array}{lll}-1.036345 & 2.891696 & 1.035228\end{array}$

$\begin{array}{lll}-0.815961 & 3.880388 & 0.623661\end{array}$

$\begin{array}{lll}-0.508407 & 2.881633 & 2.468287\end{array}$

$\begin{array}{lll}0.483025 & 3.805427 & 2.833249\end{array}$

$\begin{array}{llll}0.812530 & 4.545929 & 2.108813\end{array}$

$\begin{array}{lll}1.045152 & 3.793285 & 4.112597\end{array}$

$\begin{array}{llll}1.814164 & 4.515839 & 4.371459\end{array}$

$\begin{array}{lll}0.610163 & 2.861086 & 5.056406\end{array}$

$\begin{array}{lll}1.041385 & 2.848680 & 6.053482\end{array}$

$\begin{array}{lll}-0.398796 & 1.955682 & 4.714597\end{array}$

$\begin{array}{lll}-0.759488 & 1.241037 & 5.449608\end{array}$

$\begin{array}{llll}-0.954004 & 1.966713 & 3.433981\end{array}$

$\begin{array}{lll}-1.733977 & 1.257171 & 3.189247\end{array}$

$\begin{array}{lll}-2.549331 & 2.719438 & 0.916326\end{array}$

$\begin{array}{llll}-2.859877 & 2.795956 & -0.127339\end{array}$

$\begin{array}{lll}-2.912132 & 1.774146 & 1.315789\end{array}$

$\begin{array}{lll}-3.027777 & 3.528930 & 1.476066\end{array}$

$\begin{array}{lll}1.905817 & 0.122558 & 1.917191\end{array}$ $\begin{array}{lll}1.204647 & -0.324112 & 2.616579\end{array}$

$\begin{array}{llll}1.921656 & 1.207078 & 1.954477\end{array}$

$\begin{array}{lll}3.128553 & -0.582952 & 1.696441\end{array}$

$\begin{array}{lll}3.225378 & -1.572168 & 2.130892\end{array}$

$\begin{array}{lll}4.110677 & -0.132892 & 0.815471\end{array}$

$\begin{array}{lll}4.107030 & 0.916205 & 0.540323\end{array}$

$\begin{array}{llll}1.290830 & -1.170473 & -2.327229\end{array}$

$\begin{array}{llll}0.877895 & -0.283201 & -2.797315\end{array}$

$\begin{array}{llll}2.685791 & -1.196217 & -2.172031\end{array}$

$\begin{array}{llll}3.234521 & -0.324261 & -2.525686\end{array}$

$\begin{array}{llll}3.538519 & -2.439935 & -2.043448\end{array}$

$3.741478-2.827545 \quad-3.052583$

$\begin{array}{llll}4.505409 & -2.144177 & -1.633333\end{array}$

$2.944490 \quad-3.566674 \quad-1.170486$

$\begin{array}{llll}2.306657 & -4.217367 & -1.774927\end{array}$

$\begin{array}{llll}3.768748 & -4.188659 & -0.809271\end{array}$

$\begin{array}{llll}2.152723 & -3.086219 & 0.031129\end{array}$

$\begin{array}{llll}2.659322 & -3.140953 & 0.988343\end{array}$

$\begin{array}{llll}0.799666 & -2.869526 & 0.038176\end{array}$

$\begin{array}{llll}0.306533 & -2.795440 & 1.002711\end{array}$

$\begin{array}{lll}-0.108023 & -3.037927 & -1.159728\end{array}$

$\begin{array}{lll}-1.075442 & -2.597949 & -0.918725\end{array}$

$-0.288124-4.111533-1.309682$

$\begin{array}{llll}0.414281 & -2.414191 & -2.475363\end{array}$

$\begin{array}{llll}0.974080 & -3.158594 & -3.049905\end{array}$

$\begin{array}{lll}-0.451203 & -2.143441 & -3.085115\end{array}$

$\begin{array}{llll}5.311707 & -0.875068 & 0.427443\end{array}$

$\begin{array}{llll}5.574099 & -2.195210 & 0.846205\end{array}$

$\begin{array}{llll}6.227648 & -0.254679 & -0.443652\end{array}$

$\begin{array}{llll}6.707753 & -2.867977 & 0.398423\end{array}$

$\begin{array}{lll}4.887050 & -2.702665 & 1.515617\end{array}$

$\begin{array}{lll}7.360301 & -0.931509 & -0.893309\end{array}$

$\begin{array}{llll}6.038666 & 0.764443 & -0.771822\end{array}$

$\begin{array}{llll}7.603251 & -2.242720 & -0.476606\end{array}$

$\begin{array}{lll}6.893899 & -3.885549 & 0.729848\end{array}$

$\begin{array}{llll}8.052536 & -0.436274 & -1.568062\end{array}$

$\begin{array}{lll}8.484032 & -2.773161 & -0.826279\end{array}$

P2 exo-si

Number of imaginary frequencies : 0

The smallest frequency is : $18.4507 \mathrm{~cm}(-1)$

Electronic energy $=-2700.8703732$

Zero-point correction=

(Hartree/Particle)

Thermal correction to Energy=

Thermal correction to Enthalpy=

Thermal correction to Gibbs Free Energy=

Sum of electronic and zero-point Energies=

Sum of electronic and thermal Energies=

Sum of electronic and thermal Enthalpies=

Sum of electronic and thermal Free Energies=

0.899968

0.948162

0.949106

0.821325

$-2699.970405$

$-2699.922211$

$-2699.921267$

$-2700.049048$

Cartesian Coordinates

$1.857988-1.344926-0.888166$

$\begin{array}{lll}0.274404 & 0.273864 & -0.274770\end{array}$

$\begin{array}{lll}-0.778808 & 0.227469 & 0.992545\end{array}$

$\begin{array}{lll}-0.789369 & 0.484628 & -1.531566\end{array}$

$\begin{array}{lll}1.081495 & 1.676892 & 0.107010\end{array}$

$\begin{array}{lll}-1.916732 & -0.579766 & 0.955167\end{array}$

$\begin{array}{llll}-1.925791 & -1.674116 & 1.843671\end{array}$

$\begin{array}{lll}-1.083598 & -1.800291 & 2.510327\end{array}$

$\begin{array}{lll}-2.993552 & -2.536014 & 1.844819\end{array}$

$\begin{array}{lll}-3.015725 & -3.378452 & 2.530424\end{array}$

$\begin{array}{lll}-4.065740 & -2.355907 & 0.929561\end{array}$

$\begin{array}{llll}-5.133316 & -3.291621 & 0.858313\end{array}$

$\begin{array}{lll}-5.126844 & -4.136963 & 1.541340\end{array}$

$-6.144647-3.142378-0.063351$

$-6.952786-3.866226-0.113124$

$\begin{array}{lll}-6.121584 & -2.048572 & -0.960876\end{array}$

$\begin{array}{llll}-6.906435 & -1.946818 & -1.704864\end{array}$

$\begin{array}{lll}-5.111072 & -1.113109 & -0.905194\end{array}$

$\begin{array}{llll}-5.108365 & -0.289049 & -1.608445\end{array}$

$\begin{array}{lll}-4.061269 & -1.221093 & 0.049458\end{array}$ 
$\begin{array}{lll}1.265417 & -3.835140 & 1.738864\end{array}$

$\begin{array}{lll}0.957759 & -1.367550 & 4.701515\end{array}$

$\begin{array}{llll}2.118316 & 0.000005 & 3.516905\end{array}$

$\begin{array}{lll}0.377287 & -2.633892 & 4.783258\end{array}$

$\begin{array}{llll}0.042810 & -4.499426 & 3.748056\end{array}$

$\begin{array}{lll}0.862560 & -0.668791 & 5.525422\end{array}$

$\begin{array}{lll}-0.173614 & -2.928586 & 5.671667\end{array}$

\section{P2 endo-re}

Number of imaginary frequencies : 0

The smallest frequency is : $18.7759 \mathrm{~cm}(-1)$

Electronic energy $=-2700.8687246$

Zero-point correction $=$

(Hartree/Particle)

Thermal correction to Energy= $\quad 0.947125$

Thermal correction to Enthalpy= $\quad 0.948069$

Thermal correction to Gibbs Free Energy $=\quad 0.817844$

Sum of electronic and zero-point Energies $=\quad-2699.970282$

Sum of electronic and thermal Energies=

Sum of electronic and thermal Enthalpies=

$-2699.921599$

Sum of electronic and thermal Free Energies=

Cartesian Coordinates

$\begin{array}{crrr}77 & 1.400767 & -1.871898 & -0.598513 \\ 15 & 0.187698 & 0.144026 & -0.442390 \\ 8 & -0.710527 & 0.665341 & 0.847560 \\ 8 & -0.967475 & 0.195651 & -1.634498 \\ 7 & 1.279268 & 1.397944 & -0.574139 \\ 6 & -1.881479 & -0.016937 & 1.177065 \\ 6 & -1.842229 & -0.794230 & 2.353316 \\ 1 & -0.934137 & -0.783279 & 2.943764 \\ 6 & -2.944681 & -1.525855 & 2.718253 \\ 1 & -2.933564 & -2.119965 & 3.627829 \\ 6 & -4.102114 & -1.542885 & 1.893157 \\ 6 & -5.214256 & -2.369483 & 2.209593 \\ 1 & -5.173719 & -2.967014 & 3.116512 \\ 6 & -6.311925 & -2.426444 & 1.381043 \\ 1 & -7.153893 & -3.065879 & 1.629266 \\ 6 & -6.334649 & -1.660250 & 0.191654 \\ 1 & -7.189581 & -1.727150 & -0.474991 \\ 6 & -5.281144 & -0.833528 & -0.133895 \\ 1 & -5.315319 & -0.262610 & -1.053893 \\ 6 & -4.140536 & -0.729166 & 0.710502 \\ 6 & -3.015406 & 0.116283 & 0.392733 \\ 6 & -3.044253 & 1.068852 & -0.751402 \\ 6 & -4.078908 & 2.066659 & -0.876852 \\ 6 & -5.031659 & 2.316914 & 0.150402 \\ 1 & -4.982015 & 1.750293 & 1.072430 \\ 6 & -6.006923 & 3.279075 & -0.001123 \\ 1 & -6.716292 & 3.454352 & 0.802595 \\ 6 & -6.089395 & 4.045530 & -1.187560 \\ 1 & -6.867808 & 4.795181 & -1.295241 \\ 6 & -5.169533 & 3.848055 & -2.192297 \\ 1 & -5.205312 & 4.442689 & -3.101337 \\ 6 & -4.141977 & 2.875112 & -2.061210 \\ 6 & -3.162476 & 2.705655 & -3.076782 \\ 1 & -3.227378 & 3.306133 & -3.979642 \\ 6 & -2.126280 & 1.824336 & -2.902959 \\ 1 & -1.341277 & 1.709541 & -3.643016 \\ 6 & -2.058442 & 1.045369 & -1.726299 \\ 6 & 2.692790 & 0.952092 & -0.749778 \\ 1 & 3.164366 & 0.914523 & 0.239520 \\ 6 & 3.493034 & 1.904270 & -1.624715 \\ 6 & 4.776431 & 2.300692 & -1.233188 \\ 1 & 5.169460 & 1.969979 & -0.274724 \\ 6 & 5.550655 & 3.121207 & -2.058745 \\ 6.544790 & 3.422490 & -1.740512\end{array}$


$\begin{array}{llll}5.043484 & 3.558831 & -3.283450\end{array}$

$\begin{array}{llll}5.640664 & 4.202191 & -3.923261\end{array}$

$\begin{array}{llll}3.760107 & 3.168597 & -3.681165\end{array}$

$\begin{array}{llll}3.358135 & 3.509273 & -4.631370\end{array}$

$\begin{array}{llll}2.992835 & 2.343134 & -2.860117\end{array}$

$\begin{array}{llll}1.991604 & 2.047583 & -3.163338\end{array}$

$\begin{array}{llll}2.743748 & -0.440011 & -1.396835\end{array}$

$\begin{array}{lll}2.517782 & -0.325892 & -2.462951\end{array}$

$\begin{array}{llll}3.771589 & -0.808505 & -1.332057\end{array}$

$\begin{array}{llll}1.100544 & 2.832748 & -0.171083\end{array}$

$\begin{array}{llll}1.863009 & 3.352315 & -0.753254\end{array}$

$\begin{array}{llll}1.424947 & 3.131281 & 1.292394\end{array}$

$\begin{array}{lll}2.705754 & 3.606222 & 1.611470\end{array}$

$\begin{array}{lll}3.455698 & 3.676822 & 0.828092\end{array}$

$\begin{array}{lll}3.016357 & 4.028528 & 2.906653\end{array}$

$\begin{array}{lll}4.010868 & 4.406713 & 3.127543\end{array}$

$\begin{array}{llll}2.043517 & 3.982363 & 3.906721\end{array}$

$2.275996 \quad 4.318663 \quad 4.913351$

$\begin{array}{lll}0.770015 & 3.491569 & 3.606072\end{array}$

$\begin{array}{lll}0.009107 & 3.444239 & 4.380217\end{array}$

$\begin{array}{lll}0.463096 & 3.070102 & 2.311881\end{array}$

$\begin{array}{lll}-0.534450 & 2.709470 & 2.093372\end{array}$

$\begin{array}{llll}-0.231165 & 3.434672 & -0.614950\end{array}$

$\begin{array}{llll}-0.356157 & 3.324790 & -1.693538\end{array}$

$\begin{array}{lll}-1.093247 & 2.990415 & -0.117876\end{array}$

$\begin{array}{llll}-0.222777 & 4.502905 & -0.379986\end{array}$

$\begin{array}{llll}3.145446 & -3.117711 & -0.100216\end{array}$

$\begin{array}{llll}3.973823 & -3.057217 & -0.802142\end{array}$

$\begin{array}{llll}2.848670 & -4.134589 & 0.138027\end{array}$

$\begin{array}{llll}3.111191 & -2.134308 & 0.944801\end{array}$

$\begin{array}{lll}3.824164 & -1.316248 & 0.926011\end{array}$

$\begin{array}{llll}2.107321 & -2.139479 & 1.910886\end{array}$

$\begin{array}{lll}1.505781 & -3.038294 & 2.005425\end{array}$

$\begin{array}{llll}0.495461 & -2.092349 & -2.624019\end{array}$

$\begin{array}{llll}0.655772 & -1.152810 & -3.143561\end{array}$

$\begin{array}{llll}1.621956 & -2.944842 & -2.530335\end{array}$

$\begin{array}{llll}2.543754 & -2.587473 & -2.985723\end{array}$

$\begin{array}{llll}1.558826 & -4.454210 & -2.411562\end{array}$

$\begin{array}{llll}1.366621 & -4.866202 & -3.412587\end{array}$

$\begin{array}{llll}2.549686 & -4.812223 & -2.120892\end{array}$

$\begin{array}{llll}0.503532 & -4.991431 & -1.417653\end{array}$

$\begin{array}{llll}-0.443616 & -5.165504 & -1.935993\end{array}$

$\begin{array}{llll}0.834470 & -5.967450 & -1.050987\end{array}$

$\begin{array}{llll}0.239789 & -4.094350 & -0.219261\end{array}$

$\begin{array}{lll}0.678793 & -4.408691 & 0.723013\end{array}$

$-0.746518-3.142755-0.177519$

$\begin{array}{lll}-1.049608 & -2.761381 & 0.793677\end{array}$

$-1.658823-2.809705-1.338050$

$-2.214228 \quad-1.908825 \quad-1.084679$

$-2.402247-3.613438-1.433779$

$-0.946146-2.598258-2.694528$

$-0.947237-3.527994 \quad-3.272881$

$-1.526784-1.875625-3.272229$

$\begin{array}{llll}1.994115 & -1.217251 & 3.041541\end{array}$

$\begin{array}{lll}2.771946 & -0.051027 & 3.176958\end{array}$

$\begin{array}{lll}1.130086 & -1.575454 & 4.094876\end{array}$

$\begin{array}{llll}2.699097 & 0.711252 & 4.337536\end{array}$

$\begin{array}{llll}3.440887 & 0.261261 & 2.382197\end{array}$

$\begin{array}{llll}1.044586 & -0.797901 & 5.248755\end{array}$

$\begin{array}{llll}0.535157 & -2.481015 & 4.008054\end{array}$

$\begin{array}{lll}1.833112 & 0.347147 & 5.374338\end{array}$

$\begin{array}{llll}3.308748 & 1.602108 & 4.428595\end{array}$

$\begin{array}{llll}0.372265 & -1.091248 & 6.049579\end{array}$

$\begin{array}{llll}1.776387 & 0.954463 & 6.272859\end{array}$

13' P1 endo-re $\left[\mathrm{BF}_{4}^{-}\right]$

Number of imaginary frequencies $=0$

The smallest frequencies are $=18.322920 .596324 .1703 \mathrm{~cm}(-1)$ Electronic energy $=-3892.0338464$

Zero-point correction $=$

1.233643

(Hartree/Particle)

Thermal correction to Energy= $\quad 1.306605$

Thermal correction to Enthalpy=

1.307549

Thermal correction to Gibbs Free Energy=
Sum of electronic and zero-point Energies=

Sum of electronic and thermal Energies=

Sum of electronic and thermal Enthalpies=

Sum of electronic and thermal Free Energies=

$-3890.800203$

$-3890.727241$

$-3890.726297$

$-3890.910309$

Cartesian Coordinates

$\begin{array}{llll}-0.153630 & -0.520289 & -1.401376\end{array}$

$\begin{array}{llll}1.676133 & 0.362977 & -0.392687\end{array}$

$\begin{array}{lll}2.356939 & -0.272956 & 0.959420\end{array}$

$\begin{array}{lll}2.983135 & 0.374961 & -1.422496\end{array}$

$\begin{array}{llll}1.296273 & 1.885730 & 0.165015\end{array}$

$\begin{array}{llll}3.241984 & -1.349769 & 0.913035\end{array}$

$\begin{array}{lll}2.778813 & -2.562510 & 1.464864\end{array}$

$\begin{array}{lll}1.769548 & -2.625033 & 1.858887\end{array}$

$\begin{array}{lll}3.601645 & -3.661567 & 1.444172\end{array}$

$\begin{array}{llll}3.257575 & -4.604471 & 1.859199\end{array}$

$\begin{array}{llll}4.887481 & -3.596218 & 0.843968\end{array}$

$\begin{array}{lll}5.714289 & -4.749526 & 0.757593\end{array}$

$\begin{array}{llll}5.352039 & -5.678436 & 1.190160\end{array}$

$\begin{array}{lll}6.937760 & -4.698233 & 0.129273\end{array}$

$\begin{array}{llll}7.559451 & -5.586554 & 0.065698\end{array}$

$7.379212 \quad-3.485212 \quad-0.451438$

$\begin{array}{llll}8.333185 & -3.453196 & -0.970010\end{array}$

$\begin{array}{lll}6.608362 & -2.345632 & -0.373069\end{array}$

$\begin{array}{llll}6.960367 & -1.430325 & -0.833610\end{array}$

$\begin{array}{llll}5.349625 & -2.353806 & 0.291699\end{array}$

$\begin{array}{llll}4.516046 & -1.182936 & 0.391627\end{array}$

$\begin{array}{llll}4.973124 & 0.153366 & -0.083984\end{array}$

$\begin{array}{llll}6.175196 & 0.774508 & 0.409296\end{array}$

$\begin{array}{lll}6.932275 & 0.230944 & 1.484799\end{array}$

$\begin{array}{llll}6.597608 & -0.683237 & 1.960397\end{array}$

$\begin{array}{lll}8.072099 & 0.857216 & 1.940230\end{array}$

$\begin{array}{lll}8.626551 & 0.425989 & 2.768789\end{array}$

$\begin{array}{lll}8.523716 & 2.059523 & 1.345360\end{array}$

$\begin{array}{lll}9.427789 & 2.537830 & 1.710591\end{array}$

$\begin{array}{llll}7.804592 & 2.625560 & 0.317321\end{array}$

$\begin{array}{llll}8.127802 & 3.558636 & -0.136827\end{array}$

$\begin{array}{llll}6.614770 & 2.015345 & -0.164722\end{array}$

$\begin{array}{llll}5.843684 & 2.625743 & -1.190644\end{array}$

$\begin{array}{llll}6.194786 & 3.552259 & -1.635866\end{array}$

$\begin{array}{llll}4.652997 & 2.071138 & -1.589916\end{array}$

$\begin{array}{llll}4.029583 & 2.533773 & -2.347153\end{array}$

$\begin{array}{llll}4.218850 & 0.862055 & -1.005218\end{array}$

$\begin{array}{llll}0.072247 & 2.452609 & -0.459475\end{array}$

$\begin{array}{lll}-0.724946 & 2.444938 & 0.289367\end{array}$

$\begin{array}{llll}0.274217 & 3.885822 & -0.932184\end{array}$

$\begin{array}{lll}1.530214 & 4.361645 & -1.331105\end{array}$

$\begin{array}{llll}2.397292 & 3.717741 & -1.230955\end{array}$

$\begin{array}{lll}1.672932 & 5.656833 & -1.833038\end{array}$

$\begin{array}{llll}2.655450 & 6.013436 & -2.131443\end{array}$

$\begin{array}{llll}0.558531 & 6.491980 & -1.947077\end{array}$

$\begin{array}{llll}0.667923 & 7.500809 & -2.335593\end{array}$

$\begin{array}{llll}-0.696913 & 6.022146 & -1.551495\end{array}$

$\begin{array}{llll}-1.569436 & 6.665285 & -1.629319\end{array}$

$\begin{array}{llll}-0.839808 & 4.729464 & -1.043286\end{array}$

$\begin{array}{llll}-1.819796 & 4.376099 & -0.742779\end{array}$

$\begin{array}{llll}-0.366682 & 1.594344 & -1.656424\end{array}$

$\begin{array}{llll}0.220383 & 1.884025 & -2.535215\end{array}$

$\begin{array}{llll}-1.413070 & 1.812893 & -1.881975\end{array}$

$\begin{array}{lll}1.697729 & 2.548745 & 1.455766\end{array}$

$\begin{array}{lll}1.420735 & 3.592364 & 1.285231\end{array}$

$\begin{array}{lll}0.850516 & 2.066890 & 2.632360\end{array}$

$\begin{array}{lll}-0.347170 & 2.736684 & 2.919747\end{array}$

$\begin{array}{lll}-0.659030 & 3.582575 & 2.317256\end{array}$

$\begin{array}{lll}-1.168278 & 2.325040 & 3.972403\end{array}$

$\begin{array}{lll}-2.095730 & 2.857470 & 4.161501\end{array}$

$\begin{array}{lll}-0.795045 & 1.240680 & 4.767145\end{array}$

$\begin{array}{lll}-1.427226 & 0.920318 & 5.590826\end{array}$

$\begin{array}{llll}0.404681 & 0.572563 & 4.500254\end{array}$

$\begin{array}{lll}0.708915 & -0.267775 & 5.118553\end{array}$

$\begin{array}{lll}1.220413 & 0.982170 & 3.443781\end{array}$

$\begin{array}{lll}2.145782 & 0.452609 & 3.254754\end{array}$

$\begin{array}{lll}3.202122 & 2.552820 & 1.722242\end{array}$

$\begin{array}{llll}3.737628 & 2.974070 & 0.868831\end{array}$ 
$\begin{array}{lll}3.606012 & 1.562259 & 1.924795\end{array}$

$\begin{array}{lll}3.396871 & 3.183681 & 2.595051\end{array}$

$\begin{array}{lll}-0.867042 & -0.833241 & 0.644903\end{array}$

$\begin{array}{lll}-0.795880 & -1.898059 & 0.807401\end{array}$

$\begin{array}{lll}-0.432286 & -0.231489 & 1.437911\end{array}$

$\begin{array}{lll}-1.974640 & -0.248608 & -0.036248\end{array}$

$\begin{array}{llll}-2.185865 & 0.787885 & 0.188113\end{array}$

$\begin{array}{llll}-2.698826 & -0.863738 & -1.063177\end{array}$

$\begin{array}{llll}-2.685275 & -1.943869 & -1.148167\end{array}$

$\begin{array}{llll}0.914100 & -0.566891 & -3.403893\end{array}$

$\begin{array}{llll}1.378031 & 0.410362 & -3.494334\end{array}$

$\begin{array}{llll}-0.468807 & -0.636805 & -3.642513\end{array}$

$\begin{array}{llll}-0.980656 & 0.284970 & -3.914257\end{array}$

$\begin{array}{llll}-1.212305 & -1.898436 & -4.036605\end{array}$

$\begin{array}{llll}-1.054927 & -2.078429 & -5.110550\end{array}$

$\begin{array}{lll}-2.277824 & -1.698732 & -3.916554\end{array}$

$\begin{array}{llll}-0.837648 & -3.170983 & -3.239351\end{array}$

$\begin{array}{llll}-0.048493 & -3.721385 & -3.760950\end{array}$

$\begin{array}{llll}-1.707933 & -3.834902 & -3.221831\end{array}$

$\begin{array}{llll}-0.393457 & -2.937830 & -1.801187\end{array}$

$\begin{array}{llll}-1.071287 & -3.273445 & -1.018740\end{array}$

$\begin{array}{llll}0.909202 & -2.710251 & -1.413877\end{array}$

$\begin{array}{llll}1.128667 & -2.908991 & -0.370025\end{array}$

$\begin{array}{llll}2.103963 & -2.601399 & -2.335865\end{array}$

$\begin{array}{llll}2.934084 & -2.179078 & -1.767820\end{array}$

$\begin{array}{llll}2.413445 & -3.616027 & -2.625563\end{array}$

$\begin{array}{llll}1.868713 & -1.744320 & -3.597631\end{array}$

$\begin{array}{llll}1.490599 & -2.368443 & -4.413090\end{array}$

$\begin{array}{llll}2.832237 & -1.350823 & -3.933689\end{array}$

$\begin{array}{lll}-3.748466 & -0.172922 & -1.823719\end{array}$

$\begin{array}{llll}-4.068506 & 1.186258 & -1.620700\end{array}$

$\begin{array}{llll}-4.510419 & -0.899016 & -2.762019\end{array}$

$\begin{array}{llll}-5.111331 & 1.786026 & -2.322742\end{array}$

$\begin{array}{llll}-3.535925 & 1.782991 & -0.892877\end{array}$

$\begin{array}{lll}-5.549629 & -0.294034 & -3.469078\end{array}$

$\begin{array}{lll}-4.291507 & -1.950743 & -2.924015\end{array}$

$\begin{array}{llll}-5.855905 & 1.052760 & -3.252187\end{array}$

$\begin{array}{llll}-5.339932 & 2.828667 & -2.123324\end{array}$

$\begin{array}{llll}-6.123516 & -0.876782 & -4.184138\end{array}$

$\begin{array}{llll}-6.669722 & 1.523047 & -3.796604\end{array}$

$\begin{array}{lll}-2.229309 & -4.176975 & 0.831440\end{array}$

$\begin{array}{llll}-1.336855 & -3.896347 & 1.656464\end{array}$

$\begin{array}{lll}-0.094872 & -3.949442 & 1.576837\end{array}$

$\begin{array}{llll}-1.878532 & -3.399409 & 2.890592\end{array}$

$\begin{array}{lll}-0.930947 & -2.876557 & 3.823831\end{array}$

$\begin{array}{lll}-0.423229 & -1.990058 & 3.428988\end{array}$

$\begin{array}{llll}-1.496246 & -2.599932 & 4.719009\end{array}$

$\begin{array}{lll}-0.175652 & -3.624169 & 4.083645\end{array}$

$\begin{array}{lll}-3.554519 & -0.266387 & 3.075718\end{array}$

$\begin{array}{lll}-4.773289 & 0.635737 & 3.167797\end{array}$

$\begin{array}{lll}-2.767894 & 0.221178 & 2.495188\end{array}$

$\begin{array}{lll}-3.143500 & -0.503632 & 4.060048\end{array}$

$\begin{array}{lll}-5.464912 & 0.670580 & 1.812537\end{array}$

$\begin{array}{llll}-4.477226 & 1.652200 & 3.426895\end{array}$

$\begin{array}{lll}-5.466311 & 0.262803 & 3.930150\end{array}$

$\begin{array}{lll}-6.394513 & 1.238449 & 1.870834\end{array}$

$\begin{array}{lll}-4.823136 & 1.150822 & 1.072192\end{array}$

$\begin{array}{llll}-3.935573 & -1.527585 & 2.436147\end{array}$

$\begin{array}{lll}-4.998637 & -1.712455 & 1.660689\end{array}$

$\begin{array}{llll}-5.803134 & -0.694318 & 1.345343\end{array}$

$\begin{array}{lll}-5.215494 & -3.141753 & 1.210087\end{array}$

$\begin{array}{llll}-4.255650 & -3.651991 & 1.334514\end{array}$

$\begin{array}{llll}-5.929908 & -3.603076 & 1.903808\end{array}$

$\begin{array}{llll}-5.676803 & -3.322604 & -0.242410\end{array}$

$\begin{array}{llll}-5.144516 & -2.602157 & -0.868778\end{array}$

$\begin{array}{lll}-6.905813 & -0.742721 & 0.354236\end{array}$

$\begin{array}{llll}-7.189757 & -3.168939 & -0.436945\end{array}$

$\begin{array}{lll}-7.398764 & -3.011497 & -1.502216\end{array}$

$\begin{array}{lll}-7.769563 & -2.005553 & 0.379253\end{array}$

$\begin{array}{lll}-8.756722 & -1.738802 & -0.013540\end{array}$

$\begin{array}{lll}-7.925687 & -2.299908 & 1.423381\end{array}$

$\begin{array}{llll}-7.531514 & 0.119446 & 0.589754\end{array}$

$\begin{array}{lll}-6.487473 & -0.569060 & -0.644648\end{array}$

$\begin{array}{llll}-7.700820 & -4.095414 & -0.148298\end{array}$

$\begin{array}{llll}-5.356156 & -4.313503 & -0.580396\end{array}$

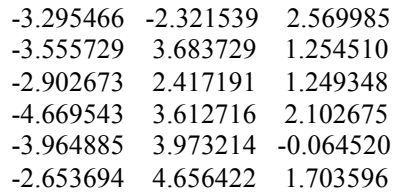

\section{$[13 \text {-14'] }]^{*}$ P1-endo-re $\left[\mathrm{BF}_{4}{ }^{-}\right]$}

Number of imaginary frequencies $=1$

The smallest frequencies are $=-262.838915 .591218 .2300 \mathrm{~cm}(-1)$ Electronic energy $=-3892.0074697$

Zero-point correction=

(Hartree/Particle)

Thermal correction to Energy= $\quad 1.301187$

Thermal correction to Enthalpy= $\quad 1.302131$

Thermal correction to Gibbs Free Energy $=\quad 1.118458$

Sum of electronic and zero-point Energies $=\quad-3890.779171$

Sum of electronic and thermal Energies=

Sum of electronic and thermal Enthalpies=

Cartesian Coordinates

\begin{tabular}{|c|c|c|c|}
\hline 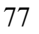 & -0.137435 & -0.452292 & -1.431226 \\
\hline & 1.675298 & 0.433438 & -0.384319 \\
\hline 8 & 2.343272 & -0.213309 & 0.970660 \\
\hline & 2.994167 & 0.457579 & -1.397355 \\
\hline & 1.275646 & 1.939538 & 0.194505 \\
\hline & 3.187186 & -1.320076 & 0.916386 \\
\hline & 2.683530 & -2.517825 & 1.465342 \\
\hline & 1.687167 & -2.524705 & 1.892089 \\
\hline & 3.455062 & -3.652506 & 1.421185 \\
\hline & 3.078826 & -4.583537 & 1.834529 \\
\hline & 4.732595 & -3.634093 & 0.799247 \\
\hline & 5.505577 & -4.821276 & 0.680869 \\
\hline & 5.108441 & -5.741018 & 1.102176 \\
\hline 6 & 6.721516 & -4.812677 & 0.036000 \\
\hline & 7.301405 & -5.726656 & -0.052875 \\
\hline & 7.210273 & -3.609883 & -0.527756 \\
\hline & 8.158357 & -3.611017 & -1.057876 \\
\hline & 6.492984 & -2.438384 & -0.417806 \\
\hline & 6.879806 & -1.530131 & -0.864457 \\
\hline 6 & 5.242837 & -2.403872 & 0.261507 \\
\hline & 4.462412 & -1.199737 & 0.387204 \\
\hline & 4.971466 & 0.127443 & -0.059851 \\
\hline D & 6.197659 & 755 & 0.448648 \\
\hline & 6.937498 & 0.081382 & 1.503023 \\
\hline 1 & 6.571285 & -0.833821 & 1.952588 \\
\hline 5 & 8.101885 & 0.649458 & 1.972486 \\
\hline 1 & 8.642523 & 0.171409 & 2.784308 \\
\hline 6 & 8.596349 & 1.852272 & 1.414044 \\
\hline 1 & 9.519002 & 2.284454 & 1.790090 \\
\hline & 7.895303 & 2.477486 & 0.408078 \\
\hline 1 & 8.251785 & 3.412156 & -0.017034 \\
\hline 6 & 6.681649 & 1.927965 & -0.087524 \\
\hline 6 & 5.929921 & 2.599858 & -1.088808 \\
\hline 1 & 6.312607 & 3.527920 & -1.503624 \\
\hline 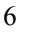 & 4.719228 & 2.102555 & -1.502797 \\
\hline 1 & 4.112092 & 2.613440 & -2.241934 \\
\hline 6 & 4.242881 & 0.890740 & -0.958224 \\
\hline 6 & 0.053911 & 2.510426 & -0.430262 \\
\hline 1 & -0.748995 & 2.488318 & 0.311960 \\
\hline 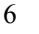 & 0.260371 & 3.950735 & -0.879060 \\
\hline & 1.513368 & 4.412986 & -1.303731 \\
\hline 1 & 2.370135 & 3.750126 & -1.245435 \\
\hline 6 & 1.666549 & 5.717508 & -1.776407 \\
\hline 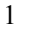 & 2.646567 & 6.064004 & -2.094241 \\
\hline 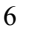 & 0.565011 & 6.575559 & -1.836308 \\
\hline 1 & 0.682549 & 7.592150 & -2.201479 \\
\hline & -0.687251 & 6.118647 & -1.417117 \\
\hline & -1.549236 & 6.779397 & -1.453428 \\
\hline & -0.840407 & 4.815951 & -0.937307 \\
\hline & -1.817646 & 4.472734 & -0.617219 \\
\hline & & & \\
\hline
\end{tabular}


$\begin{array}{lll}0.217174 & 1.977899 & -2.513474\end{array}$

$\begin{array}{llll}-1.418632 & 1.876049 & -1.869532\end{array}$

$\begin{array}{lll}1.678533 & 2.590126 & 1.491665\end{array}$

$\begin{array}{llll}1.375480 & 3.629668 & 1.342460\end{array}$

$\begin{array}{lll}0.856629 & 2.066316 & 2.667818\end{array}$

$\begin{array}{lll}-0.368606 & 2.682511 & 2.959328\end{array}$

$\begin{array}{lll}-0.714954 & 3.520945 & 2.365702\end{array}$

$\begin{array}{lll}-1.172247 & 2.227042 & 4.006996\end{array}$

$\begin{array}{lll}-2.122989 & 2.715653 & 4.198022\end{array}$

$\begin{array}{lll}-0.752990 & 1.154152 & 4.794359\end{array}$

$\begin{array}{lll}-1.373292 & 0.798138 & 5.612194\end{array}$

$\begin{array}{llll}0.475670 & 0.541934 & 4.525393\end{array}$

$\begin{array}{lll}0.815406 & -0.288855 & 5.138369\end{array}$

$\begin{array}{lll}1.273738 & 0.994209 & 3.472802\end{array}$

$\begin{array}{llll}2.222869 & 0.508760 & 3.28226\end{array}$

$\begin{array}{lll}3.186664 & 2.623459 & 1.732501\end{array}$

$\begin{array}{llll}3.694140 & 3.083922 & 0.882088\end{array}$

$\begin{array}{lll}3.619019 & 1.637426 & 1.896309\end{array}$

$\begin{array}{llll}3.383217 & 3.231977 & 2.620563\end{array}$

$\begin{array}{lll}-0.860600 & -0.843910 & 0.59777\end{array}$

$\begin{array}{lll}-0.815745 & -1.915372 & 0.739053\end{array}$

$\begin{array}{llll}-0.430767 & -0.277238 & 1.419053\end{array}$

$\begin{array}{lll}-1.966960 & -0.233170 & -0.064648\end{array}$

$\begin{array}{lll}-2.182051 & 0.793386 & 0.199342\end{array}$

$\begin{array}{lll}-2.683459 & -0.812548 & -1.116610\end{array}$

$\begin{array}{lll}-2.663002 & -1.887362 & -1.245348\end{array}$

$\begin{array}{llll}0.931834 & -0.436964 & -3.43052\end{array}$

$\begin{array}{llll}1.388265 & 0.545577 & -3.498188\end{array}$

$\begin{array}{lll}-0.450388 & -0.511080 & -3.675991\end{array}$

$\begin{array}{llll}-0.967267 & 0.414000 & -3.925713\end{array}$

$\begin{array}{lll}-1.185261 & -1.765910 & -4.105020\end{array}$

$\begin{array}{llll}-1.026916 & -1.915555 & -5.183270\end{array}$

$\begin{array}{lll}-2.252437 & -1.578570 & -3.977959\end{array}$

$\begin{array}{lll}-0.798111 & -3.053752 & -3.341248\end{array}$

$\begin{array}{llll}0.002162 & -3.580505 & -3.869492\end{array}$

$\begin{array}{lll}-1.658712 & -3.729989 & -3.343399\end{array}$

$\begin{array}{llll}-0.366369 & -2.843571 & -1.895959\end{array}$

$\begin{array}{llll}-1.059761 & -3.195416 & -1.137902\end{array}$

$\begin{array}{llll}0.933887 & -2.619735 & -1.493070\end{array}$

$\begin{array}{llll}1.163186 & -2.833101 & -0.454202\end{array}$

$\begin{array}{lll}2.130577 & -2.492252 & -2.411116\end{array}$

$2.959129 \quad-2.085404 \quad-1.830141$

$\begin{array}{llll}2.437853 & -3.499802 & -2.725958\end{array}$

$1.897348-1.601156 \quad-3.650304$

$\begin{array}{llll}1.531481 & -2.204233 & -4.486909\end{array}$

$\begin{array}{lll}2.860410 & -1.190592 & -3.966068\end{array}$

$-3.738823-0.108714-1.853815$

$\begin{array}{llll}-4.061290 & 1.243768 & -1.616495\end{array}$

$\begin{array}{llll}-4.505696 & -0.819273 & -2.799753\end{array}$

$\begin{array}{lll}-5.109654 & 1.855469 & -2.299292\end{array}$

$\begin{array}{llll}-3.528992 & 1.822900 & -0.874575\end{array}$

$\begin{array}{llll}-5.549817 & -0.201533 & -3.487831\end{array}$

$\begin{array}{llll}-4.290043 & -1.869035 & -2.978904\end{array}$

$\begin{array}{llll}-5.856307 & 1.140058 & -3.240780\end{array}$

$\begin{array}{llll}-5.341798 & 2.891907 & -2.073929\end{array}$

$\begin{array}{llll}-6.129265 & -0.770389 & -4.209483\end{array}$

$\begin{array}{llll}-6.674794 & 1.619659 & -3.769878\end{array}$

$\begin{array}{lll}-2.212068 & -4.451180 & 0.733471\end{array}$

$\begin{array}{lll}-1.288357 & -4.185535 & 1.448610\end{array}$

$-0.108438 \quad-4.210350 \quad 1.657333$

$\begin{array}{lll}-2.061391 & -3.354015 & 2.926778\end{array}$

$\begin{array}{lll}-1.140620 & -2.710906 & 3.803597\end{array}$

$\begin{array}{lll}-0.737240 & -1.785704 & 3.375626\end{array}$

$-1.638914 \quad-2.473886 \quad 4.751573$

$-0.310577 \quad-3.392605 \quad 4.004561$

$\begin{array}{lll}-3.557559 & -0.341080 & 3.079149\end{array}$

$\begin{array}{lll}-4.777184 & 0.561870 & 3.191025\end{array}$

$\begin{array}{llll}-2.793571 & 0.154344 & 2.471190\end{array}$

$\begin{array}{lll}-3.106699 & -0.518131 & 4.060929\end{array}$

$\begin{array}{lll}-5.488655 & 0.575830 & 1.847550\end{array}$

$\begin{array}{lll}-4.487832 & 1.582510 & 3.445813\end{array}$

$\begin{array}{lll}-5.456193 & 0.185687 & 3.965908\end{array}$

$\begin{array}{lll}-6.427389 & 1.132871 & 1.913269\end{array}$

$\begin{array}{lll}-4.863133 & 1.072543 & 1.099980\end{array}$

$\begin{array}{llll}-3.889438 & -1.644344 & 2.497329\end{array}$

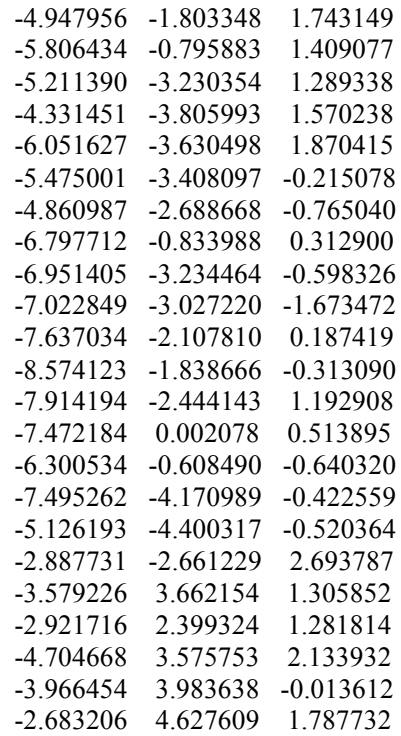

Number of imaginary frequencies $=0$

The smallest frequencies are $=16.429122 .781623 .7797 \mathrm{~cm}(-1)$ Electronic energy $=-3892.0133451$

Zero-point correction=

(Hartree/Particle)

Thermal correction to Energy= $\quad 1.304178$

Thermal correction to Enthalpy= $\quad 1.305122$

Thermal correction to Gibbs Free Energy $=\quad 1.118060$

Sum of electronic and zero-point Energies $=\quad-3890.783469$

Sum of electronic and thermal Energies $=\quad-3890.709168$

Sum of electronic and thermal Enthalpies $=\quad-3890.708223$

Sum of electronic and thermal Free Energies $=\quad-3890.895286$

Cartesian Coordinates

$77 \quad-0.181320-0.446533 \quad-1.413549$

$15 \quad \begin{array}{llll}1.619610 & 0.484387 & -0.375173\end{array}$

$\begin{array}{lll}2.302905 & -0.127847 & 0.989656\end{array}$

$2.934853 \quad 0.498729 \quad-1.394287$

$1.207884 \quad 1.996972 \quad 0.174786$

$3.150998-1.231468 \quad 0.947815$

$\begin{array}{lll}2.655579 & -2.421724 & 1.520443\end{array}$

$\begin{array}{lll}1.666667 & -2.413152 & 1.963925\end{array}$

$3.429407 \quad-3.555106 \quad 1.493367$

$3.057534 \quad-4.479123 \quad 1.924656$

$\begin{array}{llll}4.702404 & -3.543677 & 0.862242\end{array}$

$\begin{array}{lll}5.478322 & -4.730519 & 0.761188\end{array}$

$\begin{array}{llll}5.087317 & -5.643127 & 1.203251\end{array}$

$\begin{array}{llll}6.689655 & -4.730487 & 0.107715\end{array}$

$\begin{array}{llll}7.272177 & -5.644009 & 0.032611\end{array}$

$\begin{array}{llll}7.170158 & -3.537173 & -0.482581\end{array}$

$\begin{array}{llll}8.114370 & -3.545363 & -1.019480\end{array}$

$\begin{array}{llll}6.449960 & -2.365951 & -0.389714\end{array}$

$\begin{array}{llll}6.830956 & -1.465366 & -0.856263\end{array}$

$\begin{array}{llll}5.204926 & -2.321919 & 0.298307\end{array}$

$\begin{array}{llll}4.422196 & -1.117399 & 0.407456\end{array}$

$\begin{array}{llll}4.923887 & 0.202653 & -0.068436\end{array}$

$\begin{array}{lll}6.153865 & 0.774845 & 0.415869\end{array}$

$\begin{array}{lll}6.907522 & 0.191808 & 1.473020\end{array}$

$\begin{array}{lll}6.548756 & -0.715327 & 1.944437\end{array}$

$\begin{array}{lll}8.076154 & 0.771366 & 1.917254\end{array}$

$8.627629 \quad 0.310279 \quad 2.731581$

$\begin{array}{lll}8.561219 & 1.964140 & 1.329751\end{array}$

$\begin{array}{llll}9.487321 & 2.405414 & 1.686224\end{array}$

$\begin{array}{lll}7.846771 & 2.568204 & 0.320305\end{array}$

$\begin{array}{llll}8.195806 & 3.495023 & -0.127524\end{array}$

$\begin{array}{llll}6.628762 & 2.006433 & -0.150071\end{array}$

$\begin{array}{llll}5.864434 & 2.656883 & -1.155989\end{array}$

$\begin{array}{llll}6.240305 & 3.577524 & -1.592994\end{array}$ 
$\begin{array}{llll}4.650912 & 2.148408 & -1.547295\end{array}$

$\begin{array}{llll}4.034162 & 2.643142 & -2.289563\end{array}$

$\begin{array}{llll}4.184159 & 0.945972 & -0.974342\end{array}$

$\begin{array}{llll}-0.024814 & 2.539959 & -0.452966\end{array}$

$\begin{array}{lll}-0.824074 & 2.518776 & 0.293025\end{array}$

$\begin{array}{llll}0.157609 & 3.974989 & -0.927928\end{array}$

$\begin{array}{llll}1.398109 & 4.444153 & -1.381381\end{array}$

$\begin{array}{llll}2.264209 & 3.792949 & -1.324562\end{array}$

$\begin{array}{llll}1.527256 & 5.741235 & -1.880740\end{array}$

$\begin{array}{llll}2.497449 & 6.093682 & -2.221332\end{array}$

$\begin{array}{llll}0.414254 & 6.584696 & -1.938386\end{array}$

$\begin{array}{llll}0.513346 & 7.595407 & -2.324740\end{array}$

$\begin{array}{lll}-0.825179 & 6.121147 & -1.489646\end{array}$

$\begin{array}{llll}-1.695729 & 6.770660 & -1.524118\end{array}$

$\begin{array}{llll}-0.954420 & 4.825926 & -0.983026\end{array}$

$\begin{array}{llll}-1.922440 & 4.476579 & -0.641650\end{array}$

$\begin{array}{llll}-0.438571 & 1.671960 & -1.650144\end{array}$

$\begin{array}{llll}0.143703 & 1.977049 & -2.526391\end{array}$

$\begin{array}{llll}-1.489376 & 1.861673 & -1.878926\end{array}$

$\begin{array}{lll}1.620068 & 2.687539 & 1.447581\end{array}$

$\begin{array}{lll}1.315197 & 3.721852 & 1.268729\end{array}$

$\begin{array}{lll}0.808218 & 2.201615 & 2.646032\end{array}$

$\begin{array}{lll}-0.410121 & 2.832962 & 2.933590\end{array}$

$\begin{array}{lll}-0.761882 & 3.652059 & 2.316407\end{array}$

$\begin{array}{lll}-1.200616 & 2.417024 & 4.007270\end{array}$

$\begin{array}{lll}-2.146131 & 2.917016 & 4.194769\end{array}$

$\begin{array}{lll}-0.774355 & 1.369732 & 4.824584\end{array}$

$\begin{array}{lll}-1.383979 & 1.045359 & 5.663274\end{array}$

$\begin{array}{lll}0.446278 & 0.741222 & 4.557524\end{array}$

$\begin{array}{lll}0.790632 & -0.070552 & 5.192791\end{array}$

$\begin{array}{lll}1.230544 & 1.152888 & 3.478163\end{array}$

$\begin{array}{llll}2.173450 & 0.654918 & 3.288498\end{array}$

$\begin{array}{llll}3.129929 & 2.730980 & 1.676945\end{array}$

$\begin{array}{llll}3.630861 & 3.164947 & 0.808917\end{array}$

$\begin{array}{lll}3.566082 & 1.751979 & 1.870542\end{array}$

$\begin{array}{lll}3.330288 & 3.368789 & 2.543308\end{array}$

$\begin{array}{lll}-0.907679 & -0.861911 & 0.615992\end{array}$

$\begin{array}{lll}-0.904485 & -1.936592 & 0.771999\end{array}$

$\begin{array}{lll}-0.470428 & -0.302312 & 1.437801\end{array}$

$\begin{array}{lll}-2.003875 & -0.229550 & -0.041943\end{array}$

$\begin{array}{lll}-2.215889 & 0.796132 & 0.227983\end{array}$

$\begin{array}{llll}-2.720720 & -0.805937 & -1.096828\end{array}$

$-2.697888-1.880842-1.224157$

$\begin{array}{llll}0.886758 & -0.436600 & -3.412303\end{array}$

$\begin{array}{llll}1.337662 & 0.547696 & -3.488985\end{array}$

$\begin{array}{llll}-0.495603 & -0.520775 & -3.656431\end{array}$

$\begin{array}{llll}-1.017629 & 0.398495 & -3.916204\end{array}$

$\begin{array}{llll}-1.221857 & -1.784530 & -4.074380\end{array}$

$\begin{array}{llll}-1.058517 & -1.947660 & -5.149847\end{array}$

$\begin{array}{lll}-2.290721 & -1.602752 & -3.952709\end{array}$

$\begin{array}{lll}-0.826900 & -3.056372 & -3.289455\end{array}$

$\begin{array}{llll}-0.023281 & -3.588047 & -3.807308\end{array}$

$\begin{array}{lll}-1.682781 & -3.738513 & -3.276991\end{array}$

$\begin{array}{llll}-0.397365 & -2.810616 & -1.848812\end{array}$

$\begin{array}{llll}-1.092920 & -3.144708 & -1.086573\end{array}$

$\begin{array}{llll}0.904853 & -2.581296 & -1.446435\end{array}$

$\begin{array}{llll}1.140522 & -2.779595 & -0.406128\end{array}$

$\begin{array}{llll}2.099869 & -2.469536 & -2.369012\end{array}$

$\begin{array}{llll}2.928340 & -2.051083 & -1.796294\end{array}$

$\begin{array}{llll}2.408261 & -3.481379 & -2.668790\end{array}$

$\begin{array}{llll}1.859384 & -1.597407 & -3.620294\end{array}$

$\begin{array}{llll}1.495957 & -2.214220 & -4.447855\end{array}$

$\begin{array}{llll}2.819138 & -1.184987 & -3.943477\end{array}$

$-3.784520 \quad-0.110926-1.828564$

$\begin{array}{llll}-4.115312 & 1.239206 & -1.590633\end{array}$

$\begin{array}{llll}-4.555185 & -0.830665 & -2.764514\end{array}$

$\begin{array}{llll}-5.174126 & 1.841297 & -2.265776\end{array}$

$\begin{array}{llll}-3.582010 & 1.822087 & -0.852697\end{array}$

$\begin{array}{llll}-5.610826 & -0.223030 & -3.443651\end{array}$

$-4.333824-1.879682-2.941788$

$\begin{array}{llll}-5.924087 & 1.117361 & -3.198051\end{array}$

$\begin{array}{llll}-5.412053 & 2.876339 & -2.040365\end{array}$

$-6.193909-0.798356-4.157242$

$\begin{array}{lll}-6.751285 & 1.589187 & -3.720595\end{array}$

$\begin{array}{lll}-1.755123 & -5.120403 & 0.426435\end{array}$

$\begin{array}{lll}-0.715275 & -4.961725 & 0.940901\end{array}$

$\begin{array}{llll}0.370806 & -4.887938 & 1.372083\end{array}$

$\begin{array}{lll}-1.599183 & -3.291514 & 2.643583\end{array}$

$\begin{array}{lll}-0.705665 & -2.775168 & 3.607652\end{array}$

$\begin{array}{lll}-0.334679 & -1.770375 & 3.354515\end{array}$

$\begin{array}{lll}-1.157516 & -2.717057 & 4.611496\end{array}$

$\begin{array}{lll}0.158133 & -3.446480 & 3.672405\end{array}$

$\begin{array}{lll}-3.481119 & -0.323785 & 3.167058\end{array}$

$\begin{array}{lll}-4.738806 & 0.528776 & 3.277615\end{array}$

$\begin{array}{lll}-2.739567 & 0.204082 & 2.556819\end{array}$

$\begin{array}{lll}-3.016421 & -0.470916 & 4.149020\end{array}$

$\begin{array}{lll}-5.464670 & 0.486773 & 1.940802\end{array}$

$\begin{array}{lll}-4.494879 & 1.565981 & 3.515342\end{array}$

$\begin{array}{llll}-5.394541 & 0.135570 & 4.064406\end{array}$

$\begin{array}{lll}-6.423193 & 1.010130 & 2.004965\end{array}$

$\begin{array}{lll}-4.864904 & 0.994625 & 1.178197\end{array}$

$\begin{array}{lll}-3.739574 & -1.643633 & 2.592423\end{array}$

$\begin{array}{lll}-4.791652 & -1.856667 & 1.854563\end{array}$

$\begin{array}{lll}-5.732264 & -0.904888 & 1.537565\end{array}$

$\begin{array}{lll}-4.962159 & -3.297868 & 1.387490\end{array}$

$\begin{array}{lll}-4.022763 & -3.797213 & 1.629231\end{array}$

$\begin{array}{lll}-5.732774 & -3.779427 & 2.003620\end{array}$

$\begin{array}{llll}-5.290136 & -3.494063 & -0.102000\end{array}$

$\begin{array}{llll}-4.767791 & -2.728353 & -0.683155\end{array}$

$\begin{array}{lll}-6.736836 & -1.013202 & 0.463003\end{array}$

$\begin{array}{lll}-6.793340 & -3.433688 & -0.405902\end{array}$

$\begin{array}{llll}-6.936155 & -3.262622 & -1.480379\end{array}$

$\begin{array}{llll}-7.510629 & -2.333313 & 0.389833\end{array}$

$\begin{array}{llll}-8.482255 & -2.128631 & -0.074081\end{array}$

$\begin{array}{lll}-7.724233 & -2.666972 & 1.411764\end{array}$

$\begin{array}{lll}-7.450815 & -0.208747 & 0.659020\end{array}$

$\begin{array}{llll}-6.277633 & -0.786004 & -0.509837\end{array}$

$\begin{array}{llll}-7.259115 & -4.400502 & -0.177405\end{array}$

$\begin{array}{lll}-4.886242 & -4.457584 & -0.432379\end{array}$

$\begin{array}{lll}-2.419194 & -2.707264 & 2.627092\end{array}$

$\begin{array}{lll}-3.676162 & 3.666521 & 1.299164\end{array}$

$\begin{array}{lll}-2.976343 & 2.427251 & 1.266092\end{array}$

$\begin{array}{llll}-4.808726 & 3.531961 & 2.110381\end{array}$

$\begin{array}{llll}-4.057470 & 3.993958 & -0.021233\end{array}$

$\begin{array}{llll}-2.818172 & 4.654406 & 1.804741\end{array}$

[13'-14'] P1-endo-re $\left[\mathrm{BF}_{4}^{-}\right]$conf2

Number of imaginary frequencies $=1$

The smallest frequencies are $=-854.340811 .937417 .3799 \mathrm{~cm}(-1)$

Electronic energy $=-3892.0000937$

Zero-point correction=

1.226904

Hartree/Particle)

Thermal correction to Energy= $\quad 1.300235$

Thermal correction to Enthalpy=

1.301179

Thermal correction to Gibbs Free Energy=

Sum of electronic and zero-point Energies=

Sum of electronic and thermal Energies=

Sum of electronic and thermal Enthalpies=

1.115254

$-3890.773190$

$-3890.699859$

$-3890.698915$

Sum of electronic and thermal Free Energies $=\quad-3890.884840$

Cartesian Coordinates

$\begin{array}{lccc}77 & 0.135427 & -0.708930 & 1.820428 \\ 15 & 1.666294 & -0.678064 & 0.128314 \\ 8 & 1.914555 & 0.612670 & -0.858768 \\ 8 & 3.201072 & -0.885413 & 0.735623 \\ 7 & 1.252412 & -1.812919 & -1.012209 \\ 6 & 2.572785 & 1.748508 & -0.395881 \\ 6 & 1.778641 & 2.901532 & -0.223943 \\ 1 & 0.720527 & 2.843628 & -0.461012 \\ 6 & 2.362563 & 4.049659 & 0.252436 \\ 1 & 1.764024 & 4.945267 & 0.388756 \\ 6 & 3.739032 & 4.064351 & 0.608550 \\ 6 & 4.333290 & 5.222513 & 1.179581 \\ 1 & 3.716173 & 6.107203 & 1.313040 \\ 6 & 5.653795 & 5.222936 & 1.568645 \\ 1 & 6.095390 & 6.112212 & 2.008858 \\ 6 & 6.434716 & 4.053429 & 1.408792 \\ 1 & 7.469499 & 4.048445 & 1.739121\end{array}$


$\begin{array}{llll}5.894290 & 2.918297 & 0.844144\end{array}$

$\begin{array}{lll}6.505012 & 2.029422 & 0.738368\end{array}$

$\begin{array}{llll}4.539809 & 2.887941 & 0.409497\end{array}$

$\begin{array}{llll}3.940131 & 1.716096 & -0.176162\end{array}$

$\begin{array}{llll}4.726619 & 0.497241 & -0.520807\end{array}$

$\begin{array}{llll}5.864074 & 0.548257 & -1.404460\end{array}$

$\begin{array}{llll}6.242263 & 1.730207 & -2.101642\end{array}$

$\begin{array}{llll}5.655642 & 2.632275 & -1.976425\end{array}$

$\begin{array}{llll}7.330579 & 1.741206 & -2.946952\end{array}$

$\begin{array}{llll}7.591361 & 2.654915 & -3.473386\end{array}$

$\begin{array}{llll}8.106551 & 0.574097 & -3.143016\end{array}$

$\begin{array}{llll}8.965492 & 0.599941 & -3.807170\end{array}$

$\begin{array}{llll}7.756694 & -0.593379 & -2.503630\end{array}$

$\begin{array}{llll}8.329168 & -1.503860 & -2.660761\end{array}$

$\begin{array}{llll}6.629507 & -0.644198 & -1.639201\end{array}$

$6.233476-1.864504 \quad-1.028109$

$\begin{array}{llll}6.825022 & -2.758828 & -1.201971\end{array}$

$\begin{array}{llll}5.096718 & -1.921694 & -0.261293\end{array}$

$\begin{array}{llll}4.748722 & -2.848454 & 0.182669\end{array}$

$\begin{array}{llll}4.345691 & -0.747015 & -0.041133\end{array}$

$\begin{array}{llll}0.219806 & -2.775243 & -0.548009\end{array}$

$\begin{array}{llll}-0.727699 & -2.516792 & -1.028931\end{array}$

$0.573298-4.207928-0.917415$

$\begin{array}{llll}1.898995 & -4.663586 & -0.873338\end{array}$

$\begin{array}{llll}2.694218 & -3.963268 & -0.632393\end{array}$

$\begin{array}{llll}2.199179 & -5.996512 & -1.156323\end{array}$

$3.231422-6.335560 \quad-1.127076$

$\begin{array}{llll}1.175742 & -6.892893 & -1.480189\end{array}$

$\begin{array}{llll}1.408831 & -7.930469 & -1.703084\end{array}$

$-0.146830 \quad-6.445220-1.519658$

$\begin{array}{llll}-0.948551 & -7.133407 & -1.773584\end{array}$

$-0.448525 \quad-5.108953-1.243392$

$\begin{array}{lll}-1.479935 & -4.770629 & -1.272222\end{array}$

$\begin{array}{llll}0.055841 & -2.681247 & 0.974765\end{array}$

$\begin{array}{lll}0.846078 & -3.273766 & 1.447955\end{array}$

$\begin{array}{lll}-0.897619 & -3.132127 & 1.257346\end{array}$

$\begin{array}{llll}1.438009 & -1.760603 & -2.504881\end{array}$

$\begin{array}{llll}1.268614 & -2.798227 & -2.803869\end{array}$

$\begin{array}{llll}0.354586 & -0.935419 & -3.195511\end{array}$

$\begin{array}{llll}-0.835300 & -1.572000 & -3.573302\end{array}$

$\begin{array}{lll}-0.981964 & -2.625461 & -3.363564\end{array}$

$\begin{array}{llll}-1.858564 & -0.869124 & -4.212024\end{array}$

$-2.772933-1.393880 \quad-4.467144$

$\begin{array}{llll}-1.698346 & 0.486387 & -4.500549\end{array}$

$\begin{array}{llll}-2.489345 & 1.038056 & -5.001344\end{array}$

$\begin{array}{llll}-0.512599 & 1.132678 & -4.136258\end{array}$

$\begin{array}{llll}-0.377177 & 2.187268 & -4.357482\end{array}$

$\begin{array}{llll}0.504126 & 0.429460 & -3.488032\end{array}$

$\begin{array}{llll}1.415152 & 0.947532 & -3.214528\end{array}$

$\begin{array}{llll}2.861637 & -1.428420 & -2.947338\end{array}$

$\begin{array}{llll}3.570227 & -2.124168 & -2.493618\end{array}$

$\begin{array}{llll}3.167768 & -0.414183 & -2.694139\end{array}$

$\begin{array}{llll}2.920811 & -1.538481 & -4.034457\end{array}$

$\begin{array}{lll}-1.079597 & 0.537576 & 0.474133\end{array}$

$\begin{array}{lll}-1.168798 & 1.516523 & 0.929054\end{array}$

$\begin{array}{llll}-0.806826 & 0.575396 & -0.575459\end{array}$

$\begin{array}{lll}-1.936107 & -0.529511 & 0.866441\end{array}$

$\begin{array}{llll}-2.134132 & -1.297630 & 0.132136\end{array}$

$\begin{array}{lll}-2.420514 & -0.706636 & 2.168992\end{array}$

$\begin{array}{lll}-2.445690 & 0.139438 & 2.846327\end{array}$

$\begin{array}{lll}1.665766 & -1.515691 & 3.276171\end{array}$

$\begin{array}{llll}2.174939 & -2.315379 & 2.747056\end{array}$

$\begin{array}{llll}0.384086 & -1.807481 & 3.778871\end{array}$

$\begin{array}{lll}0.000007 & -2.813138 & 3.616409\end{array}$

$\begin{array}{lll}-0.287136 & -1.069699 & 4.920195\end{array}$

$\begin{array}{llll}0.123551 & -1.437280 & 5.872306\end{array}$

$\begin{array}{lll}-1.341944 & -1.350580 & 4.914258\end{array}$

$\begin{array}{lll}-0.169221 & 0.469315 & 4.858512\end{array}$

$\begin{array}{llll}0.711506 & 0.805784 & 5.413814\end{array}$

$\begin{array}{lll}-1.034075 & 0.906799 & 5.366894\end{array}$

$\begin{array}{lll}-0.103345 & 1.045594 & 3.449790\end{array}$

$\begin{array}{lll}-0.973136 & 1.605112 & 3.117807\end{array}$

$\begin{array}{lll}1.072380 & 1.277980 & 2.759067\end{array}$

$\begin{array}{lll}1.031108 & 2.007248 & 1.956996\end{array}$

$\begin{array}{lll}2.461492 & 0.945245 & 3.259330\end{array}$ $\begin{array}{lll}3.150497 & 1.034140 & 2.419064\end{array}$

$\begin{array}{lll}2.762281 & 1.708724 & 3.991023\end{array}$

$\begin{array}{lll}2.596844 & -0.463190 & 3.877507\end{array}$

$\begin{array}{llll}2.425522 & -0.423088 & 4.957802\end{array}$

$\begin{array}{lll}3.627434 & -0.802963 & 3.742983\end{array}$

$\begin{array}{lll}-3.203603 & -1.876329 & 2.584633\end{array}$

$\begin{array}{lll}-3.480581 & -2.953900 & 1.716833\end{array}$

$\begin{array}{lll}-3.729835 & -1.921548 & 3.891242\end{array}$

$-4.233895 \quad-4.040855 \quad 2.152485$

$\begin{array}{lll}-3.147496 & -2.940848 & 0.687805\end{array}$

$\begin{array}{lll}-4.476847 & -3.015303 & 4.326584\end{array}$

$\begin{array}{lll}-3.558061 & -1.083904 & 4.562568\end{array}$

$\begin{array}{lll}-4.726856 & -4.084020 & 3.460490\end{array}$

$\begin{array}{lll}-4.442516 & -4.844460 & 1.452603\end{array}$

$\begin{array}{lll}-4.870312 & -3.029920 & 5.339192\end{array}$

$\begin{array}{lll}-5.312850 & -4.934502 & 3.797274\end{array}$

$\begin{array}{llll}-1.634933 & 3.617990 & 1.637192\end{array}$

$\begin{array}{llll}-1.226930 & 4.383406 & 0.820042\end{array}$

$\begin{array}{lll}-0.758910 & 5.417468 & 0.464586\end{array}$

$\begin{array}{llll}-1.456066 & 3.344332 & -0.871569\end{array}$

$\begin{array}{llll}-1.167208 & 4.046392 & -2.064076\end{array}$

$\begin{array}{llll}-0.139493 & 3.848050 & -2.398038\end{array}$

$\begin{array}{llll}-1.851501 & 3.734904 & -2.865998\end{array}$

$\begin{array}{llll}-1.268821 & 5.131945 & -1.927282\end{array}$

$\begin{array}{llll}-3.741828 & 0.884598 & -1.543630\end{array}$

$\begin{array}{llll}-4.883815 & 0.582378 & -2.500075\end{array}$

$\begin{array}{llll}-3.871204 & 0.301545 & -0.625459\end{array}$

$-2.779153 \quad 0.600084-1.969186$

$\begin{array}{llll}-6.167411 & 1.141863 & -1.905647\end{array}$

$\begin{array}{llll}-4.985232 & -0.495196 & -2.645456\end{array}$

$\begin{array}{llll}-4.688453 & 1.048152 & -3.473115\end{array}$

$\begin{array}{llll}-6.985055 & 1.084134 & -2.630465\end{array}$

$\begin{array}{llll}-6.466901 & 0.557954 & -1.024277\end{array}$

$\begin{array}{llll}-3.694586 & 2.310279 & -1.214157\end{array}$

$\begin{array}{llll}-4.782365 & 3.040916 & -1.202116\end{array}$

$\begin{array}{llll}-6.013730 & 2.560958 & -1.524277\end{array}$

$\begin{array}{llll}-4.579211 & 4.507904 & -0.855542\end{array}$

$\begin{array}{llll}-3.553519 & 4.593198 & -0.501362\end{array}$

$\begin{array}{llll}-4.633824 & 5.095964 & -1.779848\end{array}$

$\begin{array}{llll}-5.541659 & 5.085814 & 0.196964\end{array}$

$\begin{array}{llll}-5.764397 & 4.313404 & 0.942499\end{array}$

$\begin{array}{llll}-7.300253 & 3.222746 & -1.219498\end{array}$

$\begin{array}{llll}-6.841935 & 5.624107 & -0.415858\end{array}$

$\begin{array}{lll}-7.595135 & 5.732842 & 0.374000\end{array}$

$\begin{array}{lll}-7.387396 & 4.719801 & -1.531257\end{array}$

$\begin{array}{llll}-8.440970 & 4.961602 & -1.710460\end{array}$

$\begin{array}{lll}-6.866930 & 4.907188 & -2.477276\end{array}$

$\begin{array}{lll}-8.039254 & 2.700415 & -1.831283\end{array}$

$\begin{array}{lll}-7.571970 & 3.031840 & -0.171378\end{array}$

$\begin{array}{lll}-6.670410 & 6.627093 & -0.825577\end{array}$

$\begin{array}{llll}-5.029673 & 5.888471 & 0.738190\end{array}$

$\begin{array}{lll}-2.525170 & 2.845943 & -0.983308\end{array}$

$-3.767455-3.230183-2.075601$

$-3.045470-2.140615-1.521355$

$-5.093778-2.834585-2.295773$

$-3.723311-4.304783-1.159353$

$-3.165115-3.616097-3.283483$

13' P1-exo-si $\left[\mathrm{BF}_{4}^{-}\right]$

Number of imaginary frequencies $=0$

The smallest frequencies are $=19.919324 .367225 .2023 \mathrm{~cm}(-1)$ Electronic energy $=-3892.0316167$

Zero-point correction $=$

1.234459

(Hartree/Particle)

Thermal correction to Energy $=\quad 1.307014$

Thermal correction to Enthalpy= $\quad 1.307958$

Thermal correction to Gibbs Free Energy $=\quad 1.126722$

Sum of electronic and zero-point Energies $=\quad-3890.797158$

Sum of electronic and thermal Energies=

Sum of electronic and thermal Enthalpies=

$-3890.724602$

Sum of electronic and thermal Free Energies $=\quad-3890.904895$

Cartesian Coordinates 
[13'-14'] P1-exo-si $\left[\mathrm{BF}_{4}^{-}\right]$

Number of imaginary frequencies $=1$

The smallest frequencies are $=-717.006718 .754720 .4633 \mathrm{~cm}(-1)$ Electronic energy $=-3892.0043517$

Zero-point correction= 1.226309

(Hartree/Particle)

Thermal correction to Energy= $\quad 1.299755$

Thermal correction to Enthalpy= $\quad 1.300700$

Thermal correction to Gibbs Free Energy $=\quad 1.116160$

Sum of electronic and zero-point Energies $=\quad-3890.778043$

Sum of electronic and thermal Energies $=\quad-3890.704596$

Sum of electronic and thermal Enthalpies $=\quad-3890.703652$

Sum of electronic and thermal Free Energies $=\quad-3890.888191$

Cartesian Coordinates

\begin{tabular}{|c|c|c|c|}
\hline 77 & -0.273723 & 0.148656 & \\
\hline 15 & 1.591792 & 0.637457 & -0.3465 \\
\hline 8 & 2.150735 & -0.225131 & 0.936995 \\
\hline 0 & 2.922443 & 0.575770 & -1.344915 \\
\hline 7 & 1.404121 & 2.117745 & 0.382090 \\
\hline 6 & 2.743375 & -1.471222 & 0.757825 \\
\hline 6 & 2.010980 & -2.586787 & 1.216531 \\
\hline 1 & 1.056536 & -2.418651 & 1.704881 \\
\hline 6 & 2.524349 & -3.847018 & 1.031351 \\
\hline 1 & 1.969207 & -4.713908 & 1.374875 \\
\hline 6 & 3.757513 & -4.030523 & 0.348537 \\
\hline 6 & 4.250035 & -5.334010 & 0.067643 \\
\hline 1 & 3.674556 & -6.189139 & 0.412450 \\
\hline 6 & 5.418051 & -5.512776 & -0.638549 \\
\hline 1 & 5.781093 & -6.513570 & -0.853543 \\
\hline 6 & 6.140025 & -4.386451 & -1.100657 \\
\hline 1 & 7.047994 & -4.530187 & -1.679417 \\
\hline 6 & 5.699967 & -3.108818 & -0.829715 \\
\hline 1 & 6.260198 & -2.258722 & -1.200813 \\
\hline 6 & 4.508160 & -2.885510 & -0.085305 \\
\hline 6 & 4.008255 & -1.564638 & 0.198841 \\
\hline 6 & 4.786141 & -0.332960 & -0.115151 \\
\hline 6 & 6.113494 & -0.117814 & 0.403923 \\
\hline 6 & 6.721697 & -0.9 & 1.344018 \\
\hline 1 & 6.170823 & -1.8 & 1.698963 \\
\hline 6 & 7.992579 & -0.759872 & 1.821546 \\
\hline 1 & 8.429775 & -1.4 & 946 \\
\hline 6 & 8.730705 & 0.366696 & 1.387080 \\
\hline 1 & 9.734134 & 0.535958 & 1.766550 \\
\hline 6 & 8.16 & 1.25 & 0.497949 \\
\hline 1 & 8.710678 & 2.13 & 0.171066 \\
\hline 6 & 6.8 & 73 & 1782 \\
\hline 6 & 6.2 & 277 & 8795 \\
\hline 1 & 6.815210 & 2.857919 & -1.192689 \\
\hline 6 & 4.9 & 19 & 6532 \\
\hline 1 & 4.456737 & 2.539323 & -1.938370 \\
\hline 6 & 4.229144 & 0.675730 & -0.886515 \\
\hline 6 & 0.26 & 2.913599 & -0.133193 \\
\hline 1 & -0.518376 & 2.926341 & 0.632589 \\
\hline 6 & 0.686843 & 4.343169 & -0.4 \\
\hline 6 & 1.894330 & 4.598110 & -1.102403 \\
\hline 1 & 2.544935 & 3.766037 & -1.354988 \\
\hline 6 & & & 774 \\
\hline 1 & 3.207369 & 6.086299 & -1.936369 \\
\hline 6 & 1.432663 & 6.973470 & -1.082980 \\
\hline 1 & 1.721551 & 7.991147 & -1.331139 \\
\hline 6 & 0.230933 & 6.726089 & -0.416729 \\
\hline 1 & -0.419528 & 7.552385 & -0.142357 \\
\hline 6 & -0.140986 & 5.418089 & -0.090137 \\
\hline 1 & -1.071218 & 5.233005 & 0.437233 \\
\hline 6 & -0.321825 & 2.273255 & -1.401510 \\
\hline 1 & 0.246999 & 2.637237 & -2.263826 \\
\hline 1 & -1.346351 & 2.628077 & -1.518182 \\
\hline 6 & 1.941117 & 2.586552 & 1.705056 \\
\hline 1 & 1.706464 & 3.654524 & 1.693944 \\
\hline 6 & 1.178270 & 1.989037 & 2.887103 \\
\hline 6 & -0.043982 & 2.561226 & 3.273126 \\
\hline 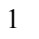 & -0.440230 & 3.418374 & 2.738584 \\
\hline
\end{tabular}

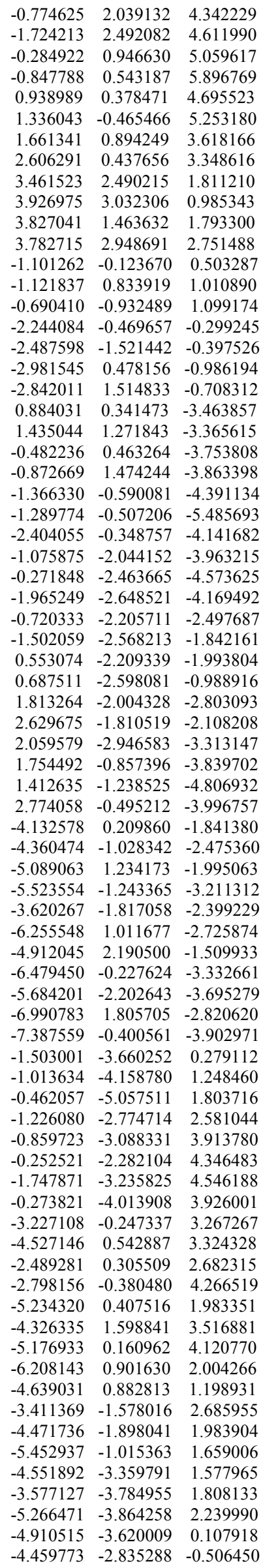




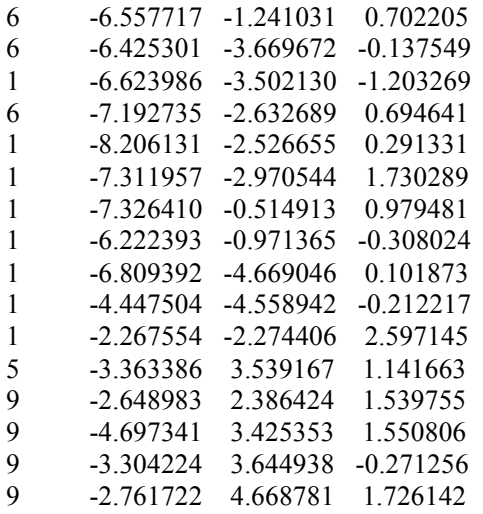

14 ' P1-exo-si $\left[\mathrm{BF}_{4}^{-}\right]$

Number of imaginary frequencies $=0$

The smallest frequencies are $=16.5970 \quad 21.3308 \quad 24.2483 \mathrm{~cm}(-$ 1)

Electronic energy $=-3892.0103178$

Zero-point correction=

(Hartree/Particle)

Thermal correction to Energy= 1.303960

Thermal correction to Enthalpy= $\quad 1.304904$

Thermal correction to Gibbs Free Energy= $\quad 1.116339$

Sum of electronic and zero-point Energies $=\quad-3890.781047$

Sum of electronic and thermal Energies $=\quad-3890.706358$

Sum of electronic and thermal Enthalpies $=\quad-3890.705414$

Sum of electronic and thermal Free Energies=

Cartesian Coordinates

\begin{tabular}{|c|c|c|c|}
\hline 77 & -0.299340 & 0.181699 & -1.493650 \\
\hline 15 & 1.577225 & 0.649564 & -0.344934 \\
\hline 8 & 2.134101 & -0.239018 & 0.920710 \\
\hline 8 & 2.902964 & 0.594215 & -1.350631 \\
\hline 7 & 1.401718 & 2.120693 & 0.404008 \\
\hline 6 & 2.739270 & -1.476329 & 0.726338 \\
\hline 6 & 2.015114 & -2.604908 & 1.169006 \\
\hline 1 & 1.049935 & -2.453560 & 1.644479 \\
\hline 6 & 2.551386 & -3.855806 & 0.981904 \\
\hline 1 & 2.017366 & -4.736920 & 1.324243 \\
\hline 6 & 3.792600 & -4.019389 & 0.308417 \\
\hline 6 & 4.307809 & -5.314047 & 0.027712 \\
\hline 1 & 3.744705 & -6.179650 & 0.366942 \\
\hline 6 & 5.483052 & -5.472065 & -0.671457 \\
\hline 1 & 5.863918 & -6.466263 & -0.886131 \\
\hline 6 & 6.189254 & -4.333117 & -1.126744 \\
\hline 1 & 7.102709 & -4.460589 & -1.700591 \\
\hline 6 & 5.727254 & -3.063524 & -0.854647 \\
\hline 1 & 6.276390 & -2.203619 & -1.219507 \\
\hline 6 & 4.528127 & -2.861359 & -0.115977 \\
\hline 6 & & -1.549230 & \\
\hline 6 & 4.774586 & -0.306798 & -0.127253 \\
\hline 6 & 6.098456 & -0.081382 & 0.396534 \\
\hline 6 & 6.710968 & -0.95 & 1.337105 \\
\hline 1 & 6.166530 & -1.824878 & 1.688043 \\
\hline 6 & 7.977457 & -0.708715 & 1.820707 \\
\hline 1 & 8.417859 & -1.389105 & 2.543908 \\
\hline 6 & 8.706923 & 0.425490 & 1.391563 \\
\hline 1 & 9.707001 & 0.603522 & 1.775807 \\
\hline 6 & 8.136714 & 1.307060 & 0.501574 \\
\hline 1 & 8.675510 & 2.194065 & 0.178627 \\
\hline 6 & 6.825975 & 1.090267 & -0.003730 \\
\hline 6 & 6.217426 & 2.027602 & -0.881159 \\
\hline 1 & 6.779977 & 2.903065 & -1.192571 \\
\hline 6 & 4.923579 & 1.849053 & -1.301606 \\
\hline 1 & 4.426180 & 2.567796 & -1.943571 \\
\hline 6 & 4.210112 & 0.700712 & -0.894695 \\
\hline 6 & 0.254567 & 2.926295 & -0.090396 \\
\hline 1 & -0.512888 & 2.937452 & 0.686493 \\
\hline
\end{tabular}

$\begin{array}{lll}0.681769 & 4.356588 & -0.386741\end{array}$

$\begin{array}{llll}1.889313 & 4.615658 & -1.052610\end{array}$

$\begin{array}{llll}2.542116 & 3.785535 & -1.305177\end{array}$

$\begin{array}{llll}2.258068 & 5.921893 & -1.372809\end{array}$

$\begin{array}{lll}3.199093 & 6.108338 & -1.883916\end{array}$

$\begin{array}{lll}1.420218 & 6.989632 & -1.033317\end{array}$

$\begin{array}{llll}1.706070 & 8.008312 & -1.280897\end{array}$

$\begin{array}{llll}0.217694 & 6.738002 & -0.369912\end{array}$

$-0.436769 \quad 7.561872-0.097751$

$\begin{array}{lll}-0.150519 & 5.428810 & -0.044340\end{array}$

$\begin{array}{lll}-1.084171 & 5.241239 & 0.476362\end{array}$

$\begin{array}{llll}-0.353630 & 2.301921 & -1.355919\end{array}$

$\begin{array}{llll}0.191895 & 2.682115 & -2.226031\end{array}$

$\begin{array}{llll}-1.382153 & 2.653888 & -1.439961\end{array}$

$\begin{array}{lll}1.943676 & 2.568138 & 1.733233\end{array}$

$\begin{array}{lll}1.719731 & 3.638466 & 1.734367\end{array}$

$\begin{array}{lll}1.174272 & 1.962995 & 2.906918\end{array}$

$\begin{array}{lll}-0.027634 & 2.560247 & 3.317882\end{array}$

$\begin{array}{lll}-0.398264 & 3.444336 & 2.808784\end{array}$

$\begin{array}{lll}-0.766308 & 2.031404 & 4.378049\end{array}$

$\begin{array}{lll}-1.700179 & 2.504134 & 4.667969\end{array}$

$\begin{array}{lll}-0.306365 & 0.902956 & 5.058484\end{array}$

$\begin{array}{lll}-0.877258 & 0.491690 & 5.886298\end{array}$

$\begin{array}{llll}0.896421 & 0.308261 & 4.668989\end{array}$

$\begin{array}{lll}1.266525 & -0.568020 & 5.193970\end{array}$

$\begin{array}{lll}1.629262 & 0.834561 & 3.604178\end{array}$

$\begin{array}{lll}2.558881 & 0.358713 & 3.316226\end{array}$

$\begin{array}{llll}3.462664 & 2.455099 & 1.840910\end{array}$

$\begin{array}{lll}3.935910 & 2.998261 & 1.020245\end{array}$

$\begin{array}{llll}3.817527 & 1.425027 & 1.816868\end{array}$

$\begin{array}{lll}3.786032 & 2.903347 & 2.785367\end{array}$

$\begin{array}{lll}-1.121113 & -0.078295 & 0.523744\end{array}$

$\begin{array}{llll}-1.167099 & 0.889121 & 1.010877\end{array}$

$\begin{array}{lll}-0.685613 & -0.858960 & 1.138933\end{array}$

$\begin{array}{llll}-2.252687 & -0.460320 & -0.276138\end{array}$

$\begin{array}{llll}2.479131 & -1.517177 & -0.367007\end{array}$

$\begin{array}{llll}-3.005670 & 0.464610 & -0.979112\end{array}$

$\begin{array}{llll}-2.884674 & 1.506455 & -0.712841\end{array}$

$\begin{array}{llll}0.849760 & 0.396867 & -3.455840\end{array}$

$\begin{array}{lll}1.395759 & 1.329734 & -3.352710\end{array}$

$\begin{array}{lll}-0.517793 & 0.512463 & -3.740317\end{array}$

$\begin{array}{llll}-0.915222 & 1.521978 & -3.838013\end{array}$

$\begin{array}{llll}-1.398143 & -0.540935 & -4.382246\end{array}$

$\begin{array}{llll}-1.326412 & -0.449822 & -5.476394\end{array}$

$\begin{array}{llll}-2.436094 & -0.308759 & -4.126053\end{array}$

$\begin{array}{lll}-1.097000 & -1.996296 & -3.966341\end{array}$

$-0.291872 \quad-2.406336 \quad-4.581650$

$-1.983170-2.604743 \quad-4.174173$

$-0.736018-2.165411-2.503136$

$\begin{array}{lll}-1.516458 & -2.535093 & -1.850951\end{array}$

$\begin{array}{llll}0.538905 & -2.167421 & -2.003869\end{array}$

$\begin{array}{llll}0.683711 & -2.564120 & -1.003606\end{array}$

$\begin{array}{llll}1.792190 & -1.953976 & -2.822217\end{array}$

$\begin{array}{llll}2.615958 & -1.771187 & -2.133209\end{array}$

$\begin{array}{llll}2.030607 & -2.890188 & -3.346943\end{array}$

$\begin{array}{lll}1.725811 & -0.794117 & -3.844723\end{array}$

$\begin{array}{llll}1.384834 & -1.165829 & -4.815935\end{array}$

$\begin{array}{lll}2.743316 & -0.425157 & -3.998389\end{array}$

$\begin{array}{lll}-4.151465 & 0.167052 & -1.832174\end{array}$

$\begin{array}{llll}-4.360787 & -1.081348 & -2.452228\end{array}$

$\begin{array}{llll}-5.123187 & 1.174966 & -1.998235\end{array}$

$\begin{array}{llll}-5.518355 & -1.321261 & -3.189081\end{array}$

$\begin{array}{llll}-3.611477 & -1.859534 & -2.365233\end{array}$

$\begin{array}{lll}-6.284700 & 0.927152 & -2.728701\end{array}$

$\begin{array}{llll}-4.962008 & 2.138673 & -1.522606\end{array}$

$\begin{array}{lll}-6.488629 & -0.321192 & -3.323659\end{array}$

$\begin{array}{llll}-5.664162 & -2.288373 & -3.662138\end{array}$

$\begin{array}{llll}-7.031849 & 1.708826 & -2.832404\end{array}$

$\begin{array}{lll}-7.392837 & -0.513603 & -3.893960\end{array}$

$\begin{array}{lll}-1.382800 & -3.971608 & 0.100605\end{array}$

$\begin{array}{lll}-0.926555 & -4.605158 & 0.973726\end{array}$

$\begin{array}{lll}-0.469711 & -5.340939 & 1.760713\end{array}$

$\begin{array}{lll}-1.001009 & -2.650896 & 2.625964\end{array}$

$\begin{array}{lll}-0.630964 & -3.014031 & 3.944041\end{array}$

$\begin{array}{lll}-0.752510 & -2.180500 & 4.649903\end{array}$ 


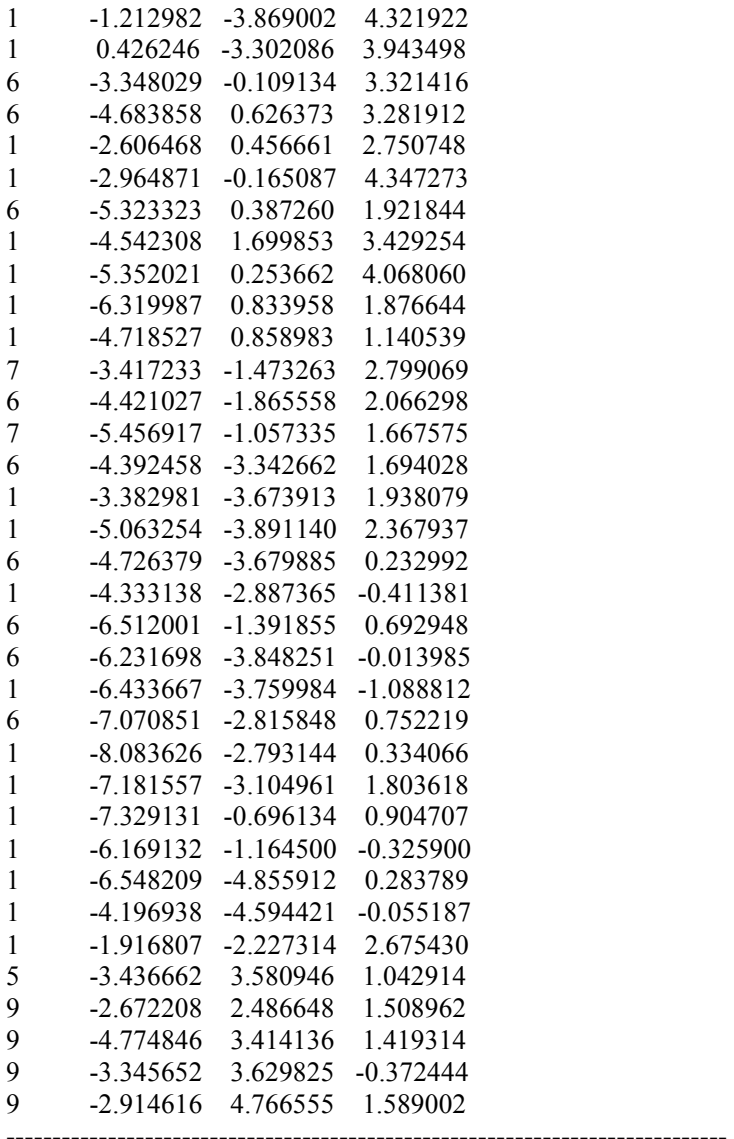

13' P1-endo-re

Number of imaginary frequencies $=0$

The smallest frequencies $=10.148820 .247923 .6708 \mathrm{~cm}(-1)$

Electronic energy $=-3467.4038844$

Zero-point correction=

(Hartree/Particle)

Thermal correction to Energy= $\quad 1.28435$

Thermal correction to Enthalpy=

1.285298

Thermal correction to Gibbs Free Energy=

Sum of electronic and zero-point Energies=

Sum of electronic and thermal Energies=

Sum of electronic and thermal Enthalpies=

Sum of electronic and thermal Free Energies=

1.113934

$-3466.186414$

$-3466.119531$

$-3466.118587$

$-3466.289950$

Cartesian Coordinates

$\begin{array}{lccc}77 & -0.447445 & -0.024230 & -1.344821 \\ 15 & 1.498812 & 0.491171 & -0.316612 \\ 8 & 2.139399 & -0.406906 & 0.895279 \\ 8 & 2.760743 & 0.534027 & -1.390018 \\ 7 & 1.309158 & 1.958447 & 0.464038 \\ 6 & 2.940335 & -1.527681 & 0.660492 \\ 6 & 2.400032 & -2.768088 & 1.057156 \\ 1 & 1.406738 & -2.805767 & 1.492409 \\ 6 & 3.129316 & -3.911429 & 0.841845 \\ 1 & 2.726343 & -4.876015 & 1.136914 \\ 6 & 4.394264 & -3.856714 & 0.197565 \\ 6 & 5.120265 & -5.044102 & -0.092297 \\ 1 & 4.696969 & -5.996518 & 0.215782 \\ 6 & 6.323407 & -4.993301 & -0.758694 \\ 1 & 6.867586 & -5.907054 & -0.978942 \\ 6 & 6.844545 & -3.744690 & -1.174497 \\ 1 & 7.781157 & -3.708959 & -1.723471 \\ 6 & 6.173039 & -2.573838 & -0.897146 \\ 1 & 6.584447 & -1.629907 & -1.234091 \\ 6 & 4.938975 & -2.585153 & -0.187758 \\ 6 & 4.207592 & -1.381771 & 0.116635 \\ 6 & 4.760819 & -0.029086 & -0.174540\end{array}$

$\begin{array}{lllll}1 & -1.702209 & -2.700200 & -1.283577 \\ 6 & 0.327074 & -2.339594 & -1.654425 \\ 1 & 0.524626 & -2.681151 & -0.642820 \\ 6 & 1.519330 & -2.254009 & -2.582744 \\ 1 & 2.401099 & -2.021208 & -1.983681 \\ 1 & 1.696612 & -3.250409 & -3.011974 \\ 6 & 1.391457 & -1.214228 & -3.718722 \\ 1 & 0.946965 & -1.676828 & -4.605173 \\ 1 & 2.396761 & -0.896924 & -4.009057\end{array}$




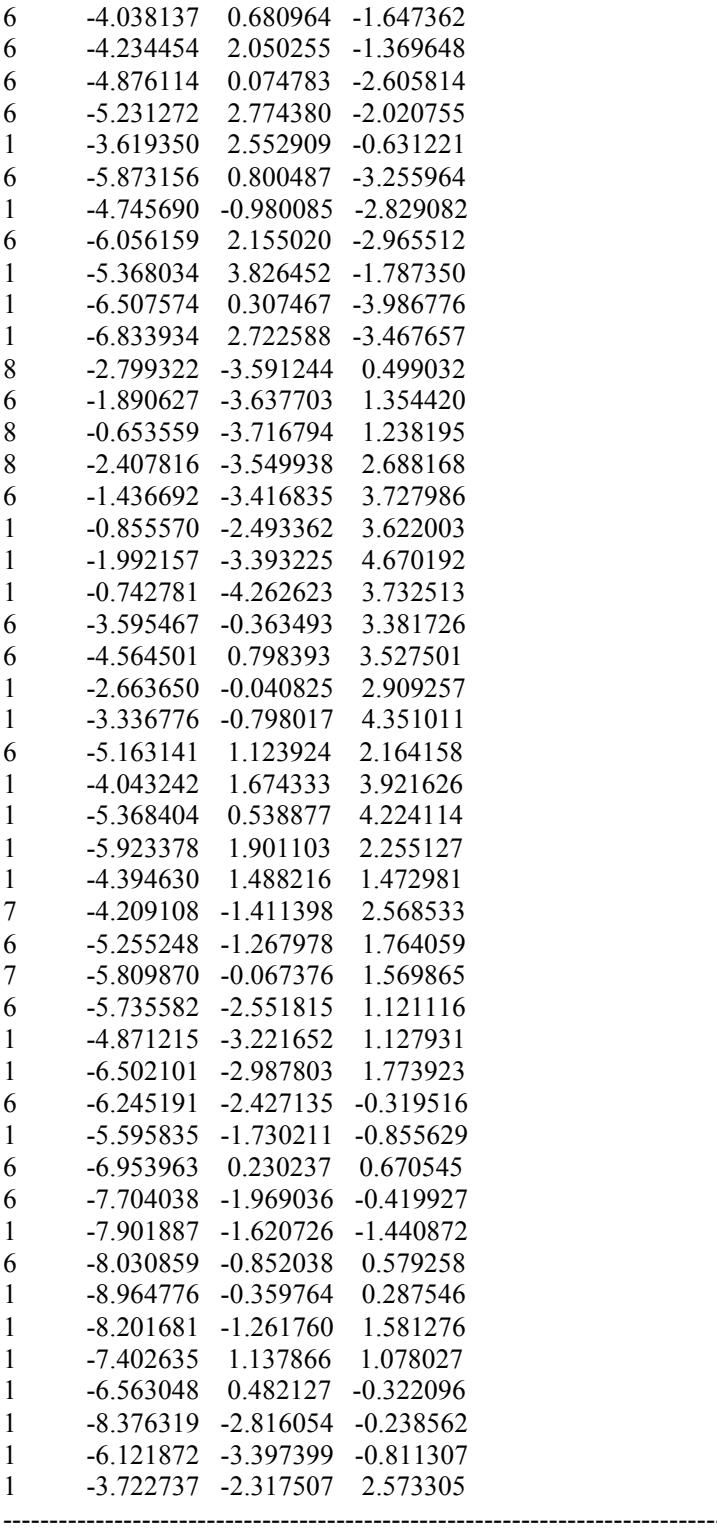

\section{$\left[13 '-14{ }^{\ddagger}\right]^{\ddagger}$ P1 endo-re}

Number of imaginary frequencies $=1$

The smallest frequencies are $=-265.8285 \quad 13.5242 \quad 16.5706$ $\mathrm{cm}(-1)$

Electronic energy $=-3467.3792646$

Zero-point correction $=$

(Hartree/Particle)

Thermal correction to Energy= $\quad 1.278715$

Thermal correction to Enthalpy= $\quad 1.279659$

Thermal correction to Gibbs Free Energy $=\quad 1.107396$

Sum of electronic and zero-point Energies=

Sum of electronic and thermal Energies=

$-3466.167579$

$-3466.100549$

Sum of electronic and thermal Enthalpies=

Sum of electronic and thermal Free Energies=

$-3466.099605$

\section{Cartesian Coordinates}

$\begin{array}{lccc}77 & -0.426616 & -0.040167 & -1.322550 \\ 15 & 1.519042 & 0.501711 & -0.305683 \\ 8 & 2.153968 & -0.366977 & 0.931694 \\ 8 & 2.777530 & 0.503339 & -1.381725 \\ 7 & 1.334800 & 1.987705 & 0.434764 \\ 6 & 2.929348 & -1.509000 & 0.722634 \\ 6 & 2.369693 & -2.725870 & 1.164292 \\ 1 & 1.392470 & -2.717485 & 1.633810\end{array}$

$\begin{array}{lll}3.065361 & -3.893412 & 0.969865\end{array}$

$\begin{array}{lll}2.647544 & -4.839335 & 1.301975\end{array}$

$\begin{array}{lll}4.319518 & -3.885176 & 0.302211\end{array}$

$\begin{array}{llll}5.011809 & -5.096994 & 0.032190\end{array}$

$\begin{array}{lll}4.570962 & -6.030731 & 0.371012\end{array}$

$6.205333-5.092253-0.653124$

$\begin{array}{llll}6.723955 & -6.024237 & -0.857841\end{array}$

$6.750279-3.867710-1.107874$

$\begin{array}{llll}7.679188 & -3.868824 & -1.670803\end{array}$

$\begin{array}{lll}6.112000 & -2.673961 & -0.850375\end{array}$

$\begin{array}{llll}6.541597 & -1.749015 & -1.215938\end{array}$

$\begin{array}{llll}4.888924 & -2.637361 & -0.123390\end{array}$

$\begin{array}{lll}4.192091 & -1.408423 & 0.159018\end{array}$

$\begin{array}{lll}4.774294 & -0.076128 & -0.169297\end{array}$

$\begin{array}{lll}6.048537 & 0.354646 & 0.345530\end{array}$

$6.785226-0.401530 \quad 1.299606$

$\begin{array}{lll}6.381274 & -1.339996 & 1.659856\end{array}$

$\begin{array}{llll}7.995268 & 0.048157 & 1.781880\end{array}$

$\begin{array}{lll}8.533864 & -0.544092 & 2.516019\end{array}$

$\begin{array}{lll}8.540136 & 1.275953 & 1.336308\end{array}$

$\begin{array}{lll}9.498030 & 1.613869 & 1.720560\end{array}$

$\begin{array}{llll}7.845431 & 2.043521 & 0.429442\end{array}$

$\begin{array}{lll}8.242858 & 2.997380 & 0.092682\end{array}$

$\begin{array}{llll}6.586696 & 1.617761 & -0.075640\end{array}$

$\begin{array}{llll}5.845006 & 2.435108 & -0.971192\end{array}$

$\begin{array}{llll}6.268983 & 3.380241 & -1.298174\end{array}$

$\begin{array}{llll}4.594283 & 2.053050 & -1.389698\end{array}$

$\begin{array}{llll}3.991644 & 2.673854 & -2.043740\end{array}$

$\begin{array}{llll}4.069129 & 0.817633 & -0.957176\end{array}$

$\begin{array}{llll}0.115766 & 2.705601 & -0.022984\end{array}$

$\begin{array}{lll}-0.652513 & 2.581069 & 0.748908\end{array}$

$\begin{array}{llll}0.353673 & 4.194222 & -0.201056\end{array}$

$\begin{array}{llll}1.535748 & 4.682945 & -0.773643\end{array}$

$\begin{array}{llll}2.324999 & 3.985878 & -1.038575\end{array}$

$\begin{array}{llll}1.708006 & 6.052501 & -0.976759\end{array}$

$\begin{array}{llll}2.631909 & 6.420835 & -1.414184\end{array}$

$\begin{array}{llll}0.698137 & 6.950066 & -0.615808\end{array}$

$\begin{array}{llll}0.834210 & 8.016230 & -0.773076\end{array}$

$\begin{array}{llll}-0.482439 & 6.469998 & -0.044265\end{array}$

$\begin{array}{lll}-1.268400 & 7.161383 & 0.246564\end{array}$

$\begin{array}{lll}-0.650075 & 5.099025 & 0.165905\end{array}$

$\begin{array}{llll}-1.564702 & 4.727090 & 0.622838\end{array}$

$\begin{array}{lll}-0.397200 & 2.091188 & -1.331299\end{array}$

$\begin{array}{llll}0.232834 & 2.432959 & -2.160758\end{array}$

$\begin{array}{lll}-1.410139 & 2.451239 & -1.526485\end{array}$

$\begin{array}{lll}1.844277 & 2.437526 & 1.779520\end{array}$

$\begin{array}{lll}1.711106 & 3.521651 & 1.735564\end{array}$

$\begin{array}{llll}0.951706 & 1.935890 & 2.913198\end{array}$

$\begin{array}{lll}-0.144831 & 2.718106 & 3.309270\end{array}$

$\begin{array}{lll}-0.312181 & 3.679211 & 2.829323\end{array}$

$\begin{array}{lll}-1.007445 & 2.291847 & 4.322734\end{array}$

$\begin{array}{lll}-1.847567 & 2.915634 & 4.615639\end{array}$

$\begin{array}{lll}-0.777617 & 1.075357 & 4.968445\end{array}$

$\begin{array}{lll}-1.442563 & 0.740872 & 5.759251\end{array}$

$\begin{array}{llll}0.322119 & 0.296566 & 4.597341\end{array}$

$\begin{array}{lll}0.517321 & -0.644465 & 5.104160\end{array}$

$\begin{array}{lll}1.178517 & 0.722191 & 3.579674\end{array}$

$\begin{array}{lll}2.025429 & 0.104290 & 3.308763\end{array}$

$\begin{array}{lll}3.338031 & 2.211741 & 2.005586\end{array}$

$\begin{array}{lll}3.914542 & 2.651647 & 1.189853\end{array}$

$3.611384 \quad 1.161620 \quad 2.091804$

$\begin{array}{lll}3.621924 & 2.713143 & 2.935906\end{array}$

$\begin{array}{lll}-1.165355 & -0.438114 & 0.687060\end{array}$

$\begin{array}{lll}-1.252009 & -1.512272 & 0.788612\end{array}$

$\begin{array}{lll}-0.656034 & 0.034491 & 1.522472\end{array}$

$\begin{array}{lll}2.226776 & 0.315835 & 0.088515\end{array}$

$\begin{array}{llll}-2.314746 & 1.356999 & 0.378440\end{array}$

$\begin{array}{lll}-3.042606 & -0.151291 & -0.939268\end{array}$

$-3.104800-1.219850-1.103102$

$\begin{array}{llll}0.595695 & -0.015000 & -3.359198\end{array}$

$\begin{array}{lll}1.164758 & 0.909615 & -3.364957\end{array}$

$\begin{array}{llll}-0.784704 & 0.099063 & -3.579366\end{array}$

$\begin{array}{lll}-1.184489 & 1.099126 & -3.741793\end{array}$

$-1.687883-1.014281-4.068146$

$\begin{array}{llll}-1.586711 & -1.089680 & -5.160883\end{array}$ 


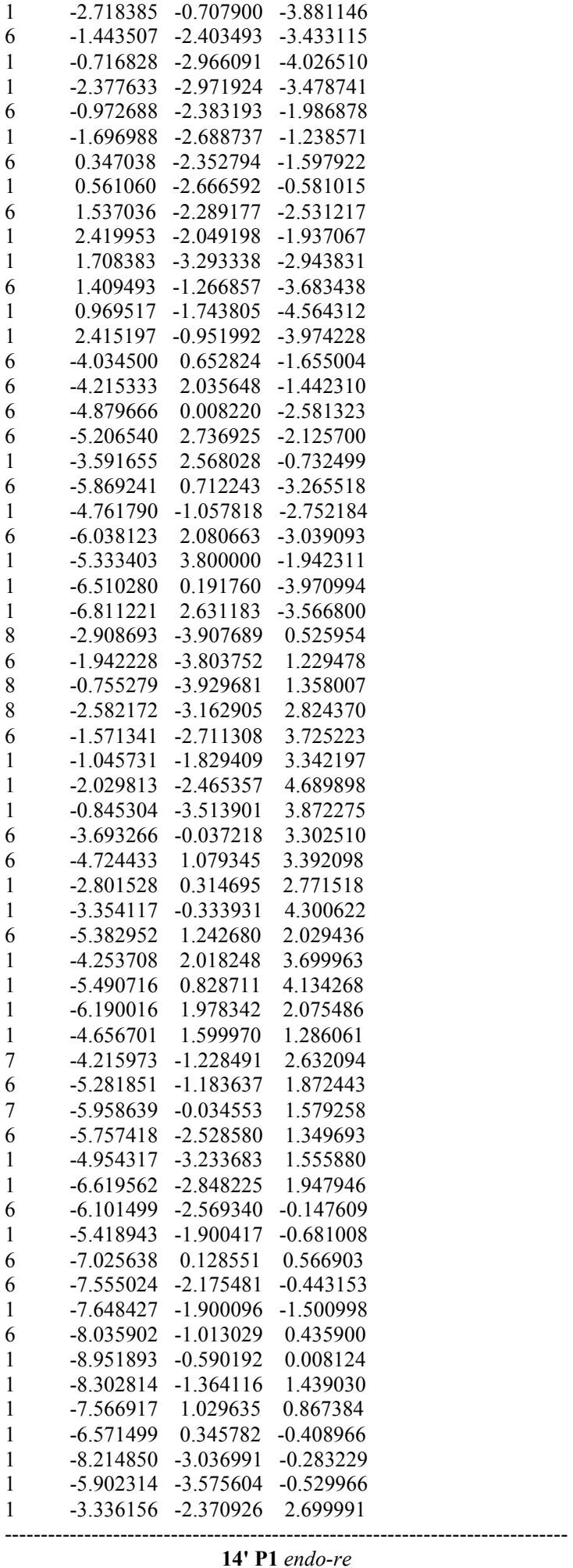

Number of imaginary frequencies $=0$

The smallest frequencies are $=17.467219 .228823 .7868 \mathrm{~cm}(-1)$ Electronic energy $=-3467.3869162$

Zero-point correction=

(Hartree/Particle)

Thermal correction to Energy=

Thermal correction to Enthalpy=

.213967
1.282193

1.283137

Thermal correction to Gibbs Free Energy=

Sum of electronic and zero-point Energies=

Sum of electronic and thermal Energies=

Sum of electronic and thermal Enthalpies=
Sum of electronic and thermal Free Energies=

$-3466.277883$ 
$\begin{array}{lll}-1.430334 & -1.226683 & 0.934365\end{array}$

$\begin{array}{lll}-0.625807 & 0.240384 & 1.641074\end{array}$

$\begin{array}{llll}-2.233142 & 0.713120 & 0.313324\end{array}$

$\begin{array}{llll}-2.193667 & 1.750138 & 0.628171\end{array}$

$\begin{array}{lll}-3.167560 & 0.356578 & -0.652802\end{array}$

$\begin{array}{llll}-3.344756 & -0.694347 & -0.843483\end{array}$

$\begin{array}{llll}0.440364 & 0.156030 & -3.285110\end{array}$

$\begin{array}{llll}1.107246 & 1.012648 & -3.296134\end{array}$

$\begin{array}{llll}-0.922673 & 0.420015 & -3.461971\end{array}$

$\begin{array}{llll}-1.218567 & 1.457975 & -3.606030\end{array}$

$\begin{array}{lll}-1.964292 & -0.592714 & -3.891922\end{array}$

$\begin{array}{llll}-1.930796 & -0.695542 & -4.986558\end{array}$

$\begin{array}{lll}-2.944047 & -0.172421 & -3.654917\end{array}$

$\begin{array}{llll}-1.835021 & -1.986593 & -3.236233\end{array}$

$\begin{array}{lll}-1.199170 & -2.634085 & -3.846836\end{array}$

$\begin{array}{llll}-2.822843 & -2.457465 & -3.222496\end{array}$

$\begin{array}{llll}-1.294950 & -1.980016 & -1.810936\end{array}$

$\begin{array}{llll}-2.005551 & -2.230555 & -1.030259\end{array}$

$\begin{array}{llll}0.040930 & -2.098179 & -1.476855\end{array}$

$\begin{array}{llll}0.261991 & -2.442551 & -0.471951\end{array}$

$\begin{array}{llll}1.188559 & -2.180346 & -2.458313\end{array}$

$\begin{array}{llll}2.116121 & -2.012116 & -1.909820\end{array}$

$1242114 \quad-3.206637 \quad-2.848187$

$\begin{array}{llll}1.104605 & -1.176014 & -3.628829\end{array}$

$\begin{array}{llll}0.572523 & -1.623438 & -4.473537\end{array}$

$\begin{array}{llll}2.119518 & -0.968990 & -3.978973\end{array}$

$\begin{array}{llll}-4.098168 & 1.275297 & -1.307129\end{array}$

$\begin{array}{lll}-4.133020 & 2.662072 & -1.048441\end{array}$

$\begin{array}{llll}-5.020934 & 0.750181 & -2.235715\end{array}$

$\begin{array}{llll}-5.056228 & 3.482198 & -1.692705\end{array}$

$\begin{array}{llll}-3.444686 & 3.102763 & -0.335067\end{array}$

$\begin{array}{llll}-5.943202 & 1.573322 & -2.879742\end{array}$

$\begin{array}{lll}-5.011653 & -0.315550 & -2.445466\end{array}$

$\begin{array}{llll}-5.965113 & 2.944097 & -2.610158\end{array}$

$\begin{array}{llll}-5.069424 & 4.546664 & -1.476616\end{array}$

$\begin{array}{llll}-6.645409 & 1.144315 & -3.588580\end{array}$

$\begin{array}{llll}-6.684150 & 3.588181 & -3.107578\end{array}$

$\begin{array}{lll}-2.686121 & -4.698655 & -0.435044\end{array}$

$\begin{array}{llll}-1.614408 & -4.832686 & 0.017343\end{array}$

$\begin{array}{lll}-0.525623 & -5.042182 & 0.391361\end{array}$

$\begin{array}{lll}-1.993885 & -3.121745 & 1.926307\end{array}$

$\begin{array}{lll}-1.159700 & -3.178007 & 3.066843\end{array}$

$\begin{array}{lll}-0.646727 & -2.221942 & 3.262537\end{array}$

$\begin{array}{lll}-1.717601 & -3.448519 & 3.976946\end{array}$

$\begin{array}{lll}-0.396669 & -3.946638 & 2.903934\end{array}$

$\begin{array}{lll}-3.697850 & -0.379050 & 3.395608\end{array}$

$\begin{array}{lll}-4.856204 & 0.590188 & 3.610839\end{array}$

$\begin{array}{llll}-2.849641 & 0.146006 & 2.939660\end{array}$

$\begin{array}{lll}-3.331176 & -0.762993 & 4.355521\end{array}$

$\begin{array}{lll}-5.540758 & 0.836367 & 2.271468\end{array}$

$\begin{array}{llll}-4.502615 & 1.535995 & 4.034839\end{array}$

$\begin{array}{llll}-5.584025 & 0.161976 & 4.309770\end{array}$

$\begin{array}{lll}-6.430184 & 1.458572 & 2.401668\end{array}$

$\begin{array}{lll}-4.867292 & 1.368770 & 1.584050\end{array}$

$\begin{array}{lll}-4.052374 & -1.523415 & 2.559973\end{array}$

$\begin{array}{lll}-5.114400 & -1.511121 & 1.804429\end{array}$

$\begin{array}{llll}-5.960835 & -0.437935 & 1.669744\end{array}$

$\begin{array}{llll}-5.382256 & -2.819906 & 1.070302\end{array}$

$\begin{array}{lll}-4.460519 & -3.397013 & 1.154068\end{array}$

$\begin{array}{llll}-6.144392 & -3.384610 & 1.622319\end{array}$

$\begin{array}{llll}-5.790876 & -2.695683 & -0.405247\end{array}$

$\begin{array}{llll}-5.250820 & -1.853588 & -0.851028\end{array}$

$\begin{array}{lll}-7.084247 & -0.306843 & 0.721125\end{array}$

$\begin{array}{llll}-7.301910 & -2.504337 & -0.595055\end{array}$

$\begin{array}{llll}-7.495842 & -2.105420 & -1.598648\end{array}$

$\begin{array}{lll}-7.909792 & -1.568166 & 0.458595\end{array}$

$\begin{array}{llll}-8.904508 & -1.249236 & 0.127352\end{array}$

$\begin{array}{lll}-8.058875 & -2.093041 & 1.408884\end{array}$

$\begin{array}{lll}-7.744921 & 0.446856 & 1.159743\end{array}$

$\begin{array}{llll}-6.723734 & 0.112364 & -0.229366\end{array}$

$\begin{array}{lll}-7.808188 & -3.475922 & -0.540891\end{array}$

$\begin{array}{llll}-5.458731 & -3.589835 & -0.943199\end{array}$

$\begin{array}{lll}-2.797418 & -2.564071 & 2.170247\end{array}$

Number of imaginary frequencies $=0$

The smallest frequencies are=14.8061 $19.266421 .2452 \mathrm{~cm}(-1)$ Electronic energy $=-3467.4003726$

$\begin{array}{lc}\text { Zero-point correction }= & 1.217318 \\ \text { (Hartree/Particle) } & 1.284245 \\ \text { Thermal correction to Energy= } & 1.285189 \\ \text { Thermal correction to Enthalpy= } & 1.113582 \\ \text { Thermal correction to Gibbs Free Energy= } & -3466.183055 \\ \text { Sum of electronic and zero-point Energies }= & -3466.116128 \\ \text { Sum of electronic and thermal Energies }= & -3466.115184 \\ \text { Sum of electronic and thermal Enthalpies }= & -3466.286790 \\ \text { Sum of electronic and thermal Free Energies }= & -3460\end{array}$

ectronic and thermal Free Energies=

Cartesian Coordinates

\begin{tabular}{|c|c|c|c|}
\hline . & -0.346039 & 0.113595 & -1.36165 \\
\hline & 1.615997 & 0.485052 & -0.312435 \\
\hline & 2.138774 & -0.416177 & 0.952664 \\
\hline & 2.892866 & 0.340858 & -1.361555 \\
\hline & 1.571644 & 2.002506 & 0.389916 \\
\hline & 2.821431 & -1.625840 & 0.794146 \\
\hline & 2.149378 & -2.777964 & 1.250448 \\
\hline & 1.151394 & -2.685071 & 1.664687 \\
\hline & 2.754851 & -4.002293 & 1.110774 \\
\hline & 2.248590 & -4.900718 & 1.452308 \\
\hline 6 & 4.023421 & -4.119180 & 0.482556 \\
\hline & 4.619536 & -5.391517 & 0.265792 \\
\hline 1 & 4.095248 & -6.275284 & 0.619919 \\
\hline 6 & 5.823533 & -5.506289 & -0.390968 \\
\hline r & 6.267496 & -6.483725 & -0.555690 \\
\hline 6 & 6.476828 & -4.345262 & -0.869201 \\
\hline 1 & 7.413673 & -4.440075 & -1.410704 \\
\hline 6 & 5.934010 & -3.095804 & -0.661278 \\
\hline 1 & 6.445199 & -2.220967 & -1.044696 \\
\hline 6 & 4.703185 & -2.936218 & 0.035457 \\
\hline 6 & 4.102647 & -1.646258 & 0.265260 \\
\hline 6 & 4.804214 & -0.380849 & -0.086662 \\
\hline 6 & 6.102703 & -0.052961 & 0.442717 \\
\hline 6 & 6.740345 & -0.836156 & 1.445239 \\
\hline 1 & 6.236337 & -1.713012 & 1.833597 \\
\hline 6 & 7.979143 & -0.487955 & 1.937920 \\
\hline 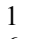 & 8.441073 & -1.097897 & 2.708909 \\
\hline 6 & 8.65 & 0.65 & 1.454607 \\
\hline 1 & 9.633097 & 0.915555 & 1.846805 \\
\hline 6 & 8.056870 & 1.452014 & 0.501063 \\
\hline 1 & 8.553986 & 2.34 & 0.134302 \\
\hline 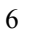 & 6.771767 & 1.131725 & -0.015312 \\
\hline 6 & 6.132016 & 1.97 & -0.962283 \\
\hline 1 & 6.654834 & 2.858209 & -1.321714 \\
\hline 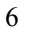 & 4.857336 & 1.701264 & -1.392501 \\
\hline 1 & 4.339305 & 2.34 & -2.094039 \\
\hline 6 & 4.200902 & 0.54 & -0.921408 \\
\hline r & 0.459394 & 2.844423 & -0.120198 \\
\hline 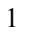 & -0.335195 & 2.853064 & 0.634956 \\
\hline 6 & 0.889556 & 4.282643 & -0.352694 \\
\hline 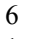 & 2.111997 & 4.584620 & -0.967908 \\
\hline 1 & 2.785817 & 3.775022 & -1.227582 \\
\hline & 2.469186 & 5.908845 & -1.221373 \\
\hline 1 & 3.422717 & 6.129622 & -1.693439 \\
\hline 6 & 1.605827 & 6.950317 & -0.865998 \\
\hline 1 & 1.885161 & 7.981673 & -1.061631 \\
\hline 6 & 0.386304 & 6.657792 & -0.251582 \\
\hline 1 & -0.286550 & 7.461457 & 0.034487 \\
\hline 6 & 0.033576 & 5.330536 & 0.007233 \\
\hline 1 & -0.912366 & 5.106012 & 0.494947 \\
\hline 6 & -0.104874 & 2.239444 & -1.408217 \\
\hline 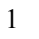 & 0.567687 & 2.485599 & -2.238582 \\
\hline 1 & -1.060006 & 2.717147 & -1.633786 \\
\hline 6 & 2.092301 & 2.433497 & 1.734443 \\
\hline 1 & 2.097768 & 3.523701 & 1.650129 \\
\hline 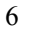 & 1.102477 & 2.087236 & 2.845046 \\
\hline & 0.115880 & 3.024508 & 3.188745 \\
\hline & 0.106565 & 3.991394 & 2.691834 \\
\hline & -0.843132 & 2.739314 & 4.164786 \\
\hline
\end{tabular}




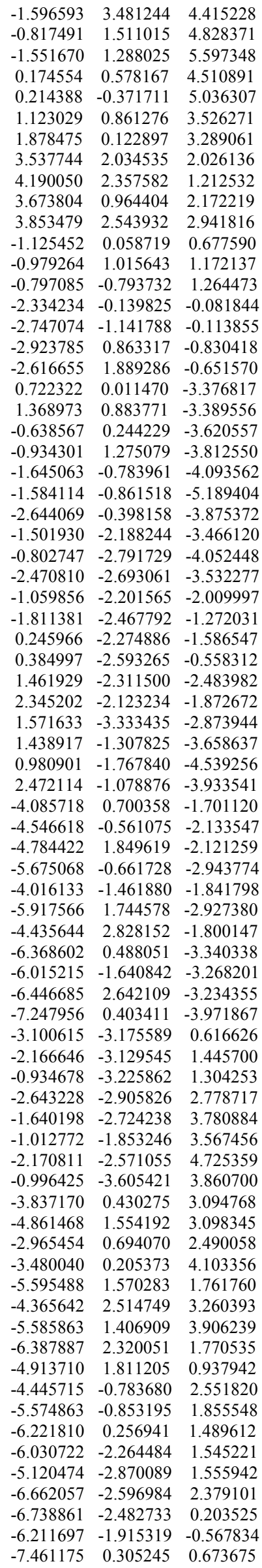

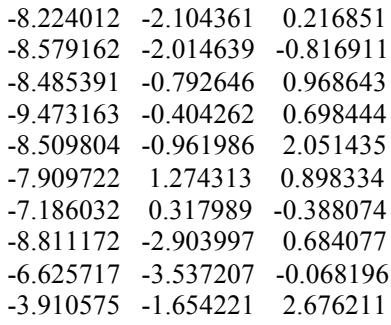

[13'-14'] P1 exo-si

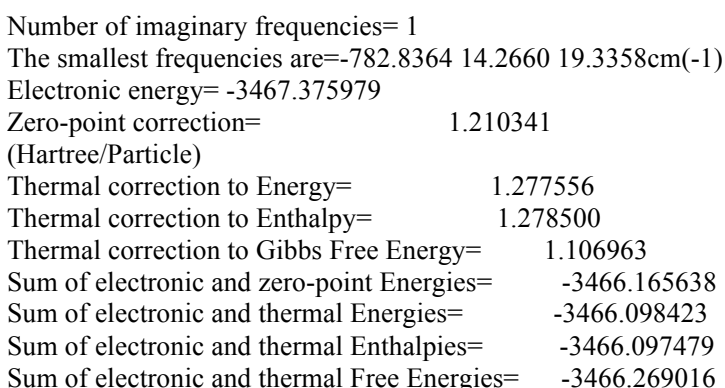

Cartesian Coordinates

\begin{tabular}{|c|c|c|c|}
\hline 77 & -0.534337 & 0.437757 & 134 \\
\hline 15 & 1.396161 & 0.738134 & -0.324585 \\
\hline 8 & 1.888787 & -0.221071 & 0.914068 \\
\hline 8 & 2.684615 & 0.597036 & -1.354812 \\
\hline f & 1.359479 & 2.210965 & 0.452066 \\
\hline 6 & 2.383590 & -1.505235 & 0.685396 \\
\hline 6 & 1.573978 & -2.569478 & 1.133169 \\
\hline 1 & 0.643823 & -2.339110 & 1.642308 \\
\hline 6 & 1.979838 & -3.861742 & 0.906404 \\
\hline 1 & 1.364064 & -4.689684 & 1.242816 \\
\hline 6 & 3.178043 & -4.125308 & 0.188988 \\
\hline 6 & 3.556846 & -5.456321 & -0.135359 \\
\hline 1 & 2.923253 & -6.271273 & 0.204475 \\
\hline 6 & 4.689073 & -5.709407 & -0.876054 \\
\hline 1 & 4.965354 & -6.730035 & -1.123929 \\
\hline 6 & 5.487146 & -4.632625 & -1.330496 \\
\hline 1 & 6.366040 & -4.833443 & -1.936383 \\
\hline 6 & 5.157891 & -3.331209 & -1.018422 \\
\hline 1 & 5.775657 & -2.520136 & -1.385264 \\
\hline 6 & 4.007175 & -3.032616 & -0.236776 \\
\hline 6 & 3.623708 & -1.682440 & 0.093108 \\
\hline 6 & 4.496190 & -0.513500 & -0.212523 \\
\hline 6 & 5.85 & -0.43 & 0.265956 \\
\hline 6 & 6.407834 & -1.38 & 1.169461 \\
\hline 1 & 5.791392 & -2.196191 & 1.529085 \\
\hline 6 & 7.710342 & -1.2 & 1.606343 \\
\hline 1 & 8.105540 & -2.007623 & 2.301712 \\
\hline 6 & 8.535977 & -0.211731 & 1.164454 \\
\hline 1 & 9.563059 & -0.14 & 1.510780 \\
\hline 6 & 8.025810 & 0.740109 & 0.311297 \\
\hline 1 & 8.640173 & 1.573013 & -0.020346 \\
\hline 6 & 6.681358 & 0.668051 & -0.144081 \\
\hline 6 & 6.139178 & 1.681866 & -0.979319 \\
\hline 1 & 6.776609 & 2.502498 & -1.295852 \\
\hline 6 & 4.818822 & 1.644059 & -1.351515 \\
\hline 1 & 4.369561 & 2.424441 & -1.956723 \\
\hline 6 & 4.011644 & 0.561907 & -0.939694 \\
\hline 6 & 0.207403 & 3.063706 & 0.070740 \\
\hline 1 & -0.524462 & 3.025563 & 0.885879 \\
\hline 6 & 0.619968 & 4.513094 & -0.125604 \\
\hline 6 & 1.784885 & 4.838514 & -0.835840 \\
\hline 1 & 2.425680 & 4.038888 & -1.198104 \\
\hline 6 & 2.128678 & 6.171811 & -1.056695 \\
\hline 1 & 3.037162 & 6.412242 & -1.602202 \\
\hline 6 & 1.308545 & 7.198133 & -0.575733 \\
\hline 1 & 1.577343 & 8.236563 & -0.747149 \\
\hline & 0.147394 & 6.881734 & 0.132239 \\
\hline
\end{tabular}




$\begin{array}{rrrrrrrr}-0.490432 & 7.673367 & 0.515524 & 1 & -4.704921 & 0.873611 & 4.605136 \\ -0.191645 & 5.544803 & 0.359550 & 1 & -5.617609 & 1.918088 & 2.566311 \\ -1.090122 & 5.299354 & 0.921311 & 1 & -4.124005 & 1.579575 & 1.679194 \\ -0.447446 & 2.540948 & -1.216391 & 7 & -3.379136 & -1.110867 & 3.010039 \\ 0.120252 & 2.925297 & -2.070645 & 6 & -4.518743 & -1.180060 & 2.362960 \\ -1.451757 & 2.965319 & -1.288296 & 7 & -5.313233 & -0.102122 & 2.136676 \\ 2.017487 & 2.609848 & 1.744688 & 6 & -4.907000 & -2.574079 & 1.904556 \\ 2.009061 & 3.701764 & 1.693355 & 1 & -4.005580 & -3.177181 & 1.997348 \\ 1.175970 & 2.232554 & 2.962626 & 1 & -5.621156 & -2.983587 & 2.629696 \\ 0.252144 & 3.164734 & 3.459687 & 6 & -5.468622 & -2.678587 & 0.480458 \\ 0.152655 & 4.128451 & 2.966418 & 1 & -4.949165 & -1.955931 & -0.153800 \\ -0.515957 & 2.886056 & 4.593729 & 6 & -6.534898 & -0.062235 & 1.302235 \\ -1.217332 & 3.627171 & 4.967319 & 6 & -6.981214 & -2.433154 & 0.410566 \\ -0.362901 & 1.666927 & 5.255956 & 1 & -7.258404 & -2.192746 & -0.623247 \\ -0.949881 & 1.448289 & 6.143332 & 6 & -7.433113 & -1.299992 & 1.341541 \\ 0.553192 & 0.729308 & 4.770140 & 1 & -8.441878 & -0.979400 & 1.058583 \\ 0.681062 & -0.220792 & 5.281544 & 1 & -7.504626 & -1.648169 & 2.378041 \\ 1.315220 & 1.008445 & 3.634655 & 1 & -7.108812 & 0.789526 & 1.676755 \\ 2.026587 & 0.272942 & 3.280197 & 1 & -6.257809 & 0.175180 & 0.265694 \\ 3.488078 & 2.214640 & 1.862061 & 1 & -7.522557 & -3.348561 & 0.678775 \\ 4.054422 & 2.605954 & 1.015001 & 1 & -5.228593 & -3.666270 & 0.074083 \\ 3.645274 & 1.138402 & 1.914687 & 1 & -2.406757 & -1.957961 & 2.782141\end{array}$

14' P1 exo-si

Number of imaginary frequencies $=0$

The smallest frequencies are $=18.627520 .804924 .3040 \mathrm{~cm}(-1)$ Electronic energy $=-3467.3838156$

Zero-point correction=

1.214032

(Hartree/Particle)

Thermal correction to Energy= $\quad 1.282318$

Thermal correction to Enthalpy $=\quad 1.283262$

Thermal correction to Gibbs Free Energy $=\quad 1.109278$

Sum of electronic and zero-point Energies $=\quad-3466.169783$

Sum of electronic and thermal Energies $=\quad-3466.101498$

Sum of electronic and thermal Enthalpies $=\quad-3466.100554$

Sum of electronic and thermal Free Energies $=\quad-3466.274538$

Cartesian Coordinates

$\begin{array}{lccc}77 & -0.456590 & 0.464939 & -1.442831 \\ 15 & 1.471454 & 0.732479 & -0.321811 \\ 8 & 1.907294 & -0.220572 & 0.944002 \\ 8 & 2.768899 & 0.519370 & -1.325448 \\ 7 & 1.474780 & 2.222557 & 0.418478 \\ 6 & 2.337152 & -1.532577 & 0.757964 \\ 6 & 1.479413 & -2.541823 & 1.246477 \\ 1 & 0.566808 & -2.248851 & 1.757769 \\ 6 & 1.827983 & -3.857708 & 1.063693 \\ 1 & 1.192366 & -4.652027 & 1.444427 \\ 6 & 3.007323 & -4.201057 & 0.347580 \\ 6 & 3.323295 & -5.557716 & 0.065270 \\ 1 & 2.654621 & -6.331695 & 0.432993 \\ 6 & 4.440510 & -5.885958 & -0.668990 \\ 1 & 4.668615 & -6.925664 & -0.884418 \\ 6 & 5.287028 & -4.862613 & -1.157844 \\ 1 & 6.154279 & -5.123029 & -1.757732 \\ 6 & 5.019644 & -3.538284 & -0.885648 \\ 1 & 5.673948 & -2.767892 & -1.276192 \\ 6 & 3.885598 & -3.162438 & -0.113464 \\ 6 & 3.569061 & -1.786410 & 0.176925 \\ 6 & 4.503775 & -0.668381 & -0.139566 \\ 6 & 5.857779 & -0.648253 & 0.357785 \\ 6 & 6.353895 & -1.615495 & 1.276256 \\ 1 & 5.694876 & -2.396788 & 1.634559 \\ 6 & 7.654175 & -1.565858 & 1.730324 \\ 1 & 8.004207 & -2.313320 & 2.436410 \\ 6 & 8.535675 & -0.549166 & 1.292756 \\ 1 & 9.559932 & -0.528169 & 1.652946 \\ 6 & 8.082990 & 0.419327 & 0.426212 \\ 1 & 8.740515 & 1.219986 & 0.097876 \\ 6 & 6.743128 & 0.407512 & -0.047940 \\ 6 & 6.262934 & 1.440587 & -0.897259 \\ 1 & 6.943541 & 2.227746 & -1.208616 \\ 6 & 4.948568 & 1.462424 & -1.290385\end{array}$




\begin{tabular}{|c|c|c|c|c|c|c|}
\hline 4.546244 & 2.258675 & -1.907828 & 8 & -1.484338 & -4.818351 & 2.037447 \\
\hline 4.084600 & 0.422537 & -0.884609 & 8 & -1.380685 & -2.032413 & 2.893842 \\
\hline 0.350103 & 3.102520 & 0.014120 & 6 & -1.114946 & -2.525666 & 4.195031 \\
\hline-0.368943 & 3.119842 & 0.840597 & 1 & -0.046233 & -2.757031 & 4.275225 \\
\hline 0.813188 & 4.527884 & -0.238179 & 1 & -1.363456 & -1.790031 & 4.972753 \\
\hline 1.984980 & 4.784486 & -0.964727 & 1 & -1.673981 & -3.448325 & 4.411832 \\
\hline 2.596281 & 3.948907 & -1.295383 & 6 & -3.444748 & 0.839681 & 3.365486 \\
\hline 2.372454 & 6.095377 & -1.241167 & 6 & -4.734230 & 1.652394 & 3.308069 \\
\hline 3.286002 & 6.282747 & -1.798923 & 1 & -2.687236 & 1.285516 & 2.708424 \\
\hline 1.589644 & 7.167586 & -0.799712 & 1 & -3.015538 & 0.866060 & 4.372959 \\
\hline 1.892116 & 8.188526 & -1.014555 & 6 & -5.448690 & 1.342232 & 1.999393 \\
\hline 0.422264 & 6.919506 & -0.074903 & 1 & -4.526532 & 2.724990 & 3.381128 \\
\hline-0.186866 & 7.747107 & 0.277918 & 1 & -5.387985 & 1.380958 & 4.145203 \\
\hline 0.039319 & 5.605198 & 0.207683 & 1 & -6.422640 & 1.838368 & 1.964378 \\
\hline-0.863950 & 5.413295 & 0.782071 & 1 & -4.862965 & 1.709564 & 1.143809 \\
\hline-0.349837 & 2.566227 & -1.245274 & 7 & -3.634001 & -0.560305 & 2.994560 \\
\hline 0.187638 & 2.937777 & -2.123848 & 6 & -4.686711 & -0.945410 & 2.329253 \\
\hline-1.354848 & 2.994368 & -1.283253 & 7 & -5.671756 & -0.105322 & 1.868502 \\
\hline 2.121144 & 2.638708 & 1.712574 & 6 & -4.789079 & -2.450311 & 2.119713 \\
\hline 2.226314 & 3.720788 & 1.596486 & 1 & -3.813464 & -2.842057 & 2.408159 \\
\hline 1.187762 & 2.416324 & 2.901229 & 1 & -5.503368 & -2.859069 & 2.845651 \\
\hline 0.438433 & 3.497922 & 3.387458 & 6 & -5.159970 & -2.912788 & 0.701721 \\
\hline 0.538860 & 4.469888 & 2.910659 & 1 & -4.708975 & -2.228637 & -0.023563 \\
\hline-0.425374 & 3.345176 & 4.475525 & 6 & -6.746707 & -0.452337 & 0.917050 \\
\hline-0.994188 & 4.197241 & 4.837667 & 6 & -6.675486 & -2.984198 & 0.472304 \\
\hline-0.546428 & 2.102320 & 5.099482 & 1 & -6.876364 & -2.997268 & -0.606088 \\
\hline-1.210317 & 1.979502 & 5.950458 & 6 & -7.423442 & -1.810462 & 1.120272 \\
\hline 0.194431 & 1.016538 & 4.623135 & 1 & -8.431266 & -1.746448 & 0.695009 \\
\hline 0.109238 & 0.046483 & 5.103236 & 1 & -7.555890 & -1.976468 & 2.195376 \\
\hline 1.052661 & 1.171602 & 3.534100 & 1 & -7.502795 & 0.327647 & 1.043310 \\
\hline 1.617094 & 0.320082 & 3.177719 & 1 & -6.377965 & -0.364017 & -0.115015 \\
\hline 3.536554 & 2.107816 & 1.936522 & 1 & -7.070067 & -3.924142 & 0.877492 \\
\hline 4.178412 & 2.358352 & 1.089641 & 1 & -4.710292 & -3.894098 & 0.513959 \\
\hline 3.575742 & 1.031717 & 2.098449 & 1 & -2.231124 & -1.492923 & 2.936473 \\
\hline
\end{tabular}

$\mathbf{P 1}$ endo-re $\left[\mathrm{BF}^{-}\right]$

Number of imaginary frequencies $=0$

The smallest frequencies are=12.8582 $21.955026 .3544 \mathrm{~cm}(-1)$ Electronic energy $=-3125.4962645$

Zero-point correction $=$

0.914550

(Hartree/Particle)

Thermal correction to Energy= $\quad 0.969328$

Thermal correction to Enthalpy= $\quad 0.970272$

Thermal correction to Gibbs Free Energy $=0.825821$

Sum of electronic and zero-point Energies $=\quad-3124.581715$

Sum of electronic and thermal Energies= $\quad-3124.526937$

Sum of electronic and thermal Enthalpies $=\quad-3124.525992$

Sum of electronic and thermal Free Energies $=\quad-3124.670443$

$\begin{array}{lccc}77 & -1.143406 & -1.367048 & -0.238216 \\ 15 & 0.592702 & 0.095956 & -0.137958 \\ 8 & 1.640024 & 0.205066 & 1.122888 \\ 8 & 1.656429 & -0.181158 & -1.389421 \\ 7 & -0.010229 & 1.640208 & -0.135852 \\ 6 & 2.710553 & -0.662991 & 1.298692 \\ 6 & 2.649576 & -1.504939 & 2.430322 \\ 1 & 1.803104 & -1.404964 & 3.101529 \\ 6 & 3.654405 & -2.414019 & 2.650767 \\ 1 & 3.624957 & -3.065077 & 3.519806 \\ 6 & 4.729562 & -2.537922 & 1.729272 \\ 6 & 5.727925 & -3.535636 & 1.897440 \\ 1 & 5.665549 & -4.190241 & 2.762757 \\ 6 & 6.743294 & -3.682207 & 0.979527 \\ 1 & 7.497265 & -4.452239 & 1.114117 \\ 6 & 6.795451 & -2.835000 & -0.152940 \\ 1 & 7.583765 & -2.968191 & -0.888294 \\ 6 & 5.853002 & -1.846438 & -0.336447 \\ 1 & 5.904764 & -1.214106 & -1.214821 \\ 6 & 4.800355 & -1.652645 & 0.601224 \\ 6 & 3.791706 & -0.638219 & 0.431518 \\ 6 & 3.874576 & 0.388027 & -0.644230 \\ 6 & 5.010146 & 1.265829 & -0.771956\end{array}$


$\begin{array}{llll}6.063035 & 1.303712 & 0.184742\end{array}$

$\begin{array}{lll}6.016499 & 0.655882 & 1.051763\end{array}$

$\begin{array}{lll}7.129281 & 2.163814 & 0.035320\end{array}$

$\begin{array}{llll}7.915619 & 2.178648 & 0.784603\end{array}$

$\begin{array}{llll}7.207109 & 3.032894 & -1.078979\end{array}$

$\begin{array}{llll}8.057010 & 3.700418 & -1.186349\end{array}$

$\begin{array}{llll}6.194854 & 3.040466 & -2.011463\end{array}$

$\begin{array}{llll}6.229551 & 3.716952 & -2.861430\end{array}$

$\begin{array}{llll}5.072453 & 2.178609 & -1.878527\end{array}$

$\begin{array}{llll}4.001733 & 2.223850 & -2.811408\end{array}$

$\begin{array}{llll}4.064760 & 2.904807 & -3.655308\end{array}$

$\begin{array}{llll}2.885841 & 1.445619 & -2.630138\end{array}$

$\begin{array}{llll}2.044281 & 1.486475 & -3.312160\end{array}$

$\begin{array}{llll}2.824829 & 0.561694 & -1.531820\end{array}$

$\begin{array}{lll}-1.422806 & 1.723442 & -0.608625\end{array}$

$\begin{array}{lll}-2.058174 & 1.905488 & 0.258410\end{array}$

$\begin{array}{llll}-1.584402 & 2.884713 & -1.580040\end{array}$

$\begin{array}{llll}-2.547015 & 3.876372 & -1.354980\end{array}$

$\begin{array}{llll}-3.175415 & 3.820868 & -0.473412\end{array}$

$\begin{array}{llll}-2.684972 & 4.939893 & -2.253010\end{array}$

$\begin{array}{llll}-3.433181 & 5.705369 & -2.065242\end{array}$

$\begin{array}{llll}-1.863306 & 5.025988 & -3.378003\end{array}$

$\begin{array}{llll}-1.969961 & 5.855755 & -4.071438\end{array}$

$\begin{array}{llll}-0.894966 & 4.041449 & -3.603861\end{array}$

$\begin{array}{llll}-0.245000 & 4.104260 & -4.472714\end{array}$

$\begin{array}{llll}-0.758639 & 2.980494 & -2.710074\end{array}$

$\begin{array}{llll}0.001388 & 2.224137 & -2.876975\end{array}$

$\begin{array}{llll}-1.845481 & 0.394193 & -1.251427\end{array}$

$\begin{array}{llll}-1.479506 & 0.352166 & -2.282386\end{array}$

$\begin{array}{llll}-2.935751 & 0.358775 & -1.294089\end{array}$

$\begin{array}{llll}0.479346 & 2.828685 & 0.650017\end{array}$

$\begin{array}{lll}-0.193978 & 3.625722 & 0.323355\end{array}$

$\begin{array}{lll}0.250778 & 2.649123 & 2.150128\end{array}$

$\begin{array}{lll}-1.046325 & 2.804976 & 2.659307\end{array}$

$\begin{array}{lll}-1.854307 & 3.128278 & 2.014144\end{array}$

$\begin{array}{lll}-1.325805 & 2.558796 & 4.005405\end{array}$

$\begin{array}{lll}-2.349326 & 2.652126 & 4.353498\end{array}$

$\begin{array}{lll}-0.299999 & 2.185772 \quad 4.875660\end{array}$

$\begin{array}{llll}-0.511476 & 1.995687 & 5.924555\end{array}$

$\begin{array}{lll}1.006040 & 2.071336 & 4.388800\end{array}$

$\begin{array}{lll}1.816194 & 1.798307 & 5.060119\end{array}$

$\begin{array}{lll}1.277231 & 2.298542 & 3.038378\end{array}$

$\begin{array}{llll}2.292887 & 2.184446 & 2.677120\end{array}$

$\begin{array}{llll}1.887350 & 3.262425 & 0.253282\end{array}$

$\begin{array}{llll}1.928429 & 3.446655 & -0.822401\end{array}$

$\begin{array}{llll}2.644945 & 2.518700 & 0.499929\end{array}$

$\begin{array}{llll}2.139193 & 4.190316 & 0.776166\end{array}$

$\begin{array}{lll}-1.568277 & -0.844508 & 1.838847\end{array}$

$\begin{array}{lll}-1.288653 & -1.697142 & 2.456207\end{array}$

$\begin{array}{lll}-1.203245 & 0.106353 & 2.216910\end{array}$

$\begin{array}{lll}-2.862544 & -0.820405 & 1.219203\end{array}$

$\begin{array}{lll}-3.300571 & 0.144262 & 0.995674\end{array}$

$\begin{array}{lll}-3.513452 & -1.977838 & 0.789035\end{array}$

$\begin{array}{lll}-3.190436 & -2.927945 & 1.198949\end{array}$

$\begin{array}{lll}-0.395876 & -2.135319 & -2.251574\end{array}$

$\begin{array}{llll}-0.199188 & -1.241114 & -2.833938\end{array}$

$\begin{array}{llll}-1.737120 & -2.518689 & -2.114060\end{array}$

$-2.490592-1.900936-2.600754$

$-2.202931 \quad-3.921825 \quad-1.777671$

$\begin{array}{llll}-2.151491 & -4.536090 & -2.688692\end{array}$

$\begin{array}{llll}-3.259595 & -3.863037 & -1.511942\end{array}$

$-1.429968-4.626582-0.636242$

$\begin{array}{llll}-0.609666 & -5.223318 & -1.047303\end{array}$

$\begin{array}{lll}-2.105788 & -5.336642 & -0.149830\end{array}$

$\begin{array}{lll}-0.862587 & -3.701035 & 0.431413\end{array}$

$\begin{array}{lll}-1.338868 & -3.734999 & 1.407015\end{array}$

$\begin{array}{llll}0.393948 & -3.137661 & 0.377410\end{array}$

$\begin{array}{lll}0.818466 & -2.789486 & 1.313577\end{array}$

$\begin{array}{llll}1.376645 & -3.304376 & -0.761925\end{array}$

$\begin{array}{llll}2.182499 & -2.584479 & -0.620799\end{array}$

$\begin{array}{llll}1.834151 & -4.300714 & -0.682620\end{array}$

$\begin{array}{llll}0.773521 & -3.114737 & -2.172080\end{array}$

$0.445781-4.077626-2.576059$

$\begin{array}{llll}1.563804 & -2.755747 & -2.836747\end{array}$

$\begin{array}{lll}-4.761883 & -2.038592 & 0.026956\end{array}$ $\begin{array}{lll}-5.399234 & -0.904694 & -0.517642\end{array}$

$-5.358231-3.301254-0.171231$

$\begin{array}{llll}-6.577667 & -1.042709 & -1.247879\end{array}$

$\begin{array}{llll}-4.996882 & 0.085964 & -0.347097\end{array}$

$-6.537502-3.433866-0.901620$

$\begin{array}{lll}-4.888481 & -4.182400 & 0.258963\end{array}$

$\begin{array}{llll}-7.149365 & -2.303485 & -1.449600\end{array}$

$-7.057283-0.157180-1.655470$

$\begin{array}{llll}-6.979074 & -4.416643 & -1.040634\end{array}$

$\begin{array}{lll}-8.068452 & -2.401647 & -2.020256\end{array}$

$\begin{array}{lll}-4.625540 & 2.553491 & 1.657912\end{array}$

$\begin{array}{lll}-4.394765 & 1.975914 & 0.374436\end{array}$

$\begin{array}{lll}5.997009 & 2.685133 & 1.868376\end{array}$

$\begin{array}{lll}-4.051506 & 1.722915 & 2.638004\end{array}$

$-3.997421 \quad 3.815990 \quad 1.686558$

P1 exo-si [BF4 $\left.{ }^{-}\right]$

Number of imaginary frequencies $=0$

The smallest frequencies are $=13.387219 .253724 .8597 \mathrm{~cm}(-1)$

Electronic energy $=-3125.4910399$

Zero-point correction=

0.914402

(Hartree/Particle)

Thermal correction to Energy=

Thermal correction to Enthalpy=

0.969248

0.970192

Thermal correction to Gibbs Free Energy $=0.825039$

Sum of electronic and zero-point Energies $=\quad-3124.576637$

Sum of electronic and thermal Energies=

Sum of electronic and thermal Enthalpies=

$-3124.521792$

$-3124.520848$

Sum of electronic and thermal Free Energies=

$-3124.666001$

$\begin{array}{lccc}77 & -1.255554 & -1.046700 & -0.340122 \\ 15 & 0.607243 & 0.237829 & -0.139153 \\ 8 & 1.624092 & 0.193146 & 1.154207 \\ 8 & 1.683399 & -0.076388 & -1.371409 \\ 7 & 0.151698 & 1.834617 & -0.082783 \\ 6 & 2.552362 & -0.829340 & 1.316434 \\ 6 & 2.317362 & -1.727555 & 2.379469 \\ 1 & 1.453394 & -1.561149 & 3.014056 \\ 6 & 3.183937 & -2.772873 & 2.583121 \\ 1 & 3.022778 & -3.468400 & 3.401733 \\ 6 & 4.284330 & -2.978158 & 1.707122 \\ 6 & 5.138539 & -4.104473 & 1.856834 \\ 1 & 4.947237 & -4.793871 & 2.675055 \\ 6 & 6.175119 & -4.327779 & 0.979186 \\ 1 & 6.818104 & -5.194733 & 1.099212 \\ 6 & 6.393561 & -3.431317 & -0.094060 \\ 1 & 7.196681 & -3.623194 & -0.799755 \\ 6 & 5.594782 & -2.320394 & -0.257274 \\ 1 & 5.772423 & -1.651062 & -1.090417 \\ 6 & 4.527677 & -2.044920 & 0.642825 \\ 6 & 3.667077 & -0.898883 & 0.496393 \\ 6 & 3.923750 & 0.161771 & -0.516833 \\ 6 & 5.166955 & 0.888141 & -0.561609 \\ 6 & 6.174098 & 0.746978 & 0.434057 \\ 1 & 6.007258 & 0.075790 & 1.268124 \\ 6 & 7.349502 & 1.462881 & 0.363539 \\ 1 & 8.098320 & 1.342646 & 1.141216 \\ 6 & 7.588154 & 2.358606 & -0.705902 \\ 1 & 8.522176 & 2.910863 & -0.751279 \\ 6 & 6.626524 & 2.537993 & -1.674195 \\ 1 & 6.786332 & 3.237472 & -2.490597 \\ 6 & 5.396087 & 1.828288 & -1.622341 \\ 6 & 4.382180 & 2.049173 & -2.593018 \\ 1 & 4.570904 & 2.744701 & -3.405775 \\ 6 & 3.167626 & 1.418400 & -2.488425 \\ 1 & 2.370795 & 1.591749 & -3.202895 \\ 6 & 2.942413 & 0.509854 & -1.431864 \\ 6 & -1.242003 & 2.080790 & -0.535795 \\ 1 & -1.847879 & 2.326429 & 0.338245 \\ 6 & -1.308310 & 3.248551 & -1.510985 \\ 6 & -2.386001 & 4.141925 & -1.464008 \\ 1 & -3.141550 & 4.023570 & -0.695604\end{array}$


$\begin{array}{lllll}6 & -2.479317 & 5.181189 & -2.393962\end{array}$

$\begin{array}{llll}-3.319008 & 5.869455 & -2.345394\end{array}$

$\begin{array}{llll}-1.498216 & 5.342903 & -3.374844\end{array}$

$\begin{array}{llll}-1.570803 & 6.155022 & -4.093138\end{array}$

$\begin{array}{llll}-0.417162 & 4.457019 & -3.421848\end{array}$

$\begin{array}{llll}0.355958 & 4.578808 & -4.176160\end{array}$

$\begin{array}{llll}-0.325674 & 3.416777 & -2.496903\end{array}$

$\begin{array}{lll}0.518712 & 2.736472 & -2.522320\end{array}$

$\begin{array}{llll}-1.833144 & 0.826938 & -1.192831\end{array}$

$\begin{array}{llll}-1.514383 & 0.799464 & -2.240298\end{array}$

$\begin{array}{llll}-2.916970 & 0.924984 & -1.183406\end{array}$

$\begin{array}{lll}0.740812 & 2.942985 & 0.750535\end{array}$

$\begin{array}{lll}0.217015 & 3.826887 & 0.376310\end{array}$

$\begin{array}{lll}0.361647 & 2.797294 & 2.223519\end{array}$

$\begin{array}{lll}-0.914515 & 3.205241 & 2.640475\end{array}$

$\begin{array}{lll}-1.600227 & 3.667932 & 1.939504\end{array}$

$\begin{array}{lll}-1.337257 & 3.012719 & 3.957412\end{array}$

$\begin{array}{llll}-2.341992 & 3.311897 & 4.241111\end{array}$

$\begin{array}{lll}-0.475084 & 2.437264 & 4.892145\end{array}$

$\begin{array}{lll}-0.798229 & 2.289505 & 5.919079\end{array}$

$\begin{array}{lll}0.814275 & 2.065479 & 4.499037\end{array}$

$\begin{array}{lll}1.501437 & 1.634579 & 5.222705\end{array}$

$\begin{array}{lll}1.226978 & 2.242002 & 3.177082\end{array}$

$\begin{array}{llll}2.225979 & 1.935505 & 2.890271\end{array}$

$\begin{array}{llll}2.224657 & 3.179805 & 0.482264\end{array}$

$\begin{array}{llll}2.387493 & 3.353668 & -0.583647\end{array}$

$\begin{array}{llll}2.852933 & 2.344447 & 0.790049\end{array}$

$\begin{array}{llll}2.547785 & 4.069188 & 1.031999\end{array}$

$\begin{array}{lll}-1.616578 & -0.321375 & 1.728000\end{array}$

$\begin{array}{lll}-0.858593 & -0.560792 & 2.469023\end{array}$

$\begin{array}{lll}-1.889101 & 0.726198 & 1.688920\end{array}$

$\begin{array}{llll}-2.640522 & -1.290627 & 1.511440\end{array}$

$\begin{array}{lll}-2.540478 & -2.258725 & 1.991221\end{array}$

$\begin{array}{llll}-3.661638 & -1.071967 & 0.586403\end{array}$

$\begin{array}{lll}-3.866706 & -0.044663 & 0.311373\end{array}$

$\begin{array}{llll}-0.582521 & -1.659501 & -2.405807\end{array}$

$\begin{array}{llll}-0.329805 & -0.731267 & -2.908663\end{array}$

$\begin{array}{llll}-1.953607 & -1.956260 & -2.297842\end{array}$

$\begin{array}{llll}-2.649207 & -1.230715 & -2.717672\end{array}$

$\begin{array}{llll}-2.538998 & -3.346165 & -2.160992\end{array}$

$\begin{array}{llll}-2.591592 & -3.802096 & -3.160827\end{array}$

$\begin{array}{llll}-3.570673 & -3.247278 & -1.820413\end{array}$

$\begin{array}{llll}-1.781198 & -4.293303 & -1.204829\end{array}$

$\begin{array}{llll}-1.002185 & -4.836677 & -1.747278\end{array}$

$\begin{array}{llll}-2.485647 & -5.046225 & -0.838552\end{array}$

$\begin{array}{llll}-1.150294 & -3.614607 & -0.003450\end{array}$

$\begin{array}{lll}-1.674676 & -3.728033 & 0.938191\end{array}$

$\begin{array}{llll}0.136525 & -3.147481 & 0.042484\end{array}$

$\begin{array}{lll}0.568033 & -2.944504 & 1.018273\end{array}$

$\begin{array}{llll}1.106515 & -3.172336 & -1.117790\end{array}$

$\begin{array}{llll}1.950053 & -2.531262 & -0.864812\end{array}$

$\begin{array}{lll}1.509788 & -4.190358 & -1.214866\end{array}$

$\begin{array}{llll}0.521224 & -2.715463 & -2.474835\end{array}$

$\begin{array}{llll}0.134892 & -3.576328 & -3.029917\end{array}$

$\begin{array}{lll}1.340190 & -2.306999 & -3.072477\end{array}$

$\begin{array}{lll}-4.687049 & -2.035151 & 0.184115\end{array}$

$\begin{array}{llll}-4.716235 & -3.377980 & 0.610643\end{array}$

$\begin{array}{lll}-5.683235 & -1.593118 & -0.708339\end{array}$

$\begin{array}{llll}-5.698747 & -4.247789 & 0.143983\end{array}$

$\begin{array}{lll}-3.969436 & -3.747341 & 1.305587\end{array}$

$-6.663384-2.466638-1.176480$

$\begin{array}{llll}-5.680098 & -0.552430 & -1.021937\end{array}$

$\begin{array}{lll}-6.671714 & -3.799504 & -0.756547\end{array}$

$\begin{array}{lll}-5.706825 & -5.280129 & 0.482517\end{array}$

$\begin{array}{lll}-7.422028 & -2.106538 & -1.865526\end{array}$

$\begin{array}{lll}-7.433594 & -4.482781 & -1.120592\end{array}$

$\begin{array}{lll}-4.582637 & 2.544256 & 1.343808\end{array}$

$\begin{array}{lll}-3.851638 & 3.754182 & 1.390241\end{array}$

$\begin{array}{lll}-3.973220 & 1.609828 & 2.202485\end{array}$

$\begin{array}{lll}-5.905690 & 2.768378 & 1.726946\end{array}$

$\begin{array}{lll}-4.550366 & 2.042617 & 0.015410\end{array}$

P1 endo-re

Number of imaginary frequencies $=0$
The smallest frequencies are $=19.030422 .084426 .5184 \mathrm{~cm}(-1)$ Electronic energy $=-2700.877626$

Zero-point correction $=$

0.898344

(Hartree/Particle)

Thermal correction to Energy $=\quad 0.946899$

Thermal correction to Enthalpy= $\quad 0.947843$

Thermal correction to Gibbs Free Energy $=0.817642$

Sum of electronic and zero-point Energies $=\quad-2699.979282$

Sum of electronic and thermal Energies $=\quad-2699.930727$

Sum of electronic and thermal Enthalpies $=\quad-2699.929783$

Sum of electronic and thermal Free Energies $=\quad-2700.059984$

Cartesian Coordinates

\begin{tabular}{|c|c|c|c|}
\hline 77 & -1.558666 & -1.024203 & $3-$ \\
\hline 15 & 0.323955 & 0.213928 & -0.022867 \\
\hline 8 & 1.416958 & 0.044447 & 1.188513 \\
\hline 8 & 1.284709 & -0.021635 & -1.351950 \\
\hline 7 & -0.093947 & 1.816913 & 0.177910 \\
\hline 6 & 2.396796 & -0.947401 & 1.180553 \\
\hline 6 & 2.289353 & -1.933277 & 2.184055 \\
\hline 1 & 1.479535 & -1.855752 & 2.901752 \\
\hline 6 & 3.208922 & -2.951572 & 2.229635 \\
\hline 1 & 3.145948 & -3.713957 & 3.000701 \\
\hline 6 & 4.237910 & -3.037578 & 1.253339 \\
\hline 6 & 5.148664 & -4.128718 & 1.244941 \\
\hline 1 & 5.056025 & -4.886030 & 2.018970 \\
\hline 6 & 6.117360 & -4.233604 & 0.272871 \\
\hline 1 & 6.805198 & -5.073984 & 0.272806 \\
\hline 6 & 6.207218 & -3.248958 & -0.739873 \\
\hline 1 & 6.956830 & -3.347032 & -1.519769 \\
\hline 6 & 5.350773 & -2.16 & -0.7 \\
\hline 1 & 5.429827 & -1.430678 & -1.539298 \\
\hline 6 & 4.351570 & -2.015968 & 0.250564 \\
\hline 6 & 3.437845 & -0.902175 & 0.267210 \\
\hline 6 & 3.575572 & 0.250901 & 5966 \\
\hline 6 & & & -0.735859 \\
\hline 6 & 5.868364 & 0.85 & 344 \\
\hline 1 & 5.801386 & 0.102776 & 0.935084 \\
\hline 6 & 6.998394 & 1.642026 & 0.073922 \\
\hline 1 & 7.812173 & 1.490204 & 0.77 \\
\hline 6 & 7.106968 & 2.647293 & -0.916364 \\
\hline 1 & 8.006993 & 3.251999 & -0.976460 \\
\hline 6 & 6.063380 & 2.865232 & -1.786809 \\
\hline 1 & 6.123749 & 3.647815 & -2.538517 \\
\hline 6 & $4.8^{\circ}$ & 2.0 & -1.7 \\
\hline 6 & 3.774987 & 2.351390 & -2.572107 \\
\hline 1 & 3.864605 & 3.134747 & -3.3 \\
\hline 6 & 2.601358 & 60 & -2.4 \\
\hline 1 & 1.736690 & 1.856401 & -3.059890 \\
\hline 6 & 2.512171 & 0.632401 & -1.466560 \\
\hline 6 & -1.526352 & 561 & -0.127668 \\
\hline 1 & -2.062940 & 2.150309 & 0.824255 \\
\hline 6 & -1.725573 & 3.37 & -0 \\
\hline 6 & -2.823100 & 4.186222 & -0.589602 \\
\hline 1 & -3.491547 & 3.906575 & 0.221565 \\
\hline 6 & -3.062314 & 5.352486 & -1.320887 \\
\hline 1 & -3.917116 & 5.977162 & -1.077029 \\
\hline 6 & -2.197994 & 5.718703 & -2.354964 \\
\hline 1 & -2.377960 & 6.628859 & -2.919952 \\
\hline 6 & -1.095050 & 4.912373 & -2.654460 \\
\hline 1 & -0.415002 & 5.196042 & -3.4 \\
\hline 6 & -0.861756 & 3.744479 & -1.927931 \\
\hline 1 & 0.001504 & 3.124006 & -2.148673 \\
\hline 6 & -2.109254 & 0.897961 & -0.920284 \\
\hline 1 & -1.786461 & 0.975944 & -1.964587 \\
\hline 1 & -3.200188 & 0.960431 & -0.913594 \\
\hline 6 & 0.540972 & 2.850550 & 1.073423 \\
\hline 1 & 0.106626 & 3.784086 & 0.705892 \\
\hline 6 & 0.069948 & 2.686570 & 2.516659 \\
\hline 6 & -1.106593 & 3.336132 & 2.922183 \\
\hline 1 & -1.619196 & 3.992534 & 2.223396 \\
\hline . & -1.621278 & 3.160898 & 4.209184 \\
\hline 1 & -2.535070 & 3.672046 & 4.499576 \\
\hline & -0.954020 & 2.340912 & 5.121244 \\
\hline
\end{tabular}




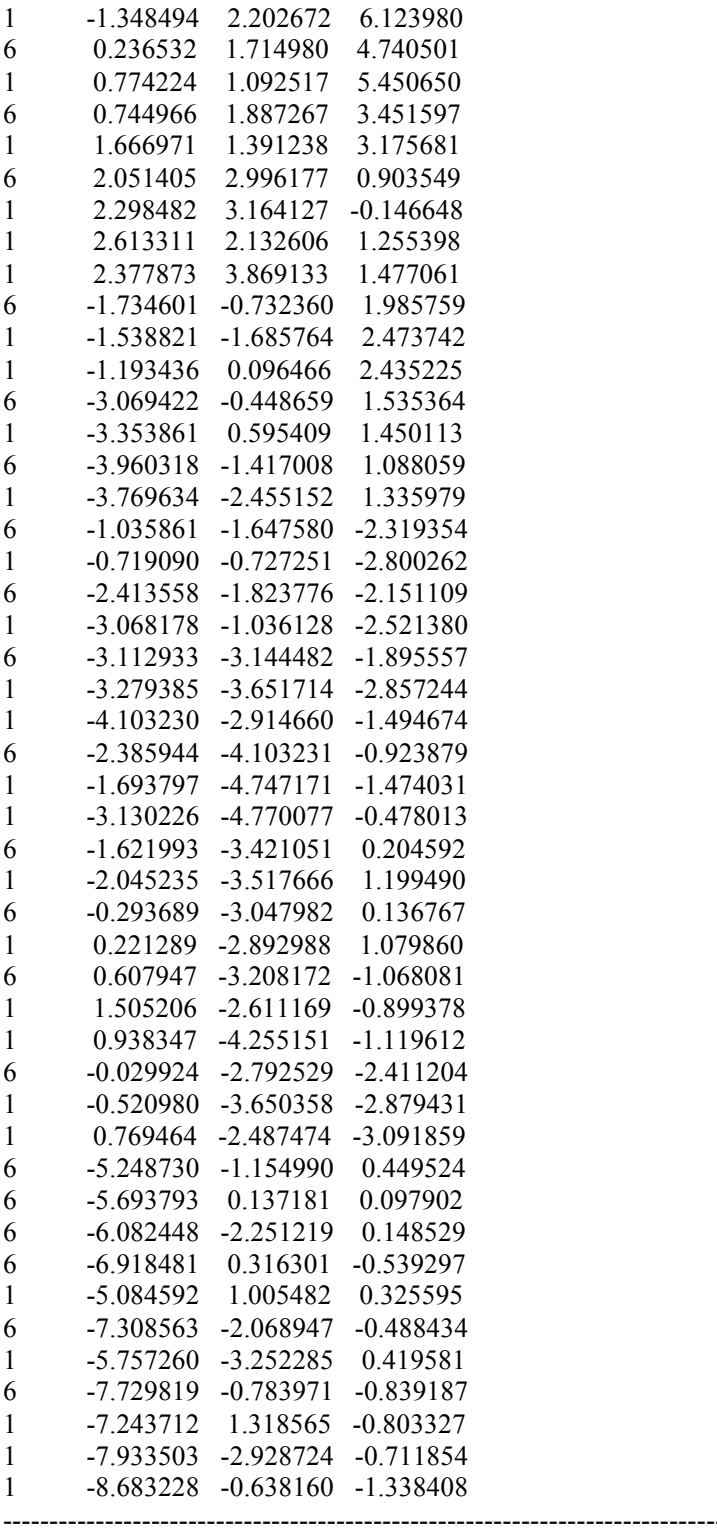

P1 exo-si

Number of imaginary frequencies $=0$

The smallest frequencies are $=10.977523 .215526 .7037 \mathrm{~cm}(-1)$ Electronic energy $=-2700.871334$

Zero-point correction $=$

(Hartree/Particle)

Thermal correction to Energy= $\quad 0.946703$

Thermal correction to Enthalpy= $\quad 0.947647$

Thermal correction to Gibbs Free Energy $=0.816297$

Sum of electronic and zero-point Energies $=\quad-2699.973374$

Sum of electronic and thermal Energies= $\quad-2699.924631$

Sum of electronic and thermal Enthalpies $=\quad-2699.923687$

Sum of electronic and thermal Free Energies $=\quad-2700.055037$

Cartesian Coordinates

$\begin{array}{lccc}77 & -1.721212 & -0.612148 & -0.215835 \\ 15 & 0.338494 & 0.299110 & -0.039200 \\ 8 & 1.357153 & -0.053155 & 1.199999 \\ 8 & 1.287281 & -0.070975 & -1.344971 \\ 7 & 0.183532 & 1.949763 & 0.154884 \\ 6 & 2.166914 & -1.187639 & 1.202910 \\ 6 & 1.872038 & -2.159149 & 2.182916 \\ 1 & 1.063147 & -1.963741 & 2.879010 \\ 6 & 2.613645 & -3.313458 & 2.232641 \\ 1 & 2.404711 & -4.068232 & 2.985228\end{array}$

$\begin{array}{lll}3.643539 & -3.549950 & 1.282549\end{array}$

$\begin{array}{lll}4.363416 & -4.775650 & 1.273881\end{array}$

$\begin{array}{lll}4.125352 & -5.521415 & 2.027689\end{array}$

$\begin{array}{llll}5.330788 & -5.020426 & 0.325873\end{array}$

$\begin{array}{lll}5.872041 & -5.961888 & 0.325171\end{array}$

$\begin{array}{llll}5.610418 & -4.046309 & -0.662002\end{array}$

$6.356994-4.252008-1.423559$

$4.942287-2.841135-0.671760$

$5.164449-2.112206-1.441777$

$3.951589-2.543894 \quad 0.305339$

$\begin{array}{llll}3.228980 & -1.297520 & 0.319679\end{array}$

$3.572405-0.172276-0.593966$

$\begin{array}{llll}4.886730 & 0.415609 & -0.623314\end{array}$

$\begin{array}{lll}5.908098 & 0.055790 & 0.299831\end{array}$

$\begin{array}{llll}5.699976 & -0.687864 & 1.059590\end{array}$

$\begin{array}{lll}7.150513 & 0.649846 & 0.251832\end{array}$

$\begin{array}{llll}7.910510 & 0.363484 & 0.973022\end{array}$

$\begin{array}{llll}7.444752 & 1.633670 & -0.722111\end{array}$

$\begin{array}{llll}8.430782 & 2.087813 & -0.751029\end{array}$

$\begin{array}{llll}6.474312 & 2.021486 & -1.617718\end{array}$

$\begin{array}{lll}6.679695 & 2.789626 & -2.358575\end{array}$

$\begin{array}{llll}5.177078 & 1.440840 & -1.585825\end{array}$

$\begin{array}{llll}4.159020 & 1.875971 & -2.477055\end{array}$

$\begin{array}{llll}4.393806 & 2.636753 & -3.215868\end{array}$

$\begin{array}{llll}2.886566 & 1.368504 & -2.383424\end{array}$

$\begin{array}{lll}2.085320 & 1.704916 & -3.031715\end{array}$

$\begin{array}{llll}2.606643 & 0.376464 & -1.420544\end{array}$

$\begin{array}{llll}-1.177275 & 2.446631 & -0.176403\end{array}$

$\begin{array}{llll}-1.704285 & 2.647311 & 0.762206\end{array}$

$\begin{array}{llll}-1.134375 & 3.744866 & -0.964085\end{array}$

$\begin{array}{llll}-2.040240 & 4.768696 & -0.661434\end{array}$

$\begin{array}{llll}-2.736970 & 4.639555 & 0.163729\end{array}$

$\begin{array}{llll}-2.050670 & 5.951181 & -1.405910\end{array}$

$\begin{array}{llll}-2.757138 & 6.738734 & -1.158983\end{array}$

$\begin{array}{llll}-1.146215 & 6.124316 & -2.455846\end{array}$

$\begin{array}{llll}-1.147071 & 7.046104 & -3.030564\end{array}$

$\begin{array}{llll}-0.233945 & 5.108395 & -2.759022\end{array}$

$\begin{array}{llll}0.477146 & 5.240047 & -3.570091\end{array}$

$\begin{array}{llll}-0.230071 & 3.925603 & -2.019562\end{array}$

$\begin{array}{llll}0.485986 & 3.141510 & -2.244013\end{array}$

$\begin{array}{llll}-1.959458 & 1.378435 & -0.948157\end{array}$

$\begin{array}{llll}-1.632226 & 1.388701 & -1.994091\end{array}$

$\begin{array}{llll}-3.016533 & 1.651202 & -0.948540\end{array}$

$\begin{array}{llll}0.962901 & 2.869337 & 1.062344\end{array}$

$\begin{array}{lll}0.717363 & 3.858552 & 0.666438\end{array}$

$\begin{array}{lll}0.417474 & 2.816329 & 2.487746\end{array}$

$\begin{array}{lll}-0.619519 & 3.689892 & 2.850035\end{array}$

$\begin{array}{lll}-0.967531 & 4.428645 & 2.132320\end{array}$

$\begin{array}{lll}-1.205609 & 3.628452 & 4.117100\end{array}$

$\begin{array}{lll}-2.010569 & 4.311632 & 4.373615\end{array}$

$\begin{array}{lll}-0.749464 & 2.696960 & 5.051707\end{array}$

$\begin{array}{lll}-1.200183 & 2.645161 & 6.038839\end{array}$

$\begin{array}{lll}0.305249 & 1.843737 & 4.713939\end{array}$

$\begin{array}{lll}0.682103 & 1.130918 & 5.442604\end{array}$

$\begin{array}{llll}0.884482 & 1.903958 & 3.445439\end{array}$

$\begin{array}{lll}1.698788 & 1.233112 & 3.203153\end{array}$

$\begin{array}{llll}2.481550 & 2.743809 & 0.958535\end{array}$

$2.801444 \quad 2.844223 \quad-0.080253$

$\begin{array}{lll}2.867902 & 1.803837 & 1.348435\end{array}$

$\begin{array}{lll}2.929827 & 3.558673 & 1.535253\end{array}$

$\begin{array}{lll}-1.863714 & -0.001862 & 1.901892\end{array}$

$\begin{array}{lll}-1.173019 & -0.490943 & 2.583444\end{array}$

$\begin{array}{lll}-1.847653 & 1.080739 & 1.983746\end{array}$

$\begin{array}{lll}-3.123664 & -0.656588 & 1.697806\end{array}$

$\begin{array}{lll}-3.243070 & -1.659546 & 2.094521\end{array}$

$\begin{array}{lll}-4.120821 & -0.129821 & 0.889266\end{array}$

$\begin{array}{llll}-4.058832 & 0.920211 & 0.622637\end{array}$

$\begin{array}{lll}-1.256574 & -1.261469 & -2.359852\end{array}$

$\begin{array}{llll}-0.850405 & -0.371777 & -2.831319\end{array}$

$\begin{array}{lll}-2.648469 & -1.291740 & -2.198089\end{array}$

$\begin{array}{llll}-3.201597 & -0.421446 & -2.549352\end{array}$

$\begin{array}{llll}-3.509202 & -2.523746 & -2.017158\end{array}$

$\begin{array}{llll}-3.773481 & -2.918277 & -3.009344\end{array}$

$\begin{array}{llll}-4.448857 & -2.208237 & -1.557297\end{array}$

$-2.887288 \quad-3.649370 \quad-1.164552$ 


\begin{tabular}{lrrr}
1 & -2.253008 & -4.288517 & -1.784578 \\
1 & -3.697354 & -4.284668 & -0.793784 \\
6 & -2.081292 & -3.163760 & 0.025926 \\
1 & -2.568707 & -3.226541 & 0.992951 \\
6 & -0.729994 & -2.936306 & 0.019727 \\
1 & -0.231500 & -2.858466 & 0.981400 \\
6 & 0.177274 & -3.090494 & -1.179590 \\
1 & 1.132602 & -2.619577 & -0.945576 \\
1 & 0.388969 & -4.159786 & -1.319523 \\
6 & -0.366818 & -2.495992 & -2.498840 \\
1 & -0.922157 & -3.256840 & -3.055403 \\
1 & 0.488481 & -2.223248 & -3.122318 \\
6 & -5.344303 & -0.812175 & 0.471363 \\
6 & -5.681489 & -2.116304 & 0.887175 \\
6 & -6.199189 & -0.156672 & -0.436296 \\
6 & -6.825304 & -2.741945 & 0.399170 \\
1 & -5.047889 & -2.642410 & 1.593848 \\
6 & -7.342047 & -0.786476 & -0.926177 \\
1 & -5.952679 & 0.850928 & -0.761863 \\
6 & -7.657698 & -2.083455 & -0.513265 \\
1 & -7.070426 & -3.747221 & 0.729659 \\
1 & -7.985495 & -0.266145 & -1.629737 \\
1 & -8.546739 & -2.577116 & -0.894454 \\
----------------------------------------------------------- \\
& & & 1 \\
\hline & & &
\end{tabular}

Number of imaginary frequencies $=0$

The smallest frequencies are $=10.071612 .637117 .3733 \mathrm{~cm}(-1)$ Electronic energy $=-5180.7406719$

Zero-point correction $=$

(Hartree/Particle)

Thermal correction to Energy=

Thermal correction to Enthalpy=

Thermal correction to Gibbs Free Energy=

Sum of electronic and zero-point Energies=

Sum of electronic and thermal Energies=

Sum of electronic and thermal Enthalpies=

Sum of electronic and thermal Free Energies=

0.862696

0.863640

0.672323

$-5179.951135$

$-5179.877976$

$-5179.877031$

$-5180.068349$

Cartesian Coordinates

$\begin{array}{lrrr}26 & 0.660887 & -3.063994 & -3.570398 \\ 15 & -1.420290 & -1.656033 & 0.986016 \\ 15 & 1.300945 & 0.514837 & -0.487597 \\ 6 & 0.188687 & -2.516170 & -1.609163 \\ 6 & 0.732123 & -1.399507 & -2.348797 \\ 6 & -0.126403 & -1.158092 & -3.464738 \\ 1 & 0.019164 & -0.394903 & -4.216422 \\ 6 & -1.172981 & -2.125289 & -3.444084 \\ 1 & -1.954709 & -2.233165 & -4.184043 \\ 6 & -0.977984 & -2.962977 & -2.308856 \\ 1 & -1.590017 & -3.807326 & -2.032099 \\ 6 & 2.578410 & -3.704751 & -4.033883 \\ 1 & 3.477672 & -3.347602 & -3.549471 \\ 6 & 1.957401 & -3.126033 & -5.183433 \\ 1 & 2.300198 & -2.251405 & -5.719930 \\ 6 & 0.776510 & -3.876392 & -5.472076 \\ 1 & 0.067551 & -3.666348 & -6.262152 \\ 6 & 0.667665 & -4.919752 & -4.502689 \\ 1 & -0.138010 & -5.638311 & -4.428367 \\ 6 & 1.780507 & -4.815139 & -3.615814 \\ 1 & 1.965712 & -5.447420 & -2.758137 \\ 6 & 0.735895 & -2.998111 & -0.308961 \\ 6 & 1.989044 & -3.628930 & -0.286552 \\ 1 & 2.487571 & -3.809688 & -1.230872 \\ 6 & 2.609232 & -3.993576 & 0.908257 \\ 1 & 3.593385 & -4.450944 & 0.891503 \\ 6 & 1.967347 & -3.739810 & 2.117848 \\ 1 & 2.439382 & -4.002908 & 3.059876 \\ 6 & 0.718592 & -3.117610 & 2.121766 \\ 1 & 0.253916 & -2.894576 & 3.074064 \\ 6 & 0.088321 & -2.730683 & 0.928363 \\ 6 & 1.916525 & -0.573256 & -1.924503 \\ 1 & 2.660718 & -1.244733 & -1.489976 \\ 6 & 2.559950 & 0.210371 & -3.071851\end{array}$

$3.461108 \quad 0.732109 \quad-2.740216$

$\begin{array}{llll}1.877779 & 0.956809 & -3.490065\end{array}$

$2.836107-0.482873-3.872676$

$\begin{array}{lll}-1.880430 & -1.562543 & 2.766537\end{array}$

$\begin{array}{lll}-1.807499 & -0.319616 & 3.408990\end{array}$

$\begin{array}{lll}-1.496722 & 0.555369 & 2.849661\end{array}$

$\begin{array}{lll}-2.148365 & -0.197940 & 4.758343\end{array}$

$\begin{array}{lll}-2.080989 & 0.770572 & 5.244847\end{array}$

$\begin{array}{lll}-2.570291 & -1.317274 & 5.475231\end{array}$

$\begin{array}{lll}-2.835393 & -1.224469 & 6.524702\end{array}$

$-2.660723-2.559734 \quad 4.838340$

$\begin{array}{lll}-2.998902 & -3.432075 & 5.390577\end{array}$

$\begin{array}{lll}-2.323872 & -2.682264 & 3.491429\end{array}$

$\begin{array}{llll}-2.408500 & -3.649334 & 3.005728\end{array}$

$\begin{array}{lll}-2.782891 & -2.659686 & 0.280115\end{array}$

$\begin{array}{lll}-2.800435 & -4.062132 & 0.303007\end{array}$

$\begin{array}{lll}-1.963513 & -4.612102 & 0.724089\end{array}$

$\begin{array}{lll}-3.882979 & -4.755450 & -0.241931\end{array}$

$\begin{array}{llll}-3.889689 & -5.841932 & -0.227463\end{array}$

$\begin{array}{llll}-4.954870 & -4.054583 & -0.805449\end{array}$

$\begin{array}{llll}-5.795013 & -4.597686 & -1.229343\end{array}$

$\begin{array}{llll}-4.944111 & -2.657598 & -0.818861\end{array}$

$\begin{array}{lll}-5.774355 & -2.101402 & -1.244476\end{array}$

$-3.860387-1.961501-0.281740$

$\begin{array}{lll}-3.857927 & -0.879329 & -0.293879\end{array}$

$\begin{array}{lll}2.350172 & 0.047378 & 0.953384\end{array}$

$\begin{array}{llll}3.648353 & -0.466636 & 0.861162\end{array}$

$\begin{array}{lll}4.137016 & -0.576891 & -0.100223\end{array}$

$\begin{array}{lll}4.332540 & -0.852959 & 2.015390\end{array}$

$\begin{array}{lll}3.744010 & -0.730989 & 3.273374\end{array}$

$\begin{array}{llll}4.277722 & -1.042375 & 4.163898\end{array}$

$\begin{array}{lll}2.455624 & -0.206310 & 3.364497\end{array}$

$\begin{array}{llll}1.760405 & 0.174036 & 2.217741\end{array}$

$\begin{array}{lll}0.748550 & 0.554523 & 2.301532\end{array}$

$\begin{array}{llll}1.835588 & 2.239597 & -0.852701\end{array}$

$\begin{array}{llll}0.832297 & 3.118586 & -1.278895\end{array}$

$\begin{array}{lll}-0.183629 & 2.758542 & -1.399212\end{array}$

$\begin{array}{llll}1.127062 & 4.460170 & -1.520813\end{array}$

$\begin{array}{llll}2.423900 & 4.944745 & -1.358169\end{array}$

$\begin{array}{llll}2.649579 & 5.988499 & -1.541117\end{array}$

$\begin{array}{llll}3.424462 & 4.064915 & -0.942760\end{array}$

$\begin{array}{llll}3.137853 & 2.723286 & -0.682038\end{array}$

$\begin{array}{llll}3.928879 & 2.067466 & -0.333653\end{array}$

$\begin{array}{llll}-0.970035 & 0.353149 & -0.015858\end{array}$

$\begin{array}{llll}4.844817 & 4.543484 & -0.807316\end{array}$

$\begin{array}{llll}-0.001060 & 5.397866 & -1.848697\end{array}$

$\begin{array}{llll}5.678299 & -1.509984 & 1.886493\end{array}$

$\begin{array}{lll}1.756590 & -0.159926 & 4.694910\end{array}$

$\begin{array}{llll}2.622849 & -0.031357 & 5.723488\end{array}$

$\begin{array}{llll}0.879454 & 0.866171 & 4.769708\end{array}$

$1.050238 \quad-1.297278 \quad 4.919258$

$\begin{array}{lll}5.556530 & -2.856659 & 1.759656\end{array}$

$6.460045 \quad-1.289476 \quad 2.965000$

$\begin{array}{lll}6.353428 & -1.077963 & 0.797550\end{array}$

$\begin{array}{llll}5.587708 & 4.206036 & -1.889852\end{array}$

$\begin{array}{llll}5.456288 & 3.993187 & 0.266815\end{array}$

$\begin{array}{llll}4.924035 & 5.884772 & -0.677039\end{array}$

$\begin{array}{llll}0.420829 & 6.513093 & -2.479295\end{array}$

$\begin{array}{llll}-0.935723 & 4.811650 & -2.630870\end{array}$

$\begin{array}{llll}-0.643199 & 5.802234 & -0.719859\end{array}$

$\begin{array}{lll}-1.881516 & 3.171368 & 1.239552\end{array}$

$\begin{array}{llll}-2.933969 & 1.177681 & -2.377993\end{array}$

$\begin{array}{llll}-2.113199 & 0.817166 & -1.646209\end{array}$

$\begin{array}{lll}-5.428473 & 1.801018 & 0.402681\end{array}$

$\begin{array}{llll}-4.324726 & 2.764765 & -0.351780\end{array}$

$\begin{array}{llll}-6.628932 & 2.627541 & -0.332146\end{array}$

$\begin{array}{lll}-5.465529 & 2.848318 & 1.664488\end{array}$

$\begin{array}{lll}-4.205516 & 0.979634 & 1.126482\end{array}$

$\begin{array}{lll}-6.516166 & 0.843824 & 1.146379\end{array}$

$\begin{array}{llll}-5.373661 & 0.758622 & -0.869766\end{array}$

$\begin{array}{lll}-1.336702 & 2.175226 & 1.009176\end{array}$

$\begin{array}{lll}-2.585562 & 4.410402 & 1.514877\end{array}$

$\begin{array}{lll}-2.564606 & 5.042568 & 0.623614\end{array}$

$\begin{array}{llll}-3.620762 & 4.172389 & 1.769177\end{array}$

$\begin{array}{llll}-2.104113 & 4.936727 & 2.343834\end{array}$ 


$\begin{array}{llll}6 & -3.968306 & 1.635969 & -3.287319 \\ 1 & -3.514834 & 2.054555 & -4.190093 \\ 1 & -4.616708 & 0.797915 & -3.555662 \\ 1 & -4.561934 & 2.396273 & -2.775685 \\ ----------------------------------------- \\ \end{array}$

Number of imaginary frequencies $=0$

The smallest frequencies are= $8.423814 .320621 .2311 \mathrm{~cm}(-1)$ Electronic energy $=-5462.9553639$

Zero-point correction=

0.888922

(Hartree/Particle)

Thermal correction to Energy= $\quad 0.966353$

Thermal correction to Enthalpy= $\quad 0.967298$

Thermal correction to Gibbs Free Energy $=0.768962$

Sum of electronic and zero-point Energies=

Sum of electronic and thermal Energies=

Sum of electronic and thermal Enthalpies=

$-5462.066442$

$-5461.989011$

$-5461.988066$

Sum of electronic and thermal Free Energies=

$-5462.186402$

\section{Cartesian Coordinates}

\begin{tabular}{|c|c|c|c|}
\hline & -1.545863 & -2.987153 & -3 \\
\hline & -1.289912 & -1.622405 & 704 \\
\hline 15 & 1.158662 & -0.078718 & -0.902259 \\
\hline 6 & -1.116474 & -2.575109 & -1.538954 \\
\hline 6 & -0.584069 & -1.555711 & -2.413338 \\
\hline U & -1.677458 & -0.975875 & -3.128306 \\
\hline 1 & -1.602857 & -0.195898 & -3.873487 \\
\hline 6 & -2.874695 & -1.644574 & -2.730965 \\
\hline 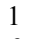 & -3.865052 & -1.463791 & -3.125325 \\
\hline 6 & -2.529483 & -2.630817 & -1.761450 \\
\hline 1 & -3.213063 & -3.317504 & -1.286487 \\
\hline 0 & -0.184769 & -4.078595 & -4.675850 \\
\hline 1 & 0.886491 & -4.066016 & -4.524284 \\
\hline 0 & -0.924226 & -3.19 & -5.515994 \\
\hline 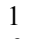 & -0.512399 & -2.384687 & -6.108210 \\
\hline 6 & -2.308381 & -3.5 & 285 \\
\hline 1 & -3.128222 & -3.010747 & -5.885793 \\
\hline 6 & -2.425183 & -4.617 & -4.489796 \\
\hline 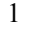 & -3.348067 & -5.0 & -4.1 \\
\hline 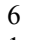 & -1.114475 & -4.961894 & -4.043364 \\
\hline 1 & -0.875446 & -5.733051 & -3.323780 \\
\hline 6 & -0.280954 & -3.3 & 1504 \\
\hline 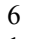 & 0.631710 & $-4.2^{\prime}$ & -1.03 \\
\hline 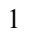 & 0.6 & -4.4 & -2.09 \\
\hline 6 & 1.546630 & -4.912948 & -0.192056 \\
\hline 1 & 2.258082 & -5.622687 & -0.602017 \\
\hline 6 & 1.55 & -4.6 & \\
\hline 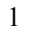 & 2.272160 & -5.071864 & 1.834493 \\
\hline 6 & 0.650492 & $-3.668 \mathrm{~S}$ & 1.66 \\
\hline 1 & 0.693605 & -3.4 & 20 \\
\hline 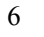 & -0.271554 & -3.01 & 0.8 \\
\hline 6 & 0.859841 & -1.13 & -2.45 \\
\hline 1 & 1.464545 & -2.032917 & -2.29 \\
\hline 6 & 1.289532 & -0.494979 & -3.775417 \\
\hline 1 & 2.354160 & -0.245535 & -3.765660 \\
\hline 1 & 0.734161 & 0.421889 & -3.991202 \\
\hline 1 & 1.105529 & -1.197372 & -4.593986 \\
\hline 6 & -1.000358 & -1.673690 & 3.329848 \\
\hline . & -0.304941 & -0.604394 & 3.910578 \\
\hline 1 & 0.004344 & 0.2373 & 3.30 \\
\hline 6 & -0.050230 & -0.592722 & 5.283069 \\
\hline 1 & 0.484189 & 0.245207 & 5.720399 \\
\hline 6 & -0.488686 & -1.645512 & 6.086018 \\
\hline 1 & -0.293541 & -1.634551 & 7.154799 \\
\hline 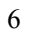 & -1.193969 & -2.709996 & 5.515233 \\
\hline 1 & -1.547915 & -3.526747 & 6.138223 \\
\hline 6 & -1.451114 & -2.724627 & 4.144891 \\
\hline 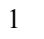 & -2.005013 & -3.552892 & 3.715696 \\
\hline 6 & -3.022401 & -2.192473 & 1.290537 \\
\hline 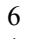 & -3.381096 & -3.546713 & 1.379477 \\
\hline 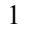 & -2.627302 & -4.298394 & 1.594347 \\
\hline 6 & -4.700343 & -3.940726 & 1.150837 \\
\hline 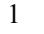 & -4.969089 & -4.991182 & 1.21929 \\
\hline
\end{tabular}

\begin{tabular}{|c|c|c|c|}
\hline 6 & -5.670286 & -2.987355 & 0.824812 \\
\hline 1 & -6.695336 & -3.296305 & 0.640164 \\
\hline 6 & -5.319280 & -1.637826 & 0.738793 \\
\hline 1 & -6.069221 & -0.892413 & 0.489541 \\
\hline 6 & -4.001296 & -1.241006 & 0.970636 \\
\hline 1 & -3.732076 & -0.192847 & 0.910497 \\
\hline 6 & 2.510762 & -0.953777 & -0.004736 \\
\hline 6 & 3.453080 & -1.789701 & -0.615598 \\
\hline 1 & 3.468448 & -1.922644 & -1.691284 \\
\hline 6 & 4.388950 & -2.475126 & 0.162066 \\
\hline 6 & 4.417469 & -2.327386 & 1.548468 \\
\hline 1 & 5.146138 & -2.864721 & 2.144728 \\
\hline 6 & 3.492032 & -1.477772 & 2.150914 \\
\hline 6 & 2.538223 & -0.807114 & 1.388397 \\
\hline 1 & 1.820763 & -0.158520 & 1.873193 \\
\hline 6 & 1.952839 & 1.474438 & -1.478692 \\
\hline 6 & 1.130679 & 2.432595 & -2.093273 \\
\hline 1 & 0.083045 & 2.218767 & -2.273850 \\
\hline 6 & 1.652401 & 3.672305 & -2.459221 \\
\hline 6 & 2.981559 & 3.997538 & -2.186865 \\
\hline 1 & 3.372456 & 4.976043 & -2.443095 \\
\hline 6 & 3.788855 & 3.052745 & -1.557261 \\
\hline 6 & 3.290093 & 1.794352 & -1.218754 \\
\hline 1 & 3.941000 & 1.079780 & -0.729682 \\
\hline 29 & -0.647890 & 0.318319 & 0.552784 \\
\hline 6 & 5.211588 & 3.418905 & -1.233650 \\
\hline 6 & 0.764774 & 4.726954 & -3.058045 \\
\hline 6 & 5.313016 & -3.467390 & -0.486740 \\
\hline 6 & 3.441248 & -1.360785 & 3.648582 \\
\hline 9 & 4.654748 & -1.539318 & 4.214899 \\
\hline 9 & 2.980666 & -0.150787 & 4.046378 \\
\hline 9 & 2.615188 & -2.290361 & 4.190297 \\
\hline 9 & 4.789742 & -4.719962 & -0.451488 \\
\hline 9 & 6.510098 & -3.530282 & 0.136432 \\
\hline 9 & 5.544683 & -3.178867 & -1.786629 \\
\hline 9 & 6.000694 & 3.423808 & -2.330960 \\
\hline 9 & 5.769132 & 2.565569 & -0.344545 \\
\hline 9 & 5.287262 & 4.661538 & -0.694726 \\
\hline 9 & 1.393268 & 5.425221 & -4.027642 \\
\hline 9 & -0.362920 & 4.210617 & -3.592165 \\
\hline 9 & 0.380219 & 5.634863 & -2.118957 \\
\hline 6 & 0.756128 & 2.918869 & 1.465384 \\
\hline 6 & -0.532466 & 3.687903 & 1.224465 \\
\hline 6 & -1.403440 & 3.274633 & 0.060281 \\
\hline 7 & 1.927692 & 3.586285 & 1.513164 \\
\hline 6 & 3.104464 & 2.952794 & 2.110186 \\
\hline 1 & 3.525753 & 3.630870 & 2.859815 \\
\hline 1 & 2.823306 & 2.018631 & 2.591455 \\
\hline 1 & 3.869314 & 2.753685 & 1.355227 \\
\hline 6 & 2.114189 & 4.973405 & 1.082184 \\
\hline 1 & 1.467898 & 5.217164 & 0.239838 \\
\hline 1 & 1.936133 & 5.677844 & 1.903074 \\
\hline 1 & 3.145836 & 5.084481 & 0.742488 \\
\hline 8 & 0.698291 & 1.695603 & 1.717606 \\
\hline 6 & -1.967843 & 4.268861 & -0.746950 \\
\hline 6 & -2.852030 & 3.933846 & -1.766945 \\
\hline 1 & -1.706433 & 5.306932 & -0.581723 \\
\hline 6 & -3.155263 & 2.587893 & -1.930712 \\
\hline 6 & -2.574810 & 1.635145 & -1.106577 \\
\hline 1 & -2.797365 & 0.581275 & -1.214944 \\
\hline 7 & -1.702428 & 1.972601 & -0.138164 \\
\hline 1 & -3.283152 & 4.698221 & -2.403502 \\
\hline 35 & -4.397366 & 2.009358 & -3.320961 \\
\hline 15 & -3.149612 & 2.058034 & +3.898785 \\
\hline 9 & -1.514625 & 2.242758 & 3.862387 \\
\hline 9 & -3.272801 & 2.947787 & 2.517597 \\
\hline 9 & -3.275955 & 3.424109 & 4.784703 \\
\hline 9 & -3.013496 & 1.164022 & 5.256016 \\
\hline 9 & -4.771576 & 1.875807 & 3.919746 \\
\hline 9 & -3.008260 & 0.703188 & 2.983662 \\
\hline 1 & -1.112118 & 3.490594 & 2.131301 \\
\hline 1 & -0.370765 & 4.762461 & 1.174334 \\
\hline
\end{tabular}


Number of imaginary frequencies $=0$

The smallest frequencies are= $12.194815 .078216 .8789 \mathrm{~cm}(-1)$ Electronic energy $=-5925.1252622$

Zero-point correction=

(Hartree/Particle)

Thermal correction to Energy $=\quad 1.226509$

Thermal correction to Enthalpy= 1.227453

Thermal correction to Gibbs Free Energy=

Sum of electronic and zero-point Energies=

Sum of electronic and thermal Energies=

Sum of electronic and thermal Enthalpies=

$-5923.987682$

$-5923.898753$

$-5923.897809$

Sum of electronic and thermal Free Energies=

$-5924.120776$

Cartesian Coordinates

\begin{tabular}{|c|c|c|c|}
\hline & 7812 & 264 & 0.805549 \\
\hline & -0.664536 & -1.201265 & \\
\hline & -1.861760 & 0.827211 & \\
\hline & -3.203889 & -2.033928 & -0.398979 \\
\hline & -3.182313 & -1.556765 & 0.966007 \\
\hline & -2.793497 & -2.646663 & 1.805074 \\
\hline & -2.699955 & -2.614138 & 2.881951 \\
\hline & -2.606442 & -3.798687 & 0.983179 \\
\hline & -2.350284 & -4.791011 & 1.327560 \\
\hline & -2.861234 & -3.423951 & -0.368093 \\
\hline & -2.830292 & -4.082174 & -1.222061 \\
\hline & -6.471456 & -2.400725 & 1.064043 \\
\hline & -6.750438 & -1.355376 & 1.076849 \\
\hline & -6.070529 & -3.176019 & 2.195528 \\
\hline & -5.983590 & -2.819392 & 3.213195 \\
\hline & -5.758189 & -4.493400 & 1.739575 \\
\hline & -5.389036 & -5.306438 & 2.350790 \\
\hline & -5.966339 & -4.533201 & 929 \\
\hline & -5.782131 & -5.380574 & -0.320210 \\
\hline 6 & -6.408029 & -3.242149 & -0.090202 \\
\hline 1 & -6.618732 & -2.944565 & -1.108567 \\
\hline & -3.465907 & -1.156200 & -1.577956 \\
\hline 6 & -4.744337 & -0.597675 & -1.734128 \\
\hline 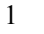 & -5.518329 & -0.890959 & -1.0 \\
\hline 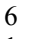 & -5.028899 & 0.333506 & -2.733131 \\
\hline 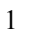 & -6.019736 & 0.77 & -2.799189 \\
\hline . & -4.026345 & 0.707129 & -3.624188 \\
\hline 1 & -4.222090 & 1.435026 & -4.4 \\
\hline 6 & -2.75 & 0.15 & -3.498022 \\
\hline 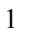 & -1.976119 & 0.483657 & -4.181117 \\
\hline 6 & -2.440471 & -0.759327 & -2.480105 \\
\hline 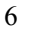 & -3.415671 & -0.12 & 1.374392 \\
\hline 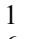 & -4.210333 & 0.271996 & 0.741313 \\
\hline 6 & -3.827218 & 0.04 & 2.83 \\
\hline 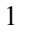 & -4.020724 & 1.097492 & 3.06 \\
\hline 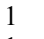 & -3.060846 & -0.312182 & 160 \\
\hline 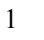 & -4.741473 & -0.5 & 332 \\
\hline & 0.237227 & -0.449388 & -3.58 \\
\hline & 0.980656 & 0.717192 & -3.360165 \\
\hline 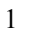 & 1.073642 & 1.11 & -2.354096 \\
\hline & 1.629159 & 1.358770 & -4.417262 \\
\hline 1 & 2.187281 & 2.269524 & -4.229561 \\
\hline 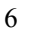 & 1.562537 & 0.829058 & -5.706276 \\
\hline & 2.073507 & 1.325556 & -6.526554 \\
\hline 6 & 0.84 & -0.347498 & 447 \\
\hline 1 & 0.797158 & -0.770607 & -6.938066 \\
\hline & 0.181719 & -0.982490 & -4.88 \\
\hline & -0.376480 & -1.89 & -5.082773 \\
\hline & -0.551049 & -3.004883 & -2.490648 \\
\hline & -1.409474 & -3.666632 & -3.383339 \\
\hline & -2.191168 & -3.114814 & -3.897598 \\
\hline 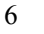 & -1.280965 & -5.040879 & -3.590896 \\
\hline 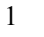 & -1.951258 & -5.547048 & -4.280151 \\
\hline 6 & -0.293630 & -5.763474 & -2.912368 \\
\hline & -0.195395 & -6.833171 & -3.075191 \\
\hline & 0.568021 & -5.106621 & -2.030802 \\
\hline & 1.347223 & -5.656264 & -1.510568 \\
\hline & 0.438868 & -3.733084 & -1.816566 \\
\hline & 1.114557 & -3.230215 & -1.138033 \\
\hline & -2.490383 & 2.172916 & -0.248797 \\
\hline
\end{tabular}

(1)

(1)

(1)

9

9

9
9
9

9
9

6

6

6

6

6

1

6

1
6
1
1

1
1
1
8

1
8
6
6

8
6
6

1
6
6

6
6
1

17

$\begin{array}{llll}-3.765173 & 2.742506 & -0.155491\end{array}$

$\begin{array}{lll}-4.447691 & 2.455827 & 0.637297\end{array}$

$\begin{array}{llll}-4.179222 & 3.685509 & -1.099392\end{array}$

$\begin{array}{llll}-3.329783 & 4.096830 & -2.126306\end{array}$

$\begin{array}{llll}-3.661099 & 4.827326 & -2.854680\end{array}$

$\begin{array}{llll}-2.050623 & 3.548099 & -2.198770\end{array}$

$\begin{array}{lll}-1.639746 & 2.581943 & -1.283057\end{array}$

$\begin{array}{llll}-0.654850 & 2.141008 & -1.368301\end{array}$

$\begin{array}{lll}-1.216136 & 1.693936 & 2.320750\end{array}$

$\begin{array}{llll}-0.579661 & 0.896232 & 3.284949\end{array}$

$\begin{array}{lll}-0.576577 & -0.182384 & 3.182954\end{array}$

$\begin{array}{lll}0.087883 & 1.484443 & 4.355311\end{array}$

$\begin{array}{lll}0.151116 & 2.873288 & 4.479179\end{array}$

$\begin{array}{lll}0.689574 & 3.329723 & 5.303104\end{array}$

$\begin{array}{lll}-0.470888 & 3.663615 & 3.513670\end{array}$

$\begin{array}{lll}-1.157756 & 3.086380 & 2.444087\end{array}$

$\begin{array}{llll}-1.622593 & 3.724220 & 1.702208\end{array}$

$\begin{array}{lll}-0.117498 & -0.354763 & -0.150150\end{array}$

$\begin{array}{lll}-0.335201 & 5.157789 & 3.621680\end{array}$

$\begin{array}{lll}0.847252 & 0.635385 & 5.336543\end{array}$

$\begin{array}{llll}-5.598594 & 4.178722 & -1.058498\end{array}$

$\begin{array}{llll}-1.129604 & 3.903602 & -3.332433\end{array}$

$\begin{array}{llll}-1.393058 & 5.124494 & -3.844930\end{array}$

$\begin{array}{llll}0.169209 & 3.897166 & -2.943398\end{array}$

$\begin{array}{llll}-1.234242 & 3.016656 & -4.352843\end{array}$

$\begin{array}{llll}-6.438112 & 3.300345 & -1.665339\end{array}$

$\begin{array}{llll}-5.750150 & 5.364047 & -1.685905\end{array}$

$\begin{array}{lll}-6.047327 & 4.327646 & 0.208804\end{array}$

$\begin{array}{llll}-0.807553 & 5.624946 & 4.798853\end{array}$

$\begin{array}{llll}-0.986374 & 5.810275 & 2.634523\end{array}$

$\begin{array}{lll}0.967423 & 5.534861 & 3.551444\end{array}$

$\begin{array}{lll}0.601478 & 0.995669 & 6.614910\end{array}$

$\begin{array}{lll}0.557167 & -0.677374 & 5.217219\end{array}$

$\begin{array}{lll}2.189264 & 0.762887 & 5.153866\end{array}$

$\begin{array}{lll}1.890108 & 1.757651 & 0.884097\end{array}$

$\begin{array}{lll}2.598730 & 0.767452 & 1.781852\end{array}$

$\begin{array}{lll}2.129852 & -0.660053 & 1.955840\end{array}$

$\begin{array}{lll}2.122492 & 3.063234 & 1.154552\end{array}$

$\begin{array}{llll}1.539035 & 4.107058 & 0.314673\end{array}$

$\begin{array}{llll}2.333662 & 4.689946 & -0.163698\end{array}$

$\begin{array}{llll}0.918610 & 3.660042 & -0.456762\end{array}$

$\begin{array}{llll}0.931688 & 4.780120 & 0.927235\end{array}$

$\begin{array}{lll}3.022013 & 3.515615 & 2.225947\end{array}$

$\begin{array}{llll}2.585921 & 3.343407 & 3.213937\end{array}$

$\begin{array}{llll}3.994022 & 3.019563 & 2.154257\end{array}$

$\begin{array}{lll}3.171104 & 4.589032 & 2.109324\end{array}$

$\begin{array}{llll}1.186231 & 1.417738 & -0.092000\end{array}$

$\begin{array}{lll}2.857091 & -1.416855 & 2.891021\end{array}$

$\begin{array}{lll}2.495450 & -2.722310 & 3.184055\end{array}$

$\begin{array}{lll}3.704549 & -0.964722 & 3.394551\end{array}$

$\begin{array}{lll}1.392667 & -3.241465 & 2.512832\end{array}$

$\begin{array}{lll}0.716701 & -2.471285 & 1.586187\end{array}$

$\begin{array}{lll}-0.129301 & -2.862104 & 1.036677\end{array}$

$\begin{array}{llll}1.077343 & -1.198614 & 1.316790\end{array}$

$\begin{array}{lll}3.051293 & -3.307742 & 3.907896\end{array}$

$\begin{array}{lll}0.790489 & -5.069230 & 2.848368\end{array}$

$\begin{array}{lll}3.981097 & -2.220880 & -1.246433\end{array}$

$\begin{array}{llll}4.274791 & -0.938685 & -0.261771\end{array}$

$\begin{array}{llll}3.163033 & -2.956101 & -0.027682\end{array}$

$\begin{array}{lll}5.373329 & -2.918582 & -0.738670\end{array}$

$\begin{array}{llll}4.789146 & -1.483399 & -2.465697\end{array}$

$\begin{array}{llll}3.673957 & -3.499526 & -2.214793\end{array}$

$\begin{array}{llll}2.598847 & -1.496498 & -1.752931\end{array}$

$\begin{array}{llll}3.638117 & 0.748867 & 1.413928\end{array}$

$\begin{array}{lll}2.670300 & 1.180259 & 2.789453\end{array}$

$\begin{array}{lll}6.422191 & 0.455642 & 1.881719\end{array}$

$\begin{array}{lll}7.787816 & 0.104342 & 1.298678\end{array}$

$\begin{array}{llll}5.803562 & -0.453268 & 1.933813\end{array}$

$\begin{array}{llll}6.523019 & 0.824627 & 2.910785\end{array}$

$\begin{array}{lll}7.608824 & -0.213920 & -0.179219\end{array}$

$\begin{array}{lll}8.235877 & -0.752200 & 1.814063\end{array}$

$\begin{array}{llll}8.470428 & 0.955937 & 1.410305\end{array}$

$\begin{array}{llll}8.582303 & -0.350157 & -0.663086\end{array}$

$\begin{array}{llll}7.043001 & -1.146297 & -0.307345\end{array}$

$\begin{array}{lll}5.706603 & 1.459707 & 1.099925\end{array}$ 


\begin{tabular}{|c|c|c|c|}
\hline 6 & 5.947446 & 1.576857 & -0.172609 \\
\hline 7 & 6.910422 & 0.880658 & -0.870904 \\
\hline 6 & 5.062279 & 2.573799 & -0.903108 \\
\hline 1 & 4.386264 & 2.964858 & -0.144373 \\
\hline 1 & 5.651072 & 3.424007 & -1.266920 \\
\hline 6 & 4.251768 & 1.940820 & -2.049888 \\
\hline 1 & 3.984558 & 0.919333 & -1.770068 \\
\hline 6 & 7.046194 & 0.745055 & -2.335781 \\
\hline 6 & 5.009123 & 1.937575 & -3.391637 \\
\hline 1 & 4.669494 & 1.087707 & -3.994350 \\
\hline 6 & 6.534406 & 1.872255 & -3.231654 \\
\hline 1 & 6.983312 & 1.707980 & -4.218887 \\
\hline 1 & 6.933606 & 2.829813 & -2.878475 \\
\hline 1 & 8.122568 & 0.638190 & -2.518690 \\
\hline 1 & 6.578364 & -0.197936 & -2.641789 \\
\hline 1 & 4.765464 & 2.842997 & -3.962872 \\
\hline 1 & 3.306130 & 2.482252 & -2.156760 \\
\hline
\end{tabular}

Number of imaginary frequencies $=1$

The smallest frequencies are $=-1562.33647 .685216 .5731 \mathrm{~cm}(-1)$ Electronic energy $=-5925.110299$

Zero-point correction $=$

1.133303

(Hartree/Particle)

Thermal correction to Energy= $\quad 1.22145$

Thermal correction to Enthalpy= $\quad 1.222396$

Thermal correction to Gibbs Free Energy $=1.002049$

Sum of electronic and zero-point Energies $=\quad-5923.976996$

Sum of electronic and thermal Energies $=\quad-5923.888848$

Sum of electronic and thermal Enthalpies $=\quad-5923.887903$

Sum of electronic and thermal Free Energies $=\quad-5924.108250$

Cartesian Coordinates

$\begin{array}{llcc}26 & -3.872270 & -3.503252 & 1.401645 \\ 15 & -0.579582 & -1.408034 & -2.047029 \\ 15 & -1.794517 & 0.738875 & 0.790199 \\ 6 & -2.833946 & -2.408074 & -0.048164 \\ 6 & -2.781662 & -1.756563 & 1.240353 \\ 6 & -2.149435 & -2.651490 & 2.157495 \\ 1 & -1.971403 & -2.461183 & 3.207004 \\ 6 & -1.842337 & -3.858806 & 1.462852 \\ 1 & -1.395866 & -4.743064 & 1.895539 \\ 6 & -2.266727 & -3.713375 & 0.110322 \\ 1 & -2.193164 & -4.465476 & -0.659852 \\ 6 & -5.873639 & -3.093024 & 1.771747 \\ 1 & -6.330013 & -2.115654 & 1.686619 \\ 6 & -5.251802 & -3.629798 & 2.940866 \\ 1 & -5.145291 & -3.126498 & 3.892781 \\ 6 & -4.752911 & -4.929047 & 2.618848 \\ 1 & -4.198713 & -5.579451 & 3.282755 \\ 6 & -5.064745 & -5.195532 & 1.250685 \\ 1 & -4.789925 & -6.082921 & 0.695896 \\ 6 & -5.758733 & -4.063579 & 0.728168 \\ 1 & -6.103376 & -3.950220 & -0.290720 \\ 6 & -3.311566 & -1.719366 & -1.282885 \\ 6 & -4.680104 & -1.440048 & -1.418940 \\ 1 & -5.356360 & -1.820004 & -0.663365 \\ 6 & -5.174005 & -0.667939 & -2.469739 \\ 1 & -6.234939 & -0.446802 & -2.529615 \\ 6 & -4.290749 & -0.158456 & -3.418507 \\ 1 & -4.653218 & 0.463502 & -4.231526 \\ 6 & -2.926745 & -0.432481 & -3.311604 \\ 1 & -2.251793 & -0.005391 & -4.043562 \\ 6 & -2.413999 & -1.204902 & -2.257588 \\ 6 & -3.201600 & -0.334293 & 1.490537 \\ 1 & -4.061642 & -0.125303 & 0.851335 \\ 6 & -3.598613 & -0.046179 & 2.940630 \\ 1 & -3.920976 & 0.991370 & 3.063889 \\ 1 & -2.778098 & -0.226447 & 3.640280 \\ 1 & -4.427325 & -0.704128 & 3.220953 \\ 6 & 0.151326 & -0.672515 & -3.571149 \\ 1 & 0.951745 & 0.471192 & -3.442110 \\ & 1.143631 & 0.893794 & -2.461683\end{array}$

$\begin{array}{lll}1.523740 & 1.059806 & -4.572562\end{array}$

$2.134176 \quad 1.949776-4.463775$

$\begin{array}{llll}1.312002 & 0.509070 & -5.836079\end{array}$

$\begin{array}{lll}1.760905 & 0.967631 & -6.712886\end{array}$

$\begin{array}{lll}0.526944 & -0.639983 & -5.971238\end{array}$

$\begin{array}{lll}0.365683 & -1.079927 & -6.951518\end{array}$

$-0.047661-1.229089-4.845980$

$-0.647748-2.125370-4.963628$

$-0.352924-3.217718-2.307443$

$-1.227623-3.973018-3.105806$

$\begin{array}{llll}-2.076986 & -3.498161 & -3.587589\end{array}$

$-1.030574-5.346422-3.258709$

$-1.715103-5.923066-3.874844$

$\begin{array}{llll}0.040808 & -5.978455 & -2.618575\end{array}$

$\begin{array}{llll}0.190586 & -7.048052 & -2.736997\end{array}$

$0.917174 \quad-5.229422-1.830273$

$\begin{array}{lll}1.758833 & -5.705836 & -1.335607\end{array}$

$\begin{array}{llll}0.719521 & -3.856518 & -1.673007\end{array}$

$\begin{array}{llll}1.402646 & -3.286186 & -1.061638\end{array}$

$\begin{array}{lll}-2.587395 & 1.796813 & -0.495154\end{array}$

$\begin{array}{lll}-3.944282 & 2.136087 & -0.522902\end{array}$

$\begin{array}{lll}-4.615451 & 1.818563 & 0.266741\end{array}$

$-4.454421 \quad 2.895272-1.578911$

$\begin{array}{llll}-3.626138 & 3.340615 & -2.607849\end{array}$

$\begin{array}{lll}-4.029471 & 3.926921 & -3.425642\end{array}$

$\begin{array}{lll}-2.270335 & 3.018138 & -2.566523\end{array}$

$\begin{array}{llll}-1.753278 & 2.246096 & -1.528587\end{array}$

$\begin{array}{llll}-0.700071 & 1.992452 & -1.510538\end{array}$

$\begin{array}{lll}-1.361234 & 1.952056 & 2.101101\end{array}$

$\begin{array}{lll}-0.634156 & 1.476629 & 3.204239\end{array}$

$\begin{array}{lll}-0.406074 & 0.420398 & 3.293048\end{array}$

$\begin{array}{lll}-0.167202 & 2.362588 & 4.171413\end{array}$

$\begin{array}{lll}-0.382315 & 3.736237 & 4.047567\end{array}$

$\begin{array}{llll}0.013328 & 4.424999 & 4.785656\end{array}$

$\begin{array}{lll}-1.079571 & 4.208253 & 2.938406\end{array}$

$\begin{array}{lll}-1.580958 & 3.328015 & 1.977837\end{array}$

$\begin{array}{llll}-2.111656 & 3.727267 & 1.121913\end{array}$

$\begin{array}{lll}0.078522 & -0.360573 & -0.137400\end{array}$

$\begin{array}{llll}-1.287741 & 5.688317 & 2.775486\end{array}$

$\begin{array}{llll}0.692385 & 1.870873 & 5.301209\end{array}$

$\begin{array}{llll}-5.932259 & 3.157380 & -1.663504\end{array}$

$\begin{array}{llll}-1.381352 & 3.404480 & -3.714852\end{array}$

$\begin{array}{llll}-1.762416 & 4.569084 & -4.285076\end{array}$

$\begin{array}{llll}-0.088239 & 3.543850 & -3.336843\end{array}$

$\begin{array}{llll}-1.405598 & 2.465420 & -4.693515\end{array}$

$\begin{array}{llll}-6.557861 & 2.216384 & -2.417058\end{array}$

$\begin{array}{lll}-6.204436 & 4.351694 & -2.234245\end{array}$

$\begin{array}{llll}-6.525683 & 3.141433 & -0.449304\end{array}$

$\begin{array}{llll}-2.365275 & 6.133666 & 3.461632\end{array}$

$\begin{array}{lll}-1.479295 & 6.032280 & 1.479176\end{array}$

$\begin{array}{lll}-0.221135 & 6.393641 & 3.221674\end{array}$

$\begin{array}{llll}0.379632 & 2.466807 & 6.472693\end{array}$

$\begin{array}{llll}0.599501 & 0.537503 & 5.482649\end{array}$

$\begin{array}{lll}2.007655 & 2.148385 & 5.073055\end{array}$

$\begin{array}{lll}1.689325 & 2.115165 & 0.767121\end{array}$

$\begin{array}{lll}2.439504 & 1.364843 & 1.779181\end{array}$

$\begin{array}{lll}2.162861 & -0.041689 & 2.120584\end{array}$

$\begin{array}{lll}1.676183 & 3.483712 & 0.872545\end{array}$

$\begin{array}{llll}1.177116 & 4.301338 & -0.228411\end{array}$

$\begin{array}{llll}1.975580 & 4.952760 & -0.605107\end{array}$

$\begin{array}{llll}0.833712 & 3.665046 & -1.038156\end{array}$

$\begin{array}{llll}0.348549 & 4.932325 & 0.111381\end{array}$

$\begin{array}{llll}2.344773 & 4.230920 & 1.935810\end{array}$

$\begin{array}{lll}2.181783 & 3.778877 & 2.913656\end{array}$

$\begin{array}{lll}3.425024 & 4.313239 & 1.758985\end{array}$

$1.918394 \quad 5.235624 \quad 1.971323$

$\begin{array}{llll}1.139093 & 1.576676 & -0.231980\end{array}$

$\begin{array}{lll}2.739214 & -0.528969 & 3.320131\end{array}$

$\begin{array}{llll}2.580544 & -1.845235 & 3.712931\end{array}$

$\begin{array}{lll}3.302733 & 0.151964 & 3.947386\end{array}$

$\begin{array}{lll}1.836058 & -2.679634 & 2.879012\end{array}$

$\begin{array}{llll}1.278109 & -2.174628 & 1.721544\end{array}$

$\begin{array}{lll}0.692074 & -2.799766 & 1.060629\end{array}$

$\begin{array}{lll}1.420501 & -0.882808 & 1.355811\end{array}$

$\begin{array}{lll}3.021490 & -2.208589 & 4.634962\end{array}$ 


\begin{tabular}{lrrr}
35 & 1.572367 & -4.565392 & 3.333795 \\
15 & 4.143633 & -2.099297 & -1.407326 \\
9 & 4.273226 & -0.827171 & -0.382250 \\
9 & 3.409681 & -2.961234 & -0.215988 \\
9 & 5.602931 & -2.650823 & -0.903787 \\
9 & 4.882039 & -1.231869 & -2.588346 \\
9 & 3.992257 & -3.369600 & -2.418884 \\
9 & 2.692361 & -1.520271 & -1.909302 \\
1 & 3.745621 & 1.453618 & 1.345946 \\
1 & 2.592773 & 1.922226 & 2.698135 \\
6 & 5.757749 & 0.506127 & 1.951757 \\
6 & 7.234209 & 0.410527 & 1.593088 \\
1 & 5.278260 & -0.474323 & 1.845502 \\
1 & 5.621427 & 0.827678 & 2.990370 \\
6 & 7.351596 & 0.146635 & 0.098793 \\
1 & 7.720147 & -0.396598 & 2.149764 \\
1 & 7.743459 & 1.348020 & 1.846260 \\
1 & 8.397069 & 0.200332 & -0.220687 \\
1 & 6.973051 & -0.850967 & -0.150816 \\
7 & 5.067550 & 1.462969 & 1.087673 \\
6 & 5.480297 & 1.693892 & -0.138924 \\
7 & 6.596649 & 1.148353 & -0.677808 \\
6 & 4.613248 & 2.639129 & -0.943661 \\
1 & 3.828599 & 2.982195 & -0.273091 \\
1 & 5.190292 & 3.529210 & -1.212588 \\
6 & 3.988294 & 1.983911 & -2.187481 \\
1 & 3.701362 & 0.962283 & -1.934698 \\
6 & 6.979829 & 1.104549 & -2.108180 \\
6 & 4.921961 & 1.983220 & -3.417268 \\
1 & 4.778548 & 1.050250 & -3.972268 \\
6 & 6.409393 & 2.141230 & -3.074914 \\
1 & 6.991559 & 2.031642 & -3.997993 \\
1 & 6.624400 & 3.150968 & -2.707903 \\
1 & 8.071875 & 1.195460 & -2.121186 \\
1 & 6.732622 & 0.101641 & -2.474260 \\
1 & 4.647550 & 2.799347 & -4.097265 \\
1 & 3.060011 & 2.514188 & -2.418005 \\
-------------------------------------------------------------------- \\
& & & 6 \\
\hline & & & \\
6 & & -1503
\end{tabular}

Number of imaginary frequencies $=0$

The smallest frequencies are= $14.700716 .483319 .9006 \mathrm{~cm}(-1)$ Electronic energy $=-5925.1275433$

Zero-point correction=

1.138909

(Hartree/Particle)

Thermal correction to Energy=

Thermal correction to Enthalpy=

1.227390

Thermal correction to Gibbs Free Energy=

Sum of electronic and zero-point Energies=

Sum of electronic and thermal Energies=

Sum of electronic and thermal Enthalpies=

1.228334

1.008422

$-5923.988634$

$-5923.900153$

$-5923.899209$

Sum of electronic and thermal Free Energies=

$-5924.119121$

\section{Cartesian Coordinates}

$\begin{array}{lrrr}26 & -3.767409 & -3.661952 & 1.332446 \\ 15 & -0.543458 & -1.356390 & -2.040361 \\ 15 & -1.863378 & 0.673844 & 0.829153 \\ 6 & -2.765384 & -2.494504 & -0.085137 \\ 6 & -2.742822 & -1.872373 & 1.218523 \\ 6 & -2.080865 & -2.764022 & 2.116965 \\ 1 & -1.913774 & -2.591758 & 3.171391 \\ 6 & -1.724556 & -3.941760 & 1.395524 \\ 1 & -1.246504 & -4.818819 & 1.809385 \\ 6 & -2.149030 & -3.780533 & 0.044763 \\ 1 & -2.044563 & -4.510743 & -0.742825 \\ 6 & -5.783796 & -3.327905 & 1.690992 \\ 1 & -6.270891 & -2.365012 & 1.612565 \\ 6 & -5.151898 & -3.855844 & 2.858794 \\ 1 & -5.068163 & -3.359181 & 3.816305 \\ 6 & -4.605573 & -5.133245 & 2.526044 \\ 1 & -4.033496 & -5.771274 & 3.186811 \\ 6 & -4.898468 & -5.395237 & 1.152808 \\ 1 & -4.590238 & -6.266910 & 0.590601 \\ 6 & -5.627974 & -4.282380 & 0.637656\end{array}$

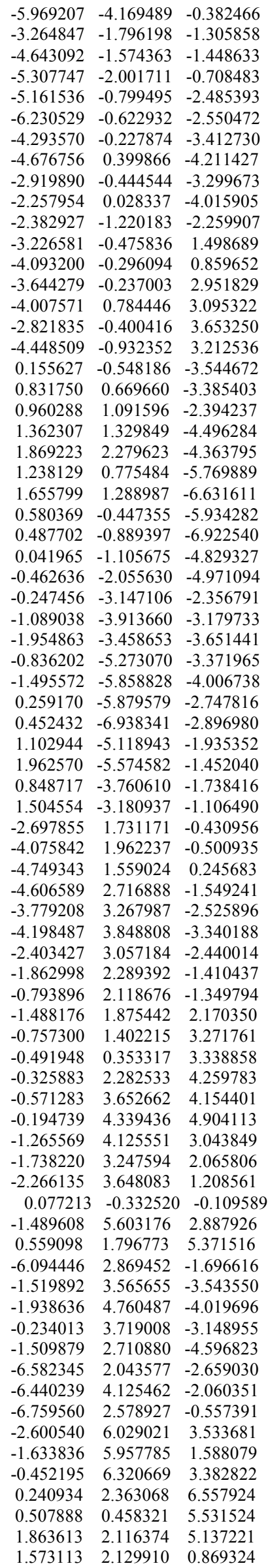




\begin{tabular}{|c|c|c|c|}
\hline & 2.178035 & 1.383606 & 1.917358 \\
\hline 6 & 2.108711 & -0.019269 & 2.152085 \\
\hline 7 & 1.570951 & 3.517780 & 0.969098 \\
\hline 6 & 1.149933 & 4.340749 & -0.153717 \\
\hline 1 & 1.979081 & 4.967913 & -0.510923 \\
\hline 1 & 0.817070 & 3.706210 & -0.968793 \\
\hline 1 & 0.326572 & 5.003099 & 0.142210 \\
\hline 6 & 2.176651 & 4.247453 & 2.072612 \\
\hline 1 & 1.975914 & 3.768225 & 3.030750 \\
\hline 1 & 3.265885 & 4.356766 & 1.954827 \\
\hline 1 & 1.736601 & 5.247118 & 2.116068 \\
\hline 8 & 1.045639 & 1.620183 & -0.175408 \\
\hline 6 & 2.796859 & -0.541817 & 3.294003 \\
\hline 6 & 2.768401 & -1.883055 & 3.604706 \\
\hline 1 & 3.339675 & 0.145619 & 3.934250 \\
\hline 6 & 2.038404 & -2.732156 & 2.762500 \\
\hline 6 & 1.373661 & -2.207673 & 1.674671 \\
\hline 1 & 0.794845 & -2.839778 & 1.013661 \\
\hline 7 & 1.388842 & -0.893538 & 1.374701 \\
\hline 1 & 3.294164 & -2.264499 & 4.474021 \\
\hline 35 & 1.955068 & -4.661603 & 3.114553 \\
\hline 15 & 4.257562 & -1.937053 & -1.399302 \\
\hline 9 & 4.285203 & -0.739456 & -0.278209 \\
\hline 9 & 3.543724 & -2.926761 & -0.304113 \\
\hline 9 & 5.731584 & -2.447580 & -0.888589 \\
\hline 9 & 4.988039 & -0.938448 & -2.478685 \\
\hline 9 & 4.210648 & -3.126311 & -2.512641 \\
\hline 9 & 2.793442 & -1.396467 & -1.901611 \\
\hline 1 & 4.224408 & 1.698494 & 1.346877 \\
\hline 1 & 2.605447 & 1.932888 & 2.743882 \\
\hline 6 & 5.941686 & 0.580024 & 1.883800 \\
\hline 6 & 7.385281 & 0.553500 & 1.405881 \\
\hline 1 & 5.475659 & -0.405381 & 1.789330 \\
\hline 1 & 5.866671 & 0.901015 & 2.926224 \\
\hline 6 & 7.408491 & 0.347567 & -0.102495 \\
\hline 1 & 7.929647 & -0.260460 & 1.891389 \\
\hline 1 & 7.883861 & 1.494872 & 1.662425 \\
\hline 1 & 8.424366 & 0.459079 & -0.488553 \\
\hline 1 & 7.044255 & -0.647620 & -0.371107 \\
\hline 7 & 5.196779 & 1.535095 & 1.064269 \\
\hline 6 & 5.481298 & 1.830430 & -0.203323 \\
\hline 7 & 6.561417 & 1.342688 & -0.802035 \\
\hline 6 & 4.492649 & 2.743205 & -0.887646 \\
\hline 1 & 3.727865 & 2.990799 & -0.152863 \\
\hline 1 & 4.988359 & 3.684723 & -1.140301 \\
\hline 6 & 3.828406 & 2.104435 & -2.118008 \\
\hline 1 & 3.540009 & 1.084719 & -1.862762 \\
\hline 6 & 6.945879 & 1.474102 & -2.229285 \\
\hline 6 & 4.714454 & 2.114768 & -3.386146 \\
\hline 1 & 4.648069 & 1.138119 & -3.874568 \\
\hline 6 & 6.188641 & 2.448161 & -3.128135 \\
\hline 1 & 6.725156 & 2.422388 & -4.084227 \\
\hline 1 & 6.303027 & 3.471020 & -2.754024 \\
\hline 1 & 8.005447 & 1.751837 & -2.226446 \\
\hline 1 & 6.869199 & 0.465415 & -2.648298 \\
\hline 1 & 4.329913 & 2.850804 & -4.101780 \\
\hline 1 & 2.897200 & 2.646575 & -2.297944 \\
\hline
\end{tabular}

Cu-enolate

Number of imaginary frequencies $=0$

The smallest frequencies are $=6.644113 .034615 .6166 \mathrm{~cm}(-1)$ Electronic energy $=-4521.7141033$

Zero-point correction $=$

(Hartree/Particle)

Thermal correction to Energy $=\quad 0.924057$

Thermal correction to Enthalpy= $\quad 0.925001$

Thermal correction to Gibbs Free Energy $=0.743664$

Sum of electronic and zero-point Energies $=\quad-4520.859488$

Sum of electronic and thermal Energies $=\quad-4520.790046$

Sum of electronic and thermal Enthalpies $=\quad-4520.789102$

Sum of electronic and thermal Free Energies $=\quad-4520.970439$$$
6
$$$$
\begin{aligned}
& 6 \\
& 1
\end{aligned}
$$$$
6
$$$$
\frac{10}{6}
$$

$$
6
$$$$
\begin{aligned}
& 6 \\
& 6 \\
& 1
\end{aligned}
$$$$
\begin{aligned}
& 6 \\
& 1 \\
& 1
\end{aligned}
$$

6
1
1

1

6

1
6
6

6
1
6

1
1

6
1
6
1
6

1
6
1
6

1
6
1
6

6
6
1

6
1
6
6

6
1
6

1
6
6
1

1
6
6
1

6
6
1

6
6
6

1
6
6 


$\begin{array}{lccl}6 & -2.991319 & 1.875971 & 1.343978 \\ 1 & -3.592381 & 0.974286 & 1.367653 \\ 29 & 0.381394 & 0.354306 & -1.187174 \\ 6 & -5.120743 & 3.192372 & 1.486329 \\ 6 & -0.715877 & 5.494806 & 1.047151 \\ 6 & -3.641312 & -3.779505 & 2.915882 \\ 6 & -4.028262 & -2.684209 & -1.928934 \\ 9 & -5.280438 & -3.193675 & -1.892219 \\ 9 & -4.048972 & -1.621779 & -2.765810 \\ 9 & -3.242931 & -3.627172 & -2.509902 \\ 9 & -3.050149 & -4.992206 & 2.752945 \\ 9 & -4.969761 & -4.013814 & 3.016169 \\ 9 & -3.223832 & -3.295823 & 4.105518 \\ 9 & -5.559076 & 3.281370 & 2.764197 \\ 9 & -5.709471 & 2.098530 & 0.946008 \\ 9 & -5.609525 & 4.272795 & 0.830818 \\ 9 & -1.082891 & 6.440377 & 1.943139 \\ 9 & 0.614972 & 5.309877 & 1.172744 \\ 9 & -0.924872 & 6.034171 & -0.183448 \\ 6 & -1.624779 & 2.370267 & -2.177475 \\ 6 & -0.634328 & 3.389007 & -2.212775 \\ 6 & 0.745985 & 3.284518 & -1.929376 \\ 1 & -0.964455 & 4.397788 & -2.417077 \\ 7 & -2.957467 & 2.742467 & -2.159758 \\ 6 & -4.010209 & 1.754270 & -2.329037 \\ 1 & -4.688325 & 2.057880 & -3.137770 \\ 1 & -3.578108 & 0.789111 & -2.577902 \\ 1 & -4.603920 & 1.650956 & -1.410498 \\ 6 & -3.416486 & 4.118752 & -2.056865 \\ 1 & -2.721812 & 4.722470 & -1.473895 \\ 1 & -3.554592 & 4.588044 & -3.042370 \\ 1 & -4.378211 & 4.133037 & -1.534482 \\ 8 & -1.373187 & 1.113819 & -2.158762 \\ 6 & 1.543084 & 4.480202 & -1.994210 \\ 6 & 2.885864 & 4.474426 & -1.704348 \\ 1 & 1.056579 & 5.411376 & -2.267110 \\ 6 & 3.471959 & 3.249998 & -1.338643 \\ 6 & 2.693494 & 2.116212 & -1.267942 \\ 1 & 3.118147 & 1.162417 & -0.980854 \\ 7 & 1.371498 & 2.116656 & -1.540930 \\ 1 & 3.471121 & 5.386969 & -1.754256 \\ 35 & 5.390550 & 3.141423 & -0.923165 \\ ------------------------------------------------------------\end{array}$

$$
8 \text { P1-C1 endo re-re }\left[\mathrm{BF}_{4}^{-}\right]
$$

Number of imaginary frequencies $=0$

The smallest frequencies are $=10.193111 .087615 .2988 \mathrm{~cm}(-1)$ Electronic energy $=-7647.26473$

Zero-point correction=

1.771428

(Hartree/Particle)

Thermal correction to Energy= $\quad 1.897342$

Thermal correction to Enthalpy=

1.898286

Thermal correction to Gibbs Free Energy $=\quad 1.598970$

Sum of electronic and zero-point Energies=

Sum of electronic and thermal Energies=

Sum of electronic and thermal Enthalpies=

$-7645.493302$

$-7645.367388$

$-7645.366444$

Sum of electronic and thermal Free Energies=

$-7645.665760$

\section{Cartesian Coordinates}

$\begin{array}{llll}26 & 4.686491 & 4.728347 & 1.989701 \\ 15 & 2.744773 & 1.766480 & -1.832938 \\ 15 & 4.687906 & 0.096269 & 0.868121 \\ 6 & 4.268027 & 3.451490 & 0.384665 \\ 6 & 4.454545 & 2.707676 & 1.608561 \\ 6 & 3.472011 & 3.154668 & 2.543881 \\ 1 & 3.357542 & 2.799769 & 3.558775 \\ 6 & 2.701368 & 4.185209 & 1.927496 \\ 1 & 1.905627 & 4.747074 & 2.396658 \\ 6 & 3.190291 & 4.369834 & 0.601323 \\ 1 & 2.827436 & 5.090179 & -0.116406 \\ 6 & 6.658163 & 5.162192 & 2.475356 \\ 1 & 7.489719 & 4.484730 & 2.332775 \\ 6 & 5.829867 & 5.241729 & 3.636928 \\ 1 & 5.917357 & 4.629447 & 4.524504\end{array}$

$\begin{array}{lll}4.832324 & 6.236478 & 3.401403\end{array}$

$\begin{array}{llll}4.031058 & 6.504547 & 4.077310\end{array}$

$5.043444 \quad 6.772734 \quad 2.094460$

$\begin{array}{lll}4.433973 & 7.522925 & 1.607914\end{array}$

$\begin{array}{lll}6.171192 & 6.111042 & 1.523203\end{array}$

$\begin{array}{lll}6.566415 & 6.281622 & 0.531174\end{array}$

$\begin{array}{llll}5.024565 & 3.191929 & -0.874111\end{array}$

$6.370960 \quad 3.576109-0.960520$

$\begin{array}{llll}6.807067 & 4.102363 & -0.120695\end{array}$

$\begin{array}{llll}7.152072 & 3.277991 & -2.077041\end{array}$

$\begin{array}{llll}8.198451 & 3.565867 & -2.099869\end{array}$

$\begin{array}{llll}6.586489 & 2.585544 & -3.145012\end{array}$

$\begin{array}{llll}7.184824 & 2.329396 & -4.013383\end{array}$

$\begin{array}{llll}5.249159 & 2.192591 & -3.082820\end{array}$

$\begin{array}{llll}4.829168 & 1.636907 & -3.913686\end{array}$

$\begin{array}{llll}4.454034 & 2.474867 & -1.960779\end{array}$

$\begin{array}{llll}5.439396 & 1.582370 & 1.783249\end{array}$

$6.340841 \quad 1.847091 \quad 1.227790$

$\begin{array}{lll}5.836681 & 1.317968 & 3.238126\end{array}$

$\begin{array}{lll}6.578597 & 0.517241 & 3.301312\end{array}$

$\begin{array}{llll}4.981788 & 1.031840 & 3.856495\end{array}$

$\begin{array}{llll}6.268621 & 2.228887 & 3.664164\end{array}$

$\begin{array}{llll}2.474861 & 0.941572 & -3.464089\end{array}$

$\begin{array}{llll}2.568668 & -0.459079 & -3.512024\end{array}$

$\begin{array}{llll}2.783326 & -1.018710 & -2.607979\end{array}$

$\begin{array}{llll}2.342566 & -1.139880 & -4.711898\end{array}$

$2.420664 \quad-2.221844 \quad-4.736563$

$\begin{array}{llll}2.008929 & -0.434242 & -5.869222\end{array}$

$\begin{array}{llll}1.820929 & -0.966576 & -6.797365\end{array}$

$\begin{array}{llll}1.912632 & 0.959987 & -5.828415\end{array}$

$\begin{array}{llll}1.651470 & 1.515271 & -6.724959\end{array}$

$2.146943 \quad 1.644648 \quad-4.635632$

$\begin{array}{llll}2.060042 & 2.725715 & -4.619860\end{array}$

$\begin{array}{lll}1.643484 & 3.236742 & -1.948352\end{array}$

$2.004873 \quad 4.409380 \quad-2.631645$

$\begin{array}{llll}2.989716 & 4.493667 & -3.081839\end{array}$

$\begin{array}{llll}1.108601 & 5.476091 & -2.722818\end{array}$

$\begin{array}{llll}1.398748 & 6.379520 & -3.251971\end{array}$

$\begin{array}{llll}-0.159410 & 5.379919 & -2.139738\end{array}$

$\begin{array}{llll}-0.861217 & 6.205750 & -2.215811\end{array}$

$\begin{array}{llll}-0.522891 & 4.218040 & -1.455463\end{array}$

$\begin{array}{llll}-1.504351 & 4.137550 & -1.001473\end{array}$

$\begin{array}{llll}0.378805 & 3.156783 & -1.351040\end{array}$

$\begin{array}{llll}0.102978 & 2.264490 & -0.801171\end{array}$

$\begin{array}{llll}5.917099 & -0.416753 & -0.405143\end{array}$

$\begin{array}{llll}5.474475 & -1.401230 & -1.302317\end{array}$

$\begin{array}{llll}4.477779 & -1.815844 & -1.177169\end{array}$

$\begin{array}{llll}6.301119 & -1.831859 & -2.340350\end{array}$

$\begin{array}{llll}7.556990 & -1.259792 & -2.535823\end{array}$

$\begin{array}{llll}8.186696 & -1.575595 & -3.359977\end{array}$

$\begin{array}{llll}7.982495 & -0.262425 & -1.658309\end{array}$

$\begin{array}{llll}7.186945 & 0.142089 & -0.586260\end{array}$

$\begin{array}{lll}7.565695 & 0.905193 & 0.080055\end{array}$

$\begin{array}{llll}4.685104 & -1.325127 & 2.037429\end{array}$

$\begin{array}{llll}3.723196 & -1.300046 & 3.060311\end{array}$

$\begin{array}{lll}3.083087 & -0.434121 & 3.182117\end{array}$

$\begin{array}{llll}3.543702 & -2.405930 & 3.885428\end{array}$

$\begin{array}{llll}4.282642 & -3.573833 & 3.687480\end{array}$

$\begin{array}{llll}4.103797 & -4.450846 & 4.299020\end{array}$

$\begin{array}{llll}5.218672 & -3.606308 & 2.657855\end{array}$

$\begin{array}{llll}5.439266 & -2.487205 & 1.851131\end{array}$

$\begin{array}{llll}6.171345 & -2.552669 & 1.055501\end{array}$

$\begin{array}{llll}2.553176 & 0.330766 & -0.099941\end{array}$

$\begin{array}{llll}5.997077 & -4.863633 & 2.389298\end{array}$

$\begin{array}{lll}2.445999 & -2.418610 & 4.912270\end{array}$

$\begin{array}{llll}5.838871 & -2.964937 & -3.213723\end{array}$

$\begin{array}{llll}9.311346 & 0.391829 & -1.915549\end{array}$

$\begin{array}{llll}9.343532 & 0.981268 & -3.137710\end{array}$

$\begin{array}{llll}9.604733 & 1.349051 & -1.007793\end{array}$

$10.326539 \quad-0.503688-1.898848$

$\begin{array}{llll}5.992614 & -4.161925 & -2.594451\end{array}$

$\begin{array}{llll}6.517776 & -3.032314 & -4.378432\end{array}$

$\begin{array}{llll}4.521702 & -2.864082 & -3.523045\end{array}$

$\begin{array}{llll}7.185087 & -4.883659 & 3.039956\end{array}$

$\begin{array}{llll}6.277583 & -5.005664 & 1.069956\end{array}$ 
$\begin{array}{llll}5.319349 & -5.969146 & 2.773813\end{array}$

$\begin{array}{llll}2.862608 & -2.960753 & 6.081679\end{array}$

$\begin{array}{llll}1.977440 & -1.181701 & 5.185270\end{array}$

$\begin{array}{llll}1.390616 & -3.162717 & 4.499017\end{array}$

$\begin{array}{lll}1.976427 & -2.600537 & 0.343102\end{array}$

$\begin{array}{lll}1.034092 & -2.282433 & 1.357794\end{array}$

$\begin{array}{lll}0.606537 & -1.019252 & 1.803723\end{array}$

$\begin{array}{lll}0.554143 & -3.108525 & 1.859147\end{array}$

$77 \quad-3.252178 \quad-0.982788-1.538013$

$\begin{array}{llll}-5.124022 & -0.252149 & -0.478483\end{array}$

$\begin{array}{llll}-5.147174 & 0.824998 & 0.765662\end{array}$

$\begin{array}{llll}-6.118051 & 0.534600 & -1.561193\end{array}$

$\begin{array}{lll}-5.847114 & -1.536081 & 0.283044\end{array}$

$\begin{array}{lll}-4.873006 & 2.169015 & 0.561740\end{array}$

$\begin{array}{lll}-3.665323 & 2.654287 & 1.108472\end{array}$

$\begin{array}{lll}-3.041595 & 1.974180 & 1.678475\end{array}$

$\begin{array}{lll}-3.315761 & 3.965356 & 0.902457\end{array}$

$\begin{array}{llll}-2.396089 & 4.356436 & 1.324832\end{array}$

$\begin{array}{llll}-4.126750 & 4.810904 & 0.096933\end{array}$

$\begin{array}{llll}-3.707807 & 6.129455 & -0.229203\end{array}$

$\begin{array}{llll}-2.766760 & 6.489089 & 0.178965\end{array}$

$\begin{array}{llll}-4.466109 & 6.927422 & -1.056457\end{array}$

$\begin{array}{llll}-4.133063 & 7.930294 & -1.30724\end{array}$

$\begin{array}{llll}-5.680230 & 6.433511 & -1.590823\end{array}$

$\begin{array}{llll}-6.266542 & 7.057857 & -2.258895\end{array}$

$\begin{array}{llll}-6.124443 & 5.166790 & -1.277335\end{array}$

$\begin{array}{llll}-7.052246 & 4.802493 & -1.702954\end{array}$

$\begin{array}{llll}-5.371875 & 4.317075 & -0.419739\end{array}$

$\begin{array}{llll}-5.785345 & 2.973900 & -0.102000\end{array}$

$\begin{array}{llll}-7.125218 & 2.437231 & -0.472897\end{array}$

$\begin{array}{llll}-8.341504 & 3.087966 & -0.053042\end{array}$

$\begin{array}{lll}-8.349924 & 4.208659 & 0.824505\end{array}$

$\begin{array}{lll}-7.411868 & 4.589610 & 1.209495\end{array}$

$\begin{array}{llll}-9.531691 & 4.806670 & 1.205285\end{array}$

$\begin{array}{lll}-9.508963 & 5.656237 & 1.881826\end{array}$

$\begin{array}{lll}-10.773655 & 4.321939 & 0.730802\end{array}$

$\begin{array}{lll}-11.696275 & 4.808570 & 1.033291\end{array}$

$\begin{array}{lll}-10.805792 & 3.224037 & -0.098548\end{array}$

$\begin{array}{llll}-11.752703 & 2.826748 & -0.454420\end{array}$

$\begin{array}{llll}-9.606574 & 2.573082 & -0.496929\end{array}$

$\begin{array}{llll}-9.643612 & 1.405914 & -1.306728\end{array}$

$\begin{array}{llll}-10.604056 & 1.028394 & -1.645701\end{array}$

$\begin{array}{llll}-8.485528 & 0.745045 & -1.629165\end{array}$

$\begin{array}{lll}-8.489844 & -0.166999 & -2.216577\end{array}$

$\begin{array}{llll}-7.244807 & 1.255037 & -1.188642\end{array}$

$\begin{array}{llll}-5.321800 & -2.860944 & -0.148189\end{array}$

$\begin{array}{llll}-4.658313 & -3.229167 & 0.635100\end{array}$

$\begin{array}{lll}-6.444430 & -3.867268 & -0.334475\end{array}$

$\begin{array}{llll}-6.325525 & -5.153533 & 0.205968\end{array}$

$\begin{array}{lll}-5.441710 & -5.405726 & 0.785001\end{array}$

$\begin{array}{lll}-7.334940 & -6.099536 & 0.003892\end{array}$

$\begin{array}{llll}-7.232636 & -7.094152 & 0.429430\end{array}$

$\begin{array}{llll}-8.473841 & -5.766364 & -0.732179\end{array}$

$\begin{array}{llll}-9.260821 & -6.500141 & -0.883222\end{array}$

$\begin{array}{llll}-8.600153 & -4.480792 & -1.270141\end{array}$

$\begin{array}{llll}-9.486389 & -4.213585 & -1.839755\end{array}$

$\begin{array}{llll}-7.590808 & -3.538788 & -1.073426\end{array}$

$\begin{array}{llll}-7.690252 & -2.536757 & -1.481753\end{array}$

$\begin{array}{llll}-4.516522 & -2.709349 & -1.444934\end{array}$

$\begin{array}{lll}-5.207501 & -2.666529 & -2.294576\end{array}$

$\begin{array}{llll}-3.894273 & -3.597849 & -1.579966\end{array}$

$\begin{array}{lll}-6.625861 & -1.522205 & 1.566334\end{array}$

$\begin{array}{lll}-6.858239 & -2.579953 & 1.719370\end{array}$

$\begin{array}{llll}-5.792735 & -1.085067 & 2.773054\end{array}$

$\begin{array}{llll}-4.722771 & -1.886513 & 3.199517\end{array}$

$\begin{array}{lll}-4.478451 & -2.809130 & 2.683298\end{array}$

$\begin{array}{llll}-3.942361 & -1.515866 & 4.296889\end{array}$

$\begin{array}{lll}-3.122995 & -2.159193 & 4.596094\end{array}$

$\begin{array}{lll}-4.224824 & -0.339785 & 4.995246\end{array}$

$\begin{array}{lll}-3.624437 & -0.055236 & 5.855399\end{array}$

$\begin{array}{lll}-5.290834 & 0.463557 & 4.582997\end{array}$

$\begin{array}{llll}-5.522032 & 1.381250 & 5.117670\end{array}$

$\begin{array}{lll}-6.066898 & 0.091426 & 3.483376\end{array}$

$\begin{array}{lll}-6.880932 & 0.738183 & 3.174420\end{array}$

$\begin{array}{lll}-7.962958 & -0.800802 & 1.414068\end{array}$ $\begin{array}{llll}-8.521036 & -1.234176 & 0.581569\end{array}$

$\begin{array}{lll}-7.840702 & 0.266782 & 1.225082\end{array}$

$\begin{array}{lll}-8.554210 & -0.919789 & 2.327124\end{array}$

$\begin{array}{lll}-2.343522 & -0.952803 & 0.446430\end{array}$

$\begin{array}{lll}-1.613305 & -0.149137 & 0.454599\end{array}$

$\begin{array}{lll}-2.997765 & -0.952774 & 1.312385\end{array}$

$-1.970098-2.236696-0.063070$

$\begin{array}{llll}-2.507779 & -3.098688 & 0.307743\end{array}$

$\begin{array}{lll}-1.015767 & -2.413573 & -1.062697\end{array}$

$\begin{array}{llll}-0.324438 & -1.605643 & -1.265270\end{array}$

$-4.152740-0.511146-3.564658$

$\begin{array}{llll}-5.164231 & -0.904838 & -3.530402\end{array}$

$\begin{array}{lll}-3.122123 & -1.444439 & -3.766049\end{array}$

$\begin{array}{llll}-3.406915 & -2.489411 & -3.878652\end{array}$

$\begin{array}{llll}-1.741049 & -1.115353 & -4.297258\end{array}$

$\begin{array}{lll}-1.782352 & -1.040458 & -5.394471\end{array}$

$\begin{array}{llll}-1.091483 & -1.965472 & -4.074238\end{array}$

$\begin{array}{llll}-1.112185 & 0.166289 & -3.711446\end{array}$

$\begin{array}{llll}-1.365145 & 1.034098 & -4.327483\end{array}$

$\begin{array}{llll}-0.027664 & 0.069486 & -3.757653\end{array}$

$\begin{array}{llll}-1.501051 & 0.461093 & -2.269397\end{array}$

$\begin{array}{llll}-0.720221 & 0.319353 & -1.529740\end{array}$

$\begin{array}{llll}-2.566283 & 1.264188 & -1.904433\end{array}$

$\begin{array}{llll}-2.537214 & 1.708203 & -0.914926\end{array}$

$\begin{array}{llll}-3.533041 & 1.907900 & -2.875797\end{array}$

$\begin{array}{lll}-4.377479 & 2.292482 & -2.304571\end{array}$

$\begin{array}{llll}-3.040995 & 2.778897 & -3.332477\end{array}$

$\begin{array}{llll}-4.060465 & 0.958824 & -3.975080\end{array}$

$\begin{array}{llll}-3.436676 & 1.031804 & -4.871161\end{array}$

$\begin{array}{llll}-5.059528 & 1.292894 & -4.268911\end{array}$

$\begin{array}{llll}-0.689029 & -3.681161 & -1.707224\end{array}$

$\begin{array}{llll}-1.362408 & -4.890377 & -1.432808\end{array}$

$\begin{array}{llll}0.368681 & -3.693709 & -2.638332\end{array}$

$\begin{array}{llll}-0.992574 & -6.061613 & -2.089707\end{array}$

$\begin{array}{llll}-2.139467 & -4.925085 & -0.678877\end{array}$

$\begin{array}{llll}0.733378 & -4.867979 & -3.293167\end{array}$

$\begin{array}{llll}0.914307 & -2.774271 & -2.823370\end{array}$

$\begin{array}{llll}0.048984 & -6.056821 & -3.025034\end{array}$

$-1.512943-6.987838 \quad-1.862530$

$\begin{array}{llll}1.558304 & -4.858703 & -4.000357\end{array}$

$\begin{array}{llll}0.331761 & -6.976144 & -3.530153\end{array}$

$\begin{array}{llll}2.446893 & -3.895160 & 0.275941\end{array}$

$\begin{array}{llll}3.268809 & -4.340236 & -0.836413\end{array}$

$\begin{array}{llll}2.902563 & -5.305531 & -1.204274\end{array}$

$3.215361 \quad-3.623264 \quad-1.649375$

$\begin{array}{llll}4.316858 & -4.463558 & -0.533883\end{array}$

$\begin{array}{lll}2.148007 & -4.922790 & 1.266207\end{array}$

$\begin{array}{llll}1.983263 & -4.484607 & 2.249802\end{array}$

$\begin{array}{llll}1.261509 & -5.507193 & 0.996054\end{array}$

$\begin{array}{lll}3.009150 & -5.594150 & 1.344131\end{array}$

$\begin{array}{llll}2.418521 & -1.768119 & -0.531539\end{array}$

$\begin{array}{lll}-0.365263 & -0.980848 & 2.866059\end{array}$

$\begin{array}{lll}-0.805837 & 0.203493 & 3.400169\end{array}$

$\begin{array}{lll}-0.763892 & -1.920741 & 3.229772\end{array}$

$\begin{array}{lll}-0.287114 & 1.397307 & 2.863658\end{array}$

$\begin{array}{lll}0.622270 & 1.347012 & 1.830918\end{array}$

$\begin{array}{lll}1.032890 & 2.251113 & 1.399539\end{array}$

$\begin{array}{llll}1.064753 & 0.184937 & 1.301098\end{array}$

$\begin{array}{lll}-1.546996 & 0.214911 & 4.191265\end{array}$

$\begin{array}{lll}-0.809050 & 3.136363 & 3.621772\end{array}$

$\begin{array}{lll}-2.179135 & -4.879906 & 2.385696\end{array}$

$\begin{array}{lll}-2.301549 & -6.031392 & 3.160293\end{array}$

$\begin{array}{lll}-2.153369 & -3.733420 & 3.206475\end{array}$

$\begin{array}{llll}-3.303286 & -4.780507 & 1.505964\end{array}$

$\begin{array}{lll}-1.010147 & -4.920101 & 1.609635\end{array}$

$[8-9]^{\ddagger} \mathrm{P1}-\mathrm{Cl}$ endo re-re $\left[\mathrm{BF}_{4}^{-}\right]$

Number of imaginary frequencies $=1$

The smallest frequencies are $=-221.751311 .314412 .4751 \mathrm{~cm}(-1)$

Electronic energy $=-7647.2600588$

Zero-point correction=

1.771281

Hartree/Particle)

Thermal correction to Energy=

Thermal correction to Enthalpy=

1.896114

1.897058 
Thermal correction to Gibbs Free Energy= Sum of electronic and zero-point Energies= Sum of electronic and thermal Energies=

Sum of electronic and thermal Enthalpies=

Sum of electronic and thermal Free Energies=

1.601868

$-7645.488778$

$-7645.363945$

$-7645.363001$

$-7645.658190$

Cartesian Coordinates

\begin{tabular}{|c|c|c|c|}
\hline 6 & 4.527102 & 4.680237 & 2.206900 \\
\hline 15 & 2.726247 & 1.866186 & -1.799305 \\
\hline 1 & 4.655960 & 0.105492 & 0.898054 \\
\hline 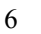 & 4.166454 & 3.457332 & 0.546751 \\
\hline 6 & 4.337096 & 2.673630 & 1.748082 \\
\hline 6 & 3.325154 & 3.071265 & 2.675171 \\
\hline 1 & 3.194206 & 2.678905 & 3.674215 \\
\hline 6 & 2.552482 & 4.111758 & 2.077363 \\
\hline 1 & 1.738233 & 4.645726 & 2.546989 \\
\hline 6 & 3.069559 & 4.350986 & 0.770844 \\
\hline 1 & 2.712500 & 5.091400 & 0.070872 \\
\hline 6 & 6.481988 & 5.123825 & 2.747443 \\
\hline 1 & 7.325398 & 4.463637 & 2.593799 \\
\hline 6 & 5.631355 & 5.145897 & 3.895184 \\
\hline 1 & 5.710635 & 4.499452 & 4.759008 \\
\hline 6 & 4.623331 & 6.134529 & 3.679280 \\
\hline 1 & 3.806199 & 6.365340 & 4.349989 \\
\hline 6 & 4.850319 & 6.724348 & 2.398444 \\
\hline 1 & 4.238474 & 7.484031 & 1.929967 \\
\hline 6 & 5.998094 & 6.101743 & 1.823547 \\
\hline 1 & 6.408336 & 6.314161 & 0.845588 \\
\hline 6 & 4.957241 & 3.269347 & -0.704298 \\
\hline 6 & 6.300274 & 3.672756 & -0.738007 \\
\hline 1 & 6.713540 & 4.148291 & 0.142307 \\
\hline 6 & 7.106560 & 3.459010 & -1.855809 \\
\hline 1 & 8.149460 & 3.758972 & -1.837542 \\
\hline 6 & 6.570330 & 2.837136 & -2.980782 \\
\hline 1 & 7.186763 & 2.65 & -3.854824 \\
\hline 6 & 5.237808 & 2.423797 & -2.971240 \\
\hline 1 & 4.837339 & 1.927005 & -3.848011 \\
\hline 6 & 4.420265 & 2.61 & -1.846299 \\
\hline 6 & 5.336892 & 1.559481 & 1.911280 \\
\hline 1 & 6.255406 & 1.865841 & 1.407361 \\
\hline 6 & 5.676347 & 1.228988 & 3.367280 \\
\hline 1 & 6.436337 & 0.444855 & 3.423031 \\
\hline 1 & 4.802108 & 0.889077 & 3.928922 \\
\hline 1 & 6.064565 & 2.126210 & 3.859442 \\
\hline 6 & 2.507967 & 1.107887 & -3.467296 \\
\hline 6 & 2.727825 & -0.273893 & -3.585921 \\
\hline 1 & 3.013503 & -0.851644 & -2.714364 \\
\hline 6 & 2.540702 & -0.916967 & -4.811702 \\
\hline 1 & 2.715674 & -1.985102 & -4.888382 \\
\hline 6 & 2.120296 & -0.190575 & -5.927620 \\
\hline 1 & 1.959915 & -0.693836 & -6.876826 \\
\hline 6 & 1.901801 & 1.185613 & -5.817938 \\
\hline 1 & 1.573713 & 1.756220 & -6.682192 \\
\hline 6 & 2.097728 & 1.833403 & -4.597496 \\
\hline 1 & 1.914471 & 2.900149 & -4.528337 \\
\hline 6 & 1.593306 & 3.312643 & -1.857759 \\
\hline 6 & 1.941543 & 4.518325 & -2.489006 \\
\hline 1 & 2.927928 & 4.636305 & -2.928034 \\
\hline 6 & 1.028341 & 5.572718 & -2.545361 \\
\hline 1 & 1.307747 & 6.501698 & -3.034435 \\
\hline 6 & -0.243463 & 5.430425 & -1.980567 \\
\hline 1 & -0.960005 & 6.245454 & -2.030770 \\
\hline 6 & -0.593923 & 4.234911 & -1.349908 \\
\hline 1 & -1.579378 & 4.118006 & -0.914386 \\
\hline 6 & 0.324430 & 3.185458 & -1.278164 \\
\hline 1 & 0.055663 & 2.266737 & -0.769672 \\
\hline 6 & 5.945718 & -0.317891 & -0.347678 \\
\hline 6 & 5.571657 & -1.286314 & -1.291010 \\
\hline 1 & 4.591229 & -1.745404 & -1.211799 \\
\hline 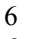 & 6.441567 & -1.647100 & -2.319155 \\
\hline 6 & 7.679774 & -1.022739 & -2.456779 \\
\hline 1 & 8.344814 & -1.284563 & -3.271975 \\
\hline & 8.038876 & -0.041423 & -1.532191 \\
\hline & 7.196218 & 0.297249 & -0.47289 \\
\hline
\end{tabular}

6

$\begin{array}{lll}7.522651 & 1.055522 & 0.225418\end{array}$

$\begin{array}{llll}4.613993 & -1.375007 & 1.991113\end{array}$

$\begin{array}{lll}3.582612 & -1.430434 & 2.942756\end{array}$

$\begin{array}{lll}2.913839 & -0.586933 & 3.068479\end{array}$

$\begin{array}{lll}3.380833 & -2.584148 & 3.696445\end{array}$

$\begin{array}{lll}4.172523 & -3.715665 & 3.495780\end{array}$

$\begin{array}{llll}3.983641 & -4.626841 & 4.052260\end{array}$

$\begin{array}{llll}5.184217 & -3.664580 & 2.540189\end{array}$

$\begin{array}{lll}5.421988 & -2.501513 & 1.805366\end{array}$

$\begin{array}{llll}6.215035 & -2.499979 & 1.067834\end{array}$

$\begin{array}{lll}2.557752 & 0.343939 & -0.138988\end{array}$

$\begin{array}{llll}6.027146 & -4.885672 & 2.298147\end{array}$

$\begin{array}{llll}2.219496 & -2.678919 & 4.647160\end{array}$

$6.037409-2.760733 \quad-3.244774$

$\begin{array}{llll}9.347349 & 0.673219 & -1.727389\end{array}$

$\begin{array}{llll}9.378752 & 1.331298 & -2.913844\end{array}$

$\begin{array}{llll}9.590336 & 1.587692 & -0.762500\end{array}$

$10.393068-0.186105-1.741046$

$\begin{array}{llll}6.202219 & -3.974080 & -2.661025\end{array}$

$\begin{array}{llll}6.751066 & -2.769578 & -4.388876\end{array}$

$\begin{array}{lll}4.725670 & -2.682669 & -3.588615\end{array}$

$\begin{array}{llll}7.033533 & -4.999894 & 3.196080\end{array}$

$\begin{array}{lll}6.596321 & -4.872275 & 1.069744\end{array}$

$\begin{array}{llll}5.295508 & -6.022048 & 2.387493\end{array}$

$\begin{array}{lll}2.561855 & -3.307672 & 5.794582\end{array}$

$\begin{array}{lll}1.727058 & -1.466090 & 4.980309\end{array}$

$\begin{array}{llll}1.195897 & -3.390200 & 4.108637\end{array}$

$\begin{array}{llll}2.034251 & -2.660873 & 0.136045\end{array}$

$\begin{array}{llll}0.871426 & -2.404637 & 0.951344\end{array}$

$\begin{array}{lll}0.524196 & -1.167832 & 1.584190\end{array}$

$\begin{array}{llll}0.400273 & -3.261406 & 1.408141\end{array}$

$\begin{array}{llll}-3.297397 & -0.941917 & -1.649865\end{array}$

$\begin{array}{lll}-5.167937 & -0.258277 & -0.540221\end{array}$

$\begin{array}{lll}-5.216634 & 0.789709 & 0.741456\end{array}$

$\begin{array}{llll}-6.205607 & 0.542435 & -1.583991\end{array}$

$\begin{array}{lll}-5.870750 & -1.571715 & 0.207437\end{array}$

$\begin{array}{llll}-4.916066 & 2.127274 & 0.563831\end{array}$

$\begin{array}{lll}-3.706747 & 2.585786 & 1.131198\end{array}$

$\begin{array}{lll}-3.098172 & 1.882384 & 1.688322\end{array}$

$\begin{array}{lll}-3.342420 & 3.898559 & 0.967725\end{array}$

$\begin{array}{llll}-2.424304 & 4.270097 & 1.411492\end{array}$

$\begin{array}{llll}-4.136390 & 4.773753 & 0.175917\end{array}$

$\begin{array}{llll}-3.704393 & 6.098078 & -0.107016\end{array}$

$\begin{array}{llll}-2.765625 & 6.438686 & 0.322251\end{array}$

$\begin{array}{llll}-4.446828 & 6.925562 & -0.919857\end{array}$

$\begin{array}{llll}-4.103675 & 7.932931 & -1.136894\end{array}$

$\begin{array}{llll}-5.657561 & 6.456361 & -1.483502\end{array}$

$\begin{array}{llll}-6.231060 & 7.104151 & -2.140325\end{array}$

$\begin{array}{llll}-6.114366 & 5.184414 & -1.212117\end{array}$

$\begin{array}{llll}-7.039929 & 4.838634 & -1.658037\end{array}$

$\begin{array}{llll}-5.378708 & 4.304677 & -0.370269\end{array}$

$\begin{array}{llll}-5.811350 & 2.960055 & -0.088560\end{array}$

$\begin{array}{lll}-7.162328 & 2.451245 & -0.459164\end{array}$

$\begin{array}{llll}-8.362729 & 3.114505 & -0.012361\end{array}$

$\begin{array}{llll}-8.342453 & 4.220079 & 0.884437\end{array}$

$\begin{array}{lll}-7.393752 & 4.580864 & 1.262493\end{array}$

$\begin{array}{llll}-9.509308 & 4.828028 & 1.294809\end{array}$

$\begin{array}{lll}-9.463279 & 5.664819 & 1.986053\end{array}$

$\begin{array}{llll}-10.765539 & 4.369477 & 0.832449\end{array}$

$\begin{array}{lll}-11.676424 & 4.862870 & 1.158755\end{array}$

$\begin{array}{llll}-10.825604 & 3.286874 & -0.015379\end{array}$

$\begin{array}{lll}-11.783505 & 2.908277 & -0.362477\end{array}$

$\begin{array}{llll}-9.642341 & 2.626303 & -0.444209\end{array}$

$\begin{array}{llll}-9.708892 & 1.472721 & -1.271554\end{array}$

$\begin{array}{lll}-10.679918 & 1.116050 & -1.603371\end{array}$

$\begin{array}{llll}-8.566037 & 0.798432 & -1.618258\end{array}$

$\begin{array}{llll}-8.593499 & -0.104974 & -2.218392\end{array}$

$\begin{array}{llll}-7.308578 & 1.278082 & -1.186956\end{array}$

$\begin{array}{llll}-5.288329 & -2.875176 & -0.218934\end{array}$

$\begin{array}{lll}-4.591764 & -3.206215 & 0.554510\end{array}$

$\begin{array}{lll}-6.363543 & -3.937827 & -0.373749\end{array}$

$\begin{array}{lll}-6.157526 & -5.220990 & 0.148235\end{array}$

$\begin{array}{llll}-5.240421 & -5.431287 & 0.691288\end{array}$

$\begin{array}{llll}-7.120057 & -6.219197 & -0.029714\end{array}$

$\begin{array}{lll}-6.948797 & -7.210444 & 0.381639\end{array}$ 


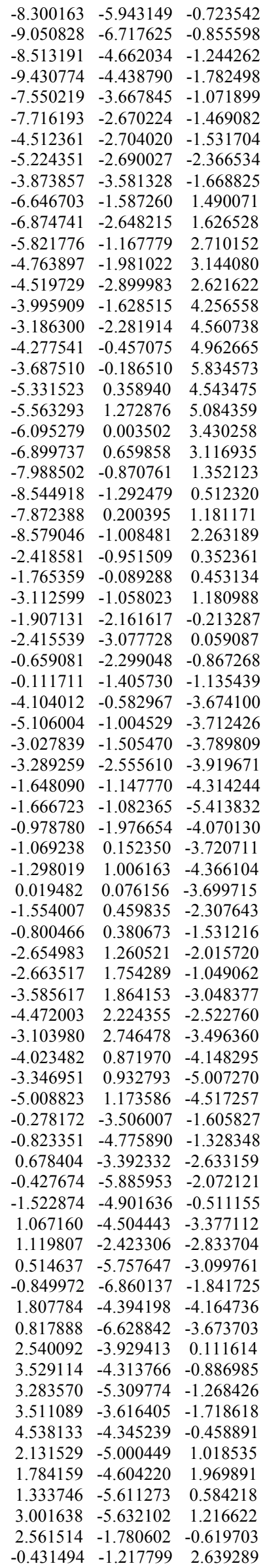

$\begin{array}{llll}6 & -0.855307 & -0.069814 & 3.276835 \\ 1 & -0.830737 & -2.178241 & 2.939963 \\ 6 & -0.325650 & 1.147240 & 2.838212 \\ 6 & 0.589937 & 1.171987 & 1.803161 \\ 1 & 1.012602 & 2.103071 & 1.447808 \\ 7 & 1.013152 & 0.049503 & 1.186891 \\ 1 & -1.590357 & -0.116863 & 4.071678 \\ 35 & -0.852423 & 2.828886 & 3.697588 \\ 5 & -2.137057 & -5.001210 & 2.366384 \\ 9 & -2.439002 & -6.151828 & 3.092114 \\ 9 & -2.209239 & -3.863181 & 3.198255 \\ 9 & -3.076601 & -4.846089 & 1.306123 \\ 9 & -0.843303 & -5.090770 & 1.819860 \\ ------------------------------------------\end{array}$

Number of imaginary frequencies $=0$

The smallest frequencies are $=11.750313 .702815 .4507 \mathrm{~cm}(-1)$ Electronic energy $=-7647.2862608$

Zero-point correction=

(Hartree/Particle)

Thermal correction to Energy= $\quad 1.899380$

Thermal correction to Enthalpy $=\quad 1.900324$

Thermal correction to Gibbs Free Energy $=\quad 1.606315$

Sum of electronic and zero-point Energies $=\quad-7645.511055$

Sum of electronic and thermal Energies $=\quad-7645.386881$

Sum of electronic and thermal Enthalpies $=\quad-7645.385937$

Sum of electronic and thermal Free Energies $=\quad-7645.679945$

Cartesian Coordinates

$\begin{array}{lccc}\ldots 26 & 4.062655 & 4.819043 & 2.026582 \\ 15 & 2.651588 & 1.789896 & -1.991157 \\ 15 & 4.398536 & 0.173199 & 0.954522 \\ 6 & 3.853617 & 3.517189 & 0.397651 \\ 6 & 3.931638 & 2.789262 & 1.642609 \\ 6 & 2.846112 & 3.218705 & 2.467753 \\ 1 & 2.636728 & 2.875028 & 3.470982 \\ 6 & 2.112225 & 4.218508 & 1.759606 \\ 1 & 1.258292 & 4.765104 & 2.135918 \\ 6 & 2.735472 & 4.407721 & 0.491923 \\ 1 & 2.431219 & 5.112144 & -0.267459 \\ 6 & 5.960564 & 5.319275 & 2.703005 \\ 1 & 6.821575 & 4.665999 & 2.650475 \\ 6 & 5.018928 & 5.380415 & 3.775737 \\ 1 & 5.037310 & 4.778495 & 4.674515 \\ 6 & 4.019295 & 6.340601 & 3.431846 \\ 1 & 3.147904 & 6.589747 & 4.023068 \\ 6 & 4.342649 & 6.874357 & 2.147160 \\ 1 & 3.761670 & 7.601094 & 1.594822 \\ 6 & 5.542120 & 6.246213 & 1.698256 \\ 1 & 6.027076 & 6.419829 & 0.747269 \\ 6 & 4.751505 & 3.292104 & -0.772705 \\ 6 & 6.083246 & 3.728405 & -0.713930 \\ 1 & 6.412921 & 4.245062 & 0.178037 \\ 6 & 6.981438 & 3.497104 & -1.755356 \\ 1 & 8.011976 & 3.825190 & -1.665170 \\ 6 & 6.551968 & 2.824002 & -2.896530 \\ 1 & 7.240427 & 2.629247 & -3.712591 \\ 6 & 5.233979 & 2.373771 & -2.976779 \\ 1 & 4.915829 & 1.836319 & -3.863167 \\ 6 & 4.325841 & 2.581656 & -1.926984 \\ 6 & 4.935445 & 1.708092 & 1.940033 \\ 1 & 5.882362 & 2.015446 & 1.493805 \\ 6 & 5.170421 & 1.465966 & 3.433589 \\ 1 & 5.942386 & 0.708126 & 3.591909 \\ 1 & 4.265035 & 1.134800 & 3.948901 \\ 1 & 5.498562 & 2.399758 & 3.900145 \\ 6 & 2.590539 & 0.989979 & -3.649908 \\ 6 & 3.005459 & -0.348661 & -3.736124 \\ 1 & 3.331264 & -0.872054 & -2.845079 \\ 6 & 2.972698 & -1.023232 & -4.957964 \\ 1 & 3.301317 & -2.055970 & -5.007483 \\ 6 & 2.503735 & -0.375015 & -6.102613 \\ 1 & 2.461005 & -0.905041 & -7.049737\end{array}$




$$
\begin{aligned}
& \begin{array}{llll}
2.084380 & 0.955496 & -6.023951
\end{array} \\
& \begin{array}{lll}
1.714834 & 1.464142 & -6.909781
\end{array} \\
& \begin{array}{llll}
2.133138 & 1.638545 & -4.807634
\end{array} \\
& \begin{array}{llll}
1.796087 & 2.668186 & -4.765570
\end{array} \\
& \begin{array}{llll}
1.497788 & 3.207588 & -2.133225
\end{array} \\
& \begin{array}{llll}
1.835450 & 4.374677 & -2.838674
\end{array} \\
& \begin{array}{llll}
2.814263 & 4.465916 & -3.300415
\end{array} \\
& \begin{array}{llll}
0.922745 & 5.425981 & -2.934103
\end{array} \\
& \begin{array}{lll}
1.192267 & 6.326281 & -3.479017
\end{array} \\
& \begin{array}{llll}
-0.336028 & 5.317612 & -2.332661
\end{array} \\
& \begin{array}{lll}
-1.051580 & 6.131407 & -2.408303
\end{array} \\
& \begin{array}{llll}
-0.674204 & 4.160303 & -1.628910
\end{array} \\
& \begin{array}{llll}
-1.648511 & 4.070933 & -1.165157
\end{array} \\
& \begin{array}{llll}
0.244066 & 3.114596 & -1.517806
\end{array} \\
& \begin{array}{llll}
-0.012039 & 2.227501 & -0.949498
\end{array} \\
& \begin{array}{lll}
5.805844 & -0.232524 & -0.168933
\end{array} \\
& \begin{array}{llll}
5.564471 & -1.253509 & -1.099373
\end{array} \\
& \begin{array}{lll}
4.603910 & -1.756592 & -1.099265
\end{array} \\
& \begin{array}{lll}
6.544574 & -1.622451 & -2.019442
\end{array} \\
& \begin{array}{lll}
7.767518 & -0.957155 & -2.064475
\end{array} \\
& \begin{array}{llll}
8.520393 & -1.229194 & -2.795643
\end{array} \\
& \begin{array}{llll}
7.997418 & 0.075059 & -1.154125
\end{array} \\
& \begin{array}{llll}
7.040803 & 0.425149 & -0.200353
\end{array} \\
& \begin{array}{lll}
7.272911 & 1.222460 & 0.492243
\end{array} \\
& \begin{array}{lll}
4.371959 & -1.256070 & 2.118498
\end{array} \\
& \begin{array}{lll}
3.326810 & -1.322236 & 3.055520
\end{array} \\
& \begin{array}{llll}
2.617316 & -0.506536 & 3.143500
\end{array} \\
& \begin{array}{lll}
3.185227 & -2.444193 & 3.871952
\end{array} \\
& \begin{array}{lll}
4.036818 & -3.540491 & 3.735826
\end{array} \\
& \begin{array}{llll}
3.895833 & -4.428078 & 4.342936
\end{array} \\
& \begin{array}{llll}
5.059382 & -3.481227 & 2.792645
\end{array} \\
& \begin{array}{llll}
5.246855 & -2.343661 & 2.008392
\end{array} \\
& \begin{array}{llll}
6.059970 & -2.331325 & 1.294463
\end{array} \\
& \begin{array}{llll}
2.411534 & 0.295462 & -0.308297
\end{array} \\
& \begin{array}{llll}
5.952463 & -4.679270 & 2.619839
\end{array} \\
& \begin{array}{llll}
2.052471 & -2.544441 & 4.856638
\end{array} \\
& \begin{array}{llll}
6.272138 & -2.793274 & -2.922162
\end{array} \\
& \begin{array}{llll}
9.294183 & 0.832089 & -1.247892
\end{array} \\
& \begin{array}{llll}
9.401360 & 1.484912 & -2.432379
\end{array} \\
& \begin{array}{llll}
9.426703 & 1.758726 & -0.273240
\end{array} \\
& \begin{array}{llll}
10.363561 & 0.006858 & -1.168850
\end{array} \\
& \begin{array}{llll}
6.443530 & -3.969841 & -2.268854
\end{array} \\
& \begin{array}{llll}
7.079051 & -2.815317 & -4.001201
\end{array} \\
& \begin{array}{lll}
4.990421 & -2.795887 & -3.372575
\end{array} \\
& \begin{array}{lll}
6.785574 & -4.850423 & 3.670800
\end{array} \\
& \begin{array}{lll}
6.720185 & -4.590714 & 1.510424
\end{array} \\
& \begin{array}{llll}
5.224732 & -5.818771 & 2.512016
\end{array} \\
& \begin{array}{llll}
2.453857 & -3.096260 & 6.023046
\end{array} \\
& \begin{array}{llll}
1.512223 & -1.339401 & 5.145115
\end{array} \\
& \begin{array}{llll}
1.053383 & -3.329644 & 4.381765
\end{array} \\
& \begin{array}{llll}
1.968146 & -2.760739 & -0.059127
\end{array} \\
& \begin{array}{llll}
0.532048 & -2.515352 & 0.386260
\end{array} \\
& \begin{array}{llll}
0.358614 & -1.346965 & 1.327151
\end{array} \\
& \begin{array}{llll}
0.119757 & -3.396566 & 0.872693
\end{array} \\
& \begin{array}{lll}
-3.285850 & -0.959771 & -1.809311
\end{array} \\
& \begin{array}{lll}
-5.108378 & -0.238812 & -0.603136
\end{array} \\
& \begin{array}{llll}
-5.070692 & 0.749109 & 0.738215
\end{array} \\
& \begin{array}{llll}
-6.179359 & 0.657499 & -1.542715
\end{array} \\
& \begin{array}{lll}
-5.833060 & -1.561948 & 0.121169
\end{array} \\
& \begin{array}{lll}
-4.703056 & 2.072152 & 0.614395
\end{array} \\
& \begin{array}{lll}
-3.447046 & 2.437869 & 1.150444
\end{array} \\
& \begin{array}{lll}
-2.854601 & 1.671426 & 1.637372
\end{array} \\
& \begin{array}{lll}
-3.021546 & 3.738373 & 1.048685
\end{array} \\
& \begin{array}{lll}
-2.065531 & 4.038786 & 1.467283
\end{array} \\
& \begin{array}{lll}
-3.804380 & 4.698673 & 0.348779
\end{array} \\
& \begin{array}{llll}
-3.319763 & 6.016936 & 0.129034
\end{array} \\
& \begin{array}{llll}
-2.349161 & 6.285909 & 0.537718
\end{array} \\
& \begin{array}{llll}
-4.049571 & 6.926837 & -0.603158
\end{array} \\
& \begin{array}{llll}
-3.665846 & 7.928860 & -0.771779
\end{array} \\
& \begin{array}{llll}
-5.300084 & 6.549019 & -1.148352
\end{array} \\
& \begin{array}{llll}
-5.864091 & 7.261233 & -1.743843
\end{array} \\
& \begin{array}{llll}
-5.806818 & 5.284672 & -0.936977
\end{array} \\
& \begin{array}{llll}
-6.762439 & 5.009182 & -1.36811
\end{array} \\
& \begin{array}{llll}
-5.086572 & 4.321700 & -0.176378
\end{array} \\
& \begin{array}{llll}
-5.577230 & 2.985656 & 0.045930
\end{array}
\end{aligned}
$$

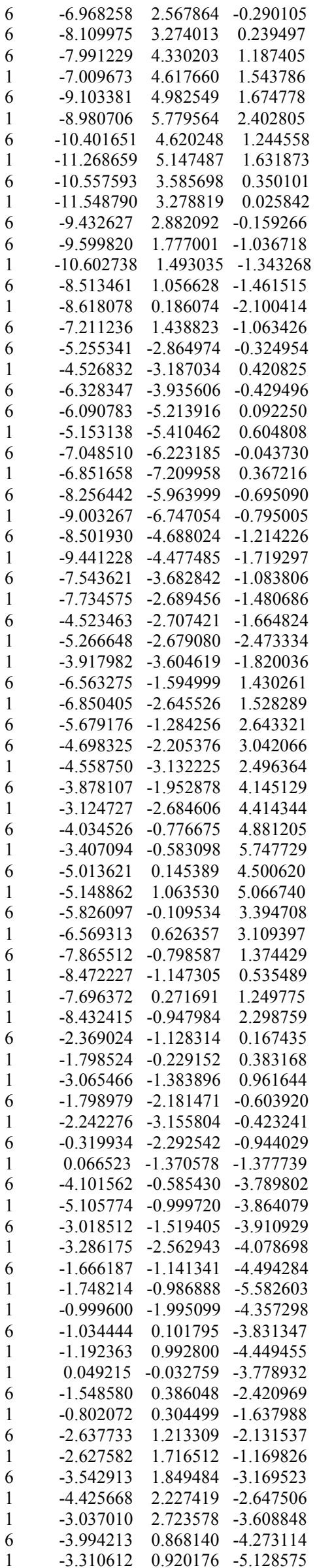




$\begin{array}{lrrr}1 & -4.970443 & 1.190108 & -4.651206 \\ 6 & -0.025485 & -3.434112 & -1.903897 \\ 6 & -0.470563 & -4.740378 & -1.646091 \\ 6 & 0.767496 & -3.208399 & -3.035763 \\ 6 & -0.140466 & -5.785588 & -2.512409 \\ 1 & -1.045829 & -4.951181 & -0.750664 \\ 6 & 1.096563 & -4.250758 & -3.904935 \\ 1 & 1.136724 & -2.208089 & -3.228960 \\ 6 & 0.640985 & -5.545634 & -3.646680 \\ 1 & -0.491248 & -6.791038 & -2.295402 \\ 1 & 1.706376 & -4.047269 & -4.781385 \\ 1 & 0.895564 & -6.361032 & -4.318340 \\ 7 & 2.544805 & -3.950498 & 0.185940 \\ 6 & 3.712521 & -4.355344 & -0.599254 \\ 1 & 3.641110 & -5.429075 & -0.790409 \\ 1 & 3.713625 & -3.830298 & -1.551807 \\ 1 & 4.649244 & -4.156514 & -0.072476 \\ 6 & 2.040319 & -4.948033 & 1.134229 \\ 1 & 1.500931 & -4.482101 & 1.955816 \\ 1 & 1.380393 & -5.665661 & 0.639963 \\ 1 & 2.901727 & -5.472329 & 1.552605 \\ 8 & 2.551041 & -1.895028 & -0.758631 \\ 6 & -0.391276 & -1.525037 & 2.495417 \\ 6 & -0.705212 & -0.434937 & 3.298935 \\ 1 & -0.759100 & -2.509666 & 2.756515 \\ 6 & -0.261922 & 0.814689 & 2.882904 \\ 6 & 0.487499 & 0.944416 & 1.722931 \\ 1 & 0.849057 & 1.906783 & 1.385301 \\ 7 & 0.808506 & -0.123294 & 0.966159 \\ 1 & -1.299788 & -0.561274 & 4.194801 \\ 35 & -0.678025 & 2.410223 & 3.929378 \\ 5 & -2.098674 & -5.162304 & 2.250988 \\ 9 & -2.516765 & -6.387444 & 2.763966 \\ 9 & -1.886748 & -4.240439 & 3.301757 \\ 9 & -3.081802 & -4.647557 & 1.369826 \\ 9 & -0.889458 & -5.316756 & 1.537279 \\ & - & \\ 6 & & \end{array}$

\section{P1-C1 endo si-re $\left[\mathrm{BF}_{4}^{-}\right]$}

Number of imaginary frequencies $=0$

The smallest frequencies are $=9.31159 .805214 .1056 \mathrm{~cm}(-1)$ Electronic energy $=-7647.2565111$

Zero-point correction=

1.772082

(Hartree/Particle)

Thermal correction to Energy=

Thermal correction to Enthalpy=

1.897640

1.898584

Thermal correction to Gibbs Free Energy=

Sum of electronic and zero-point Energies=

Sum of electronic and thermal Energies=

Sum of electronic and thermal Enthalpies=

Sum of electronic and thermal Free Energies=

1.600752

$-7645.484430$

$-7645.358871$

$-7645.357927$

$-7645.655759$

Cartesian Coordinates

$\begin{array}{llll}26 & 4.193871 & 5.079880 & 2.032509 \\ 15 & 2.465492 & 2.051622 & -1.814141 \\ 15 & 4.789256 & 0.509462 & 0.844492 \\ 6 & 3.828546 & 3.893206 & 0.344841 \\ 6 & 4.379327 & 3.109911 & 1.420386 \\ 6 & 3.484377 & 3.207010 & 2.531124 \\ 1 & 3.619643 & 2.729638 & 3.492016 \\ 6 & 2.403870 & 4.059714 & 2.161757 \\ 1 & 1.584435 & 4.358796 & 2.801473 \\ 6 & 2.617283 & 4.489482 & 0.819548 \\ 1 & 1.985891 & 5.159115 & 0.253324 \\ 6 & 6.046633 & 6.002972 & 2.222089 \\ 1 & 6.972567 & 5.616031 & 1.817981 \\ 6 & 5.506035 & 5.703752 & 3.510163 \\ 1 & 5.942863 & 5.041502 & 4.245511 \\ 6 & 4.259655 & 6.389852 & 3.635517 \\ 1 & 3.589511 & 6.339714 & 4.483694 \\ 6 & 4.028735 & 7.112196 & 2.425198 \\ 1 & 3.153038 & 7.704433 & 2.194109 \\ 6 & 5.132589 & 6.874670 & 1.552436 \\ 1 & 5.238642 & 7.262171 & 0.548345\end{array}$

\begin{tabular}{|c|c|c|}
\hline 4.416465 & 3.965958 & -1.020636 \\
\hline 5.572391 & 4.729692 & -1.238774 \\
\hline 5.988782 & 5.275825 & -0.402322 \\
\hline 6.188824 & 4.789098 & -2.488862 \\
\hline 7.088861 & 5.382636 & -2.621391 \\
\hline 5.641133 & 4.084003 & -3.557741 \\
\hline 6.103876 & 4.118924 & -4.539351 \\
\hline 4.497112 & 3.309654 & -3.360942 \\
\hline 4.097715 & 2.753291 & -4.200089 \\
\hline 3.881450 & 3.218606 & -2.101872 \\
\hline 5.560795 & 2.193303 & 1.281088 \\
\hline 6.119628 & 2.485557 & 0.387318 \\
\hline 6.515956 & 2.197606 & 2.476811 \\
\hline 7.340555 & 1.493469 & 2.330437 \\
\hline 6.006058 & 1.935135 & 3.407620 \\
\hline 6.935144 & 3.201304 & 2.595191 \\
\hline 2.275297 & 1.252492 & -3.465210 \\
\hline 3.224322 & 0.276198 & -3.814456 \\
\hline 4.038646 & 0.053335 & -3.132884 \\
\hline 3.154915 & -0.381706 & -5.040737 \\
\hline 3.904183 & -1.125594 & -5.294711 \\
\hline 2.120509 & -0.086284 & -5.934410 \\
\hline 2.057146 & -0.605275 & -6.886355 \\
\hline 1.170558 & 0.880216 & -5.597138 \\
\hline 0.365350 & 1.117880 & -6.286649 \\
\hline 1.250676 & 1.551987 & -4.372941 \\
\hline 0.517847 & 2.313977 & -4.133932 \\
\hline 1.014809 & 3.171646 & -1.658450 \\
\hline 0.847299 & 4.312310 & -2.460289 \\
\hline 1.586197 & 4.559064 & -3.217551 \\
\hline-0.264569 & 5.138292 & -2.282199 \\
\hline-0.389936 & 6.016646 & -2.909173 \\
\hline-1.210373 & 4.839900 & -1.294195 \\
\hline-2.074901 & 5.483527 & -1.155807 \\
\hline-1.035686 & 3.716316 & -0.483819 \\
\hline-1.760914 & 3.482515 & 0.287880 \\
\hline 0.070097 & 2.882159 & -0.662799 \\
\hline 0.229066 & 2.030357 & -0.006220 \\
\hline 5.987146 & -0.303160 & -0.287239 \\
\hline 6.347749 & -1.655259 & -0.186905 \\
\hline 6.029974 & -2.253436 & 0.658278 \\
\hline 7.104120 & -2.259172 & -1.192238 \\
\hline 7.487390 & -1.550576 & -2.330448 \\
\hline 8.061753 & -2.030116 & -3.113688 \\
\hline 7.099308 & -0.216568 & -2.446387 \\
\hline 6.361814 & 0.406636 & -1.439501 \\
\hline 6.057015 & 1.439439 & -1.577639 \\
\hline 4.845451 & -0.397357 & 2.441468 \\
\hline 3.654346 & -0.377657 & 3.178721 \\
\hline 2.780472 & 0.107691 & 2.760498 \\
\hline 3.599078 & -0.964530 & 4.443955 \\
\hline 4.716770 & -1.596674 & 4.984505 \\
\hline 4.668808 & -2.063759 & 5.961098 \\
\hline 5.902164 & -1.617047 & 4.247408 \\
\hline 5.977549 & -1.011433 & 2.992372 \\
\hline 6.918812 & -1.024961 & 2.455084 \\
\hline 2.701171 & 10.535956 & $5 \quad-0.143783$ \\
\hline 7.141786 & -2.227651 & 4.840291 \\
\hline 2.297441 & -0.951925 & 5.198589 \\
\hline 7.568036 & -3.679698 & -1.012204 \\
\hline 7.395729 & 0.561311 & -3.698563 \\
\hline 6.280371 & 0.709403 & -4.461225 \\
\hline 7.838317 & 1.810097 & -3.422900 \\
\hline 8.325117 & -0.036768 & -4.472497 \\
\hline 6.715099 & -4.403304 & -0.257383 \\
\hline 8.773849 & -3.724100 & -0.389849 \\
\hline 7.721332 & -4.319356 & -2.193485 \\
\hline 7.892007 & -1.300588 & 5.487965 \\
\hline 7.937089 & -2.773232 & 3.891874 \\
\hline 6.856716 & -3.195425 & 5.738172 \\
\hline 2.484205 & -1.060887 & 6.533988 \\
\hline 1.610385 & 0.196284 & 4.981036 \\
\hline 1.483689 & -1.969137 & 4.833144 \\
\hline 3.079536 & -2.199934 & -1.229624 \\
\hline ( 500 & $-x_{0}$ & 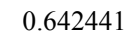 \\
\hline
\end{tabular}


$\begin{array}{llll}3.170254 & -3.573330 & -1.267672\end{array}$

$\begin{array}{llll}3.597730 & -1.589546 & -1.956394\end{array}$

$\begin{array}{llll}1.752140 & -3.671108 & 0.642311\end{array}$

$\begin{array}{llll}2.501258 & -4.337996 & -0.298333\end{array}$

$\begin{array}{lll}1.195757 & -4.236432 & 1.380436\end{array}$

$\begin{array}{llll}0.797513 & -1.634317 & 1.601203\end{array}$

$\begin{array}{lll}0.540486 & -0.259393 & 1.792534\end{array}$

$\begin{array}{lll}1.201514 & 0.701212 & 1.247658\end{array}$

$\begin{array}{llll}2.359574 & -1.535696 & -0.303625\end{array}$

$\begin{array}{lll}0.286610 & -2.336912 & 2.241577\end{array}$

$\begin{array}{lll}-0.492734 & 0.122274 & 2.633465\end{array}$

$\begin{array}{lll}-0.566908 & 1.485120 & 3.129401\end{array}$

$\begin{array}{lll}-0.359219 & 1.522255 & 4.208035\end{array}$

$\begin{array}{lll}-1.570990 & 1.900671 & 2.968811\end{array}$

$\begin{array}{lll}0.162248 & 2.102236 & 2.607943\end{array}$

$\begin{array}{lll}-1.336772 & -0.834925 & 3.328806\end{array}$

$\begin{array}{llll}-0.858500 & -1.231574 & 4.233668\end{array}$

$\begin{array}{lll}-1.595716 & -1.678545 & 2.691452\end{array}$

$\begin{array}{llll}-2.261910 & -0.334403 & 3.622347\end{array}$

$\begin{array}{llll}-3.568920 & -0.562246 & -1.627269\end{array}$

$\begin{array}{llll}-5.383073 & -0.488273 & -0.241052\end{array}$

$\begin{array}{lll}-5.489353 & 0.479723 & 1.096682\end{array}$

$\begin{array}{llll}-6.720151 & 0.028070 & -1.079738\end{array}$

$\begin{array}{llll}-5.605948 & -1.974099 & 0.465456\end{array}$

$\begin{array}{lll}-5.507917 & 1.861719 & 0.944766\end{array}$

$\begin{array}{lll}-4.362148 & 2.562594 & 1.381315\end{array}$

$\begin{array}{lll}-3.554065 & 2.002676 & 1.837315\end{array}$

$\begin{array}{lll}-4.304909 & 3.925127 & 1.223740\end{array}$

$\begin{array}{llll}-3.441948 & 4.483030 & 1.575086\end{array}$

$\begin{array}{llll}-5.353325 & 4.618194 & 0.559935\end{array}$

$\begin{array}{lll}-5.250568 & 6.008329 & 0.281698\end{array}$

$\begin{array}{llll}-4.363699 & 6.539608 & 0.617432\end{array}$

$\begin{array}{llll}-6.242792 & 6.666022 & -0.409797\end{array}$

$\begin{array}{llll}-6.150809 & 7.726552 & -0.624871\end{array}$

$\begin{array}{llll}-7.381436 & 5.952598 & -0.854336\end{array}$

$\begin{array}{lll}-8.151915 & 6.468482 & -1.420043\end{array}$

$\begin{array}{llll}-7.521798 & 4.608912 & -0.581127\end{array}$

$\begin{array}{lll}-8.397502 & 4.078138 & -0.935763\end{array}$

$\begin{array}{lll}-6.524622 & 3.899579 & 0.144450\end{array}$

$\begin{array}{llll}-6.630868 & 2.490671 & 0.431471\end{array}$

$\begin{array}{lll}-7.885941 & 1.723326 & 0.194213\end{array}$

$\begin{array}{lll}-9.138934 & 2.141878 & 0.776878\end{array}$

$\begin{array}{lll}-9.232696 & 3.211760 & 1.710754\end{array}$

$\begin{array}{lll}-8.333232 & 3.731870 & 2.016375\end{array}$

$\begin{array}{lll}-10.445734 & 3.586769 & 2.247181\end{array}$

$\begin{array}{lll}-10.485412 & 4.402217 & 2.963775\end{array}$

$\begin{array}{lll}-11.636864 & 2.916604 & 1.881709\end{array}$

$\begin{array}{lll}-12.586437 & 3.227840 & 2.307042\end{array}$

$\begin{array}{lll}-11.581253 & 1.859699 & 1.001755\end{array}$

$\begin{array}{lll}-12.483497 & 1.318996 & 0.728065\end{array}$

$\begin{array}{lll}-10.344371 & 1.436902 & 0.443924\end{array}$

$\begin{array}{llll}-10.280577 & 0.309191 & -0.418282\end{array}$

$\begin{array}{lll}-11.198404 & -0.211400 & -0.676492\end{array}$

$\begin{array}{llll}-9.072930 & -0.139678 & -0.887201\end{array}$

$\begin{array}{llll}-8.992616 & -1.022731 & -1.512183\end{array}$

$\begin{array}{llll}-7.888497 & 0.553337 & -0.551255\end{array}$

$\begin{array}{llll}-4.646388 & -3.014431 & -0.004549\end{array}$

$\begin{array}{llll}-3.837571 & -3.092427 & 0.725049\end{array}$

$\begin{array}{llll}-5.319944 & -4.371579 & -0.137347\end{array}$

$\begin{array}{llll}-4.682481 & -5.519740 & 0.346344\end{array}$

$\begin{array}{llll}-3.737310 & -5.425328 & 0.869078\end{array}$

$\begin{array}{llll}-5.270773 & -6.776553 & 0.174616\end{array}$

$\begin{array}{lll}-4.767980 & -7.659595 & 0.559612\end{array}$

$\begin{array}{llll}-6.500298 & -6.896641 & -0.475675\end{array}$

$\begin{array}{llll}-6.958509 & -7.873570 & -0.603416\end{array}$

$\begin{array}{lll}-7.144216 & -5.750872 & -0.956379\end{array}$

$\begin{array}{llll}-8.104546 & -5.835874 & -1.458178\end{array}$

$\begin{array}{llll}-6.556034 & -4.497607 & -0.789881\end{array}$

$\begin{array}{llll}-7.058616 & -3.604599 & -1.152907\end{array}$

$\begin{array}{llll}-4.068511 & -2.618597 & -1.366590\end{array}$

$\begin{array}{llll}-4.804324 & -2.869796 & -2.136684\end{array}$

$\begin{array}{llll}-3.180651 & -3.220380 & -1.566260\end{array}$

$\begin{array}{llll}-6.308980 & -2.284319 & 1.755877\end{array}$

$\begin{array}{lll}-6.462545 & -3.364442 & 1.696960\end{array}$

$\begin{array}{lll}-5.441700 & -2.048583 & 2.991920\end{array}$ $\begin{array}{llll}-4.536838 & -3.045837 & 3.384631\end{array}$

$\begin{array}{lll}-4.435240 & -3.953980 & 2.801330\end{array}$

$\begin{array}{lll}-3.756525 & -2.900702 & 4.533789\end{array}$

$\begin{array}{lll}-3.041534 & -3.675482 & 4.786395\end{array}$

$\begin{array}{lll}-3.889255 & -1.759359 & 5.325875\end{array}$

$\begin{array}{llll}-3.290022 & -1.644520 & 6.224977\end{array}$

$\begin{array}{lll}-4.787621 & -0.757246 & 4.946479\end{array}$

$\begin{array}{lll}-4.896410 & 0.136177 & 5.556038\end{array}$

$\begin{array}{lll}-5.551187 & -0.897261 & 3.785656\end{array}$

$\begin{array}{lll}-6.241853 & -0.109575 & 3.506867\end{array}$

$\begin{array}{lll}-7.703777 & -1.668909 & 1.841517\end{array}$

$\begin{array}{llll}-8.299233 & -1.969357 & 0.976796\end{array}$

$\begin{array}{lll}-7.693521 & -0.579674 & 1.889152\end{array}$

$\begin{array}{lll}-8.197952 & -2.039222 & 2.744531\end{array}$

$\begin{array}{lll}-2.366757 & -0.056189 & 0.135586\end{array}$

$\begin{array}{llll}-1.969681 & 0.941592 & -0.024562\end{array}$

$\begin{array}{lll}-2.815455 & -0.199202 & 1.111274\end{array}$

$\begin{array}{llll}-1.691140 & -1.186167 & -0.415655\end{array}$

$\begin{array}{lll}-1.821525 & -2.137346 & 0.084297\end{array}$

$\begin{array}{llll}-1.011591 & -1.140183 & -1.637108\end{array}$

$\begin{array}{llll}-0.671834 & -0.179061 & -2.006841\end{array}$

$\begin{array}{llll}-4.945728 & -0.571883 & -3.431750\end{array}$

$\begin{array}{llll}-5.706527 & -1.316461 & -3.215494\end{array}$

$\begin{array}{llll}-3.692636 & -1.063198 & -3.839135\end{array}$

$\begin{array}{lll}-3.577758 & -2.143468 & -3.911509\end{array}$

$\begin{array}{llll}-2.674275 & -0.285905 & -4.647767\end{array}$

$\begin{array}{llll}-2.954443 & -0.332208 & -5.711002\end{array}$

$\begin{array}{llll}-1.717905 & -0.805435 & -4.562484\end{array}$

$\begin{array}{llll}-2.496009 & 1.183498 & -4.218107\end{array}$

$\begin{array}{llll}-3.201987 & 1.825602 & -4.752580\end{array}$

$\begin{array}{llll}-1.499600 & 1.514271 & -4.517868\end{array}$

$\begin{array}{llll}-2.649276 & 1.423589 & -2.723634\end{array}$

$\begin{array}{llll}-1.735861 & 1.610745 & -2.170064\end{array}$

$\begin{array}{lll}-3.837840 & 1.796942 & -2.132632\end{array}$

$\begin{array}{llll}-3.787260 & 2.273314 & -1.158454\end{array}$

$\begin{array}{llll}-5.144013 & 1.963991 & -2.879963\end{array}$

$\begin{array}{llll}-5.938879 & 2.086430 & -2.146101\end{array}$

$\begin{array}{llll}-5.099696 & 2.903920 & -3.448663\end{array}$

$\begin{array}{llll}-5.506392 & 0.795043 & -3.827182\end{array}$

$\begin{array}{llll}-5.181061 & 1.018304 & -4.848250\end{array}$

$\begin{array}{lll}-6.595865 & 0.712970 & -3.864379\end{array}$

$\begin{array}{llll}-0.463499 & -2.308833 & -2.327478\end{array}$

$\begin{array}{lll}-0.644227 & -3.632692 & -1.874423\end{array}$

$\begin{array}{llll}0.285515 & -2.101620 & -3.503491\end{array}$

$\begin{array}{llll}-0.131510 & -4.703115 & -2.604882\end{array}$

$\begin{array}{llll}-1.145251 & -3.831986 & -0.934147\end{array}$

$\begin{array}{llll}0.795754 & -3.174900 & -4.229083\end{array}$

$\begin{array}{llll}0.473148 & -1.086985 & -3.837582\end{array}$

$\begin{array}{llll}0.578096 & -4.482877 & -3.789049\end{array}$

$\begin{array}{llll}-0.272886 & -5.714450 & -2.234513\end{array}$

$\begin{array}{lll}1.374607 & -2.986167 & -5.128075\end{array}$

$\begin{array}{llll}0.979469 & -5.322176 & -4.349115\end{array}$

$\begin{array}{llll}2.552733 & -5.421697 & -0.300750\end{array}$

$\begin{array}{llll}4.216194 & -4.431791 & -2.686511\end{array}$

$\begin{array}{lll}-1.430451 & -4.769122 & 2.395753\end{array}$

$\begin{array}{lll}-2.568673 & -5.453204 & 2.861472\end{array}$

$\begin{array}{lll}-0.463010 & -5.684584 & 1.957772\end{array}$

$\begin{array}{llll}-0.906350 & -3.963711 & 3.418869\end{array}$

$\begin{array}{lll}-1.816835 & -3.940817 & 1.300328\end{array}$

\section{$[8-9]^{\ddagger} \mathrm{P1}-\mathrm{C1}$ endo si-re $\left[\mathrm{BF}_{4}^{-}\right]$}

Number of imaginary frequencies $=1$

The smallest frequencies are $=-209.23807 .616110 .0335 \mathrm{~cm}(-1)$ Electronic energy $=-7647.2448607$

Zero-point correction $=$

1.771051

(Hartree/Particle)

Thermal correction to Energy= $\quad 1.896066$

Thermal correction to Enthalpy=

1.897010

Thermal correction to Gibbs Free Energy=

Sum of electronic and zero-point Energies=

Sum of electronic and thermal Energies=

Sum of electronic and thermal Enthalpies=

Sum of electronic and thermal Free Energies=

1.598936

$-7645.473809$

$-7645.348795$

$-7645.347850$

$-7645.645925$ 
Cartesian Coordinates

\begin{tabular}{|c|c|c|c|}
\hline y & 3.702977 & 4.996167 & 2.53209 \\
\hline & 2.510671 & 2.127947 & -1.614685 \\
\hline & 4.896006 & 0.613927 & 1.041874 \\
\hline 6 & 3.561194 & 3.912290 & 0.744713 \\
\hline 6 & 4.114130 & 3.101347 & 1.798887 \\
\hline 6 & 3.147003 & 3.033742 & 2.850019 \\
\hline 1 & 3.271597 & 2.508718 & 3.787545 \\
\hline 6 & 2.014514 & 3.810581 & 2.465271 \\
\hline 1 & 1.133398 & 3.990318 & 3.066674 \\
\hline 6 & 2.271203 & 4.358550 & 1.174901 \\
\hline 1 & 1.615684 & 5.008332 & 0.613190 \\
\hline 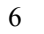 & 5.437124 & 6.075192 & 2.918652 \\
\hline 1 & 6.421337 & 5.810865 & 2.555300 \\
\hline 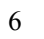 & 4.846345 & 5.631423 & 4.141078 \\
\hline 1 & 5.299406 & 4.962161 & 4.860116 \\
\hline 6 & 3.531315 & 6.182859 & 4.220630 \\
\hline 1 & 2.813466 & 6.002745 & 5.010147 \\
\hline 6 & 3.309618 & 6.968289 & 3.048363 \\
\hline 1 & 2.396281 & 7.490691 & 2.795568 \\
\hline 6 & 4.486413 & 6.902501 & 2.244443 \\
\hline 1 & 4.619505 & 7.371498 & 1.279100 \\
\hline 6 & 4.209357 & 4.152509 & -0.573908 \\
\hline 6 & 5.288939 & 5.042110 & -0.671709 \\
\hline 1 & 5.622918 & 5.543175 & 0.227713 \\
\hline 6 & 5.931895 & 5.284149 & -1.886101 \\
\hline 1 & 6.771817 & 5.971709 & -1.926251 \\
\hline 6 & 5.487016 & 4.642280 & -3.039284 \\
\hline 1 & 5.971259 & 4.821895 & -3.994189 \\
\hline 6 & 4.426363 & 3.739268 & -2.961799 \\
\hline 1 & 4.109840 & 3.230022 & -3.864042 \\
\hline 6 & 3.791308 & 3.461987 & -1.740948 \\
\hline 6 & 5.397713 & 2.328194 & 1.690596 \\
\hline 1 & 5.984453 & 2.760967 & 0.874830 \\
\hline 6 & 6.252218 & 2.333740 & 2.960665 \\
\hline 1 & 7.186337 & 1.784004 & 2.815450 \\
\hline 1 & 5.724778 & 1.893105 & 3.811093 \\
\hline 1 & 6.500542 & 3.367512 & 3.216764 \\
\hline 6 & 2.461823 & 1.415709 & -3.312033 \\
\hline 6 & 3.554157 & 0.624343 & -3.709537 \\
\hline 1 & 4.415059 & 0.529801 & -3.056213 \\
\hline 6 & 3.558205 & -0.016183 & -4.946614 \\
\hline 1 & 4.414548 & -0.617238 & -5.238616 \\
\hline 6 & 2.458120 & 0.109188 & -5.801662 \\
\hline 1 & 2.451744 & -0.401210 & -6.760278 \\
\hline 6 & 1.368772 & 0.892420 & -5.416638 \\
\hline 1 & 0.510486 & 0.994696 & -6.074479 \\
\hline 6 & 1.371345 & 1.549162 & -4.182221 \\
\hline 1 & 0.518496 & 2.157307 & -3.902277 \\
\hline 6 & 0.936636 & 3.054954 & -1.444248 \\
\hline 6 & 0.666121 & 4.211221 & -2.193451 \\
\hline 1 & 1.393189 & 4.575848 & -2.913678 \\
\hline 6 & -0.534308 & 4.898974 & -2.010594 \\
\hline 1 & -0.742119 & 5.789353 & -2.597292 \\
\hline 6 & -1.465899 & 4.444494 & -1.070761 \\
\hline 1 & -2.402089 & 4.976977 & -0.933435 \\
\hline 6 & -1.191771 & 3.303736 & -0.313585 \\
\hline 1 & -1.910787 & 2.950637 & 0.416660 \\
\hline 6 & 0.003159 & 2.605450 & -0.499713 \\
\hline 1 & 0.230731 & 1.728441 & 0.100035 \\
\hline 6 & 6.275810 & 0.124712 & -0.075605 \\
\hline 6 & 6.880171 & -1.140935 & -0.050696 \\
\hline 1 & 6.638875 & -1.856624 & 0.725295 \\
\hline 6 & 7.785427 & -1.509507 & -1.046066 \\
\hline 6 & 8.091445 & -0.646664 & -2.097477 \\
\hline 1 & 8.782618 & -0.946652 & -2.875382 \\
\hline 6 & 7.472200 & 0.601230 & -2.137185 \\
\hline 6 & 6.575228 & 0.989242 & -1.140483 \\
\hline 1 & 6.091316 & 1.958421 & -1.215917 \\
\hline 6 & 5.009794 & -0.519480 & 2.485978 \\
\hline 6 & 3.795124 & -1.003700 & 2.982820 \\
\hline 1 & 2.866238 & -0.687906 & 2.520422 \\
\hline & 3.776526 & -1.886259 & 4.065373 \\
\hline & 4.960763 & -2.298755 & 4.668430 \\
\hline
\end{tabular}

$\begin{array}{lll}4.945793 & -2.996349 & 5.497278\end{array}$

$\begin{array}{llll}6.172902 & -1.805579 & 4.179117\end{array}$

$\begin{array}{llll}6.204716 & -0.920241 & 3.102698\end{array}$

$\begin{array}{llll}7.160853 & -0.562792 & 2.738509\end{array}$

$\begin{array}{llll}2.889036 & 0.484217 & -0.096798\end{array}$

$\begin{array}{llll}7.462738 & -2.189741 & 4.851278\end{array}$

$\begin{array}{lll}2.446185 & -2.422780 & 4.517323\end{array}$

$\begin{array}{llll}8.481495 & -2.840876 & -0.952318\end{array}$

$\begin{array}{llll}7.699016 & 1.532099 & -3.297001\end{array}$

$\begin{array}{llll}6.588819 & 1.623367 & -4.075646\end{array}$

$\begin{array}{llll}7.984046 & 2.788471 & -2.884502\end{array}$

$\begin{array}{llll}8.709020 & 1.129779 & -4.095612\end{array}$

$\begin{array}{lll}7.722179 & -3.767908 & -0.330298\end{array}$

$\begin{array}{llll}9.635888 & -2.743301 & -0.246653\end{array}$

$\begin{array}{llll}8.811151 & -3.328592 & -2.169116\end{array}$

$\begin{array}{llll}7.819901 & -1.284175 & 5.795182\end{array}$

$\begin{array}{llll}8.487736 & -2.256324 & 3.971029\end{array}$

$\begin{array}{llll}7.378263 & -3.387324 & 5.469067\end{array}$

$\begin{array}{llll}2.533545 & -3.137469 & 5.657868\end{array}$

$\begin{array}{llll}1.556809 & -1.421364 & 4.736551\end{array}$

$\begin{array}{lll}1.896579 & -3.227960 & 3.577434\end{array}$

$\begin{array}{llll}3.866174 & -1.953076 & -1.503814\end{array}$

$1.954472 \quad-2.460632 \quad-0.251446$

$\begin{array}{llll}4.006801 & -3.264630 & -1.915076\end{array}$

$\begin{array}{llll}4.561651 & -1.190245 & -1.824895\end{array}$

$\begin{array}{llll}2.093682 & -3.829353 & -0.623370\end{array}$

$\begin{array}{llll}3.108326 & -4.237108 & -1.465759\end{array}$

$\begin{array}{llll}1.379377 & -4.548185 & -0.238022\end{array}$

$\begin{array}{llll}0.801769 & -2.102614 & 0.518970\end{array}$

$\begin{array}{lll}0.632348 & -0.941457 & 1.342448\end{array}$

$\begin{array}{lll}1.286177 & 0.147217 & 1.191701\end{array}$

$\begin{array}{llll}2.865070 & -1.547292 & -0.702630\end{array}$

$\begin{array}{llll}0.175329 & -2.950814 & 0.745013\end{array}$

$\begin{array}{lll}-0.319203 & -0.972335 & 2.325060\end{array}$

$\begin{array}{lll}-0.659151 & 0.246358 & 3.044270\end{array}$

$\begin{array}{lll}-0.146116 & 0.286398 & 4.014171\end{array}$

$\begin{array}{lll}-1.738639 & 0.264947 & 3.226960\end{array}$

$\begin{array}{llll}-0.370577 & 1.120728 & 2.463682\end{array}$

$\begin{array}{lll}-0.947353 & -2.179015 & 2.857287\end{array}$

$\begin{array}{lll}-0.437496 & -3.082742 & 2.537717\end{array}$

$\begin{array}{lll}-1.997017 & -2.245137 & 2.560043\end{array}$

$\begin{array}{lll}-0.902053 & -2.137834 & 3.949735\end{array}$

$\begin{array}{llll}-3.852960 & -0.741685 & -1.501797\end{array}$

$\begin{array}{lll}-5.640651 & -0.378733 & -0.138440\end{array}$

$\begin{array}{lll}-5.708252 & 0.751355 & 1.077479\end{array}$

$\begin{array}{lll}-6.964620 & 0.100862 & -1.037928\end{array}$

$\begin{array}{lll}-5.960420 & -1.746322 & 0.765176\end{array}$

$\begin{array}{llll}-5.745146 & 2.102173 & 0.775058\end{array}$

$\begin{array}{lll}-4.598030 & 2.851287 & 1.114901\end{array}$

$\begin{array}{lll}-3.781554 & 2.336043 & 1.609201\end{array}$

$\begin{array}{lll}-4.552462 & 4.191213 & 0.821804\end{array}$

$\begin{array}{lll}-3.683260 & 4.783655 & 1.090987\end{array}$

$\begin{array}{lll}-5.625641 & 4.809271 & 0.123205\end{array}$

$\begin{array}{llll}-5.547646 & 6.168592 & -0.284811\end{array}$

$\begin{array}{llll}-4.656946 & 6.735986 & -0.027126\end{array}$

$\begin{array}{llll}-6.567894 & 6.752103 & -1.001782\end{array}$

$\begin{array}{lll}-6.494254 & 7.789259 & -1.315501\end{array}$

$\begin{array}{llll}-7.711892 & 5.991804 & -1.343227\end{array}$

$\begin{array}{llll}-8.505120 & 6.447663 & -1.928759\end{array}$

$\begin{array}{llll}-7.828072 & 4.677562 & -0.944769\end{array}$

$\begin{array}{llll}-8.707475 & 4.107637 & -1.221188\end{array}$

$\begin{array}{lll}-6.801347 & 4.045628 & -0.189471\end{array}$

$\begin{array}{llll}-6.886651 & 2.670197 & 0.231140\end{array}$

$\begin{array}{lll}-8.137341 & 1.868906 & 0.108853\end{array}$

$\begin{array}{lll}-9.377895 & 2.316018 & 0.693384\end{array}$

$\begin{array}{lll}-9.468942 & 3.476604 & 1.512974\end{array}$

$\begin{array}{lll}-8.574300 & 4.048473 & 1.726995\end{array}$

$\begin{array}{lll}-10.672007 & 3.876694 & 2.053513\end{array}$

$\begin{array}{lll}-10.709335 & 4.762819 & 2.680832\end{array}$

$\begin{array}{lll}-11.855817 & 3.141759 & 1.807624\end{array}$

$\begin{array}{lll}-12.798235 & 3.473195 & 2.233722\end{array}$

$\begin{array}{lll}-11.801014 & 1.997822 & 1.044084\end{array}$

$\begin{array}{lll}-12.696806 & 1.409019 & 0.864154\end{array}$

$\begin{array}{llll}-10.573703 & 1.548844 & 0.485188\end{array}$

$\begin{array}{lll}-10.509019 & 0.338152 & -0.256255\end{array}$ 
$\begin{array}{lll}-11.420330 & -0.230048 & -0.420284\end{array}$

$\begin{array}{llll}-9.307534 & -0.124228 & -0.730172\end{array}$

$\begin{array}{llll}-9.226306 & -1.062545 & -1.268746\end{array}$

$\begin{array}{llll}-8.132256 & 0.631775 & -0.519661\end{array}$

$\begin{array}{lll}-5.142981 & -2.922063 & 0.345151\end{array}$

$\begin{array}{lll}-4.298789 & -3.017502 & 1.033750\end{array}$

$\begin{array}{llll}-5.953587 & -4.208975 & 0.397120\end{array}$

$\begin{array}{lll}-5.384319 & -5.382777 & 0.908324\end{array}$

$\begin{array}{llll}-4.372356 & -5.366217 & 1.297913\end{array}$

$\begin{array}{lll}-6.110320 & -6.577518 & 0.905901\end{array}$

$\begin{array}{lll}-5.653322 & -7.479812 & 1.303567\end{array}$

$\begin{array}{llll}-7.411691 & -6.613128 & 0.400840\end{array}$

$\begin{array}{lll}-7.975821 & -7.541827 & 0.404814\end{array}$

$\begin{array}{llll}-7.986168 & -5.443498 & -0.108139\end{array}$

$\begin{array}{llll}-8.999952 & -5.460135 & -0.499910\end{array}$

$\begin{array}{llll}-7.260335 & -4.252309 & -0.112136\end{array}$

$\begin{array}{llll}-7.708498 & -3.340212 & -0.497673\end{array}$

$\begin{array}{llll}-4.606960 & -2.708376 & -1.076704\end{array}$

$\begin{array}{llll}-5.416131 & -2.920280 & -1.785929\end{array}$

$\begin{array}{llll}-3.823038 & -3.445968 & -1.257964\end{array}$

$\begin{array}{lll}-6.539139 & -1.848475 & 2.144266\end{array}$

$\begin{array}{llll}-6.856673 & -2.892643 & 2.203419\end{array}$

$\begin{array}{lll}-5.481533 & -1.666925 & 3.233099\end{array}$

$\begin{array}{lll}-4.806488 & -2.800731 & 3.711406\end{array}$

$\begin{array}{lll}-5.044512 & -3.776379 & 3.296349\end{array}$

$\begin{array}{lll}-3.841998 & -2.696873 & 4.716321\end{array}$

$\begin{array}{llll}-3.329544 & -3.589091 & 5.064744\end{array}$

$\begin{array}{llll}-3.536023 & -1.449937 & 5.263733\end{array}$

$\begin{array}{llll}-2.785553 & -1.363567 & 6.044596\end{array}$

$\begin{array}{lll}-4.203681 & -0.312757 & 4.799700\end{array}$

$\begin{array}{lll}-3.980558 & 0.661198 & 5.227637\end{array}$

$\begin{array}{lll}-5.169477 & -0.420162 & 3.796119\end{array}$

$\begin{array}{lll}-5.684080 & 0.470167 & 3.456769\end{array}$

$\begin{array}{lll}-7.807172 & -1.022613 & 2.361734\end{array}$

$\begin{array}{lll}-8.552204 & -1.270442 & 1.602675\end{array}$

$\begin{array}{lll}-7.634996 & 0.052590 & 2.334021\end{array}$

$\begin{array}{lll}-8.221158 & -1.275909 & 3.342656\end{array}$

$\begin{array}{lll}-2.629457 & -0.468448 & 0.294839\end{array}$

$\begin{array}{llll}-2.153036 & 0.510637 & 0.289693\end{array}$

$\begin{array}{lll}-3.127349 & -0.699917 & 1.232713\end{array}$

$\begin{array}{llll}-1.989277 & -1.546714 & -0.393006\end{array}$

$\begin{array}{llll}-2.249574 & -2.552609 & -0.099911\end{array}$

$\begin{array}{llll}-0.835750 & -1.409889 & -1.201178\end{array}$

$\begin{array}{llll}-0.417136 & -0.414181 & -1.315869\end{array}$

$\begin{array}{llll}-5.068133 & -0.765905 & -3.358022\end{array}$

$\begin{array}{llll}-5.936763 & -1.390040 & -3.163390\end{array}$

$\begin{array}{llll}-3.846063 & -1.441661 & -3.615674\end{array}$

$\begin{array}{llll}-3.868803 & -2.530981 & -3.617916\end{array}$

$\begin{array}{llll}-2.708749 & -0.850816 & -4.429926\end{array}$

$\begin{array}{llll}-2.964972 & -0.882737 & -5.500685\end{array}$

$\begin{array}{llll}-1.837607 & -1.498466 & -4.307121\end{array}$

$\begin{array}{lll}-2.325533 & 0.589514 & -4.025216\end{array}$

$\begin{array}{llll}-2.831252 & 1.316334 & -4.670170\end{array}$

$\begin{array}{llll}-1.254992 & 0.723078 & -4.196923\end{array}$

$\begin{array}{llll}-2.618280 & 0.942216 & -2.569009\end{array}$

$\begin{array}{llll}-1.747441 & 1.126325 & -1.950871\end{array}$

$\begin{array}{llll}-3.809304 & 1.509550 & -2.126159\end{array}$

$\begin{array}{llll}-3.761555 & 2.097103 & -1.214136\end{array}$

$\begin{array}{llll}-5.018421 & 1.785625 & -2.999057\end{array}$

$\begin{array}{lll}-5.860439 & 2.011373 & -2.341817\end{array}$

$\begin{array}{llll}-4.832159 & 2.695009 & -3.590416\end{array}$

$\begin{array}{llll}-5.403115 & 0.613880 & -3.928497\end{array}$

$\begin{array}{llll}-4.916366 & 0.727354 & -4.903087\end{array}$

$\begin{array}{llll}-6.479470 & 0.660208 & -4.120945\end{array}$

$\begin{array}{llll}-0.379137 & -2.389301 & -2.178654\end{array}$

$\begin{array}{lll}-0.854244 & -3.715480 & -2.200934\end{array}$

$\begin{array}{llll}0.579218 & -1.998891 & -3.134840\end{array}$

$\begin{array}{llll}-0.399769 & -4.610248 & -3.165624\end{array}$

$\begin{array}{llll}-1.551891 & -4.058508 & -1.448844\end{array}$

$\begin{array}{llll}1.024946 & -2.896025 & -4.104061\end{array}$

$\begin{array}{llll}0.970415 & -0.987115 & -3.114454\end{array}$

$\begin{array}{llll}0.532404 & -4.204079 & -4.127501\end{array}$

$\begin{array}{llll}-0.764788 & -5.632914 & -3.150709\end{array}$

$\begin{array}{llll}1.759119 & -2.571363 & -4.834622\end{array}$

$\begin{array}{llll}0.882327 & -4.905666 & -4.879709\end{array}$

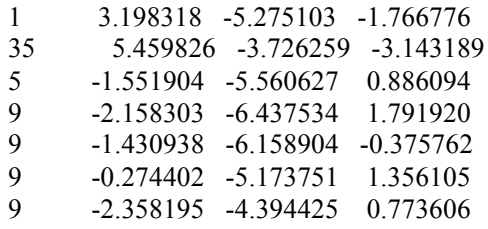

9 P1-C1 endo si-re $\left[\mathrm{BF}_{4}^{-}\right]$

Number of imaginary frequencies $=0$

The smallest frequencies are $=9.478210 .266712 .2814 \mathrm{~cm}(-1)$ Electronic energy $=-7647.2756488$

Zero-point correction=

1.774025

(Hartree/Particle)

Thermal correction to Energy= $\quad 1.898786$

Thermal correction to Enthalpy= 1.899730

Thermal correction to Gibbs Free Energy=

Sum of electronic and zero-point Energies=

Sum of electronic and thermal Energies=

1.602157

Sum of electronic and thermal Enthalpies=

$-7645.501624$

$-7645.376863$

$-7645.375918$

Sum of electronic and thermal Free Energies=

$-7645.673492$

Cartesian Coordinates

$\begin{array}{lccc}26 & 3.361765 & 4.411832 & 3.458842 \\ 15 & 2.139323 & 2.475885 & -1.207461 \\ 15 & 4.803569 & 0.570812 & 0.980305 \\ 6 & 3.194791 & 3.747351 & 1.476705 \\ 6 & 3.865135 & 2.767170 & 2.292814 \\ 6 & 2.972490 & 2.386566 & 3.342663 \\ 1 & 3.191494 & 1.674707 & 4.127313 \\ 6 & 1.765965 & 3.132521 & 3.194049 \\ 1 & 0.910561 & 3.096579 & 3.855502 \\ 6 & 1.905567 & 3.977487 & 2.054633 \\ 1 & 1.171360 & 4.679575 & 1.686987 \\ 6 & 5.022691 & 5.533315 & 4.013466 \\ 1 & 6.003075 & 5.444873 & 3.564911 \\ 6 & 4.538786 & 4.778982 & 5.125830 \\ 1 & 5.084726 & 4.013655 & 5.661086 \\ 6 & 3.190017 & 5.176912 & 5.374943 \\ 1 & 2.534334 & 4.762832 & 6.129632 \\ 6 & 2.839382 & 6.175887 & 4.416047 \\ 1 & 1.872343 & 6.650924 & 4.316150 \\ 6 & 3.971548 & 6.397670 & 3.576496 \\ 1 & 4.009658 & 7.074245 & 2.733701 \\ 6 & 3.749779 & 4.332444 & 0.223307 \\ 6 & 4.753466 & 5.309214 & 0.293498 \\ 1 & 5.091772 & 5.626745 & 1.271530 \\ 6 & 5.318435 & 5.865455 & -0.855004 \\ 1 & 6.099415 & 6.614991 & -0.764544 \\ 6 & 4.874176 & 5.455096 & -2.109360 \\ 1 & 5.301101 & 5.878125 & -3.013290 \\ 6 & 3.887735 & 4.472658 & -2.204792 \\ 1 & 3.570339 & 4.144046 & -3.187240 \\ 6 & 3.329080 & 3.888069 & -1.058016 \\ 6 & 0.547895 & 3.240312 & -0.712534 \\ 6 & 5.193147 & 2.152813 & 1.960212 \\ 1 & 5.679323 & 2.791070 & 1.215742 \\ 6 & 6.137294 & 1.996586 & 3.154461 \\ 1 & 7.116042 & 1.621935 & 2.842979 \\ 1 & 5.732339 & 1.321860 & 3.913711 \\ 1 & 6.281526 & 2.976047 & 3.617977 \\ 6 & 2.045488 & 2.175986 & -3.017826 \\ 6 & 3.099130 & 1.440111 & -3.589131 \\ 1 & 3.921797 & 1.107727 & -2.964430 \\ 6 & 3.103850 & 1.143903 & -4.950171 \\ 6 & 3.928295 & 0.580076 & -5.377004 \\ 6 & 2.042278 & 1.564772 & -5.758100 \\ 6 & 0.035574 & 1.324807 & -6.817271 \\ 6 & & 4.536638 & -1.115351\end{array}$


$\begin{array}{llll}0.815699 & 5.084924 & -1.812261\end{array}$

$\begin{array}{llll}-0.958196 & 5.137692 & -0.594326\end{array}$

$\begin{array}{llll}-1.229217 & 6.143163 & -0.903372\end{array}$

$\begin{array}{llll}-1.743864 & 4.455379 & 0.341372\end{array}$

$\begin{array}{llll}-2.617827 & 4.936642 & 0.769190\end{array}$

$\begin{array}{llll}-1.395260 & 3.161688 & 0.734484\end{array}$

$\begin{array}{lll}-1.995452 & 2.629315 & 1.466646\end{array}$

$\begin{array}{lll}-0.254505 & 2.553722 & 0.207528\end{array}$

$\begin{array}{lll}0.049046 & 1.570272 & 0.548203\end{array}$

$\begin{array}{llll}6.185025 & 0.483060 & -0.244168\end{array}$

$\begin{array}{llll}7.073361 & -0.594466 & -0.372764\end{array}$

$\begin{array}{llll}7.043351 & -1.427429 & 0.317256\end{array}$

$8.002961-0.618875-1.411565$

$\begin{array}{llll}8.059301 & 0.408414 & -2.353614\end{array}$

$\begin{array}{llll}8.774765 & 0.374134 & -3.166622\end{array}$

$\begin{array}{llll}7.164512 & 1.469407 & -2.238590\end{array}$

$\begin{array}{llll}6.235302 & 1.512168 & -1.197030\end{array}$

$\begin{array}{llll}5.536201 & 2.341770 & -1.145543\end{array}$

$\begin{array}{llll}5.083157 & -0.861900 & 2.104314\end{array}$

$\begin{array}{llll}3.989025 & -1.703438 & 2.341246\end{array}$

$\begin{array}{llll}3.032315 & -1.471851 & 1.892674\end{array}$

$\begin{array}{lll}4.127228 & -2.842429 & 3.137257\end{array}$

$\begin{array}{lll}5.353502 & -3.162682 & 3.714990\end{array}$

$\begin{array}{llll}5.463303 & -4.053526 & 4.320749\end{array}$

$\begin{array}{llll}6.442686 & -2.322800 & 3.485513\end{array}$

$\begin{array}{llll}6.314975 & -1.181073 & 2.694217\end{array}$

$\begin{array}{llll}7.187204 & -0.561051 & 2.525029\end{array}$

$\begin{array}{lll}2.764112 & 0.578058 & -0.136511\end{array}$

$\begin{array}{lll}7.792802 & -2.624551 & 4.078025\end{array}$

$\begin{array}{llll}2.922060 & -3.716041 & 3.360880\end{array}$

$\begin{array}{llll}8.999722 & -1.742897 & -1.510632\end{array}$

$\begin{array}{llll}7.161744 & 2.586280 & -3.247891\end{array}$

$\begin{array}{llll}5.951441 & 2.703951 & -3.850019\end{array}$

$\begin{array}{llll}7.422090 & 3.781428 & -2.668201\end{array}$

$\begin{array}{llll}8.075532 & 2.404501 & -4.222728\end{array}$

$\begin{array}{llll}8.657673 & -2.805239 & -0.752788\end{array}$

$10.229651 \quad-1.344690 \quad-1.104796$

$\begin{array}{llll}9.137592 & -2.177600 & -2.785745\end{array}$

$\begin{array}{llll}8.169426 & -1.668733 & 4.961269\end{array}$

$\begin{array}{lll}8.750854 & -2.661525 & 3.121188\end{array}$

$\begin{array}{lll}7.821778 & -3.805996 & 4.726307\end{array}$

$\begin{array}{lll}3.238565 & -4.898825 & 3.923532\end{array}$

$\begin{array}{lll}2.018509 & -3.115855 & 4.177227\end{array}$

$\begin{array}{llll}2.280561 & -3.976767 & 2.197587\end{array}$

$\begin{array}{llll}3.913219 & -1.685402 & -1.707365\end{array}$

$\begin{array}{llll}1.953084 & -2.404596 & -0.692809\end{array}$

$\begin{array}{llll}4.264901 & -2.987730 & -2.028509\end{array}$

$\begin{array}{llll}4.543561 & -0.849404 & -1.981587\end{array}$

$\begin{array}{llll}2.283048 & -3.741285 & -0.955339\end{array}$

$\begin{array}{llll}3.456317 & -4.048526 & -1.635329\end{array}$

$\begin{array}{lll}1.611766 & -4.530429 & -0.636092\end{array}$

$\begin{array}{llll}0.584041 & -2.099030 & -0.116300\end{array}$

$\begin{array}{lll}0.654937 & -1.273418 & 1.157581\end{array}$

$\begin{array}{lll}1.220363 & -0.148221 & 1.141356\end{array}$

$\begin{array}{llll}2.780978 & -1.398257 & -1.041357\end{array}$

$\begin{array}{llll}0.115349 & -3.060606 & 0.075799\end{array}$

$\begin{array}{llll}0.122872 & -1.719285 & 2.305643\end{array}$

$\begin{array}{lll}0.181271 & -0.808383 & 3.452619\end{array}$

$\begin{array}{lll}-0.062699 & -1.363735 & 4.355193\end{array}$

$\begin{array}{lll}-0.536223 & 0.009846 & 3.332973\end{array}$

$\begin{array}{lll}1.181471 & -0.382341 & 3.541241\end{array}$

$\begin{array}{lll}-0.649189 & -2.957891 & 2.454941\end{array}$

$\begin{array}{lll}-0.245465 & -3.757491 & 1.840905\end{array}$

$\begin{array}{lll}-1.699369 & -2.797313 & 2.198723\end{array}$

$\begin{array}{lll}-0.583231 & -3.272229 & 3.496368\end{array}$

$\begin{array}{llll}-3.657865 & -0.631366 & -1.510848\end{array}$

$\begin{array}{llll}-5.480593 & -0.272220 & -0.135864\end{array}$

$\begin{array}{lll}-5.699714 & 0.976013 & 0.957951\end{array}$

$\begin{array}{llll}-6.894505 & -0.079977 & -1.030705\end{array}$

$\begin{array}{llll}-5.658142 & -1.590271 & 0.893368\end{array}$

$\begin{array}{lll}-6.130746 & 2.221808 & 0.520750\end{array}$

$\begin{array}{llll}-5.182961 & 3.267739 & 0.551746\end{array}$

$\begin{array}{lll}-4.187025 & 3.045807 & 0.913054\end{array}$

$\begin{array}{llll}-5.534363 & 4.521365 & 0.118818\end{array}$

$\begin{array}{llll}-4.811693 & 5.331847 & 0.134495\end{array}$ $\begin{array}{llll}-6.836970 & 4.770308 & -0.389971\end{array}$

$\begin{array}{llll}-7.192259 & 6.046828 & -0.904544\end{array}$

$\begin{array}{llll}-6.450411 & 6.840906 & -0.876398\end{array}$

$\begin{array}{llll}-8.440210 & 6.272009 & -1.439791\end{array}$

$\begin{array}{llll}-8.699729 & 7.249956 & -1.834743\end{array}$

$\begin{array}{llll}-9.383068 & 5.217477 & -1.490918\end{array}$

$\begin{array}{llll}-10.358488 & 5.389380 & -1.937115\end{array}$

$\begin{array}{llll}-9.074539 & 3.972241 & -0.987776\end{array}$

$\begin{array}{llll}-9.805769 & 3.175188 & -1.047549\end{array}$

$\begin{array}{llll}-7.803059 & 3.708471 & -0.404292\end{array}$

$\begin{array}{lll}-7.446457 & 2.417635 & 0.125775\end{array}$

$\begin{array}{lll}-8.424330 & 1.297421 & 0.196535\end{array}$

$\begin{array}{lll}-9.666289 & 1.393955 & 0.914799\end{array}$

$\begin{array}{lll}-10.000432 & 2.516717 & 1.723252\end{array}$

$\begin{array}{lll}-9.295119 & 3.335043 & 1.809799\end{array}$

$\begin{array}{lll}-11.196306 & 2.572000 & 2.405715\end{array}$

$\begin{array}{lll}-11.424181 & 3.437375 & 3.021690\end{array}$

$\begin{array}{lll}-12.128036 & 1.509796 & 2.317969\end{array}$

$\begin{array}{lll}-13.069337 & 1.569549 & 2.856550\end{array}$

$\begin{array}{lll}-11.828971 & 0.398800 & 1.562091\end{array}$

$\begin{array}{lll}-12.527180 & -0.432044 & 1.499946\end{array}$

$\begin{array}{llll}-10.598088 & 0.302739 & 0.857469\end{array}$

$\begin{array}{lll}-10.267571 & -0.861658 & 0.111867\end{array}$

$\begin{array}{lll}-10.986870 & -1.673986 & 0.055641\end{array}$

$\begin{array}{lll}-9.045026 & -0.972644 & -0.503708\end{array}$

$\begin{array}{llll}-8.760184 & -1.861315 & -1.056130\end{array}$

$\begin{array}{llll}-8.123203 & 0.095577 & -0.424862\end{array}$

$\begin{array}{lll}-4.840413 & -2.755496 & 0.447022\end{array}$

$\begin{array}{lll}-3.930488 & -2.787127 & 1.059662\end{array}$

$\begin{array}{lll}-5.563246 & -4.082156 & 0.623538\end{array}$

$\begin{array}{lll}-4.820408 & -5.226402 & 0.945090\end{array}$

$\begin{array}{llll}-3.751888 & -5.146217 & 1.105521\end{array}$

$\begin{array}{lll}-5.438518 & -6.476324 & 1.019061\end{array}$

$\begin{array}{llll}-4.837412 & -7.351451 & 1.251087\end{array}$

$\begin{array}{llll}-6.811461 & -6.598356 & 0.789645\end{array}$

$\begin{array}{llll}-7.294067 & -7.569984 & 0.852451\end{array}$

$\begin{array}{llll}-7.560583 & -5.459302 & 0.478360\end{array}$

$\begin{array}{lll}-8.630222 & -5.542095 & 0.302547\end{array}$

$\begin{array}{lll}-6.939468 & -4.211872 & 0.390654\end{array}$

$\begin{array}{lll}-7.522521 & -3.326169 & 0.158486\end{array}$

$\begin{array}{llll}-4.435783 & -2.584328 & -1.025831\end{array}$

$\begin{array}{llll}-5.311748 & -2.794778 & -1.654334\end{array}$

$-3.689216-3.346030-1.256550$

$\begin{array}{llll}-6.079872 & -1.664247 & 2.324203\end{array}$

$\begin{array}{lll}-6.409308 & -2.699911 & 2.440444\end{array}$

$\begin{array}{lll}-4.904709 & -1.484529 & 3.282615\end{array}$

$\begin{array}{llll}-4.373657 & -2.608332 & 3.929457\end{array}$

$\begin{array}{llll}-4.779160 & -3.591746 & 3.704789\end{array}$

$\begin{array}{llll}-3.338145 & -2.480645 & 4.859844\end{array}$

$\begin{array}{lll}-2.945250 & -3.364498 & 5.354752\end{array}$

$\begin{array}{lll}-2.816005 & -1.220176 & 5.152352\end{array}$

$\begin{array}{lll}-2.020919 & -1.113619 & 5.884690\end{array}$

$\begin{array}{lll}-3.319610 & -0.094033 & 4.492670\end{array}$

$\begin{array}{lll}-2.912340 & 0.890067 & 4.710180\end{array}$

$\begin{array}{lll}-4.353727 & -0.224613 & 3.564611\end{array}$

$\begin{array}{lll}-4.742184 & 0.653374 & 3.061821\end{array}$

$\begin{array}{lll}-7.290256 & -0.811634 & 2.714993\end{array}$

$\begin{array}{lll}-8.134613 & -1.019313 & 2.055727\end{array}$

$\begin{array}{llll}-7.087992 & 0.257800 & 2.698263\end{array}$

$\begin{array}{llll}-7.580514 & -1.087921 & 3.733924\end{array}$

$\begin{array}{lll}-2.355391 & -0.516394 & 0.249235\end{array}$

$\begin{array}{lll}-1.882610 & 0.456532 & 0.346975\end{array}$

$\begin{array}{lll}-2.847269 & -0.845964 & 1.161490\end{array}$

$\begin{array}{llll}-1.776131 & -1.479925 & -0.628739\end{array}$

$\begin{array}{lll}-2.008146 & -2.510988 & -0.401095\end{array}$

$-0.355448-1.364518-1.170099$

$\begin{array}{llll}-0.029433 & -0.321511 & -1.219792\end{array}$

$\begin{array}{lll}-4.918727 & -0.598655 & -3.296770\end{array}$

$\begin{array}{llll}-5.807152 & -1.196238 & -3.105791\end{array}$

$\begin{array}{llll}-3.722172 & -1.312741 & -3.609178\end{array}$

$\begin{array}{lll}-3.789332 & -2.399632 & -3.651469\end{array}$

$\begin{array}{lll}-2.637221 & -0.729902 & -4.496487\end{array}$

$\begin{array}{lll}-2.995967 & -0.674654 & -5.537119\end{array}$

$\begin{array}{llll}-1.797982 & -1.423811 & -4.498412\end{array}$

$\begin{array}{llll}-2.145929 & 0.658307 & -4.035134\end{array}$ 
$\begin{array}{llll}1 & -2.621530 & 1.450203 & -4.625659 \\ 1 & -1.074831 & 0.736036 & -4.233390 \\ 6 & -2.377436 & 0.950342 & -2.551020 \\ 1 & -1.475877 & 1.072328 & -1.957507 \\ 6 & -3.526415 & 1.582052 & -2.058336 \\ 1 & -3.416513 & 2.159927 & -1.144654 \\ 6 & -4.738361 & 1.944076 & -2.902336 \\ 1 & -5.551876 & 2.202747 & -2.219406 \\ 1 & -4.524247 & 2.852756 & -3.486726 \\ 6 & -5.214471 & 0.806294 & -3.836236 \\ 1 & -4.763352 & 0.919052 & -4.828874 \\ 1 & -6.294643 & 0.906785 & -3.984119 \\ 6 & -0.142236 & -2.012066 & -2.524265 \\ 6 & -0.648315 & -3.290222 & -2.800404 \\ 6 & 0.644261 & -1.374861 & -3.491900 \\ 6 & -0.380032 & -3.909707 & -4.021696 \\ 1 & -1.231639 & -3.816526 & -2.055261 \\ 6 & 0.908216 & -1.989576 & -4.718780 \\ 1 & 1.051368 & -0.390812 & -3.285461 \\ 6 & 0.395177 & -3.261090 & -4.988637 \\ 1 & -0.770075 & -4.906545 & -4.208385 \\ 1 & 1.511143 & -1.469920 & -5.458199 \\ 1 & 0.600628 & -3.743843 & -5.940320 \\ 1 & 3.715960 & -5.077188 & -1.859543 \\ 35 & 5.909820 & -3.301773 & -3.029314 \\ 5 & -1.164972 & -5.806859 & -0.028714 \\ 9 & -1.865394 & -6.770977 & 0.704612 \\ 9 & -1.038710 & -6.187085 & -1.368527 \\ 9 & 0.121047 & -5.608405 & 0.537584 \\ 9 & -1.865635 & -4.573217 & 0.043214 \\ 6 & & & \\ 6 & & & \end{array}$

8 P1-C1 exo si-si $\left[\mathrm{BF}_{4}^{-}\right]$

Number of imaginary frequencies $=0$

The smallest frequencies are $=12.075814 .916415 .9028 \mathrm{~cm}(-1)$ Electronic energy $=-7647.2549713$

Zero-point correction=

(Hartree/Particle)

Thermal correction to Energy=

Thermal correction to Enthalpy=

1.772157

Thermal correction to Gibbs Free Energy=
Sum of electronic and zero-point Energies

Sum of electronic and zero-point Energies

Sum of electronic and thermal Energies=

1.897848

1.898792

1.600907

$-7645.482815$

$-7645.357124$

Sum of electronic and thermal Free Energies=

$-7645.356180$

$-7645.654064$ $\begin{array}{llll}3.989147 & 5.020302 & -4.210307\end{array}$

$\begin{array}{llll}2.876573 & 3.864214 & -2.778708\end{array}$

$2.357744 \quad 3.316889-3.555289$

$\begin{array}{llll}2.629698 & 3.571322 & -1.427792\end{array}$

$\begin{array}{lll}5.349808 & 2.499726 & 1.092233\end{array}$

$\begin{array}{lll}5.517052 & 2.971438 & 0.118821\end{array}$

$\begin{array}{lll}6.647155 & 2.573585 & 1.899257\end{array}$

$\begin{array}{lll}7.470206 & 2.076433 & 1.377341\end{array}$

$\begin{array}{llll}6.541900 & 2.117661 & 2.887381\end{array}$

$\begin{array}{lll}6.916917 & 3.624053 & 2.042700\end{array}$

$\begin{array}{llll}0.840858 & 1.659439 & -2.604419\end{array}$

$\begin{array}{llll}1.510711 & 0.600034 & -3.236388\end{array}$

$\begin{array}{llll}2.296709 & 0.078538 & -2.703834\end{array}$

$\begin{array}{llll}1.194132 & 0.232416 & -4.545738\end{array}$

$\begin{array}{llll}1.735850 & -0.578947 & -5.023098\end{array}$

$\begin{array}{llll}0.184474 & 0.909884 & -5.233924\end{array}$

$\begin{array}{llll}-0.068135 & 0.625662 & -6.251277\end{array}$

$\begin{array}{llll}-0.511452 & 1.944843 & -4.602139\end{array}$

$\begin{array}{llll}-1.309544 & 2.463748 & -5.125382\end{array}$

$\begin{array}{llll}-0.185251 & 2.320821 & -3.297788\end{array}$

$\begin{array}{llll}-0.719335 & 3.141430 & -2.832841\end{array}$

$\begin{array}{llll}0.094558 & 3.081986 & -0.155035\end{array}$

$\begin{array}{llll}-0.279599 & 4.381337 & -0.537521\end{array}$

$\begin{array}{llll}0.231950 & 4.875872 & -1.357814\end{array}$

$\begin{array}{lll}-1.287086 & 5.054606 & 0.154230\end{array}$

$\begin{array}{lll}-1.571673 & 6.058251 & -0.148610\end{array}$

$\begin{array}{llll}-1.929648 & 4.438200 & 1.233156\end{array}$

$\begin{array}{lll}-2.713206 & 4.964412 & 1.770926\end{array}$

$\begin{array}{lll}-1.560826 & 3.147932 & 1.618573\end{array}$

$\begin{array}{lll}-2.048645 & 2.669911 & 2.462170\end{array}$

$\begin{array}{lll}-0.549195 & 2.471206 & 0.931756\end{array}$

$\begin{array}{lll}-0.210890 & 1.496621 & 1.268361\end{array}$

$\begin{array}{llll}5.687854 & 0.323356 & -0.864685\end{array}$

$\begin{array}{llll}6.449343 & -0.843174 & -1.021394\end{array}$

$\begin{array}{llll}6.613292 & -1.516436 & -0.189862\end{array}$

$\begin{array}{llll}6.980419 & -1.174151 & -2.268750\end{array}$

$6.746694-0.375656 \quad-3.387787$

$\begin{array}{llll}7.144189 & -0.652520 & -4.356598\end{array}$

$\begin{array}{llll}5.969642 & 0.771355 & -3.237413\end{array}$

$5.448874 \quad 1.125137 \quad-1.992316$

$\begin{array}{llll}4.822804 & 2.009530 & -1.914848\end{array}$

$\begin{array}{lll}5.344994 & -0.321799 & 1.996332\end{array}$

$\begin{array}{llll}4.369218 & -0.815251 & 2.872640\end{array}$

$\begin{array}{lll}3.327838 & -0.557377 & 2.710548\end{array}$

$\begin{array}{lll}4.735937 & -1.616329 & 3.955658\end{array}$

$6.070038-1.951985 \quad 4.175905$

$\begin{array}{lll}6.351312 & -2.582676 & 5.010745\end{array}$

$7.040512-1.460195 \quad 3.302279$

$\begin{array}{lll}6.689924 & -0.642600 & 2.227250\end{array}$

$\begin{array}{lll}7.470104 & -0.266289 & 1.575878\end{array}$

$\begin{array}{lll}2.443505 & 0.509396 & 0.242613\end{array}$

$\begin{array}{llll}8.495961 & -1.751525 & 3.542288\end{array}$

$3.658417 \quad-2.154334 \quad 4.857381$

$\begin{array}{llll}7.861172 & -2.386834 & -2.403947\end{array}$

$\begin{array}{llll}5.621994 & 1.629611 & -4.422454\end{array}$

$\begin{array}{llll}4.278524 & 1.665876 & -4.623594\end{array}$

$\begin{array}{llll}6.018279 & 2.912573 & -4.248681\end{array}$

$\begin{array}{llll}6.181854 & 1.187289 & -5.567354\end{array}$

$\begin{array}{llll}7.739341 & -2.968691 & -3.619282\end{array}$

$\begin{array}{llll}7.590655 & -3.324175 & -1.471661\end{array}$

$\begin{array}{llll}9.170721 & -2.059899 & -2.262250\end{array}$

$\begin{array}{lll}9.160336 & -1.961161 & 2.381805\end{array}$

$\begin{array}{llll}8.681855 & -2.839446 & 4.319356\end{array}$

$\begin{array}{llll}9.115881 & -0.714234 & 4.158487\end{array}$

$\begin{array}{lll}4.141514 & -2.522705 & 6.065311\end{array}$

$\begin{array}{llll}2.689672 & -1.231931 & 5.075682\end{array}$

$\begin{array}{llll}3.047678 & -3.240065 & 4.329119\end{array}$

$\begin{array}{llll}3.141742 & -1.986090 & -1.227328\end{array}$

$\begin{array}{lll}1.807124 & -2.489705 & 0.648091\end{array}$

$\begin{array}{llll}3.296661 & -3.313713 & -1.556967\end{array}$

$3.618780-1.212833-1.816807$

$\begin{array}{llll}1.921260 & -3.879303 & 0.299419\end{array}$

$\begin{array}{llll}2.657925 & -4.296418 & -0.783009\end{array}$

$\begin{array}{lll}1.388372 & -4.607192 & 0.900497\end{array}$

$\begin{array}{lll}1.060339 & -2.163285 & 1.808338\end{array}$ 
$\begin{array}{lll}0.854195 & -0.894057 & 2.389706\end{array}$ $\begin{array}{llll}1.393390 & 0.199461 & 1.982191\end{array}$ $2.413744 \quad-1.563593 \quad-0.172122$ $\begin{array}{llll}0.576881 & -3.012999 & 2.265645\end{array}$ $\begin{array}{lll}0.017461 & -0.775331 & 3.497388\end{array}$ $\begin{array}{lll}0.073313 & 0.457560 & 4.274082\end{array}$ $\begin{array}{lll}0.865978 & 0.427861 & 5.037119\end{array}$ $\begin{array}{llll}-0.884623 & 0.607476 & 4.780769\end{array}$ $\begin{array}{lll}0.266395 & 1.303706 & 3.618161\end{array}$ $\begin{array}{lll}-0.417840 & -1.954278 & 4.240149\end{array}$ $\begin{array}{lll}-0.940473 & -2.659780 & 3.594057\end{array}$ $\begin{array}{lll}-1.113223 & -1.637132 & 5.021014\end{array}$ $\begin{array}{lll}0.424554 & -2.472265 & 4.718810\end{array}$ $\begin{array}{llll}-3.272084 & -1.675436 & -1.435085\end{array}$ $\begin{array}{lll}-4.614385 & -0.289422 & -0.260718\end{array}$ $\begin{array}{lll}-4.232945 & 1.290460 & 0.033493\end{array}$ $\begin{array}{lll}-6.108160 & -0.110113 & -0.974888\end{array}$ $\begin{array}{lll}-4.756719 & -0.851270 & 1.300706\end{array}$ $\begin{array}{llll}-4.507304 & 2.303548 & -0.879018\end{array}$ $\begin{array}{llll}-3.389282 & 2.919497 & -1.477627\end{array}$

$\begin{array}{llll}-2.400881 & 2.592903 & -1.180658\end{array}$ $\begin{array}{llll}-3.578787 & 3.919738 & -2.396794\end{array}$ $\begin{array}{llll}-2.725583 & 4.413675 & -2.852624\end{array}$

$\begin{array}{llll}-4.890156 & 4.310055 & -2.778216\end{array}$ $\begin{array}{lll}-5.096201 & 5.291554 & -3.785472\end{array}$ $\begin{array}{llll}-4.225069 & 5.757587 & -4.238671\end{array}$ $\begin{array}{llll}-6.365980 & 5.634888 & -4.190396\end{array}$ $\begin{array}{llll}-6.512121 & 6.382132 & -4.964890\end{array}$ $\begin{array}{llll}-7.485226 & 4.996237 & -3.605528\end{array}$ $\begin{array}{llll}-8.486040 & 5.247883 & -3.944576\end{array}$ $\begin{array}{llll}-7.320317 & 4.052307 & -2.614925\end{array}$ $\begin{array}{llll}-8.191816 & 3.568909 & -2.190901\end{array}$ $\begin{array}{llll}-6.024115 & 3.689785 & -2.15152\end{array}$ $\begin{array}{lll}-5.812400 & 2.701535 & -1.122557\end{array}$ $\begin{array}{llll}-6.950045 & 2.069182 & -0.398520\end{array}$ $\begin{array}{lll}-7.942870 & 2.838673 & 0.304614\end{array}$ $\begin{array}{llll}-7.799219 & 4.233640 & 0.545046\end{array}$ $\begin{array}{lll}-6.909398 & 4.742367 & 0.192387\end{array}$ $\begin{array}{lll}-8.762724 & 4.938339 & 1.233301\end{array}$ $\begin{array}{lll}-8.624866 & 6.000456 & 1.414483\end{array}$ $\begin{array}{lll}-9.926898 & 4.291534 & 1.713536\end{array}$ $\begin{array}{lll}-10.679778 & 4.861536 & 2.249951\end{array}$ $\begin{array}{lll}-10.092653 & 2.940139 & 1.510882\end{array}$ $\begin{array}{lll}-10.973619 & 2.427008 & 1.887724\end{array}$ $\begin{array}{lll}-9.110734 & 2.179579 & 0.818816\end{array}$ $\begin{array}{lll}-9.252974 & 0.775903 & 0.650235\end{array}$ $\begin{array}{llll}-10.149173 & 0.286741 & 1.020329\end{array}$ $\begin{array}{llll}-8.249904 & 0.038337 & 0.071857\end{array}$ $\begin{array}{llll}-8.323329 & -1.037713 & -0.036208\end{array}$ $\begin{array}{llll}-7.093949 & 0.690142 & -0.408293\end{array}$ $\begin{array}{lll}-4.334063 & -2.268566 & 1.454610\end{array}$ $\begin{array}{lll}-3.392264 & -2.287733 & 2.003168\end{array}$ $\begin{array}{lll}-5.342209 & -3.117725 & 2.212632\end{array}$ $\begin{array}{lll}-4.886077 & -4.120991 & 3.075084\end{array}$ $\begin{array}{lll}-3.821174 & -4.271862 & 3.208163\end{array}$ $\begin{array}{lll}-5.797234 & -4.930886 & 3.757950\end{array}$ $\begin{array}{llll}-5.427987 & -5.704831 & 4.425615\end{array}$ $\begin{array}{lll}-7.170950 & -4.745838 & 3.587377\end{array}$ $\begin{array}{lll}-7.878848 & -5.367431 & 4.129001\end{array}$ $\begin{array}{lll}-7.629893 & -3.754382 & 2.713662\end{array}$ $\begin{array}{lll}-8.696763 & -3.596713 & 2.578712\end{array}$ $\begin{array}{lll}-6.719822 & -2.954345 & 2.024865\end{array}$ $\begin{array}{lll}-7.072988 & -2.162718 & 1.374918\end{array}$ $\begin{array}{lll}-4.089720 & -2.925198 & 0.078518\end{array}$ $\begin{array}{llll}-5.047205 & -3.292364 & -0.307747\end{array}$ $\begin{array}{llll}-3.451483 & -3.791377 & 0.240953\end{array}$ $\begin{array}{lll}-4.710862 & 0.056365 & 2.499045\end{array}$ $\begin{array}{lll}-4.898094 & 1.053674 & 2.105643\end{array}$ $\begin{array}{lll}-5.829201 & -0.221137 & 3.494829\end{array}$ $\begin{array}{lll}-7.055291 & 0.431435 & 3.320786\end{array}$ $\begin{array}{lll}-7.170553 & 1.130132 & 2.499091\end{array}$ $\begin{array}{llll}-8.126767 & 0.192749 & 4.182538\end{array}$ $\begin{array}{lll}-9.069823 & 0.709264 & 4.024910\end{array}$ $\begin{array}{lll}-7.979766 & -0.703384 & 5.243778\end{array}$ $\begin{array}{lll}-8.811442 & -0.898911 & 5.915210\end{array}$ $\begin{array}{llll}-6.752050 & -1.341311 & 5.442395\end{array}$

$\begin{array}{llll}-6.626844 & -2.038241 & 6.266307\end{array}$

$\begin{array}{lll}-5.682793 & -1.094040 & 4.580351\end{array}$

$\begin{array}{lll}-4.743191 & -1.610428 & 4.741983\end{array}$

$\begin{array}{lll}-3.313289 & 0.077563 & 3.127327\end{array}$

$\begin{array}{lll}-2.566505 & 0.347925 & 2.379196\end{array}$

$\begin{array}{lll}-3.021786 & -0.882069 & 3.557322\end{array}$

$\begin{array}{lll}-3.291352 & 0.820566 & 3.930247\end{array}$

$\begin{array}{llll}-1.704222 & -0.967857 & -0.043673\end{array}$

$\begin{array}{llll}-1.523370 & 0.101864 & -0.007352\end{array}$

$\begin{array}{lll}-1.864189 & -1.425712 & 0.925654\end{array}$

$\begin{array}{llll}-0.969195 & -1.700920 & -1.022989\end{array}$

$\begin{array}{lll}-0.392511 & -1.134132 & -1.746171\end{array}$

$\begin{array}{llll}-1.093736 & -3.080488 & -1.167902\end{array}$

$\begin{array}{llll}-1.489947 & -3.647064 & -0.334199\end{array}$

$\begin{array}{llll}-5.035410 & -2.220459 & -2.752456\end{array}$

$\begin{array}{llll}-5.823332 & -2.493816 & -2.057342\end{array}$

$\begin{array}{llll}-4.031110 & -3.181350 & -2.959231\end{array}$

$\begin{array}{llll}-4.115746 & -4.111238 & -2.397547\end{array}$

$\begin{array}{llll}-3.162765 & -3.299949 & -4.195230\end{array}$

$\begin{array}{llll}-3.722291 & -3.860283 & -4.959136\end{array}$

$\begin{array}{llll}-2.300227 & -3.916405 & -3.939059\end{array}$

$\begin{array}{llll}-2.663090 & -1.969872 & -4.806746\end{array}$

$\begin{array}{llll}-3.399660 & -1.582487 & -5.516915\end{array}$

$\begin{array}{llll}-1.756165 & -2.180724 & -5.381998\end{array}$

$\begin{array}{llll}-2.341936 & -0.887568 & -3.798702\end{array}$

$\begin{array}{llll}-1.296978 & -0.736219 & -3.563220\end{array}$

$\begin{array}{llll}-3.228824 & 0.050571 & -3.356482\end{array}$

$\begin{array}{llll}-2.826018 & 0.909334 & -2.830160\end{array}$

$\begin{array}{llll}-4.673619 & 0.151166 & -3.791000\end{array}$

$\begin{array}{llll}-5.177451 & 0.859295 & -3.133360\end{array}$

$\begin{array}{llll}-4.705144 & 0.591910 & -4.797782\end{array}$

$\begin{array}{llll}-5.459750 & -1.182305 & -3.791836\end{array}$

$\begin{array}{llll}-5.401700 & -1.650342 & -4.779855\end{array}$

$\begin{array}{llll}-6.514293 & -0.949540 & -3.623094\end{array}$

$\begin{array}{llll}-0.459190 & -3.872354 & -2.223281\end{array}$

$\begin{array}{llll}0.272778 & -3.303310 & -3.285014\end{array}$

$\begin{array}{lll}-0.588768 & -5.273995 & -2.171918\end{array}$

$\begin{array}{llll}0.838628 & -4.110025 & -4.267647\end{array}$

$\begin{array}{llll}0.412269 & -2.229915 & -3.342398\end{array}$

$\begin{array}{lll}-0.020872 & -6.078178 & -3.159322\end{array}$

$\begin{array}{llll}-1.125515 & -5.719948 & -1.340694\end{array}$

$\begin{array}{llll}0.690042 & -5.500204 & -4.214692\end{array}$

$1.408642-3.655828-5.072688$

$\begin{array}{lll}-0.130474 & -7.157615 & -3.102190\end{array}$

$\begin{array}{llll}1.133259 & -6.125799 & -4.984393\end{array}$

$2.722935-5.348034-1.040080$

$\begin{array}{lll}4.393620 & -3.801246 & -3.106686\end{array}$

$\begin{array}{lll}-1.641388 & -5.379413 & 1.833331\end{array}$

$\begin{array}{lll}-2.301624 & -6.006572 & 2.894163\end{array}$

$\begin{array}{lll}-2.254652 & -5.730044 & 0.604783\end{array}$

$\begin{array}{lll}-0.288954 & -5.748603 & 1.813692\end{array}$

$\begin{array}{lll}-1.739169 & -3.968781 & 1.986808\end{array}$

\section{$[8-9]^{\ddagger} \mathrm{P1}-\mathrm{C1}$ exo si-si $\left[\mathrm{BF}_{4}^{-}\right]$}

Number of imaginary frequencies $=1$

The smallest frequencies are $=-240.704210 .351112 .6986 \mathrm{~cm}(-1)$ Electronic energy $=-7647.2386579$

Zero-point correction=

(Hartree/Particle)

Thermal correction to Energy= $\quad 1.896165$

Thermal correction to Enthalpy= $\quad 1.897109$

Thermal correction to Gibbs Free Energy $=\quad 1.600918$

Sum of electronic and zero-point Energies $=\quad-7645.467423$

Sum of electronic and thermal Energies=

$-7645.342493$

Sum of electronic and thermal Enthalpies=

Sum of electronic and thermal Free Energies=

Cartesian Coordinates

$\begin{array}{llll}26 & 3.610104 & 4.144078 & 3.958280 \\ 15 & 1.475687 & 2.497411 & -0.519294 \\ 15 & 4.644297 & 0.610644 & 0.908990 \\ 6 & 3.035836 & 3.628550 & 2.005252\end{array}$




\begin{tabular}{|c|c|c|c|}
\hline & 3.916291 & 2.634891 & 7684 \\
\hline & 3.295001 & 2.112916 & 3.745168 \\
\hline & 3.714325 & 1.358338 & 4.397281 \\
\hline & 2.053815 & 2.790280 & 3.929424 \\
\hline & 1.368045 & 2.648286 & 4.754078 \\
\hline 6 & 1.901427 & 3.732641 & 2.871715 \\
\hline & 1.076551 & 4.417825 & 2.748612 \\
\hline & 5.302015 & 5.323018 & 4.240113 \\
\hline 1 & 6.160717 & 5.337550 & 3.582291 \\
\hline & 5.112946 & 4.450701 & 5.355165 \\
\hline & 5.798356 & 3.681424 & 5.684361 \\
\hline 6 & 3.836938 & 4.738474 & 5.928602 \\
\hline & 3.385998 & 4.222962 & 6.766406 \\
\hline & 3.236951 & 5.788267 & 5.168219 \\
\hline 1 & 2.252680 & 6.208094 & 5.329005 \\
\hline 6 & 4.141486 & 6.149734 & 4.125645 \\
\hline 1 & 3.962635 & 6.896907 & 3.364335 \\
\hline 6 & 3.290735 & 4.323074 & 0.709750 \\
\hline ( & 4.260171 & 5.334564 & 0.653032 \\
\hline 1 & 4.756390 & 5.615907 & 1.572850 \\
\hline 6 & 4.604608 & 5.960797 & -0.545808 \\
\hline 1 & 5.367434 & 6.734111 & -0.552580 \\
\hline 6 & 3.970320 & 5.581534 & -1.725356 \\
\hline 1 & 4.232028 & 6.047982 & -2.669905 \\
\hline 6 & 3.001592 & 4.576997 & -1.695679 \\
\hline 1 & 2.529697 & 4.282406 & -2.625296 \\
\hline 6 & 2.653644 & 3.930981 & -0.499558 \\
\hline 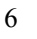 & 5.165600 & 2.138898 & 1.902219 \\
\hline 1 & 5.437505 & 2.855344 & 1.120485 \\
\hline 6 & 6.365556 & 1.964292 & 2.835199 \\
\hline 1 & 7.252499 & 1.638385 & 2.283860 \\
\hline 1 & 6.166598 & 1.240192 & 3.629980 \\
\hline 1 & 6.591948 & 2.925079 & 3.305355 \\
\hline 6 & 0.884333 & 2.450600 & -2.265991 \\
\hline 6 & 1.479853 & 1.496799 & -3.107273 \\
\hline 1 & 2.193194 & 0.794774 & -2.689471 \\
\hline 6 & 1.160428 & 1.443389 & -4.465410 \\
\hline 1 & 1.632780 & 0.701040 & -5.102312 \\
\hline 6 & 0.229888 & 2.339383 & -4.997169 \\
\hline 1 & -0.023318 & 2.300066 & -6.052402 \\
\hline 6 & -0.388953 & 3.274961 & -4.162980 \\
\hline 1 & -1.125856 & 3.962104 & -4.568740 \\
\hline 6 & -0.065685 & 3.332288 & -2.805893 \\
\hline 1 & -0.552758 & 4.067657 & -2.175668 \\
\hline 6 & 0.063123 & 3.155147 & 0.462583 \\
\hline 6 & -0.331382 & 4.502766 & 0.396370 \\
\hline 1 & 0.160822 & 5.183721 & -0.291584 \\
\hline 6 & -1.320485 & 4.988437 & 1.253205 \\
\hline 1 & -1.617185 & 6.031863 & 1.197920 \\
\hline 6 & -1.919034 & 4.137067 & 2.188511 \\
\hline 1 & -2.683022 & 4.519166 & 2.859378 \\
\hline 6 & -1.531643 & 2.797444 & 2.256721 \\
\hline 1 & -1.985400 & 2.135344 & 2.987761 \\
\hline 6 & -0.544517 & 2.306785 & 1.398346 \\
\hline 1 & -0.199553 & 1.281826 & 1.480589 \\
\hline 6 & 5.821207 & 0.660016 & -0.510273 \\
\hline 6 & 6.764462 & -0.322164 & -0.835730 \\
\hline 1 & 6.905450 & -1.192167 & -0.207728 \\
\hline 6 & 7.522276 & -0.207297 & -2.002374 \\
\hline 6 & 7.339330 & 0.859737 & -2.880408 \\
\hline 1 & 7.915748 & 0.926347 & -3.795007 \\
\hline 6 & 6.376374 & 1.819409 & -2.573146 \\
\hline 6 & 5.629788 & 1.729884 & -1.398640 \\
\hline 1 & 4.871202 & 2.479719 & -1.192489 \\
\hline 6 & 5.110239 & -0.852907 & 1.926150 \\
\hline 6 & 4.046771 & -1.648392 & 2.369579 \\
\hline 1 & 3.031940 & -1.364148 & 2.120665 \\
\hline 6 & 4.284491 & -2.793434 & 3.132376 \\
\hline 6 & 5.583126 & -3.169014 & 3.464542 \\
\hline 1 & 5.767976 & -4.065846 & 4.043727 \\
\hline 6 & 6.644501 & -2.370037 & 3.034820 \\
\hline o & 6.417862 & -1.218480 & 2.281005 \\
\hline 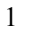 & 7.266431 & -0.622726 & 1.968497 \\
\hline 29 & 2.440416 & 0.532694 & 0.126120 \\
\hline 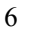 & 054102 & .716506 & 3.430 \\
\hline
\end{tabular}

\begin{tabular}{|c|c|c|c|}
\hline 6 & 3.109499 & -3.642231 & 3.538425 \\
\hline 6 & 8.586696 & -1.229113 & -2.299405 \\
\hline 6 & 6.094853 & 2.961030 & -3.511245 \\
\hline 9 & 4.769364 & 3.045751 & -3.794975 \\
\hline 9 & 6.449780 & 4.153214 & -2.975971 \\
\hline 9 & 6.746845 & 2.839587 & -4.685763 \\
\hline 9 & 8.748476 & -1.419958 & -3.628892 \\
\hline 9 & 8.313608 & -2.428991 & -1.744415 \\
\hline 9 & 9.791746 & -0.838976 & -1.813982 \\
\hline 9 & 8.943026 & -2.366098 & 2.472616 \\
\hline 9 & 8.208501 & -4.037089 & 3.664762 \\
\hline 9 & 8.427133 & -2.069317 & 4.562000 \\
\hline 9 & 3.425225 & -4.527949 & 4.506179 \\
\hline 9 & 2.087711 & -2.880772 & 4.003296 \\
\hline 9 & 2.617166 & -4.349506 & 2.494102 \\
\hline 6 & 3.602468 & -1.376230 & -1.906529 \\
\hline 6 & 2.136572 & -2.516615 & -0.484410 \\
\hline 6 & 4.103290 & -2.556617 & -2.419944 \\
\hline 1 & 3.964371 & -0.417947 & -2.259170 \\
\hline 6 & 2.667017 & -3.751713 & -0.953941 \\
\hline 6 & 3.645246 & -3.782041 & -1.926422 \\
\hline 1 & 2.257817 & -4.674452 & -0.557476 \\
\hline 6 & 0.992245 & -2.587089 & 0.384978 \\
\hline 6 & 0.638352 & -1.597743 & 1.375863 \\
\hline 8 & 1.129333 & -0.424797 & 1.422856 \\
\hline 7 & 2.646928 & -1.340125 & -0.955588 \\
\hline 1 & 0.717156 & -3.604175 & 0.622911 \\
\hline 7 & -0.256077 & -1.919294 & 2.359663 \\
\hline 6 & -0.357785 & -0.992454 & 3.486945 \\
\hline 1 & 0.625821 & -0.791496 & 3.924119 \\
\hline 1 & -0.999618 & -1.436128 & 4.247891 \\
\hline 1 & -0.791278 & -0.037748 & 3.181289 \\
\hline 6 & -0.787567 & -3.272021 & 2.517472 \\
\hline 1 & -1.224693 & -3.629045 & 1.589345 \\
\hline 1 & -1.575801 & -3.254561 & 3.270811 \\
\hline 1 & -0.018400 & -3.983243 & 2.832112 \\
\hline 77 & -3.304414 & -0.925406 & -1.905174 \\
\hline 15 & -4.838077 & -0.173505 & -0.421136 \\
\hline 8 & -4.673415 & 1.262188 & 0.406892 \\
\hline 8 & -6.343269 & 0.066418 & -1.108098 \\
\hline 7 & -4.958518 & -1.219112 & 0.884183 \\
\hline 6 & -4.998053 & 2.473580 & -0.185218 \\
\hline 6 & -3.923780 & 3.340638 & -0.483646 \\
\hline 1 & -2.918738 & 3.028107 & -0.227059 \\
\hline 6 & -4.176342 & 4.552304 & -1.076205 \\
\hline 1 & -3.359723 & 5.233698 & -1.296485 \\
\hline 6 & -5.502073 & 4.919794 & -1.431864 \\
\hline 6 & -5.764944 & 6.137435 & -2.116216 \\
\hline 1 & -4.929302 & 6.795626 & -2.339806 \\
\hline 6 & -7.043354 & 6.471385 & -2.502193 \\
\hline 1 & -7.232193 & 7.401783 & -3.029818 \\
\hline 6 & -8.113815 & 5.588324 & -2.223985 \\
\hline 1 & -9.118223 & 5.840770 & -2.551754 \\
\hline 6 & -7.895276 & 4.407468 & -1.547808 \\
\hline 1 & -8.727152 & 3.740859 & -1.355129 \\
\hline 6 & -6.591323 & 4.039471 & -1.111746 \\
\hline 6 & -6.324689 & 2.810631 & -0.407786 \\
\hline 6 & -7.416049 & 1.894310 & 0.029776 \\
\hline 6 & -8.498196 & 2.330247 & 0.873091 \\
\hline 6 & -8.521069 & 3.613078 & 1.489322 \\
\hline 1 & -7.691696 & 4.291995 & 1.328773 \\
\hline 6 & -9.570471 & 3.996321 & 2.295935 \\
\hline 1 & -9.559467 & 4.977737 & 2.761487 \\
\hline 6 & -10.659032 & 3.122134 & 2.530546 \\
\hline 1 & -11.481369 & 3.440044 & 3.164798 \\
\hline 6 & -10.662155 & 1.867148 & 1.965402 \\
\hline 1 & -11.482241 & 1.178362 & 2.151046 \\
\hline 6 & -9.589009 & 1.434285 & 1.139361 \\
\hline 6 & -9.566820 & 0.121888 & 0.594538 \\
\hline 1 & -10.402584 & -0.544728 & 0.787328 \\
\hline 6 & -8.481650 & -0.315717 & -0.123879 \\
\hline 1 & -8.421621 & -1.325939 & -0.513354 \\
\hline 6 & -7.407537 & 0.566105 & -0.372006 \\
\hline 6 & -4.267203 & -2.516245 & 0.657220 \\
\hline 1 & -3.338064 & -2515432 & 1.2 \\
\hline
\end{tabular}




\section{P1-C1 exo si-si $\left[\mathrm{BF}_{4}^{-}\right]$}

Number of imaginary frequencies $=0$

The smallest frequencies are=9.0914 $11.831815 .1039 \mathrm{~cm}(-1)$ Electronic energy $=-7647.2677172$

Zero-point correction=

1.774346

(Hartree/Particle)

Thermal correction to Energy= $\quad 1.898710$

Thermal correction to Enthalpy= $\quad 1.899654$

Thermal correction to Gibbs Free Energy $=\quad 1.605238$

Sum of electronic and zero-point Energies $=\quad-7645.493371$

Sum of electronic and thermal Energies= $\quad-7645.369008$

Sum of electronic and thermal Enthalpies $=\quad-7645.368064$

Sum of electronic and thermal Free Energies $=\quad-7645.662479$

Cartesian Coordinates

\begin{tabular}{|c|c|c|c|}
\hline 26 & -3.688441 & 58115 & \\
\hline 15 & -1.247057 & -2.696323 & \\
\hline r & -4.498341 & -0.557435 & 0.69049 \\
\hline 6 & -2.961475 & -3.232731 & 2.593200 \\
\hline & -3.873920 & -2.141137 & 2.820540 \\
\hline & -3.343329 & -1.338732 & 3.878620 \\
\hline 1 & -3.806594 & -0.451246 & 4.288462 \\
\hline 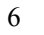 & -2.123840 & -1.934643 & 4.318837 \\
\hline 1 & -1.504298 & -1.585782 & 5.134141 \\
\hline 6 & -1.893781 & -3.105175 & 3.537884 \\
\hline 1 & -1.063194 & -3.787804 & 3.642328 \\
\hline 6 & -5.394842 & -4.372568 & 4.971779 \\
\hline 1 & -6.180258 & -4.578562 & 4.257004 \\
\hline 6 & -5.323022 & -3.237541 & 5.835692 \\
\hline 1 & -6.040524 & -2.429679 & 5.885107 \\
\hline 6 & -4.111386 & -3.330483 & 6.585811 \\
\hline 1 & -3.748918 & -2.602474 & 7.299667 \\
\hline 6 & -3.434032 & -4.52 & \\
\hline 1 & -2.470358 & -4.857868 & 6.54 \\
\hline 6 & -4.227067 & -5.167 & 5.19 \\
\hline 1 & -3.968973 & -6.0 & 4.66 \\
\hline 6 & -3.124206 & -4.228679 & 1.497355 \\
\hline 6 & -4.063459 & -5.261027 & \\
\hline 1 & -4.618135 & -5.3 & 2.5 \\
\hline 6 & -4.301306 & -6.173823 & 0.600764 \\
\hline 1 & -5.041119 & -6.957945 & 0.733921 \\
\hline 6 & -3.58 & -6.06 & -0.5 \\
\hline 1 & -3.765925 & -6.763889 & -1.404982 \\
\hline 6 & -2.653268 & -5.044729 & -0.748060 \\
\hline 1 & -2.120243 & -4.966847 & -1.687363 \\
\hline 6 & -2.415585 & -4.111935 & 0.272969 \\
\hline 6 & -5.065853 & -1.8 & 1.956509 \\
\hline 1 & -5.254579 & -2.736659 & 1.340522 \\
\hline 6 & -6.348723 & -1.513198 & 2.718245 \\
\hline 1 & -7.178388 & -1.319638 & 2.031983 \\
\hline 1 & -6.223545 & -0.641122 & 3.365439 \\
\hline 1 & -6.619979 & -2.3638 & 790 \\
\hline 6 & -0.639992 & -3.008193 & -1.704949 \\
\hline 6 & -1.392202 & -2.445574 & -2.749968 \\
\hline 1 & -2.237616 & -1.807214 & -2.511847 \\
\hline 6 & -1.073964 & -2.716060 & -4.080426 \\
\hline 1 & -1.670918 & -2.283767 & -4.878123 \\
\hline 6 & 0.017100 & -3.5 & -4.38 \\
\hline 1 & 0.274703 & -3.740526 & -5.416424 \\
\hline 6 & 0.785940 & -4.078628 & -3.349024 \\
\hline 1 & 1.647834 & -4.698430 & -3.578764 \\
\hline 6 & 0.458984 & -3.821840 & -2.016139 \\
\hline 1 & 1.068049 & -4.250730 & -1.228434 \\
\hline 6 & 0.137671 & -3.136775 & 1.127918 \\
\hline 6 & 0.519620 & -4.473237 & 1.335104 \\
\hline 1 & 0.032870 & -5.271485 & 0.783032 \\
\hline 6 & 1.502932 & -4.785395 & 2.274473 \\
\hline 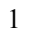 & 1.795251 & -5.820454 & 2.425617 \\
\hline 6 & 2.103796 & -3.769060 & 3.024037 \\
\hline & 2.863154 & -4.013815 & 3.760942 \\
\hline & 1.723893 & -2.440028 & 2.82602 \\
\hline
\end{tabular}


$\begin{array}{lll}2.180351 & -1.651502 & 3.415440\end{array}$

$\begin{array}{llll}0.747484 & -2.122584 & 1.879205\end{array}$

$\begin{array}{lll}0.423860 & -1.097334 & 1.744833\end{array}$

$\begin{array}{llll}-5.532325 & -0.972781 & -0.777655\end{array}$

$\begin{array}{llll}-6.515606 & -0.164060 & -1.362099\end{array}$

$\begin{array}{llll}-6.759556 & 0.806028 & -0.950967\end{array}$

$\begin{array}{lll}-7.183858 & -0.594081 & -2.508768\end{array}$

$\begin{array}{lll}-6.870033 & -1.810093 & -3.115492\end{array}$

$\begin{array}{lll}-7.382385 & -2.126990 & -4.016463\end{array}$

$\begin{array}{llll}-5.868318 & -2.598394 & -2.553739\end{array}$

$\begin{array}{llll}-5.208657 & -2.191843 & -1.393714\end{array}$

$\begin{array}{llll}-4.427644 & -2.822426 & -0.977669\end{array}$

$\begin{array}{llll}-5.076960 & 1.051346 & 1.368192\end{array}$

$\begin{array}{llll}-4.068779 & 1.881976 & 1.870108\end{array}$

$\begin{array}{lll}-3.036759 & 1.560612 & 1.811244\end{array}$

$\begin{array}{lll}-4.380678 & 3.108038 & 2.459357\end{array}$

$\begin{array}{llll}-5.700645 & 3.539463 & 2.544291\end{array}$

$\begin{array}{lll}-5.942383 & 4.499873 & 2.983469\end{array}$

$\begin{array}{lll}-6.709647 & 2.708994 & 2.051717\end{array}$

$\begin{array}{lll}-6.411213 & 1.470629 & 1.483081\end{array}$

$\begin{array}{lll}-7.225133 & 0.842927 & 1.142814\end{array}$

$\begin{array}{lll}-2.215248 & -0.638697 & 0.215954\end{array}$

$\begin{array}{lll}-8.152884 & 3.112477 & 2.190556\end{array}$

$\begin{array}{llll}-3.243662 & 3.965074 & 2.945223\end{array}$

$\begin{array}{lll}-8.284009 & 0.239216 & -3.11119\end{array}$

$\begin{array}{lll}-5.475945 & -3.908829 & -3.181294\end{array}$

$\begin{array}{llll}-4.130681 & -3.998877 & -3.332533\end{array}$

$\begin{array}{llll}-5.849581 & -4.962152 & -2.416806\end{array}$

$\begin{array}{lll}-6.028890 & -4.082592 & -4.398902\end{array}$

$\begin{array}{llll}-8.120389 & 0.391890 & -4.447707\end{array}$

$\begin{array}{llll}-8.357727 & 1.471456 & -2.568145\end{array}$

$\begin{array}{llll}-9.492432 & -0.348408 & -2.939135\end{array}$

$\begin{array}{lll}-8.886574 & 2.714588 & 1.125265\end{array}$

$\begin{array}{lll}-8.300582 & 4.448062 & 2.311047\end{array}$

$\begin{array}{lll}-8.721205 & 2.549643 & 3.284756\end{array}$

$\begin{array}{lll}-3.662288 & 5.031970 & 3.650673\end{array}$

$\begin{array}{llll}-2.398127 & 3.256251 & 3.739434\end{array}$

$\begin{array}{llll}-2.499608 & 4.422566 & 1.913830\end{array}$

$\begin{array}{llll}-3.203892 & 1.061247 & -2.163551\end{array}$

$\begin{array}{llll}-1.917141 & 2.397440 & -0.773197\end{array}$

$\begin{array}{lll}-3.880610 & 2.169339 & -2.656800\end{array}$

$\begin{array}{llll}-3.435847 & 0.061612 & -2.512192\end{array}$

$\begin{array}{llll}-2.626721 & 3.538776 & -1.177948\end{array}$

$\begin{array}{llll}-3.627218 & 3.433739 & -2.136714\end{array}$

$\begin{array}{llll}-2.351775 & 4.506963 & -0.775175\end{array}$

$\begin{array}{lll}-0.659778 & 2.620906 & 0.032277\end{array}$

$\begin{array}{lll}-0.510381 & 1.772305 & 1.287830\end{array}$

$\begin{array}{lll}-1.055964 & 0.647718 & 1.416490\end{array}$

$\begin{array}{llll}-2.245152 & 1.167666 & -1.225880\end{array}$

$\begin{array}{llll}-0.701421 & 3.662761 & 0.344473\end{array}$

$\begin{array}{lll}0.231540 & 2.246211 & 2.308302\end{array}$

$\begin{array}{lll}0.260221 & 1.450968 & 3.540809\end{array}$

$\begin{array}{lll}-0.730824 & 1.419108 & 4.003087\end{array}$

$\begin{array}{lll}0.964513 & 1.909874 & 4.234238\end{array}$

$\begin{array}{llll}0.575983 & 0.428208 & 3.335196\end{array}$

$\begin{array}{lll}0.749086 & 3.613295 & 2.412344\end{array}$

$\begin{array}{llll}0.899753 & 4.069156 & 1.441638\end{array}$

$\begin{array}{lll}1.715013 & 3.587185 & 2.920709\end{array}$

$\begin{array}{lll}0.064314 & 4.246286 & 2.981060\end{array}$

$\begin{array}{llll}3.065882 & 0.653165 & -2.068993\end{array}$

$\begin{array}{llll}4.672149 & 0.072873 & -0.542065\end{array}$

$\begin{array}{llll}4.580544 & -1.233544 & 0.499332\end{array}$

$6.160193-0.252206-1.248485$

$\begin{array}{llll}4.857351 & 1.295419 & 0.607847\end{array}$

$\begin{array}{llll}4.877646 & -2.510403 & 0.052114\end{array}$

$\begin{array}{llll}3.795035 & -3.409395 & -0.071059\end{array}$

$\begin{array}{llll}2.804117 & -3.063617 & 0.195910\end{array}$

$\begin{array}{llll}4.021706 & -4.688061 & -0.513933\end{array}$

$\begin{array}{llll}3.199116 & -5.392916 & -0.597227\end{array}$

$\begin{array}{llll}5.328571 & -5.100110 & -0.890899\end{array}$

$\begin{array}{llll}5.562950 & -6.397040 & -1.422961\end{array}$

$\begin{array}{llll}4.721620 & -7.079759 & -1.511602\end{array}$

$6.820718-6.778769-1.832100$

$\begin{array}{llll}6.986638 & -7.770407 & -2.242994\end{array}$

$\begin{array}{llll}7.898496 & -5.866996 & -1.731713\end{array}$
$8.885176-6.160291-2.078864$

$\begin{array}{llll}7.708646 & -4.607990 & -1.203987\end{array}$

$\begin{array}{llll}8.544603 & -3.921178 & -1.145448\end{array}$

$\begin{array}{llll}6.427728 & -4.185910 & -0.748980\end{array}$

$\begin{array}{llll}6.192467 & -2.875921 & -0.197146\end{array}$

$\begin{array}{llll}7.298126 & -1.911005 & 0.065982\end{array}$

$\begin{array}{llll}8.419893 & -2.231755 & 0.908802\end{array}$

$\begin{array}{lll}8.487997 & -3.430518 & 1.673386\end{array}$

$\begin{array}{lll}7.662618 & -4.131763 & 1.633074\end{array}$

$\begin{array}{llll}9.576242 & -3.704615 & 2.473003\end{array}$

$\begin{array}{lll}9.599045 & -4.623214 & 3.052508\end{array}$

$\begin{array}{llll}10.660892 & -2.798327 & 2.553787\end{array}$

$\begin{array}{lll}11.514199 & -3.030205 & 3.184467\end{array}$

$10.620734-1.619903 \quad 1.843705$

$\begin{array}{lll}11.437599 & -0.905726 & 1.909684\end{array}$

$\begin{array}{lll}9.507559 & -1.299508 & 1.019321\end{array}$

$\begin{array}{llll}9.442853 & -0.061557 & 0.324285\end{array}$

$\begin{array}{lll}10.273757 & 0.633653 & 0.404605\end{array}$

$\begin{array}{llll}8.324672 & 0.274140 & -0.398357\end{array}$

$\begin{array}{llll}8.231849 & 1.231904 & -0.899181\end{array}$

$\begin{array}{llll}7.255391 & -0.643407 & -0.498504\end{array}$

$\begin{array}{llll}4.133048 & 2.535326 & 0.223647\end{array}$

$\begin{array}{lll}3.194942 & 2.572083 & 0.790698\end{array}$

$\begin{array}{llll}4.904552 & 3.812943 & 0.503714\end{array}$

$\begin{array}{lll}4.204102 & 4.955242 & 0.906170\end{array}$

$\begin{array}{lll}3.133160 & 4.908289 & 1.072299\end{array}$

$\begin{array}{lll}4.869618 & 6.167664 & 1.100600\end{array}$

$\begin{array}{lll}4.302746 & 7.041229 & 1.411125\end{array}$

$\begin{array}{llll}6.249167 & 6.247964 & 0.899489\end{array}$

$\begin{array}{lll}6.773405 & 7.185723 & 1.062638\end{array}$

$\begin{array}{lll}6.953147 & 5.111297 & 0.484944\end{array}$

$\begin{array}{lll}8.027830 & 5.163545 & 0.330930\end{array}$

$\begin{array}{lll}6.283564 & 3.906173 & 0.276984\end{array}$

$\begin{array}{llll}6.832197 & 3.018025 & -0.020789\end{array}$

$\begin{array}{llll}3.793067 & 2.509070 & -1.281154\end{array}$

$\begin{array}{llll}4.704934 & 2.770823 & -1.833339\end{array}$

$\begin{array}{llll}3.085775 & 3.314981 & -1.477682\end{array}$

$\begin{array}{lll}5.048038 & 0.985851 & 2.064627\end{array}$

$\begin{array}{lll}5.515535 & 0.002452 & 2.083870\end{array}$

$\begin{array}{lll}6.023965 & 1.927146 & 2.760076\end{array}$

$\begin{array}{lll}7.389392 & 1.618459 & 2.733205\end{array}$

$\begin{array}{llll}7.717581 & 0.711821 & 2.236799\end{array}$

$\begin{array}{lll}8.330692 & 2.461182 & 3.325978\end{array}$

$\begin{array}{lll}9.385754 & 2.202860 & 3.286600\end{array}$

$\begin{array}{lll}7.913977 & 3.629188 & 3.969025\end{array}$

$\begin{array}{lll}8.642214 & 4.293364 & 4.426392\end{array}$

$\begin{array}{llll}6.551413 & 3.933897 & 4.026157\end{array}$

$6.215259 \quad 4.838306 \quad 4.525445$

$\begin{array}{lll}5.615090 & 3.083808 & 3.435782\end{array}$

$\begin{array}{lll}4.564347 & 3.348112 & 3.477821\end{array}$

$\begin{array}{llll}3.702213 & 0.860850 & 2.791115\end{array}$

$\begin{array}{lll}3.094549 & 0.097189 & 2.304004\end{array}$

$\begin{array}{lll}3.139585 & 1.795740 & 2.795213\end{array}$

$\begin{array}{lll}3.866062 & 0.566551 & 3.832615\end{array}$

$\begin{array}{llll}1.611050 & 0.214535 & -0.483964\end{array}$

$\begin{array}{llll}1.343145 & -0.837861 & -0.516169\end{array}$

$\begin{array}{lll}1.898290 & 0.556116 & 0.506978\end{array}$

$\begin{array}{llll}0.963797 & 1.129595 & -1.358179\end{array}$

$\begin{array}{llll}0.251178 & 0.711938 & -2.060590\end{array}$

$\begin{array}{llll}0.633166 & 2.534260 & -0.905488\end{array}$

$\begin{array}{llll}1.438812 & 2.881318 & -0.259322\end{array}$

$\begin{array}{llll}4.403483 & 0.924692 & -3.764404\end{array}$

$\begin{array}{llll}5.244446 & 1.552361 & -3.473788\end{array}$

$\begin{array}{llll}3.172119 & 1.608404 & -4.039916\end{array}$

$\begin{array}{llll}3.183040 & 2.694363 & -3.945840\end{array}$

$2.144534 \quad 1.100229 \quad-5.038435$

$\begin{array}{llll}2.503921 & 1.256717 & -6.068360\end{array}$

$\begin{array}{llll}1.249901 & 1.717048 & -4.931192\end{array}$

$\begin{array}{llll}1.768109 & -0.381146 & -4.828484\end{array}$

$\begin{array}{llll}2.351030 & -1.025056 & -5.495904\end{array}$

$\begin{array}{llll}0.720427 & -0.535175 & -5.106477\end{array}$

$\begin{array}{llll}1.953403 & -0.866870 & -3.392477\end{array}$

$\begin{array}{lll}1.035672 & -1.111843 & -2.868315\end{array}$

$\begin{array}{llll}3.112279 & -1.483722 & -2.922763\end{array}$

$2.994916 \quad-2.182234 \quad-2.101144$ 


$\begin{array}{lccl}6 & 4.391487 & -1.650705 & -3.722923 \\ 1 & 5.183723 & -1.933988 & -3.024655 \\ 1 & 4.276537 & -2.490903 & -4.425694 \\ 6 & 4.820680 & -0.370515 & -4.474745 \\ 1 & 4.416927 & -0.375446 & -5.493716 \\ 1 & 5.910102 & -0.378292 & -4.584892 \\ 6 & 0.490566 & 3.530398 & -2.047192 \\ 6 & -0.117764 & 3.178079 & -3.260132 \\ 6 & 0.934426 & 4.849877 & -1.883896 \\ 6 & -0.266138 & 4.111343 & -4.288074 \\ 1 & -0.488217 & 2.169209 & -3.406403 \\ 6 & 0.793008 & 5.785102 & -2.911709 \\ 1 & 1.369903 & 5.162744 & -0.941757 \\ 6 & 0.194626 & 5.419653 & -4.119738 \\ 1 & -0.740484 & 3.813790 & -5.219744 \\ 1 & 1.144044 & 6.802093 & -2.759605 \\ 1 & 0.082097 & 6.147315 & -4.918845 \\ 1 & -4.159935 & 4.311453 & -2.485377 \\ 35 & -5.174349 & 1.938088 & -4.096526 \\ 5 & -0.026034 & 6.711295 & 1.192165 \\ 9 & -0.385722 & 6.559348 & 2.540610 \\ 9 & -0.188406 & 8.037781 & 0.788541 \\ 9 & -0.830189 & 5.867421 & 0.387030 \\ 9 & 1.332303 & 6.326856 & 1.028811 \\ --------------------------------------------------------------------\end{array}$

\section{P1-C1 exo re-si $\left[\mathrm{BF}_{4}^{-}\right]$}

Number of imaginary frequencies $=0$

The smallest frequencies are $=11.033814 .440815 .9896 \mathrm{~cm}(-1)$ Electronic energy $=-7647.2628012$

Zero-point correction $=$

1.772049

(Hartree/Particle)

Thermal correction to Energy $=\quad 1.897722$

Thermal correction to Enthalpy= $\quad 1.898667$

Thermal correction to Gibbs Free Energy $=\quad 1.600983$

Sum of electronic and zero-point Energies $=\quad-7645.490752$

Sum of electronic and thermal Energies $=\quad-7645.365079$

Sum of electronic and thermal Enthalpies $=\quad-7645.364135$

Sum of electronic and thermal Free Energies $=\quad-7645.661818$

Cartesian Coordinates

$\begin{array}{lllc}26 & -4.166826 & -4.448765 & 3.168931 \\ 15 & -1.612583 & -2.209045 & -0.832637 \\ 15 & -4.487269 & -0.378365 & 0.706234 \\ 6 & -3.467738 & -3.541662 & 1.413839 \\ 6 & -4.128380 & -2.582765 & 2.267290 \\ 6 & -3.429382 & -2.553738 & 3.512496 \\ 1 & -3.687278 & -1.944119 & 4.367440 \\ 6 & -2.363645 & -3.501359 & 3.451377 \\ 1 & -1.675484 & -3.733975 & 4.251895 \\ 6 & -2.386691 & -4.109243 & 2.162302 \\ 1 & -1.718379 & -4.880693 & 1.809758 \\ 6 & -6.096965 & -5.213353 & 3.222871 \\ 1 & -6.931828 & -4.816174 & 2.660545 \\ 6 & -5.691903 & -4.796463 & 4.527872 \\ 1 & -6.159468 & -4.022940 & 5.122276 \\ 6 & -4.529736 & -5.543745 & 4.889708 \\ 1 & -3.961813 & -5.431898 & 5.803984 \\ 6 & -4.216475 & -6.423742 & 3.809234 \\ 1 & -3.371425 & -7.098031 & 3.761731 \\ 6 & -5.184210 & -6.221140 & 2.780579 \\ 1 & -5.200849 & -6.722659 & 1.822568 \\ 6 & -3.844135 & -3.805740 & -0.004701 \\ 6 & -5.041570 & -4.486753 & -0.274495 \\ 1 & -5.633479 & -4.826714 & 0.565219 \\ 6 & -5.487629 & -4.713655 & -1.575587 \\ 1 & -6.433042 & -5.219127 & -1.745375 \\ 6 & -4.720289 & -4.266911 & -2.648136 \\ 1 & -5.052831 & -4.426590 & -3.668782 \\ 6 & -3.529540 & -3.582444 & -2.406015 \\ 1 & -2.957123 & -3.228083 & -3.254427 \\ 6 & -3.079057 & -3.322797 & -1.100568 \\ 6 & -5.266055 & -1.697615 & 1.833972 \\ 1 & -5.913487 & -2.290911 & 1.186355\end{array}$

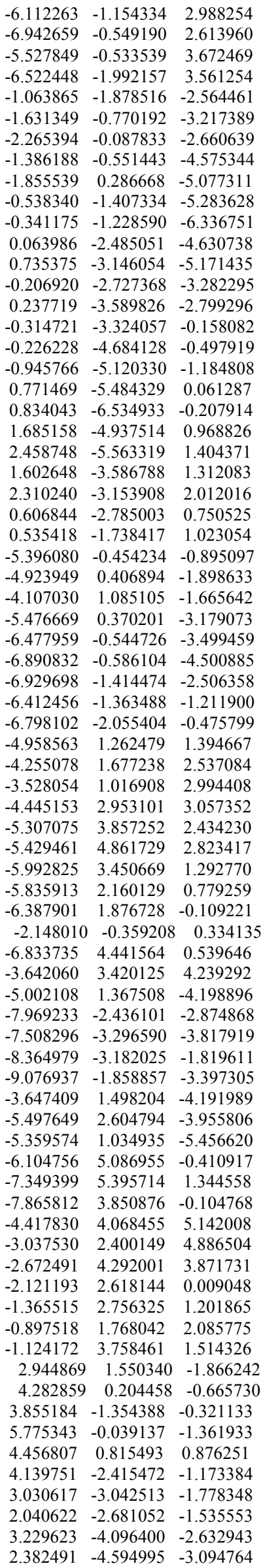




\begin{tabular}{|c|c|c|c|c|c|c|}
\hline 4.545307 & -4.533451 & -2.943746 & 1 & 3.110070 & 1.395508 & -5.944155 \\
\hline 4.765868 & -5.576202 & -3.883953 & 1 & 1.457785 & 1.975125 & -5.847701 \\
\hline 3.901537 & -6.054025 & -4.337946 & 6 & 2.028125 & 0.715829 & -4.231240 \\
\hline 6.041645 & -5.963629 & -4.225688 & 1 & 0.982218 & 0.576138 & -3.994243 \\
\hline 6.198858 & -6.757901 & -4.949552 & 6 & 2.907300 & -0.217977 & -3.765811 \\
\hline 7.153031 & -5.310006 & -3.642537 & 1 & 2.498745 & -1.063940 & -3.222333 \\
\hline 8.159475 & -5.597943 & -3.932559 & 6 & 4.357112 & -0.330475 & -4.179475 \\
\hline 6.973520 & -4.306384 & -2.715097 & 1 & 4.852525 & -1.019234 & -3.494461 \\
\hline 7.839266 & -3.813180 & -2.290376 & 1 & 4.402693 & -0.799627 & -5.172680 \\
\hline 5.669914 & -3.896049 & -2.317358 & 6 & 5.144233 & 1.002680 & -4.209412 \\
\hline 5.444025 & -2.846629 & -1.354559 & 1 & 5.094193 & 1.443714 & -5.209991 \\
\hline 6.567050 & -2.190022 & -0.629052 & 1 & 6.197175 & 0.772578 & -4.027442 \\
\hline 7.518635 & -2.929011 & 0.158068 & 6 & 0.028214 & 3.724596 & -2.632830 \\
\hline 7.348707 & -4.307658 & 0.466620 & 6 & -0.628081 & 3.130281 & -3.730351 \\
\hline 6.469804 & -4.827214 & 0.102499 & 6 & 0.047919 & 5.130247 & -2.546080 \\
\hline 8.273410 & -4.982276 & 1.233666 & 6 & -1.230110 & 3.914052 & -4.710084 \\
\hline 8.116412 & -6.031959 & 1.464827 & 1 & -0.684291 & 2.051555 & -3.810786 \\
\hline 9.422405 & -4.320475 & 1.729947 & 6 & -0.555958 & 5.912990 & -3.530282 \\
\hline 10.144489 & -4.867197 & 2.329245 & 1 & 0.515169 & 5.597831 & -1.685313 \\
\hline 9.611564 & -2.983507 & 1.463190 & 6 & -1.191403 & 5.310152 & -4.619665 \\
\hline 10.480084 & -2.458102 & 1.851721 & 1 & -1.743744 & 3.435722 & -5.539478 \\
\hline 8.670003 & -2.253287 & 0.687621 & 1 & -0.534436 & 6.995673 & -3.442674 \\
\hline 8.837192 & -0.862443 & 0.448586 & 1 & -1.662229 & 5.920288 & -5.385285 \\
\hline 9.721380 & -0.362143 & 0.832688 & 8 & -2.300860 & 1.520276 & -0.638668 \\
\hline 7.871087 & -0.148532 & -0.216640 & 6 & -0.159346 & 2.206924 & 3.244457 \\
\hline 7.961081 & 0.919178 & -0.380897 & 6 & 0.227818 & 1.329801 & 4.225289 \\
\hline 6.731396 & -0.815910 & -0.712964 & 1 & 0.059400 & 3.264372 & 3.335306 \\
\hline 4.008459 & 2.226568 & 1.008616 & 6 & -0.097203 & -0.030411 & 4.054658 \\
\hline 3.076354 & 2.239537 & 1.579600 & 6 & -0.755582 & -0.439250 & 2.918438 \\
\hline 5.013805 & 3.126824 & 1.714608 & 1 & -0.992686 & -1.481892 & 2.754237 \\
\hline 4.543197 & 4.236502 & 2.426872 & 7 & -1.146071 & 0.416048 & 1.947125 \\
\hline 3.478090 & 4.419522 & 2.489569 & 6 & -3.362568 & 3.736405 & -1.800917 \\
\hline 5.438590 & 5.112911 & 3.043159 & 1 & -3.027192 & 2.876757 & -2.370434 \\
\hline 5.052783 & 5.969459 & 3.589693 & 1 & -4.454076 & 3.695349 & -1.695318 \\
\hline 6.814842 & 4.889387 & 2.958880 & 1 & -3.105430 & 4.638330 & -2.364999 \\
\hline 7.511635 & 5.563671 & 3.449560 & 6 & -2.668807 & 5.042523 & 0.182962 \\
\hline 7.289169 & 3.788828 & 2.238330 & 1 & -2.858129 & 4.917939 & 1.251437 \\
\hline 8.357361 & 3.598518 & 2.173021 & 1 & -1.706910 & 5.555875 & 0.061285 \\
\hline 6.394070 & 2.921022 & 1.612305 & 1 & -3.462979 & 5.673103 & -0.222918 \\
\hline 6.760454 & 2.050128 & 1.082929 & 7 & -2.699124 & 3.758472 & -0.509009 \\
\hline 3.732645 & 2.846874 & -0.381919 & 1 & 0.768739 & 1.674456 & 5.100666 \\
\hline 4.678135 & 3.230102 & -0.781238 & 35 & 0.434343 & -1.357561 & 5.405057 \\
\hline 3.069520 & 3.697122 & -0.231943 & 5 & 1.057932 & 5.574609 & 1.651446 \\
\hline 4.416125 & -0.087986 & 2.081262 & 9 & 2.097705 & 6.299589 & 2.248395 \\
\hline 4.793231 & -1.046876 & 1.727150 & 9 & 0.641011 & 6.214480 & 0.465419 \\
\hline 5.363672 & 0.356333 & 3.183875 & 9 & -0.027993 & 5.469926 & 2.540764 \\
\hline 6.668622 & -0.149786 & 3.193042 & 9 & 1.523028 & 4.271495 & 1.331173 \\
\hline
\end{tabular}

$[8-9]^{\star}$ P1-C1 exo re-si $\left[\mathrm{BF}_{4}^{-}\right]$

Number of imaginary frequencies $=1$

The smallest frequencies are $=-180.178611 .729013 .0385 \mathrm{~cm}(-1)$ Electronic energy $=-7647.2560876$

Zero-point correction=

1.770967

(Hartree/Particle)

Thermal correction to Energy $=\quad 1.895815$

Thermal correction to Enthalpy= $\quad 1.896759$

Thermal correction to Gibbs Free Energy $=\quad 1.602136$

Sum of electronic and zero-point Energies $=\quad-7645.485121$

Sum of electronic and thermal Energies $=\quad-7645.360273$

Sum of electronic and thermal Enthalpies $=\quad-7645.359329$

Sum of electronic and thermal Free Energies $=\quad-7645.653951$

Cartesian Coordinates

$\begin{array}{llll}26 & -3.792829 & -4.024702 & 3.805116 \\ 15 & -1.560703 & -2.332699 & -0.619115 \\ 15 & -4.493679 & -0.410255 & 0.784259 \\ 6 & -3.245774 & -3.370065 & 1.892410 \\ 6 & -3.900806 & -2.320531 & 2.638273 \\ 6 & -3.126749 & -2.072616 & 3.813820 \\ 1 & -3.360617 & -1.351113 & 4.584335 \\ 6 & -2.021447 & -2.976629 & 3.821570 \\ 1 & -1.278120 & -3.063409 & 4.601934 \\ 6 & -2.097302 & -3.777125 & 2.644691\end{array}$


$\begin{array}{lll}-1.417456 & -4.570353 & 2.372260\end{array}$

$\begin{array}{llll}-5.677524 & -4.848318 & 4.094313\end{array}$

$\begin{array}{lll}-6.562706 & -4.573716 & 3.536008\end{array}$

$\begin{array}{llll}-5.218925 & -4.227029 & 5.296150\end{array}$

$\begin{array}{llll}-5.689557 & -3.395197 & 5.802743\end{array}$

$\begin{array}{llll}-4.002593 & -4.865274 & 5.686917\end{array}$

$\begin{array}{llll}-3.389390 & -4.597136 & 6.537207\end{array}$

$\begin{array}{llll}-3.709129 & -5.882263 & 4.727898\end{array}$

$\begin{array}{llll}-2.835911 & -6.521342 & 4.724431\end{array}$

$\begin{array}{lll}-4.743652 & -5.873175 & 3.745132\end{array}$

$\begin{array}{lll}-4.788665 & -6.506322 & 2.869443\end{array}$

$\begin{array}{llll}-3.689760 & -3.847674 & 0.550270\end{array}$

$\begin{array}{llll}-4.874915 & -4.593078 & 0.450415\end{array}$

$\begin{array}{llll}-5.403792 & -4.835779 & 1.363418\end{array}$

$\begin{array}{llll}-5.388976 & -5.003443 & -0.779031\end{array}$

$\begin{array}{llll}-6.324032 & -5.552869 & -0.820344\end{array}$

$\begin{array}{llll}-4.704549 & -4.682950 & -1.948499\end{array}$

$\begin{array}{lll}-5.092615 & -4.987235 & -2.915398\end{array}$

$\begin{array}{llll}-3.523543 & -3.943677 & -1.876563\end{array}$

$\begin{array}{llll}-3.011721 & -3.692957 & -2.798298\end{array}$

$\begin{array}{lll}-3.006409 & -3.501769 & -0.647818\end{array}$

$\begin{array}{llll}-5.110386 & -1.562790 & 2.162881\end{array}$

$\begin{array}{llll}-5.771366 & -2.277667 & 1.670443\end{array}$

$\begin{array}{lll}-5.897361 & -0.871656 & 3.279272\end{array}$

$\begin{array}{lll}-6.785876 & -0.374532 & 2.880197\end{array}$

$\begin{array}{lll}-5.299985 & -0.119664 & 3.801762\end{array}$

$\begin{array}{llll}-6.215182 & -1.619068 & 4.013003\end{array}$

$\begin{array}{lll}-1.080296 & -2.224165 & -2.397563\end{array}$

$\begin{array}{llll}-1.703481 & -1.223691 & -3.165363\end{array}$

$\begin{array}{llll}-2.352271 & -0.500126 & -2.682126\end{array}$

$\begin{array}{lll}-1.486051 & -1.152403 & -4.543155\end{array}$

$\begin{array}{llll}-1.986993 & -0.387119 & -5.125989\end{array}$

$\begin{array}{llll}-0.623414 & -2.059005 & -5.165126\end{array}$

$\begin{array}{llll}-0.448543 & -1.998187 & -6.235306\end{array}$

$\begin{array}{llll}0.029622 & -3.030712 & -4.402886\end{array}$

$\begin{array}{llll}0.717101 & -3.725472 & -4.876785\end{array}$

$\begin{array}{llll}-0.200791 & -3.117953 & -3.028083\end{array}$

$\begin{array}{llll}0.301356 & -3.889199 & -2.454985\end{array}$

$\begin{array}{llll}-0.228257 & -3.320545 & 0.176665\end{array}$

$\begin{array}{llll}-0.099051 & -4.703636 & -0.036365\end{array}$

$\begin{array}{lll}-0.774617 & -5.214079 & -0.716378\end{array}$

$\begin{array}{llll}0.872438 & -5.434668 & 0.649098\end{array}$

$\begin{array}{llll}0.965103 & -6.502999 & 0.475951\end{array}$

$\begin{array}{lll}1.715858 & -4.795711 & 1.563865\end{array}$

$\begin{array}{llll}2.466705 & -5.366997 & 2.101792\end{array}$

$\begin{array}{lll}1.592560 & -3.422469 & 1.782463\end{array}$

$\begin{array}{llll}2.244568 & -2.921560 & 2.490482\end{array}$

$\begin{array}{llll}0.628367 & -2.687839 & 1.089066\end{array}$

$\begin{array}{llll}0.529531 & -1.624043 & 1.269128\end{array}$

$\begin{array}{llll}-5.522208 & -0.774807 & -0.701426\end{array}$

$\begin{array}{llll}-5.144758 & -0.103897 & -1.874341\end{array}$

$\begin{array}{llll}-4.329598 & 0.611702 & -1.829304\end{array}$

$\begin{array}{llll}-5.781010 & -0.378064 & -3.08426\end{array}$

$\begin{array}{llll}-6.778245 & -1.348713 & -3.162470\end{array}$

$\begin{array}{llll}-7.254485 & -1.580753 & -4.108682\end{array}$

$\begin{array}{lll}-7.138649 & -2.028722 & -1.998326\end{array}$

$\begin{array}{llll}-6.536146 & -1.737264 & -0.773870\end{array}$

$\begin{array}{lll}-6.853089 & -2.287851 & 0.101194\end{array}$

$\begin{array}{lll}-4.979378 & 1.297249 & 1.267314\end{array}$

$\begin{array}{lll}-4.157054 & 1.913782 & 2.223676\end{array}$

$\begin{array}{lll}-3.343124 & 1.358438 & 2.672085\end{array}$

$\begin{array}{llll}-4.346475 & 3.248667 & 2.565269\end{array}$

$\begin{array}{lll}-5.348511 & 4.003054 & 1.952053\end{array}$

$\begin{array}{lll}-5.482434 & 5.049481 & 2.201836\end{array}$

$\begin{array}{lll}-6.164957 & 3.390439 & 1.004278\end{array}$

$\begin{array}{lll}-5.992209 & 2.045562 & 0.661476\end{array}$

$\begin{array}{llll}-6.637553 & 1.599493 & -0.086686\end{array}$

$\begin{array}{lll}-2.194616 & -0.340403 & 0.236741\end{array}$

$\begin{array}{llll}-7.184109 & 4.215268 & 0.269498\end{array}$

$\begin{array}{llll}-3.399365 & 3.922993 & 3.520405\end{array}$

$\begin{array}{llll}-5.391649 & 0.420398 & -4.297473\end{array}$

$\begin{array}{llll}-8.159077 & -3.128150 & -2.102968\end{array}$

$\begin{array}{llll}-7.718545 & -4.135669 & -2.899035\end{array}$

$\begin{array}{llll}-8.459675 & -3.676635 & -0.904837\end{array}$

$\begin{array}{llll}-9.317724 & -2.690584 & -2.648592\end{array}$ $\begin{array}{llll}-4.045840 & 0.611598 & -4.368570\end{array}$

$\begin{array}{llll}-5.943809 & 1.658512 & -4.279956\end{array}$

$\begin{array}{lll}-5.770762 & -0.169140 & -5.449059\end{array}$

$\begin{array}{llll}-6.645869 & 4.792385 & -0.839677\end{array}$

$\begin{array}{lll}-7.672835 & 5.219825 & 1.027474\end{array}$

$\begin{array}{llll}-8.232321 & 3.474794 & -0.153384\end{array}$

$\begin{array}{lll}-4.050539 & 4.741552 & 4.378288\end{array}$

$\begin{array}{lll}-2.700297 & 3.032498 & 4.260252\end{array}$

$\begin{array}{llll}-2.494545 & 4.688810 & 2.864194\end{array}$

$\begin{array}{llll}-2.380860 & 2.549283 & -0.661942\end{array}$

$\begin{array}{lll}-1.294192 & 2.913541 & 0.211112\end{array}$

$\begin{array}{lll}-0.810614 & 2.159544 & 1.324376\end{array}$

$\begin{array}{lll}-1.063519 & 3.964950 & 0.285983\end{array}$

$\begin{array}{llll}2.916766 & 1.070068 & -2.115110\end{array}$

$\begin{array}{llll}4.391995 & 0.083606 & -0.714379\end{array}$

$\begin{array}{llll}4.134204 & -1.436304 & -0.085408\end{array}$

$5.909421 \quad-0.140324-1.377063$

$\begin{array}{lll}4.519194 & 0.954361 & 0.714704\end{array}$

$\begin{array}{llll}4.468184 & -2.589247 & -0.779736\end{array}$

$\begin{array}{llll}3.395611 & -3.402383 & -1.209867\end{array}$

$\begin{array}{llll}2.385119 & -3.095598 & -0.969308\end{array}$

$\begin{array}{llll}3.655822 & -4.557147 & -1.903600\end{array}$

$2.839408 \quad-5.196833 \quad-2.226659$

$\begin{array}{llll}4.991004 & -4.919367 & -2.228314\end{array}$

$\begin{array}{llll}5.267771 & -6.074444 & -3.008683\end{array}$

$\begin{array}{llll}4.433544 & -6.690703 & -3.334240\end{array}$

$\begin{array}{llll}6.558765 & -6.400416 & -3.358246\end{array}$

$6.758553 \quad-7.283023 \quad-3.958867$

$\begin{array}{llll}7.628830 & -5.570868 & -2.946116\end{array}$

$\begin{array}{llll}8.644401 & -5.815158 & -3.244233\end{array}$

$\begin{array}{llll}7.395868 & -4.452107 & -2.175555\end{array}$

$\begin{array}{llll}8.227766 & -3.824469 & -1.878840\end{array}$

$\begin{array}{llll}6.077442 & -4.097156 & -1.773977\end{array}$

$\begin{array}{llll}5.797506 & -2.935673 & -0.968473\end{array}$

$\begin{array}{llll}6.880309 & -2.099767 & -0.377314\end{array}$

$\begin{array}{llll}7.893855 & -2.648446 & 0.485358\end{array}$

$\begin{array}{llll}7.854047 & -3.989400 & 0.960884\end{array}$

$\begin{array}{llll}7.029282 & -4.630099 & 0.671934\end{array}$

$\begin{array}{llll}8.835414 & -4.477645 & 1.795846\end{array}$

$\begin{array}{llll}8.776383 & -5.502064 & 2.152385\end{array}$

$\begin{array}{llll}9.915216 & -3.656390 & 2.200565\end{array}$

$\begin{array}{llll}10.683700 & -4.056827 & 2.855299\end{array}$

$\begin{array}{llll}9.976932 & -2.349063 & 1.774734\end{array}$

$\begin{array}{llll}10.789978 & -1.701103 & 2.091829\end{array}$

$\begin{array}{llll}8.973451 & -1.808669 & 0.924752\end{array}$

$\begin{array}{llll}9.007304 & -0.444776 & 0.527191\end{array}$

$\begin{array}{llll}9.834178 & 0.179864 & 0.852707\end{array}$

$\begin{array}{llll}7.984365 & 0.090618 & -0.216000\end{array}$

$\begin{array}{llll}7.966916 & 1.138062 & -0.495398\end{array}$

$\begin{array}{llll}6.920672 & -0.737236 & -0.634683\end{array}$

$\begin{array}{llll}3.878026 & 2.293460 & 0.631768\end{array}$

$\begin{array}{lll}2.926415 & 2.256529 & 1.175164\end{array}$

$\begin{array}{lll}4.714831 & 3.420647 & 1.219050\end{array}$

$\begin{array}{llll}4.058499 & 4.531544 & 1.761863\end{array}$

$\begin{array}{llll}2.975766 & 4.561428 & 1.78252\end{array}$

$\begin{array}{lll}4.785192 & 5.620499 & 2.245818\end{array}$

$\begin{array}{lll}4.249744 & 6.476285 & 2.647003\end{array}$

$\begin{array}{lll}6.181163 & 5.606816 & 2.20336\end{array}$

$\begin{array}{llll}6.750546 & 6.446819 & 2.592233\end{array}$

$\begin{array}{lll}6.841782 & 4.504646 & 1.650512\end{array}$

$\begin{array}{llll}7.927995 & 4.482011 & 1.615405\end{array}$

$\begin{array}{llll}6.112883 & 3.425856 & 1.149240\end{array}$

$\begin{array}{lll}6.626571 & 2.561870 & 0.742059\end{array}$

$\begin{array}{llll}3.586875 & 2.657372 & -0.842360\end{array}$

$\begin{array}{llll}4.515942 & 3.042240 & -1.279491\end{array}$

$2.884748 \quad 3.491419 \quad-0.838885$

$\begin{array}{lll}4.608229 & 0.247906 & 2.040691\end{array}$

$\begin{array}{lll}5.130229 & -0.684309 & 1.826502\end{array}$

$\begin{array}{llll}5.462088 & 0.986322 & 3.061333\end{array}$

$\begin{array}{lll}6.823967 & 0.677243 & 3.156209\end{array}$

$\begin{array}{lll}7.240149 & -0.089921 & 2.512035\end{array}$

$\begin{array}{llll}7.652387 & 1.347685 & 4.057551\end{array}$

$\begin{array}{lll}8.708263 & 1.095245 & 4.110076\end{array}$

$\begin{array}{lll}7.122529 & 2.337244 & 4.888503\end{array}$

$\begin{array}{lll}7.763684 & 2.867500 & 5.587324\end{array}$ 


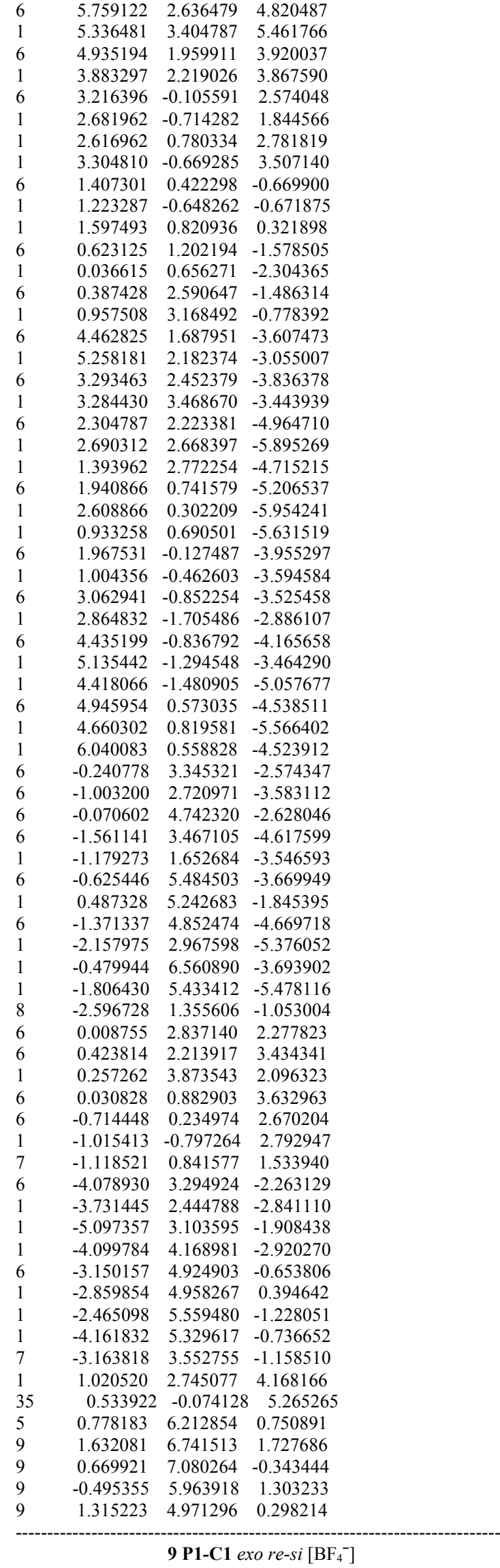

Number of imaginary frequencies $=0$

The smallest frequencies are $=10.710612 .098713 .7119 \mathrm{~cm}(-1)$
Electronic energy $=-7647.2813729$

Zero-point correction=

1.774143

(Hartree/Particle)

Thermal correction to Energy= $\quad 1.898584$

Thermal correction to Enthalpy= $\quad 1.899528$

Thermal correction to Gibbs Free Energy $=\quad 1.604842$

Sum of electronic and zero-point Energies $=\quad-7645.507230$

Sum of electronic and thermal Energies= $\quad-7645.382789$

Sum of electronic and thermal Enthalpies $=\quad-7645.381845$

Sum of electronic and thermal Free Energies $=\quad-7645.676530$ 


\begin{tabular}{|c|c|c|c|c|c|c|}
\hline-4.251611 & 0.490584 & -1.897988 & 1 & 2.771943 & 2.258012 & 1.053172 \\
\hline-5.732413 & -0.527625 & -3.084022 & 6 & 4.563769 & 3.409765 & 1.174509 \\
\hline-6.734195 & -1.495749 & -3.111837 & 6 & 3.900567 & 4.481238 & 1.782971 \\
\hline-7.234214 & -1.750242 & -4.039949 & 1 & 2.818718 & 4.482774 & 1.835068 \\
\hline-7.069304 & -2.142497 & -1.921582 & 6 & 4.615359 & 5.563637 & 2.299585 \\
\hline-6.438289 & -1.819204 & -0.719628 & 1 & 4.072174 & 6.390474 & 2.749530 \\
\hline-6.738594 & -2.342034 & 0.177956 & 6 & 6.009996 & 5.579661 & 2.228202 \\
\hline-4.895056 & 1.269644 & 1.207605 & 1 & 6.571539 & 6.413790 & 2.640440 \\
\hline-4.112488 & 1.930725 & 2.167990 & 6 & 6.679678 & 4.511762 & 1.620467 \\
\hline-3.288403 & 1.415634 & 2.646275 & 1 & 7.765411 & 4.510036 & 1.567017 \\
\hline-4.374957 & 3.256710 & 2.501173 & 6 & 5.960921 & 3.442368 & 1.086744 \\
\hline-5.393771 & 3.963603 & 1.860727 & 1 & 6.481676 & 2.603627 & 0.637734 \\
\hline-5.578481 & 5.004459 & 2.102297 & 6 & 3.510935 & 2.624270 & -0.940150 \\
\hline-6.162186 & 3.309612 & 0.900559 & 1 & 4.460700 & 2.988252 & -1.353067 \\
\hline-5.930567 & 1.969360 & 0.581043 & 1 & 2.829219 & 3.476193 & -0.974280 \\
\hline-6.549493 & 1.488091 & -0.166791 & 6 & 4.431255 & 0.287734 & 2.036847 \\
\hline-2.109534 & -0.419219 & 0.127322 & 1 & 4.904606 & -0.677241 & 1.860081 \\
\hline-7.187254 & 4.094176 & 0.129699 & 6 & 5.317726 & 1.009018 & 3.043424 \\
\hline-3.488160 & 3.977238 & 3.479669 & 6 & 6.669875 & 0.656028 & 3.121687 \\
\hline-5.373927 & 0.244015 & -4.323307 & 1 & 7.050126 & -0.127458 & 2.475566 \\
\hline-8.097814 & -3.238988 & -1.972534 & 6 & 7.532601 & 1.300276 & 4.009493 \\
\hline-7.676899 & -4.274184 & -2.742118 & 1 & 8.579973 & 1.012192 & 4.047842 \\
\hline-8.380363 & -3.744598 & -0.751732 & 6 & 7.046611 & 2.308050 & 4.845551 \\
\hline-9.262121 & -2.810783 & -2.512418 & 1 & 7.714061 & 2.818601 & 5.534438 \\
\hline-4.036405 & 0.478269 & -4.403052 & 6 & 5.692542 & 2.650869 & 4.795612 \\
\hline-5.968690 & 1.462524 & -4.338199 & 1 & 5.303932 & 3.433139 & 5.441642 \\
\hline-5.737503 & -0.392835 & -5.453546 & 6 & 4.834236 & 1.999290 & 3.908572 \\
\hline-6.608140 & 4.774045 & -0.897052 & 1 & 3.790681 & 2.292082 & 3.871527 \\
\hline-7.809996 & 5.012352 & 0.898168 & 6 & 3.021532 & 0.012514 & 2.572079 \\
\hline-8.135967 & 3.303428 & -0.416088 & 1 & 2.477727 & -0.608953 & 1.860750 \\
\hline-4.189222 & 4.822896 & 4.265546 & 1 & 2.450053 & 0.927895 & 2.732681 \\
\hline-2.825187 & 3.121234 & 4.291759 & 1 & 3.078510 & -0.514839 & 3.528975 \\
\hline-2.553362 & 4.720042 & 2.839813 & 6 & 1.258312 & 0.417877 & -0.791595 \\
\hline-2.268366 & 2.489713 & -0.897098 & 1 & 0.993609 & -0.628185 & -0.922427 \\
\hline-0.985434 & 2.872862 & -0.187346 & 1 & 1.472905 & 0.680394 & 0.238844 \\
\hline-0.729040 & 2.180462 & 1.136090 & 6 & 0.655573 & 1.380393 & -1.647490 \\
\hline-0.996770 & 3.933996 & 0.046913 & 1 & 0.028033 & 0.974580 & -2.431660 \\
\hline 2.837314 & 1.016966 & -2.192086 & 6 & 0.242244 & 2.768218 & -1.217489 \\
\hline 4.299375 & 0.042811 & -0.730902 & 1 & 1.042362 & 3.229752 & -0.646011 \\
\hline 4.085779 & -1.475289 & -0.054588 & 6 & 4.306881 & 1.627669 & -3.689434 \\
\hline 5.852785 & -0.161186 & -1.344673 & 1 & 5.121621 & 2.158066 & -3.199477 \\
\hline 4.383455 & 0.947243 & 0.689316 & 6 & 3.098931 & 2.371208 & -3.891421 \\
\hline 4.474308 & -2.624566 & -0.722728 & 1 & 3.097596 & 3.407792 & -3.554788 \\
\hline 3.443384 & -3.471075 & -1.190407 & 6 & 2.130998 & 2.110819 & -5.033710 \\
\hline 2.416022 & -3.185972 & -1.002262 & 1 & 2.543371 & 2.493532 & -5.981402 \\
\hline 3.760907 & -4.630153 & -1.851999 & 1 & 1.229066 & 2.690429 & -4.831159 \\
\hline 2.974987 & -5.293615 & -2.202130 & 6 & 1.742919 & 0.623923 & -5.193209 \\
\hline 5.117249 & -4.967371 & -2.108389 & 1 & 2.364757 & 0.145091 & -5.957504 \\
\hline 5.455296 & -6.129675 & -2.853160 & 1 & 0.712943 & 0.558173 & -5.559542 \\
\hline 4.650958 & -6.772638 & -3.201677 & 6 & 1.836265 & -0.188863 & -3.904086 \\
\hline 6.767330 & -6.429676 & -3.142370 & 1 & 0.891380 & -0.535337 & -3.502095 \\
\hline 7.013764 & -7.317828 & -3.717036 & 6 & 2.962219 & -0.899858 & -3.505319 \\
\hline 7.797666 & -5.564586 & -2.703353 & 1 & 2.795330 & -1.754964 & -2.859079 \\
\hline 8.830717 & -5.787020 & -2.955181 & 6 & 4.302419 & -0.892470 & -4.217137 \\
\hline 7.504496 & -4.437884 & -1.965533 & 1 & 5.040764 & -1.320809 & -3.534736 \\
\hline 8.307848 & -3.783306 & -1.649551 & 1 & 4.254689 & -1.561516 & -5.090550 \\
\hline 6.162088 & -4.109169 & -1.624216 & 6 & 4.772690 & 0.517103 & -4.639614 \\
\hline 5.818838 & -2.940174 & -0.854082 & 1 & 4.433545 & 0.740483 & -5.657278 \\
\hline 6.858867 & -2.053585 & -0.260202 & 1 & 5.867060 & 0.521697 & -4.681182 \\
\hline 7.873970 & -2.541149 & 0.635591 & 6 & -0.060428 & 3.658631 & -2.420351 \\
\hline 7.857651 & -3.861438 & 1.167027 & 6 & -0.894584 & 3.221693 & -3.464007 \\
\hline 7.049154 & -4.530359 & 0.895568 & 6 & 0.454956 & 4.960033 & -2.487688 \\
\hline 8.840240 & -4.293088 & 2.031304 & 6 & -1.206970 & 4.058877 & -4.535923 \\
\hline 8.799332 & -5.302733 & 2.430218 & 1 & -1.313536 & 2.221197 & -3.441445 \\
\hline 9.898534 & -3.432415 & 2.410683 & 6 & 0.154762 & 5.796020 & -3.567424 \\
\hline 10.668435 & -3.788137 & 3.089202 & 1 & 1.076112 & 5.329227 & -1.682421 \\
\hline 9.938125 & -2.143678 & 1.928539 & 6 & -0.679056 & 5.351927 & -4.595297 \\
\hline 10.735105 & -1.466072 & 2.223899 & 1 & -1.859927 & 3.697238 & -5.326143 \\
\hline 8.932805 & -1.661429 & 1.046452 & 1 & 0.567319 & 6.801087 & -3.592014 \\
\hline 8.942952 & -0.316720 & 0.586294 & 1 & -0.918215 & 6.004426 & -5.430666 \\
\hline 9.755647 & 0.338388 & 0.886876 & 8 & -2.451479 & 1.305515 & -1.276706 \\
\hline 7.913330 & 0.163662 & -0.184516 & 6 & -0.148950 & 2.946894 & 2.160565 \\
\hline 7.878379 & 1.196430 & -0.513129 & 6 & 0.126790 & 2.383428 & 3.399768 \\
\hline 6.865975 & -0.701234 & -0.572492 & 1 & 0.061174 & 3.990690 & 1.980338 \\
\hline 3.747440 & 2.286330 & 0.552556 & 6 & -0.187957 & 1.040491 & 3.574458 \\
\hline
\end{tabular}




$\begin{array}{lccc}6 & -0.755845 & 0.318830 & 2.537779 \\ 1 & -1.015730 & -0.725058 & 2.653776 \\ 7 & -1.027886 & 0.879784 & 1.341329 \\ 6 & -4.129496 & 3.201151 & -2.280575 \\ 1 & -3.770612 & 2.417771 & -2.943699 \\ 1 & -5.092022 & 2.907006 & -1.856939 \\ 1 & -4.269123 & 4.114884 & -2.862145 \\ 6 & -3.063695 & 4.852287 & -0.764705 \\ 1 & -2.679461 & 4.915574 & 0.250461 \\ 1 & -2.432433 & 5.458696 & -1.423311 \\ 1 & -4.078043 & 5.254806 & -0.766600 \\ 7 & -3.135137 & 3.464181 & -1.237391 \\ 1 & 0.563898 & 2.977690 & 4.194404 \\ 35 & 0.167680 & 0.147477 & 5.271633 \\ 5 & 0.556463 & 6.381343 & 0.873624 \\ 9 & 1.215263 & 6.937988 & 1.975650 \\ 9 & 0.554447 & 7.263137 & -0.209583 \\ 9 & -0.769722 & 6.034443 & 1.223325 \\ 9 & 1.227009 & 5.180658 & 0.499562 \\ -----------------------------------------------------------------------------\end{array}$

$[8-9]^{\ddagger}$ P1-C1 endo re-re $\left[\mathrm{BF}_{4}^{-}\right]$conf2

Number of imaginary frequencies $=1$

The smallest frequencies are $=-215.75107 .350711 .2519 \mathrm{~cm}(-1)$ Electronic energy $=-7647.2545687$

Zero-point correction $=$

1.770463

(Hartree/Particle)

Thermal correction to Energy= $\quad 1.895450$

Thermal correction to Enthalpy=

1.896395

Thermal correction to Gibbs Free Energy=

Sum of electronic and zero-point Energies=

Sum of electronic and thermal Energies=

Sum of electronic and thermal Enthalpies=

Sum of electronic and thermal Free Energies=

$-7645.484106$

$-7645.359118$

$-7645.358174$

Cartesian Coordinates

$\begin{array}{lccc}26 & 5.019122 & 4.481424 & 2.165110 \\ 15 & 2.964252 & 1.739319 & -1.765180 \\ 15 & 4.828715 & -0.109281 & 0.914472 \\ 6 & 4.551459 & 3.269858 & 0.523645 \\ 6 & 4.692987 & 2.486985 & 1.729561 \\ 6 & 3.725524 & 2.956194 & 2.670341 \\ 1 & 3.586065 & 2.581588 & 3.675176 \\ 6 & 3.010166 & 4.038785 & 2.075749 \\ 1 & 2.241675 & 4.629265 & 2.555034 \\ 6 & 3.518317 & 4.233809 & 0.758232 \\ 1 & 3.198174 & 4.991010 & 0.058420 \\ 6 & 7.008291 & 4.814255 & 2.658437 \\ 1 & 7.810534 & 4.107358 & 2.492861 \\ 6 & 6.186423 & 4.894336 & 3.824369 \\ 1 & 6.248318 & 4.251247 & 4.692050 \\ 6 & 5.232291 & 5.937703 & 3.621377 \\ 1 & 4.444558 & 6.220080 & 4.307261 \\ 6 & 5.463978 & 6.503091 & 2.330347 \\ 1 & 4.885808 & 7.292227 & 1.867919 \\ 6 & 6.560709 & 5.810463 & 1.735941 \\ 1 & 6.959510 & 5.991113 & 0.747163 \\ 6 & 5.303062 & 3.015111 & -0.739165 \\ 6 & 6.667314 & 3.334701 & -0.806274 \\ 1 & 7.125785 & 3.803939 & 0.055239 \\ 6 & 7.436293 & 3.047882 & -1.934148 \\ 1 & 8.495742 & 3.283483 & -1.942374 \\ 6 & 6.840384 & 2.436596 & -3.034740 \\ 1 & 7.427331 & 2.195283 & -3.915156 \\ 6 & 5.484796 & 2.108648 & -2.992419 \\ 1 & 5.036992 & 1.619488 & -3.850361 \\ 6 & 4.702860 & 2.375355 & -1.857475 \\ 6 & 5.624990 & 1.314792 & 1.884095 \\ 1 & 6.544277 & 1.554599 & 1.347010 \\ 6 & 5.989622 & 0.988294 & 3.334905 \\ 1 & 6.699309 & 0.157751 & 3.382250 \\ 1 & 5.115173 & 0.715458 & 3.931498 \\ 1 & 6.450722 & 1.866885 & 3.796879 \\ 6 & 2.668117 & 0.973698 & -3.416748\end{array}$

$2.817119-0.418622 \quad-3.523901$

$3.089187-1.001352-2.650703$

$2.579622-1.062983-4.740310$

$2.700931-2.138921-4.809228$

$\begin{array}{lll}2.177284 & -0.327327 & -5.856906\end{array}$

$\begin{array}{lll}1.977692 & -0.831227 & -6.798315\end{array}$

$\begin{array}{llll}2.027817 & 1.058775 & -5.757973\end{array}$

$\begin{array}{llll}1.714085 & 1.636489 & -6.622834\end{array}$

$\begin{array}{llll}2.275566 & 1.707699 & -4.547571\end{array}$

$\begin{array}{lll}2.147496 & 2.782885 & -4.486013\end{array}$

$1.921413 \quad 3.250824 \quad-1.823417$

$\begin{array}{llll}2.331047 & 4.429182 & -2.468740\end{array}$

$3.317659 \quad 4.486973 \quad-2.919212$

$\begin{array}{llll}1.478637 & 5.533473 & -2.524102\end{array}$

$\begin{array}{llll}1.805306 & 6.441483 & -3.023140\end{array}$

$\begin{array}{llll}0.206671 & 5.467670 & -1.945779\end{array}$

$\begin{array}{llll}-0.462757 & 6.321831 & -1.994139\end{array}$

$-0.204983 \quad 4.298436-1.302839$

$\begin{array}{llll}-1.191278 & 4.241065 & -0.858168\end{array}$

$\begin{array}{lll}0.653039 & 3.199314 & -1.230899\end{array}$

$\begin{array}{llll}0.338874 & 2.300496 & -0.712009\end{array}$

$\begin{array}{llll}6.064694 & -0.651444 & -0.340252\end{array}$

$\begin{array}{llll}5.611921 & -1.616410 & -1.251843\end{array}$

$\begin{array}{llll}4.606825 & -2.011175 & -1.141060\end{array}$

$\begin{array}{llll}6.434676 & -2.050363 & -2.290572\end{array}$

$\begin{array}{llll}7.703113 & -1.502798 & -2.471200\end{array}$

$8.331540 \quad-1.820670 \quad-3.295526$

$8.142208-0.525636-1.576982$

$\begin{array}{llll}7.347000 & -0.115757 & -0.506448\end{array}$

$\begin{array}{llll}7.736366 & 0.633678 & 0.168970\end{array}$

$\begin{array}{llll}4.698249 & -1.552680 & 2.046487\end{array}$

$\begin{array}{llll}3.687262 & -1.498083 & 3.019089\end{array}$

$\begin{array}{lll}3.084221 & -0.603745 & 3.124944\end{array}$

$\begin{array}{llll}3.421307 & -2.605455 & 3.820169\end{array}$

$\begin{array}{lll}4.122553 & -3.799142 & 3.644296\end{array}$

$\begin{array}{llll}3.881770 & -4.672857 & 4.239703\end{array}$

$\begin{array}{llll}5.109035 & -3.858451 & 2.662851\end{array}$

$\begin{array}{lll}5.414404 & -2.742539 & 1.881660\end{array}$

$\begin{array}{lll}6.185677 & -2.825030 & 1.125926\end{array}$

$\begin{array}{llll}2.725231 & 0.250556 & -0.083723\end{array}$

$\begin{array}{lll}5.848289 & -5.149922 & 2.447568\end{array}$

$\begin{array}{llll}2.283778 & -2.579085 & 4.802728\end{array}$

$\begin{array}{llll}5.940102 & -3.156037 & -3.181128\end{array}$

$\begin{array}{llll}9.486877 & 0.103630 & -1.815981\end{array}$

$\begin{array}{llll}9.537994 & 0.712404 & -3.027423\end{array}$

$\begin{array}{llll}9.795983 & 1.038609 & -0.890233\end{array}$

$\begin{array}{lll}10.481571 & -0.814253 & -1.808689\end{array}$

$\begin{array}{llll}6.020097 & -4.361364 & -2.564217\end{array}$

$\begin{array}{llll}6.638289 & -3.250294 & -4.330900\end{array}$

$\begin{array}{llll}4.633899 & -2.989876 & -3.515457\end{array}$

$\begin{array}{llll}6.835377 & -5.330814 & 3.355588\end{array}$

$\begin{array}{llll}6.423395 & -5.208849 & 1.223612\end{array}$

$\begin{array}{llll}5.022900 & -6.219449 & 2.553071\end{array}$

$\begin{array}{llll}2.616878 & -3.176185 & 5.969801\end{array}$

$\begin{array}{llll}1.879451 & -1.323115 & 5.090855\end{array}$

$1.203738 \quad-3.246073 \quad 4.322326$

$\begin{array}{llll}2.023163 & -2.709299 & 0.244205\end{array}$

$\begin{array}{lll}0.875612 & -2.365711 & 1.045213\end{array}$

$\begin{array}{lll}0.608684 & -1.107924 & 1.670847\end{array}$

$\begin{array}{llll}0.345543 & -3.183612 & 1.506113\end{array}$

$\begin{array}{llll}-3.245499 & -0.714164 & -1.536451\end{array}$

$\begin{array}{llll}-5.077268 & 0.088534 & -0.460704\end{array}$

$\begin{array}{lll}-5.002260 & 1.101089 & 0.863716\end{array}$

$\begin{array}{lll}-6.022166 & 1.026653 & -1.467788\end{array}$

$\begin{array}{lll}-5.968141 & -1.160274 & 0.213787\end{array}$

$\begin{array}{lll}-4.584658 & 2.411861 & 0.712634\end{array}$

$\begin{array}{lll}-3.325804 & 2.745689 & 1.260745\end{array}$

$\begin{array}{lll}-2.764374 & 1.975110 & 1.777564\end{array}$

$\begin{array}{lll}-2.850851 & 4.026406 & 1.127485\end{array}$

$\begin{array}{lll}-1.894378 & 4.303028 & 1.558684\end{array}$

$\begin{array}{lll}-3.581840 & 4.993381 & 0.384361\end{array}$

$\begin{array}{llll}-3.042674 & 6.285618 & 0.138476\end{array}$

$\begin{array}{lll}-2.071567 & 6.529759 & 0.561086\end{array}$

$\begin{array}{lll}-3.723403 & 7.200398 & -0.633417\end{array}$

$\begin{array}{llll}-3.299328 & 8.182312 & -0.822246\end{array}$ 


\begin{tabular}{|c|c|c|c|c|c|c|}
\hline-4.976465 & 6.853495 & -1.193168 & 1 & -2.481076 & 1.974333 & -1.008681 \\
\hline-5.501257 & 7.568606 & -1.820167 & 6 & -3.412185 & 2.082460 & -3.003821 \\
\hline-5.535853 & 5.616192 & -0.956013 & 1 & -4.270925 & 2.509514 & -2.482621 \\
\hline-6.492678 & 5.365632 & -1.399151 & 1 & -2.883050 & 2.920955 & -3.481301 \\
\hline-4.867701 & 4.649991 & -0.153010 & 6 & -3.913235 & 1.086141 & -4.073071 \\
\hline-5.412501 & 3.340602 & 0.100224 & 1 & -3.244228 & 1.092966 & -4.940004 \\
\hline-6.806958 & 2.967491 & -0.269492 & 1 & -4.886673 & 1.427391 & -4.438901 \\
\hline-7.941057 & 3.731155 & 0.187893 & 6 & -0.318136 & -3.417331 & -1.499725 \\
\hline-7.823216 & 4.802648 & 1.117854 & 6 & -0.897550 & -4.663265 & -1.184807 \\
\hline-6.849024 & 5.053738 & 1.519659 & 6 & 0.630497 & -3.358319 & -2.538581 \\
\hline-8.928857 & 5.515484 & 1.528730 & 6 & -0.541154 & -5.803579 & -1.902552 \\
\hline-8.809801 & 6.323550 & 2.244944 & 1 & -1.601996 & -4.746405 & -0.364763 \\
\hline-10.217478 & 5.200831 & 1.035533 & 6 & 0.980840 & -4.500339 & -3.255784 \\
\hline-11.078645 & 5.775614 & 1.363608 & 1 & 1.095254 & -2.407525 & -2.769347 \\
\hline-10.373561 & 4.154349 & 0.155374 & 6 & 0.396000 & -5.729564 & -2.940228 \\
\hline-11.358911 & 3.885559 & -0.216645 & 1 & -0.991278 & -6.758292 & -1.644931 \\
\hline-9.256103 & 3.389439 & -0.276968 & 1 & 1.717769 & -4.432428 & -4.051562 \\
\hline-9.424976 & 2.278372 & -1.145950 & 1 & 0.669479 & -6.623896 & -3.493115 \\
\hline-10.421223 & 2.033369 & -1.503277 & 7 & 2.443726 & -4.009539 & 0.261079 \\
\hline-8.349943 & 1.502457 & -1.501432 & 6 & 3.401275 & -4.493690 & -0.724458 \\
\hline-8.458430 & 0.627357 & -2.132991 & 1 & 3.084311 & -5.479383 & -1.079093 \\
\hline-7.058574 & 1.839435 & -1.038150 & 1 & 3.431236 & -3.820159 & -1.574997 \\
\hline-5.386114 & -2.503915 & -0.089518 & 1 & 4.406462 & -4.584259 & -0.296198 \\
\hline-4.744978 & -2.803030 & 0.744545 & 6 & 1.958878 & -5.011341 & 1.208697 \\
\hline-6.440886 & -3.576637 & -0.305844 & 1 & 1.704668 & -4.560029 & 2.166692 \\
\hline-6.266783 & -4.845372 & 0.257916 & 1 & 1.080134 & -5.540752 & 0.822048 \\
\hline-5.394351 & -5.037075 & 0.874817 & 1 & 2.759829 & -5.733511 & 1.385018 \\
\hline-7.205144 & -5.856306 & 0.028959 & 8 & 2.610091 & -1.884494 & -0.529169 \\
\hline-7.061733 & -6.834894 & 0.479808 & 6 & -0.347555 & -1.093373 & 2.727497 \\
\hline-8.326708 & -5.607925 & -0.763960 & 6 & -0.670545 & 0.077046 & 3.381987 \\
\hline-9.063898 & -6.388771 & -0.930578 & 1 & -0.820504 & -2.024163 & 3.012427 \\
\hline-8.497555 & -4.344706 & -1.342272 & 6 & -0.047781 & 1.253515 & 2.950564 \\
\hline-9.370855 & -4.140469 & -1.956049 & 6 & 0.850282 & 1.217660 & 1.900239 \\
\hline-7.555723 & -3.341981 & -1.122653 & 1 & 1.333592 & 2.117044 & 1.540969 \\
\hline-7.700526 & -2.353979 & -1.549076 & 7 & 1.182002 & 0.070977 & 1.272461 \\
\hline-4.531738 & -2.416693 & -1.369184 & 1 & -1.388937 & 0.078104 & 4.194596 \\
\hline-5.205176 & -2.414972 & -2.234787 & 35 & -0.405363 & 2.958234 & 3.849630 \\
\hline-3.928348 & -3.325813 & -1.437615 & 5 & -2.719139 & -4.237432 & 2.498572 \\
\hline-6.743093 & -0.952592 & 1.488020 & 9 & -1.307237 & -4.242693 & 2.498373 \\
\hline-6.971896 & 0.113892 & 1.503876 & 9 & -3.182245 & -2.917269 & 2.679835 \\
\hline-8.093947 & -1.661976 & 1.488196 & 9 & -3.204822 & -5.062013 & 3.512066 \\
\hline-9.176463 & -1.052280 & 0.841895 & 9 & -3.176264 & -4.710477 & 1.237290 \\
\hline
\end{tabular}

[8-9 $]^{\ddagger} \mathbf{P 1 - C 1}$ endo si-re $\left[\mathrm{BF}_{4}^{-}\right]$conf2

$\begin{array}{lll}033978 & -0.087825 & 0.365530\end{array}$

$\begin{array}{lll}-10.428072 & -1.667188 & 0.796743\end{array}$

$\begin{array}{lll}-11.252658 & -1.176456 & 0.285978\end{array}$

$\begin{array}{lll}-10.618768 & -2.907391 & 1.411030\end{array}$

$\begin{array}{lll}-11.589436 & -3.394453 & 1.374502\end{array}$

$\begin{array}{lll}-9.552847 & -3.513165 & 2.080608\end{array}$

$\begin{array}{llll}-9.689579 & -4.475974 & 2.565005\end{array}$

$\begin{array}{lll}-8.303979 & -2.890595 & 2.126071\end{array}$

$\begin{array}{lll}-7.486287 & -3.383249 & 2.639942\end{array}$

$\begin{array}{llll}-5.888652 & -1.253743 & 2.724702\end{array}$

$\begin{array}{lll}-5.016433 & -0.596966 & 2.738513\end{array}$

$\begin{array}{lll}-5.528724 & -2.282808 & 2.756564\end{array}$

$\begin{array}{lll}-6.476246 & -1.068413 & 3.629686\end{array}$

$\begin{array}{lll}-2.330896 & -0.744575 & 0.442289\end{array}$

$\begin{array}{llll}-1.638952 & 0.089084 & 0.527860\end{array}$

$\begin{array}{llll}-3.008657 & -0.827827 & 1.284631\end{array}$

$\begin{array}{llll}-1.880133 & -1.980191 & -0.121175\end{array}$

$\begin{array}{llll}-2.433815 & -2.866385 & 0.155070\end{array}$

$\begin{array}{lll}-0.646593 & -2.182598 & -0.782448\end{array}$

$\begin{array}{llll}-0.056496 & -1.319402 & -1.061589\end{array}$

$\begin{array}{llll}-4.059879 & -0.353081 & -3.567831\end{array}$

$\begin{array}{llll}-5.081443 & -0.726309 & -3.583155\end{array}$

$\begin{array}{llll}-3.032512 & -1.327613 & -3.673675\end{array}$

$\begin{array}{lll}-3.343896 & -2.367090 & -3.774158\end{array}$

$\begin{array}{llll}-1.641215 & -1.049711 & -4.214598\end{array}$

$\begin{array}{lll}-1.665939 & -1.005982 & -5.315044\end{array}$

$\begin{array}{llll}-1.013239 & -1.906175 & -3.957253\end{array}$

$\begin{array}{llll}-0.991288 & 0.231188 & -3.652477\end{array}$

$\begin{array}{llll}-1.177935 & 1.081181 & -4.316376\end{array}$

$\begin{array}{llll}0.092347 & 0.097200 & -3.635247\end{array}$

$\begin{array}{llll}-1.450762 & 0.595903 & -2.244002\end{array}$

$\begin{array}{llll}-0.696090 & 0.495659 & -1.470950\end{array}$

$-2.506380 \quad 1.457313-1.962633$
. 1.00868
$\begin{array}{lll}-2.883050 & 2.920955 & -3.48130\end{array}$
$\begin{array}{llll}-3.913235 & 1.086141 & -4.073071\end{array}$
$\begin{array}{lll}-3.244228 & 1.092966 & -4.940004\end{array}$
$-0.897550 \quad-4.663265-1.184807$
$\begin{array}{lll}0.630497 & -3.358319 & -2.538581\end{array}$
$-1.601996-4.746405-0.364763$
$\begin{array}{lll}6 & -0.364763\end{array}$
$1.095254-2.407525 \quad-2.769347$
$0 \quad-5.729564-2.94022$
$1.717769-4.432428-4.051562$
$-3.493115$
$3.401275-4.493690-0.724458$
$\begin{array}{lll}3.084311 & -5.479383 & -1.079093\end{array}$
$\begin{array}{lll}1.958878 & -5.011341 & 1.208697\end{array}$
$\begin{array}{lll}0.568 & -4.560029 & 2.166692\end{array}$
$\begin{array}{lll}2.759829 & -5.733511 & 1.385018\end{array}$
$884494-0.529169$
$\begin{array}{lll}-0.670545 & 0.077046 & 3.381987\end{array}$
$\begin{array}{lll}-0.820504 & -2.024163 & 3.012427\end{array}$
$\begin{array}{llll}1.333592 & 2.117044 & 1.540969\end{array}$
$\begin{array}{lll}1.182002 & 0.070977 & 1.272461\end{array}$
$\begin{array}{lll}388937 & 0.078104 & 4.194596\end{array}$
$\begin{array}{lll}-1.307237 & -4.242693 & 2.498373\end{array}$
$\begin{array}{lll}-3.176264 & -4.710477 & 1.237290\end{array}$

Number of imaginary frequencies $=1$

The smallest frequencies are $=-205.09568 .401411 .8927 \mathrm{~cm}(-1)$

Electronic energy $=-7647.2404677$

Zero-point correction=

1.770207

(Hartree/Particle)

Thermal correction to Energy $=\quad 1.895472$

Thermal correction to Enthalpy $=\quad 1.896416$

Thermal correction to Gibbs Free Energy $=\quad 1.596963$

Sum of electronic and zero-point Energies $=\quad-7645.470260$

Sum of electronic and thermal Energies $=\quad-7645.344996$

Sum of electronic and thermal Enthalpies $=\quad-7645.344052$

Sum of electronic and thermal Free Energies $=\quad-7645.643505$

Cartesian Coordinates

$\begin{array}{lccc}26 & 4.119151 & 4.767577 & 2.750452 \\ 15 & 2.801268 & 2.091587 & -1.497466 \\ 15 & 5.150260 & 0.405310 & 1.084500 \\ 6 & 3.924701 & 3.753129 & 0.928526 \\ 6 & 4.469271 & 2.887706 & 1.943010 \\ 6 & 3.516356 & 2.808642 & 3.006132 \\ 1 & 3.638787 & 2.244637 & 3.920954 \\ 6 & 2.400727 & 3.630186 & 2.667769 \\ 1 & 1.533517 & 3.811130 & 3.288572 \\ 6 & 2.653184 & 4.217263 & 1.393465 \\ 1 & 2.007184 & 4.904367 & 0.865981 \\ 6 & 5.884926 & 5.787824 & 3.150630 \\ 1 & 6.856518 & 5.509836 & 2.764424 \\ 6 & 5.297982 & 5.317503 & 4.364945\end{array}$


$\begin{array}{llll}5.742461 & 4.613689 & 5.055560\end{array}$

$\begin{array}{llll}3.998748 & 5.899366 & 4.480696\end{array}$

$\begin{array}{lll}3.286279 & 5.710517 & 5.273059\end{array}$

$\begin{array}{llll}3.782707 & 6.729499 & 3.338613\end{array}$

$\begin{array}{lll}2.879019 & 7.280830 & 3.114071\end{array}$

$\begin{array}{lll}4.947612 & 6.661786 & 2.517643\end{array}$

$\begin{array}{llll}5.080445 & 7.159945 & 1.566928\end{array}$

$\begin{array}{llll}4.569546 & 4.023276 & -0.385825\end{array}$

$\begin{array}{llll}5.677587 & 4.880141 & -0.458261\end{array}$

$\begin{array}{llll}6.031712 & 5.337894 & 0.456236\end{array}$

$\begin{array}{llll}6.325686 & 5.141406 & -1.665846\end{array}$

$\begin{array}{llll}7.187216 & 5.802635 & -1.685575\end{array}$

$\begin{array}{llll}5.858685 & 4.551750 & -2.838068\end{array}$

$\begin{array}{llll}6.346988 & 4.746000 & -3.788055\end{array}$

$\begin{array}{llll}4.768677 & 3.682595 & -2.786795\end{array}$

$\begin{array}{llll}4.434108 & 3.213657 & -3.704211\end{array}$

$\begin{array}{llll}4.125844 & 3.387533 & -1.573688\end{array}$

$\begin{array}{lll}5.724226 & 2.079681 & 1.779228\end{array}$

$\begin{array}{lll}6.305110 & 2.521033 & 0.963905\end{array}$

$\begin{array}{lll}6.608642 & 2.017481 & 3.026724\end{array}$

$\begin{array}{lll}7.521341 & 1.444518 & 2.839709\end{array}$

$\begin{array}{lll}6.088323 & 1.566931 & 3.876233\end{array}$

$\begin{array}{lll}6.894423 & 3.035040 & 3.308556\end{array}$

$\begin{array}{llll}2.760755 & 1.455333 & -3.223070\end{array}$

$\begin{array}{llll}3.794632 & 0.577645 & -3.595497\end{array}$

$\begin{array}{llll}4.586500 & 0.350614 & -2.889171\end{array}$

$\begin{array}{llll}3.830917 & 0.022038 & -4.872474\end{array}$

$\begin{array}{lll}4.644405 & -0.643210 & -5.147204\end{array}$

$\begin{array}{llll}2.815857 & 0.314732 & -5.789709\end{array}$

$\begin{array}{llll}2.831550 & -0.130725 & -6.780112\end{array}$

$\begin{array}{llll}1.782685 & 1.180845 & -5.427488\end{array}$

$\begin{array}{llll}0.991057 & 1.412084 & -6.134425\end{array}$

$\begin{array}{llll}1.759980 & 1.759321 & -4.154574\end{array}$

$\begin{array}{llll}0.958810 & 2.441133 & -3.893113\end{array}$

$\begin{array}{llll}1.261575 & 3.069385 & -1.269518\end{array}$

$\begin{array}{llll}1.021750 & 4.270613 & -1.956048\end{array}$

$\begin{array}{llll}1.737935 & 4.631955 & -2.688639\end{array}$

$-0.129333 \quad 5.013852-1.686824$

$\begin{array}{llll}-0.312108 & 5.939350 & -2.225547\end{array}$

$\begin{array}{llll}-1.038667 & 4.575179 & -0.717668\end{array}$

$\begin{array}{lll}-1.932433 & 5.155377 & -0.506762\end{array}$

$\begin{array}{llll}-0.795715 & 3.388463 & -0.023575\end{array}$

$\begin{array}{llll}-1.492888 & 3.046641 & 0.734958\end{array}$

$\begin{array}{llll}0.344731 & 2.632737 & -0.302028\end{array}$

$\begin{array}{lll}0.550304 & 1.722529 & 0.252214\end{array}$

$6.501155-0.111138-0.055324$

$\begin{array}{llll}7.069841 & -1.393747 & -0.059195\end{array}$

$\begin{array}{lll}6.823405 & -2.113476 & 0.711413\end{array}$

$\begin{array}{llll}7.943995 & -1.772807 & -1.078054\end{array}$

$\begin{array}{lll}8.257240 & -0.901653 & -2.121246\end{array}$

$\begin{array}{llll}8.925763 & -1.209117 & -2.916269\end{array}$

$\begin{array}{llll}7.678821 & 0.365970 & -2.127764\end{array}$

$\begin{array}{llll}6.809989 & 0.762790 & -1.109936\end{array}$

$\begin{array}{llll}6.354075 & 1.746807 & -1.162122\end{array}$

$\begin{array}{lll}5.214424 & -0.760086 & 2.504438\end{array}$

$3.977111-1.183229 \quad 3.001188$

$\begin{array}{llll}3.065387 & -0.816075 & 2.543052\end{array}$

$\begin{array}{llll}3.913910 & -2.066835 & 4.080579\end{array}$

$\begin{array}{llll}5.076056 & -2.545139 & 4.678384\end{array}$

$\begin{array}{llll}5.025628 & -3.242033 & 5.506281\end{array}$

$\begin{array}{llll}6.311426 & -2.115365 & 4.187308\end{array}$

$\begin{array}{llll}6.387775 & -1.226467 & 3.115611\end{array}$

$\begin{array}{lll}7.360496 & -0.912805 & 2.754507\end{array}$

$\begin{array}{llll}3.129974 & 0.372716 & -0.045592\end{array}$

$\begin{array}{lll}7.582300 & -2.566942 & 4.853904\end{array}$

$\begin{array}{llll}2.555990 & -2.523623 & 4.537559\end{array}$

$8.602685-3.125616-1.030636$

$\begin{array}{llll}7.917598 & 1.312131 & -3.272917\end{array}$

$\begin{array}{llll}6.802225 & 1.449729 & -4.036235\end{array}$

$\begin{array}{llll}8.242399 & 2.551923 & -2.839685\end{array}$

$8.907175 \quad 0.897105 \quad-4.090431$

$\begin{array}{llll}7.881116 & -4.021184 & -0.324446\end{array}$

$\begin{array}{llll}9.824727 & -3.057296 & -0.446958\end{array}$

$\begin{array}{llll}8.794300 & -3.636425 & -2.268637\end{array}$

$\begin{array}{lll}8.000262 & -1.672925 & 5.783599\end{array}$ $\begin{array}{llll}8.593512 & -2.702371 & 3.965430\end{array}$

$\begin{array}{llll}7.434347 & -3.750742 & 5.485920\end{array}$

$2.604038 \quad-3.238425 \quad 5.679716$

$\begin{array}{llll}1.728019 & -1.470429 & 4.753677\end{array}$

$\begin{array}{lll}1.957421 & -3.298052 & 3.600629\end{array}$

$\begin{array}{llll}3.916027 & -2.164103 & -1.377549\end{array}$

$\begin{array}{llll}1.943857 & -2.503088 & -0.163569\end{array}$

$\begin{array}{llll}3.952923 & -3.482990 & -1.786472\end{array}$

$\begin{array}{llll}4.679952 & -1.464226 & -1.687599\end{array}$

$\begin{array}{llll}1.956430 & -3.871630 & -0.559175\end{array}$

$2.951268-4.368562 \quad-1.376481$

$\begin{array}{llll}1.149077 & -4.513819 & -0.224586\end{array}$

$\begin{array}{lll}0.818257 & -2.050237 & 0.600998\end{array}$

$\begin{array}{lll}0.769503 & -0.870747 & 1.423930\end{array}$

$\begin{array}{lll}1.524935 & 0.144921 & 1.257943\end{array}$

$2.937799 \quad-1.670499 \quad-0.596298$

$\begin{array}{lll}0.128102 & -2.841846 & 0.848522\end{array}$

$\begin{array}{lll}-0.165907 & -0.795497 & 2.417953\end{array}$

$\begin{array}{lll}-0.366039 & 0.463212 & 3.121824\end{array}$

$\begin{array}{llll}0.199082 & 0.483057 & 4.063156\end{array}$

$\begin{array}{lll}-1.430301 & 0.574875 & 3.355281\end{array}$

$\begin{array}{lll}-0.039713 & 1.300141 & 2.507205\end{array}$

$\begin{array}{lll}0.858201 & -1.939645 & 3.007354\end{array}$

$\begin{array}{lll}-0.357080 & -2.875921 & 2.783446\end{array}$

$\begin{array}{lll}-1.891775 & -2.010931 & 2.664968\end{array}$

$-0.851434-1.821263 \quad 4.095577$

$\begin{array}{lll}-3.669512 & -0.426399 & -1.572724\end{array}$

$\begin{array}{lll}-5.475025 & 0.008226 & -0.269581\end{array}$

$\begin{array}{lll}-5.489934 & 1.093232 & 0.993705\end{array}$

$\begin{array}{llll}-6.746591 & 0.603184 & -1.175207\end{array}$

$\begin{array}{llll}-5.928156 & -1.370717 & 0.566685\end{array}$

$\begin{array}{llll}-5.650721 & 2.453339 & 0.784486\end{array}$

$\begin{array}{lll}-4.535212 & 3.267386 & 1.080373\end{array}$

$\begin{array}{lll}-3.640416 & 2.789343 & 1.463156\end{array}$

$\begin{array}{lll}-4.611306 & 4.623555 & 0.886230\end{array}$

$\begin{array}{llll}-3.764445 & 5.261605 & 1.122247\end{array}$

$\begin{array}{lll}-5.791413 & 5.207758 & 0.350872\end{array}$

$\begin{array}{lll}-5.854630 & 6.599011 & 0.067383\end{array}$

$\begin{array}{llll}-4.985681 & 7.212416 & 0.291818\end{array}$

$\begin{array}{llll}-6.982106 & 7.157216 & -0.491224\end{array}$

$\begin{array}{llll}-7.017719 & 8.220617 & -0.709135\end{array}$

$\begin{array}{llll}-8.093611 & 6.336538 & -0.797554\end{array}$

$\begin{array}{lll}-8.972935 & 6.773889 & -1.261734\end{array}$

$\begin{array}{llll}-8.070548 & 4.986668 & -0.520409\end{array}$

$\begin{array}{llll}-8.927775 & 4.374595 & -0.773654\end{array}$

$\begin{array}{lll}-6.931443 & 4.377538 & 0.077014\end{array}$

$\begin{array}{lll}-6.870927 & 2.967900 & 0.372821\end{array}$

$\begin{array}{lll}-8.051543 & 2.072763 & 0.206946\end{array}$

$\begin{array}{lll}-9.309586 & 2.325043 & 0.860274\end{array}$

$\begin{array}{lll}-9.473027 & 3.339415 & 1.844938\end{array}$

$\begin{array}{lll}-8.620359 & 3.945960 & 2.127115\end{array}$

$\begin{array}{lll}-10.690183 & 3.547955 & 2.456406\end{array}$

$\begin{array}{llll}-10.785691 & 4.322261 & 3.212336\end{array}$

$\begin{array}{lll}-11.815221 & 2.759036 & 2.115982\end{array}$

$\begin{array}{lll}-12.769121 & 2.938203 & 2.603395\end{array}$

$\begin{array}{lll}-11.690049 & 1.757783 & 1.179891\end{array}$

$\begin{array}{llll}-12.540355 & 1.132131 & 0.921018\end{array}$

$\begin{array}{lll}-10.445587 & 1.505896 & 0.540214\end{array}$

$\begin{array}{llll}-10.302374 & 0.440482 & -0.389334\end{array}$

$\begin{array}{llll}-11.170078 & -0.163713 & -0.638161\end{array}$

$\begin{array}{llll}-9.073928 & 0.145275 & -0.926181\end{array}$

$\begin{array}{llll}-8.929784 & -0.690432 & -1.601909\end{array}$

$\begin{array}{llll}-7.959186 & 0.946644 & -0.597435\end{array}$

$\begin{array}{llll}-5.263497 & -2.597831 & 0.040826\end{array}$

$\begin{array}{llll}-4.457936 & -2.869041 & 0.722279\end{array}$

$\begin{array}{lll}-6.205882 & -3.782206 & -0.089218\end{array}$

$\begin{array}{llll}-5.758348 & -5.062057 & 0.259633\end{array}$

$\begin{array}{lll}-4.762804 & -5.192152 & 0.672468\end{array}$

$\begin{array}{lll}-6.597338 & -6.169097 & 0.102152\end{array}$

$\begin{array}{lll}-6.240545 & -7.156155 & 0.384831\end{array}$

$\begin{array}{llll}-7.890876 & -6.007247 & -0.397799\end{array}$

$\begin{array}{llll}-8.548238 & -6.865930 & -0.505237\end{array}$

$\begin{array}{llll}-8.337966 & -4.730518 & -0.756027\end{array}$

$\begin{array}{llll}-9.346274 & -4.593844 & -1.138181\end{array}$

$-7.496652-3.629118-0.612861$ 


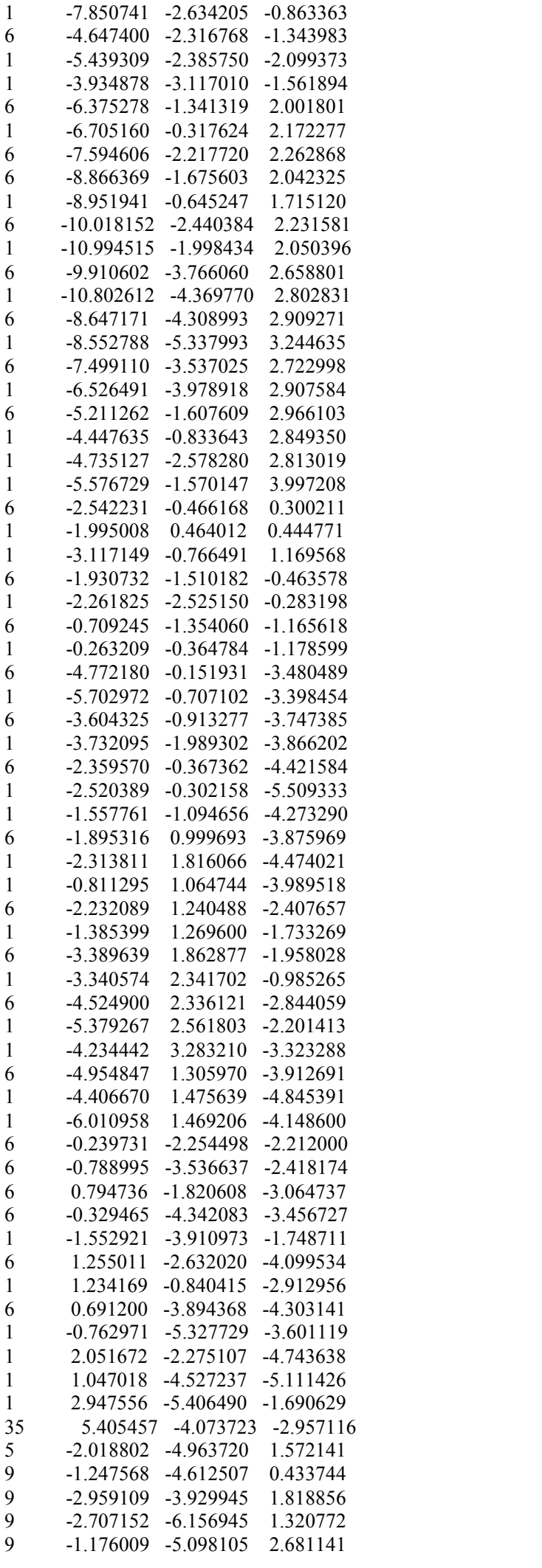

$[8-9]^{\ddagger}$ P1-C1 exo si-si $\left[\mathrm{BF}_{4}^{-}\right]$conf2

Number of imaginary frequencies $=1$

The smallest frequencies are $=-272.5272 \quad 7.8080 \quad 10.7684 \mathrm{~cm}(-$ 1)

Electronic energy $=-7647.2324557$

$\begin{array}{lc}\begin{array}{lc}\text { Zero-point correction= } \\ \text { (Hartree/Particle) }\end{array} & 1.771941 \\ \text { Thermal correction to Energy= } & 1.896556 \\ \text { Thermal correction to Enthalpy= } & 1.897500 \\ \text { Thermal correction to Gibbs Free Energy= } & 1.601958 \\ \text { Sum of electronic and zero-point Energies }= & -7645.460515 \\ \text { Sum of electronic and thermal Energies }= & -7645.335900 \\ \text { Sum of electronic and thermal Enthalpies }= & -7645.334956 \\ \text { Sum of electronic and thermal Free Energies= } & - \\ 7645.630498 & \end{array}$

Cartesian Coordinates

\begin{tabular}{|c|c|c|c|}
\hline 6 & -3.767637 & -1.273569 & 5.33209 \\
\hline & -1.316393 & -2.290113 & \\
\hline & -4.651295 & -0.161150 & 76121 \\
\hline 6 & -3.040347 & -1.897656 & 3.463517 \\
\hline 6 & -3.989283 & -0.820636 & 3.320622 \\
\hline 6 & -3.484076 & 0.310687 & 4.033318 \\
\hline 1 & -3.975255 & 1.270375 & 4.121438 \\
\hline 6 & -2.247977 & -0.062282 & 4.638117 \\
\hline 1 & -1.639119 & 0.561138 & 5.278991 \\
\hline 6 & -1.983357 & -1.421743 & 4.303504 \\
\hline 1 & -1.132595 & -1.998537 & 4.632048 \\
\hline 6 & -5.448347 & -2.203502 & 6.131974 \\
\hline 1 & -6.250890 & -2.634325 & 5.547939 \\
\hline 6 & -5.370583 & -0.849782 & 6.580361 \\
\hline 1 & -6.101371 & -0.074353 & 6.393544 \\
\hline 6 & -4.138033 & -0.688077 & 7.283446 \\
\hline 1 & -3.766787 & 0.233924 & 7.711292 \\
\hline 6 & -3.453106 & -1.941480 & 7.269588 \\
\hline 1 & -2.474117 & -2.13 & 7.687337 \\
\hline 6 & -4.262218 & -2.877925 & 6.559071 \\
\hline 1 & -4.004197 & -3.907963 & 6.353461 \\
\hline 6 & -3.172448 & -3.213 & 2.770841 \\
\hline 6 & -4.128593 & -4.132544 & 3.228888 \\
\hline 1 & -4.698225 & -3.874364 & 4.112530 \\
\hline 6 & -4.371658 & -5.338469 & 2.569607 \\
\hline 1 & -5.130207 & -6.018064 & 2.947126 \\
\hline 6 & -3.642332 & -5.655848 & 1.426948 \\
\hline 1 & -3.823638 & -6.584035 & 0.894027 \\
\hline 6 & -2.683569 & -4.760318 & 0.950828 \\
\hline 1 & -2.135078 & -5.013065 & 0.050412 \\
\hline 6 & -2.440094 & -3.5 & 1.594632 \\
\hline 6 & -5.195808 & -0.870707 & 2.433550 \\
\hline 1 & -5.398616 & -1.921351 & 2.206288 \\
\hline 6 & -6.463206 & -0.2 & 3.033446 \\
\hline 1 & -7.305259 & -0.336684 & 2.339119 \\
\hline 1 & & & 3.294546 \\
\hline 1 & -6.720242 & -0.800676 & 3.947373 \\
\hline 6 & -0.530011 & -3.206031 & -0.585640 \\
\hline 6 & -1.090030 & -3.029652 & -1.860638 \\
\hline 2 & -1.918450 & -2.342008 & -1.983351 \\
\hline 6 & -0.586165 & -3.719763 & -2.965015 \\
\hline 1 & -1.035192 & -3.573604 & -3.943333 \\
\hline 6 & 0.498817 & -4.586299 & -2.809746 \\
\hline 1 & 0.900394 & -5.116909 & -3.668001 \\
\hline 6 & 1.077927 & -4.753243 & -1.548419 \\
\hline 1 & 1.934040 & -5.410111 & -1.423454 \\
\hline 6 & 0.565765 & -4.071117 & -0.443269 \\
\hline 1 & 1.025998 & -4.213603 & 0.525985 \\
\hline $\mathrm{s}^{-}$ & 0.015356 & -2.079921 & 2.060802 \\
\hline 6 & 0.471734 & -0.779886 & 2.318993 \\
\hline 1 & 0.069645 & 0.049090 & 1.746989 \\
\hline 6 & 1.398154 & -0.548645 & 3.337690 \\
\hline 1 & 1.742014 & 0.461654 & 3.532207 \\
\hline 6 & 1.874492 & -1.612642 & 4.106179 \\
\hline 1 & 2.594914 & -1.430075 & 4.898103 \\
\hline 6 & 1.428890 & -2.914336 & 3.849368 \\
\hline 1 & 1.798869 & -3.744818 & 4.443846 \\
\hline 0 & 0.500184 & -3.148664 & 2.833689 \\
\hline 1 & 0.125418 & -4.155007 & 2.671018 \\
\hline 0 & -5.761491 & -1.055605 & -0.404259 \\
\hline & -6.635164 & -0.458176 & -1.320841 \\
\hline & -6.756098 & 0.617149 & 125500 \\
\hline
\end{tabular}




\begin{tabular}{|c|c|c|c|c|c|c|}
\hline-7.347278 & -1.245257 & -2.227903 & 6 & 8.769686 & -2.083701 & 1.273021 \\
\hline-7.186588 & -2.629431 & -2.263943 & 6 & 8.737378 & -3.053293 & 2.314834 \\
\hline-7.728132 & -3.230377 & -2.983912 & 1 & 7.797855 & -3.527760 & 2.570652 \\
\hline-6.286637 & -3.219091 & -1.378151 & 6 & 9.875954 & -3.385832 & 3.017317 \\
\hline-5.583004 & -2.447568 & -0.454168 & 1 & 9.818282 & -4.122910 & 3.813323 \\
\hline-4.870666 & -2.934927 & 0.206453 & 6 & 11.115152 & -2.771915 & 2.719373 \\
\hline-5.187808 & 1.599948 & 0.792962 & 1 & 12.005618 & -3.048711 & 3.276230 \\
\hline-4.152954 & 2.538462 & 0.890451 & 6 & 11.179623 & -1.811671 & 1.734944 \\
\hline-3.125081 & 2.196186 & 0.922714 & 1 & 12.118852 & -1.313920 & 1.507589 \\
\hline-4.433997 & 3.904075 & 0.956047 & 6 & 10.020841 & -1.434339 & 1.004145 \\
\hline-5.748525 & 4.360770 & 0.925127 & 6 & 10.076158 & -0.404581 & 0.026150 \\
\hline-5.967027 & 5.420902 & 0.969324 & 1 & 11.028840 & 0.072471 & -0.186246 \\
\hline-6.782152 & 3.425794 & 0.839327 & 6 & 8.936104 & 0.008199 & -0.612538 \\
\hline-6.513903 & 2.057519 & 0.780568 & 1 & 8.944914 & 0.821135 & -1.330837 \\
\hline-7.342373 & 1.361425 & 0.726034 & 6 & 7.700182 & -0.623109 & -0.336524 \\
\hline-2.428893 & -0.429226 & 0.137511 & 6 & 4.306922 & 2.912600 & -0.587633 \\
\hline-8.215828 & 3.879690 & 0.877663 & 1 & 3.435240 & 3.021306 & 0.068747 \\
\hline-3.276030 & 4.865589 & 1.006316 & 6 & 4.910533 & 4.292965 & -0.800089 \\
\hline-8.332480 & -0.586377 & -3.155866 & 6 & 4.205751 & 5.438509 & -0.415436 \\
\hline-6.016065 & -4.698019 & -1.416232 & 1 & 3.257876 & 5.355654 & 0.101162 \\
\hline-4.698076 & -4.948720 & -1.629047 & 6 & 4.721785 & 6.712875 & -0.666165 \\
\hline-6.335055 & -5.297797 & -0.245172 & 1 & 4.145207 & 7.579715 & -0.357654 \\
\hline-6.704096 & -5.326782 & -2.391171 & 6 & 5.960356 & 6.854657 & -1.293296 \\
\hline-8.490970 & -1.279017 & -4.306160 & 1 & 6.368424 & 7.843553 & -1.484008 \\
\hline-7.957188 & 0.669841 & -3.483521 & 6 & 6.680742 & 5.713663 & -1.667616 \\
\hline-9.559366 & -0.490492 & -2.586280 & 1 & 7.650359 & 5.816282 & -2.148007 \\
\hline-8.740082 & 3.761369 & 2.122233 & 6 & 6.157877 & 4.443318 & -1.426645 \\
\hline-8.996773 & 3.136874 & 0.058788 & 1 & 6.720118 & 3.557325 & -1.710828 \\
\hline-8.355313 & 5.171419 & 0.511436 & 6 & 3.851779 & 2.339573 & -1.943954 \\
\hline-3.654585 & 6.113185 & 1.348206 & 1 & 4.626346 & 2.569944 & -2.684429 \\
\hline-2.338458 & 4.461292 & 1.889727 & 1 & 2.962878 & 2.898622 & -2.245522 \\
\hline-2.662437 & 4.952036 & -0.203456 & 6 & 5.872034 & 2.480143 & 1.353920 \\
\hline-3.461073 & -0.331180 & -2.688011 & 1 & 5.985120 & 3.553239 & 1.182391 \\
\hline-2.224788 & 1.599534 & -2.219563 & 6 & 4.945893 & 2.344518 & 2.563536 \\
\hline-3.960200 & 0.206697 & -3.859608 & 6 & 4.009072 & 3.356899 & 2.813247 \\
\hline-3.737773 & -1.330350 & -2.372752 & 1 & 3.930322 & 4.204964 & 2.142325 \\
\hline-2.773989 & 2.207115 & -3.382812 & 6 & 3.177865 & 3.313405 & 3.935997 \\
\hline-3.633078 & 1.517248 & -4.215161 & 1 & 2.463392 & 4.115321 & 4.087666 \\
\hline-2.483436 & 3.221515 & -3.632327 & 6 & 3.286647 & 2.258216 & 4.842946 \\
\hline-1.173238 & 2.305608 & -1.533400 & 1 & 2.652583 & 2.224504 & 5.724882 \\
\hline-0.825060 & 2.161735 & -0.135294 & 6 & 4.222635 & 1.244899 & 4.609348 \\
\hline-1.240533 & 1.206044 & 0.594032 & 1 & 4.320969 & 0.422267 & 5.312825 \\
\hline-2.615685 & 0.335875 & -1.879334 & 6 & 5.038924 & 1.284176 & 3.478204 \\
\hline-1.008747 & 3.292087 & -1.946257 & 1 & 5.754106 & 0.486564 & 3.314707 \\
\hline-0.000390 & 3.079754 & 0.430565 & 6 & 7.280614 & 1.942443 & 1.594975 \\
\hline 0.303213 & 3.000228 & 1.860150 & 1 & 7.919474 & 2.165082 & 0.737621 \\
\hline-0.460503 & 2.416755 & 2.370900 & 1 & 7.303069 & 0.866729 & 1.769993 \\
\hline 0.334644 & 4.010063 & 2.267349 & 1 & 7.703088 & 2.433995 & 2.476426 \\
\hline 1.278251 & 2.527435 & 2.023417 & 6 & 2.004615 & 0.410441 & -0.490138 \\
\hline 0.515296 & 4.256908 & -0.266159 & 1 & 1.965952 & -0.432216 & 0.192228 \\
\hline 0.895411 & 4.011488 & -1.258106 & 1 & 2.050007 & 1.367515 & 0.022482 \\
\hline 1.335283 & 4.655127 & 0.320842 & 6 & 1.209765 & 0.300861 & -1.680425 \\
\hline-0.245896 & 5.036438 & -0.349182 & 1 & 0.781376 & -0.672014 & -1.888534 \\
\hline 3.528278 & 0.240722 & -2.035894 & 6 & 0.734468 & 1.380014 & -2.476098 \\
\hline 5.149019 & 0.409897 & -0.452873 & 1 & 1.195877 & 2.341088 & -2.314340 \\
\hline 5.136308 & -0.377110 & 1.015317 & 6 & 5.089536 & 0.084242 & -3.656144 \\
\hline 6.619465 & -0.129799 & -1.038522 & 1 & 5.777196 & 0.910992 & -3.495899 \\
\hline 5.273037 & 1.988755 & 0.071408 & 6 & 3.855882 & 0.402036 & -4.265043 \\
\hline 5.147693 & -1.765291 & 1.010452 & 1 & 3.681773 & 1.446289 & -4.522699 \\
\hline 3.946102 & -2.417628 & 1.364386 & 6 & 3.010875 & -0.567597 & -5.068231 \\
\hline 3.098229 & -1.815651 & 1.663969 & 1 & 3.439531 & -0.676731 & -6.076969 \\
\hline 3.885365 & -3.788111 & 1.324890 & 1 & 2.029252 & -0.112474 & -5.202535 \\
\hline 2.978206 & -4.302508 & 1.623548 & 6 & 2.828742 & -1.970192 & -4.440318 \\
\hline 4.991964 & -4.545798 & 0.855711 & 1 & 3.592193 & -2.654039 & -4.826062 \\
\hline 4.896351 & -5.953925 & 0.686321 & 1 & 1.864397 & -2.373466 & -4.766078 \\
\hline 3.965623 & -6.445763 & 0.958014 & 6 & 2.869738 & -2.018555 & -2.920504 \\
\hline 5.948791 & -6.677668 & 0.172613 & 1 & 1.927453 & -2.192353 & -2.415626 \\
\hline 5.862134 & -7.751866 & 0.037588 & 6 & 4.013176 & -2.227138 & -2.190782 \\
\hline 7.143607 & -6.014537 & -0.196503 & 1 & 3.899610 & -2.568040 & -1.167856 \\
\hline 7.963503 & -6.583270 & -0.625722 & 6 & 5.399068 & -2.355227 & -2.783817 \\
\hline 7.275986 & -4.653482 & -0.023246 & 1 & 6.119428 & -2.328234 & -1.968515 \\
\hline 8.194648 & -4.161799 & -0.320811 & 1 & 5.488603 & -3.353444 & -3.237895 \\
\hline 6.215856 & -3.875337 & 0.520057 & 6 & 5.772987 & -1.275072 & -3.826790 \\
\hline 6.311307 & -2.447059 & 0.691299 & 1 & 5.558620 & -1.642284 & -4.836756 \\
\hline 7.595302 & -1.712001 & 0.519330 & 1 & 6.855405 & -1.123346 & -3.782634 \\
\hline
\end{tabular}




$\begin{array}{lccc}6 & 0.207363 & 1.172131 & -3.829986 \\ 6 & -0.363158 & -0.047259 & -4.241888 \\ 6 & 0.270803 & 2.227118 & -4.757587 \\ 6 & -0.837295 & -0.206955 & -5.541330 \\ 1 & -0.439728 & -0.872481 & -3.544669 \\ 6 & -0.194514 & 2.063062 & -6.062470 \\ 1 & 0.694919 & 3.179213 & -4.448365 \\ 6 & -0.749715 & 0.843995 & -6.460573 \\ 1 & -1.278001 & -1.153888 & -5.839878 \\ 1 & -0.126179 & 2.887405 & -6.766703 \\ 1 & -1.115429 & 0.714697 & -7.475164 \\ 1 & -4.022782 & 1.975156 & -5.117533 \\ 35 & -5.091309 & -0.886378 & -5.019036 \\ 5 & 1.307926 & 6.801656 & 1.865493 \\ 9 & -0.049199 & 6.426134 & 1.867199 \\ 9 & 2.088722 & 5.687014 & 2.278570 \\ 9 & 1.703631 & 7.158416 & 0.558730 \\ 9 & 1.517848 & 7.868994 & 2.742817\end{array}$

$[8-9]^{+} \mathbf{P 1}-\mathbf{C 1}$ exo re-si $\left[\mathrm{BF}_{4}{ }^{-}\right]$conf2

Number of imaginary frequencies $=1$

The smallest frequencies are $=-231.113911 .506614 .2666 \mathrm{~cm}(-1)$ Electronic energy $=-7647.2535997$

$\begin{array}{lc}\begin{array}{lc}\text { Zero-point correction= } \\ \text { (Hartree/Particle) }\end{array} & 1.771838 \\ \text { Thermal correction to Energy= } & 1.896472 \\ \text { Thermal correction to Enthalpy= } & 1.603623 \\ \text { Thermal correction to Gibbs Free Energy= } & -7645.481762 \\ \text { Sum of electronic and zero-point Energies }= & -7645.357128 \\ \text { Sum of electronic and thermal Energies }= & -7645.356184 \\ \text { Sum of electronic and thermal Enthalpies }= & -7645.649977 \\ \text { Sum of electronic and thermal Free Energies }= & -7649\end{array}$

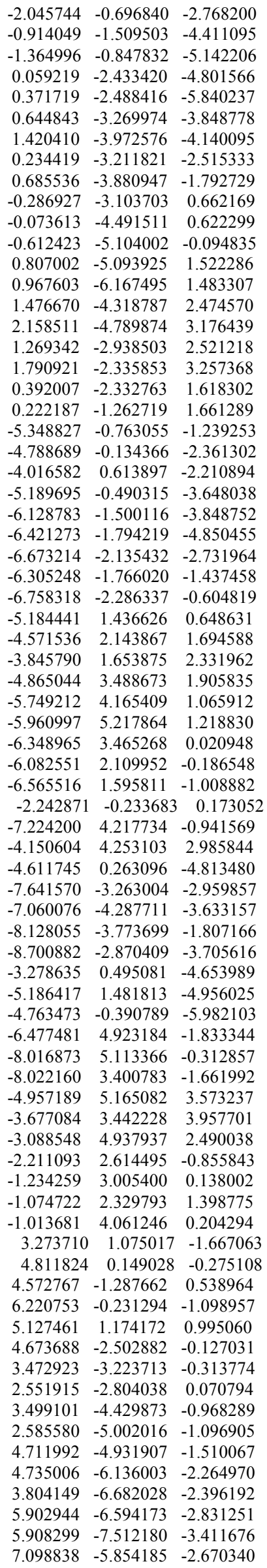


$\begin{array}{llll}8.014156 & -6.202946 & -3.139940\end{array}$

$\begin{array}{llll}7.113377 & -4.691843 & -1.929914\end{array}$

$\begin{array}{llll}8.036795 & -4.134543 & -1.826580\end{array}$

$\begin{array}{llll}5.930571 & -4.200252 & -1.309146\end{array}$

$\begin{array}{llll}5.909766 & -2.987665 & -0.530999\end{array}$

$\begin{array}{llll}7.158209 & -2.248932 & -0.193823\end{array}$

$\begin{array}{llll}8.250401 & -2.868441 & 0.510272\end{array}$

$8.170934-4.184325 \quad 1.047156$

$\begin{array}{llll}7.253087 & -4.748920 & 0.933684\end{array}$

$\begin{array}{llll}9.234555 & -4.744417 & 1.720893\end{array}$

$\begin{array}{lll}9.144370 & -5.748083 & 2.126695\end{array}$

$\begin{array}{llll}10.440054 & -4.023671 & 1.896049\end{array}$

$\begin{array}{lll}11.272466 & -4.480638 & 2.423192\end{array}$

$\begin{array}{lll}10.544528 & -2.739733 & 1.410976\end{array}$

$11.456587 \quad-2.166203 \quad 1.554973$

$\begin{array}{lll}9.460901 & -2.126156 & 0.725319\end{array}$

$\begin{array}{llll}9.549240 & -0.782214 & 0.270585\end{array}$

$10.473682 \quad-0.232724 \quad 0.424575$

$\begin{array}{llll}8.470588 & -0.174560 & -0.322427\end{array}$

$\begin{array}{llll}8.499562 & 0.860867 & -0.644749\end{array}$

$\begin{array}{llll}7.278467 & -0.906946 & -0.520148\end{array}$

$\begin{array}{llll}4.529584 & 2.533121 & 0.826499\end{array}$

$\begin{array}{llll}3.687446 & 2.608646 & 1.517879\end{array}$

$\begin{array}{lll}5.528632 & 3.626632 & 1.173405\end{array}$

$\begin{array}{lll}5.101715 & 4.767199 & 1.863223\end{array}$

$\begin{array}{lll}4.069099 & 4.843599 & 2.181062\end{array}$

$\begin{array}{llll}5.993114 & 5.812261 & 2.120724\end{array}$

$\begin{array}{llll}5.641571 & 6.695335 & 2.647744\end{array}$

$\begin{array}{llll}7.322849 & 5.725237 & 1.702402\end{array}$

$\begin{array}{lll}8.016347 & 6.536326 & 1.907632\end{array}$

$\begin{array}{llll}7.756817 & 4.585425 & 1.017098\end{array}$

$\begin{array}{llll}8.790857 & 4.506293 & 0.691002\end{array}$

$\begin{array}{lll}6.864028 & 3.546889 & 0.750732\end{array}$

$\begin{array}{lll}7.200498 & 2.656001 & 0.226811\end{array}$

$\begin{array}{llll}4.010801 & 2.769723 & -0.604369\end{array}$

$\begin{array}{llll}4.835912 & 3.175704 & -1.200792\end{array}$

$\begin{array}{llll}3.256677 & 3.551540 & -0.542920\end{array}$

$\begin{array}{lll}5.603091 & 0.925615 & 2.390171\end{array}$

$\begin{array}{lll}6.301569 & 1.743220 & 2.591571\end{array}$

$\begin{array}{lll}4.447745 & 1.072041 & 3.371966\end{array}$

$\begin{array}{lll}4.502577 & 2.053553 & 4.366703\end{array}$

$\begin{array}{lll}5.361961 & 2.717328 & 4.414298\end{array}$

$\begin{array}{lll}3.458277 & 2.199125 & 5.283191\end{array}$

$\begin{array}{llll}3.512137 & 2.969131 & 6.047947\end{array}$

$\begin{array}{lll}2.343172 & 1.365485 & 5.204207\end{array}$

$\begin{array}{lll}1.525366 & 1.479728 & 5.907629\end{array}$

$\begin{array}{llll}2.275808 & 0.388377 & 4.205371\end{array}$

$\begin{array}{lll}1.398120 & -0.245639 & 4.132013\end{array}$

$\begin{array}{lll}3.322078 & 0.239530 & 3.295726\end{array}$

$\begin{array}{lll}3.266585 & -0.513463 & 2.515755\end{array}$

$\begin{array}{lll}6.383264 & -0.365373 & 2.654383\end{array}$

$\begin{array}{lll}7.257951 & -0.442859 & 2.009165\end{array}$

$\begin{array}{lll}5.772844 & -1.260178 & 2.540244\end{array}$

$\begin{array}{lll}6.736074 & -0.322513 & 3.689712\end{array}$

$\begin{array}{llll}1.834417 & 0.685066 & -0.034541\end{array}$

$\begin{array}{llll}1.701090 & -0.369837 & 0.185973\end{array}$

$\begin{array}{llll}2.072747 & 1.287778 & 0.838061\end{array}$

$\begin{array}{llll}1.002343 & 1.240410 & -1.051988\end{array}$

$\begin{array}{llll}0.428897 & 0.540403 & -1.646243\end{array}$

$\begin{array}{llll}0.680602 & 2.614117 & -1.200080\end{array}$

$\begin{array}{llll}1.191421 & 3.316796 & -0.556839\end{array}$

$\begin{array}{llll}4.822163 & 1.464370 & -3.209670\end{array}$

$\begin{array}{llll}5.649050 & 1.963304 & -2.710806\end{array}$

$\begin{array}{llll}3.691688 & 2.268427 & -3.508489\end{array}$

$\begin{array}{llll}3.735622 & 3.315664 & -3.209740\end{array}$

$2.740568 \quad 2.008263 \quad-4.659427$

$\begin{array}{llll}3.212567 & 2.321216 & -5.604387\end{array}$

$\begin{array}{llll}1.871882 & 2.654024 & -4.525898\end{array}$

$\begin{array}{llll}2.267254 & 0.542475 & -4.781724\end{array}$

$2.903744 \quad-0.002706 \quad-5.486783$

$\begin{array}{llll}1.259678 & 0.531448 & -5.208553\end{array}$

$\begin{array}{llll}2.228993 & -0.230569 & -3.471473\end{array}$

$\begin{array}{lll}1.245542 & -0.451727 & -3.081275\end{array}$

$\begin{array}{llll}3.270541 & -0.989721 & -2.987416\end{array}$

$3.027570 \quad-1.772190 \quad-2.277300$ $\begin{array}{llll}4.632334 & -1.092937 & -3.638994\end{array}$

$\begin{array}{llll}5.304348 & -1.589727 & -2.939156\end{array}$

$\begin{array}{lll}4.551199 & -1.752673 & -4.516155\end{array}$

$\begin{array}{llll}5.248845 & 0.263209 & -4.058907\end{array}$

$\begin{array}{llll}5.011833 & 0.474255 & -5.107852\end{array}$

$\begin{array}{llll}6.337758 & 0.173209 & -4.005708\end{array}$

$\begin{array}{llll}0.151072 & 3.155399 & -2.460062\end{array}$

$-0.462068 \quad 2.344154 \quad-3.436113$

$\begin{array}{llll}0.277051 & 4.533237 & -2.718084\end{array}$

$\begin{array}{llll}-0.922170 & 2.891481 & -4.630572\end{array}$

$\begin{array}{llll}-0.593019 & 1.285342 & -3.255486\end{array}$

$\begin{array}{llll}-0.177876 & 5.076971 & -3.919707\end{array}$

$\begin{array}{llll}0.745237 & 5.166869 & -1.972551\end{array}$

$\begin{array}{llll}-0.778910 & 4.260119 & -4.882080\end{array}$

$\begin{array}{lll}-1.410679 & 2.250962 & -5.359182\end{array}$

$\begin{array}{llll}-0.062867 & 6.141694 & -4.103828\end{array}$

$\begin{array}{llll}-1.135620 & 4.685001 & -5.816251\end{array}$

$\begin{array}{llll}-2.407868 & 1.405833 & -1.202527\end{array}$

$\begin{array}{lll}-0.611416 & 3.089608 & 2.511161\end{array}$

$\begin{array}{lll}-0.618476 & 2.564276 & 3.785691\end{array}$

$\begin{array}{lll}-0.304298 & 4.112393 & 2.343197\end{array}$

$\begin{array}{lll}-1.059517 & 1.244015 & 3.939042\end{array}$

$\begin{array}{lll}-1.401038 & 0.498050 & 2.831686\end{array}$

$\begin{array}{lll}-1.695626 & -0.538954 & 2.920411\end{array}$

$\begin{array}{lll}-1.408985 & 1.017574 & 1.584514\end{array}$

$\begin{array}{llll}-3.662000 & 3.299424 & -2.709926\end{array}$

$\begin{array}{llll}-3.280197 & 2.393499 & -3.167279\end{array}$

$\begin{array}{llll}-4.730057 & 3.182319 & -2.498168\end{array}$

$\begin{array}{lll}-3.537010 & 4.119715 & -3.422681\end{array}$

$\begin{array}{llll}-2.947985 & 4.987824 & -1.050440\end{array}$

$\begin{array}{lll}-2.890812 & 5.048788 & 0.036119\end{array}$

$\begin{array}{llll}-2.136940 & 5.583685 & -1.485510\end{array}$

$\begin{array}{llll}-3.904695 & 5.416890 & -1.359026\end{array}$

$\begin{array}{lll}-2.903018 & 3.598791 & -1.501676\end{array}$

$\begin{array}{llll}-0.310935 & 3.163679 & 4.634865\end{array}$

$\begin{array}{lll}-1.238045 & 0.443558 & 5.720399\end{array}$

$\begin{array}{llll}1.703422 & 5.560664 & 0.934216\end{array}$

$\begin{array}{llll}2.182463 & 5.650636 & -0.391822\end{array}$

$\begin{array}{lll}0.315303 & 5.822300 & 0.946961\end{array}$

$\begin{array}{llll}2.374551 & 6.474401 & 1.752374\end{array}$

$\begin{array}{llll}1.924949 & 4.244762 & 1.408607\end{array}$

8 P1-C2 endo si-re $\left[\mathrm{BF}_{4}^{-}\right]$

Number of imaginary frequencies $=0$

The smallest frequencies are $=11.913214 .081115 .6683 \mathrm{~cm}(-1)$ Electronic energy $=-7647.2667522$

Zero-point correction=

(Hartree/Particle)

Thermal correction to Energy= $\quad 1.897448$

Thermal correction to Enthalpy=

1.898392

Thermal correction to Gibbs Free Energy $=\quad 1.601068$

Sum of electronic and zero-point Energies $=\quad-7645.495005$

Sum of electronic and thermal Energies

Sum of electronic and thermal Enthalpies

$-7645.369304$

-7645.369304
-7645.368360

Sum of electronic and thermal Free Energies $=\quad-7645.665684$

Cartesian Coordinates

$\begin{array}{llrc}26 & 7.920044 & -0.029804 & -1.234545 \\ 15 & 2.921525 & 1.387491 & -2.050180 \\ 15 & 4.092551 & 0.790777 & 1.466934 \\ 6 & 6.003907 & 0.814471 & -1.344478 \\ 6 & 6.211234 & 0.145267 & -0.082932 \\ 6 & 6.487956 & -1.229212 & -0.359243 \\ 1 & 6.693932 & -1.992945 & 0.377967 \\ 6 & 6.493104 & -1.409439 & -1.773879 \\ 1 & 6.705010 & -2.332298 & -2.295008 \\ 6 & 6.193475 & -0.154352 & -2.381307 \\ 1 & 6.141289 & 0.043947 & -3.441479 \\ 6 & 9.485035 & 1.056902 & -0.405838 \\ 1 & 9.376476 & 1.785706 & 0.386540 \\ 6 & 9.704480 & -0.343997 & -0.230866 \\ 1 & 9.783711 & -0.862359 & 0.715467 \\ 6 & 9.754222 & -0.948190 & -1.524192\end{array}$


$\begin{array}{llll}9.872479 & -2.004331 & -1.727896\end{array}$

$\begin{array}{llll}9.564680 & 0.078341 & -2.498959\end{array}$

$\begin{array}{llll}9.517634 & -0.062668 & -3.570756\end{array}$

$\begin{array}{llll}9.399962 & 1.316629 & -1.809144\end{array}$

$\begin{array}{llll}9.209947 & 2.275905 & -2.271101\end{array}$

$\begin{array}{llll}5.600697 & 2.242246 & -1.480478\end{array}$

$\begin{array}{llll}6.527114 & 3.243534 & -1.147571\end{array}$

$\begin{array}{llll}7.515628 & 2.936690 & -0.830753\end{array}$

$\begin{array}{llll}6.203827 & 4.598304 & -1.202153\end{array}$

$\begin{array}{llll}6.938608 & 5.343813 & -0.914606\end{array}$

$\begin{array}{llll}4.931385 & 4.981029 & -1.619308\end{array}$

$\begin{array}{llll}4.659028 & 6.030176 & -1.670186\end{array}$

$3.990930 \quad 4.004443 \quad-1.945485$

$\begin{array}{llll}3.000263 & 4.322269 & -2.246483\end{array}$

$\begin{array}{llll}4.289271 & 2.633154 & -1.862248\end{array}$

$\begin{array}{lll}5.981063 & 0.780303 & 1.261189\end{array}$

$\begin{array}{llll}6.276721 & 1.828561 & 1.189867\end{array}$

$\begin{array}{lll}6.761987 & 0.136759 & 2.409532\end{array}$

$\begin{array}{llll}6.566405 & 0.654217 & 3.353126\end{array}$

$\begin{array}{lll}6.501998 & -0.916333 & 2.545871\end{array}$

$\begin{array}{lll}7.834119 & 0.194830 & 2.195690\end{array}$

$\begin{array}{llll}1.456865 & 2.463879 & -2.363784\end{array}$

$\begin{array}{llll}0.740372 & 2.920712 & -1.246377\end{array}$

$\begin{array}{llll}1.011617 & 2.568425 & -0.257405\end{array}$

$\begin{array}{llll}-0.314351 & 3.819059 & -1.405212\end{array}$

$\begin{array}{llll}-0.846072 & 4.178854 & -0.534843\end{array}$

$\begin{array}{llll}-0.696364 & 4.237260 & -2.682319\end{array}$

$\begin{array}{llll}-1.529384 & 4.924617 & -2.801069\end{array}$

$\begin{array}{lll}-0.011784 & 3.758643 & -3.801855\end{array}$

$\begin{array}{llll}-0.308756 & 4.071129 & -4.799116\end{array}$

$\begin{array}{llll}1.067283 & 2.885095 & -3.644244\end{array}$

$\begin{array}{llll}1.605582 & 2.539563 & -4.520674\end{array}$

$\begin{array}{llll}3.177018 & 0.597275 & -3.688773\end{array}$

$\begin{array}{llll}3.992544 & 1.136514 & -4.693941\end{array}$

$\begin{array}{llll}4.539094 & 2.057557 & -4.514172\end{array}$

$\begin{array}{llll}4.111799 & 0.485999 & -5.924432\end{array}$

$\begin{array}{llll}4.751063 & 0.906137 & -6.695862\end{array}$

$3.410845-0.699605-6.164426$

$\begin{array}{llll}3.507902 & -1.204558 & -7.121232\end{array}$

$\begin{array}{llll}2.586762 & -1.235311 & -5.170707\end{array}$

$2.042754 \quad-2.158399 \quad-5.349983$

$\begin{array}{llll}2.473702 & -0.591212 & -3.937818\end{array}$

$\begin{array}{llll}1.852175 & -1.015554 & -3.154897\end{array}$

$\begin{array}{lll}3.596820 & 2.510713 & 1.899225\end{array}$

$\begin{array}{lll}2.221835 & 2.707899 & 2.102317\end{array}$

$\begin{array}{lll}1.556544 & 1.849471 & 2.062512\end{array}$

$\begin{array}{lll}1.709765 & 3.992833 & 2.281570\end{array}$

$\begin{array}{lll}2.543830 & 5.108437 & 2.239499\end{array}$

$\begin{array}{llll}2.140043 & 6.107710 & 2.357684\end{array}$

$\begin{array}{lll}3.908523 & 4.911270 & 2.020925\end{array}$

$\begin{array}{lll}4.438218 & 3.630005 & 1.862526\end{array}$

$\begin{array}{llll}5.500833 & 3.527181 & 1.687377\end{array}$

$\begin{array}{lll}3.728797 & -0.181923 & 2.984586\end{array}$

$\begin{array}{lll}3.807298 & -1.576776 & 2.847552\end{array}$

$\begin{array}{lll}4.114751 & -2.008996 & 1.902292\end{array}$

$3.447943 \quad-2.409612 \quad 3.902243$

$\begin{array}{llll}2.961080 & -1.877148 & 5.097201\end{array}$

$2.646344 \quad-2.528687 \quad 5.904525$

$\begin{array}{lll}2.864515 & -0.493734 & 5.224518\end{array}$

$\begin{array}{llll}3.261023 & 0.354806 & 4.186615\end{array}$

$\begin{array}{llll}3.177297 & 1.427030 & 4.321150\end{array}$

$\begin{array}{llll}2.711715 & 0.015328 & -0.274347\end{array}$

$\begin{array}{llll}2.221244 & 0.101152 & 6.443864\end{array}$

$\begin{array}{lll}3.466696 & -3.903488 & 3.731527\end{array}$

$\begin{array}{lll}0.233964 & 4.143930 & 2.524483\end{array}$

$\begin{array}{lll}4.793185 & 6.123744 & 1.924219\end{array}$

$\begin{array}{llll}4.441073 & 6.911930 & 0.877603\end{array}$

$\begin{array}{llll}6.097159 & 5.809262 & 1.762950\end{array}$

$\begin{array}{lll}4.701080 & 6.898432 & 3.031334\end{array}$

$\begin{array}{lll}-0.494356 & 3.360466 & 1.677683\end{array}$

$\begin{array}{lll}-0.119218 & 3.770395 & 3.775308\end{array}$

$\begin{array}{lll}-0.198343 & 5.410785 & 2.344268\end{array}$

$\begin{array}{llll}2.745188 & 1.307550 & 6.760177\end{array}$

$\begin{array}{llll}0.888697 & 0.300743 & 6.248424\end{array}$

$\begin{array}{lll}2.338349 & -0.691000 & 7.530726\end{array}$ $\begin{array}{llll}4.020548 & -4.518755 & 4.804589\end{array}$

$\begin{array}{llll}4.164571 & -4.288651 & 2.641059\end{array}$

$2.216466 \quad-4.408831 \quad 3.602948$

$\begin{array}{lll}0.717095 & -1.019649 & 1.723135\end{array}$

$\begin{array}{lll}1.004665 & -2.359676 & 1.361311\end{array}$

$\begin{array}{lll}1.860244 & -2.839681 & 0.348957\end{array}$

$\begin{array}{lll}0.496826 & -3.140061 & 1.906378\end{array}$

$\begin{array}{llll}-3.060097 & -0.826585 & -1.717200\end{array}$

$\begin{array}{lll}-4.917394 & -0.179210 & -0.540863\end{array}$

$\begin{array}{lll}-4.918732 & 0.971937 & 0.641579\end{array}$

$\begin{array}{llll}-6.041055 & 0.463615 & -1.582553\end{array}$

$\begin{array}{lll}-5.504892 & -1.468152 & 0.332332\end{array}$

$\begin{array}{lll}-4.746192 & 2.317694 & 0.343065\end{array}$

$\begin{array}{lll}-3.557964 & 2.917670 & 0.815273\end{array}$

$\begin{array}{lll}-2.862561 & 2.315535 & 1.389149\end{array}$

$\begin{array}{lll}-3.326128 & 4.245464 & 0.554647\end{array}$

$\begin{array}{llll}-2.442815 & 4.735727 & 0.949168\end{array}$

$\begin{array}{llll}-4.223930 & 4.986356 & -0.260184\end{array}$

$\begin{array}{llll}-3.926452 & 6.320649 & -0.649640\end{array}$

$\begin{array}{llll}-3.008555 & 6.773976 & -0.284293\end{array}$

$\begin{array}{llll}-4.770176 & 7.018136 & -1.484764\end{array}$

$\begin{array}{llll}-4.528963 & 8.033901 & -1.783985\end{array}$

$\begin{array}{llll}-5.950965 & 6.403024 & -1.965419\end{array}$

$\begin{array}{llll}-6.603508 & 6.947541 & -2.641859\end{array}$

$\begin{array}{llll}-6.279340 & 5.118624 & -1.58827\end{array}$

$\begin{array}{llll}-7.184086 & 4.660437 & -1.970491\end{array}$

$\begin{array}{llll}-5.438054 & 4.372303 & -0.716999\end{array}$

$\begin{array}{llll}-5.742941 & 3.022541 & -0.314882\end{array}$

$\begin{array}{llll}-7.071192 & 2.397924 & -0.573539\end{array}$

$\begin{array}{llll}-8.294111 & 3.020974 & -0.127194\end{array}$

$\begin{array}{llll}-8.311211 & 4.192261 & 0.681713\end{array}$

$\begin{array}{llll}-7.374753 & 4.637666 & 0.994182\end{array}$

$\begin{array}{llll}-9.498646 & 4.760207 & 1.090249\end{array}$

$\begin{array}{lll}-9.480496 & 5.649452 & 1.713891\end{array}$

$\begin{array}{lll}-10.739324 & 4.193841 & 0.713851\end{array}$

$\begin{array}{lll}-11.666952 & 4.657138 & 1.036883\end{array}$

$\begin{array}{llll}-10.761846 & 3.045455 & -0.044392\end{array}$

$\begin{array}{llll}-11.705770 & 2.584205 & -0.322736\end{array}$

$\begin{array}{llll}-9.555324 & 2.424720 & -0.467835\end{array}$

$\begin{array}{llll}-9.580796 & 1.208089 & -1.201550\end{array}$

$\begin{array}{llll}-10.539144 & 0.769817 & -1.465082\end{array}$

$\begin{array}{llll}-8.411768 & 0.576119 & -1.541126\end{array}$

$\begin{array}{lll}-8.404217 & -0.372201 & -2.067906\end{array}$

$\begin{array}{llll}-7.174788 & 1.164773 & -1.199065\end{array}$

$\begin{array}{lll}-4.789823 & -2.743491 & 0.041806\end{array}$

$\begin{array}{llll}-4.033121 & -2.892055 & 0.813719\end{array}$

$\begin{array}{llll}-5.725393 & -3.940238 & 0.052118\end{array}$

$\begin{array}{llll}-5.292555 & -5.146004 & 0.618634\end{array}$

$\begin{array}{lll}-4.317106 & -5.201411 & 1.090896\end{array}$

$\begin{array}{lll}-6.113485 & -6.276337 & 0.576657\end{array}$

$\begin{array}{lll}-5.765868 & -7.204605 & 1.022055\end{array}$

$\begin{array}{llll}-7.373046 & -6.211782 & -0.023414\end{array}$

$\begin{array}{lll}-8.012672 & -7.089805 & -0.048855\end{array}$

$\begin{array}{llll}-7.810389 & -5.008271 & -0.586941\end{array}$

$\begin{array}{llll}-8.791452 & -4.949054 & -1.051005\end{array}$

$-6.989465-3.880766-0.552795$

$\begin{array}{llll}-7.329710 & -2.941555 & -0.981357\end{array}$

$\begin{array}{lll}-4.106704 & -2.653863 & -1.324858\end{array}$

$\begin{array}{lll}-4.868309 & -2.775611 & -2.102943\end{array}$

$\begin{array}{llll}-3.405636 & -3.483130 & -1.428198\end{array}$

$\begin{array}{lll}-6.247024 & -1.436383 & 1.639753\end{array}$

$\begin{array}{lll}-6.620625 & -2.459159 & 1.730629\end{array}$

$\begin{array}{lll}-5.328742 & -1.212128 & 2.840313\end{array}$

$\begin{array}{lll}-4.644604 & -2.310408 & 3.382654\end{array}$

$\begin{array}{lll}-4.765095 & -3.293422 & 2.938688\end{array}$

$\begin{array}{lll}-3.807967 & -2.164989 & 4.490885\end{array}$

$\begin{array}{lll}-3.268887 & -3.029882 & 4.862136\end{array}$

$\begin{array}{lll}-3.655594 & -0.912420 & 5.087888\end{array}$

$\begin{array}{lll}-3.007630 & -0.793214 & 5.951894\end{array}$

$\begin{array}{lll}-4.338820 & 0.189389 & 4.564374\end{array}$

$\begin{array}{llll}-4.229480 & 1.167470 & 5.025727\end{array}$

$\begin{array}{llll}-5.165715 & 0.041028 & 3.448974\end{array}$

$\begin{array}{lll}-5.686377 & 0.907071 & 3.057291\end{array}$

$\begin{array}{lll}-7.484058 & -0.541779 & 1.612675\end{array}$

$\begin{array}{lll}-8.143238 & -0.838358 & 0.794091\end{array}$ 
$\begin{array}{lll}-7.248498 & 0.515791 & 1.495522\end{array}$

$\begin{array}{lll}-8.027732 & -0.664553 & 2.554200\end{array}$

$\begin{array}{lll}-1.893600 & -0.410936 & 0.108749\end{array}$

$\begin{array}{llll}-1.223189 & 0.412520 & -0.113582\end{array}$

$\begin{array}{lll}-2.424815 & -0.308120 & 1.049941\end{array}$

$\begin{array}{lll}-1.533064 & -1.735316 & -0.261993\end{array}$

$\begin{array}{lll}-1.921078 & -2.541527 & 0.345048\end{array}$

$\begin{array}{lll}-0.871939 & -2.040761 & -1.462661\end{array}$

$\begin{array}{llll}-0.235068 & -1.289955 & -1.914442\end{array}$

$\begin{array}{lll}-4.231189 & -0.682505 & -3.650526\end{array}$

$\begin{array}{llll}-5.179295 & -1.176068 & -3.460536\end{array}$

$\begin{array}{llll}-3.119099 & -1.514821 & -3.873792\end{array}$

$\begin{array}{llll}-3.282161 & -2.590588 & -3.845412\end{array}$

$\begin{array}{llll}-1.875843 & -1.096173 & -4.634177\end{array}$

$\begin{array}{llll}-2.095193 & -1.118151 & -5.711985\end{array}$

$\begin{array}{llll}-1.115781 & -1.859112 & -4.462398\end{array}$

$\begin{array}{llll}-1.309962 & 0.284433 & -4.238286\end{array}$

$\begin{array}{llll}-1.701977 & 1.064714 & -4.898010\end{array}$

$\begin{array}{llll}-0.227057 & 0.279525 & -4.391727\end{array}$

$\begin{array}{llll}-1.582218 & 0.688380 & -2.795300\end{array}$

$\begin{array}{llll}-0.713834 & 0.728749 & -2.147337\end{array}$

$\begin{array}{llll}-2.686725 & 1.419215 & -2.391429\end{array}$

$\begin{array}{llll}-2.598238 & 1.977913 & -1.465618\end{array}$

$\begin{array}{llll}-3.812441 & 1.851465 & -3.305096\end{array}$

$\begin{array}{llll}-4.623728 & 2.231456 & -2.684782\end{array}$

$\begin{array}{llll}-3.460186 & 2.697326 & -3.912882\end{array}$

$\begin{array}{llll}-4.355235 & 0.731092 & -4.220757\end{array}$

$\begin{array}{llll}-3.850622 & 0.756824 & -5.191812\end{array}$

$\begin{array}{lll}-5.412707 & 0.926862 & -4.418061\end{array}$

$\begin{array}{llll}-0.624626 & -3.419662 & -1.906851\end{array}$

$\begin{array}{lll}-1.112636 & -4.548856 & -1.214400\end{array}$

$\begin{array}{llll}0.177875 & -3.636415 & -3.042693\end{array}$

$\begin{array}{lll}-0.823061 & -5.835347 & -1.662608\end{array}$

$\begin{array}{llll}-1.677849 & -4.432897 & -0.298435\end{array}$

$\begin{array}{llll}0.463643 & -4.924342 & -3.491730\end{array}$

$\begin{array}{llll}0.600317 & -2.782739 & -3.560704\end{array}$

$\begin{array}{llll}-0.041150 & -6.031956 & -2.805413\end{array}$

$\begin{array}{llll}-1.196771 & -6.686358 & -1.100348\end{array}$

$\begin{array}{llll}1.092410 & -5.062890 & -4.366475\end{array}$

$\begin{array}{llll}0.185371 & -7.037730 & -3.147727\end{array}$

$\begin{array}{llll}1.958093 & -4.264737 & 0.178631\end{array}$

$\begin{array}{llll}2.768490 & -4.822192 & -0.777504\end{array}$

$\begin{array}{lll}1.352140 & -4.897001 & 0.816953\end{array}$

$\begin{array}{llll}3.507449 & -3.948849 & -1.597590\end{array}$

$\begin{array}{llll}3.408919 & -2.589441 & -1.416435\end{array}$

$\begin{array}{llll}3.968188 & -1.898725 & -2.032916\end{array}$

$2.619994 \quad-2.031750 \quad-0.470189$

$\begin{array}{lll}1.058059 & 0.025207 & 1.055005\end{array}$

$\begin{array}{lll}-0.009324 & -0.811051 & 2.879389\end{array}$

$\begin{array}{lll}-0.594436 & 0.487303 & 3.169204\end{array}$

$\begin{array}{lll}-1.647470 & 0.369411 & 3.449829\end{array}$

$\begin{array}{lll}-0.078230 & 0.988560 & 3.998339\end{array}$

$\begin{array}{lll}-0.537309 & 1.118235 & 2.287938\end{array}$

$\begin{array}{lll}-0.364343 & -1.888077 & 3.796298\end{array}$

$\begin{array}{llll}-1.251222 & -2.436247 & 3.467781\end{array}$

$\begin{array}{lll}0.457821 & -2.596174 & 3.903521\end{array}$

$\begin{array}{llll}-0.561273 & -1.453900 & 4.779853\end{array}$

$\begin{array}{llll}2.822292 & -5.897801 & -0.905184\end{array}$

$\begin{array}{llll}4.663727 & -4.660584 & -3.021564\end{array}$

$\begin{array}{llll}-1.843833 & -5.078996 & 2.576569\end{array}$

$\begin{array}{lll}-0.499595 & -5.261391 & 2.210208\end{array}$

$\begin{array}{llll}-2.592213 & -6.219716 & 2.252439\end{array}$

$\begin{array}{llll}-2.372108 & -3.970446 & 1.848145\end{array}$

$\begin{array}{lll}-1.939345 & -4.800528 & 3.949042\end{array}$

\section{$[8-9]^{\ddagger} \mathrm{P1}-\mathrm{C} 2$ endo si-re $\left[\mathrm{BF}_{4}^{-}\right]$}

Number of imaginary frequencies $=1$

The smallest frequencies are=-200.2306 $12.633215 .8549 \mathrm{~cm}(-1)$

Electronic energy $=-7647.2593186$

Zero-point correction=

.772212

(Hartree/Particle)

Thermal correction to Energy=

Thermal correction to Enthalpy=

1.896726

1.897670
Thermal correction to Gibbs Free Energy=

Sum of electronic and zero-point Energies= Sum of electronic and thermal Energies=

Sum of electronic and thermal Enthalpies=

Sum of electronic and thermal Free Energies=

1.603398

$-7645.487107$

$-7645.362593$

$-7645.361649$

$-7645.655921$

Cartesian Coordinates

$\begin{array}{llll}26 & 8.216916 & 0.349719 & -0.767204\end{array}$

$\begin{array}{lllll}15 & 3.256633 & 1.430368 & -2.098114\end{array}$

$\begin{array}{lllll}15 & 4.055078 & 0.709317 & 1.520691\end{array}$

$\begin{array}{lll}6.259398 & 1.041268 & -1.052938\end{array}$

$\begin{array}{lll}6.369270 & 0.261700 & 0.158069\end{array}$

$\begin{array}{lll}6.810321 & -1.048145 & -0.204870\end{array}$

$\begin{array}{lll}7.000315 & -1.863232 & 0.480028\end{array}$

$\begin{array}{llll}7.007105 & -1.079515 & -1.618008\end{array}$

$\begin{array}{llll}7.371059 & -1.921979 & -2.189003\end{array}$

$\begin{array}{llll}6.667018 & 0.202654 & -2.139922\end{array}$

$\begin{array}{llll}6.728315 & 0.504679 & -3.174715\end{array}$

$\begin{array}{llll}9.544373 & 1.495567 & 0.343908\end{array}$

$\begin{array}{lll}9.258981 & 2.143241 & 1.162493\end{array}$

$\begin{array}{llll}9.876014 & 0.109900 & 0.448943\end{array}$

$\begin{array}{llll}9.879514 & -0.476834 & 1.357839\end{array}$

$10.153388-0.378971 \quad-0.864238$

$\begin{array}{llll}10.399705 & -1.400467 & -1.122566\end{array}$

$\begin{array}{llll}9.993595 & 0.704017 & -1.781501\end{array}$

$\begin{array}{lll}10.099816 & 0.648180 & -2.856867\end{array}$

$\begin{array}{llll}9.618874 & 1.861644 & -1.036276\end{array}$

$\begin{array}{llll}9.396297 & 2.834887 & -1.451927\end{array}$

$\begin{array}{llll}5.750454 & 2.441688 & -1.119746\end{array}$

$\begin{array}{llll}6.535244 & 3.478876 & -0.591975\end{array}$

$\begin{array}{llll}7.502567 & 3.226053 & -0.177375\end{array}$

$\begin{array}{llll}6.098474 & 4.803082 & -0.578600\end{array}$

$\begin{array}{llll}6.719771 & 5.575285 & -0.136051\end{array}$

$\begin{array}{lll}4.856379 & 5.119621 & -1.123010\end{array}$

$\begin{array}{llll}4.499220 & 6.144444 & -1.123819\end{array}$

$\begin{array}{llll}4.054963 & 4.105680 & -1.647196\end{array}$

$\begin{array}{llll}3.083318 & 4.367730 & -2.048952\end{array}$

$\begin{array}{llll}4.466197 & 2.762256 & -1.637404\end{array}$

$\begin{array}{llll}5.952595 & 0.740620 & 1.522619\end{array}$

$\begin{array}{llll}6.223921 & 1.795225 & 1.595204\end{array}$

$\begin{array}{lll}6.615371 & -0.006990 & 2.682602\end{array}$

$\begin{array}{lll}6.303263 & 0.407011 & 3.645472\end{array}$

$\begin{array}{lll}6.369868 & -1.072351 & 2.680287\end{array}$

$\begin{array}{llll}7.702674 & 0.087978 & 2.598948\end{array}$

$\begin{array}{llll}1.746627 & 2.383295 & -2.558322\end{array}$

$\begin{array}{lll}0.862304 & 2.732329 & -1.523991\end{array}$

$\begin{array}{llll}1.041025 & 2.364011 & -0.520057\end{array}$

$-0.245764 \quad 3.538035-1.786261$

$\begin{array}{llll}-0.917676 & 3.807952 & -0.981129\end{array}$

$\begin{array}{llll}-0.506475 & 3.972653 & -3.088089\end{array}$

$\begin{array}{llll}-1.378177 & 4.589307 & -3.288863\end{array}$

$\begin{array}{llll}0.347927 & 3.599652 & -4.128286\end{array}$

$\begin{array}{llll}0.145836 & 3.924959 & -5.144950\end{array}$

$\begin{array}{llll}1.474990 & 2.816810 & -3.865066\end{array}$

$\begin{array}{llll}2.142032 & 2.552996 & -4.679070\end{array}$

$\begin{array}{llll}3.843545 & 0.760866 & -3.702034\end{array}$

$\begin{array}{llll}4.626186 & 1.501377 & -4.600736\end{array}$

$\begin{array}{llll}4.921989 & 2.516875 & -4.353665\end{array}$

$\begin{array}{llll}5.036923 & 0.930195 & -5.806697\end{array}$

$\begin{array}{llll}5.646907 & 1.506956 & -6.496263\end{array}$

$\begin{array}{llll}4.666433 & -0.380254 & -6.126047\end{array}$

$\begin{array}{llll}4.992099 & -0.823465 & -7.062790\end{array}$

$\begin{array}{llll}3.878150 & -1.118571 & -5.239573\end{array}$

$\begin{array}{llll}3.588609 & -2.136983 & -5.482582\end{array}$

$\begin{array}{llll}3.470081 & -0.550099 & -4.031722\end{array}$

$\begin{array}{llll}2.874487 & -1.127112 & -3.331450\end{array}$

$\begin{array}{lll}3.495801 & 2.404324 & 1.975188\end{array}$

$\begin{array}{lll}2.107117 & 2.599682 & 1.963785\end{array}$

$\begin{array}{lll}1.460194 & 1.758110 & 1.738551\end{array}$

$\begin{array}{lll}1.566397 & 3.862930 & 2.201226\end{array}$

$\begin{array}{llll}2.392273 & 4.966000 & 2.408905\end{array}$

$\begin{array}{lll}1.970598 & 5.951986 & 2.569262\end{array}$

$\begin{array}{lll}3.774692 & 4.774545 & 2.392035\end{array}$

$\begin{array}{llll}4.327547 & 3.508063 & 2.199349\end{array}$ 
$\begin{array}{llll}5.404418 & 3.409976 & 2.207640\end{array}$

$\begin{array}{llll}3.514513 & -0.336397 & 2.937156\end{array}$

$\begin{array}{llll}3.635456 & -1.723741 & 2.755111\end{array}$

$\begin{array}{lll}4.095071 & -2.113772 & 1.854510\end{array}$

$\begin{array}{llll}3.131734 & -2.607378 & 3.704990\end{array}$

$\begin{array}{llll}2.469530 & -2.133376 & 4.838764\end{array}$

$\begin{array}{llll}2.053023 & -2.824314 & 5.563123\end{array}$

$\begin{array}{lll}2.338182 & -0.757747 & 5.012816\end{array}$

$\begin{array}{llll}2.865746 & 0.140628 & 4.080711\end{array}$

$\begin{array}{llll}2.748237 & 1.204563 & 4.248817\end{array}$

$\begin{array}{llll}2.944271 & -0.021386 & -0.405327\end{array}$

$\begin{array}{llll}1.535546 & -0.239632 & 6.173736\end{array}$

$\begin{array}{lll}3.199727 & -4.092291 & 3.470077\end{array}$

$\begin{array}{llll}0.070814 & 3.996841 & 2.267441\end{array}$

$\begin{array}{lll}4.663632 & 5.977235 & 2.552904\end{array}$

$\begin{array}{llll}4.496102 & 6.856935 & 1.534033\end{array}$

$\begin{array}{llll}5.975504 & 5.653598 & 2.584308\end{array}$

$\begin{array}{lll}4.388254 & 6.655795 & 3.691238\end{array}$

$\begin{array}{lll}-0.541623 & 3.279226 & 1.283023\end{array}$

$\begin{array}{lll}-0.426384 & 3.525469 & 3.434874\end{array}$

$\begin{array}{lll}-0.348004 & 5.273087 & 2.137522\end{array}$

$\begin{array}{llll}1.888609 & 1.017621 & 6.520437\end{array}$

$\begin{array}{lll}0.206135 & -0.199815 & 5.878343\end{array}$

$\begin{array}{llll}1.661569 & -1.017169 & 7.269885\end{array}$

$\begin{array}{lll}3.529714 & -4.760643 & 4.599276\end{array}$

$\begin{array}{lll}4.108100 & -4.420214 & 2.524823\end{array}$

$\begin{array}{llll}2.005718 & -4.588563 & 3.062323\end{array}$

$\begin{array}{lll}0.661408 & -1.101223 & 1.211266\end{array}$

$\begin{array}{llll}0.902014 & -2.432625 & 0.715784\end{array}$

$\begin{array}{llll}2.034101 & -2.906010 & -0.004512\end{array}$

$\begin{array}{lll}0.319858 & -3.226435 & 1.158053\end{array}$

$\begin{array}{lll}-3.359084 & -0.846837 & -1.773469\end{array}$

$\begin{array}{lll}-5.166432 & -0.134518 & -0.584035\end{array}$

$\begin{array}{llll}-5.122364 & 1.026389 & 0.600537\end{array}$

$\begin{array}{llll}-6.310801 & 0.542448 & -1.595150\end{array}$

$\begin{array}{lll}-5.791248 & -1.406023 & 0.307420\end{array}$

$\begin{array}{lll}-4.916255 & 2.357202 & 0.265349\end{array}$

$\begin{array}{lll}-3.678049 & 2.924052 & 0.641615\end{array}$

$\begin{array}{lll}-2.964949 & 2.307294 & 1.177325\end{array}$

$\begin{array}{llll}-3.410266 & 4.232508 & 0.321993\end{array}$

$\begin{array}{lll}-2.476088 & 4.691676 & 0.627316\end{array}$

$\begin{array}{llll}-4.338995 & 4.992179 & -0.439751\end{array}$

$\begin{array}{llll}-4.025152 & 6.308961 & -0.872972\end{array}$

$\begin{array}{llll}-3.066130 & 6.732824 & -0.586156\end{array}$

$\begin{array}{llll}-4.905784 & 7.026805 & -1.650506\end{array}$

$\begin{array}{llll}-4.652979 & 8.029640 & -1.982196\end{array}$

$\begin{array}{llll}-6.140696 & 6.448700 & -2.030677\end{array}$

$\begin{array}{llll}-6.824357 & 7.008564 & -2.662460\end{array}$

$\begin{array}{llll}-6.482938 & 5.180744 & -1.612531\end{array}$

$\begin{array}{llll}-7.429347 & 4.750665 & -1.919208\end{array}$

$\begin{array}{llll}-5.604745 & 4.415382 & -0.795575\end{array}$

$\begin{array}{llll}-5.923860 & 3.082789 & -0.352649\end{array}$

$\begin{array}{llll}-7.274456 & 2.479361 & -0.536783\end{array}$

$\begin{array}{llll}-8.463399 & 3.114413 & -0.025172\end{array}$

$\begin{array}{llll}-8.424273 & 4.293376 & 0.771677\end{array}$

$\begin{array}{lll}-7.467335 & 4.736464 & 1.019182\end{array}$

$\begin{array}{llll}-9.581128 & 4.870977 & 1.248625\end{array}$

$\begin{array}{lll}-9.520481 & 5.766186 & 1.861014\end{array}$

$\begin{array}{lll}-10.845447 & 4.306659 & 0.956548\end{array}$

$\begin{array}{lll}-11.748952 & 4.776662 & 1.333837\end{array}$

$\begin{array}{lll}-10.920770 & 3.152768 & 0.210038\end{array}$

$\begin{array}{lll}-11.882610 & 2.694451 & -0.005097\end{array}$

$\begin{array}{llll}-9.746239 & 2.522555 & -0.283809\end{array}$

$\begin{array}{llll}-9.821969 & 1.301118 & -1.006298\end{array}$

$\begin{array}{llll}-10.796975 & 0.865941 & -1.206869\end{array}$

$\begin{array}{llll}-8.678833 & 0.661608 & -1.414475\end{array}$

$\begin{array}{llll}-8.708395 & -0.289755 & -1.935123\end{array}$

$\begin{array}{llll}-7.419420 & 1.244903 & -1.152414\end{array}$

$\begin{array}{llll}-5.140275 & -2.705889 & -0.032141\end{array}$

$\begin{array}{llll}-4.347545 & -2.889842 & 0.697629\end{array}$

$\begin{array}{lll}-6.116306 & -3.868070 & 0.022940\end{array}$

$\begin{array}{llll}-5.691583 & -5.103837 & 0.530713\end{array}$

$\begin{array}{llll}-4.686483 & -5.203477 & 0.930622\end{array}$

$\begin{array}{lll}-6.554842 & -6.202911 & 0.520120\end{array}$

$\begin{array}{lll}-6.214237 & -7.155171 & 0.917926\end{array}$ $\begin{array}{llll}-7.849455 & -6.078682 & 0.010462\end{array}$

$\begin{array}{llll}-8.520962 & -6.932977 & 0.008776\end{array}$

$\begin{array}{lll}-8.279021 & -4.846425 & -0.493096\end{array}$

$\begin{array}{llll}-9.286613 & -4.740412 & -0.886819\end{array}$

$-7.416491 \quad-3.749708 \quad-0.489182$

$\begin{array}{llll}-7.749109 & -2.787898 & -0.870752\end{array}$

$\begin{array}{llll}-4.512864 & -2.623856 & -1.428476\end{array}$

$\begin{array}{lll}-5.309482 & -2.686798 & -2.179980\end{array}$

$\begin{array}{llll}-3.866898 & -3.494353 & -1.572734\end{array}$

$\begin{array}{lll}-6.429946 & -1.355992 & 1.666643\end{array}$

$\begin{array}{lll}-6.848614 & -2.359692 & 1.777144\end{array}$

$\begin{array}{llll}-5.407275 & -1.196987 & 2.791872\end{array}$

$\begin{array}{lll}-4.734998 & -2.336456 & 3.260644\end{array}$

$\begin{array}{llll}-4.955588 & -3.307368 & 2.828461\end{array}$

$\begin{array}{lll}-3.781087 & -2.248535 & 4.276344\end{array}$

$\begin{array}{lll}-3.246704 & -3.142983 & 4.579996\end{array}$

$\begin{array}{llll}-3.497485 & -1.012204 & 4.859745\end{array}$

$\begin{array}{lll}-2.749382 & -0.933753 & 5.644207\end{array}$

$\begin{array}{lll}-4.172414 & 0.128992 & 4.415786\end{array}$

$\begin{array}{lll}-3.962858 & 1.095090 & 4.867815\end{array}$

$\begin{array}{lll}-5.115438 & 0.038027 & 3.389266\end{array}$

$\begin{array}{lll}-5.622323 & 0.935135 & 3.054158\end{array}$

$\begin{array}{lll}-7.616778 & -0.397856 & 1.747699\end{array}$

$\begin{array}{lll}-8.353659 & -0.648926 & 0.981821\end{array}$

$\begin{array}{lll}-7.335585 & 0.647073 & 1.619634\end{array}$

$\begin{array}{lll}-8.090248 & -0.504911 & 2.728579\end{array}$

$\begin{array}{lll}-2.323092 & -0.716570 & 0.146756\end{array}$

$\begin{array}{lll}-1.652986 & 0.138133 & 0.137402\end{array}$

$\begin{array}{llll}-2.947252 & -0.770045 & 1.034965\end{array}$

$\begin{array}{llll}-1.862991 & -1.959530 & -0.389866\end{array}$

$\begin{array}{lll}-2.347492 & -2.858492 & -0.037617\end{array}$

$\begin{array}{lll}-0.682788 & -2.130891 & -1.139754\end{array}$

$\begin{array}{llll}-0.108312 & -1.253473 & -1.409340\end{array}$

$\begin{array}{llll}-4.331824 & -0.665584 & -3.764457\end{array}$

$\begin{array}{llll}-5.324665 & -1.099761 & -3.67620\end{array}$

$\begin{array}{llll}-3.250099 & -1.575387 & -3.884133\end{array}$

$\begin{array}{llll}-3.491839 & -2.637475 & -3.897377\end{array}$

$\begin{array}{lll}-1.919652 & -1.241324 & -4.537506\end{array}$

$\begin{array}{llll}-2.030658 & -1.247992 & -5.633345\end{array}$

$\begin{array}{llll}-1.228501 & -2.051644 & -4.295312\end{array}$

$\begin{array}{llll}-1.302355 & 0.097139 & -4.076932\end{array}$

$\begin{array}{llll}-1.565008 & 0.903663 & -4.769460\end{array}$

$\begin{array}{llll}-0.210781 & 0.017832 & -4.112551\end{array}$

$\begin{array}{llll}-1.701063 & 0.518977 & -2.665328\end{array}$

$\begin{array}{llll}-0.891544 & 0.548144 & -1.943874\end{array}$

$\begin{array}{llll}-2.801014 & 1.319397 & -2.365689\end{array}$

$\begin{array}{llll}-2.740162 & 1.900645 & -1.452088\end{array}$

$\begin{array}{llll}-3.823130 & 1.823205 & -3.363990\end{array}$

$\begin{array}{llll}-4.680106 & 2.196038 & -2.798876\end{array}$

$\begin{array}{lll}-3.402780 & 2.683593 & -3.906278\end{array}$

$\begin{array}{lll}-4.308070 & 0.745522 & -4.356518\end{array}$

$\begin{array}{llll}-3.682011 & 0.747528 & -5.255128\end{array}$

$\begin{array}{llll}-5.318319 & 1.003297 & -4.689628\end{array}$

$\begin{array}{llll}-0.351725 & -3.355491 & -1.861553\end{array}$

$\begin{array}{llll}-0.898007 & -4.610779 & -1.526145\end{array}$

$\begin{array}{llll}0.572087 & -3.283292 & -2.921972\end{array}$

$\begin{array}{llll}-0.542979 & -5.746572 & -2.250782\end{array}$

$\begin{array}{llll}-1.563209 & -4.701771 & -0.675427\end{array}$

$\begin{array}{llll}0.921514 & -4.421013 & -3.646887\end{array}$

$\begin{array}{llll}1.003779 & -2.320311 & -3.178144\end{array}$

$\begin{array}{llll}0.361297 & -5.657917 & -3.315414\end{array}$

$\begin{array}{llll}-0.964402 & -6.708838 & -1.974208\end{array}$

$\begin{array}{llll}1.630719 & -4.344271 & -4.466290\end{array}$

$\begin{array}{llll}0.634449 & -6.548399 & -3.874435\end{array}$

$\begin{array}{llll}2.192696 & -4.320507 & -0.122315\end{array}$

$\begin{array}{llll}3.227230 & -4.862447 & -0.851533\end{array}$

$\begin{array}{lll}1.475301 & -4.961923 & 0.375625\end{array}$

$\begin{array}{llll}4.124002 & -3.976242 & -1.463827\end{array}$

$\begin{array}{llll}3.957907 & -2.614092 & -1.320602\end{array}$

$\begin{array}{llll}4.637883 & -1.911018 & -1.782094\end{array}$

$\begin{array}{llll}2.942065 & -2.079274 & -0.611356\end{array}$

$\begin{array}{llll}1.061462 & -0.039789 & 0.623731\end{array}$

$\begin{array}{lll}-0.110831 & -0.975573 & 2.328791\end{array}$

$\begin{array}{lll}-0.597026 & 0.331381 & 2.751152\end{array}$

$\begin{array}{lll}-1.625135 & 0.236597 & 3.109803\end{array}$ 


\begin{tabular}{|c|c|c|c|}
\hline 1 & 0.012592 & 0.741391 & 3.563428 \\
\hline 1 & -0.580185 & 1.021598 & 1.913719 \\
\hline 6 & -0.562789 & -2.113303 & 3.130394 \\
\hline 1 & -1.492908 & -2.530744 & 2.743390 \\
\hline 1 & 0.188978 & -2.898455 & 3.161008 \\
\hline 1 & -0.728381 & -1.768301 & 4.151467 \\
\hline 1 & 3.338107 & -5.937086 & -0.946424 \\
\hline 35 & 5.610953 & -4.657593 & -2.548101 \\
\hline 5 & -1.878126 & -5.247618 & 2.242608 \\
\hline 9 & -0.593755 & -5.269740 & 1.656681 \\
\hline 9 & -2.497156 & -6.492890 & 2.105932 \\
\hline 9 & -2.665925 & -4.271068 & 1.565150 \\
\hline 9 & -1.779320 & -4.889825 & 3.597844 \\
\hline
\end{tabular}

Number of imaginary frequencies $=0$

The smallest frequencies are $=13.875616 .765919 .3367 \mathrm{~cm}(-1)$ Electronic energy $=-7647.2886223$

Zero-point correction $=$

(Hartree/Particle)

$\begin{array}{lc}\text { Thermal correction to Energy= } & 1.899770 \\ \text { Thermal correction to Enthalpy= }= & 1.900714 \\ \text { Thermal correction to Gibbs Free Energy= } & 1.608276 \\ \text { Sum of electronic and zero-point Energies }= & -7645.512810 \\ \text { Sum of electronic and thermal Energies }= & -7645.388853 \\ \text { Sum of electronic and thermal Enthalpies }= & -7645.387908 \\ \text { Sum of electronic and thermal Free Energies }= & -7645.680347\end{array}$

\begin{tabular}{|c|c|c|c|}
\hline 6 & 0.114560 & 3.853584 & -3.897664 \\
\hline 1 & -0.127792 & 4.227343 & -4.888429 \\
\hline 6 & 1.257652 & 3.070289 & -3.716450 \\
\hline 1 & 1.894979 & 2.854455 & -4.567269 \\
\hline 6 & 3.727584 & 1.074048 & -3.712399 \\
\hline 6 & 4.387430 & 1.929086 & -4.608602 \\
\hline 1 & 4.554637 & 2.969329 & -4.344177 \\
\hline 6 & 4.844816 & 1.439120 & -5.832979 \\
\hline 1 & 5.357566 & 2.103974 & -6.522243 \\
\hline 6 & 4.647312 & 0.095814 & -6.170855 \\
\hline 1 & 5.009333 & -0.282734 & -7.122432 \\
\hline 6 & 3.987776 & -0.758432 & -5.283603 \\
\hline 1 & 3.835905 & -1.803219 & -5.539361 \\
\hline 6 & 3.532387 & -0.270639 & -4.057449 \\
\hline 1 & 3.040601 & -0.935933 & -3.355496 \\
\hline 6 & 3.434061 & 2.240598 & 2.065955 \\
\hline 6 & 2.043820 & 2.416867 & 2.094027 \\
\hline 1 & 1.401676 & 1.593125 & 1.803794 \\
\hline 6 & 1.488928 & 3.644826 & 2.451195 \\
\hline 6 & 2.303427 & 4.735799 & 2.746036 \\
\hline 1 & 1.872156 & 5.697377 & 3.001484 \\
\hline 6 & 3.687799 & 4.567300 & 2.688637 \\
\hline 6 & 4.255537 & 3.332280 & 2.371181 \\
\hline 1 & 5.333690 & 3.252823 & 2.344492 \\
\hline 6 & 3.550543 & -0.566236 & 2.821932 \\
\hline 6 & 3.710493 & -1.938035 & 2.563631 \\
\hline 1 & 4.156423 & -2.270994 & 1.633736 \\
\hline 6 & 3.282400 & -2.884408 & 3.492352 \\
\hline 6 & 2.646285 & -2.491665 & 4.670248 \\
\hline 1 & 2.284051 & -3.232862 & 5.374147 \\
\hline 6 & 2.473551 & -1.132291 & 4.918511 \\
\hline 6 & 2.937974 & -0.172136 & 4.017008 \\
\hline 1 & 2.795707 & 0.877269 & 4.244626 \\
\hline 29 & 2.851961 & 0.048322 & -0.499550 \\
\hline 6 & 1.679792 & -0.714881 & 6.126323 \\
\hline 6 & 3.407234 & -4.354904 & 3.200228 \\
\hline 6 & -0.007959 & 3.750065 & 2.547343 \\
\hline 6 & 4.560702 & 5.767634 & 2.935710 \\
\hline 9 & 4.371249 & 6.719727 & 1.988407 \\
\hline 9 & 5.876912 & 5.462255 & 2.932135 \\
\hline 9 & 4.284351 & 6.352382 & 4.124206 \\
\hline 9 & -0.623332 & 3.076060 & 1.535414 \\
\hline 9 & -0.477799 & 3.209122 & 3.696142 \\
\hline 9 & -0.445765 & 5.025305 & 2.493809 \\
\hline 9 & 1.834033 & 0.593458 & 6.418964 \\
\hline 9 & 0.347894 & -0.916593 & 5.926475 \\
\hline 9 & 2.013177 & -1.424565 & 7.225358 \\
\hline 9 & 3.765876 & -5.053727 & 4.299288 \\
\hline 9 & 4.323967 & -4.611547 & 2.239994 \\
\hline 9 & 2.228491 & -4.876193 & 2.776789 \\
\hline 6 & 0.511596 & -1.141301 & 1.059426 \\
\hline 6 & 0.526347 & -2.374878 & 0.167358 \\
\hline 6 & 1.897661 & -2.875013 & -0.220935 \\
\hline 1 & 0.011930 & -3.203187 & 0.649082 \\
\hline 77 & -3.350840 & -0.762691 & -1.853825 \\
\hline 15 & -5.166875 & -0.125446 & -0.582006 \\
\hline 8 & -5.143661 & 0.965516 & 0.683480 \\
\hline 8 & -6.369669 & 0.588394 & -1.516580 \\
\hline 7 & -5.752232 & -1.460720 & 0.253884 \\
\hline 6 & -4.945557 & 2.309234 & 0.417913 \\
\hline 6 & -3.709058 & 2.865211 & 0.816120 \\
\hline 1 & -2.988819 & 2.224287 & 1.311521 \\
\hline 6 & -3.454224 & 4.192452 & 0.574497 \\
\hline 1 & -2.525050 & 4.642164 & 0.908561 \\
\hline 6 & -4.391927 & 4.987216 & -0.139023 \\
\hline 6 & -4.092538 & 6.329709 & -0.497230 \\
\hline 1 & -3.135455 & 6.745341 & -0.192040 \\
\hline 6 & -4.984229 & 7.083407 & -1.227080 \\
\hline 1 & -4.742027 & 8.105948 & -1.501866 \\
\hline 6 & -6.216575 & 6.517197 & -1.632870 \\
\hline 1 & -6.909000 & 7.106331 & -2.227463 \\
\hline 6 & -6.544760 & 5.224108 & -1.286485 \\
\hline 1 & -7.489229 & 4.803021 & -1.611472 \\
\hline 6 & -5.654916 & 4.420611 & -0.520258 \\
\hline 6 & -5.962429 & 3.063938 & -0.149008 \\
\hline
\end{tabular}


$\begin{array}{llll}-1.278156 & -4.611846 & -1.071105\end{array}$

$\begin{array}{llll}0.919274 & -3.933518 & -4.193047\end{array}$

$\begin{array}{llll}1.087458 & -1.921808 & -3.438515\end{array}$

$\begin{array}{llll}0.367342 & -5.200836 & -3.991722\end{array}$

$\begin{array}{llll}-0.862535 & -6.415923 & -2.699186\end{array}$

$\begin{array}{llll}1.541250 & -3.739039 & -5.062969\end{array}$

$\begin{array}{llll}0.561921 & -6.001840 & -4.699565\end{array}$

$\begin{array}{llll}2.148181 & -4.252286 & -0.158097\end{array}$

$\begin{array}{llll}3.332845 & -4.772630 & -0.663510\end{array}$

$\begin{array}{llll}1.404103 & -4.906855 & 0.276747\end{array}$

$\begin{array}{llll}4.233059 & -3.877125 & -1.230216\end{array}$

$3.952423 \quad-2.520053 \quad-1.254174$

$\begin{array}{llll}4.640210 & -1.805554 & -1.684696\end{array}$

$\begin{array}{llll}2.806897 & -2.025225 & -0.744771\end{array}$

$\begin{array}{lll}0.910856 & -0.040513 & 0.598795\end{array}$

$\begin{array}{lll}-0.036326 & -1.217717 & 2.281598\end{array}$

$\begin{array}{llll}-0.435180 & 0.019811 & 2.955863\end{array}$

$\begin{array}{lll}-1.329111 & -0.182249 & 3.545985\end{array}$

$\begin{array}{lll}0.347504 & 0.394363 & 3.618387\end{array}$

$\begin{array}{lll}-0.667977 & 0.780918 & 2.214777\end{array}$

$\begin{array}{lll}-0.440942 & -2.471414 & 2.929145\end{array}$

$\begin{array}{lll}-1.454666 & -2.748419 & 2.644081\end{array}$

$\begin{array}{llll}0.234861 & -3.284954 & 2.677732\end{array}$

$\begin{array}{lll}-0.395319 & -2.319775 & 4.007517\end{array}$

$\begin{array}{llll}3.534827 & -5.836993 & -0.621655\end{array}$

$\begin{array}{llll}5.906552 & -4.514543 & -2.010732\end{array}$

$\begin{array}{lll}-1.813832 & -5.374023 & 1.849609\end{array}$

$\begin{array}{lll}-0.475690 & -5.386392 & 1.383565\end{array}$

$\begin{array}{llll}-2.451996 & -6.572040 & 1.519771\end{array}$

$\begin{array}{lll}-2.494508 & -4.298857 & 1.220000\end{array}$

$\begin{array}{lll}-1.821713 & -5.172248 & 3.240489\end{array}$

$\begin{array}{llll}-4.952105 & -3.560691 & 2.662350\end{array}$

$\begin{array}{llll}-3.725115 & -2.621522 & 4.151198\end{array}$

$\begin{array}{lll}-3.219630 & -3.548344 & 4.402313\end{array}$

$\begin{array}{llll}-3.395356 & -1.427674 & 4.796187\end{array}$

$\begin{array}{lll}-2.641203 & -1.416743 & 5.579038\end{array}$

$\begin{array}{lll}-4.033279 & -0.241982 & 4.417987\end{array}$

$\begin{array}{lll}-3.787132 & 0.691279 & 4.918231\end{array}$

$\begin{array}{lll}-4.986244 & -0.246161 & 3.396903\end{array}$

$\begin{array}{lll}-5.463428 & 0.683679 & 3.112012\end{array}$

$\begin{array}{llll}-7.549335 & -0.549870 & 1.797561\end{array}$

$\begin{array}{lll}-8.299442 & -0.735678 & 1.025494\end{array}$

$\begin{array}{lll}-7.266488 & 0.500964 & 1.748795\end{array}$

$\begin{array}{lll}-8.008967 & -0.734850 & 2.773658\end{array}$

$\begin{array}{lll}-2.275925 & -0.768209 & 0.061037\end{array}$

$\begin{array}{lll}-1.678676 & 0.134243 & 0.168702\end{array}$

$\begin{array}{lll}-2.906241 & -0.976239 & 0.922926\end{array}$

$\begin{array}{llll}-1.776118 & -1.864036 & -0.697559\end{array}$

$\begin{array}{llll}-2.196856 & -2.822413 & -0.422247\end{array}$

$\begin{array}{llll}-0.329671 & -2.022016 & -1.131125\end{array}$

$\begin{array}{llll}0.082218 & -1.092857 & -1.528258\end{array}$

$\begin{array}{lll}-4.313910 & -0.555213 & -3.794293\end{array}$

$\begin{array}{llll}-5.295370 & -1.025732 & -3.768526\end{array}$

$\begin{array}{llll}-3.189196 & -1.435237 & -3.936429\end{array}$

$\begin{array}{lll}-3.405070 & -2.500591 & -4.020819\end{array}$

$\begin{array}{llll}-1.906172 & -1.021010 & -4.639455\end{array}$

$\begin{array}{llll}-2.079047 & -0.931877 & -5.724537\end{array}$

$\begin{array}{llll}-1.187516 & -1.831056 & -4.508773\end{array}$

$\begin{array}{llll}-1.298189 & 0.286435 & -4.088989\end{array}$

$\begin{array}{llll}-1.549877 & 1.134524 & -4.735911\end{array}$

$\begin{array}{llll}-0.204921 & 0.215196 & -4.107841\end{array}$

$\begin{array}{llll}-1.728281 & 0.617908 & -2.659401\end{array}$

$\begin{array}{llll}-0.919361 & 0.640749 & -1.935531\end{array}$

$\begin{array}{llll}-2.843468 & 1.402786 & -2.339146\end{array}$

$\begin{array}{llll}-2.787925 & 1.969850 & -1.415703\end{array}$

$\begin{array}{llll}-3.859031 & 1.929699 & -3.336502\end{array}$

$\begin{array}{llll}-4.727980 & 2.269694 & -2.766866\end{array}$

$\begin{array}{llll}-3.448945 & 2.814685 & -3.848289\end{array}$

$\begin{array}{llll}-4.320018 & 0.870810 & -4.360716\end{array}$

$\begin{array}{llll}-3.691642 & 0.912083 & -5.258123\end{array}$

\section{P1-C2 endo re-re $\left[\mathrm{BF}_{4}^{-}\right]$}

Number of imaginary frequencies $=0$

The smallest frequencies are $=13.327413 .865916 .4012 \mathrm{~cm}(-1)$ Electronic energy $=-7647.2606003$

Zero-point correction $=$

1.771808

(Hartree/Particle)

Thermal correction to Energy= $\quad 1.897604$

Thermal correction to Enthalpy=

1.898549

Thermal correction to Gibbs Free Energy=

Sum of electronic and zero-point Energies=

Sum of electronic and thermal Energies=

Sum of electronic and thermal Enthalpies=

Sum of electronic and thermal Free Energies=

1.601354

$-7645.488792$

$-7645.362996$

$-7645.362052$

$-7645.659246$

Cartesian Coordinates

$\begin{array}{llcc}26 & 7.607984 & -1.768224 & -1.645119 \\ 15 & 3.005969 & 0.607669 & -2.335866 \\ 15 & 4.385803 & 0.405589 & 1.136393 \\ 6 & 5.930109 & -0.524559 & -1.795444 \\ 6 & 6.058125 & -1.068170 & -0.464605 \\ 6 & 5.997338 & -2.489334 & -0.566951 \\ 1 & 6.072702 & -3.184928 & 0.256978 \\ 6 & 5.870188 & -2.834333 & -1.943266 \\ 1 & 5.843145 & -3.837098 & -2.346634 \\ 6 & 5.831127 & -1.628699 & -2.700301 \\ 1 & 5.754461 & -1.550938 & -3.774986 \\ 6 & 9.424725 & -0.959951 & -1.045890 \\ 1 & 9.532553 & -0.118688 & -0.373916 \\ 6 & 9.340888 & -2.333677 & -0.661579 \\ 1 & 9.365955 & -2.714470 & 0.350673 \\ 6 & 9.169882 & -3.113840 & -1.845956 \\ 1 & 9.038512 & -4.187238 & -1.885841 \\ 6 & 9.148507 & -2.223445 & -2.962626 \\ 1 & 9.001023 & -2.502960 & -3.997519 \\ 6 & 9.307484 & -0.894127 & -2.469390 \\ 1 & 9.306750 & 0.005676 & -3.069313\end{array}$


$\begin{array}{llll}5.837717 & 0.927961 & -2.117644\end{array}$

$\begin{array}{llll}6.989290 & 1.725427 & -2.038988\end{array}$

$\begin{array}{llll}7.921078 & 1.248732 & -1.763056\end{array}$

$\begin{array}{llll}6.956585 & 3.096294 & -2.291099\end{array}$

$\begin{array}{llll}7.861640 & 3.687474 & -2.196319\end{array}$

$\begin{array}{llll}5.752606 & 3.698114 & -2.648543\end{array}$

$\begin{array}{llll}5.707413 & 4.763904 & -2.849204\end{array}$

$\begin{array}{llll}4.591712 & 2.928232 & -2.724236\end{array}$

$\begin{array}{llll}3.660721 & 3.416630 & -2.987503\end{array}$

$\begin{array}{llll}4.602832 & 1.551657 & -2.444579\end{array}$

$\begin{array}{lll}6.141181 & -0.233420 & 0.783858\end{array}$

$\begin{array}{llll}6.709703 & 0.659556 & 0.522933\end{array}$

$\begin{array}{lll}6.852765 & -0.911281 & 1.958418\end{array}$

$\begin{array}{llll}6.887219 & -0.251675 & 2.830262\end{array}$

$\begin{array}{lll}6.365087 & -1.841073 & 2.260003\end{array}$

$\begin{array}{llll}7.878935 & -1.152352 & 1.664682\end{array}$

$\begin{array}{llll}1.743559 & 1.875213 & -2.785103\end{array}$

$\begin{array}{llll}1.325389 & 2.738948 & -1.758312\end{array}$

$\begin{array}{llll}1.754563 & 2.637143 & -0.766892\end{array}$

$\begin{array}{llll}0.383794 & 3.736402 & -2.004404\end{array}$

$\begin{array}{llll}0.085373 & 4.402916 & -1.205529\end{array}$

$\begin{array}{llll}-0.175886 & 3.871499 & -3.277731\end{array}$

$\begin{array}{llll}-0.923864 & 4.637508 & -3.461081\end{array}$

$\begin{array}{llll}0.226601 & 3.013561 & -4.303135\end{array}$

$\begin{array}{llll}-0.203888 & 3.110690 & -5.29596\end{array}$

$\begin{array}{lll}1.185276 & 2.025118 & -4.062208\end{array}$

$\begin{array}{llll}1.485422 & 1.369613 & -4.871750\end{array}$

$\begin{array}{lll}3.049234 & -0.537054 & -3.770543\end{array}$

$\begin{array}{lll}3.570826 & -0.167663 & -5.020115\end{array}$

$\begin{array}{llll}3.979102 & 0.828525 & -5.165708\end{array}$

$\begin{array}{llll}3.578973 & -1.083219 & -6.074032\end{array}$

$\begin{array}{llll}3.982963 & -0.791903 & -7.039547\end{array}$

$3.071629-2.373636 \quad-5.886287$

$3.081157 \quad-3.084564 \quad-6.707824$

$\begin{array}{llll}2.565031 & -2.749923 & -4.639841\end{array}$

$\begin{array}{llll}2.185263 & -3.756312 & -4.486303\end{array}$

$2.557611 \quad-1.837772 \quad-3.582066$

$\begin{array}{llll}2.220899 & -2.138519 & -2.593979\end{array}$

$\begin{array}{lll}4.545434 & 2.238723 & 1.309375\end{array}$

$\begin{array}{lll}3.355362 & 2.960965 & 1.485835\end{array}$

$\begin{array}{lll}2.433695 & 2.432146 & 1.687195\end{array}$

$\begin{array}{llll}3.332071 & 4.351087 & 1.372352\end{array}$

$\begin{array}{lll}4.497329 & 5.061655 & 1.095607\end{array}$

$\begin{array}{llll}4.475608 & 6.139404 & 0.979800\end{array}$

$\begin{array}{lll}5.689111 & 4.351849 & 0.951633\end{array}$

$\begin{array}{lll}5.720808 & 2.961667 & 1.057351\end{array}$

$\begin{array}{lll}6.668442 & 2.462091 & 0.913176\end{array}$

$\begin{array}{lll}3.908042 & -0.233068 & 2.791292\end{array}$

$\begin{array}{llll}3.745586 & -1.625782 & 2.874374\end{array}$

$\begin{array}{llll}3.977603 & -2.251351 & 2.020912\end{array}$

$3.227350 \quad-2.206178 \quad 4.026590$

$\begin{array}{llll}2.808659 & -1.413440 & 5.095837\end{array}$

$2.365485 \quad-1.865719 \quad 5.976753$

$\begin{array}{lll}2.948069 & -0.029577 & 5.002453\end{array}$

$\begin{array}{lll}3.509863 & 0.563469 & 3.869704\end{array}$

$3.606845 \quad 1.641630 \quad 3.831383$

$2.660853 \quad-0.337656-0.302858$

$\begin{array}{llll}2.401263 & 0.817769 & 6.117126\end{array}$

$\begin{array}{llll}3.028981 & -3.694432 & 4.113739\end{array}$

$\begin{array}{lll}2.022728 & 5.074078 & 1.531511\end{array}$

$\begin{array}{lll}6.938093 & 5.127236 & 0.630255\end{array}$

$\begin{array}{llll}6.810758 & 5.809662 & -0.535036\end{array}$

$8.027650 \quad 4.337609 \quad 0.511257$

$\begin{array}{llll}7.210993 & 6.048744 & 1.583346\end{array}$

$\begin{array}{llll}0.998424 & 4.353172 & 0.995750\end{array}$

$\begin{array}{lll}1.706779 & 5.287809 & 2.827371\end{array}$

$\begin{array}{llll}2.019226 & 6.274989 & 0.916886\end{array}$

$\begin{array}{lll}2.717820 & 2.124444 & 5.980677\end{array}$

$\begin{array}{lll}1.048650 & 0.738338 & 6.173062\end{array}$

$\begin{array}{llll}2.858543 & 0.416995 & 7.327537\end{array}$

$3.648973-4.210660 \quad 5.204340$

$\begin{array}{llll}3.515485 & -4.348095 & 3.034511\end{array}$

$\begin{array}{lll}1.721226 & -4.021152 & 4.229392\end{array}$

$\begin{array}{llll}0.601568 & 0.744018 & 1.544646\end{array}$

$\begin{array}{lll}0.713662 & -1.605208 & 1.685315\end{array}$ $\begin{array}{lll}-0.102364 & 0.840832 & 2.725302\end{array}$

$\begin{array}{lll}0.830765 & 1.627649 & 0.962661\end{array}$

$\begin{array}{lll}-0.114245 & -1.532518 & 2.860749\end{array}$

$\begin{array}{lll}-0.489272 & -0.330043 & 3.402542\end{array}$

$\begin{array}{lll}1.118832 & -2.886515 & 1.264886\end{array}$

$\begin{array}{llll}2.008821 & -3.230856 & 0.218993\end{array}$

$\begin{array}{llll}2.704622 & -2.392979 & -0.458484\end{array}$

$\begin{array}{lll}1.037451 & -0.430076 & 1.035027\end{array}$

$\begin{array}{llll}0.682294 & -3.688353 & 1.837014\end{array}$

$\begin{array}{llll}-3.183331 & -1.155262 & -1.563686\end{array}$

$\begin{array}{lll}-4.920382 & -0.027117 & -0.627406\end{array}$

$\begin{array}{lll}-4.761580 & 1.157156 & 0.500201\end{array}$

$\begin{array}{lll}-5.776710 & 0.796060 & -1.793218\end{array}$

$\begin{array}{lll}-5.841557 & -1.092697 & 0.254827\end{array}$

$\begin{array}{lll}-4.209517 & 2.385849 & 0.174138\end{array}$

$\begin{array}{lll}-2.939728 & 2.666608 & 0.720498\end{array}$

$\begin{array}{lll}-2.479992 & 1.929418 & 1.367601\end{array}$

$\begin{array}{lll}-2.330098 & 3.859455 & 0.424134\end{array}$

$\begin{array}{llll}-1.363276 & 4.098806 & 0.852303\end{array}$

$\begin{array}{llll}-2.940871 & 4.771888 & -0.479248\end{array}$

$\begin{array}{llll}-2.271106 & 5.960521 & -0.878146\end{array}$

$\begin{array}{llll}-1.300552 & 6.178638 & -0.439918\end{array}$

$\begin{array}{llll}-2.831505 & 6.811102 & -1.805152\end{array}$

$\begin{array}{llll}-2.307060 & 7.712030 & -2.109781\end{array}$

$\begin{array}{llll}-4.093261 & 6.504308 & -2.369287\end{array}$

$\begin{array}{llll}-4.522881 & 7.165694 & -3.116218\end{array}$

$\begin{array}{llll}-4.782859 & 5.375725 & -1.980229\end{array}$

$\begin{array}{llll}-5.748011 & 5.155167 & -2.421959\end{array}$

$\begin{array}{llll}-4.240565 & 4.480599 & -1.017052\end{array}$

$\begin{array}{llll}-4.925530 & 3.283139 & -0.600226\end{array}$

$\begin{array}{llll}-6.345843 & 2.992850 & -0.949909\end{array}$

$\begin{array}{llll}-7.400907 & 3.922808 & -0.624777\end{array}$

$\begin{array}{lll}-7.174212 & 5.110475 & 0.126954\end{array}$

$\begin{array}{lll}-6.176694 & 5.332084 & 0.485365\end{array}$

$\begin{array}{lll}-8.205842 & 5.974143 & 0.425824\end{array}$

$\begin{array}{lll}-8.003660 & 6.868082 & 1.008940\end{array}$

$\begin{array}{llll}-9.524749 & 5.705067 & -0.009965\end{array}$

$\begin{array}{lll}-10.326297 & 6.398535 & 0.226953\end{array}$

$\begin{array}{llll}-9.786963 & 4.553115 & -0.715847\end{array}$

$\begin{array}{llll}-10.798307 & 4.319191 & -1.038084\end{array}$

$\begin{array}{lll}-8.749120 & 3.632328 & -1.025151\end{array}$

$\begin{array}{llll}-9.033639 & 2.413588 & -1.697573\end{array}$

$\begin{array}{llll}-10.054417 & 2.205777 & -2.005566\end{array}$

$\begin{array}{llll}-8.039947 & 1.495476 & -1.922015\end{array}$

$\begin{array}{llll}-8.238096 & 0.541262 & -2.398593\end{array}$

$\begin{array}{llll}-6.715857 & 1.783588 & -1.523862\end{array}$

$\begin{array}{llll}-5.500722 & -2.520715 & 0.012397\end{array}$

$\begin{array}{lll}-4.885468 & -2.867500 & 0.843764\end{array}$

$\begin{array}{lll}-6.744190 & -3.390351 & -0.051629\end{array}$

$\begin{array}{lll}-6.773338 & -4.610662 & 0.634717\end{array}$

$\begin{array}{lll}-5.918650 & -4.899712 & 1.239988\end{array}$

$\begin{array}{llll}-7.892388 & -5.443550 & 0.544901\end{array}$

$\begin{array}{lll}-7.904958 & -6.387132 & 1.083668\end{array}$

$\begin{array}{llll}-8.993666 & -5.061042 & -0.223993\end{array}$

$\begin{array}{llll}-9.865767 & -5.705961 & -0.287914\end{array}$

$\begin{array}{llll}-8.972026 & -3.840001 & -0.906869\end{array}$

$\begin{array}{llll}-9.828187 & -3.534267 & -1.502488\end{array}$

$\begin{array}{llll}-7.853193 & -3.011417 & -0.822429\end{array}$

$\begin{array}{lll}-7.836404 & -2.058789 & -1.344906\end{array}$

$\begin{array}{lll}-4.693186 & -2.649603 & -1.282698\end{array}$

$\begin{array}{llll}-5.377067 & -2.601404 & -2.137180\end{array}$

$-4.212850-3.630408-1.30722$

$\begin{array}{lll}-6.599692 & -0.803103 & 1.519298\end{array}$

$\begin{array}{lll}-7.027084 & -1.776800 & 1.774862\end{array}$

$\begin{array}{lll}-5.692382 & -0.404837 & 2.686148\end{array}$

$\begin{array}{lll}4.803150 & -1.346868 & 3.226462\end{array}$

$\begin{array}{lll}-4.763249 & -2.359697 & 2.838450\end{array}$

$\begin{array}{lll}-3.941897 & -1.003349 & 4.271356\end{array}$

$\begin{array}{lll}-3.259170 & -1.753439 & 4.652831\end{array}$

$\begin{array}{lll}-3.966174 & 0.286014 & 4.806063\end{array}$

$\begin{array}{lll}-3.297824 & 0.555146 & 5.619526\end{array}$

$\begin{array}{lll}-4.860065 & 1.226915 & 4.289519\end{array}$

$\begin{array}{lll}-4.891455 & 2.233069 & 4.699536\end{array}$

$\begin{array}{lll}-5.714092 & 0.882039 & 3.240807\end{array}$

$\begin{array}{lll}-6.385185 & 1.634632 & 2.841906\end{array}$ 


\begin{tabular}{|c|c|c|c|}
\hline 6 & -7.775980 & 0.137155 & 1.266742 \\
\hline 1 & -8.408483 & -0.270912 & 0.475197 \\
\hline 1 & -7.454758 & 1.135791 & 0.966168 \\
\hline 1 & -8.376648 & 0.232714 & 2.176379 \\
\hline 6 & -2.292533 & -0.996239 & 0.432002 \\
\hline 1 & -1.455636 & -0.313301 & 0.349526 \\
\hline 1 & -2.944081 & -0.789989 & 1.275540 \\
\hline 6 & -2.128073 & -2.372148 & 0.089108 \\
\hline 1 & -2.781796 & -3.085507 & 0.570673 \\
\hline 6 & -1.243252 & -2.823017 & -0.891671 \\
\hline 1 & -0.430625 & -2.175086 & -1.193279 \\
\hline 6 & -4.001258 & -0.862768 & -3.645943 \\
\hline 1 & -5.056836 & -1.112004 & -3.584903 \\
\hline 6 & -3.100522 & -1.945301 & -3.681547 \\
\hline 1 & -3.519717 & -2.950066 & -3.652725 \\
\hline 6 & -1.693381 & -1.873663 & -4.241490 \\
\hline 1 & -1.741816 & -1.937581 & -5.339271 \\
\hline 1 & -1.160551 & -2.767439 & -3.910579 \\
\hline 6 & -0.892226 & -0.619080 & -3.832652 \\
\hline 1 & -1.026170 & 0.175295 & -4.571269 \\
\hline 1 & 0.171364 & -0.864983 & -3.850498 \\
\hline 6 & -1.226696 & -0.054616 & -2.457185 \\
\hline 1 & -0.468450 & -0.176492 & -1.687995 \\
\hline 6 & -2.180054 & 0.921798 & -2.240444 \\
\hline 1 & -2.097880 & 1.498983 & -1.325648 \\
\hline 6 & -3.067792 & 1.526337 & -3.308944 \\
\hline 1 & -3.854518 & 2.091365 & -2.809434 \\
\hline 1 & -2.474884 & 2.253665 & -3.878389 \\
\hline 6 & -3.711797 & 0.502460 & -4.268141 \\
\hline 1 & -3.079339 & 0.357489 & -5.149647 \\
\hline 1 & -4.655296 & 0.917751 & -4.633768 \\
\hline 6 & -1.156807 & -4.202002 & -1.369884 \\
\hline 6 & -2.019726 & -5.229629 & -0.933304 \\
\hline 6 & -0.146868 & -4.525715 & -2.297660 \\
\hline 6 & -1.880389 & -6.524080 & -1.429323 \\
\hline 1 & -2.764859 & -5.031659 & -0.173054 \\
\hline 6 & -0.013080 & -5.820865 & -2.794137 \\
\hline 1 & 0.544171 & -3.751747 & -2.614589 \\
\hline 6 & -0.883542 & -6.826106 & -2.363808 \\
\hline 1 & -2.546642 & -7.305318 & -1.074514 \\
\hline 1 & 0.772925 & -6.046985 & -3.509293 \\
\hline 1 & -0.780755 & -7.838504 & -2.744164 \\
\hline 7 & 2.153132 & -4.567299 & -0.104633 \\
\hline 6 & 3.183840 & -4.984810 & -1.036941 \\
\hline 1 & 4.042624 & -5.438756 & -0.518324 \\
\hline 1 & 2.776485 & -5.727584 & -1.732861 \\
\hline 1 & 3.533729 & -4.121386 & -1.596498 \\
\hline 6 & 1.479726 & -5.629186 & 0.632004 \\
\hline 1 & 1.572508 & -6.558117 & 0.065025 \\
\hline 1 & 1.921499 & -5.783289 & 1.625845 \\
\hline 1 & 0.416382 & -5.419233 & 0.754865 \\
\hline 1 & -1.084845 & -0.284333 & 4.305852 \\
\hline 1 & -0.441196 & -2.456651 & 3.323587 \\
\hline 35 & -0.498173 & 2.598089 & 3.498033 \\
\hline 5 & -2.596648 & -4.729279 & 2.818636 \\
\hline 9 & -1.449563 & -4.702927 & 2.004694 \\
\hline 9 & -2.747934 & -5.980731 & 3.412228 \\
\hline 9 & -2.492674 & -3.725319 & 3.798074 \\
\hline 9 & -3.738601 & -4.457882 & 1.998339 \\
\hline
\end{tabular}

\section{$[8-9]^{\ddagger} \mathrm{P1}-\mathrm{C} 2$ endo re-re $\left[\mathrm{BF}_{4}^{-}\right]$}

Number of imaginary frequencies $=1$

The smallest frequencies are $=-249.305013 .419315 .5271 \mathrm{~cm}(-1)$ Electronic energy $=-7647.2526318$

Zero-point correction $=$

1.772247

(Hartree/Particle)

Thermal correction to Energy= $\quad 1.896862$

Thermal correction to Enthalpy= $\quad 1.897806$

Thermal correction to Gibbs Free Energy $=\quad 1.604410$

Sum of electronic and zero-point Energies $=\quad-7645.480385$

Sum of electronic and thermal Energies $=\quad-7645.355770$

Sum of electronic and thermal Enthalpies $=\quad-7645.354826$

Sum of electronic and thermal Free Energies $=\quad-7645.648221$
Cartesian Coordinates

\begin{tabular}{|c|c|c|c|}
\hline 26 & 7.611726 & -1.586968 & -1.728655 \\
\hline & 2.986467 & 0.740081 & -2.364246 \\
\hline & 4.328100 & 0.390446 & 1.138366 \\
\hline 6 & 5.915759 & -0.366522 & -1.834847 \\
\hline 6 & 6.028085 & -0.989833 & -0.537017 \\
\hline & 5.997998 & -2.402323 & -0.726755 \\
\hline & 6.073469 & -3.145396 & 0.054457 \\
\hline 6 & 5.901720 & -2.663846 & -2.124815 \\
\hline 1 & 5.900611 & -3.640026 & -2.590212 \\
\hline & 5.855853 & -1.414174 & -2.807801 \\
\hline 1 & 5.798026 & -1.273622 & -3.877110 \\
\hline 6 & 9.399457 & -0.796227 & -1.032063 \\
\hline 1 & 9.480148 & -0.008511 & -0.294711 \\
\hline 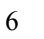 & 9.337884 & -2.197585 & -0.759882 \\
\hline 1 & 9.351752 & -2.657163 & 0.219341 \\
\hline 6 & 9.203742 & -2.883786 & -2.005418 \\
\hline 1 & 9.095424 & -3.952841 & -2.133694 \\
\hline 6 & 9.184527 & -1.907884 & -3.048088 \\
\hline 1 & 9.062240 & -2.106938 & -4.104652 \\
\hline 6 & 9.307901 & -0.619397 & -2.447995 \\
\hline 1 & 9.301532 & 0.324752 & -2.975347 \\
\hline 6 & 5.799770 & 1.103553 & -2.052847 \\
\hline 6 & 6.926525 & 1.917349 & -1.859903 \\
\hline 1 & 7.860837 & 1.438964 & -1.595282 \\
\hline 6 & 6.863584 & 3.305072 & -1.976905 \\
\hline 1 & 7.746201 & 3.906874 & -1.786313 \\
\hline 6 & 5.656235 & 3.908778 & -2.320257 \\
\hline 1 & 5.588053 & 4.988001 & -2.417863 \\
\hline 6 & 4.521437 & 3.121321 & -2.518065 \\
\hline 1 & 3.587732 & 3.610268 & -2.771009 \\
\hline 6 & 4.560993 & 1.725246 & -2.366804 \\
\hline 6 & 6.085247 & -0.227709 & 0.757741 \\
\hline 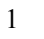 & & & \\
\hline 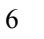 & 6.797871 & -0.9 & 1.900078 \\
\hline 1 & 6.819211 & -0.343605 & 2.805469 \\
\hline 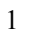 & 6.320432 & -1.907310 & 2.149991 \\
\hline 1 & 7.828386 & -1.169443 & 1.600081 \\
\hline 6 & 1.688661 & 1.977452 & -2.787752 \\
\hline 6 & 1.266516 & 2.833014 & -1.755133 \\
\hline 1 & 1.728000 & 2.759295 & -0.775640 \\
\hline 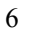 & 0.281287 & 3.791413 & -1.981579 \\
\hline 1 & -0.016613 & 4.454866 & -1.179980 \\
\hline 6 & -0.322022 & 3.891407 & -3.238266 \\
\hline 1 & -1.104182 & 4.625988 & -3.406016 \\
\hline 6 & 0.083079 & 3.0 & -4.267473 \\
\hline 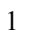 & -0.381634 & 3.109322 & -5.246894 \\
\hline 6 & 1.087412 & 2.092621 & -4.048633 \\
\hline 1 & 1.385072 & 1.440210 & -4.861240 \\
\hline 6 & 3.097747 & -0.350828 & -3.831921 \\
\hline 6 & 3.599090 & 0.083616 & -5.068304 \\
\hline 1 & 3.942670 & 1.107401 & -5.187053 \\
\hline 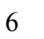 & 3.670387 & -0.804201 & -6.143207 \\
\hline 1 & 4.058684 & -0.464565 & -7.099221 \\
\hline 6 & 3.245800 & -2.128954 & -5.989735 \\
\hline 1 & 3.304001 & -2.816875 & -6.828537 \\
\hline 6 & 2.756947 & -2.568213 & -4.756948 \\
\hline 1 & 2.438168 & -3.599005 & -4.629560 \\
\hline 6 & 2.688568 & -1.682993 & -3.679603 \\
\hline 1 & 2.363250 & -2.024993 & -2.702109 \\
\hline 6 & 4.510013 & 2.204288 & 1.446435 \\
\hline 6 & 3.326424 & 2.949904 & 1.544423 \\
\hline 1 & 2.378024 & 2.438004 & 1.611121 \\
\hline 6 & 3.346292 & 4.343964 & 1.523863 \\
\hline 6 & 4.551633 & 5.033545 & 1.415273 \\
\hline 1 & 4.567565 & 6.116650 & 1.365377 \\
\hline 6 & 5.734794 & 4.298329 & 1.351091 \\
\hline 6 & 5.723323 & 2.903740 & 1.374211 \\
\hline 1 & 6.669473 & 2.384119 & 1.312529 \\
\hline & 3.829942 & -0.371114 & 2.732694 \\
\hline & 3.694005 & -1.769887 & 2.717116 \\
\hline & 3.961441 & -2.327440 & 1.827291 \\
\hline & 3.173272 & -2.439077 & 3.819048 \\
\hline & 2.733190 & -1.730784 & 4.939116 \\
\hline
\end{tabular}


$\begin{array}{llll}2.300136 & -2.251170 & 5.786299\end{array}$

$\begin{array}{lll}2.849034 & -0.342382 & 4.943700\end{array}$

$\begin{array}{lll}3.403431 & 0.341079 & 3.857302\end{array}$

$\begin{array}{llll}3.482255 & 1.421650 & 3.892781\end{array}$

$\begin{array}{llll}2.624261 & -0.270999 & -0.376123\end{array}$

$\begin{array}{lll}2.277302 & 0.429258 & 6.100872\end{array}$

$\begin{array}{llll}2.986875 & -3.931382 & 3.781604\end{array}$

$\begin{array}{lll}2.048062 & 5.101540 & 1.597761\end{array}$

$\begin{array}{llll}7.027364 & 5.049555 & 1.179846\end{array}$

$\begin{array}{lll}7.036801 & 5.749369 & 0.016941\end{array}$

$\begin{array}{lll}8.103832 & 4.234411 & 1.160264\end{array}$

$\begin{array}{llll}7.219129 & 5.951123 & 2.169691\end{array}$

$\begin{array}{lll}1.040953 & 4.416193 & 0.988794\end{array}$

$\begin{array}{lll}1.652042 & 5.319099 & 2.871364\end{array}$

$\begin{array}{llll}2.126146 & 6.305650 & 0.995192\end{array}$

$\begin{array}{llll}2.810900 & 1.666848 & 6.207093\end{array}$

$\begin{array}{llll}0.936415 & 0.589871 & 5.969274\end{array}$

$2.477003 \quad-0.202101 \quad 7.279651$

$\begin{array}{llll}3.413544 & -4.516021 & 4.924708\end{array}$

$\begin{array}{lll}3.662566 & -4.505556 & 2.758198\end{array}$

$\begin{array}{lll}1.683857 & -4.271278 & 3.637056\end{array}$

$\begin{array}{llll}0.566952 & 0.786383 & 1.455995\end{array}$

$\begin{array}{llll}0.562486 & -1.550874 & 1.516763\end{array}$

$\begin{array}{lll}-0.123567 & 0.870289 & 2.651185\end{array}$

$\begin{array}{lll}0.819132 & 1.676092 & 0.894469\end{array}$

$\begin{array}{lll}-0.214172 & -1.500596 & 2.707074\end{array}$

$\begin{array}{lll}-0.532928 & -0.296515 & 3.301236\end{array}$

$\begin{array}{lll}0.801485 & -2.848345 & 0.946875\end{array}$

$\begin{array}{llll}1.953157 & -3.207332 & 0.162669\end{array}$

$\begin{array}{llll}2.702485 & -2.350992 & -0.410829\end{array}$

$\begin{array}{lll}0.947738 & -0.386274 & 0.911936\end{array}$

$\begin{array}{llll}0.326817 & -3.632349 & 1.510969\end{array}$

$-3.225896-1.129652-1.655556$

$\begin{array}{lll}-4.957064 & -0.045806 & -0.643766\end{array}$

$\begin{array}{lll}-4.817798 & 1.140950 & 0.502115\end{array}$

$\begin{array}{lll}-5.889863 & 0.777600 & -1.767185\end{array}$

$\begin{array}{llll}-5.831545 & -1.143078 & 0.259618\end{array}$

$\begin{array}{lll}-4.281279 & 2.369735 & 0.165162\end{array}$

$\begin{array}{lll}-2.998649 & 2.664883 & 0.676775\end{array}$

$\begin{array}{lll}-2.516156 & 1.929751 & 1.309432\end{array}$

$\begin{array}{lll}-2.409853 & 3.865904 & 0.370300\end{array}$

$\begin{array}{llll}-1.433288 & 4.116158 & 0.772158\end{array}$

$\begin{array}{llll}-3.055837 & 4.776478 & -0.511113\end{array}$

$\begin{array}{llll}-2.416025 & 5.978661 & -0.918124\end{array}$

$\begin{array}{lll}-1.438042 & 6.209972 & -0.504212\end{array}$

$\begin{array}{lll}-3.013134 & 6.828481 & -1.822770\end{array}$

$\begin{array}{llll}-2.510328 & 7.739907 & -2.132787\end{array}$

$\begin{array}{llll}4.284088 & 6.506806 & -2.356836\end{array}$

$\begin{array}{llll}-4.743280 & 7.167211 & -3.086887\end{array}$

$\begin{array}{llll}-4.945321 & 5.364569 & -1.958945\end{array}$

$\begin{array}{llll}-5.918424 & 5.132157 & -2.376503\end{array}$

$\begin{array}{llll}-4.364849 & 4.470262 & -1.017208\end{array}$

$\begin{array}{llll}-5.024119 & 3.263674 & -0.588128\end{array}$

$\begin{array}{llll}-6.451718 & 2.962367 & -0.894746\end{array}$

$\begin{array}{llll}-7.504103 & 3.879949 & -0.529095\end{array}$

$\begin{array}{llll}-7.264947 & 5.064053 & 0.224883\end{array}$

$\begin{array}{llll}-6.258382 & 5.292368 & 0.552758\end{array}$

$\begin{array}{lll}-8.294477 & 5.915139 & 0.564796\end{array}$

$\begin{array}{lll}-8.081927 & 6.806136 & 1.148881\end{array}$

$\begin{array}{lll}-9.624322 & 5.636666 & 0.170003\end{array}$

$\begin{array}{lll}-10.424594 & 6.319820 & 0.439251\end{array}$

$\begin{array}{llll}-9.897744 & 4.488092 & -0.537427\end{array}$

$\begin{array}{llll}-10.916767 & 4.246754 & -0.828839\end{array}$

$\begin{array}{llll}-8.861726 & 3.580413 & -0.888416\end{array}$

$\begin{array}{llll}-9.155077 & 2.364752 & -1.562896\end{array}$

$\begin{array}{llll}-10.182799 & 2.150154 & -1.842212\end{array}$

$\begin{array}{llll}-8.160415 & 1.458034 & -1.825054\end{array}$

$\begin{array}{llll}-8.365276 & 0.506661 & -2.304568\end{array}$

$\begin{array}{llll}-6.825426 & 1.751268 & -1.464081\end{array}$

$\begin{array}{lll}-5.432502 & -2.559105 & 0.024626\end{array}$

$\begin{array}{lll}-4.779640 & -2.869051 & 0.842711\end{array}$

$\begin{array}{lll}-6.640133 & -3.481028 & 0.008096\end{array}$

$\begin{array}{lll}-6.586784 & -4.710200 & 0.677518\end{array}$

$\begin{array}{llll}-5.693458 & -4.969671 & 1.238512\end{array}$

$\begin{array}{lll}-7.670920 & -5.591040 & 0.624936\end{array}$ $\begin{array}{lll}-7.617810 & -6.540900 & 1.150334\end{array}$

$\begin{array}{llll}-8.821166 & -5.249498 & -0.089815\end{array}$

$-9.666187 \quad-5.931778 \quad-0.124472$

$\begin{array}{llll}-8.882234 & -4.020960 & -0.756549\end{array}$

$-9.775868-3.746306-1.311054$

$\begin{array}{lll}-7.797864 & -3.144713 & -0.709310\end{array}$

$\begin{array}{llll}-7.844813 & -2.186567 & -1.219528\end{array}$

$-4.650598-2.685476-1.290055$

$\begin{array}{llll}-5.362577 & -2.707674 & -2.124228\end{array}$

$\begin{array}{lll}-4.130622 & -3.647211 & -1.286234\end{array}$

$\begin{array}{lll}-6.570071 & -0.877108 & 1.538043\end{array}$

$\begin{array}{lll}-6.971332 & -1.859877 & 1.801317\end{array}$

$\begin{array}{lll}-5.656551 & -0.461485 & 2.695713\end{array}$

$\begin{array}{lll}-4.747551 & -1.387255 & 3.231480\end{array}$

$\begin{array}{lll}-4.688377 & -2.397182 & 2.838809\end{array}$

$\begin{array}{llll}-3.893041 & -1.031649 & 4.278211\end{array}$

$\begin{array}{llll}-3.198061 & -1.770987 & 4.658585\end{array}$

$\begin{array}{lll}-3.941034 & 0.254592 & 4.818649\end{array}$

$\begin{array}{lll}-3.280206 & 0.531853 & 5.635771\end{array}$

$\begin{array}{llll}-4.850948 & 1.181169 & 4.303641\end{array}$

$\begin{array}{lll}-4.901107 & 2.185306 & 4.716873\end{array}$

$\begin{array}{llll}-5.698934 & 0.823546 & 3.254493\end{array}$

$\begin{array}{lll}-6.383890 & 1.564966 & 2.858624\end{array}$

$\begin{array}{lll}-7.773099 & 0.037025 & 1.314054\end{array}$

$\begin{array}{lll}-8.410749 & -0.380801 & 0.531734\end{array}$

$\begin{array}{lll}-7.480280 & 1.044021 & 1.013079\end{array}$

$\begin{array}{lll}-8.359452 & 0.114062 & 2.234950\end{array}$

$\begin{array}{lll}-2.311139 & -0.959841 & 0.328847\end{array}$

$\begin{array}{lll}-1.555216 & -0.184653 & 0.288940\end{array}$

$\begin{array}{lll}-2.991688 & -0.851739 & 1.168192\end{array}$

$\begin{array}{llll}-2.000582 & -2.295620 & -0.063701\end{array}$

$\begin{array}{lll}-2.618802 & -3.067723 & 0.373588\end{array}$

$\begin{array}{lll}-0.828277 & -2.738443 & -0.736343\end{array}$

$\begin{array}{llll}-0.178013 & -1.995734 & -1.177566\end{array}$

$\begin{array}{lll}-4.006149 & -0.993522 & -3.712393\end{array}$

$\begin{array}{llll}-5.051571 & -1.295237 & -3.697946\end{array}$

$\begin{array}{lll}-3.046717 & -2.045836 & -3.667097\end{array}$

$\begin{array}{llll}-3.432495 & -3.064924 & -3.639429\end{array}$

$\begin{array}{llll}-1.639355 & -1.943244 & -4.228930\end{array}$

$\begin{array}{llll}-1.659379 & -2.061345 & -5.324343\end{array}$

$\begin{array}{lll}-1.073789 & -2.793690 & -3.841410\end{array}$

$\begin{array}{lll}-0.905529 & -0.640334 & -3.854706\end{array}$

$\begin{array}{llll}-1.046067 & 0.118113 & -4.629967\end{array}$

$\begin{array}{llll}0.170050 & -0.833848 & -3.823790\end{array}$

$\begin{array}{llll}-1.324913 & -0.048164 & -2.511743\end{array}$

$\begin{array}{llll}-0.574171 & -0.081647 & -1.726378\end{array}$

$\begin{array}{llll}-2.322391 & 0.910210 & -2.363747\end{array}$

$\begin{array}{llll}-2.263385 & 1.548597 & -1.488468\end{array}$

$\begin{array}{llll}-3.200689 & 1.440000 & -3.479623\end{array}$

$\begin{array}{llll}-4.036223 & 1.966902 & -3.015313\end{array}$

$\begin{array}{llll}-2.635595 & 2.186920 & -4.054470\end{array}$

$\begin{array}{llll}-3.757007 & 0.345211 & -4.413716\end{array}$

$\begin{array}{llll}-3.081303 & 0.188144 & -5.261319\end{array}$

$\begin{array}{llll}-4.701036 & 0.697649 & -4.841586\end{array}$

$\begin{array}{llll}-0.731378 & -4.088452 & -1.303406\end{array}$

$\begin{array}{llll}-1.584272 & -5.143684 & -0.917901\end{array}$

$\begin{array}{llll}0.245305 & -4.347434 & -2.284320\end{array}$

$\begin{array}{llll}-1.467721 & -6.398210 & -1.511934\end{array}$

$\begin{array}{llll}-2.315536 & -4.997241 & -0.133477\end{array}$

$\begin{array}{llll}0.359548 & -5.602908 & -2.878181\end{array}$

$\begin{array}{llll}0.913077 & -3.548171 & -2.583476\end{array}$

$-0.499718-6.636195 \quad-2.494529$

$\begin{array}{llll}-2.131111 & -7.199416 & -1.198464\end{array}$

$\begin{array}{llll}1.117502 & -5.773737 & -3.637857\end{array}$

$\begin{array}{llll}1.0 .415200 & -7.617608 & -2.952680\end{array}$

$\begin{array}{llll}2.252157 & -4.530482 & -0.023443\end{array}$

$\begin{array}{llll}3.393977 & -4.898950 & -0.846293\end{array}$

$\begin{array}{llll}4.241228 & -5.213592 & -0.220944\end{array}$

$3.116991 \quad-5.737083 \quad-1.495123$

$3.701140 \quad-4.051739-1.452551$

$\begin{array}{lll}1.600227 & -5.666770 & 0.617084\end{array}$

$\begin{array}{llll}1.307372 & -6.389125 & -0.152022\end{array}$

$\begin{array}{llll}2.296710 & -6.154055 & 1.309736\end{array}$

$\begin{array}{lll}0.706581 & -5.389180 & 1.164120\end{array}$

$\begin{array}{lll}-1.096048 & -0.260613 & 4.224653\end{array}$ 


$\begin{array}{llll}1 & -0.559060 & -2.427135 & 3.149781 \\ 35 & -0.470260 & 2.619206 & 3.446516 \\ 5 & -2.497515 & -4.782187 & 2.849892 \\ 9 & -1.272130 & -4.984560 & 2.180995 \\ 9 & -2.845180 & -5.920219 & 3.573885 \\ 9 & -2.375170 & -3.670951 & 3.706875 \\ 9 & -3.507072 & -4.507338 & 1.881972\end{array}$

9 P1-C2 endo re-re $\left[\mathrm{BF}_{4}^{-}\right]$

Number of imaginary frequencies $=0$

The smallest frequencies are= $11.861914 .024716 .9918 \mathrm{~cm}(-1)$ Electronic energy $=-7647.2794543$

Zero-point correction=

1.775431

(Hartree/Particle)

Thermal correction to Energy=
Thermal correction to Enthalpy=

1.899614

1.900559

Thermal correction to Gibbs Free Energy=

Sum of electronic and zero-point Energies=

Sum of electronic and thermal Energies=

1.607571

$-7645.504023$

$-7645.379840$

Sum of electronic and thermal Enthalpies=

$-7645.378896$

Sum of electronic and thermal Free Energies=

$-7645.671884$

Cartesian Coordinates

\begin{tabular}{|c|c|c|c|}
\hline 26 & 7.578807 & -1.642230 & -1.53 \\
\hline 15 & 2.989710 & 0.654340 & -2.447610 \\
\hline 15 & 4.171059 & 0.422766 & 1.131911 \\
\hline 6 & 5.890055 & -0.425481 & -1.755560 \\
\hline 6 & 5.942113 & -1.010177 & -0.436289 \\
\hline 6 & 5.923330 & -2.428140 & -0.586507 \\
\hline 1 & 5.966561 & -3.147871 & 0.218092 \\
\hline 6 & 5.886342 & -2.730876 & -1.979400 \\
\hline 1 & 5.904350 & -3.720378 & -2.415681 \\
\hline 6 & 5.873666 & -1.501437 & -2.699120 \\
\hline 1 & 5.862090 & -1.390187 & -3.773363 \\
\hline 6 & 9.339506 & -0.819033 & -0.807130 \\
\hline 1 & 9.393324 & 0.002992 & -0.105642 \\
\hline 6 & 9.263748 & -2.205539 & -0.470535 \\
\hline 1 & 9.240823 & -2.617557 & 0.529465 \\
\hline 6 & 9.178477 & -2.950781 & -1.685758 \\
\hline 1 & 9.072330 & -4.024645 & -1.766685 \\
\hline 6 & 9.203635 & -2.026233 & -2.774097 \\
\hline 1 & 9.122040 & -2.27 & -3.823896 \\
\hline 6 & 9.305897 & -0.710565 & -2.232279 \\
\hline 1 & 9.321018 & 0.207 & -2.804212 \\
\hline 6 & 5.785 & 1.0 & -2.0 \\
\hline 6 & 6.911173 & 1.854068 & -1.855329 \\
\hline 1 & 7.836903 & 1.387520 & -1.544189 \\
\hline 6 & 6.857626 & 3.233 & -2.051391 \\
\hline 1 & 7.739724 & 3.8 & -1.877355 \\
\hline 6 & 5.660867 & 3.8 & -2.450495 \\
\hline 1 & 5.601112 & 4.896 & 6982 \\
\hline 6 & 4.526072 & 3.031771 & -2.630038 \\
\hline 1 & 3.599622 & 3.508549 & -2.928971 \\
\hline 6 & 4.558609 & 1.645414 & -2.409504 \\
\hline 6 & 5.940089 & -0.219688 & 0.842114 \\
\hline 1 & 6.519935 & 0.681848 & 0.646851 \\
\hline 6 & 6.584765 & -0.935469 & 2.032594 \\
\hline 1 & 6.571893 & -0.305070 & 2.926119 \\
\hline 1 & 6.084177 & -1.875346 & 2.276869 \\
\hline 1 & 7.625250 & -1.165511 & 1.784934 \\
\hline 6 & 1.695965 & 1.874041 & -2.920564 \\
\hline 6 & 1.233275 & 2.736559 & -1.911384 \\
\hline 1 & 1.663670 & 2.681117 & -0.916428 \\
\hline 6 & 0.240856 & 3.676239 & -2.179157 \\
\hline 1 & -0.094754 & 4.341065 & -1.394331 \\
\hline 6 & -0.325725 & 3.752370 & -3.454448 \\
\hline 1 & -1.113959 & 4.472457 & -3.653802 \\
\hline 6 & 0.121050 & 2.894636 & -4.460522 \\
\hline 1 & -0.316753 & 2.943527 & -5.453377 \\
\hline 6 & 1.131195 & 1.964132 & -4.200138 \\
\hline 1 & 1.459944 & 1.303897 & -4.994322 \\
\hline & 3.179203 & -0.455465 & -3.889119 \\
\hline & 3.704874 & -0.026935 & -5.117378 \\
\hline
\end{tabular}

\begin{tabular}{|c|c|c|c|}
\hline & 4.003527 & 988 & -5.250695 \\
\hline 0 & 3.859944 & -0.937213 & -6.164124 \\
\hline 1 & 4.266363 & -0.602880 & -7.114370 \\
\hline 6 & 3.497348 & -2.277736 & -5.989412 \\
\hline 1 & 3.622044 & -2.983274 & -6.805925 \\
\hline 6 & 2.985438 & -2.710121 & -4.763745 \\
\hline 1 & 2.716961 & -3.752392 & -4.617374 \\
\hline 6 & 2.832819 & -1.801440 & -3.715406 \\
\hline 1 & 2.486921 & -2.133757 & -2.741814 \\
\hline 6 & 4.357832 & 2.254692 & 1.321618 \\
\hline 6 & 3.182740 & 3.013321 & 1.405544 \\
\hline 1 & 2.229930 & 2.517986 & 1.508938 \\
\hline 6 & 3.212687 & 4.405890 & 1.336587 \\
\hline 6 & 4.420908 & 5.083562 & 1.200430 \\
\hline 1 & 4.444879 & 6.164810 & 1.120451 \\
\hline 6 & 5.597429 & 4.336496 & 1.151218 \\
\hline 6 & 5.574643 & 2.943575 & 1.211451 \\
\hline 1 & 6.516572 & 2.417504 & 1.147866 \\
\hline 6 & 3.649542 & -0.212014 & 2.773214 \\
\hline 6 & 3.457074 & -1.600350 & 2.844569 \\
\hline 1 & 3.671303 & -2.218454 & 1.979469 \\
\hline 6 & 2.976175 & -2.185941 & 4.012820 \\
\hline 6 & 2.615827 & -1.399237 & 5.105652 \\
\hline 1 & 2.207900 & -1.854969 & 6.001210 \\
\hline 6 & 2.775883 & -0.015788 & 5.019959 \\
\hline 6 & 3.305853 & 0.580487 & 3.874639 \\
\hline 1 & 3.432277 & 1.656112 & 3.843694 \\
\hline 29 & 2.561950 & -0.327872 & -0.451923 \\
\hline 6 & 2.294250 & 0.826814 & 6.169500 \\
\hline 6 & 2.729338 & -3.668653 & 4.045780 \\
\hline 6 & 1.916513 & 5.166991 & 1.395461 \\
\hline 6 & 6.897856 & 5.073646 & 0.975065 \\
\hline 9 & 6.911795 & 5.776425 & -0.184717 \\
\hline 9 & 7.964839 & 4.245938 & 0.951378 \\
\hline 9 & 7.101933 & 5.968989 & 1.968012 \\
\hline 9 & 0.915541 & 4.471151 & 0.786640 \\
\hline 9 & 1.510077 & 5.394254 & 2.664313 \\
\hline 9 & 1.995845 & 6.363700 & 0.781647 \\
\hline 9 & 2.621972 & 2.129105 & 6.031388 \\
\hline 9 & 0.942994 & 0.763252 & 6.282421 \\
\hline 9 & 2.798642 & 0.404014 & 7.350861 \\
\hline 9 & 2.857176 & -4.178687 & 5.287392 \\
\hline 9 & 3.583743 & -4.341998 & 3.237365 \\
\hline 9 & 1.476196 & -3.971803 & 3.622624 \\
\hline 6 & 0.499137 & 0.742053 & 1.366656 \\
\hline 6 & 0.379425 & -1.572321 & 1.395885 \\
\hline 6 & -0.048468 & 0.822264 & 2.638791 \\
\hline 1 & 0.726806 & 1.629598 & 0.792053 \\
\hline 6 & -0.190289 & -1.547147 & 2.674569 \\
\hline 6 & -0.382501 & -0.336316 & 3.330714 \\
\hline 6 & 0.418409 & -2.878699 & 0.635212 \\
\hline 6 & 1.832601 & -3.289984 & 0.243616 \\
\hline 8 & 2.600890 & -2.451756 & -0.296655 \\
\hline 7 & 0.765831 & -0.435380 & 0.776385 \\
\hline 1 & -0.049252 & -3.624803 & 1.270515 \\
\hline 77 & -3.233500 & -1.211518 & -1.728583 \\
\hline 15 & -4.937248 & -0.065731 & $1-0.690200$ \\
\hline 8 & -4.767943 & 1.123481 & 0.460309 \\
\hline 8 & -5.894226 & 0.785143 & -1.785301 \\
\hline 7 & -5.816555 & -1.142405 & 0.241838 \\
\hline 6 & -4.220870 & 2.343392 & 0.127776 \\
\hline 6 & -2.936021 & 2.625087 & 0.643959 \\
\hline 1 & -2.462962 & 1.880131 & 1.273282 \\
\hline 6 & -2.338986 & 3.825570 & 0.353835 \\
\hline 1 & -1.366654 & 4.069397 & 0.766196 \\
\hline 6 & -2.972528 & 4.747838 & -0.524119 \\
\hline 6 & -2.318229 & 5.945884 & -0.920622 \\
\hline 1 & -1.338514 & 6.162860 & -0.501792 \\
\hline 6 & -2.902705 & 6.806079 & -1.823842 \\
\hline 1 & -2.389252 & 7.713586 & -2.127972 \\
\hline 6 & -4.174905 & 6.499852 & -2.364314 \\
\hline 1 & -4.624297 & 7.168431 & -3.093097 \\
\hline 6 & -4.849621 & 5.362303 & -1.975013 \\
\hline 1 & -5.823485 & 5.141846 & -2.397484 \\
\hline 6 & -4.281993 & 4.456366 & -1.036573 \\
\hline
\end{tabular}




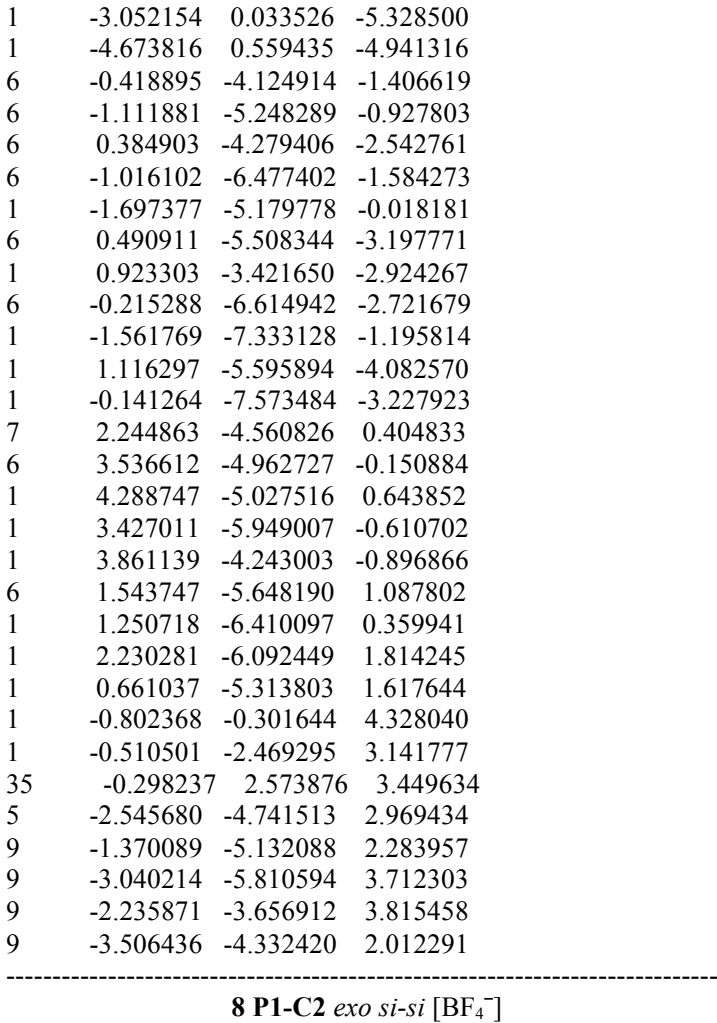

Number of imaginary frequencies $=0$

The smallest frequencies are $=12.773514 .994517 .4871 \mathrm{~cm}(-1)$ Electronic energy $=-7647.262549$

Zero-point correction=

(Hartree/Particle)

Thermal correction to Energy= $\quad 1.898486$

Thermal correction to Enthalpy=

1.899431

Thermal correction to Gibbs Free Energy=

Sum of electronic and zero-point Energies=

Sum of electronic and thermal Energies=

Sum of electronic and thermal Enthalpies=

Sum of electronic and thermal Free Energies=

1.603479

$-7645.489351$

$-7645.364063$

$-7645.363118$

$-7645.659070$ 
$\begin{array}{llll}3.905970 & 6.113346 & -0.602866\end{array}$

$\begin{array}{llll}3.773679 & 4.089693 & -1.324574\end{array}$

$\begin{array}{llll}2.781673 & 4.242590 & -1.734919\end{array}$

$\begin{array}{llll}4.377132 & 2.825305 & -1.419980\end{array}$

$\begin{array}{llll}5.955501 & 0.862667 & 1.683428\end{array}$

$\begin{array}{llll}6.134679 & 1.930067 & 1.822131\end{array}$

$\begin{array}{lll}6.617380 & 0.109423 & 2.840176\end{array}$

$\begin{array}{lll}6.228830 & 0.451748 & 3.803415\end{array}$

$\begin{array}{llll}6.451839 & -0.969610 & 2.778228\end{array}$

$\begin{array}{llll}7.697470 & 0.286703 & 2.817864\end{array}$

$\begin{array}{llll}1.813532 & 2.121338 & -2.619614\end{array}$

$\begin{array}{llll}0.715868 & 2.033123 & -1.748075\end{array}$

$\begin{array}{llll}0.815408 & 1.496513 & -0.810648\end{array}$

$\begin{array}{llll}-0.509509 & 2.602820 & -2.099803\end{array}$

$\begin{array}{lll}-1.351394 & 2.528129 & -1.422164\end{array}$

$\begin{array}{lll}-0.659853 & 3.251954 & -3.327130\end{array}$

$\begin{array}{llll}-1.617686 & 3.687371 & -3.597705\end{array}$

$\begin{array}{llll}0.422200 & 3.325548 & -4.208088\end{array}$

$\begin{array}{llll}0.311539 & 3.821144 & -5.168602\end{array}$

$\begin{array}{llll}1.652778 & 2.766397 & -3.856633\end{array}$

$\begin{array}{llll}2.484272 & 2.836455 & -4.549945\end{array}$

$\begin{array}{llll}4.239490 & 0.936560 & -3.632724\end{array}$

$\begin{array}{llll}4.942071 & 1.870284 & -4.411305\end{array}$

$\begin{array}{llll}5.013197 & 2.904655 & -4.087467\end{array}$

$\begin{array}{llll}5.573926 & 1.468186 & -5.589389\end{array}$

$\begin{array}{llll}6.120197 & 2.195581 & -6.183482\end{array}$

$\begin{array}{llll}5.508280 & 0.132732 & -6.001380\end{array}$

$6.007048 \quad-0.179046 \quad-6.914741$

$\begin{array}{llll}4.802299 & -0.798346 & -5.235663\end{array}$

$\begin{array}{llll}4.745398 & -1.836969 & -5.548991\end{array}$

$\begin{array}{llll}4.172709 & -0.397793 & -4.055357\end{array}$

$3.632454-1.122652-3.457400$

$\begin{array}{lll}3.345031 & 2.289788 & 2.055962\end{array}$

$\begin{array}{lll}1.961081 & 2.407936 & 1.859268\end{array}$

$\begin{array}{lll}1.415776 & 1.548166 & 1.482259\end{array}$

$\begin{array}{lll}1.308390 & 3.612970 & 2.119322\end{array}$

$\begin{array}{llll}2.021546 & 4.735510 & 2.536852\end{array}$

$\begin{array}{llll}1.518323 & 5.680068 & 2.711481\end{array}$

$\begin{array}{llll}3.401961 & 4.622512 & 2.706493\end{array}$

$\begin{array}{lll}4.060742 & 3.411028 & 2.491040\end{array}$

$\begin{array}{lll}5.130586 & 3.370019 & 2.644749\end{array}$

$\begin{array}{lll}3.575200 & -0.462813 & 2.930514\end{array}$

$\begin{array}{lll}3.756386 & -1.831547 & 2.677107\end{array}$

$\begin{array}{lll}4.186859 & -2.151627 & 1.735585\end{array}$

$\begin{array}{lll}3.337838 & -2.781990 & 3.601648\end{array}$

$\begin{array}{lll}2.697879 & -2.393844 & 4.780444\end{array}$

$\begin{array}{llll}2.343258 & -3.135926 & 5.486553\end{array}$

$2.497945 \quad-1.036077 \quad 5.020667$

$2.940594 \quad-0.069481 \quad 4.111320$

$\begin{array}{lll}2.768808 & 0.979950 & 4.324353\end{array}$

$\begin{array}{llll}3.133599 & -0.168459 & -0.424854\end{array}$

$\begin{array}{llll}1.706154 & -0.595731 & 6.219006\end{array}$

$\begin{array}{lll}3.466664 & -4.246657 & 3.283214\end{array}$

$\begin{array}{lll}-0.185236 & 3.673445 & 1.959942\end{array}$

$\begin{array}{lll}4.178963 & 5.858568 & 3.064659\end{array}$

$\begin{array}{lll}4.120809 & 6.783143 & 2.071516\end{array}$

$\begin{array}{llll}5.487050 & 5.605162 & 3.288566\end{array}$

$\begin{array}{lll}3.688123 & 6.460693 & 4.173001\end{array}$

$\begin{array}{lll}-0.598434 & 2.958984 & 0.877683\end{array}$

$\begin{array}{lll}-0.837866 & 3.147396 & 3.026160\end{array}$

$\begin{array}{lll}-0.643396 & 4.932910 & 1.796831\end{array}$

$\begin{array}{llll}2.166161 & 0.565438 & 6.736829\end{array}$

$\begin{array}{llll}0.399466 & -0.376274 & 5.893446\end{array}$

$\begin{array}{lll}1.708665 & -1.512872 & 7.208113\end{array}$

$\begin{array}{llll}3.890497 & -4.954059 & 4.357805\end{array}$

$\begin{array}{llll}4.337941 & -4.477525 & 2.276874\end{array}$

$\begin{array}{lll}2.281394 & -4.788292 & 2.909423\end{array}$

$\begin{array}{lll}0.853602 & -1.384573 & 1.123069\end{array}$

$\begin{array}{lll}1.193436 & -2.687458 & 0.671656\end{array}$

$\begin{array}{llll}2.239223 & -3.067380 & -0.192785\end{array}$

$\begin{array}{llll}0.588723 & -3.506926 & 1.028340\end{array}$

$77 \quad-3.714420 \quad-1.126396 \quad-1.568170$

$\begin{array}{lll}-5.303728 & -0.272093 & -0.212846\end{array}$

$\begin{array}{lll}-5.091975 & 1.099167 & 0.689984\end{array}$

$\begin{array}{lll}-6.653257 & 0.155672 & -1.079585\end{array}$ $\begin{array}{llll}-5.644338 & -1.352567 & 1.003247\end{array}$

$\begin{array}{lll}-4.921635 & 2.324301 & 0.060223\end{array}$

$\begin{array}{lll}-3.646562 & 2.918111 & 0.175197\end{array}$

$\begin{array}{lll}-2.898792 & 2.430991 & 0.787596\end{array}$

$\begin{array}{llll}-3.391687 & 4.102077 & -0.470790\end{array}$

$\begin{array}{llll}-2.421705 & 4.578892 & -0.371977\end{array}$

$\begin{array}{llll}-4.381811 & 4.689900 & -1.304651\end{array}$

$\begin{array}{llll}-4.091462 & 5.841396 & -2.085542\end{array}$

$\begin{array}{llll}-3.098903 & 6.278231 & -2.012285\end{array}$

$\begin{array}{llll}-5.036312 & 6.380026 & -2.929812\end{array}$

$\begin{array}{llll}-4.800672 & 7.254123 & -3.529740\end{array}$

$\begin{array}{llll}-6.316069 & 5.783095 & -3.026069\end{array}$

$\begin{array}{lll}-7.051872 & 6.196622 & -3.709784\end{array}$

$\begin{array}{llll}-6.637058 & 4.678849 & -2.266044\end{array}$

$\begin{array}{llll}-7.619028 & 4.229467 & -2.358555\end{array}$

$\begin{array}{llll}-5.690559 & 4.101963 & -1.374691\end{array}$

$\begin{array}{llll}-5.985156 & 2.930295 & -0.588382\end{array}$

$\begin{array}{llll}-7.364279 & 2.381291 & -0.448657\end{array}$

$\begin{array}{lll}-8.451434 & 3.211081 & 0.015800\end{array}$

$\begin{array}{llll}-8.259847 & 4.544509 & 0.475417\end{array}$

$\begin{array}{lll}-7.260671 & 4.961456 & 0.499494\end{array}$

$\begin{array}{lll}-9.320742 & 5.310434 & 0.908942\end{array}$

$\begin{array}{lll}-9.141906 & 6.322502 & 1.260712\end{array}$

$\begin{array}{lll}-10.636598 & 4.791074 & 0.908766\end{array}$

$\begin{array}{lll}-11.463933 & 5.408894 & 1.245376\end{array}$

$\begin{array}{lll}-10.856497 & 3.495607 & 0.499204\end{array}$

$\begin{array}{lll}-11.857469 & 3.072342 & 0.514585\end{array}$

$\begin{array}{llll}-9.782231 & 2.673931 & 0.062548\end{array}$

$\begin{array}{llll}-10.004192 & 1.318927 & -0.303046\end{array}$

$\begin{array}{llll}-11.015325 & 0.922858 & -0.276547\end{array}$

$\begin{array}{llll}-8.951651 & 0.512345 & -0.651156\end{array}$

$\begin{array}{llll}-9.089169 & -0.534379 & -0.900977\end{array}$

$\begin{array}{llll}-7.643319 & 1.044309 & -0.694412\end{array}$

$\begin{array}{llll}-4.953951 & -2.661947 & 0.860618\end{array}$

$\begin{array}{lll}-4.108474 & -2.675879 & 1.552590\end{array}$

$\begin{array}{lll}-5.868141 & -3.826329 & 1.205686\end{array}$

$\begin{array}{lll}-5.328219 & -4.961230 & 1.822974\end{array}$

$\begin{array}{lll}-4.273930 & -4.982973 & 2.073025\end{array}$

$\begin{array}{llll}-6.133556 & -6.073080 & 2.081570\end{array}$

$\begin{array}{llll}-5.697539 & -6.950476 & 2.551626\end{array}$

$\begin{array}{lll}-7.486825 & -6.059489 & 1.735591\end{array}$

$\begin{array}{lll}-8.113359 & -6.922804 & 1.942529\end{array}$

$\begin{array}{lll}-8.031122 & -4.927596 & 1.120134\end{array}$

$\begin{array}{llll}-9.083580 & -4.907890 & 0.849285\end{array}$

$\begin{array}{llll}-7.224962 & -3.820410 & 0.851808\end{array}$

$\begin{array}{lll}-7.646234 & -2.937629 & 0.378153\end{array}$

$\begin{array}{llll}-4.420607 & -2.861136 & -0.566742\end{array}$

$\begin{array}{llll}-5.231807 & -3.263954 & -1.182220\end{array}$

$\begin{array}{llll}-3.633298 & -3.611644 & -0.525239\end{array}$

$\begin{array}{lll}-6.146085 & -1.054092 & 2.386991\end{array}$

$\begin{array}{lll}-6.530879 & -2.019751 & 2.724479\end{array}$

$\begin{array}{lll}-5.017813 & -0.685139 & 3.351695\end{array}$

$\begin{array}{lll}-4.355295 & -1.713802 & 4.039758\end{array}$

$\begin{array}{lll}-4.656255 & -2.745312 & 3.874969\end{array}$

$\begin{array}{llll}-3.324993 & -1.432863 & 4.940983\end{array}$

$\begin{array}{lll}-2.823719 & -2.247105 & 5.456234\end{array}$

$\begin{array}{llll}-2.942992 & -0.111166 & 5.176898\end{array}$

$\begin{array}{lll}-2.129978 & 0.108335 & 5.862623\end{array}$

$\begin{array}{lll}-3.599742 & 0.923374 & 4.503654\end{array}$

$\begin{array}{lll}-3.304194 & 1.955581 & 4.672627\end{array}$

$\begin{array}{lll}-4.629177 & 0.639537 & 3.603645\end{array}$

$\begin{array}{llll}-5.130104 & 1.455864 & 3.097798\end{array}$

$\begin{array}{lll}-7.338596 & -0.099248 & 2.413704\end{array}$

$\begin{array}{lll}-8.143595 & -0.486255 & 1.784383\end{array}$

$\begin{array}{lll}-7.094090 & 0.907782 & 2.076136\end{array}$

$\begin{array}{lll}-7.710203 & -0.031205 & 3.440528\end{array}$

$\begin{array}{lll}-2.412080 & -0.715623 & 0.149035\end{array}$

$\begin{array}{lll}-2.429990 & 0.293562 & 0.541676\end{array}$

$\begin{array}{llll}-2.567977 & -1.480047 & 0.902536\end{array}$

$\begin{array}{llll}-1.466511 & -0.976739 & -0.888590\end{array}$

$\begin{array}{lll}-0.956116 & -0.126332 & -1.321461\end{array}$

$\begin{array}{llll}-1.224642 & -2.248505 & -1.394168\end{array}$

$\begin{array}{lll}-1.604114 & -3.100496 & -0.844898\end{array}$

$\begin{array}{llll}-5.270106 & -1.506282 & -3.186062\end{array}$

$\begin{array}{lll}-6.077230 & -2.024896 & -2.675923\end{array}$ 


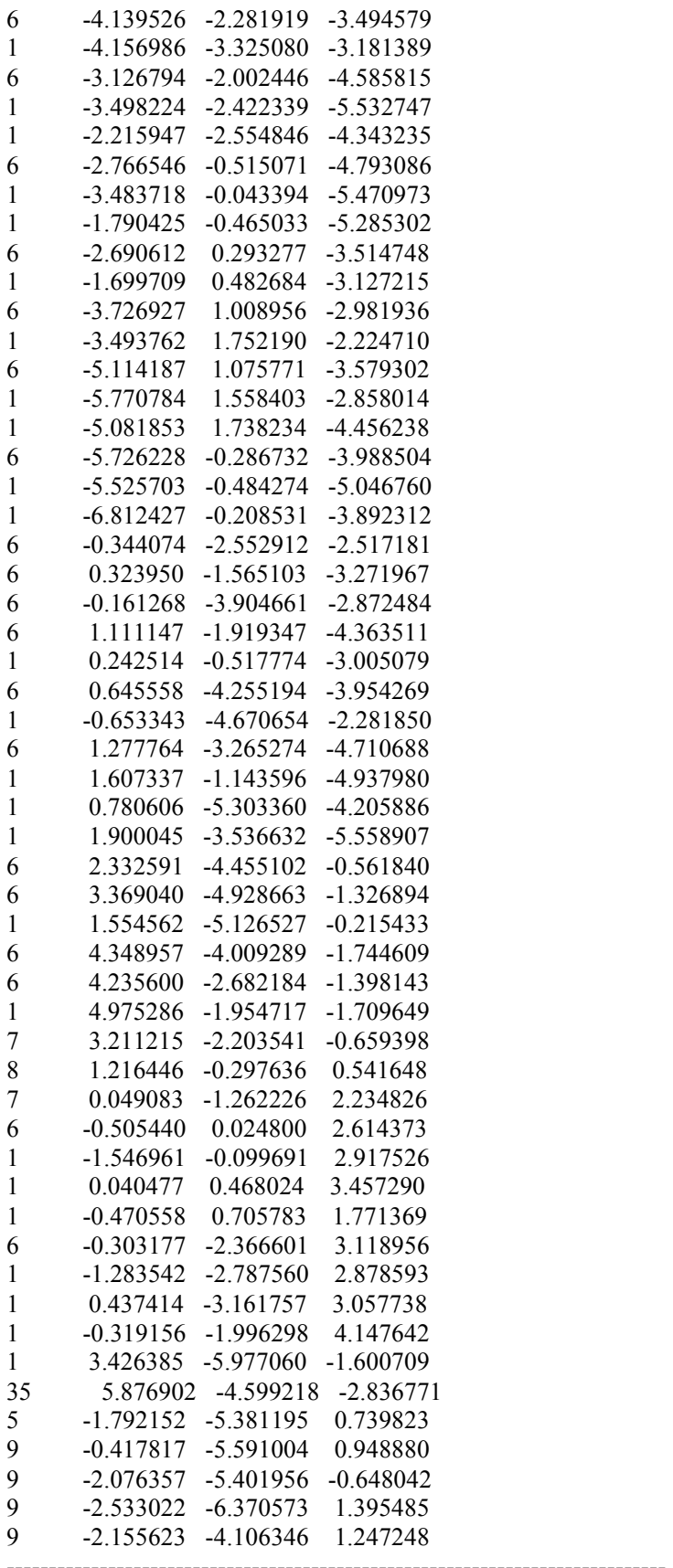

$[8-9]^{\ddagger}$ P1-C2 exo si-si $\left[\mathrm{BF}_{4}^{-}\right]$

Number of imaginary frequencies $=1$

The smallest frequencies are $=-162.465610 .607012 .7531 \mathrm{~cm}(-1)$ Electronic energy $=-7647.2559268$

Zero-point correction=

(Hartree/Particle)

Thermal correction to Energy= $\quad 1.896472$

Thermal correction to Enthalpy= $\quad 1.897416$

Thermal correction to Gibbs Free Energy $=\quad 1.602470$

Sum of electronic and zero-point Energies $=\quad-7645.484104$

Sum of electronic and thermal Energies $=\quad-7645.359455$

Sum of electronic and thermal Enthalpies $=\quad-7645.358510$

Sum of electronic and thermal Free Energies $=\quad-7645.653457$

Cartesian Coordinates

$\begin{array}{llll}26 & 8.187235 & 0.694637 & -0.695070 \\ 15 & 3.196836 & 1.523967 & -2.041619 \\ 15 & 3.988762 & 0.691339 & 1.546762 \\ 6 & 6.197312 & 1.283858 & -0.971458\end{array}$

$\begin{array}{lll}6.338900 & 0.443481 & 0.194554\end{array}$

$\begin{array}{lll}6.859621 & -0.815714 & -0.235252\end{array}$

$\begin{array}{lll}7.091546 & -1.655785 & 0.404975\end{array}$

$7.073911-0.754263-1.644740$

$7.498349-1.538242-2.256359$

$\begin{array}{llll}6.669113 & 0.535256 & -2.097850\end{array}$

$6.728319 \quad 0.898629 \quad-3.112524$

$\begin{array}{lll}9.432098 & 1.843932 & 0.504263\end{array}$

$\begin{array}{lll}9.106272 & 2.421783 & 1.358994\end{array}$

$\begin{array}{llll}9.846589 & 0.476942 & 0.524920\end{array}$

$\begin{array}{lll}9.875586 & -0.165746 & 1.394783\end{array}$

$\begin{array}{llll}10.165352 & 0.091789 & -0.813029\end{array}$

$10.476378-0.893901-1.133290$

$\begin{array}{llll}9.949088 & 1.220491 & -1.661118\end{array}$

$\begin{array}{llll}10.070047 & 1.241162 & -2.736196\end{array}$

$\begin{array}{llll}9.498064 & 2.302856 & -0.848251\end{array}$

$\begin{array}{llll}9.223664 & 3.287251 & -1.202240\end{array}$

$5.591845 \quad 2.647136-0.953041$

$\begin{array}{llll}6.294826 & 3.699448 & -0.345910\end{array}$

$\begin{array}{lll}7.278033 & 3.490830 & 0.055902\end{array}$

$\begin{array}{lll}5.754986 & 4.979906 & -0.231112\end{array}$

$\begin{array}{lll}6.314734 & 5.762230 & 0.271791\end{array}$

$\begin{array}{llll}4.487639 & 5.237631 & -0.747584\end{array}$

$\begin{array}{llll}4.048658 & 6.226564 & -0.663320\end{array}$

$3.767357 \quad 4.207993-1.352922$

$\begin{array}{lll}2.775694 & 4.422167 & -1.734010\end{array}$

$\begin{array}{llll}4.286316 & 2.906525 & -1.451578\end{array}$

$\begin{array}{llll}5.882568 & 0.820001 & 1.577979\end{array}$

$\begin{array}{llll}6.098464 & 1.880196 & 1.719047\end{array}$

$\begin{array}{llll}6.570615 & 0.038313 & 2.700608\end{array}$

$\begin{array}{lll}6.232936 & 0.382548 & 3.682138\end{array}$

$\begin{array}{lll}6.374997 & -1.035511 & 2.637184\end{array}$

$\begin{array}{lll}7.652834 & 0.188134 & 2.632722\end{array}$

$\begin{array}{llll}1.635130 & 2.387167 & -2.498416\end{array}$

$\begin{array}{llll}0.645192 & 2.490770 & -1.507218\end{array}$

$\begin{array}{llll}0.779922 & 1.981263 & -0.559186\end{array}$

$\begin{array}{llll}-0.512285 & 3.232812 & -1.745417\end{array}$

$\begin{array}{llll}-1.271152 & 3.305353 & -0.977899\end{array}$

$\begin{array}{llll}-0.710994 & 3.851006 & -2.982080\end{array}$

$\begin{array}{llll}-1.620377 & 4.416707 & -3.164780\end{array}$

$\begin{array}{llll}0.251726 & 3.719845 & -3.985767\end{array}$

$0.098143 \quad 4.186435 \quad-4.954793$

$\begin{array}{llll}1.422860 & 2.997366 & -3.744718\end{array}$

$\begin{array}{llll}2.171711 & 2.920816 & -4.526063\end{array}$

$\begin{array}{llll}3.911758 & 1.028075 & -3.657951\end{array}$

$\begin{array}{llll}4.633902 & 1.912248 & -4.475036\end{array}$

$\begin{array}{llll}4.790313 & 2.939819 & -4.159960\end{array}$

$\begin{array}{llll}5.172888 & 1.467926 & -5.683756\end{array}$

$\begin{array}{llll}5.734406 & 2.156457 & -6.308947\end{array}$

$\begin{array}{llll}4.996248 & 0.139989 & -6.086367\end{array}$

$\begin{array}{llll}5.424788 & -0.205313 & -7.022883\end{array}$

$\begin{array}{llll}4.269843 & -0.741371 & -5.281649\end{array}$

$\begin{array}{llll}4.131653 & -1.774786 & -5.586667\end{array}$

$\begin{array}{llll}3.729195 & -0.297949 & -4.073172\end{array}$

$\begin{array}{lll}3.175429 & -0.984814 & -3.442746\end{array}$

$\begin{array}{lll}3.339830 & 2.330829 & 2.083751\end{array}$

$\begin{array}{llll}1.945929 & 2.473317 & 2.016774\end{array}$

$\begin{array}{lll}1.347932 & 1.623129 & 1.706276\end{array}$

$\begin{array}{lll}1.342656 & 3.694782 & 2.314235\end{array}$

$\begin{array}{lll}2.112342 & 4.809020 & 2.644277\end{array}$

$\begin{array}{lll}1.644118 & 5.765042 & 2.850839\end{array}$

$\begin{array}{lll}3.500162 & 4.671065 & 2.687027\end{array}$

$\begin{array}{lll}4.113975 & 3.444618 & 2.428378\end{array}$

$\begin{array}{llll}5.192926 & 3.387759 & 2.475580\end{array}$

$\begin{array}{lll}3.508069 & -0.444336 & 2.912390\end{array}$

$\begin{array}{llll}3.678293 & -1.813822 & 2.654177\end{array}$

$\begin{array}{llll}4.116445 & -2.137087 & 1.717559\end{array}$

$\begin{array}{lll}3.250806 & -2.765069 & 3.574394\end{array}$

$\begin{array}{llll}2.619571 & -2.377143 & 4.757823\end{array}$

$\begin{array}{lll}2.263311 & -3.119828 & 5.462413\end{array}$

$\begin{array}{llll}2.438075 & -1.018998 & 5.007549\end{array}$

$\begin{array}{llll}2.884147 & -0.052468 & 4.100294\end{array}$

$\begin{array}{llll}2.726462 & 0.996623 & 4.323147\end{array}$

$2.931674-0.027596 \quad-0.422410$

$\begin{array}{llll}1.666317 & -0.583807 & 6.222283\end{array}$ 
$\begin{array}{lll}3.363026 & -4.230012 & 3.250462\end{array}$

$\begin{array}{lll}-0.158715 & 3.777002 & 2.308282\end{array}$

$\begin{array}{lll}4.329392 & 5.893613 & 2.969712\end{array}$

$\begin{array}{llll}4.191941 & 6.823104 & 1.990492\end{array}$

$\begin{array}{lll}5.648460 & 5.616443 & 3.067326\end{array}$

$\begin{array}{lll}3.956583 & 6.497282 & 4.122091\end{array}$

$\begin{array}{lll}-0.696543 & 3.044223 & 1.294127\end{array}$

$\begin{array}{lll}-0.698709 & 3.286214 & 3.449123\end{array}$

$\begin{array}{lll}-0.611649 & 5.040222 & 2.162106\end{array}$

$\begin{array}{lll}2.091769 & 0.607692 & 6.697005\end{array}$

$\begin{array}{lll}0.341384 & -0.432693 & 5.938626\end{array}$

$\begin{array}{lll}1.745262 & -1.477483 & 7.229617\end{array}$

$\begin{array}{llll}3.738805 & -4.953322 & 4.330238\end{array}$

$\begin{array}{llll}4.260843 & -4.469851 & 2.268460\end{array}$

$\begin{array}{lll}2.177922 & -4.739322 & 2.835066\end{array}$

$\begin{array}{llll}0.677969 & -1.270567 & 1.177577\end{array}$

$\begin{array}{lll}0.917451 & -2.569293 & 0.594411\end{array}$

$\begin{array}{llll}2.068477 & -2.962543 & -0.158578\end{array}$

$\begin{array}{llll}0.401173 & -3.403180 & 1.042831\end{array}$

$\begin{array}{lll}-3.455070 & -0.845779 & -1.773057\end{array}$

$\begin{array}{lll}-5.152038 & -0.274979 & -0.373986\end{array}$

$\begin{array}{lll}-5.103258 & 0.977913 & 0.723397\end{array}$

$\begin{array}{llll}-6.511378 & 0.166886 & -1.240667\end{array}$

$\begin{array}{llll}-5.478535 & -1.522390 & 0.679468\end{array}$

$\begin{array}{llll}-4.982487 & 2.279268 & 0.266646\end{array}$

$\begin{array}{lll}-3.764266 & 2.939177 & 0.542556\end{array}$

$\begin{array}{lll}-3.017786 & 2.423006 & 1.133344\end{array}$

$\begin{array}{llll}-3.559997 & 4.210561 & 0.066761\end{array}$

$\begin{array}{llll}-2.640349 & 4.739585 & 0.297018\end{array}$

$\begin{array}{llll}-4.533804 & 4.829949 & -0.763739\end{array}$

$\begin{array}{llll}-4.282972 & 6.086848 & -1.378311\end{array}$

$\begin{array}{llll}-3.334012 & 6.579124 & -1.181327\end{array}$

$\begin{array}{lll}-5.210292 & 6.661182 & -2.218847\end{array}$

$\begin{array}{llll}-5.003686 & 7.616954 & -2.691570\end{array}$

$\begin{array}{llll}-6.432642 & 5.996196 & -2.478376\end{array}$

$\begin{array}{llll}-7.152865 & 6.441056 & -3.159028\end{array}$

$\begin{array}{llll}-6.716031 & 4.787015 & -1.880621\end{array}$

$\begin{array}{llll}-7.653252 & 4.286263 & -2.094800\end{array}$

$\begin{array}{llll}-5.786642 & 4.168523 & -0.999090\end{array}$

$\begin{array}{llll}-6.044267 & 2.894135 & -0.378574\end{array}$

$\begin{array}{llll}-7.390633 & 2.254535 & -0.391172\end{array}$

$\begin{array}{lll}-8.549856 & 2.958615 & 0.106226\end{array}$

$\begin{array}{llll}-8.466384 & 4.233380 & 0.734882\end{array}$

$\begin{array}{llll}-7.497939 & 4.699824 & 0.866687\end{array}$

$\begin{array}{llll}-9.593536 & 4.878291 & 1.197494\end{array}$

$\begin{array}{llll}-9.495995 & 5.846561 & 1.680312\end{array}$

$\begin{array}{lll}-10.872564 & 4.290161 & 1.058545\end{array}$

$\begin{array}{lll}-11.752938 & 4.813270 & 1.420371\end{array}$

$\begin{array}{lll}-10.989454 & 3.045903 & 0.481498\end{array}$

$\begin{array}{lll}-11.961281 & 2.568097 & 0.387366\end{array}$

$\begin{array}{lll}-9.845194 & 2.347005 & 0.010490\end{array}$

$\begin{array}{llll}-9.962250 & 1.037666 & -0.529227\end{array}$

$\begin{array}{lll}-10.946573 & 0.585105 & -0.609709\end{array}$

$\begin{array}{llll}-8.844794 & 0.340985 & -0.909590\end{array}$

$\begin{array}{llll}-8.904126 & -0.673962 & -1.288201\end{array}$

$\begin{array}{llll}-7.568830 & 0.943354 & -0.811789\end{array}$

$\begin{array}{lll}-4.688655 & -2.758181 & 0.413115\end{array}$

$\begin{array}{lll}-3.868470 & -2.799478 & 1.134627\end{array}$

$\begin{array}{llll}-5.553733 & -3.998839 & 0.590178\end{array}$

$\begin{array}{lll}-5.075513 & -5.100293 & 1.308630\end{array}$

$\begin{array}{lll}-4.097410 & -5.056494 & 1.769836\end{array}$

$\begin{array}{llll}-5.849039 & -6.258667 & 1.427660\end{array}$

$\begin{array}{lll}-5.455772 & -7.105641 & 1.983611\end{array}$

$\begin{array}{llll}-7.112441 & -6.326521 & 0.837912\end{array}$

$\begin{array}{llll}-7.715084 & -7.225673 & 0.933785\end{array}$

$\begin{array}{lll}-7.599735 & -5.226124 & 0.123352\end{array}$

$\begin{array}{lll}-8.584252 & -5.267746 & -0.335592\end{array}$

$\begin{array}{lll}-6.824441 & -4.073465 & -0.001403\end{array}$

$\begin{array}{llll}-7.204642 & -3.215657 & -0.550155\end{array}$

$\begin{array}{llll}-4.098039 & -2.741130 & -1.008027\end{array}$

$\begin{array}{llll}-4.869632 & -3.097677 & -1.699926\end{array}$

$\begin{array}{llll}-3.293460 & -3.476966 & -1.035094\end{array}$

$\begin{array}{llll}-6.093957 & -1.451687 & 2.042815\end{array}$

$\begin{array}{lll}-6.424105 & -2.478272 & 2.222550\end{array}$

$\begin{array}{lll}-5.074180 & -1.139223 & 3.140576\end{array}$ $\begin{array}{lll}-4.401815 & -2.203140 & 3.761162\end{array}$

$\begin{array}{lll}-4.608270 & -3.221994 & 3.445277\end{array}$

$\begin{array}{lll}-3.480712 & -1.976508 & 4.787211\end{array}$

$\begin{array}{lll}-2.970035 & -2.818235 & 5.245967\end{array}$

$\begin{array}{lll}-3.218392 & -0.674926 & 5.217317\end{array}$

$\begin{array}{lll}-2.489237 & -0.493354 & 6.002138\end{array}$

$\begin{array}{llll}-3.887403 & 0.394015 & 4.612675\end{array}$

$\begin{array}{lll}-3.692134 & 1.412076 & 4.939564\end{array}$

$\begin{array}{lll}-4.806163 & 0.164269 & 3.586363\end{array}$

$\begin{array}{lll}-5.316323 & 1.005621 & 3.133791\end{array}$

$\begin{array}{lll}-7.358678 & -0.595863 & 2.107796\end{array}$

$\begin{array}{lll}-8.085157 & -0.943936 & 1.370109\end{array}$

$\begin{array}{lll}-7.174538 & 0.464175 & 1.933813\end{array}$

$\begin{array}{lll}-7.803475 & -0.702915 & 3.101927\end{array}$

$\begin{array}{lll}-2.122793 & -0.430775 & -0.106784\end{array}$

$\begin{array}{lll}-2.001373 & 0.630646 & 0.089702\end{array}$

$\begin{array}{llll}-2.370429 & -1.004123 & 0.783463\end{array}$

$\begin{array}{llll}-1.225164 & -1.017665 & -1.059909\end{array}$

$\begin{array}{lll}-0.617277 & -0.345212 & -1.650650\end{array}$

$\begin{array}{lll}-0.854964 & -2.383825 & -1.070468\end{array}$

$\begin{array}{lll}-1.401070 & -3.051295 & -0.418343\end{array}$

$\begin{array}{llll}-4.800575 & -1.163012 & -3.522799\end{array}$

$\begin{array}{lll}-5.614555 & -1.806495 & -3.195735\end{array}$

$\begin{array}{llll}-3.563427 & -1.808932 & -3.779114\end{array}$

$\begin{array}{llll}-3.533373 & -2.888391 & -3.632036\end{array}$

$\begin{array}{llll}-2.488294 & -1.301972 & -4.722688\end{array}$

$\begin{array}{llll}-2.747464 & -1.559427 & -5.761902\end{array}$

$\begin{array}{llll}-1.569055 & -1.845685 & -4.490727\end{array}$

$\begin{array}{llll}-2.212016 & 0.211876 & -4.612421\end{array}$

$\begin{array}{llll}-2.849455 & 0.766843 & -5.308004\end{array}$

$\begin{array}{llll}-1.180214 & 0.409859 & -4.921582\end{array}$

$\begin{array}{llll}-2.403853 & 0.780013 & -3.211971\end{array}$

$\begin{array}{llll}-1.497713 & 1.051311 & -2.687118\end{array}$

$\begin{array}{llll}-3.574266 & 1.358478 & -2.762647\end{array}$

$\begin{array}{llll}-3.500153 & 2.051160 & -1.929849\end{array}$

$\begin{array}{llll}-4.856543 & 1.431744 & -3.565241\end{array}$

$\begin{array}{llll}-5.656032 & 1.752912 & -2.897460\end{array}$

$\begin{array}{llll}-4.749810 & 2.224124 & -4.321429\end{array}$

$\begin{array}{llll}-5.264786 & 0.104424 & -4.246032\end{array}$

$\begin{array}{llll}-4.890564 & 0.078095 & -5.275415\end{array}$

$\begin{array}{llll}-6.356180 & 0.079093 & -4.320462\end{array}$

$\begin{array}{llll}-0.161582 & -3.013753 & -2.188666\end{array}$

$\begin{array}{llll}0.622143 & -2.287560 & -3.109824\end{array}$

$\begin{array}{llll}-0.283358 & -4.406300 & -2.361685\end{array}$

$\begin{array}{llll}1.232636 & -2.931438 & -4.182596\end{array}$

$\begin{array}{llll}0.755095 & -1.218195 & -2.982738\end{array}$

$\begin{array}{llll}0.327320 & -5.046170 & -3.440620\end{array}$

$\begin{array}{llll}0.858279 & -4.981974 & -1.643094\end{array}$

$\begin{array}{llll}1.084260 & -4.312904 & -4.356964\end{array}$

$\begin{array}{llll}1.821161 & -2.355452 & -4.890058\end{array}$

$\begin{array}{llll}0.215406 & -6.120152 & -3.560565\end{array}$

$\begin{array}{llll}1.558834 & -4.809960 & -5.198426\end{array}$

$\begin{array}{llll}2.256502 & -4.357732 & -0.385367\end{array}$

$\begin{array}{llll}3.316609 & -4.824139 & -1.132972\end{array}$

$\begin{array}{llll}1.546605 & -5.051274 & 0.049046\end{array}$

$\begin{array}{llll}4.209800 & -3.879352 & -1.649771\end{array}$

$\begin{array}{llll}4.017152 & -2.535850 & -1.398721\end{array}$

$\begin{array}{llll}4.702999 & -1.790058 & -1.778191\end{array}$

$\begin{array}{lll}2.971413 & -2.076170 & -0.682067\end{array}$

$\begin{array}{lll}1.060425 & -0.181368 & 0.641162\end{array}$

$\begin{array}{lll}-0.074454 & -1.205164 & 2.314990\end{array}$

$\begin{array}{lll}-0.588994 & 0.074654 & 2.783076\end{array}$

$\begin{array}{lll}-1.613008 & -0.058446 & 3.138648\end{array}$

$\begin{array}{lll}0.011529 & 0.469559 & 3.610156\end{array}$

$\begin{array}{lll}-0.594615 & 0.788997 & 1.967627\end{array}$

$\begin{array}{lll}-0.417888 & -2.353322 & 3.153058\end{array}$

$\begin{array}{lll}-1.407236 & -2.747180 & 2.911794\end{array}$

$\begin{array}{lll}0.312269 & -3.152187 & 3.043420\end{array}$

$\begin{array}{lll}-0.408217 & -2.027380 & 4.195259\end{array}$

$\begin{array}{llll}3.447746 & -5.886020 & -1.310372\end{array}$

$\begin{array}{llll}5.739176 & -4.440601 & -2.740875\end{array}$

$\begin{array}{lll}-1.524003 & -5.572301 & 1.262093\end{array}$

$\begin{array}{lll}-0.137276 & -5.569175 & 1.517887\end{array}$

$\begin{array}{llll}-1.782006 & -6.153421 & 0.009164\end{array}$

$\begin{array}{lll}-2.194153 & -6.270788 & 2.274195\end{array}$ 
9 P1-C2 exo si-si $\left[\mathrm{BF}_{4}{ }^{-}\right]$

Number of imaginary frequencies $=0$

The smallest frequencies are $=11.348813 .478014 .7365 \mathrm{~cm}(-1)$ Electronic energy $=-7647.2823841$

Zero-point correction $=$

1.774828

(Hartree/Particle)

Thermal correction to Energy= $\quad 1.898964$

Thermal correction to Enthalpy=

1.899908

Thermal correction to Gibbs Free Energy=

Sum of electronic and zero-point Energies=

Sum of electronic and thermal Energies=

Sum of electronic and thermal Enthalpies=

Sum of electronic and thermal Free Energies=

1.606673

$-7645.507556$

$-7645.383420$

$-7645.382476$

Cartesian Coordinates

\begin{tabular}{|c|c|c|c|}
\hline 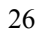 & 7.809167 & 23151 & -1 \\
\hline & 2.737231 & 1.862724 & -1.897025 \\
\hline & 3.973813 & 0.595449 & 1.437647 \\
\hline 6 & 5.853603 & 1.277276 & -1.357709 \\
\hline 6 & 6.088644 & 0.362088 & -0.267855 \\
\hline 6 & 6.441353 & -0.904161 & -0.829109 \\
\hline 1 & 6.695101 & -1.795510 & -0.271609 \\
\hline S & 6.457515 & -0.772249 & -2.250773 \\
\hline 1 & 6.730350 & -1.543284 & -2.955660 \\
\hline 6 & 6.091744 & 0.566163 & -2.576717 \\
\hline 1 & 6.030334 & 0.985831 & -3.569877 \\
\hline 6 & 9.321613 & 1.514625 & -0.385075 \\
\hline 1 & 9.181660 & 2.083129 & 0.524718 \\
\hline 6 & 9.607100 & 0.117218 & -0.463274 \\
\hline 1 & 9.709951 & -0.559682 & 0.374409 \\
\hline 6 & 9.685308 & -0.241010 & -1.843539 \\
\hline 1 & 9.852303 & -1.236712 & -2.232846 \\
\hline 6 & 9.446152 & 0.93 & -2.618962 \\
\hline 1 & 9.402209 & 0.985375 & -3.698885 \\
\hline 6 & 9.222348 & 2.018624 & -1.719129 \\
\hline 1 & 8.984200 & 3.03 & -2.001936 \\
\hline 6 & 5.396966 & 2.684774 & -1.191722 \\
\hline 6 & 6.292859 & 3.633723 & -0.673786 \\
\hline 1 & 7.297306 & 3.30 & 272 \\
\hline 6 & 5.921134 & 4.959063 & -0.455300 \\
\hline 1 & 6.633539 & 5.660643 & -0.032507 \\
\hline 6 & 4.630479 & 5.36 & 0801 \\
\hline 1 & 4.320874 & 6.396240 & -0.625760 \\
\hline 6 & 3.719672 & 4.440438 & -1.284108 \\
\hline 1 & 2.716030 & 4.777612 & -1.510262 \\
\hline 6 & 4.066779 & 3.091183 & -1.474821 \\
\hline 6 & 5.852409 & 0.695078 & 1.179265 \\
\hline 1 & 6.102537 & 1.748237 & 1.317606 \\
\hline 6 & 6.690025 & -0.130116 & 2.159941 \\
\hline 1 & 6.495118 & 0.168336 & 3.193723 \\
\hline 1 & 6.488947 & -1.201461 & 2.076333 \\
\hline 1 & 7.750836 & 0.030763 & 1.944808 \\
\hline 6 & 1.253311 & 2.943222 & -2.005470 \\
\hline 6 & 0.541707 & 3.174701 & -0.818429 \\
\hline 1 & 0.820739 & 2.645373 & 0.085026 \\
\hline 6 & -0.519136 & 4.078623 & -0.796249 \\
\hline 1 & -1.050404 & 4.254605 & 0.129029 \\
\hline 6 & -0.906790 & 4.733467 & -1.967651 \\
\hline 1 & -1.746579 & 5.422500 & -1.951598 \\
\hline 6 & -0.221649 & 4.486084 & -3.159650 \\
\hline 1 & -0.525041 & 4.983255 & -4.076545 \\
\hline 6 & 0.861358 & 3.603705 & -3.178791 \\
\hline 1 & 1.395807 & 3.435244 & -4.107780 \\
\hline 6 & 3.024923 & 1.343413 & -3.630603 \\
\hline 6 & 3.742338 & 2.109393 & -4.561081 \\
\hline 1 & 4.187965 & 3.053308 & -4.260677 \\
\hline 6 & 3.893740 & 1.652695 & -5.872202 \\
\hline 1 & 4.454226 & 2.247056 & -6.588213 \\
\hline 6 & 3.325881 & 0.436229 & -6.264175 \\
\hline 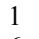 & 3.447888 & 0.084148 & -7.284436 \\
\hline & 2.602861 & -0.326853 & -5.34319 \\
\hline
\end{tabular}

$2.160188-1.272694 \quad-5.642084$

$\begin{array}{llll}2.456558 & 0.124038 & -4.030279\end{array}$

$\begin{array}{llll}1.909934 & -0.472548 & -3.306423\end{array}$

$\begin{array}{lll}3.441488 & 2.200068 & 2.171098\end{array}$

$\begin{array}{lll}2.062041 & 2.326950 & 2.389931\end{array}$

$\begin{array}{lll}1.414714 & 1.482314 & 2.180331\end{array}$

$\begin{array}{lll}1.515897 & 3.536653 & 2.814832\end{array}$

$2.325110 \quad 4.654390 \quad 3.006536$

$\begin{array}{lll}1.898282 & 5.602973 & 3.312632\end{array}$

$\begin{array}{llll}3.696820 & 4.530890 & 2.775962\end{array}$

$\begin{array}{lll}4.259987 & 3.318026 & 2.374510\end{array}$

$\begin{array}{lll}5.327712 & 3.276161 & 2.202190\end{array}$

$\begin{array}{lll}3.705343 & -0.635881 & 2.773595\end{array}$

$\begin{array}{lll}3.851935 & -1.984849 & 2.409914\end{array}$

$\begin{array}{lll}4.147338 & -2.245644 & 1.399606\end{array}$

$\begin{array}{llll}3.608954 & -2.993069 & 3.338763\end{array}$

$\begin{array}{lll}3.172944 & -2.684563 & 4.628427\end{array}$

$\begin{array}{lll}2.959067 & -3.472761 & 5.341034\end{array}$

$\begin{array}{llll}3.010505 & -1.347516 & 4.980468\end{array}$

$\begin{array}{lll}3.287756 & -0.323882 & 4.070417\end{array}$

$\begin{array}{lll}3.164987 & 0.707722 & 4.378674\end{array}$

$\begin{array}{llll}2.561818 & 0.163241 & -0.400475\end{array}$

$\begin{array}{llll}2.444727 & -1.007084 & 6.330951\end{array}$

$\begin{array}{lll}3.726618 & -4.439335 & 2.942540\end{array}$

$\begin{array}{llll}0.034046 & 3.595636 & 3.067533\end{array}$

$\begin{array}{lll}4.554499 & 5.757846 & 2.928343\end{array}$

$\begin{array}{llll}4.212711 & 6.714124 & 2.029640\end{array}$

$\begin{array}{lll}5.868574 & 5.498878 & 2.752687\end{array}$

$\begin{array}{llll}4.413828 & 6.317165 & 4.152810\end{array}$

$\begin{array}{lll}-0.663353 & 2.913101 & 2.115644\end{array}$

$\begin{array}{lll}-0.300034 & 3.031851 & 4.251043\end{array}$

$\begin{array}{lll}-0.443449 & 4.858532 & 3.071069\end{array}$

$\begin{array}{lll}2.849669 & 0.206434 & 6.764535\end{array}$

$\begin{array}{llll}1.084641 & -0.973888 & 6.296798\end{array}$

$\begin{array}{lll}2.786052 & -1.913186 & 7.270720\end{array}$

$\begin{array}{lll}4.275655 & -5.184412 & 3.927277\end{array}$

$\begin{array}{llll}4.485874 & -4.606725 & 1.835886\end{array}$

$\begin{array}{llll}2.511540 & -4.977585 & 2.677829\end{array}$

$\begin{array}{llll}0.529160 & -1.303316 & 1.451053\end{array}$

$\begin{array}{lll}0.491645 & -2.512552 & 0.535818\end{array}$

$\begin{array}{llll}1.774088 & -2.836049 & -0.193475\end{array}$

$\begin{array}{llll}0.245106 & -3.401883 & 1.107448\end{array}$

$\begin{array}{lll}-3.067192 & -0.452141 & -1.861412\end{array}$

$\begin{array}{lll}-4.944706 & -0.200371 & -0.556083\end{array}$

$\begin{array}{lll}-5.069482 & 0.766573 & 0.803361\end{array}$

$-6.219674 \quad 0.436536-1.446711$

$\begin{array}{lll}-5.376813 & -1.661944 & 0.139572\end{array}$

$\begin{array}{lll}-4.927091 & 2.134554 & 0.670208\end{array}$

$\begin{array}{lll}-3.764767 & 2.712958 & 1.228457\end{array}$

$\begin{array}{lll}-3.066121 & 2.069292 & 1.749745\end{array}$

$\begin{array}{lll}-3.558547 & 4.064557 & 1.112179\end{array}$

$\begin{array}{llll}-2.695888 & 4.528939 & 1.579098\end{array}$

$\begin{array}{lll}-4.454634 & 4.864708 & 0.352153\end{array}$

$\begin{array}{lll}-4.179678 & 6.236088 & 0.098758\end{array}$

$\begin{array}{lll}-3.278746 & 6.670502 & 0.524859\end{array}$

$\begin{array}{llll}-5.022472 & 6.994554 & -0.683056\end{array}$

$\begin{array}{llll}-4.797288 & 8.038902 & -0.878910\end{array}$

$\begin{array}{llll}-6.181593 & 6.405964 & -1.243508\end{array}$

$\begin{array}{llll}-6.833653 & 7.000465 & -1.877094\end{array}$

$\begin{array}{llll}-6.488558 & 5.084880 & -0.997822\end{array}$

$\begin{array}{llll}-7.376258 & 4.646739 & -1.439631\end{array}$

$\begin{array}{llll}-5.646077 & 4.273121 & -0.188268\end{array}$

$\begin{array}{llll}-5.929089 & 2.884990 & 0.072441\end{array}$

$\begin{array}{lll}-7.246985 & 2.263253 & -0.241674\end{array}$

$\begin{array}{lll}-8.473843 & 2.826329 & 0.272800\end{array}$

$\begin{array}{lll}-8.497609 & 3.915910 & 1.189229\end{array}$

$\begin{array}{llll}-7.563438 & 4.340965 & 1.534685\end{array}$

$\begin{array}{lll}-9.686705 & 4.428538 & 1.662260\end{array}$

$\begin{array}{llll}-9.670679 & 5.255301 & 2.366937\end{array}$

$\begin{array}{lll}-10.925269 & 3.885395 & 1.247415\end{array}$

$\begin{array}{lll}-11.854766 & 4.304017 & 1.622211\end{array}$

$\begin{array}{lll}-10.941543 & 2.813070 & 0.384465\end{array}$

$\begin{array}{lll}-11.882770 & 2.366849 & 0.073486\end{array}$

$\begin{array}{llll}-9.732860 & 2.250226 & -0.107479\end{array}$

$\begin{array}{llll}-9.751542 & 1.104997 & -0.948578\end{array}$ 
$\begin{array}{lrlllll}-10.707447 & 0.680975 & -1.243258 & 6 & 2.127499 & -4.191683 & -0.275767 \\ -8.578945 & 0.521799 & -1.352872 & 6 & 3.148817 & -4.601596 & -1.120768 \\ -8.566703 & -0.375038 & -1.963470 & 1 & 1.569378 & -4.919444 & 0.299182 \\ -7.337777 & 1.086119 & -0.973882 & 6 & 3.796925 & -3.618187 & -1.858959 \\ -4.490015 & -2.786182 & -0.276726 & 6 & 3.463206 & -2.282297 & -1.695571 \\ -3.738426 & -2.935106 & 0.505316 & 1 & 3.976449 & -1.498633 & -2.236398 \\ -5.267006 & -4.083035 & -0.444410 & 7 & 2.472542 & -1.894941 & -0.865455 \\ -4.708150 & -5.291782 & -0.010737 & 8 & 0.918664 & -0.188183 & 1.027578 \\ -3.754148 & -5.289201 & 0.502748 & 7 & 0.023959 & -1.416273 & 2.696438 \\ -5.373884 & -6.500287 & -0.233256 & 6 & -0.324970 & -0.201477 & 3.433940 \\ -4.921878 & -7.426409 & 0.112255 & 1 & -1.192247 & -0.419764 & 4.060925 \\ -6.609287 & -6.515182 & -0.884289 & 1 & 0.495730 & 0.134324 & 4.073791 \\ -7.129924 & -7.454190 & -1.052255 & 1 & -0.587181 & 0.589007 & 2.734620 \\ -7.175523 & -5.310283 & -1.315477 & 6 & -0.167576 & -2.674658 & 3.427275 \\ -8.138626 & -5.311637 & -1.819700 & 1 & -1.194931 & -3.024333 & 3.347420 \\ -6.507458 & -4.104476 & -1.099951 & 1 & 0.501336 & -3.451332 & 3.064814 \\ -6.947841 & -3.166642 & -1.429191 & 1 & 0.073932 & -2.486575 & 4.475720 \\ -3.778917 & -2.458793 & -1.598183 & 1 & 3.408641 & -5.650228 & -1.211364 \\ -4.488591 & -2.648576 & -2.413249 & 35 & 5.169165 & -4.124038 & -3.154642 \\ -2.971166 & -3.177244 & -1.725410 & 5 & -1.443388 & -5.546525 & 2.095143 \\ -6.132991 & -1.898680 & 1.410968 & 9 & -0.050147 & -5.512520 & 1.847705 \\ -6.453759 & -2.940247 & 1.324562 & 9 & -2.017197 & -6.626240 & 1.411755 \\ -5.235408 & -1.838718 & 2.648364 & 9 & -1.677148 & -5.637292 & 3.474485 \\ -4.566838 & -3.004736 & 3.049848 & 9 & -2.019026 & -4.342926 & 1.606013\end{array}$

$\begin{array}{lll}03152 & -3.920580 & 2.483465\end{array}$

$\begin{array}{lll}-3.723656 & -3.011804 & 4.162712\end{array}$

$\begin{array}{llll}-3.197626 & -3.924881 & 4.423137\end{array}$

$\begin{array}{llll}-3.546298 & -1.843970 & 4.907038\end{array}$

$\begin{array}{lll}-2.891838 & -1.841622 & 5.774712\end{array}$

$\begin{array}{lll}-4.218012 & -0.676864 & 4.528280\end{array}$

$\begin{array}{lll}-4.093386 & 0.234748 & 5.107266\end{array}$

$\begin{array}{llll}-5.053629 & -0.673972 & 3.409247\end{array}$

$\begin{array}{lll}-5.563324 & 0.239781 & 3.128946\end{array}$

$\begin{array}{lll}-7.418210 & -1.079829 & 1.523887\end{array}$

$\begin{array}{lll}-8.056112 & -1.262976 & 0.656083\end{array}$

$\begin{array}{lll}-7.242054 & -0.007053 & 1.596835\end{array}$

$\begin{array}{lll}-7.960734 & -1.397159 & 2.419924\end{array}$

$\begin{array}{lll}-1.897152 & -0.192854 & -0.027378\end{array}$

$\begin{array}{lll}-1.677096 & 0.862166 & 0.115747\end{array}$

$\begin{array}{llll}-2.303314 & -0.670183 & 0.863502\end{array}$

$\begin{array}{llll}-1.083974 & -0.974413 & -0.897089\end{array}$

$\begin{array}{llll}-0.281297 & -0.461429 & -1.419320\end{array}$

$\begin{array}{lll}-0.763794 & -2.394519 & -0.491535\end{array}$

$\begin{array}{llll}-1.591683 & -2.762097 & 0.111032\end{array}$

$\begin{array}{lll}-4.104434 & -0.435110 & -3.777362\end{array}$

$\begin{array}{llll}-4.946481 & -1.124974 & -3.748837\end{array}$

$\begin{array}{llll}-2.810751 & -1.031526 & -3.961387\end{array}$

$\begin{array}{llll}-2.780037 & -2.116683 & -4.059004\end{array}$

$\begin{array}{lll}-1.666615 & -0.332670 & -4.677717\end{array}$

$\begin{array}{lll}-1.847022 & -0.315288 & -5.764872\end{array}$

$\begin{array}{llll}-0.764377 & -0.932771 & -4.531394\end{array}$

$\begin{array}{llll}-1.410246 & 1.095634 & -4.158996\end{array}$

$\begin{array}{llll}-1.916014 & 1.833269 & -4.791299\end{array}$

$\begin{array}{llll}-0.342536 & 1.322350 & -4.234393\end{array}$

$\begin{array}{llll}-1.842061 & 1.308231 & -2.709487\end{array}$

$\begin{array}{llll}-1.030017 & 1.464734 & -2.009094\end{array}$

$\begin{array}{llll}-3.086115 & 1.814722 & -2.334479\end{array}$

$\begin{array}{llll}-3.140676 & 2.347448 & -1.389347\end{array}$

$\begin{array}{llll}-4.214962 & 2.101741 & -3.307423\end{array}$

$\begin{array}{llll}-5.121252 & 2.274465 & -2.723605\end{array}$

$\begin{array}{llll}-4.002209 & 3.041430 & -3.840858\end{array}$

$\begin{array}{llll}-4.472244 & 0.955965 & -4.313104\end{array}$

$\begin{array}{llll}-3.929494 & 1.142463 & -5.247314\end{array}$

$\begin{array}{llll}-5.534328 & 0.959244 & -4.579828\end{array}$

$\begin{array}{llll}-0.543788 & -3.372535 & -1.632488\end{array}$

$\begin{array}{llll}0.050465 & -2.969383 & -2.834635\end{array}$

$\begin{array}{llll}-0.884491 & -4.724709 & -1.476360\end{array}$

$\begin{array}{llll}0.310235 & -3.888182 & -3.853153\end{array}$

$\begin{array}{llll}0.310824 & -1.928141 & -2.974124\end{array}$

$\begin{array}{llll}-0.631853 & -5.646154 & -2.494654\end{array}$

$\begin{array}{lll}-1.346724 & -5.055334 & -0.554012\end{array}$

$\begin{array}{llll}-0.028304 & -5.234207 & -3.686017\end{array}$

$\begin{array}{llll}0.773480 & -3.552127 & -4.777431\end{array}$

$\begin{array}{llll}-0.906643 & -6.687959 & -2.353109\end{array}$

$\begin{array}{llll}0.171221 & -5.951697 & -4.477277\end{array}$

Cartesian Coordinates
8 P1-C2 exo re-si $\left[\mathrm{BF}_{4}^{-}\right]$

Number of imaginary frequencies $=0$

The smallest frequencies are=9.4809 $13.040316 .2326 \mathrm{~cm}(-1)$ Electronic energy $=-7647.2412462$

Zero-point correction=

1.770572

(Hartree/Particle)

Thermal correction to Energy= $\quad 1.896360$

Thermal correction to Enthalpy= $\quad 1.897304$

Thermal correction to Gibbs Free Energy $=\quad 1.599230$

Sum of electronic and zero-point Energies $=\quad-7645.470674$

Sum of electronic and thermal Energies $=\quad-7645.344886$

Sum of electronic and thermal Enthalpies $=\quad-7645.343942$

Sum of electronic and thermal Free Energies $=\quad-7645.642016$

$\begin{array}{lccc}77 & -4.141716 & 0.269213 & 1.775798 \\ 15 & -5.896433 & 0.711160 & 0.437980 \\ 8 & -6.270248 & -0.134354 & -0.920838 \\ 8 & -7.321327 & 0.551700 & 1.284219 \\ 7 & -5.734748 & 2.245503 & -0.211171 \\ 6 & -6.923900 & -1.362609 & -0.891686 \\ 6 & -6.172041 & -2.463641 & -1.345137 \\ 1 & -5.167802 & -2.287622 & -1.703182 \\ 6 & -6.735463 & -3.713884 & -1.333537 \\ 1 & -6.161656 & -4.562909 & -1.691673 \\ 6 & -8.051048 & -3.903291 & -0.828754 \\ 6 & -8.618320 & -5.202826 & -0.731700 \\ 1 & -8.027132 & -6.050134 & -1.069022 \\ 6 & -9.879210 & -5.389539 & -0.211407 \\ 1 & -10.299209 & -6.388488 & -0.137642 \\ 6 & -10.625036 & -4.275655 & 0.242709 \\ 1 & -11.610630 & -4.427519 & 0.673672 \\ 6 & -10.111359 & -3.000093 & 0.149006 \\ 1 & -10.693564 & -2.159609 & 0.508741 \\ 6 & -8.820080 & -2.766511 & -0.401274 \\ 6 & -8.252701 & -1.446484 & -0.508288 \\ 6 & -9.032194 & -0.207558 & -0.229465 \\ 6 & -10.258471 & 0.095661 & -0.922688 \\ 6 & -10.758619 & -0.708181 & -1.985688 \\ 1 & -10.200158 & -1.581320 & -2.300131 \\ 6 & -11.930921 & -0.384214 & -2.633322 \\ 1 & -12.284867 & -1.011076 & -3.446786 \\ 6 & -12.673389 & 0.760143 & -2.256279 \\ 1 & -13.599353 & 0.998788 & -2.771392 \\ 6 & -12.206969 & 1.575601 & -1.250486 \\ 1 & -12.754409 & 2.470115 & -0.964982 \\ 6 & -10.992235 & 1.280080 & -0.573474 \\ 6 & -10.480403 & 2.154963 & 0.422644\end{array}$




\begin{tabular}{|c|c|c|c|c|c|c|}
\hline-11.047122 & 3.042983 & 0.687882 & 26 & 7.177306 & 3.404227 & -2.216491 \\
\hline-9.269100 & 1.900890 & 1.016535 & 15 & 5.161368 & 1.148804 & 2.011169 \\
\hline-8.839997 & 2.570871 & 1.753886 & 15 & 4.736900 & -0.607663 & -1.314567 \\
\hline-8.552228 & 0.736739 & 0.664254 & 6 & 6.602436 & 2.210473 & -0.597654 \\
\hline-4.666073 & 3.028543 & 0.463979 & 6 & 5.909589 & 1.820966 & -1.803841 \\
\hline-3.768059 & 2.987636 & -0.165481 & 6 & 5.159889 & 2.946245 & -2.258744 \\
\hline-5.050879 & 4.486998 & 0.637547 & 1 & 4.540522 & 2.973072 & -3.144875 \\
\hline-4.111467 & 5.490975 & 0.375028 & 6 & 5.403333 & 4.034985 & -1.372796 \\
\hline-3.129544 & 5.216754 & -0.003899 & 1 & 5.008929 & 5.036906 & -1.473538 \\
\hline-4.428864 & 6.835362 & 0.587693 & 6 & 6.294908 & 3.587754 & -0.356199 \\
\hline-3.690250 & 7.604495 & 0.378894 & 1 & 6.686128 & 4.182705 & 0.455537 \\
\hline-5.695852 & 7.189144 & 1.056632 & 6 & 8.774708 & 2.797737 & -3.396402 \\
\hline-5.947285 & 8.234018 & 1.215598 & 1 & 9.048500 & 1.767459 & -3.581114 \\
\hline-6.642826 & 6.191677 & 1.312691 & 6 & 7.854650 & 3.572031 & -4.167669 \\
\hline-7.633250 & 6.461181 & 1.669674 & 1 & 7.303865 & 3.229926 & -5.033601 \\
\hline-6.321291 & 4.850125 & 1.107235 & 6 & 7.753423 & 4.864550 & -3.567794 \\
\hline-7.059285 & 4.073740 & 1.289091 & 1 & 7.108389 & 5.668656 & -3.897033 \\
\hline-4.351129 & 2.398969 & 1.825522 & 6 & 8.611342 & 4.890490 & -2.426049 \\
\hline-5.156473 & 2.654770 & 2.525508 & 1 & 8.730218 & 5.716581 & -1.737424 \\
\hline-3.439172 & 2.845749 & 2.225591 & 6 & 9.242936 & 3.615210 & -2.320379 \\
\hline-6.079863 & 2.757149 & -1.580648 & 1 & 9.928200 & 3.313684 & -1.539996 \\
\hline-6.207587 & 3.831628 & -1.421736 & 6 & 7.403323 & 1.288105 & 0.259398 \\
\hline-4.909838 & 2.591359 & -2.548378 & 6 & 8.659000 & 0.847028 & -0.184226 \\
\hline-4.207257 & 3.723000 & -2.984425 & 1 & 9.026714 & 1.219381 & -1.132409 \\
\hline-4.510848 & 4.706948 & -2.634677 & 6 & 9.423430 & -0.060154 & 0.549558 \\
\hline-3.117706 & 3.599475 & -3.852680 & 1 & 10.378424 & -0.401434 & 0.163223 \\
\hline-2.584712 & 4.488645 & -4.178759 & 6 & 8.942767 & -0.535960 & 1.767159 \\
\hline-2.703888 & 2.336358 & -4.282004 & 1 & 9.522963 & -1.245668 & 2.348658 \\
\hline-1.839601 & 2.232568 & -4.931537 & 6 & 7.695836 & -0.113265 & 2.229773 \\
\hline-3.398208 & 1.201393 & -3.853766 & 1 & 7.328975 & -0.502171 & 3.173105 \\
\hline-3.077264 & 0.210371 & -4.152396 & 6 & 6.903536 & 0.777777 & 1.489743 \\
\hline-4.495436 & 1.330657 & -3.003264 & 6 & 5.922352 & 0.431579 & -2.378725 \\
\hline-5.014091 & 0.439878 & -2.675593 & 1 & 6.910189 & 0.020548 & -2.171276 \\
\hline-7.408703 & 2.266440 & -2.160089 & 6 & 5.704153 & 0.365935 & -3.893099 \\
\hline-8.222824 & 2.422031 & -1.449311 & 1 & 5.729938 & -0.667501 & -4.249851 \\
\hline-7.388417 & 1.217463 & -2.450756 & 1 & 4.750855 & 0.803058 & -4.198193 \\
\hline-7.621010 & 2.860967 & -3.054285 & 1 & 6.501198 & 0.926753 & -4.390813 \\
\hline-3.001061 & 0.081161 & -0.053545 & 6 & 4.937219 & 0.267595 & 3.615544 \\
\hline-3.111750 & -0.888476 & -0.525390 & 6 & 4.733906 & -1.122673 & 3.572693 \\
\hline-3.138924 & 0.903864 & -0.752453 & 1 & 4.798548 & -1.645532 & 2.624918 \\
\hline-1.908139 & 0.212289 & 0.885237 & 6 & 4.456903 & -1.841982 & 4.734627 \\
\hline-1.448014 & -0.684310 & 1.273998 & 1 & 4.315274 & -2.916159 & 4.678128 \\
\hline-1.368270 & 1.432499 & 1.241842 & 6 & 4.355386 & -1.176167 & 5.960280 \\
\hline-1.773644 & 2.314289 & 0.753180 & 1 & 4.125164 & -1.731443 & 6.865120 \\
\hline-5.358103 & 0.135314 & 3.687371 & 6 & 4.551563 & 0.204953 & 6.014286 \\
\hline-6.013812 & 1.002279 & 3.681913 & 1 & 4.476116 & 0.729931 & 6.962562 \\
\hline-4.011191 & 0.375440 & 4.019032 & 6 & 4.847870 & 0.923140 & 4.852046 \\
\hline-3.741784 & 1.400811 & 4.272600 & 1 & 4.996938 & 1.995143 & 4.915424 \\
\hline-3.008320 & -0.655751 & 4.508159 & 6 & 5.213467 & 2.920925 & 2.484912 \\
\hline-3.100380 & -0.770858 & 5.599058 & 6 & 6.318849 & 3.485198 & 3.141478 \\
\hline-2.008289 & -0.245535 & 4.330118 & 1 & 7.171826 & 2.864481 & 3.401753 \\
\hline-3.111309 & -2.037638 & 3.826578 & 6 & 6.332343 & 4.847327 & 3.444466 \\
\hline-3.837070 & -2.666138 & 4.350358 & 1 & 7.190990 & 5.280841 & 3.949657 \\
\hline-2.146228 & -2.546097 & 3.919719 & 6 & 5.243097 & 5.653377 & 3.093275 \\
\hline-3.477812 & -1.983022 & 2.348574 & 1 & 5.258246 & 6.715086 & 3.324008 \\
\hline-2.685651 & -2.228066 & 1.648112 & 6 & 4.141171 & 5.093060 & 2.442796 \\
\hline-4.760676 & -2.051684 & 1.840825 & 1 & 3.297655 & 5.717834 & 2.161605 \\
\hline-4.847788 & -2.347986 & 0.800294 & 6 & 4.123743 & 3.730047 & 2.136193 \\
\hline-6.036241 & -2.132056 & 2.650055 & 1 & 3.290980 & 3.294541 & 1.593780 \\
\hline-6.867793 & -1.882583 & 1.988182 & 6 & 5.752100 & -2.032678 & -0.716601 \\
\hline-6.189428 & -3.175125 & 2.962774 & 6 & 5.162290 & -2.872391 & 0.239744 \\
\hline-6.076286 & -1.199607 & 3.880077 & 1 & 4.123279 & -2.732966 & 0.512572 \\
\hline-5.655681 & -1.705023 & 4.754358 & 6 & 5.902113 & -3.878709 & 0.862159 \\
\hline-7.123494 & -0.994050 & 4.121562 & 6 & 7.250496 & -4.060127 & 0.564272 \\
\hline-0.340865 & 1.689114 & 2.229690 & 1 & 7.832151 & -4.820629 & 1.073134 \\
\hline 0.302667 & 0.666816 & 2.961754 & 6 & 7.838713 & -3.229381 & -0.388334 \\
\hline 0.034311 & 3.028279 & 2.474805 & 6 & 7.100876 & -2.239239 & -1.035216 \\
\hline 1.287432 & 0.976550 & 3.894627 & 1 & 7.605310 & -1.624840 & -1.767148 \\
\hline 0.043029 & -0.370574 & 2.787873 & 6 & 3.482029 & -1.362222 & -2.433794 \\
\hline 1.009936 & 3.334865 & 3.418507 & 6 & 2.613992 & -0.474753 & -3.095444 \\
\hline-0.444617 & 3.822534 & 1.908238 & 1 & 2.774286 & 0.593914 & -3.029486 \\
\hline 1.642563 & 2.309645 & 4.128375 & 6 & 1.505073 & -0.955455 & -3.781864 \\
\hline 1.790609 & 0.179664 & 4.434383 & 6 & 1.198265 & -2.318825 & -3.789974 \\
\hline 1.292004 & 4.368809 & 3.591394 & 1 & 0.281528 & -2.680283 & -4.239592 \\
\hline 2.418670 & 2.543748 & 4.849733 & 6 & 2.062571 & -3.196293 & -3.145544 \\
\hline
\end{tabular}




\begin{tabular}{|c|c|c|c|}
\hline 6 & 3.204273 & -2.731447 & -2.483341 \\
\hline 1 & 3.847425 & -3.448738 & -1.986121 \\
\hline 29 & 3.662128 & 0.519851 & 0.437594 \\
\hline 6 & 1.717127 & -4.655047 & -3.039790 \\
\hline 6 & 0.529525 & -0.009282 & -4.420887 \\
\hline 6 & 5.224090 & -4.780163 & 1.856698 \\
\hline 6 & 9.305570 & -3.401788 & -0.674721 \\
\hline 9 & 10.049613 & -3.243232 & 0.448381 \\
\hline 9 & 9.767301 & -2.515143 & -1.583937 \\
\hline 9 & 9.581928 & -4.640566 & -1.144573 \\
\hline 9 & 4.291128 & -4.111356 & 2.579839 \\
\hline 9 & 4.582943 & -5.805371 & 1.250213 \\
\hline 9 & 6.097444 & -5.321238 & 2.733114 \\
\hline 9 & 2.813514 & -5.438899 & -3.200986 \\
\hline 9 & 1.210715 & -4.956791 & -1.819427 \\
\hline 9 & 0.807025 & -5.040692 & -3.955995 \\
\hline 9 & 0.335657 & -0.285439 & -5.731431 \\
\hline 9 & 0.919541 & 1.285181 & -4.341917 \\
\hline 9 & -0.689679 & -0.092499 & -3.830264 \\
\hline 6 & 1.874585 & -1.789367 & 1.191959 \\
\hline 6 & 0.907024 & -0.338292 & -0.388054 \\
\hline 6 & 0.977811 & -2.795576 & 0.889656 \\
\hline 1 & 2.616393 & -1.913452 & 1.973061 \\
\hline 6 & 0.032940 & -1.400789 & -0.784025 \\
\hline 6 & 0.060809 & -2.624650 & -0.155763 \\
\hline 1 & -0.674470 & -1.231693 & -1.585849 \\
\hline 6 & 0.684815 & 0.945378 & -0.945098 \\
\hline 6 & 1.431993 & 2.131726 & -0.722503 \\
\hline 8 & 2.574363 & 2.173371 & -0.157539 \\
\hline 7 & 1.876185 & -0.601501 & 0.554092 \\
\hline 1 & -0.161002 & 0.993937 & -1.613735 \\
\hline 7 & 0.891450 & 3.345345 & -1.126835 \\
\hline 6 & 1.751863 & 4.516693 & -1.211156 \\
\hline 1 & 2.072683 & 4.709165 & -2.247087 \\
\hline 1 & 1.213303 & 5.402347 & -0.854509 \\
\hline 1 & 2.635365 & 4.361635 & -0.596486 \\
\hline 6 & -0.348116 & 3.421908 & -1.885994 \\
\hline 1 & -1.141917 & 2.844549 & -1.407850 \\
\hline 1 & -0.673388 & 4.464574 & -1.928358 \\
\hline 1 & -0.233799 & 3.055416 & -2.914012 \\
\hline 1 & -0.620298 & -3.405540 & -0.461675 \\
\hline 35 & 0.982782 & -4.467808 & 1.918132 \\
\hline 5 & -2.835452 & -3.105356 & -2.258942 \\
\hline 9 & -3.784591 & -3.982221 & -2.803202 \\
\hline 9 & -1.538565 & -3.464082 & -2.662857 \\
\hline 9 & -2.924079 & -3.150083 & -0.843303 \\
\hline 9 & -3.114385 & -1.782955 & -2.684941 \\
\hline
\end{tabular}

$[8-9]^{\ddagger} \mathbf{P 1}-\mathrm{C2}$ exo re-si $\left[\mathrm{BF}_{4}^{-}\right]$

Number of imaginary frequencies $=1$

The smallest frequencies are : $-36.10058 .439711 .3461 \mathrm{~cm}(-1)$ Electronic energy $=-7647.2408933$

Zero-point correction=

1.769999

(Hartree/Particle)

Thermal correction to Energy=

1.895171

Thermal correction to Enthalpy=

1.896115

Thermal correction to Gibbs Free Energy=

Sum of electronic and zero-point Energies=

Sum of electronic and thermal Energies=

1.599819

Sum of electronic and thermal Enthalpies=

$-7645.470895$

$-7645.345723$

$-7645.344778$

Sum of electronic and thermal Free Energies=

$-7645.641074$

Cartesian Coordinates

$\begin{array}{llll}77 & -4.138918 & 0.209671 & 1.785793 \\ 15 & -5.846176 & 0.797364 & 0.424896 \\ 8 & -6.258386 & 0.011679 & -0.966521 \\ 8 & -7.299465 & 0.706495 & 1.238304 \\ 7 & -5.571567 & 2.332004 & -0.193111 \\ 6 & -6.876697 & -1.232643 & -0.946152 \\ 6 & -6.105527 & -2.313913 & -1.418234 \\ 1 & -5.110165 & -2.115469 & -1.793979 \\ 6 & -6.636168 & -3.578579 & -1.392857 \\ 1 & -6.045133 & -4.411987 & -1.758632\end{array}$

$\begin{array}{lll}-7.933167 & -3.801194 & -0.853811\end{array}$

$\begin{array}{lll}-8.459351 & -5.115932 & -0.732792\end{array}$

$\begin{array}{llll}-7.852718 & -5.948023 & -1.080431\end{array}$

$\begin{array}{lll}-9.698891 & -5.335961 & -0.175283\end{array}$

$\begin{array}{lll}-10.086469 & -6.346353 & -0.082209\end{array}$

$\begin{array}{lll}-10.464429 & -4.241142 & 0.292173\end{array}$

$\begin{array}{lll}-11.432140 & -4.418811 & 0.752875\end{array}$

$\begin{array}{lll}-9.991827 & -2.951640 & 0.174715\end{array}$

$\begin{array}{lll}-10.588032 & -2.125656 & 0.545231\end{array}$

$\begin{array}{lll}-8.723559 & -2.684439 & -0.412139\end{array}$

$\begin{array}{lll}-8.195179 & -1.349953 & -0.537068\end{array}$

$\begin{array}{lll}-9.000665 & -0.127510 & -0.254306\end{array}$

$\begin{array}{llll}-10.250046 & 0.129195 & -0.926781\end{array}$

$\begin{array}{lll}-10.748825 & -0.705401 & -1.966723\end{array}$

$\begin{array}{lll}-10.169993 & -1.564443 & -2.282941\end{array}$

$\begin{array}{lll}-11.946521 & -0.430255 & -2.590313\end{array}$

$\begin{array}{llll}-12.298285 & -1.081002 & -3.385816\end{array}$

$\begin{array}{llll}-12.717916 & 0.694176 & -2.211919\end{array}$

$\begin{array}{llll}-13.663632 & 0.894179 & -2.707236\end{array}$

$\begin{array}{llll}-12.254220 & 1.539425 & -1.229742\end{array}$

$\begin{array}{lll}-12.823588 & 2.419892 & -0.943198\end{array}$

$\begin{array}{lll}-11.014813 & 1.293996 & -0.578154\end{array}$

$\begin{array}{lll}-10.509695 & 2.200356 & 0.392729\end{array}$

$\begin{array}{lll}-11.098814 & 3.074034 & 0.657108\end{array}$

$\begin{array}{lll}-9.279490 & 1.992331 & 0.964119\end{array}$

$\begin{array}{lll}-8.855167 & 2.686475 & 1.682118\end{array}$

$\begin{array}{llll}-8.532110 & 0.844961 & 0.616981\end{array}$

$\begin{array}{lll}-4.406620 & 2.999043 & 0.451295\end{array}$

$\begin{array}{llll}-3.535264 & 2.858968 & -0.201382\end{array}$

$\begin{array}{lll}-4.620932 & 4.492116 & 0.622766\end{array}$

$\begin{array}{llll}-3.579805 & 5.380347 & 0.327863\end{array}$

$\begin{array}{llll}-2.646084 & 4.992895 & -0.073628\end{array}$

$\begin{array}{llll}-3.733959 & 6.753056 & 0.540105\end{array}$

$\begin{array}{llll}-2.917676 & 7.431035 & 0.305694\end{array}$

$\begin{array}{lll}-4.938128 & 7.252479 & 1.041356\end{array}$

$\begin{array}{lll}-5.063088 & 8.319925 & 1.200191\end{array}$

$\begin{array}{lll}-5.985765 & 6.371706 & 1.331256\end{array}$

$\begin{array}{lll}-6.927593 & 6.754727 & 1.714916\end{array}$

$\begin{array}{lll}-5.826554 & 5.001064 & 1.126570\end{array}$

$\begin{array}{lll}-6.642749 & 4.314934 & 1.337408\end{array}$

$\begin{array}{lll}-4.121559 & 2.350633 & 1.811573\end{array}$

$\begin{array}{lll}-4.882138 & 2.692294 & 2.525887\end{array}$

$\begin{array}{lll}-3.162333 & 2.716405 & 2.182974\end{array}$

$\begin{array}{llll}-5.923759 & 2.904145 & -1.534256\end{array}$

$\begin{array}{llll}-5.973244 & 3.980489 & -1.347864\end{array}$

$\begin{array}{llll}-4.806410 & 2.690704 & -2.554913\end{array}$

$\begin{array}{llll}-4.101052 & 3.797679 & -3.045494\end{array}$

$\begin{array}{llll}-4.352918 & 4.792772 & -2.686713\end{array}$

$\begin{array}{llll}-3.073052 & 3.637082 & -3.980559\end{array}$

$\begin{array}{llll}-2.537390 & 4.508703 & -4.347204\end{array}$

$\begin{array}{llll}-2.723166 & 2.360295 & -4.424863\end{array}$

$\begin{array}{llll}-1.906247 & 2.226788 & -5.128424\end{array}$

$\begin{array}{llll}-3.419883 & 1.249271 & -3.941274\end{array}$

$\begin{array}{llll}-3.146964 & 0.247451 & -4.253224\end{array}$

$\begin{array}{llll}-4.454602 & 1.414869 & -3.021921\end{array}$

$\begin{array}{llll}-4.979922 & 0.543081 & -2.657409\end{array}$

$\begin{array}{llll}-7.301819 & 2.519234 & -2.080098\end{array}$

$\begin{array}{lll}-8.080990 & 2.712777 & -1.339470\end{array}$

$\begin{array}{llll}-7.362232 & 1.478036 & -2.392258\end{array}$

$\begin{array}{lll}-7.501689 & 3.147748 & -2.953621\end{array}$

$\begin{array}{lll}-2.969313 & -0.094161 & -0.004338\end{array}$

$\begin{array}{llll}-3.119356 & -1.077021 & -0.435260\end{array}$

$\begin{array}{llll}-3.044990 & 0.703353 & -0.741107\end{array}$

$\begin{array}{llll}-1.931653 & 0.034606 & 0.991935\end{array}$

$\begin{array}{lll}-1.530367 & -0.860526 & 1.446983\end{array}$

$\begin{array}{llll}-1.253996 & 1.233655 & 1.219876\end{array}$

$\begin{array}{lll}-1.593933 & 2.104422 & 0.670103\end{array}$

$\begin{array}{lll}-5.371269 & 0.215089 & 3.674504\end{array}$

$\begin{array}{lll}-5.946525 & 1.137964 & 3.664991\end{array}$

$\begin{array}{lll}-4.008345 & 0.335378 & 4.023921\end{array}$

$\begin{array}{lll}-3.652862 & 1.333691 & 4.279784\end{array}$

$\begin{array}{llll}-3.115907 & -0.780063 & 4.543169\end{array}$

$\begin{array}{lll}-3.247156 & -0.890766 & 5.630949\end{array}$

$\begin{array}{lll}-2.078611 & -0.461283 & 4.391752\end{array}$

$\begin{array}{lll}-3.324966 & -2.143590 & 3.848962\end{array}$ 
$\begin{array}{lll}-4.105096 & -2.714746 & 4.360970\end{array}$

$\begin{array}{lll}-2.406502 & -2.731893 & 3.945620\end{array}$

$\begin{array}{lll}-3.673729 & -2.044405 & 2.366822\end{array}$

$\begin{array}{lll}-2.899770 & -2.360050 & 1.672996\end{array}$

$\begin{array}{lll}-4.961578 & -2.018014 & 1.858859\end{array}$

$\begin{array}{lll}-5.078330 & -2.319362 & 0.823023\end{array}$

$\begin{array}{lll}-6.235168 & -2.004664 & 2.676729\end{array}$

$\begin{array}{lll}-7.050967 & -1.713356 & 2.01368\end{array}$

$\begin{array}{lll}-6.453869 & -3.030355 & 3.008207\end{array}$

$\begin{array}{lll}-6.206764 & -1.048244 & 3.888272\end{array}$

$\begin{array}{llll}-5.838517 & -1.570596 & 4.776665\end{array}$

$\begin{array}{lll}-7.234246 & -0.748628 & 4.116596\end{array}$

$\begin{array}{lll}-0.297213 & 1.499410 & 2.273134\end{array}$

$\begin{array}{lll}0.282825 & 0.480811 & 3.061866\end{array}$

$\begin{array}{lll}0.074003 & 2.838268 & 2.525735\end{array}$

$\begin{array}{lll}1.202793 & 0.794640 & 4.057919\end{array}$

$\begin{array}{llll}0.026584 & -0.556224 & 2.880618\end{array}$

$\begin{array}{lll}0.976719 & 3.149145 & 3.538087\end{array}$

$\begin{array}{lll}-0.355000 & 3.628687 & 1.915898\end{array}$

$\begin{array}{lll}1.550615 & 2.127874 & 4.301737\end{array}$

$\begin{array}{lll}1.661258 & 0.000797 & 4.640409\end{array}$

$\begin{array}{lll}1.250859 & 4.183437 & 3.721104\end{array}$

$\begin{array}{lll}2.274360 & 2.365471 & 5.074822\end{array}$

$\begin{array}{llll}6.958836 & 3.500910 & -2.218676\end{array}$

$\begin{array}{lll}5.105058 & 1.147570 & 2.035187\end{array}$

$\begin{array}{llll}4.654419 & -0.587653 & -1.304054\end{array}$

$\begin{array}{llll}6.451843 & 2.280096 & -0.596960\end{array}$

$\begin{array}{llll}5.740069 & 1.885194 & -1.790663\end{array}$

$\begin{array}{llll}4.952474 & 2.995440 & -2.217489\end{array}$

$\begin{array}{llll}4.315492 & 3.016630 & -3.091274\end{array}$

$\begin{array}{llll}5.191303 & 4.082183 & -1.327402\end{array}$

$\begin{array}{llll}4.771029 & 5.075278 & -1.409692\end{array}$

$6.116786 \quad 3.647403 \quad-0.335442$

$\begin{array}{lll}6.512018 & 4.244831 & 0.472515\end{array}$

$\begin{array}{llll}8.537814 & 2.937790 & -3.443822\end{array}$

$8.826814 \quad 1.914832-3.644653$

$\begin{array}{llll}7.583744 & 3.700083 & -4.184972\end{array}$

$\begin{array}{llll}7.019097 & 3.354862 & -5.040675\end{array}$

$\begin{array}{llll}7.471928 & 4.985070 & -3.571285\end{array}$

$\begin{array}{llll}6.804931 & 5.779684 & -3.879010\end{array}$

$\begin{array}{llll}8.357265 & 5.018503 & -2.451007\end{array}$

$8.477408 \quad 5.841258 \quad-1.758635$

$\begin{array}{llll}9.016405 & 3.755359 & -2.372445\end{array}$

$\begin{array}{llll}9.727585 & 3.461449 & -1.612677\end{array}$

$\begin{array}{llll}7.295321 & 1.365250 & 0.227865\end{array}$

$\begin{array}{llll}8.547600 & 0.957385 & -0.255492\end{array}$

$\begin{array}{llll}8.881484 & 1.352906 & -1.206391\end{array}$

$\begin{array}{llll}9.348639 & 0.052141 & 0.440581\end{array}$

$\begin{array}{lll}10.299027 & -0.263573 & 0.022545\end{array}$

$\begin{array}{lll}8.909740 & -0.455023 & 1.661245\end{array}$

$9.518560-1.163679 \quad 2.214214$

$\begin{array}{lll}7.668152 & -0.064524 & 2.164519\end{array}$

$\begin{array}{llll}7.334656 & -0.476941 & 3.11022\end{array}$

$\begin{array}{llll}6.839283 & 0.823527 & 1.461882\end{array}$

$\begin{array}{llll}5.780091 & 0.503298 & -2.380282\end{array}$

$\begin{array}{llll}6.783634 & 0.121494 & -2.194932\end{array}$

$\begin{array}{llll}5.535388 & 0.445121 & -3.890663\end{array}$

$\begin{array}{llll}5.585055 & -0.583572 & -4.258395\end{array}$

$\begin{array}{llll}4.564127 & 0.856576 & -4.174229\end{array}$

$\begin{array}{llll}6.306058 & 1.034327 & -4.396745\end{array}$

$\begin{array}{lll}4.937504 & 0.240799 & 3.630957\end{array}$

$\begin{array}{llll}4.783471 & -1.155611 & 3.577101\end{array}$

$\begin{array}{llll}4.855220 & -1.667987 & 2.623872\end{array}$

$\begin{array}{llll}4.548659 & -1.894501 & 4.735866\end{array}$

$\begin{array}{llll}4.445035 & -2.972623 & 4.671318\end{array}$

$\begin{array}{llll}4.440930 & -1.243367 & 5.968967\end{array}$

$\begin{array}{llll}4.243983 & -1.814420 & 6.871795\end{array}$

$\begin{array}{llll}4.587728 & 0.143450 & 6.033322\end{array}$

$\begin{array}{llll}4.506576 & 0.656767 & 6.987473\end{array}$

$\begin{array}{llll}4.841102 & 0.882437 & 4.874003\end{array}$

$\begin{array}{llll}4.949517 & 1.958919 & 4.944298\end{array}$

$\begin{array}{lll}5.127266 & 2.914822 & 2.525822\end{array}$

$\begin{array}{lll}6.238528 & 3.501521 & 3.151868\end{array}$

$\begin{array}{lll}7.113961 & 2.900560 & 3.381663\end{array}$

$\begin{array}{llll}6.228526 & 4.862044 & 3.462077\end{array}$
$7.091796 \quad 5.313616 \quad 3.942914$

$\begin{array}{lll}5.110074 & 5.643603 & 3.149410\end{array}$

$\begin{array}{lll}5.107415 & 6.704258 & 3.385214\end{array}$

$\begin{array}{lll}4.001412 & 5.060244 & 2.531479\end{array}$

$\begin{array}{lll}3.134295 & 5.665721 & 2.281721\end{array}$

$\begin{array}{lll}4.007810 & 3.698897 & 2.217676\end{array}$

$\begin{array}{llll}3.168013 & 3.244726 & 1.702928\end{array}$

$\begin{array}{lll}5.727055 & -1.995028 & -0.768366\end{array}$

$\begin{array}{llll}5.203799 & -2.861451 & 0.202539\end{array}$

$\begin{array}{llll}4.173781 & -2.757068 & 0.520122\end{array}$

$\begin{array}{llll}6.000179 & -3.848678 & 0.784602\end{array}$

$\begin{array}{llll}7.339194 & -3.986443 & 0.426934\end{array}$

$\begin{array}{lll}7.966259 & -4.730151 & 0.905928\end{array}$

$\begin{array}{llll}7.859389 & -3.133335 & -0.545204\end{array}$

$\begin{array}{llll}7.065101 & -2.160803 & -1.150332\end{array}$

$\begin{array}{llll}7.518268 & -1.528852 & -1.901017\end{array}$

$\begin{array}{llll}3.388776 & -1.345629 & -2.404326\end{array}$

$\begin{array}{llll}2.488119 & -0.461315 & -3.023945\end{array}$

$\begin{array}{llll}2.627618 & 0.608690 & -2.930705\end{array}$

$\begin{array}{llll}1.381978 & -0.949636 & -3.709491\end{array}$

$\begin{array}{llll}1.110439 & -2.319792 & -3.754224\end{array}$

$\begin{array}{llll}0.199798 & -2.693072 & -4.205897\end{array}$

$2.004426-3.193220 \quad-3.144871$

$\begin{array}{llll}3.144108 & -2.719336 & -2.485686\end{array}$

$\begin{array}{llll}3.814116 & -3.433017 & -2.019571\end{array}$

$\begin{array}{llll}3.592168 & 0.489857 & 0.488194\end{array}$

$\begin{array}{llll}1.700684 & -4.664747 & -3.084319\end{array}$

$\begin{array}{llll}0.372018 & -0.006056 & -4.296542\end{array}$

$\begin{array}{llll}5.396980 & -4.777094 & 1.802720\end{array}$

$\begin{array}{llll}9.317717 & -3.255797 & -0.894226\end{array}$

$10.100594 \quad-3.087603 \quad 0.200546$

$\begin{array}{llll}9.712998 & -2.342409 & -1.808188\end{array}$

$\begin{array}{llll}9.613718 & -4.478232 & -1.393033\end{array}$

$\begin{array}{lll}4.454193 & -4.151236 & 2.550964\end{array}$

$\begin{array}{llll}4.790488 & -5.836368 & 1.220247\end{array}$

$\begin{array}{llll}6.322275 & -5.269153 & 2.654499\end{array}$

$2.821141 \quad-5.409846 \quad-3.264324$

$1.200134 \quad-5.018319-1.875974$

$\begin{array}{llll}0.807065 & -5.048614 & -4.016431\end{array}$

$\begin{array}{llll}0.085243 & -0.298116 & -5.585337\end{array}$

$\begin{array}{llll}0.780494 & 1.286036 & -4.262770\end{array}$

$\begin{array}{llll}-0.802355 & -0.067633 & -3.621260\end{array}$

$\begin{array}{lll}1.913791 & -1.902400 & 1.207493\end{array}$

$\begin{array}{llll}0.828406 & -0.445491 & -0.277713\end{array}$

$\begin{array}{lll}1.057618 & -2.940544 & 0.887495\end{array}$

$\begin{array}{llll}2.684276 & -2.018161 & 1.961411\end{array}$

$\begin{array}{llll}-0.014706 & -1.522540 & -0.684399\end{array}$

$\begin{array}{llll}0.092124 & -2.770546 & -0.109775\end{array}$

$\begin{array}{llll}-0.757418 & -1.354096 & -1.453086\end{array}$

$\begin{array}{llll}0.511265 & 0.856949 & -0.758488\end{array}$

$\begin{array}{llll}1.261696 & 2.060018 & -0.588549\end{array}$

$\begin{array}{llll}2.408959 & 2.115419 & -0.045789\end{array}$

$\begin{array}{lll}1.834344 & -0.690847 & 0.623074\end{array}$

$\begin{array}{llll}-0.325435 & 0.879103 & -1.438411\end{array}$

$\begin{array}{llll}0.697988 & 3.253602 & -1.005667\end{array}$

$\begin{array}{llll}1.526380 & 4.451622 & -1.049133\end{array}$

$\begin{array}{llll}1.899111 & 4.646083 & -2.066253\end{array}$

$\begin{array}{llll}0.936896 & 5.318996 & -0.732171\end{array}$

$\begin{array}{llll}2.378182 & 4.330525 & -0.384637\end{array}$

$\begin{array}{llll}-0.509378 & 3.305031 & -1.822666\end{array}$

$\begin{array}{lll}-1.307059 & 2.689624 & -1.404735\end{array}$

$\begin{array}{llll}-0.869482 & 4.335708 & -1.855307\end{array}$

$\begin{array}{llll}-0.327962 & 2.971855 & -2.850805\end{array}$

$\begin{array}{llll}-0.566953 & -3.567534 & -0.423599\end{array}$

$\begin{array}{lll}1.189766 & -4.653730 & 1.831457\end{array}$

$\begin{array}{llll}-2.780039 & -3.230597 & -2.259127\end{array}$

$\begin{array}{lll}-3.780242 & -4.036149 & -2.823726\end{array}$

$\begin{array}{lll}-1.505662 & -3.656518 & -2.674348\end{array}$

$-2.866462-3.305113-0.846759$

$\begin{array}{llll}-2.974932 & -1.884901 & -2.658534\end{array}$

9 P1-C2 exo re-si $\left[\mathrm{BF}_{4}^{-}\right]$

Number of imaginary frequencies $=0$

The smallest frequencies are $=5.71868 .198412 .4651 \mathrm{~cm}(-1)$ 
Electronic energy $=-7647.2701262$

Zero-point correction $=$

(Hartree/Particle)

Thermal correction to Energy=

Thermal correction to Enthalpy=

1.773081

1.897970

1.898914

Thermal correction to Gibbs Free Energy=

Sum of electronic and zero-point Energies=

Sum of electronic and thermal Energies=

1.600729

$-7645.497045$

$-7645.372156$

Sum of electronic and thermal Enthalpies=

$-7645.371212$

Sum of electronic and thermal Free Energies=

$-7645.669398$

Cartesian Coordinates

\begin{tabular}{|c|c|c|c|}
\hline 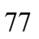 & -3.995098 & 0 & \\
\hline & -5.763899 & 0.799999 & 0.370302 \\
\hline & -6.214703 & -0.092475 & -0.962086 \\
\hline & -7.235918 & 0.795504 & 1.183392 \\
\hline & -5.486396 & 2.271402 & -0.394256 \\
\hline & -6.747038 & -1.360780 & -0.793652 \\
\hline & -5.928385 & -2.444155 & -1.176705 \\
\hline & -4.956307 & -2.233532 & -1.603791 \\
\hline & -6.386493 & -3.726084 & -1.006241 \\
\hline & -5.764324 & -4.561335 & -1.311672 \\
\hline & -7.651831 & -3.961018 & -0.400291 \\
\hline & -8.099871 & -5.282268 & -0.128499 \\
\hline & -7.458459 & -6.112750 & -0.411976 \\
\hline & -9.307778 & -5.509037 & 0.492432 \\
\hline 1 & -9.635125 & -6.523685 & 0.700373 \\
\hline & -10.119087 & -4.413923 & 0.874651 \\
\hline & -11.060898 & -4.594364 & 1.385346 \\
\hline 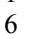 & -9.723159 & -3.120344 & 0.610535 \\
\hline 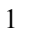 & -10.351828 & -2.291739 & 0.916216 \\
\hline 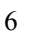 & -8.490290 & -2.848409 & -0.045322 \\
\hline - & -8.04 & -1.50 & 474 \\
\hline 6 & -8.910808 & -0.307059 & -0.160659 \\
\hline 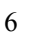 & -10.188054 & -0.201619 & -0.822713 \\
\hline & -10.658135 & $-1.1^{\prime}$ & -1.747285 \\
\hline . & -10.034499 & -2.031440 & -1.980541 \\
\hline 6 & -11.883674 & -1.045908 & -2.364118 \\
\hline , & -12.211671 & -1.803162 & -3.070652 \\
\hline 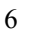 & -12.714315 & 0.067429 & -2.094163 \\
\hline 1 & -13.680780 & 0.15 & -2.582213 \\
\hline 6 & -12.281368 & 5117 & -1.227452 \\
\hline 1 & -12.896716 & 3392 & -1.0 \\
\hline . & -11.01 & 0.9 & -0.58 \\
\hline & -10.545618 & 1.98 & 0.260068 \\
\hline & 913 & 2.8 & 707 \\
\hline & -9.29 & 1.91 & 363 \\
\hline & -8.897300 & 2.71 & 1.43 \\
\hline 6 & -8.478199 & 0.78 & 0.57 \\
\hline & -4.30 & 2.99 & 086 \\
\hline & -3.480586 & 2.861630 & -0.564524 \\
\hline 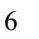 & -4.581168 & 4.49 & 0.275586 \\
\hline & -3.578769 & 5.420986 & -0.022582 \\
\hline 1 & -2.622467 & 5.071387 & -0.401670 \\
\hline & -3.79 & 6.78 & 0.167674 \\
\hline & -3.000603 & 7.496355 & -0.069769 \\
\hline 6 & -5.017937 & 7.24 & 176 \\
\hline 1 & -5.188722 & 8.309691 & 0.795886 \\
\hline & -6.027791 & 6.324859 & 0.949974 \\
\hline 1 & -6.987368 & 6.671768 & 1.324267 \\
\hline & -5.809416 & 4.959564 & 0.765908 \\
\hline & -6.596275 & 4.243506 & 0.986928 \\
\hline 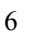 & -3.876169 & 2.449181 & 1.518133 \\
\hline & -4.523066 & 2.903233 & 2.280054 \\
\hline T. & -2.867845 & 2.814273 & 1.724312 \\
\hline 6 & -5.889461 & 2.745533 & -1.754530 \\
\hline & -5.919883 & 3.834530 & -1.655725 \\
\hline & -4.826218 & 2.440274 & -2.811822 \\
\hline 6 & -4.194084 & 3.497709 & -3.480230 \\
\hline & -4.456435 & 4.522398 & -3.226585 \\
\hline & -3.228324 & 3.252747 & -4.462904 \\
\hline & -2.749986 & 4.088143 & -4.967633 \\
\hline & -2.866340 & 1.941338 & -4.778572 \\
\hline & -2.095906 & 1.743875 & -5.51904 \\
\hline
\end{tabular}

$\begin{array}{llll}-3.488443 & 0.879305 & -4.115936\end{array}$

$\begin{array}{lll}-3.203248 & -0.145798 & -4.325473\end{array}$

$\begin{array}{lll}-4.460232 & 1.127595 & -3.147457\end{array}$

$\begin{array}{llll}-4.927747 & 0.294792 & -2.641379\end{array}$

$\begin{array}{lll}-7.294656 & 2.3388553 & -2.209298\end{array}$

$\begin{array}{lll}-8.038216 & 2.609354 & -1.455856\end{array}$

$\begin{array}{llll}-7.380869 & 1.274617 & -2.421814\end{array}$

$\begin{array}{llll}-7.525059 & 2.890936 & -3.126031\end{array}$

$\begin{array}{llll}-2.910835 & -0.219864 & -0.050551\end{array}$

$\begin{array}{lll}-3.110559 & -1.249160 & -0.320626\end{array}$

$\begin{array}{lll}-3.019447 & 0.469824 & -0.886365\end{array}$

$\begin{array}{lll}-1.939210 & 0.085844 & 0.947641\end{array}$

$\begin{array}{lll}-1.479704 & -0.752053 & 1.460525\end{array}$

$\begin{array}{lll}-0.968875 & 1.225750 & 0.696271\end{array}$

$\begin{array}{lll}-1.519373 & 2.052136 & 0.242447\end{array}$

$\begin{array}{lll}-4.984176 & 0.661862 & 3.677361\end{array}$

$\begin{array}{lll}-5.488729 & 1.627103 & 3.675726\end{array}$

$\begin{array}{lll}-3.559801 & 0.696787 & 3.862551\end{array}$

$\begin{array}{lll}-3.109636 & 1.680521 & 4.001709\end{array}$

$\begin{array}{lll}-2.762823 & -0.416771 & 4.525801\end{array}$

$\begin{array}{lll}-2.903115 & -0.394426 & 5.618739\end{array}$

$\begin{array}{lll}-1.704811 & -0.205989 & 4.349168\end{array}$

$\begin{array}{lll}-3.092478 & -1.824201 & 3.982560\end{array}$

$\begin{array}{lll}-3.843470 & -2.310807 & 4.613540\end{array}$

$\begin{array}{lll}-2.197667 & -2.453291 & 4.043826\end{array}$

$\begin{array}{lll}-3.583427 & -1.832094 & 2.536397\end{array}$

$\begin{array}{lll}-2.904469 & -2.275937 & 1.811787\end{array}$

$\begin{array}{lll}-4.922605 & -1.780849 & 2.166611\end{array}$

$\begin{array}{lll}-5.180256 & -2.210105 & 1.204174\end{array}$

$-6.088451 \quad-1.594950 \quad 3.119648$

$\begin{array}{lll}-6.961256 & -1.332065 & 2.518512\end{array}$

$\begin{array}{lll}-6.317668 & -2.556790 & 3.603710\end{array}$

$\begin{array}{lll}-5.863154 & -0.492791 & 4.176274\end{array}$

$\begin{array}{lll}-5.425261 & -0.920064 & 5.085669\end{array}$

$\begin{array}{lll}-6.838058 & -0.092664 & 4.475114\end{array}$

$\begin{array}{lll}-0.275476 & 1.767011 & 1.929635\end{array}$

$\begin{array}{lll}0.249334 & 0.931642 & 2.927466\end{array}$

$\begin{array}{lll}-0.154228 & 3.155127 & 2.097737\end{array}$

$\begin{array}{llll}0.881831 & 1.465974 & 4.051568\end{array}$

$\begin{array}{lll}0.162480 & -0.143405 & 2.832946\end{array}$

$\begin{array}{lll}0.461272 & 3.694981 & 3.229583\end{array}$

$\begin{array}{rrr}0.556197 & 3.819339 & 1.336618\end{array}$

$\begin{array}{lll}0.988409 & 2.850336 & 4.208666\end{array}$

$\begin{array}{lll}1.290807 & 0.799506 & 4.805312\end{array}$

$\begin{array}{lll}0.535826 & 4.772716 & 3.341709\end{array}$

$\begin{array}{llll}1.478461 & 3.265215 & 5.084771\end{array}$

$\begin{array}{llll}6.760541 & 3.437716 & -2.279128\end{array}$

$\begin{array}{llll}4.829607 & 1.344279 & 2.062250\end{array}$

$\begin{array}{llll}4.590684 & -0.693777 & -1.163678\end{array}$

$\begin{array}{llll}6.229412 & 2.322296 & -0.593033\end{array}$

$\begin{array}{llll}5.566682 & 1.822560 & -1.775131\end{array}$

$\begin{array}{llll}4.769542 & 2.877413 & -2.308813\end{array}$

$\begin{array}{llll}4.163923 & 2.819122 & -3.202333\end{array}$

$\begin{array}{llll}4.949862 & 4.031266 & -1.491828\end{array}$

$\begin{array}{llll}4.507479 & 5.003971 & -1.658624\end{array}$

$\begin{array}{llll}5.851199 & 3.694488 & -0.441496\end{array}$

$\begin{array}{llll}6.202810 & 4.359042 & 0.333258\end{array}$

$\begin{array}{llll}8.386555 & 2.838295 & -3.423090\end{array}$

$\begin{array}{llll}8.701569 & 1.811983 & -3.558108\end{array}$

$\begin{array}{llll}7.442278 & 3.539808 & -4.233968\end{array}$

$\begin{array}{lll}6.910554 & 3.136623 & -5.085488\end{array}$

$\begin{array}{llll}7.288700 & 4.854031 & -3.696046\end{array}$

$\begin{array}{llll}6.616278 & 5.617142 & -4.065583\end{array}$

$\begin{array}{llll}8.138124 & 4.966183 & -2.553184\end{array}$

$8.221798 \quad 5.827866-1.904173$

$\begin{array}{llll}8.817278 & 3.722622 & -2.385225\end{array}$

$\begin{array}{llll}9.509980 & 3.484505 & -1.589631\end{array}$

$\begin{array}{lll}7.069813 & 1.491044 & 0.316315\end{array}$

$\begin{array}{llll}8.335056 & 1.061174 & -0.111596\end{array}$

$\begin{array}{llll}8.683463 & 1.384210 & -1.084761\end{array}$

$\begin{array}{lll}9.129950 & 0.219704 & 0.666592\end{array}$

$\begin{array}{lll}10.087381 & -0.124243 & 0.288711\end{array}$

$\begin{array}{lll}8.677592 & -0.185277 & 1.920306\end{array}$

$\begin{array}{lll}9.286680 & -0.834244 & 2.542571\end{array}$

$\begin{array}{lll}7.424044 & 0.231912 & 2.370760\end{array}$ 
$\begin{array}{lll}7.081369 & -0.102702 & 3.343306\end{array}$ $\begin{array}{llll}6.595059 & 1.042400 & 1.579078\end{array}$ $\begin{array}{lll}5.681335 & 0.408352 & -2.267109\end{array}$ $\begin{array}{llll}6.693747 & 0.088916 & -2.023755\end{array}$ $\begin{array}{llll}5.485432 & 0.238366 & -3.775727\end{array}$ $\begin{array}{llll}5.606100 & -0.806856 & -4.074453\end{array}$ $\begin{array}{llll}4.499291 & 0.569972 & -4.109757\end{array}$ $\begin{array}{llll}6.235237 & 0.839653 & -4.298074\end{array}$ $\begin{array}{llll}4.619922 & 0.549345 & 3.708661\end{array}$ $\begin{array}{lll}4.594298 & -0.855866 & 3.761655\end{array}$ $\begin{array}{lll}4.787965 & -1.431972 & 2.862591\end{array}$ $\begin{array}{llll}4.340092 & -1.519120 & 4.960881\end{array}$ $\begin{array}{llll}4.338440 & -2.604036 & 4.983772\end{array}$ $\begin{array}{llll}4.085689 & -0.784810 & 6.123897\end{array}$ $\begin{array}{lll}3.874913 & -1.299163 & 7.057110\end{array}$ $\begin{array}{lll}4.103856 & 0.610140 & 6.079997\end{array}$ $\begin{array}{llll}3.906733 & 1.186471 & 6.979594\end{array}$ $\begin{array}{llll}4.373957 & 1.276766 & 4.881195\end{array}$ $\begin{array}{llll}4.377767 & 2.360801 & 4.864597\end{array}$ $\begin{array}{lll}4.723987 & 3.140385 & 2.394613\end{array}$ $\begin{array}{lll}5.758533 & 3.844189 & 3.031456\end{array}$ $\begin{array}{llll}6.647773 & 3.318413 & 3.367753\end{array}$ $\begin{array}{llll}5.654341 & 5.223218 & 3.214934\end{array}$ $\begin{array}{lll}6.457994 & 5.765676 & 3.704786\end{array}$ $\begin{array}{lll}4.518069 & 5.905918 & 2.765050\end{array}$ $\begin{array}{lll}4.441866 & 6.980730 & 2.903996\end{array}$ $\begin{array}{llll}3.483594 & 5.206098 & 2.140275\end{array}$ $\begin{array}{lll}2.599074 & 5.732417 & 1.793267\end{array}$ $\begin{array}{llll}3.584768 & 3.825797 & 1.954059\end{array}$ $2.794434 \quad 3.281517 \quad 1.448315$ $\begin{array}{llll}5.719734 & -2.044989 & -0.599542\end{array}$ $\begin{array}{llll}5.293007 & -2.843568 & 0.471080\end{array}$ $\begin{array}{lll}4.278576 & -2.759572 & 0.836233\end{array}$ $\begin{array}{llll}6.173072 & -3.723567 & 1.102958\end{array}$ $\begin{array}{lll}7.499538 & -3.820508 & 0.689919\end{array}$ $\begin{array}{llll}8.194845 & -4.468839 & 1.211186\end{array}$ $\begin{array}{llll}7.921157 & -3.046111 & -0.389365\end{array}$ $\begin{array}{llll}7.042695 & -2.184658 & -1.043599\end{array}$ $\begin{array}{llll}7.420641 & -1.608772 & -1.877028\end{array}$ $\begin{array}{llll}3.348727 & -1.467239 & -2.26931\end{array}$ $\begin{array}{lll}2.436947 & -0.575657 & -2.861820\end{array}$ $\begin{array}{llll}2.556623 & 0.491288 & -2.710466\end{array}$ $\begin{array}{llll}1.362241 & -1.056911 & -3.598467\end{array}$ $\begin{array}{llll}1.138309 & -2.431164 & -3.725891\end{array}$ $\begin{array}{llll}0.255735 & -2.806486 & -4.228554\end{array}$ $2.041697 \quad-3.310106-3.137170$ $\begin{array}{llll}3.149465 & -2.840827 & -2.419715\end{array}$ $\begin{array}{llll}3.833652 & -3.551939 & -1.968516\end{array}$ $\begin{array}{llll}3.442286 & 0.461645 & 0.518318\end{array}$ $\begin{array}{llll}1.794390 & -4.794181 & -3.188371\end{array}$ $\begin{array}{llll}0.345009 & -0.105984 & -4.160544\end{array}$ $\begin{array}{llll}5.690288 & -4.564718 & 2.253836\end{array}$ $\begin{array}{llll}9.369910 & -3.111722 & -0.791383\end{array}$ $10.178575 \quad-2.8704890 .270196$ $\begin{array}{llll}9.684670 & -2.207036 & -1.744245\end{array}$ $\begin{array}{llll}9.708936 & -4.331633 & -1.265833\end{array}$ $\begin{array}{llll}4.728483 & -3.927295 & 2.968026\end{array}$ $\begin{array}{lll}5.155317 & -5.734336 & 1.835973\end{array}$ $\begin{array}{llll}6.688067 & -4.870059 & 3.109983\end{array}$ $\begin{array}{llll}2.943091 & -5.476970 & -3.421887\end{array}$ $\begin{array}{llll}1.310333 & -5.259142 & -2.010769\end{array}$ $\begin{array}{llll}0.915762 & -5.139518 & -4.147628\end{array}$ $0.098118 \quad-0.324744 \quad-5.470278$ $\begin{array}{llll}0.721645 & 1.190250 & -4.034466\end{array}$ $\begin{array}{llll}-0.842149 & -0.235876 & -3.519162\end{array}$ $\begin{array}{lll}1.941105 & -1.940444 & 1.251011\end{array}$ $\begin{array}{llll}0.645210 & -0.593495 & -0.124866\end{array}$ $\begin{array}{llll}1.294648 & -3.082202 & 0.799179\end{array}$ $\begin{array}{lll}2.703393 & -1.982906 & 2.019358\end{array}$ $\begin{array}{llll}-0.007802 & -1.722468 & -0.637967\end{array}$ $\begin{array}{llll}0.322958 & -2.994593 & -0.190048\end{array}$ $-0.783018-1.607772-1.382360$ $\begin{array}{lll}0.050572 & 0.758155 & -0.452586\end{array}$ $\begin{array}{llll}1.010453 & 1.881312 & -0.802802\end{array}$ $\begin{array}{llll}2.218607 & 1.864896 & -0.479097\end{array}$ $\begin{array}{lll}1.651682 & -0.718129 & 0.765627\end{array}$

$\begin{array}{llll}-0.586444 & 0.583790 & -1.317243\end{array}$

$\begin{array}{llll}0.512984 & 2.965971 & -1.439679\end{array}$

$\begin{array}{llll}1.398213 & 4.107135 & -1.669437\end{array}$

$\begin{array}{llll}1.907378 & 4.017479 & -2.636275\end{array}$

$\begin{array}{llll}0.797131 & 5.020157 & -1.671710\end{array}$

$2.148499 \quad 4.161874 \quad-0.883495$

$\begin{array}{lll}-0.772550 & 3.058599 & -2.130709\end{array}$

$\begin{array}{llll}-1.365833 & 2.155001 & -2.035490\end{array}$

$\begin{array}{llll}-1.348274 & 3.899551 & -1.736824\end{array}$

$\begin{array}{llll}-0.595953 & 3.229361 & -3.195596\end{array}$

$\begin{array}{llll}-0.180608 & -3.858343 & -0.598690\end{array}$

$\begin{array}{lll}1.794534 & -4.817749 & 1.533214\end{array}$

$\begin{array}{lll}-2.632873 & -3.437126 & -2.155269\end{array}$

$\begin{array}{lll}-3.649599 & -4.263465 & -2.650176\end{array}$

$-1.373083-3.852876-2.637641$

$\begin{array}{llll}-2.629239 & -3.487633 & -0.743626\end{array}$

$-2.862365-2.100384-2.568740$

$[8-9]^{\ddagger}$ P1-C2 endo si-re $\left[\mathrm{BF}_{4}{ }^{-}\right]$conf2

Number of imaginary frequencies $=1$

The smallest frequencies are $=-191.032511 .876813 .7626 \mathrm{~cm}(-1)$ Electronic energy $=-7647.2550994$

Zero-point correction $=$

1.771637

(Hartree/Particle)

Thermal correction to Energy $=\quad 1.896247$

Thermal correction to Enthalpy=

1.897191

Thermal correction to Gibbs Free Energy=

Sum of electronic and zero-point Energies=

Sum of electronic and thermal Energies=

Sum of electronic and thermal Enthalpies=

1.602906

$-7645.483463$

$-7645.358852$

$-7645.357908$

Sum of electronic and thermal Free Energies $=\quad-7645.652193$

$\begin{array}{lccc}\text { Cartesian Coordinates } & & \\ \ldots \ldots \ldots \ldots \ldots \ldots \ldots \ldots \ldots \ldots \ldots \ldots \ldots \ldots \ldots \ldots \ldots \ldots \\ 26 & 8.463431 & 0.029180 & -0.575056 \\ 15 & 3.558421 & 1.029441 & -2.129554 \\ 15 & 4.260951 & 0.715919 & 1.562992 \\ 6 & 6.526149 & 0.719231 & -0.979293 \\ 6 & 6.589651 & 0.056988 & 0.302681 \\ 6 & 7.019970 & -1.286944 & 0.079230 \\ 1 & 7.175293 & -2.037139 & 0.842306 \\ 6 & 7.251474 & -1.456274 & -1.319710 \\ 1 & 7.621259 & -2.352829 & -1.797311 \\ 6 & 6.949761 & -0.224177 & -1.969949 \\ 1 & 7.044129 & -0.022780 & -3.026627 \\ 6 & 9.784715 & 1.252223 & 0.460276 \\ 1 & 9.491028 & 1.976406 & 1.208606 \\ 6 & 10.088356 & -0.123081 & 0.700361 \\ 1 & 10.060439 & -0.623767 & 1.658943 \\ 6 & 10.389225 & -0.734711 & -0.554715 \\ 1 & 10.624150 & -1.779175 & -0.712249 \\ 6 & 10.271020 & 0.261424 & -1.571276 \\ 1 & 10.401379 & 0.105000 & -2.633972 \\ 6 & 9.899377 & 1.488607 & -0.945166 \\ 1 & 9.703883 & 2.422720 & -1.453905 \\ 6 & 6.034843 & 2.111990 & -1.186223 \\ 6 & 6.819950 & 3.185295 & -0.737330 \\ 1 & 7.774929 & 2.961353 & -0.279491 \\ 6 & 6.396346 & 4.508809 & -0.848934 \\ 1 & 7.016344 & 5.311912 & -0.463343 \\ 6 & 5.167382 & 4.785574 & -1.442399 \\ 1 & 4.819555 & 5.809076 & -1.539034 \\ 6 & 4.365246 & 3.735812 & -1.889738 \\ 1 & 3.404341 & 3.969901 & -2.332173 \\ 6 & 4.762998 & 2.395522 & -1.753663 \\ 6 & 6.158698 & 0.678816 & 1.603746 \\ 1 & 6.470100 & 1.724316 & 1.581755 \\ 6 & 6.769374 & 0.022597 & 2.845005 \\ 1 & 6.459757 & 0.541436 & 3.756519 \\ 1 & 6.478871 & -1.027129 & 2.941539 \\ 1 & 7.860689 & 0.065037 & 2.773188 \\ 6 & 2.081188 & 1.945029 & -2.743085 \\ 6 & 1.201827 & 2.462891 & -1.775473\end{array}$


$\begin{array}{lll}1.361851 & 2.232579 & -0.728113\end{array}$

$\begin{array}{llll}0.126935 & 3.265683 & -2.155898\end{array}$

$\begin{array}{llll}-0.537987 & 3.669109 & -1.401210\end{array}$

$\begin{array}{llll}-0.109175 & 3.527319 & -3.507999\end{array}$

$\begin{array}{llll}-0.955123 & 4.142002 & -3.80209\end{array}$

$\begin{array}{llll}0.734724 & 2.982348 & -4.477687\end{array}$

$\begin{array}{llll}0.549282 & 3.170661 & -5.531439\end{array}$

$\begin{array}{lll}1.831550 & 2.203322 & -4.098980\end{array}$

$\begin{array}{llll}2.490910 & 1.807551 & -4.863734\end{array}$

$\begin{array}{llll}4.246484 & 0.213193 & -3.623166\end{array}$

$\begin{array}{llll}4.971483 & 0.916406 & -4.598623\end{array}$

$\begin{array}{llll}5.136459 & 1.984349 & -4.488741\end{array}$

$\begin{array}{llll}5.498893 & 0.243048 & -5.701625\end{array}$

$\begin{array}{llll}6.061265 & 0.793135 & -6.450847\end{array}$

$\begin{array}{llll}5.308973 & -1.136132 & -5.840129\end{array}$

$\begin{array}{llll}5.727072 & -1.658470 & -6.695868\end{array}$

$\begin{array}{llll}4.586146 & -1.840501 & -4.874381\end{array}$

$\begin{array}{llll}4.442186 & -2.912866 & -4.971657\end{array}$

$\begin{array}{llll}4.058586 & -1.167923 & -3.770590\end{array}$

$\begin{array}{llll}3.519048 & -1.719076 & -3.009725\end{array}$

$\begin{array}{lll}3.758304 & 2.464521 & 1.850721\end{array}$

$\begin{array}{lll}2.379778 & 2.713155 & 1.777042\end{array}$

$\begin{array}{lll}1.703123 & 1.881341 & 1.607322\end{array}$

$\begin{array}{llll}1.886947 & 4.013641 & 1.880651\end{array}$

$\begin{array}{lll}2.752939 & 5.096259 & 2.021235\end{array}$

$\begin{array}{lll}2.370093 & 6.109154 & 2.075523\end{array}$

$\begin{array}{llll}4.125377 & 4.848757 & 2.075333\end{array}$

$\begin{array}{lll}4.629126 & 3.548912 & 2.010736\end{array}$

$\begin{array}{llll}5.699821 & 3.408316 & 2.065098\end{array}$

$\begin{array}{lll}3.667405 & -0.169150 & 3.062003\end{array}$

$\begin{array}{lll}3.722519 & -1.571107 & 3.007634\end{array}$

$\begin{array}{lll}4.157642 & -2.061817 & 2.145665\end{array}$

$\begin{array}{llll}3.184538 & -2.338921 & 4.034837\end{array}$

$\begin{array}{llll}2.559112 & -1.732860 & 5.126358\end{array}$

$\begin{array}{llll}2.119158 & -2.333365 & 5.914213\end{array}$

$\begin{array}{lll}2.498856 & -0.342721 & 5.175734\end{array}$

$\begin{array}{llll}3.054386 & 0.440776 & 4.159634\end{array}$

$2.989891 \quad 1.520450 \quad 4.230137$

$\begin{array}{llll}3.148684 & -0.207007 & -0.284262\end{array}$

$\begin{array}{lll}1.744119 & 0.333534 & 6.285500\end{array}$

$\begin{array}{llll}3.172979 & -3.838610 & 3.923811\end{array}$

$\begin{array}{lll}0.397481 & 4.215656 & 1.868916\end{array}$

$\begin{array}{llll}5.060642 & 6.025026 & 2.151559\end{array}$

$\begin{array}{lll}5.005882 & 6.774000 & 1.021076\end{array}$

$\begin{array}{lll}6.349633 & 5.653841 & 2.315622\end{array}$

$\begin{array}{lll}4.744485 & 6.853591 & 3.173500\end{array}$

$\begin{array}{llll}-0.205224 & 3.442261 & 0.920614\end{array}$

$\begin{array}{lll}-0.170241 & 3.868623 & 3.047336\end{array}$

$\begin{array}{lll}0.043941 & 5.492586 & 1.613374\end{array}$

$\begin{array}{llll}2.276282 & 1.534686 & 6.603557\end{array}$

$\begin{array}{llll}0.451376 & 0.568599 & 5.929093\end{array}$

$\begin{array}{lll}1.706900 & -0.408856 & 7.411442\end{array}$

$3.439710 \quad-4.432139 \quad 5.109051$

$\begin{array}{llll}4.077378 & -4.291970 & 3.028107\end{array}$

$\begin{array}{llll}1.958898 & -4.297024 & 3.526476\end{array}$

$\begin{array}{lll}0.734024 & -0.969403 & 1.356788\end{array}$

$\begin{array}{llll}0.875771 & -2.352270 & 0.970827\end{array}$

$\begin{array}{llll}2.016763 & -2.971448 & 0.385016\end{array}$

$\begin{array}{lll}0.197292 & -3.051749 & 1.435025\end{array}$

$\begin{array}{lll}-3.305158 & -0.854540 & -1.733786\end{array}$

$\begin{array}{llll}-5.013561 & 0.098633 & -0.603344\end{array}$

$\begin{array}{llll}-4.787765 & 1.312701 & 0.515780\end{array}$

$\begin{array}{llll}-6.122620 & 0.838501 & -1.608889\end{array}$

$\begin{array}{lll}-5.765050 & -1.029705 & 0.380556\end{array}$

$\begin{array}{lll}-4.610569 & 2.632542 & 0.133541\end{array}$

$\begin{array}{lll}-3.337120 & 3.196459 & 0.375261\end{array}$

$\begin{array}{lll}-2.579404 & 2.585195 & 0.852549\end{array}$

$\begin{array}{llll}-3.089088 & 4.495277 & 0.006351\end{array}$

$\begin{array}{llll}-2.122290 & 4.946254 & 0.204561\end{array}$

$\begin{array}{llll}-4.087192 & 5.257498 & -0.659144\end{array}$

$\begin{array}{llll}-3.813078 & 6.571686 & -1.125599\end{array}$

$\begin{array}{llll}-2.826688 & 6.989917 & -0.941920\end{array}$

$\begin{array}{llll}-4.766659 & 7.294838 & -1.805983\end{array}$

$\begin{array}{llll}-4.544897 & 8.296361 & -2.162924\end{array}$

$\begin{array}{llll}-6.037318 & 6.722829 & -2.053653\end{array}$ $\begin{array}{lll}-6.780785 & 7.285425 & -2.611147\end{array}$

$\begin{array}{llll}-6.340525 & 5.456934 & -1.600639\end{array}$

$\begin{array}{llll}-7.315795 & 5.033419 & -1.809290\end{array}$

$\begin{array}{llll}-5.387223 & 4.687385 & -0.876551\end{array}$

$\begin{array}{llll}-5.667315 & 3.358731 & -0.395309\end{array}$

$\begin{array}{llll}-7.028048 & 2.754782 & -0.473584\end{array}$

$\begin{array}{lll}-8.182631 & 3.386679 & 0.110854\end{array}$

$\begin{array}{lll}-8.091635 & 4.550987 & 0.924467\end{array}$

$\begin{array}{llll}-7.118267 & 4.982147 & 1.126348\end{array}$

$\begin{array}{lll}-9.217524 & 5.126496 & 1.472129\end{array}$

$\begin{array}{lll}-9.119164 & 6.009734 & 2.096829\end{array}$

$\begin{array}{lll}-10.499662 & 4.574807 & 1.236452\end{array}$

$\begin{array}{lll}-11.378297 & 5.042523 & 1.671006\end{array}$

$\begin{array}{lll}-10.624081 & 3.438037 & 0.470685\end{array}$

$\begin{array}{lll}-11.599758 & 2.991644 & 0.296671\end{array}$

$\begin{array}{llll}-9.481947 & 2.810406 & -0.097764\end{array}$

$\begin{array}{llll}-9.604176 & 1.609307 & -0.847367\end{array}$

$\begin{array}{lll}-10.591472 & 1.186810 & -1.011250\end{array}$

$\begin{array}{llll}-8.487005 & 0.965422 & -1.319023\end{array}$

$\begin{array}{llll}-8.551348 & 0.027043 & -1.858704\end{array}$

$\begin{array}{llll}-7.212410 & 1.532488 & -1.102700\end{array}$

$\begin{array}{llll}-5.351306 & -2.430057 & 0.078357\end{array}$

$\begin{array}{lll}-4.668288 & -2.764987 & 0.860948\end{array}$

$\begin{array}{lll}-6.519743 & -3.400821 & 0.001118\end{array}$

$\begin{array}{llll}-6.353986 & -4.716150 & 0.451742\end{array}$

$\begin{array}{lll}-5.405285 & -5.016383 & 0.885322\end{array}$

$\begin{array}{llll}-7.400316 & -5.636446 & 0.348675\end{array}$

$\begin{array}{lll}-7.260301 & -6.651493 & 0.711447\end{array}$

$\begin{array}{llll}-8.623882 & -5.253291 & -0.205067\end{array}$

$\begin{array}{llll}-9.442555 & -5.964806 & -0.271681\end{array}$

$\begin{array}{llll}-8.788859 & -3.944507 & -0.671085\end{array}$

$\begin{array}{lll}-9.739057 & -3.633510 & -1.097585\end{array}$

$\begin{array}{llll}-7.740434 & -3.030305 & -0.577265\end{array}$

$\begin{array}{llll}-7.875875 & -2.006478 & -0.910568\end{array}$

$\begin{array}{lll}-4.608822 & -2.489575 & -1.275766\end{array}$

$\begin{array}{lll}-5.356714 & -2.553174 & -2.074910\end{array}$

$\begin{array}{lll}-4.043612 & -3.423538 & -1.293810\end{array}$

$\begin{array}{lll}-6.248191 & -0.676902 & 1.760825\end{array}$

$\begin{array}{lll}-6.476787 & 0.387572 & 1.714940\end{array}$

$\begin{array}{llll}-7.554317 & -1.367304 & 2.129371\end{array}$

$\begin{array}{lll}-8.764154 & -0.735230 & 1.818759\end{array}$

$\begin{array}{lll}-8.746178 & 0.236615 & 1.336912\end{array}$

$\begin{array}{llll}-9.987622 & -1.339515 & 2.110983\end{array}$

$\begin{array}{lll}-10.914612 & -0.832392 & 1.855996\end{array}$

$\begin{array}{lll}-10.015495 & -2.590733 & 2.731298\end{array}$

$\begin{array}{lll}-10.964328 & -3.070388 & 2.955975\end{array}$

$\begin{array}{llll}-8.813838 & -3.219806 & 3.067881\end{array}$

$\begin{array}{lll}-8.825130 & -4.192435 & 3.551743\end{array}$

$\begin{array}{llll}-7.593357 & -2.608258 & 2.777642\end{array}$

$\begin{array}{lll}-6.669992 & -3.117071 & 3.032824\end{array}$

$\begin{array}{lll}-5.146076 & -0.864729 & 2.813410\end{array}$

$\begin{array}{lll}-4.319503 & -0.180448 & 2.606811\end{array}$

$\begin{array}{lll}-4.748088 & -1.881179 & 2.837449\end{array}$

$\begin{array}{llll}-5.542155 & -0.630424 & 3.806735\end{array}$

$\begin{array}{lll}-2.306360 & -0.806565 & 0.194948\end{array}$

$\begin{array}{lll}-1.640425 & 0.049970 & 0.255597\end{array}$

$\begin{array}{lll}-2.950109 & -0.917066 & 1.060537\end{array}$

$\begin{array}{llll}-1.804472 & -2.017687 & -0.383831\end{array}$

$\begin{array}{llll}-2.297402 & -2.944107 & -0.128136\end{array}$

$\begin{array}{lll}-0.542643 & -2.101140 & -1.009060\end{array}$

$\begin{array}{llll}0.001490 & -1.179051 & -1.167234\end{array}$

$\begin{array}{llll}-4.263908 & -0.714934 & -3.745872\end{array}$

$\begin{array}{llll}-5.282785 & -1.077575 & -3.635404\end{array}$

$\begin{array}{llll}-3.244269 & -1.696623 & -3.807030\end{array}$

$\begin{array}{lll}-3.551703 & -2.740701 & -3.757042\end{array}$

$\begin{array}{llll}-1.885328 & -1.483518 & -4.452514\end{array}$

$\begin{array}{llll}-1.977870 & -1.550676 & -5.548127\end{array}$

$\begin{array}{llll}-1.245375 & -2.315371 & -4.147985\end{array}$

$\begin{array}{llll}-1.191475 & -0.156350 & -4.067734\end{array}$

$\begin{array}{llll}-1.397669 & 0.617282 & -4.814781\end{array}$

$\begin{array}{lll}-0.107545 & -0.306583 & -4.086844\end{array}$

$\begin{array}{llll}-1.569888 & 0.384682 & -2.690916\end{array}$

$\begin{array}{llll}-0.764913 & 0.421978 & -1.966965\end{array}$

$\begin{array}{llll}-2.623084 & 1.258062 & -2.449269\end{array}$

$\begin{array}{llll}-2.535960 & 1.884234 & -1.568015\end{array}$ 


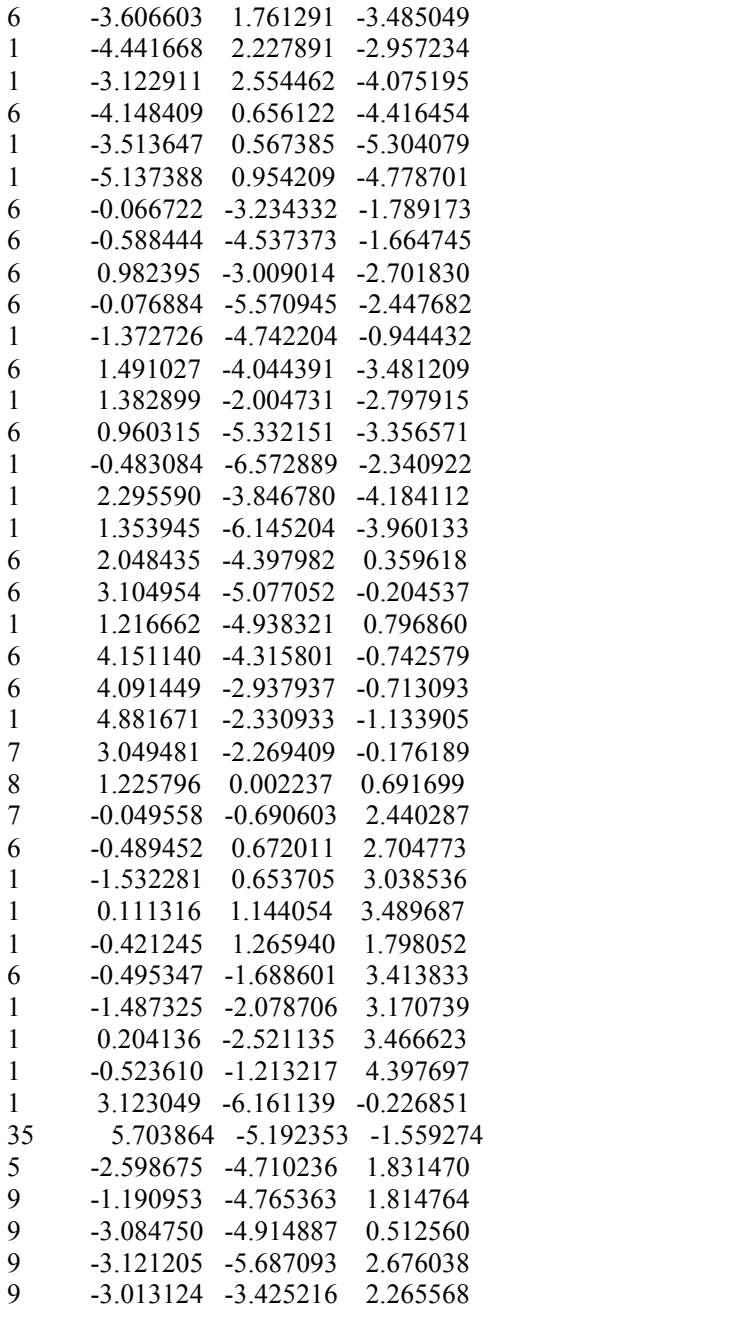

$[8-9]^{\ddagger}$ P1-C2 endo re-re $\left[\mathrm{BF}_{4}^{-}\right]$conf2

Number of imaginary frequencies $=1$

The smallest frequencies are $=-241.683310 .617712 .7392 \mathrm{~cm}(-1)$ Electronic energy $=-7647.2463107$

Zero-point correction $=$

1.771399

(Hartree/Particle)

Thermal correction to Energy=

Thermal correction to Enthalpy=

1.896249

1.897193

Thermal correction to Gibbs Free Energy=

Sum of electronic and zero-point Energies=

Sum of electronic and thermal Energies=

Sum of electronic and thermal Enthalpies=

Sum of electronic and thermal Free Energies=

1.601928

$-7645.474912$

$-7645.350061$

$-7645.349117$

$-7645.644383$

Cartesian Coordinates

$\begin{array}{lllll}26 & 7.650844 & -2.088963 & -1.462913 \\ 15 & & 3.218711 & 0.490143 & -2.333527 \\ 15 & 4.463365 & 0.277974 & 1.204344 \\ 6 & 6.055729 & -0.753252 & -1.683725 \\ 6 & 6.093966 & -1.302894 & -0.349086 \\ 6 & 5.956910 & -2.718367 & -0.457064 \\ 1 & 5.957001 & -3.415986 & 0.368530 \\ 6 & 5.869206 & -3.056226 & -1.838062 \\ 1 & 5.801530 & -4.056046 & -2.244325 \\ 6 & 5.935239 & -1.851417 & -2.593780 \\ 1 & 5.908063 & -1.769663 & -3.670510 \\ 6 & 9.480592 & -1.375065 & -0.791198 \\ 1 & 9.602128 & -0.534979 & -0.120094 \\ 6 & 9.307986 & -2.739404 & -0.403222 \\ 1 & 9.270985 & -3.113338 & 0.611213 \\ 6 & 9.145457 & -3.518659 & -1.589165\end{array}$

$8.958392-4.583731-1.628445$

$9.218908-2.637189-2.710723$

$\begin{array}{llll}9.100546 & -2.916601 & -3.749370\end{array}$

$9.428220 \quad-1.314440 \quad-2.218745$

$\begin{array}{llll}9.500543 & -0.420810 & -2.823444\end{array}$

$\begin{array}{llll}6.045060 & 0.700685 & -2.012560\end{array}$

$\begin{array}{llll}7.228408 & 1.446136 & -1.907996\end{array}$

$\begin{array}{llll}8.131746 & 0.932757 & -1.603805\end{array}$

$\begin{array}{llll}7.262000 & 2.815015 & -2.172573\end{array}$

$\begin{array}{llll}8.189719 & 3.366359 & -2.060239\end{array}$

$\begin{array}{llll}6.095239 & 3.467800 & -2.562847\end{array}$

$\begin{array}{llll}6.103393 & 4.533264 & -2.770496\end{array}$

$\begin{array}{llll}4.903039 & 2.749940 & -2.665093\end{array}$

$\begin{array}{llll}4.000572 & 3.275372 & -2.956324\end{array}$

$\begin{array}{llll}4.849231 & 1.376605 & -2.379404\end{array}$

$\begin{array}{llll}6.181207 & -0.480358 & 0.907133\end{array}$

$\begin{array}{llll}6.821617 & 0.371180 & 0.677400\end{array}$

$\begin{array}{llll}6.792066 & -1.213823 & 2.105011\end{array}$

$\begin{array}{llll}6.837045 & -0.563500 & 2.983167\end{array}$

$\begin{array}{llll}6.227696 & -2.108847 & 2.377998\end{array}$

$\begin{array}{lll}7.809768 & -1.525596 & 1.851652\end{array}$

$\begin{array}{llll}1.993735 & 1.781707 & -2.809368\end{array}$

$1.599946 \quad 2.686231-1.807815$

$\begin{array}{llll}2.041820 & 2.617838 & -0.818926\end{array}$

$\begin{array}{llll}0.660636 & 3.679978 & -2.075149\end{array}$

$\begin{array}{llll}0.378222 & 4.375725 & -1.296194\end{array}$

$\begin{array}{llll}0.078901 & 3.771338 & -3.342580\end{array}$

$\begin{array}{lll}-0.667440 & 4.534915 & -3.541645\end{array}$

$\begin{array}{llll}0.457608 & 2.873267 & -4.341719\end{array}$

$\begin{array}{llll}0.009187 & 2.935126 & -5.329218\end{array}$

$\begin{array}{llll}1.413492 & 1.887168 & -4.081156\end{array}$

$\begin{array}{llll}1.691298 & 1.197966 & -4.870138\end{array}$

$\begin{array}{llll}3.285936 & -0.646438 & -3.770567\end{array}$

$3.830946-0.273200 \quad-5.008766$

$\begin{array}{llll}4.229923 & 0.727471 & -5.148844\end{array}$

$\begin{array}{llll}3.873070 & -1.192221 & -6.058588\end{array}$

$\begin{array}{llll}4.294929 & -0.899443 & -7.015902\end{array}$

$\begin{array}{llll}3.377036 & -2.488294 & -5.877952\end{array}$

$\begin{array}{llll}3.413265 & -3.201146 & -6.696973\end{array}$

$\begin{array}{llll}2.845885 & -2.867320 & -4.642772\end{array}$

$\begin{array}{llll}2.473416 & -3.877042 & -4.494579\end{array}$

$\begin{array}{llll}2.806094 & -1.950601 & -3.590386\end{array}$

$\begin{array}{llll}2.450724 & -2.247978 & -2.608893\end{array}$

$\begin{array}{lll}4.739568 & 2.102859 & 1.332044\end{array}$

$\begin{array}{llll}3.599363 & 2.909534 & 1.454320\end{array}$

$\begin{array}{lll}2.636212 & 2.452551 & 1.629197\end{array}$

$\begin{array}{llll}3.678372 & 4.296397 & 1.330379\end{array}$

$\begin{array}{lll}4.900840 & 4.920163 & 1.096506\end{array}$

$\begin{array}{llll}4.960842 & 5.995845 & 0.973882\end{array}$

$\begin{array}{llll}6.044038 & 4.125967 & 1.007355\end{array}$

$\begin{array}{lll}5.972486 & 2.738164 & 1.124559\end{array}$

$\begin{array}{lll}6.887843 & 2.172024 & 1.028048\end{array}$

$\begin{array}{lll}3.923910 & -0.265360 & 2.872936\end{array}$

$\begin{array}{lll}3.630400 & -1.632820 & 2.995959\end{array}$

$\begin{array}{lll}3.794825 & -2.299802 & 2.157225\end{array}$

$\begin{array}{lll}3.100680 & -2.136684 & 4.180038\end{array}$

$\begin{array}{llll}2.797353 & -1.285188 & 5.242542\end{array}$

$\begin{array}{llll}2.353206 & -1.673943 & 6.152615\end{array}$

$\begin{array}{llll}3.064807 & 0.076788 & 5.108401\end{array}$

$\begin{array}{lll}3.640793 & 0.588635 & 3.943919\end{array}$

$\begin{array}{lll}3.845812 & 1.650189 & 3.876208\end{array}$

$\begin{array}{llll}2.780936 & -0.431093 & -0.304979\end{array}$

$\begin{array}{lll}2.663441 & 0.994163 & 6.230607\end{array}$

$\begin{array}{llll}2.756743 & -3.596830 & 4.285252\end{array}$

$\begin{array}{lll}2.417017 & 5.108307 & 1.438225\end{array}$

$\begin{array}{lll}7.359240 & 4.806551 & 0.737814\end{array}$

$\begin{array}{llll}7.326584 & 5.501144 & -0.426370\end{array}$

$\begin{array}{llll}8.389191 & 3.936697 & 0.653715\end{array}$

$\begin{array}{lll}7.664951 & 5.699755 & 1.707273\end{array}$

$\begin{array}{llll}1.365788 & 4.452411 & 0.870530\end{array}$

$\begin{array}{lll}2.070266 & 5.350974 & 2.721395\end{array}$

$\begin{array}{llll}2.518009 & 6.301162 & 0.818941\end{array}$

$\begin{array}{llll}3.086128 & 2.262876 & 6.039852\end{array}$

$\begin{array}{llll}1.315511 & 1.037133 & 6.366423\end{array}$

$\begin{array}{lll}3.156988 & 0.578087 & 7.420426\end{array}$ 


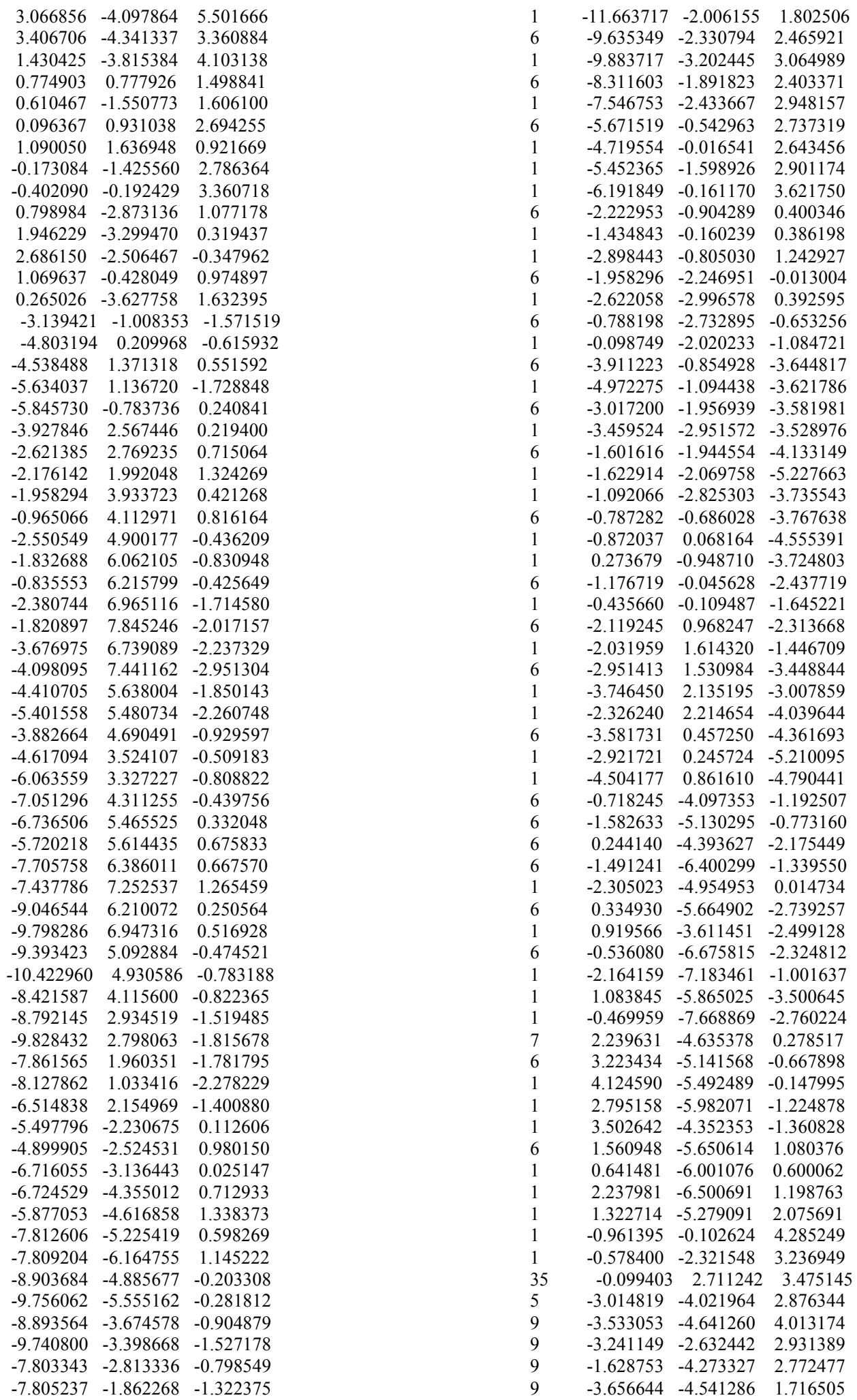

$\begin{array}{llll}-11.663717 & -2.006155 & 1.802506\end{array}$

$\begin{array}{lll}-9.635349 & -2.330794 & 2.465921\end{array}$

$\begin{array}{lll}-9.883717 & -3.202445 & 3.064989\end{array}$

$\begin{array}{lll}-8.311603 & -1.891823 & 2.403371\end{array}$

$\begin{array}{lll}-7.546753 & -2.433667 & 2.948157\end{array}$

$\begin{array}{lll}-5.671519 & -0.542963 & 2.737319\end{array}$

$\begin{array}{lll}-4.719554 & -0.016541 & 2.643456\end{array}$

$\begin{array}{lll}-5.452365 & -1.598926 & 2.901174\end{array}$

$\begin{array}{lll}-6.191849 & -0.161170 & 3.621750\end{array}$

$\begin{array}{lll}-2.222953 & -0.904289 & 0.400346\end{array}$

$\begin{array}{lll}-1.434843 & -0.160239 & 0.386198\end{array}$

$\begin{array}{llll}-2.898443 & -0.805030 & 1.242927\end{array}$

$\begin{array}{llll}-1.958296 & -2.246951 & -0.013004\end{array}$

$\begin{array}{lll}-2.622058 & -2.996578 & 0.392595\end{array}$

$\begin{array}{lll}-0.788198 & -2.732895 & -0.653256\end{array}$

$\begin{array}{llll}-0.098749 & -2.020233 & -1.084721\end{array}$

$\begin{array}{lll}-3.911223 & -0.854928 & -3.644817\end{array}$

$\begin{array}{llll}-4.972275 & -1.094438 & -3.621786\end{array}$

$\begin{array}{llll}-3.017200 & -1.956939 & -3.581981\end{array}$

$\begin{array}{lll}-3.459524 & -2.951572 & -3.528976\end{array}$

$-1.601616-1.944554-4.133149$

$\begin{array}{llll}-1.622914 & -2.069758 & -5.227663\end{array}$

$\begin{array}{llll}-1.092066 & -2.825303 & -3.735543\end{array}$

$\begin{array}{lll}-0.787282 & -0.686028 & -3.767638\end{array}$

$\begin{array}{llll}-0.872037 & 0.068164 & -4.555391\end{array}$

$\begin{array}{llll}0.273679 & -0.948710 & -3.724803\end{array}$

$\begin{array}{llll}-1.176719 & -0.045628 & -2.437719\end{array}$

$\begin{array}{llll}-0.435660 & -0.109487 & -1.645221\end{array}$

$\begin{array}{llll}-2.119245 & 0.968247 & -2.313668\end{array}$

$\begin{array}{llll}-2.031959 & 1.614320 & -1.446709\end{array}$

$\begin{array}{llll}-2.951413 & 1.530984 & -3.448844\end{array}$

$\begin{array}{llll}-3.746450 & 2.135195 & -3.007859\end{array}$

$\begin{array}{llll}-2.326240 & 2.214654 & -4.039644\end{array}$

$\begin{array}{llll}-3.581731 & 0.457250 & -4.361693\end{array}$

$\begin{array}{llll}-2.921721 & 0.245724 & -5.210095\end{array}$

$\begin{array}{llll}-4.504177 & 0.861610 & -4.790441\end{array}$

$\begin{array}{lll}-0.718245 & -4.097353 & -1.192507\end{array}$

$\begin{array}{llll}-1.582633 & -5.130295 & -0.773160\end{array}$

$\begin{array}{llll}0.244140 & -4.393627 & -2.175449\end{array}$

$\begin{array}{llll}-1.491241 & -6.400299 & -1.339550\end{array}$

$\begin{array}{lll}-2.305023 & -4.954953 & 0.014734\end{array}$

$\begin{array}{llll}0.334930 & -5.664902 & -2.739257\end{array}$

$\begin{array}{llll}0.919566 & -3.611451 & -2.499128\end{array}$

$\begin{array}{llll}-0.536080 & -6.675815 & -2.324812\end{array}$

$\begin{array}{llll}-2.164159 & -7.183461 & -1.001637\end{array}$

$\begin{array}{lll}1.083845 & -5.865025 & -3.500645\end{array}$

$\begin{array}{lll}-0.469959 & -7.668869 & -2.760224\end{array}$

$\begin{array}{llll}2.239631 & -4.635378 & 0.278517\end{array}$

$\begin{array}{llll}3.223434 & -5.141568 & -0.667898\end{array}$

$\begin{array}{llll}4.124590 & -5.492489 & -0.147995\end{array}$

$\begin{array}{llll}2.795158 & -5.982071 & -1.224878\end{array}$

$\begin{array}{llll}3.502642 & -4.352353 & -1.360828\end{array}$

$\begin{array}{llll}1.560948 & -5.650614 & 1.080376\end{array}$

$\begin{array}{lll}0.641481 & -6.001076 & 0.600062\end{array}$

$\begin{array}{llll}2.237981 & -6.500691 & 1.198763\end{array}$

$\begin{array}{llll}1.322714 & -5.279091 & 2.075691\end{array}$

$\begin{array}{lll}-0.961395 & -0.102624 & 4.285249\end{array}$

$\begin{array}{lll}-0.578400 & -2.321548 & 3.236949\end{array}$

$\begin{array}{lll}-0.099403 & 2.711242 & 3.475145\end{array}$

$\begin{array}{lll}-3.014819 & -4.021964 & 2.876344\end{array}$

$\begin{array}{lll}-3.533053 & -4.641260 & 4.013174\end{array}$

$\begin{array}{lll}-3.241149 & -2.632442 & 2.931389\end{array}$

$\begin{array}{llll}-1.628753 & -4.273327 & 2.772477\end{array}$

$\begin{array}{lll}-3.656644 & -4.541286 & 1.716505\end{array}$

$[8-9]^{\ddagger}$ P1-C2 exo re-si $\left[\mathrm{BF}_{4}{ }^{-}\right]$conf2

Number of imaginary frequencies $=1$

The smallest frequencies are $=-69.81846 .97837 .7797 \mathrm{~cm}(-1)$ Electronic energy $=-7647.2255649$

Zero-point correction $=\quad 1.769579$

(Hartree/Particle)

Thermal correction to Energy $=\quad 1.894763$

Thermal correction to Enthalpy= $\quad 1.895708$

Thermal correction to Gibbs Free Energy $=\quad 1.596992$ 
Sum of electronic and zero-point Energies= Sum of electronic and thermal Energies= Sum of electronic and thermal Enthalpies= Sum of electronic and thermal Free Energies=

\section{Cartesian Coordinates}

\begin{tabular}{|c|c|c|c|}
\hline 77 & 4.083621 & -0.739790 & -1.5775 \\
\hline 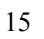 & 5.823060 & -0.164991 & 0250003 \\
\hline 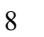 & 6.186270 & -0.945256 & 1.168125 \\
\hline & 7.281177 & -0.307216 & -1.051900 \\
\hline 7 & 5.608832 & 1.383067 & 0.349440 \\
\hline 6 & 6.933018 & -2.115040 & 1.197935 \\
\hline 6 & 6.252456 & -3.280829 & 1.613597 \\
\hline 1 & 5.205548 & -3.197911 & 1.886557 \\
\hline 6 & 6.923573 & -4.476811 & 1.664576 \\
\hline 1 & 6.413851 & -5.379266 & 1.989925 \\
\hline 6 & 8.284992 & -4.556499 & 1.265099 \\
\hline 6 & 8.968137 & -5.802716 & 1.243795 \\
\hline 1 & 8.431956 & -6.691822 & 1.565296 \\
\hline 6 & 10.272868 & -5.887522 & 0.813795 \\
\hline 1 & 10.784290 & -6.845458 & 0.797245 \\
\hline 6 & 10.944045 & -4.721672 & 0.374780 \\
\hline 1 & 11.964776 & -4.793898 & 0.010338 \\
\hline 6 & 10.315132 & -3.495617 & 0.397283 \\
\hline 1 & 10.844577 & -2.618506 & 0.045694 \\
\hline 6 & 8.975332 & -3.364210 & 0.859599 \\
\hline 6 & 8.280 & -2.0 & 0.896273 \\
\hline 6 & 8.977 & -0.8 & 0.574031 \\
\hline 6 & 10.167658 & 367 & 1.262097 \\
\hline 6 & 10.6 & -1. & 2.423136 \\
\hline 1 & 10.1 & -1. & 2.816275 \\
\hline 6 & 11.799356 & -0.6 & 3.061763 \\
\hline 1 & & & 3.952405 \\
\hline 6 & 12.510967 & 0.5 & 2.574757 \\
\hline 1 & 13.40 & 0.8 & 3.086753 \\
\hline 6 & 12.053342 & 919 & 1.463165 \\
\hline 1 & 12.579277 & 2.064731 & 1.087208 \\
\hline 6 & 10.875434 & 0.768490 & 0.788414 \\
\hline 6 & 10.3 & 1.48 & -0.330359 \\
\hline 1 & 10.925407 & 2.349413 & -0.694693 \\
\hline 6 & 9.183824 & 1.128806 & -0.911973 \\
\hline 1 & 8.7 & 1.6 & 1497 \\
\hline 6 & 8.48 & 0.00 & -0.428449 \\
\hline 6 & & & \\
\hline 1 & & & 0.245408 \\
\hline 6 & 5.022226 & 3.552865 & -0.777936 \\
\hline 6 & 4.089700 & 4.594334 & -0.777964 \\
\hline 1 & 3.069497 & 4.4 & -0.457032 \\
\hline 6 & 4.459945 & 5.879481 & -1.184124 \\
\hline 1 & 3.712644 & 6.665996 & -1.178216 \\
\hline 6 & 5.771829 & 6.134190 & -1.588277 \\
\hline 1 & 6.066504 & 7.135459 & -1.891179 \\
\hline 6 & 6.706391 & 5.09 & -1.600793 \\
\hline 1 & 7.731921 & 5.283079 & -1.906498 \\
\hline 6 & 6.329828 & 3.809014 & -1.209565 \\
\hline 1 & 7.060047 & 3.00 & -1.188952 \\
\hline 6 & 4.186847 & 1.404782 & -1.685657 \\
\hline 1 & 4.927983 & 1.652759 & -2.453941 \\
\hline 1 & 3.235465 & 1.805524 & -2.040487 \\
\hline 6 & 5.932421 & 1.759015 & 1.768843 \\
\hline 1 & 6.629571 & 0.99 & 2.110043 \\
\hline 6 & 6.675546 & 3.083804 & 1.878168 \\
\hline 6 & 8.072286 & 3.073862 & 1.783100 \\
\hline 1 & 8.587708 & 2.126926 & 1.662417 \\
\hline 6 & 8.804304 & 4.260847 & 1.829227 \\
\hline 1 & 9.887590 & 4.229625 & 1.747502 \\
\hline 6 & 8.142239 & 5.481307 & 1.982937 \\
\hline 1 & 8.705405 & 6.410137 & 2.011626 \\
\hline 6 & 6.750568 & 5.500129 & 2.109299 \\
\hline 1 & 6.226823 & 6.443880 & 2.232122 \\
\hline 6 & 6.024487 & 4.308948 & 2.067903 \\
\hline 1 & 4.943940 & 4.344937 & 2.151505 \\
\hline 6 & 4.692663 & 1.669375 & 2.668156 \\
\hline 1 & 4.287423 & 0.654273 & 2.644111 \\
\hline
\end{tabular}

$-7645.455986$ $-7645.330801$ $-7645.329857$ $-7645.628572$ $\begin{array}{lll}3.900924 & 2.363633 & 2.375023\end{array}$ $\begin{array}{lll}4.971409 & 1.903815 & 3.700107\end{array}$ $\begin{array}{llll}2.946390 & -0.977471 & 0.249431\end{array}$ $\begin{array}{lll}3.070044 & -1.971793 & 0.677221\end{array}$ $\begin{array}{lll}3.080637 & -0.185045 & 0.983665\end{array}$ $\begin{array}{lll}1.890147 & -0.793575 & -0.715814\end{array}$ $\begin{array}{llll}1.417638 & -1.663301 & -1.152016\end{array}$ $\begin{array}{llll}1.283802 & 0.452921 & -0.926434\end{array}$ $\begin{array}{llll}1.709917 & 1.299601 & -0.401163\end{array}$ $\begin{array}{lll}5.274959 & -0.818315 & -3.477298\end{array}$ $\begin{array}{llll}5.883942 & 0.081856 & -3.507790\end{array}$ $\begin{array}{llll}3.908110 & -0.656075 & -3.802539\end{array}$ $3.584144 \quad 0.346446-4.081052$ $2.974739-1.752012-4.290789$ $\begin{array}{llll}3.094142 & -1.891198 & -5.376449\end{array}$ $\begin{array}{llll}1.950540 & -1.394577 & -4.138972\end{array}$ $\begin{array}{llll}3.146464 & -3.103775 & -3.565021\end{array}$ $\begin{array}{lll}3.898050 & -3.716310 & -4.071703\end{array}$ $\begin{array}{llll}2.206810 & -3.662083 & -3.631087\end{array}$ $\begin{array}{llll}3.522518 & -2.970844 & -2.091629\end{array}$ $\begin{array}{llll}2.743383 & -3.241717 & -1.383592\end{array}$ $\begin{array}{llll}4.821353 & -2.973459 & -1.600126\end{array}$ $\begin{array}{llll}4.951365 & -3.254695 & -0.559452\end{array}$ $\begin{array}{llll}6.085768 & -3.028678 & -2.431846\end{array}$ $\begin{array}{llll}6.915893 & -2.729430 & -1.788052\end{array}$ $\begin{array}{lll}6.276484 & -4.070777 & -2.727838\end{array}$ $\begin{array}{llll}6.061745 & -2.115158 & -3.675188\end{array}$ $\begin{array}{llll}5.654043 & -2.653435 & -4.536070\end{array}$ $\begin{array}{llll}7.092602 & -1.858685 & -3.937745\end{array}$ $\begin{array}{llll}0.366993 & 0.789413 & -1.994075\end{array}$ $\begin{array}{lll}-0.269065 & -0.183581 & -2.798324\end{array}$ $\begin{array}{llll}0.140560 & 2.154561 & -2.271341\end{array}$ $\begin{array}{llll}-1.089308 & 0.203167 & -3.852998\end{array}$ $\begin{array}{lll}-0.127539 & -1.238230 & -2.592458\end{array}$ $\begin{array}{llll}-0.663781 & 2.534478 & -3.341956\end{array}$ $\begin{array}{lll}0.592992 & 2.917102 & -1.646605\end{array}$ $\begin{array}{llll}-1.280181 & 1.562089 & -4.133342\end{array}$ $\begin{array}{llll}-1.589662 & -0.549571 & -4.455352\end{array}$ $\begin{array}{llll}-0.812782 & 3.589666 & -3.535399\end{array}$ $\begin{array}{llll}-1.919895 & 1.857245 & -4.958770\end{array}$ $\begin{array}{lll}-6.821179 & 3.666494 & 2.113766\end{array}$ $\begin{array}{llll}-4.834843 & 1.033138 & -1.947097\end{array}$ $\begin{array}{lll}-4.891703 & -0.654912 & 1.409581\end{array}$ $\begin{array}{lll}-6.288957 & 2.381061 & 0.549232\end{array}$ $\begin{array}{lll}-5.721870 & 1.941110 & 1.801901\end{array}$ $\begin{array}{lll}-4.878416 & 2.983318 & 2.289111\end{array}$ $\begin{array}{llll}-4.318098 & 2.962726 & 3.213314\end{array}$ $\begin{array}{lll}-4.940693 & 4.073746 & 1.373876\end{array}$ $\begin{array}{lll}-4.441418 & 5.026562 & 1.484878\end{array}$ $\begin{array}{lll}-5.811875 & 3.708425 & 0.308517\end{array}$ $\begin{array}{lll}-6.076488 & 4.326240 & -0.536641\end{array}$ $\begin{array}{llll}-8.565262 & 3.285985 & 3.178220\end{array}$ $\begin{array}{lll}-8.987320 & 2.303546 & 3.343463\end{array}$ $\begin{array}{lll}-7.607966 & 3.938968 & 4.013979\end{array}$ $\begin{array}{lll}-7.170883 & 3.535292 & 4.917362\end{array}$ $\begin{array}{lll}-7.294785 & 5.201379 & 3.424376\end{array}$ $\begin{array}{llll}-6.574255 & 5.916344 & 3.799257\end{array}$ $\begin{array}{lll}-8.058939 & 5.330507 & 2.224863\end{array}$ $\begin{array}{lll}-8.021317 & 6.161473 & 1.532802\end{array}$ $\begin{array}{llll}-8.844197 & 4.148971 & 2.072641\end{array}$ $\begin{array}{llll}-9.508884 & 3.934591 & 1.246803\end{array}$ $\begin{array}{llll}-7.142774 & 1.535651 & -0.335041\end{array}$ $\begin{array}{lll}-8.469695 & 1.275372 & 0.040142\end{array}$ $\begin{array}{lll}-8.840427 & 1.731158 & 0.949200\end{array}$ $\begin{array}{llll}-9.301014 & 0.443602 & -0.709100\end{array}$ $\begin{array}{llll}-10.313194 & 0.242020 & -0.373582\end{array}$ $\begin{array}{llll}-8.813693 & -0.136575 & -1.877464\end{array}$ $\begin{array}{llll}-9.444779 & -0.787586 & -2.474650\end{array}$ $\begin{array}{lll}-7.497722 & 0.106640 & -2.272362\end{array}$ $\begin{array}{llll}-7.133819 & -0.360320 & -3.180322\end{array}$ $\begin{array}{llll}-6.638705 & 0.917157 & -1.512480\end{array}$ $\begin{array}{llll}-5.947168 & 0.579256 & 2.398289\end{array}$ $\begin{array}{lll}-6.974846 & 0.304081 & 2.159026\end{array}$ $\begin{array}{lll}-5.787986 & 0.515449 & 3.920059\end{array}$ $\begin{array}{lll}-5.972506 & -0.495854 & 4.293500\end{array}$ 
$\begin{array}{llll}-4.790901 & 0.818651 & 4.247751\end{array}$

$\begin{array}{llll}-6.510596 & 1.193977 & 4.382869\end{array}$

$\begin{array}{lll}-4.691932 & 0.090070 & -3.524158\end{array}$

$\begin{array}{llll}-4.650491 & -1.312068 & -3.429894\end{array}$

$\begin{array}{llll}-4.768861 & -1.787721 & -2.461516\end{array}$

$\begin{array}{llll}-4.471673 & -2.100278 & -4.565809\end{array}$

$\begin{array}{llll}-4.455505 & -3.181174 & -4.470853\end{array}$

$\begin{array}{llll}-4.310287 & -1.494074 & -5.815925\end{array}$

$\begin{array}{llll}-4.156107 & -2.103681 & -6.701751\end{array}$

$\begin{array}{lll}-4.350417 & -0.102275 & -5.920471\end{array}$

$\begin{array}{llll}-4.227527 & 0.375508 & -6.888483\end{array}$

$\begin{array}{llll}-4.548229 & 0.687013 & -4.783693\end{array}$

$\begin{array}{llll}-4.572034 & 1.766237 & -4.883499\end{array}$

$\begin{array}{llll}-4.593954 & 2.791967 & -2.401377\end{array}$

$\begin{array}{llll}-5.536902 & 3.504680 & -3.160616\end{array}$

$\begin{array}{llll}-6.426459 & 3.004920 & -3.534240\end{array}$

$\begin{array}{llll}-5.343071 & 4.862418 & -3.416718\end{array}$

$\begin{array}{llll}-6.074441 & 5.411588 & -4.003482\end{array}$

$\begin{array}{llll}-4.213347 & 5.516448 & -2.909813\end{array}$

$\begin{array}{llll}-4.070457 & 6.576337 & -3.102647\end{array}$

$\begin{array}{llll}-3.273453 & 4.813523 & -2.152679\end{array}$

$\begin{array}{llll}-2.403593 & 5.317769 & -1.744440\end{array}$

$\begin{array}{llll}-3.464190 & 3.452454 & -1.899457\end{array}$

$\begin{array}{llll}-2.758387 & 2.909909 & -1.279868\end{array}$

$\begin{array}{lll}-6.063697 & -1.969663 & 0.855504\end{array}$

$\begin{array}{llll}-5.556405 & -2.930158 & -0.031584\end{array}$

$\begin{array}{llll}-4.495331 & -2.959655 & -0.243833\end{array}$

$\begin{array}{llll}-6.404237 & -3.834160 & -0.671608\end{array}$

$\begin{array}{llll}-7.778083 & -3.803877 & -0.445449\end{array}$

$\begin{array}{llll}-8.440329 & -4.486065 & -0.966531\end{array}$

$\begin{array}{lll}-8.284283 & -2.861964 & 0.449757\end{array}$

$\begin{array}{llll}-7.443572 & -1.961580 & 1.102245\end{array}$

$\begin{array}{lll}-7.889097 & -1.245323 & 1.778313\end{array}$

$\begin{array}{llll}-3.767216 & -1.470233 & 2.612824\end{array}$

$\begin{array}{lll}-2.791137 & -0.642398 & 3.195873\end{array}$

$\begin{array}{lll}-2.790590 & 0.420137 & 2.984871\end{array}$

$\begin{array}{lll}-1.797311 & -1.185623 & 4.001224\end{array}$

$\begin{array}{lll}-1.723433 & -2.564858 & 4.212461\end{array}$

$\begin{array}{lll}-0.928512 & -2.990294 & 4.814574\end{array}$

$\begin{array}{lll}-2.678174 & -3.384797 & 3.618904\end{array}$

$\begin{array}{lll}-3.704034 & -2.848118 & 2.833824\end{array}$

$\begin{array}{llll}-4.433655 & -3.516175 & 2.390185\end{array}$

$\begin{array}{lll}-3.519767 & 0.188829 & -0.311025\end{array}$

$\begin{array}{lll}-2.548059 & -4.878401 & 3.718586\end{array}$

$\begin{array}{lll}-0.718658 & -0.314389 & 4.580950\end{array}$

$\begin{array}{lll}-5.812822 & -4.841156 & -1.618911\end{array}$

$\begin{array}{lll}-9.773360 & -2.808611 & 0.659414\end{array}$

$\begin{array}{llll}-10.427607 & -2.585433 & -0.507366\end{array}$

$\begin{array}{lll}-10.143538 & -1.831443 & 1.515796\end{array}$

$\begin{array}{lll}-10.252281 & -3.975703 & 1.149410\end{array}$

$\begin{array}{llll}-4.793186 & -4.303517 & -2.337804\end{array}$

$\begin{array}{llll}-5.302903 & -5.909974 & -0.965291\end{array}$

$\begin{array}{llll}-6.720107 & -5.309336 & -2.500989\end{array}$

$\begin{array}{lll}-3.751374 & -5.492998 & 3.765070\end{array}$

$\begin{array}{llll}-1.897138 & -5.383849 & 2.638471\end{array}$

$\begin{array}{lll}-1.851056 & -5.262991 & 4.809924\end{array}$

$\begin{array}{lll}-0.602397 & -0.479552 & 5.918228\end{array}$

$\begin{array}{lll}-0.930425 & 0.999720 & 4.351825\end{array}$

$\begin{array}{lll}0.501168 & -0.616900 & 4.056378\end{array}$

$\begin{array}{llll}-2.090011 & -2.405742 & -0.790908\end{array}$

$\begin{array}{lll}-0.870439 & -0.999718 & 0.636079\end{array}$

$\begin{array}{llll}-1.366388 & -3.518465 & -0.400883\end{array}$

$\begin{array}{llll}-2.854568 & -2.478277 & -1.555337\end{array}$

$\begin{array}{llll}-0.155755 & -2.144760 & 1.110412\end{array}$

$\begin{array}{llll}-0.398590 & -3.402147 & 0.604987\end{array}$

$\begin{array}{lll}0.581338 & -2.006446 & 1.892347\end{array}$

$\begin{array}{llll}-0.414877 & 0.276728 & 1.061724\end{array}$

$\begin{array}{lll}-1.133333 & 1.519535 & 1.00512\end{array}$

$\begin{array}{lll}-2.317495 & 1.624809 & 0.557265\end{array}$

$\begin{array}{llll}-1.890319 & -1.180536 & -0.266018\end{array}$

$\begin{array}{lll}0.425072 & 0.232961 & 1.738013\end{array}$

$\begin{array}{llll}-0.527143 & 2.653696 & 1.480464\end{array}$

$\begin{array}{lll}-1.326836 & 3.854216 & 1.697106\end{array}$

$\begin{array}{lll}-1.584465 & 3.958383 & 2.762077\end{array}$

$\begin{array}{lll}-0.770026 & 4.731152 & 1.370597\end{array}$

$\begin{array}{llll}-2.243661 & 3.783855 & 1.118801\end{array}$

$\begin{array}{llll}0.802413 & 2.672295 & 2.074243\end{array}$

$\begin{array}{lll}1.485487 & 2.017945 & 1.532780\end{array}$

$\begin{array}{lll}1.195090 & 3.687317 & 2.001259\end{array}$

$\begin{array}{lll}0.786404 & 2.365666 & 3.128398\end{array}$

$\begin{array}{llll}0.139485 & -4.267955 & 0.974354\end{array}$

$\begin{array}{llll}-1.696787 & -5.256988 & -1.238116\end{array}$

$\begin{array}{llll}0.448269 & 5.783154 & -1.024556\end{array}$

$\begin{array}{llll}-0.752750 & 6.247650 & -0.452656\end{array}$

$\begin{array}{llll}0.205207 & 5.329489 & -2.330672\end{array}$

$\begin{array}{llll}1.396815 & 6.818200 & -1.039645\end{array}$

$0.942500 \quad 4.704663 \quad-0.240401$

8 P1-C1 endo re-re

Number of imaginary frequencies $=0$

The smallest frequencies are $=0.2961 \quad 8.2522 \quad 13.4052 \mathrm{~cm}(-1)$ Electronic energy $=-7222.6420682$

Zero-point correction=

1.756572

(Hartree/Particle)

Thermal correction to Energy= $\quad 1.876002$

Thermal correction to Enthalpy= $\quad 1.876946$

Thermal correction to Gibbs Free Energy $=\quad 1.588710$

Sum of electronic and zero-point Energies $=\quad-7220.885497$

Sum of electronic and thermal Energies $=\quad-7220.766066$

Sum of electronic and thermal Enthalpies $=\quad-7220.765122$

Sum of electronic and thermal Free Energies $=\quad-7221.053358$

Cartesian Coordinates

\begin{tabular}{|c|c|c|c|}
\hline 26 & 4.429627 & 4.784835 & 1.752745 \\
\hline & 2.590342 & 1.576280 & -1.898661 \\
\hline & 4.602001 & 0.105116 & 0.881224 \\
\hline & 4.058127 & 3.409803 & 0.222031 \\
\hline & 4.282766 & 2.740668 & 1.481915 \\
\hline & 3.286950 & 3.193210 & 2.400907 \\
\hline & 3.195513 & 2.889401 & 3.434645 \\
\hline & 2.468162 & 4.154404 & 1.736278 \\
\hline & 1.653248 & 4.708212 & 2.181614 \\
\hline & 2.941715 & 4.290370 & 0.398397 \\
\hline & 2.547383 & 4.959991 & -0.350934 \\
\hline & 6.387461 & 5.334302 & 2.177258 \\
\hline & 7.247219 & 4.688763 & 2.055202 \\
\hline & 5.576842 & 5.438264 & 3.349077 \\
\hline & 5.712598 & 4.883587 & 4.267963 \\
\hline & 4.529659 & 6.371776 & 3.080763 \\
\hline & 3.729220 & 6.640227 & 3.757511 \\
\hline & 4.692548 & 6.845414 & 1.743174 \\
\hline & 4.039921 & 7.538988 & 1.229463 \\
\hline & 5.840014 & 6.206554 & 1.185665 \\
\hline & 6.207374 & 6.338448 & 0.177204 \\
\hline & 4.823263 & 3.114347 & -1.023670 \\
\hline & 6.156047 & 3.537682 & -1.133844 \\
\hline & 6.575015 & 4.124271 & -0.325947 \\
\hline & 6.945607 & 3.203946 & -2.234373 \\
\hline & 7.982186 & 3.523598 & -2.275117 \\
\hline & 6.403011 & 2.436025 & -3.262102 \\
\hline & 7.009707 & 2.152374 & -4.115973 \\
\hline & 5.079231 & 2.003982 & -3.176312 \\
\hline & 4.676325 & 1.390599 & -3.974602 \\
\hline b & 4.277522 & 2.321180 & -2.068713 \\
\hline & 5.311762 & 1.665006 & 1.702135 \\
\hline & 6.195031 & 1.932920 & 1.119886 \\
\hline & 5.741425 & 1.497135 & 3.162162 \\
\hline & 6.513279 & 0.728719 & 3.258736 \\
\hline & 4.907801 & 1.219161 & 3.812326 \\
\hline & 6.147100 & 2.445601 & 3.527622 \\
\hline & 2.340015 & 0.630535 & -3.464856 \\
\hline & 2.436833 & -0.769312 & -3.408219 \\
\hline & 2.660529 & -1.257820 & -2.466904 \\
\hline & 2.194144 & -1.540609 & -4.548341 \\
\hline & 2.267172 & -2.621722 & -4.489606 \\
\hline & 1.847709 & -0.925228 & -5.752259 \\
\hline & 1.646810 & -1.526148 & -6.634663 \\
\hline & 1.755070 & 0.468482 & -5.817815 \\
\hline
\end{tabular}


$\begin{array}{llll}1.486710 & 0.954185 & -6.751767\end{array}$

$2.001364 \quad 1.242389 \quad-4.683525$

$\begin{array}{llll}1.915005 & 2.321455 & -4.749913\end{array}$

$\begin{array}{llll}1.451740 & 3.007862 & -2.119362\end{array}$

$\begin{array}{llll}1.794065 & 4.143464 & -2.871530\end{array}$

$\begin{array}{llll}2.781371 & 4.224107 & -3.316477\end{array}$

$\begin{array}{llll}0.873703 & 5.179698 & -3.041511\end{array}$

$\begin{array}{llll}1.149789 & 6.054488 & -3.623480\end{array}$

$\begin{array}{llll}-0.399517 & 5.090832 & -2.469208\end{array}$

$\begin{array}{llll}-1.118340 & 5.893632 & -2.606695\end{array}$

$\begin{array}{llll}-0.744949 & 3.966230 & -1.716842\end{array}$

$\begin{array}{llll}-1.730603 & 3.890378 & -1.270114\end{array}$

$\begin{array}{llll}0.180796 & 2.936787 & -1.534190\end{array}$

$\begin{array}{llll}-0.083454 & 2.075123 & -0.933647\end{array}$

$\begin{array}{llll}5.826783 & -0.422556 & -0.391120\end{array}$

$\begin{array}{llll}5.403211 & -1.453128 & -1.243773\end{array}$

$\begin{array}{llll}4.416789 & -1.883115 & -1.100669\end{array}$

$\begin{array}{llll}6.239508 & -1.916899 & -2.259664\end{array}$

$\begin{array}{llll}7.483766 & -1.329653 & -2.481802\end{array}$

$\begin{array}{llll}8.120430 & -1.671085 & -3.290261\end{array}$

$\begin{array}{llll}7.888441 & -0.284003 & -1.652132\end{array}$

$\begin{array}{llll}7.085546 & 0.151819 & -0.598308\end{array}$

$\begin{array}{llll}7.449592 & 0.951695 & 0.031707\end{array}$

$\begin{array}{llll}4.697055 & -1.254314 & 2.120914\end{array}$

$3.773803 \quad-1.217656 \quad 3.178397$

$\begin{array}{lll}3.095685 & -0.378490 & 3.280709\end{array}$

$\begin{array}{llll}3.683608 & -2.281769 & 4.070779\end{array}$

$\begin{array}{lll}4.468017 & -3.424380 & 3.904225\end{array}$

$\begin{array}{llll}4.357853 & -4.272329 & 4.570855\end{array}$

$\begin{array}{lll}5.360287 & -3.473325 & 2.836858\end{array}$

$\begin{array}{lll}5.496579 & -2.391145 & 1.964523\end{array}$

$\begin{array}{llll}6.200587 & -2.465906 & 1.144869\end{array}$

$\begin{array}{llll}2.452209 & 0.246879 & -0.065844\end{array}$

$\begin{array}{lll}6.172487 & -4.716672 & 2.602352\end{array}$

$\begin{array}{llll}2.630989 & -2.289917 & 5.142475\end{array}$

$\begin{array}{lll}5.805147 & -3.101894 & -3.076560\end{array}$

$\begin{array}{llll}9.201214 & 0.389314 & -1.943189\end{array}$

$9.207959 \quad 0.942043 \quad-3.182368$

$\begin{array}{llll}9.482251 & 1.378983 & -1.067327\end{array}$

$10.233937-0.484998-1.910256$

$\begin{array}{llll}6.012574 & -4.265374 & -2.409796\end{array}$

$\begin{array}{llll}6.467402 & -3.193534 & -4.248534\end{array}$

$\begin{array}{lll}4.480576 & -3.062842 & -3.365653\end{array}$

$\begin{array}{llll}7.344363 & -4.706807 & 3.278699\end{array}$

$\begin{array}{llll}6.479691 & -4.874928 & 1.292021\end{array}$

$\begin{array}{lll}5.504624 & -5.829089 & 2.991730\end{array}$

$\begin{array}{lll}3.119784 & -2.734223 & 6.323004\end{array}$

$\begin{array}{lll}2.095721 & -1.069833 & 5.357335\end{array}$

$\begin{array}{llll}1.603704 & -3.122126 & 4.819280\end{array}$

$\begin{array}{lll}1.993048 & -2.687579 & 0.573036\end{array}$

$\begin{array}{lll}1.100542 & -2.340917 & 1.624053\end{array}$

$\begin{array}{lll}0.616464 & -1.068276 & 1.986428\end{array}$

$\begin{array}{lll}0.768402 & -3.133790 & 2.277425\end{array}$

$\begin{array}{llll}-3.333619 & -1.316452 & -1.274286\end{array}$

$\begin{array}{llll}-5.227325 & -0.514560 & -0.312508\end{array}$

$\begin{array}{lll}-5.225833 & 0.663190 & 0.834052\end{array}$

$\begin{array}{llll}-6.232070 & 0.160544 & -1.444266\end{array}$

$\begin{array}{lll}-5.957234 & -1.738883 & 0.553017\end{array}$

$\begin{array}{lll}-5.021092 & 2.000576 & 0.512166\end{array}$

$\begin{array}{llll}-3.825929 & 2.579375 & 0.989476\end{array}$

$\begin{array}{lll}-3.155789 & 1.972373 & 1.587789\end{array}$

$\begin{array}{llll}-3.545741 & 3.888170 & 0.685641\end{array}$

$\begin{array}{llll}-2.637660 & 4.353008 & 1.056388\end{array}$

$\begin{array}{llll}-4.417352 & 4.634606 & -0.153373\end{array}$

$\begin{array}{llll}-4.077449 & 5.949465 & -0.572369\end{array}$

$\begin{array}{llll}-3.148258 & 6.383476 & -0.212453\end{array}$

$\begin{array}{llll}-4.897367 & 6.652682 & -1.425963\end{array}$

$\begin{array}{llll}-4.625047 & 7.653999 & -1.746232\end{array}$

$\begin{array}{llll}-6.095551 & 6.063601 & -1.895934\end{array}$

$\begin{array}{llll}-6.730569 & 6.613885 & -2.584150\end{array}$

$\begin{array}{llll}-6.463354 & 4.797517 & -1.493698\end{array}$

$\begin{array}{llll}-7.381136 & 4.359264 & -1.868142\end{array}$

$\begin{array}{llll}-5.646451 & 4.044810 & -0.605180\end{array}$

$\begin{array}{llll}-5.986740 & 2.709129 & -0.185267\end{array}$

$\begin{array}{llll}-7.310654 & 2.085286 & -0.471625\end{array}$ $\begin{array}{llll}-8.544852 & 2.700529 & -0.050916\end{array}$

$\begin{array}{lll}-8.585219 & 3.889425 & 0.730889\end{array}$

$\begin{array}{lll}-7.657998 & 4.358730 & 1.036114\end{array}$

$\begin{array}{lll}-9.784554 & 4.444979 & 1.120836\end{array}$

$\begin{array}{lll}-9.785773 & 5.349470 & 1.722300\end{array}$

$\begin{array}{lll}-11.013129 & 3.846660 & 0.753520\end{array}$

$\begin{array}{lll}-11.950118 & 4.300168 & 1.062949\end{array}$

$\begin{array}{lll}-11.012575 & 2.681687 & 0.020836\end{array}$

$\begin{array}{llll}-11.947028 & 2.198010 & -0.251081\end{array}$

$\begin{array}{llll}-9.793382 & 2.073935 & -0.385032\end{array}$

$\begin{array}{llll}-9.795059 & 0.842834 & -1.094512\end{array}$

$\begin{array}{lll}-10.744811 & 0.382049 & -1.350654\end{array}$

$\begin{array}{llll}-8.613674 & 0.226434 & -1.423243\end{array}$

$\begin{array}{llll}-8.587494 & -0.729370 & -1.935361\end{array}$

$\begin{array}{llll}-7.391499 & 0.845964 & -1.087397\end{array}$

$\begin{array}{lll}-5.409692 & -3.082683 & 0.231014\end{array}$

$\begin{array}{lll}-4.744694 & -3.376236 & 1.050279\end{array}$

$\begin{array}{llll}-6.512360 & -4.120836 & 0.118370\end{array}$

$\begin{array}{lll}-6.324561 & -5.399726 & 0.655035\end{array}$

$\begin{array}{lll}-5.405171 & -5.625922 & 1.190646\end{array}$

$\begin{array}{llll}-7.307189 & -6.382701 & 0.508651\end{array}$

$\begin{array}{llll}-7.150252 & -7.370918 & 0.932009\end{array}$

$\begin{array}{lll}-8.491919 & -6.091455 & -0.170841\end{array}$

$\begin{array}{llll}-9.259851 & -6.852095 & -0.279506\end{array}$

$\begin{array}{llll}-8.688494 & -4.813377 & -0.704564\end{array}$

$\begin{array}{llll}-9.610697 & -4.579184 & -1.229502\end{array}$

$\begin{array}{llll}-7.704651 & -3.835034 & -0.562151\end{array}$

$\begin{array}{llll}-7.860006 & -2.837758 & -0.964412\end{array}$

$\begin{array}{llll}-4.594450 & -3.034818 & -1.066989\end{array}$

$\begin{array}{llll}-5.281691 & -3.057237 & -1.920097\end{array}$

$\begin{array}{llll}-3.974083 & -3.931492 & -1.133713\end{array}$

$\begin{array}{lll}-6.646965 & -1.651896 & 1.890013\end{array}$

$\begin{array}{lll}-7.135499 & -2.626578 & 1.974217\end{array}$

$\begin{array}{lll}-5.635029 & -1.570035 & 3.032359\end{array}$

$\begin{array}{lll}-5.095710 & -2.759874 & 3.547485\end{array}$

$\begin{array}{lll}-5.457505 & -3.714693 & 3.174684\end{array}$

$\begin{array}{lll}-4.116641 & -2.737775 & 4.544420\end{array}$

$\begin{array}{lll}-3.711930 & -3.671741 & 4.924497\end{array}$

$\begin{array}{llll}-3.676121 & -1.518468 & 5.062438\end{array}$

$\begin{array}{llll}-2.918603 & -1.495802 & 5.840981\end{array}$

$\begin{array}{lll}-4.234238 & -0.328191 & 4.586935\end{array}$

$\begin{array}{llll}-3.916845 & 0.625232 & 5.000405\end{array}$

$\begin{array}{lll}-5.202801 & -0.353575 & 3.582148\end{array}$

$\begin{array}{lll}-5.619193 & 0.580518 & 3.227047\end{array}$

$\begin{array}{lll}-7.766453 & -0.614834 & 1.942828\end{array}$

$\begin{array}{lll}-8.487968 & -0.802393 & 1.144930\end{array}$

$\begin{array}{lll}-7.411106 & 0.410208 & 1.847424\end{array}$

$\begin{array}{lll}-8.283323 & -0.707916 & 2.902708\end{array}$

$\begin{array}{llll}-2.493795 & -1.105737 & 0.742453\end{array}$

$\begin{array}{llll}-1.777093 & -0.290168 & 0.719088\end{array}$

$\begin{array}{lll}-3.181962 & -1.054177 & 1.580741\end{array}$

$\begin{array}{lll}-2.084231 & -2.411513 & 0.327130\end{array}$

$\begin{array}{lll}-2.619365 & -3.264868 & 0.731016\end{array}$

$\begin{array}{llll}-1.105427 & -2.626561 & -0.641960\end{array}$

$\begin{array}{lll}-0.415460 & -1.824249 & -0.866106\end{array}$

$\begin{array}{llll}-4.192531 & -1.063301 & -3.348139\end{array}$

$\begin{array}{llll}-5.190917 & -1.488166 & -3.299794\end{array}$

$\begin{array}{llll}-3.125366 & -1.977854 & -3.431889\end{array}$

$\begin{array}{lll}-3.374308 & -3.038106 & -3.452819\end{array}$

$\begin{array}{lll}-1.750226 & -1.649389 & -3.978054\end{array}$

$\begin{array}{llll}-1.784206 & -1.675919 & -5.077318\end{array}$

$\begin{array}{lll}-1.071437 & -2.449458 & -3.674826\end{array}$

$\begin{array}{lll}-1.178861 & -0.296954 & -3.506350\end{array}$

$\begin{array}{llll}-1.458063 & 0.501613 & -4.199005\end{array}$

$\begin{array}{lll}-0.091927 & -0.353955 & -3.532561\end{array}$

$\begin{array}{llll}-1.597745 & 0.109086 & -2.101846\end{array}$

$\begin{array}{llll}-0.824440 & 0.057900 & -1.341784\end{array}$

$\begin{array}{llll}-2.694215 & 0.905384 & -1.830151\end{array}$

$\begin{array}{llll}-2.698577 & 1.439127 & -0.885792\end{array}$

$\begin{array}{llll}-3.656336 & 1.427722 & -2.875417\end{array}$

$\begin{array}{llll}-4.520518 & 1.847428 & -2.360596\end{array}$

$\begin{array}{llll}-3.172060 & 2.261230 & -3.403926\end{array}$

$\begin{array}{lll}-4.140074 & 0.365819 & -3.889722\end{array}$

$\begin{array}{llll}-3.508166 & 0.376883 & -4.782925\end{array}$

$\begin{array}{llll}-5.145129 & 0.639903 & -4.221343\end{array}$ 


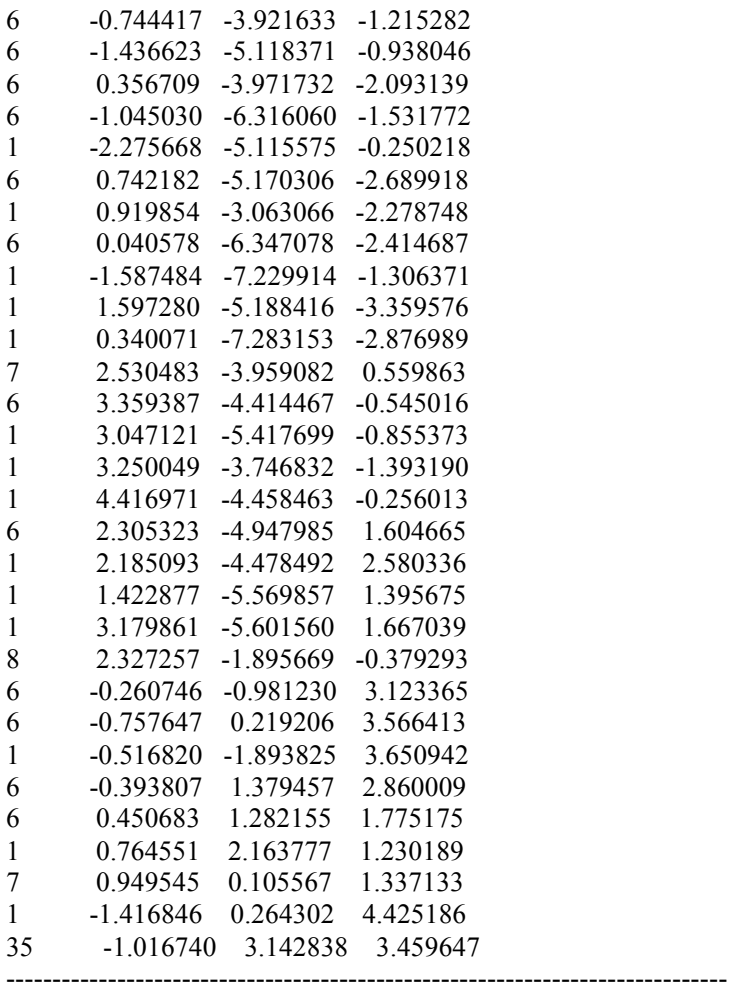

$[8-9]^{\ddagger}$ P1-C1 endo re-re

Number of imaginary frequencies $=1$

The smallest frequencies are $=-247.70245 .095710 .4338 \mathrm{~cm}(-1)$ Electronic energy $=-7222.6348462$

Zero-point correction=

(Hartree/Particle)

Thermal correction to Energy=

Thermal correction to Enthalpy=

Thermal correction to Gibbs Free Energy=
Sum of electronic and zero-point Energies=

Sum of electronic and thermal Energies=

Sum of electronic and thermal Enthalpies=

1.874115

1.875059

1.593083

$-7220.879146$

$-7220.760731$

Sum of electronic and thermal Free Energies=

$-7220.759787$

$-7221.041763$

Cartesian Coordinates

$\begin{array}{lllc}\ldots 6 & 4.295437 & 4.787355 & 1.873202 \\ 15 & 2.562805 & 1.624327 & -1.886450 \\ 15 & 4.540775 & 0.128166 & 0.933392 \\ 6 & 3.967400 & 3.424797 & 0.318842 \\ 6 & 4.154449 & 2.746058 & 1.580220 \\ 6 & 3.132327 & 3.194430 & 2.473067 \\ 1 & 3.009849 & 2.884580 & 3.501734 \\ 6 & 2.334999 & 4.162811 & 1.791665 \\ 1 & 1.508954 & 4.716069 & 2.216440 \\ 6 & 2.848848 & 4.307323 & 0.469963 \\ 1 & 2.478862 & 4.983288 & -0.285896 \\ 6 & 6.237760 & 5.325940 & 2.375800 \\ 1 & 7.098839 & 4.677139 & 2.283312 \\ 6 & 5.382516 & 5.426388 & 3.515834 \\ 1 & 5.479243 & 4.865002 & 4.435574 \\ 6 & 4.350403 & 6.365820 & 3.212671 \\ 1 & 3.525224 & 6.633413 & 3.859406 \\ 6 & 4.567478 & 6.847204 & 1.885567 \\ 1 & 3.938634 & 7.547022 & 1.351141 \\ 6 & 5.733355 & 6.207169 & 1.369596 \\ 1 & 6.141457 & 6.344865 & 0.377657 \\ 6 & 4.764269 & 3.153492 & -0.912977 \\ 6 & 6.097323 & 3.584280 & -0.982639 \\ 1 & 6.500778 & 4.139337 & -0.145298 \\ 6 & 6.906087 & 3.300278 & -2.082949 \\ 1 & 7.941613 & 3.625013 & -2.092786 \\ 6 & 6.383592 & 2.576754 & -3.152403\end{array}$

$\begin{array}{llll}7.004122 & 2.336950 & -4.009854\end{array}$

$\begin{array}{llll}5.061542 & 2.133443 & -3.105789\end{array}$

$\begin{array}{lll}4.672316 & 1.556624 & -3.937792\end{array}$

$\begin{array}{llll}4.241815 & 2.397477 & -1.997422\end{array}$

$\begin{array}{lll}5.179769 & 1.672470 & 1.830733\end{array}$

$\begin{array}{lll}6.093160 & 1.961786 & 1.308082\end{array}$

$\begin{array}{llll}5.519495 & 1.464681 & 3.309230\end{array}$

$\begin{array}{lll}6.298243 & 0.706930 & 3.429948\end{array}$

$\begin{array}{lll}4.650642 & 1.149189 & 3.893039\end{array}$

$\begin{array}{llll}5.881822 & 2.407557 & 3.730235\end{array}$

$\begin{array}{lll}2.354461 & 0.721721 & -3.482111\end{array}$

$\begin{array}{llll}2.616243 & -0.658015 & -3.490617\end{array}$

$2.930053-1.152734 \quad-2.579016$

$2.435227 \quad-1.405154 \quad-4.656733$

$\begin{array}{llll}2.642167 & -2.470234 & -4.648829\end{array}$

$\begin{array}{llll}1.979184 & -0.786494 & -5.822554\end{array}$

$\begin{array}{llll}1.824055 & -1.370575 & -6.725138\end{array}$

$\begin{array}{lll}1.719168 & 0.586530 & -5.822771\end{array}$

$\begin{array}{llll}1.363613 & 1.074301 & -6.725932\end{array}$

$\begin{array}{llll}1.908661 & 1.338316 & -4.662386\end{array}$

$\begin{array}{lll}1.692248 & 2.400819 & -4.679778\end{array}$

$\begin{array}{llll}1.403676 & 3.038178 & -2.070656\end{array}$

$\begin{array}{llll}1.740556 & 4.197606 & -2.788378\end{array}$

$\begin{array}{llll}2.733256 & 4.302541 & -3.215998\end{array}$

$\begin{array}{llll}0.806428 & 5.222696 & -2.947956\end{array}$

$\begin{array}{lll}1.077442 & 6.116537 & -3.502721\end{array}$

$\begin{array}{llll}-0.475220 & 5.095145 & -2.402608\end{array}$

$\begin{array}{llll}-1.208071 & 5.886350 & -2.533051\end{array}$

$\begin{array}{llll}-0.814284 & 3.945084 & -1.687034\end{array}$

$\begin{array}{llll}-1.807464 & 3.839152 & -1.267659\end{array}$

$\begin{array}{llll}0.124988 & 2.927386 & -1.509523\end{array}$

$\begin{array}{llll}-0.137097 & 2.044720 & -0.936918\end{array}$

$\begin{array}{llll}5.833954 & -0.351351 & -0.288109\end{array}$

$\begin{array}{llll}5.486642 & -1.402292 & -1.149405\end{array}$

$\begin{array}{llll}4.518843 & -1.879066 & -1.036746\end{array}$

$\begin{array}{llll}6.371623 & -1.829989 & -2.138243\end{array}$

$\begin{array}{llll}7.598250 & -1.194274 & -2.319206\end{array}$

$\begin{array}{llll}8.275893 & -1.511055 & -3.103890\end{array}$

$\begin{array}{llll}7.930661 & -0.131925 & -1.478082\end{array}$

$\begin{array}{llll}7.072228 & 0.277409 & -0.457198\end{array}$

$\begin{array}{lll}7.378658 & 1.096619 & 0.178492\end{array}$

$\begin{array}{llll}4.568586 & -1.271452 & 2.131450\end{array}$

$\begin{array}{llll}3.559513 & -1.295134 & 3.107628\end{array}$

$\begin{array}{lll}2.867673 & -0.465451 & 3.195798\end{array}$

$3.410474 \quad-2.401824 \quad 3.940594$

$\begin{array}{llll}4.233691 & -3.519354 & 3.798021\end{array}$

$\begin{array}{llll}4.087756 & -4.396385 & 4.418651\end{array}$

$\begin{array}{llll}5.223171 & -3.501141 & 2.818428\end{array}$

$\begin{array}{llll}5.409585 & -2.382662 & 2.005045\end{array}$

$\begin{array}{lll}6.188526 & -2.406511 & 1.253458\end{array}$

$\begin{array}{llll}2.440281 & 0.234724 & -0.102190\end{array}$

$\begin{array}{llll}6.091969 & -4.714890 & 2.637310\end{array}$

$\begin{array}{llll}2.273122 & -2.466927 & 4.920975\end{array}$

$\begin{array}{llll}5.997085 & -3.025715 & -2.969085\end{array}$

$\begin{array}{llll}9.225234 & 0.592960 & -1.725516\end{array}$

$\begin{array}{llll}9.228151 & 1.197511 & -2.940401\end{array}$

$\begin{array}{llll}9.467207 & 1.552928 & -0.806092\end{array}$

$10.282676-0.251335-1.717126$

$\begin{array}{llll}6.178690 & -4.181980 & -2.282707\end{array}$

$\begin{array}{llll}6.720677 & -3.117967 & -4.102424\end{array}$

$\begin{array}{llll}4.686754 & -3.002237 & -3.326825\end{array}$

$\begin{array}{llll}7.068699 & -4.789599 & 3.569641\end{array}$

$\begin{array}{llll}6.695617 & -4.731925 & 1.427184\end{array}$

$\begin{array}{lll}5.369560 & -5.858128 & 2.739829\end{array}$

$\begin{array}{llll}2.644887 & -3.031518 & 6.090498\end{array}$

$\begin{array}{llll}1.757515 & -1.251404 & 5.200191\end{array}$

$\begin{array}{llll}1.250767 & -3.226987 & 4.437728\end{array}$

$\begin{array}{lll}1.975894 & -2.751384 & 0.404035\end{array}$

$\begin{array}{lll}0.812390 & -2.465302 & 1.213731\end{array}$

$\begin{array}{llll}0.453886 & -1.190357 & 1.765515\end{array}$

$\begin{array}{lll}0.409642 & -3.292585 & 1.779814\end{array}$

$\begin{array}{llll}-3.400058 & -1.296214 & -1.447055\end{array}$

$\begin{array}{lll}-5.267032 & -0.538264 & -0.377953\end{array}$

$\begin{array}{lll}-5.260515 & 0.604632 & 0.820413\end{array}$

$\begin{array}{lll}-6.330094 & 0.174129 & -1.442135\end{array}$ 


\begin{tabular}{|c|c|c|c|c|c|c|}
\hline-5.966540 & -1.799001 & 0.479356 & 6 & -3.126479 & -2.048729 & -3.521760 \\
\hline-4.982080 & 1.930143 & 0.525274 & 1 & -3.365222 & -3.111893 & -3.550807 \\
\hline-3.757894 & 2.439098 & 1.010716 & 6 & -1.761944 & -1.710569 & -4.097577 \\
\hline-3.119051 & 1.781975 & 1.589817 & 1 & -1.805699 & -1.728966 & -5.197714 \\
\hline-3.415964 & 3.740371 & 0.740351 & 1 & -1.073388 & -2.507899 & -3.807469 \\
\hline-2.486805 & 4.152909 & 1.120380 & 6 & -1.196652 & -0.358774 & -3.615835 \\
\hline-4.248649 & 4.546652 & -0.083622 & 1 & -1.429525 & 0.434488 & -4.332820 \\
\hline-3.843320 & 5.851210 & -0.476787 & 1 & -0.107346 & -0.422356 & -3.582652 \\
\hline-2.895142 & 6.232401 & -0.106594 & 6 & -1.691489 & 0.068035 & -2.237650 \\
\hline-4.622758 & 6.608483 & -1.322358 & 1 & -0.934668 & 0.084646 & -1.459797 \\
\hline-4.300125 & 7.600830 & -1.623550 & 6 & -2.806514 & 0.873821 & -2.022098 \\
\hline-5.844831 & 6.086323 & -1.809761 & 1 & -2.816425 & 1.461214 & -1.109695 \\
\hline-6.447826 & 6.678270 & -2.492188 & 6 & -3.753039 & 1.359069 & -3.101273 \\
\hline-6.275796 & 4.832860 & -1.431574 & 1 & -4.645283 & 1.750086 & -2.607389 \\
\hline-7.211195 & 4.445828 & -1.818952 & 1 & -3.289387 & 2.204787 & -3.630586 \\
\hline-5.501727 & 4.025512 & -0.552583 & 6 & -4.170800 & 0.260249 & -4.102568 \\
\hline-5.909897 & 2.701986 & -0.155887 & 1 & -3.492722 & 0.252237 & -4.962362 \\
\hline-7.269139 & 2.156938 & -0.436261 & 1 & -5.160724 & 0.506430 & -4.498882 \\
\hline-8.460106 & 2.845577 & -0.001972 & 6 & -0.336645 & -3.784997 & -1.225085 \\
\hline-8.420903 & 4.023158 & 0.796991 & 6 & -0.871124 & -5.040118 & -0.873180 \\
\hline-7.464244 & 4.422286 & 1.110883 & 6 & 0.626971 & -3.732315 & -2.250610 \\
\hline-9.579451 & 4.655925 & 1.192965 & 6 & -0.457207 & -6.196872 & -1.530475 \\
\hline-9.518930 & 5.549286 & 1.807975 & 1 & -1.601547 & -5.116434 & -0.074047 \\
\hline-10.845783 & 4.150826 & 0.814544 & 6 & 1.036553 & -4.889497 & -2.909893 \\
\hline-11.749891 & 4.664936 & 1.127243 & 1 & 1.058532 & -2.774681 & -2.515986 \\
\hline-10.923652 & 2.997935 & 0.066874 & 6 & 0.497635 & -6.127752 & -2.551846 \\
\hline-11.888643 & 2.583726 & -0.213333 & 1 & -0.877255 & -7.156874 & -1.244075 \\
\hline-9.748662 & 2.311610 & -0.344190 & 1 & 1.782672 & -4.824824 & -3.697000 \\
\hline-9.832602 & 1.090689 & -1.066300 & 1 & 0.817501 & -7.032100 & -3.061287 \\
\hline-10.811106 & 0.699068 & -1.329435 & 7 & 2.527335 & -3.998332 & 0.475409 \\
\hline-8.695431 & 0.398867 & -1.398607 & 6 & 3.530308 & -4.417710 & -0.497322 \\
\hline-8.734292 & -0.551473 & -1.920471 & 1 & 3.343462 & -5.458859 & -0.776613 \\
\hline-7.430840 & 0.928255 & -1.060056 & 1 & 3.460363 & -3.806065 & -1.391307 \\
\hline-5.328337 & -3.110858 & 0.180972 & 1 & 4.542655 & -4.343739 & -0.084232 \\
\hline-4.591423 & -3.316290 & 0.967031 & 6 & 2.172731 & -5.004927 & 1.472704 \\
\hline-6.342526 & -4.240820 & 0.175098 & 1 & 1.857177 & -4.544831 & 2.407745 \\
\hline-6.026818 & -5.466230 & 0.773385 & 1 & 1.379541 & -5.669547 & 1.108073 \\
\hline-5.072363 & -5.581304 & 1.282332 & 1 & 3.059622 & -5.605100 & 1.688340 \\
\hline-6.925397 & -6.535560 & 0.723005 & 8 & 2.451419 & -1.919421 & -0.431553 \\
\hline-6.667750 & -7.480499 & 1.193528 & 6 & -0.453527 & -1.173928 & 2.861533 \\
\hline-8.155374 & -6.385415 & 0.079134 & 6 & -0.885357 & 0.012338 & 3.418690 \\
\hline-8.858562 & -7.212779 & 0.045011 & 1 & -0.794742 & -2.114293 & 3.277071 \\
\hline-8.480070 & -5.162129 & -0.517078 & 6 & -0.417740 & 1.201507 & 2.853854 \\
\hline-9.437494 & -5.037574 & -1.015792 & 6 & 0.455954 & 1.160391 & 1.781903 \\
\hline-7.578937 & -4.098569 & -0.471435 & 1 & 0.835530 & 2.067905 & 1.329475 \\
\hline-7.831501 & -3.144311 & -0.925590 & 7 & 0.890375 & 0.000915 & 1.248314 \\
\hline-4.594830 & -3.051752 & -1.163554 & 1 & -1.574634 & 0.013224 & 4.253959 \\
\hline-5.333876 & -3.113068 & -1.971371 & 35 & -0.962326 & 2.928041 & 3.596268 \\
\hline
\end{tabular}

$333876-3.113068-1.971$

$\begin{array}{llll}-3.953525 & -3.933830 & -1.249482\end{array}$

$\begin{array}{lll}-6.667729 & -1.732629 & 1.807972\end{array}$

$\begin{array}{lll}-7.141378 & -2.714903 & 1.886702\end{array}$

$\begin{array}{llll}-5.678308 & -1.640575 & 2.970885\end{array}$

$\begin{array}{lll}-5.126431 & -2.826175 & 3.482675\end{array}$

$\begin{array}{lll}-5.452995 & -3.781295 & 3.079979\end{array}$

$\begin{array}{lll}-4.182184 & -2.800242 & 4.512444\end{array}$

$-3.769148 \quad-3.731660 \quad 4.890196$

$\begin{array}{llll}-3.790282 & -1.580866 & 5.068470\end{array}$

$\begin{array}{lll}-3.064029 & -1.554831 & 5.876307\end{array}$

$\begin{array}{lll}-4.357260 & -0.394938 & 4.591550\end{array}$

$\begin{array}{llll}-4.076297 & 0.558110 & 5.032312\end{array}$

$\begin{array}{lll}-5.290662 & -0.424603 & 3.553663\end{array}$

$\begin{array}{lll}-5.716327 & 0.505727 & 3.199489\end{array}$

$\begin{array}{lll}-7.806830 & -0.715833 & 1.852804\end{array}$

$\begin{array}{lll}-8.514428 & -0.911722 & 1.044022\end{array}$

$\begin{array}{lll}-7.468556 & 0.315852 & 1.765797\end{array}$

$\begin{array}{lll}-8.334977 & -0.822796 & 2.805277\end{array}$

$\begin{array}{llll}-2.525378 & -1.128243 & 0.544821\end{array}$

$\begin{array}{lll}-1.882608 & -0.253504 & 0.590611\end{array}$

$\begin{array}{lll}-3.221230 & -1.183407 & 1.377318\end{array}$

$\begin{array}{llll}-1.992560 & -2.367340 & 0.062609\end{array}$

$\begin{array}{lll}-2.496886 & -3.272067 & 0.385508\end{array}$

$\begin{array}{lll}-0.734650 & -2.534722 & -0.568683\end{array}$

$-0.209022-1.652832-0.907242$

$\begin{array}{lll}-4.223052 & -1.143972 & -3.493042\end{array}$

$\begin{array}{llll}-5.215202 & -1.589672 & -3.481890\end{array}$

\section{P1-C1 endo re-re}

Number of imaginary frequencies $=0$

The smallest frequencies are $=11.093911 .900415 .4503 \mathrm{~cm}(-1)$ Electronic energy $=-7222.6615389$

Zero-point correction=

(Hartree/Particle)

Thermal correction to Energy= $\quad 1.877378$

Thermal correction to Enthalpy= $\quad 1.878322$

Thermal correction to Gibbs Free Energy= 1.600701

Sum of electronic and zero-point Energies $=\quad-7220.901568$

Sum of electronic and thermal Energies $=\quad-7220.784161$

Sum of electronic and thermal Enthalpies $=\quad-7220.783217$

Sum of electronic and thermal Free Energies $=\quad-7221.060837$

Cartesian Coordinates

$\begin{array}{llll}26 & 3.898852 & 4.895652 & 1.574662 \\ 15 & 2.505678 & 1.493604 & -2.125655 \\ 15 & 4.288697 & 0.175760 & 0.959066 \\ 6 & 3.705985 & 3.444438 & 0.076183 \\ 6 & 3.795150 & 2.839976 & 1.385025 \\ 6 & 2.704916 & 3.329922 & 2.168481 \\ 1 & 2.504626 & 3.081424 & 3.200907 \\ 6 & 1.956141 & 4.247582 & 1.370366 \\ 1 & 1.097177 & 4.818407 & 1.696106\end{array}$


$\begin{array}{llll}2.575495 & 4.324161 & 0.088722\end{array}$

$\begin{array}{llll}2.264191 & 4.953707 & -0.730918\end{array}$

$\begin{array}{llll}5.792080 & 5.494294 & 2.188071\end{array}$

$\begin{array}{llll}6.669873 & 4.861403 & 2.187112\end{array}$

$\begin{array}{lll}4.856658 & 5.629564 & 3.259116\end{array}$

$\begin{array}{lll}4.895020 & 5.110323 & 4.207423\end{array}$

$\begin{array}{llll}3.833127 & 6.532902 & 2.840018\end{array}$

$\begin{array}{lll}2.959487 & 6.812483 & 3.413998\end{array}$

$\begin{array}{llll}4.136058 & 6.958018 & 1.510580\end{array}$

$\begin{array}{llll}3.534624 & 7.619056 & 0.900597\end{array}$

$\begin{array}{lll}5.346139 & 6.318416 & 1.108535\end{array}$

$\begin{array}{llll}5.822201 & 6.417649 & 0.142497\end{array}$

$\begin{array}{llll}4.601876 & 3.115393 & -1.070793\end{array}$

$\begin{array}{llll}5.931854 & 3.561327 & -1.059641\end{array}$

$\begin{array}{llll}6.261421 & 4.171234 & -0.227766\end{array}$

$\begin{array}{llll}6.829249 & 3.225715 & -2.073325\end{array}$

$\begin{array}{llll}7.859437 & 3.562799 & -2.019111\end{array}$

$6.400546 \quad 2.437933-3.139203$

$\begin{array}{llll}7.088314 & 2.163539 & -3.932731\end{array}$

$\begin{array}{llll}5.082946 & 1.980105 & -3.172868\end{array}$

$\begin{array}{llll}4.764083 & 1.355996 & -4.000352\end{array}$

$\begin{array}{llll}4.176842 & 2.291200 & -2.147363\end{array}$

$\begin{array}{lll}4.811713 & 1.805365 & 1.784469\end{array}$

$\begin{array}{llll}5.752783 & 2.076861 & 1.304325\end{array}$

$\begin{array}{lll}5.057391 & 1.721512 & 3.293739\end{array}$

$\begin{array}{lll}5.830874 & 0.984693 & 3.525912\end{array}$

$\begin{array}{llll}4.156839 & 1.451760 & 3.851920\end{array}$

$\begin{array}{lll}5.390093 & 2.700158 & 3.652133\end{array}$

$\begin{array}{llll}2.428632 & 0.521864 & -3.688723\end{array}$

$\begin{array}{llll}2.852147 & -0.816242 & -3.642761\end{array}$

$3.198788-1.241976-2.708631$

$\begin{array}{llll}2.802474 & -1.614770 & -4.786679\end{array}$

$\begin{array}{llll}3.139747 & -2.644643 & -4.734638\end{array}$

$\begin{array}{llll}2.307189 & -1.092385 & -5.983579\end{array}$

$2.251864-1.718018-6.869740$

$\begin{array}{llll}1.879338 & 0.236563 & -6.036056\end{array}$

$\begin{array}{llll}1.490742 & 0.648092 & -6.963134\end{array}$

$\begin{array}{llll}1.945102 & 1.043281 & -4.898745\end{array}$

$\begin{array}{llll}1.603394 & 2.070385 & -4.957956\end{array}$

$\begin{array}{llll}1.336508 & 2.875912 & -2.409276\end{array}$

$\begin{array}{llll}1.672124 & 3.982612 & -3.206747\end{array}$

$\begin{array}{llll}2.662318 & 4.055669 & -3.646738\end{array}$

$\begin{array}{llll}0.740997 & 4.999371 & -3.423022\end{array}$

$\begin{array}{llll}1.009247 & 5.854494 & -4.036807\end{array}$

$\begin{array}{llll}-0.534241 & 4.914402 & -2.854057\end{array}$

$\begin{array}{lll}-1.263253 & 5.701658 & -3.022807\end{array}$

$\begin{array}{llll}-0.871397 & 3.815359 & -2.061827\end{array}$

$\begin{array}{llll}-1.858525 & 3.744447 & -1.623095\end{array}$

$\begin{array}{llll}0.064321 & 2.806106 & -1.828621\end{array}$

$\begin{array}{llll}-0.194066 & 1.965707 & -1.193434\end{array}$

$\begin{array}{llll}5.697310 & -0.323147 & -0.121857\end{array}$

$\begin{array}{llll}5.459598 & -1.417378 & -0.964535\end{array}$

$4.499379-1.918269-0.922542$

$\begin{array}{llll}6.443022 & -1.861064 & -1.847170\end{array}$

$\begin{array}{llll}7.664916 & -1.198737 & -1.942312\end{array}$

$\begin{array}{llll}8.420315 & -1.526745 & -2.647662\end{array}$

$\begin{array}{llll}7.891725 & -0.096161 & -1.117536\end{array}$

$6.932187 \quad 0.330273-0.199030$

$\begin{array}{llll}7.159065 & 1.180042 & 0.430233\end{array}$

$\begin{array}{llll}4.284222 & -1.120836 & 2.267199\end{array}$

$3.261048-1.083798 \quad 3.229720$

$\begin{array}{lll}2.543213 & -0.270423 & 3.240426\end{array}$

$3.156952 \quad-2.100436 \quad 4.179169$

$\begin{array}{llll}4.022675 & -3.194066 & 4.157214\end{array}$

$\begin{array}{llll}3.913604 & -3.997554 & 4.877271\end{array}$

$\begin{array}{llll}5.017017 & -3.242328 & 3.183799\end{array}$

$\begin{array}{llll}5.166913 & -2.207576 & 2.261673\end{array}$

$\begin{array}{lll}5.958559 & -2.272909 & 1.526397\end{array}$

$\begin{array}{llll}2.304474 & 0.180445 & -0.293679\end{array}$

$\begin{array}{llll}5.923673 & -4.441784 & 3.133663\end{array}$

$2.045863 \quad-2.101369 \quad 5.191645$

$\begin{array}{llll}6.179031 & -3.107291 & -2.645813\end{array}$

$9.187503 \quad 0.652943-1.270096$

$\begin{array}{llll}9.300671 & 1.193357 & -2.508789\end{array}$

$\begin{array}{llll}9.309511 & 1.665366 & -0.383888\end{array}$ $\begin{array}{llll}10.257425 & -0.158557 & -1.106329\end{array}$

$\begin{array}{llll}6.381976 & -4.222665 & -1.900409\end{array}$

$\begin{array}{llll}6.969383 & -3.204166 & -3.732114\end{array}$

$\begin{array}{llll}4.890161 & -3.170284 & -3.072659\end{array}$

$\begin{array}{lll}6.812859 & -4.453036 & 4.150498\end{array}$

$\begin{array}{llll}6.628989 & -4.501331 & 1.981996\end{array}$

$\begin{array}{llll}5.211436 & -5.593426 & 3.225971\end{array}$

$\begin{array}{llll}2.466653 & -2.530160 & 6.399824\end{array}$

$\begin{array}{lll}1.495784 & -0.879552 & 5.361999\end{array}$

$\begin{array}{llll}1.042401 & -2.941324 & 4.815327\end{array}$

$\begin{array}{llll}1.904720 & -2.855651 & 0.275697\end{array}$

$\begin{array}{llll}0.471302 & -2.584441 & 0.720069\end{array}$

$\begin{array}{llll}0.293470 & -1.321795 & 1.528937\end{array}$

$\begin{array}{llll}0.094693 & -3.410216 & 1.320553\end{array}$

$\begin{array}{lll}-3.398990 & -1.374753 & -1.577203\end{array}$

$\begin{array}{lll}-5.202687 & -0.547572 & -0.412545\end{array}$

$\begin{array}{llll}-5.095100 & 0.556870 & 0.829907\end{array}$

$\begin{array}{lll}-6.311413 & 0.250549 & -1.379204\end{array}$

$\begin{array}{lll}-5.911439 & -1.805403 & 0.452277\end{array}$

$\begin{array}{lll}-4.739953 & 1.865575 & 0.562120\end{array}$

$\begin{array}{lll}-3.463728 & 2.280947 & 1.005437\end{array}$

$\begin{array}{lll}-2.848336 & 1.564393 & 1.537783\end{array}$

$\begin{array}{llll}-3.048163 & 3.565601 & 0.759888\end{array}$

$\begin{array}{lll}-2.076417 & 3.905325 & 1.105124\end{array}$

$\begin{array}{llll}-3.865358 & 4.454340 & 0.007362\end{array}$

$\begin{array}{llll}-3.398937 & 5.747892 & -0.354242\end{array}$

$\begin{array}{llll}-2.414650 & 6.057170 & -0.012221\end{array}$

$\begin{array}{llll}-4.163181 & 6.581445 & -1.139814\end{array}$

$\begin{array}{llll}-3.793667 & 7.564225 & -1.417728\end{array}$

$\begin{array}{llll}-5.430961 & 6.149392 & -1.598070\end{array}$

$\begin{array}{llll}-6.022370 & 6.800009 & -2.235776\end{array}$

$\begin{array}{llll}-5.920968 & 4.910030 & -1.247307\end{array}$

$\begin{array}{llll}-6.891611 & 4.593281 & -1.610658\end{array}$

$\begin{array}{llll}-5.165169 & 4.026385 & -0.427398\end{array}$

$\begin{array}{llll}-5.641663 & 2.718697 & -0.054722\end{array}$

$\begin{array}{lll}-7.046725 & 2.275014 & -0.283614\end{array}$

$\begin{array}{lll}-8.163596 & 3.037382 & 0.221348\end{array}$

$\begin{array}{lll}-8.003136 & 4.187366 & 1.045704\end{array}$

$\begin{array}{llll}-7.007585 & 4.504579 & 1.330384\end{array}$

$\begin{array}{lll}-9.092460 & 4.897434 & 1.502718\end{array}$

$\begin{array}{lll}-8.937739 & 5.767693 & 2.134236\end{array}$

$\begin{array}{lll}-10.408124 & 4.501622 & 1.164997\end{array}$

$\begin{array}{lll}-11.256769 & 5.076019 & 1.524781\end{array}$

$\begin{array}{llll}-10.603783 & 3.376563 & 0.396724\end{array}$

$\begin{array}{lll}-11.608453 & 3.044916 & 0.147213\end{array}$

$\begin{array}{llll}-9.502507 & 2.612313 & -0.076179\end{array}$

$\begin{array}{llll}-9.709209 & 1.420851 & -0.821847\end{array}$

$\begin{array}{llll}-10.724875 & 1.111694 & -1.052178\end{array}$

$\begin{array}{llll}-8.642771 & 0.655417 & -1.219175\end{array}$

$\begin{array}{lll}-8.776182 & -0.275106 & -1.760695\end{array}$

$\begin{array}{llll}-7.325580 & 1.075824 & -0.926222\end{array}$

$\begin{array}{llll}-5.318610 & -3.134465 & 0.126799\end{array}$

$\begin{array}{lll}-4.566352 & -3.366158 & 0.891324\end{array}$

$\begin{array}{lll}-6.371224 & -4.229289 & 0.147882\end{array}$

$\begin{array}{lll}-6.094524 & -5.461548 & 0.751570\end{array}$

$\begin{array}{llll}-5.137585 & -5.608883 & 1.247618\end{array}$

$\begin{array}{llll}-7.032498 & -6.497591 & 0.720948\end{array}$

$\begin{array}{lll}-6.803539 & -7.448006 & 1.195536\end{array}$

$\begin{array}{lll}-8.264214 & -6.307308 & 0.091318\end{array}$

$\begin{array}{lll}-8.997881 & -7.108355 & 0.071659\end{array}$

$\begin{array}{lll}-8.550879 & -5.076750 & -0.509846\end{array}$

$\begin{array}{llll}-9.508885 & -4.920830 & -0.998773\end{array}$

$\begin{array}{llll}-7.610995 & -4.046813 & -0.482562\end{array}$

$\begin{array}{lll}-7.833035 & -3.087152 & -0.941240\end{array}$

$\begin{array}{llll}-4.613402 & -3.106013 & -1.235594\end{array}$

$\begin{array}{llll}-5.374847 & -3.161841 & -2.023754\end{array}$

$\begin{array}{llll}-4.007562 & -4.013187 & -1.318606\end{array}$

$\begin{array}{lll}-6.566210 & -1.734907 & 1.802624\end{array}$

$\begin{array}{lll}-7.062251 & -2.705112 & 1.892971\end{array}$

$\begin{array}{lll}-5.542587 & -1.674544 & 2.940006\end{array}$

$\begin{array}{lll}-4.995473 & -2.874662 & 3.422018\end{array}$

$\begin{array}{llll}-5.344589 & -3.819636 & 3.014814\end{array}$

$\begin{array}{llll}-4.027176 & -2.876948 & 4.430088\end{array}$

$\begin{array}{llll}-3.620992 & -3.819991 & 4.786061\end{array}$

$\begin{array}{lll}-3.603492 & -1.671633 & 4.993211\end{array}$ 


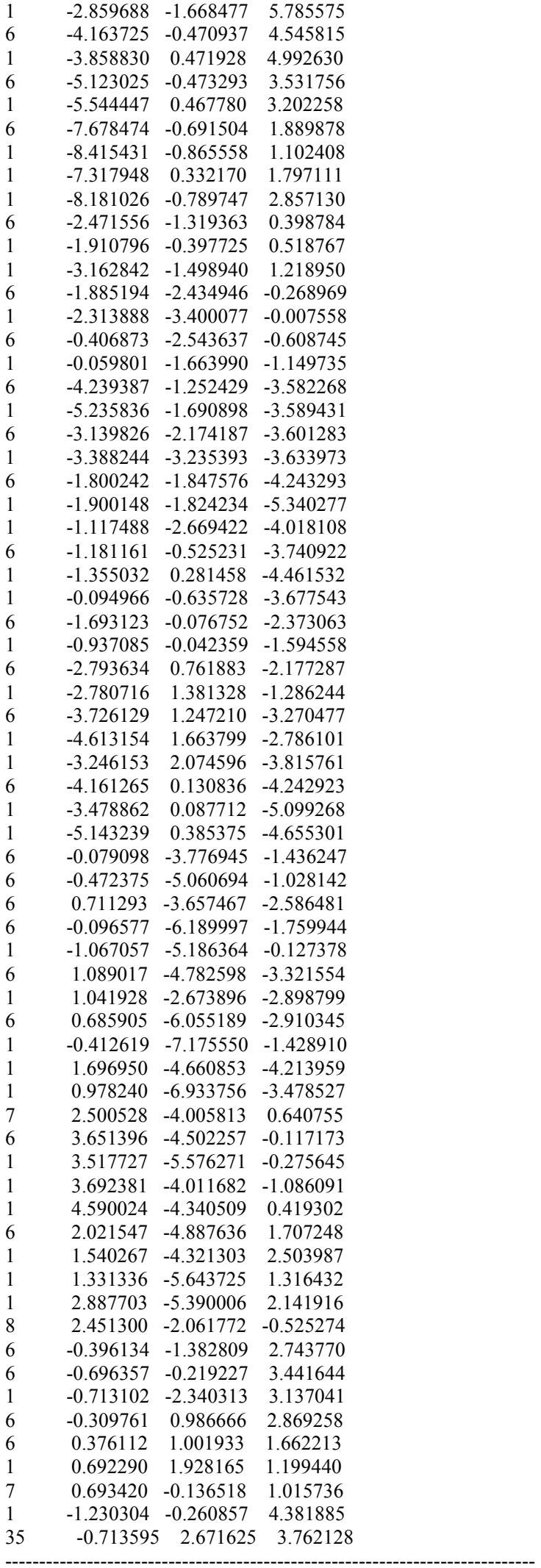

8 P1-C1 endo si-re

Number of imaginary frequencies $=0$

The smallest frequencies are $=6.7103 \quad 10.7191 \quad 12.5515 \mathrm{~cm}(-1)$ Electronic energy $=-7222.6313862$

Zero-point correction=

1.754774

(Hartree/Particle)

Thermal correction to Energy=
Thermal correction to Enthalpy=

1.875699

Thermal correction to Gibbs Free Energy $=\quad 1.587896$

Sum of electronic and zero-point Energies $=\quad-7220.876612$

Sum of electronic and thermal Energies $=\quad-7220.756632$

Sum of electronic and thermal Enthalpies $=\quad-7220.755688$

Sum of electronic and thermal Free Energies $=\quad-7221.043491$

Cartesian Coordinates

\begin{tabular}{|c|c|c|c|}
\hline 26 & 3.664540 & 4.915752 & 2.324858 \\
\hline 15 & 2.301603 & 1.884705 & -1.677665 \\
\hline 15 & 4.847618 & 0.531832 & 0.895544 \\
\hline 6 & 3.428097 & 3.790679 & 0.568043 \\
\hline 6 & 4.081870 & 3.022424 & 1.595888 \\
\hline ( & 3.195018 & 2.946413 & 2.715012 \\
\hline 1 & 3.399770 & 2.437762 & 3.647361 \\
\hline 6 & 2.013720 & 3.680525 & 2.398279 \\
\hline 1 & 1.173539 & 3.848301 & 3.058804 \\
\hline 6 & 2.159878 & 4.208570 & 1.081812 \\
\hline 1 & 1.445466 & 4.826642 & 0.557252 \\
\hline 6 & 5.390318 & 6.052408 & 2.566718 \\
\hline 1 & 6.349728 & 5.812212 & 2.128320 \\
\hline 6 & 4.910786 & 5.599213 & 3.833425 \\
\hline 1 & 5.439383 & 4.948964 & 4.517517 \\
\hline 6 & 3.589786 & 6.111536 & 4.013532 \\
\hline 1 & 2.942158 & 5.914584 & 4.857785 \\
\hline 6 & 3.252460 & 6.880402 & 2.858136 \\
\hline 1 & 2.306952 & 7.374220 & 2.675869 \\
\hline 6 & 4.364599 & 6.845272 & 1.964886 \\
\hline 1 & 4.408193 & 7.311676 & 0.990014 \\
\hline 6 & 3.985413 & 4.006117 & -0.795795 \\
\hline 6 & 5.023659 & 4.930415 & -0.982547 \\
\hline 1 & 5.371874 & 5.487664 & -0.122557 \\
\hline 6 & 5.613217 & 5.131353 & -2.230776 \\
\hline 1 & 6.422968 & 5.847235 & -2.338262 \\
\hline 6 & 5.154874 & 4.409390 & -3.329920 \\
\hline 1 & 5.598649 & 4.552289 & -4.310460 \\
\hline 6 & 4.127302 & 3.479696 & -3.166203 \\
\hline 1 & 3.795783 & 2.917532 & -4.030651 \\
\hline 6 & 3.544962 & 3.245332 & -1.910031 \\
\hline 6 & 5.375220 & 2.283247 & 1.401850 \\
\hline 1 & 5.864259 & 2.688986 & 0.511549 \\
\hline 6 & 6.350335 & 2.371261 & 2.578213 \\
\hline 1 & 7.247545 & 1.772090 & 2.397046 \\
\hline 1 & 5.894725 & 2.029300 & 3.511184 \\
\hline 1 & 6.655361 & 3.412838 & 2.714922 \\
\hline 6 & 2.203467 & 1.130534 & -3.356081 \\
\hline 6 & 3.297277 & 0.348027 & -3.764475 \\
\hline 1 & 4.177224 & 0.288068 & -3.133206 \\
\hline 6 & 3.285071 & -0.319558 & -4.986880 \\
\hline 1 & 4.144817 & -0.913260 & -5.283302 \\
\hline 6 & 2.167608 & -0.223663 & -5.822599 \\
\hline 1 & 2.148892 & -0.750610 & -6.771988 \\
\hline 6 & 1.080730 & 0.561460 & -5.433932 \\
\hline 1 & 0.214780 & 0.651195 & -6.083695 \\
\hline 6 & 1.097587 & 1.238521 & -4.209835 \\
\hline 1 & 0.248125 & 1.849850 & -3.927337 \\
\hline 6 & 0.718655 & 2.797272 & -1.462523 \\
\hline 6 & 0.375262 & 3.893661 & -2.270458 \\
\hline 1 & 1.035060 & 4.202114 & -3.076509 \\
\hline 6 & -0.806247 & 4.598530 & -2.035503 \\
\hline 1 & -1.067925 & 5.441515 & -2.668708 \\
\hline 6 & -1.641022 & 4.230628 & -0.974747 \\
\hline 1 & -2.555562 & 4.784773 & -0.784909 \\
\hline 6 & -1.293223 & 3.152783 & -0.157946 \\
\hline 1 & -1.926990 & 2.883348 & 0.681504 \\
\hline 6 & -0.124231 & 2.426153 & -0.403892 \\
\hline 1 & 0.157092 & 1.595413 & 0.239850 \\
\hline 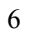 & 6.160757 & -0.043198 & -0.254063 \\
\hline 6 & 6.761141 & -1.308826 & -0.192164 \\
\hline 1 & 6.555445 & -1.982364 & 0.630726 \\
\hline 6 & 7.619142 & -1.731518 & -1.208797 \\
\hline 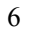 & 7.872389 & -0.928289 & -2.319476 \\
\hline 1 & 8.528815 & -1.268984 & -3.110545 \\
\hline & & & \\
\hline
\end{tabular}


$\begin{array}{llll}6.401401 & 0.758317 & -1.381675\end{array}$

$\begin{array}{llll}5.907470 & 1.719413 & -1.489860\end{array}$

$\begin{array}{lll}5.018653 & -0.432753 & 2.451119\end{array}$

$\begin{array}{llll}3.824046 & -0.650368 & 3.150695\end{array}$

$\begin{array}{lll}2.891447 & -0.290985 & 2.730415\end{array}$

$\begin{array}{llll}3.838115 & -1.311438 & 4.380124\end{array}$

$\begin{array}{llll}5.032820 & -1.778134 & 4.924911\end{array}$

$\begin{array}{lll}5.041372 & -2.300157 & 5.874271\end{array}$

$\begin{array}{llll}6.221846 & -1.555918 & 4.229222\end{array}$

$\begin{array}{lll}6.223839 & -0.880159 & 3.007840\end{array}$

$\begin{array}{lll}7.166122 & -0.708384 & 2.500789\end{array}$

$\begin{array}{llll}2.778057 & 0.308790 & -0.123307\end{array}$

$\begin{array}{lll}7.536708 & -1.984624 & 4.820174\end{array}$

$\begin{array}{llll}2.533140 & -1.566892 & 5.082463\end{array}$

$\begin{array}{llll}8.325242 & -3.052707 & -1.064398\end{array}$

$\begin{array}{llll}7.408498 & 1.181108 & -3.618254\end{array}$

$\begin{array}{llll}6.274653 & 1.192214 & -4.368515\end{array}$

$\begin{array}{llll}7.660244 & 2.469165 & -3.289297\end{array}$

$\begin{array}{llll}8.408725 & 0.764755 & -4.421629\end{array}$

$\begin{array}{llll}7.572548 & -3.959306 & -0.403415\end{array}$

$\begin{array}{llll}9.477231 & -2.918073 & -0.359941\end{array}$

$\begin{array}{llll}8.660198 & -3.587904 & -2.258501\end{array}$

$\begin{array}{lll}8.172205 & -0.949133 & 5.422037\end{array}$

$\begin{array}{llll}8.379675 & -2.455898 & 3.872558\end{array}$

$\begin{array}{lll}7.389723 & -2.951333 & 5.751251\end{array}$

$\begin{array}{llll}2.697905 & -1.788422 & 6.405051\end{array}$

$\begin{array}{lll}1.683220 & -0.520562 & 4.950946\end{array}$

$\begin{array}{llll}1.891764 & -2.652147 & 4.581568\end{array}$

$\begin{array}{llll}3.717082 & -2.199699 & -1.422304\end{array}$

$\begin{array}{llll}2.266743 & -2.689207 & 0.364976\end{array}$

$\begin{array}{llll}4.055059 & -3.523701 & -1.597412\end{array}$

$\begin{array}{llll}4.141850 & -1.433780 & -2.055417\end{array}$

$\begin{array}{llll}2.631776 & -4.070579 & 0.225988\end{array}$

$\begin{array}{llll}3.511252 & -4.494081 & -0.742528\end{array}$

$\begin{array}{llll}2.182039 & -4.796429 & 0.896772\end{array}$

$\begin{array}{llll}1.300919 & -2.349744 & 1.344383\end{array}$

$\begin{array}{lll}0.784759 & -1.072936 & 1.671260\end{array}$

$\begin{array}{lll}1.253062 & 0.044140 & 1.243250\end{array}$

$\begin{array}{llll}2.858204 & -1.774707 & -0.476334\end{array}$

$\begin{array}{lll}0.943827 & -3.192133 & 1.919768\end{array}$

$\begin{array}{lll}-0.293757 & -0.985039 & 2.534911\end{array}$

$\begin{array}{lll}-0.644058 & 0.283469 & 3.151514\end{array}$

$\begin{array}{llll}-0.622359 & 0.191916 & 4244120\end{array}$

$\begin{array}{lll}-1.654239 & 0.599007 & 2.860340\end{array}$

$\begin{array}{lll}0.068291 & 1.046418 & 2.843759\end{array}$

$\begin{array}{lll}-1.016273 & -2.135229 & 3.045918\end{array}$

$\begin{array}{lll}-0.590099 & -2.507096 & 3.987782\end{array}$

$\begin{array}{lll}-1.017986 & -2.950923 & 2.322186\end{array}$

$\begin{array}{lll}-2.053266 & -1.848256 & 3.231155\end{array}$

$\begin{array}{lll}-3.752460 & -0.928732 & -1.451154\end{array}$

$\begin{array}{llll}-5.568317 & -0.654832 & -0.122233\end{array}$

$\begin{array}{lll}-5.731716 & 0.592528 & 0.941835\end{array}$

$\begin{array}{llll}-6.926857 & -0.450872 & -1.046731\end{array}$

$\begin{array}{lll}-5.679788 & -1.946209 & 0.923955\end{array}$

$\begin{array}{lll}-6.079094 & 1.876305 & 0.526745\end{array}$

$\begin{array}{lll}-5.087889 & 2.867382 & 0.687864\end{array}$

$\begin{array}{lll}-4.140805 & 2.581623 & 1.132009\end{array}$

$\begin{array}{lll}-5.346841 & 4.153791 & 0.284855\end{array}$

$\begin{array}{llll}-4.601682 & 4.932741 & 0.417204\end{array}$

$\begin{array}{llll}-6.582679 & 4.476536 & -0.338784\end{array}$

$\begin{array}{llll}-6.826075 & 5.781049 & -0.847378\end{array}$

$\begin{array}{llll}-6.054912 & 6.537066 & -0.724863\end{array}$

$\begin{array}{llll}-8.002950 & 6.078022 & -1.49653\end{array}$

$\begin{array}{llll}-8.176155 & 7.076086 & -1.887882\end{array}$

$\begin{array}{llll}-8.984907 & 5.073404 & -1.668016\end{array}$

$\begin{array}{llll}-9.901727 & 5.304026 & -2.202776\end{array}$

$\begin{array}{llll}-8.788405 & 3.803777 & -1.169419\end{array}$

$\begin{array}{llll}-9.548683 & 3.046233 & -1.317589\end{array}$

$\begin{array}{llll}-7.593422 & 3.465421 & -0.475537\end{array}$

$\begin{array}{lll}-7.351341 & 2.144541 & 0.048377\end{array}$

$\begin{array}{lll}-8.406894 & 1.093197 & 0.060834\end{array}$

$\begin{array}{llll}-9.680334 & 1.300592 & 0.702366\end{array}$

$\begin{array}{lll}-9.970510 & 2.458885 & 1.476432\end{array}$

$\begin{array}{lll}-9.209141 & 3.219356 & 1.601462\end{array}$

$\begin{array}{lll}-11.197327 & 2.619835 & 2.083137\end{array}$ $\begin{array}{lll}-11.390402 & 3.509556 & 2.675509\end{array}$

$\begin{array}{lll}-12.205999 & 1.636350 & 1.948323\end{array}$

$\begin{array}{lll}-13.169816 & 1.780543 & 2.427542\end{array}$

$\begin{array}{lll}-11.953325 & 0.493192 & 1.224820\end{array}$

$\begin{array}{lll}-12.710481 & -0.280633 & 1.129219\end{array}$

$\begin{array}{lll}-10.693423 & 0.288080 & 0.599397\end{array}$

$\begin{array}{lll}-10.414014 & -0.916840 & -0.100733\end{array}$

$\begin{array}{lll}-11.192078 & -1.669867 & -0.186801\end{array}$

$\begin{array}{lll}-9.167061 & -1.142740 & -0.628427\end{array}$

$\begin{array}{llll}-8.915217 & -2.069759 & -1.132232\end{array}$

$-8.174915 \quad-0.146216-0.511464$

$\begin{array}{llll}-4.738522 & -3.051055 & 0.586947\end{array}$

$\begin{array}{lll}-3.876222 & -2.972082 & 1.260974\end{array}$

$\begin{array}{lll}-5.368371 & -4.419596 & 0.781810\end{array}$

$\begin{array}{lll}-4.646732 & -5.435110 & 1.418531\end{array}$

$\begin{array}{llll}-3.659501 & -5.218212 & 1.819758\end{array}$

$\begin{array}{llll}-5.188082 & -6.717544 & 1.546711\end{array}$

$\begin{array}{llll}-4.617786 & -7.497316 & 2.043692\end{array}$

$\begin{array}{lll}-6.462396 & -6.991841 & 1.046206\end{array}$

$\begin{array}{lll}-6.887641 & -7.985867 & 1.150775\end{array}$

$\begin{array}{llll}-7.192411 & -5.978904 & 0.415068\end{array}$

$\begin{array}{llll}-8.187311 & -6.184951 & 0.030081\end{array}$

$\begin{array}{lll}-6.647353 & -4.702444 & 0.280632\end{array}$

$\begin{array}{llll}-7.217708 & -3.910452 & -0.197595\end{array}$

$\begin{array}{llll}-4.273235 & -2.910014 & -0.866731\end{array}$

$\begin{array}{llll}-5.077190 & -3.256898 & -1.525495\end{array}$

$\begin{array}{llll}-3.415942 & -3.564283 & -1.038747\end{array}$

$\begin{array}{lll}-6.245449 & -2.023774 & 2.311838\end{array}$

$\begin{array}{lll}-6.536004 & -3.073029 & 2.404208\end{array}$

$\begin{array}{lll}-5.199301 & -1.771074 & 3.396031\end{array}$

$\begin{array}{lll}-4.730148 & -2.854228 & 4.152685\end{array}$

$\begin{array}{llll}-5.066943 & -3.858410 & 3.908813\end{array}$

$\begin{array}{lll}-3.853357 & -2.658591 & 5.223467\end{array}$

$\begin{array}{lll}-3.508043 & -3.511452 & 5.801195\end{array}$

$\begin{array}{lll}-3.427313 & -1.370232 & 5.550493\end{array}$

$\begin{array}{llll}-2.748147 & -1.212812 & 6.383439\end{array}$

$\begin{array}{lll}-3.871571 & -0.284645 & 4.789882\end{array}$

$\begin{array}{lll}-3.541240 & 0.720885 & 5.034889\end{array}$

$\begin{array}{lll}-4.748873 & -0.482022 & 3.721490\end{array}$

$\begin{array}{lll}-5.100088 & 0.369712 & 3.151930\end{array}$

$\begin{array}{lll}-7.526802 & -1.222171 & 2.549566\end{array}$

$\begin{array}{llll}-8.291583 & -1.490990 & 1.819569\end{array}$

$\begin{array}{lll}-7.374226 & -0.144176 & 2.521288\end{array}$

$\begin{array}{lll}-7.902556 & -1.482962 & 3.543408\end{array}$

$\begin{array}{lll}-2.587962 & -0.315513 & 0.281443\end{array}$

$\begin{array}{llll}-2.213057 & 0.684827 & 0.093668\end{array}$

$\begin{array}{llll}-3.033954 & -0.436279 & 1.264608\end{array}$

$\begin{array}{llll}-1.838612 & -1.435361 & -0.208268\end{array}$

$\begin{array}{llll}-1.963779 & -2.380301 & 0.305461\end{array}$

$\begin{array}{llll}-1.054309 & -1.400529 & -1.353484\end{array}$

$\begin{array}{llll}-0.769945 & -0.433889 & -1.750436\end{array}$

$\begin{array}{llll}-5.087949 & -1.187663 & -3.283040\end{array}$

$\begin{array}{llll}-5.859481 & -1.883561 & -2.967586\end{array}$

$\begin{array}{llll}-3.832697 & -1.739550 & -3.576889\end{array}$

$\begin{array}{lll}-3.724840 & -2.818683 & -3.479497\end{array}$

$\begin{array}{llll}-2.766276 & -1.084494 & -4.433055\end{array}$

$\begin{array}{llll}-2.994582 & -1.271927 & -5.492751\end{array}$

$\begin{array}{llll}-1.819954 & -1.591755 & -4.230155\end{array}$

$\begin{array}{llll}-2.585718 & 0.432214 & -4.203801\end{array}$

$\begin{array}{llll}-3.258172 & 0.997209 & -4.855299\end{array}$

$\begin{array}{llll}-1.573146 & 0.703137 & -4.505793\end{array}$

$\begin{array}{llll}-2.794553 & 0.894742 & -2.768890\end{array}$

$\begin{array}{llll}-1.906989 & 1.181492 & -2.212768\end{array}$

$\begin{array}{llll}-4.001973 & 1.337800 & -2.272086\end{array}$

$\begin{array}{llll}-3.971677 & 1.948454 & -1.374298\end{array}$

$\begin{array}{llll}-5.298016 & 1.389930 & -3.053668\end{array}$

$\begin{array}{llll}-6.108918 & 1.570225 & -2.349049\end{array}$

$\begin{array}{llll}-5.266403 & 2.263832 & -3.719837\end{array}$

$\begin{array}{llll}-5.616504 & 0.118110 & -3.874450\end{array}$

$\begin{array}{llll}-5.231825 & 0.217675 & -4.893678\end{array}$

$\begin{array}{llll}-6.702811 & 0.033394 & -3.963986\end{array}$

$\begin{array}{llll}-0.403031 & -2.548862 & -1.975238\end{array}$

$-0.623965-3.881078-1.566788$

$\begin{array}{llll}0.491117 & -2.314106 & -3.039071\end{array}$

$\begin{array}{llll}0.016455 & -4.933820 & -2.213585\end{array}$ 


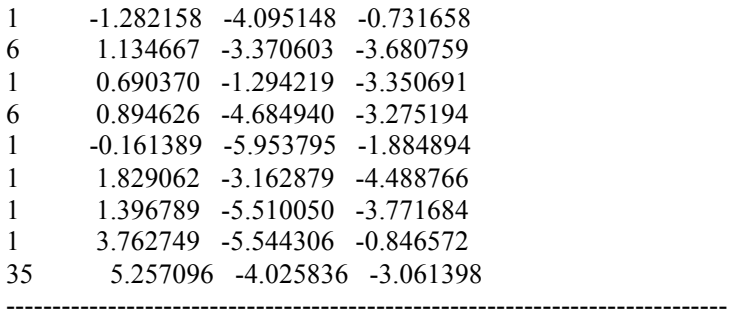

[8-9 ${ }^{\star}-\mathbf{P 1 - C 1}$ endo si-re

Number of imaginary frequencies $=1$

The smallest frequencies are $=-207.64349 .175212 .4396 \mathrm{~cm}(-1)$ Electronic energy $=-7222.6226241$

Zero-point correction $=$

1.754801

(Hartree/Particle)

Thermal correction to Energy=

Thermal correction to Enthalpy=

1.873691

1.874635

Thermal correction to Gibbs Free Energy= $\quad 1.591070$

Sum of electronic and zero-point Energies=

Sum of electronic and thermal Energies $=$

$-7220.867823$

Sum of electronic and thermal Enthalpies=

$-7220.748933$

Sum of electronic and thermal Free Energies=

$-7220.747989$

Cartesian Coordinates

\begin{tabular}{|c|c|c|c|}
\hline 26 & 3.537559 & 4.933678 & 2.365887 \\
\hline 15 & 2.430775 & 1.875807 & $7-1.665330$ \\
\hline 15 & 4.905493 & 0.554700 & 1.039527 \\
\hline 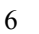 & 3.429227 & 3.780081 & 0.619380 \\
\hline 0 & 4.030930 & 3.035572 & 1.696227 \\
\hline 6 & 3.078478 & 2.960040 & 2.760166 \\
\hline 1 & 3.236207 & 2.472982 & 3.713164 \\
\hline 6 & 1.906457 & 3.668378 & 2.361093 \\
\hline 1 & 1.023868 & 3.829111 & 2.965776 \\
\hline 6 & 2.124278 & 4.180751 & 1.048957 \\
\hline 1 & 1.433778 & 4.779267 & 0.472552 \\
\hline 6 & 5.226402 & 6.096288 & 2.712956 \\
\hline 1 & 6.222867 & 5.856508 & 2.366250 \\
\hline 6 & 4.645625 & 5.680501 & 3.950010 \\
\hline 1 & 5.121395 & 5.062442 & 4.699766 \\
\hline 6 & 3.309717 & 6.183 & 4.000569 \\
\hline 1 & 2.594658 & 6.009075 & 4.793896 \\
\hline 6 & 3.064627 & 6.909198 & 2.795041 \\
\hline 1 & 2.133867 & 7.385865 & 2.517082 \\
\hline 6 & 4.248422 & 6.856829 & 2.000515 \\
\hline 1 & 4.368959 & 7.289547 & 1.016704 \\
\hline 6 & 4.054965 & 3.999464 & -0.713703 \\
\hline 6 & 5.099945 & 4.924240 & -0.851466 \\
\hline 1 & 5.417968 & 5.471254 & 0.026979 \\
\hline 6 & 5.728715 & 5.142413 & -2.077845 \\
\hline 1 & 6.542167 & 5.858646 & -2.149143 \\
\hline 6 & 5.304417 & 4.439891 & -3.203170 \\
\hline 1 & 5.778730 & 4.600162 & -4.166453 \\
\hline 6 & 4.276812 & 3.503235 & -3.086551 \\
\hline 1 & 3.975055 & 2.948696 & -3.967044 \\
\hline 6 & 3.657427 & 3.251235 & -1.852439 \\
\hline 6 & 5.345107 & 2.315403 & 1.597922 \\
\hline 1 & 5.895256 & 2.73 & 0.747999 \\
\hline 6 & 6.227529 & 2.414701 & 2.844478 \\
\hline 1 & 7.176422 & 1.890035 & 2.701335 \\
\hline 1 & 5.735577 & 1.999638 & 3.728511 \\
\hline 1 & 6.446755 & 3.467535 & 3.042745 \\
\hline 6 & 2.391338 & 1.092887 & -3.332333 \\
\hline 6 & 3.507054 & 0.326948 & -3.714362 \\
\hline 1 & 4.378408 & 0.285580 & -3.070463 \\
\hline 6 & 3.523078 & -0.356459 & -4.928375 \\
\hline 1 & 4.398868 & -0.934860 & -5.208336 \\
\hline 6 & 2.411772 & -0.299652 & -5.775985 \\
\hline 1 & 2.415471 & -0.840972 & -6.717503 \\
\hline 6 & 1.298533 & 0.457033 & -5.406221 \\
\hline 1 & 0.432020 & 0.507170 & -6.059319 \\
\hline 6 & 1.288060 & 1.155219 & -4.194790 \\
\hline & 0.416130 & 1.741368 & -3.926972 \\
\hline
\end{tabular}

$\begin{array}{llll}0.821357 & 2.743792 & -1.509722\end{array}$

$\begin{array}{llll}0.499895 & 3.859966 & -2.297940\end{array}$

$\begin{array}{llll}1.203277 & 4.220662 & -3.043107\end{array}$

$\begin{array}{lll}-0.720276 & 4.513852 & -2.121386\end{array}$

$\begin{array}{lll}-0.966645 & 5.373972 & -2.737444\end{array}$

$\begin{array}{llll}-1.620643 & 4.066426 & -1.148682\end{array}$

$\begin{array}{llll}-2.570886 & 4.573649 & -1.015495\end{array}$

$\begin{array}{lll}-1.297192 & 2.964945 & -0.353680\end{array}$

$\begin{array}{lll}-1.992309 & 2.616367 & 0.403092\end{array}$

$\begin{array}{llll}-0.083017 & 2.299438 & -0.534314\end{array}$

$\begin{array}{llll}0.182698 & 1.454867 & 0.094702\end{array}$

$\begin{array}{llll}6.293799 & 0.064811 & -0.065657\end{array}$

$\begin{array}{lll}6.960642 & -1.165479 & 0.020071\end{array}$

$\begin{array}{llll}6.765570 & -1.846434 & 0.839220\end{array}$

$\begin{array}{llll}7.864569 & -1.546509 & -0.972241\end{array}$

$\begin{array}{llll}8.116221 & -0.726633 & -2.071256\end{array}$

$\begin{array}{llll}8.809893 & -1.035511 & -2.843436\end{array}$

$\begin{array}{llll}7.438380 & 0.487649 & -2.166800\end{array}$

$\begin{array}{llll}6.533986 & 0.883633 & -1.180464\end{array}$

$\begin{array}{llll}5.999009 & 1.821470 & -1.298940\end{array}$

$\begin{array}{llll}5.057403 & -0.507350 & 2.534476\end{array}$

$\begin{array}{llll}3.860544 & -1.028522 & 3.037527\end{array}$

$\begin{array}{lll}2.924592 & -0.778098 & 2.550358\end{array}$

$\begin{array}{llll}3.868006 & -1.864717 & 4.156400\end{array}$

$\begin{array}{lll}5.061056 & -2.191017 & 4.793940\end{array}$

$\begin{array}{llll}5.067263 & -2.851295 & 5.652848\end{array}$

$\begin{array}{llll}6.254839 & -1.660486 & 4.299044\end{array}$

$6.260726-0.824539 \quad 3.183111$

$\begin{array}{lll}7.205553 & -0.440327 & 2.816814\end{array}$

$\begin{array}{llll}2.903091 & 0.325791 & -0.082593\end{array}$

$\begin{array}{llll}7.552954 & -1.950421 & 5.003010\end{array}$

$\begin{array}{llll}2.559615 & -2.451160 & 4.607937\end{array}$

$\begin{array}{llll}8.610223 & -2.846017 & -0.825761\end{array}$

$\begin{array}{llll}7.612303 & 1.370148 & -3.373227\end{array}$

$\begin{array}{llll}6.488334 & 1.382427 & -4.136914\end{array}$

$\begin{array}{llll}7.853824 & 2.654152 & -3.023934\end{array}$

$\begin{array}{llll}8.625305 & 0.968745 & -4.167670\end{array}$

$\begin{array}{llll}7.881874 & -3.773729 & -0.165950\end{array}$

$\begin{array}{llll}9.758568 & -2.681039 & -0.124801\end{array}$

$\begin{array}{llll}8.955130 & -3.371586 & -2.022036\end{array}$

$\begin{array}{lll}7.861216 & -0.978438 & 5.895487\end{array}$

$\begin{array}{llll}8.587481 & -2.024083 & 4.135013\end{array}$

$\begin{array}{llll}7.511463 & -3.114038 & 5.686928\end{array}$

$\begin{array}{llll}2.666246 & -3.146959 & 5.757204\end{array}$

$\begin{array}{llll}1.620683 & -1.492507 & 4.801179\end{array}$

$\begin{array}{lll}2.056466 & -3.297205 & 3.671727\end{array}$

$\begin{array}{llll}3.928713 & -2.124015 & -1.402345\end{array}$

$\begin{array}{llll}2.015091 & -2.667492 & -0.167948\end{array}$

$\begin{array}{llll}4.113810 & -3.435447 & -1.798819\end{array}$

$\begin{array}{llll}4.608779 & -1.345105 & -1.717953\end{array}$

$\begin{array}{llll}2.191444 & -4.030893 & -0.536374\end{array}$

$\begin{array}{llll}3.229056 & -4.423284 & -1.359430\end{array}$

$\begin{array}{llll}1.485060 & -4.768909 & -0.172994\end{array}$

$\begin{array}{llll}0.853930 & -2.324108 & 0.603451\end{array}$

$\begin{array}{llll}0.674063 & -1.140536 & 1.400386\end{array}$

$\begin{array}{lll}1.299026 & -0.049094 & 1.194626\end{array}$

$\begin{array}{llll}2.905959 & -1.737101 & -0.620815\end{array}$

$\begin{array}{llll}0.261572 & -3.184943 & 0.877316\end{array}$

$\begin{array}{lll}-0.251755 & -1.153567 & 2.407642\end{array}$

$\begin{array}{lll}-0.606222 & 0.087336 & 3.083678\end{array}$

$\begin{array}{lll}-0.164225 & 0.121637 & 4.087243\end{array}$

$\begin{array}{lll}-1.693910 & 0.147711 & 3.184867\end{array}$

$\begin{array}{lll}-0.242410 & 0.939027 & 2.513169\end{array}$

$\begin{array}{lll}-0.863160 & -2.350847 & 2.972582\end{array}$

$\begin{array}{lll}-0.252655 & -3.232761 & 2.791335\end{array}$

$\begin{array}{lll}-1.872361 & -2.515991 & 2.580924\end{array}$

$\begin{array}{lll}-0.937626 & -2.223208 & 4.055264\end{array}$

$\begin{array}{lll}-3.863156 & -1.086179 & -1.381528\end{array}$

$\begin{array}{lll}-5.660694 & -0.580324 & -0.078168\end{array}$

$\begin{array}{llll}-5.764089 & 0.699321 & 0.970763\end{array}$

$\begin{array}{lll}-6.994161 & -0.288662 & -1.032086\end{array}$

$\begin{array}{llll}-5.940295 & -1.833221 & 0.996289\end{array}$

$\begin{array}{lll}-6.023332 & 1.989752 & 0.524749\end{array}$

$\begin{array}{lll}-4.982236 & 2.927151 & 0.699716\end{array}$

$\begin{array}{llll}-4.070876 & 2.596501 & 1.185279\end{array}$ 
$\begin{array}{lll}-5.155612 & 4.221412 & 0.276660\end{array}$

$\begin{array}{llll}-4.372862 & 4.959210 & 0.427031\end{array}$

$\begin{array}{lll}-6.351918 & 4.606615 & -0.387009\end{array}$

$\begin{array}{llll}-6.506081 & 5.915526 & -0.918754\end{array}$

$\begin{array}{llll}-5.694320 & 6.626445 & -0.787958\end{array}$

$\begin{array}{llll}-7.648784 & 6.272747 & -1.598019\end{array}$

$\begin{array}{llll}-7.753676 & 7.273583 & -2.006419\end{array}$

$\begin{array}{llll}-8.685425 & 5.325937 & -1.777275\end{array}$

$\begin{array}{lll}-9.576029 & 5.602894 & -2.334010\end{array}$

$\begin{array}{llll}-8.575038 & 4.054329 & -1.257603\end{array}$

$\begin{array}{llll}-9.375763 & 3.340798 & -1.411988\end{array}$

$\begin{array}{llll}-7.416561 & 3.654500 & -0.534672\end{array}$

$\begin{array}{llll}-7.266996 & 2.330411 & 0.013692\end{array}$

$\begin{array}{lll}-8.389006 & 1.349658 & 0.034046\end{array}$

$\begin{array}{llll}-9.651965 & 1.646237 & 0.660397\end{array}$

$\begin{array}{lll}-9.883795 & 2.841636 & 1.397450\end{array}$

$\begin{array}{lll}-9.083810 & 3.564312 & 1.503616\end{array}$

$\begin{array}{lll}-11.102911 & 3.086672 & 1.991477\end{array}$

$\begin{array}{lll}-11.250782 & 4.003801 & 2.554477\end{array}$

$\begin{array}{lll}-12.161850 & 2.154315 & 1.881543\end{array}$

$\begin{array}{lll}-13.119209 & 2.364828 & 2.349166\end{array}$

$\begin{array}{lll}-11.966485 & 0.976865 & 1.196084\end{array}$

$\begin{array}{lll}-12.763594 & 0.241918 & 1.119630\end{array}$

$\begin{array}{lll}-10.716413 & 0.684867 & 0.585522\end{array}$

$\begin{array}{lll}-10.498919 & -0.555040 & -0.074825\end{array}$

$\begin{array}{llll}-11.315085 & -1.269218 & -0.137580\end{array}$

$\begin{array}{llll}-9.265665 & -0.860971 & -0.594493\end{array}$

$\begin{array}{llll}-9.061650 & -1.814821 & -1.069605\end{array}$

$\begin{array}{llll}-8.221940 & 0.085857 & -0.507502\end{array}$

$\begin{array}{lll}-5.148494 & -3.050361 & 0.667266\end{array}$

$\begin{array}{lll}-4.274257 & -3.076900 & 1.332119\end{array}$

$\begin{array}{lll}-5.952390 & -4.320737 & 0.883090\end{array}$

$\begin{array}{llll}-5.363304 & -5.428237 & 1.503569\end{array}$

$\begin{array}{lll}-4.345521 & -5.351860 & 1.879360\end{array}$

$\begin{array}{lll}-6.073078 & -6.623823 & 1.649178\end{array}$

$\begin{array}{lll}-5.603174 & -7.475081 & 2.134108\end{array}$

$\begin{array}{llll}-7.385453 & -6.719331 & 1.182243\end{array}$

$\begin{array}{llll}-7.941236 & -7.645164 & 1.300535\end{array}$

$\begin{array}{llll}-7.983683 & -5.614065 & 0.567314\end{array}$

$\begin{array}{llll}-9.006904 & -5.679937 & 0.207361\end{array}$

$\begin{array}{lll}-7.271194 & -4.424888 & 0.416038\end{array}$

$\begin{array}{llll}-7.737621 & -3.560843 & -0.049895\end{array}$

$\begin{array}{llll}-4.668892 & -2.983773 & -0.788970\end{array}$

$\begin{array}{llll}-5.514834 & -3.228570 & -1.441948\end{array}$

$\begin{array}{llll}-3.919481 & -3.764315 & -0.945969\end{array}$

$\begin{array}{lll}-6.466387 & -1.803597 & 2.400550\end{array}$

$\begin{array}{llll}-6.834796 & -2.821696 & 2.550373\end{array}$

$\begin{array}{llll}-5.354566 & -1.598058 & 3.427242\end{array}$

$\begin{array}{lll}-4.813040 & -2.718634 & 4.074407\end{array}$

$\begin{array}{lll}-5.182605 & -3.708342 & 3.818319\end{array}$

$\begin{array}{lll}-3.822834 & -2.578556 & 5.050816\end{array}$

$\begin{array}{llll}-3.419601 & -3.459926 & 5.541945\end{array}$

$\begin{array}{lll}-3.360748 & -1.308146 & 5.398238\end{array}$

$\begin{array}{llll}-2.595761 & -1.192598 & 6.160886\end{array}$

$\begin{array}{lll}-3.890203 & -0.183843 & 4.757231\end{array}$

$\begin{array}{lll}-3.540262 & 0.809085 & 5.026517\end{array}$

$\begin{array}{lll}-4.875696 & -0.326617 & 3.778253\end{array}$

$\begin{array}{lll}-5.280152 & 0.553372 & 3.293144\end{array}$

$\begin{array}{lll}-7.677613 & -0.898516 & 2.632618\end{array}$

$\begin{array}{lll}-8.480922 & -1.151682 & 1.938827\end{array}$

$\begin{array}{lll}-7.453995 & 0.161838 & 2.531777\end{array}$

$\begin{array}{lll}-8.039494 & -1.074507 & 3.650323\end{array}$

$\begin{array}{lll}-2.631299 & -0.764792 & 0.388900\end{array}$

$\begin{array}{llll}-2.128352 & 0.199897 & 0.350129\end{array}$

$\begin{array}{lll}-3.124181 & -0.949664 & 1.340076\end{array}$

$\begin{array}{lll}-1.997034 & -1.875979 & -0.260801\end{array}$

$\begin{array}{llll}-2.272389 & -2.873770 & 0.060821\end{array}$

$\begin{array}{lll}-0.841397 & -1.762637 & -1.071611\end{array}$

$\begin{array}{llll}-0.438422 & -0.765154 & -1.216620\end{array}$

$\begin{array}{llll}-5.101135 & -1.217233 & -3.236439\end{array}$

$\begin{array}{lll}-5.980052 & -1.803700 & -2.984296\end{array}$

$\begin{array}{llll}-3.900060 & -1.932990 & -3.457096\end{array}$

$-3.942798-3.018770-3.376505$

$\begin{array}{llll}-2.756579 & -1.417307 & -4.312387\end{array}$

$\begin{array}{lll}-3.025622 & -1.507390 & -5.376257\end{array}$

$\begin{array}{llll}-1.898401 & -2.075686 & -4.160284\end{array}$

$\begin{array}{llll}-2.338351 & 0.037730 & -4.001485\end{array}$

$\begin{array}{lll}-2.829440 & 0.731607 & -4.692108\end{array}$

$\begin{array}{llll}-1.266187 & 0.133308 & -4.185037\end{array}$

$\begin{array}{llll}-2.613605 & 0.498739 & -2.572049\end{array}$

$\begin{array}{lll}-1.736100 & 0.712050 & -1.972295\end{array}$

$\begin{array}{llll}-3.790272 & 1.115759 & -2.160972\end{array}$

$-3.725881 \quad 1.760556-1.289438$

$\begin{array}{llll}-5.003718 & 1.352505 & -3.039129\end{array}$

$\begin{array}{llll}-5.838780 & 1.631439 & -2.392722\end{array}$

$\begin{array}{llll}-4.808522 & 2.219146 & -3.688235\end{array}$

$\begin{array}{llll}-5.411623 & 0.130254 & -3.891019\end{array}$

$\begin{array}{lll}-4.924819 & 0.172242 & -4.870717\end{array}$

$\begin{array}{llll}-6.487289 & 0.183527 & -4.083905\end{array}$

$-0.393054-2.748970 \quad-2.047445$

$\begin{array}{llll}-0.905429 & -4.060459 & -2.105855\end{array}$

$\begin{array}{llll}0.580355 & -2.365351 & -2.990852\end{array}$

$-0.466008-4.948210-3.084969$

$\begin{array}{llll}-1.657338 & -4.383180 & -1.392787\end{array}$

$\begin{array}{llll}1.017984 & -3.255759 & -3.969447\end{array}$

$\begin{array}{lll}0.986841 & -1.359952 & -2.955231\end{array}$

$\begin{array}{llll}0.494723 & -4.550225 & -4.022320\end{array}$

$-0.874943-5.954068-3.120554$

$\begin{array}{llll}1.764446 & -2.933662 & -4.688414\end{array}$

$\begin{array}{llll}0.831715 & -5.245333 & -4.785899\end{array}$

$3.345944-5.460889-1.652616$

$35 \quad 5.605669 \quad-3.870390 \quad-2.983613$

9 P1-C1 endo si-re

Number of imaginary frequencies $=0$

The smallest frequencies are=7.3520 $12.687417 .7843 \mathrm{~cm}(-1)$ Electronic energy $=-7222.6534935$

Zero-point correction=

1.758428

(Hartree/Particle)

Thermal correction to Energy=

1.876614

Thermal correction to Enthalpy=

1.877558

Thermal correction to Gibbs Free Energy $=\quad 1.596829$

Sum of electronic and zero-point Energies $=\quad-7220.895065$

Sum of electronic and thermal Energies=

$-7220.776880$

Sum of electronic and thermal Enthalpies=

$-7220.775935$

Sum of electronic and thermal Free Energies $=\quad-7221.056665$

Cartesian Coordinates

$\begin{array}{llll}26 & 3.256921 & 4.238150 & 3.458309 \\ 15 & 2.093331 & 2.283848 & -1.218822 \\ 15 & 4.758477 & 0.407260 & 0.999729 \\ 6 & 3.109452 & 3.569550 & 1.475231 \\ 6 & 3.783381 & 2.597172 & 2.297717 \\ 6 & 2.887626 & 2.210082 & 3.342722 \\ 1 & 3.109085 & 1.503464 & 4.131503 \\ 6 & 1.674997 & 2.944680 & 3.184545 \\ 1 & 0.816771 & 2.904446 & 3.842099 \\ 6 & 1.814435 & 3.788870 & 2.044388 \\ 1 & 1.076660 & 4.484231 & 1.670853 \\ 6 & 4.903573 & 5.374287 & 4.028178 \\ 1 & 5.888972 & 5.295373 & 3.589028 \\ 6 & 4.416070 & 4.614778 & 5.135373 \\ 1 & 4.963193 & 3.852928 & 5.674277 \\ 6 & 3.061786 & 5.001072 & 5.372578 \\ 1 & 2.402666 & 4.580953 & 6.120906 \\ 6 & 2.711392 & 5.997782 & 4.411340 \\ 1 & 1.741650 & 6.465515 & 4.303389 \\ 6 & 3.849201 & 6.230043 & 3.582594 \\ 1 & 3.889298 & 6.908543 & 2.741451 \\ 6 & 3.667416 & 4.160287 & 0.225683 \\ 6 & 4.657531 & 5.150194 & 0.303030 \\ 1 & 4.983501 & 5.472798 & 1.283455 \\ 6 & 5.224244 & 5.713082 & -0.841397 \\ 1 & 5.994320 & 6.473051 & -0.745449 \\ 6 & 4.795596 & 5.296460 & -2.099054 \\ 1 & 5.224132 & 5.724827 & -2.999647 \\ 6 & 3.822609 & 4.301467 & -2.201892 \\ 1 & 3.516998 & 3.969163 & -3.186749\end{array}$


$\begin{array}{llll}3.263037 & 3.710249 & -1.059008\end{array}$

$\begin{array}{llll}5.120424 & 1.996016 & 1.977274\end{array}$

$\begin{array}{lll}5.605115 & 2.635922 & 1.23315\end{array}$

$\begin{array}{lll}6.057962 & 1.858720 & 3.178987\end{array}$

$\begin{array}{lll}7.043648 & 1.494495 & 2.877499\end{array}$

$\begin{array}{lll}5.655039 & 1.186528 & 3.941472\end{array}$

$\begin{array}{llll}6.187879 & 2.843758 & 3.634533\end{array}$

$\begin{array}{llll}2.014290 & 1.982817 & -3.029288\end{array}$

$\begin{array}{llll}3.082671 & 1.265690 & -3.597401\end{array}$

$\begin{array}{llll}3.912196 & 0.951445 & -2.972439\end{array}$

$\begin{array}{llll}3.097414 & 0.971644 & -4.958967\end{array}$

$\begin{array}{llll}3.934240 & 0.425132 & -5.384271\end{array}$

$\begin{array}{lll}2.032312 & 1.376392 & -5.770619\end{array}$

$\begin{array}{llll}2.034407 & 1.139143 & -6.830407\end{array}$

$\begin{array}{llll}0.965250 & 2.083343 & -5.213850\end{array}$

$\begin{array}{llll}0.132868 & 2.394598 & -5.837976\end{array}$

$\begin{array}{llll}0.954402 & 2.390374 & -3.849571\end{array}$

$\begin{array}{llll}0.114750 & 2.935892 & -3.432911\end{array}$

$\begin{array}{llll}0.487746 & 3.019227 & -0.728007\end{array}$

$\begin{array}{llll}0.111785 & 4.313032 & -1.123026\end{array}$

$\begin{array}{llll}0.731841 & 4.873375 & -1.816808\end{array}$

$\begin{array}{llll}-1.040279 & 4.897297 & -0.594805\end{array}$

$\begin{array}{llll}-1.323620 & 5.901728 & -0.895859\end{array}$

$\begin{array}{llll}-1.814140 & 4.200844 & 0.339969\end{array}$

$\begin{array}{lll}-2.690004 & 4.670794 & 0.775487\end{array}$

$\begin{array}{lll}-1.451544 & 2.907641 & 0.722072\end{array}$

$\begin{array}{lll}-2.042621 & 2.364368 & 1.453750\end{array}$

$\begin{array}{lll}-0.306107 & 2.316346 & 0.187568\end{array}$

$\begin{array}{llll}0.008214 & 1.333924 & 0.521061\end{array}$

$\begin{array}{llll}6.147063 & 0.335922 & -0.216317\end{array}$

$\begin{array}{llll}7.055272 & -0.726202 & -0.330067\end{array}$

$\begin{array}{llll}7.034965 & -1.553645 & 0.367306\end{array}$

$7.990288-0.741984-1.364472$

$\begin{array}{llll}8.035874 & 0.281507 & -2.311324\end{array}$

$\begin{array}{llll}8.757508 & 0.254711 & -3.119152\end{array}$

$\begin{array}{llll}7.122393 & 1.327894 & -2.209193\end{array}$

$\begin{array}{llll}6.184985 & 1.360166 & -1.174920\end{array}$

$\begin{array}{llll}5.470901 & 2.177478 & -1.132471\end{array}$

$\begin{array}{llll}5.044799 & -1.026725 & 2.120036\end{array}$

$\begin{array}{llll}3.969875 & -1.903469 & 2.303155\end{array}$

$3.025134 \quad-1.695930 \quad 1.819432$

$\begin{array}{llll}4.111812 & -3.050481 & 3.088243\end{array}$

$\begin{array}{llll}5.319242 & -3.334233 & 3.719492\end{array}$

$\begin{array}{llll}5.433415 & -4.228136 & 4.320876\end{array}$

$\begin{array}{lll}6.390054 & -2.454853 & 3.547799\end{array}$

$6.263388-1.315278 \quad 2.755045$

$\begin{array}{llll}7.124757 & -0.673899 & 2.618096\end{array}$

$2.732381 \quad 0.396551 \quad-0.139466$

$\begin{array}{llll}7.690862 & -2.709674 & 4.261892\end{array}$

$\begin{array}{llll}2.930897 & -3.969351 & 3.241419\end{array}$

$\begin{array}{llll}9.003616 & -1.852202 & -1.454813\end{array}$

$\begin{array}{llll}7.110715 & 2.441226 & -3.222804\end{array}$

$\begin{array}{llll}5.897878 & 2.550016 & -3.821698\end{array}$

$\begin{array}{llll}7.366193 & 3.639360 & -2.648149\end{array}$

$\begin{array}{llll}8.022210 & 2.259695 & -4.199455\end{array}$

$8.666398 \quad-2.920713-0.702192$

$10.223813-1.440348 \quad-1.036569$

$\begin{array}{llll}9.158084 & -2.283174 & -2.729365\end{array}$

$\begin{array}{lll}7.731152 & -2.071461 & 5.455902\end{array}$

$\begin{array}{llll}8.749037 & -2.267195 & 3.546294\end{array}$

$\begin{array}{llll}7.884800 & -4.022112 & 4.509771\end{array}$

$\begin{array}{llll}3.267959 & -5.163880 & 3.764045\end{array}$

$\begin{array}{lll}1.978592 & -3.436432 & 4.046645\end{array}$

$\begin{array}{llll}2.333437 & -4.205308 & 2.044170\end{array}$

$\begin{array}{llll}3.910965 & -1.837961 & -1.725700\end{array}$

$\begin{array}{llll}1.948717 & -2.604274 & -0.751934\end{array}$

$\begin{array}{llll}4.285657 & -3.127152 & -2.075528\end{array}$

$\begin{array}{llll}4.533819 & -0.987988 & -1.971444\end{array}$

$2.298084-3.927036-1.051880$

$\begin{array}{llll}3.482502 & -4.206538 & -1.723777\end{array}$

$\begin{array}{llll}1.632706 & -4.735864 & -0.771892\end{array}$

$\begin{array}{llll}0.573425 & -2.334681 & -0.171872\end{array}$

$\begin{array}{llll}0.624331 & -1.519190 & 1.111551\end{array}$

$\begin{array}{lll}1.177802 & -0.391440 & 1.108697\end{array}$

$\begin{array}{llll}2.767822 & -1.580875 & -1.066750\end{array}$ $\begin{array}{lll}0.135688 & -3.312888 & 0.014798\end{array}$

$\begin{array}{llll}0.085890 & -1.983067 & 2.251400\end{array}$

$\begin{array}{llll}0.140514 & -1.088219 & 3.412924\end{array}$

$\begin{array}{llll}0.124934 & -1.650263 & 4.305338\end{array}$

$\begin{array}{lll}-0.561268 & -0.256993 & 3.293325\end{array}$

$\begin{array}{lll}1.146228 & -0.679981 & 3.521454\end{array}$

$\begin{array}{lll}-0.676060 & -3.229624 & 2.370068\end{array}$

$-0.171850 \quad-4.045480 \quad 1.853426$

$\begin{array}{lll}-1.690464 & -3.112159 & 1.978651\end{array}$

$\begin{array}{lll}-0.736527 & -3.496500 & 3.423844\end{array}$

$\begin{array}{llll}-3.685349 & -0.895944 & -1.529037\end{array}$

$\begin{array}{lll}-5.520861 & -0.537279 & -0.164444\end{array}$

$\begin{array}{lll}-5.720302 & 0.691081 & 0.949121\end{array}$

$\begin{array}{lll}-6.924264 & -0.315653 & -1.058694\end{array}$

$\begin{array}{lll}-5.704047 & -1.876460 & 0.838124\end{array}$

$\begin{array}{llll}-6.166479 & 1.948579 & 0.555692\end{array}$

$\begin{array}{lll}-5.227548 & 3.000369 & 0.625464\end{array}$

$\begin{array}{lll}-4.228390 & 2.774149 & 0.974706\end{array}$

$\begin{array}{llll}-5.592050 & 4.268243 & 0.248349\end{array}$

$\begin{array}{llll}-4.877555 & 5.084445 & 0.298004\end{array}$

$\begin{array}{llll}-6.898083 & 4.526993 & -0.245559\end{array}$

$\begin{array}{llll}-7.266827 & 5.822141 & -0.700645\end{array}$

$\begin{array}{llll}-6.533759 & 6.622056 & -0.635185\end{array}$

$\begin{array}{llll}-8.517197 & 6.057963 & -1.225293\end{array}$

$\begin{array}{llll}-8.788264 & 7.050328 & -1.573615\end{array}$

$\begin{array}{llll}-9.447674 & 4.996185 & -1.326671\end{array}$

$\begin{array}{lll}-10.424443 & 5.177392 & -1.766082\end{array}$

$\begin{array}{lll}-9.126052 & 3.732632 & -0.880644\end{array}$

$\begin{array}{llll}-9.849441 & 2.932132 & -0.977325\end{array}$

$\begin{array}{llll}-7.852515 & 3.456691 & -0.307350\end{array}$

$\begin{array}{lll}-7.484748 & 2.148632 & 0.172240\end{array}$

$\begin{array}{lll}-8.459381 & 1.024539 & 0.206576\end{array}$

$\begin{array}{lll}-9.703786 & 1.098191 & 0.924453\end{array}$

$\begin{array}{lll}-10.039492 & 2.192707 & 1.769977\end{array}$

$\begin{array}{lll}-9.332595 & 3.005191 & 1.890435\end{array}$

$\begin{array}{lll}-11.239454 & 2.227439 & 2.446429\end{array}$

$\begin{array}{lll}-11.468630 & 3.071347 & 3.090907\end{array}$

$\begin{array}{lll}-12.174466 & 1.172776 & 2.314398\end{array}$

$\begin{array}{lll}-13.119515 & 1.217374 & 2.847715\end{array}$

$\begin{array}{lll}-11.873710 & 0.087698 & 1.522588\end{array}$

$\begin{array}{lll}-12.574403 & -0.737702 & 1.426383\end{array}$

$\begin{array}{llll}-10.638138 & 0.012374 & 0.823766\end{array}$

$\begin{array}{lll}-10.305462 & -1.127428 & 0.042177\end{array}$

$\begin{array}{llll}-11.026188 & -1.935646 & -0.045415\end{array}$

$\begin{array}{lll}-9.079670 & -1.220530 & -0.569718\end{array}$

$\begin{array}{lll}-8.795562 & -2.089639 & -1.152436\end{array}$

$\begin{array}{llll}-8.158128 & -0.156471 & -0.452286\end{array}$

$\begin{array}{lll}-4.877380 & -3.032223 & 0.383229\end{array}$

$\begin{array}{llll}-3.967190 & -3.061436 & 0.998242\end{array}$

$\begin{array}{llll}-5.596365 & -4.357888 & 0.569246\end{array}$

$\begin{array}{llll}-4.920855 & -5.448204 & 1.130497\end{array}$

$\begin{array}{lll}-3.899486 & -5.320104 & 1.481635\end{array}$

$\begin{array}{llll}-5.548305 & -6.691703 & 1.251187\end{array}$

$\begin{array}{lll}-5.010925 & -7.527823 & 1.690527\end{array}$

$\begin{array}{lll}-6.866124 & -6.855189 & 0.819394\end{array}$

$\begin{array}{llll}-7.358790 & -7.818308 & 0.918808\end{array}$

$\begin{array}{llll}-7.550924 & -5.768913 & 0.264170\end{array}$

$\begin{array}{llll}-8.578890 & -5.886371 & -0.068373\end{array}$

$\begin{array}{llll}-6.919175 & -4.532097 & 0.138011\end{array}$

$\begin{array}{llll}-7.453121 & -3.684224 & -0.278439\end{array}$

$\begin{array}{llll}-4.475049 & -2.847314 & -1.087529\end{array}$

$\begin{array}{llll}-5.351144 & -3.045045 & -1.719713\end{array}$

$-3.735207-3.612973-1.337559$

$\begin{array}{lll}-6.152112 & -1.988814 & 2.259341\end{array}$

$\begin{array}{lll}-6.519869 & -3.015513 & 2.335321\end{array}$

$\begin{array}{lll}-4.982205 & -1.878899 & 3.234862\end{array}$

$-4.559456-3.019458 \quad 3.929755$

$\begin{array}{lll}-5.051270 & -3.970029 & 3.739369\end{array}$

$\begin{array}{llll}-3.523125 & -2.948725 & 4.865746\end{array}$

$\begin{array}{lll}-3.215641 & -3.843303 & 5.400654\end{array}$

$\begin{array}{lll}-2.891091 & -1.729677 & 5.114720\end{array}$

$\begin{array}{lll}-2.093840 & -1.666942 & 5.849852\end{array}$

$\begin{array}{lll}-3.293898 & -0.587535 & 4.414478\end{array}$

$\begin{array}{lll}-2.805975 & 0.364961 & 4.603310\end{array}$

$\begin{array}{lll}-4.329257 & -0.660920 & 3.481534\end{array}$ 


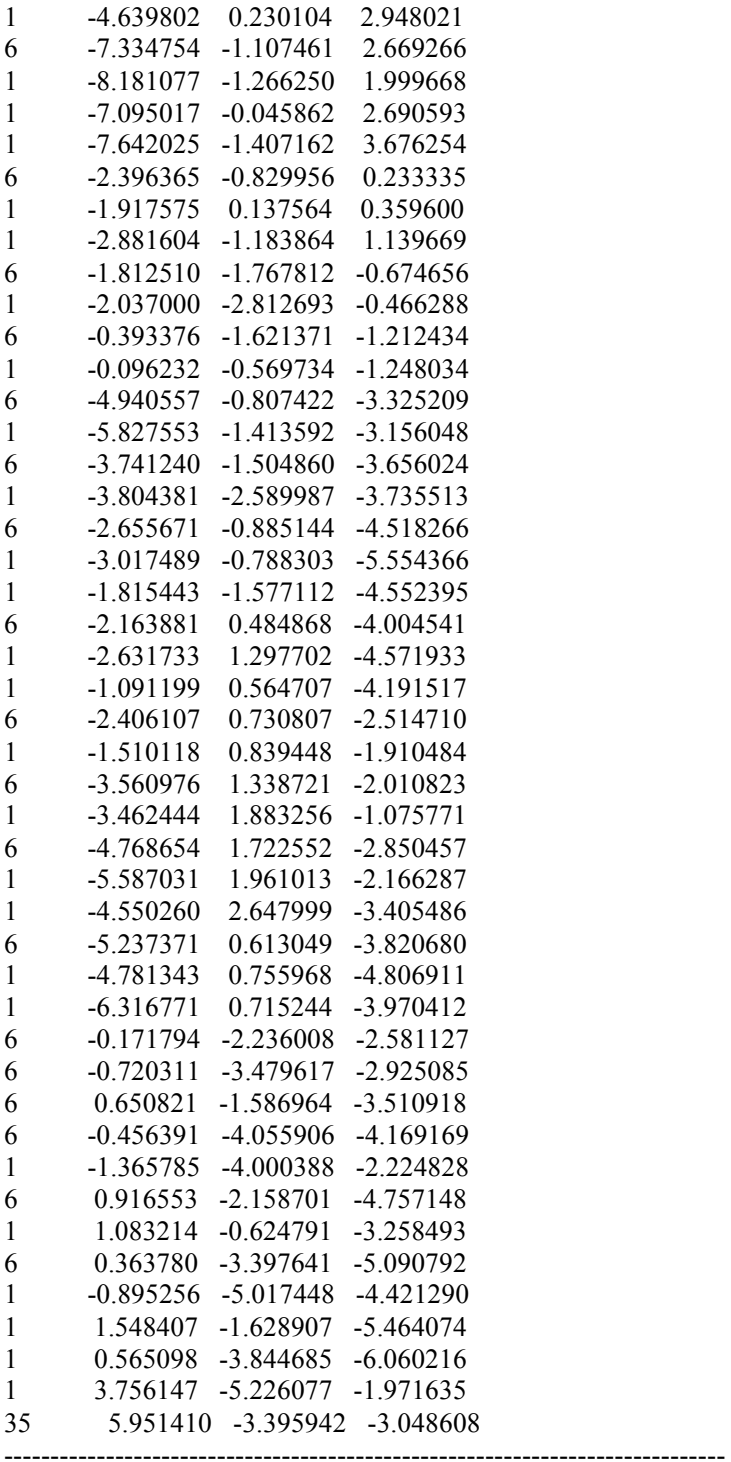

8 P1-C1 exo si-si

Number of imaginary frequencies $=0$

The smallest frequencies are= $12.500014 .061615 .2317 \mathrm{~cm}(-1)$ Electronic energy $=-7222.6326144$

Zero-point correction=

(Hartree/Particle)

Thermal correction to Energy=

Thermal correction to Enthalpy=

1.756627

Thermal correction to Gibbs Free Energy=
Sum of electronic and zero-point Energies=

Sum of electronic and thermal Energies=

Sum of electronic and thermal Enthalpies=

Sum of electronic and thermal Free Energies=

1.875892

1.876836

1.593717

$-7220.875988$

$-7220.756723$

$-7220.755779$

$-7221.038898$

Cartesian Coordinates

$\begin{array}{lccc}26 & 3.647586 & 5.476001 & 1.344250 \\ 15 & 1.261867 & 1.776080 & -1.425452 \\ 15 & 4.554397 & 0.877020 & 0.440065 \\ 6 & 2.936411 & 4.040812 & -0.007006 \\ 6 & 3.915793 & 3.470882 & 0.882603 \\ 6 & 3.430884 & 3.618329 & 2.219074 \\ 1 & 3.946389 & 3.297904 & 3.114446 \\ 6 & 2.173494 & 4.289281 & 2.165269 \\ 1 & 1.574055 & 4.584372 & 3.016111 \\ 6 & 1.870938 & 4.556784 & 0.797710 \\ 1 & 0.997735 & 5.072822 & 0.425436 \\ 6 & 5.279053 & 6.640996 & 0.788594\end{array}$

$\begin{array}{llll}6.057013 & 6.321308 & 0.108280\end{array}$

$\begin{array}{llll}5.269557 & 6.442862 & 2.202927\end{array}$

$\begin{array}{lll}6.036778 & 5.945216 & 2.780683\end{array}$

$\begin{array}{llll}4.048172 & 6.977150 & 2.714957\end{array}$

$\begin{array}{lll}3.726304 & 6.949793 & 3.747731\end{array}$

$\begin{array}{lll}3.301872 & 7.504567 & 1.617470\end{array}$

$\begin{array}{lll}2.316525 & 7.948300 & 1.672521\end{array}$

$\begin{array}{lll}4.061845 & 7.297685 & 0.427645\end{array}$

$\begin{array}{llll}3.752654 & 7.563071 & -0.574344\end{array}$

$\begin{array}{llll}3.045063 & 3.994614 & -1.491778\end{array}$

$\begin{array}{llll}3.931706 & 4.859447 & -2.149214\end{array}$

$\begin{array}{llll}4.482983 & 5.574095 & -1.552978\end{array}$

$\begin{array}{llll}4.123053 & 4.807217 & -3.530280\end{array}$

$\begin{array}{llll}4.824992 & 5.486415 & -4.005442\end{array}$

$\begin{array}{llll}3.412961 & 3.878737 & -4.286501\end{array}$

$\begin{array}{llll}3.551914 & 3.817369 & -5.361344\end{array}$

$\begin{array}{llll}2.527771 & 3.005055 & -3.653655\end{array}$

$\begin{array}{lll}1.997813 & 2.279322 & -4.257347\end{array}$

$\begin{array}{llll}2.339781 & 3.034191 & -2.262606\end{array}$

$\begin{array}{lll}5.115598 & 2.692307 & 0.425270\end{array}$

$\begin{array}{llll}5.266869 & 2.901143 & -0.638379\end{array}$

$\begin{array}{llll}6.412420 & 3.013122 & 1.170805\end{array}$

$\begin{array}{lll}7.251821 & 2.432628 & 0.777225\end{array}$

$\begin{array}{lll}6.330110 & 2.815959 & 2.243221\end{array}$

$\begin{array}{lll}6.643062 & 4.074339 & 1.040363\end{array}$

$\begin{array}{lll}0.669682 & 0.777792 & -2.861585\end{array}$

$\begin{array}{llll}1.480237 & -0.301093 & -3.248935\end{array}$

$\begin{array}{llll}2.371630 & -0.525959 & -2.674285\end{array}$

$\begin{array}{llll}1.170978 & -1.059491 & -4.378777\end{array}$

$\begin{array}{llll}1.821416 & -1.877857 & -4.673458\end{array}$

$\begin{array}{llll}0.028307 & -0.760893 & -5.126171\end{array}$

$-0.220974-1.352376-6.002255$

$\begin{array}{llll}-0.793586 & 0.302229 & -4.741398\end{array}$

$\begin{array}{lll}-1.683241 & 0.538448 & -5.317870\end{array}$

$\begin{array}{llll}-0.472815 & 1.071526 & -3.620421\end{array}$

$\begin{array}{lll}-1.101734 & 1.911804 & -3.355180\end{array}$

$\begin{array}{llll}-0.182623 & 2.793637 & -0.908188\end{array}$

$\begin{array}{llll}-0.653021 & 3.864107 & -1.687564\end{array}$

$\begin{array}{llll}-0.176153 & 4.099093 & -2.634460\end{array}$

$\begin{array}{llll}-1.718494 & 4.645530 & -1.239060\end{array}$

$\begin{array}{llll}-2.078390 & 5.468593 & -1.849627\end{array}$

$\begin{array}{llll}-2.323193 & 4.366633 & -0.008890\end{array}$

$\begin{array}{lll}-3.154275 & 4.974488 & 0.337230\end{array}$

$\begin{array}{lll}-1.851781 & 3.311895 & 0.775498\end{array}$

$\begin{array}{lll}-2.308661 & 3.102242 & 1.737867\end{array}$

$\begin{array}{lll}-0.779355 & 2.531412 & 0.334684\end{array}$

$\begin{array}{lll}-0.368037 & 1.749545 & 0.966363\end{array}$

$\begin{array}{llll}5.508389 & 0.116236 & -0.942907\end{array}$

$\begin{array}{llll}6.348541 & -1.001049 & -0.829889\end{array}$

$\begin{array}{llll}6.562244 & -1.442955 & 0.134638\end{array}$

$\begin{array}{llll}6.909987 & -1.577099 & -1.969634\end{array}$

$\begin{array}{llll}6.629157 & -1.080636 & -3.242885\end{array}$

$\begin{array}{llll}7.057496 & -1.542534 & -4.124237\end{array}$

$\begin{array}{llll}5.771499 & 0.010841 & -3.357843\end{array}$

$\begin{array}{llll}5.218579 & 0.609393 & -2.224741\end{array}$

$\begin{array}{llll}4.539173 & 1.447572 & -2.348171\end{array}$

$\begin{array}{llll}5.238470 & 0.165879 & 1.993355\end{array}$

$\begin{array}{llll}4.299245 & -0.175505 & 2.976195\end{array}$

$\begin{array}{lll}3.244622 & -0.022332 & 2.781556\end{array}$

$\begin{array}{llll}4.717563 & -0.696533 & 4.201462\end{array}$

$\begin{array}{lll}6.070904 & -0.903429 & 4.464192\end{array}$

$\begin{array}{llll}6.393517 & -1.315328 & 5.412587\end{array}$

$\begin{array}{lll}7.004693 & -0.561553 & 3.487270\end{array}$

$\begin{array}{lll}6.600574 & -0.022045 & 2.264890\end{array}$

$\begin{array}{lll}7.354721 & 0.246625 & 1.534152\end{array}$

$\begin{array}{llll}2.265836 & 0.483744 & 0.155578\end{array}$

$\begin{array}{llll}8.474385 & -0.791064 & 3.709951\end{array}$

$\begin{array}{lll}3.683360 & -1.087610 & 5.221211\end{array}$

$\begin{array}{llll}7.883518 & -2.717386 & -1.833746\end{array}$

$\begin{array}{llll}5.390718 & 0.556677 & -4.706772\end{array}$

$\begin{array}{llll}4.047828 & 0.492159 & -4.901366\end{array}$

$\begin{array}{llll}5.734316 & 1.859659 & -4.833942\end{array}$

$\begin{array}{llll}5.971932 & -0.114224 & -5.722184\end{array}$

$\begin{array}{llll}7.721660 & -3.639634 & -2.811148\end{array}$

$\begin{array}{llll}7.771724 & -3.353908 & -0.649078\end{array}$ 
$\begin{array}{llll}9.164003 & -2.279787 & -1.926973\end{array}$

$\begin{array}{llll}8.949713 & -1.760071 & 2.890219\end{array}$

$\begin{array}{llll}8.755462 & -1.163051 & 4.975781\end{array}$

$\begin{array}{llll}9.197035 & 0.323201 & 3.444089\end{array}$

$\begin{array}{llll}4.190944 & -1.130048 & 6.472755\end{array}$

$\begin{array}{lll}2.643628 & -0.218332 & 5.237849\end{array}$

$\begin{array}{llll}3.161052 & -2.312526 & 4.969276\end{array}$

$\begin{array}{llll}3.054481 & -2.293972 & -0.577314\end{array}$

$\begin{array}{llll}1.890357 & -2.312607 & 1.466400\end{array}$

$\begin{array}{lll}3.436620 & -3.612470 & -0.449214\end{array}$

$\begin{array}{llll}3.364723 & -1.712909 & -1.436701\end{array}$

$\begin{array}{llll}2.265910 & -3.688554 & 1.618045\end{array}$

$3.034749 \quad-4.339229 \quad 0.681229$

$\begin{array}{llll}1.936784 & -4.220641 & 2.505485\end{array}$

$\begin{array}{lll}1.101949 & -1.724924 & 2.490903\end{array}$

$\begin{array}{lll}0.775514 & -0.361951 & 2.666078\end{array}$

$\begin{array}{llll}1.203012 & 0.595960 & 1.931030\end{array}$

$\begin{array}{llll}2.307657 & -1.643993 & 0.338535\end{array}$

$\begin{array}{lll}0.840303 & -2.409315 & 3.284688\end{array}$

$\begin{array}{lll}-0.060181 & 0.007867 & 3.721310\end{array}$

$\begin{array}{lll}0.016631 & 1.382433 & 4.206788\end{array}$

$\begin{array}{llll}0.808574 & 1.499770 & 4.961756\end{array}$

$\begin{array}{lll}-0.937216 & 1.662243 & 4.662268\end{array}$

$\begin{array}{lll}0.228879 & 2.055396 & 3.378719\end{array}$

$\begin{array}{lll}-0.481847 & -0.965722 & 4.718075\end{array}$

$\begin{array}{llll}-0.923356 & -1.842361 & 4.238478\end{array}$

$\begin{array}{lll}-1.246341 & -0.511408 & 5.352752\end{array}$

$\begin{array}{lll}0.347400 & -1.296312 & 5.359400\end{array}$

$\begin{array}{llll}-3.122743 & -2.219695 & -0.659717\end{array}$

$\begin{array}{lll}-4.579835 & -0.667152 & 0.132497\end{array}$

$\begin{array}{lll}-4.409199 & 0.945899 & -0.163624\end{array}$

$\begin{array}{llll}-6.116859 & -0.917317 & -0.43326\end{array}$

$\begin{array}{llll}-4.518265 & -0.655107 & 1.797901\end{array}$

$\begin{array}{llll}-4.786179 & 1.510886 & -1.376929\end{array}$

$\begin{array}{llll}-3.735662 & 1.998201 & -2.179845\end{array}$

$\begin{array}{llll}-2.726795 & 1.937629 & -1.792276\end{array}$

$\begin{array}{llll}-4.019760 & 2.545521 & -3.405325\end{array}$

$\begin{array}{llll}-3.222104 & 2.939613 & -4.028676\end{array}$

$\begin{array}{llll}-5.357091 & 2.579305 & -3.884774\end{array}$

$\begin{array}{llll}-5.653607 & 3.060010 & -5.189019\end{array}$

$\begin{array}{llll}-4.835547 & 3.425607 & -5.804295\end{array}$

$\begin{array}{llll}-6.942895 & 3.049324 & -5.670739\end{array}$

$\begin{array}{llll}-7.157809 & 3.412330 & -6.671431\end{array}$

$\begin{array}{llll}-7.990599 & 2.546818 & -4.862994\end{array}$

$\begin{array}{llll}-9.003294 & 2.515498 & -5.254243\end{array}$

$\begin{array}{lll}-7.739786 & 2.090551 & -3.586981\end{array}$

$\begin{array}{lll}-8.555524 & 1.700829 & -2.990318\end{array}$

$\begin{array}{lll}-6.423856 & 2.107029 & -3.045747\end{array}$

$\begin{array}{llll}-6.123881 & 1.624653 & -1.719986\end{array}$

$\begin{array}{lll}-7.190046 & 1.216407 & -0.763125\end{array}$

$\begin{array}{llll}-8.265441 & 2.101426 & -0.392347\end{array}$

$\begin{array}{llll}-8.293452 & 3.473709 & -0.768515\end{array}$

$\begin{array}{llll}-7.479231 & 3.879853 & -1.356782\end{array}$

$\begin{array}{lll}-9.328495 & 4.296652 & -0.380578\end{array}$

$\begin{array}{llll}-9.320267 & 5.342961 & -0.671995\end{array}$

$\begin{array}{lll}-10.397553 & 3.795625 & 0.400000\end{array}$

$\begin{array}{llll}-11.208766 & 4.455139 & 0.693796\end{array}$

$\begin{array}{lll}-10.395830 & 2.476943 & 0.795057\end{array}$

$\begin{array}{lll}-11.200336 & 2.081601 & 1.409526\end{array}$

$\begin{array}{llll}-9.335212 & 1.604962 & 0.426541\end{array}$

$\begin{array}{lll}-9.297212 & 0.264478 & 0.893955\end{array}$

$\begin{array}{lll}-10.112888 & -0.103657 & 1.509046\end{array}$

$\begin{array}{lll}-8.216882 & -0.536556 & 0.624006\end{array}$

$\begin{array}{llll}-8.142556 & -1.546401 & 1.011566\end{array}$

$\begin{array}{llll}-7.168674 & -0.041218 & -0.179208\end{array}$

$\begin{array}{lll}-3.825442 & -1.829133 & 2.384951\end{array}$

$\begin{array}{llll}-2.875587 & -1.483442 & 2.802190\end{array}$

$\begin{array}{lll}-4.627977 & -2.485685 & 3.495034\end{array}$

$\begin{array}{lll}-4.006550 & -2.851905 & 4.692943\end{array}$

$\begin{array}{lll}-2.953214 & -2.630889 & 4.840060\end{array}$

$\begin{array}{lll}4.735714 & -3.479263 & 5.708085\end{array}$

$\begin{array}{llll}-4.243060 & -3.749640 & 6.637972\end{array}$

$\begin{array}{llll}-6.094014 & -3.748050 & 5.529868\end{array}$

$\begin{array}{lll}-6.664121 & -4.227536 & 6.320478\end{array}$

$\begin{array}{llll}-6.718139 & -3.394901 & 4.328231\end{array}$ $\begin{array}{llll}-7.776339 & -3.595459 & 4.185269\end{array}$

$\begin{array}{lll}-5.988800 & -2.771283 & 3.318342\end{array}$

$\begin{array}{lll}-6.478947 & -2.470549 & 2.397260\end{array}$

$\begin{array}{lll}-3.523042 & -2.897884 & 1.310358\end{array}$

$\begin{array}{lll}-4.387614 & -3.562980 & 1.230568\end{array}$

$\begin{array}{llll}-2.695117 & -3.505450 & 1.681346\end{array}$

$\begin{array}{lll}-4.649906 & 0.608023 & 2.611493\end{array}$

$\begin{array}{lll}-4.754342 & 1.399465 & 1.873607\end{array}$

$\begin{array}{lll}-5.894017 & 0.697453 & 3.491989\end{array}$

$\begin{array}{lll}-6.977439 & 1.458505 & 3.035615\end{array}$

$\begin{array}{llll}-6.929101 & 1.917611 & 2.052910\end{array}$

$\begin{array}{lll}-8.119574 & 1.636251 & 3.819329\end{array}$

$\begin{array}{lll}-8.944895 & 2.229440 & 3.436009\end{array}$

$\begin{array}{lll}-8.194840 & 1.049921 & 5.083412\end{array}$

$\begin{array}{lll}-9.081843 & 1.181881 & 5.697103\end{array}$

$\begin{array}{lll}-7.115748 & 0.297901 & 5.557384\end{array}$

$\begin{array}{lll}-7.159818 & -0.160548 & 6.541466\end{array}$

$\begin{array}{lll}-5.973019 & 0.133310 & 4.774107\end{array}$

$\begin{array}{lll}-5.148104 & -0.449209 & 5.165816\end{array}$

$\begin{array}{lll}-3.361255 & 0.901420 & 3.387078\end{array}$

$\begin{array}{lll}-2.490416 & 0.859372 & 2.728535\end{array}$

$\begin{array}{lll}-3.197698 & 0.207140 & 4.213734\end{array}$

$\begin{array}{lll}-3.430358 & 1.906857 & 3.811935\end{array}$

$\begin{array}{llll}-1.582023 & -0.850621 & 0.114874\end{array}$

$\begin{array}{llll}-1.586311 & 0.157056 & -0.285238\end{array}$

$\begin{array}{llll}-1.574240 & -0.896873 & 1.199461\end{array}$

$\begin{array}{llll}-0.843294 & -1.822430 & -0.623220\end{array}$

$-0.436948-1.521823-1.582666$

$\begin{array}{lll}-0.785133 & -3.164254 & -0.244897\end{array}$

$\begin{array}{lll}-0.997962 & -3.408868 & 0.789445\end{array}$

$\begin{array}{llll}-4.908649 & -3.420802 & -1.388926\end{array}$

$\begin{array}{llll}-5.570197 & -3.488475 & -0.530145\end{array}$

$\begin{array}{llll}-3.792889 & -4.274889 & -1.379420\end{array}$

$\begin{array}{lll}-3.680619 & -4.924942 & -0.512310\end{array}$

$\begin{array}{llll}-3.069573 & -4.781222 & -2.610473\end{array}$

$\begin{array}{llll}-3.637280 & -5.631734 & -3.015610\end{array}$

$\begin{array}{lll}-2.106062 & -5.183820 & -2.297305\end{array}$

$\begin{array}{lll}-2.840764 & -3.739355 & -3.727718\end{array}$

$\begin{array}{llll}-3.694119 & -3.725529 & -4.411492\end{array}$

$\begin{array}{lll}-1.974251 & -4.054525 & -4.317065\end{array}$

$\begin{array}{lll}-2.586163 & -2.329852 & -3.237612\end{array}$

$\begin{array}{llll}-1.555360 & -1.999517 & -3.237596\end{array}$

$\begin{array}{llll}-3.549857 & -1.377522 & -3.064611\end{array}$

$\begin{array}{llll}-3.227652 & -0.344819 & -2.971759\end{array}$

$\begin{array}{lll}-5.029194 & -1.608227 & -3.278136\end{array}$

$\begin{array}{lll}-5.569212 & -0.757970 & -2.864418\end{array}$

$\begin{array}{llll}-5.230048 & -1.603737 & -4.358847\end{array}$

$\begin{array}{llll}-5.593748 & -2.911252 & -2.659105\end{array}$

$\begin{array}{llll}-5.573832 & -3.717894 & -3.398889\end{array}$

$-6.646451 \quad-2.738240-2.423088$

$-0.155034-4.238022-1.013849$

$\begin{array}{llll}0.424175 & -4.042547 & -2.282306\end{array}$

$-0.137690 \quad-5.532669-0.461715$

$0.982738-5.111072-2.977921$

$\begin{array}{llll}0.445686 & -3.054057 & -2.725684\end{array}$

$0.421499-6.601661-1.159717$

$\begin{array}{lll}-0.569174 & -5.692527 & 0.523089\end{array}$

$0.980118-6.395363-2.424211$

$1.432995-4.941369-3.951556$

$\begin{array}{llll}0.422764 & -7.594001 & -0.717992\end{array}$

$1.417393 \quad-7.225969-2.970434$

$\begin{array}{llll}3.311472 & -5.380007 & 0.810723\end{array}$

$4.540285-4.439700-1.834570$

$[8-9]^{\ddagger} \mathbf{P 1}-\mathrm{C1}$ exo si-si

Number of imaginary frequencies $=1$

The smallest frequencies are $=-121.05288 .839511 .1131 \mathrm{~cm}(-1)$

Electronic energy $=-7222.6230214$

Zero-point correction=

(Hartree/Particle)

Thermal correction to Energy= $\quad 1.874307$

Thermal correction to Enthalpy= $\quad 1.875251$

Thermal correction to Gibbs Free Energy $=\quad 1.593526$

Sum of electronic and zero-point Energies $=\quad-7220.867221$ 
Sum of electronic and thermal Energies=

Sum of electronic and thermal Enthalpies=

Sum of electronic and thermal Free Energies=

$-7220.748715$

$-7220.747770$

$-7221.029495$

Cartesian Coordinates

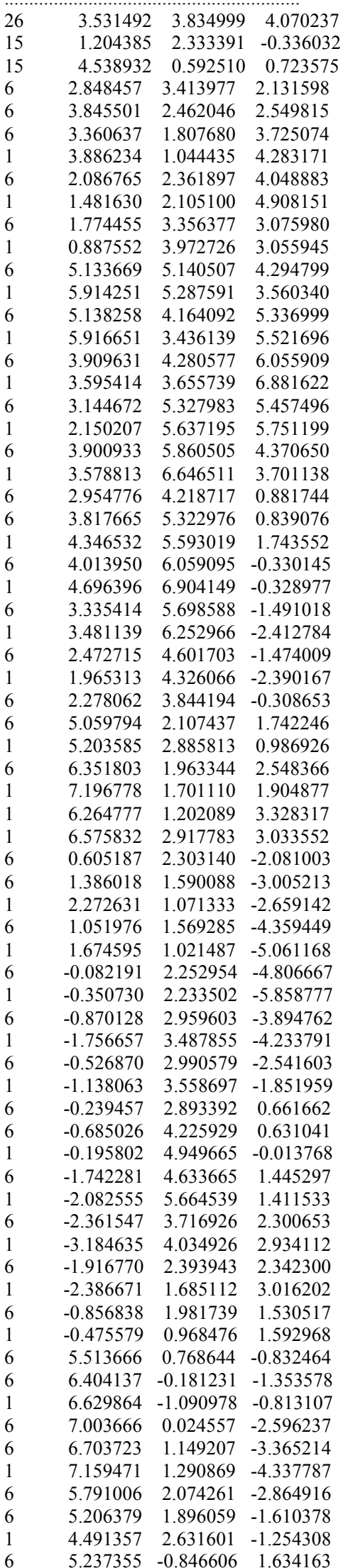

$\begin{array}{lll}4.308727 & -1.642738 & 2.317280\end{array}$

$\begin{array}{llll}3.257596 & -1.386138 & 2.277964\end{array}$

$\begin{array}{llll}4.731340 & -2.749286 & 3.054128\end{array}$

$\begin{array}{llll}6.081189 & -3.092212 & 3.114813\end{array}$

$\begin{array}{llll}6.407036 & -3.958480 & 3.678356\end{array}$

$\begin{array}{lll}7.007874 & -2.289976 & 2.449923\end{array}$

$\begin{array}{lll}6.598048 & -1.168927 & 1.725869\end{array}$

$\begin{array}{lll}7.348350 & -0.552485 & 1.245676\end{array}$

$\begin{array}{llll}2.244787 & 0.393514 & 0.262345\end{array}$

$\begin{array}{lll}8.470759 & -2.642510 & 2.455539\end{array}$

$\begin{array}{lll}3.704757 & -3.612675 & 3.733489\end{array}$

$\begin{array}{llll}8.034122 & -0.946624 & -3.107855\end{array}$

$\begin{array}{llll}5.386769 & 3.278450 & -3.670971\end{array}$

$\begin{array}{llll}4.044630 & 3.300098 & -3.878062\end{array}$

$\begin{array}{llll}5.696535 & 4.432646 & -3.035494\end{array}$

$\begin{array}{llll}5.977311 & 3.315014 & -4.882614\end{array}$

$\begin{array}{llll}7.911559 & -1.155065 & -4.439618\end{array}$

$\begin{array}{llll}7.960667 & -2.147468 & -2.496628\end{array}$

$\begin{array}{llll}9.290684 & -0.478206 & -2.906294\end{array}$

$\begin{array}{llll}8.844594 & -3.202721 & 1.279392\end{array}$

$\begin{array}{llll}8.786995 & -3.518004 & 3.432138\end{array}$

$\begin{array}{llll}9.246728 & -1.546404 & 2.623675\end{array}$

$\begin{array}{llll}4.211668 & -4.267394 & 4.799269\end{array}$

$\begin{array}{llll}2.647114 & -2.885702 & 4.170163\end{array}$

$\begin{array}{lll}3.204795 & -4.556725 & 2.895740\end{array}$

$\begin{array}{llll}3.205686 & -1.527663 & -1.867108\end{array}$

$\begin{array}{llll}2.205759 & -2.725324 & -0.123677\end{array}$

$\begin{array}{llll}3.792158 & -2.665837 & -2.388565\end{array}$

$\begin{array}{llll}3.364641 & -0.565015 & -2.335352\end{array}$

$\begin{array}{lll}2.847115 & -3.906713 & -0.599066\end{array}$

$\begin{array}{llll}3.637822 & -3.889236 & -1.729161\end{array}$

$\begin{array}{llll}2.688845 & -4.838406 & -0.066331\end{array}$

$\begin{array}{llll}1.275955 & -2.865293 & 0.951660\end{array}$

$\begin{array}{llll}0.804221 & -1.831906 & 1.820954\end{array}$

$\begin{array}{lll}1.124408 & -0.606321 & 1.713349\end{array}$

$\begin{array}{llll}2.436952 & -1.534497 & -0.761305\end{array}$

$\begin{array}{rrr}1.175508 & -3.880124 & 1.304127\end{array}$

$\begin{array}{lll}-0.050102 & -2.165798 & 2.857597\end{array}$

$\begin{array}{llll}-0.148977 & -1.226058 & 3.974838\end{array}$

$\begin{array}{lll}0.729309 & -1.294560 & 4.631928\end{array}$

$\begin{array}{lll}-1.040713 & -1.459052 & 4.559198\end{array}$

$\begin{array}{lll}-0.222208 & -0.205120 & 3.606026\end{array}$

$\begin{array}{lll}-0.354158 & -3.556379 & 3.181326\end{array}$

$\begin{array}{lll}-0.651562 & -4.109343 & 2.288345\end{array}$

$\begin{array}{lll}-1.191659 & -3.577505 & 3.881993\end{array}$ $\begin{array}{lll}0.497986 & -4.071292 & 3.643495\end{array}$

$\begin{array}{llll}-3.072523 & -1.482067 & -1.762259\end{array}$

$\begin{array}{llll}-4.593017 & -0.536388 & -0.363487\end{array}$

$\begin{array}{lll}-4.502152 & 0.999769 & 0.242329\end{array}$

$\begin{array}{lll}-6.085709 & -0.476398 & -1.100183\end{array}$

$\begin{array}{lll}-4.654660 & -1.387404 & 1.081199\end{array}$

$\begin{array}{rrr}-4.845274 & 2.106467 & -0.525428\end{array}$

$\begin{array}{llll}-3.785910 & 2.969572 & -0.873613\end{array}$

$\begin{array}{lll}-2.794503 & 2.733561 & -0.508281\end{array}$

$\begin{array}{llll}-4.039569 & 4.075601 & -1.644550\end{array}$

$\begin{array}{llll}-3.236204 & 4.757703 & -1.908940\end{array}$

$\begin{array}{llll}-5.351045 & 4.329203 & -2.129627\end{array}$

$\begin{array}{llll}-5.610642 & 5.423522 & -2.998638\end{array}$

$\begin{array}{lll}-4.785727 & 6.079637 & -3.264306 \\ -6.871656 & 5.641488 & -3.505446\end{array}$

$\begin{array}{lll}-6.871656 & 5.641488 & -3.505446\end{array}$

$\begin{array}{llll}-7.057620 & 6.477431 & -4.173366\end{array}$

$\begin{array}{llll}-7.926892 & 4.761656 & -3.166128\end{array}$

$\begin{array}{llll}-8.915711 & 4.920313 & -3.586694\end{array}$

$\begin{array}{llll}-7.712510 & 3.701110 & -2.312342\end{array}$

$\begin{array}{lll}-8.532002 & 3.034112 & -2.072887\end{array}$

$\begin{array}{lll}-6.427784 & 3.456833 & -1.751125\end{array}$

$\begin{array}{llll}-6.167418 & 2.353462 & -0.860006\end{array}$

$\begin{array}{llll}-7.255938 & 1.482953 & -0.334198\end{array}$

$\begin{array}{lll}-8.391157 & 2.018356 & 0.373801\end{array}$

$\begin{array}{lll}-8.476676 & 3.382885 & 0.769780\end{array}$

$\begin{array}{llll}-7.659562 & 4.055471 & 0.537305\end{array}$

$\begin{array}{lll}-9.571448 & 3.856670 & 1.459746\end{array}$

$\begin{array}{lll}-9.606527 & 4.900177 & 1.759344\end{array}$

$\begin{array}{lll}-10.646507 & 2.997111 & 1.789393\end{array}$

$\begin{array}{lll}-11.505319 & 3.386489 & 2.328146\end{array}$ 
$\begin{array}{lll}-10.589955 & 1.667174 & 1.438501\end{array}$

$\begin{array}{lll}-11.399041 & 0.990254 & 1.700093\end{array}$

$\begin{array}{lll}-9.467514 & 1.141786 & 0.741739\end{array}$

$\begin{array}{llll}-9.378166 & -0.243845 & 0.441834\end{array}$

$\begin{array}{lll}-10.199994 & -0.897634 & 0.718558\end{array}$

$\begin{array}{llll}-8.247662 & -0.758718 & -0.140257\end{array}$

$\begin{array}{llll}-8.136842 & -1.818987 & -0.338912\end{array}$

$\begin{array}{llll}-7.192065 & 0.106991 & -0.496913\end{array}$

$\begin{array}{lll}-4.014210 & -2.721951 & 0.966406\end{array}$

$\begin{array}{lll}-3.021473 & -2.664961 & 1.427028\end{array}$

$\begin{array}{lll}-4.800652 & -3.818560 & 1.659715\end{array}$

$\begin{array}{lll}-4.146928 & -4.726092 & 2.499890\end{array}$

$\begin{array}{llll}-3.081727 & -4.611201 & 2.685892\end{array}$

$\begin{array}{lll}-4.854585 & -5.764551 & 3.113160\end{array}$

$\begin{array}{lll}-4.336556 & -6.457548 & 3.770637\end{array}$

$\begin{array}{lll}-6.225486 & -5.903995 & 2.887547\end{array}$

$\begin{array}{llll}-6.779531 & -6.704869 & 3.368982\end{array}$

$\begin{array}{lll}-6.883107 & -5.004124 & 2.041237\end{array}$

$\begin{array}{lll}-7.951489 & -5.101013 & 1.868851\end{array}$

$\begin{array}{lll}-6.174653 & -3.971254 & 1.431252\end{array}$

$\begin{array}{lll}-6.689752 & -3.251649 & 0.802408\end{array}$

$\begin{array}{llll}-3.835335 & -3.078667 & -0.516376\end{array}$

$\begin{array}{llll}-4.805388 & -3.389817 & -0.920576\end{array}$

$\begin{array}{lll}-3.184986 & -3.952822 & -0.588457\end{array}$

$\begin{array}{lll}-4.764888 & -0.740720 & 2.433597\end{array}$

$\begin{array}{lll}-4.836852 & 0.325708 & 2.229592\end{array}$

$\begin{array}{lll}-6.031192 & -1.100313 & 3.208690\end{array}$

$\begin{array}{lll}-7.163389 & -0.290813 & 3.050790\end{array}$

$\begin{array}{llll}-7.124545 & 0.549550 & 2.364963\end{array}$

$\begin{array}{lll}-8.339950 & -0.547086 & 3.757392\end{array}$

$\begin{array}{lll}-9.203007 & 0.096005 & 3.611079\end{array}$

$\begin{array}{llll}-8.399285 & -1.621806 & 4.646368\end{array}$

$\begin{array}{lll}-9.312147 & -1.826509 & 5.199242\end{array}$

$\begin{array}{lll}-7.272696 & -2.429608 & 4.824113\end{array}$

$\begin{array}{lll}-7.306109 & -3.269182 & 5.513109\end{array}$

$\begin{array}{lll}-6.098908 & -2.166854 & 4.116501\end{array}$

$\begin{array}{llll}-5.241756 & -2.813580 & 4.262570\end{array}$

$\begin{array}{lll}-3.482950 & -0.948661 & 3.247645\end{array}$

$\begin{array}{llll}-2.610891 & -0.627871 & 2.673601\end{array}$

$\begin{array}{llll}-3.330173 & -1.989597 & 3.541560\end{array}$

$\begin{array}{lll}-3.537127 & -0.351248 & 4.162354\end{array}$

$\begin{array}{llll}-1.536525 & -0.659593 & -0.465404\end{array}$

$\begin{array}{llll}-1.264995 & 0.357011 & -0.735547\end{array}$

$\begin{array}{llll}-1.737925 & -0.779387 & 0.596011\end{array}$

$\begin{array}{llll}-0.825932 & -1.726175 & -1.120392\end{array}$

$\begin{array}{lll}-0.199617 & -1.507489 & -1.975864\end{array}$

$\begin{array}{lll}-0.769596 & -3.011739 & -0.564728\end{array}$

$\begin{array}{llll}-1.314767 & -3.158435 & 0.358684\end{array}$

$\begin{array}{llll}-4.587814 & -2.077999 & -3.304566\end{array}$

$\begin{array}{llll}-5.414749 & -2.541833 & -2.77358\end{array}$

$\begin{array}{llll}-3.430371 & -2.870847 & -3.480559\end{array}$

$\begin{array}{lll}-3.464148 & -3.887291 & -3.089254\end{array}$

$\begin{array}{llll}-2.387237 & -2.664396 & -4.566415\end{array}$

$\begin{array}{llll}-2.744592 & -3.098951 & -5.512725\end{array}$

$\begin{array}{llll}-1.502962 & -3.241964 & -4.281952\end{array}$

$\begin{array}{llll}-1.977873 & -1.191168 & -4.782645\end{array}$

$\begin{array}{llll}-2.605200 & -0.728456 & -5.550859\end{array}$

$-0.954460-1.159146-5.170183$

$\begin{array}{llll}-2.035127 & -0.337858 & -3.519244\end{array}$

$\begin{array}{lll}-1.076260 & -0.002497 & -3.137606\end{array}$

$\begin{array}{llll}-3.140361 & 0.406229 & -3.124232\end{array}$

$\begin{array}{llll}-2.945417 & 1.258788 & -2.483072\end{array}$

$\begin{array}{lll}-4.473617 & 0.441221 & -3.841827\end{array}$

$\begin{array}{llll}-5.198079 & 0.919992 & -3.180503\end{array}$

$\begin{array}{llll}-4.382228 & 1.088835 & -4.726344\end{array}$

$\begin{array}{lll}-5.003883 & -0.950466 & -4.250491\end{array}$

$\begin{array}{llll}-4.679375 & -1.198846 & -5.265901\end{array}$

$\begin{array}{lll}-6.096777 & -0.911165 & -4.282039\end{array}$

$-0.363186-4.228522-1.248620$

$\begin{array}{llll}0.274984 & -4.226665 & -2.504901\end{array}$

$-0.632705-5.468420-0.633835$

$\begin{array}{llll}0.608046 & -5.424998 & -3.132050\end{array}$

$0.512988-3.286786-2.990956$

$\begin{array}{llll}-0.292959 & -6.665230 & -1.259127\end{array}$

$\begin{array}{lll}-1.115247 & -5.481073 & 0.340406\end{array}$

$\begin{array}{lll}0.325687 & -6.647292 & -2.514108\end{array}$

$\begin{array}{llll}1.096809 & -5.405318 & -4.101853\end{array}$

$-0.511619-7.611107-0.772140$

$\begin{array}{llll}0.589276 & -7.579420 & -3.005276\end{array}$

$\begin{array}{llll}4.104807 & -4.796132 & -2.097680\end{array}$

$35 \quad 4.823869-2.546664-4.044691$

9 P1-C1 exo si-si

Number of imaginary frequencies $=0$

The smallest frequencies are $=8.7113 \quad 13.9586 \quad 16.0075 \mathrm{~cm}(-1)$ Electronic energy $=-7222.6529593$

Zero-point correction $=$

1.759678

(Hartree/Particle)

Thermal correction to Energy= $\quad 1.877518$

Thermal correction to Enthalpy= $\quad 1.878462$

Thermal correction to Gibbs Free Energy $=\quad \begin{aligned} & 1.600172 \\ & \text { Sum of }\end{aligned}$

Sum of electronic and zero-point Energies $=\quad-7220.893282$

Sum of electronic and thermal Energies $=\quad-7220.775441$

Sum of electronic and thermal Enthalpies $=\quad-7220.774497$

Sum of electronic and thermal Free Energies $=\quad-7221.052787$

Cartesian Coordinates

\begin{tabular}{|c|c|c|c|}
\hline U & 3.611430 & 3.253681 & 4.433987 \\
\hline & 1.188826 & 2.471207 & -0.065753 \\
\hline 15 & 4.504369 & 0.442604 & 0.665531 \\
\hline 6 & 2.902062 & 3.139132 & 2.468253 \\
\hline 6 & 3.844826 & 2.083074 & 2.738077 \\
\hline 0 & 3.328948 & 1.301665 & 3.819378 \\
\hline 1 & 3.814498 & 0.442414 & 4.262317 \\
\hline 6 & 2.089015 & 1.875094 & 4.230893 \\
\hline 1 & 1.475564 & 1.537013 & 5.055329 \\
\hline 6 & 1.828863 & 3.009498 & 3.406969 \\
\hline 1 & 0.979090 & 3.672562 & 3.480910 \\
\hline 6 & 5.283996 & 4.428628 & 4.817483 \\
\hline 1 & 6.073975 & 4.621311 & 4.103995 \\
\hline 6 & 5.228095 & 3.331965 & 5.730614 \\
\hline 1 & 5.967700 & 2.548575 & 5.828997 \\
\hline 6 & 4.004308 & 3.427693 & 6.460317 \\
\hline 1 & 3.650507 & 2.724689 & 7.202991 \\
\hline 6 & 3.303049 & 4.582722 & 5.997942 \\
\hline 1 & 2.326135 & 4.909218 & 6.329416 \\
\hline 6 & 4.093525 & 5.201721 & 4.984090 \\
\hline 1 & 3.820197 & 6.082594 & 4.419214 \\
\hline 6 & 3.033531 & 4.104152 & 1.342432 \\
\hline 6 & 3.946390 & 5.164341 & 1.427487 \\
\hline 1 & 4.517134 & 5.278813 & 2.340155 \\
\hline 6 & 4.133344 & 6.055606 & 0.369610 \\
\hline 1 & 4.852846 & 6.863714 & 0.464781 \\
\hline 6 & 3.397623 & 5.899243 & -0.802266 \\
\hline 1 & 3.533861 & 6.581123 & -1.635643 \\
\hline 6 & 2.491091 & 4.844078 & -0.913971 \\
\hline 1 & 1.942027 & 4.722230 & -1.839591 \\
\hline 6 & 2.306987 & 3.932448 & 0.136282 \\
\hline 6 & 5.047322 & 1.792160 & 1.888296 \\
\hline 1 & 5.224946 & 2.660026 & 1.245287 \\
\hline 6 & 6.329173 & 1.493347 & 2.667489 \\
\hline 1 & 7.167914 & 1.295592 & 1.993767 \\
\hline 1 & 6.211799 & 0.635570 & 3.335023 \\
\hline 1 & 6.581180 & 2.363571 & 3.279296 \\
\hline 6 & 0.626107 & 2.620122 & -1.810236 \\
\hline 6 & 1.431328 & 2.003800 & -2.782718 \\
\hline 1 & 2.313145 & 1.452124 & -2.471160 \\
\hline 6 & 1.123007 & 2.117846 & -4.137648 \\
\hline 1 & 1.760296 & 1.644942 & -4.879275 \\
\hline 6 & -0.007919 & 2.836992 & -4.535260 \\
\hline 1 & -0.258309 & 2.918166 & -5.588869 \\
\hline 6 & -0.819932 & 3.444046 & -3.574532 \\
\hline 1 & -1.705823 & 3.994324 & -3.877864 \\
\hline 6 & -0.503583 & 3.342904 & -2.218127 \\
\hline 1 & -1.137993 & 3.826935 & -1.485381 \\
\hline 6 & -0.247263 & 2.939769 & 0.981672 \\
\hline 6 & -0.675845 & 4.273584 & 1.088775 \\
\hline & -0.181497 & 5.053571 & 0.517758 \\
\hline
\end{tabular}




$$
\begin{aligned}
& \begin{array}{lll}
-1.723966 & 4.607611 & 1.947332
\end{array} \\
& \begin{array}{lll}
-2.052880 & 5.640115 & 2.019927
\end{array} \\
& \begin{array}{lll}
-2.346786 & 3.616389 & 2.712133
\end{array} \\
& \begin{array}{llll}
-3.161427 & 3.878176 & 3.381028
\end{array} \\
& \begin{array}{lll}
-1.917878 & 2.290678 & 2.617521
\end{array} \\
& \begin{array}{lll}
-2.392686 & 1.522075 & 3.218437
\end{array} \\
& \begin{array}{lll}
-0.870582 & 1.952622 & 1.757874
\end{array} \\
& \begin{array}{lll}
-0.509928 & 0.932538 & 1.704739
\end{array} \\
& \begin{array}{llll}
5.481700 & 0.842180 & -0.845900
\end{array} \\
& \begin{array}{llll}
6.460994 & 0.036413 & -1.442592
\end{array} \\
& 6.737813-0.918409-1.017361 \\
& \begin{array}{llll}
7.088467 & 0.451313 & -2.616939
\end{array} \\
& \begin{array}{llll}
6.736920 & 1.648615 & -3.239933
\end{array} \\
& \begin{array}{llll}
7.222203 & 1.956251 & -4.158894
\end{array} \\
& \begin{array}{llll}
5.737906 & 2.432085 & -2.667532
\end{array} \\
& \begin{array}{llll}
5.117733 & 2.040567 & -1.480166
\end{array} \\
& \begin{array}{llll}
4.337823 & 2.666564 & -1.056295
\end{array} \\
& \begin{array}{llll}
5.158692 & -1.122290 & 1.378799
\end{array} \\
& \begin{array}{llll}
4.204157 & -1.949806 & 1.982323
\end{array} \\
& \begin{array}{lll}
3.163670 & -1.653356 & 1.975384
\end{array} \\
& \begin{array}{lll}
4.584541 & -3.132879 & 2.617605
\end{array} \\
& \begin{array}{llll}
5.918785 & -3.531625 & 2.640175
\end{array} \\
& \begin{array}{lll}
6.214141 & -4.454226 & 3.125387
\end{array} \\
& \begin{array}{llll}
6.872958 & -2.703380 & 2.048006
\end{array} \\
& \begin{array}{llll}
6.507385 & -1.501617 & 1.440673
\end{array} \\
& \begin{array}{lll}
7.285701 & -0.864616 & 1.040506
\end{array} \\
& \begin{array}{lll}
2.215412 & 0.468549 & 0.287701
\end{array} \\
& \begin{array}{llll}
8.322485 & -3.109171 & 2.017690
\end{array} \\
& \begin{array}{llll}
3.515495 & -3.983371 & 3.244858
\end{array} \\
& \begin{array}{llll}
8.180286 & -0.377646 & -3.240373
\end{array} \\
& \begin{array}{llll}
5.308527 & 3.722160 & -3.314355
\end{array} \\
& \begin{array}{llll}
3.960313 & 3.780461 & -3.448979
\end{array} \\
& \begin{array}{llll}
5.671073 & 4.796997 & -2.575570
\end{array} \\
& \begin{array}{llll}
5.841004 & 3.883100 & -4.542773
\end{array} \\
& 7.923418-0.638401-4.545591 \\
& \begin{array}{llll}
8.354089 & -1.561710 & -2.618104
\end{array} \\
& \begin{array}{llll}
9.366954 & 0.272705 & -3.205831
\end{array} \\
& \begin{array}{llll}
8.659703 & -3.634165 & 0.814752
\end{array} \\
& \begin{array}{llll}
8.615355 & -4.035760 & 2.953016
\end{array} \\
& \begin{array}{llll}
9.139351 & -2.050261 & 2.220340
\end{array} \\
& \begin{array}{llll}
4.011707 & -4.891904 & 4.103946
\end{array} \\
& \begin{array}{llll}
2.613645 & -3.228160 & 3.920635
\end{array} \\
& \begin{array}{llll}
2.801590 & -4.669718 & 2.310289
\end{array} \\
& \begin{array}{llll}
3.176540 & -1.457683 & -1.989493
\end{array} \\
& 2.001531 \quad-2.716098 \quad-0.437508 \\
& \begin{array}{llll}
3.918320 & -2.566090 & -2.381129
\end{array} \\
& \begin{array}{llll}
3.338235 & -0.488375 & -2.445470
\end{array} \\
& \begin{array}{llll}
2.778476 & -3.844600 & -0.738246
\end{array} \\
& \begin{array}{llll}
3.754797 & -3.780676 & -1.725693
\end{array} \\
& \begin{array}{llll}
2.597718 & -4.779231 & -0.221322
\end{array} \\
& \begin{array}{lll}
0.783463 & -2.908867 & 0.436956
\end{array} \\
& \begin{array}{lll}
0.624631 & -1.933467 & 1.596302
\end{array} \\
& \begin{array}{lll}
1.114398 & -0.781988 & 1.584785
\end{array} \\
& \begin{array}{llll}
2.240722 & -1.526781 & -1.028086
\end{array} \\
& \begin{array}{lll}
0.889876 & -3.902855 & 0.870711
\end{array} \\
& \begin{array}{lll}
-0.084697 & -2.340619 & 2.674480
\end{array} \\
& \begin{array}{lll}
-0.137752 & -1.444440 & 3.834895
\end{array} \\
& \begin{array}{lll}
0.770466 & -1.545234 & 4.439642
\end{array} \\
& \begin{array}{lll}
-1.005069 & -1.704763 & 4.441987
\end{array} \\
& \begin{array}{lll}
-0.224942 & -0.410613 & 3.507287
\end{array} \\
& \begin{array}{lll}
-0.477951 & -3.733445 & 2.917065
\end{array} \\
& \begin{array}{lll}
-0.911177 & -4.193022 & 2.029218
\end{array} \\
& \begin{array}{lll}
-1.237472 & -3.743863 & 3.699720
\end{array} \\
& \begin{array}{llll}
0.377117 & -4.329366 & 3.251563
\end{array} \\
& \begin{array}{llll}
-2.956652 & -1.233969 & -1.857037
\end{array} \\
& \begin{array}{lll}
-4.569201 & -0.418380 & -0.443435
\end{array} \\
& \begin{array}{lll}
-4.564976 & 1.046118 & 0.354383
\end{array} \\
& \begin{array}{lll}
-6.046114 & -0.297710 & -1.232367
\end{array} \\
& \begin{array}{lll}
-4.689256 & -1.423133 & 0.905061
\end{array} \\
& \begin{array}{llll}
-4.890501 & 2.217564 & -0.311048
\end{array} \\
& \begin{array}{llll}
-3.836008 & 3.131445 & -0.524289
\end{array} \\
& \begin{array}{llll}
-2.851621 & 2.872399 & -0.155039
\end{array} \\
& \begin{array}{llll}
-4.082307 & 4.313516 & -1.175610
\end{array} \\
& \begin{array}{llll}
-3.282448 & 5.032870 & -1.328075
\end{array} \\
& \begin{array}{llll}
-5.380133 & 4.601714 & -1.678526
\end{array}
\end{aligned}
$$

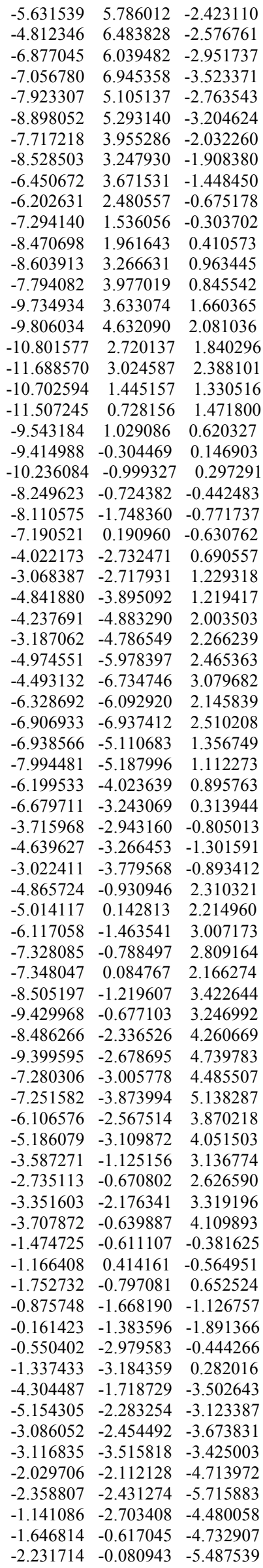




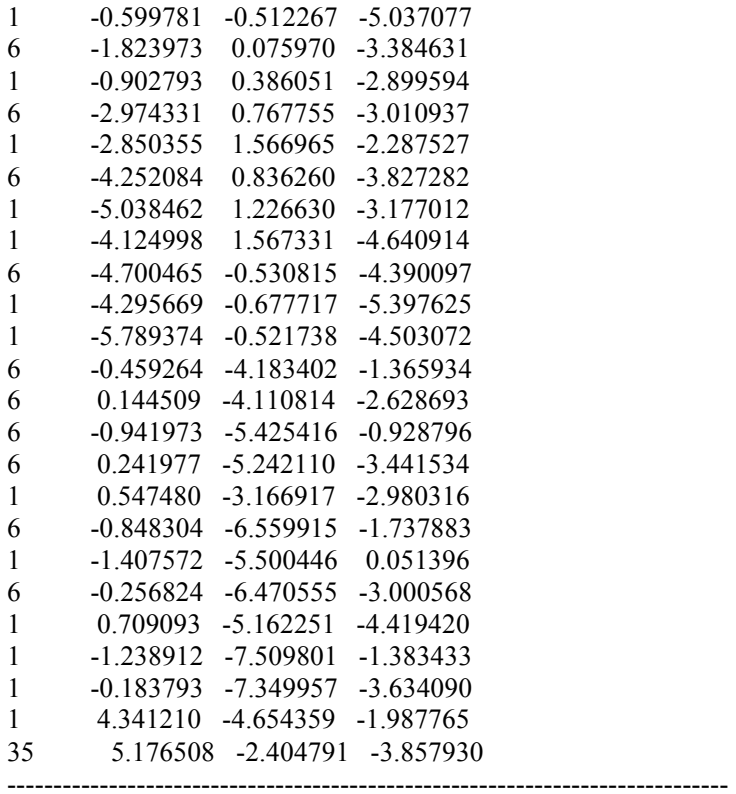

8 P1-C1 exo re-si

Number of imaginary frequencies $=0$

The smallest frequencies are=12.7618 $14.895915 .3289 \mathrm{~cm}(-1)$ Electronic energy $=-7222.6420137$

Zero-point correction $=$

1.756579

(Hartree/Particle)

Thermal correction to Energy $=\quad 1.875873$

Thermal correction to Enthalpy= $\quad 1.876817$

Thermal correction to Gibbs Free Energy $=\quad 1.594748$

Sum of electronic and zero-point Energies $=\quad-7220.885435$

Sum of electronic and thermal Energies $=\quad-7220.766141$

Sum of electronic and thermal Enthalpies $=\quad-7220.765197$

Sum of electronic and thermal Free Energies $=\quad-7221.047266$

Cartesian Coordinates

$\begin{array}{lccc}26 & 3.853418 & 5.372567 & 0.852098 \\ 15 & 1.398131 & 1.457485 & -1.626879 \\ 15 & 4.427099 & 0.662786 & 0.401833 \\ 6 & 3.190009 & 3.755324 & -0.306676 \\ 6 & 3.935204 & 3.303941 & 0.844421 \\ 6 & 3.260966 & 3.778266 & 2.011538 \\ 1 & 3.575576 & 3.618977 & 3.033482 \\ 6 & 2.126389 & 4.539519 & 1.596367 \\ 1 & 1.436775 & 5.056365 & 2.248873 \\ 6 & 2.082918 & 4.526674 & 0.172000 \\ 1 & 1.354200 & 5.027948 & -0.447785 \\ 6 & 5.729581 & 6.188412 & 0.497437 \\ 1 & 6.580485 & 5.633929 & 0.123935 \\ 6 & 5.369196 & 6.346420 & 1.870954 \\ 1 & 5.891225 & 5.923004 & 2.718640 \\ 6 & 4.166259 & 7.114461 & 1.926389 \\ 1 & 3.616617 & 7.370232 & 2.822706 \\ 6 & 3.783192 & 7.432602 & 0.587724 \\ 1 & 2.897127 & 7.978980 & 0.292366 \\ 6 & 4.749021 & 6.863178 & -0.294533 \\ 1 & 4.721821 & 6.908780 & -1.374787 \\ 6 & 3.510462 & 3.392927 & -1.716261 \\ 6 & 4.629087 & 3.973867 & -2.333541 \\ 1 & 5.207494 & 4.693393 & -1.768087 \\ 6 & 5.012620 & 3.640316 & -3.631791 \\ 1 & 5.897410 & 4.091391 & -4.069518 \\ 6 & 4.260659 & 2.714423 & -4.350144 \\ 1 & 4.544190 & 2.438307 & -5.360543 \\ 6 & 3.148010 & 2.119117 & -3.756059 \\ 1 & 2.586364 & 1.390166 & -4.327535 \\ 6 & 2.762323 & 2.426237 & -2.440490 \\ 6 & 5.128056 & 2.387356 & 0.784462 \\ 1 & 5.718107 & 2.667833 & -0.090156\end{array}$

$6.036320 \quad 2.457321 \quad 2.015318$

$6.901959 \quad 1.799247 \quad 1.900235$

$\begin{array}{lll}5.513907 & 2.167374 & 2.931192\end{array}$

$\begin{array}{lll}6.393680 & 3.484151 & 2.141629\end{array}$

$\begin{array}{lll}0.840703 & 0.351400 & -2.999411\end{array}$

$1.501340-0.880596-3.146143$

$2.245757-1.180621-2.416081$

$1.201262-1.720004-4.221689$

$1.740922-2.652475-4.340681$

$\begin{array}{lll}0.210351 & -1.361049 & -5.139578\end{array}$

$-0.031000 \quad-2.021800 \quad-5.967114$

$\begin{array}{lll}-0.469645 & -0.151063 & -4.986029\end{array}$

$\begin{array}{lll}-1.245556 & 0.134306 & -5.690461\end{array}$

$\begin{array}{lll}-0.144807 & 0.707171 & -3.933013\end{array}$

$\begin{array}{lll}-0.652460 & 1.660737 & -3.855027\end{array}$

$\begin{array}{lll}0.022882 & 2.669535 & -1.458995\end{array}$

$\begin{array}{llll}-0.187005 & 3.706747 & -2.382759\end{array}$

$0.485854 \quad 3.825213 \quad-3.227141$

$\begin{array}{llll}-1.248385 & 4.596747 & -2.211716\end{array}$

$\begin{array}{llll}-1.404804 & 5.393664 & -2.933114\end{array}$

$\begin{array}{llll}-2.106492 & 4.465654 & -1.114394\end{array}$

$\begin{array}{llll}-2.931858 & 5.159550 & -0.984405\end{array}$

$\begin{array}{llll}-1.898656 & 3.443184 & -0.186681\end{array}$

$\begin{array}{llll}-2.557817 & 3.335150 & 0.668514\end{array}$

$\begin{array}{llll}-0.837706 & 2.551815 & -0.358291\end{array}$

$\begin{array}{lll}-0.670478 & 1.766943 & 0.369936\end{array}$

$\begin{array}{llll}5.275565 & 0.063074 & -1.119878\end{array}$

$\begin{array}{llll}4.864734 & -1.196160 & -1.584729\end{array}$

$\begin{array}{llll}4.127287 & -1.751279 & -1.012593\end{array}$

$\begin{array}{llll}5.370473 & -1.709237 & -2.779557\end{array}$

$\begin{array}{llll}6.264745 & -0.969656 & -3.551135\end{array}$

$\begin{array}{llll}6.642848 & -1.361526 & -4.488329\end{array}$

$\begin{array}{llll}6.654150 & 0.291681 & -3.098370\end{array}$

$\begin{array}{llll}6.181085 & 0.802875 & -1.889777\end{array}$

$\begin{array}{llll}6.508956 & 1.785320 & -1.579040\end{array}$

$\begin{array}{llll}5.042131 & -0.469601 & 1.715587\end{array}$

$\begin{array}{lll}4.376432 & -0.396033 & 2.949400\end{array}$

$\begin{array}{lll}3.592255 & 0.336713 & 3.096065\end{array}$

$\begin{array}{llll}4.683864 & -1.288056 & 3.972074\end{array}$

$\begin{array}{llll}5.633473 & -2.293084 & 3.779851\end{array}$

$\begin{array}{llll}5.855313 & -3.003964 & 4.567749\end{array}$

$\begin{array}{lll}6.281874 & -2.374466 & 2.550309\end{array}$

$\begin{array}{llll}6.005249 & -1.464726 & 1.526011\end{array}$

$\begin{array}{llll}6.532868 & -1.556642 & 0.583949\end{array}$

$\begin{array}{lll}2.091232 & 0.344708 & 0.218255\end{array}$

$\begin{array}{lll}7.220296 & -3.516182 & 2.282441\end{array}$

$\begin{array}{llll}3.918456 & -1.252089 & 5.265274\end{array}$

$\begin{array}{llll}4.959146 & -3.093351 & -3.200873\end{array}$

$\begin{array}{llll}7.580395 & 1.100576 & -3.964369\end{array}$

$\begin{array}{llll}7.016924 & 1.381936 & -5.165976\end{array}$

$\begin{array}{llll}7.923372 & 2.280105 & -3.402003\end{array}$

$\begin{array}{llll}8.727807 & 0.432347 & -4.229634\end{array}$

$\begin{array}{llll}3.617167 & -3.287047 & -3.070835\end{array}$

$\begin{array}{llll}5.548048 & -4.044408 & -2.435898\end{array}$

$\begin{array}{llll}5.272652 & -3.361405 & -4.484349\end{array}$

$\begin{array}{llll}6.555336 & -4.586017 & 1.765466\end{array}$

$\begin{array}{llll}7.836283 & -3.949313 & 3.403089\end{array}$

$\begin{array}{llll}8.179139 & -3.190821 & 1.388241\end{array}$

$\begin{array}{llll}4.732738 & -1.406272 & 6.334820\end{array}$

$\begin{array}{lll}3.241674 & -0.096370 & 5.433163\end{array}$

$\begin{array}{llll}3.006453 & -2.258219 & 5.332959\end{array}$

$\begin{array}{lll}2.262119 & -2.480738 & 1.274115\end{array}$

$\begin{array}{lll}1.472450 & -2.147900 & 2.411011\end{array}$

$\begin{array}{llll}0.991437 & -0.884209 & 2.807089\end{array}$

$\begin{array}{llll}1.305139 & -2.930083 & 3.138836\end{array}$

$\begin{array}{lll}-2.852630 & -2.440422 & -0.622471\end{array}$

$\begin{array}{lll}-4.235835 & -0.727928 & -0.090766\end{array}$

$\begin{array}{llll}-4.028270 & 0.806435 & -0.654326\end{array}$

$\begin{array}{llll}-5.782404 & -1.032241 & -0.596138\end{array}$

$\begin{array}{lll}-4.159612 & -0.435619 & 1.550100\end{array}$

$\begin{array}{llll}-4.427344 & 1.195876 & -1.927992\end{array}$

$\begin{array}{llll}-3.386715 & 1.544212 & -2.812804\end{array}$

$\begin{array}{llll}-2.372184 & 1.516687 & -2.437627\end{array}$

$\begin{array}{llll}-3.686565 & 1.912638 & -4.099078\end{array}$

$\begin{array}{lll}-2.895135 & 2.194436 & -4.787616\end{array}$ 


\begin{tabular}{|c|c|c|c|c|c|c|}
\hline-5.033472 & 1.900631 & -4.553329 & 1 & -3.377178 & -4.469950 & -4.070037 \\
\hline-5.350035 & 2.191878 & -5.907766 & 1 & -1.666156 & -4.791471 & -3.864574 \\
\hline-4.539138 & 2.452898 & -6.582772 & 6 & -2.300391 & -2.923612 & -3.064779 \\
\hline-6.648451 & 2.129880 & -6.360525 & 1 & -1.272567 & -2.589655 & -3.128792 \\
\hline-6.878310 & 2.348417 & -7.399193 & 6 & -3.269750 & -1.955585 & -3.062633 \\
\hline-7.685406 & 1.761882 & -5.470823 & 1 & -2.945103 & -0.922839 & -3.145601 \\
\hline-8.705646 & 1.686914 & -5.835825 & 6 & -4.743177 & -2.210009 & -3.288798 \\
\hline-7.414733 & 1.491163 & -4.146720 & 1 & -5.288147 & -1.304536 & -3.025790 \\
\hline-8.222298 & 1.201708 & -3.485213 & 1 & -4.904608 & -2.366893 & -4.364730 \\
\hline-6.088823 & 1.568461 & -3.636440 & 6 & -5.336221 & -3.402405 & -2.498853 \\
\hline-5.770097 & 1.281693 & -2.258770 & 1 & -5.306857 & -4.309474 & -3.110866 \\
\hline-6.825829 & 1.043586 & -1.234860 & 1 & -6.392254 & -3.190444 & -2.313575 \\
\hline-7.881282 & 1.994028 & -0.989151 & 6 & 0.170420 & -4.609388 & -0.583210 \\
\hline-7.891154 & 3.296108 & -1.563538 & 6 & 0.712739 & -4.625952 & -1.884119 \\
\hline-7.075015 & 3.598252 & -2.209174 & 6 & 0.277007 & -5.773590 & 0.201308 \\
\hline-8.910262 & 4.183995 & -1.295142 & 6 & 1.338337 & -5.766944 & -2.377554 \\
\hline-8.887993 & 5.176003 & -1.736965 & 1 & 0.656494 & -3.741333 & -2.506901 \\
\hline-9.980867 & 3.821092 & -0.443433 & 6 & 0.900746 & -6.917297 & -0.295853 \\
\hline-10.779721 & 4.529886 & -0.246537 & 1 & -0.129982 & -5.771666 & 1.209256 \\
\hline-9.995232 & 2.576351 & 0.144141 & 6 & 1.432135 & -6.919080 & -1.588766 \\
\hline-10.800495 & 2.289278 & 0.815229 & 1 & 1.763975 & -5.755585 & -3.376825 \\
\hline-8.950328 & 1.642793 & -0.096523 & 1 & 0.974410 & -7.805140 & 0.325474 \\
\hline-8.927178 & 0.387549 & 0.567809 & 1 & 1.921008 & -7.807751 & -1.977057 \\
\hline-9.741715 & 0.129810 & 1.238015 & 8 & 2.364090 & -1.765481 & 0.216017 \\
\hline-7.861075 & -0.461975 & 0.413503 & 6 & 0.321254 & -0.784866 & 4.077767 \\
\hline-7.792524 & -1.400828 & 0.952106 & 6 & -0.037493 & 0.427210 & 4.613966 \\
\hline-6.816836 & -0.110958 & -0.467165 & 1 & 0.145801 & -1.695506 & 4.641624 \\
\hline-3.460205 & -1.504167 & 2.309144 & 6 & 0.240996 & 1.582816 & 3.862831 \\
\hline-2.470585 & -1.130850 & 2.585170 & 6 & 0.811648 & 1.462614 & 2.615206 \\
\hline-4.183524 & -1.887802 & 3.586081 & 1 & 0.996970 & 2.333881 & 2.001437 \\
\hline-3.469374 & -1.998191 & 4.783904 & 7 & 1.175266 & 0.276513 & 2.080541 \\
\hline-2.405440 & -1.772827 & 4.793522 & 6 & 3.590441 & -4.184142 & 0.093044 \\
\hline-4.119210 & -2.371579 & 5.964207 & 1 & 3.136705 & -3.731161 & -0.782077 \\
\hline-3.556981 & -2.443866 & 6.891202 & 1 & 4.668896 & -3.984591 & 0.085951 \\
\hline-5.489582 & -2.640025 & 5.952441 & 1 & 3.441877 & -5.266872 & 0.033920 \\
\hline-5.998659 & -2.921088 & 6.870059 & 6 & 3.112161 & -4.493898 & 2.488948 \\
\hline-6.205950 & -2.541706 & 4.754366 & 1 & 3.140010 & -3.879831 & 3.389661 \\
\hline-7.273763 & -2.741920 & 4.741443 & 1 & 2.307195 & -5.236951 & 2.589288 \\
\hline-5.555832 & -2.170450 & 3.579203 & 1 & 4.064171 & -5.027556 & 2.423737 \\
\hline-6.115828 & -2.063047 & 2.654202 & 7 & 2.956788 & -3.672178 & 1.297902 \\
\hline-3.277078 & -2.761592 & 1.438243 & 1 & -0.516614 & 0.487740 & 5.585458 \\
\hline-4.200457 & -3.349817 & 1.476331 & 35 & -0.232046 & 3.356751 & 4.555772 \\
\hline
\end{tabular}

$[8-9]^{\ddagger}-\mathbf{P 1}-\mathrm{C1}$ exo re-si

Number of imaginary frequencies $=1$

The smallest frequencies are $=-148.650313 .376616 .1508 \mathrm{~cm}(-1)$ Electronic energy $=-7222.6391298$

Zero-point correction=

1.757111

(Hartree/Particle)

Thermal correction to Energy= $\quad 1.874980$

Thermal correction to Enthalpy= $\quad 1.875924$

Thermal correction to Gibbs Free Energy $=\quad 1.598871$

Sum of electronic and zero-point Energies $=\quad-7220.882019$

Sum of electronic and thermal Energies $=\quad-7220.764150$

Sum of electronic and thermal Enthalpies $=\quad-7220.763206$

Sum of electronic and thermal Free Energies $=\quad-7221.040258$

Cartesian Coordinates

$\begin{array}{lll}2.659961 & 0.779520 & 3.670276\end{array}$

$\begin{array}{lll}-2.912685 & 2.401942 & 3.036608\end{array}$

$\begin{array}{lll}-1.235960 & -1.065855 & -0.073046\end{array}$

$\begin{array}{llll}-1.161585 & -0.146373 & -0.643158\end{array}$

$\begin{array}{lll}-1.224566 & -0.912449 & 1.000628\end{array}$

$-0.526996-2.183122-0.611266$

$\begin{array}{llll}-0.091912 & -2.063222 & -1.593851\end{array}$

$-0.483070 \quad-3.434999-0.009506$

$\begin{array}{lll}-0.739848 & -3.509540 & 1.039724\end{array}$

$-4.677283-3.714920-1.155793$

$\begin{array}{llll}-5.335302 & -3.611987 & -0.298172\end{array}$

$\begin{array}{llll}-3.586949 & -4.581296 & -0.998290\end{array}$

$\begin{array}{lll}-3.485799 & -5.074125 & -0.032064\end{array}$

$\begin{array}{llll}-2.829582 & -5.264784 & -2.115575\end{array}$

$-3.386438-6.164150-2.416970$

$\begin{array}{llll}-1.879960 & -5.615892 & -1.708945\end{array}$

$\begin{array}{llll}-2.550005 & -4.391730 & -3.358467\end{array}$

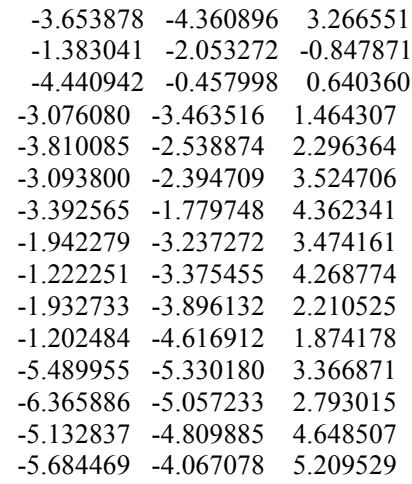


$\begin{array}{lll}-3.895822 & -5.406903 & 5.038699\end{array}$

$\begin{array}{lll}-3.344346 & -5.192082 & 5.944569\end{array}$

$\begin{array}{lll}-3.487569 & -6.296659 & 3.998840\end{array}$

$\begin{array}{llll}-2.573913 & -6.875998 & 3.979624\end{array}$

$\begin{array}{lll}-4.471753 & -6.250432 & 2.966942\end{array}$

$\begin{array}{lll}-4.433242 & -6.792561 & 2.031889\end{array}$

$\begin{array}{llll}-3.440056 & -3.817858 & 0.062447\end{array}$

$\begin{array}{lll}-4.561156 & -4.629448 & -0.165449\end{array}$

$\begin{array}{llll}-5.108841 & -4.991610 & 0.694856\end{array}$

$\begin{array}{llll}-4.985213 & -4.958175 & -1.452369\end{array}$

$\begin{array}{llll}-5.872661 & -5.566750 & -1.593171\end{array}$

$\begin{array}{llll}-4.274075 & -4.480977 & -2.550553\end{array}$

$\begin{array}{lll}-4.592571 & -4.720773 & -3.559921\end{array}$

$\begin{array}{llll}-3.159602 & -3.666068 & -2.350471\end{array}$

$\begin{array}{llll}-2.628614 & -3.288814 & -3.216688\end{array}$

$\begin{array}{llll}-2.733161 & -3.309407 & -1.060950\end{array}$

$\begin{array}{llll}-5.038292 & -1.781562 & 1.865779\end{array}$

$\begin{array}{lll}-5.661370 & -2.462054 & 1.282888\end{array}$

$\begin{array}{llll}-5.878306 & -1.243277 & 3.027763\end{array}$

$\begin{array}{lll}-6.779410 & -0.743895 & 2.661544\end{array}$

$\begin{array}{lll}-5.323838 & -0.527809 & 3.640975\end{array}$

$\begin{array}{lll}-6.179398 & -2.075281 & 3.671907\end{array}$

$\begin{array}{llll}-0.864089 & -1.690596 & -2.582618\end{array}$

$\begin{array}{llll}-1.483406 & -0.606509 & -3.229072\end{array}$

$\begin{array}{llll}-2.176634 & 0.022329 & -2.681572\end{array}$

$-1.200716-0.328170 \quad-4.568233$

$\begin{array}{llll}-1.697918 & 0.499879 & -5.061661\end{array}$

$\begin{array}{lll}-0.275729 & -1.107235 & -5.268024\end{array}$

$\begin{array}{llll}-0.049570 & -0.882841 & -6.306451\end{array}$

$\begin{array}{llll}0.363588 & -2.169856 & -4.625129\end{array}$

$\begin{array}{llll}1.091876 & -2.773494 & -5.159163\end{array}$

$\begin{array}{llll}0.064780 & -2.467144 & -3.294009\end{array}$

$\begin{array}{lll}0.553828 & -3.310859 & -2.820458\end{array}$

$\begin{array}{llll}0.016253 & -3.013115 & -0.145669\end{array}$

$\begin{array}{llll}0.245190 & -4.360945 & -0.468981\end{array}$

$\begin{array}{llll}-0.419639 & -4.876051 & -1.156135\end{array}$

$\begin{array}{llll}1.314236 & -5.048680 & 0.106484\end{array}$

$\begin{array}{llll}1.487035 & -6.089744 & -0.150811\end{array}$

$\begin{array}{llll}2.158394 & -4.401156 & 1.014813\end{array}$

$\begin{array}{llll}2.988992 & -4.938639 & 1.462875\end{array}$

$\begin{array}{lll}1.932651 & -3.063520 & 1.344879\end{array}$

$\begin{array}{llll}2.584725 & -2.558209 & 2.048636\end{array}$

$\begin{array}{lll}0.865870 & -2.372660 & 0.766780\end{array}$

$\begin{array}{llll}0.684208 & -1.336242 & 1.026700\end{array}$

$\begin{array}{llll}-5.348557 & -0.750464 & -0.935305\end{array}$

$\begin{array}{llll}-4.990358 & 0.084664 & -2.003972\end{array}$

$\begin{array}{llll}-4.245637 & 0.858108 & -1.843578\end{array}$

$\begin{array}{lll}-5.573194 & -0.086379 & -3.259797\end{array}$

$\begin{array}{lll}-6.489833 & -1.110656 & -3.489010\end{array}$

$\begin{array}{llll}-6.927204 & -1.252196 & -4.470849\end{array}$

$\begin{array}{lll}-6.825690 & -1.954819 & -2.430503\end{array}$

$\begin{array}{llll}-6.278061 & -1.771907 & -1.160882\end{array}$

$\begin{array}{llll}-6.572408 & -2.448106 & -0.370422\end{array}$

$\begin{array}{lll}-5.098907 & 1.163491 & 1.212507\end{array}$

$\begin{array}{lll}-4.422534 & 1.765941 & 2.285526\end{array}$

$\begin{array}{lll}-3.619074 & 1.236811 & 2.783612\end{array}$

$\begin{array}{lll}-4.747878 & 3.057251 & 2.689119\end{array}$

$\begin{array}{lll}-5.730776 & 3.788890 & 2.019127\end{array}$

$\begin{array}{lll}-5.962413 & 4.805840 & 2.314677\end{array}$

$\begin{array}{llll}-6.396954 & 3.192313 & 0.952083\end{array}$

$\begin{array}{lll}-6.098298 & 1.886120 & 0.554477\end{array}$

$\begin{array}{llll}-6.636999 & 1.453258 & -0.279932\end{array}$

$\begin{array}{lll}-2.137676 & -0.227144 & 0.246064\end{array}$

$\begin{array}{lll}-7.370805 & 3.998126 & 0.139045\end{array}$

$\begin{array}{lll}-3.961186 & 3.727293 & 3.780690\end{array}$

$\begin{array}{llll}-5.231261 & 0.890555 & -4.349281\end{array}$

$\begin{array}{llll}-7.765291 & -3.098870 & -2.69829\end{array}$

$\begin{array}{llll}-7.223158 & -3.994689 & -3.56124\end{array}$

$\begin{array}{llll}-8.088002 & -3.779071 & -1.575784\end{array}$

$\begin{array}{llll}-8.920887 & -2.677514 & -3.262059\end{array}$

$\begin{array}{llll}-3.897042 & 1.146304 & -4.397955\end{array}$

$\begin{array}{llll}-5.835301 & 2.088491 & -4.148205\end{array}$

$\begin{array}{llll}-5.599281 & 0.458639 & -5.571338\end{array}$

$\begin{array}{llll}-6.743598 & 4.614021 & -0.902446\end{array}$

$\begin{array}{llll}-7.964825 & 4.970172 & 0.861773\end{array}$ $\begin{array}{llll}-8.344061 & 3.232398 & -0.399772\end{array}$

$\begin{array}{llll}-4.755117 & 4.437565 & 4.612130\end{array}$

$\begin{array}{lll}-3.266868 & 2.846538 & 4.531968\end{array}$

$\begin{array}{llll}-3.057379 & 4.607781 & 3.269836\end{array}$

$\begin{array}{llll}-2.379747 & 2.744898 & -0.332892\end{array}$

$\begin{array}{lll}-1.531176 & 3.067129 & 0.783648\end{array}$

$\begin{array}{lll}-1.065097 & 2.191917 & 1.808382\end{array}$

$\begin{array}{lll}-1.459548 & 4.110600 & 1.050365\end{array}$

$\begin{array}{llll}2.905887 & 1.854924 & -1.750349\end{array}$

$\begin{array}{llll}4.264555 & 0.521611 & -0.508946\end{array}$

$\begin{array}{llll}4.048181 & -1.105551 & -0.291991\end{array}$

$\begin{array}{llll}5.810405 & 0.537698 & -1.122666\end{array}$

$\begin{array}{lll}4.241061 & 1.015910 & 1.096226\end{array}$

$\begin{array}{llll}4.414845 & -2.026443 & -1.266261\end{array}$

$\begin{array}{llll}3.357614 & -2.719450 & -1.892951\end{array}$

$\begin{array}{llll}2.346609 & -2.516875 & -1.564736\end{array}$

$3.634409-3.628371 \quad-2.881731$

$\begin{array}{llll}2.829111 & -4.175107 & -3.364110\end{array}$

$\begin{array}{llll}4.973609 & -3.844230 & -3.306002\end{array}$

$\begin{array}{llll}5.267075 & -4.721531 & -4.384825\end{array}$

$\begin{array}{llll}4.444104 & -5.246734 & -4.862730\end{array}$

$\begin{array}{llll}6.559349 & -4.892488 & -4.827193\end{array}$

$\begin{array}{llll}6.771657 & -5.561106 & -5.656291\end{array}$

$\begin{array}{llll}7.613471 & -4.179048 & -4.208816\end{array}$

$\begin{array}{llll}8.628995 & -4.294095 & -4.576658\end{array}$

$\begin{array}{llll}7.364946 & -3.333745 & -3.148935\end{array}$

$\begin{array}{llll}8.184772 & -2.788799 & -2.696557\end{array}$

$\begin{array}{llll}6.045754 & -3.150595 & -2.647771\end{array}$

$\begin{array}{llll}5.750937 & -2.269599 & -1.544695\end{array}$

$\begin{array}{llll}6.824494 & -1.611695 & -0.747447\end{array}$

$\begin{array}{llll}7.869804 & -2.362657 & -0.099884\end{array}$

$\begin{array}{llll}7.864238 & -3.784322 & -0.029844\end{array}$

$\begin{array}{llll}7.044294 & -4.334534 & -0.476135\end{array}$

$\begin{array}{llll}8.874092 & -4.469350 & 0.610393\end{array}$

$\begin{array}{llll}8.840398 & -5.554046 & 0.657945\end{array}$

$\begin{array}{llll}9.950377 & -3.775156 & 1.212927\end{array}$

$\begin{array}{lll}10.741899 & -4.329089 & 1.708978\end{array}$

$\begin{array}{llll}9.979899 & -2.399287 & 1.179899\end{array}$

$\begin{array}{llll}10.790014 & -1.851290 & 1.653845\end{array}$

$\begin{array}{llll}8.945409 & -1.660735 & 0.542864\end{array}$

$\begin{array}{llll}8.939305 & -0.240547 & 0.571908\end{array}$

$\begin{array}{llll}9.758102 & 0.280591 & 1.059078\end{array}$

$\begin{array}{llll}7.885768 & 0.461921 & 0.044011\end{array}$

$\begin{array}{llll}7.832199 & 1.543864 & 0.098982\end{array}$

$\begin{array}{llll}6.834093 & -0.233933 & -0.588971\end{array}$

$\begin{array}{lll}3.595397 & 2.346732 & 1.256224\end{array}$

$\begin{array}{lll}2.561065 & 2.193140 & 1.583428\end{array}$

$\begin{array}{lll}4.280111 & 3.221683 & 2.288118\end{array}$

$\begin{array}{lll}3.517534 & 3.849185 & 3.279648\end{array}$

$\begin{array}{lll}2.445766 & 3.666620 & 3.322773\end{array}$

$\begin{array}{lll}4.124902 & 4.686046 & 4.220595\end{array}$

$\begin{array}{llll}3.523580 & 5.160346 & 4.991516\end{array}$

$\begin{array}{llll}5.503509 & 4.903876 & 4.173436\end{array}$

$\begin{array}{llll}5.979871 & 5.547828 & 4.907323\end{array}$

$\begin{array}{lll}6.269526 & 4.286485 & 3.178318\end{array}$

$\begin{array}{lll}7.343596 & 4.446322 & 3.142207\end{array}$

$\begin{array}{llll}5.661219 & 3.452650 & 2.242021\end{array}$

$\begin{array}{lll}6.259158 & 2.946896 & 1.489340\end{array}$

$\begin{array}{llll}3.568952 & 3.064996 & -0.098918\end{array}$

$\begin{array}{llll}4.583155 & 3.415181 & -0.324231\end{array}$

$\begin{array}{llll}2.955759 & 3.963572 & -0.007125\end{array}$

$\begin{array}{llll}4.242362 & 0.055995 & 2.254266\end{array}$

$\begin{array}{lll}4.338412 & -0.927545 & 1.799578\end{array}$

$\begin{array}{llll}5.435897 & 0.187799 & 3.198267\end{array}$

$\begin{array}{lll}6.562237 & -0.609627 & 2.958712\end{array}$

$\begin{array}{llll}6.570579 & -1.270787 & 2.098034\end{array}$

$\begin{array}{llll}7.670778 & -0.571786 & 3.806424\end{array}$

$\begin{array}{lll}8.530897 & -1.199586 & 3.591180\end{array}$

$\begin{array}{llll}7.665235 & 0.266332 & 4.922802\end{array}$

$\begin{array}{lll}8.524413 & 0.300652 & 5.587276\end{array}$

$\begin{array}{lll}6.540875 & 1.054335 & 5.184670\end{array}$

$\begin{array}{llll}6.522958 & 1.708948 & 6.051783\end{array}$

$\begin{array}{lll}5.435228 & 1.010666 & 4.33392\end{array}$

$\begin{array}{llll}4.579508 & 1.638130 & 4.553622\end{array}$

$\begin{array}{lll}2.899790 & 0.071323 & 2.987751\end{array}$ 


\begin{tabular}{lrrr}
1 & 2.077811 & -0.105543 & 2.290446 \\
1 & 2.706006 & 1.012686 & 3.503998 \\
1 & 2.888842 & -0.721838 & 3.738899 \\
6 & 1.251863 & 0.866989 & -0.741813 \\
1 & 0.992129 & -0.092264 & -1.180617 \\
1 & 1.365308 & 0.820317 & 0.337944 \\
6 & 0.601067 & 2.023844 & -1.296258 \\
1 & 0.035989 & 1.906934 & -2.211097 \\
6 & 0.505463 & 3.248135 & -0.615137 \\
1 & 1.006435 & 3.315970 & 0.340987 \\
6 & 4.578084 & 2.719314 & -2.955389 \\
1 & 5.358820 & 3.012501 & -2.258156 \\
6 & 3.458668 & 3.581200 & -3.043075 \\
1 & 3.473956 & 4.475664 & -2.421006 \\
6 & 2.527592 & 3.684904 & -4.239885 \\
1 & 2.981907 & 4.325833 & -5.011241 \\
1 & 1.624153 & 4.203800 & -3.905923 \\
6 & 2.126689 & 2.326047 & -4.854733 \\
1 & 2.828885 & 2.042634 & -5.644504 \\
1 & 1.151424 & 2.431961 & -5.341585 \\
6 & 2.031909 & 1.187735 & -3.844595 \\
1 & 1.032523 & 0.824520 & -3.636464 \\
6 & 3.067846 & 0.319797 & -3.538073 \\
1 & 2.790673 & -0.646160 & -3.130294 \\
6 & 4.474811 & 0.403844 & -4.091351 \\
1 & 5.111281 & -0.241447 & -3.483607 \\
1 & 4.481598 & -0.017097 & -5.107601 \\
6 & 5.066193 & 1.830497 & -4.101034 \\
1 & 4.849468 & 2.325622 & -5.052705 \\
1 & 6.155815 & 1.753923 & -4.039343 \\
6 & 0.134119 & 4.526725 & -1.213548 \\
6 & -0.533802 & 4.632475 & -2.449558 \\
6 & 0.453550 & 5.710821 & -0.519358 \\
6 & -0.865451 & 5.881353 & -2.969850 \\
1 & -0.805080 & 3.738493 & -2.999813 \\
6 & 0.128926 & 6.959357 & -1.045353 \\
1 & 0.960016 & 5.640217 & 0.440193 \\
6 & -0.535097 & 7.049079 & -2.272891 \\
1 & -1.384295 & 5.945725 & -3.921968 \\
1 & 0.389101 & 7.860946 & -0.498341 \\
1 & -0.794424 & 8.020392 & -2.683780 \\
8 & -2.412381 & 1.597784 & -0.884579 \\
6 & -0.414134 & 2.778216 & 2.936893 \\
6 & -0.020418 & 2.016017 & 4.015671 \\
1 & -0.270068 & 3.853312 & 2.956414 \\
6 & -0.262051 & 0.637234 & 3.959010 \\
6 & -0.830976 & 0.086812 & 2.827584 \\
1 & -0.994355 & -0.979419 & 2.743840 \\
7 & -1.221094 & 0.830633 & 1.771799 \\
6 & -3.774168 & 3.568413 & -2.175580 \\
1 & -3.305298 & 2.749982 & -2.715187 \\
1 & -4.843042 & 3.358191 & -2.062092 \\
1 & -3.666976 & 4.484989 & -2.764343 \\
6 & -3.344549 & 5.055493 & -0.261721 \\
1 & -3.300235 & 4.985033 & 0.824248 \\
1 & -2.615766 & 5.797709 & -0.609586 \\
7 & -4.347859 & 5.398417 & -0.527145 \\
1 & -3.126062 & 3.753475 & -0.883963 \\
35 & 0.450890 & 2.473348 & 4.878783 \\
-------------------------------------------------------------- \\
\hline
\end{tabular}

9 P1-C1 exo re-si

Number of imaginary frequencies $=0$

The smallest frequencies are=9.8610 $14.1888 \quad 19.4395 \mathrm{~cm}(-1)$ Electronic energy $=-7222.6660768$

Zero-point correction=

(Hartree/Particle)

Thermal correction to Energy=

Thermal correction to Enthalpy=

Thermal correction to Gibbs Free Energy=
Sum of electronic and zero-point Energies=

Sum of electronic and thermal Energies=

Sum of electronic and thermal Enthalpies=

.877368

1.878312

1.601625

$-7220.906218$

$-7220.788709$

Sum of electronic and thermal Free Energies=
Cartesian Coordinates

\begin{tabular}{|c|c|c|c|}
\hline 26 & -3.675495 & -4.160212 & 3.4787 \\
\hline 15 & -1.401574 & -2.160062 & -0.795818 \\
\hline 15 & -4.409120 & -0.373140 & 0.660570 \\
\hline 6 & -3.098860 & -3.375124 & 1.624613 \\
\hline 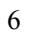 & -3.801072 & -2.389738 & 2.412879 \\
\hline 6 & -3.068807 & -2.198163 & 3.625788 \\
\hline 1 & -3.347462 & -1.535902 & 4.433687 \\
\hline 6 & -1.936951 & -3.069884 & 3.608267 \\
\hline 1 & -1.213015 & -3.186044 & 4.402755 \\
\hline 6 & -1.958013 & -3.795642 & 2.381548 \\
\hline 1 & -1.250110 & -4.552480 & 2.078221 \\
\hline 6 & -5.531870 & -5.078427 & 3.660635 \\
\hline 1 & -6.414108 & -4.812172 & 3.093704 \\
\hline 6 & -5.136781 & -4.509744 & 4.909975 \\
\hline 1 & -5.659561 & -3.729863 & 5.447580 \\
\hline 6 & -3.905871 & -5.117440 & 5.302366 \\
\hline 1 & -3.330536 & -4.875164 & 6.186215 \\
\hline 6 & -3.540274 & -6.063427 & 4.296974 \\
\hline 1 & -2.640180 & -6.663864 & 4.285550 \\
\hline 6 & -4.544380 & -6.040688 & 3.283973 \\
\hline 1 & -4.537450 & -6.624578 & 2.373589 \\
\hline 6 & -3.494838 & -3.8 & 0.251823 \\
\hline 6 & -4.653237 & -4.5 & 0.086659 \\
\hline 1 & -5.206836 & -4.8 & 0.972569 \\
\hline 6 & -5.105669 & -4.9 & -1.172494 \\
\hline 1 & -6.021074 & -5.5 & -1.265923 \\
\hline 6 & -4.383904 & -4.5 & -2.305112 \\
\hline 1 & -4.720479 & -4.8 & -3.293673 \\
\hline 6 & -3.233902 & -3.821346 & -2.168100 \\
\hline 1 & -2.695274 & -3.527739 & -3.061411 \\
\hline 6 & -2.7 & -3.3 & -0.9 \\
\hline 6 & -5.014165 & -1.629056 & 1.951943 \\
\hline 1 & -5.648357 & -2.323719 & 1.398571 \\
\hline 6 & -5.8 & -1.0 & 3.0 \\
\hline 1 & -6.737582 & -0.527 & 2.699271 \\
\hline 1 & -5.281260 & & 3.675055 \\
\hline 1 & -6.16 & -1.8 & 3.761982 \\
\hline 6 & -0.905238 & -1.921505 & -2.552419 \\
\hline 6 & -1.600277 & -0.9 & -3.288118 \\
\hline 1 & -2.329053 & -0.315123 & -2.791173 \\
\hline 6 & -1.355319 & -0.781874 & -4.652162 \\
\hline 1 & -1.91 & -0.0 & 2442 \\
\hline 6 & -0.387718 & -1.564175 & -5.288281 \\
\hline 1 & -0.187877 & -1.428400 & -6.347135 \\
\hline 6 & 0.33 & -2.5 & -4.555639 \\
\hline 1 & 1.09 & -3.1 & -5.040432 \\
\hline 6 & & & \\
\hline 1 & 0.627194 & -3.447283 & -2.647982 \\
\hline 6 & -0.014234 & -3.072251 & -0.014608 \\
\hline 6 & & & -0.2 \\
\hline 1 & -0.424373 & -4.990161 & -0.929031 \\
\hline 6 & 1.257206 & -5.094807 & 0.410365 \\
\hline 1 & 1.432842 & -6.150029 & 0.222494 \\
\hline 6 & 2.069339 & -4.398512 & 1.311844 \\
\hline 1 & 2.877317 & -4.912994 & 1.823504 \\
\hline 6 & 1.844187 & -3.041122 & 1.549476 \\
\hline 1 & 2.475517 & -2.494916 & 2.242173 \\
\hline 6 & 0.806440 & -2.381787 & 0.888729 \\
\hline 1 & 0.625842 & -1.329780 & 1.074691 \\
\hline 6 & -5.359605 & -0.709067 & -0.880348 \\
\hline 6 & -5.002021 & 0.062668 & -1.994089 \\
\hline 1 & -4.229018 & 0.815843 & -1.892631 \\
\hline 6 & -5.614229 & -0.148434 & -3.228930 \\
\hline 6 & -6.559920 & -1.158276 & -3.392280 \\
\hline 1 & -7.019749 & -1.335202 & -4.358141 \\
\hline 6 & -6.895281 & -1.943244 & -2.288186 \\
\hline 6 & -6.319279 & -1.715229 & -1.038332 \\
\hline 1 & -6.614731 & -2.344668 & -0.210704 \\
\hline 6 & -4.999368 & 1.285744 & 1.195453 \\
\hline 6 & -4.273489 & 1.903433 & 2.226576 \\
\hline 1 & -3.471918 & 1.367717 & 2.721527 \\
\hline & -4.561147 & 3.212068 & 2.605684 \\
\hline
\end{tabular}




\begin{tabular}{|c|c|c|c|c|c|c|}
\hline-5.556817 & 3.941455 & 1.953816 & 1 & 6.250296 & 3.002480 & 1.112822 \\
\hline-5.763802 & 4.967381 & 2.236418 & 6 & 3.469725 & 2.988874 & -0.396630 \\
\hline-6.273012 & 3.328209 & 0.928542 & 1 & 4.457212 & 3.364459 & -0.695186 \\
\hline-6.009961 & 2.008353 & 0.553953 & 1 & 2.821539 & 3.865478 & -0.351720 \\
\hline-6.585512 & 1.561565 & -0.247790 & 6 & 4.281850 & 0.241589 & 2.233521 \\
\hline-2.123634 & -0.264415 & 0.197414 & 1 & 4.433119 & -0.777839 & 1.884437 \\
\hline-7.268018 & 4.137118 & 0.142614 & 6 & 5.458599 & 0.532243 & 3.164204 \\
\hline-3.713676 & 3.892739 & 3.643373 & 6 & 6.643599 & -0.191746 & 2.982937 \\
\hline-5.261455 & 0.771596 & -4.364214 & 1 & 6.701734 & -0.919548 & 2.180333 \\
\hline-7.859712 & -3.081740 & -2.485684 & 6 & 7.747098 & 0.003777 & 3.815454 \\
\hline-7.337634 & -4.036605 & -3.295068 & 1 & 8.653992 & -0.570071 & 3.645843 \\
\hline-8.190096 & -3.687678 & -1.323713 & 6 & 7.677618 & 0.928742 & 4.858814 \\
\hline-9.008162 & -2.670773 & -3.069936 & 1 & 8.533176 & 1.087189 & 5.509763 \\
\hline-3.920671 & 0.993561 & -4.433863 & 6 & 6.494521 & 1.643914 & 5.064532 \\
\hline-5.832309 & 1.991917 & -4.205368 & 1 & 6.426689 & 2.365874 & 5.873819 \\
\hline-5.652613 & 0.297641 & -5.561464 & 6 & 5.394829 & 1.442233 & 4.229046 \\
\hline-6.649539 & 4.826338 & -0.856382 & 1 & 4.494484 & 2.020723 & 4.400095 \\
\hline-7.901376 & 5.050382 & 0.906908 & 6 & 2.933444 & 0.256226 & 2.962486 \\
\hline-8.207032 & 3.366432 & -0.444248 & 1 & 2.127843 & -0.026276 & 2.280231 \\
\hline-4.426061 & 4.745858 & 4.405790 & 1 & 2.688697 & 1.230940 & 3.388429 \\
\hline-3.103783 & 3.012835 & 4.466421 & 1 & 2.957326 & -0.464583 & 3.784216 \\
\hline-2.724713 & 4.628894 & 3.059635 & 6 & 1.152724 & 0.808443 & -0.683952 \\
\hline-2.243518 & 2.732333 & -0.578339 & 1 & 0.845571 & -0.165191 & -1.053855 \\
\hline-1.082697 & 3.093847 & 0.329604 & 1 & 1.331693 & 0.824107 & 0.386286 \\
\hline-0.855027 & 2.244747 & 1.562946 & 6 & 0.638318 & 1.977304 & -1.316450 \\
\hline-1.229686 & 4.100892 & 0.712219 & 1 & 0.004465 & 1.812600 & -2.180116 \\
\hline 2.787953 & 1.610609 & -1.888202 & 6 & 0.265896 & 3.217508 & -0.534558 \\
\hline 4.235345 & 0.413204 & -0.569212 & 1 & 1.018713 & 3.395046 & 0.233933 \\
\hline 4.107453 & -1.199728 & -0.158144 & 6 & 4.293133 & 2.408654 & -3.259966 \\
\hline 5.785760 & 0.404804 & -1.206764 & 1 & 5.108899 & 2.848280 & -2.688739 \\
\hline 4.246448 & 1.073998 & 0.985300 & 6 & 3.101098 & 3.195515 & -3.367824 \\
\hline 4.489652 & -2.195882 & -1.044835 & 1 & 3.113099 & 4.172998 & -2.884519 \\
\hline 3.453296 & -2.985527 & -1.590507 & 6 & 2.145071 & 3.119680 & -4.548447 \\
\hline 2.437112 & -2.785822 & -1.276732 & 1 & 2.576792 & 3.629477 & -5.424584 \\
\hline 3.754485 & -3.979538 & -2.486245 & 1 & 1.250225 & 3.683153 & -4.275618 \\
\hline 2.965412 & -4.599752 & -2.902433 & 6 & 1.734945 & 1.678630 & -4.924894 \\
\hline 5.097039 & -4.193701 & -2.900601 & 1 & 2.363169 & 1.300023 & -5.738049 \\
\hline 5.412680 & -5.165758 & -3.888147 & 1 & 0.711456 & 1.683270 & -5.315344 \\
\hline 4.604650 & -5.762621 & -4.303436 & 6 & 1.793646 & 0.694852 & -3.759246 \\
\hline 6.707275 & -5.339999 & -4.322459 & 1 & 0.835589 & 0.312388 & -3.424130 \\
\hline 6.936404 & -6.081793 & -5.081977 & 6 & 2.903289 & -0.089038 & -3.456734 \\
\hline 7.740770 & -4.534052 & -3.788684 & 1 & 2.713107 & -1.025236 & -2.943027 \\
\hline 8.757573 & -4.653104 & -4.151830 & 6 & 4.251048 & -0.005509 & -4.149634 \\
\hline 7.470518 & -3.593839 & -2.817807 & 1 & 4.971191 & -0.546597 & -3.531135 \\
\hline 8.275148 & -2.979666 & -2.431866 & 1 & 4.199671 & -0.538224 & -5.111652 \\
\hline 6.148814 & -3.402287 & -2.325444 & 6 & 4.756330 & 1.439140 & -4.354434 \\
\hline 5.830971 & -2.424062 & -1.315027 & 1 & 4.442374 & 1.817013 & -5.333966 \\
\hline 6.886449 & -1.645187 & -0.607792 & 1 & 5.850944 & 1.425965 & -4.376459 \\
\hline 7.966316 & -2.279570 & 0.104031 & 6 & 0.169077 & 4.475032 & -1.380824 \\
\hline 8.017660 & -3.683997 & 0.330115 & 6 & -0.509604 & 4.496754 & -2.608372 \\
\hline 7.215970 & -4.310070 & -0.043784 & 6 & 0.696567 & 5.678232 & -0.891463 \\
\hline 9.058409 & -4.255327 & 1.029818 & 6 & -0.657325 & 5.687367 & -3.322633 \\
\hline 9.067615 & -5.328599 & 1.197491 & 1 & -0.932808 & 3.583751 & -3.014223 \\
\hline 10.111269 & -3.458125 & 1.539381 & 6 & 0.561198 & 6.870100 & -1.607433 \\
\hline 10.927553 & -3.923327 & 2.084173 & 1 & 1.219179 & 5.680590 & 0.062386 \\
\hline 10.087137 & -2.094257 & 1.353126 & 6 & -0.120923 & 6.878870 & -2.826293 \\
\hline 10.879844 & -1.466538 & 1.752002 & 1 & -1.189259 & 5.682374 & -4.270062 \\
\hline 9.020392 & -1.470595 & 0.649875 & 1 & 0.985604 & 7.788685 & -1.211815 \\
\hline 8.961089 & -0.057404 & 0.515034 & 1 & -0.233878 & 7.803954 & -3.384347 \\
\hline 9.765436 & 0.545813 & 0.925853 & 8 & -2.341542 & 1.579577 & -1.060805 \\
\hline 7.877082 & 0.540811 & -0.075952 & 6 & -0.287117 & 2.898921 & 2.669441 \\
\hline 7.786117 & 1.619277 & -0.148124 & 6 & -0.006372 & 2.207103 & 3.838919 \\
\hline 6.841430 & -0.258278 & -0.608144 & 1 & -0.083471 & 3.962168 & 2.613800 \\
\hline 3.583102 & 2.404948 & 1.025739 & 6 & -0.305315 & 0.848799 & 3.859508 \\
\hline 2.566918 & 2.270162 & 1.415972 & 6 & -0.848582 & 0.238421 & 2.740941 \\
\hline 4.298574 & 3.393576 & 1.925729 & 1 & -1.077454 & -0.818942 & 2.734009 \\
\hline 3.569936 & 4.151681 & 2.848909 & 7 & -1.122022 & 0.924265 & 1.610348 \\
\hline 2.499260 & 3.984797 & 2.947956 & 6 & -3.938220 & 3.499643 & -2.138326 \\
\hline 4.207224 & 5.102735 & 3.651753 & 1 & -3.498994 & 2.744605 & -2.785972 \\
\hline 3.630907 & 5.678734 & 4.371019 & 1 & -4.942369 & 3.186710 & -1.843691 \\
\hline 5.583908 & 5.304651 & 3.534451 & 1 & -4.014693 & 4.438771 & -2.691179 \\
\hline 6.083809 & 6.037845 & 4.161205 & 6 & -3.161376 & 5.056926 & -0.381635 \\
\hline 6.317416 & 4.554756 & 2.607679 & 1 & -2.929855 & 5.038404 & 0.681154 \\
\hline 7.390309 & 4.701425 & 2.517934 & 1 & -2.493218 & 5.755182 & -0.896826 \\
\hline 5.678528 & 3.608421 & 1.809454 & 1 & -4.190256 & 5.406068 & -0.483662 \\
\hline
\end{tabular}


$\begin{array}{cccc}7 & -3.081420 & 3.716803 & -0.970433 \\ 1 & 0.424379 & 2.711207 & 4.696547\end{array}$

$\begin{array}{lllll}35 & 0.051938 & -0.216441 & 5.449740\end{array}$

\section{$[8-9]^{\ddagger}-\mathbf{P 1}-\mathrm{C} 1$ endo re-re conf2}

Number of imaginary frequencies $=1$

The smallest frequencies are $=-255.28709 .890213 .3757 \mathrm{~cm}(-1)$ Electronic energy $=-7222.6284099$

\begin{tabular}{|c|c|}
\hline $\begin{array}{l}\text { Zero-point correction= } \\
\text { (Hartree/Particle) }\end{array}$ & 400 \\
\hline Thermal correction to Energy= & 1.873024 \\
\hline Thermal correction to Enthalpy= & 1.873969 \\
\hline Thermal correction to Gibbs Free Energy= & 1.592776 \\
\hline ad zero-point Energies $=$ & -7220.8740 \\
\hline and thermal Energies $=$ & -7220.75538 \\
\hline II II) & -7220.75444 \\
\hline and thermal Free En & -7221.03563 \\
\hline
\end{tabular}

Sum of electronic and thermal Free Energies $=-7221.035634$

Cartesian Coordinates

\begin{tabular}{|c|c|c|c|}
\hline 26 & 4.734876 & 4.665648 & 1.790828 \\
\hline 15 & 2.778976 & 1.496143 & -1.852997 \\
\hline & 4.720415 & -0.025016 & 0.973603 \\
\hline & 4.311286 & 3.285680 & 0.276447 \\
\hline b & 4.471768 & 2.629714 & 1.554062 \\
\hline & 3.486268 & 3.159440 & 2.443433 \\
\hline & 3.357523 & 2.884734 & 3.481337 \\
\hline$b$ & 2.739763 & 4.155098 & 1.744204 \\
\hline & 1.952984 & 4.767889 & 2.161824 \\
\hline & 3.247655 & 4.235424 & 0.414901 \\
\hline & 2.908977 & 4.911343 & -0.355475 \\
\hline & 6.712328 & 5.103573 & 2.249086 \\
\hline & 7.534788 & 4.406082 & 2.160754 \\
\hline & 5.884480 & 5.281748 & 3.399768 \\
\hline & 5.965066 & 4.739122 & 4.332252 \\
\hline & 4.903276 & 6.271747 & 3.089069 \\
\hline & 4.107226 & 6.604164 & 3.742301 \\
\hline & 5.124386 & 6.706628 & 1.746834 \\
\hline & 4.527708 & 7.428255 & 1.204558 \\
\hline & 6.242283 & 5.987554 & 1.228673 \\
\hline 1 & 6.639690 & 6.077519 & 0.227101 \\
\hline & 5.073361 & 2.936457 & -0.957505 \\
\hline & 6.425866 & 3.293670 & -1.059945 \\
\hline & 6.869296 & 3.852986 & -0.245951 \\
\hline & 7.201259 & 2.932575 & -2.161579 \\
\hline & 8.252026 & 3.201832 & -2.196677 \\
\hline & 6.624981 & 2.202506 & -3.198459 \\
\hline & 7.218922 & 1.901502 & -4.055510 \\
\hline & 5.281961 & 1.832979 & -3.119432 \\
\hline & 4.849837 & 1.249479 & -3.924896 \\
\hline & 4.493837 & 2.177866 & -2.010336 \\
\hline & 5.440212 & 1.509746 & 1.827295 \\
\hline & 6.364481 & 1.738298 & 1.294295 \\
\hline & 5.781844 & 1.324266 & 3.308722 \\
\hline & 6.520698 & 0.529810 & 3.443456 \\
\hline & 4.904319 & 1.071833 & 3.910538 \\
\hline & 6.198353 & 2.257802 & 3.699520 \\
\hline & 2.495753 & 0.578051 & -3.427557 \\
\hline & 2.670048 & -0.815338 & -3.410763 \\
\hline & 2.964223 & -1.310811 & -2.493196 \\
\hline & 2.423256 & -1.573056 & -4.557882 \\
\hline & 2.562388 & -2.648807 & -4.530720 \\
\hline & 1.987530 & -0.950144 & -5.729121 \\
\hline & 1.780427 & -1.541180 & -6.616637 \\
\hline & 1.814441 & 0.436439 & -5.754474 \\
\hline & 1.474782 & 0.927147 & -6.662151 \\
\hline & 2.070009 & 1.198042 & -4.613388 \\
\hline & 1.917692 & 2.271150 & -4.648788 \\
\hline & 1.681455 & 2.957763 & -2.032188 \\
\hline & 2.044408 & 4.089534 & -2.780267 \\
\hline & 3.025720 & 4.142965 & -3.242472 \\
\hline & 1.152634 & 5.154235 & -2.922007 \\
\hline & 1.443336 & 6.027021 & -3.499819 \\
\hline & -0.112128 & 5.094536 & -2.327153 \\
\hline
\end{tabular}

$-0.811319 \quad 5.918001 \quad-2.441125$

$\begin{array}{llll}-0.477433 & 3.971542 & -1.582082\end{array}$

$\begin{array}{lll}-1.457338 & 3.918817 & -1.122687\end{array}$

$\begin{array}{llll}0.420052 & 2.913593 & -1.424861\end{array}$

$\begin{array}{lll}0.139551 & 2.050393 & -0.831819\end{array}$

$\begin{array}{llll}5.971003 & -0.589798 & -0.256482\end{array}$

$\begin{array}{llll}5.553791 & -1.626147 & -1.104339\end{array}$

$\begin{array}{lll}4.561945 & -2.045645 & -0.974622\end{array}$

$\begin{array}{llll}6.401443 & -2.111379 & -2.099470\end{array}$

$7.659829-1.547551-2.299872$

$8.308401-1.906805-3.091011$

$8.062994-0.500968-1.470279$

$7.242515 \quad-0.036803-0.441901$

$\begin{array}{llll}7.604516 & 0.766071 & 0.185457\end{array}$

$\begin{array}{lll}4.702065 & -1.400400 & 2.197776\end{array}$

$3.709644-1.360011 \quad 3.190328$

$\begin{array}{lll}3.061362 & -0.495234 & 3.278179\end{array}$

$3.521745 \quad-2.447015 \quad 4.041263$

$\begin{array}{lll}4.285451 & -3.606439 & 3.900939\end{array}$

$\begin{array}{lll}4.105483 & -4.466153 & 4.536604\end{array}$

$\begin{array}{llll}5.258688 & -3.650347 & 2.906114\end{array}$

$\begin{array}{llll}5.486404 & -2.552863 & 2.074913\end{array}$

$\begin{array}{lll}6.251931 & -2.624110 & 1.312639\end{array}$

$\begin{array}{llll}2.608439 & 0.155047 & -0.035119\end{array}$

$\begin{array}{lll}6.064355 & -4.907344 & 2.727315\end{array}$

$\begin{array}{llll}2.402325 & -2.444623 & 5.043561\end{array}$

$\begin{array}{llll}5.953960 & -3.292336 & -2.914854\end{array}$

$\begin{array}{llll}9.394121 & 0.146051 & -1.738017\end{array}$

$\begin{array}{llll}9.430555 & 0.703246 & -2.974403\end{array}$

$\begin{array}{llll}9.682281 & 1.126412 & -0.853913\end{array}$

$\begin{array}{llll}10.407182 & -0.749805 & -1.692741\end{array}$

$\begin{array}{llll}6.097249 & -4.451938 & -2.224925\end{array}$

$6.648349-3.424210-4.062660$

$\begin{array}{llll}4.639346 & -3.210586 & -3.245790\end{array}$

$\begin{array}{lll}7.051545 & -5.017031 & 3.644752\end{array}$

$\begin{array}{lll}6.646343 & -4.968888 & 1.507978\end{array}$

$\begin{array}{llll}5.289495 & -6.013049 & 2.856370\end{array}$

$\begin{array}{llll}2.771513 & -3.007454 & 6.214737\end{array}$

$\begin{array}{llll}1.947293 & -1.203105 & 5.312761\end{array}$

$\begin{array}{lll}1.338807 & -3.166055 & 4.590925\end{array}$

$2.009404 \quad-2.805056 \quad 0.520106$

$\begin{array}{lll}0.863432 & -2.457954 & 1.334344\end{array}$

$\begin{array}{llll}0.586916 & -1.161900 & 1.886880\end{array}$

$\begin{array}{lll}0.430912 & -3.259962 & 1.915220\end{array}$

$\begin{array}{llll}-3.322384 & -1.086574 & -1.263464\end{array}$

$\begin{array}{llll}-5.180379 & -0.221177 & -0.276298\end{array}$

$\begin{array}{llll}-5.130235 & 0.890185 & 0.963847\end{array}$

$\begin{array}{llll}-6.128182 & 0.604624 & -1.364921\end{array}$

$\begin{array}{lll}-6.046986 & -1.433860 & 0.500085\end{array}$

$\begin{array}{lll}-4.741202 & 2.196185 & 0.704787\end{array}$

$\begin{array}{lll}-3.487864 & 2.600235 & 1.216052\end{array}$

$\begin{array}{lll}-2.907060 & 1.885957 & 1.789243\end{array}$

$\begin{array}{lll}-3.039587 & 3.874474 & 0.972015\end{array}$

$\begin{array}{lll}-2.085309 & 4.205274 & 1.368787\end{array}$

$\begin{array}{lll}-3.795160 & 4.760783 & 0.156473\end{array}$

$\begin{array}{llll}-3.284483 & 6.038911 & -0.199631\end{array}$

$\begin{array}{llll}-2.316676 & 6.337410 & 0.194550\end{array}$

$\begin{array}{llll}-3.988888 & 6.871899 & -1.039730\end{array}$

$\begin{array}{llll}-3.587029 & 7.843374 & -1.312195\end{array}$

$\begin{array}{llll}-5.237117 & 6.453771 & -1.560084\end{array}$

$\begin{array}{llll}-5.780550 & 7.103705 & -2.239794\end{array}$

$\begin{array}{llll}-5.768606 & 5.228910 & -1.218230\end{array}$

$\begin{array}{llll}-6.721960 & 4.923497 & -1.632906\end{array}$

$\begin{array}{llll}-5.075786 & 4.346913 & -0.342845\end{array}$

$\begin{array}{lll}-5.589839 & 3.050355 & 0.018595\end{array}$

$\begin{array}{lll}-6.971883 & 2.613923 & -0.328568\end{array}$

$\begin{array}{lll}-8.127960 & 3.381716 & 0.061258\end{array}$

$\begin{array}{lll}-8.043676 & 4.517441 & 0.914972\end{array}$

$\begin{array}{lll}-7.079099 & 4.819624 & 1.304820\end{array}$

$\begin{array}{lll}-9.170086 & 5.229009 & 1.267304\end{array}$

$\begin{array}{lll}-9.077932 & 6.086915 & 1.927181\end{array}$

$\begin{array}{lll}-10.445787 & 4.849572 & 0.786234\end{array}$

$\begin{array}{lll}-11.323605 & 5.423823 & 1.067622\end{array}$

$\begin{array}{llll}-10.568429 & 3.743489 & -0.023494\end{array}$

$\begin{array}{llll}-11.543205 & 3.427105 & -0.385488\end{array}$ 
$\begin{array}{llll}-9.428917 & 2.978401 & -0.393426\end{array}$

$\begin{array}{llll}-9.561130 & 1.808581 & -1.188721\end{array}$

$\begin{array}{llll}-10.546331 & 1.519230 & -1.543383\end{array}$

$\begin{array}{llll}-8.465132 & 1.033292 & -1.475770\end{array}$

$\begin{array}{llll}-8.546792 & 0.116087 & -2.048937\end{array}$

$\begin{array}{llll}-7.189765 & 1.428639 & -1.015716\end{array}$

$\begin{array}{llll}-5.442938 & -2.781203 & 0.300783\end{array}$

$\begin{array}{llll}-4.790720 & -2.998431 & 1.155926\end{array}$

$\begin{array}{llll}-6.472364 & -3.893572 & 0.199576\end{array}$

$\begin{array}{lll}-6.274070 & -5.090790 & 0.893887\end{array}$

$\begin{array}{lll}-5.410773 & -5.192679 & 1.548194\end{array}$

$\begin{array}{llll}-7.182515 & -6.146043 & 0.768515\end{array}$

$\begin{array}{lll}-7.019263 & -7.068470 & 1.319337\end{array}$

$\begin{array}{lll}-8.303154 & -6.008365 & -0.051822\end{array}$

$\begin{array}{llll}-9.018595 & -6.821068 & -0.140955\end{array}$

$\begin{array}{llll}-8.503235 & -4.815446 & -0.756362\end{array}$

$\begin{array}{llll}-9.377475 & -4.698558 & -1.390684\end{array}$

$\begin{array}{lll}-7.589015 & -3.771233 & -0.638620\end{array}$

$\begin{array}{llll}-7.754702 & -2.836197 & -1.164787\end{array}$

$\begin{array}{lll}-4.592917 & -2.785321 & -0.981220\end{array}$

$\begin{array}{lll}-5.270255 & -2.851645 & -1.840252\end{array}$

$\begin{array}{llll}-3.984551 & -3.694256 & -0.987588\end{array}$

$\begin{array}{lll}-6.809579 & -1.156680 & 1.767566\end{array}$

$\begin{array}{lll}-7.022820 & -0.087728 & 1.739561\end{array}$

$\begin{array}{lll}-8.168874 & -1.849830 & 1.808171\end{array}$

$-9.226728-1.293036 \quad 1.078526$

$\begin{array}{lll}-9.059276 & -0.381243 & 0.515453\end{array}$

$\begin{array}{lll}-10.485268 & -1.893670 & 1.061738\end{array}$

$\begin{array}{lll}-11.289853 & -1.444396 & 0.485453\end{array}$

$\begin{array}{lll}-10.709360 & -3.065143 & 1.789839\end{array}$

$\begin{array}{lll}-11.686381 & -3.540249 & 1.777687\end{array}$

$\begin{array}{llll}-9.668577 & -3.616460 & 2.540364\end{array}$

$\begin{array}{lll}-9.831680 & -4.525534 & 3.112485\end{array}$

$\begin{array}{lll}-8.411284 & -3.008653 & 2.554762\end{array}$

$\begin{array}{lll}-7.614804 & -3.461522 & 3.133835\end{array}$

$\begin{array}{lll}-5.942115 & -1.411747 & 3.006053\end{array}$

$\begin{array}{lll}-5.035254 & -0.804049 & 2.954251\end{array}$

$\begin{array}{lll}-5.648206 & -2.459737 & 3.107965\end{array}$

$\begin{array}{lll}-6.494011 & -1.131852 & 3.908470\end{array}$

$\begin{array}{lll}-2.423526 & -0.942308 & 0.712994\end{array}$

$\begin{array}{llll}-1.732806 & -0.103841 & 0.747850\end{array}$

$\begin{array}{lll}-3.113382 & -0.949671 & 1.551921\end{array}$

$\begin{array}{lll}-1.958862 & -2.216281 & 0.247681\end{array}$

$\begin{array}{lll}-2.509278 & -3.086050 & 0.590555\end{array}$

$\begin{array}{llll}-0.717999 & -2.463590 & -0.395898\end{array}$

$\begin{array}{llll}-0.157444 & -1.617306 & -0.768159\end{array}$

$\begin{array}{llll}-4.132566 & -0.907129 & -3.324558\end{array}$

$\begin{array}{llll}-5.147622 & -1.297974 & -3.310912\end{array}$

$\begin{array}{llll}-3.087863 & -1.867625 & -3.343275\end{array}$

$\begin{array}{llll}-3.379627 & -2.917487 & -3.360667\end{array}$

$\begin{array}{llll}-1.703626 & -1.603257 & -3.908452\end{array}$

$\begin{array}{llll}-1.739189 & -1.625115 & -5.008761\end{array}$

$\begin{array}{llll}-1.060923 & -2.434164 & -3.608493\end{array}$

$\begin{array}{lll}-1.071831 & -0.279898 & -3.428919\end{array}$

$\begin{array}{llll}-1.254022 & 0.519770 & -4.153522\end{array}$

$\begin{array}{lll}0.011288 & -0.402818 & -3.385258\end{array}$

$\begin{array}{llll}-1.553349 & 0.184129 & -2.057720\end{array}$

$\begin{array}{llll}-0.801674 & 0.170190 & -1.274931\end{array}$

$\begin{array}{llll}-2.623135 & 1.052701 & -1.861170\end{array}$

$\begin{array}{llll}-2.611944 & 1.650899 & -0.955842\end{array}$

$\begin{array}{llll}-3.525804 & 1.575691 & -2.959091\end{array}$

$\begin{array}{llll}-4.395086 & 2.033533 & -2.483674\end{array}$

$\begin{array}{llll}-3.000766 & 2.377920 & -3.498656\end{array}$

$\begin{array}{llll}-4.001828 & 0.486802 & -3.946223\end{array}$

$\begin{array}{llll}-3.322140 & 0.429538 & -4.802825\end{array}$

$\begin{array}{llll}-4.975615 & 0.781597 & -4.348778\end{array}$

$\begin{array}{llll}-0.402729 & -3.745395 & -1.03918\end{array}$

$\begin{array}{llll}-1.009892 & -4.961363 & -0.668111\end{array}$

$\begin{array}{llll}0.556393 & -3.765279 & -2.070143\end{array}$

$\begin{array}{llll}-0.668875 & -6.150014 & -1.310659\end{array}$

$\begin{array}{lll}-1.742089 & -4.983548 & 0.132718\end{array}$

$\begin{array}{llll}0.894530 & -4.954242 & -2.713181\end{array}$

$\begin{array}{llll}1.042081 & -2.838933 & -2.352651\end{array}$

$\begin{array}{llll}0.284747 & -6.153041 & -2.335231\end{array}$

$\begin{array}{lll}-1.145863 & -7.078559 & -1.010034\end{array}$

$\begin{array}{llll}1.639552 & -4.945298 & -3.503928\end{array}$

$\begin{array}{llll}0.547972 & -7.082085 & -2.832511\end{array}$

$\begin{array}{llll}2.504851 & -4.074127 & 0.599916\end{array}$

$3.479588-4.544778 \quad-0.378463$

$\begin{array}{llll}3.255080 & -5.584320 & -0.634380\end{array}$

$\begin{array}{llll}3.417061 & -3.947691 & -1.282892\end{array}$

$\begin{array}{llll}4.499727 & -4.496321 & 0.018635\end{array}$

$2.123175 \quad-5.051184 \quad 1.616552$

$\begin{array}{lll}1.837413 & -4.564482 & 2.547970\end{array}$

$\begin{array}{lll}1.300225 & -5.689286 & 1.271348\end{array}$

$\begin{array}{lll}2.988540 & -5.682263 & 1.831596\end{array}$

$\begin{array}{llll}2.516492 & -2.003332 & -0.325648\end{array}$

$\begin{array}{lll}-0.286717 & -1.091165 & 3.007704\end{array}$

$\begin{array}{lll}-0.634782 & 0.118956 & 3.573551\end{array}$

$\begin{array}{lll}-0.658035 & -2.011796 & 3.44105\end{array}$

$\begin{array}{lll}-0.116292 & 1.278133 & 2.989209\end{array}$

$\begin{array}{lll}0.717805 & 1.183299 & 1.889420\end{array}$

$\begin{array}{lll}1.130387 & 2.066699 & 1.418588\end{array}$

$\begin{array}{lll}1.071509 & 0.000310 & 1.348849\end{array}$

$\begin{array}{lll}-1.286227 & 0.162370 & 4.439816\end{array}$

$\begin{array}{lll}-0.517184 & 3.037021 & 3.743722\end{array}$

[8-9] $]^{\ddagger}-\mathbf{P 1 - C 1}$ endo si-re conf2

Number of imaginary frequencies $=$

The smallest frequencies are $=-216.80886 .92389 .5093 \mathrm{~cm}(-1)$ Electronic energy $=-7222.6176097$

Zero-point correction=

(Hartree/Particle)

Thermal correction to Energy=

Thermal correction to Enthalpy=

1.754258

1.873240

1.874184

Thermal correction to Gibbs Free Energy=

Sum of electronic and zero-point Energies=

Sum of electronic and thermal Energies=

Sum of electronic and thermal Enthalpies=

Sum of electronic and thermal Free Energies=

$-7220.863352$

$-7220.744370$

$-7220.743425$

$-7221.027898$

Cartesian Coordinates

$\begin{array}{lccc}26 & 3.751073 & 4.706004 & 2.839634 \\ 15 & 2.590871 & 1.976120 & -1.414747 \\ 15 & 5.010192 & 0.394924 & 1.200239 \\ 6 & 3.620888 & 3.680124 & 1.019391 \\ 6 & 4.188951 & 2.841943 & 2.044171 \\ 6 & 3.226776 & 2.723733 & 3.094967 \\ 1 & 3.363400 & 2.170661 & 4.014529 \\ 6 & 2.081347 & 3.496316 & 2.740173 \\ 1 & 1.199599 & 3.642823 & 3.349587 \\ 6 & 2.325683 & 4.092038 & 1.468420 \\ 1 & 1.657964 & 4.750164 & 0.931297 \\ 6 & 5.469358 & 5.801223 & 3.255929 \\ 1 & 6.458124 & 5.562305 & 2.887453 \\ 6 & 4.884835 & 5.317391 & 4.465972 \\ 1 & 5.348323 & 4.638281 & 5.168862 \\ 6 & 3.561812 & 5.846961 & 4.558718 \\ 1 & 2.847305 & 5.638679 & 5.344324 \\ 6 & 3.328870 & 6.658415 & 3.406776 \\ 1 & 2.408191 & 7.173955 & 3.166807 \\ 6 & 4.506572 & 6.630737 & 2.602318 \\ 1 & 4.632577 & 7.126218 & 1.649375 \\ 6 & 4.264866 & 3.978616 & -0.289369 \\ 6 & 5.340876 & 4.875934 & -0.351368 \\ 1 & 5.674287 & 5.339422 & 0.568029 \\ 6 & 5.981356 & 5.170980 & -1.555234 \\ 1 & 6.818555 & 5.862848 & -1.567468 \\ 6 & 5.537393 & 4.576642 & -2.734017 \\ 1 & 6.019379 & 4.798521 & -3.681114 \\ 6 & 4.481361 & 3.666050 & -2.693064 \\ 1 & 4.166721 & 3.193099 & -3.615471 \\ 6 & 3.849524 & 3.335221 & -1.483675 \\ 6 & 5.483723 & 2.092680 & 1.907476 \\ 1 & 6.059566 & 2.559394 & 1.102636 \\ 6 & 6.343566 & 2.073070 & 3.173575 \\ 1 & 7.289992 & 1.551775 & 3.006294 \\ 1 & 5.829853 & 1.592549 & 4.010651\end{array}$


$\begin{array}{lll}6.568743 & 3.103056 & 3.463729\end{array}$

$\begin{array}{llll}2.563170 & 1.346159 & -3.143306\end{array}$

$\begin{array}{llll}3.655102 & 0.556909 & -3.546052\end{array}$

$\begin{array}{llll}4.483885 & 0.401820 & -2.864129\end{array}$

$\begin{array}{llll}3.699821 & -0.001787 & -4.821352\end{array}$

$\begin{array}{llll}4.556534 & -0.599669 & -5.117885\end{array}$

$\begin{array}{llll}2.640442 & 0.205346 & -5.710905\end{array}$

$\begin{array}{llll}2.665818 & -0.238256 & -6.701857\end{array}$

$\begin{array}{llll}1.551742 & 0.986605 & -5.320387\end{array}$

$\begin{array}{llll}0.726921 & 1.153883 & -6.007058\end{array}$

$\begin{array}{lll}1.513758 & 1.561270 & -4.046016\end{array}$

$\begin{array}{llll}0.662554 & 2.170128 & -3.763065\end{array}$

$\begin{array}{llll}0.997666 & 2.855443 & -1.170792\end{array}$

$\begin{array}{llll}0.676543 & 4.036867 & -1.856945\end{array}$

$\begin{array}{llll}1.360528 & 4.438020 & -2.599331\end{array}$

$\begin{array}{llll}-0.514336 & 4.708722 & -1.574507\end{array}$

$\begin{array}{llll}-0.760172 & 5.620585 & -2.111221\end{array}$

$\begin{array}{lll}-1.381860 & 4.217113 & -0.592860\end{array}$

$\begin{array}{llll}-2.304505 & 4.744656 & -0.372579\end{array}$

$\begin{array}{llll}-1.059992 & 3.047090 & 0.098072\end{array}$

$\begin{array}{lll}-1.721861 & 2.665496 & 0.869728\end{array}$

$\begin{array}{llll}0.120628 & 2.361939 & -0.193696\end{array}$

$\begin{array}{lll}0.388540 & 1.466474 & 0.358343\end{array}$

$\begin{array}{llll}6.402601 & -0.039012 & 0.075946\end{array}$

$\begin{array}{llll}7.045743 & -1.285096 & 0.079880\end{array}$

$\begin{array}{llll}6.828537 & -2.020060 & 0.845048\end{array}$

$\begin{array}{llll}7.958317 & -1.610717 & -0.924096\end{array}$

$8.236329-0.721384-1.960948$

$\begin{array}{llll}8.933056 & -0.987920 & -2.745966\end{array}$

$\begin{array}{llll}7.580258 & 0.508168 & -1.978452\end{array}$

$\begin{array}{llll}6.673676 & 0.852189 & -0.974755\end{array}$

$\begin{array}{llll}6.160254 & 1.807386 & -1.035321\end{array}$

$\begin{array}{lll}5.124265 & -0.793778 & 2.599171\end{array}$

$\begin{array}{llll}3.916950 & -1.351470 & 3.034081\end{array}$

$\begin{array}{llll}2.988864 & -1.053041 & 2.559096\end{array}$

$3.904218 \quad-2.286428 \quad 4.071335$

$\begin{array}{llll}5.086535 & -2.675735 & 4.694698\end{array}$

$5.076258-3.409265 \quad 5.492204$

$\begin{array}{llll}6.290004 & -2.109823 & 4.268205\end{array}$

$\begin{array}{lll}6.316214 & -1.175389 & 3.233135\end{array}$

$\begin{array}{lll}7.267203 & -0.765949 & 2.912543\end{array}$

$\begin{array}{lll}3.025412 & 0.278329 & 0.036066\end{array}$

$\begin{array}{lll}7.577864 & -2.467329 & 4.960123\end{array}$

$\begin{array}{llll}2.590167 & -2.913776 & 4.444332\end{array}$

$\begin{array}{llll}8.684891 & -2.927683 & -0.857230\end{array}$

$\begin{array}{llll}7.775141 & 1.465982 & -3.122472\end{array}$

$\begin{array}{llll}6.654398 & 1.546948 & -3.886887\end{array}$

$\begin{array}{llll}8.036089 & 2.719821 & -2.688038\end{array}$

$\begin{array}{llll}8.784788 & 1.100960 & -3.938537\end{array}$

$\begin{array}{llll}7.938747 & -3.886637 & -0.265652\end{array}$

$9.829363-2.824230 \quad-0.138282$

$\begin{array}{llll}9.033840 & -3.378549 & -2.082505\end{array}$

$7.901575 \quad-1.552956 \quad 5.906290$

$\begin{array}{llll}8.615080 & -2.512499 & 4.092971\end{array}$

$\begin{array}{llll}7.510148 & -3.666286 & 5.576818\end{array}$

$\begin{array}{llll}2.651102 & -3.607767 & 5.597933\end{array}$

$\begin{array}{llll}1.612107 & -1.985991 & 4.578342\end{array}$

$\begin{array}{lll}2.168245 & -3.776665 & 3.481457\end{array}$

$\begin{array}{llll}4.049175 & -2.089442 & -1.454655\end{array}$

$\begin{array}{llll}2.090797 & -2.680569 & -0.317761\end{array}$

$\begin{array}{llll}4.210990 & -3.368778 & -1.953269\end{array}$

$\begin{array}{llll}4.759072 & -1.308506 & -1.690380\end{array}$

$\begin{array}{llll}2.243621 & -4.014033 & -0.790050\end{array}$

$\begin{array}{llll}3.292751 & -4.365737 & -1.616481\end{array}$

$\begin{array}{llll}1.510822 & -4.760360 & -0.504530\end{array}$

$\begin{array}{llll}0.912549 & -2.370385 & 0.442902\end{array}$

$\begin{array}{llll}0.730679 & -1.257918 & 1.337533\end{array}$

$\begin{array}{lll}1.395580 & -0.173633 & 1.268476\end{array}$

$3.014696-1.739160 \quad-0.670109$

$\begin{array}{llll}0.293217 & -3.236036 & 0.628905\end{array}$

$\begin{array}{lll}-0.246219 & -1.348552 & 2.291811\end{array}$

$\begin{array}{lll}-0.582328 & -0.187670 & 3.105816\end{array}$

$\begin{array}{lll}-0.175924 & -0.288549 & 4.119572\end{array}$

$\begin{array}{lll}-1.671280 & -0.091788 & 3.174989\end{array}$

$\begin{array}{lll}-0.169301 & 0.712060 & 2.654622\end{array}$ $\begin{array}{lll}-0.877956 & -2.609003 & 2.678339\end{array}$

$\begin{array}{lll}-0.137793 & -3.404908 & 2.780525\end{array}$

$\begin{array}{lll}-1.648122 & -2.922084 & 1.965969\end{array}$

$\begin{array}{lll}-1.351463 & -2.470585 & 3.652094\end{array}$

$\begin{array}{lll}-3.683484 & -0.732043 & -1.549594\end{array}$

$\begin{array}{llll}-5.455104 & -0.250026 & -0.211656\end{array}$

$\begin{array}{lll}-5.522295 & 0.981458 & 0.900597\end{array}$

$\begin{array}{llll}-6.806198 & 0.093143 & -1.122734\end{array}$

$\begin{array}{llll}-5.748516 & -1.550620 & 0.814325\end{array}$

$\begin{array}{llll}-5.890401 & 2.277136 & 0.555093\end{array}$

$\begin{array}{llll}-4.889503 & 3.263828 & 0.692393\end{array}$

$\begin{array}{lll}-3.915934 & 2.961726 & 1.059824\end{array}$

$\begin{array}{lll}-5.168888 & 4.568230 & 0.371546\end{array}$

$\begin{array}{llll}-4.413062 & 5.338897 & 0.491685\end{array}$

$\begin{array}{llll}-6.442317 & 4.924797 & -0.146738\end{array}$

$\begin{array}{llll}-6.714966 & 6.256182 & -0.562612\end{array}$

$\begin{array}{llll}-5.933738 & 7.003414 & -0.449661\end{array}$

$\begin{array}{llll}-7.932524 & 6.590886 & -1.110021\end{array}$

$\begin{array}{llll}-8.129145 & 7.609851 & -1.430121\end{array}$

$\begin{array}{llll}-8.925369 & 5.595737 & -1.272480\end{array}$

$\begin{array}{llll}-9.875664 & 5.854203 & -1.730744\end{array}$

$\begin{array}{llll}-8.698383 & 4.299537 & -0.863007\end{array}$

$\begin{array}{llll}-9.469227 & 3.552797 & -1.008746\end{array}$

$\begin{array}{llll}-7.461413 & 3.921029 & -0.268829\end{array}$

$\begin{array}{lll}-7.189991 & 2.574029 & 0.170486\end{array}$

$\begin{array}{lll}-8.247205 & 1.523930 & 0.160216\end{array}$

$\begin{array}{llll}-9.508998 & 1.687730 & 0.835259\end{array}$

$\begin{array}{lll}-9.769974 & 2.768234 & 1.723478\end{array}$

$\begin{array}{lll}-8.992299 & 3.499194 & 1.911522\end{array}$

$\begin{array}{lll}-10.987264 & 2.888929 & 2.357869\end{array}$

$\begin{array}{lll}-11.158021 & 3.717933 & 3.038665\end{array}$

$\begin{array}{lll}-12.015004 & 1.940567 & 2.138066\end{array}$

$\begin{array}{lll}-12.970730 & 2.052357 & 2.641541\end{array}$

$\begin{array}{lll}-11.792672 & 0.873757 & 1.297338\end{array}$

$\begin{array}{lll}-12.566347 & 0.128754 & 1.130908\end{array}$

$\begin{array}{lll}-10.543787 & 0.710956 & 0.637674\end{array}$

$\begin{array}{lll}-10.295388 & -0.416112 & -0.191665\end{array}$

$\begin{array}{llll}-11.088795 & -1.140667 & -0.349835\end{array}$

$\begin{array}{llll}-9.055240 & -0.612969 & -0.746329\end{array}$

$\begin{array}{llll}-8.830704 & -1.483941 & -1.351481\end{array}$

$\begin{array}{llll}-8.039255 & 0.343496 & -0.535315\end{array}$

$\begin{array}{lll}-5.042474 & -2.781200 & 0.366018\end{array}$

$\begin{array}{lll}-4.141882 & -2.906006 & 0.979552\end{array}$

$\begin{array}{llll}-5.877488 & -4.042494 & 0.495429\end{array}$

$\begin{array}{lll}-5.294910 & -5.211065 & 0.996125\end{array}$

$\begin{array}{llll}-4.262507 & -5.189699 & 1.339239\end{array}$

$\begin{array}{llll}-6.032816 & -6.395169 & 1.082126\end{array}$

$\begin{array}{llll}-5.569582 & -7.292970 & 1.482468\end{array}$

$\begin{array}{llll}-7.366035 & -6.418310 & 0.669057\end{array}$

$\begin{array}{llll}-7.946882 & -7.333004 & 0.747388\end{array}$

$\begin{array}{lll}-7.951588 & -5.254763 & 0.157206\end{array}$

$\begin{array}{lll}-8.991152 & -5.262546 & -0.158627\end{array}$

$\begin{array}{lll}-7.209347 & -4.079828 & 0.062391\end{array}$

$\begin{array}{llll}-7.670501 & -3.169804 & -0.305597\end{array}$

$\begin{array}{lll}-4.609324 & -2.613560 & -1.103893\end{array}$

$\begin{array}{llll}-5.488934 & -2.746347 & -1.746792\end{array}$

$\begin{array}{llll}-3.919997 & -3.424826 & -1.354332\end{array}$

$\begin{array}{lll}-6.097375 & -1.380472 & 2.266505\end{array}$

$\begin{array}{lll}-6.470220 & -0.360721 & 2.345387\end{array}$

$\begin{array}{lll}-7.246331 & -2.276329 & 2.714887\end{array}$

$\begin{array}{lll}-8.556772 & -1.839316 & 2.487061\end{array}$

$\begin{array}{lll}-8.716470 & -0.875939 & 2.016677\end{array}$

$\begin{array}{lll}-9.651055 & -2.623532 & 2.852142\end{array}$

$\begin{array}{lll}-10.658809 & -2.264337 & 2.661697\end{array}$

$\begin{array}{lll}-9.446426 & -3.860082 & 3.469249\end{array}$

$\begin{array}{llll}-10.294104 & -4.477511 & 3.753561\end{array}$

$\begin{array}{llll}-8.143422 & -4.292671 & 3.729662\end{array}$

$\begin{array}{lll}-7.974250 & -5.249824 & 4.215089\end{array}$

$\begin{array}{lll}-7.052692 & -3.502657 & 3.362005\end{array}$

$\begin{array}{lll}-6.048995 & -3.861246 & 3.560845\end{array}$

$\begin{array}{lll}-4.856259 & -1.474872 & 3.163211\end{array}$

$\begin{array}{llll}-4.138042 & -0.696877 & 2.890865\end{array}$

$\begin{array}{lll}-4.355756 & -2.444252 & 3.096129\end{array}$

$\begin{array}{llll}-5.145266 & -1.322213 & 4.207351\end{array}$

$\begin{array}{lll}-2.439592 & -0.677393 & 0.229737\end{array}$ 


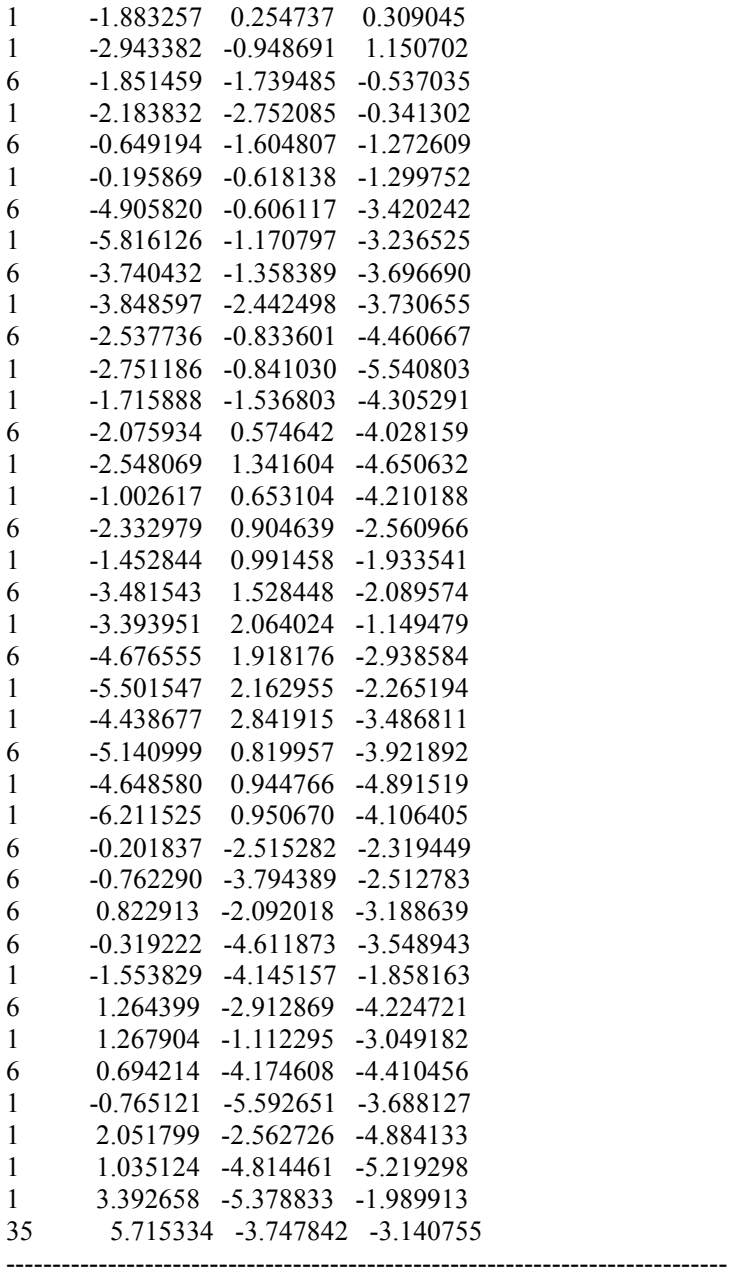

\section{$[8-9]^{\ddagger}-\mathbf{P} 1-\mathrm{C} 1$ exo si-si conf2}

Number of imaginary frequencies $=1$

The smallest frequencies are $=-153.75358 .528610 .6626 \mathrm{~cm}(-1)$ Electronic energy $=-7222.6206504$

Zero-point correction=

1.754800

(Hartree/Particle)

Thermal correction to Energy $=\quad 1.873746$

Thermal correction to Enthalpy= $\quad 1.874690$

Thermal correction to Gibbs Free Energy $=\quad 1.591401$

Sum of electronic and zero-point Energies $=\quad-7220.865850$

Sum of electronic and thermal Energies $=\quad-7220.746904$

Sum of electronic and thermal Enthalpies $=\quad-7220.745960$

Sum of electronic and thermal Free Energies $=\quad-7221.029250$

Cartesian Coordinates

$\begin{array}{lccc}26 & 3.848815 & 4.474038 & 3.163571 \\ 15 & 1.178196 & 2.281163 & -0.762619 \\ 15 & 4.500735 & 0.586678 & 0.479637 \\ 6 & 3.024899 & 3.723646 & 1.383359 \\ 6 & 3.993749 & 2.811162 & 1.934884 \\ 6 & 3.547074 & 2.432492 & 3.239977 \\ 1 & 4.065125 & 1.762936 & 3.913485 \\ 6 & 2.327904 & 3.122425 & 3.509484 \\ 1 & 1.763928 & 3.081824 & 4.431821 \\ 6 & 2.013816 & 3.927019 & 2.375719 \\ 1 & 1.163357 & 4.585570 & 2.281329 \\ 6 & 5.547678 & 5.667846 & 3.062478 \\ 1 & 6.305508 & 5.590952 & 2.294592 \\ 6 & 5.522980 & 4.940855 & 4.291703 \\ 1 & 6.255351 & 4.213774 & 4.615529 \\ 6 & 4.335308 & 5.306043 & 4.996047 \\ 1 & 4.009366 & 4.900646 & 5.944925 \\ 6 & 3.625250 & 6.257669 & 4.202055\end{array}$

$\begin{array}{llll}2.669105 & 6.702386 & 4.444690\end{array}$

$\begin{array}{lll}4.374341 & 6.482383 & 3.008691\end{array}$

$\begin{array}{lll}4.082891 & 7.128999 & 2.192228\end{array}$

$\begin{array}{llll}3.093660 & 4.259107 & -0.006428\end{array}$

$\begin{array}{lll}4.022763 & 5.264815 & -0.310069\end{array}$

$\begin{array}{lll}4.632675 & 5.654343 & 0.494407\end{array}$

$\begin{array}{llll}4.183964 & 5.753562 & -1.606999\end{array}$

$4.919114 \quad 6.527997-1.805896$

$\begin{array}{llll}3.399995 & 5.239397 & -2.635937\end{array}$

$\begin{array}{lll}3.512157 & 5.602520 & -3.652811\end{array}$

$2.472360 \quad 4.233875 \quad-2.360348$

$\begin{array}{llll}1.882781 & 3.835751 & -3.176872\end{array}$

$2.311541 \quad 3.720787-1.063363$

$\begin{array}{lll}5.144042 & 2.234635 & 1.164052\end{array}$

$\begin{array}{lll}5.296655 & 2.843947 & 0.267845\end{array}$

$\begin{array}{lll}6.465569 & 2.166542 & 1.932385\end{array}$

$\begin{array}{llll}7.256091 & 1.716614 & 1.324572\end{array}$

$\begin{array}{lll}6.372459 & 1.589336 & 2.855956\end{array}$

$\begin{array}{lll}6.774958 & 3.180889 & 2.199178\end{array}$

$\begin{array}{llll}0.393806 & 2.041237 & -2.411425\end{array}$

$\begin{array}{llll}1.067586 & 1.203547 & -3.315764\end{array}$

$\begin{array}{llll}1.980172 & 0.709364 & -3.000198\end{array}$

$\begin{array}{llll}0.596199 & 1.033860 & -4.617521\end{array}$

$\begin{array}{llll}1.136267 & 0.393218 & -5.308890\end{array}$

$\begin{array}{llll}-0.568838 & 1.689520 & -5.027015\end{array}$

$\begin{array}{llll}-0.942708 & 1.555005 & -6.037694\end{array}$

$\begin{array}{llll}-1.257600 & 2.507392 & -4.128354\end{array}$

$\begin{array}{llll}-2.172798 & 3.006321 & -4.434356\end{array}$

$\begin{array}{llll}-0.779130 & 2.685885 & -2.828666\end{array}$

$\begin{array}{llll}-1.326732 & 3.322496 & -2.144992\end{array}$

$\begin{array}{lll}-0.125625 & 3.020655 & 0.301035\end{array}$

$\begin{array}{lll}-0.593189 & 2.270094 & 1.388423\end{array}$

$\begin{array}{lll}-0.219855 & 1.263219 & 1.539828\end{array}$

$\begin{array}{lll}-1.487399 & 2.836209 & 2.298789\end{array}$

$\begin{array}{lll}-1.831309 & 2.247395 & 3.141354\end{array}$

$\begin{array}{lll}-1.927402 & 4.150313 & 2.127637\end{array}$

$\begin{array}{lll}-2.621316 & 4.591014 & 2.837972\end{array}$

$\begin{array}{lll}-1.472402 & 4.901078 & 1.038012\end{array}$

$\begin{array}{llll}-1.812584 & 5.923383 & 0.900178\end{array}$

$\begin{array}{llll}-0.571297 & 4.342086 & 0.130308\end{array}$

$\begin{array}{llll}-0.190964 & 4.944910 & -0.689166\end{array}$

$\begin{array}{llll}5.435537 & 0.398634 & -1.092956\end{array}$

$\begin{array}{llll}6.286910 & -0.660847 & -1.430276\end{array}$

$\begin{array}{llll}6.507661 & -1.449004 & -0.722168\end{array}$

$\begin{array}{llll}6.846374 & -0.729863 & -2.707556\end{array}$

$\begin{array}{llll}6.547295 & 0.223174 & -3.680227\end{array}$

$\begin{array}{llll}6.969846 & 0.148552 & -4.674646\end{array}$

$\begin{array}{llll}5.669387 & 1.255628 & -3.355214\end{array}$

$\begin{array}{llll}5.123109 & 1.350992 & -2.075786\end{array}$

$\begin{array}{llll}4.428766 & 2.157069 & -1.853612\end{array}$

$\begin{array}{lll}5.123842 & -0.668699 & 1.669124\end{array}$

$\begin{array}{lll}4.155195 & -1.205260 & 2.527467\end{array}$

$\begin{array}{lll}3.124080 & -0.891268 & 2.423723\end{array}$

$\begin{array}{lll}4.512586 & -2.123313 & 3.515007\end{array}$

$\begin{array}{lll}5.836875 & -2.533597 & 3.658500\end{array}$

$6.114379 \quad-3.252783 \quad 4.419869$

$\begin{array}{llll}6.803057 & -1.994975 & 2.808349\end{array}$

$\begin{array}{lll}6.459847 & -1.060188 & 1.829654\end{array}$

$\begin{array}{lll}7.241192 & -0.641463 & 1.206850\end{array}$

$\begin{array}{lll}2.216633 & 0.413509 & 0.037266\end{array}$

$\begin{array}{lll}8.252356 & -2.363175 & 2.977041\end{array}$

$\begin{array}{llll}3.437891 & -2.713807 & 4.385625\end{array}$

$\begin{array}{llll}7.829741 & -1.824940 & -3.022557\end{array}$

$\begin{array}{llll}5.253989 & 2.275056 & -4.380508\end{array}$

$\begin{array}{llll}3.903318 & 2.292336 & -4.532239\end{array}$

$\begin{array}{llll}5.614490 & 3.528138 & -4.017635\end{array}$

$\begin{array}{llll}5.788076 & 2.037435 & -5.595171\end{array}$

$\begin{array}{llll}7.806155 & -2.171272 & -4.328782\end{array}$

$\begin{array}{llll}7.596066 & -2.941334 & -2.297754\end{array}$

$\begin{array}{llll}9.099940 & -1.439115 & -2.745267\end{array}$

$\begin{array}{llll}8.874275 & -2.494767 & 1.782384\end{array}$

$\begin{array}{llll}8.411508 & -3.523133 & 3.648435\end{array}$

$\begin{array}{llll}8.929436 & -1.410381 & 3.663059\end{array}$

$\begin{array}{llll}3.919686 & -3.145366 & 5.570564\end{array}$

$\begin{array}{llll}2.453997 & -1.821131 & 4.646779\end{array}$ 
$\begin{array}{llll}2.842248 & -3.782257 & 3.793898\end{array}$

$\begin{array}{llll}3.022076 & -1.777692 & -1.904706\end{array}$

$\begin{array}{lll}2.167523 & -2.715210 & 0.063567\end{array}$

$\begin{array}{llll}3.602389 & -2.973499 & -2.286588\end{array}$

$\begin{array}{llll}3.122605 & -0.893822 & -2.522858\end{array}$

$\begin{array}{llll}2.824287 & -3.936930 & -0.260382\end{array}$

$\begin{array}{llll}3.536480 & -4.078617 & -1.434278\end{array}$

$\begin{array}{lll}2.729286 & -4.779147 & 0.416711\end{array}$

$\begin{array}{lll}1.270084 & -2.718815 & 1.177994\end{array}$

$\begin{array}{lll}0.781542 & -1.577089 & 1.893775\end{array}$

$\begin{array}{lll}1.078883 & -0.377610 & 1.590708\end{array}$

$\begin{array}{llll}2.330323 & -1.630305 & -0.759229\end{array}$

$\begin{array}{lll}1.212009 & -3.671856 & 1.683712\end{array}$

$\begin{array}{lll}-0.061431 & -1.764290 & 2.958049\end{array}$

$\begin{array}{lll}-0.414954 & -0.627611 & 3.802938\end{array}$

$\begin{array}{lll}0.248955 & 0.209716 & 3.597411\end{array}$

$\begin{array}{lll}-0.315969 & -0.909920 & 4.855133\end{array}$

$\begin{array}{lll}-1.449552 & -0.315441 & 3.628521\end{array}$

$\begin{array}{lll}-0.448599 & -3.070399 & 3.475583\end{array}$

$\begin{array}{lll}-0.628499 & -3.786038 & 2.672937\end{array}$

$\begin{array}{lll}-1.379684 & -2.958743 & 4.036294\end{array}$

$\begin{array}{llll}0.317685 & -3.481829 & 4.144105\end{array}$

$\begin{array}{llll}-3.324811 & -1.621256 & -1.294941\end{array}$

$\begin{array}{lll}-4.944766 & -0.732630 & 0.043854\end{array}$

$\begin{array}{lll}-4.932245 & 0.801502 & 0.679756\end{array}$

$\begin{array}{llll}-6.379665 & -0.682495 & -0.807385\end{array}$

$\begin{array}{llll}-5.080214 & -1.627383 & 1.451612\end{array}$

$\begin{array}{llll}-5.100838 & 1.896575 & -0.167456\end{array}$

$\begin{array}{llll}-3.964235 & 2.704101 & -0.389256\end{array}$

$\begin{array}{lll}-3.040795 & 2.450732 & 0.116040\end{array}$

$\begin{array}{lll}-4.056707 & 3.784010 & -1.231606\end{array}$

$\begin{array}{llll}-3.197319 & 4.427611 & -1.389014\end{array}$

$\begin{array}{llll}-5.263702 & 4.053917 & -1.929924\end{array}$

$\begin{array}{llll}-5.336925 & 5.103715 & -2.885262\end{array}$

$\begin{array}{lll}-4.455271 & 5.718571 & -3.046830\end{array}$

$\begin{array}{llll}-6.490004 & 5.327817 & -3.602766\end{array}$

$\begin{array}{llll}-6.533677 & 6.128024 & -4.335789\end{array}$

$\begin{array}{llll}-7.618439 & 4.499258 & -3.394996\end{array}$

$\begin{array}{llll}-8.518939 & 4.660584 & -3.980560\end{array}$

$\begin{array}{llll}-7.585904 & 3.485363 & -2.461972\end{array}$

$\begin{array}{llll}-8.457290 & 2.856171 & -2.326920\end{array}$

$\begin{array}{llll}-6.419077 & 3.237573 & -1.685893\end{array}$

$\begin{array}{llll}-6.344408 & 2.175751 & -0.713059\end{array}$

$\begin{array}{llll}-7.542586 & 1.375698 & -0.335797\end{array}$

$\begin{array}{llll}-8.743531 & 1.995997 & 0.164634\end{array}$

$\begin{array}{lll}-8.814779 & 3.380647 & 0.485518\end{array}$

$\begin{array}{llll}-7.935058 & 4.000756 & 0.360325\end{array}$

$\begin{array}{lll}-9.977994 & 3.940962 & 0.967788\end{array}$

$\begin{array}{lll}-10.002459 & 4.998912 & 1.213003\end{array}$

$\begin{array}{lll}-11.138276 & 3.152282 & 1.152900\end{array}$

$\begin{array}{lll}-12.049693 & 3.609327 & 1.526916\end{array}$

$\begin{array}{lll}-11.100024 & 1.805214 & 0.872124\end{array}$

$\begin{array}{lll}-11.977294 & 1.182364 & 1.026578\end{array}$

$\begin{array}{llll}-9.911774 & 1.192526 & 0.389816\end{array}$

$\begin{array}{lll}-9.856604 & -0.206691 & 0.146074\end{array}$

$\begin{array}{lll}-10.751065 & -0.804453 & 0.297176\end{array}$

$\begin{array}{lll}-8.680610 & -0.800107 & -0.237672\end{array}$

$\begin{array}{llll}-8.601088 & -1.871379 & -0.389816\end{array}$

$\begin{array}{lll}-7.528643 & -0.006530 & -0.436996\end{array}$

$\begin{array}{lll}-4.212014 & -2.840196 & 1.444922\end{array}$

$\begin{array}{lll}-3.276873 & -2.596094 & 1.966660\end{array}$

$\begin{array}{lll}-4.851920 & -4.024925 & 2.148608\end{array}$

$\begin{array}{lll}-4.095539 & -4.801663 & 3.033718\end{array}$

$\begin{array}{lll}-3.071960 & -4.511962 & 3.258111\end{array}$

$\begin{array}{lll}-4.644722 & -5.940405 & 3.630441\end{array}$

$\begin{array}{lll}-4.045129 & -6.533053 & 4.315980\end{array}$

$\begin{array}{llll}-5.962397 & -6.309191 & 3.352890\end{array}$

$\begin{array}{lll}-6.393802 & -7.189344 & 3.821095\end{array}$

$\begin{array}{lll}-6.726421 & -5.535923 & 2.471974\end{array}$

$\begin{array}{lll}-7.753905 & -5.814785 & 2.254706\end{array}$

$\begin{array}{lll}-6.173321 & -4.405679 & 1.870770\end{array}$

$\begin{array}{lll}-6.767864 & -3.798736 & 1.192831\end{array}$

$\begin{array}{lll}-3.910916 & -3.232060 & -0.005204\end{array}$

$\begin{array}{llll}-4.821703 & -3.673642 & -0.427915\end{array}$

$\begin{array}{lll}-3.159548 & -4.022976 & -0.015008\end{array}$ $\begin{array}{lll}-5.650712 & -1.249653 & 2.781851\end{array}$

$\begin{array}{lll}-5.949852 & -2.208256 & 3.210562\end{array}$

$\begin{array}{lll}-4.639131 & -0.661463 & 3.766330\end{array}$

$\begin{array}{lll}-4.098088 & -1.498204 & 4.754998\end{array}$

$\begin{array}{lll}-4.330772 & -2.559683 & 4.735497\end{array}$

$\begin{array}{lll}-3.302029 & -0.982936 & 5.781079\end{array}$

$\begin{array}{llll}-2.902687 & -1.649006 & 6.541054\end{array}$

$\begin{array}{lll}-3.038774 & 0.386632 & 5.837928\end{array}$

$\begin{array}{llll}-2.436971 & 0.796838 & 6.643903\end{array}$

$\begin{array}{llll}-3.554812 & 1.226927 & 4.847461\end{array}$

$\begin{array}{lll}-3.362003 & 2.295375 & 4.890236\end{array}$

$\begin{array}{lll}-4.337319 & 0.707763 & 3.812668\end{array}$

$\begin{array}{lll}-4.736629 & 1.375023 & 3.057162\end{array}$

$\begin{array}{lll}-6.934130 & -0.420856 & 2.715089\end{array}$

$\begin{array}{lll}-7.699123 & -0.947687 & 2.142923\end{array}$

$\begin{array}{lll}-6.788751 & 0.565660 & 2.276511\end{array}$

$\begin{array}{lll}-7.306909 & -0.286089 & 3.734718\end{array}$

$\begin{array}{lll}-1.821838 & -0.703978 & -0.028123\end{array}$

$\begin{array}{llll}-1.635621 & 0.332736 & -0.295925\end{array}$

$\begin{array}{lll}-1.996199 & -0.841426 & 1.037346\end{array}$

$\begin{array}{llll}-1.037369 & -1.703501 & -0.709668\end{array}$

$\begin{array}{llll}-0.457764 & -1.410292 & -1.575978\end{array}$

$\begin{array}{llll}-0.824305 & -2.989951 & -0.189307\end{array}$

$\begin{array}{lll}-1.320596 & -3.219461 & 0.745023\end{array}$

$\begin{array}{lll}-4.850463 & -2.360752 & -2.784668\end{array}$

$\begin{array}{llll}-5.639243 & -2.831294 & -2.204316\end{array}$

$\begin{array}{llll}-3.665032 & -3.103617 & -2.959079\end{array}$

$\begin{array}{llll}-3.634376 & -4.099957 & -2.518856\end{array}$

$-2.672642-2.900151-4.089186$

$\begin{array}{llll}-3.057071 & -3.370951 & -5.007206\end{array}$

$\begin{array}{llll}-1.763747 & -3.446973 & -3.826480\end{array}$

$\begin{array}{llll}-2.312991 & -1.424482 & -4.364393\end{array}$

$\begin{array}{llll}-2.961366 & -1.011535 & -5.144122\end{array}$

$-1.295404-1.374193-4.763490$

$\begin{array}{llll}-2.385797 & -0.517547 & -3.139691\end{array}$

$\begin{array}{llll}-1.437332 & -0.119822 & -2.796214\end{array}$

$\begin{array}{llll}-3.514431 & 0.199931 & -2.769956\end{array}$

$\begin{array}{llll}-3.352193 & 1.095514 & -2.180559\end{array}$

$\begin{array}{llll}-4.853958 & 0.129482 & -3.472526\end{array}$

$\begin{array}{llll}-5.589780 & 0.629686 & -2.843537\end{array}$

$\begin{array}{llll}-4.791951 & 0.712250 & -4.403690\end{array}$

$\begin{array}{llll}-5.339124 & -1.305301 & -3.775909\end{array}$

$\begin{array}{llll}-5.035379 & -1.603521 & -4.784789\end{array}$

$\begin{array}{llll}-6.433030 & -1.309260 & -3.773348\end{array}$

$-0.353554-4.139609-0.950390$

$\begin{array}{llll}0.199482 & -4.025599 & -2.240705\end{array}$

$\begin{array}{llll}-0.458892 & -5.422511 & -0.37716\end{array}$

$\begin{array}{llll}0.612432 & -5.157345 & -2.938996\end{array}$

$\begin{array}{llll}0.318525 & -3.047913 & -2.693548\end{array}$

$-0.043634 \quad-6.554039 \quad-1.076074$

$\begin{array}{lll}-0.871623 & -5.522964 & 0.623680\end{array}$

$\begin{array}{llll}0.490027 & -6.425734 & -2.362749\end{array}$

$\begin{array}{llll}1.039111 & -5.048856 & -3.931915\end{array}$

$\begin{array}{llll}-0.137091 & -7.535417 & -0.620051\end{array}$

$\begin{array}{llll}0.813350 & -7.306753 & -2.909456\end{array}$

$\begin{array}{llll}4.006063 & -5.021744 & -1.691069\end{array}$

$35 \quad 4.469991 \quad-3.113521 \quad-4.032577$

[8-9 $]^{\ddagger}-\mathbf{P 1 - C 1}$ exo re-si conf2

Number of imaginary frequencies $=1$

The smallest frequencies are $=-68.12748 .584810 .3975 \mathrm{~cm}(-1)$ Electronic energy $=-7222.6318937$

Zero-point correction $=$

1.754511

(Hartree/Particle)

Thermal correction to Energy= $\quad 1.873363$

Thermal correction to Enthalpy= $\quad 1.874307$

Thermal correction to Gibbs Free Energy $=\quad 1.591971$

Sum of electronic and zero-point Energies $=\quad-7220.877383$

Sum of electronic and thermal Energies=

$-7220.758531$

Sum of electronic and thermal Enthalpies $=\quad-7220.757587$

Sum of electronic and thermal Free Energies $=\quad-7221.039922$

Cartesian Coordinates 


$$
\begin{aligned}
& \begin{array}{lll}
3.394848 & 3.907272 & -0.422804
\end{array} \\
& \begin{array}{lll}
5.495369 & 0.999139 & 2.517477
\end{array} \\
& \begin{array}{llll}
6.155411 & 1.821469 & 2.806663
\end{array} \\
& \begin{array}{llll}
4.334637 & 0.989186 & 3.501868
\end{array} \\
& \begin{array}{llll}
4.402932 & 1.776620 & 4.655722
\end{array} \\
& \begin{array}{lll}
5.267771 & 2.415376 & 4.814835
\end{array} \\
& \begin{array}{llll}
3.369017 & 1.754245 & 5.594863
\end{array} \\
& \begin{array}{lll}
3.434960 & 2.368992 & 6.488372
\end{array} \\
& \begin{array}{llll}
2.250126 & 0.950215 & 5.377913
\end{array} \\
& \begin{array}{llll}
1.443003 & 0.932405 & 6.102070
\end{array} \\
& \begin{array}{llll}
2.169679 & 0.168440 & 4.220550
\end{array} \\
& \begin{array}{llll}
1.293545 & -0.446877 & 4.044645
\end{array} \\
& \begin{array}{lll}
3.207278 & 0.184449 & 3.288530
\end{array} \\
& \begin{array}{llll}
3.149142 & -0.428148 & 2.394230
\end{array} \\
& \begin{array}{lll}
6.327502 & -0.278554 & 2.663330
\end{array} \\
& \begin{array}{lll}
7.211387 & -0.257486 & 2.027502
\end{array} \\
& \begin{array}{llll}
5.755599 & -1.182002 & 2.456253
\end{array} \\
& \begin{array}{lll}
6.667118 & -0.321393 & 3.702757
\end{array} \\
& \begin{array}{llll}
1.673532 & 0.729137 & -0.303819
\end{array} \\
& \begin{array}{lll}
1.382752 & -0.298403 & -0.512495
\end{array} \\
& \begin{array}{lll}
1.852961 & 0.910124 & 0.754894
\end{array} \\
& \begin{array}{llll}
0.981058 & 1.757141 & -1.047737
\end{array} \\
& \begin{array}{llll}
0.378097 & 1.482459 & -1.902983
\end{array} \\
& \begin{array}{llll}
0.960355 & 3.083604 & -0.622297
\end{array} \\
& \begin{array}{llll}
1.425172 & 3.294493 & 0.336343
\end{array} \\
& \begin{array}{llll}
4.851659 & 1.956497 & -3.139731
\end{array} \\
& \begin{array}{llll}
5.683236 & 2.389801 & -2.591274
\end{array} \\
& \begin{array}{lll}
3.729723 & 2.784673 & -3.336541
\end{array} \\
& \begin{array}{llll}
3.791002 & 3.800197 & -2.946548
\end{array} \\
& \begin{array}{llll}
2.686832 & 2.606290 & -4.424901
\end{array} \\
& \begin{array}{llll}
3.064031 & 3.026680 & -5.369707
\end{array} \\
& \begin{array}{llll}
1.821692 & 3.213371 & -4.145778
\end{array} \\
& \begin{array}{llll}
2.228070 & 1.148270 & -4.638002
\end{array} \\
& \begin{array}{llll}
2.844639 & 0.658098 & -5.398034
\end{array} \\
& \begin{array}{llll}
1.207869 & 1.151000 & -5.033810
\end{array} \\
& \begin{array}{llll}
2.240201 & 0.300960 & -3.371344
\end{array} \\
& \begin{array}{llll}
1.269506 & 0.009478 & -2.992471
\end{array} \\
& \begin{array}{llll}
3.308694 & -0.479707 & -2.961863
\end{array} \\
& \begin{array}{llll}
3.078743 & -1.316907 & -2.312718
\end{array} \\
& \begin{array}{llll}
4.641917 & -0.564868 & -3.671184
\end{array} \\
& \begin{array}{llll}
5.340494 & -1.087258 & -3.017698
\end{array} \\
& \begin{array}{llll}
4.519351 & -1.194298 & -4.564833
\end{array} \\
& \begin{array}{llll}
5.239791 & 0.804209 & -4.064726
\end{array} \\
& \begin{array}{llll}
4.953092 & 1.064195 & -5.088765
\end{array} \\
& \begin{array}{llll}
6.330081 & 0.718273 & -4.066006
\end{array} \\
& \begin{array}{llll}
0.598451 & 4.252504 & -1.406709
\end{array} \\
& \begin{array}{llll}
-0.043682 & 4.172334 & -2.659135
\end{array} \\
& \begin{array}{llll}
0.931817 & 5.526788 & -0.902730
\end{array} \\
& \begin{array}{llll}
-0.332129 & 5.326825 & -3.381068
\end{array} \\
& \begin{array}{llll}
-0.331269 & 3.205526 & -3.056622
\end{array} \\
& \begin{array}{llll}
0.649063 & 6.680492 & -1.630343
\end{array} \\
& \begin{array}{llll}
1.419764 & 5.600600 & 0.065986
\end{array} \\
& \begin{array}{llll}
0.014666 & 6.584513 & -2.872999
\end{array} \\
& \begin{array}{llll}
-0.831728 & 5.247554 & -4.342062
\end{array} \\
& \begin{array}{llll}
0.919398 & 7.652801 & -1.229024
\end{array} \\
& \begin{array}{llll}
-0.210919 & 7.482085 & -3.441384
\end{array} \\
& \begin{array}{llll}
-2.144660 & 1.599995 & -1.036464
\end{array} \\
& \begin{array}{lll}
-0.775259 & 2.944424 & 3.003491
\end{array} \\
& \begin{array}{lll}
-0.766464 & 2.262086 & 4.197768
\end{array} \\
& \begin{array}{lll}
-0.526297 & 4.000297 & 2.984421
\end{array} \\
& \begin{array}{lll}
-1.142839 & 0.910296 & 4.176874
\end{array} \\
& \begin{array}{lll}
-1.417018 & 0.295481 & 2.973816
\end{array} \\
& \begin{array}{lll}
-1.639363 & -0.761325 & 2.921081
\end{array} \\
& \begin{array}{llll}
-1.441065 & 0.963647 & 1.801198
\end{array} \\
& \begin{array}{llll}
-3.296622 & 3.510944 & -2.573860
\end{array} \\
& \begin{array}{llll}
-2.772745 & 2.674595 & -3.027898
\end{array} \\
& \begin{array}{lll}
-4.368428 & 3.286752 & -2.541379
\end{array} \\
& \begin{array}{llll}
-3.150289 & 4.395460 & -3.200523
\end{array} \\
& \begin{array}{llll}
-2.975560 & 5.122925 & -0.733714
\end{array} \\
& \begin{array}{lll}
-3.086949 & 5.119344 & 0.350539
\end{array} \\
& \begin{array}{llll}
-2.154759 & 5.795955 & -1.011726
\end{array} \\
& \begin{array}{llll}
-3.904103 & 5.510999 & -1.159274
\end{array} \\
& \begin{array}{llll}
-2.762648 & 3.773027 & -1.244347
\end{array} \\
& \begin{array}{lll}
-0.491289 & 2.758444 & 5.121748
\end{array} \\
& \begin{array}{llll}
35 & -1.287029 & -0.113802 & 5.841464
\end{array}
\end{aligned}
$$

\begin{tabular}{|c|c|}
\hline \multicolumn{2}{|c|}{$\begin{array}{l}\text { Number of imaginary frequencies }=1 \\
\text { The smallest frequencies are }=-133.54837 .607011 .1405 \mathrm{~cm}(-1) \\
\text { Electronic energy }=-7222.6211169\end{array}$} \\
\hline $\begin{array}{l}\text { Zero-point correction }= \\
\text { (Hartree/Particle) }\end{array}$ & 5465 \\
\hline & .87 \\
\hline thalpy $=$ & 1.874798 \\
\hline Free Energy= & 1.594441 \\
\hline Energies $=$ & \\
\hline & -7220.7 \\
\hline thermal Enthalpies= & -7220.746319 \\
\hline & -7221.02667 \\
\hline
\end{tabular}

[8-9]-P1-C1 exo re-re 


$\begin{array}{llll}6 & -1.623486 & 4.306705 & 1.096786 \\ 1 & -0.933636 & 5.148147 & 1.217972 \\ 1 & -1.575642 & 3.965366 & 0.067045 \\ 1 & -2.639694 & 4.656186 & 1.313740 \\ 6 & -0.901595 & 3.670702 & 3.356350 \\ 1 & -1.069120 & 2.876920 & 4.082416 \\ 1 & 0.137118 & 4.014803 & 3.426575 \\ 1 & -1.561933 & 4.500514 & 3.623727 \\ 8 & -1.721907 & 1.674917 & 0.431896 \\ 6 & -0.599409 & -1.178342 & 3.655655 \\ 6 & -0.782843 & -2.543024 & 3.748118 \\ 1 & -0.183767 & -0.634436 & 4.495089 \\ 6 & -1.325597 & -3.199102 & 2.640356 \\ 6 & -1.719129 & -2.469576 & 1.531761 \\ 1 & -2.174580 & -2.950999 & 0.676075 \\ 7 & -1.567336 & -1.133600 & 1.452623 \\ 1 & -0.499961 & -3.083261 & 4.645202 \\ 35 & -1.550509 & -5.143510 & 2.650606\end{array}$

[8-9]

Number of imaginary frequencies $=1$

The smallest frequencies are $=-161.12687 .316911 .9812 \mathrm{~cm}(-1)$ Electronic energy $=-7222.6073152$

$\begin{array}{lc}\begin{array}{l}\text { Zero-point correction }= \\ \text { (Hartree/Particle) }\end{array} & 1.755306 \\ \text { Thermal correction to Energy= } & 1.875023 \\ \text { Thermal correction to Enthalpy= } & 1.591574 \\ \text { Thermal correction to Gibbs Free Energy }= & -7220.852010 \\ \text { Sum of electronic and zero-point Energies }= & -7220.733236 \\ \text { Sum of electronic and thermal Energies }= & -7220.732292 \\ \text { Sum of electronic and thermal Enthalpies }= & -7221.015741\end{array}$

Sum of electronic and thermal Free Energies $=\quad-7221.015741$

Cartesian Coordinates

$\begin{array}{llll}26 & -5.598755 & 4.384651 & -1.540507 \\ 15 & -2.492914 & 1.716035 & 1.652616 \\ 15 & -5.125201 & -0.215715 & -0.407475 \\ 6 & -4.622327 & 3.311225 & -0.027686 \\ 6 & -5.282422 & 2.423259 & -0.949316 \\ 6 & -4.728400 & 2.650792 & -2.247237 \\ 1 & -5.017325 & 2.137587 & -3.154649 \\ 6 & -3.751328 & 3.682801 & -2.139058 \\ 1 & -3.184383 & 4.107802 & -2.956178 \\ 6 & -3.689611 & 4.096783 & -0.776287 \\ 1 & -3.058339 & 4.874657 & -0.371441 \\ 6 & -7.560140 & 4.998947 & -1.219100 \\ 1 & -8.269627 & 4.488539 & -0.581488 \\ 6 & -7.339899 & 4.739644 & -2.606361 \\ 1 & -7.845813 & 3.991904 & -3.202055 \\ 6 & -6.299136 & 5.607899 & -3.057096 \\ 1 & -5.877683 & 5.630316 & -4.053550 \\ 6 & -5.874600 & 6.402111 & -1.948646 \\ 1 & -5.076199 & 7.132457 & -1.957344 \\ 6 & -6.653995 & 6.027289 & -0.813804 \\ 1 & -6.551643 & 6.431173 & 0.184282 \\ 6 & -4.860042 & 3.284818 & 1.441345 \\ 6 & -6.016166 & 3.875169 & 1.970508 \\ 1 & -6.681872 & 4.392976 & 1.292234 \\ 6 & -6.325542 & 3.795384 & 3.328996 \\ 1 & -7.232877 & 4.258870 & 3.705517 \\ 6 & -5.469675 & 3.112963 & 4.189156 \\ 1 & -5.698740 & 3.028261 & 5.246841 \\ 6 & -4.312746 & 2.517233 & 3.684372 \\ 1 & -3.670656 & 1.975066 & 4.366597 \\ 6 & -3.993257 & 2.583417 & 2.319164 \\ 6 & -6.223341 & 1.327984 & -0.534443 \\ 1 & -6.534428 & 1.518855 & 0.497296 \\ 6 & -7.476712 & 1.197092 & -1.402119 \\ 1 & -8.122948 & 0.388055 & -1.050316 \\ 1 & -7.231415 & 1.010516 & -2.451067 \\ 1 & -8.040023 & 2.132957 & -1.349599 \\ 6 & -1.790514 & 0.938753 & 3.167069 \\ 6 & -2.119513 & -0.403945 & 3.401761\end{array}$

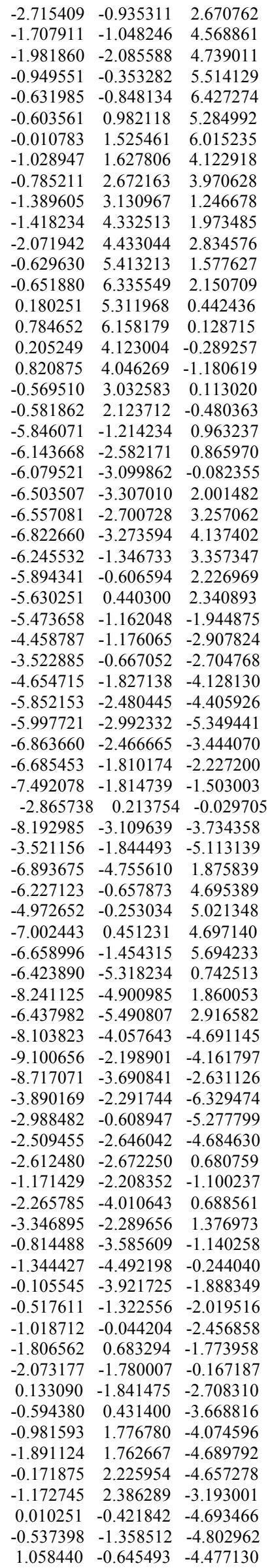




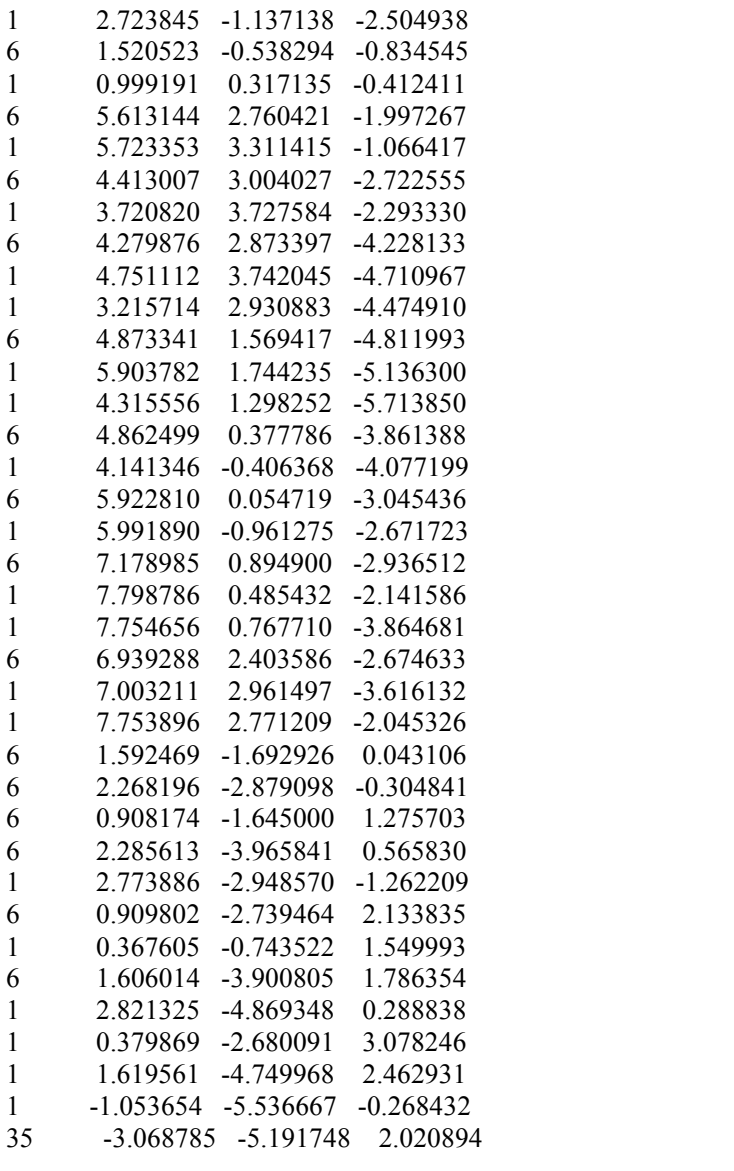

$[8-9]^{\dagger}-\mathbf{P 1}-\mathrm{C} 1$ endo si-si

Number of imaginary frequencies $=1$

The smallest frequencies are $=-150.10398 .239310 .3348 \mathrm{~cm}(-1)$ Electronic energy $=-7222.6218129$

Zero-point correction $=\quad 1.754670$

(Hartree/Particle)

Thermal correction to Energy= $\quad 1.873731$

Thermal correction to Enthalpy= $\quad 1.874675$

Thermal correction to Gibbs Free Energy $=\quad \begin{array}{r}1.590704 \\ \text { Sum }\end{array}$

Sum of electronic and zero-point Energies $=\quad-7220.867143$

Sum of electronic and thermal Energies= $\quad-7220.748082$

Sum of electronic and thermal Enthalpies $=\quad-7220.747138$

Sum of electronic and thermal Free Energies $=\quad-7221.031109$

Cartesian Coordinates

$\begin{array}{llrc}26 & -7.001877 & -3.547878 & -0.911926 \\ 15 & -2.597776 & -1.191541 & -2.403530 \\ 15 & -4.668373 & 0.354317 & 0.508115 \\ 6 & -5.432445 & -2.383361 & -1.681650 \\ 6 & -5.877104 & -1.880038 & -0.408055 \\ 6 & -5.700299 & -2.919611 & 0.558840 \\ 1 & -5.947674 & -2.852849 & 1.609846 \\ 6 & -5.171293 & -4.063825 & -0.110533 \\ 1 & -4.968395 & -5.026358 & 0.339846 \\ 6 & -5.016720 & -3.738768 & -1.490258 \\ 1 & -4.656505 & -4.398710 & -2.266346 \\ 6 & -8.957429 & -3.056239 & -1.430306 \\ 1 & -9.297133 & -2.059188 & -1.676927 \\ 6 & -8.916943 & -3.627384 & -0.121918 \\ 1 & -9.211696 & -3.135055 & 0.795035 \\ 6 & -8.371960 & -4.942899 & -0.231965 \\ 1 & -8.180463 & -5.622240 & 0.588279 \\ 6 & -8.073385 & -5.183921 & -1.607776 \\ 1 & -7.617244 & -6.077396 & -2.013352 \\ 6 & -8.436084 & -4.019385 & -2.348276 \\ 1 & -8.306122 & -3.881913 & -3.413065\end{array}$


$\begin{array}{llll}-5.375322 & -1.563749 & -2.923210\end{array}$

$\begin{array}{llll}-6.559403 & -1.284632 & -3.620584\end{array}$

$\begin{array}{lll}-7.480616 & -1.714005 & -3.249823\end{array}$

$\begin{array}{llll}-6.571841 & -0.471599 & -4.754022\end{array}$

$\begin{array}{llll}-7.508546 & -0.268547 & -5.265110\end{array}$

$\begin{array}{llll}-5.377461 & 0.071689 & -5.220135\end{array}$

$\begin{array}{llll}-5.363375 & 0.703183 & -6.103336\end{array}$

$\begin{array}{lll}-4.187325 & -0.189648 & -4.539879\end{array}$

$\begin{array}{llll}-3.271320 & 0.249425 & -4.915018\end{array}$

$\begin{array}{lll}-4.164061 & -0.983531 & -3.381402\end{array}$

$\begin{array}{llll}-6.252858 & -0.448407 & -0.155022\end{array}$

$\begin{array}{llll}-6.413210 & 0.034967 & -1.122685\end{array}$

$\begin{array}{lll}-7.506615 & -0.252026 & 0.700172\end{array}$

$\begin{array}{llll}-7.706751 & 0.808934 & 0.877748\end{array}$

$\begin{array}{lll}-7.423051 & -0.751533 & 1.669030\end{array}$

$\begin{array}{lll}-8.364482 & -0.680176 & 0.174231\end{array}$

$\begin{array}{lll}-1.380998 & -0.214840 & -3.384318\end{array}$

$\begin{array}{lll}-1.488529 & 1.186276 & -3.321175\end{array}$

$\begin{array}{llll}-2.291770 & 1.639041 & -2.748370\end{array}$

$\begin{array}{llll}-0.609712 & 2.002506 & -4.029741\end{array}$

$\begin{array}{llll}-0.711770 & 3.081370 & -3.974379\end{array}$

$\begin{array}{llll}0.403803 & 1.430891 & -4.804933\end{array}$

$\begin{array}{lll}1.105332 & 2.067130 & -5.335613\end{array}$

$\begin{array}{llll}0.518348 & 0.042170 & -4.873917\end{array}$

$\begin{array}{llll}1.304325 & -0.409685 & -5.472366\end{array}$

$\begin{array}{llll}-0.367215 & -0.779635 & -4.169450\end{array}$

$\begin{array}{llll}-0.259634 & -1.856670 & -4.232894\end{array}$

$\begin{array}{llll}-2.144091 & -2.950891 & -2.677511\end{array}$

$\begin{array}{llll}-1.704256 & -3.705078 & -1.579553\end{array}$

$\begin{array}{llll}-1.635214 & -3.238743 & -0.602087\end{array}$

$\begin{array}{llll}-1.397149 & -5.058744 & -1.737902\end{array}$

$\begin{array}{llll}-1.070409 & -5.640048 & -0.879932\end{array}$

$\begin{array}{llll}-1.520955 & -5.665288 & -2.989934\end{array}$

$\begin{array}{llll}-1.282926 & -6.718176 & -3.112345\end{array}$

$\begin{array}{llll}-1.962827 & -4.917309 & -4.086931\end{array}$

$\begin{array}{llll}-2.067368 & -5.387520 & -5.060585\end{array}$

$\begin{array}{llll}-2.279534 & -3.566968 & -3.931826\end{array}$

$\begin{array}{llll}-2.641090 & -2.996741 & -4.782688\end{array}$

$\begin{array}{llll}-4.746756 & 2.100570 & -0.055424\end{array}$

$\begin{array}{llll}-4.456715 & 3.195344 & 0.768045\end{array}$

$\begin{array}{lll}-4.284469 & 3.061327 & 1.829215\end{array}$

$\begin{array}{lll}-4.361904 & 4.479335 & 0.227860\end{array}$

$\begin{array}{llll}-4.519084 & 4.698844 & -1.138974\end{array}$

$\begin{array}{llll}-4.433699 & 5.695422 & -1.554508\end{array}$

$\begin{array}{llll}-4.770636 & 3.602117 & -1.964521\end{array}$

$\begin{array}{llll}-4.885680 & 2.317170 & -1.436208\end{array}$

$\begin{array}{llll}-5.059058 & 1.485955 & -2.113725\end{array}$

$\begin{array}{lll}-4.935075 & 0.366944 & 2.325913\end{array}$

$\begin{array}{lll}-4.297743 & -0.653070 & 3.041849\end{array}$

$\begin{array}{lll}-3.656527 & -1.349544 & 2.516596\end{array}$

$\begin{array}{lll}-4.498239 & -0.781103 & 4.417551\end{array}$

$\begin{array}{lll}-5.319474 & 0.110773 & 5.103617\end{array}$

$\begin{array}{llll}-5.464645 & 0.017061 & 6.173352\end{array}$

$\begin{array}{llll}-5.956604 & 1.125987 & 4.388128\end{array}$

$\begin{array}{lll}-5.781002 & 1.250894 & 3.010118\end{array}$

$\begin{array}{lll}-6.306691 & 2.036534 & 2.480657\end{array}$

$\begin{array}{lll}-2.671216 & -0.522967 & -0.226427\end{array}$

$\begin{array}{lll}-6.906219 & 2.058356 & 5.090742\end{array}$

$\begin{array}{lll}-3.758050 & -1.858407 & 5.160005\end{array}$

$\begin{array}{lll}-4.130359 & 5.632400 & 1.167131\end{array}$

$\begin{array}{llll}-4.828272 & 3.767861 & -3.458341\end{array}$

$\begin{array}{llll}-3.657817 & 3.384003 & -4.035672\end{array}$

$\begin{array}{lll}-5.796502 & 3.005918 & -4.015600\end{array}$

$\begin{array}{llll}-5.049031 & 5.045266 & -3.828446\end{array}$

$\begin{array}{llll}-3.649086 & 6.723996 & 0.536158\end{array}$

$\begin{array}{lll}-3.257203 & 5.307248 & 2.149800\end{array}$

$\begin{array}{llll}-5.279454 & 6.004967 & 1.782181\end{array}$

$\begin{array}{lll}-6.902983 & 3.289665 & 4.531387\end{array}$

$\begin{array}{lll}-6.602948 & 2.204304 & 6.398593\end{array}$

$\begin{array}{lll}-8.181814 & 1.605236 & 5.029660\end{array}$

$\begin{array}{lll}-4.320475 & -2.141232 & 6.353484\end{array}$

$\begin{array}{llll}-3.707153 & -3.011171 & 4.451471\end{array}$

$\begin{array}{lll}-2.472754 & -1.496969 & 5.407532\end{array}$

$\begin{array}{lll}-1.266739 & 2.088448 & 0.199442\end{array}$

$\begin{array}{lll}-0.937964 & 0.689809 & 2.052098\end{array}$ $\begin{array}{lll}-0.803961 & 3.198179 & 0.885120\end{array}$

$\begin{array}{llll}-1.586757 & 2.164885 & -0.831388\end{array}$

$\begin{array}{lll}-0.538362 & 1.829018 & 2.810988\end{array}$

$\begin{array}{llll}-0.459305 & 3.083516 & 2.234354\end{array}$

$\begin{array}{lll}-0.264167 & 1.697624 & 3.851534\end{array}$

$\begin{array}{lll}-0.770436 & -0.600790 & 2.648316\end{array}$

$\begin{array}{lll}-1.279368 & -1.854022 & 2.150290\end{array}$

$\begin{array}{lll}-1.895739 & -1.968311 & 1.045465\end{array}$

$\begin{array}{lll}-1.346183 & 0.867784 & 0.754920\end{array}$

$\begin{array}{lll}-0.493010 & -0.561639 & 3.690653\end{array}$

$\begin{array}{lll}-1.122850 & -3.002491 & 2.882601\end{array}$

$\begin{array}{llll}-1.686826 & -4.247034 & 2.372637\end{array}$

$\begin{array}{lll}-2.551844 & -4.041884 & 1.744378\end{array}$

$\begin{array}{llll}-1.992920 & -4.869390 & 3.217852\end{array}$

$\begin{array}{lll}-0.949189 & -4.807371 & 1.780849\end{array}$

$\begin{array}{llll}-0.329133 & -3.152921 & 4.092195\end{array}$

$\begin{array}{llll}0.070220 & -2.205009 & 4.437313\end{array}$

$\begin{array}{llll}0.513305 & -3.832939 & 3.909085\end{array}$

$\begin{array}{lll}-0.945477 & -3.575212 & 4.892087\end{array}$

$\begin{array}{llll}3.025390 & -1.225949 & -0.871594\end{array}$

$\begin{array}{llll}4.935058 & 0.113242 & -0.557546\end{array}$

$\begin{array}{llll}5.743252 & 0.487099 & 0.854109\end{array}$

$\begin{array}{llll}6.210483 & -0.534728 & -1.428717\end{array}$

$\begin{array}{llll}4.586144 & 1.665662 & -1.082294\end{array}$

$\begin{array}{llll}6.372502 & -0.548638 & 1.535972\end{array}$

$\begin{array}{lll}5.763048 & -0.982818 & 2.731954\end{array}$

$\begin{array}{llll}4.880296 & -0.460797 & 3.074790\end{array}$

$\begin{array}{llll}6.304056 & -2.035488 & 3.426449\end{array}$

$\begin{array}{llll}5.847806 & -2.372765 & 4.352988\end{array}$

$\begin{array}{llll}7.445318 & -2.718533 & 2.923752\end{array}$

$\begin{array}{lll}7.960192 & -3.869271 & 3.580225\end{array}$

$\begin{array}{llll}7.478512 & -4.201209 & 4.496316\end{array}$

$\begin{array}{llll}9.032822 & -4.559640 & 3.062460\end{array}$

$\begin{array}{llll}9.413608 & -5.441718 & 3.568964\end{array}$

$\begin{array}{llll}9.630678 & -4.126490 & 1.855066\end{array}$

$10.459989-4.687525 \quad 1.433927$

$\begin{array}{llll}9.168585 & -3.002729 & 1.204644\end{array}$

$\begin{array}{llll}9.633638 & -2.691747 & 0.276763\end{array}$

$\begin{array}{llll}8.076823 & -2.250689 & 1.721645\end{array}$

$\begin{array}{llll}7.558808 & -1.080809 & 1.056844\end{array}$

$\begin{array}{lll}8.240408 & -0.470368 & -0.117376\end{array}$

$\begin{array}{llll}9.619278 & -0.051611 & -0.053417\end{array}$

$\begin{array}{lll}10.365453 & -0.051221 & 1.158708\end{array}$

$\begin{array}{llll}9.885308 & -0.364296 & 2.077967\end{array}$

$\begin{array}{llll}11.682191 & 0.355355 & 1.186189\end{array}$

$\begin{array}{lll}12.224908 & 0.352462 & 2.127203\end{array}$

$\begin{array}{lll}12.331842 & 0.782968 & 0.004185\end{array}$

$\begin{array}{llll}13.371845 & 1.093877 & 0.039350\end{array}$

$\begin{array}{llll}11.634286 & 0.818923 & -1.182079\end{array}$

$\begin{array}{lll}12.112832 & 1.164090 & -2.095006\end{array}$

$\begin{array}{llll}10.270906 & 0.422020 & -1.241247\end{array}$

$\begin{array}{llll}9.535499 & 0.506722 & -2.454433\end{array}$

$\begin{array}{lll}10.039473 & 0.847717 & -3.354436\end{array}$

$\begin{array}{llll}8.199699 & 0.198134 & -2.480787\end{array}$

$\begin{array}{llll}7.606970 & 0.295382 & -3.384312\end{array}$

$\begin{array}{llll}7.556066 & -0.252378 & -1.304950\end{array}$

$\begin{array}{llll}3.189292 & 1.823585 & -1.569880\end{array}$

$\begin{array}{llll}2.570531 & 2.183448 & -0.737798\end{array}$

$\begin{array}{llll}3.082955 & 2.834396 & -2.700678\end{array}$

$\begin{array}{lll}2.051120 & 3.777648 & -2.692660\end{array}$

$\begin{array}{llll}1.372021 & 3.818000 & -1.846652\end{array}$

$\begin{array}{llll}1.877524 & 4.656943 & -3.764839\end{array}$

$\begin{array}{llll}1.069006 & 5.382934 & -3.741576\end{array}$

$2.741101 \quad 4.601655-4.860560$

$\begin{array}{llll}2.612713 & 5.286468 & -5.693974\end{array}$

$3.780139 \quad 3.663296-4.875024$

$\begin{array}{llll}4.460149 & 3.619034 & -5.721478\end{array}$

$\begin{array}{lll}3.947650 & 2.785245 & -3.804275\end{array}$

$\begin{array}{llll}4.757492 & 2.060406 & -3.811372\end{array}$

$\begin{array}{llll}2.634258 & 0.483321 & -2.072003\end{array}$

$\begin{array}{llll}3.054368 & 0.296089 & -3.066398\end{array}$

$\begin{array}{llll}1.558240 & 0.610641 & -2.210588\end{array}$

$\begin{array}{llll}5.295981 & 2.939737 & -0.740282\end{array}$

$\begin{array}{llll}4.930984 & 3.640007 & -1.494559\end{array}$

$\begin{array}{llll}4.887969 & 3.540403 & 0.605556\end{array}$ 


\begin{tabular}{|c|c|c|}
\hline 3100 & 2776 & 58 \\
\hline 3.143755 & 4.493482 & -0.227765 \\
\hline 3.372668 & 4.990010 & 1.851486 \\
\hline 2.483232 & 5.613743 & 1.873244 \\
\hline 4.171498 & 4.863960 & 2.989631 \\
\hline 3.905892 & 5.383989 & 3.906027 \\
\hline 5.316564 & 4.062997 & 2.941247 \\
\hline 5.940499 & 3.953672 & 3.824130 \\
\hline 5.665341 & 3.399744 & 1.764002 \\
\hline 6.554920 & 2.781127 & 1.746488 \\
\hline 6.806996 & 2.867898 & -0.946874 \\
\hline 7.029169 & 2.555016 & -1.968775 \\
\hline 7.303562 & 2.181230 & -0.261942 \\
\hline 7.231963 & 3.864266 & -0.792913 \\
\hline 0.893744 & -1.240541 & -0.543256 \\
\hline 0.334473 & -0.767065 & -1.341586 \\
\hline 0.468024 & -2.193405 & -0.245975 \\
\hline 1.416447 & -0.395205 & 0.503704 \\
\hline 1.396331 & 0.681451 & 0.378241 \\
\hline 1.574380 & -0.905827 & 1.81 \\
\hline 1.441416 & -1.975227 & 1.93 \\
\hline 4.070067 & -2.185618 & -2.573614 \\
\hline 4.487615 & -1.398139 & -3.195694 \\
\hline 2.668702 & -2.391584 & -2.697299 \\
\hline 2.147235 & -1.754911 & -3.408280 \\
\hline 1.946129 & -3.695890 & -2.434987 \\
\hline 2.053025 & -4.361246 & -3.305043 \\
\hline 0.880762 & -3.469716 & -2.351267 \\
\hline 2.408797 & -4.422138 & -1.155477 \\
\hline 3.230126 & -5.107725 & -1.384801 \\
\hline 1.586484 & -5.044458 & -0.787432 \\
\hline 2.843953 & -3.484664 & -0.035018 \\
\hline 2.151680 & -3.402362 & 0.795083 \\
\hline 4.149196 & -3.091448 & 0.191754 \\
\hline 4.405777 & -2.729518 & 1.183846 \\
\hline 5.319722 & -3.469169 & -0.694401 \\
\hline 6.173790 & -2.859397 & -0.401634 \\
\hline 5.598135 & -4.512178 & -0.483771 \\
\hline 5.067744 & -3.287901 & -2.210051 \\
\hline 4.723405 & -4.229387 & -2.651197 \\
\hline 6.023354 & -3.056447 & -2.688353 \\
\hline 2.199150 & -0.253758 & 2.951750 \\
\hline 2.484181 & 1.125176 & 2.992446 \\
\hline 2.518094 & -1.041770 & 4.081195 \\
\hline 3.028640 & 1.693854 & 4.144043 \\
\hline 2.284209 & 1.751008 & 2.131060 \\
\hline 3.071439 & -0.472351 & 5.221632 \\
\hline 2.334312 & -2.110874 & 4.045772 \\
\hline 3.318846 & 0.905344 & 5.259445 \\
\hline 3.230467 & 2.757317 & 4.164571 \\
\hline 3.312699 & -1.096014 & 6.077522 \\
\hline 3.744578 & 1.359141 & 6.149675 \\
\hline-0.125574 & 3.940289 & 2.809546 \\
\hline-0.65985 & 4.90916 & -0.0501 \\
\hline
\end{tabular}

\section{$[8-9]^{\ddagger}-\mathbf{P 1}-\mathrm{C} 1$ endo re-si}

Number of imaginary frequencies $=1$

The smallest frequencies are $=-212.02518 .767814 .5321 \mathrm{~cm}(-1)$ Electronic energy $=-7222.6286595$

Zero-point correction=

1.756482

(Hartree/Particle)

Thermal correction to Energy= $\quad 1.874688$

Thermal correction to Enthalpy=

1.875632

Thermal correction to Gibbs Free Energy=

Sum of electronic and zero-point Energies=

Sum of electronic and thermal Energies=

1.596626

$-7220.872177$

Sum of electronic and thermal Enthalpies=

$-7220.753971$

Sum of electronic and thermal Free Energies=

$-7220.753027$

$-7221.032033$

\section{Cartesian Coordinates}

$\begin{array}{llll}77 & 3.617031 & 1.685173 & -1.313260 \\ 15 & 5.234705 & 0.304245 & -0.330767 \\ 8 & 5.060197 & -0.987404 & 0.709001\end{array}$

$\begin{array}{lll}6.088017 & -0.464985 & -1.550010\end{array}$

$\begin{array}{lll}6.251110 & 1.261166 & 0.592513\end{array}$

$\begin{array}{lll}4.452208 & -2.155591 & 0.264830\end{array}$

$\begin{array}{llll}3.178730 & -2.440023 & 0.801847\end{array}$

$\begin{array}{lll}2.771582 & -1.752139 & 1.530148\end{array}$

$\begin{array}{llll}2.510297 & -3.576281 & 0.421677\end{array}$

$\begin{array}{llll}1.542024 & -3.813657 & 0.852552\end{array}$

$\begin{array}{llll}3.066956 & -4.435911 & -0.563268\end{array}$

$\begin{array}{llll}2.354312 & -5.573853 & -1.029917\end{array}$

$\begin{array}{lll}1.394125 & -5.804400 & -0.575717\end{array}$

$\begin{array}{llll}2.860763 & -6.360904 & -2.039928\end{array}$

$\begin{array}{llll}2.304581 & -7.223641 & -2.394769\end{array}$

$\begin{array}{llll}4.106744 & -6.033633 & -2.626881\end{array}$

$\begin{array}{llll}4.490968 & -6.636366 & -3.444744\end{array}$

$\begin{array}{llll}4.839731 & -4.959512 & -2.169212\end{array}$

$\begin{array}{llll}5.792435 & -4.725714 & -2.629592\end{array}$

$\begin{array}{llll}4.361028 & -4.139203 & -1.109617\end{array}$

$\begin{array}{llll}5.110549 & -3.014428 & -0.601918\end{array}$

$\begin{array}{llll}6.530260 & -2.774031 & -0.980317\end{array}$

$\begin{array}{llll}7.534204 & -3.799848 & -0.820415\end{array}$

$\begin{array}{lll}7.276465 & -5.032125 & -0.155718\end{array}$

$\begin{array}{llll}6.294902 & -5.215391 & 0.263864\end{array}$

$\begin{array}{llll}8.258486 & -5.989766 & -0.018433\end{array}$

$\begin{array}{llll}8.033662 & -6.916401 & 0.502159\end{array}$

$\begin{array}{llll}9.556077 & -5.776018 & -0.539726\end{array}$

$10.317468 \quad-6.542999 \quad-0.431845$

$\begin{array}{llll}9.850229 & -4.585105 & -1.164347\end{array}$

$10.848423 \quad-4.393944-1.549776$

$\begin{array}{llll}8.865845 & -3.569811 & -1.304628\end{array}$

$9.188534-2.317643 \quad-1.894116$

$10.193061 \quad-2.156064 \quad-2.274909$

$8.256344 \quad-1.313895 \quad-1.944155$

$\begin{array}{llll}8.490535 & -0.334075 & -2.347093\end{array}$

$\begin{array}{llll}6.948280 & -1.537908 & -1.454469\end{array}$

$\begin{array}{llll}5.867962 & 2.702601 & 0.594392\end{array}$

$\begin{array}{llll}5.232144 & 2.886296 & 1.470279\end{array}$

$\begin{array}{lll}7.081866 & 3.613322 & 0.692818\end{array}$

$\begin{array}{lll}7.066818 & 4.710164 & 1.561662\end{array}$

$\begin{array}{llll}6.203899 & 4.869230 & 2.204232\end{array}$

$\begin{array}{llll}8.148041 & 5.595251 & 1.612120\end{array}$

$\begin{array}{lll}8.121619 & 6.441669 & 2.292981\end{array}$

$\begin{array}{llll}9.261975 & 5.385882 & 0.797128\end{array}$

$\begin{array}{lll}10.106478 & 6.067903 & 0.839651\end{array}$

$\begin{array}{llll}9.286809 & 4.289695 & -0.072264\end{array}$

$10.152262 \quad 4.118260 \quad-0.706651$

$\begin{array}{llll}8.203372 & 3.413878 & -0.127094\end{array}$

$\begin{array}{llll}8.224426 & 2.556507 & -0.794883\end{array}$

$\begin{array}{llll}5.089628 & 3.069255 & -0.684988\end{array}$

$\begin{array}{llll}5.815095 & 3.191310 & -1.498584\end{array}$

$\begin{array}{lll}4.637417 & 4.053591 & -0.517788\end{array}$

$\begin{array}{lll}7.177636 & 0.838054 & 1.691401\end{array}$

$\begin{array}{llll}7.854773 & 1.688357 & 1.790707\end{array}$

$\begin{array}{lll}6.514167 & 0.684171 & 3.061145\end{array}$

$\begin{array}{lll}6.490380 & 1.790650 & 3.923662\end{array}$

$\begin{array}{lll}6.885469 & 2.741696 & 3.576178\end{array}$

$\begin{array}{llll}6.006763 & 1.678091 & 5.229597\end{array}$

$\begin{array}{llll}6.009964 & 2.546023 & 5.883754\end{array}$

$\begin{array}{llll}5.542914 & 0.447364 & 5.696897\end{array}$

$\begin{array}{llll}5.176456 & 0.350489 & 6.715224\end{array}$

$\begin{array}{llll}5.546166 & -0.658554 & 4.842620\end{array}$

$\begin{array}{llll}5.180137 & -1.618810 & 5.195634\end{array}$

$\begin{array}{llll}6.025847 & -0.541676 & 3.537589\end{array}$

$\begin{array}{lll}6.032016 & -1.413154 & 2.894487\end{array}$

$\begin{array}{lll}8.068968 & -0.346826 & 1.322488\end{array}$

$\begin{array}{lll}8.623367 & -0.130729 & 0.407322\end{array}$

$\begin{array}{lll}7.516788 & -1.275162 & 1.179877\end{array}$

$\begin{array}{lll}8.790704 & -0.504828 & 2.129639\end{array}$

$\begin{array}{llll}4.644301 & 1.414179 & -3.257535\end{array}$

$\begin{array}{llll}5.700390 & 1.627803 & -3.112943\end{array}$

$\begin{array}{llll}3.787857 & 2.547038 & -3.326181\end{array}$

$\begin{array}{llll}4.265895 & 3.522347 & -3.237950\end{array}$

$\begin{array}{llll}2.465991 & 2.596001 & -4.068468\end{array}$

$\begin{array}{lll}2.655019 & 2.742674 & -5.142598\end{array}$

$\begin{array}{llll}1.926585 & 3.486305 & -3.729987\end{array}$

$\begin{array}{llll}1.575212 & 1.353696 & -3.859259\end{array}$ 
$\begin{array}{llll}1.795802 & 0.598769 & -4.618416\end{array}$

$\begin{array}{llll}0.531043 & 1.635058 & -4.015185\end{array}$

$\begin{array}{llll}1.699830 & 0.713289 & -2.483250\end{array}$

$\begin{array}{llll}0.864901 & 0.882942 & -1.810564\end{array}$

$\begin{array}{llll}2.548278 & -0.333631 & -2.198881\end{array}$

$\begin{array}{llll}2.328202 & -0.940035 & -1.323732\end{array}$

$\begin{array}{llll}3.521125 & -0.932356 & -3.195976\end{array}$

$\begin{array}{llll}4.185788 & -1.606512 & -2.657640\end{array}$

$2.951881 \quad-1.557904 \quad-3.895403$

$\begin{array}{llll}4.371182 & 0.093951 & -3.982750\end{array}$

$\begin{array}{llll}3.897869 & 0.318172 & -4.945057\end{array}$

$\begin{array}{llll}5.332591 & -0.371519 & -4.216033\end{array}$

$\begin{array}{lll}-2.244775 & -0.069496 & 3.242668\end{array}$

$\begin{array}{lll}-1.153237 & 1.807847 & 2.357390\end{array}$

$\begin{array}{lll}-1.994735 & 0.334465 & 4.539300\end{array}$

$\begin{array}{lll}-2.775325 & -0.988103 & 3.033932\end{array}$

$\begin{array}{lll}-0.881926 & 2.247482 & 3.682681\end{array}$

$\begin{array}{lll}-1.296297 & 1.521248 & 4.778572\end{array}$

$\begin{array}{lll}-0.348713 & 3.179613 & 3.825615\end{array}$

$\begin{array}{lll}-0.646871 & 2.599179 & 1.272709\end{array}$

$\begin{array}{lll}-1.114118 & 2.538599 & -0.099406\end{array}$

$\begin{array}{llll}-1.408978 & 1.447744 & -0.683074\end{array}$

$\begin{array}{lll}-1.835708 & 0.639019 & 2.171117\end{array}$

$\begin{array}{lll}-0.242074 & 3.547107 & 1.597720\end{array}$

$\begin{array}{lll}-1.160482 & 3.699815 & -0.819422\end{array}$

$\begin{array}{llll}-1.310144 & 3.663555 & -2.269311\end{array}$

$\begin{array}{llll}-2.313544 & 3.978669 & -2.571981\end{array}$

$\begin{array}{llll}-0.586219 & 4.348500 & -2.725587\end{array}$

$\begin{array}{llll}-1.123889 & 2.657531 & -2.634180\end{array}$

$\begin{array}{lll}-1.020050 & 5.035656 & -0.247361\end{array}$

$\begin{array}{lll}-1.377699 & 5.064673 & 0.780589\end{array}$

$\begin{array}{llll}0.021153 & 5.381874 & -0.280167\end{array}$

$\begin{array}{llll}-1.634508 & 5.724611 & -0.832478\end{array}$

$\begin{array}{llll}2.160936 & 3.238104 & -0.941991\end{array}$

$\begin{array}{llll}1.230727 & 3.097509 & -1.477018\end{array}$

$\begin{array}{llll}2.568720 & 4.240896 & -1.059206\end{array}$

$\begin{array}{llll}2.323147 & 2.616935 & 0.347598\end{array}$

$\begin{array}{lll}2.966607 & 3.120054 & 1.062806\end{array}$

$\begin{array}{llll}1.476249 & 1.590445 & 0.858822\end{array}$

$\begin{array}{llll}0.911595 & 0.997217 & 0.152200\end{array}$

$\begin{array}{lll}1.682524 & 0.967117 & 2.163286\end{array}$

$\begin{array}{llll}2.367595 & 1.598057 & 3.219490\end{array}$

$\begin{array}{lll}1.097285 & -0.289931 & 2.404664\end{array}$

$\begin{array}{llll}2.467347 & 0.983657 & 4.464301\end{array}$

$\begin{array}{lll}2.809498 & 2.578500 & 3.076368\end{array}$

$\begin{array}{lll}1.206809 & -0.908777 & 3.647151\end{array}$

$\begin{array}{lll}0.551681 & -0.769494 & 1.599278\end{array}$

$\begin{array}{lll}1.895058 & -0.273285 & 4.683058\end{array}$

$\begin{array}{lll}2.999152 & 1.483875 & 5.265313\end{array}$

$\begin{array}{llll}0.750967 & -1.880674 & 3.807800\end{array}$

$\begin{array}{lll}1.982488 & -0.749831 & 5.655097\end{array}$

$\begin{array}{llll}-1.084385 & 1.862322 & 5.785877\end{array}$

$\begin{array}{lll}-2.599275 & -0.779716 & 6.029734\end{array}$

$\begin{array}{lll}-6.539922 & -2.911334 & 2.398187\end{array}$

$\begin{array}{lll}-2.221957 & -2.221189 & -0.490513\end{array}$

$\begin{array}{llll}-4.611280 & 0.673053 & -0.111081\end{array}$

$\begin{array}{llll}-5.036001 & -2.534740 & 0.984262\end{array}$

$\begin{array}{lll}-5.531721 & -1.306012 & 1.557194\end{array}$

$\begin{array}{lll}-5.311250 & -1.364139 & 2.968217\end{array}$

$\begin{array}{lll}-5.584913 & -0.600804 & 3.682873\end{array}$

$\begin{array}{lll}-4.705053 & -2.620121 & 3.277232\end{array}$

$\begin{array}{llll}-4.448898 & -2.974155 & 4.265978\end{array}$

$\begin{array}{llll}-4.536955 & -3.339374 & 2.058488\end{array}$

$\begin{array}{lll}-4.124989 & -4.332520 & 1.954006\end{array}$

$\begin{array}{lll}-8.525171 & -2.978125 & 1.781222\end{array}$

$\begin{array}{lll}-8.934801 & -2.418656 & 0.950718\end{array}$

$\begin{array}{lll}-8.440209 & -2.527693 & 3.134110\end{array}$

$\begin{array}{lll}-8.764220 & -1.564539 & 3.505445\end{array}$

$\begin{array}{lll}-7.811598 & -3.554195 & 3.902450\end{array}$

$\begin{array}{llll}-7.569139 & -3.501305 & 4.955712\end{array}$

$\begin{array}{lll}-7.507993 & -4.639395 & 3.025106\end{array}$

$\begin{array}{llll}-6.997996 & -5.554274 & 3.296786\end{array}$

$\begin{array}{llll}-7.950247 & -4.285131 & 1.716056\end{array}$

$\begin{array}{lll}-7.837364 & -4.889692 & 0.826509\end{array}$

$\begin{array}{lll}-5.010912 & -2.864216 & -0.469195\end{array}$ $\begin{array}{llll}-6.214934 & -3.168737 & -1.122582\end{array}$

$\begin{array}{llll}-7.128242 & -3.152396 & -0.542607\end{array}$

$-6.259521 \quad-3.477936-2.480946$

$\begin{array}{llll}-7.211249 & -3.682556 & -2.960508\end{array}$

$\begin{array}{lll}-5.075998 & -3.508984 & -3.213948\end{array}$

$-5.088363-3.753213-4.271424$

$\begin{array}{llll}-3.868462 & -3.197644 & -2.589023\end{array}$

$-2.961811 \quad-3.211514-3.181326$

$\begin{array}{llll}-3.811923 & -2.847667 & -1.229644\end{array}$

$\begin{array}{lll}-6.084234 & -0.147198 & 0.769380\end{array}$

$\begin{array}{lll}-6.703670 & -0.558748 & -0.028646\end{array}$

$\begin{array}{lll}-6.944441 & 0.813140 & 1.595553\end{array}$

$\begin{array}{lll}-7.370151 & 1.598332 & 0.965115\end{array}$

$\begin{array}{lll}-6.375300 & 1.294945 & 2.394847\end{array}$

$\begin{array}{lll}-7.763511 & 0.253315 & 2.057377\end{array}$

$-1.068379-2.287438-1.926668$

$\begin{array}{llll}-1.188787 & -1.276047 & -2.895517\end{array}$

$\begin{array}{lll}-1.862260 & -0.444070 & -2.724878\end{array}$

$-0.465199 \quad-1.349721 \quad-4.085156$

$\begin{array}{lll}-0.592257 & -0.581028 & -4.838062\end{array}$

$\begin{array}{llll}0.425242 & -2.405067 & -4.300818\end{array}$

$\begin{array}{llll}0.988693 & -2.459736 & -5.227813\end{array}$

$\begin{array}{llll}0.603092 & -3.375620 & -3.313633\end{array}$

$\begin{array}{llll}1.317393 & -4.181465 & -3.453833\end{array}$

$\begin{array}{llll}1.0 .148557 & -3.324111 & -2.137667\end{array}$

$\begin{array}{llll}-0.018462 & -4.102378 & -1.396460\end{array}$

$\begin{array}{llll}-1.673868 & -3.586771 & 0.607505\end{array}$

$\begin{array}{lll}-1.852819 & -4.936226 & 0.262301\end{array}$

$\begin{array}{lll}-2.329221 & -5.197022 & -0.678316\end{array}$

$\begin{array}{llll}-1.428062 & -5.945091 & 1.128386\end{array}$

$\begin{array}{llll}-1.569850 & -6.986639 & 0.854590\end{array}$

$\begin{array}{llll}-0.827883 & -5.615696 & 2.348820\end{array}$

$\begin{array}{lll}-0.502074 & -6.402129 & 3.023324\end{array}$

$\begin{array}{lll}-0.655375 & -4.275292 & 2.701692\end{array}$

$\begin{array}{lll}-0.198608 & -4.017289 & 3.652650\end{array}$

$\begin{array}{lll}-1.077587 & -3.265023 & 1.834210\end{array}$

$\begin{array}{llll}-0.965899 & -2.225091 & 2.117222\end{array}$

$\begin{array}{llll}-4.970649 & 0.608936 & -1.917294\end{array}$

$\begin{array}{llll}-3.975824 & 1.140893 & -2.750355\end{array}$

$\begin{array}{llll}-3.083388 & 1.568427 & -2.304349\end{array}$

$\begin{array}{lll}-4.120814 & 1.106069 & -4.137874\end{array}$

$\begin{array}{llll}-5.228500 & 0.498604 & -4.724301\end{array}$

$\begin{array}{llll}-5.329145 & 0.454812 & -5.802618\end{array}$

$\begin{array}{llll}-6.201043 & -0.059499 & -3.894004\end{array}$

$\begin{array}{llll}-6.090501 & 0.009766 & -2.505643\end{array}$

$\begin{array}{lll}-6.876543 & -0.425801 & -1.905235\end{array}$

$\begin{array}{llll}-4.676696 & 2.478191 & 0.260570\end{array}$

$\begin{array}{lll}-4.247204 & 2.877306 & 1.537241\end{array}$

$\begin{array}{llll}-3.985688 & 2.133894 & 2.280228\end{array}$

$\begin{array}{lll}-4.122192 & 4.228609 & 1.848706\end{array}$

$\begin{array}{llll}-4.392156 & 5.211555 & 0.895149\end{array}$

$\begin{array}{llll}-4.267569 & 6.261979 & 1.132108\end{array}$

$\begin{array}{lll}-4.816115 & 4.815319 & -0.369809\end{array}$

$\begin{array}{llll}-4.972593 & 3.463824 & -0.686872\end{array}$

$\begin{array}{llll}-5.309166 & 3.197044 & -1.681079\end{array}$

$\begin{array}{lll}-2.465066 & -0.139101 & 0.349486\end{array}$

$\begin{array}{llll}-5.001200 & 5.844480 & -1.449287\end{array}$

$\begin{array}{lll}-3.579665 & 4.651342 & 3.185120\end{array}$

$\begin{array}{llll}-3.073419 & 1.770510 & -4.985791\end{array}$

$\begin{array}{lll}-7.361062 & -0.774419 & -4.532514\end{array}$

$\begin{array}{lll}-6.952409 & -1.883110 & -5.198030\end{array}$

$\begin{array}{llll}-8.285453 & -1.172413 & -3.630637\end{array}$

$\begin{array}{lll}-7.991782 & 0.005992 & -5.440214\end{array}$

$\begin{array}{llll}-1.816483 & 1.413972 & -4.600903\end{array}$

$\begin{array}{lll}-3.127571 & 3.120383 & -4.876202\end{array}$

$\begin{array}{llll}-3.189308 & 1.468000 & -6.292270\end{array}$

$\begin{array}{llll}-3.844810 & 6.027950 & -2.146837\end{array}$

$\begin{array}{llll}-5.351741 & 7.049987 & -0.956347\end{array}$

$\begin{array}{llll}-5.938337 & 5.479501 & -2.350185\end{array}$

$\begin{array}{lll}-4.201122 & 5.753969 & 3.657123\end{array}$

$\begin{array}{lll}-3.695264 & 3.684946 & 4.119524\end{array}$

$\begin{array}{lll}-2.256775 & 4.964472 & 3.100598\end{array}$ 
Number of imaginary frequencies $=0$

The smallest frequencies are $=12.227313 .994316 .3898 \mathrm{~cm}(-1)$ Electronic energy $=-7222.6449288$

Zero-point correction=

1.757633

(Hartree/Particle)

Thermal correction to Energy=
Thermal correction to Enthalpy

1.876644

1.877588

Thermal correction to Gibbs Free Energy=

1.595936

Sum of electronic and zero-point Energies=

$-7220.887296$

$-7220.768285$

Sum of electronic and thermal Energies

$-7220.767341$

Sum of electronic and thermal Enthalpies=

$-7221.048993$

Cartesian Coordinates

\begin{tabular}{|c|c|c|c|}
\hline 6 & 7.925814 & -0.325224 & -1.1 \\
\hline & 2.922148 & 0.805089 & -2.273970 \\
\hline 15 & 4.030165 & 0.950354 & 1.306089 \\
\hline 6 & 5.998236 & 0.434263 & -1.456759 \\
\hline 6 & 6.181396 & 0.039836 & -0.081598 \\
\hline 6 & 6.494505 & -1.354101 & -0.064761 \\
\hline 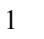 & 6.703414 & -1.945704 & 0.816129 \\
\hline 6 & 6.539492 & -1.817961 & -1.413029 \\
\hline 1 & 6.786649 & -2.821197 & -1.729566 \\
\hline 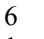 & 6.231513 & -0.720903 & -2.270311 \\
\hline 1 & 6.204419 & -0.743662 & -3.349671 \\
\hline 6 & 9.449517 & 0.946475 & -0.519666 \\
\hline 1 & 9.302701 & 1.824772 & 0.094856 \\
\hline 6 & 9.684464 & -0.379525 & -0.042923 \\
\hline 1 & 9.742400 & -0.681957 & 0.994222 \\
\hline 6 & 9.781060 & -1.245800 & -1.174419 \\
\hline 1 & 9.919926 & -2.318443 & -1.143464 \\
\hline 6 & 9.604910 & -0.456108 & -2.351115 \\
\hline 1 & 9.590076 & -0.823746 & -3.368586 \\
\hline 6 & 9.402240 & 0.897433 & -1.947626 \\
\hline 1 & 9.214803 & 1.731169 & -2.610324 \\
\hline 6 & 5.581058 & $1.7 \mathrm{~S}$ & -1.884723 \\
\hline 6 & 6.496532 & 2.857024 & -1.769087 \\
\hline 1 & 7.487919 & 2.632083 & -1.398927 \\
\hline 6 & 6.158489 & 4.167144 & -2.104324 \\
\hline 1 & 6.886855 & 4.963352 & -1.985809 \\
\hline 6 & 4.880405 & 4.441331 & -2.584728 \\
\hline 1 & 4.596885 & 5.45 & -2.853684 \\
\hline 6 & 3.949029 & 3.409321 & -2.693197 \\
\hline 1 & 2.954232 & 3.648722 & -3.047782 \\
\hline 6 & 4.264640 & 2.08 & -2.330954 \\
\hline 6 & 5.919411 & 0.938114 & 1.094939 \\
\hline 1 & 6.184337 & 1.955500 & 0.801017 \\
\hline 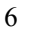 & 6.718367 & 0.58 & 2.352443 \\
\hline 1 & 6.495671 & 1.277847 & 3.168714 \\
\hline 1 & 6.509126 & -0.427933 & 2.706243 \\
\hline 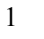 & 7.787432 & 0.646327 & 2.125911 \\
\hline 6 & 1.448430 & 1.769654 & -2.817881 \\
\hline 6 & 0.689774 & 2.406650 & -1.824343 \\
\hline 2 & 0.916632 & 2.224623 & -0.779689 \\
\hline 6 & -0.357839 & 3.259972 & -2.173672 \\
\hline 1 & -0.930258 & 3.753986 & -1.398155 \\
\hline 6 & -0.681650 & 3.457819 & -3.517713 \\
\hline 1 & -1.502779 & 4.116767 & -3.785825 \\
\hline 6 & 0.044160 & 2.795651 & -4.512405 \\
\hline 1 & -0.210690 & 2.936256 & -5.559079 \\
\hline 6 & 1.109999 & 1.962801 & -4.166295 \\
\hline 1 & 1.680649 & 1.473063 & -4.948597 \\
\hline 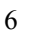 & 3.246914 & -0.299572 & -3.705513 \\
\hline 6 & 4.024431 & 0.075192 & -4.811141 \\
\hline 1 & 4.489441 & 1.056046 & -4.841388 \\
\hline 6 & 4.214063 & -0.816806 & -5.869087 \\
\hline . & 4.822328 & -0.521990 & -6.719524 \\
\hline 6 & 3.623844 & -2.084206 & -5.836469 \\
\hline 1 & 3.776506 & -2.776069 & -6.659737 \\
\hline 6 & 2.839779 & -2.460217 & -4.742703 \\
\hline- & 2.379132 & -3.443346 & -4.710467 \\
\hline & 2.657067 & -1.571959 & -3.682046 \\
\hline & 2.064445 & -1.864330 & -2.820951 \\
\hline & 3.500772 & 2.713180 & 1.32806 \\
\hline
\end{tabular}

$\begin{array}{lll}2.121969 & 2.921969 & 1.479330\end{array}$

$\begin{array}{lll}1.469818 & 2.065264 & 1.618908\end{array}$

$\begin{array}{lll}1.585456 & 4.207387 & 1.398662\end{array}$

$\begin{array}{llll}2.400871 & 5.305844 & 1.134333\end{array}$

$\begin{array}{lll}1.980961 & 6.301780 & 1.051005\end{array}$

$\begin{array}{lll}3.769925 & 5.092640 & 0.962481\end{array}$

$\begin{array}{lll}4.323109 & 3.816256 & 1.067320\end{array}$

$\begin{array}{lll}5.388602 & 3.701662 & 0.919449\end{array}$

$\begin{array}{lll}3.713243 & 0.379189 & 3.025056\end{array}$

$\begin{array}{llll}3.852063 & -1.000539 & 3.246024\end{array}$

$\begin{array}{lll}4.161940 & -1.648693 & 2.434777\end{array}$

$\begin{array}{llll}3.565036 & -1.545552 & 4.493093\end{array}$

$\begin{array}{lll}3.087450 & -0.741836 & 5.530667\end{array}$

$\begin{array}{llll}2.830596 & -1.174408 & 6.490813\end{array}$

$\begin{array}{lll}2.924830 & 0.620937 & 5.301693\end{array}$

$\begin{array}{lll}3.249737 & 1.187842 & 4.065021\end{array}$

$\begin{array}{lll}3.119609 & 2.254864 & 3.923956\end{array}$

$2.700866 \quad-0.193534 \quad-0.250734$

$\begin{array}{lll}2.287902 & 1.491938 & 6.345678\end{array}$

$\begin{array}{llll}3.628336 & -3.032096 & 4.701745\end{array}$

$\begin{array}{lll}0.108236 & 4.377453 & 1.616582\end{array}$

$\begin{array}{llll}4.637740 & 6.275913 & 0.629617\end{array}$

$\begin{array}{llll}4.287974 & 6.826188 & -0.559149\end{array}$

$\begin{array}{llll}5.947621 & 5.955068 & 0.550604\end{array}$

$\begin{array}{lll}4.518696 & 7.257098 & 1.554576\end{array}$

$\begin{array}{lll}-0.609107 & 3.450289 & 0.917727\end{array}$

$\begin{array}{lll}-0.237014 & 4.213746 & 2.914325\end{array}$

$\begin{array}{lll}-0.344545 & 5.587847 & 1.228843\end{array}$

$\begin{array}{lll}2.855183 & 2.717586 & 6.403121\end{array}$

$\begin{array}{llll}0.968579 & 1.693079 & 6.071378\end{array}$

$\begin{array}{llll}2.351297 & 0.953628 & 7.580985\end{array}$

$\begin{array}{lll}4.179514 & -3.350635 & 5.895001\end{array}$

$\begin{array}{lll}4.339859 & -3.660831 & 3.743098\end{array}$

$\begin{array}{llll}2.384706 & -3.585362 & 4.696763\end{array}$

$\begin{array}{lll}0.708598 & -0.836216 & 1.902132\end{array}$

$\begin{array}{lll}1.145430 & -2.184002 & 1.953592\end{array}$

$\begin{array}{lll}2.019429 & -2.861564 & 1.076952\end{array}$

$\begin{array}{lll}0.782115 & -2.794423 & 2.766981\end{array}$

$\begin{array}{llll}-3.211574 & -1.482140 & -1.122462\end{array}$

$\begin{array}{llll}-5.072355 & -0.469072 & -0.309144\end{array}$

$\begin{array}{lll}-5.041297 & 0.887456 & 0.620831\end{array}$

$\begin{array}{llll}-6.067192 & 0.015503 & -1.543218\end{array}$

$\begin{array}{lll}-5.835475 & -1.520125 & 0.736256\end{array}$

$\begin{array}{lll}-4.903476 & 2.165492 & 0.082417\end{array}$

$\begin{array}{lll}-3.720457 & 2.860830 & 0.416048\end{array}$

$\begin{array}{lll}-3.005546 & 2.390050 & 1.081903\end{array}$

$\begin{array}{llll}-3.504110 & 4.111937 & -0.106891\end{array}$

$\begin{array}{llll}-2.610258 & 4.667407 & 0.154640\end{array}$

$\begin{array}{llll}-4.429819 & 4.680661 & -1.021813\end{array}$

$\begin{array}{llll}-4.159948 & 5.923329 & -1.656847\end{array}$

$\begin{array}{llll}-3.240188 & 6.444947 & -1.405280\end{array}$

$\begin{array}{llll}-5.034120 & 6.448223 & -2.581740\end{array}$

$\begin{array}{llll}-4.815395 & 7.394207 & -3.068253\end{array}$

$\begin{array}{lll}-6.217086 & 5.744013 & -2.910712\end{array}$

$\begin{array}{llll}-6.894207 & 6.149833 & -3.656696\end{array}$

$\begin{array}{llll}-6.517188 & 4.546090 & -2.299105\end{array}$

$\begin{array}{llll}-7.422795 & 4.017107 & -2.571679\end{array}$

$\begin{array}{llll}-5.644538 & 3.980085 & -1.328379\end{array}$

$\begin{array}{lll}-5.915939 & 2.718918 & -0.686572\end{array}$

$\begin{array}{llll}-7.217351 & 2.012640 & -0.854442\end{array}$

$\begin{array}{lll}-8.471583 & 2.637452 & -0.518945\end{array}$

$\begin{array}{lll}-8.551240 & 3.915745 & 0.101137\end{array}$

$\begin{array}{lll}-7.639412 & 4.448055 & 0.343129\end{array}$

$\begin{array}{lll}-9.769058 & 4.481302 & 0.411080\end{array}$

$\begin{array}{lll}-9.801597 & 5.455367 & 0.890550\end{array}$

$\begin{array}{lll}-10.976781 & 3.804320 & 0.118374\end{array}$

$\begin{array}{llll}-11.928772 & 4.266696 & 0.361934\end{array}$

$\begin{array}{llll}-10.937582 & 2.556030 & -0.459983\end{array}$

$\begin{array}{llll}-11.856219 & 2.015696 & -0.672888\end{array}$

$\begin{array}{llll}-9.698168 & 1.936224 & -0.777212\end{array}$

$\begin{array}{llll}-9.655857 & 0.626591 & -1.327494\end{array}$

$\begin{array}{llll}-10.588673 & 0.109889 & -1.534727\end{array}$

$\begin{array}{llll}-8.453258 & 0.008042 & -1.564177\end{array}$

$\begin{array}{llll}-8.393949 & -1.002366 & -1.953490\end{array}$

$\begin{array}{llll}-7.253863 & 0.701769 & -1.298145\end{array}$ 
$\begin{array}{lll}-5.336342 & -2.916192 & 0.620096\end{array}$

$\begin{array}{lll}-4.666604 & -3.102881 & 1.466805\end{array}$

$\begin{array}{lll}-6.467569 & -3.927946 & 0.683626\end{array}$

$\begin{array}{lll}-6.284294 & -5.130019 & 1.377392\end{array}$

$\begin{array}{lll}-5.350172 & -5.305772 & 1.906690\end{array}$

$\begin{array}{lll}-7.289861 & -6.100122 & 1.395407\end{array}$

$\begin{array}{lll}-7.135114 & -7.028251 & 1.938636\end{array}$

$\begin{array}{lll}-8.493789 & -5.872026 & 0.725328\end{array}$

$\begin{array}{llll}-9.279300 & -6.622170 & 0.743970\end{array}$

$\begin{array}{lll}-8.685896 & -4.670458 & 0.035597\end{array}$

$\begin{array}{llll}-9.622637 & -4.484669 & -0.482959\end{array}$

$\begin{array}{llll}-7.678144 & -3.706268 & 0.011943\end{array}$

$\begin{array}{lll}-7.829178 & -2.767961 & -0.513660\end{array}$

$\begin{array}{llll}-4.544356 & -3.093554 & -0.683091\end{array}$

$\begin{array}{llll}-5.249358 & -3.204875 & -1.514899\end{array}$

$\begin{array}{llll}-3.972165 & -4.021951 & -0.627821\end{array}$

$\begin{array}{llll}-6.526263 & -1.209345 & 2.036833\end{array}$

$\begin{array}{lll}-7.041500 & -2.145703 & 2.266541\end{array}$

$\begin{array}{lll}-5.523556 & -0.976156 & 3.165555\end{array}$

$\begin{array}{lll}-5.028534 & -2.082952 & 3.873462\end{array}$

$\begin{array}{lll}-5.397760 & -3.077251 & 3.636084\end{array}$

$\begin{array}{llll}-4.088861 & -1.925666 & 4.895517\end{array}$

$\begin{array}{lll}-3.720538 & -2.797213 & 5.429609\end{array}$

$\begin{array}{lll}-3.641106 & -0.649228 & 5.240888\end{array}$

$\begin{array}{lll}-2.915134 & -0.520817 & 6.038165\end{array}$

$\begin{array}{lll}-4.143465 & 0.463192 & 4.559930\end{array}$

$\begin{array}{llll}-3.813305 & 1.461817 & 4.832560\end{array}$

$\begin{array}{lll}-5.073870 & 0.301304 & 3.531425\end{array}$

$\begin{array}{lll}-5.450135 & 1.177166 & 3.017821\end{array}$

$\begin{array}{lll}-7.619972 & -0.149005 & 1.926030\end{array}$

$\begin{array}{lll}-8.332792 & -0.426279 & 1.146904\end{array}$

$\begin{array}{lll}-7.237701 & 0.845788 & 1.701689\end{array}$

$\begin{array}{lll}-8.154188 & -0.098597 & 2.879699\end{array}$

$\begin{array}{lll}-2.362657 & -1.104933 & 0.863865\end{array}$

$\begin{array}{lll}-1.614309 & -0.331488 & 0.736158\end{array}$

$\begin{array}{llll}-3.045478 & -0.943347 & 1.694039\end{array}$

$\begin{array}{lll}-2.010206 & -2.460870 & 0.580295\end{array}$

$\begin{array}{llll}-2.572059 & -3.246636 & 1.072994\end{array}$

$\begin{array}{llll}-1.065027 & -2.808574 & -0.384659\end{array}$

$\begin{array}{lll}-0.338560 & -2.059441 & -0.672341\end{array}$

$\begin{array}{lll}-4.067174 & -1.434412 & -3.227217\end{array}$

$\begin{array}{llll}-5.082178 & -1.805557 & -3.125945\end{array}$

$\begin{array}{llll}-3.040762 & -2.391668 & -3.199937\end{array}$

$\begin{array}{llll}-3.332660 & -3.435448 & -3.092064\end{array}$

$\begin{array}{llll}-1.649344 & -2.185603 & -3.765441\end{array}$

$\begin{array}{llll}-1.681757 & -2.344268 & -4.853669\end{array}$

$\begin{array}{llll}-1.009794 & -2.973774 & -3.363024\end{array}$

$\begin{array}{llll}-1.014042 & -0.810886 & -3.461598\end{array}$

$\begin{array}{llll}-1.258610 & -0.093658 & -4.249446\end{array}$

$\begin{array}{llll}0.072165 & -0.914731 & -3.487306\end{array}$

$\begin{array}{lll}-1.402849 & -0.203269 & -2.120457\end{array}$

$\begin{array}{lll}-0.632285 & -0.174569 & -1.354500\end{array}$

$\begin{array}{llll}-2.474868 & 0.647652 & -1.948413\end{array}$

$\begin{array}{llll}-2.458766 & 1.284001 & -1.069642\end{array}$

$\begin{array}{llll}-3.438540 & 1.069741 & -3.037405\end{array}$

$\begin{array}{llll}-4.291752 & 1.555954 & -2.564765\end{array}$

$\begin{array}{llll}-2.945924 & 1.832806 & -3.653656\end{array}$

$\begin{array}{llll}-3.950668 & -0.082373 & -3.929422\end{array}$

$\begin{array}{lll}-3.305348 & -0.200219 & -4.805016\end{array}$

$\begin{array}{llll}-4.938933 & 0.189764 & -4.309865\end{array}$

$\begin{array}{lll}-0.760032 & -4.170959 & -0.822647\end{array}$

$\begin{array}{llll}-1.506417 & -5.303644 & -0.437704\end{array}$

$\begin{array}{llll}0.349161 & -4.362239 & -1.669755\end{array}$

$\begin{array}{llll}-1.156086 & -6.572150 & -0.893974\end{array}$

$\begin{array}{llll}-2.354830 & -5.198931 & 0.230317\end{array}$

$\begin{array}{llll}0.698207 & -5.631245 & -2.126058\end{array}$

$\begin{array}{llll}0.951638 & -3.503591 & -1.945698\end{array}$

$\begin{array}{llll}-0.056581 & -6.742560 & -1.742888\end{array}$

$\begin{array}{llll}-1.741279 & -7.433624 & -0.584811\end{array}$

$\begin{array}{llll}1.566098 & -5.754269 & -2.766924\end{array}$

$\begin{array}{llll}0.212762 & -7.734347 & -2.094270\end{array}$

$\begin{array}{llll}2.256449 & -4.260521 & 1.302117\end{array}$

$\begin{array}{llll}3.092895 & -4.991072 & 0.494639\end{array}$

$\begin{array}{lll}1.754882 & -4.743240 & 2.134381\end{array}$

$\begin{array}{llll}3.725217 & -4.321054 & -0.566510\end{array}$ $\begin{array}{llll}3.511412 & -2.973262 & -0.747986\end{array}$

$\begin{array}{llll}4.004475 & -2.431397 & -1.543307\end{array}$

$\begin{array}{llll}2.689339 & -2.247853 & 0.040284\end{array}$

$\begin{array}{llll}0.877319 & -0.044638 & 0.901305\end{array}$

$\begin{array}{lll}0.025860 & -0.333891 & 2.997855\end{array}$

$\begin{array}{lll}-0.640796 & 0.957006 & 2.946396\end{array}$

$\begin{array}{lll}-1.696931 & 0.851147 & 3.222895\end{array}$

$\begin{array}{lll}-0.177926 & 1.666894 & 3.642681\end{array}$

$\begin{array}{lll}-0.580844 & 1.359081 & 1.940471\end{array}$

$\begin{array}{lll}-0.207341 & -1.091612 & 4.219022\end{array}$

$\begin{array}{lll}1.108677 & -1.716421 & 4.153651\end{array}$

$\begin{array}{llll}0.644840 & -1.724226 & 4.463700\end{array}$

$\begin{array}{lll}-0.340337 & -0.386551 & 5.043792\end{array}$

$\begin{array}{lll}3.250486 & -6.050471 & 0.666791\end{array}$

$\begin{array}{llll}4.892871 & -5.296552 & -1.807416\end{array}$

[8-9] ${ }^{\ddagger}-\mathbf{P 1 - C 2}$ endo si-re

Number of imaginary frequencies $=1$

The smallest frequencies are $=-255.83779 .994911 .5913 \mathrm{~cm}(-1)$ Electronic energy $=-7222.6359279$

Zero-point correction=

1.754830

Hartree/Particle)

Thermal correction to Energy=

Thermal correction to Enthalpy=

1.873567

Thermal correction to Gibbs Free Energy $=\quad 1.591721$

Sum of electronic and zero-point Energies $=\quad-7220.881098$

Sum of electronic and thermal Energies=

$-7220.762361$

Sum of electronic and thermal Enthalpies=

$-7220.761417$

Sum of electronic and thermal Free Energies $=\quad-7221.044206$

Cartesian Coordinates

$\begin{array}{lccc}26 & 8.013585 & 0.343897 & -1.088066 \\ 15 & 2.922532 & 1.119765 & -2.182064 \\ 15 & 3.990229 & 0.789981 & 1.429510 \\ 6 & 6.009727 & 0.919138 & -1.309794 \\ 6 & 6.234266 & 0.288003 & -0.031298 \\ 6 & 6.714893 & -1.035525 & -0.275536 \\ 1 & 6.991852 & -1.757614 & 0.480056 \\ 6 & 6.821167 & -1.218896 & -1.687297 \\ 1 & 7.193866 & -2.102310 & -2.185261 \\ 6 & 6.384704 & -0.020045 & -2.323710 \\ 1 & 6.365875 & 0.166428 & -3.387146 \\ 6 & 9.369801 & 1.645924 & -0.202897 \\ 1 & 9.121733 & 2.363341 & 0.567879 \\ 6 & 9.768064 & 0.290874 & 0.011963 \\ 1 & 9.868212 & -0.198103 & 0.971781 \\ 6 & 9.968867 & -0.318377 & -1.263998 \\ 1 & 10.240037 & -1.351161 & -1.439402 \\ 6 & 9.694377 & 0.659312 & -2.268013 \\ 1 & 9.724054 & 0.498671 & -3.337631 \\ 6 & 9.326245 & 1.872438 & -1.613604 \\ 1 & 9.033500 & 2.790205 & -2.104997 \\ 6 & 5.448299 & 2.287370 & -1.493808 \\ 6 & 6.228200 & 3.396000 & -1.127490 \\ 1 & 7.220695 & 3.213810 & -0.736441 \\ 6 & 5.757374 & 4.702772 & -1.243501 \\ 1 & 6.378555 & 5.534264 & -0.925750 \\ 6 & 4.484610 & 4.926807 & -1.762333 \\ 1 & 4.101643 & 5.936520 & -1.869047 \\ 6 & 3.685297 & 3.841621 & -2.119483 \\ 1 & 2.690234 & 4.036899 & -2.499706 \\ 6 & 4.129307 & 2.516357 & -1.968797 \\ 6 & 5.879962 & 0.906477 & 1.293815 \\ 1 & 6.095716 & 1.973722 & 1.226218 \\ 6 & 6.650607 & 0.334890 & 2.486675 \\ 1 & 6.373280 & 0.846161 & 3.412751 \\ 1 & 6.465570 & -0.733535 & 2.626049 \\ 1 & 7.723876 & 0.471584 & 2.321711 \\ 6 & 1.372942 & 2.004486 & -2.636755 \\ 6 & 0.596619 & 2.521408 & -1.588057 \\ 1 & 0.871447 & 2.306826 & -0.562153 \\ 6 & -0.516855 & 3.315024 & -1.857986 \\ 1 & -1.097015 & 3.719966 & -1.039079\end{array}$


$\begin{array}{llll}-0.894043 & 3.567458 & -3.179151\end{array}$

$\begin{array}{llll}-1.768850 & 4.177171 & -3.386267\end{array}$

$\begin{array}{llll}-0.150596 & 3.022693 & -4.228866\end{array}$

$\begin{array}{llll}-0.444707 & 3.205400 & -5.258558\end{array}$

$\begin{array}{llll}0.984828 & 2.253225 & -3.961556\end{array}$

$\begin{array}{llll}1.567529 & 1.858243 & -4.786949\end{array}$

$\begin{array}{llll}3.388234 & 0.262077 & -3.734089\end{array}$

$\begin{array}{llll}4.120953 & 0.871566 & -4.763020\end{array}$

$\begin{array}{llll}4.466335 & 1.895325 & -4.651753\end{array}$

$\begin{array}{llll}4.416914 & 0.159295 & -5.927190\end{array}$

$\begin{array}{llll}4.989510 & 0.634668 & -6.718488\end{array}$

$3.977008-1.159910 \quad-6.076442$

$\begin{array}{lll}4.211704 & -1.711110 & -6.982506\end{array}$

$\begin{array}{llll}3.236075 & -1.768112 & -5.059595\end{array}$

$\begin{array}{llll}2.890969 & -2.792220 & -5.170375\end{array}$

$2.946676-1.059899-3.892315$

$\begin{array}{llll}2.386229 & -1.532306 & -3.091901\end{array}$

$\begin{array}{lll}3.365067 & 2.493353 & 1.742100\end{array}$

$\begin{array}{lll}1.969775 & 2.608751 & 1.822245\end{array}$

$\begin{array}{lll}1.363782 & 1.711991 & 1.747155\end{array}$

$\begin{array}{lll}1.366465 & 3.859593 & 1.950376\end{array}$

$\begin{array}{lll}2.134041 & 5.022455 & 1.965849\end{array}$

$\begin{array}{lll}1.664559 & 5.996572 & 2.043932\end{array}$

$\begin{array}{llll}3.521414 & 4.906831 & 1.862576\end{array}$

$\begin{array}{lll}4.139376 & 3.659304 & 1.767062\end{array}$

$\begin{array}{llll}5.217730 & 3.623620 & 1.691951\end{array}$

$\begin{array}{llll}3.620326 & -0.110043 & 2.992437\end{array}$

$\begin{array}{lll}3.817723 & -1.500192 & 2.965952\end{array}$

$\begin{array}{llll}4.221766 & -1.973060 & 2.078184\end{array}$

$\begin{array}{llll}3.469084 & -2.282548 & 4.063264\end{array}$

$\begin{array}{llll}2.887458 & -1.707057 & 5.193904\end{array}$

$\begin{array}{llll}2.597672 & -2.320527 & 6.039191\end{array}$

$\begin{array}{llll}2.675268 & -0.331111 & 5.211879\end{array}$

$\begin{array}{llll}3.047389 & 0.469972 & 4.128846\end{array}$

$\begin{array}{lll}2.871896 & 1.538234 & 4.177447\end{array}$

$\begin{array}{lll}2.774266 & -0.168179 & -0.329656\end{array}$

$\begin{array}{llll}1.945819 & 0.286375 & 6.372373\end{array}$

$\begin{array}{lll}3.605167 & -3.777839 & 4.000112\end{array}$

$\begin{array}{lll}-0.129155 & 3.921549 & 2.091867\end{array}$

$\begin{array}{lll}4.340426 & 6.168202 & 1.808339\end{array}$

$\begin{array}{lll}4.074819 & 6.881200 & 0.686067\end{array}$

$\begin{array}{llll}5.669524 & 5.925061 & 1.817700\end{array}$

$\begin{array}{lll}4.070787 & 6.984207 & 2.853582\end{array}$

$\begin{array}{lll}-0.749568 & 3.062411 & 1.232615\end{array}$

$\begin{array}{lll}-0.537272 & 3.564982 & 3.331985\end{array}$

$\begin{array}{lll}-0.626428 & 5.150700 & 1.840889\end{array}$

$\begin{array}{lll}2.296359 & 1.574532 & 6.571417\end{array}$

$\begin{array}{lll}0.598981 & 0.279052 & 6.162938\end{array}$

$\begin{array}{llll}2.158141 & -0.380955 & 7.525952\end{array}$

$\begin{array}{llll}4.008173 & -4.298975 & 5.179179\end{array}$

$\begin{array}{llll}4.481473 & -4.176891 & 3.054785\end{array}$

$\begin{array}{llll}2.412686 & -4.368264 & 3.707710\end{array}$

$\begin{array}{lll}0.684270 & -1.248018 & 1.603274\end{array}$

$\begin{array}{lll}0.979343 & -2.614031 & 1.228778\end{array}$

$\begin{array}{llll}2.125789 & -3.069664 & 0.497687\end{array}$

$\begin{array}{lll}0.548422 & -3.383922 & 1.852395\end{array}$

$\begin{array}{llll}-3.285994 & -1.400841 & -1.340864\end{array}$

$\begin{array}{lll}-5.130050 & -0.468749 & -0.389346\end{array}$

$\begin{array}{lll}-5.129980 & 0.850851 & 0.608671\end{array}$

$\begin{array}{llll}-6.198061 & 0.045254 & -1.559467\end{array}$

$\begin{array}{llll}-5.836775 & -1.580625 & 0.647523\end{array}$

$\begin{array}{lll}-4.988865 & 2.141733 & 0.117587\end{array}$

$\begin{array}{lll}-3.809202 & 2.825907 & 0.484014\end{array}$

$\begin{array}{lll}-3.095744 & 2.325615 & 1.128465\end{array}$

$\begin{array}{llll}-3.598915 & 4.102882 & 0.026441\end{array}$

$\begin{array}{lll}-2.713554 & 4.653171 & 0.328165\end{array}$

$\begin{array}{llll}-4.521105 & 4.706591 & -0.870386\end{array}$

$\begin{array}{llll}-4.255578 & 5.977447 & -1.448944\end{array}$

$\begin{array}{llll}-3.340923 & 6.493310 & -1.168274\end{array}$

$\begin{array}{llll}-5.126170 & 6.536262 & -2.357599\end{array}$

$\begin{array}{llll}-4.909479 & 7.503637 & -2.801145\end{array}$

$\begin{array}{llll}-6.302575 & 5.840109 & -2.725166\end{array}$

$\begin{array}{llll}-6.976644 & 6.273494 & -3.458403\end{array}$

$\begin{array}{llll}-6.599625 & 4.615603 & -2.166711\end{array}$

$\begin{array}{llll}-7.500876 & 4.093093 & -2.465823\end{array}$ $\begin{array}{llll}-5.729997 & 4.013015 & -1.215613\end{array}$

$\begin{array}{llll}-6.002414 & 2.727491 & -0.626094\end{array}$

$\begin{array}{llll}-7.311827 & 2.035310 & -0.793625\end{array}$

$\begin{array}{llll}-8.554317 & 2.660744 & -0.415695\end{array}$

$\begin{array}{lll}-8.613518 & 3.927716 & 0.230415\end{array}$

$\begin{array}{llll}-7.693760 & 4.451075 & 0.461742\end{array}$

$\begin{array}{llll}-9.820338 & 4.493587 & 0.580772\end{array}$

$\begin{array}{llll}-9.835357 & 5.458598 & 1.079266\end{array}$

$\begin{array}{lll}-11.038694 & 3.828579 & 0.305626\end{array}$

$\begin{array}{lll}-11.982209 & 4.290564 & 0.580991\end{array}$

$\begin{array}{lll}-11.019193 & 2.590539 & -0.295379\end{array}$

$\begin{array}{lll}-11.945164 & 2.057694 & -0.495514\end{array}$

$\begin{array}{llll}-9.791362 & 1.971174 & -0.655083\end{array}$

$\begin{array}{llll}-9.770946 & 0.669846 & -1.225856\end{array}$

$\begin{array}{lll}-10.712231 & 0.161365 & -1.414815\end{array}$

$\begin{array}{llll}-8.578302 & 0.050053 & -1.503615\end{array}$

$\begin{array}{llll}-8.533575 & -0.955622 & -1.907759\end{array}$

$\begin{array}{llll}-7.365993 & 0.731728 & -1.260776\end{array}$

$\begin{array}{llll}-5.257581 & -2.943055 & 0.493732\end{array}$

$\begin{array}{lll}-4.516197 & -3.087677 & 1.290022\end{array}$

$\begin{array}{llll}-6.315481 & -4.022913 & 0.632083\end{array}$

$\begin{array}{llll}-6.025570 & -5.193709 & 1.342471\end{array}$

$\begin{array}{lll}-5.058859 & -5.295613 & 1.830990\end{array}$

$\begin{array}{lll}-6.964063 & -6.225820 & 1.428305\end{array}$

$\begin{array}{lll}-6.725267 & -7.128468 & 1.984138\end{array}$

$\begin{array}{llll}-8.208923 & -6.091871 & 0.809744\end{array}$

$\begin{array}{lll}-8.942888 & -6.889639 & 0.880865\end{array}$

$\begin{array}{llll}-8.508546 & -4.921993 & 0.103573\end{array}$

$\begin{array}{llll}-9.477716 & -4.808921 & -0.374839\end{array}$

$\begin{array}{lll}-7.567748 & -3.896147 & 0.013387\end{array}$

$\begin{array}{llll}-7.802952 & -2.981983 & -0.524445\end{array}$

$\begin{array}{llll}-4.544156 & -3.068651 & -0.859606\end{array}$

$\begin{array}{llll}-5.297704 & -3.190653 & -1.647305\end{array}$

$\begin{array}{llll}-3.944224 & -3.983004 & -0.850979\end{array}$

$\begin{array}{lll}-6.497243 & -1.329322 & 1.973705\end{array}$

$\begin{array}{lll}-6.976835 & -2.286640 & 2.194633\end{array}$

$\begin{array}{lll}-5.476687 & -1.096454 & 3.088429\end{array}$

$\begin{array}{lll}-4.933107 & -2.208517 & 3.751555\end{array}$

$\begin{array}{lll}-5.273828 & -3.205540 & 3.484266\end{array}$

$\begin{array}{lll}-3.981754 & -2.053968 & 4.763393\end{array}$

$\begin{array}{lll}-3.576546 & -2.930020 & 5.262337\end{array}$

$\begin{array}{llll}-3.568680 & -0.775393 & 5.143393\end{array}$

$\begin{array}{lll}-2.831533 & -0.647594 & 5.931251\end{array}$

$\begin{array}{llll}-4.119314 & 0.341204 & 4.507372\end{array}$

$\begin{array}{llll}-3.815321 & 1.341034 & 4.805624\end{array}$

$\begin{array}{lll}-5.062545 & 0.182258 & 3.490057\end{array}$

$\begin{array}{lll}-5.477042 & 1.060834 & 3.011866\end{array}$

$\begin{array}{lll}-7.628632 & -0.303576 & 1.924271\end{array}$

$\begin{array}{lll}-8.357645 & -0.587921 & 1.162658\end{array}$

$\begin{array}{lll}-7.287443 & 0.707665 & 1.708018\end{array}$

$\begin{array}{lll}-8.132257 & -0.292434 & 2.895927\end{array}$

$\begin{array}{llll}-2.348044 & -1.109401 & 0.602813\end{array}$

$\begin{array}{lll}-1.665523 & -0.267453 & 0.545778\end{array}$

$\begin{array}{lll}-3.012226 & -1.072223 & 1.463453\end{array}$

$\begin{array}{llll}-1.876670 & -2.401500 & 0.201076\end{array}$

$\begin{array}{llll}-2.397768 & -3.262137 & 0.607984\end{array}$

$\begin{array}{lll}-0.652580 & -2.660400 & -0.467897\end{array}$

$\begin{array}{llll}-0.109245 & -1.817138 & -0.872079\end{array}$

$\begin{array}{llll}-4.157221 & -1.403955 & -3.385837\end{array}$

$\begin{array}{llll}-5.163595 & -1.804488 & -3.299028\end{array}$

$\begin{array}{llll}-3.092997 & -2.339778 & -3.364876\end{array}$

$\begin{array}{llll}-3.354414 & -3.394594 & -3.286062\end{array}$

$\begin{array}{lll}-1.731807 & -2.083852 & -3.989331\end{array}$

$\begin{array}{llll}-1.804121 & -2.178119 & -5.084213\end{array}$

$\begin{array}{lll}-1.066468 & -2.883897 & -3.659208\end{array}$

$\begin{array}{lll}-1.103537 & -0.720021 & -3.622120\end{array}$

$\begin{array}{llll}-1.306962 & 0.020496 & -4.402154\end{array}$

$\begin{array}{lll}-0.014181 & -0.826433 & -3.595175\end{array}$

$\begin{array}{llll}-1.559941 & -0.148565 & -2.280616\end{array}$

$\begin{array}{lll}-0.786661 & -0.057986 & -1.523445\end{array}$

$\begin{array}{llll}-2.654492 & 0.696163 & -2.123149\end{array}$

$\begin{array}{llll}-2.636668 & 1.358649 & -1.263653\end{array}$

$\begin{array}{llll}-3.612026 & 1.106893 & -3.223777\end{array}$

$\begin{array}{llll}-4.486996 & 1.559363 & -2.753048\end{array}$

$\begin{array}{llll}-3.137817 & 1.890867 & -3.830470\end{array}$ 


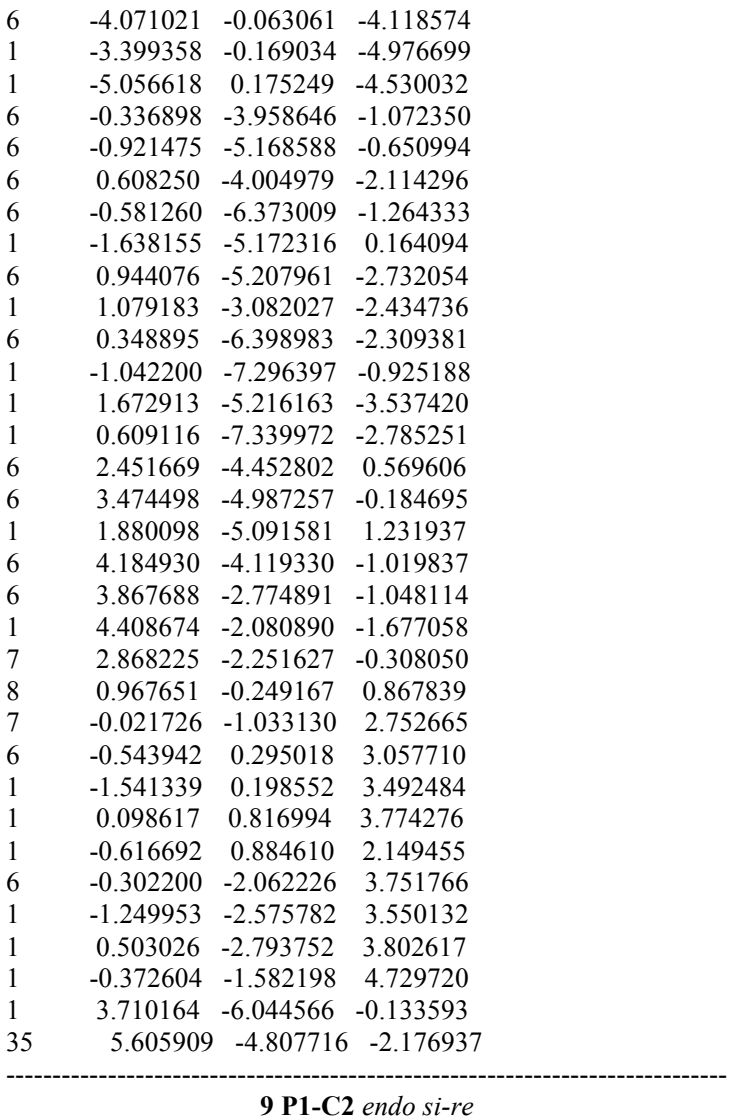

Number of imaginary frequencies $=0$

The smallest frequencies are $=7.7509 \quad 11.5710 \quad 14.5476 \mathrm{~cm}(-1)$ Electronic energy $=-7222.663312$

Zero-point correction $=$

(Hartree/Particle)

Thermal correction to Energy $=\quad 1.876584$

Thermal correction to Enthalpy= $\quad 1.877528$

Thermal correction to Gibbs Free Energy $=\quad 1.596755$

Sum of electronic and zero-point Energies $=\quad-7220.904733$

Sum of electronic and thermal Energies $=\quad-7220.786728$

Sum of electronic and thermal Enthalpies $=\quad-7220.785783$

Sum of electronic and thermal Free Energies $=\quad-7221.066556$

Cartesian Coordinates

$\begin{array}{lccc}26 & 8.026783 & 0.590559 & -1.013377 \\ 15 & 2.955995 & 1.270479 & -2.189525 \\ 15 & 3.932007 & 0.691861 & 1.445553 \\ 6 & 6.013767 & 1.125994 & -1.251033 \\ 6 & 6.219869 & 0.384003 & -0.029235 \\ 6 & 6.754721 & -0.894584 & -0.379394 \\ 1 & 7.033625 & -1.675725 & 0.313851 \\ 6 & 6.906369 & -0.942665 & -1.799463 \\ 1 & 7.327217 & -1.762200 & -2.364849 \\ 6 & 6.453570 & 0.298757 & -2.334101 \\ 1 & 6.461868 & 0.583925 & -3.375410 \\ 6 & 9.310548 & 1.844252 & 0.035117 \\ 1 & 9.012997 & 2.484498 & 0.855115 \\ 6 & 9.738808 & 0.485776 & 0.146496 \\ 1 & 9.809943 & -0.084890 & 1.062871 \\ 6 & 10.004068 & -0.002674 & -1.169118 \\ 1 & 10.308914 & -1.009204 & -1.423700 \\ 6 & 9.739399 & 1.053094 & -2.093777 \\ 1 & 9.812348 & 0.988666 & -3.171384 \\ 6 & 9.312893 & 2.193734 & -1.350822 \\ 1 & 9.012512 & 3.144036 & -1.770335 \\ 6 & 5.401252 & 2.483517 & -1.327653 \\ 6 & 6.127555 & 3.585925 & -0.851519 \\ 1 & 7.123301 & 3.412595 & -0.463869\end{array}$

$\begin{array}{lll}5.598355 & 4.875665 & -0.853217\end{array}$

$\begin{array}{llll}6.176457 & 5.700403 & -0.448905\end{array}$

$\begin{array}{lll}4.321826 & 5.090593 & -1.366231\end{array}$

$\begin{array}{llll}3.894618 & 6.088153 & -1.382083\end{array}$

$\begin{array}{lll}3.576425 & 4.009928 & -1.837897\end{array}$

$\begin{array}{lll}2.578114 & 4.195239 & -2.215498\end{array}$

$\begin{array}{llll}4.080134 & 2.698946 & -1.804320\end{array}$

$\begin{array}{lll}5.820773 & 0.863283 & 1.339394\end{array}$

$\begin{array}{lll}6.010643 & 1.937025 & 1.376020\end{array}$

$\begin{array}{lll}6.590118 & 0.202254 & 2.486324\end{array}$

$\begin{array}{lll}6.283261 & 0.611169 & 3.452800\end{array}$

$\begin{array}{lll}6.438946 & -0.880148 & 2.515657\end{array}$

$\begin{array}{lll}7.660173 & 0.388548 & 2.353682\end{array}$

$\begin{array}{llll}1.376747 & 2.097030 & -2.641610\end{array}$

$\begin{array}{lll}0.551808 & 2.519301 & -1.585992\end{array}$

$\begin{array}{lll}0.818118 & 2.274000 & -0.564547\end{array}$

$\begin{array}{lll}-0.598399 & 3.261635 & -1.843443\end{array}$

$-1.216751 \quad 3.593775-1.019688$

$\begin{array}{llll}-0.965340 & 3.553172 & -3.159744\end{array}$

$\begin{array}{lll}-1.870736 & 4.120229 & -3.356245\end{array}$

$\begin{array}{llll}-0.174289 & 3.098601 & -4.216891\end{array}$

$\begin{array}{llll}-0.460661 & 3.309302 & -5.243310\end{array}$

$\begin{array}{llll}0.999260 & 2.383338 & -3.961471\end{array}$

$\begin{array}{llll}1.616356 & 2.058533 & -4.792320\end{array}$

$\begin{array}{llll}3.581388 & 0.565554 & -3.761511\end{array}$

$\begin{array}{llll}4.189636 & 1.351908 & -4.752406\end{array}$

$\begin{array}{llll}4.305579 & 2.421523 & -4.603164\end{array}$

$\begin{array}{llll}4.661759 & 0.757291 & -5.923611\end{array}$

$\begin{array}{llll}5.135616 & 1.369259 & -6.685740\end{array}$

$\begin{array}{llll}4.529044 & -0.622327 & -6.115125\end{array}$

$\begin{array}{llll}4.902949 & -1.081799 & -7.025507\end{array}$

$\begin{array}{llll}3.918834 & -1.408363 & -5.134618\end{array}$

$\begin{array}{llll}3.815775 & -2.480358 & -5.277869\end{array}$

$\begin{array}{llll}3.449721 & -0.815463 & -3.961123\end{array}$

$\begin{array}{llll}2.994734 & -1.425337 & -3.188497\end{array}$

$\begin{array}{lll}3.275164 & 2.361345 & 1.864862\end{array}$

$\begin{array}{lll}1.878412 & 2.472238 & 1.881083\end{array}$

$\begin{array}{lll}1.275313 & 1.591813 & 1.692170\end{array}$

$\begin{array}{lll}1.265097 & 3.705880 & 2.093926\end{array}$

$\begin{array}{llll}2.025540 & 4.862235 & 2.252417\end{array}$

$\begin{array}{lll}1.548907 & 5.827083 & 2.387491\end{array}$

$\begin{array}{llll}3.416872 & 4.753522 & 2.213215\end{array}$

$\begin{array}{lll}4.043215 & 3.518364 & 2.043142\end{array}$

$\begin{array}{lll}5.124336 & 3.487428 & 2.029303\end{array}$

$\begin{array}{lll}3.570882 & -0.328697 & 2.934317\end{array}$

$\begin{array}{llll}3.811507 & -1.708378 & 2.824895\end{array}$

$\begin{array}{lll}4.257008 & -2.113599 & 1.923353\end{array}$

$\begin{array}{llll}3.468305 & -2.567308 & 3.866968\end{array}$

$\begin{array}{llll}2.840251 & -2.081313 & 5.014394\end{array}$

$\begin{array}{llll}2.548311 & -2.755474 & 5.811749\end{array}$

$\begin{array}{lll}2.585235 & -0.715917 & 5.112492\end{array}$

$\begin{array}{lll}2.964383 & 0.162759 & 4.095629\end{array}$

$2.763555 \quad 1.221144 \quad 4.209568$

$2.796704 \quad-0.131348 \quad-0.425302$

$\begin{array}{llll}1.797222 & -0.214070 & 6.291477\end{array}$

$\begin{array}{llll}3.666350 & -4.051137 & 3.725434\end{array}$

$\begin{array}{lll}-0.235694 & 3.747218 & 2.173731\end{array}$

$\begin{array}{lll}4.233642 & 6.013100 & 2.319443\end{array}$

$\begin{array}{lll}3.964836 & 6.861213 & 1.296610\end{array}$

$\begin{array}{lll}5.562986 & 5.771851 & 2.297648\end{array}$

$\begin{array}{lll}3.964431 & 6.688614 & 3.460084\end{array}$

$\begin{array}{lll}-0.805638 & 2.915393 & 1.255609\end{array}$

$\begin{array}{lll}-0.689640 & 3.334397 & 3.380452\end{array}$

$\begin{array}{lll}-0.736200 & 4.980210 & 1.953793\end{array}$

$\begin{array}{lll}1.893978 & 1.121479 & 6.446514\end{array}$

$\begin{array}{lll}0.471562 & -0.497526 & 6.143061\end{array}$

$\begin{array}{llll}2.184753 & -0.793980 & 7.446675\end{array}$

$\begin{array}{llll}4.052835 & -4.623803 & 4.883885\end{array}$

$\begin{array}{lll}4.585873 & -4.362074 & 2.786884\end{array}$

$\begin{array}{llll}2.506038 & -4.666252 & 3.361363\end{array}$

$\begin{array}{llll}0.533026 & -1.292173 & 1.326855\end{array}$

$\begin{array}{llll}0.591575 & -2.608455 & 0.563982\end{array}$

$\begin{array}{lll}1.980789 & -3.075217 & 0.191306\end{array}$

$\begin{array}{llll}0.132993 & -3.406397 & 1.145408\end{array}$

$\begin{array}{llll}-3.367146 & -1.333923 & -1.544716\end{array}$ 


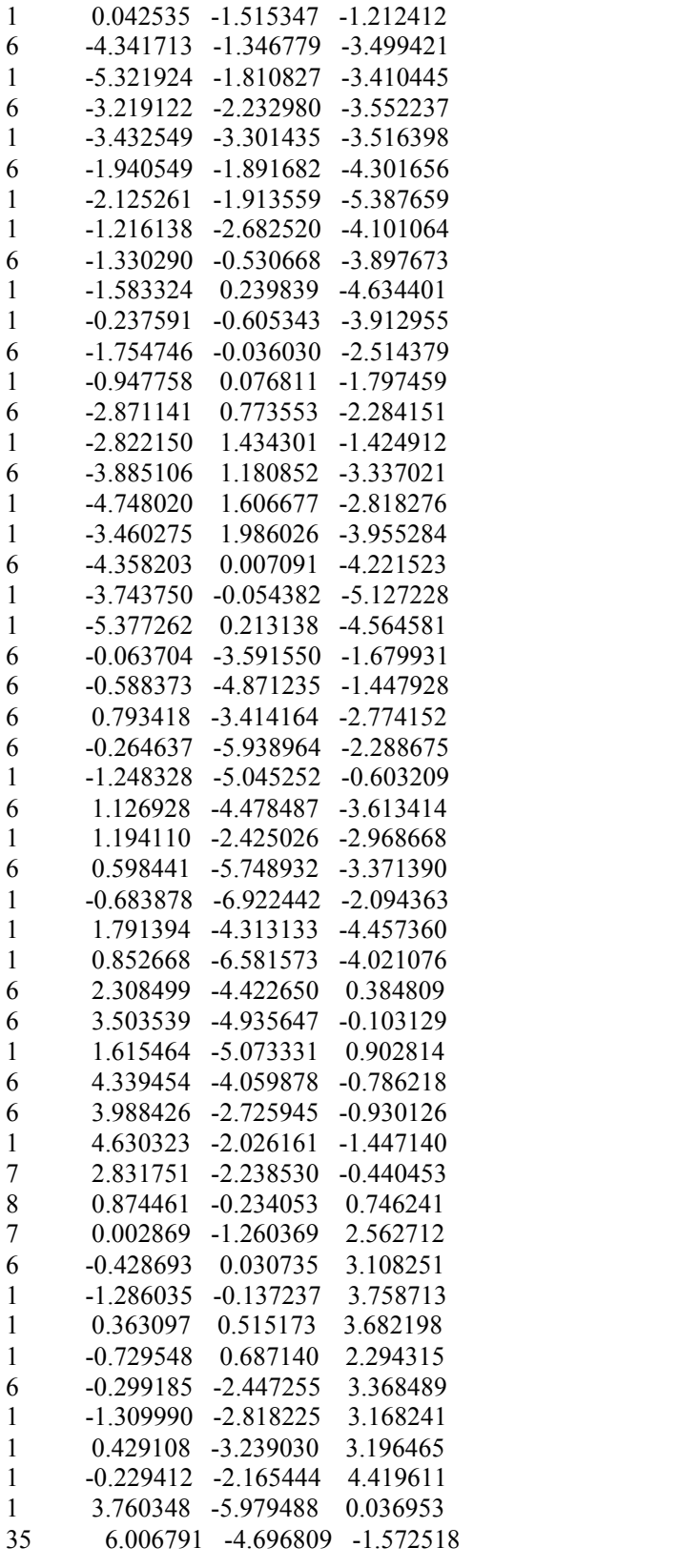
35

8 P1-C2 endo re-re

Number of imaginary frequencies $=0$

The smallest frequencies are $=12.205115 .973517 .6675 \mathrm{~cm}(-1)$ Electronic energy $=-7222.6397511$

Zero-point correction=

(Hartree/Particle)

Thermal correction to Energy= $\quad 1.875620$

Thermal correction to Enthalpy= $\quad 1.876564$

Thermal correction to Gibbs Free Energy $=\quad 1.594294$

Sum of electronic and zero-point Energies $=\quad-7220.883414$

Sum of electronic and thermal Energies $=\quad-7220.764131$

Sum of electronic and thermal Enthalpies $=\quad-7220.763187$

Sum of electronic and thermal Free Energies $=\quad-7221.045457$

Cartesian Coordinates

$\begin{array}{llll}26 & 7.441012 & -1.991164 & -1.488528 \\ 15 & 2.808648 & 0.278055 & -2.408758 \\ 15 & 4.217532 & 0.443973 & 1.081482 \\ 6 & 5.747600 & -0.784360 & -1.768330 \\ 6 & 5.890754 & -1.174439 & -0.386168 \\ 6 & 5.845281 & -2.599618 & -0.327343\end{array}$


$\begin{array}{llll}5.935154 & -3.197316 & 0.569395\end{array}$

$\begin{array}{llll}5.713553 & -3.097234 & -1.657291\end{array}$

$\begin{array}{llll}5.693384 & -4.138947 & -1.947086\end{array}$

$\begin{array}{llll}5.658625 & -1.983541 & -2.544030\end{array}$

$\begin{array}{llll}5.574007 & -2.027901 & -3.619718\end{array}$

$\begin{array}{llll}9.258882 & -1.100962 & -1.014756\end{array}$

$\begin{array}{llll}9.367275 & -0.179530 & -0.457816\end{array}$

$\begin{array}{llll}9.188315 & -2.414188 & -0.457135\end{array}$

$\begin{array}{llll}9.227255 & -2.662026 & 0.595137\end{array}$

$\begin{array}{llll}9.012458 & -3.340617 & -1.529870\end{array}$

$8.888847-4.411034-1.430536$

$8.974074 \quad-2.600948 \quad-2.750994$

$\begin{array}{llll}8.819039 & -3.011814 & -3.739957\end{array}$

$\begin{array}{llll}9.127706 & -1.218486 & -2.433748\end{array}$

$\begin{array}{llll}9.113821 & -0.403109 & -3.144180\end{array}$

$\begin{array}{llll}5.642337 & 0.627068 & -2.239032\end{array}$

$\begin{array}{llll}6.792283 & 1.431057 & -2.248418\end{array}$

$\begin{array}{llll}7.728157 & 0.982587 & -1.941370\end{array}$

$\begin{array}{llll}6.753190 & 2.774155 & -2.620160\end{array}$

$\begin{array}{llll}7.658263 & 3.372242 & -2.590190\end{array}$

$\begin{array}{llll}5.542518 & 3.339974 & -3.011497\end{array}$

$\begin{array}{llll}5.490854 & 4.384646 & -3.301680\end{array}$

$\begin{array}{llll}4.383670 & 2.562884 & -3.007115\end{array}$

$\begin{array}{llll}3.449265 & 3.024998 & -3.303276\end{array}$

$\begin{array}{llll}4.401819 & 1.216472 & -2.607759\end{array}$

$\begin{array}{lll}5.977131 & -0.201861 & 0.756348\end{array}$

$\begin{array}{llll}6.512831 & 0.668156 & 0.377911\end{array}$

$\begin{array}{lll}6.743615 & -0.717472 & 1.977623\end{array}$

$\begin{array}{lll}6.776953 & 0.035556 & 2.769903\end{array}$

$\begin{array}{lll}6.305764 & -1.626336 & 2.397200\end{array}$

$\begin{array}{lll}7.769436 & -0.952002 & 1.677817\end{array}$

$\begin{array}{llll}1.535708 & 1.461717 & -3.027719\end{array}$

$\begin{array}{llll}1.110655 & 2.460364 & -2.134466\end{array}$

$\begin{array}{llll}1.535135 & 2.503206 & -1.137262\end{array}$

$\begin{array}{llll}0.169587 & 3.413049 & -2.522955\end{array}$

$\begin{array}{llll}-0.125808 & 4.190594 & -1.829107\end{array}$

$\begin{array}{llll}-0.387325 & 3.363445 & -3.803669\end{array}$

$\begin{array}{lll}-1.134154 & 4.094882 & -4.098555\end{array}$

$\begin{array}{llll}0.013823 & 2.363975 & -4.692755\end{array}$

$\begin{array}{lll}-0.417703 & 2.315082 & -5.688606\end{array}$

$\begin{array}{llll}0.974469 & 1.422227 & -4.312297\end{array}$

$\begin{array}{llll}1.275610 & 0.658486 & -5.019963\end{array}$

$2.909805-1.026610-3.698306$

$3.425793 \quad-0.773818-4.979266$

$\begin{array}{llll}3.772387 & 0.222245 & -5.240059\end{array}$

$3.511201-1.804985-5.916315$

$\begin{array}{llll}3.911529 & -1.603335 & -6.905861\end{array}$

$\begin{array}{llll}3.087846 & -3.095757 & -5.580352\end{array}$

$\begin{array}{llll}3.158965 & -3.896633 & -6.311061\end{array}$

$\begin{array}{llll}2.585393 & -3.353828 & -4.302749\end{array}$

$\begin{array}{llll}271406 & -4.358293 & -4.031464\end{array}$

$2.500354 \quad-2.325496 \quad-3.361474$

$\begin{array}{llll}2.166465 & -2.530693 & -2.348847\end{array}$

$\begin{array}{lll}4.360593 & 2.286304 & 0.974393\end{array}$

$\begin{array}{llll}3.161074 & 3.013615 & 0.988590\end{array}$

$2.228038 \quad 2.506158 \quad 1.189316$

$\begin{array}{llll}3.146251 & 4.382170 & 0.719880\end{array}$

$\begin{array}{lll}4.327378 & 5.062468 & 0.431774\end{array}$

$\begin{array}{llll}4.314256 & 6.119908 & 0.191480\end{array}$

$\begin{array}{llll}5.524302 & 4.348152 & 0.438180\end{array}$

$\begin{array}{llll}5.549431 & 2.981391 & 0.714194\end{array}$

$\begin{array}{lll}6.505190 & 2.476370 & 0.698020\end{array}$

$\begin{array}{lll}3.845361 & 0.094028 & 2.847545\end{array}$

$\begin{array}{lll}3.759848 & -1.261476 & 3.206327\end{array}$

$\begin{array}{llll}3.974078 & -2.032556 & 2.476597\end{array}$

$3.358574 \quad-1.625839 \quad 4.486913$

$\begin{array}{lll}2.990919 & -0.657570 & 5.423030\end{array}$

$\begin{array}{llll}2.651737 & -0.945306 & 6.412193\end{array}$

$\begin{array}{lll}3.054270 & 0.685022 & 5.058925\end{array}$

$\begin{array}{lll}3.487566 & 1.066467 & 3.786159\end{array}$

$3.528178 \quad 2.120434 \quad 3.537416$

$\begin{array}{llll}2.479149 & -0.419308 & -0.265898\end{array}$

$\begin{array}{lll}2.539847 & 1.725197 & 6.013853\end{array}$

$\begin{array}{lll}3.217934 & -3.074792 & 4.859036\end{array}$

$\begin{array}{lll}1.840917 & 5.129600 & 0.732945\end{array}$ $\begin{array}{lll}6.790316 & 5.085385 & 0.094369\end{array}$

$\begin{array}{llll}6.704945 & 5.666181 & -1.127869\end{array}$

$\begin{array}{lll}7.874772 & 4.280210 & 0.07765\end{array}$

$\begin{array}{llll}7.040627 & 6.081134 & 0.975574\end{array}$

$\begin{array}{llll}0.801699 & 4.320225 & 0.390573\end{array}$

$\begin{array}{lll}1.551574 & 5.636269 & 1.951810\end{array}$

$\begin{array}{llll}1.837226 & 6.162238 & -0.136395\end{array}$

$\begin{array}{lll}3.062050 & 2.947930 & 5.772963\end{array}$

$\begin{array}{lll}1.190526 & 1.851492 & 5.920550\end{array}$

$\begin{array}{lll}2.809072 & 1.412388 & 7.301352\end{array}$

$\begin{array}{lll}3.846065 & -3.357515 & 6.023138\end{array}$

$\begin{array}{lll}3.719759 & -3.904193 & 3.916721\end{array}$

$\begin{array}{llll}1.915394 & -3.422111 & 5.036767\end{array}$

$\begin{array}{lll}0.378127 & 0.889630 & 1.451379\end{array}$

$\begin{array}{llll}0.743636 & -1.355641 & 2.071081\end{array}$

$\begin{array}{lll}-0.174600 & 1.201046 & 2.677177\end{array}$

$\begin{array}{llll}0.449915 & 1.633310 & 0.665363\end{array}$

$\begin{array}{lll}0.130624 & -1.062016 & 3.337357\end{array}$

$\begin{array}{lll}-0.298220 & 0.199964 & 3.656895\end{array}$

$\begin{array}{lll}1.192299 & -2.677426 & 1.861085\end{array}$

$\begin{array}{lll}1.887491 & -3.192162 & 0.740012\end{array}$

$\begin{array}{llll}2.282920 & -2.486138 & -0.252795\end{array}$

$\begin{array}{lll}0.863996 & -0.335852 & 1.147344\end{array}$

$\begin{array}{llll}1.029060 & -3.339242 & 2.698511\end{array}$

$\begin{array}{llll}-3.268545 & -1.641923 & -1.104670\end{array}$

$\begin{array}{llll}-4.969048 & -0.353688 & -0.357085\end{array}$

$\begin{array}{lll}-4.758287 & 1.005189 & 0.538919\end{array}$

$\begin{array}{llll}-5.865984 & 0.245678 & -1.613990\end{array}$

$\begin{array}{llll}-5.895745 & -1.248926 & 0.703625\end{array}$

$\begin{array}{lll}-4.348034 & 2.205928 & -0.031564\end{array}$

$\begin{array}{lll}-3.067403 & 2.659432 & 0.343418\end{array}$

$\begin{array}{lll}-2.503986 & 2.073846 & 1.059445\end{array}$

$\begin{array}{llll}-2.573766 & 3.815788 & -0.205470\end{array}$

$\begin{array}{lll}-1.594225 & 4.182342 & 0.081944\end{array}$

$\begin{array}{llll}-3.321612 & 4.514618 & -1.191541\end{array}$

$\begin{array}{lll}-2.769888 & 5.639704 & -1.861899\end{array}$

$\begin{array}{llll}-1.776242 & 5.975370 & -1.576902\end{array}$

$\begin{array}{llll}-3.469466 & 6.281406 & -2.859483\end{array}$

$\begin{array}{llll}-3.034775 & 7.134688 & -3.371689\end{array}$

$\begin{array}{llll}-4.757132 & 5.820385 & -3.224627\end{array}$

$\begin{array}{llll}-5.298065 & 6.316828 & -4.025033\end{array}$

$\begin{array}{llll}-5.330340 & 4.746122 & -2.578532\end{array}$

$\begin{array}{llll}-6.314749 & 4.402798 & -2.875454\end{array}$

$\begin{array}{llll}-4.640692 & 4.063416 & -1.538414\end{array}$

$\begin{array}{lll}-5.195289 & 2.914162 & -0.868624\end{array}$

$\begin{array}{llll}-6.606564 & 2.470519 & -1.053685\end{array}$

$\begin{array}{llll}-7.719195 & 3.349509 & -0.793480\end{array}$

$\begin{array}{llll}-7.557077 & 4.661602 & -0.265887\end{array}$

$\begin{array}{llll}-6.562920 & 5.024525 & -0.035652\end{array}$

$\begin{array}{lll}-8.645288 & 5.472785 & -0.027624\end{array}$

$\begin{array}{lll}-8.493085 & 6.467507 & 0.381287\end{array}$

$\begin{array}{llll}-9.958319 & 5.022019 & -0.301760\end{array}$

$\begin{array}{llll}-10.805286 & 5.675483 & -0.114743\end{array}$

$\begin{array}{llll}-10.155602 & 3.749176 & -0.786312\end{array}$

$\begin{array}{llll}-11.159087 & 3.379858 & -0.980865\end{array}$

$\begin{array}{llll}-9.056509 & 2.880958 & -1.028851\end{array}$

$\begin{array}{llll}-9.266879 & 1.548312 & -1.474685\end{array}$

$\begin{array}{llll}-10.281880 & 1.206308 & -1.655709\end{array}$

$\begin{array}{llll}-8.205779 & 0.694462 & -1.644043\end{array}$

$\begin{array}{llll}-8.342303 & -0.337231 & -1.949976\end{array}$

$\begin{array}{llll}-6.895083 & 1.163506 & -1.414545\end{array}$

$\begin{array}{llll}-5.594514 & -2.703771 & 0.640130\end{array}$

$\begin{array}{lll}-4.961242 & -2.953551 & 1.498192\end{array}$

$\begin{array}{lll}-6.859229 & -3.539599 & 0.721629\end{array}$

$\begin{array}{lll}-6.886814 & -4.689997 & 1.518317\end{array}$

$\begin{array}{lll}-6.012149 & -4.952415 & 2.109284\end{array}$

$\begin{array}{lll}-8.026690 & -5.497718 & 1.559305\end{array}$

$\begin{array}{lll}-8.036405 & -6.387582 & 2.182589\end{array}$

$\begin{array}{llll}-9.153611 & -5.155073 & 0.809238\end{array}$

$\begin{array}{lll}-10.043090 & -5.777617 & 0.845084\end{array}$

$\begin{array}{lll}-9.135009 & -4.002612 & 0.016204\end{array}$

$\begin{array}{llll}-10.011292 & -3.728408 & -0.564954\end{array}$

$\begin{array}{lll}-7.994649 & -3.201015 & -0.028230\end{array}$

$\begin{array}{llll}-7.979940 & -2.298960 & -0.633597\end{array}$

$\begin{array}{llll}-4.821957 & -3.032438 & -0.643201\end{array}$ 
$\begin{array}{lll}-5.521696 & -3.061716 & -1.486394\end{array}$

$\begin{array}{llll}-4.388260 & -4.031349 & -0.555524\end{array}$

$\begin{array}{lll}-6.520081 & -0.798842 & 1.999463\end{array}$

$\begin{array}{lll}-7.181215 & -1.632967 & 2.250046\end{array}$

$\begin{array}{lll}-5.475830 & -0.719264 & 3.112413\end{array}$

$\begin{array}{lll}-5.174296 & -1.882581 & 3.838499\end{array}$

$\begin{array}{lll}-5.740503 & -2.790532 & 3.646369\end{array}$

$\begin{array}{lll}-4.169908 & -1.888175 & 4.809965\end{array}$

$\begin{array}{lll}-3.952873 & -2.801398 & 5.357407\end{array}$

$\begin{array}{lll}-3.457886 & -0.718842 & 5.082469\end{array}$

$\begin{array}{lll}-2.674901 & -0.717511 & 5.835530\end{array}$

$\begin{array}{llll}-3.771976 & 0.455034 & 4.391758\end{array}$

$\begin{array}{lll}-3.232400 & 1.373359 & 4.605257\end{array}$

$\begin{array}{lll}-4.773887 & 0.456211 & 3.420088\end{array}$

$\begin{array}{lll}-4.999602 & 1.375973 & 2.896051\end{array}$

$\begin{array}{lll}-7.421447 & 0.427047 & 1.865827\end{array}$

$\begin{array}{lll}-8.181033 & 0.253080 & 1.100453\end{array}$

$\begin{array}{lll}-6.881155 & 1.337254 & 1.609597\end{array}$

$\begin{array}{lll}-7.929343 & 0.590781 & 2.821204\end{array}$

$\begin{array}{llll}-2.408034 & -1.329227 & 0.879479\end{array}$

$\begin{array}{lll}-1.583040 & -0.638538 & 0.752365\end{array}$

$\begin{array}{lll}-3.071837 & -1.075092 & 1.701160\end{array}$

$\begin{array}{lll}-2.201170 & -2.724121 & 0.633458\end{array}$

$\begin{array}{lll}-2.858614 & -3.427441 & 1.133954\end{array}$

$\begin{array}{llll}-1.284628 & -3.206409 & -0.299696\end{array}$

$\begin{array}{llll}-0.482138 & -2.550413 & -0.612371\end{array}$

$\begin{array}{llll}-4.084531 & -1.537359 & -3.229709\end{array}$

$\begin{array}{llll}-5.140955 & -1.765904 & -3.127117\end{array}$

$\begin{array}{lll}-3.200261 & -2.624503 & -3.170928\end{array}$

$\begin{array}{llll}-3.631084 & -3.614686 & -3.028326\end{array}$

$\begin{array}{lll}-1.789899 & -2.634442 & -3.723970\end{array}$

$\begin{array}{llll}-1.835898 & -2.838455 & -4.804358\end{array}$

$\begin{array}{llll}-1.266480 & -3.482715 & -3.278384\end{array}$

$\begin{array}{llll}-0.970276 & -1.347578 & -3.481726\end{array}$

$\begin{array}{llll}-1.128403 & -0.638723 & -4.298055\end{array}$

$\begin{array}{llll}0.089822 & -1.604209 & -3.510739\end{array}$

$\begin{array}{lll}-1.251398 & -0.634449 & -2.169729\end{array}$

$\begin{array}{lll}-0.492925 & -0.710157 & -1.394526\end{array}$

$\begin{array}{llll}-2.192278 & 0.358693 & -2.029986\end{array}$

$\begin{array}{llll}-2.099754 & 1.010049 & -1.167905\end{array}$

$\begin{array}{llll}-3.098834 & 0.863176 & -3.132949\end{array}$

$\begin{array}{llll}-3.861552 & 1.495966 & -2.680662\end{array}$

$\begin{array}{llll}-2.507429 & 1.514523 & -3.787813\end{array}$

$\begin{array}{llll}-3.788379 & -0.235566 & -3.972121\end{array}$

$\begin{array}{llll}-3.187224 & -0.471563 & -4.855834\end{array}$

$\begin{array}{llll}-4.735503 & 0.163796 & -4.344615\end{array}$

$\begin{array}{llll}-1.132407 & -4.605678 & -0.698578\end{array}$

$\begin{array}{llll}-1.994584 & -5.637450 & -0.274323\end{array}$

$\begin{array}{llll}-0.060202 & -4.940803 & -1.549972\end{array}$

$\begin{array}{llll}-1.795650 & -6.949182 & -0.699332\end{array}$

$\begin{array}{llll}-2.816691 & -5.419695 & 0.399163\end{array}$

$\begin{array}{llll}0.129918 & -6.251844 & -1.982759\end{array}$

$\begin{array}{llll}0.631562 & -4.162254 & -1.855233\end{array}$

$\begin{array}{llll}-0.737385 & -7.262433 & -1.559358\end{array}$

$\begin{array}{llll}-2.468243 & -7.731117 & -0.358410\end{array}$

$\begin{array}{llll}0.958573 & -6.486411 & -2.644666\end{array}$

$\begin{array}{llll}-0.589310 & -8.285698 & -1.891886\end{array}$

$\begin{array}{lll}2.158470 & -4.545926 & 0.695966\end{array}$

$\begin{array}{llll}3.106355 & -5.079581 & -0.266505\end{array}$

$\begin{array}{lll}4.002412 & -5.468575 & 0.238243\end{array}$

$\begin{array}{llll}2.654204 & -5.899617 & -0.836185\end{array}$

$\begin{array}{llll}3.401083 & -4.289129 & -0.951607\end{array}$

$\begin{array}{lll}1.629918 & -5.481947 & 1.675559\end{array}$

$\begin{array}{llll}0.563686 & -5.305847 & 1.845333\end{array}$

$\begin{array}{lll}1.738968 & -6.496459 & 1.284950\end{array}$

$\begin{array}{llll}2.154402 & -5.424434 & 2.638341\end{array}$

$\begin{array}{lll}-0.715158 & 0.418342 & 4.631925\end{array}$

$\begin{array}{lll}0.050027 & -1.858649 & 4.069159\end{array}$

35
[8-9] $]^{\ddagger}-\mathbf{P 1 - C 2}$ endo re-re

Number of imaginary frequencies $=1$

The smallest frequencies are $=-265.722712 .673915 .6523 \mathrm{~cm}(-1)$ Electronic energy $=-7222.6279167$

$\begin{array}{lc}\begin{array}{lc}\text { Zero-point correction= } \\ \text { (Hartree/Particle) }\end{array} & 1.756220 \\ \text { Thermal correction to Energy= } & 1.874726 \\ \text { Thermal correction to Enthalpy= } & 1.875671 \\ \text { Thermal correction to Gibbs Free Energy= } & 1.594484 \\ \text { Sum of electronic and zero-point Energies }= & -7220.871696 \\ \text { Sum of electronic and thermal Energies }= & -7220.753190 \\ \text { Sum of electronic and thermal Enthalpies }= & -7220.752246 \\ \text { Sum of electronic and thermal Free Energies }= & -7221.033432\end{array}$

Cartesian Coordinates

\begin{tabular}{|c|c|c|c|}
\hline 26 & 7.446044 & -1.994494 & - \\
\hline & 2.804950 & 0.143088 & -2.3 \\
\hline 15 & 4.203109 & 0.516716 & 1.047348 \\
\hline 6 & 5.747914 & -0.813568 & -1.748292 \\
\hline 6 & 5.882726 & -1.165584 & -0.355363 \\
\hline 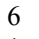 & 5.849050 & -2.589418 & -0.263148 \\
\hline 1 & 5.934535 & -3.164080 & 0.648204 \\
\hline 6 & 5.727533 & -3.122546 & -1.578879 \\
\hline 1 & 5.718781 & -4.171674 & -1.841401 \\
\hline 6 & 5.668578 & -2.032931 & -2.493508 \\
\hline 1 & 5.587293 & -2.102442 & -3.568512 \\
\hline 6 & 9.252220 & -1.048363 & -1.025935 \\
\hline 1 & 9.352218 & -0.090783 & -0.532183 \\
\hline 6 & 9.205148 & -2.322816 & -0.382560 \\
\hline 1 & 9.254811 & -2.499891 & 0.683494 \\
\hline 6 & 9.038339 & -3.320495 & -1.390670 \\
\hline 1 & 8.931335 & -4.383728 & -1.220351 \\
\hline 6 & 8.983960 & -2.663530 & -2.657679 \\
\hline 1 & 8.829995 & -3.140891 & -3.616514 \\
\hline 6 & 9.117520 & -1.260977 & -2.433216 \\
\hline 1 & 9.087675 & -0.494236 & -3.195470 \\
\hline 6 & 5.621999 & 0.571472 & -2.281292 \\
\hline 6 & 6.757307 & 1.389503 & -2.371197 \\
\hline 1 & 7.707429 & 0.983335 & -2.048889 \\
\hline 6 & 6.685108 & 2.696897 & -2.850800 \\
\hline 1 & 7.578574 & 3.311338 & -2.885909 \\
\hline 6 & 5.457933 & 3.210072 & -3.263944 \\
\hline 1 & 5.384107 & 4.225799 & -3.639454 \\
\hline 6 & 4.312361 & & -3.174661 \\
\hline 1 & 3.363445 & 2.83 & -3.489659 \\
\hline 6 & 4.366319 & 1.108805 & -2.672054 \\
\hline 6 & 5.956553 & -0.177670 & 0.777243 \\
\hline 1 & 6.548166 & 0.669756 & 0.429730 \\
\hline 6 & 6.629559 & -0.724864 & 2.040262 \\
\hline 1 & 6.686009 & 0.040193 & 2.819342 \\
\hline 1 & 6.100690 & -1.587148 & 2.453888 \\
\hline 1 & 7.645943 & -1.043673 & 1.790931 \\
\hline 6 & 1.487041 & 1.296524 & -2.960167 \\
\hline 6 & 1.109569 & 2.308575 & -2.060583 \\
\hline 1 & 1.610314 & 2.385381 & -1.099675 \\
\hline 6 & 0.119866 & 3.227778 & -2.401179 \\
\hline 1 & -0.144987 & 4.012078 & -1.705292 \\
\hline 6 & -0.527714 & 3.135162 & -3.636364 \\
\hline 1 & -1.312095 & 3.842316 & -3.889593 \\
\hline 6 & -0.164586 & 2.128526 & -4.532462 \\
\hline 1 & -0.663203 & 2.0484 & -5.494149 \\
\hline 6 & 0.841744 & 1.216534 & -4.201431 \\
\hline 1 & 1.109436 & 0.440922 & -4.909535 \\
\hline 6 & 2.861782 & -1.184534 & -3.657816 \\
\hline 6 & 3.298512 & -0.966106 & -4.973336 \\
\hline 1 & 3.620201 & 0.024148 & -5.283107 \\
\hline 6 & 3.331096 & -2.024897 & -5.882485 \\
\hline 1 & 3.668357 & -1.852378 & -6.900543 \\
\hline 6 & 2.933351 & -3.305989 & -5.483693 \\
\hline 1 & 2.960535 & -4.127218 & -6.194326 \\
\hline 6 & 2.513045 & -3.530270 & -4.170420 \\
\hline 1 & 2.216585 & -4.526117 & -3.853125 \\
\hline 6 & 2.484168 & -2.473774 & -3.258524 \\
\hline 1 & 2.213633 & -2.642363 & -2.221235 \\
\hline 6 & 4.348849 & 2.351175 & 0.875952 \\
\hline 6 & 3.169297 & 3.098664 & 1.005255 \\
\hline 1 & 2.260813 & 2.621492 & 1.342477 \\
\hline & 3.139340 & 4.457900 & 0.694463 \\
\hline
\end{tabular}


$\begin{array}{llll}4.289658 & 5.115354 & 0.266391\end{array}$

$\begin{array}{llll}4.263460 & 6.168201 & 0.008473\end{array}$

$\begin{array}{llll}5.472183 & 4.383030 & 0.164358\end{array}$

$\begin{array}{llll}5.507908 & 3.020472 & 0.458456\end{array}$

$\begin{array}{lll}6.444536 & 2.497795 & 0.330791\end{array}$

$\begin{array}{llll}3.807646 & 0.223484 & 2.816342\end{array}$

$\begin{array}{lll}3.513278 & -1.109823 & 3.144628\end{array}$

$\begin{array}{lll}3.555803 & -1.874530 & 2.376686\end{array}$

$\begin{array}{llll}3.157207 & -1.454231 & 4.444657\end{array}$

$\begin{array}{lll}3.028718 & -0.473998 & 5.430895\end{array}$

$\begin{array}{lll}2.733988 & -0.740213 & 6.439490\end{array}$

$\begin{array}{llll}3.293767 & 0.852703 & 5.096000\end{array}$

$\begin{array}{lll}3.698219 & 1.205245 & 3.804507\end{array}$

$\begin{array}{llll}3.917066 & 2.243870 & 3.582765\end{array}$

$2.513651 \quad-0.501220-0.231243$

$\begin{array}{lll}3.077099 & 1.938411 & 6.115120\end{array}$

$\begin{array}{llll}2.836308 & -2.887883 & 4.766774\end{array}$

$\begin{array}{llll}1.838007 & 5.200874 & 0.824593\end{array}$

$\begin{array}{llll}6.711796 & 5.100955 & -0.298180\end{array}$

$\begin{array}{llll}6.537839 & 5.653900 & -1.522893\end{array}$

$\begin{array}{llll}7.787634 & 4.287733 & -0.375703\end{array}$

$\begin{array}{llll}7.034223 & 6.114539 & 0.538308\end{array}$

$\begin{array}{llll}0.791232 & 4.416825 & 0.440551\end{array}$

$\begin{array}{lll}1.587458 & 5.576626 & 2.098416\end{array}$

$\begin{array}{lll}1.798984 & 6.311577 & 0.063049\end{array}$

$\begin{array}{lll}3.921634 & 2.978261 & 5.933632\end{array}$

$\begin{array}{lll}1.819216 & 2.440377 & 6.037026\end{array}$

$\begin{array}{lll}3.243272 & 1.494344 & 7.379928\end{array}$

$\begin{array}{llll}3.197567 & -3.217582 & 6.024826\end{array}$

$\begin{array}{llll}3.458011 & -3.745259 & 3.924082\end{array}$

$1.505448 \quad-3.146589 \quad 4.666306$

$\begin{array}{lll}0.469370 & 0.806528 & 1.480102\end{array}$

$\begin{array}{llll}0.454674 & -1.490388 & 1.908432\end{array}$

$\begin{array}{lll}-0.205455 & 1.083661 & 2.656755\end{array}$

$\begin{array}{lll}0.723520 & 1.595311 & 0.784173\end{array}$

$\begin{array}{lll}-0.309157 & -1.247762 & 3.081496\end{array}$

$\begin{array}{lll}-0.617118 & 0.036005 & 3.482922\end{array}$

$\begin{array}{lll}0.709586 & -2.861920 & 1.552949\end{array}$

$\begin{array}{llll}1.877914 & -3.314470 & 0.833054\end{array}$

$\begin{array}{llll}2.536509 & -2.573405 & 0.038962\end{array}$

$\begin{array}{llll}0.829135 & -0.440287 & 1.117159\end{array}$

$\begin{array}{llll}0.285698 & -3.561034 & 2.259382\end{array}$

$\begin{array}{llll}-3.381035 & -1.624164 & -1.235990\end{array}$

$\begin{array}{lll}-5.087347 & -0.367642 & -0.417419\end{array}$

$\begin{array}{llll}-4.888227 & 0.976658 & 0.526153\end{array}$

$\begin{array}{llll}-6.016392 & 0.279243 & -1.636951\end{array}$

$\begin{array}{llll}-5.991633 & -1.292386 & 0.653223\end{array}$

$\begin{array}{llll}-4.361369 & 2.143310 & -0.002330\end{array}$

$\begin{array}{llll}-3.080734 & 2.516895 & 0.459481\end{array}$

$\begin{array}{lll}-2.594630 & 1.883158 & 1.191648\end{array}$

$\begin{array}{lll}-2.500661 & 3.665615 & -0.016327\end{array}$

$\begin{array}{llll}-1.530579 & 3.983523 & 0.348483\end{array}$

$\begin{array}{llll}-3.146844 & 4.429288 & -1.026428\end{array}$

$\begin{array}{llll}-2.510477 & 5.562165 & -1.603278\end{array}$

$\begin{array}{llll}-1.537536 & 5.859761 & -1.220101\end{array}$

$\begin{array}{llll}-3.104275 & 6.258441 & -2.632397\end{array}$

$\begin{array}{llll}-2.605528 & 7.116426 & -3.073468\end{array}$

$-4.366266 \quad 5.846293 \quad-3.124430$

$\begin{array}{llll}-4.821335 & 6.382280 & -3.952317\end{array}$

$\begin{array}{llll}-5.024960 & 4.773437 & -2.562765\end{array}$

$\begin{array}{llll}-5.992008 & 4.473086 & -2.949769\end{array}$

$\begin{array}{llll}-4.449081 & 4.039267 & -1.489201\end{array}$

$\begin{array}{llll}-5.109265 & 2.912505 & -0.879280\end{array}$

$\begin{array}{llll}-6.542923 & 2.583650 & -1.124844\end{array}$

$\begin{array}{llll}-7.580814 & 3.565625 & -0.914700\end{array}$

$\begin{array}{llll}-7.323066 & 4.855998 & -0.370709\end{array}$

$\begin{array}{llll}-6.313460 & 5.121787 & -0.083367\end{array}$

$\begin{array}{llll}-8.338065 & 5.769947 & -0.185972\end{array}$

$\begin{array}{lll}-8.110598 & 6.744085 & 0.237540\end{array}$

$\begin{array}{llll}-9.671945 & 5.450874 & -0.532807\end{array}$

$\begin{array}{lll}-10.460182 & 6.184109 & -0.389451\end{array}$

$\begin{array}{llll}-9.964632 & 4.202130 & -1.031860\end{array}$

$\begin{array}{lll}-10.987432 & 3.931237 & -1.280672\end{array}$

$\begin{array}{llll}-8.943865 & 3.230847 & -1.218816\end{array}$

$\begin{array}{llll}-9.259327 & 1.923192 & -1.676044\end{array}$ $\begin{array}{llll}-10.291336 & 1.681268 & -1.913784\end{array}$

$\begin{array}{llll}-8.279549 & 0.969539 & -1.783217\end{array}$

$\begin{array}{llll}-8.499451 & -0.045557 & -2.096510\end{array}$

$\begin{array}{llll}-6.939771 & 1.302639 & -1.484173\end{array}$

$\begin{array}{lll}-5.555086 & -2.715488 & 0.684967\end{array}$

$\begin{array}{lll}-4.859778 & -2.837853 & 1.524549\end{array}$

$\begin{array}{lll}-6.723534 & -3.661216 & 0.896774\end{array}$

$\begin{array}{lll}-6.581554 & -4.763091 & 1.748565\end{array}$

$\begin{array}{lll}-5.648647 & -4.900494 & 2.291035\end{array}$

$\begin{array}{lll}-7.622962 & -5.681904 & 1.904918\end{array}$

$\begin{array}{lll}-7.498775 & -6.531378 & 2.570961\end{array}$

$\begin{array}{llll}-8.823712 & -5.501488 & 1.215110\end{array}$

$\begin{array}{lll}-9.637540 & -6.210096 & 1.340398\end{array}$

$\begin{array}{llll}-8.974876 & -4.400622 & 0.365284\end{array}$

$\begin{array}{llll}-9.908307 & -4.252340 & -0.171063\end{array}$

$\begin{array}{lll}-7.931444 & -3.488723 & 0.205452\end{array}$

$\begin{array}{llll}-8.049292 & -2.628004 & -0.447050\end{array}$

$-4.816873-3.076028-0.609576$

$\begin{array}{llll}-5.554688 & -3.209580 & -1.410053\end{array}$

$\begin{array}{lll}-4.320248 & -4.041046 & -0.470628\end{array}$

$\begin{array}{lll}-6.687144 & -0.820372 & 1.900070\end{array}$

$\begin{array}{llll}-7.304621 & -1.677850 & 2.180686\end{array}$

$\begin{array}{lll}-5.708752 & -0.599119 & 3.056181\end{array}$

$\begin{array}{llll}-5.322093 & -1.701802 & 3.835227\end{array}$

$\begin{array}{lll}-5.765438 & -2.674077 & 3.637555\end{array}$

$\begin{array}{lll}-4.396331 & -1.566532 & 4.873279\end{array}$

$\begin{array}{lll}-4.114139 & -2.435558 & 5.461670\end{array}$

$\begin{array}{lll}-3.854562 & -0.313291 & 5.165571\end{array}$

$\begin{array}{llll}-3.142771 & -0.199885 & 5.978863\end{array}$

$\begin{array}{lll}-4.254530 & 0.799018 & 4.418977\end{array}$

$\begin{array}{lll}-3.853841 & 1.782272 & 4.649988\end{array}$

$\begin{array}{llll}-5.173338 & 0.657220 & 3.377678\end{array}$

$\begin{array}{lll}-5.470451 & 1.532803 & 2.814330\end{array}$

$\begin{array}{lll}-7.659685 & 0.331102 & 1.650978\end{array}$

$\begin{array}{lll}-8.373334 & 0.052732 & 0.871982\end{array}$

$\begin{array}{lll}-7.166296 & 1.253049 & 1.345094\end{array}$

$\begin{array}{lll}-8.215429 & 0.531048 & 2.572242\end{array}$

$\begin{array}{llll}-2.452207 & -1.181895 & 0.686477\end{array}$

$\begin{array}{lll}-1.713838 & -0.400637 & 0.548203\end{array}$

$\begin{array}{lll}-3.134608 & -0.982242 & 1.508629\end{array}$

$\begin{array}{lll}-2.100657 & -2.552783 & 0.469627\end{array}$

$\begin{array}{lll}-2.717120 & -3.278232 & 0.988707\end{array}$

$\begin{array}{lll}-0.903254 & -3.061023 & -0.105510\end{array}$

$\begin{array}{llll}-0.268341 & -2.382314 & -0.659922\end{array}$

$\begin{array}{llll}-4.172185 & -1.837046 & -3.290237\end{array}$

$\begin{array}{lll}-5.211824 & -2.147287 & -3.213561\end{array}$

$\begin{array}{lll}-3.198676 & -2.852511 & -3.075322\end{array}$

$\begin{array}{llll}-3.570877 & -3.856545 & -2.870240\end{array}$

$\begin{array}{llll}-1.790596 & -2.825968 & -3.645304\end{array}$

$\begin{array}{lll}-1.806681 & -3.136735 & -4.701947\end{array}$

$-1.207177-3.581885-3.113141$

$\begin{array}{llll}-1.083331 & -1.462430 & -3.511302\end{array}$

$\begin{array}{lll}-1.253695 & -0.852629 & -4.402620\end{array}$

$\begin{array}{llll}-0.003855 & -1.625622 & -3.467458\end{array}$

$\begin{array}{llll}-1.497153 & -0.658300 & -2.283643\end{array}$

$\begin{array}{llll}-0.743315 & -0.554863 & -1.507221\end{array}$

$\begin{array}{llll}-2.505240 & 0.295932 & -2.290322\end{array}$

$\begin{array}{llll}-2.446241 & 1.075217 & -1.537933\end{array}$

$\begin{array}{llll}-3.394406 & 0.616464 & -3.474781\end{array}$

$\begin{array}{llll}-4.227392 & 1.220703 & -3.110241\end{array}$

$\begin{array}{llll}-2.829981 & 1.248368 & -4.173938\end{array}$

$\begin{array}{llll}-3.950369 & -0.626693 & -4.202224\end{array}$

$\begin{array}{llll}-3.285690 & -0.914928 & -5.023533\end{array}$

$\begin{array}{lll}-4.905749 & -0.360551 & -4.665147\end{array}$

$\begin{array}{llll}-0.774866 & -4.484594 & -0.457120\end{array}$

$\begin{array}{lll}-1.629415 & -5.480426 & 0.059293\end{array}$

$\begin{array}{llll}0.234923 & -4.882465 & -1.352812\end{array}$

$\begin{array}{llll}-1.476539 & -6.814711 & -0.310915\end{array}$

$\begin{array}{lll}-2.414923 & -5.216339 & 0.758855\end{array}$

$\begin{array}{llll}0.388035 & -6.216974 & -1.724388\end{array}$

$\begin{array}{llll}0.902373 & -4.132516 & -1.758563\end{array}$

$\begin{array}{llll}-0.466544 & -7.191393 & -1.203141\end{array}$

$\begin{array}{lll}-2.146912 & -7.564718 & 0.099175\end{array}$

$1.175488-6.495093 \quad-2.419006$

$\begin{array}{llll}-0.350017 & -8.232701 & -1.488848\end{array}$ 


$\begin{array}{lccc}7 & 2.268113 & -4.615425 & 0.977785 \\ 6 & 3.309758 & -5.166752 & 0.120480 \\ 1 & 4.215880 & -5.384135 & 0.699985 \\ 1 & 2.955687 & -6.097873 & -0.334786 \\ 1 & 3.553604 & -4.450484 & -0.659128 \\ 6 & 1.655765 & -5.548460 & 1.920821 \\ 1 & 0.693704 & -5.923014 & 1.554922 \\ 1 & 2.330276 & -6.397675 & 2.047013 \\ 1 & 1.519495 & -5.088848 & 2.900639 \\ 1 & -1.172250 & 0.218686 & 4.394973 \\ 1 & -0.621810 & -2.088751 & 3.688999 \\ 35 & -0.523342 & 2.932769 & 3.188581 \\ ---------------------------------------------------\end{array}$

9 P1-C2 endo re-re

Number of imaginary frequencies $=0$

The smallest frequencies are=13.1436 $14.884215 .7376 \mathrm{~cm}(-1)$ Electronic energy $=-7222.6543802$

Zero-point correction=

1.760436

(Hartree/Particle)

Thermal correction to Energy=

Thermal correction to Enthalpy=

1.878042

1.878986

Thermal correction to Gibbs Free Energy=

Sum of electronic and zero-point Energies=

1.601965

Sum of electronic and thermal Energies=

$-7220.893945$

$-7220.776338$

Sum of electronic and thermal Enthalpies=

$-7220.775394$

Sum of electronic and thermal Free Energies $=\quad-7221.052416$

\section{Cartesian Coordinates}

$\begin{array}{lrrr}26 & 7.402476 & -2.049259 & -1.281595 \\ 15 & 2.782095 & 0.037806 & -2.463580 \\ 15 & 4.090251 & 0.550071 & 1.024472 \\ 6 & 5.707118 & -0.889985 & -1.684451 \\ 6 & 5.803571 & -1.193073 & -0.276911 \\ 6 & 5.780459 & -2.612806 & -0.135914 \\ 1 & 5.851971 & -3.154758 & 0.795570 \\ 6 & 5.694461 & -3.192871 & -1.435079 \\ 1 & 5.699453 & -4.250682 & -1.661105 \\ 6 & 5.658118 & -2.135346 & -2.388473 \\ 1 & 5.609495 & -2.244295 & -3.462116 \\ 6 & 9.199477 & -1.089190 & -0.858616 \\ 1 & 9.290583 & -0.118420 & -0.389649 \\ 6 & 9.137822 & -2.345250 & -0.181313 \\ 1 & 9.163606 & -2.492796 & 0.890018 \\ 6 & 8.994720 & -3.370647 & -1.164683 \\ 1 & 8.883953 & -4.428835 & -0.967550 \\ 6 & 8.969840 & -2.749370 & -2.450376 \\ 1 & 8.837927 & -3.253492 & -3.398675 \\ 6 & 9.098029 & -1.341120 & -2.262108 \\ 1 & 9.087682 & -0.596422 & -3.046353 \\ 6 & 5.592954 & 0.477797 & -2.264234 \\ 6 & 6.729462 & 1.295228 & -2.343729 \\ 1 & 7.669591 & 0.899994 & -1.981282 \\ 6 & 6.671051 & 2.587612 & -2.863680 \\ 1 & 7.565610 & 3.200637 & -2.892126 \\ 6 & 5.455942 & 3.087833 & -3.325244 \\ 1 & 5.392759 & 4.092360 & -3.731557 \\ 6 & 4.308521 & 2.297761 & -3.246481 \\ 1 & 3.368935 & 2.705810 & -3.600433 \\ 6 & 4.349289 & 1.002470 & -2.706652 \\ 6 & 5.845636 & -0.168323 & 0.822113 \\ 1 & 6.450317 & 0.663451 & 0.461199 \\ 6 & 6.483669 & -0.671410 & 2.120807 \\ 1 & 6.520277 & 0.119596 & 2.874546 \\ 1 & 5.945135 & -1.519888 & 2.550131 \\ 1 & 7.505572 & -0.997581 & 1.906890 \\ 6 & 1.475696 & 1.164077 & -3.101259 \\ 6 & 1.066322 & 2.207001 & -2.251600 \\ 1 & 1.541927 & 2.330613 & -1.283360 \\ 6 & 0.075748 & 3.100742 & -2.651672 \\ 1 & -0.219979 & 3.905643 & -1.992367 \\ 6 & -0.536892 & 2.954408 & -3.899318 \\ & -1.321958 & 3.641951 & -4.199985 \\ & -0.139760 & 1.919452 & -4.747312\end{array}$

$\begin{array}{llll}-0.612883 & 1.796987 & -5.717299\end{array}$

$\begin{array}{llll}0.864967 & 1.030368 & -4.355073\end{array}$

$\begin{array}{llll}1.155315 & 0.229635 & -5.024944\end{array}$

$2.909180-1.333997-3.666918$

$\begin{array}{llll}3.368986 & -1.152464 & -4.980234\end{array}$

$3.641733-0.161151-5.330606$

$3.493714-2.250587 \quad-5.832610$

$3.848027-2.107506 \quad-6.849362$

$3.170839-3.533908-5.376962$

$\begin{array}{lll}3.274186 & -4.386253 & -6.042335\end{array}$

$\begin{array}{llll}2.727814 & -3.719903 & -4.065442\end{array}$

$\begin{array}{llll}2.493245 & -4.715770 & -3.700927\end{array}$

$\begin{array}{llll}2.601924 & -2.622411 & -3.212055\end{array}$

$2.306586 \quad-2.758726 \quad-2.176656$

$\begin{array}{lll}4.265837 & 2.376289 & 0.779549\end{array}$

$\begin{array}{llll}3.096474 & 3.147979 & 0.834707\end{array}$

$\begin{array}{lll}2.166526 & 2.699572 & 1.149306\end{array}$

$\begin{array}{llll}3.102926 & 4.496092 & 0.476576\end{array}$

$\begin{array}{llll}4.281071 & 5.119545 & 0.076437\end{array}$

$\begin{array}{llll}4.283922 & 6.162261 & -0.220890\end{array}$

$\begin{array}{llll}5.453965 & 4.365630 & 0.050996\end{array}$

$\begin{array}{llll}5.453623 & 3.014075 & 0.392784\end{array}$

$\begin{array}{llll}6.387158 & 2.475367 & 0.325827\end{array}$

$\begin{array}{llll}3.666950 & 0.335805 & 2.798319\end{array}$

$\begin{array}{lll}3.384504 & -0.982872 & 3.184448\end{array}$

$\begin{array}{llll}3.439728 & -1.781114 & 2.451975\end{array}$

$\begin{array}{llll}3.041296 & -1.274916 & 4.501813\end{array}$

$\begin{array}{llll}2.916702 & -0.256830 & 5.446640\end{array}$

$\begin{array}{lll}2.636476 & -0.482523 & 6.468735\end{array}$

$\begin{array}{lll}3.173306 & 1.057345 & 5.054257\end{array}$

$\begin{array}{llll}3.559607 & 1.359179 & 3.745993\end{array}$

$\begin{array}{llll}3.772448 & 2.388366 & 3.479739\end{array}$

$\begin{array}{llll}2.436171 & -0.509549 & -0.288472\end{array}$

$\begin{array}{lll}2.973567 & 2.176165 & 6.041485\end{array}$

$\begin{array}{lll}2.744914 & -2.702735 & 4.867250\end{array}$

$\begin{array}{lll}1.811040 & 5.265302 & 0.519431\end{array}$

$\begin{array}{llll}6.722335 & 5.047839 & -0.388979\end{array}$

$\begin{array}{llll}6.600981 & 5.559096 & -1.637973\end{array}$

$\begin{array}{llll}7.786105 & 4.215387 & -0.399040\end{array}$

$\begin{array}{llll}7.030188 & 6.085019 & 0.423081\end{array}$

$\begin{array}{lll}0.769278 & 4.484852 & 0.113203\end{array}$

$\begin{array}{lll}1.506828 & 5.691084 & 1.765829\end{array}$

$\begin{array}{llll}1.828490 & 6.347288 & -0.281450\end{array}$

$\begin{array}{lll}3.767333 & 3.235931 & 5.773328\end{array}$

$\begin{array}{lll}1.695215 & 2.628735 & 6.019122\end{array}$

$\begin{array}{llll}3.226860 & 1.786147 & 7.309622\end{array}$

$\begin{array}{llll}2.846363 & -2.929400 & 6.190635\end{array}$

$\begin{array}{llll}3.583400 & -3.561985 & 4.236958\end{array}$

$\begin{array}{llll}1.485554 & -3.063974 & 4.501548\end{array}$

$\begin{array}{lll}0.428575 & 0.869373 & 1.359105\end{array}$

$\begin{array}{llll}0.245546 & -1.396386 & 1.804903\end{array}$

$\begin{array}{lll}-0.101693 & 1.194917 & 2.600379\end{array}$

$\begin{array}{llll}0.676056 & 1.629471 & 0.630899\end{array}$

$\begin{array}{lll}-0.312724 & -1.123194 & 3.059516\end{array}$

$\begin{array}{lll}-0.467534 & 0.189116 & 3.488323\end{array}$

$\begin{array}{llll}0.275827 & -2.822096 & 1.305970\end{array}$

$\begin{array}{llll}1.697643 & -3.295141 & 1.023801\end{array}$

$\begin{array}{llll}2.438091 & -2.610251 & 0.275810\end{array}$

$\begin{array}{llll}0.652224 & -0.402605 & 0.986205\end{array}$

$\begin{array}{lll}-0.186394 & -3.433268 & 2.076677\end{array}$

$\begin{array}{llll}-3.414678 & -1.677748 & -1.298318\end{array}$

$\begin{array}{llll}-5.099732 & -0.382800 & -0.428104\end{array}$

$\begin{array}{lll}-4.869179 & 0.955115 & 0.532503\end{array}$

$\begin{array}{lll}-6.079792 & 0.301702 & -1.598950\end{array}$

$\begin{array}{lll}-5.979805 & -1.306550 & 0.669735\end{array}$

$\begin{array}{lll}-4.326972 & 2.109617 & 0.001348\end{array}$

$\begin{array}{lll}-3.027912 & 2.453625 & 0.435643\end{array}$

$\begin{array}{lll}-2.543223 & 1.805620 & 1.156547\end{array}$

$\begin{array}{llll}-2.432470 & 3.591335 & -0.047187\end{array}$

$\begin{array}{lll}-1.449986 & 3.887787 & 0.299910\end{array}$

$\begin{array}{llll}-3.082786 & 4.375321 & -1.039052\end{array}$

$\begin{array}{llll}-2.433823 & 5.496644 & -1.625124\end{array}$

$\begin{array}{llll}-1.445323 & 5.770120 & -1.263969\end{array}$

$\begin{array}{llll}-3.035989 & 6.211564 & -2.636606\end{array}$

$\begin{array}{llll}-2.528164 & 7.059999 & -3.085835\end{array}$ 


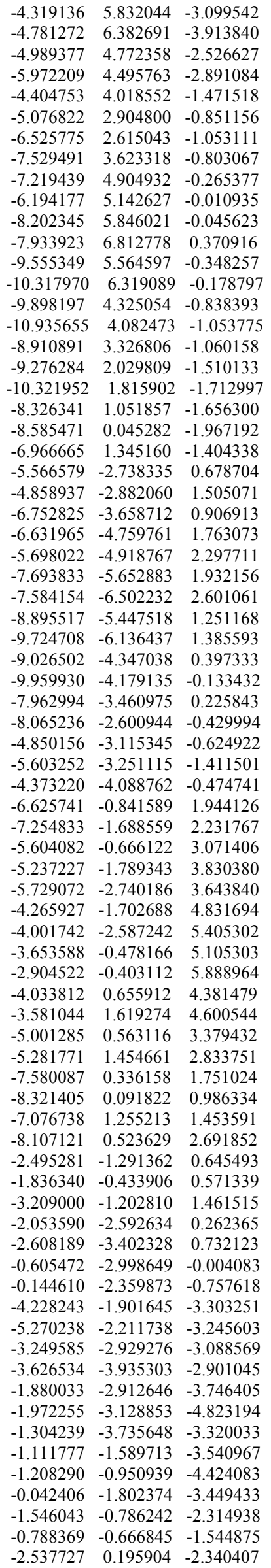

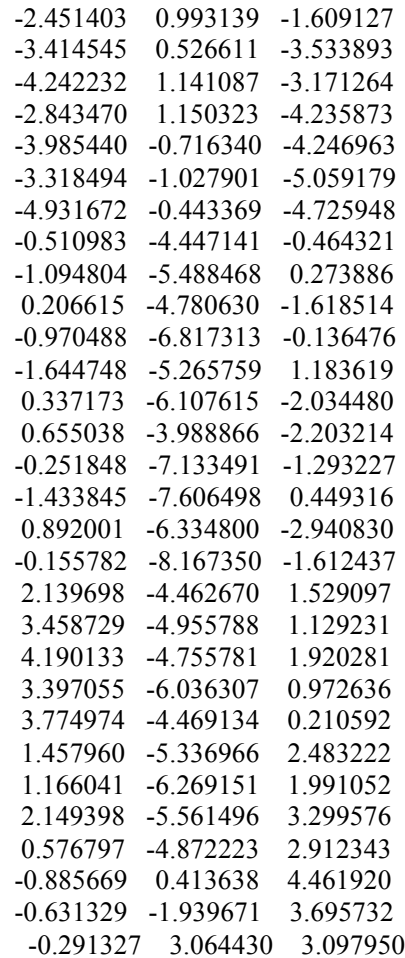

P1-C2 exo si-si

Number of imaginary frequencies $=0$

The smallest frequencies are $=9.2056 \quad 11.6531 \quad 13.9144 \mathrm{~cm}(-1)$

Electronic energy $=-7222.6394745$

Zero-point correction=

(Hartree/Particle)

Thermal correction to Energy=

Thermal correction to Enthalpy=

1.756770

1.875959

1.876903

ectronic and zero-point Energies $=\quad-7220.882704$

Sum of electronic and thermal Energies=

Sum of electronic and thermal Enthalpies=

$-7220.763515$

$-7220.762571$

Sum of electronic and thermal Free Energies $=\quad-7221.045806$

Cartesian Coordinates

$\begin{array}{lccc}26 & 8.187524 & 0.983036 & -0.259123 \\ 15 & 3.230664 & 1.495266 & -1.840112 \\ 15 & 3.898022 & 0.473492 & 1.718423 \\ 6 & 6.170438 & 1.424102 & -0.600683 \\ 6 & 6.327879 & 0.525223 & 0.519112 \\ 6 & 6.972486 & -0.657414 & 0.042980 \\ 1 & 7.242147 & -1.515317 & 0.643488 \\ 6 & 7.246294 & -0.489628 & -1.347408 \\ 1 & 7.762968 & -1.195636 & -1.982733 \\ 6 & 6.754608 & 0.788428 & -1.743901 \\ 1 & 6.831828 & 1.219145 & -2.730681 \\ 6 & 9.267155 & 2.177105 & 1.051200 \\ 1 & 8.843625 & 2.681547 & 1.909685 \\ 6 & 9.798432 & 0.850942 & 1.036719 \\ 1 & 9.844794 & 0.174387 & 1.879719 \\ 6 & 10.215285 & 0.556450 & -0.297216 \\ 1 & 10.627345 & -0.383066 & -0.641353 \\ 6 & 9.940989 & 1.699424 & -1.108363 \\ 1 & 10.109605 & 1.778375 & -2.174266 \\ 6 & 9.355953 & 2.700455 & -0.276372 \\ 1 & 9.007195 & 3.670261 & -0.604401 \\ 6 & 5.452702 & 2.730609 & -0.535000 \\ 6 & 6.036142 & 3.795724 & 0.168797 \\ 1 & 7.011567 & 3.640969 & 0.612533 \\ 6 & 5.391386 & 5.022926 & 0.319797 \\ 1 & 5.860954 & 5.815573 & 0.893730\end{array}$


$\begin{array}{llll}4.138181 & 5.214054 & -0.256964\end{array}$

$\begin{array}{llll}3.620219 & 6.161292 & -0.147510\end{array}$

$\begin{array}{llll}3.535067 & 4.169697 & -0.957800\end{array}$

$\begin{array}{llll}2.553271 & 4.330767 & -1.388181\end{array}$

$\begin{array}{llll}4.158720 & 2.918810 & -1.093597\end{array}$

$\begin{array}{lll}5.764109 & 0.767876 & 1.893405\end{array}$

$\begin{array}{llll}5.878225 & 1.830228 & 2.115760\end{array}$

$6.445758 \quad-0.033421 \quad 3.005552$

$\begin{array}{llll}6.010496 & 0.203241 & 3.980486\end{array}$

$\begin{array}{llll}6.355051 & -1.112028 & 2.85139\end{array}$

$\begin{array}{lll}7.511645 & 0.214383 & 3.030269\end{array}$

$\begin{array}{llll}1.614340 & 2.243166 & -2.326951\end{array}$

$\begin{array}{llll}0.616172 & 2.318586 & -1.342086\end{array}$

$\begin{array}{llll}0.802275 & 1.901322 & -0.359183\end{array}$

$\begin{array}{llll}-0.617508 & 2.906304 & -1.626148\end{array}$

$\begin{array}{lll}-1.372025 & 2.968751 & -0.851643\end{array}$

$\begin{array}{llll}-0.884214 & 3.395642 & -2.907713\end{array}$

$\begin{array}{lll}-1.849264 & 3.842540 & -3.129469\end{array}$

$\begin{array}{llll}0.092261 & 3.297377 & -3.902299\end{array}$

$\begin{array}{llll}-0.108816 & 3.668438 & -4.903325\end{array}$

$\begin{array}{llll}1.337929 & 2.733860 & -3.612439\end{array}$

$\begin{array}{llll}2.091133 & 2.680916 & -4.391144\end{array}$

$\begin{array}{llll}4.062528 & 1.216245 & -3.454551\end{array}$

$\begin{array}{llll}4.757982 & 2.231379 & -4.131656\end{array}$

$\begin{array}{llll}4.824384 & 3.226118 & -3.701152\end{array}$

$\begin{array}{llll}5.380605 & 1.962454 & -5.351505\end{array}$

$\begin{array}{llll}5.920788 & 2.751915 & -5.866509\end{array}$

$\begin{array}{llll}5.310708 & 0.680639 & -5.908074\end{array}$

$\begin{array}{llll}5.801844 & 0.471831 & -6.854342\end{array}$

$\begin{array}{llll}4.607250 & -0.328071 & -5.246412\end{array}$

$4.541988-1.323620 \quad-5.675329$

$\begin{array}{llll}3.986240 & -0.061419 & -4.024292\end{array}$

$3.436924-0.845024 \quad-3.515967$

$\begin{array}{lll}3.054070 & 1.991046 & 2.331746\end{array}$

$\begin{array}{lll}1.656357 & 1.977460 & 2.215156\end{array}$

$\begin{array}{lll}1.175605 & 1.079298 & 1.840104\end{array}$

$\begin{array}{llll}0.905335 & 3.106554 & 2.537732\end{array}$

$\begin{array}{lll}1.527991 & 4.284461 & 2.946753\end{array}$

$\begin{array}{llll}0.945172 & 5.170527 & 3.172596\end{array}$

$\begin{array}{llll}2.920424 & 4.303207 & 3.041713\end{array}$

$\begin{array}{lll}3.681818 & 3.168431 & 2.756112\end{array}$

$\begin{array}{lll}4.757724 & 3.232358 & 2.845471\end{array}$

$\begin{array}{llll}3.432538 & -0.823292 & 2.939488\end{array}$

$3.749749-2.139484 \quad 2.569900$

$\begin{array}{lll}4.294519 & -2.325282 & 1.651436\end{array}$

$\begin{array}{lll}3.303986 & -3.212537 & 3.334164\end{array}$

$\begin{array}{lll}2.502136 & -3.002718 & 4.457327\end{array}$

$\begin{array}{llll}2.112619 & -3.843649 & 5.021395\end{array}$

$\begin{array}{llll}2.167951 & -1.696566 & 4.810177\end{array}$

$\begin{array}{llll}2.648310 & -0.608075 & 4.076897\end{array}$

$\begin{array}{lll}2.365771 & 0.395546 & 4.371042\end{array}$

$\begin{array}{llll}3.007436 & -0.199281 & -0.355098\end{array}$

$\begin{array}{lll}1.246371 & -1.480091 & 5.979942\end{array}$

$\begin{array}{lll}3.598086 & -4.624889 & 2.911536\end{array}$

$\begin{array}{lll}-0.592102 & 3.015159 & 2.464429\end{array}$

$\begin{array}{lll}3.588810 & 5.597526 & 3.418474\end{array}$

$\begin{array}{llll}3.359479 & 6.560673 & 2.490531\end{array}$

$\begin{array}{lll}4.928735 & 5.475982 & 3.539801\end{array}$

$\begin{array}{lll}3.119766 & 6.081075 & 4.592584\end{array}$

$\begin{array}{llll}-0.991947 & 2.274105 & 1.388533\end{array}$

$\begin{array}{lll}-1.115464 & 2.398781 & 3.551450\end{array}$

$\begin{array}{lll}-1.191089 & 4.216566 & 2.355615\end{array}$

$\begin{array}{llll}0.734468 & -0.226742 & 6.006067\end{array}$

$\begin{array}{llll}0.190846 & -2.334699 & 5.941830\end{array}$

$\begin{array}{lll}1.862127 & -1.682732 & 7.166582\end{array}$

$\begin{array}{llll}4.252485 & -5.310404 & 3.878730\end{array}$

$\begin{array}{lll}4.350059 & -4.687267 & 1.793119\end{array}$

$\begin{array}{llll}2.455802 & -5.318014 & 2.663095\end{array}$

$\begin{array}{lll}0.835102 & -1.845575 & 0.965364\end{array}$

$\begin{array}{lll}1.297741 & -3.030179 & 0.332265\end{array}$

$\begin{array}{llll}2.405011 & -3.193620 & -0.529573\end{array}$

$\begin{array}{llll}0.792663 & -3.954536 & 0.577158\end{array}$

$\begin{array}{lll}-3.526199 & -1.155425 & -1.720107\end{array}$

$\begin{array}{lll}-5.160722 & -0.635875 & -0.235522\end{array}$

$\begin{array}{llll}-5.120270 & 0.728081 & 0.701079\end{array}$ $\begin{array}{lll}-6.617188 & -0.439884 & -0.992042\end{array}$

$\begin{array}{lll}-5.212627 & -1.800641 & 0.953090\end{array}$

$\begin{array}{lll}-5.217915 & 1.982055 & 0.105056\end{array}$

$\begin{array}{lll}-4.064864 & 2.795385 & 0.152080\end{array}$

$\begin{array}{lll}-3.202428 & 2.446563 & 0.704507\end{array}$

$\begin{array}{lll}-4.070574 & 4.012263 & -0.483454\end{array}$

$\begin{array}{llll}-3.195249 & 4.654411 & -0.443596\end{array}$

$\begin{array}{llll}-5.207234 & 4.426724 & -1.229993\end{array}$

$\begin{array}{llll}-5.186118 & 5.625279 & -1.993435\end{array}$

$\begin{array}{llll}-4.284796 & 6.232584 & -1.978575\end{array}$

$\begin{array}{llll}-6.274710 & 6.001917 & -2.747314\end{array}$

$\begin{array}{llll}-6.245763 & 6.915566 & -3.333599\end{array}$

$\begin{array}{llll}-7.432465 & 5.188040 & -2.768197\end{array}$

$\begin{array}{llll}-8.281289 & 5.476754 & -3.381265\end{array}$

$\begin{array}{llll}-7.493251 & 4.030743 & -2.022213\end{array}$

$\begin{array}{llll}-8.385002 & 3.415937 & -2.055829\end{array}$

$\begin{array}{llll}-6.393079 & 3.616589 & -1.221549\end{array}$

$\begin{array}{lll}-6.415399 & 2.398040 & -0.451673\end{array}$

$\begin{array}{llll}-7.662718 & 1.610065 & -0.239352\end{array}$

$\begin{array}{lll}-8.841494 & 2.223015 & 0.326648\end{array}$

$\begin{array}{lll}-8.850482 & 3.555671 & 0.826463\end{array}$

$\begin{array}{llll}-7.939905 & 4.141190 & 0.800486\end{array}$

$\begin{array}{lll}-9.993793 & 4.109451 & 1.361257\end{array}$

$\begin{array}{lll}-9.968525 & 5.126279 & 1.742405\end{array}$

$\begin{array}{lll}-11.196015 & 3.366774 & 1.426386\end{array}$

$\begin{array}{lll}-12.090784 & 3.819303 & 1.843488\end{array}$

$\begin{array}{lll}-11.218241 & 2.066072 & 0.976894\end{array}$

$\begin{array}{lll}-12.127330 & 1.473673 & 1.039142\end{array}$

$\begin{array}{lll}-10.052321 & 1.459710 & 0.435815\end{array}$

$\begin{array}{lll}-10.062959 & 0.099607 & 0.024904\end{array}$

$\begin{array}{lll}-10.986825 & -0.467098 & 0.096477\end{array}$

$\begin{array}{llll}-8.913787 & -0.500104 & -0.422530\end{array}$

$\begin{array}{lll}-8.884729 & -1.547163 & -0.704512\end{array}$

$\begin{array}{llll}-7.723159 & 0.253609 & -0.519646\end{array}$

$\begin{array}{lll}-4.284708 & -2.940146 & 0.735651\end{array}$

$\begin{array}{lll}-3.415554 & -2.791459 & 1.387272\end{array}$

$\begin{array}{lll}-4.921252 & -4.270525 & 1.104383\end{array}$

$\begin{array}{lll}-4.181305 & -5.224628 & 1.811517\end{array}$

$\begin{array}{lll}-3.172767 & -4.982692 & 2.137720\end{array}$

$\begin{array}{lll}-4.730523 & -6.476490 & 2.102781\end{array}$

$\begin{array}{lll}-4.145316 & -7.208126 & 2.652983\end{array}$

$\begin{array}{lll}-6.031213 & -6.781344 & 1.696579\end{array}$

$\begin{array}{llll}-6.462564 & -7.750923 & 1.928567\end{array}$

$\begin{array}{llll}-6.779123 & -5.829771 & 0.994744\end{array}$

$\begin{array}{llll}-7.793988 & -6.058986 & 0.681500\end{array}$

$\begin{array}{lll}-6.226106 & -4.584572 & 0.697094\end{array}$

$\begin{array}{llll}-6.808117 & -3.839228 & 0.161146\end{array}$

$\begin{array}{lll}-3.819522 & -3.006625 & -0.729418\end{array}$

$\begin{array}{llll}-4.578219 & -3.545475 & -1.306070\end{array}$

$\begin{array}{llll}-2.909909 & -3.611275 & -0.760536\end{array}$

$\begin{array}{lll}-5.729374 & -1.685564 & 2.359470\end{array}$

$\begin{array}{lll}-5.989988 & -2.715528 & 2.614605\end{array}$

$\begin{array}{lll}-4.652812 & -1.260095 & 3.360097\end{array}$

$\begin{array}{llll}-3.996105 & -2.248774 & 4.109561\end{array}$

$\begin{array}{lll}-4.241143 & -3.294181 & 3.939027\end{array}$

$\begin{array}{llll}-3.043337 & -1.909491 & 5.074629\end{array}$

$\begin{array}{llll}-2.539644 & -2.689821 & 5.637695\end{array}$

$\begin{array}{lll}-2.732429 & -0.569069 & 5.308511\end{array}$

$\begin{array}{lll}-1.972565 & -0.305321 & 6.037802\end{array}$

$\begin{array}{lll}-3.379729 & 0.425224 & 4.569419\end{array}$

$\begin{array}{llll}-3.136488 & 1.470180 & 4.734388\end{array}$

$\begin{array}{llll}-4.330025 & 0.083564 & 3.604732\end{array}$

$\begin{array}{lll}-4.825946 & 0.867099 & 3.045322\end{array}$

$\begin{array}{lll}-7.027001 & -0.888720 & 2.485315\end{array}$

$\begin{array}{lll}-7.797416 & -1.314816 & 1.839317\end{array}$

$\begin{array}{lll}-6.913227 & 0.166785 & 2.239299\end{array}$

$\begin{array}{lll}-7.374767 & -0.959132 & 3.520013\end{array}$

$\begin{array}{llll}-2.211515 & -0.518671 & -0.104994\end{array}$

$\begin{array}{lll}-2.331834 & 0.484742 & 0.285238\end{array}$

$\begin{array}{llll}-2.219207 & -1.285205 & 0.659866\end{array}$

$\begin{array}{lll}-1.297337 & -0.665490 & -1.192112\end{array}$

$\begin{array}{llll}-0.921441 & 0.237032 & -1.656016\end{array}$

$\begin{array}{llll}-0.993179 & -1.894913 & -1.764829\end{array}$

$\begin{array}{llll}-1.203755 & -2.794421 & -1.200770\end{array}$

$\begin{array}{lll}-5.092367 & -1.766676 & -3.244718\end{array}$ 


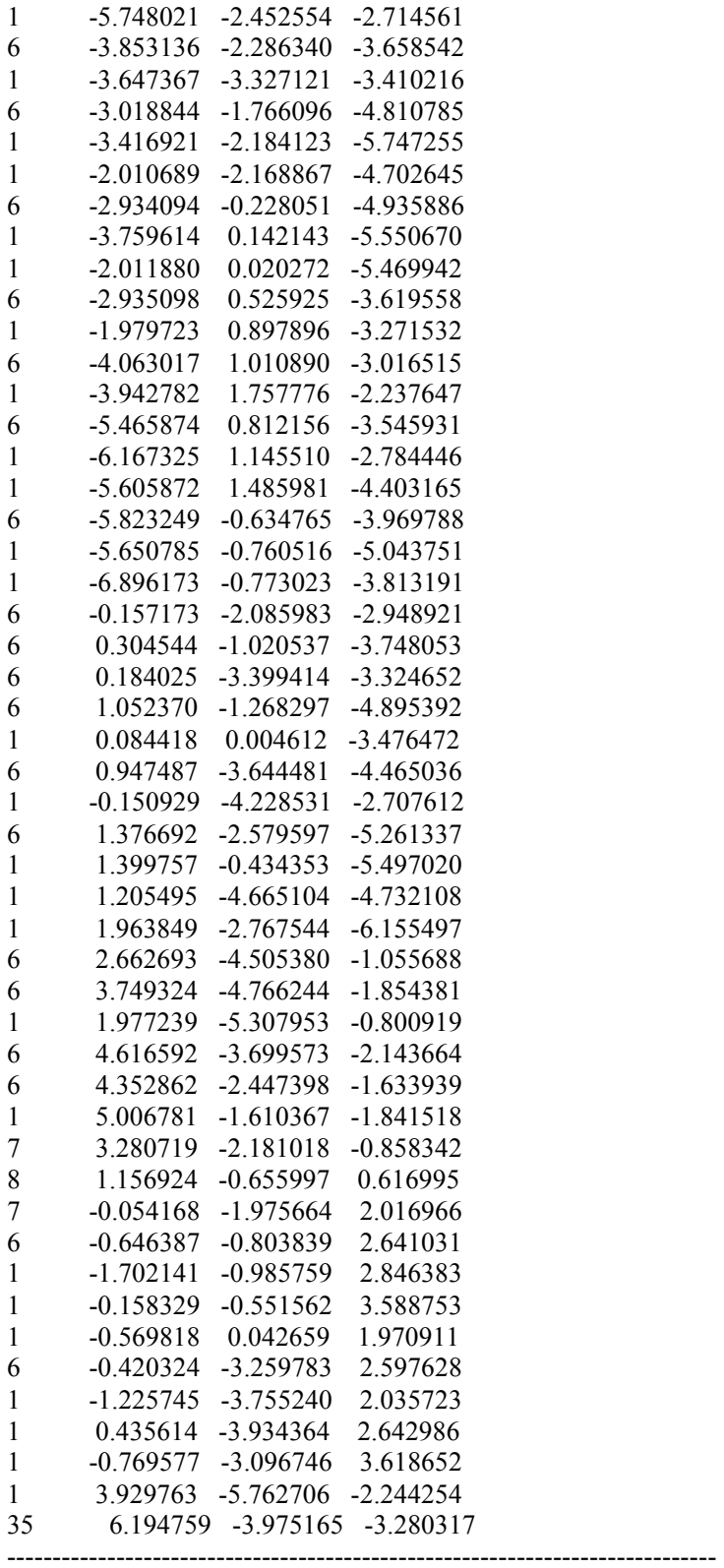

\section{$[8-9]^{\ddagger}-\mathbf{P 1}-\mathrm{C} 2$ exo si-si}

Number of imaginary frequencies $=1$

The smallest frequencies are $=-129.62148 .720213 .0915 \mathrm{~cm}(-1)$ Electronic energy $=-7222.6324643$

$\begin{array}{lc}\text { Zero-point correction= } & 1.754828 \\ \text { (Hartree/Particle) } & 1.873410 \\ \text { Thermal correction to Energy= } & 1.874354 \\ \text { Thermal correction to Enthalpy= } & 1.592590 \\ \text { Thermal correction to Gibbs Free Energy= } & -7220.877637 \\ \text { Sum of electronic and zero-point Energies }= & -7220.759054 \\ \text { Sum of electronic and thermal Energies }= & -7220.758110 \\ \text { Sum of electronic and thermal Enthalpies }= & -7221.039875 \\ \text { Sum of electronic and thermal Free Energies }= & -725\end{array}$

Cartesian Coordinates

$\begin{array}{llcc}26 & 8.003890 & 0.535566 & -0.858794 \\ 15 & 2.937988 & 1.172384 & -2.105429 \\ 15 & 3.903793 & 0.834210 & 1.536138 \\ 6 & 5.990347 & 1.055563 & -1.115708 \\ 6 & 6.207648 & 0.424180 & 0.164300 \\ 6 & 6.729963 & -0.884036 & -0.076119 \\ 1 & 7.012639 & -1.601361 & 0.681993 \\ 6 & 6.868040 & -1.058283 & -1.486177\end{array}$

$\begin{array}{llll}7.273277 & -1.929953 & -1.980245\end{array}$

$\begin{array}{llll}6.410565 & 0.131149 & -2.126277\end{array}$

$\begin{array}{llll}6.407013 & 0.320672 & -3.189396\end{array}$

$\begin{array}{llll}9.301507 & 1.883611 & 0.045256\end{array}$

$\begin{array}{lll}9.014316 & 2.596080 & 0.807040\end{array}$

$\begin{array}{lll}9.738124 & 0.543182 & 0.275880\end{array}$

$\begin{array}{lll}9.835801 & 0.063437 & 1.240657\end{array}$

$\begin{array}{llll}9.982438 & -0.066493 & -0.992189\end{array}$

$10.289443-1.091121-1.155838$

$\begin{array}{llll}9.695783 & 0.896155 & -2.007336\end{array}$

$\begin{array}{llll}9.749415 & 0.730002 & -3.075186\end{array}$

$\begin{array}{llll}9.276435 & 2.100579 & -1.367427\end{array}$

$8.961294 \quad 3.005025-1.869508$

$\begin{array}{llll}5.392609 & 2.409612 & -1.299378\end{array}$

$\begin{array}{llll}6.133043 & 3.535728 & -0.904898\end{array}$

$\begin{array}{llll}7.122845 & 3.377599 & -0.497121\end{array}$

$\begin{array}{llll}5.628771 & 4.830260 & -1.016791\end{array}$

$\begin{array}{llll}6.222067 & 5.674824 & -0.680954\end{array}$

$\begin{array}{llll}4.358914 & 5.025010 & -1.553914\end{array}$

$\begin{array}{llll}3.949256 & 6.024757 & -1.653976\end{array}$

$3.597294 \quad 3.922174 \quad-1.938852$

$\begin{array}{llll}2.604470 & 4.094589 & -2.335989\end{array}$

$\begin{array}{llll}4.077699 & 2.608912 & -1.800000\end{array}$

$\begin{array}{lll}5.792712 & 1.016022 & 1.484079\end{array}$

$\begin{array}{llll}5.982368 & 2.089455 & 1.442124\end{array}$

$\begin{array}{llll}6.534867 & 0.445288 & 2.695471\end{array}$

$\begin{array}{llll}6.206978 & 0.929446 & 3.619615\end{array}$

$\begin{array}{llll}6.378539 & -0.630937 & 2.806653\end{array}$

$\begin{array}{llll}7.609044 & 0.618500 & 2.575339\end{array}$

$\begin{array}{llll}1.365084 & 2.011567 & -2.575314\end{array}$

$\begin{array}{llll}0.536934 & 2.457240 & -1.531021\end{array}$

$\begin{array}{llll}0.782039 & 2.203854 & -0.506001\end{array}$

$\begin{array}{llll}-0.586182 & 3.237088 & -1.805196\end{array}$

$\begin{array}{llll}-1.204690 & 3.590108 & -0.990616\end{array}$

$\begin{array}{llll}-0.923816 & 3.543122 & -3.125953\end{array}$

$\begin{array}{llll}-1.808134 & 4.138311 & -3.335526\end{array}$

$\begin{array}{llll}-0.132947 & 3.064127 & -4.172045\end{array}$

$\begin{array}{lll}-0.397336 & 3.284584 & -5.202323\end{array}$

$\begin{array}{llll}1.012603 & 2.310695 & -3.900001\end{array}$

$\begin{array}{llll}1.635356 & 1.973547 & -4.722064\end{array}$

$\begin{array}{llll}3.531091 & 0.436053 & -3.679490\end{array}$

$\begin{array}{llll}4.233159 & 1.176010 & -4.643879\end{array}$

$\begin{array}{llll}4.443656 & 2.227942 & -4.475262\end{array}$

$\begin{array}{llll}4.676108 & 0.558584 & -5.814982\end{array}$

$\begin{array}{llll}5.222360 & 1.136239 & -6.555264\end{array}$

$\begin{array}{llll}4.422340 & -0.799447 & -6.033291\end{array}$

$\begin{array}{llll}4.776719 & -1.279233 & -6.941087\end{array}$

$\begin{array}{llll}3.715673 & -1.538401 & -5.081356\end{array}$

$\begin{array}{llll}3.523036 & -2.594971 & -5.242819\end{array}$

$\begin{array}{llll}3.270681 & -0.921861 & -3.910648\end{array}$

$\begin{array}{llll}2.735752 & -1.497083 & -3.163707\end{array}$

$\begin{array}{lll}3.203815 & 2.503927 & 1.868286\end{array}$

$\begin{array}{lll}1.803611 & 2.563888 & 1.922557\end{array}$

$\begin{array}{llll}1.236118 & 1.643447 & 1.834558\end{array}$

$\begin{array}{llll}1.148962 & 3.790224 & 2.034938\end{array}$

$\begin{array}{lll}1.868441 & 4.982606 & 2.066686\end{array}$

$\begin{array}{llll}1.359302 & 5.937613 & 2.131572\end{array}$

$\begin{array}{lll}3.261061 & 4.922266 & 1.993397\end{array}$

$\begin{array}{lll}3.929960 & 3.700669 & 1.907468\end{array}$

$\begin{array}{lll}5.009462 & 3.709763 & 1.846349\end{array}$

$3.500589-0.138279 \quad 3.045923$

$\begin{array}{llll}3.727933 & -1.520681 & 2.955165\end{array}$

$\begin{array}{lll}4.171269 & -1.936818 & 2.057899\end{array}$

$\begin{array}{lll}3.351204 & -2.367602 & 3.993031\end{array}$

$\begin{array}{llll}2.712721 & -1.863475 & 5.127957\end{array}$

$\begin{array}{llll}2.398448 & -2.526595 & 5.925894\end{array}$

$\begin{array}{llll}2.471183 & -0.494005 & 5.208861\end{array}$

$\begin{array}{llll}2.870839 & 0.370616 & 4.185713\end{array}$

$2.670480 \quad 1.431386 \quad 4.280891$

$\begin{array}{lll}2.787150 & -0.157308 & -0.283877\end{array}$

$\begin{array}{lll}1.683282 & 0.045914 & 6.370248\end{array}$

$\begin{array}{llll}3.524710 & -3.854620 & 3.853161\end{array}$

$\begin{array}{lll}-0.350981 & 3.792703 & 2.129852\end{array}$

$\begin{array}{lll}4.026792 & 6.217386 & 1.965405\end{array}$

$\begin{array}{llll}3.731786 & 6.942228 & 0.857819\end{array}$ 
$\begin{array}{llll}5.364877 & 6.031830 & 1.973323\end{array}$

$\begin{array}{lll}3.720977 & 7.000060 & 3.026524\end{array}$

$\begin{array}{lll}-0.904734 & 2.912419 & 1.246604\end{array}$

$\begin{array}{lll}-0.787025 & 3.414231 & 3.353145\end{array}$

$\begin{array}{lll}-0.890289 & 5.001200 & 1.864338\end{array}$

$\begin{array}{lll}1.964132 & 1.341726 & 6.622703\end{array}$

$\begin{array}{lll}0.343271 & -0.019432 & 6.128443\end{array}$

$\begin{array}{llll}1.901361 & -0.651043 & 7.505477\end{array}$

$\begin{array}{llll}3.955865 & -4.422762 & 5.000824\end{array}$

$\begin{array}{lll}4.400600 & -4.182316 & 2.880021\end{array}$

$\begin{array}{llll}2.347092 & -4.464357 & 3.543223\end{array}$

$\begin{array}{llll}0.664618 & -1.343779 & 1.568125\end{array}$

$\begin{array}{lll}0.978067 & -2.683055 & 1.144436\end{array}$

$\begin{array}{llll}2.105648 & -3.099675 & 0.371665\end{array}$

$\begin{array}{llll}0.534831 & -3.488568 & 1.711424\end{array}$

$\begin{array}{lll}-3.317360 & -1.168261 & -1.663083\end{array}$

$\begin{array}{lll}-5.086051 & -0.635548 & -0.337471\end{array}$

$\begin{array}{lll}-5.147114 & 0.646869 & 0.712297\end{array}$

$\begin{array}{llll}-6.419382 & -0.301638 & -1.273955\end{array}$

$\begin{array}{lll}-5.382708 & -1.872892 & 0.752307\end{array}$

$\begin{array}{lll}-5.093938 & 1.941340 & 0.214706\end{array}$

$\begin{array}{lll}-3.922669 & 2.675242 & 0.499373\end{array}$

$\begin{array}{lll}-3.159375 & 2.211649 & 1.111422\end{array}$

$\begin{array}{llll}-3.785653 & 3.947113 & 0.002123\end{array}$

$\begin{array}{llll}-2.908543 & 4.538430 & 0.245215\end{array}$

$\begin{array}{llll}-4.776293 & 4.488919 & -0.861866\end{array}$

$\begin{array}{llll}-4.587239 & 5.747248 & -1.495212\end{array}$

$\begin{array}{llll}-3.677494 & 6.302471 & -1.281546\end{array}$

$\begin{array}{llll}-5.523394 & 6.245101 & -2.373624\end{array}$

$\begin{array}{llll}-5.363959 & 7.202950 & -2.860077\end{array}$

$\begin{array}{llll}-6.692360 & 5.498157 & -2.654394\end{array}$

$\begin{array}{llll}-7.418263 & 5.882659 & -3.365069\end{array}$

$\begin{array}{lll}-6.917166 & 4.285452 & -2.039016\end{array}$

$\begin{array}{llll}-7.814683 & 3.723395 & -2.270132\end{array}$

$\begin{array}{llll}-5.978206 & 3.745382 & -1.117032\end{array}$

$\begin{array}{llll}-6.173879 & 2.470569 & -0.473410\end{array}$

$\begin{array}{llll}-7.473496 & 1.741497 & -0.517906\end{array}$

$\begin{array}{lll}-8.698688 & 2.375843 & -0.089723\end{array}$

$\begin{array}{llll}-8.729461 & 3.667587 & 0.507785\end{array}$

$\begin{array}{llll}-7.801889 & 4.202851 & 0.668885\end{array}$

$\begin{array}{lll}-9.917447 & 4.244819 & 0.902321\end{array}$

$\begin{array}{lll}-9.907776 & 5.229156 & 1.361672\end{array}$

$\begin{array}{lll}-11.146448 & 3.567788 & 0.722654\end{array}$

$\begin{array}{lll}-12.075404 & 4.038674 & 1.030497\end{array}$

$\begin{array}{lll}-11.153327 & 2.305334 & 0.174465\end{array}$

$\begin{array}{lll}-12.085789 & 1.760982 & 0.049612\end{array}$

$\begin{array}{llll}-9.944378 & 1.675075 & -0.226614\end{array}$

$\begin{array}{lll}-9.950186 & 0.349627 & -0.738300\end{array}$

$\begin{array}{llll}-10.897883 & -0.169175 & -0.851536\end{array}$

$\begin{array}{llll}-8.772557 & -0.278082 & -1.052212\end{array}$

$\begin{array}{llll}-8.745614 & -1.302187 & -1.409395\end{array}$

$\begin{array}{llll}-7.547319 & 0.412849 & -0.911950\end{array}$

$\begin{array}{lll}-4.478865 & -3.038698 & 0.557847\end{array}$

$\begin{array}{lll}-3.622325 & -2.931166 & 1.237613\end{array}$

$\begin{array}{lll}-5.160225 & -4.360359 & 0.866996\end{array}$

$\begin{array}{llll}-4.476101 & -5.342630 & 1.592576\end{array}$

$\begin{array}{llll}-3.485519 & -5.122596 & 1.984952\end{array}$

$\begin{array}{llll}-5.055329 & -6.594453 & 1.818986\end{array}$

$\begin{array}{lll}-4.512542 & -7.347034 & 2.384430\end{array}$

$\begin{array}{lll}-6.332526 & -6.872698 & 1.327951\end{array}$

$\begin{array}{lll}-6.788051 & -7.842213 & 1.508564\end{array}$

$\begin{array}{llll}-7.025434 & -5.894084 & 0.607110\end{array}$

$\begin{array}{llll}-8.021626 & -6.102745 & 0.226598\end{array}$

$\begin{array}{lll}-6.441547 & -4.648920 & 0.374838\end{array}$

$\begin{array}{llll}-6.979722 & -3.884697 & -0.180005\end{array}$

$\begin{array}{lll}-3.981610 & -3.056184 & -0.891386\end{array}$

$\begin{array}{llll}-4.810426 & -3.394618 & -1.525733\end{array}$

$\begin{array}{llll}-3.196513 & -3.808029 & -0.987752\end{array}$

$\begin{array}{llll}-6.060015 & -1.787521 & 2.086950\end{array}$

$\begin{array}{lll}-6.349542 & -2.821694 & 2.288701\end{array}$

$\begin{array}{lll}-5.113751 & -1.387694 & 3.220964\end{array}$

$\begin{array}{llll}-4.455679 & -2.398772 & 3.938012\end{array}$

$\begin{array}{lll}-4.621061 & -3.437361 & 3.663530\end{array}$

$\begin{array}{lll}-3.611561 & -2.093375 & 5.008966\end{array}$

$\begin{array}{lll}-3.114559 & -2.894232 & 5.549218\end{array}$ $\begin{array}{lll}-3.412058 & -0.764114 & 5.385051\end{array}$

$\begin{array}{lll}-2.747936 & -0.520000 & 6.209711\end{array}$

$\begin{array}{lll}-4.068313 & 0.252366 & 4.684259\end{array}$

$\begin{array}{lll}-3.926018 & 1.290361 & 4.972860\end{array}$

$\begin{array}{lll}-4.911577 & -0.055917 & 3.614710\end{array}$

$\begin{array}{lll}-5.416562 & 0.745463 & 3.090342\end{array}$

$\begin{array}{lll}-7.366059 & -0.993532 & 2.066696\end{array}$

$\begin{array}{lll}-8.037607 & -1.391153 & 1.302667\end{array}$

$\begin{array}{lll}-7.225259 & 0.070737 & 1.879485\end{array}$

$\begin{array}{lll}-7.854068 & -1.102902 & 3.039908\end{array}$

$\begin{array}{lll}-2.037822 & -0.563759 & -0.021653\end{array}$

$\begin{array}{llll}-1.867713 & 0.509758 & -0.005942\end{array}$

$\begin{array}{lll}-2.339479 & -0.958567 & 0.946512\end{array}$

$\begin{array}{llll}-1.126730 & -1.359133 & -0.802591\end{array}$

$-0.457311-0.854996-1.487689$

$\begin{array}{lll}-0.890079 & -2.720818 & -0.540821\end{array}$

$\begin{array}{llll}-1.492548 & -3.178405 & 0.236813\end{array}$

$-4.591862-1.533292 \quad-3.475866$

$\begin{array}{llll}-5.426017 & -2.154432 & -3.158489\end{array}$

$\begin{array}{llll}-3.355285 & -2.194074 & -3.655959\end{array}$

$\begin{array}{llll}-3.341394 & -3.269576 & -3.479775\end{array}$

$\begin{array}{llll}-2.221618 & -1.710671 & -4.542860\end{array}$

$\begin{array}{lll}-2.429265 & -1.974615 & -5.591614\end{array}$

$\begin{array}{llll}-1.325087 & -2.270086 & -4.259044\end{array}$

$\begin{array}{llll}-1.930652 & -0.200554 & -4.426890\end{array}$

$\begin{array}{llll}-2.514331 & 0.358217 & -5.165222\end{array}$

$\begin{array}{llll}-0.879790 & -0.018640 & -4.672220\end{array}$

$\begin{array}{llll}-2.200958 & 0.382911 & -3.042860\end{array}$

$\begin{array}{llll}-1.321846 & 0.684430 & -2.485682\end{array}$

$\begin{array}{llll}-3.398028 & 0.973716 & -2.666098\end{array}$

$\begin{array}{llll}-3.358076 & 1.692024 & -1.853416\end{array}$

$\begin{array}{llll}-4.635510 & 1.051792 & -3.535880\end{array}$

$\begin{array}{llll}-5.470005 & 1.367339 & -2.908603\end{array}$

$\begin{array}{llll}-4.490378 & 1.848229 & -4.281138\end{array}$

$\begin{array}{llll}-5.002847 & -0.273467 & -4.240052\end{array}$

$\begin{array}{lll}-4.558562 & -0.307561 & -5.240192\end{array}$

$\begin{array}{lll}-6.086551 & -0.297610 & -4.388563\end{array}$

$-0.253048 \quad-3.644818-1.466985$

$\begin{array}{llll}0.551408 & -3.206530 & -2.538361\end{array}$

$\begin{array}{llll}-0.447676 & -5.029189 & -1.298871\end{array}$

$\begin{array}{llll}1.130086 & -4.121177 & -3.412224\end{array}$

$\begin{array}{llll}0.726742 & -2.146634 & -2.678783\end{array}$

$\begin{array}{llll}0.124863 & -5.944817 & -2.182095\end{array}$

$\begin{array}{llll}-1.056937 & -5.381590 & -0.470229\end{array}$

$\begin{array}{llll}0.916524 & -5.494688 & -3.241414\end{array}$

$\begin{array}{llll}1.746639 & -3.763945 & -4.231734\end{array}$

$\begin{array}{llll}-0.043494 & -7.008489 & -2.040384\end{array}$

$\begin{array}{llll}1.366453 & -6.205604 & -3.928249\end{array}$

$\begin{array}{llll}2.375532 & -4.497008 & 0.298040\end{array}$

$\begin{array}{llll}3.405259 & -4.991793 & -0.472884\end{array}$

$\begin{array}{lll}1.751333 & -5.179032 & 0.863015\end{array}$

$\begin{array}{llll}4.191349 & -4.068646 & -1.170036\end{array}$

$\begin{array}{llll}3.927347 & -2.717494 & -1.060514\end{array}$

$\begin{array}{llll}4.529179 & -1.983958 & -1.579772\end{array}$

$2.910818-2.233947-0.318995$

$\begin{array}{lll}1.010437 & -0.304093 & 0.925976\end{array}$

$\begin{array}{lll}-0.123647 & -1.187449 & 2.678108\end{array}$

$\begin{array}{lll}-0.677401 & 0.121984 & 3.001388\end{array}$

$\begin{array}{lll}-1.694729 & 0.002151 & 3.380026\end{array}$

$\begin{array}{lll}-0.083392 & 0.630983 & 3.768268\end{array}$

$\begin{array}{lll}-0.710999 & 0.735585 & 2.107635\end{array}$

$-0.433338-2.264960 \quad 3.611711$

$\begin{array}{lll}-1.335075 & -2.818157 & 3.319425\end{array}$

$\begin{array}{llll}0.401576 & -2.959606 & 3.701103\end{array}$

$\begin{array}{lll}-0.610287 & -1.829356 & 4.596274\end{array}$

$\begin{array}{llll}3.594101 & -6.058022 & -0.533278\end{array}$

$35 \quad 5.659027 \quad-4.674031 \quad-2.315731$

9 P1-C2 exo si-si

Number of imaginary frequencies $=0$

The smallest frequencies are $=10.803613 .398614 .8641 \mathrm{~cm}(-1)$ Electronic energy $=-7222.6577931$

Zero-point correction=

1.759193

(Hartree/Particle) 


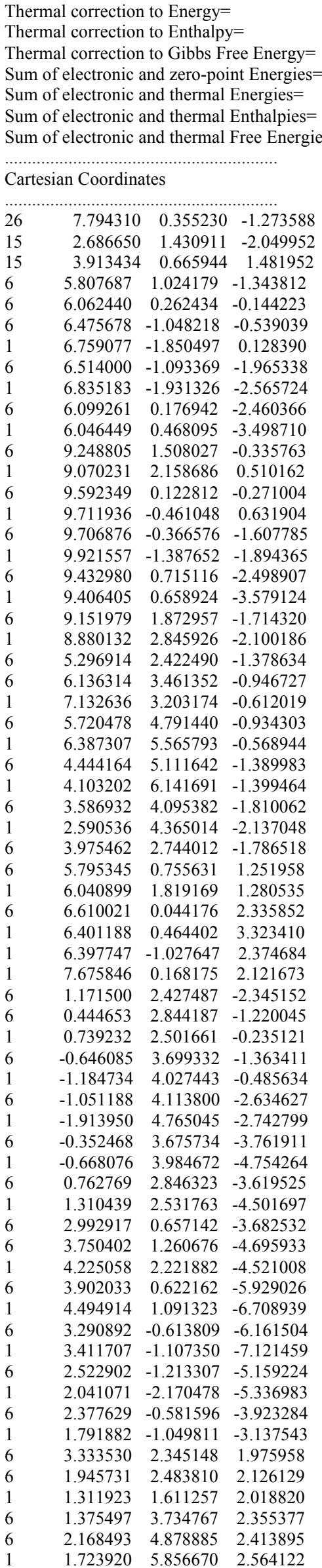

1.876944

1.877888

1.599335

$-7220.898600$

$-7220.780849$

$-7220.779905$

$-7221.058458$
6

6

6

1

(1)

(2)

(20)

(n)

(n)

(n)

9.

9

(n)

(2)

9

6

0

(1)

1
77
15

77

8

8
8
7

6

6
6
1

6
6
1

$$
\begin{aligned}
& 6 \\
& 1 \\
& 1
\end{aligned}
$$

$$
\begin{aligned}
& 1 \\
& 6 \\
& 6
\end{aligned}
$$

6
6
6
6

6

$$
1
$$

1
6
1
6

6

6

6
6
6

6
6
6

6
1
6
1

6

6

6

6

6

6
1
6
6

6

6

1
6
6
1 $\begin{array}{lll}3.548684 & 4.741092 & 2.253987\end{array}$

$\begin{array}{lll}4.134541 & 3.490609 & 2.050447\end{array}$

$\begin{array}{lll}5.207787 & 3.436212 & 1.927366\end{array}$

$\begin{array}{lll}3.595424 & -0.383520 & 2.960947\end{array}$

$\begin{array}{lll}3.747534 & -1.770033 & 2.794226\end{array}$

$\begin{array}{lll}4.121657 & -2.167974 & 1.857340\end{array}$

$\begin{array}{lll}3.397674 & -2.644475 & 3.821552\end{array}$

$\begin{array}{lll}2.859229 & -2.163886 & 5.015868\end{array}$

$\begin{array}{llll}2.564372 & -2.848718 & 5.803322\end{array}$

$\begin{array}{lll}2.698800 & -0.789664 & 5.174460\end{array}$

$\begin{array}{lll}3.078002 & 0.100132 & 4.167487\end{array}$

$\begin{array}{lll}2.946397 & 1.163522 & 4.325955\end{array}$

$\begin{array}{llll}2.575400 & -0.013384 & -0.320871\end{array}$

$\begin{array}{llll}2.018857 & -0.284107 & 6.417204\end{array}$

$\begin{array}{lll}3.492116 & -4.131863 & 3.625756\end{array}$

$\begin{array}{lll}-0.115316 & 3.813331 & 2.542335\end{array}$

$\begin{array}{lll}4.392610 & 5.987060 & 2.271657\end{array}$

$\begin{array}{llll}4.048581 & 6.832084 & 1.269546\end{array}$

$\begin{array}{llll}5.710945 & 5.724202 & 2.136701\end{array}$

$\begin{array}{lll}4.234535 & 6.675875 & 3.425729\end{array}$

$\begin{array}{lll}-0.772294 & 2.976810 & 1.688830\end{array}$

$\begin{array}{lll}-0.489603 & 3.442489 & 3.788535\end{array}$

$\begin{array}{lll}-0.601829 & 5.052789 & 2.323775\end{array}$

$\begin{array}{llll}2.185321 & 1.041968 & 6.594986\end{array}$

$\begin{array}{lll}0.677144 & -0.508610 & 6.359959\end{array}$

$\begin{array}{lll}2.464380 & -0.907756 & 7.528696\end{array}$

$\begin{array}{llll}3.938117 & -4.761347 & 4.731978\end{array}$

$\begin{array}{llll}4.305585 & -4.469967 & 2.604306\end{array}$

$\begin{array}{llll}2.266959 & -4.667802 & 3.348964\end{array}$

$\begin{array}{lll}0.537416 & -1.307282 & 1.677552\end{array}$

$\begin{array}{llll}0.583063 & -2.637404 & 0.949269\end{array}$

$\begin{array}{lll}1.862488 & -2.990332 & 0.220002\end{array}$

$\begin{array}{llll}0.432895 & -3.445949 & 1.660331\end{array}$

$\begin{array}{llll}-3.071862 & -1.029253 & -1.637402\end{array}$

$\begin{array}{lll}-4.941596 & -0.548568 & -0.384647\end{array}$

$\begin{array}{lll}-5.074949 & 0.658906 & 0.762327\end{array}$

$\begin{array}{lll}-6.236870 & -0.136064 & -1.358032\end{array}$

$\begin{array}{llll}-5.323497 & -1.860067 & 0.596315\end{array}$

$\begin{array}{llll}-4.975997 & 1.981871 & 0.369363\end{array}$

$\begin{array}{llll}-3.835294 & 2.689221 & 0.809596\end{array}$

$\begin{array}{lll}-3.119132 & 2.176441 & 1.440498\end{array}$

$\begin{array}{lll}-3.671335 & 4.002167 & 0.446149\end{array}$

$\begin{array}{llll}-2.829207 & 4.572743 & 0.824309\end{array}$

$\begin{array}{llll}-4.582098 & 4.615082 & -0.456846\end{array}$

$\begin{array}{llll}-4.341788 & 5.918716 & -0.970190\end{array}$

$\begin{array}{llll}-3.459173 & 6.454636 & -0.630262\end{array}$

$\begin{array}{llll}-5.193617 & 6.483274 & -1.893571\end{array}$

$\begin{array}{llll}-4.994626 & 7.475240 & -2.288404\end{array}$

$\begin{array}{llll}-6.327044 & 5.761600 & -2.338848\end{array}$

$\begin{array}{llll}-6.985335 & 6.200380 & -3.083115\end{array}$

$\begin{array}{llll}-6.601897 & 4.505868 & -1.841371\end{array}$

$\begin{array}{llll}-7.471413 & 3.964420 & -2.196320\end{array}$

$\begin{array}{llll}-5.748799 & 3.894033 & -0.881406\end{array}$

$\begin{array}{llll}-5.997827 & 2.575096 & -0.356999\end{array}$

$\begin{array}{llll}-7.301457 & 1.874890 & -0.536821\end{array}$

$\begin{array}{lll}-8.541648 & 2.506202 & -0.147211\end{array}$

$\begin{array}{lll}-8.591950 & 3.760983 & 0.523893\end{array}$

$\begin{array}{llll}-7.669099 & 4.266865 & 0.778562\end{array}$

$\begin{array}{llll}-9.793650 & 4.339340 & 0.872922\end{array}$

$\begin{array}{lll}-9.797595 & 5.294648 & 1.390130\end{array}$

$\begin{array}{lll}-11.019192 & 3.699553 & 0.574060\end{array}$

$\begin{array}{lll}-11.958698 & 4.171115 & 0.847065\end{array}$

$\begin{array}{llll}-11.009858 & 2.470596 & -0.045778\end{array}$

$\begin{array}{llll}-11.940704 & 1.952520 & -0.261889\end{array}$

$\begin{array}{lll}-9.787607 & 1.839836 & -0.403347\end{array}$

$\begin{array}{llll}-9.781279 & 0.546579 & -0.992001\end{array}$

$\begin{array}{lll}-10.727615 & 0.052564 & -1.194315\end{array}$

$\begin{array}{llll}-8.595989 & -0.083359 & -1.269408\end{array}$

$\begin{array}{llll}-8.563052 & -1.085298 & -1.684484\end{array}$

$\begin{array}{llll}-7.368899 & 0.573213 & -1.015814\end{array}$

$\begin{array}{lll}-4.390759 & -3.004214 & 0.407327\end{array}$

$\begin{array}{lll}-3.600654 & -2.928453 & 1.167347\end{array}$

$\begin{array}{lll}-5.080014 & -4.347443 & 0.576869\end{array}$

$\begin{array}{llll}-4.438857 & -5.379831 & 1.272348\end{array}$

$\begin{array}{lll}-3.481943 & -5.185779 & 1.751650\end{array}$ 
$\begin{array}{lll}-5.016590 & -6.649589 & 1.359470\end{array}$

$\begin{array}{lll}-4.505288 & -7.440182 & 1.902106\end{array}$

$\begin{array}{lll}-6.252070 & -6.898261 & 0.758067\end{array}$

$\begin{array}{llll}-6.706992 & -7.882155 & 0.829556\end{array}$

$\begin{array}{lll}-6.903592 & -5.870600 & 0.067702\end{array}$

$\begin{array}{llll}-7.867255 & -6.055390 & -0.399487\end{array}$

$\begin{array}{llll}-6.319878 & -4.607233 & -0.025627\end{array}$

$\begin{array}{lll}-6.826046 & -3.806608 & -0.558591\end{array}$

$\begin{array}{llll}-3.753031 & -2.948111 & -0.988443\end{array}$

$\begin{array}{llll}-4.506165 & -3.294052 & -1.708219\end{array}$

$\begin{array}{llll}-2.947630 & -3.679519 & -1.021218\end{array}$

$\begin{array}{lll}-6.071530 & -1.860603 & 1.893970\end{array}$

$\begin{array}{lll}-6.391988 & -2.899638 & 2.005104\end{array}$

$\begin{array}{lll}-5.176676 & -1.567856 & 3.101800\end{array}$

$\begin{array}{lll}-4.588350 & -2.644880 & 3.783533\end{array}$

$\begin{array}{lll}-4.776370 & -3.657638 & 3.437799\end{array}$

$\begin{array}{llll}-3.785001 & -2.438358 & 4.908127\end{array}$

$\begin{array}{lll}-3.344789 & -3.289405 & 5.420675\end{array}$

$\begin{array}{llll}-3.557888 & -1.142586 & 5.376009\end{array}$

$\begin{array}{lll}-2.936766 & -0.975376 & 6.251801\end{array}$

$\begin{array}{lll}-4.144828 & -0.061292 & 4.711656\end{array}$

$\begin{array}{llll}-3.985496 & 0.950807 & 5.074165\end{array}$

$\begin{array}{lll}-4.946760 & -0.271779 & 3.587840\end{array}$

$\begin{array}{llll}-5.399001 & 0.577974 & 3.093086\end{array}$

$\begin{array}{llll}-7.359059 & -1.036950 & 1.868338\end{array}$

$\begin{array}{lll}-7.993496 & -1.355485 & 1.038281\end{array}$

$\begin{array}{lll}-7.186138 & 0.034603 & 1.774688\end{array}$

$\begin{array}{lll}-7.905059 & -1.211535 & 2.800510\end{array}$

$\begin{array}{lll}-1.881241 & -0.484106 & 0.106923\end{array}$

$\begin{array}{lll}-1.656728 & 0.579711 & 0.088293\end{array}$

$\begin{array}{lll}-2.275259 & -0.817695 & 1.066302\end{array}$

$\begin{array}{llll}-1.075900 & -1.393570 & -0.645687\end{array}$

$\begin{array}{lll}-0.282224 & -0.966599 & -1.252997\end{array}$

$\begin{array}{llll}-0.715221 & -2.728031 & -0.024141\end{array}$

$\begin{array}{lll}-1.522653 & -3.004822 & 0.659633\end{array}$

$-4.133344-1.350628-3.519073$

$\begin{array}{llll}-4.971067 & -2.026159 & -3.358068\end{array}$

$\begin{array}{llll}-2.839230 & -1.963278 & -3.607421\end{array}$

$\begin{array}{llll}-2.804781 & -3.049652 & -3.518731\end{array}$

$\begin{array}{llll}-1.698979 & -1.392024 & -4.436893\end{array}$

$\begin{array}{lll}-1.874909 & -1.575012 & -5.508989\end{array}$

$\begin{array}{llll}-0.793354 & -1.946757 & -4.178664\end{array}$

$\begin{array}{llll}-1.451975 & 0.108980 & -4.186626\end{array}$

$\begin{array}{llll}-1.973289 & 0.716501 & -4.933660\end{array}$

$\begin{array}{llll}-0.387735 & 0.327864 & -4.316780\end{array}$

$\begin{array}{lll}-1.869428 & 0.571937 & -2.793265\end{array}$

$\begin{array}{llll}-1.051578 & 0.854148 & -2.139234\end{array}$

$\begin{array}{lll}-3.113996 & 1.125433 & -2.500489\end{array}$

$\begin{array}{llll}-3.164722 & 1.815720 & -1.664089\end{array}$

$\begin{array}{lll}-4.257091 & 1.228350 & -3.492559\end{array}$

$\begin{array}{lll}-5.159174 & 1.488119 & -2.935292\end{array}$

$\begin{array}{llll}-4.061384 & 2.065828 & -4.179760\end{array}$

$\begin{array}{lll}-4.511507 & -0.074677 & -4.284865\end{array}$

$\begin{array}{llll}-3.971074 & -0.051421 & -5.237978\end{array}$

$\begin{array}{llll}-5.573973 & -0.122402 & -4.544698\end{array}$

$-0.506825 \quad-3.876585-0.992527$

$\begin{array}{llll}0.082969 & -3.678898 & -2.247025\end{array}$

$-0.845213-5.182368-0.611797$

$\begin{array}{lll}0.331317 & -4.755978 & -3.099191\end{array}$

$\begin{array}{llll}0.355545 & -2.677413 & -2.555687\end{array}$

$\begin{array}{lll}-0.602609 & -6.264151 & -1.460938\end{array}$

$\begin{array}{lll}-1.313231 & -5.350287 & 0.355370\end{array}$

$\begin{array}{llll}-0.008707 & -6.054607 & -2.708396\end{array}$

$\begin{array}{llll}0.790392 & -4.581714 & -4.068655\end{array}$

$\begin{array}{llll}-0.880081 & -7.267595 & -1.15009\end{array}$

$0.182941-6.893390 \quad-3.371463$

$\begin{array}{llll}2.272542 & -4.330386 & 0.278423\end{array}$

$\begin{array}{lll}3.287719 & -4.797853 & -0.543847\end{array}$

$\begin{array}{llll}1.768904 & -5.016515 & 0.945904\end{array}$

$3.880069-3.882566 \quad-1.404965$

$3.500522-2.548508-1.374859$

$\begin{array}{llll}3.977694 & -1.809583 & -2.004813\end{array}$

$2.510570-2.108378-0.570692$

$\begin{array}{lll}0.949977 & -0.259417 & 1.134215\end{array}$

$\begin{array}{lll}-0.089282 & -1.249722 & 2.871657\end{array}$

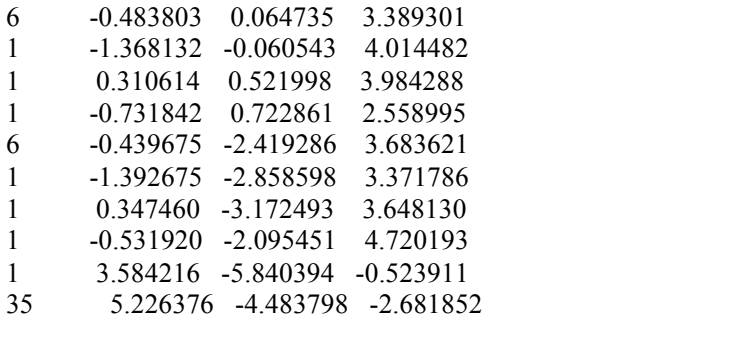

8 P1-C2 exo re-si

Number of imaginary frequencies $=0$

The smallest frequencies are $=5.18926 .494513 .2689 \mathrm{~cm}(-1)$

Electronic energy $=-7222.617113$

Zero-point correction=

1.754125

Hartree/Particle)

Thermal correction to Energy=

Thermal correction to Enthalpy=

1.874073

1.875017

Thermal correction to Gibbs Free Energy $=1.587486$

Sum of electronic and zero-point Energies $=\quad-7220.862988$

Sum of electronic and thermal Energies=

$-7220.743040$

Sum of electronic and thermal Enthalpies=

$-7220.742096$

Sum of electronic and thermal Free Energies=

$-7221.029627$

\begin{tabular}{|c|c|c|c|}
\hline 77 & 4.362977 & 5342 & -1.638825 \\
\hline 15 & 6.061223 & 0.435774 & -0.265409 \\
\hline 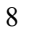 & 6.503065 & -0.481815 & 1.032417 \\
\hline 8 & 7.494338 & 0.505473 & -1.100923 \\
\hline 7 & 5.712911 & 1.883777 & 0.489262 \\
\hline 6 & 7.299690 & -1.616994 & 0.894089 \\
\hline 6 & 6.682831 & -2.849902 & 1.197718 \\
\hline 1 & 5.650958 & -2.843865 & 1.532949 \\
\hline 6 & 7.395706 & -4.014967 & 1.060236 \\
\hline 1 & 6.936163 & -4.970675 & 1.295321 \\
\hline 0 & 8.731783 & -3.988812 & 0.577217 \\
\hline 6 & 9.450131 & -5.193555 & 0.347276 \\
\hline 1 & 8.962316 & -6.138197 & 0.572789 \\
\hline 6 & 10.728083 & -5.167977 & -0.163123 \\
\hline 1 & 11.266305 & -6.094361 & -0.340775 \\
\hline 6 & 11.337841 & -3.928504 & -0.471311 \\
\hline 1 & 12.338065 & -3.912237 & -0.894367 \\
\hline 6 & 10.674774 & -2.741643 & -0.245498 \\
\hline 1 & 11.155959 & -1.803847 & -0.496471 \\
\hline 6 & 9.360033 & -2.727952 & 0.29 \\
\hline 6 & 8.633641 & -1.50 & 0.53 \\
\hline 6 & 9.266651 & -0.166735 & 0.378691 \\
\hline 6 & 10.446277 & 0.212895 & 1.111772 \\
\hline 6 & 11.021667 & -0.60 & 395 \\
\hline 1 & 10.558773 & -1.559504 & 2.360227 \\
\hline 6 & 12.147384 & -0.210208 & 2.807176 \\
\hline 1 & 12.562366 & -0.853754 & 3.577510 \\
\hline 6 & 12.764706 & 1.031642 & 2.524458 \\
\hline 1 & 13.655960 & 1.328722 & 3.069211 \\
\hline 6 & 12.221946 & 1.863927 & 1.572143 \\
\hline 1 & 12.674046 & 2.828789 & 1.358248 \\
\hline 6 & 11.051218 & 1.490358 & 0.857270 \\
\hline 6 & 10.458639 & 2.372745 & -0.086286 \\
\hline 1 & 10.931597 & 3.330678 & -0.282264 \\
\hline 6 & 9.287383 & 2.034616 & -0.718083 \\
\hline 1 & 8.798980 & 2.703334 & -1.418368 \\
\hline 6 & 8.695975 & 0.780736 & -0.454544 \\
\hline 6 & 4.566114 & 2.593487 & -0.138411 \\
\hline 1 & 3.684034 & 2.434995 & 0.494120 \\
\hline 6 & 4.805924 & 4.089060 & -0.258226 \\
\hline 6 & 3.757939 & 4.982323 & -0.008464 \\
\hline 1 & 2.797835 & 4.598189 & 0.324138 \\
\hline 6 & 3.939280 & 6.357067 & -0.182033 \\
\hline 1 & 3.117459 & 7.039139 & 0.018028 \\
\hline 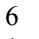 & 5.175707 & 6.852740 & -0.601758 \\
\hline 1 & 5.320920 & 7.921575 & -0.730226 \\
\hline & 6.228463 & 5.965859 & -0.850199 \\
\hline
\end{tabular}


$\begin{array}{llll}7.194664 & 6.344664 & -1.172244\end{array}$

$\begin{array}{llll}6.043214 & 4.593354 & -0.683787\end{array}$

$\begin{array}{llll}6.862969 & 3.903325 & -0.862600\end{array}$

$\begin{array}{llll}4.299457 & 2.011651 & -1.528492\end{array}$

$\begin{array}{llll}5.057944 & 2.404330 & -2.216120\end{array}$

$\begin{array}{llll}3.338380 & 2.375164 & -1.894519\end{array}$

$\begin{array}{llll}6.053022 & 2.393975 & 1.859304\end{array}$

$6.143068 \quad 3.474270 \quad 1.716979$

$\begin{array}{lll}4.893219 & 2.170680 & 2.824906\end{array}$

$\begin{array}{lll}4.179380 & 3.271664 & 3.313805\end{array}$

$\begin{array}{lll}4.455829 & 4.272043 & 2.990456\end{array}$

$\begin{array}{lll}3.113428 & 3.097253 & 4.201692\end{array}$

$2.571272 \quad 3.963175 \quad 4.571354$

$\begin{array}{llll}2.739411 & 1.813320 & 4.600405\end{array}$

$\begin{array}{lll}1.904274 & 1.671410 & 5.278361\end{array}$

$3.443933 \quad 0.707230 \quad 4.114058$

$\begin{array}{llll}3.151309 & -0.293995 & 4.416025\end{array}$

$\begin{array}{llll}4.515393 & 0.883971 & 3.237544\end{array}$

$\begin{array}{llll}5.059892 & 0.021884 & 2.867780\end{array}$

$\begin{array}{llll}7.392298 & 1.943411 & 2.446567\end{array}$

$\begin{array}{lll}8.214945 & 2.167742 & 1.766301\end{array}$

$\begin{array}{llll}7.416073 & 0.884683 & 2.699645\end{array}$

$\begin{array}{lll}7.554209 & 2.514187 & 3.366152\end{array}$

$\begin{array}{lll}3.267822 & -0.591032 & 0.172735\end{array}$

$\begin{array}{lll}3.532804 & -1.544663 & 0.627579\end{array}$

$\begin{array}{lll}3.280632 & 0.225838 & 0.892716\end{array}$

$\begin{array}{llll}2.168545 & -0.619805 & -0.768411\end{array}$

$\begin{array}{llll}1.822249 & -1.574831 & -1.142396\end{array}$

$\begin{array}{llll}1.555755 & 0.534202 & -1.222423\end{array}$

$\begin{array}{llll}1.843008 & 1.469041 & -0.755753\end{array}$

$\begin{array}{llll}5.591913 & 0.112824 & -3.544468\end{array}$

$\begin{array}{llll}6.114286 & 1.059137 & -3.437406\end{array}$

$\begin{array}{llll}4.226245 & 0.189460 & -3.869587\end{array}$

$\begin{array}{llll}3.809398 & 1.185646 & -4.013969\end{array}$

$\begin{array}{lll}3.379385 & -0.917205 & -4.472310\end{array}$

$\begin{array}{llll}3.469676 & -0.892180 & -5.568590\end{array}$

$\begin{array}{llll}2.332930 & -0.684285 & -4.248165\end{array}$

$\begin{array}{llll}3.693102 & -2.336964 & -3.953521\end{array}$

$\begin{array}{llll}4.509020 & -2.782607 & -4.529079\end{array}$

$\begin{array}{llll}2.817519 & -2.970112 & -4.127106\end{array}$

$\begin{array}{llll}4.040246 & -2.398158 & -2.471847\end{array}$

$\begin{array}{llll}3.281111 & -2.822225 & -1.821610\end{array}$

$\begin{array}{llll}5.316932 & -2.324415 & -1.952663\end{array}$

$\begin{array}{llll}5.460110 & -2.706972 & -0.947349\end{array}$

$\begin{array}{llll}6.589967 & -2.134722 & -2.748215\end{array}$

$\begin{array}{llll}7.380691 & -1.852684 & -2.050916\end{array}$

$6.883784-3.104567 \quad-3.174630$

$6.500319-1.072026-3.864714$

$\begin{array}{llll}6.166856 & -1.529002 & -4.800737\end{array}$

$\begin{array}{llll}7.506919 & -0.688580 & -4.054482\end{array}$

$\begin{array}{llll}0.590941 & 0.647250 & -2.299690\end{array}$

$\begin{array}{llll}-0.031752 & -0.466345 & -2.902280\end{array}$

$\begin{array}{llll}0.294460 & 1.934968 & -2.791052\end{array}$

$-0.911066-0.292173 \quad-3.967091$

$\begin{array}{llll}0.160814 & -1.464921 & -2.526018\end{array}$

$\begin{array}{llll}-0.562135 & 2.102916 & -3.875105\end{array}$

$\begin{array}{llll}0.749534 & 2.799520 & -2.315045\end{array}$

$\begin{array}{lll}-1.169450 & 0.990451 & -4.463591\end{array}$

$-1.405203-1.152524-4.408909$

$\begin{array}{llll}-0.776039 & 3.098881 & -4.248649\end{array}$

$\begin{array}{lll}-1.855092 & 1.120540 & -5.294083\end{array}$

$\begin{array}{llll}-6.675169 & 4.080018 & 1.291181\end{array}$

$\begin{array}{lll}-4.800380 & 0.777871 & -2.260978\end{array}$

$\begin{array}{lll}-4.733392 & -0.294642 & 1.350875\end{array}$

$\begin{array}{llll}-6.192573 & 2.519307 & -0.018802\end{array}$

$\begin{array}{lll}-5.594742 & 2.312947 & 1.279263\end{array}$

$\begin{array}{llll}-4.731185 & 3.418759 & 1.541834\end{array}$

$\begin{array}{lll}-4.149269 & 3.564368 & 2.441513\end{array}$

$\begin{array}{llll}-4.811855 & 4.321374 & 0.442473\end{array}$

$\begin{array}{llll}-4.304519 & 5.273095 & 0.362978\end{array}$

$\begin{array}{lll}-5.715085 & 3.775109 & -0.513358\end{array}$

$\begin{array}{llll}-6.004259 & 4.229251 & -1.449345\end{array}$

$\begin{array}{lll}-8.369070 & 3.923278 & 2.484995\end{array}$

$\begin{array}{lll}-8.772225 & 2.994077 & 2.865762\end{array}$

$\begin{array}{llll}-7.383706 & 4.736086 & 3.124987\end{array}$ $\begin{array}{lll}-6.903593 & 4.528129 & 4.071786\end{array}$

$\begin{array}{lll}-7.109626 & 5.850326 & 2.274250\end{array}$

$\begin{array}{lll}-6.381266 & 6.628537 & 2.460953\end{array}$

$\begin{array}{lll}-7.925440 & 5.727487 & 1.108353\end{array}$

$\begin{array}{lll}-7.924701 & 6.395822 & 0.257458\end{array}$

$\begin{array}{llll}-8.704105 & 4.538986 & 1.238762\end{array}$

$\begin{array}{lll}-9.399640 & 4.157929 & 0.503570\end{array}$

$\begin{array}{llll}-7.071762 & 1.540345 & -0.721047\end{array}$

$\begin{array}{llll}-8.392937 & 1.361324 & -0.286271\end{array}$

$\begin{array}{lll}-8.741904 & 1.961379 & 0.544546\end{array}$

$\begin{array}{lll}-9.245362 & 0.432456 & -0.882716\end{array}$

$\begin{array}{llll}-10.254072 & 0.301381 & -0.505023\end{array}$

$\begin{array}{lll}-8.785070 & -0.335187 & -1.949917\end{array}$

$\begin{array}{llll}-9.434622 & -1.064419 & -2.423891\end{array}$

$\begin{array}{lll}-7.472056 & -0.180463 & -2.396591\end{array}$

$-7.124688-0.794416 \quad-3.219794$

$\begin{array}{llll}-6.595173 & 0.731686 & -1.789453\end{array}$

$\begin{array}{llll}-5.794994 & 1.084930 & 2.122593\end{array}$

$\begin{array}{llll}-6.825369 & 0.763361 & 1.972016\end{array}$

$\begin{array}{lll}-5.592035 & 1.308571 & 3.623905\end{array}$

$\begin{array}{lll}-5.783868 & 0.391265 & 4.187321\end{array}$

$\begin{array}{llll}-4.579700 & 1.643192 & 3.863835\end{array}$

$\begin{array}{lll}-6.289577 & 2.079376 & 3.966066\end{array}$

$\begin{array}{llll}-4.646104 & -0.438061 & -3.64024\end{array}$

$\begin{array}{llll}-4.639187 & -1.804046 & -3.307682\end{array}$

$\begin{array}{llll}-4.802745 & -2.107674 & -2.279409\end{array}$

$\begin{array}{lll}-4.442269 & -2.777886 & -4.286082\end{array}$

$\begin{array}{lll}-4.454480 & -3.826545 & -4.008295\end{array}$

$\begin{array}{llll}-4.227443 & -2.398981 & -5.614946\end{array}$

$\begin{array}{llll}-4.062598 & -3.154766 & -6.377577\end{array}$

$\begin{array}{llll}-4.230725 & -1.045431 & -5.956400\end{array}$

$\begin{array}{llll}-4.068421 & -0.742576 & -6.987181\end{array}$

$\begin{array}{llll}-4.445410 & -0.070074 & -4.978320\end{array}$

$\begin{array}{llll}-4.443869 & 0.975438 & -5.264603\end{array}$

$\begin{array}{llll}-4.613038 & 2.412810 & -3.074879\end{array}$

$\begin{array}{llll}-5.598384 & 2.934508 & -3.928783\end{array}$

$\begin{array}{llll}-6.492075 & 2.356052 & -4.145477\end{array}$

$\begin{array}{llll}-5.441577 & 4.203963 & -4.486234\end{array}$

$\begin{array}{llll}-6.207300 & 4.603907 & -5.145008\end{array}$

$\begin{array}{llll}-4.301921 & 4.961540 & -4.191848\end{array}$

$\begin{array}{llll}-4.184638 & 5.952756 & -4.621269\end{array}$

$\begin{array}{llll}-3.321175 & 4.444766 & -3.342372\end{array}$

$\begin{array}{llll}-2.440034 & 5.034706 & -3.104575\end{array}$

$\begin{array}{llll}-3.473911 & 3.173766 & -2.782361\end{array}$

$\begin{array}{llll}-2.735811 & 2.782634 & -2.089993\end{array}$

$\begin{array}{lll}-5.919553 & -1.657642 & 0.958211\end{array}$

$\begin{array}{lll}-5.400415 & -2.760275 & 0.264239\end{array}$

$\begin{array}{lll}-4.337008 & -2.820786 & 0.064454\end{array}$

$\begin{array}{lll}-6.237894 & -3.784092 & -0.181014\end{array}$

$\begin{array}{lll}-7.611513 & -3.726144 & 0.039508\end{array}$

$\begin{array}{llll}-8.264801 & -4.508405 & -0.329589\end{array}$

$\begin{array}{llll}-8.129609 & -2.633584 & 0.733921\end{array}$

$\begin{array}{lll}-7.298792 & -1.616645 & 1.201958\end{array}$

$\begin{array}{lll}-7.752242 & -0.793138 & 1.735215\end{array}$

$\begin{array}{lll}-3.711151 & -0.999943 & 2.712850\end{array}$

$\begin{array}{lll}-2.663964 & -0.196506 & 3.196074\end{array}$

$\begin{array}{llll}-2.522488 & 0.804216 & 2.807394\end{array}$

$\begin{array}{lll}-1.775320 & -0.694701 & 4.143846\end{array}$

$\begin{array}{lll}-1.879440 & -2.009335 & 4.604662\end{array}$

$\begin{array}{lll}-1.170028 & -2.401227 & 5.324539\end{array}$

$\begin{array}{lll}-2.910045 & -2.807074 & 4.118167\end{array}$

$\begin{array}{lll}-3.829160 & -2.308875 & 3.189127\end{array}$

$\begin{array}{lll}-4.625695 & -2.956780 & 2.842036\end{array}$

$\begin{array}{llll}-3.433097 & 0.309399 & -0.508783\end{array}$

$\begin{array}{lll}-3.002339 & -4.251207 & 4.524943\end{array}$

$\begin{array}{lll}-0.609077 & 0.134458 & 4.600637\end{array}$

$\begin{array}{llll}-5.631305 & -4.963752 & -0.889885\end{array}$

$\begin{array}{lll}-9.618924 & -2.565222 & 0.938718\end{array}$

$\begin{array}{llll}-10.280415 & -2.579544 & -0.244856\end{array}$

$\begin{array}{lll}-10.003931 & -1.449257 & 1.595946\end{array}$

$\begin{array}{lll}-10.075331 & -3.625946 & 1.644516\end{array}$

$\begin{array}{llll}-4.640221 & -4.577931 & -1.733731\end{array}$

$\begin{array}{llll}-5.077868 & -5.839789 & -0.020354\end{array}$

$\begin{array}{llll}-6.537291 & -5.645284 & -1.621471\end{array}$

$\begin{array}{lll}-4.285202 & -4.657263 & 4.661604\end{array}$ 
$\begin{array}{lrrr}9 & -2.437388 & -5.060767 & 3.593823 \\ 9 & -2.371561 & -4.497138 & 5.693713 \\ 9 & -0.396627 & 0.030510 & 5.932273 \\ 9 & -0.760201 & 1.448112 & 4.320773 \\ 9 & 0.545807 & -0.267882 & 4.003376 \\ 6 & -1.939592 & -2.300606 & -0.689398 \\ 6 & -0.794978 & -0.696871 & 0.598237 \\ 6 & -1.166184 & -3.333445 & -0.195288 \\ 1 & -2.705065 & -2.482723 & -1.435584 \\ 6 & -0.020998 & -1.759753 & 1.173280 \\ 6 & -0.200835 & -3.067674 & 0.788259 \\ 1 & 0.716787 & -1.515670 & 1.928457 \\ 6 & -0.451814 & 0.630198 & 0.947978 \\ 6 & -1.054894 & 1.836470 & 0.496223 \\ 8 & -2.131598 & 1.896274 & -0.180866 \\ 7 & -1.794430 & -1.021180 & -0.291341 \\ 1 & 0.365821 & 0.703550 & 1.648568 \\ 7 & -0.424101 & 3.036902 & 0.788981 \\ 6 & -1.119482 & 4.293715 & 0.546543 \\ 1 & -1.508250 & 4.725455 & 1.480897 \\ 1 & -0.433411 & 5.021147 & 0.097241 \\ 1 & -1.953733 & 4.121076 & -0.129065 \\ 6 & 0.723941 & 3.107093 & 1.684724 \\ 1 & 1.546178 & 2.477942 & 1.333454 \\ 1 & 1.083509 & 4.136999 & 1.717346 \\ 1 & 0.475685 & 2.804505 & 2.708642 \\ 1 & 0.390692 & -3.864681 & 1.226544 \\ 35 & -1.415692 & -5.163356 & -0.852197 \\ -------------------------------------------------------\end{array}$

$[8-9]^{\ddagger}-\mathrm{P} 1-\mathrm{C} 2$ exo re-si

Number of imaginary frequencies $=1$

The smallest frequencies are $=-132.96344 .45649 .8735 \mathrm{~cm}(-1)$ Electronic energy $=-7222.6148776$

Zero-point correction $=$

(Hartree/Particle)

Thermal correction to Energy=

1.754242

Thermal correction to Enthalpy=

Thermal correction to Gibbs Free Energy=

Sum of electronic and zero-point Energies=

Sum of electronic and thermal Energies=

Sum of electronic and thermal Enthalpies=

Sum of electronic and thermal Free Energies=

1.873142

1.874086

1.589201

$-7220.860636$

$-7220.741736$

$-7220.740791$

$-7221.025677$

Cartesian Coordinates

$\begin{array}{lccc}77 & 4.332396 & -0.374629 & -1.603431 \\ 15 & 6.025750 & 0.348253 & -0.273900 \\ 8 & 6.544391 & -0.442426 & 1.085853 \\ 8 & 7.452148 & 0.436447 & -1.130948 \\ 7 & 5.630408 & 1.837402 & 0.383436 \\ 6 & 7.404842 & -1.534014 & 1.018542 \\ 6 & 6.870200 & -2.775879 & 1.425842 \\ 1 & 5.847254 & -2.805383 & 1.786433 \\ 6 & 7.648829 & -3.904255 & 1.357368 \\ 1 & 7.252601 & -4.865284 & 1.672470 \\ 6 & 8.971623 & -3.836502 & 0.842834 \\ 6 & 9.756983 & -5.010419 & 0.684014 \\ 1 & 9.331298 & -5.963224 & 0.987590 \\ 6 & 11.021358 & -4.947369 & 0.143451 \\ 1 & 11.610485 & -5.851316 & 0.019907 \\ 6 & 11.549151 & -3.700160 & -0.267815 \\ 1 & 12.537704 & -3.656312 & -0.715803 \\ 6 & 10.820523 & -2.540978 & -0.110980 \\ 1 & 11.237540 & -1.596890 & -0.440850 \\ 6 & 9.518059 & -2.564841 & 0.461554 \\ 6 & 8.725103 & -1.373626 & 0.626852 \\ 6 & 9.276406 & -0.014953 & 0.364525 \\ 6 & 10.442101 & 0.484438 & 1.046144 \\ 6 & 11.083222 & -0.229751 & 2.097135 \\ 1 & 10.682692 & -1.186344 & 2.410760 \\ 6 & 12.194677 & 0.282497 & 2.730439 \\ 1 & 12.661434 & -0.279839 & 3.534059 \\ 6 & 12.730814 & 1.535990 & 2.350071 \\ 1 & 13.611743 & 1.923086 & 2.853633\end{array}$

$\begin{array}{lll}12.122640 & 2.265270 & 1.353828\end{array}$

$\begin{array}{lll}12.511373 & 3.237822 & 1.063215\end{array}$ $\begin{array}{lll}10.965422 & 1.773674 & 0.690608\end{array}$ $\begin{array}{llll}10.309336 & 2.547756 & -0.304550\end{array}$ $\begin{array}{llll}10.723091 & 3.513625 & -0.580065\end{array}$ $\begin{array}{llll}9.153135 & 2.095002 & -0.890820\end{array}$ $\begin{array}{llll}8.621576 & 2.677890 & -1.634862\end{array}$ $\begin{array}{llll}8.638216 & 0.832171 & -0.525981\end{array}$ $\begin{array}{llll}4.473202 & 2.462424 & -0.310995\end{array}$ $\begin{array}{lll}3.588658 & 2.322733 & 0.322535\end{array}$ $\begin{array}{llll}4.655760 & 3.953650 & -0.538056\end{array}$ $\begin{array}{llll}3.539685 & 4.797784 & -0.479353\end{array}$ $\begin{array}{llll}2.570385 & 4.383320 & -0.215657\end{array}$ $\begin{array}{llll}3.661243 & 6.160184 & -0.762446\end{array}$ $\begin{array}{llll}2.785919 & 6.802278 & -0.711671\end{array}$ $\begin{array}{llll}4.905957 & 6.696658 & -1.101082\end{array}$ $\begin{array}{llll}5.004377 & 7.757290 & -1.314332\end{array}$ $\begin{array}{llll}6.025382 & 5.860726 & -1.159122\end{array}$ $\begin{array}{llll}6.997962 & 6.271211 & -1.416976\end{array}$ $\begin{array}{llll}5.899904 & 4.498332 & -0.883901\end{array}$ $\begin{array}{llll}6.769918 & 3.849430 & -0.915470\end{array}$ $\begin{array}{lll}4.239226 & 1.769942 & -1.660766\end{array}$ $\begin{array}{llll}4.999801 & 2.125685 & -2.367627\end{array}$ $\begin{array}{llll}3.276996 & 2.095506 & -2.060404\end{array}$ $\begin{array}{lll}5.919074 & 2.430463 & 1.727104\end{array}$ $\begin{array}{lll}5.917368 & 3.507670 & 1.539505\end{array}$ $\begin{array}{lll}4.792885 & 2.161493 & 2.722883\end{array}$ $\begin{array}{lll}4.082311 & 3.239366 & 3.266005\end{array}$ $\begin{array}{llll}4.326163 & 4.251238 & 2.951341\end{array}$ $\begin{array}{llll}3.065153 & 3.030753 & 4.202556\end{array}$ $\begin{array}{lll}2.526899 & 3.880977 & 4.612188\end{array}$ $\begin{array}{lll}2.729035 & 1.733808 & 4.593410\end{array}$ $\begin{array}{lll}1.924223 & 1.563003 & 5.301609\end{array}$ $\begin{array}{llll}3.428765 & 0.650610 & 4.053264\end{array}$ $\begin{array}{lll}3.167690 & -0.361708 & 4.347880\end{array}$ $\begin{array}{lll}4.457565 & 0.862301 & 3.134232\end{array}$ $\begin{array}{lll}5.002695 & 0.016739 & 2.731616\end{array}$ $\begin{array}{lll}7.295553 & 2.120943 & 2.324049\end{array}$ $\begin{array}{llll}8.091833 & 2.359130 & 1.616779\end{array}$ $\begin{array}{lll}7.398614 & 1.084453 & 2.641683\end{array}$ $\begin{array}{lll}7.423705 & 2.759140 & 3.203909\end{array}$ $\begin{array}{lll}3.162798 & -0.687350 & 0.185106\end{array}$ $\begin{array}{lll}3.357187 & -1.657387 & 0.642379\end{array}$ $\begin{array}{llll}3.208281 & 0.128467 & 0.902990\end{array}$ $\begin{array}{llll}2.130595 & -0.625976 & -0.821616\end{array}$ $\begin{array}{llll}1.746505 & -1.549882 & -1.234773\end{array}$ $\begin{array}{llll}1.417210 & 0.550553 & -1.111116\end{array}$ $\begin{array}{llll}1.761653 & 1.465697 & -0.647745\end{array}$ $\begin{array}{llll}5.572529 & -0.319746 & -3.491331\end{array}$ $\begin{array}{llll}6.097222 & 0.632047 & -3.487794\end{array}$ $\begin{array}{lll}4.208452 & -0.280733 & -3.848576\end{array}$ $\begin{array}{llll}3.795032 & 0.692537 & -4.111720\end{array}$ $\begin{array}{llll}3.389898 & -1.453872 & -4.362016\end{array}$ $\begin{array}{llll}3.553540 & -1.579979 & -5.443430\end{array}$ $\begin{array}{lll}2.333374 & -1.191502 & -4.241446\end{array}$ $\begin{array}{lll}3.661411 & -2.787008 & -3.631675\end{array}$ $\begin{array}{llll}4.476042 & -3.329679 & -4.120183\end{array}$ $\begin{array}{lll}2.776195 & -3.424857 & -3.722590\end{array}$ $\begin{array}{llll}3.988902 & -2.628396 & -2.148659\end{array}$ $\begin{array}{lll}3.224379 & -2.977866 & -1.459155\end{array}$ $\begin{array}{llll}5.274710 & -2.522152 & -1.634638\end{array}$ $\begin{array}{llll}5.412421 & -2.808339 & -0.596587\end{array}$ $\begin{array}{llll}6.548563 & -2.471991 & -2.453460\end{array}$ $\begin{array}{llll}7.353730 & -2.136574 & -1.797015\end{array}$ $\begin{array}{lll}6.809180 & -3.492988 & -2.768310\end{array}$ $\begin{array}{llll}6.477416 & -1.538100 & -3.680881\end{array}$ $\begin{array}{llll}6.145473 & -2.093894 & -4.563300\end{array}$ $\begin{array}{llll}7.488193 & -1.185584 & -3.906788\end{array}$ $\begin{array}{llll}0.539571 & 0.718805 & -2.256941\end{array}$ $\begin{array}{lll}-0.049480 & -0.371389 & -2.931896\end{array}$ $\begin{array}{llll}0.295966 & 2.021280 & -2.738852\end{array}$ $\begin{array}{lll}-0.848977 & -0.161993 & -4.052044\end{array}$ $\begin{array}{llll}0.105626 & -1.381169 & -2.569131\end{array}$ $\begin{array}{llll}-0.477659 & 2.224115 & -3.878663\end{array}$ $\begin{array}{llll}0.730346 & 2.868524 & -2.215372\end{array}$ 


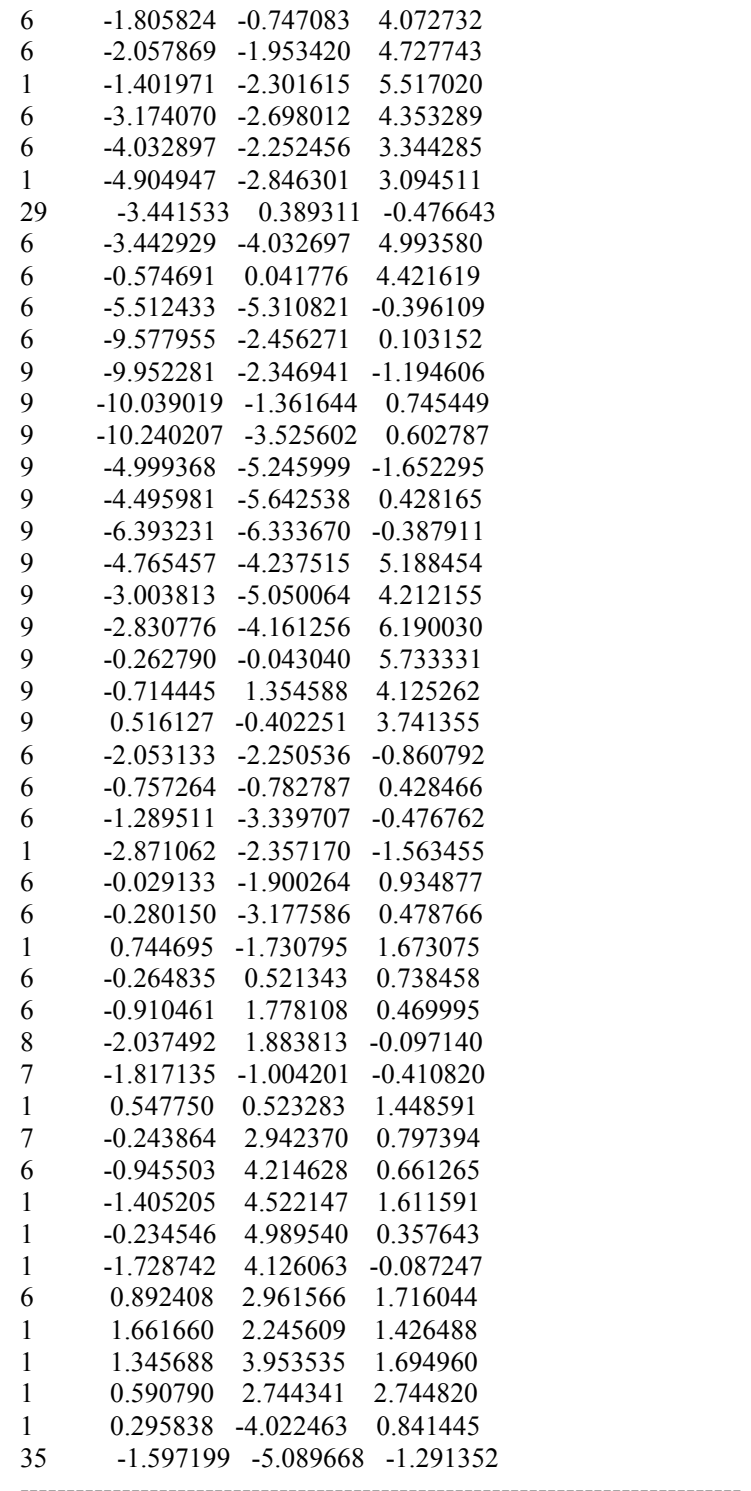

9 P1-C2 exo re-si

Number of imaginary frequencies $=0$

The smallest frequencies are $=5.5973 \quad 13.7396 \quad 15.8178 \mathrm{~cm}(-1)$ Electronic energy $=-7222.6463069$

Zero-point correction=

(Hartree/Particle)

Thermal correction to Energy= $\quad 1.876913$

Thermal correction to Enthalpy= $\quad 1.877857$

Thermal correction to Gibbs Free Energy $=\quad \begin{array}{r}1.598010 \\ -7220.8873\end{array}$

Sum of electronic and zero-point Energies $=\quad-7220.887376$

Sum of electronic and thermal Energies $=\quad-7220.769394$

Sum of electronic and thermal Enthalpies $=\quad-7220.768450$

Sum of electronic and thermal Free Energies $=\quad-7221.048297$

Cartesian Coordinates

$\begin{array}{lccc}77 & 4.127270 & -0.436316 & -1.492146 \\ 15 & 5.934451 & 0.283089 & -0.262997 \\ 8 & 6.574711 & -0.494770 & 1.067507 \\ 8 & 7.320159 & 0.414034 & -1.198779 \\ 7 & 5.574352 & 1.772875 & 0.431295 \\ 6 & 7.433909 & -1.575326 & 0.919210 \\ 6 & 6.942321 & -2.834121 & 1.331236 \\ 1 & 5.941592 & -2.886251 & 1.747045 \\ 6 & 7.730402 & -3.949975 & 1.198352 \\ 1 & 7.365047 & -4.922065 & 1.517159 \\ 6 & 9.021202 & -3.854366 & 0.611540\end{array}$


$\begin{array}{llll}9.812943 & -5.013479 & 0.388753\end{array}$

$\begin{array}{llll}9.419131 & -5.977404 & 0.700527\end{array}$

$\begin{array}{llll}11.044326 & -4.922737 & -0.219980\end{array}$

$\begin{array}{llll}11.638143 & -5.815670 & -0.391932\end{array}$

$\begin{array}{llll}11.530325 & -3.661008 & -0.638590\end{array}$

$\begin{array}{llll}12.491495 & -3.594648 & -1.140271\end{array}$

$\begin{array}{llll}10.794592 & -2.515884 & -0.422407\end{array}$

$\begin{array}{lll}11.178949 & -1.560460 & -0.759360\end{array}$

$\begin{array}{llll}9.525702 & -2.568385 & 0.220134\end{array}$

$\begin{array}{llll}8.726387 & -1.392660 & 0.450786\end{array}$

$\begin{array}{lll}9.237911 & -0.021228 & 0.175780\end{array}$

$\begin{array}{lll}10.430828 & 0.492646 & 0.795482\end{array}$

$\begin{array}{lll}11.144210 & -0.221023 & 1.799457\end{array}$

$\begin{array}{lll}10.780694 & -1.189435 & 2.122071\end{array}$

$\begin{array}{lll}12.279164 & 0.305650 & 2.376985\end{array}$

$\begin{array}{lll}12.800541 & -0.256683 & 3.146432\end{array}$

$\begin{array}{llll}12.768721 & 1.574141 & 1.983656\end{array}$

$\begin{array}{lll}13.668297 & 1.973662 & 2.442760\end{array}$

$\begin{array}{lll}12.091997 & 2.302249 & 1.031610\end{array}$

$\begin{array}{lll}12.445576 & 3.285740 & 0.732930\end{array}$

$\begin{array}{lll}10.909433 & 1.795539 & 0.426845\end{array}$

$\begin{array}{llll}10.183632 & 2.566889 & -0.521005\end{array}$

$\begin{array}{llll}10.562968 & 3.543553 & -0.808662\end{array}$

$\begin{array}{llll}9.004139 & 2.098077 & -1.044978\end{array}$

$\begin{array}{llll}8.418678 & 2.678950 & -1.749452\end{array}$

$\begin{array}{llll}8.530013 & 0.822508 & -0.665227\end{array}$

$\begin{array}{llll}4.364374 & 2.389874 & -0.173420\end{array}$

$\begin{array}{lll}3.540523 & 2.258735 & 0.538805\end{array}$

$\begin{array}{llll}4.524228 & 3.879921 & -0.432242\end{array}$

$\begin{array}{llll}3.390842 & 4.703659 & -0.400203\end{array}$

$\begin{array}{llll}2.427615 & 4.274745 & -0.136375\end{array}$

$\begin{array}{llll}3.482721 & 6.059990 & -0.718777\end{array}$

$\begin{array}{llll}2.592785 & 6.683216 & -0.688530\end{array}$

$\begin{array}{llll}4.716337 & 6.614757 & -1.070003\end{array}$

$\begin{array}{llll}4.792271 & 7.671006 & -1.312219\end{array}$

$\begin{array}{llll}5.851933 & 5.800439 & -1.105652\end{array}$

$\begin{array}{llll}6.816013 & 6.222936 & -1.376062\end{array}$

$\begin{array}{llll}5.755238 & 4.443002 & -0.793340\end{array}$

$\begin{array}{llll}6.637671 & 3.811062 & -0.813335\end{array}$

$\begin{array}{llll}4.001566 & 1.697581 & -1.499738\end{array}$

$\begin{array}{llll}4.682775 & 2.077177 & -2.272664\end{array}$

$\begin{array}{lll}3.005425 & 2.025193 & -1.796085\end{array}$

$\begin{array}{lll}5.936460 & 2.356788 & 1.759240\end{array}$

$\begin{array}{llll}5.903962 & 3.436681 & 1.588769\end{array}$

$\begin{array}{lll}4.883569 & 2.057814 & 2.826696\end{array}$

$\begin{array}{llll}4.205713 & 3.117148 & 3.443862\end{array}$

$\begin{array}{llll}4.421494 & 4.138278 & 3.138433\end{array}$

$\begin{array}{llll}3.257403 & 2.879856 & 4.444341\end{array}$

$\begin{array}{llll}2.744954 & 3.716938 & 4.910564\end{array}$

$\begin{array}{llll}2.956682 & 1.571910 & 4.827710\end{array}$

$\begin{array}{lll}2.200200 & 1.378604 & 5.582568\end{array}$

$\begin{array}{llll}3.625516 & 0.507171 & 4.215932\end{array}$

$\begin{array}{lll}3.395907 & -0.514618 & 4.505804\end{array}$

$\begin{array}{llll}4.585923 & 0.747376 & 3.231986\end{array}$

$\begin{array}{llll}5.106571 & -0.084100 & 2.772203\end{array}$

$\begin{array}{lll}7.353391 & 2.068937 & 2.267331\end{array}$

$\begin{array}{lll}8.099336 & 2.323493 & 1.512552\end{array}$

$\begin{array}{lll}7.494075 & 1.032516 & 2.569635\end{array}$

$\begin{array}{lll}7.527517 & 2.704920 & 3.141169\end{array}$

$\begin{array}{lll}3.035720 & -0.751934 & 0.373660\end{array}$

$\begin{array}{lll}3.233828 & -1.745074 & 0.777508\end{array}$

$\begin{array}{lll}3.150436 & 0.035823 & 1.114756\end{array}$

$\begin{array}{llll}2.059634 & -0.579915 & -0.658265\end{array}$

$\begin{array}{llll}1.586015 & -1.475516 & -1.048861\end{array}$

$\begin{array}{llll}1.115682 & 0.611617 & -0.610984\end{array}$

$\begin{array}{llll}1.683353 & 1.487640 & -0.298314\end{array}$

$\begin{array}{llll}5.092262 & -0.333014 & -3.455431\end{array}$

$\begin{array}{llll}5.575985 & 0.635361 & -3.570272\end{array}$

$\begin{array}{llll}3.671842 & -0.353768 & -3.632944\end{array}$

$\begin{array}{llll}3.193032 & 0.594216 & -3.879068\end{array}$

$\begin{array}{llll}2.896016 & -1.562936 & -4.133051\end{array}$

$\begin{array}{llll}3.030045 & -1.680622 & -5.220031\end{array}$

$\begin{array}{llll}1.835135 & -1.354768 & -3.976032\end{array}$

$\begin{array}{llll}3.264661 & -2.876821 & -3.411146\end{array}$

$\begin{array}{lll}4.033642 & -3.418186 & -3.971259\end{array}$ $\begin{array}{llll}2.389861 & -3.535833 & -3.390193\end{array}$

$\begin{array}{llll}3.750989 & -2.674061 & -1.977229\end{array}$

$\begin{array}{llll}3.076165 & -3.029895 & -1.200998\end{array}$

$\begin{array}{llll}5.092360 & -2.545081 & -1.623880\end{array}$

$\begin{array}{llll}5.369688 & -2.835291 & -0.615762\end{array}$

$\begin{array}{llll}6.248906 & -2.474264 & -2.606978\end{array}$

$\begin{array}{llll}7.127756 & -2.130352 & -2.055388\end{array}$

$\begin{array}{llll}6.483086 & -3.487901 & -2.966152\end{array}$

$\begin{array}{llll}5.998166 & -1.521995 & -3.797988\end{array}$

$\begin{array}{llll}5.570060 & -2.074013 & -4.641882\end{array}$

$6.963043-1.141664 \quad-4.148371$

$\begin{array}{llll}0.473520 & 0.947838 & -1.943332\end{array}$

$-0.078293 \quad-0.033017 \quad-2.781212$

$\begin{array}{llll}0.453998 & 2.280116 & -2.382297\end{array}$

$\begin{array}{llll}-0.630601 & 0.306676 & -4.017168\end{array}$

$\begin{array}{llll}-0.070722 & -1.071695 & -2.475189\end{array}$

$\begin{array}{llll}-0.074925 & 2.621883 & -3.629322\end{array}$

$\begin{array}{llll}0.869913 & 3.056465 & -1.745161\end{array}$

$\begin{array}{llll}-0.623741 & 1.635267 & -4.449895\end{array}$

$\begin{array}{llll}-1.063361 & -0.466999 & -4.644452\end{array}$

$\begin{array}{llll}-0.069596 & 3.658557 & -3.952051\end{array}$

$\begin{array}{llll}-1.047746 & 1.897697 & -5.414590\end{array}$

$\begin{array}{lll}-6.479467 & 4.008003 & 1.490923\end{array}$

$\begin{array}{llll}-4.505868 & 1.026240 & -2.300025\end{array}$

$\begin{array}{lll}-4.693422 & -0.434886 & 1.233552\end{array}$

$\begin{array}{llll}-5.956740 & 2.577324 & 0.053624\end{array}$

$\begin{array}{lll}-5.433177 & 2.226975 & 1.353427\end{array}$

$\begin{array}{lll}-4.566660 & 3.277885 & 1.776019\end{array}$

$\begin{array}{llll}-4.033707 & 3.314108 & 2.715723\end{array}$

$\begin{array}{lll}-4.569892 & 4.289783 & 0.771986\end{array}$

$\begin{array}{llll}-4.044496 & 5.233909 & 0.818946\end{array}$

$\begin{array}{llll}-5.428084 & 3.864449 & -0.282939\end{array}$

$\begin{array}{llll}-5.654396 & 4.419327 & -1.180995\end{array}$

$\begin{array}{lll}-8.243476 & 3.772956 & 2.562936\end{array}$

$\begin{array}{lll}-8.686112 & 2.820163 & 2.821259\end{array}$

$\begin{array}{lll}-7.283573 & 4.490393 & 3.340425\end{array}$

$\begin{array}{llll}-6.867401 & 4.174203 & 4.287640\end{array}$

$\begin{array}{lll}-6.936612 & 5.678208 & 2.627051\end{array}$

$\begin{array}{lll}-6.206597 & 6.414356 & 2.936766\end{array}$

$\begin{array}{llll}-7.683150 & 5.696677 & 1.409358\end{array}$

$\begin{array}{lll}-7.617774 & 6.447943 & 0.633399\end{array}$

$\begin{array}{llll}-8.490766 & 4.521267 & 1.369942\end{array}$

$\begin{array}{llll}-9.146416 & 4.232916 & 0.559656\end{array}$

$\begin{array}{llll}-6.820414 & 1.686884 & -0.776314\end{array}$

$\begin{array}{llll}-8.151847 & 1.465428 & -0.392664\end{array}$

$\begin{array}{lll}-8.526705 & 1.984647 & 0.480240\end{array}$

$\begin{array}{llll}-8.984384 & 0.588320 & -1.087565\end{array}$

$\begin{array}{llll}-9.998422 & 0.414213 & -0.742399\end{array}$

$\begin{array}{llll}-8.498829 & -0.070195 & -2.214695\end{array}$

$\begin{array}{llll}-9.135054 & -0.751364 & -2.771861\end{array}$

$\begin{array}{llll}-7.178797 & 0.134414 & -2.619455\end{array}$

$\begin{array}{lll}-6.813822 & -0.394654 & -3.492500\end{array}$

$\begin{array}{llll}-6.317538 & 0.982412 & -1.905063\end{array}$

$\begin{array}{llll}-5.727021 & 0.933897 & 2.057221\end{array}$

$\begin{array}{lll}-6.752282 & 0.673668 & 1.796132\end{array}$

$\begin{array}{lll}-5.633668 & 1.004609 & 3.583665\end{array}$

$\begin{array}{llll}-5.867988 & 0.039029 & 4.040720\end{array}$

$\begin{array}{lll}-4.641439 & 1.307515 & 3.927271\end{array}$

$\begin{array}{lll}-6.352520 & 1.744787 & 3.947575\end{array}$

$\begin{array}{llll}-4.301775 & -0.043154 & -3.782010\end{array}$

$\begin{array}{llll}-4.361177 & -1.435234 & -3.593129\end{array}$

$\begin{array}{llll}-4.609824 & -1.837426 & -2.616262\end{array}$

$\begin{array}{llll}-4.130267 & -2.309152 & -4.654186\end{array}$

$\begin{array}{llll}-4.195115 & -3.379973 & -4.490266\end{array}$

$\begin{array}{llll}-3.816967 & -1.801769 & -5.919431\end{array}$

$\begin{array}{lll}-3.624326 & -2.479902 & -6.745689\end{array}$

$\begin{array}{llll}-3.755942 & -0.421134 & -6.116571\end{array}$

$\begin{array}{lll}-3.515623 & -0.020569 & -7.097320\end{array}$

$\begin{array}{llll}-4.001622 & 0.456300 & -5.056553\end{array}$

$\begin{array}{llll}-3.945702 & 1.525371 & -5.227916\end{array}$

$\begin{array}{llll}-4.197481 & 2.730743 & -2.888443\end{array}$

$\begin{array}{llll}-5.106651 & 3.409330 & -3.715721\end{array}$

$\begin{array}{llll}-6.007738 & 2.909010 & -4.058986\end{array}$

$\begin{array}{llll}-4.863860 & 4.733983 & -4.079885\end{array}$

$\begin{array}{llll}-5.570607 & 5.257791 & -4.717114\end{array}$ 


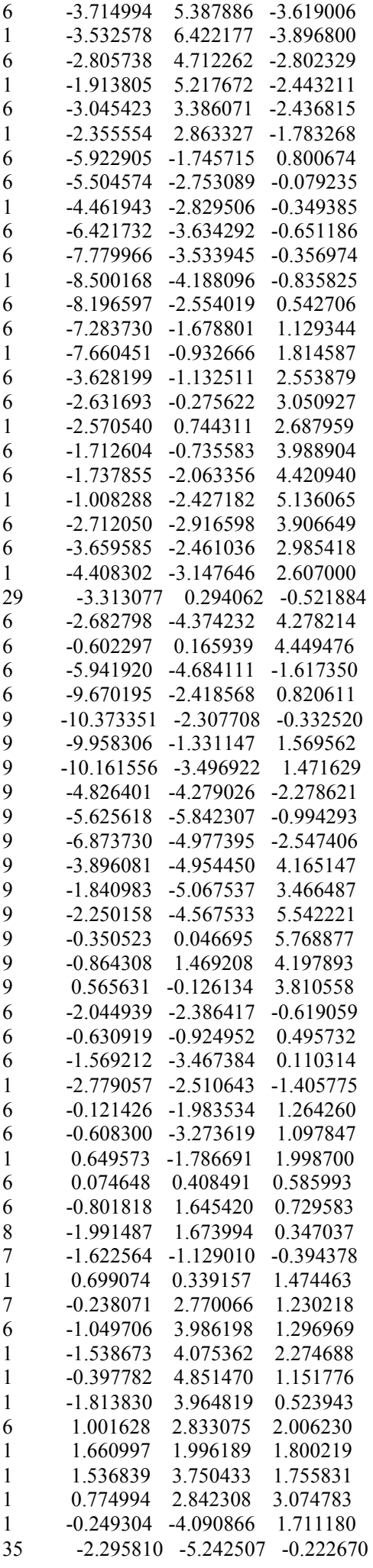

[8-9] $]^{\ddagger}-\mathbf{P 1 - C 2}$ endo si-re conf2

Number of imaginary frequencies $=1$

The smallest frequencies are $=-267.97277 .678211 .0818 \mathrm{~cm}(-1)$ Electronic energy $=-7222.6289103$

Zero-point correction=

1.754635

(Hartree/Particle)

Thermal correction to Energy $=\quad 1.873395$

Thermal correction to Enthalpy= $\quad 1.874339$

Thermal correction to Gibbs Free Energy= $\quad 1.590919$
Sum of electronic and zero-point Energies=

Sum of electronic and thermal Energies=

Sum of electronic and thermal Enthalpies=

Sum of electronic and thermal Free Energies=

$-7220.874275$

$-7220.755515$

$-7220.754571$

$-7221.037991$

Cartesian Coordinates

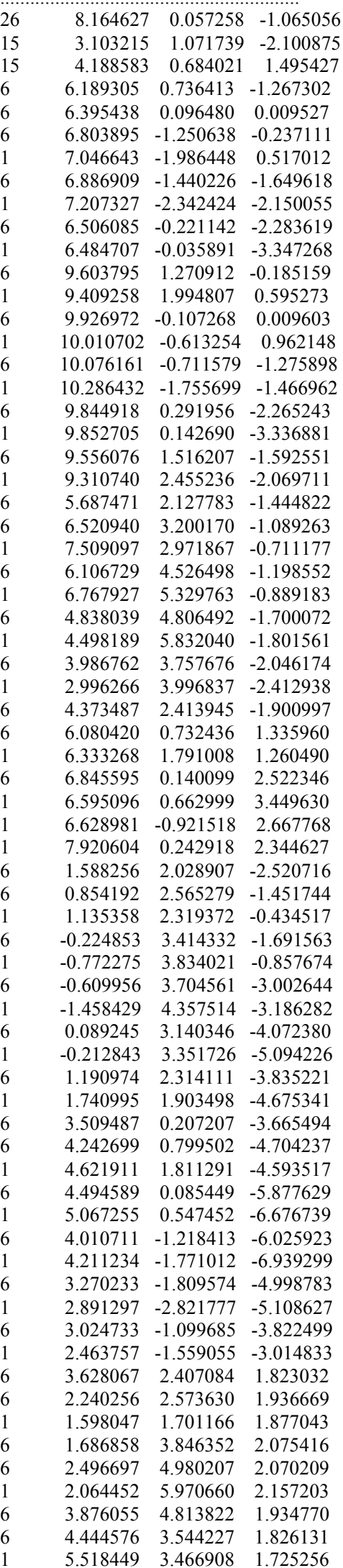


$\begin{array}{lll}3.813247 & -0.208535 & 3.060639\end{array}$

$\begin{array}{llll}3.953177 & -1.605412 & 3.026630\end{array}$

$\begin{array}{lll}4.319934 & -2.091735 & 2.130204\end{array}$

$\begin{array}{llll}3.596890 & -2.375974 & 4.129803\end{array}$

$\begin{array}{lll}3.066368 & -1.780576 & 5.275698\end{array}$

$\begin{array}{llll}2.771185 & -2.384507 & 6.125832\end{array}$

$\begin{array}{llll}2.916692 & -0.396811 & 5.303276\end{array}$

$\begin{array}{lll}3.295856 & 0.390940 & 4.212801\end{array}$

$\begin{array}{llll}3.170008 & 1.465730 & 4.269138\end{array}$

$\begin{array}{llll}2.914389 & -0.222419 & -0.250930\end{array}$

$\begin{array}{lll}2.249939 & 0.256051 & 6.481960\end{array}$

$\begin{array}{llll}3.672285 & -3.875360 & 4.060073\end{array}$

$\begin{array}{lll}0.197558 & 3.963067 & 2.247629\end{array}$

$\begin{array}{llll}4.738377 & 6.045317 & 1.864166\end{array}$

$\begin{array}{lll}4.463300 & 6.776960 & 0.756422\end{array}$

$\begin{array}{llll}6.057682 & 5.755554 & 1.830887\end{array}$

$\begin{array}{lll}4.530439 & 6.861124 & 2.923739\end{array}$

$\begin{array}{lll}-0.470335 & 3.133043 & 1.394911\end{array}$

$\begin{array}{lll}-0.198654 & 3.612299 & 3.492956\end{array}$

$\begin{array}{lll}-0.259078 & 5.210987 & 2.013743\end{array}$

$\begin{array}{lll}2.716304 & 1.503103 & 6.706278\end{array}$

$\begin{array}{llll}0.908081 & 0.375665 & 6.278115\end{array}$

$2.405996 \quad-0.451977 \quad 7.619593$

$\begin{array}{llll}4.076246 & -4.416492 & 5.229632\end{array}$

$\begin{array}{lll}4.514138 & -4.305467 & 3.097178\end{array}$

$\begin{array}{llll}2.452208 & -4.416622 & 3.787833\end{array}$

$\begin{array}{llll}0.794431 & -1.230090 & 1.704803\end{array}$

$\begin{array}{lll}1.032434 & -2.604850 & 1.318073\end{array}$

$\begin{array}{lll}2.160810 & -3.099280 & 0.581783\end{array}$

$\begin{array}{lll}0.578073 & -3.361320 & 1.941823\end{array}$

$-3.150248-1.209094-1.279168$

$\begin{array}{lll}-4.969673 & -0.206197 & -0.363083\end{array}$

$\begin{array}{lll}-4.906103 & 1.091553 & 0.671777\end{array}$

$\begin{array}{llll}-5.977806 & 0.396016 & -1.542326\end{array}$

$\begin{array}{lll}-5.781249 & -1.285238 & 0.636034\end{array}$

$\begin{array}{lll}-4.737106 & 2.390790 & 0.215375\end{array}$

$\begin{array}{llll}-3.534557 & 3.030063 & 0.588299\end{array}$

$\begin{array}{llll}-2.825818 & 2.487869 & 1.203111\end{array}$

$\begin{array}{llll}-3.295559 & 4.317217 & 0.177371\end{array}$

$\begin{array}{llll}-2.390918 & 4.830795 & 0.487255\end{array}$

$\begin{array}{llll}-4.215290 & 4.980298 & -0.678977\end{array}$

$\begin{array}{llll}-3.925776 & 6.268675 & -1.204750\end{array}$

$\begin{array}{llll}-2.995146 & 6.748843 & -0.913348\end{array}$

$\begin{array}{llll}-4.793704 & 6.888768 & -2.075136\end{array}$

$\begin{array}{llll}-4.559551 & 7.869942 & -2.477539\end{array}$

$\begin{array}{llll}-5.990066 & 6.236662 & -2.457820\end{array}$

$\begin{array}{llll}-6.662314 & 6.717237 & -3.162781\end{array}$

$\begin{array}{llll}-6.309535 & 4.995453 & -1.950757\end{array}$

$\begin{array}{llll}-7.225643 & 4.508827 & -2.263904\end{array}$

$\begin{array}{llll}-5.445243 & 4.330559 & -1.036623\end{array}$

$\begin{array}{llll}-5.742922 & 3.027629 & -0.496323\end{array}$

$\begin{array}{llll}-7.065426 & 2.369024 & -0.700156\end{array}$

$\begin{array}{llll}-8.303484 & 3.008170 & -0.332868\end{array}$

$\begin{array}{llll}-8.351307 & 4.234255 & 0.388649\end{array}$

$\begin{array}{lll}-7.425616 & 4.710223 & 0.689592\end{array}$

$\begin{array}{lll}-9.554354 & 4.816360 & 0.724215\end{array}$

$\begin{array}{llll}-9.562232 & 5.747676 & 1.283257\end{array}$

$\begin{array}{llll}-10.779386 & 4.210049 & 0.356587\end{array}$

$\begin{array}{lll}-11.719380 & 4.683912 & 0.623714\end{array}$

$\begin{array}{llll}-10.772150 & 3.013970 & -0.324112\end{array}$

$\begin{array}{llll}-11.704305 & 2.526230 & -0.597187\end{array}$

$\begin{array}{llll}-9.549171 & 2.379077 & -0.674232\end{array}$

$\begin{array}{llll}-9.542772 & 1.120606 & -1.334249\end{array}$

$\begin{array}{llll}-10.489076 & 0.657902 & -1.599548\end{array}$

$\begin{array}{llll}-8.358714 & 0.473582 & -1.587626\end{array}$

$\begin{array}{lll}-8.328875 & -0.509454 & -2.045150\end{array}$

$\begin{array}{llll}-7.139114 & 1.094955 & -1.242775\end{array}$

$\begin{array}{lll}-5.288977 & -2.680555 & 0.489171\end{array}$

$\begin{array}{lll}-4.625244 & -2.904366 & 1.333081\end{array}$

$\begin{array}{llll}-6.406358 & -3.709268 & 0.471281\end{array}$

$\begin{array}{llll}-6.247922 & -4.922587 & 1.148004\end{array}$

$\begin{array}{llll}-5.344924 & -5.096874 & 1.729607\end{array}$

$\begin{array}{lll}-7.245138 & -5.900790 & 1.098804\end{array}$

$\begin{array}{llll}-7.112177 & -6.835860 & 1.636321\end{array}$

$\begin{array}{lll}-8.414162 & -5.670503 & 0.371596\end{array}$ $\begin{array}{llll}-9.197211 & -6.423017 & 0.342538\end{array}$

$\begin{array}{llll}-8.574169 & -4.462231 & -0.316409\end{array}$

$\begin{array}{llll}-9.485282 & -4.271920 & -0.877004\end{array}$

$\begin{array}{llll}-7.573663 & -3.493785 & -0.273904\end{array}$

$-7.708082 \quad-2.543396 \quad-0.781634$

$\begin{array}{llll}-4.481752 & -2.823384 & -0.818203\end{array}$

$\begin{array}{llll}-5.189316 & -2.934270 & -1.648145\end{array}$

$\begin{array}{llll}-3.915436 & -3.757515 & -0.765689\end{array}$

$\begin{array}{lll}-6.412671 & -0.852692 & 1.931326\end{array}$

$\begin{array}{lll}-6.571832 & 0.218423 & 1.817575\end{array}$

$\begin{array}{lll}-7.794520 & -1.452908 & 2.161222\end{array}$

$\begin{array}{lll}-8.903473 & -0.808050 & 1.600233\end{array}$

$\begin{array}{lll}-8.755903 & 0.106445 & 1.037050\end{array}$

$\begin{array}{lll}-10.190381 & -1.325304 & 1.751519\end{array}$

$\begin{array}{lll}-11.034508 & -0.809937 & 1.300956\end{array}$

$\begin{array}{lll}-10.388724 & -2.496572 & 2.486437\end{array}$

$\begin{array}{lll}-11.387626 & -2.907417 & 2.604529\end{array}$

$\begin{array}{lll}-9.293547 & -3.130036 & 3.079569\end{array}$

$\begin{array}{lll}-9.437447 & -4.037735 & 3.658924\end{array}$

$\begin{array}{lll}-8.008772 & -2.606538 & 2.925138\end{array}$

$\begin{array}{lll}-7.170992 & -3.119755 & 3.383866\end{array}$

$\begin{array}{llll}-5.452989 & -1.041281 & 3.112248\end{array}$

$\begin{array}{lll}-4.539112 & -0.465571 & 2.943836\end{array}$

$\begin{array}{llll}-5.178459 & -2.087017 & 3.273580\end{array}$

$\begin{array}{lll}-5.923841 & -0.676867 & 4.030315\end{array}$

$\begin{array}{lll}-2.211349 & -0.949093 & 0.664911\end{array}$

$\begin{array}{lll}-1.486568 & -0.142814 & 0.610226\end{array}$

$\begin{array}{lll}-2.877946 & -0.874836 & 1.519657\end{array}$

$\begin{array}{lll}-1.803542 & -2.266255 & 0.277710\end{array}$

$\begin{array}{lll}-2.370507 & -3.095565 & 0.687714\end{array}$

$\begin{array}{llll}-0.583919 & -2.588666 & -0.374943\end{array}$

$\begin{array}{llll}-0.002701 & -1.773371 & -0.784455\end{array}$

$\begin{array}{lll}-4.003435 & -1.173992 & -3.334234\end{array}$

$\begin{array}{llll}-5.026108 & -1.534114 & -3.259055\end{array}$

$\begin{array}{lll}-2.980269 & -2.154369 & -3.305783\end{array}$

$\begin{array}{llll}-3.288377 & -3.196737 & -3.231164\end{array}$

$\begin{array}{llll}-1.603539 & -1.956038 & -3.915390\end{array}$

$-1.666414-2.055688-5.010388$

$\begin{array}{lll}-0.973590 & -2.778802 & -3.572018\end{array}$

$\begin{array}{lll}-0.928652 & -0.614885 & -3.549963\end{array}$

$\begin{array}{llll}-1.105923 & 0.131550 & -4.330499\end{array}$

$\begin{array}{llll}0.155780 & -0.759474 & -3.523157\end{array}$

$\begin{array}{lll}-1.364575 & -0.027204 & -2.208830\end{array}$

$\begin{array}{llll}-0.591475 & 0.027085 & -1.448133\end{array}$

$\begin{array}{llll}-2.424440 & 0.861223 & -2.055503\end{array}$

$\begin{array}{llll}-2.387926 & 1.519427 & -1.193202\end{array}$

$\begin{array}{llll}-3.353834 & 1.313672 & -3.163631\end{array}$

$\begin{array}{llll}-4.210623 & 1.806001 & -2.699853\end{array}$

$\begin{array}{llll}-2.839314 & 2.074057 & -3.767211\end{array}$

$\begin{array}{llll}-3.858263 & 0.164662 & -4.061951\end{array}$

$\begin{array}{llll}-3.188775 & 0.032729 & -4.918127\end{array}$

$\begin{array}{llll}-4.831859 & 0.444521 & -4.475569\end{array}$

$\begin{array}{lll}-0.332417 & -3.902849 & -0.976818\end{array}$

$\begin{array}{llll}-0.980416 & -5.079744 & -0.554989\end{array}$

$\begin{array}{llll}0.609486 & -3.999896 & -2.018315\end{array}$

$\begin{array}{llll}-0.707569 & -6.300270 & -1.170134\end{array}$

$\begin{array}{llll}-1.695585 & -5.045282 & 0.260748\end{array}$

$\begin{array}{llll}0.878579 & -5.218901 & -2.637400\end{array}$

$\begin{array}{llll}1.132495 & -3.104430 & -2.336763\end{array}$

$\begin{array}{llll}0.218407 & -6.375738 & -2.216531\end{array}$

$\begin{array}{llll}-1.218099 & -7.197441 & -0.831647\end{array}$

$\begin{array}{llll}1.606273 & -5.266146 & -3.442538\end{array}$

$\begin{array}{llll}0.425975 & -7.328863 & -2.694156\end{array}$

$\begin{array}{llll}2.439914 & -4.491991 & 0.654309\end{array}$

$\begin{array}{llll}3.434671 & -5.063463 & -0.110404\end{array}$

$\begin{array}{lll}1.853719 & -5.109753 & 1.323792\end{array}$

$\begin{array}{llll}4.162758 & -4.222890 & -0.957634\end{array}$

$\begin{array}{llll}3.892060 & -2.868232 & -0.985269\end{array}$

$\begin{array}{llll}4.447336 & -2.195952 & -1.625001\end{array}$

$\begin{array}{llll}2.921474 & -2.308020 & -0.233194\end{array}$

$\begin{array}{lll}1.106842 & -0.239353 & 0.971772\end{array}$

$\begin{array}{lll}0.110066 & -0.996949 & 2.865573\end{array}$

$\begin{array}{lll}-0.376830 & 0.341672 & 3.180703\end{array}$

$\begin{array}{lll}-1.377473 & 0.267450 & 3.618817\end{array}$

$\begin{array}{llll}0.277053 & 0.842017 & 3.902282\end{array}$ 


$\begin{array}{lccc}1 & -0.435635 & 0.940033 & 2.277296 \\ 6 & -0.169849 & -2.010177 & 3.880411 \\ 1 & -1.149658 & -2.480153 & 3.723726 \\ 1 & 0.599318 & -2.780768 & 3.897645 \\ 1 & -0.170951 & -1.524820 & 4.859250 \\ 1 & 3.634474 & -6.128068 & -0.059188 \\ 35 & 5.540006 & -4.964556 & -2.134005\end{array}$

[8-9] $]^{\dagger}-\mathbf{P 1 - C 2}$ endo re-re conf2

Number of imaginary frequencies $=1$

The smallest frequencies are $=-266.922714 .603016 .4956 \mathrm{~cm}(-1)$ Electronic energy $=-7222.6225041$

Zero-point correction=

1.755305

(Hartree/Particle)

$\begin{array}{lc}\text { Thermal correction to Energy= } & 1.873789 \\ \text { Thermal correction to Enthalpy= } & 1.874733 \\ \text { Thermal correction to Gibbs Free Energy= } & 1.596155 \\ \text { Sum of electronic and zero-point Energies }= & -7220.867200 \\ \text { Sum of electronic and thermal Energies= } & -7220.748715 \\ \text { Sum of electronic and thermal Enthalpies }= & -7220.747771 \\ \text { Sum of electronic and thermal Free Energies }= & -7221.026349\end{array}$

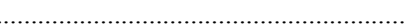

Cartesian Coordinates

\begin{tabular}{|c|c|c|c|}
\hline 26 & 7.587737 & -2.030120 & -1 \\
\hline 15 & 2.982145 & 0.290134 & -2.301438 \\
\hline 15 & 4.408503 & 0.363313 & 1.158495 \\
\hline 6 & 5.910194 & -0.797811 & -1.737637 \\
\hline 6 & 6.045487 & -1.241406 & -0.371125 \\
\hline 6 & 5.984273 & -2.666595 & -0.366827 \\
\hline 1 & 6.061588 & -3.298816 & 0.506460 \\
\hline 6 & 5.847183 & -3.111998 & -1.714095 \\
\hline 1 & 5.816015 & -4.141703 & -2.043405 \\
\hline 6 & 5.805672 & -1.965233 & -2.558266 \\
\hline 1 & 5.719254 & -1.966488 & -3.634902 \\
\hline 6 & 9.41 & -1.1 & -1.007964 \\
\hline 1 & 9.524467 & -0.257497 & -0.428184 \\
\hline 6 & 9.332159 & -2.490786 & -0.483910 \\
\hline 1 & 071 & -2.76 & 0.561692 \\
\hline 6 & 9.150577 & -3.388404 & -1.579896 \\
\hline 1 & 9.020587 & -4.460268 & -1.508050 \\
\hline 6 & 9.116668 & -2.6 & 1714 \\
\hline 1 & 8.958973 & -3.002187 & -3.780748 \\
\hline 6 & 9.278997 & -1.244724 & -2.429334 \\
\hline 1 & 9.270492 & -0.41 & -3.118186 \\
\hline 6 & 5.813259 & 0.628040 & -2.160974 \\
\hline 6 & 6.966849 & 1.425874 & -2.171613 \\
\hline 1 & 7.9066 & 0.967310 & -1.8 \\
\hline 6 & 6.926067 & 2.776475 & -2.516030 \\
\hline 1 & 7.83 & 3.37 & -2.489856 \\
\hline 6 & 5.711640 & 3.356397 & -2.874325 \\
\hline 1 & 5.660840 & 4.406631 & -3.1 \\
\hline 6 & 4.548595 & 2.58 & -2.8 \\
\hline 1 & 3.610231 & 3.056927 & -3.13 \\
\hline 6 & 4.570425 & 1.231128 & 5048 \\
\hline 6 & 6.147653 & -0.3 & 0.8 \\
\hline 1 & 6.710589 & 0.554532 & 0.4 \\
\hline 6 & 6.880430 & -0.900879 & 2.0 \\
\hline 1 & 6.922259 & -0.181175 & 2.841372 \\
\hline 1 & 6.407743 & -1.812402 & 2.392121 \\
\hline 1 & 7.903848 & -1.152778 & 1.725778 \\
\hline 6 & 1.696044 & 1.506233 & -2.806632 \\
\hline 6 & 1.308245 & 2.452313 & -1.842264 \\
\hline 1 & 1.780838 & 2.450113 & -0.865178 \\
\hline 6 & 0.340765 & 3.411024 & -2.134609 \\
\hline 1 & 0.063706 & 4.138879 & -1.38 \\
\hline 6 & -0.272839 & 3.426692 & -3.389954 \\
\hline 1 & -1.040171 & 4.163886 & -3.607896 \\
\hline 6 & 0.102141 & 2.488160 & -4.352854 \\
\hline 1 & -0.369765 & 2.492138 & -5.331237 \\
\hline 6 & 1.084434 & 1.535572 & -4.067389 \\
\hline 1 & 1.358886 & 0.813545 & -4.827830 \\
\hline 6 & 3.024373 & -0.958034 & -3.641947 \\
\hline & 3.483239 & -0.670641 & -4.936489 \\
\hline
\end{tabular}

$\begin{array}{llll}3.835003 & 0.327737 & -5.180319\end{array}$

$\begin{array}{lll}3.500509 & -1.672787 & -5.908177\end{array}$

$\begin{array}{llll}3.855396 & -1.447454 & -6.909791\end{array}$

$3.064349-2.964904-5.593817$

$\begin{array}{llll}3.079468 & -3.741132 & -6.353684\end{array}$

$\begin{array}{llll}2.621482 & -3.258475 & -4.301722\end{array}$

$\begin{array}{llll}2.294495 & -4.263382 & -4.049774\end{array}$

$\begin{array}{llll}2.609322 & -2.259187 & -3.327461\end{array}$

$\begin{array}{llll}2.323342 & -2.483730 & -2.305137\end{array}$

$\begin{array}{lll}4.586523 & 2.202559 & 1.171447\end{array}$

$\begin{array}{lll}3.406763 & 2.947169 & 1.308168\end{array}$

$\begin{array}{llll}2.486690 & 2.444570 & 1.568588\end{array}$

$\begin{array}{llll}3.392252 & 4.323874 & 1.083888\end{array}$

$\begin{array}{llll}4.559426 & 4.998675 & 0.735291\end{array}$

$\begin{array}{llll}4.544650 & 6.063574 & 0.531420\end{array}$

$\begin{array}{llll}5.743208 & 4.268481 & 0.633010\end{array}$

$\begin{array}{llll}5.765116 & 2.891021 & 0.849402\end{array}$

$\begin{array}{lll}6.707336 & 2.373713 & 0.735995\end{array}$

$\begin{array}{lll}3.984023 & -0.127869 & 2.874739\end{array}$

$\begin{array}{llll}3.800150 & -1.506259 & 3.073924\end{array}$

$\begin{array}{lll}3.981371 & -2.198174 & 2.259017\end{array}$

$\begin{array}{llll}3.351382 & -1.985637 & 4.299730\end{array}$

$3.023056-1.105273 \quad 5.331860$

$\begin{array}{llll}2.644004 & -1.478063 & 6.277408\end{array}$

$\begin{array}{lll}3.175939 & 0.263913 & 5.120270\end{array}$

$\begin{array}{llll}3.668938 & 0.757962 & 3.909861\end{array}$

$\begin{array}{llll}3.786002 & 1.827315 & 3.780416\end{array}$

$2.671094 \quad-0.489416 \quad-0.185857$

$\begin{array}{llll}2.712818 & 1.206199 & 6.197233\end{array}$

$\begin{array}{llll}3.121335 & -3.457736 & 4.496390\end{array}$

$\begin{array}{lll}2.092172 & 5.070769 & 1.207198\end{array}$

$\begin{array}{lll}6.996213 & 5.003425 & 0.237303\end{array}$

$\begin{array}{llll}6.842756 & 5.633724 & -0.952664\end{array}$

$\begin{array}{llll}8.065468 & 4.186331 & 0.121469\end{array}$

$\begin{array}{lll}7.316338 & 5.959702 & 1.139242\end{array}$

$\begin{array}{llll}1.048641 & 4.307505 & 0.774004\end{array}$

$\begin{array}{llll}1.814905 & 5.412257 & 2.484499\end{array}$

$\begin{array}{llll}2.076139 & 6.203968 & 0.477696\end{array}$

$\begin{array}{lll}3.100815 & 2.479518 & 5.975162\end{array}$

$\begin{array}{llll}1.357473 & 1.211062 & 6.283723\end{array}$

$\begin{array}{lll}3.174111 & 0.845838 & 7.416237\end{array}$

$\begin{array}{llll}3.629667 & -3.895178 & 5.668971\end{array}$

$3.670476-4.201419 \quad 3.510756$

$\begin{array}{lll}1.793668 & -3.755215 & 4.519455\end{array}$

$\begin{array}{lll}0.655877 & 0.743710 & 1.630039\end{array}$

$\begin{array}{lll}0.638721 & -1.572463 & 1.927870\end{array}$

$\begin{array}{lll}0.065667 & 0.958541 & 2.864419\end{array}$

$\begin{array}{llll}0.869166 & 1.568309 & 0.961855\end{array}$

$\begin{array}{lll}-0.020897 & -1.391874 & 3.172509\end{array}$

$\begin{array}{lll}-0.281009 & -0.129956 & 3.668636\end{array}$

$\begin{array}{lll}0.830053 & -2.923155 & 1.463339\end{array}$

$\begin{array}{llll}1.981571 & -3.354441 & 0.703627\end{array}$

$\begin{array}{llll}2.674443 & -2.568196 & -0.014418\end{array}$

$\begin{array}{lll}0.983157 & -0.482844 & 1.177981\end{array}$

$\begin{array}{llll}0.396854 & -3.657551 & 2.127216\end{array}$

$\begin{array}{llll}-3.249702 & -1.353953 & -1.191222\end{array}$

$\begin{array}{lll}-4.980383 & -0.111354 & -0.400278\end{array}$

$\begin{array}{lll}-4.786750 & 1.132620 & 0.694008\end{array}$

$\begin{array}{llll}-5.791872 & 0.691865 & -1.604590\end{array}$

$\begin{array}{lll}-6.025333 & -1.086679 & 0.486867\end{array}$

$\begin{array}{lll}-4.165387 & 2.307615 & 0.299515\end{array}$

$\begin{array}{lll}-2.885259 & 2.561738 & 0.839178\end{array}$

$\begin{array}{lll}-2.460850 & 1.840555 & 1.528158\end{array}$

$\begin{array}{llll}-2.218962 & 3.709108 & 0.488588\end{array}$

$\begin{array}{lll}-1.248093 & 3.929903 & 0.915264\end{array}$

$\begin{array}{llll}-2.775601 & 4.600843 & -0.467735\end{array}$

$\begin{array}{llll}-2.052354 & 5.743244 & -0.908393\end{array}$

$\begin{array}{llll}-1.083531 & 5.947966 & -0.459549\end{array}$

$\begin{array}{llll}-2.558960 & 6.563232 & -1.891720\end{array}$

$\begin{array}{llll}-1.995281 & 7.428200 & -2.228564\end{array}$

$\begin{array}{llll}-3.815955 & 6.269698 & -2.473442\end{array}$

$\begin{array}{llll}-4.201616 & 6.903069 & -3.267098\end{array}$

$\begin{array}{llll}-4.557010 & 5.190135 & -2.042460\end{array}$

$\begin{array}{llll}-5.517664 & 4.981791 & -2.498886\end{array}$

$\begin{array}{llll}-4.074030 & 4.330988 & -1.016403\end{array}$ 
$\begin{array}{llll}-4.821494 & 3.193713 & -0.539746\end{array}$

$\begin{array}{llll}-6.247008 & 2.965437 & -0.908242\end{array}$

$\begin{array}{llll}-7.249032 & 3.982496 & -0.696563\end{array}$

$\begin{array}{lll}-6.982310 & 5.187734 & 0.012310\end{array}$

$\begin{array}{lll}-5.996154 & 5.353524 & 0.428541\end{array}$

$\begin{array}{llll}-7.962015 & 6.139720 & 0.195545\end{array}$

$\begin{array}{llll}-7.731517 & 7.045817 & 0.748646\end{array}$

$\begin{array}{llll}-9.265030 & 5.946545 & -0.321017\end{array}$

$\begin{array}{llll}-10.024430 & 6.709253 & -0.175741\end{array}$

$\begin{array}{llll}-9.566726 & 4.782288 & -0.990153\end{array}$

$\begin{array}{lll}-10.568243 & 4.607887 & -1.374807\end{array}$

$\begin{array}{llll}-8.584255 & 3.773004 & -1.180717\end{array}$

$\begin{array}{lll}-8.909765 & 2.551198 & -1.827940\end{array}$

$\begin{array}{llll}-9.914821 & 2.408384 & -2.214461\end{array}$

$\begin{array}{llll}-7.976183 & 1.551583 & -1.935139\end{array}$

$\begin{array}{llll}-8.207728 & 0.599236 & -2.400263\end{array}$

$\begin{array}{llll}-6.669172 & 1.753820 & -1.437371\end{array}$

$\begin{array}{lll}-5.503862 & -2.482518 & 0.608196\end{array}$

$\begin{array}{lll}-4.826803 & -2.526032 & 1.471196\end{array}$

$\begin{array}{lll}-6.594349 & -3.515506 & 0.822127\end{array}$

$\begin{array}{lll}-6.500817 & -4.410780 & 1.892276\end{array}$

$\begin{array}{llll}-5.669207 & -4.324837 & 2.588272\end{array}$

$\begin{array}{lll}-7.469792 & -5.400752 & 2.082399\end{array}$

$\begin{array}{lll}-7.386643 & -6.085136 & 2.922383\end{array}$

$\begin{array}{lll}-8.546678 & -5.499720 & 1.200720\end{array}$

$\begin{array}{llll}-9.308449 & -6.259650 & 1.350951\end{array}$

$\begin{array}{llll}-8.643363 & -4.611633 & 0.122885\end{array}$

$\begin{array}{llll}-9.484150 & -4.677443 & -0.561974\end{array}$

$\begin{array}{llll}-7.670748 & -3.633504 & -0.068620\end{array}$

$\begin{array}{llll}-7.762044 & -2.929414 & -0.889730\end{array}$

$\begin{array}{llll}-4.701377 & -2.822897 & -0.653531\end{array}$

$\begin{array}{llll}-5.400111 & -2.951205 & -1.488719\end{array}$

$\begin{array}{lll}-4.204870 & -3.785707 & -0.50465\end{array}$

$\begin{array}{lll}-6.838547 & -0.514542 & 1.613808\end{array}$

$\begin{array}{lll}-6.918846 & 0.551492 & 1.391314\end{array}$

$\begin{array}{llll}-8.271917 & -1.044484 & 1.628640\end{array}$

$\begin{array}{lll}-9.136787 & -0.659239 & 0.596078\end{array}$

$\begin{array}{lll}-8.769013 & 0.004679 & -0.177798\end{array}$

$\begin{array}{llll}-10.453131 & -1.114706 & 0.548968\end{array}$

$\begin{array}{lll}-11.103648 & -0.803250 & -0.264173\end{array}$

$\begin{array}{lll}-10.934077 & -1.964616 & 1.549127\end{array}$

$\begin{array}{lll}-11.957839 & -2.326918 & 1.516299\end{array}$

$\begin{array}{lll}-10.088629 & -2.337583 & 2.595309\end{array}$

$\begin{array}{lll}-10.450863 & -2.995556 & 3.380435\end{array}$

$\begin{array}{lll}-8.770401 & -1.875485 & 2.638058\end{array}$

$\begin{array}{lll}-8.131015 & -2.190353 & 3.454715\end{array}$

$\begin{array}{lll}-6.105061 & -0.643980 & 2.952757\end{array}$

$\begin{array}{lll}-5.121362 & -0.174050 & 2.880776\end{array}$

$\begin{array}{lll}-5.971500 & -1.686373 & 3.253771\end{array}$

$\begin{array}{lll}-6.671785 & -0.137031 & 3.739421\end{array}$

$\begin{array}{lll}-2.318315 & -1.070200 & 0.755855\end{array}$

$\begin{array}{lll}-1.559398 & -0.299935 & 0.679539\end{array}$

$\begin{array}{llll}-3.003253 & -0.913907 & 1.58573\end{array}$

$\begin{array}{llll}-1.998348 & -2.432440 & 0.445753\end{array}$

$\begin{array}{lll}-2.635957 & -3.175261 & 0.912855\end{array}$

$\begin{array}{llll}-0.817255 & -2.930609 & -0.174793\end{array}$

$\begin{array}{llll}-0.168654 & -2.225006 & -0.676007\end{array}$

$\begin{array}{lll}-4.022496 & -1.428697 & -3.273842\end{array}$

$\begin{array}{llll}-5.069154 & -1.720004 & -3.222733\end{array}$

$\begin{array}{lll}-3.074783 & -2.474430 & -3.115846\end{array}$

$\begin{array}{llll}-3.468441 & -3.480273 & -2.970482\end{array}$

$\begin{array}{llll}-1.662516 & -2.437511 & -3.672419\end{array}$

$\begin{array}{llll}-1.680858 & -2.665149 & -4.749895\end{array}$

$\begin{array}{llll}-1.101479 & -3.246424 & -3.199723\end{array}$

$\begin{array}{llll}-0.923776 & -1.105375 & -3.433387\end{array}$

$\begin{array}{llll}-1.067264 & -0.431602 & -4.282261\end{array}$

$\begin{array}{llll}0.150876 & -1.297224 & -3.390687\end{array}$

$\begin{array}{lll}-1.332603 & -0.373143 & -2.159149\end{array}$

$\begin{array}{llll}-0.588904 & -0.340278 & -1.367119\end{array}$

$\begin{array}{llll}-2.317779 & 0.603716 & -2.123063\end{array}$

$\begin{array}{llll}-2.263352 & 1.332004 & -1.320746\end{array}$

$\begin{array}{llll}-3.167382 & 1.014023 & -3.309561\end{array}$

$\begin{array}{llll}-3.978624 & 1.641595 & -2.937885\end{array}$

$\begin{array}{llll}-2.558247 & 1.645894 & -3.969469\end{array}$

$\begin{array}{llll}-3.763633 & -0.168918 & -4.105441\end{array}$ $\begin{array}{lll}-3.109604 & -0.426838 & -4.945559\end{array}$

$\begin{array}{llll}-4.711027 & 0.153960 & -4.547676\end{array}$

$\begin{array}{lll}-0.732928 & -4.322475 & -0.646696\end{array}$

$\begin{array}{llll}-1.632388 & -5.328497 & -0.237527\end{array}$

$\begin{array}{llll}0.281065 & -4.677026 & -1.556531\end{array}$

$\begin{array}{llll}-1.516381 & -6.630094 & -0.720953\end{array}$

$\begin{array}{lll}-2.426262 & -5.100209 & 0.464569\end{array}$

$\begin{array}{llll}0.398245 & -5.978573 & -2.040027\end{array}$

$\begin{array}{llll}0.980711 & -3.918388 & -1.884172\end{array}$

$\begin{array}{llll}-0.499392 & -6.963978 & -1.622039\end{array}$

$\begin{array}{llll}-2.221314 & -7.388102 & -0.391630\end{array}$

$\begin{array}{llll}1.190821 & -6.222555 & -2.741667\end{array}$

$-0.411355 \quad-7.980124-1.995193$

$\begin{array}{llll}2.319757 & -4.676802 & 0.726304\end{array}$

$\begin{array}{llll}3.368129 & -5.177616 & -0.152904\end{array}$

$\begin{array}{llll}4.255889 & -5.464131 & 0.425316\end{array}$

$\begin{array}{llll}3.004638 & -6.058515 & -0.692500\end{array}$

$3.645772-4.403945-0.863112$

$\begin{array}{llll}1.663159 & -5.672476 & 1.570751\end{array}$

$\begin{array}{lll}0.686093 & -5.961851 & 1.171121\end{array}$

$2.297112-6.560357 \quad 1.606387$

$\begin{array}{llll}1.545581 & -5.312153 & 2.593206\end{array}$

$\begin{array}{lll}-0.731780 & 0.007933 & 4.644936\end{array}$

$-0.271878-2.266163 \quad 3.762167$

$\begin{array}{lll}-0.201993 & 2.776895 & 3.513522\end{array}$

[8-9] $]^{\ddagger}-\mathbf{P 1}-\mathrm{C} 2$ exo si-si conf2

Number of imaginary frequencies $=1$

The smallest frequencies are $=-137.35137 .26628 .6309 \mathrm{~cm}(-1)$

Electronic energy $=-7222.6278115$

Zero-point correction=

(Hartree/Particle)

Thermal correction to Energy=

Thermal correction to Enthalpy=

1.754809

Thermal correction to Gibbs Free Energy=
Sum of electronic and zero-point Energies=

Sum of electronic and thermal Energies=

Sum of electronic and thermal Enthalpies=

Sum of electronic and thermal Free Energies=

1.873359

1.874303

1.591806

$-7220.873003$

$-7220.754453$

$-7220.753509$

$-7221.036005$

Cartesian Coordinates

$\begin{array}{lccc}26 & 8.123816 & 0.204442 & -0.683955 \\ 15 & 3.132810 & 1.198662 & -1.972731 \\ 15 & 4.000928 & 0.629832 & 1.659858 \\ 6 & 6.156735 & 0.873616 & -0.966352 \\ 6 & 6.291601 & 0.146301 & 0.273613 \\ 6 & 6.735765 & -1.174944 & -0.041473 \\ 1 & 6.940851 & -1.960815 & 0.672408 \\ 6 & 6.909649 & -1.263471 & -1.455759 \\ 1 & 7.271915 & -2.125593 & -1.997291 \\ 6 & 6.552780 & -0.006101 & -2.024642 \\ 1 & 6.595303 & 0.253525 & -3.071855 \\ 6 & 9.466962 & 1.393982 & 0.361338 \\ 1 & 9.192959 & 2.071497 & 1.159293 \\ 6 & 9.802185 & 0.013380 & 0.513001 \\ 1 & 9.819586 & -0.539353 & 1.442767 \\ 6 & 10.059706 & -0.523777 & -0.785284 \\ 1 & 10.303390 & -1.553912 & -1.009533 \\ 6 & 9.883634 & 0.523902 & -1.739685 \\ 1 & 9.973178 & 0.428006 & -2.813733 \\ 6 & 9.519567 & 1.708256 & -1.032429 \\ 1 & 9.289825 & 2.665246 & -1.480752 \\ 6 & 5.636403 & 2.266411 & -1.076911 \\ 6 & 6.429857 & 3.327957 & -0.614881 \\ 1 & 7.410048 & 3.096796 & -0.218372 \\ 6 & 5.987407 & 4.649693 & -0.645587 \\ 1 & 6.618355 & 5.442517 & -0.256671 \\ 6 & 4.728644 & 4.937369 & -1.166654 \\ 1 & 4.365751 & 5.959468 & -1.200870 \\ 6 & 3.917556 & 3.898767 & -1.623591 \\ 1 & 2.935155 & 4.142773 & -2.009012 \\ 6 & 4.336169 & 2.558577 & -1.570047 \\ 6 & 5.896694 & 0.683226 & 1.622907\end{array}$


$\begin{array}{lll}6.155978 & 1.743103 & 1.643536\end{array}$ $\begin{array}{lll}6.590079 & -0.005930 & 2.801370\end{array}$ $\begin{array}{lll}6.291556 & 0.445426 & 3.751619\end{array}$ $\begin{array}{llll}6.357761 & -1.073461 & 2.848293\end{array}$ $\begin{array}{lll}7.674059 & 0.098261 & 2.691776\end{array}$ $\begin{array}{llll}1.621021 & 2.143083 & -2.443718\end{array}$ $\begin{array}{llll}0.769851 & 2.550895 & -1.402421\end{array}$ $\begin{array}{llll}0.958775 & 2.208899 & -0.391093\end{array}$ $\begin{array}{llll}-0.304121 & 3.402474 & -1.661729\end{array}$ $\begin{array}{lll}-0.942332 & 3.724083 & -0.849313\end{array}$ $\begin{array}{llll}-0.567222 & 3.823139 & -2.967586\end{array}$ $\begin{array}{llll}-1.412709 & 4.475377 & -3.167053\end{array}$ $\begin{array}{llll}0.246194 & 3.385331 & -4.014498\end{array}$ $\begin{array}{llll}0.038502 & 3.695760 & -5.034483\end{array}$ $\begin{array}{llll}1.341372 & 2.556771 & -3.754943\end{array}$ $\begin{array}{llll}1.981961 & 2.249277 & -4.574726\end{array}$ $\begin{array}{llll}3.732023 & 0.499314 & -3.560843\end{array}$ $\begin{array}{llll}4.464915 & 1.253884 & -4.490384\end{array}$ $\begin{array}{llll}4.701303 & 2.293063 & -4.281400\end{array}$ $\begin{array}{llll}4.905683 & 0.666850 & -5.677663\end{array}$ $\begin{array}{llll}5.474969 & 1.255919 & -6.391067\end{array}$ $4.620403 \quad-0.675906-5.946365$ $\begin{array}{llll}4.972158 & -1.131973 & -6.867301\end{array}$ $\begin{array}{llll}3.886058 & -1.430036 & -5.027974\end{array}$ $\begin{array}{llll}3.668577 & -2.475109 & -5.228772\end{array}$ $\begin{array}{llll}3.443306 & -0.843657 & -3.840690\end{array}$ $\begin{array}{llll}2.886366 & -1.431735 & -3.120347\end{array}$ $\begin{array}{lll}3.415880 & 2.330178 & 2.055388\end{array}$ $\begin{array}{lll}2.023206 & 2.495491 & 2.067662\end{array}$ $\begin{array}{lll}1.387575 & 1.629686 & 1.911685\end{array}$ $\begin{array}{lll}1.460151 & 3.760108 & 2.235633\end{array}$ $\begin{array}{llll}2.265560 & 4.889999 & 2.361761\end{array}$ $\begin{array}{lll}1.826439 & 5.875919 & 2.466776\end{array}$ $\begin{array}{lll}3.651380 & 4.724869 & 2.334540\end{array}$ $\begin{array}{lll}4.228110 & 3.461492 & 2.199776\end{array}$ $\begin{array}{lll}5.306873 & 3.386915 & 2.186304\end{array}$ $\begin{array}{lll}3.510529 & -0.363082 & 3.129093\end{array}$ $3.637443 \quad-1.755559 \quad 3.002582$ $\begin{array}{llll}4.068762 & -2.180401 & 2.103742\end{array}$ $\begin{array}{llll}3.176955 & -2.599786 & 4.008644\end{array}$ $\begin{array}{lll}2.557702 & -2.081335 & 5.147177\end{array}$ $\begin{array}{llll}2.181739 & -2.741959 & 5.920170\end{array}$ $2.419954 \quad-0.700448 \quad 5.265042$ $\begin{array}{llll}2.900642 & 0.159323 & 4.273764\end{array}$ $\begin{array}{llll}2.780079 & 1.228842 & 4.399057\end{array}$

$2.839669-0.191748 \quad-0.212912$ $\begin{array}{lll}1.651498 & -0.137346 & 6.427632\end{array}$ $3.240867 \quad-4.091124 \quad 3.829625$ $\begin{array}{lll}-0.037633 & 3.866968 & 2.295731\end{array}$ $\begin{array}{lll}4.515686 & 5.952941 & 2.424772\end{array}$ $\begin{array}{llll}4.284020 & 6.798037 & 1.390016\end{array}$ $\begin{array}{llll}5.835279 & 5.662441 & 2.413470\end{array}$ $\begin{array}{lll}4.267249 & 6.655281 & 3.554832\end{array}$ $\begin{array}{lll}-0.627740 & 3.065491 & 1.360624\end{array}$ $\begin{array}{lll}-0.527579 & 3.462704 & 3.490621\end{array}$ $\begin{array}{lll}-0.486137 & 5.118578 & 2.075401\end{array}$ $\begin{array}{llll}2.008727 & 1.132572 & 6.713329\end{array}$ $\begin{array}{llll}0.315331 & -0.115704 & 6.164066\end{array}$ $\begin{array}{llll}1.809306 & -0.870987 & 7.549838\end{array}$ $\begin{array}{llll}3.569313 & -4.723409 & 4.977460\end{array}$ $\begin{array}{llll}4.135009 & -4.459054 & 2.888356\end{array}$ $\begin{array}{llll}2.035589 & -4.596987 & 3.446103\end{array}$ $\begin{array}{lll}0.597146 & -1.310859 & 1.558994\end{array}$ $\begin{array}{llll}0.840214 & -2.647740 & 1.083441\end{array}$ $\begin{array}{llll}1.978550 & -3.108201 & 0.349186\end{array}$ $\begin{array}{llll}0.324523 & -3.444471 & 1.600192\end{array}$ $\begin{array}{lll}-3.154510 & -0.810413 & -1.893736\end{array}$ $\begin{array}{lll}-4.874625 & -0.232858 & -0.533667\end{array}$ $\begin{array}{lll}-4.808645 & 1.011541 & 0.566522\end{array}$ $\begin{array}{llll}-6.215701 & 0.219663 & -1.404793\end{array}$ $\begin{array}{llll}-5.231873 & -1.484887 & 0.534976\end{array}$ $\begin{array}{llll}-4.834384 & 2.332076 & 0.143947\end{array}$ $\begin{array}{llll}-3.650123 & 3.074387 & 0.337131\end{array}$ $\begin{array}{lll}-2.810203 & 2.588381 & 0.815615\end{array}$ $\begin{array}{llll}-3.598397 & 4.385009 & -0.066113\end{array}$ $\begin{array}{lll}-2.704424 & 4.976359 & 0.111510\end{array}$

$\begin{array}{llll}-4.705298 & 4.972065 & -0.738197\end{array}$

$\begin{array}{llll}4.629997 & 6.292293 & -1.258589\end{array}$

$\begin{array}{llll}-3.710568 & 6.853565 & -1.113002\end{array}$

$\begin{array}{llll}-5.688789 & 6.845021 & -1.943290\end{array}$

$\begin{array}{llll}-5.617823 & 7.852751 & -2.341933\end{array}$

$\begin{array}{llll}-6.869770 & 6.090427 & -2.140183\end{array}$

$\begin{array}{lll}-7.695009 & 6.520200 & -2.700609\end{array}$

$\begin{array}{llll}-6.982623 & 4.814012 & -1.632311\end{array}$

$\begin{array}{llll}-7.892140 & 4.248524 & -1.798618\end{array}$

$\begin{array}{llll}-5.916363 & 4.216566 & -0.904321\end{array}$

$\begin{array}{llll}-5.999614 & 2.882091 & -0.365447\end{array}$

$\begin{array}{lll}-7.272408 & 2.106585 & -0.332079\end{array}$

$\begin{array}{llll}-8.464171 & 2.635660 & 0.284542\end{array}$

$\begin{array}{lll}-8.474041 & 3.855788 & 1.017436\end{array}$

$\begin{array}{lll}-7.552102 & 4.411654 & 1.137341\end{array}$

$\begin{array}{llll}-9.631994 & 4.333009 & 1.592500\end{array}$

$\begin{array}{lll}-9.608174 & 5.262765 & 2.153694\end{array}$

$\begin{array}{lll}-10.848791 & 3.621437 & 1.467576\end{array}$

$\begin{array}{lll}-11.754661 & 4.013637 & 1.920344\end{array}$

$\begin{array}{lll}-10.872265 & 2.425062 & 0.787752\end{array}$

$\begin{array}{lll}-11.793853 & 1.855363 & 0.701672\end{array}$

$\begin{array}{lll}-9.692385 & 1.896292 & 0.197031\end{array}$

$\begin{array}{llll}-9.706340 & 0.635447 & -0.457560\end{array}$

$\begin{array}{llll}-10.642182 & 0.088971 & -0.533008\end{array}$

$\begin{array}{llll}-8.545759 & 0.096264 & -0.953946\end{array}$

$\begin{array}{llll}-8.522835 & -0.882238 & -1.421599\end{array}$

$\begin{array}{llll}-7.339458 & 0.824287 & -0.857885\end{array}$

$\begin{array}{llll}-4.400367 & -2.691751 & 0.281392\end{array}$

$\begin{array}{llll}-3.515882 & -2.649081 & 0.929544\end{array}$

$\begin{array}{lll}-5.113073 & -4.002842 & 0.558404\end{array}$

$\begin{array}{lll}-4.455682 & -5.008262 & 1.274582\end{array}$

$\begin{array}{llll}-3.465111 & -4.815265 & 1.681826\end{array}$

$\begin{array}{lll}-5.065919 & -6.247769 & 1.488149\end{array}$

$\begin{array}{lll}-4.545851 & -7.017068 & 2.052634\end{array}$

$\begin{array}{lll}-6.345979 & -6.489785 & 0.987844\end{array}$

$\begin{array}{lll}-6.829544 & -7.446836 & 1.162316\end{array}$

$\begin{array}{lll}-7.006713 & -5.490350 & 0.263942\end{array}$

$\begin{array}{llll}-8.006956 & -5.669006 & -0.120718\end{array}$

$\begin{array}{lll}-6.390460 & -4.260799 & 0.042986\end{array}$

$\begin{array}{lll}-6.911501 & -3.476974 & -0.498102\end{array}$

$\begin{array}{llll}-3.937165 & -2.681535 & -1.180723\end{array}$

$\begin{array}{llll}-4.794666 & -2.948261 & -1.809519\end{array}$

$\begin{array}{llll}-3.197460 & -3.471797 & -1.318506\end{array}$

$\begin{array}{lll}-5.700346 & -1.204214 & 1.936245\end{array}$

$\begin{array}{lll}-6.162596 & -0.218115 & 1.886488\end{array}$

$\begin{array}{lll}-6.802940 & -2.156882 & 2.388866\end{array}$

$\begin{array}{lll}-8.101863 & -1.950034 & 1.906875\end{array}$

$\begin{array}{lll}-8.291759 & -1.115806 & 1.240055\end{array}$

$\begin{array}{lll}-9.146449 & -2.802569 & 2.262131\end{array}$

$\begin{array}{lll}-10.144962 & -2.624941 & 1.871402\end{array}$

$\begin{array}{lll}-8.907720 & -3.877998 & 3.122068\end{array}$

$\begin{array}{llll}-9.716166 & -4.548819 & 3.399247\end{array}$

$\begin{array}{lll}-7.622496 & -4.078556 & 3.629706\end{array}$

$\begin{array}{lll}-7.425944 & -4.908962 & 4.302166\end{array}$

$\begin{array}{lll}-6.580453 & -3.220075 & 3.271500\end{array}$

$\begin{array}{lll}-5.588310 & -3.404061 & 3.668035\end{array}$

$\begin{array}{lll}-4.517737 & -1.101550 & 2.906447\end{array}$

$\begin{array}{lll}-3.825470 & -0.331141 & 2.559695\end{array}$

$\begin{array}{lll}-3.968153 & -2.041920 & 3.001299\end{array}$

$\begin{array}{lll}-4.875232 & -0.824122 & 3.902667\end{array}$

$\begin{array}{llll}-1.893456 & -0.285120 & -0.200779\end{array}$

$\begin{array}{llll}-1.666373 & 0.777359 & -0.166937\end{array}$

$\begin{array}{lll}-2.242174 & -0.672986 & 0.754081\end{array}$

$\begin{array}{llll}-1.006502 & -1.118792 & -0.963638\end{array}$

$-0.284026-0.647084-1.616820$

$\begin{array}{llll}-0.870825 & -2.499055 & -0.722476\end{array}$

$\begin{array}{llll}-1.539057 & -2.932239 & 0.011057\end{array}$

$-4.420274-1.061213-3.710464$

$\begin{array}{lll}-5.288135 & -1.650420 & -3.425474\end{array}$

$\begin{array}{llll}-3.212391 & -1.779691 & -3.897265\end{array}$

$\begin{array}{lll}-3.256700 & -2.859764 & -3.758216\end{array}$

$-2.048120 \quad-1.327633-4.762364$

$\begin{array}{llll}-2.251572 & -1.561522 & -5.819029\end{array}$

$\begin{array}{llll}-1.179007 & -1.928879 & -4.477630\end{array}$ 


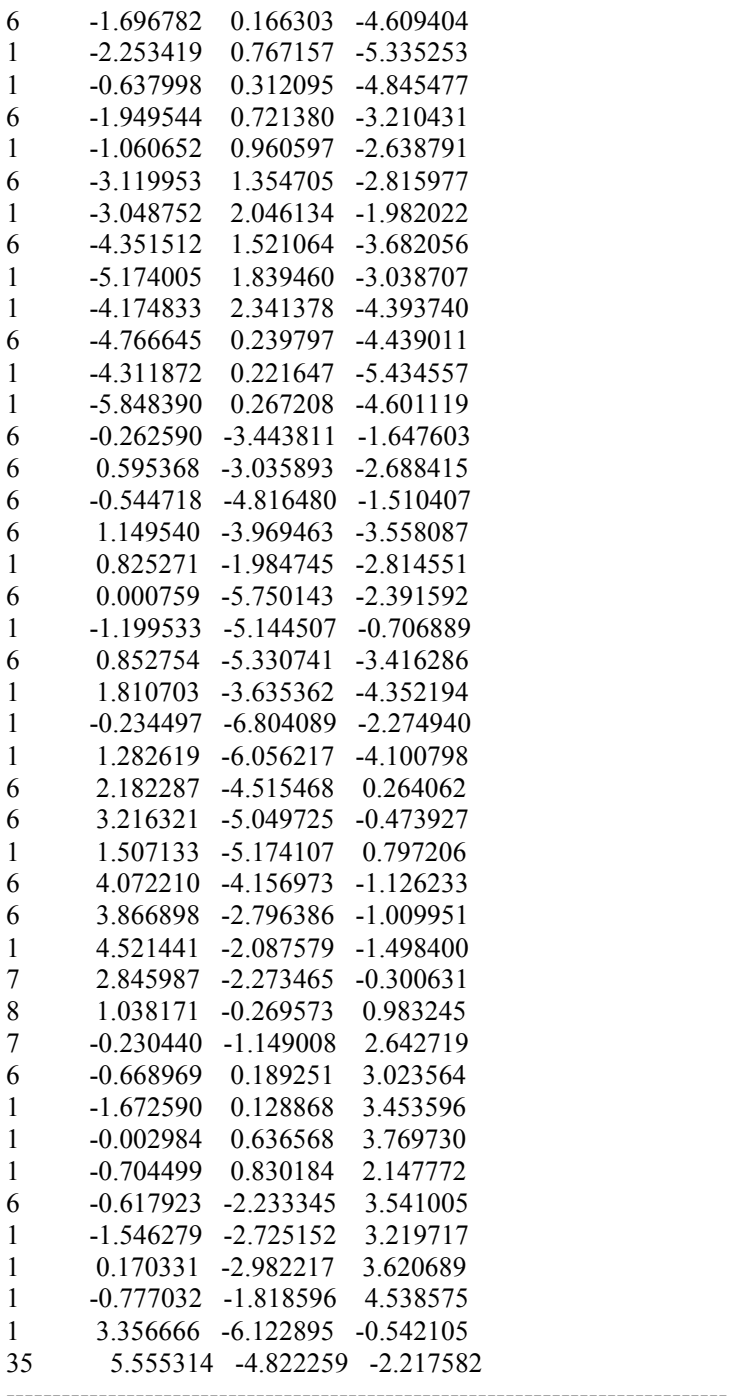

$[8-9]^{\ddagger}-\mathbf{P 1}-\mathrm{C} 2$ exo re-si conf2

Number of imaginary frequencies $=1$

The smallest frequencies are $=-133.97854 .37477 .6585 \mathrm{~cm}(-1)$ Electronic energy $=-7222.6089123$

Zero-point correction=

(Hartree/Particle)

Thermal correction to Energy= $\quad 1.873225$

Thermal correction to Enthalpy= $\quad 1.874170$

Thermal correction to Gibbs Free Energy $=\quad 1.588051$

Sum of electronic and zero-point Energies $=\quad-7220.854713$

Sum of electronic and thermal Energies $=\quad-7220.735687$

Sum of electronic and thermal Enthalpies $=\quad-7220.734743$

Sum of electronic and thermal Free Energies $=\quad-7221.020862$

Cartesian Coordinates

$\begin{array}{llcc}77 & -4.163394 & -0.905691 & 1.466574 \\ 15 & -5.888732 & -0.077176 & 0.255889 \\ 8 & -6.427972 & -0.741237 & -1.163095 \\ 8 & -7.297192 & -0.051063 & 1.141311 \\ 7 & -5.526030 & 1.472669 & -0.289678 \\ 6 & -7.373590 & -1.759853 & -1.201906 \\ 6 & -6.930404 & -3.001340 & -1.708319 \\ 1 & -5.902321 & -3.086230 & -2.044108 \\ 6 & -7.803080 & -4.058401 & -1.773576 \\ 1 & -7.477209 & -5.015994 & -2.169464 \\ 6 & -9.135200 & -3.923533 & -1.298271 \\ 6 & -10.023451 & -5.033099 & -1.288988 \\ 1 & -9.668713 & -5.983292 & -1.679548 \\ 6 & -11.298091 & -4.914661 & -0.783544\end{array}$

$-11.967334-5.769982-0.776304$

$-11.731230-3.675381-0.255291$

$\begin{array}{lll}-12.728349 & -3.589994 & 0.167011\end{array}$

$-10.900216-2.575970-0.264725$

$\begin{array}{lll}-11.249247 & -1.640940 & 0.156110\end{array}$

$-9.584273-2.653321 \quad-0.801413$

$\begin{array}{lll}-8.686878 & -1.524912 & -0.823998\end{array}$

$-9.131060-0.165153-0.405705$

$\begin{array}{lll}-10.259841 & 0.499136 & -1.003412\end{array}$

$\begin{array}{lll}-10.915591 & 0.001273 & -2.163882\end{array}$

$-10.555157-0.910042-2.626731$

$\begin{array}{llll}-11.987146 & 0.669155 & -2.715540\end{array}$

$\begin{array}{lll}-12.464602 & 0.274149 & -3.607647\end{array}$

$\begin{array}{llll}-12.468408 & 1.868040 & -2.136575\end{array}$

$\begin{array}{lll}-13.316937 & 2.380022 & -2.580770\end{array}$

$\begin{array}{lll}-11.849689 & 2.386489 & -1.021775\end{array}$

$\begin{array}{lll}-12.197906 & 3.314493 & -0.575657\end{array}$

$\begin{array}{lll}-10.732372 & 1.731089 & -0.435400\end{array}$

$\begin{array}{lll}-10.060291 & 2.289718 & 0.685321\end{array}$

$\begin{array}{lll}-10.435007 & 3.211361 & 1.120941\end{array}$

$\begin{array}{lll}-8.922668 & 1.701285 & 1.180411\end{array}$

$\begin{array}{lll}-8.369978 & 2.130370 & 2.008630\end{array}$

$\begin{array}{lll}-8.456944 & 0.499467 & 0.606157\end{array}$

$\begin{array}{llll}-4.345464 & 2.037661 & 0.409395\end{array}$

$\begin{array}{llll}-3.475895 & 1.937405 & -0.248412\end{array}$

$\begin{array}{lll}-4.479223 & 3.501004 & 0.794688\end{array}$

$\begin{array}{llll}-3.349177 & 4.325700 & 0.743645\end{array}$

$\begin{array}{lll}-2.408198 & 3.923255 & 0.376287\end{array}$

$\begin{array}{lll}-3.421402 & 5.659652 & 1.152933\end{array}$

$\begin{array}{lll}-2.537528 & 6.289938 & 1.099395\end{array}$

$\begin{array}{lll}-4.629058 & 6.182399 & 1.619946\end{array}$

$\begin{array}{lll}-4.692686 & 7.222365 & 1.927488\end{array}$

$\begin{array}{llll}-5.758075 & 5.358610 & 1.686749\end{array}$

$\begin{array}{lll}-6.703280 & 5.759160 & 2.042741\end{array}$

$\begin{array}{lll}-5.680374 & 4.026139 & 1.285875\end{array}$

$\begin{array}{lll}-6.561794 & 3.395180 & 1.308350\end{array}$

$\begin{array}{lll}-4.090902 & 1.230695 & 1.694764\end{array}$

$\begin{array}{lll}-4.846156 & 1.521440 & 2.435655\end{array}$

$\begin{array}{lll}-3.129220 & 1.533692 & 2.111887\end{array}$

$\begin{array}{llll}-5.851346 & 1.915907 & -1.689169\end{array}$

$\begin{array}{llll}-6.709416 & 1.311991 & -1.980335\end{array}$

$\begin{array}{llll}-6.320023 & 3.363303 & -1.753660\end{array}$

$\begin{array}{llll}-7.675308 & 3.635094 & -1.531301\end{array}$

$\begin{array}{llll}-8.355308 & 2.811640 & -1.343467\end{array}$

$\begin{array}{llll}-8.157419 & 4.943962 & -1.539939\end{array}$

$\begin{array}{llll}-9.212871 & 5.130450 & -1.360139\end{array}$

$\begin{array}{llll}-7.283810 & 6.006417 & -1.785454\end{array}$

$\begin{array}{llll}-7.651586 & 7.028748 & -1.788952\end{array}$

$\begin{array}{llll}-5.934698 & 5.744749 & -2.038152\end{array}$

$\begin{array}{llll}-5.248907 & 6.563868 & -2.235720\end{array}$

$\begin{array}{llll}-5.459112 & 4.432178 & -2.030150\end{array}$

$\begin{array}{llll}-4.406439 & 4.249913 & -2.217050\end{array}$

$\begin{array}{llll}-4.716799 & 1.588847 & -2.667699\end{array}$

$\begin{array}{llll}-4.525778 & 0.512819 & -2.668453\end{array}$

$\begin{array}{llll}-3.784830 & 2.105667 & -2.426314\end{array}$

$\begin{array}{llll}-5.006847 & 1.887374 & -3.679864\end{array}$

$\begin{array}{llll}-3.037761 & -1.080624 & -0.376645\end{array}$

$\begin{array}{llll}-3.250356 & -2.010803 & -0.903345\end{array}$

$\begin{array}{llll}-3.087164 & -0.209065 & -1.026858\end{array}$

$\begin{array}{lll}-1.984389 & -1.105518 & 0.609651\end{array}$

$\begin{array}{lll}-1.588149 & -2.058477 & 0.936665\end{array}$

$\begin{array}{lll}-1.304706 & 0.055352 & 1.014936\end{array}$

$\begin{array}{lll}-1.665432 & 0.999067 & 0.627751\end{array}$

$\begin{array}{lll}-5.355750 & -1.009332 & 3.368924\end{array}$

$\begin{array}{lll}-5.874425 & -0.059149 & 3.466968\end{array}$

$\begin{array}{lll}-3.980141 & -1.004088 & 3.690594\end{array}$

$\begin{array}{lll}-3.560276 & -0.059059 & 4.035427\end{array}$

$\begin{array}{lll}-3.157283 & -2.218362 & 4.088608\end{array}$

$\begin{array}{lll}-3.287247 & -2.422849 & 5.162474\end{array}$

$\begin{array}{lll}-2.103019 & -1.955203 & 3.952852\end{array}$

$\begin{array}{lll}-3.466178 & -3.489944 & 3.270072\end{array}$

$\begin{array}{lll}-4.279888 & -4.054185 & 3.734456\end{array}$

$\begin{array}{lll}-2.590283 & -4.146401 & 3.293166\end{array}$

$\begin{array}{lll}-3.819448 & -3.212938 & 1.811765\end{array}$

$\begin{array}{lll}-3.066339 & -3.501265 & 1.083016\end{array}$ 


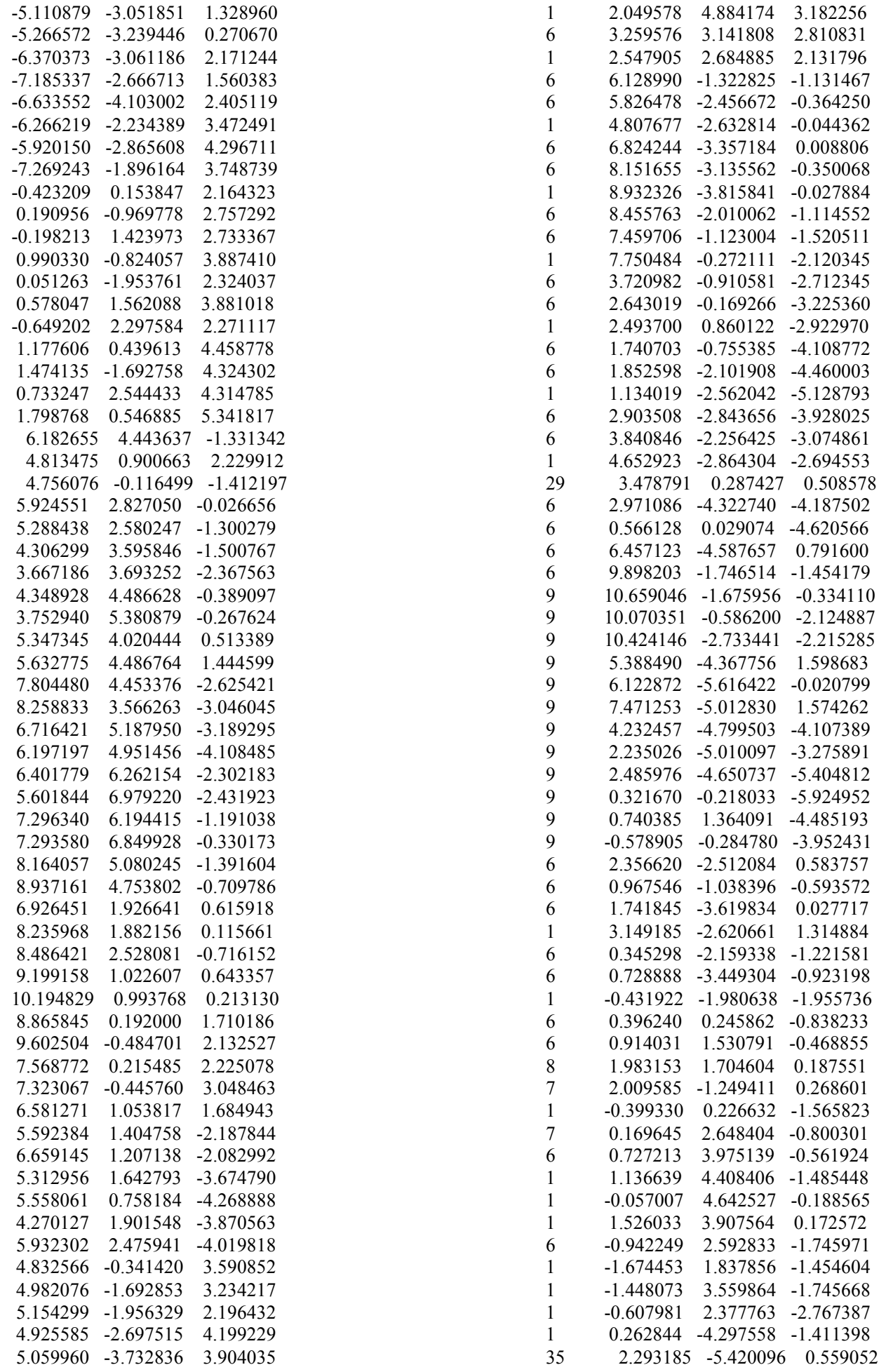

Zero-point correction=
[8-9] ${ }^{\star}-\mathbf{P 1 - C 2}$ exo si-re

Number of imaginary frequencies $=1$

The smallest frequencies are $=-203.72119 .500913 .8096 \mathrm{~cm}(-1)$ Electronic energy $=-7222.6216665$

(Hartree/Particle)

Thermal correction to Energy= $\quad 1.874396$

Thermal correction to Enthalpy= $\quad 1.875340$

Thermal correction to Gibbs Free Energy $=\quad 1.596320$

Sum of electronic and zero-point Energies $=\quad-7220.865442$

Sum of electronic and thermal Energies $=\quad-7220.747271$

Sum of electronic and thermal Enthalpies $=\quad-7220.746327$ 
Sum of electronic and thermal Free Energies=

Cartesian Coordinates

\begin{tabular}{|c|c|c|c|}
\hline & -7.113289 & 3.462019 & 0.4 \\
\hline & -3.294569 & 1.186117 & 58655 \\
\hline & -4.516794 & +-0.539422 & 0.74811 \\
\hline 6 & -5.817688 & 2.297932 & -0.761513 \\
\hline & -5.808180 & 1.873496 & 0.618379 \\
\hline & -5.381577 & 2.981080 & 1.413904 \\
\hline 1 & -5.284851 & 2.987554 & 2.490781 \\
\hline 6 & -5.161228 & 4.093375 & 0.548122 \\
\hline 1 & -4.872171 & 5.087794 & 0.855571 \\
\hline 6 & -5.429424 & 3.675797 & -0.787767 \\
\hline 1 & -5.378129 & 4.297133 & -1.668693 \\
\hline 6 & -9.084764 & 2.853777 & 0.650119 \\
\hline 1 & -9.414112 & 1.823231 & 0.660160 \\
\hline 6 & -8.651419 & 3.607975 & 1.783474 \\
\hline 1 & -8.587958 & 3.248362 & 2.801656 \\
\hline 6 & -8.271432 & 4.907923 & 1.329888 \\
\hline 1 & -7.865470 & 5.701372 & 1.943523 \\
\hline 6 & -8.470419 & 4.958391 & -0.083261 \\
\hline 1 & -8.243046 & 5.796859 & -0.728357 \\
\hline 6 & -8.974061 & 3.691426 & -0.503194 \\
\hline 1 & -9.197917 & 3.40 & -1.5 \\
\hline 6 & -6.110564 & 1.387222 & -1.904513 \\
\hline 6 & -7.427513 & 0.946648 & -2.107691 \\
\hline 1 & -8.200494 & 1.3 & 400 \\
\hline 6 & -7.749940 & 0.013892 & -3.092670 \\
\hline 1 & -8.774463 & -0.327728 & -3.203290 \\
\hline 6 & -6.744000 & -0.489231 & 3760 \\
\hline 1 & -6.972202 & -1.225689 & -4.676832 \\
\hline 6 & -5.427741 & -0.066814 & -3.731137 \\
\hline 1 & -4.657237 & -0.486660 & 6205 \\
\hline 6 & -5.083580 & 0.857629 & -2.730227 \\
\hline 6 & -6.096490 & 0.47 & \\
\hline 1 & -6.850968 & 0.06 & 965 \\
\hline 6 & -6.635096 & 0.383578 & 2.5 \\
\hline 1 & -6.867460 & -0.64 & 011 \\
\hline 1 & -5.926605 & 0.773530 & 3.24 \\
\hline 1 & -7.550783 & 0.977648 & 2.5 \\
\hline 6 & -2.418552 & 0.304218 & -3.7 \\
\hline 6 & -1.743448 & -0.887547 & -3.429769 \\
\hline 1 & -1.705953 & -1.243341 & -2.405420 \\
\hline 6 & -1.109325 & -1.605933 & -4.448414 \\
\hline 1 & -0.590894 & -2.527835 & -4.216817 \\
\hline 6 & -1.132006 & -1.134373 & -5.761376 \\
\hline 1 & -0.629218 & -1.691085 & -6.546956 \\
\hline 6 & -1.793698 & 0.060161 & -6.062395 \\
\hline 1 & -1.808742 & 0.4 & 1618 \\
\hline 6 & -2.433770 & 0.775907 & -5.051802 \\
\hline 1 & -2.945571 & 1.700765 & -5.296840 \\
\hline 6 & -2.989122 & 2.943440 & -2.805489 \\
\hline 6 & -3.785939 & 3.679768 & -3.694126 \\
\hline 1 & -4.669593 & 3.227844 & -4.135315 \\
\hline 6 & -3.455226 & 5.002268 & -3.997251 \\
\hline 1 & -4.078955 & 5.569888 & -4.682046 \\
\hline 6 & -2.327035 & 5.595 & -3.4 \\
\hline 1 & -2.076180 & 6.626148 & -3.656634 \\
\hline 6 & -1.524902 & 4.862287 & -2.542943 \\
\hline 1 & -0.647663 & 5.315706 & -2.090311 \\
\hline 0 & -1.856820 & 3.541800 & -2.235774 \\
\hline 1 & -1.240073 & 2.969924 & -1.549749 \\
\hline 6 & -5.003566 & -1.921488 & -0.371936 \\
\hline 0 & -3.937194 & -2.650708 & -0.918195 \\
\hline 1 & -2.919078 & -2.381869 & -0.656002 \\
\hline 6 & -4.180086 & -3.695129 & -1.810800 \\
\hline 6 & -5.479480 & -3.998223 & -2.214304 \\
\hline 1 & -5.664855 & -4.789424 & -2.932442 \\
\hline 6 & -6.535770 & -3.252371 & -1.690442 \\
\hline 6 & -6.310400 & -2.239668 & -0.757743 \\
\hline 1 & -7.160662 & -1.700557 & -0.362031 \\
\hline 6 & -4.169643 & -1.407179 & 2.334201 \\
\hline 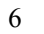 & -3.563514 & -0.656441 & 3.353409 \\
\hline & & 0391607 & 3.196682 \\
\hline
\end{tabular}

$-7221.025346$

9

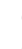

6

6

1

15

8

8

7

6

6
1

1

6

1
6

6

6
1

6

1

6

1

1

6

6
6

6

6

1

6

1
6

1

6
1

1
6
6

6

1

6
1

6

6

1

6

6

1
6

1

6

1
6 $\begin{array}{lll}-3.238070 & -1.253953 & 4.569188\end{array}$

$\begin{array}{lll}-3.490187 & -2.608509 & 4.791772\end{array}$

$\begin{array}{lll}-3.215875 & -3.075336 & 5.730600\end{array}$

$\begin{array}{lll}-4.083278 & -3.353153 & 3.775736\end{array}$

$\begin{array}{lll}-4.425520 & -2.763903 & 2.555737\end{array}$

$\begin{array}{lll}-4.889454 & -3.370733 & 1.786272\end{array}$

$\begin{array}{llll}-2.716793 & 0.572549 & -0.263089\end{array}$

$\begin{array}{lll}-4.260257 & -4.837292 & 3.933247\end{array}$

$\begin{array}{llll}-2.479705 & -0.469666 & 5.601981\end{array}$

$\begin{array}{lll}-3.024910 & -4.527612 & -2.294605\end{array}$

$\begin{array}{lll}-7.926812 & -3.541827 & -2.185924\end{array}$

$-8.023494 \quad-3.342798 \quad-3.523800$

$\begin{array}{llll}-8.859789 & -2.760006 & -1.600201\end{array}$

$\begin{array}{lll}-8.280936 & -4.828893 & -1.964607\end{array}$

$\begin{array}{llll}-1.906255 & -3.780060 & -2.483691\end{array}$

$\begin{array}{llll}-2.691042 & -5.485215 & -1.394201\end{array}$

$\begin{array}{llll}-3.286343 & -5.152917 & -3.459353\end{array}$

$\begin{array}{lll}-5.404719 & -5.276438 & 3.365112\end{array}$

$\begin{array}{lll}-3.245533 & -5.510980 & 3.326349\end{array}$

$\begin{array}{lll}-4.269467 & -5.222023 & 5.225608\end{array}$

$\begin{array}{lll}-2.760937 & -0.869522 & 6.858901\end{array}$

$\begin{array}{llll}-2.725622 & 0.855341 & 5.526289\end{array}$

$\begin{array}{lll}-1.132828 & -0.624942 & 5.440021\end{array}$

$\begin{array}{lll}-0.812623 & -1.269109 & 1.160874\end{array}$

$\begin{array}{lll}-0.435409 & -0.241353 & 2.111134\end{array}$

$\begin{array}{lll}-0.962047 & 1.091585 & 2.183471\end{array}$

$\begin{array}{lll}-0.031320 & -0.584251 & 3.050978\end{array}$

$\begin{array}{lll}4.378743 & -1.572575 & 1.493309\end{array}$

$\begin{array}{llll}5.232450 & -0.710391 & -0.519941\end{array}$

$\begin{array}{llll}5.342302 & 0.893987 & -0.974416\end{array}$

$\begin{array}{llll}6.834860 & -1.147767 & -0.713109\end{array}$

$\begin{array}{llll}4.339376 & -1.320950 & -1.796537\end{array}$

$\begin{array}{llll}6.061571 & 1.712187 & -0.111926\end{array}$

$\begin{array}{llll}5.317806 & 2.584518 & 0.710684\end{array}$

$\begin{array}{llll}4.244132 & 2.625339 & 0.579902\end{array}$

$\begin{array}{llll}5.969081 & 3.352834 & 1.642861\end{array}$

$\begin{array}{llll}5.410999 & 4.036097 & 2.277086\end{array}$

$\begin{array}{llll}7.375454 & 3.232554 & 1.818750\end{array}$

$\begin{array}{llll}8.048313 & 3.941380 & 2.850901\end{array}$

$\begin{array}{lll}7.467053 & 4.604925 & 3.486095\end{array}$

$\begin{array}{llll}9.400078 & 3.780228 & 3.055956\end{array}$

$\begin{array}{llll}9.902338 & 4.321049 & 3.852714\end{array}$

$\begin{array}{lll}10.134431 & 2.891486 & 2.235012\end{array}$

$\begin{array}{lll}11.195450 & 2.744787 & 2.415749\end{array}$

$\begin{array}{lll}9.516389 & 2.205698 & 1.211636\end{array}$

$\begin{array}{lll}10.093180 & 1.523425 & 0.598562\end{array}$

$\begin{array}{lll}8.125594 & 2.363445 & 0.955982\end{array}$

$\begin{array}{llll}7.444363 & 1.644891 & -0.092288\end{array}$

$\begin{array}{llll}8.174203 & 0.843096 & -1.113260\end{array}$

$\begin{array}{llll}9.214791 & 1.440977 & -1.917437\end{array}$

$\begin{array}{llll}9.487826 & 2.837943 & -1.901392\end{array}$

$8.887440 \quad 3.490076-1.279006$

$\begin{array}{llll}10.490875 & 3.379482 & -2.676659\end{array}$

$\begin{array}{llll}10.670391 & 4.450529 & -2.648902\end{array}$

$\begin{array}{lll}11.282590 & 2.558041 & -3.512950\end{array}$

$12.074505 \quad 2.997306-4.112493$

$\begin{array}{lll}11.029890 & 1.206105 & -3.574600\end{array}$

$\begin{array}{llll}11.613862 & 0.562834 & -4.227750\end{array}$

$\begin{array}{llll}9.992489 & 0.620051 & -2.800446\end{array}$

$\begin{array}{llll}9.697142 & -0.766410 & -2.902150\end{array}$

$10.301234-1.389694 \quad-3.555517$

$\begin{array}{llll}8.641669 & -1.303157 & -2.212983\end{array}$

$\begin{array}{llll}8.371872 & -2.349693 & -2.305734\end{array}$

$\begin{array}{llll}7.863997 & -0.489855 & -1.353472\end{array}$

$\begin{array}{llll}3.116661 & -2.051609 & -1.376582\end{array}$

$2.276208-1.345268-1.391141$

$\begin{array}{llll}2.773681 & -3.202315 & -2.310587\end{array}$

$\begin{array}{llll}1.431191 & -3.493484 & -2.576642\end{array}$

$\begin{array}{llll}0.660748 & -2.831298 & -2.193545\end{array}$

$\begin{array}{llll}1.078250 & -4.621405 & -3.323497\end{array}$

$\begin{array}{llll}0.030254 & -4.837684 & -3.505340\end{array}$

$2.071521 \quad-5.461685-3.830331$

$\begin{array}{llll}1.801287 & -6.334883 & -4.417412\end{array}$

$3.416974 \quad-5.171943 \quad-3.579186$

$\begin{array}{llll}4.195546 & -5.818955 & -3.974305\end{array}$ 
$\begin{array}{lll}3.764622 & -4.055914 & -2.817712\end{array}$

$\begin{array}{llll}4.809358 & -3.832784 & -2.617215\end{array}$

$\begin{array}{lll}3.268688 & -2.650546 & 0.039740\end{array}$

$\begin{array}{llll}3.759063 & -3.624376 & -0.072248\end{array}$

$\begin{array}{lll}2.254870 & -2.855998 & 0.398827\end{array}$

$\begin{array}{llll}4.341048 & -0.829296 & -3.212161\end{array}$

$\begin{array}{llll}3.904145 & -1.659749 & -3.771130\end{array}$

$\begin{array}{llll}3.423224 & 0.368951 & -3.461994\end{array}$

$\begin{array}{llll}2.076054 & 0.137056 & -3.776471\end{array}$

$\begin{array}{llll}1.699812 & -0.881700 & -3.804451\end{array}$

$\begin{array}{llll}1.214766 & 1.193597 & -4.085104\end{array}$

$\begin{array}{llll}0.180068 & 0.990756 & -4.331606\end{array}$

$\begin{array}{llll}1.695879 & 2.503231 & -4.101586\end{array}$

$\begin{array}{llll}1.030565 & 3.324863 & -4.353155\end{array}$

$\begin{array}{llll}3.035082 & 2.748511 & -3.785162\end{array}$

$\begin{array}{llll}3.418123 & 3.765449 & -3.789566\end{array}$

$3.888657 \quad 1.692452 \quad-3.465091$

$\begin{array}{lll}4.923735 & 1.902656 & -3.224022\end{array}$

$\begin{array}{llll}5.748703 & -0.644493 & -3.773679\end{array}$

$\begin{array}{llll}6.325100 & -1.564197 & -3.653928\end{array}$

$\begin{array}{lll}6.296816 & 0.170011 & -3.300012\end{array}$

$\begin{array}{llll}5.676308 & -0.420114 & -4.841900\end{array}$

$\begin{array}{llll}2.635942 & -1.759777 & 2.746889\end{array}$

$\begin{array}{lll}1.922428 & -2.455555 & 2.320947\end{array}$

$\begin{array}{llll}2.819967 & -1.930831 & 3.804452\end{array}$

$\begin{array}{llll}2.585304 & -0.390834 & 2.308729\end{array}$

$\begin{array}{llll}2.939609 & 0.358796 & 3.009370\end{array}$

$\begin{array}{lll}1.774222 & 0.093493 & 1.241015\end{array}$

$\begin{array}{llll}1.498392 & -0.598996 & 0.458667\end{array}$

$\begin{array}{lll}6.011918 & -3.076039 & 1.406075\end{array}$

$\begin{array}{lll}6.009092 & -3.525103 & 0.415696\end{array}$

$\begin{array}{llll}4.997875 & -3.527201 & 2.294042\end{array}$

$\begin{array}{lll}4.314779 & -4.283682 & 1.910175\end{array}$

$\begin{array}{llll}5.118877 & -3.552583 & 3.806536\end{array}$

$\begin{array}{llll}5.741587 & -4.408391 & 4.106439\end{array}$

$\begin{array}{llll}4.122576 & -3.743953 & 4.215572\end{array}$

$\begin{array}{llll}5.683906 & -2.253073 & 4.420374\end{array}$

$6.770047 \quad-2.331577 \quad 4.528350$

$\begin{array}{llll}5.287744 & -2.135898 & 5.433947\end{array}$

$\begin{array}{lll}5.361231 & -0.992639 & 3.627341\end{array}$

$\begin{array}{lll}4.612913 & -0.333946 & 4.059224\end{array}$

$\begin{array}{lll}6.207153 & -0.456749 & 2.679846\end{array}$

$6.089274 \quad 0.591149 \quad 2.421603$

$\begin{array}{llll}7.504785 & -1.108685 & 2.250672\end{array}$

$\begin{array}{lll}7.896417 & -0.554954 & 1.400025\end{array}$

$\begin{array}{lll}8.238006 & -0.982863 & 3.060304\end{array}$

$\begin{array}{lll}7.392772 & -2.608083 & 1.876114\end{array}$

$\begin{array}{lll}7.693427 & -3.231091 & 2.726326\end{array}$

$\begin{array}{lll}8.111407 & -2.811731 & 1.078144\end{array}$

$\begin{array}{llll}1.725695 & 1.510969 & 0.894056\end{array}$

$\begin{array}{lll}1.986961 & 2.518942 & 1.848668\end{array}$

$\begin{array}{llll}1.379341 & 1.905147 & -0.412630\end{array}$

$\begin{array}{lll}1.916860 & 3.865666 & 1.505725\end{array}$

$\begin{array}{lll}2.232782 & 2.250752 & 2.869247\end{array}$

$\begin{array}{llll}1.326139 & 3.255177 & -0.755756\end{array}$

$\begin{array}{llll}1.172089 & 1.150050 & -1.164593\end{array}$

$\begin{array}{llll}1.588046 & 4.239926 & 0.199873\end{array}$

$\begin{array}{lll}2.113704 & 4.623926 & 2.258054\end{array}$

$\begin{array}{llll}1.081445 & 3.536980 & -1.771690\end{array}$

$\begin{array}{llll}1.539606 & 5.290783 & -0.070777\end{array}$

$\begin{array}{lll}-0.687987 & 1.849057 & 3.355103\end{array}$

$\begin{array}{lll}-1.065283 & 3.174054 & 3.455694\end{array}$

$\begin{array}{lll}-0.180637 & 1.369331 & 4.183568\end{array}$

$\begin{array}{lll}-1.717920 & 3.745246 & 2.361923\end{array}$

$\begin{array}{lll}-2.015759 & 2.969979 & 1.254802\end{array}$

$\begin{array}{lll}-2.555692 & 3.379774 & 0.411247\end{array}$

$\begin{array}{lll}-1.666570 & 1.673435 & 1.165754\end{array}$

$\begin{array}{lll}-1.192714 & -1.005700 & -0.022379\end{array}$

$\begin{array}{lll}-0.657308 & -2.586558 & 1.523968\end{array}$

$-0.795394-3.640980 \quad 0.526456$

$\begin{array}{lll}-0.010802 & -4.389855 & 0.681815\end{array}$

$-1.766228-4.143754 \quad 0.611745$

$\begin{array}{llll}-0.700356 & -3.229317 & -0.472527\end{array}$

$\begin{array}{lll}-0.590105 & -3.098866 & 2.889800\end{array}$

$0.358894-3.617109 \quad 3.068685$ $\begin{array}{llll}-0.705580 & -2.310326 & 3.627461\end{array}$

$\begin{array}{lll}-1.397452 & -3.823023 & 3.037536\end{array}$

$\begin{array}{lll}-0.845161 & 3.749108 & 4.348772\end{array}$

$\begin{array}{llll}35 & -2.211992 & 5.638486 & 2.380165\end{array}$

Number of imaginary frequencies $=$

The smallest frequencies are $=-159.321010 .579411 .5867 \mathrm{~cm}(-1)$ Electronic energy $=-7222.6196948$

Zero-point correction $=$

1.754881

Hartree/Particle)

Thermal correction to Energy= $\quad 1.873735$

Thermal correction to Enthalpy= $\quad 1.874679$

Thermal correction to Gibbs Free Energy $=\quad 1.591336$

Sum of electronic and zero-point Energies $=\quad-7220.864813$

Sum of electronic and thermal Energies $=\quad-7220.745960$

Sum of electronic and thermal Enthalpies $=\quad-7220.745015$

Sum of electronic and thermal Free Energies $=\quad-7221.028359$

Cartesian Coordinates

\begin{tabular}{|c|c|c|c|}
\hline & & & \\
\hline 26 & -5.900050 & 4.224129 & 5207 \\
\hline & -2.101593 & 1.905616 & 5708 \\
\hline & -4.755698 & -0.305295 & 0316320 \\
\hline & -4.648665 & 3.154903 & 0.058699 \\
\hline & -5.257507 & 2.284577 & 1.038382 \\
\hline & -4.855019 & 2.735537 & 2.332422 \\
\hline & -5.154540 & 2.298033 & 3.274762 \\
\hline & -4.033203 & 3.887775 & 2.169275 \\
\hline & -3.607818 & 4.483107 & 2.965784 \\
\hline & -3.911088 & 4.151245 & 0.774693 \\
\hline & -3.372055 & 4.972890 & 0.328210 \\
\hline & -7.911616 & 4.428106 & 0.880804 \\
\hline & -8.490539 & 3.695835 & 0.333540 \\
\hline 6 & -7.729743 & 4.464456 & 2.297389 \\
\hline & -8.140344 & 3.761202 & 3.009676 \\
\hline & -6.870307 & 5.563551 & 2.603734 \\
\hline 1 & -6.515327 & 5.837735 & 3.588566 \\
\hline & -6.520632 & 6.206493 & 1.377162 \\
\hline & -5.855107 & 7.052840 & 1.269658 \\
\hline 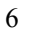 & -7.163919 & 5.506557 & 0.313319 \\
\hline 1 & -7.070713 & 5.734444 & -0.739796 \\
\hline 6 & -4.755888 & 2.965790 & -1.417509 \\
\hline 6 & -5.993861 & 3.180335 & -2.042831 \\
\hline & -6.819458 & 3.520893 & -1.431422 \\
\hline 6 & -6.184583 & 2.953111 & -3.406067 \\
\hline 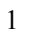 & -7.161475 & 3.120512 & -3.850630 \\
\hline 6 & -5.11 & 2.511939 & -4.184529 \\
\hline 1 & -5.244789 & 2.322637 & -5.245498 \\
\hline 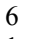 & -3.87 & 2.286461 & -3.586194 \\
\hline 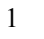 & -3.06 & 1.914683 & -4.199637 \\
\hline 6 & -3.676279 & 2.490547 & -2.211075 \\
\hline 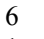 & -6.035742 & 1.045992 & 0.698723 \\
\hline 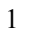 & -6.498996 & 1.196498 & -0.281282 \\
\hline 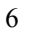 & -7.13 & 0.667676 & 293 \\
\hline & -7.653692 & -0.240961 & 1.378160 \\
\hline 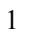 & -6.740795 & 0.498574 & 2.700952 \\
\hline 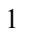 & -7.863771 & 1.480852 & 1.752022 \\
\hline 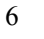 & -1.094374 & 1.337320 & -2.857293 \\
\hline 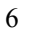 & -1.101431 & -0.038351 & -3.133559 \\
\hline 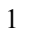 & -1.65 & -0.707651 & -2.481032 \\
\hline & -0.420615 & -0.545732 & -4.240182 \\
\hline & -0.446686 & -1.611163 & -4.449517 \\
\hline 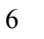 & 0.301621 & 0.318376 & -5.068102 \\
\hline 1 & 0.842616 & -0.074730 & -5.924147 \\
\hline 6 & 0.332699 & 1.687462 & -4.788430 \\
\hline 1 & 0.905707 & 2.362108 & -5.417030 \\
\hline 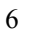 & -0.369528 & 2.197123 & -3.694875 \\
\hline 2 & -0.340555 & 3.261539 & -3.496851 \\
\hline 6 & -1.267410 & 3.419329 & -0.810670 \\
\hline & -1.375552 & 4.664334 & -1.449094 \\
\hline & -1.951335 & 4.758880 & -2.364990 \\
\hline & -0.771882 & 5.793058 & -0.890848 \\
\hline & -0.864475 & 6.755040 & -1.386730 \\
\hline
\end{tabular}




\begin{tabular}{|c|c|c|c|c|c|c|}
\hline-0.053415 & 5.685842 & 0.305135 & 6 & 10.447709 & -0.110106 & 1.119642 \\
\hline 0.413558 & 6.565965 & 0.738030 & 1 & 9.962917 & -0.603303 & 1.953394 \\
\hline 0.060128 & 4.447175 & 0.942918 & 6 & 11.775873 & 0.244399 & 1.223775 \\
\hline 0.616400 & 4.362196 & 1.870905 & 1 & 12.320203 & 0.020186 & 2.136807 \\
\hline-0.547225 & 3.318236 & 0.389373 & 6 & 12.434768 & 0.902169 & 0.158775 \\
\hline-0.514598 & 2.367170 & 0.910832 & 1 & 13.483657 & 1.168312 & 0.251282 \\
\hline-5.534548 & -1.181061 & -1.097637 & 6 & 11.734339 & 1.216567 & -0.984069 \\
\hline-4.931838 & -1.000425 & -2.350711 & 1 & 12.218999 & 1.740282 & -1.804022 \\
\hline-4.018654 & -0.421489 & -2.427892 & 6 & 10.360034 & 0.880383 & -1.115418 \\
\hline-5.496598 & -1.562324 & -3.493232 & 6 & 9.622941 & 1.249820 & -2.273242 \\
\hline-6.663374 & -2.324491 & -3.407493 & 1 & 10.132550 & 1.764054 & -3.083229 \\
\hline-7.093928 & -2.774692 & -4.294228 & 6 & 8.279196 & 0.992854 & -2.351620 \\
\hline-7.261320 & -2.504628 & -2.162228 & 1 & 7.687034 & 1.300340 & -3.207226 \\
\hline-6.713243 & -1.930185 & -1.011758 & 6 & 7.621676 & 0.312777 & -1.297848 \\
\hline-7.198811 & -2.086352 & -0.053647 & 6 & 3.022071 & 2.087417 & -1.140195 \\
\hline-4.783241 & -1.488293 & 1.716564 & 1 & 2.461619 & 2.546021 & -0.316825 \\
\hline-4.491002 & -0.971675 & 2.990214 & 6 & 2.972244 & 3.034044 & -2.331809 \\
\hline-4.347275 & 0.093616 & 3.124520 & 6 & 2.098103 & 4.122858 & -2.338109 \\
\hline-4.339359 & -1.827818 & 4.076643 & 1 & 1.480398 & 4.311978 & -1.468422 \\
\hline-4.431134 & -3.212816 & 3.918022 & 6 & 2.008422 & 4.961590 & -3.453639 \\
\hline-4.301093 & -3.876888 & 4.765266 & 1 & 1.318583 & 5.801084 & -3.439129 \\
\hline-4.684671 & -3.724376 & 2.648221 & 6 & 2.802633 & 4.720373 & -4.575623 \\
\hline-4.866455 & -2.875496 & 1.552890 & 1 & 2.738648 & 5.371090 & -5.443247 \\
\hline-5.060408 & -3.310811 & 0.580202 & 6 & 3.693596 & 3.639401 & -4.571385 \\
\hline-2.523547 & 0.315019 & 0.095579 & 1 & 4.323062 & 3.450709 & -5.436997 \\
\hline-4.693140 & -5.214008 & 2.437726 & 6 & 3.778160 & 2.805021 & -3.457900 \\
\hline-4.097915 & -1.275914 & 5.453458 & 1 & 4.478987 & 1.974335 & -3.451428 \\
\hline-4.881125 & -1.313871 & -4.843372 & 6 & 2.333903 & 0.745458 & -1.525633 \\
\hline-8.527211 & -3.306715 & -2.022602 & 1 & 2.251802 & 0.716852 & -2.614126 \\
\hline-9.569373 & -2.521809 & -1.657506 & 1 & 1.308723 & 0.792978 & -1.151153 \\
\hline-8.405658 & -4.254621 & -1.063867 & 6 & 5.075495 & 3.228530 & -0.288437 \\
\hline-8.874682 & -3.929105 & -3.167220 & 1 & 4.712773 & 3.937671 & -1.036857 \\
\hline-3.598616 & -0.894777 & -4.751326 & 6 & 4.603443 & 3.759795 & 1.062845 \\
\hline-4.889024 & -2.428554 & -5.608971 & 6 & 5.151712 & 3.317304 & 2.276095 \\
\hline-5.559197 & -0.364048 & -5.532330 & 1 & 5.882561 & 2.517416 & 2.274848 \\
\hline-5.455070 & -5.574071 & 1.381576 & 6 & 4.770606 & 3.904409 & 3.482342 \\
\hline-3.444247 & -5.689210 & 2.198593 & 1 & 5.210378 & 3.554810 & 4.412041 \\
\hline-5.158082 & -5.875764 & 3.520472 & 6 & 3.825905 & 4.935608 & 3.500493 \\
\hline-5.186622 & -1.416809 & 6.245949 & 1 & 3.536948 & 5.395452 & 4.441614 \\
\hline-3.789556 & 0.041081 & 5.436795 & 6 & 3.256726 & 5.367856 & 2.302047 \\
\hline-3.082679 & -1.920831 & 6.078901 & 1 & 2.520759 & 6.167323 & 2.302049 \\
\hline-2.115199 & -2.561640 & -0.489835 & 6 & 3.647240 & 4.783908 & 1.093372 \\
\hline-1.138647 & -2.016016 & 1.567477 & 1 & 3.216071 & 5.137597 & 0.160873 \\
\hline-1.818389 & -3.903714 & -0.339991 & 6 & 6.598026 & 3.233875 & -0.420983 \\
\hline-2.645632 & -2.206488 & -1.365869 & 1 & 6.888807 & 2.997365 & -1.445692 \\
\hline-0.797420 & -3.387495 & 1.741085 & 1 & 7.093273 & 2.535234 & 0.254002 \\
\hline-1.135128 & -4.339184 & 0.798234 & 1 & 6.961318 & 4.239503 & -0.189788 \\
\hline-0.728252 & -1.087575 & 2.577777 & 6 & 1.257567 & -1.798598 & -0.445155 \\
\hline-1.322495 & 0.195566 & 2.854698 & 1 & 0.481249 & -1.141927 & -0.827731 \\
\hline-1.970872 & 0.877256 & 2.001210 & 1 & 1.058884 & -2.842039 & -0.662770 \\
\hline-1.777547 & -1.634273 & 0.420767 & 6 & 1.798312 & -1.484601 & 0.856338 \\
\hline-0.193173 & -1.554510 & 3.391758 & 1 & 2.269234 & -2.278936 & 1.429088 \\
\hline 3.228805 & -1.095254 & -0.952156 & 6 & 1.417541 & -0.322011 & 1.562033 \\
\hline 4.973146 & 0.384320 & -0.473046 & 1 & 0.874777 & 0.431942 & 0.999991 \\
\hline 5.809958 & 0.533671 & 0.969356 & 6 & 4.073396 & -1.347319 & -2.978981 \\
\hline 6.274228 & 0.106374 & -1.491721 & 1 & 4.203057 & -0.358698 & -3.413956 \\
\hline 4.426655 & 1.938578 & -0.671215 & 6 & 2.737913 & -1.837477 & -2.960144 \\
\hline 6.450976 & -0.617149 & 1.416169 & 1 & 1.977899 & -1.183202 & -3.377346 \\
\hline 5.844842 & -1.305049 & 2.488761 & 6 & 2.347521 & -3.301314 & -3.014804 \\
\hline 4.961931 & -0.870562 & 2.939933 & 1 & 2.467806 & -3.667848 & -4.044827 \\
\hline 6.382999 & -2.488625 & 2.928066 & 1 & 1.278146 & -3.369238 & -2.793755 \\
\hline 5.933043 & -3.022527 & 3.760768 & 6 & 3.131174 & -4.213177 & -2.042963 \\
\hline 7.510913 & -3.052217 & 2.270616 & 1 & 3.996074 & -4.645364 & -2.555942 \\
\hline 8.014508 & -4.327871 & 2.643900 & 1 & 2.495945 & -5.057353 & -1.756552 \\
\hline 7.539037 & -4.850137 & 3.470094 & 6 & 3.616812 & -3.522859 & -0.775545 \\
\hline 9.067085 & -4.899660 & 1.965856 & 1 & 3.088179 & -3.766853 & 0.142089 \\
\hline 9.439131 & -5.878202 & 2.254979 & 6 & 4.858962 & -2.944884 & -0.661278 \\
\hline 9.654719 & -4.215205 & 0.875354 & 1 & 5.254798 & -2.778648 & 0.334869 \\
\hline 10.466823 & -4.679898 & 0.323636 & 6 & 5.852479 & -2.860026 & -1.800205 \\
\hline 9.204100 & -2.967335 & 0.502134 & 1 & 6.703482 & -2.271034 & -1.464391 \\
\hline 9.660960 & -2.463585 & -0.341153 & 1 & 6.238164 & -3.871681 & -1.991630 \\
\hline 8.133781 & -2.334284 & 1.193904 & 6 & 5.296293 & -2.259313 & -3.116709 \\
\hline 7.626568 & -1.037617 & 0.816302 & 1 & 5.039478 & -3.065900 & -3.813760 \\
\hline 8.306385 & -0.185009 & -0.196937 & 1 & 6.097526 & -1.687501 & -3.591675 \\
\hline 9.697540 & 0.175478 & -0.055810 & 6 & 1.966632 & 0.124978 & 2.835560 \\
\hline
\end{tabular}




$\begin{array}{lrrr}6 & 2.629175 & -0.742799 & 3.729046 \\ 6 & 1.793415 & 1.470771 & 3.211096 \\ 6 & 3.117794 & -0.271214 & 4.943568 \\ 1 & 2.749981 & -1.791187 & 3.475327 \\ 6 & 2.274189 & 1.937982 & 4.432226 \\ 1 & 1.283437 & 2.148503 & 2.533045 \\ 6 & 2.942387 & 1.071734 & 5.301026 \\ 1 & 3.629572 & -0.950855 & 5.618893 \\ 1 & 2.136208 & 2.979533 & 4.699660 \\ 1 & 3.319820 & 1.436323 & 6.251865 \\ 7 & -1.138921 & 0.750963 & 4.092877 \\ 6 & -1.525852 & 2.136578 & 4.323209 \\ 1 & -2.488137 & 2.194715 & 4.847900 \\ 1 & -0.763761 & 2.622725 & 4.941368 \\ 1 & -1.614191 & 2.659892 & 3.373627 \\ 6 & -0.684936 & 0.031324 & 5.277596 \\ 1 & 0.384467 & 0.177893 & 5.454727 \\ 1 & -1.236784 & 0.406879 & 6.143380 \\ 1 & -0.899561 & -1.032987 & 5.199698 \\ 1 & -0.889474 & -5.384268 & 0.947858 \\ 1 & -0.281075 & -3.688718 & 2.646717 \\ 35 & -2.392121 & -5.164067 & -1.720141 \\ ------------------------------------------------------------\end{array}$

$[8-9]^{\star}-\mathbf{P 1 - C 2}$ endo si-si

Number of imaginary frequencies $=1$

The smallest frequencies are $=-180.705411 .613414 .5069 \mathrm{~cm}(-1)$ Electronic energy $=-7222.6307633$

Zero-point correction $=$

1.755896

(Hartree/Particle)

Thermal correction to Energy=

1.874226

1.875170

Thermal correction to Enthalpy=

1.595804

Thermal correction to Gibbs Free Energy=

Sum of electronic and zero-point Energies=

Sum of electronic and thermal Energies=

$-7220.874868$

$-7220.756538$

Sum of electronic and thermal Enthalpies=

$-7220.755593$

Sum of electronic and thermal Free Energies=

$-7221.034960$

$\begin{array}{llcc}\ldots 6 & 6.791719 & -3.245451 & 1.102479 \\ 15 & 2.484432 & -1.598787 & -1.497132 \\ 15 & 4.666575 & 0.928280 & 0.262182 \\ 6 & 5.290347 & -2.398989 & -0.098147 \\ 6 & 5.750121 & -1.469810 & 0.906060 \\ 6 & 5.506037 & -2.055669 & 2.187051 \\ 1 & 5.750597 & -1.610958 & 3.142034 \\ 6 & 4.940489 & -3.350403 & 1.985900 \\ 1 & 4.681185 & -4.056998 & 2.760891 \\ 6 & 4.803044 & -3.560542 & 0.581945 \\ 1 & 4.423843 & -4.452474 & 0.105142 \\ 6 & 8.789636 & -3.000150 & 0.583499 \\ 1 & 9.188678 & -2.137679 & 0.066236 \\ 6 & 8.655818 & -3.139565 & 1.998880 \\ 1 & 8.930624 & -2.400486 & 2.739457 \\ 6 & 8.054385 & -4.407723 & 2.263170 \\ 1 & 7.788564 & -4.793818 & 3.238424 \\ 6 & 7.815832 & -5.052520 & 1.011629 \\ 1 & 7.340908 & -6.014667 & 0.871700 \\ 6 & 8.270729 & -4.184695 & -0.025335 \\ 1 & 8.201858 & -4.380436 & -1.086654 \\ 6 & 5.306564 & -2.143071 & -1.565560 \\ 6 & 6.544419 & -2.098118 & -2.226996 \\ 1 & 7.438873 & -2.261026 & -1.640461 \\ 6 & 6.645586 & -1.847063 & -3.593949 \\ 1 & 7.621455 & -1.798540 & -4.066545 \\ 6 & 5.485671 & -1.654299 & -4.340015 \\ 1 & 5.539380 & -1.465037 & -5.407036 \\ 6 & 4.246271 & -1.673024 & -3.701859 \\ 1 & 3.359168 & -1.492471 & -4.296000 \\ 6 & 4.128289 & -1.887664 & -2.316912 \\ 6 & 6.235190 & -0.074812 & 0.616566 \\ 1 & 6.780854 & -0.098025 & -0.328430 \\ 6 & 7.154321 & 0.514226 & 1.689997 \\ 1 & 7.486221 & 1.518779 & 1.412539\end{array}$

$\begin{array}{lll}6.660960 & 0.581544 & 2.663212\end{array}$

$\begin{array}{llll}8.035592 & -0.124992 & 1.802005\end{array}$

$1.429974-1.094231-2.919876$

$\begin{array}{llll}1.370369 & 0.270482 & -3.241121\end{array}$

$\begin{array}{llll}1.883332 & 0.990985 & -2.614229\end{array}$

$\begin{array}{llll}0.651174 & 0.703490 & -4.356391\end{array}$

$\begin{array}{llll}0.620879 & 1.760643 & -4.596460\end{array}$

$\begin{array}{lll}-0.033862 & -0.222205 & -5.148910\end{array}$

$\begin{array}{llll}-0.603236 & 0.115705 & -6.010213\end{array}$

$\begin{array}{llll}0.009037 & -1.580771 & -4.827543\end{array}$

$\begin{array}{lll}-0.534920 & -2.305455 & -5.426030\end{array}$

$\begin{array}{llll}0.746396 & -2.016428 & -3.725098\end{array}$

$\begin{array}{lll}0.780101 & -3.074418 & -3.495705\end{array}$

$1.814738-3.244191 \quad-1.037764$

$\begin{array}{llll}2.241983 & -4.447969 & -1.616287\end{array}$

$3.013208-4.439423 \quad-2.381067$

$\begin{array}{llll}1.684882 & -5.660978 & -1.202963\end{array}$

$2.021814-6.589674 \quad-1.654597$

$\begin{array}{lll}0.702389 & -5.681473 & -0.207867\end{array}$

$\begin{array}{llll}0.278269 & -6.627165 & 0.117194\end{array}$

$\begin{array}{llll}0.266459 & -4.483825 & 0.366195\end{array}$

$\begin{array}{lll}-0.499281 & -4.490663 & 1.134341\end{array}$

$\begin{array}{llll}0.813774 & -3.270826 & -0.053422\end{array}$

$\begin{array}{llll}0.473581 & -2.340779 & 0.393744\end{array}$

$\begin{array}{llll}4.903041 & 1.770350 & -1.356921\end{array}$

$\begin{array}{llll}3.846741 & 2.596220 & -1.767914\end{array}$

$3.006957 \quad 2.750554 \quad-1.099172$

$3.840169 \quad 3.162210 \quad-3.042051$

$\begin{array}{llll}4.868314 & 2.899632 & -3.944059\end{array}$

$\begin{array}{llll}4.854002 & 3.323671 & -4.941613\end{array}$

$\begin{array}{llll}5.910369 & 2.061796 & -3.540512\end{array}$

$\begin{array}{llll}5.940246 & 1.505707 & -2.261131\end{array}$

$\begin{array}{llll}6.761835 & 0.852221 & -1.999268\end{array}$

$\begin{array}{lll}4.603176 & 2.287840 & 1.499487\end{array}$

$\begin{array}{lll}4.164374 & 1.920283 & 2.781384\end{array}$

$\begin{array}{llll}3.950982 & 0.880705 & 3.002756\end{array}$

$\begin{array}{lll}3.957215 & 2.887213 & 3.760541\end{array}$

$\begin{array}{lll}4.147068 & 4.240679 & 3.476659\end{array}$

$\begin{array}{llll}3.961543 & 4.996858 & 4.230953\end{array}$

$\begin{array}{lll}4.571292 & 4.604430 & 2.200834\end{array}$

$\begin{array}{llll}4.815606 & 3.640662 & 1.218338\end{array}$

$\begin{array}{llll}5.150248 & 3.956936 & 0.237084\end{array}$

$\begin{array}{lll}2.591822 & -0.137994 & 0.227299\end{array}$

$\begin{array}{lll}4.669204 & 6.063252 & 1.852757\end{array}$

$\begin{array}{lll}3.423968 & 2.493220 & 5.109691\end{array}$

$\begin{array}{llll}2.684477 & 4.050562 & -3.411273\end{array}$

$\begin{array}{llll}6.989147 & 1.742766 & -4.539614\end{array}$

$\begin{array}{llll}6.497643 & 1.045347 & -5.593267\end{array}$

$\begin{array}{llll}7.991433 & 1.008432 & -4.009205\end{array}$

$\begin{array}{llll}7.541089 & 2.868402 & -5.050435\end{array}$

$\begin{array}{llll}1.487844 & 3.447137 & -3.146618\end{array}$

$\begin{array}{llll}2.683451 & 5.200272 & -2.695051\end{array}$

$\begin{array}{llll}2.676580 & 4.383957 & -4.715144\end{array}$

$\begin{array}{llll}5.538138 & 6.295750 & 0.846770\end{array}$

$\begin{array}{lll}3.462906 & 6.547974 & 1.446161\end{array}$

$\begin{array}{lll}5.049723 & 6.815854 & 2.906934\end{array}$

$\begin{array}{llll}4.020125 & 3.179425 & 6.108833\end{array}$

$\begin{array}{lll}3.585233 & 1.177944 & 5.363854\end{array}$

$\begin{array}{lll}2.090441 & 2.752640 & 5.210600\end{array}$

$\begin{array}{lll}1.161085 & 2.353613 & 1.308430\end{array}$

$\begin{array}{lll}0.671555 & 1.613459 & 2.445856\end{array}$

$\begin{array}{llll}1.134287 & 0.333988 & 2.904920\end{array}$

$\begin{array}{lll}0.217091 & 2.197515 & 3.233295\end{array}$

$\begin{array}{llll}-3.042671 & 1.137995 & -1.161454\end{array}$

$\begin{array}{lll}-4.798710 & -0.358150 & -0.763792\end{array}$

$\begin{array}{lll}-5.554637 & -0.753470 & 0.671734\end{array}$

$\begin{array}{llll}-6.144025 & 0.146826 & -1.629879\end{array}$

$-4.321434-1.886650-1.243961$

$\begin{array}{lll}-6.225826 & 0.255868 & 1.349674\end{array}$

$\begin{array}{lll}-5.609648 & 0.753813 & 2.517443\end{array}$

$\begin{array}{lll}-4.686131 & 0.294541 & 2.845531\end{array}$

$\begin{array}{lll}-6.191270 & 1.791570 & 3.201494\end{array}$

$\begin{array}{lll}-5.731217 & 2.177589 & 4.106967\end{array}$

$\begin{array}{lll}-7.381381 & 2.397729 & 2.713091\end{array}$

$\begin{array}{lll}-7.941379 & 3.536255 & 3.354017\end{array}$ 
$\begin{array}{lll}-7.454829 & 3.918250 & 4.247917\end{array}$

$\begin{array}{llll}-9.063521 & 4.153235 & 2.848575\end{array}$

$\begin{array}{llll}-9.478945 & 5.026646 & 3.342721\end{array}$

$\begin{array}{llll}-9.667775 & 3.655955 & 1.669477\end{array}$

$\begin{array}{lll}-10.537177 & 4.159783 & 1.256912\end{array}$

$\begin{array}{lll}-9.161679 & 2.541799 & 1.035492\end{array}$

$\begin{array}{lll}-9.633491 & 2.181992 & 0.129014\end{array}$

$\begin{array}{lll}-8.017189 & 1.864079 & 1.541036\end{array}$

$\begin{array}{lll}-7.452778 & 0.707137 & 0.891092\end{array}$

$\begin{array}{llll}-8.129629 & 0.027017 & -0.247591\end{array}$

$\begin{array}{lll}-9.483869 & -0.458946 & -0.132071\end{array}$

$\begin{array}{lll}-10.194548 & -0.464090 & 1.101306\end{array}$

$\begin{array}{lll}-9.705582 & -0.103446 & 1.998175\end{array}$

$\begin{array}{lll}-11.487770 & -0.935082 & 1.178922\end{array}$

$\begin{array}{lll}-12.002300 & -0.933615 & 2.135647\end{array}$

$\begin{array}{lll}-12.148746 & -1.427139 & 0.028921\end{array}$

$\begin{array}{lll}-13.170150 & -1.788732 & 0.103344\end{array}$

$\begin{array}{llll}-11.484157 & -1.460575 & -1.176226\end{array}$

$\begin{array}{llll}-11.969971 & -1.854564 & -2.065233\end{array}$

$\begin{array}{lll}-10.145261 & -0.997190 & -1.286402\end{array}$

$\begin{array}{llll}-9.441585 & -1.079204 & -2.518501\end{array}$

$\begin{array}{llll}-9.952888 & -1.469182 & -3.394155\end{array}$

$\begin{array}{llll}-8.124199 & -0.707722 & -2.591689\end{array}$

$\begin{array}{llll}-7.552454 & -0.801917 & -3.508933\end{array}$

$\begin{array}{lll}-7.467840 & -0.193529 & -1.448512\end{array}$

$\begin{array}{llll}-2.914144 & -1.942319 & -1.737372\end{array}$

$\begin{array}{llll}-2.269233 & -2.230337 & -0.896288\end{array}$

$\begin{array}{llll}-2.751985 & -2.984528 & -2.834817\end{array}$

$\begin{array}{llll}-1.752970 & -3.958191 & -2.742303\end{array}$

$\begin{array}{llll}-1.121887 & -3.991081 & -1.860895\end{array}$

$\begin{array}{llll}-1.552801 & -4.879588 & -3.774454\end{array}$

$\begin{array}{llll}-0.768542 & -5.626028 & -3.681684\end{array}$

$\begin{array}{llll}-2.358632 & -4.839282 & -4.913435\end{array}$

$\begin{array}{llll}-2.209075 & -5.555633 & -5.716387\end{array}$

$\begin{array}{llll}-3.369634 & -3.875250 & -5.010190\end{array}$

$\begin{array}{lll}-4.007355 & -3.843404 & -5.889505\end{array}$

$\begin{array}{llll}-3.563055 & -2.955822 & -3.979650\end{array}$

$\begin{array}{llll}-4.354517 & -2.214431 & -4.052262\end{array}$

$\begin{array}{llll}-2.463519 & -0.569638 & -2.277113\end{array}$

$\begin{array}{llll}-2.865893 & -0.456094 & -3.289813\end{array}$

$\begin{array}{llll}-1.375981 & -0.603608 & -2.377659\end{array}$

$\begin{array}{llll}-4.945904 & -3.201616 & -0.897742\end{array}$

$-4.531382-3.881998-1.643900$

$\begin{array}{llll}-4.529869 & -3.779568 & 0.457858\end{array}$

$\begin{array}{lll}-3.435847 & -4.655839 & 0.510134\end{array}$

$\begin{array}{lll}-2.868274 & -4.852669 & -0.394716\end{array}$

$\begin{array}{lll}-3.091403 & -5.311297 & 1.696238\end{array}$

$\begin{array}{llll}-2.254242 & -6.003935 & 1.706978\end{array}$

$\begin{array}{llll}-3.839137 & -5.097816 & 2.855587\end{array}$

$\begin{array}{lll}-3.582536 & -5.614115 & 3.776595\end{array}$

$\begin{array}{llll}-4.920974 & -4.213330 & 2.820685\end{array}$

$\begin{array}{llll}-5.506693 & -4.035055 & 3.718366\end{array}$

$\begin{array}{llll}-5.263779 & -3.563501 & 1.634483\end{array}$

$\begin{array}{lll}-6.115668 & -2.895139 & 1.624109\end{array}$

$\begin{array}{llll}-6.458075 & -3.228182 & -1.115233\end{array}$

$\begin{array}{lll}-6.694749 & -2.932485 & -2.139225\end{array}$

$\begin{array}{llll}-7.001305 & -2.574518 & -0.433113\end{array}$

$\begin{array}{lll}-6.818577 & -4.249573 & -0.960772\end{array}$

$\begin{array}{llll}-0.936590 & 1.508277 & -0.892753\end{array}$

$\begin{array}{llll}-0.341104 & 1.140620 & -1.717205\end{array}$

$\begin{array}{llll}-0.712302 & 2.534698 & -0.635712\end{array}$

$\begin{array}{llll}-1.224348 & 0.592974 & 0.177770\end{array}$

$\begin{array}{lll}-0.990769 & -0.456613 & 0.025535\end{array}$

$\begin{array}{lll}-1.463234 & 1.032592 & 1.505218\end{array}$

$\begin{array}{lll}-1.673083 & 2.085005 & 1.644547\end{array}$

$\begin{array}{llll}-4.154368 & 1.947704 & -2.889895\end{array}$

$\begin{array}{llll}-4.476851 & 1.112694 & -3.506458\end{array}$

$\begin{array}{llll}-2.780991 & 2.306932 & -2.999207\end{array}$

$\begin{array}{llll}-2.174891 & 1.720204 & -3.688609\end{array}$

$\begin{array}{llll}-2.226844 & 3.699355 & -2.760619\end{array}$

$\begin{array}{llll}-2.427095 & 4.326736 & -3.642172\end{array}$

$\begin{array}{llll}-1.138307 & 3.619308 & -2.687114\end{array}$

$\begin{array}{llll}-2.777008 & 4.393339 & -1.493597\end{array}$

$\begin{array}{llll}-3.661375 & 4.986429 & -1.744357\end{array}$

$-2.029416 \quad 5.104482-1.127153$ $\begin{array}{llll}-3.131962 & 3.445106 & -0.354829\end{array}$

$\begin{array}{llll}-2.442697 & 3.442267 & 0.482677\end{array}$

$\begin{array}{lll}-4.386912 & 2.917093 & -0.144287\end{array}$

$\begin{array}{lll}-4.623619 & 2.541180 & 0.846833\end{array}$

$\begin{array}{llll}-5.568743 & 3.146664 & -1.066430\end{array}$

$\begin{array}{llll}-6.361889 & 2.461072 & -0.773424\end{array}$

$\begin{array}{llll}-5.956410 & 4.160911 & -0.891924\end{array}$

$\begin{array}{llll}-5.268024 & 2.950143 & -2.571931\end{array}$

$\begin{array}{llll}-5.015382 & 3.910134 & -3.035861\end{array}$

$\begin{array}{lll}-6.185847 & 2.608090 & -3.058018\end{array}$

$\begin{array}{lll}-1.895354 & 0.168460 & 2.600578\end{array}$

$\begin{array}{llll}-1.855033 & -1.235791 & 2.520608\end{array}$

$\begin{array}{lll}-2.341589 & 0.758977 & 3.799326\end{array}$

$\begin{array}{lll}-2.261744 & -2.017833 & 3.598926\end{array}$

$\begin{array}{lll}-1.508269 & -1.721050 & 1.614747\end{array}$

$\begin{array}{lll}-2.753915 & -0.024185 & 4.875434\end{array}$

$\begin{array}{lll}-2.366800 & 1.843141 & 3.875323\end{array}$

$\begin{array}{lll}-2.718673 & -1.418509 & 4.776632\end{array}$

$\begin{array}{lll}-2.232880 & -3.097714 & 3.514127\end{array}$

$\begin{array}{lll}-3.104505 & 0.451108 & 5.787031\end{array}$

$\begin{array}{lll}-3.042044 & -2.034496 & 5.610689\end{array}$

$\begin{array}{llll}0.838382 & -0.028262 & 4.247816\end{array}$

$\begin{array}{llll}1.112930 & -1.292974 & 4.726293\end{array}$

$\begin{array}{llll}0.381073 & 0.704976 & 4.899684\end{array}$

$\begin{array}{lll}1.718610 & -2.195356 & 3.849709\end{array}$

$\begin{array}{llll}2.052836 & -1.796686 & 2.568860\end{array}$

$\begin{array}{llll}2.547990 & -2.469809 & 1.883034\end{array}$

$\begin{array}{lll}1.772918 & -0.564697 & 2.096823\end{array}$

$\begin{array}{lll}1.674104 & 1.808810 & 0.287759\end{array}$

$\begin{array}{llll}0.972430 & 3.714271 & 1.291416\end{array}$

$\begin{array}{lll}1.217231 & 4.464024 & 0.064152\end{array}$

$\begin{array}{lll}0.498030 & 5.286852 & -0.001248\end{array}$

$\begin{array}{llll}2.226064 & 4.890761 & 0.043804\end{array}$

$\begin{array}{llll}1.088119 & 3.817320 & -0.798996\end{array}$

$\begin{array}{lll}0.697465 & 4.520821 & 2.477999\end{array}$

$\begin{array}{lll}-0.376133 & 4.710259 & 2.610176\end{array}$

$\begin{array}{lll}1.090541 & 4.047591 & 3.377469\end{array}$

$\begin{array}{lll}1.205795 & 5.482425 & 2.368033\end{array}$

$\begin{array}{lll}0.861907 & -1.570456 \quad 5.744138\end{array}$

$\begin{array}{llll}35 & 2.073399 & -4.034667 & 4.414989\end{array}$

[8-9] $]^{\dagger}-\mathbf{P 1 - C 2}$ endo re-si

Number of imaginary frequencies $=1$

The smallest frequencies are $=-73.874213 .137815 .6372 \mathrm{~cm}(-1)$ Electronic energy $=-7222.6203364$

Zero-point correction=

(Hartree/Particle)

Thermal correction to Energy=

Thermal correction to Enthalpy=

Thermal correction to Gibbs Free Energy=

Sum of electronic and zero-point Energies=

Sum of electronic and thermal Energies=

Sum of electronic and thermal Enthalpies=

Sum of electronic and thermal Free Energies=

1.756912

1.875122

1.876066

1.597000

$-7220.863425$

$-7220.745215$

$-7220.744271$

$-7221.023337$

Cartesian Coordinates

$77-3.917185-0.752632-1.985459$

$\begin{array}{lll}-5.573902 & -0.442159 & -0.362628\end{array}$

$\begin{array}{lll}-5.396538 & 0.083956 & 1.208338\end{array}$

$\begin{array}{llll}-6.674955 & 0.691943 & -0.904556\end{array}$

$\begin{array}{lll}-6.329944 & -1.906327 & -0.076414\end{array}$

$\begin{array}{lll}-4.845095 & 1.344519 & 1.396746\end{array}$

$\begin{array}{lll}-3.526753 & 1.386139 & 1.894599\end{array}$

$\begin{array}{lll}-3.053740 & 0.453513 & 2.170332\end{array}$

$\begin{array}{lll}-2.885181 & 2.592239 & 2.012691\end{array}$

$\begin{array}{lll}-1.871963 & 2.635206 & 2.396408\end{array}$

$\begin{array}{lll}-3.526447 & 3.789391 & 1.591125\end{array}$

$\begin{array}{lll}-2.833571 & 5.029538 & 1.592579\end{array}$

$\begin{array}{lll}-1.807855 & 5.049280 & 1.948992\end{array}$

$\begin{array}{lll}-3.434458 & 6.177246 & 1.126905\end{array}$

$\begin{array}{lll}-2.888768 & 7.116194 & 1.118634\end{array}$

$\begin{array}{lll}-4.761758 & 6.126764 & 0.638743\end{array}$

$\begin{array}{llll}-5.226501 & 7.028217 & 0.249667\end{array}$ 


\begin{tabular}{|c|c|c|c|c|c|c|}
\hline-5.470489 & 4.944192 & 0.646582 & 6 & 1.404153 & -2.424841 & 0.601752 \\
\hline-6.483194 & 4.922999 & 0.261367 & 1 & 0.407932 & -3.240558 & -1.064053 \\
\hline-4.886495 & 3.741199 & 1.132036 & 6 & -2.233271 & -1.987347 & -2.511728 \\
\hline-5.586442 & 2.480370 & 1.116625 & 1 & -1.400107 & -1.436930 & -2.932244 \\
\hline-7.039618 & 2.373537 & 0.806850 & 1 & -2.508864 & -2.860426 & -3.099419 \\
\hline-8.015458 & 3.128319 & 1.558095 & 6 & -2.290680 & -2.113243 & -1.074955 \\
\hline-7.672778 & 3.900080 & 2.704143 & 1 & -2.768470 & -2.989603 & -0.648156 \\
\hline-6.645382 & 3.917837 & 3.046402 & 6 & -1.468272 & -1.331014 & -0.228810 \\
\hline-8.627675 & 4.611754 & 3.398500 & 1 & -0.970134 & -0.475180 & -0.662271 \\
\hline-8.336841 & 5.185399 & 4.274001 & 6 & -1.418993 & -1.387063 & 1.217019 \\
\hline-9.981649 & 4.596498 & 2.989217 & 6 & -2.074598 & -2.378058 & 1.976783 \\
\hline-10.722089 & 5.167792 & 3.541419 & 6 & -0.644065 & -0.419696 & 1.895182 \\
\hline-10.354974 & 3.839566 & 1.901713 & 6 & -1.960169 & -2.392044 & 3.363540 \\
\hline-11.394307 & 3.798032 & 1.586172 & 1 & -2.678290 & -3.133987 & 1.485230 \\
\hline-9.397601 & 3.082099 & 1.174280 & 6 & -0.539731 & -0.432402 & 3.281573 \\
\hline-9.792541 & 2.258346 & 0.085699 & 1 & -0.116428 & 0.334692 & 1.319716 \\
\hline-10.837433 & 2.238708 & -0.210781 & 6 & -1.196470 & -1.420694 & 4.020297 \\
\hline-8.873515 & 1.472322 & -0.559007 & 1 & -2.472616 & -3.158155 & 3.933517 \\
\hline-9.156372 & 0.806289 & -1.367263 & 1 & 0.058252 & 0.317188 & 3.784744 \\
\hline-7.512572 & 1.511635 & -0.174436 & 1 & -1.113419 & -1.434541 & 5.103323 \\
\hline-5.716970 & -3.040971 & -0.820484 & 6 & 1.156392 & -3.621889 & 1.337543 \\
\hline-4.950583 & -3.499250 & -0.181582 & 6 & 1.708457 & -3.824599 & 2.583178 \\
\hline-6.737888 & -4.109460 & -1.177807 & 1 & 0.544160 & -4.394746 & 0.886475 \\
\hline-6.422056 & -5.462777 & -1.012980 & 6 & 2.519248 & -2.809691 & 3.104814 \\
\hline-5.466123 & -5.737754 & -0.573070 & 6 & 2.712511 & -1.647458 & 2.384145 \\
\hline-7.321668 & -6.458143 & -1.405956 & 1 & 3.328743 & -0.850678 & 2.777052 \\
\hline-7.061987 & -7.504460 & -1.270420 & 7 & 2.160749 & -1.437168 & 1.175152 \\
\hline-8.553477 & -6.107709 & -1.961549 & 8 & 1.395968 & -0.100014 & -1.385670 \\
\hline-9.257218 & -6.879158 & -2.260928 & 7 & 1.103936 & -1.649580 & -3.018313 \\
\hline-8.878816 & -4.756938 & -2.126862 & 6 & 1.160846 & -0.615596 & -4.043610 \\
\hline-9.837499 & -4.476980 & -2.555056 & 1 & 0.247079 & -0.636940 & -4.652481 \\
\hline-7.976007 & -3.766314 & -1.742295 & 1 & 2.016038 & -0.788024 & -4.706252 \\
\hline-8.229676 & -2.716328 & -1.862808 & 1 & 1.262204 & 0.360002 & -3.573702 \\
\hline-5.060987 & -2.540475 & -2.119933 & 6 & 0.935777 & -3.006861 & -3.525748 \\
\hline-5.857260 & -2.377467 & -2.856271 & 1 & -0.114618 & -3.232048 & -3.747738 \\
\hline-4.440248 & -3.356888 & -2.503414 & 1 & 1.316139 & -3.743383 & -2.820486 \\
\hline-7.170835 & -2.269447 & 1.109174 & 1 & 1.509077 & -3.107846 & -4.450300 \\
\hline-7.698707 & -3.167553 & 0.781997 & 1 & 1.525432 & -4.741745 & 3.132437 \\
\hline-6.362555 & -2.686742 & 2.339380 & 35 & 3.399699 & -3.022492 & 4.838745 \\
\hline-5.948258 & -4.022521 & 2.453958 & 26 & 5.074278 & 3.714224 & -3.249707 \\
\hline-6.184969 & -4.723417 & 1.658274 & 15 & 2.341285 & 2.486228 & 1.047457 \\
\hline-5.270317 & -4.473509 & 3.589724 & 15 & 4.776693 & -0.191232 & -0.489234 \\
\hline-4.967383 & -5.514753 & 3.660155 & 6 & 4.279343 & 3.215126 & -1.370158 \\
\hline-5.007006 & -3.592383 & 4.640196 & 6 & 4.813978 & 2.053119 & -2.038669 \\
\hline-4.493382 & -3.940641 & 5.532123 & 6 & 4.104197 & 1.901946 & -3.273389 \\
\hline-5.409612 & -2.257981 & 4.535897 & 1 & 4.274675 & 1.140494 & -4.021327 \\
\hline-5.206993 & -1.564119 & 5.347207 & 6 & 3.157378 & 2.963232 & -3.379760 \\
\hline-6.080078 & -1.810180 & 3.397071 & 1 & 2.499633 & 3.145071 & -4.219227 \\
\hline-6.390391 & -0.773944 & 3.338630 & 6 & 3.271890 & 3.777272 & -2.216249 \\
\hline-8.257194 & -1.236652 & 1.403631 & 1 & 2.708055 & 4.674423 & -2.005830 \\
\hline-8.858197 & -1.061950 & 0.508621 & 6 & 7.072125 & 4.274698 & -3.378685 \\
\hline-7.862770 & -0.279205 & 1.743858 & 1 & 7.852366 & 3.936134 & -2.710265 \\
\hline-8.913496 & -1.626249 & 2.187528 & 6 & 6.658850 & 3.623887 & -4.581235 \\
\hline-5.232656 & 0.209663 & -3.485237 & 1 & 7.063740 & 2.701300 & -4.975357 \\
\hline-6.213188 & -0.255211 & -3.421791 & 6 & 5.584225 & 4.378050 & -5.143382 \\
\hline-4.230921 & -0.549036 & -4.152777 & 1 & 5.029963 & 4.124600 & -6.037606 \\
\hline-4.535177 & -1.518745 & -4.544672 & 6 & 5.332038 & 5.493657 & -4.288065 \\
\hline-3.029390 & 0.035762 & -4.871978 & 1 & 4.555401 & 6.235186 & -4.421940 \\
\hline-3.337878 & 0.402237 & -5.862326 & 6 & 6.251437 & 5.431026 & -3.198893 \\
\hline-2.322134 & -0.780064 & -5.052805 & 1 & 6.292848 & 6.119724 & -2.366149 \\
\hline-2.318622 & 1.158413 & -4.088918 & 6 & 4.693689 & 3.698040 & -0.022046 \\
\hline-2.746727 & 2.130620 & -4.351120 & 6 & 5.936807 & 4.330271 & 0.127547 \\
\hline-1.267886 & 1.196622 & -4.391817 & 1 & 6.560505 & 4.443199 & -0.749167 \\
\hline-2.375687 & 0.986268 & -2.576508 & 6 & 6.377391 & 4.809970 & 1.361214 \\
\hline-1.443305 & 0.689820 & -2.107918 & 1 & 7.348553 & 5.290109 & 1.439636 \\
\hline-3.332608 & 1.577630 & -1.773928 & 6 & 5.558120 & 4.679676 & 2.478877 \\
\hline-3.092709 & 1.730007 & -0.726409 & 1 & 5.872862 & 5.062078 & 3.445274 \\
\hline-4.485256 & 2.401397 & -2.311895 & 6 & 4.326000 & 4.035481 & 2.359099 \\
\hline-5.169333 & 2.607509 & -1.490760 & 1 & 3.713356 & 3.924028 & 3.244963 \\
\hline-4.090346 & 3.376765 & -2.632696 & 6 & 3.886720 & 3.513239 & 1.132199 \\
\hline-5.268281 & 1.739841 & -3.470021 & 6 & 5.798510 & 1.112171 & -1.397601 \\
\hline-4.900126 & 2.104760 & -4.435238 & 1 & 6.232374 & 1.642156 & -0.542331 \\
\hline-6.312371 & 2.056692 & -3.401657 & 6 & 6.962047 & 0.633452 & -2.271989 \\
\hline 1.152120 & -1.312638 & -1.688559 & 1 & 7.573775 & -0.103405 & -1.741731 \\
\hline 0.848882 & -2.322046 & -0.706223 & 1 & 6.633846 & 0.189502 & -3.212571 \\
\hline
\end{tabular}


$\begin{array}{llll}7.594570 & 1.491173 & -2.519237\end{array}$

$\begin{array}{lll}1.763176 & 2.463799 & 2.799330\end{array}$

$\begin{array}{lll}2.485427 & 1.682687 & 3.717925\end{array}$

$\begin{array}{lll}3.363013 & 1.139397 & 3.390566\end{array}$

$\begin{array}{lll}2.107064 & 1.619275 & 5.057617\end{array}$

$\begin{array}{llll}2.696339 & 1.026860 & 5.750359\end{array}$

$\begin{array}{llll}0.975973 & 2.315430 & 5.496458\end{array}$

$\begin{array}{lll}0.669601 & 2.258039 & 6.536779\end{array}$

$\begin{array}{lll}0.242754 & 3.084159 & 4.590239\end{array}$

$\begin{array}{lll}-0.638203 & 3.627017 & 4.920857\end{array}$

$\begin{array}{llll}0.640576 & 3.169010 & 3.252440\end{array}$

$\begin{array}{llll}0.074839 & 3.788510 & 2.566465\end{array}$

$\begin{array}{lll}1.144650 & 3.571514 & 0.176976\end{array}$

$\begin{array}{lll}1.127983 & 4.960243 & 0.389131\end{array}$

$\begin{array}{lll}1.814251 & 5.409100 & 1.101303\end{array}$

$\begin{array}{llll}0.243874 & 5.768691 & -0.324879\end{array}$

$\begin{array}{llll}0.234067 & 6.841448 & -0.154205\end{array}$

$\begin{array}{llll}-0.625550 & 5.197299 & -1.259902\end{array}$

$\begin{array}{llll}-1.313554 & 5.826578 & -1.816898\end{array}$

$\begin{array}{llll}-0.611114 & 3.818015 & -1.474891\end{array}$

$\begin{array}{llll}-1.280102 & 3.376441 & -2.204843\end{array}$

$\begin{array}{llll}0.268202 & 3.001797 & -0.757744\end{array}$

$\begin{array}{llll}0.305611 & 1.932216 & -0.948105\end{array}$

$\begin{array}{llll}5.861286 & -0.637407 & 0.930688\end{array}$

$\begin{array}{lll}5.729328 & 0.177259 & 2.064860\end{array}$

$\begin{array}{lll}5.055526 & 1.027493 & 2.038603\end{array}$

$\begin{array}{llll}6.437723 & -0.108744 & 3.229800\end{array}$

$\begin{array}{llll}7.296897 & -1.206309 & 3.286503\end{array}$

$\begin{array}{llll}7.837168 & -1.438120 & 4.196804\end{array}$

$\begin{array}{llll}7.448135 & -1.999989 & 2.150401\end{array}$

$\begin{array}{llll}6.740973 & -1.724040 & 0.976928\end{array}$

$\begin{array}{llll}6.873413 & -2.367916 & 0.114713\end{array}$

$\begin{array}{llll}4.669619 & -1.724526 & -1.492276\end{array}$

$\begin{array}{llll}4.344389 & -2.930929 & -0.847881\end{array}$

$\begin{array}{llll}4.279443 & -2.975995 & 0.232124\end{array}$

$\begin{array}{llll}4.074510 & -4.076616 & -1.591904\end{array}$

$\begin{array}{llll}4.120774 & -4.058198 & -2.986950\end{array}$

$\begin{array}{llll}3.900489 & -4.951707 & -3.559879\end{array}$

$\begin{array}{llll}4.416456 & -2.857163 & -3.624866\end{array}$

$\begin{array}{llll}4.677785 & -1.700243 & -2.890956\end{array}$

$\begin{array}{lll}4.867510 & -0.784199 & -3.427371\end{array}$

$\begin{array}{llll}2.651684 & 0.413008 & 0.220353\end{array}$

$\begin{array}{llll}4.476063 & -2.800238 & -5.125847\end{array}$

$\begin{array}{llll}3.596995 & -5.325520 & -0.905090\end{array}$

$\begin{array}{lll}6.166719 & 0.703341 & 4.466096\end{array}$

$\begin{array}{llll}8.316279 & -3.227942 & 2.206914\end{array}$

$\begin{array}{lll}9.283921 & -3.127155 & 3.142575\end{array}$

$8.919124-3.473609 \quad 1.021771$

$\begin{array}{lll}7.586538 & -4.330017 & 2.509172\end{array}$

$\begin{array}{llll}5.076715 & 0.225747 & 5.126815\end{array}$

$\begin{array}{llll}7.194973 & 0.678059 & 5.337258\end{array}$

$\begin{array}{llll}5.901531 & 1.997203 & 4.177706\end{array}$

$4.198191-1.556990-5.595301$

$\begin{array}{llll}5.699213 & -3.129225 & -5.598776\end{array}$

$\begin{array}{llll}3.592071 & -3.649881 & -5.699807\end{array}$

$2.245177-5.449703-1.013160$

$\begin{array}{llll}3.887742 & -5.339858 & 0.412170\end{array}$

$4.126840-6.440713-1.454028$

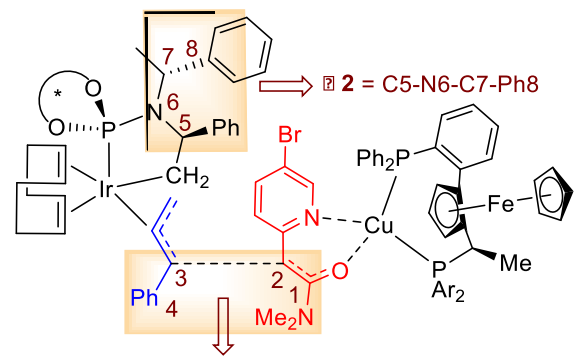

[] $1=\mathrm{C} 1-\mathrm{C} 2-\mathrm{C} 3-\mathrm{Ph} 4$

C1-P1 re-re_1

Number of imaginary frequencies $=1$
The smallest frequencies are $=-208.32737 .681212 .1104 \mathrm{~cm}(-1)$ Electronic energy $=-7222.6357129$

Zero-point correction $=$

1.756033

(Hartree/Particle)

Thermal correction to Energy= $\quad 1.874272$

Thermal correction to Enthalpy= $\quad 1.875216$

Thermal correction to Gibbs Free Energy $=\quad 1.594952$

Sum of electronic and zero-point Energies $=\quad-7220.879680$

Sum of electronic and thermal Energies $=\quad-7220.761441$

Sum of electronic and thermal Enthalpies $=\quad-7220.760497$

Sum of electronic and thermal Free Energies $=\quad-7221.040761$

Cartesian Coordinates

$\begin{array}{lll}-3.649716 & -3.884826 & 3.789579\end{array}$

$\begin{array}{llll}-1.482765 & -2.060473 & -0.606649\end{array}$

$\begin{array}{lll}-4.596284 & -0.382582 & 0.700313\end{array}$

$\begin{array}{lll}-3.112134 & -3.223545 & 1.876075\end{array}$

$\begin{array}{lll}-3.859689 & -2.214117 & 2.590619\end{array}$

$\begin{array}{lll}-3.132328 & -1.889180 & 3.776957\end{array}$

$\begin{array}{lll}-3.437348 & -1.177099 & 4.531464\end{array}$

$\begin{array}{lll}-1.961947 & -2.706301 & 3.822712\end{array}$

$\begin{array}{lll}-1.231703 & -2.724702 & 4.619890\end{array}$

$\begin{array}{llll}-1.951245 & -3.528639 & 2.658434\end{array}$

$\begin{array}{lll}-1.207938 & -4.272036 & 2.413123\end{array}$

$\begin{array}{llll}-5.483411 & -4.813437 & 4.086595\end{array}$

$\begin{array}{lll}-6.382623 & -4.591045 & 3.527574\end{array}$

$\begin{array}{lll}-5.058256 & -4.161469 & 5.284436\end{array}$

$\begin{array}{llll}-5.573746 & -3.353758 & 5.786635\end{array}$

$\begin{array}{lll}-3.807116 & -4.728154 & 5.675508\end{array}$

$\begin{array}{lll}-3.208154 & -4.421987 & 6.523122\end{array}$

$\begin{array}{lll}-3.459293 & -5.732043 & 4.721147\end{array}$

$\begin{array}{llll}-2.553133 & -6.323420 & 4.720771\end{array}$

$\begin{array}{lll}-4.494627 & -5.786372 & 3.741221\end{array}$

$\begin{array}{llll}-4.506715 & -6.429105 & 2.871632\end{array}$

$\begin{array}{lll}-3.487137 & -3.751378 & 0.531344\end{array}$

$\begin{array}{llll}-4.599695 & -4.598156 & 0.411167\end{array}$

$\begin{array}{llll}-5.125622 & -4.881431 & 1.313965\end{array}$

$\begin{array}{llll}-5.050761 & -5.054906 & -0.826891\end{array}$

$\begin{array}{lll}-5.933937 & -5.683152 & -0.884366\end{array}$

$\begin{array}{llll}-4.375036 & -4.677926 & -1.984722\end{array}$

$\begin{array}{llll}-4.717250 & -5.016956 & -2.957451\end{array}$

$\begin{array}{llll}-3.264530 & -3.838481 & -1.892812\end{array}$

$\begin{array}{llll}-2.757172 & -3.542564 & -2.803861\end{array}$

$\begin{array}{llll}-2.812807 & -3.353056 & -0.655229\end{array}$

$\begin{array}{llll}-5.122014 & -1.570116 & 2.084573\end{array}$

$\begin{array}{lll}-5.704578 & -2.348662 & 1.589603\end{array}$

$\begin{array}{lll}-5.988507 & -0.939632 & 3.177768\end{array}$

$\begin{array}{lll}-6.911916 & -0.531037 & 2.758062\end{array}$

$\begin{array}{lll}-5.470746 & -0.130063 & 3.700070\end{array}$

$\begin{array}{lll}-6.248948 & -1.703773 & 3.916410\end{array}$

$\begin{array}{lll}-0.961158 & -1.899025 & -2.367365\end{array}$

$\begin{array}{lll}-1.568255 & -0.891421 & -3.135663\end{array}$

$\begin{array}{lll}-2.273830 & -0.210875 & -2.672079\end{array}$

$\begin{array}{lll}-1.250959 & -0.749187 & -4.488110\end{array}$

$\begin{array}{llll}-1.734225 & 0.025954 & -5.073911\end{array}$

$\begin{array}{llll}-0.308457 & -1.592940 & -5.080574\end{array}$

$\begin{array}{llll}-0.052871 & -1.472457 & -6.129446\end{array}$

$\begin{array}{llll}0.308749 & -2.589376 & -4.319491\end{array}$

$\begin{array}{llll}1.048841 & -3.243476 & -4.771453\end{array}$

$\begin{array}{lll}-0.022155 & -2.748020 & -2.973068\end{array}$

$\begin{array}{lll}0.451827 & -3.533515 & -2.396960\end{array}$

$\begin{array}{lll}-0.069806 & -2.901280 & 0.216256\end{array}$

$\begin{array}{llll}0.171732 & -4.277998 & 0.072593\end{array}$

$\begin{array}{llll}-0.482513 & -4.883233 & -0.547922\end{array}$

$\begin{array}{llll}1.235238 & -4.878864 & 0.747353\end{array}$

$\begin{array}{llll}1.416308 & -5.943185 & 0.628054\end{array}$

$\begin{array}{lll}2.062597 & -4.115718 & 1.577885\end{array}$

$\begin{array}{lll}2.887707 & -4.586882 & 2.103843\end{array}$

$\begin{array}{lll}1.827556 & -2.747714 & 1.727479\end{array}$

$\begin{array}{llll}2.466874 & -2.149698 & 2.368136\end{array}$

$\begin{array}{llll}0.766715 & -2.144034 & 1.048874\end{array}$

$\begin{array}{lll}0.578766 & -1.084999 & 1.174393\end{array}$

$\begin{array}{llll}-5.537348 & -0.879096 & -0.801354\end{array}$

$\begin{array}{llll}-5.177935 & -0.222388 & -1.987088\end{array}$ 
$\begin{array}{llll}-4.420313 & 0.554059 & -1.953974\end{array}$

$\begin{array}{llll}-5.774319 & -0.574027 & -3.197618\end{array}$

$\begin{array}{llll}-6.702892 & -1.611080 & -3.261587\end{array}$

$\begin{array}{llll}-7.141875 & -1.904676 & -4.208664\end{array}$

$\begin{array}{llll}-7.043764 & -2.275524 & -2.082898\end{array}$

$\begin{array}{lll}-6.488838 & -1.903575 & -0.858536\end{array}$

$\begin{array}{llll}-6.793552 & -2.437080 & 0.031534\end{array}$

$\begin{array}{lll}-5.276353 & 1.277269 & 1.108922\end{array}$

$\begin{array}{lll}-4.571776 & 2.008548 & 2.078421\end{array}$

$\begin{array}{lll}-3.730979 & 1.556074 & 2.589661\end{array}$

$\begin{array}{lll}-4.915684 & 3.327360 & 2.356145\end{array}$

$\begin{array}{llll}-5.950197 & 3.956596 & 1.660289\end{array}$

$\begin{array}{lll}-6.198181 & 4.992913 & 1.858779\end{array}$

$\begin{array}{llll}-6.643454 & 3.232474 & 0.694077\end{array}$

$\begin{array}{lll}-6.322368 & 1.899050 & 0.421581\end{array}$

$\begin{array}{llll}-6.882393 & 1.365220 & -0.337699\end{array}$

$\begin{array}{lll}-2.316360 & -0.137261 & 0.241172\end{array}$

$\begin{array}{llll}-7.675411 & 3.918225 & -0.156376\end{array}$

$\begin{array}{lll}-4.091183 & 4.131173 & 3.322459\end{array}$

$\begin{array}{llll}-5.421418 & 0.217830 & -4.425393\end{array}$

$\begin{array}{llll}-7.987452 & -3.443507 & -2.168686\end{array}$

$\begin{array}{llll}-7.467307 & -4.439418 & -2.929289\end{array}$

$\begin{array}{llll}-8.266363 & -3.975950 & -0.958330\end{array}$

$\begin{array}{llll}-9.164610 & -3.099930 & -2.740184\end{array}$

$\begin{array}{llll}-4.089643 & 0.480331 & -4.489269\end{array}$

$\begin{array}{llll}-6.042960 & 1.423970 & -4.434930\end{array}$

$\begin{array}{llll}-5.761250 & -0.413537 & -5.566359\end{array}$

$\begin{array}{llll}-7.120605 & 4.382847 & -1.311315\end{array}$

$\begin{array}{lll}-8.232090 & 4.980135 & 0.461069\end{array}$

$\begin{array}{lll}-8.672693 & 3.083733 & -0.521016\end{array}$

$\begin{array}{llll}-4.853926 & 4.946647 & 4.082379\end{array}$

$\begin{array}{llll}-3.367893 & 3.351529 & 4.155072\end{array}$

$\begin{array}{llll}-3.205775 & 4.931987 & 2.668783\end{array}$

$\begin{array}{llll}-2.675215 & 2.730787 & -0.735925\end{array}$

$\begin{array}{llll}-1.737037 & 3.213478 & 0.249897\end{array}$

$\begin{array}{lll}-1.231080 & 2.503283 & 1.384655\end{array}$

$\begin{array}{llll}-1.667853 & 4.285573 & 0.360771\end{array}$

$\begin{array}{llll}2.926648 & 1.743362 & -1.610752\end{array}$

$\begin{array}{llll}4.401385 & 0.391576 & -0.503008\end{array}$

$\begin{array}{llll}4.192610 & -1.208945 & -0.132411\end{array}$

$\begin{array}{llll}5.835260 & 0.310493 & -1.354219\end{array}$

$\begin{array}{lll}4.651121 & 1.007078 & 1.040555\end{array}$

$\begin{array}{llll}4.414873 & -2.210427 & -1.075077\end{array}$

$\begin{array}{llll}3.278069 & -2.921201 & -1.514091\end{array}$

$\begin{array}{llll}2.315095 & -2.668185 & -1.091628\end{array}$

$3.418599 \quad-3.912610 \quad-2.451511$

$\begin{array}{llll}2.552977 & -4.475887 & -2.787270\end{array}$

$\begin{array}{llll}4.689634 & -4.199003 & -3.016088\end{array}$

$\begin{array}{llll}4.831283 & -5.167645 & -4.046551\end{array}$

$\begin{array}{lll}3.946473 & -5.708320 & -4.372813\end{array}$

$\begin{array}{llll}6.054735 & -5.405707 & -4.630276\end{array}$

$\begin{array}{llll}6.151548 & -6.143655 & -5.421292\end{array}$

$\begin{array}{llll}7.188269 & -4.670748 & -4.208376\end{array}$

$\begin{array}{llll}8.147776 & -4.839667 & -4.688735\end{array}$

$\begin{array}{llll}7.087693 & -3.737724 & -3.198966\end{array}$

$\begin{array}{llll}7.966512 & -3.179268 & -2.900238\end{array}$

$\begin{array}{llll}5.844546 & -3.482251 & -2.554211\end{array}$

$5.701046-2.510362-1.498335$

$\begin{array}{llll}6.878004 & -1.809743 & -0.915507\end{array}$

$\begin{array}{llll}7.996553 & -2.520328 & -0.351515\end{array}$

$\begin{array}{llll}7.982376 & -3.926995 & -0.135171\end{array}$

$\begin{array}{llll}7.097945 & -4.494774 & -0.399504\end{array}$

$\begin{array}{llll}9.065223 & -4.571462 & 0.422412\end{array}$

$\begin{array}{llll}9.025188 & -5.644319 & 0.587691\end{array}$

$\begin{array}{llll}10.226077 & -3.849603 & 0.790211\end{array}$

$\begin{array}{llll}11.073625 & -4.372366 & 1.223594\end{array}$

$\begin{array}{llll}10.268683 & -2.485401 & 0.610261\end{array}$

$\begin{array}{llll}11.146396 & -1.914966 & 0.902762\end{array}$

$\begin{array}{llll}9.162510 & -1.786847 & 0.053835\end{array}$

$\begin{array}{llll}9.179979 & -0.372208 & -0.073651\end{array}$

$\begin{array}{lll}10.070734 & 0.173584 & 0.223220\end{array}$

$\begin{array}{llll}8.070097 & 0.301405 & -0.517769\end{array}$

$8.048371 \quad 1.383006 \quad-0.587409$

$\begin{array}{llll}6.922773 & -0.424175 & -0.903688\end{array}$

$\begin{array}{lll}4.218312 & 2.428225 & 1.136931\end{array}$ $\begin{array}{lll}3.239927 & 2.455688 & 1.629039\end{array}$

$\begin{array}{lll}5.167578 & 3.287810 & 1.949581\end{array}$

$\begin{array}{lll}4.667321 & 4.109723 & 2.965206\end{array}$

$\begin{array}{llll}3.602308 & 4.094472 & 3.188624\end{array}$

$\begin{array}{lll}5.524378 & 4.932758 & 3.702142\end{array}$

$\begin{array}{lll}5.123959 & 5.560053 & 4.493945\end{array}$

$\begin{array}{lll}6.893146 & 4.939795 & 3.426319\end{array}$

$\begin{array}{lll}7.563071 & 5.571277 & 4.003104\end{array}$

$\begin{array}{lll}7.398311 & 4.126765 & 2.405412\end{array}$

$\begin{array}{lll}8.463483 & 4.121561 & 2.191008\end{array}$

$\begin{array}{llll}6.540329 & 3.310545 & 1.671766\end{array}$

$\begin{array}{llll}6.934202 & 2.655311 & 0.902334\end{array}$

$\begin{array}{llll}4.057027 & 3.005749 & -0.280041\end{array}$

$\begin{array}{llll}5.049881 & 3.193230 & -0.707041\end{array}$

$\begin{array}{llll}3.563162 & 3.978043 & -0.201398\end{array}$

$\begin{array}{lll}4.713377 & 0.172690 & 2.287413\end{array}$

$\begin{array}{llll}4.660512 & -0.857871 & 1.939430\end{array}$

$\begin{array}{lll}6.034956 & 0.291195 & 3.046524\end{array}$

$\begin{array}{llll}7.103204 & -0.530220 & 2.665106\end{array}$

$\begin{array}{lll}6.973851 & -1.218528 & 1.836596\end{array}$

$\begin{array}{lll}8.328396 & -0.479325 & 3.332087\end{array}$

$\begin{array}{llll}9.138933 & -1.128081 & 3.012700\end{array}$

$\begin{array}{llll}8.503077 & 0.398177 & 4.404094\end{array}$

$\begin{array}{llll}9.455023 & 0.443764 & 4.926160\end{array}$

$\begin{array}{lll}7.440205 & 1.211259 & 4.806175\end{array}$

$\begin{array}{lll}7.562432 & 1.897305 & 5.639863\end{array}$

$\begin{array}{lll}6.216129 & 1.151949 & 4.138107\end{array}$

$\begin{array}{llll}5.410868 & 1.800470 & 4.462475\end{array}$

$\begin{array}{lll}3.488327 & 0.400901 & 3.180660\end{array}$

$\begin{array}{llll}2.563925 & 0.226610 & 2.625494\end{array}$

$\begin{array}{lll}3.443898 & 1.409191 & 3.597734\end{array}$

$\begin{array}{llll}3.518097 & -0.300227 & 4.018737\end{array}$

$\begin{array}{llll}1.405672 & 1.187666 & -0.177037\end{array}$

$\begin{array}{llll}0.696157 & 0.472618 & -0.591579\end{array}$

$\begin{array}{llll}1.766019 & 0.898060 & 0.804943\end{array}$

$\begin{array}{llll}1.159219 & 2.586241 & -0.406895\end{array}$

$\begin{array}{lll}1.585692 & 3.299293 & 0.289329\end{array}$

$0.130179 \quad 3.060528-1.253751$

$\begin{array}{llll}-0.411077 & 2.313733 & -1.821374\end{array}$

$\begin{array}{llll}4.407298 & 2.161353 & -3.248681\end{array}$

$\begin{array}{llll}5.354987 & 2.369794 & -2.761105\end{array}$

$\begin{array}{llll}3.447780 & 3.195949 & -3.241972\end{array}$

$3.734034 \quad 4.137894 \quad-2.774567$

$\begin{array}{llll}2.315131 & 3.319958 & -4.247936\end{array}$

$\begin{array}{llll}2.708127 & 3.707765 & -5.200463\end{array}$

$\begin{array}{llll}1.622697 & 4.075345 & -3.870576\end{array}$

$\begin{array}{llll}1.535426 & 2.008026 & -4.498139\end{array}$

$\begin{array}{llll}1.938397 & 1.483543 & -5.370893\end{array}$

$\begin{array}{llll}0.499332 & 2.258052 & -4.749114\end{array}$

$\begin{array}{llll}1.519613 & 1.046121 & -3.312257\end{array}$

$\begin{array}{llll}0.549847 & 0.871725 & -2.863041\end{array}$

$\begin{array}{llll}2.464825 & 0.043896 & -3.103966\end{array}$

$\begin{array}{llll}2.139520 & -0.812964 & -2.522927\end{array}$

$\begin{array}{llll}3.682117 & -0.201598 & -3.974063\end{array}$

$\begin{array}{llll}4.342032 & -0.890030 & -3.443156\end{array}$

$\begin{array}{llll}3.365617 & -0.720196 & -4.891027\end{array}$

$\begin{array}{llll}4.469707 & 1.078445 & -4.326640\end{array}$

$\begin{array}{llll}4.109717 & 1.496447 & -5.272258\end{array}$

$\begin{array}{llll}5.517751 & 0.810495 & -4.491120\end{array}$

$\begin{array}{llll}0.012186 & 4.429541 & -1.747950\end{array}$

$\begin{array}{llll}0.717887 & 5.510814 & -1.184201\end{array}$

$\begin{array}{llll}-0.843939 & 4.679135 & -2.838789\end{array}$

$\begin{array}{llll}0.561432 & 6.797992 & -1.691694\end{array}$

$\begin{array}{llll}1.389893 & 5.341415 & -0.348890\end{array}$

$\begin{array}{llll}-0.997596 & 5.968025 & -3.345872\end{array}$

$\begin{array}{llll}-1.384861 & 3.850750 & -3.286144\end{array}$

$\begin{array}{llll}-0.298113 & 7.033750 & -2.771400\end{array}$

$\begin{array}{llll}1.111510 & 7.622212 & -1.246523\end{array}$

$\begin{array}{llll}-1.660075 & 6.141337 & -4.189109\end{array}$

$\begin{array}{llll}-0.416191 & 8.039463 & -3.164018\end{array}$

$\begin{array}{llll}-2.706355 & 1.521089 & -1.130457\end{array}$

$\begin{array}{lll}-0.505546 & 3.244922 & 2.360275\end{array}$

$\begin{array}{lll}0.003430 & 2.639266 & 3.492163\end{array}$

$\begin{array}{lll}-0.378535 & 4.312662 & 2.217493\end{array}$

$\begin{array}{lll}-0.216923 & 1.266509 & 3.645712\end{array}$ 


$\begin{array}{lccc}6 & -0.895930 & 0.566451 & 2.665950 \\ 1 & -1.066549 & -0.498312 & 2.757059 \\ 7 & -1.378022 & 1.155286 & 1.554492 \\ 6 & -4.213165 & 3.261992 & -2.560918 \\ 1 & -3.690467 & 2.459764 & -3.077638 \\ 1 & -5.232315 & 2.932475 & -2.332747 \\ 1 & -4.274070 & 4.130279 & -3.223748 \\ 6 & -3.750787 & 4.989251 & -0.857768 \\ 1 & -3.581521 & 5.053029 & 0.215722 \\ 1 & -3.127736 & 5.733708 & -1.368103 \\ 1 & -4.802120 & 5.223880 & -1.040952 \\ 7 & -3.487412 & 3.640072 & -1.353026 \\ 1 & 0.540399 & 3.214325 & 4.239019 \\ 35 & 0.402353 & 0.321820 & 5.239910 \\ --------------------------------------------------------------------\end{array}$

\section{C1-P1 re-re_2}

Number of imaginary frequencies $=1$

The smallest frequencies are $=-204.74516 .092612 .6994 \mathrm{~cm}(-1)$ Electronic energy $=-7222.6357296$

Zero-point correction $=$

1.756081

(Hartree/Particle)

Thermal correction to Energy= $\quad 1.874315$

Thermal correction to Enthalpy= $\quad 1.875259$

Thermal correction to Gibbs Free Energy= 1.595318

Sum of electronic and zero-point Energies $=\quad-7220.879649$

Sum of electronic and thermal Energies $=\quad-7220.761415$

Sum of electronic and thermal Enthalpies $=\quad-7220.760470$

Sum of electronic and thermal Free Energies $=\quad-7221.040412$

Cartesian Coordinates

$\begin{array}{llll}26 & -3.590371 & -3.885361 & 3.799579 \\ 15 & -1.457814 & -2.062168 & -0.611400 \\ 15 & -4.570627 & -0.396323 & 0.705988 \\ 6 & -3.066686 & -3.227495 & 1.880959 \\ 6 & -3.810546 & -2.217526 & 2.598450 \\ 6 & -3.075182 & -1.888830 & 3.778864 \\ 1 & -3.375648 & -1.175409 & 4.533926 \\ 6 & -1.903685 & -2.704660 & 3.818314 \\ 1 & -1.168941 & -2.722338 & 4.611395 \\ 6 & -1.900223 & -3.529770 & 2.655968 \\ 1 & -1.157862 & -4.272866 & 2.406808 \\ 6 & -5.420004 & -4.818231 & 4.109312 \\ 1 & -6.322729 & -4.600426 & 3.554122 \\ 6 & -4.990545 & -4.160879 & 5.302668 \\ 1 & -5.505826 & -3.353052 & 5.804888 \\ 6 & -3.735809 & -4.722661 & 5.689290 \\ 1 & -3.133341 & -4.411813 & 6.532710 \\ 6 & -3.390099 & -5.728941 & 4.736716 \\ 1 & -2.482380 & -6.317918 & 4.733891 \\ 6 & -4.430265 & -5.789606 & 3.762306 \\ 1 & -4.444977 & -6.435651 & 2.895240 \\ 6 & -3.449443 & -3.758148 & 0.539536 \\ 6 & -4.557405 & -4.611891 & 0.428364 \\ 1 & -5.074981 & -4.897454 & 1.335234 \\ 6 & -5.014197 & -5.072935 & -0.806006 \\ 1 & -5.893314 & -5.707424 & -0.856441 \\ 6 & -4.350186 & -4.691561 & -1.969181 \\ 1 & -4.697983 & -5.033183 & -2.939011 \\ 6 & -3.244734 & -3.844409 & -1.886301 \\ 1 & -2.746368 & -3.544967 & -2.801222 \\ 6 & -2.786417 & -3.356490 & -0.652272 \\ 6 & -5.078983 & -1.578623 & 2.100900 \\ 1 & -5.664463 & -2.361024 & 1.615569 \\ 6 & -5.936610 & -0.944784 & 3.199130 \\ 1 & -6.866715 & -0.543950 & 2.786847 \\ 1 & -5.417818 & -0.128842 & 3.710329 \\ 1 & -6.185162 & -1.704962 & 3.945875 \\ 6 & -0.945050 & -1.898348 & -2.375136 \\ 6 & -1.549628 & -0.883712 & -3.136070 \\ 1 & -2.248950 & -0.201337 & -2.666093 \\ 6 & -1.236642 & -0.735743 & -4.488971 \\ 6 & -1.717573 & 0.045530 & -5.068557 \\ & -0.301070 & -1.581057 & -5.089826\end{array}$

$-0.048811-1.456218-6.138995$

$0.313057-2.585256-4.336505$

$1.047612-3.241124-4.794851$

$-0.013475-2.749411-2.989689$

$0.458283 \quad-3.541521-2.420831$

$\begin{array}{lll}-0.042068 & -2.909125 & 0.200232\end{array}$

$\begin{array}{lll}0.195456 & -4.285280 & 0.044266\end{array}$

$-0.464902-4.884460-0.575553$

$\begin{array}{llll}1.262979 & -4.893773 & 0.705434\end{array}$

$\begin{array}{llll}1.440857 & -5.957468 & 0.576171\end{array}$

$2.098322-4.138709 \quad 1.535178$

$\begin{array}{llll}2.926970 & -4.615188 & 2.050686\end{array}$

$1.866574 \quad-2.771735 \quad 1.697922$

$\begin{array}{llll}2.512767 & -2.181304 & 2.338296\end{array}$

$\begin{array}{lll}0.801702 & -2.160009 & 1.032766\end{array}$

$\begin{array}{lll}0.616493 & -1.101443 & 1.168451\end{array}$

$\begin{array}{lll}-5.516896 & -0.911005 & -0.786813\end{array}$

$-5.166459-0.263716-1.980404$

$\begin{array}{llll}-4.410359 & 0.514535 & -1.958709\end{array}$

$-5.770928-0.625822-3.183875$

$-6.698371-1.664597-3.232843$

$-7.142961-1.967096-4.174450$

$-7.029992-2.319966-2.046585$

$\begin{array}{lll}-6.467614 & -1.936996 & -0.829043\end{array}$

$\begin{array}{lll}-6.765359 & -2.463973 & 0.067311\end{array}$

$\begin{array}{lll}-5.266634 & 1.259013 & 1.105166\end{array}$

$\begin{array}{lll}-4.567164 & 2.007039 & 2.065722\end{array}$

$\begin{array}{lll}-3.719124 & 1.568676 & 2.577604\end{array}$

$\begin{array}{llll}-4.927156 & 3.323757 & 2.333394\end{array}$

$\begin{array}{llll}-5.972653 & 3.933610 & 1.636531\end{array}$

$\begin{array}{lll}-6.233073 & 4.968259 & 1.827509\end{array}$

$\begin{array}{lll}-6.660714 & 3.192763 & 0.679592\end{array}$

$\begin{array}{lll}-6.323387 & 1.861463 & 0.417077\end{array}$

$\begin{array}{llll}-6.879807 & 1.314050 & -0.335172\end{array}$

$\begin{array}{lll}-2.292198 & -0.138168 & 0.238162\end{array}$

$\begin{array}{llll}-7.706188 & 3.855264 & -0.172820\end{array}$

$\begin{array}{lll}-4.110427 & 4.148770 & 3.288318\end{array}$

$\begin{array}{llll}-5.428108 & 0.155653 & -4.421128\end{array}$

$\begin{array}{llll}-7.971326 & -3.490731 & -2.117847\end{array}$

$\begin{array}{llll}-7.456418 & -4.487642 & -2.880798\end{array}$

$\begin{array}{llll}-8.235651 & -4.019933 & -0.902831\end{array}$

$\begin{array}{llll}-9.155444 & -3.152560 & -2.678077\end{array}$

$\begin{array}{llll}-4.098095 & 0.423361 & -4.494709\end{array}$

$\begin{array}{llll}-6.055638 & 1.358727 & -4.439756\end{array}$

$\begin{array}{llll}-5.771268 & -0.488566 & -5.554043\end{array}$

$\begin{array}{llll}-7.166590 & 4.301648 & -1.342086\end{array}$

$\begin{array}{lll}-8.265090 & 4.924244 & 0.429998\end{array}$

$\begin{array}{llll}-8.700488 & 3.006245 & -0.511386\end{array}$

$\begin{array}{lll}-4.881993 & 4.959076 & 4.044934\end{array}$

$\begin{array}{lll}-3.370709 & 3.388453 & 4.124252\end{array}$

$\begin{array}{lll}-3.241677 & 4.957834 & 2.622366\end{array}$

$\begin{array}{llll}-2.688127 & 2.718667 & -0.768294\end{array}$

$\begin{array}{llll}-1.757673 & 3.221047 & 0.214034\end{array}$

$\begin{array}{lll}-1.243961 & 2.528303 & 1.355541\end{array}$

$\begin{array}{llll}-1.701428 & 4.294975 & 0.314217\end{array}$

$\begin{array}{llll}2.928883 & 1.759844 & -1.618323\end{array}$

$\begin{array}{llll}4.386573 & 0.398025 & -0.502155\end{array}$

$\begin{array}{llll}4.177905 & -1.206392 & -0.150434\end{array}$

$\begin{array}{llll}5.832590 & 0.329479 & -1.334011\end{array}$

$\begin{array}{lll}4.610688 & 0.997478 & 1.051336\end{array}$

$\begin{array}{llll}4.413931 & -2.197939 & -1.099995\end{array}$

$3.284111-2.906786 \quad-1.559309$

$\begin{array}{llll}2.316296 & -2.661429 & -1.143937\end{array}$

$\begin{array}{llll}3.436926 & -3.886161 & -2.507233\end{array}$

$\begin{array}{llll}2.575831 & -4.446331 & -2.859389\end{array}$

$\begin{array}{llll}4.714822 & -4.162569 & -3.061322\end{array}$

$\begin{array}{llll}4.870110 & -5.117543 & -4.102518\end{array}$

$\begin{array}{llll}3.990156 & -5.655848 & -4.445463\end{array}$

$\begin{array}{llll}6.100486 & -5.345355 & -4.675699\end{array}$

$\begin{array}{llll}6.207709 & -6.072714 & -5.475125\end{array}$

$\begin{array}{llll}7.227588 & -4.613538 & -4.231695\end{array}$

$8.192741-4.774137 \quad-4.703523$

$\begin{array}{llll}7.113734 & -3.693935 & -3.211423\end{array}$

$\begin{array}{llll}7.987968 & -3.137527 & -2.895867\end{array}$

$\begin{array}{llll}5.862920 & -3.449233 & -2.577455\end{array}$ 


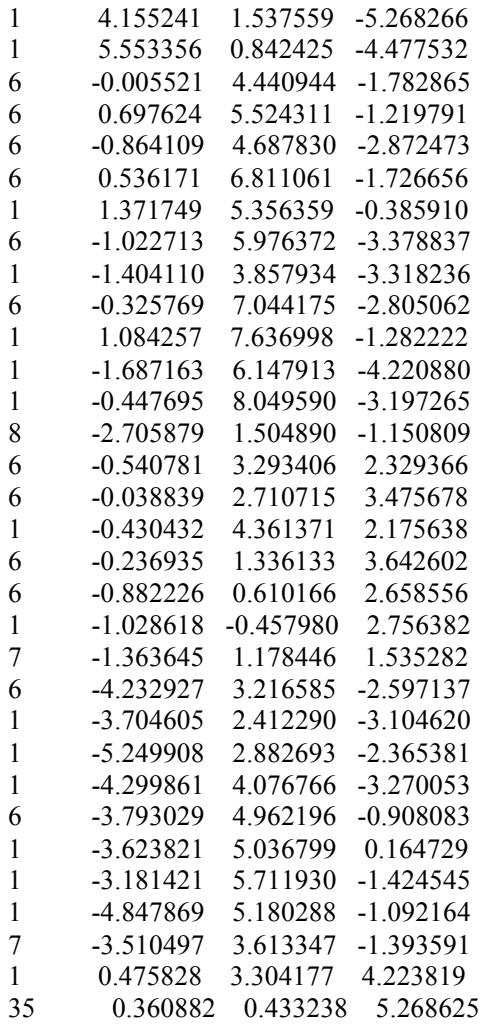

C1-P1 re-re 3

Number of imaginary frequencies $=1$

The smallest frequencies are $=-255.28709 .890213 .3757 \mathrm{~cm}(-1)$ Electronic energy $=-7222.6284099$

Zero-point correction $=$

1.754400

(Hartree/Particle)

Thermal correction to Energy= $\quad 1.873024$

Thermal correction to Enthalpy= $\quad 1.873969$

Thermal correction to Gibbs Free Energy $=\quad 1.592776$

Sum of electronic and zero-point Energies $=\quad-7220.874010$

Sum of electronic and thermal Energies $=\quad-7220.755386$

Sum of electronic and thermal Enthalpies $=\quad-7220.754441$

Sum of electronic and thermal Free Energies $=\quad-7221.035634$

Cartesian Coordinates

$\begin{array}{lllc}26 & 4.734876 & 4.665648 & 1.790828 \\ 15 & 2.778976 & 1.496143 & -1.852997 \\ 15 & 4.720415 & -0.025016 & 0.973603 \\ 6 & 4.311286 & 3.285680 & 0.276447 \\ 6 & 4.471768 & 2.629714 & 1.554062 \\ 6 & 3.486268 & 3.159440 & 2.443433 \\ 1 & 3.357523 & 2.884734 & 3.481337 \\ 6 & 2.739763 & 4.155098 & 1.744204 \\ 1 & 1.952984 & 4.767889 & 2.161824 \\ 6 & 3.247655 & 4.235424 & 0.414901 \\ 1 & 2.908977 & 4.911343 & -0.355475 \\ 6 & 6.712328 & 5.103573 & 2.249086 \\ 1 & 7.534788 & 4.406082 & 2.160754 \\ 6 & 5.884480 & 5.281748 & 3.399768 \\ 1 & 5.965066 & 4.739122 & 4.332252 \\ 6 & 4.903276 & 6.271747 & 3.089069 \\ 1 & 4.107226 & 6.604164 & 3.742301 \\ 6 & 5.124386 & 6.706628 & 1.746834 \\ 1 & 4.527708 & 7.428255 & 1.204558 \\ 6 & 6.242283 & 5.987554 & 1.228673 \\ 1 & 6.639690 & 6.077519 & 0.227101 \\ 6 & 5.073361 & 2.936457 & -0.957505 \\ 6 & 6.425866 & 3.293670 & -1.059945 \\ 1 & 6.869296 & 3.852986 & -0.245951 \\ 6 & 7.201259 & 2.932575 & -2.161579\end{array}$


$\begin{array}{llll}8.252026 & 3.201832 & -2.196677\end{array}$

$\begin{array}{llll}6.624981 & 2.202506 & -3.198459\end{array}$

$\begin{array}{llll}7.218922 & 1.901502 & -4.055510\end{array}$

$\begin{array}{llll}5.281961 & 1.832979 & -3.119432\end{array}$

$\begin{array}{llll}4.849837 & 1.249479 & -3.924896\end{array}$

$\begin{array}{llll}4.493837 & 2.177866 & -2.010336\end{array}$

$\begin{array}{llll}5.440212 & 1.509746 & 1.827295\end{array}$

$\begin{array}{lll}6.364481 & 1.738298 & 1.294295\end{array}$

$\begin{array}{llll}5.781844 & 1.324266 & 3.308722\end{array}$

$\begin{array}{lll}6.520698 & 0.529810 & 3.443456\end{array}$

$\begin{array}{lll}4.904319 & 1.071833 & 3.910538\end{array}$

$\begin{array}{llll}6.198353 & 2.257802 & 3.699520\end{array}$

$\begin{array}{llll}2.495753 & 0.578051 & -3.427557\end{array}$

$2.670048 \quad-0.815338 \quad-3.410763$

$\begin{array}{llll}2.964223 & -1.310811 & -2.493196\end{array}$

$\begin{array}{llll}2.423256 & -1.573056 & -4.557882\end{array}$

$2.562388 \quad-2.648807 \quad-4.530720$

$\begin{array}{llll}1.987530 & -0.950144 & -5.72912\end{array}$

$\begin{array}{llll}1.780427 & -1.541180 & -6.616637\end{array}$

$\begin{array}{llll}1.814441 & 0.436439 & -5.754474\end{array}$

$\begin{array}{llll}1.474782 & 0.927147 & -6.662151\end{array}$

$\begin{array}{llll}2.070009 & 1.198042 & -4.613388\end{array}$

$\begin{array}{llll}1.917692 & 2.271150 & -4.648788\end{array}$

$\begin{array}{llll}1.681455 & 2.957763 & -2.032188\end{array}$

$\begin{array}{llll}2.044408 & 4.089534 & -2.780267\end{array}$

$\begin{array}{llll}3.025720 & 4.142965 & -3.242472\end{array}$

$\begin{array}{llll}1.152634 & 5.154235 & -2.922007\end{array}$

$\begin{array}{llll}1.443336 & 6.027021 & -3.499819\end{array}$

$-0.112128 \quad 5.094536 \quad-2.327153$

$\begin{array}{llll}-0.811319 & 5.918001 & -2.441125\end{array}$

$\begin{array}{lll}-0.477433 & 3.971542 & -1.582082\end{array}$

$\begin{array}{llll}-1.457338 & 3.918817 & -1.122687\end{array}$

$\begin{array}{llll}0.420052 & 2.913593 & -1.424861\end{array}$

$\begin{array}{llll}0.139551 & 2.050393 & -0.831819\end{array}$

$\begin{array}{llll}5.971003 & -0.589798 & -0.256482\end{array}$

$\begin{array}{llll}5.553791 & -1.626147 & -1.104339\end{array}$

$\begin{array}{llll}4.561945 & -2.045645 & -0.974622\end{array}$

$6.401443-2.111379-2.099470$

$\begin{array}{llll}7.659829 & -1.547551 & -2.299872\end{array}$

$\begin{array}{llll}8.308401 & -1.906805 & -3.091011\end{array}$

$8.062994-0.500968-1.470279$

$\begin{array}{llll}7.242515 & -0.036803 & -0.441901\end{array}$

$\begin{array}{llll}7.604516 & 0.766071 & 0.185457\end{array}$

$\begin{array}{llll}4.702065 & -1.400400 & 2.197776\end{array}$

$\begin{array}{lll}3.709644 & -1.360011 & 3.190328\end{array}$

$\begin{array}{lll}3.061362 & -0.495234 & 3.278179\end{array}$

$\begin{array}{lll}3.521745 & -2.447015 & 4.041263\end{array}$

$\begin{array}{llll}4.285451 & -3.606439 & 3.900939\end{array}$

$\begin{array}{lll}4.105483 & -4.466153 & 4.536604\end{array}$

$\begin{array}{lll}5.258688 & -3.650347 & 2.906114\end{array}$

$\begin{array}{llll}5.486404 & -2.552863 & 2.074913\end{array}$

$\begin{array}{llll}6.251931 & -2.624110 & 1.312639\end{array}$

$\begin{array}{llll}2.608439 & 0.155047 & -0.035119\end{array}$

$\begin{array}{lll}6.064355 & -4.907344 & 2.727315\end{array}$

$\begin{array}{llll}2.402325 & -2.444623 & 5.043561\end{array}$

$\begin{array}{llll}5.953960 & -3.292336 & -2.914854\end{array}$

$\begin{array}{llll}9.394121 & 0.146051 & -1.738017\end{array}$

$\begin{array}{llll}9.430555 & 0.703246 & -2.974403\end{array}$

$\begin{array}{llll}9.682281 & 1.126412 & -0.853913\end{array}$

$10.407182 \quad-0.749805 \quad-1.692741$

$6.097249-4.451938-2.224925$

$\begin{array}{llll}6.648349 & -3.424210 & -4.062660\end{array}$

$\begin{array}{llll}4.639346 & -3.210586 & -3.245790\end{array}$

$\begin{array}{llll}7.051545 & -5.017031 & 3.644752\end{array}$

$\begin{array}{lll}6.646343 & -4.968888 & 1.507978\end{array}$

$\begin{array}{llll}5.289495 & -6.013049 & 2.856370\end{array}$

$\begin{array}{lll}2.771513 & -3.007454 & 6.214737\end{array}$

$1.947293-1.203105 \quad 5.31276$

$\begin{array}{lll}1.338807 & -3.166055 & 4.590925\end{array}$

$\begin{array}{llll}2.009404 & -2.805056 & 0.520106\end{array}$

$\begin{array}{lll}0.863432 & -2.457954 & 1.334344\end{array}$

$\begin{array}{llll}0.586916 & -1.161900 & 1.886880\end{array}$

$\begin{array}{lll}0.430912 & -3.259962 & 1.915220\end{array}$

$\begin{array}{lllll}77 & -3.322384 & -1.086574 & -1.263464\end{array}$

$\begin{array}{lllll}15 & -5.180379 & -0.221177 & -0.276298\end{array}$ $\begin{array}{lll}-5.130235 & 0.890185 & 0.963847\end{array}$

$\begin{array}{lll}-6.128182 & 0.604624 & -1.364921\end{array}$

$\begin{array}{lll}-6.046986 & -1.433860 & 0.500085\end{array}$

$\begin{array}{lll}-4.741202 & 2.196185 & 0.704787\end{array}$

$\begin{array}{lll}-3.487864 & 2.600235 & 1.216052\end{array}$

$\begin{array}{lll}-2.907060 & 1.885957 & 1.789243\end{array}$

$\begin{array}{lll}-3.039587 & 3.874474 & 0.972015\end{array}$

$\begin{array}{lll}-2.085309 & 4.205274 & 1.368787\end{array}$

$\begin{array}{lll}-3.795160 & 4.760783 & 0.156473\end{array}$

$\begin{array}{llll}-3.284483 & 6.038911 & -0.199631\end{array}$

$\begin{array}{llll}-2.316676 & 6.337410 & 0.194550\end{array}$

$\begin{array}{llll}-3.988888 & 6.871899 & -1.039730\end{array}$

$\begin{array}{lll}-3.587029 & 7.843374 & -1.312195\end{array}$

$\begin{array}{llll}-5.237117 & 6.453771 & -1.560084\end{array}$

$\begin{array}{lll}-5.780550 & 7.103705 & -2.239794\end{array}$

$\begin{array}{llll}-5.768606 & 5.228910 & -1.218230\end{array}$

$\begin{array}{llll}-6.721960 & 4.923497 & -1.632906\end{array}$

$\begin{array}{llll}-5.075786 & 4.346913 & -0.342845\end{array}$

$\begin{array}{lll}-5.589839 & 3.050355 & 0.018595\end{array}$

$\begin{array}{lll}-6.971883 & 2.613923 & -0.328568\end{array}$

$\begin{array}{lll}-8.127960 & 3.381716 & 0.061258\end{array}$

$\begin{array}{llll}-8.043676 & 4.517441 & 0.914972\end{array}$

$\begin{array}{lll}-7.079099 & 4.819624 & 1.304820\end{array}$

$\begin{array}{lll}-9.170086 & 5.229009 & 1.267304\end{array}$

$\begin{array}{lll}-9.077932 & 6.086915 & 1.927181\end{array}$

$\begin{array}{lll}-10.445787 & 4.849572 & 0.786234\end{array}$

$\begin{array}{lll}-11.323605 & 5.423823 & 1.067622\end{array}$

$\begin{array}{lll}-10.568429 & 3.743489 & -0.023494\end{array}$

$\begin{array}{llll}-11.543205 & 3.427105 & -0.385488\end{array}$

$\begin{array}{llll}-9.428917 & 2.978401 & -0.393426\end{array}$

$\begin{array}{llll}-9.561130 & 1.808581 & -1.188721\end{array}$

$\begin{array}{lll}-10.546331 & 1.519230 & -1.543383\end{array}$

$\begin{array}{lll}-8.465132 & 1.033292 & -1.475770\end{array}$

$\begin{array}{llll}-8.546792 & 0.116087 & -2.048937\end{array}$

$\begin{array}{llll}-7.189765 & 1.428639 & -1.015716\end{array}$

$\begin{array}{lll}-5.442938 & -2.781203 & 0.300783\end{array}$

$\begin{array}{lll}-4.790720 & -2.998431 & 1.155926\end{array}$

$\begin{array}{lll}-6.472364 & -3.893572 & 0.199576\end{array}$

$\begin{array}{lll}-6.274070 & -5.090790 & 0.893887\end{array}$

$\begin{array}{lll}-5.410773 & -5.192679 & 1.548194\end{array}$

$\begin{array}{lll}-7.182515 & -6.146043 & 0.768515\end{array}$

$\begin{array}{lll}-7.019263 & -7.068470 & 1.319337\end{array}$

$\begin{array}{lll}-8.303154 & -6.008365 & -0.051822\end{array}$

$\begin{array}{llll}-9.018595 & -6.821068 & -0.140955\end{array}$

$\begin{array}{llll}-8.503235 & -4.815446 & -0.756362\end{array}$

$\begin{array}{llll}-9.377475 & -4.698558 & -1.390684\end{array}$

$\begin{array}{llll}-7.589015 & -3.771233 & -0.638620\end{array}$

$\begin{array}{llll}-7.754702 & -2.836197 & -1.164787\end{array}$

$\begin{array}{llll}-4.592917 & -2.785321 & -0.981220\end{array}$

$\begin{array}{llll}-5.270255 & -2.851645 & -1.840252\end{array}$

$\begin{array}{llll}-3.984551 & -3.694256 & -0.987588\end{array}$

$\begin{array}{lll}-6.809579 & -1.156680 & 1.767566\end{array}$

$\begin{array}{lll}-7.022820 & -0.087728 & 1.739561\end{array}$

$\begin{array}{lll}-8.168874 & -1.849830 & 1.808171\end{array}$

$-9.226728-1.293036 \quad 1.078526$

$\begin{array}{lll}-9.059276 & -0.381243 & 0.515453\end{array}$

$\begin{array}{lll}-10.485268 & -1.893670 & 1.061738\end{array}$

$\begin{array}{lll}-11.289853 & -1.444396 & 0.485453\end{array}$

$\begin{array}{lll}-10.709360 & -3.065143 & 1.789839\end{array}$

$\begin{array}{lll}-11.686381 & -3.540249 & 1.777687\end{array}$

$\begin{array}{lll}-9.668577 & -3.616460 & 2.540364\end{array}$

$\begin{array}{lll}-9.831680 & -4.525534 & 3.112485\end{array}$

$\begin{array}{lll}-8.411284 & -3.008653 & 2.554762\end{array}$

$\begin{array}{lll}-7.614804 & -3.461522 & 3.133835\end{array}$

$\begin{array}{llll}-5.942115 & -1.411747 & 3.006053\end{array}$

$\begin{array}{lll}-5.035254 & -0.804049 & 2.954251\end{array}$

$\begin{array}{lll}-5.648206 & -2.459737 & 3.107965\end{array}$

$\begin{array}{lll}-6.494011 & -1.131852 & 3.908470\end{array}$

$\begin{array}{lll}-2.423526 & -0.942308 & 0.712994\end{array}$

$\begin{array}{lll}-1.732806 & -0.103841 & 0.747850\end{array}$

$\begin{array}{lll}-3.113382 & -0.949671 & 1.551921\end{array}$

$\begin{array}{llll}-1.958862 & -2.216281 & 0.247681\end{array}$

$\begin{array}{llll}-2.509278 & -3.086050 & 0.590555\end{array}$

$\begin{array}{lll}-0.717999 & -2.463590 & -0.395898\end{array}$

$\begin{array}{llll}-0.157444 & -1.617306 & -0.768159\end{array}$ 


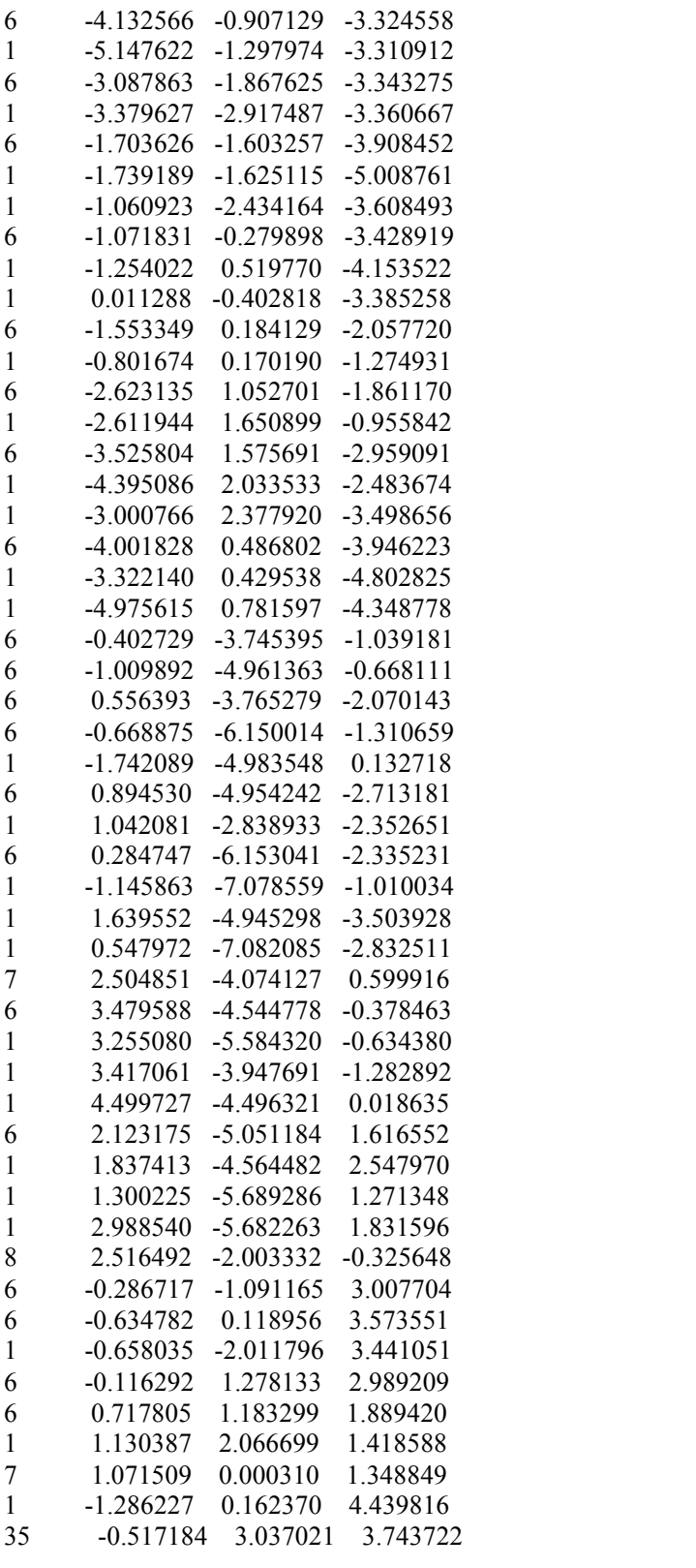

C1-P1 re-re 4

Number of imaginary frequencies $=1$

The smallest frequencies are $=-280.681010 .242114 .1723 \mathrm{~cm}(-1)$ Electronic energy $=-7222.6274683$

Zero-point correction=

(Hartree/Particle)

Thermal correction to Energy=

Thermal correction to Enthalpy=

1.756318

Thermal correction to Gibbs Free Energy=
Sum of electronic and zero-point Energies

Sum of electronic and zero-point Energies=

Sum of electronic and thermal Enthalpies=

Sum of electronic and thermal Free Energies=

1.874582

1.875527

1.595160

$-7220.871151$

$-7220.752886$

$-7220.751942$

$-7221.032308$

\section{Cartesian Coordinates}

$\begin{array}{llll}26 & -8.485868 & -1.159941 & -0.400074 \\ 15 & -3.890269 & 0.488585 & -2.289694 \\ 15 & -4.442076 & 0.587633 & 1.414697 \\ 6 & -6.733614 & -0.183022 & -1.004959 \\ 6 & -6.624601 & -0.668396 & 0.351238 \\ 6 & -6.791957 & -2.087148 & 0.323926 \\ 1 & -6.779286 & -2.741573 & 1.184732\end{array}$

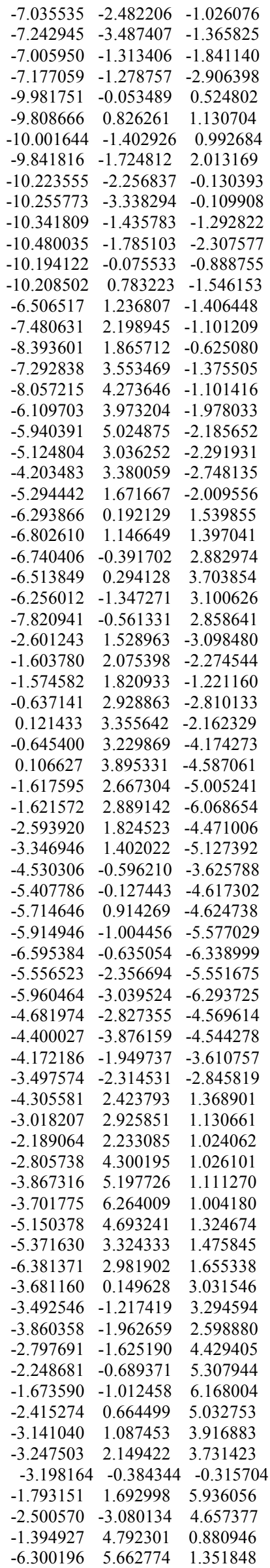




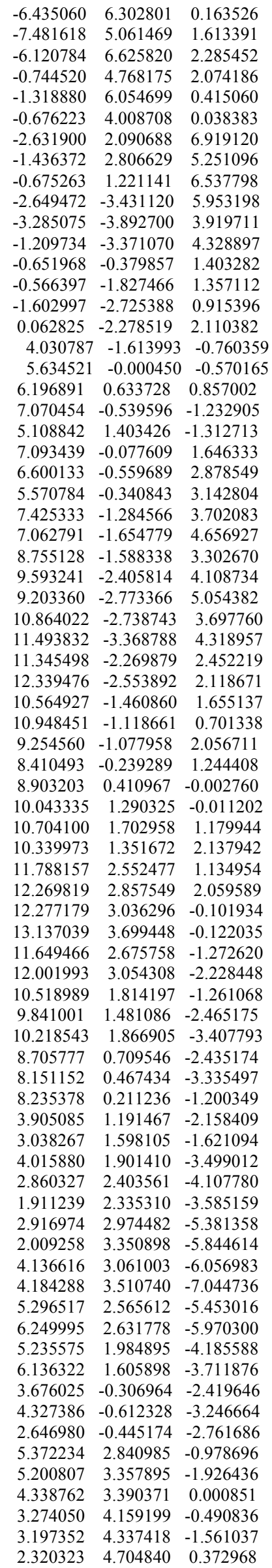

$\begin{array}{lll}1.501075 & 5.294872 & -0.025074\end{array}$

$\begin{array}{llll}2.422142 & 4.488733 & 1.748149\end{array}$

$\begin{array}{lll}1.679115 & 4.905628 & 2.419606\end{array}$

$\begin{array}{lll}3.477202 & 3.720803 & 2.249738\end{array}$

$\begin{array}{lll}3.564098 & 3.551493 & 3.319881\end{array}$

$\begin{array}{lll}4.425734 & 3.172472 & 1.384374\end{array}$

$\begin{array}{llll}5.239632 & 2.580225 & 1.784984\end{array}$

$\begin{array}{llll}6.811456 & 3.179401 & -0.584913\end{array}$

$\begin{array}{llll}7.509970 & 2.834727 & -1.349127\end{array}$

$\begin{array}{lll}7.107949 & 2.757394 & 0.373588\end{array}$

$\begin{array}{llll}6.893300 & 4.268678 & -0.516546\end{array}$

$\begin{array}{llll}2.926676 & -0.615817 & 0.846786\end{array}$

$\begin{array}{lll}2.993476 & -1.215428 & 1.752253\end{array}$

$\begin{array}{lll}3.164330 & 0.432742 & 1.010726\end{array}$

$\begin{array}{llll}1.917899 & -0.892317 & -0.124069\end{array}$

$\begin{array}{lll}1.643844 & -0.052239 & -0.751865\end{array}$

$\begin{array}{llll}1.061163 & -2.034169 & -0.219269\end{array}$

$\begin{array}{lll}1.348697 & -2.928086 & 0.315880\end{array}$

$\begin{array}{llll}5.264864 & -2.857789 & -2.116680\end{array}$

$\begin{array}{llll}5.684853 & -2.200070 & -2.872810\end{array}$

$3.906860 \quad-3.239476 \quad-2.287151$

$\begin{array}{llll}3.389236 & -2.863216 & -3.168949\end{array}$

$3.327445-4.532241-1.740553$

$\begin{array}{llll}3.670242 & -5.379199 & -2.355137\end{array}$

$\begin{array}{llll}2.245395 & -4.485023 & -1.868699\end{array}$

$\begin{array}{llll}3.655098 & -4.793340 & -0.253916\end{array}$

$\begin{array}{llll}4.524851 & -5.452348 & -0.164650\end{array}$

$\begin{array}{lll}2.821413 & -5.335157 & 0.205465\end{array}$

$\begin{array}{llll}3.912031 & -3.529543 & 0.565482\end{array}$

$\begin{array}{llll}3.172403 & -3.319730 & 1.334513\end{array}$

$\begin{array}{lll}5.171799 & -2.962004 & 0.750669\end{array}$

$\begin{array}{llll}5.319139 & -2.371437 & 1.649633\end{array}$

$\begin{array}{lll}6.449352 & -3.447982 & 0.094156\end{array}$

$\begin{array}{llll}7.209239 & -2.676304 & 0.234683\end{array}$

$\begin{array}{llll}6.808935 & -4.342907 & 0.623375\end{array}$

$6.302384 \quad-3.738831 \quad-1.415053$

$\begin{array}{llll}6.048299 & -4.791774 & -1.577419\end{array}$

$\begin{array}{llll}7.272928 & -3.583834 & -1.895816\end{array}$

$\begin{array}{llll}0.284153 & -2.272376 & -1.446400\end{array}$

$\begin{array}{lll}-0.064988 & -1.240757 & -2.340847\end{array}$

$\begin{array}{llll}-0.101093 & -3.587302 & -1.769861\end{array}$

$-0.712386-1.527749-3.540311$

$\begin{array}{llll}0.155492 & -0.208213 & -2.101151\end{array}$

$\begin{array}{llll}0.753469 & -3.874476 & -2.968477\end{array}$

$\begin{array}{llll}0.127084 & -4.389910 & -1.076206\end{array}$

$\begin{array}{lll}-1.042913 & -2.846668 & -3.868857\end{array}$

$\begin{array}{llll}-0.971582 & -0.715908 & -4.212699\end{array}$

$\begin{array}{llll}-1.024493 & -4.900294 & -3.201793\end{array}$

$-1.546262-3.064038-4.805628$

$\begin{array}{llll}0.072989 & 0.273749 & 2.360595\end{array}$

$\begin{array}{llll}0.277422 & 1.714731 & 2.266188\end{array}$

$\begin{array}{lll}1.296480 & 1.949106 & 2.583656\end{array}$

$\begin{array}{lll}0.149137 & 2.047440 & 1.239595\end{array}$

$\begin{array}{lll}-0.417366 & 2.262472 & 2.913052\end{array}$

$\begin{array}{lll}0.647502 & -0.340194 & 3.554104\end{array}$

$\begin{array}{llll}0.149707 & -1.274884 & 3.804807\end{array}$

$\begin{array}{lll}1.722352 & -0.520895 & 3.435897\end{array}$

$\begin{array}{lll}0.505169 & 0.339463 & 4.399052\end{array}$

$\begin{array}{llll}-1.280881 & 0.303097 & 0.540744\end{array}$

$\begin{array}{lll}-1.472848 & -4.097325 & 1.263375\end{array}$

$\begin{array}{lll}-2.393275 & -5.036639 & 0.842807\end{array}$

$\begin{array}{lll}-0.640566 & -4.403161 & 1.887403\end{array}$

$\begin{array}{lll}-3.462671 & -4.586276 & 0.064591\end{array}$

$\begin{array}{lll}-3.569419 & -3.244665 & -0.250116\end{array}$

$\begin{array}{llll}-4.399563 & -2.873953 & -0.836929\end{array}$

$-2.664988-2.330089 \quad 0.153660$

$-2.288557-6.081230 \quad 1.115485$

$\begin{array}{llll}-4.814999 & -5.836447 & -0.594539\end{array}$

C1-P1 re-re_5

Number of imaginary frequencies $=1$

The smallest frequencies are $=-180.77428 .680412 .4610 \mathrm{~cm}(-1)$ Electronic energy $=-7222.6357815$ 


\begin{tabular}{|c|c|c|c|}
\hline \multicolumn{4}{|c|}{ Zero-point correction $=$} \\
\hline \multicolumn{4}{|c|}{ (Hartree/Particle) } \\
\hline \multicolumn{4}{|c|}{ Thermal correction to Energy= } \\
\hline \multicolumn{4}{|c|}{ Thermal correction to Enthalpy= } \\
\hline \multicolumn{4}{|c|}{ Thermal correction to Gibbs Free Energy= } \\
\hline \multicolumn{4}{|c|}{ Sum of electronic and zero-point Energies $=$} \\
\hline \multicolumn{4}{|c|}{ Sum of electronic and thermal Energies $=$} \\
\hline \multicolumn{4}{|c|}{ Sum of electronic and thermal Enthalpies $=$} \\
\hline \multicolumn{4}{|c|}{ Sum of electronic and thermal Free Energie } \\
\hline \multicolumn{4}{|c|}{ Cartesian Coordinates } \\
\hline 20 & -8.542937 & -0.875966 & 0.241093 \\
\hline 15 & -4.045284 & -0.270153 & -2.340607 \\
\hline 15 & -4.260155 & 0.966463 & 1.202010 \\
\hline 6 & -6.786609 & -0.271264 & -0.722541 \\
\hline 6 & -6.602300 & -0.346793 & 0.708562 \\
\hline 6 & -6.860546 & -1.692856 & 1.112893 \\
\hline 1 & -6.823933 & -2.067641 & 2.126402 \\
\hline 6 & -7.234877 & -2.442534 & -0.042280 \\
\hline 1 & -7.534578 & -3.481146 & -0.055788 \\
\hline 6 & -7.192531 & -1.570530 & -1.168540 \\
\hline 1 & -7.444417 & -1.834749 & -2.184071 \\
\hline 6 & -9.884203 & 0.562252 & 0.912364 \\
\hline 1 & -9.606972 & 1.551338 & 1.252997 \\
\hline 6 & -9.967906 & -0.611388 & 1.722440 \\
\hline 1 & -9.758911 & -0.669171 & 2.782318 \\
\hline 6 & -10.332222 & -1.704997 & 0.879109 \\
\hline 1 & -10.442475 & -2.736295 & 1.187738 \\
\hline 6 & -10.473766 & -1.208110 & -0.452337 \\
\hline 1 & -10.711621 & -1.795436 & -1.329512 \\
\hline 6 & -10.198493 & 0.191532 & -0.432070 \\
\hline 1 & -10.196059 & 0.846369 & -1.292761 \\
\hline 6 & -6.499909 & 0.945189 & -1.538176 \\
\hline 6 & -7.352371 & 2.054686 & -1.436167 \\
\hline 1 & -8.228177 & 1.967602 & -0.806029 \\
\hline 6 & -7.085986 & 3.253023 & -2.098037 \\
\hline 1 & -7.751235 & 4.101549 & -1.973245 \\
\hline 6 & -5.948307 & 3.359138 & -2.894654 \\
\hline 1 & -5.718911 & 4.289191 & -3.405274 \\
\hline 6 & -5.085226 & 2.269362 & -3.014265 \\
\hline 1 & -4.194451 & 2.370443 & -3.624278 \\
\hline 6 & -5.331899 & 1.062694 & -2.340455 \\
\hline 6 & -6.115377 & 0.787352 & 1.568631 \\
\hline 1 & -6.576327 & 1.702462 & 1.193693 \\
\hline 6 & -6.476914 & 0.647632 & 3.050139 \\
\hline 1 & -6.141965 & 1.518815 & 3.619668 \\
\hline 1 & -6.033678 & -0.241817 & 3.505643 \\
\hline 1 & -7.563985 & 0.565649 & 3.145380 \\
\hline 6 & -2.747429 & 0.328732 & -3.505344 \\
\hline 6 & -1.589659 & 0.898895 & -2.955619 \\
\hline 1 & -1.486186 & 0.973205 & -1.879557 \\
\hline 6 & -0.550380 & 1.328917 & -3.784248 \\
\hline 1 & 0.338764 & 1.773913 & -3.348720 \\
\hline 6 & -0.653392 & 1.184201 & -5.169048 \\
\hline 1 & 0.159382 & 1.511058 & -5.811667 \\
\hline 6 & -1.805287 & 0.618425 & -5.725210 \\
\hline 1 & -1.891683 & 0.504227 & -6.802165 \\
\hline 6 & -2.848567 & 0.195963 & -4.900069 \\
\hline 1 & -3.732809 & -0.247924 & -5.345159 \\
\hline 6 & -4.826410 & -1.659380 & -3.252476 \\
\hline 6 & -5.801861 & -1.465223 & -4.243472 \\
\hline 1 & -6.127042 & -0.460688 & -4.498341 \\
\hline 6 & -6.378946 & -2.562924 & -4.884016 \\
\hline 1 & -7.136836 & -2.405290 & -5.646231 \\
\hline 6 & -5.987460 & -3.861780 & -4.541791 \\
\hline 1 & -6.444156 & -4.714449 & -5.036152 \\
\hline 6 & -5.009122 & -4.059782 & -3.564455 \\
\hline 1 & -4.697514 & -5.065619 & -3.296933 \\
\hline 6 & -4.431492 & -2.962555 & -2.922612 \\
\hline 1 & -3.670555 & -3.115378 & -2.165873 \\
\hline 6 & -4.008114 & 2.692548 & 0.608198 \\
\hline 6 & -2.738174 & 2.972834 & 0.085864 \\
\hline 1 & -1.984989 & 2.191432 & 0.074560 \\
\hline 6 & 11 & 4.240637 & -0.420 \\
\hline
\end{tabular}

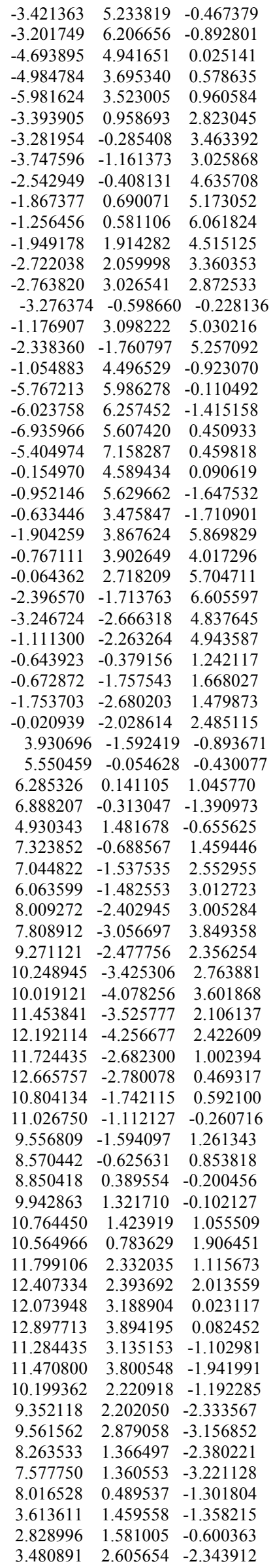


$\begin{array}{llll}2.358758 & 3.436176 & -2.281071\end{array}$

$\begin{array}{llll}1.638119 & 3.287469 & -1.486567\end{array}$

$\begin{array}{llll}2.167354 & 4.456239 & -3.217168\end{array}$

$\begin{array}{llll}1.282458 & 5.083054 & -3.151863\end{array}$

$\begin{array}{llll}3.118201 & 4.665244 & -4.218023\end{array}$

$\begin{array}{llll}2.980467 & 5.459796 & -4.946047\end{array}$

$\begin{array}{llll}4.256782 & 3.852141 & -4.274567\end{array}$

$\begin{array}{llll}5.004654 & 4.019289 & -5.045104\end{array}$

$\begin{array}{llll}4.436569 & 2.826869 & -3.345656\end{array}$

$\begin{array}{llll}5.326177 & 2.203290 & -3.381690\end{array}$

$\begin{array}{llll}3.412577 & 0.115812 & -2.072814\end{array}$

$\begin{array}{llll}4.009476 & 0.109509 & -2.993340\end{array}$

$\begin{array}{llll}2.366217 & 0.042921 & -2.381544\end{array}$

$\begin{array}{llll}5.222324 & 2.783125 & 0.026392\end{array}$

$\begin{array}{llll}5.006195 & 3.530357 & -0.741772\end{array}$

$\begin{array}{lll}4.264734 & 3.063587 & 1.184901\end{array}$

$\begin{array}{llll}3.357399 & 4.127935 & 1.087307\end{array}$

$\begin{array}{lll}3.308654 & 4.702099 & 0.166125\end{array}$

$\begin{array}{llll}2.514824 & 4.456211 & 2.153540\end{array}$

$\begin{array}{lll}1.806932 & 5.273482 & 2.048205\end{array}$

$\begin{array}{lll}2.566066 & 3.718888 & 3.337859\end{array}$

$\begin{array}{llll}1.898091 & 3.955234 & 4.160152\end{array}$

$3.460426 \quad 2.648918 \quad 3.442210$

$\begin{array}{lll}3.503386 & 2.065982 & 4.358253\end{array}$

$\begin{array}{lll}4.300792 & 2.321578 & 2.375458\end{array}$

$\begin{array}{lll}4.994919 & 1.493723 & 2.469399\end{array}$

$\begin{array}{llll}6.678278 & 3.024747 & 0.434700\end{array}$

$\begin{array}{lll}7.346954 & 2.910689 & -0.418962\end{array}$

$\begin{array}{lll}7.011552 & 2.370623 & 1.238901\end{array}$

$\begin{array}{llll}6.754281 & 4.058641 & 0.785543\end{array}$

$\begin{array}{llll}2.938583 & -0.939173 & 0.913684\end{array}$

$\begin{array}{lll}3.116285 & -1.627343 & 1.741336\end{array}$

$\begin{array}{lll}3.111430 & 0.099200 & 1.183702\end{array}$

$\begin{array}{llll}1.845119 & -1.220466 & 0.024937\end{array}$

$\begin{array}{llll}1.434888 & -0.391137 & -0.540010\end{array}$

$\begin{array}{lll}1.051096 & -2.395389 & 0.071081\end{array}$

$\begin{array}{llll}1.349402 & -3.164556 & 0.774441\end{array}$

$\begin{array}{llll}5.054064 & -2.427093 & -2.641065\end{array}$

$\begin{array}{llll}5.394560 & -1.575074 & -3.222426\end{array}$

$\begin{array}{lll}3.691848 & -2.784084 & -2.769676\end{array}$

$\begin{array}{llll}3.086130 & -2.199300 & -3.461669\end{array}$

$\begin{array}{llll}3.135039 & -4.169446 & -2.485647\end{array}$

$\begin{array}{llll}3.359767 & -4.839103 & -3.330162\end{array}$

$\begin{array}{llll}2.047250 & -4.083894 & -2.443760\end{array}$

$\begin{array}{llll}3.636866 & -4.804918 & -1.168952\end{array}$

$\begin{array}{llll}4.516057 & -5.428581 & -1.358795\end{array}$

$\begin{array}{llll}2.863037 & -5.480371 & -0.789780\end{array}$

$\begin{array}{llll}3.979255 & -3.802453 & -0.069259\end{array}$

$\begin{array}{llll}3.326400 & -3.807393 & 0.798280\end{array}$

$\begin{array}{llll}5.239951 & -3.249612 & 0.119616\end{array}$

$\begin{array}{llll}5.472060 & -2.887397 & 1.116456\end{array}$

$\begin{array}{llll}6.449586 & -3.499030 & -0.760525\end{array}$

$7.203936-2.751125-0.509699$

$\begin{array}{llll}6.881731 & -4.477826 & -0.505423\end{array}$

$\begin{array}{llll}6.156216 & -3.421875 & -2.274221\end{array}$

$\begin{array}{llll}5.887917 & -4.410578 & -2.660176\end{array}$

$\begin{array}{llll}7.075279 & -3.132041 & -2.792327\end{array}$

$\begin{array}{llll}0.201301 & -2.853434 & -1.020858\end{array}$

$-0.087608-2.046454-2.138176$

$-0.284655-4.176640-1.00475$

$\begin{array}{llll}-0.801100 & -2.557881 & -3.217377\end{array}$

$\begin{array}{llll}0.257459 & -1.021841 & -2.167254\end{array}$

$-1.006964-4.685646-2.082808$

$-0.072733-4.808613-0.147515$

$\begin{array}{llll}-1.255002 & -3.881893 & -3.199319\end{array}$

$\begin{array}{llll}-1.007793 & -1.921521 & -4.071688\end{array}$

$\begin{array}{llll}-1.363563 & -5.711448 & -2.058282\end{array}$

$\begin{array}{llll}-1.810424 & -4.277109 & -4.044123\end{array}$

$\begin{array}{lll}0.169155 & 0.492020 & 1.913129\end{array}$

$\begin{array}{lll}0.487452 & 1.786917 & 1.326189\end{array}$

$\begin{array}{lll}1.560385 & 1.966009 & 1.411832\end{array}$

$\begin{array}{lll}0.206353 & 1.796545 & 0.276283\end{array}$

$\begin{array}{lll}-0.035046 & 2.597141 & 1.844554\end{array}$

$\begin{array}{lll}0.795179 & 0.234947 & 3.204861\end{array}$

$\begin{array}{lll}0.254472 & -0.524569 & 3.764103\end{array}$ $\begin{array}{llll}1.839726 & -0.074411 & 3.096391\end{array}$

$\begin{array}{lll}0.777293 & 1.155071 & 3.793182\end{array}$

$\begin{array}{lll}-1.253336 & 0.038444 & 0.208263\end{array}$

$\begin{array}{lll}-1.674381 & -3.935691 & 2.146557\end{array}$

$\begin{array}{lll}-2.663535 & -4.887860 & 2.007221\end{array}$

$\begin{array}{lll}-0.819675 & -4.137392 & 2.783597\end{array}$

$\begin{array}{lll}-3.752965 & -4.572464 & 1.189034\end{array}$

$\begin{array}{lll}-3.811166 & -3.341383 & 0.563837\end{array}$

$\begin{array}{llll}-4.653031 & -3.068896 & -0.059436\end{array}$

$\begin{array}{lll}-2.842553 & -2.413495 & 0.694775\end{array}$

$\begin{array}{lll}2.595169 & -5.841680 & 2.519306\end{array}$

$\begin{array}{llll}35 & -5.201254 & -5.860062 & 0.914978\end{array}$

C1-P1 si-re_1

Number of imaginary frequencies $=1$

The smallest frequencies are $=-209.39746 .234612 .4896 \mathrm{~cm}(-1)$ Electronic energy $=-7222.6227613$

Zero-point correction=

1.755738

(Hartree/Particle)

Thermal correction to Energy=

1.874328

Thermal correction to Enthalpy=

1.875272

Thermal correction to Gibbs Free Energy=

Sum of electronic and zero-point Energies=

Sum of electronic and thermal Energies=

.592693

Sum of electronic and thermal Enthalpies=

$-7220.867023$ $-7220.748433$

$-7220.747489$

Sum of electronic and thermal Free Energies $=\quad-7221.030068$

Cartesian Coordinates

$\begin{array}{llcc}26 & 3.572952 & 4.879751 & 2.501907 \\ 15 & 2.418129 & 1.927009 & -1.598467 \\ 15 & 4.924159 & 0.536289 & 1.045293 \\ 6 & 3.445587 & 3.768283 & 0.729847 \\ 6 & 4.054105 & 2.996938 & 1.783670 \\ 6 & 3.110520 & 2.898802 & 2.853375 \\ 1 & 3.275385 & 2.390576 & 3.793998 \\ 6 & 1.937416 & 3.619918 & 2.481491 \\ 1 & 1.060388 & 3.768378 & 3.097246 \\ 6 & 2.145605 & 4.162631 & 1.180128 \\ 1 & 1.451774 & 4.776030 & 0.623643 \\ 6 & 5.269727 & 6.026613 & 2.861980 \\ 1 & 6.262474 & 5.790685 & 2.502144 \\ 6 & 4.696892 & 5.586050 & 4.094140 \\ 1 & 5.175506 & 4.948440 & 4.825530 \\ 6 & 3.363717 & 6.093255 & 4.166478 \\ 1 & 2.654370 & 5.905167 & 4.961777 \\ 6 & 3.112633 & 6.847192 & 2.979564 \\ 1 & 2.182021 & 7.334474 & 2.720013 \\ 6 & 4.289656 & 6.807100 & 2.174429 \\ 1 & 4.404676 & 7.261566 & 1.199888 \\ 6 & 4.059044 & 4.019560 & -0.603018 \\ 6 & 5.110630 & 4.938984 & -0.725743 \\ 1 & 5.445815 & 5.454597 & 0.165162 \\ 6 & 5.724185 & 5.191531 & -1.953134 \\ 1 & 6.543335 & 5.902305 & -2.012683 \\ 6 & 5.276805 & 4.530560 & -3.094518 \\ 1 & 5.738356 & 4.718428 & -4.058980 \\ 6 & 4.244288 & 3.597735 & -2.992832 \\ 1 & 3.927271 & 3.072808 & -3.885969 \\ 6 & 3.640977 & 3.309816 & -1.758606 \\ 6 & 5.366543 & 2.277138 & 1.659822 \\ 1 & 5.917807 & 2.719475 & 0.824558 \\ 6 & 6.246311 & 2.331862 & 2.911026 \\ 1 & 7.193943 & 1.809206 & 2.754124 \\ 1 & 5.750575 & 1.888277 & 3.778871 \\ 1 & 6.467296 & 3.376836 & 3.145718 \\ 6 & 2.369558 & 1.197541 & -3.287919 \\ 6 & 3.478061 & 0.428191 & -3.683506 \\ 1 & 4.338910 & 0.351849 & -3.028763 \\ 6 & 3.499079 & -0.211653 & -4.920690 \\ 6 & 4.369666 & -0.792629 & -5.211132 \\ 6 & 2.398558 & -0.108088 & -5.778077 \\ & 2.405282 & -0.615977 & -6.737998 \\ 6.292273 & 0.651920 & -5.394490\end{array}$


$\begin{array}{llll}0.434607 & 0.738265 & -6.055365\end{array}$ $\begin{array}{llll}1.277971 & 1.308257 & -4.159682\end{array}$ $\begin{array}{llll}0.412164 & 1.899247 & -3.882431\end{array}$ $\begin{array}{llll}0.805375 & 2.778847 & -1.388601\end{array}$ $\begin{array}{llll}0.454534 & 3.913388 & -2.136827\end{array}$ $\begin{array}{llll}1.133030 & 4.297866 & -2.892995\end{array}$ $\begin{array}{llll}-0.761467 & 4.557750 & -1.903205\end{array}$ $\begin{array}{llll}-1.029769 & 5.433150 & -2.487763\end{array}$ $\begin{array}{llll}-1.627352 & 4.084186 & -0.911502\end{array}$ $\begin{array}{llll}-2.571521 & 4.588601 & -0.731488\end{array}$ $\begin{array}{lll}-1.275906 & 2.962428 & -0.157669\end{array}$ $\begin{array}{llll}-1.939538 & 2.595623 & 0.619344\end{array}$ $\begin{array}{lll}-0.067242 & 2.305514 & -0.397687\end{array}$ $\begin{array}{llll}0.221891 & 1.446807 & 0.200695\end{array}$ $\begin{array}{llll}6.302347 & 0.083958 & -0.089659\end{array}$ $\begin{array}{llll}6.973271 & -1.146744 & -0.047335\end{array}$ $\begin{array}{llll}6.787933 & -1.852863 & 0.752444\end{array}$ $\begin{array}{llll}7.870788 & -1.494951 & -1.057264\end{array}$ $8.108461 \quad-0.643251 \quad-2.134968$ $\begin{array}{llll}8.796303 & -0.926994 & -2.921864\end{array}$ $\begin{array}{llll}7.424003 & 0.569468 & -2.190180\end{array}$ $\begin{array}{llll}6.528688 & 0.934800 & -1.183641\end{array}$ $\begin{array}{llll}5.990910 & 1.874590 & -1.272099\end{array}$ $\begin{array}{llll}5.091444 & -0.574631 & 2.502196\end{array}$ $\begin{array}{lll}3.898581 & -1.101886 & 3.008657\end{array}$ $\begin{array}{lll}2.956523 & -0.825242 & 2.548126\end{array}$ $\begin{array}{llll}3.918209 & -1.978544 & 4.095818\end{array}$ $\begin{array}{llll}5.120044 & -2.341445 & 4.696586\end{array}$ $\begin{array}{llll}5.136134 & -3.034345 & 5.529258\end{array}$ $\begin{array}{llll}6.309715 & -1.804809 & 4.198581\end{array}$ $\begin{array}{lll}6.303166 & -0.925601 & 3.116342\end{array}$ $\begin{array}{llll}7.244282 & -0.535400 & 2.746323\end{array}$ $\begin{array}{llll}2.917459 & 0.333519 & -0.067233\end{array}$ $\begin{array}{lll}7.619610 & -2.139922 & 4.859317\end{array}$ $\begin{array}{llll}2.612289 & -2.569372 & 4.549458\end{array}$ $\begin{array}{llll}8.627671 & -2.792355 & -0.955207\end{array}$ $\begin{array}{llll}7.579848 & 1.484385 & -3.374779\end{array}$ $\begin{array}{llll}6.443221 & 1.519470 & -4.118413\end{array}$ $\begin{array}{llll}7.830663 & 2.757781 & -2.994438\end{array}$ $\begin{array}{llll}8.577926 & 1.102979 & -4.197636\end{array}$ $\begin{array}{lll}7.923550 & -3.737762 & -0.294633\end{array}$ $\begin{array}{llll}9.794008 & -2.632646 & -0.283303\end{array}$ $\begin{array}{llll}8.944335 & -3.292032 & -2.170568\end{array}$ $\begin{array}{llll}7.973446 & -1.198373 & 5.767257\end{array}$ $\begin{array}{lll}8.628379 & -2.212012 & 3.961181\end{array}$ $\begin{array}{lll}7.571792 & -3.320200 & 5.513414\end{array}$ $\begin{array}{llll}2.729858 & -3.293780 & 5.679977\end{array}$ $\begin{array}{llll}1.681109 & -1.610992 & 4.777625\end{array}$ $\begin{array}{lll}2.092325 & -3.390298 & 3.599886\end{array}$ $\begin{array}{lll}3.935070 & -2.087640 & -1.451342\end{array}$ $\begin{array}{llll}2.028659 & -2.656797 & -0.217100\end{array}$ $\begin{array}{llll}4.117984 & -3.390747 & -1.875394\end{array}$ $\begin{array}{llll}4.613519 & -1.302627 & -1.755690\end{array}$ $2.202224 \quad-4.012011 \quad-0.615471$ $\begin{array}{llll}3.235552 & -4.387396 & -1.451653\end{array}$ $\begin{array}{llll}1.497935 & -4.757520 & -0.263523\end{array}$ $\begin{array}{lll}0.871990 & -2.329912 & 0.568414\end{array}$ $\begin{array}{lll}0.703758 & -1.169883 & 1.400876\end{array}$ $\begin{array}{lll}1.338591 & -0.078509 & 1.226974\end{array}$ $\begin{array}{llll}2.916537 & -1.716903 & -0.656347\end{array}$ $\begin{array}{lll}0.276156 & -3.195034 & 0.820516\end{array}$ $\begin{array}{lll}-0.218480 & -1.205434 & 2.410666\end{array}$ $\begin{array}{lll}-0.561927 & 0.017185 & 3.124358\end{array}$ $\begin{array}{lll}-0.141610 & 0.004317 & 4.137638\end{array}$ $\begin{array}{lll}-1.649964 & 0.098671 & 3.204479\end{array}$ $\begin{array}{lll}-0.167028 & 0.880529 & 2.593673\end{array}$ $\begin{array}{lll}-0.841092 & -2.414260 & 2.937350\end{array}$ $\begin{array}{lll}-0.232257 & -3.293802 & 2.738444\end{array}$ $\begin{array}{lll}-1.847280 & -2.564235 & 2.532012\end{array}$ $\begin{array}{llll}-0.925952 & -2.314369 & 4.022175\end{array}$ $\begin{array}{lll}-3.841043 & -1.026078 & -1.389123\end{array}$ $\begin{array}{llll}-5.659637 & -0.552855 & -0.100806\end{array}$ $\begin{array}{lll}-5.795202 & 0.731242 & 0.939830\end{array}$ $\begin{array}{lll}-6.997396 & -0.294416 & -1.061569\end{array}$ $\begin{array}{llll}-5.924545 & -1.815150 & 0.967997\end{array}$

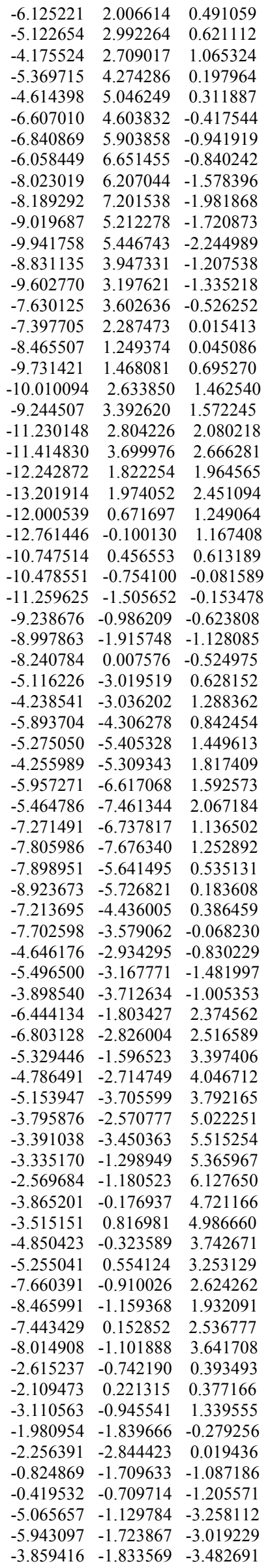


$\begin{array}{lrrr}1 & -3.893845 & -2.920790 & -3.419875 \\ 6 & -2.717103 & -1.291392 & -4.323186 \\ 1 & -2.988725 & -1.350614 & -5.388475 \\ 1 & -1.858142 & -1.953019 & -4.193314 \\ 6 & -2.299038 & 0.155558 & -3.972001 \\ 1 & -2.778001 & 0.866864 & -4.653685 \\ 1 & -1.224439 & 0.250748 & -4.139361 \\ 6 & -2.591230 & 0.588115 & -2.536868 \\ 1 & -1.721152 & 0.798499 & -1.924994 \\ 6 & -3.775526 & 1.189506 & -2.124735 \\ 1 & -3.719894 & 1.818317 & -1.241008 \\ 6 & -4.985510 & 1.434146 & -3.005544 \\ 1 & -5.828139 & 1.688104 & -2.358558 \\ 1 & -4.796178 & 2.318446 & -3.632164 \\ 6 & -5.375320 & 0.228719 & -3.888871 \\ 1 & -4.875397 & 0.294755 & -4.860610 \\ 1 & -6.448567 & 0.280791 & -4.095115 \\ 6 & -0.378734 & -2.670246 & -2.089277 \\ 6 & -0.895893 & -3.977548 & -2.185546 \\ 6 & 0.597722 & -2.263610 & -3.019823 \\ 6 & -0.459966 & -4.838127 & -3.190162 \\ 1 & -1.648623 & -4.317867 & -1.481533 \\ 6 & 1.032396 & -3.127056 & -4.023493 \\ 1 & 1.009304 & -1.261856 & -2.953623 \\ 6 & 0.502884 & -4.416848 & -4.115088 \\ 1 & -0.873082 & -5.840787 & -3.255370 \\ 1 & 1.781570 & -2.787804 & -4.731578 \\ 1 & 0.836979 & -5.090499 & -4.898920 \\ 1 & 3.351171 & -5.418830 & -1.766153 \\ 35 & 5.603698 & -3.802582 & -3.076109 \\ --------------------------------------------------------------- \\ 1\end{array}$

C1-P1 si-re 2

Number of imaginary frequencies $=1$

The smallest frequencies are $=-216.81396 .51309 .5023 \mathrm{~cm}(-1)$

Electronic energy $=-7222.6176109$

Zero-point correction=

(Hartree/Particle)

Thermal correction to Energy=

Thermal correction to Enthalpy=

Thermal correction to Gibbs Free Energy=
Sum of electronic and zero-point Energies

Sum of electronic and zero-point Energies

Sum of electronic and thermal Energies=

Sum of electronic and thermal Enthalpies=

Sum of electronic and thermal Free Energies=

1.873228

1.874173

1.589602

$-7220.863371$

$-7220.744382$

$-7220.743438$

$-7221.028008$

Cartesian Coordinates

$\begin{array}{lllc}\ldots 6 & 3.750607 & 4.705403 & 2.840742 \\ 15 & 2.590679 & 1.976356 & -1.414242 \\ 15 & 5.010277 & 0.394881 & 1.200302 \\ 6 & 3.620554 & 3.679845 & 1.020323 \\ 6 & 4.188778 & 2.841560 & 2.044930 \\ 6 & 3.226651 & 2.722991 & 3.095725 \\ 1 & 3.363379 & 2.169748 & 4.015168 \\ 6 & 2.081083 & 3.495447 & 2.741104 \\ 1 & 1.199318 & 3.641675 & 3.350562 \\ 6 & 2.325284 & 4.091448 & 1.469456 \\ 1 & 1.657445 & 4.749574 & 0.932483 \\ 6 & 5.468728 & 5.800839 & 3.257170 \\ 1 & 6.457527 & 5.562139 & 2.888641 \\ 6 & 4.884311 & 5.316723 & 4.467151 \\ 1 & 5.347931 & 4.637585 & 5.169928 \\ 6 & 3.561208 & 5.846068 & 4.560015 \\ 1 & 2.846762 & 5.637566 & 5.345618 \\ 6 & 3.328105 & 6.657660 & 3.408205 \\ 1 & 2.407338 & 7.173091 & 3.168343 \\ 6 & 4.505791 & 6.630297 & 2.603711 \\ 1 & 4.631689 & 7.125949 & 1.650842 \\ 6 & 4.264480 & 3.978728 & -0.288373 \\ 6 & 5.340427 & 4.876139 & -0.350146 \\ 1 & 5.673812 & 5.339397 & 0.569376 \\ 6 & 5.980872 & 5.171550 & -1.553940 \\ 1 & 6.818023 & 5.863480 & -1.566000 \\ 6 & 5.536939 & 4.577496 & -2.732878\end{array}$

$\begin{array}{llll}6.018896 & 4.799667 & -3.679921\end{array}$

$\begin{array}{llll}4.480979 & 3.666809 & -2.692154\end{array}$

$\begin{array}{llll}4.166363 & 3.194072 & -3.614680\end{array}$

$\begin{array}{llll}3.849183 & 3.335608 & -1.482846\end{array}$

$\begin{array}{lll}5.483642 & 2.092489 & 1.908031\end{array}$

$\begin{array}{lll}6.059425 & 2.559482 & 1.103312\end{array}$

$\begin{array}{llll}6.343499 & 2.072651 & 3.174114\end{array}$

$\begin{array}{lll}7.289940 & 1.551425 & 3.006720\end{array}$

$\begin{array}{llll}5.829820 & 1.591946 & 4.011104\end{array}$

$\begin{array}{lll}6.568638 & 3.102590 & 3.464470\end{array}$

$\begin{array}{llll}2.563007 & 1.346779 & -3.142949\end{array}$

$\begin{array}{llll}3.655126 & 0.557941 & -3.545999\end{array}$

$\begin{array}{llll}4.484058 & 0.402985 & -2.864226\end{array}$

$\begin{array}{llll}3.699833 & -0.000518 & -4.821403\end{array}$

$\begin{array}{llll}4.556680 & -0.598096 & -5.118155\end{array}$

$\begin{array}{llll}2.640278 & 0.206472 & -5.710779\end{array}$

$2.665661 \quad-0.236921 \quad-6.701823$

$\begin{array}{llll}1.551396 & 0.987324 & -5.319962\end{array}$

$\begin{array}{llll}0.726444 & 1.154497 & -6.006501\end{array}$

$\begin{array}{lll}1.513388 & 1.561714 & -4.045466\end{array}$

$\begin{array}{llll}0.662007 & 2.170215 & -3.762268\end{array}$

$\begin{array}{llll}0.997360 & 2.855389 & -1.170097\end{array}$

$\begin{array}{llll}0.676025 & 4.036921 & -1.855951\end{array}$

$\begin{array}{llll}1.359978 & 4.438421 & -2.598180\end{array}$

$\begin{array}{llll}-0.515034 & 4.708414 & -1.573413\end{array}$

$\begin{array}{lll}-0.761033 & 5.620379 & -2.109879\end{array}$

$\begin{array}{llll}-1.382550 & 4.216302 & -0.592007\end{array}$

$\begin{array}{llll}-2.305339 & 4.743577 & -0.371687\end{array}$

$\begin{array}{lll}-1.060471 & 3.046150 & 0.098612\end{array}$

$\begin{array}{lll}-1.722333 & 2.664073 & 0.870031\end{array}$

$\begin{array}{llll}0.120361 & 2.361403 & -0.193223\end{array}$

$\begin{array}{lll}0.388458 & 1.465855 & 0.358583\end{array}$

$\begin{array}{llll}6.402732 & -0.038677 & 0.075922\end{array}$

$\begin{array}{llll}7.045867 & -1.284766 & 0.079510\end{array}$

$\begin{array}{llll}6.828656 & -2.019939 & 0.844477\end{array}$

$\begin{array}{llll}7.958435 & -1.610131 & -0.924557\end{array}$

$\begin{array}{llll}8.236436 & -0.720522 & -1.961174\end{array}$

$\begin{array}{llll}8.933155 & -0.986840 & -2.746273\end{array}$

$\begin{array}{llll}7.580386 & 0.509047 & -1.978327\end{array}$

$\begin{array}{llll}6.673814 & 0.852813 & -0.974535\end{array}$

$\begin{array}{llll}6.160414 & 1.808037 & -1.034872\end{array}$

$\begin{array}{llll}5.124426 & -0.794139 & 2.598954\end{array}$

$\begin{array}{lll}3.917159 & -1.352025 & 3.033755\end{array}$

$\begin{array}{llll}2.989047 & -1.053618 & 2.558807\end{array}$

$\begin{array}{llll}3.904508 & -2.287124 & 4.070881\end{array}$

$\begin{array}{llll}5.086857 & -2.676387 & 4.694217\end{array}$

$\begin{array}{llll}5.076638 & -3.410010 & 5.491640\end{array}$

$\begin{array}{llll}6.290276 & -2.110304 & 4.267813\end{array}$

$\begin{array}{lll}6.316408 & -1.175733 & 3.232861\end{array}$

$\begin{array}{lll}7.267359 & -0.766147 & 2.912328\end{array}$

$\begin{array}{lll}3.025509 & 0.278342 & 0.036196\end{array}$

$\begin{array}{lll}7.578183 & -2.467740 & 4.959681\end{array}$

$\begin{array}{llll}2.590505 & -2.914631 & 4.443787\end{array}$

$\begin{array}{llll}8.684988 & -2.927129 & -0.858040\end{array}$

$\begin{array}{llll}7.775248 & 1.467142 & -3.122112\end{array}$

$\begin{array}{llll}6.654586 & 1.548057 & -3.886655\end{array}$

$\begin{array}{llll}8.035907 & 2.720939 & -2.687389\end{array}$

$\begin{array}{llll}8.785067 & 1.102468 & -3.938119\end{array}$

$\begin{array}{llll}7.938797 & -3.886247 & -0.266784\end{array}$

$\begin{array}{llll}9.829422 & -2.823902 & -0.138997\end{array}$

$\begin{array}{llll}9.033997 & -3.377644 & -2.083427\end{array}$

$\begin{array}{llll}7.902000 & -1.553217 & 5.905667\end{array}$

$\begin{array}{lll}8.615321 & -2.513065 & 4.092442\end{array}$

$\begin{array}{llll}7.510502 & -3.666596 & 5.576570\end{array}$

$\begin{array}{lll}2.651475 & -3.608716 & 5.597330\end{array}$

$\begin{array}{llll}1.612359 & -1.986944 & 4.577849\end{array}$

$\begin{array}{llll}2.168680 & -3.777474 & 3.480828\end{array}$

$\begin{array}{llll}4.049268 & -2.088867 & -1.455307\end{array}$

$\begin{array}{llll}2.091015 & -2.680425 & -0.318430\end{array}$

$\begin{array}{llll}4.211158 & -3.368067 & -1.954244\end{array}$

$\begin{array}{llll}4.759060 & -1.307810 & -1.690927\end{array}$

$\begin{array}{llll}2.243936 & -4.013768 & -0.791021\end{array}$

$\begin{array}{llll}3.293048 & -4.365192 & -1.617604\end{array}$

$\begin{array}{llll}1.511216 & -4.760223 & -0.505634\end{array}$

$\begin{array}{lll}0.912740 & -2.370477 & 0.442310\end{array}$ 


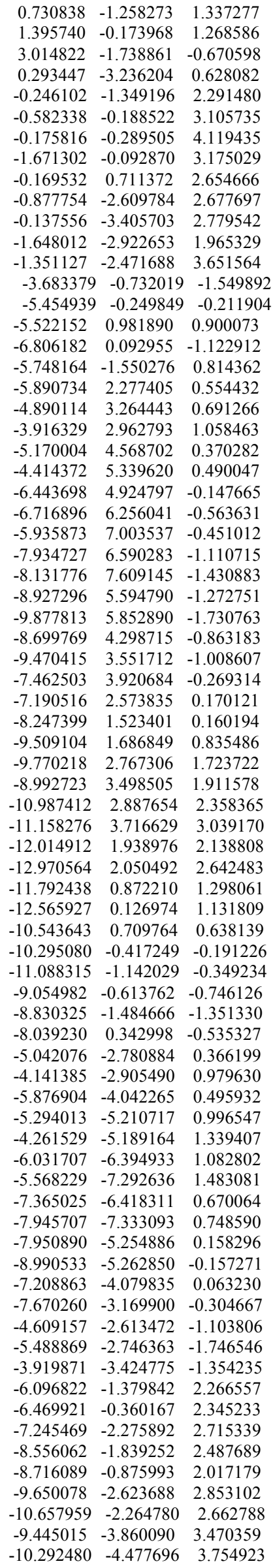

$\begin{array}{lll}-8.141851 & -4.292306 & 3.730595\end{array}$

$\begin{array}{lll}-7.972344 & -5.249342 & 4.216136\end{array}$

$\begin{array}{lll}-7.051392 & -3.502067 & 3.362617\end{array}$

$\begin{array}{lll}-6.047562 & -3.860363 & 3.561318\end{array}$

$\begin{array}{lll}-4.855535 & -1.473690 & 3.163088\end{array}$

$\begin{array}{lll}-4.137636 & -0.695475 & 2.890526\end{array}$

$\begin{array}{llll}-4.354707 & -2.442907 & 3.096047\end{array}$

$\begin{array}{lll}-5.144427 & -1.320998 & 4.207255\end{array}$

$\begin{array}{llll}-2.439417 & -0.677362 & 0.229388\end{array}$

$\begin{array}{llll}-1.883119 & 0.254780 & 0.308715\end{array}$

$\begin{array}{lll}-2.943192 & -0.948719 & 1.150341\end{array}$

$\begin{array}{llll}-1.851271 & -1.739400 & -0.537441\end{array}$

$-2.183576-2.752028-0.341733$

$\begin{array}{llll}-0.648991 & -1.604648 & -1.273002\end{array}$

$\begin{array}{llll}-0.195699 & -0.617963 & -1.300069\end{array}$

$\begin{array}{lll}-4.905902 & -0.606344 & -3.420442\end{array}$

$\begin{array}{llll}-5.816163 & -1.171041 & -3.236556\end{array}$

$\begin{array}{lll}-3.740513 & -1.358594 & -3.696931\end{array}$

$\begin{array}{llll}-3.848630 & -2.442711 & -3.730760\end{array}$

$\begin{array}{lll}-2.537924 & -0.833824 & -4.461085\end{array}$

$-2.751500 \quad-0.841353 \quad-5.541196$

$\begin{array}{llll}-1.716024 & -1.536972 & -4.305749\end{array}$

$\begin{array}{lll}-2.076148 & 0.574482 & -4.028760\end{array}$

$\begin{array}{llll}-2.548434 & 1.341353 & -4.651227\end{array}$

$\begin{array}{llll}-1.002867 & 0.653017 & -4.210995\end{array}$

$\begin{array}{llll}-2.333014 & 0.904589 & -2.561554\end{array}$

$\begin{array}{llll}-1.452816 & 0.991498 & -1.934225\end{array}$

$\begin{array}{llll}-3.481563 & 1.528403 & -2.090116\end{array}$

$\begin{array}{llll}-3.393917 & 2.064061 & -1.150073\end{array}$

$\begin{array}{lll}-4.676652 & 1.918013 & -2.939080\end{array}$

$\begin{array}{llll}-5.501585 & 2.162886 & -2.265652\end{array}$

$\begin{array}{llll}-4.438823 & 2.841681 & -3.487448\end{array}$

$\begin{array}{llll}-5.141196 & 0.819670 & -3.922206\end{array}$

$\begin{array}{llll}-4.648925 & 0.944396 & -4.891919\end{array}$

$\begin{array}{llll}-6.211754 & 0.950326 & -4.106574\end{array}$

$\begin{array}{llll}-0.201671 & -2.515000 & -2.319968\end{array}$

$\begin{array}{llll}-0.762089 & -3.794108 & -2.51339\end{array}$

$\begin{array}{llll}0.822982 & -2.091602 & -3.189204\end{array}$

$\begin{array}{llll}0.319094 & -4.611452 & -3.549693\end{array}$

$\begin{array}{llll}-1.553553 & -4.144979 & -1.858732\end{array}$

$\begin{array}{llll}1.264397 & -2.912312 & -4.225428\end{array}$

$\begin{array}{llll}1.267942 & -1.111874 & -3.049695\end{array}$

$\begin{array}{llll}0.694239 & -4.174049 & -4.411258\end{array}$

$\begin{array}{llll}-0.764971 & -5.592231 & -3.688947\end{array}$

$\begin{array}{llll}2.051722 & -2.562053 & -4.884872\end{array}$

$1.035089-4.813793 \quad-5.220212$

$3.393029-5.378199-1.991261$

$5.715417-3.746642-3.141995$

\section{C1-P1 si-re 3}

Number of imaginary frequencies $=1$

The smallest frequencies are $=-197.19898 .608513 .7259 \mathrm{~cm}(-1)$ Electronic energy $=-7222.6148556$

Zero-point correction=

(Hartree/Particle)

Thermal correction to Energy= $\quad 1.873794$

Thermal correction to Enthalpy= $\quad 1.874739$

Thermal correction to Gibbs Free Energy $=\quad 1.591463$

Sum of electronic and zero-point Energies $=\quad-7220.859906$

Sum of electronic and thermal Energies $=\quad-7220.741061$

Sum of electronic and thermal Enthalpies $=\quad-7220.740117$

Sum of electronic and thermal Free Energies $=\quad-7221.023393$

Cartesian Coordinates

$\begin{array}{llll}26 & 3.528601 & 4.903890 & 2.540814 \\ 15 & 2.483930 & 1.882572 & -1.541068 \\ 15 & 5.013984 & 0.585536 & 1.145216 \\ 6 & 3.448653 & 3.768440 & 0.781108 \\ 6 & 4.073219 & 3.028227 & 1.848084 \\ 6 & 3.125227 & 2.912800 & 2.911998 \\ 1 & 3.298121 & 2.418099 & 3.858415 \\ 6 & 1.933266 & 3.593364 & 2.524110 \\ 1 & 1.046845 & 3.719385 & 3.131448\end{array}$


$\begin{array}{llll}2.134050 & 4.127768 & 1.218251\end{array}$

$\begin{array}{llll}1.425762 & 4.713804 & 0.650702\end{array}$

$\begin{array}{llll}5.188166 & 6.103891 & 2.899930\end{array}$

$\begin{array}{llll}6.190541 & 5.890923 & 2.552695\end{array}$

$\begin{array}{lll}4.616213 & 5.663516 & 4.132721\end{array}$

$\begin{array}{lll}5.106094 & 5.051265 & 4.878127\end{array}$

$\begin{array}{llll}3.268422 & 6.133268 & 4.185969\end{array}$

$\begin{array}{llll}2.557588 & 5.936634 & 4.977864\end{array}$

$\begin{array}{llll}3.006866 & 6.863034 & 2.986272\end{array}$

$\begin{array}{llll}2.064946 & 7.318847 & 2.711043\end{array}$

$\begin{array}{lll}4.192414 & 6.846262 & 2.192717\end{array}$

$\begin{array}{llll}4.302511 & 7.289831 & 1.212583\end{array}$

$\begin{array}{llll}4.064568 & 4.023735 & -0.550071\end{array}$

$\begin{array}{llll}5.092343 & 4.969741 & -0.672666\end{array}$

$\begin{array}{llll}5.404663 & 5.502762 & 0.216326\end{array}$

$\begin{array}{llll}5.710212 & 5.227093 & -1.896897\end{array}$

$\begin{array}{llll}6.510352 & 5.959258 & -1.956082\end{array}$

$\begin{array}{llll}5.291411 & 4.543388 & -3.035693\end{array}$

$\begin{array}{llll}5.756624 & 4.734493 & -3.997772\end{array}$

$\begin{array}{llll}4.282557 & 3.584847 & -2.934412\end{array}$

$\begin{array}{llll}3.987348 & 3.043941 & -3.825475\end{array}$

$\begin{array}{llll}3.675642 & 3.292660 & -1.703027\end{array}$

$\begin{array}{lll}5.405997 & 2.344848 & 1.741505\end{array}$

$\begin{array}{llll}5.949252 & 2.788938 & 0.901867\end{array}$

$\begin{array}{llll}6.278879 & 2.442914 & 2.994967\end{array}$

$\begin{array}{llll}7.243003 & 1.948478 & 2.846670\end{array}$

$\begin{array}{llll}5.794361 & 1.995467 & 3.867185\end{array}$

$\begin{array}{llll}6.466682 & 3.497241 & 3.216606\end{array}$

$\begin{array}{llll}2.458898 & 1.141285 & -3.226528\end{array}$

$\begin{array}{llll}3.588554 & 0.399374 & -3.615339\end{array}$

$\begin{array}{llll}4.449462 & 0.348947 & -2.958318\end{array}$

$\begin{array}{llll}3.631534 & -0.242093 & -4.851130\end{array}$

$\begin{array}{llll}4.517125 & -0.802345 & -5.136686\end{array}$

$2.534872 \quad-0.163840 \quad-5.715750$

$\begin{array}{llll}2.560175 & -0.669795 & -6.676353\end{array}$

$\begin{array}{llll}1.409063 & 0.570555 & -5.339793\end{array}$

$\begin{array}{llll}0.556327 & 0.639571 & -6.008860\end{array}$

$\begin{array}{llll}1.368272 & 1.222832 & -4.103199\end{array}$

$\begin{array}{llll}0.484481 & 1.788692 & -3.829084\end{array}$

$\begin{array}{llll}0.854191 & 2.703911 & -1.346888\end{array}$

$\begin{array}{llll}0.490797 & 3.836069 & -2.092431\end{array}$

$\begin{array}{llll}1.165203 & 4.230735 & -2.846981\end{array}$

$-0.730650 \quad 4.468223-1.853575$

$\begin{array}{llll}-1.007527 & 5.344601 & -2.432616\end{array}$

$\begin{array}{llll}-1.589410 & 3.983436 & -0.860994\end{array}$

$\begin{array}{llll}-2.533186 & 4.483991 & -0.670865\end{array}$

$\begin{array}{llll}-1.228796 & 2.858923 & -0.115627\end{array}$

$\begin{array}{lll}-1.886286 & 2.484749 & 0.663632\end{array}$

$\begin{array}{llll}-0.013811 & 2.215874 & -0.359709\end{array}$

$\begin{array}{lll}0.289045 & 1.361490 & 0.237712\end{array}$

$\begin{array}{lll}6.417499 & 0.158062 & 0.032015\end{array}$

$\begin{array}{llll}7.136464 & -1.043375 & 0.106526\end{array}$

$\begin{array}{llll}6.974793 & -1.737246 & 0.922185\end{array}$

$\begin{array}{llll}8.051274 & -1.379395 & -0.892220\end{array}$

$\begin{array}{llll}8.261526 & -0.543294 & -1.987607\end{array}$

$\begin{array}{llll}8.963643 & -0.817800 & -2.765096\end{array}$

$\begin{array}{llll}7.533314 & 0.642051 & -2.071677\end{array}$

$6.618334 \quad 0.993995-1.078396$

$\begin{array}{llll}6.046509 & 1.910991 & -1.189091\end{array}$

$\begin{array}{llll}5.187639 & -0.512282 & 2.611220\end{array}$

$\begin{array}{lll}4.004047 & -1.090312 & 3.083195\end{array}$

$\begin{array}{llll}3.065068 & -0.854450 & 2.595107\end{array}$

$\begin{array}{lll}4.028068 & -1.964439 & 4.171965\end{array}$

$\begin{array}{llll}5.225045 & -2.272317 & 4.811926\end{array}$

$\begin{array}{llll}5.244443 & -2.960839 & 5.648212\end{array}$

$6.404569-1.683652 \quad 4.350160$

$\begin{array}{llll}6.393803 & -0.809768 & 3.263291\end{array}$

$\begin{array}{lll}7.329054 & -0.381549 & 2.921582\end{array}$

$\begin{array}{lll}3.020806 & 0.320595 & 0.012771\end{array}$

$\begin{array}{lll}7.705114 & -1.949253 & 5.058983\end{array}$

$\begin{array}{llll}2.736340 & -2.612401 & 4.586006\end{array}$

$\begin{array}{llll}8.854931 & -2.645324 & -0.758537\end{array}$

$\begin{array}{llll}7.667459 & 1.541841 & -3.270395\end{array}$

$\begin{array}{llll}6.530542 & 1.539584 & -4.014499\end{array}$

$\begin{array}{llll}7.888079 & 2.826402 & -2.909186\end{array}$ $\begin{array}{llll}8.674464 & 1.171599 & -4.087157\end{array}$

$\begin{array}{llll}8.176930 & -3.605835 & -0.092135\end{array}$

$10.004381 \quad-2.431117 \quad-0.072563$

$\begin{array}{llll}9.207404 & -3.152249 & -1.960810\end{array}$

$\begin{array}{lll}7.981350 & -0.981704 & 5.966528\end{array}$

$\begin{array}{llll}8.746707 & -1.982149 & 4.196686\end{array}$

$\begin{array}{llll}7.691622 & -3.123162 & 5.725827\end{array}$

$\begin{array}{llll}2.843333 & -3.303483 & 5.737972\end{array}$

$\begin{array}{lll}1.747547 & -1.700288 & 4.749733\end{array}$

$\begin{array}{lll}2.300075 & -3.481883 & 3.636345\end{array}$

$\begin{array}{llll}4.141254 & -2.086212 & -1.323601\end{array}$

$\begin{array}{llll}2.232969 & -2.697387 & -0.113160\end{array}$

$\begin{array}{llll}4.370083 & -3.388354 & -1.727640\end{array}$

$\begin{array}{llll}4.801408 & -1.285844 & -1.628287\end{array}$

$\begin{array}{llll}2.454334 & -4.052126 & -0.490192\end{array}$

$\begin{array}{llll}3.511766 & -4.406310 & -1.304436\end{array}$

$\begin{array}{llll}1.766791 & -4.813916 & -0.139551\end{array}$

$\begin{array}{llll}1.052305 & -2.397424 & 0.644060\end{array}$

$\begin{array}{llll}0.814467 & -1.232284 & 1.449890\end{array}$

$\begin{array}{lll}1.419684 & -0.122472 & 1.289580\end{array}$

$\begin{array}{llll}3.099657 & -1.735882 & -0.548592\end{array}$

$\begin{array}{llll}0.471687 & -3.276111 & 0.885870\end{array}$

$\begin{array}{lll}-0.148077 & -1.293612 & 2.422726\end{array}$

$\begin{array}{lll}-0.539432 & -0.085927 & 3.137139\end{array}$

$\begin{array}{lll}-0.095029 & -0.064641 & 4.140077\end{array}$

$\begin{array}{lll}-1.629740 & -0.058915 & 3.239534\end{array}$

$\begin{array}{lll}-0.206532 & 0.794205 & 2.591207\end{array}$

$\begin{array}{lll}-0.736250 & -2.534792 & 2.917014\end{array}$

$\begin{array}{lll}-0.011582 & -3.348525 & 2.905114\end{array}$

$\begin{array}{lll}-1.621472 & -2.829390 & 2.341241\end{array}$

$\begin{array}{lll}-1.037454 & -2.384613 & 3.956597\end{array}$

$-3.637088-1.138790 \quad-1.433773$

$\begin{array}{lll}-5.408193 & -0.561772 & -0.132310\end{array}$

$\begin{array}{llll}-5.580272 & 0.833721 & 0.737650\end{array}$

$\begin{array}{llll}-6.801111 & -0.551163 & -1.034384\end{array}$

$\begin{array}{lll}-5.530560 & -1.667629 & 1.143465\end{array}$

$\begin{array}{lll}-6.035123 & 2.010905 & 0.153211\end{array}$

$\begin{array}{lll}-5.110653 & 3.076647 & 0.100524\end{array}$

$\begin{array}{lll}-4.125354 & 2.926702 & 0.525776\end{array}$

$\begin{array}{llll}-5.476666 & 4.266797 & -0.475588\end{array}$

$\begin{array}{llll}-4.780665 & 5.099918 & -0.509264\end{array}$

$\begin{array}{llll}-6.760236 & 4.417151 & -1.064455\end{array}$

$\begin{array}{llll}-7.117044 & 5.613843 & -1.743228\end{array}$

$\begin{array}{llll}-6.394037 & 6.424755 & -1.776949\end{array}$

$\begin{array}{llll}-8.341837 & 5.739591 & -2.358122\end{array}$

$\begin{array}{llll}-8.602899 & 6.655525 & -2.880069\end{array}$

$\begin{array}{llll}-9.257256 & 4.660840 & -2.324888\end{array}$

$\begin{array}{llll}-10.212221 & 4.750595 & -2.834623\end{array}$

$\begin{array}{llll}-8.949170 & 3.494700 & -1.658225\end{array}$

$\begin{array}{llll}-9.661425 & 2.678608 & -1.655748\end{array}$

$\begin{array}{llll}-7.702451 & 3.336794 & -0.989287\end{array}$

$\begin{array}{llll}-7.345873 & 2.129267 & -0.285338\end{array}$

$\begin{array}{lll}-8.331375 & 1.033900 & -0.070724\end{array}$

$\begin{array}{lll}-9.604409 & 1.262615 & 0.561499\end{array}$

$\begin{array}{lll}-9.935181 & 2.492780 & 1.194305\end{array}$

$\begin{array}{lll}-9.202965 & 3.291015 & 1.215842\end{array}$

$\begin{array}{lll}-11.158860 & 2.674713 & 1.800956\end{array}$

$\begin{array}{lll}-11.381916 & 3.619802 & 2.287843\end{array}$

$\begin{array}{lll}-12.123383 & 1.638910 & 1.806933\end{array}$

$\begin{array}{lll}-13.085248 & 1.797632 & 2.285766\end{array}$

$\begin{array}{lll}-11.830347 & 0.429163 & 1.219071\end{array}$

$\begin{array}{lll}-12.552920 & -0.382664 & 1.233189\end{array}$

$\begin{array}{lll}-10.572912 & 0.204677 & 0.596189\end{array}$

$\begin{array}{lll}-10.250201 & -1.059497 & 0.035100\end{array}$

$\begin{array}{lll}-10.997394 & -1.847941 & 0.043745\end{array}$

$\begin{array}{lll}-8.999350 & -1.294117 & -0.478556\end{array}$

$\begin{array}{llll}-8.723817 & -2.255726 & -0.896691\end{array}$

$\begin{array}{lll}-8.046164 & -0.254063 & -0.491018\end{array}$

$\begin{array}{lll}-4.819007 & -2.933488 & 0.813426\end{array}$

$\begin{array}{lll}-3.865837 & -2.954218 & 1.351589\end{array}$

$\begin{array}{lll}-5.596904 & -4.182054 & 1.190564\end{array}$

$-4.929656-5.272086 \quad 1.762428$

$\begin{array}{lll}-3.869922 & -5.188808 & 1.994390\end{array}$

$\begin{array}{lll}-5.614378 & -6.455354 & 2.050865\end{array}$

$\begin{array}{llll}-5.083847 & -7.290404 & 2.500295\end{array}$ 


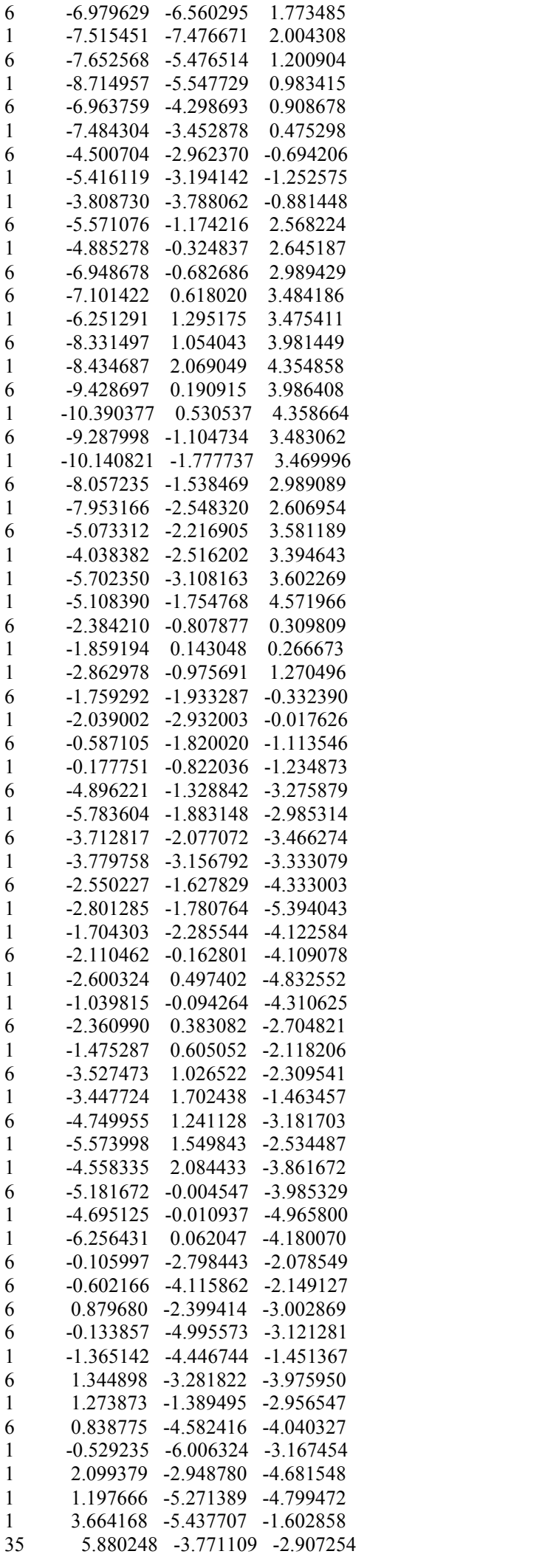

C1-P1 si-si_1

Number of imaginary frequencies $=1$

The smallest frequencies are $=-123.24306 .59769 .6602 \mathrm{~cm}(-1)$ Electronic energy $=-7222.6139549$

Zero-point correction $=$

1.754755

(Hartree/Particle)

Thermal correction to Energy=
Thermal correction to Enthalpy=

1.874152

Thermal correction to Gibbs Free Energy $=\quad 1.591107$

Sum of electronic and zero-point Energies $=\quad-7220.859199$

Sum of electronic and thermal Energies $=\quad-7220.740747$

Sum of electronic and thermal Enthalpies $=\quad-7220.739803$

Sum of electronic and thermal Free Energies $=\quad-7221.022848$

Cartesian Coordinates

$\begin{array}{llll}26 & 5.035540 & 4.254583 & 2.966468\end{array}$

$\begin{array}{llll}15 & 3.561049 & 2.295706 & -1.620015\end{array}$

$15 \quad 4.951451 \quad-0.123056 \quad 1.058023$

$\begin{array}{llll}6 & 4.786274 & 3.443273 & 1.052508\end{array}$

$\begin{array}{lll}4.926098 & 2.403798 & 2.039746\end{array}$

$\begin{array}{lll}3.863602 & 2.556667 & 2.983544\end{array}$

$\begin{array}{lll}3.698047 & 1.934565 & 3.852886\end{array}$

$\begin{array}{llll}3.085930 & 3.687663 & 2.601372\end{array}$

$2.237095 \quad 4.085076 \quad 3.139354$

$\begin{array}{llll}3.650688 & 4.234979 & 1.413943\end{array}$

$\begin{array}{llll}3.304397 & 5.110035 & 0.882210\end{array}$

$\begin{array}{lll}6.955507 & 4.669372 & 3.651684\end{array}$

$\begin{array}{llll}7.846447 & 4.133736 & 3.352093\end{array}$

$\begin{array}{lll}6.099384 & 4.329154 & 4.743521\end{array}$

$\begin{array}{llll}6.223790 & 3.485593 & 5.409207\end{array}$

$\begin{array}{lll}5.025574 & 5.270791 & 4.771618\end{array}$

$\begin{array}{lll}4.190823 & 5.261747 & 5.460152\end{array}$

$\begin{array}{llll}5.216943 & 6.191408 & 3.696725\end{array}$

$4.555637 \quad 7.005357 \quad 3.430404$

$\begin{array}{llll}6.409951 & 5.822127 & 3.006985\end{array}$

$\begin{array}{llll}6.809845 & 6.312328 & 2.129722\end{array}$

$\begin{array}{llll}5.641668 & 3.583221 & -0.154849\end{array}$

$\begin{array}{llll}6.946651 & 4.081872 & -0.031134\end{array}$

$\begin{array}{lll}7.292206 & 4.370947 & 0.952957\end{array}$

$\begin{array}{llll}7.793013 & 4.201483 & -1.133400\end{array}$

$8.801487 \quad 4.583466 \quad-1.003407$

$\begin{array}{lll}7.335055 & 3.826959 & -2.394035\end{array}$

$\begin{array}{lll}7.978829 & 3.910666 & -3.264049\end{array}$

$\begin{array}{lll}6.046781 & 3.311399 & -2.536465\end{array}$

$\begin{array}{llll}5.720612 & 2.995985 & -3.519532\end{array}$

$\begin{array}{llll}5.191082 & 3.164061 & -1.432438\end{array}$

$\begin{array}{lll}5.902412 & 1.265634 & 1.943467\end{array}$

$\begin{array}{llll}6.689924 & 1.548025 & 1.238468\end{array}$

$\begin{array}{lll}6.556394 & 0.875020 & 3.271241\end{array}$

$\begin{array}{llll}7.262531 & 0.050067 & 3.140221\end{array}$

$\begin{array}{llll}5.816887 & 0.576471 & 4.019308\end{array}$

$\begin{array}{lll}7.103212 & 1.737149 & 3.663580\end{array}$

$\begin{array}{llll}3.644415 & 1.727885 & -3.369028\end{array}$

$\begin{array}{llll}4.236069 & 0.476326 & -3.598783\end{array}$

$\begin{array}{llll}4.598305 & -0.104717 & -2.755973\end{array}$

$\begin{array}{llll}4.376696 & -0.017410 & -4.894934\end{array}$

$\begin{array}{llll}4.840491 & -0.986365 & -5.056209\end{array}$

$\begin{array}{llll}3.917334 & 0.734662 & -5.979834\end{array}$

$\begin{array}{llll}4.018587 & 0.350436 & -6.990736\end{array}$

$\begin{array}{llll}3.329514 & 1.983976 & -5.761235\end{array}$

$\begin{array}{llll}2.974349 & 2.572925 & -6.602171\end{array}$

$\begin{array}{llll}3.194802 & 2.481864 & -4.462659\end{array}$

$\begin{array}{llll}2.737237 & 3.453448 & -4.307392\end{array}$

$\begin{array}{llll}2.311328 & 3.644733 & -1.623630\end{array}$

$\begin{array}{llll}1.019500 & 3.338959 & -1.167521\end{array}$

$\begin{array}{llll}0.817847 & 2.348136 & -0.778363\end{array}$

$\begin{array}{llll}0.022472 & 4.316253 & -1.165737\end{array}$

$\begin{array}{llll}-0.971341 & 4.071513 & -0.801145\end{array}$

$\begin{array}{llll}0.308625 & 5.609572 & -1.611276\end{array}$

$\begin{array}{llll}-0.464912 & 6.372421 & -1.604432\end{array}$

$\begin{array}{llll}1.597083 & 5.924040 & -2.054844\end{array}$

$\begin{array}{llll}1.825360 & 6.930166 & -2.394999\end{array}$

$\begin{array}{llll}2.595897 & 4.947490 & -2.061285\end{array}$

$\begin{array}{llll}3.595821 & 5.201641 & -2.400735\end{array}$

$\begin{array}{lll}6.191891 & -0.944994 & -0.030792\end{array}$

$\begin{array}{llll}6.356181 & -2.337801 & -0.111769\end{array}$

$\begin{array}{llll}5.822683 & -3.000961 & 0.556448\end{array}$

$\begin{array}{llll}7.209643 & -2.891945 & -1.064236\end{array}$

$\begin{array}{llll}7.888125 & -2.086438 & -1.980407\end{array}$

$\begin{array}{llll}8.543286 & -2.525103 & -2.724029\end{array}$

$\begin{array}{lll}7.702099 & -0.708193 & -1.921904\end{array}$ 
$\begin{array}{lll}6.867243 & -0.136824 & -0.958832\end{array}$ $\begin{array}{llll}6.733250 & 0.940434 & -0.957584\end{array}$ $\begin{array}{llll}4.553942 & -1.348013 & 2.375641\end{array}$ $\begin{array}{llll}3.221528 & -1.386129 & 2.803457\end{array}$ $\begin{array}{lll}2.500771 & -0.711965 & 2.358390\end{array}$ $\begin{array}{lll}2.821409 & -2.283402 & 3.797150\end{array}$ $\begin{array}{lll}3.738443 & -3.157788 & 4.375241\end{array}$ $\begin{array}{llll}3.425685 & -3.862541 & 5.136499\end{array}$ $\begin{array}{llll}5.069793 & -3.111322 & 3.957944\end{array}$ $\begin{array}{lll}5.482497 & -2.210714 & 2.976170\end{array}$ $\begin{array}{llll}6.524351 & -2.196499 & 2.677850\end{array}$

$\begin{array}{llll}3.130558 & 0.589568 & -0.176657\end{array}$ $\begin{array}{llll}6.091965 & -4.000740 & 4.612839\end{array}$

$\begin{array}{llll}1.370356 & -2.337081 & 4.192284\end{array}$

$\begin{array}{llll}7.458273 & -4.376472 & -1.104799\end{array}$

$\begin{array}{llll}8.358066 & 0.196827 & -2.930266\end{array}$

$\begin{array}{llll}7.441768 & 0.751447 & -3.763384\end{array}$

$\begin{array}{llll}9.008955 & 1.222356 & -2.333682\end{array}$

$\begin{array}{llll}9.252364 & -0.453903 & -3.702994\end{array}$

$\begin{array}{llll}7.260205 & -4.882364 & -2.345572\end{array}$

$\begin{array}{llll}6.666840 & -5.061059 & -0.254296\end{array}$

$8.741338 \quad-4.664113 \quad-0.776241$

$\begin{array}{lll}7.067758 & -4.361860 & 3.748901\end{array}$

$\begin{array}{llll}5.541081 & -5.132761 & 5.101390\end{array}$

$\begin{array}{lll}6.700882 & -3.377719 & 5.650520\end{array}$

$\begin{array}{lll}1.184025 & -2.883061 & 5.412533\end{array}$

$\begin{array}{llll}0.817977 & -1.101425 & 4.215207\end{array}$

$\begin{array}{lll}0.633974 & -3.075433 & 3.321343\end{array}$

$\begin{array}{lll}2.936145 & -2.189322 & -1.185528\end{array}$

$\begin{array}{llll}1.108732 & -1.780339 & 0.221149\end{array}$

$\begin{array}{llll}2.688471 & -3.549797 & -1.205471\end{array}$

$3.771343-1.767187 \quad-1.729747$

$\begin{array}{llll}0.837690 & -3.178241 & 0.237508\end{array}$

$\begin{array}{llll}1.620029 & -4.069870 & -0.469736\end{array}$

$\begin{array}{llll}-0.001258 & -3.541417 & 0.821201\end{array}$

$\begin{array}{lll}0.191428 & -0.914324 & 0.902779\end{array}$

$\begin{array}{lll}0.450775 & 0.437412 & 1.327051\end{array}$

$\begin{array}{lll}1.470026 & 1.100761 & 0.968828\end{array}$

$2.179468 \quad-1.318970 \quad-0.495263$

$\begin{array}{lll}-0.584421 & -1.449580 & 1.428747\end{array}$

$\begin{array}{lll}-0.468249 & 1.095314 & 2.116119\end{array}$

$\begin{array}{lll}-0.199867 & 2.480752 & 2.482453\end{array}$

$\begin{array}{lll}0.374090 & 2.974139 & 1.701803\end{array}$

$\begin{array}{llll}0.365357 & 2.544156 & 3.422785\end{array}$

$\begin{array}{lll}-1.154695 & 2.998174 & 2.621518\end{array}$

$\begin{array}{lll}-1.442158 & 0.445319 & 2.980932\end{array}$

$\begin{array}{lll}-1.688445 & -0.551582 & 2.637987\end{array}$

$\begin{array}{lll}-2.366310 & 1.029308 & 2.997148\end{array}$

$\begin{array}{lll}-1.064198 & 0.372541 & 4.007812\end{array}$

$\begin{array}{llll}-4.207658 & 0.693955 & -1.558242\end{array}$

$\begin{array}{llll}-5.861341 & -0.304227 & -0.354238\end{array}$

$\begin{array}{lll}-6.293499 & 0.131352 & 1.185835\end{array}$

$\begin{array}{lll}-7.326813 & -0.145424 & -1.133122\end{array}$

$\begin{array}{llll}-5.497212 & -1.919441 & -0.089014\end{array}$

$\begin{array}{lll}-7.074101 & 1.261574 & 1.407926\end{array}$

$\begin{array}{lll}-6.438235 & 2.348372 & 2.046740\end{array}$

$\begin{array}{lll}-5.398192 & 2.239450 & 2.336237\end{array}$

$\begin{array}{lll}-7.143095 & 3.502667 & 2.282421\end{array}$

$\begin{array}{lll}-6.669335 & 4.344254 & 2.779741\end{array}$

$\begin{array}{llll}-8.491924 & 3.623394 & 1.851481\end{array}$

$\begin{array}{lll}-9.207757 & 4.839864 & 2.019542\end{array}$

$\begin{array}{lll}-8.707420 & 5.671556 & 2.508688\end{array}$

$\begin{array}{llll}-10.499398 & 4.969435 & 1.562003\end{array}$

$\begin{array}{lll}-11.035365 & 5.905184 & 1.690704\end{array}$

$\begin{array}{llll}-11.126113 & 3.882206 & 0.907373\end{array}$

$\begin{array}{llll}-12.137030 & 3.994677 & 0.526292\end{array}$

$\begin{array}{lll}-10.465827 & 2.683809 & 0.743066\end{array}$

$\begin{array}{lll}-10.959663 & 1.865841 & 0.23209\end{array}$

$\begin{array}{lll}-9.137439 & 2.505844 & 1.221606\end{array}$

$\begin{array}{lll}-8.416795 & 1.268899 & 1.061412\end{array}$

$\begin{array}{lll}-9.060602 & 0.038223 & 0.520593\end{array}$

$\begin{array}{lll}-10.226836 & -0.548762 & 1.127181\end{array}$

$\begin{array}{lll}-10.781283 & -0.070042 & 2.347485\end{array}$

$\begin{array}{lll}-10.312374 & 0.766074 & 2.852401\end{array}$

$\begin{array}{lll}-11.894970 & -0.661255 & 2.903536\end{array}$ $\begin{array}{llll}-12.293973 & -0.280571 & 3.839400\end{array}$

$\begin{array}{lll}-12.520305 & -1.762763 & 2.272109\end{array}$

$\begin{array}{lll}-13.401391 & -2.214032 & 2.718806\end{array}$

$\begin{array}{lll}-11.998221 & -2.266677 & 1.102497\end{array}$

$\begin{array}{lll}-12.457091 & -3.123553 & 0.616083\end{array}$

$\begin{array}{lll}-10.841388 & -1.690762 & 0.510182\end{array}$

$\begin{array}{lll}-10.272682 & -2.242196 & -0.669925\end{array}$

$-10.753185 \quad-3.096386-1.138524$

$\begin{array}{lll}-9.112984 & -1.725135 & -1.192355\end{array}$

$\begin{array}{lll}-8.638361 & -2.150063 & -2.070284\end{array}$

$\begin{array}{lll}-8.509113 & -0.609243 & -0.573306\end{array}$

$\begin{array}{llll}-4.362469 & -2.378411 & -0.927135\end{array}$

$\begin{array}{lll}-3.468882 & -2.401763 & -0.292395\end{array}$

$\begin{array}{llll}-4.571226 & -3.777322 & -1.478889\end{array}$

$\begin{array}{llll}-3.493235 & -4.669855 & -1.521897\end{array}$

$\begin{array}{llll}-2.536281 & -4.368950 & -1.100706\end{array}$

$\begin{array}{llll}-3.638931 & -5.935077 & -2.096418\end{array}$

$\begin{array}{llll}-2.793796 & -6.617530 & -2.123583\end{array}$

$\begin{array}{llll}-4.871780 & -6.324011 & -2.625270\end{array}$

$\begin{array}{llll}-4.990215 & -7.309672 & -3.066277\end{array}$

$\begin{array}{llll}-5.955345 & -5.440453 & -2.578777\end{array}$

$\begin{array}{llll}-6.918314 & -5.740081 & -2.983466\end{array}$

$\begin{array}{llll}-5.805380 & -4.174493 & -2.011978\end{array}$

$\begin{array}{llll}-6.646823 & -3.488383 & -1.966545\end{array}$

$\begin{array}{llll}-4.132932 & -1.399923 & -2.083708\end{array}$

$\begin{array}{llll}-4.898127 & -1.583535 & -2.847170\end{array}$

$\begin{array}{llll}-3.170379 & -1.618595 & -2.548435\end{array}$

$\begin{array}{lll}-5.767722 & -2.792761 & 1.102326\end{array}$

$\begin{array}{lll}-5.686977 & -3.802735 & 0.691128\end{array}$

$\begin{array}{lll}-4.667143 & -2.676504 & 2.157564\end{array}$

$\begin{array}{llll}-3.579407 & -3.562967 & 2.105613\end{array}$

$\begin{array}{lll}-3.545227 & -4.314203 & 1.320967\end{array}$

$\begin{array}{lll}-2.553556 & -3.506196 & 3.053635\end{array}$

$\begin{array}{llll}-1.716385 & -4.195401 & 2.992603\end{array}$

$\begin{array}{llll}-2.612531 & -2.572121 & 4.089613\end{array}$

$\begin{array}{lll}-1.815121 & -2.524610 & 4.825641\end{array}$

$\begin{array}{llll}-3.697102 & -1.691803 & 4.159339\end{array}$

$\begin{array}{lll}-3.751662 & -0.962104 & 4.962692\end{array}$

$\begin{array}{lll}-4.708269 & -1.734244 & 3.196410\end{array}$

$\begin{array}{lll}-5.535739 & -1.036848 & 3.258129\end{array}$

$\begin{array}{lll}-7.182593 & -2.699028 & 1.673551\end{array}$

$\begin{array}{llll}-7.919072 & -2.889571 & 0.891282\end{array}$

$\begin{array}{lll}-7.406083 & -1.736359 & 2.129281\end{array}$

$\begin{array}{lll}-7.291307 & -3.472567 & 2.440098\end{array}$

$\begin{array}{lll}-3.112255 & 0.735374 & 0.313012\end{array}$

$\begin{array}{llll}-3.304507 & 1.659261 & 0.855627\end{array}$

$\begin{array}{lll}-3.241877 & -0.148475 & 0.934244\end{array}$

$\begin{array}{llll}-2.022546 & 0.712566 & -0.626499\end{array}$

$\begin{array}{llll}-1.551600 & 1.638780 & -0.938844\end{array}$

$\begin{array}{llll}-1.401323 & -0.504861 & -0.972567\end{array}$

$\begin{array}{lll}-1.854168 & -1.393270 & -0.553646\end{array}$

$\begin{array}{llll}-5.403607 & 1.018238 & -3.411100\end{array}$

$\begin{array}{llll}-5.938327 & 0.093811 & -3.615758\end{array}$

$\begin{array}{llll}-4.027546 & 1.034328 & -3.754119\end{array}$

$\begin{array}{llll}-3.624370 & 0.132318 & -4.214090\end{array}$

$\begin{array}{llll}-3.205224 & 2.281227 & -4.039908\end{array}$

$\begin{array}{llll}-3.372558 & 2.616156 & -5.075358\end{array}$

$\begin{array}{llll}-2.150087 & 1.993530 & -3.978462\end{array}$

$\begin{array}{llll}-3.465308 & 3.443644 & -3.059015\end{array}$

$\begin{array}{llll}-4.264266 & 4.090300 & -3.434259\end{array}$

$\begin{array}{llll}-2.568380 & 4.069457 & -3.003719\end{array}$

$\begin{array}{llll}-3.817627 & 2.986304 & -1.645366\end{array}$

$\begin{array}{llll}-3.060540 & 3.186768 & -0.890792\end{array}$

$\begin{array}{llll}-5.115394 & 2.802882 & -1.171233\end{array}$

$\begin{array}{llll}-5.261409 & 2.884149 & -0.098444\end{array}$

$\begin{array}{llll}-6.382015 & 2.930437 & -1.994277\end{array}$

$\begin{array}{llll}-7.192856 & 2.468283 & -1.426060\end{array}$

$\begin{array}{llll}-6.638923 & 3.995043 & -2.098033\end{array}$

$\begin{array}{llll}-6.293493 & 2.262153 & -3.382556\end{array}$

$\begin{array}{llll}-5.934746 & 2.978110 & -4.128746\end{array}$

$\begin{array}{llll}-7.301948 & 1.976292 & -3.696770\end{array}$

$\begin{array}{llll}-0.578593 & -0.755700 & -2.143216\end{array}$

$\begin{array}{llll}0.018410 & 0.264859 & -2.910124\end{array}$

$\begin{array}{llll}-0.419719 & -2.092319 & -2.565615\end{array}$

$0.744046 \quad-0.047816 \quad-4.054811$ 


$\begin{array}{lrrr}1 & -0.086814 & 1.300788 & -2.610139 \\ 6 & 0.323251 & -2.403898 & -3.701939 \\ 1 & -0.888884 & -2.886624 & -1.995184 \\ 6 & 0.908718 & -1.380766 & -4.450360 \\ 1 & 1.195550 & 0.745068 & -4.638359 \\ 1 & 0.441440 & -3.440553 & -4.003353 \\ 1 & 1.489391 & -1.612846 & -5.338043 \\ 1 & 1.406114 & -5.133352 & -0.455857 \\ 35 & 3.836318 & -4.714453 & -2.273222\end{array}$

\section{C1-P1 si-si_2}

Number of imaginary frequencies $=1$

The smallest frequencies are $=-137.40568 .968411 .7779 \mathrm{~cm}(-1)$ Electronic energy $=-7222.6224907$

Zero-point correction $=$

1.755417

(Hartree/Particle)

Thermal correction to Energy=

Thermal correction to Enthalpy=

1.873917

Thermal correction to Gibbs Free Energy=

Sum of electronic and zero-point Energies=

Sum of electronic and thermal Energies=

Sum of electronic and thermal Enthalpies=

1.593551

$-7220.867073$

$-7220.748573$

$-7220.747629$

Sum of electronic and thermal Free Energies=

$-7221.028940$

Cartesian Coordinates

\begin{tabular}{|c|c|c|c|}
\hline 26 & 3.553249 & 5.046322 & 2.2 \\
\hline 15 & 2.258195 & 1.915635 & \\
\hline 15 & 4.679298 & 0.568674 & \\
\hline 6 & 3.361399 & 3.846684 & 0.52300 \\
\hline 6 & 3.934571 & 3.106577 & 1.617797 \\
\hline 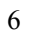 & 2.990953 & 3.116651 & 2.692892 \\
\hline 1 & 3.133540 & 2.653601 & 3.660178 \\
\hline 6 & 1.854544 & 3.875379 & 2.282060 \\
\hline 1 & 0.989414 & 4.104864 & 2.889879 \\
\hline 6 & 2.086282 & 4.333540 & 0.952099 \\
\hline 1 & 1.424204 & 4.951502 & 0.362846 \\
\hline 6 & 5.306005 & 6.129042 & 2.522573 \\
\hline 1 & 6.280392 & 5.829949 & 2.159826 \\
\hline 6 & 4.731437 & 5.769021 & 3.779708 \\
\hline 1 & 5.190080 & 5.140755 & 4.531609 \\
\hline 6 & 3.424136 & 6.340 & 3.846259 \\
\hline 1 & 2.717830 & 6.217210 & 4.656730 \\
\hline 6 & 3.190429 & 7.052857 & 2.630590 \\
\hline 1 & 2.278984 & 7.569998 & 2.360966 \\
\hline 6 & 4.353037 & 6.923893 & 1.813835 \\
\hline 1 & 4.475812 & 7.329184 & 0.818644 \\
\hline 6 & 3.977802 & 3.999845 & -0.823701 \\
\hline 6 & 5.055791 & 4.879659 & -0.999139 \\
\hline 1 & 5.412577 & 5.428629 & -0.137251 \\
\hline 6 & 5.665906 & 5.054341 & -2.241334 \\
\hline 1 & 6.506498 & 5.734862 & -2.340937 \\
\hline 6 & 5.183203 & 4.358651 & -3.347292 \\
\hline 1 & 5.635472 & 4.491773 & -4.325365 \\
\hline 6 & 4.121655 & 3.466567 & -3.193119 \\
\hline 1 & 3.773054 & $2.920^{7}$ & -4.061386 \\
\hline 6 & 3.526791 & 3.247655 & -1.940098 \\
\hline 6 & 5.210459 & 2.319627 & 1.531356 \\
\hline 1 & 5.775351 & 2.688006 & 0.670026 \\
\hline 6 & 6.104315 & 2.404763 & 2.770820 \\
\hline 1 & 7.019406 & 1.819882 & 2.640895 \\
\hline 1 & 5.593599 & 2.046556 & 3.668887 \\
\hline 1 & 6.385601 & 3.448386 & 2.935991 \\
\hline 6 & 2.188332 & 1.061324 & -3.328139 \\
\hline 6 & 3.346713 & 0.419328 & -3.801677 \\
\hline 1 & 4.281234 & 0.517127 & -3.260231 \\
\hline 6 & 3.321995 & -0.311742 & -4.987617 \\
\hline 1 & 4.232029 & -0.785271 & -5.344190 \\
\hline 6 & 2.131037 & -0.433269 & -5.710826 \\
\hline 1 & 2.108311 & -1.007168 & -6.632617 \\
\hline 6 & 0.971302 & 0.184725 & -5.239812 \\
\hline 1 & 0.040000 & 0.090528 & -5.790640 \\
\hline 6 & 0.999831 & 0.931406 & -4.058383 \\
\hline 1 & 0.093600 & 1.410436 & -3.707246 \\
\hline
\end{tabular}

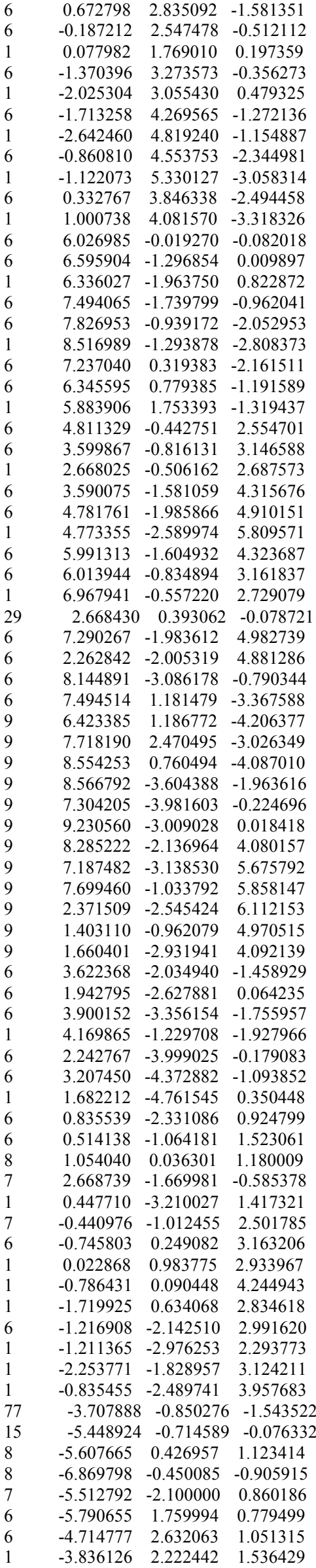




$\begin{array}{llllllll}-4.807767 & 3.954689 & 0.696516 & 1 & -1.732271 & -1.265556 & -4.312520 \\ -3.992761 & 4.640081 & 0.909499 & 6 & -2.712851 & 0.659854 & -4.252819 \\ -5.954423 & 4.432297 & 0.004880 & 1 & -3.480782 & 1.155419 & -4.852598 \\ -6.019960 & 5.771232 & -0.467986 & 1 & -1.761236 & 1.074769 & -4.593088 \\ -5.183158 & 6.434326 & -0.263841 & 6 & -2.911798 & 1.025805 & -2.787287 \\ -7.109974 & 6.216688 & -1.181111 & 1 & -2.028262 & 1.355916 & -2.249450 \\ -7.146439 & 7.239304 & -1.544836 & 6 & -4.140183 & 1.381395 & -2.256233 \\ -8.181912 & 5.333375 & -1.452945 & 1 & -4.137643 & 1.982577 & -1.353050 \\ -9.030074 & 5.680807 & -2.035753 & 6 & -5.441042 & 1.376231 & -3.034584 \\ -8.159321 & 4.035510 & -0.989887 & 1 & -6.257101 & 1.504336 & -2.323848 \\ -8.986007 & 3.371189 & -1.212720 & 1 & -5.459655 & 2.258655 & -3.690997 \\ -7.057074 & 3.544319 & -0.236513 & 6 & -5.691587 & 0.093028 & -3.863322 \\ -6.996040 & 2.188092 & 0.247676 & 1 & -5.368527 & 0.237585 & -4.899464 \\ -8.166413 & 1.267524 & 0.194369 & 1 & -6.770852 & -0.080248 & -3.904612 \\ -9.430453 & 1.628646 & 0.789898 & 6 & -0.457270 & -3.051854 & -1.722450 \\ -9.606276 & 2.808934 & 1.566171 & 6 & 0.291787 & -2.484568 & -2.773272 \\ -8.761897 & 3.465585 & 1.736120 & 6 & -0.610582 & -4.452514 & -1.684501 \\ -10.828545 & 3.123513 & 2.120183 & 6 & 0.852440 & -3.294878 & -3.756096 \\ -10.930841 & 4.027358 & 2.713973 & 1 & 0.448403 & -1.413375 & -2.813282 \\ -11.947972 & 2.279291 & 1.930952 & 6 & -0.062559 & -5.259682 & -2.680552 \\ -12.906616 & 2.543988 & 2.367317 & 1 & -1.170748 & -4.903515 & -0.869257 \\ -11.809031 & 1.115784 & 1.208791 & 6 & 0.672324 & -4.682903 & -3.719268 \\ -12.652828 & 0.444431 & 1.071993 & 1 & 1.433945 & -2.840353 & -4.551264 \\ -10.558742 & 0.754182 & 0.637913 & 1 & -0.201887 & -6.336155 & -2.641063 \\ -10.404490 & -0.473038 & -0.061694 & 1 & 1.109308 & -5.308525 & -4.492277 \\ -11.266415 & -1.122437 & -0.185676 & 1 & 3.408223 & -5.419594 & -1.293729 \\ -9.176444 & -0.849646 & -0.542530 & 35 & 5.243145 & -3.767622 & -3.113830\end{array}$

C1-P1 si-si 3

Number of imaginary frequencies $=1$

The smallest frequencies are $=-153.68818 .377510 .6465 \mathrm{~cm}(-1)$ Electronic energy $=-7222.62065$

Zero-point correction $=$

1.754803

(Hartree/Particle)

Thermal correction to Energy= $\quad 1.873748$

Thermal correction to Enthalpy= $\quad 1.874692$

Thermal correction to Gibbs Free Energy $=\quad 1.591384$

Sum of electronic and zero-point Energies $=\quad-7220.865847$

Sum of electronic and thermal Energies $=\quad-7220.746902$

Sum of electronic and thermal Enthalpies $=\quad-7220.745958$

Sum of electronic and thermal Free Energies $=\quad-7221.029266$

Cartesian Coordinates

$4.089888-2.884641-1.000589$

$\begin{array}{llll}-4.911856 & -3.235071 & -1.635955\end{array}$

$-3.234954-3.518607-1.237735$

$-6.123175-2.312939 \quad 2.210306$

$\begin{array}{lll}-6.355628 & -3.380393 & 2.222160\end{array}$

$\begin{array}{lll}-5.147451 & -2.084111 & 3.365651\end{array}$

$\begin{array}{lll}-4.611870 & -3.197659 & 4.029655\end{array}$

$\begin{array}{lll}-4.852347 & -4.195276 & 3.672202\end{array}$

$\begin{array}{lll}-3.791357 & -3.044072 & 5.150712\end{array}$

$\begin{array}{lll}-3.394122 & -3.921854 & 5.653014\end{array}$

$\begin{array}{lll}-3.487745 & -1.766524 & 5.624259\end{array}$

$\begin{array}{llll}-2.852972 & -1.641965 & 6.497006\end{array}$

$\begin{array}{lll}-4.002776 & -0.648065 & 4.962436\end{array}$

$\begin{array}{lll}-3.771648 & 0.350791 & 5.322621\end{array}$

$\begin{array}{lll}-4.824772 & -0.804546 & 3.844175\end{array}$

$\begin{array}{llll}-5.228227 & 0.069976 & 3.349837\end{array}$

$\begin{array}{lll}-7.461572 & -1.604149 & 2.426798\end{array}$

$\begin{array}{lll}-8.172297 & -1.884760 & 1.647135\end{array}$

$\begin{array}{lll}-7.377345 & -0.518128 & 2.448044\end{array}$

$\begin{array}{lll}-7.867784 & -1.931238 & 3.388568\end{array}$

$\begin{array}{lll}-2.409812 & -0.296112 & 0.108386\end{array}$

$\begin{array}{llll}-2.356429 & 0.779221 & 0.246792\end{array}$

$\begin{array}{lll}-2.632504 & -0.831062 & 1.026802\end{array}$

$\begin{array}{lll}-1.469224 & -0.900134 & -0.799432\end{array}$

$\begin{array}{llll}-0.893359 & -0.264651 & -1.462908\end{array}$

$\begin{array}{lll}-1.091118 & -2.255970 & -0.684132\end{array}$

$\begin{array}{lll}-1.618536 & -2.825742 & 0.070706\end{array}$

$\begin{array}{llll}-5.027942 & -1.169314 & -3.307416\end{array}$

$\begin{array}{llll}-5.735886 & -1.951828 & -3.043774\end{array}$

$\begin{array}{llll}-3.717441 & -1.608239 & -3.632754\end{array}$

$\begin{array}{llll}-3.543473 & -2.684087 & -3.608405\end{array}$

$\begin{array}{llll}-2.729067 & -0.857784 & -4.510125\end{array}$

$\begin{array}{lll}-2.939581 & -1.059692 & -5.572099\end{array}$

$\begin{array}{ccc}3.848819 & 4.474283 & 3.163302 \\ 1.178209 & 2.281145 & -0.762769 \\ 4.500708 & 0.586735 & 0.479643 \\ 3.024928 & 3.723749 & 1.383135 \\ 3.993776 & 2.811314 & 1.934741 \\ 3.547088 & 2.432744 & 3.239859 \\ 4.065136 & 1.763240 & 3.913422 \\ 2.327912 & 3.122690 & 3.509300 \\ 1.763926 & 3.082151 & 4.431633 \\ 2.013831 & 3.927192 & 2.375468 \\ 1.163368 & 4.585731 & 2.281021 \\ 5.547755 & 5.667987 & 3.062239 \\ 6.305671 & 5.590942 & 2.294452 \\ 5.522873 & 4.941171 & 4.291565 \\ 6.255172 & 4.214109 & 4.615597 \\ 4.335137 & 5.306523 & 4.995717 \\ 4.009062 & 4.901281 & 5.944615 \\ 3.625225 & 6.258076 & 4.201508 \\ 2.669078 & 6.702885 & 4.443966 \\ 4.374470 & 6.482581 & 3.008201 \\ 4.083149 & 7.129097 & 2.191613 \\ 3.093700 & 4.259112 & -0.006689 \\ 4.022822 & 5.264784 & -0.310391 \\ 4.632757 & 5.654333 & 0.494057 \\ 4.184005 & 5.753477 & -1.607344 \\ 4.919165 & 6.527891 & -1.806286 \\ 3.400000 & 5.239290 & -2.636242 \\ 3.512140 & 5.602377 & -3.653132 \\ 2.472362 & 4.233786 & -2.360597 \\ 1.882765 & 3.835637 & -3.177095\end{array}$


$\begin{array}{llll}2.311564 & 3.720749 & -1.063589\end{array}$

$\begin{array}{lll}5.144069 & 2.234710 & 1.163966\end{array}$

$\begin{array}{llll}5.296733 & 2.843966 & 0.267729\end{array}$

$\begin{array}{lll}6.465568 & 2.166602 & 1.932345\end{array}$

$\begin{array}{lll}7.256095 & 1.716619 & 1.324578\end{array}$

$\begin{array}{lll}6.372406 & 1.589436 & 2.855936\end{array}$

$\begin{array}{llll}6.774983 & 3.180950 & 2.199109\end{array}$

$\begin{array}{llll}0.393841 & 2.041141 & -2.411573\end{array}$

$\begin{array}{llll}1.067668 & 1.203452 & -3.315877\end{array}$

$\begin{array}{llll}1.980277 & 0.709333 & -3.000282\end{array}$

$\begin{array}{llll}0.596304 & 1.033693 & -4.617632\end{array}$

$\begin{array}{llll}1.136412 & 0.393055 & -5.308972\end{array}$

$\begin{array}{llll}-0.568762 & 1.689279 & -5.027160\end{array}$

$\begin{array}{llll}-0.942618 & 1.554707 & -6.037836\end{array}$

$\begin{array}{lll}-1.257566 & 2.507159 & -4.128538\end{array}$

$\begin{array}{llll}-2.172781 & 3.006040 & -4.434568\end{array}$

$\begin{array}{llll}-0.779114 & 2.685727 & -2.828853\end{array}$

$\begin{array}{lll}-1.326746 & 3.322348 & -2.145215\end{array}$

$\begin{array}{lll}-0.125620 & 3.020705 & 0.300825\end{array}$

$\begin{array}{lll}-0.593197 & 2.270210 & 1.388253\end{array}$

$\begin{array}{lll}-0.219851 & 1.263351 & 1.539739\end{array}$

$\begin{array}{lll}-1.487436 & 2.836374 & 2.298559\end{array}$

$\begin{array}{lll}-1.831361 & 2.247610 & 3.141152\end{array}$

$\begin{array}{llll}-1.927450 & 4.150463 & 2.127312\end{array}$

$\begin{array}{lll}-2.621388 & 4.591200 & 2.837600\end{array}$

$\begin{array}{lll}-1.472429 & 4.901163 & 1.037651\end{array}$

$\begin{array}{lll}-1.812616 & 5.923456 & 0.899746\end{array}$

$\begin{array}{lll}-0.571297 & 4.342122 & 0.130005\end{array}$

$\begin{array}{llll}-0.190951 & 4.944895 & -0.689500\end{array}$

$\begin{array}{llll}5.435463 & 0.398553 & -1.092958\end{array}$

$6.286664-0.661066-1.430276$

$\begin{array}{llll}6.507317 & -1.449239 & -0.722155\end{array}$

$6.846088-0.730199 \quad-2.707567$

$\begin{array}{llll}6.547120 & 0.222850 & -3.680259\end{array}$

$\begin{array}{llll}6.969642 & 0.148144 & -4.674684\end{array}$

$\begin{array}{llll}5.669381 & 1.255448 & -3.355247\end{array}$

$\begin{array}{llll}5.123158 & 1.350936 & -2.075805\end{array}$

$\begin{array}{llll}4.428959 & 2.157139 & -1.853630\end{array}$

$\begin{array}{lll}5.123819 & -0.668581 & 1.669191\end{array}$

$\begin{array}{llll}4.155203 & -1.205005 & 2.527652\end{array}$

$3.124104 \quad-0.890941 \quad 2.423965$

$\begin{array}{llll}4.512604 & -2.123019 & 3.515225\end{array}$

$\begin{array}{lll}5.836872 & -2.533404 & 3.658627\end{array}$

$\begin{array}{llll}6.114385 & -3.252565 & 4.420016\end{array}$

$\begin{array}{lll}6.803026 & -1.994915 & 2.808357\end{array}$

$6.459806-1.060160 \quad 1.829635$

$\begin{array}{lll}7.241128 & -0.641538 & 1.206732\end{array}$

$\begin{array}{llll}2.216608 & 0.413531 & 0.037248\end{array}$

$\begin{array}{lll}8.252313 & -2.363202 & 2.976967\end{array}$

$\begin{array}{llll}3.437939 & -2.713367 & 4.385979\end{array}$

$\begin{array}{llll}7.829324 & -1.825408 & -3.022511\end{array}$

$\begin{array}{llll}5.254087 & 2.274895 & -4.380564\end{array}$

$3.903434 \quad 2.292144 \quad-4.532466$

$\begin{array}{llll}5.614509 & 3.527973 & -4.017604\end{array}$

$\begin{array}{llll}5.788331 & 2.037324 & -5.595167\end{array}$

$\begin{array}{llll}7.805924 & -2.171607 & -4.328772\end{array}$

$\begin{array}{llll}7.595332 & -2.941836 & -2.297859\end{array}$

$\begin{array}{llll}9.099534 & -1.439822 & -2.744936\end{array}$

$\begin{array}{lll}8.874177 & -2.494772 & 1.782280\end{array}$

$\begin{array}{llll}8.411429 & -3.523197 & 3.648303\end{array}$

$\begin{array}{lll}8.929473 & -1.410475 & 3.663001\end{array}$

$\begin{array}{llll}3.919794 & -3.144814 & 5.570935\end{array}$

$\begin{array}{lll}2.454102 & -1.820618 & 4.647097\end{array}$

$\begin{array}{lll}2.842209 & -3.781858 & 3.794415\end{array}$

$\begin{array}{llll}3.021907 & -1.777740 & -1.904670\end{array}$

$2.167577 \quad-2.715146 \quad 0.063756$

$3.602165 \quad-2.973571 \quad-2.286561$

$3.122378-0.893907 \quad-2.522884$

$\begin{array}{llll}2.824318 & -3.936880 & -0.260199\end{array}$

$\begin{array}{llll}3.536372 & -4.078634 & -1.434171\end{array}$

$\begin{array}{llll}2.729397 & -4.779059 & 0.416954\end{array}$

$\begin{array}{llll}1.270267 & -2.718700 & 1.178280\end{array}$

$\begin{array}{lll}0.781649 & -1.576942 & 1.893963\end{array}$

$\begin{array}{lll}1.078908 & -0.377469 & 1.590791\end{array}$

$\begin{array}{lll}2.330292 & -1.630285 & -0.759120\end{array}$ $\begin{array}{lll}1.212286 & -3.671699 & 1.684084\end{array}$

$\begin{array}{lll}-0.061312 & -1.764092 & 2.958255\end{array}$

$\begin{array}{lll}-0.414931 & -0.627346 & 3.803012\end{array}$

$\begin{array}{lll}0.248974 & 0.209982 & 3.597473\end{array}$

$\begin{array}{lll}-0.316030 & -0.909569 & 4.855238\end{array}$

$\begin{array}{lll}-1.449523 & -0.315214 & 3.628489\end{array}$

$\begin{array}{lll}-0.448460 & -3.070141 & 3.475949\end{array}$

$\begin{array}{llll}-0.627818 & -3.786026 & 2.673404\end{array}$

$\begin{array}{lll}-1.379810 & -2.958540 & 4.036234\end{array}$

$\begin{array}{llll}0.317624 & -3.481235 & 4.144909\end{array}$

$\begin{array}{lll}-3.324642 & -1.621299 & -1.294851\end{array}$

$\begin{array}{lll}-4.944643 & -0.732540 & 0.043809\end{array}$

$\begin{array}{lll}-4.932145 & 0.801641 & 0.679589\end{array}$

$\begin{array}{lll}-6.379497 & -0.682462 & -0.807514\end{array}$

$\begin{array}{lll}-5.080180 & -1.627181 & 1.451633\end{array}$

$\begin{array}{llll}-5.100774 & 1.896646 & -0.167704\end{array}$

$\begin{array}{lll}-3.964191 & 2.704181 & -0.389575\end{array}$

$\begin{array}{lll}-3.040737 & 2.450861 & 0.115718\end{array}$

$\begin{array}{llll}-4.056706 & 3.784046 & -1.231977\end{array}$

$\begin{array}{llll}-3.197338 & 4.427660 & -1.389436\end{array}$

$\begin{array}{llll}-5.263724 & 4.053896 & -1.930278\end{array}$

$\begin{array}{llll}-5.336995 & 5.103659 & -2.885651\end{array}$

$\begin{array}{llll}-4.455362 & 5.718532 & -3.047263\end{array}$

$\begin{array}{llll}-6.490098 & 5.327708 & -3.603134\end{array}$

$\begin{array}{llll}-6.533811 & 6.127889 & -4.336182\end{array}$

$\begin{array}{llll}-7.618508 & 4.499129 & -3.395307\end{array}$

$\begin{array}{llll}-8.519027 & 4.660412 & -3.980854\end{array}$

$\begin{array}{llll}-7.585925 & 3.485267 & -2.462249\end{array}$

$\begin{array}{llll}-8.457292 & 2.856058 & -2.327152\end{array}$

$\begin{array}{llll}-6.419073 & 3.237535 & -1.686188\end{array}$

$\begin{array}{llll}-6.344359 & 2.175761 & -0.713306\end{array}$

$\begin{array}{llll}-7.542507 & 1.375686 & -0.335997\end{array}$

$\begin{array}{lll}-8.743468 & 1.995955 & 0.164429\end{array}$

$\begin{array}{lll}-8.814764 & 3.380612 & 0.485271\end{array}$

$\begin{array}{llll}-7.935068 & 4.000750 & 0.360040\end{array}$

$\begin{array}{llll}-9.977991 & 3.940896 & 0.967546\end{array}$

$\begin{array}{lll}-10.002493 & 4.998852 & 1.212731\end{array}$

$\begin{array}{lll}-11.138238 & 3.152175 & 1.152705\end{array}$

$\begin{array}{lll}-12.049665 & 3.609194 & 1.526728\end{array}$

$\begin{array}{lll}-11.099940 & 1.805100 & 0.871968\end{array}$

$\begin{array}{llll}-11.977183 & 1.182221 & 1.026458\end{array}$

$\begin{array}{llll}-9.911675 & 1.192444 & 0.389654\end{array}$

$\begin{array}{lll}-9.856455 & -0.206779 & 0.145953\end{array}$

$\begin{array}{lll}-10.750890 & -0.804571 & 0.297085\end{array}$

$\begin{array}{llll}-8.680442 & -0.800162 & -0.237790\end{array}$

$\begin{array}{lll}-8.600879 & -1.871435 & -0.389903\end{array}$

$\begin{array}{lll}-7.528510 & -0.006543 & -0.437150\end{array}$

$\begin{array}{llll}-4.212015 & -2.840017 & 1.445056\end{array}$

$\begin{array}{lll}-3.276879 & -2.595896 & 1.966795\end{array}$

$\begin{array}{lll}-4.851954 & -4.024690 & 2.148805\end{array}$

$\begin{array}{lll}-4.095567 & -4.801431 & 3.033908\end{array}$

$\begin{array}{lll}-3.071968 & -4.511759 & 3.258255\end{array}$

$\begin{array}{lll}-4.644765 & -5.940140 & 3.630678\end{array}$

$\begin{array}{lll}-4.045167 & -6.532792 & 4.316210\end{array}$

$\begin{array}{llll}-5.962462 & -6.308890 & 3.353181\end{array}$

$\begin{array}{lll}-6.393880 & -7.189018 & 3.821423\end{array}$

$\begin{array}{llll}-6.726491 & -5.535619 & 2.472273\end{array}$

$\begin{array}{lll}-7.753992 & -5.814453 & 2.255047\end{array}$

$\begin{array}{lll}-6.173375 & -4.405407 & 1.871021\end{array}$

$\begin{array}{lll}-6.767922 & -3.798463 & 1.193087\end{array}$

$\begin{array}{lll}-3.910899 & -3.231993 & -0.005033\end{array}$

$\begin{array}{llll}-4.821695 & -3.673559 & -0.427742\end{array}$

$\begin{array}{llll}-3.159564 & -4.022940 & -0.014769\end{array}$

$\begin{array}{lll}-5.650701 & -1.249343 & 2.781834\end{array}$

$\begin{array}{llll}-5.949910 & -2.207906 & 3.210586\end{array}$

$\begin{array}{lll}-4.639110 & -0.661164 & 3.766310\end{array}$

$\begin{array}{llll}-4.098134 & -1.497888 & 4.755029\end{array}$

$\begin{array}{lll}-4.330872 & -2.559357 & 4.735570\end{array}$

$\begin{array}{lll}-3.302073 & -0.982616 & 5.781105\end{array}$

$\begin{array}{lll}-2.902781 & -1.648672 & 6.541119\end{array}$

$\begin{array}{llll}-3.038756 & 0.386943 & 5.837904\end{array}$

$\begin{array}{llll}-2.436956 & 0.797155 & 6.643878\end{array}$

$\begin{array}{llll}-3.554729 & 1.227220 & 4.847388\end{array}$

$\begin{array}{lll}-3.361872 & 2.295660 & 4.890126\end{array}$

$\begin{array}{lll}-4.337230 & 0.708048 & 3.812593\end{array}$ 


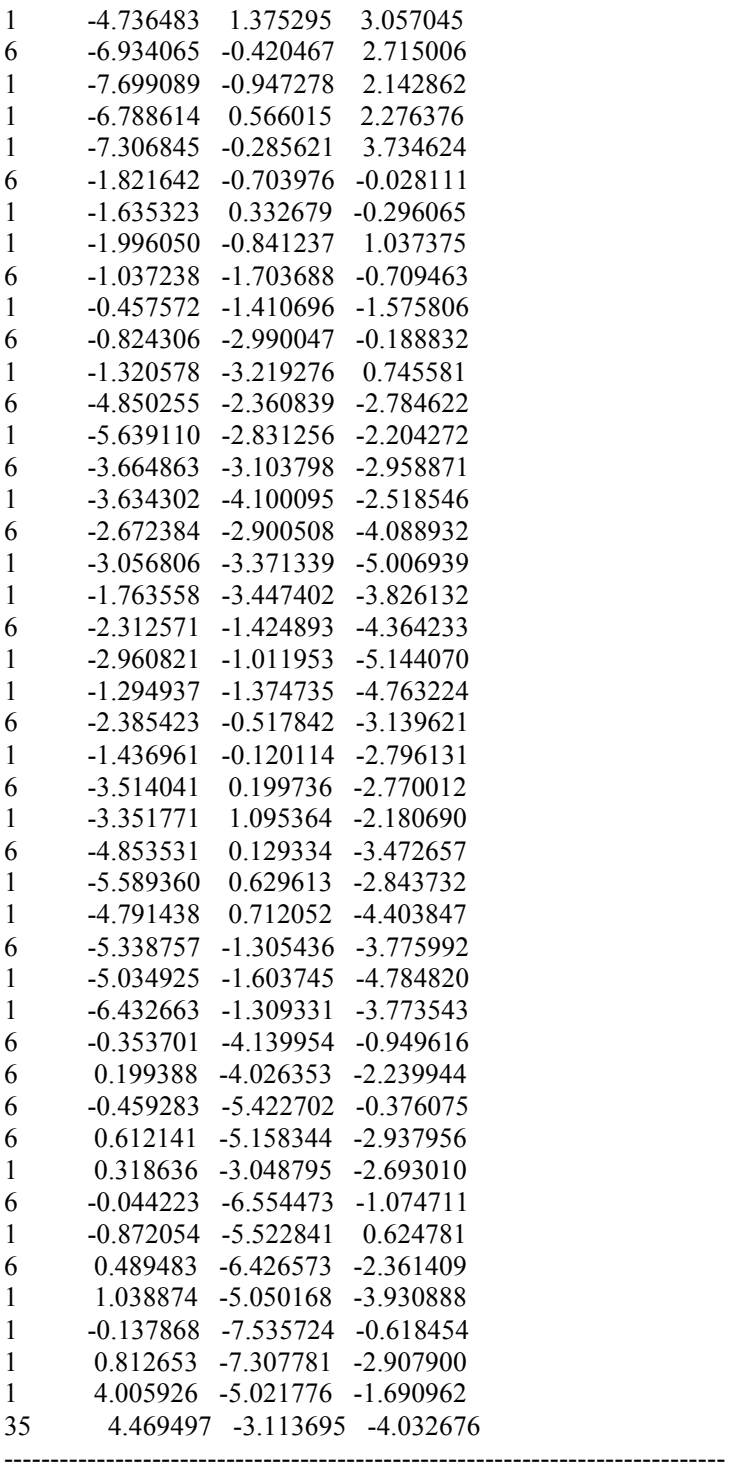

C1-P1 si-si 4

Number of imaginary frequencies $=2$

The smallest frequencies are $=-150.0093 \quad-2.310311 .0100 \mathrm{~cm}(-1)$ Electronic energy $=-7222.6173492$

Zero-point correction=

(Hartree/Particle)

Thermal correction to Energy=

Thermal correction to Enthalpy=

Thermal correction to Gibbs Free Energy=
Sum of electronic and zero-point Energies

Sum of electronic and zero-point Energies

Sum of electronic and thermal Enthalpies=

Sum of electronic and thermal Free Energies=

1.872225

1.873170

1.594755

$-7220.863009$

$-7220.745124$

$-7220.744180$

$-7221.022594$

Cartesian Coordinates

$\begin{array}{llll}26 & -7.190961 & -2.567863 & 2.869730 \\ 15 & -5.003359 & -1.675470 & -1.741316 \\ 15 & -4.423367 & 0.892806 & 1.129024 \\ 6 & -6.554572 & -1.928662 & 0.980697 \\ 6 & -5.978186 & -1.122548 & 2.026955 \\ 6 & -5.213391 & -1.988421 & 2.871215 \\ 1 & -4.658873 & -1.686093 & 3.748886 \\ 6 & -5.330740 & -3.317976 & 2.371319 \\ 1 & -4.900280 & -4.204496 & 2.817133 \\ 6 & -6.159240 & -3.284643 & 1.212650 \\ 1 & -6.459225 & -4.134294 & 0.616324 \\ 6 & -8.908072 & -1.730530 & 3.686032\end{array}$

$\begin{array}{lll}-9.253091 & -0.724528 & 3.488603\end{array}$

$\begin{array}{lll}-8.002711 & -2.118288 & 4.721000\end{array}$

$\begin{array}{lll}-7.538141 & -1.456622 & 5.439830\end{array}$

$\begin{array}{lll}-7.781590 & -3.525054 & 4.608781\end{array}$

$\begin{array}{lll}-7.118533 & -4.115375 & 5.227599\end{array}$

$\begin{array}{lll}-8.547825 & -4.006290 & 3.503568\end{array}$

$\begin{array}{lll}-8.568940 & -5.024784 & 3.139005\end{array}$

$\begin{array}{llll}-9.244680 & -2.898726 & 2.934735\end{array}$

$\begin{array}{lll}-9.888733 & -2.934862 & 2.066658\end{array}$

$\begin{array}{llll}-7.345279 & -1.390897 & -0.160247\end{array}$

$\begin{array}{lll}-8.653426 & -0.931305 & 0.050845\end{array}$

$\begin{array}{llll}-9.063573 & -0.995168 & 1.050450\end{array}$

$\begin{array}{lll}-9.419836 & -0.399845 & -0.986696\end{array}$

$\begin{array}{lll}-10.426930 & -0.044630 & -0.788318\end{array}$

$\begin{array}{llll}-8.886949 & -0.332766 & -2.271884\end{array}$

$\begin{array}{llll}-9.470615 & 0.073170 & -3.092200\end{array}$

$\begin{array}{llll}-7.580744 & -0.765367 & -2.500662\end{array}$

$\begin{array}{llll}-7.171041 & -0.682047 & -3.500164\end{array}$

$\begin{array}{llll}-6.787788 & -1.272250 & -1.459241\end{array}$

$\begin{array}{lll}-6.009272 & 0.378744 & 2.048138\end{array}$

$\begin{array}{lll}-6.825645 & 0.715712 & 1.402887\end{array}$

$\begin{array}{lll}-6.186120 & 1.004762 & 3.433190\end{array}$

$\begin{array}{lll}-6.149363 & 2.097269 & 3.381888\end{array}$

$\begin{array}{llll}-5.413577 & 0.671946 & 4.131783\end{array}$

$\begin{array}{lll}-7.158495 & 0.711020 & 3.839812\end{array}$

$\begin{array}{lll}-4.710028 & -1.116748 & -3.470629\end{array}$

$\begin{array}{llll}-4.585035 & 0.266165 & -3.688544\end{array}$

$\begin{array}{llll}-4.724606 & 0.955018 & -2.860708\end{array}$

$\begin{array}{llll}-4.307574 & 0.764084 & -4.960400\end{array}$

$\begin{array}{llll}-4.223660 & 1.836383 & -5.113605\end{array}$

$\begin{array}{llll}-4.137853 & -0.118117 & -6.032855\end{array}$

$\begin{array}{llll}-3.911786 & 0.265991 & -7.023396\end{array}$

$\begin{array}{llll}-4.266959 & -1.493256 & -5.826415\end{array}$

$\begin{array}{llll}-4.142673 & -2.182869 & -6.656650\end{array}$

$\begin{array}{llll}-4.557269 & -1.992495 & -4.553246\end{array}$

$\begin{array}{llll}-4.655374 & -3.062756 & -4.406639\end{array}$

$\begin{array}{llll}-4.977965 & -3.508255 & -1.828976\end{array}$

$\begin{array}{llll}-3.828382 & -4.173134 & -1.380227\end{array}$

$\begin{array}{llll}-2.994790 & -3.595887 & -0.996666\end{array}$

$\begin{array}{llll}-3.774902 & -5.568638 & -1.396948\end{array}$

$\begin{array}{llll}-2.882679 & -6.076292 & -1.041100\end{array}$

$\begin{array}{llll}-4.866560 & -6.306979 & -1.859695\end{array}$

$-4.827756-7.392780 \quad-1.864982$

$\begin{array}{llll}-6.013517 & -5.647182 & -2.314558\end{array}$

$\begin{array}{llll}-6.863597 & -6.218532 & -2.676761\end{array}$

$\begin{array}{llll}-6.071039 & -4.252734 & -2.300094\end{array}$

$\begin{array}{llll}-6.968404 & -3.746314 & -2.643575\end{array}$

$\begin{array}{lll}-4.878985 & 2.418297 & 0.204021\end{array}$

$\begin{array}{lll}-4.120192 & 3.598672 & 0.182584\end{array}$

$\begin{array}{llll}-3.230508 & 3.702376 & 0.787962\end{array}$

$\begin{array}{llll}-4.495382 & 4.663664 & -0.635814\end{array}$

$\begin{array}{llll}-5.589685 & 4.561724 & -1.494808\end{array}$

$\begin{array}{llll}-5.868407 & 5.388504 & -2.137173\end{array}$

$\begin{array}{llll}-6.309226 & 3.369602 & -1.517493\end{array}$

$\begin{array}{llll}-5.970430 & 2.310714 & -0.673224\end{array}$

$\begin{array}{llll}-6.547140 & 1.392104 & -0.724754\end{array}$

$\begin{array}{lll}-3.288957 & 1.364788 & 2.500010\end{array}$

$\begin{array}{lll}-2.505807 & 0.309347 & 2.988611\end{array}$

$\begin{array}{lll}-2.580418 & -0.666226 & 2.525222\end{array}$

$\begin{array}{lll}-1.651209 & 0.505133 & 4.073010\end{array}$

$\begin{array}{llll}-1.520783 & 1.762201 & 4.657386\end{array}$

$\begin{array}{llll}-0.839738 & 1.918296 & 5.486438\end{array}$

$\begin{array}{lll}-2.286626 & 2.816989 & 4.161276\end{array}$

$\begin{array}{lll}-3.191783 & 2.619145 & 3.117457\end{array}$

$\begin{array}{lll}-3.814226 & 3.447061 & 2.803661\end{array}$

$\begin{array}{lll}-3.541061 & -0.713997 & -0.308693\end{array}$

$\begin{array}{lll}-2.061160 & 4.195686 & 4.716991\end{array}$

$\begin{array}{lll}-0.801278 & -0.628452 & 4.570886\end{array}$

$\begin{array}{llll}-3.770520 & 5.979714 & -0.542237\end{array}$

$\begin{array}{llll}-7.441102 & 3.176530 & -2.490214\end{array}$

$\begin{array}{llll}-7.119580 & 2.260724 & -3.439363\end{array}$

$\begin{array}{llll}-8.557781 & 2.722315 & -1.876644\end{array}$

$\begin{array}{lll}-7.768900 & 4.315832 & -3.133905\end{array}$

$\begin{array}{llll}-3.595023 & 6.549521 & -1.756278\end{array}$

$\begin{array}{llll}-2.557307 & 5.863304 & 0.037347\end{array}$ 


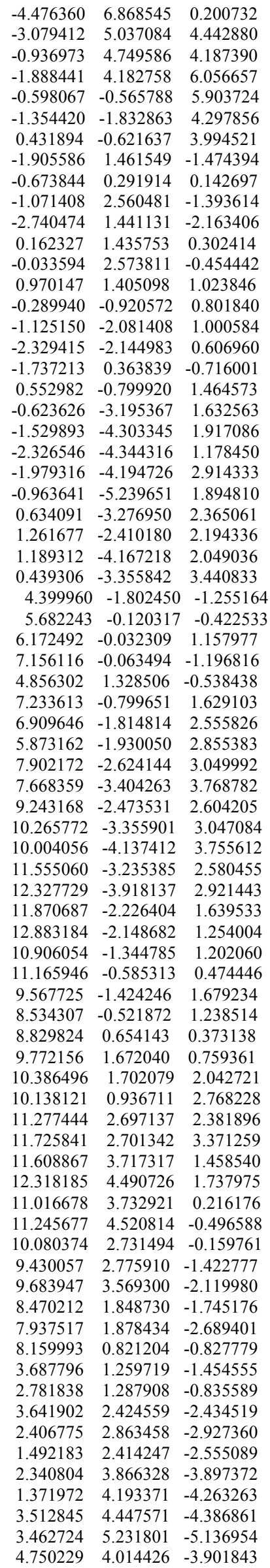

$\begin{array}{llll}5.668071 & 4.462178 & -4.273479\end{array}$

$\begin{array}{llll}4.813646 & 3.007709 & -2.937241\end{array}$

$\begin{array}{llll}5.774904 & 2.678460 & -2.555904\end{array}$

$\begin{array}{llll}3.711630 & -0.046377 & -2.269053\end{array}$

$\begin{array}{llll}4.372977 & 0.109412 & -3.129279\end{array}$

$\begin{array}{llll}2.714865 & -0.214408 & -2.681675\end{array}$

$\begin{array}{lll}4.878944 & 2.556088 & 0.316255\end{array}$

$\begin{array}{lll}4.674234 & 3.368111 & -0.386378\end{array}$

$\begin{array}{lll}3.747593 & 2.580078 & 1.343445\end{array}$

$\begin{array}{lll}2.854696 & 3.660474 & 1.34592\end{array}$

$\begin{array}{lll}2.947128 & 4.429349 & 0.582204\end{array}$

$\begin{array}{lll}1.855618 & 3.766055 & 2.317858\end{array}$

$\begin{array}{lll}1.170870 & 4.609399 & 2.307989\end{array}$

$\begin{array}{lll}1.725932 & 2.777420 & 3.294209\end{array}$

$\begin{array}{lll}0.946746 & 2.861333 & 4.043835\end{array}$

$\begin{array}{lll}2.600170 & 1.686061 & 3.293201\end{array}$

$\begin{array}{llll}2.493159 & 0.907105 & 4.042235\end{array}$

$\begin{array}{lll}3.609323 & 1.592945 & 2.331045\end{array}$

$\begin{array}{lll}4.302360 & 0.759693 & 2.352986\end{array}$

$\begin{array}{lll}6.220326 & 2.909021 & 0.968011\end{array}$

$\begin{array}{lll}7.016118 & 2.963561 & 0.223984\end{array}$

$\begin{array}{lll}6.508949 & 2.208430 & 1.750074\end{array}$

$\begin{array}{lll}6.114795 & 3.899412 & 1.421321\end{array}$

$\begin{array}{lll}3.259947 & -1.589228 & 0.567926\end{array}$

$\begin{array}{lll}3.702031 & -2.135946 & 1.400507\end{array}$

$\begin{array}{lll}3.022576 & -0.558452 & 0.822645\end{array}$

$\begin{array}{llll}2.302009 & -2.291337 & -0.247800\end{array}$

$\begin{array}{llll}2.213209 & -3.364976 & -0.138883\end{array}$

$\begin{array}{llll}1.288295 & -1.622210 & -0.958881\end{array}$

$\begin{array}{lll}1.399200 & -0.551277 & -1.061035\end{array}$

$\begin{array}{llll}5.735013 & -2.201645 & -3.006380\end{array}$

$\begin{array}{llll}5.973499 & -1.239122 & -3.451637\end{array}$

$\begin{array}{llll}4.445665 & -2.716378 & -3.284061\end{array}$

$\begin{array}{llll}3.811397 & -2.122207 & -3.941613\end{array}$

$\begin{array}{llll}4.051979 & -4.184820 & -3.228481\end{array}$

$\begin{array}{llll}4.354377 & -4.689094 & -4.159258\end{array}$

$\begin{array}{llll}2.957768 & -4.232250 & -3.196916\end{array}$

$\begin{array}{llll}4.622226 & -4.946399 & -2.012736\end{array}$

$\begin{array}{llll}5.601076 & -5.370296 & -2.254650\end{array}$

$\begin{array}{llll}3.970437 & -5.797209 & -1.789255\end{array}$

$\begin{array}{llll}4.750261 & -4.096061 & -0.752808\end{array}$

$\begin{array}{llll}4.044189 & -4.322128 & 0.042266\end{array}$

$\begin{array}{llll}5.896473 & -3.410442 & -0.378329\end{array}$

$\begin{array}{llll}6.002897 & -3.171697 & 0.675189\end{array}$

$7.176286-3.330286-1.185738$

$\begin{array}{llll}7.786198 & -2.527990 & -0.765892\end{array}$

$\begin{array}{llll}7.744840 & -4.261205 & -1.044615\end{array}$

$6.959617 \quad-3.062558 \quad-2.691378$

$\begin{array}{llll}6.881565 & -4.007143 & -3.238712\end{array}$

$7.847281 \quad-2.557214 \quad-3.083313$

$\begin{array}{llll}0.376964 & -2.243454 & -1.906272\end{array}$

$\begin{array}{llll}0.117826 & -3.631020 & -1.923392\end{array}$

$\begin{array}{llll}0.269719 & -1.425224 & -2.854602\end{array}$

$-0.750466-4.172866-2.865544$

$\begin{array}{llll}0.589536 & -4.279554 & -1.192484\end{array}$

$\begin{array}{llll}-1.143680 & -1.970874 & -3.792499\end{array}$

$\begin{array}{llll}-0.077962 & -0.357412 & -2.848969\end{array}$

$\begin{array}{lll}-1.387192 & -3.346090 & -3.799502\end{array}$

$\begin{array}{lll}-0.947046 & -5.240687 & -2.866852\end{array}$

$-1.642338-1.326070-4.509202$

$\begin{array}{llll}-2.077185 & -3.771271 & -4.521533\end{array}$

$0.612974 \quad 3.434284-0.332364$

$-1.348273 \quad 4.067460-2.605480$

C1-P1 si-si 5

Number of imaginary frequencies $=1$

The smallest frequencies are $=-120.63648 .836411 .1071 \mathrm{~cm}(-1)$ Electronic energy $=-7222.6230217$

Zero-point correction=

(Hartree/Particle)

Thermal correction to Energy= $\quad 1.874309$

Thermal correction to Enthalpy= $\quad 1.875253$

Thermal correction to Gibbs Free Energy $=\quad 1.593538$

Sum of electronic and zero-point Energies $=\quad-7220.867219$ 
Sum of electronic and thermal Energies=

Sum of electronic and thermal Enthalpies=

Sum of electronic and thermal Free Energies=

$-7220.748713$

$-7220.747769$

$-7221.029484$

Cartesian Coordinates

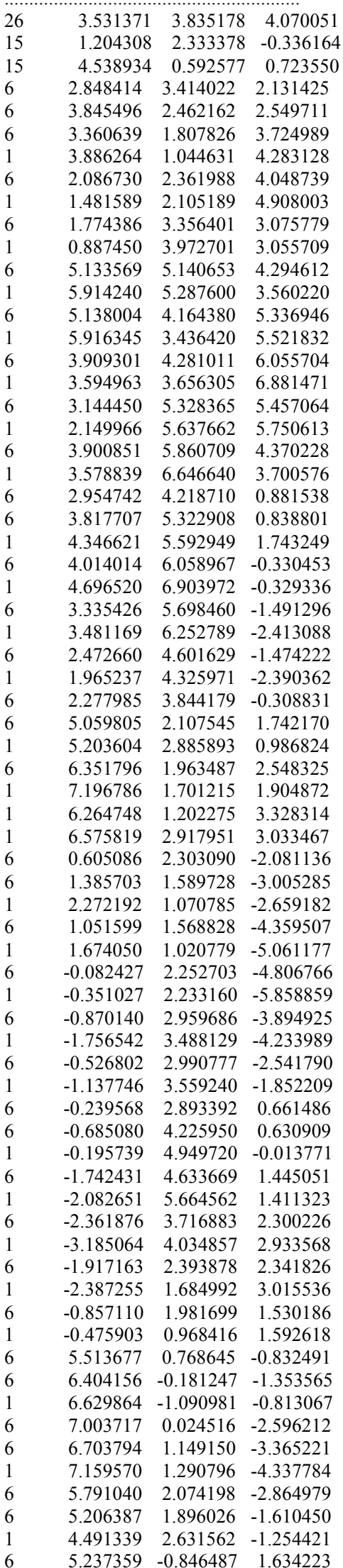

$4.308741-1.642550 \quad 2.317432$

$\begin{array}{llll}3.257608 & -1.385956 & 2.278107\end{array}$

$\begin{array}{lll}4.731367 & -2.749019 & 3.054395\end{array}$

$\begin{array}{lll}6.081218 & -3.091925 & 3.115108\end{array}$

$\begin{array}{llll}6.407079 & -3.958121 & 3.678754\end{array}$

$\begin{array}{lll}7.007896 & -2.289755 & 2.450125\end{array}$

$\begin{array}{llll}6.598057 & -1.168790 & 1.725950\end{array}$

$\begin{array}{lll}7.348354 & -0.552391 & 1.245698\end{array}$

$\begin{array}{llll}2.244759 & 0.393549 & 0.262353\end{array}$

$\begin{array}{lll}8.470777 & -2.642307 & 2.455794\end{array}$

$3.704808-3.612338 \quad 3.733885$

$8.034164 \quad-0.946685-3.107813$

$\begin{array}{llll}5.386815 & 3.278364 & -3.671072\end{array}$

$\begin{array}{llll}4.044655 & 3.300106 & -3.878014\end{array}$

$\begin{array}{llll}5.696744 & 4.432582 & -3.035711\end{array}$

$\begin{array}{llll}5.977226 & 3.314799 & -4.882782\end{array}$

$7.911474-1.155298 \quad-4.439537$

$\begin{array}{llll}7.960829 & -2.147455 & -2.496429\end{array}$

$\begin{array}{llll}9.290727 & -0.478180 & -2.906448\end{array}$

$\begin{array}{lll}8.844510 & -3.203169 & 1.279926\end{array}$

$\begin{array}{llll}8.787077 & -3.517283 & 3.432841\end{array}$

$\begin{array}{llll}9.246778 & -1.546126 & 2.623280\end{array}$

$\begin{array}{llll}4.211755 & -4.266906 & 4.799745\end{array}$

$\begin{array}{llll}2.647159 & -2.885336 & 4.170482\end{array}$

$\begin{array}{lll}3.204860 & -4.556517 & 2.896275\end{array}$

$\begin{array}{llll}3.205760 & -1.527776 & -1.866993\end{array}$

$2.205912 \quad-2.725323 \quad-0.123421$

$\begin{array}{llll}3.792239 & -2.665970 & -2.388385\end{array}$

$\begin{array}{lll}3.364695 & -0.565156 & -2.335303\end{array}$

$\begin{array}{llll}2.847273 & -3.906743 & -0.598771\end{array}$

$3.637931-3.889333-1.728891$

$\begin{array}{llll}2.689036 & -4.838404 & -0.065969\end{array}$

$\begin{array}{lll}1.276265 & -2.865214 & 0.952023\end{array}$

$\begin{array}{llll}0.804309 & -1.831780 & 1.821076\end{array}$

$\begin{array}{lll}1.124359 & -0.606149 & 1.713383\end{array}$

$\begin{array}{llll}2.437058 & -1.534537 & -0.761165\end{array}$

$\begin{array}{lll}1.175793 & -3.880022 & 1.304549\end{array}$

$\begin{array}{lll}-0.050095 & -2.165616 & 2.857691\end{array}$

$\begin{array}{lll}-0.149016 & -1.225787 & 3.974853\end{array}$

$\begin{array}{llll}0.729476 & -1.293851 & 4.631713\end{array}$

$\begin{array}{lll}-1.040500 & -1.459071 & 4.559481\end{array}$

$\begin{array}{lll}-0.222766 & -0.204915 & 3.605958\end{array}$

$\begin{array}{llll}-0.354166 & -3.556181 & 3.181448\end{array}$

$\begin{array}{lll}-0.651518 & -4.109159 & 2.288452\end{array}$

$\begin{array}{lll}-1.191703 & -3.577298 & 3.882071\end{array}$

$\begin{array}{llll}0.497948 & -4.071101 & 3.643672\end{array}$

$\begin{array}{lll}-3.072559 & -1.482184 & -1.762288\end{array}$

$\begin{array}{llll}-4.593006 & -0.536469 & -0.363508\end{array}$

$\begin{array}{lll}-4.502112 & 0.999706 & 0.242214\end{array}$

$\begin{array}{lll}-6.085708 & -0.476523 & -1.100167\end{array}$

$\begin{array}{lll}-4.654591 & -1.387416 & 1.081214\end{array}$

$\begin{array}{llll}-4.845296 & 2.106381 & -0.525557\end{array}$

$\begin{array}{llll}-3.785954 & 2.969463 & -0.873859\end{array}$

$\begin{array}{lll}-2.794513 & 2.733389 & -0.508664\end{array}$

$\begin{array}{llll}-4.039676 & 4.075496 & -1.644773\end{array}$

$\begin{array}{llll}-3.236343 & 4.757605 & -1.909244\end{array}$

$\begin{array}{llll}-5.351201 & 4.329110 & -2.129712\end{array}$

$\begin{array}{llll}-5.610876 & 5.423448 & -2.998677\end{array}$

$\begin{array}{lll}-4.785985 & 6.079570 & -3.264400\end{array}$

$\begin{array}{llll}-6.871935 & 5.641422 & -3.505372\end{array}$

$\begin{array}{llll}-7.057959 & 6.477382 & -4.173253\end{array}$

$\begin{array}{llll}-7.927137 & 4.761574 & -3.165991\end{array}$

$\begin{array}{llll}-8.915990 & 4.920233 & -3.586474\end{array}$

$\begin{array}{lll}-7.712678 & 3.701009 & -2.312248\end{array}$

$\begin{array}{llll}-8.532146 & 3.034000 & -2.072745\end{array}$

$\begin{array}{llll}-6.427907 & 3.456731 & -1.751135\end{array}$

$\begin{array}{llll}-6.167468 & 2.353361 & -0.860034\end{array}$

$\begin{array}{llll}-7.255939 & 1.482832 & -0.334160\end{array}$

$\begin{array}{lll}-8.391136 & 2.018220 & 0.373889\end{array}$

$\begin{array}{llll}-8.476654 & 3.382747 & 0.769876\end{array}$

$\begin{array}{lll}-7.659558 & 4.055344 & 0.537368\end{array}$

$\begin{array}{lll}-9.571399 & 3.856517 & 1.459895\end{array}$

$\begin{array}{lll}-9.606476 & 4.900021 & 1.759501\end{array}$

$\begin{array}{lll}-10.646435 & 2.996946 & 1.789584\end{array}$

$\begin{array}{llll}-11.505228 & 3.386311 & 2.328377\end{array}$ 
$\begin{array}{llll}-10.589884 & 1.667010 & 1.438685\end{array}$

$\begin{array}{lll}-11.398949 & 0.990080 & 1.700311\end{array}$

$\begin{array}{lll}-9.467465 & 1.141637 & 0.741875\end{array}$

$\begin{array}{lll}-9.378110 & -0.243994 & 0.441976\end{array}$

$\begin{array}{lll}-10.199917 & -0.897793 & 0.718735\end{array}$

$\begin{array}{lll}-8.247621 & -0.758853 & -0.140156\end{array}$

$\begin{array}{lll}-8.136796 & -1.819121 & -0.338815\end{array}$

$\begin{array}{lll}-7.192052 & 0.106869 & -0.496860\end{array}$

$\begin{array}{lll}-4.014179 & -2.721980 & 0.966435\end{array}$

$\begin{array}{lll}-3.021417 & -2.664996 & 1.427004\end{array}$

$\begin{array}{lll}-4.800603 & -3.818563 & 1.659807\end{array}$

$\begin{array}{lll}-4.146850 & -4.726077 & 2.499978\end{array}$

$\begin{array}{lll}-3.081642 & -4.611190 & 2.685933\end{array}$

$\begin{array}{lll}-4.854491 & -5.764514 & 3.113306\end{array}$

$\begin{array}{lll}-4.336439 & -6.457497 & 3.770778\end{array}$

$\begin{array}{lll}-6.225403 & -5.903951 & 2.887754\end{array}$

$\begin{array}{lll}-6.779434 & -6.704807 & 3.369235\end{array}$

$\begin{array}{lll}-6.883053 & -5.004095 & 2.041451\end{array}$

$\begin{array}{llll}-7.951443 & -5.100978 & 1.869114\end{array}$

$\begin{array}{lll}-6.174616 & -3.971248 & 1.431408\end{array}$

$\begin{array}{lll}-6.689736 & -3.251652 & 0.802573\end{array}$

$\begin{array}{llll}-3.835395 & -3.078736 & -0.516347\end{array}$

$\begin{array}{llll}-4.805475 & -3.389860 & -0.920502\end{array}$

$\begin{array}{llll}-3.185078 & -3.952911 & -0.588442\end{array}$

$\begin{array}{lll}-4.764769 & -0.740686 & 2.433601\end{array}$

$\begin{array}{lll}-4.836704 & 0.325739 & 2.229565\end{array}$

$\begin{array}{lll}-6.031062 & -1.100224 & 3.208737\end{array}$

$\begin{array}{lll}-7.163233 & -0.290681 & 3.050880\end{array}$

$\begin{array}{llll}-7.124389 & 0.549671 & 2.365041\end{array}$

$\begin{array}{lll}-8.339771 & -0.546897 & 3.757542\end{array}$

$\begin{array}{llll}-9.202808 & 0.096230 & 3.611267\end{array}$

$\begin{array}{llll}-8.399109 & -1.621606 & 4.646531\end{array}$

$\begin{array}{lll}-9.311952 & -1.826264 & 5.199451\end{array}$

$\begin{array}{llll}-7.272547 & -2.429455 & 4.824227\end{array}$

$\begin{array}{lll}-7.305962 & -3.269022 & 5.513233\end{array}$

$\begin{array}{lll}-6.098780 & -2.166755 & 4.116561\end{array}$

$\begin{array}{llll}-5.241646 & -2.813513 & 4.262598\end{array}$

$\begin{array}{lll}-3.482826 & -0.948653 & 3.247631\end{array}$

$\begin{array}{lll}-2.610765 & -0.627822 & 2.673613\end{array}$

$\begin{array}{lll}-3.330040 & -1.989603 & 3.541492\end{array}$

$\begin{array}{lll}-3.537007 & -0.351293 & 4.162374\end{array}$

$\begin{array}{llll}-1.536550 & -0.659667 & -0.465503\end{array}$

$\begin{array}{llll}-1.265005 & 0.356929 & -0.735661\end{array}$

$\begin{array}{lll}-1.737896 & -0.779453 & 0.595922\end{array}$

$\begin{array}{llll}-0.825926 & -1.726227 & -1.120511\end{array}$

$\begin{array}{llll}-0.199629 & -1.507520 & -1.975991\end{array}$

$\begin{array}{lll}-0.769742 & -3.011812 & -0.564998\end{array}$

$\begin{array}{llll}-1.314810 & -3.158530 & 0.358473\end{array}$

$\begin{array}{lll}-4.587873 & -2.078126 & -3.304613\end{array}$

$\begin{array}{lll}-5.414798 & -2.541972 & -2.773623\end{array}$

$\begin{array}{lll}-3.430433 & -2.870966 & -3.480598\end{array}$

$\begin{array}{lll}-3.464214 & -3.887405 & -3.089277\end{array}$

$\begin{array}{lll}-2.387264 & -2.664514 & -4.566418\end{array}$

$\begin{array}{lll}-2.744589 & -3.099100 & -5.512724\end{array}$

$\begin{array}{llll}-1.502987 & -3.242063 & -4.281916\end{array}$

$\begin{array}{llll}-1.977916 & -1.191286 & -4.782683\end{array}$

$\begin{array}{lll}-2.605250 & -0.728599 & -5.550905\end{array}$

$\begin{array}{lll}-0.954505 & -1.159269 & -5.170225\end{array}$

$\begin{array}{llll}-2.035162 & -0.337945 & -3.519305\end{array}$

$\begin{array}{llll}-1.076297 & -0.002593 & -3.137662\end{array}$

$\begin{array}{llll}-3.140396 & 0.406128 & -3.124288\end{array}$

$\begin{array}{llll}-2.945455 & 1.258674 & -2.483111\end{array}$

$\begin{array}{llll}-4.473654 & 0.441110 & -3.841876\end{array}$

$\begin{array}{llll}-5.198109 & 0.919918 & -3.180571\end{array}$

$\begin{array}{lll}-4.382249 & 1.088694 & -4.726412\end{array}$

$\begin{array}{lll}-5.003950 & -0.950577 & -4.250512\end{array}$

$-4.679487-1.198965-5.265935$

$\begin{array}{lll}-6.096844 & -0.911259 & -4.282015\end{array}$

$\begin{array}{llll}-0.363084 & -4.228535 & -1.248810\end{array}$

$\begin{array}{lll}0.275234 & -4.226617 & -2.505021\end{array}$

$-0.632504 \quad-5.468456-0.634025$

$\begin{array}{lll}0.608531 & -5.424916 & -3.132102\end{array}$

$\begin{array}{llll}0.513179 & -3.286715 & -2.991061\end{array}$

$\begin{array}{lll}-0.292519 & -6.665233 & -1.259250\end{array}$

$\begin{array}{lll}-1.115156 & -5.481151 & 0.340161\end{array}$ $\begin{array}{llll}0.326267 & -6.647235 & -2.514161\end{array}$

$1.097409-5.405194-4.101845$

$\begin{array}{lll}-0.511099 & -7.611130 & -0.772266\end{array}$

$0.590043-7.579338-3.005276$

$4.104913-4.796248-2.097368$

$35 \quad 4.823940 \quad-2.546896 \quad-4.044531$

C1-P1 si-si 6

Number of imaginary frequencies $=1$

The smallest frequencies are $=-184.195210 .000914 .2259 \mathrm{~cm}(-1)$ Electronic energy $=-7222.619327$

Zero-point correction $=$

1.755410

(Hartree/Particle)

Thermal correction to Energy $=\quad 1.874062$

Thermal correction to Enthalpy= $\quad 1.875006$

Thermal correction to Gibbs Free Energy $=\quad 1.592536$

Sum of electronic and zero-point Energies $=\quad-7220.863917$

Sum of electronic and thermal Energies $=\quad-7220.745265$

Sum of electronic and thermal Enthalpies $=\quad-7220.744321$

Sum of electronic and thermal Free Energies $=\quad-7221.026791$

Cartesian Coordinates

$\begin{array}{llll}15 & 2.513187 & 2.139896 & -1.283945\end{array}$

$\begin{array}{lllll}15 & 4.846396 & 0.307284 & 1.178649\end{array}$

$\begin{array}{llll}6 & 3.493798 & 3.601526 & 1.316702\end{array}$

$\begin{array}{lll}4.041921 & 2.659524 & 2.257781\end{array}$

$\begin{array}{lll}3.060378 & 2.435633 & 3.272750\end{array}$

$3.173363 \quad 1.782124 \quad 4.127290$

$\begin{array}{lll}1.923664 & 3.246980 & 2.981676\end{array}$

$1.031538 \quad 3.331785 \quad 3.587898$

$\begin{array}{llll}2.193276 & 3.972421 & 1.784195\end{array}$

$\begin{array}{lll}1.539695 & 4.689650 & 1.309078\end{array}$

$5.303740 \quad 5.480681 \quad 3.788546$

$\begin{array}{lll}6.295134 & 5.292940 & 3.398571\end{array}$

$\begin{array}{lll}4.719765 & 4.852495 & 4.930794\end{array}$

$\begin{array}{llll}5.186484 & 4.100137 & 5.551983\end{array}$

$\begin{array}{llll}3.392612 & 5.357916 & 5.081176\end{array}$

$2.678180 \quad 5.050904 \quad 5.833597$

$\begin{array}{llll}3.155296 & 6.297179 & 4.031919\end{array}$

$2.229476 \quad 6.826605 \quad 3.848752$

$\begin{array}{llll}4.335589 & 6.374004 & 3.233804\end{array}$

$\begin{array}{lll}4.459842 & 6.978168 & 2.345392\end{array}$

$\begin{array}{lll}4.158511 & 4.028240 & 0.054734\end{array}$

$\begin{array}{llll}5.216517 & 4.947848 & 0.101612\end{array}$

$\begin{array}{lll}5.521760 & 5.327828 & 1.068290\end{array}$

$\begin{array}{llll}5.870446 & 5.371973 & -1.055206\end{array}$

$\begin{array}{llll}6.693356 & 6.077369 & -0.984647\end{array}$

$\begin{array}{llll}5.455962 & 4.890302 & -2.294837\end{array}$

$\begin{array}{llll}5.945750 & 5.217113 & -3.206973\end{array}$

$\begin{array}{llll}4.417336 & 3.961517 & -2.363261\end{array}$

$\begin{array}{llll}4.124185 & 3.583340 & -3.335021\end{array}$

$\begin{array}{llll}3.777030 & 3.495066 & -1.203948\end{array}$

$\begin{array}{llll}5.333853 & 1.921501 & 2.057546\end{array}$

$\begin{array}{llll}5.921935 & 2.468283 & 1.315194\end{array}$

$\begin{array}{lll}6.181066 & 1.762956 & 3.322235\end{array}$

$\begin{array}{lll}7.117858 & 1.240047 & 3.111471\end{array}$

$\begin{array}{llll}5.652144 & 1.215986 & 4.107242\end{array}$

$\begin{array}{lll}6.424002 & 2.756563 & 3.709346\end{array}$

$\begin{array}{llll}2.570147 & 1.571698 & -3.033952\end{array}$

$\begin{array}{lll}3.775700 & 1.041892 & -3.527948\end{array}$

$\begin{array}{llll}4.673581 & 1.079571 & -2.920658\end{array}$

$\begin{array}{llll}3.844665 & 0.500992 & -4.809947\end{array}$

$\begin{array}{llll}4.790334 & 0.113309 & -5.177270\end{array}$

$\begin{array}{llll}2.701173 & 0.458932 & -5.614195\end{array}$

$2.750452 \quad 0.032172-6.611700$

$\begin{array}{llll}1.496930 & 0.972021 & -5.129031\end{array}$

$\begin{array}{llll}0.604216 & 0.943129 & -5.747207\end{array}$

$\begin{array}{llll}1.431014 & 1.530673 & -3.849042\end{array}$

$\begin{array}{llll}0.491522 & 1.933872 & -3.488391\end{array}$

$\begin{array}{llll}0.920837 & 3.040903 & -1.116819\end{array}$

$\begin{array}{llll}0.623307 & 4.174259 & -1.890901\end{array}$

$\begin{array}{llll}1.317418 & 4.505513 & -2.658294\end{array}$ 
$\begin{array}{llll}-0.557677 & 4.884004 & -1.670438\end{array}$

$\begin{array}{llll}-0.785134 & 5.756798 & -2.275740\end{array}$

$\begin{array}{llll}-1.436977 & 4.482880 & -0.658135\end{array}$

$\begin{array}{llll}-2.349941 & 5.043156 & -0.477660\end{array}$

$\begin{array}{lll}-1.139242 & 3.361212 & 0.117240\end{array}$

$\begin{array}{lll}-1.810298 & 3.055631 & 0.913408\end{array}$

$\begin{array}{llll}0.027948 & 2.629610 & -0.117656\end{array}$

$\begin{array}{llll}0.260064 & 1.753585 & 0.480894\end{array}$

$\begin{array}{llll}6.223359 & -0.021452 & -0.000339\end{array}$

$\begin{array}{llll}6.784053 & -1.295257 & -0.176124\end{array}$

$\begin{array}{llll}6.499171 & -2.120650 & 0.464434\end{array}$

$\begin{array}{llll}7.709307 & -1.525371 & -1.194138\end{array}$

$\begin{array}{llll}8.080868 & -0.508966 & -2.073307\end{array}$

$\begin{array}{llll}8.793601 & -0.697019 & -2.867133\end{array}$

$\begin{array}{llll}7.503997 & 0.750027 & -1.916403\end{array}$

$\begin{array}{llll}6.585107 & 0.997862 & -0.894841\end{array}$

$\begin{array}{llll}6.141938 & 1.985802 & -0.819720\end{array}$

$\begin{array}{lll}4.990113 & -1.006216 & 2.459291\end{array}$

$\begin{array}{llll}3.791567 & -1.553057 & 2.926144\end{array}$

$\begin{array}{lll}2.850950 & -1.203177 & 2.517493\end{array}$

$\begin{array}{lll}3.804891 & -2.541772 & 3.914326\end{array}$

$\begin{array}{llll}5.005689 & -3.002702 & 4.443271\end{array}$

$\begin{array}{lll}5.013907 & -3.777640 & 5.201364\end{array}$

$\begin{array}{lll}6.204104 & -2.453734 & 3.977569\end{array}$

$\begin{array}{llll}6.203425 & -1.462327 & 2.999632\end{array}$

$\begin{array}{lll}7.146836 & -1.057684 & 2.653533\end{array}$

$\begin{array}{llll}2.829484 & 0.343387 & 0.071229\end{array}$

$\begin{array}{llll}7.499803 & -2.911938 & 4.591384\end{array}$

$\begin{array}{llll}2.496209 & -3.142355 & 4.344222\end{array}$

$\begin{array}{llll}8.363635 & -2.876460 & -1.303737\end{array}$

$\begin{array}{llll}7.809639 & 1.854332 & -2.892236\end{array}$

$\begin{array}{llll}6.783613 & 2.039673 & -3.765420\end{array}$

$\begin{array}{llll}8.000432 & 3.038057 & -2.267470\end{array}$

$\begin{array}{llll}8.910141 & 1.600599 & -3.628728\end{array}$

$\begin{array}{llll}8.696851 & -3.176318 & -2.578476\end{array}$

$\begin{array}{llll}7.567042 & -3.865708 & -0.843198\end{array}$

$\begin{array}{llll}9.508727 & -2.927786 & -0.579227\end{array}$

$\begin{array}{llll}7.708417 & -2.329609 & 5.796663\end{array}$

$\begin{array}{llll}8.565552 & -2.612534 & 3.817869\end{array}$

$\begin{array}{llll}7.509797 & -4.247897 & 4.799101\end{array}$

$\begin{array}{llll}2.601085 & -3.864418 & 5.477226\end{array}$

$\begin{array}{llll}1.554971 & -2.190845 & 4.553353\end{array}$

$\begin{array}{lll}1.998799 & -3.972509 & 3.388491\end{array}$

$\begin{array}{llll}3.714725 & -1.885271 & -1.657351\end{array}$

$\begin{array}{llll}1.969809 & -2.640610 & -0.289756\end{array}$

$\begin{array}{llll}3.954651 & -3.155510 & -2.147341\end{array}$

$\begin{array}{llll}4.299086 & -1.038829 & -1.991755\end{array}$

$\begin{array}{llll}2.224826 & -3.967140 & -0.735600\end{array}$

$\begin{array}{llll}3.206197 & -4.233977 & -1.670485\end{array}$

$\begin{array}{lll}1.618513 & -4.778364 & -0.347766\end{array}$

$\begin{array}{llll}0.838896 & -2.430269 & 0.573695\end{array}$

$\begin{array}{llll}0.596089 & -1.269328 & 1.393384\end{array}$

$\begin{array}{lll}1.164765 & -0.146727 & 1.217102\end{array}$

$2.748574 \quad-1.622858 \quad-0.759747$

$\begin{array}{llll}0.395914 & -3.356306 & 0.911672\end{array}$

$\begin{array}{lll}-0.326216 & -1.369109 & 2.399054\end{array}$

$\begin{array}{lll}-0.601506 & -0.226268 & 3.259965\end{array}$

$\begin{array}{lll}-1.657703 & 0.060300 & 3.193348\end{array}$

$\begin{array}{lll}0.016176 & 0.614967 & 2.953443\end{array}$

$\begin{array}{lll}-0.373905 & -0.476260 & 4.302307\end{array}$

$\begin{array}{lll}-1.004241 & -2.616887 & 2.735106\end{array}$

$\begin{array}{lll}-1.640352 & -2.964818 & 1.914883\end{array}$

$\begin{array}{lll}-1.641763 & -2.440224 & 3.601352\end{array}$

$\begin{array}{lll}-0.293740 & -3.405679 & 2.993941\end{array}$

$\begin{array}{llll}-3.489748 & -0.393341 & -1.687887\end{array}$

$\begin{array}{llll}-5.254890 & -0.206432 & -0.270345\end{array}$

$\begin{array}{lll}-5.409334 & 0.954127 & 0.909670\end{array}$

$\begin{array}{llll}-6.670767 & 0.039246 & -1.114022\end{array}$

$\begin{array}{lll}-5.374173 & -1.577328 & 0.694085\end{array}$

$\begin{array}{lll}-5.905830 & 2.223541 & 0.642316\end{array}$

$\begin{array}{lll}-4.993808 & 3.290766 & 0.795158\end{array}$

$\begin{array}{llll}-3.984646 & 3.061962 & 1.117507\end{array}$

$\begin{array}{lll}-5.400583 & 4.576332 & 0.542098\end{array}$

$\begin{array}{llll}-4.710432 & 5.405983 & 0.667592\end{array}$

$\begin{array}{lll}-6.721104 & 4.835861 & 0.086362\end{array}$ $\begin{array}{lll}-7.129705 & 6.153544 & -0.255080\end{array}$

$\begin{array}{llll}-6.415327 & 6.963846 & -0.134660\end{array}$

$\begin{array}{lll}-8.394261 & 6.398922 & -0.739909\end{array}$

$\begin{array}{llll}-8.695024 & 7.408933 & -1.002366\end{array}$

$\begin{array}{lll}-9.299574 & 5.325281 & -0.912978\end{array}$

$\begin{array}{llll}-10.287967 & 5.515817 & -1.321154\end{array}$

$\begin{array}{llll}-8.939705 & 4.038243 & -0.576131\end{array}$

$\begin{array}{llll}-9.645343 & 3.230800 & -0.728031\end{array}$

$\begin{array}{llll}-7.650120 & 3.748793 & -0.047659\end{array}$

$\begin{array}{lll}-7.239687 & 2.413586 & 0.312756\end{array}$

$\begin{array}{llll}-8.192058 & 1.266970 & 0.282709\end{array}$

$\begin{array}{lll}-9.437504 & 1.275505 & 1.005785\end{array}$

$\begin{array}{lll}-9.765535 & 2.281846 & 1.957025\end{array}$

$\begin{array}{lll}-9.053395 & 3.073659 & 2.158393\end{array}$

$\begin{array}{lll}-10.964205 & 2.254431 & 2.636095\end{array}$

$\begin{array}{lll}-11.186264 & 3.028918 & 3.364606\end{array}$

$\begin{array}{lll}-11.906168 & 1.224423 & 2.400041\end{array}$

$\begin{array}{lll}-12.848407 & 1.219235 & 2.940005\end{array}$

$\begin{array}{lll}-11.616433 & 0.226083 & 1.497891\end{array}$

$\begin{array}{lll}-12.322460 & -0.580597 & 1.318308\end{array}$

$\begin{array}{lll}-10.382752 & 0.215062 & 0.791163\end{array}$

$\begin{array}{lll}-10.058424 & -0.844154 & -0.099271\end{array}$

$-10.784360 \quad-1.634523 \quad-0.266835$

$\begin{array}{llll}-8.823973 & -0.897693 & -0.697471\end{array}$

$\begin{array}{llll}-8.534214 & -1.719432 & -1.342958\end{array}$

$\begin{array}{llll}-7.897847 & 0.143736 & -0.474769\end{array}$

$\begin{array}{lll}-4.511344 & -2.684135 & 0.207620\end{array}$

$\begin{array}{lll}-3.610782 & -2.722619 & 0.832326\end{array}$

$\begin{array}{llll}-5.185272 & -4.043430 & 0.260412\end{array}$

$\begin{array}{lll}-4.459078 & -5.162168 & 0.681024\end{array}$

$\begin{array}{llll}-3.434088 & -5.037008 & 1.024239\end{array}$

$\begin{array}{lll}-5.044523 & -6.431558 & 0.685245\end{array}$

$\begin{array}{llll}-4.471225 & -7.290849 & 1.022680\end{array}$

$\begin{array}{lll}-6.367499 & -6.591105 & 0.269240\end{array}$

$\begin{array}{llll}-6.829872 & -7.574057 & 0.282778\end{array}$

$\begin{array}{llll}-7.096767 & -5.476686 & -0.160753\end{array}$

$\begin{array}{llll}-8.129909 & -5.591968 & -0.476882\end{array}$

$\begin{array}{llll}-6.506922 & -4.214759 & -0.173003\end{array}$

$\begin{array}{llll}-7.080874 & -3.345272 & -0.476656\end{array}$

$\begin{array}{llll}-4.083647 & -2.408359 & -1.247183\end{array}$

$\begin{array}{llll}-4.929180 & -2.656565 & -1.899996\end{array}$

$\begin{array}{llll}-3.288614 & -3.108467 & -1.508765\end{array}$

$\begin{array}{lll}-5.743280 & -1.507747 & 2.149520\end{array}$

$\begin{array}{lll}-6.241960 & -0.547145 & 2.263779\end{array}$

$\begin{array}{lll}-6.765424 & -2.557577 & 2.570084\end{array}$

$\begin{array}{lll}-8.124383 & -2.263485 & 2.407684\end{array}$

$\begin{array}{llll}-8.410718 & -1.297237 & 2.009001\end{array}$

$\begin{array}{lll}-9.109248 & -3.190071 & 2.751405\end{array}$

$\begin{array}{lll}-10.157308 & -2.939019 & 2.611865\end{array}$

$\begin{array}{lll}-8.744052 & -4.429876 & 3.281160\end{array}$

$\begin{array}{lll}-9.505214 & -5.158125 & 3.547231\end{array}$

$\begin{array}{llll}-7.391889 & -4.722311 & 3.478930\end{array}$

$\begin{array}{lll}-7.097162 & -5.680812 & 3.896948\end{array}$

$\begin{array}{lll}-6.412006 & -3.789808 & 3.134327\end{array}$

$\begin{array}{llll}-5.368481 & -4.042232 & 3.284963\end{array}$

$\begin{array}{lll}-4.495122 & -1.476722 & 3.037163\end{array}$

$\begin{array}{llll}-3.850887 & -0.644705 & 2.742173\end{array}$

$\begin{array}{lll}-3.914229 & -2.398680 & 2.977361\end{array}$

$\begin{array}{lll}-4.787962 & -1.335512 & 4.082028\end{array}$

$\begin{array}{lll}-2.207084 & -0.135959 & 0.047335\end{array}$

$\begin{array}{lll}-2.098328 & 0.902087 & 0.340777\end{array}$

$\begin{array}{llll}-2.468891 & -0.786098 & 0.877306\end{array}$

$\begin{array}{lll}-1.284776 & -0.655600 & -0.925722\end{array}$

$\begin{array}{llll}-0.649156 & 0.031635 & -1.470647\end{array}$

$\begin{array}{llll}-0.970673 & -2.035027 & -1.004763\end{array}$

$\begin{array}{llll}-1.568425 & -2.691593 & -0.384602\end{array}$

$\begin{array}{llll}-4.796352 & -0.467904 & -3.490031\end{array}$

$\begin{array}{llll}-5.575783 & -1.198752 & -3.290918\end{array}$

$\begin{array}{llll}-3.524766 & -0.991620 & -3.835234\end{array}$

$\begin{array}{llll}-3.437889 & -2.077099 & -3.886804\end{array}$

$\begin{array}{llll}-2.465807 & -0.252496 & -4.635823\end{array}$

$\begin{array}{llll}-2.686416 & -0.330978 & -5.711984\end{array}$

$\begin{array}{llll}-1.512277 & -0.767384 & -4.482362\end{array}$

$\begin{array}{llll}-2.310091 & 1.229551 & -4.240813\end{array}$

$\begin{array}{llll}-3.008626 & 1.849909 & -4.808928\end{array}$ 


$\begin{array}{lccc}1 & -1.312933 & 1.571852 & -4.526133 \\ 6 & -2.509301 & 1.496407 & -2.752887 \\ 1 & -1.615159 & 1.716739 & -2.178496 \\ 6 & -3.716035 & 1.899704 & -2.203719 \\ 1 & -3.676637 & 2.424964 & -1.254256 \\ 6 & -5.008482 & 2.063268 & -2.980610 \\ 1 & -5.821018 & 2.160469 & -2.258218 \\ 1 & -4.969647 & 3.010643 & -3.538114 \\ 6 & -5.332134 & 0.893907 & -3.941672 \\ 1 & -4.957289 & 1.110295 & -4.946908 \\ 1 & -6.419588 & 0.819532 & -4.033631 \\ 6 & -0.358463 & -2.672062 & -2.164599 \\ 6 & 0.458469 & -1.969336 & -3.072332 \\ 6 & -0.593112 & -4.041501 & -2.392411 \\ 6 & 1.008398 & -2.615064 & -4.175677 \\ 1 & 0.675079 & -0.920985 & -2.906152 \\ 6 & -0.056604 & -4.682495 & -3.509030 \\ 1 & -1.206917 & -4.599333 & -1.689634 \\ 6 & 0.747477 & -3.971630 & -4.403442 \\ 1 & 1.643913 & -2.057405 & -4.856062 \\ 1 & -0.258851 & -5.736657 & -3.675661 \\ 1 & 1.174617 & -4.470335 & -5.268749 \\ 1 & 3.376695 & -5.244071 & -2.026056 \\ 35 & 5.326223 & -3.409244 & -3.513751 \\ -----------------------------------------------------------------------\end{array}$

C1-P1 si-si_7

Number of imaginary frequencies $=1$

The smallest frequencies are $=-187.61246 .963611 .0676 \mathrm{~cm}(-1)$ Electronic energy $=-7222.6193586$

Zero-point correction=

1.755050

(Hartree/Particle)

Thermal correction to Energy $=\quad 1.873850$

Thermal correction to Enthalpy= $\quad 1.874795$

Thermal correction to Gibbs Free Energy $=\quad 1.591033$

Sum of electronic and zero-point Energies $=\quad-7220.864308$

Sum of electronic and thermal Energies $=\quad-7220.745508$

Sum of electronic and thermal Enthalpies $=\quad-7220.744564$

Sum of electronic and thermal Free Energies $=\quad-7221.028326$

Cartesian Coordinates

$\begin{array}{llll}26 & 3.550937 & 4.404932 & 3.282371 \\ 15 & 2.504507 & 2.147182 & -1.267645 \\ 15 & 4.838037 & 0.309906 & 1.189014 \\ 6 & 3.464854 & 3.593595 & 1.349670 \\ 6 & 4.013463 & 2.646947 & 2.285808 \\ 6 & 3.027443 & 2.408371 & 3.293169 \\ 1 & 3.139243 & 1.748394 & 4.142879 \\ 6 & 1.887972 & 3.216045 & 3.003156 \\ 1 & 0.992177 & 3.290458 & 3.605360 \\ 6 & 2.160005 & 3.953453 & 1.813549 \\ 1 & 1.505012 & 4.671099 & 1.341013 \\ 6 & 5.251306 & 5.458734 & 3.852579 \\ 1 & 6.246306 & 5.279006 & 3.468073 \\ 6 & 4.661636 & 4.816815 & 4.984227 \\ 1 & 5.126931 & 4.060345 & 5.601485 \\ 6 & 3.331048 & 5.314710 & 5.129488 \\ 1 & 2.612291 & 4.996598 & 5.873119 \\ 6 & 3.097286 & 6.263095 & 4.087642 \\ 1 & 2.170448 & 6.790138 & 3.902742 \\ 6 & 4.283247 & 6.353097 & 3.299304 \\ 1 & 4.411313 & 6.966245 & 2.417623 \\ 6 & 4.132848 & 4.034169 & 0.094275 \\ 6 & 5.184612 & 4.960208 & 0.153473 \\ 1 & 5.482614 & 5.334662 & 1.124585 \\ 6 & 5.840853 & 5.398156 & -0.996866 \\ 1 & 6.658688 & 6.108430 & -0.916819 \\ 6 & 5.435057 & 4.923971 & -2.242240 \\ 1 & 5.926618 & 5.261594 & -3.149464 \\ 6 & 4.402789 & 3.989111 & -2.323001 \\ 1 & 4.116409 & 3.617271 & -3.299200 \\ 6 & 3.760543 & 3.508736 & -1.170455 \\ 6 & 5.310979 & 1.918160 & 2.087057 \\ 1 & 5.901995 & 2.476156 & 1.355445\end{array}$

$6.148487 \quad 1.749355 \quad 3.356982$

$\begin{array}{llll}7.089093 & 1.232293 & 3.148647\end{array}$

$\begin{array}{llll}5.615201 & 1.191674 & 4.131545\end{array}$

$\begin{array}{llll}6.384184 & 2.739605 & 3.756909\end{array}$

$\begin{array}{lll}2.574630 & 1.591986 & -3.021124\end{array}$

$3.786745 \quad 1.073701-3.511025$

$\begin{array}{lll}4.680009 & 1.113460 & -2.897174\end{array}$

$3.867288 \quad 0.541155 \quad-4.795800$

$\begin{array}{llll}4.817701 & 0.161999 & -5.159753\end{array}$

$2.728971 \quad 0.496467 \quad-5.607184$

$\begin{array}{lll}2.787190 & 0.076352 & -6.607030\end{array}$

$\begin{array}{llll}1.518307 & 0.998316 & -5.126238\end{array}$

$\begin{array}{llll}0.629897 & 0.966850 & -5.750477\end{array}$

$\begin{array}{llll}1.440568 & 1.548400 & -3.843141\end{array}$

$\begin{array}{lll}0.495781 & 1.941696 & -3.485174\end{array}$

$\begin{array}{lll}0.906744 & 3.038677 & -1.102917\end{array}$

$\begin{array}{llll}0.604982 & 4.174843 & -1.871176\end{array}$

$1.299972 \quad 4.515544 \quad-2.633605$

$\begin{array}{llll}-0.581505 & 4.875505 & -1.650948\end{array}$

$\begin{array}{llll}-0.812219 & 5.750567 & -2.251719\end{array}$

$\begin{array}{llll}-1.462452 & 4.462331 & -0.644846\end{array}$

$\begin{array}{llll}-2.380077 & 5.015083 & -0.464929\end{array}$

$\begin{array}{lll}-1.160373 & 3.337870 & 0.124668\end{array}$

$\begin{array}{lll}-1.832746 & 3.021132 & 0.915465\end{array}$

$\begin{array}{llll}0.012680 & 2.615771 & -0.109950\end{array}$

$\begin{array}{lll}0.248288 & 1.737542 & 0.483880\end{array}$

$\begin{array}{llll}6.221009 & 0.000835 & 0.011254\end{array}$

$6.787422-1.268911-0.176052$

$\begin{array}{llll}6.503833 & -2.102416 & 0.454537\end{array}$

$\begin{array}{llll}7.717723 & -1.484476 & -1.192544\end{array}$

$\begin{array}{lll}8.088317 & -0.457479 & -2.059734\end{array}$

$\begin{array}{llll}8.804862 & -0.634261 & -2.852734\end{array}$

$\begin{array}{llll}7.505834 & 0.797441 & -1.891714\end{array}$

$\begin{array}{llll}6.582487 & 1.031080 & -0.870743\end{array}$

$\begin{array}{llll}6.135496 & 2.016582 & -0.787057\end{array}$

$\begin{array}{llll}4.987517 & -1.015906 & 2.456272\end{array}$

$\begin{array}{llll}3.790666 & -1.575062 & 2.916770\end{array}$

$2.848392-1.224580 \quad 2.512320$

$\begin{array}{llll}3.808799 & -2.575743 & 3.890993\end{array}$

$\begin{array}{llll}5.012607 & -3.038742 & 4.415424\end{array}$

$\begin{array}{llll}5.025166 & -3.823223 & 5.162662\end{array}$

$\begin{array}{llll}6.206362 & -2.479060 & 3.956553\end{array}$

$\begin{array}{llll}6.200980 & -1.473726 & 2.990356\end{array}$

$\begin{array}{llll}7.143944 & -1.062629 & 2.648310\end{array}$

$\begin{array}{llll}2.823378 & 0.342269 & 0.076018\end{array}$

$\begin{array}{lll}7.520880 & -2.916985 & 4.544457\end{array}$

$\begin{array}{llll}2.503684 & -3.190118 & 4.312009\end{array}$

$\begin{array}{llll}8.379525 & -2.831050 & -1.312436\end{array}$

$\begin{array}{llll}7.810207 & 1.912950 & -2.855063\end{array}$

$\begin{array}{llll}6.788095 & 2.100307 & -3.732352\end{array}$

$\begin{array}{llll}7.989935 & 3.091681 & -2.217786\end{array}$

$8.916549 \quad 1.673195 \quad-3.587542$

$\begin{array}{llll}8.717639 & -3.117600 & -2.588979\end{array}$

$\begin{array}{lll}7.587389 & -3.828733 & -0.862701\end{array}$

$\begin{array}{llll}9.523123 & -2.882380 & -0.585589\end{array}$

$\begin{array}{llll}7.444452 & -4.139462 & 5.111489\end{array}$

$\begin{array}{llll}7.943065 & -2.059414 & 5.504772\end{array}$

$\begin{array}{lll}8.496166 & -2.961199 & 3.608410\end{array}$

$\begin{array}{llll}2.609869 & -3.915539 & 5.442844\end{array}$

$\begin{array}{lll}1.552262 & -2.248532 & 4.520089\end{array}$

$\begin{array}{llll}2.018349 & -4.021542 & 3.351054\end{array}$

$3.725554-1.868663-1.667216$

$\begin{array}{lll}1.976385 & -2.642192 & -0.315705\end{array}$

$\begin{array}{llll}3.970547 & -3.133400 & -2.168909\end{array}$

$\begin{array}{lll}4.309284 & -1.017670 & -1.991299\end{array}$

$\begin{array}{llll}2.235558 & -3.963374 & -0.774679\end{array}$

$\begin{array}{llll}3.221907 & -4.218390 & -1.707495\end{array}$

$\begin{array}{llll}1.628655 & -4.779679 & -0.398622\end{array}$

$\begin{array}{lll}0.840704 & -2.443486 & 0.544284\end{array}$

$\begin{array}{lll}0.592852 & -1.294001 & 1.378619\end{array}$

$\begin{array}{lll}1.161259 & -0.168492 & 1.221485\end{array}$

$2.754778 \quad-1.617714 \quad-0.771093$

$\begin{array}{llll}0.397771 & -3.374437 & 0.868332\end{array}$

$\begin{array}{lll}-0.336897 & -1.409057 & 2.375798\end{array}$

$\begin{array}{lll}0.623974 & -0.277019 & 3.247043\end{array}$ 
$\begin{array}{lll}-0.309666 & -0.497412 & 4.274059\end{array}$

$\begin{array}{lll}-1.698470 & -0.064402 & 3.250257\end{array}$

$\begin{array}{lll}-0.085437 & 0.598952 & 2.892500\end{array}$

$\begin{array}{lll}-0.995175 & -2.668760 & 2.706754\end{array}$

$\begin{array}{lll}-1.642432 & -3.012266 & 1.893237\end{array}$

$\begin{array}{lll}-1.617421 & -2.511879 & 3.587864\end{array}$

$\begin{array}{llll}-0.272155 & -3.453366 & 2.941689\end{array}$

$\begin{array}{lll}-3.486366 & -0.396203 & -1.707225\end{array}$

$\begin{array}{lll}-5.254054 & -0.194251 & -0.298755\end{array}$

$\begin{array}{lll}-5.400796 & 0.977635 & 0.870916\end{array}$

$\begin{array}{llll}-6.672339 & 0.046508 & -1.139904\end{array}$

$\begin{array}{llll}-5.380209 & -1.557168 & 0.677257\end{array}$

$\begin{array}{lll}-5.919742 & 2.238743 & 0.608503\end{array}$

$\begin{array}{llll}-5.022602 & 3.320256 & 0.750542\end{array}$

$\begin{array}{lll}-4.006109 & 3.108302 & 1.061051\end{array}$

$\begin{array}{lll}-5.452829 & 4.599502 & 0.504377\end{array}$

$\begin{array}{llll}-4.773937 & 5.439568 & 0.621733\end{array}$

$\begin{array}{lll}-6.783433 & 4.839312 & 0.067604\end{array}$

$\begin{array}{llll}-7.217562 & 6.151077 & -0.265083\end{array}$

$\begin{array}{lll}-6.514151 & 6.972205 & -0.153489\end{array}$

$\begin{array}{llll}-8.492835 & 6.377537 & -0.730667\end{array}$

$\begin{array}{llll}-8.813225 & 7.383215 & -0.986454\end{array}$

$\begin{array}{llll}-9.383675 & 5.290145 & -0.892598\end{array}$

$\begin{array}{llll}-10.380958 & 5.465856 & -1.285524\end{array}$

$\begin{array}{llll}-8.998727 & 4.008244 & -0.563993\end{array}$

$\begin{array}{llll}-9.694002 & 3.190265 & -0.707195\end{array}$

$\begin{array}{llll}-7.697051 & 3.738004 & -0.055311\end{array}$

$\begin{array}{llll}-7.260612 & 2.408897 & 0.296885\end{array}$

$\begin{array}{llll}-8.195777 & 1.248016 & 0.276706\end{array}$

$\begin{array}{lll}-9.431307 & 1.235433 & 1.016400\end{array}$

$\begin{array}{lll}-9.760084 & 2.232235 & 1.977280\end{array}$

$\begin{array}{lll}-9.056324 & 3.032987 & 2.172849\end{array}$

$\begin{array}{lll}-10.948494 & 2.184044 & 2.673035\end{array}$

$\begin{array}{lll}-11.171256 & 2.951354 & 3.408876\end{array}$

$\begin{array}{lll}-11.878968 & 1.141920 & 2.444575\end{array}$

$\begin{array}{lll}-12.813380 & 1.120583 & 2.997595\end{array}$

$\begin{array}{lll}-11.587893 & 0.152408 & 1.533155\end{array}$

$\begin{array}{lll}-12.284794 & -0.663405 & 1.359305\end{array}$

$\begin{array}{lll}-10.364265 & 0.162679 & 0.809248\end{array}$

$\begin{array}{lll}-10.037212 & -0.887181 & -0.091355\end{array}$

$-10.754596-1.686318-0.254246$

$\begin{array}{llll}-8.809832 & -0.920622 & -0.705537\end{array}$

$\begin{array}{llll}-8.517752 & -1.734003 & -1.360466\end{array}$

$\begin{array}{llll}-7.895313 & 0.132244 & -0.488944\end{array}$

$\begin{array}{llll}-4.514627 & -2.668445 & 0.205559\end{array}$

$\begin{array}{llll}-3.615739 & -2.699734 & 0.832772\end{array}$

$\begin{array}{lll}-5.181433 & -4.031035 & 0.270345\end{array}$

$\begin{array}{lll}-4.437685 & -5.143897 & 0.676478\end{array}$

$\begin{array}{lll}-3.407436 & -5.009415 & 1.000159\end{array}$

$\begin{array}{lll}-5.011011 & -6.418408 & 0.690299\end{array}$

$\begin{array}{lll}-4.423626 & -7.272715 & 1.016030\end{array}$

$\begin{array}{llll}-6.340137 & -6.589599 & 0.299084\end{array}$

$\begin{array}{lll}-6.793434 & -7.576641 & 0.320561\end{array}$

$\begin{array}{llll}-7.087107 & -5.481414 & -0.115947\end{array}$

$\begin{array}{llll}-8.124987 & -5.605584 & -0.412605\end{array}$

$\begin{array}{lll}-6.508796 & -4.213992 & -0.139026\end{array}$

$\begin{array}{llll}-7.095731 & -3.349805 & -0.432039\end{array}$

$\begin{array}{llll}-4.084779 & -2.407245 & -1.251955\end{array}$

$\begin{array}{llll}-4.930887 & -2.659656 & -1.902673\end{array}$

$\begin{array}{llll}-3.291507 & -3.112071 & -1.506410\end{array}$

$\begin{array}{lll}-5.744090 & -1.458844 & 2.132482\end{array}$

$\begin{array}{llll}-6.286757 & -0.519291 & 2.219246\end{array}$

$\begin{array}{lll}-6.715200 & -2.540490 & 2.588127\end{array}$

$\begin{array}{llll}-8.086865 & -2.310437 & 2.428818\end{array}$

$\begin{array}{llll}-8.418581 & -1.368008 & 2.008905\end{array}$

$\begin{array}{lll}-9.027359 & -3.271707 & 2.799702\end{array}$

$\begin{array}{lll}-10.086426 & -3.070407 & 2.662227\end{array}$

$\begin{array}{lll}-8.603941 & -4.482623 & 3.352474\end{array}$

$\begin{array}{lll}-9.329880 & -5.238424 & 3.639270\end{array}$

$\begin{array}{llll}-7.238889 & -4.710821 & 3.545949\end{array}$

$\begin{array}{lll}-6.899652 & -5.646499 & 3.981523\end{array}$

$\begin{array}{lll}-6.303417 & -3.743189 & 3.175431\end{array}$

$\begin{array}{llll}-5.248592 & -3.944890 & 3.325064\end{array}$

$\begin{array}{lll}-4.493918 & -1.340619 & 3.010155\end{array}$

$\begin{array}{lll}-3.903819 & -0.476208 & 2.695393\end{array}$ $\begin{array}{llll}-3.860467 & -2.228298 & 2.960477\end{array}$

$\begin{array}{lll}-4.785849 & -1.198925 & 4.055310\end{array}$

$\begin{array}{lll}-2.206016 & -0.143103 & 0.029955\end{array}$

$\begin{array}{lll}-2.098138 & 0.893756 & 0.326879\end{array}$

$\begin{array}{lll}-2.467879 & -0.795501 & 0.858029\end{array}$

$\begin{array}{llll}-1.281740 & -0.658883 & -0.943487\end{array}$

$\begin{array}{llll}-0.646643 & 0.030836 & -1.485919\end{array}$

$\begin{array}{lll}-0.965059 & -2.037646 & -1.027908\end{array}$

$\begin{array}{llll}-1.562817 & -2.698114 & -0.411732\end{array}$

$\begin{array}{llll}-4.789581 & -0.477286 & -3.513144\end{array}$

$\begin{array}{llll}-5.569959 & -1.206570 & -3.311946\end{array}$

$\begin{array}{llll}-3.517664 & -1.002572 & -3.852946\end{array}$

$\begin{array}{lll}-3.429739 & -2.088187 & -3.898690\end{array}$

$\begin{array}{llll}-2.456149 & -0.265944 & -4.652569\end{array}$

$\begin{array}{llll}-2.674305 & -0.346455 & -5.729067\end{array}$

$\begin{array}{lll}-1.503817 & -0.781716 & -4.495558\end{array}$

$\begin{array}{llll}-2.298581 & 1.217150 & -4.260940\end{array}$

$\begin{array}{llll}-2.992852 & 1.837665 & -4.834166\end{array}$

$\begin{array}{llll}-1.299118 & 1.555605 & -4.542657\end{array}$

$\begin{array}{lll}-2.503522 & 1.490013 & -2.774873\end{array}$

$\begin{array}{lll}-1.611869 & 1.713442 & -2.197684\end{array}$

$\begin{array}{llll}-3.712416 & 1.895125 & -2.231908\end{array}$

$-3.676301 \quad 2.424540-1.284585$

$\begin{array}{llll}-5.002187 & 2.055790 & -3.013947\end{array}$

$\begin{array}{lll}-5.817515 & 2.155589 & -2.294967\end{array}$

$\begin{array}{lll}-4.961188 & 3.001240 & -3.574550\end{array}$

$\begin{array}{llll}-5.322134 & 0.883241 & -3.972260\end{array}$

$\begin{array}{lll}-4.941706 & 1.095664 & -4.976207\end{array}$

$\begin{array}{llll}-6.409146 & 0.809531 & -4.069779\end{array}$

$-0.350814-2.666356-2.191670$

$\begin{array}{llll}0.469140 & -1.956543 & -3.091034\end{array}$

$\begin{array}{llll}-0.584901 & -4.033698 & -2.431364\end{array}$

$1.023015-2.593163-4.197725$

$\begin{array}{lll}0.685029 & -0.909593 & -2.915485\end{array}$

$\begin{array}{lll}-0.044456 & -4.665630 & -3.551363\end{array}$

$-1.201381-4.597240-1.735571$

$\begin{array}{llll}0.762822 & -3.947801 & -4.437303\end{array}$

$1.660858-2.029859-4.871314$

$\begin{array}{llll}-0.246283 & -5.718375 & -3.727238\end{array}$

$\begin{array}{llll}1.192902 & -4.439682 & -5.305056\end{array}$

$3.396181-5.224224-2.073195$

$\begin{array}{lllll}35 & 5.349575 & -3.371245 & -3.530345\end{array}$

C1-P1 si-si_8

Number of imaginary frequencies $=1$

The smallest frequencies are $=-153.75358 .528610 .6626 \mathrm{~cm}(-1)$ Electronic energy $=-7222.6206504$

Zero-point correction=

1.754800

(Hartree/Particle)

Thermal correction to Energy= $\quad 1.873746$

Thermal correction to Enthalpy= $\quad 1.874690$

Thermal correction to Gibbs Free Energy $=\quad 1.591401$

Sum of electronic and zero-point Energies $=\quad-7220.865850$

Sum of electronic and thermal Energies $=\quad-7220.746904$

Sum of electronic and thermal Enthalpies $=\quad-7220.745960$

Sum of electronic and thermal Free Energies $=\quad-7221.029250$

Cartesian Coordinates

$\begin{array}{lclc}26 & 3.848815 & 4.474038 & 3.163571 \\ 15 & 1.178196 & 2.281163 & -0.762619 \\ 15 & 4.500735 & 0.586678 & 0.479637 \\ 6 & 3.024899 & 3.723646 & 1.383359 \\ 6 & 3.993749 & 2.811162 & 1.934884 \\ 6 & 3.547074 & 2.432492 & 3.239977 \\ 1 & 4.065125 & 1.762936 & 3.913485 \\ 6 & 2.327904 & 3.122425 & 3.509484 \\ 1 & 1.763928 & 3.081824 & 4.431821 \\ 6 & 2.013816 & 3.927019 & 2.375719 \\ 1 & 1.163357 & 4.585570 & 2.281329 \\ 6 & 5.547678 & 5.667846 & 3.062478 \\ 1 & 6.305508 & 5.590952 & 2.294592 \\ 6 & 5.522980 & 4.940855 & 4.291703 \\ 1 & 6.255351 & 4.213774 & 4.615529\end{array}$


$\begin{array}{llll}4.335308 & 5.306043 & 4.996047\end{array}$

$\begin{array}{llll}4.009366 & 4.900646 & 5.944925\end{array}$

$\begin{array}{lll}3.625250 & 6.257669 & 4.202055\end{array}$

$\begin{array}{lll}2.669105 & 6.702386 & 4.444690\end{array}$

$\begin{array}{lll}4.374341 & 6.482383 & 3.008691\end{array}$

$\begin{array}{lll}4.082891 & 7.128999 & 2.192228\end{array}$

$\begin{array}{llll}3.093660 & 4.259107 & -0.006428\end{array}$

$\begin{array}{llll}4.022763 & 5.264815 & -0.310069\end{array}$

$\begin{array}{llll}4.632675 & 5.654343 & 0.494407\end{array}$

$\begin{array}{llll}4.183964 & 5.753562 & -1.606999\end{array}$

$\begin{array}{llll}4.919114 & 6.527997 & -1.805896\end{array}$

$\begin{array}{llll}3.399995 & 5.239397 & -2.635937\end{array}$

$3.512157 \quad 5.602520 \quad-3.652811$

$\begin{array}{llll}2.472360 & 4.233875 & -2.360348\end{array}$

$\begin{array}{llll}1.882781 & 3.835751 & -3.176872\end{array}$

$\begin{array}{llll}2.311541 & 3.720787 & -1.063363\end{array}$

$\begin{array}{llll}5.144042 & 2.234635 & 1.164052\end{array}$

$\begin{array}{llll}5.296655 & 2.843947 & 0.267845\end{array}$

$\begin{array}{lll}6.465569 & 2.166542 & 1.932385\end{array}$

$\begin{array}{lll}7.256091 & 1.716614 & 1.324572\end{array}$

$\begin{array}{lll}6.372459 & 1.589336 & 2.855956\end{array}$

$\begin{array}{llll}6.774958 & 3.180889 & 2.199178\end{array}$

$\begin{array}{llll}0.393806 & 2.041237 & -2.411425\end{array}$

$\begin{array}{llll}1.067586 & 1.203547 & -3.315764\end{array}$

$\begin{array}{llll}1.980172 & 0.709364 & -3.000198\end{array}$

$\begin{array}{llll}0.596199 & 1.033860 & -4.617521\end{array}$

$\begin{array}{lll}1.136267 & 0.393218 & -5.308890\end{array}$

$\begin{array}{llll}-0.568838 & 1.689520 & -5.027015\end{array}$

$\begin{array}{llll}-0.942708 & 1.555005 & -6.037694\end{array}$

$\begin{array}{lll}-1.257600 & 2.507392 & -4.128354\end{array}$

$\begin{array}{llll}-2.172798 & 3.006321 & -4.434356\end{array}$

$\begin{array}{llll}-0.779130 & 2.685885 & -2.828666\end{array}$

$\begin{array}{llll}-1.326732 & 3.322496 & -2.144992\end{array}$

$\begin{array}{lll}-0.125625 & 3.020655 & 0.301035\end{array}$

$\begin{array}{lll}-0.593189 & 2.270094 & 1.388423\end{array}$

$\begin{array}{lll}-0.219855 & 1.263219 & 1.539828\end{array}$

$\begin{array}{llll}-1.487399 & 2.836209 & 2.298789\end{array}$

$\begin{array}{lll}-1.831309 & 2.247395 & 3.141354\end{array}$

$\begin{array}{lll}-1.927402 & 4.150313 & 2.127637\end{array}$

$\begin{array}{llll}-2.621316 & 4.591014 & 2.837972\end{array}$

$\begin{array}{lll}-1.472402 & 4.901078 & 1.038012\end{array}$

$\begin{array}{llll}-1.812584 & 5.923383 & 0.900178\end{array}$

$\begin{array}{lll}-0.571297 & 4.342086 & 0.130308\end{array}$

$\begin{array}{llll}-0.190964 & 4.944910 & -0.689166\end{array}$

$\begin{array}{llll}5.435537 & 0.398634 & -1.092956\end{array}$

$\begin{array}{llll}6.286910 & -0.660847 & -1.430276\end{array}$

$\begin{array}{llll}6.507661 & -1.449004 & -0.722168\end{array}$

$6.846374-0.729863 \quad-2.707556$

$\begin{array}{llll}6.547295 & 0.223174 & -3.680227\end{array}$

$\begin{array}{llll}6.969846 & 0.148552 & -4.674646\end{array}$

$\begin{array}{llll}5.669387 & 1.255628 & -3.355214\end{array}$

$\begin{array}{llll}5.123109 & 1.350992 & -2.075786\end{array}$

$\begin{array}{llll}4.428766 & 2.157069 & -1.853612\end{array}$

$\begin{array}{llll}5.123842 & -0.668699 & 1.669124\end{array}$

$\begin{array}{llll}4.155195 & -1.205260 & 2.527467\end{array}$

$\begin{array}{lll}3.124080 & -0.891268 & 2.423723\end{array}$

$\begin{array}{lll}4.512586 & -2.123313 & 3.515007\end{array}$

$\begin{array}{llll}5.836875 & -2.533597 & 3.658500\end{array}$

$\begin{array}{lll}6.114379 & -3.252783 & 4.419869\end{array}$

$\begin{array}{lll}6.803057 & -1.994975 & 2.808349\end{array}$

$\begin{array}{llll}6.459847 & -1.060188 & 1.829654\end{array}$

$\begin{array}{llll}7.241192 & -0.641463 & 1.206850\end{array}$

$\begin{array}{llll}2.216633 & 0.413509 & 0.037266\end{array}$

$\begin{array}{lll}8.252356 & -2.363175 & 2.977041\end{array}$

$\begin{array}{llll}3.437891 & -2.713807 & 4.385625\end{array}$

$\begin{array}{llll}7.829741 & -1.824940 & -3.022557\end{array}$

$\begin{array}{llll}5.253989 & 2.275056 & -4.380508\end{array}$

$\begin{array}{llll}3.903318 & 2.292336 & -4.532239\end{array}$

$\begin{array}{llll}5.614490 & 3.528138 & -4.017635\end{array}$

$\begin{array}{llll}5.788076 & 2.037435 & -5.595171\end{array}$

$\begin{array}{llll}7.806155 & -2.171272 & -4.328782\end{array}$

$\begin{array}{llll}7.596066 & -2.941334 & -2.297754\end{array}$

$\begin{array}{llll}9.099940 & -1.439115 & -2.745267\end{array}$

$\begin{array}{llll}8.874275 & -2.494767 & 1.782384\end{array}$

$\begin{array}{lll}8.411508 & -3.523133 & 3.648435\end{array}$ $\begin{array}{llll}8.929436 & -1.410381 & 3.663059\end{array}$

$\begin{array}{llll}3.919686 & -3.145366 & 5.570564\end{array}$

$\begin{array}{llll}2.453997 & -1.821131 & 4.646779\end{array}$

$\begin{array}{lll}2.842248 & -3.782257 & 3.793898\end{array}$

$\begin{array}{llll}3.022076 & -1.777692 & -1.904706\end{array}$

$\begin{array}{llll}2.167523 & -2.715210 & 0.063567\end{array}$

$\begin{array}{llll}3.602389 & -2.973499 & -2.286588\end{array}$

$\begin{array}{llll}3.122605 & -0.893822 & -2.522858\end{array}$

$\begin{array}{llll}2.824287 & -3.936930 & -0.260382\end{array}$

$\begin{array}{llll}3.536480 & -4.078617 & -1.434278\end{array}$

$\begin{array}{llll}2.729286 & -4.779147 & 0.416711\end{array}$

$\begin{array}{llll}1.270084 & -2.718815 & 1.177994\end{array}$

$\begin{array}{llll}0.781542 & -1.577089 & 1.893775\end{array}$

$\begin{array}{lll}1.078883 & -0.377610 & 1.590708\end{array}$

$\begin{array}{llll}2.330323 & -1.630305 & -0.759229\end{array}$

$\begin{array}{lll}1.212009 & -3.671856 & 1.683712\end{array}$

$\begin{array}{lll}-0.061431 & -1.764290 & 2.958049\end{array}$

$\begin{array}{lll}-0.414954 & -0.627611 & 3.802938\end{array}$

$\begin{array}{lll}0.248955 & 0.209716 & 3.597411\end{array}$

$\begin{array}{lll}-0.315969 & -0.909920 & 4.855133\end{array}$

$\begin{array}{lll}-1.449552 & -0.315441 & 3.628521\end{array}$

$\begin{array}{lll}-0.448599 & -3.070399 & 3.475583\end{array}$

$\begin{array}{lll}-0.628499 & -3.786038 & 2.672937\end{array}$

$\begin{array}{lll}-1.379684 & -2.958743 & 4.036294\end{array}$

$\begin{array}{llll}0.317685 & -3.481829 & 4.144105\end{array}$

$\begin{array}{lll}-3.324811 & -1.621256 & -1.294941\end{array}$

$\begin{array}{llll}-4.944766 & -0.732630 & 0.043854\end{array}$

$\begin{array}{lll}-4.932245 & 0.801502 & 0.679756\end{array}$

$\begin{array}{llll}-6.379665 & -0.682495 & -0.807385\end{array}$

$\begin{array}{lll}-5.080214 & -1.627383 & 1.451612\end{array}$

$\begin{array}{llll}-5.100838 & 1.896575 & -0.167456\end{array}$

$\begin{array}{llll}-3.964235 & 2.704101 & -0.389256\end{array}$

$\begin{array}{lll}-3.040795 & 2.450732 & 0.116040\end{array}$

$\begin{array}{llll}-4.056707 & 3.784010 & -1.231606\end{array}$

$\begin{array}{llll}-3.197319 & 4.427611 & -1.389014\end{array}$

$\begin{array}{llll}-5.263702 & 4.053917 & -1.929924\end{array}$

$\begin{array}{llll}-5.336925 & 5.103715 & -2.885262\end{array}$

$\begin{array}{llll}-4.455271 & 5.718571 & -3.046830\end{array}$

$\begin{array}{llll}-6.490004 & 5.327817 & -3.602766\end{array}$

$\begin{array}{llll}-6.533677 & 6.128024 & -4.335789\end{array}$

$\begin{array}{llll}-7.618439 & 4.499258 & -3.394996\end{array}$

$\begin{array}{llll}-8.518939 & 4.660584 & -3.980560\end{array}$

$\begin{array}{llll}-7.585904 & 3.485363 & -2.461972\end{array}$

$\begin{array}{llll}-8.457290 & 2.856171 & -2.326920\end{array}$

$\begin{array}{llll}-6.419077 & 3.237573 & -1.685893\end{array}$

$\begin{array}{llll}-6.344408 & 2.175751 & -0.713059\end{array}$

$\begin{array}{llll}-7.542586 & 1.375698 & -0.335797\end{array}$

$\begin{array}{llll}-8.743531 & 1.995997 & 0.164634\end{array}$

$\begin{array}{llll}-8.814779 & 3.380647 & 0.485518\end{array}$

$\begin{array}{llll}-7.935058 & 4.000756 & 0.360325\end{array}$

$\begin{array}{lll}-9.977994 & 3.940962 & 0.967788\end{array}$

$\begin{array}{lll}-10.002459 & 4.998912 & 1.213003\end{array}$

$\begin{array}{lll}-11.138276 & 3.152282 & 1.152900\end{array}$

$\begin{array}{lll}-12.049693 & 3.609327 & 1.526916\end{array}$

$\begin{array}{lll}-11.100024 & 1.805214 & 0.872124\end{array}$

$\begin{array}{lll}-11.977294 & 1.182364 & 1.026578\end{array}$

$\begin{array}{llll}-9.911774 & 1.192526 & 0.389816\end{array}$

$\begin{array}{lll}-9.856604 & -0.206691 & 0.146074\end{array}$

$\begin{array}{lll}-10.751065 & -0.804453 & 0.297176\end{array}$

$\begin{array}{lll}-8.680610 & -0.800107 & -0.237672\end{array}$

$\begin{array}{lll}-8.601088 & -1.871379 & -0.389816\end{array}$

$\begin{array}{lll}-7.528643 & -0.006530 & -0.436996\end{array}$

$\begin{array}{lll}-4.212014 & -2.840196 & 1.444922\end{array}$

$\begin{array}{lll}-3.276873 & -2.596094 & 1.966660\end{array}$

$\begin{array}{lll}-4.851920 & -4.024925 & 2.148608\end{array}$

$\begin{array}{lll}-4.095539 & -4.801663 & 3.033718\end{array}$

$\begin{array}{lll}-3.071960 & -4.511962 & 3.258111\end{array}$

$\begin{array}{lll}-4.644722 & -5.940405 & 3.630441\end{array}$

$\begin{array}{lll}-4.045129 & -6.533053 & 4.315980\end{array}$

$\begin{array}{lll}-5.962397 & -6.309191 & 3.352890\end{array}$

$\begin{array}{lll}-6.393802 & -7.189344 & 3.821095\end{array}$

$\begin{array}{llll}-6.726421 & -5.535923 & 2.471974\end{array}$

$\begin{array}{lll}-7.753905 & -5.814785 & 2.254706\end{array}$

$\begin{array}{lll}-6.173321 & -4.405679 & 1.870770\end{array}$

$\begin{array}{lll}-6.767864 & -3.798736 & 1.192831\end{array}$ 


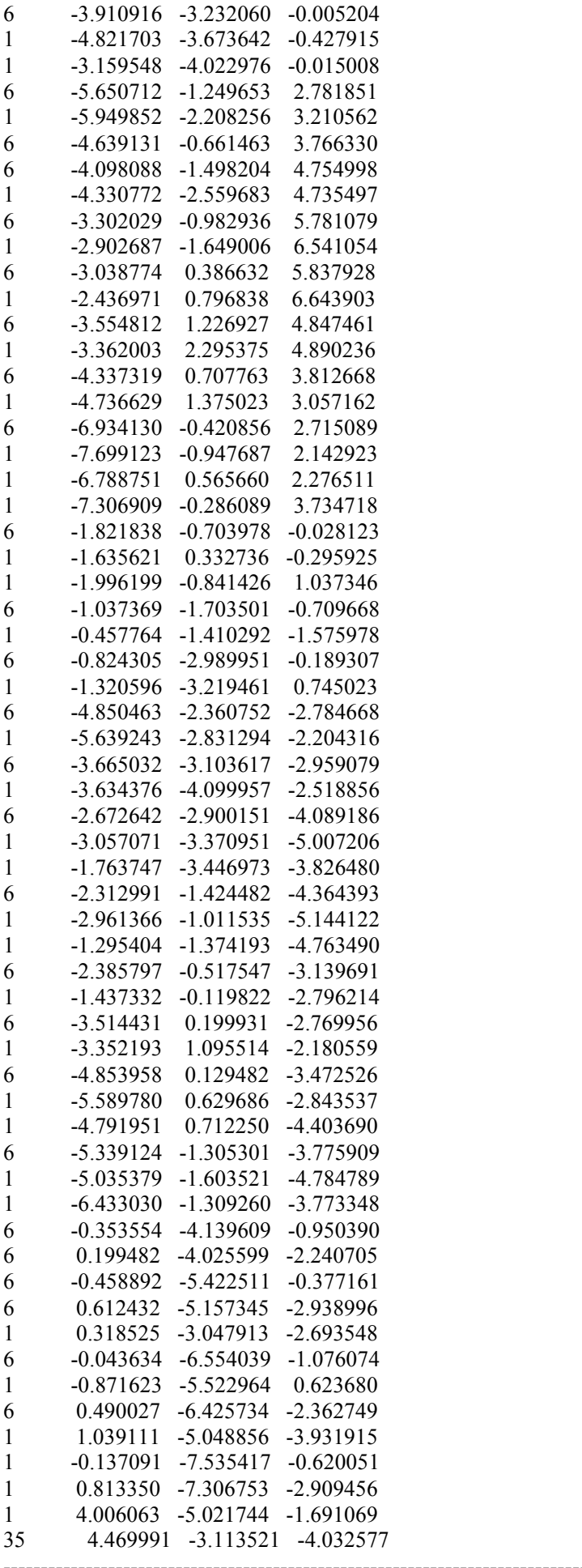

C1-P1 re-si 1

Number of imaginary frequencies $=1$

The smallest frequencies are $=-138.38557 .83769 .4099 \mathrm{~cm}(-1)$ Electronic energy $=-7222.6241764$

Zero-point correction= (Hartree/Particle)

Thermal correction to Energy=

Thermal correction to Enthalpy=

1.754258

Thermal correction to Gibbs Free Energy=
Sum of electronic and zero-point Energies=

Sum of electronic and thermal Energies=

Sum of electronic and thermal Enthalpies=

1.873058

1.874002

1.591132

$-7220.869918$

$-7220.751118$

Sum of electronic and thermal Free Energies=
$-7220.750174$

$-7221.033045$

\section{Cartesian Coordinates}

\begin{tabular}{|c|c|c|c|}
\hline 6 & -8.571311 & -1.286216 & \\
\hline & -4.315801 & -0.218965 & \\
\hline & -4.433480 & 0.950588 & 1.25854 \\
\hline 6 & -6.949038 & -0.504018 & -0.534920 \\
\hline 6 & -6.673923 & -0.553744 & 0.882388 \\
\hline 6 & -6.762362 & -1.917191 & 1.299706 \\
\hline 1 & -6.622789 & -2.280161 & 2.308873 \\
\hline 6 & -7.122195 & -2.707738 & 0.167372 \\
\hline 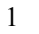 & -7.308350 & -3.772834 & 0.170028 \\
\hline 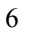 & -7.242069 & -1.841039 & -0.957864 \\
\hline 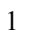 & -7.526420 & -2.136075 & -1.956070 \\
\hline 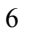 & -10.007696 & 0.005358 & 1.287063 \\
\hline 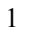 & -9.810015 & 1.015820 & 1.620078 \\
\hline 6 & -9.928029 & -1.173829 & 2.089992 \\
\hline 1 & -9.653550 & -1.214732 & 3.135652 \\
\hline 6 & -10.227823 & -2.295677 & 1.258238 \\
\hline 1 & -10.214620 & -3.333738 & 1.563189 \\
\hline 6 & -10.493390 & -1.810862 & -0.058636 \\
\hline 1 & -10.720876 & -2.415971 & -0.926386 \\
\hline 6 & -10.359930 & -0.390479 & -0.040894 \\
\hline 1 & -10.472966 & 0.264724 & -0.893966 \\
\hline 6 & -6.834008 & 0.737182 & -1.356043 \\
\hline 6 & -7.792121 & 1.751514 & -1.204733 \\
\hline 1 & -8.624798 & 1.572 & -0.535987 \\
\hline 6 & -7.682571 & 2.97 & -1.866163 \\
\hline 1 & -8.425364 & 3.749032 & -1.701700 \\
\hline 6 & -6.603616 & 3.201 & -2.717246 \\
\hline 1 & -6.49 & 4.15 & 539 \\
\hline 6 & -5.639840 & 2.20 & -2.888634 \\
\hline 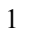 & -4.797360 & 2.40 & \\
\hline 6 & -5.725656 & 0.9 & -2.2 \\
\hline 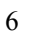 & -6.250309 & 0.630 & 1.704936 \\
\hline 1 & -6.794337 & 1.499990 & 1.331969 \\
\hline 6 & -6.538231 & 0.49 & 3.202518 \\
\hline 1 & -6.248179 & 1.399995 & 3.741954 \\
\hline 1 & -6.005706 & -0.348281 & 3.652190 \\
\hline 1 & -7.610521 & 0.330563 & 3.347153 \\
\hline 6 & -3.165843 & 0.505432 & -3.551167 \\
\hline 6 & -2.017649 & 1.15 & 967 \\
\hline 1 & -1.835731 & 1.220820 & -2.009660 \\
\hline 6 & -1.085852 & 1.688731 & -3.972232 \\
\hline 1 & -0.195636 & 2.18 & 058 \\
\hline 6 & -1.290176 & 1.570994 & -5.348078 \\
\hline 1 & & & \\
\hline 6 & -2.434335 & 0.927334 & -5.828814 \\
\hline 1 & -2.599328 & 0.833020 & -6.898498 \\
\hline 6 & -3.368142 & 0.35 & -4.937188 \\
\hline- & -4.245342 & -0.107430 & -5.326420 \\
\hline 6 & -5.032043 & -1.668418 & -3.181211 \\
\hline 6 & -6.078064 & -1.548066 & -4.110489 \\
\hline 1 & -6.498043 & -0.571986 & -4.334433 \\
\hline 6 & -6.604296 & -2.684468 & -4.726682 \\
\hline 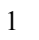 & -7.416332 & -2.582834 & -5.441160 \\
\hline 6 & -6.093660 & -3.950349 & -4.420083 \\
\hline 2 & -6.510536 & -4.833903 & -4.895024 \\
\hline 6 & -5.048750 & -4.075255 & -3.501532 \\
\hline 1 & -4.645862 & -5.054641 & -3.259094 \\
\hline 6 & -4.521778 & -2.93 & -2.88 \\
\hline 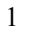 & -3.712971 & -3.037668 & -2.170794 \\
\hline 6 & -4.338658 & 2.696390 & 0.680530 \\
\hline 6 & -3.111259 & 3.066853 & 0.114264 \\
\hline 1 & -2.306536 & 2.339776 & 0.085369 \\
\hline 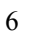 & -2.936717 & 4.340126 & -0.427115 \\
\hline 6 & -3.989202 & 5.252551 & -0.456320 \\
\hline & -3.862795 & 6.231614 & -0.905134 \\
\hline 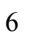 & -5.218206 & 4.871097 & 0.083992 \\
\hline 6 & -5.393838 & 3.615961 & 0.667563 \\
\hline & -6.362557 & 3.367538 & 1.080326 \\
\hline & -3.516795 & 0.965093 & 2.850436 \\
\hline & -3.212361 & -0.290929 & 3.398049 \\
\hline & -3.543194 & -1.193406 & 2.897807 \\
\hline & -2.460424 & -0.385908 & 4.56482 \\
\hline
\end{tabular}


$\begin{array}{llll}5.344199 & 2.260536 & -2.561914\end{array}$ $3.403116 \quad-0.226048 \quad-1.731938$ $\begin{array}{llll}3.973419 & -0.087898 & -2.658113\end{array}$ $\begin{array}{llll}2.363837 & -0.350710 & -2.041600\end{array}$ $\begin{array}{lll}5.090114 & 2.141102 & 0.847552\end{array}$ $\begin{array}{lll}6.088838 & 1.912579 & 1.215669\end{array}$ $\begin{array}{llll}5.164521 & 3.559744 & 0.300666\end{array}$ $\begin{array}{llll}6.403021 & 4.040412 & -0.139968\end{array}$ $\begin{array}{llll}7.274496 & 3.399485 & -0.066663\end{array}$ $\begin{array}{llll}6.528335 & 5.323347 & -0.673747\end{array}$ $\begin{array}{llll}7.499639 & 5.673506 & -1.013020\end{array}$ $\begin{array}{llll}5.407679 & 6.152422 & -0.763096\end{array}$ $\begin{array}{llll}5.496868 & 7.151171 & -1.181554\end{array}$ $\begin{array}{llll}4.171172 & 5.692791 & -0.301259\end{array}$ $\begin{array}{llll}3.294576 & 6.331647 & -0.362788\end{array}$ $\begin{array}{lll}4.053271 & 4.409357 & 0.234088\end{array}$ $\begin{array}{llll}3.082419 & 4.065105 & 0.574213\end{array}$ $\begin{array}{lll}4.121055 & 1.986442 & 2.023844\end{array}$ $\begin{array}{lll}4.219818 & 0.987632 & 2.456546\end{array}$ $\begin{array}{lll}3.081247 & 2.130760 & 1.725272\end{array}$ $\begin{array}{lll}4.350211 & 2.724980 & 2.798070\end{array}$ $\begin{array}{llll}3.170187 & -1.741846 & 1.119350\end{array}$ $\begin{array}{llll}3.672942 & -2.257750 & 1.937276\end{array}$ $\begin{array}{lll}2.955285 & -0.700249 & 1.348658\end{array}$ $\begin{array}{llll}2.161683 & -2.482785 & 0.399174\end{array}$ $\begin{array}{llll}2.092111 & -3.553781 & 0.551045\end{array}$ $\begin{array}{llll}1.123965 & -1.863413 & -0.321079\end{array}$ $\begin{array}{llll}1.181017 & -0.788374 & -0.428704\end{array}$ $\begin{array}{llll}5.274973 & -2.476102 & -2.691216\end{array}$ $\begin{array}{llll}5.485987 & -1.519559 & -3.161651\end{array}$ $\begin{array}{lll}3.959911 & -2.963816 & -2.828070\end{array}$ $\begin{array}{llll}3.263042 & -2.353602 & -3.401625\end{array}$ $\begin{array}{llll}3.525316 & -4.410282 & -2.678228\end{array}$ $\begin{array}{llll}3.681967 & -4.948881 & -3.625451\end{array}$ $2.445475 \quad-4.403738 \quad-2.502915$ $\begin{array}{llll}4.212476 & -5.168579 & -1.522868\end{array}$ $\begin{array}{llll}5.143134 & -5.629070 & -1.867142\end{array}$ $\begin{array}{llll}3.564965 & -5.994628 & -1.211244\end{array}$ $\begin{array}{llll}4.506574 & -4.304722 & -0.299882\end{array}$ $\begin{array}{llll}3.893327 & -4.502097 & 0.575075\end{array}$ $\begin{array}{llll}5.703290 & -3.643086 & -0.067982\end{array}$ $\begin{array}{llll}5.935679 & -3.393047 & 0.963162\end{array}$ $\begin{array}{llll}6.884786 & -3.606105 & -1.015348\end{array}$ $\begin{array}{llll}7.557753 & -2.812977 & -0.683212\end{array}$ $\begin{array}{llll}7.443689 & -4.548530 & -0.920825\end{array}$ $\begin{array}{llll}6.507049 & -3.359619 & -2.492346\end{array}$ $\begin{array}{llll}6.348071 & -4.311514 & -3.007838\end{array}$ $\begin{array}{llll}7.355916 & -2.882299 & -2.990503\end{array}$ $\begin{array}{llll}0.264461 & -2.542879 & -1.277869\end{array}$ $0.080383 \quad-3.940956-1.285651$ $\begin{array}{lll}-0.377497 & -1.772292 & -2.265828\end{array}$ $\begin{array}{llll}-0.693742 & -4.545147 & -2.273947\end{array}$ $\begin{array}{llll}0.545143 & -4.553007 & -0.520539\end{array}$ $\begin{array}{lll}-1.134498 & -2.380269 & -3.263959\end{array}$ $\begin{array}{llll}-0.273552 & -0.693864 & -2.243762\end{array}$ $\begin{array}{lll}-1.289162 & -3.769757 & -3.274957\end{array}$ $\begin{array}{lll}-0.826649 & -5.623252 & -2.269911\end{array}$ $\begin{array}{llll}-1.614413 & -1.769470 & -4.021270\end{array}$ $\begin{array}{lll}-1.886591 & -4.243180 & -4.048009\end{array}$ $\begin{array}{lll}-1.382403 & 0.429426 & 0.142007\end{array}$ $\begin{array}{llll}-1.226200 & -3.670449 & 1.884064\end{array}$ $\begin{array}{lll}-2.113186 & -4.721602 & 1.783504\end{array}$ $\begin{array}{lll}-0.300898 & -3.796820 & 2.435980\end{array}$ $\begin{array}{lll}-3.306603 & -4.497485 & 1.088021\end{array}$ $\begin{array}{llll}-3.554223 & -3.258903 & 0.529123\end{array}$ $\begin{array}{llll}-4.475741 & -3.059798 & -0.002932\end{array}$ $\begin{array}{lll}-2.681831 & -2.234458 & 0.614558\end{array}$ $\begin{array}{lll}0.106080 & 2.330465 & 1.364831\end{array}$ $\begin{array}{lll}-0.639699 & 2.905716 & 1.926483\end{array}$ $\begin{array}{lll}1.086457 & 2.776968 & 1.550698\end{array}$ $\begin{array}{lll}-0.118629 & 2.404291 & 0.305463\end{array}$ $\begin{array}{lll}0.767899 & 0.668019 & 3.061690\end{array}$ $\begin{array}{lll}0.291395 & -0.159777 & 3.582859\end{array}$ $\begin{array}{llll}1.833852 & 0.446633 & 2.944512\end{array}$ $\begin{array}{lll}0.667907 & 1.554762 & 3.691986\end{array}$ 
$\begin{array}{cccc}7 & 0.119933 & 0.931946 & 1.776571 \\ 1 & -1.892311 & -5.682449 & 2.236076\end{array}$

$35 \quad-4.635338 \quad-5.922098 \quad 0.891954$

C1-P1 re-si_2

Number of imaginary frequencies $=1$

The smallest frequencies are $=-212.384711 .326512 .5086 \mathrm{~cm}(-1)$ Electronic energy $=-7222.6317145$

Zero-point correction=

1.755524

(Hartree/Particle)

Thermal correction to Energy=

1.874043

Thermal correction to Enthalpy=

1.874987

Thermal correction to Gibbs Free Energy=

Sum of electronic and zero-point Energies=

Sum of electronic and thermal Energies=

Sum of electronic and thermal Enthalpies=

1.595134

$-7220.876191$

$-7220.757672$

Sum of electronic and thermal Free Energies=

$-7220.756728$

$-7221.036581$

Cartesian Coordinates

\begin{tabular}{|c|c|c|c|}
\hline 26 & 4.283280 & 3.820783 & 3.51752 \\
\hline & 1.367622 & 2.086474 & 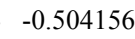 \\
\hline 15 & 4.554921 & 0.254983 & 0.36041 \\
\hline 6 & 3.413989 & 3.165503 & 1.724286 \\
\hline 6 & 4.236201 & 2.135908 & 2.315575 \\
\hline 6 & 3.709391 & 1.845850 & 3.611834 \\
\hline 1 & 4.113380 & 1.128493 & 4.312666 \\
\hline 6 & 2.588343 & 2.701323 & 3.840437 \\
\hline 1 & 1.999086 & 2.746820 & 4.745786 \\
\hline 6 & 2.410637 & 3.515145 & 2.683688 \\
\hline 1 & 1.660849 & 4.281327 & 2.555308 \\
\hline 6 & 6.145631 & 4.745422 & 3.461426 \\
\hline 1 & 6.918194 & 4.540359 & 2.731789 \\
\hline 6 & 5.965814 & 4.065049 & 4.704266 \\
\hline 1 & 6.569880 & 3.247666 & 5.075361 \\
\hline 6 & 4.820218 & 4.621654 & 5.350475 \\
\hline 1 & 4.402093 & 4.296106 & 6.293933 \\
\hline 6 & 4.291023 & 5.645609 & 4.506843 \\
\hline 1 & 3.402993 & 6.232960 & 4.699679 \\
\hline 6 & 5.109144 & 5.722340 & 3.340419 \\
\hline 1 & 4.951398 & 6.383588 & 2.499288 \\
\hline 6 & 3.594343 & 3.68 & 0.337685 \\
\hline 6 & 4.715873 & 4.481531 & 0.056668 \\
\hline 1 & 5.381678 & 4.726830 & 0.874064 \\
\hline 6 & 4.997378 & 4.9 & -1.230617 \\
\hline 1 & 5.891488 & 5.523422 & -1.416554 \\
\hline 6 & 4.131936 & 4.615731 & -2.273104 \\
\hline 1 & 4.334601 & 4.956877 & -3.283219 \\
\hline 6 & 3.010074 & 3.826384 & -2.020208 \\
\hline 1 & 2.357922 & 3.576244 & -2.848319 \\
\hline 6 & 2.729363 & 3.334272 & -0.734486 \\
\hline 6 & 5.363663 & 1.437073 & 1.606221 \\
\hline 1 & 5.873289 & 2.185954 & 0.997958 \\
\hline 6 & 6.390492 & 0.789113 & 2.538595 \\
\hline 1 & 7.211479 & 0.344130 & 1.969733 \\
\hline 1 & 5.947361 & 0.003673 & 3.157055 \\
\hline 1 & 6.802668 & 1.551837 & 3.206695 \\
\hline 6 & 0.613818 & 2.010127 & -2.183147 \\
\hline 6 & 1.152179 & 1.069274 & -3.079132 \\
\hline 1 & 1.886440 & 0.351923 & -2.727617 \\
\hline 6 & 0.752290 & 1.059600 & -4.417267 \\
\hline 1 & 1.192989 & 0.344459 & -5.103176 \\
\hline 6 & -0.211103 & 1.966765 & -4.867312 \\
\hline 1 & -0.525861 & 1.955289 & -5.906619 \\
\hline 6 & -0.783624 & 2.873800 & -3.972193 \\
\hline 1 & -1.550126 & 3.565621 & -4.309571 \\
\hline 6 & -0.369368 & 2.900957 & -2.638878 \\
\hline 1 & -0.808483 & 3.624318 & -1.962434 \\
\hline 6 & 0.143573 & 2.954343 & 0.556064 \\
\hline 6 & -0.099880 & 4.333060 & 0.442533 \\
\hline 1 & 0.414956 & 4.915287 & -0.316123 \\
\hline 6 & -0.976874 & 4.967840 & 1.323605 \\
\hline 1 & -1.160057 & 6.034138 & 1.227770 \\
\hline & -1.611640 & 4.234658 & 2.331064 \\
\hline
\end{tabular}

$\begin{array}{lll}-2.289278 & 4.730878 & 3.019505\end{array}$

$\begin{array}{lll}-1.374464 & 2.863383 & 2.450970\end{array}$

$\begin{array}{lll}-1.870638 & 2.294734 & 3.230515\end{array}$

$\begin{array}{lll}-0.501865 & 2.224898 & 1.566395\end{array}$

$\begin{array}{lll}-0.309732 & 1.162211 & 1.665180\end{array}$

$\begin{array}{llll}5.289975 & 0.648870 & -1.282057\end{array}$

$\begin{array}{llll}4.727116 & -0.034795 & -2.369688\end{array}$

$3.963152-0.781663-2.181047$

$\begin{array}{llll}5.109101 & 0.272063 & -3.674691\end{array}$

$\begin{array}{llll}6.028581 & 1.288196 & -3.927926\end{array}$

$\begin{array}{lll}6.303926 & 1.546422 & -4.944339\end{array}$

$\begin{array}{llll}6.574341 & 1.979209 & -2.845496\end{array}$

$6.228618 \quad 1.656848-1.532493$

$\begin{array}{llll}6.685093 & 2.216755 & -0.727556\end{array}$

$\begin{array}{lll}5.167931 & -1.434382 & 0.754298\end{array}$

$\begin{array}{lll}4.531871 & -2.078331 & 1.827282\end{array}$

$\begin{array}{llll}3.784092 & -1.552797 & 2.406895\end{array}$

$\begin{array}{llll}4.823585 & -3.404168 & 2.131380\end{array}$

$\begin{array}{lll}5.746530 & -4.121760 & 1.369336\end{array}$

$\begin{array}{llll}5.962452 & -5.159788 & 1.595160\end{array}$

$\begin{array}{lll}6.380118 & -3.481059 & 0.306628\end{array}$

$\begin{array}{llll}6.102817 & -2.146137 & -0.003080\end{array}$

$\begin{array}{llll}6.605916 & -1.679336 & -0.842297\end{array}$

$\begin{array}{llll}2.209171 & 0.121090 & 0.194019\end{array}$

$\begin{array}{llll}7.301334 & -4.273180 & -0.578115\end{array}$

$\begin{array}{lll}4.055173 & -4.108980 & 3.214424\end{array}$

$\begin{array}{llll}4.532555 & -0.535199 & -4.804150\end{array}$

$\begin{array}{llll}7.511973 & 3.119903 & -3.131351\end{array}$

$\begin{array}{llll}6.891114 & 4.107458 & -3.824536\end{array}$

$\begin{array}{llll}8.009654 & 3.679259 & -2.006828\end{array}$

$\begin{array}{lll}8.563368 & 2.726791 & -3.887137\end{array}$

$\begin{array}{llll}3.216067 & -0.823265 & -4.600693\end{array}$

$\begin{array}{llll}5.157968 & -1.730076 & -4.936276\end{array}$

$\begin{array}{llll}4.621595 & 0.096468 & -5.990963\end{array}$

$\begin{array}{llll}6.606667 & -4.912165 & -1.559183\end{array}$

$\begin{array}{llll}7.969409 & -5.227429 & 0.104728\end{array}$

$\begin{array}{llll}8.215289 & -3.494781 & -1.195305\end{array}$

$\begin{array}{llll}4.827786 & -4.976466 & 3.902654\end{array}$

$\begin{array}{llll}3.512002 & -3.248000 & 4.102515\end{array}$

$\begin{array}{llll}3.026662 & -4.834914 & 2.696901\end{array}$

$\begin{array}{llll}2.234627 & -2.769580 & -0.736163\end{array}$

$\begin{array}{lll}1.264677 & -3.148446 & 0.269404\end{array}$

$\begin{array}{lll}1.004508 & -2.415215 & 1.471809\end{array}$

$\begin{array}{llll}1.073769 & -4.208544 & 0.371407\end{array}$

$\begin{array}{llll}-3.304172 & -1.406542 & -1.558923\end{array}$

$\begin{array}{llll}-4.875441 & -0.536030 & -0.169564\end{array}$

$\begin{array}{lll}-4.718079 & 0.942160 & 0.580147\end{array}$

$\begin{array}{llll}-6.314048 & -0.302192 & -0.975279\end{array}$

$\begin{array}{lll}-5.082606 & -1.534858 & 1.150487\end{array}$

$\begin{array}{lll}-4.818303 & 2.111261 & -0.169251\end{array}$

$\begin{array}{llll}-3.627470 & 2.848369 & -0.351341\end{array}$

$\begin{array}{lll}-2.717807 & 2.485189 & 0.108819\end{array}$

$\begin{array}{llll}-3.649605 & 3.998815 & -1.099441\end{array}$

$\begin{array}{llll}-2.745230 & 4.585570 & -1.225459\end{array}$

$\begin{array}{llll}-4.843480 & 4.415588 & -1.746044\end{array}$

$\begin{array}{llll}-4.852088 & 5.545067 & -2.608776\end{array}$

$\begin{array}{llll}-3.928438 & 6.103412 & -2.738475\end{array}$

$\begin{array}{llll}-5.995774 & 5.913104 & -3.280382\end{array}$

$\begin{array}{llll}-5.990277 & 6.772960 & -3.943846\end{array}$

$\begin{array}{llll}-7.180435 & 5.156064 & -3.116785\end{array}$

$\begin{array}{llll}-8.075222 & 5.432748 & -3.666974\end{array}$

$\begin{array}{llll}-7.210279 & 4.067616 & -2.271742\end{array}$

$\begin{array}{llll}-8.125053 & 3.496332 & -2.168454\end{array}$

$\begin{array}{llll}-6.053113 & 3.668831 & -1.545520\end{array}$

$\begin{array}{lll}-6.045160 & 2.527613 & -0.664078\end{array}$

$\begin{array}{llll}-7.295447 & 1.797295 & -0.313885\end{array}$

$\begin{array}{llll}-8.428714 & 2.468207 & 0.271514\end{array}$

$\begin{array}{llll}-8.385613 & 3.829481 & 0.685136\end{array}$

$\begin{array}{lll}-7.465572 & 4.390309 & 0.571838\end{array}$

$\begin{array}{lll}-9.488286 & 4.440504 & 1.241933\end{array}$

$\begin{array}{lll}-9.424912 & 5.478418 & 1.556127\end{array}$

$\begin{array}{llll}-10.699328 & 3.729144 & 1.415124\end{array}$

$\begin{array}{lll}-11.562354 & 4.226215 & 1.848238\end{array}$

$\begin{array}{lll}-10.770649 & 2.404221 & 1.048968\end{array}$

$\begin{array}{lll}-11.687457 & 1.838826 & 1.194079\end{array}$ 
$\begin{array}{lll}-9.646785 & 1.739272 & 0.487555\end{array}$

$\begin{array}{llll}-9.704852 & 0.357961 & 0.157860\end{array}$

$\begin{array}{lll}-10.634920 & -0.182401 & 0.309745\end{array}$

$\begin{array}{llll}-8.591713 & -0.292580 & -0.311655\end{array}$

$\begin{array}{llll}-8.596180 & -1.355103 & -0.530914\end{array}$

$\begin{array}{llll}-7.393112 & 0.428749 & -0.511025\end{array}$

$\begin{array}{lll}-4.245485 & -2.770860 & 1.088624\end{array}$

$\begin{array}{llll}-3.343729 & -2.589769 & 1.685313\end{array}$

$\begin{array}{lll}-4.956726 & -3.977497 & 1.677653\end{array}$

$\begin{array}{lll}-4.296195 & -4.795737 & 2.600460\end{array}$

$\begin{array}{llll}-3.297047 & -4.524357 & 2.932739\end{array}$

$\begin{array}{llll}-4.914283 & -5.943456 & 3.104957\end{array}$

$\begin{array}{llll}-4.389134 & -6.568955 & 3.821692\end{array}$

$\begin{array}{llll}-6.207993 & -6.277172 & 2.699064\end{array}$

$\begin{array}{lll}-6.693547 & -7.164304 & 3.095841\end{array}$

$\begin{array}{lll}-6.878639 & -5.459958 & 1.782474\end{array}$

$\begin{array}{llll}-7.887817 & -5.710679 & 1.467013\end{array}$

$\begin{array}{llll}-6.255055 & -4.321411 & 1.272925\end{array}$

$\begin{array}{llll}-6.777893 & -3.680253 & 0.567783\end{array}$

$\begin{array}{llll}-3.838925 & -3.079915 & -0.358189\end{array}$

$\begin{array}{lll}-4.688087 & -3.563722 & -0.854875\end{array}$

$\begin{array}{llll}-3.041069 & -3.825041 & -0.322626\end{array}$

$\begin{array}{lll}-5.652536 & -1.266219 & 2.506394\end{array}$

$\begin{array}{lll}-6.270729 & -2.140855 & 2.727405\end{array}$

$\begin{array}{lll}-4.554955 & -1.219180 & 3.561976\end{array}$

$\begin{array}{lll}-4.652476 & -2.047266 & 4.685710\end{array}$

$\begin{array}{llll}-5.484111 & -2.742497 & 4.763861\end{array}$

$\begin{array}{llll}-3.691360 & -1.994630 & 5.697573\end{array}$

$\begin{array}{llll}-3.782479 & -2.641836 & 6.565597\end{array}$

$\begin{array}{llll}-2.613150 & -1.116501 & 5.586543\end{array}$

$\begin{array}{lll}-1.860390 & -1.073448 & 6.366486\end{array}$

$\begin{array}{lll}-2.500220 & -0.296876 & 4.458685\end{array}$

$\begin{array}{lll}-1.652839 & 0.373865 & 4.366700\end{array}$

$\begin{array}{lll}-3.465695 & -0.343446 & 3.452446\end{array}$

$\begin{array}{lll}-3.379704 & 0.299211 & 2.581440\end{array}$

$\begin{array}{lll}-6.576292 & -0.052500 & 2.650681\end{array}$

$\begin{array}{lll}-7.422695 & -0.107450 & 1.966412\end{array}$

$\begin{array}{lll}-6.055641 & 0.893147 & 2.505778\end{array}$

$\begin{array}{lll}-6.973023 & -0.070472 & 3.670373\end{array}$

$\begin{array}{llll}-1.774056 & -0.770874 & -0.135122\end{array}$

$\begin{array}{llll}-1.639020 & 0.306303 & -0.085842\end{array}$

$\begin{array}{llll}-1.908542 & -1.225735 & 0.844688\end{array}$

$-0.989638-1.463911-1.118575$

$\begin{array}{llll}-0.471288 & -0.854845 & -1.846909\end{array}$

$\begin{array}{llll}-0.669796 & -2.839567 & -1.108360\end{array}$

$\begin{array}{lll}-1.158600 & -3.465170 & -0.373627\end{array}$

$\begin{array}{llll}-4.895384 & -2.025464 & -3.031028\end{array}$

$\begin{array}{llll}-5.653388 & -2.549649 & -2.454569\end{array}$

$\begin{array}{llll}-3.716524 & -2.745259 & -3.319606\end{array}$

$\begin{array}{llll}-3.656400 & -3.768588 & -2.950802\end{array}$

$\begin{array}{llll}-2.798108 & -2.467609 & -4.493378\end{array}$

$\begin{array}{llll}-3.238457 & -2.888184 & -5.410731\end{array}$

$\begin{array}{llll}-1.870939 & -3.014785 & -4.319908\end{array}$

$\begin{array}{llll}-2.460878 & -0.976397 & -4.717846\end{array}$

$\begin{array}{llll}-3.153114 & -0.536988 & -5.443557\end{array}$

$\begin{array}{llll}-1.464639 & -0.904784 & -5.165262\end{array}$

$\begin{array}{llll}-2.471791 & -0.115985 & -3.461567\end{array}$

$\begin{array}{llll}-1.508032 & 0.231966 & -3.115148\end{array}$

$\begin{array}{llll}-3.572608 & 0.577382 & -3.012186\end{array}$

$\begin{array}{llll}-3.393845 & 1.420098 & -2.353648\end{array}$

$\begin{array}{llll}-4.954720 & 0.504467 & -3.620531\end{array}$

$\begin{array}{llll}-5.651775 & 0.978039 & -2.929928\end{array}$

$\begin{array}{llll}-4.962079 & 1.113659 & -4.536724\end{array}$

$\begin{array}{lll}-5.447089 & -0.925420 & -3.939362\end{array}$

$\begin{array}{llll}-5.206727 & -1.182140 & -4.976879\end{array}$

$\begin{array}{lll}-6.538512 & -0.934980 & -3.868016\end{array}$

$\begin{array}{llll}-0.148547 & -3.532532 & -2.290866\end{array}$

$\begin{array}{lll}0.488069 & -2.849102 & -3.346465\end{array}$

$\begin{array}{llll}-0.297789 & -4.928022 & -2.391502\end{array}$

$\begin{array}{llll}0.949722 & -3.539356 & -4.463449\end{array}$

$\begin{array}{llll}0.641640 & -1.779198 & -3.284035\end{array}$

$\begin{array}{llll}0.156571 & -5.617069 & -3.515749\end{array}$

$-0.780852 \quad-5.469053-1.581540$

$\begin{array}{llll}0.783146 & -4.925205 & -4.556978\end{array}$

$\begin{array}{llll}1.455516 & -2.994057 & -5.255209\end{array}$ $\begin{array}{llll}0.022714 & -6.693215 & -3.579203\end{array}$

$\begin{array}{llll}1.141340 & -5.461094 & -5.431158\end{array}$

$\begin{array}{llll}2.366246 & -1.574454 & -1.147083\end{array}$

$\begin{array}{lll}0.373152 & -3.091323 & 2.557611\end{array}$

$\begin{array}{llll}0.295016 & -2.517105 & 3.809775\end{array}$

$\begin{array}{llll}0.011510 & -4.103251 & 2.405059\end{array}$

$\begin{array}{lll}0.827751 & -1.230745 & 3.964737\end{array}$

$\begin{array}{lll}1.306928 & -0.550417 & 2.864410\end{array}$

$\begin{array}{lll}1.662714 & 0.468707 & 2.945253\end{array}$

$\begin{array}{llll}1.382555 & -1.110985 & 1.639886\end{array}$

$\begin{array}{llll}3.734447 & -3.472671 & -2.541552\end{array}$

$3.294559-2.631130 \quad-3.065213$

$\begin{array}{llll}4.784737 & -3.258781 & -2.317507\end{array}$

$\begin{array}{llll}3.690317 & -4.345762 & -3.198671\end{array}$

$\begin{array}{llll}3.095450 & -5.113346 & -0.812001\end{array}$

$\begin{array}{llll}2.981170 & -5.133372 & 0.271037\end{array}$

$\begin{array}{llll}2.359060 & -5.786275 & -1.268731\end{array}$

$\begin{array}{llll}4.097521 & -5.481544 & -1.046531\end{array}$

$2.974411 \quad-3.752659-1.328486$

$\begin{array}{lll}-0.143322 & -3.053391 & 4.643799\end{array}$

$\begin{array}{lll}0.980977 & -0.401965 \quad 5.730726\end{array}$

C1-P1 re-si 3

Number of imaginary frequencies $=1$

The smallest frequencies are $=-50.100411 .087412 .5338 \mathrm{~cm}(-1)$ Electronic energy $=-7222.6312683$

Zero-point correction $=$

1.755056

(Hartree/Particle)

Thermal correction to Energy=

Thermal correction to Enthalpy=

1.873591

1.874536

Thermal correction to Gibbs Free Energy=

Sum of electronic and zero-point Energies=

Sum of electronic and thermal Energies=

Sum of electronic and thermal Enthalpies=

Sum of electronic and thermal Free Energies=

1.593392

$-7220.876212$

$-7220.757677$

$-7220.756733$

$-7221.037876$

Cartesian Coordinates

$\begin{array}{lccc}26 & 4.332351 & 4.770046 & 2.089876 \\ 15 & 2.533156 & 1.718804 & -1.760630 \\ 15 & 4.617029 & 0.151016 & 0.938765 \\ 6 & 3.961879 & 3.466281 & 0.493366 \\ 6 & 4.234704 & 2.736842 & 1.709765 \\ 6 & 3.250870 & 3.114733 & 2.675203 \\ 1 & 3.192475 & 2.753293 & 3.692495 \\ 6 & 2.392574 & 4.089297 & 2.083088 \\ 1 & 1.574135 & 4.596753 & 2.574150 \\ 6 & 2.827845 & 4.305085 & 0.742987 \\ 1 & 2.396790 & 5.000257 & 0.038482 \\ 6 & 6.282628 & 5.384433 & 2.463682 \\ 1 & 7.166321 & 4.795065 & 2.257341 \\ 6 & 5.527297 & 5.366389 & 3.675822 \\ 1 & 5.735245 & 4.758479 & 4.546170 \\ 6 & 4.426019 & 6.262815 & 3.523687 \\ 1 & 3.648165 & 6.443087 & 4.253976 \\ 6 & 4.499963 & 6.835416 & 2.217479 \\ 1 & 3.790079 & 7.528346 & 1.785257 \\ 6 & 5.646426 & 6.294161 & 1.562944 \\ 1 & 5.956060 & 6.509383 & 0.549207 \\ 6 & 4.706655 & 3.295584 & -0.788248 \\ 6 & 6.018429 & 3.783570 & -0.890428 \\ 1 & 6.440782 & 4.297187 & -0.036358 \\ 6 & 6.781453 & 3.611990 & -2.045155 \\ 1 & 7.802093 & 3.979462 & -2.079938 \\ 6 & 6.229485 & 2.953919 & -3.141562 \\ 1 & 6.809755 & 2.809047 & -4.046932 \\ 6 & 4.929267 & 2.455001 & -3.063383 \\ 1 & 4.519325 & 1.930911 & -3.919271 \\ 6 & 4.159022 & 2.595814 & -1.897858 \\ 6 & 5.289270 & 1.672610 & 1.853985 \\ 1 & 6.163367 & 1.994129 & 1.285428 \\ 6 & 5.726697 & 1.412216 & 3.298241 \\ 1 & 6.519877 & 0.660546 & 3.336474 \\ 1 & 4.901122 & 1.060722 & 3.923234\end{array}$


$\begin{array}{llll}6.105947 & 2.341866 & 3.733607\end{array}$

$\begin{array}{llll}2.319982 & 0.911784 & -3.404858\end{array}$

$\begin{array}{llll}2.873231 & -0.367684 & -3.579755\end{array}$

$\begin{array}{llll}3.358028 & -0.863903 & -2.746159\end{array}$

$\begin{array}{llll}2.789558 & -1.008370 & -4.817236\end{array}$

$\begin{array}{llll}3.247413 & -1.982180 & -4.947910\end{array}$

$\begin{array}{llll}2.115890 & -0.400180 & -5.879518\end{array}$

$\begin{array}{llll}2.039165 & -0.907128 & -6.837094\end{array}$

$\begin{array}{llll}1.540562 & 0.860806 & -5.705519\end{array}$

$\begin{array}{llll}1.013174 & 1.339080 & -6.525956\end{array}$

$\begin{array}{llll}1.650809 & 1.519719 & -4.478752\end{array}$

$\begin{array}{llll}1.213309 & 2.505816 & -4.365475\end{array}$

$1.265143 \quad 3.041943-1.791166$

$\begin{array}{llll}1.461241 & 4.293076 & -2.396218\end{array}$

$\begin{array}{llll}2.419431 & 4.535793 & -2.846253\end{array}$

$\begin{array}{llll}0.427318 & 5.231605 & -2.413007\end{array}$

$\begin{array}{llll}0.585769 & 6.199051 & -2.881062\end{array}$

$\begin{array}{llll}-0.809809 & 4.926284 & -1.834739\end{array}$

$\begin{array}{llll}-1.615380 & 5.654890 & -1.853193\end{array}$

$\begin{array}{llll}-1.009154 & 3.680815 & -1.235208\end{array}$

$\begin{array}{llll}-1.965656 & 3.434307 & -0.790260\end{array}$

$\begin{array}{llll}0.026619 & 2.746597 & -1.208716\end{array}$

$\begin{array}{lll}-0.120180 & 1.780248 & -0.737520\end{array}$

$\begin{array}{llll}5.875585 & -0.317319 & -0.321252\end{array}$

$\begin{array}{llll}5.537229 & -1.408022 & -1.136127\end{array}$

$\begin{array}{llll}4.606195 & -1.936883 & -0.954232\end{array}$

$\begin{array}{llll}6.369028 & -1.790800 & -2.188417\end{array}$

$\begin{array}{llll}7.530026 & -1.076612 & -2.476168\end{array}$

$\begin{array}{llll}8.164135 & -1.361294 & -3.308055\end{array}$

$\begin{array}{llll}7.854023 & 0.020945 & -1.677318\end{array}$

$\begin{array}{llll}7.050005 & 0.392304 & -0.599811\end{array}$

$\begin{array}{llll}7.344941 & 1.247422 & -0.007588\end{array}$

$\begin{array}{lll}4.642449 & -1.257250 & 2.125069\end{array}$

$\begin{array}{lll}3.619114 & -1.275196 & 3.087225\end{array}$

$2.921654 \quad-0.448283 \quad 3.151194$

$\begin{array}{lll}3.464603 & -2.369410 & 3.933114\end{array}$

$\begin{array}{lll}4.304292 & -3.479752 & 3.824468\end{array}$

$\begin{array}{llll}4.167216 & -4.342008 & 4.466611\end{array}$

$\begin{array}{llll}5.312427 & -3.464867 & 2.864956\end{array}$

$\begin{array}{lll}5.494658 & -2.361077 & 2.026587\end{array}$

$\begin{array}{lll}6.290606 & -2.383905 & 1.291254\end{array}$

$\begin{array}{llll}2.497905 & 0.252365 & -0.051837\end{array}$

$\begin{array}{llll}6.154676 & -4.690536 & 2.647740\end{array}$

$2.312053 \quad-2.423640 \quad 4.896801$

$\begin{array}{llll}5.996614 & -3.010920 & -2.983703\end{array}$

$\begin{array}{llll}9.080354 & 0.818716 & -2.027922\end{array}$

$\begin{array}{llll}8.964816 & 1.392962 & -3.251222\end{array}$

$\begin{array}{llll}9.325175 & 1.812290 & -1.145383\end{array}$

$\begin{array}{llll}10.186864 & 0.040457 & -2.075234\end{array}$

$\begin{array}{lll}4.683110 & -2.999431 & -3.339016\end{array}$

$\begin{array}{llll}6.178517 & -4.146560 & -2.265694\end{array}$

$6.714719-3.134476 \quad-4.116947$

$5.632338 \quad-5.473240 \quad 1.662280$

$\begin{array}{lll}6.238033 & -5.462076 & 3.751533\end{array}$

$\begin{array}{lll}7.413010 & -4.383619 & 2.265163\end{array}$

$\begin{array}{llll}2.668818 & -2.967273 & 6.081643\end{array}$

$\begin{array}{llll}1.788928 & -1.205197 & 5.147958\end{array}$

$\begin{array}{llll}1.298736 & -3.194259 & 4.413073\end{array}$

$\begin{array}{llll}1.983263 & -2.685062 & 0.409219\end{array}$

$\begin{array}{llll}0.882735 & -2.394831 & 1.284453\end{array}$

$\begin{array}{llll}0.478187 & -1.129213 & 1.793844\end{array}$

$\begin{array}{llll}0.452182 & -3.230533 & 1.816490\end{array}$

$-3.361313-1.003969-1.721116$

$\begin{array}{lll}-5.130744 & -0.723169 & -0.316345\end{array}$

$\begin{array}{llll}-5.244770 & 0.434580 & 0.865852\end{array}$

$\begin{array}{llll}-6.488733 & -0.365632 & -1.208248\end{array}$

$\begin{array}{llll}-5.343109 & -2.086801 & 0.632569\end{array}$

$\begin{array}{lll}-5.291511 & 1.776469 & 0.511263\end{array}$

$\begin{array}{lll}-4.167975 & 2.559825 & 0.856574\end{array}$

$\begin{array}{lll}-3.356349 & 2.089255 & 1.400062\end{array}$

$\begin{array}{lll}-4.142730 & 3.889387 & 0.513689\end{array}$

$\begin{array}{llll}-3.300378 & 4.514522 & 0.794741\end{array}$

$\begin{array}{llll}-5.195075 & 4.453895 & -0.257749\end{array}$

$\begin{array}{llll}-5.120147 & 5.792011 & -0.730569\end{array}$

$\begin{array}{llll}-4.252285 & 6.388429 & -0.460936\end{array}$ $\begin{array}{lll}-6.114228 & 6.317648 & -1.524742\end{array}$

$\begin{array}{llll}-6.042986 & 7.338172 & -1.889194\end{array}$

$\begin{array}{lll}-7.227983 & 5.519020 & -1.878479\end{array}$

$\begin{array}{llll}-7.999341 & 5.928438 & -2.524361\end{array}$

$\begin{array}{llll}-7.342896 & 4.226057 & -1.415260\end{array}$

$\begin{array}{llll}-8.200445 & 3.627602 & -1.699764\end{array}$

$\begin{array}{lll}-6.341878 & 3.653763 & -0.582167\end{array}$

$\begin{array}{lll}-6.423708 & 2.300522 & -0.092571\end{array}$

$\begin{array}{llll}-7.669476 & 1.490258 & -0.203205\end{array}$

$\begin{array}{lll}-8.925585 & 1.979931 & 0.314739\end{array}$

$\begin{array}{lll}-9.031535 & 3.183502 & 1.067734\end{array}$

$\begin{array}{lll}-8.139023 & 3.757565 & 1.284428\end{array}$

$\begin{array}{llll}-10.248126 & 3.624261 & 1.542762\end{array}$

$\begin{array}{lll}-10.296214 & 4.542869 & 2.120509\end{array}$

$\begin{array}{lll}-11.431590 & 2.890328 & 1.293845\end{array}$

$\begin{array}{lll}-12.384325 & 3.254400 & 1.666863\end{array}$

$\begin{array}{lll}-11.363644 & 1.706206 & 0.595454\end{array}$

$\begin{array}{lll}-12.259411 & 1.117834 & 0.414211\end{array}$

$\begin{array}{lll}-10.122781 & 1.215526 & 0.106387\end{array}$

$-10.047778-0.034170 \quad-0.566005$

$\begin{array}{lll}-10.959813 & -0.600296 & -0.732755\end{array}$

$\begin{array}{lll}-8.836490 & -0.534801 & -0.968326\end{array}$

$\begin{array}{lll}-8.746027 & -1.504046 & -1.447286\end{array}$

$\begin{array}{llll}-7.659707 & 0.218112 & -0.756355\end{array}$

$\begin{array}{lll}-4.354894 & -3.155201 & 0.325541\end{array}$

$\begin{array}{lll}-3.502304 & -3.043463 & 1.008838\end{array}$

$\begin{array}{llll}-4.929104 & -4.548760 & 0.512250\end{array}$

$\begin{array}{lll}-4.162272 & -5.539332 & 1.136667\end{array}$

$\begin{array}{lll}-3.186184 & -5.281776 & 1.542016\end{array}$

$\begin{array}{llll}-4.641385 & -6.847931 & 1.245657\end{array}$

$\begin{array}{lll}-4.035115 & -7.606593 & 1.732922\end{array}$

$\begin{array}{llll}-5.900096 & -7.175817 & 0.738029\end{array}$

$\begin{array}{llll}-6.277795 & -8.190367 & 0.827564\end{array}$

$\begin{array}{lll}-6.675518 & -6.189589 & 0.118707\end{array}$

$\begin{array}{lll}-7.658118 & -6.437389 & -0.273416\end{array}$

$\begin{array}{llll}-6.191595 & -4.886761 & 0.003228\end{array}$

$\begin{array}{llll}-6.795036 & -4.116662 & -0.470184\end{array}$

$\begin{array}{llll}-3.881275 & -2.999703 & -1.121352\end{array}$

$\begin{array}{llll}-4.695050 & -3.331885 & -1.776516\end{array}$

$\begin{array}{lll}-3.045211 & -3.677280 & -1.300872\end{array}$

$\begin{array}{lll}-6.016058 & -2.180043 & 1.968140\end{array}$

$\begin{array}{lll}-6.179158 & -3.253269 & 2.091725\end{array}$

$\begin{array}{lll}-5.124121 & -1.757856 & 3.136616\end{array}$

$\begin{array}{lll}-4.262227 & -2.708093 & 3.706323\end{array}$

$\begin{array}{lll}-4.230278 & -3.712203 & 3.292046\end{array}$

$\begin{array}{lll}-3.477181 & -2.395884 & 4.819444\end{array}$

$\begin{array}{llll}-2.823756 & -3.150167 & 5.249508\end{array}$

$\begin{array}{lll}-3.558609 & -1.126308 & 5.395901\end{array}$

$\begin{array}{lll}-2.966034 & -0.884030 & 6.273749\end{array}$

$\begin{array}{lll}-4.411640 & -0.169852 & 4.836163\end{array}$

$\begin{array}{llll}-4.483997 & 0.819730 & 5.279330\end{array}$

$\begin{array}{llll}-5.181647 & -0.480930 & 3.713972\end{array}$

$\begin{array}{lll}-5.840607 & 0.271878 & 3.298319\end{array}$

$\begin{array}{lll}-7.405441 & -1.546139 & 1.987778\end{array}$

$\begin{array}{lll}-8.026724 & -1.982477 & 1.202783\end{array}$

$\begin{array}{lll}-7.387663 & -0.465343 & 1.849357\end{array}$

$\begin{array}{llll}-7.875706 & -1.754733 & 2.953310\end{array}$

$\begin{array}{lll}-2.087303 & -0.379894 & -0.087680\end{array}$

$\begin{array}{llll}-1.974383 & 0.700945 & -0.046493\end{array}$

$\begin{array}{llll}-2.358569 & -0.803448 & 0.875211\end{array}$

$\begin{array}{llll}-1.139897 & -1.120783 & -0.887210\end{array}$

$\begin{array}{llll}-0.496290 & -0.595049 & -1.583062\end{array}$

$\begin{array}{llll}-0.889051 & -2.477274 & -0.644559\end{array}$

$\begin{array}{lll}-1.471169 & -2.939071 & 0.143973\end{array}$

$\begin{array}{llll}-4.648179 & -1.314924 & -3.532399\end{array}$

$\begin{array}{lll}-5.428063 & -2.018144 & -3.249736\end{array}$

$\begin{array}{llll}-3.370536 & -1.869480 & -3.780063\end{array}$

$\begin{array}{lll}-3.279122 & -2.951562 & -3.688296\end{array}$

$\begin{array}{llll}-2.284409 & -1.239880 & -4.636569\end{array}$

$\begin{array}{llll}-2.472823 & -1.456965 & -5.699378\end{array}$

$\begin{array}{lll}-1.340430 & -1.735019 & -4.386422\end{array}$

$\begin{array}{llll}-2.125130 & 0.278053 & -4.425178\end{array}$

$\begin{array}{llll}-2.783613 & 0.829769 & -5.102497\end{array}$

$\begin{array}{llll}-1.103383 & 0.567850 & -4.687792\end{array}$

$\begin{array}{llll}-2.393972 & 0.724747 & -2.992458\end{array}$ 


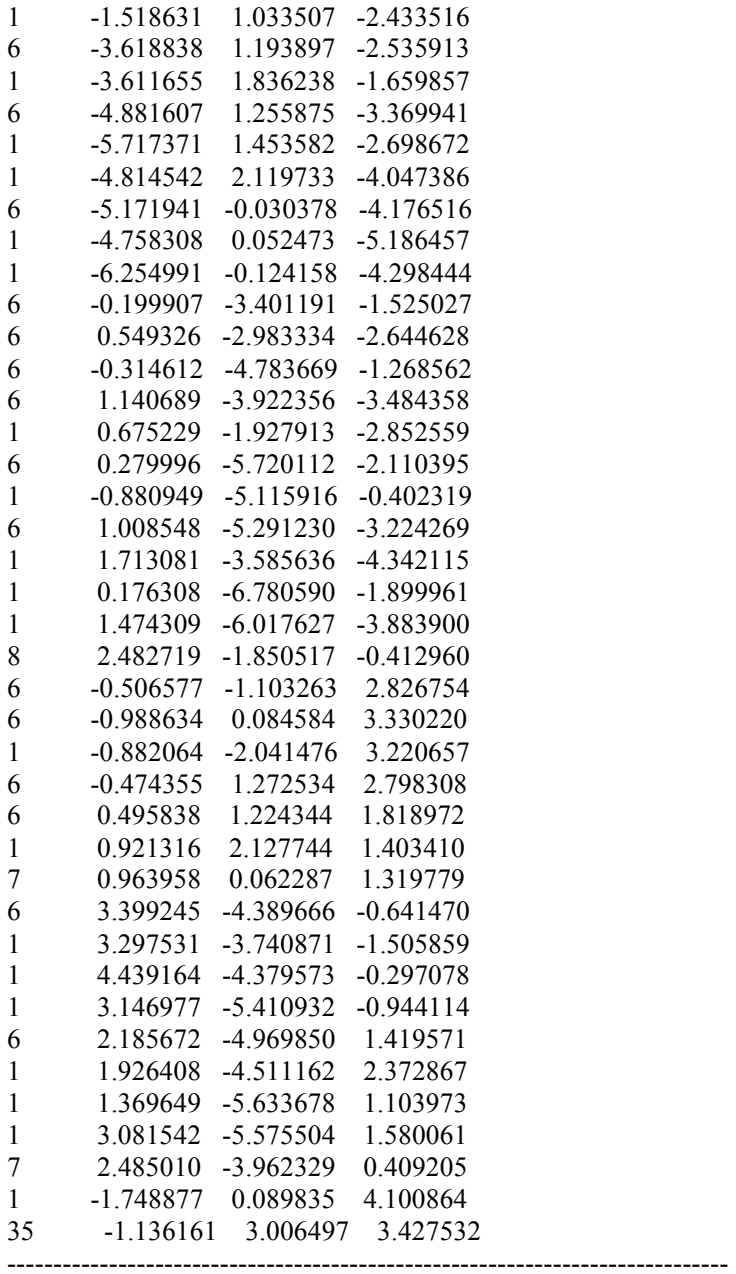

\section{C1-P1 re-si_4}

Number of imaginary frequencies $=1$

The smallest frequencies are $=-68.15908 .530910 .3557 \mathrm{~cm}(-1)$ Electronic energy $=-7222.6318939$

\section{Zero-point correction=}

1.754512

(Hartree/Particle)

Thermal correction to Energy= $\quad 1.873365$

Thermal correction to Enthalpy= $\quad 1.874310$

Thermal correction to Gibbs Free Energy $=\quad 1.591948$

Sum of electronic and zero-point Energies $=\quad-7220.877382$

Sum of electronic and thermal Energies $=\quad-7220.758529$

Sum of electronic and thermal Enthalpies $=\quad-7220.757584$

Sum of electronic and thermal Free Energies $=\quad-7221.039946$

\section{Cartesian Coordinates}

$\begin{array}{llll}26 & -4.418478 & -3.968074 & 3.286596 \\ 15 & -1.345928 & -2.104418 & -0.576348 \\ 15 & -4.499164 & -0.254368 & 0.279527 \\ 6 & -3.472622 & -3.243938 & 1.557089 \\ 6 & -4.283432 & -2.222275 & 2.175738 \\ 6 & -3.794582 & -2.020454 & 3.502773 \\ 1 & -4.198909 & -1.327494 & 4.227267 \\ 6 & -2.706533 & -2.920232 & 3.720557 \\ 1 & -2.150412 & -3.030775 & 4.640544 \\ 6 & -2.509971 & -3.673038 & 2.526104 \\ 1 & -1.777094 & -4.452164 & 2.377555 \\ 6 & -6.312933 & -4.812345 & 3.147567 \\ 1 & -7.062282 & -4.530791 & 2.419796 \\ 6 & -6.126885 & -4.215376 & 4.431922 \\ 1 & -6.703098 & -3.396962 & 4.842789 \\ 6 & -5.016244 & -4.856984 & 5.059998 \\ 1 & -4.600949 & -4.605738 & 6.027130 \\ 6 & -4.514213 & -5.849033 & 4.163544\end{array}$

$\begin{array}{lll}-3.654470 & -6.483625 & 4.333743\end{array}$

$\begin{array}{lll}-5.315831 & -5.823528 & 2.983750\end{array}$

$\begin{array}{lll}-5.169407 & -6.440373 & 2.107726\end{array}$

$\begin{array}{lll}-3.623390 & -3.698928 & 0.145155\end{array}$

$-4.750230-4.462084-0.199490$

$\begin{array}{lll}-5.452338 & -4.714246 & 0.584292\end{array}$

$\begin{array}{lll}-4.992611 & -4.878295 & -1.507243\end{array}$

$-5.890645-5.441130-1.741311$

$\begin{array}{lll}-4.081102 & -4.549724 & -2.506818\end{array}$

$-4.249303-4.863221 \quad-3.532160$

$-2.957453-3.786193-2.191782$

$-2.272844-3.528072-2.989943$

$-2.715571-3.330155-0.884270$

$\begin{array}{lll}-5.369288 & -1.455787 & 1.468251\end{array}$

$\begin{array}{lll}-5.893256 & -2.161889 & 0.822379\end{array}$

$\begin{array}{lll}-6.395673 & -0.809622 & 2.403033\end{array}$

$\begin{array}{lll}-7.184853 & -0.310884 & 1.833821\end{array}$

$\begin{array}{lll}-5.942586 & -0.068232 & 3.066925\end{array}$

$\begin{array}{lll}-6.853908 & -1.583986 & 3.026426\end{array}$

$\begin{array}{llll}-0.561417 & -1.999952 & -2.240337\end{array}$

$\begin{array}{lll}-1.113132 & -1.077713 & -3.147125\end{array}$

$\begin{array}{lll}-1.865883 & -0.376447 & -2.803920\end{array}$

$\begin{array}{lll}-0.705169 & -1.071562 & -4.482449\end{array}$

$\begin{array}{llll}-1.157882 & -0.375093 & -5.179148\end{array}$

$\begin{array}{llll}0.287824 & -1.954689 & -4.915695\end{array}$

$\begin{array}{llll}0.610809 & -1.943439 & -5.952548\end{array}$

$\begin{array}{llll}0.876262 & -2.838501 & -4.008507\end{array}$

$\begin{array}{llll}1.664969 & -3.511446 & -4.332858\end{array}$

$\begin{array}{llll}0.447066 & -2.869348 & -2.680299\end{array}$

$\begin{array}{llll}0.894045 & -3.578874 & -1.994690\end{array}$

$\begin{array}{lll}-0.138010 & -3.018894 & 0.464067\end{array}$

$\begin{array}{llll}0.078545 & -4.399042 & 0.318423\end{array}$

$\begin{array}{llll}-0.465917 & -4.956693 & -0.438099\end{array}$

$\begin{array}{llll}0.973185 & -5.064314 & 1.158128\end{array}$

$\begin{array}{lll}1.135538 & -6.131445 & 1.036920\end{array}$

$\begin{array}{lll}1.653857 & -4.359207 & 2.155316\end{array}$

$\begin{array}{llll}2.346488 & -4.877851 & 2.811623\end{array}$

$\begin{array}{llll}1.443762 & -2.986704 & 2.307326\end{array}$

$\begin{array}{llll}1.975260 & -2.441834 & 3.079968\end{array}$

$\begin{array}{lll}0.553008 & -2.316914 & 1.464245\end{array}$

$\begin{array}{lll}0.384196 & -1.251426 & 1.585291\end{array}$

$\begin{array}{llll}-5.172675 & -0.606801 & -1.398651\end{array}$

$\begin{array}{llll}-4.577395 & 0.111938 & -2.446293\end{array}$

$\begin{array}{llll}-3.810130 & 0.844620 & -2.213885\end{array}$

$\begin{array}{llll}-4.950033 & -0.125960 & -3.769136\end{array}$

$\begin{array}{llll}-5.882743 & -1.112877 & -4.081066\end{array}$

$\begin{array}{llll}-6.152125 & -1.315374 & -5.111639\end{array}$

$\begin{array}{llll}-6.454870 & -1.843820 & -3.039394\end{array}$

$\begin{array}{llll}-6.125963 & -1.583909 & -1.708834\end{array}$

$\begin{array}{lll}-6.610714 & -2.165492 & -0.936439\end{array}$

$\begin{array}{lll}-5.135943 & 1.433242 & 0.650329\end{array}$

$\begin{array}{lll}-4.622838 & 2.058110 & 1.799310\end{array}$

$\begin{array}{lll}-3.949339 & 1.520421 & 2.455684\end{array}$

$\begin{array}{lll}-4.948457 & 3.380270 & 2.086406\end{array}$

$\begin{array}{lll}-5.767267 & 4.117733 & 1.229161\end{array}$

$\begin{array}{llll}-6.002345 & 5.153431 & 1.445079\end{array}$

$\begin{array}{llll}-6.269225 & 3.500045 & 0.087421\end{array}$

$\begin{array}{llll}-5.967813 & 2.166270 & -0.201802\end{array}$

$\begin{array}{llll}-6.377003 & 1.716526 & -1.098890\end{array}$

$\begin{array}{lll}-2.134978 & -0.147374 & 0.195299\end{array}$

$\begin{array}{llll}-7.067266 & 4.301944 & -0.901818\end{array}$

$\begin{array}{llll}-4.324565 & 4.072438 & 3.265658\end{array}$

$\begin{array}{llll}-4.357409 & 0.732385 & -4.850808\end{array}$

$\begin{array}{llll}-7.407735 & -2.954264 & -3.387579\end{array}$

$\begin{array}{llll}-6.788464 & -3.934047 & -4.093123\end{array}$

$\begin{array}{lll}-7.949279 & -3.536524 & -2.295314\end{array}$

$\begin{array}{llll}-8.428321 & -2.515007 & -4.159793\end{array}$

$\begin{array}{llll}-3.028848 & 0.951958 & -4.651466\end{array}$

$\begin{array}{llll}-4.934964 & 1.958581 & -4.886833\end{array}$

$\begin{array}{lll}-4.490826 & 0.190389 & -6.077140\end{array}$

$\begin{array}{llll}-6.263753 & 4.822644 & -1.870365\end{array}$

$\begin{array}{llll}-7.703367 & 5.343846 & -0.326060\end{array}$

$\begin{array}{llll}-7.995582 & 3.553606 & -1.536303\end{array}$

$\begin{array}{llll}-5.192957 & 4.906340 & 3.879798\end{array}$

$\begin{array}{llll}-3.868659 & 3.202747 & 4.192423\end{array}$ 


\begin{tabular}{|c|c|c|c|c|c|c|}
\hline-3.265021 & 4.835697 & 2.884130 & 6 & 1.673541 & 0.729213 & -0.303939 \\
\hline-2.121897 & 2.779638 & -0.546769 & 1 & 1.382780 & -0.298324 & -0.512659 \\
\hline-1.424876 & 3.138515 & 0.654046 & 1 & 1.852922 & 0.910167 & 0.754789 \\
\hline-1.201330 & 2.316438 & 1.788711 & 6 & 0.981092 & 1.757239 & -1.047847 \\
\hline-1.290792 & 4.193216 & 0.844555 & 1 & 0.378188 & 1.482581 & -1.903141 \\
\hline 3.275870 & 1.440995 & -1.593615 & 6 & 0.960256 & 3.083677 & -0.622302 \\
\hline 4.791238 & 0.444259 & -0.228509 & 1 & 1.425045 & 3.294549 & 0.336354 \\
\hline 4.587728 & -1.062123 & 0.432929 & 6 & 4.851818 & 1.956627 & -3.139655 \\
\hline 6.218589 & 0.217387 & -1.054648 & 1 & 5.683373 & 2.389885 & -2.591129 \\
\hline 5.022151 & 1.361945 & 1.146046 & 6 & 3.729921 & 2.784844 & -3.336489 \\
\hline 4.774129 & -2.217863 & -0.324162 & 1 & 3.791211 & 3.800347 & -2.946443 \\
\hline 3.619408 & -2.990020 & -0.578785 & 6 & 2.687071 & 2.606560 & -4.424904 \\
\hline 2.674327 & -2.659347 & -0.166372 & 1 & 3.064307 & 3.027012 & -5.369668 \\
\hline 3.720530 & -4.133850 & -1.330377 & 1 & 1.821928 & 3.213634 & -4.145766 \\
\hline 2.843995 & -4.747592 & -1.513797 & 6 & 2.228300 & 1.148563 & -4.638117 \\
\hline 4.963522 & -4.513391 & -1.903248 & 1 & 2.844914 & 0.658424 & -5.398134 \\
\hline 5.059387 & -5.639676 & -2.764683 & 1 & 1.208124 & 1.151323 & -5.033990 \\
\hline 4.162832 & -6.224545 & -2.953717 & 6 & 2.240341 & 0.301199 & -3.371499 \\
\hline 6.253844 & -5.973460 & -3.361705 & 1 & 1.269620 & 0.009733 & -2.992685 \\
\hline 6.314959 & -6.831415 & -4.024770 & 6 & 3.308778 & -0.479534 & -2.961998 \\
\hline 7.403774 & -5.183950 & -3.121902 & 1 & 3.078760 & -1.316753 & -2.312905 \\
\hline 8.339604 & -5.434380 & -3.613221 & 6 & 4.642038 & -0.564707 & -3.671243 \\
\hline 7.348449 & -4.096843 & -2.276617 & 1 & 5.340559 & -1.087151 & -3.017741 \\
\hline 8.238488 & -3.500960 & -2.113778 & 1 & 4.519507 & -1.194086 & -4.564932 \\
\hline 6.135997 & -3.732531 & -1.626421 & 6 & 5.239978 & 0.804372 & -4.064680 \\
\hline 6.040901 & -2.595084 & -0.745874 & 1 & 4.953350 & 1.064417 & -5.088724 \\
\hline 7.245968 & -1.828179 & -0.322727 & 1 & 6.330265 & 0.718405 & -4.065896 \\
\hline 8.361145 & -2.455511 & 0.338564 & 6 & 0.598341 & 4.252586 & -1.406703 \\
\hline 8.346205 & -3.819579 & 0.744382 & 6 & -0.043795 & 4.172420 & -2.659128 \\
\hline 7.461413 & -4.417826 & 0.562989 & 6 & 0.931688 & 5.526867 & -0.902708 \\
\hline 9.431938 & -4.386443 & 1.375773 & 6 & -0.332271 & 5.326916 & -3.381041 \\
\hline 9.391651 & -5.428166 & 1.680855 & 1 & -0.331368 & 3.205612 & -3.056629 \\
\hline 10.595650 & -3.624625 & 1.637683 & 6 & 0.648912 & 6.680576 & -1.630306 \\
\hline 11.446166 & -4.087397 & 2.129524 & 1 & 1.419639 & 5.600675 & 0.066007 \\
\hline 10.635904 & -2.295445 & 1.283004 & 6 & 0.014504 & 6.584603 & -2.872957 \\
\hline 11.514262 & -1.691946 & 1.496585 & 1 & -0.831880 & 5.247650 & -4.342031 \\
\hline 9.527611 & -1.675277 & 0.644319 & 1 & 0.919234 & 7.652885 & -1.228977 \\
\hline 9.549366 & -0.289768 & 0.327942 & 1 & -0.211102 & 7.482179 & -3.441326 \\
\hline 10.439998 & 0.289313 & 0.554670 & 8 & -2.144635 & 1.600043 & -1.036421 \\
\hline 8.447841 & 0.316170 & -0.223320 & 6 & -0.775076 & 2.944202 & 3.003565 \\
\hline 8.422774 & 1.379303 & -0.437780 & 6 & -0.766321 & 2.261796 & 4.197806 \\
\hline 7.304619 & -0.458133 & -0.517977 & 1 & -0.526083 & 4.000068 & 2.984560 \\
\hline 4.307366 & 2.672593 & 1.078506 & 6 & -1.142750 & 0.910024 & 4.176833 \\
\hline 3.346309 & 2.558081 & 1.595183 & 6 & -1.416920 & 0.295279 & 2.973736 \\
\hline 5.079480 & 3.791393 & 1.756381 & 1 & -1.639312 & -0.761516 & 2.920940 \\
\hline 4.426793 & 4.642268 & 2.655232 & 7 & -1.440919 & 0.963513 & 1.801158 \\
\hline 3.386608 & 4.452114 & 2.908753 & 6 & -3.296820 & 3.511003 & -2.573568 \\
\hline 5.103711 & 5.718295 & 3.236027 & 1 & -2.772897 & 2.674756 & -3.027742 \\
\hline 4.584251 & 6.370433 & 3.932817 & 1 & -4.368588 & 3.286651 & -2.540947 \\
\hline 6.447292 & 5.946276 & 2.931304 & 1 & -3.150705 & 4.395588 & -3.200181 \\
\hline 6.977581 & 6.777275 & 3.387763 & 6 & -2.975535 & 5.122945 & -0.733406 \\
\hline 7.109438 & 5.095101 & 2.039957 & 1 & -3.086848 & 5.119322 & 0.350856 \\
\hline 8.156708 & 5.263154 & 1.803807 & 1 & -2.154741 & 5.795968 & -1.011448 \\
\hline 6.428683 & 4.028403 & 1.453688 & 1 & -3.904103 & 5.511050 & -1.158881 \\
\hline 6.945467 & 3.360788 & 0.768853 & 7 & -2.762685 & 3.773063 & -1.244112 \\
\hline 4.054641 & 3.039443 & -0.383389 & 1 & -0.491142 & 2.758092 & 5.121818 \\
\hline 5.005089 & 3.359696 & -0.825516 & 35 & -1.287056 & -0.114156 & 5.841357 \\
\hline
\end{tabular}

C2-P1 re-re 1

Number of imaginary frequencies $=1$

The smallest frequencies are $=-185.29168 .165611 .5906 \mathrm{~cm}(-1)$ Electronic energy $=-7222.6155244$

Zero-point correction=

1.755258

(Hartree/Particle)

Thermal correction to Energy $=\quad 1.873470$

Thermal correction to Enthalpy= $\quad 1.874414$

Thermal correction to Gibbs Free Energy $=\quad 1.594351$

Sum of electronic and zero-point Energies $=\quad-7220.860266$

Sum of electronic and thermal Energies $=\quad-7220.742054$

Sum of electronic and thermal Enthalpies $=\quad-7220.741110$

Sum of electronic and thermal Free Energies $=\quad-7221.021174$

Cartesian Coordinates 
$\begin{array}{llll}1.234213 & -2.319093 & -0.152169\end{array}$ $\begin{array}{llll}1.491786 & -3.229285 & 0.380140\end{array}$ $\begin{array}{llll}5.141929 & -1.625488 & -2.911372\end{array}$ $\begin{array}{llll}5.491959 & -0.649903 & -3.237613\end{array}$ $\begin{array}{llll}3.767278 & -1.904197 & -3.094563\end{array}$ $3.160336-1.131388-3.566404$ $\begin{array}{llll}3.195173 & -3.305499 & -3.223389\end{array}$ $\begin{array}{llll}3.429710 & -3.707230 & -4.221074\end{array}$ $\begin{array}{llll}2.108207 & -3.226737 & -3.173601\end{array}$ $\begin{array}{llll}3.676132 & -4.294789 & -2.136100\end{array}$ $\begin{array}{llll}4.522879 & -4.882841 & -2.505568\end{array}$ $\begin{array}{llll}2.872820 & -5.011228 & -1.936280\end{array}$ $\begin{array}{llll}4.077970 & -3.646996 & -0.811761\end{array}$ $\begin{array}{llll}3.453535 & -3.889668 & 0.042081\end{array}$ $\begin{array}{llll}5.366815 & -3.211069 & -0.515959\end{array}$ $\begin{array}{lll}5.638066 & -3.164780 & 0.534392\end{array}$ $6.543047 \quad-3.239759-1.473311$ $\begin{array}{llll}7.339030 & -2.629231 & -1.042459\end{array}$ $\begin{array}{llll}6.933543 & -4.266537 & -1.530655\end{array}$ $\begin{array}{llll}6.218996 & -2.711359 & -2.887462\end{array}$ $\begin{array}{llll}5.910215 & -3.535349 & -3.539203\end{array}$ $\begin{array}{llll}7.135575 & -2.304751 & -3.325238\end{array}$ $\begin{array}{llll}0.418390 & -2.508723 & -1.339935\end{array}$ $\begin{array}{llll}0.126068 & -1.452205 & -2.220805\end{array}$ $\begin{array}{llll}-0.080170 & -3.791258 & -1.641428\end{array}$ $\begin{array}{llll}-0.654850 & -1.664679 & -3.350239\end{array}$ $\begin{array}{llll}0.523294 & -0.467282 & -2.023820\end{array}$ $\begin{array}{lll}-0.866828 & -4.002810 & -2.771908\end{array}$ $\begin{array}{llll}0.153947 & -4.619648 & -0.979802\end{array}$ $\begin{array}{llll}-1.165835 & -2.937896 & -3.626994\end{array}$ $\begin{array}{llll}-0.870329 & -0.833001 & -4.010983\end{array}$ $\begin{array}{llll}-1.250720 & -4.995940 & -2.984665\end{array}$ $\begin{array}{llll}-1.791595 & -3.094509 & -4.500493\end{array}$ $\begin{array}{llll}-2.993647 & 4.930987 & 1.168654\end{array}$ $\begin{array}{lll}-3.273113 & 1.106713 & -2.310361\end{array}$ $\begin{array}{lll}-4.619759 & 0.408625 & 1.114931\end{array}$ $\begin{array}{llll}-3.469534 & 3.456386 & -0.239197\end{array}$ $\begin{array}{lll}-3.539761 & 2.929593 & 1.099016\end{array}$ $\begin{array}{lll}-2.247895 & 3.077370 & 1.690460\end{array}$ $\begin{array}{lll}-1.977853 & 2.780210 & 2.693922\end{array}$ $\begin{array}{lll}-1.390496 & 3.710323 & 0.744723\end{array}$ $\begin{array}{lll}-0.361348 & 3.995636 & 0.912948\end{array}$ $\begin{array}{llll}-2.140148 & 3.947053 & -0.442970\end{array}$ $\begin{array}{llll}-1.781799 & 4.423859 & -1.344153\end{array}$ $\begin{array}{llll}-4.524196 & 6.137605 & 1.895263\end{array}$ $\begin{array}{llll}-5.559382 & 5.833468 & 1.978126\end{array}$ $\begin{array}{lll}-3.516086 & 5.962661 & 2.892139\end{array}$ $\begin{array}{lll}-3.652467 & 5.500704 & 3.860875\end{array}$ $\begin{array}{lll}-2.285933 & 6.465934 & 2.369668\end{array}$ $\begin{array}{lll}-1.327567 & 6.446827 & 2.871931\end{array}$ $\begin{array}{lll}-2.533060 & 6.952898 & 1.050187\end{array}$ $\begin{array}{llll}-1.795570 & 7.367205 & 0.375283\end{array}$ $\begin{array}{lll}-3.914864 & 6.752456 & 0.757613\end{array}$ $\begin{array}{llll}-4.403888 & 6.996724 & -0.175610\end{array}$ $\begin{array}{llll}-4.564207 & 3.407823 & -1.245188\end{array}$ $\begin{array}{llll}-5.614194 & 4.335235 & -1.186665\end{array}$ $\begin{array}{llll}-5.609451 & 5.063426 & -0.386279\end{array}$ $\begin{array}{llll}-6.641272 & 4.336038 & -2.129688\end{array}$ $\begin{array}{llll}-7.443679 & 5.063874 & -2.056941\end{array}$ $\begin{array}{llll}-6.629320 & 3.398735 & -3.160313\end{array}$ $\begin{array}{llll}-7.418356 & 3.388594 & -3.904681\end{array}$ $\begin{array}{llll}-5.613647 & 2.444629 & -3.214826\end{array}$ $\begin{array}{llll}-5.634060 & 1.704371 & -4.006320\end{array}$ $\begin{array}{llll}-4.584197 & 2.419411 & -2.260300\end{array}$ $\begin{array}{lll}-4.734979 & 2.228580 & 1.682930\end{array}$ $\begin{array}{lll}-5.630272 & 2.636044 & 1.208534\end{array}$ $\begin{array}{lll}-4.874199 & 2.406897 & 3.198682\end{array}$ $\begin{array}{lll}-5.790341 & 1.942241 & 3.571431\end{array}$ $\begin{array}{lll}-4.030583 & 1.973679 & 3.744051\end{array}$ $\begin{array}{llll}-4.909601 & 3.476192 & 3.425347\end{array}$ $\begin{array}{llll}-3.936426 & -0.062156 & -3.574345\end{array}$ $\begin{array}{llll}-5.027873 & -0.857256 & -3.181968\end{array}$ $\begin{array}{llll}-5.465315 & -0.718898 & -2.198753\end{array}$ $\begin{array}{llll}-5.563804 & -1.814192 & -4.041439\end{array}$ $\begin{array}{lll}-6.401871 & -2.419140 & -3.710547\end{array}$ 


\begin{tabular}{|c|c|}
\hline \multicolumn{2}{|l|}{ C2-P1 re-re_2 } \\
\hline \multicolumn{2}{|l|}{ Number of imaginary frequencies $=1$} \\
\hline \multicolumn{2}{|c|}{ The smallest frequencies are $=-227.296911 .519913 .3150 \mathrm{~cm}(-1)$} \\
\hline \multicolumn{2}{|c|}{ Electronic energy $=-7222.6110077$} \\
\hline Zero-point correction $=$ & 4688 \\
\hline \multicolumn{2}{|l|}{ (Hartree/Particle) } \\
\hline Thermal correction to Energy= & 1.873132 \\
\hline Thermal correction to Enthalpy= & 1.874076 \\
\hline Thermal correction to Gibbs Free Energy= & 1.593819 \\
\hline Sum of electronic and zero-point Energies $=$ & -7220.856320 \\
\hline Sum of electronic and thermal Energies $=$ & -7220.737876 \\
\hline Sum of electronic and thermal Enthalpies $=$ & -7220.736932 \\
\hline Sum of electronic and thermal Free Energies & -7221.017189 \\
\hline
\end{tabular}

\begin{tabular}{|c|c|c|c|}
\hline \multicolumn{4}{|c|}{ Cartesian Coordinates } \\
\hline 26 & -3.617782 & -3.632523 & -4.194816 \\
\hline 15 & -1.785632 & -2.510082 & 0.520230 \\
\hline 15 & -4.661583 & -0.460481 & -0.751923 \\
\hline 6 & -3.187839 & -3.334846 & -2.168828 \\
\hline 6 & -3.930512 & -2.225032 & -2.709383 \\
\hline 6 & -3.159054 & -1.663254 & -3.773247 \\
\hline 1 & -3.448091 & -0.821035 & -4.387136 \\
\hline 6 & -1.966038 & -2.430046 & -3.913246 \\
\hline 1 & -1.199791 & -2.280681 & -4.660782 \\
\hline 6 & -1.981116 & -3.458557 & -2.927848 \\
\hline 1 & -1.225922 & -4.217909 & -2.781764 \\
\hline 6 & -5.369217 & -4.633010 & -4.696005 \\
\hline 1 & -6.269423 & -4.632380 & -4.095812 \\
\hline 6 & -5.035162 & -3.679537 & -5.705870 \\
\hline 1 & -5.634958 & -2.828524 & -5.999691 \\
\hline 6 & -3.748776 & -4.01 & -6.225493 \\
\hline 1 & -3.203130 & -3.468192 & -6.980349 \\
\hline 6 & -3.286875 & -5.181607 & -5.536438 \\
\hline 1 & -2.330206 & -5.66 & 6924 \\
\hline 6 & -4.288156 & -5.562460 & -4.593944 \\
\hline 1 & -4.221507 & -6.391277 & -3.902360 \\
\hline 6 & -3.586953 & -4.127097 & -0.974302 \\
\hline 6 & -4.584028 & -5.107541 & -1.081781 \\
\hline 1 & -5.042000 & -5.270188 & -2.048821 \\
\hline 6 & -4.987373 & -5.863628 & 0.018826 \\
\hline 1 & -5.767888 & -6.610066 & -0.0 \\
\hline 6 & -4.387299 & -5.6 & 078 \\
\hline 1 & -4.691625 & -6.22 & 2.124020 \\
\hline 6 & & -4.6 & \\
\hline 1 & -2.971277 & -4.49 & 2.368241 \\
\hline 6 & -3.009537 & -3.88 & 0.296999 \\
\hline 6 & & & 2871 \\
\hline 1 & -5.694807 & -2.503615 & -1.587260 \\
\hline 6 & -6.187821 & -1.112280 & -3.133131 \\
\hline 1 & -7.083571 & -0.722807 & -2.641061 \\
\hline 1 & -5.748526 & -0.303482 & -3.722782 \\
\hline 1 & -6.485882 & -1.908137 & -3.821549 \\
\hline 6 & -1.635200 & -2.410763 & 2.352531 \\
\hline 6 & -2.626992 & -1.661083 & 3.009300 \\
\hline 1 & -3.414313 & -1.193165 & 2.426861 \\
\hline 6 & -2.615505 & -1.516404 & 4.395777 \\
\hline 1 & -3.395616 & -0.936124 & 4.879202 \\
\hline 6 & & & \\
\hline 1 & -1.573809 & -1.992553 & 6.225457 \\
\hline 6 & -0.608654 & -2.862346 & 4.503935 \\
\hline 1 & 0.185590 & -3.325153 & 5.082151 \\
\hline 6 & -0.632219 & -3.023095 & 3.115028 \\
\hline 1 & 0.130283 & -3.623433 & 2.634101 \\
\hline 6 & -0.224201 & -3.235165 & -0.117639 \\
\hline 6 & 0.144208 & -4.569949 & 0.111204 \\
\hline 1 & -0.488473 & -5.213987 & 0.715074 \\
\hline 6 & 1.317349 & -5.078747 & -0.449781 \\
\hline 1 & 1.600846 & -6.110760 & -0.264349 \\
\hline 6 & 2.120809 & -4.264867 & -1.255376 \\
\hline 1 & 3.029497 & -4.665685 & -1.694967 \\
\hline 6 & 1.748145 & -2.940423 & -1.500047 \\
\hline 1 & 2.363254 & -2.309348 & -2.135410 \\
\hline
\end{tabular}


$\begin{array}{lll}0.580637 & -2.425093 & -0.932976\end{array}$

$\begin{array}{llll}0.267851 & -1.408979 & -1.151936\end{array}$

$\begin{array}{lll}-5.628147 & -0.894174 & 0.757207\end{array}$

$\begin{array}{llll}-5.709802 & 0.052980 & 1.793707\end{array}$

$\begin{array}{lll}-5.290849 & 1.045465 & 1.667912\end{array}$

$\begin{array}{lll}-6.341399 & -0.268280 & 2.994543\end{array}$

$\begin{array}{llll}-6.878116 & -1.537167 & 3.204656\end{array}$

$\begin{array}{lll}-7.378317 & -1.777099 & 4.136039\end{array}$

$\begin{array}{lll}-6.769709 & -2.485677 & 2.191310\end{array}$

$\begin{array}{lll}-6.145550 & -2.178404 & 0.981285\end{array}$

$\begin{array}{lll}-6.067259 & -2.955311 & 0.231301\end{array}$

$\begin{array}{llll}-5.352952 & 1.158287 & -1.295740\end{array}$

$\begin{array}{llll}-4.607247 & 1.834214 & -2.273097\end{array}$

$\begin{array}{llll}-3.698286 & 1.389467 & -2.657599\end{array}$

$\begin{array}{llll}-5.019212 & 3.078917 & -2.742011\end{array}$

$\begin{array}{llll}-6.168524 & 3.687027 & -2.234129\end{array}$

$\begin{array}{llll}-6.479296 & 4.663096 & -2.588913\end{array}$

$\begin{array}{llll}-6.913776 & 3.010820 & -1.271125\end{array}$

$\begin{array}{llll}-6.522365 & 1.750392 & -0.810279\end{array}$

$\begin{array}{llll}-7.138232 & 1.246178 & -0.074764\end{array}$

$\begin{array}{lll}-2.382876 & -0.482635 & -0.324266\end{array}$

$\begin{array}{llll}-8.125799 & 3.661034 & -0.660893\end{array}$

$\begin{array}{lll}-4.178003 & 3.821585 & -3.741682\end{array}$

$\begin{array}{llll}-6.407129 & 0.739070 & 4.109870\end{array}$

$\begin{array}{lll}-7.312611 & -3.866700 & 2.445997\end{array}$

$\begin{array}{lll}-6.478592 & -4.588831 & 3.233946\end{array}$

$\begin{array}{llll}-7.495516 & -4.568812 & 1.307641\end{array}$

$\begin{array}{lll}-8.502783 & -3.820562 & 3.087357\end{array}$

$\begin{array}{llll}-5.398366 & 0.554938 & 5.001139\end{array}$

$\begin{array}{lll}-6.314975 & 2.005517 & 3.656287\end{array}$

$\begin{array}{lll}-7.559396 & 0.639127 & 4.808905\end{array}$

$\begin{array}{llll}-9.092713 & 2.757699 & -0.381887\end{array}$

$\begin{array}{lll}-7.817833 & 4.274483 & 0.508109\end{array}$

$\begin{array}{llll}-8.665377 & 4.598810 & -1.468276\end{array}$

$\begin{array}{llll}-4.929051 & 4.500721 & -4.633236\end{array}$

$\begin{array}{llll}-3.362242 & 2.997607 & -4.436825\end{array}$

$\begin{array}{llll}-3.376313 & 4.743281 & -3.135696\end{array}$

$\begin{array}{lll}-2.385630 & 1.653475 & 1.860233\end{array}$

$\begin{array}{llll}-2.136748 & 2.534262 & -0.293105\end{array}$

$\begin{array}{llll}-2.903496 & 2.846087 & 2.336067\end{array}$

$\begin{array}{lll}-2.239195 & 0.804156 & 2.516702\end{array}$

$\begin{array}{llll}-2.804724 & 3.717213 & 0.125890\end{array}$

$\begin{array}{lll}-3.177752 & 3.887798 & 1.445303\end{array}$

$\begin{array}{llll}-2.978215 & 4.507194 & -0.595040\end{array}$

$\begin{array}{lll}-1.470502 & 2.546662 & -1.565060\end{array}$

$\begin{array}{llll}-1.035428 & 1.384111 & -2.303260\end{array}$

$\begin{array}{llll}-1.117977 & 0.207745 & -1.843694\end{array}$

$\begin{array}{llll}-2.034516 & 1.479016 & 0.572016\end{array}$

$\begin{array}{llll}-1.639582 & 3.452702 & -2.123755\end{array}$

$\begin{array}{llll}3.523850 & 2.347193 & -0.180770\end{array}$

$\begin{array}{llll}4.757479 & 0.485705 & 0.286328\end{array}$

$\begin{array}{llll}5.123518 & -0.743399 & -0.760790\end{array}$

$\begin{array}{llll}6.277304 & 0.935270 & 0.780174\end{array}$

$\begin{array}{llll}3.983686 & -0.422548 & 1.481700\end{array}$

$\begin{array}{llll}6.163174 & -0.640903 & -1.676444\end{array}$

$\begin{array}{llll}5.788392 & -0.616738 & -3.037822\end{array}$

$\begin{array}{llll}4.734433 & -0.694895 & -3.283661\end{array}$

$\begin{array}{llll}6.752312 & -0.499471 & -4.007248\end{array}$

$\begin{array}{llll}6.478086 & -0.489183 & -5.058341\end{array}$

$8.119584-0.357123 \quad-3.647756$

$9.117855 \quad-0.155020 \quad-4.639150$

$\begin{array}{llll}8.814457 & -0.139459 & -5.682689\end{array}$

$\begin{array}{llll}10.435857 & 0.030371 & -4.288980\end{array}$

$\begin{array}{llll}11.190337 & 0.187634 & -5.054147\end{array}$

$\begin{array}{llll}10.804660 & 0.033275 & -2.922825\end{array}$

$\begin{array}{llll}11.840376 & 0.207776 & -2.645833\end{array}$

$\begin{array}{llll}9.863379 & -0.175825 & -1.938238\end{array}$

$\begin{array}{llll}0.167530 & -0.159100 & -0.898892\end{array}$

$\begin{array}{llll}8.495383 & -0.399475 & -2.262467\end{array}$

$\begin{array}{llll}7.485910 & -0.623766 & -1.256639\end{array}$

$\begin{array}{llll}7.836451 & -0.793655 & 0.181680\end{array}$

$\begin{array}{llll}8.778464 & -1.785910 & 0.630608\end{array}$

$\begin{array}{llll}9.305956 & -2.788101 & -0.230056\end{array}$

$\begin{array}{llll}8.985099 & -2.821452 & -1.264329\end{array}$

$\begin{array}{lll}10.197953 & -3.729744 & 0.235448\end{array}$ $\begin{array}{lll}10.577312 & -4.490560 & -0.440796\end{array}$

$\begin{array}{lll}10.617049 & -3.721937 & 1.587066\end{array}$

$\begin{array}{lll}11.324635 & -4.466820 & 1.939303\end{array}$

$\begin{array}{llll}10.109847 & -2.779982 & 2.453351\end{array}$

$\begin{array}{llll}10.403442 & -2.775801 & 3.499837\end{array}$

$\begin{array}{llll}9.175533 & -1.805813 & 2.009490\end{array}$

$\begin{array}{lll}8.599584 & -0.880896 & 2.920315\end{array}$

$\begin{array}{llll}8.913142 & -0.899149 & 3.960130\end{array}$

$\begin{array}{lll}7.627761 & -0.007029 & 2.502317\end{array}$

$\begin{array}{lll}7.146316 & 0.686431 & 3.183155\end{array}$

$\begin{array}{lll}7.245004 & 0.007320 & 1.144645\end{array}$

$\begin{array}{lll}2.942325 & 0.395742 & 2.164536\end{array}$

$\begin{array}{lll}1.964165 & 0.149089 & 1.736882\end{array}$

$\begin{array}{llll}2.870461 & 0.165641 & 3.663146\end{array}$

$\begin{array}{lll}1.621207 & 0.060499 & 4.287015\end{array}$

$\begin{array}{lll}0.717380 & 0.072486 & 3.682277\end{array}$

$\begin{array}{llll}1.528000 & -0.086407 & 5.673061\end{array}$

$\begin{array}{lll}0.550619 & -0.180834 & 6.137509\end{array}$

$\begin{array}{llll}2.686881 & -0.132238 & 6.451962\end{array}$

$\begin{array}{llll}2.617015 & -0.254070 & 7.529243\end{array}$

$\begin{array}{llll}3.938047 & -0.025008 & 5.836041\end{array}$

$\begin{array}{llll}4.844425 & -0.060789 & 6.434621\end{array}$

$\begin{array}{lll}4.028114 & 0.127280 & 4.451398\end{array}$

$\begin{array}{llll}4.998477 & 0.201982 & 3.970932\end{array}$

$\begin{array}{llll}3.213002 & 1.885127 & 1.892655\end{array}$

$\begin{array}{lll}4.081266 & 2.205096 & 2.482206\end{array}$

$\begin{array}{lll}2.361418 & 2.459596 & 2.262171\end{array}$

$\begin{array}{llll}3.764048 & -1.901818 & 1.284735\end{array}$

$\begin{array}{llll}3.527847 & -2.059576 & 0.231878\end{array}$

$\begin{array}{llll}4.980391 & -2.757276 & 1.604821\end{array}$

$\begin{array}{lll}5.504056 & -3.605592 & 0.621614\end{array}$

$\begin{array}{llll}5.111130 & -3.551498 & -0.389772\end{array}$

$\begin{array}{llll}6.517883 & -4.516359 & 0.927675\end{array}$

$\begin{array}{llll}6.914317 & -5.165442 & 0.152156\end{array}$

$\begin{array}{llll}7.026241 & -4.585179 & 2.225900\end{array}$

$\begin{array}{lll}7.820809 & -5.285137 & 2.465616\end{array}$

$\begin{array}{lll}6.522574 & -3.729131 & 3.209210\end{array}$

$\begin{array}{lll}6.923529 & -3.766091 & 4.218551\end{array}$

$\begin{array}{llll}5.506868 & -2.823613 & 2.901473\end{array}$

$\begin{array}{lll}5.109629 & -2.172466 & 3.673452\end{array}$

$\begin{array}{lll}2.560612 & -2.438823 & 2.067498\end{array}$

$\begin{array}{lll}1.639965 & -1.913284 & 1.814804\end{array}$

$\begin{array}{lll}2.709285 & -2.396282 & 3.147078\end{array}$

$\begin{array}{lll}2.429008 & -3.484688 & 1.779633\end{array}$

$\begin{array}{llll}1.978586 & 1.037701 & -0.997120\end{array}$

$\begin{array}{llll}1.975303 & 1.070297 & -2.085480\end{array}$

$\begin{array}{llll}1.958300 & 0.025842 & -0.603590\end{array}$

$\begin{array}{llll}1.237828 & 2.028025 & -0.270808\end{array}$

$\begin{array}{lll}0.896185 & 1.761429 & 0.723096\end{array}$

$\begin{array}{lll}0.678371 & 3.206494 & -0.829612\end{array}$

$\begin{array}{llll}0.916121 & 3.428686 & -1.859917\end{array}$

$\begin{array}{lll}5.172938 & 3.726614 & 0.414563\end{array}$

$\begin{array}{llll}5.566884 & 3.354054 & 1.355572\end{array}$

$\begin{array}{llll}3.957110 & 4.447270 & 0.471724\end{array}$

$\begin{array}{llll}3.507956 & 4.594657 & 1.453716\end{array}$

$\begin{array}{llll}3.532366 & 5.470870 & -0.567134\end{array}$

$\begin{array}{llll}4.111563 & 6.396570 & -0.427752\end{array}$

$\begin{array}{llll}2.491038 & 5.730797 & -0.369730\end{array}$

$\begin{array}{llll}3.663113 & 4.989230 & -2.031319\end{array}$

$\begin{array}{llll}4.609721 & 5.332133 & -2.461872\end{array}$

$\begin{array}{llll}2.874495 & 5.459790 & -2.627858\end{array}$

$\begin{array}{llll}3.563080 & 3.475005 & -2.218582\end{array}$

$\begin{array}{llll}2.703637 & 3.127494 & -2.785244\end{array}$

$\begin{array}{llll}4.655746 & 2.609721 & -2.185588\end{array}$

$\begin{array}{llll}4.555374 & 1.672900 & -2.726667\end{array}$

$\begin{array}{llll}6.086773 & 3.015001 & -1.884062\end{array}$

$\begin{array}{llll}6.659782 & 2.104406 & -1.697487\end{array}$

$\begin{array}{llll}6.524414 & 3.480575 & -2.779500\end{array}$

$\begin{array}{llll}6.229119 & 3.954707 & -0.667704\end{array}$

$\begin{array}{llll}6.191841 & 5.000446 & -0.989744\end{array}$

$\begin{array}{llll}7.219675 & 3.807223 & -0.226976\end{array}$

$\begin{array}{llll}0.252565 & 4.361496 & -0.033562\end{array}$

$\begin{array}{lll}0.241257 & 4.354775 & 1.372565\end{array}$

$\begin{array}{llll}-0.157444 & 5.533729 & -0.697730\end{array}$

$\begin{array}{lll}-0.153419 & 5.484035 & 2.087199\end{array}$ 


$\begin{array}{lccc}1 & 0.539896 & 3.465259 & 1.913868 \\ 6 & -0.555638 & 6.661899 & 0.015854 \\ 1 & -0.161905 & 5.550888 & -1.784708 \\ 6 & -0.552456 & 6.642612 & 1.414312 \\ 1 & -0.154552 & 5.456506 & 3.173054 \\ 1 & -0.865081 & 7.556465 & -0.517054 \\ 1 & -0.858720 & 7.521654 & 1.973872 \\ 7 & -0.456773 & 1.551306 & -3.538852 \\ 6 & 0.130667 & 0.408446 & -4.225269 \\ 1 & -0.387648 & 0.221217 & -5.174085 \\ 1 & 1.186324 & 0.610827 & -4.449508 \\ 1 & 0.055330 & -0.474031 & -3.596456 \\ 6 & -0.268021 & 2.816815 & -4.235410 \\ 1 & 0.779405 & 3.145322 & -4.182116 \\ 1 & -0.523207 & 2.682838 & -5.291973 \\ 1 & -0.906129 & 3.604285 & -3.845734 \\ 1 & -3.636723 & 4.811942 & 1.778506 \\ 35 & -3.161731 & 3.087895 & 4.258275\end{array}$

C2-P1 re-re_3

Number of imaginary frequencies $=1$

The smallest frequencies are $=-243.36335 .192911 .3286 \mathrm{~cm}(-1)$ Electronic energy $=-7222.6143593$

Zero-point correction $=$

(Hartree/Particle)

$\begin{array}{lc}\text { Thermal correction to Energy= } & 1.873982 \\ \text { Thermal correction to Enthalpy= } & 1.874926 \\ \text { Thermal correction to Gibbs Free Energy= } & 1.595009 \\ \text { Sum of electronic and zero-point Energies }= & -7220.858606 \\ \text { Sum of electronic and thermal Energies= } & -7220.740377 \\ \text { Sum of electronic and thermal Enthalpies }= & -7220.739433 \\ \text { Sum of electronic and thermal Free Energies }= & -7221.019351\end{array}$

1.755753

1.873982
1.874926
1.595009
-7220.858606
-7220.740377
-7220.739433
-7221.019351

Cartesian Coordinates

$\begin{array}{lrrr}26 & 3.098552 & 4.346842 & -2.946487 \\ 15 & 2.723955 & 1.778242 & 1.595644 \\ 15 & 4.662810 & 0.171975 & -1.198096 \\ 6 & 3.306451 & 3.357167 & -1.108541 \\ 6 & 3.463878 & 2.443095 & -2.214772 \\ 6 & 2.252988 & 2.461451 & -2.969471 \\ 1 & 2.062134 & 1.889919 & -3.866411 \\ 6 & 1.363192 & 3.397739 & -2.368806 \\ 1 & 0.375680 & 3.659591 & -2.723591 \\ 6 & 2.013460 & 3.957349 & -1.232335 \\ 1 & 1.604568 & 4.704531 & -0.568390 \\ 6 & 4.787638 & 5.141194 & -3.860085 \\ 1 & 5.786850 & 4.737222 & -3.762289 \\ 6 & 3.822969 & 4.764758 & -4.844341 \\ 1 & 3.960524 & 4.023414 & -5.620177 \\ 6 & 2.627981 & 5.507249 & -4.598358 \\ 1 & 1.701955 & 5.422780 & -5.151875 \\ 6 & 2.853511 & 6.344472 & -3.463473 \\ 1 & 2.129974 & 7.006535 & -3.006327 \\ 6 & 4.187102 & 6.120458 & -3.008917 \\ 1 & 4.647275 & 6.591305 & -2.151244 \\ 6 & 4.292657 & 3.552661 & -0.006946 \\ 6 & 5.445922 & 4.313135 & -0.247322 \\ 1 & 5.573347 & 4.745836 & -1.231144 \\ 6 & 6.418071 & 4.509936 & 0.732880 \\ 1 & 7.308668 & 5.086430 & 0.505792 \\ 6 & 6.244019 & 3.943881 & 1.993062 \\ 1 & 6.991600 & 4.083066 & 2.767876 \\ 6 & 5.108893 & 3.175373 & 2.254982 \\ 1 & 4.995236 & 2.734632 & 3.238670 \\ 6 & 4.131175 & 2.952557 & 1.272790 \\ 6 & 4.682566 & 1.596176 & -2.459617 \\ 1 & 5.546982 & 2.199633 & -2.180450 \\ 6 & 4.866719 & 1.168543 & -3.918547 \\ 1 & 5.785689 & 0.590083 & -4.046769 \\ 1 & 4.033525 & 0.562498 & -4.283916 \\ 1 & 4.928771 & 2.063543 & -4.544285 \\ 6 & 2.935230 & 1.277019 & 3.354536 \\ 6 & 3.958183 & 0.357405 & 3.646158\end{array}$

$\begin{array}{lll}4.620305 & 0.020327 & 2.855882\end{array}$

$\begin{array}{lll}4.147086 & -0.112732 & 4.944300\end{array}$

$\begin{array}{lll}4.950287 & -0.813393 & 5.147889\end{array}$

$\begin{array}{llll}3.300226 & 0.316311 & 5.971205\end{array}$

$\begin{array}{llll}3.437614 & -0.055818 & 6.982309\end{array}$

$\begin{array}{llll}2.275013 & 1.221826 & 5.690777\end{array}$

$\begin{array}{lll}1.611610 & 1.556659 & 6.483182\end{array}$

$\begin{array}{lll}2.092782 & 1.704175 & 4.390818\end{array}$

$\begin{array}{lll}1.289258 & 2.405020 & 4.192815\end{array}$

$\begin{array}{lll}1.257298 & 2.875700 & 1.574121\end{array}$

$\begin{array}{lll}1.276705 & 4.136089 & 2.193073\end{array}$

$\begin{array}{llll}2.137339 & 4.438712 & 2.782723\end{array}$

$\begin{array}{llll}0.206569 & 5.014809 & 2.024279\end{array}$

$\begin{array}{lll}0.224789 & 5.989068 & 2.504304\end{array}$

$\begin{array}{lll}-0.876164 & 4.647634 & 1.219210\end{array}$

$\begin{array}{lll}-1.699759 & 5.336812 & 1.068344\end{array}$

$\begin{array}{llll}-0.901289 & 3.392740 & 0.606487\end{array}$

$\begin{array}{lll}-1.732637 & 3.120962 & -0.033055\end{array}$

$\begin{array}{lll}0.154665 & 2.499478 & 0.793959\end{array}$

$\begin{array}{llll}0.163945 & 1.544056 & 0.277598\end{array}$

$6.242684 \quad 0.327386 \quad-0.251892$

$\begin{array}{llll}6.405520 & -0.541350 & 0.836230\end{array}$

$\begin{array}{lll}5.678619 & -1.322724 & 1.013041\end{array}$

$\begin{array}{llll}7.482372 & -0.402261 & 1.710972\end{array}$

$\begin{array}{lll}8.426650 & 0.603474 & 1.520542\end{array}$

$\begin{array}{llll}9.248513 & 0.730054 & 2.216386\end{array}$

$\begin{array}{llll}8.278039 & 1.457683 & 0.428674\end{array}$

$\begin{array}{llll}7.207504 & 1.322638 & -0.454743\end{array}$

$\begin{array}{llll}7.132597 & 2.021026 & -1.276505\end{array}$

$\begin{array}{llll}4.871468 & -1.383548 & -2.156494\end{array}$

$\begin{array}{llll}3.768799 & -1.794599 & -2.922655\end{array}$

$\begin{array}{llll}2.875694 & -1.180804 & -2.960475\end{array}$

$3.811956-2.989566-3.634851$

$\begin{array}{llll}4.923678 & -3.830732 & -3.554262\end{array}$

$\begin{array}{llll}4.946259 & -4.770575 & -4.093006\end{array}$

$\begin{array}{llll}6.004674 & -3.435024 & -2.771346\end{array}$

$\begin{array}{llll}5.994071 & -2.214180 & -2.089876\end{array}$

$6.864618-1.926988-1.510735$

$\begin{array}{llll}2.753746 & 0.037544 & 0.146468\end{array}$

$\begin{array}{llll}7.188241 & -4.346665 & -2.594247\end{array}$

$\begin{array}{llll}2.613713 & -3.416221 & -4.434464\end{array}$

$\begin{array}{lll}7.613051 & -1.356852 & 2.865633\end{array}$

$\begin{array}{llll}9.287522 & 2.559200 & 0.248888\end{array}$

$\begin{array}{llll}9.352550 & 3.351123 & 1.347280\end{array}$

$\begin{array}{llll}9.004365 & 3.358367 & -0.802951\end{array}$

$\begin{array}{lll}10.532938 & 2.066720 & 0.056962\end{array}$

$\begin{array}{lll}6.396003 & -1.690817 & 3.369814\end{array}$

$\begin{array}{llll}8.209479 & -2.513682 & 2.498303\end{array}$

$\begin{array}{llll}8.337522 & -0.838027 & 3.877862\end{array}$

$\begin{array}{llll}8.355066 & -3.662927 & -2.606535\end{array}$

$7.131954-4.994732-1.404132$

$\begin{array}{llll}7.259561 & -5.291470 & -3.555416\end{array}$

$\begin{array}{lll}2.953432 & -4.157459 & -5.508580\end{array}$

$\begin{array}{llll}1.899622 & -2.355661 & -4.878540\end{array}$

$\begin{array}{lll}1.757426 & -4.175338 & -3.695669\end{array}$

$\begin{array}{lll}3.496618 & -2.626341 & 1.184771\end{array}$

$\begin{array}{llll}1.812732 & -2.842681 & -0.417829\end{array}$

$\begin{array}{llll}3.837452 & -3.964946 & 1.080434\end{array}$

$\begin{array}{lll}3.982458 & -1.978916 & 1.904378\end{array}$

$\begin{array}{lll}2.134308 & -4.216389 & -0.553049\end{array}$

$\begin{array}{llll}3.163573 & -4.784331 & 0.174985\end{array}$

$\begin{array}{llll}1.572200 & -4.821336 & -1.255099\end{array}$

$\begin{array}{llll}0.640538 & -2.349851 & -1.107672\end{array}$

$\begin{array}{llll}0.501247 & -1.004525 & -1.631432\end{array}$

$\begin{array}{llll}1.051091 & 0.003734 & -1.103951\end{array}$

$\begin{array}{llll}2.543447 & -2.058763 & 0.425200\end{array}$

$\begin{array}{llll}0.171513 & -3.124017 & -1.698212\end{array}$

$\begin{array}{llll}-3.976706 & -1.556195 & 0.982391\end{array}$

$\begin{array}{lll}-5.584482 & -0.021394 & 0.433059\end{array}$

$\begin{array}{llll}-6.115986 & 0.323675 & -1.104779\end{array}$

$\begin{array}{lll}-7.035782 & -0.448497 & 1.144644\end{array}$

$\begin{array}{lll}-5.090929 & 1.506450 & 0.900726\end{array}$

$\begin{array}{llll}-6.994253 & -0.525274 & -1.769483\end{array}$

$\begin{array}{llll}-6.472886 & -1.218475 & -2.884478\end{array}$

$\begin{array}{lll}-5.439179 & -1.043874 & -3.164104\end{array}$ 
$\begin{array}{lll}-7.275670 & -2.086682 & -3.581334\end{array}$

$\begin{array}{llll}-6.890019 & -2.619471 & -4.445921\end{array}$

$\begin{array}{llll}-8.612514 & -2.325148 & -3.162304\end{array}$

$\begin{array}{llll}-9.428069 & -3.281482 & -3.826063\end{array}$

$\begin{array}{llll}-9.014629 & -3.809989 & -4.681100\end{array}$

$\begin{array}{lll}-10.706495 & -3.545480 & -3.389532\end{array}$

$\begin{array}{lll}-11.319433 & -4.282340 & -3.900263\end{array}$

$-11.217440 \quad-2.865058 \quad-2.258685$

$\begin{array}{llll}-12.217095 & -3.093376 & -1.900319\end{array}$

$\begin{array}{llll}-10.458519 & -1.919868 & -1.603038\end{array}$

$\begin{array}{llll}-10.864447 & -1.416822 & -0.733612\end{array}$

$\begin{array}{llll}-9.141511 & -1.603120 & -2.039681\end{array}$

$\begin{array}{lll}-8.319817 & -0.624005 & -1.373791\end{array}$

$\begin{array}{llll}-8.849527 & 0.232424 & -0.275626\end{array}$

$\begin{array}{llll}-9.997895 & 1.082956 & -0.450557\end{array}$

$\begin{array}{lll}-10.626833 & 1.273899 & -1.712860\end{array}$

$\begin{array}{llll}-10.229100 & 0.769684 & -2.585520\end{array}$

$\begin{array}{llll}-11.721433 & 2.100633 & -1.844882\end{array}$

$\begin{array}{lll}-12.177994 & 2.234842 & -2.821395\end{array}$

$\begin{array}{llll}-12.252950 & 2.781658 & -0.723740\end{array}$

$\begin{array}{llll}-13.120834 & 3.423775 & -0.841753\end{array}$

$\begin{array}{lll}-11.655680 & 2.639310 & 0.508191\end{array}$

$\begin{array}{lll}-12.040161 & 3.171338 & 1.374546\end{array}$

$\begin{array}{lll}-10.515026 & 1.807958 & 0.676200\end{array}$

$\begin{array}{lll}-9.866565 & 1.701877 & 1.936777\end{array}$

$\begin{array}{lll}-10.274929 & 2.240223 & 2.787432\end{array}$

$\begin{array}{lll}-8.719909 & 0.958664 & 2.070063\end{array}$

$\begin{array}{llll}-8.183647 & 0.893037 & 3.010800\end{array}$

$\begin{array}{lll}-8.210442 & 0.259542 & 0.953273\end{array}$

$\begin{array}{lll}-3.901159 & 1.474834 & 1.798216\end{array}$

$\begin{array}{lll}-3.030917 & 1.776889 & 1.204227\end{array}$

$\begin{array}{lll}-4.035669 & 2.418411 & 2.984259\end{array}$

$\begin{array}{lll}-2.887116 & 3.004654 & 3.530630\end{array}$

$\begin{array}{lll}-1.925157 & 2.834814 & 3.058379\end{array}$

$\begin{array}{lll}-2.969857 & 3.802552 & 4.673939\end{array}$

$\begin{array}{llll}-2.068678 & 4.255333 & 5.078892\end{array}$

$\begin{array}{llll}-4.205014 & 4.023980 & 5.287828\end{array}$

$\begin{array}{llll}-4.271780 & 4.649418 & 6.173626\end{array}$

$\begin{array}{llll}-5.355571 & 3.436355 & 4.752834\end{array}$

$\begin{array}{llll}-6.321001 & 3.602665 & 5.223431\end{array}$

$\begin{array}{lll}-5.270680 & 2.635814 & 3.612664\end{array}$

$\begin{array}{lll}-6.166143 & 2.184265 & 3.194337\end{array}$

$\begin{array}{lll}-3.664854 & 0.055280 & 2.348232\end{array}$

$\begin{array}{lll}-4.336739 & -0.093389 & 3.201688\end{array}$

$\begin{array}{lll}-2.645128 & 0.010783 & 2.740255\end{array}$

$\begin{array}{lll}-5.417417 & 2.857846 & 0.356311\end{array}$

$\begin{array}{llll}-5.338270 & 3.517162 & 1.224321\end{array}$

$\begin{array}{llll}-4.402690 & 3.373576 & -0.662743\end{array}$

$\begin{array}{llll}-3.932527 & 4.687420 & -0.525658\end{array}$

$\begin{array}{lll}-4.244388 & 5.275857 & 0.334021\end{array}$

$\begin{array}{llll}-3.069496 & 5.246853 & -1.470307\end{array}$

$\begin{array}{llll}-2.713109 & 6.264821 & -1.340749\end{array}$

$\begin{array}{llll}-2.656400 & 4.493406 & -2.571224\end{array}$

$\begin{array}{llll}-1.985072 & 4.923379 & -3.309436\end{array}$

$\begin{array}{llll}-3.123988 & 3.184938 & -2.721298\end{array}$

$\begin{array}{llll}-2.821907 & 2.599395 & -3.584550\end{array}$

$\begin{array}{llll}-3.992396 & 2.629315 & -1.777518\end{array}$

$\begin{array}{llll}-4.369314 & 1.623910 & -1.919148\end{array}$

$\begin{array}{llll}-6.843202 & 3.053655 & -0.174444\end{array}$

$\begin{array}{lll}-7.583886 & 2.780054 & 0.577142\end{array}$

$\begin{array}{llll}-7.033273 & 2.491965 & -1.087741\end{array}$

$\begin{array}{llll}-6.967790 & 4.117546 & -0.398130\end{array}$

$-2.755634-0.843256-0.667956$

$\begin{array}{llll}-2.840742 & -1.515738 & -1.518320\end{array}$

$\begin{array}{llll}-2.885841 & 0.201107 & -0.936061\end{array}$

$\begin{array}{lll}-1.788942 & -1.116307 & 0.353746\end{array}$

$\begin{array}{llll}-1.448779 & -0.250349 & 0.911351\end{array}$

$\begin{array}{lll}-0.985791 & -2.296782 & 0.500758\end{array}$

$\begin{array}{lll}-1.344958 & -3.199652 & 0.030013\end{array}$

$\begin{array}{lll}-5.262764 & -2.528004 & 2.524103\end{array}$

$\begin{array}{lll}-5.685333 & -1.735112 & 3.136015\end{array}$

$\begin{array}{lll}-3.914886 & -2.880029 & 2.786021\end{array}$

$\begin{array}{lll}-3.410984 & -2.348868 & 3.593502\end{array}$

$\begin{array}{llll}-3.315218 & -4.240353 & 2.477239\end{array}$

$\begin{array}{lll}-3.639899 & -4.971324 & 3.233952\end{array}$

\begin{abstract}
$\begin{array}{lll}-2.232935 & -4.150061 & 2.583097\end{array}$
$\begin{array}{lll}-3.638167 & -4.770056 & 1.062978\end{array}$

$\begin{array}{lll}-4.519233 & -5.419234 & 1.090494\end{array}$

$\begin{array}{lll}-2.809900 & -5.401555 & 0.723792\end{array}$

$\begin{array}{llll}-3.868792 & -3.678147 & 0.019773\end{array}$

$-3.123988-3.614270 \quad-0.768792$

$\begin{array}{llll}-5.119131 & -3.145299 & -0.282437\end{array}$

$-5.246545 \quad-2.719350 \quad-1.272921$

$\begin{array}{lll}-6.411231 & -3.498924 & 0.428390\end{array}$

$\begin{array}{lll}-7.161128 & -2.762613 & 0.135671\end{array}$

$\begin{array}{lll}-6.766559 & -4.473767 & 0.062926\end{array}$

$\begin{array}{lll}-6.295456 & -3.511851 & 1.968420\end{array}$

$\begin{array}{lll}-6.054838 & -4.519904 & 2.322316\end{array}$

$\begin{array}{lll}-7.273890 & -3.266283 & 2.392041\end{array}$

$\begin{array}{lll}-0.188202 & -2.516683 & 1.714152\end{array}$

$\begin{array}{lll}0.220852 & -1.471957 & 2.565717\end{array}$

$\begin{array}{lll}0.214367 & -3.828388 & 2.035472\end{array}$

$\begin{array}{lll}0.987071 & -1.735693 & 3.699512\end{array}$

$\begin{array}{lll}-0.043973 & -0.444624 & 2.340402\end{array}$

$\begin{array}{lll}0.995558 & -4.089935 & 3.158072\end{array}$

$\begin{array}{lll}-0.087340 & -4.645087 & 1.386040\end{array}$

$\begin{array}{lll}1.384351 & -3.041966 & 3.997229\end{array}$

$\begin{array}{lll}1.287645 & -0.917963 & 4.343911\end{array}$

$\begin{array}{lll}1.301200 & -5.108684 & 3.377847\end{array}$

$\begin{array}{lll}1.993782 & -3.238780 & 4.874548\end{array}$

$\begin{array}{lll}-0.314673 & -0.807853 & -2.712395\end{array}$

$\begin{array}{llll}-0.725240 & 0.542841 & -3.076130\end{array}$

$\begin{array}{lll}-0.231616 & 0.869728 & -3.999901\end{array}$

$\begin{array}{lll}-1.806783 & 0.551213 & -3.24106\end{array}$

$\begin{array}{llll}-0.471942 & 1.235857 & -2.278557\end{array}$

$-0.867063-1.856158-3.563136$

$-1.888785-2.125738-3.266678$

$-0.900588-1.484476-4.591396$

$\begin{array}{lll}-0.246469 & -2.748210 & -3.563089\end{array}$

$\begin{array}{llll}3.420218 & -5.831527 & 0.057872\end{array}$

$\begin{array}{llll}5.254624 & -4.692806 & 2.205930\end{array}$
\end{abstract}

C2-P1 re-re 4

Number of imaginary frequencies $=1$

The smallest frequencies are $=-266.922714 .603016 .4956 \mathrm{~cm}(-1)$ Electronic energy $=-7222.6225041$

Zero-point correction=

(Hartree/Particle)

Thermal correction to Energy= $\quad 1.873789$

Thermal correction to Enthalpy= $\quad 1.874733$

Thermal correction to Gibbs Free Energy $=\quad 1.596155$

Sum of electronic and zero-point Energies $=\quad-7220.867200$

Sum of electronic and thermal Energies $=\quad-7220.748715$

Sum of electronic and thermal Enthalpies $=\quad-7220.747771$

Sum of electronic and thermal Free Energies $=\quad-7221.026349$

Cartesian Coordinates

$\begin{array}{llll}26 & 7.587737 & -2.030120 & -1.503578 \\ 15 & 2.982145 & 0.290134 & -2.301438 \\ 15 & 4.408503 & 0.363313 & 1.158495 \\ 6 & 5.910194 & -0.797811 & -1.737637 \\ 6 & 6.045487 & -1.241406 & -0.371125 \\ 6 & 5.984273 & -2.666595 & -0.366827 \\ 1 & 6.061588 & -3.298816 & 0.506460 \\ 6 & 5.847183 & -3.111998 & -1.714095 \\ 1 & 5.816015 & -4.141703 & -2.043405 \\ 6 & 5.805672 & -1.965233 & -2.558266 \\ 1 & 5.719254 & -1.966488 & -3.634902 \\ 6 & 9.411110 & -1.164151 & -1.007964 \\ 1 & 9.524467 & -0.257497 & -0.428184 \\ 6 & 9.332159 & -2.490786 & -0.483910 \\ 1 & 9.370071 & -2.765494 & 0.561692 \\ 6 & 9.150577 & -3.388404 & -1.579896 \\ 1 & 9.020587 & -4.460268 & -1.508050 \\ 6 & 9.116668 & -2.617626 & -2.781714 \\ 1 & 8.958973 & -3.002187 & -3.780748 \\ 6 & 9.278997 & -1.244724 & -2.429334 \\ 1 & 9.270492 & -0.411021 & -3.118186\end{array}$


$\begin{array}{llll}5.813259 & 0.628040 & -2.160974\end{array}$

$\begin{array}{llll}6.966849 & 1.425874 & -2.171613\end{array}$

$\begin{array}{llll}7.906619 & 0.967310 & -1.892626\end{array}$

$\begin{array}{llll}6.926067 & 2.776475 & -2.516030\end{array}$

$\begin{array}{llll}7.833657 & 3.370536 & -2.489856\end{array}$

$\begin{array}{llll}5.711640 & 3.356397 & -2.874325\end{array}$

$\begin{array}{llll}5.660840 & 4.406631 & -3.143498\end{array}$

$\begin{array}{llll}4.548595 & 2.585868 & -2.864101\end{array}$

$\begin{array}{llll}3.610231 & 3.056927 & -3.132745\end{array}$

$\begin{array}{llll}4.570425 & 1.231128 & -2.495048\end{array}$

$\begin{array}{lll}6.147653 & -0.315184 & 0.808645\end{array}$

$\begin{array}{lll}6.710589 & 0.554532 & 0.470750\end{array}$

$\begin{array}{lll}6.880430 & -0.900879 & 2.019068\end{array}$

$\begin{array}{llll}6.922259 & -0.181175 & 2.841372\end{array}$

$\begin{array}{lll}6.407743 & -1.812402 & 2.392121\end{array}$

$\begin{array}{lll}7.903848 & -1.152778 & 1.725778\end{array}$

$\begin{array}{llll}1.696044 & 1.506233 & -2.806632\end{array}$

$\begin{array}{llll}1.308245 & 2.452313 & -1.842264\end{array}$

$\begin{array}{llll}1.780838 & 2.450113 & -0.865178\end{array}$

$\begin{array}{llll}0.340765 & 3.411024 & -2.134609\end{array}$

$\begin{array}{llll}0.063706 & 4.138879 & -1.384071\end{array}$

$\begin{array}{llll}-0.272839 & 3.426692 & -3.389954\end{array}$

$\begin{array}{llll}-1.040171 & 4.163886 & -3.607896\end{array}$

$\begin{array}{llll}0.102141 & 2.488160 & -4.352854\end{array}$

$\begin{array}{llll}-0.369765 & 2.492138 & -5.331237\end{array}$

$\begin{array}{llll}1.084434 & 1.535572 & -4.067389\end{array}$

$\begin{array}{llll}1.358886 & 0.813545 & -4.827830\end{array}$

$\begin{array}{llll}3.024373 & -0.958034 & -3.641947\end{array}$

$\begin{array}{lll}3.483239 & -0.670641 & -4.936489\end{array}$

$\begin{array}{llll}3.835003 & 0.327737 & -5.180319\end{array}$

$\begin{array}{llll}3.500509 & -1.672787 & -5.908177\end{array}$

$3.855396-1.447454 \quad-6.909791$

$\begin{array}{llll}3.064349 & -2.964904 & -5.593817\end{array}$

$3.079468 \quad-3.741132 \quad-6.353684$

$\begin{array}{llll}2.621482 & -3.258475 & -4.301722\end{array}$

$\begin{array}{llll}2.294495 & -4.263382 & -4.049774\end{array}$

$\begin{array}{llll}2.609322 & -2.259187 & -3.327461\end{array}$

$\begin{array}{llll}2.323342 & -2.483730 & -2.305137\end{array}$

$\begin{array}{lll}4.586523 & 2.202559 & 1.171447\end{array}$

$\begin{array}{lll}3.406763 & 2.947169 & 1.308168\end{array}$

$\begin{array}{lll}2.486690 & 2.444570 & 1.568588\end{array}$

$\begin{array}{llll}3.392252 & 4.323874 & 1.083888\end{array}$

$\begin{array}{llll}4.559426 & 4.998675 & 0.735291\end{array}$

$\begin{array}{llll}4.544650 & 6.063574 & 0.531420\end{array}$

$\begin{array}{llll}5.743208 & 4.268481 & 0.633010\end{array}$

$\begin{array}{llll}5.765116 & 2.891021 & 0.849402\end{array}$

$\begin{array}{llll}6.707336 & 2.373713 & 0.735995\end{array}$

$3.984023 \quad-0.127869 \quad 2.874739$

$\begin{array}{lll}3.800150 & -1.506259 & 3.073924\end{array}$

$\begin{array}{llll}3.981371 & -2.198174 & 2.259017\end{array}$

$\begin{array}{llll}3.351382 & -1.985637 & 4.299730\end{array}$

$\begin{array}{llll}3.023056 & -1.105273 & 5.331860\end{array}$

$2.644004 \quad-1.478063 \quad 6.277408$

$\begin{array}{lll}3.175939 & 0.263913 & 5.120270\end{array}$

$\begin{array}{lll}3.668938 & 0.757962 & 3.909861\end{array}$

$\begin{array}{lll}3.786002 & 1.827315 & 3.780416\end{array}$

$2.671094-0.489416 \quad-0.185857$

$\begin{array}{llll}2.712818 & 1.206199 & 6.197233\end{array}$

$\begin{array}{lll}3.121335 & -3.457736 & 4.496390\end{array}$

$\begin{array}{lll}2.092172 & 5.070769 & 1.207198\end{array}$

$\begin{array}{lll}6.996213 & 5.003425 & 0.237303\end{array}$

$\begin{array}{llll}6.842756 & 5.633724 & -0.952664\end{array}$

$\begin{array}{lll}8.065468 & 4.186331 & 0.121469\end{array}$

$\begin{array}{lll}7.316338 & 5.959702 & 1.139242\end{array}$

$\begin{array}{lll}1.048641 & 4.307505 & 0.774004\end{array}$

$\begin{array}{lll}1.814905 & 5.412257 & 2.484499\end{array}$

$\begin{array}{llll}2.076139 & 6.203968 & 0.477696\end{array}$

$\begin{array}{lll}3.100815 & 2.479518 & 5.975162\end{array}$

$\begin{array}{lll}1.357473 & 1.211062 & 6.283723\end{array}$

$\begin{array}{llll}3.174111 & 0.845838 & 7.416237\end{array}$

$\begin{array}{llll}3.629667 & -3.895178 & 5.668971\end{array}$

$\begin{array}{lll}3.670476 & -4.201419 & 3.510756\end{array}$

$\begin{array}{lll}1.793668 & -3.755215 & 4.519455\end{array}$

$\begin{array}{llll}0.655877 & 0.743710 & 1.630039\end{array}$

$\begin{array}{lll}0.638721 & -1.572463 & 1.927870\end{array}$ $\begin{array}{llll}0.065667 & 0.958541 & 2.864419\end{array}$

$\begin{array}{llll}0.869166 & 1.568309 & 0.961855\end{array}$

$\begin{array}{lll}-0.020897 & -1.391874 & 3.172509\end{array}$

$\begin{array}{lll}-0.281009 & -0.129956 & 3.668636\end{array}$

$\begin{array}{lll}0.830053 & -2.923155 & 1.463339\end{array}$

$\begin{array}{llll}1.981571 & -3.354441 & 0.703627\end{array}$

$\begin{array}{llll}2.674443 & -2.568196 & -0.014418\end{array}$

$\begin{array}{lll}0.983157 & -0.482844 & 1.177981\end{array}$

$\begin{array}{llll}0.396854 & -3.657551 & 2.127216\end{array}$

$\begin{array}{llll}-3.249702 & -1.353953 & -1.191222\end{array}$

$\begin{array}{lll}-4.980383 & -0.111354 & -0.400278\end{array}$

$\begin{array}{lll}-4.786750 & 1.132620 & 0.694008\end{array}$

$\begin{array}{lll}-5.791872 & 0.691865 & -1.604590\end{array}$

$\begin{array}{lll}-6.025333 & -1.086679 & 0.486867\end{array}$

$\begin{array}{lll}-4.165387 & 2.307615 & 0.299515\end{array}$

$\begin{array}{lll}-2.885259 & 2.561738 & 0.839178\end{array}$

$\begin{array}{lll}-2.460850 & 1.840555 & 1.528158\end{array}$

$\begin{array}{lll}-2.218962 & 3.709108 & 0.488588\end{array}$

$\begin{array}{lll}-1.248093 & 3.929903 & 0.915264\end{array}$

$\begin{array}{llll}-2.775601 & 4.600843 & -0.467735\end{array}$

$\begin{array}{llll}-2.052354 & 5.743244 & -0.908393\end{array}$

$\begin{array}{llll}-1.083531 & 5.947966 & -0.459549\end{array}$

$\begin{array}{llll}2.558960 & 6.563232 & -1.891720\end{array}$

$\begin{array}{llll}-1.995281 & 7.428200 & -2.228564\end{array}$

$\begin{array}{llll}-3.815955 & 6.269698 & -2.473442\end{array}$

$\begin{array}{llll}-4.201616 & 6.903069 & -3.267098\end{array}$

$\begin{array}{llll}-4.557010 & 5.190135 & -2.042460\end{array}$

$\begin{array}{llll}-5.517664 & 4.981791 & -2.498886\end{array}$

$\begin{array}{llll}-4.074030 & 4.330988 & -1.016403\end{array}$

$\begin{array}{llll}-4.821494 & 3.193713 & -0.539746\end{array}$

$\begin{array}{llll}-6.247008 & 2.965437 & -0.908242\end{array}$

$\begin{array}{llll}-7.249032 & 3.982496 & -0.696563\end{array}$

$\begin{array}{lll}-6.982310 & 5.187734 & 0.012310\end{array}$

$\begin{array}{lll}-5.996154 & 5.353524 & 0.428541\end{array}$

$\begin{array}{lll}-7.962015 & 6.139720 & 0.195545\end{array}$

$\begin{array}{lll}-7.731517 & 7.045817 & 0.748646\end{array}$

$\begin{array}{llll}-9.265030 & 5.946545 & -0.321017\end{array}$

$\begin{array}{lll}-10.024430 & 6.709253 & -0.175741\end{array}$

$\begin{array}{llll}-9.566726 & 4.782288 & -0.990153\end{array}$

$\begin{array}{llll}-10.568243 & 4.607887 & -1.374807\end{array}$

$\begin{array}{llll}-8.584255 & 3.773004 & -1.180717\end{array}$

$\begin{array}{llll}-8.909765 & 2.551198 & -1.827940\end{array}$

$\begin{array}{llll}-9.914821 & 2.408384 & -2.214461\end{array}$

$\begin{array}{llll}-7.976183 & 1.551583 & -1.935139\end{array}$

$\begin{array}{llll}-8.207728 & 0.599236 & -2.400263\end{array}$

$\begin{array}{llll}-6.669172 & 1.753820 & -1.437371\end{array}$

$\begin{array}{llll}-5.503862 & -2.482518 & 0.608196\end{array}$

$\begin{array}{lll}-4.826803 & -2.526032 & 1.471196\end{array}$

$\begin{array}{lll}-6.594349 & -3.515506 & 0.822127\end{array}$

$\begin{array}{lll}-6.500817 & -4.410780 & 1.892276\end{array}$

$\begin{array}{lll}-5.669207 & -4.324837 & 2.588272\end{array}$

$\begin{array}{llll}-7.469792 & -5.400752 & 2.082399\end{array}$

$\begin{array}{lll}-7.386643 & -6.085136 & 2.922383\end{array}$

$\begin{array}{lll}-8.546678 & -5.499720 & 1.200720\end{array}$

$\begin{array}{lll}-9.308449 & -6.259650 & 1.350951\end{array}$

$\begin{array}{lll}-8.643363 & -4.611633 & 0.122885\end{array}$

$\begin{array}{llll}-9.484150 & -4.677443 & -0.561974\end{array}$

$\begin{array}{llll}-7.670748 & -3.633504 & -0.068620\end{array}$

$\begin{array}{llll}-7.762044 & -2.929414 & -0.889730\end{array}$

$\begin{array}{llll}-4.701377 & -2.822897 & -0.653531\end{array}$

$\begin{array}{llll}-5.400111 & -2.951205 & -1.488719\end{array}$

$\begin{array}{lll}-4.204870 & -3.785707 & -0.504651\end{array}$

$\begin{array}{lll}-6.838547 & -0.514542 & 1.613808\end{array}$

$\begin{array}{lll}-6.918846 & 0.551492 & 1.391314\end{array}$

$\begin{array}{lll}-8.271917 & -1.044484 & 1.628640\end{array}$

$\begin{array}{lll}-9.136787 & -0.659239 & 0.596078\end{array}$

$\begin{array}{llll}-8.769013 & 0.004679 & -0.177798\end{array}$

$\begin{array}{lll}-10.453131 & -1.114706 & 0.548968\end{array}$

$-11.103648 \quad-0.803250 \quad-0.264173$

$\begin{array}{lll}-10.934077 & -1.964616 & 1.549127\end{array}$

$\begin{array}{lll}-11.957839 & -2.326918 & 1.516299\end{array}$

$\begin{array}{lll}-10.088629 & -2.337583 & 2.595309\end{array}$

$\begin{array}{lll}-10.450863 & -2.995556 & 3.380435\end{array}$

$\begin{array}{lll}-8.770401 & -1.875485 & 2.638058\end{array}$

$\begin{array}{lll}-8.131015 & -2.190353 & 3.454715\end{array}$ 


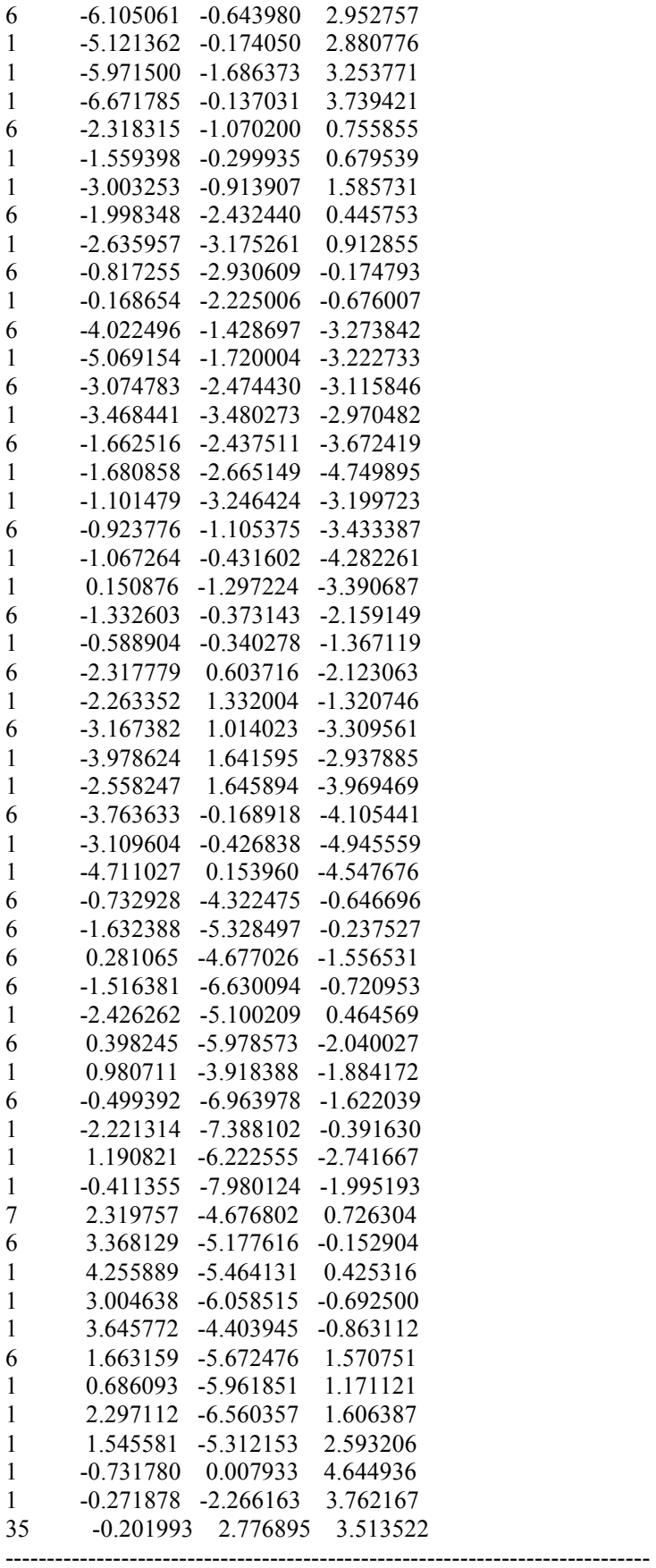

C2-P1 si-re_1

Number of imaginary frequencies $=1$

The smallest frequencies are $=-266.57828 .969412 .2158 \mathrm{~cm}(-1)$ Electronic energy $=-7222.6288434$

Zero-point correction=

1.755010

(Hartree/Particle)

Thermal correction to Energy= $\quad 1.873675$

Thermal correction to Enthalpy= $\quad 1.874619$

Thermal correction to Gibbs Free Energy $=\quad 1.591955$

Sum of electronic and zero-point Energies $=\quad-7220.873833$

Sum of electronic and thermal Energies $=\quad-7220.755168$

Sum of electronic and thermal Enthalpies $=\quad-7220.754224$

Sum of electronic and thermal Free Energies $=\quad-7221.036889$

Cartesian Coordinates

$\begin{array}{llll}26 & 8.173479 & 0.126622 & -1.046102 \\ 15 & 3.105130 & 1.081939 & -2.104116 \\ 15 & 4.181241 & 0.712969 & 1.497141\end{array}$

$6.191743 \quad 0.782250-1.260198$

$\begin{array}{lll}6.398708 & 0.147056 & 0.018896\end{array}$

$\begin{array}{llll}6.823828 & -1.195723 & -0.222994\end{array}$

$\begin{array}{llll}7.071930 & -1.927036 & 0.533746\end{array}$

$6.916169-1.387003-1.634717$

$7.249864-2.286256-2.131767$

$\begin{array}{lll}6.524832 & -0.173516 & -2.273013\end{array}$

$6.507144 \quad 0.009607 \quad-3.337064$

$\begin{array}{llll}9.591264 & 1.359005 & -0.158807\end{array}$

$\begin{array}{lll}9.381651 & 2.080819 & 0.619551\end{array}$

$\begin{array}{llll}9.930463 & -0.014732 & 0.040055\end{array}$

$\begin{array}{lll}10.014025 & -0.518305 & 0.993879\end{array}$

$10.095671-0.618956-1.243534$

$10.319878 \quad-1.660725 \quad-1.431581$

$\begin{array}{llll}9.858569 & 0.380250 & -2.235851\end{array}$

$\begin{array}{lll}9.875228 & 0.229649 & -3.307201\end{array}$

$\begin{array}{llll}9.549981 & 1.601765 & -1.566885\end{array}$

$9.296348 \quad 2.536986-2.047165$

$\begin{array}{llll}5.675318 & 2.167851 & -1.441739\end{array}$

$\begin{array}{llll}6.495671 & 3.249989 & -1.085088\end{array}$

$\begin{array}{llll}7.485757 & 3.033720 & -0.704963\end{array}$

$\begin{array}{llll}6.066322 & 4.571439 & -1.195369\end{array}$

$6.717849 \quad 5.382326-0.885284$

$\begin{array}{llll}4.795154 & 4.836642 & -1.698553\end{array}$

$\begin{array}{llll}4.443382 & 5.858151 & -1.800035\end{array}$

$\begin{array}{lll}3.956792 & 3.778016 & -2.046262\end{array}$

$\begin{array}{llll}2.964125 & 4.005815 & -2.414387\end{array}$

$4.359042 \quad 2.438885-1.900829$

$\begin{array}{llll}6.072221 & 0.783363 & 1.342356\end{array}$

$\begin{array}{lll}6.311817 & 1.844760 & 1.263598\end{array}$

$\begin{array}{lll}6.841574 & 0.204931 & 2.532790\end{array}$

$\begin{array}{llll}6.581689 & 0.727523 & 3.457658\end{array}$

$6.638014-0.858867 \quad 2.681092$

$\begin{array}{llll}7.915675 & 0.320898 & 2.357787\end{array}$

$\begin{array}{llll}1.582349 & 2.020357 & -2.538139\end{array}$

$\begin{array}{llll}0.833090 & 2.550727 & -1.476866\end{array}$

$\begin{array}{lll}1.108614 & 2.311522 & -0.456536\end{array}$

$\begin{array}{llll}-0.254064 & 3.386100 & -1.727871\end{array}$

$\begin{array}{llll}-0.813345 & 3.801012 & -0.899507\end{array}$

$\begin{array}{llll}-0.631651 & 3.668381 & -3.042823\end{array}$

$\begin{array}{llll}-1.486103 & 4.310838 & -3.235468\end{array}$

$\begin{array}{llll}0.083578 & 3.110371 & -4.105236\end{array}$

$\begin{array}{llll}-0.212270 & 3.315851 & -5.130111\end{array}$

$\begin{array}{llll}1.192997 & 2.297991 & -3.856712\end{array}$

$\begin{array}{llll}1.754873 & 1.892089 & -4.691224\end{array}$

$\begin{array}{llll}3.529597 & 0.216634 & -3.663587\end{array}$

$\begin{array}{llll}4.264158 & 0.812188 & -4.699472\end{array}$

$\begin{array}{llll}4.634804 & 1.827179 & -4.589085\end{array}$

$\begin{array}{llll}4.528760 & 0.097300 & -5.869547\end{array}$

$\begin{array}{llll}5.102528 & 0.561901 & -6.666359\end{array}$

$4.056094-1.210692-6.017512$

$\begin{array}{llll}4.266487 & -1.763964 & -6.928261\end{array}$

$\begin{array}{llll}3.313909 & -1.805027 & -4.993443\end{array}$

$\begin{array}{llll}2.943505 & -2.820380 & -5.103146\end{array}$

$\begin{array}{llll}3.055640 & -1.094244 & -3.820432\end{array}$

$\begin{array}{llll}2.493088 & -1.556040 & -3.015237\end{array}$

$\begin{array}{llll}3.600033 & 2.430466 & 1.817849\end{array}$

$\begin{array}{lll}2.209408 & 2.580771 & 1.918846\end{array}$

$\begin{array}{lll}1.578904 & 1.700044 & 1.855346\end{array}$

$\begin{array}{llll}1.639579 & 3.847114 & 2.048749\end{array}$

$\begin{array}{lll}2.436161 & 4.990382 & 2.048330\end{array}$

$\begin{array}{llll}1.991663 & 5.975992 & 2.128357\end{array}$

$\begin{array}{lll}3.818629 & 4.839929 & 1.926249\end{array}$

$\begin{array}{llll}4.403297 & 3.576947 & 1.825817\end{array}$

$\begin{array}{lll}5.478949 & 3.512512 & 1.734470\end{array}$

$\begin{array}{lll}3.812556 & -0.179726 & 3.063736\end{array}$

$\begin{array}{llll}3.969735 & -1.574882 & 3.033955\end{array}$

$\begin{array}{llll}4.342472 & -2.059214 & 2.138861\end{array}$

$3.622966-2.346443 \quad 4.139455$

$\begin{array}{lll}3.085032 & -1.754213 & 5.283477\end{array}$

$\begin{array}{llll}2.797592 & -2.359048 & 6.135636\end{array}$

$\begin{array}{lll}2.916973 & -0.372468 & 5.306322\end{array}$

$\begin{array}{lll}3.286058 & 0.416648 & 4.213403\end{array}$

$\begin{array}{llll}3.145515 & 1.489863 & 4.265852\end{array}$

$2.923102 \quad-0.209205 \quad-0.251598$ 
$\begin{array}{llll}2.240972 & 0.275285 & 6.482516\end{array}$

$3.715418-3.844998 \quad 4.073674$

$\begin{array}{lll}0.147429 & 3.947550 & 2.206310\end{array}$

$\begin{array}{llll}4.667083 & 6.081259 & 1.860284\end{array}$

$\begin{array}{llll}4.390252 & 6.809289 & 0.750577\end{array}$

$\begin{array}{llll}5.989773 & 5.806473 & 1.834923\end{array}$

$\begin{array}{llll}4.443569 & 6.894973 & 2.918274\end{array}$

$\begin{array}{llll}-0.503238 & 3.100586 & 1.356959\end{array}$

$\begin{array}{lll}-0.255872 & 3.605936 & 3.451877\end{array}$

$\begin{array}{lll}-0.321638 & 5.187488 & 1.954720\end{array}$

$\begin{array}{lll}2.692111 & 1.528378 & 6.704283\end{array}$

$\begin{array}{llll}0.897943 & 0.378418 & 6.276718\end{array}$

$\begin{array}{llll}2.403932 & -0.428076 & 7.622065\end{array}$

$\begin{array}{llll}4.121452 & -4.378816 & 5.245827\end{array}$

$\begin{array}{lll}4.565315 & -4.267963 & 3.114631\end{array}$

$\begin{array}{llll}2.502229 & -4.400354 & 3.798675\end{array}$

$\begin{array}{llll}0.812662 & -1.236523 & 1.705658\end{array}$

$\begin{array}{lll}1.067586 & -2.609704 & 1.324877\end{array}$

$\begin{array}{llll}2.200684 & -3.093095 & 0.588749\end{array}$

$\begin{array}{llll}0.622624 & -3.369309 & 1.951598\end{array}$

$\begin{array}{llll}-3.134078 & -1.268589 & -1.268067\end{array}$

$\begin{array}{lll}-4.959371 & -0.293010 & -0.325044\end{array}$

$\begin{array}{lll}-4.920781 & 1.024690 & 0.684221\end{array}$

$\begin{array}{llll}-5.992180 & 0.261247 & -1.505768\end{array}$

$\begin{array}{lll}-5.710316 & -1.383800 & 0.706052\end{array}$

$\begin{array}{lll}-4.764172 & 2.316091 & 0.199809\end{array}$

$\begin{array}{lll}-3.571888 & 2.977413 & 0.567449\end{array}$

$\begin{array}{llll}-2.862557 & 2.458604 & 1.201564\end{array}$

$\begin{array}{lll}-3.341709 & 4.255875 & 0.125525\end{array}$

$\begin{array}{lll}-2.445102 & 4.786152 & 0.430562\end{array}$

$\begin{array}{llll}-4.258447 & 4.886110 & -0.758370\end{array}$

$\begin{array}{llll}-3.974767 & 6.160767 & -1.319543\end{array}$

$\begin{array}{lll}-3.051818 & 6.658379 & -1.033060\end{array}$

$\begin{array}{llll}-4.838385 & 6.745674 & -2.218264\end{array}$

$\begin{array}{lll}-4.608338 & 7.716065 & -2.648252\end{array}$

$\begin{array}{llll}-6.024637 & 6.071310 & -2.593842\end{array}$

$\begin{array}{llll}-6.693005 & 6.523811 & -3.320723\end{array}$

$\begin{array}{llll}-6.338925 & 4.843540 & -2.052018\end{array}$

$\begin{array}{llll}-7.247207 & 4.339013 & -2.359781\end{array}$

$\begin{array}{llll}-5.478579 & 4.214901 & -1.109115\end{array}$

$\begin{array}{lll}-5.770684 & 2.925427 & -0.534683\end{array}$

$\begin{array}{llll}-7.088322 & 2.254637 & -0.725316\end{array}$

$\begin{array}{llll}-8.330292 & 2.898471 & -0.376846\end{array}$

$\begin{array}{lll}-8.385579 & 4.145329 & 0.307508\end{array}$

$\begin{array}{llll}-7.462989 & 4.640091 & 0.586600\end{array}$

$\begin{array}{lll}-9.591982 & 4.724153 & 0.637321\end{array}$

$\begin{array}{lll}-9.604783 & 5.671259 & 1.169053\end{array}$

$\begin{array}{lll}-10.813490 & 4.093808 & 0.300569\end{array}$

$\begin{array}{llll}-11.756209 & 4.564609 & 0.563419\end{array}$

$\begin{array}{llll}-10.798929 & 2.878735 & -0.346126\end{array}$

$\begin{array}{lll}-11.727882 & 2.373270 & -0.597142\end{array}$

$\begin{array}{llll}-9.572087 & 2.246890 & -0.688366\end{array}$

$\begin{array}{llll}-9.558758 & 0.964294 & -1.298944\end{array}$

$\begin{array}{llll}-10.502809 & 0.482222 & -1.535432\end{array}$

$\begin{array}{llll}-8.371860 & 0.318987 & -1.536332\end{array}$

$\begin{array}{lll}-8.335960 & -0.680577 & -1.955552\end{array}$

$\begin{array}{llll}-7.155538 & 0.962548 & -1.223769\end{array}$

$\begin{array}{lll}-5.228741 & -2.777456 & 0.508417\end{array}$

$\begin{array}{lll}-4.540905 & -3.016814 & 1.326605\end{array}$

$\begin{array}{llll}-6.358851 & -3.790670 & 0.520366\end{array}$

$\begin{array}{lll}-6.239949 & -4.969182 & 1.263888\end{array}$

$\begin{array}{llll}-5.350204 & -5.133268 & 1.868198\end{array}$

$\begin{array}{llll}-7.260142 & -5.925468 & 1.249219\end{array}$

$\begin{array}{lll}-7.159140 & -6.833090 & 1.838129\end{array}$

$\begin{array}{lll}-8.409013 & -5.709745 & 0.485713\end{array}$

$\begin{array}{lll}-9.206908 & -6.447045 & 0.479244\end{array}$

$\begin{array}{llll}-8.529596 & -4.536698 & -0.267888\end{array}$

$\begin{array}{llll}-9.425153 & -4.357949 & -0.856626\end{array}$

$\begin{array}{llll}-7.510719 & -3.586831 & -0.251857\end{array}$

$\begin{array}{llll}-7.617203 & -2.660309 & -0.808483\end{array}$

$\begin{array}{llll}-4.453598 & -2.897375 & -0.817897\end{array}$

$\begin{array}{llll}-5.173833 & -2.997943 & -1.637835\end{array}$

$\begin{array}{llll}-3.880450 & -3.828576 & -0.789866\end{array}$

$\begin{array}{lll}-6.310065 & -1.000710 & 2.031657\end{array}$

$\begin{array}{lll}-6.197399 & 0.079875 & 2.087405\end{array}$ $\begin{array}{lll}-7.810641 & -1.260609 & 2.148750\end{array}$

$\begin{array}{lll}-8.693384 & -0.238845 & 1.776531\end{array}$

$\begin{array}{lll}-8.292940 & 0.696244 & 1.397975\end{array}$

$\begin{array}{lll}-10.075679 & -0.401347 & 1.885751\end{array}$

$\begin{array}{lll}-10.735336 & 0.408308 & 1.586469\end{array}$

$\begin{array}{lll}-10.599671 & -1.596441 & 2.382140\end{array}$

$\begin{array}{lll}-11.674585 & -1.730054 & 2.469017\end{array}$

$\begin{array}{lll}-9.729678 & -2.617365 & 2.774825\end{array}$

$\begin{array}{lll}-10.124686 & -3.551545 & 3.164590\end{array}$

$\begin{array}{lll}-8.348789 & -2.447278 & 2.665610\end{array}$

$\begin{array}{lll}-7.694955 & -3.257511 & 2.966230\end{array}$

$\begin{array}{llll}-5.505225 & -1.583609 & 3.199836\end{array}$

$\begin{array}{lll}-4.451718 & -1.297092 & 3.116680\end{array}$

$\begin{array}{llll}-5.561408 & -2.673272 & 3.259287\end{array}$

$\begin{array}{lll}-5.897007 & -1.179904 & 4.138071\end{array}$

$\begin{array}{lll}-2.203071 & -1.009234 & 0.680503\end{array}$

$\begin{array}{llll}-1.489875 & -0.192447 & 0.633349\end{array}$

$\begin{array}{lll}-2.875870 & -0.949863 & 1.532407\end{array}$

$\begin{array}{lll}-1.775661 & -2.317946 & 0.285333\end{array}$

$\begin{array}{lll}-2.329427 & -3.159498 & 0.688435\end{array}$

$\begin{array}{llll}-0.551485 & -2.617812 & -0.369162\end{array}$

$\begin{array}{llll}0.017503 & -1.792389 & -0.775284\end{array}$

$\begin{array}{llll}-3.987196 & -1.235922 & -3.320249\end{array}$

$\begin{array}{llll}-5.005765 & -1.607438 & -3.246520\end{array}$

$\begin{array}{llll}-2.951259 & -2.203768 & -3.294128\end{array}$

$-3.246098 \quad-3.250407 \quad-3.224828$

$\begin{array}{llll}-1.578505 & -1.985461 & -3.906596\end{array}$

$\begin{array}{llll}-1.644032 & -2.076406 & -5.002184\end{array}$

$\begin{array}{llll}-0.938357 & -2.804121 & -3.572350\end{array}$

$\begin{array}{lll}-0.917243 & -0.639824 & -3.531694\end{array}$

$\begin{array}{lll}-1.097714 & 0.108859 & -4.309373\end{array}$

$\begin{array}{lll}0.168427 & -0.774548 & -3.500559\end{array}$

$\begin{array}{lll}-1.364443 & -0.062442 & -2.189772\end{array}$

$\begin{array}{llll}-0.592999 & 0.001359 & -1.427943\end{array}$

$\begin{array}{llll}-2.435762 & 0.812254 & -2.034454\end{array}$

$\begin{array}{llll}-2.405596 & 1.469078 & -1.170925\end{array}$

$\begin{array}{llll}-3.372899 & 1.256783 & -3.139337\end{array}$

$\begin{array}{llll}-4.238971 & 1.728791 & -2.671708\end{array}$

$\begin{array}{llll}-2.873072 & 2.031798 & -3.736695\end{array}$

$\begin{array}{llll}-3.856397 & 0.105530 & -4.045147\end{array}$

$\begin{array}{llll}-3.180312 & -0.014758 & -4.897853\end{array}$

$\begin{array}{llll}-4.831253 & 0.373730 & -4.463694\end{array}$

$\begin{array}{llll}-0.278320 & -3.925986 & -0.974505\end{array}$

$\begin{array}{llll}-0.905256 & -5.115139 & -0.555109\end{array}$

$\begin{array}{llll}0.664891 & -4.004078 & -2.016361\end{array}$

$-0.609577 \quad-6.329599-1.171741$

$\begin{array}{llll}-1.621408 & -5.095133 & 0.260248\end{array}$

$\begin{array}{llll}0.956520 & -5.216988 & -2.637135\end{array}$

$\begin{array}{llll}1.170936 & -3.098660 & -2.333693\end{array}$

$\begin{array}{llll}0.318184 & -6.386550 & -2.217668\end{array}$

$\begin{array}{lll}-1.103622 & -7.236460 & -0.834639\end{array}$

$\begin{array}{llll}1.684749 & -5.249722 & -3.442456\end{array}$

$\begin{array}{llll}0.543660 & -7.335043 & -2.696382\end{array}$

$\begin{array}{llll}2.496027 & -4.482333 & 0.664891\end{array}$

$\begin{array}{llll}3.497859 & -5.043931 & -0.097873\end{array}$

$\begin{array}{lll}1.917235 & -5.105073 & 1.336152\end{array}$

$\begin{array}{llll}4.217018 & -4.196946 & -0.946439\end{array}$

$\begin{array}{llll}3.930382 & -2.845671 & -0.977806\end{array}$

$\begin{array}{llll}4.478808 & -2.168269 & -1.618111\end{array}$

$\begin{array}{llll}2.952163 & -2.295276 & -0.228367\end{array}$

$\begin{array}{lll}1.111520 & -0.245169 & 0.967572\end{array}$

$\begin{array}{llll}0.126791 & -1.006379 & 2.866197\end{array}$

$\begin{array}{lll}-0.375934 & 0.327560 & 3.176243\end{array}$

$\begin{array}{lll}-1.375529 & 0.242963 & 3.614864\end{array}$

$\begin{array}{lll}0.272218 & 0.838046 & 3.895856\end{array}$

$\begin{array}{lll}-0.441983 & 0.922338 & 2.270895\end{array}$

$\begin{array}{lll}-0.143418 & -2.019043 & 3.884079\end{array}$

$\begin{array}{lll}-1.118971 & -2.498385 & 3.728890\end{array}$

$\begin{array}{lll}0.632763 & -2.782448 & 3.903088\end{array}$

$\begin{array}{lll}-0.148687 & -1.530994 & 4.861539\end{array}$

$3.710091-6.105990-0.043765$

$\begin{array}{llll}5.604807 & -4.925348 & -2.118787\end{array}$

C2-P1 si-re 2 
Number of imaginary frequencies $=1$

The smallest frequencies are $=-267.94207 .645911 .1107 \mathrm{~cm}(-1)$ Electronic energy $=-7222.6289103$

Zero-point correction=

(Hartree/Particle)

Thermal correction to Energy=

Thermal correction to Enthalpy=

1.754635

Thermal correction to Gibbs Free Energy

1.873394

1.874339

1.590922

Sum of electronic and thermal Energies=

$-7220.874276$

$-7220.755516$

Sum of electronic and thermal Enthalpies=

$-7220.754572$

Sum of electronic and thermal Free Energies=

$-7221.037988$

\begin{tabular}{|c|c|c|c|}
\hline 26 & 8.164596 & 0.057089 & -1.06502 \\
\hline ; & 3.103223 & 1.071702 & -2.100878 \\
\hline 15 & 4.188561 & 0.684018 & 1.495436 \\
\hline 6 & 6.189305 & 0.736313 & -1.267302 \\
\hline 6 & 6.395395 & 0.096392 & 0.009540 \\
\hline 6 & 6.803807 & -1.250745 & -0.237072 \\
\hline 1 & 7.046516 & -1.986556 & 0.517061 \\
\hline 6 & 6.886832 & -1.440358 & -1.649576 \\
\hline 1 & 7.207218 & -2.342574 & -2.149999 \\
\hline 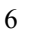 & 6.506061 & -0.221269 & -2.283599 \\
\hline 1 & 6.484705 & -0.036040 & -3.347252 \\
\hline 0 & 9.603761 & 1.270656 & -0.185019 \\
\hline 1 & 9.409197 & 1.994515 & 0.595439 \\
\hline 6 & 9.926895 & -0.107543 & 0.009688 \\
\hline 1 & 10.010555 & -0.613585 & 0.962210 \\
\hline 6 & 10.076116 & -0.711796 & -1.275836 \\
\hline 1 & 10.286363 & -1.755911 & -1.466947 \\
\hline 6 & 9.844939 & 0.291794 & -2.265142 \\
\hline 1 & 9.852769 & 0.142583 & -3.336788 \\
\hline 6 & 9.556107 & 1.516021 & -1.592401 \\
\hline 1 & 9.310821 & 2.455078 & -2.069532 \\
\hline 6 & 5.687507 & 2.127693 & -1.444852 \\
\hline 6 & 6.521001 & 3.200066 & -1.089314 \\
\hline 1 & 7.509152 & 2.971749 & -0.711222 \\
\hline 6 & 6.106821 & 4.52 & -1.1 \\
\hline 1 & 6.768038 & 5.329658 & -0.889273 \\
\hline 6 & 4.838139 & 4.80 & -1.700158 \\
\hline 1 & 4.498315 & 5.83 & -1.801671 \\
\hline 6 & 3.986838 & 3.757615 & -2.046240 \\
\hline 1 & & 3.996 & -2.413012 \\
\hline 6 & 4.373531 & 2.41 & -1.90 \\
\hline 6 & 6.080397 & 0.732386 & 1.335960 \\
\hline 1 & 6.333263 & 1.790951 & 1.260462 \\
\hline 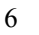 & 6.845569 & 0.140065 & 2.522356 \\
\hline 1 & 6.595070 & 0.662979 & 3.449632 \\
\hline 1 & 6.628949 & -0.921550 & 2.667793 \\
\hline 1 & 7.920578 & 0.242877 & 2.344635 \\
\hline 6 & 1.588280 & 2.028895 & -2.520715 \\
\hline 6 & 0.854220 & 2.56 & -1.451744 \\
\hline 1 & 1.135384 & 2.319370 & -0.434517 \\
\hline 6 & -0.224819 & 3.414335 & -1.691568 \\
\hline 1 & -0.772237 & 3.834032 & -0.857681 \\
\hline 6 & -0.609920 & 3.704560 & -3.002651 \\
\hline 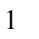 & -1.458390 & 4.357516 & -3.186291 \\
\hline 6 & 0.089280 & 3.140339 & -4.072384 \\
\hline 1 & -0.212805 & 3.351720 & -5.094232 \\
\hline 6 & 1.191005 & 2.314099 & -3.835222 \\
\hline 2 & 1.741030 & 1.903483 & -4.675338 \\
\hline 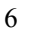 & 3.509439 & 0.207130 & -3.665490 \\
\hline 6 & 4.242777 & 0.799321 & -4.704202 \\
\hline 1 & 4.622138 & 1.811052 & -4.593463 \\
\hline 6 & 4.494598 & 0.085240 & -5.877593 \\
\hline 1 & 5.067364 & 0.547162 & -6.676677 \\
\hline 6 & 4.010517 & -1.218543 & -6.025919 \\
\hline 1 & 4.210982 & -1.771163 & -6.939296 \\
\hline 6 & 3.269900 & -1.809595 & -4.998815 \\
\hline 1 & 2.890790 & -2.821728 & -5.108692 \\
\hline 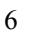 & 3.024478 & -1.099682 & -3.822531 \\
\hline & 2.463354 & -1.558946 & -3.014907 \\
\hline & 3.628086 & 2.407097 & 1.823019 \\
\hline
\end{tabular}


$\begin{array}{lll}-5.288916 & -2.680451 & 0.489108\end{array}$

$\begin{array}{lll}-4.625090 & -2.904031 & 1.333007\end{array}$

$\begin{array}{lll}-6.406104 & -3.709375 & 0.471441\end{array}$

$\begin{array}{lll}-6.247398 & -4.922542 & 1.148375\end{array}$

$\begin{array}{lll}-5.344342 & -5.096541 & 1.729974\end{array}$

$\begin{array}{llll}-7.244412 & -5.900959 & 1.099385\end{array}$

$\begin{array}{llll}-7.111238 & -6.835906 & 1.637062\end{array}$

$\begin{array}{lll}-8.413510 & -5.671041 & 0.372179\end{array}$

$\begin{array}{llll}-9.196407 & -6.423720 & 0.343283\end{array}$

$\begin{array}{lll}-8.573788 & -4.462925 & -0.316036\end{array}$

$\begin{array}{llll}-9.484958 & -4.272903 & -0.876635\end{array}$

$\begin{array}{llll}-7.573476 & -3.494269 & -0.273745\end{array}$

$\begin{array}{lll}-7.708096 & -2.544006 & -0.781655\end{array}$

$\begin{array}{llll}-4.481756 & -2.823316 & -0.818293\end{array}$

$\begin{array}{llll}-5.189365 & -2.934201 & -1.648198\end{array}$

$\begin{array}{llll}-3.915443 & -3.757449 & -0.765796\end{array}$

$\begin{array}{lll}-6.412893 & -0.852560 & 1.931022\end{array}$

$\begin{array}{lll}-6.572347 & 0.218491 & 1.817066\end{array}$

$\begin{array}{llll}-7.794571 & -1.453105 & 2.161082\end{array}$

$\begin{array}{lll}-8.903723 & -0.808695 & 1.599969\end{array}$

$\begin{array}{lll}-8.756435 & 0.105708 & 1.036563\end{array}$

$\begin{array}{lll}-10.190481 & -1.326272 & 1.751424\end{array}$

$\begin{array}{lll}-11.034769 & -0.811252 & 1.300764\end{array}$

$\begin{array}{lll}-10.388473 & -2.497416 & 2.486633\end{array}$

$\begin{array}{lll}-11.387256 & -2.908512 & 2.604857\end{array}$

$\begin{array}{lll}-9.293101 & -3.130427 & 3.079885\end{array}$

$\begin{array}{lll}-9.436728 & -4.038024 & 3.659469\end{array}$

$\begin{array}{lll}-8.008477 & -2.606604 & 2.925292\end{array}$

$\begin{array}{lll}-7.170541 & -3.119467 & 3.384134\end{array}$

$\begin{array}{lll}-5.453105 & -1.040678 & 3.111932\end{array}$

$\begin{array}{lll}-4.539489 & -0.464576 & 2.943454\end{array}$

$\begin{array}{llll}-5.178114 & -2.086289 & 3.273283\end{array}$

$\begin{array}{lll}-5.924080 & -0.676436 & 4.030004\end{array}$

$\begin{array}{lll}-2.211414 & -0.948938 & 0.664811\end{array}$

$\begin{array}{lll}-1.486620 & -0.142668 & 0.610105\end{array}$

$\begin{array}{lll}-2.878030 & -0.874641 & 1.519539\end{array}$

$\begin{array}{llll}-1.803609 & -2.266116 & 0.277670\end{array}$

$\begin{array}{llll}-2.370587 & -3.095407 & 0.687690\end{array}$

$\begin{array}{llll}-0.583987 & -2.588567 & -0.374959\end{array}$

$\begin{array}{llll}-0.002725 & -1.773293 & -0.784453\end{array}$

$\begin{array}{lll}-4.003453 & -1.173954 & -3.334358\end{array}$

$\begin{array}{llll}-5.026124 & -1.534087 & -3.259187\end{array}$

$\begin{array}{llll}-2.980285 & -2.154330 & -3.305886\end{array}$

$\begin{array}{llll}-3.288398 & -3.196696 & -3.231257\end{array}$

$\begin{array}{llll}-1.603541 & -1.956016 & -3.915466\end{array}$

$\begin{array}{llll}-1.666374 & -2.055741 & -5.010459\end{array}$

$\begin{array}{llll}-0.973587 & -2.778745 & -3.572015\end{array}$

$\begin{array}{lll}-0.928697 & -0.614830 & -3.550096\end{array}$

$\begin{array}{llll}-1.106033 & 0.131585 & -4.330637\end{array}$

$\begin{array}{lll}0.155741 & -0.759368 & -3.523330\end{array}$

$\begin{array}{llll}-1.364605 & -0.027155 & -2.208957\end{array}$

$\begin{array}{llll}-0.591504 & 0.027126 & -1.448263\end{array}$

$\begin{array}{llll}-2.424463 & 0.861284 & -2.055641\end{array}$

$\begin{array}{llll}-2.387954 & 1.519497 & -1.193345\end{array}$

$\begin{array}{llll}-3.353845 & 1.313721 & -3.163783\end{array}$

$\begin{array}{llll}-4.210627 & 1.806076 & -2.700019\end{array}$

$\begin{array}{llll}-2.839309 & 2.074082 & -3.767379\end{array}$

$\begin{array}{llll}-3.858290 & 0.164698 & -4.062084\end{array}$

$\begin{array}{llll}-3.188817 & 0.032754 & -4.918270\end{array}$

$\begin{array}{llll}-4.831893 & 0.444553 & -4.475688\end{array}$

$\begin{array}{lll}-0.332517 & -3.902762 & -0.976821\end{array}$

$\begin{array}{llll}-0.980602 & -5.079626 & -0.555033\end{array}$

$\begin{array}{llll}0.609420 & -3.999850 & -2.018281\end{array}$

$\begin{array}{llll}-0.707810 & -6.300157 & -1.170191\end{array}$

$\begin{array}{llll}-1.695800 & -5.045136 & 0.260676\end{array}$

$\begin{array}{llll}0.878459 & -5.218860 & -2.637381\end{array}$

$\begin{array}{llll}1.132501 & -3.104412 & -2.336690\end{array}$

$\begin{array}{llll}0.218197 & -6.375663 & -2.216559\end{array}$

$\begin{array}{llll}-1.218409 & -7.197302 & -0.831740\end{array}$

$\begin{array}{llll}1.606178 & -5.266137 & -3.442493\end{array}$

$\begin{array}{llll}0.425720 & -7.328792 & -2.694197\end{array}$

$\begin{array}{llll}2.439817 & -4.491969 & 0.654367\end{array}$

$\begin{array}{llll}3.434545 & -5.063464 & -0.110365\end{array}$

$\begin{array}{lll}1.853635 & -5.109713 & 1.323878\end{array}$

$\begin{array}{lll}4.162617 & -4.222916 & -0.957633\end{array}$ $\begin{array}{llll}3.891941 & -2.868253 & -0.985277\end{array}$

$\begin{array}{llll}4.447199 & -2.195988 & -1.625042\end{array}$

$\begin{array}{llll}2.921393 & -2.308018 & -0.233172\end{array}$

$\begin{array}{lll}1.106818 & -0.239302 & 0.971804\end{array}$

$\begin{array}{lll}0.109987 & -0.996879 & 2.865584\end{array}$

$\begin{array}{lll}-0.376885 & 0.341754 & 3.180696\end{array}$

$\begin{array}{lll}-1.377492 & 0.267545 & 3.618895\end{array}$

$\begin{array}{lll}0.277053 & 0.842137 & 3.902199\end{array}$

$\begin{array}{lll}-0.435768 & 0.940081 & 2.277270\end{array}$

$\begin{array}{lll}-0.169926 & -2.010091 & 3.880438\end{array}$

$\begin{array}{lll}-1.149738 & -2.480066 & 3.723768\end{array}$

$\begin{array}{lll}0.599238 & -2.780685 & 3.897675\end{array}$

$\begin{array}{lll}0.171017 & -1.524723 & 4.859270\end{array}$

$\begin{array}{llll}3.634336 & -6.128071 & -0.059138\end{array}$

$\begin{array}{llll}35 & 5.539805 & -4.964628 & -2.134047\end{array}$

C2-P1 si-re_3

Number of imaginary frequencies $=1$

The smallest frequencies are $=-151.489110 .414212 .5611 \mathrm{~cm}(-1)$ Electronic energy $=-7222.6271114$

Zero-point correction=

(Hartree/Particle)

Thermal correction to Energy=

Thermal correction to Enthalpy=

1.873703

Thermal correction to Gibbs Free Energy=
Sum of electronic and zero-point Energies=

Sum of electronic and thermal Energies=

Sum of electronic and thermal Enthalpies=

Sum of electronic and thermal Free Energies=

874647

1.595076

$-7220.871636$

$-7220.753408$

$-7220.752464$

Cartesian Coordinates

$\begin{array}{llcc}26 & -4.703316 & 4.864637 & 1.510872 \\ 15 & -1.968104 & 1.590501 & -1.525797 \\ 15 & -4.729643 & 0.191844 & 0.600289 \\ 6 & -3.935303 & 3.477491 & 0.144183 \\ 6 & -4.502374 & 2.819748 & 1.298288 \\ 6 & -3.793209 & 3.270937 & 2.453908 \\ 1 & -3.991948 & 2.975056 & 3.474842 \\ 6 & -2.816633 & 4.223855 & 2.033908 \\ 1 & -2.151718 & 4.779338 & 2.680581 \\ 6 & -2.907912 & 4.356301 & 0.617848 \\ 1 & -2.318943 & 5.021697 & 0.006026 \\ 6 & -6.697907 & 5.430963 & 1.378593 \\ 1 & -7.497552 & 4.792330 & 1.027115 \\ 6 & -6.241528 & 5.537042 & 2.727980 \\ 1 & -6.629706 & 4.988742 & 3.575910 \\ 6 & -5.152271 & 6.460379 & 2.754751 \\ 1 & -4.567214 & 6.727332 & 3.625046 \\ 6 & -4.936020 & 6.927212 & 1.422389 \\ 1 & -4.161066 & 7.612525 & 1.105284 \\ 6 & -5.890831 & 6.293266 & 0.572913 \\ 1 & -5.964697 & 6.420128 & -0.498729 \\ 6 & -4.336940 & 3.173893 & -1.261579 \\ 6 & -5.591825 & 3.600843 & -1.722503 \\ 1 & -6.206097 & 4.193377 & -1.056625 \\ 6 & -6.069345 & 3.261593 & -2.988096 \\ 1 & -7.058917 & 3.581746 & -3.298624 \\ 6 & -5.279701 & 2.487920 & -3.834924 \\ 1 & -5.641183 & 2.203732 & -4.818011 \\ 6 & -4.026171 & 2.054113 & -3.403365 \\ 1 & -3.427372 & 1.442011 & -4.068281 \\ 6 & -3.541975 & 2.370220 & -2.124247 \\ 6 & -5.575977 & 1.768811 & 1.236742 \\ 1 & -6.274682 & 2.062380 & 0.451861 \\ 6 & -6.361406 & 1.600409 & 2.539857 \\ 1 & -7.156872 & 0.858761 & 2.425783 \\ 1 & -5.723779 & 1.283256 & 3.369438 \\ 1 & -6.814218 & 2.558527 & 2.812495 \\ 6 & -1.303626 & 0.720716 & -3.007530 \\ 6 & -1.575619 & -0.651670 & -3.130450 \\ 1 & -2.129089 & -1.166211 & -2.351844 \\ 6 & -1.120971 & -1.361376 & -4.244500 \\ 1 & -1.356819 & -2.415664 & -4.339946\end{array}$




\begin{tabular}{|c|c|c|c|c|c|c|}
\hline-0.363396 & -0.719420 & -5.227307 & 6 & 8.911891 & -0.787361 & 1.583295 \\
\hline 0.001689 & -1.277548 & -6.084841 & 6 & 7.791170 & -0.137280 & 0.949531 \\
\hline-0.078895 & 0.642960 & -5.104481 & 6 & 7.955982 & 0.683040 & -0.282071 \\
\hline 0.517662 & $1.150089-$ & -5.857220 & 6 & 8.874174 & 1.791179 & -0.342303 \\
\hline-0.559281 & 1.362149 & -4.009563 & 6 & 9.529738 & 2.303463 & 0.812669 \\
\hline-0.339163 & 2.419514 & -3.938761 & 1 & 9.331908 & 1.851779 & 1.777636 \\
\hline-0.848529 & 3.033208 & -1.303808 & 6 & 10.391547 & 3.375129 & 0.723709 \\
\hline-0.950400 & 4.179677 & -2.109555 & 1 & 10.870609 & 3.755131 & 1.621500 \\
\hline-1.682088 & 4.218041 & -2.910598 & 6 & 10.653940 & 3.990153 & -0.524059 \\
\hline-0.136735 & 5.287275 & -1.866941 & 1 & 11.341773 & 4.828827 & -0.579512 \\
\hline-0.223194 & 6.168231 & -2.496321 & 6 & 10.022021 & 3.533366 & -1.658432 \\
\hline 0.777947 & $5.265986-$ & -0.808986 & 1 & 10.197374 & 4.009688 & -2.619564 \\
\hline 1.401651 & 6.132510 & -0.610350 & 6 & 9.110352 & 2.444071 & -1.599026 \\
\hline 0.889670 & 4.127395 & -0.007621 & 6 & 8.404534 & 2.012714 & -2.754882 \\
\hline 1.600082 & 4.107201 & 0.812216 & 1 & 8.600041 & 2.500282 & -3.705783 \\
\hline 0.087312 & $3.011289-$ & -0.260346 & 6 & 7.448075 & 1.032974 & -2.660622 \\
\hline 0.173955 & 2.127107 & 0.364180 & 1 & 6.852022 & 0.732276 & -3.514389 \\
\hline-5.656583 & -0.327273 & -0.904822 & 6 & 7.210449 & 0.405772 & -1.417843 \\
\hline-5.073225 & -1.370307 & -1.639001 & 6 & 2.879476 & 0.918995 & -1.907172 \\
\hline-4.163158 & -1.830312 & -1.267536 & 1 & 2.000895 & 1.294922 & -1.370313 \\
\hline-5.643988 & -1.797643 & -2.837543 & 6 & 3.050484 & 1.752888 & -3.173317 \\
\hline-6.779162 & -1.171666 & -3.349170 & 6 & 2.306581 & 2.922709 & -3.351593 \\
\hline-7.207877 & -1.485016 & -4.294573 & 1 & 1.590576 & 3.215663 & -2.594262 \\
\hline-7.345003 & -0.120153 & -2.627141 & 6 & 2.474135 & 3.717648 & -4.489066 \\
\hline-6.808311 & 0.289937 & -1.406219 & 1 & 1.881657 & 4.621232 & -4.604201 \\
\hline-7.289190 & 1.101427 & -0.877107 & 6 & 3.400916 & 3.351730 & -5.466034 \\
\hline-5.106239 & -1.121589 & 1.829520 & 1 & 3.536127 & 3.965571 & -6.352141 \\
\hline-4.323940 & -1.113691 & 2.995191 & 6 & 4.161114 & 2.189129 & -5.292892 \\
\hline-3.588366 & -0.332861 & 3.147230 & 1 & 4.891010 & 1.899668 & -6.044122 \\
\hline-4.461197 & -2.122588 & 3.942379 & 6 & 3.988348 & 1.400314 & -4.155961 \\
\hline-5.362171 & -3.171055 & 3.742249 & 1 & 4.592051 & 0.506997 & -4.029292 \\
\hline-5.452944 & -3.968166 & 4.471265 & 6 & 2.660961 & -0.578968 & -2.231307 \\
\hline-6.130306 & -3.181469 & 2.581277 & 1 & 3.178585 & -0.827243 & -3.160888 \\
\hline-6.014884 & -2.163326 & 1.629217 & 1 & 1.595055 & -0.715565 & -2.433425 \\
\hline-6.625787 & -2.198555 & 0.734008 & 6 & 4.243649 & 2.538587 & -0.515868 \\
\hline-2.410679 & 0.194977 & 0.199909 & 1 & 3.291252 & 3.014606 & -0.767358 \\
\hline-7.028666 & -4.349347 & 2.286546 & 6 & 4.371382 & 2.647097 & 1.002162 \\
\hline-3.550362 & -2.155515 & 5.137089 & 6 & 3.292981 & 2.246199 & 1.803111 \\
\hline-5.036179 & -2.976563 & -3.546011 & 1 & 2.380841 & 1.896727 & 1.329948 \\
\hline-8.520776 & 0.603681 & -3.223502 & 6 & 3.386845 & 2.255634 & 3.193467 \\
\hline-8.180125 & 1.230263 & -4.378452 & 1 & 2.544702 & 1.924756 & 3.791751 \\
\hline-9.023315 & 1.546798 & -2.396964 & 6 & 4.562012 & 2.692815 & 3.812519 \\
\hline-9.529534 & -0.243418 & -3.532701 & 1 & 4.640451 & 2.698674 & 4.896228 \\
\hline-3.678800 & -2.955679 & -3.491640 & 6 & 5.629591 & 3.127245 & 3.025395 \\
\hline-5.418356 & -4.148176 & -2.978065 & 1 & 6.547265 & 3.473306 & 3.493406 \\
\hline-5.383987 & -3.035980 & -4.847354 & 6 & 5.533812 & 3.106095 & 1.631327 \\
\hline-8.157753 & -3.973222 & 1.647519 & 1 & 6.389046 & 3.418257 & 1.042615 \\
\hline-6.407953 & -5.249646 & 1.473970 & 6 & 5.331362 & 3.288454 & -1.283946 \\
\hline-7.390500 & -5.017087 & 3.401108 & 1 & 5.178353 & 3.183004 & -2.358051 \\
\hline-4.195490 & -2.549668 & 6.256319 & 1 & 6.327856 & 2.920214 & -1.044391 \\
\hline-2.988930 & -0.953336 & 5.388893 & 1 & 5.291072 & 4.353120 & -1.030385 \\
\hline-2.526930 & -3.034169 & 4.953178 & 6 & 2.090734 & -1.092918 & 0.762709 \\
\hline-2.239519 & -2.787264 & 0.831445 & 1 & 2.335236 & -1.437122 & 1.768004 \\
\hline-1.262323 & -2.573214 & 1.870209 & 1 & 2.022854 & -0.012126 & 0.696658 \\
\hline-0.905633 & -1.314236 & 2.446267 & 6 & 1.132684 & -1.856395 & 0.004651 \\
\hline-1.048546 & -3.412630 & 2.516390 & 1 & 0.573925 & -1.361009 & -0.782314 \\
\hline 3.322308 & -1.995212 & -0.770188 & 6 & 0.680288 & -3.127364 & 0.418321 \\
\hline 4.789639 & -0.281638 & -0.528835 & 1 & 1.111090 & -3.521200 & 1.331057 \\
\hline 5.402195 & 0.186997 & 0.922334 & 6 & 4.650842 & -2.953625 & -2.332594 \\
\hline 6.205136 & -0.550804 & -1.381854 & 1 & 4.879320 & -2.165605 & -3.044115 \\
\hline 4.044075 & 1.128132 & -0.993369 & 6 & 3.372498 & -3.537570 & -2.414784 \\
\hline 6.539651 & -0.327423 & 1.514012 & 1 & 2.704489 & -3.177251 & -3.196870 \\
\hline 6.348015 & -0.973142 & 2.756465 & 6 & 3.040698 & -4.928861 & -1.905193 \\
\hline 5.344141 & -1.009446 & 3.166090 & 1 & 3.438309 & -5.677030 & -2.607927 \\
\hline 7.422929 & -1.516876 & 3.412185 & 1 & 1.956113 & -5.040085 & -1.925442 \\
\hline 7.290817 & -2.007705 & 4.372253 & 6 & 3.550405 & -5.241959 & -0.477946 \\
\hline 8.720301 & -1.467712 & 2.833127 & 1 & 4.517081 & -5.754236 & -0.526644 \\
\hline 9.827579 & -2.094860 & 3.465684 & 1 & 2.855442 & -5.947044 & -0.012260 \\
\hline 9.665206 & -2.595085 & 4.416964 & 6 & 3.693759 & -4.032779 & 0.441727 \\
\hline 11.075233 & -2.083800 & 2.883951 & 1 & 3.024084 & -3.995715 & 1.293137 \\
\hline 11.913512 & -2.570567 & 3.373707 & 6 & 4.846780 & -3.275097 & 0.546219 \\
\hline 11.260108 & -1.449086 & 1.632998 & 1 & 4.983371 & -2.710499 & 1.463660 \\
\hline 12.238865 & -1.463321 & 1.162097 & 6 & 6.107918 & -3.496730 & -0.264776 \\
\hline 10.210205 & -0.817696 & 1.001247 & 1 & 6.765259 & -2.639987 & -0.112429 \\
\hline 10.372068 & -0.347734 & 0.038678 & 1 & 6.634776 & -4.370406 & 0.145989 \\
\hline
\end{tabular}




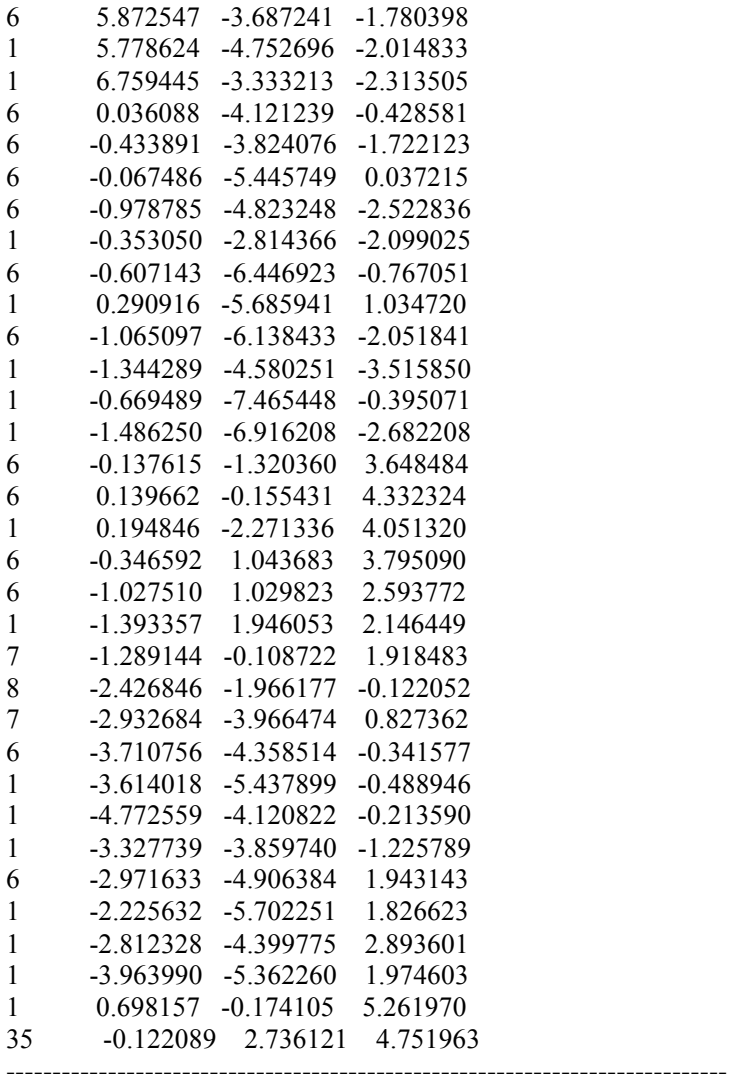

\section{C2-P1 si-re_4}

Number of imaginary frequencies $=1$

The smallest frequencies are $=-192.11846 .934212 .9296 \mathrm{~cm}(-1)$ Electronic energy $=-7222.6277096$

Zero-point correction $=$

(Hartree/Particle)

Thermal correction to Energy $=\quad 1.874298$

Thermal correction to Enthalpy= $\quad 1.875242$

Thermal correction to Gibbs Free Energy $=\quad 1.594238$

Sum of electronic and zero-point Energies $=\quad-7220.871633$

Sum of electronic and thermal Energies $=\quad-7220.753412$

Sum of electronic and thermal Enthalpies $=\quad-7220.752467$

Sum of electronic and thermal Free Energies $=\quad-7221.033471$

Cartesian Coordinates

$\begin{array}{llcc}26 & -7.069352 & -3.098020 & -2.145295 \\ 15 & -5.001012 & -0.832388 & 2.068271 \\ 15 & -4.596823 & 0.882428 & -1.284847 \\ 6 & -6.456923 & -1.892342 & -0.548376 \\ 6 & -5.801999 & -1.508007 & -1.777220 \\ 6 & -5.060745 & -2.635115 & -2.248521 \\ 1 & -4.470866 & -2.668476 & -3.154440 \\ 6 & -5.275857 & -3.720710 & -1.348342 \\ 1 & -4.881926 & -4.721641 & -1.455647 \\ 6 & -6.132651 & -3.266141 & -0.303916 \\ 1 & -6.499091 & -3.859569 & 0.519964 \\ 6 & -8.679830 & -2.502214 & -3.311441 \\ 1 & -8.945602 & -1.473048 & -3.513338 \\ 6 & -7.778414 & -3.301065 & -4.079737 \\ 1 & -7.236549 & -2.981903 & -4.959955 \\ 6 & -7.680330 & -4.581712 & -3.454775 \\ 1 & -7.047559 & -5.398624 & -3.776066 \\ 6 & -8.520667 & -4.575308 & -2.300108 \\ 1 & -8.636745 & -5.385661 & -1.592596 \\ 6 & -9.139235 & -3.292538 & -2.212048 \\ 1 & -9.809580 & -2.968739 & -1.427692 \\ 6 & -7.247223 & -0.967541 & 0.315217 \\ 6 & -8.502307 & -0.516555 & -0.121051 \\ 1 & -8.878311 & -0.882615 & -1.068337\end{array}$

$\begin{array}{lll}-9.257730 & 0.390953 & 0.621804\end{array}$

$\begin{array}{lll}-10.213136 & 0.738894 & 0.242081\end{array}$

$\begin{array}{lll}-8.769316 & 0.858278 & 1.839834\end{array}$

$\begin{array}{lll}-9.342550 & 1.567671 & 2.427707\end{array}$

$\begin{array}{lll}-7.520900 & 0.430099 & 2.291704\end{array}$

$\begin{array}{lll}-7.142699 & 0.815080 & 3.232270\end{array}$

$\begin{array}{lll}-6.738910 & -0.462805 & 1.542690\end{array}$

$-5.808919-0.117356-2.349968$

$\begin{array}{lll}-6.791978 & 0.312846 & -2.154151\end{array}$

$-5.554482-0.054956-3.858648$

$\begin{array}{lll}-5.603143 & 0.975052 & -4.222436\end{array}$

$\begin{array}{lll}-4.577362 & -0.462144 & -4.131888\end{array}$

$-6.319367-0.643684-4.374751$

$\begin{array}{lll}-4.770992 & 0.137592 & 3.617053\end{array}$

$\begin{array}{lll}-4.293409 & 1.452863 & 3.500282\end{array}$

$\begin{array}{lll}-4.054642 & 1.851356 & 2.521055\end{array}$

$\begin{array}{llll}-4.092010 & 2.234251 & 4.638819\end{array}$

$-3.733431 \quad 3.252554 \quad 4.536585$

$\begin{array}{lll}-4.338342 & 1.701024 & 5.906968\end{array}$

$\begin{array}{lll}-4.168091 & 2.304934 & 6.793866\end{array}$

$\begin{array}{llll}-4.797967 & 0.387629 & 6.031880\end{array}$

$\begin{array}{lll}-4.987473 & -0.033325 & 7.015329\end{array}$

$\begin{array}{llll}-5.021996 & -0.388938 & 4.893132\end{array}$

$\begin{array}{llll}-5.385317 & -1.405028 & 5.004427\end{array}$

$\begin{array}{lll}-5.058881 & -2.567209 & 2.665016\end{array}$

$\begin{array}{lll}-6.238066 & -3.175452 & 3.122630\end{array}$

$\begin{array}{lll}-7.171188 & -2.620028 & 3.126900\end{array}$

$\begin{array}{lll}-6.218278 & -4.501404 & 3.559376\end{array}$

$\begin{array}{lll}-7.135737 & -4.967795 & 3.907165\end{array}$

$\begin{array}{lll}-5.022894 & -5.227540 & 3.549119\end{array}$

$\begin{array}{lll}-5.012338 & -6.260715 & 3.884340\end{array}$

$\begin{array}{lll}-3.843010 & -4.620856 & 3.111147\end{array}$

$\begin{array}{lll}-2.910229 & -5.177784 & 3.106883\end{array}$

$\begin{array}{llll}-3.861744 & -3.296147 & 2.670886\end{array}$

$\begin{array}{llll}-2.946352 & -2.821638 & 2.335720\end{array}$

$\begin{array}{llll}-5.539436 & 2.319685 & -0.619003\end{array}$

$\begin{array}{lll}-4.825082 & 3.165998 & 0.241664\end{array}$

$\begin{array}{llll}-3.779117 & 2.957163 & 0.443540\end{array}$

$\begin{array}{llll}-5.454864 & 4.251293 & 0.850998\end{array}$

$\begin{array}{llll}-6.812755 & 4.491611 & 0.653517\end{array}$

$\begin{array}{llll}-7.304773 & 5.322927 & 1.145167\end{array}$

$\begin{array}{llll}-7.527941 & 3.632747 & -0.181661\end{array}$

$\begin{array}{llll}-6.901167 & 2.568089 & -0.829118\end{array}$

$\begin{array}{llll}-7.496123 & 1.935167 & -1.472754\end{array}$

$\begin{array}{llll}-3.386763 & 1.676055 & -2.430178\end{array}$

$\begin{array}{llll}-2.381799 & 0.852336 & -2.964862\end{array}$

$\begin{array}{lll}-2.386178 & -0.211662 & -2.760483\end{array}$

$\begin{array}{llll}-1.354547 & 1.398782 & -3.730310\end{array}$

$\begin{array}{llll}-1.281372 & 2.775604 & -3.951135\end{array}$

$\begin{array}{llll}-0.462519 & 3.201517 & -4.519795\end{array}$

$\begin{array}{llll}-2.269215 & 3.592373 & -3.409834\end{array}$

$\begin{array}{llll}-3.323886 & 3.052469 & -2.668958\end{array}$

$\begin{array}{llll}-4.077735 & 3.720273 & -2.269396\end{array}$

$\begin{array}{lll}-3.527326 & -0.247511 & 0.453859\end{array}$

$\begin{array}{llll}-2.145457 & 5.086018 & -3.514967\end{array}$

$\begin{array}{llll}-0.222105 & 0.536057 & -4.213613\end{array}$

$\begin{array}{lll}-4.624223 & 5.180588 & 1.690310\end{array}$

$\begin{array}{llll}-9.005270 & 3.866291 & -0.345581\end{array}$

$\begin{array}{llll}-9.666595 & 3.701638 & 0.826675\end{array}$

$\begin{array}{llll}-9.569181 & 3.024910 & -1.239791\end{array}$

$\begin{array}{llll}-9.267741 & 5.128625 & -0.757656\end{array}$

$\begin{array}{llll}-3.796330 & 4.499921 & 2.526259\end{array}$

$\begin{array}{llll}-3.821985 & 5.957638 & 0.919973\end{array}$

$\begin{array}{llll}-5.369505 & 6.005885 & 2.450259\end{array}$

$\begin{array}{llll}-3.347817 & 5.695020 & -3.595207\end{array}$

$\begin{array}{llll}-1.525708 & 5.598283 & -2.415040\end{array}$

$\begin{array}{llll}-1.417728 & 5.469050 & -4.584451\end{array}$

$\begin{array}{llll}0.157485 & 0.845755 & -5.472055\end{array}$

$\begin{array}{llll}-0.523607 & -0.779281 & -4.191144\end{array}$

$\begin{array}{lll}0.886039 & 0.700128 & -3.434040\end{array}$

$\begin{array}{lll}-1.117908 & 1.561780 & 0.138246\end{array}$

$\begin{array}{llll}-0.352904 & 0.469250 & -0.405170\end{array}$

$-0.749334-0.889744-0.61161$

$\begin{array}{llll}0.517635 & 0.736270 & -0.979135\end{array}$

$\begin{array}{lll}4.234866 & 0.147758 & 1.712140\end{array}$ 


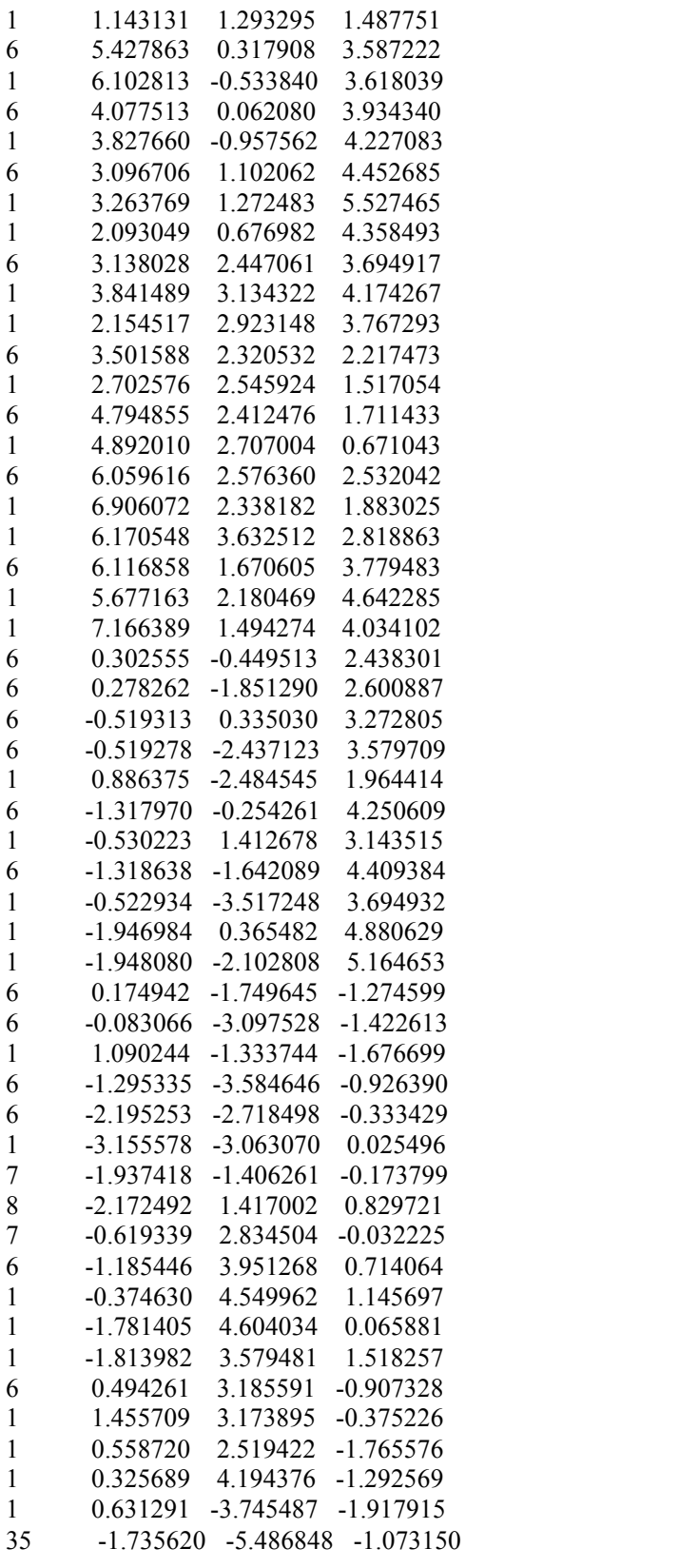

C2-P1 si-re_5

Number of imaginary frequencies $=1$

The smallest frequencies are $=-199.81914 .917111 .4236 \mathrm{~cm}(-1)$ Electronic energy $=-7222.624432$

Zero-point correction=

(Hartree/Particle)

Thermal correction to Energy= $\quad 1.873743$

Thermal correction to Enthalpy= $\quad 1.874687$

Thermal correction to Gibbs Free Energy $=\quad 1.590645$

Sum of electronic and zero-point Energies $=\quad-7220.869313$

Sum of electronic and thermal Energies $=\quad-7220.750689$

Sum of electronic and thermal Enthalpies $=\quad-7220.749745$

Sum of electronic and thermal Free Energies $=\quad-7221.033787$

Cartesian Coordinates

$\begin{array}{lllc}26 & 8.310019 & -1.881266 & 1.008163 \\ 15 & 4.672251 & -0.243612 & -2.339006 \\ 15 & 4.497913 & 0.963224 & 1.230877 \\ 6 & 6.976428 & -0.872883 & -0.250214 \\ 6 & 6.506934 & -0.881995 & 1.115899 \\ 6 & 6.341480 & -2.244389 & 1.513670\end{array}$




$$
\begin{aligned}
& \begin{array}{lll}
6.015386 & -2.583150 & 2.487609
\end{array} \\
& \begin{array}{llll}
6.735937 & -3.078443 & 0.424854
\end{array} \\
& \begin{array}{lll}
6.769344 & -4.159226 & 0.432550
\end{array} \\
& \begin{array}{llll}
7.131387 & -2.237843 & -0.656168
\end{array} \\
& \begin{array}{llll}
7.504230 & -2.569360 & -1.613048
\end{array} \\
& \begin{array}{lll}
9.790536 & -0.824787 & 2.010162
\end{array} \\
& \begin{array}{lll}
9.689775 & 0.195126 & 2.357369
\end{array} \\
& \begin{array}{llll}
9.435283 & -1.998996 & 2.742638
\end{array} \\
& \begin{array}{llll}
9.012508 & -2.025185 & 3.738124
\end{array} \\
& \begin{array}{llll}
9.692008 & -3.132357 & 1.912157
\end{array} \\
& \begin{array}{llll}
9.493261 & -4.165015 & 2.167207
\end{array} \\
& \begin{array}{lll}
10.205637 & -2.659796 & 0.666110
\end{array} \\
& \begin{array}{llll}
10.467032 & -3.270709 & -0.187844
\end{array} \\
& \begin{array}{llll}
10.269186 & -1.235780 & 0.727177
\end{array} \\
& \begin{array}{lll}
10.595106 & -0.584326 & -0.072310
\end{array} \\
& \begin{array}{llll}
7.146473 & 0.368053 & -1.061202
\end{array} \\
& \begin{array}{lll}
8.205606 & 1.240642 & -0.771091
\end{array} \\
& \begin{array}{lll}
8.905621 & 0.952797 & 0.003117
\end{array} \\
& \begin{array}{llll}
8.356907 & 2.460725 & -1.429979
\end{array} \\
& \begin{array}{llll}
9.169915 & 3.126802 & -1.158985
\end{array} \\
& \begin{array}{llll}
7.443216 & 2.827556 & -2.415268
\end{array} \\
& \begin{array}{llll}
7.540359 & 3.778964 & -2.928464
\end{array} \\
& \begin{array}{llll}
6.382048 & 1.975827 & -2.724834
\end{array} \\
& \begin{array}{llll}
5.669072 & 2.278339 & -3.483995
\end{array} \\
& \begin{array}{lll}
6.206384 & 0.755079 & -2.054983
\end{array} \\
& \begin{array}{lll}
6.164698 & 0.351275 & 1.905135
\end{array} \\
& \begin{array}{lll}
6.888454 & 1.120566 & 1.631822
\end{array} \\
& \begin{array}{lll}
6.218055 & 0.159133 & 3.423257
\end{array} \\
& \begin{array}{llll}
5.996461 & 1.093632 & 3.945798
\end{array} \\
& \begin{array}{llll}
5.507552 & -0.596220 & 3.770329
\end{array} \\
& \begin{array}{lll}
7.222755 & -0.168930 & 3.706807
\end{array} \\
& \begin{array}{llll}
3.783444 & 0.636374 & -3.696024
\end{array} \\
& \begin{array}{llll}
2.775470 & 1.544709 & -3.333549
\end{array} \\
& \begin{array}{lll}
2.538645 & 1.698393 & -2.287606
\end{array} \\
& \begin{array}{llll}
2.049240 & 2.224618 & -4.313711
\end{array} \\
& \begin{array}{llll}
1.277404 & 2.928173 & -4.017678
\end{array} \\
& \begin{array}{llll}
2.311594 & 1.995108 & -5.666004
\end{array} \\
& \begin{array}{llll}
1.739527 & 2.515526 & -6.428955
\end{array} \\
& \begin{array}{llll}
3.310810 & 1.090637 & -6.035322
\end{array} \\
& \begin{array}{llll}
3.519502 & 0.907261 & -7.085610
\end{array} \\
& \begin{array}{llll}
4.046408 & 0.417527 & -5.058150
\end{array} \\
& \begin{array}{llll}
4.817101 & -0.282268 & -5.362876
\end{array} \\
& \begin{array}{llll}
5.295562 & -1.771570 & -3.149551
\end{array} \\
& \begin{array}{lll}
6.447557 & -1.775813 & -3.952818
\end{array} \\
& \begin{array}{llll}
6.999875 & -0.855509 & -4.117912
\end{array} \\
& \begin{array}{llll}
6.903728 & -2.966244 & -4.521058
\end{array} \\
& \begin{array}{llll}
7.798266 & -2.961037 & -5.137407
\end{array} \\
& \begin{array}{llll}
6.215633 & -4.162538 & -4.292806
\end{array} \\
& \begin{array}{llll}
6.577109 & -5.089073 & -4.729635
\end{array} \\
& \begin{array}{llll}
5.064235 & -4.162957 & -3.50184
\end{array} \\
& \begin{array}{llll}
4.524504 & -5.088245 & -3.320570
\end{array} \\
& \begin{array}{llll}
4.607821 & -2.972709 & -2.932273
\end{array} \\
& \begin{array}{lll}
3.716237 & -2.976729 & -2.317504
\end{array} \\
& \begin{array}{llll}
4.772165 & 2.695198 & 0.664352
\end{array} \\
& \begin{array}{llll}
3.712462 & 3.275060 & -0.047950
\end{array} \\
& \begin{array}{llll}
2.800731 & 2.706722 & -0.199131
\end{array} \\
& \begin{array}{llll}
3.833383 & 4.561771 & -0.572749
\end{array} \\
& \begin{array}{llll}
5.019091 & 5.280575 & -0.433251
\end{array} \\
& \begin{array}{llll}
5.121381 & 6.269935 & -0.864973
\end{array} \\
& \begin{array}{lll}
6.078834 & 4.693093 & 0.258445
\end{array} \\
& \begin{array}{lll}
5.957153 & 3.422612 & 0.821613
\end{array} \\
& \begin{array}{lll}
6.801681 & 3.011921 & 1.357934
\end{array} \\
& \begin{array}{lll}
3.390004 & 1.170975 & 2.685800
\end{array} \\
& \begin{array}{llll}
2.798743 & 0.004149 & 3.195874
\end{array} \\
& \begin{array}{lll}
3.042668 & -0.957311 & 2.760489
\end{array} \\
& \begin{array}{lll}
1.877239 & 0.076560 & 4.237018
\end{array} \\
& \begin{array}{lll}
1.502799 & 1.310533 & 4.773196
\end{array} \\
& \begin{array}{lll}
0.767603 & 1.368983 & 5.567312
\end{array} \\
& \begin{array}{llll}
2.078707 & 2.467308 & 4.255672
\end{array} \\
& \begin{array}{lll}
3.023455 & 2.406260 & 3.227081
\end{array} \\
& \begin{array}{lll}
3.454015 & 3.326437 & 2.847702
\end{array} \\
& 29 \quad \begin{array}{lllll}
3.460688 & -0.328538 & -0.419233
\end{array} \\
& \begin{array}{lllll}
6 & 1.612668 & 3.818481 & 4.718776
\end{array} \\
& \begin{array}{lllll}
6 & 1.177562 & -1.169110 & 4.704683
\end{array} \\
& \begin{array}{lllll}
6 & 2.644815 & 5.174667 & -1.259499
\end{array}
\end{aligned}
$$

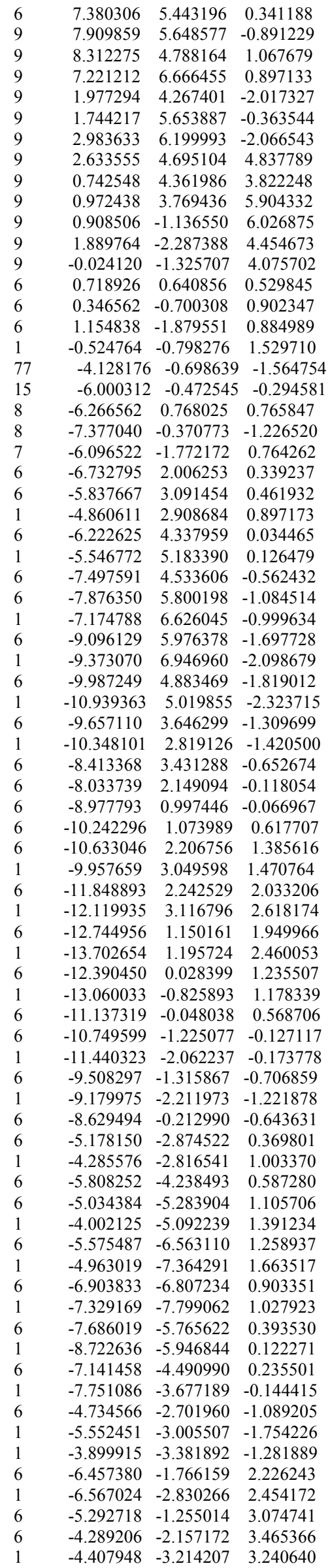




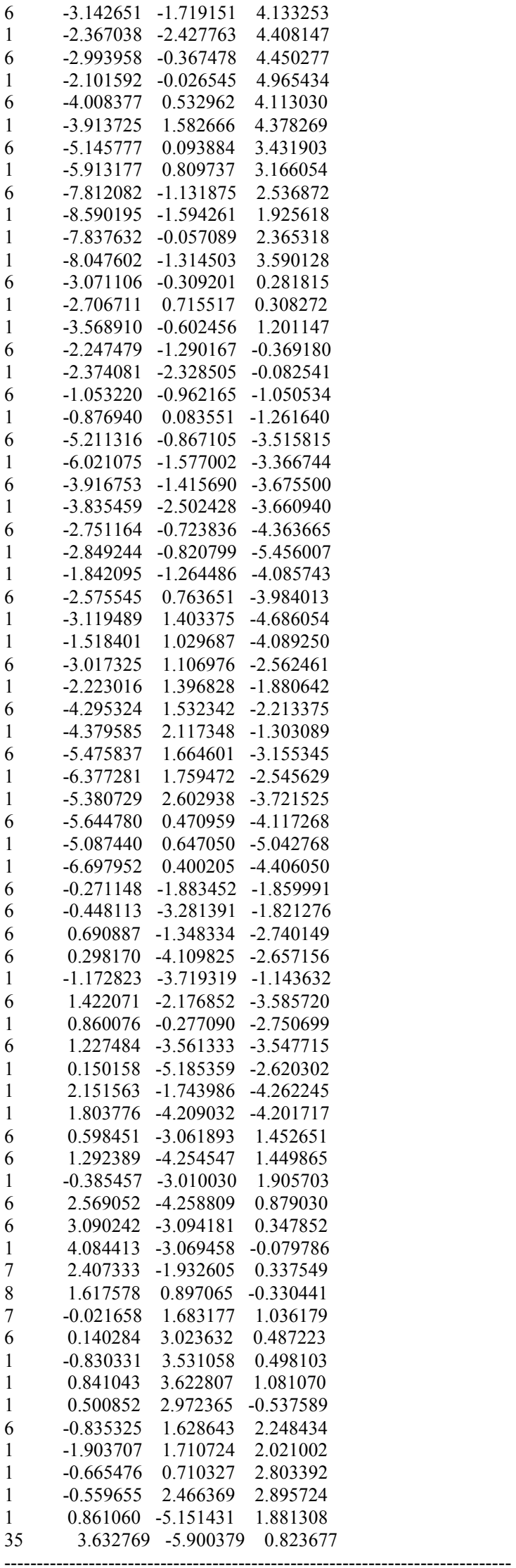

C2-P1 si-re_6

Number of imaginary frequencies $=1$

The smallest frequencies are $=-248.500510 .000013 .1047 \mathrm{~cm}(-1)$ Electronic energy $=-7222.6257487$

$\begin{array}{lc}\text { Zero-point correction= } & 1.754465 \\ \text { (Hartree/Particle) } & 1.873243 \\ \text { Thermal correction to Energy= } & 1.874187 \\ \text { Thermal correction to Enthalpy= } & 1.591464 \\ \text { Thermal correction to Gibbs Free Energy= } & -7220.871284 \\ \text { Sum of electronic and zero-point Energies }= & -7220.752506 \\ \text { Sum of electronic and thermal Energies }= & -7220.751562 \\ \text { Sum of electronic and thermal Enthalpies }= & -7221.034284 \\ \text { Sum of electronic and thermal Free Energies }= & -728\end{array}$

Cartesian Coordinates

\begin{tabular}{|c|c|c|c|}
\hline 26 & 8.171352 & 0.400264 & -0 \\
\hline $1-$ & 3.085816 & 1.016431 & -2.164600 \\
\hline 15 & 4.107150 & 0.913566 & 1.472824 \\
\hline 6 & 6.163942 & 0.929946 & -1.249987 \\
\hline 6 & 6.376207 & 0.381543 & 0.067724 \\
\hline 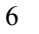 & 6.877733 & -0.947986 & -0.086649 \\
\hline 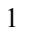 & 7.152668 & -1.617624 & 0.716925 \\
\hline 6 & 7.012186 & -1.214371 & -1.482496 \\
\hline 1 & 7.406374 & -2.120317 & -1.919606 \\
\hline 6 & 6.569918 & -0.063265 & -2.198315 \\
\hline 1 & 6.565983 & 0.056778 & -3.271434 \\
\hline 0 & 9.487853 & 1.772900 & -0.122959 \\
\hline 1 & 9.211619 & 2.531625 & 0.596958 \\
\hline 0 & 9.900238 & 0.439638 & 0.181717 \\
\hline 1 & 9.984676 & 0.010357 & 1.171166 \\
\hline 6 & 10.139559 & -0.241567 & -1.050424 \\
\hline 1 & 10.429511 & -1.278614 & -1.157079 \\
\hline 6 & 9.875125 & 0.669894 & -2.117425 \\
\hline 1 & 9.932438 & 0.446702 & -3.174669 \\
\hline 6 & 9.474562 & 1.914018 & -1.545475 \\
\hline 1 & 9.182024 & 2.797107 & -2.097082 \\
\hline 6 & 5.580578 & 2.273591 & -1.525755 \\
\hline 6 & 6.336946 & 3.417716 & -1.226608 \\
\hline 1 & 7.327854 & 3.278995 & -0.814767 \\
\hline 6 & 5.844621 & 4.704996 & -1.434722 \\
\hline 1 & 6.448499 & 5.566850 & -1.168819 \\
\hline 6 & 4.573318 & 4.870 & 8747 \\
\hline 1 & 4.174318 & 5.864279 & -2.156035 \\
\hline 6 & 3.796291 & 3.750287 & -2.268522 \\
\hline 1 & 2.801785 & 3.900 & -2.670334 \\
\hline 6 & 4.262421 & 2.446620 & -2.025279 \\
\hline 6 & 5.993165 & 1.076572 & 1.345210 \\
\hline- & 6.183098 & 2.14 & 734 \\
\hline 6 & 6.767818 & 0.606401 & 2.579257 \\
\hline 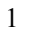 & 6.464181 & 1.164472 & 3.469342 \\
\hline 1 & 6.613545 & -0.456716 & 2.781545 \\
\hline 1 & 7.837920 & 0.766972 & 2.415564 \\
\hline 6 & 1.517469 & 1.839410 & -2.670439 \\
\hline 6 & 0.728505 & 2.402337 & -1.654965 \\
\hline 1 & 1.010280 & 2.262250 & -0.617739 \\
\hline 6 & -0.407259 & 3.145283 & -1.972804 \\
\hline 1 & -0.999438 & 3.585229 & -1.180855 \\
\hline 6 & -0.792865 & 3.300697 & -3.306472 \\
\hline 1 & -1.684878 & 3.870860 & -3.549642 \\
\hline 6 & -0.035736 & 2.709891 & -4.320717 \\
\hline 1 & -0.336082 & 2.816969 & -5.359216 \\
\hline 6 & 1.121104 & 1.991270 & -4.007411 \\
\hline 1 & 1.713490 & 1.559432 & -4.807137 \\
\hline 6 & 3.576269 & 0.089231 & -3.668282 \\
\hline 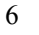 & 4.329449 & 0.650123 & -4.709874 \\
\hline 1 & 4.678474 & 1.675969 & -4.637139 \\
\hline 6 & 4.640975 & -0.113630 & -5.836812 \\
\hline 1 & 5.229790 & 0.324141 & -6.637848 \\
\hline 6 & 4.195391 & -1.435455 & -5.936981 \\
\hline 1 & 4.441366 & -2.026124 & -6.814722 \\
\hline 6 & 3.433901 & -1.995437 & -4.907591 \\
\hline 1 & 3.083520 & -3.021099 & -4.981052 \\
\hline 6 & 3.130215 & -1.236219 & -3.776723 \\
\hline 1 & 2.555117 & -1.671193 & -2.965615 \\
\hline 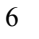 & 3.426446 & 2.612038 & 1.682206 \\
\hline ( & 2.029308 & 2.686222 & 1.773477 \\
\hline & 1.452539 & 1.767133 & 1.772268 \\
\hline & 1.385068 & 3.922731 & 1.811706 \\
\hline
\end{tabular}


$\begin{array}{lll}2.111703 & 5.108299 & 1.729414\end{array}$

$\begin{array}{llll}1.608471 & 6.068431 & 1.737739\end{array}$

$\begin{array}{lll}3.501144 & 5.031969 & 1.618546\end{array}$

$\begin{array}{lll}4.160524 & 3.802259 & 1.608403\end{array}$

$\begin{array}{lll}5.238168 & 3.796635 & 1.518459\end{array}$

$\begin{array}{lll}3.754814 & 0.084734 & 3.077701\end{array}$

$\begin{array}{llll}3.974664 & -1.301763 & 3.111190\end{array}$

$\begin{array}{llll}4.375163 & -1.806536 & 2.239554\end{array}$

$\begin{array}{llll}3.653867 & -2.039654 & 4.246913\end{array}$

$3.073836-1.423474 \quad 5.356711$

$\begin{array}{lll}2.802449 & -2.002192 & 6.232183\end{array}$

$\begin{array}{lll}2.838021 & -0.051749 & 5.314677\end{array}$

$\begin{array}{lll}3.185936 & 0.706819 & 4.193346\end{array}$

$\begin{array}{llll}2.993390 & 1.773627 & 4.196537\end{array}$

$\begin{array}{lll}2.935672 & -0.167548 & -0.242190\end{array}$

$\begin{array}{lll}2.096885 & 0.602942 & 6.446470\end{array}$

$\begin{array}{llll}3.821607 & -3.533398 & 4.250155\end{array}$

$\begin{array}{lll}-0.110929 & 3.942683 & 1.954101\end{array}$

$\begin{array}{llll}4.274385 & 6.314049 & 1.465368\end{array}$

$\begin{array}{llll}3.977678 & 6.933805 & 0.296380\end{array}$

$\begin{array}{llll}5.611065 & 6.119761 & 1.483587\end{array}$

$\begin{array}{llll}3.979329 & 7.194268 & 2.449877\end{array}$

$\begin{array}{lll}-0.701200 & 3.035790 & 1.122548\end{array}$

$\begin{array}{lll}-0.507282 & 3.611638 & 3.204838\end{array}$

$\begin{array}{lll}-0.648911 & 5.145104 & 1.663801\end{array}$

$\begin{array}{llll}2.433071 & 1.901235 & 6.598045\end{array}$

$\begin{array}{llll}0.752320 & 0.573420 & 6.225238\end{array}$

$2.306594 \quad-0.017628 \quad 7.625950$

$\begin{array}{llll}4.238274 & -3.992021 & 5.449971\end{array}$

$\begin{array}{lll}4.704078 & -3.954907 & 3.320541\end{array}$

$\begin{array}{llll}2.642169 & -4.162869 & 3.987426\end{array}$

$\begin{array}{llll}0.843076 & -1.212898 & 1.716931\end{array}$

$\begin{array}{lll}1.172598 & -2.587651 & 1.405314\end{array}$

$\begin{array}{llll}2.336716 & -3.048117 & 0.702959\end{array}$

$\begin{array}{lll}0.761949 & -3.337349 & 2.066485\end{array}$

$\begin{array}{llll}-3.139462 & -1.581887 & -1.194792\end{array}$

$\begin{array}{lll}-4.969431 & -0.610966 & -0.264716\end{array}$

$\begin{array}{lll}-4.979220 & 0.775639 & 0.634258\end{array}$

$\begin{array}{llll}-6.064701 & -0.233332 & -1.451510\end{array}$

$\begin{array}{lll}-5.629202 & -1.640161 & 0.899673\end{array}$

$\begin{array}{lll}-4.890346 & 2.031346 & 0.052830\end{array}$

$\begin{array}{lll}-3.733276 & 2.775026 & 0.370819\end{array}$

$\begin{array}{lll}-3.008751 & 2.341172 & 1.049676\end{array}$

$\begin{array}{llll}-3.554325 & 4.020548 & -0.175329\end{array}$

$\begin{array}{llll}-2.684410 & 4.613533 & 0.088907\end{array}$

$-4.490012 \quad 4.531535-1.114558$

$\begin{array}{llll}-4.256254 & 5.764617 & -1.781629\end{array}$

$\begin{array}{llll}-3.358345 & 6.324684 & -1.533463\end{array}$

$\begin{array}{llll}-5.134509 & 6.232224 & -2.733106\end{array}$

$\begin{array}{llll}-4.941875 & 7.171311 & -3.243566\end{array}$

$\begin{array}{llll}-6.285801 & 5.476005 & -3.057426\end{array}$

$\begin{array}{llll}-6.965224 & 5.833794 & -3.825696\end{array}$

$\begin{array}{llll}-6.552362 & 4.286887 & -2.413284\end{array}$

$\begin{array}{llll}-7.434875 & 3.719203 & -2.683341\end{array}$

$\begin{array}{llll}-5.676694 & 3.779574 & -1.412918\end{array}$

$\begin{array}{llll}-5.919948 & 2.531166 & -0.731398\end{array}$

$\begin{array}{llll}-7.212508 & 1.800430 & -0.864513\end{array}$

$\begin{array}{lll}-8.474074 & 2.440304 & -0.587280\end{array}$

$\begin{array}{lll}-8.564881 & 3.736231 & -0.007832\end{array}$

$\begin{array}{llll}-7.656789 & 4.269613 & 0.245464\end{array}$

$\begin{array}{llll}-9.786970 & 4.312121 & 0.263952\end{array}$

$\begin{array}{llll}-9.826525 & 5.297745 & 0.718902\end{array}$

$\begin{array}{llll}-10.989973 & 3.629375 & -0.034376\end{array}$

$\begin{array}{lll}-11.946266 & 4.097774 & 0.179525\end{array}$

$\begin{array}{llll}-10.939950 & 2.366218 & -0.579386\end{array}$

$\begin{array}{llll}-11.854792 & 1.819659 & -0.793331\end{array}$

$\begin{array}{llll}-9.695889 & 1.736462 & -0.853888\end{array}$

$\begin{array}{lll}-9.645882 & 0.403632 & -1.342319\end{array}$

$\begin{array}{lll}-10.575626 & -0.117594 & -1.552326\end{array}$

$\begin{array}{llll}-8.441371 & -0.233712 & -1.498600\end{array}$

$\begin{array}{lll}-8.375392 & -1.264482 & -1.830055\end{array}$

$\begin{array}{llll}-7.244230 & 0.465443 & -1.233612\end{array}$

$\begin{array}{llll}-5.122406 & -3.030967 & 0.742061\end{array}$

$\begin{array}{lll}-4.373200 & -3.218922 & 1.520211\end{array}$

$\begin{array}{llll}-6.227649 & -4.067346 & 0.855829\end{array}$ $\begin{array}{lll}-6.005568 & -5.264879 & 1.544582\end{array}$

$\begin{array}{lll}-5.055854 & -5.422032 & 2.051320\end{array}$

$\begin{array}{lll}-6.995519 & -6.249665 & 1.598900\end{array}$

$\begin{array}{lll}-6.810052 & -7.171806 & 2.143118\end{array}$

$\begin{array}{lll}-8.222865 & -6.045431 & 0.964125\end{array}$

$\begin{array}{lll}-8.996461 & -6.806648 & 1.011853\end{array}$

$\begin{array}{llll}-8.450183 & -4.853407 & 0.268527\end{array}$

$-9.401883-4.686392 \quad-0.229004$

$\begin{array}{llll}-7.457979 & -3.874137 & 0.211612\end{array}$

$\begin{array}{lll}-7.634761 & -2.945289 & -0.322202\end{array}$

$\begin{array}{llll}-4.424006 & -3.198730 & -0.622416\end{array}$

$\begin{array}{llll}-5.186161 & -3.335963 & -1.398683\end{array}$

$\begin{array}{lll}-3.842523 & -4.124675 & -0.591178\end{array}$

$\begin{array}{llll}-5.948064 & -1.126449 & 2.282981\end{array}$

$\begin{array}{lll}-5.101361 & -0.514843 & 2.611211\end{array}$

$\begin{array}{lll}-7.191498 & -0.249358 & 2.326483\end{array}$

$\begin{array}{llll}-7.112778 & 1.048046 & 2.846741\end{array}$

$\begin{array}{lll}-6.142458 & 1.454416 & 3.119409\end{array}$

$\begin{array}{lll}-8.262485 & 1.823384 & 3.010654\end{array}$

$\begin{array}{lll}-8.182890 & 2.831964 & 3.406490\end{array}$

$\begin{array}{lll}-9.509881 & 1.307397 & 2.654092\end{array}$

$\begin{array}{lll}-10.404592 & 1.912072 & 2.767012\end{array}$

$\begin{array}{lll}-9.595920 & 0.017916 & 2.123833\end{array}$

$\begin{array}{lll}-10.560993 & -0.384614 & 1.829149\end{array}$

$\begin{array}{lll}-8.445403 & -0.754754 & 1.961320\end{array}$

$\begin{array}{lll}-8.519538 & -1.758229 & 1.554576\end{array}$

$\begin{array}{lll}-6.104609 & -2.247305 & 3.323402\end{array}$

$\begin{array}{lll}-5.206331 & -2.862148 & 3.415323\end{array}$

$\begin{array}{lll}-6.955020 & -2.894860 & 3.106043\end{array}$

$\begin{array}{llll}-6.280471 & -1.769971 & 4.291898\end{array}$

$\begin{array}{llll}-2.181762 & -1.249102 & 0.720077\end{array}$

$\begin{array}{llll}-1.517403 & -0.394114 & 0.648731\end{array}$

$\begin{array}{llll}-2.833506 & -1.215138 & 1.590779\end{array}$

$\begin{array}{lll}-1.679832 & -2.540552 & 0.340723\end{array}$

$\begin{array}{llll}-2.180655 & -3.406355 & 0.763046\end{array}$

$\begin{array}{llll}-0.434432 & -2.777130 & -0.296953\end{array}$

$\begin{array}{llll}0.075481 & -1.930303 & -0.736024\end{array}$

$\begin{array}{lll}-4.022457 & -1.671812 & -3.240999\end{array}$

$\begin{array}{llll}-5.029824 & -2.063254 & -3.127690\end{array}$

$\begin{array}{llll}-2.962806 & -2.608451 & -3.179946\end{array}$

$\begin{array}{llll}-3.229203 & -3.656944 & -3.048479\end{array}$

$\begin{array}{llll}-1.597978 & -2.389820 & -3.808486\end{array}$

$\begin{array}{lll}-1.661746 & -2.546523 & -4.896701\end{array}$

$\begin{array}{llll}-0.936443 & -3.170124 & -3.427088\end{array}$

$\begin{array}{llll}-0.968387 & -1.008286 & -3.516506\end{array}$

$\begin{array}{llll}-1.178041 & -0.310212 & -4.332977\end{array}$

$\begin{array}{llll}0.121301 & -1.113356 & -3.492165\end{array}$

$\begin{array}{llll}-1.415064 & -0.366395 & -2.203245\end{array}$

$\begin{array}{llll}-0.639246 & -0.240905 & -1.453538\end{array}$

$\begin{array}{llll}-2.511444 & 0.480157 & -2.077790\end{array}$

$\begin{array}{llll}-2.491145 & 1.181892 & -1.249710\end{array}$

$\begin{array}{llll}-3.478618 & 0.839667 & -3.188270\end{array}$

$\begin{array}{llll}-4.353825 & 1.304456 & -2.730944\end{array}$

$\begin{array}{llll}-3.014485 & 1.602514 & -3.828422\end{array}$

$\begin{array}{llll}-3.936163 & -0.366317 & -4.032658\end{array}$

$\begin{array}{lll}-3.264168 & -0.509222 & -4.884992\end{array}$

$\begin{array}{lll}-4.921706 & -0.146129 & -4.454367\end{array}$

$\begin{array}{lll}-0.058808 & -4.084712 & -0.844712\end{array}$

$\begin{array}{llll}-0.556959 & -5.301701 & -0.341610\end{array}$

$\begin{array}{llll}0.862803 & -4.131509 & -1.907224\end{array}$

$\begin{array}{llll}-0.159063 & -6.515939 & -0.898290\end{array}$

$\begin{array}{llll}-1.245260 & -5.300789 & 0.497762\end{array}$

$\begin{array}{llll}1.256397 & -5.344707 & -2.468369\end{array}$

$\begin{array}{llll}1.264920 & -3.200658 & -2.291735\end{array}$

$\begin{array}{llll}0.745003 & -6.543726 & -1.965918\end{array}$

$\begin{array}{lll}-0.552147 & -7.445289 & -0.496032\end{array}$

$\begin{array}{llll}1.964674 & -5.354620 & -3.291843\end{array}$

$\begin{array}{llll}1.051270 & -7.492413 & -2.396928\end{array}$

$\begin{array}{llll}2.695018 & -4.417577 & 0.842188\end{array}$

$\begin{array}{llll}3.730470 & -4.963552 & 0.113460\end{array}$

$\begin{array}{llll}2.137090 & -5.037426 & 1.533418\end{array}$

$\begin{array}{llll}4.422086 & -4.119547 & -0.760605\end{array}$

$\begin{array}{llll}4.075532 & -2.784985 & -0.850776\end{array}$

$\begin{array}{llll}4.604556 & -2.108988 & -1.508905\end{array}$

$3.062404-2.251105-0.137151$ 


\begin{tabular}{|c|c|c|c|}
\hline 8 & 1.100745 & -0.242518 & 0.936661 \\
\hline 7 & 0.131004 & -0.965683 & 2.858689 \\
\hline 6 & -0.460183 & 0.346315 & 3.096538 \\
\hline 1 & -1.473536 & 0.220276 & 3.492329 \\
\hline 1 & 0.123488 & 0.916169 & 3.826576 \\
\hline 1 & -0.516880 & 0.904594 & 2.167675 \\
\hline 6 & -0.107884 & -1.948062 & 3.912827 \\
\hline 1 & -1.046612 & -2.494707 & 3.753149 \\
\hline 1 & 0.713674 & -2.658506 & 3.986010 \\
\hline 1 & -0.172364 & -1.419482 & 4.866890 \\
\hline 1 & 3.989890 & -6.011654 & 0.213961 \\
\hline 35 & 5.857954 & -4.829666 & -1.885014 \\
\hline
\end{tabular}

C2-P1 si-re_7

Number of imaginary frequencies $=1$

The smallest frequencies are $=-267.97277 .678211 .0818 \mathrm{~cm}(-1)$ Electronic energy $=-7222.6289103$

Zero-point correction=

(Hartree/Particle)

Thermal correction to Energy=

Thermal correction to Enthalpy=

1.873395

Thermal correction to Gibbs Free Energy= $\quad 1.590919$

Sum of electronic and zero-point Energies=

Sum of electronic and thermal Energies=

$-7220.874275$

$-7220.755515$

Sum of electronic and thermal Enthalpies=

$-7220.754571$

Sum of electronic and thermal Free Energies=

$-7221.037991$

\begin{tabular}{|c|c|c|c|}
\hline 26 & 8.164627 & 0.057258 & -1.065056 \\
\hline 15 & 3.103215 & 1.071739 & -2.100875 \\
\hline 15 & 4.188583 & 0.684021 & 1.495427 \\
\hline 6 & 6.189305 & 0.736413 & -1.267302 \\
\hline 6 & 6.395438 & 0.096480 & 0.009527 \\
\hline 6 & 6.803895 & -1.250638 & -0.237111 \\
\hline 1 & 7.046643 & -1.986448 & 0.517012 \\
\hline 6 & 6.886909 & -1.440226 & -1.649618 \\
\hline 1 & 7.207327 & -2.342424 & -2.150055 \\
\hline 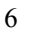 & 6.506085 & -0.221142 & -2.283619 \\
\hline 1 & 6.484707 & -0.035891 & -3.347268 \\
\hline 6 & 9.603795 & 1.270912 & -0.185159 \\
\hline 1 & 9.409258 & 1.994807 & 0.595273 \\
\hline 6 & 9.926972 & -0.107268 & 0.009603 \\
\hline 1 & 10.010702 & -0.613254 & 0.962148 \\
\hline J & 10.076161 & -0.711579 & -1.275898 \\
\hline 1 & 10.286432 & -1.755699 & -1.466962 \\
\hline 6 & 9.844918 & 0.291956 & -2.265243 \\
\hline 1 & 9.852705 & 0.142690 & -3.336881 \\
\hline 6 & 9.556076 & 1.516207 & -1.592551 \\
\hline 1 & 9.310740 & 2.455236 & -2.069711 \\
\hline 6 & 5.687471 & 2.127783 & -1.444822 \\
\hline 6 & 6.520940 & 3.200170 & -1.089263 \\
\hline 1 & 7.509097 & 2.971867 & -0.711177 \\
\hline 6 & 6.106729 & 4.526498 & -1.198552 \\
\hline 1 & 6.767927 & 5.329763 & -0.889183 \\
\hline 6 & 4.838039 & 4.806492 & -1.700072 \\
\hline 1 & 4.498189 & 5.832040 & -1.801561 \\
\hline 6 & 3.986762 & 3.757676 & -2.046174 \\
\hline 1 & 2.996266 & 3.996837 & -2.412938 \\
\hline 6 & 4.373487 & 2.413945 & -1.900997 \\
\hline 6 & 6.080420 & 0.732436 & 1.335960 \\
\hline 1 & 6.333268 & 1.791008 & 1.260490 \\
\hline 6 & 6.845595 & 0.140099 & 2.522346 \\
\hline 1 & 6.595096 & 0.662999 & 3.449630 \\
\hline 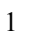 & 6.628981 & -0.921518 & 2.667768 \\
\hline 1 & 7.920604 & 0.242918 & 2.344627 \\
\hline 0 & 1.588256 & 2.028907 & -2.520716 \\
\hline 6 & 0.854192 & 2.565279 & -1.451744 \\
\hline 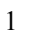 & 1.135358 & 2.319372 & -0.434517 \\
\hline ס & -0.224853 & 3.414332 & -1.691563 \\
\hline 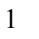 & -0.772275 & 3.834021 & -0.857674 \\
\hline 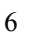 & -0.609956 & 3.704561 & -3.002644 \\
\hline & -1.458429 & 4.357514 & -3.186282 \\
\hline & 0.089245 & 3.140346 & -4.072380 \\
\hline
\end{tabular}

\begin{tabular}{|c|c|c|}
\hline & & \\
\hline & & \\
\hline & & \\
\hline 487 & 207 & \\
\hline & & \\
\hline & & \\
\hline & & \\
\hline & & \\
\hline 711 & 13 & 923 \\
\hline & & 299 \\
\hline & & \\
\hline 297 & -2.8 & -5 \\
\hline & & \\
\hline & -1. & -3 \\
\hline & & \\
\hline & & \\
\hline 1.5 & & \\
\hline & & \\
\hline & & \\
\hline & & \\
\hline & & \\
\hline & & \\
\hline & & \\
\hline & & \\
\hline & & \\
\hline & -2 & \\
\hline & & \\
\hline & -1 . & \\
\hline 85 & -2.3 & \\
\hline & -0. & \\
\hline & & \\
\hline 08 & 1.4 & \\
\hline & & \\
\hline & & \\
\hline 285 & -3.8 & \\
\hline & & \\
\hline & & \\
\hline & & \\
\hline & & \\
\hline & & \\
\hline & & \\
\hline & & \\
\hline 78 & & \\
\hline & & \\
\hline & & \\
\hline 2.4 & & \\
\hline & & \\
\hline & & \\
\hline 2.4 & & \\
\hline & & \\
\hline & & \\
\hline 2.1 & -3 . & \\
\hline 0.5 & -3.3 & \\
\hline & & \\
\hline & & \\
\hline & & \\
\hline & & \\
\hline & -1 & \\
\hline & & \\
\hline & & \\
\hline-2.8 & 2. & 1. \\
\hline & & \\
\hline & & \\
\hline & & -0 \\
\hline-3.9 & 6.2 & \\
\hline-2.9 & 6.7 & \\
\hline & & \\
\hline & 7.8 & \\
\hline & 6.2 & -2 \\
\hline-6.6 & 6.7 & -3 \\
\hline & 4.9 & \\
\hline & 4.5 & -2.2 \\
\hline-5.445243 & 4.33 & -1.036623 \\
\hline & & \\
\hline & & \\
\hline
\end{tabular}

$\begin{array}{llll}-0.212843 & 3.351726 & -5.094226\end{array}$

$1111-3.83522$

$\begin{array}{lll}3.509487 & 0.207207 & -3.665494\end{array}$

$\begin{array}{lll}4.242699 & 0.799502 & -4.704237\end{array}$

$\begin{array}{lll}4.494589 & 0.085449 & -5.877629\end{array}$

$\begin{array}{lll}5.067255 & 0.547452 & -6.676739\end{array}$

$\begin{array}{llll}4.010711 & -1.218413 & -6.025923\end{array}$

$\begin{array}{lll}4.211234 & -1.771012 & -6.939299\end{array}$

$\begin{array}{llll}2.891297 & -2.821777 & -5.108627\end{array}$

$3.024733-1.099685-3.822499$

$\begin{array}{llll}3.628067 & 2.407084 & 1.823032\end{array}$

$\begin{array}{llll}2.240256 & 2.573630 & 1.936669\end{array}$

$\begin{array}{lll}1.598047 & 1.701166 & 1.877043\end{array}$

$\begin{array}{lll}1.686858 & 3.846352 & 2.075416\end{array}$

$\begin{array}{llll}2.496697 & 4.980207 & 2.070209\end{array}$

$\begin{array}{lll}2.064452 & 5.970660 & 2.157203\end{array}$

$\begin{array}{llll}4.444576 & 3.544227 & 1.826131\end{array}$

$\begin{array}{llll}5.518449 & 3.466908 & 1.725256\end{array}$

$\begin{array}{lll}3.813247 & -0.208535 & 3.060639\end{array}$

$\begin{array}{lll}3.953177 & -1.605412 & 3.026630\end{array}$

$\begin{array}{llll}3.596890 & -2.375974 & 4.129803\end{array}$

$\begin{array}{lll}3.066368 & -1.780576 & 5.275698\end{array}$

$\begin{array}{llll}2.771185 & -2.384507 & 6.125832\end{array}$

$\begin{array}{lll}2.916692 & -0.396811 & 5.303276\end{array}$

$\begin{array}{lll}3.170008 & 1.465730 & 4.269138\end{array}$

$\begin{array}{llll}3.672285 & -3.875360 & 4.060073\end{array}$

$\begin{array}{lll}0.197558 & 3.963067 & 2.247629\end{array}$

$\begin{array}{llll}4.738377 & 6.045317 & 1.864166\end{array}$

$\begin{array}{lll}4.463300 & 6.776960 & 0.756422\end{array}$

$\begin{array}{llll}6.057682 & 5.755554 & 1.830887\end{array}$

$\begin{array}{lll}6.861124 & 2.923739\end{array}$

$\begin{array}{lll}-0.198654 & 3.612299 & 3.492956\end{array}$

$\begin{array}{lll}-0.259078 & 5.210987 & 2.013743\end{array}$

$\begin{array}{lll}2.716304 & 1.503103 & 6.706278\end{array}$

$\begin{array}{lll}.908081 & 0.375665 & 6.278115\end{array}$

$\begin{array}{lll}4.076246 & -4.416492 & 5.229632\end{array}$

$\begin{array}{lll}4.514138 & -4.305467 & 3.097178\end{array}$

$\begin{array}{lll}2.452208 & -4.416622 & 3.787833\end{array}$

$\begin{array}{lll}7.230090 & 1.704803\end{array}$

$\begin{array}{lll}2.160810 & -3.099280 & 0.581783\end{array}$

$\begin{array}{llll}0.578073 & -3.361320 & 1.941823\end{array}$

$\begin{array}{llll}-4.969673 & -0.206197 & -0.363083\end{array}$

$\begin{array}{lll}-4.906103 & 1.091553 & 0.671777\end{array}$

$\begin{array}{llll}-5.977806 & 0.396016 & -1.542326\end{array}$

$\begin{array}{llll}-5.781249 & -1.285238 & 0.636034\end{array}$

$\begin{array}{lll}-4.737106 & 2.390790 & 0.215375\end{array}$

$\begin{array}{llll}-3.534557 & 3.030063 & 0.588299\end{array}$

$\begin{array}{lll}-2.825818 & 2.487869 & 1.203111\end{array}$

$\begin{array}{llll}-4.215290 & 4.980298 & -0.678977\end{array}$

$\begin{array}{llll}-3.925776 & 6.268675 & -1.204750\end{array}$

$\begin{array}{llll}-2.995146 & 6.748843 & -0.913348\end{array}$

$-4.559551$

$\begin{array}{llll}-5.990066 & 6.236662 & -2.457820\end{array}$

$\begin{array}{llll}-6.662314 & 6.717237 & -3.162781\end{array}$

$\begin{array}{llll}-6.309535 & 4.995453 & -1.950757\end{array}$

$\begin{array}{llll}-7.065426 & 2.369024 & -0.700156\end{array}$ 


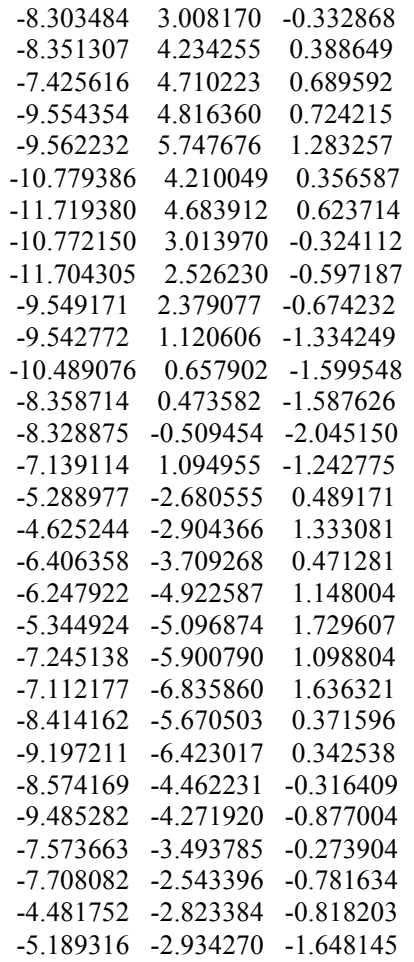

Number of imaginary frequencies $=1$

The smallest frequencies are $=-140.202611 .871612 .8133 \mathrm{~cm}(-1)$ Electronic energy $=-7222.6277013$

Zero-point correction=

(Hartree/Particle)

Thermal correction to Energy= $\quad 1.874655$

Thermal correction to Enthalpy=

1.875599

Thermal correction to Gibbs Free Energy $=\quad 1.595400$

Sum of electronic and zero-point Energies $=\quad-7220.871182$

Sum of electronic and thermal Energies $=\quad-7220.753046$

Sum of electronic and thermal Enthalpies $=\quad-7220.752102$

Sum of electronic and thermal Free Energies $=\quad-7221.032301$

Cartesian Coordinates

$\begin{array}{lccc}26 & 8.099631 & 0.502906 & -0.733919 \\ 15 & 3.044641 & 1.442877 & -1.834633 \\ 15 & 4.010285 & 0.578992 & 1.700866 \\ 6 & 6.108555 & 1.130246 & -0.930538 \\ 6 & 6.291835 & 0.319628 & 0.249556 \\ 6 & 6.764823 & -0.962213 & -0.169932 \\ 1 & 7.011537 & -1.790213 & 0.480553 \\ 6 & 6.912653 & -0.941280 & -1.589442 \\ 1 & 7.290771 & -1.748986 & -2.199538 \\ 6 & 6.507640 & 0.342893 & -2.058169 \\ 1 & 6.521958 & 0.678906 & -3.084115 \\ 6 & 9.429658 & 1.657482 & 0.368282 \\ 1 & 9.153607 & 2.271831 & 1.215259 \\ 6 & 9.805420 & 0.280150 & 0.419181 \\ 1 & 9.852366 & -0.333334 & 1.309118 \\ 6 & 10.052640 & -0.160105 & -0.917000 \\ 1 & 10.320525 & -1.165192 & -1.215162 \\ 6 & 9.829910 & 0.944626 & -1.794212 \\ 1 & 9.902758 & 0.924853 & -2.873594 \\ 6 & 9.446226 & 2.067059 & -1.001341 \\ 1 & 9.182504 & 3.045690 & -1.378421 \\ 6 & 5.544381 & 2.510249 & -0.919997 \\ 6 & 6.317704 & 3.556830 & -0.392774 \\ 1 & 7.315816 & 3.326226 & -0.043619 \\ 6 & 5.833449 & 4.860760 & -0.300554 \\ 1 & 6.450843 & 5.639397 & 0.136117 \\ 6 & 4.552509 & 5.148798 & -0.764247\end{array}$


$\begin{array}{llll}4.157628 & 6.157674 & -0.703396\end{array}$

$\begin{array}{llll}3.762429 & 4.125028 & -1.286357\end{array}$

$\begin{array}{llll}2.763621 & 4.367699 & -1.627764\end{array}$

$\begin{array}{llll}4.222406 & 2.798519 & -1.352913\end{array}$

$\begin{array}{lll}5.898551 & 0.744085 & 1.637553\end{array}$

$\begin{array}{llll}6.096490 & 1.813915 & 1.722373\end{array}$

$\begin{array}{lll}6.654260 & 0.023328 & 2.756709\end{array}$

$\begin{array}{lll}6.344756 & 0.390415 & 3.739304\end{array}$

$\begin{array}{llll}6.490697 & -1.057415 & 2.734885\end{array}$

$\begin{array}{lll}7.727456 & 0.203276 & 2.640273\end{array}$

$\begin{array}{llll}1.500462 & 2.380076 & -2.201644\end{array}$

$\begin{array}{llll}0.649035 & 2.673276 & -1.122987\end{array}$

$\begin{array}{llll}0.859722 & 2.257767 & -0.143781\end{array}$

$\begin{array}{llll}-0.452305 & 3.511066 & -1.301605\end{array}$

$\begin{array}{llll}-1.079248 & 3.760330 & -0.455022\end{array}$

$\begin{array}{llll}-0.745713 & 4.024521 & -2.567031\end{array}$

$\begin{array}{llll}-1.614118 & 4.662066 & -2.705770\end{array}$

$\begin{array}{llll}0.068756 & 3.699667 & -3.653640\end{array}$

$\begin{array}{llll}-0.161108 & 4.084907 & -4.642918\end{array}$

$\begin{array}{lll}1.194189 & 2.892090 & -3.471680\end{array}$

$\begin{array}{llll}1.835469 & 2.674854 & -4.319271\end{array}$

$\begin{array}{llll}3.601617 & 0.887524 & -3.493175\end{array}$

$\begin{array}{llll}4.335039 & 1.704784 & -4.367093\end{array}$

$\begin{array}{llll}4.614612 & 2.709845 & -4.065349\end{array}$

$\begin{array}{llll}4.716705 & 1.223413 & -5.620834\end{array}$

$\begin{array}{llll}5.288476 & 1.859412 & -6.290604\end{array}$

$\begin{array}{llll}4.366598 & -0.072612 & -6.013702\end{array}$

$\begin{array}{llll}4.669999 & -0.445244 & -6.987842\end{array}$

$\begin{array}{llll}3.628713 & -0.887390 & -5.151384\end{array}$

$\begin{array}{llll}3.357806 & -1.896698 & -5.448472\end{array}$

$\begin{array}{llll}3.250341 & -0.408201 & -3.896190\end{array}$

$2.697034 \quad-1.044997-3.215360$

$\begin{array}{lll}3.339975 & 2.203247 & 2.255286\end{array}$

$\begin{array}{lll}1.940415 & 2.283870 & 2.305323\end{array}$

$\begin{array}{llll}1.354707 & 1.398757 & 2.079805\end{array}$

$\begin{array}{lll}1.306360 & 3.491186 & 2.595133\end{array}$

$\begin{array}{llll}2.047976 & 4.652707 & 2.800130\end{array}$

$\begin{array}{lll}1.556512 & 5.598370 & 2.999695\end{array}$

$\begin{array}{llll}3.440162 & 4.576562 & 2.723709\end{array}$

$\begin{array}{llll}4.088766 & 3.366469 & 2.473670\end{array}$

$\begin{array}{lll}5.169720 & 3.359839 & 2.425814\end{array}$

$\begin{array}{lll}3.625457 & -0.569335 & 3.084647\end{array}$

$3.816813 \quad-1.935162 \quad 2.821856$

$\begin{array}{lll}4.227610 & -2.250926 & 1.870237\end{array}$

$\begin{array}{lll}3.443306 & -2.893147 & 3.759604\end{array}$

$\begin{array}{lll}2.843695 & -2.516807 & 4.962913\end{array}$

$\begin{array}{llll}2.527922 & -3.265347 & 5.680834\end{array}$

$2.644009-1.162361 \quad 5.217284$

$\begin{array}{lll}3.042455 & -0.188882 & 4.296615\end{array}$

$2.874657 \quad 0.856888 \quad 4.526245$

$\begin{array}{llll}2.833047 & -0.122771 & -0.215867\end{array}$

$\begin{array}{lll}1.886924 & -0.750351 & 6.448369\end{array}$

$\begin{array}{llll}3.575263 & -4.356711 & 3.442463\end{array}$

$\begin{array}{lll}-0.193852 & 3.500344 & 2.700452\end{array}$

$\begin{array}{lll}4.228912 & 5.847659 & 2.882676\end{array}$

$\begin{array}{lll}3.901053 & 6.753351 & 1.927872\end{array}$

$\begin{array}{llll}5.562192 & 5.646621 & 2.799495\end{array}$

$3.984473 \quad 6.442877 \quad 4.073422$

$\begin{array}{lll}-0.773535 & 2.787610 & 1.689030\end{array}$

$\begin{array}{lll}-0.620864 & 2.922770 & 3.848647\end{array}$

$\begin{array}{lll}-0.713877 & 4.741289 & 2.653013\end{array}$

$\begin{array}{llll}2.215039 & 0.492226 & 6.862206\end{array}$

$\begin{array}{lll}0.545945 & -0.740602 & 6.212608\end{array}$

$\begin{array}{llll}2.091325 & -1.594547 & 7.481743\end{array}$

$\begin{array}{llll}4.025904 & -5.062662 & 4.502893\end{array}$

$\begin{array}{lll}4.413535 & -4.589334 & 2.410929\end{array}$

$\begin{array}{llll}2.373279 & -4.900894 & 3.103966\end{array}$

$0.719404-1.518585 \quad 1.531865$

$\begin{array}{lll}1.013475 & -2.790757 & 0.929927\end{array}$

$\begin{array}{llll}2.138110 & -3.117931 & 0.109775\end{array}$

$\begin{array}{llll}0.555089 & -3.658185 & 1.383041\end{array}$

$77 \quad-2.997266-0.810485-1.913630$

$\begin{array}{lll}-4.703352 & -0.241234 & -0.528701\end{array}$

$\begin{array}{lll}-4.722996 & 1.083782 & 0.470185\end{array}$

$\begin{array}{lll}-6.081842 & 0.037773 & -1.416609\end{array}$ $\begin{array}{lll}-4.940296 & -1.436800 & 0.627163\end{array}$

$\begin{array}{lll}-4.966311 & 2.358140 & -0.025738\end{array}$

$\begin{array}{llll}-3.884208 & 3.262344 & 0.031762\end{array}$

$\begin{array}{lll}-2.959516 & 2.929436 & 0.488475\end{array}$

$\begin{array}{lll}-4.030711 & 4.528382 & -0.476454\end{array}$

$\begin{array}{llll}-3.208331 & 5.236983 & -0.432137\end{array}$

$\begin{array}{llll}-5.250638 & 4.918170 & -1.093411\end{array}$

$\begin{array}{llll}-5.386676 & 6.193641 & -1.705364\end{array}$

$\begin{array}{llll}-4.541472 & 6.876377 & -1.671762\end{array}$

$\begin{array}{llll}-6.554929 & 6.554187 & -2.338012\end{array}$

$\begin{array}{llll}-6.646386 & 7.529396 & -2.807147\end{array}$

$\begin{array}{llll}-7.636579 & 5.643046 & -2.389321\end{array}$

$\begin{array}{llll}-8.548571 & 5.919984 & -2.910321\end{array}$

$\begin{array}{llll}-7.543182 & 4.404913 & -1.791151\end{array}$

$\begin{array}{llll}-8.378492 & 3.717487 & -1.850955\end{array}$

$\begin{array}{lll}-6.359146 & 4.004474 & -1.111009\end{array}$

$\begin{array}{llll}-6.224910 & 2.711464 & -0.488284\end{array}$

$\begin{array}{lll}-7.371947 & 1.767940 & -0.360071\end{array}$

$\begin{array}{lll}-8.603783 & 2.138534 & 0.289369\end{array}$

$\begin{array}{lll}-8.766778 & 3.369195 & 0.985620\end{array}$

$\begin{array}{llll}-7.936351 & 4.063257 & 1.038926\end{array}$

$\begin{array}{lll}-9.955084 & 3.682163 & 1.609530\end{array}$

$\begin{array}{lll}-10.049254 & 4.623594 & 2.143150\end{array}$

$\begin{array}{lll}-11.050988 & 2.787421 & 1.569674\end{array}$

$\begin{array}{lll}-11.982537 & 3.050236 & 2.062252\end{array}$

$\begin{array}{lll}-10.924355 & 1.580824 & 0.919183\end{array}$

$\begin{array}{lll}-11.750941 & 0.875413 & 0.893917\end{array}$

$\begin{array}{lll}-9.707572 & 1.219161 & 0.278895\end{array}$

$\begin{array}{llll}-9.557136 & -0.053567 & -0.333997\end{array}$

$\begin{array}{lll}-10.399322 & -0.739292 & -0.339124\end{array}$

$\begin{array}{lll}-8.349352 & -0.433483 & -0.862014\end{array}$

$\begin{array}{llll}-8.193034 & -1.417173 & -1.291772\end{array}$

$\begin{array}{llll}-7.268911 & 0.473686 & -0.846418\end{array}$

$\begin{array}{lll}-4.225476 & -2.692818 & 0.282646\end{array}$

$\begin{array}{lll}-3.308940 & -2.739129 & 0.882269\end{array}$

$\begin{array}{llll}-5.046994 & -3.935731 & 0.567299\end{array}$

$\begin{array}{lll}-4.474810 & -5.014748 & 1.249197\end{array}$

$\begin{array}{lll}-3.452108 & -4.937586 & 1.612225\end{array}$

$\begin{array}{lll}-5.210978 & -6.180671 & 1.481698\end{array}$

$\begin{array}{lll}-4.757407 & -7.008496 & 2.019987\end{array}$

$\begin{array}{lll}-6.528797 & -6.275549 & 1.030161\end{array}$

$\begin{array}{lll}-7.106032 & -7.176772 & 1.216751\end{array}$

$\begin{array}{lll}-7.103984 & -5.202665 & 0.339779\end{array}$

$\begin{array}{llll}-8.132008 & -5.267090 & -0.005931\end{array}$

$\begin{array}{lll}-6.367161 & -4.043099 & 0.109040\end{array}$

$\begin{array}{lll}-6.822179 & -3.196355 & -0.396768\end{array}$

$\begin{array}{llll}-3.826968 & -2.670626 & -1.200279\end{array}$

$\begin{array}{llll}-4.714354 & -2.898095 & -1.801972\end{array}$

$\begin{array}{llll}-3.120621 & -3.482465 & -1.381694\end{array}$

$\begin{array}{llll}-5.270899 & -1.153588 & 2.067018\end{array}$

$\begin{array}{lll}-5.365971 & -0.071506 & 2.124093\end{array}$

$\begin{array}{lll}-6.616280 & -1.706228 & 2.533924\end{array}$

$\begin{array}{lll}-7.745545 & -0.883763 & 2.430201\end{array}$

$\begin{array}{lll}-7.644015 & 0.106144 & 1.996275\end{array}$

$\begin{array}{llll}-8.997438 & -1.313219 & 2.874492\end{array}$

$\begin{array}{lll}-9.854803 & -0.653081 & 2.778247\end{array}$

$\begin{array}{lll}-9.138177 & -2.580798 & 3.442314\end{array}$

$\begin{array}{lll}-10.109537 & -2.921163 & 3.790846\end{array}$

$\begin{array}{lll}-8.017330 & -3.406551 & 3.565254\end{array}$

$\begin{array}{llll}-8.113564 & -4.395201 & 4.005770\end{array}$

$\begin{array}{lll}-6.768105 & -2.970083 & 3.121714\end{array}$

$\begin{array}{lll}-5.915958 & -3.632252 & 3.220106\end{array}$

$\begin{array}{lll}-4.108029 & -1.537515 & 2.990308\end{array}$

$\begin{array}{lll}-3.186352 & -1.060170 & 2.649953\end{array}$

$\begin{array}{lll}-3.940640 & -2.615826 & 3.044122\end{array}$

$\begin{array}{lll}-4.324345 & -1.185313 & 4.003263\end{array}$

$\begin{array}{llll}-1.691874 & -0.340174 & -0.242144\end{array}$

$\begin{array}{llll}-1.432081 & 0.713696 & -0.181073\end{array}$

$\begin{array}{lll}-2.026754 & -0.749874 & 0.707945\end{array}$

$\begin{array}{llll}-0.841222 & -1.177512 & -1.044647\end{array}$

$-0.119443-0.712602-1.704053$

$\begin{array}{llll}-0.745448 & -2.565619 & -0.841083\end{array}$

$\begin{array}{llll}-1.405564 & -2.996916 & -0.098812\end{array}$

$\begin{array}{llll}-4.284287 & -1.029999 & -3.728597\end{array}$

$\begin{array}{llll}-5.152810 & -1.615975 & -3.440678\end{array}$ 


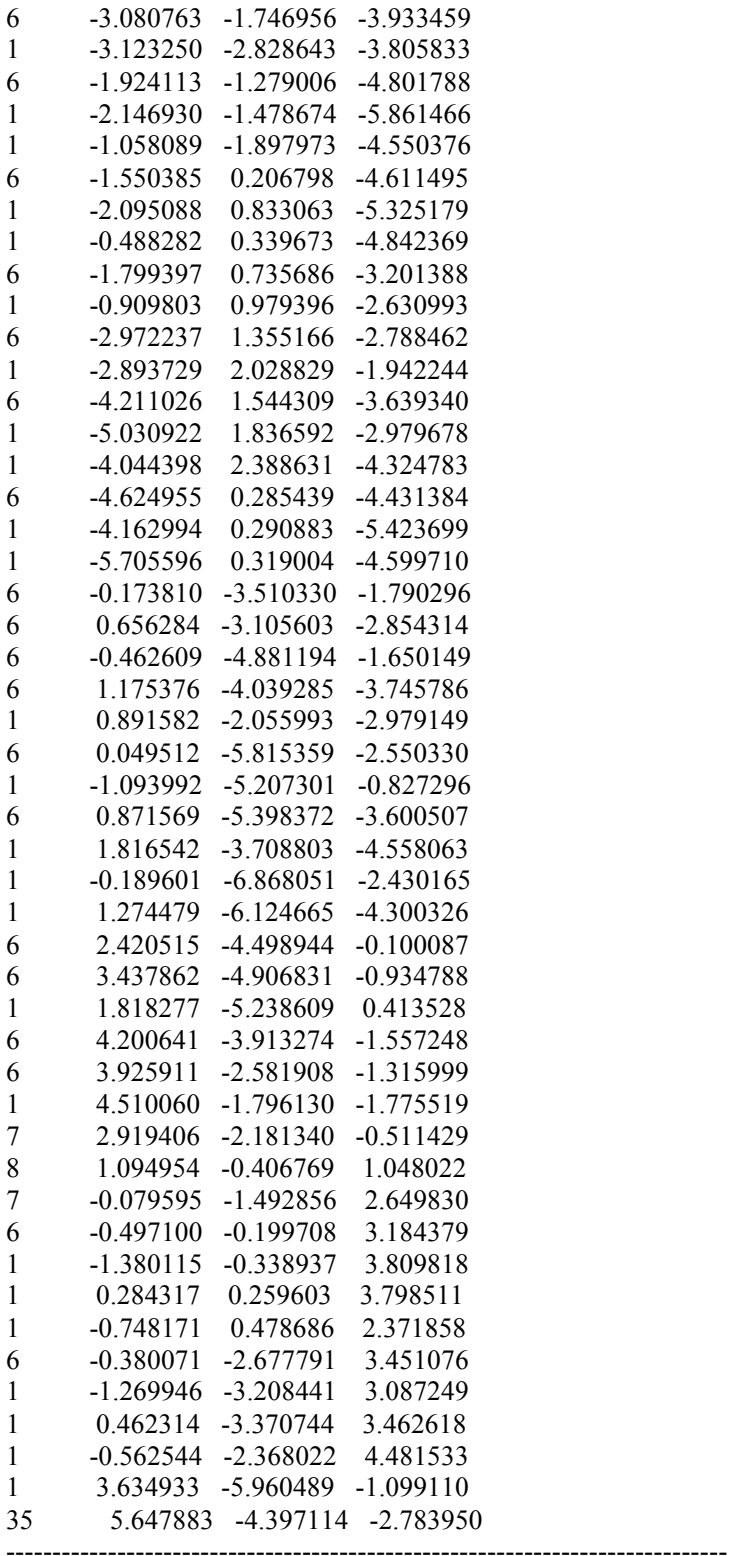

C2-P1 si-si_2

Number of imaginary frequencies $=1$

The smallest frequencies are $=-129.61608 .720513 .0915 \mathrm{~cm}(-1)$ Electronic energy $=-7222.6324644$

Zero-point correction $=$

(Hartree/Particle)

Thermal correction to Energy= $\quad 1.873410$

Thermal correction to Enthalpy= $\quad 1.874354$

Thermal correction to Gibbs Free Energy $=\quad 1.592590$

Sum of electronic and zero-point Energies $=\quad-7220.877637$

Sum of electronic and thermal Energies $=\quad-7220.759054$

Sum of electronic and thermal Enthalpies $=\quad-7220.758110$

Sum of electronic and thermal Free Energies $=\quad-7221.039874$

Cartesian Coordinates

$\begin{array}{lccc}26 & 8.003884 & 0.535572 & -0.858814 \\ 15 & 2.937978 & 1.172396 & -2.105426 \\ 15 & 3.903795 & 0.834200 & 1.536135 \\ 6 & 5.990341 & 1.055570 & -1.115717 \\ 6 & 6.207646 & 0.424178 & 0.164285 \\ 6 & 6.729960 & -0.884036 & -0.076144 \\ 1 & 7.012639 & -1.601366 & 0.681961 \\ 6 & 6.868033 & -1.058273 & -1.486205 \\ 1 & 7.273268 & -1.929939 & -1.980279\end{array}$

$6.410555 \quad 0.131163-2.126295$

$\begin{array}{llll}6.407000 & 0.320694 & -3.189412\end{array}$

$\begin{array}{llll}9.301505 & 1.883611 & 0.045241\end{array}$

$\begin{array}{llll}9.014316 & 2.596076 & 0.807030\end{array}$

$\begin{array}{lll}9.738121 & 0.543180 & 0.275857\end{array}$

$\begin{array}{lll}9.835801 & 0.063431 & 1.240631\end{array}$

$9.982431-0.066488-0.992217$

$10.289436-1.091115-1.155872$

$\begin{array}{llll}9.695775 & 0.896166 & -2.007358\end{array}$

$\begin{array}{llll}9.749405 & 0.730018 & -3.075208\end{array}$

$\begin{array}{llll}9.276429 & 2.100586 & -1.367441\end{array}$

$8.961288 \quad 3.005036-1.869516$

$\begin{array}{llll}5.392602 & 2.409621 & -1.299377\end{array}$

$\begin{array}{lll}6.133038 & 3.535734 & -0.904894\end{array}$

$\begin{array}{lll}7.122842 & 3.377603 & -0.497125\end{array}$

$\begin{array}{llll}5.628766 & 4.830266 & -1.016776\end{array}$

$\begin{array}{llll}6.222063 & 5.674828 & -0.680939\end{array}$

$4.358904 \quad 5.025020-1.553889$

$\begin{array}{llll}3.949245 & 6.024768 & -1.653941\end{array}$

$\begin{array}{llll}3.597282 & 3.922186 & -1.938830\end{array}$

$\begin{array}{lll}2.604456 & 4.094603 & -2.335960\end{array}$

$\begin{array}{llll}4.077689 & 2.608923 & -1.799990\end{array}$

$\begin{array}{llll}5.792714 & 1.016011 & 1.484070\end{array}$

$\begin{array}{llll}5.982371 & 2.089444 & 1.442123\end{array}$

$\begin{array}{lll}6.534874 & 0.445267 & 2.695455\end{array}$

$\begin{array}{lll}6.206988 & 0.929418 & 3.619604\end{array}$

$\begin{array}{llll}6.378547 & -0.630959 & 2.806629\end{array}$

$\begin{array}{llll}7.609050 & 0.618480 & 2.575321\end{array}$

$\begin{array}{llll}1.365072 & 2.011582 & -2.575301\end{array}$

$\begin{array}{llll}0.536925 & 2.457247 & -1.531003\end{array}$

$\begin{array}{llll}0.782031 & 2.203854 & -0.505985\end{array}$

$\begin{array}{llll}-0.586191 & 3.237098 & -1.805170\end{array}$

$\begin{array}{llll}-1.204698 & 3.590112 & -0.990586\end{array}$

$\begin{array}{llll}-0.923828 & 3.543141 & -3.125924\end{array}$

$\begin{array}{llll}-1.808147 & 4.138332 & -3.335491\end{array}$

$\begin{array}{llll}-0.132961 & 3.064154 & -4.172022\end{array}$

$\begin{array}{llll}-0.397353 & 3.284618 & -5.202298\end{array}$

$\begin{array}{llll}1.012589 & 2.310720 & -3.899985\end{array}$

$\begin{array}{llll}1.635340 & 1.973577 & -4.722052\end{array}$

$\begin{array}{llll}3.531077 & 0.436076 & -3.679493\end{array}$

$\begin{array}{llll}4.233139 & 1.176041 & -4.643880\end{array}$

$\begin{array}{llll}4.443634 & 2.227973 & -4.475258\end{array}$

$\begin{array}{llll}4.676087 & 0.558623 & -5.814987\end{array}$

$\begin{array}{llll}5.222334 & 1.136283 & -6.555269\end{array}$

$\begin{array}{llll}4.422323 & -0.799408 & -6.033303\end{array}$

$\begin{array}{llll}4.776701 & -1.279187 & -6.941104\end{array}$

$3.715663 \quad-1.538370 \quad-5.081370$

$\begin{array}{llll}3.523029 & -2.594940 & -5.242838\end{array}$

$\begin{array}{llll}3.270672 & -0.921838 & -3.910657\end{array}$

$\begin{array}{lll}2.735748 & -1.497067 & -3.163718\end{array}$

$\begin{array}{lll}3.203819 & 2.503915 & 1.868294\end{array}$

$\begin{array}{llll}1.803615 & 2.563877 & 1.922569\end{array}$

$\begin{array}{lll}1.236121 & 1.643437 & 1.834567\end{array}$

$\begin{array}{llll}1.148967 & 3.790213 & 2.034958\end{array}$

$\begin{array}{lll}1.868448 & 4.982594 & 2.066710\end{array}$

$\begin{array}{lll}1.359310 & 5.937601 & 2.131603\end{array}$

$\begin{array}{lll}3.261068 & 4.922253 & 1.993418\end{array}$

$\begin{array}{lll}3.929965 & 3.700657 & 1.907480\end{array}$

$\begin{array}{llll}5.009467 & 3.709750 & 1.846357\end{array}$

$\begin{array}{lll}3.500598 & -0.138297 & 3.045917\end{array}$

$\begin{array}{llll}3.727943 & -1.520698 & 2.955153\end{array}$

$\begin{array}{llll}4.171277 & -1.936831 & 2.057884\end{array}$

$\begin{array}{lll}3.351217 & -2.367624 & 3.993016\end{array}$

$\begin{array}{llll}2.712737 & -1.863502 & 5.127946\end{array}$

$\begin{array}{lll}2.398466 & -2.526625 & 5.925881\end{array}$

$\begin{array}{llll}2.471198 & -0.494033 & 5.208857\end{array}$

$\begin{array}{llll}2.870850 & 0.370593 & 4.185711\end{array}$

$\begin{array}{llll}2.670491 & 1.431362 & 4.280895\end{array}$

$\begin{array}{llll}2.787146 & -0.157307 & -0.283882\end{array}$

$\begin{array}{llll}1.683300 & 0.045881 & 6.370248\end{array}$

$\begin{array}{llll}3.524724 & -3.854641 & 3.853140\end{array}$

$\begin{array}{lll}-0.350975 & 3.792693 & 2.129877\end{array}$

$\begin{array}{lll}4.026799 & 6.217373 & 1.965437\end{array}$

$\begin{array}{llll}3.731767 & 6.942241 & 0.857875\end{array}$

$\begin{array}{lll}5.364884 & 6.031817 & 1.973319\end{array}$ 
$\begin{array}{lll}3.721010 & 7.000022 & 3.026581\end{array}$

$\begin{array}{lll}-0.904732 & 2.912411 & 1.246629\end{array}$

$\begin{array}{lll}-0.787016 & 3.414220 & 3.353170\end{array}$

$\begin{array}{llll}-0.890283 & 5.001191 & 1.864365\end{array}$

$\begin{array}{lll}1.964150 & 1.341692 & 6.622708\end{array}$

$\begin{array}{lll}0.343289 & -0.019464 & 6.128446\end{array}$

$\begin{array}{lll}1.901382 & -0.651080 & 7.505473\end{array}$

$\begin{array}{llll}3.955881 & -4.422788 & 5.000799\end{array}$

$\begin{array}{llll}4.400611 & -4.182333 & 2.879996\end{array}$

$\begin{array}{llll}2.347104 & -4.464377 & 3.543201\end{array}$

$\begin{array}{llll}0.664620 & -1.343787 & 1.568122\end{array}$

$\begin{array}{llll}0.978069 & -2.683061 & 1.144427\end{array}$

$\begin{array}{lll}2.105648 & -3.099677 & 0.37165\end{array}$

$\begin{array}{llll}0.534834 & -3.488576 & 1.711411\end{array}$

$\begin{array}{llll}-3.317360 & -1.168254 & -1.663083\end{array}$

$\begin{array}{lll}-5.086051 & -0.635547 & -0.337468\end{array}$

$\begin{array}{llll}-5.147113 & 0.646866 & 0.712306\end{array}$

$\begin{array}{llll}-6.419381 & -0.301632 & -1.273951\end{array}$

$\begin{array}{llll}-5.382708 & -1.872896 & 0.752305\end{array}$

$\begin{array}{llll}-5.093938 & 1.941339 & 0.214721\end{array}$

$\begin{array}{lll}-3.922670 & 2.675241 & 0.499392\end{array}$

$\begin{array}{lll}-3.159375 & 2.211645 & 1.111439\end{array}$

$\begin{array}{lll}-3.785654 & 3.947114 & 0.002149\end{array}$

$\begin{array}{llll}-2.908545 & 4.538430 & 0.245245\end{array}$

$\begin{array}{llll}-4.776295 & 4.488924 & -0.861837\end{array}$

$\begin{array}{llll}-4.587242 & 5.747256 & -1.495177\end{array}$

$\begin{array}{llll}-3.677497 & 6.302479 & -1.281508\end{array}$

$\begin{array}{llll}-5.523396 & 6.245112 & -2.373587\end{array}$

$\begin{array}{llll}-5.363961 & 7.202964 & -2.860036\end{array}$

$\begin{array}{llll}-6.692362 & 5.498169 & -2.654362\end{array}$

$\begin{array}{llll}-7.418264 & 5.882674 & -3.365035\end{array}$

$\begin{array}{llll}-6.917166 & 4.285461 & -2.038989\end{array}$

$\begin{array}{llll}-7.814683 & 3.723404 & -2.270109\end{array}$

$\begin{array}{llll}-5.978207 & 3.745387 & -1.117008\end{array}$

$\begin{array}{llll}-6.173879 & 2.470571 & -0.473392\end{array}$

$\begin{array}{llll}-7.473496 & 1.741498 & -0.517892\end{array}$

$\begin{array}{llll}-8.698688 & 2.375843 & -0.089706\end{array}$

$\begin{array}{lll}-8.729462 & 3.667583 & 0.507809\end{array}$

$\begin{array}{llll}-7.801889 & 4.202847 & 0.668911\end{array}$

$\begin{array}{llll}-9.917448 & 4.244813 & 0.902347\end{array}$

$\begin{array}{lll}-9.907777 & 5.229148 & 1.361703\end{array}$

$\begin{array}{lll}-11.146448 & 3.567782 & 0.722677\end{array}$

$\begin{array}{lll}-12.075404 & 4.038667 & 1.030522\end{array}$

$\begin{array}{lll}-11.153327 & 2.305331 & 0.174481\end{array}$

$\begin{array}{lll}-12.085789 & 1.760980 & 0.049625\end{array}$

$\begin{array}{llll}-9.944378 & 1.675074 & -0.226600\end{array}$

$\begin{array}{llll}-9.950185 & 0.349629 & -0.738293\end{array}$

$\begin{array}{lll}-10.897882 & -0.169173 & -0.851531\end{array}$

$\begin{array}{llll}-8.772557 & -0.278079 & -1.052207\end{array}$

$\begin{array}{llll}-8.745613 & -1.302182 & -1.409395\end{array}$

$\begin{array}{lll}-7.547319 & 0.412852 & -0.911943\end{array}$

$\begin{array}{llll}-4.478865 & -3.038700 & 0.557839\end{array}$

$\begin{array}{lll}-3.622324 & -2.931172 & 1.237606\end{array}$

$\begin{array}{lll}-5.160225 & -4.360363 & 0.866982\end{array}$

$\begin{array}{lll}-4.476100 & -5.342638 & 1.592556\end{array}$

$\begin{array}{lll}-3.485518 & -5.122606 & 1.984933\end{array}$

$\begin{array}{llll}-5.055328 & -6.594462 & 1.818960\end{array}$

$\begin{array}{lll}-4.512540 & -7.347046 & 2.384400\end{array}$

$\begin{array}{lll}-6.332525 & -6.872705 & 1.327925\end{array}$

$\begin{array}{llll}-6.788050 & -7.842221 & 1.508533\end{array}$

$\begin{array}{lll}-7.025433 & -5.894087 & 0.607089\end{array}$

$\begin{array}{llll}-8.021626 & -6.102746 & 0.226577\end{array}$

$\begin{array}{lll}-6.441547 & -4.648921 & 0.374824\end{array}$

$\begin{array}{llll}-6.979723 & -3.884696 & -0.180016\end{array}$

$\begin{array}{lll}-3.981609 & -3.056180 & -0.891394\end{array}$

$\begin{array}{lll}-4.810425 & -3.394612 & -1.525742\end{array}$

$\begin{array}{llll}-3.196512 & -3.808024 & -0.987763\end{array}$

$\begin{array}{lll}-6.060014 & -1.787530 & 2.086948\end{array}$

$\begin{array}{lll}-6.349541 & -2.821704 & 2.288694\end{array}$

$\begin{array}{llll}-5.113749 & -1.387708 & 3.220963\end{array}$

$\begin{array}{lll}-4.455675 & -2.398789 & 3.938005\end{array}$

$\begin{array}{llll}-4.621056 & -3.437377 & 3.663518\end{array}$

$\begin{array}{lll}-3.611556 & -2.093396 & 5.008959\end{array}$

$\begin{array}{lll}-3.114552 & -2.894256 & 5.549206\end{array}$

$\begin{array}{lll}-3.412055 & -0.764138 & 5.385051\end{array}$ $\begin{array}{llll}-2.747932 & -0.520027 & 6.209711\end{array}$

$\begin{array}{llll}-4.068312 & 0.252346 & 4.684265\end{array}$

$\begin{array}{lll}-3.926018 & 1.290340 & 4.972872\end{array}$

$\begin{array}{lll}-4.911577 & -0.055932 & 3.614715\end{array}$

$\begin{array}{lll}-5.416563 & 0.745449 & 3.090352\end{array}$

$\begin{array}{lll}-7.366058 & -0.993542 & 2.066698\end{array}$

$\begin{array}{lll}-8.037607 & -1.391159 & 1.302667\end{array}$

$\begin{array}{lll}-7.225258 & 0.070728 & 1.879491\end{array}$

$\begin{array}{lll}-7.854067 & -1.102915 & 3.039909\end{array}$

$\begin{array}{lll}-2.037822 & -0.563758 & -0.021649\end{array}$

$\begin{array}{llll}-1.867714 & 0.509759 & -0.005933\end{array}$

$\begin{array}{llll}-2.339479 & -0.958571 & 0.946514\end{array}$

$\begin{array}{lll}-1.126730 & -1.359128 & -0.802592\end{array}$

$\begin{array}{llll}-0.457311 & -0.854987 & -1.487687\end{array}$

$\begin{array}{llll}-0.890079 & -2.720814 & -0.540829\end{array}$

$\begin{array}{lll}-1.492548 & -3.178405 & 0.236803\end{array}$

$\begin{array}{llll}-4.591862 & -1.533278 & -3.475866\end{array}$

$\begin{array}{lll}-5.426016 & -2.154420 & -3.158492\end{array}$

$\begin{array}{llll}-3.355284 & -2.194058 & -3.655963\end{array}$

$\begin{array}{llll}-3.341393 & -3.269562 & -3.479783\end{array}$

$\begin{array}{llll}-2.221617 & -1.710651 & -4.542862\end{array}$

$\begin{array}{lll}-2.429264 & -1.974592 & -5.591617\end{array}$

$\begin{array}{llll}-1.325087 & -2.270066 & -4.259048\end{array}$

$\begin{array}{llll}-1.930652 & -0.200535 & -4.426885\end{array}$

$\begin{array}{llll}-2.514332 & 0.358239 & -5.165215\end{array}$

$\begin{array}{lll}-0.879791 & -0.018619 & -4.672216\end{array}$

$\begin{array}{llll}-2.200959 & 0.382924 & -3.042853\end{array}$

$\begin{array}{llll}-1.321846 & 0.684441 & -2.485674\end{array}$

$\begin{array}{llll}-3.398029 & 0.973727 & -2.666088\end{array}$

$\begin{array}{llll}-3.358076 & 1.692032 & -1.853403\end{array}$

$\begin{array}{llll}-4.635510 & 1.051807 & -3.535869\end{array}$

$\begin{array}{llll}-5.470006 & 1.367351 & -2.908592\end{array}$

$\begin{array}{llll}-4.490379 & 1.848247 & -4.281124\end{array}$

$\begin{array}{llll}-5.002847 & -0.273450 & -4.240047\end{array}$

$\begin{array}{llll}-4.558563 & -0.307539 & -5.240188\end{array}$

$\begin{array}{llll}-6.086551 & -0.297592 & -4.388558\end{array}$

$\begin{array}{llll}-0.253049 & -3.644810 & -1.466998\end{array}$

$\begin{array}{llll}0.551407 & -3.206516 & -2.538373\end{array}$

$\begin{array}{llll}-0.447678 & -5.029182 & -1.298891\end{array}$

$\begin{array}{llll}1.130084 & -4.121159 & -3.412241\end{array}$

$\begin{array}{llll}0.726740 & -2.146620 & -2.678790\end{array}$

$\begin{array}{llll}0.124860 & -5.944805 & -2.182121\end{array}$

$\begin{array}{llll}-1.056939 & -5.381587 & -0.470251\end{array}$

$\begin{array}{llll}0.916521 & -5.494671 & -3.241438\end{array}$

$\begin{array}{llll}1.746636 & -3.763923 & -4.231749\end{array}$

$\begin{array}{llll}-0.043497 & -7.008477 & -2.040415\end{array}$

$\begin{array}{llll}1.366449 & -6.205583 & -3.928277\end{array}$

$\begin{array}{llll}2.375532 & -4.497009 & 0.298019\end{array}$

$\begin{array}{llll}3.405258 & -4.991791 & -0.472909\end{array}$

$\begin{array}{llll}1.751336 & -5.179037 & 0.862994\end{array}$

$\begin{array}{llll}4.191345 & -4.068640 & -1.170060\end{array}$

$\begin{array}{llll}3.927343 & -2.717489 & -1.060531\end{array}$

$\begin{array}{llll}4.529173 & -1.983950 & -1.579787\end{array}$

$\begin{array}{llll}2.910815 & -2.233945 & -0.319008\end{array}$

$\begin{array}{llll}1.010437 & -0.304098 & 0.925978\end{array}$

$\begin{array}{lll}-0.123642 & -1.187463 & 2.678109\end{array}$

$\begin{array}{lll}-0.677395 & 0.121969 & 3.001396\end{array}$

$\begin{array}{lll}-1.694722 & 0.002134 & 3.380036\end{array}$

$\begin{array}{lll}-0.083385 & 0.630964 & 3.768277\end{array}$

$\begin{array}{lll}-0.710996 & 0.735574 & 2.107646\end{array}$

$\begin{array}{lll}-0.433329 & -2.264978 & 3.611708\end{array}$

$\begin{array}{lll}-1.335066 & -2.818175 & 3.319422\end{array}$

$\begin{array}{lll}0.401586 & -2.959623 & 3.701094\end{array}$

$\begin{array}{lll}-0.610275 & -1.829378 & 4.596273\end{array}$

$\begin{array}{llll}3.594100 & -6.058020 & -0.533308\end{array}$

$\begin{array}{llll}5.659020 & -4.674019 & -2.315761\end{array}$

C2-P1 si-si 3

Number of imaginary frequencies $=1$

The smallest frequencies are $=-144.518111 .538811 .9627 \mathrm{~cm}(-1)$ Electronic energy $=-7222.6282241$

Zero-point correction=

1.756203

(Hartree/Particle)

Thermal correction to Energy=

1.874354 
Thermal correction to Enthalpy=

Thermal correction to Gibbs Free Energy= Sum of electronic and zero-point Energies= Sum of electronic and thermal Energies= Sum of electronic and thermal Enthalpies= Sum of electronic and thermal Free Energies=

.875298 1.595172 $-7220.872021$ $-7220.753870$ $-7220.752926$

Cartesian Coordinates

\begin{tabular}{|c|c|c|c|}
\hline y & 8.092456 & 0.520782 & -0.649762 \\
\hline & 3.054031 & 1.500962 & -1.795088 \\
\hline & 3.972654 & 0.521829 & 1.730185 \\
\hline 6 & 6.103351 & 1.153527 & -0.851510 \\
\hline & 6.273186 & 0.306954 & 0.305064 \\
\hline 6 & 6.751376 & -0.961422 & -0.148749 \\
\hline 1 & 6.991041 & -1.809095 & 0.478660 \\
\hline 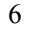 & 6.916825 & -0.896084 & -1.564898 \\
\hline 1 & 7.302180 & -1.684618 & -2.195259 \\
\hline 6 & 6.516845 & 0.402161 & -1.998324 \\
\hline 1 & 6.542510 & 0.769819 & -3.013192 \\
\hline 6 & 9.407516 & 1.637457 & 0.508017 \\
\hline 1 & 9.122472 & 2.222290 & 1.372716 \\
\hline 6 & 9.785035 & 0.259813 & 0.515243 \\
\hline 1 & 9.823720 & -0.384103 & 1.383864 \\
\hline 6 & 10.048776 & -0.133152 & -0.832455 \\
\hline 1 & 10.322523 & -1.126684 & -1.162385 \\
\hline 6 & 9.834717 & 1.001345 & -1.673139 \\
\hline 1 & 9.921046 & 1.019619 & -2.751557 \\
\hline 6 & 9.440038 & 2.094843 & -0.846014 \\
\hline 1 & 9.179285 & 3.086140 & -1.190849 \\
\hline 6 & 5.539586 & 2.532587 & -0.806571 \\
\hline 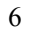 & 6.303127 & 3.560121 & -0.229996 \\
\hline 1 & 7.291265 & 3.3 & 0.1 \\
\hline 6 & 5.821107 & 4.862579 & -0.110494 \\
\hline 1 & 6.430155 & 5.625525 & 0.363981 \\
\hline 6 & 4.552992 & 5.169464 & -0.597232 \\
\hline 1 & 4.160690 & 6.178038 & -0.517938 \\
\hline 6 & 3.771704 & 4.164137 & -1.165873 \\
\hline 1 & 2.782108 & 4.420370 & -1.523842 \\
\hline 6 & 4.228231 & 2.837973 & -1.258847 \\
\hline 6 & 5.861336 & 0.688781 & 1.700006 \\
\hline 1 & 6.057034 & 1.755641 & 1.820109 \\
\hline 6 & 6.602726 & -0.063039 & 556 \\
\hline 1 & 6.280341 & 0.276919 & 3.796807 \\
\hline 1 & 6.440400 & -1.142866 & 2.755692 \\
\hline 1 & 7.677353 & 0.120181 & 2.710981 \\
\hline 6 & 1.514434 & 2.450703 & -2.149022 \\
\hline 6 & 0.660769 & 2.724590 & -1.066949 \\
\hline 1 & 0.866836 & 2.287623 & -0.095991 \\
\hline 6 & -0.434864 & 3.572894 & -1.230695 \\
\hline 1 & -1.059769 & 3.811669 & -0.379412 \\
\hline 6 & -0.721782 & 4.114685 & -2.485895 \\
\hline 1 & -1.585514 & 4.760875 & -2.613119 \\
\hline 6 & 0.093965 & 3.808168 & -3.576963 \\
\hline 1 & -0.130747 & 4.215931 & -4.558360 \\
\hline 6 & 1.215147 & 2.991626 & -3.408701 \\
\hline 1 & 1.859325 & 2.790456 & -4.258090 \\
\hline 6 & 3.625865 & 0.991762 & -3.463452 \\
\hline 6 & 4.375644 & 1.828605 & -4.304315 \\
\hline 1 & 4.660248 & 2.821821 & -3.969715 \\
\hline 6 & 4.766973 & 1.382136 & -5.568036 \\
\hline 1 & 5.351637 & 2.033039 & -6.211862 \\
\hline 6 & 4.409491 & 0.102175 & -6.004370 \\
\hline 1 & 4.719893 & -0.243129 & -6.986357 \\
\hline 6 & 3.654931 & -0.731601 & -5.175293 \\
\hline 1 & 3.377419 & -1.728696 & -5.505812 \\
\hline 6 & 3.267812 & -0.287707 & -3.909912 \\
\hline 1 & 2.701009 & -0.939560 & -3.255387 \\
\hline 6 & 3.293739 & 2.124502 & 2.335622 \\
\hline 6 & 1.893619 & 2.203115 & 2.374426 \\
\hline 1 & 1.310151 & 1.325244 & 2.116467 \\
\hline 6 & 1.256507 & 3.402180 & 2.690686 \\
\hline & 1.995129 & 4.557564 & 2.937097 \\
\hline & 1.501105 & 5.497054 & 3.158632 \\
\hline & 3.388098 & 4.483941 & 2.872382 \\
\hline
\end{tabular}

$\begin{array}{llll}4.039455 & 3.281855 & 2.592286\end{array}$

$\begin{array}{lll}5.120539 & 3.276874 & 2.550506\end{array}$

$\begin{array}{llll}3.571900 & -0.683951 & 3.059237\end{array}$

$\begin{array}{lll}3.764982 & -2.037282 & 2.738932\end{array}$

$\begin{array}{lll}4.182717 & -2.311440 & 1.777460\end{array}$

$\begin{array}{lll}3.384149 & -3.034215 & 3.632092\end{array}$

$\begin{array}{llll}2.776737 & -2.709408 & 4.846470\end{array}$

$2.457331-3.487954 \quad 5.530123$

$\begin{array}{llll}2.575653 & -1.367047 & 5.157617\end{array}$

$\begin{array}{lll}2.980340 & -0.355356 & 4.281981\end{array}$

$\begin{array}{lll}2.810953 & 0.679788 & 4.554167\end{array}$

$\begin{array}{llll}2.817728 & -0.106381 & -0.220078\end{array}$

$\begin{array}{llll}1.813696 & -1.006381 & 6.401870\end{array}$

$\begin{array}{llll}3.518824 & -4.482971 & 3.254496\end{array}$

$\begin{array}{lll}-0.245089 & 3.408484 & 2.772806\end{array}$

$\begin{array}{lll}4.174646 & 5.750804 & 3.071949\end{array}$

$\begin{array}{llll}3.870882 & 6.672916 & 2.124751\end{array}$

$\begin{array}{lll}5.509374 & 5.549479 & 3.017106\end{array}$

$\begin{array}{llll}3.902190 & 6.326470 & 4.266125\end{array}$

$\begin{array}{lll}-0.807711 & 2.732192 & 1.726861\end{array}$

$\begin{array}{lll}-0.691134 & 2.791286 & 3.892362\end{array}$

$\begin{array}{lll}-0.764665 & 4.650522 & 2.760208\end{array}$

$\begin{array}{lll}2.147653 & 0.214002 & 6.873210\end{array}$

$\begin{array}{llll}0.474146 & -0.976646 & 6.159889\end{array}$

$\begin{array}{llll}2.006680 & -1.897424 & 7.397452\end{array}$

$\begin{array}{llll}3.967663 & -5.231883 & 4.285829\end{array}$

$\begin{array}{llll}4.360361 & -4.671288 & 2.216647\end{array}$

$\begin{array}{llll}2318372 & -5.013766 & 2.890636\end{array}$

$\begin{array}{llll}0.683793 & -1.563762 & 1.447691\end{array}$

$\begin{array}{llll}0.977509 & -2.808047 & 0.791128\end{array}$

$\begin{array}{llll}2.107544 & -3.107001 & -0.032029\end{array}$

$\begin{array}{llll}0.507316 & -3.692094 & 1.197816\end{array}$

$-3.030878-0.701125-1.967298$

$\begin{array}{lll}-4.737343 & -0.154320 & -0.582609\end{array}$

$\begin{array}{lll}-4.719668 & 1.136120 & 0.461512\end{array}$

$\begin{array}{lll}-6.124743 & 0.177434 & -1.438508\end{array}$

$\begin{array}{llll}-5.011786 & -1.380061 & 0.537298\end{array}$

$\begin{array}{lll}-4.965277 & 2.428488 & 0.018642\end{array}$

$\begin{array}{llll}-3.875520 & 3.323078 & 0.074713\end{array}$

$\begin{array}{lll}-2.940211 & 2.967223 & 0.490051\end{array}$

$\begin{array}{llll}-4.029911 & 4.607904 & -0.381679\end{array}$

$\begin{array}{llll}-3.201947 & 5.310121 & -0.337886\end{array}$

$\begin{array}{llll}-5.267138 & 5.027792 & -0.942049\end{array}$

$\begin{array}{llll}-5.415223 & 6.327424 & -1.497689\end{array}$

$\begin{array}{llll}-4.564169 & 7.003039 & -1.467747\end{array}$

$\begin{array}{llll}-6.602953 & 6.720864 & -2.071686\end{array}$

$\begin{array}{llll}-6.703645 & 7.714981 & -2.497233\end{array}$

$\begin{array}{llll}-7.693195 & 5.819737 & -2.119348\end{array}$

$\begin{array}{llll}-8.621770 & 6.124093 & -2.593643\end{array}$

$\begin{array}{llll}-7.587901 & 4.557629 & -1.575849\end{array}$

$\begin{array}{llll}-8.430815 & 3.879089 & -1.631133\end{array}$

$\begin{array}{lll}-6.382708 & 4.122282 & -0.956975\end{array}$

$\begin{array}{llll}-6.234807 & 2.805150 & -0.391802\end{array}$

$\begin{array}{llll}-7.378465 & 1.857202 & -0.261430\end{array}$

$\begin{array}{lll}-8.584490 & 2.198623 & 0.447952\end{array}$

$\begin{array}{lll}-8.726234 & 3.406510 & 1.187583\end{array}$

$\begin{array}{llll}-7.896282 & 4.101571 & 1.234050\end{array}$

$\begin{array}{llll}-9.894653 & 3.697711 & 1.857498\end{array}$

$\begin{array}{lll}-9.973512 & 4.622836 & 2.421269\end{array}$

$\begin{array}{lll}-10.990045 & 2.801773 & 1.825202\end{array}$

$\begin{array}{lll}-11.906212 & 3.048068 & 2.353886\end{array}$

$\begin{array}{lll}-10.881895 & 1.614896 & 1.136983\end{array}$

$\begin{array}{lll}-11.707499 & 0.908252 & 1.118618\end{array}$

$\begin{array}{lll}-9.686183 & 1.276224 & 0.446403\end{array}$

$\begin{array}{llll}-9.557631 & 0.028588 & -0.221622\end{array}$

$\begin{array}{llll}-10.399315 & -0.658178 & -0.224502\end{array}$

$\begin{array}{llll}-8.370992 & -0.325052 & -0.815281\end{array}$

$\begin{array}{llll}-8.230934 & -1.289315 & -1.292321\end{array}$

$\begin{array}{llll}-7.292852 & 0.585175 & -0.806650\end{array}$

$\begin{array}{lll}-4.255427 & -2.614532 & 0.208628\end{array}$

$\begin{array}{llll}-3.348959 & -2.642446 & 0.826575\end{array}$

$\begin{array}{llll}-5.032172 & -3.897516 & 0.445661\end{array}$

$\begin{array}{lll}-4.373604 & -5.014787 & 0.970107\end{array}$

$\begin{array}{lll}-3.329465 & -4.931693 & 1.265045\end{array}$

$\begin{array}{llll}-5.049607 & -6.226328 & 1.138438\end{array}$ 


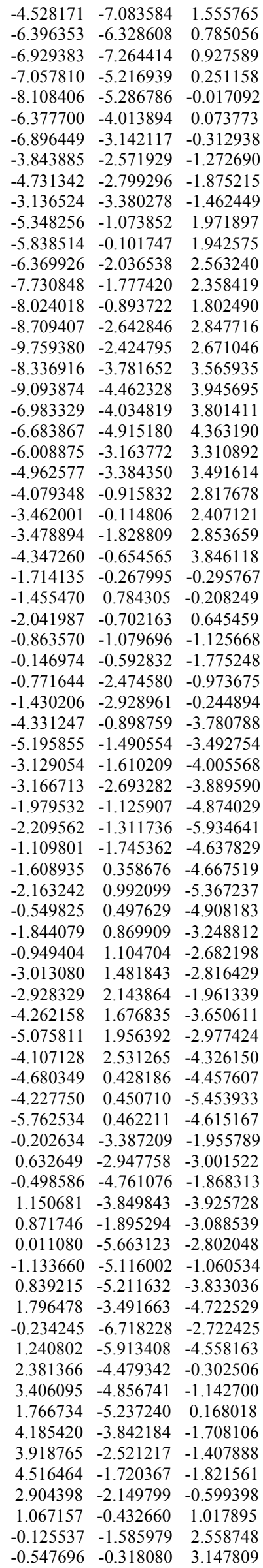

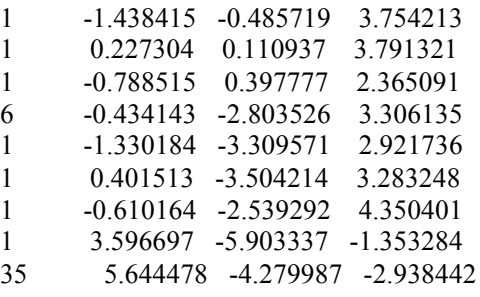

C2-P1 si-si_4

Number of imaginary frequencies $=1$

The smallest frequencies are $=-85.0501 \quad 8.768412 .6547 \mathrm{~cm}(-1)$ Electronic energy $=-7222.6286078$

Zero-point correction $=$

1.755409

(Hartree/Particle)

Thermal correction to Energy= $\quad 1.873899$

Thermal correction to Enthalpy= $\quad 1.874843$

Thermal correction to Gibbs Free Energy $=\quad 1.593613$

Sum of electronic and zero-point Energies $=\quad-7220.873199$

Sum of electronic and thermal Energies $=\quad-7220.754709$

Sum of electronic and thermal Enthalpies $=\quad-7220.753764$

Sum of electronic and thermal Free Energies $=\quad-7221.034995$

\begin{tabular}{|c|c|c|c|}
\hline 26 & -7.835704 & 2.630500 & 0.942235 \\
\hline 15 & -4.584026 & 0.210632 & -2.343153 \\
\hline 15 & -4.518735 & -0.774960 & 1.278003 \\
\hline 6 & -6.704372 & 1.355528 & -0.269583 \\
\hline 6 & -6.230632 & 1.338663 & 1.093900 \\
\hline 6 & -5.831146 & 2.666715 & 1.436144 \\
\hline 1 & -5.446908 & 2.985409 & 2.395498 \\
\hline 6 & -6.083841 & 3.511506 & 0.313705 \\
\hline 1 & -5.927799 & 4.580754 & 0.276613 \\
\hline 6 & -6.621451 & 2.707917 & -0.734318 \\
\hline 1 & -6.936455 & 3.058807 & -1.705457 \\
\hline 6 & -9.510846 & 1.869082 & 1.909937 \\
\hline 1 & -9.620620 & 0.844945 & 2.241378 \\
\hline 6 & -8.942605 & 2.938145 & 2.668127 \\
\hline 1 & -8.537790 & 2.8628 & 3.6 \\
\hline 6 & -8.959195 & 4.113 & 1.8 \\
\hline 1 & -8.565551 & 5.08 & 200 \\
\hline 6 & & & \\
\hline 1 & -9.661281 & 4.4 & 467 \\
\hline 6 & -9.878570 & 2.385852 & 0.628683 \\
\hline 1 & -10.310918 & 1.822942 & -0.187302 \\
\hline 6 & -7.112118 & 0.132592 & -1.020443 \\
\hline 6 & -8.317473 & -0.506259 & -0.692479 \\
\hline 1 & -8.940640 & -0.058401 & 0.070759 \\
\hline 6 & -8.712537 & -1.695404 & 5105 \\
\hline 1 & -9.639377 & -2.176703 & -1.009111 \\
\hline 6 & -7.900637 & -2.266217 & -2.282212 \\
\hline 1 & -8.188914 & -3.194535 & -2.764493 \\
\hline 6 & -6.695532 & -1.651847 & -2.622919 \\
\hline 1 & -6.067636 & -2.11 & -3.375748 \\
\hline 6 & -6.274171 & -0.467618 & -1.997700 \\
\hline 6 & -6.0851 & 0.091 & 1.919550 \\
\hline 1 & -6.910028 & -0.570532 & 1.650449 \\
\hline 6 & -6.138570 & 0.325875 & 3.431427 \\
\hline 1 & -6.053005 & -0.616297 & 3.979800 \\
\hline 1 & -5.341740 & 0.989343 & 3.777771 \\
\hline 1 & -7.095489 & 0.791494 & 3.686274 \\
\hline 6 & -3.919774 & -0.926969 & -3.630842 \\
\hline 6 & -3.139596 & -2.010707 & -3.198147 \\
\hline 1 & -2.922182 & -2.127791 & -2.142506 \\
\hline 6 & -2.609040 & -2.911984 & -4.122694 \\
\hline 1 & -2.015284 & -3.750941 & -3.775903 \\
\hline 6 & -2.833784 & -2.727613 & -5.489496 \\
\hline 1 & -2.411547 & -3.422726 & -6.209803 \\
\hline 6 & -3.599871 & -1.644104 & -5.928010 \\
\hline 1 & -3.775440 & -1.493670 & -6.989569 \\
\hline 6 & -4.145989 & -0.750843 & -5.004140 \\
\hline 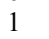 & -4.738902 & 0.085285 & -5.359322 \\
\hline
\end{tabular}


$\begin{array}{lll}-4.929514 & 1.764748 & -3.261880\end{array}$

$\begin{array}{llll}-6.085804 & 1.938413 & -4.039508\end{array}$

$-6.812040 \quad 1.135166 \quad-4.120764$

$\begin{array}{llll}-6.317020 & 3.149924 & -4.693146\end{array}$

$\begin{array}{llll}-7.216123 & 3.277840 & -5.289296\end{array}$

$\begin{array}{llll}-5.397272 & 4.197581 & -4.577035\end{array}$

$\begin{array}{llll}-5.583494 & 5.142061 & -5.080461\end{array}$

$\begin{array}{llll}-4.240130 & 4.026784 & -3.813390\end{array}$

$\begin{array}{llll}-3.520901 & 4.835866 & -3.720859\end{array}$

$\begin{array}{llll}-4.008922 & 2.815633 & -3.158332\end{array}$

$\begin{array}{llll}-3.112555 & 2.683600 & -2.564817\end{array}$

$\begin{array}{llll}-5.028409 & -2.478078 & 0.792060\end{array}$

$\begin{array}{lll}-4.028594 & -3.261167 & 0.196156\end{array}$

$\begin{array}{llll}-3.029410 & -2.850828 & 0.086485\end{array}$

$\begin{array}{llll}-4.318463 & -4.545392 & -0.265191\end{array}$

$\begin{array}{llll}-5.610971 & -5.059318 & -0.180565\end{array}$

$\begin{array}{llll}-5.839025 & -6.049232 & -0.559226\end{array}$

$\begin{array}{lll}-6.608041 & -4.269216 & 0.391806\end{array}$

$\begin{array}{lll}-6.323863 & -2.998881 & 0.892401\end{array}$

$\begin{array}{lll}-7.128412 & -2.427770 & 1.334976\end{array}$

$\begin{array}{lll}-3.460087 & -1.067567 & 2.756483\end{array}$

$\begin{array}{lll}-2.805824 & 0.046916 & 3.303721\end{array}$

$\begin{array}{lll}-2.942720 & 1.029534 & 2.864392\end{array}$

$\begin{array}{lll}-1.964430 & -0.101548 & 4.405434\end{array}$

$\begin{array}{lll}-1.707523 & -1.361439 & 4.947387\end{array}$

$\begin{array}{lll}-1.032169 & -1.475378 & 5.786477\end{array}$

$\begin{array}{lll}-2.325439 & -2.470932 & 4.375507\end{array}$

$\begin{array}{lll}-3.210045 & -2.329772 & 3.304357\end{array}$

$\begin{array}{lll}-3.691365 & -3.213022 & 2.901252\end{array}$

$\begin{array}{lll}-3.315129 & 0.259136 & -0.459285\end{array}$

$\begin{array}{lll}-1.987499 & -3.857662 & 4.845237\end{array}$

$\begin{array}{llll}-1.354371 & 1.124833 & 5.022988\end{array}$

$\begin{array}{llll}-3.199369 & -5.388240 & -0.808822\end{array}$

$\begin{array}{lll}-8.010743 & -4.810221 & 0.428779\end{array}$

$\begin{array}{llll}-8.496268 & -5.012669 & -0.821399\end{array}$

$\begin{array}{lll}-8.871933 & -3.981961 & 1.059046\end{array}$

$\begin{array}{lll}-8.067533 & -6.007045 & 1.058123\end{array}$

$\begin{array}{lll}-2.345686 & -4.662827 & -1.577053\end{array}$

$\begin{array}{llll}-2.448210 & -5.918106 & 0.189738\end{array}$

$\begin{array}{llll}-3.640591 & -6.415435 & -1.561559\end{array}$

$\begin{array}{lll}-3.072651 & -4.662666 & 4.870178\end{array}$

$\begin{array}{llll}-1.091511 & -4.451351 & 4.008193\end{array}$

$\begin{array}{llll}-1.438923 & -3.869707 & 6.076795\end{array}$

$\begin{array}{llll}-0.252072 & 0.837426 & 5.754444\end{array}$

$\begin{array}{lll}-2.220756 & 1.756266 & 5.848219\end{array}$

$\begin{array}{lll}-0.985811 & 2.030722 & 4.083637\end{array}$

$\begin{array}{lll}-0.749904 & -0.946408 & 0.607561\end{array}$

$\begin{array}{lll}-0.253737 & 0.349790 & 0.976208\end{array}$

$\begin{array}{llll}-0.876572 & 1.619441 & 0.813514\end{array}$

$\begin{array}{lll}0.625481 & 0.376314 & 1.604438\end{array}$

$\begin{array}{llll}4.180867 & -0.260119 & -1.753589\end{array}$

$\begin{array}{llll}5.855185 & 0.321354 & -0.341730\end{array}$

$\begin{array}{llll}6.401547 & -0.635434 & 0.889732\end{array}$

$\begin{array}{llll}7.274701 & 0.588151 & -1.171072\end{array}$

$\begin{array}{llll}5.410008 & 1.685301 & 0.527965\end{array}$

$\begin{array}{llll}7.305140 & -1.670013 & 0.664967\end{array}$

$\begin{array}{llll}6.818481 & -2.980302 & 0.864055\end{array}$

$\begin{array}{llll}5.791088 & -3.107310 & 1.189124\end{array}$

$\begin{array}{llll}7.648870 & -4.051320 & 0.645782\end{array}$

$\begin{array}{llll}7.290073 & -5.064957 & 0.799849\end{array}$

$\begin{array}{llll}8.979001 & -3.850739 & 0.187476\end{array}$

$\begin{array}{llll}9.823017 & -4.953711 & -0.114754\end{array}$

$\begin{array}{llll}9.435182 & -5.958142 & 0.033469\end{array}$

$\begin{array}{llll}11.097414 & -4.757847 & -0.596391\end{array}$

$\begin{array}{llll}11.732432 & -5.607665 & -0.828810\end{array}$

$\begin{array}{llll}11.576071 & -3.442266 & -0.804204\end{array}$

$\begin{array}{llll}12.573654 & -3.290702 & -1.206286\end{array}$

$10.788411-2.351076 \quad-0.507762$

$\begin{array}{llll}11.170349 & -1.352372 & -0.682758\end{array}$

$\begin{array}{llll}9.473594 & -2.514023 & 0.012016\end{array}$

$\begin{array}{llll}8.621747 & -1.395926 & 0.327720\end{array}$

$\begin{array}{llll}9.107388 & 0.012004 & 0.273368\end{array}$

$\begin{array}{lll}10.237245 & 0.465646 & 1.041969\end{array}$

$\begin{array}{lll}10.897901 & -0.358101 & 1.996110\end{array}$

$\begin{array}{lll}10.541712 & -1.367475 & 2.163336\end{array}$ $\begin{array}{lll}11.972379 & 0.112078 & 2.719473\end{array}$

$\begin{array}{lll}12.454200 & -0.535201 & 3.446651\end{array}$

$\begin{array}{llll}12.450323 & 1.430766 & 2.530136\end{array}$

$\begin{array}{llll}13.303081 & 1.784591 & 3.102035\end{array}$

$\begin{array}{lll}11.821022 & 2.263547 & 1.633002\end{array}$

$\begin{array}{lll}12.164963 & 3.284749 & 1.490874\end{array}$

$\begin{array}{lll}10.699699 & 1.815935 & 0.882506\end{array}$

$\begin{array}{lll}10.017760 & 2.691419 & -0.005563\end{array}$

$\begin{array}{llll}10.384581 & 3.706113 & -0.131103\end{array}$

$\begin{array}{llll}8.891687 & 2.272389 & -0.670009\end{array}$

$\begin{array}{llll}8.335501 & 2.930277 & -1.328788\end{array}$

$\begin{array}{llll}8.437551 & 0.947037 & -0.498627\end{array}$

$\begin{array}{llll}4.218096 & 2.351965 & -0.057398\end{array}$

$\begin{array}{llll}3.342704 & 2.060445 & 0.535962\end{array}$

$\begin{array}{llll}4.324054 & 3.866171 & -0.010877\end{array}$

$\begin{array}{llll}3.190470 & 4.629263 & 0.295759\end{array}$

$\begin{array}{llll}2.259274 & 4.126887 & 0.544232\end{array}$

$\begin{array}{llll}3.245906 & 6.025179 & 0.280370\end{array}$

$\begin{array}{llll}2.358185 & 6.604408 & 0.520487\end{array}$

$\begin{array}{llll}4.442670 & 6.673941 & -0.032050\end{array}$

$\begin{array}{llll}4.490579 & 7.759164 & -0.037639\end{array}$

$\begin{array}{llll}5.581060 & 5.918368 & -0.331257\end{array}$

$\begin{array}{llll}6.517057 & 6.416898 & -0.568522\end{array}$

$\begin{array}{llll}5.521376 & 4.524381 & -0.323360\end{array}$

$\begin{array}{llll}6.407665 & 3.936410 & -0.541789\end{array}$

$\begin{array}{llll}4.014587 & 1.873464 & -1.498009\end{array}$

$\begin{array}{llll}4.765491 & 2.358507 & -2.133778\end{array}$

$\begin{array}{llll}3.042220 & 2.213240 & -1.858397\end{array}$

$\begin{array}{llll}5.635317 & 2.001231 & 1.979757\end{array}$

$\begin{array}{lll}5.433488 & 3.074820 & 2.030037\end{array}$

$\begin{array}{llll}4.588008 & 1.323786 & 2.861364\end{array}$

$\begin{array}{lll}3.397000 & 2.009880 & 3.145626\end{array}$

$\begin{array}{llll}3.264467 & 3.020101 & 2.765788\end{array}$

$\begin{array}{llll}2.390063 & 1.423490 & 3.917603\end{array}$

$\begin{array}{lll}1.478247 & 1.972157 & 4.127092\end{array}$

$\begin{array}{llll}2.566753 & 0.136105 & 4.428543\end{array}$

$\begin{array}{lll}1.790403 & -0.318671 & 5.034613\end{array}$

$3.752924 \quad-0.553928 \quad 4.162414$

$3.902537-1.551636 \quad 4.566425$

$\begin{array}{llll}4.754472 & 0.033709 & 3.386719\end{array}$

$\begin{array}{llll}5.667069 & -0.515866 & 3.191051\end{array}$

$\begin{array}{llll}7.074820 & 1.829354 & 2.463000\end{array}$

$\begin{array}{lll}7.758190 & 2.390306 & 1.822524\end{array}$

$\begin{array}{llll}7.402362 & 0.791847 & 2.489002\end{array}$

$\begin{array}{llll}7.145754 & 2.234407 & 3.477307\end{array}$

$\begin{array}{lll}3.147713 & -0.927215 & 0.044990\end{array}$

$\begin{array}{llll}3.425085 & -1.943337 & 0.321863\end{array}$

$\begin{array}{lll}3.216513 & -0.232782 & 0.880892\end{array}$

$\begin{array}{llll}2.040015 & -0.770183 & -0.86176\end{array}$

$\begin{array}{lll}1.652012 & -1.637480 & -1.382002\end{array}$

$\begin{array}{llll}1.339323 & 0.440204 & -0.978846\end{array}$

$\begin{array}{llll}1.707965 & 1.270318 & -0.392369\end{array}$

$\begin{array}{llll}5.351743 & 0.137442 & -3.618577\end{array}$

$\begin{array}{llll}5.834681 & 1.102404 & -3.488288\end{array}$

$\begin{array}{llll}3.975385 & 0.166890 & -3.943427\end{array}$

$\begin{array}{llll}3.516534 & 1.149452 & -4.052612\end{array}$

$\begin{array}{llll}3.203874 & -0.943846 & -4.634687\end{array}$

$\begin{array}{llll}3.350065 & -0.878428 & -5.723959\end{array}$

$\begin{array}{llll}2.140050 & -0.756576 & -4.458238\end{array}$

$\begin{array}{llll}3.551921 & -2.363810 & -4.141348\end{array}$

$\begin{array}{lll}4.381150 & -2.777600 & -4.722786\end{array}$

$2.696061 \quad-3.021359-4.324861$

$\begin{array}{llll}3.903320 & -2.436154 & -2.658524\end{array}$

$\begin{array}{llll}3.168030 & -2.921599 & -2.022737\end{array}$

$\begin{array}{llll}5.191975 & -2.349151 & -2.149013\end{array}$

$\begin{array}{llll}5.363149 & -2.788284 & -1.171390\end{array}$

$6.448324 \quad-2.101525 \quad-2.959124$

$\begin{array}{llll}7.244565 & -1.830725 & -2.261571\end{array}$

$\begin{array}{llll}6.757962 & -3.043044 & -3.435724\end{array}$

$\begin{array}{llll}6.307119 & -0.988213 & -4.018854\end{array}$

$\begin{array}{llll}5.980736 & -1.410180 & -4.974238\end{array}$

$\begin{array}{llll}7.295220 & -0.554895 & -4.199996\end{array}$

$\begin{array}{llll}0.450573 & 0.807886 & -2.067877\end{array}$

$-0.095783 \quad-0.128623 \quad-2.968019$

$\begin{array}{llll}0.200584 & 2.178993 & -2.288304\end{array}$ 


$\begin{array}{lrrr}6 & -0.823855 & 0.298061 & -4.074460 \\ 1 & 0.048632 & -1.189738 & -2.802006 \\ 6 & -0.527884 & 2.602489 & -3.397242 \\ 1 & 0.596508 & 2.909004 & -1.589138 \\ 6 & -1.034789 & 1.662781 & -4.299358 \\ 1 & -1.235031 & -0.433871 & -4.760710 \\ 1 & -0.701608 & 3.662762 & -3.556685 \\ 1 & -1.607854 & 1.988241 & -5.162156 \\ 6 & -0.162756 & 2.769337 & 1.271717 \\ 6 & -0.681080 & 4.038715 & 1.135546 \\ 1 & 0.803511 & 2.627636 & 1.744382 \\ 6 & -1.940748 & 4.163067 & 0.534469 \\ 6 & -2.612330 & 3.035264 & 0.105739 \\ 1 & -3.591063 & 3.104995 & -0.350453 \\ 7 & -2.102514 & 1.793288 & 0.228018 \\ 8 & -1.661839 & -1.142855 & -0.257991 \\ 7 & -0.128635 & -2.042106 & 1.160630 \\ 6 & -0.353827 & -3.371423 & 0.613356 \\ 1 & 0.596039 & -3.918030 & 0.588421 \\ 1 & -1.058251 & -3.949205 & 1.224527 \\ 1 & -0.745277 & -3.296977 & -0.398155 \\ 6 & 0.686261 & -1.999942 & 2.369411 \\ 1 & 1.759265 & -1.979638 & 2.147061 \\ 1 & 0.438646 & -1.131307 & 2.976429 \\ 1 & 0.469558 & -2.889505 & 2.966875 \\ 1 & -0.132424 & 4.906190 & 1.486921 \\ 35 & -2.758559 & 5.925349 & 0.285171\end{array}$

C2-P1 si-si_5

Number of imaginary frequencies $=1$

The smallest frequencies are $=-137.35137 .26628 .6309 \mathrm{~cm}(-1)$ Electronic energy $=-7222.6278115$

Zero-point correction=

1.754809

(Hartree/Particle)

Thermal correction to Energy=

Thermal correction to Enthalpy=

1.874303

Thermal correction to Gibbs Free Energy=

Sum of electronic and zero-point Energies=

Sum of electronic and thermal Energies=

1.591806

Sum of electronic and thermal Enthalpies=

$-7220.873003$

$-7220.754453$

Sum of electronic and thermal Free Energies=

$-7220.753509$

Cartesian Coordinates

$\begin{array}{lccc}26 & 8.123816 & 0.204442 & -0.683955 \\ 15 & 3.132810 & 1.198662 & -1.972731 \\ 15 & 4.000928 & 0.629832 & 1.659858 \\ 6 & 6.156735 & 0.873616 & -0.966352 \\ 6 & 6.291601 & 0.146301 & 0.273613 \\ 6 & 6.735765 & -1.174944 & -0.041473 \\ 1 & 6.940851 & -1.960815 & 0.672408 \\ 6 & 6.909649 & -1.263471 & -1.455759 \\ 1 & 7.271915 & -2.125593 & -1.997291 \\ 6 & 6.552780 & -0.006101 & -2.024642 \\ 1 & 6.595303 & 0.253525 & -3.071855 \\ 6 & 9.466962 & 1.393982 & 0.361338 \\ 1 & 9.192959 & 2.071497 & 1.159293 \\ 6 & 9.802185 & 0.013380 & 0.513001 \\ 1 & 9.819586 & -0.539353 & 1.442767 \\ 6 & 10.059706 & -0.523777 & -0.785284 \\ 1 & 10.303390 & -1.553912 & -1.009533 \\ 6 & 9.883634 & 0.523902 & -1.739685 \\ 1 & 9.973178 & 0.428006 & -2.813733 \\ 6 & 9.519567 & 1.708256 & -1.032429 \\ 1 & 9.289825 & 2.665246 & -1.480752 \\ 6 & 5.636403 & 2.266411 & -1.076911 \\ 6 & 6.429857 & 3.327957 & -0.614881 \\ 1 & 7.410048 & 3.096796 & -0.218372 \\ 6 & 5.987407 & 4.649693 & -0.645587 \\ 1 & 6.618355 & 5.442517 & -0.256671 \\ 6 & 4.728644 & 4.937369 & -1.166654 \\ 1 & 4.365751 & 5.959468 & -1.200870 \\ 6 & 3.917556 & 3.898767 & -1.623591 \\ 1 & 2.935155 & 4.142773 & -2.009012\end{array}$

$\begin{array}{llll}4.336169 & 2.558577 & -1.570047\end{array}$

$\begin{array}{lll}5.896694 & 0.683226 & 1.622907\end{array}$

$\begin{array}{lll}6.155978 & 1.743103 & 1.643536\end{array}$

$\begin{array}{lll}6.590079 & -0.005930 & 2.801370\end{array}$

$\begin{array}{lll}6.291556 & 0.445426 & 3.751619\end{array}$

$\begin{array}{llll}6.357761 & -1.073461 & 2.848293\end{array}$

$\begin{array}{lll}7.674059 & 0.098261 & 2.691776\end{array}$

$\begin{array}{llll}1.621021 & 2.143083 & -2.443718\end{array}$

$\begin{array}{llll}0.769851 & 2.550895 & -1.402421\end{array}$

$\begin{array}{llll}0.958775 & 2.208899 & -0.391093\end{array}$

$\begin{array}{llll}-0.304121 & 3.402474 & -1.661729\end{array}$

$\begin{array}{llll}-0.942332 & 3.724083 & -0.849313\end{array}$

$\begin{array}{llll}-0.567222 & 3.823139 & -2.967586\end{array}$

$\begin{array}{llll}-1.412709 & 4.475377 & -3.167053\end{array}$

$\begin{array}{llll}0.246194 & 3.385331 & -4.014498\end{array}$

$\begin{array}{llll}0.038502 & 3.695760 & -5.034483\end{array}$

$\begin{array}{llll}1.341372 & 2.556771 & -3.754943\end{array}$

$\begin{array}{llll}1.981961 & 2.249277 & -4.574726\end{array}$

$\begin{array}{llll}3.732023 & 0.499314 & -3.560843\end{array}$

$\begin{array}{llll}4.464915 & 1.253884 & -4.490384\end{array}$

$\begin{array}{llll}4.701303 & 2.293063 & -4.281400\end{array}$

$\begin{array}{llll}4.905683 & 0.666850 & -5.677663\end{array}$

$\begin{array}{llll}5.474969 & 1.255919 & -6.391067\end{array}$

$\begin{array}{llll}4.620403 & -0.675906 & -5.946365\end{array}$

$\begin{array}{llll}4.972158 & -1.131973 & -6.867301\end{array}$

$\begin{array}{llll}3.886058 & -1.430036 & -5.027974\end{array}$

$\begin{array}{llll}3.668577 & -2.475109 & -5.228772\end{array}$

$\begin{array}{llll}3.443306 & -0.843657 & -3.840690\end{array}$

$\begin{array}{llll}2.886366 & -1.431735 & -3.120347\end{array}$

$\begin{array}{lll}3.415880 & 2.330178 & 2.055388\end{array}$

$\begin{array}{lll}2.023206 & 2.495491 & 2.067662\end{array}$

$\begin{array}{lll}1.387575 & 1.629686 & 1.911685\end{array}$

$\begin{array}{lll}1.460151 & 3.760108 & 2.235633\end{array}$

$\begin{array}{llll}2.265560 & 4.889999 & 2.361761\end{array}$

$\begin{array}{llll}1.826439 & 5.875919 & 2.466776\end{array}$

$\begin{array}{lll}3.651380 & 4.724869 & 2.334540\end{array}$

$\begin{array}{lll}4.228110 & 3.461492 & 2.199776\end{array}$

$\begin{array}{llll}5.306873 & 3.386915 & 2.186304\end{array}$

$\begin{array}{lll}3.510529 & -0.363082 & 3.129093\end{array}$

$\begin{array}{lll}3.637443 & -1.755559 & 3.002582\end{array}$

$\begin{array}{lll}4.068762 & -2.180401 & 2.103742\end{array}$

$\begin{array}{llll}3.176955 & -2.599786 & 4.008644\end{array}$

$\begin{array}{llll}2.557702 & -2.081335 & 5.147177\end{array}$

$\begin{array}{llll}2.181739 & -2.741959 & 5.920170\end{array}$

$2.419954 \quad-0.700448 \quad 5.265042$

$\begin{array}{llll}2.900642 & 0.159323 & 4.273764\end{array}$

$\begin{array}{lll}2.780079 & 1.228842 & 4.399057\end{array}$

$\begin{array}{llll}2.839669 & -0.191748 & -0.212912\end{array}$

$\begin{array}{llll}1.651498 & -0.137346 & 6.427632\end{array}$

$\begin{array}{lll}3.240867 & -4.091124 & 3.829625\end{array}$

$\begin{array}{lll}-0.037633 & 3.866968 & 2.295731\end{array}$

$\begin{array}{lll}4.515686 & 5.952941 & 2.424772\end{array}$

$\begin{array}{lll}4.284020 & 6.798037 & 1.390016\end{array}$

$\begin{array}{llll}5.835279 & 5.662441 & 2.413470\end{array}$

$\begin{array}{lll}4.267249 & 6.655281 & 3.554832\end{array}$

$\begin{array}{llll}-0.627740 & 3.065491 & 1.360624\end{array}$

$\begin{array}{llll}-0.527579 & 3.462704 & 3.490621\end{array}$

$\begin{array}{lll}-0.486137 & 5.118578 & 2.075401\end{array}$

$\begin{array}{llll}2.008727 & 1.132572 & 6.713329\end{array}$

$\begin{array}{lll}0.315331 & -0.115704 & 6.164066\end{array}$

$\begin{array}{llll}1.809306 & -0.870987 & 7.549838\end{array}$

$\begin{array}{llll}3.569313 & -4.723409 & 4.977460\end{array}$

$\begin{array}{llll}4.135009 & -4.459054 & 2.888356\end{array}$

$\begin{array}{llll}2.035589 & -4.596987 & 3.446103\end{array}$

$\begin{array}{llll}0.597146 & -1.310859 & 1.558994\end{array}$

$\begin{array}{llll}0.840214 & -2.647740 & 1.083441\end{array}$

$\begin{array}{llll}1.978550 & -3.108201 & 0.349186\end{array}$

$\begin{array}{llll}0.324523 & -3.444471 & 1.600192\end{array}$

$\begin{array}{llll}-3.154510 & -0.810413 & -1.893736\end{array}$

$\begin{array}{lll}-4.874625 & -0.232858 & -0.533667\end{array}$

$\begin{array}{lll}-4.808645 & 1.011541 & 0.566522\end{array}$

$\begin{array}{llll}-6.215701 & 0.219663 & -1.404793\end{array}$

$\begin{array}{llll}-5.231873 & -1.484887 & 0.534976\end{array}$

$\begin{array}{lll}-4.834384 & 2.332076 & 0.143947\end{array}$

$\begin{array}{lll}-3.650123 & 3.074387 & 0.337131\end{array}$ 


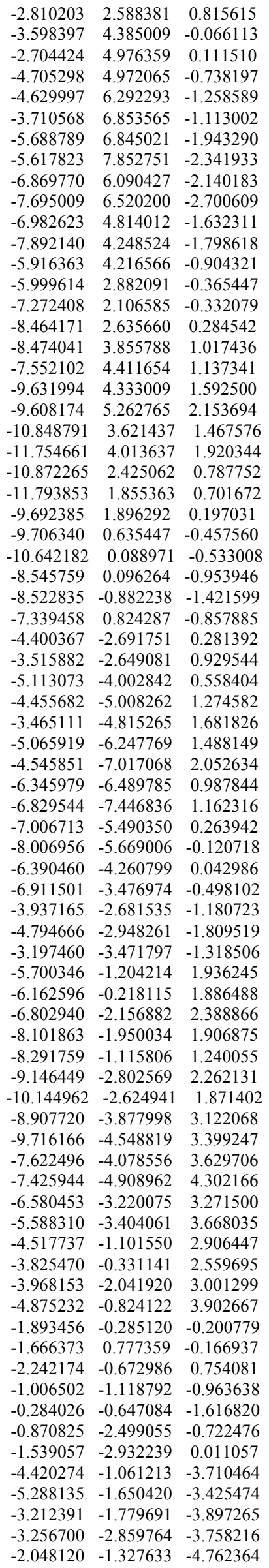

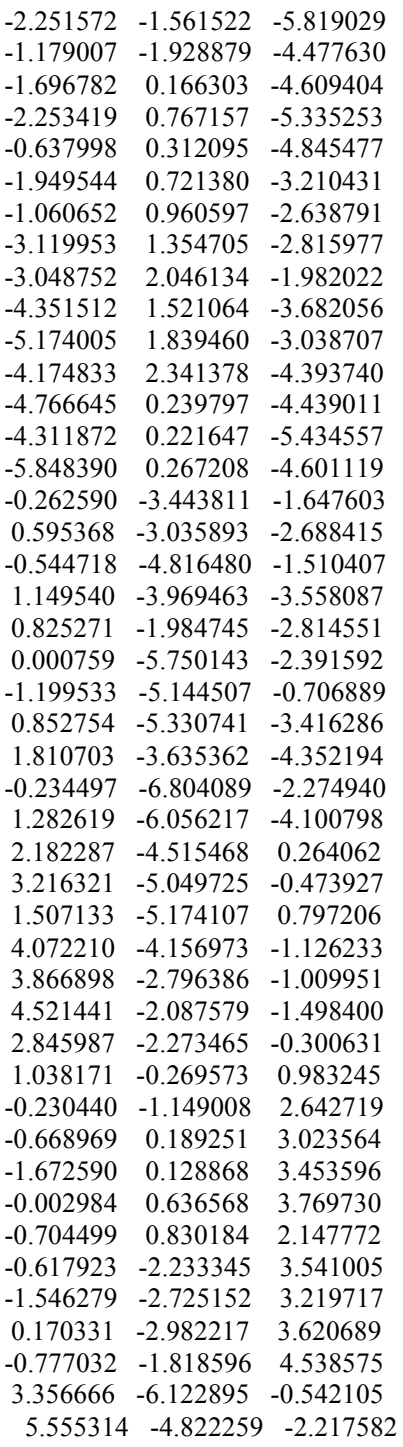

C2-P1 re-si 1

Number of imaginary frequencies $=1$

The smallest frequencies are $=-109.75887 .216810 .9356 \mathrm{~cm}(-1)$

Electronic energy $=-7222.6051215$

Zero-point correction=

(Hartree/Particle)

Thermal correction to Energy= $\quad 1.874136$

Thermal correction to Enthalpy= $\quad 1.875080$

Thermal correction to Gibbs Free Energy $=\quad \begin{array}{r}1.592739 \\ \text { S }\end{array}$

Sum of electronic and zero-point Energies $=\quad-7220.849369$

Sum of electronic and thermal Energies $=\quad-7220.730985$

Sum of electronic and thermal Enthalpies $=\quad-7220.730041$

Sum of electronic and thermal Free Energies $=\quad-7221.012382$

Cartesian Coordinates

$\begin{array}{lccc}26 & 3.691301 & 4.212964 & -3.467863 \\ 15 & 2.121518 & 2.499022 & 1.191326 \\ 15 & 4.584788 & 0.388958 & -0.705458 \\ 6 & 3.367073 & 3.634746 & -1.481144 \\ 6 & 3.912833 & 2.548935 & -2.253005 \\ 6 & 2.998932 & 2.275090 & -3.317970 \\ 1 & 3.126766 & 1.518776 & -4.080419 \\ 6 & 1.916060 & 3.197483 & -3.227072 \\ 1 & 1.089270 & 3.277743 & -3.917293 \\ 6 & 2.138889 & 4.033012 & -2.096807 \\ 1 & 1.508595 & 4.845156 & -1.762138\end{array}$


$\begin{array}{llll}5.523459 & 5.055217 & -3.977729\end{array}$

$\begin{array}{llll}6.457493 & 4.845652 & -3.473402\end{array}$

$\begin{array}{llll}4.985357 & 4.327894 & -5.083272\end{array}$

$\begin{array}{llll}5.435306 & 3.468351 & -5.560947\end{array}$

$\begin{array}{llll}3.724280 & 4.906676 & -5.421069\end{array}$

$\begin{array}{llll}3.052773 & 4.558855 & -6.195061\end{array}$

$3.481959 \quad 5.991298 \quad-4.524910$

$\begin{array}{llll}2.595526 & 6.611537 & -4.500636\end{array}$

$\begin{array}{llll}4.593326 & 6.085034 & -3.635427\end{array}$

$\begin{array}{llll}4.696748 & 6.796479 & -2.827529\end{array}$

$\begin{array}{llll}3.934622 & 4.187906 & -0.224863\end{array}$

$\begin{array}{llll}5.012557 & 5.082709 & -0.277977\end{array}$

$\begin{array}{llll}5.428106 & 5.324574 & -1.247229\end{array}$

$\begin{array}{llll}5.540902 & 5.659172 & 0.876399\end{array}$

$\begin{array}{llll}6.379995 & 6.344842 & 0.805344\end{array}$

$\begin{array}{lll}4.983826 & 5.349750 & 2.114887\end{array}$

$\begin{array}{llll}5.378584 & 5.792671 & 3.023195\end{array}$

$\begin{array}{llll}3.935803 & 4.433146 & 2.192037\end{array}$

$\begin{array}{llll}3.537945 & 4.180111 & 3.167489\end{array}$

$\begin{array}{llll}3.408121 & 3.826253 & 1.039772\end{array}$

$\begin{array}{llll}5.149069 & 1.773783 & -1.887819\end{array}$

$\begin{array}{llll}5.795894 & 2.425603 & -1.296124\end{array}$

$\begin{array}{llll}5.951728 & 1.275861 & -3.095079\end{array}$

$\begin{array}{llll}6.847110 & 0.732711 & -2.781709\end{array}$

$\begin{array}{llll}5.361738 & 0.611666 & -3.733218\end{array}$

$\begin{array}{llll}6.261616 & 2.136248 & -3.694328\end{array}$

$\begin{array}{lll}2.134599 & 2.183430 & 3.009259\end{array}$

$\begin{array}{lll}3.125346 & 1.314950 & 3.497037\end{array}$

$\begin{array}{llll}3.824939 & 0.860235 & 2.804463\end{array}$

$\begin{array}{llll}3.227522 & 1.042407 & 4.861009\end{array}$

$\begin{array}{lll}3.999772 & 0.367043 & 5.215562\end{array}$

$\begin{array}{llll}2.327886 & 1.630086 & 5.755666\end{array}$

$\begin{array}{llll}2.397399 & 1.414733 & 6.818019\end{array}$

$\begin{array}{lll}1.333027 & 2.486006 & 5.278074\end{array}$

$\begin{array}{llll}0.625494 & 2.936331 & 5.968366\end{array}$

$\begin{array}{lll}1.235164 & 2.765221 & 3.912294\end{array}$

$\begin{array}{lll}0.454370 & 3.429495 & 3.556511\end{array}$

$\begin{array}{lll}0.511647 & 3.320428 & 0.880364\end{array}$

$\begin{array}{llll}0.279869 & 4.686940 & 1.100816\end{array}$

$\begin{array}{lll}1.092873 & 5.331074 & 1.423647\end{array}$

$\begin{array}{lll}-0.997907 & 5.218698 & 0.909536\end{array}$

$\begin{array}{lll}-1.171233 & 6.277697 & 1.079116\end{array}$

$\begin{array}{lll}-2.053920 & 4.389258 & 0.516601\end{array}$

$\begin{array}{lll}-3.050951 & 4.799916 & 0.382665\end{array}$

$\begin{array}{llll}-1.824617 & 3.029501 & 0.292663\end{array}$

$\begin{array}{lll}-2.641207 & 2.374385 & 0.008858\end{array}$

$\begin{array}{lll}-0.543782 & 2.500986 & 0.453724\end{array}$

$\begin{array}{llll}-0.354294 & 1.454846 & 0.237094\end{array}$

$\begin{array}{llll}5.638734 & 0.511542 & 0.804963\end{array}$

$\begin{array}{lll}5.674183 & -0.605176 & 1.659946\end{array}$

$\begin{array}{llll}5.170006 & -1.524696 & 1.383298\end{array}$

$\begin{array}{lll}6.360600 & -0.546533 & 2.870513\end{array}$

$\begin{array}{lll}7.008660 & 0.622157 & 3.268724\end{array}$

$\begin{array}{llll}7.549616 & 0.659144 & 4.207603\end{array}$

$\begin{array}{lll}6.957240 & 1.734397 & 2.433225\end{array}$

$\begin{array}{lll}6.272534 & 1.691689 & 1.215827\end{array}$

$\begin{array}{lll}6.241696 & 2.586963 & 0.607581\end{array}$

$\begin{array}{llll}5.192815 & -1.172180 & -1.483561\end{array}$

$\begin{array}{llll}4.347900 & -1.802958 & -2.405281\end{array}$

$\begin{array}{llll}3.375292 & -1.379334 & -2.628613\end{array}$

$\begin{array}{llll}4.748322 & -2.981300 & -3.039762\end{array}$

$\begin{array}{llll}5.982942 & -3.561750 & -2.757118\end{array}$

$6.286801 \quad-4.479903-3.244840$

$\begin{array}{llll}6.827750 & -2.927539 & -1.847058\end{array}$

$\begin{array}{llll}6.447776 & -1.739372 & -1.222676\end{array}$

$\begin{array}{llll}7.139452 & -1.263864 & -0.536595\end{array}$

$\begin{array}{llll}2.386573 & 0.560797 & 0.015571\end{array}$

$\begin{array}{llll}8.152575 & -3.541921 & -1.483594\end{array}$

$\begin{array}{llll}3.810726 & -3.632903 & -4.017928\end{array}$

$\begin{array}{lll}6.362061 & -1.727584 & 3.801660\end{array}$

$\begin{array}{lll}7.627760 & 3.002174 & 2.891514\end{array}$

$\begin{array}{lll}6.874711 & 3.657538 & 3.808392\end{array}$

$\begin{array}{lll}7.858651 & 3.860325 & 1.875368\end{array}$

$\begin{array}{llll}8.817889 & 2.748310 & 3.482733\end{array}$

$\begin{array}{lll}5.438341 & -1.576593 & 4.786652\end{array}$
$6.079781-2.881285 \quad 3.163260$

$\begin{array}{llll}7.555868 & -1.880857 & 4.416115\end{array}$

$9.120596-2.604528 \quad-1.360031$

$\begin{array}{llll}8.085729 & -4.187913 & -0.294416\end{array}$

$\begin{array}{llll}8.571016 & -4.439335 & -2.400659\end{array}$

$\begin{array}{llll}4.415290 & -4.576583 & -4.765138\end{array}$

$3.273578 \quad-2.723776 \quad-4.867132$

$\begin{array}{lll}2.762040 & -4.237261 & -3.394882\end{array}$

$\begin{array}{llll}2.251231 & -1.841940 & 1.826107\end{array}$

$\begin{array}{llll}1.627823 & -2.381280 & -0.368792\end{array}$

$\begin{array}{llll}2.402396 & -3.176413 & 2.164731\end{array}$

$\begin{array}{llll}2.420424 & -1.055900 & 2.551930\end{array}$

$\begin{array}{lll}1.899107 & -3.751884 & -0.088906\end{array}$

$\begin{array}{llll}2.266632 & -4.158960 & 1.178933\end{array}$

$\begin{array}{lll}1.791874 & -4.482086 & -0.881917\end{array}$

$\begin{array}{llll}1.056887 & -2.062135 & -1.642974\end{array}$

$\begin{array}{llll}0.801814 & -0.741872 & -2.160172\end{array}$

$\begin{array}{llll}0.853676 & 0.303247 & -1.445392\end{array}$

$\begin{array}{llll}1.887447 & -1.441865 & 0.595102\end{array}$

$\begin{array}{llll}1.091564 & -2.880196 & -2.340384\end{array}$

$-4.323843-1.620371-1.577345$

$\begin{array}{llll}-5.103615 & -0.406037 & 0.183200\end{array}$

$\begin{array}{lll}-4.905920 & 1.238930 & 0.305267\end{array}$

$\begin{array}{lll}-6.770405 & -0.501013 & 0.283098\end{array}$

$\begin{array}{lll}-4.387236 & -0.930633 & 1.598785\end{array}$

$\begin{array}{lll}-5.723178 & 2.114512 & -0.405669\end{array}$

$\begin{array}{llll}-5.105680 & 2.858354 & -1.436915\end{array}$

$\begin{array}{llll}-4.046002 & 2.720590 & -1.618133\end{array}$

$\begin{array}{llll}-5.851021 & 3.732672 & -2.188684\end{array}$

$\begin{array}{llll}-5.382059 & 4.314197 & -2.977363\end{array}$

$\begin{array}{llll}-7.248609 & 3.862077 & -1.970682\end{array}$

$\begin{array}{llll}-8.046415 & 4.701162 & -2.795117\end{array}$

$\begin{array}{lll}-7.558488 & 5.265873 & -3.585345\end{array}$

$\begin{array}{llll}-9.407545 & 4.786873 & -2.609122\end{array}$

$\begin{array}{llll}-10.009383 & 5.426465 & -3.248027\end{array}$

$\begin{array}{llll}-10.027489 & 4.024781 & -1.590023\end{array}$

$\begin{array}{lll}-11.104774 & 4.075483 & -1.460756\end{array}$

$\begin{array}{llll}-9.280002 & 3.214423 & -0.763281\end{array}$

$\begin{array}{llll}-9.773817 & 2.632106 & 0.005663\end{array}$

$\begin{array}{llll}-7.867873 & 3.117272 & -0.911485\end{array}$

$\begin{array}{lll}-7.059268 & 2.276822 & -0.066441\end{array}$

$\begin{array}{lll}-7.621916 & 1.579402 & 1.122767\end{array}$

$\begin{array}{lll}-8.258264 & 2.287983 & 2.203143\end{array}$

$\begin{array}{lll}-8.332118 & 3.708432 & 2.256667\end{array}$

$\begin{array}{lll}-7.892940 & 4.294522 & 1.458559\end{array}$

$\begin{array}{lll}-8.939638 & 4.350292 & 3.313966\end{array}$

$\begin{array}{llll}-8.977250 & 5.435765 & 3.332301\end{array}$

$\begin{array}{lll}-9.508213 & 3.611987 & 4.379188\end{array}$

$\begin{array}{llll}-9.990196 & 4.132001 & 5.201813\end{array}$

$\begin{array}{llll}-9.431570 & 2.237909 & 4.374531\end{array}$

$\begin{array}{llll}-9.845601 & 1.657491 & 5.194875\end{array}$

$\begin{array}{llll}-8.798718 & 1.544498 & 3.307139\end{array}$

$\begin{array}{lll}-8.671096 & 0.129248 & 3.331440\end{array}$

$\begin{array}{lll}-9.092136 & -0.423634 & 4.166427\end{array}$

$\begin{array}{lll}-8.002044 & -0.528860 & 2.329538\end{array}$

$\begin{array}{lll}-7.870812 & -1.605217 & 2.337366\end{array}$

$\begin{array}{llll}-7.468471 & 0.208165 & 1.250608\end{array}$

$\begin{array}{llll}-3.680292 & -2.236831 & 1.420532\end{array}$

$\begin{array}{lll}-2.610406 & -2.023343 & 1.356031\end{array}$

$\begin{array}{lll}-3.884503 & -3.201366 & 2.575560\end{array}$

$\begin{array}{lll}-2.786686 & -3.909614 & 3.079104\end{array}$

$\begin{array}{lll}-1.793266 & -3.701559 & 2.688426\end{array}$

$\begin{array}{llll}-2.953440 & -4.856647 & 4.091352\end{array}$

$\begin{array}{lll}-2.090721 & -5.395806 & 4.473332\end{array}$

$\begin{array}{llll}-4.223315 & -5.096670 & 4.622675\end{array}$

$\begin{array}{lll}-4.354218 & -5.825265 & 5.417851\end{array}$

$\begin{array}{llll}-5.324327 & -4.389939 & 4.129160\end{array}$

$\begin{array}{lll}-6.314264 & -4.567269 & 4.540860\end{array}$

$\begin{array}{lll}-5.155678 & -3.453956 & 3.107558\end{array}$

$\begin{array}{lll}-6.009644 & -2.901456 & 2.726443\end{array}$

$\begin{array}{lll}-4.125419 & -2.909243 & 0.122030\end{array}$

$\begin{array}{llll}-5.099170 & -3.382976 & 0.290624\end{array}$

$\begin{array}{llll}-3.423198 & -3.710637 & -0.112270\end{array}$

$\begin{array}{lll}-4.229610 & -0.303502 & 2.952666\end{array}$

$\begin{array}{lll}-4.822820 & -0.912083 & 3.642992\end{array}$ 


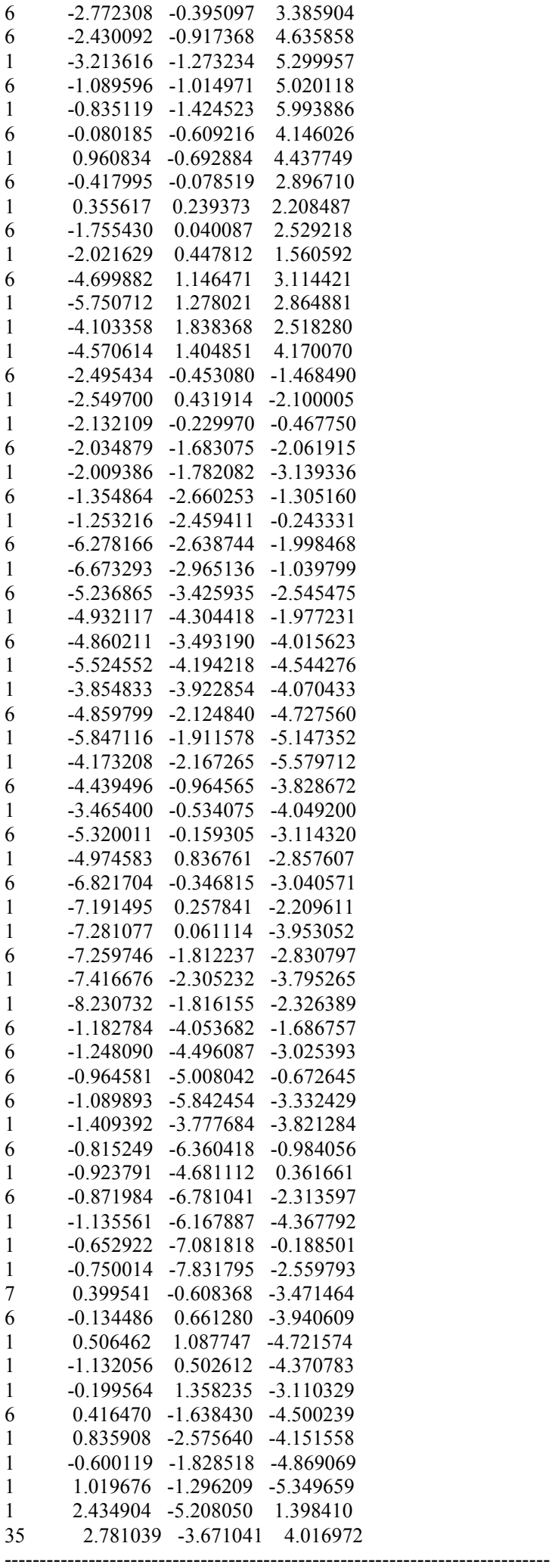

C2-P1 re-si 2

Number of imaginary frequencies $=1$

The smallest frequencies are $=-157.42976 .60908 .1764 \mathrm{~cm}(-1)$ Electronic energy $=-7222.6051024$

Zero-point correction=

1.753731

(Hartree/Particle)

Thermal correction to Energy= $\quad 1.872606$

Thermal correction to Enthalpy=

1.873551

Thermal correction to Gibbs Free Energy=
Sum of electronic and zero-point Energies=

Sum of electronic and thermal Energies=

Sum of electronic and thermal Enthalpies=

Sum of electronic and thermal Free Energies=

$-7220.851372$

$-7220.732496$

$-7220.731552$

$-7221.016359$

Cartesian Coordinates

\begin{tabular}{|c|c|c|c|}
\hline 7 & 4.093730 & -0.613844 & -1.540 \\
\hline & 5.812917 & 0.197980 & 300611 \\
\hline 8 & 6.418242 & -0.503008 & 1.065429 \\
\hline & 7.175259 & 0.322794 & -1.234544 \\
\hline 7 & 5.393970 & 1.701232 & 0.353534 \\
\hline 6 & 7.319171 & -1.560745 & 1.010103 \\
\hline 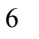 & 6.856398 & -2.793739 & 1.520111 \\
\hline 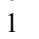 & 5.857108 & -2.835907 & 1.940508 \\
\hline 6 & 7.669345 & -3.898143 & 1.473038 \\
\hline 1 & 7.327003 & -4.850158 & 1.868425 \\
\hline 6 & 8.954853 & -3.818982 & 0.873598 \\
\hline 0 & 9.769843 & -4.975449 & 0.737405 \\
\hline 1 & 9.398249 & -5.919200 & 1.127859 \\
\hline 0 & 10.993986 & -4.908175 & 0.111886 \\
\hline 1 & 11.606679 & -5.798790 & 0.007649 \\
\hline 6 & 11.446714 & -3.674653 & -0.413460 \\
\hline 1 & 12.400137 & -3.628663 & -0.931835 \\
\hline 6 & 10.688237 & -2.531570 & -0.281731 \\
\hline 1 & 11.049549 & -1.600361 & -0.700724 \\
\hline 6 & 9.428676 & -2.556476 & 0.380941 \\
\hline 6 & 8.608337 & -1.379181 & 0.530213 \\
\hline 6 & 9.107494 & -0.02 & 0.154684 \\
\hline 6 & 10.328916 & 0.514281 & 0.693390 \\
\hline 6 & 11.048605 & -0.116794 & 1.745592 \\
\hline 1 & 10.666372 & -1.0 & 374 \\
\hline 6 & 12.206203 & 0.435833 & 2.248405 \\
\hline 1 & 12.729392 & -0.060653 & 3.060634 \\
\hline 6 & 12.713606 & 1.649479 & 1.726236 \\
\hline 1 & 13.629530 & 2.071619 & 2.129338 \\
\hline 6 & 12.033124 & $2.29^{\prime}$ & 0.720284 \\
\hline 1 & 12.398967 & 3.240913 & 0.323724 \\
\hline 6 & 10.828069 & 1.761961 & 0.189863 \\
\hline 6 & 10.092074 & 2.464329 & -0.801067 \\
\hline 1 & 10.485036 & 3.400710 & -1.186770 \\
\hline 6 & 8.880865 & 1.988089 & -1.236033 \\
\hline 1 & 8.28 & 2.518 & -1.971387 \\
\hline 6 & 8.394882 & 0.763594 & -0.731530 \\
\hline 6 & & & \\
\hline 1 & 3.343431 & 2.16 & 0.208563 \\
\hline 6 & 4.416152 & 3.768372 & -0.693198 \\
\hline 6 & 3.291745 & 4.603656 & -0.683370 \\
\hline 1 & 2.323959 & 4.195062 & -0.402653 \\
\hline 6 & 3.403918 & 5.953212 & -1.025049 \\
\hline 1 & 2.523084 & 6.589620 & -1.006464 \\
\hline 6 & 4.646317 & 6.485102 & -1.380179 \\
\hline 1 & 4.737510 & 7.536304 & -1.638443 \\
\hline 6 & 5.771485 & 5.655199 & -1.399446 \\
\hline 1 & 6.741461 & 6.059720 & -1.676109 \\
\hline 6 & 5.655504 & 4.305319 & -1.062863 \\
\hline 1 & 6.527898 & 3.661 & -1.071528 \\
\hline 6 & 4.044171 & 1.531334 & -1.715046 \\
\hline 1 & 4.819964 & 1.843488 & -2.425490 \\
\hline 1 & 3.093883 & 1.849029 & -2.149290 \\
\hline 6 & 5.502595 & 1.939746 & 1.840895 \\
\hline 1 & 5.107434 & 1.054230 & 2.349622 \\
\hline 6 & 6.934641 & 2.141491 & 2.317449 \\
\hline 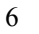 & 7.463820 & 1.306325 & 3.308649 \\
\hline 1 & 6.883813 & 0.454078 & 3.652313 \\
\hline 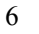 & 8.725116 & 1.553886 & 3.854012 \\
\hline 1 & 9.123651 & 0.890845 & 4.616885 \\
\hline 6 & 9.475768 & 2.644861 & 3.412081 \\
\hline 1 & 10.462605 & 2.833099 & 3.823618 \\
\hline 6 & 8.960656 & 3.476316 & 2.414639 \\
\hline 1 & 9.544632 & 4.318676 & 2.055055 \\
\hline 6 & 7.699866 & 3.226199 & 1.871128 \\
\hline 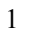 & 7.302064 & 3.882521 & 1.104250 \\
\hline & 4.658974 & 3.132383 & 2.319650 \\
\hline & 3.596542 & 3.009879 & 2.102175 \\
\hline
\end{tabular}


$\begin{array}{llll}4.999366 & 4.075949 & 1.890377\end{array}$

$\begin{array}{llll}4.769112 & 3.195217 & 3.406081\end{array}$

$\begin{array}{llll}2.983054 & -0.810706 & 0.312751\end{array}$

$\begin{array}{lll}3.190403 & -1.752556 & 0.819986\end{array}$

$\begin{array}{llll}3.046733 & 0.047474 & 0.981244\end{array}$

$\begin{array}{lll}1.921939 & -0.809531 & -0.666133\end{array}$

$\begin{array}{llll}1.525237 & -1.757212 & -1.007654\end{array}$

$\begin{array}{llll}1.224996 & 0.353182 & -1.037947\end{array}$

$\begin{array}{llll}1.582650 & 1.293141 & -0.640676\end{array}$

$\begin{array}{llll}5.257813 & -0.678653 & -3.458012\end{array}$

$\begin{array}{llll}5.777136 & 0.272373 & -3.542776\end{array}$

$\begin{array}{llll}3.876480 & -0.666350 & -3.757934\end{array}$

$\begin{array}{llll}3.451692 & 0.285509 & -4.076858\end{array}$

$\begin{array}{llll}3.048497 & -1.872126 & -4.172316\end{array}$

$\begin{array}{llll}3.169409 & -2.057419 & -5.250771\end{array}$

$\begin{array}{llll}1.996005 & -1.609464 & -4.023144\end{array}$

$\begin{array}{llll}3.360559 & -3.159002 & -3.378902\end{array}$

$\begin{array}{llll}4.163444 & -3.721545 & -3.863978\end{array}$

$\begin{array}{llll}2.479876 & -3.809159 & -3.399379\end{array}$

$\begin{array}{lll}3.737920 & -2.907552 & -1.921854\end{array}$

$\begin{array}{llll}2.997699 & -3.211435 & -1.185806\end{array}$

$\begin{array}{llll}5.037705 & -2.755149 & -1.457706\end{array}$

$\begin{array}{llll}5.209311 & -2.968606 & -0.407037\end{array}$

$6.285291 \quad-2.749431 \quad-2.317658$

$\begin{array}{llll}7.105459 & -2.357601 & -1.712983\end{array}$

$\begin{array}{llll}6.551324 & -3.786814 & -2.567474\end{array}$

$\begin{array}{llll}6.161070 & -1.904400 & -3.603862\end{array}$

$5.795917 \quad-2.520546 \quad-4.431225$

$\begin{array}{llll}7.160180 & -1.566146 & -3.894551\end{array}$

$\begin{array}{llll}0.334107 & 0.468425 & -2.179486\end{array}$

$\begin{array}{llll}-0.271583 & -0.645743 & -2.797955\end{array}$

$\begin{array}{llll}0.090159 & 1.750354 & -2.713630\end{array}$

$\begin{array}{lll}-1.084807 & -0.479104 & -3.915605\end{array}$

$\begin{array}{llll}-0.114676 & -1.639582 & -2.394804\end{array}$

$\begin{array}{lll}-0.699518 & 1.910218 & -3.848997\end{array}$

$\begin{array}{llll}0.536272 & 2.616781 & -2.233356\end{array}$

$\begin{array}{llll}-1.293388 & 0.796996 & -4.450037\end{array}$

$\begin{array}{llll}-1.563848 & -1.341317 & -4.370289\end{array}$

$\begin{array}{llll}-0.869881 & 2.901762 & -4.255334\end{array}$

$\begin{array}{llll}-1.926010 & 0.921133 & -5.322766\end{array}$

$\begin{array}{lll}-6.495305 & 4.278278 & 1.436723\end{array}$

$\begin{array}{llll}-4.933990 & 0.895355 & -2.199234\end{array}$

$\begin{array}{lll}-4.825224 & -0.204469 & 1.417687\end{array}$

$\begin{array}{lll}-6.147213 & 2.707414 & 0.097168\end{array}$

$\begin{array}{lll}-5.520753 & 2.454518 & 1.373771\end{array}$

$\begin{array}{llll}-4.587516 & 3.505767 & 1.618009\end{array}$

$\begin{array}{lll}-3.967612 & 3.606308 & 2.498306\end{array}$

$\begin{array}{llll}-4.650391 & 4.421923 & 0.527909\end{array}$

$\begin{array}{llll}-4.091282 & 5.343371 & 0.438975\end{array}$

$\begin{array}{llll}-5.613539 & 3.937598 & -0.403100\end{array}$

$\begin{array}{llll}-5.904862 & 4.415861 & -1.326347\end{array}$

$\begin{array}{llll}-8.136677 & 4.189989 & 2.701655\end{array}$

$\begin{array}{llll}-8.561377 & 3.276583 & 3.096266\end{array}$

$\begin{array}{lll}-7.090609 & 4.956875 & 3.300879\end{array}$

$\begin{array}{llll}-6.577953 & 4.722303 & 4.224213\end{array}$

$\begin{array}{lll}-6.806447 & 6.062805 & 2.442991\end{array}$

$\begin{array}{llll}-6.038353 & 6.808440 & 2.601281\end{array}$

$\begin{array}{lll}-7.678079 & 5.982453 & 1.314500\end{array}$

$\begin{array}{lll}-7.687324 & 6.655706 & 0.467569\end{array}$

$\begin{array}{llll}-8.501394 & 4.828500 & 1.475202\end{array}$

$\begin{array}{lll}-9.246643 & 4.483988 & 0.771587\end{array}$

$\begin{array}{llll}-7.101757 & 1.778499 & -0.575321\end{array}$

$\begin{array}{llll}-8.409863 & 1.659177 & -0.082968\end{array}$

$\begin{array}{lll}-8.696432 & 2.274447 & 0.760591\end{array}$

$\begin{array}{llll}-9.326988 & 0.764659 & -0.634173\end{array}$

$\begin{array}{llll}-10.321909 & 0.677379 & -0.209954\end{array}$

$\begin{array}{llll}-8.948434 & -0.025985 & -1.716381\end{array}$

$\begin{array}{lll}-9.649029 & -0.729087 & -2.156030\end{array}$

$\begin{array}{llll}-7.651595 & 0.071214 & -2.222688\end{array}$

$\begin{array}{lll}-7.369032 & -0.560123 & -3.057612\end{array}$

$\begin{array}{llll}-6.709297 & 0.945447 & -1.659404\end{array}$

$\begin{array}{llll}-5.780526 & 1.235308 & 2.213274\end{array}$

$\begin{array}{lll}-6.829085 & 0.977597 & 2.063482\end{array}$

$\begin{array}{lll}-5.566033 & 1.437864 & 3.716048\end{array}$

$\begin{array}{lll}-5.770003 & 0.517819 & 4.270962\end{array}$ $\begin{array}{lll}-4.549031 & 1.757180 & 3.955338\end{array}$

$\begin{array}{lll}-6.250502 & 2.215609 & 4.067583\end{array}$

$-4.880057 \quad-0.325755 \quad-3.577612$

$\begin{array}{llll}-4.954377 & -1.689520 & -3.243980\end{array}$

$\begin{array}{lll}-5.121720 & -1.980890 & -2.212375\end{array}$

$\begin{array}{llll}-4.834729 & -2.672278 & -4.225764\end{array}$

$\begin{array}{llll}-4.910954 & -3.718639 & -3.949422\end{array}$

$\begin{array}{lll}-4.614691 & -2.305121 & -5.557278\end{array}$

$-4.509364-3.068701-6.322688$

$\begin{array}{lll}-4.533643 & -0.953831 & -5.898176\end{array}$

$\begin{array}{llll}-4.365058 & -0.661079 & -6.930792\end{array}$

$\begin{array}{llll}-4.671628 & 0.032211 & -4.916990\end{array}$

$\begin{array}{llll}-4.604740 & 1.076336 & -5.200599\end{array}$

$\begin{array}{llll}-4.679799 & 2.521090 & -3.010341\end{array}$

$\begin{array}{llll}-5.660196 & 3.100118 & -3.832144\end{array}$

$\begin{array}{llll}-6.587658 & 2.569496 & -4.028839\end{array}$

$\begin{array}{llll}-5.453134 & 4.365076 & -4.383270\end{array}$

$\begin{array}{llll}-6.215194 & 4.810603 & -5.016433\end{array}$

$-4.267777 \quad 5.059816-4.115586$

$\begin{array}{llll}-4.111619 & 6.047754 & -4.539945\end{array}$

$\begin{array}{llll}-3.290493 & 4.484955 & -3.299928\end{array}$

$\begin{array}{llll}-2.372958 & 5.025915 & -3.084329\end{array}$

$\begin{array}{lll}-3.494356 & 3.218922 & -2.745707\end{array}$

$\begin{array}{lll}-2.756629 & 2.781724 & -2.081948\end{array}$

$\begin{array}{lll}-6.096789 & -1.511704 & 1.110320\end{array}$

$\begin{array}{lll}-5.695453 & -2.624800 & 0.358185\end{array}$

$\begin{array}{lll}-4.655971 & -2.738382 & 0.078924\end{array}$

$\begin{array}{llll}-6.621667 & -3.583609 & -0.053495\end{array}$

$\begin{array}{lll}-7.972845 & -3.445744 & 0.253096\end{array}$

$\begin{array}{llll}-8.696146 & -4.173550 & -0.097302\end{array}$

$\begin{array}{lll}-8.375194 & -2.341692 & 1.003722\end{array}$

$\begin{array}{lll}-7.452552 & -1.395182 & 1.445975\end{array}$

$\begin{array}{llll}-7.817077 & -0.562960 & 2.030855\end{array}$

$\begin{array}{lll}-3.726973 & -0.910685 & 2.714879\end{array}$

$\begin{array}{lll}-2.706059 & -0.078756 & 3.205476\end{array}$

$\begin{array}{lll}-2.634420 & 0.950853 & 2.874751\end{array}$

$\begin{array}{lll}-1.761838 & -0.575683 & 4.099046\end{array}$

$\begin{array}{lll}-1.773921 & -1.918560 & 4.480860\end{array}$

$\begin{array}{lll}-1.021142 & -2.308118 & 5.157187\end{array}$

$\begin{array}{lll}-2.765810 & -2.750064 & 3.967782\end{array}$

$\begin{array}{lll}-3.745952 & -2.253675 & 3.104716\end{array}$

$\begin{array}{llll}-4.510865 & -2.927373 & 2.737040\end{array}$

$\begin{array}{lll}-3.567222 & 0.335787 & -0.476213\end{array}$

$\begin{array}{lll}-2.718954 & -4.224014 & 4.261965\end{array}$

$\begin{array}{llll}-0.650365 & 0.306127 & 4.592950\end{array}$

$\begin{array}{llll}-6.143012 & -4.779246 & -0.829779\end{array}$

$\begin{array}{lll}-9.843307 & -2.171475 & 1.288378\end{array}$

$\begin{array}{lll}-10.562306 & -2.115390 & 0.140102\end{array}$

$\begin{array}{lll}-10.110913 & -1.042258 & 1.980148\end{array}$

$\begin{array}{lll}-10.338929 & -3.209194 & 2.000910\end{array}$

$\begin{array}{lll}-5.124893 & -4.451429 & -1.666146\end{array}$

$\begin{array}{llll}-5.676374 & -5.752875 & -0.014951\end{array}$

$\begin{array}{llll}-7.120755 & -5.324016 & -1.582444\end{array}$

$\begin{array}{llll}-3.936973 & -4.801960 & 4.180491\end{array}$

$\begin{array}{lll}-1.918990 & -4.871317 & 3.375097\end{array}$

$\begin{array}{lll}-2.224142 & -4.483768 & 5.491963\end{array}$

$\begin{array}{llll}-0.408272 & 0.124818 & 5.908464\end{array}$

$\begin{array}{llll}-0.914351 & 1.619945 & 4.405333\end{array}$

$\begin{array}{lll}0.522839 & 0.047225 & 3.950971\end{array}$

$\begin{array}{llll}-2.274010 & -2.397065 & -0.570818\end{array}$

$\begin{array}{lll}-0.961514 & -0.847993 & 0.596274\end{array}$

$\begin{array}{lll}-1.611249 & -3.468539 & 0.002575\end{array}$

$\begin{array}{llll}-3.054636 & -2.549171 & -1.307836\end{array}$

$\begin{array}{lll}-0.289537 & -1.930409 & 1.239079\end{array}$

$\begin{array}{lll}-0.613125 & -3.240077 & 0.956564\end{array}$

$\begin{array}{lll}0.475463 & -1.707063 & 1.973579\end{array}$

$\begin{array}{lll}-0.447929 & 0.464226 & 0.823338\end{array}$

$\begin{array}{lll}-1.054221 & 1.717707 & 0.477995\end{array}$

$\begin{array}{lll}-2.152934 & 1.825854 & -0.141923\end{array}$

$\begin{array}{llll}-1.989453 & -1.116084 & -0.266487\end{array}$

$\begin{array}{lll}0.352283 & 0.487984 & 1.545910\end{array}$

$\begin{array}{llll}-0.374299 & 2.878975 & 0.793760\end{array}$

$\begin{array}{lll}-1.032730 & 4.165220 & 0.591893\end{array}$

$\begin{array}{llll}-1.458957 & 4.547171 & 1.530429\end{array}$

$\begin{array}{llll}-0.304748 & 4.895969 & 0.224149\end{array}$ 


$\begin{array}{lccc}1 & -1.835067 & 4.055288 & -0.133251 \\ 6 & 0.764168 & 2.897858 & 1.708628 \\ 1 & 1.519071 & 2.165101 & 1.419249 \\ 1 & 1.229545 & 3.884209 & 1.665430 \\ 1 & 0.466186 & 2.699840 & 2.744877 \\ 1 & -0.113725 & -4.060079 & 1.459612 \\ 35 & -2.074867 & -5.299841 & -0.501215\end{array}$

C2-P1 re-si_3

Number of imaginary frequencies $=1$

The smallest frequencies are $=-180.25009 .10619 .9596 \mathrm{~cm}(-1)$ Electronic energy $=-7222.6127823$

Zero-point correction=

1.754790

(Hartree/Particle)

Thermal correction to Energy=

1.873573

Thermal correction to Enthalpy=

1.874518

Thermal correction to Gibbs Free Energy=

Sum of electronic and zero-point Energies=

Sum of electronic and thermal Energies=

1.590856

Sum of electronic and thermal Enthalpies=

$-7220.857992$

$-7220.739209$

Sum of electronic and thermal Free Energies=

$-7220.738265$

$-7221.021926$

Cartesian Coordinates

\begin{tabular}{|c|c|c|c|}
\hline 77 & -4.080831 & 3505 & 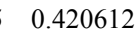 \\
\hline 15 & -4.859186 & 0.258181 & 1606 \\
\hline 8 & -5.131437 & -0.959346 & 1.010266 \\
\hline 0 & -6.369988 & 0.384241 & -0.770509 \\
\hline 7 & -3.796341 & -0.531158 & -1.113528 \\
\hline 6 & -6.294699 & -1.032248 & 1.767119 \\
\hline 6 & -6.136206 & -0.864607 & 3.160440 \\
\hline 1 & -5.136027 & -0.708465 & 3.550917 \\
\hline 6 & -7.235369 & -0.91 & 3.980269 \\
\hline 1 & -7.124889 & -0.792 & 5.054443 \\
\hline 6 & -8.534850 & -1.0 & 3.4 \\
\hline 6 & -9.687246 & -1.0 & \\
\hline 1 & -9.548958 & -0.920443 & 5.333737 \\
\hline 6 & -10.949507 & -1.172133 & 3.728477 \\
\hline 1 & -11.823909 & -1.1 & 4.371971 \\
\hline 6 & -11.106982 & -1.3 & 2.329636 \\
\hline 1 & -12.104576 & -1.383033 & 1.905142 \\
\hline 6 & -10.00 & -1.3 & 830 \\
\hline 1 & -10.155761 & -1.464305 & 0.429200 \\
\hline 6 & -8.687125 & -1.27 & 959 \\
\hline 6 & -7.5 & & \\
\hline 6 & -7.611940 & -1.6 & 3411 \\
\hline 6 & -8.253370 & -2.79 & 3461 \\
\hline 6 & -8.67 & -3.8 & \\
\hline 1 & -8.501100 & -3.79 & 1948 \\
\hline 6 & -9.269723 & -4.992481 & -0.477727 \\
\hline 1 & -9.56 & -5.7 & 565 \\
\hline 6 & -9.491057 & -5.11 & 0766 \\
\hline 1 & -9.969397 & -6.00 & 8732 \\
\hline 6 & -9.084616 & -4.1 & 894 \\
\hline 1 & -9.232634 & -4.187268 & -3.789435 \\
\hline 6 & -8.447994 & -2.9 & -2.210050 \\
\hline & -7.964179 & -1.93 & -3.088409 \\
\hline 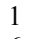 & -8.119641 & -2.0 & 268 \\
\hline 6 & -7.258305 & -0.86 & 8894 \\
\hline 1 & -6.833396 & -0.106206 & -3.252170 \\
\hline 6 & -7.073 & -0.72 & -1.2 \\
\hline & -2.787039 & 0.41 & -1.6 \\
\hline 1 & -1.8 & 0.3 & -1.0 \\
\hline 6 & -2.4 & 0.1 & 838 \\
\hline 6 & -1.053921 & -0.014998 & -3.433492 \\
\hline 1 & -0.311057 & 0.0 & -2.6 \\
\hline 6 & -0.661315 & -0.255644 & -4.752711 \\
\hline 1 & 0.392271 & -0.364833 & -4.993310 \\
\hline 6 & -1.626728 & -0.369615 & -5.755158 \\
\hline 1 & -1.328306 & -0.564952 & -6.781371 \\
\hline 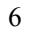 & -2.982424 & -0.238349 & -5.431025 \\
\hline 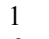 & -3.737341 & -0.337580 & -6.206212 \\
\hline 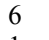 & -3.369754 & 0.004030 & -4.114259 \\
\hline & -4.422997 & 0.072432 & -3.857627 \\
\hline
\end{tabular}

$\begin{array}{llll}-3.330548 & 1.841986 & -1.541444\end{array}$

$\begin{array}{lll}-4.139650 & 1.978061 & -2.269616\end{array}$

$\begin{array}{llll}-2.546987 & 2.544290 & -1.825034\end{array}$

$\begin{array}{llll}-3.447550 & -1.988175 & -0.987367\end{array}$

$-4.167863-2.386152 \quad-0.274923$

$-3.653739-2.791328 \quad-2.268841$

$\begin{array}{lll}-4.923573 & -3.326262 & -2.520178\end{array}$

$\begin{array}{llll}-5.720682 & -3.151744 & -1.806330\end{array}$

$\begin{array}{llll}-5.176650 & -4.085192 & -3.663272\end{array}$

$\begin{array}{lll}-6.172219 & -4.486952 & -3.830584\end{array}$

$\begin{array}{llll}-4.148257 & -4.332644 & -4.575552\end{array}$

$\begin{array}{llll}-4.337632 & -4.921478 & -5.469102\end{array}$

$\begin{array}{llll}2.870256 & -3.828255 & -4.322250\end{array}$

$\begin{array}{llll}-2.057866 & -4.021484 & -5.016414\end{array}$

$\begin{array}{lll}-2.623555 & -3.071529 & -3.175503\end{array}$

$\begin{array}{lll}-1.625077 & -2.690103 & -3.005705\end{array}$

$\begin{array}{lll}-2.063290 & -2.170515 & -0.360994\end{array}$

$\begin{array}{llll}-2.023347 & -1.656719 & 0.601002\end{array}$

$\begin{array}{llll}-1.250993 & -1.790967 & -0.984767\end{array}$

$\begin{array}{lll}-1.876263 & -3.233251 & -0.188893\end{array}$

$\begin{array}{lll}-2.482386 & 1.403334 & 1.572744\end{array}$

$\begin{array}{llll}-2.787071 & 1.130937 & 2.582843\end{array}$

$\begin{array}{lll}-1.971196 & 0.594842 & 1.057735\end{array}$

$\begin{array}{lll}-1.971241 & 2.736943 & 1.386347\end{array}$

$\begin{array}{lll}-2.101692 & 3.460639 & 2.181483\end{array}$

$\begin{array}{lll}-1.166505 & 3.122359 & 0.294736\end{array}$

$\begin{array}{llll}-1.015523 & 2.388193 & -0.485251\end{array}$

$\begin{array}{llll}-5.813978 & 3.370996 & -0.569310\end{array}$

$\begin{array}{llll}-5.950909 & 2.890216 & -1.533780\end{array}$

$\begin{array}{llll}-4.742975 & 4.285437 & -0.469210\end{array}$

$\begin{array}{llll}-4.150687 & 4.453905 & -1.368622\end{array}$

$\begin{array}{llll}-4.628348 & 5.396697 & 0.559821\end{array}$

$\begin{array}{llll}-5.218556 & 6.268046 & 0.236731\end{array}$

$\begin{array}{llll}-3.583981 & 5.721662 & 0.572494\end{array}$

$\begin{array}{lll}-5.041544 & 4.984025 & 1.989564\end{array}$

$\begin{array}{lll}-6.109407 & 5.164443 & 2.144569\end{array}$

$\begin{array}{llll}-4.519261 & 5.626214 & 2.706235\end{array}$

$\begin{array}{lll}-4.730821 & 3.529289 & 2.332479\end{array}$

$\begin{array}{llll}-3.936686 & 3.375845 & 3.058033\end{array}$

$\begin{array}{lll}-5.626411 & 2.478186 & 2.174562\end{array}$

$\begin{array}{lll}-5.470236 & 1.598746 & 2.792230\end{array}$

$\begin{array}{lll}-7.016029 & 2.595435 & 1.579555\end{array}$

$\begin{array}{lll}-7.377497 & 1.587029 & 1.369802\end{array}$

$\begin{array}{lll}-7.691924 & 3.016031 & 2.338460\end{array}$

$\begin{array}{llll}-7.080868 & 3.435179 & 0.284817\end{array}$

$\begin{array}{lll}-7.298836 & 4.481258 & 0.521722\end{array}$

$\begin{array}{llll}-7.921404 & 3.078204 & -0.317530\end{array}$

$\begin{array}{llll}-0.925955 & 4.511467 & -0.090470\end{array}$

$\begin{array}{llll}-0.927089 & 5.573136 & 0.837712\end{array}$

$\begin{array}{llll}-0.687025 & 4.806287 & -1.446173\end{array}$

$\begin{array}{lll}-0.690540 & 6.879020 & 0.420410\end{array}$

$\begin{array}{lll}-1.097456 & 5.369812 & 1.890040\end{array}$

$\begin{array}{lll}-0.469807 & 6.120429 & -1.865197\end{array}$

$\begin{array}{llll}-0.680519 & 3.998221 & -2.171059\end{array}$

$\begin{array}{llll}-0.464497 & 7.159588 & -0.933972\end{array}$

$\begin{array}{lll}-0.682658 & 7.684064 & 1.149770\end{array}$

$\begin{array}{llll}-0.293906 & 6.327081 & -2.916970\end{array}$

$\begin{array}{llll}-0.283812 & 8.181234 & -1.255277\end{array}$

$\begin{array}{llll}4.515873 & -2.243117 & 4.828356\end{array}$

$\begin{array}{lll}1.952821 & -2.722825 & 0.278429\end{array}$

$\begin{array}{lll}4.496174 & 0.107281 & 0.655196\end{array}$

$\begin{array}{llll}3.810208 & -2.491199 & 2.870783\end{array}$

$\begin{array}{lll}4.236290 & -1.131445 & 3.107834\end{array}$

$\begin{array}{llll}3.446047 & -0.602796 & 4.173687\end{array}$

$\begin{array}{llll}3.535954 & 0.389250 & 4.594941\end{array}$

$\begin{array}{llll}2.561762 & -1.628135 & 4.622676\end{array}$

$\begin{array}{llll}1.864935 & -1.551177 & 5.445981\end{array}$

$2.791244 \quad-2.790004 \quad 3.830729$

$\begin{array}{llll}2.291768 & -3.740295 & 3.941082\end{array}$

$\begin{array}{lll}6.538778 & -2.487782 & 5.235433\end{array}$

$\begin{array}{llll}7.336844 & -2.262385 & 4.540499\end{array}$

$\begin{array}{llll}5.953209 & -1.575586 & 6.165619\end{array}$

$6.223080 \quad-0.536007 \quad 6.294151$

$\begin{array}{llll}4.913125 & -2.262395 & 6.862778\end{array}$

$\begin{array}{llll}4.257764 & -1.833545 & 7.609488\end{array}$ 
$\begin{array}{llll}4.857104 & -3.600157 & 6.366490\end{array}$

$\begin{array}{llll}4.155555 & -4.364694 & 6.673219\end{array}$

$\begin{array}{llll}5.861407 & -3.740289 & 5.363412\end{array}$

$\begin{array}{llll}6.054541 & -4.633112 & 4.784413\end{array}$

$\begin{array}{llll}4.324233 & -3.341347 & 1.756153\end{array}$

$\begin{array}{llll}5.628021 & -3.854648 & 1.836130\end{array}$

$\begin{array}{llll}6.194336 & -3.664247 & 2.738890\end{array}$

$\begin{array}{lll}6.210716 & -4.562357 & 0.785473\end{array}$

$\begin{array}{llll}7.234518 & -4.913219 & 0.868238\end{array}$

$\begin{array}{llll}5.477479 & -4.789534 & -0.376503\end{array}$

$\begin{array}{llll}5.918018 & -5.329018 & -1.209319\end{array}$

$\begin{array}{llll}4.175737 & -4.297777 & -0.478793\end{array}$

$3.625258-4.469163-1.397037$

$\begin{array}{llll}3.585150 & -3.561760 & 0.561570\end{array}$

$\begin{array}{llll}5.290142 & -0.425471 & 2.302596\end{array}$

$\begin{array}{lll}6.012922 & -1.187861 & 2.011129\end{array}$

$\begin{array}{llll}6.050820 & 0.661378 & 3.069102\end{array}$

$\begin{array}{lll}6.800840 & 1.142796 & 2.436030\end{array}$

$\begin{array}{llll}5.392452 & 1.439611 & 3.462151\end{array}$

$\begin{array}{lll}6.561349 & 0.199677 & 3.918888\end{array}$

$\begin{array}{llll}1.363901 & -3.421179 & -1.324618\end{array}$

$\begin{array}{llll}1.743627 & -2.734713 & -2.487912\end{array}$

$\begin{array}{llll}2.329089 & -1.829159 & -2.398117\end{array}$

$\begin{array}{llll}1.395524 & -3.211920 & -3.751917\end{array}$

$\begin{array}{llll}1.719523 & -2.675125 & -4.636752\end{array}$

$\begin{array}{llll}0.638790 & -4.379125 & -3.866283\end{array}$

$\begin{array}{llll}0.359990 & -4.753986 & -4.846471\end{array}$

$\begin{array}{llll}0.229788 & -5.056647 & -2.714650\end{array}$

$\begin{array}{lll}-0.378222 & -5.952506 & -2.798429\end{array}$

$\begin{array}{llll}0.592844 & -4.587325 & -1.451053\end{array}$

$\begin{array}{lll}0.263683 & -5.128682 & -0.571957\end{array}$

$\begin{array}{lll}0.844839 & -3.454671 & 1.544078\end{array}$

$\begin{array}{lll}0.895989 & -4.817119 & 1.881897\end{array}$

$\begin{array}{lll}1.606020 & -5.475759 & 1.390102\end{array}$

$\begin{array}{llll}0.055329 & -5.325610 & 2.872891\end{array}$

$\begin{array}{llll}0.099394 & -6.380465 & 3.128578\end{array}$

$\begin{array}{lll}-0.834613 & -4.477219 & 3.540969\end{array}$

$\begin{array}{lll}-1.484007 & -4.874756 & 4.315894\end{array}$

$\begin{array}{lll}-0.879803 & -3.119807 & 3.215635\end{array}$

$\begin{array}{lll}-1.560401 & -2.454808 & 3.740518\end{array}$

$\begin{array}{lll}-0.042267 & -2.607554 & 2.222199\end{array}$

$\begin{array}{lll}-0.049828 & -1.547693 & 1.991377\end{array}$

$\begin{array}{llll}5.530746 & -0.724263 & -0.631135\end{array}$

$\begin{array}{llll}4.938330 & -0.879034 & -1.890899\end{array}$

$\begin{array}{llll}3.966134 & -0.443297 & -2.068929\end{array}$

$\begin{array}{llll}5.573174 & -1.601716 & -2.897442\end{array}$

$6.810999 \quad-2.199794 \quad-2.663864$

$\begin{array}{llll}7.292143 & -2.794675 & -3.433745\end{array}$

$\begin{array}{llll}7.408028 & -2.038834 & -1.415377\end{array}$

$\begin{array}{llll}6.788092 & -1.296555 & -0.409276\end{array}$

$\begin{array}{lll}7.291832 & -1.197929 & 0.543276\end{array}$

$\begin{array}{llll}4.890486 & 1.893108 & 0.452287\end{array}$

$\begin{array}{lll}4.312634 & 2.788295 & 1.367537\end{array}$

$\begin{array}{lll}3.709457 & 2.417071 & 2.187108\end{array}$

$\begin{array}{llll}4.485800 & 4.160896 & 1.217619\end{array}$

$\begin{array}{llll}5.201840 & 4.676473 & 0.135702\end{array}$

$\begin{array}{llll}5.318059 & 5.746901 & 0.008885\end{array}$

$\begin{array}{llll}5.756575 & 3.789207 & -0.783082\end{array}$

$\begin{array}{llll}5.614773 & 2.407510 & -0.627489\end{array}$

$\begin{array}{llll}6.062813 & 1.745395 & -1.359624\end{array}$

$\begin{array}{llll}2.233927 & -0.457934 & 0.284737\end{array}$

$\begin{array}{llll}6.428924 & 4.324876 & -2.016491\end{array}$

$\begin{array}{llll}3.804163 & 5.108078 & 2.164549\end{array}$

$\begin{array}{llll}4.896875 & -1.843855 & -4.218214\end{array}$

$\begin{array}{llll}8.713212 & -2.740390 & -1.153639\end{array}$

$8.604333-4.072928-1.382179$

$\begin{array}{llll}9.136578 & -2.589275 & 0.119955\end{array}$

$\begin{array}{llll}9.697981 & -2.289475 & -1.963401\end{array}$

$3.751033-1.134418-4.351346$

$\begin{array}{llll}5.693445 & -1.519953 & -5.260148\end{array}$

$\begin{array}{llll}4.570921 & -3.150918 & -4.366949\end{array}$

$\begin{array}{llll}7.375812 & 3.484391 & -2.487714\end{array}$

$\begin{array}{llll}5.532843 & 4.504751 & -3.022045\end{array}$

$\begin{array}{llll}7.015651 & 5.521857 & -1.799890\end{array}$

$\begin{array}{lll}4.563745 & 6.187868 & 2.440443\end{array}$
$3.485686 \quad 4.518479 \quad 3.338956$

$\begin{array}{llll}2.636315 & 5.584263 & 1.643083\end{array}$

$\begin{array}{llll}2.094641 & 1.194154 & -2.371828\end{array}$

$\begin{array}{llll}1.678299 & 2.468190 & -0.453986\end{array}$

$\begin{array}{llll}2.518241 & 2.303983 & -3.082375\end{array}$

$\begin{array}{llll}2.051278 & 0.220712 & -2.847186\end{array}$

$\begin{array}{llll}2.129225 & 3.627181 & -1.150110\end{array}$

$\begin{array}{llll}2.563704 & 3.553240 & -2.456850\end{array}$

$\begin{array}{llll}2.145623 & 4.576592 & -0.630150\end{array}$

$\begin{array}{lll}1.163844 & 2.661611 & 0.869799\end{array}$

$\begin{array}{lll}0.850279 & 1.638239 & 1.829057\end{array}$

$\begin{array}{lll}0.795710 & 0.405336 & 1.526305\end{array}$

$\begin{array}{lll}1.714908 & 1.250183 & -1.079884\end{array}$

$\begin{array}{lll}1.335613 & 3.656612 & 1.242373\end{array}$

$\begin{array}{lll}0.538778 & 1.995398 & 3.117649\end{array}$

$\begin{array}{lll}0.165247 & 0.972497 & 4.085502\end{array}$

$\begin{array}{llll}0.698908 & 1.146061 & 5.026653\end{array}$

$\begin{array}{lll}-0.912910 & 1.010473 & 4.292016\end{array}$

$\begin{array}{lll}0.421000 & -0.011590 & 3.703730\end{array}$

$\begin{array}{lll}0.489482 & 3.351881 & 3.648536\end{array}$

$\begin{array}{lll}0.611299 & 4.105237 & 2.876854\end{array}$

$\begin{array}{lll}-0.479187 & 3.518804 & 4.134379\end{array}$

$\begin{array}{lll}1.276361 & 3.498522 & 4.398016\end{array}$

$2.927644 \quad 4.433331 \quad-2.973347$

$\begin{array}{llll}35 & 3.072554 & 2.123120 & -4.949532\end{array}$

C2-P1 re-si 4

Number of imaginary frequencies $=1$

The smallest frequencies are $=-133.97854 .37477 .6585 \mathrm{~cm}(-1)$

Electronic energy $=-7222.6089123$

Zero-point correction=

1.754200

Hartree/Particle)

Thermal correction to Energy=

Thermal correction to Enthalpy=

1.873225

1.874170

Thermal correction to Gibbs Free Energy $=\quad 1.588051$

Sum of electronic and zero-point Energies $=\quad-7220.854713$

Sum of electronic and thermal Energies=

$-7220.735687$

Sum of electronic and thermal Enthalpies=

$-7220.734743$

Sum of electronic and thermal Free Energies=

$-7221.020862$

$\begin{array}{lccc}\ldots \ldots \ldots \ldots \ldots \ldots \ldots \\ 77 & -4.163394 & -0.905691 & 1.466574 \\ 15 & -5.888732 & -0.077176 & 0.255889 \\ 8 & -6.427972 & -0.741237 & -1.163095 \\ 8 & -7.297192 & -0.051063 & 1.141311 \\ 7 & -5.526030 & 1.472669 & -0.289678 \\ 6 & -7.373590 & -1.759853 & -1.201906 \\ 6 & -6.930404 & -3.001340 & -1.708319 \\ 1 & -5.902321 & -3.086230 & -2.044108 \\ 6 & -7.803080 & -4.058401 & -1.773576 \\ 1 & -7.477209 & -5.015994 & -2.169464 \\ 6 & -9.135200 & -3.923533 & -1.298271 \\ 6 & -10.023451 & -5.033099 & -1.288988 \\ 1 & -9.668713 & -5.983292 & -1.679548 \\ 6 & -11.298091 & -4.914661 & -0.783544 \\ 1 & -11.967334 & -5.769982 & -0.776304 \\ 6 & -11.731230 & -3.675381 & -0.255291 \\ 1 & -12.728349 & -3.589994 & 0.167011 \\ 6 & -10.900216 & -2.575970 & -0.264725 \\ 1 & -11.249247 & -1.640940 & 0.156110 \\ 6 & -9.584273 & -2.653321 & -0.801413 \\ 6 & -8.686878 & -1.524912 & -0.823998 \\ 6 & -9.131060 & -0.165153 & -0.405705 \\ 6 & -10.259841 & 0.499136 & -1.003412 \\ 6 & -10.915591 & 0.001273 & -2.163882 \\ 1 & -10.555157 & -0.910042 & -2.626731 \\ 6 & -11.987146 & 0.669155 & -2.715540 \\ 1 & -12.464602 & 0.274149 & -3.607647 \\ 6 & -12.468408 & 1.868040 & -2.136575 \\ 1 & -13.316937 & 2.380022 & -2.580770 \\ 6 & -11.849689 & 2.386489 & -1.021775 \\ 1 & -12.197906 & 3.314493 & -0.575657 \\ 6 & -10.732372 & 1.731089 & -0.435400\end{array}$


$$
6
$$$$
6
$$$$
6
$$$$
16
$$

$$
\text { 1 }
$$$$
\begin{aligned}
& 6 \\
& 1
\end{aligned}
$$$$
6
$$

6
6
1

1

6

1

6

6

6
6

6
1
6

6
1
6

6

6

1
6
6

6

6

6

6
1
6
1

6
1
6
1

6
1
6

1
6
6
1

6
1
6
6
1

6
6
1

6
1
6
6

1
6
6

6

1
6
6
1

6

1
6
6
1 $\begin{array}{rrrr}6.182655 & 4.443637 & -1.331342\end{array}$ $\begin{array}{lll}4.813475 & 0.900663 & 2.229912\end{array}$ $\begin{array}{llll}4.756076 & -0.116499 & -1.412197\end{array}$ $\begin{array}{llll}5.924551 & 2.827050 & -0.026656\end{array}$ $\begin{array}{llll}5.288438 & 2.580247 & -1.300279\end{array}$ $\begin{array}{lll}4.306299 & 3.595846 & -1.500767\end{array}$ $\begin{array}{lll}3.667186 & 3.693252 & -2.367563\end{array}$ $\begin{array}{llll}4.348928 & 4.486628 & -0.389097\end{array}$ $\begin{array}{llll}3.752940 & 5.380879 & -0.267624\end{array}$ $\begin{array}{llll}5.347345 & 4.020444 & 0.513389\end{array}$ $\begin{array}{llll}5.632775 & 4.486764 & 1.444599\end{array}$ $\begin{array}{llll}7.804480 & 4.453376 & -2.625421\end{array}$ $\begin{array}{llll}8.258833 & 3.566263 & -3.046045\end{array}$ $\begin{array}{lll}6.716421 & 5.187950 & -3.189295\end{array}$ $\begin{array}{llll}6.197197 & 4.951456 & -4.108485\end{array}$ $\begin{array}{llll}6.401779 & 6.262154 & -2.302183\end{array}$ $\begin{array}{llll}5.601844 & 6.979220 & -2.431923\end{array}$ $\begin{array}{llll}7.296340 & 6.194415 & -1.191038\end{array}$ $\begin{array}{llll}7.293580 & 6.849928 & -0.330173\end{array}$ $\begin{array}{llll}8.164057 & 5.080245 & -1.391604\end{array}$ $\begin{array}{llll}8.937161 & 4.753802 & -0.709786\end{array}$ $\begin{array}{llll}6.926451 & 1.926641 & 0.615918\end{array}$ $\begin{array}{llll}8.235968 & 1.882156 & 0.115661\end{array}$ $\begin{array}{llll}8.486421 & 2.528081 & -0.716152\end{array}$ $\begin{array}{llll}9.199158 & 1.022607 & 0.643357\end{array}$ $\begin{array}{ccc}10.194829 & 0.993768 & 0.213130\end{array}$ $\begin{array}{lll}8.865845 & 0.192000 & 1.710186\end{array}$ $\begin{array}{lll}9.602504 & -0.484701 & 2.132527\end{array}$ $\begin{array}{llll}7.568772 & 0.215485 & 2.225078\end{array}$ $\begin{array}{lll}7.323067 & -0.445760 & 3.048463\end{array}$ $\begin{array}{lll}6.581271 & 1.053817 & 1.684943\end{array}$ $\begin{array}{llll}5.592384 & 1.404758 & -2.187844\end{array}$ $\begin{array}{lll}6.659145 & 1.207138 & -2.082992\end{array}$ $\begin{array}{lll}5.312956 & 1.642793 & -3.674790\end{array}$ $\begin{array}{llll}5.558061 & 0.758184 & -4.268888\end{array}$ $\begin{array}{llll}4.270127 & 1.901548 & -3.870563\end{array}$ $\begin{array}{llll}5.932302 & 2.475941 & -4.019818\end{array}$ $\begin{array}{lll}4.832566 & -0.341420 & 3.590852\end{array}$ $\begin{array}{lll}4.982076 & -1.692853 & 3.234217\end{array}$ $\begin{array}{llll}5.154299 & -1.956329 & 2.196432\end{array}$ $\begin{array}{llll}4.925585 & -2.697515 & 4.199229\end{array}$ $\begin{array}{lll}5.059960 & -3.732836 & 3.904035\end{array}$ $\begin{array}{lll}4.693236 & -2.366047 & 5.537861\end{array}$ $\begin{array}{lll}4.636775 & -3.147090 & 6.290717\end{array}$ $\begin{array}{lll}4.536101 & -1.027543 & 5.901859\end{array}$ $\begin{array}{lll}4.356510 & -0.762363 & 6.940056\end{array}$ $\begin{array}{lll}4.611110 & -0.018733 & 4.937015\end{array}$ $\begin{array}{llll}4.484713 & 1.014691 & 5.238777\end{array}$ $\begin{array}{lll}4.476299 & 2.496678 & 3.068280\end{array}$ $\begin{array}{lll}5.423281 & 3.105152 & 3.907920\end{array}$ $\begin{array}{lll}6.374780 & 2.616378 & 4.098643\end{array}$ $\begin{array}{lll}5.151979 & 4.346145 & 4.484839\end{array}$ $\begin{array}{lll}5.888353 & 4.814377 & 5.131856\end{array}$ $\begin{array}{llll}3.935081 & 4.987469 & 4.225671\end{array}$ $\begin{array}{llll}3.728014 & 5.956514 & 4.671261\end{array}$ $\begin{array}{llll}2.991291 & 4.383776 & 3.391554\end{array}$ $\begin{array}{llll}2.049578 & 4.884174 & 3.182256\end{array}$ $\begin{array}{lll}3.259576 & 3.141808 & 2.810831\end{array}$ $\begin{array}{lll}2.547905 & 2.684885 & 2.131796\end{array}$ $\begin{array}{llll}6.128990 & -1.322825 & -1.131467\end{array}$ $\begin{array}{llll}5.826478 & -2.456672 & -0.364250\end{array}$ $\begin{array}{llll}4.807677 & -2.632814 & -0.044362\end{array}$ $\begin{array}{lll}6.824244 & -3.357184 & 0.008806\end{array}$ $\begin{array}{lll}8.151655 & -3.135562 & -0.350068\end{array}$ $\begin{array}{llll}8.932326 & -3.815841 & -0.027884\end{array}$ $\begin{array}{llll}8.455763 & -2.010062 & -1.114552\end{array}$ $\begin{array}{llll}7.459706 & -1.123004 & -1.520511\end{array}$ $\begin{array}{llll}7.750484 & -0.272111 & -2.120345\end{array}$ $\begin{array}{llll}3.720982 & -0.910581 & -2.712345\end{array}$ $\begin{array}{llll}2.643019 & -0.169266 & -3.225360\end{array}$ $\begin{array}{lll}2.493700 & 0.860122 & -2.922970\end{array}$ $\begin{array}{lll}1.740703 & -0.755385 & -4.108772\end{array}$ $\begin{array}{lll}1.852598 & -2.101908 & -4.460003\end{array}$ $\begin{array}{lll}1.134019 & -2.562042 & -5.128793\end{array}$ 


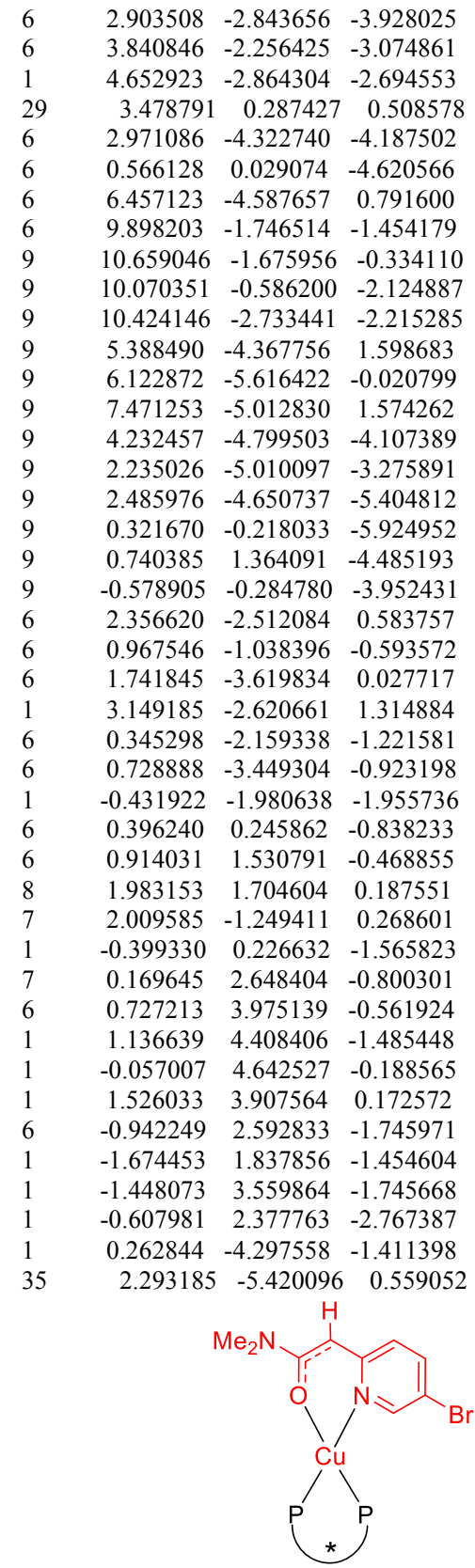

$\mathrm{Cu}$-enolate

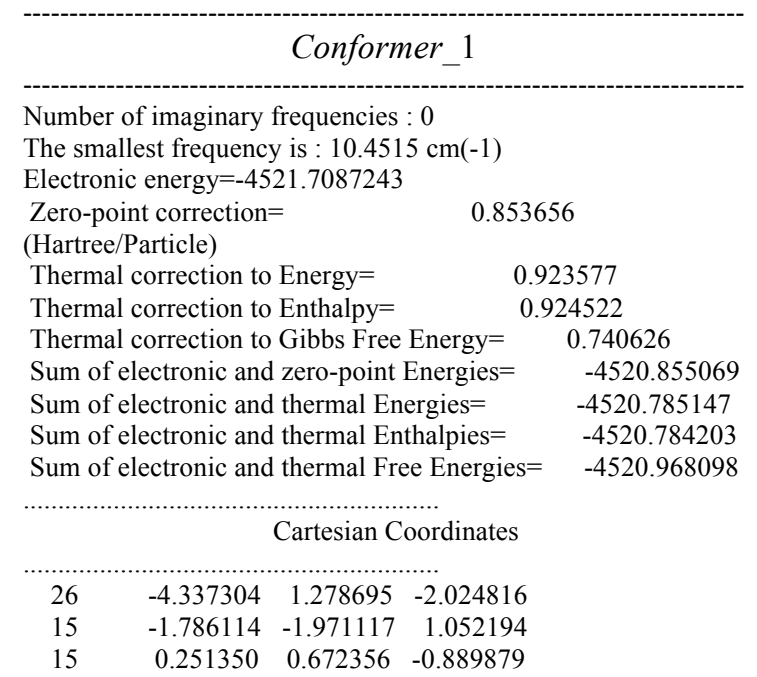

$\begin{array}{lll}-3.167803 & -0.194116 & -1.108795\end{array}$

$\begin{array}{llll}-2.372501 & 0.955442 & -1.458104\end{array}$

$\begin{array}{llll}-2.912546 & 2.083650 & -0.766055\end{array}$

$\begin{array}{llll}-2.537351 & 3.096425 & -0.824103\end{array}$

$\begin{array}{llll}-4.042255 & 1.649549 & -0.013941\end{array}$

$\begin{array}{lll}-4.688193 & 2.279447 & 0.582874\end{array}$

$\begin{array}{llll}-4.203706 & 0.250009 & -0.226239\end{array}$

$\begin{array}{lll}-4.980335 & -0.376180 & 0.189051\end{array}$

$\begin{array}{llll}-4.550142 & 1.261739 & -4.091546\end{array}$

$\begin{array}{llll}-3.802772 & 0.897337 & -4.783889\end{array}$

$\begin{array}{llll}-4.651652 & 2.596591 & -3.592623\end{array}$

$\begin{array}{llll}-3.992820 & 3.418985 & -3.837426\end{array}$

$\begin{array}{llll}-5.749775 & 2.644358 & -2.680257\end{array}$

$\begin{array}{llll}-6.061543 & 3.507437 & -2.106840\end{array}$

$\begin{array}{llll}-6.326926 & 1.339369 & -2.614495\end{array}$

$\begin{array}{llll}-7.154955 & 1.040437 & -1.985255\end{array}$

$\begin{array}{llll}-5.587389 & 0.485739 & -3.486688\end{array}$

$\begin{array}{lll}-5.762549 & -0.571185 & -3.635466\end{array}$

$\begin{array}{llll}-2.883863 & -1.592547 & -1.530571\end{array}$

$\begin{array}{llll}-3.119845 & -1.987608 & -2.855357\end{array}$

$\begin{array}{llll}-3.510050 & -1.252277 & -3.547971\end{array}$

$\begin{array}{llll}-2.864014 & -3.289785 & -3.286105\end{array}$

$-3.046734 \quad-3.563730 \quad-4.321235$

$\begin{array}{llll}-2.382688 & -4.232260 & -2.379737\end{array}$

$\begin{array}{llll}-2.189717 & -5.253271 & -2.694692\end{array}$

$-2.121273 \quad-3.854236 \quad-1.062345$

$\begin{array}{llll}-1.721320 & -4.592575 & -0.377511\end{array}$

$\begin{array}{llll}-2.333719 & -2.537111 & -0.625323\end{array}$

$\begin{array}{llll}-1.083351 & 0.898866 & -2.226859\end{array}$

$\begin{array}{lll}-1.063252 & -0.035158 & -2.795616\end{array}$

$\begin{array}{llll}-0.867923 & 2.065095 & -3.193680\end{array}$

$\begin{array}{llll}0.076752 & 1.964174 & -3.735435\end{array}$

$\begin{array}{llll}-0.862627 & 3.028700 & -2.677120\end{array}$

$\begin{array}{llll}-1.683778 & 2.077819 & -3.922790\end{array}$

$\begin{array}{lll}-0.843379 & -3.401707 & 1.726084\end{array}$

$\begin{array}{llll}0.320474 & -3.805586 & 1.047409\end{array}$

$\begin{array}{llll}0.580709 & -3.345030 & 0.100249\end{array}$

$\begin{array}{lll}1.132826 & -4.812677 & 1.563101\end{array}$

$\begin{array}{llll}2.018850 & -5.120011 & 1.015147\end{array}$

$\begin{array}{llll}0.810222 & -5.414935 & 2.783242\end{array}$

$\begin{array}{llll}1.447857 & -6.193185 & 3.192915\end{array}$

$\begin{array}{lll}1.0 .335446 & -5.011503 & 3.471394\end{array}$

$\begin{array}{lll}-0.593307 & -5.475810 & 4.419303\end{array}$

$\begin{array}{lll}-1.163455 & -4.015035 & 2.945448\end{array}$

$\begin{array}{lll}-2.054878 & -3.718577 & 3.489001\end{array}$

$\begin{array}{lll}-3.333697 & -1.927589 & 2.039236\end{array}$

$\begin{array}{lll}-4.354646 & -2.876022 & 1.872939\end{array}$

$\begin{array}{llll}-4.239045 & -3.678801 & 1.150002\end{array}$

$\begin{array}{llll}-5.525737 & -2.782087 & 2.625920\end{array}$

$\begin{array}{lll}-6.315230 & -3.516355 & 2.491287\end{array}$

$\begin{array}{lll}-5.683436 & -1.743554 & 3.550358\end{array}$

$\begin{array}{lll}-6.597963 & -1.670774 & 4.132543\end{array}$

$\begin{array}{lll}-4.667813 & -0.799583 & 3.720602\end{array}$

$\begin{array}{lll}-4.790222 & 0.012775 & 4.431816\end{array}$

$\begin{array}{lll}-3.495269 & -0.887382 & 2.966354\end{array}$

$\begin{array}{lll}-2.718604 & -0.134072 & 3.061396\end{array}$

$\begin{array}{llll}1.493787 & -0.463231 & -1.635063\end{array}$

$\begin{array}{llll}2.876771 & -0.262901 & -1.507560\end{array}$

$\begin{array}{llll}3.266684 & 0.652634 & -1.079693\end{array}$

$\begin{array}{llll}3.772897 & -1.254524 & -1.906573\end{array}$

$\begin{array}{llll}3.320487 & -2.468460 & -2.422507\end{array}$

$\begin{array}{llll}4.021852 & -3.238295 & -2.720748\end{array}$

$\begin{array}{lll}1.946468 & -2.675595 & -2.532614\end{array}$

$\begin{array}{llll}1.037572 & -1.690235 & -2.143145\end{array}$

$\begin{array}{lll}-0.025671 & -1.899687 & -2.211893\end{array}$

$\begin{array}{llll}1.091662 & 2.303587 & -0.750509\end{array}$

$\begin{array}{llll}0.822027 & 3.027991 & 0.416390\end{array}$

$\begin{array}{lll}0.154456 & 2.607520 & 1.161594\end{array}$

$\begin{array}{llll}1.401757 & 4.282574 & 0.616740\end{array}$

$\begin{array}{llll}2.259565 & 4.830554 & -0.333246\end{array}$

$\begin{array}{llll}2.716910 & 5.799471 & -0.171157\end{array}$

$\begin{array}{llll}2.525355 & 4.107546 & -1.498949\end{array}$

$\begin{array}{llll}1.945706 & 2.857329 & -1.715159\end{array}$

$\begin{array}{llll}2.174176 & 2.317001 & -2.626789\end{array}$

$\begin{array}{lll}-0.472046 & -0.133068 & 1.129753\end{array}$ 


\begin{abstract}
$\begin{array}{llll}3.397484 & 4.709921 & -2.566184\end{array}$
$\begin{array}{llll}1.126803 & 4.997862 & 1.911251\end{array}$

$\begin{array}{llll}5.252940 & -0.992287 & -1.830461\end{array}$

$\begin{array}{llll}1.414870 & -4.001919 & -3.003340\end{array}$

$\begin{array}{llll}0.946383 & -4.743152 & -1.965185\end{array}$

$\begin{array}{llll}0.383740 & -3.853420 & -3.865993\end{array}$

$\begin{array}{llll}2.356654 & -4.744902 & -3.621723\end{array}$

$\begin{array}{llll}5.563416 & -0.097276 & -0.868695\end{array}$

$\begin{array}{llll}5.725208 & -0.490331 & -2.999325\end{array}$

$\begin{array}{llll}5.954818 & -2.121164 & -1.583737\end{array}$

$\begin{array}{llll}2.667056 & 5.410540 & -3.468667\end{array}$

$\begin{array}{llll}4.070397 & 3.764695 & -3.261329\end{array}$

$\begin{array}{llll}4.310189 & 5.564804 & -2.054813\end{array}$

$\begin{array}{lll}1.445559 & 6.309207 & 1.853760\end{array}$

$\begin{array}{lll}-0.180501 & 4.913722 & 2.258324\end{array}$

$\begin{array}{llll}1.829963 & 4.463751 & 2.939843\end{array}$

$\begin{array}{llll}2.223036 & -1.091574 & 1.850512\end{array}$

$\begin{array}{lll}1.499044 & 0.522387 & 3.410540\end{array}$

$\begin{array}{llll}3.441334 & -1.244700 & 2.477152\end{array}$

$\begin{array}{llll}1.978267 & -1.657713 & 0.961047\end{array}$

$\begin{array}{lll}2.759103 & 0.375026 & 4.083010\end{array}$

$\begin{array}{llll}3.724365 & -0.493999 & 3.629368\end{array}$

$\begin{array}{lll}2.948200 & 0.972155 & 4.970154\end{array}$

$\begin{array}{llll}0.561392 & 1.449142 & 3.935083\end{array}$

$\begin{array}{lll}-0.705054 & 1.810484 & 3.418232\end{array}$

$\begin{array}{lll}-1.214484 & 1.369486 & 2.324927\end{array}$

$\begin{array}{lll}1.273044 & -0.245149 & 2.291487\end{array}$

$\begin{array}{lll}0.895361 & 1.947476 & 4.834380\end{array}$

$\begin{array}{llll}-1.489903 & 2.718072 & 4.121031\end{array}$

$\begin{array}{lll}-2.685339 & 3.241914 & 3.470501\end{array}$

$\begin{array}{llll}-2.458350 & 4.079305 & 2.794178\end{array}$

$\begin{array}{llll}-3.383726 & 3.594637 & 4.235028\end{array}$

$\begin{array}{lll}-3.163765 & 2.456393 & 2.887593\end{array}$

$\begin{array}{lll}-0.926738 & 3.525718 & 5.195529\end{array}$

$\begin{array}{lll}-0.099455 & 4.159573 & 4.848988\end{array}$

$\begin{array}{lll}-0.563974 & 2.898118 & 6.015756\end{array}$

$\begin{array}{lll}-1.708378 & 4.172686 & 5.599084\end{array}$

$\begin{array}{llll}4.673240 & -0.591963 & 4.146680\end{array}$

$\begin{array}{llll}4.759327 & -2.498923 & 1.741425\end{array}$
\end{abstract}

\section{Conformer_2}

Number of imaginary frequencies : 0

The smallest frequency is : $14.9561 \mathrm{~cm}(-1)$

Electronic energy :

$\mathrm{HF}=-4521.7138307$

Zero-point correction=

0.854728

(Hartree/Particle)

Thermal correction to Energy=

0.924099

0.925044

. Enthalpy

0.744563

Thermal correction to Gibbs Free Energy=

Sum of electronic and zero-point Energies=

Sum of electronic and thermal Energies=

$-4520.859102$

$-4520.789731$

Sum of electronic and thermal Enthalpies=

$-4520.788787$

Sum of electronic and thermal Free Energies=

$-4520.969268$

\begin{tabular}{crrr} 
& \multicolumn{3}{c}{ Cartesian Coordinates } \\
\hline 26 & -3.560983 & -1.209726 & -2.713418 \\
15 & -1.139370 & -1.582697 & 1.924464 \\
15 & 0.658137 & 0.064658 & -0.920207 \\
6 & -2.336719 & -1.538462 & -1.043363 \\
6 & -1.845501 & -0.443897 & -1.848875 \\
6 & -2.865995 & 0.553835 & -1.901284 \\
1 & -2.806982 & 1.490563 & -2.437989 \\
6 & -3.994239 & 0.078799 & -1.168492 \\
1 & -4.939469 & 0.591455 & -1.056699 \\
6 & -3.670889 & -1.206242 & -0.642642 \\
1 & -4.326663 & -1.835584 & -0.060865 \\
6 & -3.126653 & -2.158465 & -4.509579 \\
1 & -2.129468 & -2.416451 & -4.841425 \\
6 & -3.796518 & -0.919936 & -4.750861 \\
1 & -3.393865 & -0.073814 & -5.291615 \\
6 & -5.072241 & -0.975225 & -4.110545 \\
1 & -5.800997 & -0.176068 & -4.076766 \\
6 & -5.191383 & -2.247564 & -3.472426
\end{tabular}

$\begin{array}{llll}-6.027690 & -2.582909 & -2.873282\end{array}$

$\begin{array}{llll}-3.991318 & -2.978934 & -3.719755\end{array}$

$\begin{array}{lll}-3.765473 & -3.967537 & -3.343618\end{array}$

$\begin{array}{lll}-1.515668 & -2.727922 & -0.670425\end{array}$

$\begin{array}{lll}-1.179305 & -3.658437 & -1.666308\end{array}$

$\begin{array}{lll}-1.585098 & -3.511774 & -2.659305\end{array}$

$-0.325071-4.731880-1.416790$

$\begin{array}{lll}-0.058429 & -5.412442 & -2.219397\end{array}$

$\begin{array}{lll}0.201559 & -4.905477 & -0.139218\end{array}$

$\begin{array}{lll}0.878502 & -5.727653 & 0.070538\end{array}$

$\begin{array}{lll}-0.122785 & -3.998673 & 0.869748\end{array}$

$\begin{array}{lll}0.310192 & -4.137644 & 1.853735\end{array}$

$\begin{array}{lll}-0.963695 & -2.899229 & 0.628994\end{array}$

$\begin{array}{llll}-0.450042 & -0.353189 & -2.404243\end{array}$

$\begin{array}{lll}-0.157359 & -1.357671 & -2.715801\end{array}$

$\begin{array}{lll}-0.310501 & 0.586261 & -3.604537\end{array}$

$\begin{array}{llll}0.712451 & 0.577659 & -3.991369\end{array}$

$\begin{array}{llll}-0.563519 & 1.619458 & -3.351433\end{array}$

$\begin{array}{lll}-0.986563 & 0.259000 & -4.400757\end{array}$

$\begin{array}{lll}-0.242516 & -2.256977 & 3.387172\end{array}$

$\begin{array}{lll}1.022189 & -1.724104 & 3.681001\end{array}$

$\begin{array}{lll}1.421240 & -0.917235 & 3.075155\end{array}$

$\begin{array}{lll}1.755779 & -2.210033 & 4.766574\end{array}$

$\begin{array}{lll}2.735982 & -1.794833 & 4.979297\end{array}$

$\begin{array}{lll}1.230253 & -3.221257 & 5.572191\end{array}$

$\begin{array}{llll}1.799412 & -3.594940 & 6.418951\end{array}$

$\begin{array}{llll}-0.034557 & -3.748522 & 5.292267\end{array}$

$\begin{array}{llll}-0.450240 & -4.531847 & 5.920113\end{array}$

$\begin{array}{lll}-0.767998 & -3.270725 & 4.206357\end{array}$

$\begin{array}{lll}-1.749168 & -3.687102 & 4.001048\end{array}$

$\begin{array}{lll}-2.896428 & -1.664477 & 2.456124\end{array}$

$\begin{array}{lll}-3.686254 & -2.816427 & 2.317966\end{array}$

$\begin{array}{lll}-3.268184 & -3.711035 & 1.865550\end{array}$

$\begin{array}{lll}-5.017966 & -2.809421 & 2.737061\end{array}$

$\begin{array}{lll}-5.624150 & -3.703671 & 2.621597\end{array}$

$\begin{array}{lll}-5.570573 & -1.654974 & 3.300611\end{array}$

$\begin{array}{lll}-6.608848 & -1.649350 & 3.620033\end{array}$

$\begin{array}{lll}-4.785057 & -0.509581 & 3.453739\end{array}$

$\begin{array}{llll}-5.208410 & 0.389181 & 3.893219\end{array}$

$\begin{array}{lll}-3.453606 & -0.513920 & 3.033226\end{array}$

$\begin{array}{llll}-2.845655 & 0.379721 & 3.142619\end{array}$

$\begin{array}{llll}1.960028 & -1.235860 & -0.854886\end{array}$

$\begin{array}{llll}2.137127 & -2.258344 & -1.793188\end{array}$

$\begin{array}{llll}1.532515 & -2.306841 & -2.689513\end{array}$

$\begin{array}{llll}3.096961 & -3.248465 & -1.576711\end{array}$

$\begin{array}{llll}3.920192 & -3.219738 & -0.450932\end{array}$

$\begin{array}{llll}4.669910 & -3.987225 & -0.294917\end{array}$

$\begin{array}{llll}3.753599 & -2.189474 & 0.473508\end{array}$

$\begin{array}{llll}2.771515 & -1.216852 & 0.289947\end{array}$

$\begin{array}{lll}2.617062 & -0.436620 & 1.030692\end{array}$

$\begin{array}{llll}1.592959 & 1.591393 & -1.344512\end{array}$

$\begin{array}{llll}0.878285 & 2.797897 & -1.271193\end{array}$

$\begin{array}{llll}-0.182111 & 2.786530 & -1.047400\end{array}$

$\begin{array}{llll}1.535509 & 4.014662 & -1.421851\end{array}$

$\begin{array}{llll}2.918599 & 4.061524 & -1.607171\end{array}$

$3.433543 \quad 5.012861-1.678112$

$\begin{array}{llll}3.630013 & 2.865497 & -1.650568\end{array}$

$\begin{array}{llll}2.975804 & 1.635464 & -1.543388\end{array}$

$\begin{array}{llll}3.559328 & 0.723171 & -1.573759\end{array}$

$\begin{array}{lll}-0.307080 & 0.377506 & 1.179366\end{array}$

$\begin{array}{llll}5.125655 & 2.895790 & -1.798332\end{array}$

$\begin{array}{llll}0.788620 & 5.307640 & -1.251390\end{array}$

$\begin{array}{llll}3.221859 & -4.404855 & -2.529279\end{array}$

$\begin{array}{lll}4.670379 & -2.075120 & 1.659522\end{array}$

$\begin{array}{llll}5.647676 & -1.158219 & 1.443341\end{array}$

$\begin{array}{lll}4.010848 & -1.671498 & 2.773225\end{array}$

$\begin{array}{llll}5.282319 & -3.241124 & 1.955215\end{array}$

$\begin{array}{llll}2.706966 & -5.544873 & -2.000344\end{array}$

$\begin{array}{llll}4.513782 & -4.673949 & -2.827291\end{array}$

$\begin{array}{llll}2.573694 & -4.187859 & -3.694993\end{array}$

$\begin{array}{llll}5.516187 & 2.907034 & -3.094458\end{array}$

$\begin{array}{llll}5.708809 & 1.816453 & -1.223845\end{array}$

$\begin{array}{llll}5.664790 & 3.995576 & -1.218779\end{array}$

$\begin{array}{llll}1.111971 & 6.203309 & -2.213821\end{array}$

$-0.550224 \quad 5.141797 \quad-1.283475$ 


$\begin{array}{rrrr}9 & 1.086532 & 5.900366 & -0.065211 \\ 6 & 1.812729 & 2.347376 & 2.029694 \\ 6 & 0.859350 & 3.399281 & 2.098343 \\ 6 & -0.526318 & 3.352928 & 1.830206 \\ 1 & 1.231606 & 4.390094 & 2.319710 \\ 7 & 3.154777 & 2.677467 & 1.979169 \\ 6 & 4.177772 & 1.654659 & 2.131158 \\ 1 & 4.985496 & 2.034226 & 2.768283 \\ 1 & 3.756744 & 0.767980 & 2.598798 \\ 1 & 4.615408 & 1.371063 & 1.164674 \\ 6 & 3.653261 & 4.028395 & 1.771103 \\ 1 & 2.933955 & 4.629684 & 1.216311 \\ 1 & 3.886188 & 4.537484 & 2.718064 \\ 1 & 4.569549 & 3.982096 & 1.173972 \\ 8 & 1.523249 & 1.097910 & 2.021357 \\ 6 & -1.276608 & 4.576678 & 1.923648 \\ 6 & -2.615428 & 4.631531 & 1.623192 \\ 1 & -0.756039 & 5.480563 & 2.224070 \\ 6 & -3.246258 & 3.440890 & 1.221095 \\ 6 & -2.515910 & 2.276381 & 1.140608 \\ 1 & -2.977028 & 1.344699 & 0.838207 \\ 7 & -1.198270 & 2.216520 & 1.428568 \\ 1 & -3.164230 & 5.565194 & 1.691658 \\ 35 & -5.162335 & 3.423250 & 0.780462 \\ ------------------------------------------------------------\end{array}$

\section{Conformer_3}

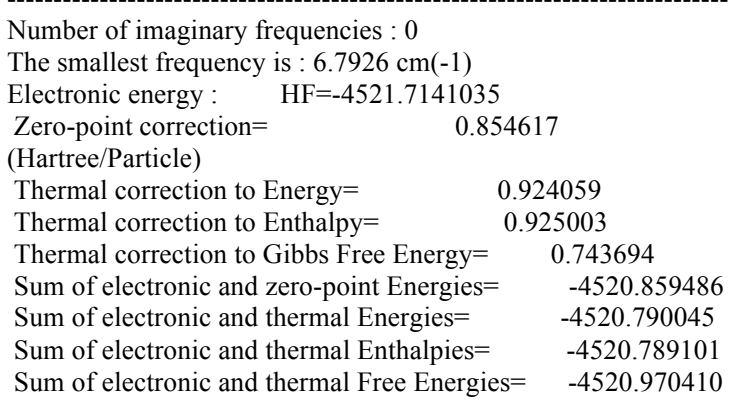

\begin{tabular}{|c|c|c|c|}
\hline \multicolumn{4}{|c|}{ Cartesian Coordinates } \\
\hline 26 & -3.417808 & -1.167275 & -2.827133 \\
\hline 15 & -1.016607 & -1.727843 & 1.778315 \\
\hline 15 & 0.692692 & 0.242161 & -0.871092 \\
\hline 6 & -2.190875 & -1.556902 & -1.174311 \\
\hline 6 & -1.759094 & -0.371412 & -1.879982 \\
\hline 6 & -2.839039 & 0.563333 & -1.862890 \\
\hline 1 & -2.833751 & 1.543976 & -2.318600 \\
\hline 6 & -3.941847 & -0.037883 & -1.186967 \\
\hline 1 & -4.917596 & 0.406059 & -1.046043 \\
\hline 6 & -3.545775 & -1.340339 & -0.764921 \\
\hline 1 & -4.166088 & -2.054541 & -0.245542 \\
\hline 6 & -2.915636 & -1.946039 & -4.686635 \\
\hline 1 & -1.904436 & -2.126435 & -5.026897 \\
\hline 6 & -3.648434 & -0.728938 & -4.838605 \\
\hline 1 & -3.285372 & 0.177639 & -5.304210 \\
\hline 6 & -4.925334 & -0.902048 & -4.222345 \\
\hline 1 & -5.695750 & -0.147325 & -4.134599 \\
\hline 6 & -4.982810 & -2.226138 & -3.689664 \\
\hline 1 & -5.806560 & -2.653674 & -3.133368 \\
\hline 6 & -3.743253 & -2.871514 & -3.977491 \\
\hline 1 & -3.470192 & -3.875030 & -3.680542 \\
\hline 6 & -1.293428 & -2.713496 & -0.885331 \\
\hline 6 & -0.903933 & -3.549066 & -1.943453 \\
\hline 1 & -1.341651 & -3.373701 & -2.918026 \\
\hline 6 & 0.043064 & -4.558615 & -1.776391 \\
\hline 1 & 0.344231 & -5.167088 & -2.623349 \\
\hline 6 & 0.622506 & -4.753078 & -0.524927 \\
\hline 1 & 1.382571 & -5.515072 & -0.382436 \\
\hline 6 & 0.243351 & -3.943647 & 0.546090 \\
\hline 1 & 0.731004 & -4.086544 & 1.502902 \\
\hline 6 & -0.708914 & -2.922415 & 0.393973 \\
\hline 6 & -0.365140 & -0.148115 & -2.403289 \\
\hline 1 & 0.005019 & -1.106786 & -2.771936 \\
\hline
\end{tabular}

$\begin{array}{llll}-0.275754 & 0.878006 & -3.535568\end{array}$

$\begin{array}{llll}0.750437 & 0.970208 & -3.902212\end{array}$

$\begin{array}{llll}-0.607005 & 1.870887 & -3.219482\end{array}$

$\begin{array}{llll}-0.914548 & 0.559781 & -4.365446\end{array}$

$\begin{array}{lll}-0.095508 & -2.410930 & 3.223351\end{array}$

$\begin{array}{lll}0.942963 & -1.641012 & 3.768407\end{array}$

$\begin{array}{lll}1.186218 & -0.674754 & 3.336414\end{array}$

$\begin{array}{llll}1.660608 & -2.109479 & 4.872855\end{array}$

$\begin{array}{llll}2.469785 & -1.509197 & 5.278489\end{array}$

$\begin{array}{lll}1.346935 & -3.343125 & 5.442320\end{array}$

$\begin{array}{lll}1.906741 & -3.707272 & 6.299410\end{array}$

$\begin{array}{llll}0.305223 & -4.111360 & 4.910354\end{array}$

$\begin{array}{lll}0.052785 & -5.070099 & 5.354997\end{array}$

$\begin{array}{lll}-0.415521 & -3.647462 & 3.811135\end{array}$

$\begin{array}{lll}-1.226382 & -4.248432 & 3.410756\end{array}$

$\begin{array}{lll}-2.764268 & -2.021164 & 2.271571\end{array}$

$\begin{array}{lll}-3.436515 & -3.231305 & 2.043178\end{array}$

$\begin{array}{lll}-2.931438 & -4.046223 & 1.533151\end{array}$

$\begin{array}{lll}-4.764854 & -3.382675 & 2.445293\end{array}$

$\begin{array}{llll}-5.280609 & -4.320841 & 2.259662\end{array}$

$\begin{array}{lll}-5.430968 & -2.330095 & 3.081160\end{array}$

$\begin{array}{lll}-6.466598 & -2.448260 & 3.387203\end{array}$

$\begin{array}{lll}-4.762267 & -1.127165 & 3.323046\end{array}$

$\begin{array}{lll}-5.273846 & -0.306979 & 3.818924\end{array}$

$\begin{array}{lll}-3.434579 & -0.973293 & 2.919399\end{array}$

$\begin{array}{lll}-2.915687 & -0.035628 & 3.098566\end{array}$

$\begin{array}{llll}2.015545 & -1.041748 & -0.841237\end{array}$

$2.397543 \quad-1.823030 \quad-1.937716$

$\begin{array}{llll}1.982225 & -1.647568 & -2.922643\end{array}$

$3.317133-2.860228-1.771753$

$\begin{array}{llll}3.881994 & -3.125561 & -0.525507\end{array}$

$\begin{array}{llll}4.584053 & -3.942746 & -0.400861\end{array}$

$\begin{array}{llll}3.520214 & -2.329175 & 0.560119\end{array}$

$\begin{array}{llll}2.592822 & -1.299173 & 0.411867\end{array}$

$\begin{array}{lll}2.304843 & -0.690713 & 1.262990\end{array}$

$\begin{array}{llll}1.602366 & 1.801157 & -1.207407\end{array}$

$\begin{array}{llll}0.859592 & 2.990159 & -1.130108\end{array}$

$\begin{array}{llll}-0.208783 & 2.951357 & -0.950631\end{array}$

$\begin{array}{llll}1.496111 & 4.223212 & -1.227995\end{array}$

$\begin{array}{llll}2.883811 & 4.301275 & -1.358739\end{array}$

$\begin{array}{llll}3.381568 & 5.263892 & -1.389593\end{array}$

$\begin{array}{llll}3.622579 & 3.121430 & -1.399105\end{array}$

$\begin{array}{llll}2.991614 & 1.875931 & -1.344197\end{array}$

$\begin{array}{llll}3.592682 & 0.974247 & -1.367850\end{array}$

$\begin{array}{lll}-0.381384 & 0.354409 & 1.186763\end{array}$

$\begin{array}{llll}5.121071 & 3.192235 & -1.486222\end{array}$

$\begin{array}{llll}0.716164 & 5.494773 & -1.047519\end{array}$

$3.641131 \quad-3.780424 \quad-2.914989$

$\begin{array}{lll}4.028156 & -2.683881 & 1.929483\end{array}$

$\begin{array}{llll}5.280450 & -3.193080 & 1.892916\end{array}$

$\begin{array}{llll}4.048578 & -1.621368 & 2.766257\end{array}$

$\begin{array}{llll}3.242990 & -3.626967 & 2.510465\end{array}$

$\begin{array}{llll}3.050485 & -4.993268 & -2.751274\end{array}$

$\begin{array}{llll}4.969633 & -4.014284 & -3.015665\end{array}$

$\begin{array}{llll}3.223039 & -3.297432 & -4.104691\end{array}$

$\begin{array}{llll}5.559735 & 3.280102 & -2.764049\end{array}$

$\begin{array}{llll}5.709602 & 2.098800 & -0.944831\end{array}$

$\begin{array}{llll}5.609757 & 4.273163 & -0.831483\end{array}$

$\begin{array}{llll}1.083180 & 6.440315 & -1.943538\end{array}$

$\begin{array}{llll}-0.614684 & 5.309835 & -1.173108\end{array}$

$\begin{array}{lll}0.925152 & 6.034189 & 0.183061\end{array}$

$\begin{array}{lll}1.624693 & 2.370404 & 2.177221\end{array}$

$\begin{array}{lll}0.634255 & 3.389161 & 2.212260\end{array}$

$\begin{array}{lll}-0.746083 & 3.284577 & 1.929025\end{array}$

$\begin{array}{llll}0.964365 & 4.397979 & 2.416401\end{array}$

$\begin{array}{lll}2.957400 & 2.742567 & 2.159545\end{array}$

$\begin{array}{llll}4.010090 & 1.754407 & 2.329371\end{array}$

$\begin{array}{lll}4.687853 & 2.058086 & 3.138380\end{array}$

$\begin{array}{llll}3.577898 & 0.789262 & 2.578118\end{array}$

$\begin{array}{llll}4.604201 & 1.651054 & 1.411101\end{array}$

$3.416487 \quad 4.118818 \quad 2.056569$

$\begin{array}{llll}2.721822 & 4.722549 & 1.473609\end{array}$

$\begin{array}{lll}3.554675 & 4.588142 & 3.042049\end{array}$

$\begin{array}{llll}4.378189 & 4.133027 & 1.534135\end{array}$

$\begin{array}{lll}1.373067 & 1.113961 & 2.158722\end{array}$ 


$\begin{array}{cccc}6 & -1.543252 & 4.480214 & 1.993884 \\ 6 & -2.886115 & 4.474289 & 1.704402 \\ 1 & -1.056752 & 5.411456 & 2.266563 \\ 6 & -3.472207 & 3.249772 & 1.338992 \\ 6 & -2.693642 & 2.116065 & 1.268103 \\ 1 & -3.118271 & 1.162218 & 0.981142 \\ 7 & -1.371577 & 2.116648 & 1.540747 \\ 1 & -3.471440 & 5.386784 & 1.754392 \\ 35 & -5.390942 & 3.140939 & 0.924243\end{array}$

\section{Conformer_4}

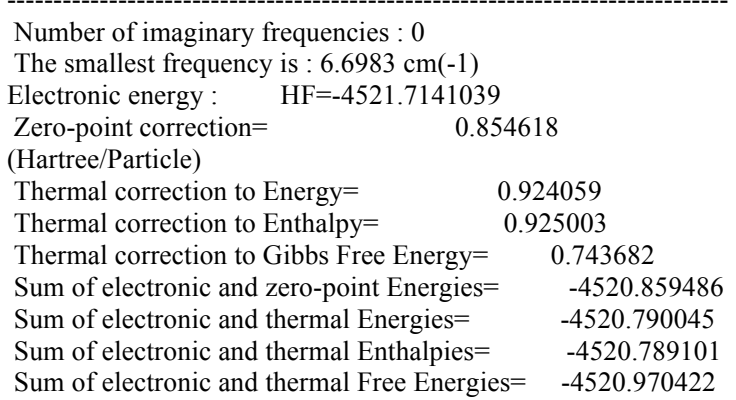

Cartesian Coordinates $\begin{array}{lll}-2.764502 & -2.020903 & 2.271702\end{array}$

$\begin{array}{lll}-3.436814 & -3.231027 & 2.043411\end{array}$

$\begin{array}{lll}-2.931767 & -4.046021 & 1.533476\end{array}$

$\begin{array}{lll}-4.765176 & -3.382274 & 2.445498\end{array}$

$\begin{array}{lll}-5.280982 & -4.320428 & 2.259949\end{array}$

$\begin{array}{lll}-5.431248 & -2.329584 & 3.081227\end{array}$

$\begin{array}{lll}-6.466896 & -2.447655 & 3.387247\end{array}$

$-4.762487-1.126663 \quad 3.322996$

$\begin{array}{lll}-5.274038 & -0.306390 & 3.818759\end{array}$

$\begin{array}{lll}-3.434776 & -0.972915 & 2.919377\end{array}$

$\begin{array}{lll}-2.915838 & -0.035254 & 3.098433\end{array}$

$\begin{array}{llll}2.015228 & -1.041940 & -0.841249\end{array}$

$\begin{array}{llll}2.397226 & -1.823025 & -1.937871\end{array}$

$\begin{array}{llll}1.982008 & -1.647319 & -2.922796\end{array}$

$\begin{array}{llll}3.316718 & -2.860332 & -1.772061\end{array}$

$\begin{array}{llll}3.881501 & -3.125958 & -0.525842\end{array}$

$\begin{array}{llll}4.583483 & -3.943228 & -0.401327\end{array}$

$\begin{array}{llll}3.519756 & -2.329743 & 0.559918\end{array}$

$\begin{array}{llll}2.592456 & -1.299631 & 0.411821\end{array}$

$\begin{array}{lll}2.304535 & -0.691274 & 1.263037\end{array}$

$\begin{array}{llll}1.602558 & 1.800993 & -1.207253\end{array}$

$\begin{array}{llll}0.860039 & 2.990155 & -1.130009\end{array}$

$\begin{array}{lll}-0.208359 & 2.951588 & -0.950616\end{array}$

$\begin{array}{llll}1.496835 & 4.223072 & -1.227850\end{array}$

$\begin{array}{llll}2.884557 & 4.300831 & -1.358527\end{array}$

$\begin{array}{llll}3.382533 & 5.263337 & -1.389377\end{array}$

$\begin{array}{llll}3.623070 & 3.120823 & -1.398845\end{array}$

$\begin{array}{llll}2.991837 & 1.875465 & -1.343934\end{array}$

$\begin{array}{llll}3.592716 & 0.973654 & -1.367508\end{array}$

$\begin{array}{lll}-0.381433 & 0.354460 & 1.186953\end{array}$

$\begin{array}{llll}5.121569 & 3.191373 & -1.486006\end{array}$

$\begin{array}{llll}0.717156 & 5.494805 & -1.047439\end{array}$

$3.640689 \quad-3.780312 \quad-2.915477$

$\begin{array}{lll}4.027586 & -2.684766 & 1.929240\end{array}$

$\begin{array}{lll}5.279731 & -3.194325 & 1.892626\end{array}$

$\begin{array}{llll}4.048297 & -1.622365 & 2.766145\end{array}$

$\begin{array}{llll}3.242140 & -3.627699 & 2.510102\end{array}$

$\begin{array}{llll}3.049815 & -4.993094 & -2.752119\end{array}$

$\begin{array}{llll}4.969160 & -4.014371 & -3.016064\end{array}$

$\begin{array}{llll}3.222805 & -3.296963 & -4.105107\end{array}$

$\begin{array}{llll}5.560155 & 3.279802 & -2.763825\end{array}$

$\begin{array}{llll}5.709952 & 2.097584 & -0.945191\end{array}$

$\begin{array}{llll}5.610477 & 4.271915 & -0.830789\end{array}$

$\begin{array}{llll}1.084426 & 6.440251 & -1.943454\end{array}$

$\begin{array}{lll}-0.613725 & 5.310161 & -1.173100\end{array}$

$\begin{array}{lll}0.926191 & 6.034195 & 0.183146\end{array}$

$\begin{array}{lll}1.624924 & 2.370211 & 2.177300\end{array}$

$\begin{array}{lll}0.634621 & 3.389100 & 2.212364\end{array}$

$\begin{array}{lll}-0.745721 & 3.284718 & 1.929064\end{array}$

$\begin{array}{llll}0.964870 & 4.397875 & 2.416495\end{array}$

$\begin{array}{lll}2.957679 & 2.742193 & 2.159626\end{array}$

$\begin{array}{lll}4.010238 & 1.753871 & 2.329311\end{array}$

$\begin{array}{lll}4.687977 & 2.057270 & 3.138447\end{array}$

$\begin{array}{lll}3.577914 & 0.788714 & 2.577792\end{array}$

$\begin{array}{llll}4.604402 & 1.650664 & 1.411059\end{array}$

$\begin{array}{llll}3.416941 & 4.118398 & 2.056782\end{array}$

$\begin{array}{llll}2.722409 & 4.722246 & 1.473784\end{array}$

$\begin{array}{llll}3.555080 & 4.587647 & 3.042305\end{array}$

$\begin{array}{llll}4.378697 & 4.132520 & 1.534449\end{array}$

$\begin{array}{lll}1.373130 & 1.113803 & 2.158758\end{array}$

$\begin{array}{lll}-1.542705 & 4.480482 & 1.993839\end{array}$

$\begin{array}{lll}-2.885535 & 4.474777 & 1.704203\end{array}$

$\begin{array}{lll}-1.056079 & 5.411649 & 2.266549\end{array}$

$\begin{array}{lll}-3.471788 & 3.250352 & 1.338746\end{array}$

$\begin{array}{lll}-2.693412 & 2.116507 & 1.267997\end{array}$

$\begin{array}{llll}-3.118177 & 1.162722 & 0.981034\end{array}$

$\begin{array}{lll}-1.371375 & 2.116876 & 1.540779\end{array}$

$\begin{array}{lll}-3.470712 & 5.387372 & 1.754106\end{array}$

$\begin{array}{lll}-5.390480 & 3.141847 & 0.923712\end{array}$

\section{Conformer 5}

Number of imaginary frequencies : 0

The smallest frequency is : $6.6441 \mathrm{~cm}(-1)$

Electronic energy : $\quad H F=-4521.7141033$ 
$\begin{array}{lll}-3.882139 & -3.125293 & 0.526188\end{array}$

$\begin{array}{llll}-4.584325 & -3.942403 & 0.401777\end{array}$

$\begin{array}{lll}-3.520264 & -2.329254 & -0.559657\end{array}$

$\begin{array}{llll}-2.592721 & -1.299342 & -0.411698\end{array}$

$\begin{array}{lll}-2.304723 & -0.691109 & -1.262976\end{array}$

$\begin{array}{lll}-1.602071 & 1.801196 & 1.207144\end{array}$

$\begin{array}{lll}-0.859299 & 2.990193 & 1.129801\end{array}$

$\begin{array}{lll}0.209073 & 2.951389 & 0.950305\end{array}$

$\begin{array}{lll}-1.495820 & 4.223250 & 1.227675\end{array}$

$\begin{array}{lll}-2.883508 & 4.301313 & 1.358506\end{array}$

$\begin{array}{lll}-3.381268 & 5.263930 & 1.389398\end{array}$

$\begin{array}{lll}-3.622274 & 3.121465 & 1.398943\end{array}$

$\begin{array}{lll}-2.991319 & 1.875971 & 1.343978\end{array}$

$\begin{array}{lll}-3.592381 & 0.974286 & 1.367653\end{array}$

$0.381394 \quad 0.354306 \quad-1.187174$

$\begin{array}{lll}-5.120743 & 3.192372 & 1.486329\end{array}$

$\begin{array}{lll}-0.715877 & 5.494806 & 1.047151\end{array}$

$\begin{array}{lll}-3.641312 & -3.779505 & 2.915882\end{array}$

$\begin{array}{llll}-4.028262 & -2.684209 & -1.928934\end{array}$

$\begin{array}{llll}-5.280438 & -3.193675 & -1.892219\end{array}$

$\begin{array}{llll}-4.048972 & -1.621779 & -2.765810\end{array}$

$\begin{array}{llll}-3.242931 & -3.627172 & -2.509902\end{array}$

$\begin{array}{lll}-3.050149 & -4.992206 & 2.752945\end{array}$

$\begin{array}{lll}-4.969761 & -4.013814 & 3.016169\end{array}$

$\begin{array}{lll}-3.223832 & -3.295823 & 4.105518\end{array}$

$\begin{array}{llll}-5.559076 & 3.281370 & 2.764197\end{array}$

$\begin{array}{lll}-5.709471 & 2.098530 & 0.946008\end{array}$

$\begin{array}{lll}-5.609525 & 4.272795 & 0.830818\end{array}$

$\begin{array}{llll}-1.082891 & 6.440377 & 1.943139\end{array}$

$\begin{array}{llll}0.614972 & 5.309877 & 1.172744\end{array}$

$\begin{array}{llll}-0.924872 & 6.034171 & -0.183448\end{array}$

$\begin{array}{llll}-1.624779 & 2.370267 & -2.177475\end{array}$

$\begin{array}{llll}-0.634328 & 3.389007 & -2.212775\end{array}$

$\begin{array}{llll}0.745985 & 3.284518 & -1.929376\end{array}$

$\begin{array}{llll}-0.964455 & 4.397788 & -2.417077\end{array}$

$\begin{array}{llll}-2.957467 & 2.742467 & -2.159758\end{array}$

$\begin{array}{llll}-4.010209 & 1.754270 & -2.329037\end{array}$

$\begin{array}{lll}-4.688325 & 2.057880 & -3.137770\end{array}$

$\begin{array}{llll}-3.578108 & 0.789111 & -2.577902\end{array}$

$\begin{array}{lll}-4.603920 & 1.650956 & -1.410498\end{array}$

$\begin{array}{llll}-3.416486 & 4.118752 & -2.056865\end{array}$

$\begin{array}{llll}-2.721812 & 4.722470 & -1.473895\end{array}$

$\begin{array}{llll}-3.554592 & 4.588044 & -3.042370\end{array}$

$\begin{array}{llll}-4.378211 & 4.133037 & -1.534482\end{array}$

$\begin{array}{llll}-1.373187 & 1.113819 & -2.158762\end{array}$

$\begin{array}{llll}1.543084 & 4.480202 & -1.994210\end{array}$

$\begin{array}{llll}2.885864 & 4.474426 & -1.704348\end{array}$

$\begin{array}{llll}1.056579 & 5.411376 & -2.267110\end{array}$

$\begin{array}{llll}3.471959 & 3.249998 & -1.338643\end{array}$

$\begin{array}{llll}2.693494 & 2.116212 & -1.267942\end{array}$

$\begin{array}{llll}3.118147 & 1.162417 & -0.980854\end{array}$

$\begin{array}{llll}1.371498 & 2.116656 & -1.540930\end{array}$

$\begin{array}{llll}3.471121 & 5.386969 & -1.754256\end{array}$

$\begin{array}{llll}5.390550 & 3.141423 & -0.923165\end{array}$

\section{Conformer_6}

Number of imaginary frequencies : 0

The smallest frequency is : $14.2033 \mathrm{~cm}(-1)$

Electronic energy : $\quad \mathrm{HF}=-4521.7143606$

Zero-point correction $=\quad 0.854526$

(Hartree/Particle)

Thermal correction to Energy $=\quad 0.923890$

Thermal correction to Enthalpy= $\quad 0.924834$

Thermal correction to Gibbs Free Energy $=0.745303$

Sum of electronic and zero-point Energies $=\quad-4520.859834$

Sum of electronic and thermal Energies $=\quad-4520.790471$

Sum of electronic and thermal Enthalpies $=\quad-4520.789526$

Sum of electronic and thermal Free Energies $=\quad-4520.969057$

Cartesian Coordinates

$\begin{array}{cccc}26 & -3.571972 & -1.118214 & -2.718328 \\ 15 & -1.130748 & -1.605321 & 1.892634 \\ 15 & 0.668341 & 0.090975 & -0.918947\end{array}$

$\begin{array}{lllll}15 & 0.668341 & 0.090975 & -0.918947\end{array}$ 
$\begin{array}{llll}5.155545 & 2.861693 & -1.842365\end{array}$

$\begin{array}{llll}0.859761 & 5.333392 & -1.212167\end{array}$

$\begin{array}{llll}3.079512 & -4.477867 & -2.486964\end{array}$

$\begin{array}{llll}4.702532 & -2.081679 & 1.595511\end{array}$

$\begin{array}{lll}5.675111 & -1.167551 & 1.349038\end{array}$

$\begin{array}{lll}4.073626 & -1.671284 & 2.724662\end{array}$

$\begin{array}{llll}5.319345 & -3.247291 & 1.881334\end{array}$

$\begin{array}{llll}2.837641 & -5.637586 & -1.825402\end{array}$

$\begin{array}{llll}4.307077 & -4.605241 & -3.043683\end{array}$

$\begin{array}{llll}2.185776 & -4.406320 & -3.498267\end{array}$

$\begin{array}{llll}5.602627 & 1.847780 & -2.616579\end{array}$

$\begin{array}{llll}5.748434 & 2.705931 & -0.625878\end{array}$

$\begin{array}{llll}5.632234 & 4.012993 & -2.361223\end{array}$

$\begin{array}{llll}1.175462 & 6.231059 & -2.174993\end{array}$

$\begin{array}{llll}-0.481210 & 5.181898 & -1.221558\end{array}$

$\begin{array}{llll}1.184200 & 5.915959 & -0.027626\end{array}$

$\begin{array}{lll}1.816453 & 2.319511 & 2.058409\end{array}$

$\begin{array}{lll}0.872970 & 3.381014 & 2.103157\end{array}$

$\begin{array}{lll}-0.514840 & 3.340675 & 1.844456\end{array}$

$\begin{array}{llll}1.253164 & 4.371891 & 2.310009\end{array}$

$\begin{array}{lll}3.161838 & 2.637757 & 2.022831\end{array}$

$\begin{array}{lll}4.172096 & 1.617076 & 2.256644\end{array}$

$\begin{array}{lll}4.934480 & 2.002655 & 2.944840\end{array}$

$\begin{array}{llll}3.717160 & 0.736033 & 2.702152\end{array}$

$\begin{array}{lll}4.674905 & 1.323314 & 1.326761\end{array}$

$\begin{array}{lll}3.670430 & 3.987632 & 1.834794\end{array}$

$\begin{array}{llll}2.993113 & 4.578327 & 1.218341\end{array}$

$\begin{array}{llll}3.829117 & 4.512855 & 2.788724\end{array}$

$\begin{array}{llll}4.629106 & 3.931348 & 1.311246\end{array}$

$\begin{array}{lll}1.517195 & 1.072016 & 2.057274\end{array}$

$\begin{array}{lll}-1.257707 & 4.569115 & 1.932089\end{array}$

$\begin{array}{lll}-2.601087 & 4.625766 & 1.652166\end{array}$

$\begin{array}{lll}-0.728785 & 5.474341 & 2.213386\end{array}$

$\begin{array}{lll}-3.242657 & 3.432841 & 1.275298\end{array}$

$\begin{array}{lll}-2.517359 & 2.265152 & 1.193301\end{array}$

$\begin{array}{lll}-2.986681 & 1.332864 & 0.906199\end{array}$

$\begin{array}{lll}-1.195372 & 2.203669 & 1.459613\end{array}$

$\begin{array}{lll}-3.145206 & 5.562372 & 1.717746\end{array}$

$\begin{array}{llll}-5.166425 & 3.416350 & 0.869340\end{array}$

\section{Conformer_7}

Number of imaginary frequencies : 0

The smallest frequency is : $13.2622 \mathrm{~cm}(-1)$

Electronic energy : $\quad \mathrm{HF}=-4521.7138804$

Zero-point correction $=\quad 0.854510$

(Hartree/Particle)

Thermal correction to Energy= $\quad 0.923920$

Thermal correction to Enthalpy=

0.924864

Thermal correction to Gibbs Free Energy $=0.744401$

Sum of electronic and zero-point Energies $=\quad-4520.859371$

Sum of electronic and thermal Energies=

Sum of electronic and thermal Enthalpies=

Sum of electronic and thermal Free Energies=

$-4520.789960$

$-4520.789016$

$-4520.969480$

Cartesian Coordinates

$\begin{array}{lll}-3.598204 & -1.129862 & -2.692285\end{array}$

$\begin{array}{lll}-1.125875 & -1.576421 & 1.911722\end{array}$

$\begin{array}{lll}0.662679 & 0.066215 & -0.938195\end{array}$

$\begin{array}{lll}-2.360109 & -1.491858 & -1.039700\end{array}$

$\begin{array}{lll}-1.858735 & -0.400131 & -1.842537\end{array}$

$\begin{array}{lll}-2.862344 & 0.615400 & -1.876299\end{array}$

$\begin{array}{lll}-2.793095 & 1.554987 & -2.406788\end{array}$

$\begin{array}{lll}-3.990085 & 0.154949 & -1.133625\end{array}$

$\begin{array}{lll}-4.924562 & 0.683363 & -1.006612\end{array}$

$\begin{array}{lll}-3.683184 & -1.139069 & -0.620388\end{array}$

$\begin{array}{llll}-4.342826 & -1.761004 & -0.034962\end{array}$

$\begin{array}{llll}-3.205720 & -2.086556 & -4.493928\end{array}$

$\begin{array}{lll}-2.219308 & -2.371572 & -4.835923\end{array}$

$\begin{array}{lll}-3.843014 & -0.829559 & -4.727355\end{array}$

$\begin{array}{llll}-3.421849 & 0.006000 & -5.270365\end{array}$

$\begin{array}{lll}-5.114184 & -0.850771 & -4.076124\end{array}$

$\begin{array}{llll}-5.819977 & -0.031616 & -4.034173\end{array}$

$\begin{array}{lll}-5.263128 & -2.120552 & -3.439293\end{array}$ 


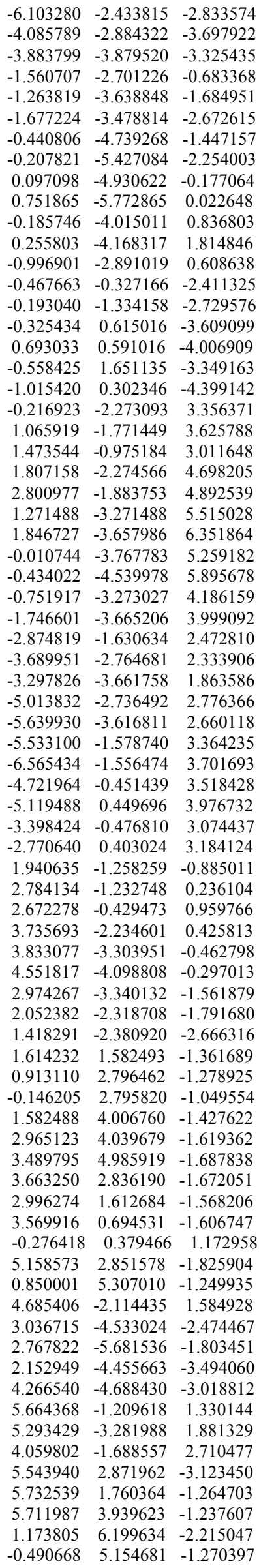

$\begin{array}{lll}1.164037 & 5.896555 & -0.066315\end{array}$

$\begin{array}{lll}1.867798 & 2.331385 & 2.008043\end{array}$

$\begin{array}{lll}0.921994 & 3.389104 & 2.090132\end{array}$

$\begin{array}{lll}-0.466708 & 3.354285 & 1.836531\end{array}$

$\begin{array}{lll}1.303316 & 4.376267 & 2.312140\end{array}$

$\begin{array}{llll}3.211613 & 2.654590 & 1.952260\end{array}$

$\begin{array}{llll}4.230020 & 1.626509 & 2.098968\end{array}$

$\begin{array}{lll}5.037789 & 1.997578 & 2.741171\end{array}$

$\begin{array}{llll}3.803858 & 0.738163 & 2.558573\end{array}$

$\begin{array}{lll}4.668902 & 1.348417 & 1.131474\end{array}$

$\begin{array}{lll}3.716054 & 4.004838 & 1.754827\end{array}$

$\begin{array}{llll}3.001451 & 4.612414 & 1.200757\end{array}$

$\begin{array}{lll}3.947166 & 4.507440 & 2.705762\end{array}$

$\begin{array}{llll}4.634483 & 3.958278 & 1.161151\end{array}$

$\begin{array}{lll}1.571587 & 1.083607 & 1.990879\end{array}$

$\begin{array}{lll}-1.205726 & 4.584205 & 1.939238\end{array}$

$\begin{array}{lll}-2.548252 & 4.649551 & 1.657969\end{array}$

$\begin{array}{lll}-0.673788 & 5.484109 & 2.231625\end{array}$

$\begin{array}{lll}-3.194112 & 3.463642 & 1.266081\end{array}$

$\begin{array}{lll}-2.473895 & 2.293660 & 1.174496\end{array}$

$\begin{array}{lll}-2.946976 & 1.365818 & 0.879203\end{array}$

$\begin{array}{lll}-1.152578 & 2.223542 & 1.442727\end{array}$

$\begin{array}{lll}-3.088644 & 5.587572 & 1.733581\end{array}$

$\begin{array}{llll}-5.117007 & 3.460200 & 0.855823\end{array}$

\section{Conformer_8}

Number of imaginary frequencies : 0

The smallest frequency is : $13.1635 \mathrm{~cm}(-1)$

Electronic energy :

$\mathrm{HF}=-4521.713881$

Zero-point correction=

(Hartree/Particle)

Thermal correction to Energy=

Thermal correction to Enthalpy=

0.923922

Thermal correction to Gibbs Free Energy $=0.744386$

Sum of electronic and zero-point Energies $=\quad-4520.859368$

Sum of electronic and thermal Energies=

Sum of electronic and thermal Enthalpies=

$-4520.789959$

Sum of electronic and thermal Free Energies=

$-4520.789015$

$-4520.969495$

$\begin{array}{crrrr} & & & & \text { Cartesian Coordinates } \\ \ldots \ldots \ldots \ldots \ldots \ldots \ldots \ldots \ldots \ldots \ldots \ldots \ldots \ldots \ldots . . & \\ 26 & -3.599567 & -1.130466 & -2.691043 \\ 15 & -1.125634 & -1.574536 & 1.912621 \\ 15 & 0.662416 & 0.065006 & -0.939422 \\ 6 & -2.360927 & -1.491429 & -1.038626 \\ 6 & -1.859321 & -0.400764 & -1.842766 \\ 6 & -2.862432 & 0.615242 & -1.877151 \\ 1 & -2.792925 & 1.554272 & -2.408591 \\ 6 & -3.990099 & 0.156120 & -1.133551 \\ 1 & -4.924255 & 0.685124 & -1.006681 \\ 6 & -3.683629 & -1.137513 & -0.619094 \\ 1 & -4.343355 & -1.758512 & -0.032763 \\ 6 & -3.208711 & -2.090064 & -4.491528 \\ 1 & -2.222766 & -2.376660 & -4.833562 \\ 6 & -3.844716 & -0.832701 & -4.726472 \\ 1 & -3.422849 & 0.001624 & -5.270831 \\ 6 & -5.115688 & -0.851652 & -4.074780 \\ 1 & -5.820590 & -0.031684 & -4.033718 \\ 6 & -5.265775 & -2.120384 & -3.436131 \\ 1 & -6.106059 & -2.431908 & -2.829697 \\ 6 & -4.089332 & -2.885771 & -3.694080 \\ 1 & -3.888226 & -3.880646 & -3.320249 \\ 6 & -1.562113 & -2.700980 & -0.681529 \\ 6 & -1.266099 & -3.639558 & -1.682485 \\ 1 & -1.679685 & -3.479999 & -2.670148 \\ 6 & -0.443768 & -4.740354 & -1.444092 \\ 1 & -0.211454 & -5.428921 & -2.250493 \\ 6 & 0.094299 & -4.931148 & -0.173985 \\ 1 & 0.748518 & -5.773702 & 0.026208 \\ 6 & -0.187655 & -4.014582 & 0.839260 \\ 1 & 0.254033 & -4.167482 & 1.817302 \\ 6 & -0.998098 & -2.890194 & 0.610484 \\ 6 & -0.468368 & -0.328865 & -2.411994 \\ 1 & -0.194252 & -1.336201 & -2.729562 \\ & & & \end{array}$


$\begin{array}{lll}-0.325964 & 0.612382 & -3.610478\end{array}$

$\begin{array}{llll}0.692372 & 0.587493 & -4.008569\end{array}$

$\begin{array}{llll}-0.558269 & 1.648826 & -3.351214\end{array}$

$\begin{array}{llll}-1.016375 & 0.299540 & -4.400083\end{array}$

$\begin{array}{lll}-0.216411 & -2.270684 & 3.357345\end{array}$

$\begin{array}{llll}1.067082 & -1.769980 & 3.625409\end{array}$

$\begin{array}{lll}1.475018 & -0.974682 & 3.010215\end{array}$

$\begin{array}{llll}1.808585 & -2.272771 & 4.697792\end{array}$

$\begin{array}{llll}2.802902 & -1.882718 & 4.891084\end{array}$

$\begin{array}{lll}1.272512 & -3.268398 & 5.515937\end{array}$

$\begin{array}{lll}1.847952 & -3.654630 & 6.352759\end{array}$

$\begin{array}{lll}-0.010356 & -3.763737 & 5.261440\end{array}$

$\begin{array}{lll}-0.433922 & -4.534922 & 5.898968\end{array}$

$\begin{array}{lll}-0.751784 & -3.269328 & 4.188430\end{array}$

$\begin{array}{lll}-1.746945 & -3.660773 & 4.002350\end{array}$

$\begin{array}{llll}-2.874305 & -1.627141 & 2.474689\end{array}$

$\begin{array}{lll}-3.690441 & -2.760569 & 2.336626\end{array}$

$\begin{array}{lll}-3.299322 & -3.658121 & 1.866377\end{array}$

$\begin{array}{lll}-5.014036 & -2.731154 & 2.779866\end{array}$

$\begin{array}{lll}-5.640927 & -3.610994 & 2.664273\end{array}$

$\begin{array}{lll}-5.531996 & -1.572794 & 3.367692\end{array}$

$\begin{array}{lll}-6.564108 & -1.549578 & 3.705766\end{array}$

$\begin{array}{lll}-4.719848 & -0.446104 & 3.521041\end{array}$

$\begin{array}{llll}-5.116369 & 0.455496 & 3.979296\end{array}$

$\begin{array}{lll}-3.396600 & -0.472686 & 3.076256\end{array}$

$\begin{array}{lll}-2.768038 & 0.406679 & 3.185258\end{array}$

$\begin{array}{lll}1.940101 & -1.259728 & -0.885721\end{array}$

$\begin{array}{llll}2.784334 & -1.233130 & 0.234812\end{array}$

$\begin{array}{llll}2.673240 & -0.428896 & 0.957515\end{array}$

$\begin{array}{llll}3.735641 & -2.235081 & 0.425202\end{array}$

$\begin{array}{llll}3.832066 & -3.305607 & -0.462088\end{array}$

$\begin{array}{llll}4.550624 & -4.100513 & -0.295769\end{array}$

$\begin{array}{llll}2.972551 & -3.342843 & -1.560588\end{array}$

$\begin{array}{llll}2.050873 & -2.321387 & -1.791105\end{array}$

$\begin{array}{lll}1.416238 & -2.384556 & -2.665279\end{array}$

$\begin{array}{llll}1.614357 & 1.580901 & -1.363460\end{array}$

$\begin{array}{llll}0.913724 & 2.795145 & -1.280857\end{array}$

$\begin{array}{llll}-0.145618 & 2.794916 & -1.051619\end{array}$

$\begin{array}{llll}1.583630 & 4.005172 & -1.429490\end{array}$

$\begin{array}{llll}2.966337 & 4.037553 & -1.620813\end{array}$

$\begin{array}{llll}3.491409 & 4.983592 & -1.689155\end{array}$

$\begin{array}{llll}3.663971 & 2.833753 & -1.673287\end{array}$

$\begin{array}{llll}2.996453 & 1.610547 & -1.569718\end{array}$

$\begin{array}{llll}3.569747 & 0.692194 & -1.608242\end{array}$

$\begin{array}{llll}-0.275183 & 0.380268 & 1.172060\end{array}$

$\begin{array}{llll}5.159388 & 2.848663 & -1.826368\end{array}$

$\begin{array}{llll}0.851551 & 5.305737 & -1.252352\end{array}$

$\begin{array}{llll}4.686043 & -2.113717 & 1.583624\end{array}$

$\begin{array}{llll}3.034078 & -4.536879 & -2.471736\end{array}$

$\begin{array}{lll}2.764648 & -5.684418 & -1.799277\end{array}$

$2.150148 \quad-4.460244 \quad-3.491242$

$\begin{array}{llll}4.263702 & -4.693674 & -3.016140\end{array}$

$\begin{array}{lll}5.664500 & -1.208723 & 1.327579\end{array}$

$\begin{array}{llll}5.294648 & -3.280825 & 1.880540\end{array}$

$\begin{array}{lll}4.060964 & -1.687202 & 2.709243\end{array}$

$\begin{array}{llll}5.545426 & 2.872443 & -3.123648\end{array}$

$\begin{array}{llll}5.732523 & 1.755645 & -1.267804\end{array}$

$\begin{array}{llll}5.713071 & 3.934866 & -1.234881\end{array}$

$\begin{array}{llll}1.175746 & 6.197850 & -2.217811\end{array}$

$\begin{array}{rrrr}-0.489162 & 5.153845 & -1.272899\end{array}$

$\begin{array}{llll}1.165634 & 5.895700 & -0.068952\end{array}$

$\begin{array}{llll}1.869042 & 2.332627 & 2.005732\end{array}$

$\begin{array}{lll}0.922975 & 3.390078 & 2.088314\end{array}$

$\begin{array}{lll}-0.465828 & 3.355084 & 1.835220\end{array}$

$\begin{array}{lll}1.304177 & 4.377318 & 2.310144\end{array}$

$\begin{array}{lll}3.212699 & 2.656338 & 1.949589\end{array}$

$\begin{array}{llll}4.231615 & 1.628459 & 2.094261\end{array}$

$\begin{array}{lll}5.039574 & 1.998845 & 2.736608\end{array}$

$\begin{array}{lll}3.806059 & 0.739310 & 2.552852\end{array}$

$\begin{array}{lll}4.670060 & 1.351994 & 1.126088\end{array}$

$\begin{array}{lll}3.716135 & 4.007280 & 1.754201\end{array}$

$\begin{array}{llll}3.002655 & 4.614109 & 1.197820\end{array}$

$\begin{array}{lll}3.943362 & 4.510037 & 2.705995\end{array}$

$\begin{array}{llll}4.636635 & 3.961881 & 1.163725\end{array}$

$\begin{array}{lll}1.573197 & 1.084755 & 1.988095\end{array}$

$\begin{array}{lll}-1.204856 & 4.584988 & 1.938066\end{array}$

$\begin{array}{lll}-2.547357 & 4.650294 & 1.656660\end{array}$

$\begin{array}{lll}-0.672967 & 5.484931 & 2.230428\end{array}$

$\begin{array}{lll}-3.193187 & 3.464399 & 1.264682\end{array}$

$\begin{array}{lll}-2.472975 & 2.294406 & 1.173268\end{array}$

$\begin{array}{lll}-2.946050 & 1.366521 & 0.878109\end{array}$

$\begin{array}{lll}-1.151686 & 2.224256 & 1.441639\end{array}$

$\begin{array}{lll}-3.087771 & 5.588307 & 1.732251\end{array}$

$\begin{array}{llll}35 & -5.116040 & 3.461025 & 0.854246\end{array}$

\section{Conformer_9}

Number of imaginary frequencies : 0

The smallest frequency is : $13.2755 \mathrm{~cm}(-1)$

Electronic energy : $\quad H F=-4521.7138802$

Zero-point correction $=\quad 0.854513$

(Hartree/Particle)

Thermal correction to Energy $=\quad 0.923921$

Thermal correction to Enthalpy= $\quad 0.924866$

Thermal correction to Gibbs Free Energy $=0.744415$

Sum of electronic and zero-point Energies $=\quad-4520.859368$

Sum of electronic and thermal Energies $=\quad-4520.789959$

Sum of electronic and thermal Enthalpies $=\quad-4520.789015$

Sum of electronic and thermal Free Energies $=\quad-4520.969465$

\begin{tabular}{|c|c|c|c|}
\hline & & \multicolumn{2}{|c|}{ Cartesian Coordinates } \\
\hline 26 & -3.597999 & -1.129357 & -2.692651 \\
\hline 15 & -1.126052 & -1.576745 & 1.911428 \\
\hline 15 & 0.662697 & 0.066459 & -0.937906 \\
\hline 6 & -2.360079 & -1.491728 & -1.040028 \\
\hline 6 & -1.858641 & -0.399802 & -1.842546 \\
\hline 6 & -2.862262 & 0.615723 & -1.876157 \\
\hline 1 & -2.792971 & 1.555438 & -2.406417 \\
\hline 6 & -3.990069 & 0.155068 & -1.133707 \\
\hline 1 & -4.924565 & 0.683440 & -1.006652 \\
\hline 6 & -3.683204 & -1.139076 & -0.620766 \\
\hline 1 & -4.342882 & -1.761168 & -0.035550 \\
\hline 6 & -3.205194 & -2.085261 & -4.494625 \\
\hline 1 & -2.218685 & -2.369904 & -4.836647 \\
\hline 6 & -3.842771 & -0.828324 & -4.727606 \\
\hline 1 & -3.421755 & 0.007555 & -5.270238 \\
\hline 6 & -5.113973 & -0.850080 & -4.076459 \\
\hline 1 & -5.819959 & -0.031105 & -4.034235 \\
\hline 6 & -5.262668 & -2.120147 & -3.440142 \\
\hline 1 & -6.102785 & -2.433839 & -2.834597 \\
\hline 6 & -4.085141 & -2.883546 & -3.699001 \\
\hline 1 & -3.882968 & -3.878856 & -3.326910 \\
\hline 6 & -1.560657 & -2.701136 & -0.683887 \\
\hline 6 & -1.263652 & -3.638567 & -1.685612 \\
\hline 1 & -1.676963 & -3.478378 & -2.673290 \\
\hline 6 & -0.440619 & -4.738998 & -1.447938 \\
\hline 1 & -0.207546 & -5.426671 & -2.254882 \\
\hline 6 & 0.097214 & -4.930541 & -0.177844 \\
\hline 1 & 0.752006 & -5.772789 & 0.021769 \\
\hline 6 & -0.185725 & -4.015106 & 0.836156 \\
\hline 1 & 0.255777 & -4.168544 & 1.814198 \\
\hline 6 & -0.996915 & -2.891113 & 0.608120 \\
\hline 6 & -0.467518 & -0.326717 & -2.411188 \\
\hline 1 & -0.192817 & -1.333657 & -2.729526 \\
\hline 6 & -0.325198 & 0.615599 & -3.608848 \\
\hline 1 & 0.693317 & 0.591702 & -4.006541 \\
\hline 1 & -0.558289 & 1.651676 & -3.348841 \\
\hline 1 & -1.015066 & 0.302968 & -4.399009 \\
\hline 6 & -0.217246 & -2.273676 & 3.356052 \\
\hline 6 & 1.065525 & -1.772008 & 3.625764 \\
\hline 1 & 1.473196 & -0.975581 & 3.011869 \\
\hline 6 & 1.806643 & -2.275305 & 4.698179 \\
\hline 1 & 2.800409 & -1.884464 & 4.892730 \\
\hline 6 & 1.270925 & -3.272431 & 5.514719 \\
\hline 1 & 1.846069 & -3.659065 & 6.351557 \\
\hline 6 & -0.011238 & -3.768755 & 5.258583 \\
\hline 1 & -0.434560 & -4.541113 & 5.894853 \\
\hline 6 & -0.752290 & -3.273819 & 4.185559 \\
\hline 1 & -1.746923 & -3.666026 & 3.998281 \\
\hline
\end{tabular}


$\begin{array}{lll}-2.875055 & -1.631101 & 2.472315\end{array}$

$\begin{array}{lll}-3.690110 & -2.765179 & 2.333220\end{array}$

$\begin{array}{lll}-3.297889 & -3.662186 & 1.862845\end{array}$

$\begin{array}{lll}-5.014032 & -2.737116 & 2.775563\end{array}$

$\begin{array}{lll}-5.640067 & -3.617461 & 2.659164\end{array}$

$\begin{array}{lll}-5.533424 & -1.579456 & 3.363506\end{array}$

$\begin{array}{lll}-6.565788 & -1.557288 & 3.700875\end{array}$

$\begin{array}{lll}-4.722367 & -0.452125 & 3.517888\end{array}$

$\begin{array}{llll}-5.119982 & 0.448940 & 3.976252\end{array}$

$\begin{array}{lll}-3.398786 & -0.477372 & 3.074011\end{array}$

$\begin{array}{lll}-2.771068 & 0.402490 & 3.183849\end{array}$

$\begin{array}{llll}1.940567 & -1.258102 & -0.884796\end{array}$

$\begin{array}{lll}2.783926 & -1.232808 & 0.236428\end{array}$

$\begin{array}{lll}2.671979 & -0.429672 & 0.960232\end{array}$

$\begin{array}{lll}3.735480 & -2.234681 & 0.426049\end{array}$

$\begin{array}{llll}3.832995 & -3.303844 & -0.462774\end{array}$

$\begin{array}{llll}4.551740 & -4.098716 & -0.297073\end{array}$

$\begin{array}{llll}2.974319 & -3.339814 & -1.561968\end{array}$

$\begin{array}{llll}2.052436 & -2.318366 & -1.791668\end{array}$

$\begin{array}{llll}1.418437 & -2.380433 & -2.666383\end{array}$

$\begin{array}{llll}1.614417 & 1.582672 & -1.361266\end{array}$

$\begin{array}{llll}0.913376 & 2.796706 & -1.278696\end{array}$

$\begin{array}{llll}-0.145975 & 2.796179 & -1.049472\end{array}$

$\begin{array}{llll}1.582859 & 4.006934 & -1.427490\end{array}$

$\begin{array}{llll}2.965511 & 4.039734 & -1.619103\end{array}$

$\begin{array}{llll}3.490256 & 4.985927 & -1.687628\end{array}$

$\begin{array}{llll}3.663555 & 2.836192 & -1.671598\end{array}$

$\begin{array}{llll}2.996480 & 1.612748 & -1.567649\end{array}$

$\begin{array}{llll}3.570063 & 0.694550 & -1.606038\end{array}$

$\begin{array}{lll}-0.276569 & 0.379361 & 1.173231\end{array}$

$\begin{array}{llll}5.158884 & 2.851402 & -1.825399\end{array}$

$\begin{array}{llll}0.850437 & 5.307245 & -1.250030\end{array}$

$\begin{array}{lll}4.685083 & -2.114711 & 1.585272\end{array}$

$\begin{array}{llll}3.036920 & -4.532497 & -2.474820\end{array}$

$\begin{array}{llll}2.768093 & -5.681182 & -1.804074\end{array}$

$\begin{array}{llll}2.153205 & -4.454984 & -3.494446\end{array}$

$\begin{array}{llll}4.266787 & -4.687678 & -3.019134\end{array}$

$\begin{array}{llll}5.664172 & -1.209986 & 1.330650\end{array}$

$\begin{array}{llll}5.292949 & -3.282354 & 1.881647\end{array}$

$\begin{array}{llll}4.059417 & -1.688841 & 2.710789\end{array}$

$\begin{array}{llll}5.544307 & 2.870672 & -3.122945\end{array}$

$\begin{array}{llll}5.732758 & 1.760590 & -1.263300\end{array}$

$\begin{array}{llll}5.712334 & 3.939878 & -1.237955\end{array}$

$\begin{array}{llll}1.175009 & 6.200012 & -2.214744\end{array}$

$\begin{array}{llll}-0.490238 & 5.155100 & -1.271437\end{array}$

$\begin{array}{lll}1.163738 & 5.896498 & -0.066066\end{array}$

$\begin{array}{llll}1.867631 & 2.331178 & 2.008493\end{array}$

$\begin{array}{lll}0.921819 & 3.388885 & 2.090599\end{array}$

$\begin{array}{lll}-0.466901 & 3.354068 & 1.837098\end{array}$

$\begin{array}{lll}1.303143 & 4.376051 & 2.312582\end{array}$

$\begin{array}{lll}3.211439 & 2.654412 & 1.952502\end{array}$

$\begin{array}{llll}4.229950 & 1.626485 & 2.099552\end{array}$

$\begin{array}{lll}5.037310 & 1.997544 & 2.742291\end{array}$

$\begin{array}{llll}3.803683 & 0.737960 & 2.558706\end{array}$

$\begin{array}{lll}4.669409 & 1.348725 & 1.132229\end{array}$

$\begin{array}{lll}3.715849 & 4.004658 & 1.755022\end{array}$

$\begin{array}{llll}3.001123 & 4.612295 & 1.201182\end{array}$

$\begin{array}{lll}3.947242 & 4.507183 & 2.705931\end{array}$

$\begin{array}{llll}4.634122 & 3.958112 & 1.161093\end{array}$

$\begin{array}{lll}1.571429 & 1.083392 & 1.991488\end{array}$

$\begin{array}{lll}-1.205946 & 4.583954 & 1.940035\end{array}$

$\begin{array}{llll}-2.548476 & 4.649323 & 1.658786\end{array}$

$\begin{array}{lll}-0.674030 & 5.483816 & 2.232595\end{array}$

$\begin{array}{llll}-3.194312 & 3.463474 & 1.266683\end{array}$

$\begin{array}{lll}-2.474077 & 2.293517 & 1.174913\end{array}$

$\begin{array}{lll}-2.947143 & 1.365726 & 0.879437\end{array}$

$\begin{array}{lll}-1.152765 & 2.223370 & 1.443153\end{array}$

$\begin{array}{lll}-3.088888 & 5.587319 & 1.734582\end{array}$

$\begin{array}{lll}-5.117183 & 3.460078 & 0.856318\end{array}$

Conformer_10

Number of imaginary frequencies : 0

The smallest frequency is : $9.3144 \mathrm{~cm}(-1)$

Electronic energy : $\quad \mathrm{HF}=-4521.7140753$
Zero-point correction $=$

(Hartree/Particle)

Thermal correction to Energy= $\quad 0.923958$

Thermal correction to Enthalpy=

0.924902

Thermal correction to Gibbs Free Energy=

Sum of electronic and zero-point Energies=

Sum of electronic and thermal Energies=

Sum of electronic and thermal Enthalpies=

Sum of electronic and thermal Free Energies=

0.743994

$-4520.859567$

$-4520.790118$

$-4520.789173$

Cartesian Coordinates

$\begin{array}{lll}-3.396779 & -1.177541 & -2.850045\end{array}$

$\begin{array}{lll}-1.034088 & -1.734788 & 1.777324\end{array}$

$\begin{array}{llll}0.696493 & 0.237149 & -0.861382\end{array}$

$\begin{array}{llll}-2.182110 & -1.563207 & -1.186699\end{array}$

$-1.744441-0.379956-1.892531$

$\begin{array}{llll}-2.824196 & 0.555233 & -1.886360\end{array}$

$\begin{array}{llll}-2.814951 & 1.534596 & -2.344706\end{array}$

$\begin{array}{lll}-3.932337 & -0.043513 & -1.217016\end{array}$

$\begin{array}{llll}-4.908991 & 0.401131 & -1.084844\end{array}$

$\begin{array}{llll}-3.540016 & -1.344958 & -0.788328\end{array}$

$-4.164693-2.057388-0.271740$

$\begin{array}{llll}-2.882470 & -1.960871 & -4.704539\end{array}$

$\begin{array}{llll}-1.868932 & -2.142144 & -5.037300\end{array}$

$\begin{array}{llll}-3.614048 & -0.743983 & -4.863990\end{array}$

$\begin{array}{llll}-3.247817 & 0.161524 & -5.329205\end{array}$

$-4.894998-0.915578-4.255740$

$\begin{array}{lll}-5.665944 & -0.160642 & -4.174795\end{array}$

$\begin{array}{llll}-4.956098 & -2.238444 & -3.720452\end{array}$

$\begin{array}{llll}-5.783602 & -2.664739 & -3.168785\end{array}$

$\begin{array}{llll}-3.714810 & -2.884633 & -3.998780\end{array}$

$\begin{array}{llll}-3.443950 & -3.887515 & -3.697753\end{array}$

$\begin{array}{lll}-1.287612 & -2.719746 & -0.888820\end{array}$

$\begin{array}{lll}-0.890776 & -3.556570 & -1.943014\end{array}$

$-1.321534-3.382381-2.920870$

$\begin{array}{llll}0.053539 & -4.567202 & -1.767833\end{array}$

$\begin{array}{llll}0.357622 & -5.177915 & -2.611726\end{array}$

$\begin{array}{llll}0.625283 & -4.759352 & -0.512467\end{array}$

$\begin{array}{llll}1.381795 & -5.523690 & -0.361964\end{array}$

$\begin{array}{llll}0.238519 & -3.948595 & 0.554940\end{array}$

$\begin{array}{llll}0.718954 & -4.090501 & 1.515446\end{array}$

$\begin{array}{lll}-0.713205 & -2.928160 & 0.395140\end{array}$

$\begin{array}{llll}-0.345912 & -0.156297 & -2.403491\end{array}$

$\begin{array}{llll}0.031130 & -1.114675 & -2.766389\end{array}$

$\begin{array}{llll}-0.247203 & 0.868621 & -3.536091\end{array}$

$\begin{array}{llll}0.782314 & 0.961944 & -3.893093\end{array}$

$\begin{array}{llll}-0.582684 & 1.861458 & -3.224168\end{array}$

$\begin{array}{llll}-0.877592 & 0.548762 & -4.371791\end{array}$

$\begin{array}{lll}-0.124962 & -2.420036 & 3.228965\end{array}$

$\begin{array}{llll}0.911233 & -1.652773 & 3.782093\end{array}$

$\begin{array}{llll}1.157786 & -0.685373 & 3.354431\end{array}$

$\begin{array}{llll}1.622753 & -2.125634 & 4.888646\end{array}$

$\begin{array}{lll}2.430382 & -1.527490 & 5.300475\end{array}$

$\begin{array}{lll}1.304876 & -3.360795 & 5.452481\end{array}$

$\begin{array}{llll}1.860055 & -3.728396 & 6.311105\end{array}$

$\begin{array}{llll}0.264723 & -4.125927 & 4.913055\end{array}$

$\begin{array}{llll}0.008796 & -5.085691 & 5.353473\end{array}$

$\begin{array}{lll}-0.449700 & -3.657796 & 3.811529\end{array}$

$\begin{array}{lll}-1.258828 & -4.256814 & 3.404792\end{array}$

$\begin{array}{lll}-2.786124 & -2.028705 & 2.254065\end{array}$

$\begin{array}{lll}-3.463202 & -0.980561 & 2.894358\end{array}$

$\begin{array}{lll}-2.946429 & -0.042503 & 3.077580\end{array}$

$\begin{array}{lll}-4.794795 & -1.134555 & 3.284847\end{array}$

$\begin{array}{lll}-5.311503 & -0.314196 & 3.775081\end{array}$

$\begin{array}{lll}-5.460716 & -2.337836 & 3.037080\end{array}$

$\begin{array}{lll}-6.499312 & -2.456107 & 3.332860\end{array}$

$\begin{array}{lll}-4.788083 & -3.390578 & 2.408353\end{array}$

$\begin{array}{lll}-5.301793 & -4.328906 & 2.217934\end{array}$

$\begin{array}{llll}-3.455825 & -3.239088 & 2.019467\end{array}$

$\begin{array}{lll}-2.945605 & -4.053971 & 1.514519\end{array}$

$\begin{array}{llll}2.026410 & -1.039262 & -0.828061\end{array}$

$\begin{array}{llll}2.444881 & -1.788554 & -1.932701\end{array}$

$\begin{array}{llll}2.051170 & -1.593492 & -2.923452\end{array}$

$3.380844 \quad-2.813453 \quad-1.770848$ 
$\begin{array}{llll}3.919424 & -3.101057 & -0.518687\end{array}$

$\begin{array}{llll}4.634683 & -3.906686 & -0.398502\end{array}$

$\begin{array}{lll}3.520146 & -2.335555 & 0.576873\end{array}$

$\begin{array}{llll}2.581707 & -1.316116 & 0.431104\end{array}$

$\begin{array}{lll}2.271497 & -0.727305 & 1.287862\end{array}$

$\begin{array}{llll}1.601846 & 1.800706 & -1.188758\end{array}$

$\begin{array}{llll}0.853798 & 2.986486 & -1.113091\end{array}$

$\begin{array}{llll}-0.215354 & 2.942731 & -0.939341\end{array}$

$\begin{array}{llll}1.485781 & 4.222289 & -1.204921\end{array}$

$\begin{array}{llll}2.873826 & 4.306290 & -1.328367\end{array}$

$\begin{array}{llll}3.367797 & 5.270965 & -1.354524\end{array}$

$\begin{array}{llll}3.617591 & 3.129610 & -1.367370\end{array}$

$\begin{array}{llll}2.991452 & 1.881379 & -1.318065\end{array}$

$\begin{array}{llll}3.596562 & 0.982395 & -1.340482\end{array}$

$\begin{array}{lll}-0.393426 & 0.345696 & 1.188494\end{array}$

$\begin{array}{llll}5.116242 & 3.205963 & -1.446187\end{array}$

$\begin{array}{llll}0.699993 & 5.490285 & -1.024882\end{array}$

$\begin{array}{llll}3.735856 & -3.687519 & -2.940752\end{array}$

$\begin{array}{lll}4.007436 & -2.705756 & 1.949602\end{array}$

$\begin{array}{llll}5.258019 & -3.219394 & 1.925818\end{array}$

$\begin{array}{llll}4.020404 & -1.650212 & 2.795706\end{array}$

$\begin{array}{llll}3.210362 & -3.649909 & 2.512383\end{array}$

$\begin{array}{llll}2.934056 & -4.782670 & -3.001488\end{array}$

$\begin{array}{llll}5.005960 & -4.143575 & -2.868790\end{array}$

$\begin{array}{llll}3.600785 & -3.042225 & -4.120799\end{array}$

$\begin{array}{llll}5.562156 & 3.287937 & -2.721878\end{array}$

$\begin{array}{llll}5.705592 & 2.117616 & -0.895207\end{array}$

$\begin{array}{llll}5.597123 & 4.292180 & -0.794647\end{array}$

$\begin{array}{llll}1.068117 & 6.440011 & -1.916047\end{array}$

$\begin{array}{llll}-0.629447 & 5.300643 & -1.158154\end{array}$

$\begin{array}{llll}0.900510 & 6.026511 & 0.208418\end{array}$

$\begin{array}{lll}1.604602 & 2.357069 & 2.198215\end{array}$

$\begin{array}{lll}0.614238 & 3.375980 & 2.232680\end{array}$

$\begin{array}{lll}-0.764026 & 3.273239 & 1.938755\end{array}$

$\begin{array}{llll}0.943281 & 4.383670 & 2.444050\end{array}$

$\begin{array}{lll}2.937414 & 2.729283 & 2.185312\end{array}$

$\begin{array}{lll}3.990063 & 1.741783 & 2.359021\end{array}$

$\begin{array}{lll}4.651475 & 2.032477 & 3.186410\end{array}$

$\begin{array}{lll}3.556269 & 0.770932 & 2.581567\end{array}$

$\begin{array}{llll}4.601765 & 1.657463 & 1.450571\end{array}$

$\begin{array}{lll}3.395548 & 4.106423 & 2.090798\end{array}$

$\begin{array}{llll}2.703879 & 4.711428 & 1.505436\end{array}$

$\begin{array}{lll}3.526641 & 4.572286 & 3.078906\end{array}$

$\begin{array}{llll}4.360692 & 4.123736 & 1.574867\end{array}$

$\begin{array}{llll}1.352569 & 1.100857 & 2.175645\end{array}$

$\begin{array}{lll}-1.561854 & 4.468276 & 2.005997\end{array}$

$\begin{array}{lll}-2.901856 & 4.464827 & 1.703612\end{array}$

$\begin{array}{lll}-1.078009 & 5.397262 & 2.290781\end{array}$

$\begin{array}{lll}-3.484467 & 3.243307 & 1.322923\end{array}$

$\begin{array}{lll}-2.705526 & 2.109889 & 1.251490\end{array}$

$\begin{array}{lll}-3.127615 & 1.158234 & 0.953714\end{array}$

$\begin{array}{lll}-1.386142 & 2.108124 & 1.536816\end{array}$

$\begin{array}{lll}-3.487552 & 5.376993 & 1.755106\end{array}$

$\begin{array}{lll}-5.398914 & 3.138368 & 0.887795\end{array}$

\section{Conformer_11}

Number of imaginary frequencies : 0

The smallest frequency is : $9.2302 \mathrm{~cm}(-1)$

Electronic energy :

$\mathrm{HF}=-4521.7140754$

Zero-point correction $=$

0.854510

(Hartree/Particle)

Thermal correction to Energy=

Thermal correction to Enthalpy=

0.923958

0.924903

Thermal correction to Gibbs Free Energy=
Sum of electronic and zero-point Energies=

Sum of electronic and zero-point Energies
Sum of electronic and thermal Energies=

0.743990

Sum of electronic and thermal Enthalpies=

$-4520.859566$

$-4520.790117$

$-4520.789173$

Sum of electronic and thermal Free Energies=

$-4520.970085$

Cartesian Coordinates

\begin{tabular}{|c|c|c|c|}
\hline \multicolumn{4}{|c|}{ Cartesian Coordinates } \\
\hline 26 & 3.397355 & -1.177171 & 2.849504 \\
\hline 15 & 1.033948 & -1.734894 & -1.777429 \\
\hline 15 & -0.696279 & 0.237179 & 0.861372 \\
\hline
\end{tabular}

6 $\begin{array}{llll}2.182436 & -1.563059 & 1.186401\end{array}$

$\begin{array}{lll}1.744856 & -0.379729 & 1.892157\end{array}$

$\begin{array}{lll}2.824595 & 0.555474 & 1.885687\end{array}$

$\begin{array}{llll}2.815413 & 1.534903 & 2.343896\end{array}$

$\begin{array}{llll}3.932639 & -0.043345 & 1.216244\end{array}$

$\begin{array}{llll}4.909254 & 0.401311 & 1.083836\end{array}$

$\begin{array}{llll}3.540271 & -1.344849 & 0.787782\end{array}$

$\begin{array}{lll}4.164859 & -2.057347 & 0.271179\end{array}$

$\begin{array}{llll}2.883297 & -1.960232 & 4.704168\end{array}$

$\begin{array}{llll}1.869809 & -2.141449 & 5.037107\end{array}$

$\begin{array}{llll}3.614918 & -0.743336 & 4.863358\end{array}$

$\begin{array}{llll}3.248761 & 0.162240 & 5.328498\end{array}$

$\begin{array}{lll}4.895779 & -0.915027 & 4.254949\end{array}$

$\begin{array}{llll}5.666725 & -0.160112 & 4.173800\end{array}$

$\begin{array}{llll}4.956788 & -2.237967 & 3.719832\end{array}$

$\begin{array}{llll}5.784208 & -2.664344 & 3.168102\end{array}$

$\begin{array}{llll}3.715529 & -2.884102 & 3.998419\end{array}$

$\begin{array}{lll}3.444610 & -3.887019 & 3.697561\end{array}$

$\begin{array}{llll}1.287923 & -2.719643 & 0.888769\end{array}$

$\begin{array}{lll}0.891243 & -3.556369 & 1.943098\end{array}$

$\begin{array}{llll}1.322087 & -3.382054 & 2.920893\end{array}$

$\begin{array}{lll}-0.053030 & -4.567075 & 1.768126\end{array}$

$\begin{array}{llll}-0.356996 & -5.177706 & 2.612119\end{array}$

$\begin{array}{llll}-0.624867 & -4.759415 & 0.512832\end{array}$

$\begin{array}{lll}-1.381320 & -5.523840 & 0.362477\end{array}$

$-0.238273 \quad-3.948743 \quad-0.554700$

$\begin{array}{llll}-0.718793 & -4.090811 & -1.515140\end{array}$

$\begin{array}{llll}0.713383 & -2.928211 & -0.395105\end{array}$

$\begin{array}{llll}0.346402 & -0.156087 & 2.403336\end{array}$

$\begin{array}{lll}-0.030530 & -1.114468 & 2.766343\end{array}$

$\begin{array}{lll}0.247825 & 0.868885 & 3.535894\end{array}$

$\begin{array}{rrr}-0.781640 & 0.962178 & 3.893050\end{array}$

$\begin{array}{lll}0.583213 & 1.861717 & 3.223865\end{array}$

$\begin{array}{llll}0.878358 & 0.549099 & 4.371513\end{array}$

$\begin{array}{llll}0.124485 & -2.420235 & -3.228822\end{array}$

$\begin{array}{llll}-0.911916 & -1.653054 & -3.781680\end{array}$

$\begin{array}{llll}-1.158408 & -0.685650 & -3.353988\end{array}$

$\begin{array}{llll}-1.623719 & -2.125983 & -4.888021\end{array}$

$-2.431508-1.527901 \quad-5.299624$

$\begin{array}{llll}-1.305914 & -3.361128 & -5.451928\end{array}$

$\begin{array}{llll}-1.861310 & -3.728784 & -6.310388\end{array}$

$\begin{array}{llll}-0.265545 & -4.126170 & -4.912793\end{array}$

$\begin{array}{llll}-0.009665 & -5.085916 & -5.353276\end{array}$

$\begin{array}{llll}0.449156 & -3.657974 & -3.811472\end{array}$

$\begin{array}{lll}1.258462 & -4.256918 & -3.404978\end{array}$

$\begin{array}{llll}2.785885 & -2.028808 & -2.254547\end{array}$

$\begin{array}{llll}3.462890 & -0.980696 & -2.894968\end{array}$

$\begin{array}{llll}2.946148 & -0.042599 & -3.078071\end{array}$

$\begin{array}{llll}4.794394 & -1.134759 & -3.285736\end{array}$

$\begin{array}{lll}5.311044 & -0.314422 & -3.776069\end{array}$

$\begin{array}{llll}5.460308 & -2.338071 & -3.038112\end{array}$

$\begin{array}{lll}6.498837 & -2.456392 & -3.334108\end{array}$

$\begin{array}{llll}4.787753 & -3.390780 & -2.409246\end{array}$

$\begin{array}{llll}5.301455 & -4.329135 & -2.218936\end{array}$

$\begin{array}{llll}3.455583 & -3.239225 & -2.020086\end{array}$

$\begin{array}{llll}2.945433 & -4.054092 & -1.515043\end{array}$

$\begin{array}{lll}-2.026095 & -1.039348 & 0.828377\end{array}$

$\begin{array}{lll}-2.444355 & -1.788558 & 1.933155\end{array}$

$\begin{array}{lll}-2.050469 & -1.593406 & 2.923821\end{array}$

$\begin{array}{lll}-3.380355 & -2.813466 & 1.771556\end{array}$

$\begin{array}{lll}-3.919166 & -3.101158 & 0.519517\end{array}$

$\begin{array}{lll}-4.634473 & -3.906776 & 0.399531\end{array}$

$\begin{array}{llll}-3.520085 & -2.335754 & -0.576182\end{array}$

$\begin{array}{llll}-2.581608 & -1.316316 & -0.430667\end{array}$

$-2.271536 \quad-0.727583-1.287526$

$\begin{array}{lll}-1.601745 & 1.800674 & 1.188750\end{array}$

$\begin{array}{lll}-0.853792 & 2.986515 & 1.113043\end{array}$

$\begin{array}{lll}0.215370 & 2.942848 & 0.939326\end{array}$

$\begin{array}{lll}-1.485880 & 4.222269 & 1.204782\end{array}$

$\begin{array}{lll}-2.873937 & 4.306162 & 1.328183\end{array}$

$\begin{array}{llll}-3.367983 & 5.270797 & 1.354284\end{array}$

$\begin{array}{lll}-3.617604 & 3.129425 & 1.367230\end{array}$

$\begin{array}{lll}-2.991362 & 1.881241 & 1.318000\end{array}$

$\begin{array}{llll}-3.596400 & 0.982207 & 1.340427\end{array}$

$\begin{array}{lll}0.393283 & 0.345617 & -1.188678\end{array}$ 


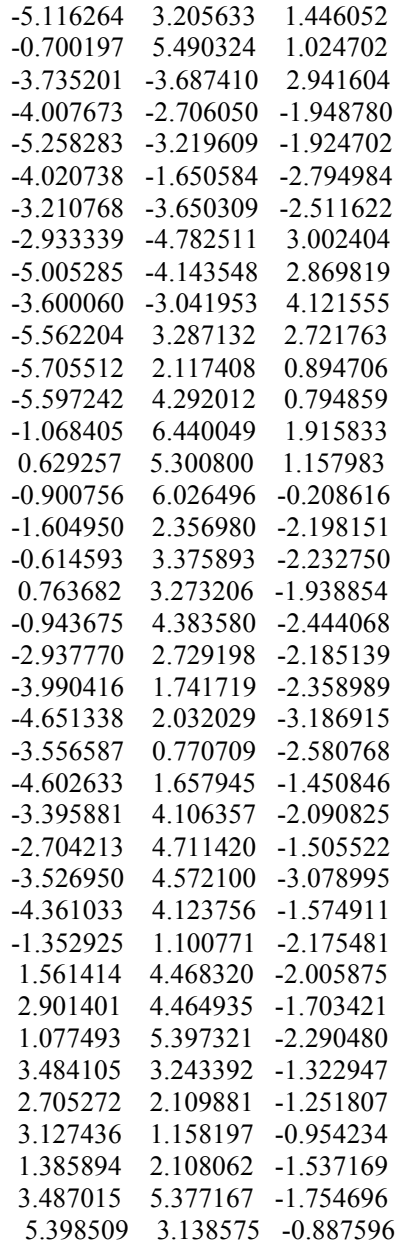

\section{Conformer_12}

Number of imaginary frequencies : 0

The smallest frequency is : $13.2444 \mathrm{~cm}(-1)$

Electronic energy :

$\mathrm{HF}=-4521.7138803$

Zero-point correction=

(Hartree/Particle)

Thermal correction to Energy=

Thermal correction to Enthalpy=

0.923919

0.924863

Thermal correction to Gibbs Free Energy=

Sum of electronic and zero-point Energies=

Sum of electronic and thermal Energies=

0.744393

Sum of electronic and thermal Enthalpies=

$-4520.859373$

$-4520.789961$

Sum of electronic and thermal Free Energies=

$-4520.789017$

$-4520.969488$

Cartesian Coordinates

$\begin{array}{lll}-3.598034 & -1.129679 & -2.692613\end{array}$

$\begin{array}{lll}-1.126135 & -1.576365 & 1.911678\end{array}$

$\begin{array}{llll}0.662736 & 0.066266 & -0.938142\end{array}$

$-2.360153-1.491731-1.039886$

$\begin{array}{lll}-1.858673 & -0.399969 & -1.842602\end{array}$

$\begin{array}{lll}-2.862285 & 0.615558 & -1.876461\end{array}$

$\begin{array}{llll}-2.792968 & 1.555173 & -2.406893\end{array}$

$\begin{array}{llll}-3.990125 & 0.155075 & -1.133955\end{array}$

$\begin{array}{llll}-4.924620 & 0.683481 & -1.007044\end{array}$

$\begin{array}{llll}-3.683291 & -1.138964 & -0.620738\end{array}$

$-4.343004-1.760940-0.035427$

$\begin{array}{lll}-3.205300 & -2.086270 & -4.494262\end{array}$

$-2.218834-2.371244-4.836135$

$\begin{array}{lll}-3.842592 & -0.829274 & -4.727703\end{array}$

$\begin{array}{lll}-3.421378 & 0.006325 & -5.270611\end{array}$

$\begin{array}{lll}-5.113844 & -0.850551 & -4.076635\end{array}$

$\begin{array}{lll}-5.819671 & -0.031421 & -4.034746\end{array}$

$\begin{array}{lll}-5.262845 & -2.120372 & -3.439900\end{array}$

$\begin{array}{lll}-6.103070 & -2.433692 & -2.834313\end{array}$

$\begin{array}{llll}-4.085457 & -2.884101 & -3.698421\end{array}$

$\begin{array}{lll}-3.883509 & -3.879324 & -3.325978\end{array}$

$\begin{array}{llll}-1.560841 & -2.701138 & -0.683478\end{array}$

$\begin{array}{lll}-1.263928 & -3.638793 & -1.685019\end{array}$

$\begin{array}{lll}-1.677175 & -3.478712 & -2.672740\end{array}$

$-0.441064 \quad-4.739301 \quad-1.447119$

$\begin{array}{llll}-0.208041 & -5.427138 & -2.253937\end{array}$

$\begin{array}{llll}0.096650 & -4.930710 & -0.176956\end{array}$

$\begin{array}{lll}0.751292 & -5.773026 & 0.022852\end{array}$

$\begin{array}{llll}-0.186223 & -4.015061 & 0.836868\end{array}$

$\begin{array}{lll}0.255179 & -4.168423 & 1.814966\end{array}$

$\begin{array}{lll}-0.997242 & -2.890986 & 0.608610\end{array}$

$\begin{array}{llll}-0.467566 & -0.327042 & -2.411314\end{array}$

$\begin{array}{lll}-0.192951 & -1.334039 & -2.729550\end{array}$

$\begin{array}{llll}-0.325226 & 0.615135 & -3.609082\end{array}$

$\begin{array}{llll}0.693270 & 0.591105 & -4.006815\end{array}$

$\begin{array}{llll}-0.558209 & 1.651260 & -3.349166\end{array}$

$\begin{array}{llll}-1.015157 & 0.302484 & -4.399178\end{array}$

$\begin{array}{lll}-0.217310 & -2.273197 & 3.356357\end{array}$

$\begin{array}{llll}-0.752346 & -3.273146 & 4.186101\end{array}$

$\begin{array}{lll}-1.747050 & -3.665265 & 3.999038\end{array}$

$\begin{array}{llll}-0.011189 & -3.768009 & 5.259085\end{array}$

$\begin{array}{lll}-0.434513 & -4.540215 & 5.895540\end{array}$

$\begin{array}{lll}1.271081 & -3.271819 & 5.514944\end{array}$

$\begin{array}{lll}1.846303 & -3.658403 & 6.351753\end{array}$

$\begin{array}{llll}1.806801 & -2.274892 & 4.698166\end{array}$

$\begin{array}{lll}2.800650 & -1.884154 & 4.892501\end{array}$

$\begin{array}{llll}1.065575 & -1.771669 & 3.625789\end{array}$

$\begin{array}{lll}1.473249 & -0.975396 & 3.011699\end{array}$

$\begin{array}{lll}-2.875116 & -1.630359 & 2.472763\end{array}$

$\begin{array}{lll}-3.398463 & -0.476509 & 3.074576\end{array}$

$\begin{array}{lll}-2.770499 & 0.403186 & 3.184346\end{array}$

$\begin{array}{lll}-4.721948 & -0.450941 & 3.518711\end{array}$

$\begin{array}{lll}-5.119246 & 0.450214 & 3.977170\end{array}$

$\begin{array}{llll}-5.533311 & -1.578071 & 3.364476\end{array}$

$-6.565603 \quad-1.555658 \quad 3.702055$

$\begin{array}{lll}-5.014320 & -2.735840 & 2.776403\end{array}$

$\begin{array}{lll}-5.640594 & -3.616028 & 2.660108\end{array}$

$\begin{array}{lll}-3.690491 & -2.764226 & 2.333792\end{array}$

$\begin{array}{lll}-3.298605 & -3.661336 & 1.863335\end{array}$

$\begin{array}{llll}1.940615 & -1.258286 & -0.884933\end{array}$

$\begin{array}{llll}2.784155 & -1.232759 & 0.236152\end{array}$

$\begin{array}{llll}2.672374 & -0.429442 & 0.959782\end{array}$

$\begin{array}{lll}3.735639 & -2.234675 & 0.425909\end{array}$

$\begin{array}{llll}3.832926 & -3.304090 & -0.462630\end{array}$

$\begin{array}{llll}4.551615 & -4.098985 & -0.296807\end{array}$

$\begin{array}{llll}2.974086 & -3.340280 & -1.561689\end{array}$

$2.052254 \quad-2.318815 \quad-1.791525$

$\begin{array}{lll}1.418129 & -2.381059 & -2.666135\end{array}$

$\begin{array}{llll}1.614434 & 1.582461 & -1.361622\end{array}$

$\begin{array}{llll}0.913406 & 2.796488 & -1.278915\end{array}$

$\begin{array}{llll}-0.145922 & 2.795929 & -1.049590\end{array}$

$\begin{array}{llll}1.582886 & 4.006728 & -1.427635\end{array}$

$\begin{array}{llll}2.965526 & 4.039536 & -1.619347\end{array}$

$\begin{array}{llll}3.490276 & 4.985733 & -1.687814\end{array}$

$\begin{array}{llll}3.663555 & 2.835990 & -1.671997\end{array}$

$\begin{array}{llll}2.996481 & 1.612540 & -1.568117\end{array}$

$\begin{array}{llll}3.570059 & 0.694346 & -1.606643\end{array}$

$\begin{array}{lll}-0.276394 & 0.379467 & 1.172980\end{array}$

$\begin{array}{llll}5.158875 & 2.851219 & -1.825865\end{array}$

$\begin{array}{llll}0.850489 & 5.307031 & -1.249995\end{array}$

$\begin{array}{lll}4.685299 & -2.114525 & 1.585069\end{array}$

$\begin{array}{llll}3.036440 & -4.533233 & -2.474205\end{array}$

$\begin{array}{llll}2.767360 & -5.681675 & -1.803145\end{array}$

$\begin{array}{llll}2.152751 & -4.455821 & -3.493862\end{array}$

$\begin{array}{llll}4.266282 & -4.688829 & -3.018460\end{array}$

$\begin{array}{lll}5.664144 & -1.209539 & 1.330443\end{array}$

$\begin{array}{lll}5.293469 & -3.282032 & 1.881348\end{array}$

$\begin{array}{llll}4.059583 & -1.688878 & 2.710641\end{array}$

$\begin{array}{llll}5.544238 & 2.871037 & -3.123420\end{array}$

$\begin{array}{llll}5.732753 & 1.760179 & -1.264231\end{array}$

$\begin{array}{llll}5.712364 & 3.939457 & -1.238009\end{array}$

$\begin{array}{llll}1.174694 & 6.199752 & -2.214880\end{array}$

$\begin{array}{llll}-0.490187 & 5.154849 & -1.270914\end{array}$ 
$\begin{array}{ccc}1.164204 & 5.896369 & -0.066184 \\ 1.867769 & 2.331306 & 2.008206 \\ 0.921982 & 3.389036 & 2.090351 \\ -0.466729 & 3.354235 & 1.836784 \\ 1.303313 & 4.376180 & 2.312426 \\ 3.211592 & 2.654478 & 1.952409 \\ 4.229975 & 1.626374 & 2.099108 \\ 5.037778 & 1.997447 & 2.741269 \\ 3.803809 & 0.738052 & 2.558759 \\ 4.668819 & 1.348242 & 1.131610 \\ 3.716087 & 4.004687 & 1.754849 \\ 3.001400 & 4.612320 & 1.200952 \\ 3.947478 & 4.507268 & 2.705727 \\ 4.634371 & 3.958057 & 1.160947 \\ 1.571537 & 1.083531 & 1.991038 \\ -1.205772 & 4.584123 & 1.939701 \\ -2.548281 & 4.649503 & 1.658360 \\ -0.673864 & 5.483977 & 2.232299 \\ -3.194100 & 3.463665 & 1.266192 \\ -2.473870 & 2.293699 & 1.174469 \\ -2.946917 & 1.365904 & 0.878979 \\ -1.152574 & 2.223544 & 1.442788 \\ -3.088691 & 5.587503 & 1.734119 \\ -5.116937 & 3.460324 & 0.855669\end{array}$

Conformer_13

Number of imaginary frequencies : 0

The smallest frequency is : $13.3028 \mathrm{~cm}(-1)$

Electronic energy :

$\mathrm{HF}=-4521.7138802$

Zero-point correction=

0.854510

(Hartree/Particle)

Thermal correction to Energy=

Thermal correction to Enthalpy=

0.923920

0.924864

Thermal correction to Gibbs Free Energy=

Sum of electronic and zero-point Energies=

Sum of electronic and thermal Energies=

Sum of electronic and thermal Enthalpies=

0.744407

$-4520.859370$

$-4520.789960$

$-4520.789016$

Sum of electronic and thermal Free Energies=

$-4520.969473$
Cartesian Coordinates

$\begin{array}{lll}-3.597906 & -1.129440 & -2.692820\end{array}$

$\begin{array}{lll}-1.126175 & -1.576374 & 1.911453\end{array}$

$\begin{array}{llll}0.662824 & 0.066321 & -0.938138\end{array}$

$\begin{array}{lll}-2.360078 & -1.491628 & -1.040074\end{array}$

$\begin{array}{lll}-1.858517 & -0.399887 & -1.842774\end{array}$

$\begin{array}{llll}-2.862047 & 0.615719 & -1.876610\end{array}$

$\begin{array}{llll}-2.792652 & 1.555334 & -2.407031\end{array}$

$\begin{array}{lll}-3.989929 & 0.155297 & -1.134134\end{array}$

$\begin{array}{llll}-4.924390 & 0.683770 & -1.007241\end{array}$

$\begin{array}{llll}-3.683194 & -1.138777 & -0.620938\end{array}$

$\begin{array}{lll}-4.342959 & -1.760702 & -0.035639\end{array}$

$\begin{array}{lll}-3.205184 & -2.085854 & -4.494557\end{array}$

$\begin{array}{lll}-2.218714 & -2.370772 & -4.836464\end{array}$

$\begin{array}{lll}-3.842507 & -0.828849 & -4.727868\end{array}$

$\begin{array}{lll}-3.421314 & 0.006816 & -5.270692\end{array}$

$\begin{array}{llll}-5.113748 & -0.850211 & -4.076779\end{array}$

$\begin{array}{llll}-5.819581 & -0.031092 & -4.034789\end{array}$

$\begin{array}{llll}-5.262712 & -2.120095 & -3.440159\end{array}$

$\begin{array}{llll}-6.102917 & -2.433484 & -2.834580\end{array}$

$\begin{array}{lll}-4.085317 & -2.883779 & -3.698779\end{array}$

$\begin{array}{lll}-3.883343 & -3.879032 & -3.326431\end{array}$

$\begin{array}{lll}-1.560821 & -2.701079 & -0.683698\end{array}$

$-1.263912-3.638705-1.685268$

$-1.677166-3.478603-2.672983$

$-0.441061 \quad-4.739229-1.447392$

$-0.208048 \quad-5.427050-2.254226$

$\begin{array}{llll}0.096650 & -4.930687 & -0.177234\end{array}$

$\begin{array}{llll}0.751277 & -5.773022 & 0.022547\end{array}$

$\begin{array}{llll}-0.186211 & -4.015066 & 0.836619\end{array}$

$\begin{array}{lll}0.255178 & -4.168460 & 1.814720\end{array}$

$\begin{array}{llll}-0.997193 & -2.890967 & 0.608371\end{array}$

$\begin{array}{llll}-0.467370 & -0.326995 & -2.411388\end{array}$

$\begin{array}{llll}-0.192745 & -1.334004 & -2.729579\end{array}$ $\begin{array}{lll}-0.324939 & 0.615154 & -3.609166\end{array}$

$\begin{array}{llll}0.693580 & 0.591101 & -4.006840\end{array}$

$\begin{array}{llll}-0.557925 & 1.651288 & -3.349287\end{array}$

$\begin{array}{lll}-1.014829 & 0.302494 & -4.399296\end{array}$

$\begin{array}{lll}-0.217383 & -2.273107 & 3.356173\end{array}$

$\begin{array}{lll}-0.752444 & -3.273184 & 4.185748\end{array}$

$\begin{array}{lll}-1.747084 & -3.665384 & 3.998484\end{array}$

$-0.011396 \quad-3.768058 \quad 5.258803$

$\begin{array}{lll}-0.434725 & -4.540368 & 5.895126\end{array}$

$\begin{array}{lll}1.270775 & -3.271735 & 5.514898\end{array}$

$\begin{array}{llll}1.845919 & -3.658329 & 6.351755\end{array}$

$\begin{array}{lll}1.806502 & -2.274658 & 4.698303\end{array}$

$\begin{array}{llll}2.800269 & -1.883816 & 4.892845\end{array}$

$\begin{array}{lll}1.065391 & -1.771424 & 3.625851\end{array}$

$\begin{array}{lll}1.473042 & -0.975020 & 3.011909\end{array}$

$\begin{array}{lll}-2.875147 & -1.630426 & 2.472504\end{array}$

$\begin{array}{llll}-3.398547 & -0.476675 & 3.074463\end{array}$

$\begin{array}{lll}-2.770624 & 0.403034 & 3.184363\end{array}$

$\begin{array}{lll}-4.722055 & -0.451211 & 3.518538\end{array}$

$\begin{array}{lll}-5.119406 & 0.449869 & 3.977100\end{array}$

$\begin{array}{lll}-5.533380 & -1.578343 & 3.364098\end{array}$

$\begin{array}{lll}-6.565690 & -1.556004 & 3.701624\end{array}$

$\begin{array}{lll}-5.014325 & -2.736020 & 2.775897\end{array}$

$\begin{array}{lll}-5.640564 & -3.616214 & 2.659459\end{array}$

$\begin{array}{lll}-3.690471 & -2.764305 & 2.333360\end{array}$

$\begin{array}{lll}-3.298504 & -3.661338 & 1.862824\end{array}$

$\begin{array}{llll}1.940619 & -1.258306 & -0.884796\end{array}$

$\begin{array}{lll}2.784020 & -1.232833 & 0.236392\end{array}$

$\begin{array}{llll}2.672172 & -0.429526 & 0.960019\end{array}$

$\begin{array}{llll}3.735463 & -2.234768 & 0.426236\end{array}$

$\begin{array}{llll}3.832835 & -3.304158 & -0.462328\end{array}$

$\begin{array}{llll}4.551493 & -4.099070 & -0.296445\end{array}$

$\begin{array}{llll}2.974121 & -3.340302 & -1.561486\end{array}$

$\begin{array}{llll}2.052346 & -2.318805 & -1.791410\end{array}$

$\begin{array}{llll}1.418323 & -2.381010 & -2.666098\end{array}$

$\begin{array}{llll}1.614630 & 1.582457 & -1.361592\end{array}$

$\begin{array}{llll}0.913645 & 2.796519 & -1.279017\end{array}$

$\begin{array}{lll}-0.145705 & 2.796023 & -1.049786\end{array}$

$\begin{array}{llll}1.583187 & 4.006721 & -1.427779\end{array}$

$\begin{array}{llll}2.965843 & 4.039458 & -1.619375\end{array}$

$\begin{array}{llll}3.490637 & 4.985627 & -1.687882\end{array}$

$\begin{array}{llll}3.663830 & 2.835881 & -1.671866\end{array}$

$2.996698 \quad 1.612466-1.567960$

$\begin{array}{llll}3.570243 & 0.694247 & -1.606375\end{array}$

$\begin{array}{lll}-0.276481 & 0.379497 & 1.172879\end{array}$

$\begin{array}{llll}5.159167 & 2.851056 & -1.825570\end{array}$

$\begin{array}{llll}0.850833 & 5.307067 & -1.250285\end{array}$

$\begin{array}{lll}4.685052 & -2.114633 & 1.585455\end{array}$

$\begin{array}{llll}3.036569 & -4.533217 & -2.474046\end{array}$

$\begin{array}{llll}2.767668 & -5.681713 & -1.803008\end{array}$

$\begin{array}{llll}2.152811 & -4.455875 & -3.493650\end{array}$

$\begin{array}{lll}4.266396 & -4.688641 & -3.018382\end{array}$

$\begin{array}{llll}5.664106 & -1.209895 & 1.330749\end{array}$

$\begin{array}{llll}5.292955 & -3.282218 & 1.881972\end{array}$

$\begin{array}{lll}4.059344 & -1.688661 & 2.710910\end{array}$

$\begin{array}{llll}5.544669 & 2.870927 & -3.123083\end{array}$

$\begin{array}{llll}5.732943 & 1.759969 & -1.263926\end{array}$

$\begin{array}{llll}5.712631 & 3.939247 & -1.237602\end{array}$

$\begin{array}{llll}1.175257 & 6.199753 & -2.215127\end{array}$

$\begin{array}{rrrr}-0.489848 & 5.154964 & -1.271431\end{array}$

$\begin{array}{llll}1.164376 & 5.896405 & -0.066427\end{array}$

$\begin{array}{llll}1.867570 & 2.331327 & 2.008273\end{array}$

$\begin{array}{lll}0.921785 & 3.389077 & 2.090216\end{array}$

$\begin{array}{lll}-0.466934 & 3.354226 & 1.836697\end{array}$

$\begin{array}{lll}1.303106 & 4.376255 & 2.312154\end{array}$

$\begin{array}{lll}3.211394 & 2.654493 & 1.952439\end{array}$

$\begin{array}{lll}4.229804 & 1.626450 & 2.099398\end{array}$

$\begin{array}{llll}5.037412 & 1.997547 & 2.741793\end{array}$

$\begin{array}{lll}3.803562 & 0.738093 & 2.558903\end{array}$

$\begin{array}{lll}4.668925 & 1.348378 & 1.132007\end{array}$

$\begin{array}{lll}3.715861 & 4.004725 & 1.754966\end{array}$

$\begin{array}{llll}3.001313 & 4.612278 & 1.200802\end{array}$

$\begin{array}{llll}3.946909 & 4.507380 & 2.705889\end{array}$

$\begin{array}{llll}4.634344 & 3.958115 & 1.161371\end{array}$

$\begin{array}{lll}1.571317 & 1.083551 & 1.991314\end{array}$ 


$\begin{array}{cccc}6 & -1.206022 & 4.584089 & 1.939585 \\ 6 & -2.548572 & 4.649380 & 1.658413 \\ 1 & -0.674126 & 5.483989 & 2.232068 \\ 6 & -3.194382 & 3.463481 & 1.266416 \\ 6 & -2.474090 & 2.293558 & 1.174628 \\ 1 & -2.947121 & 1.365730 & 0.879211 \\ 7 & -1.152758 & 2.223493 & 1.442784 \\ 1 & -3.089023 & 5.587355 & 1.734189 \\ 35 & -5.117296 & 3.459970 & 0.856249\end{array}$

\section{Conformer_14}

\begin{tabular}{|c|c|}
\hline \multicolumn{2}{|l|}{$\begin{array}{l}\text { Number of imaginary frequencies : } 0 \\
\text { The smallest frequency is : } 13.2433 \mathrm{~cm}(-1)\end{array}$} \\
\hline Electronic energy : $\quad \mathrm{HF}=-4521.7138804$ & \\
\hline $\begin{array}{l}\text { Zero-point correction= } \\
\text { (Hartree/Particle) }\end{array}$ & 4507 \\
\hline Thermal correction to Energy= & .923919 \\
\hline Thermal correction to Enthalpy $=$ & 0.924863 \\
\hline Thermal correction to Gibbs Free Energy= & 0.744389 \\
\hline Sum of electronic and zero-point Energies $=$ & -4520.859373 \\
\hline Sum of electronic and thermal Energies $=$ & -4520.789961 \\
\hline Sum of electronic and thermal Enthalpies= & -4520.789017 \\
\hline Sum of electronic and thermal Free Energies & -4520.969492 \\
\hline
\end{tabular}

\begin{tabular}{|c|c|c|c|}
\hline \multicolumn{4}{|c|}{ Cartesian Coordinates } \\
\hline 26 & -3.598294 & -1.128994 & -2.692458 \\
\hline 15 & -1.126176 & -1.576370 & 1.911506 \\
\hline 15 & 0.662753 & 0.066265 & -0.938174 \\
\hline 6 & -2.360251 & -1.491322 & -1.039917 \\
\hline 6 & -1.858664 & -0.399652 & -1.842692 \\
\hline 6 & -2.862072 & 0.616079 & -1.876385 \\
\hline 1 & -2.792636 & 1.555685 & -2.406817 \\
\hline 6 & -3.989897 & 0.155811 & -1.133726 \\
\hline 1 & -4.924268 & 0.684406 & -1.006679 \\
\hline 6 & -3.683247 & -1.138294 & -0.620557 \\
\hline 1 & -4.342994 & -1.760127 & -0.035136 \\
\hline 6 & -3.206042 & -2.085668 & -4.494163 \\
\hline 1 & -2.219698 & -2.370870 & -4.836196 \\
\hline 6 & -3.843082 & -0.828526 & -4.727506 \\
\hline 1 & -3.421752 & 0.006979 & -5.270471 \\
\hline 6 & -5.114243 & -0.849514 & -4.076250 \\
\hline 1 & -5.819866 & -0.030215 & -4.034237 \\
\hline 6 & -5.263438 & -2.119302 & -3.439493 \\
\hline 1 & -6.103643 & -2.432427 & -2.833778 \\
\hline 6 & -4.086261 & -2.883300 & -3.698189 \\
\hline 1 & -3.884472 & -3.878564 & -3.325768 \\
\hline 6 & -1.561142 & -2.700902 & -0.683666 \\
\hline 6 & -1.264502 & -3.638571 & -1.685275 \\
\hline 1 & -1.677792 & -3.478368 & -2.672959 \\
\hline 6 & -0.441891 & -4.739288 & -1.447463 \\
\hline 1 & -0.209097 & -5.427159 & -2.254318 \\
\hline 6 & 0.095854 & -4.930892 & -0.177340 \\
\hline 1 & 0.750285 & -5.773390 & 0.022391 \\
\hline 6 & -0.186710 & -4.015206 & 0.836537 \\
\hline 1 & 0.254704 & -4.168709 & 1.814609 \\
\hline 6 & -0.997442 & -2.890916 & 0.608350 \\
\hline 6 & -0.467547 & -0.326880 & -2.411395 \\
\hline 1 & -0.193008 & -1.333890 & -2.729647 \\
\hline 6 & -0.325103 & 0.615312 & -3.609136 \\
\hline 1 & 0.693385 & 0.591178 & -4.006882 \\
\hline 1 & -0.557969 & 1.651457 & -3.349193 \\
\hline 1 & -1.015080 & 0.302756 & -4.399233 \\
\hline 6 & -0.217171 & -2.273271 & 3.356014 \\
\hline 6 & -0.752245 & -3.273208 & 4.185749 \\
\hline 1 & -1.746972 & -3.665282 & 3.998673 \\
\hline 6 & -0.011071 & -3.768165 & 5.258679 \\
\hline 1 & -0.434412 & -4.540367 & 5.895125 \\
\hline 6 & 1.271247 & -3.272075 & 5.514477 \\
\hline 1 & 1.846492 & -3.658727 & 6.351238 \\
\hline 6 & 1.807007 & -2.275170 & 4.697693 \\
\hline 1 & 2.800900 & -1.884530 & 4.891988 \\
\hline 6 & 1.065769 & -1.771849 & 3.625370 \\
\hline 1 & 1.473475 & -0.975599 & 3.011264 \\
\hline
\end{tabular}

$\begin{array}{lll}-2.875100 & -1.630518 & 2.472682\end{array}$

$\begin{array}{llll}-3.398617 & -0.476761 & 3.074503\end{array}$

$\begin{array}{lll}-2.770769 & 0.402998 & 3.184370\end{array}$

$\begin{array}{lll}-4.722161 & -0.451356 & 3.518479\end{array}$

$\begin{array}{lll}-5.119611 & 0.449733 & 3.976940\end{array}$

$\begin{array}{llll}-5.533400 & -1.578551 & 3.364054\end{array}$

$\begin{array}{lll}-6.565736 & -1.556256 & 3.701504\end{array}$

$\begin{array}{lll}-5.014237 & -2.736224 & 2.775940\end{array}$

$\begin{array}{llll}-5.640421 & -3.616454 & 2.659477\end{array}$

$\begin{array}{lll}-3.690351 & -2.764440 & 2.333496\end{array}$

$\begin{array}{lll}-3.298307 & -3.661414 & 1.862918\end{array}$

$\begin{array}{llll}1.940425 & -1.258492 & -0.884931\end{array}$

$\begin{array}{llll}2.784086 & -1.232937 & 0.236064\end{array}$

$\begin{array}{lll}2.672481 & -0.429514 & 0.959600\end{array}$

$\begin{array}{llll}3.735485 & -2.234931 & 0.425830\end{array}$

$\begin{array}{llll}3.832566 & -3.304452 & -0.462606\end{array}$

$\begin{array}{llll}4.551205 & -4.099393 & -0.296788\end{array}$

$\begin{array}{llll}2.973592 & -3.340679 & -1.561557\end{array}$

$\begin{array}{llll}2.051833 & -2.319151 & -1.791402\end{array}$

$\begin{array}{llll}1.417578 & -2.381487 & -2.665912\end{array}$

$\begin{array}{llll}1.614680 & 1.582332 & -1.361613\end{array}$

$\begin{array}{llll}0.913794 & 2.796447 & -1.278962\end{array}$

$\begin{array}{llll}-0.145544 & 2.796030 & -1.049683\end{array}$

$\begin{array}{llll}1.583420 & 4.006605 & -1.427706\end{array}$

$\begin{array}{llll}2.966070 & 4.039243 & -1.619362\end{array}$

$\begin{array}{llll}3.490934 & 4.985375 & -1.687852\end{array}$

$\begin{array}{llll}3.663960 & 2.835613 & -1.671936\end{array}$

$\begin{array}{llll}2.996739 & 1.612245 & -1.568052\end{array}$

$\begin{array}{llll}3.570213 & 0.693985 & -1.606530\end{array}$

$\begin{array}{lll}-0.276482 & 0.379521 & 1.172922\end{array}$

$\begin{array}{llll}5.159293 & 2.850675 & -1.825693\end{array}$

$\begin{array}{llll}0.851166 & 5.307000 & -1.250140\end{array}$

$\begin{array}{lll}4.685367 & -2.114732 & 1.584802\end{array}$

$\begin{array}{llll}3.035795 & -4.533692 & -2.474006\end{array}$

$\begin{array}{llll}2.766836 & -5.682097 & -1.802839\end{array}$

$\begin{array}{llll}2.151948 & -4.456343 & -3.493532\end{array}$

$\begin{array}{llll}4.265552 & -4.689305 & -3.018451\end{array}$

$\begin{array}{llll}5.664359 & -1.210010 & 1.329801\end{array}$

$\begin{array}{llll}5.293339 & -3.282303 & 1.881226\end{array}$

$\begin{array}{llll}4.059954 & -1.688698 & 2.710399\end{array}$

$\begin{array}{llll}5.544755 & 2.870527 & -3.123219\end{array}$

$\begin{array}{llll}5.733004 & 1.759538 & -1.264078\end{array}$

$\begin{array}{llll}5.712865 & 3.938816 & -1.237734\end{array}$

$\begin{array}{llll}1.175577 & 6.199675 & -2.214996\end{array}$

$\begin{array}{lll}-0.489528 & 5.154986 & -1.271186\end{array}$

$\begin{array}{llll}1.164835 & 5.896305 & -0.066299\end{array}$

$\begin{array}{lll}1.867929 & 2.331192 & 2.008126\end{array}$

$\begin{array}{lll}0.922244 & 3.389017 & 2.090226\end{array}$

$\begin{array}{lll}-0.466479 & 3.354329 & 1.836726\end{array}$

$\begin{array}{lll}1.303670 & 4.376134 & 2.312248\end{array}$

$\begin{array}{lll}3.211781 & 2.654264 & 1.952314\end{array}$

$\begin{array}{lll}4.230097 & 1.626134 & 2.099300\end{array}$

$\begin{array}{lll}5.037719 & 1.997161 & 2.741719\end{array}$

$\begin{array}{lll}3.803756 & 0.737821 & 2.558799\end{array}$

$\begin{array}{lll}4.669225 & 1.348009 & 1.131928\end{array}$

$\begin{array}{lll}3.716356 & 4.004463 & 1.754914\end{array}$

$3.001849 \quad 4.612104 \quad 1.200792$

$3.947459 \quad 4.507050 \quad 2.705861$

$\begin{array}{llll}4.634826 & 3.957811 & 1.161302\end{array}$

$\begin{array}{lll}1.571587 & 1.083445 & 1.990982\end{array}$

$\begin{array}{lll}-1.205418 & 4.584281 & 1.939645\end{array}$

$\begin{array}{lll}-2.547968 & 4.649737 & 1.658519\end{array}$

$\begin{array}{lll}-0.673402 & 5.484115 & 2.232110\end{array}$

$\begin{array}{llll}-3.193925 & 3.463917 & 1.266526\end{array}$

$\begin{array}{lll}-2.473771 & 2.293914 & 1.174693\end{array}$

$\begin{array}{lll}-2.946921 & 1.366159 & 0.879256\end{array}$

$\begin{array}{lll}-1.152439 & 2.223677 & 1.442813\end{array}$

$\begin{array}{lll}-3.088305 & 5.587776 & 1.734315\end{array}$

$\begin{array}{lll}-5.116858 & 3.460617 & 0.856446\end{array}$

\section{Conformer 15}

Number of imaginary frequencies : 0

The smallest frequency is : $14.9981 \mathrm{~cm}(-1)$

Electronic energy : $\quad H F=-4521.7138308$ 
$\begin{array}{llll}3.920686 & -3.219201 & -0.451240\end{array}$

$\begin{array}{llll}4.670562 & -3.986567 & -0.295365\end{array}$

$\begin{array}{llll}3.097409 & -3.247967 & -1.576987\end{array}$

$\begin{array}{llll}2.137399 & -2.257988 & -1.793330\end{array}$

$\begin{array}{llll}1.532742 & -2.306486 & -2.689628\end{array}$

$\begin{array}{llll}1.592660 & 1.591633 & -1.344507\end{array}$

$\begin{array}{llll}0.877769 & 2.798005 & -1.271158\end{array}$

$\begin{array}{lll}-0.182619 & 2.786445 & -1.047349\end{array}$

$\begin{array}{llll}1.534761 & 4.014897 & -1.421804\end{array}$

$\begin{array}{llll}2.917842 & 4.062010 & -1.607130\end{array}$

$\begin{array}{llll}3.432628 & 5.013431 & -1.678067\end{array}$

$\begin{array}{llll}3.629474 & 2.866114 & -1.650563\end{array}$

$\begin{array}{llll}2.975495 & 1.635956 & -1.543409\end{array}$

$\begin{array}{llll}3.559174 & 0.723760 & -1.573817\end{array}$

$\begin{array}{lll}-0.306958 & 0.377363 & 1.179446\end{array}$

$\begin{array}{llll}5.125104 & 2.896718 & -1.798369\end{array}$

$\begin{array}{llll}0.787620 & 5.307733 & -1.251349\end{array}$

$\begin{array}{lll}4.670772 & -2.074636 & 1.659310\end{array}$

$\begin{array}{llll}3.222450 & -4.404259 & -2.529644\end{array}$

$\begin{array}{llll}2.707780 & -5.544417 & -2.000782\end{array}$

$\begin{array}{llll}2.574208 & -4.187272 & -3.695314\end{array}$

$\begin{array}{llll}4.514414 & -4.673120 & -2.827704\end{array}$

$\begin{array}{llll}5.647905 & -1.157560 & 1.443090\end{array}$

$\begin{array}{llll}5.282920 & -3.240551 & 1.954922\end{array}$

$\begin{array}{lll}4.011256 & -1.671148 & 2.773061\end{array}$

$\begin{array}{llll}5.515595 & 2.908179 & -3.094508\end{array}$

$\begin{array}{lll}5.708496 & 1.817446 & -1.224009\end{array}$

$\begin{array}{llll}5.664035 & 3.996561 & -1.218727\end{array}$

$\begin{array}{llll}1.110679 & 6.203418 & -2.213865\end{array}$

$\begin{array}{llll}-0.551192 & 5.141605 & -1.283277\end{array}$

$\begin{array}{llll}1.085536 & 5.900596 & -0.065238\end{array}$

$\begin{array}{lll}1.812370 & 2.347615 & 2.029743\end{array}$

$\begin{array}{lll}0.858720 & 3.399265 & 2.098478\end{array}$

$\begin{array}{lll}-0.526942 & 3.352605 & 1.830319\end{array}$

$\begin{array}{lll}1.230744 & 4.390165 & 2.319850\end{array}$

$\begin{array}{llll}3.154337 & 2.678018 & 1.979289\end{array}$

$\begin{array}{lll}4.177544 & 1.655376 & 2.131009\end{array}$

$\begin{array}{lll}4.985307 & 2.035046 & 2.768018\end{array}$

$\begin{array}{lll}3.756766 & 0.768581 & 2.598664\end{array}$

$\begin{array}{llll}4.615046 & 1.371938 & 1.164419\end{array}$

$\begin{array}{lll}3.652516 & 4.029064 & 1.771250\end{array}$

$\begin{array}{llll}2.933072 & 4.630202 & 1.216470\end{array}$

$\begin{array}{llll}3.885320 & 4.538189 & 2.718222\end{array}$

$\begin{array}{llll}4.568817 & 3.982986 & 1.174123\end{array}$

$\begin{array}{lll}1.523209 & 1.098074 & 2.021271\end{array}$

$\begin{array}{lll}-1.277470 & 4.576211 & 1.923715\end{array}$

$\begin{array}{lll}-2.616282 & 4.630813 & 1.623180\end{array}$

$\begin{array}{lll}-0.757088 & 5.480201 & 2.224148\end{array}$

$\begin{array}{lll}-3.246878 & 3.440043 & 1.221102\end{array}$

$\begin{array}{lll}-2.516326 & 2.275657 & 1.140716\end{array}$

$\begin{array}{llll}-2.977285 & 1.343879 & 0.838375\end{array}$

$\begin{array}{lll}-1.198681 & 2.216051 & 1.428724\end{array}$

$\begin{array}{llll}-3.165253 & 5.564381 & 1.691584\end{array}$

$\begin{array}{lll}-5.162928 & 3.422066 & 0.780349\end{array}$

Conformer_16

Number of imaginary frequencies : 0

The smallest frequency is : $13.2538 \mathrm{~cm}(-1)$

Electronic energy : $\quad \mathrm{HF}=-4521.7138804$

$\begin{array}{lr}\text { Zero-point correction }= & 0.854519\end{array}$

(Hartree/Particle)

Thermal correction to Energy $=\quad 0.923926$

Thermal correction to Enthalpy=

0.924871

Thermal correction to Gibbs Free Energy $=0.744439$

Sum of electronic and zero-point Energies $=\quad-4520.859361$

Sum of electronic and thermal Energies $=\quad-4520.789954$

Sum of electronic and thermal Enthalpies $=\quad-4520.789010$

Sum of electronic and thermal Free Energies $=\quad-4520.969442$

Cartesian Coordinates

$-3.598708 \quad-1.128678-2.691959$

$\begin{array}{lll}-1.125589 & -1.577395 & 1.911490\end{array}$

$\begin{array}{llll}0.662446 & 0.066819 & -0.937563\end{array}$ 
$\begin{array}{llll}3.036436 & -4.531452 & -2.476541\end{array}$

$\begin{array}{llll}2.768320 & -5.680519 & -1.806174\end{array}$

$\begin{array}{llll}2.152160 & -4.453739 & -3.495663\end{array}$

$\begin{array}{llll}4.266055 & -4.686006 & -3.021601\end{array}$

$\begin{array}{llll}5.664305 & -1.211190 & 1.330135\end{array}$

$\begin{array}{llll}5.292365 & -3.283539 & 1.880743\end{array}$

$\begin{array}{llll}4.059441 & -1.689747 & 2.710244\end{array}$

$\begin{array}{llll}5.543442 & 2.871211 & -3.123688\end{array}$

$\begin{array}{llll}5.732307 & 1.760726 & -1.264315\end{array}$

$\begin{array}{llll}5.712025 & 3.940011 & -1.238531\end{array}$

$\begin{array}{llll}1.174536 & 6.200640 & -2.213547\end{array}$

$\begin{array}{llll}-0.490465 & 5.155621 & -1.269920\end{array}$

$\begin{array}{llll}1.163993 & 5.896593 & -0.064939\end{array}$

$\begin{array}{lll}1.868269 & 2.330517 & 2.008928\end{array}$

$\begin{array}{lll}0.922684 & 3.388406 & 2.091373\end{array}$

$\begin{array}{lll}-0.466061 & 3.353854 & 1.837970\end{array}$

$\begin{array}{llll}1.304221 & 4.375473 & 2.313441\end{array}$

$\begin{array}{lll}3.212136 & 2.653420 & 1.952731\end{array}$

$\begin{array}{lll}4.230354 & 1.625074 & 2.098931\end{array}$

$\begin{array}{lll}5.038597 & 1.996009 & 2.740613\end{array}$

$\begin{array}{llll}3.804241 & 0.736877 & 2.558876\end{array}$

$\begin{array}{lll}4.668596 & 1.346767 & 1.131206\end{array}$

$\begin{array}{lll}3.716863 & 4.003508 & 1.754920\end{array}$

$\begin{array}{llll}3.001934 & 4.611399 & 1.201622\end{array}$

$\begin{array}{lll}3.949098 & 4.505912 & 2.705683\end{array}$

$\begin{array}{llll}4.634695 & 3.956702 & 1.160331\end{array}$

$\begin{array}{lll}1.571776 & 1.082802 & 1.991851\end{array}$

$\begin{array}{lll}-1.204883 & 4.583870 & 1.940967\end{array}$

$\begin{array}{lll}-2.547374 & 4.649524 & 1.659607\end{array}$

$\begin{array}{lll}-0.672803 & 5.483610 & 2.233603\end{array}$

$\begin{array}{llll}-3.193408 & 3.463827 & 1.267362\end{array}$

$\begin{array}{lll}-2.473411 & 2.293716 & 1.175651\end{array}$

$\begin{array}{llll}-2.946652 & 1.366039 & 0.880096\end{array}$

$\begin{array}{lll}-1.152137 & 2.223285 & 1.444017\end{array}$

$\begin{array}{lll}-3.087604 & 5.587625 & 1.735402\end{array}$

$\begin{array}{llll}35 & -5.116221 & 3.460847 & 0.856698\end{array}$

\section{Conformer_17}

Number of imaginary frequencies : 0

The smallest frequency is : $13.2979 \mathrm{~cm}(-1)$

Electronic energy : $\quad H F=-4521.7138803$

Zero-point correction $=\quad 0.854510$

(Hartree/Particle)

Thermal correction to Energy= $\quad 0.923920$

Thermal correction to Enthalpy=

0.924864

Thermal correction to Gibbs Free Energy $=\quad 0.744404$

Sum of electronic and zero-point Energies $=\quad-4520.859371$

Sum of electronic and thermal Energies=

$-4520.789961$

Sum of electronic and thermal Enthalpies=

Sum of electronic and thermal Free Energies=

$-4520.789016$

Cartesian Coordinates

$-3.597981-1.129408-2.692757$

$\begin{array}{lll}-1.126147 & -1.576253 & 1.911509\end{array}$

$\begin{array}{llll}0.662837 & 0.066230 & -0.938193\end{array}$

$\begin{array}{llll}-2.360124 & -1.491531 & -1.040009\end{array}$

$\begin{array}{llll}-1.858509 & -0.399888 & -1.842809\end{array}$

$\begin{array}{llll}-2.861980 & 0.615773 & -1.876720\end{array}$

$\begin{array}{llll}-2.792531 & 1.555338 & -2.407223\end{array}$

$\begin{array}{lll}-3.989874 & 0.155489 & -1.134180\end{array}$

$\begin{array}{lll}-4.924302 & 0.684030 & -1.007331\end{array}$

$\begin{array}{llll}-3.683205 & -1.138559 & -0.620872\end{array}$

$-4.342995-1.760393-0.035500$

$\begin{array}{llll}-3.205488 & -2.086310 & -4.494303\end{array}$

$-2.219096-2.371563 \quad-4.836159$

$\begin{array}{llll}-3.842469 & -0.829185 & -4.727884\end{array}$

$\begin{array}{llll}-3.421047 & 0.006248 & -5.270888\end{array}$

$\begin{array}{llll}-5.113723 & -0.850069 & -4.076804\end{array}$

$\begin{array}{llll}-5.819335 & -0.030751 & -4.034994\end{array}$

$\begin{array}{llll}-5.263031 & -2.119776 & -3.439912\end{array}$ 
$\begin{array}{lll}-6.103323 & -2.432805 & -2.834267\end{array}$

$\begin{array}{llll}-4.085831 & -2.883826 & -3.698350\end{array}$

$\begin{array}{lll}-3.884107 & -3.879047 & -3.325776\end{array}$

$\begin{array}{llll}-1.560969 & -2.701037 & -0.683588\end{array}$

$-1.264190-3.638748-1.685120$

$\begin{array}{llll}-1.677506 & -3.478681 & -2.672814\end{array}$

$\begin{array}{llll}-0.441389 & -4.739307 & -1.447237\end{array}$

$-0.208483 \quad-5.427195 \quad-2.254046$

$\begin{array}{llll}0.096406 & -4.930716 & -0.177107\end{array}$

$\begin{array}{llll}0.751009 & -5.773068 & 0.022677\end{array}$

$\begin{array}{lll}-0.186340 & -4.015020 & 0.836711\end{array}$

$\begin{array}{llll}0.255113 & -4.168381 & 1.814787\end{array}$

$\begin{array}{lll}-0.997278 & -2.890889 & 0.608457\end{array}$

$\begin{array}{llll}-0.467362 & -0.327082 & -2.411434\end{array}$

$\begin{array}{llll}-0.192779 & -1.334111 & -2.729591\end{array}$

$\begin{array}{llll}-0.324887 & 0.615035 & -3.609232\end{array}$

$\begin{array}{llll}0.693618 & 0.590884 & -4.006935\end{array}$

$\begin{array}{llll}-0.557771 & 1.651193 & -3.349360\end{array}$

$\begin{array}{llll}-1.014830 & 0.302421 & -4.399335\end{array}$

$\begin{array}{lll}-0.217393 & -2.273051 & 3.356217\end{array}$

$\begin{array}{lll}1.065429 & -1.771476 & 3.625847\end{array}$

$\begin{array}{lll}1.473156 & -0.975166 & 3.011835\end{array}$

$\begin{array}{lll}1.806510 & -2.274727 & 4.698312\end{array}$

$\begin{array}{llll}2.800319 & -1.883972 & 4.892815\end{array}$

$\begin{array}{llll}1.270697 & -3.271708 & 5.514967\end{array}$

$\begin{array}{llll}1.845816 & -3.658310 & 6.351839\end{array}$

$\begin{array}{lll}-0.011520 & -3.767933 & 5.258910\end{array}$

$\begin{array}{lll}-0.434907 & -4.540178 & 5.895273\end{array}$

$\begin{array}{llll}-0.752535 & -3.273042 & 4.185842\end{array}$

$\begin{array}{lll}-1.747209 & -3.665164 & 3.998588\end{array}$

$\begin{array}{llll}-2.875112 & -1.630276 & 2.472534\end{array}$

$\begin{array}{lll}-3.398524 & -0.476531 & 3.074481\end{array}$

$\begin{array}{lll}-2.770615 & 0.403187 & 3.184391\end{array}$

$\begin{array}{lll}-4.722041 & -0.451089 & 3.518538\end{array}$

$\begin{array}{lll}-5.119411 & 0.449983 & 3.977099\end{array}$

$\begin{array}{lll}-5.533359 & -1.578219 & 3.364061\end{array}$

$\begin{array}{lll}-6.565677 & -1.555886 & 3.701563\end{array}$

$\begin{array}{lll}-5.014287 & -2.735890 & 2.775858\end{array}$

$\begin{array}{lll}-5.640523 & -3.616083 & 2.659390\end{array}$

$\begin{array}{lll}-3.690423 & -2.764160 & 2.333356\end{array}$

$\begin{array}{lll}-3.298446 & -3.661179 & 1.862791\end{array}$

$\begin{array}{llll}1.940620 & -1.258409 & -0.884830\end{array}$

$\begin{array}{llll}2.784066 & -1.232875 & 0.236324\end{array}$

$\begin{array}{llll}2.672270 & -0.429505 & 0.959891\end{array}$

$\begin{array}{lll}3.735488 & -2.234822 & 0.426208\end{array}$

$\begin{array}{llll}3.832791 & -3.304289 & -0.462271\end{array}$

$\begin{array}{llll}4.551433 & -4.099208 & -0.296358\end{array}$

$\begin{array}{llll}2.974032 & -3.340492 & -1.561393\end{array}$

$\begin{array}{llll}2.052277 & -2.318987 & -1.791363\end{array}$

$\begin{array}{lll}1.418223 & -2.381264 & -2.666024\end{array}$

$\begin{array}{llll}1.614641 & 1.582368 & -1.361651\end{array}$

$\begin{array}{llll}0.913662 & 2.796430 & -1.279010\end{array}$

$\begin{array}{llll}-0.145677 & 2.795928 & -1.049729\end{array}$

$\begin{array}{llll}1.583200 & 4.006636 & -1.427768\end{array}$

$\begin{array}{llll}2.965846 & 4.039374 & -1.619431\end{array}$

$\begin{array}{llll}3.490638 & 4.985544 & -1.687937\end{array}$

$\begin{array}{llll}3.663827 & 2.835796 & -1.671996\end{array}$

$\begin{array}{llll}2.996698 & 1.612379 & -1.568094\end{array}$

$\begin{array}{llll}3.570237 & 0.694159 & -1.606564\end{array}$

$\begin{array}{lll}-0.276409 & 0.379545 & 1.172837\end{array}$

$\begin{array}{llll}5.159157 & 2.850970 & -1.825779\end{array}$

$\begin{array}{llll}0.850857 & 5.306980 & -1.250209\end{array}$

$\begin{array}{lll}4.685125 & -2.114622 & 1.585382\end{array}$

$\begin{array}{llll}3.036423 & -4.533473 & -2.473869\end{array}$

$\begin{array}{llll}2.767510 & -5.681913 & -1.802742\end{array}$

$\begin{array}{llll}2.152644 & -4.456182 & -3.493459\end{array}$

$\begin{array}{llll}4.266234 & -4.688964 & -3.018225\end{array}$

$\begin{array}{llll}5.664134 & -1.209854 & 1.330610\end{array}$

$\begin{array}{llll}5.293084 & -3.282177 & 1.881903\end{array}$

$\begin{array}{lll}4.059454 & -1.688644 & 2.710857\end{array}$

$\begin{array}{llll}5.544589 & 2.870842 & -3.123313\end{array}$

$\begin{array}{llll}5.732958 & 1.759879 & -1.264169\end{array}$

$\begin{array}{llll}5.712657 & 3.939157 & -1.237840\end{array}$

$\begin{array}{llll}1.175230 & 6.199681 & -2.215054\end{array}$

$\begin{array}{llll}-0.489825 & 5.154878 & -1.271282\end{array}$ $\begin{array}{llll}1.164464 & 5.896303 & -0.066360\end{array}$

$\begin{array}{lll}1.867718 & 2.331366 & 2.008141\end{array}$

$\begin{array}{lll}0.921919 & 3.389092 & 2.090216\end{array}$

$\begin{array}{lll}-0.466803 & 3.354263 & 1.836707\end{array}$

$\begin{array}{llll}1.303240 & 4.376251 & 2.312237\end{array}$

$\begin{array}{lll}3.211535 & 2.654558 & 1.952311\end{array}$

$\begin{array}{lll}4.229953 & 1.626505 & 2.099145\end{array}$

$\begin{array}{lll}5.037649 & 1.997608 & 2.741423\end{array}$

$\begin{array}{llll}3.803769 & 0.738158 & 2.558726\end{array}$

$\begin{array}{lll}4.668942 & 1.348410 & 1.131700\end{array}$

$\begin{array}{llll}3.715984 & 4.004796 & 1.754843\end{array}$

$\begin{array}{llll}3.001384 & 4.612366 & 1.200766\end{array}$

$\begin{array}{lll}3.947120 & 4.507417 & 2.705763\end{array}$

$\begin{array}{llll}4.634410 & 3.958208 & 1.161158\end{array}$

$\begin{array}{lll}1.571499 & 1.083584 & 1.991050\end{array}$

$\begin{array}{lll}-1.205876 & 4.584129 & 1.939669\end{array}$

$\begin{array}{lll}-2.548419 & 4.649456 & 1.658480\end{array}$

$\begin{array}{lll}-0.673972 & 5.484004 & 2.232212\end{array}$

$\begin{array}{lll}-3.194245 & 3.463592 & 1.266403\end{array}$

$\begin{array}{lll}-2.473976 & 2.293657 & 1.174572\end{array}$

$\begin{array}{lll}-2.947016 & 1.365845 & 0.879112\end{array}$

$\begin{array}{lll}-1.152646 & 2.223560 & 1.442742\end{array}$

$\begin{array}{lll}-3.088854 & 5.587436 & 1.734301\end{array}$

$\begin{array}{llll}-5.117151 & 3.460184 & 0.856180\end{array}$

Conformer_18

Number of imaginary frequencies : 0

The smallest frequency is : $13.2386 \mathrm{~cm}(-1)$

Electronic energy :

Zero-point correction $=$

$\mathrm{HF}=-4521.7138803$

(Hartree/Particle)

Thermal correction to Energy=

Thermal correction to Enthalpy=

0.854508

Thermal correction to Gibbs Free Energy=
Sum of electronic and zero-point Energies=

Sum of electronic and thermal Energies=

Sum of electronic and thermal Enthalpies=

Sum of electronic and thermal Free Energies=

0.923919

0.924863

0.744396

$-4520.859372$

$-4520.789961$

$-4520.789017$

$-4520.969484$

Cartesian Coordinates

$-3.598109-1.129640-2.692546$

$\begin{array}{lll}-1.126140 & -1.576301 & 1.911642\end{array}$

$\begin{array}{lll}0.662697 & 0.066235 & -0.938135\end{array}$

$\begin{array}{lll}-2.360172 & -1.491685 & -1.039859\end{array}$

$\begin{array}{lll}-1.858689 & -0.399975 & -1.842649\end{array}$

$\begin{array}{lll}-2.862257 & 0.615596 & -1.876487\end{array}$

$\begin{array}{lll}-2.792925 & 1.555183 & -2.406965\end{array}$

$\begin{array}{lll}-3.990077 & 0.155183 & -1.133910\end{array}$

$\begin{array}{lll}-4.924547 & 0.683631 & -1.006984\end{array}$

$\begin{array}{llll}-3.683269 & -1.138851 & -0.620658\end{array}$

$\begin{array}{llll}-4.342982 & -1.760763 & -0.035286\end{array}$

$\begin{array}{llll}-3.205487 & -2.086335 & -4.494161\end{array}$

$\begin{array}{lll}-2.219047 & -2.371377 & -4.836053\end{array}$

$\begin{array}{llll}-3.842713 & -0.829313 & -4.727637\end{array}$

$\begin{array}{llll}-3.421456 & 0.006238 & -5.270587\end{array}$

$\begin{array}{lll}-5.113946 & -0.850482 & -4.076527\end{array}$

$\begin{array}{lll}-5.819713 & -0.031302 & -4.034640\end{array}$

$\begin{array}{lll}-5.263001 & -2.120265 & -3.439727\end{array}$

$\begin{array}{llll}-6.103223 & -2.433501 & -2.834091\end{array}$

$\begin{array}{llll}-4.085665 & -2.884076 & -3.698251\end{array}$

$\begin{array}{llll}-3.883749 & -3.879289 & -3.325761\end{array}$

$\begin{array}{lll}-1.560849 & -2.701090 & -0.683474\end{array}$

$\begin{array}{lll}-1.263942 & -3.638731 & -1.685032\end{array}$

$\begin{array}{lll}-1.677266 & -3.478681 & -2.672726\end{array}$

$\begin{array}{lll}-0.441003 & -4.739192 & -1.447176\end{array}$

$\begin{array}{llll}-0.207994 & -5.427021 & -2.254005\end{array}$

$\begin{array}{llll}0.096803 & -4.930571 & -0.177046\end{array}$

$\begin{array}{lll}0.751509 & -5.772849 & 0.022717\end{array}$

$\begin{array}{lll}-0.186063 & -4.014941 & 0.836797\end{array}$

$\begin{array}{lll}0.255405 & -4.168270 & 1.814871\end{array}$

$\begin{array}{lll}-0.997143 & -2.890907 & 0.608570\end{array}$

$\begin{array}{lll}-0.467575 & -0.327075 & -2.411345\end{array}$

$\begin{array}{llll}-0.192988 & -1.334079 & -2.729593\end{array}$ 


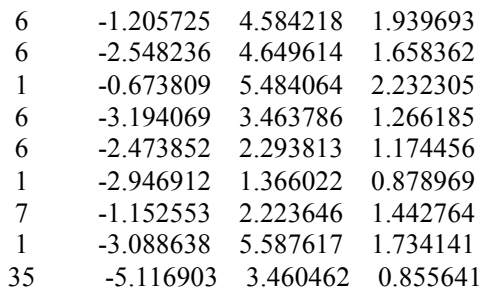

\begin{tabular}{|c|c|}
\hline \multicolumn{2}{|l|}{ Conformer_19 } \\
\hline \multicolumn{2}{|l|}{ Number of imaginary frequencies : 0} \\
\hline \multicolumn{2}{|l|}{ The smallest frequency is : $13.2488 \mathrm{~cm}(-1)$} \\
\hline \multicolumn{2}{|l|}{ Electronic energy : $\quad H F=-4521.7138804$} \\
\hline \multicolumn{2}{|l|}{$\begin{array}{l}\text { Zero-point correction= } \\
(\text { Hartree/Particle })\end{array}$} \\
\hline Thermal correction to Energy= & .923919 \\
\hline Thermal correction to Enthalpy $=$ & 0.924863 \\
\hline Thermal correction to Gibbs Free Energy= & 0.744393 \\
\hline Sum of electronic and zero-point Energies $=$ & -4520.859372 \\
\hline Sum of electronic and thermal Energies $=$ & -4520.789961 \\
\hline Sum of electronic and thermal Enthalpies= & -4520.789017 \\
\hline Sum of electronic and thermal Free Energies= & -4520.969488 \\
\hline
\end{tabular}


$\begin{array}{lll}-2.874920 & -1.629991 & 2.472952\end{array}$

$\begin{array}{lll}-3.398117 & -0.476095 & 3.074801\end{array}$

$\begin{array}{lll}-2.770093 & 0.403570 & 3.184450\end{array}$

$\begin{array}{lll}-4.721552 & -0.450437 & 3.519080\end{array}$

$\begin{array}{lll}-5.118750 & 0.450749 & 3.977563\end{array}$

$\begin{array}{llll}-5.533005 & -1.577519 & 3.364950\end{array}$

$\begin{array}{lll}-6.565257 & -1.555032 & 3.702643\end{array}$

$\begin{array}{lll}-5.014158 & -2.735333 & 2.776836\end{array}$

$\begin{array}{lll}-5.640502 & -3.615482 & 2.660625\end{array}$

$\begin{array}{lll}-3.690376 & -2.763809 & 2.334094\end{array}$

$\begin{array}{lll}-3.298580 & -3.660940 & 1.863620\end{array}$

$\begin{array}{llll}1.940606 & -1.258429 & -0.884937\end{array}$

$\begin{array}{llll}2.784211 & -1.232699 & 0.236094\end{array}$

$\begin{array}{lll}2.672473 & -0.429239 & 0.959569\end{array}$

$\begin{array}{llll}3.735705 & -2.234577 & 0.425987\end{array}$

$\begin{array}{llll}3.832942 & -3.304156 & -0.462364\end{array}$

$\begin{array}{llll}4.551651 & -4.099014 & -0.296450\end{array}$

$\begin{array}{llll}2.974027 & -3.340556 & -1.561356\end{array}$

$\begin{array}{llll}2.052173 & -2.319140 & -1.791329\end{array}$

$\begin{array}{llll}1.417978 & -2.381591 & -2.665876\end{array}$

$\begin{array}{llll}1.614411 & 1.582334 & -1.361806\end{array}$

$\begin{array}{llll}0.913340 & 2.796339 & -1.279102\end{array}$

$\begin{array}{llll}-0.145981 & 2.795745 & -1.049744\end{array}$

$\begin{array}{llll}1.582771 & 4.006601 & -1.427862\end{array}$

$\begin{array}{llll}2.965405 & 4.039452 & -1.619607\end{array}$

$\begin{array}{llll}3.490120 & 4.985666 & -1.688106\end{array}$

$\begin{array}{llll}3.663477 & 2.835930 & -1.672247\end{array}$

$\begin{array}{llll}2.996450 & 1.612456 & -1.568338\end{array}$

$\begin{array}{llll}3.570059 & 0.694281 & -1.606864\end{array}$

$\begin{array}{lll}-0.276360 & 0.379565 & 1.172821\end{array}$

$\begin{array}{llll}5.158797 & 2.851223 & -1.826110\end{array}$

$\begin{array}{llll}0.850332 & 5.306887 & -1.250258\end{array}$

$\begin{array}{lll}4.685488 & -2.114191 & 1.585022\end{array}$

$\begin{array}{llll}3.036354 & -4.533669 & -2.473664\end{array}$

$\begin{array}{llll}2.767297 & -5.681995 & -1.802398\end{array}$

$\begin{array}{llll}2.152638 & -4.456438 & -3.493313\end{array}$

$\begin{array}{llll}4.266182 & -4.689352 & -3.017923\end{array}$

$\begin{array}{llll}5.664375 & -1.209349 & 1.330050\end{array}$

$\begin{array}{llll}5.293596 & -3.281664 & 1.881554\end{array}$

$\begin{array}{llll}4.059927 & -1.688185 & 2.710551\end{array}$

$\begin{array}{llll}5.544164 & 2.871138 & -3.123663\end{array}$

$\begin{array}{llll}5.732715 & 1.760172 & -1.264539\end{array}$

$\begin{array}{llll}5.712245 & 3.939446 & -1.238187\end{array}$

$\begin{array}{llll}1.174380 & 6.199526 & -2.215273\end{array}$

$\begin{array}{llll}-0.490340 & 5.154641 & -1.270998\end{array}$

$\begin{array}{llll}1.164161 & 5.896366 & -0.066547\end{array}$

$\begin{array}{llll}1.867741 & 2.331564 & 2.007923\end{array}$

$\begin{array}{lll}0.921887 & 3.389234 & 2.090105\end{array}$

$\begin{array}{lll}-0.466824 & 3.354346 & 1.836557\end{array}$

$\begin{array}{llll}1.303159 & 4.376397 & 2.312195\end{array}$

$\begin{array}{lll}3.211539 & 2.654833 & 1.952075\end{array}$

$\begin{array}{lll}4.230002 & 1.626809 & 2.098786\end{array}$

$\begin{array}{lll}5.037772 & 1.997945 & 2.740952\end{array}$

$\begin{array}{llll}3.803899 & 0.738459 & 2.558436\end{array}$

$\begin{array}{lll}4.668874 & 1.348707 & 1.131290\end{array}$

$\begin{array}{lll}3.715920 & 4.005093 & 1.754576\end{array}$

$\begin{array}{llll}3.001227 & 4.612657 & 1.200611\end{array}$

$\begin{array}{lll}3.947165 & 4.507694 & 2.705479\end{array}$

$\begin{array}{llll}4.634269 & 3.958551 & 1.160769\end{array}$

$\begin{array}{lll}1.571595 & 1.083770 & 1.990759\end{array}$

$\begin{array}{lll}-1.205962 & 4.584172 & 1.939538\end{array}$

$\begin{array}{lll}-2.548484 & 4.649459 & 1.658237\end{array}$

$\begin{array}{lll}-0.674118 & 5.484054 & 2.232165\end{array}$

$\begin{array}{lll}-3.194220 & 3.463585 & 1.266040\end{array}$

$\begin{array}{lll}-2.473897 & 2.293684 & 1.174239\end{array}$

$\begin{array}{llll}-2.946877 & 1.365874 & 0.878696\end{array}$

$\begin{array}{lll}-1.152590 & 2.223620 & 1.442522\end{array}$

$\begin{array}{lll}-3.088966 & 5.587413 & 1.734056\end{array}$

$\begin{array}{llll}-5.117073 & 3.460078 & 0.855596\end{array}$

Conformer_20

Number of imaginary frequencies : 0

The smallest frequency is : $15.0427 \mathrm{~cm}(-1)$

Electronic energy : $\quad \mathrm{HF}=-4521.713831$
Zero-point correction $=$

(Hartree/Particle)

Thermal correction to Energy= $\quad 0.924103$

Thermal correction to Enthalpy=

0.925047

Thermal correction to Gibbs Free Energy=

Sum of electronic and zero-point Energies=

Sum of electronic and thermal Energies=

Sum of electronic and thermal Enthalpies=

Sum of electronic and thermal Free Energies=

0.744598

$-4520.859096$

$-4520.789728$

$-4520.788784$

Cartesian Coordinates

$\begin{array}{rrr}-3.560730 & -1.211117 & -2.713246\end{array}$

$\begin{array}{lll}-1.139101 & -1.582565 & 1.924984\end{array}$

$\begin{array}{llll}0.658045 & 0.064578 & -0.920077\end{array}$

$\begin{array}{lll}-2.336466 & -1.539148 & -1.042933\end{array}$

$\begin{array}{lll}-1.845496 & -0.444722 & -1.848768\end{array}$

$\begin{array}{lll}-2.866204 & 0.552782 & -1.901480\end{array}$

$\begin{array}{llll}-2.807379 & 1.489377 & -2.438437\end{array}$

$\begin{array}{lll}-3.994372 & 0.077683 & -1.168623\end{array}$

$\begin{array}{lll}-4.939728 & 0.590149 & -1.057015\end{array}$

$\begin{array}{llll}-3.670739 & -1.207133 & -0.642406\end{array}$

$\begin{array}{lll}-4.326406 & -1.836484 & -0.060518\end{array}$

$\begin{array}{lll}-3.126103 & -2.160180 & -4.509168\end{array}$

$\begin{array}{lll}-2.128840 & -2.417992 & -4.840915\end{array}$

$\begin{array}{lll}-3.796268 & -0.921873 & -4.750762\end{array}$

$\begin{array}{llll}-3.393801 & -0.075773 & -5.291692\end{array}$

$\begin{array}{lll}-5.071998 & -0.977314 & -4.110464\end{array}$

$\begin{array}{llll}-5.800935 & -0.178313 & -4.076864\end{array}$

$\begin{array}{llll}-5.190849 & -2.249534 & -3.472050\end{array}$

$\begin{array}{llll}-6.027127 & -2.584971 & -2.872918\end{array}$

$\begin{array}{lll}-3.990597 & -2.980669 & -3.719177\end{array}$

$\begin{array}{llll}-3.764533 & -3.969130 & -3.342802\end{array}$

$\begin{array}{llll}-1.515134 & -2.728331 & -0.669712\end{array}$

$\begin{array}{llll}-1.178505 & -3.658951 & -1.665397\end{array}$

$\begin{array}{llll}-1.584312 & -3.512571 & -2.658433\end{array}$

$-0.323997-4.732121-1.415698$

$-0.057214 \quad-5.412736-2.218205$

$\begin{array}{llll}0.202689 & -4.905356 & -0.138101\end{array}$

$\begin{array}{lll}0.879864 & -5.727303 & 0.071819\end{array}$

$\begin{array}{lll}-0.121961 & -3.998473 & 0.870697\end{array}$

$\begin{array}{llll}0.311074 & -4.137121 & 1.854704\end{array}$

$\begin{array}{lll}-0.963135 & -2.899267 & 0.629740\end{array}$

$\begin{array}{lll}-0.450023 & -0.353795 & -2.404031\end{array}$

$\begin{array}{lll}-0.157053 & -1.358236 & -2.715461\end{array}$

$\begin{array}{lll}-0.310659 & 0.585528 & -3.604457\end{array}$

$\begin{array}{llll}0.712307 & 0.577117 & -3.991258\end{array}$

$\begin{array}{llll}-0.563926 & 1.618702 & -3.351499\end{array}$

$\begin{array}{lll}-0.986618 & 0.258001 & -4.400656\end{array}$

$\begin{array}{lll}-0.242222 & -2.256450 & 3.387847\end{array}$

$\begin{array}{lll}1.022575 & -1.723620 & 3.681370\end{array}$

$\begin{array}{lll}1.421646 & -0.916995 & 3.075220\end{array}$

$\begin{array}{lll}1.756237 & -2.209300 & 4.767002\end{array}$

$\begin{array}{llll}2.736514 & -1.794150 & 4.979484\end{array}$

$\begin{array}{llll}1.230677 & -3.220201 & 5.573008\end{array}$

$\begin{array}{llll}1.799894 & -3.593688 & 6.419816\end{array}$

$\begin{array}{lll}-0.034241 & -3.747380 & 5.293422\end{array}$

$\begin{array}{lll}-0.449955 & -4.530438 & 5.921581\end{array}$

$\begin{array}{lll}-0.767748 & -3.269846 & 4.207438\end{array}$

$\begin{array}{lll}-1.748991 & -3.686155 & 4.002347\end{array}$

$\begin{array}{lll}-2.896226 & -1.664372 & 2.456353\end{array}$

$\begin{array}{lll}-3.453684 & -0.513623 & 3.032806\end{array}$

$\begin{array}{lll}-2.845853 & 0.380132 & 3.141933\end{array}$

$\begin{array}{lll}-4.785254 & -0.509250 & 3.452945\end{array}$

$\begin{array}{llll}-5.208846 & 0.389664 & 3.891883\end{array}$

$\begin{array}{lll}-5.570592 & -1.654804 & 3.300110\end{array}$

$\begin{array}{llll}-6.608960 & -1.649154 & 3.619231\end{array}$

$\begin{array}{lll}-5.017691 & -2.809452 & 2.737259\end{array}$

$\begin{array}{lll}-5.623735 & -3.703830 & 2.622057\end{array}$

$\begin{array}{lll}-3.685866 & -2.816490 & 2.318532\end{array}$

$\begin{array}{lll}-3.267549 & -3.711294 & 1.866720\end{array}$

$\begin{array}{llll}1.960278 & -1.235597 & -0.854760\end{array}$

$\begin{array}{llll}2.771646 & -1.216574 & 0.290160\end{array}$

$\begin{array}{lll}2.616915 & -0.436444 & 1.030953\end{array}$

$\begin{array}{lll}3.754015 & -2.188926 & 0.473621\end{array}$ 
$\begin{array}{lll}3.920978 & -3.218952 & -0.451022\end{array}$

$\begin{array}{llll}4.670959 & -3.986211 & -0.295148\end{array}$

$\begin{array}{llll}3.097849 & -3.247728 & -1.576872\end{array}$

$\begin{array}{llll}2.137701 & -2.257878 & -1.793230\end{array}$

$\begin{array}{llll}1.533184 & -2.306365 & -2.689631\end{array}$

$\begin{array}{llll}1.592504 & 1.591476 & -1.344615\end{array}$

$\begin{array}{llll}0.877611 & 2.797846 & -1.271277\end{array}$

$\begin{array}{lll}-0.182765 & 2.786283 & -1.047413\end{array}$

$\begin{array}{llll}1.534595 & 4.014735 & -1.421970\end{array}$

$\begin{array}{llll}2.917667 & 4.061849 & -1.607360\end{array}$

$\begin{array}{llll}3.432449 & 5.013270 & -1.678329\end{array}$

$\begin{array}{llll}3.629300 & 2.865953 & -1.650810\end{array}$

$\begin{array}{llll}2.975330 & 1.635796 & -1.543588\end{array}$

$\begin{array}{llll}3.559009 & 0.723598 & -1.573981\end{array}$

$\begin{array}{lll}-0.307185 & 0.377595 & 1.179427\end{array}$

$\begin{array}{llll}5.124924 & 2.896546 & -1.798673\end{array}$

$\begin{array}{llll}0.787461 & 5.307573 & -1.251498\end{array}$

$\begin{array}{lll}4.670747 & -2.074482 & 1.659659\end{array}$

$\begin{array}{llll}3.223130 & -4.403905 & -2.529629\end{array}$

$\begin{array}{llll}2.707711 & -5.543959 & -2.001264\end{array}$

$\begin{array}{llll}2.575736 & -4.186503 & -3.695689\end{array}$

$\begin{array}{llll}4.515191 & -4.673162 & -2.826899\end{array}$

$\begin{array}{llll}5.647944 & -1.157466 & 1.443466\end{array}$

$\begin{array}{llll}5.282823 & -3.240416 & 1.955346\end{array}$

$\begin{array}{llll}4.011190 & -1.670923 & 2.773356\end{array}$

$\begin{array}{llll}5.515368 & 2.907917 & -3.094826\end{array}$

$\begin{array}{llll}5.708331 & 1.817313 & -1.224258\end{array}$

$\begin{array}{llll}5.663880 & 3.996427 & -1.219125\end{array}$

$\begin{array}{llll}1.110429 & 6.203225 & -2.214076\end{array}$

$\begin{array}{llll}-0.551353 & 5.141434 & -1.283306\end{array}$

$\begin{array}{llll}1.085479 & 5.900479 & -0.065436\end{array}$

$\begin{array}{lll}1.812261 & 2.347722 & 2.029680\end{array}$

$\begin{array}{lll}0.858700 & 3.399464 & 2.098388\end{array}$

$\begin{array}{lll}-0.526934 & 3.352923 & 1.830111\end{array}$

$\begin{array}{llll}1.230793 & 4.390320 & 2.319846\end{array}$

$\begin{array}{lll}3.154250 & 2.678017 & 1.979135\end{array}$

$\begin{array}{lll}4.177369 & 1.655287 & 2.130833\end{array}$

$\begin{array}{lll}4.985318 & 2.034999 & 2.767579\end{array}$

$\begin{array}{lll}3.756590 & 0.768644 & 2.598770\end{array}$

$\begin{array}{lll}4.614630 & 1.371582 & 1.164209\end{array}$

$\begin{array}{lll}3.652529 & 4.028995 & 1.770886\end{array}$

$\begin{array}{llll}2.933033 & 4.630165 & 1.216211\end{array}$

$\begin{array}{lll}3.885581 & 4.538165 & 2.717772\end{array}$

$\begin{array}{llll}4.568700 & 3.982781 & 1.173569\end{array}$

$\begin{array}{llll}1.522985 & 1.098211 & 2.021346\end{array}$

$\begin{array}{lll}-1.277414 & 4.576554 & 1.923568\end{array}$

$\begin{array}{lll}-2.616167 & 4.631279 & 1.622794\end{array}$

$\begin{array}{lll}-0.757031 & 5.480468 & 2.224232\end{array}$

$\begin{array}{llll}-3.246747 & 3.440610 & 1.220381\end{array}$

$\begin{array}{lll}-2.516253 & 2.276190 & 1.139989\end{array}$

$\begin{array}{lll}-2.977184 & 1.344470 & 0.837424\end{array}$

$\begin{array}{lll}-1.198681 & 2.216455 & 1.428284\end{array}$

$\begin{array}{lll}-3.165101 & 5.564865 & 1.691242\end{array}$

$\begin{array}{lll}-5.162693 & 3.422809 & 0.779180\end{array}$

Conformer_21

Number of imaginary frequencies : 0

The smallest frequency is : $13.2479 \mathrm{~cm}(-1)$

Electronic energy :

$\mathrm{HF}=-4521.7138804$

Zero-point correction=

(Hartree/Particle)

Thermal correction to Energy=

Thermal correction to Enthalpy=

0.923920

0.924865

Thermal correction to Gibbs Free Energy=

Sum of electronic and zero-point Energies=

Sum of electronic and thermal Energies=

0.744403

$-4520.859370$

$-4520.789960$

Sum of electronic and thermal Enthalpies=

$-4520.789016$

Sum of electronic and thermal Free Energies=

$-4520.969478$

Cartesian Coordinates

$\begin{array}{lllr}26 & -3.598254 & -1.129946 & -2.692206 \\ 15 & -1.125885 & -1.576382 & 1.911808 \\ 15 & 0.662600 & 0.066133 & -0.938115\end{array}$

$\begin{array}{llll}-2.360176 & -1.491914 & -1.039594\end{array}$

$\begin{array}{llll}-1.858792 & -0.400212 & -1.842454\end{array}$

$\begin{array}{llll}-2.862392 & 0.615326 & -1.876256\end{array}$

$\begin{array}{llll}-2.793123 & 1.554900 & -2.406766\end{array}$

$\begin{array}{llll}-3.990146 & 0.154904 & -1.133581\end{array}$

$\begin{array}{llll}-4.924629 & 0.683314 & -1.006592\end{array}$

$\begin{array}{llll}-3.683263 & -1.139109 & -0.620325\end{array}$

$\begin{array}{lll}-4.342941 & -1.761045 & -0.034941\end{array}$

$\begin{array}{llll}-3.205777 & -2.086766 & -4.493789\end{array}$

$-2.219372 \quad-2.371848 \quad-4.835751$

$\begin{array}{lll}-3.843011 & -0.829756 & -4.727304\end{array}$

$\begin{array}{llll}-3.421802 & 0.005749 & -5.270361\end{array}$

$\begin{array}{llll}-5.114195 & -0.850874 & -4.076095\end{array}$

$\begin{array}{llll}-5.819953 & -0.031687 & -4.034208\end{array}$

$\begin{array}{llll}-5.263206 & -2.120610 & -3.439191\end{array}$

$\begin{array}{lll}-6.103382 & -2.433800 & -2.833468\end{array}$

$\begin{array}{lll}-4.085894 & -2.884446 & -3.697749\end{array}$

$\begin{array}{lll}-3.883951 & -3.879630 & -3.325196\end{array}$

$\begin{array}{llll}-1.560782 & -2.701279 & -0.683212\end{array}$

$\begin{array}{llll}-1.263902 & -3.638939 & -1.684762\end{array}$

$\begin{array}{lll}-1.677311 & -3.478942 & -2.672431\end{array}$

$\begin{array}{lll}-0.440885 & -4.739348 & -1.446933\end{array}$

$\begin{array}{llll}-0.207892 & -5.427187 & -2.253760\end{array}$

$\begin{array}{llll}0.097030 & -4.930662 & -0.176840\end{array}$

$\begin{array}{llll}0.751804 & -5.772894 & 0.022893\end{array}$

$\begin{array}{lll}-0.185802 & -4.015017 & 0.836997\end{array}$

$\begin{array}{llll}0.255767 & -4.168273 & 1.815038\end{array}$

$\begin{array}{llll}-0.996956 & -2.891032 & 0.608795\end{array}$

$\begin{array}{lll}-0.467722 & -0.327292 & -2.411244\end{array}$

$\begin{array}{llll}-0.193122 & -1.334309 & -2.729433\end{array}$

$\begin{array}{llll}-0.325465 & 0.614805 & -3.609081\end{array}$

$\begin{array}{llll}0.693010 & 0.590771 & -4.006868\end{array}$

$\begin{array}{llll}-0.558458 & 1.650945 & -3.349225\end{array}$

$\begin{array}{llll}-1.015436 & 0.302080 & -4.399115\end{array}$

$\begin{array}{lll}-0.216758 & -2.272866 & 3.356445\end{array}$

$\begin{array}{llll}1.065961 & -1.770936 & 3.625926\end{array}$

$\begin{array}{lll}1.473509 & -0.974678 & 3.011728\end{array}$

$\begin{array}{lll}1.807190 & -2.273740 & 4.698497\end{array}$

$\begin{array}{llll}2.800920 & -1.882710 & 4.892851\end{array}$

$\begin{array}{lll}1.271623 & -3.270601 & 5.515460\end{array}$

$\begin{array}{llll}1.846848 & -3.656834 & 6.352429\end{array}$

$\begin{array}{rrr}-0.010494 & -3.767170 & 5.259578\end{array}$

$\begin{array}{lll}-0.433705 & -4.539322 & 5.896169\end{array}$

$\begin{array}{llll}-0.751628 & -3.272753 & 4.186373\end{array}$

$\begin{array}{lll}-1.746220 & -3.665176 & 3.999270\end{array}$

$\begin{array}{llll}-2.874823 & -1.630491 & 2.472928\end{array}$

$\begin{array}{lll}-3.690080 & -2.764442 & 2.333986\end{array}$

$\begin{array}{lll}-3.298007 & -3.661574 & 1.863744\end{array}$

$\begin{array}{lll}-5.014028 & -2.736062 & 2.776237\end{array}$

$\begin{array}{lll}-5.640215 & -3.616311 & 2.659943\end{array}$

$\begin{array}{lll}-5.533244 & -1.578210 & 3.363947\end{array}$

$\begin{array}{lll}-6.565627 & -1.555792 & 3.701245\end{array}$

$\begin{array}{lll}-4.721991 & -0.450998 & 3.518182\end{array}$

$\begin{array}{lll}-5.119473 & 0.450220 & 3.976360\end{array}$

$\begin{array}{lll}-3.398391 & -0.476557 & 3.074392\end{array}$

$\begin{array}{llll}-2.770545 & 0.403228 & 3.184113\end{array}$

$\begin{array}{lll}1.940523 & -1.258381 & -0.884910\end{array}$

$\begin{array}{llll}2.784058 & -1.232824 & 0.236179\end{array}$

$\begin{array}{llll}2.672246 & -0.429509 & 0.959802\end{array}$

$\begin{array}{lll}3.735623 & -2.234663 & 0.425910\end{array}$

$\begin{array}{llll}3.832991 & -3.304052 & -0.462657\end{array}$

$\begin{array}{llll}4.551746 & -4.098894 & -0.296864\end{array}$

$\begin{array}{llll}2.974155 & -3.340277 & -1.561718\end{array}$

$\begin{array}{llll}2.052252 & -2.318870 & -1.791533\end{array}$

$\begin{array}{lll}1.418150 & -2.381122 & -2.666159\end{array}$

$\begin{array}{llll}1.614236 & 1.582336 & -1.361708\end{array}$

$\begin{array}{llll}0.913185 & 2.796355 & -1.279061\end{array}$

$\begin{array}{llll}-0.146138 & 2.795798 & -1.049706\end{array}$

$\begin{array}{llll}1.582636 & 4.006599 & -1.427899\end{array}$

$\begin{array}{llll}2.965268 & 4.039421 & -1.619648\end{array}$

$\begin{array}{llll}3.489998 & 4.985622 & -1.688210\end{array}$

$\begin{array}{lll}3.663322 & 2.835886 & -1.672234\end{array}$

$\begin{array}{llll}2.996276 & 1.612432 & -1.568259\end{array}$

$\begin{array}{llll}3.569863 & 0.694243 & -1.606751\end{array}$

$\begin{array}{lll}-0.276349 & 0.379523 & 1.173168\end{array}$ 
$\begin{array}{rrr}5.158637 & 2.851156 & -1.826140 \\ 0.850210 & 5.306903 & -1.250380 \\ 4.685359 & -2.114423 & 1.584999 \\ 3.036590 & -4.533201 & -2.474264 \\ 2.767463 & -5.681667 & -1.803260 \\ 2.152978 & -4.455764 & -3.493986 \\ 4.266475 & -4.688772 & -3.018424 \\ 5.664313 & -1.209618 & 1.330140 \\ 5.293394 & -3.281956 & 1.881456 \\ 4.059778 & -1.688476 & 2.710534 \\ 5.543965 & 2.871086 & -3.123706 \\ 5.732559 & 1.760091 & -1.264603 \\ 5.712119 & 3.939368 & -1.238224 \\ 1.174494 & 6.199583 & -2.215278 \\ -0.490463 & 5.154707 & -1.271416 \\ 1.163810 & 5.896305 & -0.066571 \\ 1.867853 & 2.331615 & 2.007908 \\ 0.921985 & 3.389260 & 2.090164 \\ -0.466733 & 3.354361 & 1.836640 \\ 1.303262 & 4.376433 & 2.312205 \\ 3.211641 & 2.654895 & 1.951995 \\ 4.230110 & 1.626853 & 2.098544 \\ 5.038006 & 1.997997 & 2.740543 \\ 3.804080 & 0.738525 & 2.558310 \\ 4.668789 & 1.348704 & 1.130975 \\ 3.715992 & 4.005159 & 1.754443 \\ 3.001247 & 4.612708 & 1.200526 \\ 3.947300 & 4.507773 & 2.705324 \\ 4.634293 & 3.958624 & 1.160565 \\ 1.571738 & 1.083811 & 1.990736 \\ -1.205847 & 4.584205 & 1.939541 \\ -2.548363 & 4.649497 & 1.658214 \\ -0.673991 & 5.484098 & 2.232112 \\ -3.194121 & 3.463609 & 1.266098 \\ -2.473834 & 2.293673 & 1.174430 \\ -2.946829 & 1.365824 & 0.879040 \\ -1.152527 & 2.223614 & 1.442713 \\ -3.088827 & 5.587468 & 1.733953 \\ -5.116963 & 3.460147 & 0.855599\end{array}$

Conformer_22

Number of imaginary frequencies : 0

The smallest frequency is : $13.2641 \mathrm{~cm}(-1)$

Electronic energy :

$\mathrm{HF}=-4521.7138803$

Zero-point correction=

(Hartree/Particle)

Thermal correction to Energy=

Thermal correction to Enthalpy=

0.923917

0.924861

Thermal correction to Gibbs Free Energy=

Sum of electronic and zero-point Energies=

Sum of electronic and thermal Energies=

0.744391

Sum of electronic and thermal Enthalpies=

$-4520.859374$

$-4520.789963$

$-4520.789019$

Sum of electronic and thermal Free Energies=

$-4520.969489$

Cartesian Coordinates

$\begin{array}{lll}-3.598107 & -1.129979 & -2.692357\end{array}$

$\begin{array}{lll}-1.125952 & -1.576019 & 1.911885\end{array}$

$\begin{array}{llll}0.662785 & 0.066022 & -0.938391\end{array}$

$\begin{array}{llll}-2.359937 & -1.491581 & -1.039729\end{array}$

$\begin{array}{lll}-1.858479 & -0.400176 & -1.842955\end{array}$

$\begin{array}{llll}-2.861970 & 0.615470 & -1.876974\end{array}$

$\begin{array}{llll}-2.792626 & 1.554876 & -2.407771\end{array}$

$\begin{array}{llll}-3.989738 & 0.155389 & -1.134115\end{array}$

$\begin{array}{lll}-4.924160 & 0.683943 & -1.007270\end{array}$

$\begin{array}{llll}-3.682973 & -1.138501 & -0.620467\end{array}$

$\begin{array}{llll}-4.342759 & -1.760247 & -0.035005\end{array}$

$\begin{array}{llll}-3.206058 & -2.087466 & -4.493690\end{array}$

$\begin{array}{lll}-2.219767 & -2.372789 & -4.835781\end{array}$

$\begin{array}{llll}-3.843214 & -0.830473 & -4.727523\end{array}$

$\begin{array}{llll}-3.422022 & 0.004797 & -5.270958\end{array}$

$\begin{array}{llll}-5.114273 & -0.851244 & -4.076063\end{array}$

$\begin{array}{llll}-5.819938 & -0.031968 & -4.034313\end{array}$

$\begin{array}{lll}-5.263280 & -2.120740 & -3.438679\end{array}$

$\begin{array}{lll}-6.103366 & -2.433636 & -2.832679\end{array}$

$\begin{array}{llll}-4.086093 & -2.884778 & -3.697194\end{array}$

$\begin{array}{lll}-3.884158 & -3.879844 & -3.324321\end{array}$

$\begin{array}{llll}-1.560692 & -2.700988 & -0.683100\end{array}$

$\begin{array}{lll}-1.263823 & -3.638746 & -1.684568\end{array}$

$-1.677143-3.478753 \quad-2.672273$

$\begin{array}{lll}-0.440991 & -4.739270 & -1.446622\end{array}$

$\begin{array}{lll}-0.208027 & -5.427177 & -2.253397\end{array}$

$\begin{array}{llll}0.096772 & -4.930599 & -0.176465\end{array}$

$\begin{array}{lll}0.751392 & -5.772923 & 0.023390\end{array}$

$\begin{array}{lll}-0.186029 & -4.014843 & 0.837286\end{array}$

$\begin{array}{llll}0.255392 & -4.168119 & 1.815394\end{array}$

$\begin{array}{lll}-0.996978 & -2.890749 & 0.608943\end{array}$

$-0.467350 \quad-0.327354-2.411665$

$\begin{array}{llll}-0.192732 & -1.334378 & -2.729812\end{array}$

$\begin{array}{lll}-0.324946 & 0.614745 & -3.609480\end{array}$

$\begin{array}{llll}0.693538 & 0.590600 & -4.007237\end{array}$

$\begin{array}{llll}-0.557834 & 1.650904 & -3.349613\end{array}$

$\begin{array}{llll}-1.014928 & 0.302106 & -4.399537\end{array}$

$\begin{array}{lll}-0.217226 & -2.272468 & 3.356740\end{array}$

$\begin{array}{lll}1.065724 & -1.771060 & 3.626084\end{array}$

$\begin{array}{llll}1.473541 & -0.975027 & 3.011768\end{array}$

$\begin{array}{llll}1.806783 & -2.274098 & 4.698662\end{array}$

$\begin{array}{llll}2.800694 & -1.883500 & 4.892950\end{array}$

$\begin{array}{lll}1.270811 & -3.270667 & 5.515720\end{array}$

$\begin{array}{llll}1.845914 & -3.657095 & 6.352682\end{array}$

$\begin{array}{llll}1.0 .011540 & -3.766694 & 5.259959\end{array}$

$\begin{array}{lll}-0.435046 & -4.538606 & 5.896647\end{array}$

$\begin{array}{lll}-0.752532 & -3.272036 & 4.186765\end{array}$

$\begin{array}{lll}-1.747308 & -3.664003 & 3.999726\end{array}$

$\begin{array}{lll}-2.875038 & -1.630282 & 2.472583\end{array}$

$\begin{array}{lll}-3.398822 & -0.476490 & 3.074116\end{array}$

$\begin{array}{lll}-2.771038 & 0.403309 & 3.184111\end{array}$

$\begin{array}{lll}-4.722522 & -0.451086 & 3.517602\end{array}$

$\begin{array}{lll}-5.120181 & 0.450041 & 3.975807\end{array}$

$\begin{array}{lll}-5.533649 & -1.578340 & 3.363015\end{array}$

$\begin{array}{lll}-6.566122 & -1.556039 & 3.700043\end{array}$

$\begin{array}{lll}-5.014185 & -2.736103 & 2.775349\end{array}$

$\begin{array}{lll}-5.640263 & -3.616406 & 2.658873\end{array}$

$\begin{array}{lll}-3.690123 & -2.764334 & 2.333417\end{array}$

$\begin{array}{lll}-3.297818 & -3.661438 & 1.863321\end{array}$

$\begin{array}{lll}1.940648 & -1.258533 & -0.884876\end{array}$

$\begin{array}{llll}2.784066 & -1.232860 & 0.236300\end{array}$

$\begin{array}{llll}2.672186 & -0.429445 & 0.959802\end{array}$

$\begin{array}{llll}3.735574 & -2.234715 & 0.426251\end{array}$

$\begin{array}{llll}3.832989 & -3.304218 & -0.462177\end{array}$

$\begin{array}{llll}4.551693 & -4.099068 & -0.296198\end{array}$

$\begin{array}{llll}2.974272 & -3.340545 & -1.561324\end{array}$

$2.052441 \quad-2.319126 \quad-1.791369$

$\begin{array}{llll}1.418438 & -2.381490 & -2.666058\end{array}$

$\begin{array}{llll}1.614556 & 1.582182 & -1.361889\end{array}$

$\begin{array}{llll}0.913560 & 2.796228 & -1.279253\end{array}$

$\begin{array}{llll}-0.145776 & 2.795699 & -1.049967\end{array}$

$\begin{array}{llll}1.583072 & 4.006448 & -1.428008\end{array}$

$\begin{array}{llll}2.965720 & 4.039205 & -1.619658\end{array}$

$\begin{array}{llll}3.490499 & 4.985381 & -1.688174\end{array}$

$\begin{array}{llll}3.663724 & 2.835641 & -1.672210\end{array}$

$\begin{array}{llll}2.996616 & 1.612213 & -1.568320\end{array}$

$3.570163 \quad 0.693996-1.606773$

$\begin{array}{lll}-0.276576 & 0.379715 & 1.172530\end{array}$

$\begin{array}{llll}5.159056 & 2.850863 & -1.825955\end{array}$

$\begin{array}{llll}0.850685 & 5.306778 & -1.250510\end{array}$

$\begin{array}{lll}4.685219 & -2.114396 & 1.585407\end{array}$

$\begin{array}{llll}3.036787 & -4.533562 & -2.473745\end{array}$

$\begin{array}{llll}2.767999 & -5.682000 & -1.802568\end{array}$

$\begin{array}{llll}2.152992 & -4.456410 & -3.493332\end{array}$

$\begin{array}{llll}4.266610 & -4.688946 & -3.018103\end{array}$

$\begin{array}{llll}5.664239 & -1.209669 & 1.330538\end{array}$

$\begin{array}{lll}5.293168 & -3.281931 & 1.882037\end{array}$

$\begin{array}{lll}4.059569 & -1.688305 & 2.710851\end{array}$

$\begin{array}{llll}5.544529 & 2.870919 & -3.123474\end{array}$

$\begin{array}{llll}5.732866 & 1.759710 & -1.264474\end{array}$

$\begin{array}{llll}5.712520 & 3.938985 & -1.237851\end{array}$

$\begin{array}{llll}1.175021 & 6.199440 & -2.215407\end{array}$

$\begin{array}{llll}-0.489990 & 5.154617 & -1.271564\end{array}$ 


$\begin{array}{cccc}9 & 1.164278 & 5.896180 & -0.066701 \\ 6 & 1.867690 & 2.331622 & 2.007839 \\ 6 & 0.921850 & 3.389304 & 2.090048 \\ 6 & -0.466864 & 3.354469 & 1.836518 \\ 1 & 1.303153 & 4.376454 & 2.312150 \\ 7 & 3.211491 & 2.654893 & 1.952013 \\ 6 & 4.229943 & 1.626849 & 2.098676 \\ 1 & 5.037762 & 1.997981 & 2.740780 \\ 1 & 3.803855 & 0.738520 & 2.558385 \\ 1 & 4.668734 & 1.348708 & 1.131157 \\ 6 & 3.715861 & 4.005179 & 1.754655 \\ 1 & 3.001252 & 4.612734 & 1.200568 \\ 1 & 3.946894 & 4.507765 & 2.705619 \\ 1 & 4.634329 & 3.958689 & 1.161033 \\ 8 & 1.571534 & 1.083834 & 1.990630 \\ 6 & -1.205931 & 4.584336 & 1.939512 \\ 6 & -2.548449 & 4.649707 & 1.658231 \\ 1 & -0.674031 & 5.484188 & 2.232132 \\ 6 & -3.194271 & 3.463870 & 1.266064 \\ 6 & -2.474033 & 2.293915 & 1.174286 \\ 1 & -2.947101 & 1.366111 & 0.878847 \\ 7 & -1.152713 & 2.223774 & 1.442529 \\ 1 & -3.088871 & 5.587695 & 1.734051 \\ 35 & -5.117138 & 3.460535 & 0.855667 \\ -------------------------------------------------------\end{array}$

\section{Conformer_23}

Number of imaginary frequencies : 0

The smallest frequency is : $13.2967 \mathrm{~cm}(-1)$

Electronic energy :

$\mathrm{HF}=-4521.7138801$

Zero-point correction=

(Hartree/Particle)

Thermal correction to Energy=

Thermal correction to Enthalpy=

Thermal correction to Gibbs Free Energy=
Sum of electronic and zero-point Energies

Sum of electronic and thermal Energies=

Sum of electronic and thermal Enthalpies=

0.923921

0.924865

0.744416

$-4520.859369$

$-4520.789960$

$-4520.789015$

Sum of electronic and thermal Free Energies=

$-4520.969464$

Cartesian Coordinates

$\begin{array}{lll}-3.597807 & -1.130067 & -2.692668\end{array}$

$\begin{array}{lll}-1.125919 & -1.576613 & 1.911561\end{array}$

$\begin{array}{llll}0.662729 & 0.066411 & -0.937955\end{array}$

$\begin{array}{llll}-2.359934 & -1.492131 & -1.039922\end{array}$

$\begin{array}{lll}-1.858543 & -0.400272 & -1.842570\end{array}$

$\begin{array}{lll}-2.862234 & 0.615172 & -1.876377\end{array}$

$\begin{array}{llll}-2.792984 & 1.554817 & -2.406764\end{array}$

$\begin{array}{llll}-3.990046 & 0.154536 & -1.133924\end{array}$

$\begin{array}{llll}-4.924594 & 0.682852 & -1.007007\end{array}$

$\begin{array}{llll}-3.683112 & -1.139517 & -0.620790\end{array}$

$-4.342788-1.761573-0.035534$

$\begin{array}{llll}-3.204938 & -2.086411 & -4.494416\end{array}$

$-2.218431-2.371228 \quad-4.836301$

$-3.842374-0.829463-4.727720$

$\begin{array}{lll}-3.421243 & 0.006250 & -5.270518\end{array}$

$\begin{array}{lll}-5.113632 & -0.850957 & -4.076668\end{array}$

$\begin{array}{llll}-5.819537 & -0.031901 & -4.034674\end{array}$

$\begin{array}{llll}-5.262492 & -2.120867 & -3.440075\end{array}$

$\begin{array}{llll}-6.102684 & -2.434344 & -2.834523\end{array}$

$\begin{array}{llll}-4.085015 & -2.884434 & -3.698675\end{array}$

$\begin{array}{llll}-3.882954 & -3.879676 & -3.326342\end{array}$

$\begin{array}{llll}-1.560389 & -2.701408 & -0.683589\end{array}$

$\begin{array}{llll}-1.263301 & -3.638948 & -1.685189\end{array}$

$\begin{array}{llll}-1.676737 & -3.478986 & -2.672853\end{array}$

$\begin{array}{llll}-0.440004 & -4.739164 & -1.447427\end{array}$

$\begin{array}{llll}-0.206856 & -5.426907 & -2.254290\end{array}$

$\begin{array}{llll}0.097994 & -4.930390 & -0.177355\end{array}$

$\begin{array}{llll}0.753012 & -5.772448 & 0.022315\end{array}$

$\begin{array}{lll}-0.185089 & -4.014888 & 0.836546\end{array}$

$\begin{array}{lll}0.256526 & -4.168096 & 1.814574\end{array}$

$\begin{array}{llll}-0.996555 & -2.891112 & 0.608417\end{array}$

$-0.467414-0.327198 \quad-2.411191$

$\begin{array}{llll}-0.192693 & -1.334209 & -2.729316\end{array}$

$\begin{array}{lll}-0.325123 & 0.614894 & -3.609029\end{array}$

$\begin{array}{llll}0.693405 & 0.590979 & -4.006690\end{array}$

$\begin{array}{llll}-0.558273 & 1.651009 & -3.349215\end{array}$

$\begin{array}{lll}-1.014954 & 0.302079 & -4.399149\end{array}$

$\begin{array}{lll}-0.217187 & -2.273313 & 3.356330\end{array}$

$\begin{array}{lll}1.065510 & -1.771510 & 3.626138\end{array}$

$\begin{array}{llll}1.473166 & -0.975081 & 3.012235\end{array}$

$\begin{array}{lll}1.806548 & -2.274669 & 4.698678\end{array}$

$\begin{array}{llll}2.800248 & -1.883719 & 4.893335\end{array}$

$\begin{array}{lll}1.270833 & -3.271800 & 5.515213\end{array}$

$\begin{array}{lll}1.845920 & -3.658324 & 6.352142\end{array}$

$\begin{array}{llll}-0.011247 & -3.768276 & 5.258957\end{array}$

$\begin{array}{lll}-0.434558 & -4.540648 & 5.895217\end{array}$

$\begin{array}{lll}-0.752224 & -3.273470 & 4.185824\end{array}$

$\begin{array}{lll}-1.746788 & -3.665800 & 3.998429\end{array}$

$\begin{array}{lll}-2.874955 & -1.631066 & 2.472357\end{array}$

$\begin{array}{lll}-3.689935 & -2.765202 & 2.333289\end{array}$

$\begin{array}{llll}-3.297639 & -3.662210 & 1.862977\end{array}$

$\begin{array}{lll}-5.013877 & -2.737200 & 2.775577\end{array}$

$\begin{array}{lll}-5.639850 & -3.617590 & 2.659201\end{array}$

$\begin{array}{lll}-5.533363 & -1.579542 & 3.363440\end{array}$

$\begin{array}{lll}-6.565742 & -1.557419 & 3.700768\end{array}$

$\begin{array}{lll}-4.722381 & -0.452155 & 3.517801\end{array}$

$\begin{array}{llll}-5.120072 & 0.448907 & 3.976106\end{array}$

$\begin{array}{lll}-3.398779 & -0.477341 & 3.073981\end{array}$

$\begin{array}{lll}-2.771114 & 0.402561 & 3.183809\end{array}$

$\begin{array}{llll}1.940820 & -1.257925 & -0.884677\end{array}$

$\begin{array}{llll}2.784059 & -1.232581 & 0.236636\end{array}$

$\begin{array}{lll}2.671974 & -0.429473 & 0.960445\end{array}$

$\begin{array}{lll}3.735702 & -2.234365 & 0.426293\end{array}$

$\begin{array}{llll}3.833379 & -3.303507 & -0.462541\end{array}$

$\begin{array}{llll}4.552156 & -4.098334 & -0.296764\end{array}$

$\begin{array}{llll}2.974828 & -3.339516 & -1.561828\end{array}$

$\begin{array}{llll}2.052929 & -2.318102 & -1.791608\end{array}$

$\begin{array}{llll}1.419105 & -2.380061 & -2.666454\end{array}$

$\begin{array}{llll}1.614212 & 1.582727 & -1.361473\end{array}$

$\begin{array}{llll}0.913042 & 2.796675 & -1.278788\end{array}$

$\begin{array}{llll}-0.146286 & 2.796000 & -1.049466\end{array}$

$\begin{array}{llll}1.582386 & 4.006987 & -1.427523\end{array}$

$\begin{array}{llll}2.965019 & 4.039945 & -1.619259\end{array}$

$\begin{array}{llll}3.489663 & 4.986194 & -1.687749\end{array}$

$\begin{array}{llll}3.663190 & 2.836483 & -1.671886\end{array}$

$\begin{array}{llll}2.996259 & 1.612960 & -1.567948\end{array}$

$\begin{array}{llll}3.569938 & 0.694823 & -1.606406\end{array}$

$\begin{array}{llll}-0.276619 & 0.379466 & 1.173131\end{array}$

$\begin{array}{llll}5.158506 & 2.851842 & -1.825791\end{array}$

$\begin{array}{llll}0.849869 & 5.307213 & -1.249816\end{array}$

$\begin{array}{lll}4.685247 & -2.114341 & 1.585561\end{array}$

$3.037554 \quad-4.532194 \quad-2.474675$

$\begin{array}{llll}2.768944 & -5.680910 & -1.803897\end{array}$

$\begin{array}{llll}2.153745 & -4.454819 & -3.494230\end{array}$

$\begin{array}{llll}4.267401 & -4.687195 & -3.019086\end{array}$

$\begin{array}{llll}5.664404 & -1.209695 & 1.330913\end{array}$

$\begin{array}{llll}5.293035 & -3.281995 & 1.882063\end{array}$

$\begin{array}{llll}4.059554 & -1.688348 & 2.711007\end{array}$

$\begin{array}{llll}5.543839 & 2.871021 & -3.123366\end{array}$

$\begin{array}{llll}5.732526 & 1.761153 & -1.263617\end{array}$

$\begin{array}{llll}5.711880 & 3.940444 & -1.238506\end{array}$

$\begin{array}{llll}1.173959 & 6.200002 & -2.214677\end{array}$

$\begin{array}{llll}-0.490794 & 5.154904 & -1.270680\end{array}$

$\begin{array}{llll}1.163576 & 5.896538 & -0.065994\end{array}$

$\begin{array}{llll}1.867337 & 2.331433 & 2.008396\end{array}$

$\begin{array}{lll}0.921472 & 3.389102 & 2.090447\end{array}$

$\begin{array}{lll}-0.467232 & 3.354190 & 1.836859\end{array}$

$\begin{array}{llll}1.302731 & 4.376284 & 2.312477\end{array}$

$\begin{array}{lll}3.211141 & 2.654679 & 1.952567\end{array}$

$\begin{array}{llll}4.229578 & 1.626658 & 2.099487\end{array}$

$\begin{array}{lll}5.037370 & 1.997935 & 2.741543\end{array}$

$\begin{array}{lll}3.803465 & 0.738425 & 2.559358\end{array}$

$\begin{array}{llll}4.668423 & 1.348317 & 1.132048\end{array}$

$3.715608 \quad 4.004860 \quad 1.754762$

$\begin{array}{llll}3.000732 & 4.612503 & 1.201128\end{array}$

$\begin{array}{lll}3.947388 & 4.507453 & 2.705539\end{array}$

$\begin{array}{llll}4.633663 & 3.958186 & 1.160500\end{array}$

$\begin{array}{lll}1.571183 & 1.083638 & 1.991359\end{array}$ 


$\begin{array}{cccc}6 & -1.206365 & 4.584029 & 1.939718 \\ 6 & -2.548880 & 4.649300 & 1.658386 \\ 1 & -0.674520 & 5.483935 & 2.232272 \\ 6 & -3.194615 & 3.463399 & 1.266270 \\ 6 & -2.474299 & 2.293485 & 1.174575 \\ 1 & -2.947283 & 1.365649 & 0.879105 \\ 7 & -1.152997 & 2.223435 & 1.442895 \\ 1 & -3.089359 & 5.587263 & 1.734116 \\ 35 & -5.117467 & 3.459890 & 0.855803\end{array}$

\section{Conformer_24}

\begin{tabular}{|c|c|}
\hline \multicolumn{2}{|l|}{$\begin{array}{l}\text { Number of imaginary frequencies : } 0 \\
\text { The smallest frequency is : } 14.4527 \mathrm{~cm}(-1)\end{array}$} \\
\hline Electronic energy : $\quad \mathrm{HF}=-4521.7142872$ & \\
\hline $\begin{array}{l}\text { Zero-point correction= } \\
\text { (Hartree/Particle) }\end{array}$ & 4354 \\
\hline Thermal correction to Energy= & .923813 \\
\hline Thermal correction to Enthalpy $=$ & 0.924757 \\
\hline Thermal correction to Gibbs Free Energy= & 0.744829 \\
\hline Sum of electronic and zero-point Energies $=$ & -4520.859933 \\
\hline Sum of electronic and thermal Energies $=$ & -4520.790474 \\
\hline Sum of electronic and thermal Enthalpies= & -4520.789530 \\
\hline Sum of electronic and thermal Free Energies & -4520.969458 \\
\hline
\end{tabular}

\begin{tabular}{|c|c|c|c|}
\hline \multicolumn{4}{|c|}{ Cartesian Coordinates } \\
\hline 26 & -3.560698 & -1.115072 & -2.732050 \\
\hline 15 & -1.138262 & -1.606845 & 1.888657 \\
\hline 15 & 0.670509 & 0.093662 & -0.911215 \\
\hline 6 & -2.339905 & -1.495191 & -1.070741 \\
\hline 6 & -1.838590 & -0.385418 & -1.848037 \\
\hline 6 & -2.850777 & 0.621823 & -1.876105 \\
\hline 1 & -2.782978 & 1.571190 & -2.389108 \\
\hline 6 & -3.983425 & 0.138354 & -1.155870 \\
\hline 1 & -4.923871 & 0.656692 & -1.030848 \\
\hline 6 & -3.671333 & -1.161974 & -0.661957 \\
\hline 1 & -4.331876 & -1.800056 & -0.095224 \\
\hline 6 & -3.134425 & -2.027323 & -4.548601 \\
\hline 1 & -2.139981 & -2.291407 & -4.883771 \\
\hline 6 & -3.786407 & -0.774342 & -4.762623 \\
\hline 1 & -3.370291 & 0.078781 & -5.281679 \\
\hline 6 & -5.064508 & -0.826632 & -4.126941 \\
\hline 1 & -5.781743 & -0.018036 & -4.075564 \\
\hline 6 & -5.203502 & -2.111971 & -3.519725 \\
\hline 1 & -6.046249 & -2.449718 & -2.931055 \\
\hline 6 & -4.013162 & -2.854184 & -3.781267 \\
\hline 1 & -3.802728 & -3.854876 & -3.428766 \\
\hline 6 & -1.534329 & -2.702892 & -0.724056 \\
\hline 6 & -1.220459 & -3.625514 & -1.734098 \\
\hline 1 & -1.624069 & -3.455986 & -2.724265 \\
\hline 6 & -0.392755 & -4.723447 & -1.500660 \\
\hline 1 & -0.146612 & -5.399922 & -2.313154 \\
\hline 6 & 0.133053 & -4.926815 & -0.227356 \\
\hline 1 & 0.791040 & -5.767319 & -0.030937 \\
\hline 6 & -0.166316 & -4.025484 & 0.794592 \\
\hline 1 & 0.265982 & -4.187623 & 1.775319 \\
\hline 6 & -0.982637 & -2.904395 & 0.571183 \\
\hline 6 & -0.442307 & -0.291881 & -2.400721 \\
\hline 1 & -0.155016 & -1.291639 & -2.730638 \\
\hline 6 & -0.296201 & 0.669407 & -3.582949 \\
\hline 1 & 0.726449 & 0.660634 & -3.970435 \\
\hline 1 & -0.541257 & 1.699317 & -3.309434 \\
\hline 1 & -0.974735 & 0.362937 & -4.385258 \\
\hline 6 & -0.235191 & -2.313237 & 3.332505 \\
\hline 6 & 1.043448 & -1.808342 & 3.615805 \\
\hline 1 & 1.450071 & -1.001963 & 3.014756 \\
\hline 6 & 1.780687 & -2.320495 & 4.686699 \\
\hline 1 & 2.771259 & -1.926939 & 4.891792 \\
\hline 6 & 1.245271 & -3.329611 & 5.488582 \\
\hline 1 & 1.817477 & -3.723096 & 6.324247 \\
\hline 6 & -0.032958 & -3.828945 & 5.219270 \\
\hline 1 & -0.456331 & -4.610576 & 5.844079 \\
\hline 6 & -0.770097 & -3.325290 & 4.147556 \\
\hline 1 & -1.761604 & -3.720352 & 3.950008 \\
\hline
\end{tabular}

$\begin{array}{lll}-2.891599 & -1.686842 & 2.432732\end{array}$

$\begin{array}{lll}-3.693569 & -2.827484 & 2.273339\end{array}$

$\begin{array}{lll}-3.287821 & -3.714810 & 1.796044\end{array}$

$\begin{array}{lll}-5.021180 & -2.818613 & 2.705372\end{array}$

$\begin{array}{lll}-5.636910 & -3.704018 & 2.573383\end{array}$

$\begin{array}{lll}-5.557359 & -1.673769 & 3.303258\end{array}$

$\begin{array}{lll}-6.592462 & -1.666583 & 3.632785\end{array}$

$\begin{array}{lll}-4.759400 & -0.540077 & 3.477941\end{array}$

$\begin{array}{lll}-5.169890 & 0.351045 & 3.944363\end{array}$

$\begin{array}{lll}-3.432187 & -0.546199 & 3.044346\end{array}$

$\begin{array}{lll}-2.814330 & 0.338458 & 3.170347\end{array}$

$\begin{array}{llll}1.954284 & -1.224883 & -0.859196\end{array}$

$\begin{array}{llll}2.791484 & -1.202850 & 0.266624\end{array}$

$\begin{array}{lll}2.667710 & -0.409120 & 0.998935\end{array}$

$\begin{array}{lll}3.750526 & -2.198656 & 0.451341\end{array}$

$\begin{array}{llll}3.862513 & -3.257507 & -0.448202\end{array}$

$\begin{array}{llll}4.587780 & -4.047296 & -0.286618\end{array}$

$3.010131-3.290143-1.552276$

$2.080230-2.274776 \quad-1.776095$

$\begin{array}{llll}1.451267 & -2.333413 & -2.654682\end{array}$

$\begin{array}{llll}1.626286 & 1.610566 & -1.324149\end{array}$

$\begin{array}{llll}0.933128 & 2.827639 & -1.215516\end{array}$

$\begin{array}{llll}-0.120485 & 2.829737 & -0.961657\end{array}$

$\begin{array}{llll}1.601304 & 4.035315 & -1.385788\end{array}$

$\begin{array}{llll}2.976386 & 4.063369 & -1.630113\end{array}$

$\begin{array}{llll}3.500764 & 5.006635 & -1.731281\end{array}$

$\begin{array}{llll}3.664971 & 2.857393 & -1.719326\end{array}$

$\begin{array}{llll}2.999088 & 1.635160 & -1.582497\end{array}$

$\begin{array}{llll}3.565355 & 0.713798 & -1.657574\end{array}$

$\begin{array}{lll}-0.305303 & 0.370252 & 1.185403\end{array}$

$\begin{array}{llll}5.160373 & 2.853264 & -1.858712\end{array}$

$\begin{array}{llll}0.876121 & 5.337377 & -1.192229\end{array}$

$\begin{array}{lll}4.693242 & -2.083847 & 1.616691\end{array}$

$\begin{array}{llll}3.089546 & -4.471452 & -2.478653\end{array}$

$\begin{array}{llll}2.833563 & -5.631119 & -1.822125\end{array}$

$\begin{array}{llll}2.207284 & -4.392702 & -3.499499\end{array}$

$\begin{array}{llll}4.322424 & -4.604901 & -3.021939\end{array}$

$\begin{array}{lll}5.668113 & -1.170550 & 1.375775\end{array}$

$\begin{array}{lll}5.307370 & -3.250374 & 1.904565\end{array}$

$\begin{array}{llll}4.059249 & -1.673419 & 2.742903\end{array}$

$\begin{array}{llll}5.595620 & 1.841548 & -2.642509\end{array}$

$\begin{array}{llll}5.764844 & 2.688759 & -0.649058\end{array}$

$\begin{array}{llll}5.636320 & 4.005295 & -2.376386\end{array}$

$\begin{array}{llll}1.176073 & 6.227326 & -2.167266\end{array}$

$\begin{array}{llll}-0.464923 & 5.187247 & -1.177541\end{array}$

$\begin{array}{llll}1.221395 & 5.929219 & -0.018142\end{array}$

$\begin{array}{llll}1.806721 & 2.320496 & 2.075396\end{array}$

$\begin{array}{lll}0.859142 & 3.378856 & 2.128110\end{array}$

$\begin{array}{lll}-0.526801 & 3.338157 & 1.861123\end{array}$

$\begin{array}{lll}1.234597 & 4.367615 & 2.352982\end{array}$

$\begin{array}{lll}3.151671 & 2.640869 & 2.049247\end{array}$

$\begin{array}{llll}4.161428 & 1.612957 & 2.247367\end{array}$

$\begin{array}{lll}4.957873 & 2.001828 & 2.893271\end{array}$

$\begin{array}{lll}3.721199 & 0.741141 & 2.725232\end{array}$

$\begin{array}{llll}4.619081 & 1.302653 & 1.299287\end{array}$

$\begin{array}{llll}3.668229 & 3.982234 & 1.824398\end{array}$

$\begin{array}{llll}2.941713 & 4.592118 & 1.289801\end{array}$

$\begin{array}{llll}3.932867 & 4.489076 & 2.764367\end{array}$

$\begin{array}{llll}4.568463 & 3.916004 & 1.205649\end{array}$

$\begin{array}{lll}1.510779 & 1.072563 & 2.063716\end{array}$

$\begin{array}{lll}-1.271848 & 4.565177 & 1.953332\end{array}$

$\begin{array}{llll}-2.613538 & 4.622229 & 1.666047\end{array}$

$\begin{array}{lll}-0.745358 & 5.469082 & 2.243604\end{array}$

$\begin{array}{lll}-3.251292 & 3.431332 & 1.275992\end{array}$

$\begin{array}{lll}-2.524091 & 2.265235 & 1.189696\end{array}$

$\begin{array}{lll}-2.990362 & 1.334642 & 0.892417\end{array}$

$\begin{array}{lll}-1.203748 & 2.203008 & 1.464215\end{array}$

$\begin{array}{lll}-3.159178 & 5.557710 & 1.735086\end{array}$

$\begin{array}{lll}-5.172198 & 3.415892 & 0.856491\end{array}$

Conformer 25

Number of imaginary frequencies : 0

The smallest frequency is : $13.2528 \mathrm{~cm}(-1)$

Electronic energy : $\quad H F=-4521.7138804$ 
$\begin{array}{llll}3.832549 & -3.304457 & -0.462527\end{array}$

$\begin{array}{llll}4.551166 & -4.099410 & -0.296668\end{array}$

$\begin{array}{llll}2.973762 & -3.340573 & -1.561630\end{array}$

$\begin{array}{llll}2.052031 & -2.319028 & -1.791516\end{array}$

$\begin{array}{llll}1.417928 & -2.381234 & -2.666145\end{array}$

$\begin{array}{llll}1.614613 & 1.582308 & -1.361718\end{array}$

$\begin{array}{llll}0.913717 & 2.796413 & -1.279035\end{array}$

$\begin{array}{llll}-0.145617 & 2.795974 & -1.049733\end{array}$

$\begin{array}{llll}1.583334 & 4.006578 & -1.427756\end{array}$

$\begin{array}{llll}2.965981 & 4.039230 & -1.619434\end{array}$

$\begin{array}{llll}3.490838 & 4.985368 & -1.687904\end{array}$

$\begin{array}{llll}3.663880 & 2.835604 & -1.672054\end{array}$

$\begin{array}{llll}2.996668 & 1.612231 & -1.568184\end{array}$

$\begin{array}{llll}3.570144 & 0.693973 & -1.606696\end{array}$

$\begin{array}{lll}-0.276378 & 0.379537 & 1.172872\end{array}$

$\begin{array}{llll}5.159209 & 2.850700 & -1.825853\end{array}$

$\begin{array}{lll}0.851080 & 5.306969 & -1.250165\end{array}$

$\begin{array}{lll}4.684905 & -2.114956 & 1.585223\end{array}$

$\begin{array}{llll}3.036079 & -4.533518 & -2.474159\end{array}$

$\begin{array}{llll}2.767038 & -5.681966 & -1.803095\end{array}$

$2.152341 \quad-4.456103 \quad-3.493775$

$\begin{array}{llll}4.265894 & -4.689097 & -3.018477\end{array}$

$\begin{array}{lll}5.663910 & -1.210143 & 1.330594\end{array}$

$\begin{array}{llll}5.292873 & -3.282537 & 1.881628\end{array}$

$\begin{array}{lll}4.059177 & -1.689125 & 2.710721\end{array}$

$\begin{array}{llll}5.544626 & 2.870882 & -3.123387\end{array}$

$\begin{array}{llll}5.732947 & 1.759432 & -1.264524\end{array}$

$\begin{array}{llll}5.712791 & 3.938704 & -1.237647\end{array}$

$\begin{array}{llll}1.175353 & 6.199598 & -2.215112\end{array}$

$\begin{array}{llll}-0.489612 & 5.154932 & -1.271034\end{array}$

$\begin{array}{llll}1.164894 & 5.896345 & -0.066398\end{array}$

$\begin{array}{lll}1.867910 & 2.331284 & 2.008078\end{array}$

$\begin{array}{lll}0.922187 & 3.389075 & 2.090188\end{array}$

$\begin{array}{lll}-0.466534 & 3.354328 & 1.836662\end{array}$

$\begin{array}{lll}1.303576 & 4.376212 & 2.312191\end{array}$

$\begin{array}{llll}3.211753 & 2.654370 & 1.952271\end{array}$

$\begin{array}{lll}4.230080 & 1.626211 & 2.099006\end{array}$

$\begin{array}{llll}5.037826 & 1.997192 & 2.741292\end{array}$

$\begin{array}{llll}3.803825 & 0.737868 & 2.558534\end{array}$

$\begin{array}{llll}4.669019 & 1.348144 & 1.131530\end{array}$

$\begin{array}{llll}3.716306 & 4.004579 & 1.754867\end{array}$

$\begin{array}{llll}3.001793 & 4.612203 & 1.200732\end{array}$

$\begin{array}{lll}3.947386 & 4.507177 & 2.705813\end{array}$

$\begin{array}{llll}4.634783 & 3.957939 & 1.161268\end{array}$

$\begin{array}{lll}1.571599 & 1.083528 & 1.990923\end{array}$

$\begin{array}{lll}-1.205528 & 4.584250 & 1.939527\end{array}$

$\begin{array}{lll}-2.548052 & 4.649659 & 1.658255\end{array}$

$\begin{array}{lll}-0.673574 & 5.484104 & 2.232041\end{array}$

$\begin{array}{lll}-3.193937 & 3.463818 & 1.266208\end{array}$

$\begin{array}{lll}-2.473750 & 2.293824 & 1.174513\end{array}$

$\begin{array}{lll}-2.946846 & 1.366028 & 0.879102\end{array}$

$\begin{array}{lll}-1.152441 & 2.223643 & 1.442758\end{array}$

$\begin{array}{lll}-3.088423 & 5.587684 & 1.733982\end{array}$

$\begin{array}{llll}-5.116804 & 3.460494 & 0.855805\end{array}$

Conformer_26

Number of imaginary frequencies : 0

The smallest frequency is : $13.2912 \mathrm{~cm}(-1)$

Electronic energy : $\quad \mathrm{HF}=-4521.7138804$

Zero-point correction $=\quad 0.854508$

(Hartree/Particle)

Thermal correction to Energy=

Thermal correction to Enthalpy=

0.923919

0.924863

Thermal correction to Gibbs Free Energy $=0.744401$

Sum of electronic and zero-point Energies $=\quad-4520.859372$

Sum of electronic and thermal Energies $=$

Sum of electronic and thermal Enthalpies=

Sum of electronic and thermal Free Energies=

$-4520.789962$

$-4520.789017$

$-4520.969479$

\begin{tabular}{rrrr}
\multicolumn{4}{c}{ Cartesian Coordinates } \\
\hline 26 & -3.598124 & -1.129599 & -2.692571 \\
15 & -1.126024 & -1.576161 & 1.911562 \\
15 & 0.662800 & 0.066132 & -0.938353
\end{tabular}


$\begin{array}{llll}3.036434 & -4.533662 & -2.473628\end{array}$

$\begin{array}{llll}2.767375 & -5.682012 & -1.802406\end{array}$

$2.152744 \quad-4.456408 \quad-3.493297$

$\begin{array}{llll}4.266278 & -4.689311 & -3.017863\end{array}$

$\begin{array}{llll}5.664286 & -1.209529 & 1.330360\end{array}$

$\begin{array}{llll}5.293363 & -3.281843 & 1.881770\end{array}$

$\begin{array}{llll}4.059714 & -1.688333 & 2.710729\end{array}$

$\begin{array}{llll}5.544503 & 2.870812 & -3.123365\end{array}$

$\begin{array}{llll}5.732853 & 1.760075 & -1.264088\end{array}$

$\begin{array}{llll}5.712422 & 3.939353 & -1.238003\end{array}$

$\begin{array}{llll}1.174818 & 6.199569 & -2.215271\end{array}$

$\begin{array}{llll}-0.490096 & 5.154689 & -1.271322\end{array}$

$\begin{array}{llll}1.164252 & 5.896258 & -0.066570\end{array}$

$\begin{array}{llll}1.867754 & 2.331533 & 2.007814\end{array}$

$\begin{array}{lll}0.921923 & 3.389217 & 2.090010\end{array}$

$\begin{array}{lll}-0.466802 & 3.354361 & 1.836526\end{array}$

$\begin{array}{llll}1.303221 & 4.376366 & 2.312123\end{array}$

$\begin{array}{lll}3.211562 & 2.654762 & 1.952005\end{array}$

$\begin{array}{lll}4.229988 & 1.626719 & 2.098850\end{array}$

$\begin{array}{lll}5.037673 & 1.997834 & 2.741133\end{array}$

$\begin{array}{llll}3.803800 & 0.738376 & 2.558440\end{array}$

$\begin{array}{lll}4.668986 & 1.348614 & 1.131414\end{array}$

$\begin{array}{lll}3.715995 & 4.005013 & 1.754590\end{array}$

$\begin{array}{llll}3.001343 & 4.612628 & 1.200630\end{array}$

$\begin{array}{lll}3.947224 & 4.507560 & 2.705527\end{array}$

$\begin{array}{llll}4.634363 & 3.958466 & 1.160812\end{array}$

$\begin{array}{lll}1.571576 & 1.083747 & 1.990599\end{array}$

$\begin{array}{lll}-1.205906 & 4.584199 & 1.939595\end{array}$

$\begin{array}{lll}-2.548467 & 4.649504 & 1.658486\end{array}$

$\begin{array}{lll}-0.674012 & 5.484071 & 2.232163\end{array}$

$\begin{array}{llll}-3.194274 & 3.463641 & 1.266375\end{array}$

$\begin{array}{lll}-2.473971 & 2.293738 & 1.174426\end{array}$

$\begin{array}{llll}-2.947007 & 1.365929 & 0.878960\end{array}$

$\begin{array}{lll}-1.152622 & 2.223660 & 1.442510\end{array}$

$\begin{array}{lll}-3.088928 & 5.587461 & 1.734396\end{array}$

$\begin{array}{llll}35 & -5.117215 & 3.460154 & 0.856322\end{array}$

Conformer_27

Number of imaginary frequencies : 0

The smallest frequency is : $13.2559 \mathrm{~cm}(-1)$

Electronic energy : $\quad H F=-4521.7138804$

$\begin{array}{lr}\text { Zero-point correction }= & 0.854508\end{array}$

(Hartree/Particle)

Thermal correction to Energy $=\quad 0.923919$

Thermal correction to Enthalpy= $\quad 0.924863$

Thermal correction to Gibbs Free Energy $=0.744393$

Sum of electronic and zero-point Energies $=\quad-4520.859372$

Sum of electronic and thermal Energies=

$-4520.789961$

Sum of electronic and thermal Enthalpies $=\quad-4520.789017$

Sum of electronic and thermal Free Energies $=\quad-4520.969487$

\begin{tabular}{crrr} 
& \multicolumn{3}{c}{ Cartesian Coordinates } \\
\hline 26 & -3.598230 & -1.129615 & -2.692417 \\
15 & -1.125955 & -1.576320 & 1.911651 \\
15 & 0.662711 & 0.066192 & -0.938236 \\
6 & -2.360189 & -1.491652 & -1.039795 \\
6 & -1.858706 & -0.399994 & -1.842659 \\
6 & -2.862234 & 0.615616 & -1.876482 \\
1 & -2.792893 & 1.555181 & -2.406998 \\
6 & -3.990029 & 0.155284 & -1.133816 \\
1 & -4.924469 & 0.683775 & -1.006845 \\
6 & -3.683242 & -1.138739 & -0.620523 \\
1 & -4.342946 & -1.760595 & -0.035082 \\
6 & -3.205852 & -2.086507 & -4.493990 \\
1 & -2.219479 & -2.371710 & -4.835942 \\
6 & -3.842933 & -0.829423 & -4.727522 \\
1 & -3.421616 & 0.006029 & -5.270576 \\
6 & -5.114123 & -0.850382 & -4.076320 \\
1 & -5.819784 & -0.031109 & -4.034447 \\
6 & -5.263291 & -2.120093 & -3.439403
\end{tabular}




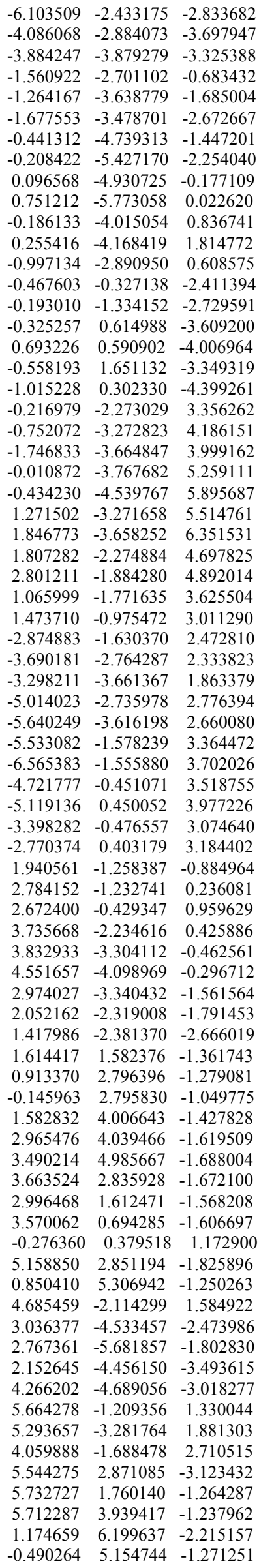

Conformer_28

Number of imaginary frequencies : 0

The smallest frequency is : $13.3063 \mathrm{~cm}(-1)$

Electronic energy : $\quad H F=-4521.7138803$

Zero-point correction $=\quad 0.854508$

(Hartree/Particle)

Thermal correction to Energy $=\quad 0.923919$

Thermal correction to Enthalpy $=\quad 0.924863$

Thermal correction to Gibbs Free Energy $=0.744396$

Sum of electronic and zero-point Energies $=\quad-4520.859372$

Sum of electronic and thermal Energies $=\quad-4520.789961$

Sum of electronic and thermal Enthalpies $=\quad-4520.789017$

Sum of electronic and thermal Free Energies $=\quad-4520.969484$

\begin{tabular}{|c|c|c|c|}
\hline & & \multicolumn{2}{|c|}{ Cartesian Coordinates } \\
\hline 26 & -3.597887 & -1.129199 & -2.692939 \\
\hline 15 & -1.126429 & -1.576097 & 1.911481 \\
\hline 15 & 0.662960 & 0.066110 & -0.938260 \\
\hline 6 & -2.360065 & -1.491272 & -1.040171 \\
\hline 6 & -1.858354 & -0.399748 & -1.843068 \\
\hline 6 & -2.861732 & 0.616005 & -1.877060 \\
\hline 1 & -2.792195 & 1.555523 & -2.407636 \\
\hline 6 & -3.989667 & 0.155888 & -1.134483 \\
\hline 1 & -4.924047 & 0.684522 & -1.007668 \\
\hline 6 & -3.683115 & -1.138139 & -0.621061 \\
\hline 1 & -4.342968 & -1.759861 & -0.035647 \\
\hline 6 & -3.205443 & -2.086171 & -4.494446 \\
\hline 1 & -2.219061 & -2.371404 & -4.836344 \\
\hline 6 & -3.842493 & -0.829085 & -4.728063 \\
\hline 1 & -3.421132 & & -5.271131 \\
\hline 6 & -5.113713 & -0.849993 & -4.076920 \\
\hline 1 & -5.819362 & -0.030707 & -4.035115 \\
\hline 6 & -5.262935 & -2.119676 & -3.439959 \\
\hline 1 & -6.103185 & -2.432719 & -2.834265 \\
\hline 6 & -4.085715 & -2.883688 & -3.698416 \\
\hline 1 & -3.883931 & -3.878882 & -3.325805 \\
\hline 6 & -1.561093 & -2.700864 & -0.683640 \\
\hline 6 & -1.264242 & -3.638624 & -1.685103 \\
\hline 1 & -1.677252 & -3.478465 & -2.672912 \\
\hline 6 & -0.441757 & -4.739371 & -1.446992 \\
\hline 1 & -0.208757 & -5.427289 & -2.253746 \\
\hline 6 & 0.095594 & -4.930954 & -0.176697 \\
\hline 1 & 0.749885 & -5.773504 & 0.023275 \\
\hline 6 & -0.187194 & -4.015188 & 0.837044 \\
\hline 1 & 0.253889 & -4.168667 & 1.815272 \\
\hline 6 & -0.997741 & -2.890830 & 0.608532 \\
\hline 6 & -0.467160 & -0.327078 & -2.411591 \\
\hline 1 & -0.192622 & -1.334126 & -2.729718 \\
\hline
\end{tabular}




$\begin{array}{cccc}6 & -1.205701 & 4.584208 & 1.939497 \\ 6 & -2.548256 & 4.649552 & 1.658366 \\ 1 & -0.673770 & 5.484083 & 2.231994 \\ 6 & -3.194117 & 3.463687 & 1.266346 \\ 6 & -2.473863 & 2.293747 & 1.174493 \\ 1 & -2.946927 & 1.365943 & 0.879057 \\ 7 & -1.152525 & 2.223630 & 1.442612 \\ 1 & -3.088674 & 5.587543 & 1.734190 \\ 35 & -5.117046 & 3.460252 & 0.856249\end{array}$

Conformer_ 29

Number of imaginary frequencies : 0

The smallest frequency is : $14.3747 \mathrm{~cm}(-1)$

Electronic energy : $\quad \mathrm{HF}=-4521.714362$

Zero-point correction $=\quad 0.854529$

(Hartree/Particle)

Thermal correction to Energy $=\quad 0.923892$

Thermal correction to Enthalpy $=\quad 0.924836$

Thermal correction to Gibbs Free Energy $=\quad 0.745317$

Sum of electronic and zero-point Energies $=\quad-4520.859833$

Sum of electronic and thermal Energies= $\quad-4520.790470$

Sum of electronic and thermal Enthalpies $=\quad-4520.789526$

Sum of electronic and thermal Free Energies $=\quad-4520.969045$

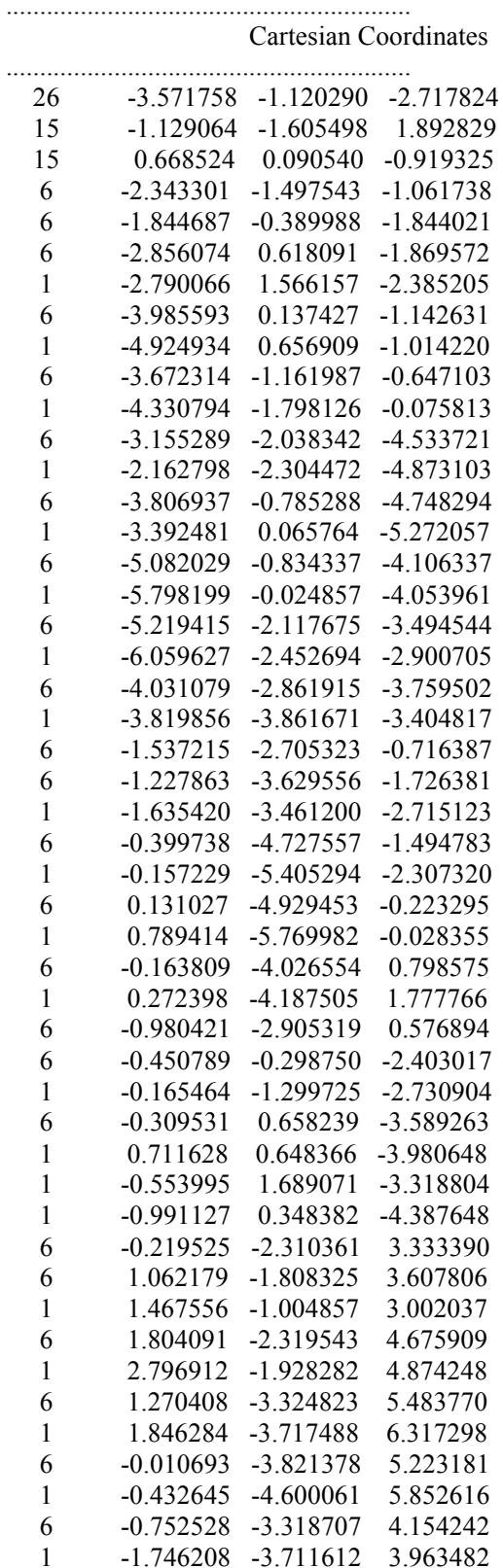


$\begin{array}{lll}-2.879741 & -1.683323 & 2.445668\end{array}$

$\begin{array}{lll}-3.683871 & -2.823048 & 2.290669\end{array}$

$\begin{array}{lll}-3.281557 & -3.711076 & 1.811792\end{array}$

$\begin{array}{lll}-5.009384 & -2.812320 & 2.729086\end{array}$

$\begin{array}{lll}-5.626863 & -3.696997 & 2.600442\end{array}$

$\begin{array}{lll}-5.541240 & -1.666543 & 3.329034\end{array}$

$\begin{array}{lll}-6.574751 & -1.657894 & 3.663484\end{array}$

$\begin{array}{lll}-4.741024 & -0.533778 & 3.499425\end{array}$

$\begin{array}{lll}-5.148184 & 0.358025 & 3.967461\end{array}$

$\begin{array}{lll}-3.415926 & -0.541747 & 3.059440\end{array}$

$\begin{array}{lll}-2.796300 & 0.342172 & 3.182035\end{array}$

$\begin{array}{llll}1.954145 & -1.226260 & -0.870283\end{array}$

$\begin{array}{llll}2.796739 & -1.200833 & 0.251478\end{array}$

$\begin{array}{llll}2.676604 & -0.404545 & 0.981730\end{array}$

$\begin{array}{llll}3.756271 & -2.196489 & 0.434761\end{array}$

$\begin{array}{llll}3.863679 & -3.258118 & -0.462040\end{array}$

$\begin{array}{llll}4.589430 & -4.047657 & -0.301511\end{array}$

$\begin{array}{llll}3.006448 & -3.293704 & -1.562248\end{array}$

$2.075743 \quad-2.278844 \quad-1.784723$

$\begin{array}{llll}1.442874 & -2.339791 & -2.660297\end{array}$

$\begin{array}{llll}1.619367 & 1.609326 & -1.336792\end{array}$

$\begin{array}{llll}0.920727 & 2.823924 & -1.235529\end{array}$

$\begin{array}{lll}-0.134369 & 2.822657 & -0.987528\end{array}$

$\begin{array}{llll}1.585245 & 4.033901 & -1.403282\end{array}$

$\begin{array}{llll}2.961892 & 4.067077 & -1.637797\end{array}$

$\begin{array}{llll}3.483332 & 5.012201 & -1.736600\end{array}$

$\begin{array}{llll}3.655898 & 2.863687 & -1.719683\end{array}$

$\begin{array}{llll}2.994000 & 1.639107 & -1.585215\end{array}$

$3.564965 \quad 0.720174 \quad-1.653483$

$\begin{array}{lll}-0.298208 & 0.369413 & 1.181759\end{array}$

$\begin{array}{llll}5.152575 & 2.866107 & -1.844526\end{array}$

$\begin{array}{llll}0.854535 & 5.333053 & -1.211973\end{array}$

$\begin{array}{lll}4.704583 & -2.078958 & 1.595278\end{array}$

$\begin{array}{llll}3.081720 & -4.477598 & -2.485637\end{array}$

$\begin{array}{llll}2.826206 & -5.635192 & -1.825289\end{array}$

$\begin{array}{llll}2.196709 & -4.400512 & -3.504194\end{array}$

$\begin{array}{llll}4.312929 & -4.613977 & -3.031973\end{array}$

$\begin{array}{lll}5.676757 & -1.164450 & 1.348576\end{array}$

$\begin{array}{llll}5.321920 & -3.244249 & 1.881289\end{array}$

$\begin{array}{llll}4.075575 & -1.668598 & 2.724364\end{array}$

$\begin{array}{lll}5.600662 & 1.852188 & -2.618150\end{array}$

$\begin{array}{llll}5.746074 & 2.712013 & -0.628145\end{array}$

$\begin{array}{llll}5.627630 & 4.017618 & -2.364423\end{array}$

$\begin{array}{llll}1.168016 & 6.230803 & -2.175463\end{array}$

$\begin{array}{llll}-0.486270 & 5.180050 & -1.219586\end{array}$

$\begin{array}{llll}1.179879 & 5.916320 & -0.028039\end{array}$

$\begin{array}{llll}1.815997 & 2.320925 & 2.057673\end{array}$

$\begin{array}{lll}0.871684 & 3.381657 & 2.103493\end{array}$

$\begin{array}{lll}-0.516145 & 3.340492 & 1.845070\end{array}$

$\begin{array}{lll}1.251196 & 4.372662 & 2.311011\end{array}$

$\begin{array}{lll}3.161090 & 2.640287 & 2.022063\end{array}$

$\begin{array}{llll}4.172155 & 1.619950 & 2.253868\end{array}$

$\begin{array}{llll}4.935995 & 2.006026 & 2.940122\end{array}$

$\begin{array}{lll}3.718530 & 0.738941 & 2.700814\end{array}$

$\begin{array}{llll}4.672921 & 1.325908 & 1.322951\end{array}$

$\begin{array}{lll}3.668495 & 3.990639 & 1.834106\end{array}$

$\begin{array}{llll}2.990410 & 4.580886 & 1.218046\end{array}$

$\begin{array}{llll}3.827083 & 4.515804 & 2.788077\end{array}$

$\begin{array}{llll}4.626997 & 3.935319 & 1.310159\end{array}$

$\begin{array}{lll}1.517774 & 1.073196 & 2.055683\end{array}$

$\begin{array}{llll}-1.259874 & 4.568337 & 1.933794\end{array}$

$\begin{array}{llll}-2.603307 & 4.624300 & 1.654036\end{array}$

$\begin{array}{lll}-0.731541 & 5.473702 & 2.215748\end{array}$

$\begin{array}{lll}-3.244098 & 3.431216 & 1.276314\end{array}$

$\begin{array}{lll}-2.518048 & 2.264063 & 1.193493\end{array}$

$\begin{array}{lll}-2.986802 & 1.331647 & 0.905904\end{array}$

$\begin{array}{lll}-1.195974 & 2.203271 & 1.459597\end{array}$

$\begin{array}{lll}-3.148053 & 5.560488 & 1.720377\end{array}$

$\begin{array}{lll}-5.167854 & 3.413756 & 0.870336\end{array}$

Conformer_30

Number of imaginary frequencies : 0

The smallest frequency is : $13.1870 \mathrm{~cm}(-1)$

Electronic energy : $\quad H F=-4521.7138805$
Zero-point correction $=$

0.854507

(Hartree/Particle)

Thermal correction to Energy=

Thermal correction to Enthalpy=

0.924863

Thermal correction to Gibbs Free Energy=

Sum of electronic and zero-point Energies=

Sum of electronic and thermal Energies=

Sum of electronic and thermal Enthalpies=

Sum of electronic and thermal Free Energies=

0.74438

$-4520.859373$

$-4520.78996$

$-4520.789017$

Cartesian Coordinates

$-3.598383 \quad-1.129884 \quad-2.692084$

$\begin{array}{lll}1.125989 & -1.576165 & 1.911979\end{array}$

$\begin{array}{llll}0.662576 & 0.066124 & -0.938094\end{array}$

$\begin{array}{llll}-2.360319 & -1.491814 & -1.039471\end{array}$

$\begin{array}{llll}-1.858922 & -0.400110 & -1.842329\end{array}$

$\begin{array}{llll}-2.862534 & 0.615414 & -1.876165\end{array}$

$\begin{array}{llll}-2.793268 & 1.554980 & -2.406690\end{array}$

$\begin{array}{llll}-3.990288 & 0.154998 & -1.133486\end{array}$

$\begin{array}{llll}-4.924766 & 0.683420 & -1.006501\end{array}$

$\begin{array}{llll}-3.683402 & -1.139002 & -0.620199\end{array}$

$\begin{array}{llll}-4.343056 & -1.760907 & -0.034755\end{array}$

$\begin{array}{llll}-3.205899 & -2.086790 & -4.493619\end{array}$

$-2.219494 \quad-2.371925 \quad-4.835536$

$\begin{array}{llll}-3.843063 & -0.829757 & -4.727200\end{array}$

$\begin{array}{llll}-3.421788 & 0.005710 & -5.270264\end{array}$

$\begin{array}{llll}-5.114266 & -0.850783 & -4.076027\end{array}$

$\begin{array}{lll}-5.819978 & -0.031553 & -4.034187\end{array}$

$-5.263364-2.120488-3.439084$

$\begin{array}{llll}-6.103571 & -2.433611 & -2.833370\end{array}$

$-4.086084 \quad-2.884396 \quad-3.697582$

$\begin{array}{llll}-3.884206 & -3.879581 & -3.324996\end{array}$

$\begin{array}{lll}-1.560936 & -2.701176 & -0.683050\end{array}$

$\begin{array}{llll}-1.264156 & -3.638958 & -1.684515\end{array}$

$-1.677637-3.479078-2.672172$

$\begin{array}{llll}-0.441131 & -4.739349 & -1.446622\end{array}$

$\begin{array}{llll}-0.208246 & -5.427303 & -2.253380\end{array}$

$\begin{array}{llll}0.096936 & -4.930489 & -0.176566\end{array}$

$\begin{array}{llll}0.751747 & -5.772687 & 0.023193\end{array}$

$\begin{array}{llll}-0.185799 & -4.014716 & 0.837187\end{array}$

$\begin{array}{lll}0.255880 & -4.167827 & 1.815201\end{array}$

$\begin{array}{llll}-0.997005 & -2.890788 & 0.608927\end{array}$

$\begin{array}{llll}-0.467865 & -0.327189 & -2.411159\end{array}$

$\begin{array}{llll}-0.193301 & -1.334187 & -2.729424\end{array}$

$\begin{array}{llll}-0.325628 & 0.614982 & -3.608942\end{array}$

$\begin{array}{llll}0.692824 & 0.590929 & -4.006784\end{array}$

$\begin{array}{llll}-0.558565 & 1.651111 & -3.349007\end{array}$

$\begin{array}{llll}-1.015652 & 0.302342 & -4.398963\end{array}$

$\begin{array}{lll}-0.216929 & -2.272905 & 3.356531\end{array}$

$\begin{array}{lll}-0.751984 & -3.272573 & 4.186592\end{array}$

$\begin{array}{lll}-1.746787 & -3.664568 & 3.999788\end{array}$

$\begin{array}{lll}-0.010722 & -3.767313 & 5.259564\end{array}$

$\begin{array}{lll}-0.434062 & -4.539302 & 5.896270\end{array}$

$\begin{array}{lll}1.271662 & -3.271260 & 5.515095\end{array}$

$\begin{array}{llll}1.846965 & -3.657729 & 6.351901\end{array}$

$\begin{array}{llll}1.807411 & -2.274616 & 4.697985\end{array}$

$\begin{array}{llll}2.801356 & -1.884002 & 4.892061\end{array}$

$\begin{array}{llll}1.066104 & -1.771529 & 3.625602\end{array}$

$\begin{array}{llll}1.473836 & -0.975471 & 3.011254\end{array}$

$\begin{array}{lll}-2.874993 & -1.630486 & 2.472886\end{array}$

$\begin{array}{llll}-3.689927 & -2.764698 & 2.334128\end{array}$

$\begin{array}{llll}-3.297599 & -3.661822 & 1.864067\end{array}$

$\begin{array}{lll}-5.013877 & -2.736624 & 2.776384\end{array}$

$\begin{array}{lll}-5.639811 & -3.617075 & 2.660251\end{array}$

$\begin{array}{lll}-5.533426 & -1.578817 & 3.363899\end{array}$

$\begin{array}{lll}-6.565812 & -1.556642 & 3.701201\end{array}$

$\begin{array}{lll}-4.722498 & -0.451348 & 3.517941\end{array}$

$\begin{array}{lll}-5.120238 & 0.449834 & 3.975966\end{array}$

$\begin{array}{lll}-3.398886 & -0.476606 & 3.074156\end{array}$

$\begin{array}{lll}-2.771280 & 0.403366 & 3.183737\end{array}$

$\begin{array}{llll}1.940449 & -1.258441 & -0.884993\end{array}$

$\begin{array}{llll}2.784121 & -1.232859 & 0.235991\end{array}$

$\begin{array}{lll}2.672377 & -0.429533 & 0.959617\end{array}$

$\begin{array}{llll}3.735732 & -2.234683 & 0.425604\end{array}$ 
$\begin{array}{lll}3.832989 & -3.304084 & -0.462956\end{array}$

$\begin{array}{llll}4.551778 & -4.098915 & -0.297257\end{array}$

$2.973997-3.340346-1.561892$

$\begin{array}{llll}2.052066 & -2.318948 & -1.791616\end{array}$

$\begin{array}{llll}1.417854 & -2.381248 & -2.666159\end{array}$

$\begin{array}{llll}1.614254 & 1.582308 & -1.361676\end{array}$

$\begin{array}{llll}0.913235 & 2.796340 & -1.278947\end{array}$

$-0.146080 \quad 2.795793-1.049565$

$\begin{array}{llll}1.582713 & 4.006576 & -1.427706\end{array}$

$2.965341 \quad 4.039376 \quad-1.619505$

$\begin{array}{llll}3.490091 & 4.985571 & -1.688005\end{array}$

$\begin{array}{llll}3.663359 & 2.835825 & -1.672198\end{array}$

$\begin{array}{lll}2.996288 & 1.612380 & -1.568261\end{array}$

$\begin{array}{llll}3.569858 & 0.694183 & -1.606816\end{array}$

$\begin{array}{lll}-0.276379 & 0.379630 & 1.173066\end{array}$

$\begin{array}{llll}5.158670 & 2.851064 & -1.826161\end{array}$

$\begin{array}{lll}0.850337 & 5.306892 & -1.250068\end{array}$

$\begin{array}{lll}4.685643 & -2.114418 & 1.584545\end{array}$

$\begin{array}{llll}3.036292 & -4.533318 & -2.474387\end{array}$

$\begin{array}{llll}2.767354 & -5.681752 & -1.803251\end{array}$

$\begin{array}{llll}2.152461 & -4.455979 & -3.493925\end{array}$

$\begin{array}{llll}4.266068 & -4.688870 & -3.018798\end{array}$

$\begin{array}{llll}5.664380 & -1.209395 & 1.329632\end{array}$

$\begin{array}{llll}5.293947 & -3.281872 & 1.880752\end{array}$

$\begin{array}{llll}4.060174 & -1.688737 & 2.710240\end{array}$

$\begin{array}{llll}5.543946 & 2.871089 & -3.123739\end{array}$

$\begin{array}{llll}5.732583 & 1.759939 & -1.264732\end{array}$

$\begin{array}{llll}5.712205 & 3.939212 & -1.238178\end{array}$

$\begin{array}{llll}1.174306 & 6.199483 & -2.215154\end{array}$

$\begin{array}{llll}-0.490342 & 5.154697 & -1.270656\end{array}$

$\begin{array}{llll}1.164325 & 5.896399 & -0.066414\end{array}$

$\begin{array}{lll}1.867939 & 2.331404 & 2.008093\end{array}$

$\begin{array}{lll}0.922189 & 3.389160 & 2.090400\end{array}$

$\begin{array}{lll}-0.466523 & 3.354426 & 1.836849\end{array}$

$\begin{array}{llll}1.303567 & 4.376274 & 2.312527\end{array}$

$\begin{array}{llll}3.211761 & 2.654554 & 1.952172\end{array}$

$\begin{array}{lll}4.230126 & 1.626412 & 2.098724\end{array}$

$\begin{array}{lll}5.038046 & 1.997462 & 2.740748\end{array}$

$\begin{array}{llll}3.803990 & 0.738121 & 2.558463\end{array}$

$\begin{array}{lll}4.668800 & 1.348231 & 1.131162\end{array}$

$\begin{array}{lll}3.716252 & 4.004758 & 1.754571\end{array}$

$\begin{array}{llll}3.001532 & 4.612387 & 1.200709\end{array}$

$\begin{array}{lll}3.947698 & 4.507350 & 2.705430\end{array}$

$\begin{array}{llll}4.634499 & 3.958116 & 1.160617\end{array}$

$\begin{array}{lll}1.571678 & 1.083640 & 1.990891\end{array}$

$\begin{array}{lll}-1.205530 & 4.584332 & 1.939825\end{array}$

$\begin{array}{lll}-2.548010 & 4.649794 & 1.658371\end{array}$

$\begin{array}{lll}-0.673606 & 5.484142 & 2.232530\end{array}$

$\begin{array}{lll}-3.193846 & 3.464015 & 1.266047\end{array}$

$\begin{array}{lll}-2.473666 & 2.294019 & 1.174335\end{array}$

$\begin{array}{llll}-2.946723 & 1.366267 & 0.878723\end{array}$

$\begin{array}{lll}-1.152403 & 2.223778 & 1.442791\end{array}$

$\begin{array}{lll}-3.088385 & 5.587812 & 1.734157\end{array}$

$\begin{array}{llll}-5.116629 & 3.460793 & 0.855265\end{array}$

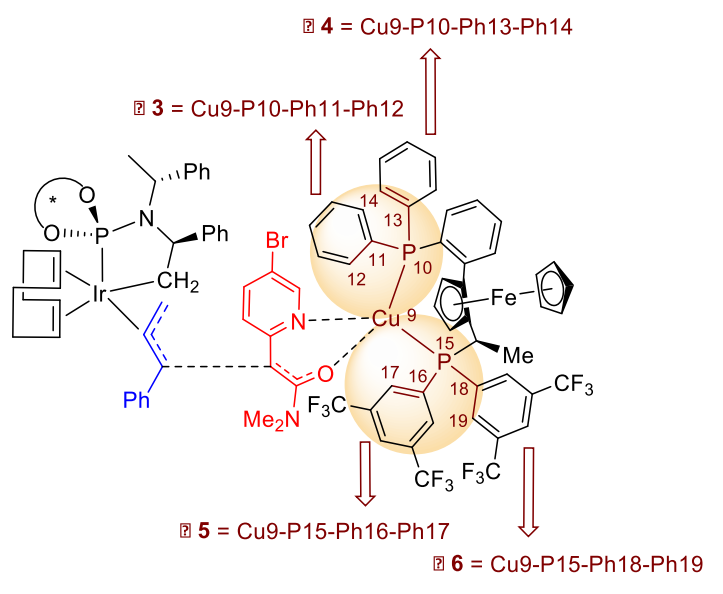

Conformer_1
Number of imaginary frequencies : 1

The smallest frequency is : $-247.7050 \mathrm{~cm}(-1)$

Electronic energy :

$\mathrm{HF}=-7222.6348461$

Zero-point correction $=$

1.755699

(Hartree/Particle)

Thermal correction to Energy= $\quad 1.874114$

Thermal correction to Enthalpy= $\quad 1.875058$

Thermal correction to Gibbs Free Energy $=\quad 1.593069$

Sum of electronic and zero-point Energies $=\quad-7220.879147$

Sum of electronic and thermal Energies $=\quad-7220.760732$

Sum of electronic and thermal Enthalpies $=\quad-7220.759788$

Sum of electronic and thermal Free Energies $=\quad-7221.041777$

Cartesian Coordinates

$2.562756 \quad 1.624351-1.886440$

$\begin{array}{lll}4.540846 & 0.128139 & 0.933294\end{array}$

$\begin{array}{lll}3.967410 & 3.424777 & 0.318854\end{array}$

$\begin{array}{llll}4.154545 & 2.746011 & 1.580203\end{array}$

$\begin{array}{lll}3.132457 & 3.194330 & 2.473117\end{array}$

$\begin{array}{lll}3.010044 & 2.884449 & 3.501783\end{array}$

$\begin{array}{lll}2.335062 & 4.162702 & 1.791781\end{array}$

$\begin{array}{lll}1.509022 & 4.715925 & 2.216611\end{array}$

$\begin{array}{llll}2.848836 & 4.307262 & 0.470055\end{array}$

$\begin{array}{llll}2.478789 & 4.983235 & -0.285769\end{array}$

$\begin{array}{lll}6.237819 & 5.325975 & 2.375695\end{array}$

$\begin{array}{llll}7.098923 & 4.677221 & 2.283117\end{array}$

$\begin{array}{llll}5.382659 & 5.426340 & 3.515800\end{array}$

$\begin{array}{llll}5.479489 & 4.864930 & 4.435514\end{array}$

$\begin{array}{lll}4.350475 & 6.365732 & 3.212751\end{array}$

$\begin{array}{lll}3.525333 & 6.633260 & 3.859560\end{array}$

$\begin{array}{llll}4.567421 & 6.847172 & 1.885647\end{array}$

$\begin{array}{llll}3.938500 & 7.546977 & 1.351295\end{array}$

$\begin{array}{llll}5.733290 & 6.207213 & 1.369562\end{array}$

$\begin{array}{llll}6.141305 & 6.344961 & 0.377594\end{array}$

$\begin{array}{llll}4.764225 & 3.153541 & -0.913015\end{array}$

$\begin{array}{llll}6.097254 & 3.584398 & -0.982741\end{array}$

$\begin{array}{llll}6.500731 & 4.139446 & -0.145405\end{array}$

$\begin{array}{llll}6.905965 & 3.300484 & -2.083113\end{array}$

$\begin{array}{llll}7.941472 & 3.625280 & -2.093003\end{array}$

$\begin{array}{llll}6.383442 & 2.576975 & -3.152563\end{array}$

$\begin{array}{llll}7.003929 & 2.337237 & -4.010062\end{array}$

$\begin{array}{llll}5.061419 & 2.133590 & -3.105883\end{array}$

$\begin{array}{llll}4.672171 & 1.556787 & -3.937886\end{array}$

$\begin{array}{llll}4.241745 & 2.397541 & -1.997457\end{array}$

$\begin{array}{llll}5.179891 & 1.672429 & 1.830628\end{array}$

$\begin{array}{llll}6.093248 & 1.961759 & 1.307923\end{array}$

$\begin{array}{llll}5.519720 & 1.464613 & 3.309098\end{array}$

$\begin{array}{lll}6.298475 & 0.706859 & 3.429748\end{array}$

$\begin{array}{llll}4.650907 & 1.149113 & 3.892963\end{array}$

$\begin{array}{llll}5.882077 & 2.407481 & 3.730094\end{array}$

$\begin{array}{llll}2.354388 & 0.721764 & -3.482108\end{array}$

$\begin{array}{llll}2.616264 & -0.657954 & -3.490658\end{array}$

$\begin{array}{llll}2.930154 & -1.152676 & -2.579085\end{array}$

$\begin{array}{llll}2.435238 & -1.405079 & -4.656781\end{array}$

$\begin{array}{llll}2.642253 & -2.470144 & -4.648914\end{array}$

$\begin{array}{llll}1.979091 & -0.786424 & -5.822565\end{array}$

$\begin{array}{llll}1.823954 & -1.370497 & -6.725154\end{array}$

$\begin{array}{llll}1.718981 & 0.586581 & -5.822737\end{array}$

$\begin{array}{llll}1.363344 & 1.074348 & -6.725869\end{array}$

$\begin{array}{llll}1.908484 & 1.338355 & -4.662346\end{array}$

$\begin{array}{llll}1.691997 & 2.400842 & -4.679702\end{array}$

$\begin{array}{llll}1.403612 & 3.038197 & -2.070606\end{array}$

$\begin{array}{llll}1.740478 & 4.197636 & -2.788318\end{array}$

$\begin{array}{llll}2.733170 & 4.302579 & -3.215953\end{array}$

$\begin{array}{llll}0.806347 & 5.222726 & -2.947869\end{array}$

$\begin{array}{llll}1.077352 & 6.116575 & -3.502627\end{array}$

$\begin{array}{llll}-0.475294 & 5.095166 & -2.402505\end{array}$

$\begin{array}{llll}-1.208148 & 5.886371 & -2.532928\end{array}$

$\begin{array}{llll}-0.814345 & 3.945094 & -1.686943\end{array}$

$\begin{array}{llll}-1.807520 & 3.839153 & -1.267558\end{array}$

$\begin{array}{llll}0.124931 & 2.927395 & -1.509458\end{array}$

$\begin{array}{llll}-0.137151 & 2.044722 & -0.936863\end{array}$ 
$\begin{array}{llll}5.833965 & -0.351382 & -0.288271\end{array}$

$\begin{array}{llll}5.486637 & -1.402383 & -1.149489\end{array}$

$\begin{array}{llll}4.518877 & -1.879209 & -1.036718\end{array}$

$\begin{array}{llll}6.371537 & -1.830052 & -2.138412\end{array}$

$\begin{array}{llll}7.598093 & -1.194248 & -2.319539\end{array}$

$\begin{array}{llll}8.275671 & -1.510998 & -3.104292\end{array}$

$\begin{array}{llll}7.930518 & -0.131841 & -1.478494\end{array}$

$\begin{array}{lll}7.072175 & 0.277465 & -0.457524\end{array}$

$\begin{array}{llll}7.378611 & 1.096731 & 0.178092\end{array}$

$\begin{array}{lll}4.568674 & -1.271479 & 2.131350\end{array}$

$\begin{array}{lll}3.559625 & -1.295138 & 3.107554\end{array}$

$\begin{array}{llll}2.867800 & -0.465444 & 3.195734\end{array}$

$3.410590 \quad-2.401818 \quad 3.940533$

$\begin{array}{llll}4.233786 & -3.519362 & 3.797948\end{array}$

$\begin{array}{llll}4.087853 & -4.396385 & 4.418590\end{array}$

$\begin{array}{llll}5.223241 & -3.501172 & 2.818331\end{array}$

$\begin{array}{lll}5.409653 & -2.382703 & 2.004934\end{array}$

$\begin{array}{lll}6.188576 & -2.406568 & 1.253329\end{array}$

$\begin{array}{llll}2.440299 & 0.234709 & -0.102205\end{array}$

$\begin{array}{lll}6.092016 & -4.714936 & 2.637202\end{array}$

$\begin{array}{llll}2.273267 & -2.466890 & 4.920951\end{array}$

$\begin{array}{llll}5.996979 & -3.025825 & -2.969177\end{array}$

$\begin{array}{llll}9.225013 & 0.593122 & -1.726107\end{array}$

$\begin{array}{llll}9.227801 & 1.197511 & -2.941072\end{array}$

$\begin{array}{llll}9.466965 & 1.553228 & -0.806824\end{array}$

$10.282529 \quad-0.251083 \quad-1.717675$

$\begin{array}{llll}6.178599 & -4.182051 & -2.282737\end{array}$

$6.720536-3.118143 \quad-4.102531$

$\begin{array}{llll}4.686637 & -3.002365 & -3.326882\end{array}$

$\begin{array}{llll}7.068756 & -4.789663 & 3.569521\end{array}$

$\begin{array}{lll}6.695649 & -4.731981 & 1.427069\end{array}$

$\begin{array}{llll}5.369588 & -5.858161 & 2.739730\end{array}$

$\begin{array}{llll}2.645063 & -3.031461 & 6.090473\end{array}$

$\begin{array}{llll}1.757680 & -1.251357 & 5.200159\end{array}$

$\begin{array}{llll}1.250890 & -3.226951 & 4.437751\end{array}$

$\begin{array}{lll}1.975914 & -2.751405 & 0.404028\end{array}$

$\begin{array}{lll}0.812416 & -2.465320 & 1.213732\end{array}$

$\begin{array}{lll}0.453925 & -1.190375 & 1.765525\end{array}$

$\begin{array}{llll}0.409664 & -3.292603 & 1.779811\end{array}$

$\begin{array}{llll}-3.400074 & -1.296233 & -1.446980\end{array}$

$\begin{array}{lll}-5.267027 & -0.538266 & -0.377852\end{array}$

$\begin{array}{llll}-5.260491 & 0.604631 & 0.820512\end{array}$

$\begin{array}{lll}-6.330099 & 0.174126 & -1.442024\end{array}$

$\begin{array}{llll}-5.966526 & -1.799001 & 0.479468\end{array}$

$\begin{array}{lll}-4.982081 & 1.930148 & 0.525376\end{array}$

$\begin{array}{lll}-3.757901 & 2.439120 & 1.010815\end{array}$

$\begin{array}{lll}-3.119048 & 1.782006 & 1.589915\end{array}$

$\begin{array}{lll}-3.415991 & 3.740399 & 0.740454\end{array}$

$\begin{array}{llll}-2.486837 & 4.152949 & 1.120483\end{array}$

$\begin{array}{llll}-4.248689 & 4.546670 & -0.083515\end{array}$

$\begin{array}{llll}-3.843378 & 5.851233 & -0.476680\end{array}$

$\begin{array}{llll}-2.895203 & 6.232436 & -0.106490\end{array}$

$\begin{array}{llll}-4.622828 & 6.608497 & -1.322249\end{array}$

$\begin{array}{llll}-4.300209 & 7.600847 & -1.623443\end{array}$

$\begin{array}{llll}-5.844896 & 6.086321 & -1.809648\end{array}$

$\begin{array}{llll}-6.447900 & 6.678260 & -2.492075\end{array}$

$\begin{array}{llll}-6.275844 & 4.832853 & -1.431460\end{array}$

$\begin{array}{llll}-7.211239 & 4.445808 & -1.818835\end{array}$

$\begin{array}{llll}-5.501762 & 4.025514 & -0.552471\end{array}$

$\begin{array}{llll}-5.909913 & 2.701982 & -0.155776\end{array}$

$\begin{array}{llll}-7.269153 & 2.156923 & -0.436138\end{array}$

$\begin{array}{llll}-8.460122 & 2.845551 & -0.001838\end{array}$

$\begin{array}{lll}-8.420922 & 4.023133 & 0.797125\end{array}$

$\begin{array}{llll}-7.464264 & 4.422269 & 1.111009\end{array}$

$\begin{array}{lll}-9.579472 & 4.655891 & 1.193107\end{array}$

$\begin{array}{lll}-9.518953 & 5.549253 & 1.808116\end{array}$

$\begin{array}{lll}-10.845803 & 4.150782 & 0.814696\end{array}$

$\begin{array}{lll}-11.749912 & 4.664885 & 1.127401\end{array}$

$\begin{array}{lll}-10.923669 & 2.997890 & 0.067028\end{array}$

$\begin{array}{llll}-11.888658 & 2.583673 & -0.213172\end{array}$

$\begin{array}{llll}-9.748677 & 2.311574 & -0.344045\end{array}$

$\begin{array}{llll}-9.832613 & 1.090652 & -1.066154\end{array}$

$\begin{array}{lll}-10.811116 & 0.699023 & -1.32928\end{array}$

$\begin{array}{llll}-8.695439 & 0.398840 & -1.398472\end{array}$

$\begin{array}{lll}-8.734297 & -0.551501 & -1.920336\end{array}$ $\begin{array}{llll}-7.430849 & 0.928239 & -1.059933\end{array}$

$\begin{array}{lll}-5.328328 & -3.110859 & 0.181070\end{array}$

$\begin{array}{lll}-4.591384 & -3.316281 & 0.967104\end{array}$

$\begin{array}{lll}-6.342514 & -4.240822 & 0.175232\end{array}$

$\begin{array}{lll}-6.026794 & -5.466224 & 0.773531\end{array}$

$\begin{array}{lll}-5.072330 & -5.581289 & 1.282463\end{array}$

$\begin{array}{llll}-6.925372 & -6.535557 & 0.723178\end{array}$

$\begin{array}{lll}-6.667715 & -7.480489 & 1.193710\end{array}$

$\begin{array}{lll}-8.155359 & -6.385423 & 0.079323\end{array}$

$\begin{array}{lll}-8.858545 & -7.212789 & 0.045222\end{array}$

$\begin{array}{llll}-8.480066 & -5.162146 & -0.516900\end{array}$

$\begin{array}{llll}-9.437497 & -5.037600 & -1.015602\end{array}$

$\begin{array}{lll}-7.578935 & -4.098583 & -0.471284\end{array}$

$\begin{array}{llll}-7.831509 & -3.144332 & -0.925449\end{array}$

$\begin{array}{llll}-4.594867 & -3.051751 & -1.163480\end{array}$

$\begin{array}{llll}-5.333941 & -3.113043 & -1.971274\end{array}$

$\begin{array}{llll}-3.953584 & -3.933841 & -1.249441\end{array}$

$\begin{array}{lll}-6.667696 & -1.732628 & 1.808093\end{array}$

$\begin{array}{lll}-7.141346 & -2.714901 & 1.886829\end{array}$

$\begin{array}{lll}-5.678261 & -1.640576 & 2.970994\end{array}$

$\begin{array}{lll}-5.126373 & -2.826177 & 3.482772\end{array}$

$\begin{array}{lll}-5.452940 & -3.781296 & 3.080077\end{array}$

$\begin{array}{lll}-4.182113 & -2.800245 & 4.512528\end{array}$

$\begin{array}{lll}-3.769070 & -3.731663 & 4.890271\end{array}$

$\begin{array}{lll}-3.790207 & -1.580870 & 5.068553\end{array}$

$-3.063943-1.554835 \quad 5.876380$

$\begin{array}{lll}-4.357194 & -0.394942 & 4.591645\end{array}$

$\begin{array}{lll}-4.076226 & 0.558106 & 5.032405\end{array}$

$\begin{array}{llll}-5.290609 & -0.424605 & 3.553769\end{array}$

$\begin{array}{lll}-5.716280 & 0.505726 & 3.199603\end{array}$

$\begin{array}{lll}-7.806795 & -0.715831 & 1.852940\end{array}$

$\begin{array}{lll}-8.514404 & -0.911719 & 1.044168\end{array}$

$\begin{array}{lll}-7.468521 & 0.315854 & 1.765928\end{array}$

$\begin{array}{lll}-8.334929 & -0.822792 & 2.805420\end{array}$

$\begin{array}{llll}-2.525395 & -1.128371 & 0.544898\end{array}$

$\begin{array}{lll}-1.882648 & -0.253616 & 0.590751\end{array}$

$\begin{array}{lll}-3.221247 & -1.183612 & 1.377390\end{array}$

$\begin{array}{lll}-1.992538 & -2.367425 & 0.062609\end{array}$

$\begin{array}{lll}-2.496844 & -3.272189 & 0.385435\end{array}$

$\begin{array}{llll}-0.734615 & -2.534737 & -0.568679\end{array}$

$\begin{array}{llll}-0.209016 & -1.652817 & -0.907203\end{array}$

$\begin{array}{llll}-4.223093 & -1.143940 & -3.492962\end{array}$

$\begin{array}{lll}-5.215254 & -1.589616 & -3.481799\end{array}$

$\begin{array}{lll}-3.126537 & -2.048714 & -3.521705\end{array}$

$\begin{array}{llll}-3.365293 & -3.111875 & -3.550763\end{array}$

$-1.762002-1.710558-4.097524$

$\begin{array}{llll}-1.805768 & -1.728915 & -5.197661\end{array}$

$\begin{array}{llll}-1.073460 & -2.507913 & -3.807452\end{array}$

$\begin{array}{llll}-1.196672 & -0.358791 & -3.615743\end{array}$

$\begin{array}{llll}-1.429506 & 0.434494 & -4.332715\end{array}$

$\begin{array}{lll}-0.107368 & -0.422413 & -3.582549\end{array}$

$\begin{array}{llll}-1.691505 & 0.068016 & -2.237555\end{array}$

$\begin{array}{llll}-0.934684 & 0.084613 & -1.459702\end{array}$

$\begin{array}{llll}-2.806519 & 0.873812 & -2.021990\end{array}$

$\begin{array}{llll}-2.816421 & 1.461192 & -1.109578\end{array}$

$\begin{array}{llll}-3.753045 & 1.359085 & -3.101152\end{array}$

$\begin{array}{llll}-4.645288 & 1.750094 & -2.607258\end{array}$

$\begin{array}{lll}-3.289392 & 2.204813 & -3.630447\end{array}$

$\begin{array}{llll}-4.170809 & 0.260288 & -4.102468\end{array}$

$\begin{array}{llll}-3.492715 & 0.252276 & -4.962250\end{array}$

$\begin{array}{llll}-5.160721 & 0.506491 & -4.498797\end{array}$

$\begin{array}{llll}-0.336566 & -3.784974 & -1.225126\end{array}$

$\begin{array}{lll}-0.870963 & -5.040132 & -0.873228\end{array}$

$\begin{array}{llll}0.627007 & -3.732217 & -2.250687\end{array}$

$\begin{array}{lll}-0.457009 & -6.196849 & -1.530564\end{array}$

$\begin{array}{llll}-1.601347 & -5.116504 & -0.074064\end{array}$

$\begin{array}{llll}1.036626 & -4.889362 & -2.910012\end{array}$

$\begin{array}{llll}1.058507 & -2.774554 & -2.516059\end{array}$

$\begin{array}{llll}0.497790 & -6.127654 & -2.551971\end{array}$

$\begin{array}{llll}-0.876991 & -7.156881 & -1.244167\end{array}$

$\begin{array}{lll}1.782713 & -4.824630 & -3.697145\end{array}$

$\begin{array}{llll}0.817686 & -7.031973 & -3.061444\end{array}$

$\begin{array}{llll}2.527339 & -3.998361 & 0.475398\end{array}$

$\begin{array}{llll}3.530290 & -4.417758 & -0.497346\end{array}$

$\begin{array}{llll}3.343419 & -5.458902 & -0.776638\end{array}$ 


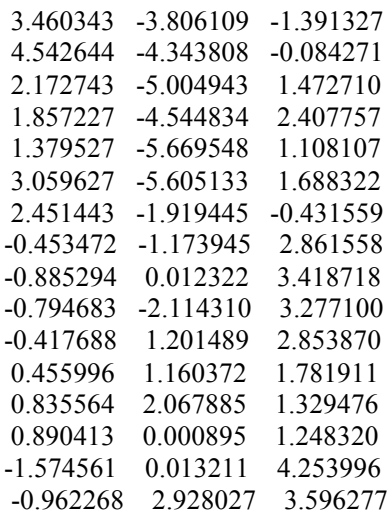

\section{Conformer_2}

Number of imaginary frequencies : 1

The smallest frequency is : $-247.7139 \mathrm{~cm}(-1)$

Electronic energy :

$\mathrm{HF}=-7222.6348455$

$\begin{array}{lc}\text { Zero-point correction }= & 1.755698 \\ \text { (Hartree/Particle) } & 1.874114 \\ \text { Thermal correction to Energy= } & 1.875058 \\ \text { Thermal correction to Enthalpy= } & 1.593085 \\ \text { Thermal correction to Gibbs Free Energy= } & -7220.879148 \\ \text { Sum of electronic and zero-point Energies }= & -7220.760732 \\ \text { Sum of electronic and thermal Energies }= & -7220.759787 \\ \text { Sum of electronic and thermal Enthalpies }= & -7221.041761 \\ \text { Sum of electronic and thermal Free Energies }= & -7204\end{array}$

Cartesian Coordinates

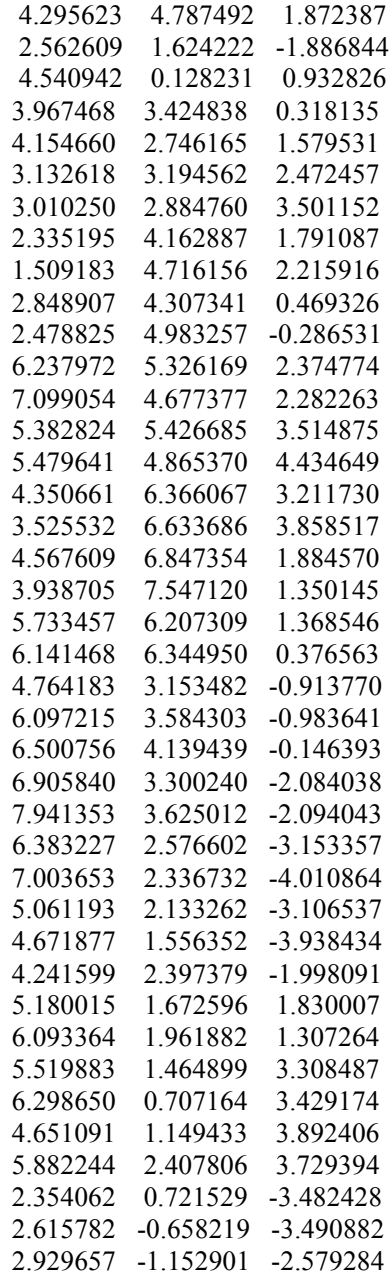

$\begin{array}{llll}2.641470 & -2.470510 & -4.648989\end{array}$

$\begin{array}{llll}1.978419 & -0.786810 & -5.822730\end{array}$

$\begin{array}{llll}1.823142 & -1.370941 & -6.725257\end{array}$

$\begin{array}{llll}1.718464 & 0.586225 & -5.822998\end{array}$

$\begin{array}{llll}1.362811 & 1.073956 & -6.726142\end{array}$

$\begin{array}{llll}1.908138 & 1.338073 & -4.662683\end{array}$

$\begin{array}{llll}1.691750 & 2.400580 & -4.680106\end{array}$

$\begin{array}{llll}1.403399 & 3.038015 & -2.070967\end{array}$

$\begin{array}{llll}1.740109 & 4.197411 & -2.788820\end{array}$

$\begin{array}{llll}2.732744 & 4.302372 & -3.216587\end{array}$

$\begin{array}{llll}0.805900 & 5.222436 & -2.948334\end{array}$

$\begin{array}{llll}1.076782 & 6.116255 & -3.503200\end{array}$

$\begin{array}{llll}-0.475666 & 5.094848 & -2.402797\end{array}$

$\begin{array}{llll}-1.208582 & 5.885999 & -2.533197\end{array}$

$\begin{array}{llll}-0.814561 & 3.944820 & -1.687091\end{array}$

$\begin{array}{llll}-1.807678 & 3.838848 & -1.267574\end{array}$

$\begin{array}{llll}0.124798 & 2.927192 & -1.509640\end{array}$

$\begin{array}{lll}-0.137151 & 2.044552 & -0.936934\end{array}$

$\begin{array}{llll}5.833938 & -0.351367 & -0.288838\end{array}$

$\begin{array}{llll}5.486480 & -1.402366 & -1.150004\end{array}$

$\begin{array}{llll}4.518701 & -1.879129 & -1.037136\end{array}$

$\begin{array}{llll}6.371280 & -1.830115 & -2.138981\end{array}$

$\begin{array}{llll}7.597866 & -1.194396 & -2.320209\end{array}$

$\begin{array}{llll}8.275368 & -1.511209 & -3.105003\end{array}$

$\begin{array}{llll}7.930409 & -0.131974 & -1.479227\end{array}$

$\begin{array}{llll}7.072167 & 0.277407 & -0.458200\end{array}$

$\begin{array}{llll}7.378693 & 1.096672 & 0.177374\end{array}$

$\begin{array}{llll}4.568902 & -1.271331 & 2.130945\end{array}$

$\begin{array}{llll}3.559919 & -1.294997 & 3.107218\end{array}$

$\begin{array}{lll}2.868059 & -0.465336 & 3.195416\end{array}$

$\begin{array}{lll}3.410979 & -2.401658 & 3.940242\end{array}$

$\begin{array}{lll}4.234190 & -3.519186 & 3.797623\end{array}$

$\begin{array}{llll}4.088322 & -4.396198 & 4.418296\end{array}$

$\begin{array}{llll}5.223581 & -3.500989 & 2.817940\end{array}$

$\begin{array}{llll}5.409909 & -2.382533 & 2.004508\end{array}$

$\begin{array}{lll}6.188789 & -2.406394 & 1.252857\end{array}$

$\begin{array}{llll}2.440313 & 0.234733 & -0.102469\end{array}$

$\begin{array}{llll}6.092378 & -4.714728 & 2.636762\end{array}$

$\begin{array}{llll}2.273746 & -2.466725 & 4.920768\end{array}$

$\begin{array}{llll}5.996575 & -3.025872 & -2.969706\end{array}$

$\begin{array}{llll}9.224913 & 0.592926 & -1.726976\end{array}$

$\begin{array}{llll}9.227679 & 1.197136 & -2.942030\end{array}$

$\begin{array}{llll}9.466905 & 1.553165 & -0.807841\end{array}$

$10.282414 \quad-0.251294-1.718444$

$\begin{array}{llll}6.178280 & -4.182112 & -2.283313\end{array}$

$\begin{array}{llll}6.719959 & -3.118181 & -4.103173\end{array}$

$\begin{array}{lll}4.686184 & -3.002385 & -3.327216\end{array}$

$\begin{array}{lll}7.069189 & -4.789413 & 3.569010\end{array}$

$\begin{array}{lll}6.695920 & -4.731765 & 1.426585\end{array}$

$\begin{array}{lll}5.369994 & -5.857976 & 2.739356\end{array}$

$\begin{array}{llll}2.645663 & -3.031272 & 6.090263\end{array}$

$\begin{array}{lll}1.758173 & -1.251193 & 5.200007\end{array}$

$\begin{array}{lll}1.251330 & -3.226804 & 4.437680\end{array}$

$\begin{array}{llll}1.975938 & -2.751414 & 0.403912\end{array}$

$\begin{array}{lll}0.812506 & -2.465310 & 1.213713\end{array}$

$\begin{array}{llll}0.454112 & -1.190336 & 1.765512\end{array}$

$\begin{array}{lll}0.409808 & -3.292570 & 1.779866\end{array}$

$\begin{array}{llll}-3.400234 & -1.296174 & -1.446510\end{array}$

$\begin{array}{lll}-5.267086 & -0.538255 & -0.377160\end{array}$

$\begin{array}{llll}-5.260470 & 0.604660 & 0.821190\end{array}$

$\begin{array}{lll}-6.330314 & 0.174058 & -1.441230\end{array}$

$\begin{array}{lll}-5.966438 & -1.799016 & 0.480245\end{array}$

$\begin{array}{lll}-4.982086 & 1.930171 & 0.525982\end{array}$

$\begin{array}{lll}-3.757872 & 2.439171 & 1.011303\end{array}$

$\begin{array}{lll}-3.119001 & 1.782107 & 1.590437\end{array}$

$\begin{array}{lll}-3.415949 & 3.740415 & 0.740790\end{array}$

$\begin{array}{llll}-2.486755 & 4.152976 & 1.120707\end{array}$

$\begin{array}{llll}-4.248687 & 4.546626 & -0.083197\end{array}$

$\begin{array}{llll}-3.843371 & 5.851138 & -0.476529\end{array}$

$\begin{array}{llll}-2.895150 & 6.232347 & -0.106463\end{array}$

$\begin{array}{llll}-4.622876 & 6.608340 & -1.322102\end{array}$

$\begin{array}{llll}-4.300251 & 7.600649 & -1.623428\end{array}$

$\begin{array}{llll}-5.845004 & 6.086154 & -1.809338\end{array}$

$\begin{array}{llll}-6.448053 & 6.678045 & -2.491767\end{array}$

$-6.275955 \quad 4.832736-1.430989$ 
$\begin{array}{llll}-7.211396 & 4.445681 & -1.818245\end{array}$

$\begin{array}{llll}-5.501815 & 4.025460 & -0.551994\end{array}$

$\begin{array}{llll}-5.909961 & 2.701970 & -0.155150\end{array}$

$\begin{array}{llll}-7.269236 & 2.156915 & -0.435348\end{array}$

$\begin{array}{lll}-8.460149 & 2.845592 & -0.000970\end{array}$

$\begin{array}{lll}-8.420846 & 4.023231 & 0.797902\end{array}$

$\begin{array}{lll}-7.464150 & 4.422372 & 1.111661\end{array}$

$\begin{array}{lll}-9.579345 & 4.656040 & 1.193954\end{array}$

$\begin{array}{llll}-9.518747 & 5.549448 & 1.808889\end{array}$

$\begin{array}{lll}-10.845723 & 4.150924 & 0.815712\end{array}$

$\begin{array}{lll}-11.749792 & 4.665065 & 1.128472\end{array}$

$\begin{array}{lll}-10.923686 & 2.997979 & 0.068135\end{array}$

$\begin{array}{lll}-11.888710 & 2.583757 & -0.211936\end{array}$

$\begin{array}{llll}-9.748747 & 2.311615 & -0.343011\end{array}$

$\begin{array}{llll}-9.832777 & 1.090649 & -1.065035\end{array}$

$\begin{array}{lll}-10.811314 & 0.699024 & -1.328042\end{array}$

$\begin{array}{llll}-8.695649 & 0.398798 & -1.397428\end{array}$

$-8.734578-0.551571-1.919234$

$\begin{array}{llll}-7.431015 & 0.928198 & -1.059053\end{array}$

$\begin{array}{lll}-5.328274 & -3.110862 & 0.181718\end{array}$

$\begin{array}{lll}-4.591216 & -3.316322 & 0.967637\end{array}$

$\begin{array}{lll}-6.342464 & -4.240823 & 0.175982\end{array}$

$\begin{array}{lll}-6.026689 & -5.466228 & 0.774243\end{array}$

$\begin{array}{llll}-5.072166 & -5.581302 & 1.283064\end{array}$

$\begin{array}{lll}-6.925278 & -6.535556 & 0.723982\end{array}$

$\begin{array}{lll}-6.667575 & -7.480491 & 1.194482\end{array}$

$\begin{array}{llll}-8.155332 & -6.385413 & 0.080258\end{array}$

$\begin{array}{lll}-8.858525 & -7.212776 & 0.046225\end{array}$

$\begin{array}{llll}-8.480097 & -5.162130 & -0.515923\end{array}$

$\begin{array}{llll}-9.437582 & -5.037577 & -1.014521\end{array}$

$\begin{array}{llll}-7.578957 & -4.098572 & -0.470395\end{array}$

$\begin{array}{llll}-7.831576 & -3.144314 & -0.924523\end{array}$

$\begin{array}{llll}-4.594997 & -3.051697 & -1.162929\end{array}$

$\begin{array}{llll}-5.334177 & -3.112961 & -1.970627\end{array}$

$\begin{array}{llll}-3.953725 & -3.933785 & -1.249007\end{array}$

$\begin{array}{lll}-6.667450 & -1.732679 & 1.808953\end{array}$

$\begin{array}{lll}-7.141101 & -2.714949 & 1.887719\end{array}$

$\begin{array}{lll}-5.677859 & -1.640676 & 2.971724\end{array}$

$\begin{array}{lll}-5.125934 & -2.826302 & 3.483404\end{array}$

$\begin{array}{lll}-5.452590 & -3.781408 & 3.080746\end{array}$

$\begin{array}{lll}-4.181513 & -2.800411 & 4.513015\end{array}$

$\begin{array}{llll}-3.768443 & -3.731847 & 4.890684\end{array}$

$\begin{array}{lll}-3.789470 & -1.581054 & 5.068985\end{array}$

$\begin{array}{lll}-3.063075 & -1.555052 & 5.876694\end{array}$

$\begin{array}{lll}-4.356494 & -0.395103 & 4.592179\end{array}$

$\begin{array}{lll}-4.075423 & 0.557933 & 5.032900\end{array}$

$\begin{array}{lll}-5.290074 & -0.424724 & 3.554450\end{array}$

$\begin{array}{lll}-5.715768 & 0.505623 & 3.200356\end{array}$

$\begin{array}{lll}-7.806535 & -0.715873 & 1.853966\end{array}$

$\begin{array}{lll}-8.514244 & -0.911732 & 1.045272\end{array}$

$\begin{array}{lll}-7.468267 & 0.315812 & 1.766939\end{array}$

$\begin{array}{lll}-8.334557 & -0.822858 & 2.806506\end{array}$

$\begin{array}{lll}-2.525330 & -1.128326 & 0.545249\end{array}$

$\begin{array}{lll}-1.882521 & -0.253615 & 0.591033\end{array}$

$\begin{array}{lll}-3.221098 & -1.183503 & 1.377814\end{array}$

$\begin{array}{lll}-1.992585 & -2.367429 & 0.062946\end{array}$

$\begin{array}{llll}-2.496920 & -3.272146 & 0.385869\end{array}$

$\begin{array}{llll}-0.734736 & -2.534814 & -0.568473\end{array}$

$\begin{array}{lll}-0.209166 & -1.652923 & -0.907126\end{array}$

$\begin{array}{llll}-4.223446 & -1.143893 & -3.492437\end{array}$

$-5.215604-1.589573-3.481180$

$\begin{array}{lll}-3.126887 & -2.048656 & -3.521275\end{array}$

$\begin{array}{lll}-3.365632 & -3.111820 & -3.550308\end{array}$

$\begin{array}{llll}-1.762395 & -1.710482 & -4.097188\end{array}$

$\begin{array}{llll}-1.806237 & -1.728822 & -5.197322\end{array}$

$-1.073826-2.507836-3.807175$

$\begin{array}{llll}-1.197049 & -0.358716 & -3.615417\end{array}$

$\begin{array}{llll}-1.429926 & 0.434583 & -4.332360\end{array}$

$\begin{array}{lll}-0.107745 & -0.422327 & -3.582268\end{array}$

$\begin{array}{llll}-1.691816 & 0.068050 & -2.237190\end{array}$

$\begin{array}{llll}-0.934936 & 0.084645 & -1.459396\end{array}$

$\begin{array}{llll}-2.806808 & 0.873864 & -2.021551\end{array}$

$\begin{array}{llll}-2.816638 & 1.461267 & -1.109154\end{array}$

$\begin{array}{llll}-3.753409 & 1.359134 & -3.100648\end{array}$

$\begin{array}{llll}-4.645627 & 1.750118 & -2.606691\end{array}$ $\begin{array}{llll}-3.289805 & 2.204874 & -3.629966\end{array}$

$\begin{array}{lll}-4.171213 & 0.260335 & -4.101946\end{array}$

$\begin{array}{llll}-3.493158 & 0.252329 & -4.961759\end{array}$

$\begin{array}{llll}-5.161147 & 0.506527 & -4.498227\end{array}$

$\begin{array}{llll}-0.336805 & -3.785097 & -1.224912\end{array}$

$\begin{array}{llll}-0.871252 & -5.040215 & -0.872944\end{array}$

$\begin{array}{llll}0.626705 & -3.732429 & -2.250537\end{array}$

$-0.457389-6.196979-1.530256$

$\begin{array}{lll}-1.601609 & -5.116522 & -0.073750\end{array}$

$\begin{array}{llll}1.036239 & -4.889622 & -2.909832\end{array}$

$\begin{array}{llll}1.058229 & -2.774799 & -2.515983\end{array}$

$\begin{array}{llll}0.497368 & -6.127874 & -2.551707\end{array}$

$\begin{array}{llll}-0.877411 & -7.156977 & -1.243805\end{array}$

$\begin{array}{llll}1.782285 & -4.824960 & -3.697009\end{array}$

$\begin{array}{llll}0.817197 & -7.032230 & -3.061157\end{array}$

$\begin{array}{llll}2.527397 & -3.998354 & 0.475280\end{array}$

$\begin{array}{llll}3.530277 & -4.417740 & -0.497543\end{array}$

$\begin{array}{llll}3.343464 & -5.458919 & -0.776739\end{array}$

$\begin{array}{llll}3.460179 & -3.806158 & -1.391558\end{array}$

$\begin{array}{llll}4.542672 & -4.343676 & -0.084589\end{array}$

$\begin{array}{llll}2.172939 & -5.004906 & 1.472669\end{array}$

$\begin{array}{llll}1.857476 & -4.544767 & 2.407720\end{array}$

$\begin{array}{lll}1.379726 & -5.669570 & 1.108166\end{array}$

$\begin{array}{llll}3.059874 & -5.605038 & 1.688239\end{array}$

$\begin{array}{llll}2.451366 & -1.919479 & -0.431755\end{array}$

$\begin{array}{lll}-0.453161 & -1.173844 & 2.861646\end{array}$

$\begin{array}{lll}-0.884894 & 0.012454 & 3.418815\end{array}$

$\begin{array}{lll}-0.794345 & -2.114184 & 3.277264\end{array}$

$\begin{array}{lll}-0.417329 & 1.201587 & 2.853865\end{array}$

$\begin{array}{llll}0.456225 & 1.160407 & 1.781803\end{array}$

$\begin{array}{lll}0.835742 & 2.067896 & 1.329277\end{array}$

$\begin{array}{lll}0.890569 & 0.000905 & 1.248214\end{array}$

$\begin{array}{lll}-1.574064 & 0.013389 & 4.254175\end{array}$

$\begin{array}{lll}-0.961760 & 2.928178 & 3.596240\end{array}$

\section{Conformer_3}

Number of imaginary frequencies : 1

The smallest frequency is : $-247.6851 \mathrm{~cm}(-1)$

Electronic energy :

Zero-point correction=

$\mathrm{HF}=-7222.6348466$

(Hartree/Particle)

Thermal correction to Energy=

1.755697

Thermal correction to Enthalpy=

1.874112

1.875056

Thermal correction to Gibbs Free Energy $=1.593051$

Sum of electronic and zero-point Energies $=\quad-7220.879150$

Sum of electronic and thermal Energies=

Sum of electronic and thermal Enthalpies=

$-7220.760735$

Sum of electronic and thermal Free Energies $=\quad-7221.041796$

Cartesian Coordinates

$\begin{array}{rrr}4.294958 & 4.787330 & 1.873779 \\ 2.562885 & 1.624402 & -1.886218 \\ 4.540623 & 0.128186 & 0.933775 \\ 3.967157 & 3.424827 & 0.319317 \\ 4.154106 & 2.746036 & 1.580680 \\ 3.131870 & 3.194315 & 2.473442 \\ 3.009300 & 2.884410 & 3.502081 \\ 2.334563 & 4.162687 & 1.792003 \\ 1.508442 & 4.715879 & 2.216717 \\ 2.848545 & 4.307291 & 0.470363 \\ 2.478603 & 4.983273 & -0.285504 \\ 6.237185 & 5.325973 & 2.376683 \\ 7.098302 & 4.677205 & 2.284305 \\ 5.381765 & 5.426356 & 3.516591 \\ 5.478367 & 4.864942 & 4.436327 \\ 4.349662 & 6.365758 & 3.213300 \\ 3.524376 & 6.633302 & 3.859918 \\ 4.566920 & 6.847188 & 1.886243 \\ 3.938132 & 7.547000 & 1.351741 \\ 5.732900 & 6.207214 & 1.370430 \\ 6.141151 & 6.344956 & 0.378559 \\ 4.764171 & 3.153624 & -0.912430 \\ 6.097214 & 3.584475 & -0.981923\end{array}$


$\begin{array}{llll}6.500558 & 4.139492 & -0.144503\end{array}$

$\begin{array}{llll}6.906105 & 3.300581 & -2.082167\end{array}$

$\begin{array}{llll}7.941618 & 3.625362 & -2.091874\end{array}$

$\begin{array}{llll}6.383752 & 2.577108 & -3.151724\end{array}$

$\begin{array}{llll}7.004379 & 2.337393 & -4.009129\end{array}$

$\begin{array}{llll}5.061719 & 2.133727 & -3.105275\end{array}$

$\begin{array}{llll}4.672605 & 1.556947 & -3.937355\end{array}$

$\begin{array}{llll}4.241868 & 2.397646 & -1.996972\end{array}$

$\begin{array}{llll}5.179449 & 1.672485 & 1.831247\end{array}$

$\begin{array}{lll}6.092875 & 1.961865 & 1.308696\end{array}$

$\begin{array}{lll}5.519036 & 1.464650 & 3.309769\end{array}$

$\begin{array}{lll}6.297820 & 0.706942 & 3.430532\end{array}$

$\begin{array}{lll}4.650144 & 1.149075 & 3.893474\end{array}$

$\begin{array}{llll}5.881260 & 2.407529 & 3.730854\end{array}$

$\begin{array}{llll}2.354751 & 0.721876 & -3.481954\end{array}$

$\begin{array}{llll}2.616715 & -0.657825 & -3.490535\end{array}$

$\begin{array}{llll}2.930536 & -1.152566 & -2.578949\end{array}$

$\begin{array}{llll}2.435866 & -1.404912 & -4.656709\end{array}$

$\begin{array}{llll}2.642955 & -2.469964 & -4.648863\end{array}$

$\begin{array}{llll}1.979804 & -0.786238 & -5.822516\end{array}$

$\begin{array}{llll}1.824805 & -1.370282 & -6.725147\end{array}$

$\begin{array}{llll}1.719603 & 0.586750 & -5.822657\end{array}$

$\begin{array}{llll}1.364032 & 1.074533 & -6.725805\end{array}$

$\begin{array}{llll}1.908934 & 1.338487 & -4.662213\end{array}$

$\begin{array}{llll}1.692385 & 2.400963 & -4.679551\end{array}$

$\begin{array}{llll}1.403723 & 3.038220 & -2.070468\end{array}$

$\begin{array}{llll}1.740632 & 4.197680 & -2.788125\end{array}$

$\begin{array}{llll}2.733362 & 4.302653 & -3.215666\end{array}$

$\begin{array}{llll}0.806494 & 5.222753 & -2.947743\end{array}$

$\begin{array}{lll}1.077530 & 6.116620 & -3.502457\end{array}$

$\begin{array}{llll}-0.475194 & 5.095154 & -2.402498\end{array}$

$\begin{array}{lll}-1.208052 & 5.886348 & -2.532968\end{array}$

$\begin{array}{llll}-0.814287 & 3.945060 & -1.686992\end{array}$

$\begin{array}{llll}-1.807496 & 3.839092 & -1.267696\end{array}$

$\begin{array}{llll}0.124994 & 2.927377 & -1.509441\end{array}$

$\begin{array}{llll}-0.137115 & 2.044688 & -0.936884\end{array}$

$\begin{array}{llll}5.833958 & -0.351192 & -0.287616\end{array}$

$\begin{array}{llll}5.486806 & -1.402125 & -1.148990\end{array}$

$\begin{array}{llll}4.519031 & -1.878974 & -1.036443\end{array}$

$\begin{array}{llll}6.371917 & -1.829723 & -2.137754\end{array}$

$\begin{array}{llll}7.598521 & -1.193918 & -2.318565\end{array}$

$\begin{array}{llll}8.276267 & -1.510620 & -3.103191\end{array}$

$\begin{array}{llll}7.930777 & -0.131587 & -1.477359\end{array}$

$\begin{array}{llll}7.072211 & 0.277652 & -0.456548\end{array}$

$\begin{array}{llll}7.378523 & 1.096853 & 0.179209\end{array}$

$\begin{array}{llll}4.568405 & -1.271487 & 2.131768\end{array}$

$\begin{array}{llll}3.559253 & -1.295272 & 3.107862\end{array}$

$\begin{array}{lll}2.867355 & -0.465634 & 3.196010\end{array}$

$\begin{array}{llll}3.410223 & -2.402002 & 3.940776\end{array}$

$\begin{array}{lll}4.233523 & -3.519474 & 3.798226\end{array}$

$\begin{array}{llll}4.087597 & -4.396538 & 4.418812\end{array}$

$\begin{array}{lll}5.223071 & -3.501165 & 2.818705\end{array}$

$\begin{array}{lll}5.409479 & -2.382643 & 2.005380\end{array}$

$\begin{array}{llll}6.188478 & -2.406418 & 1.253851\end{array}$

$\begin{array}{lll}2.440234 & 0.234668 & -0.102059\end{array}$

$\begin{array}{lll}6.091955 & -4.714855 & 2.637605\end{array}$

$\begin{array}{llll}2.272794 & -2.467220 & 4.921060\end{array}$

$\begin{array}{llll}5.997555 & -3.025447 & -2.968678\end{array}$

$\begin{array}{llll}9.225336 & 0.593380 & -1.724623\end{array}$

$\begin{array}{llll}9.228344 & 1.197998 & -2.939474\end{array}$

$\begin{array}{llll}9.467170 & 1.553307 & -0.805121\end{array}$

$\begin{array}{lll}10.282818 & -0.250865 & -1.716178\end{array}$

$\begin{array}{llll}6.179155 & -4.181721 & -2.282314\end{array}$

$\begin{array}{llll}6.721289 & -3.117617 & -4.101932\end{array}$

$\begin{array}{lll}4.687264 & -3.002046 & -3.326574\end{array}$

$\begin{array}{lll}7.068643 & -4.789525 & 3.569983\end{array}$

$\begin{array}{lll}6.695666 & -4.731826 & 1.427509\end{array}$

$\begin{array}{llll}5.369611 & -5.858140 & 2.740061\end{array}$

$\begin{array}{llll}2.644496 & -3.031834 & 6.090591\end{array}$

$\begin{array}{llll}1.757085 & -1.251741 & 5.200282\end{array}$

$\begin{array}{lll}1.250530 & -3.227328 & 4.437695\end{array}$

$\begin{array}{llll}1.975987 & -2.751479 & 0.404006\end{array}$

$\begin{array}{lll}0.812394 & -2.465505 & 1.213612\end{array}$

$\begin{array}{llll}0.453769 & -1.190609 & 1.765429\end{array}$

$\begin{array}{lll}0.409643 & -3.292839 & 1.779616\end{array}$ $\begin{array}{llll}-3.399949 & -1.296318 & -1.447397\end{array}$

$\begin{array}{llll}-5.266925 & -0.538237 & -0.378408\end{array}$

$\begin{array}{lll}-5.260414 & 0.604596 & 0.820015\end{array}$

$\begin{array}{llll}-6.329826 & 0.174291 & -1.442661\end{array}$

$\begin{array}{lll}-5.966617 & -1.798955 & 0.478771\end{array}$

$\begin{array}{lll}-4.981987 & 1.930136 & 0.524989\end{array}$

$\begin{array}{lll}-3.757844 & 2.439078 & 1.010553\end{array}$

$\begin{array}{lll}-3.119024 & 1.781930 & 1.589650\end{array}$

$\begin{array}{llll}-3.415919 & 3.740378 & 0.740319\end{array}$

$\begin{array}{lll}-2.486791 & 4.152895 & 1.120446\end{array}$

$\begin{array}{llll}-4.248559 & 4.546714 & -0.083645\end{array}$

$\begin{array}{llll}-3.843220 & 5.851306 & -0.476684\end{array}$

$\begin{array}{llll}-2.895072 & 6.232481 & -0.106398\end{array}$

$\begin{array}{llll}-4.622612 & 6.608632 & -1.322251\end{array}$

$\begin{array}{llll}-4.299973 & 7.601005 & -1.623348\end{array}$

$\begin{array}{llll}-5.844644 & 6.086491 & -1.809775\end{array}$

$\begin{array}{llll}-6.447601 & 6.678480 & -2.492200\end{array}$

$\begin{array}{llll}-6.275617 & 4.832993 & -1.431712\end{array}$

$\begin{array}{llll}-7.210984 & 4.445977 & -1.819185\end{array}$

$\begin{array}{llll}-5.501595 & 4.025589 & -0.552731\end{array}$

$\begin{array}{llll}-5.909771 & 2.702024 & -0.156169\end{array}$

$\begin{array}{llll}-7.268986 & 2.156991 & -0.436698\end{array}$

$\begin{array}{llll}-8.459996 & 2.845579 & -0.002451\end{array}$

$\begin{array}{llll}-8.420872 & 4.023080 & 0.796634\end{array}$

$\begin{array}{lll}-7.464243 & 4.422181 & 1.110652\end{array}$

$\begin{array}{llll}-9.579460 & 4.655801 & 1.192564\end{array}$

$\begin{array}{lll}-9.519002 & 5.549100 & 1.807670\end{array}$

$\begin{array}{lll}-10.845754 & 4.150733 & 0.813972\end{array}$

$\begin{array}{lll}-11.749894 & 4.664807 & 1.126637\end{array}$

$\begin{array}{lll}-10.923548 & 2.997917 & 0.066178\end{array}$

$\begin{array}{llll}-11.888511 & 2.583735 & -0.214163\end{array}$

$\begin{array}{llll}-9.748516 & 2.311639 & -0.344844\end{array}$

$\begin{array}{llll}-9.832381 & 1.090790 & -1.067085\end{array}$

$\begin{array}{llll}-10.810859 & 0.699191 & -1.330353\end{array}$

$\begin{array}{lll}-8.695175 & 0.399007 & -1.399355\end{array}$

$\begin{array}{lll}-8.733981 & -0.551281 & -1.921319\end{array}$

$\begin{array}{llll}-7.430620 & 0.928369 & -1.060628\end{array}$

$\begin{array}{llll}-5.328537 & -3.110859 & 0.180333\end{array}$

$\begin{array}{llll}-4.591710 & -3.316429 & 0.966435\end{array}$

$\begin{array}{lll}-6.342864 & -4.240697 & 0.174294\end{array}$

$\begin{array}{lll}-6.027401 & -5.466162 & 0.772599\end{array}$

$\begin{array}{lll}-5.073024 & -5.581375 & 1.281661\end{array}$

$\begin{array}{lll}-6.926122 & -6.535367 & 0.722088\end{array}$

$\begin{array}{lll}-6.668663 & -7.480350 & 1.192627\end{array}$

$\begin{array}{lll}-8.155998 & -6.385041 & 0.078065\end{array}$

$\begin{array}{lll}-8.859296 & -7.212306 & 0.043841\end{array}$

$\begin{array}{llll}-8.480448 & -5.161700 & -0.518169\end{array}$

$\begin{array}{llll}-9.437791 & -5.037005 & -1.017004\end{array}$

$\begin{array}{llll}-7.579174 & -4.098266 & -0.472393\end{array}$

$\begin{array}{llll}-7.831548 & -3.143964 & -0.926565\end{array}$

$\begin{array}{llll}-4.594914 & -3.051733 & -1.164129\end{array}$

$\begin{array}{llll}-5.333900 & -3.112909 & -1.972013\end{array}$

$\begin{array}{llll}-3.953705 & -3.933881 & -1.250066\end{array}$

$\begin{array}{lll}-6.667910 & -1.732617 & 1.807334\end{array}$

$\begin{array}{lll}-7.141656 & -2.714853 & 1.885947\end{array}$

$\begin{array}{lll}-5.678581 & -1.640743 & 2.970338\end{array}$

$\begin{array}{lll}-5.126858 & -2.826430 & 3.482094\end{array}$

$\begin{array}{lll}-5.453476 & -3.781494 & 3.079308\end{array}$

$\begin{array}{llll}-4.182695 & -2.800655 & 4.511944\end{array}$

$\begin{array}{lll}-3.769777 & -3.732136 & 4.889666\end{array}$

$\begin{array}{lll}-3.790726 & -1.581353 & 5.068086\end{array}$

$\begin{array}{llll}-3.064538 & -1.555440 & 5.875985\end{array}$

$\begin{array}{lll}-4.357553 & -0.395341 & 4.591198\end{array}$

$\begin{array}{lll}-4.076539 & 0.557653 & 5.032048\end{array}$

$\begin{array}{lll}-5.290867 & -0.424847 & 3.553228\end{array}$

$\begin{array}{lll}-5.716414 & 0.505547 & 3.199079\end{array}$

$\begin{array}{lll}-7.806921 & -0.715722 & 1.852157\end{array}$

$\begin{array}{lll}-8.514477 & -0.911484 & 1.043308\end{array}$

$\begin{array}{lll}-7.468547 & 0.315939 & 1.765255\end{array}$

$\begin{array}{lll}-8.335148 & -0.822710 & 2.804584\end{array}$

$\begin{array}{llll}-2.525478 & -1.128834 & 0.544591\end{array}$

$\begin{array}{lll}-1.882771 & -0.254063 & 0.590686\end{array}$

$\begin{array}{lll}-3.221423 & -1.184265 & 1.376996\end{array}$

$\begin{array}{lll}-1.992482 & -2.367782 & 0.062158\end{array}$

$\begin{array}{lll}-2.496764 & -3.272643 & 0.384755\end{array}$ 
$\begin{array}{llll}-0.734470 & -2.534926 & -0.568990\end{array}$

$\begin{array}{llll}-0.208889 & -1.652937 & -0.907357\end{array}$

$-4.222766-1.143785 \quad-3.493461$

$\begin{array}{llll}-5.214958 & -1.589400 & -3.482439\end{array}$

$\begin{array}{lll}-3.126270 & -2.048629 & -3.522160\end{array}$

$\begin{array}{lll}-3.365101 & -3.111771 & -3.551335\end{array}$

$\begin{array}{llll}-1.761641 & -1.710517 & -4.097784\end{array}$

$-1.805264-1.728805-5.197928$

$-1.073186-2.507930-3.807666$

$\begin{array}{lll}-1.196282 & -0.358810 & -3.615857\end{array}$

$\begin{array}{llll}-1.429011 & 0.434533 & -4.332799\end{array}$

$\begin{array}{lll}-0.106983 & -0.422492 & -3.582572\end{array}$

$\begin{array}{lll}-1.691220 & 0.067941 & -2.237687\end{array}$

$\begin{array}{llll}-0.934483 & 0.084433 & -1.459751\end{array}$

$\begin{array}{llll}-2.806213 & 0.873769 & -2.022172\end{array}$

$\begin{array}{llll}-2.816183 & 1.461068 & -1.109709\end{array}$

$\begin{array}{llll}-3.752604 & 1.359180 & -3.101388\end{array}$

$\begin{array}{lll}-4.644873 & 1.750201 & -2.607555\end{array}$

$\begin{array}{llll}-3.288844 & 2.204923 & -3.630567\end{array}$

$\begin{array}{lll}-4.170329 & 0.260491 & -4.102840\end{array}$

$\begin{array}{llll}-3.492142 & 0.252508 & -4.962549\end{array}$

$\begin{array}{llll}-5.160183 & 0.506790 & -4.499253\end{array}$

$\begin{array}{lll}-0.336216 & -3.785072 & -1.225482\end{array}$

$\begin{array}{llll}-0.870450 & -5.040323 & -0.873671\end{array}$

$\begin{array}{llll}0.627417 & -3.732126 & -2.250976\end{array}$

$-0.456288-6.196949-1.531035$

$\begin{array}{llll}-1.600859 & -5.116835 & -0.074543\end{array}$

$\begin{array}{llll}1.037240 & -4.889180 & -2.910334\end{array}$

$\begin{array}{llll}1.058799 & -2.774389 & -2.516269\end{array}$

$\begin{array}{llll}0.498560 & -6.127566 & -2.552384\end{array}$

$\begin{array}{llll}-0.876143 & -7.157056 & -1.244703\end{array}$

$\begin{array}{llll}1.783365 & -4.824305 & -3.697418\end{array}$

$\begin{array}{llll}0.818618 & -7.031816 & -3.061879\end{array}$

$\begin{array}{llll}2.527494 & -3.998398 & 0.475385\end{array}$

$\begin{array}{llll}3.530581 & -4.417691 & -0.497265\end{array}$

$\begin{array}{llll}3.343786 & -5.458825 & -0.776645\end{array}$

$\begin{array}{llll}3.460718 & -3.805989 & -1.391217\end{array}$

$\begin{array}{llll}4.542879 & -4.343724 & -0.084058\end{array}$

$\begin{array}{llll}2.172855 & -5.005047 & 1.472615\end{array}$

$\begin{array}{llll}1.857223 & -4.545000 & 2.407654\end{array}$

$\begin{array}{llll}1.379711 & -5.669678 & 1.107903\end{array}$

$\begin{array}{llll}3.059751 & -5.605201 & 1.688282\end{array}$

$2.451543 \quad-1.919452 \quad-0.431500$

$\begin{array}{lll}-0.453691 & -1.174294 & 2.861410\end{array}$

$\begin{array}{lll}-0.885590 & 0.011917 & 3.418628\end{array}$

$\begin{array}{lll}-0.794881 & -2.114701 & 3.276873\end{array}$

$\begin{array}{lll}-0.418003 & 1.201144 & 2.853890\end{array}$

$\begin{array}{lll}0.455721 & 1.160138 & 1.781958\end{array}$

$\begin{array}{lll}0.835270 & 2.067699 & 1.329601\end{array}$

$\begin{array}{lll}0.890210 & 0.000715 & 1.248307\end{array}$

$\begin{array}{lll}-1.574897 & 0.012721 & 4.253873\end{array}$

$\begin{array}{lll}-0.962660 & 2.927600 & 3.596441\end{array}$

\section{Conformer_ 4}

Number of imaginary frequencies : 1

The smallest frequency is : $-252.7213 \mathrm{~cm}(-1)$

Electronic energy :

$\mathrm{HF}=-7222.6349087$

Zero-point correction=

1.756167

(Hartree/Particle)

Thermal correction to Energy=

1.874507

1.875451

Thermal correction to Enthalpy=

Thermal correction to Gibbs Free Energy=

Sum of electronic and zero-point Energies=

Sum of electronic and thermal Energies=

$-7220.878742$

$-7220.760402$

Sum of electronic and thermal Enthalpies=

$-7220.759457$

Sum of electronic and thermal Free Energies $=\quad-7221.039945$

Cartesian Coordinates

$\begin{array}{cccc}26 & 4.279443 & 4.714743 & 1.902900 \\ 15 & 2.584093 & 1.561554 & -1.887574 \\ 15 & 4.512562 & 0.053563 & 0.959528 \\ 6 & 3.962428 & 3.356736 & 0.340511 \\ 6 & 4.115765 & 2.675469 & 1.606312\end{array}$

$\begin{array}{lll}3.082682 & 3.138934 & 2.478415\end{array}$

$\begin{array}{llll}2.935092 & 2.831405 & 3.504439\end{array}$

$\begin{array}{llll}2.312697 & 4.118418 & 1.781568\end{array}$

$\begin{array}{llll}1.485939 & 4.682808 & 2.190160\end{array}$

$\begin{array}{llll}2.854697 & 4.255518 & 0.470325\end{array}$

$2.508408 \quad 4.935806 \quad-0.292841$

$\begin{array}{lll}6.217270 & 5.218765 & 2.453717\end{array}$

$\begin{array}{llll}7.068151 & 4.553981 & 2.382973\end{array}$

$\begin{array}{llll}5.335442 & 5.335381 & 3.571771\end{array}$

$\begin{array}{lll}5.397471 & 4.771862 & 4.493205\end{array}$

$\begin{array}{lll}4.328455 & 6.293043 & 3.242430\end{array}$

$\begin{array}{llll}3.492240 & 6.575861 & 3.868204\end{array}$

$\begin{array}{lll}4.587634 & 6.769771 & 1.921197\end{array}$

$\begin{array}{llll}3.985461 & 7.480781 & 1.371063\end{array}$

$\begin{array}{llll}5.754520 & 6.108458 & 1.434979\end{array}$

$\begin{array}{llll}6.190645 & 6.238793 & 0.454008\end{array}$

$\begin{array}{llll}4.782276 & 3.065903 & -0.871988\end{array}$

$\begin{array}{llll}6.127074 & 3.463383 & -0.902595\end{array}$

$\begin{array}{llll}6.513237 & 4.026288 & -0.062347\end{array}$

$\begin{array}{llll}6.969482 & 3.130395 & -1.962470\end{array}$

$\begin{array}{llll}8.012839 & 3.427349 & -1.940330\end{array}$

$\begin{array}{llll}6.470726 & 2.387139 & -3.029722\end{array}$

$\begin{array}{lll}7.118678 & 2.102944 & -3.853186\end{array}$

$\begin{array}{llll}5.135404 & 1.982792 & -3.026248\end{array}$

$\begin{array}{llll}4.769243 & 1.378405 & -3.848754\end{array}$

$\begin{array}{lll}4.278849 & 2.303740 & -1.961194\end{array}$

$\begin{array}{lll}5.128276 & 1.595390 & 1.882334\end{array}$

$\begin{array}{llll}6.055243 & 1.880944 & 1.382341\end{array}$

$\begin{array}{llll}5.427540 & 1.383871 & 3.368701\end{array}$

$\begin{array}{lll}6.201683 & 0.624441 & 3.508340\end{array}$

$\begin{array}{lll}4.543326 & 1.067400 & 3.928582\end{array}$

$\begin{array}{llll}5.780022 & 2.324843 & 3.802246\end{array}$

$\begin{array}{llll}2.379352 & 0.668740 & -3.490959\end{array}$

$\begin{array}{llll}2.507679 & -0.729138 & -3.486238\end{array}$

$\begin{array}{llll}2.714660 & -1.248673 & -2.558664\end{array}$

$2.329809 \quad-1.462964 \quad-4.661081$

$\begin{array}{llll}2.446030 & -2.542062 & -4.639743\end{array}$

$\begin{array}{llll}2.000971 & -0.811244 & -5.850157\end{array}$

$\begin{array}{llll}1.848683 & -1.381558 & -6.761981\end{array}$

$\begin{array}{llll}1.865461 & 0.580436 & -5.863451\end{array}$

$\begin{array}{llll}1.607695 & 1.094005 & -6.785375\end{array}$

$\begin{array}{llll}2.058223 & 1.317841 & -4.694824\end{array}$

$\begin{array}{lll}1.943671 & 2.395948 & -4.723637\end{array}$

$\begin{array}{llll}1.445516 & 2.990630 & -2.078621\end{array}$

$\begin{array}{lll}1.797616 & 4.146836 & -2.793846\end{array}$

$\begin{array}{llll}2.793699 & 4.241772 & -3.216081\end{array}$

$\begin{array}{llll}0.875848 & 5.183006 & -2.953635\end{array}$

$\begin{array}{llll}1.158344 & 6.074645 & -3.506217\end{array}$

$\begin{array}{llll}-0.408078 & 5.070117 & -2.410159\end{array}$

$\begin{array}{lll}-1.131020 & 5.870400 & -2.540484\end{array}$

$\begin{array}{llll}-0.761816 & 3.923694 & -1.695707\end{array}$

$\begin{array}{llll}-1.756350 & 3.829015 & -1.276618\end{array}$

$\begin{array}{llll}0.165212 & 2.894915 & -1.518344\end{array}$

$\begin{array}{llll}-0.106135 & 2.015368 & -0.945227\end{array}$

$\begin{array}{llll}5.839277 & -0.369948 & -0.248826\end{array}$

$\begin{array}{llll}5.448174 & -1.173274 & -1.329906\end{array}$

$\begin{array}{llll}4.434633 & -1.553073 & -1.364871\end{array}$

$\begin{array}{llll}6.351295 & -1.480806 & -2.345488\end{array}$

$\begin{array}{llll}7.654149 & -0.985862 & -2.311800\end{array}$

$\begin{array}{llll}8.349880 & -1.205863 & -3.114245\end{array}$

$\begin{array}{llll}8.044493 & -0.194689 & -1.232937\end{array}$

$\begin{array}{llll}7.153561 & 0.108243 & -0.201896\end{array}$

$\begin{array}{lll}7.499244 & 0.721549 & 0.620871\end{array}$

$\begin{array}{llll}4.535120 & -1.365383 & 2.128618\end{array}$

$\begin{array}{lll}3.530442 & -1.407681 & 3.108620\end{array}$

$\begin{array}{lll}2.841248 & -0.578098 & 3.217310\end{array}$

$\begin{array}{llll}3.383115 & -2.532634 & 3.916747\end{array}$

$\begin{array}{lll}4.205853 & -3.646855 & 3.746908\end{array}$

$\begin{array}{llll}4.063975 & -4.535117 & 4.352527\end{array}$

$\begin{array}{llll}5.187769 & -3.611238 & 2.759924\end{array}$

$\begin{array}{llll}5.367220 & -2.478307 & 1.964792\end{array}$

$\begin{array}{llll}6.133228 & -2.487944 & 1.199219\end{array}$

$\begin{array}{llll}2.425119 & 0.190344 & -0.099040\end{array}$

$\begin{array}{llll}6.052324 & -4.823978 & 2.553574\end{array}$

$\begin{array}{llll}2.246514 & -2.620873 & 4.895952\end{array}$ 
$\begin{array}{llll}5.885245 & -2.239265 & -3.556692\end{array}$

$\begin{array}{llll}9.425626 & 0.400355 & -1.248975\end{array}$

$\begin{array}{llll}9.545134 & 1.333709 & -2.228470\end{array}$

$\begin{array}{llll}9.742972 & 1.007139 & -0.085268\end{array}$

$10.373249-0.532574 \quad-1.493889$

$\begin{array}{llll}6.856309 & -3.022900 & -4.073291\end{array}$

$\begin{array}{llll}5.498556 & -1.393685 & -4.544421\end{array}$

$\begin{array}{llll}4.822122 & -3.036271 & -3.288578\end{array}$

$\begin{array}{llll}7.014733 & -4.932838 & 3.497261\end{array}$

$\begin{array}{llll}6.673731 & -4.809056 & 1.352095\end{array}$

$\begin{array}{lll}5.322509 & -5.965893 & 2.610606\end{array}$

$\begin{array}{llll}2.618694 & -3.211071 & 6.052469\end{array}$

$\begin{array}{lll}1.729012 & -1.412721 & 5.202072\end{array}$

$\begin{array}{llll}1.225001 & -3.371228 & 4.395068\end{array}$

$\begin{array}{lll}1.958396 & -2.789158 & 0.365403\end{array}$

$\begin{array}{lll}0.794800 & -2.517053 & 1.182390\end{array}$

$\begin{array}{llll}0.441405 & -1.247219 & 1.752365\end{array}$

$\begin{array}{llll}0.402266 & -3.349501 & 1.747949\end{array}$

$\begin{array}{llll}-3.411373 & -1.270432 & -1.453762\end{array}$

$\begin{array}{llll}-5.265342 & -0.498284 & -0.370937\end{array}$

$\begin{array}{llll}-5.239470 & 0.633901 & 0.837985\end{array}$

$\begin{array}{llll}-6.323099 & 0.237507 & -1.425117\end{array}$

$\begin{array}{lll}-5.979199 & -1.757030 & 0.476674\end{array}$

$\begin{array}{lll}-4.940948 & 1.957127 & 0.550977\end{array}$

$\begin{array}{lll}-3.705874 & 2.443028 & 1.032161\end{array}$

$\begin{array}{lll}-3.074976 & 1.772168 & 1.603804\end{array}$

$\begin{array}{llll}-3.343053 & 3.739565 & 0.766199\end{array}$

$\begin{array}{llll}-2.404078 & 4.133532 & 1.141913\end{array}$

$\begin{array}{llll}-4.167333 & 4.564871 & -0.047309\end{array}$

$\begin{array}{llll}-3.743032 & 5.865092 & -0.435020\end{array}$

$\begin{array}{llll}-2.786094 & 6.227965 & -0.068930\end{array}$

$\begin{array}{llll}-4.515618 & 6.640696 & -1.270206\end{array}$

$\begin{array}{llll}-4.178624 & 7.629518 & -1.567237\end{array}$

$\begin{array}{llll}-5.749465 & 6.142019 & -1.752533\end{array}$

$\begin{array}{llll}-6.347276 & 6.748400 & -2.426796\end{array}$

$\begin{array}{llll}-6.198490 & 4.893340 & -1.379635\end{array}$

$\begin{array}{llll}-7.142692 & 4.524443 & -1.763349\end{array}$

$\begin{array}{llll}-5.432030 & 4.067582 & -0.511121\end{array}$

$\begin{array}{llll}-5.859265 & 2.748237 & -0.120676\end{array}$

$\begin{array}{lll}-7.227697 & 2.226182 & -0.399437\end{array}$

$\begin{array}{lll}-8.405996 & 2.929267 & 0.045752\end{array}$

$\begin{array}{lll}-8.345023 & 4.098302 & 0.855760\end{array}$

$\begin{array}{lll}-7.380882 & 4.479415 & 1.169223\end{array}$

$\begin{array}{lll}-9.491918 & 4.744990 & 1.263019\end{array}$

$\begin{array}{lll}-9.414951 & 5.631144 & 1.886552\end{array}$

$\begin{array}{lll}-10.767510 & 4.263340 & 0.885090\end{array}$

$\begin{array}{lll}-11.662240 & 4.788127 & 1.206896\end{array}$

$\begin{array}{lll}-10.866452 & 3.119504 & 0.126076\end{array}$

$\begin{array}{llll}-11.838955 & 2.723254 & -0.154231\end{array}$

$\begin{array}{llll}-9.704006 & 2.419042 & -0.296678\end{array}$

$\begin{array}{llll}-9.809575 & 1.206982 & -1.030762\end{array}$

$\begin{array}{lll}-10.795042 & 0.833839 & -1.294847\end{array}$

$\begin{array}{llll}-8.684626 & 0.500264 & -1.373390\end{array}$

$\begin{array}{llll}-8.740566 & -0.444079 & -1.904511\end{array}$

$\begin{array}{llll}-7.410556 & 1.005756 & -1.033457\end{array}$

$\begin{array}{lll}-5.363707 & -3.075860 & 0.162961\end{array}$

$\begin{array}{lll}-4.634784 & -3.305735 & 0.949423\end{array}$

$\begin{array}{lll}-6.399136 & -4.186587 & 0.138795\end{array}$

$\begin{array}{lll}-6.109952 & -5.424735 & 0.724192\end{array}$

$\begin{array}{lll}-5.160076 & -5.563892 & 1.235829\end{array}$

$\begin{array}{lll}-7.028645 & -6.475895 & 0.657410\end{array}$

$\begin{array}{llll}-6.791339 & -7.431036 & 1.117935\end{array}$

$\begin{array}{lll}-8.252464 & -6.294674 & 0.009811\end{array}$

$\begin{array}{llll}-8.971213 & -7.107909 & -0.037262\end{array}$

$\begin{array}{llll}-8.550786 & -5.058482 & -0.573461\end{array}$

$-9.503269-4.909791-1.075045$

$\begin{array}{llll}-7.629649 & -4.013040 & -0.511332\end{array}$

$\begin{array}{lll}-7.861742 & -3.048813 & -0.955340\end{array}$

$-4.624352-3.014716-1.178447$

$\begin{array}{llll}-5.361360 & -3.064071 & -1.988868\end{array}$

$-3.991613-3.902693-1.267493$

$\begin{array}{lll}-6.679162 & -1.695426 & 1.805877\end{array}$

$\begin{array}{lll}-7.171428 & -2.669443 & 1.871404\end{array}$

$\begin{array}{lll}-5.687805 & -1.638299 & 2.969429\end{array}$

$\begin{array}{lll}-5.164092 & -2.841459 & 3.469855\end{array}$ $\begin{array}{lll}-5.511241 & -3.784801 & 3.056632\end{array}$

$-4.221344-2.847542 \quad 4.501305$

$\begin{array}{lll}-3.830344 & -3.792019 & 4.869973\end{array}$

$\begin{array}{lll}-3.802725 & -1.643230 & 5.070518\end{array}$

$\begin{array}{lll}-3.077672 & -1.641979 & 5.879847\end{array}$

$\begin{array}{lll}-4.341214 & -0.439847 & 4.604502\end{array}$

$\begin{array}{llll}-4.039137 & 0.502086 & 5.055194\end{array}$

$\begin{array}{llll}-5.272760 & -0.437397 & 3.564485\end{array}$

$\begin{array}{lll}-5.675988 & 0.505913 & 3.218466\end{array}$

$\begin{array}{lll}-7.799131 & -0.658375 & 1.866349\end{array}$

$\begin{array}{lll}-8.511250 & -0.829743 & 1.055943\end{array}$

$\begin{array}{lll}-7.442229 & 0.368069 & 1.793485\end{array}$

$\begin{array}{lll}-8.328066 & -0.769259 & 2.817928\end{array}$

$\begin{array}{lll}-2.522744 & -1.109980 & 0.533199\end{array}$

$\begin{array}{lll}-1.863615 & -0.247375 & 0.569963\end{array}$

$\begin{array}{llll}-3.215320 & -1.146255 & 1.369288\end{array}$

$\begin{array}{lll}-2.017129 & -2.362489 & 0.058122\end{array}$

$\begin{array}{lll}-2.540575 & -3.252212 & 0.391740\end{array}$

$\begin{array}{lll}-0.765301 & -2.565525 & -0.576229\end{array}$

$\begin{array}{llll}-0.222763 & -1.700377 & -0.932512\end{array}$

$-4.241411-1.100126-3.494009$

$\begin{array}{llll}-5.236794 & -1.538726 & -3.481979\end{array}$

$\begin{array}{llll}-3.153606 & -2.016023 & -3.533997\end{array}$

$\begin{array}{llll}-3.405053 & -3.076131 & -3.569257\end{array}$

$\begin{array}{llll}-1.785335 & -1.691684 & -4.109343\end{array}$

$-1.823648-1.723164-5.209449$

$\begin{array}{llll}-1.101475 & -2.488315 & -3.805038\end{array}$

$\begin{array}{llll}-1.219700 & -0.337200 & -3.639353\end{array}$

$\begin{array}{llll}-1.475438 & 0.455139 & -4.349202\end{array}$

$\begin{array}{lll}-0.129527 & -0.390382 & -3.630479\end{array}$

$\begin{array}{llll}-1.692064 & 0.077354 & -2.249743\end{array}$

$\begin{array}{llll}-0.927489 & 0.072675 & -1.479117\end{array}$

$\begin{array}{llll}-2.795992 & 0.894192 & -2.018268\end{array}$

$\begin{array}{llll}-2.793932 & 1.475870 & -1.102333\end{array}$

$\begin{array}{lll}-3.742430 & 1.397672 & -3.089519\end{array}$

$\begin{array}{llll}-4.624609 & 1.800924 & -2.587722\end{array}$

$\begin{array}{llll}-3.269365 & 2.237705 & -3.619492\end{array}$

$\begin{array}{llll}-4.186045 & 0.309148 & -4.092352\end{array}$

$\begin{array}{llll}-3.523882 & 0.302331 & -4.964380\end{array}$

$\begin{array}{llll}-5.180709 & 0.566750 & -4.468937\end{array}$

$-0.407947-3.834304-1.222803$

$\begin{array}{llll}-0.985060 & -5.068523 & -0.863212\end{array}$

$\begin{array}{llll}0.557746 & -3.822777 & -2.247296\end{array}$

$\begin{array}{llll}-0.609787 & -6.243482 & -1.511471\end{array}$

$\begin{array}{llll}-1.720021 & -5.115346 & -0.066048\end{array}$

$\begin{array}{llll}0.929718 & -4.998046 & -2.897119\end{array}$

$\begin{array}{llll}1.017885 & -2.881606 & -2.521838\end{array}$

$\begin{array}{llll}0.348832 & -6.214833 & -2.531087\end{array}$

$\begin{array}{llll}-1.063717 & -7.186218 & -1.219440\end{array}$

$\begin{array}{llll}1.676637 & -4.964184 & -3.685390\end{array}$

$\begin{array}{llll}0.638014 & -7.133128 & -3.033866\end{array}$

$\begin{array}{llll}2.526019 & -4.029143 & 0.429151\end{array}$

$\begin{array}{llll}3.532636 & -4.437266 & -0.543013\end{array}$

$\begin{array}{llll}3.243040 & -5.395601 & -0.987554\end{array}$

$\begin{array}{llll}3.610981 & -3.695479 & -1.330836\end{array}$

$\begin{array}{llll}4.510537 & -4.555895 & -0.062086\end{array}$

$\begin{array}{lll}2.137220 & -5.066963 & 1.380596\end{array}$

$\begin{array}{lll}1.835474 & -4.638752 & 2.335405\end{array}$

$\begin{array}{llll}1.324199 & -5.689303 & 0.986652\end{array}$

$\begin{array}{lll}3.004940 & -5.703280 & 1.568065\end{array}$

$\begin{array}{llll}2.421561 & -1.943783 & -0.462058\end{array}$

$\begin{array}{lll}-0.457564 & -1.242659 & 2.854911\end{array}$

$\begin{array}{lll}-0.882433 & -0.062124 & 3.430219\end{array}$

$\begin{array}{lll}-0.797498 & -2.187182 & 3.262036\end{array}$

$\begin{array}{lll}-0.418197 & 1.133163 & 2.876199\end{array}$

$\begin{array}{lll}0.444755 & 1.103502 & 1.795077\end{array}$

$\begin{array}{llll}0.819598 & 2.015208 & 1.347446\end{array}$

$\begin{array}{lll}0.873893 & -0.050268 & 1.245628\end{array}$

$\begin{array}{lll}-1.564955 & -0.070435 & 4.270811\end{array}$

$\begin{array}{lll}-0.951533 & 2.851643 & 3.645191\end{array}$

Conformer 5

Number of imaginary frequencies : 1

The smallest frequency is : $-247.7157 \mathrm{~cm}(-1)$ 
$\begin{array}{lr}\text { Electronic energy : } & \mathrm{HF}=-7222.634846 \\ \text { Zero-point correction }= & 1.75\end{array}$ (Hartree/Particle)

$\begin{array}{lc}\text { Thermal correction to Energy= } & 1.874114 \\ \text { Thermal correction to Enthalpy= } & 1.875059 \\ \text { Thermal correction to Gibbs Free Energy= } & 1.593069 \\ \text { Sum of electronic and zero-point Energies= } & -7220.879147 \\ \text { Sum of electronic and thermal Energies }= & -7220.760732 \\ \text { Sum of electronic and thermal Enthalpies }= & -7220.759787 \\ \text { Sum of electronic and thermal Free Energies }= & -7221.041777\end{array}$

Cartesian Coordinates

$\begin{array}{llll}4.295578 & 4.787372 & 1.872975\end{array}$

$\begin{array}{llll}2.562747 & 1.624284 & -1.886532\end{array}$

$\begin{array}{llll}4.540877 & 0.128166 & 0.933214\end{array}$

$\begin{array}{lll}3.967464 & 3.424790 & 0.318652\end{array}$

$\begin{array}{lll}4.154607 & 2.746067 & 1.580024\end{array}$

$\begin{array}{lll}3.132537 & 3.194431 & 2.472935\end{array}$

$\begin{array}{lll}3.010134 & 2.884590 & 3.501615\end{array}$

$\begin{array}{llll}2.335146 & 4.162790 & 1.791576\end{array}$

$\begin{array}{lll}1.509121 & 4.716042 & 2.216398\end{array}$

$\begin{array}{llll}2.848905 & 4.307296 & 0.469838\end{array}$

$\begin{array}{llll}2.478856 & 4.983247 & -0.286005\end{array}$

$\begin{array}{lll}6.237932 & 5.326028 & 2.375384\end{array}$

$\begin{array}{lll}7.099025 & 4.677259 & 2.282806\end{array}$

$\begin{array}{llll}5.382798 & 5.426437 & 3.515504\end{array}$

$\begin{array}{llll}5.479641 & 4.865052 & 4.435232\end{array}$

$\begin{array}{lll}4.350619 & 6.365833 & 3.212451\end{array}$

$\begin{array}{lll}3.525495 & 6.633390 & 3.859270\end{array}$

$\begin{array}{lll}4.567543 & 6.847233 & 1.885329\end{array}$

$\begin{array}{lll}3.938620 & 7.547031 & 1.350970\end{array}$

$\begin{array}{lll}5.733392 & 6.207244 & 1.369236\end{array}$

$\begin{array}{lll}6.141387 & 6.344961 & 0.377256\end{array}$

$\begin{array}{llll}4.764251 & 3.153497 & -0.913222\end{array}$

$\begin{array}{llll}6.097280 & 3.584345 & -0.982998\end{array}$

$\begin{array}{llll}6.500776 & 4.139432 & -0.145696\end{array}$

$\begin{array}{llll}6.905965 & 3.300378 & -2.083376\end{array}$

$\begin{array}{llll}7.941472 & 3.625169 & -2.093305\end{array}$

$\begin{array}{llll}6.383415 & 2.576818 & -3.152778\end{array}$

$\begin{array}{llll}7.003883 & 2.337034 & -4.010279\end{array}$

$\begin{array}{lll}5.061390 & 2.133443 & -3.106049\end{array}$

$\begin{array}{llll}4.672122 & 1.556600 & -3.938015\end{array}$

$\begin{array}{llll}4.241742 & 2.397452 & -1.997619\end{array}$

$\begin{array}{lll}5.179947 & 1.672484 & 1.830478\end{array}$

$\begin{array}{llll}6.093302 & 1.961785 & 1.307754\end{array}$

$\begin{array}{lll}5.519791 & 1.464722 & 3.308953\end{array}$

$\begin{array}{lll}6.298542 & 0.706966 & 3.429623\end{array}$

$\begin{array}{llll}4.650983 & 1.149251 & 3.892842\end{array}$

$\begin{array}{lll}5.882162 & 2.407603 & 3.729907\end{array}$

$\begin{array}{llll}2.354335 & 0.721630 & -3.482156\end{array}$

$\begin{array}{lll}2.616162 & -0.658098 & -3.490644\end{array}$

$\begin{array}{llll}2.930042 & -1.152788 & -2.579051\end{array}$

$\begin{array}{llll}2.435092 & -1.405271 & -4.656730\end{array}$

$\begin{array}{llll}2.642068 & -2.470344 & -4.648816\end{array}$

$\begin{array}{llll}1.978951 & -0.786654 & -5.822536\end{array}$

$\begin{array}{llll}1.823779 & -1.370764 & -6.725095\end{array}$

$\begin{array}{llll}1.82378899 & 0.586360 & -5.822770\end{array}$

$\begin{array}{llll}1.363256 & 1.074097 & -6.725919\end{array}$

$\begin{array}{llll}1.908435 & 1.338181 & -4.662416\end{array}$

$\begin{array}{llll}1.691981 & 2.400675 & -4.679817\end{array}$

$\begin{array}{llll}1.403602 & 3.038125 & -2.070729\end{array}$

$\begin{array}{llll}1.740452 & 4.197538 & -2.788490\end{array}$

$\begin{array}{llll}2.733137 & 4.302470 & -3.216144\end{array}$

$\begin{array}{llll}0.806313 & 5.222618 & -2.948065\end{array}$

$\begin{array}{llll}1.077305 & 6.116447 & -3.502860\end{array}$

$\begin{array}{llll}-0.475319 & 5.095071 & -2.402678\end{array}$

$\begin{array}{lll}-1.208180 & 5.886267 & -2.533120\end{array}$

$\begin{array}{llll}-0.814355 & 3.945024 & -1.687067\end{array}$

$\begin{array}{llll}-1.807523 & 3.839093 & -1.267664\end{array}$

$\begin{array}{llll}0.124930 & 2.927338 & -1.509558\end{array}$

$\begin{array}{llll}-0.137137 & 2.044684 & -0.936926\end{array}$

$\begin{array}{llll}5.833960 & -0.351414 & -0.288366\end{array}$

$\begin{array}{llll}5.486597 & -1.402444 & -1.149534\end{array}$

$\begin{array}{llll}4.518834 & -1.879252 & -1.036718\end{array}$ $\begin{array}{llll}6.371467 & -1.830163 & -2.138462\end{array}$

$\begin{array}{llll}7.598025 & -1.194380 & -2.319647\end{array}$

$\begin{array}{lll}7.275579 & -1.511167 & -3.104405\end{array}$

$\begin{array}{lll}7.930482 & -0.131940 & -1.478655\end{array}$

$\begin{array}{lll}7.072172 & 0.277414 & -0.457677\end{array}$

$\begin{array}{lll}7.378632 & 1.096703 & 0.177898\end{array}$

$\begin{array}{lll}4.568723 & -1.271414 & 2.131317\end{array}$

$\begin{array}{lll}3.559692 & -1.295038 & 3.107541\end{array}$

$\begin{array}{lll}2.867872 & -0.465339 & 3.195712\end{array}$

$3.410664 \quad-2.401694 \quad 3.940554$

$\begin{array}{lll}4.233847 & -3.519249 & 3.797983\end{array}$

$\begin{array}{lll}4.087918 & -4.396255 & 4.418651\end{array}$

$\begin{array}{lll}5.223286 & -3.501093 & 2.818349\end{array}$

$\begin{array}{lll}5.409693 & -2.382647 & 2.004919\end{array}$

$\begin{array}{lll}6.188603 & -2.406538 & 1.253301\end{array}$

$\begin{array}{llll}2.440312 & 0.234719 & -0.102233\end{array}$

$\begin{array}{lll}6.092050 & -4.714867 & 2.637234\end{array}$

$\begin{array}{llll}2.273362 & -2.466727 & 4.921000\end{array}$

$\begin{array}{llll}5.996870 & -3.025962 & -2.969173\end{array}$

$\begin{array}{llll}9.224975 & 0.593002 & -1.726338\end{array}$

$\begin{array}{llll}9.227747 & 1.197305 & -2.941345\end{array}$

$\begin{array}{lll}9.466942 & 1.553174 & -0.807125\end{array}$

$\begin{array}{lll}10.282490 & -0.251203 & -1.717860\end{array}$

$\begin{array}{llll}6.178481 & -4.182165 & -2.282692\end{array}$

$\begin{array}{llll}6.720402 & -3.118338 & -4.102538\end{array}$

$\begin{array}{lll}4.686522 & -3.002490 & -3.326853\end{array}$

$\begin{array}{lll}7.068807 & -4.789575 & 3.569536\end{array}$

$\begin{array}{lll}6.695659 & -4.731946 & 1.427090\end{array}$

$\begin{array}{lll}5.369617 & -5.858086 & 2.739806\end{array}$

$\begin{array}{llll}2.645178 & -3.031276 & 6.090526\end{array}$

$\begin{array}{lll}1.757799 & -1.251179 & 5.200191\end{array}$

$\begin{array}{lll}1.250963 & -3.226783 & 4.437841\end{array}$

$\begin{array}{lll}1.975895 & -2.751381 & 0.404109\end{array}$

$\begin{array}{lll}0.812414 & -2.465256 & 1.213827\end{array}$

$\begin{array}{lll}0.453953 & -1.190285 & 1.765583\end{array}$

$\begin{array}{llll}0.409668 & -3.292514 & 1.779947\end{array}$

$\begin{array}{lll}-3.400098 & -1.296252 & -1.446855\end{array}$

$\begin{array}{lll}-5.267051 & -0.538277 & -0.377729\end{array}$

$\begin{array}{lll}-5.260519 & 0.604662 & 0.820594\end{array}$

$\begin{array}{llll}-6.330148 & 0.174060 & -1.441914\end{array}$

$\begin{array}{lll}-5.966521 & -1.798994 & 0.479642\end{array}$

$\begin{array}{lll}-4.982104 & 1.930166 & 0.525403\end{array}$

$\begin{array}{lll}-3.757919 & 2.439152 & 1.010814\end{array}$

$\begin{array}{lll}-3.119070 & 1.782058 & 1.589942\end{array}$

$\begin{array}{lll}-3.415999 & 3.740414 & 0.740388\end{array}$

$\begin{array}{lll}-2.486839 & 4.152975 & 1.120389\end{array}$

$\begin{array}{lll}-4.248696 & 4.546653 & -0.083616\end{array}$

$\begin{array}{llll}-3.843377 & 5.851194 & -0.476845\end{array}$

$\begin{array}{lll}-2.895196 & 6.232406 & -0.106679\end{array}$

$\begin{array}{llll}-4.622826 & 6.608424 & -1.322444\end{array}$

$\begin{array}{llll}-4.300200 & 7.600757 & -1.623687\end{array}$

$\begin{array}{llll}-5.844902 & 6.086236 & -1.809810\end{array}$

$\begin{array}{llll}-6.447906 & 6.678147 & -2.492261\end{array}$

$\begin{array}{llll}-6.275857 & 4.832789 & -1.431560\end{array}$

$\begin{array}{llll}-7.211259 & 4.445733 & -1.818911\end{array}$

$\begin{array}{llll}-5.501776 & 4.025485 & -0.552539\end{array}$

$\begin{array}{llll}-5.909935 & 2.701975 & -0.155779\end{array}$

$\begin{array}{lll}-7.269179 & 2.156909 & -0.436107\end{array}$

$\begin{array}{lll}-8.460141 & 2.845564 & -0.001828\end{array}$

$\begin{array}{lll}-8.420928 & 4.023184 & 0.797077\end{array}$

$\begin{array}{llll}-7.464265 & 4.422330 & 1.110934\end{array}$

$\begin{array}{lll}-9.579470 & 4.655970 & 1.193037\end{array}$

$\begin{array}{llll}-9.518941 & 5.549363 & 1.808000\end{array}$

$\begin{array}{lll}-10.845807 & 4.150849 & 0.814662\end{array}$

$\begin{array}{lll}-11.749911 & 4.664973 & 1.127349\end{array}$

$\begin{array}{lll}-10.923687 & 2.997920 & 0.067052\end{array}$

$\begin{array}{llll}-11.888681 & 2.583694 & -0.213118\end{array}$

$\begin{array}{llll}-9.748702 & 2.311577 & -0.343997\end{array}$

$\begin{array}{llll}-9.832651 & 1.090621 & -1.066047\end{array}$

$\begin{array}{llll}-10.811159 & 0.698985 & -1.329145\end{array}$

$\begin{array}{llll}-8.695485 & 0.398787 & -1.398343\end{array}$

$\begin{array}{lll}-8.734353 & -0.551578 & -1.920161\end{array}$

$\begin{array}{llll}-7.430888 & 0.928196 & -1.059843\end{array}$

$\begin{array}{lll}-5.328309 & -3.110854 & 0.181280\end{array}$

$\begin{array}{lll}-4.591355 & -3.316242 & 0.967314\end{array}$ 


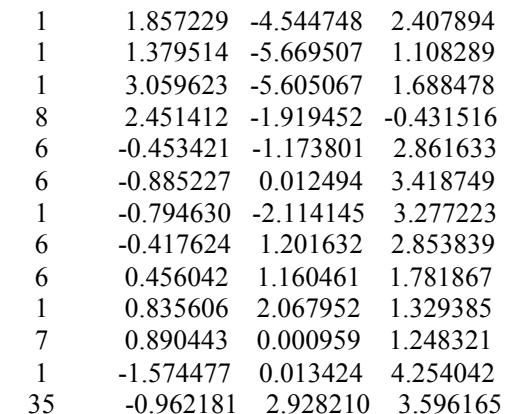

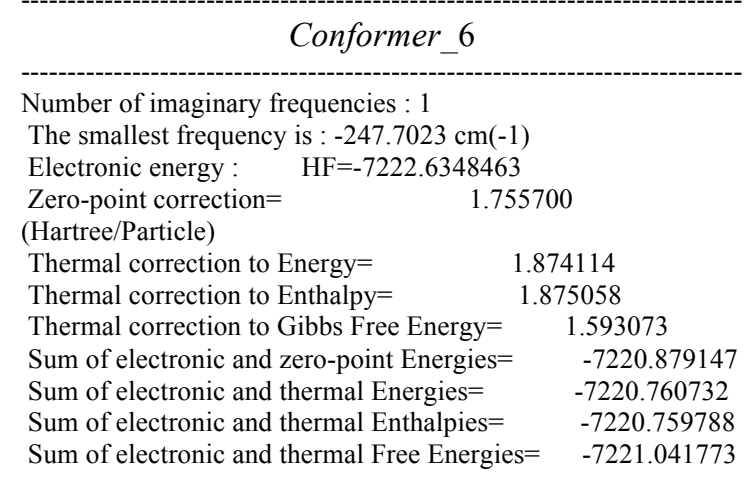

\begin{tabular}{|c|c|c|c|}
\hline & & \multicolumn{2}{|c|}{ Cartesian Coordinates } \\
\hline 26 & 4.295336 & 4.787275 & 1.873550 \\
\hline 15 & 2.562844 & 1.624410 & -1.886305 \\
\hline 15 & 4.540754 & 0.128128 & 0.933530 \\
\hline 6 & 3.967358 & 3.424788 & 0.319114 \\
\hline 6 & 4.154391 & 2.745989 & 1.580461 \\
\hline 6 & 3.132243 & 3.194299 & 2.473309 \\
\hline 1 & 3.009751 & 2.884396 & 3.501959 \\
\hline 6 & 2.334912 & 4.162700 & 1.791939 \\
\hline 1 & 1.508847 & 4.715922 & 2.216722 \\
\hline 6 & 2.848787 & 4.307286 & 0.470255 \\
\hline 1 & 2.478803 & 4.983280 & -0.285581 \\
\hline 6 & 6.237636 & 5.325868 & 2.376228 \\
\hline 1 & 7.098728 & 4.677085 & 2.283727 \\
\hline 6 & 5.382360 & 5.426240 & 3.516244 \\
\hline 1 & 5.479070 & 4.864805 & 4.435956 \\
\hline 6 & 4.350238 & 6.365670 & 3.213105 \\
\hline 1 & 3.525037 & 6.633213 & 3.859832 \\
\hline 6 & 4.567340 & 6.847129 & 1.886033 \\
\hline 1 & 3.938499 & 7.546966 & 1.351627 \\
\hline 6 & 5.733242 & 6.207143 & 1.370059 \\
\hline 1 & 6.141370 & 6.344900 & 0.378138 \\
\hline 6 & 4.764258 & 3.153566 & -0.912703 \\
\hline 6 & 6.097302 & 3.584394 & -0.982319 \\
\hline 1 & 6.500729 & 4.139412 & -0.144938 \\
\hline 6 & 6.906090 & 3.300485 & -2.082635 \\
\hline 1 & 7.941606 & 3.625252 & -2.092438 \\
\hline 6 & 6.383630 & 2.577013 & -3.152141 \\
\hline 1 & 7.004176 & 2.337280 & -4.009599 \\
\hline 6 & 5.061592 & 2.133657 & -3.105571 \\
\hline 1 & 4.672394 & 1.556880 & -3.937615 \\
\hline 6 & 4.241843 & 2.397599 & -1.997199 \\
\hline 6 & 5.179719 & 1.672402 & 1.830944 \\
\hline 1 & 6.093114 & 1.961746 & 1.308317 \\
\hline 6 & 5.519427 & 1.464557 & 3.309437 \\
\hline 1 & 6.298184 & 0.706811 & 3.430134 \\
\hline 1 & 4.650571 & 1.149028 & 3.893221 \\
\hline 1 & 5.881735 & 2.407420 & 3.730485 \\
\hline 6 & 2.354563 & 0.721873 & -3.482015 \\
\hline 6 & 2.616435 & -0.657846 & -3.490592 \\
\hline 1 & 2.930268 & -1.152596 & -2.579015 \\
\hline 6 & 2.435479 & -1.404934 & -4.656750 \\
\hline 1 & 2.642493 & -2.469999 & -4.648902 \\
\hline 6 & 1.979405 & -0.786241 & -5.822542 \\
\hline 1 & 1.824324 & -1.370284 & -6.725159 \\
\hline
\end{tabular}


$\begin{array}{llll}1.719297 & 0.586765 & -5.822687\end{array}$

$\begin{array}{llll}1.363717 & 1.074561 & -6.725824\end{array}$

$\begin{array}{llll}1.908732 & 1.338502 & -4.662260\end{array}$

$\begin{array}{llll}1.692251 & 2.400991 & -4.679598\end{array}$

$\begin{array}{llll}1.403705 & 3.038257 & -2.070491\end{array}$

$\begin{array}{llll}1.740606 & 4.197716 & -2.788154\end{array}$

$\begin{array}{llll}2.733321 & 4.302673 & -3.215734\end{array}$

$\begin{array}{llll}0.806479 & 5.222807 & -2.947729\end{array}$

$\begin{array}{llll}1.077509 & 6.116671 & -3.502449\end{array}$

$\begin{array}{llll}-0.475190 & 5.095226 & -2.402437\end{array}$

$\begin{array}{llll}-1.208041 & 5.886432 & -2.532878\end{array}$

$\begin{array}{llll}-0.814276 & 3.945133 & -1.686925\end{array}$

$\begin{array}{llll}-1.807472 & 3.839179 & -1.267594\end{array}$

$\begin{array}{llll}0.124995 & 2.927434 & -1.509416\end{array}$

$\begin{array}{llll}-0.137112 & 2.044744 & -0.936857\end{array}$

$\begin{array}{llll}5.833962 & -0.351335 & -0.287962\end{array}$

$\begin{array}{llll}5.486692 & -1.402281 & -1.149271\end{array}$

$\begin{array}{llll}4.518914 & -1.879098 & -1.036609\end{array}$

$\begin{array}{llll}6.371678 & -1.829918 & -2.138130\end{array}$

$\begin{array}{llll}7.598269 & -1.194137 & -2.319101\end{array}$

$8.275916-1.510865-3.103803$

$\begin{array}{llll}7.930640 & -0.131787 & -1.477963\end{array}$

$\begin{array}{llll}7.072205 & 0.277488 & -0.457058\end{array}$

$\begin{array}{llll}7.378601 & 1.096706 & 0.178638\end{array}$

$\begin{array}{llll}4.568535 & -1.271529 & 2.131542\end{array}$

$\begin{array}{llll}3.559431 & -1.295242 & 3.107688\end{array}$

$\begin{array}{llll}2.867581 & -0.465566 & 3.195855\end{array}$

$\begin{array}{llll}3.410379 & -2.401951 & 3.940626\end{array}$

$\begin{array}{llll}4.233609 & -3.519471 & 3.798052\end{array}$

$\begin{array}{llll}4.087663 & -4.396517 & 4.418659\end{array}$

$\begin{array}{llll}5.223113 & -3.501230 & 2.818485\end{array}$

$\begin{array}{llll}5.409543 & -2.382731 & 2.005133\end{array}$

$\begin{array}{lll}6.188505 & -2.406558 & 1.253567\end{array}$

$\begin{array}{llll}2.440286 & 0.234713 & -0.102117\end{array}$

$\begin{array}{llll}6.091922 & -4.714970 & 2.637361\end{array}$

$\begin{array}{llll}2.272998 & -2.467083 & 4.920974\end{array}$

$\begin{array}{llll}5.997181 & -3.025644 & -2.968991\end{array}$

$\begin{array}{llll}9.225179 & 0.593156 & -1.725406\end{array}$

$\begin{array}{llll}9.228083 & 1.197661 & -2.940313\end{array}$

$\begin{array}{llll}9.467091 & 1.553168 & -0.806012\end{array}$

$10.282664 \quad-0.251087 \quad-1.716970$

$\begin{array}{llll}6.178786 & -4.181910 & -2.282617\end{array}$

$\begin{array}{llll}6.720802 & -3.117875 & -4.102312\end{array}$

$\begin{array}{llll}4.686858 & -3.002184 & -3.326764\end{array}$

$\begin{array}{lll}7.068630 & -4.789695 & 3.569713\end{array}$

$\begin{array}{lll}6.695598 & -4.731976 & 1.427249\end{array}$

$\begin{array}{llll}5.369515 & -5.858214 & 2.739839\end{array}$

$\begin{array}{llll}2.644734 & -3.031693 & 6.090496\end{array}$

$\begin{array}{llll}1.757374 & -1.251569 & 5.200198\end{array}$

$\begin{array}{llll}1.250664 & -3.227143 & 4.437681\end{array}$

$\begin{array}{lll}1.975913 & -2.751417 & 0.404002\end{array}$

$\begin{array}{llll}0.812373 & -2.465370 & 1.213659\end{array}$

$\begin{array}{llll}0.453836 & -1.190447 & 1.765472\end{array}$

$\begin{array}{llll}0.409606 & -3.292675 & 1.779695\end{array}$

$\begin{array}{lll}-3.400018 & -1.296220 & -1.447199\end{array}$

$\begin{array}{lll}-5.266998 & -0.538254 & -0.378120\end{array}$

$\begin{array}{llll}-5.260494 & 0.604608 & 0.820277\end{array}$

$\begin{array}{lll}-6.330016 & 0.174183 & -1.442317\end{array}$

$\begin{array}{llll}-5.966549 & -1.799003 & 0.479135\end{array}$

$\begin{array}{lll}-4.982072 & 1.930135 & 0.525191\end{array}$

$\begin{array}{lll}-3.757907 & 2.439090 & 1.010687\end{array}$

$\begin{array}{lll}-3.119073 & 1.781959 & 1.589787\end{array}$

$\begin{array}{lll}-3.415987 & 3.740377 & 0.740380\end{array}$

$\begin{array}{llll}-2.486845 & 4.152911 & 1.120453\end{array}$

$\begin{array}{llll}-4.248656 & 4.546677 & -0.083591\end{array}$

$\begin{array}{llll}-3.843329 & 5.851251 & -0.476701\end{array}$

$\begin{array}{llll}-2.895166 & 6.232440 & -0.106466\end{array}$

$\begin{array}{llll}-4.622750 & 6.608543 & -1.322271\end{array}$

$\begin{array}{llll}-4.300119 & 7.600903 & -1.623423\end{array}$

$\begin{array}{llll}-5.844802 & 6.086385 & -1.809727\end{array}$

$\begin{array}{llll}-6.447783 & 6.678347 & -2.492155\end{array}$

$\begin{array}{llll}-6.275765 & 4.832906 & -1.431594\end{array}$

$\begin{array}{llll}-7.211148 & 4.445875 & -1.819013\end{array}$

$\begin{array}{llll}-5.501713 & 4.025537 & -0.552606\end{array}$

$\begin{array}{llll}-5.909880 & 2.701993 & -0.155967\end{array}$ $\begin{array}{lll}-7.269109 & 2.156945 & -0.436402\end{array}$

$\begin{array}{llll}-8.460095 & 2.845561 & -0.002128\end{array}$

$\begin{array}{llll}-8.420926 & 4.023111 & 0.796883\end{array}$

$\begin{array}{llll}-7.464280 & 4.422231 & 1.110825\end{array}$

$\begin{array}{lll}-9.579491 & 4.655858 & 1.192839\end{array}$

$\begin{array}{lll}-9.518996 & 5.549196 & 1.807886\end{array}$

$\begin{array}{lll}-10.845806 & 4.150768 & 0.814351\end{array}$

$\begin{array}{lll}-11.749928 & 4.664863 & 1.127035\end{array}$

$\begin{array}{llll}-10.923644 & 2.997906 & 0.066633\end{array}$

$\begin{array}{llll}-11.888623 & 2.583705 & -0.213626\end{array}$

$\begin{array}{llll}-9.748636 & 2.311601 & -0.344413\end{array}$

$\begin{array}{llll}-9.832544 & 1.090708 & -1.066574\end{array}$

$\begin{array}{lll}-10.811037 & 0.699093 & -1.329761\end{array}$

$\begin{array}{llll}-8.695358 & 0.398904 & -1.398867\end{array}$

$\begin{array}{llll}-8.734196 & -0.551416 & -1.920771\end{array}$

$\begin{array}{llll}-7.430782 & 0.928286 & -1.060250\end{array}$

$\begin{array}{llll}-5.328363 & -3.110863 & 0.180722\end{array}$

$\begin{array}{lll}-4.591461 & -3.316326 & 0.966783\end{array}$

$\begin{array}{lll}-6.342574 & -4.240804 & 0.174800\end{array}$

$\begin{array}{lll}-6.026918 & -5.466228 & 0.773087\end{array}$

$\begin{array}{lll}-5.072484 & -5.581329 & 1.282066\end{array}$

$\begin{array}{lll}-6.925522 & -6.535536 & 0.722665\end{array}$

$\begin{array}{lll}-6.667914 & -7.480485 & 1.193190\end{array}$

$\begin{array}{lll}-8.155472 & -6.385355 & 0.078751\end{array}$

$\begin{array}{lll}-8.858679 & -7.212701 & 0.044595\end{array}$

$\begin{array}{llll}-8.480115 & -5.162056 & -0.517464\end{array}$

$\begin{array}{llll}-9.437517 & -5.037474 & -1.016213\end{array}$

$\begin{array}{lll}-7.578958 & -4.098519 & -0.471778\end{array}$

$\begin{array}{llll}-7.831482 & -3.144250 & -0.925936\end{array}$

$-4.594840 \quad-3.051726 \quad-1.163792$

$\begin{array}{llll}-5.333878 & -3.112987 & -1.971621\end{array}$

$\begin{array}{lll}-3.953564 & -3.933823 & -1.249747\end{array}$

$\begin{array}{lll}-6.667778 & -1.732663 & 1.807731\end{array}$

$\begin{array}{lll}-7.141447 & -2.714931 & 1.886412\end{array}$

$\begin{array}{llll}-5.678395 & -1.640666 & 2.970680\end{array}$

$\begin{array}{lll}-5.126553 & -2.826292 & 3.482448\end{array}$

$\begin{array}{lll}-5.453117 & -3.781394 & 3.079709\end{array}$

$\begin{array}{lll}-4.182343 & -2.800409 & 4.512252\end{array}$

$\begin{array}{llll}-3.769335 & -3.731846 & 4.889986\end{array}$

$\begin{array}{lll}-3.790444 & -1.581058 & 5.068336\end{array}$

$\begin{array}{lll}-3.064221 & -1.555062 & 5.876200\end{array}$

$\begin{array}{llll}-4.357387 & -0.395106 & 4.591436\end{array}$

$\begin{array}{lll}-4.076426 & 0.557924 & 5.032241\end{array}$

$\begin{array}{lll}-5.290750 & -0.424720 & 3.553512\end{array}$

$\begin{array}{lll}-5.716387 & 0.505629 & 3.199354\end{array}$

$\begin{array}{lll}-7.806862 & -0.715849 & 1.852562\end{array}$

$\begin{array}{lll}-8.514440 & -0.911698 & 1.043753\end{array}$

$\begin{array}{lll}-7.468568 & 0.315834 & 1.765599\end{array}$

$\begin{array}{lll}-8.335039 & -0.822834 & 2.805016\end{array}$

$\begin{array}{lll}-2.525423 & -1.128453 & 0.544722\end{array}$

$\begin{array}{lll}-1.882685 & -0.253696 & 0.590649\end{array}$

$\begin{array}{lll}-3.221311 & -1.183744 & 1.377181\end{array}$

$\begin{array}{lll}-1.992530 & -2.367480 & 0.062398\end{array}$

$\begin{array}{lll}-2.496838 & -3.272266 & 0.385161\end{array}$

$\begin{array}{lll}-0.734576 & -2.534752 & -0.568836\end{array}$

$-0.208969-1.652813-0.907300$

$\begin{array}{lll}-4.222955 & -1.143859 & -3.493210\end{array}$

$\begin{array}{llll}-5.215119 & -1.589531 & -3.482104\end{array}$

$-3.126405-2.048642-3.521932$

$\begin{array}{lll}-3.365173 & -3.111799 & -3.551035\end{array}$

$\begin{array}{llll}-1.761839 & -1.710484 & -4.097678\end{array}$

$\begin{array}{llll}-1.805546 & -1.728829 & -5.197817\end{array}$

$\begin{array}{lll}-1.073316 & -2.507845 & -3.807575\end{array}$

$\begin{array}{llll}-1.196527 & -0.358724 & -3.615853\end{array}$

$\begin{array}{llll}-1.429346 & 0.434573 & -4.332816\end{array}$

$\begin{array}{lll}-0.107223 & -0.422342 & -3.582632\end{array}$

$\begin{array}{llll}-1.691403 & 0.068053 & -2.237671\end{array}$

$\begin{array}{llll}-0.934613 & 0.084618 & -1.459787\end{array}$

$\begin{array}{llll}-2.806422 & 0.873845 & -2.022124\end{array}$

$\begin{array}{llll}-2.816359 & 1.461196 & -1.109695\end{array}$

$\begin{array}{llll}-3.752905 & 1.359153 & -3.101308\end{array}$

$\begin{array}{llll}-4.645163 & 1.750154 & -2.607437\end{array}$

$\begin{array}{lll}-3.289226 & 2.204892 & -3.630563\end{array}$

$\begin{array}{llll}-4.170640 & 0.260387 & -4.102672\end{array}$

$\begin{array}{llll}-3.492515 & 0.252396 & -4.962430\end{array}$ 


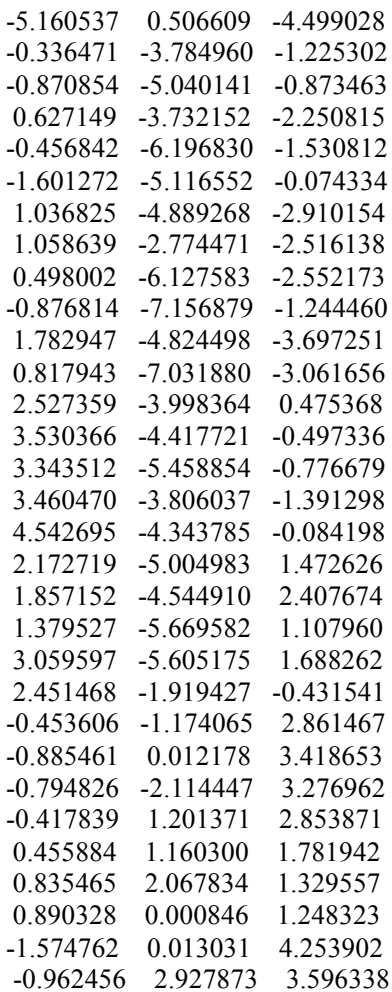

\section{Conformer_ 7}

Number of imaginary frequencies :

The smallest frequency is : $-247.6951 \mathrm{~cm}(-1)$

Electronic energy :

$\mathrm{HF}=-7222.6348457$

Zero-point correction=

1.755696

(Hartree/Particle)

Thermal correction to Energy=

Thermal correction to Enthalpy=

1.874112

1.875056

Thermal correction to Gibbs Free Energy $=\quad 1.593068$

Sum of electronic and zero-point Energies=

Sum of electronic and thermal Energies=

$-7220.879150$

Sum of electronic and thermal Enthalpies=

$-7220.760734$

$-7220.759789$

Sum of electronic and thermal Free Energies=

$-7221.041778$
Cartesian Coordinates

$$
\begin{array}{ccc}
4.295435 & 4.787495 & 1.872566 \\
2.562609 & 1.624221 & -1.886776 \\
4.540837 & 0.128255 & 0.932967 \\
3.967333 & 3.424868 & 0.318278 \\
4.154465 & 2.746181 & 1.579673 \\
3.132385 & 3.194574 & 2.472558 \\
3.009967 & 2.884764 & 3.501245 \\
2.335001 & 4.162913 & 1.791163 \\
1.508972 & 4.716180 & 2.215962 \\
2.848776 & 4.307382 & 0.469428 \\
2.478733 & 4.983307 & -0.286440 \\
6.237781 & 5.326139 & 2.375006 \\
7.098864 & 4.677351 & 2.282472 \\
5.382618 & 5.426599 & 3.515100 \\
5.479424 & 4.865240 & 4.434848 \\
4.350462 & 6.366001 & 3.211990 \\
3.525325 & 6.633590 & 3.858778 \\
4.567430 & 6.847355 & 1.884858 \\
3.938533 & 7.547148 & 1.350459 \\
5.733282 & 6.207332 & 1.368817 \\
6.141308 & 6.345018 & 0.376845 \\
4.764110 & 3.153521 & -0.913590 \\
6.097136 & 3.584372 & -0.983402 \\
6.500631 & 4.139515 & -0.146137 \\
6.905815 & 3.300330 & -2.083765 \\
7.941322 & 3.625123 & -2.093723
\end{array}
$$$$
\begin{array}{llll}
7.941322 & 3.625123 & -2.093723
\end{array}
$$

$\begin{array}{llll}6.383263 & 2.576689 & -3.153112\end{array}$

$\begin{array}{llll}7.003730 & 2.336842 & -4.010596\end{array}$

$\begin{array}{lll}5.061237 & 2.133318 & -3.106349\end{array}$

$\begin{array}{llll}4.671968 & 1.556409 & -3.938269\end{array}$

$\begin{array}{lll}4.241592 & 2.397407 & -1.997935\end{array}$

$\begin{array}{lll}5.179827 & 1.672631 & 1.830186\end{array}$

$\begin{array}{lll}6.093188 & 1.961945 & 1.307479\end{array}$

$\begin{array}{lll}5.519640 & 1.464934 & 3.308678\end{array}$

$\begin{array}{lll}6.298416 & 0.707212 & 3.429395\end{array}$

$\begin{array}{llll}4.650829 & 1.149450 & 3.892557\end{array}$

$\begin{array}{lll}5.881965 & 2.407845 & 3.729606\end{array}$

$\begin{array}{llll}2.354153 & 0.721543 & -3.482381\end{array}$

$\begin{array}{llll}1.908192 & 1.338068 & -4.662631\end{array}$

$\begin{array}{llll}1.691717 & 2.400557 & -4.680041\end{array}$

$\begin{array}{lll}1.718596 & 0.586222 & -5.822961\end{array}$

$\begin{array}{llll}1.362912 & 1.073937 & -6.726101\end{array}$

$\begin{array}{llll}1.978670 & -0.786790 & -5.822713\end{array}$

$\begin{array}{llll}1.823458 & -1.370919 & -6.725253\end{array}$

$\begin{array}{llll}2.434875 & -1.405380 & -4.656918\end{array}$

$\begin{array}{llll}2.641860 & -2.470451 & -4.648992\end{array}$

$\begin{array}{llll}2.615993 & -0.658182 & -3.490856\end{array}$

$\begin{array}{llll}2.929908 & -1.152852 & -2.579265\end{array}$

$\begin{array}{llll}1.403412 & 3.038022 & -2.070936\end{array}$

$\begin{array}{llll}1.740161 & 4.197411 & -2.788781\end{array}$

$\begin{array}{llll}2.732807 & 4.302354 & -3.216526\end{array}$

$\begin{array}{llll}0.805976 & 5.222455 & -2.948318\end{array}$

$\begin{array}{llll}1.076889 & 6.116268 & -3.503177\end{array}$

$\begin{array}{llll}-0.475603 & 5.094893 & -2.402806\end{array}$

$\begin{array}{llll}-1.208500 & 5.886060 & -2.533219\end{array}$

$\begin{array}{llll}-0.814537 & 3.944870 & -1.687110\end{array}$

$\begin{array}{llll}-1.807664 & 3.838922 & -1.267611\end{array}$

$\begin{array}{llll}0.124796 & 2.927221 & -1.509641\end{array}$

$\begin{array}{llll}-0.137188 & 2.044587 & -0.936943\end{array}$

$\begin{array}{llll}5.833922 & -0.351281 & -0.288629\end{array}$

$\begin{array}{llll}5.486559 & -1.402285 & -1.149827\end{array}$

$\begin{array}{llll}4.518788 & -1.879085 & -1.037033\end{array}$

$\begin{array}{llll}6.371447 & -1.830005 & -2.138738\end{array}$

$\begin{array}{llll}7.598028 & -1.194249 & -2.319871\end{array}$

$\begin{array}{llll}8.275599 & -1.511042 & -3.104613\end{array}$

$\begin{array}{llll}7.930482 & -0.131830 & -1.478852\end{array}$

$\begin{array}{llll}7.072149 & 0.277524 & -0.457892\end{array}$

$\begin{array}{llll}7.378609 & 1.096790 & 0.177713\end{array}$

$\begin{array}{llll}4.568805 & -1.271311 & 2.131083\end{array}$

$\begin{array}{llll}3.559810 & -1.295002 & 3.107342\end{array}$

$\begin{array}{lll}2.867938 & -0.465350 & 3.195538\end{array}$

$\begin{array}{lll}3.410883 & -2.401669 & 3.940359\end{array}$

$\begin{array}{llll}4.234127 & -3.519175 & 3.797755\end{array}$

$\begin{array}{lll}4.088273 & -4.396190 & 4.418426\end{array}$

$\begin{array}{llll}5.223531 & -3.500953 & 2.818086\end{array}$

$\begin{array}{llll}5.409841 & -2.382493 & 2.004655\end{array}$

$\begin{array}{lll}6.188730 & -2.406334 & 1.253015\end{array}$

$\begin{array}{llll}2.440256 & 0.234688 & -0.102444\end{array}$ $\begin{array}{llll}6.092365 & -4.714670 & 2.636932\end{array}$

$\begin{array}{llll}2.273629 & -2.466774 & 4.920856\end{array}$

$\begin{array}{llll}5.996852 & -3.025779 & -2.969486\end{array}$

$\begin{array}{llll}9.224991 & 0.593101 & -1.726488\end{array}$

$\begin{array}{llll}9.227796 & 1.197431 & -2.941483\end{array}$

$\begin{array}{llll}9.466948 & 1.553251 & -0.807251\end{array}$

$\begin{array}{llll}10.282494 & -0.251117 & -1.718005\end{array}$

$\begin{array}{llll}6.178545 & -4.182008 & -2.283071\end{array}$

$\begin{array}{llll}6.720333 & -3.118071 & -4.102893\end{array}$

$\begin{array}{llll}4.686488 & -3.002342 & -3.327102\end{array}$

$\begin{array}{lll}7.069158 & -4.789325 & 3.569201\end{array}$

$\begin{array}{lll}6.695934 & -4.731700 & 1.426768\end{array}$

$\begin{array}{llll}5.370008 & -5.857936 & 2.739517\end{array}$

$\begin{array}{llll}2.645532 & -3.031312 & 6.090361\end{array}$

$\begin{array}{llll}1.758008 & -1.251260 & 5.200084\end{array}$

$\begin{array}{llll}1.251252 & -3.226887 & 4.437738\end{array}$

$\begin{array}{lll}1.975986 & -2.751445 & 0.403928\end{array}$

$\begin{array}{lll}0.812531 & -2.465379 & 1.213706\end{array}$

$\begin{array}{lll}0.454070 & -1.190423 & 1.765496\end{array}$

$\begin{array}{llll}0.409850 & -3.292656 & 1.779845\end{array}$

$\begin{array}{llll}-3.400184 & -1.296268 & -1.446648\end{array}$

$\begin{array}{lll}-5.267035 & -0.538250 & -0.377380\end{array}$

$\begin{array}{lll}-5.260409 & 0.604666 & 0.820965\end{array}$ 
$\begin{array}{llll}-1.073749 & -2.507964 & -3.807261\end{array}$

$-1.196880 \quad-0.358842 \quad-3.615465$

$\begin{array}{llll}-1.429700 & 0.434477 & -4.332405\end{array}$

$\begin{array}{llll}-0.107579 & -0.422506 & -3.582293\end{array}$

$\begin{array}{llll}-1.691663 & 0.067938 & -2.237249\end{array}$

$\begin{array}{llll}-0.934811 & 0.084496 & -1.459427\end{array}$

$\begin{array}{llll}-2.806636 & 0.873776 & -2.021628\end{array}$

$\begin{array}{llll}-2.816480 & 1.461148 & -1.109211\end{array}$

$\begin{array}{lll}-3.753188 & 1.359102 & -3.100741\end{array}$

$\begin{array}{llll}-4.645404 & 1.750115 & -2.606804\end{array}$

$\begin{array}{llll}-3.289532 & 2.204833 & -3.630028\end{array}$

$\begin{array}{llll}-4.171009 & 0.260342 & -4.102075\end{array}$

$\begin{array}{llll}-3.492930 & 0.252328 & -4.961868\end{array}$

$\begin{array}{llll}-5.160922 & 0.506586 & -4.498378\end{array}$

$\begin{array}{llll}-0.336612 & -3.785144 & -1.225017\end{array}$

$\begin{array}{llll}-0.870930 & -5.040314 & -0.873043\end{array}$

$\begin{array}{llll}0.626886 & -3.732377 & -2.250647\end{array}$

$\begin{array}{llll}-0.456966 & -6.197035 & -1.530368\end{array}$

$\begin{array}{llll}-1.601257 & -5.116693 & -0.073828\end{array}$

$\begin{array}{llll}1.036518 & -4.889526 & -2.909957\end{array}$

$\begin{array}{llll}1.058322 & -2.774705 & -2.516085\end{array}$

$\begin{array}{llll}0.497766 & -6.127831 & -2.551836\end{array}$

$-0.876886 \quad-7.157076 \quad-1.243911$

$\begin{array}{llll}1.782548 & -4.824788 & -3.697144\end{array}$

$\begin{array}{llll}0.817673 & -7.032154 & -3.061296\end{array}$

$\begin{array}{llll}2.527478 & -3.998370 & 0.475303\end{array}$

$\begin{array}{llll}3.530397 & -4.417731 & -0.497489\end{array}$

$\begin{array}{llll}3.343600 & -5.458906 & -0.776712\end{array}$

$\begin{array}{llll}3.460332 & -3.806133 & -1.391496\end{array}$

$\begin{array}{llll}4.542775 & -4.343668 & -0.084491\end{array}$

$\begin{array}{lll}2.173018 & -5.004933 & 1.472682\end{array}$

$\begin{array}{lll}1.857522 & -4.544804 & 2.407727\end{array}$

$\begin{array}{llll}1.379830 & -5.669614 & 1.108158\end{array}$

$\begin{array}{llll}3.059960 & -5.605046 & 1.688271\end{array}$

$\begin{array}{lll}2.451413 & -1.919492 & -0.431723\end{array}$

$\begin{array}{lll}-0.453211 & -1.173973 & 2.861624\end{array}$

$\begin{array}{llll}-0.884982 & 0.012304 & 3.418804\end{array}$

$\begin{array}{lll}-0.794364 & -2.114329 & 3.277231\end{array}$

$\begin{array}{lll}-0.417452 & 1.201460 & 2.853870\end{array}$

$\begin{array}{lll}0.456104 & 1.160322 & 1.781808\end{array}$

$\begin{array}{lll}0.835598 & 2.067827 & 1.329296\end{array}$

$\begin{array}{lll}0.890482 & 0.000838 & 1.248205\end{array}$

$\begin{array}{lll}-1.574152 & 0.013209 & 4.254163\end{array}$

35

$\begin{array}{lll}-0.961938 & 2.928020 & 3.596285\end{array}$

\section{Conformer_8}

Number of imaginary frequencies : 1

The smallest frequency is : $-247.6994 \mathrm{~cm}(-1)$

Electronic energy : $\quad \mathrm{HF}=-7222.6348461$

Zero-point correction $=\quad 1.755697$

(Hartree/Particle)

Thermal correction to Energy=

Thermal correction to Enthalpy=

1.874113

1.875057

Thermal correction to Gibbs Free Energy $=\quad 1.593060$

Sum of electronic and zero-point Energies $=\quad-7220.879149$

Sum of electronic and thermal Energies=

$\begin{array}{ll}\text { Sum of electronic and thermal Enthalpies }= & -7220.759789 \\ \text { Sum of electronic and thermal Free Energies }= & -7221.041786\end{array}$

$-7220.760734$

\begin{tabular}{cccc} 
& \multicolumn{3}{c}{ Cartesian Coordinates } \\
\hline 26 & 4.295245 & 4.787336 & 1.873320 \\
15 & 2.562727 & 1.624358 & -1.886421 \\
15 & 4.540791 & 0.128175 & 0.933353 \\
6 & 3.967263 & 3.424792 & 0.318939 \\
6 & 4.154394 & 2.746028 & 1.580291 \\
6 & 3.132271 & 3.194307 & 2.473183 \\
1 & 3.009845 & 2.884417 & 3.501845 \\
6 & 2.334853 & 4.162650 & 1.791832
\end{tabular}


$\begin{array}{lll}1.508781 & 4.715839 & 2.216645\end{array}$

$\begin{array}{llll}2.848651 & 4.307232 & 0.470117\end{array}$

$\begin{array}{llll}2.478598 & 4.983198 & -0.285710\end{array}$

$\begin{array}{llll}6.237545 & 5.326095 & 2.375829\end{array}$

$\begin{array}{lll}7.098682 & 4.677379 & 2.283279\end{array}$

$\begin{array}{lll}5.382354 & 5.426435 & 3.515912\end{array}$

$\begin{array}{llll}5.479187 & 4.865038 & 4.435634\end{array}$

$\begin{array}{lll}4.350134 & 6.365775 & 3.212828\end{array}$

$\begin{array}{llll}3.524965 & 6.633273 & 3.859614\end{array}$

$\begin{array}{llll}4.567090 & 6.847210 & 1.885723\end{array}$

$\begin{array}{llll}3.938149 & 7.546980 & 1.351348\end{array}$

$\begin{array}{llll}5.733000 & 6.207299 & 1.369673\end{array}$

$\begin{array}{lll}6.141033 & 6.345052 & 0.377713\end{array}$

$\begin{array}{llll}4.764124 & 3.153592 & -0.912908\end{array}$

$\begin{array}{llll}6.097146 & 3.584480 & -0.982583\end{array}$

$\begin{array}{llll}6.500583 & 4.139525 & -0.145226\end{array}$

$\begin{array}{llll}6.905900 & 3.300600 & -2.082931\end{array}$

$\begin{array}{llll}7.941400 & 3.625417 & -2.092781\end{array}$

$\begin{array}{llll}6.383429 & 2.577096 & -3.152410\end{array}$

$\begin{array}{llll}7.003950 & 2.337385 & -4.009892\end{array}$

$\begin{array}{llll}5.061414 & 2.133680 & -3.105781\end{array}$

$\begin{array}{llll}4.672206 & 1.556881 & -3.937805\end{array}$

$\begin{array}{llll}4.241699 & 2.397594 & -1.997377\end{array}$

$\begin{array}{lll}5.179764 & 1.672472 & 1.830729\end{array}$

$\begin{array}{lll}6.093126 & 1.961835 & 1.308053\end{array}$

$\begin{array}{lll}5.519556 & 1.464645 & 3.309205\end{array}$

$\begin{array}{llll}6.298333 & 0.706915 & 3.429868\end{array}$

$\begin{array}{llll}4.650736 & 1.149107 & 3.893039\end{array}$

$\begin{array}{lll}5.881869 & 2.407519 & 3.730227\end{array}$

$\begin{array}{lll}2.354428 & 0.721799 & -3.482115\end{array}$

$\begin{array}{llll}1.908498 & 1.338390 & -4.662342\end{array}$

$\begin{array}{llll}1.691950 & 2.400866 & -4.679678\end{array}$

$\begin{array}{llll}1.719046 & 0.586631 & -5.822752\end{array}$

$\begin{array}{llll}1.363388 & 1.074399 & -6.725875\end{array}$

$\begin{array}{lll}1.979236 & -0.786358 & -5.822609\end{array}$

$\begin{array}{llll}1.824140 & -1.370420 & -6.725212\end{array}$

$2.435411 \quad-1.405013 \quad-4.656836$

$\begin{array}{llll}2.642490 & -2.470066 & -4.648990\end{array}$

$2.616384 \quad-0.657904 \quad-3.490695$

$\begin{array}{llll}2.930294 & -1.152628 & -2.579130\end{array}$

$\begin{array}{lll}1.403558 & 3.038183 & -2.070585\end{array}$

$\begin{array}{llll}1.740407 & 4.197626 & -2.788297\end{array}$

$\begin{array}{llll}2.733096 & 4.302580 & -3.215940\end{array}$

$\begin{array}{llll}0.806265 & 5.222708 & -2.947839\end{array}$

$\begin{array}{lll}1.077256 & 6.116561 & -3.502597\end{array}$

$\begin{array}{llll}-0.475370 & 5.095134 & -2.402463\end{array}$

$\begin{array}{llll}-1.208233 & 5.886333 & -2.532876\end{array}$

$\begin{array}{llll}-0.814404 & 3.945057 & -1.686901\end{array}$

$\begin{array}{llll}-1.807572 & 3.839106 & -1.267505\end{array}$

$\begin{array}{llll}0.124883 & 2.927367 & -1.509427\end{array}$

$\begin{array}{llll}-0.137183 & 2.044692 & -0.936828\end{array}$

$\begin{array}{llll}5.833964 & -0.351279 & -0.288182\end{array}$

$\begin{array}{llll}5.486699 & -1.402269 & -1.149439\end{array}$

$\begin{array}{llll}4.518948 & -1.879126 & -1.036717\end{array}$

$\begin{array}{llll}6.371654 & -1.829896 & -2.138330\end{array}$

$\begin{array}{llll}7.598205 & -1.194062 & -2.319387\end{array}$

$\begin{array}{llll}8.275826 & -1.510782 & -3.104115\end{array}$

$\begin{array}{llll}7.930569 & -0.131668 & -1.478302\end{array}$

$\begin{array}{llll}7.072168 & 0.277598 & -0.457364\end{array}$

$\begin{array}{llll}7.378559 & 1.096854 & 0.178287\end{array}$

$\begin{array}{llll}4.568642 & -1.271466 & 2.131383\end{array}$

$\begin{array}{llll}3.559576 & -1.295180 & 3.107569\end{array}$

$\begin{array}{llll}2.867721 & -0.465511 & 3.195754\end{array}$

$\begin{array}{llll}3.410569 & -2.401881 & 3.940525\end{array}$

$\begin{array}{llll}4.233808 & -3.519392 & 3.797932\end{array}$

$\begin{array}{llll}4.087897 & -4.396433 & 4.418556\end{array}$

$\begin{array}{llll}5.223278 & -3.501149 & 2.818331\end{array}$

$\begin{array}{llll}5.409663 & -2.382657 & 2.004959\end{array}$

$\begin{array}{llll}6.188599 & -2.406481 & 1.253366\end{array}$

$\begin{array}{llll}2.440265 & 0.234673 & -0.102217\end{array}$

$\begin{array}{llll}6.092100 & -4.714878 & 2.637194\end{array}$

$\begin{array}{llll}2.273233 & -2.467018 & 4.920922\end{array}$

$\begin{array}{llll}5.997167 & -3.025664 & -2.969135\end{array}$

$\begin{array}{llll}9.225060 & 0.593328 & -1.725836\end{array}$

$\begin{array}{llll}9.227875 & 1.197788 & -2.940766\end{array}$ $\begin{array}{llll}9.466972 & 1.553386 & -0.806491\end{array}$

$10.282587-0.250862-1.717422$

$6.178808-4.181899-2.282716$

$\begin{array}{llll}6.720767 & -3.117929 & -4.102466\end{array}$

$\begin{array}{llll}4.686836 & -3.002245 & -3.326885\end{array}$

$\begin{array}{lll}7.068833 & -4.789582 & 3.569521\end{array}$

$\begin{array}{llll}6.695745 & -4.731885 & 1.427066\end{array}$

$\begin{array}{lll}5.369712 & -5.858132 & 2.739699\end{array}$

$\begin{array}{llll}2.645032 & -3.031594 & 6.090441\end{array}$

$\begin{array}{llll}1.757591 & -1.251510 & 5.200143\end{array}$

$\begin{array}{lll}1.250897 & -3.227113 & 4.437690\end{array}$

$\begin{array}{lll}1.975980 & -2.751462 & 0.403953\end{array}$

$\begin{array}{lll}0.812459 & -2.465437 & 1.213644\end{array}$

$\begin{array}{llll}0.453923 & -1.190519 & 1.765468\end{array}$

$\begin{array}{llll}0.409725 & -3.292748 & 1.779694\end{array}$

$\begin{array}{llll}-3.400055 & -1.296266 & -1.447053\end{array}$

$\begin{array}{llll}-5.266979 & -0.538251 & -0.377916\end{array}$

$\begin{array}{lll}-5.260415 & 0.604610 & 0.820481\end{array}$

$\begin{array}{llll}-6.330012 & 0.174205 & -1.442085\end{array}$

$\begin{array}{llll}-5.966536 & -1.798985 & 0.479354\end{array}$

$\begin{array}{lll}-4.982021 & 1.930143 & 0.525395\end{array}$

$\begin{array}{llll}-3.757849 & 2.439111 & 1.010859\end{array}$

$\begin{array}{lll}-3.118990 & 1.781987 & 1.589941\end{array}$

$\begin{array}{llll}-3.415952 & 3.740403 & 0.740552\end{array}$

$\begin{array}{lll}-2.486803 & 4.152945 & 1.120603\end{array}$

$\begin{array}{llll}-4.248653 & 4.546700 & -0.083389\end{array}$

$\begin{array}{llll}-3.843350 & 5.851279 & -0.476506\end{array}$

$\begin{array}{llll}-2.895180 & 6.232476 & -0.106300\end{array}$

$\begin{array}{llll}-4.622804 & 6.608567 & -1.322050\end{array}$

$\begin{array}{llll}-4.300192 & 7.600931 & -1.623209\end{array}$

$\begin{array}{llll}-5.844866 & 6.086399 & -1.809471\end{array}$

$\begin{array}{llll}-6.447874 & 6.678357 & -2.491878\end{array}$

$\begin{array}{llll}-6.275805 & 4.832914 & -1.431329\end{array}$

$\begin{array}{llll}-7.211196 & 4.445876 & -1.818722\end{array}$

$\begin{array}{llll}-5.501719 & 4.025549 & -0.552369\end{array}$

$\begin{array}{llll}-5.909859 & 2.701998 & -0.155726\end{array}$

$\begin{array}{llll}-7.269094 & 2.156944 & -0.436121\end{array}$

$\begin{array}{lll}-8.460071 & 2.845544 & -0.001800\end{array}$

$\begin{array}{llll}-8.420886 & 4.023086 & 0.797220\end{array}$

$\begin{array}{lll}-7.464233 & 4.422214 & 1.111132\end{array}$

$\begin{array}{lll}-9.579444 & 4.655817 & 1.193223\end{array}$

$\begin{array}{lll}-9.518937 & 5.549150 & 1.808276\end{array}$

$\begin{array}{lll}-10.845767 & 4.150718 & 0.814775\end{array}$

$\begin{array}{lll}-11.749883 & 4.664800 & 1.127496\end{array}$

$\begin{array}{lll}-10.923619 & 2.997862 & 0.067048\end{array}$

$\begin{array}{lll}-11.888603 & 2.583654 & -0.213182\end{array}$

$\begin{array}{llll}-9.748618 & 2.311574 & -0.344047\end{array}$

$\begin{array}{lll}-9.832539 & 1.090687 & -1.066217\end{array}$

$\begin{array}{llll}-10.811037 & 0.699065 & -1.329373\end{array}$

$\begin{array}{llll}-8.695357 & 0.398898 & -1.398557\end{array}$

$\begin{array}{llll}-8.734203 & -0.551417 & -1.920468\end{array}$

$\begin{array}{llll}-7.430775 & 0.928290 & -1.059977\end{array}$

$\begin{array}{lll}-5.328407 & -3.110862 & 0.180895\end{array}$

$\begin{array}{lll}-4.591474 & -3.316360 & 0.966919\end{array}$

$\begin{array}{lll}-6.342657 & -4.240768 & 0.175001\end{array}$

$\begin{array}{lll}-6.027025 & -5.466205 & 0.773274\end{array}$

$\begin{array}{lll}-5.072578 & -5.581342 & 1.282221\end{array}$

$\begin{array}{lll}-6.925669 & -6.535481 & 0.722875\end{array}$

$\begin{array}{llll}-6.668080 & -7.480441 & 1.193387\end{array}$

$\begin{array}{lll}-8.155633 & -6.385254 & 0.078999\end{array}$

$\begin{array}{lll}-8.858869 & -7.212576 & 0.044861\end{array}$

$\begin{array}{llll}-8.480252 & -5.161941 & -0.517199\end{array}$

$\begin{array}{llll}-9.437665 & -5.037322 & -1.015918\end{array}$

$\begin{array}{lll}-7.579057 & -4.098435 & -0.471536\end{array}$

$\begin{array}{llll}-7.831562 & -3.144155 & -0.925680\end{array}$

$-4.594939-3.051727-1.163650$

$\begin{array}{llll}-5.334012 & -3.112940 & -1.971451\end{array}$

$\begin{array}{llll}-3.953704 & -3.933849 & -1.249650\end{array}$

$\begin{array}{lll}-6.667712 & -1.732641 & 1.807978\end{array}$

$\begin{array}{lll}-7.141406 & -2.714896 & 1.886665\end{array}$

$\begin{array}{lll}-5.678281 & -1.640687 & 2.970889\end{array}$

$\begin{array}{lll}-5.126455 & -2.826336 & 3.482622\end{array}$

$\begin{array}{lll}-5.453062 & -3.781424 & 3.079885\end{array}$

$\begin{array}{lll}-4.182203 & -2.800491 & 4.512389\end{array}$

$\begin{array}{lll}-3.769208 & -3.731944 & 4.890097\end{array}$ 
$\begin{array}{lll}-3.790243 & -1.581158 & 5.068468\end{array}$

$\begin{array}{llll}-3.063986 & -1.555191 & 5.876303\end{array}$

$\begin{array}{lll}-4.357168 & -0.395183 & 4.591602\end{array}$

$\begin{array}{llll}-4.076157 & 0.557834 & 5.032402\end{array}$

$\begin{array}{lll}-5.290573 & -0.424759 & 3.553715\end{array}$

$\begin{array}{lll}-5.716195 & 0.505607 & 3.199581\end{array}$

$\begin{array}{lll}-7.806766 & -0.715796 & 1.852869\end{array}$

$\begin{array}{lll}-8.514383 & -0.911614 & 1.044087\end{array}$

$\begin{array}{llll}-7.468447 & 0.315878 & 1.765907\end{array}$

$\begin{array}{lll}-8.334906 & -0.822780 & 2.805344\end{array}$

$\begin{array}{llll}-2.525414 & -1.128651 & 0.544850\end{array}$

$\begin{array}{lll}-1.882683 & -0.253892 & 0.590832\end{array}$

$\begin{array}{lll}-3.221287 & -1.184009 & 1.377319\end{array}$

$\begin{array}{lll}-1.992503 & -2.367642 & 0.062441\end{array}$

$\begin{array}{lll}-2.496794 & -3.272461 & 0.385143\end{array}$

$\begin{array}{llll}-0.734552 & -2.534855 & -0.568814\end{array}$

$\begin{array}{llll}-0.208977 & -1.652893 & -0.907263\end{array}$

$-4.223037-1.143824-3.493053$

$\begin{array}{llll}-5.215218 & -1.589457 & -3.481931\end{array}$

$\begin{array}{lll}-3.126521 & -2.048642 & -3.521816\end{array}$

$\begin{array}{lll}-3.365324 & -3.111792 & -3.550934\end{array}$

$\begin{array}{llll}-1.761954 & -1.710512 & -4.097576\end{array}$

$\begin{array}{lll}-1.805687 & -1.728807 & -5.197714\end{array}$

$\begin{array}{llll}-1.073455 & -2.507911 & -3.807525\end{array}$

$\begin{array}{lll}-1.196576 & -0.358792 & -3.615709\end{array}$

$\begin{array}{llll}-1.429354 & 0.434539 & -4.332649\end{array}$

$\begin{array}{llll}-0.107276 & -0.422466 & -3.582489\end{array}$

$\begin{array}{lll}-1.691426 & 0.067976 & -2.237514\end{array}$

$\begin{array}{llll}-0.934626 & 0.084511 & -1.459640\end{array}$

$\begin{array}{llll}-2.806416 & 0.873798 & -2.021935\end{array}$

$\begin{array}{llll}-2.816320 & 1.461130 & -1.109493\end{array}$

$\begin{array}{llll}-3.752902 & 1.359160 & -3.101091\end{array}$

$\begin{array}{llll}-4.645144 & 1.750169 & -2.607197\end{array}$

$\begin{array}{llll}-3.289208 & 2.204903 & -3.630327\end{array}$

$\begin{array}{llll}-4.170676 & 0.260434 & -4.102481\end{array}$

$\begin{array}{llll}-3.492551 & 0.252441 & -4.962238\end{array}$

$\begin{array}{llll}-5.160564 & 0.506699 & -4.498831\end{array}$

$\begin{array}{llll}-0.336411 & -3.785037 & -1.225308\end{array}$

$\begin{array}{lll}-0.870703 & -5.040249 & -0.873446\end{array}$

$\begin{array}{llll}0.627159 & -3.732168 & -2.250865\end{array}$

$\begin{array}{lll}-0.456655 & -6.196912 & -1.530817\end{array}$

$\begin{array}{llll}-1.601074 & -5.116704 & -0.074277\end{array}$

$\begin{array}{llll}1.036871 & -4.889260 & -2.910227\end{array}$

$\begin{array}{llll}1.058583 & -2.774463 & -2.516205\end{array}$

$0.498136-6.127606-2.552224$

$-0.876555-7.156987-1.244446$

$\begin{array}{llll}1.782952 & -4.824443 & -3.697359\end{array}$

$\begin{array}{llll}0.818107 & -7.031884 & -3.061724\end{array}$

$\begin{array}{llll}2.527448 & -3.998400 & 0.475308\end{array}$

$\begin{array}{llll}3.530436 & -4.417742 & -0.497422\end{array}$

$\begin{array}{llll}3.343605 & -5.458885 & -0.776743\end{array}$

$\begin{array}{llll}3.460490 & -3.806075 & -1.391391\end{array}$

$\begin{array}{llll}4.542777 & -4.343767 & -0.084320\end{array}$

$\begin{array}{llll}2.172872 & -5.005010 & 1.472598\end{array}$

$\begin{array}{llll}1.857333 & -4.544926 & 2.407651\end{array}$

$\begin{array}{lll}1.379679 & -5.669633 & 1.107977\end{array}$

$\begin{array}{llll}3.059772 & -5.605178 & 1.688208\end{array}$

$2.451497-1.919466-0.431606$

$\begin{array}{lll}-0.453470 & -1.174150 & 2.861504\end{array}$

$\begin{array}{lll}-0.885311 & 0.012087 & 3.418713\end{array}$

$\begin{array}{lll}-0.794659 & -2.114537 & 3.277012\end{array}$

$\begin{array}{lll}-0.417733 & 1.201285 & 2.853907\end{array}$

$\begin{array}{lll}0.455935 & 1.160227 & 1.781933\end{array}$

$\begin{array}{lll}0.835476 & 2.067766 & 1.329526\end{array}$

$\begin{array}{lll}0.890373 & 0.000779 & 1.248295\end{array}$

$\begin{array}{lll}-1.574570 & 0.012930 & 4.253997\end{array}$

$\begin{array}{lll}-0.962330 & 2.927780 & 3.596405\end{array}$

\section{Conformer_9}

Number of imaginary frequencies : 1

The smallest frequency is : $-247.7215 \mathrm{~cm}(-1)$

Electronic energy :

$\mathrm{HF}=-7222.6348462$

Zero-point correction=

1.75570

(Hartree/Particle)
Thermal correction to Energy=

Thermal correction to Enthalpy=

1.874116

1.875060

Thermal correction to Gibbs Free Energy $=\quad 1.593080$

Sum of electronic and zero-point Energies $=\quad-7220.879145$

Sum of electronic and thermal Energies=

$-7220.760731$

Sum of electronic and thermal Enthalpies=

$-7220.759786$

Sum of electronic and thermal Free Energies=

$-7221.041767$

Cartesian Coordinates

$\begin{array}{lll}4.295540 & 4.787317 & 1.873314\end{array}$

$2.562877 \quad 1.624340 \quad-1.886366$

$\begin{array}{lll}4.540811 & 0.128129 & 0.933481\end{array}$

$\begin{array}{lll}3.967490 & 3.424777 & 0.318941\end{array}$

$\begin{array}{lll}4.154538 & 2.746024 & 1.580311\end{array}$

$\begin{array}{lll}3.132422 & 3.194394 & 2.473167\end{array}$

$\begin{array}{lll}3.009949 & 2.884537 & 3.501833\end{array}$

$\begin{array}{llll}2.335097 & 4.162787 & 1.791777\end{array}$

$\begin{array}{llll}1.509058 & 4.716049 & 2.216559\end{array}$

$\begin{array}{llll}2.848943 & 4.307307 & 0.470074\end{array}$

$\begin{array}{llll}2.478957 & 4.983280 & -0.285779\end{array}$

$\begin{array}{llll}6.237875 & 5.325898 & 2.375873\end{array}$

$\begin{array}{lll}7.098954 & 4.677102 & 2.283341\end{array}$

$\begin{array}{llll}5.382663 & 5.426307 & 3.515934\end{array}$

$\begin{array}{llll}5.479419 & 4.864894 & 4.435655\end{array}$

$\begin{array}{lll}4.350536 & 6.365743 & 3.212830\end{array}$

$\begin{array}{llll}3.525375 & 6.633311 & 3.859597\end{array}$

$\begin{array}{llll}4.567571 & 6.847168 & 1.885735\end{array}$

$\begin{array}{lll}3.938708 & 7.547000 & 1.351348\end{array}$

$\begin{array}{lll}5.733436 & 6.207154 & 1.369710\end{array}$

$\begin{array}{lll}6.141509 & 6.344881 & 0.377763\end{array}$

$\begin{array}{llll}4.764350 & 3.153490 & -0.912888\end{array}$

$\begin{array}{llll}6.097397 & 3.584297 & -0.982563\end{array}$

$\begin{array}{llll}6.500855 & 4.139349 & -0.145221\end{array}$

$\begin{array}{llll}6.906147 & 3.300327 & -2.082892\end{array}$

$\begin{array}{llll}7.941667 & 3.625082 & -2.092742\end{array}$

$\begin{array}{llll}6.383646 & 2.576813 & -3.152349\end{array}$

$\begin{array}{llll}7.004164 & 2.337031 & -4.009814\end{array}$

$\begin{array}{llll}5.061603 & 2.133480 & -3.105721\end{array}$

$\begin{array}{llll}4.672372 & 1.556671 & -3.937727\end{array}$

$\begin{array}{llll}4.241889 & 2.397486 & -1.997337\end{array}$

$\begin{array}{llll}5.179844 & 1.672421 & 1.830813\end{array}$

$\begin{array}{llll}6.093235 & 1.961720 & 1.308151\end{array}$

$\begin{array}{llll}5.519588 & 1.464627 & 3.309305\end{array}$

$\begin{array}{lll}6.298325 & 0.706862 & 3.430012\end{array}$

$\begin{array}{llll}4.650738 & 1.149148 & 3.893128\end{array}$

$\begin{array}{llll}5.881936 & 2.407496 & 3.730305\end{array}$

$\begin{array}{llll}2.354537 & 0.721721 & -3.482021\end{array}$

$\begin{array}{llll}1.908740 & 1.338311 & -4.662300\end{array}$

$\begin{array}{llll}1.692327 & 2.400813 & -4.679697\end{array}$

$\begin{array}{lll}1.719254 & 0.586520 & -5.822683\end{array}$

$\begin{array}{llll}1.363702 & 1.074287 & -6.725848\end{array}$

$\begin{array}{llll}1.979271 & -0.786503 & -5.822458\end{array}$

$\begin{array}{llll}1.824147 & -1.370589 & -6.725041\end{array}$

$2.435308 \quad-1.405158 \quad-4.656632$

$\begin{array}{llll}2.642249 & -2.470238 & -4.648723\end{array}$

$2.616318-0.658014 \quad-3.490518$

$\begin{array}{llll}2.930121 & -1.152732 & -2.578913\end{array}$

$\begin{array}{llll}1.403757 & 3.038197 & -2.070601\end{array}$

$\begin{array}{llll}1.740667 & 4.197630 & -2.788299\end{array}$

$\begin{array}{llll}2.733384 & 4.302568 & -3.215878\end{array}$

$\begin{array}{llll}0.806547 & 5.222721 & -2.947911\end{array}$

$\begin{array}{llll}1.077585 & 6.116567 & -3.502658\end{array}$

$\begin{array}{llll}-0.475126 & 5.095166 & -2.402622\end{array}$

$\begin{array}{llll}-1.207972 & 5.886370 & -2.533093\end{array}$

$\begin{array}{llll}-0.814222 & 3.945098 & -1.687074\end{array}$

$\begin{array}{llll}-1.807423 & 3.839162 & -1.267748\end{array}$

$\begin{array}{llll}0.125042 & 2.927399 & -1.509527\end{array}$

$\begin{array}{llll}-0.137071 & 2.044728 & -0.936943\end{array}$

$\begin{array}{llll}5.833967 & -0.351431 & -0.288030\end{array}$

$\begin{array}{llll}5.486639 & -1.402416 & -1.149267\end{array}$

$\begin{array}{llll}4.518854 & -1.879203 & -1.036543\end{array}$

$\begin{array}{llll}6.371575 & -1.830125 & -2.138140\end{array}$

$\begin{array}{llll}7.598171 & -1.194377 & -2.319198\end{array}$

$\begin{array}{llll}8.275778 & -1.511159 & -3.103912\end{array}$ 
$\begin{array}{llll}7.930596 & -0.131984 & -1.478135\end{array}$

$\begin{array}{llll}7.072213 & 0.277361 & -0.457213\end{array}$

$\begin{array}{llll}7.378651 & 1.096611 & 0.178422\end{array}$

$\begin{array}{llll}4.568575 & -1.271479 & 2.131549\end{array}$

$\begin{array}{lll}3.559489 & -1.295123 & 3.107715\end{array}$

$\begin{array}{lll}2.867667 & -0.465422 & 3.195864\end{array}$

$3.410411 \quad-2.401796 \quad 3.940695$

$\begin{array}{lll}4.233598 & -3.519351 & 3.798147\end{array}$

$\begin{array}{llll}4.087632 & -4.396370 & 4.418786\end{array}$

$\begin{array}{llll}5.223089 & -3.501178 & 2.818564\end{array}$

$\begin{array}{lll}5.409545 & -2.382714 & 2.005169\end{array}$

$\begin{array}{llll}6.188495 & -2.406595 & 1.253592\end{array}$

$\begin{array}{lll}2.440322 & 0.234743 & -0.102100\end{array}$

$\begin{array}{llll}6.091854 & -4.714953 & 2.637468\end{array}$

$\begin{array}{lll}2.273046 & -2.466849 & 4.921067\end{array}$

$\begin{array}{llll}5.997013 & -3.025885 & -2.968923\end{array}$

$\begin{array}{llll}9.225136 & 0.592923 & -1.725677\end{array}$

$\begin{array}{llll}9.228011 & 1.197325 & -2.940635\end{array}$

$\begin{array}{llll}9.467086 & 1.553009 & -0.806372\end{array}$

$10.282612-0.251331 \quad-1.717202$

$6.178578-4.182118-2.282481$

$6.720611-3.118218-4.102250$

$\begin{array}{llll}4.686686 & -3.002388 & -3.326675\end{array}$

$\begin{array}{llll}7.068574 & -4.789680 & 3.569807\end{array}$

$\begin{array}{llll}6.695511 & -4.732019 & 1.427347\end{array}$

$\begin{array}{llll}5.369411 & -5.858170 & 2.739994\end{array}$

$\begin{array}{llll}2.644781 & -3.031436 & 6.090601\end{array}$

$\begin{array}{llll}1.757477 & -1.251304 & 5.200261\end{array}$

$\begin{array}{llll}1.250670 & -3.226880 & 4.437821\end{array}$

$\begin{array}{lll}1.975847 & -2.751355 & 0.404153\end{array}$

$\begin{array}{lll}0.812329 & -2.465232 & 1.213816\end{array}$

$\begin{array}{lll}0.453839 & -1.190268 & 1.765570\end{array}$

$\begin{array}{lll}0.409546 & -3.292495 & 1.779903\end{array}$

$\begin{array}{llll}-3.400033 & -1.296222 & -1.447085\end{array}$

$\begin{array}{llll}-5.267046 & -0.538279 & -0.378041\end{array}$

$\begin{array}{lll}-5.260577 & 0.604647 & 0.820297\end{array}$

$\begin{array}{llll}-6.330097 & 0.174070 & -1.442264\end{array}$

$\begin{array}{llll}-5.966545 & -1.799009 & 0.479287\end{array}$

$\begin{array}{lll}-4.982131 & 1.930149 & 0.525132\end{array}$

$\begin{array}{lll}-3.757960 & 2.439114 & 1.010600\end{array}$

$\begin{array}{lll}-3.119140 & 1.782003 & 1.589740\end{array}$

$\begin{array}{llll}-3.416017 & 3.740377 & 0.740208\end{array}$

$\begin{array}{lll}-2.486869 & 4.152924 & 1.120254\end{array}$

$\begin{array}{llll}-4.248672 & 4.546637 & -0.083818\end{array}$

$\begin{array}{llll}-3.843326 & 5.851182 & -0.477005\end{array}$

$\begin{array}{llll}-2.895159 & 6.232380 & -0.106790\end{array}$

$\begin{array}{lll}-4.622732 & 6.608433 & -1.322625\end{array}$

$\begin{array}{llll}-4.300086 & 7.600770 & -1.623836\end{array}$

$\begin{array}{llll}-5.844789 & 6.086262 & -1.810056\end{array}$

$\begin{array}{llll}-6.447759 & 6.678191 & -2.492522\end{array}$

$\begin{array}{llll}-6.275771 & 4.832812 & -1.431847\end{array}$

$\begin{array}{llll}-7.211158 & 4.445771 & -1.819247\end{array}$

$\begin{array}{llll}-5.501736 & 4.025487 & -0.552805\end{array}$

$\begin{array}{llll}-5.909924 & 2.701974 & -0.156083\end{array}$

$\begin{array}{llll}-7.269158 & 2.156921 & -0.436483\end{array}$

$\begin{array}{lll}-8.460136 & 2.845583 & -0.002257\end{array}$

$\begin{array}{lll}-8.420953 & 4.023192 & 0.796666\end{array}$

$\begin{array}{lll}-7.464302 & 4.422322 & 1.110580\end{array}$

$\begin{array}{llll}-9.579509 & 4.655984 & 1.192573\end{array}$

$\begin{array}{lll}-9.519002 & 5.549368 & 1.807552\end{array}$

$\begin{array}{llll}-10.845832 & 4.150883 & 0.814123\end{array}$

$\begin{array}{llll}-11.749946 & 4.665014 & 1.126768\end{array}$

$\begin{array}{lll}-10.923684 & 2.997965 & 0.066495\end{array}$

$\begin{array}{llll}-11.888668 & 2.583754 & -0.213733\end{array}$

$\begin{array}{lll}-9.748685 & 2.311614 & -0.344501\end{array}$

$\begin{array}{llll}-9.832609 & 1.090668 & -1.066570\end{array}$

$\begin{array}{llll}-10.811107 & 0.699045 & -1.329725\end{array}$

$\begin{array}{llll}-8.695432 & 0.398826 & -1.398814\end{array}$

$\begin{array}{lll}-8.734283 & -0.551532 & -1.920646\end{array}$

$\begin{array}{llll}-7.430847 & 0.928216 & -1.060240\end{array}$

$\begin{array}{lll}-5.328293 & -3.110859 & 0.180965\end{array}$

$\begin{array}{lll}-4.591386 & -3.316233 & 0.967045\end{array}$

$\begin{array}{lll}-6.342446 & -4.240853 & 0.175114\end{array}$

$\begin{array}{llll}-6.026715 & -5.466229 & 0.773460\end{array}$

$\begin{array}{lll}-5.072269 & -5.581252 & 1.282435\end{array}$ $\begin{array}{lll}-6.925260 & -6.535589 & 0.723101\end{array}$

$\begin{array}{lll}-6.667595 & -7.480501 & 1.193670\end{array}$

$\begin{array}{lll}-8.155225 & -6.385509 & 0.079192\end{array}$

$\begin{array}{lll}-8.858386 & -7.212896 & 0.045086\end{array}$

$\begin{array}{llll}-8.479943 & -5.162258 & -0.517080\end{array}$

$\begin{array}{llll}-9.437357 & -5.037754 & -1.015825\end{array}$

$\begin{array}{llll}-7.578844 & -4.098668 & -0.471457\end{array}$

$\begin{array}{llll}-7.831426 & -3.144437 & -0.925661\end{array}$

$\begin{array}{llll}-4.594767 & -3.051779 & -1.163550\end{array}$

$\begin{array}{llll}-5.333799 & -3.113137 & -1.971376\end{array}$

$\begin{array}{llll}-3.953444 & -3.933848 & -1.249442\end{array}$

$\begin{array}{lll}-6.667775 & -1.732612 & 1.807879\end{array}$

$\begin{array}{lll}-7.141403 & -2.714894 & 1.886628\end{array}$

$\begin{array}{lll}-5.678392 & -1.640495 & 2.970820\end{array}$

$\begin{array}{lll}-5.126496 & -2.826065 & 3.482660\end{array}$

$\begin{array}{lll}-5.453026 & -3.781205 & 3.079986\end{array}$

$\begin{array}{llll}-4.182275 & -2.800077 & 4.512451\end{array}$

$\begin{array}{lll}-3.769224 & -3.731472 & 4.890241\end{array}$

$\begin{array}{lll}-3.790419 & -1.580673 & 5.068450\end{array}$

$\begin{array}{lll}-3.064186 & -1.554594 & 5.876303\end{array}$

$\begin{array}{lll}-4.357419 & -0.394775 & 4.591482\end{array}$

$\begin{array}{llll}-4.076494 & 0.558294 & 5.032224\end{array}$

$\begin{array}{lll}-5.290794 & -0.424496 & 3.553572\end{array}$

$\begin{array}{lll}-5.716477 & 0.505813 & 3.199363\end{array}$

$\begin{array}{lll}-7.806901 & -0.715841 & 1.852638\end{array}$

$\begin{array}{lll}-8.514468 & -0.911775 & 1.043839\end{array}$

$\begin{array}{lll}-7.468648 & 0.315849 & 1.765606\end{array}$

$\begin{array}{lll}-8.335078 & -0.822782 & 2.805097\end{array}$

$\begin{array}{lll}-2.525406 & -1.128159 & 0.544810\end{array}$

$\begin{array}{llll}-1.882652 & -0.253405 & 0.590587\end{array}$

$\begin{array}{lll}-3.221276 & -1.183307 & 1.377292\end{array}$

$\begin{array}{lll}-1.992571 & -2.367262 & 0.062642\end{array}$

$\begin{array}{lll}-2.496897 & -3.271984 & 0.385554\end{array}$

$\begin{array}{llll}-0.734643 & -2.534654 & -0.568619\end{array}$

$\begin{array}{llll}-0.209013 & -1.652766 & -0.907184\end{array}$

$\begin{array}{llll}-4.223001 & -1.144052 & -3.493083\end{array}$

$\begin{array}{llll}-5.215144 & -1.589767 & -3.481931\end{array}$

$\begin{array}{llll}-3.126408 & -2.048789 & -3.521764\end{array}$

$\begin{array}{lll}-3.365127 & -3.111959 & -3.550786\end{array}$

$\begin{array}{llll}-1.761873 & -1.710613 & -4.097571\end{array}$

$\begin{array}{llll}-1.805611 & -1.729037 & -5.197708\end{array}$

$\begin{array}{llll}-1.073307 & -2.507923 & -3.807433\end{array}$

$\begin{array}{llll}-1.196613 & -0.358796 & -3.615851\end{array}$

$\begin{array}{llll}-1.429484 & 0.434444 & -4.332861\end{array}$

$\begin{array}{lll}-0.107306 & -0.422360 & -3.582645\end{array}$

$\begin{array}{llll}-1.691484 & 0.068041 & -2.237687\end{array}$

$\begin{array}{llll}-0.934677 & 0.084685 & -1.459821\end{array}$

$\begin{array}{llll}-2.806527 & 0.873814 & -2.022177\end{array}$

$\begin{array}{llll}-2.816466 & 1.461231 & -1.109789\end{array}$

$\begin{array}{llll}-3.753043 & 1.359011 & -3.101383\end{array}$

$\begin{array}{llll}-4.645304 & 1.750022 & -2.607526\end{array}$

$\begin{array}{lll}-3.289400 & 2.204723 & -3.630712\end{array}$

$\begin{array}{llll}-4.170762 & 0.260151 & -4.102652\end{array}$

$\begin{array}{llll}-3.492662 & 0.252124 & -4.962429\end{array}$

$\begin{array}{llll}-5.160680 & 0.506301 & -4.498998\end{array}$

$-0.336638-3.784936-1.225009$

$\begin{array}{llll}-0.871132 & -5.040051 & -0.873106\end{array}$

$\begin{array}{llll}0.626986 & -3.732266 & -2.250527\end{array}$

$\begin{array}{llll}-0.457219 & -6.196811 & -1.530393\end{array}$

$\begin{array}{llll}-1.601565 & -5.116357 & -0.073981\end{array}$

$\begin{array}{llll}1.036563 & -4.889454 & -2.909803\end{array}$

$\begin{array}{llll}1.058555 & -2.774637 & -2.515905\end{array}$

$\begin{array}{llll}0.497633 & -6.127703 & -2.551755\end{array}$

$\begin{array}{llll}-0.877279 & -7.156808 & -1.243993\end{array}$

$\begin{array}{llll}1.782690 & -4.824790 & -3.696903\end{array}$

$\begin{array}{llll}0.817496 & -7.032055 & -3.061190\end{array}$

$\begin{array}{llll}2.527250 & -3.998319 & 0.475558\end{array}$

$\begin{array}{llll}3.530226 & -4.417743 & -0.497150\end{array}$

$\begin{array}{llll}3.343345 & -5.458887 & -0.776438\end{array}$

$\begin{array}{llll}3.460319 & -3.806102 & -1.391140\end{array}$

$\begin{array}{llll}4.542567 & -4.343807 & -0.084042\end{array}$

$\begin{array}{llll}2.172597 & -5.004890 & 1.472859\end{array}$

$\begin{array}{lll}1.857049 & -4.544772 & 2.407891\end{array}$

$\begin{array}{llll}1.379387 & -5.669485 & 1.108226\end{array}$

$\begin{array}{lll}3.059464 & -5.605093 & 1.688510\end{array}$ 


$\begin{array}{cccc}8 & 2.451414 & -1.919418 & -0.431435 \\ 6 & -0.453600 & -1.173799 & 2.861566 \\ 6 & -0.885435 & 0.012489 & 3.418674 \\ 1 & -0.794837 & -2.114148 & 3.277122 \\ 6 & -0.417791 & 1.201636 & 2.853815 \\ 6 & 0.455940 & 1.160479 & 1.781896 \\ 1 & 0.835541 & 2.067976 & 1.329456 \\ 7 & 0.890364 & 0.000983 & 1.248355 \\ 1 & -1.574738 & 0.013408 & 4.253923 \\ 35 & -0.962390 & 2.928201 & 3.596146\end{array}$

\section{Conformer_10}

Number of imaginary frequencies : 1

The smallest frequency is : $-247.7141 \mathrm{~cm}(-1)$

Electronic energy : $\quad \mathrm{HF}=-7222.6348463$

Zero-point correction

1.755701

(Hartree/Particle)

Thermal correction to Energy $=\quad 1.874115$

Thermal correction to Enthalpy= $\quad 1.875059$

Thermal correction to Gibbs Free Energy $=\quad 1.593081$

Sum of electronic and zero-point Energies $=\quad-7220.879146$

Sum of electronic and thermal Energies=

Sum of electronic and thermal Enthalpies=

$-7220.760731$

Cartesian Coordinates

$\begin{array}{rrr}4.295523 & 4.787283 & 1.873415 \\ 2.562859 & 1.624389 & -1.886344 \\ 4.540779 & 0.128116 & 0.933480 \\ 3.967467 & 3.424790 & 0.318999 \\ 4.154487 & 2.746003 & 1.580354 \\ 3.132366 & 3.194366 & 2.473206 \\ 3.009873 & 2.884484 & 3.501862 \\ 2.335068 & 4.162790 & 1.791831 \\ 1.509031 & 4.716054 & 2.216616 \\ 2.848935 & 4.307338 & 0.470140 \\ 2.478968 & 4.983332 & -0.285704 \\ 6.237859 & 5.325784 & 2.376050 \\ 7.098917 & 4.676959 & 2.283529 \\ 5.382612 & 5.426193 & 3.516086 \\ 5.479316 & 4.864752 & 4.435795 \\ 4.350528 & 6.365674 & 3.212972 \\ 3.525355 & 6.633254 & 3.859719 \\ 4.567624 & 6.847126 & 1.885897 \\ 3.938804 & 7.546994 & 1.351507 \\ 5.733484 & 6.207085 & 1.369894 \\ 6.141595 & 6.344828 & 0.377965 \\ 4.764335 & 3.153514 & -0.912827 \\ 6.097389 & 3.584304 & -0.982483 \\ 6.500846 & 4.139335 & -0.145127 \\ 6.906147 & 3.300340 & -2.082807 \\ 7.941671 & 3.625079 & -2.092641 \\ 6.383646 & 2.576849 & -3.152280 \\ 7.004169 & 2.337072 & -4.009743 \\ 5.061597 & 2.133532 & -3.105672 \\ 4.672367 & 1.556740 & -3.937690 \\ 4.241875 & 2.397531 & -1.997292 \\ 5.179790 & 1.672394 & 1.830849 \\ 6.093188 & 1.961706 & 1.308207 \\ 5.519512 & 1.464574 & 3.309343 \\ 6.298249 & 0.706809 & 3.430047 \\ 4.650655 & 1.149083 & 3.893147 \\ 5.881852 & 2.407437 & 3.730364 \\ 2.354520 & 0.721816 & -3.482025 \\ 1.908698 & 1.338433 & -4.662281 \\ 1.692262 & 2.400931 & -4.679642 \\ 1.719216 & 0.586674 & -5.822685 \\ 1.363644 & 1.074462 & -6.725831 \\ 1.979265 & -0.786343 & -5.822507 \\ 2.616331 & -0.657914 & -3.490569\end{array}$

$2.616331-0.657914-3.490569$

$2.930153-1.152654-2.578983$

$\begin{array}{llll}1.403740 & 3.038250 & -2.070541\end{array}$

$1.740649 \quad 4.197698-2.788216$

$2.733367 \quad 4.302644 \quad-3.215793$

$\begin{array}{lll}0.806529 & 5.222792 & -2.947806\end{array}$

$\begin{array}{lll}1.077566 & 6.116649 & -3.502535\end{array}$

$\begin{array}{llll}-0.475144 & 5.095225 & -2.402520\end{array}$

$-1.207990 \quad 5.886432-2.532974$

$\begin{array}{llll}-0.814239 & 3.945142 & -1.686995\end{array}$

$\begin{array}{lll}-1.807439 & 3.839196 & -1.267670\end{array}$

$\begin{array}{llll}0.125025 & 2.927440 & -1.509470\end{array}$

$\begin{array}{llll}-0.137089 & 2.044756 & -0.936905\end{array}$

$\begin{array}{llll}5.833953 & -0.351407 & -0.288025\end{array}$

$\begin{array}{llll}5.486635 & -1.402359 & -1.149307\end{array}$

$\begin{array}{llll}4.518845 & -1.879143 & -1.036618\end{array}$

$\begin{array}{llll}6.371590 & -1.830042 & -2.138174\end{array}$

$\begin{array}{llll}7.598198 & -1.194303 & -2.319180\end{array}$

$\begin{array}{llll}8.275821 & -1.511066 & -3.103888\end{array}$

$\begin{array}{llll}7.930614 & -0.131943 & -1.478071\end{array}$

$\begin{array}{llll}7.072210 & 0.277377 & -0.457157\end{array}$

$\begin{array}{llll}7.378641 & 1.096600 & 0.178516\end{array}$

$\begin{array}{llll}4.568549 & -1.271515 & 2.131522\end{array}$

$\begin{array}{llll}3.559458 & -1.295186 & 3.107682\end{array}$

$\begin{array}{llll}2.867626 & -0.465495 & 3.195841\end{array}$

$\begin{array}{lll}3.410391 & -2.401874 & 3.940645\end{array}$

$\begin{array}{llll}4.233594 & -3.519415 & 3.798083\end{array}$

$\begin{array}{llll}4.087636 & -4.396446 & 4.418709\end{array}$

$\begin{array}{llll}5.223087 & -3.501216 & 2.818504\end{array}$

$\begin{array}{lll}5.409531 & -2.382738 & 2.005126\end{array}$

$\begin{array}{lll}6.188483 & -2.406598 & 1.253550\end{array}$

$\begin{array}{llll}2.440300 & 0.234739 & -0.102121\end{array}$

$\begin{array}{llll}6.091867 & -4.714978 & 2.637393\end{array}$

$\begin{array}{llll}2.273022 & -2.466957 & 4.921009\end{array}$

$\begin{array}{llll}5.997038 & -3.025770 & -2.969008\end{array}$

$\begin{array}{llll}9.225167 & 0.592959 & -1.725555\end{array}$

$\begin{array}{llll}9.228067 & 1.197427 & -2.940480\end{array}$

$\begin{array}{llll}9.467118 & 1.552993 & -0.806194\end{array}$

$10.282632-0.251309-1.717111$

$\begin{array}{llll}6.178623 & -4.182032 & -2.282621\end{array}$

$\begin{array}{llll}6.720629 & -3.118040 & -4.102345\end{array}$

$\begin{array}{llll}4.686707 & -3.002276 & -3.326751\end{array}$

$\begin{array}{llll}7.068584 & -4.789707 & 3.569735\end{array}$

$\begin{array}{llll}6.695530 & -4.732018 & 1.427274\end{array}$

$\begin{array}{llll}5.369438 & -5.858205 & 2.739898\end{array}$

$\begin{array}{llll}2.644757 & -3.031557 & 6.090536\end{array}$

$\begin{array}{llll}1.757434 & -1.251424 & 5.200219\end{array}$

$\begin{array}{llll}1.250659 & -3.226996 & 4.437745\end{array}$

$\begin{array}{llll}1.975859 & -2.751371 & 0.404067\end{array}$

$\begin{array}{llll}0.812338 & -2.465278 & 1.213737\end{array}$

$\begin{array}{llll}0.453834 & -1.190330 & 1.765518\end{array}$

$\begin{array}{lll}0.409561 & -3.292558 & 1.779802\end{array}$

$\begin{array}{lll}-3.400038 & -1.296205 & -1.447131\end{array}$

$\begin{array}{llll}-5.267035 & -0.538264 & -0.378057\end{array}$

$\begin{array}{lll}-5.260548 & 0.604631 & 0.820310\end{array}$

$\begin{array}{llll}-6.330084 & 0.174120 & -1.442259\end{array}$

$\begin{array}{lll}-5.966543 & -1.799009 & 0.479241\end{array}$

$\begin{array}{lll}-4.982113 & 1.930144 & 0.525179\end{array}$

$\begin{array}{lll}-3.757942 & 2.439104 & 1.010653\end{array}$

$\begin{array}{lll}-3.119114 & 1.781985 & 1.589774\end{array}$

$\begin{array}{llll}-3.416010 & 3.740377 & 0.740294\end{array}$

$\begin{array}{lll}-2.486862 & 4.152919 & 1.120347\end{array}$

$\begin{array}{llll}-4.248674 & 4.546654 & -0.083704\end{array}$

$\begin{array}{llll}-3.843337 & 5.851211 & -0.476862\end{array}$

$\begin{array}{llll}-2.895170 & 6.232405 & -0.106643\end{array}$

$\begin{array}{llll}-4.622754 & 6.608480 & -1.322457\end{array}$

$-4.300115 \quad 7.600826-1.623645$

$\begin{array}{llll}-5.844812 & 6.086314 & -1.809892\end{array}$

$\begin{array}{llll}-6.447789 & 6.678257 & -2.492339\end{array}$

$\begin{array}{llll}-6.275784 & 4.832852 & -1.431712\end{array}$

$\begin{array}{llll}-7.211172 & 4.445816 & -1.819115\end{array}$

$\begin{array}{llll}-5.501738 & 4.025509 & -0.552697\end{array}$

$\begin{array}{llll}-5.909915 & 2.701983 & -0.156008\end{array}$

$\begin{array}{llll}-7.269150 & 2.156934 & -0.436413\end{array}$

$\begin{array}{lll}-8.460127 & 2.845576 & -0.002156\end{array}$

$\begin{array}{lll}-8.420942 & 4.023159 & 0.796804\end{array}$ 
$\begin{array}{llll}1.036639 & -4.889373 & -2.909968\end{array}$

$\begin{array}{llll}1.058567 & -2.774563 & -2.516023\end{array}$

$\begin{array}{llll}0.497750 & -6.127646 & -2.551945\end{array}$

$\begin{array}{llll}-0.877121 & -7.156824 & -1.244196\end{array}$

$\begin{array}{llll}1.782761 & -4.824668 & -3.697070\end{array}$

$\begin{array}{llll}0.817639 & -7.031978 & -3.061400\end{array}$

$\begin{array}{llll}2.527278 & -3.998329 & 0.475452\end{array}$

$\begin{array}{llll}3.530260 & -4.417727 & -0.497261\end{array}$

$\begin{array}{llll}3.343383 & -5.458864 & -0.776575\end{array}$

$\begin{array}{llll}3.460356 & -3.806064 & -1.391236\end{array}$

$\begin{array}{llll}4.542598 & -4.343798 & -0.084145\end{array}$

$\begin{array}{llll}2.172633 & -5.004921 & 1.472735\end{array}$

$\begin{array}{llll}1.857080 & -4.544823 & 2.407776\end{array}$

$\begin{array}{llll}1.379429 & -5.669517 & 1.108090\end{array}$

$\begin{array}{llll}3.059505 & -5.605121 & 1.688376\end{array}$

$2.451415 \quad-1.919413-0.431506$

$\begin{array}{lll}-0.453604 & -1.173896 & 2.861516\end{array}$

$\begin{array}{lll}-0.885440 & 0.012374 & 3.418660\end{array}$

$\begin{array}{lll}-0.794838 & -2.114258 & 3.277044\end{array}$

$\begin{array}{lll}-0.417800 & 1.201539 & 2.853837\end{array}$

$\begin{array}{lll}0.455926 & 1.160417 & 1.781912\end{array}$

$\begin{array}{lll}0.835523 & 2.067929 & 1.329498\end{array}$

$\begin{array}{lll}0.890350 & 0.000937 & 1.248334\end{array}$

$\begin{array}{lll}-1.574741 & 0.013267 & 4.253910\end{array}$

$\begin{array}{llll}35 & -0.962394 & 2.928080 & 3.596228\end{array}$

\section{Conformer_11}

Number of imaginary frequencies : 1

The smallest frequency is : $-247.7126 \mathrm{~cm}(-1)$

Eectronic energy : HF=-7222.6348461

Zero-point correction $=\quad 1.755699$

(Hartree/Particle)

Thermal correction to Energy= $\quad 1.874114$

Thermal correction to Enthalpy= $\quad 1.875059$

Thermal correction to Gibbs Free Energy $=\quad 1.593073$

Sum of electronic and zero-point Energies $=\quad-7220.879147$

Sum of electronic and thermal Energies $=\quad-7220.760732$

Sum of electronic and thermal Enthalpies $=\quad-7220.759788$

Sum of electronic and thermal Free Energies $=\quad-7221.041773$

\begin{tabular}{|c|c|c|c|}
\hline \multicolumn{4}{|c|}{ Cartesian Coordinates } \\
\hline 26 & 4.295529 & 4.787371 & 1.873113 \\
\hline 15 & 2.562813 & 1.624303 & -1.886472 \\
\hline 15 & 4.540798 & 0.128166 & 0.933365 \\
\hline 6 & 3.967455 & 3.424815 & 0.318756 \\
\hline 6 & 4.154497 & 2.746078 & 1.580136 \\
\hline 6 & 3.132386 & 3.194474 & 2.472985 \\
\hline 1 & 3.009909 & 2.884634 & 3.501656 \\
\hline 6 & 2.335074 & 4.162868 & 1.791584 \\
\hline 1 & 1.509043 & 4.716147 & 2.216359 \\
\hline 6 & 2.848922 & 4.307364 & 0.469879 \\
\hline 1 & 2.478941 & 4.983328 & -0.285986 \\
\hline 6 & 6.237873 & 5.325909 & 2.375676 \\
\hline 1 & 7.098935 & 4.677091 & 2.283153 \\
\hline 6 & 5.382657 & 5.426347 & 3.515732 \\
\hline 1 & 5.479393 & 4.864939 & 4.435457 \\
\hline 6 & 4.350555 & 6.365808 & 3.212617 \\
\hline 1 & 3.525397 & 6.633400 & 3.859377 \\
\hline 6 & 4.567609 & 6.847218 & 1.885520 \\
\hline 1 & 3.938767 & 7.547062 & 1.351124 \\
\hline 6 & 5.733461 & 6.207172 & 1.369505 \\
\hline 1 & 6.141543 & 6.344887 & 0.377561 \\
\hline 6 & 4.764298 & 3.153492 & -0.913075 \\
\hline 6 & 6.097343 & 3.584301 & -0.982785 \\
\hline 1 & 6.500810 & 4.139382 & -0.145466 \\
\hline 6 & 6.906078 & 3.300295 & -2.083116 \\
\hline 1 & 7.941597 & 3.625053 & -2.092993 \\
\hline 6 & 6.383563 & 2.576739 & -3.152538 \\
\hline 1 & 7.004070 & 2.336927 & -4.010002 \\
\hline 6 & 5.061522 & 2.133405 & -3.105876 \\
\hline
\end{tabular}




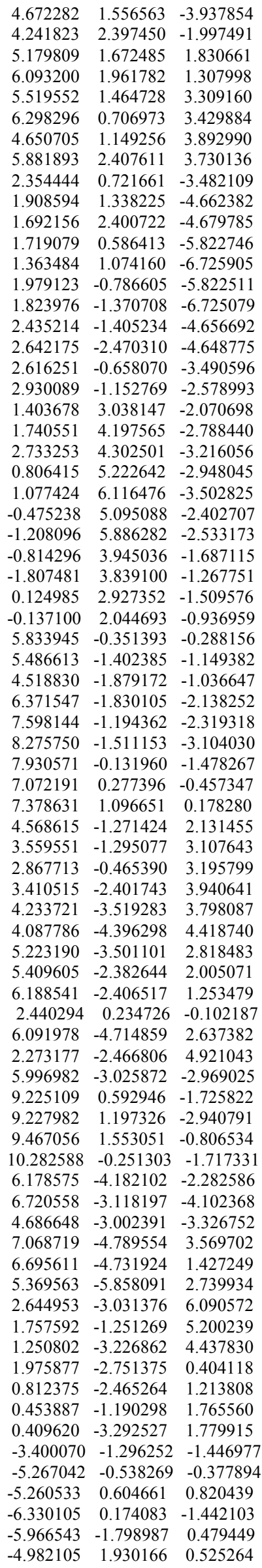

$\begin{array}{lll}-3.757929 & 2.439144 & 1.010706\end{array}$

$\begin{array}{lll}-3.119096 & 1.782045 & 1.589845\end{array}$

$\begin{array}{lll}-3.415995 & 3.740405 & 0.740292\end{array}$

$\begin{array}{lll}-2.486840 & 4.152958 & 1.120316\end{array}$

$\begin{array}{lll}-4.248668 & 4.546653 & -0.083725\end{array}$

$\begin{array}{llll}-3.843333 & 5.851193 & -0.476939\end{array}$

$\begin{array}{llll}-2.895158 & 6.232398 & -0.106751\end{array}$

$\begin{array}{lll}-4.622760 & 6.608433 & -1.322551\end{array}$

$\begin{array}{llll}-4.300122 & 7.600766 & -1.623782\end{array}$

$\begin{array}{lll}-5.844828 & 6.086254 & -1.809946\end{array}$

$\begin{array}{lll}-6.447814 & 6.678172 & -2.492406\end{array}$

$\begin{array}{llll}-6.275799 & 4.832807 & -1.431713\end{array}$

$\begin{array}{lll}-7.211194 & 4.445759 & -1.819086\end{array}$

$\begin{array}{llll}-5.501741 & 4.025494 & -0.552679\end{array}$

$\begin{array}{llll}-5.909916 & 2.701984 & -0.155936\end{array}$

$\begin{array}{llll}-7.269155 & 2.156926 & -0.436303\end{array}$

$\begin{array}{lll}-8.460126 & 2.845583 & -0.002050\end{array}$

$\begin{array}{lll}-8.420928 & 4.023194 & 0.796868\end{array}$

$\begin{array}{lll}-7.464271 & 4.422331 & 1.110756\end{array}$

$\begin{array}{lll}-9.579478 & 4.655982 & 1.192802\end{array}$

$\begin{array}{lll}-9.518961 & 5.549368 & 1.807776\end{array}$

$\begin{array}{llll}-10.845807 & 4.150873 & 0.814385\end{array}$

$\begin{array}{lll}-11.749917 & 4.664999 & 1.127052\end{array}$

$\begin{array}{lll}-10.923672 & 2.997952 & 0.066761\end{array}$

$\begin{array}{lll}-11.888660 & 2.583735 & -0.213442\end{array}$

$\begin{array}{lll}-9.748679 & 2.311607 & -0.344262\end{array}$

$\begin{array}{llll}-9.832615 & 1.090659 & -1.066328\end{array}$

$\begin{array}{lll}-10.811117 & 0.699032 & -1.329460\end{array}$

$\begin{array}{lll}-8.695442 & 0.398823 & -1.398599\end{array}$

$\begin{array}{llll}-8.734301 & -0.551536 & -1.920430\end{array}$

$\begin{array}{llll}-7.430853 & 0.928221 & -1.060056\end{array}$

$\begin{array}{llll}-5.328337 & -3.110851 & 0.181092\end{array}$

$\begin{array}{lll}-4.591409 & -3.316256 & 0.967144\end{array}$

$\begin{array}{lll}-6.342523 & -4.240815 & 0.175262\end{array}$

$\begin{array}{lll}-6.026821 & -5.466197 & 0.773609\end{array}$

$\begin{array}{lll}-5.072368 & -5.581248 & 1.282566\end{array}$

$\begin{array}{lll}-6.925400 & -6.535530 & 0.723271\end{array}$

$\begin{array}{lll}-6.667757 & -7.480447 & 1.193840\end{array}$

$\begin{array}{llll}-8.155371 & -6.385414 & 0.079382\end{array}$

$\begin{array}{lll}-8.858559 & -7.212779 & 0.045292\end{array}$

$-8.480061 \quad-5.162156-0.516890$

$-9.437480-5.037624-1.015620$

$\begin{array}{lll}-7.578929 & -4.098594 & -0.471288\end{array}$

$\begin{array}{llll}-7.831489 & -3.144357 & -0.925491\end{array}$

$\begin{array}{lll}-4.594850 & -3.051773 & -1.163446\end{array}$

$\begin{array}{llll}-5.333907 & -3.113096 & -1.971252\end{array}$

$\begin{array}{llll}-3.953558 & -3.933862 & -1.249368\end{array}$

$\begin{array}{llll}-6.667735 & -1.732586 & 1.808062\end{array}$

$\begin{array}{lll}-7.141384 & -2.714858 & 1.886814\end{array}$

$\begin{array}{lll}-5.678314 & -1.640505 & 2.970973\end{array}$

$\begin{array}{lll}-5.126434 & -2.826093 & 3.482788\end{array}$

$\begin{array}{lll}-5.453003 & -3.781223 & 3.080119\end{array}$

$\begin{array}{lll}-4.182176 & -2.800135 & 4.512546\end{array}$

$\begin{array}{lll}-3.769138 & -3.731544 & 4.890317\end{array}$

$\begin{array}{llll}-3.790265 & -1.580745 & 5.068537\end{array}$

$\begin{array}{lll}-3.064002 & -1.554691 & 5.876363\end{array}$

$\begin{array}{lll}-4.357249 & -0.394830 & 4.591595\end{array}$

$\begin{array}{llll}-4.076281 & 0.558230 & 5.032329\end{array}$

$\begin{array}{lll}-5.290662 & -0.424519 & 3.553717\end{array}$

$\begin{array}{lll}-5.716330 & 0.505803 & 3.199525\end{array}$

$\begin{array}{lll}-7.806836 & -0.715789 & 1.852865\end{array}$

$\begin{array}{lll}-8.514432 & -0.911699 & 1.044086\end{array}$

$\begin{array}{lll}-7.468562 & 0.315894 & 1.765832\end{array}$

$\begin{array}{lll}-8.334985 & -0.822727 & 2.805340\end{array}$

$\begin{array}{llll}-2.525412 & -1.128298 & 0.544905\end{array}$

$\begin{array}{lll}-1.882660 & -0.253545 & 0.590721\end{array}$

$\begin{array}{llll}-3.221273 & -1.183491 & 1.377392\end{array}$

$\begin{array}{lll}-1.992565 & -2.367380 & 0.062683\end{array}$

$\begin{array}{lll}-2.496881 & -3.272122 & 0.385555\end{array}$

$\begin{array}{llll}-0.734642 & -2.534735 & -0.568595\end{array}$

$-0.209035-1.652834-0.907161$

$-4.223068-1.144028-3.492971$

$-5.215223-1.589716-3.481808$

$\begin{array}{llll}-3.126499 & -2.048789 & -3.521677\end{array}$

$\begin{array}{llll}-3.365240 & -3.111953 & -3.550709\end{array}$ 
$\begin{array}{lll}-1.761962 & -1.710629 & -4.097490\end{array}$

$\begin{array}{llll}-1.805713 & -1.729018 & -5.197627\end{array}$

$\begin{array}{lll}-1.073412 & -2.507966 & -3.807385\end{array}$

$\begin{array}{lll}-1.196658 & -0.358840 & -3.615738\end{array}$

$\begin{array}{llll}-1.429496 & 0.434423 & -4.332733\end{array}$

$\begin{array}{lll}-0.107354 & -0.422443 & -3.582525\end{array}$

$\begin{array}{llll}-1.691518 & 0.067991 & -2.237567\end{array}$

$\begin{array}{lll}-0.934706 & 0.084614 & -1.459705\end{array}$

$\begin{array}{llll}-2.806541 & 0.873785 & -2.022040\end{array}$

$\begin{array}{llll}-2.816461 & 1.461193 & -1.109647\end{array}$

$\begin{array}{llll}-3.753056 & 1.359017 & -3.101231\end{array}$

$\begin{array}{llll}-4.645309 & 1.750032 & -2.607362\end{array}$

$\begin{array}{lll}-3.289402 & 2.204731 & -3.630547\end{array}$

$\begin{array}{llll}-4.170794 & 0.260183 & -4.102519\end{array}$

$\begin{array}{llll}-3.492687 & 0.252152 & -4.962291\end{array}$

$\begin{array}{llll}-5.160703 & 0.506361 & -4.498869\end{array}$

$-0.336613-3.785004-1.224996$

$\begin{array}{lll}-0.871041 & -5.040139 & -0.873062\end{array}$

$\begin{array}{llll}0.626972 & -3.732303 & -2.250548\end{array}$

$\begin{array}{llll}-0.457102 & -6.196888 & -1.530352\end{array}$

$\begin{array}{llll}-1.601439 & -5.116469 & -0.073907\end{array}$

$\begin{array}{llll}1.036576 & -4.889480 & -2.909827\end{array}$

$\begin{array}{llll}1.058493 & -2.774658 & -2.515949\end{array}$

$\begin{array}{llll}0.497712 & -6.127748 & -2.551748\end{array}$

$\begin{array}{llll}-0.877109 & -7.156900 & -1.243928\end{array}$

$\begin{array}{llll}1.782673 & -4.824791 & -3.696954\end{array}$

$\begin{array}{llll}0.817596 & -7.032092 & -3.061185\end{array}$

$\begin{array}{llll}2.527307 & -3.998327 & 0.475526\end{array}$

$\begin{array}{llll}3.530271 & -4.417740 & -0.497199\end{array}$

$\begin{array}{llll}3.343410 & -5.458892 & -0.776467\end{array}$

$\begin{array}{llll}3.460327 & -3.806113 & -1.391196\end{array}$

$\begin{array}{llll}4.542620 & -4.343770 & -0.084117\end{array}$

$\begin{array}{llll}2.172707 & -5.004889 & 1.472854\end{array}$

$\begin{array}{llll}1.857166 & -4.544763 & 2.407885\end{array}$

$\begin{array}{llll}1.379507 & -5.669514 & 1.108252\end{array}$

$\begin{array}{lll}3.059596 & -5.605062 & 1.688497\end{array}$

$\begin{array}{llll}2.451405 & -1.919439 & -0.431494\end{array}$

$\begin{array}{lll}-0.453510 & -1.173827 & 2.861591\end{array}$

$\begin{array}{lll}-0.885329 & 0.012463 & 3.418710\end{array}$

$\begin{array}{lll}-0.794723 & -2.114175 & 3.277168\end{array}$

$\begin{array}{lll}-0.417717 & 1.201608 & 2.853821\end{array}$

$\begin{array}{lll}0.455968 & 1.160449 & 1.781864\end{array}$

$\begin{array}{lll}0.835540 & 2.067945 & 1.329399\end{array}$

$\begin{array}{lll}0.890380 & 0.000952 & 1.248315\end{array}$

$\begin{array}{lll}-1.574596 & 0.013384 & 4.253988\end{array}$

$\begin{array}{lll}-0.962283 & 2.928177 & 3.596162\end{array}$

Conformer_12

Number of imaginary frequencies : 1

The smallest frequency is : $-247.7381 \mathrm{~cm}(-1)$

Electronic energy :

$\mathrm{HF}=-7222.6348463$

Zero-point correction=

1.755705

(Hartree/Particle)

Thermal correction to Energy=

1.874118

Thermal correction to Enthalpy=

1.875062

Thermal correction to Gibbs Free Energy $=\quad 1.593102$

Sum of electronic and zero-point Energies=

Sum of electronic and thermal Energies=

$-7220.879141$

Sum of electronic and thermal Enthalpies=

$-7220.760728$

Sum of electronic and thermal Free Energies=

20.759784

Cartesian Coordinates

$$
\begin{array}{ccc}
4.295840 & 4.787191 & 1.873517 \\
2.562936 & 1.624433 & -1.886248 \\
4.540873 & 0.128032 & 0.933513 \\
3.967682 & 3.424716 & 0.319109 \\
4.154744 & 2.745915 & 1.580451 \\
3.132675 & 3.194295 & 2.473355 \\
3.010226 & 2.884407 & 3.502015 \\
2.335368 & 4.162744 & 1.792024 \\
1.509366 & 4.716025 & 2.216851 \\
2.849175 & 4.307286 & 0.470308 \\
2.479190 & 4.983298 & -0.285512
\end{array}
$$

$6.238211 \quad 5.325654 \quad 2.376061$

$\begin{array}{llll}7.099252 & 4.676813 & 2.283505\end{array}$

$5.383019 \quad 5.426088 \quad 3.516135$

$\begin{array}{llll}5.479755 & 4.864652 & 4.435844\end{array}$

$\begin{array}{lll}4.350940 & 6.365588 & 3.213063\end{array}$

$\begin{array}{lll}3.525802 & 6.633189 & 3.859846\end{array}$

$\begin{array}{llll}4.567985 & 6.847028 & 1.885975\end{array}$

$3.939154 \quad 7.546905 \quad 1.351610$

$\begin{array}{lll}5.733808 & 6.206960 & 1.369923\end{array}$

$\begin{array}{lll}6.141875 & 6.344685 & 0.377973\end{array}$

$\begin{array}{llll}4.764499 & 3.153440 & -0.912750\end{array}$

$\begin{array}{llll}6.097570 & 3.584175 & -0.982432\end{array}$

$\begin{array}{llll}6.501077 & 4.139163 & -0.145071\end{array}$

$\begin{array}{llll}6.906284 & 3.300213 & -2.082789\end{array}$

$\begin{array}{llll}7.941822 & 3.624910 & -2.092643\end{array}$

$\begin{array}{lll}6.383722 & 2.576779 & -3.152270\end{array}$

$\begin{array}{llll}7.004209 & 2.337003 & -4.009759\end{array}$

$\begin{array}{llll}5.061656 & 2.133515 & -3.105636\end{array}$

$\begin{array}{llll}4.672377 & 1.556767 & -3.937662\end{array}$

$\begin{array}{llll}4.241978 & 2.397512 & -1.997223\end{array}$

$\begin{array}{lll}5.180012 & 1.672259 & 1.830880\end{array}$

$\begin{array}{llll}6.093398 & 1.961534 & 1.308197\end{array}$

$\begin{array}{lll}5.519798 & 1.464396 & 3.309353\end{array}$

$\begin{array}{lll}6.298500 & 0.706588 & 3.430005\end{array}$

$\begin{array}{lll}4.650952 & 1.148942 & 3.893195\end{array}$

$\begin{array}{lll}5.882209 & 2.407233 & 3.730373\end{array}$

$\begin{array}{llll}2.354531 & 0.721870 & -3.481925\end{array}$

$\begin{array}{llll}2.616239 & -0.657880 & -3.490466\end{array}$

$\begin{array}{llll}2.930017 & -1.152644 & -2.578878\end{array}$

$\begin{array}{llll}2.435195 & -1.404974 & -4.656607\end{array}$

$\begin{array}{llll}2.642079 & -2.470065 & -4.648734\end{array}$

$\begin{array}{llll}1.979202 & -0.786254 & -5.822416\end{array}$

$\begin{array}{llll}1.824055 & -1.370301 & -6.725020\end{array}$

$\begin{array}{llll}1.719257 & 0.586783 & -5.822595\end{array}$

$\begin{array}{llll}1.363740 & 1.074600 & -6.725747\end{array}$

$\begin{array}{llll}1.908775 & 1.338524 & -4.662186\end{array}$

$\begin{array}{llll}1.692424 & 2.401039 & -4.679548\end{array}$

$1.403865 \quad 3.038338-2.070432$

$\begin{array}{llll}1.740834 & 4.197802 & -2.788054\end{array}$

$2.733569 \quad 4.302734 \quad-3.215592$

$\begin{array}{llll}0.806747 & 5.222927 & -2.947647\end{array}$

$\begin{array}{llll}1.077831 & 6.116794 & -3.502336\end{array}$

$\begin{array}{llll}-0.474950 & 5.095375 & -2.402416\end{array}$

$\begin{array}{llll}-1.207771 & 5.886606 & -2.532875\end{array}$

$\begin{array}{llll}-0.814105 & 3.945277 & -1.686945\end{array}$

$\begin{array}{llll}-1.807326 & 3.839346 & -1.267665\end{array}$

$\begin{array}{lll}0.125125 & 2.927543 & -1.509415\end{array}$

$\begin{array}{llll}-0.137039 & 2.044845 & -0.936894\end{array}$

$\begin{array}{llll}5.833981 & -0.351575 & -0.288029\end{array}$

$\begin{array}{llll}5.486580 & -1.402521 & -1.149284\end{array}$

$\begin{array}{llll}4.518775 & -1.879263 & -1.036548\end{array}$

$\begin{array}{llll}6.371466 & -1.830242 & -2.138196\end{array}$

$\begin{array}{llll}7.598084 & -1.194543 & -2.319277\end{array}$

$\begin{array}{llll}8.275652 & -1.511333 & -3.104022\end{array}$

$\begin{array}{llll}7.930583 & -0.132188 & -1.478195\end{array}$

$\begin{array}{llll}7.072250 & 0.277167 & -0.457235\end{array}$

$\begin{array}{llll}7.378742 & 1.096389 & 0.178411\end{array}$

$\begin{array}{llll}4.568555 & -1.271600 & 2.131554\end{array}$

$\begin{array}{llll}3.559461 & -1.295196 & 3.107713\end{array}$

$\begin{array}{lll}2.867686 & -0.465457 & 3.195864\end{array}$

$\begin{array}{llll}3.410313 & -2.401870 & 3.940679\end{array}$

$\begin{array}{lll}4.233439 & -3.519469 & 3.798124\end{array}$

$\begin{array}{llll}4.087419 & -4.396487 & 4.418753\end{array}$

$\begin{array}{llll}5.222937 & -3.501342 & 2.818548\end{array}$

$\begin{array}{llll}5.409461 & -2.382882 & 2.005164\end{array}$

$\begin{array}{lll}6.188410 & -2.406798 & 1.253587\end{array}$

$\begin{array}{llll}2.440369 & 0.234799 & -0.102018\end{array}$

$\begin{array}{lll}6.091632 & -4.715167 & 2.637445\end{array}$

$\begin{array}{llll}2.272933 & -2.466866 & 4.921037\end{array}$

$\begin{array}{llll}5.996820 & -3.025958 & -2.969004\end{array}$

$\begin{array}{llll}9.225145 & 0.592671 & -1.725759\end{array}$

$\begin{array}{llll}9.228005 & 1.197109 & -2.940699\end{array}$

$\begin{array}{llll}9.467166 & 1.552721 & -0.806433\end{array}$

$10.282586-0.251626-1.717343$

$\begin{array}{llll}6.178356 & -4.182222 & -2.282607\end{array}$ 
$\begin{array}{llll}6.720371 & -3.118287 & -4.102362\end{array}$

$\begin{array}{llll}4.686480 & -3.002390 & -3.326708\end{array}$

$\begin{array}{lll}7.068336 & -4.789964 & 3.569796\end{array}$

$\begin{array}{llll}6.695303 & -4.732252 & 1.427331\end{array}$

$\begin{array}{llll}5.369120 & -5.858342 & 2.739946\end{array}$

$\begin{array}{llll}2.644623 & -3.031480 & 6.090572\end{array}$

$\begin{array}{llll}1.757429 & -1.251294 & 5.200231\end{array}$

$\begin{array}{lll}1.250519 & -3.226836 & 4.437775\end{array}$

$\begin{array}{llll}1.975746 & -2.751283 & 0.404165\end{array}$

$\begin{array}{llll}0.812237 & -2.465111 & 1.213824\end{array}$

$\begin{array}{llll}0.453788 & -1.190138 & 1.765586\end{array}$

$\begin{array}{llll}0.409406 & -3.292362 & 1.779894\end{array}$

$\begin{array}{llll}-3.400029 & -1.296117 & -1.447162\end{array}$

$\begin{array}{lll}-5.267098 & -0.538292 & -0.378116\end{array}$

$\begin{array}{lll}-5.260678 & 0.604628 & 0.820229\end{array}$

$\begin{array}{llll}-6.330194 & 0.174020 & -1.442320\end{array}$

$\begin{array}{lll}-5.966522 & -1.799062 & 0.479218\end{array}$

$\begin{array}{lll}-4.982207 & 1.930123 & 0.525061\end{array}$

$\begin{array}{lll}-3.758030 & 2.439071 & 1.010534\end{array}$

$\begin{array}{lll}-3.119220 & 1.781943 & 1.589666\end{array}$

$\begin{array}{lll}-3.416077 & 3.740332 & 0.740146\end{array}$

$\begin{array}{llll}-2.486929 & 4.152874 & 1.120199\end{array}$

$\begin{array}{llll}-4.248722 & 4.546600 & -0.083881\end{array}$

$\begin{array}{llll}-3.843367 & 5.851147 & -0.477054\end{array}$

$\begin{array}{llll}-2.895203 & 6.232340 & -0.106826\end{array}$

$\begin{array}{llll}-4.622760 & 6.608408 & -1.322677\end{array}$

$\begin{array}{llll}-4.300107 & 7.600746 & -1.623876\end{array}$

$\begin{array}{llll}-5.844814 & 6.086246 & -1.810126\end{array}$

$\begin{array}{llll}-6.447772 & 6.678181 & -2.492596\end{array}$

$\begin{array}{llll}-6.275806 & 4.832795 & -1.431931\end{array}$

$\begin{array}{llll}-7.211191 & 4.445760 & -1.819341\end{array}$

$\begin{array}{llll}-5.501785 & 4.025461 & -0.552884\end{array}$

$\begin{array}{lll}-5.909987 & 2.701952 & -0.156166\end{array}$

$\begin{array}{llll}-7.269225 & 2.156906 & -0.436563\end{array}$

$\begin{array}{lll}-8.460196 & 2.845585 & -0.002345\end{array}$

$\begin{array}{lll}-8.421000 & 4.023203 & 0.796565\end{array}$

$\begin{array}{lll}-7.464346 & 4.422327 & 1.110476\end{array}$

$\begin{array}{lll}-9.579549 & 4.656013 & 1.192465\end{array}$

$\begin{array}{lll}-9.519032 & 5.549403 & 1.807435\end{array}$

$\begin{array}{lll}-10.845878 & 4.150922 & 0.814022\end{array}$

$\begin{array}{llll}-11.749987 & 4.665067 & 1.126661\end{array}$

$\begin{array}{lll}-10.923742 & 2.997995 & 0.066407\end{array}$

$\begin{array}{llll}-11.888731 & 2.583791 & -0.213815\end{array}$

$\begin{array}{llll}-9.748751 & 2.311627 & -0.344580\end{array}$

$\begin{array}{llll}-9.832690 & 1.090670 & -1.066630\end{array}$

$\begin{array}{llll}-10.811192 & 0.699055 & -1.329779\end{array}$

$\begin{array}{llll}-8.695521 & 0.398811 & -1.398863\end{array}$

$\begin{array}{llll}-8.734383 & -0.551555 & -1.920679\end{array}$

$\begin{array}{llll}-7.430928 & 0.928191 & -1.060299\end{array}$

$\begin{array}{lll}-5.328157 & -3.110867 & 0.180947\end{array}$

$\begin{array}{lll}-4.591238 & -3.316151 & 0.967040\end{array}$

$\begin{array}{lll}-6.342203 & -4.240956 & 0.175121\end{array}$

$\begin{array}{lll}-6.026330 & -5.466304 & 0.773450\end{array}$

$\begin{array}{lll}-5.071863 & -5.581232 & 1.282406\end{array}$

$\begin{array}{lll}-6.924765 & -6.535758 & 0.723102\end{array}$

$\begin{array}{lll}-6.666992 & -7.480646 & 1.193657\end{array}$

$\begin{array}{llll}-8.154760 & -6.385800 & 0.079221\end{array}$

$\begin{array}{lll}-8.857836 & -7.213260 & 0.045124\end{array}$

$\begin{array}{llll}-8.479619 & -5.162578 & -0.517034\end{array}$

$\begin{array}{llll}-9.437057 & -5.038169 & -1.015757\end{array}$

$\begin{array}{llll}-7.578629 & -4.098896 & -0.471424\end{array}$

$\begin{array}{llll}-7.831318 & -3.144688 & -0.925616\end{array}$

$\begin{array}{llll}-4.594631 & -3.051768 & -1.163566\end{array}$

$\begin{array}{llll}-5.333659 & -3.113207 & -1.971389\end{array}$

$\begin{array}{lll}-3.953234 & -3.933784 & -1.249436\end{array}$

$\begin{array}{lll}-6.667775 & -1.732679 & 1.807799\end{array}$

$\begin{array}{lll}-7.141333 & -2.714994 & 1.886569\end{array}$

$\begin{array}{lll}-5.678417 & -1.640456 & 2.970754\end{array}$

$\begin{array}{lll}-5.126428 & -2.825972 & 3.482622\end{array}$

$\begin{array}{lll}-5.452867 & -3.781145 & 3.079951\end{array}$

$\begin{array}{lll}-4.182236 & -2.799890 & 4.512436\end{array}$

$\begin{array}{llll}-3.769111 & -3.731244 & 4.890246\end{array}$

$\begin{array}{lll}-3.790506 & -1.580446 & 5.068435\end{array}$

$\begin{array}{lll}-3.064298 & -1.554294 & 5.876309\end{array}$

$\begin{array}{lll}-4.357598 & -0.394603 & 4.591440\end{array}$ $\begin{array}{lll}-4.076772 & 0.558495 & 5.032182\end{array}$

$\begin{array}{lll}-5.290943 & -0.424418 & 3.553505\end{array}$

$\begin{array}{lll}-5.716700 & 0.505850 & 3.199277\end{array}$

$\begin{array}{lll}-7.806973 & -0.715987 & 1.852506\end{array}$

$\begin{array}{lll}-8.514510 & -0.911996 & 1.043700\end{array}$

$\begin{array}{lll}-7.468790 & 0.315725 & 1.765449\end{array}$

$\begin{array}{lll}-8.335161 & -0.822935 & 2.804958\end{array}$

$\begin{array}{lll}-2.525386 & -1.127790 & 0.544722\end{array}$

$\begin{array}{lll}-1.882619 & -0.253039 & 0.590377\end{array}$

$\begin{array}{lll}-3.221241 & -1.182825 & 1.377221\end{array}$

$\begin{array}{lll}-1.992613 & -2.366952 & 0.062665\end{array}$

$\begin{array}{lll}-2.496962 & -3.271616 & 0.385698\end{array}$

$\begin{array}{llll}-0.734709 & -2.534447 & -0.568618\end{array}$

$\begin{array}{llll}-0.209033 & -1.652600 & -0.907221\end{array}$

$-4.223013-1.144094-3.493141$

$\begin{array}{llll}-5.215129 & -1.589869 & -3.481965\end{array}$

$\begin{array}{llll}-3.126364 & -2.048769 & -3.521799\end{array}$

$\begin{array}{lll}-3.365020 & -3.111954 & -3.550775\end{array}$

$\begin{array}{llll}-1.761865 & -1.710544 & -4.097661\end{array}$

$\begin{array}{llll}-1.805635 & -1.729022 & -5.197796\end{array}$

$\begin{array}{lll}-1.073244 & -2.507800 & -3.807507\end{array}$

$\begin{array}{lll}-1.196675 & -0.358674 & -3.616017\end{array}$

$\begin{array}{llll}-1.429613 & 0.434520 & -4.333055\end{array}$

$\begin{array}{llll}-0.107364 & -0.422171 & -3.582835\end{array}$

$\begin{array}{lll}-1.691540 & 0.068186 & -2.237859\end{array}$

$\begin{array}{llll}-0.934715 & 0.084899 & -1.460011\end{array}$

$\begin{array}{llll}-2.806624 & 0.873910 & -2.022358\end{array}$

$\begin{array}{llll}-2.816575 & 1.461365 & -1.109995\end{array}$

$\begin{array}{llll}-3.753188 & 1.359012 & -3.101568\end{array}$

$\begin{array}{lll}-4.645460 & 1.749996 & -2.607707\end{array}$

$\begin{array}{llll}-3.289605 & 2.204727 & -3.630945\end{array}$

$\begin{array}{llll}-4.170871 & 0.260085 & -4.102777\end{array}$

$\begin{array}{llll}-3.492797 & 0.252060 & -4.962575\end{array}$

$\begin{array}{llll}-5.160814 & 0.506160 & -4.499106\end{array}$

$\begin{array}{llll}-0.336806 & -3.784774 & -1.224986\end{array}$

$\begin{array}{llll}-0.871455 & -5.039831 & -0.873108\end{array}$

$\begin{array}{llll}0.626857 & -3.732210 & -2.250472\end{array}$

$\begin{array}{llll}-0.457651 & -6.196635 & -1.530387\end{array}$

$\begin{array}{llll}-1.601932 & -5.116060 & -0.074017\end{array}$

$\begin{array}{llll}1.036327 & -4.889441 & -2.909739\end{array}$

$\begin{array}{llll}1.058542 & -2.774627 & -2.515834\end{array}$

$0.497244-6.127631-2.551716$

$\begin{array}{llll}-0.877832 & -7.156585 & -1.244009\end{array}$

$\begin{array}{llll}1.782489 & -4.824858 & -3.696813\end{array}$

$\begin{array}{llll}0.817021 & -7.032017 & -3.061146\end{array}$

$\begin{array}{llll}2.527083 & -3.998277 & 0.475554\end{array}$

$\begin{array}{llll}3.530030 & -4.417748 & -0.497163\end{array}$

$\begin{array}{llll}3.343079 & -5.458873 & -0.776472\end{array}$

$\begin{array}{llll}3.460165 & -3.806084 & -1.391140\end{array}$

$\begin{array}{lll}4.542376 & -4.343889 & -0.084053\end{array}$

$\begin{array}{lll}2.172359 & -5.004850 & 1.472828\end{array}$

$\begin{array}{llll}1.856833 & -4.544734 & 2.407869\end{array}$

$\begin{array}{lll}1.379109 & -5.669386 & 1.108173\end{array}$

$\begin{array}{llll}3.059186 & -5.605114 & 1.688471\end{array}$

$\begin{array}{lll}2.451358 & -1.919359 & -0.431410\end{array}$

$\begin{array}{lll}-0.453705 & -1.173643 & 2.861536\end{array}$

$\begin{array}{lll}-0.885532 & 0.012658 & 3.418625\end{array}$

$\begin{array}{lll}-0.795000 & -2.113983 & 3.277067\end{array}$

$\begin{array}{lll}-0.417814 & 1.201791 & 2.853801\end{array}$

$\begin{array}{lll}0.455986 & 1.160610 & 1.781938\end{array}$

$\begin{array}{lll}0.835653 & 2.068097 & 1.329532\end{array}$

$\begin{array}{lll}0.890393 & 0.001100 & 1.248412\end{array}$

$\begin{array}{lll}-1.574887 & 0.013594 & 4.253832\end{array}$

$\begin{array}{lll}-0.962417 & 2.928371 & 3.596094\end{array}$

Conformer_13

Number of imaginary frequencies : 1

The smallest frequency is : $-247.7265 \mathrm{~cm}(-1)$

Electronic energy : $\quad H F=-7222.6348462$

Zero-point correctio

(Hartree/Particle)

Thermal correction to Energy=
Thermal correction to Enthalpy=

1.755703

1.874117

Thermal correction to Gibbs Free Energy $=\quad 1.593091$ 
Sum of electronic and zero-point Energies= Sum of electronic and thermal Energies= Sum of electronic and thermal Enthalpies= Sum of electronic and thermal Free Energies=

Cartesian Coordinates

$\begin{array}{lll}4.295686 & 4.787218 & 1.873484\end{array}$

$\begin{array}{llll}2.562882 & 1.624418 & -1.886287\end{array}$

$\begin{array}{llll}4.540861 & 0.128064 & 0.933464\end{array}$

$\begin{array}{llll}3.967576 & 3.424733 & 0.319076\end{array}$

$\begin{array}{lll}4.154664 & 2.745935 & 1.580415\end{array}$

$\begin{array}{lll}3.132583 & 3.194277 & 2.473323\end{array}$

$\begin{array}{lll}3.010146 & 2.884381 & 3.501982\end{array}$

$\begin{array}{llll}2.335239 & 4.162698 & 1.791996\end{array}$

$\begin{array}{lll}1.509217 & 4.715948 & 2.216826\end{array}$

$\begin{array}{lll}2.849036 & 4.307261 & 0.470279\end{array}$

$\begin{array}{llll}2.479027 & 4.983263 & -0.285537\end{array}$

$\begin{array}{lll}6.238038 & 5.325774 & 2.376005\end{array}$

$\begin{array}{lll}7.099110 & 4.676976 & 2.283435\end{array}$

$\begin{array}{lll}5.382857 & 5.426162 & 3.516092\end{array}$

$\begin{array}{lll}5.479634 & 4.864727 & 4.435797\end{array}$

$\begin{array}{lll}4.350728 & 6.365612 & 3.213037 \\ 3.525586 & 6.633171 & 3.859832\end{array}$

$\begin{array}{lll}3.525586 & 6.633171 & 3.859832\end{array}$

$\begin{array}{lll}4.567731 & 6.847067 & 1.885948\end{array}$

$\begin{array}{lll}3.938858 & 7.546915 & 1.351594\end{array}$

$\begin{array}{lll}5.733578 & 6.207057 & 1.369877\end{array}$

$\begin{array}{llll}6.7331624 & 6.344803 & 0.377922\end{array}$

$\begin{array}{llll}4.764400 & 3.153488 & -0.912786\end{array}$

$\begin{array}{llll}6.097453 & 3.584275 & -0.982472\end{array}$

$\begin{array}{llll}6.500942 & 4.139279 & -0.145113\end{array}$

$\begin{array}{llll}6.906173 & 3.300347 & -2.082834\end{array}$

$\begin{array}{llll}7.941698 & 3.625085 & -2.092692\end{array}$

$\begin{array}{llll}6.383634 & 2.576895 & -3.152315\end{array}$

$\begin{array}{llll}7.004127 & 2.337147 & -4.009807\end{array}$

$\begin{array}{lll}5.061586 & 2.133581 & -3.105675\end{array}$

$\begin{array}{llll}4.672325 & 1.556821 & -3.937702\end{array}$

$\begin{array}{llll}4.241903 & 2.397543 & -1.997259\end{array}$

$\begin{array}{lll}5.179963 & 1.672306 & 1.830835\end{array}$

$\begin{array}{lll}6.093339 & 1.961607 & 1.308148\end{array}$

$\begin{array}{llll}5.519762 & 1.464447 & 3.309306\end{array}$

$\begin{array}{lll}6.298485 & 0.706658 & 3.429952\end{array}$

$\begin{array}{lll}4.650927 & 1.148969 & 3.893151\end{array}$

$\begin{array}{lll}5.882151 & 2.407291 & 3.730327\end{array}$

$\begin{array}{llll}2.354504 & 0.721857 & -3.481969\end{array}$

$\begin{array}{lll}2.616279 & -0.657880 & -3.490522\end{array}$

$\begin{array}{llll}2.930094 & -1.152635 & -2.578941\end{array}$

$\begin{array}{llll}2.435253 & -1.404977 & -4.656664\end{array}$

$\begin{array}{llll}2.642189 & -2.470058 & -4.648799\end{array}$

$\begin{array}{lll}1.979210 & -0.786274 & -5.822462\end{array}$

$\begin{array}{llll}1.824075 & -1.370323 & -6.725066\end{array}$

$\begin{array}{llll}1.719199 & 0.586751 & -5.822630\end{array}$

$\begin{array}{lll}1.363643 & 1.074555 & -6.725773\end{array}$

$\begin{array}{llll}1.908700 & 1.338495 & -4.662220\end{array}$

$\begin{array}{llll}1.692297 & 2.401000 & -4.679573\end{array}$

$\begin{array}{llll}1.403785 & 3.038302 & -2.070464\end{array}$

$\begin{array}{llll}1.740725 & 4.197763 & -2.788106\end{array}$

$\begin{array}{llll}2.733448 & 4.302702 & -3.215670\end{array}$

$\begin{array}{llll}0.806624 & 5.222878 & -2.947683\end{array}$

$\begin{array}{llll}1.077685 & 6.116743 & -3.502387\end{array}$

$\begin{array}{lll}1.07475057 & 5.095320 & -2.402416\end{array}$

$\begin{array}{llll}-1.207888 & 5.886543 & -2.532862\end{array}$

$\begin{array}{llll}-0.814182 & 3.945225 & -1.686925\end{array}$

$\begin{array}{llll}-1.807390 & 3.839288 & -1.267617\end{array}$

$\begin{array}{llll}0.125062 & 2.927502 & -1.509412\end{array}$

$\begin{array}{llll}0.1257078 & 2.044808 & -0.936873\end{array}$

$\begin{array}{llll}5.833980 & -0.351502 & -0.288083\end{array}$

$\begin{array}{llll}5.486614 & -1.402466 & -1.149331\end{array}$

$\begin{array}{llll}4.518828 & -1.879244 & -1.036588\end{array}$

$\begin{array}{llll}6.371511 & -1.830157 & -2.138246\end{array}$

$\begin{array}{llll}7.598103 & -1.194412 & -2.319337\end{array}$

$\begin{array}{llll}8.275679 & -1.511180 & -3.104084\end{array}$

$\begin{array}{llll}7.930567 & -0.132041 & -1.478261\end{array}$

$\begin{array}{lll}7.072224 & 0.277287 & -0.457299\end{array}$

$\begin{array}{llll}7.378689 & 1.096523 & 0.178341\end{array}$
$-7220.879143$

$-7220.760729$

$-7220.759785$

$-7221.041755$
29

9

6

15

8

6 $\begin{array}{lll}4.568586 & -1.271572 & 2.131500\end{array}$

$\begin{array}{llll}3.559502 & -1.295196 & 3.107668\end{array}$

$\begin{array}{lll}2.867709 & -0.465473 & 3.195831\end{array}$

$\begin{array}{llll}3.410389 & -2.401877 & 3.940632\end{array}$

$\begin{array}{lll}4.233540 & -3.519456 & 3.798065\end{array}$

$\begin{array}{llll}4.087547 & -4.396480 & 4.418692\end{array}$

$\begin{array}{llll}5.223028 & -3.501302 & 2.818479\end{array}$

$\begin{array}{lll}5.409518 & -2.382833 & 2.005099\end{array}$

$\begin{array}{llll}6.188462 & -2.406727 & 1.253516\end{array}$

$\begin{array}{llll}2.440346 & 0.234767 & -0.102067\end{array}$

$\begin{array}{lll}6.091752 & -4.715104 & 2.637365\end{array}$

$\begin{array}{llll}2.273022 & -2.466906 & 4.921003\end{array}$

$\begin{array}{llll}5.996907 & -3.025893 & -2.969045\end{array}$

$\begin{array}{llll}9.225102 & 0.592863 & -1.725833\end{array}$

$\begin{array}{llll}9.227936 & 1.197297 & -2.940775\end{array}$

$\begin{array}{llll}9.467094 & 1.552924 & -0.806512\end{array}$

$10.282573 \quad-0.251397 \quad-1.717420$

$\begin{array}{llll}6.178469 & -4.182144 & -2.282633\end{array}$

$6.720471 \quad-3.118214 \quad-4.102395$

$\begin{array}{llll}4.686569 & -3.002366 & -3.326761\end{array}$

$\begin{array}{lll}7.068463 & -4.789883 & 3.569709\end{array}$

$\begin{array}{llll}6.695416 & -4.732169 & 1.427248\end{array}$

$\begin{array}{llll}5.369267 & -5.858297 & 2.739865\end{array}$

$\begin{array}{llll}2.644742 & -3.031511 & 6.090533\end{array}$

$\begin{array}{llll}1.757485 & -1.251350 & 5.200204\end{array}$

$\begin{array}{lll}1.250625 & -3.226908 & 4.437752\end{array}$

$\begin{array}{lll}1.975807 & -2.751330 & 0.404110\end{array}$

$\begin{array}{lll}0.812296 & -2.465194 & 1.213779\end{array}$

$\begin{array}{llll}0.453827 & -1.190236 & 1.765558\end{array}$

$\begin{array}{llll}0.409490 & -3.292461 & 1.779843\end{array}$

$\begin{array}{llll}-3.400034 & -1.296155 & -1.447131\end{array}$

$\begin{array}{lll}-5.267067 & -0.538283 & -0.378061\end{array}$

$\begin{array}{llll}-5.260610 & 0.604623 & 0.820296\end{array}$

$\begin{array}{llll}-6.330148 & 0.174064 & -1.442256\end{array}$

$\begin{array}{lll}-5.966522 & -1.799044 & 0.479259\end{array}$

$\begin{array}{lll}-4.982159 & 1.930127 & 0.525147\end{array}$

$\begin{array}{lll}-3.757984 & 2.439081 & 1.010618\end{array}$

$\begin{array}{lll}-3.119160 & 1.781954 & 1.589736\end{array}$

$\begin{array}{lll}-3.416046 & 3.740351 & 0.740252\end{array}$

$\begin{array}{lll}-2.486899 & 4.152894 & 1.120304\end{array}$

$\begin{array}{llll}-4.248707 & 4.546626 & -0.083752\end{array}$

$\begin{array}{llll}-3.843366 & 5.851182 & -0.476910\end{array}$

$\begin{array}{llll}-2.895201 & 6.232376 & -0.106687\end{array}$

$\begin{array}{llll}-4.622776 & 6.608450 & -1.322512\end{array}$

$\begin{array}{lll}-4.300134 & 7.600795 & -1.623700\end{array}$

$\begin{array}{lll}-5.844831 & 6.086284 & -1.809955\end{array}$

$\begin{array}{llll}-6.447802 & 6.678226 & -2.492409\end{array}$

$\begin{array}{llll}-6.275808 & 4.832824 & -1.431774\end{array}$

$\begin{array}{llll}-7.211194 & 4.445788 & -1.819181\end{array}$

$\begin{array}{llll}-5.501770 & 4.025483 & -0.552750\end{array}$

$\begin{array}{llll}-5.909955 & 2.701963 & -0.156051\end{array}$

$\begin{array}{lll}-7.269192 & 2.156913 & -0.436447\end{array}$

$\begin{array}{lll}-8.460165 & 2.845572 & -0.002202\end{array}$

$\begin{array}{lll}-8.420972 & 4.023172 & 0.796735\end{array}$

$\begin{array}{lll}-7.464318 & 4.422297 & 1.110645\end{array}$

$\begin{array}{lll}-9.579523 & 4.655961 & 1.192661\end{array}$

$\begin{array}{llll}-9.519009 & 5.549337 & 1.807652\end{array}$

$\begin{array}{lll}-10.845850 & 4.150867 & 0.814218\end{array}$

$\begin{array}{lll}-11.749961 & 4.664996 & 1.126878\end{array}$

$\begin{array}{lll}-10.923712 & 2.997958 & 0.066576\end{array}$

$\begin{array}{llll}-11.888699 & 2.583752 & -0.213647\end{array}$

$\begin{array}{lll}-9.748718 & 2.311610 & -0.344439\end{array}$

$\begin{array}{llll}-9.832652 & 1.090670 & -1.066518\end{array}$

$\begin{array}{llll}-10.811153 & 0.699052 & -1.329667\end{array}$

$\begin{array}{llll}-8.695480 & 0.398829 & -1.398778\end{array}$

$-8.734337-0.551525 \quad-1.920618$

$\begin{array}{llll}-7.430890 & 0.928213 & -1.060212\end{array}$

$\begin{array}{llll}-5.328213 & -3.110866 & 0.180943\end{array}$

$\begin{array}{lll}-4.591289 & -3.316200 & 0.967018\end{array}$

$\begin{array}{lll}-6.342307 & -4.240912 & 0.175104\end{array}$

$\begin{array}{lll}-6.026489 & -5.466281 & 0.773418\end{array}$

$\begin{array}{llll}-5.072025 & -5.581259 & 1.282369\end{array}$

$\begin{array}{lll}-6.924973 & -6.535693 & 0.723060\end{array}$

$\begin{array}{lll}-6.667241 & -7.480599 & 1.193604\end{array}$

$\begin{array}{lll}-8.154962 & -6.385673 & 0.079184\end{array}$ 
$\begin{array}{lll}-8.858076 & -7.213100 & 0.045079\end{array}$

$\begin{array}{llll}-8.479767 & -5.162429 & -0.517056\end{array}$

$\begin{array}{llll}-9.437200 & -5.037971 & -1.015775\end{array}$

$\begin{array}{lll}-7.578729 & -4.098788 & -0.471436\end{array}$

$\begin{array}{lll}-7.831377 & -3.144563 & -0.925616\end{array}$

$\begin{array}{llll}-4.594704 & -3.051759 & -1.163580\end{array}$

$\begin{array}{llll}-5.333745 & -3.113144 & -1.971396\end{array}$

$\begin{array}{lll}-3.953347 & -3.933802 & -1.249482\end{array}$

$\begin{array}{lll}-6.667755 & -1.732669 & 1.807851\end{array}$

$\begin{array}{lll}-7.141345 & -2.714970 & 1.886602\end{array}$

$\begin{array}{lll}-5.678379 & -1.640510 & 2.970794\end{array}$

$\begin{array}{lll}-5.126429 & -2.826057 & 3.482630\end{array}$

$\begin{array}{lll}-5.452907 & -3.781209 & 3.079944\end{array}$

$\begin{array}{lll}-4.182223 & -2.800031 & 4.512434\end{array}$

$\begin{array}{lll}-3.769129 & -3.731409 & 4.890220\end{array}$

$-3.790438-1.580613 \quad 5.068452$

$\begin{array}{lll}-3.064219 & -1.554506 & 5.876316\end{array}$

$\begin{array}{lll}-4.357489 & -0.394739 & 4.591486\end{array}$

$\begin{array}{lll}-4.076619 & 0.558340 & 5.032241\end{array}$

$\begin{array}{lll}-5.290849 & -0.424497 & 3.553562\end{array}$

$\begin{array}{lll}-5.716573 & 0.505794 & 3.199356\end{array}$

$\begin{array}{lll}-7.806919 & -0.715940 & 1.852603\end{array}$

$\begin{array}{lll}-8.514476 & -0.911904 & 1.043803\end{array}$

$\begin{array}{lll}-7.468703 & 0.315762 & 1.765567\end{array}$

$\begin{array}{lll}-8.335094 & -0.822897 & 2.805061\end{array}$

$\begin{array}{lll}-2.525389 & -1.128000 & 0.544757\end{array}$

$\begin{array}{lll}-1.882631 & -0.253247 & 0.590490\end{array}$

$\begin{array}{lll}-3.221247 & -1.183113 & 1.377250\end{array}$

$\begin{array}{lll}-1.992583 & -2.367120 & 0.062613\end{array}$

$\begin{array}{lll}-2.496918 & -3.271821 & 0.385566\end{array}$

$\begin{array}{llll}-0.734668 & -2.534547 & -0.568665\end{array}$

$\begin{array}{llll}-0.209017 & -1.652671 & -0.907230\end{array}$

$\begin{array}{llll}-4.223020 & -1.144029 & -3.493114\end{array}$

$\begin{array}{llll}-5.215152 & -1.589769 & -3.481949\end{array}$

$\begin{array}{lll}-3.126404 & -2.048740 & -3.521800\end{array}$

$-3.365096-3.111916-3.550810$

$-1.761889-1.710538-4.097640$

$-1.805653-1.728971-5.197775$

$\begin{array}{llll}-1.073298 & -2.507830 & -3.807513\end{array}$

$\begin{array}{lll}-1.196651 & -0.358705 & -3.615942\end{array}$

$\begin{array}{llll}-1.429551 & 0.434523 & -4.332955\end{array}$

$\begin{array}{llll}-0.107343 & -0.422247 & -3.582752\end{array}$

$\begin{array}{llll}-1.691510 & 0.068133 & -2.237774\end{array}$

$\begin{array}{llll}-0.934689 & 0.084802 & -1.459920\end{array}$

$\begin{array}{llll}-2.806571 & 0.873881 & -2.022251\end{array}$

$\begin{array}{llll}-2.816508 & 1.461305 & -1.109869\end{array}$

$\begin{array}{llll}-3.753117 & 1.359047 & -3.101446\end{array}$

$\begin{array}{llll}-4.645380 & 1.750038 & -2.607576\end{array}$

$\begin{array}{llll}-3.289507 & 2.204768 & -3.630790\end{array}$

$\begin{array}{lll}-4.170825 & 0.260169 & -4.102699\end{array}$

$\begin{array}{llll}-3.492740 & 0.252152 & -4.962489\end{array}$

$\begin{array}{llll}-5.160756 & 0.506292 & -4.499031\end{array}$

$\begin{array}{llll}-0.336709 & -3.784837 & -1.225066\end{array}$

$\begin{array}{llll}-0.871271 & -5.039932 & -0.873193\end{array}$

$\begin{array}{llll}0.626930 & -3.732198 & -2.250571\end{array}$

$-0.457410-6.196701-1.530497$

$\begin{array}{llll}-1.601721 & -5.116217 & -0.074081\end{array}$

$\begin{array}{llll}1.036456 & -4.889395 & -2.909864\end{array}$

$\begin{array}{llll}1.058552 & -2.774585 & -2.515927\end{array}$

$\begin{array}{llll}0.497457 & -6.127622 & -2.551847\end{array}$

$\begin{array}{lll}-0.877524 & -7.156682 & -1.244122\end{array}$

$\begin{array}{llll}1.782596 & -4.824754 & -3.696954\end{array}$

$\begin{array}{llll}0.817279 & -7.031982 & -3.061296\end{array}$

$\begin{array}{llll}2.527174 & -3.998311 & 0.475487\end{array}$

$\begin{array}{llll}3.530129 & -4.417751 & -0.497235\end{array}$

$\begin{array}{llll}3.343205 & -5.458879 & -0.776548\end{array}$

$\begin{array}{llll}3.460242 & -3.806085 & -1.391210\end{array}$

$\begin{array}{llll}4.542475 & -4.343865 & -0.084130\end{array}$

$\begin{array}{llll}2.172487 & -5.004893 & 1.472765\end{array}$

$\begin{array}{llll}1.856961 & -4.544787 & 2.407810\end{array}$

$\begin{array}{llll}1.379249 & -5.669449 & 1.108119\end{array}$

$\begin{array}{llll}3.059331 & -5.605136 & 1.688397\end{array}$

$\begin{array}{llll}2.451396 & -1.919387 & -0.431460\end{array}$

$\begin{array}{lll}-0.453641 & -1.173774 & 2.861530\end{array}$

$\begin{array}{lll}-0.885473 & 0.012510 & 3.418650\end{array}$
$-0.794908-2.114126 \quad 3.277055$

$\begin{array}{lll}-0.417792 & 1.201660 & 2.853830\end{array}$

$\begin{array}{lll}0.455975 & 1.160512 & 1.781939\end{array}$

$\begin{array}{lll}0.835609 & 2.068012 & 1.329533\end{array}$

$\begin{array}{lll}0.890392 & 0.001018 & 1.248385\end{array}$

$\begin{array}{lll}-1.574803 & 0.013422 & 4.253876\end{array}$

$\begin{array}{lll}-0.962399 & 2.928218 & 3.596174\end{array}$

\section{Conformer 14}

Number of imaginary frequencies : 1

The smallest frequency is : $-247.7215 \mathrm{~cm}(-1)$

Electronic energy :

$\mathrm{HF}=-7222.6348463$

Zero-point correction=

(Hartree/Particle)

Thermal correction to Energy=

Thermal correction to Enthalpy=

Thermal correction to Gibbs Free Energy $=\quad 1.593090$

Sum of electronic and zero-point Energies $=\quad-7220.879144$

Sum of electronic and thermal Energies

Sum of electronic and thermal Enthalpies=

$-7220.760730$

Sum of electronic and thermal Free Energies= 
$\begin{array}{llll}2.733434 & 4.302757 & -3.215584\end{array}$

$\begin{array}{llll}0.806609 & 5.222924 & -2.947584\end{array}$

$\begin{array}{llll}1.077669 & 6.116800 & -3.502272\end{array}$

$\begin{array}{llll}-0.475074 & 5.095354 & -2.402322\end{array}$

$\begin{array}{llll}-1.207906 & 5.886578 & -2.532754\end{array}$

$-0.814198 \quad 3.945246 \quad-1.686853$

$\begin{array}{llll}-1.807406 & 3.839300 & -1.267547\end{array}$

$\begin{array}{llll}0.125047 & 2.927521 & -1.509356\end{array}$

$\begin{array}{llll}-0.137092 & 2.044817 & -0.936833\end{array}$

$\begin{array}{llll}5.833982 & -0.351470 & -0.288049\end{array}$

$\begin{array}{llll}5.486638 & -1.402417 & -1.149325\end{array}$

$\begin{array}{llll}4.518855 & -1.879209 & -1.036605\end{array}$

$\begin{array}{llll}6.371551 & -1.830077 & -2.138239\end{array}$

$\begin{array}{llll}7.598140 & -1.194317 & -2.319299\end{array}$

$\begin{array}{llll}8.275729 & -1.511060 & -3.104044\end{array}$

$\begin{array}{llll}7.930583 & -0.131963 & -1.478193\end{array}$

$\begin{array}{llll}7.072223 & 0.277334 & -0.457233\end{array}$

$\begin{array}{lll}7.378672 & 1.096558 & 0.178430\end{array}$

$\begin{array}{llll}4.568570 & -1.271602 & 2.131502\end{array}$

$\begin{array}{llll}3.559476 & -1.295253 & 3.107661\end{array}$

$\begin{array}{llll}2.867675 & -0.465538 & 3.195830\end{array}$

$\begin{array}{llll}3.410368 & -2.401950 & 3.940604\end{array}$

$\begin{array}{lll}4.233532 & -3.519518 & 3.798025\end{array}$

$\begin{array}{llll}4.087543 & -4.396554 & 4.418636\end{array}$

$\begin{array}{llll}5.223029 & -3.501337 & 2.818448\end{array}$

$\begin{array}{llll}5.409514 & -2.382852 & 2.005088\end{array}$

$\begin{array}{llll}6.188464 & -2.406726 & 1.253511\end{array}$

$\begin{array}{llll}2.440337 & 0.234757 & -0.102062\end{array}$

$\begin{array}{llll}6.091765 & -4.715128 & 2.637321\end{array}$

$\begin{array}{llll}2.272992 & -2.467010 & 4.920962\end{array}$

$\begin{array}{llll}5.996971 & -3.025798 & -2.969069\end{array}$

$\begin{array}{llll}9.225117 & 0.592957 & -1.725728\end{array}$

$\begin{array}{llll}9.227958 & 1.197435 & -2.940648\end{array}$

$\begin{array}{llll}9.467095 & 1.552986 & -0.806370\end{array}$

$10.282592-0.251298-1.717336$

$\begin{array}{llll}6.178546 & -4.182063 & -2.282684\end{array}$

$6.720546 \quad-3.118081-4.102415$

$\begin{array}{lll}4.686636 & -3.002283 & -3.326795\end{array}$

$\begin{array}{llll}7.068469 & -4.789913 & 3.569673\end{array}$

$\begin{array}{llll}6.695440 & -4.732168 & 1.427209\end{array}$

$\begin{array}{llll}5.369291 & -5.858329 & 2.739796\end{array}$

$\begin{array}{lll}2.644708 & -3.031626 & 6.090488\end{array}$

$\begin{array}{lll}1.757436 & -1.251465 & 5.200176\end{array}$

$\begin{array}{llll}1.250612 & -3.227019 & 4.437689\end{array}$

$\begin{array}{lll}1.975830 & -2.751352 & 0.404049\end{array}$

$\begin{array}{llll}0.812309 & -2.465246 & 1.213714\end{array}$

$\begin{array}{llll}0.453821 & -1.190305 & 1.765519\end{array}$

$\begin{array}{lll}0.409505 & -3.292531 & 1.779754\end{array}$

$\begin{array}{llll}-3.400025 & -1.296147 & -1.447187\end{array}$

$\begin{array}{lll}-5.267049 & -0.538273 & -0.378105\end{array}$

$\begin{array}{lll}-5.260583 & 0.604606 & 0.820278\end{array}$

$\begin{array}{llll}-6.330118 & 0.174108 & -1.442288\end{array}$

$\begin{array}{llll}-5.966521 & -1.799044 & 0.479185\end{array}$

$\begin{array}{lll}-4.982139 & 1.930120 & 0.525162\end{array}$

$\begin{array}{lll}-3.757968 & 2.439068 & 1.010647\end{array}$

$\begin{array}{lll}-3.119140 & 1.781932 & 1.589750\end{array}$

$\begin{array}{llll}-3.416039 & 3.740348 & 0.740318\end{array}$

$\begin{array}{lll}-2.486894 & 4.152886 & 1.120384\end{array}$

$\begin{array}{llll}-4.248703 & 4.546641 & -0.083665\end{array}$

$\begin{array}{llll}-3.843369 & 5.851209 & -0.476789\end{array}$

$\begin{array}{llll}-2.895207 & 6.232399 & -0.106555\end{array}$

$\begin{array}{llll}-4.622783 & 6.608494 & -1.322372\end{array}$

$\begin{array}{llll}-4.300147 & 7.600849 & -1.623534\end{array}$

$\begin{array}{llll}-5.844835 & 6.086335 & -1.809829\end{array}$

$\begin{array}{llll}-6.447809 & 6.678291 & -2.492267\end{array}$

$\begin{array}{llll}-6.275805 & 4.832862 & -1.431681\end{array}$

$\begin{array}{llll}-7.211189 & 4.445831 & -1.819098\end{array}$

$\begin{array}{llll}-5.501762 & 4.025503 & -0.552677\end{array}$

$\begin{array}{llll}-5.909940 & 2.701969 & -0.156015\end{array}$

$\begin{array}{lll}-7.269175 & 2.156923 & -0.436426\end{array}$

$\begin{array}{lll}-8.460151 & 2.845564 & -0.002165\end{array}$

$\begin{array}{llll}-8.420964 & 4.023139 & 0.796808\end{array}$

$\begin{array}{lll}-7.464312 & 4.422260 & 1.110731\end{array}$

$\begin{array}{llll}-9.579519 & 4.655912 & 1.192752\end{array}$

$\begin{array}{lll}-9.519010 & 5.549269 & 1.807770\end{array}$ $\begin{array}{lll}-10.845843 & 4.150824 & 0.814291\end{array}$

$\begin{array}{lll}-11.749957 & 4.664938 & 1.126965\end{array}$

$\begin{array}{lll}-10.923698 & 2.997937 & 0.066613\end{array}$

$\begin{array}{llll}-11.888684 & 2.583736 & -0.213624\end{array}$

$\begin{array}{llll}-9.748701 & 2.311606 & -0.344420\end{array}$

$\begin{array}{llll}-9.832628 & 1.090688 & -1.066536\end{array}$

$\begin{array}{lll}-10.811127 & 0.699074 & -1.329699\end{array}$

$\begin{array}{llll}-8.695452 & 0.398861 & -1.398814\end{array}$

$\begin{array}{llll}-8.734304 & -0.551477 & -1.920682\end{array}$

$\begin{array}{llll}-7.430866 & 0.928240 & -1.060228\end{array}$

$\begin{array}{lll}-5.328232 & -3.110868 & 0.180835\end{array}$

$\begin{array}{lll}-4.591310 & -3.316233 & 0.966905\end{array}$

$\begin{array}{lll}-6.342343 & -4.240899 & 0.174966\end{array}$

$\begin{array}{lll}-6.026548 & -5.466286 & 0.773255\end{array}$

$\begin{array}{llll}-5.072088 & -5.581290 & 1.282207\end{array}$

$\begin{array}{lll}-6.925049 & -6.535682 & 0.722869\end{array}$

$\begin{array}{lll}-6.667335 & -7.480603 & 1.193394\end{array}$

$\begin{array}{lll}-8.155033 & -6.385627 & 0.078991\end{array}$

$\begin{array}{lll}-8.858161 & -7.213042 & 0.044866\end{array}$

$\begin{array}{llll}-8.479815 & -5.162365 & -0.517223\end{array}$

$\begin{array}{llll}-9.437245 & -5.037880 & -1.015943\end{array}$

$\begin{array}{llll}-7.578760 & -4.098740 & -0.471575\end{array}$

$\begin{array}{llll}-7.831390 & -3.144501 & -0.925735\end{array}$

$\begin{array}{llll}-4.594723 & -3.051737 & -1.163686\end{array}$

$\begin{array}{llll}-5.333765 & -3.113086 & -1.971504\end{array}$

$\begin{array}{llll}-3.953380 & -3.933787 & -1.249612\end{array}$

$\begin{array}{lll}-6.667757 & -1.732695 & 1.807777\end{array}$

$\begin{array}{lll}-7.141361 & -2.714991 & 1.886500\end{array}$

$\begin{array}{lll}-5.678384 & -1.640580 & 2.970726\end{array}$

$\begin{array}{lll}-5.126454 & -2.826147 & 3.482535\end{array}$

$\begin{array}{lll}-5.452942 & -3.781286 & 3.079822\end{array}$

$\begin{array}{lll}-4.182256 & -2.800161 & 4.512347\end{array}$

$\begin{array}{lll}-3.769177 & -3.731554 & 4.890113\end{array}$

$\begin{array}{lll}-3.790459 & -1.580763 & 5.068400\end{array}$

$\begin{array}{lll}-3.064247 & -1.554686 & 5.876272\end{array}$

$\begin{array}{lll}-4.357490 & -0.394869 & 4.591459\end{array}$

$\begin{array}{lll}-4.076610 & 0.558195 & 5.032241\end{array}$

$\begin{array}{lll}-5.290841 & -0.424587 & 3.553527\end{array}$

$\begin{array}{lll}-5.716549 & 0.505719 & 3.199340\end{array}$

$\begin{array}{lll}-7.806908 & -0.715953 & 1.852554\end{array}$

$\begin{array}{lll}-8.514467 & -0.911885 & 1.043749\end{array}$

$\begin{array}{lll}-7.468679 & 0.315748 & 1.765548\end{array}$

$\begin{array}{lll}-8.335085 & -0.822929 & 2.805009\end{array}$

$\begin{array}{llll}-2.525393 & -1.128096 & 0.544711\end{array}$

$\begin{array}{lll}-1.882641 & -0.253342 & 0.590498\end{array}$

$\begin{array}{lll}-3.221259 & -1.183261 & 1.377195\end{array}$

$\begin{array}{lll}-1.992568 & -2.367189 & 0.062510\end{array}$

$\begin{array}{lll}-2.496898 & -3.271913 & 0.385410\end{array}$

$\begin{array}{llll}-0.734643 & -2.534573 & -0.568756\end{array}$

$\begin{array}{lll}-0.208998 & -1.652680 & -0.907281\end{array}$

$\begin{array}{llll}-4.222998 & -1.143952 & -3.493175\end{array}$

$\begin{array}{llll}-5.215135 & -1.589679 & -3.482029\end{array}$

$\begin{array}{llll}-3.126395 & -2.048677 & -3.521875\end{array}$

$\begin{array}{llll}-3.365103 & -3.111849 & -3.550916\end{array}$

$\begin{array}{llll}-1.761869 & -1.710479 & -4.097691\end{array}$

$\begin{array}{lll}-1.805620 & -1.728885 & -5.197828\end{array}$

$\begin{array}{llll}-1.073291 & -2.507788 & -3.807577\end{array}$

$\begin{array}{lll}-1.196616 & -0.358666 & -3.615956\end{array}$

$\begin{array}{llll}-1.429499 & 0.434582 & -4.332952\end{array}$

$\begin{array}{lll}-0.107308 & -0.422224 & -3.582762\end{array}$

$\begin{array}{llll}-1.691477 & 0.068148 & -2.237781\end{array}$

$\begin{array}{llll}-0.934664 & 0.084789 & -1.459920\end{array}$

$\begin{array}{llll}-2.806531 & 0.873900 & -2.022245\end{array}$

$\begin{array}{llll}-2.816470 & 1.461300 & -1.109847\end{array}$

$\begin{array}{llll}-3.753066 & 1.359106 & -3.101432\end{array}$

$\begin{array}{llll}-4.645329 & 1.750091 & -2.607557\end{array}$

$\begin{array}{llll}-3.289445 & 2.204838 & -3.630748\end{array}$

$\begin{array}{lll}-4.170778 & 0.260262 & -4.102721\end{array}$

$\begin{array}{llll}-3.492684 & 0.252260 & -4.962503\end{array}$

$\begin{array}{llll}-5.160701 & 0.506408 & -4.499056\end{array}$

$\begin{array}{llll}-0.336649 & -3.784837 & -1.225187\end{array}$

$\begin{array}{llll}-0.871179 & -5.039955 & -0.873344\end{array}$

$\begin{array}{llll}0.626993 & -3.732147 & -2.250686\end{array}$

$\begin{array}{llll}-0.457283 & -6.196697 & -1.530673\end{array}$

$\begin{array}{llll}-1.601627 & -5.116277 & -0.074235\end{array}$ 
$\begin{array}{llll}1.036553 & -4.889317 & -2.910004\end{array}$

$\begin{array}{llll}1.058591 & -2.774517 & -2.516016\end{array}$

$0.497586-6.127568-2.552019$

$\begin{array}{llll}-0.877371 & -7.156696 & -1.244321\end{array}$

$\begin{array}{llll}1.782695 & -4.824637 & -3.697089\end{array}$

$\begin{array}{llll}0.817434 & -7.031906 & -3.061488\end{array}$

$\begin{array}{llll}2.527209 & -3.998329 & 0.475409\end{array}$

$\begin{array}{lll}3.530177 & -4.417743 & -0.497311\end{array}$

$\begin{array}{llll}3.343262 & -5.458867 & -0.776649\end{array}$

$\begin{array}{llll}3.460298 & -3.806059 & -1.391274\end{array}$

$\begin{array}{lll}4.542518 & -4.343861 & -0.084192\end{array}$

$\begin{array}{llll}2.172521 & -5.004933 & 1.472665\end{array}$

$\begin{array}{lll}1.856988 & -4.544845 & 2.407718\end{array}$

$\begin{array}{llll}1.379288 & -5.669485 & 1.108003\end{array}$

$\begin{array}{llll}3.059367 & -5.605176 & 1.688290\end{array}$

$\begin{array}{llll}2.451419 & -1.919387 & -0.431498\end{array}$

$\begin{array}{lll}-0.453653 & -1.173880 & 2.861486\end{array}$

$\begin{array}{lll}-0.885495 & 0.012386 & 3.418636\end{array}$

$\begin{array}{lll}-0.794917 & -2.114245 & 3.276983\end{array}$

$\begin{array}{lll}-0.417819 & 1.201555 & 2.853853\end{array}$

$\begin{array}{lll}0.455952 & 1.160443 & 1.781964\end{array}$

$\begin{array}{lll}0.835582 & 2.067958 & 1.329584\end{array}$

$\begin{array}{lll}0.890378 & 0.000967 & 1.248378\end{array}$

$\begin{array}{lll}-1.574830 & 0.013270 & 4.253859\end{array}$

$\begin{array}{lll}-0.962438 & 2.928086 & 3.596251\end{array}$

Conformer_15

Number of imaginary frequencies : 1

The smallest frequency is : $-247.7358 \mathrm{~cm}(-1)$

Electronic energy : $\quad \mathrm{HF}=-7222.6348463$

Zero-point correction=

1.755702

(Hartree/Particle)

Thermal correction to Energy $=\quad 1.874116$

Thermal correction to Enthalpy= $\quad 1.875060$

Thermal correction to Gibbs Free Energy $=\quad \begin{array}{r}1.593081 \\ \hline\end{array}$

Sum of electronic and zero-point Energies $=\quad-7220.879144$

Sum of electronic and thermal Energies=

Sum of electronic and thermal Enthalpies=

$-7220.760730$

Sum of electronic and thermal Free Energies $=\quad-7221.041766$

Cartesian Coordinates

$\begin{array}{ccc}4.295574 & 4.787382 & 1.873216 \\ 2.562959 & 1.624264 & -1.886363 \\ 4.540792 & 0.128152 & 0.933596 \\ 3.967542 & 3.424784 & 0.318890 \\ 4.154535 & 2.746081 & 1.580296 \\ 3.132400 & 3.194512 & 2.473099 \\ 3.009886 & 2.884705 & 3.501775 \\ 2.335118 & 4.162892 & 1.791643 \\ 1.509079 & 4.716194 & 2.216374 \\ 2.849010 & 4.307345 & 0.469951 \\ 2.479065 & 4.983294 & -0.285945 \\ 6.237919 & 5.325962 & 2.375738 \\ 7.098994 & 4.677163 & 2.283195 \\ 5.382728 & 5.426383 & 3.515813 \\ 5.479502 & 4.864978 & 4.435537 \\ 4.350599 & 6.365819 & 3.212720 \\ 3.525449 & 6.633393 & 3.859500 \\ 4.567611 & 6.847232 & 1.885616 \\ 3.938738 & 7.547061 & 1.351237 \\ 5.733464 & 6.207210 & 1.369576 \\ 6.141517 & 6.344924 & 0.377618 \\ 4.764429 & 3.153431 & -0.912907 \\ 6.097481 & 3.584227 & -0.982576 \\ 6.500922 & 4.139322 & -0.145254 \\ 6.906253 & 3.300194 & -2.082872 \\ 7.941775 & 3.624941 & -2.092717 \\ 6.383769 & 2.576628 & -3.152303 \\ 7.004305 & 2.336796 & -4.009741 \\ 5.061721 & 2.133311 & -3.105684 \\ 4.672503 & 1.556463 & -3.937669 \\ 4.241984 & 2.397382 & -1.997333 \\ 5.179816 & 1.672471 & 1.830881\end{array}$

$6.093230 \quad 1.961735 \quad 1.308239$

$\begin{array}{lll}5.519505 & 1.464734 & 3.309395\end{array}$

$6.298226 \quad 0.706963 \quad 3.430158$

$\begin{array}{llll}4.650631 & 1.149293 & 3.893201\end{array}$

$\begin{array}{lll}5.881855 & 2.407616 & 3.730366\end{array}$

$\begin{array}{llll}2.354635 & 0.721569 & -3.481977\end{array}$

$2.616353-0.658179-3.490390$

$\begin{array}{llll}2.930101 & -1.152861 & -2.578747\end{array}$

$2.435343-1.405378-4.656469$

$\begin{array}{llll}2.642232 & -2.470467 & -4.648496\end{array}$

$\begin{array}{llll}1.979371 & -0.786765 & -5.822343\end{array}$

$\begin{array}{llll}1.824246 & -1.370893 & -6.724898\end{array}$

$\begin{array}{llll}1.719416 & 0.586270 & -5.822650\end{array}$

$\begin{array}{llll}1.363914 & 1.074004 & -6.725852\end{array}$

$\begin{array}{llll}1.908902 & 1.338116 & -4.662303\end{array}$

$\begin{array}{llll}1.692537 & 2.400626 & -4.679763\end{array}$

$\begin{array}{llll}1.403852 & 3.038119 & -2.070688\end{array}$

$\begin{array}{llll}1.740783 & 4.197526 & -2.788419\end{array}$

$\begin{array}{llll}2.733515 & 4.302451 & -3.215967\end{array}$

$\begin{array}{llll}0.806664 & 5.222608 & -2.948102\end{array}$

$\begin{array}{llll}1.077718 & 6.116433 & -3.502873\end{array}$

$\begin{array}{llll}-0.475027 & 5.095068 & -2.402853\end{array}$

$\begin{array}{lll}-1.207872 & 5.886265 & -2.533380\end{array}$

$\begin{array}{llll}-0.814144 & 3.945027 & -1.687271\end{array}$

$\begin{array}{llll}-1.807359 & 3.839103 & -1.267977\end{array}$

$\begin{array}{llll}0.125119 & 2.927339 & -1.509652\end{array}$

$\begin{array}{llll}-0.137009 & 2.044688 & -0.937043\end{array}$

$\begin{array}{llll}5.833966 & -0.351467 & -0.287872\end{array}$

$\begin{array}{llll}5.486642 & -1.402478 & -1.149078\end{array}$

$\begin{array}{llll}4.518847 & -1.879246 & -1.036357\end{array}$

$\begin{array}{llll}6.371598 & -1.830243 & -2.137909\end{array}$

$\begin{array}{llll}7.598209 & -1.194522 & -2.318960\end{array}$

$\begin{array}{llll}8.275834 & -1.511348 & -3.103641\end{array}$

$\begin{array}{llll}7.930630 & -0.132102 & -1.477929\end{array}$

$\begin{array}{lll}7.072228 & 0.277296 & -0.457046\end{array}$

$\begin{array}{llll}7.378663 & 1.096565 & 0.178567\end{array}$

$\begin{array}{lll}4.568523 & -1.271422 & 2.131708\end{array}$

$\begin{array}{llll}3.559417 & -1.295034 & 3.107855\end{array}$

$\begin{array}{llll}2.867598 & -0.465329 & 3.195972\end{array}$

$\begin{array}{lll}3.410312 & -2.401687 & 3.940858\end{array}$

$\begin{array}{lll}4.233491 & -3.519253 & 3.798350\end{array}$

$\begin{array}{llll}4.087503 & -4.396258 & 4.419005\end{array}$

$\begin{array}{llll}5.223000 & -3.501112 & 2.818787\end{array}$

$\begin{array}{llll}5.409485 & -2.382669 & 2.005372\end{array}$

$\begin{array}{lll}6.188450 & -2.406575 & 1.253811\end{array}$

$\begin{array}{llll}2.440337 & 0.234755 & -0.102031\end{array}$

$\begin{array}{lll}6.091758 & -4.714899 & 2.637730\end{array}$

$\begin{array}{lll}2.272927 & -2.466703 & 4.921209\end{array}$

$\begin{array}{llll}5.997037 & -3.026030 & -2.968653\end{array}$

$\begin{array}{llll}9.225191 & 0.592770 & -1.725464\end{array}$

$\begin{array}{llll}9.228115 & 1.197117 & -2.940448\end{array}$

$\begin{array}{llll}9.467132 & 1.552893 & -0.806193\end{array}$

$\begin{array}{lll}10.282650 & -0.251503 & -1.716918\end{array}$

$\begin{array}{llll}6.178541 & -4.182237 & -2.282149\end{array}$

$\begin{array}{llll}6.720680 & -3.118437 & -4.101946\end{array}$

$\begin{array}{lll}4.686726 & -3.002513 & -3.326461\end{array}$

$\begin{array}{llll}7.068482 & -4.789597 & 3.570067\end{array}$

$\begin{array}{llll}6.695410 & -4.732009 & 1.427607\end{array}$

$\begin{array}{llll}5.369310 & -5.858108 & 2.740299\end{array}$

$\begin{array}{llll}2.644629 & -3.031279 & 6.090759\end{array}$

$\begin{array}{lll}1.757376 & -1.251145 & 5.200374\end{array}$

$\begin{array}{llll}1.250545 & -3.226722 & 4.437956\end{array}$

$\begin{array}{lll}1.975795 & -2.751320 & 0.404315\end{array}$

$\begin{array}{llll}0.812267 & -2.465150 & 1.213949\end{array}$

$\begin{array}{llll}0.453785 & -1.190158 & 1.765646\end{array}$

$\begin{array}{llll}0.409467 & -3.292385 & 1.780066\end{array}$

$\begin{array}{llll}-3.400008 & -1.296253 & -1.447098\end{array}$

$\begin{array}{lll}-5.267064 & -0.538294 & -0.378136\end{array}$

$\begin{array}{lll}-5.260639 & 0.604679 & 0.820157\end{array}$

$\begin{array}{llll}-6.330093 & 0.174003 & -1.442415\end{array}$

$\begin{array}{lll}-5.966569 & -1.798999 & 0.479225\end{array}$

$\begin{array}{llll}-4.982155 & 1.930162 & 0.524942\end{array}$

$\begin{array}{lll}-3.757987 & 2.439123 & 1.010423\end{array}$

$\begin{array}{lll}-3.119200 & 1.782023 & 1.589611\end{array}$

$\begin{array}{lll}-3.416008 & 3.740366 & 0.739976\end{array}$ 
$\begin{array}{lll}-2.486861 & 4.152911 & 1.120027\end{array}$

$\begin{array}{llll}-4.248621 & 4.546603 & -0.084112\end{array}$

$\begin{array}{llll}-3.843237 & 5.851123 & -0.477346\end{array}$

$\begin{array}{llll}-2.895074 & 6.232319 & -0.107119\end{array}$

$\begin{array}{llll}-4.622602 & 6.608352 & -1.323024\end{array}$

$\begin{array}{llll}-4.299927 & 7.600668 & -1.624270\end{array}$

$\begin{array}{llll}-5.844654 & 6.086182 & -1.810470\end{array}$

$\begin{array}{llll}-6.447590 & 6.678092 & -2.492983\end{array}$

$\begin{array}{llll}-6.275673 & 4.832758 & -1.432219\end{array}$

$\begin{array}{llll}-7.211055 & 4.445718 & -1.819631\end{array}$

$\begin{array}{llll}-5.501681 & 4.025458 & -0.553115\end{array}$

$\begin{array}{llll}-5.909909 & 2.701972 & -0.156343\end{array}$

$\begin{array}{lll}-7.269144 & 2.156929 & -0.436760\end{array}$

$\begin{array}{lll}-8.460123 & 2.845636 & -0.002609\end{array}$

$\begin{array}{lll}-8.420941 & 4.023284 & 0.796257\end{array}$

$\begin{array}{lll}-7.464294 & 4.422408 & 1.110188\end{array}$

$\begin{array}{lll}-9.579498 & 4.656124 & 1.192088\end{array}$

$\begin{array}{lll}-9.518994 & 5.549537 & 1.807024\end{array}$

$\begin{array}{lll}-10.845818 & 4.151033 & 0.813616\end{array}$

$\begin{array}{lll}-11.749933 & 4.665201 & 1.126200\end{array}$

$\begin{array}{lll}-10.923669 & 2.998078 & 0.066044\end{array}$

$\begin{array}{llll}-11.888652 & 2.583874 & -0.214199\end{array}$

$\begin{array}{lll}-9.748670 & 2.311680 & -0.344873\end{array}$

$\begin{array}{llll}-9.832595 & 1.090699 & -1.066884\end{array}$

$\begin{array}{llll}-10.811093 & 0.699086 & -1.330056\end{array}$

$\begin{array}{llll}-8.695422 & 0.398816 & -1.399053\end{array}$

$\begin{array}{llll}-8.734276 & -0.551567 & -1.920839\end{array}$

$\begin{array}{lll}-7.430837 & 0.928195 & -1.060462\end{array}$

$\begin{array}{lll}-5.328287 & -3.110853 & 0.180986\end{array}$

$\begin{array}{lll}-4.591412 & -3.316182 & 0.967107\end{array}$

$\begin{array}{lll}-6.342426 & -4.240860 & 0.175147\end{array}$

$\begin{array}{lll}-6.026704 & -5.466204 & 0.773563\end{array}$

$\begin{array}{lll}-5.072276 & -5.581193 & 1.282579\end{array}$

$\begin{array}{lll}-6.925236 & -6.535576 & 0.723221\end{array}$

$\begin{array}{lll}-6.667580 & -7.480463 & 1.193845\end{array}$

$\begin{array}{lll}-8.155179 & -6.385539 & 0.079258\end{array}$

$\begin{array}{llll}-8.858331 & -7.212935 & 0.045166\end{array}$

$\begin{array}{llll}-8.479886 & -5.162321 & -0.517086\end{array}$

$-9.437282 \quad-5.037850 \quad-1.015874$

$\begin{array}{lll}-7.578801 & -4.098720 & -0.471479\end{array}$

$\begin{array}{lll}-7.831373 & -3.144513 & -0.925739\end{array}$

$\begin{array}{llll}-4.594706 & -3.051828 & -1.163502\end{array}$

$\begin{array}{llll}-5.333704 & -3.113251 & -1.971353\end{array}$

$\begin{array}{llll}-3.953357 & -3.933886 & -1.249319\end{array}$

$\begin{array}{lll}-6.667845 & -1.732550 & 1.807790\end{array}$

$\begin{array}{lll}-7.141462 & -2.714835 & 1.886568\end{array}$

$\begin{array}{lll}-5.678498 & -1.640365 & 2.970757\end{array}$

$\begin{array}{lll}-5.126601 & -2.825904 & 3.482668\end{array}$

$\begin{array}{lll}-5.453114 & -3.781068 & 3.080033\end{array}$

$\begin{array}{lll}-4.182402 & -2.799856 & 4.512477\end{array}$

$\begin{array}{llll}-3.769348 & -3.731228 & 4.890320\end{array}$

$\begin{array}{lll}-3.790569 & -1.580421 & 5.068425\end{array}$

$\begin{array}{lll}-3.064352 & -1.554295 & 5.876290\end{array}$

$\begin{array}{lll}-4.357577 & -0.394553 & 4.591391\end{array}$

$\begin{array}{lll}-4.076675 & 0.558540 & 5.032094\end{array}$

$\begin{array}{lll}-5.290931 & -0.424335 & 3.553463\end{array}$

$\begin{array}{lll}-5.716620 & 0.505952 & 3.199204\end{array}$

$\begin{array}{lll}-7.806985 & -0.715791 & 1.852459\end{array}$

$\begin{array}{lll}-8.514520 & -0.911774 & 1.043644\end{array}$

$\begin{array}{lll}-7.468741 & 0.315899 & 1.765387\end{array}$

$\begin{array}{lll}-8.335195 & -0.822691 & 2.804905\end{array}$

$\begin{array}{lll}-2.525418 & -1.127996 & 0.544805\end{array}$

$\begin{array}{lll}-1.882657 & -0.253243 & 0.590503\end{array}$

$\begin{array}{llll}-3.221301 & -1.183053 & 1.377282\end{array}$

$\begin{array}{lll}-1.992601 & -2.367149 & 0.062759\end{array}$

$\begin{array}{lll}-2.496944 & -3.271831 & 0.385756\end{array}$

$-0.734673-2.534618-0.568485$

$\begin{array}{lll}-0.209022 & -1.652764 & -0.907107\end{array}$

$-4.222941-1.144214-3.493111$

$\begin{array}{llll}-5.215075 & -1.589950 & -3.481956\end{array}$

$\begin{array}{llll}-3.126325 & -2.048925 & -3.521729\end{array}$

$\begin{array}{lll}-3.365015 & -3.112103 & -3.550699\end{array}$

$\begin{array}{llll}-1.761793 & -1.710745 & -4.097542\end{array}$

$\begin{array}{lll}-1.805523 & -1.729220 & -5.197678\end{array}$

$\begin{array}{llll}-1.073211 & -2.508026 & -3.807362\end{array}$ $\begin{array}{lll}-1.196569 & -0.358894 & -3.615877\end{array}$

$\begin{array}{llll}-1.429439 & 0.434307 & -4.332930\end{array}$

$\begin{array}{lll}-0.107262 & -0.422434 & -3.582641\end{array}$

$\begin{array}{llll}-1.691481 & 0.067998 & -2.237744\end{array}$

$\begin{array}{llll}-0.934686 & 0.084697 & -1.459867\end{array}$

$\begin{array}{llll}-2.806542 & 0.873764 & -2.022298\end{array}$

$\begin{array}{lll}-2.816507 & 1.461231 & -1.109943\end{array}$

$\begin{array}{llll}-3.753046 & 1.358883 & -3.101550\end{array}$

$\begin{array}{llll}-4.645324 & 1.749906 & -2.607732\end{array}$

$\begin{array}{llll}-3.289407 & 2.204572 & -3.630919\end{array}$

$\begin{array}{llll}-4.170723 & 0.259956 & -4.102762\end{array}$

$\begin{array}{llll}-3.492607 & 0.251893 & -4.962526\end{array}$

$\begin{array}{lll}-5.160639 & 0.506061 & -4.499141\end{array}$

$-0.336708-3.784949-1.224807$

$\begin{array}{llll}-0.871273 & -5.040024 & -0.872865\end{array}$

$\begin{array}{llll}0.626940 & -3.732370 & -2.250306\end{array}$

$\begin{array}{llll}-0.457399 & -6.196832 & -1.530091\end{array}$

$\begin{array}{lll}-1.601733 & -5.116262 & -0.073758\end{array}$

$\begin{array}{llll}1.036479 & -4.889606 & -2.909521\end{array}$

$\begin{array}{lll}1.058558 & -2.774772 & -2.515716\end{array}$

$\begin{array}{llll}0.497482 & -6.127814 & -2.551433\end{array}$

$\begin{array}{llll}-0.877513 & -7.156797 & -1.243662\end{array}$

$\begin{array}{llll}1.782626 & -4.825012 & -3.696609\end{array}$

$\begin{array}{llll}0.817313 & -7.032204 & -3.060822\end{array}$

$\begin{array}{llll}2.527182 & -3.998287 & 0.475769\end{array}$

$\begin{array}{llll}3.530168 & -4.417751 & -0.496911\end{array}$

$\begin{array}{llll}3.343282 & -5.458901 & -0.776170\end{array}$

$\begin{array}{llll}3.460282 & -3.806135 & -1.390920\end{array}$

$\begin{array}{llll}4.542503 & -4.343810 & -0.083791\end{array}$

$\begin{array}{lll}2.172498 & -5.004828 & 1.473089\end{array}$

$\begin{array}{lll}1.856933 & -4.544683 & 2.408101\end{array}$

$\begin{array}{lll}1.379291 & -5.669427 & 1.108456\end{array}$

$\begin{array}{lll}3.059356 & -5.605032 & 1.688776\end{array}$

$\begin{array}{llll}2.451385 & -1.919417 & -0.431295\end{array}$

$\begin{array}{lll}-0.453674 & -1.173629 & 2.861625\end{array}$

$\begin{array}{lll}-0.885511 & 0.012690 & 3.418669\end{array}$

$\begin{array}{lll}-0.794925 & -2.113956 & 3.277220\end{array}$

$\begin{array}{lll}-0.417845 & 1.201805 & 2.853763\end{array}$

$\begin{array}{lll}0.455908 & 1.160590 & 1.781864\end{array}$

$\begin{array}{lll}0.835527 & 2.068063 & 1.329389\end{array}$

$\begin{array}{lll}0.890333 & 0.001065 & 1.248386\end{array}$

$\begin{array}{lll}-1.574829 & 0.013654 & 4.253906\end{array}$

$\begin{array}{lll}-0.962440 & 2.928412 & 3.595999\end{array}$

\section{Conformer 16}

Number of imaginary frequencies : 1

The smallest frequency is : $-247.6887 \mathrm{~cm}(-1)$

Electronic energy : $\quad \mathrm{HF}=-7222.6348464$

Zero-point correction=

1.755698

(Hartree/Particle)

Thermal correction to Energy $=\quad 1.874113$

Thermal correction to Enthalpy= $\quad 1.875057$

Thermal correction to Gibbs Free Energy $=\quad 1.593067$

Sum of electronic and zero-point Energies $=\quad-7220.879148$

Sum of electronic and thermal Energies $=\quad-7220.760733$

Sum of electronic and thermal Enthalpies $=\quad-7220.759789$

Sum of electronic and thermal Free Energies $=\quad-7221.041779$

\begin{tabular}{cccc} 
& \multicolumn{3}{c}{ Cartesian Coordinates } \\
\hline 26 & 4.295208 & 4.787275 & 1.873634 \\
15 & 2.562826 & 1.624424 & -1.886287 \\
15 & 4.540717 & 0.128141 & 0.933561 \\
6 & 3.967274 & 3.424801 & 0.319177 \\
6 & 4.154296 & 2.745989 & 1.580519 \\
6 & 3.132127 & 3.194275 & 2.473356 \\
1 & 3.009622 & 2.884359 & 3.502000 \\
6 & 2.334793 & 4.162672 & 1.791983 \\
1 & 1.508713 & 4.715877 & 2.216759 \\
6 & 2.848688 & 4.307282 & 0.470310 \\
1 & 2.478708 & 4.983279 & -0.285524 \\
6 & 6.237494 & 5.325896 & 2.376333 \\
1 & 7.098599 & 4.677132 & 2.283826 \\
6 & 5.382209 & 5.426231 & 3.516346
\end{tabular}


$\begin{array}{llll}5.478925 & 4.864783 & 4.436049\end{array}$

$\begin{array}{llll}4.350071 & 6.365647 & 3.213217\end{array}$

$\begin{array}{llll}3.524862 & 6.633163 & 3.859944\end{array}$

$\begin{array}{llll}4.567172 & 6.847133 & 1.886155\end{array}$

$\begin{array}{llll}3.938319 & 7.546967 & 1.351758\end{array}$

$\begin{array}{llll}5.733089 & 6.207178 & 1.370176\end{array}$

$\begin{array}{lll}6.141220 & 6.344958 & 0.378260\end{array}$

$\begin{array}{llll}4.764199 & 3.153605 & -0.912629\end{array}$

$\begin{array}{llll}6.097237 & 3.584458 & -0.982219\end{array}$

$\begin{array}{llll}6.500642 & 4.139472 & -0.144826\end{array}$

$\begin{array}{llll}6.906047 & 3.300574 & -2.082525\end{array}$

$\begin{array}{llll}7.941558 & 3.625359 & -2.092308\end{array}$

$\begin{array}{llll}6.383616 & 2.577108 & -3.152049\end{array}$

$\begin{array}{llll}7.004180 & 2.337397 & -4.009500\end{array}$

$\begin{array}{llll}5.061585 & 2.133729 & -3.105505\end{array}$

$\begin{array}{llll}4.672410 & 1.556957 & -3.937563\end{array}$

$\begin{array}{llll}4.241814 & 2.397642 & -1.997142\end{array}$

$\begin{array}{llll}5.179636 & 1.672416 & 1.831005\end{array}$

$\begin{array}{lll}6.093035 & 1.961783 & 1.308397\end{array}$

$\begin{array}{lll}5.519323 & 1.464559 & 3.309502\end{array}$

$\begin{array}{lll}6.298090 & 0.706824 & 3.430203\end{array}$

$\begin{array}{llll}4.650462 & 1.149009 & 3.893267\end{array}$

$\begin{array}{llll}5.881608 & 2.407423 & 3.730568\end{array}$

$\begin{array}{llll}2.354585 & 0.721912 & -3.482015\end{array}$

$\begin{array}{llll}2.616517 & -0.657795 & -3.490620\end{array}$

$\begin{array}{llll}2.930372 & -1.152550 & -2.579054\end{array}$

$2.435594 \quad-1.404868 \quad-4.656793$

$\begin{array}{llll}2.642657 & -2.469924 & -4.648966\end{array}$

$\begin{array}{llll}1.979492 & -0.786172 & -5.822572\end{array}$

$\begin{array}{llll}1.824437 & -1.370205 & -6.725201\end{array}$

$\begin{array}{llll}1.719324 & 0.586821 & -5.822690\end{array}$

$\begin{array}{llll}1.363722 & 1.074621 & -6.725818\end{array}$

$\begin{array}{llll}1.908727 & 1.338544 & -4.662248\end{array}$

$\begin{array}{llll}1.692200 & 2.401025 & -4.679566\end{array}$

$\begin{array}{llll}1.403674 & 3.038261 & -2.070464\end{array}$

$\begin{array}{llll}1.740566 & 4.197726 & -2.788121\end{array}$

$\begin{array}{llll}2.733279 & 4.302692 & -3.215703\end{array}$

$\begin{array}{llll}0.806433 & 5.222813 & -2.947686\end{array}$

$\begin{array}{llll}1.077456 & 6.116682 & -3.502401\end{array}$

$\begin{array}{lll}-0.475234 & 5.095222 & -2.402390\end{array}$

$\begin{array}{lll}-1.208089 & 5.886424 & -2.532823\end{array}$

$\begin{array}{llll}-0.814310 & 3.945122 & -1.686884\end{array}$

$\begin{array}{llll}-1.807504 & 3.839160 & -1.267550\end{array}$

$\begin{array}{llll}0.124966 & 2.927427 & -1.509385\end{array}$

$\begin{array}{llll}-0.137133 & 2.044733 & -0.936831\end{array}$

$\begin{array}{llll}5.833959 & -0.351275 & -0.287914\end{array}$

$\begin{array}{llll}5.486730 & -1.402216 & -1.149245\end{array}$

$\begin{array}{llll}4.518961 & -1.879057 & -1.036612\end{array}$

$\begin{array}{llll}6.371749 & -1.829822 & -2.138088\end{array}$

$\begin{array}{llll}7.598333 & -1.194016 & -2.319020\end{array}$

$\begin{array}{llll}8.276006 & -1.510721 & -3.103709\end{array}$

$\begin{array}{llll}7.930664 & -0.131672 & -1.477858\end{array}$

$\begin{array}{llll}7.072194 & 0.277573 & -0.456969\end{array}$

$\begin{array}{llll}7.378560 & 1.096787 & 0.178746\end{array}$

$\begin{array}{lll}4.568512 & -1.271532 & 2.131554\end{array}$

$\begin{array}{llll}3.559400 & -1.295279 & 3.107691\end{array}$

$\begin{array}{llll}2.867533 & -0.465618 & 3.195862\end{array}$

$\begin{array}{llll}3.410364 & -2.402001 & 3.940614\end{array}$

$\begin{array}{lll}4.233620 & -3.519502 & 3.798033\end{array}$

$\begin{array}{llll}4.087689 & -4.396559 & 4.418628\end{array}$

$\begin{array}{lll}5.223132 & -3.501228 & 2.818474\end{array}$

$\begin{array}{llll}5.409545 & -2.382715 & 2.005138\end{array}$

$\begin{array}{lll}6.188513 & -2.406516 & 1.253578\end{array}$

$\begin{array}{llll}2.440262 & 0.234690 & -0.102128\end{array}$

$\begin{array}{llll}6.091968 & -4.714948 & 2.637344\end{array}$

$2.272977 \quad-2.467173 \quad 4.920949$

$\begin{array}{llll}5.997298 & -3.025545 & -2.968974\end{array}$

$\begin{array}{llll}9.225197 & 0.593296 & -1.725254\end{array}$

$\begin{array}{llll}9.228112 & 1.197847 & -2.940138\end{array}$

$\begin{array}{llll}9.467084 & 1.553276 & -0.805821\end{array}$

$10.282692-0.250934-1.716835$

$\begin{array}{llll}6.178913 & -4.181816 & -2.282610\end{array}$

$\begin{array}{llll}6.720948 & -3.117746 & -4.102279\end{array}$

$\begin{array}{llll}4.686982 & -3.002111 & -3.326778\end{array}$

$\begin{array}{lll}7.068672 & -4.789661 & 3.569701\end{array}$ $\begin{array}{lll}6.695653 & -4.731928 & 1.427235\end{array}$

$\begin{array}{llll}5.369585 & -5.858208 & 2.739804\end{array}$

$\begin{array}{llll}2.644715 & -3.031788 & 6.090469\end{array}$

$\begin{array}{llll}1.757320 & -1.251675 & 5.200183\end{array}$

$\begin{array}{lll}1.250667 & -3.227252 & 4.437638\end{array}$

$\begin{array}{lll}1.975952 & -2.751452 & 0.403946\end{array}$

$\begin{array}{lll}0.812400 & -2.465443 & 1.213598\end{array}$

$\begin{array}{llll}0.453834 & -1.190537 & 1.765432\end{array}$

$\begin{array}{llll}0.409642 & -3.292766 & 1.779613\end{array}$

$\begin{array}{llll}-3.400009 & -1.296237 & -1.447248\end{array}$

$\begin{array}{lll}-5.266971 & -0.538242 & -0.378163\end{array}$

$\begin{array}{llll}-5.260448 & 0.604596 & 0.820257\end{array}$

$\begin{array}{llll}-6.329965 & 0.174236 & -1.442355\end{array}$

$\begin{array}{lll}-5.966557 & -1.798992 & 0.479062\end{array}$

$\begin{array}{lll}-4.982038 & 1.930132 & 0.525204\end{array}$

$\begin{array}{lll}-3.757879 & 2.439086 & 1.010715\end{array}$

$\begin{array}{lll}-3.119039 & 1.781948 & 1.589802\end{array}$

$\begin{array}{lll}-3.415968 & 3.740382 & 0.740444\end{array}$

$\begin{array}{lll}-2.486829 & 4.152912 & 1.120531\end{array}$

$\begin{array}{lll}-4.248641 & 4.546698 & -0.083506\end{array}$

$\begin{array}{lll}-3.843322 & 5.851285 & -0.476585\end{array}$

$\begin{array}{llll}-2.895161 & 6.232471 & -0.106340\end{array}$

$\begin{array}{llll}-4.622747 & 6.608592 & -1.322137\end{array}$

$\begin{array}{llll}-4.300123 & 7.600961 & -1.623265\end{array}$

$\begin{array}{llll}-5.844796 & 6.086438 & -1.809607\end{array}$

$\begin{array}{lll}-6.447780 & 6.678413 & -2.492020\end{array}$

$\begin{array}{llll}-6.275751 & 4.832946 & -1.431504\end{array}$

$\begin{array}{llll}-7.211131 & 4.445920 & -1.818934\end{array}$

$\begin{array}{llll}-5.501694 & 4.025561 & -0.552536\end{array}$

$\begin{array}{llll}-5.909851 & 2.702003 & -0.155933\end{array}$

$\begin{array}{llll}-7.269077 & 2.156958 & -0.436386\end{array}$

$\begin{array}{lll}-8.460069 & 2.845553 & -0.002096\end{array}$

$\begin{array}{lll}-8.420909 & 4.023078 & 0.796952\end{array}$

$\begin{array}{lll}-7.464267 & 4.422194 & 1.110909\end{array}$

$\begin{array}{llll}-9.579480 & 4.655805 & 1.192924\end{array}$

$\begin{array}{lll}-9.518993 & 5.549123 & 1.807999\end{array}$

$\begin{array}{lll}-10.845791 & 4.150719 & 0.814415\end{array}$

$\begin{array}{lll}-11.749917 & 4.664799 & 1.127112\end{array}$

$\begin{array}{lll}-10.923618 & 2.997881 & 0.066659\end{array}$

$\begin{array}{llll}-11.888594 & 2.583684 & -0.213618\end{array}$

$\begin{array}{llll}-9.748605 & 2.311597 & -0.344405\end{array}$

$\begin{array}{llll}-9.832502 & 1.090726 & -1.066605\end{array}$

$\begin{array}{lll}-10.810992 & 0.699114 & -1.329809\end{array}$

$\begin{array}{llll}-8.695311 & 0.398939 & -1.398916\end{array}$

$\begin{array}{lll}-8.734140 & -0.551364 & -1.920850\end{array}$

$\begin{array}{llll}-7.430740 & 0.928318 & -1.060274\end{array}$

$\begin{array}{lll}-5.328412 & -3.110863 & 0.180610\end{array}$

$\begin{array}{lll}-4.591513 & -3.316370 & 0.966663\end{array}$

$\begin{array}{lll}-6.342659 & -4.240772 & 0.174656\end{array}$

$\begin{array}{lll}-6.027050 & -5.466217 & 0.772924\end{array}$

$\begin{array}{lll}-5.072623 & -5.581360 & 1.281909\end{array}$

$\begin{array}{lll}-6.925690 & -6.535493 & 0.722475\end{array}$

$\begin{array}{lll}-6.668118 & -7.480460 & 1.192984\end{array}$

$\begin{array}{lll}-8.155629 & -6.385259 & 0.078553\end{array}$

$\begin{array}{lll}-8.858864 & -7.212580 & 0.044377\end{array}$

$-8.480226-5.161938-0.517642$

$\begin{array}{llll}-9.437619 & -5.037314 & -1.016397\end{array}$

$\begin{array}{llll}-7.579033 & -4.098432 & -0.471929\end{array}$

$\begin{array}{lll}-7.831520 & -3.144146 & -0.926070\end{array}$

$-4.594888-3.051708-1.163903$

$-5.333929-3.112918-1.971734$

$\begin{array}{llll}-3.953644 & -3.933825 & -1.249886\end{array}$

$\begin{array}{lll}-6.667788 & -1.732671 & 1.807657\end{array}$

$\begin{array}{lll}-7.141483 & -2.714928 & 1.886308\end{array}$

$\begin{array}{lll}-5.678407 & -1.640734 & 2.970613\end{array}$

$\begin{array}{lll}-5.126600 & -2.826389 & 3.482352\end{array}$

$\begin{array}{llll}-5.453186 & -3.781471 & 3.079584\end{array}$

$-4.182396 \quad-2.800558 \quad 4.512162$

$\begin{array}{lll}-3.769414 & -3.732016 & 4.889873\end{array}$

$\begin{array}{lll}-3.790468 & -1.581233 & 5.068281\end{array}$

$\begin{array}{lll}-3.064249 & -1.555277 & 5.876151\end{array}$

$\begin{array}{lll}-4.357374 & -0.395252 & 4.591408\end{array}$

$\begin{array}{lll}-4.076390 & 0.557758 & 5.032240\end{array}$

$\begin{array}{lll}-5.290731 & -0.424814 & 3.553478\end{array}$

$\begin{array}{lll}-5.716340 & 0.505557 & 3.199340\end{array}$ 
$\begin{array}{lll}-7.806846 & -0.715829 & 1.852518\end{array}$

$\begin{array}{lll}-8.514429 & -0.911636 & 1.043703\end{array}$

$\begin{array}{lll}-7.468526 & 0.315847 & 1.765585\end{array}$

$\begin{array}{lll}-8.335025 & -0.822828 & 2.804969\end{array}$

$\begin{array}{lll}-2.525439 & -1.128634 & 0.544690\end{array}$

$\begin{array}{lll}-1.882714 & -0.253873 & 0.590703\end{array}$

$\begin{array}{lll}-3.221340 & -1.184006 & 1.377135\end{array}$

$\begin{array}{lll}-1.992510 & -2.367617 & 0.062283\end{array}$

$\begin{array}{llll}-2.496805 & -3.272441 & 0.384961\end{array}$

$\begin{array}{lll}-0.734538 & -2.534820 & -0.568931\end{array}$

$\begin{array}{llll}-0.208947 & -1.652852 & -0.907342\end{array}$

$\begin{array}{llll}-4.222924 & -1.143779 & -3.493271\end{array}$

$\begin{array}{lll}-5.215102 & -1.589422 & -3.482190\end{array}$

$\begin{array}{lll}-3.126400 & -2.048590 & -3.522007\end{array}$

$-3.365197 \quad-3.111740 \quad-3.551147$

$-1.761815-1.710448-4.097717$

$\begin{array}{llll}-1.805506 & -1.728746 & -5.197857\end{array}$

$\begin{array}{lll}-1.073320 & -2.507840 & -3.807636\end{array}$

$\begin{array}{lll}-1.196466 & -0.358722 & -3.615832\end{array}$

$\begin{array}{llll}-1.429241 & 0.434607 & -4.332774\end{array}$

$\begin{array}{llll}-0.107165 & -0.422381 & -3.582590\end{array}$

$\begin{array}{llll}-1.691355 & 0.068033 & -2.237647\end{array}$

$\begin{array}{llll}-0.934579 & 0.084562 & -1.459749\end{array}$

$\begin{array}{llll}-2.806359 & 0.873840 & -2.022092\end{array}$

$\begin{array}{llll}-2.816297 & 1.461161 & -1.109643\end{array}$

$\begin{array}{lll}-3.752818 & 1.359205 & -3.101271\end{array}$

$\begin{array}{llll}-4.645077 & 1.750202 & -2.607398\end{array}$

$\begin{array}{lll}-3.289116 & 2.204957 & -3.630486\end{array}$

$\begin{array}{llll}-4.170556 & 0.260486 & -4.102684\end{array}$

$\begin{array}{llll}-3.492408 & 0.252506 & -4.962423\end{array}$

$\begin{array}{lll}-5.160435 & 0.506748 & -4.499058\end{array}$

$\begin{array}{llll}-0.336371 & -3.784990 & -1.225432\end{array}$

$\begin{array}{lll}-0.870682 & -5.040208 & -0.873617\end{array}$

$\begin{array}{llll}0.627245 & -3.732103 & -2.250945\end{array}$

$-0.456607-6.196858-1.530993$

$\begin{array}{llll}-1.601088 & -5.116676 & -0.074483\end{array}$

$\begin{array}{llll}1.036983 & -4.889182 & -2.910312\end{array}$

$\begin{array}{lll}1.058683 & -2.774393 & -2.516246\end{array}$

$\begin{array}{llll}0.498229 & -6.127535 & -2.552357\end{array}$

$\begin{array}{lll}-0.876522 & -7.156938 & -1.244660\end{array}$

$\begin{array}{llll}1.783099 & -4.824352 & -3.697409\end{array}$

$\begin{array}{llll}0.818220 & -7.031803 & -3.061861\end{array}$

$\begin{array}{llll}2.527421 & -3.998390 & 0.475299\end{array}$

$\begin{array}{llll}3.530450 & -4.417712 & -0.497396\end{array}$

$\begin{array}{llll}3.343619 & -5.458845 & -0.776760\end{array}$

$\begin{array}{llll}3.460556 & -3.806016 & -1.391350\end{array}$

$\begin{array}{llll}4.542772 & -4.343765 & -0.084242\end{array}$

$\begin{array}{llll}2.172795 & -5.005027 & 1.472545\end{array}$

$\begin{array}{llll}1.857213 & -4.544970 & 2.407596\end{array}$

$\begin{array}{llll}1.379617 & -5.669637 & 1.107867\end{array}$

$\begin{array}{llll}3.059683 & -5.605204 & 1.688178\end{array}$

$\begin{array}{llll}2.451500 & -1.919439 & -0.431579\end{array}$

$-0.453607 \quad-1.174195 \quad 2.861429$

$\begin{array}{lll}-0.885472 & 0.012028 & 3.418647\end{array}$

$\begin{array}{lll}-0.794812 & -2.114593 & 3.276901\end{array}$

$\begin{array}{lll}-0.417868 & 1.201241 & 2.853894\end{array}$

$\begin{array}{llll}0.455847 & 1.160210 & 1.781957\end{array}$

$\begin{array}{lll}0.835412 & 2.067759 & 1.329591\end{array}$

$\begin{array}{lll}0.890303 & 0.000774 & 1.248306\end{array}$

$\begin{array}{lll}-1.574769 & 0.012851 & 4.253900\end{array}$

$-0.962496 \quad 2.927716 \quad 3.596420$

\section{Conformer_17}

Number of imaginary frequencies : 1

The smallest frequency is : $-247.6882 \mathrm{~cm}(-1)$

Electronic energy :

$\mathrm{HF}=-7222.6348457$

Zero-point correction=

1.755694

(Hartree/Particle)

Thermal correction to Energy=

1.874111

Thermal correction to Enthalpy=

1.875055

Thermal correction to Gibbs Free Energy=

Sum of electronic and zero-point Energies=

Sum of electronic and thermal Energies=

Sum of electronic and thermal Enthalpies=
Sum of electronic and thermal Free Energies=

$-7221.041786$

Cartesian Coordinates

$\begin{array}{lll}4.295363 & 4.787509 & 1.872529\end{array}$

$\begin{array}{llll}2.562566 & 1.624211 & -1.886802\end{array}$

$\begin{array}{lll}4.540844 & 0.128272 & 0.932918\end{array}$

$\begin{array}{llll}3.967277 & 3.424873 & 0.318246\end{array}$

$\begin{array}{llll}4.154439 & 2.746190 & 1.579638\end{array}$

$\begin{array}{lll}3.132360 & 3.194559 & 2.472537\end{array}$

$\begin{array}{lll}3.009963 & 2.884745 & 3.501225\end{array}$

$\begin{array}{llll}2.334945 & 4.162879 & 1.791151\end{array}$

$\begin{array}{lll}1.508908 & 4.716126 & 2.215959\end{array}$

$\begin{array}{llll}2.848700 & 4.307360 & 0.469410\end{array}$

$\begin{array}{llll}2.478634 & 4.983279 & -0.286453\end{array}$

$\begin{array}{lll}6.237702 & 5.326212 & 2.374939\end{array}$

$\begin{array}{llll}7.098804 & 4.677450 & 2.282395\end{array}$

$\begin{array}{lll}5.382553 & 5.426650 & 3.515046\end{array}$

$\begin{array}{llll}5.479391 & 4.865296 & 4.434794\end{array}$

$\begin{array}{llll}4.350365 & 6.366020 & 3.211949\end{array}$

$\begin{array}{lll}3.525229 & 6.633586 & 3.858749\end{array}$

$\begin{array}{llll}4.567298 & 6.847376 & 1.884812\end{array}$

$\begin{array}{llll}3.938372 & 7.547149 & 1.350421\end{array}$

$\begin{array}{llll}5.733162 & 6.207387 & 1.368755\end{array}$

$\begin{array}{lll}6.141167 & 6.345081 & 0.376776\end{array}$

$\begin{array}{llll}4.764047 & 3.153548 & -0.913631\end{array}$

$\begin{array}{llll}6.097061 & 3.584434 & -0.983459\end{array}$

$\begin{array}{llll}6.500554 & 4.139582 & -0.146196\end{array}$

$\begin{array}{llll}6.905732 & 3.300421 & -2.083835\end{array}$

$\begin{array}{llll}7.941230 & 3.625242 & -2.093806\end{array}$

$\begin{array}{llll}6.383184 & 2.576774 & -3.153179\end{array}$

$\begin{array}{llll}7.003645 & 2.336950 & -4.010674\end{array}$

$\begin{array}{llll}5.061171 & 2.133366 & -3.106400\end{array}$

$\begin{array}{llll}4.671905 & 1.556454 & -3.938319\end{array}$

$\begin{array}{llll}4.241535 & 2.397427 & -1.997973\end{array}$

$\begin{array}{lll}5.179823 & 1.672655 & 1.830135\end{array}$

$\begin{array}{llll}6.093173 & 1.961984 & 1.307418\end{array}$

$\begin{array}{lll}5.519655 & 1.464959 & 3.308622\end{array}$

$\begin{array}{lll}6.298443 & 0.707247 & 3.429328\end{array}$

$\begin{array}{llll}4.650856 & 1.149462 & 3.892510\end{array}$

$\begin{array}{lll}5.881973 & 2.407873 & 3.729549\end{array}$

$\begin{array}{llll}2.354118 & 0.721534 & -3.482409\end{array}$

$\begin{array}{llll}2.616006 & -0.658182 & -3.490895\end{array}$

$\begin{array}{llll}2.929955 & -1.152845 & -2.579312\end{array}$

$\begin{array}{llll}2.434895 & -1.405380 & -4.656958\end{array}$

$\begin{array}{llll}2.641918 & -2.470444 & -4.649040\end{array}$

$\begin{array}{llll}1.978647 & -0.786802 & -5.822742\end{array}$

$\begin{array}{llll}1.823439 & -1.370933 & -6.725281\end{array}$

$\begin{array}{llll}1.718524 & 0.586201 & -5.822978\end{array}$

$\begin{array}{llll}1.362807 & 1.073908 & -6.726111\end{array}$

$\begin{array}{llll}1.908114 & 1.338048 & -4.662649\end{array}$

$\begin{array}{llll}1.691601 & 2.400529 & -4.680051\end{array}$

$\begin{array}{llll}1.403351 & 3.037998 & -2.070951\end{array}$

$\begin{array}{llll}1.740078 & 4.197386 & -2.788811\end{array}$

$\begin{array}{llll}2.732714 & 4.302333 & -3.216577\end{array}$

$\begin{array}{llll}0.805883 & 5.222423 & -2.948333\end{array}$

$\begin{array}{llll}1.076778 & 6.116234 & -3.503204\end{array}$

$\begin{array}{llll}-0.475684 & 5.094857 & -2.402793\end{array}$

$\begin{array}{llll}-1.208588 & 5.886018 & -2.533194\end{array}$

$\begin{array}{llll}-0.814594 & 3.944836 & -1.687082\end{array}$

$\begin{array}{llll}-1.807711 & 3.838884 & -1.267560\end{array}$

$\begin{array}{llll}0.124749 & 2.927194 & -1.509627\end{array}$

$\begin{array}{lll}-0.137217 & 2.044562 & -0.936917\end{array}$

$\begin{array}{llll}5.833924 & -0.351243 & -0.288692\end{array}$

$\begin{array}{llll}5.486577 & -1.402266 & -1.149874\end{array}$

$\begin{array}{llll}4.518822 & -1.879093 & -1.037060\end{array}$

$\begin{array}{llll}6.371459 & -1.829967 & -2.138799\end{array}$

$\begin{array}{llll}7.598016 & -1.194175 & -2.319962\end{array}$

$\begin{array}{llll}8.275581 & -1.510952 & -3.104715\end{array}$

$\begin{array}{llll}7.930454 & -0.131738 & -1.478959\end{array}$

$\begin{array}{llll}7.072129 & 0.277598 & -0.457985\end{array}$

$\begin{array}{llll}7.378576 & 1.096880 & 0.177606\end{array}$

$\begin{array}{lll}4.568841 & -1.271296 & 2.131032\end{array}$

$\begin{array}{llll}3.559856 & -1.294998 & 3.107301\end{array}$

$\begin{array}{lll}2.867977 & -0.465353 & 3.195505\end{array}$ 
$\begin{array}{lll}3.410950 & -2.401666 & 3.940320\end{array}$

$\begin{array}{lll}4.234205 & -3.519163 & 3.797707\end{array}$

$\begin{array}{llll}4.088368 & -4.396179 & 4.418381\end{array}$

$\begin{array}{llll}5.223600 & -3.500930 & 2.818030\end{array}$

$\begin{array}{llll}5.409888 & -2.382467 & 2.004597\end{array}$

$\begin{array}{llll}6.188771 & -2.406299 & 1.252949\end{array}$

$\begin{array}{llll}2.440246 & 0.234668 & -0.102474\end{array}$

$\begin{array}{llll}6.092447 & -4.714636 & 2.636869\end{array}$

$\begin{array}{llll}2.273708 & -2.466785 & 4.920831\end{array}$

$\begin{array}{llll}5.996883 & -3.025760 & -2.969529\end{array}$

$\begin{array}{llll}9.224937 & 0.593228 & -1.726626\end{array}$

$\begin{array}{llll}9.227710 & 1.197532 & -2.941633\end{array}$

$\begin{array}{llll}9.466876 & 1.553404 & -0.807411\end{array}$

$10.282467-0.250958-1.718138$

$\begin{array}{llll}6.178593 & -4.181976 & -2.283096\end{array}$

$\begin{array}{llll}6.720365 & -3.118057 & -4.102934\end{array}$

$\begin{array}{llll}4.686518 & -3.002348 & -3.327145\end{array}$

$\begin{array}{lll}7.069249 & -4.789279 & 3.569129\end{array}$

$\begin{array}{llll}6.696006 & -4.731659 & 1.426699\end{array}$

$\begin{array}{llll}5.370105 & -5.857911 & 2.739460\end{array}$

$\begin{array}{llll}2.645634 & -3.031313 & 6.090332\end{array}$

$\begin{array}{llll}1.758073 & -1.251277 & 5.200061\end{array}$

$\begin{array}{llll}1.251338 & -3.226915 & 4.437727\end{array}$

$\begin{array}{llll}1.976024 & -2.751473 & 0.403897\end{array}$

$\begin{array}{llll}0.812572 & -2.465427 & 1.213684\end{array}$

$\begin{array}{llll}0.454101 & -1.190479 & 1.765486\end{array}$

$\begin{array}{llll}0.409904 & -3.292713 & 1.779821\end{array}$

$\begin{array}{llll}-3.400191 & -1.296293 & -1.446609\end{array}$

$\begin{array}{lll}-5.267019 & -0.538246 & -0.377325\end{array}$

$\begin{array}{llll}-5.260369 & 0.604663 & 0.821026\end{array}$

$\begin{array}{llll}-6.330157 & 0.174140 & -1.441436\end{array}$

$\begin{array}{lll}-5.966491 & -1.798959 & 0.480047\end{array}$

$\begin{array}{llll}-4.982011 & 1.930187 & 0.525853\end{array}$

$\begin{array}{lll}-3.757809 & 2.439194 & 1.011200\end{array}$

$\begin{array}{lll}-3.118919 & 1.782121 & 1.590304\end{array}$

$\begin{array}{llll}-3.415920 & 3.740461 & 0.740758\end{array}$

$\begin{array}{lll}-2.486739 & 4.153026 & 1.120705\end{array}$

$\begin{array}{llll}-4.248673 & 4.546693 & -0.083194\end{array}$

$\begin{array}{llll}-3.843387 & 5.851233 & -0.476458\end{array}$

$\begin{array}{llll}-2.895181 & 6.232451 & -0.106363\end{array}$

$\begin{array}{llll}-4.622902 & 6.608456 & -1.322004\end{array}$

$\begin{array}{llll}-4.300302 & 7.600789 & -1.623278\end{array}$

$\begin{array}{llll}-5.845011 & 6.086261 & -1.809278\end{array}$

$\begin{array}{llll}-6.448069 & 6.678168 & -2.491686\end{array}$

$\begin{array}{llll}-6.275933 & 4.832813 & -1.430993\end{array}$

$\begin{array}{lll}-7.211360 & 4.445752 & -1.818278\end{array}$

$\begin{array}{llll}-5.501781 & 4.025515 & -0.552029\end{array}$

$\begin{array}{llll}-5.909899 & 2.701996 & -0.155251\end{array}$

$\begin{array}{llll}-7.269158 & 2.156926 & -0.435499\end{array}$

$\begin{array}{lll}-8.460097 & 2.845553 & -0.001116\end{array}$

$\begin{array}{lll}-8.420843 & 4.023152 & 0.797815\end{array}$

$\begin{array}{lll}-7.464163 & 4.422304 & 1.111612\end{array}$

$\begin{array}{lll}-9.579367 & 4.655908 & 1.193877\end{array}$

$\begin{array}{lll}-9.518807 & 5.549286 & 1.808859\end{array}$

$\begin{array}{lll}-10.845723 & 4.150777 & 0.815582\end{array}$

$\begin{array}{lll}-11.749813 & 4.664877 & 1.128349\end{array}$

$\begin{array}{lll}-10.923639 & 2.997868 & 0.067944\end{array}$

$\begin{array}{llll}-11.888647 & 2.583635 & -0.212169\end{array}$

$\begin{array}{llll}-9.748674 & 2.311556 & -0.343212\end{array}$

$\begin{array}{lll}-9.832655 & 1.090623 & -1.065296\end{array}$

$\begin{array}{llll}-10.811176 & 0.698982 & -1.328340\end{array}$

$\begin{array}{llll}-8.695502 & 0.398817 & -1.397697\end{array}$

$\begin{array}{lll}-8.734393 & -0.551530 & -1.919546\end{array}$

$\begin{array}{llll}-7.430891 & 0.928236 & -1.059267\end{array}$

$\begin{array}{lll}-5.328414 & -3.110849 & 0.181524\end{array}$

$\begin{array}{lll}-4.591395 & -3.316363 & 0.967464\end{array}$

$\begin{array}{lll}-6.342687 & -4.240734 & 0.175750\end{array}$

$\begin{array}{lll}-6.027028 & -5.466161 & 0.774028\end{array}$

$\begin{array}{llll}-5.072534 & -5.581306 & 1.282886\end{array}$

$\begin{array}{lll}-6.925698 & -6.535420 & 0.723739\end{array}$

$\begin{array}{lll}-6.668086 & -7.480373 & 1.194252\end{array}$

$\begin{array}{llll}-8.155717 & -6.385185 & 0.079969\end{array}$

$\begin{array}{lll}-8.858973 & -7.212494 & 0.045915\end{array}$

$\begin{array}{llll}-8.480366 & -5.161880 & -0.516230\end{array}$

$\begin{array}{llll}-9.437822 & -5.037255 & -1.014864\end{array}$ $\begin{array}{lll}-7.579145 & -4.098391 & -0.470674\end{array}$

$\begin{array}{llll}-7.831674 & -3.144116 & -0.924815\end{array}$

$\begin{array}{lll}-4.595090 & -3.051726 & -1.163102\end{array}$

$\begin{array}{llll}-5.334249 & -3.112922 & -1.970825\end{array}$

$\begin{array}{lll}-3.953886 & -3.933864 & -1.249168\end{array}$

$\begin{array}{lll}-6.667528 & -1.732592 & 1.808742\end{array}$

$\begin{array}{lll}-7.141236 & -2.714836 & 1.887487\end{array}$

$\begin{array}{lll}-5.677965 & -1.640654 & 2.971541\end{array}$

$\begin{array}{lll}-5.126125 & -2.826315 & 3.483232\end{array}$

$\begin{array}{lll}-5.452820 & -3.781399 & 3.080556\end{array}$

$\begin{array}{lll}-4.181740 & -2.800484 & 4.512878\end{array}$

$\begin{array}{llll}-3.768737 & -3.731946 & 4.890556\end{array}$

$\begin{array}{lll}-3.789651 & -1.581153 & 5.068872\end{array}$

$\begin{array}{llll}-3.063285 & -1.555198 & 5.876609\end{array}$

$\begin{array}{lll}-4.356587 & -0.395166 & 4.592051\end{array}$

$\begin{array}{lll}-4.075475 & 0.557851 & 5.032787\end{array}$

$\begin{array}{lll}-5.290130 & -0.424728 & 3.554287\end{array}$

$\begin{array}{lll}-5.715757 & 0.505646 & 3.200183\end{array}$

$\begin{array}{lll}-7.806557 & -0.715723 & 1.853746\end{array}$

$\begin{array}{lll}-8.514263 & -0.911532 & 1.045039\end{array}$

$\begin{array}{lll}-7.468229 & 0.315943 & 1.766740\end{array}$

$\begin{array}{lll}-8.334600 & -0.822691 & 2.806277\end{array}$

$\begin{array}{lll}-2.525373 & -1.128708 & 0.545200\end{array}$

$\begin{array}{lll}-1.882607 & -0.253972 & 0.591132\end{array}$

$\begin{array}{llll}-3.221178 & -1.184036 & 1.377727\end{array}$

$\begin{array}{lll}-1.992522 & -2.367724 & 0.062771\end{array}$

$\begin{array}{lll}-2.496812 & -3.272525 & 0.385527\end{array}$

$\begin{array}{llll}-0.734626 & -2.534965 & -0.568588\end{array}$

$-0.209091-1.653018-0.907140$

$\begin{array}{llll}-4.223337 & -1.143861 & -3.492563\end{array}$

$\begin{array}{llll}-5.215525 & -1.589478 & -3.481351\end{array}$

$\begin{array}{lll}-3.126837 & -2.048690 & -3.521402\end{array}$

$\begin{array}{llll}-3.365649 & -3.111838 & -3.550484\end{array}$

$\begin{array}{llll}-1.762304 & -1.710572 & -4.097252\end{array}$

$-1.806113-1.728855-5.197388$

$\begin{array}{lll}-1.073796 & -2.507986 & -3.807257\end{array}$

$\begin{array}{llll}-1.196877 & -0.358865 & -3.615406\end{array}$

$\begin{array}{llll}-1.429672 & 0.434477 & -4.332329\end{array}$

$-0.107578 \quad-0.422558 \quad-3.582231$

$\begin{array}{llll}-1.691652 & 0.067903 & -2.237182\end{array}$

$\begin{array}{llll}-0.934801 & 0.084436 & -1.459359\end{array}$

$\begin{array}{llll}-2.806610 & 0.873755 & -2.021545\end{array}$

$\begin{array}{llll}-2.816443 & 1.461109 & -1.109117\end{array}$

$\begin{array}{llll}-3.753155 & 1.359121 & -3.100647\end{array}$

$\begin{array}{llll}-4.645364 & 1.750139 & -2.606702\end{array}$

$\begin{array}{lll}-3.289484 & 2.204855 & -3.629915\end{array}$

$\begin{array}{llll}-4.170995 & 0.260391 & -4.102006\end{array}$

$\begin{array}{llll}-3.492912 & 0.252381 & -4.961796\end{array}$

$\begin{array}{llll}-5.160900 & 0.506664 & -4.498308\end{array}$

$\begin{array}{llll}-0.336546 & -3.785178 & -1.225063\end{array}$

$\begin{array}{lll}-0.870804 & -5.040375 & -0.873092\end{array}$

$\begin{array}{llll}0.626933 & -3.732360 & -2.250709\end{array}$

$\begin{array}{lll}-0.456802 & -6.197072 & -1.530435\end{array}$

$\begin{array}{llll}-1.601110 & -5.116793 & -0.073861\end{array}$

$\begin{array}{llll}1.036602 & -4.889485 & -2.910038\end{array}$

$\begin{array}{llll}1.058325 & -2.774667 & -2.516143\end{array}$

$\begin{array}{llll}0.497909 & -6.127816 & -2.551919\end{array}$

$\begin{array}{llll}-0.876674 & -7.157134 & -1.243979\end{array}$

$\begin{array}{llll}1.782616 & -4.824707 & -3.697236\end{array}$

$\begin{array}{llll}0.817846 & -7.032120 & -3.061394\end{array}$

$\begin{array}{llll}2.527533 & -3.998391 & 0.475266\end{array}$

$\begin{array}{llll}3.530451 & -4.417738 & -0.497534\end{array}$

$\begin{array}{llll}3.343664 & -5.458915 & -0.776758\end{array}$

$\begin{array}{llll}3.460372 & -3.806139 & -1.391539\end{array}$

$\begin{array}{llll}4.542830 & -4.343664 & -0.084543\end{array}$

$\begin{array}{lll}2.173098 & -5.004957 & 1.472651\end{array}$

$\begin{array}{llll}1.857606 & -4.544830 & 2.407698\end{array}$

$\begin{array}{llll}1.379914 & -5.669649 & 1.108137\end{array}$

$\begin{array}{llll}3.060051 & -5.605057 & 1.688231\end{array}$

$\begin{array}{lll}2.451436 & -1.919511 & -0.431754\end{array}$

$\begin{array}{lll}-0.453164 & -1.174047 & 2.861627\end{array}$

$\begin{array}{lll}-0.884938 & 0.012220 & 3.418824\end{array}$

$\begin{array}{lll}-0.794302 & -2.114410 & 3.277230\end{array}$

$\begin{array}{lll}-0.417429 & 1.201386 & 2.853893\end{array}$

$\begin{array}{lll}0.456109 & 1.160265 & 1.781816\end{array}$ 
$\begin{array}{lll}-11.888662 & 2.583690 & -0.213095\end{array}$

$\begin{array}{llll}-9.748682 & 2.311580 & -0.343976\end{array}$

$\begin{array}{llll}-9.832628 & 1.090638 & -1.066049\end{array}$

$\begin{array}{llll}-10.811135 & 0.699005 & -1.329157\end{array}$

$\begin{array}{llll}-8.695459 & 0.398813 & -1.398357\end{array}$

$\begin{array}{llll}-8.734324 & -0.551542 & -1.920194\end{array}$

$\begin{array}{llll}-7.430865 & 0.928219 & -1.059844\end{array}$

$\begin{array}{lll}-5.328343 & -3.110853 & 0.181213\end{array}$

$\begin{array}{lll}-4.591391 & -3.316276 & 0.967238\end{array}$

$\begin{array}{lll}-6.342543 & -4.240805 & 0.175401\end{array}$

$\begin{array}{lll}-6.026838 & -5.466197 & 0.773726\end{array}$

$\begin{array}{lll}-5.072372 & -5.581265 & 1.282654\end{array}$

$\begin{array}{lll}-6.925431 & -6.535519 & 0.723402\end{array}$

$\begin{array}{llll}-6.667785 & -7.480444 & 1.193953\end{array}$

$\begin{array}{lll}-8.155419 & -6.385383 & 0.079549\end{array}$

$\begin{array}{lll}-8.858616 & -7.212739 & 0.045469\end{array}$

$\begin{array}{llll}-8.480112 & -5.162114 & -0.516700\end{array}$

$\begin{array}{lll}-9.437543 & -5.037565 & -1.015401\end{array}$

$\begin{array}{llll}-7.578966 & -4.098562 & -0.471111\end{array}$

$\begin{array}{llll}-7.831528 & -3.144317 & -0.925295\end{array}$

$-4.594898-3.051766-1.163347$

$\begin{array}{llll}-5.333981 & -3.113063 & -1.971132\end{array}$

$\begin{array}{llll}-3.953623 & -3.933863 & -1.249304\end{array}$

$\begin{array}{lll}-6.667677 & -1.732592 & 1.808240\end{array}$

$\begin{array}{lll}-7.141335 & -2.714860 & 1.886995\end{array}$

$\begin{array}{lll}-5.678221 & -1.640536 & 2.971123\end{array}$

$\begin{array}{lll}-5.126341 & -2.826137 & 3.482909\end{array}$

$\begin{array}{lll}-5.452933 & -3.781258 & 3.080239\end{array}$

$\begin{array}{llll}-4.182054 & -2.800201 & 4.512641\end{array}$

$\begin{array}{llll}-3.769017 & -3.731619 & 4.890391\end{array}$

$\begin{array}{lll}-3.790111 & -1.580822 & 5.068632\end{array}$

$\begin{array}{lll}-3.063825 & -1.554784 & 5.876438\end{array}$

$\begin{array}{lll}-4.357092 & -0.394894 & 4.591717\end{array}$

$\begin{array}{llll}-4.076097 & 0.558158 & 5.032451\end{array}$

$\begin{array}{lll}-5.290535 & -0.424561 & 3.553867\end{array}$

$\begin{array}{lll}-5.716200 & 0.505770 & 3.199695\end{array}$

$\begin{array}{lll}-7.806766 & -0.715784 & 1.853091\end{array}$

$\begin{array}{lll}-8.514389 & -0.911675 & 1.044331\end{array}$

$\begin{array}{lll}-7.468485 & 0.315897 & 1.766061\end{array}$

$\begin{array}{lll}-8.334888 & -0.822730 & 2.805580\end{array}$

$\begin{array}{llll}-2.525395 & -1.128381 & 0.544984\end{array}$

$\begin{array}{llll}-1.882641 & -0.253631 & 0.590820\end{array}$

$\begin{array}{lll}-3.221239 & -1.183605 & 1.377483\end{array}$

$\begin{array}{llll}-1.992547 & -2.367447 & 0.062713\end{array}$

$\begin{array}{lll}-2.496855 & -3.272204 & 0.385561\end{array}$

$\begin{array}{lll}-0.734632 & -2.534775 & -0.568587\end{array}$

$\begin{array}{llll}-0.209042 & -1.652862 & -0.907147\end{array}$

$\begin{array}{llll}-4.223137 & -1.143990 & -3.492859\end{array}$

$\begin{array}{llll}-5.215299 & -1.589662 & -3.481680\end{array}$

$\begin{array}{llll}-3.126583 & -2.048766 & -3.521601\end{array}$

$\begin{array}{llll}-3.365340 & -3.111927 & -3.550642\end{array}$

$\begin{array}{llll}-1.762051 & -1.710616 & -4.097432\end{array}$

$\begin{array}{lll}-1.805824 & -1.728980 & -5.197568\end{array}$

$\begin{array}{llll}-1.073509 & -2.507971 & -3.807358\end{array}$

$\begin{array}{lll}-1.196716 & -0.358846 & -3.615662\end{array}$

$\begin{array}{llll}-1.429548 & 0.434435 & -4.332639\end{array}$

$\begin{array}{lll}-0.107413 & -0.422470 & -3.582465\end{array}$

$\begin{array}{lll}-1.691548 & 0.067972 & -2.237476\end{array}$

$\begin{array}{llll}-0.934723 & 0.084576 & -1.459628\end{array}$

$\begin{array}{llll}-2.806556 & 0.873778 & -2.021917\end{array}$

$\begin{array}{llll}-2.816451 & 1.461173 & -1.109515\end{array}$

$\begin{array}{llll}-3.753084 & 1.359040 & -3.101082\end{array}$

$\begin{array}{llll}-4.645324 & 1.750057 & -2.607191\end{array}$

$\begin{array}{llll}-3.289429 & 2.204759 & -3.630390\end{array}$

$\begin{array}{lll}-4.170852 & 0.260230 & -4.102383\end{array}$

$\begin{array}{llll}-3.492758 & 0.252205 & -4.962165\end{array}$

$\begin{array}{llll}-5.160764 & 0.506430 & -4.498715\end{array}$

$\begin{array}{llll}-0.336594 & -3.785028 & -1.225012\end{array}$

$\begin{array}{llll}-0.870983 & -5.040178 & -0.873074\end{array}$

$\begin{array}{llll}0.626961 & -3.732295 & -2.250590\end{array}$

$-0.457036-6.196912-1.530386$

$\begin{array}{llll}-1.601355 & -5.116531 & -0.073897\end{array}$

$\begin{array}{llll}1.036574 & -4.889457 & -2.909890\end{array}$

$\begin{array}{llll}1.058453 & -2.774639 & -2.515996\end{array}$

$\begin{array}{llll}0.497749 & -6.127741 & -2.551807\end{array}$ $\begin{array}{llll}-0.877011 & -7.156937 & -1.243957\end{array}$

$\begin{array}{llll}1.782648 & -4.824745 & -3.697038\end{array}$

$\begin{array}{llll}0.817640 & -7.032073 & -3.061260\end{array}$

$\begin{array}{llll}2.527355 & -3.998351 & 0.475459\end{array}$

$\begin{array}{llll}3.530298 & -4.417757 & -0.497290\end{array}$

$\begin{array}{llll}3.343443 & -5.458912 & -0.776549\end{array}$

$\begin{array}{llll}3.460322 & -3.806134 & -1.391288\end{array}$

$\begin{array}{llll}4.542658 & -4.343773 & -0.084236\end{array}$

$\begin{array}{lll}2.172789 & -5.004913 & 1.472801\end{array}$

$\begin{array}{llll}1.857273 & -4.544785 & 2.407839\end{array}$

$\begin{array}{llll}1.379583 & -5.669544 & 1.108225\end{array}$

$\begin{array}{llll}3.059688 & -5.605079 & 1.688421\end{array}$

$\begin{array}{llll}2.451426 & -1.919459 & -0.431549\end{array}$

$\begin{array}{lll}-0.453416 & -1.173882 & 2.861607\end{array}$

$\begin{array}{lll}-0.885226 & 0.012401 & 3.418745\end{array}$

$\begin{array}{lll}-0.794614 & -2.114235 & 3.277186\end{array}$

$\begin{array}{lll}-0.417636 & 1.201552 & 2.853849\end{array}$

$\begin{array}{lll}0.456018 & 1.160404 & 1.781867\end{array}$

$\begin{array}{lll}0.835569 & 2.067905 & 1.329394\end{array}$

$\begin{array}{lll}0.890425 & 0.000913 & 1.248301\end{array}$

$\begin{array}{lll}-1.574470 & 0.013313 & 4.254043\end{array}$

$\begin{array}{lll}-0.962192 & 2.928114 & 3.596214\end{array}$

C2-P2 si-si conf1

Number of imaginary frequencies : 1

The smallest frequency is : $-247.7047 \mathrm{~cm}(-1)$

Electronic energy : $\quad \mathrm{HF}=-7222.6348462$

Zero-point correction $=\quad 1.755700$

(Hartree/Particle)

Thermal correction to Energy= $\quad 1.874115$

Thermal correction to Enthalpy= $\quad 1.875059$

Thermal correction to Gibbs Free Energy $=1.593083$

Sum of electronic and zero-point Energies $=\quad-7220.879146$

Sum of electronic and thermal Energies $=\quad-7220.760731$

Sum of electronic and thermal Enthalpies $=\quad-7220.759787$

Sum of electronic and thermal Free Energies $=\quad-7221.041763$

Cartesian Coordinates

$\begin{array}{lll}-4.295437 & 4.787355 & 1.873202\end{array}$

$\begin{array}{lll}-2.562805 & 1.624327 & -1.886450\end{array}$

$\begin{array}{lll}-4.540775 & 0.128166 & 0.933392\end{array}$

$\begin{array}{lll}-3.967400 & 3.424797 & 0.318842\end{array}$

$\begin{array}{lll}-4.154449 & 2.746058 & 1.580220\end{array}$

$\begin{array}{lll}-3.132327 & 3.194430 & 2.473067\end{array}$

$\begin{array}{lll}-3.009849 & 2.884580 & 3.501734\end{array}$

$\begin{array}{lll}-2.334999 & 4.162811 & 1.791665\end{array}$

$\begin{array}{lll}-1.508954 & 4.716069 & 2.216440\end{array}$

$\begin{array}{lll}-2.848848 & 4.307323 & 0.469963\end{array}$

$\begin{array}{lll}-2.478862 & 4.983288 & -0.285896\end{array}$

$\begin{array}{lll}-6.237760 & 5.325940 & 2.375800\end{array}$

$\begin{array}{lll}-7.098839 & 4.677139 & 2.283312\end{array}$

$\begin{array}{lll}-5.382516 & 5.426388 & 3.515834\end{array}$

$\begin{array}{lll}-5.479243 & 4.865002 & 4.435574\end{array}$

$\begin{array}{lll}-4.350403 & 6.365820 & 3.212671\end{array}$

$\begin{array}{lll}-3.525224 & 6.633413 & 3.859406\end{array}$

$\begin{array}{lll}-4.567478 & 6.847204 & 1.885567\end{array}$

$\begin{array}{lll}-3.938634 & 7.547022 & 1.351141\end{array}$

$\begin{array}{lll}-5.733355 & 6.207169 & 1.369596\end{array}$

$\begin{array}{lll}-6.141457 & 6.344865 & 0.377657\end{array}$

$\begin{array}{llll}-4.764269 & 3.153492 & -0.912977\end{array}$

$\begin{array}{llll}-6.097323 & 3.584280 & -0.982639\end{array}$

$\begin{array}{llll}-6.500778 & 4.139337 & -0.145298\end{array}$

$\begin{array}{llll}-6.906087 & 3.300278 & -2.082949\end{array}$

$\begin{array}{lll}-7.941613 & 3.625013 & -2.092786\end{array}$

$\begin{array}{llll}-6.383592 & 2.576754 & -3.152403\end{array}$

$\begin{array}{lll}-7.004122 & 2.336950 & -4.009854\end{array}$

$\begin{array}{llll}-5.061542 & 2.133443 & -3.105789\end{array}$

$\begin{array}{llll}-4.672316 & 1.556624 & -3.937792\end{array}$

$\begin{array}{llll}-4.241815 & 2.397477 & -1.997422\end{array}$

$\begin{array}{lll}-5.179769 & 1.672470 & 1.830733\end{array}$

$\begin{array}{lll}-6.093160 & 1.961786 & 1.308082\end{array}$

$\begin{array}{lll}-5.519495 & 1.464681 & 3.309230\end{array}$

$\begin{array}{lll}-6.298243 & 0.706930 & 3.429948\end{array}$ 
$\begin{array}{lll}-4.650642 & 1.149189 & 3.893039\end{array}$

$\begin{array}{lll}-5.881822 & 2.407557 & 3.730235\end{array}$

$\begin{array}{lll}-2.354461 & 0.721721 & -3.482111\end{array}$

$\begin{array}{llll}-2.616243 & -0.658015 & -3.490617\end{array}$

$\begin{array}{lll}-2.930053 & -1.152734 & -2.579016\end{array}$

$\begin{array}{llll}-2.435227 & -1.405154 & -4.656733\end{array}$

$\begin{array}{llll}-2.642167 & -2.470234 & -4.648829\end{array}$

$\begin{array}{lll}-1.979184 & -0.786494 & -5.822554\end{array}$

$-1.824055-1.370575-6.725138$

$\begin{array}{lll}-1.719168 & 0.586530 & -5.822771\end{array}$

$\begin{array}{llll}-1.363613 & 1.074301 & -6.725932\end{array}$

$\begin{array}{llll}-1.908661 & 1.338316 & -4.662386\end{array}$

$\begin{array}{lll}-1.692248 & 2.400819 & -4.679778\end{array}$

$\begin{array}{llll}-1.403676 & 3.038178 & -2.070656\end{array}$

$\begin{array}{llll}-1.740556 & 4.197606 & -2.788378\end{array}$

$\begin{array}{llll}-2.733256 & 4.302541 & -3.215998\end{array}$

$\begin{array}{llll}-0.806428 & 5.222696 & -2.947956\end{array}$

$\begin{array}{lll}-1.077442 & 6.116537 & -3.502721\end{array}$

$\begin{array}{llll}0.475220 & 5.095145 & -2.402608\end{array}$

$\begin{array}{llll}1.208071 & 5.886350 & -2.533051\end{array}$

$\begin{array}{llll}0.814284 & 3.945084 & -1.687034\end{array}$

$\begin{array}{llll}1.807464 & 3.839152 & -1.267659\end{array}$

$-0.124988 \quad 2.927386-1.509523$

$\begin{array}{llll}0.137097 & 2.044720 & -0.936918\end{array}$

$\begin{array}{llll}-5.833954 & -0.351351 & -0.288109\end{array}$

$\begin{array}{llll}-5.486642 & -1.402292 & -1.149405\end{array}$

$-4.518843-1.879066-1.036746$

$-6.371623-1.829989-2.138243$

$\begin{array}{llll}-7.598250 & -1.194274 & -2.319206\end{array}$

$-8.275893-1.511055-3.103890$

$\begin{array}{lll}-7.930661 & -0.131925 & -1.478082\end{array}$

$\begin{array}{llll}-7.072228 & 0.277409 & -0.457198\end{array}$

$\begin{array}{lll}-7.378658 & 1.096619 & 0.178492\end{array}$

$\begin{array}{lll}-4.568586 & -1.271452 & 2.131450\end{array}$

$\begin{array}{lll}-3.559513 & -1.295134 & 3.107628\end{array}$

$\begin{array}{lll}-2.867673 & -0.465451 & 3.195798\end{array}$

$\begin{array}{lll}-3.410474 & -2.401824 & 3.940594\end{array}$

$\begin{array}{lll}-4.233691 & -3.519354 & 3.798021\end{array}$

$\begin{array}{llll}-4.087756 & -4.396385 & 4.418651\end{array}$

$\begin{array}{lll}-5.223171 & -3.501141 & 2.818428\end{array}$

$\begin{array}{lll}-5.409585 & -2.382662 & 2.005045\end{array}$

$\begin{array}{lll}-6.188526 & -2.406511 & 1.253458\end{array}$

$\begin{array}{lll}-2.440281 & 0.234724 & -0.102190\end{array}$

$\begin{array}{lll}-6.091969 & -4.714890 & 2.637310\end{array}$

$\begin{array}{lll}-2.273122 & -2.466927 & 4.920975\end{array}$

$\begin{array}{llll}-5.997085 & -3.025715 & -2.969085\end{array}$

$\begin{array}{llll}-9.225234 & 0.592960 & -1.725516\end{array}$

$\begin{array}{llll}-9.228151 & 1.197511 & -2.940401\end{array}$

$\begin{array}{llll}-9.467207 & 1.552928 & -0.806092\end{array}$

$-10.282676-0.251335-1.717126$

$\begin{array}{llll}-6.178690 & -4.181980 & -2.282707\end{array}$

$\begin{array}{llll}-6.720677 & -3.117967 & -4.102424\end{array}$

$-4.686754-3.002237 \quad-3.326825$

$\begin{array}{lll}-7.068699 & -4.789599 & 3.569641\end{array}$

$\begin{array}{lll}-6.695617 & -4.731925 & 1.427184\end{array}$

$\begin{array}{lll}-5.369560 & -5.858128 & 2.739829\end{array}$

$\begin{array}{lll}-2.644887 & -3.031518 & 6.090498\end{array}$

$\begin{array}{lll}-1.757515 & -1.251404 & 5.200191\end{array}$

$\begin{array}{lll}-1.250767 & -3.226987 & 4.437728\end{array}$

$\begin{array}{lll}-1.975894 & -2.751384 & 0.404035\end{array}$

$\begin{array}{lll}-0.812390 & -2.465302 & 1.213731\end{array}$

$\begin{array}{lll}-0.453886 & -1.190357 & 1.765515\end{array}$

$\begin{array}{lll}-0.409642 & -3.292585 & 1.779814\end{array}$

$\begin{array}{llll}3.400058 & -1.296214 & -1.447055\end{array}$

$\begin{array}{llll}5.267032 & -0.538264 & -0.377953\end{array}$

$\begin{array}{llll}5.260515 & 0.604632 & 0.820413\end{array}$

$\begin{array}{llll}6.330094 & 0.174129 & -1.442135\end{array}$

$\begin{array}{llll}5.966540 & -1.799001 & 0.479356\end{array}$

$\begin{array}{lll}4.982080 & 1.930143 & 0.525274\end{array}$

$\begin{array}{lll}3.757894 & 2.439098 & 1.010716\end{array}$

$\begin{array}{lll}3.119051 & 1.781975 & 1.589817\end{array}$

$\begin{array}{llll}3.415964 & 3.740371 & 0.740351\end{array}$

$\begin{array}{llll}2.486805 & 4.152909 & 1.120380\end{array}$

$\begin{array}{lll}4.248649 & 4.546652 & -0.083622\end{array}$

$\begin{array}{llll}3.843320 & 5.851210 & -0.476787\end{array}$ $\begin{array}{llll}2.895142 & 6.232401 & -0.106594\end{array}$

$\begin{array}{llll}4.622758 & 6.608483 & -1.322358\end{array}$

$\begin{array}{lll}4.300125 & 7.600830 & -1.623550\end{array}$

$\begin{array}{llll}5.844831 & 6.086323 & -1.809761\end{array}$

$\begin{array}{llll}6.447826 & 6.678270 & -2.492188\end{array}$

$\begin{array}{llll}6.275796 & 4.832860 & -1.431574\end{array}$

$\begin{array}{llll}7.211195 & 4.445828 & -1.818952\end{array}$

$\begin{array}{llll}5.501727 & 4.025512 & -0.552583\end{array}$

$\begin{array}{llll}5.909897 & 2.701986 & -0.155887\end{array}$

$\begin{array}{llll}7.269139 & 2.156938 & -0.436261\end{array}$

$\begin{array}{llll}8.460106 & 2.845577 & -0.001972\end{array}$

$\begin{array}{llll}8.420903 & 4.023158 & 0.796991\end{array}$

$\begin{array}{lll}7.464244 & 4.422286 & 1.110883\end{array}$

$\begin{array}{llll}9.579451 & 4.655925 & 1.192965\end{array}$

$\begin{array}{llll}9.518930 & 5.549286 & 1.807975\end{array}$

$\begin{array}{llll}10.845783 & 4.150826 & 0.814544\end{array}$

$\begin{array}{llll}11.749891 & 4.664936 & 1.127243\end{array}$

$\begin{array}{lll}10.923652 & 2.997935 & 0.066874\end{array}$

$11.888643 \quad 2.583726 \quad-0.213333$

$\begin{array}{llll}9.748662 & 2.311610 & -0.344190\end{array}$

$\begin{array}{llll}9.832602 & 1.090689 & -1.066300\end{array}$

$\begin{array}{llll}10.811106 & 0.699068 & -1.329435\end{array}$

$\begin{array}{llll}8.695431 & 0.398867 & -1.398607\end{array}$

$\begin{array}{llll}8.734292 & -0.551473 & -1.920471\end{array}$

$\begin{array}{llll}7.430840 & 0.928255 & -1.060056\end{array}$

$\begin{array}{llll}5.328337 & -3.110858 & 0.180972\end{array}$

$\begin{array}{llll}4.591423 & -3.316290 & 0.967031\end{array}$

$\begin{array}{llll}6.342526 & -4.240820 & 0.175098\end{array}$

$\begin{array}{lll}6.026818 & -5.466230 & 0.773385\end{array}$

$\begin{array}{llll}5.072363 & -5.581304 & 1.282332\end{array}$

$6.925397 \quad-6.535560 \quad 0.723005$

$\begin{array}{llll}6.667750 & -7.480499 & 1.193528\end{array}$

$\begin{array}{lll}8.155374 & -6.385415 & 0.079134\end{array}$

$\begin{array}{lll}8.858562 & -7.212779 & 0.045011\end{array}$

$\begin{array}{llll}8.480070 & -5.162129 & -0.517078\end{array}$

$\begin{array}{llll}9.437494 & -5.037574 & -1.015792\end{array}$

$\begin{array}{llll}7.578937 & -4.098569 & -0.471435\end{array}$

$\begin{array}{llll}7.831501 & -3.144311 & -0.925590\end{array}$

$\begin{array}{llll}4.594830 & -3.051752 & -1.163554\end{array}$

$\begin{array}{llll}5.333876 & -3.113068 & -1.971371\end{array}$

$\begin{array}{llll}3.953525 & -3.933830 & -1.249482\end{array}$

$\begin{array}{lll}6.667729 & -1.732629 & 1.807972\end{array}$

$\begin{array}{lll}7.141378 & -2.714903 & 1.886702\end{array}$

$\begin{array}{llll}5.678308 & -1.640575 & 2.970885\end{array}$

$\begin{array}{lll}5.126431 & -2.826175 & 3.482675\end{array}$

$\begin{array}{lll}5.452995 & -3.781295 & 3.079979\end{array}$

$\begin{array}{llll}4.182184 & -2.800242 & 4.512444\end{array}$

$3.769148 \quad-3.731660 \quad 4.890196$

$\begin{array}{llll}3.790282 & -1.580866 & 5.068470\end{array}$

$\begin{array}{llll}3.064029 & -1.554831 & 5.876307\end{array}$

$\begin{array}{lll}4.357260 & -0.394938 & 4.591550\end{array}$

$\begin{array}{llll}4.076297 & 0.558110 & 5.032312\end{array}$

$\begin{array}{lll}5.290662 & -0.424603 & 3.553663\end{array}$

$\begin{array}{lll}5.716327 & 0.505727 & 3.199489\end{array}$

$\begin{array}{lll}7.806830 & -0.715833 & 1.852804\end{array}$

$\begin{array}{lll}8.514428 & -0.911722 & 1.044022\end{array}$

$\begin{array}{llll}7.468556 & 0.315852 & 1.765797\end{array}$

$\begin{array}{lll}8.334977 & -0.822796 & 2.805277\end{array}$

$\begin{array}{llll}2.525378 & -1.128243 & 0.544821\end{array}$

$\begin{array}{llll}1.882608 & -0.253504 & 0.590611\end{array}$

$\begin{array}{llll}3.221230 & -1.183407 & 1.377318\end{array}$

$\begin{array}{llll}1.992560 & -2.367340 & 0.062609\end{array}$

$\begin{array}{llll}2.496886 & -3.272067 & 0.385508\end{array}$

$\begin{array}{lll}0.734650 & -2.534722 & -0.568683\end{array}$

$\begin{array}{llll}0.209022 & -1.652832 & -0.907242\end{array}$

$\begin{array}{llll}4.223052 & -1.143972 & -3.493042\end{array}$

$\begin{array}{llll}5.215202 & -1.589672 & -3.481890\end{array}$

$\begin{array}{llll}3.126479 & -2.048729 & -3.521760\end{array}$

$\begin{array}{llll}3.365222 & -3.111893 & -3.550807\end{array}$

$\begin{array}{llll}1.761944 & -1.710569 & -4.097577\end{array}$

$\begin{array}{llll}1.805699 & -1.728966 & -5.197714\end{array}$

$\begin{array}{llll}1.073388 & -2.507899 & -3.807469\end{array}$

$\begin{array}{llll}1.196652 & -0.358774 & -3.615835\end{array}$

$\begin{array}{llll}1.429525 & 0.434488 & -4.332820\end{array}$

$\begin{array}{llll}0.107346 & -0.422356 & -3.582652\end{array}$ 
$\begin{array}{llll}1.691489 & 0.068035 & -2.237650\end{array}$

$\begin{array}{llll}0.934668 & 0.084646 & -1.459797\end{array}$

$\begin{array}{llll}2.806514 & 0.873821 & -2.022098\end{array}$

$\begin{array}{llll}2.816425 & 1.461214 & -1.109695\end{array}$

$\begin{array}{lll}3.753039 & 1.359069 & -3.101273\end{array}$

$\begin{array}{llll}4.645283 & 1.750086 & -2.607389\end{array}$

$3.289387 \quad 2.204787 \quad-3.630586$

$\begin{array}{lll}4.170800 & 0.260249 & -4.102568\end{array}$

$\begin{array}{llll}3.492722 & 0.252237 & -4.962362\end{array}$

$\begin{array}{llll}5.160724 & 0.506430 & -4.498882\end{array}$

$\begin{array}{llll}0.336645 & -3.784997 & -1.225085\end{array}$

$\begin{array}{llll}0.871124 & -5.040118 & -0.873180\end{array}$

$-0.626971 \quad-3.732315-2.250610$

$\begin{array}{llll}0.457207 & -6.196872 & -1.530475\end{array}$

$\begin{array}{llll}1.601547 & -5.116434 & -0.074047\end{array}$

$\begin{array}{llll}-1.036553 & -4.889497 & -2.909893\end{array}$

$\begin{array}{llll}-1.058532 & -2.774681 & -2.515986\end{array}$

$-0.497635-6.127752 \quad-2.551846$

$\begin{array}{llll}0.877255 & -7.156874 & -1.244075\end{array}$

$\begin{array}{llll}-1.782672 & -4.824824 & -3.697000\end{array}$

$\begin{array}{llll}-0.817501 & -7.032100 & -3.061287\end{array}$

$\begin{array}{lll}-2.527335 & -3.998332 & 0.475409\end{array}$

$\begin{array}{lll}-3.530308 & -4.417710 & -0.497322\end{array}$

$\begin{array}{llll}-3.343462 & -5.458859 & -0.776613\end{array}$

$\begin{array}{llll}-3.460363 & -3.806065 & -1.391307\end{array}$

$\begin{array}{llll}-4.542655 & -4.343739 & -0.084232\end{array}$

$\begin{array}{llll}-2.172731 & -5.004927 & 1.472704\end{array}$

$\begin{array}{lll}-1.857177 & -4.544831 & 2.407745\end{array}$

$\begin{array}{llll}-1.379541 & -5.669547 & 1.108073\end{array}$

$\begin{array}{lll}-3.059622 & -5.605100 & 1.688340\end{array}$

$\begin{array}{lll}-2.451419 & -1.919421 & -0.431553\end{array}$

$\begin{array}{lll}0.453527 & -1.173928 & 2.861533\end{array}$

$\begin{array}{lll}0.885357 & 0.012338 & 3.418690\end{array}$

$\begin{array}{lll}0.794742 & -2.114293 & 3.277071\end{array}$

$\begin{array}{lll}0.417740 & 1.201507 & 2.853854\end{array}$

$\begin{array}{lll}-0.455954 & 1.160391 & 1.781903\end{array}$

$\begin{array}{lll}-0.835530 & 2.067905 & 1.329475\end{array}$

$\begin{array}{lll}-0.890375 & 0.000915 & 1.248314\end{array}$

$\begin{array}{lll}1.574634 & 0.013224 & 4.253959\end{array}$

$\begin{array}{lll}0.962326 & 2.928041 & 3.596268\end{array}$

C2-P2 re-si conf1

Number of imaginary frequencies : 1

The smallest frequency is : $-207.6432 \mathrm{~cm}(-1)$

Electronic energy :

$\mathrm{HF}=-7222.6226241$

Zero-point correction=

(Hartree/Particle)

Thermal correction to Energy=

Thermal correction to Enthalpy=

1.873691

1.874635

Thermal correction to Gibbs Free Energy=

Sum of electronic and zero-point Energies=

Sum of electronic and thermal Energies=

1.591070

Sum of electronic and thermal Enthalpies=

$-7220.867823$

$-7220.748933$

Sum of electronic and thermal Free Energies=

$-7220.747989$

$-7221.031554$

Cartesian Coordinates

$\begin{array}{lll}-3.537559 & 4.933678 & 2.365887\end{array}$

$\begin{array}{llll}-2.430775 & 1.875807 & -1.665330\end{array}$

$\begin{array}{lll}-4.905493 & 0.554700 & 1.039527\end{array}$

$\begin{array}{lll}-3.429227 & 3.780081 & 0.619380\end{array}$

$\begin{array}{lll}-4.030930 & 3.035572 & 1.696227\end{array}$

$\begin{array}{lll}-3.078478 & 2.960040 & 2.760166\end{array}$

$\begin{array}{lll}-3.236207 & 2.472982 & 3.713164\end{array}$

$\begin{array}{lll}-1.906457 & 3.668378 & 2.361093\end{array}$

$\begin{array}{lll}-1.023868 & 3.829111 & 2.965776\end{array}$

$\begin{array}{lll}-2.124278 & 4.180751 & 1.048957\end{array}$

$\begin{array}{lll}-1.433778 & 4.779267 & 0.472552\end{array}$

$\begin{array}{lll}-5.226402 & 6.096288 & 2.712956\end{array}$

$\begin{array}{lll}-6.222867 & 5.856508 & 2.366250\end{array}$

$\begin{array}{lll}-4.645625 & 5.680501 & 3.950010\end{array}$

$\begin{array}{lll}-5.121395 & 5.062442 & 4.699766\end{array}$

$\begin{array}{lll}-3.309717 & 6.183300 & 4.000569\end{array}$

$\begin{array}{lll}-2.594658 & 6.009075 & 4.793896\end{array}$

$\begin{array}{llll}-3.064627 & 6.909198 & 2.795041\end{array}$

$\begin{array}{lll}-2.133867 & 7.385865 & 2.517082\end{array}$

$\begin{array}{lll}-4.248422 & 6.856829 & 2.000515\end{array}$

$\begin{array}{llll}-4.368959 & 7.289547 & 1.016704\end{array}$

$\begin{array}{llll}-4.054965 & 3.999464 & -0.713703\end{array}$

$\begin{array}{llll}-5.099945 & 4.924240 & -0.851466\end{array}$

$\begin{array}{lll}-5.417968 & 5.471254 & 0.026979\end{array}$

$\begin{array}{lll}-5.728715 & 5.142413 & -2.077845\end{array}$

$\begin{array}{llll}-6.542167 & 5.858646 & -2.149143\end{array}$

$\begin{array}{llll}-5.304417 & 4.439891 & -3.203170\end{array}$

$\begin{array}{llll}-5.778730 & 4.600162 & -4.166453\end{array}$

$\begin{array}{llll}-4.276812 & 3.503235 & -3.086551\end{array}$

$\begin{array}{llll}-3.975055 & 2.948696 & -3.967044\end{array}$

$\begin{array}{llll}-3.657427 & 3.251235 & -1.852439\end{array}$

$\begin{array}{lll}-5.345107 & 2.315403 & 1.597922\end{array}$

$\begin{array}{lll}-5.895256 & 2.730304 & 0.747999\end{array}$

$\begin{array}{lll}-6.227529 & 2.414701 & 2.844478\end{array}$

$\begin{array}{lll}-7.176422 & 1.890035 & 2.701335\end{array}$

$\begin{array}{lll}-5.735577 & 1.999638 & 3.728511\end{array}$

$\begin{array}{lll}-6.446755 & 3.467535 & 3.042745\end{array}$

$\begin{array}{llll}-2.391338 & 1.092887 & -3.332333\end{array}$

$\begin{array}{llll}-3.507054 & 0.326948 & -3.714362\end{array}$

$\begin{array}{llll}-4.378408 & 0.285580 & -3.070463\end{array}$

$\begin{array}{llll}-3.523078 & -0.356459 & -4.928375\end{array}$

$\begin{array}{lll}-4.398868 & -0.934860 & -5.208336\end{array}$

$\begin{array}{llll}-2.411772 & -0.299652 & -5.775985\end{array}$

$\begin{array}{llll}-2.415471 & -0.840972 & -6.717503\end{array}$

$\begin{array}{llll}-1.298533 & 0.457033 & -5.406221\end{array}$

$\begin{array}{llll}-0.432020 & 0.507170 & -6.059319\end{array}$

$\begin{array}{llll}-1.288060 & 1.155219 & -4.194790\end{array}$

$\begin{array}{llll}-0.416130 & 1.741368 & -3.926972\end{array}$

$\begin{array}{lll}-0.821357 & 2.743792 & -1.509722\end{array}$

$\begin{array}{llll}-0.499895 & 3.859966 & -2.297940\end{array}$

$\begin{array}{llll}-1.203277 & 4.220662 & -3.043107\end{array}$

$\begin{array}{llll}0.720276 & 4.513852 & -2.121386\end{array}$

$\begin{array}{llll}0.966645 & 5.373972 & -2.737444\end{array}$

$\begin{array}{lll}1.620643 & 4.066426 & -1.148682\end{array}$

$\begin{array}{llll}2.570886 & 4.573649 & -1.015495\end{array}$

$\begin{array}{llll}1.297192 & 2.964945 & -0.353680\end{array}$

$\begin{array}{lll}1.992309 & 2.616367 & 0.403092\end{array}$

$\begin{array}{llll}0.083017 & 2.299438 & -0.534314\end{array}$

$\begin{array}{rrr}-0.182698 & 1.454867 & 0.094702\end{array}$

$\begin{array}{llll}-6.293799 & 0.064811 & -0.065657\end{array}$

$\begin{array}{llll}-6.960642 & -1.165479 & 0.020071\end{array}$

$\begin{array}{lll}-6.765570 & -1.846434 & 0.839220\end{array}$

$\begin{array}{llll}-7.864569 & -1.546509 & -0.972241\end{array}$

$\begin{array}{lll}-8.116221 & -0.726633 & -2.071256\end{array}$

$\begin{array}{llll}-8.809893 & -1.035511 & -2.843436\end{array}$

$\begin{array}{lll}-7.438380 & 0.487649 & -2.166800\end{array}$

$\begin{array}{llll}-6.533986 & 0.883633 & -1.180464\end{array}$

$\begin{array}{llll}-5.999009 & 1.821470 & -1.298940\end{array}$

$\begin{array}{lll}-5.057403 & -0.507350 & 2.534476\end{array}$

$\begin{array}{lll}-3.860544 & -1.028522 & 3.037527\end{array}$

$\begin{array}{lll}-2.924592 & -0.778098 & 2.550358\end{array}$

$\begin{array}{lll}-3.868006 & -1.864717 & 4.156400\end{array}$

$\begin{array}{lll}-5.061056 & -2.191017 & 4.793940\end{array}$

$\begin{array}{lll}-5.067263 & -2.851295 & 5.652848\end{array}$

$\begin{array}{lll}-6.254839 & -1.660486 & 4.299044\end{array}$

$\begin{array}{lll}-6.260726 & -0.824539 & 3.183111\end{array}$

$\begin{array}{lll}-7.205553 & -0.440327 & 2.816814\end{array}$

$\begin{array}{llll}-2.903091 & 0.325791 & -0.082593\end{array}$

$\begin{array}{lll}-7.552954 & -1.950421 & 5.003010\end{array}$

$\begin{array}{lll}-2.559615 & -2.451160 & 4.607937\end{array}$

$\begin{array}{llll}-8.610223 & -2.846017 & -0.825761\end{array}$

$\begin{array}{lll}-7.612303 & 1.370148 & -3.373227\end{array}$

$\begin{array}{lll}-6.488334 & 1.382427 & -4.136914\end{array}$

$\begin{array}{lll}-7.853824 & 2.654152 & -3.023934\end{array}$

$\begin{array}{llll}-8.625305 & 0.968745 & -4.167670\end{array}$

$\begin{array}{llll}-7.881874 & -3.773729 & -0.165950\end{array}$

$\begin{array}{llll}-9.758568 & -2.681039 & -0.124801\end{array}$

$\begin{array}{lll}-8.955130 & -3.371586 & -2.022036\end{array}$

$\begin{array}{lll}-7.861216 & -0.978438 & 5.895487\end{array}$

$\begin{array}{lll}-8.587481 & -2.024083 & 4.135013\end{array}$

$\begin{array}{lll}-7.511463 & -3.114038 & 5.686928\end{array}$

$\begin{array}{lll}-2.666246 & -3.146959 & 5.757204\end{array}$ 
$\begin{array}{llll}5.354566 & -1.598058 & 3.427242\end{array}$

$\begin{array}{lll}4.813040 & -2.718634 & 4.074407\end{array}$

$\begin{array}{llll}5.182605 & -3.708342 & 3.818319\end{array}$

$\begin{array}{lll}3.822834 & -2.578556 & 5.050816\end{array}$

$\begin{array}{llll}3.419601 & -3.459926 & 5.541945\end{array}$

$\begin{array}{llll}3.360748 & -1.308146 & 5.398238\end{array}$

$\begin{array}{llll}2.595761 & -1.192598 & 6.160886\end{array}$

$\begin{array}{llll}3.890203 & -0.183843 & 4.757231\end{array}$

$\begin{array}{lll}3.540262 & 0.809085 & 5.026517\end{array}$

$\begin{array}{llll}4.875696 & -0.326617 & 3.778253\end{array}$

$\begin{array}{llll}5.280152 & 0.553372 & 3.293144\end{array}$

$\begin{array}{lll}7.677613 & -0.898516 & 2.632618\end{array}$

$\begin{array}{lll}8.480922 & -1.151682 & 1.938827\end{array}$

$\begin{array}{lll}7.453995 & 0.161838 & 2.531777\end{array}$

$\begin{array}{llll}8.039494 & -1.074507 & 3.650323\end{array}$

$\begin{array}{llll}2.631299 & -0.764792 & 0.388900\end{array}$

$\begin{array}{llll}2.128352 & 0.199897 & 0.350129\end{array}$

$\begin{array}{llll}3.124181 & -0.949664 & 1.340076\end{array}$

$\begin{array}{llll}1.997034 & -1.875979 & -0.260801\end{array}$

$\begin{array}{llll}2.272389 & -2.873770 & 0.060821\end{array}$

$\begin{array}{llll}0.841397 & -1.762637 & -1.071611\end{array}$

$\begin{array}{llll}0.438422 & -0.765154 & -1.216620\end{array}$

$\begin{array}{llll}5.101135 & -1.217233 & -3.236439\end{array}$

$\begin{array}{llll}5.980052 & -1.803700 & -2.984296\end{array}$

$\begin{array}{llll}3.900060 & -1.932990 & -3.457096\end{array}$

$\begin{array}{llll}3.942798 & -3.018770 & -3.376505\end{array}$

$\begin{array}{llll}2.756579 & -1.417307 & -4.312387\end{array}$

$\begin{array}{llll}3.025622 & -1.507390 & -5.376257\end{array}$

$\begin{array}{llll}1.898401 & -2.075686 & -4.160284\end{array}$

$\begin{array}{llll}2.338351 & 0.037730 & -4.001485\end{array}$

$\begin{array}{llll}2.829440 & 0.731607 & -4.692108\end{array}$

$\begin{array}{lll}1.266187 & 0.133308 & -4.185037\end{array}$

$\begin{array}{llll}2.613605 & 0.498739 & -2.572049\end{array}$

$\begin{array}{lll}1.736100 & 0.712050 & -1.972295\end{array}$

$\begin{array}{llll}3.790272 & 1.115759 & -2.160972\end{array}$

$\begin{array}{llll}3.725881 & 1.760556 & -1.289438\end{array}$

$\begin{array}{llll}5.003718 & 1.352505 & -3.039129\end{array}$

$\begin{array}{llll}5.838780 & 1.631439 & -2.392722\end{array}$

$\begin{array}{llll}4.808522 & 2.219146 & -3.688235\end{array}$

$\begin{array}{lll}5.411623 & 0.130254 & -3.891019\end{array}$

$\begin{array}{llll}4.924819 & 0.172242 & -4.870717\end{array}$

$\begin{array}{llll}6.487289 & 0.183527 & -4.083905\end{array}$

$\begin{array}{llll}0.393054 & -2.748970 & -2.047445\end{array}$

$\begin{array}{llll}0.905429 & -4.060459 & -2.105855\end{array}$

$\begin{array}{lll}-0.580355 & -2.365351 & -2.990852\end{array}$

$\begin{array}{llll}0.466008 & -4.948210 & -3.084969\end{array}$

$\begin{array}{llll}1.657338 & -4.383180 & -1.392787\end{array}$

$\begin{array}{llll}-1.017984 & -3.255759 & -3.969447\end{array}$

$-0.986841-1.359952-2.955231$

$\begin{array}{lll}-0.494723 & -4.550225 & -4.022320\end{array}$

$\begin{array}{llll}0.874943 & -5.954068 & -3.120554\end{array}$

$\begin{array}{llll}-1.764446 & -2.933662 & -4.688414\end{array}$

$\begin{array}{lll}-0.831715 & -5.245333 & -4.785899\end{array}$

$\begin{array}{lll}-3.345944 & -5.460889 & -1.652616\end{array}$

$\begin{array}{lll}-5.605669 & -3.870390 & -2.983613\end{array}$

C2-P2 re-re conf1

Number of imaginary frequencies : 1

The smallest frequency is : $-153.7490 \mathrm{~cm}(-1)$

Electronic energy : $\quad H F=-7222.6206504$

Zero-point correction $=\quad 1.754801$

(Hartree/Particle)

Thermal correction to Energy= $\quad 1.873746$

Thermal correction to Enthalpy $=\quad 1.874690$

Thermal correction to Gibbs Free Energy $=\quad 1.591401$

Sum of electronic and zero-point Energies $=\quad-7220.865850$

Sum of electronic and thermal Energies $=\quad-7220.746904$

Sum of electronic and thermal Enthalpies $=\quad-7220.745960$

Sum of electronic and thermal Free Energies $=\quad-7221.029250$

Cartesian Coordinates 


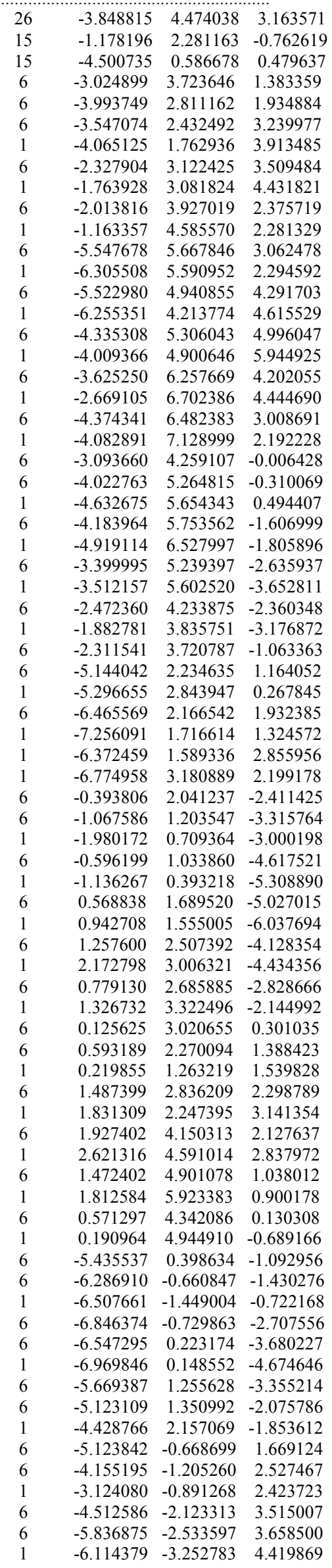

$\begin{array}{lll}-6.803057 & -1.994975 & 2.808349\end{array}$

$\begin{array}{lll}-6.459847 & -1.060188 & 1.829654\end{array}$

$\begin{array}{lll}-7.241192 & -0.641463 & 1.206850\end{array}$

$\begin{array}{lll}-2.216633 & 0.413509 & 0.037266\end{array}$

$\begin{array}{lll}-8.252356 & -2.363175 & 2.977041\end{array}$

$\begin{array}{llll}-3.437891 & -2.713807 & 4.385625\end{array}$

$\begin{array}{lll}-7.829741 & -1.824940 & -3.022557\end{array}$

$\begin{array}{llll}-5.253989 & 2.275056 & -4.380508\end{array}$

$\begin{array}{llll}-3.903318 & 2.292336 & -4.532239\end{array}$

$\begin{array}{llll}-5.614490 & 3.528138 & -4.017635\end{array}$

$\begin{array}{lll}-5.788076 & 2.037435 & -5.595171\end{array}$

$\begin{array}{llll}-7.806155 & -2.171272 & -4.328782\end{array}$

$\begin{array}{llll}-7.596066 & -2.941334 & -2.297754\end{array}$

$\begin{array}{llll}-9.099940 & -1.439115 & -2.745267\end{array}$

$\begin{array}{llll}-8.874275 & -2.494767 & 1.782384\end{array}$

$\begin{array}{lll}-8.411508 & -3.523133 & 3.648435\end{array}$

$\begin{array}{lll}-8.929436 & -1.410381 & 3.663059\end{array}$

$\begin{array}{lll}-3.919686 & -3.145366 & 5.570564\end{array}$

$\begin{array}{llll}-2.453997 & -1.821131 & 4.646779\end{array}$

$\begin{array}{lll}-2.842248 & -3.782257 & 3.793898\end{array}$

$\begin{array}{lll}-3.022076 & -1.777692 & -1.904706\end{array}$

$\begin{array}{lll}-2.167523 & -2.715210 & 0.063567\end{array}$

$\begin{array}{lll}-3.602389 & -2.973499 & -2.286588\end{array}$

$\begin{array}{llll}-3.122605 & -0.893822 & -2.522858\end{array}$

$\begin{array}{llll}-2.824287 & -3.936930 & -0.260382\end{array}$

$\begin{array}{llll}-3.536480 & -4.078617 & -1.434278\end{array}$

$\begin{array}{llll}-2.729286 & -4.779147 & 0.416711\end{array}$

$\begin{array}{lll}-1.270084 & -2.718815 & 1.177994\end{array}$

$\begin{array}{lll}-0.781542 & -1.577089 & 1.893775\end{array}$

$\begin{array}{lll}-1.078883 & -0.377610 & 1.590708\end{array}$

$-2.330323-1.630305-0.759229$

$\begin{array}{lll}-1.212009 & -3.671856 & 1.683712\end{array}$

$\begin{array}{lll}0.061431 & -1.764290 & 2.958049\end{array}$

$\begin{array}{llll}0.414954 & -0.627611 & 3.802938\end{array}$

$\begin{array}{llll}-0.248955 & 0.209716 & 3.597411\end{array}$

$\begin{array}{llll}0.315969 & -0.909920 & 4.855133\end{array}$

$\begin{array}{llll}1.449552 & -0.315441 & 3.628521\end{array}$

$\begin{array}{lll}0.448599 & -3.070399 & 3.475583\end{array}$

$\begin{array}{llll}0.628499 & -3.786038 & 2.672937\end{array}$

$\begin{array}{lll}1.379684 & -2.958743 & 4.036294\end{array}$

$\begin{array}{llll}-0.317685 & -3.481829 & 4.144105\end{array}$

$3.324811-1.621256-1.294941$

$\begin{array}{lll}4.944766 & -0.732630 & 0.043854\end{array}$

$\begin{array}{llll}4.932245 & 0.801502 & 0.679756\end{array}$

$\begin{array}{lll}6.379665 & -0.682495 & -0.807385\end{array}$

$\begin{array}{lll}5.080214 & -1.627383 & 1.451612\end{array}$

$\begin{array}{lll}5.100838 & 1.896575 & -0.167456\end{array}$

$\begin{array}{llll}3.964235 & 2.704101 & -0.389256\end{array}$

$\begin{array}{lll}3.040795 & 2.450732 & 0.116040\end{array}$

$\begin{array}{lll}4.056707 & 3.784010 & -1.231606\end{array}$

$\begin{array}{llll}3.197319 & 4.427611 & -1.389014\end{array}$

$\begin{array}{llll}5.263702 & 4.053917 & -1.929924\end{array}$

$\begin{array}{llll}5.336925 & 5.103715 & -2.885262\end{array}$

$\begin{array}{llll}4.455271 & 5.718571 & -3.046830\end{array}$

$\begin{array}{lll}6.490004 & 5.327817 & -3.602766\end{array}$

$\begin{array}{llll}6.533677 & 6.128024 & -4.335789\end{array}$

$\begin{array}{llll}7.618439 & 4.499258 & -3.394996\end{array}$

$\begin{array}{llll}8.518939 & 4.660584 & -3.980560\end{array}$

$\begin{array}{llll}7.585904 & 3.485363 & -2.461972\end{array}$

$\begin{array}{lll}8.457290 & 2.856171 & -2.326920\end{array}$

$\begin{array}{llll}6.419077 & 3.237573 & -1.685893\end{array}$

$\begin{array}{llll}6.344408 & 2.175751 & -0.713059\end{array}$

$\begin{array}{llll}7.542586 & 1.375698 & -0.335797\end{array}$

$\begin{array}{llll}8.743531 & 1.995997 & 0.164634\end{array}$

$\begin{array}{llll}8.814779 & 3.380647 & 0.485518\end{array}$

$\begin{array}{lll}7.935058 & 4.000756 & 0.360325\end{array}$

$\begin{array}{llll}9.977994 & 3.940962 & 0.967788\end{array}$

$\begin{array}{lll}10.002459 & 4.998912 & 1.213003\end{array}$

$\begin{array}{llll}11.138276 & 3.152282 & 1.152900\end{array}$

$\begin{array}{lll}12.049693 & 3.609327 & 1.526916\end{array}$

$\begin{array}{lll}11.100024 & 1.805214 & 0.872124\end{array}$

$\begin{array}{lll}11.977294 & 1.182364 & 1.026578\end{array}$

$\begin{array}{llll}9.911774 & 1.192526 & 0.389816\end{array}$

$\begin{array}{llll}9.856604 & -0.206691 & 0.146074\end{array}$

$\begin{array}{lll}10.751065 & -0.804453 & 0.297176\end{array}$ 
$\begin{array}{llll}8.680610 & -0.800107 & -0.237672\end{array}$

$\begin{array}{llll}8.601088 & -1.871379 & -0.389816\end{array}$

$\begin{array}{llll}7.528643 & -0.006530 & -0.436996\end{array}$

$\begin{array}{llll}4.212014 & -2.840196 & 1.444922\end{array}$

$\begin{array}{lll}3.276873 & -2.596094 & 1.966660\end{array}$

$\begin{array}{lll}4.851920 & -4.024925 & 2.148608\end{array}$

$\begin{array}{llll}4.095539 & -4.801663 & 3.033718\end{array}$

$3.071960 \quad-4.511962 \quad 3.258111$

$\begin{array}{llll}4.644722 & -5.940405 & 3.630441\end{array}$

$\begin{array}{llll}4.045129 & -6.533053 & 4.315980\end{array}$

$\begin{array}{llll}5.962397 & -6.309191 & 3.352890\end{array}$

$\begin{array}{llll}6.393802 & -7.189344 & 3.821095\end{array}$

$\begin{array}{lll}6.726421 & -5.535923 & 2.471974\end{array}$

$\begin{array}{lll}7.753905 & -5.814785 & 2.254706\end{array}$

$\begin{array}{lll}6.173321 & -4.405679 & 1.870770\end{array}$

$\begin{array}{lll}6.767864 & -3.798736 & 1.192831\end{array}$

$\begin{array}{llll}3.910916 & -3.232060 & -0.005204\end{array}$

$\begin{array}{llll}4.821703 & -3.673642 & -0.427915\end{array}$

$\begin{array}{llll}3.159548 & -4.022976 & -0.015008\end{array}$

$\begin{array}{lll}5.650712 & -1.249653 & 2.781851\end{array}$

$\begin{array}{lll}5.949852 & -2.208256 & 3.210562\end{array}$

$\begin{array}{llll}4.639131 & -0.661463 & 3.766330\end{array}$

$\begin{array}{llll}4.098088 & -1.498204 & 4.754998\end{array}$

$\begin{array}{llll}4.330772 & -2.559683 & 4.735497\end{array}$

$\begin{array}{lll}3.302029 & -0.982936 & 5.781079\end{array}$

$\begin{array}{llll}2.902687 & -1.649006 & 6.541054\end{array}$

$\begin{array}{llll}3.038774 & 0.386632 & 5.837928\end{array}$

$\begin{array}{llll}2.436971 & 0.796838 & 6.643903\end{array}$

$\begin{array}{llll}3.554812 & 1.226927 & 4.847461\end{array}$

$\begin{array}{lll}3.362003 & 2.295375 & 4.890236\end{array}$

$\begin{array}{lll}4.337319 & 0.707763 & 3.812668\end{array}$

$\begin{array}{llll}4.736629 & 1.375023 & 3.057162\end{array}$

$\begin{array}{lll}6.934130 & -0.420856 & 2.715089\end{array}$

$\begin{array}{llll}7.699123 & -0.947687 & 2.142923\end{array}$

$\begin{array}{lll}6.788751 & 0.565660 & 2.276511\end{array}$

$\begin{array}{lll}7.306909 & -0.286089 & 3.734718\end{array}$

$\begin{array}{llll}1.821838 & -0.703978 & -0.028123\end{array}$

$\begin{array}{llll}1.635621 & 0.332736 & -0.295925\end{array}$

$\begin{array}{llll}1.996199 & -0.841426 & 1.037346\end{array}$

$\begin{array}{llll}1.037369 & -1.703501 & -0.709668\end{array}$

$\begin{array}{llll}0.457764 & -1.410292 & -1.575978\end{array}$

$\begin{array}{llll}0.824305 & -2.989951 & -0.189307\end{array}$

$\begin{array}{llll}1.320596 & -3.219461 & 0.745023\end{array}$

$\begin{array}{llll}4.850463 & -2.360752 & -2.784668\end{array}$

$\begin{array}{llll}5.639243 & -2.831294 & -2.204316\end{array}$

$\begin{array}{llll}3.665032 & -3.103617 & -2.959079\end{array}$

$\begin{array}{llll}3.634376 & -4.099957 & -2.518856\end{array}$

$\begin{array}{llll}2.672642 & -2.900151 & -4.089186\end{array}$

$\begin{array}{llll}3.057071 & -3.370951 & -5.007206\end{array}$

$\begin{array}{llll}1.763747 & -3.446973 & -3.826480\end{array}$

$\begin{array}{llll}2.312991 & -1.424482 & -4.364393\end{array}$

$\begin{array}{llll}2.961366 & -1.011535 & -5.144122\end{array}$

$\begin{array}{lll}1.295404 & -1.374193 & -4.763490\end{array}$

$\begin{array}{llll}2.385797 & -0.517547 & -3.139691\end{array}$

$\begin{array}{llll}1.437332 & -0.119822 & -2.796214\end{array}$

$\begin{array}{llll}3.514431 & 0.199931 & -2.769956\end{array}$

$\begin{array}{llll}3.352193 & 1.095514 & -2.180559\end{array}$

$\begin{array}{llll}4.853958 & 0.129482 & -3.472526\end{array}$

$\begin{array}{llll}5.589780 & 0.629686 & -2.843537\end{array}$

$\begin{array}{llll}4.791951 & 0.712250 & -4.403690\end{array}$

$\begin{array}{llll}5.339124 & -1.305301 & -3.775909\end{array}$

$\begin{array}{llll}5.035379 & -1.603521 & -4.784789\end{array}$

$\begin{array}{llll}6.433030 & -1.309260 & -3.773348\end{array}$

$\begin{array}{llll}0.353554 & -4.139609 & -0.950390\end{array}$

$\begin{array}{llll}-0.199482 & -4.025599 & -2.240705\end{array}$

$\begin{array}{llll}0.458892 & -5.422511 & -0.377161\end{array}$

$\begin{array}{llll}-0.612432 & -5.157345 & -2.938996\end{array}$

$\begin{array}{llll}-0.318525 & -3.047913 & -2.693548\end{array}$

$\begin{array}{llll}0.043634 & -6.554039 & -1.076074\end{array}$

$\begin{array}{llll}0.871623 & -5.522964 & 0.623680\end{array}$

$\begin{array}{llll}-0.490027 & -6.425734 & -2.362749\end{array}$

$\begin{array}{llll}-1.039111 & -5.048856 & -3.931915\end{array}$

$\begin{array}{llll}0.137091 & -7.535417 & -0.620051\end{array}$

$\begin{array}{lll}-0.813350 & -7.306753 & -2.909456\end{array}$

$-4.006063-5.021744-1.691069$ $\begin{array}{llll}-4.469991 & -3.113521 & -4.032577\end{array}$

C2-P2 si-re conf1

Number of imaginary frequencies : 1

The smallest frequency is : $-68.1152 \mathrm{~cm}(-1)$

Electronic energy : $\mathrm{HF}=-7222.6318937$

Zero-point correction=

1.754510

Hartree/Particle)

Thermal correction to Energy= $\quad 1.873363$

Thermal correction to Enthalpy= $\quad 1.874307$

Thermal correction to Gibbs Free Energy $=\quad 1.591959$

Sum of electronic and zero-point Energies $=\quad-7220.877384$

$-7220.758531$

$-7220.757587$

Sum of electronic and thermal Free Energies $=\quad-7221.039934$

Cartesian Coordinates

\begin{tabular}{|c|c|c|c|}
\hline 26 & 4.418781 & -3.967516 & 7034 \\
\hline 15 & 1.345940 & -2.104493 & -0.575991 \\
\hline 15 & 4.499202 & -0.254366 & 0.279287 \\
\hline 6 & 3.472727 & -3.243735 & 1.557490 \\
\hline 6 & 4.283628 & -2.221959 & 2.175827 \\
\hline 6 & 3.794937 & -2.019844 & 3.502876 \\
\hline 1 & 4.199355 & -1.326740 & 4.227183 \\
\hline 6 & 2.706899 & -2.919562 & 3.720973 \\
\hline 1 & 2.150878 & -3.029909 & 4.641044 \\
\hline 6 & 2.510178 & -3.672610 & 2.526699 \\
\hline 1 & 1.777261 & -4.451744 & 2.378378 \\
\hline 6 & 6.312883 & -4.812526 & 3.147372 \\
\hline 1 & 7.061892 & -4.531699 & 2.418971 \\
\hline 6 & 6.127850 & -4.214662 & 4.431453 \\
\hline 1 & 6.704607 & -3.396176 & 4.841416 \\
\hline 6 & 5.017383 & -4.855476 & 5.060638 \\
\hline 1 & 4.602786 & -4.603468 & 6.027871 \\
\hline 6 & 4.514442 & -5.847925 & 4.165137 \\
\hline 1 & 3.654572 & -6.482088 & 4.336296 \\
\hline 6 & 5.315310 & -5.823449 & 2.984809 \\
\hline 1 & 5.168102 & -6.440757 & 2.109241 \\
\hline 6 & 3.623348 & -3.699034 & 0.145642 \\
\hline 6 & 4.750051 & -4.462437 & -0.198902 \\
\hline 1 & 5.452121 & -4.714621 & 0.584907 \\
\hline 6 & 4.992324 & -4.878896 & -1.506599 \\
\hline 1 & 5.890254 & -5.441924 & -1.740604 \\
\hline 6 & 4.080835 & -4.550345 & -2.506199 \\
\hline 1 & 4.248951 & -4.864034 & -3.531496 \\
\hline 6 & 2.957298 & -3.786612 & -2.191249 \\
\hline 1 & 2.272691 & -3.528540 & -2.989427 \\
\hline 6 & 2.715530 & -3.330322 & -0.883805 \\
\hline 6 & 5.369427 & -1.455682 & 1.468028 \\
\hline 1 & 5.893169 & -2.161949 & 0.822150 \\
\hline 6 & 6.396073 & -0.809492 & 2.402503 \\
\hline 1 & 7.185170 & -0.310891 & 1.833055 \\
\hline 1 & 5.943192 & -0.067982 & 3.066402 \\
\hline 1 & 6.854376 & -1.583819 & 3.025889 \\
\hline 6 & 0.561395 & -2.000206 & -2.239975 \\
\hline 6 & 1.113160 & -1.078150 & -3.146919 \\
\hline 1 & 1.866005 & -0.376916 & -2.803853 \\
\hline 6 & 0.705139 & -1.072152 & -4.482226 \\
\hline 1 & 1.157886 & -0.375823 & -5.179042 \\
\hline 6 & -0.287952 & -1.955251 & -4.915305 \\
\hline 1 & -0.610979 & -1.944120 & -5.952146 \\
\hline 6 & -0.876433 & -2.838878 & -4.007965 \\
\hline 1 & -1.665217 & -3.511795 & -4.332186 \\
\hline 6 & -0.447184 & -2.869571 & -2.679772 \\
\hline 1 & -0.894202 & -3.578943 & -1.994031 \\
\hline 6 & 0.138044 & -3.018860 & 0.464544 \\
\hline 6 & -0.078478 & -4.399032 & 0.319065 \\
\hline 1 & 0.466006 & -4.956762 & -0.437383 \\
\hline 6 & -0.973130 & -5.064219 & 1.158823 \\
\hline 1 & -1.135458 & -6.131368 & 1.037740 \\
\hline 6 & -1.653849 & -4.359003 & 2.155901 \\
\hline 1 & -2.346492 & -4.877579 & 2.812249 \\
\hline 6 & -1.443782 & -2.986477 & 2.307753 \\
\hline
\end{tabular}




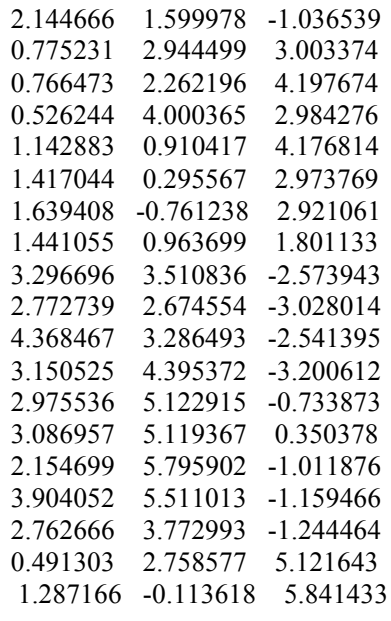

C2-P2 si-si conf2

Number of imaginary frequencies : 1

The smallest frequency is : $-255.0249 \mathrm{~cm}(-1)$

Electronic energy :

$\mathrm{HF}=-7222.6284099$

Zero-point correction $=$

1.754397

(Hartree/Particle)

Thermal correction to Energy $=\quad 1.873023$

Thermal correction to Enthalpy= $\quad 1.873967$

Thermal correction to Gibbs Free Energy= $\quad 1.592763$

Sum of electronic and zero-point Energies $=\quad-7220.874013$

Sum of electronic and thermal Energies $=\quad-7220.755387$

Sum of electronic and thermal Enthalpies $=\quad-7220.754443$

Sum of electronic and thermal Free Energies $=\quad-7221.035647$

Cartesian Coordinates

$\begin{array}{lll}-4.735526 & 4.665373 & 1.791059\end{array}$

$\begin{array}{lll}-2.779367 & 1.496281 & -1.852960\end{array}$

$\begin{array}{lll}-4.720730 & -0.025239 & 0.973444\end{array}$

$\begin{array}{lll}-4.311830 & 3.285601 & 0.276525\end{array}$

$\begin{array}{lll}-4.472359 & 2.629466 & 1.554048\end{array}$

$\begin{array}{lll}-3.486915 & 3.159103 & 2.443532\end{array}$

$\begin{array}{lll}-3.358205 & 2.884261 & 3.481404\end{array}$

$\begin{array}{lll}-2.740405 & 4.154872 & 1.744466\end{array}$

$\begin{array}{lll}-1.953665 & 4.767635 & 2.162197\end{array}$

$\begin{array}{lll}-3.248231 & 4.235351 & 0.415148\end{array}$

$\begin{array}{lll}-2.909530 & 4.911371 & -0.355130\end{array}$

$\begin{array}{lll}-6.713017 & 5.103259 & 2.249197\end{array}$

$\begin{array}{lll}-7.535475 & 4.405793 & 2.160640\end{array}$

$\begin{array}{lll}-5.885277 & 5.281187 & 3.399995\end{array}$

$\begin{array}{llll}-5.965963 & 4.738366 & 4.332357\end{array}$

$-4.904017 \quad 6.271220 \quad 3.089588$

$\begin{array}{lll}-4.108014 & 6.603472 & 3.742961\end{array}$

$\begin{array}{lll}-5.124981 & 6.706369 & 1.747414\end{array}$

$\begin{array}{lll}-4.528228 & 7.428088 & 1.205344\end{array}$

$\begin{array}{llll}-6.242843 & 5.987421 & 1.229001\end{array}$

$\begin{array}{lll}-6.640157 & 6.077598 & 0.227411\end{array}$

$\begin{array}{llll}-5.073820 & 2.936500 & -0.957512\end{array}$

$\begin{array}{llll}-6.426326 & 3.293695 & -1.059997\end{array}$

$\begin{array}{llll}-6.869819 & 3.852934 & -0.245983\end{array}$

$\begin{array}{llll}-7.201644 & 2.932676 & -2.161712\end{array}$

$\begin{array}{llll}-8.252417 & 3.201907 & -2.196849\end{array}$

$\begin{array}{llll}-6.625285 & 2.202715 & -3.198624\end{array}$

$\begin{array}{llll}-7.219168 & 1.901770 & -4.055735\end{array}$

$\begin{array}{llll}-5.282261 & 1.833211 & -3.119552\end{array}$

$\begin{array}{llll}-4.850080 & 1.249785 & -3.925041\end{array}$

$\begin{array}{llll}-4.494213 & 2.178012 & -2.010374\end{array}$

$\begin{array}{lll}-5.440781 & 1.509428 & 1.827072\end{array}$

$\begin{array}{lll}-6.364961 & 1.737946 & 1.293901\end{array}$

$\begin{array}{lll}-5.782674 & 1.323878 & 3.308429\end{array}$

$\begin{array}{lll}-6.521497 & 0.529364 & 3.443000\end{array}$

$\begin{array}{lll}-4.905246 & 1.071489 & 3.910403\end{array}$

$\begin{array}{lll}-6.199325 & 2.257376 & 3.699168\end{array}$

$\begin{array}{llll}-2.495996 & 0.578242 & -3.427521\end{array}$ $\begin{array}{lll}-2.670034 & -0.815179 & -3.410733\end{array}$

$\begin{array}{llll}-2.964176 & -1.310709 & -2.493184\end{array}$

$\begin{array}{llll}-2.423001 & -1.572857 & -4.557826\end{array}$

$\begin{array}{llll}-2.561924 & -2.648635 & -4.530662\end{array}$

$\begin{array}{lll}-1.987294 & -0.949870 & -5.729031\end{array}$

$\begin{array}{llll}-1.779998 & -1.540871 & -6.616525\end{array}$

$\begin{array}{llll}-1.814474 & 0.436749 & -5.754383\end{array}$

$\begin{array}{lll}-1.474843 & 0.927516 & -6.662039\end{array}$

$\begin{array}{llll}-2.070288 & 1.198311 & -4.613325\end{array}$

$\begin{array}{lll}-1.918188 & 2.271451 & -4.648716\end{array}$

$\begin{array}{llll}-1.681790 & 2.957877 & -2.032004\end{array}$

$\begin{array}{llll}-2.044603 & 4.089834 & -2.779855\end{array}$

$\begin{array}{llll}-3.025906 & 4.143487 & -3.242056\end{array}$

$\begin{array}{llll}-1.152673 & 5.154441 & -2.921353\end{array}$

$\begin{array}{llll}-1.443243 & 6.027389 & -3.498989\end{array}$

$\begin{array}{llll}0.112075 & 5.094453 & -2.326491\end{array}$

$\begin{array}{llll}0.811369 & 5.917856 & -2.440284\end{array}$

$\begin{array}{llll}0.477247 & 3.971249 & -1.581665\end{array}$

$\begin{array}{llll}1.457157 & 3.918282 & -1.122299\end{array}$

$\begin{array}{llll}-0.420394 & 2.913401 & -1.424697\end{array}$

$\begin{array}{llll}-0.140133 & 2.049927 & -0.831957\end{array}$

$\begin{array}{llll}-5.971124 & -0.590150 & -0.256777\end{array}$

$\begin{array}{llll}-5.553733 & -1.626509 & -1.104533\end{array}$

$\begin{array}{llll}-4.561892 & -2.045965 & -0.974635\end{array}$

$\begin{array}{llll}-6.401191 & -2.111789 & -2.099807\end{array}$

$\begin{array}{llll}-7.659553 & -1.547997 & -2.300452\end{array}$

$\begin{array}{llll}-8.307977 & -1.907293 & -3.091693\end{array}$

$\begin{array}{llll}-8.062892 & -0.501393 & -1.470966\end{array}$

$\begin{array}{lll}-7.242612 & -0.037181 & -0.442451\end{array}$

$\begin{array}{lll}-7.604743 & 0.765705 & 0.184817\end{array}$

$\begin{array}{lll}-4.702302 & -1.400594 & 2.197640\end{array}$

$\begin{array}{lll}-3.709971 & -1.360019 & 3.190275\end{array}$

$\begin{array}{lll}-3.061790 & -0.495164 & 3.278111\end{array}$

$\begin{array}{lll}-3.522024 & -2.446938 & 4.041308\end{array}$

$\begin{array}{lll}-4.285565 & -3.606469 & 3.900975\end{array}$

$\begin{array}{lll}-4.105550 & -4.466124 & 4.536707\end{array}$

$\begin{array}{lll}-5.258689 & -3.650572 & 2.906048\end{array}$

$\begin{array}{llll}-5.486475 & -2.553167 & 2.074761\end{array}$

$\begin{array}{lll}-6.251910 & -2.624563 & 1.312407\end{array}$

$\begin{array}{llll}-2.608735 & 0.155127 & -0.035102\end{array}$

$\begin{array}{lll}-6.064137 & -4.907704 & 2.727220\end{array}$

$\begin{array}{lll}-2.402696 & -2.444343 & 5.043711\end{array}$

$\begin{array}{llll}-5.953504 & -3.292743 & -2.915088\end{array}$

$\begin{array}{llll}-9.394006 & 0.145557 & -1.738945\end{array}$

$\begin{array}{llll}-9.430300 & 0.702638 & -2.975386\end{array}$

$\begin{array}{llll}-9.682326 & 1.125983 & -0.854966\end{array}$

$-10.407037-0.750338-1.693726$

$\begin{array}{lll}-6.096681 & -4.452319 & -2.225093\end{array}$

$\begin{array}{llll}-6.647804 & -3.424782 & -4.062928\end{array}$

$\begin{array}{lll}-4.638879 & -3.210841 & -3.245950\end{array}$

$\begin{array}{lll}-7.051453 & -5.017477 & 3.644513\end{array}$

$\begin{array}{lll}-6.645932 & -4.969422 & 1.507798\end{array}$

$\begin{array}{lll}-5.289128 & -6.013279 & 2.856471\end{array}$

$\begin{array}{lll}-2.771955 & -3.007066 & 6.214919\end{array}$

$\begin{array}{lll}-1.947789 & -1.202758 & 5.312811\end{array}$

$\begin{array}{lll}-1.339078 & -3.165744 & 4.591268\end{array}$

$\begin{array}{lll}-2.009369 & -2.804850 & 0.520170\end{array}$

$\begin{array}{lll}-0.863612 & -2.457614 & 1.334614\end{array}$

$\begin{array}{lll}-0.587109 & -1.161505 & 1.886983\end{array}$

$\begin{array}{lll}-0.431043 & -3.259570 & 1.915526\end{array}$

$\begin{array}{llll}3.322569 & -1.086486 & -1.263050\end{array}$

$\begin{array}{llll}5.180741 & -0.221194 & -0.276149\end{array}$

$\begin{array}{llll}5.130757 & 0.890244 & 0.963923\end{array}$

$\begin{array}{llll}6.128459 & 0.604470 & -1.364928\end{array}$

$\begin{array}{llr}6.047339 & -1.433883 & 0.500206\end{array}$

$\begin{array}{lll}4.741712 & 2.196245 & 0.704864\end{array}$

$\begin{array}{lll}3.488507 & 2.600376 & 1.216391\end{array}$

$\begin{array}{lll}2.907855 & 1.886198 & 1.789858\end{array}$

$\begin{array}{llll}3.040148 & 3.874567 & 0.972245\end{array}$

$\begin{array}{lll}2.085956 & 4.205411 & 1.369188\end{array}$

$\begin{array}{llll}3.795509 & 4.760745 & 0.156365\end{array}$

$\begin{array}{llll}3.284723 & 6.038795 & -0.199861\end{array}$

$\begin{array}{llll}2.316988 & 6.337337 & 0.194465\end{array}$

$\begin{array}{llll}3.988936 & 6.871655 & -1.040248\end{array}$

$\begin{array}{llll}3.586993 & 7.843070 & -1.312803\end{array}$ 
$\begin{array}{llll}2.612674 & 1.651102 & -0.955295\end{array}$

$\begin{array}{llll}3.526156 & 1.575835 & -2.958708\end{array}$

$\begin{array}{llll}4.395580 & 2.033549 & -2.483435\end{array}$

$\begin{array}{llll}3.001121 & 2.378148 & -3.498149\end{array}$

$\begin{array}{llll}4.001859 & 0.486921 & -3.945952\end{array}$

$\begin{array}{llll}3.321991 & 0.429744 & -4.802419\end{array}$

$\begin{array}{llll}4.975598 & 0.781614 & -4.348699\end{array}$

$\begin{array}{llll}0.402899 & -3.745088 & -1.038908\end{array}$

$\begin{array}{llll}1.009774 & -4.961164 & -0.667729\end{array}$

$\begin{array}{lll}-0.556184 & -3.764833 & -2.069909\end{array}$

$\begin{array}{llll}0.668558 & -6.149779 & -1.310239\end{array}$

$\begin{array}{llll}1.741888 & -4.983459 & 0.133170\end{array}$

$\begin{array}{lll}-0.894512 & -4.953758 & -2.712918\end{array}$

$\begin{array}{llll}-1.041721 & -2.838417 & -2.352446\end{array}$

$\begin{array}{llll}-0.284986 & -6.152660 & -2.334881\end{array}$

$\begin{array}{llll}1.145326 & -7.078409 & -1.009525\end{array}$

$\begin{array}{llll}-1.639499 & -4.944708 & -3.503696\end{array}$

$\begin{array}{llll}-0.548365 & -7.081673 & -2.832138\end{array}$

$\begin{array}{llll}-2.504675 & -4.073995 & 0.599919\end{array}$

$\begin{array}{lll}-3.479229 & -4.544784 & -0.378568\end{array}$

$\begin{array}{llll}-3.254481 & -5.584254 & -0.634568\end{array}$

$\begin{array}{llll}-3.416757 & -3.947610 & -1.282943\end{array}$

$\begin{array}{lll}-4.499417 & -4.496580 & 0.018440\end{array}$

$\begin{array}{lll}-2.122847 & -5.051000 & 1.616548\end{array}$

$\begin{array}{lll}-1.837670 & -4.564269 & 2.548136\end{array}$

$\begin{array}{lll}-1.299424 & -5.688620 & 1.271567\end{array}$

$\begin{array}{lll}-2.987954 & -5.682567 & 1.831190\end{array}$

$\begin{array}{llll}-2.516461 & -2.003190 & -0.325657\end{array}$

$\begin{array}{lll}0.286472 & -1.090641 & 3.007860\end{array}$

$\begin{array}{lll}0.634528 & 0.119535 & 3.573574\end{array}$

$\begin{array}{llll}0.657762 & -2.011226 & 3.441329\end{array}$

$\begin{array}{lll}0.116064 & 1.278655 & 2.989075\end{array}$

$\begin{array}{lll}-0.718021 & 1.183704 & 1.889292\end{array}$

$\begin{array}{lll}-1.130629 & 2.067056 & 1.418394\end{array}$

$\begin{array}{lll}-1.071701 & 0.000661 & 1.348813\end{array}$

$\begin{array}{lll}1.285951 & 0.163052 & 4.439853\end{array}$

$\begin{array}{lll}0.516986 & 3.037621 & 3.743411\end{array}$

\section{C2-P2 re-si conf2}

Number of imaginary frequencies : 1

The smallest frequency is : $-216.8088 \mathrm{~cm}(-1)$

Electronic energy :

Zero-point correction $=$ $\mathrm{HF}=-7222.6176097$

(Hartree/Particle)

Thermal correction to Energy=

Thermal correction to Enthalpy=

1.754258

Thermal correction to Gibbs Free Energy=
Sum of electronic and zero-point Energies

Sum of electronic and zero-point Energies
Sum of electronic and thermal Energies=

Sum of electronic and thermal Enthalpies=

Sum of electronic and thermal Free Energies=

1.873240

1.874184

1.589712

$-7220.863352$ $-7220.744369$

$-7220.743425$

$-7221.027898$

Cartesian Coordinates

$\begin{array}{lll}-3.751073 & 4.706004 & 2.839634 \\ -2.590871 & 1.976120 & -1.414747 \\ -5.010192 & 0.394924 & 1.200239 \\ -3.620888 & 3.680124 & 1.019391 \\ -4.188951 & 2.841943 & 2.044171 \\ -3.226776 & 2.723733 & 3.094967 \\ -3.363400 & 2.170661 & 4.014529 \\ -2.081347 & 3.496316 & 2.740173 \\ -1.199599 & 3.642823 & 3.349587 \\ -2.325683 & 4.092038 & 1.468420 \\ -1.657964 & 4.750164 & 0.931297 \\ -5.469358 & 5.801223 & 3.255929 \\ -6.458124 & 5.562305 & 2.887453 \\ -4.884835 & 5.317391 & 4.465972 \\ -5.348323 & 4.638281 & 5.168862 \\ -3.561812 & 5.846961 & 4.558718 \\ -2.847305 & 5.638679 & 5.344324 \\ -3.328870 & 6.658415 & 3.406776 \\ -2.408191 & 7.173955 & 3.166807 \\ -4.506572 & 6.630737 & 2.602318\end{array}$




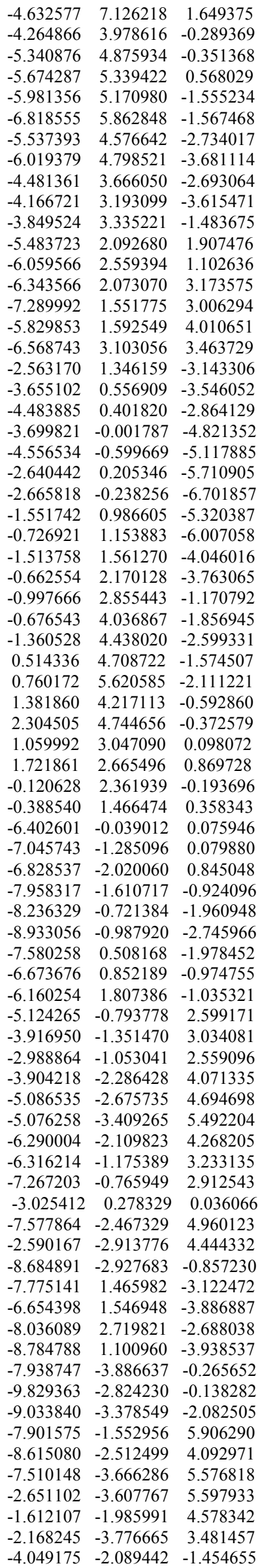

$\begin{array}{llll}-2.090797 & -2.680569 & -0.317761\end{array}$

$\begin{array}{llll}-4.210990 & -3.368778 & -1.953269\end{array}$

$-4.759072-1.308506-1.690380$

$\begin{array}{llll}-2.243621 & -4.014033 & -0.790050\end{array}$

$\begin{array}{llll}-3.292751 & -4.365737 & -1.616481\end{array}$

$\begin{array}{llll}-1.510822 & -4.760360 & -0.504530\end{array}$

$\begin{array}{lll}-0.912549 & -2.370385 & 0.442902\end{array}$

$\begin{array}{lll}-0.730679 & -1.257918 & 1.337533\end{array}$

$\begin{array}{lll}-1.395580 & -0.173633 & 1.268476\end{array}$

$\begin{array}{lll}-3.014696 & -1.739160 & -0.670109\end{array}$

$\begin{array}{lll}-0.293217 & -3.236036 & 0.628905\end{array}$

$\begin{array}{lll}0.246219 & -1.348552 & 2.291811\end{array}$

$\begin{array}{lll}0.582328 & -0.187670 & 3.105816\end{array}$

$\begin{array}{llll}0.175924 & -0.288549 & 4.119572\end{array}$

$\begin{array}{lll}1.671280 & -0.091788 & 3.174989\end{array}$

$\begin{array}{lll}0.169301 & 0.712060 & 2.654622\end{array}$

$\begin{array}{llll}0.877956 & -2.609003 & 2.678339\end{array}$

$\begin{array}{lll}0.137793 & -3.404908 & 2.780525\end{array}$

$\begin{array}{lll}1.648122 & -2.922084 & 1.965969\end{array}$

$\begin{array}{lll}1.351463 & -2.470585 & 3.652094\end{array}$

$\begin{array}{llll}3.683484 & -0.732043 & -1.549594\end{array}$

$\begin{array}{llll}5.455104 & -0.250026 & -0.211656\end{array}$

$\begin{array}{llll}5.522295 & 0.981458 & 0.900597\end{array}$

$\begin{array}{llll}6.806198 & 0.093143 & -1.122734\end{array}$

$\begin{array}{llll}5.748516 & -1.550620 & 0.814325\end{array}$

$\begin{array}{llll}5.890401 & 2.277136 & 0.555093\end{array}$

$\begin{array}{llll}4.889503 & 3.263828 & 0.692393\end{array}$

$\begin{array}{lll}3.915934 & 2.961726 & 1.059824\end{array}$

$\begin{array}{llll}5.168888 & 4.568230 & 0.371546\end{array}$

$\begin{array}{llll}4.413062 & 5.338897 & 0.491685\end{array}$

$\begin{array}{llll}6.442317 & 4.924797 & -0.146738\end{array}$

$\begin{array}{llll}6.714966 & 6.256182 & -0.562612\end{array}$

$\begin{array}{llll}5.933738 & 7.003414 & -0.449661\end{array}$

$\begin{array}{llll}7.932524 & 6.590886 & -1.110021\end{array}$

$\begin{array}{llll}8.129145 & 7.609851 & -1.430121\end{array}$

$\begin{array}{llll}8.925369 & 5.595737 & -1.272480\end{array}$

$\begin{array}{llll}9.875664 & 5.854203 & -1.730744\end{array}$

$\begin{array}{llll}8.698383 & 4.299537 & -0.863007\end{array}$

$\begin{array}{llll}9.469227 & 3.552797 & -1.008746\end{array}$

$\begin{array}{llll}7.461413 & 3.921029 & -0.268829\end{array}$

$\begin{array}{lll}7.189991 & 2.574029 & 0.170486\end{array}$

$\begin{array}{llll}8.247205 & 1.523930 & 0.160216\end{array}$

$\begin{array}{llll}9.508998 & 1.687730 & 0.835259\end{array}$

$\begin{array}{llll}9.769974 & 2.768234 & 1.723478\end{array}$

$\begin{array}{lll}8.992299 & 3.499194 & 1.911522\end{array}$

$\begin{array}{lll}10.987264 & 2.888929 & 2.357869\end{array}$

$\begin{array}{lll}11.158021 & 3.717933 & 3.038665\end{array}$

$\begin{array}{lll}12.015004 & 1.940567 & 2.138066\end{array}$

$\begin{array}{lll}12.970730 & 2.052357 & 2.641541\end{array}$

$\begin{array}{lll}11.792672 & 0.873757 & 1.297338\end{array}$

$\begin{array}{lll}12.566347 & 0.128754 & 1.130908\end{array}$

$\begin{array}{llll}10.543787 & 0.710956 & 0.637674\end{array}$

$10.295388-0.416112-0.191665$

$11.088795-1.140667-0.349835$

$\begin{array}{llll}9.055240 & -0.612969 & -0.746329\end{array}$

$\begin{array}{llll}8.830704 & -1.483941 & -1.351481\end{array}$

$\begin{array}{llll}8.039255 & 0.343496 & -0.535315\end{array}$

$\begin{array}{llll}5.042474 & -2.781200 & 0.366018\end{array}$

$\begin{array}{llll}4.141882 & -2.906006 & 0.979552\end{array}$

$\begin{array}{llll}5.877488 & -4.042494 & 0.495429\end{array}$

$\begin{array}{llll}5.294910 & -5.211065 & 0.996125\end{array}$

$\begin{array}{llll}4.262507 & -5.189699 & 1.339239\end{array}$

$\begin{array}{lll}6.032816 & -6.395169 & 1.082126\end{array}$

$\begin{array}{llll}5.569582 & -7.292970 & 1.482468\end{array}$

$\begin{array}{llll}7.366035 & -6.418310 & 0.669057\end{array}$

$\begin{array}{llll}7.946882 & -7.333004 & 0.747388\end{array}$

$\begin{array}{llll}7.951588 & -5.254763 & 0.157206\end{array}$

$\begin{array}{llll}8.991152 & -5.262546 & -0.158627\end{array}$

$\begin{array}{llll}7.209347 & -4.079828 & 0.062391\end{array}$

$\begin{array}{llll}7.670501 & -3.169804 & -0.305597\end{array}$

$\begin{array}{llll}4.609324 & -2.613560 & -1.103893\end{array}$

$\begin{array}{llll}5.488934 & -2.746347 & -1.746792\end{array}$

$\begin{array}{llll}3.919997 & -3.424826 & -1.354332\end{array}$

$\begin{array}{lll}6.097375 & -1.380472 & 2.266505\end{array}$

$\begin{array}{lll}6.470220 & -0.360721 & 2.345387\end{array}$ 
$\begin{array}{llll}7.246331 & -2.276329 & 2.714887\end{array}$

$\begin{array}{llll}8.556772 & -1.839316 & 2.487061\end{array}$

$\begin{array}{lll}8.716470 & -0.875939 & 2.016677\end{array}$

$\begin{array}{llll}9.651055 & -2.623532 & 2.852142\end{array}$

$\begin{array}{llll}10.658809 & -2.264337 & 2.661697\end{array}$

$\begin{array}{lll}9.446426 & -3.860082 & 3.469249\end{array}$

$10.294104 \quad-4.477511 \quad 3.753561$

$\begin{array}{lll}8.143422 & -4.292671 & 3.729662\end{array}$

$\begin{array}{llll}7.974250 & -5.249824 & 4.215089\end{array}$

$\begin{array}{lll}7.052692 & -3.502657 & 3.362005\end{array}$

$\begin{array}{lll}6.048995 & -3.861246 & 3.560845\end{array}$

$\begin{array}{llll}4.856259 & -1.474872 & 3.163211\end{array}$

$\begin{array}{lll}4.138042 & -0.696877 & 2.890865\end{array}$

$\begin{array}{llll}4.355756 & -2.444252 & 3.096129\end{array}$

$\begin{array}{llll}5.145266 & -1.322213 & 4.207351\end{array}$

$\begin{array}{lll}2.439592 & -0.677393 & 0.229737\end{array}$

$\begin{array}{llll}1.883257 & 0.254737 & 0.309045\end{array}$

$\begin{array}{lll}2.943382 & -0.948691 & 1.150702\end{array}$

$\begin{array}{llll}1.851459 & -1.739485 & -0.537035\end{array}$

$\begin{array}{llll}2.183832 & -2.752085 & -0.341302\end{array}$

$\begin{array}{llll}0.649194 & -1.604807 & -1.272609\end{array}$

$\begin{array}{llll}0.195869 & -0.618138 & -1.299752\end{array}$

$\begin{array}{llll}4.905820 & -0.606117 & -3.420242\end{array}$

$\begin{array}{llll}5.816126 & -1.170797 & -3.236525\end{array}$

$\begin{array}{llll}3.740432 & -1.358389 & -3.696690\end{array}$

$\begin{array}{llll}3.848597 & -2.442498 & -3.730655\end{array}$

$\begin{array}{llll}2.537736 & -0.833601 & -4.460667\end{array}$

$\begin{array}{llll}2.751186 & -0.841030 & -5.540803\end{array}$

$\begin{array}{llll}1.715888 & -1.536803 & -4.305291\end{array}$

$\begin{array}{llll}2.075934 & 0.574642 & -4.028159\end{array}$

$\begin{array}{llll}2.548069 & 1.341604 & -4.650632\end{array}$

$\begin{array}{llll}1.002617 & 0.653104 & -4.210188\end{array}$

$\begin{array}{llll}2.332979 & 0.904639 & -2.560966\end{array}$

$\begin{array}{llll}1.452844 & 0.991458 & -1.933541\end{array}$

$\begin{array}{llll}3.481543 & 1.528448 & -2.089574\end{array}$

$\begin{array}{llll}3.393951 & 2.064024 & -1.149479\end{array}$

$\begin{array}{llll}4.676555 & 1.918176 & -2.938584\end{array}$

$\begin{array}{llll}5.501547 & 2.162955 & -2.265194\end{array}$

$\begin{array}{llll}4.438677 & 2.841915 & -3.486811\end{array}$

$\begin{array}{llll}5.140999 & 0.819957 & -3.921892\end{array}$

$\begin{array}{llll}4.648580 & 0.944766 & -4.891519\end{array}$

$\begin{array}{llll}6.211525 & 0.950670 & -4.106405\end{array}$

$\begin{array}{llll}0.201837 & -2.515282 & -2.319449\end{array}$

$\begin{array}{llll}0.762290 & -3.794389 & -2.512783\end{array}$

$-0.822913-2.092018-3.188639$

$\begin{array}{llll}0.319222 & -4.611873 & -3.548943\end{array}$

$\begin{array}{lll}1.553829 & -4.145157 & -1.858163\end{array}$

$\begin{array}{lll}-1.264399 & -2.912869 & -4.224721\end{array}$

$\begin{array}{llll}-1.267904 & -1.112295 & -3.049182\end{array}$

$-0.694214-4.174608-4.410456$

$\begin{array}{lll}0.765121 & -5.592651 & -3.688127\end{array}$

$\begin{array}{llll}-2.051799 & -2.562726 & -4.884133\end{array}$

$-1.035124-4.814461-5.219298$

$\begin{array}{llll}-3.392658 & -5.378833 & -1.989913\end{array}$

$\begin{array}{lll}-5.715334 & -3.747842 & -3.140755\end{array}$

C2-P2 re-re conf2

Number of imaginary frequencies : 1

The smallest frequency is : $-121.0242 \mathrm{~cm}(-1)$

Electronic energy : $\quad \mathrm{HF}=-7222.6230214$

Zero-point correction $=\quad 1.755800$

(Hartree/Particle)

Thermal correction to Energy=

Thermal correction to Enthalpy=

1.874307

1.875251

Thermal correction to Gibbs Free Energy $=\quad 1.593527$

Sum of electronic and zero-point Energies $=\quad-7220.867221$

Sum of electronic and thermal Energies=

$-7220.748715$

Sum of electronic and thermal Enthalpies=

$-7220.747770$

Sum of electronic and thermal Free Energies $=\quad-7221.029495$

Cartesian Coordinates

$\begin{array}{llll}26 & -3.531492 & 3.834999 & 4.070237 \\ 15 & -1.204385 & 2.333391 & -0.336032\end{array}$ $\begin{array}{llll}-4.538932 & 0.592510 & 0.723575\end{array}$

$\begin{array}{llll}-2.848457 & 3.413977 & 2.131598\end{array}$

$\begin{array}{lll}-3.845501 & 2.462046 & 2.549815\end{array}$

$\begin{array}{lll}-3.360637 & 1.807680 & 3.725074\end{array}$

$\begin{array}{lll}-3.886234 & 1.044435 & 4.283171\end{array}$

$\begin{array}{lll}-2.086765 & 2.361897 & 4.048883\end{array}$

$\begin{array}{lll}-1.481630 & 2.105100 & 4.908151\end{array}$

$\begin{array}{lll}-1.774455 & 3.356377 & 3.075980\end{array}$

$\begin{array}{lll}-0.887552 & 3.972726 & 3.055945\end{array}$

$\begin{array}{llll}-5.133669 & 5.140507 & 4.294799\end{array}$

$\begin{array}{lll}-5.914251 & 5.287591 & 3.560340\end{array}$

$\begin{array}{lll}-5.138258 & 4.164092 & 5.336999\end{array}$

$\begin{array}{lll}-5.916651 & 3.436139 & 5.521696\end{array}$

$\begin{array}{lll}-3.909631 & 4.280577 & 6.055909\end{array}$

$\begin{array}{llll}-3.595414 & 3.655739 & 6.881622\end{array}$

$\begin{array}{lll}-3.144672 & 5.327983 & 5.457496\end{array}$

$\begin{array}{lll}-2.150207 & 5.637195 & 5.751199\end{array}$

$\begin{array}{lll}-3.900933 & 5.860505 & 4.370650\end{array}$

$\begin{array}{lll}-3.578813 & 6.646511 & 3.701138\end{array}$

$\begin{array}{llll}-2.954776 & 4.218717 & 0.881744\end{array}$

$\begin{array}{lll}-3.817665 & 5.322976 & 0.839076\end{array}$

$\begin{array}{lll}-4.346532 & 5.593019 & 1.743552\end{array}$

$\begin{array}{llll}-4.013950 & 6.059095 & -0.330145\end{array}$

$\begin{array}{llll}-4.696396 & 6.904149 & -0.328977\end{array}$

$\begin{array}{llll}-3.335414 & 5.698588 & -1.491018\end{array}$

$\begin{array}{llll}-3.481139 & 6.252966 & -2.412784\end{array}$

$\begin{array}{llll}-2.472715 & 4.601703 & -1.474009\end{array}$

$\begin{array}{llll}-1.965313 & 4.326066 & -2.390167\end{array}$

$\begin{array}{llll}-2.278062 & 3.844194 & -0.308653\end{array}$

$\begin{array}{lll}-5.059794 & 2.107437 & 1.742246\end{array}$

$\begin{array}{lll}-5.203585 & 2.885813 & 0.986926\end{array}$

$\begin{array}{lll}-6.351803 & 1.963344 & 2.548366\end{array}$

$\begin{array}{lll}-7.196778 & 1.701110 & 1.904877\end{array}$

$\begin{array}{lll}-6.264777 & 1.202089 & 3.328317\end{array}$

$\begin{array}{lll}-6.575832 & 2.917783 & 3.033552\end{array}$

$\begin{array}{llll}-0.605187 & 2.303140 & -2.081003\end{array}$

$\begin{array}{llll}-1.386018 & 1.590088 & -3.005213\end{array}$

$\begin{array}{llll}-2.272631 & 1.071333 & -2.659142\end{array}$

$\begin{array}{llll}-1.051976 & 1.569285 & -4.359449\end{array}$

$\begin{array}{llll}-1.674595 & 1.021487 & -5.061168\end{array}$

$\begin{array}{llll}0.082191 & 2.252954 & -4.806667\end{array}$

$\begin{array}{llll}0.350730 & 2.233502 & -5.858777\end{array}$

$\begin{array}{llll}0.870128 & 2.959603 & -3.894762\end{array}$

$\begin{array}{llll}1.756657 & 3.487855 & -4.233791\end{array}$

$\begin{array}{llll}0.526870 & 2.990579 & -2.541603\end{array}$

$\begin{array}{llll}1.138063 & 3.558697 & -1.851959\end{array}$

$\begin{array}{llll}0.239457 & 2.893392 & 0.661662\end{array}$

$\begin{array}{llll}0.685026 & 4.225929 & 0.631041\end{array}$

$\begin{array}{llll}0.195802 & 4.949665 & -0.013768\end{array}$

$\begin{array}{llll}1.742281 & 4.633665 & 1.445297\end{array}$

$\begin{array}{llll}2.082555 & 5.664539 & 1.411533\end{array}$

$\begin{array}{llll}2.361547 & 3.716926 & 2.300653\end{array}$

$\begin{array}{llll}3.184635 & 4.034926 & 2.934112\end{array}$

$\begin{array}{lll}1.916770 & 2.393943 & 2.342300\end{array}$

$\begin{array}{lll}2.386671 & 1.685112 & 3.016202\end{array}$

$\begin{array}{lll}0.856838 & 1.981739 & 1.530517\end{array}$

$\begin{array}{llll}0.475579 & 0.968476 & 1.592968\end{array}$

$\begin{array}{llll}-5.513666 & 0.768644 & -0.832464\end{array}$

$\begin{array}{llll}-6.404137 & -0.181231 & -1.353578\end{array}$

$\begin{array}{llll}-6.629864 & -1.090978 & -0.813107\end{array}$

$\begin{array}{llll}-7.003666 & 0.024557 & -2.596237\end{array}$

$\begin{array}{llll}-6.703723 & 1.149207 & -3.365214\end{array}$

$\begin{array}{llll}-7.159471 & 1.290869 & -4.337787\end{array}$

$\begin{array}{llll}-5.791006 & 2.074261 & -2.864916\end{array}$

$\begin{array}{llll}-5.206379 & 1.896059 & -1.610378\end{array}$

$\begin{array}{llll}-4.491357 & 2.631601 & -1.254308\end{array}$

$\begin{array}{llll}-5.237355 & -0.846606 & 1.634163\end{array}$

$\begin{array}{lll}-4.308727 & -1.642738 & 2.317280\end{array}$

$\begin{array}{lll}-3.257596 & -1.386138 & 2.277964\end{array}$

$\begin{array}{lll}-4.731340 & -2.749286 & 3.054128\end{array}$

$\begin{array}{lll}-6.081189 & -3.092212 & 3.114813\end{array}$

$\begin{array}{lll}-6.407036 & -3.958480 & 3.678356\end{array}$

$\begin{array}{lll}-7.007874 & -2.289976 & 2.449923\end{array}$

$\begin{array}{lll}-6.598048 & -1.168927 & 1.725869\end{array}$

$\begin{array}{lll}-7.348350 & -0.552485 & 1.245676\end{array}$ 
$\begin{array}{lll}4.014210 & -2.721951 & 0.966406\end{array}$

$\begin{array}{lll}3.021473 & -2.664961 & 1.427028\end{array}$

$\begin{array}{lll}4.800652 & -3.818560 & 1.659715\end{array}$

$\begin{array}{lll}4.146928 & -4.726092 & 2.499890\end{array}$

$\begin{array}{llll}3.081727 & -4.611201 & 2.685892\end{array}$

$\begin{array}{llll}4.854585 & -5.764551 & 3.113160\end{array}$

$\begin{array}{llll}4.336556 & -6.457548 & 3.770637\end{array}$

$\begin{array}{lll}6.225486 & -5.903995 & 2.887547\end{array}$

$\begin{array}{llll}6.779531 & -6.704869 & 3.368982\end{array}$

$\begin{array}{llll}6.883107 & -5.004124 & 2.041237\end{array}$

$\begin{array}{lll}7.951489 & -5.101013 & 1.868851\end{array}$

$\begin{array}{llll}6.174653 & -3.971254 & 1.431252\end{array}$

$\begin{array}{llll}6.689752 & -3.251649 & 0.802408\end{array}$

$\begin{array}{llll}3.835335 & -3.078667 & -0.516376\end{array}$

$\begin{array}{llll}4.805388 & -3.389817 & -0.920576\end{array}$

$\begin{array}{llll}3.184986 & -3.952822 & -0.588457\end{array}$

$\begin{array}{lll}4.764888 & -0.740720 & 2.433597\end{array}$

$\begin{array}{lll}4.836852 & 0.325708 & 2.229592\end{array}$

$\begin{array}{llll}6.031192 & -1.100313 & 3.208690\end{array}$

$\begin{array}{lll}7.163389 & -0.290813 & 3.050790\end{array}$

$\begin{array}{llll}7.124545 & 0.549550 & 2.364963\end{array}$

$\begin{array}{lll}8.339950 & -0.547086 & 3.757392\end{array}$

$\begin{array}{llll}9.203007 & 0.096005 & 3.611079\end{array}$

$\begin{array}{llll}8.399285 & -1.621806 & 4.646368\end{array}$

$\begin{array}{llll}9.312147 & -1.826509 & 5.199242\end{array}$

$\begin{array}{llll}7.272696 & -2.429608 & 4.824113\end{array}$

$\begin{array}{llll}7.306109 & -3.269182 & 5.513109\end{array}$

$\begin{array}{lll}6.098908 & -2.166854 & 4.116501\end{array}$

$\begin{array}{llll}5.241756 & -2.813580 & 4.262570\end{array}$

$\begin{array}{lll}3.482950 & -0.948661 & 3.247645\end{array}$

$\begin{array}{llll}2.610891 & -0.627871 & 2.673601\end{array}$

$\begin{array}{lll}3.330173 & -1.989597 & 3.541560\end{array}$

$\begin{array}{llll}3.537127 & -0.351248 & 4.162354\end{array}$

$\begin{array}{llll}1.536525 & -0.659593 & -0.465404\end{array}$

$\begin{array}{llll}1.264995 & 0.357011 & -0.735547\end{array}$

$\begin{array}{llll}1.737925 & -0.779387 & 0.596011\end{array}$

$\begin{array}{llll}0.825932 & -1.726175 & -1.120392\end{array}$

$\begin{array}{llll}0.199617 & -1.507489 & -1.975864\end{array}$

$\begin{array}{llll}0.769596 & -3.011739 & -0.564728\end{array}$

$\begin{array}{lll}1.314767 & -3.158435 & 0.358684\end{array}$

$\begin{array}{llll}4.587814 & -2.077999 & -3.304566\end{array}$

$\begin{array}{llll}5.414749 & -2.541833 & -2.773581\end{array}$

$\begin{array}{llll}3.430371 & -2.870847 & -3.480559\end{array}$

$\begin{array}{llll}3.464148 & -3.887291 & -3.089254\end{array}$

$\begin{array}{llll}2.387237 & -2.664396 & -4.566415\end{array}$

$\begin{array}{llll}2.744592 & -3.098951 & -5.512725\end{array}$

$\begin{array}{llll}1.502962 & -3.241964 & -4.281952\end{array}$

$\begin{array}{llll}1.977873 & -1.191168 & -4.782645\end{array}$

$\begin{array}{llll}2.605200 & -0.728456 & -5.550859\end{array}$

$\begin{array}{llll}0.954460 & -1.159146 & -5.170183\end{array}$

$\begin{array}{llll}2.035127 & -0.337858 & -3.519244\end{array}$

$\begin{array}{llll}1.076260 & -0.002497 & -3.137606\end{array}$

$\begin{array}{llll}3.140361 & 0.406229 & -3.124232\end{array}$

$\begin{array}{llll}2.945417 & 1.258788 & -2.483072\end{array}$

$\begin{array}{llll}4.473617 & 0.441221 & -3.841827\end{array}$

$\begin{array}{llll}5.198079 & 0.919992 & -3.180503\end{array}$

$\begin{array}{llll}4.382228 & 1.088835 & -4.726344\end{array}$

$\begin{array}{llll}5.003883 & -0.950466 & -4.250491\end{array}$

$\begin{array}{llll}4.679375 & -1.198846 & -5.265901\end{array}$

$\begin{array}{lll}6.096777 & -0.911165 & -4.282039\end{array}$

$\begin{array}{llll}0.363186 & -4.228522 & -1.248620\end{array}$

$\begin{array}{llll}-0.274984 & -4.226665 & -2.504901\end{array}$

$\begin{array}{llll}0.632705 & -5.468420 & -0.633835\end{array}$

$-0.608046-5.424998-3.132050$

$-0.512988-3.286786-2.990956$

$\begin{array}{llll}0.292959 & -6.665230 & -1.259127\end{array}$

$\begin{array}{llll}1.115247 & -5.481073 & 0.340406\end{array}$

$\begin{array}{lll}1.0 .325687 & -6.647292 & -2.514108\end{array}$

$\begin{array}{llll}-1.096809 & -5.405318 & -4.101853\end{array}$

$\begin{array}{llll}0.511619 & -7.611107 & -0.772140\end{array}$

$\begin{array}{lll}-0.589276 & -7.579420 & -3.005276\end{array}$

$\begin{array}{llll}-4.104807 & -4.796132 & -2.097680\end{array}$

$\begin{array}{llll}-4.823869 & -2.546664 & -4.044691\end{array}$

C2-P2 si-re conf2 
Number of imaginary frequencies : 1

The smallest frequency is : $-148.7219 \mathrm{~cm}(-1)$

Electronic energy : $\quad \mathrm{HF}=-7222.6391298$

$\begin{array}{lc}\text { Zero-point correction= } & 1.757111 \\ \text { (Hartree/Particle) } & 1.874979 \\ \text { Thermal correction to Energy= } & 1.875924 \\ \text { Thermal correction to Enthalpy= } & 1.598885 \\ \text { Thermal correction to Gibbs Free Energy }= & -7220.882019 \\ \text { Sum of electronic and zero-point Energies }= & -7220.764150 \\ \text { Sum of electronic and thermal Energies }= & -7220.763206 \\ \text { Sum of electronic and thermal Enthalpies }= & -7221.040245\end{array}$

Cartesian Coordinates

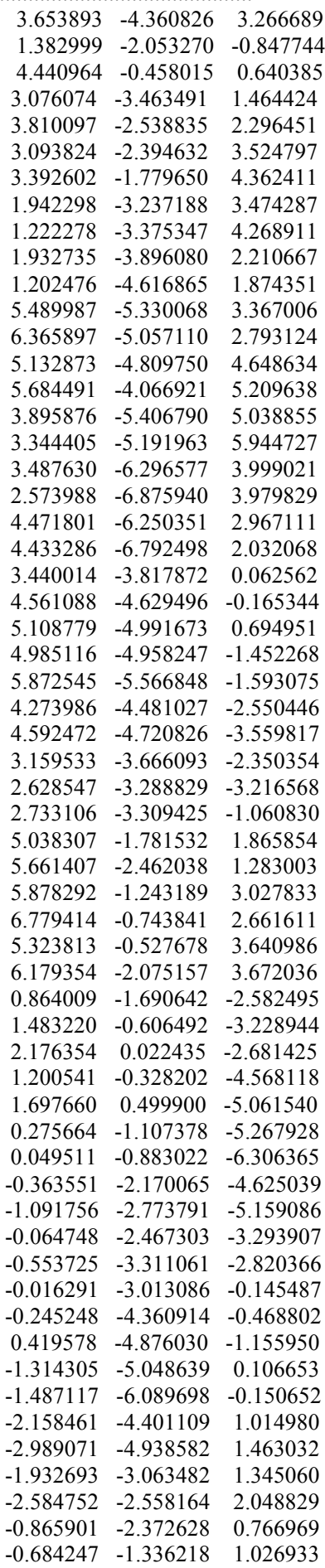

$\begin{array}{llll}5.348522 & -0.750570 & -0.935296\end{array}$

$\begin{array}{llll}4.990268 & 0.084493 & -2.003995\end{array}$

$\begin{array}{llll}4.245550 & 0.857943 & -1.843612\end{array}$

$\begin{array}{llll}5.573049 & -0.086616 & -3.259835\end{array}$

$\begin{array}{llll}6.489670 & -1.110910 & -3.489036\end{array}$

$\begin{array}{llll}6.926983 & -1.252512 & -4.470891\end{array}$

$\begin{array}{llll}6.825586 & -1.955006 & -2.430494\end{array}$

$6.278027-1.772016-1.160854$

$\begin{array}{llll}6.572417 & -2.448166 & -0.370367\end{array}$

$\begin{array}{llll}5.098980 & 1.163508 & 1.212392\end{array}$

$\begin{array}{lll}4.422668 & 1.766076 & 2.285380\end{array}$

$\begin{array}{llll}3.619229 & 1.237008 & 2.783566\end{array}$

$\begin{array}{lll}4.748027 & 3.057437 & 2.688805\end{array}$

$\begin{array}{llll}5.730865 & 3.789013 & 2.018661\end{array}$

$\begin{array}{lll}5.962504 & 4.806003 & 2.314077\end{array}$

$\begin{array}{lll}6.396982 & 3.192320 & 0.951640\end{array}$

$\begin{array}{llll}6.098326 & 1.886074 & 0.554221\end{array}$

$\begin{array}{llll}6.636994 & 1.453129 & -0.280165\end{array}$

$\begin{array}{lll}2.137728 & -0.227137 & 0.246146\end{array}$

$\begin{array}{llll}7.370728 & 3.998091 & 0.138431\end{array}$

$\begin{array}{lll}3.961409 & 3.727591 & 3.780360\end{array}$

$\begin{array}{llll}5.231075 & 0.890267 & -4.349352\end{array}$

$\begin{array}{llll}7.765218 & -3.099036 & -2.698256\end{array}$

$\begin{array}{llll}7.223213 & -3.994761 & -3.561381\end{array}$

$\begin{array}{llll}8.087761 & -3.779352 & -1.575768\end{array}$

$\begin{array}{lll}8.920903 & -2.677625 & -3.261803\end{array}$

$\begin{array}{llll}3.896862 & 1.146050 & -4.397957\end{array}$

$\begin{array}{llll}5.835161 & 2.088198 & -4.148377\end{array}$

$\begin{array}{llll}5.599010 & 0.458274 & -5.571408\end{array}$

$\begin{array}{llll}6.743291 & 4.614369 & -0.902693\end{array}$

$\begin{array}{llll}7.965219 & 4.969842 & 0.861174\end{array}$

$\begin{array}{llll}8.343619 & 3.232274 & -0.400909\end{array}$

$\begin{array}{llll}4.755413 & 4.437840 & 4.611750\end{array}$

$\begin{array}{llll}3.267038 & 2.846929 & 4.531695\end{array}$

$\begin{array}{llll}3.057658 & 4.608126 & 3.269484\end{array}$

$\begin{array}{llll}2.379755 & 2.744893 & -0.332962\end{array}$

$\begin{array}{lll}1.531224 & 3.067185 & 0.783596\end{array}$

$\begin{array}{lll}1.065175 & 2.192012 & 1.808382\end{array}$

$\begin{array}{lll}1.459646 & 4.110665 & 1.050290\end{array}$

$\begin{array}{llll}-2.905918 & 1.854984 & -1.750265\end{array}$

$\begin{array}{llll}-4.264534 & 0.521607 & -0.508885\end{array}$

$\begin{array}{llll}-4.048164 & -1.105561 & -0.291959\end{array}$

$\begin{array}{llll}-5.810395 & 0.537706 & -1.122586\end{array}$

$\begin{array}{lll}-4.240999 & 1.015847 & 1.096297\end{array}$

$-4.414767-2.026434 \quad-1.266267$

$\begin{array}{lll}-3.357500 & -2.719432 & -1.892901\end{array}$

$-2.346524-2.516884-1.564591$

$\begin{array}{llll}-3.634232 & -3.628301 & -2.881748\end{array}$

$\begin{array}{llll}-2.828907 & -4.175026 & -3.364093\end{array}$

$\begin{array}{llll}-4.973402 & -3.844113 & -3.306137\end{array}$

$\begin{array}{llll}-5.266797 & -4.721332 & -4.385045\end{array}$

$-4.443796-5.246508 \quad-4.862928$

$-6.559040 \quad-4.892245 \quad-4.827521$

$-6.771294-5.560798-5.656684$

$-7.613202-4.178846-4.209163$

$\begin{array}{lll}-8.628702 & -4.293863 & -4.577083\end{array}$

$\begin{array}{llll}-7.364747 & -3.333623 & -3.149202\end{array}$

$-8.184601 \quad-2.788708-2.696837$

$-6.045588-3.150512-2.647938$

$\begin{array}{llll}-5.750841 & -2.269578 & -1.544794\end{array}$

$-6.824445-1.611743-0.747555$

$-7.869758-2.362775-0.100075$

$-7.864184-3.784446-0.030168$

$\begin{array}{lll}-7.044228 & -4.334613 & -0.476493\end{array}$

$\begin{array}{lll}-8.874047 & -4.469544 & 0.609981\end{array}$

$\begin{array}{lll}-8.840346 & -5.554244 & 0.657428\end{array}$

$\begin{array}{lll}-9.950348 & -3.775415 & 1.212560\end{array}$

$\begin{array}{lll}-10.741876 & -4.329402 & 1.708542\end{array}$

$\begin{array}{lll}-9.979880 & -2.399544 & 1.179661\end{array}$

$\begin{array}{lll}-10.790010 & -1.851598 & 1.653640\end{array}$

$\begin{array}{lll}-8.945383 & -1.660923 & 0.542716\end{array}$

$\begin{array}{lll}-8.939296 & -0.240737 & 0.571887\end{array}$

$\begin{array}{lll}-9.758102 & 0.280349 & 1.059097\end{array}$

$\begin{array}{lll}-7.885763 & 0.461790 & 0.044062\end{array}$

$\begin{array}{lll}-7.832195 & 1.543728 & 0.099143\end{array}$ 
$\begin{array}{lll}-6.834070 & -0.233995 & -0.588970\end{array}$

$\begin{array}{lll}-3.595405 & 2.346698 & 1.256321\end{array}$

$\begin{array}{lll}-2.561062 & 2.193151 & 1.583512\end{array}$

$\begin{array}{lll}-4.280180 & 3.221572 & 2.288242\end{array}$

$\begin{array}{lll}-3.517678 & 3.849058 & 3.279838\end{array}$

$\begin{array}{llll}-2.445902 & 3.666560 & 3.322994\end{array}$

$\begin{array}{lll}-4.125130 & 4.685828 & 4.220814\end{array}$

$\begin{array}{lll}-3.523864 & 5.160116 & 4.991785\end{array}$

$\begin{array}{llll}-5.503747 & 4.903580 & 4.173619\end{array}$

$\begin{array}{llll}-5.980174 & 5.547457 & 4.907530\end{array}$

$\begin{array}{lll}-6.269691 & 4.286209 & 3.178433\end{array}$

$\begin{array}{lll}-7.343769 & 4.445985 & 3.142291\end{array}$

$\begin{array}{lll}-5.661301 & 3.452466 & 2.242108\end{array}$

$\begin{array}{lll}-6.259182 & 2.946729 & 1.489369\end{array}$

$\begin{array}{llll}-3.568991 & 3.064998 & -0.098803\end{array}$

$\begin{array}{llll}-4.583202 & 3.415170 & -0.324100\end{array}$

$\begin{array}{llll}-2.955814 & 3.963582 & -0.006981\end{array}$

$\begin{array}{lll}-4.242269 & 0.055917 & 2.254323\end{array}$

$\begin{array}{lll}-4.338198 & -0.927631 & 1.799624\end{array}$

$\begin{array}{lll}-5.435865 & 0.187614 & 3.198260\end{array}$

$\begin{array}{lll}-6.562151 & -0.609859 & 2.958613\end{array}$

$\begin{array}{lll}-6.570422 & -1.270977 & 2.097901\end{array}$

$\begin{array}{lll}-7.670736 & -0.572113 & 3.806272\end{array}$

$\begin{array}{lll}-8.530813 & -1.199945 & 3.590954\end{array}$

$\begin{array}{lll}-7.665293 & 0.265954 & 4.922688\end{array}$

$\begin{array}{lll}-8.524505 & 0.300197 & 5.587121\end{array}$

$\begin{array}{llll}-6.540990 & 1.054010 & 5.184643\end{array}$

$\begin{array}{lll}-6.523153 & 1.708590 & 6.051783\end{array}$

$\begin{array}{lll}-5.435301 & 1.010442 & 4.333943\end{array}$

$\begin{array}{lll}-4.579636 & 1.637962 & 4.553705\end{array}$

$\begin{array}{lll}-2.899729 & 0.071366 & 2.987868\end{array}$

$\begin{array}{lll}-2.077674 & -0.105372 & 2.290606\end{array}$

$\begin{array}{lll}-2.706079 & 1.012749 & 3.504126\end{array}$

$\begin{array}{lll}-2.888724 & -0.721797 & 3.739012\end{array}$

$\begin{array}{llll}-1.251841 & 0.867068 & -0.741771\end{array}$

$\begin{array}{llll}-0.992107 & -0.092142 & -1.180659\end{array}$

$\begin{array}{llll}-1.365287 & 0.820291 & 0.337983\end{array}$

$\begin{array}{llll}-0.601085 & 2.023977 & -1.296140\end{array}$

$\begin{array}{llll}-0.036050 & 1.907165 & -2.211018\end{array}$

$\begin{array}{llll}-0.505486 & 3.248233 & -0.614953\end{array}$

$\begin{array}{lll}-1.006443 & 3.316014 & 0.341183\end{array}$

$\begin{array}{llll}-4.578105 & 2.719336 & -2.955338\end{array}$

$\begin{array}{llll}-5.358854 & 3.012516 & -2.258116\end{array}$

$\begin{array}{llll}-3.458717 & 3.581261 & -3.043018\end{array}$

$\begin{array}{llll}-3.474054 & 4.475720 & -2.420944\end{array}$

$\begin{array}{llll}-2.527591 & 3.684986 & -4.239784\end{array}$

$\begin{array}{llll}-2.981874 & 4.325927 & -5.011151\end{array}$

$\begin{array}{llll}-1.624167 & 4.203876 & -3.905775\end{array}$

$\begin{array}{llll}-2.126661 & 2.326139 & -4.854627\end{array}$

$\begin{array}{llll}-2.828852 & 2.042711 & -5.644397\end{array}$

$\begin{array}{llll}-1.151396 & 2.432068 & -5.341476\end{array}$

$\begin{array}{llll}-2.031866 & 1.187838 & -3.844480\end{array}$

$\begin{array}{lll}-1.032472 & 0.824659 & -3.636325\end{array}$

$\begin{array}{llll}-3.067782 & 0.319862 & -3.537987\end{array}$

$\begin{array}{llll}-2.790593 & -0.646085 & -3.130195\end{array}$

$\begin{array}{llll}-4.474727 & 0.403866 & -4.091325\end{array}$

$\begin{array}{llll}-5.111192 & -0.241496 & -3.483650\end{array}$

$\begin{array}{llll}-4.481435 & -0.017018 & -5.107599\end{array}$

$\begin{array}{llll}-5.066197 & 1.830486 & -4.100966\end{array}$

$\begin{array}{llll}-4.849557 & 2.325629 & -5.052648\end{array}$

$\begin{array}{llll}-6.155811 & 1.753839 & -4.039220\end{array}$

$\begin{array}{llll}-0.134202 & 4.526861 & -1.213322\end{array}$

$\begin{array}{llll}0.533717 & 4.632663 & -2.449327\end{array}$

$\begin{array}{llll}-0.453695 & 5.710924 & -0.519107\end{array}$

$\begin{array}{llll}0.865298 & 5.881567 & -2.969599\end{array}$

$\begin{array}{llll}0.805047 & 3.738690 & -2.999573\end{array}$

$\begin{array}{llll}-0.129134 & 6.959486 & -1.045082\end{array}$

$\begin{array}{lll}-0.960154 & 5.640274 & 0.440445\end{array}$

$\begin{array}{llll}0.534882 & 7.049262 & -2.272620\end{array}$

$\begin{array}{llll}1.384143 & 5.945983 & -3.921714\end{array}$

$\begin{array}{lll}-0.389352 & 7.861053 & -0.498054\end{array}$

$\begin{array}{llll}0.794158 & 8.020596 & -2.683491\end{array}$

$\begin{array}{llll}2.412353 & 1.597754 & -0.884598\end{array}$

$\begin{array}{llll}0.414283 & 2.778366 & 2.936902\end{array}$

$\begin{array}{lll}0.020584 & 2.016207 & 4.015713\end{array}$ $\begin{array}{lll}0.270264 & 3.853469 & 2.956399\end{array}$

$\begin{array}{llll}0.262151 & 0.637412 & 3.959083\end{array}$

$\begin{array}{lll}0.831023 & 0.086946 & 2.827649\end{array}$

$\begin{array}{llll}0.994359 & -0.979294 & 2.743927\end{array}$

$\begin{array}{lll}1.221125 & 0.830724 & 1.771828\end{array}$

$\begin{array}{llll}3.774136 & 3.568279 & -2.175722\end{array}$

$\begin{array}{llll}3.305195 & 2.749858 & -2.715282\end{array}$

$\begin{array}{llll}4.842999 & 3.357988 & -2.062262\end{array}$

$\begin{array}{llll}3.666992 & 4.484837 & -2.764518\end{array}$

$\begin{array}{llll}3.344534 & 5.055497 & -0.261934\end{array}$

$\begin{array}{lll}3.300421 & 4.985072 & 0.824045\end{array}$

$\begin{array}{llll}2.615607 & 5.797619 & -0.609695\end{array}$

$\begin{array}{llll}4.347756 & 5.398517 & -0.527548\end{array}$

$\begin{array}{llll}3.126084 & 3.753428 & -0.884087\end{array}$

$\begin{array}{lll}-0.450669 & 2.473582 & 4.878830\end{array}$

$\begin{array}{lll}-0.211994 & -0.518593 & 5.463298\end{array}$

P2-C1 re-si conf1

Number of imaginary frequencies : 1

The smallest frequency is : $-255.8618 \mathrm{~cm}(-1)$

Electronic energy :

Zero-point correction=

$\mathrm{HF}=-7222.6359279$

(Hartree/Particle)

Thermal correction to Energy= $\quad 1.873567$

Thermal correction to Enthalpy= $\quad 1.874511$

Thermal correction to Gibbs Free Energy $=\quad 1.591721$

Sum of electronic and zero-point Energies $=\quad-7220.881098$

Sum of electronic and thermal Energies=

Sum of electronic and thermal Enthalpies=

Sum of electronic and thermal Free Energies=

$-7220.762361$

$-7220.761417$

$-7221.044207$

Cartesian Coordinates

$\begin{array}{lll}-8.013585 & 0.343897 & -1.088066\end{array}$

$\begin{array}{lll}-2.922532 & 1.119765 & -2.182064\end{array}$

$\begin{array}{lll}-3.990229 & 0.789981 & 1.429510\end{array}$

$\begin{array}{lll}-6.009727 & 0.919138 & -1.309794\end{array}$

$\begin{array}{lll}-6.234266 & 0.288003 & -0.031298\end{array}$

$-6.714893-1.035525-0.275536$

$-6.991852-1.757614 \quad 0.480056$

$-6.821167-1.218896-1.687297$

$-7.193866-2.102310-2.185261$

$-6.384704-0.020045-2.323710$

$\begin{array}{llll}-6.365875 & 0.166428 & -3.387146\end{array}$

$\begin{array}{llll}-9.369801 & 1.645924 & -0.202897\end{array}$

$\begin{array}{llll}-9.121733 & 2.363341 & 0.567879\end{array}$

$\begin{array}{lll}-9.768064 & 0.290874 & 0.011963\end{array}$

$\begin{array}{lll}-9.868212 & -0.198103 & 0.971781\end{array}$

$\begin{array}{lll}-9.968867 & -0.318377 & -1.263998\end{array}$

$-10.240037-1.351161-1.439402$

$\begin{array}{llll}-9.694377 & 0.659312 & -2.268013\end{array}$

$\begin{array}{llll}-9.724054 & 0.498671 & -3.337631\end{array}$

$\begin{array}{llll}-9.326245 & 1.872438 & -1.613604\end{array}$

$\begin{array}{llll}-9.033500 & 2.790205 & -2.104997\end{array}$

$\begin{array}{lll}-5.448299 & 2.287370 & -1.493808\end{array}$

$\begin{array}{llll}-6.228200 & 3.396000 & -1.127490\end{array}$

$\begin{array}{llll}-7.220695 & 3.213810 & -0.736441\end{array}$

$\begin{array}{llll}-5.757374 & 4.702772 & -1.243501\end{array}$

$\begin{array}{llll}-6.378555 & 5.534264 & -0.925750\end{array}$

$\begin{array}{llll}-4.484610 & 4.926807 & -1.762333\end{array}$

$\begin{array}{llll}-4.101643 & 5.936520 & -1.869047\end{array}$

$\begin{array}{llll}-3.685297 & 3.841621 & -2.119483\end{array}$

$\begin{array}{llll}-2.690234 & 4.036899 & -2.499706\end{array}$

$\begin{array}{llll}-4.129307 & 2.516357 & -1.968797\end{array}$

$\begin{array}{lll}-5.879962 & 0.906477 & 1.293815\end{array}$

$\begin{array}{lll}-6.095716 & 1.973722 & 1.226218\end{array}$

$\begin{array}{lll}-6.650607 & 0.334890 & 2.486675\end{array}$

$\begin{array}{lll}-6.373280 & 0.846161 & 3.412751\end{array}$

$\begin{array}{lll}-6.465570 & -0.733535 & 2.626049\end{array}$

$\begin{array}{lll}-7.723876 & 0.471584 & 2.321711\end{array}$

$\begin{array}{llll}-1.372942 & 2.004486 & -2.636755\end{array}$

$\begin{array}{llll}-0.596619 & 2.521408 & -1.588057\end{array}$

$\begin{array}{llll}-0.871447 & 2.306826 & -0.562153\end{array}$

$\begin{array}{llll}0.516855 & 3.315024 & -1.857986\end{array}$ 
$\begin{array}{llll}1.097015 & 3.719966 & -1.039079\end{array}$

$\begin{array}{llll}0.894043 & 3.567458 & -3.179151\end{array}$

$\begin{array}{llll}1.768850 & 4.177171 & -3.386267\end{array}$

$\begin{array}{llll}0.150596 & 3.022693 & -4.228866\end{array}$

$\begin{array}{llll}0.444707 & 3.205400 & -5.258558\end{array}$

$\begin{array}{llll}-0.984828 & 2.253225 & -3.961556\end{array}$

$\begin{array}{llll}-1.567529 & 1.858243 & -4.786949\end{array}$

$\begin{array}{llll}-3.388234 & 0.262077 & -3.734089\end{array}$

$\begin{array}{llll}-4.120953 & 0.871566 & -4.763020\end{array}$

$\begin{array}{llll}-4.466335 & 1.895325 & -4.651753\end{array}$

$\begin{array}{lll}-4.416914 & 0.159295 & -5.927190\end{array}$

$\begin{array}{llll}-4.989510 & 0.634668 & -6.718488\end{array}$

$-3.977008-1.159910 \quad-6.076442$

$-4.211704-1.711110-6.982506$

$\begin{array}{lll}-3.236075 & -1.768112 & -5.059595\end{array}$

$\begin{array}{llll}-2.890969 & -2.792220 & -5.170375\end{array}$

$\begin{array}{llll}-2.946676 & -1.059899 & -3.892315\end{array}$

$\begin{array}{llll}-2.386229 & -1.532306 & -3.091901\end{array}$

$\begin{array}{lll}-3.365067 & 2.493353 & 1.742100\end{array}$

$\begin{array}{lll}-1.969775 & 2.608751 & 1.822245\end{array}$

$\begin{array}{lll}-1.363782 & 1.711991 & 1.747155\end{array}$

$\begin{array}{lll}-1.366465 & 3.859593 & 1.950376\end{array}$

$\begin{array}{lll}-2.134041 & 5.022455 & 1.965849\end{array}$

$\begin{array}{lll}-1.664559 & 5.996572 & 2.043932\end{array}$

$\begin{array}{lll}-3.521414 & 4.906831 & 1.862576\end{array}$

$\begin{array}{lll}-4.139376 & 3.659304 & 1.767062\end{array}$

$\begin{array}{llll}-5.217730 & 3.623620 & 1.691951\end{array}$

$\begin{array}{lll}-3.620326 & -0.110043 & 2.992437\end{array}$

$\begin{array}{lll}-3.817723 & -1.500192 & 2.965952\end{array}$

$\begin{array}{lll}-4.221766 & -1.973060 & 2.078184\end{array}$

$\begin{array}{lll}-3.469084 & -2.282548 & 4.063264\end{array}$

$\begin{array}{lll}-2.887458 & -1.707057 & 5.193904\end{array}$

$\begin{array}{llll}-2.597672 & -2.320527 & 6.039191\end{array}$

$\begin{array}{lll}-2.675268 & -0.331111 & 5.211879\end{array}$

$\begin{array}{lll}-3.047389 & 0.469972 & 4.128846\end{array}$

$\begin{array}{llll}-2.871896 & 1.538234 & 4.177447\end{array}$

$-2.774266 \quad-0.168179 \quad-0.329656$

$\begin{array}{lll}-1.945819 & 0.286375 & 6.372373\end{array}$

$\begin{array}{lll}-3.605167 & -3.777839 & 4.000112\end{array}$

$\begin{array}{lll}0.129155 & 3.921549 & 2.091867\end{array}$

$\begin{array}{lll}-4.340426 & 6.168202 & 1.808339\end{array}$

$\begin{array}{llll}-4.074819 & 6.881200 & 0.686067\end{array}$

$\begin{array}{lll}-5.669524 & 5.925061 & 1.817700\end{array}$

$\begin{array}{lll}-4.070787 & 6.984207 & 2.853582\end{array}$

$\begin{array}{lll}0.749568 & 3.062411 & 1.232615\end{array}$

$\begin{array}{lll}0.537272 & 3.564982 & 3.331985\end{array}$

$\begin{array}{llll}0.626428 & 5.150700 & 1.840889\end{array}$

$\begin{array}{llll}-2.296359 & 1.574532 & 6.571417\end{array}$

$\begin{array}{llll}-0.598981 & 0.279052 & 6.162938\end{array}$

$\begin{array}{lll}-2.158141 & -0.380955 & 7.525952\end{array}$

$\begin{array}{lll}-4.008173 & -4.298975 & 5.179179\end{array}$

$\begin{array}{llll}-4.481473 & -4.176891 & 3.054785\end{array}$

$\begin{array}{lll}-2.412686 & -4.368264 & 3.707710\end{array}$

$\begin{array}{lll}-0.684270 & -1.248018 & 1.603274\end{array}$

$\begin{array}{lll}-0.979343 & -2.614031 & 1.228778\end{array}$

$\begin{array}{lll}-2.125789 & -3.069664 & 0.497687\end{array}$

$\begin{array}{lll}-0.548422 & -3.383922 & 1.852395\end{array}$

$\begin{array}{llll}3.285994 & -1.400841 & -1.340864\end{array}$

$\begin{array}{llll}5.130050 & -0.468749 & -0.389346\end{array}$

$\begin{array}{llll}5.129980 & 0.850851 & 0.608671\end{array}$

$\begin{array}{llll}6.198061 & 0.045254 & -1.559467\end{array}$

$\begin{array}{llll}5.836775 & -1.580625 & 0.647523\end{array}$

$\begin{array}{lll}4.988865 & 2.141733 & 0.117587\end{array}$

$\begin{array}{llll}3.809202 & 2.825907 & 0.484014\end{array}$

$\begin{array}{lll}3.095744 & 2.325615 & 1.128465\end{array}$

$\begin{array}{lll}3.598915 & 4.102882 & 0.026441\end{array}$

$\begin{array}{llll}2.713554 & 4.653171 & 0.328165\end{array}$

$\begin{array}{llll}4.521105 & 4.706591 & -0.870386\end{array}$

$\begin{array}{llll}4.255578 & 5.977447 & -1.448944\end{array}$

$\begin{array}{llll}3.340923 & 6.493310 & -1.168274\end{array}$

$\begin{array}{llll}5.126170 & 6.536262 & -2.357599\end{array}$

$\begin{array}{llll}4.909479 & 7.503637 & -2.801145\end{array}$

$\begin{array}{llll}6.302575 & 5.840109 & -2.725166\end{array}$

$\begin{array}{llll}6.976644 & 6.273494 & -3.458403\end{array}$

$\begin{array}{llll}6.599625 & 4.615603 & -2.166711\end{array}$ $\begin{array}{llll}7.500876 & 4.093093 & -2.465823\end{array}$

$\begin{array}{llll}5.729997 & 4.013015 & -1.215613\end{array}$

$\begin{array}{llll}6.002414 & 2.727491 & -0.626094\end{array}$

$\begin{array}{llll}7.311827 & 2.035310 & -0.793625\end{array}$

$\begin{array}{llll}8.554317 & 2.660744 & -0.415695\end{array}$

$\begin{array}{llll}8.613518 & 3.927716 & 0.230415\end{array}$

$\begin{array}{lll}7.693760 & 4.451075 & 0.461742\end{array}$

$\begin{array}{llll}9.820338 & 4.493587 & 0.580772\end{array}$

$\begin{array}{llll}9.835357 & 5.458598 & 1.079266\end{array}$

$\begin{array}{lll}11.038694 & 3.828579 & 0.305626\end{array}$

$\begin{array}{llll}11.982209 & 4.290564 & 0.580991\end{array}$

$\begin{array}{llll}11.019193 & 2.590539 & -0.295379\end{array}$

$\begin{array}{llll}11.945164 & 2.057694 & -0.495514\end{array}$

$\begin{array}{llll}9.791362 & 1.971174 & -0.655083\end{array}$

$9.770946 \quad 0.669846 \quad-1.225856$

$\begin{array}{llll}10.712231 & 0.161365 & -1.414815\end{array}$

$\begin{array}{llll}8.578302 & 0.050053 & -1.503615\end{array}$

$\begin{array}{llll}8.533575 & -0.955622 & -1.907759\end{array}$

$\begin{array}{llll}7.365993 & 0.731728 & -1.260776\end{array}$

$\begin{array}{llll}5.257581 & -2.943055 & 0.493732\end{array}$

$\begin{array}{llll}4.516197 & -3.087677 & 1.290022\end{array}$

$\begin{array}{llll}6.315481 & -4.022913 & 0.632083\end{array}$

$\begin{array}{llll}6.025570 & -5.193709 & 1.342471\end{array}$

$\begin{array}{llll}5.058859 & -5.295613 & 1.830990\end{array}$

$\begin{array}{llll}6.964063 & -6.225820 & 1.428305\end{array}$

$\begin{array}{llll}6.725267 & -7.128468 & 1.984138\end{array}$

$\begin{array}{llll}8.208923 & -6.091871 & 0.809744\end{array}$

$\begin{array}{llll}8.942888 & -6.889639 & 0.880865\end{array}$

$\begin{array}{llll}8.508546 & -4.921993 & 0.103573\end{array}$

$\begin{array}{llll}9.477716 & -4.808921 & -0.374839\end{array}$

$\begin{array}{llll}7.567748 & -3.896147 & 0.013387\end{array}$

$\begin{array}{lll}7.802952 & -2.981983 & -0.524445\end{array}$

$\begin{array}{llll}4.544156 & -3.068651 & -0.859606\end{array}$

$\begin{array}{llll}5.297704 & -3.190653 & -1.647305\end{array}$

$\begin{array}{llll}3.944224 & -3.983004 & -0.850979\end{array}$

$\begin{array}{llll}6.497243 & -1.329322 & 1.973705\end{array}$

$\begin{array}{llll}6.976835 & -2.286640 & 2.194633\end{array}$

$\begin{array}{llll}5.476687 & -1.096454 & 3.088429\end{array}$

$\begin{array}{llll}4.933107 & -2.208517 & 3.751555\end{array}$

$\begin{array}{llll}5.273828 & -3.205540 & 3.484266\end{array}$

$\begin{array}{llll}3.981754 & -2.053968 & 4.763393\end{array}$

$\begin{array}{llll}3.576546 & -2.930020 & 5.262337\end{array}$

$\begin{array}{lll}3.568680 & -0.775393 & 5.143393\end{array}$

$\begin{array}{llll}2.831533 & -0.647594 & 5.931251\end{array}$

$\begin{array}{lll}4.119314 & 0.341204 & 4.507372\end{array}$

$\begin{array}{llll}3.815321 & 1.341034 & 4.805624\end{array}$

$\begin{array}{llll}5.062545 & 0.182258 & 3.490057\end{array}$

$\begin{array}{lll}5.477042 & 1.060834 & 3.011866\end{array}$

$\begin{array}{llll}7.628632 & -0.303576 & 1.924271\end{array}$

$\begin{array}{lll}8.357645 & -0.587921 & 1.162658\end{array}$

$\begin{array}{lll}7.287443 & 0.707665 & 1.708018\end{array}$

$\begin{array}{llll}8.132257 & -0.292434 & 2.895927\end{array}$

$\begin{array}{llll}2.348044 & -1.109401 & 0.602813\end{array}$

$\begin{array}{llll}1.665523 & -0.267453 & 0.545778\end{array}$

$\begin{array}{llll}3.012226 & -1.072223 & 1.463453\end{array}$

$\begin{array}{lll}1.876670 & -2.401500 & 0.201076\end{array}$

$\begin{array}{llll}2.397768 & -3.262137 & 0.607984\end{array}$

$\begin{array}{lll}0.652580 & -2.660400 & -0.467897\end{array}$

$\begin{array}{llll}0.109245 & -1.817138 & -0.872079\end{array}$

$\begin{array}{llll}4.157221 & -1.403955 & -3.385837\end{array}$

$\begin{array}{llll}5.163595 & -1.804488 & -3.299028\end{array}$

$\begin{array}{llll}3.092997 & -2.339778 & -3.364876\end{array}$

$\begin{array}{llll}3.354414 & -3.394594 & -3.286062\end{array}$

$\begin{array}{llll}1.731807 & -2.083852 & -3.989331\end{array}$

$\begin{array}{lll}1.804121 & -2.178119 & -5.084213\end{array}$

$1.066468 \quad-2.883897 \quad-3.659208$

$\begin{array}{llll}1.103537 & -0.720021 & -3.622120\end{array}$

$\begin{array}{llll}1.306962 & 0.020496 & -4.402154\end{array}$

$\begin{array}{llll}0.014181 & -0.826433 & -3.595175\end{array}$

$\begin{array}{llll}1.559941 & -0.148565 & -2.280616\end{array}$

$\begin{array}{llll}0.786661 & -0.057986 & -1.523445\end{array}$

$\begin{array}{llll}2.654492 & 0.696163 & -2.123149\end{array}$

$\begin{array}{llll}2.636668 & 1.358649 & -1.263653\end{array}$

$\begin{array}{llll}3.612026 & 1.106893 & -3.223777\end{array}$

$\begin{array}{llll}4.486996 & 1.559363 & -2.753048\end{array}$ 
$\begin{array}{llll}3.137817 & 1.890867 & -3.830470\end{array}$

$\begin{array}{llll}4.071021 & -0.063061 & -4.118574\end{array}$

$\begin{array}{lll}3.399358 & -0.169034 & -4.976699\end{array}$

$\begin{array}{llll}5.056618 & 0.175249 & -4.530032\end{array}$

$\begin{array}{llll}0.336898 & -3.958646 & -1.072350\end{array}$

$\begin{array}{llll}0.921475 & -5.168588 & -0.650994\end{array}$

$\begin{array}{llll}-0.608250 & -4.004979 & -2.114296\end{array}$

$\begin{array}{lll}0.581260 & -6.373009 & -1.264333\end{array}$

$\begin{array}{llll}1.638155 & -5.172316 & 0.164094\end{array}$

$\begin{array}{llll}-0.944076 & -5.207961 & -2.732054\end{array}$

$-1.079183-3.082027-2.434736$

$\begin{array}{llll}-0.348895 & -6.398983 & -2.309381\end{array}$

$\begin{array}{lll}1.042200 & -7.296397 & -0.925188\end{array}$

$\begin{array}{llll}-1.672913 & -5.216163 & -3.537420\end{array}$

$\begin{array}{llll}-0.609116 & -7.339972 & -2.785251\end{array}$

$\begin{array}{lll}-2.451669 & -4.452802 & 0.569606\end{array}$

$\begin{array}{lll}-3.474498 & -4.987257 & -0.184695\end{array}$

$-1.880098-5.091581 \quad 1.231937$

$-4.184930 \quad-4.119330-1.019837$

$\begin{array}{llll}-3.867688 & -2.774891 & -1.048114\end{array}$

$-4.408674-2.080890-1.677058$

$\begin{array}{llll}-2.868225 & -2.251627 & -0.308050\end{array}$

$\begin{array}{lll}-0.967651 & -0.249167 & 0.867839\end{array}$

$\begin{array}{llll}0.021726 & -1.033130 & 2.752665\end{array}$

$\begin{array}{llll}0.543942 & 0.295018 & 3.057710\end{array}$

$\begin{array}{lll}1.541339 & 0.198552 & 3.492484\end{array}$

$\begin{array}{lll}-0.098617 & 0.816994 & 3.774276\end{array}$

$\begin{array}{lll}0.616692 & 0.884610 & 2.149455\end{array}$

$\begin{array}{lll}0.302200 & -2.062226 & 3.751766\end{array}$

$\begin{array}{lll}1.249953 & -2.575782 & 3.550132\end{array}$

$\begin{array}{lll}-0.503026 & -2.793752 & 3.802617\end{array}$

$\begin{array}{lll}0.372604 & -1.582198 & 4.729720\end{array}$

$-3.710164-6.044566-0.133593$

$\begin{array}{lll}-5.605909 & -4.807716 & -2.176937\end{array}$

P2-C1 si-si conf1

Number of imaginary frequencies : 1

The smallest frequency is : $-265.7217 \mathrm{~cm}(-1)$

Electronic energy : $\quad \mathrm{HF}=-7222.6279167$

Zero-point correction=

1.756220

(Hartree/Particle)

Thermal correction to Energy= 1874726

Thermal correction to Enthalpy= $\quad 1.875671$

Thermal correction to Gibbs Free Energy= 1.594484

Sum of electronic and zero-point Energies $=\quad-7220.871696$

Sum of electronic and thermal Energies $=\quad-7220.753190$

Sum of electronic and thermal Enthalpies $=\quad-7220.752246$

Sum of electronic and thermal Free Energies $=\quad-7221.033432$

Cartesian Coordinates

$-7.446044-1.994494-1.432371$

$\begin{array}{lll}-2.804950 & 0.143088 & -2.396528\end{array}$

$\begin{array}{lll}-4.203109 & 0.516716 & 1.047348\end{array}$

$-5.747914-0.813568-1.748292$

$\begin{array}{llll}-5.882726 & -1.165584 & -0.355363\end{array}$

$\begin{array}{llll}-5.849050 & -2.589418 & -0.263148\end{array}$

$\begin{array}{llll}-5.934535 & -3.164080 & 0.648204\end{array}$

$\begin{array}{llll}-5.727533 & -3.122546 & -1.578879\end{array}$

$\begin{array}{llll}-5.718781 & -4.171674 & -1.841401\end{array}$

$\begin{array}{llll}-5.668578 & -2.032931 & -2.493508\end{array}$

$\begin{array}{lll}-5.587293 & -2.102442 & -3.568512\end{array}$

$\begin{array}{llll}-9.252220 & -1.048363 & -1.025935\end{array}$

$\begin{array}{llll}-9.352218 & -0.090783 & -0.532183\end{array}$

$-9.205148-2.322816 \quad-0.382560$

$\begin{array}{llll}-9.254811 & -2.499891 & 0.683494\end{array}$

$\begin{array}{llll}-9.038339 & -3.320495 & -1.390670\end{array}$

$\begin{array}{llll}-8.931335 & -4.383728 & -1.220351\end{array}$

$\begin{array}{llll}-8.983960 & -2.663530 & -2.657679\end{array}$

$\begin{array}{llll}-8.829995 & -3.140891 & -3.616514\end{array}$

$\begin{array}{llll}-9.117520 & -1.260977 & -2.433216\end{array}$

$\begin{array}{lll}-9.087675 & -0.494236 & -3.195470\end{array}$

$\begin{array}{llll}-5.621999 & 0.571472 & -2.281292\end{array}$

$\begin{array}{llll}-6.757307 & 1.389503 & -2.371197\end{array}$ $\begin{array}{lll}-7.707429 & 0.983335 & -2.048889\end{array}$

$\begin{array}{llll}-6.685108 & 2.696897 & -2.850800\end{array}$

$\begin{array}{llll}-7.578574 & 3.311338 & -2.885909\end{array}$

$\begin{array}{llll}-5.457933 & 3.210072 & -3.263944\end{array}$

$\begin{array}{llll}-5.384107 & 4.225799 & -3.639454\end{array}$

$\begin{array}{llll}-4.312361 & 2.418467 & -3.174661\end{array}$

$\begin{array}{llll}-3.363445 & 2.836736 & -3.489659\end{array}$

$\begin{array}{lll}-4.366319 & 1.108805 & -2.672054\end{array}$

$\begin{array}{lll}-5.956553 & -0.177670 & 0.777243\end{array}$

$\begin{array}{lll}-6.548166 & 0.669756 & 0.429730\end{array}$

$\begin{array}{lll}-6.629559 & -0.724864 & 2.040262\end{array}$

$\begin{array}{llll}-6.686009 & 0.040193 & 2.819342\end{array}$

$\begin{array}{lll}-6.100690 & -1.587148 & 2.453888\end{array}$

$\begin{array}{lll}-7.645943 & -1.043673 & 1.790931\end{array}$

$\begin{array}{llll}-1.487041 & 1.296524 & -2.960167\end{array}$

$\begin{array}{lll}-1.109569 & 2.308575 & -2.060583\end{array}$

$\begin{array}{lll}-1.610314 & 2.385381 & -1.099675\end{array}$

$\begin{array}{llll}-0.119866 & 3.227778 & -2.401179\end{array}$

$\begin{array}{llll}0.144987 & 4.012078 & -1.705292\end{array}$

$\begin{array}{llll}0.527714 & 3.135162 & -3.636364\end{array}$

$\begin{array}{llll}1.312095 & 3.842316 & -3.889593\end{array}$

$\begin{array}{llll}0.164586 & 2.128526 & -4.532462\end{array}$

$\begin{array}{llll}0.663203 & 2.048413 & -5.494149\end{array}$

$\begin{array}{llll}-0.841744 & 1.216534 & -4.201431\end{array}$

$\begin{array}{lll}-1.109436 & 0.440922 & -4.909535\end{array}$

$-2.861782-1.184534 \quad-3.657816$

$\begin{array}{llll}-3.298512 & -0.966106 & -4.973336\end{array}$

$\begin{array}{llll}-3.620201 & 0.024148 & -5.283107\end{array}$

$\begin{array}{llll}-3.331096 & -2.024897 & -5.882485\end{array}$

$\begin{array}{llll}-3.668357 & -1.852378 & -6.900543\end{array}$

$\begin{array}{llll}-2.933351 & -3.305989 & -5.483693\end{array}$

$\begin{array}{llll}-2.960535 & -4.127218 & -6.194326\end{array}$

$\begin{array}{llll}-2.513045 & -3.530270 & -4.170420\end{array}$

$\begin{array}{llll}-2.216585 & -4.526117 & -3.853125\end{array}$

$\begin{array}{llll}-2.484168 & -2.473774 & -3.258524\end{array}$

$\begin{array}{llll}-2.213633 & -2.642363 & -2.221235\end{array}$

$\begin{array}{lll}-4.348849 & 2.351175 & 0.875952\end{array}$

$\begin{array}{lll}-3.169297 & 3.098664 & 1.005255\end{array}$

$\begin{array}{lll}-2.260813 & 2.621492 & 1.342477\end{array}$

$\begin{array}{llll}-3.139340 & 4.457900 & 0.694463\end{array}$

$\begin{array}{llll}-4.289658 & 5.115354 & 0.266391\end{array}$

$\begin{array}{lll}-4.263460 & 6.168201 & 0.008473\end{array}$

$\begin{array}{llll}-5.472183 & 4.383030 & 0.164358\end{array}$

$\begin{array}{llll}-5.507908 & 3.020472 & 0.458456\end{array}$

$\begin{array}{lll}-6.444536 & 2.497795 & 0.330791\end{array}$

$\begin{array}{lll}-3.807646 & 0.223484 & 2.816342\end{array}$

$\begin{array}{lll}-3.513278 & -1.109823 & 3.144628\end{array}$

$\begin{array}{lll}-3.555803 & -1.874530 & 2.376686\end{array}$

$\begin{array}{llll}-3.157207 & -1.454231 & 4.444657\end{array}$

$\begin{array}{lll}-3.028718 & -0.473998 & 5.430895\end{array}$

$\begin{array}{lll}-2.733988 & -0.740213 & 6.439490\end{array}$

$\begin{array}{lll}-3.293767 & 0.852703 & 5.096000\end{array}$

$\begin{array}{lll}-3.698219 & 1.205245 & 3.804507\end{array}$

$\begin{array}{lll}-3.917066 & 2.243870 & 3.582765\end{array}$

$\begin{array}{lll}-2.513651 & -0.501220 & -0.231243\end{array}$

$\begin{array}{lll}-3.077099 & 1.938411 & 6.115120\end{array}$

$\begin{array}{lll}-2.836308 & -2.887883 & 4.766774\end{array}$

$\begin{array}{lll}-1.838007 & 5.200874 & 0.824593\end{array}$

$\begin{array}{llll}-6.711796 & 5.100955 & -0.298180\end{array}$

$\begin{array}{llll}-6.537839 & 5.653900 & -1.522893\end{array}$

$\begin{array}{llll}-7.787634 & 4.287733 & -0.375703\end{array}$

$\begin{array}{lll}-7.034223 & 6.114539 & 0.538308\end{array}$

$\begin{array}{lll}-0.791232 & 4.416825 & 0.440551\end{array}$

$\begin{array}{lll}-1.587458 & 5.576626 & 2.098416\end{array}$

$\begin{array}{lll}-1.798984 & 6.311577 & 0.063049\end{array}$

$\begin{array}{lll}-3.921634 & 2.978261 & 5.933632\end{array}$

$\begin{array}{llll}-1.819216 & 2.440377 & 6.037026\end{array}$

$\begin{array}{llll}-3.243272 & 1.494344 & 7.379928\end{array}$

$\begin{array}{lll}-3.197567 & -3.217582 & 6.024826\end{array}$

$\begin{array}{lll}-3.458011 & -3.745259 & 3.924082\end{array}$

$-1.505448-3.1465894 .666306$

$\begin{array}{lll}-0.469370 & 0.806528 & 1.480102\end{array}$

$\begin{array}{lll}-0.454674 & -1.490388 & 1.908432\end{array}$

$\begin{array}{lll}0.205455 & 1.083661 & 2.656755\end{array}$

$\begin{array}{llll}-0.723520 & 1.595311 & 0.784173\end{array}$ 
$\begin{array}{lll}0.309157 & -1.247762 & 3.081496\end{array}$

$\begin{array}{lll}0.617118 & 0.036005 & 3.482922\end{array}$

$\begin{array}{lll}-0.709586 & -2.861920 & 1.552949\end{array}$

$\begin{array}{llll}-1.877914 & -3.314470 & 0.833054\end{array}$

$\begin{array}{lll}-2.536509 & -2.573405 & 0.038962\end{array}$

$\begin{array}{llll}-0.829135 & -0.440287 & 1.117159\end{array}$

$\begin{array}{lll}-0.285698 & -3.561034 & 2.259382\end{array}$

$\begin{array}{llll}3.381035 & -1.624164 & -1.235990\end{array}$

$\begin{array}{llll}5.087347 & -0.367642 & -0.417419\end{array}$

$\begin{array}{llll}4.888227 & 0.976658 & 0.526153\end{array}$

$\begin{array}{llll}6.016392 & 0.279243 & -1.636951\end{array}$

$\begin{array}{llll}5.991633 & -1.292386 & 0.653223\end{array}$

$\begin{array}{llll}4.361369 & 2.143310 & -0.002330\end{array}$

$\begin{array}{llll}3.080734 & 2.516895 & 0.459481\end{array}$

$\begin{array}{lll}2.594630 & 1.883158 & 1.191648\end{array}$

$\begin{array}{llll}2.500661 & 3.665615 & -0.016327\end{array}$

$\begin{array}{llll}1.530579 & 3.983523 & 0.348483\end{array}$

$\begin{array}{llll}3.146844 & 4.429288 & -1.026428\end{array}$

$\begin{array}{llll}2.510477 & 5.562165 & -1.603278\end{array}$

$\begin{array}{llll}1.537536 & 5.859761 & -1.220101\end{array}$

$\begin{array}{llll}3.104275 & 6.258441 & -2.632397\end{array}$

$\begin{array}{llll}2.605528 & 7.116426 & -3.073468\end{array}$

$\begin{array}{llll}4.366266 & 5.846293 & -3.124430\end{array}$

$\begin{array}{llll}4.821335 & 6.382280 & -3.952317\end{array}$

$\begin{array}{llll}5.024960 & 4.773437 & -2.562765\end{array}$

$\begin{array}{llll}5.992008 & 4.473086 & -2.949769\end{array}$

$\begin{array}{llll}4.449081 & 4.039267 & -1.489201\end{array}$

$\begin{array}{llll}5.109265 & 2.912505 & -0.879280\end{array}$

$\begin{array}{llll}6.542923 & 2.583650 & -1.124844\end{array}$

$\begin{array}{llll}7.580814 & 3.565625 & -0.914700\end{array}$

$\begin{array}{llll}7.323066 & 4.855998 & -0.370709\end{array}$

$\begin{array}{llll}6.313460 & 5.121787 & -0.083367\end{array}$

$\begin{array}{llll}8.338065 & 5.769947 & -0.185972\end{array}$

$\begin{array}{llll}8.110598 & 6.744085 & 0.237540\end{array}$

$\begin{array}{llll}9.671945 & 5.450874 & -0.532807\end{array}$

$10.460182 \quad 6.184109 \quad-0.389451$

$\begin{array}{cccc}9.964632 & 4.202130 & -1.031860\end{array}$

$\begin{array}{llll}10.987432 & 3.931237 & -1.280672\end{array}$

$\begin{array}{llll}8.943865 & 3.230847 & -1.218816\end{array}$

$\begin{array}{llll}9.259327 & 1.923192 & -1.676044\end{array}$

$\begin{array}{llll}10.291336 & 1.681268 & -1.913784\end{array}$

$\begin{array}{llll}8.279549 & 0.969539 & -1.783217\end{array}$

$\begin{array}{llll}8.499451 & -0.045557 & -2.096510\end{array}$

$\begin{array}{llll}6.939771 & 1.302639 & -1.484173\end{array}$

$\begin{array}{llll}5.555086 & -2.715488 & 0.684967\end{array}$

$\begin{array}{lll}4.859778 & -2.837853 & 1.524549\end{array}$

$\begin{array}{llll}6.723534 & -3.661216 & 0.896774\end{array}$

$\begin{array}{llll}6.581554 & -4.763091 & 1.748565\end{array}$

$\begin{array}{llll}5.648647 & -4.900494 & 2.291035\end{array}$

$\begin{array}{lll}7.622962 & -5.681904 & 1.904918\end{array}$

$\begin{array}{lll}7.498775 & -6.531378 & 2.570961\end{array}$

$\begin{array}{llll}8.823712 & -5.501488 & 1.215110\end{array}$

$\begin{array}{llll}9.637540 & -6.210096 & 1.340398\end{array}$

$\begin{array}{llll}8.974876 & -4.400622 & 0.365284\end{array}$

$\begin{array}{llll}9.908307 & -4.252340 & -0.171063\end{array}$

$\begin{array}{llll}7.931444 & -3.488723 & 0.205452\end{array}$

$\begin{array}{llll}8.049292 & -2.628004 & -0.447050\end{array}$

$\begin{array}{llll}4.816873 & -3.076028 & -0.609576\end{array}$

$\begin{array}{llll}5.554688 & -3.209580 & -1.410053\end{array}$

$\begin{array}{lll}4.320248 & -4.041046 & -0.470628\end{array}$

$\begin{array}{lll}6.687144 & -0.820372 & 1.900070\end{array}$

$\begin{array}{lll}7.304621 & -1.677850 & 2.180686\end{array}$

$\begin{array}{lll}5.708752 & -0.599119 & 3.056181\end{array}$

$\begin{array}{llll}5.322093 & -1.701802 & 3.835227\end{array}$

$\begin{array}{lll}5.765438 & -2.674077 & 3.637555\end{array}$

$\begin{array}{llll}4.396331 & -1.566532 & 4.873279\end{array}$

$\begin{array}{lll}4.114139 & -2.435558 & 5.461670\end{array}$

$\begin{array}{lll}3.854562 & -0.313291 & 5.165571\end{array}$

$\begin{array}{llll}3.142771 & -0.199885 & 5.978863\end{array}$

$\begin{array}{llll}4.254530 & 0.799018 & 4.418977\end{array}$

$\begin{array}{llll}3.853841 & 1.782272 & 4.649988\end{array}$

$\begin{array}{llll}5.173338 & 0.657220 & 3.377678\end{array}$

$\begin{array}{lll}5.470451 & 1.532803 & 2.814330\end{array}$

$\begin{array}{llll}7.659685 & 0.331102 & 1.650978\end{array}$

$\begin{array}{lll}8.373334 & 0.052732 & 0.871982\end{array}$

$\begin{array}{llll}7.166296 & 1.253049 & 1.345094\end{array}$

$\begin{array}{llll}8.215429 & 0.531048 & 2.572242\end{array}$

$\begin{array}{llll}2.452207 & -1.181895 & 0.686477\end{array}$

$\begin{array}{llll}1.713838 & -0.400637 & 0.548203\end{array}$

$\begin{array}{lll}3.134608 & -0.982242 & 1.508629\end{array}$

$\begin{array}{llll}2.100657 & -2.552783 & 0.469627\end{array}$

$\begin{array}{llll}2.717120 & -3.278232 & 0.988707\end{array}$

$\begin{array}{llll}0.903254 & -3.061023 & -0.105510\end{array}$

$\begin{array}{llll}0.268341 & -2.382314 & -0.659922\end{array}$

$\begin{array}{llll}4.172185 & -1.837046 & -3.290237\end{array}$

$\begin{array}{llll}5.211824 & -2.147287 & -3.213561\end{array}$

$\begin{array}{llll}3.198676 & -2.852511 & -3.075322\end{array}$

$\begin{array}{llll}3.570877 & -3.856545 & -2.870240\end{array}$

$\begin{array}{llll}1.790596 & -2.825968 & -3.645304\end{array}$

$\begin{array}{llll}1.806681 & -3.136735 & -4.701947\end{array}$

$\begin{array}{llll}1.207177 & -3.581885 & -3.113141\end{array}$

$\begin{array}{llll}1.083331 & -1.462430 & -3.511302\end{array}$

$\begin{array}{lll}1.253695 & -0.852629 & -4.402620\end{array}$

$\begin{array}{llll}0.003855 & -1.625622 & -3.467458\end{array}$

$\begin{array}{llll}1.497153 & -0.658300 & -2.283643\end{array}$

$\begin{array}{llll}0.743315 & -0.554863 & -1.507221\end{array}$

$\begin{array}{llll}2.505240 & 0.295932 & -2.290322\end{array}$

$\begin{array}{llll}2.446241 & 1.075217 & -1.537933\end{array}$

$\begin{array}{llll}3.394406 & 0.616464 & -3.474781\end{array}$

$\begin{array}{llll}4.227392 & 1.220703 & -3.110241\end{array}$

$\begin{array}{llll}2.829981 & 1.248368 & -4.173938\end{array}$

$\begin{array}{lll}3.950369 & -0.626693 & -4.202224\end{array}$

$\begin{array}{lll}3.285690 & -0.914928 & -5.023533\end{array}$

$\begin{array}{llll}4.905749 & -0.360551 & -4.665147\end{array}$

$\begin{array}{llll}0.774866 & -4.484594 & -0.457120\end{array}$

$\begin{array}{llll}1.629415 & -5.480426 & 0.059293\end{array}$

$\begin{array}{llll}-0.234923 & -4.882465 & -1.352812\end{array}$

$\begin{array}{llll}1.476539 & -6.814711 & -0.310915\end{array}$

$\begin{array}{llll}2.414923 & -5.216339 & 0.758855\end{array}$

$\begin{array}{lll}-0.388035 & -6.216974 & -1.724388\end{array}$

$-0.902373-4.132516-1.758563$

$\begin{array}{llll}0.466544 & -7.191393 & -1.203141\end{array}$

$\begin{array}{llll}2.146912 & -7.564718 & 0.099175\end{array}$

$-1.175488-6.495093 \quad-2.419006$

$\begin{array}{llll}0.350017 & -8.232701 & -1.488848\end{array}$

$\begin{array}{lll}-2.268113 & -4.615425 & 0.977785\end{array}$

$\begin{array}{lll}-3.309758 & -5.166752 & 0.120480\end{array}$

$\begin{array}{lll}-4.215880 & -5.384135 & 0.699985\end{array}$

$\begin{array}{lll}-2.955687 & -6.097873 & -0.334786\end{array}$

$\begin{array}{lll}-3.553604 & -4.450484 & -0.659128\end{array}$

$\begin{array}{lll}-1.655765 & -5.548460 & 1.920821\end{array}$

$\begin{array}{lll}-0.693704 & -5.923014 & 1.554922\end{array}$

$\begin{array}{lll}-2.330276 & -6.397675 & 2.047013\end{array}$

$\begin{array}{lll}-1.519495 & -5.088848 & 2.900639\end{array}$

$\begin{array}{lll}1.172250 & 0.218686 & 4.394973\end{array}$

$\begin{array}{lll}0.621810 & -2.088751 & 3.688999\end{array}$

$\begin{array}{lll}0.523342 & 2.932769 & 3.188581\end{array}$

\section{P2-C1 re-re conf1}

Number of imaginary frequencies : 1

The smallest frequency is : $-129.6199 \mathrm{~cm}(-1)$

Electronic energy : $\quad \mathrm{HF}=-7222.6324643$

Zero-point correction $=\quad 1.754828$

(Hartree/Particle)

Thermal correction to Energy= $\quad 1.873410$

Thermal correction to Enthalpy= $\quad 1.874354$

Thermal correction to Gibbs Free Energy $=\quad 1.592590$

Sum of electronic and zero-point Energies $=\quad-7220.877637$

Sum of electronic and thermal Energies $=\quad-7220.759054$

Sum of electronic and thermal Enthalpies $=\quad-7220.758110$

Sum of electronic and thermal Free Energies $=\quad-7221.039874$

\begin{tabular}{cccc}
\multicolumn{4}{c}{ Cartesian Coordinates } \\
26 & -8.003890 & 0.535566 & -0.858794 \\
15 & -2.937988 & 1.172384 & -2.105429 \\
15 & -3.903793 & 0.834210 & 1.536138 \\
6 & -5.990347 & 1.055563 & -1.115708 \\
6 & -6.207648 & 0.424180 & 0.164300
\end{tabular}


$\begin{array}{lll}-6.729963 & -0.884036 & -0.076119\end{array}$

$\begin{array}{llll}-7.012639 & -1.601361 & 0.681993\end{array}$

$-6.868040-1.058283-1.486177$

$\begin{array}{llll}-7.273277 & -1.929953 & -1.980245\end{array}$

$\begin{array}{lll}-6.410565 & 0.131149 & -2.126277\end{array}$

$\begin{array}{llll}-6.407013 & 0.320672 & -3.189396\end{array}$

$\begin{array}{llll}-9.301507 & 1.883611 & 0.045256\end{array}$

$\begin{array}{lll}-9.014316 & 2.596080 & 0.807040\end{array}$

$\begin{array}{lll}-9.738124 & 0.543182 & 0.275880\end{array}$

$\begin{array}{llll}-9.835801 & 0.063437 & 1.240657\end{array}$

$\begin{array}{lll}-9.982438 & -0.066493 & -0.992189\end{array}$

$\begin{array}{llll}-10.289443 & -1.091121 & -1.155838\end{array}$

$\begin{array}{lll}-9.695783 & 0.896155 & -2.007336\end{array}$

$\begin{array}{llll}-9.749415 & 0.730002 & -3.075186\end{array}$

$\begin{array}{llll}-9.276435 & 2.100579 & -1.367427\end{array}$

$\begin{array}{llll}-8.961294 & 3.005025 & -1.869508\end{array}$

$\begin{array}{llll}-5.392609 & 2.409612 & -1.299378\end{array}$

$\begin{array}{llll}-6.133043 & 3.535728 & -0.904898\end{array}$

$\begin{array}{llll}-7.122845 & 3.377599 & -0.497121\end{array}$

$\begin{array}{llll}-5.628771 & 4.830260 & -1.016791\end{array}$

$\begin{array}{lll}-6.222067 & 5.674824 & -0.680954\end{array}$

$\begin{array}{llll}-4.358914 & 5.025010 & -1.553914\end{array}$

$\begin{array}{llll}-3.949256 & 6.024757 & -1.653976\end{array}$

$\begin{array}{llll}-3.597294 & 3.922174 & -1.938852\end{array}$

$\begin{array}{llll}-2.604470 & 4.094589 & -2.335989\end{array}$

$\begin{array}{lll}-4.077699 & 2.608912 & -1.800000\end{array}$

$\begin{array}{lll}-5.792712 & 1.016022 & 1.484079\end{array}$

$\begin{array}{lll}-5.982368 & 2.089455 & 1.442124\end{array}$

$\begin{array}{lll}-6.534867 & 0.445288 & 2.695471\end{array}$

$\begin{array}{lll}-6.206978 & 0.929446 & 3.619615\end{array}$

$\begin{array}{lll}-6.378539 & -0.630937 & 2.806653\end{array}$

$\begin{array}{lll}-7.609044 & 0.618500 & 2.575339\end{array}$

$\begin{array}{lll}-1.365084 & 2.011567 & -2.575314\end{array}$

$\begin{array}{lll}-0.536934 & 2.457240 & -1.531021\end{array}$

$\begin{array}{lll}-0.782039 & 2.203854 & -0.506001\end{array}$

$\begin{array}{llll}0.586182 & 3.237088 & -1.805196\end{array}$

$\begin{array}{llll}1.204690 & 3.590108 & -0.990616\end{array}$

$\begin{array}{llll}0.923816 & 3.543122 & -3.125953\end{array}$

$\begin{array}{llll}1.808134 & 4.138311 & -3.335526\end{array}$

$\begin{array}{llll}0.132947 & 3.064127 & -4.172045\end{array}$

$\begin{array}{llll}0.397336 & 3.284584 & -5.202323\end{array}$

$\begin{array}{llll}-1.012603 & 2.310695 & -3.900001\end{array}$

$\begin{array}{llll}-1.635356 & 1.973547 & -4.722064\end{array}$

$\begin{array}{llll}-3.531091 & 0.436053 & -3.679490\end{array}$

$\begin{array}{llll}-4.233159 & 1.176010 & -4.643879\end{array}$

$\begin{array}{llll}-4.443656 & 2.227942 & -4.475262\end{array}$

$\begin{array}{llll}-4.676108 & 0.558584 & -5.814982\end{array}$

$\begin{array}{llll}-5.222360 & 1.136239 & -6.555264\end{array}$

$\begin{array}{llll}-4.422340 & -0.799447 & -6.033291\end{array}$

$\begin{array}{llll}-4.776719 & -1.279233 & -6.941087\end{array}$

$\begin{array}{llll}-3.715673 & -1.538401 & -5.081356\end{array}$

$\begin{array}{llll}-3.523036 & -2.594971 & -5.242819\end{array}$

$\begin{array}{lll}-3.270681 & -0.921861 & -3.910648\end{array}$

$\begin{array}{lll}-2.735752 & -1.497083 & -3.163707\end{array}$

$\begin{array}{lll}-3.203815 & 2.503927 & 1.868286\end{array}$

$\begin{array}{lll}-1.803611 & 2.563888 & 1.922557\end{array}$

$\begin{array}{lll}-1.236118 & 1.643447 & 1.834558\end{array}$

$\begin{array}{lll}-1.148962 & 3.790224 & 2.034938\end{array}$

$\begin{array}{lll}-1.868441 & 4.982606 & 2.066686\end{array}$

$\begin{array}{lll}-1.359302 & 5.937613 & 2.131572\end{array}$

$\begin{array}{lll}-3.261061 & 4.922266 & 1.993397\end{array}$

$\begin{array}{lll}-3.929960 & 3.700669 & 1.907468\end{array}$

$\begin{array}{lll}-5.009462 & 3.709763 & 1.846349\end{array}$

$\begin{array}{lll}-3.500589 & -0.138279 & 3.045923\end{array}$

$\begin{array}{llll}-3.727933 & -1.520681 & 2.955165\end{array}$

$\begin{array}{lll}-4.171269 & -1.936818 & 2.057899\end{array}$

$\begin{array}{lll}-3.351204 & -2.367602 & 3.993031\end{array}$

$\begin{array}{lll}-2.712721 & -1.863475 & 5.127957\end{array}$

$\begin{array}{lll}-2.398448 & -2.526595 & 5.925894\end{array}$

$\begin{array}{lll}-2.471183 & -0.494005 & 5.208861\end{array}$

$\begin{array}{llll}-2.870839 & 0.370616 & 4.185713\end{array}$

$\begin{array}{llll}-2.670480 & 1.431386 & 4.280891\end{array}$

$\begin{array}{lll}-2.787150 & -0.157308 & -0.283877\end{array}$

$\begin{array}{lll}-1.683282 & 0.045914 & 6.370248\end{array}$

$\begin{array}{lll}-3.524710 & -3.854620 & 3.853161\end{array}$ $\begin{array}{lll}0.350981 & 3.792703 & 2.129852\end{array}$

$\begin{array}{lll}-4.026792 & 6.217386 & 1.965405\end{array}$

$\begin{array}{lll}-3.731786 & 6.942228 & 0.857819\end{array}$

$\begin{array}{llll}-5.364877 & 6.031830 & 1.973323\end{array}$

$\begin{array}{lll}-3.720977 & 7.000060 & 3.026524\end{array}$

$\begin{array}{lll}0.904734 & 2.912419 & 1.246604\end{array}$

$\begin{array}{lll}0.787025 & 3.414231 & 3.353145\end{array}$

$\begin{array}{llll}0.890289 & 5.001200 & 1.864338\end{array}$

$\begin{array}{llll}-1.964132 & 1.341726 & 6.622703\end{array}$

$\begin{array}{lll}-0.343271 & -0.019432 & 6.128443\end{array}$

$\begin{array}{lll}-1.901361 & -0.651043 & 7.505477\end{array}$

$\begin{array}{lll}-3.955865 & -4.422762 & 5.000824\end{array}$

$\begin{array}{lll}-4.400600 & -4.182316 & 2.880021\end{array}$

$\begin{array}{llll}-2.347092 & -4.464357 & 3.543223\end{array}$

$\begin{array}{lll}-0.664618 & -1.343779 & 1.568125\end{array}$

$\begin{array}{lll}-0.978067 & -2.683055 & 1.144436\end{array}$

$\begin{array}{llll}-2.105648 & -3.099675 & 0.371665\end{array}$

$\begin{array}{lll}-0.534831 & -3.488568 & 1.711424\end{array}$

$\begin{array}{llll}3.317360 & -1.168261 & -1.663083\end{array}$

$\begin{array}{llll}5.086051 & -0.635548 & -0.337471\end{array}$

$\begin{array}{llll}5.147114 & 0.646869 & 0.712297\end{array}$

$\begin{array}{llll}6.419382 & -0.301638 & -1.273955\end{array}$

$\begin{array}{llll}5.382708 & -1.872892 & 0.752307\end{array}$

$\begin{array}{llll}5.093938 & 1.941340 & 0.214706\end{array}$

$\begin{array}{lll}3.922669 & 2.675242 & 0.499373\end{array}$

$\begin{array}{lll}3.159375 & 2.211649 & 1.111422\end{array}$

$\begin{array}{lll}3.785653 & 3.947113 & 0.002123\end{array}$

$2.908543 \quad 4.538430 \quad 0.245215$

$\begin{array}{llll}4.776293 & 4.488919 & -0.861866\end{array}$

$\begin{array}{llll}4.587239 & 5.747248 & -1.495212\end{array}$

$\begin{array}{llll}3.677494 & 6.302471 & -1.281546\end{array}$

$\begin{array}{llll}5.523394 & 6.245101 & -2.373624\end{array}$

$\begin{array}{llll}5.363959 & 7.202950 & -2.860077\end{array}$

$\begin{array}{llll}6.692360 & 5.498157 & -2.654394\end{array}$

$\begin{array}{llll}7.418263 & 5.882659 & -3.365069\end{array}$

$\begin{array}{llll}6.917166 & 4.285452 & -2.039016\end{array}$

$\begin{array}{llll}7.814683 & 3.723395 & -2.270132\end{array}$

$\begin{array}{llll}5.978206 & 3.745382 & -1.117032\end{array}$

$\begin{array}{llll}6.173879 & 2.470569 & -0.473410\end{array}$

$\begin{array}{llll}7.473496 & 1.741497 & -0.517906\end{array}$

$\begin{array}{llll}8.698688 & 2.375843 & -0.089723\end{array}$

$\begin{array}{llll}8.729461 & 3.667587 & 0.507785\end{array}$

$\begin{array}{lll}7.801889 & 4.202851 & 0.668885\end{array}$

$\begin{array}{llll}9.917447 & 4.244819 & 0.902321\end{array}$

$\begin{array}{llll}9.907776 & 5.229156 & 1.361672\end{array}$

$\begin{array}{lll}11.146448 & 3.567788 & 0.722654\end{array}$

$\begin{array}{lll}12.075404 & 4.038674 & 1.030497\end{array}$

$\begin{array}{lll}11.153327 & 2.305334 & 0.174465\end{array}$

$\begin{array}{lll}12.085789 & 1.760982 & 0.049612\end{array}$

$\begin{array}{llll}9.944378 & 1.675075 & -0.226614\end{array}$

$\begin{array}{llll}9.950186 & 0.349627 & -0.738300\end{array}$

$10.897883-0.169175 \quad-0.851536$

$\begin{array}{llll}8.772557 & -0.278082 & -1.052212\end{array}$

$\begin{array}{llll}8.745614 & -1.302187 & -1.409395\end{array}$

$\begin{array}{llll}7.547319 & 0.412849 & -0.911950\end{array}$

$\begin{array}{llll}4.478865 & -3.038698 & 0.557847\end{array}$

$\begin{array}{llll}3.622325 & -2.931166 & 1.237613\end{array}$

$\begin{array}{llll}5.160225 & -4.360359 & 0.866996\end{array}$

$\begin{array}{llll}4.476101 & -5.342630 & 1.592576\end{array}$

$\begin{array}{lll}3.485519 & -5.122596 & 1.984952\end{array}$

$\begin{array}{llll}5.055329 & -6.594453 & 1.818986\end{array}$

$\begin{array}{llll}4.512542 & -7.347034 & 2.384430\end{array}$

$\begin{array}{lll}6.332526 & -6.872698 & 1.327951\end{array}$

$\begin{array}{lll}6.788051 & -7.842213 & 1.508564\end{array}$

$\begin{array}{llll}7.025434 & -5.894084 & 0.607110\end{array}$

$\begin{array}{llll}8.021626 & -6.102745 & 0.226598\end{array}$

$\begin{array}{llll}6.441547 & -4.648920 & 0.374838\end{array}$

$\begin{array}{llll}6.979722 & -3.884697 & -0.180005\end{array}$

$\begin{array}{llll}3.981610 & -3.056184 & -0.891386\end{array}$

$\begin{array}{llll}4.810426 & -3.394618 & -1.525733\end{array}$

$\begin{array}{llll}3.196513 & -3.808029 & -0.987752\end{array}$

$\begin{array}{llll}6.060015 & -1.787521 & 2.086950\end{array}$

$\begin{array}{lll}6.349542 & -2.821694 & 2.288701\end{array}$

$\begin{array}{llll}5.113751 & -1.387694 & 3.220964\end{array}$

$\begin{array}{llll}4.455679 & -2.398772 & 3.938012\end{array}$ 
$\begin{array}{lll}4.621061 & -3.437361 & 3.663530\end{array}$

$\begin{array}{llll}3.611561 & -2.093375 & 5.008966\end{array}$

$\begin{array}{lll}3.114559 & -2.894232 & 5.549218\end{array}$

$\begin{array}{llll}3.412058 & -0.764114 & 5.385051\end{array}$

$\begin{array}{lll}2.747936 & -0.520000 & 6.209711\end{array}$

$\begin{array}{llll}4.068313 & 0.252366 & 4.684259\end{array}$

$\begin{array}{llll}3.926018 & 1.290361 & 4.972860\end{array}$

$\begin{array}{lll}4.911577 & -0.055917 & 3.614710\end{array}$

$\begin{array}{llll}5.416562 & 0.745463 & 3.090342\end{array}$

$\begin{array}{lll}7.366059 & -0.993532 & 2.066696\end{array}$

$\begin{array}{lll}8.037607 & -1.391153 & 1.302667\end{array}$

$\begin{array}{llll}7.225259 & 0.070737 & 1.879485\end{array}$

$\begin{array}{lll}7.854068 & -1.102902 & 3.039908\end{array}$

$\begin{array}{llll}2.037822 & -0.563759 & -0.021653\end{array}$

$\begin{array}{llll}1.867713 & 0.509758 & -0.005942\end{array}$

$\begin{array}{llll}2.339479 & -0.958567 & 0.946512\end{array}$

$\begin{array}{llll}1.126730 & -1.359133 & -0.802591\end{array}$

$\begin{array}{llll}0.457311 & -0.854996 & -1.487689\end{array}$

$\begin{array}{llll}0.890079 & -2.720818 & -0.540821\end{array}$

$\begin{array}{lll}1.492548 & -3.178405 & 0.236813\end{array}$

$\begin{array}{llll}4.591862 & -1.533292 & -3.475866\end{array}$

$\begin{array}{llll}5.426017 & -2.154432 & -3.158489\end{array}$

$\begin{array}{llll}3.355285 & -2.194074 & -3.655959\end{array}$

$\begin{array}{llll}3.341394 & -3.269576 & -3.479775\end{array}$

$\begin{array}{llll}2.221618 & -1.710671 & -4.542860\end{array}$

$\begin{array}{llll}2.429265 & -1.974615 & -5.591614\end{array}$

$\begin{array}{llll}1.325087 & -2.270086 & -4.259044\end{array}$

$\begin{array}{lll}1.930652 & -0.200554 & -4.426890\end{array}$

$\begin{array}{llll}2.514331 & 0.358217 & -5.165222\end{array}$

$\begin{array}{llll}0.879790 & -0.018640 & -4.672220\end{array}$

$\begin{array}{llll}2.200958 & 0.382911 & -3.042860\end{array}$

$\begin{array}{llll}1.321846 & 0.684430 & -2.485682\end{array}$

$\begin{array}{llll}3.398028 & 0.973716 & -2.666098\end{array}$

$\begin{array}{llll}3.358076 & 1.692024 & -1.853416\end{array}$

$\begin{array}{lll}4.635510 & 1.051792 & -3.535880\end{array}$

$\begin{array}{llll}5.470005 & 1.367339 & -2.908603\end{array}$

$\begin{array}{llll}4.490378 & 1.848229 & -4.281138\end{array}$

$\begin{array}{llll}5.002847 & -0.273467 & -4.240052\end{array}$

$\begin{array}{llll}4.558562 & -0.307561 & -5.240192\end{array}$

$\begin{array}{llll}6.086551 & -0.297610 & -4.388563\end{array}$

$\begin{array}{llll}0.253048 & -3.644818 & -1.466985\end{array}$

$-0.551408 \quad-3.206530 \quad-2.538361$

$\begin{array}{llll}0.447676 & -5.029189 & -1.298871\end{array}$

$\begin{array}{llll}-1.130086 & -4.121177 & -3.412224\end{array}$

$-0.726742 \quad-2.146634-2.678783$

$\begin{array}{llll}-0.124863 & -5.944817 & -2.182095\end{array}$

$\begin{array}{llll}1.056937 & -5.381590 & -0.470229\end{array}$

$\begin{array}{llll}-0.916524 & -5.494688 & -3.241414\end{array}$

$\begin{array}{llll}-1.746639 & -3.763945 & -4.231734\end{array}$

$\begin{array}{llll}0.043494 & -7.008489 & -2.040384\end{array}$

$\begin{array}{llll}-1.366453 & -6.205604 & -3.928249\end{array}$

$\begin{array}{llll}-2.375532 & -4.497008 & 0.298040\end{array}$

$\begin{array}{llll}-3.405259 & -4.991793 & -0.472884\end{array}$

$\begin{array}{lll}-1.751333 & -5.179032 & 0.863015\end{array}$

$-4.191349-4.068646-1.170036$

$\begin{array}{llll}-3.927347 & -2.717494 & -1.060514\end{array}$

$\begin{array}{llll}-4.529179 & -1.983958 & -1.579772\end{array}$

$\begin{array}{llll}-2.910818 & -2.233947 & -0.318995\end{array}$

$\begin{array}{lll}-1.010437 & -0.304093 & 0.925976\end{array}$

$\begin{array}{llll}0.123647 & -1.187449 & 2.678108\end{array}$

$\begin{array}{lll}0.677401 & 0.121984 & 3.001388\end{array}$

$\begin{array}{lll}1.694729 & 0.002151 & 3.380026\end{array}$

$\begin{array}{lll}0.083392 & 0.630983 & 3.768268\end{array}$

$\begin{array}{lll}0.710999 & 0.735585 & 2.107635\end{array}$

$\begin{array}{llll}0.433338 & -2.264960 & 3.611711\end{array}$

$\begin{array}{lll}1.335075 & -2.818157 & 3.319425\end{array}$

$\begin{array}{llll}-0.401576 & -2.959606 & 3.701103\end{array}$

$\begin{array}{llll}0.610287 & -1.829356 & 4.596274\end{array}$

$\begin{array}{llll}-3.594101 & -6.058022 & -0.533278\end{array}$

$-5.659027-4.674031 \quad-2.315731$

P2-C1 si-re conf

Number of imaginary frequencies : 1

The smallest frequency is : $-132.9607 \mathrm{~cm}(-1)$
Electronic energy : $\quad H F=-7222.6148776$

Zero-point correction=

(Hartree/Particle)

Thermal correction to Energy=

Thermal correction to Enthalpy=

1.873142

Thermal correction to Gibbs Free Energy $=\quad 1.589201$

Sum of electronic and zero-point Energies $=\quad-7220.860636$

Sum of electronic and thermal Energies

$-7220.741736$

Sum of electronic and thermal Enthalpies=

$-7220.740791$

Sum of electronic and thermal Free Energies $=\quad-7221.025677$

Cartesian Coordinates

$-4.332396-0.374629-1.603431$

$\begin{array}{lll}-6.025750 & 0.348253 & -0.273900\end{array}$

$-6.544391-0.442426 \quad 1.085853$

$\begin{array}{lll}-7.452148 & 0.436447 & -1.130948\end{array}$

$\begin{array}{lll}-5.630408 & 1.837402 & 0.383436\end{array}$

$-7.404842-1.534014 \quad 1.018542$

$\begin{array}{lll}-6.870200 & -2.775879 & 1.425842\end{array}$

$-5.847254-2.805383 \quad 1.786433$

$\begin{array}{lll}-7.648829 & -3.904255 & 1.357368\end{array}$

$\begin{array}{lll}-7.252601 & -4.865284 & 1.672470\end{array}$

$\begin{array}{lll}-8.971623 & -3.836502 & 0.842834\end{array}$

$\begin{array}{lll}-9.756983 & -5.010419 & 0.684014\end{array}$

$-9.331298-5.963224 \quad 0.987590$

$\begin{array}{llll}-11.021358 & -4.947369 & 0.143451\end{array}$

$\begin{array}{lll}-11.610485 & -5.851316 & 0.019907\end{array}$

$\begin{array}{llll}-11.549151 & -3.700160 & -0.267815\end{array}$

$-12.537704 \quad-3.656312-0.715803$

$-10.820523-2.540978-0.110980$

$\begin{array}{llll}-11.237540 & -1.596890 & -0.440850\end{array}$

$\begin{array}{lll}-9.518059 & -2.564841 & 0.461554\end{array}$

$\begin{array}{lll}-8.725103 & -1.373626 & 0.626852\end{array}$

$\begin{array}{lll}-9.276406 & -0.014953 & 0.364525\end{array}$

$\begin{array}{lll}-10.442101 & 0.484438 & 1.046144\end{array}$

$\begin{array}{lll}-11.083222 & -0.229751 & 2.097135\end{array}$

$\begin{array}{lll}-10.682692 & -1.186344 & 2.410760\end{array}$

$\begin{array}{lll}-12.194677 & 0.282497 & 2.730439\end{array}$

$\begin{array}{lll}-12.661434 & -0.279839 & 3.534059\end{array}$

$\begin{array}{llll}-12.730814 & 1.535990 & 2.350071\end{array}$

$\begin{array}{lll}-13.611743 & 1.923086 & 2.853633\end{array}$

$\begin{array}{lll}-12.122640 & 2.265270 & 1.353828\end{array}$

$\begin{array}{lll}-12.511373 & 3.237822 & 1.063215\end{array}$

$\begin{array}{lll}-10.965422 & 1.773674 & 0.690608\end{array}$

$\begin{array}{lll}-10.309336 & 2.547756 & -0.304550\end{array}$

$\begin{array}{llll}-10.723091 & 3.513625 & -0.580065\end{array}$

$\begin{array}{llll}-9.153135 & 2.095002 & -0.890820\end{array}$

$\begin{array}{llll}-8.621576 & 2.677890 & -1.634862\end{array}$

$\begin{array}{llll}-8.638216 & 0.832171 & -0.525981\end{array}$

$\begin{array}{llll}-4.473202 & 2.462424 & -0.310995\end{array}$

$\begin{array}{lll}-3.588658 & 2.322733 & 0.322535\end{array}$

$\begin{array}{llll}-4.655760 & 3.953650 & -0.538056\end{array}$

$\begin{array}{llll}-3.539685 & 4.797784 & -0.479353\end{array}$

$\begin{array}{llll}-2.570385 & 4.383320 & -0.215657\end{array}$

$-3.661243 \quad 6.160184 \quad-0.762446$

$\begin{array}{llll}-2.785919 & 6.802278 & -0.711671\end{array}$

$-4.905957 \quad 6.696658-1.101082$

$\begin{array}{llll}-5.004377 & 7.757290 & -1.314332\end{array}$

$\begin{array}{llll}-6.025382 & 5.860726 & -1.159122\end{array}$

$\begin{array}{llll}-6.997962 & 6.271211 & -1.416976\end{array}$

$\begin{array}{llll}-5.899904 & 4.498332 & -0.883901\end{array}$

$\begin{array}{llll}-6.769918 & 3.849430 & -0.915470\end{array}$

$\begin{array}{llll}-4.239226 & 1.769942 & -1.660766\end{array}$

$\begin{array}{llll}-4.999801 & 2.125685 & -2.367627\end{array}$

$\begin{array}{llll}-3.276996 & 2.095506 & -2.060404\end{array}$

$\begin{array}{lll}-5.919074 & 2.430463 & 1.727104\end{array}$

$\begin{array}{lll}-5.917368 & 3.507670 & 1.539505\end{array}$

$\begin{array}{lll}-4.792885 & 2.161493 & 2.722883\end{array}$

$\begin{array}{lll}-4.082311 & 3.239366 & 3.266005\end{array}$

$\begin{array}{lll}-4.326163 & 4.251238 & 2.951341\end{array}$

$\begin{array}{llll}-3.065153 & 3.030753 & 4.202556\end{array}$

$\begin{array}{lll}-2.526899 & 3.880977 & 4.612188\end{array}$

$\begin{array}{lll}-2.729035 & 1.733808 & 4.593410\end{array}$

$\begin{array}{lll}-1.924223 & 1.563003 & 5.301609\end{array}$ 
$\begin{array}{lll}-3.428765 & 0.650610 & 4.053264\end{array}$

$\begin{array}{lll}-3.167690 & -0.361708 & 4.347880\end{array}$

$\begin{array}{lll}-4.457565 & 0.862301 & 3.134232\end{array}$

$\begin{array}{lll}-5.002695 & 0.016739 & 2.731616\end{array}$

$\begin{array}{lll}-7.295553 & 2.120943 & 2.324049\end{array}$

$\begin{array}{lll}-8.091833 & 2.359130 & 1.616779\end{array}$

$\begin{array}{lll}-7.398614 & 1.084453 & 2.641683\end{array}$

$\begin{array}{lll}-7.423705 & 2.759140 & 3.203909\end{array}$

$\begin{array}{lll}-3.162798 & -0.687350 & 0.185106\end{array}$

$\begin{array}{lll}-3.357187 & -1.657387 & 0.642379\end{array}$

$\begin{array}{lll}-3.208281 & 0.128467 & 0.902990\end{array}$

$\begin{array}{llll}-2.130595 & -0.625976 & -0.821616\end{array}$

$\begin{array}{llll}-1.746505 & -1.549882 & -1.234773\end{array}$

$\begin{array}{lll}-1.417210 & 0.550553 & -1.111116\end{array}$

$\begin{array}{llll}-1.761653 & 1.465697 & -0.647745\end{array}$

$\begin{array}{lll}-5.572529 & -0.319746 & -3.491331\end{array}$

$\begin{array}{llll}-6.097222 & 0.632047 & -3.487794\end{array}$

$\begin{array}{lll}-4.208452 & -0.280733 & -3.848576\end{array}$

$\begin{array}{llll}-3.795032 & 0.692537 & -4.111720\end{array}$

$\begin{array}{lll}-3.389898 & -1.453872 & -4.362016\end{array}$

$\begin{array}{llll}-3.553540 & -1.579979 & -5.443430\end{array}$

$-2.333374-1.191502-4.241446$

$\begin{array}{lll}-3.661411 & -2.787008 & -3.631675\end{array}$

$\begin{array}{llll}-4.476042 & -3.329679 & -4.120183\end{array}$

$\begin{array}{llll}-2.776195 & -3.424857 & -3.722590\end{array}$

$\begin{array}{llll}-3.988902 & -2.628396 & -2.148659\end{array}$

$\begin{array}{llll}-3.224379 & -2.977866 & -1.459155\end{array}$

$-5.274710-2.522152-1.634638$

$\begin{array}{llll}-5.412421 & -2.808339 & -0.596587\end{array}$

$\begin{array}{llll}-6.548563 & -2.471991 & -2.453460\end{array}$

$\begin{array}{lll}-7.353730 & -2.136574 & -1.797015\end{array}$

$\begin{array}{llll}-6.809180 & -3.492988 & -2.768310\end{array}$

$-6.477416-1.538100-3.680881$

$\begin{array}{llll}-6.145473 & -2.093894 & -4.563300\end{array}$

$-7.488193-1.185584-3.906788$

$\begin{array}{llll}-0.539571 & 0.718805 & -2.256941\end{array}$

$\begin{array}{lll}0.049480 & -0.371389 & -2.931896\end{array}$

$\begin{array}{llll}-0.295966 & 2.021280 & -2.738852\end{array}$

$\begin{array}{llll}0.848977 & -0.161993 & -4.052044\end{array}$

$\begin{array}{llll}-0.105626 & -1.381169 & -2.569131\end{array}$

$\begin{array}{llll}0.477659 & 2.224115 & -3.878663\end{array}$

$\begin{array}{llll}-0.730346 & 2.868524 & -2.215372\end{array}$

$\begin{array}{llll}1.057791 & 1.134590 & -4.534571\end{array}$

$\begin{array}{llll}1.317705 & -1.007151 & -4.547229\end{array}$

$\begin{array}{llll}0.646583 & 3.230840 & -4.245962\end{array}$

$\begin{array}{lll}1.680333 & 1.294533 & -5.409291\end{array}$

$\begin{array}{llll}6.436005 & 4.125323 & 1.552940\end{array}$

$\begin{array}{llll}4.788566 & 0.969086 & -2.212482\end{array}$

$\begin{array}{llll}4.760229 & -0.355784 & 1.288420\end{array}$

$\begin{array}{lll}6.075154 & 2.650657 & 0.112248\end{array}$

$\begin{array}{lll}5.529620 & 2.272533 & 1.391793\end{array}$

$\begin{array}{llll}4.571111 & 3.263789 & 1.765445\end{array}$

$3.999476 \quad 3.273139 \quad 2.683413$

$\begin{array}{llll}4.539956 & 4.261994 & 0.748898\end{array}$

$\begin{array}{llll}3.941690 & 5.162490 & 0.761645\end{array}$

$\begin{array}{llll}5.466567 & 3.889206 & -0.266531\end{array}$

$\begin{array}{llll}5.687643 & 4.442688 & -1.167726\end{array}$

$\begin{array}{llll}8.181348 & 4.016208 & 2.675849\end{array}$

$\begin{array}{llll}8.692739 & 3.096320 & 2.927461\end{array}$

$\begin{array}{llll}7.139714 & 4.631182 & 3.436033\end{array}$

$\begin{array}{llll}6.720922 & 4.257803 & 4.361001\end{array}$

$\begin{array}{llll}6.716157 & 5.803004 & 2.737856\end{array}$

$\begin{array}{llll}5.917868 & 6.468589 & 3.039113\end{array}$

$\begin{array}{llll}7.495468 & 5.913357 & 1.546196\end{array}$

$\begin{array}{llll}7.392553 & 6.676581 & 0.786131\end{array}$

$\begin{array}{llll}8.401884 & 4.812218 & 1.508783\end{array}$

$\begin{array}{llll}9.108815 & 4.603894 & 0.717369\end{array}$

$\begin{array}{llll}7.027495 & 1.835287 & -0.691129\end{array}$

$\begin{array}{llll}8.384210 & 1.798518 & -0.339825\end{array}$

$\begin{array}{lll}8.702660 & 2.368055 & 0.523534\end{array}$

$\begin{array}{llll}9.310617 & 1.053962 & -1.070027\end{array}$

$10.352428 \quad 1.036270 \quad-0.765903$

$\begin{array}{llll}8.887562 & 0.332725 & -2.184542\end{array}$

$\begin{array}{llll}9.596209 & -0.245877 & -2.767539\end{array}$

$\begin{array}{llll}7.538210 & 0.336223 & -2.537883\end{array}$ $\begin{array}{llll}7.220348 & -0.243913 & -3.396951\end{array}$

$\begin{array}{llll}6.591903 & 1.060790 & -1.797027\end{array}$

$\begin{array}{lll}5.844068 & 0.986073 & 2.101867\end{array}$

$\begin{array}{llll}6.873190 & 0.717856 & 1.857606\end{array}$

$\begin{array}{lll}5.727521 & 1.064050 & 3.627042\end{array}$

$\begin{array}{llll}5.982542 & 0.107772 & 4.092229\end{array}$

$\begin{array}{lll}4.719424 & 1.336996 & 3.950217\end{array}$

$\begin{array}{lll}6.419089 & 1.826962 & 3.996330\end{array}$

$\begin{array}{llll}4.704496 & -0.335888 & -3.504852\end{array}$

$\begin{array}{llll}4.883674 & -1.664064 & -3.076371\end{array}$

$\begin{array}{llll}5.128530 & -1.863508 & -2.036563\end{array}$

$\begin{array}{llll}4.755131 & -2.725475 & -3.969750\end{array}$

$\begin{array}{llll}4.903708 & -3.741618 & -3.617714\end{array}$

$\begin{array}{llll}4.427299 & -2.473744 & -5.306761\end{array}$

$\begin{array}{llll}4.315892 & -3.298527 & -6.004750\end{array}$

$\begin{array}{llll}4.246011 & -1.158931 & -5.740526\end{array}$

$\begin{array}{llll}3.995069 & -0.957947 & -6.778363\end{array}$

$\begin{array}{llll}4.389006 & -0.092028 & -4.847090\end{array}$

$\begin{array}{llll}4.246410 & 0.924323 & -5.198044\end{array}$

$\begin{array}{llll}4.491816 & 2.565707 & -3.066405\end{array}$

$\begin{array}{llll}5.420651 & 3.114857 & -3.965143\end{array}$

$\begin{array}{llll}6.327042 & 2.569409 & -4.212343\end{array}$

$\begin{array}{llll}5.192234 & 4.370907 & -4.527888\end{array}$

$\begin{array}{llll}5.914128 & 4.791573 & -5.222222\end{array}$

$\begin{array}{llll}4.039945 & 5.090236 & -4.190505\end{array}$

$\begin{array}{llll}3.868907 & 6.072503 & -4.622252\end{array}$

$\begin{array}{llll}3.115352 & 4.547354 & -3.295679\end{array}$

$\begin{array}{llll}2.222806 & 5.106177 & -3.027665\end{array}$

$\begin{array}{llll}3.337872 & 3.287812 & -2.733611\end{array}$

$\begin{array}{llll}2.636787 & 2.871186 & -2.018067\end{array}$

$\begin{array}{llll}5.903843 & -1.728206 & 0.819084\end{array}$

$\begin{array}{llll}5.338711 & -2.961397 & 0.453130\end{array}$

$\begin{array}{llll}4.267887 & -3.117777 & 0.530870\end{array}$

$\begin{array}{llll}6.147964 & -4.007963 & 0.006237\end{array}$

$\begin{array}{llll}7.526621 & -3.849476 & -0.104770\end{array}$

$\begin{array}{llll}8.150582 & -4.668864 & -0.441707\end{array}$

$\begin{array}{llll}8.087558 & -2.621686 & 0.240923\end{array}$

$\begin{array}{llll}7.292062 & -1.568266 & 0.690627\end{array}$

$\begin{array}{llll}7.773930 & -0.629674 & 0.929115\end{array}$

$\begin{array}{llll}3.763388 & -1.056926 & 2.671284\end{array}$

$\begin{array}{lll}2.636050 & -0.308996 & 3.043079\end{array}$

$\begin{array}{llll}2.403537 & 0.610962 & 2.518671\end{array}$

$\begin{array}{lll}1.805824 & -0.747083 & 4.072732\end{array}$

$\begin{array}{llll}2.057869 & -1.953420 & 4.727743\end{array}$

$\begin{array}{lll}1.401971 & -2.301615 & 5.517020\end{array}$

$\begin{array}{lll}3.174070 & -2.698012 & 4.353289\end{array}$

$\begin{array}{llll}4.032897 & -2.252456 & 3.344285\end{array}$

$\begin{array}{llll}4.904947 & -2.846301 & 3.094511\end{array}$

$\begin{array}{llll}3.441533 & 0.389311 & -0.476643\end{array}$

$\begin{array}{llll}3.442929 & -4.032697 & 4.993580\end{array}$

$\begin{array}{lll}0.574691 & 0.041776 & 4.421619\end{array}$

$\begin{array}{llll}5.512433 & -5.310821 & -0.396109\end{array}$

$\begin{array}{llll}9.577955 & -2.456271 & 0.103152\end{array}$

$\begin{array}{llll}9.952281 & -2.346941 & -1.194606\end{array}$

$\begin{array}{lll}10.039019 & -1.361644 & 0.745449\end{array}$

$10.240207 \quad-3.525602 \quad 0.602787$

$\begin{array}{llll}4.999368 & -5.245999 & -1.652295\end{array}$

$\begin{array}{llll}4.495981 & -5.642538 & 0.428165\end{array}$

$\begin{array}{llll}6.393231 & -6.333670 & -0.387911\end{array}$

$\begin{array}{lll}4.765457 & -4.237515 & 5.188454\end{array}$

$\begin{array}{llll}3.003813 & -5.050064 & 4.212155\end{array}$

$\begin{array}{llll}2.830776 & -4.161256 & 6.190030\end{array}$

$\begin{array}{lll}0.262790 & -0.043040 & 5.733331\end{array}$

$\begin{array}{llll}0.714445 & 1.354588 & 4.125262\end{array}$

$\begin{array}{llll}-0.516127 & -0.402251 & 3.741355\end{array}$

$\begin{array}{llll}2.053133 & -2.250536 & -0.860792\end{array}$

$\begin{array}{llll}0.757264 & -0.782787 & 0.428466\end{array}$

$\begin{array}{llll}1.289511 & -3.339707 & -0.476762\end{array}$

$\begin{array}{llll}2.871062 & -2.357170 & -1.563455\end{array}$

$\begin{array}{lll}0.029133 & -1.900264 & 0.934877\end{array}$

$\begin{array}{lll}0.280150 & -3.177586 & 0.478766\end{array}$

$\begin{array}{lll}-0.744695 & -1.730795 & 1.673075\end{array}$

$\begin{array}{lll}0.264835 & 0.521343 & 0.738458\end{array}$

$\begin{array}{llll}0.910461 & 1.778108 & 0.469995\end{array}$

$\begin{array}{llll}2.037492 & 1.883813 & -0.097140\end{array}$ 
$\begin{array}{llll}1.817135 & -1.004201 & -0.410820\end{array}$

$\begin{array}{llll}-0.547750 & 0.523283 & 1.448591\end{array}$

$\begin{array}{lll}0.243864 & 2.942370 & 0.797394\end{array}$

$\begin{array}{llll}0.945503 & 4.214628 & 0.661265\end{array}$

$\begin{array}{lll}1.405205 & 4.522147 & 1.611591\end{array}$

$\begin{array}{llll}0.234546 & 4.989540 & 0.357643\end{array}$

$\begin{array}{llll}1.728742 & 4.126063 & -0.087247\end{array}$

$\begin{array}{lll}-0.892408 & 2.961566 & 1.716044\end{array}$

$\begin{array}{lll}-1.661660 & 2.245609 & 1.426488\end{array}$

$\begin{array}{lll}-1.345688 & 3.953535 & 1.694960\end{array}$

$\begin{array}{lll}-0.590790 & 2.744341 & 2.744820\end{array}$

$\begin{array}{llll}-0.295838 & -4.022463 & 0.841445\end{array}$

$\begin{array}{llll}35 & 1.597199 & -5.089668 & -1.291352\end{array}$

P2-C1 re-si conf2

Number of imaginary frequencies : 1

The smallest frequency is : $-267.9767 \mathrm{~cm}(-1)$

Electronic energy :

$\mathrm{HF}=-7222.6289103$

Zero-point correction=

1.754635

(Hartree/Particle)

Thermal correction to Energy= $\quad 1.873395$

Thermal correction to Enthalpy= $\quad 1.874339$

Thermal correction to Gibbs Free Energy= $\quad 1.590919$

Sum of electronic and zero-point Energies $=\quad-7220.874275$

Sum of electronic and thermal Energies=

Sum of electronic and thermal Enthalpies=

$-7220.755515$

Sum of electronic and thermal Free Energies=

$-7220.75457$

$-7221.037991$

Cartesian Coordinates

$\begin{array}{lll}-8.164627 & 0.057258 & -1.065056\end{array}$

$\begin{array}{lll}-3.103215 & 1.071739 & -2.100875\end{array}$

$\begin{array}{lll}-4.188583 & 0.684021 & 1.495427\end{array}$

$\begin{array}{llll}-6.189305 & 0.736413 & -1.267302\end{array}$

$\begin{array}{lll}-6.395438 & 0.096480 & 0.009527\end{array}$

$\begin{array}{lll}-6.803895 & -1.250638 & -0.237111\end{array}$

$\begin{array}{lll}-7.046643 & -1.986448 & 0.517012\end{array}$

$\begin{array}{lll}-6.886909 & -1.440226 & -1.649618\end{array}$

$\begin{array}{llll}-7.207327 & -2.342424 & -2.150055\end{array}$

$\begin{array}{lll}-6.506085 & -0.221142 & -2.283619\end{array}$

$\begin{array}{lll}-6.484707 & -0.035891 & -3.347268\end{array}$

$\begin{array}{lll}-9.603795 & 1.270912 & -0.185159\end{array}$

$\begin{array}{lll}-9.409258 & 1.994807 & 0.595273\end{array}$

$\begin{array}{lll}-9.926972 & -0.107268 & 0.009603\end{array}$

$\begin{array}{lll}-10.010702 & -0.613254 & 0.962148\end{array}$

$\begin{array}{lll}-10.076161 & -0.711579 & -1.275898\end{array}$

$\begin{array}{lll}-10.286432 & -1.755699 & -1.466962\end{array}$

$\begin{array}{llll}-9.844918 & 0.291956 & -2.265243\end{array}$

$\begin{array}{llll}-9.852705 & 0.142690 & -3.336881\end{array}$

$\begin{array}{llll}-9.556076 & 1.516207 & -1.592551\end{array}$

$\begin{array}{lll}-9.310740 & 2.455236 & -2.069711\end{array}$

$\begin{array}{llll}-5.687471 & 2.127783 & -1.444822\end{array}$

$\begin{array}{llll}-6.520940 & 3.200170 & -1.089263\end{array}$

$\begin{array}{llll}-7.509097 & 2.971867 & -0.711177\end{array}$

$\begin{array}{llll}-6.106729 & 4.526498 & -1.198552\end{array}$

$\begin{array}{llll}-6.767927 & 5.329763 & -0.889183\end{array}$

$\begin{array}{llll}-4.838039 & 4.806492 & -1.700072\end{array}$

$\begin{array}{llll}-4.498189 & 5.832040 & -1.801561\end{array}$

$\begin{array}{llll}-3.986762 & 3.757676 & -2.046174\end{array}$

$\begin{array}{llll}-2.996266 & 3.996837 & -2.412938\end{array}$

$\begin{array}{lll}-4.373487 & 2.413945 & -1.900997\end{array}$

$\begin{array}{lll}-6.080420 & 0.732436 & 1.335960\end{array}$

$\begin{array}{lll}-6.333268 & 1.791008 & 1.260490\end{array}$

$\begin{array}{lll}-6.845595 & 0.140099 & 2.522346\end{array}$

$\begin{array}{lll}-6.595096 & 0.662999 & 3.449630\end{array}$

$\begin{array}{lll}-6.628981 & -0.921518 & 2.667768\end{array}$

$\begin{array}{lll}-7.920604 & 0.242918 & 2.344627\end{array}$

$\begin{array}{lll}-1.588256 & 2.028907 & -2.520716\end{array}$

$\begin{array}{lll}-0.854192 & 2.565279 & -1.451744\end{array}$

$\begin{array}{lll}-1.135358 & 2.319372 & -0.434517\end{array}$

$\begin{array}{llll}0.224853 & 3.414332 & -1.691563\end{array}$

$\begin{array}{llll}0.772275 & 3.834021 & -0.857674\end{array}$

$\begin{array}{llll}0.609956 & 3.704561 & -3.002644\end{array}$

$\begin{array}{llll}1.458429 & 4.357514 & -3.186282\end{array}$ $\begin{array}{llll}-0.089245 & 3.140346 & -4.072380\end{array}$

$0.212843 \quad 3.351726-5.094226$

$\begin{array}{llll}-1.190974 & 2.314111 & -3.835221\end{array}$

$\begin{array}{lll}-1.740995 & 1.903498 & -4.675341\end{array}$

$\begin{array}{llll}-3.509487 & 0.207207 & -3.665494\end{array}$

$\begin{array}{llll}-4.242699 & 0.799502 & -4.704237\end{array}$

$\begin{array}{lll}-4.621911 & 1.811291 & -4.593517\end{array}$

$\begin{array}{llll}-4.494589 & 0.085449 & -5.877629\end{array}$

$\begin{array}{llll}-5.067255 & 0.547452 & -6.676739\end{array}$

$-4.010711-1.218413-6.025923$

$-4.211234-1.771012-6.939299$

$-3.270233-1.809574-4.998783$

$\begin{array}{lll}-2.891297 & -2.821777 & -5.108627\end{array}$

$\begin{array}{llll}-3.024733 & -1.099685 & -3.822499\end{array}$

$\begin{array}{llll}-2.463757 & -1.559055 & -3.014833\end{array}$

$\begin{array}{llll}-3.628067 & 2.407084 & 1.823032\end{array}$

$\begin{array}{lll}-2.240256 & 2.573630 & 1.936669\end{array}$

$\begin{array}{lll}-1.598047 & 1.701166 & 1.877043\end{array}$

$\begin{array}{lll}-1.686858 & 3.846352 & 2.075416\end{array}$

$\begin{array}{lll}-2.496697 & 4.980207 & 2.070209\end{array}$

$\begin{array}{lll}-2.064452 & 5.970660 & 2.157203\end{array}$

$\begin{array}{lll}-3.876055 & 4.813822 & 1.934770\end{array}$

$\begin{array}{lll}-4.444576 & 3.544227 & 1.826131\end{array}$

$\begin{array}{llll}-5.518449 & 3.466908 & 1.725256\end{array}$

$\begin{array}{lll}-3.813247 & -0.208535 & 3.060639\end{array}$

$\begin{array}{lll}-3.953177 & -1.605412 & 3.026630\end{array}$

$\begin{array}{lll}-4.319934 & -2.091735 & 2.130204\end{array}$

$\begin{array}{llll}-3.596890 & -2.375974 & 4.129803\end{array}$

$\begin{array}{lll}-3.066368 & -1.780576 & 5.275698\end{array}$

$\begin{array}{llll}-2.771185 & -2.384507 & 6.125832\end{array}$

$\begin{array}{lll}-2.916692 & -0.396811 & 5.303276\end{array}$

$\begin{array}{lll}-3.295856 & 0.390940 & 4.212801\end{array}$

$\begin{array}{llll}-3.170008 & 1.465730 & 4.269138\end{array}$

$\begin{array}{lll}-2.914389 & -0.222419 & -0.250930\end{array}$

$\begin{array}{lll}-2.249939 & 0.256051 & 6.481960\end{array}$

$\begin{array}{llll}-3.672285 & -3.875360 & 4.060073\end{array}$

$\begin{array}{llll}-0.197558 & 3.963067 & 2.247629\end{array}$

$\begin{array}{llll}-4.738377 & 6.045317 & 1.864166\end{array}$

$\begin{array}{llll}-4.463300 & 6.776960 & 0.756422\end{array}$

$\begin{array}{lll}-6.057682 & 5.755554 & 1.830887\end{array}$

$\begin{array}{llll}-4.530439 & 6.861124 & 2.923739\end{array}$

$\begin{array}{llll}0.470335 & 3.133043 & 1.394911\end{array}$

$\begin{array}{lll}0.198654 & 3.612299 & 3.492956\end{array}$

$\begin{array}{lll}0.259078 & 5.210987 & 2.013743\end{array}$

$\begin{array}{lll}-2.716304 & 1.503103 & 6.706278\end{array}$

$\begin{array}{lll}-0.908081 & 0.375665 & 6.278115\end{array}$

$\begin{array}{lll}-2.405996 & -0.451977 & 7.619593\end{array}$

$\begin{array}{lll}-4.076246 & -4.416492 & 5.229632\end{array}$

$\begin{array}{lll}-4.514138 & -4.305467 & 3.097178\end{array}$

$\begin{array}{lll}-2.452208 & -4.416622 & 3.787833\end{array}$

$\begin{array}{llll}-0.794431 & -1.230090 & 1.704803\end{array}$

$\begin{array}{lll}-1.032434 & -2.604850 & 1.318073\end{array}$

$\begin{array}{lll}-2.160810 & -3.099280 & 0.581783\end{array}$

$\begin{array}{lll}-0.578073 & -3.361320 & 1.941823\end{array}$

$\begin{array}{llll}3.150248 & -1.209094 & -1.279168\end{array}$

$\begin{array}{llll}4.969673 & -0.206197 & -0.363083\end{array}$

$\begin{array}{llll}4.906103 & 1.091553 & 0.671777\end{array}$

$\begin{array}{llll}5.977806 & 0.396016 & -1.542326\end{array}$

$\begin{array}{llll}5.781249 & -1.285238 & 0.636034\end{array}$

$\begin{array}{llll}4.737106 & 2.390790 & 0.215375\end{array}$

$\begin{array}{llll}3.534557 & 3.030063 & 0.588299\end{array}$

$\begin{array}{llll}2.825818 & 2.487869 & 1.203111\end{array}$

$\begin{array}{llll}3.295559 & 4.317217 & 0.177371\end{array}$

$\begin{array}{llll}2.390918 & 4.830795 & 0.487255\end{array}$

$\begin{array}{llll}4.215290 & 4.980298 & -0.678977\end{array}$

$\begin{array}{llll}3.925776 & 6.268675 & -1.204750\end{array}$

$\begin{array}{llll}2.995146 & 6.748843 & -0.913348\end{array}$

$\begin{array}{llll}4.793704 & 6.888768 & -2.075136\end{array}$

$\begin{array}{llll}4.559551 & 7.869942 & -2.477539\end{array}$

$\begin{array}{llll}5.990066 & 6.236662 & -2.457820\end{array}$

$\begin{array}{llll}6.662314 & 6.717237 & -3.162781\end{array}$

$\begin{array}{llll}6.309535 & 4.995453 & -1.950757\end{array}$

$\begin{array}{llll}7.225643 & 4.508827 & -2.263904\end{array}$

$\begin{array}{llll}5.445243 & 4.330559 & -1.036623\end{array}$

$\begin{array}{llll}5.742922 & 3.027629 & -0.496323\end{array}$ 
$\begin{array}{llll}-3.434671 & -5.063463 & -0.110404\end{array}$

$\begin{array}{llll}-1.853719 & -5.109753 & 1.323792\end{array}$

$-4.162758 \quad-4.222890 \quad-0.957634$

$\begin{array}{llll}-3.892060 & -2.868232 & -0.985269\end{array}$

$\begin{array}{lll}-4.447336 & -2.195952 & -1.625001\end{array}$

$\begin{array}{lll}-2.921474 & -2.308020 & -0.233194\end{array}$

$\begin{array}{lll}-1.106842 & -0.239353 & 0.971772\end{array}$

$\begin{array}{lll}-0.110066 & -0.996949 & 2.865573\end{array}$

$\begin{array}{lll}0.376830 & 0.341672 & 3.180703\end{array}$

$\begin{array}{lll}1.377473 & 0.267450 & 3.618817\end{array}$

$\begin{array}{lll}-0.277053 & 0.842017 & 3.902282\end{array}$

$\begin{array}{lll}0.435635 & 0.940033 & 2.277296\end{array}$

$\begin{array}{llll}0.169849 & -2.010177 & 3.880411\end{array}$

$\begin{array}{lll}1.149658 & -2.480153 & 3.723726\end{array}$

$\begin{array}{lll}-0.599318 & -2.780768 & 3.897645\end{array}$

$\begin{array}{llll}0.170951 & -1.524820 & 4.859250\end{array}$

$\begin{array}{lll}-3.634474 & -6.128068 & -0.059188\end{array}$

$35 \quad-5.540006 \quad-4.964556-2.134005$

\section{P2-C1 si-si conf2}

Number of imaginary frequencies : 1

The smallest frequency is : $-266.9252 \mathrm{~cm}(-1)$

Electronic energy : $\quad \mathrm{HF}=-7222.6225041$

Zero-point correction=

(Hartree/Particle)

Thermal correction to Energy $=\quad 1.873789$

Thermal correction to Enthalpy= $\quad 1.874733$

Thermal correction to Gibbs Free Energy $=\quad 1.596156$

Sum of electronic and zero-point Energies $=\quad-7220.867200$

Sum of electronic and thermal Energies $=\quad-7220.748715$

Sum of electronic and thermal Enthalpies $=\quad-7220.747771$

Sum of electronic and thermal Free Energies $=\quad-7221.026348$

Cartesian Coordinates

$\begin{array}{ccccc}26 & -7.587737 & -2.030120 & -1.503578 \\ 15 & -2.982145 & 0.290134 & -2.301438 \\ 15 & -4.408503 & 0.363313 & 1.158495 \\ 6 & -5.910194 & -0.797811 & -1.737637 \\ 6 & -6.045487 & -1.241406 & -0.371125 \\ 6 & -5.984273 & -2.666595 & -0.366827 \\ 1 & -6.061588 & -3.298816 & 0.506460 \\ 6 & -5.847183 & -3.111998 & -1.714095 \\ 1 & -5.816015 & -4.141703 & -2.043405 \\ 6 & -5.805672 & -1.965233 & -2.558266 \\ 1 & -5.719254 & -1.966488 & -3.634902 \\ 6 & -9.411110 & -1.164151 & -1.007964 \\ 1 & -9.524467 & -0.257497 & -0.428184 \\ 6 & -9.332159 & -2.490786 & -0.483910 \\ 1 & -9.370071 & -2.765494 & 0.561692 \\ 6 & -9.150577 & -3.388404 & -1.579896 \\ 1 & -9.020587 & -4.460268 & -1.508050 \\ 6 & -9.116668 & -2.617626 & -2.781714 \\ 1 & -8.958973 & -3.002187 & -3.780748 \\ 6 & -9.278997 & -1.244724 & -2.429334 \\ 1 & -9.270492 & -0.411021 & -3.118186 \\ 6 & -5.813259 & 0.628040 & -2.160974 \\ 6 & -6.966849 & 1.425874 & -2.171613 \\ 1 & -7.906619 & 0.967310 & -1.892626 \\ 6 & -6.926067 & 2.776475 & -2.516030 \\ 1 & -7.833657 & 3.370536 & -2.489856\end{array}$


$\begin{array}{llll}-5.711640 & 3.356397 & -2.874325\end{array}$

$\begin{array}{llll}-5.660840 & 4.406631 & -3.143498\end{array}$

$\begin{array}{llll}-4.548595 & 2.585868 & -2.864101\end{array}$

$\begin{array}{llll}-3.610231 & 3.056927 & -3.132745\end{array}$

$\begin{array}{llll}-4.570425 & 1.231128 & -2.495048\end{array}$

$\begin{array}{lll}-6.147653 & -0.315184 & 0.808645\end{array}$

$\begin{array}{lll}-6.710589 & 0.554532 & 0.470750\end{array}$

$\begin{array}{lll}-6.880430 & -0.900879 & 2.019068\end{array}$

$\begin{array}{lll}-6.922259 & -0.181175 & 2.841372\end{array}$

$\begin{array}{lll}-6.407743 & -1.812402 & 2.392121\end{array}$

$\begin{array}{lll}-7.903848 & -1.152778 & 1.725778\end{array}$

$\begin{array}{llll}-1.696044 & 1.506233 & -2.806632\end{array}$

$\begin{array}{lll}-1.308245 & 2.452313 & -1.842264\end{array}$

$\begin{array}{llll}-1.780838 & 2.450113 & -0.865178\end{array}$

$\begin{array}{llll}-0.340765 & 3.411024 & -2.134609\end{array}$

$\begin{array}{llll}-0.063706 & 4.138879 & -1.384071\end{array}$

$\begin{array}{llll}0.272839 & 3.426692 & -3.389954\end{array}$

$\begin{array}{llll}1.040171 & 4.163886 & -3.607896\end{array}$

$\begin{array}{llll}-0.102141 & 2.488160 & -4.352854\end{array}$

$\begin{array}{llll}0.369765 & 2.492138 & -5.331237\end{array}$

$\begin{array}{lll}-1.084434 & 1.535572 & -4.067389\end{array}$

$\begin{array}{llll}-1.358886 & 0.813545 & -4.827830\end{array}$

$\begin{array}{llll}-3.024373 & -0.958034 & -3.641947\end{array}$

$\begin{array}{llll}-3.483239 & -0.670641 & -4.936489\end{array}$

$\begin{array}{llll}-3.835003 & 0.327737 & -5.180319\end{array}$

$\begin{array}{llll}-3.500509 & -1.672787 & -5.908177\end{array}$

$\begin{array}{llll}-3.855396 & -1.447454 & -6.909791\end{array}$

$\begin{array}{lll}-3.064349 & -2.964904 & -5.593817\end{array}$

$\begin{array}{llll}-3.079468 & -3.741132 & -6.353684\end{array}$

$\begin{array}{llll}-2.621482 & -3.258475 & -4.301722\end{array}$

$\begin{array}{llll}-2.294495 & -4.263382 & -4.049774\end{array}$

$\begin{array}{llll}-2.609322 & -2.259187 & -3.327461\end{array}$

$\begin{array}{llll}-2.323342 & -2.483730 & -2.305137\end{array}$

$\begin{array}{lll}-4.586523 & 2.202559 & 1.171447\end{array}$

$\begin{array}{lll}-3.406763 & 2.947169 & 1.308168\end{array}$

$\begin{array}{lll}-2.486690 & 2.444570 & 1.568588\end{array}$

$\begin{array}{llll}-3.392252 & 4.323874 & 1.083888\end{array}$

$\begin{array}{lll}-4.559426 & 4.998675 & 0.735291\end{array}$

$\begin{array}{lll}-4.544650 & 6.063574 & 0.531420\end{array}$

$\begin{array}{llll}-5.743208 & 4.268481 & 0.633010\end{array}$

$\begin{array}{lll}-5.765116 & 2.891021 & 0.849402\end{array}$

$\begin{array}{lll}-6.707336 & 2.373713 & 0.735995\end{array}$

$\begin{array}{lll}-3.984023 & -0.127869 & 2.874739\end{array}$

$\begin{array}{lll}-3.800150 & -1.506259 & 3.073924\end{array}$

$\begin{array}{lll}-3.981371 & -2.198174 & 2.259017\end{array}$

$\begin{array}{lll}-3.351382 & -1.985637 & 4.299730\end{array}$

$\begin{array}{lll}-3.023056 & -1.105273 & 5.331860\end{array}$

$\begin{array}{lll}-2.644004 & -1.478063 & 6.277408\end{array}$

$\begin{array}{lll}-3.175939 & 0.263913 & 5.120270\end{array}$

$\begin{array}{lll}-3.668938 & 0.757962 & 3.909861\end{array}$

$\begin{array}{lll}-3.786002 & 1.827315 & 3.780416\end{array}$

$\begin{array}{lll}-2.671094 & -0.489416 & -0.185857\end{array}$

$\begin{array}{llll}-2.712818 & 1.206199 & 6.197233\end{array}$

$\begin{array}{lll}-3.121335 & -3.457736 & 4.496390\end{array}$

$\begin{array}{lll}-2.092172 & 5.070769 & 1.207198\end{array}$

$\begin{array}{lll}-6.996213 & 5.003425 & 0.237303\end{array}$

$\begin{array}{llll}-6.842756 & 5.633724 & -0.952664\end{array}$

$\begin{array}{lll}-8.065468 & 4.186331 & 0.121469\end{array}$

$\begin{array}{lll}-7.316338 & 5.959702 & 1.139242\end{array}$

$\begin{array}{lll}-1.048641 & 4.307505 & 0.774004\end{array}$

$\begin{array}{lll}-1.814905 & 5.412257 & 2.484499\end{array}$

$\begin{array}{lll}-2.076139 & 6.203968 & 0.477696\end{array}$

$\begin{array}{lll}-3.100815 & 2.479518 & 5.975162\end{array}$

$\begin{array}{lll}-1.357473 & 1.211062 & 6.283723\end{array}$

$\begin{array}{lll}-3.174111 & 0.845838 & 7.416237\end{array}$

$\begin{array}{lll}-3.629667 & -3.895178 & 5.668971\end{array}$

$\begin{array}{lll}-3.670476 & -4.201419 & 3.510756\end{array}$

$\begin{array}{lll}-1.793668 & -3.755215 & 4.519455\end{array}$

$\begin{array}{lll}-0.655877 & 0.743710 & 1.630039\end{array}$

$\begin{array}{lll}-0.638721 & -1.572463 & 1.927870\end{array}$

$\begin{array}{lll}-0.065667 & 0.958541 & 2.864419\end{array}$

$\begin{array}{llll}-0.869166 & 1.568309 & 0.961855\end{array}$

$\begin{array}{lll}0.020897 & -1.391874 & 3.172509\end{array}$

$\begin{array}{llll}0.281009 & -0.129956 & 3.668636\end{array}$

$\begin{array}{lll}-0.830053 & -2.923155 & 1.463339\end{array}$ $\begin{array}{lll}-1.981571 & -3.354441 & 0.703627\end{array}$

$\begin{array}{lll}-2.674443 & -2.568196 & -0.014418\end{array}$

$\begin{array}{lll}-0.983157 & -0.482844 & 1.177981\end{array}$

$\begin{array}{lll}-0.396854 & -3.657551 & 2.127216\end{array}$

$\begin{array}{llll}3.249702 & -1.353953 & -1.191222\end{array}$

$\begin{array}{lll}4.980383 & -0.111354 & -0.400278\end{array}$

$\begin{array}{llll}4.786750 & 1.132620 & 0.694008\end{array}$

$\begin{array}{llll}5.791872 & 0.691865 & -1.604590\end{array}$

$\begin{array}{llll}6.025333 & -1.086679 & 0.486867\end{array}$

$\begin{array}{lll}4.165387 & 2.307615 & 0.299515\end{array}$

$\begin{array}{lll}2.885259 & 2.561738 & 0.839178\end{array}$

$\begin{array}{lll}2.460850 & 1.840555 & 1.528158\end{array}$

$\begin{array}{llll}2.218962 & 3.709108 & 0.488588\end{array}$

$\begin{array}{llll}1.248093 & 3.929903 & 0.915264\end{array}$

$\begin{array}{llll}2.775601 & 4.600843 & -0.467735\end{array}$

$\begin{array}{llll}2.052354 & 5.743244 & -0.908393\end{array}$

$\begin{array}{llll}1.083531 & 5.947966 & -0.459549\end{array}$

$\begin{array}{llll}2.558960 & 6.563232 & -1.891720\end{array}$

$\begin{array}{llll}1.995281 & 7.428200 & -2.228564\end{array}$

$\begin{array}{llll}3.815955 & 6.269698 & -2.473442\end{array}$

$\begin{array}{llll}4.201616 & 6.903069 & -3.267098\end{array}$

$\begin{array}{llll}4.557010 & 5.190135 & -2.042460\end{array}$

$\begin{array}{llll}5.517664 & 4.981791 & -2.498886\end{array}$

$\begin{array}{llll}4.074030 & 4.330988 & -1.016403\end{array}$

$\begin{array}{llll}4.821494 & 3.193713 & -0.539746\end{array}$

$\begin{array}{llll}6.247008 & 2.965437 & -0.908242\end{array}$

$\begin{array}{lll}7.249032 & 3.982496 & -0.696563\end{array}$

$\begin{array}{llll}6.982310 & 5.187734 & 0.012310\end{array}$

$\begin{array}{llll}5.996154 & 5.353524 & 0.428541\end{array}$

$\begin{array}{llll}7.962015 & 6.139720 & 0.195545\end{array}$

$\begin{array}{llll}7.731517 & 7.045817 & 0.748646\end{array}$

$\begin{array}{llll}9.265030 & 5.946545 & -0.321017\end{array}$

$\begin{array}{llll}10.024430 & 6.709253 & -0.175741\end{array}$

$\begin{array}{llll}9.566726 & 4.782288 & -0.990153\end{array}$

$\begin{array}{llll}10.568243 & 4.607887 & -1.374807\end{array}$

$\begin{array}{llll}8.584255 & 3.773004 & -1.180717\end{array}$

$\begin{array}{llll}8.909765 & 2.551198 & -1.827940\end{array}$

$\begin{array}{llll}9.914821 & 2.408384 & -2.214461\end{array}$

$\begin{array}{llll}7.976183 & 1.551583 & -1.935139\end{array}$

$\begin{array}{llll}8.207728 & 0.599236 & -2.400263\end{array}$

$\begin{array}{llll}6.669172 & 1.753820 & -1.437371\end{array}$

$\begin{array}{lll}5.503862 & -2.482518 & 0.608196\end{array}$

$\begin{array}{lll}4.826803 & -2.526032 & 1.471196\end{array}$

$\begin{array}{llll}6.594349 & -3.515506 & 0.822127\end{array}$

$\begin{array}{lll}6.500817 & -4.410780 & 1.892276\end{array}$

$\begin{array}{llll}5.669207 & -4.324837 & 2.588272\end{array}$

$\begin{array}{llll}7.469792 & -5.400752 & 2.082399\end{array}$

$\begin{array}{llll}7.386643 & -6.085136 & 2.922383\end{array}$

$8.546678 \quad-5.499720 \quad 1.200720$

$\begin{array}{llll}9.308449 & -6.259650 & 1.350951\end{array}$

$\begin{array}{llll}8.643363 & -4.611633 & 0.122885\end{array}$

$\begin{array}{llll}9.484150 & -4.677443 & -0.561974\end{array}$

$\begin{array}{llll}7.670748 & -3.633504 & -0.068620\end{array}$

$\begin{array}{llll}7.762044 & -2.929414 & -0.889730\end{array}$

$\begin{array}{llll}4.701377 & -2.822897 & -0.653531\end{array}$

$\begin{array}{llll}5.400111 & -2.951205 & -1.488719\end{array}$

$\begin{array}{llll}4.204870 & -3.785707 & -0.504651\end{array}$

$\begin{array}{lll}6.838547 & -0.514542 & 1.613808\end{array}$

$\begin{array}{llll}6.918846 & 0.551492 & 1.391314\end{array}$

$\begin{array}{llll}8.271917 & -1.044484 & 1.628640\end{array}$

$\begin{array}{lll}9.136787 & -0.659239 & 0.596078\end{array}$

$\begin{array}{llll}8.769013 & 0.004679 & -0.177798\end{array}$

$10.453131 \quad-1.114706 \quad 0.548968$

$11.103648 \quad-0.803250 \quad-0.264173$

$\begin{array}{lll}10.934077 & -1.964616 & 1.549127\end{array}$

$\begin{array}{lll}11.957839 & -2.326918 & 1.516299\end{array}$

$\begin{array}{lll}10.088629 & -2.337583 & 2.595309\end{array}$

$\begin{array}{lll}10.450863 & -2.995556 & 3.380435\end{array}$

$\begin{array}{llll}8.770401 & -1.875485 & 2.638058\end{array}$

$\begin{array}{lll}8.131015 & -2.190353 & 3.454715\end{array}$

$\begin{array}{lll}6.105061 & -0.643980 & 2.952757\end{array}$

$\begin{array}{llll}5.121362 & -0.174050 & 2.880776\end{array}$

$\begin{array}{lll}5.971500 & -1.686373 & 3.253771\end{array}$

$\begin{array}{llll}6.671785 & -0.137031 & 3.739421\end{array}$

$\begin{array}{llll}2.318315 & -1.070200 & 0.755855\end{array}$ 
$\begin{array}{llll}1.559398 & -0.299935 & 0.679539\end{array}$

$\begin{array}{llll}3.003253 & -0.913907 & 1.585731\end{array}$

$\begin{array}{lll}1.998348 & -2.432440 & 0.445753\end{array}$

$\begin{array}{llll}2.635957 & -3.175261 & 0.912855\end{array}$

$\begin{array}{llll}0.817255 & -2.930609 & -0.174793\end{array}$

$\begin{array}{llll}0.168654 & -2.225006 & -0.676007\end{array}$

$\begin{array}{llll}4.022496 & -1.428697 & -3.273842\end{array}$

$\begin{array}{llll}5.069154 & -1.720004 & -3.222733\end{array}$

$\begin{array}{llll}3.074783 & -2.474430 & -3.115846\end{array}$

$\begin{array}{llll}3.468441 & -3.480273 & -2.970482\end{array}$

$\begin{array}{llll}1.662516 & -2.437511 & -3.672419\end{array}$

$\begin{array}{llll}1.680858 & -2.665149 & -4.749895\end{array}$

$\begin{array}{lll}1.101479 & -3.246424 & -3.199723\end{array}$

$\begin{array}{llll}0.923776 & -1.105375 & -3.433387\end{array}$

$\begin{array}{llll}1.067264 & -0.431602 & -4.282261\end{array}$

$\begin{array}{llll}-0.150876 & -1.297224 & -3.390687\end{array}$

$\begin{array}{llll}1.332603 & -0.373143 & -2.159149\end{array}$

$\begin{array}{llll}0.588904 & -0.340278 & -1.367119\end{array}$

$\begin{array}{llll}2.317779 & 0.603716 & -2.123063\end{array}$

$\begin{array}{llll}2.263352 & 1.332004 & -1.320746\end{array}$

$\begin{array}{lll}3.167382 & 1.014023 & -3.309561\end{array}$

$\begin{array}{llll}3.978624 & 1.641595 & -2.937885\end{array}$

$\begin{array}{llll}2.558247 & 1.645894 & -3.969469\end{array}$

$\begin{array}{llll}3.763633 & -0.168918 & -4.105441\end{array}$

$\begin{array}{llll}3.109604 & -0.426838 & -4.945559\end{array}$

$\begin{array}{llll}4.711027 & 0.153960 & -4.547676\end{array}$

$\begin{array}{llll}0.732928 & -4.322475 & -0.646696\end{array}$

$\begin{array}{llll}1.632388 & -5.328497 & -0.237527\end{array}$

$-0.281065 \quad-4.677026-1.556531$

$\begin{array}{llll}1.516381 & -6.630094 & -0.720953\end{array}$

$\begin{array}{llll}2.426262 & -5.100209 & 0.464569\end{array}$

$-0.398245 \quad-5.978573 \quad-2.040027$

$-0.980711-3.918388-1.884172$

$\begin{array}{llll}0.499392 & -6.963978 & -1.622039\end{array}$

$\begin{array}{llll}2.221314 & -7.388102 & -0.391630\end{array}$

$\begin{array}{llll}-1.190821 & -6.222555 & -2.741667\end{array}$

$\begin{array}{llll}0.411355 & -7.980124 & -1.995193\end{array}$

$\begin{array}{lll}-2.319757 & -4.676802 & 0.726304\end{array}$

$\begin{array}{llll}-3.368129 & -5.177616 & -0.152904\end{array}$

$\begin{array}{llll}-4.255889 & -5.464131 & 0.425316\end{array}$

$\begin{array}{llll}-3.004638 & -6.058515 & -0.692500\end{array}$

$\begin{array}{llll}-3.645772 & -4.403945 & -0.863112\end{array}$

$\begin{array}{lll}-1.663159 & -5.672476 & 1.570751\end{array}$

$\begin{array}{lll}-0.686093 & -5.961851 & 1.171121\end{array}$

$\begin{array}{lll}-2.297112 & -6.560357 & 1.606387\end{array}$

$\begin{array}{lll}-1.545581 & -5.312153 & 2.593206\end{array}$

$\begin{array}{llll}0.731780 & 0.007933 & 4.644936\end{array}$

$\begin{array}{lll}0.271878 & -2.266163 & 3.762167\end{array}$
35

$\begin{array}{llll}0.201993 & 2.776895 & 3.513522\end{array}$

P2-C1 re-re conf2

Number of imaginary frequencies :

The smallest frequency is : $-137.3962 \mathrm{~cm}(-1)$

Electronic energy : $\quad \mathrm{HF}=-7222.6278114$

Zero-point correction $=\quad 1.754810$

(Hartree/Particle)

Thermal correction to Energy= $\quad 1873359$

Thermal correction to Enthalpy= $\quad 1.874303$

Thermal correction to Gibbs Free Energy $=\quad 1.591878$

Sum of electronic and zero-point Energies $=\quad-7220.873001$

Sum of electronic and thermal Energies $=\quad-7220.754453$

Sum of electronic and thermal Enthalpies $=\quad-7220.753508$

Sum of electronic and thermal Free Energies $=\quad-7221.035933$

Cartesian Coordinates

$\begin{array}{cccc}26 & -8.123830 & 0.204817 & -0.683927 \\ 15 & -3.132747 & 1.198590 & -1.972785 \\ 15 & -4.000833 & 0.629849 & 1.659803 \\ 6 & -6.156690 & 0.873804 & -0.966363 \\ 6 & -6.291582 & 0.146445 & 0.273574 \\ 6 & -6.735904 & -1.174737 & -0.041560 \\ 1 & -6.941051 & -1.960623 & 0.672289 \\ 6 & -6.909847 & -1.263180 & -1.455847\end{array}$

$-7.272232-2.125234-1.997412$

$\begin{array}{llll}-6.552872 & -0.005819 & -2.024679\end{array}$

$\begin{array}{lll}-6.595405 & 0.253864 & -3.071878\end{array}$

$\begin{array}{lll}-9.466827 & 1.394445 & 0.361458\end{array}$

$\begin{array}{lll}-9.192734 & 2.071904 & 1.159431\end{array}$

$\begin{array}{lll}-9.802175 & 0.013870 & 0.513080\end{array}$

$\begin{array}{lll}-9.819592 & -0.538898 & 1.442826\end{array}$

$-10.059790-0.523214-0.785216$

$\begin{array}{llll}-10.303576 & -1.553319 & -1.009495\end{array}$

$\begin{array}{lll}-9.883652 & 0.524484 & -1.739583\end{array}$

$\begin{array}{lll}-9.973240 & 0.428638 & -2.813632\end{array}$

$\begin{array}{llll}-9.519449 & 1.708777 & -1.032295\end{array}$

$-9.289638 \quad 2.665763-1.480591$

$\begin{array}{llll}-5.636227 & 2.266554 & -1.076882\end{array}$

$\begin{array}{lll}-6.429587 & 3.328160 & -0.614828\end{array}$

$\begin{array}{llll}-7.409802 & 3.097080 & -0.218331\end{array}$

$\begin{array}{llll}-5.987012 & 4.649856 & -0.645487\end{array}$

$\begin{array}{lll}-6.617885 & 5.442727 & -0.256545\end{array}$

$\begin{array}{llll}-4.728217 & 4.937427 & -1.166532\end{array}$

$\begin{array}{lll}-4.365227 & 5.959493 & -1.200712\end{array}$

$\begin{array}{llll}-3.917224 & 3.898765 & -1.623499\end{array}$

$\begin{array}{llll}-2.934798 & 4.142698 & -2.008904\end{array}$

$\begin{array}{lll}-4.335965 & 2.558612 & -1.570007\end{array}$

$\begin{array}{lll}-5.896603 & 0.683288 & 1.622884\end{array}$

$\begin{array}{lll}-6.155847 & 1.743175 & 1.643569\end{array}$

$\begin{array}{lll}-6.589979 & -0.005894 & 2.801336\end{array}$

$\begin{array}{lll}-6.291410 & 0.445409 & 3.751596\end{array}$

$\begin{array}{lll}-6.357697 & -1.073434 & 2.848205\end{array}$

$\begin{array}{lll}-7.673959 & 0.098343 & 2.691782\end{array}$

$\begin{array}{llll}-1.620922 & 2.142911 & -2.443869\end{array}$

$\begin{array}{lll}-0.769686 & 2.550694 & -1.402612\end{array}$

$\begin{array}{lll}-0.958555 & 2.208684 & -0.391279\end{array}$

$\begin{array}{llll}0.304277 & 3.402272 & -1.661963\end{array}$

$\begin{array}{llll}0.942535 & 3.723868 & -0.849577\end{array}$

$\begin{array}{llll}0.567313 & 3.822954 & -2.967828\end{array}$

$\begin{array}{llll}1.412791 & 4.475193 & -3.167330\end{array}$

$\begin{array}{rrr}-0.246152 & 3.385154 & -4.014705\end{array}$

$\begin{array}{llll}-0.038506 & 3.695587 & -5.034697\end{array}$

$\begin{array}{lll}-1.341324 & 2.556600 & -3.755104\end{array}$

$\begin{array}{llll}-1.981951 & 2.249119 & -4.574863\end{array}$

$\begin{array}{lll}-3.732211 & 0.499302 & -3.560832\end{array}$

$\begin{array}{llll}-4.464886 & 1.254067 & -4.490390\end{array}$

$\begin{array}{llll}-4.700886 & 2.293347 & -4.281471\end{array}$

$\begin{array}{llll}-4.905950 & 0.667107 & -5.677594\end{array}$

$\begin{array}{llll}-5.475059 & 1.256333 & -6.391010\end{array}$

$\begin{array}{lll}-4.621204 & -0.675782 & -5.946196\end{array}$

$\begin{array}{llll}-4.973197 & -1.131797 & -6.867066\end{array}$

$\begin{array}{llll}-3.887095 & -1.430113 & -5.027782\end{array}$

$\begin{array}{llll}-3.670054 & -2.475292 & -5.228498\end{array}$

$\begin{array}{llll}-3.444029 & -0.843807 & -3.840577\end{array}$

$\begin{array}{llll}-2.887264 & -1.432059 & -3.120240\end{array}$

$\begin{array}{lll}-3.415752 & 2.330194 & 2.055306\end{array}$

$\begin{array}{lll}-2.023077 & 2.495509 & 2.067452\end{array}$

$\begin{array}{lll}-1.387458 & 1.629709 & 1.911397\end{array}$

$\begin{array}{llll}-1.460009 & 3.760121 & 2.235426\end{array}$

$\begin{array}{llll}-2.265409 & 4.890007 & 2.361671\end{array}$

$\begin{array}{lll}-1.826279 & 5.875922 & 2.466685\end{array}$

$\begin{array}{lll}-3.651230 & 4.724875 & 2.334576\end{array}$

$\begin{array}{llll}-4.227969 & 3.461500 & 2.199829\end{array}$

$\begin{array}{lll}-5.306733 & 3.386918 & 2.186479\end{array}$

$\begin{array}{lll}-3.510402 & -0.363061 & 3.129032\end{array}$

$\begin{array}{lll}-3.637351 & -1.755537 & 3.002554\end{array}$

$\begin{array}{lll}-4.068750 & -2.180391 & 2.103758\end{array}$

$\begin{array}{lll}-3.176788 & -2.599758 & 4.008590\end{array}$

$\begin{array}{lll}-2.557424 & -2.081300 & 5.147057\end{array}$

$\begin{array}{lll}-2.181401 & -2.741917 & 5.920028\end{array}$

$\begin{array}{lll}-2.419641 & -0.700412 & 5.264888\end{array}$

$\begin{array}{llll}-2.900411 & 0.159351 & 4.273648\end{array}$

$\begin{array}{llll}-2.779819 & 1.228868 & 4.398918\end{array}$

$\begin{array}{lll}-2.839595 & -0.191799 & -0.212967\end{array}$

$\begin{array}{lll}-1.651058 & -0.137351 & 6.427414\end{array}$

$\begin{array}{lll}-3.240742 & -4.091098 & 3.829599\end{array}$

$\begin{array}{lll}0.037777 & 3.866988 & 2.295458\end{array}$

$\begin{array}{lll}-4.515539 & 5.952937 & 2.424933\end{array}$

$\begin{array}{lll}-4.283961 & 6.798085 & 1.390200\end{array}$ 


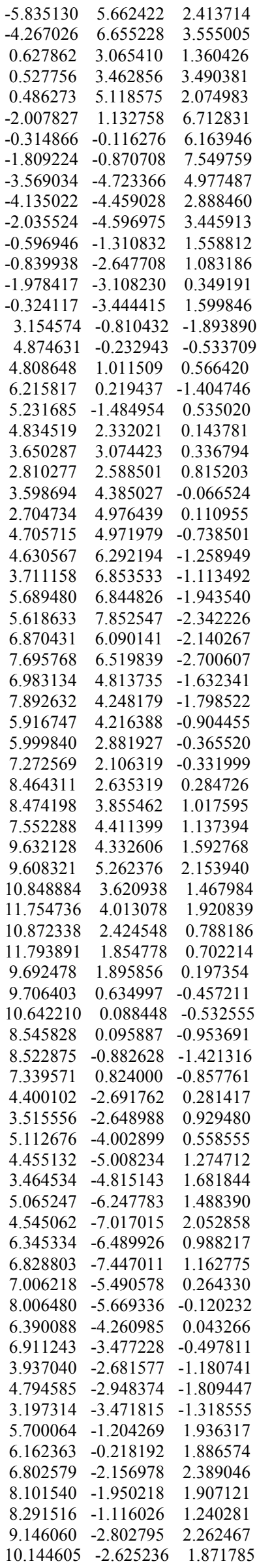

$\begin{array}{lll}8.907226 & -3.878177 & 3.122434\end{array}$

$\begin{array}{llll}9.715622 & -4.549031 & 3.399683\end{array}$

$\begin{array}{llll}7.621965 & -4.078644 & 3.630011\end{array}$

$\begin{array}{llll}7.425330 & -4.909013 & 4.302493\end{array}$

$\begin{array}{lll}6.579986 & -3.220121 & 3.271713\end{array}$

$\begin{array}{lll}5.587813 & -3.404039 & 3.668203\end{array}$

$\begin{array}{llll}4.517388 & -1.101523 & 2.906428\end{array}$

$\begin{array}{lll}3.825162 & -0.331123 & 2.559577\end{array}$

$\begin{array}{llll}3.967779 & -2.041876 & 3.001307\end{array}$

$\begin{array}{lll}4.874820 & -0.824035 & 3.902655\end{array}$

$\begin{array}{llll}1.893470 & -0.285061 & -0.200998\end{array}$

$\begin{array}{llll}1.666502 & 0.777440 & -0.167093\end{array}$

$\begin{array}{lll}2.242065 & -0.673042 & 0.753858\end{array}$

$\begin{array}{llll}1.006479 & -1.118566 & -0.963988\end{array}$

$\begin{array}{llll}0.284058 & -0.646689 & -1.617112\end{array}$

$\begin{array}{llll}0.870653 & -2.498851 & -0.722995\end{array}$

$\begin{array}{llll}1.538935 & -2.932227 & 0.010376\end{array}$

$\begin{array}{lll}4.420417 & -1.061445 & -3.710510\end{array}$

$\begin{array}{llll}5.288197 & -1.650741 & -3.425455\end{array}$

$\begin{array}{llll}3.212461 & -1.779803 & -3.897356\end{array}$

$\begin{array}{llll}3.256647 & -2.859875 & -3.758256\end{array}$

$2.048303 \quad-1.327666 \quad-4.762564$

$\begin{array}{llll}2.251803 & -1.561639 & -5.819201\end{array}$

$\begin{array}{llll}1.179096 & -1.928789 & -4.477856\end{array}$

$\begin{array}{llll}1.697133 & 0.166319 & -4.609713\end{array}$

$\begin{array}{llll}2.253915 & 0.767067 & -5.335537\end{array}$

$\begin{array}{llll}0.638389 & 0.312232 & -4.845891\end{array}$

$\begin{array}{llll}1.949838 & 0.721423 & -3.210744\end{array}$

$\begin{array}{llll}1.060928 & 0.960727 & -2.639171\end{array}$

$\begin{array}{llll}3.120274 & 1.354659 & -2.816236\end{array}$

$\begin{array}{llll}3.049086 & 2.046132 & -1.982317\end{array}$

$\begin{array}{llll}4.351912 & 1.520850 & -3.682239\end{array}$

$\begin{array}{llll}5.174390 & 1.839216 & -3.038853\end{array}$

$\begin{array}{llll}4.175358 & 2.341134 & -4.393988\end{array}$

$\begin{array}{llll}4.766985 & 0.239497 & -4.439088\end{array}$

$\begin{array}{llll}4.312308 & 0.221344 & -5.434678\end{array}$

$\begin{array}{llll}5.848748 & 0.266793 & -4.601092\end{array}$

$\begin{array}{llll}0.262198 & -3.443384 & -1.648214\end{array}$

$\begin{array}{llll}-0.595839 & -3.035189 & -2.688853\end{array}$

$\begin{array}{llll}0.544177 & -4.816110 & -1.511291\end{array}$

$\begin{array}{lll}-1.150214 & -3.968543 & -3.558631\end{array}$

$\begin{array}{llll}-0.825637 & -1.983994 & -2.814789\end{array}$

$\begin{array}{lll}-0.001512 & -5.749557 & -2.392574\end{array}$

$\begin{array}{llll}1.199047 & -5.144362 & -0.707909\end{array}$

$\begin{array}{llll}-0.853573 & -5.329879 & -3.417100\end{array}$

$-1.811414-3.634225-4.352615$

$\begin{array}{lll}0.233634 & -6.803551 & -2.276130\end{array}$

$-1.283594-6.055186-4.101692$

$\begin{array}{llll}-2.182133 & -4.515505 & 0.264164\end{array}$

$\begin{array}{llll}-3.216414 & -5.049806 & -0.473445\end{array}$

$\begin{array}{lll}-1.506797 & -5.174110 & 0.797120\end{array}$

$-4.072537-4.157089-1.125492$

$-3.867160-2.796496-1.009406$

$-4.521848-2.087723-1.497711$

$\begin{array}{lll}-2.846025 & -2.273531 & -0.300442\end{array}$

$\begin{array}{lll}-1.038046 & -0.269545 & 0.983126\end{array}$

$\begin{array}{lll}0.230638 & -1.148996 & 2.642535\end{array}$

$\begin{array}{lll}0.669024 & 0.189289 & 3.023469\end{array}$

$\begin{array}{lll}1.672535 & 0.128951 & 3.453752\end{array}$

$\begin{array}{lll}0.002836 & 0.636616 & 3.769449\end{array}$

$\begin{array}{llll}0.704748 & 0.830204 & 2.147669\end{array}$

$\begin{array}{llll}0.618167 & -2.233399 & 3.540728\end{array}$

$\begin{array}{lll}1.546396 & -2.725316 & 3.219247\end{array}$

$\begin{array}{lll}-0.170166 & -2.982179 & 3.620539\end{array}$

$\begin{array}{llll}0.777517 & -1.818695 & 4.538273\end{array}$

$\begin{array}{llll}-3.356787 & -6.122981 & -0.541506\end{array}$

$\begin{array}{lll}-5.556135 & -4.822422 & -2.216132\end{array}$

P2-C1 si-re conf2

Number of imaginary frequencies : 1

The smallest frequency is : $-133.9792 \mathrm{~cm}(-1)$

Electronic energy :

Zero-point correction= $\mathrm{HF}=-7222.6089123$

(Hartree/Particle) 


\begin{tabular}{|c|c|c|c|}
\hline & \multicolumn{3}{|c|}{$\begin{array}{l}\text { Thermal correction to Gibbs Free Energy= } \\
\text { Sum of electronic and zero-point Energies= } \\
\text { Sum of electronic and thermal Energies= } \\
\text { Sum of electronic and thermal Enthalpies= } \\
\text { Sum of electronic and thermal Free Energies }\end{array}$} \\
\hline 77 & 4.163394 & -0.905691 & 1.466574 \\
\hline 15 & 5.888732 & -0.077176 & 0.255889 \\
\hline 8 & 6.427972 & -0.741237 & -1.163095 \\
\hline 8 & 7.297192 & -0.051063 & 1.141311 \\
\hline 7 & 5.526030 & 1.472669 & -0.289678 \\
\hline 6 & 7.373590 & -1.759853 & -1.201906 \\
\hline 6 & 6.930404 & -3.001340 & -1.708319 \\
\hline 1 & 5.902321 & -3.086230 & -2.044108 \\
\hline 6 & 7.803080 & -4.058401 & -1.773576 \\
\hline 1 & 7.477209 & -5.015994 & -2.169464 \\
\hline 6 & 9.135200 & -3.923533 & -1.298271 \\
\hline 6 & 10.023451 & -5.033099 & -1.288988 \\
\hline 1 & 9.668713 & -5.983292 & -1.679548 \\
\hline 6 & 11.298091 & -4.914661 & -0.783544 \\
\hline 1 & 11.967334 & -5.769982 & -0.776304 \\
\hline 6 & 11.731230 & -3.675381 & -0.255291 \\
\hline 1 & 12.728349 & -3.589994 & 0.167011 \\
\hline 6 & 10.900216 & -2.575970 & -0.264725 \\
\hline 1 & 11.249247 & -1.640940 & 0.156110 \\
\hline 6 & 9.584273 & -2.653321 & -0.801413 \\
\hline 6 & & -1.5 & -0.823998 \\
\hline 6 & 9.131060 & -0.165153 & -0.405705 \\
\hline 6 & 10.259841 & 0.499136 & -1.003412 \\
\hline 6 & 10.5 & 1273 & 63882 \\
\hline 1 & 10.555157 & -0.910042 & -2.626731 \\
\hline 6 & 11.987146 & 0.669155 & -2.715540 \\
\hline 1 & 12.464602 & 0.274149 & -3.607647 \\
\hline 6 & 12.468408 & 1.868040 & -2.136575 \\
\hline 1 & 13.316937 & 2.380022 & -2.580770 \\
\hline 6 & 11.8496 & 2.386489 & -1.021775 \\
\hline 1 & 12.197906 & 3.314493 & -0.575657 \\
\hline 6 & 10.732372 & 1.731089 & -0.435400 \\
\hline 6 & 10.060291 & 2.289718 & 0.685321 \\
\hline 1 & 10.435007 & 3.211361 & 1.120941 \\
\hline 6 & 8.922668 & 1.701285 & 1.180411 \\
\hline 1 & 8.369978 & 2.130370 & 2.008630 \\
\hline 6 & 8.456944 & 0.499467 & 0.606157 \\
\hline 6 & 4.345464 & 2.037661 & 0.409395 \\
\hline 1 & 3.475895 & 1.937405 & -0.248412 \\
\hline 6 & 4.479223 & 3.501004 & 0.794688 \\
\hline 6 & 3.349177 & 4.325700 & 0.743645 \\
\hline 1 & 2.408198 & 3.923255 & 0.376287 \\
\hline 6 & 3.421402 & 5.659652 & 1.152933 \\
\hline 1 & 2.537528 & 6.289938 & 1.099395 \\
\hline 6 & 4.629058 & 6.182399 & 1.619946 \\
\hline 1 & 4.692686 & 7.222365 & 1.927488 \\
\hline 6 & 5.758075 & 5.358610 & 1.686749 \\
\hline 1 & 6.703280 & 5.759160 & 2.042741 \\
\hline 6 & 5.680374 & 4.026139 & 1.285875 \\
\hline 1 & 6.561794 & 3.395180 & 1.308350 \\
\hline 6 & 4.090902 & 1.230695 & 1.694764 \\
\hline 1 & 4.846156 & 1.521440 & 2.435655 \\
\hline 1 & 3.129220 & 1.533692 & 2.111887 \\
\hline 6 & 5.851346 & 1.915907 & -1.689169 \\
\hline 1 & 6.709416 & 1.311991 & -1.980335 \\
\hline 6 & 6.320023 & 3.363303 & -1.753660 \\
\hline 6 & 7.675308 & 3.635094 & -1.531301 \\
\hline 1 & 8.355308 & 2.811640 & -1.343467 \\
\hline 6 & 8.157419 & 4.943962 & -1.539939 \\
\hline 1 & 9.212871 & 5.130450 & -1.360139 \\
\hline 6 & 7.283810 & 6.006417 & -1.785454 \\
\hline 1 & 7.651586 & 7.028748 & -1.788952 \\
\hline 6 & 5.934698 & 5.744749 & -2.038152 \\
\hline 1 & 5.248907 & 6.563868 & -2.235720 \\
\hline 6 & 5.459112 & 4.432178 & -2.030150 \\
\hline
\end{tabular}

1.873225
1.874170
1.588051
-7220.854713
-7220.735687
-7220.734743
$=\quad-7221.020861$

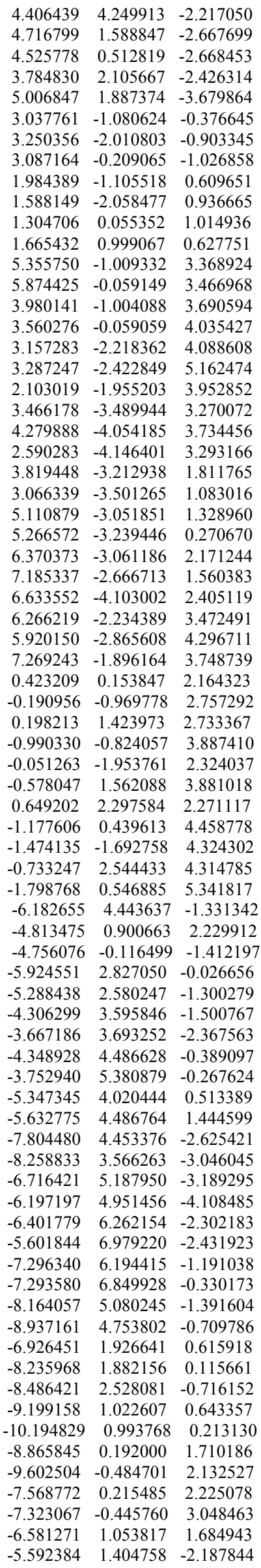




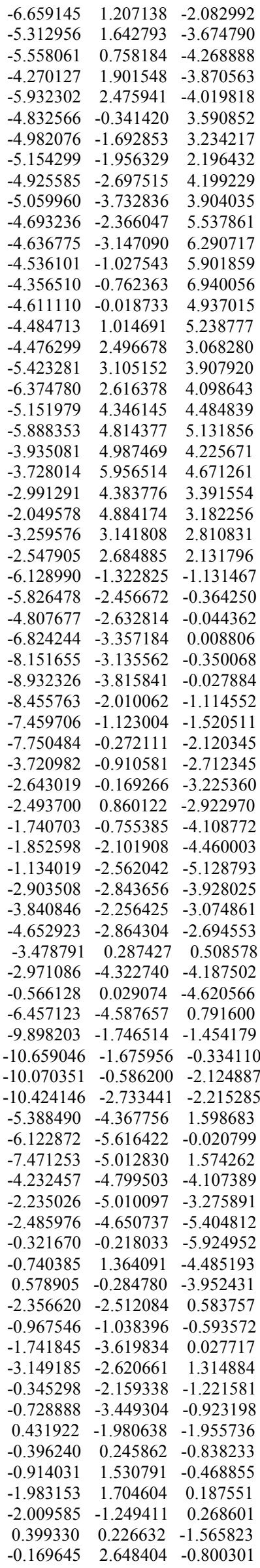


End of Supporting Information 